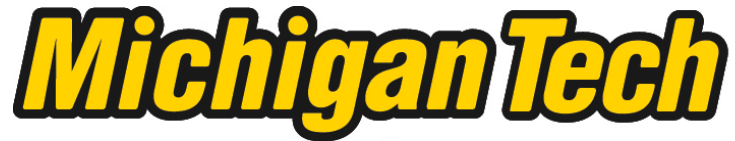 \\ Michigan Technological University Create the Future Digital Commons @ Michigan Tech
}

Dissertations, Master's Theses and Master's Reports - Open

Dissertations, Master's Theses and Master's

Reports

2004

Examining patterns of Italian immigration to Michigan's Houghton County, 1860-1930

Cristina Menghini

Michigan Technological University

Follow this and additional works at: https://digitalcommons.mtu.edu/etds

Part of the Archaeological Anthropology Commons

Copyright 2004 Cristina Menghini

\section{Recommended Citation}

Menghini, Cristina, "Examining patterns of Italian immigration to Michigan's Houghton County, 1860-1930

", Master's Thesis, Michigan Technological University, 2004.

https://doi.org/10.37099/mtu.dc.etds/292

Follow this and additional works at: https://digitalcommons.mtu.edu/etds

Part of the Archaeological Anthropology Commons 


\title{
EXAMINING PATTERNS OF ITALIAN IMMIGRATION TO MICHIGAN'S \\ HOUGHTON COUNTY, 1860-1930
}

\author{
By \\ CRISTINA MENGHINI
}

\begin{abstract}
A THESIS
Submitted in partial fulfillment

for the degree of

MASTER OF SCIENCE IN INDUSTRIAL ARCHAEOLOGY
\end{abstract}

MICHIGAN TECHNOLOGICAL UNIVERSITY

2004 


\section{Table of Contents}

\section{Introduction}

Chapter I - Italian Emigration I

Chapter II - Provenience 1

Chapter III - Occupations 11

Chapter IV - Settlements

Chapter V - Organizations $\quad 54$

$\begin{array}{ll}\text { Chapter VI - Conclusions } & 67\end{array}$

Bibliography 86

$\begin{array}{ll}\text { Appendixes } & 89\end{array}$

Appendix I - Bylaws of the Colonial Union 98

Appendix II - Bylaws of the Italian-American 99

$\begin{array}{ll}\text { Federation } & 107\end{array}$

Appendix III - Satiric Poems from the Miners’ Bulletin 117

$\begin{array}{ll}\text { Appendix IV - Municipalities in Lucca } & 121\end{array}$

$\begin{array}{ll}\text { Appendix V - Main villages of Canavese } & 123\end{array}$

$\begin{array}{ll}\text { Appendix VI - Census of } 1870 & 125\end{array}$

$\begin{array}{ll}\text { Appendix VII - Census of } 1880 & 127\end{array}$

Appendix VIII - Census of $1900 \quad 132$

$\begin{array}{ll}\text { Appendix IX - Census of } 1910 & 178\end{array}$

$\begin{array}{ll}\text { Appendix X - Census of } 1920 & 249\end{array}$

$\begin{array}{ll}\text { Appendix XI - Census of } 1930 & 320\end{array}$ 


\section{Introduction}

Italians, as well as immigrants from all over Europe, came to Houghton County to work in the expanding mining industry. Michigan's copper mining district, opened in the 1840 s, was the first metal mining rush in United States history. During the 1840s and early 1850s the Copper Country was still a frontier mining community. The demand for labor was seasonal, in fact it was high during the spring when shipping was possible and the harsh weather did not impede exploration of the territory. During the winter the demand was considerably lower. ${ }^{1}$ The census of 1850 indicates that there were 708 people living in Houghton County. By the 1860s seasonal fluctuations became less significant because mining companies started to focus on underground progress during the winter and they were also able to have supply of labor at rates not much higher than those for mining in the East. The census of 1860 indicates that the population of Houghton County had reached 9,253. Serious shortages in the supply of labor appeared during the Civil War and when many mines opened at the same time during the $1860 \mathrm{~s}$. At this point some mining companies working through foreign agents and the Mine Emigrant Society started to import workers from abroad. The Copper Country entered its great period of growth during the $1870 \mathrm{~s}$. The population of the area grew accordingly. The censuses indicate that the population of Houghton County reached 13,882 in 1870, 22,473 in 1880, and 35,389 in 1890. Irishmen, Englishmen, Canadians, Germans, and Scandinavians were the more numerous immigrants during the 1870 s and 1880s. Starting from the 1890s the great bulk of immigrants came from Finland, Austria, and Italy. From 1890 until 1910 the population of Houghton County more than doubled. It reached 66,063 in 1900 and 88,098 in 1910. During the 1910s the mining industry started a steady decline and the population declined as a consequence. In fact it decreased to 71,930 in 1920 and dropped to 52,851 in $1930 .^{2}$ The first Italians arrived in Houghton County around 1860 and were very important in the early history of the region. Even before the mass migration of Italians to America in the 1890s, Michigan had 3,000 Italian immigrants, $75 \%$ of whom lived in the remote Upper Peninsula. By 1910 over 7,000 Italians lived in the Upper Peninsula.

\footnotetext{
${ }^{1}$ Gates, B. William Jr. Michigan Copper and Boston Dollars. Cambridge, Massachusetts: Harvard University Press, 1951, pp. 96-98.

${ }^{2}$ Ibiden, pp. 105-105 and 166-166.
} 
Italians concentrated in the Houghton County communities of Laurium, Calumet, South Range, and Hancock. Their community was of national significance as shown by their symbolic involvement in the famous Italian Hall disaster of 1913 that linked this region to the larger issue of American labor strife.

They left a rich documentary record in the form of newspapers, diaries, benevolent society papers and other materials. The purpose of this work is to identify and characterize the Italians that came to Michigan's Houghton County during the years between 1870 and 1930 . The following chapters explain the regions of origin of these Italians, their jobs in Houghton County, the areas where they preferred to settle and the relations, if any, among origins, jobs, and patterns of settlement.

The starting point for this thesis is the work of Professor Rudolph J. Vecoli - who wrote extensively on the topic of Italian immigration and is also one of the first to have written specifically about Italian immigrants to the mines of Lake Superior - and Professor Russell M. Magnaghi - who wrote particularly on the Italians of Upper Michigan and interviewed some of them in the 1980s.

Historic documents concerning Italian immigrants to the Upper Peninsula of Michigan may be found at three main repositories. The Michigan Technological University and Copper Country Archives in Houghton maintains collections of primary sources on the Italian immigrants of the region, including census records, mining company records, newspapers, diaries, and benevolent society papers. Databases of the Italians living in Houghton County in the years 1870, 1880, 1900, 1910, 1920, and 1930 have been extracted from the censuses of the same years. Mining company records such as employees' cards and job applications have been useful in tracking provenience and places of residence of the Italian workers. Newspapers such as Il Minatore Italiano and Miners' Bulletin have been helpful to know more about everyday life of the Italians in Houghton County.

The Northern Michigan University Archives in Marquette maintains a collection of oral interviews of Italians living in the Upper Peninsula that Professor Russell Magnaghi of Northern Michigan University made during the 1980s. The tapes of the interviews helped to make more sense out of the data previously collected, and to form an idea of how Italians perceived themselves within their new environment. Besides the tapes, Professor Magnaghi's papers represented a rich source for each part of this work. 


\section{The Immigration History Research Center at University of Minnesota in}

Minneapolis keeps the country's largest collection about Italian immigrants in general and also of Italians in Upper Michigan. Most important, the only available issues of newspapers and other publications such as L'Indipendente and Pro Nobis are preserved there.

Chapter I, Italian Emigration, is a general introduction to the history of Italian emigration, with a particular focus on Italian emigration to the United States. It also includes an overview of the different stages that emigrants had to go through to reach their destinations: recruitment, travel, laws of emigration in Italy and laws of immigration in the United States.

Chapter II, Provenience, examines the number and the provenience of the Italians who settled in Houghton County from 1870 until 1930. It also gives a general description of the Italian provinces from which most of them came: Torino and Lucca.

Chapter III, Occupations, considers the occupations that Italians had when they first arrived in Houghton County and examines how these changed through the years. It tries to verify previously assumed ideas about the occupations in which most of the Italians were involved. It compares the occupations of the two main groups of Italians - Torinesi and Lucchesi - to find out if there is a relation between provenience and occupation.

Chapter IV, Settlements, analyzes the patterns of settlement of Italians in the townships and villages of Houghton County through the years. It also shows a comparison in the pattern of settlements of the Torinesi and Lucchesi.

Chapter V, Organizations, discusses Italian organizations of different kind - cultural, business, religious, and social - established in Houghton County and in the Upper Peninsula of Michigan.

Chapter VI, Conclusion, sums up what it has been possible to discover about Italian immigrants to Houghton County and suggests further research. 


\section{Chapter I - Italian Emigration}

\subsection{Italian emigration: an introduction.}

Italians have been the protagonists of the biggest migratory exodus in modern history. ${ }^{3}$ From 1861 - year of the birth of the Italian nation - until 1988, the year of almost complete exhaustion of the flows, about twenty-six million Italians left their country. ${ }^{4}$ This emigration evolved in four phases.

The first phase extended from 1876 until 1900 and saw a steady increase in the number of emigrants. During the second phase, 1900-1914, the so-called years of the "great emigration", Italian emigration reached its peak. It ceased during World War I. The third phase, between the two world wars, was characterized by a decrease in the number of emigrants because of the global economic crisis and because of the restrictions imposed by several foreign countries (an example is the earlier 1921 Immigration Act in the U.S.). Fascism, which was hostile to emigration, and World War II almost eliminated emigration. The fourth phase extended from the end of World War II until the 1960s. Immediately after the end of the war there was an increase, but the pattern of emigration changed after Italy's entrance into the European Economic Community in 1957; the average of people going abroad dropped severely. ${ }^{5}$

Figures I, II, and III show the number of emigrants from each Italian region during the first three phases of emigration. Although there are significant differences in time of departure and in final destinations, people from every Italian region contributed to this emigration. Between 1876 and 1900 there was a prevalence of emigrants from the northern regions: Veneto, Piemonte, Friuli Venezia Giulia. During the following decades the trend was inverted with the biggest number of departures from Sicilia, Campania, and Calabria. As for the countries of destination, Italians almost equally divided between European countries and American ones, with France and United States as the principal recipients (see Table I). Italians emigrating towards America also showed particular patterns. Most Southern Italians

\footnotetext{
3 Tirabassi, Maddalena. L'emigrazione Italiana. www.comune.torino.it/infocultura/intercultura/12/12f3.html, 0130-2004.

${ }^{4}$ The net difference between the number of emigrants and those that returned is between 12 and 14 million.

${ }^{5}$ Collier's Encyclopedia. New York: MacMillan Educational Company, 1991.
} 
immigrated to North America. Northern Italians preferred Latin America. Central Italians equally divided between North and South America.

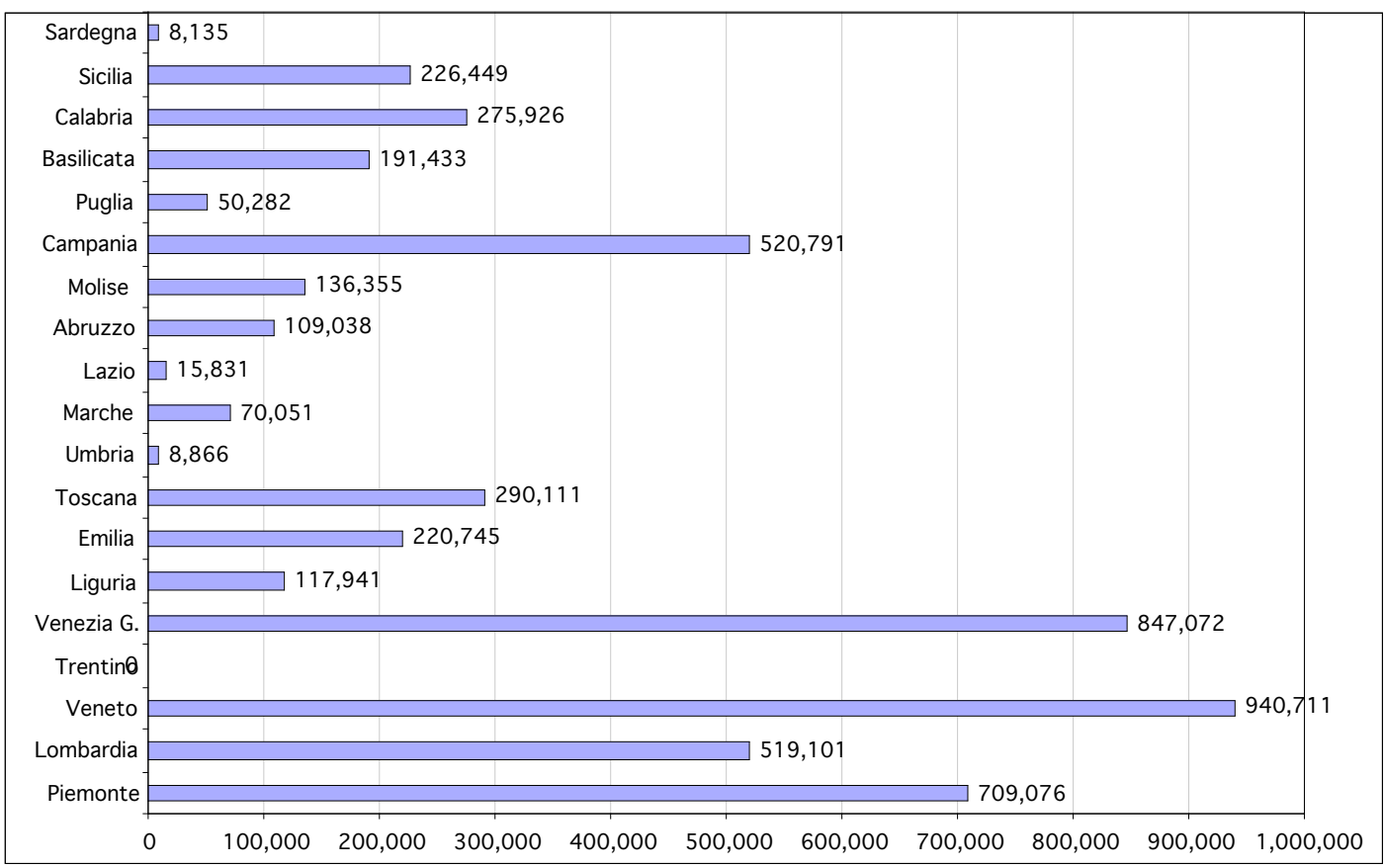

Figure I. Emigration from Italian regions 1876-1900 (Total emigrants 5,257,830). Source: Centro Studi Emigrazione, Roma 1978.

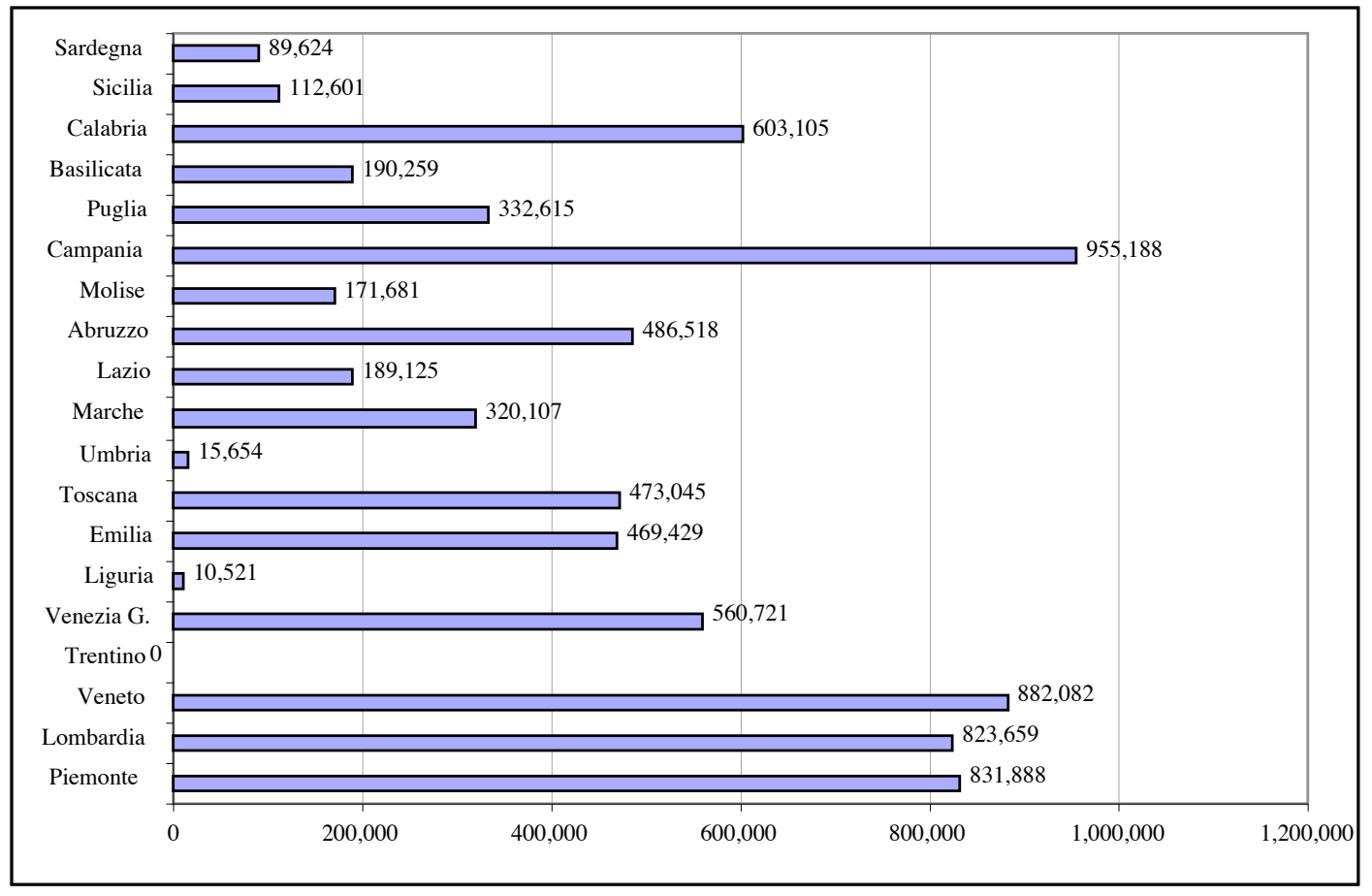

Figure II. Emigration from Italian regions 1901-1915 (Total emigrants 8,768,680). Source: Centro Studi Emigrazione, Roma 1978. 


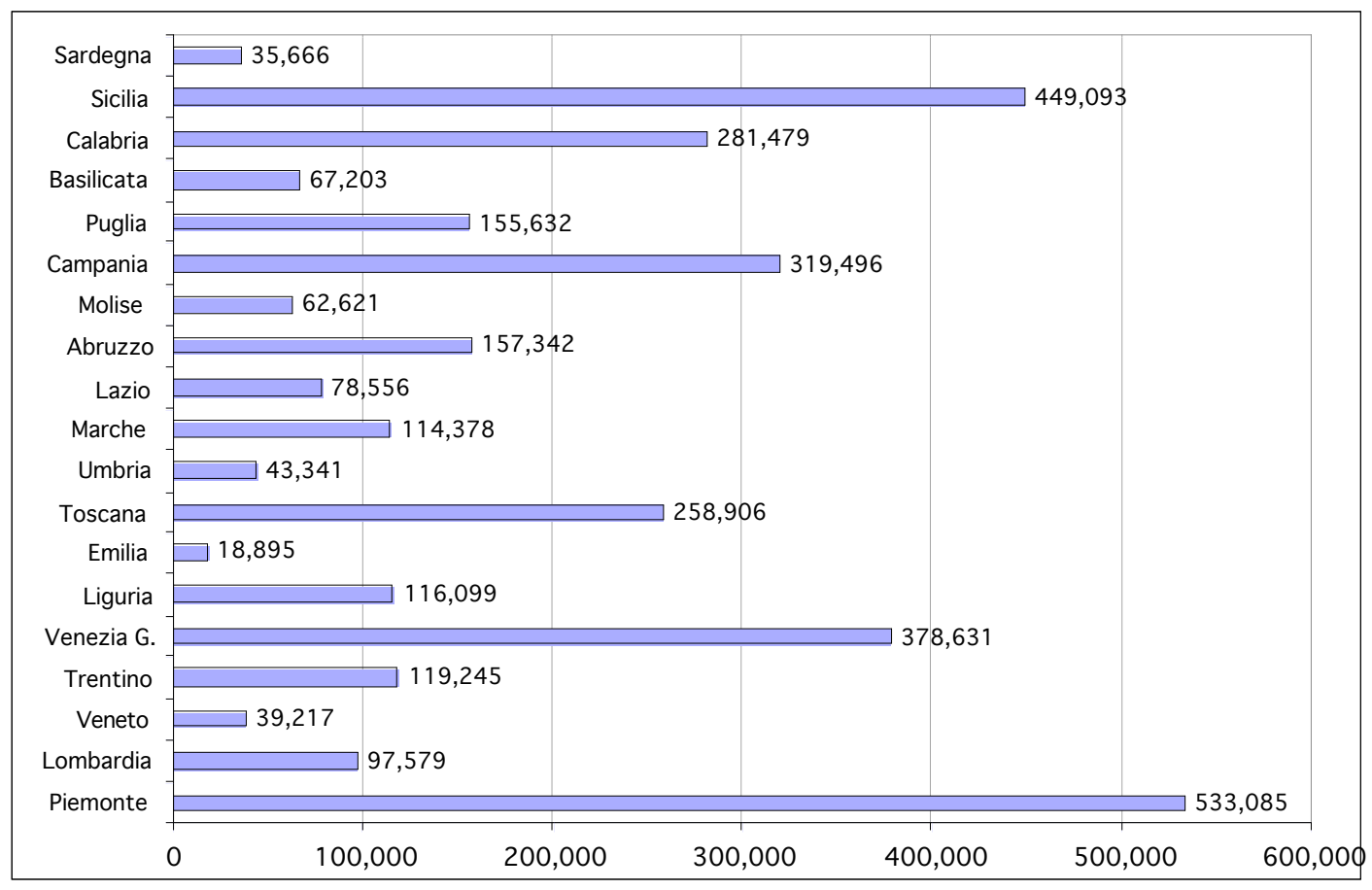

Table I. Countries of Destination of Italian Emigration, 1876-1976

\begin{tabular}{|l|c|l|c|}
\hline European Country & Italian emigrants & American country & Italian emigrants \\
\hline France & $4,117,394$ & United States & $5,691,404$ \\
\hline Switzerland & $3,989,813$ & Argentina & $2,969,402$ \\
\hline Germany & $2,452,587$ & Brazil & $1,456,914$ \\
\hline Belgium & 535,031 & Canada & 650,358 \\
\hline Great Britain & 263,598 & Australia & 428,289 \\
\hline Others & $1,188,135$ & Venezuela & 285,014 \\
\hline TOTAL & $\mathbf{1 2 , 5 4 6 , 5 5 8}$ & TOTAL & $\mathbf{1 1 , 4 8 1 , 3 8 1}$ \\
\hline
\end{tabular}

Sources: Elaboration of Data from Istat in Gianfausto Rosoli, Un secolo di emigrazione italiana 1876-1976, Roma, Cser, 1978.

\subsection{Characteristics of Italian emigration}

The characteristics of Italian emigration included a prevalence of men - about $80 \%$ during the first period. The percentage of women was very low during the first years; it almost 
equaled that of men during the 1930s. The emigrants were very young; most of them were between 15 and 40 years old. It was a temporary emigration, at least at the beginning. It began as a seasonal migration of Northern Italians to Europe and Northern Africa, but soon developed into a permanent immigration overseas. Only 1/3 of the Italians who left between 1861 and 1940 decided to stay abroad. It was a proletarian emigration. Most of the emigrants were landless countrymen, craftsmen, and building workers. They were mostly illiterate; in 1871 the percentage of illiteracy in Italy was $67.5 \%$. In Southern Italy it was around $90 \%$. The number of professional people was extremely small.

\subsection{The reasons: factors of expulsion and factors of attraction}

The reasons for the mass emigration of the Italians were many, and there were also differences in the reasons that made people emigrate from the south and north of Italy. There were both factors of expulsion (push factors) and factors of attraction (pull factors).

The factors of expulsion were the crisis in agriculture of the 1880s, the increase in taxes in the country after the Italian unification of 1861, and the decline of the traditional trades and in the domestic industries. To make things worse for the countrymen of Southern Italy, because of the latifondo system ${ }^{6}$, they could count only on small lands that could not provide enough to survive. Between 1870 and 1900 the standard of living became worse in the whole of Italy, especially in the countryside. This led to the spreading of diseases and starvation. Food had become the biggest cost for an Italian family. Many peasant families spent about $75 \%$ of their money on food. Despite the high cost, this food often did not contain enough nutrition to sustain a person. ${ }^{7}$

In the North, the population suffered from pellagra. In the South malaria was the main plague. At first, malaria only struck in the coastal areas, but with deforestation, erosion and

\footnotetext{
${ }^{6}$ The latifondo system was a way of managing the land originating during the Roman period because of the surplus of land compared to the men available to work it. It remained during the Middle Ages and, in recent times, evolved into a form of exploitative income. The latifondo is a large landed estate owned by a single owner who rents small pieces of it to several countrymen. Because neither the owner of the land nor the men that work it have any interests in investing in the land itself (systems of irrigation, rational fertilization, mechanization, etc.) the productivity never increases.

${ }^{7}$ Lyttelton, A. Politics and Society 1870-1915. The Oxford Illustrated History of Italy. New York: Oxford University Press Inc., 1997.
} 
flooding it spread all over. The conditions which people endured in these areas were exceedingly harsh as 2 million Italians died each year. ${ }^{8}$

The agricultural system of Italy was not modernized, and there was little hope of improving the situation. Moreover, increasing numbers of products from America invaded Italian markets and made prices fall. There was no alternative other than to emigrate, and several factors of attraction - pull factors - opened the way to it: the request for labor and the decrease in the cost of travel.

\subsection{The ways of emigration}

The first to leave were the so-called pioneers that left Italy looking for opportunities. Then emigration developed following the pattern of chain migration. These chains followed familial, regional, and professional connections. In the case of regional connections, people decided to emigrate to places where people from the same region were already living. Professional connections led people with specific labor skills to reach places where suitable jobs were available. In the case of family connections, relatives, friends or people from the same village joined the first emigrants because of the news received by letters. These letters often included prepaid tickets, the main form of emigration support during the 1890s.

Another way of financing the travel overseas was credit, and the agents of emigration played a fundamental role. Before the Italian law of 1901 that established that the intermediaries of emigration had to be employees of the navigation companies, the agents of emigration were private citizens embarking in this activity to increase their incomes. They were usually usurers, people in prominent positions such as mayors, priests, notaries, and town council employees (or people in close contact with them) aiming at exploiting their connections. Emigrants applying at the town council to obtain documents to expatriate and/or to ask for advice from other important persons in their village sooner or later got in touch with these agents. The agents did not always have the best intentions and numerous emigrants were cheated in many ways: higher priced tickets, steamships that reached places they did not want to go, false promises of fantastic places and jobs. With the law of 1901 on emigration, the Italian state tried to solve this problem. Starting from that date, in fact, navigation companies directly appointed their intermediaries to sell tickets. In most cases the navigation companies

\footnotetext{
${ }^{8}$ Jones, A. Maldwin. The Italian Exodus. London, U.K.: Thames Television Ltd., 1976.
} 
chose the same agents, but instructed them on their new positions and professional duties. Still, these representatives of navigation companies were able to take advantage of the emigrants in many ways. In fact, it took a while before the new laws started to work properly and the agents remained the main referees for people to get information and to speed up the process of getting passports.

The navigation companies both favored and took advantage of the big boom in emigration. The shift from sail to steam navigation considerably shortened the duration of travels and allowed a considerable decrease in the price of tickets. For example, in 1902 the Societa' di Navigazione Generale Italiana Florio and Rubattino had nine steamships that were able to reach New York from Genoa in 16 days, and 110 vessels which made the same trip in one month. A new market was ready to pay for these services. European companies with headquarters in sea cities like Palermo, Naples, Genoa, and Marseilles flourished. In 1905 there were six navigation companies - Navigazione Generale Italiana with fourteen ships, La Veloce with thirteen ships, La Patria with six ships, Anglo-Italiana with four ships, HamburgAmerika Linie with four ships, Giuseppe Fornari with five ships- travelling from the Italian ports of Genoa, Naples, and Palermo to New York. The Compagnie Générale Transatlantique, a French company with ships travelling from Havre to New York, was the only foreign one authorized to sell tickets in Northern Italy through its representatives residing in Italy. The prices of the tickets sold by these companies ranged from a minimum of 143 to a maximum of 190 liras. People leaving from Havre had to add the cost of the train ticket from Italy, 40-60 liras according to the city of departure. ${ }^{9}$

\subsection{Italian emigration to the United States}

From 1857 to 1869 the total Italian emigration to the U.S. never exceeded one thousand per year. Starting in 1870 it steadily increased, but it stayed within five thousand per year until 1879. In 1880 the number jumped to reach about 12,000 Italian emigrants. ${ }^{10}$ According to the 1880 census there were 44,000 Italians in the U.S.

\footnotetext{
${ }^{9}$ Noli massimi per il trasporto degli emigranti. In Bollettino dell'Emigrazione Vol. 9 (1905), pp. 56-57.

${ }^{10}$ Beccherini, Francesco. Il Fenomeno dell'Emigrazione negli Stati Uniti. Sansepolcro: Tipografia Boncompagni, 1906. Beccherini was a priest at the church of Saint Francis in Detroit and was asked by Monsignor Scalabrini, Vescovo of Piacenza and founder of the Congregation of the Missioners of San Carlo for the Italian Emigrants to write a report.
} 
Between 1880 and 1890 about 268,000 Italians came to the U.S., but many of them returned and only 183,000 were enumerated in the 1890 census.

\begin{tabular}{|l|c|l|c|}
\hline \multicolumn{5}{|c|}{ Table II. Italian Emigrants departing for the United States between $\mathbf{1 8 7 6}$ and $\mathbf{1 9 2 5}}$. \\
\hline $\mathbf{1 8 7 6 - 1 8 7 9}$ & $7,618^{*}$ & $\mathbf{1 9 1 5 - 1 9 1 9}$ & 214,759 \\
\hline $\mathbf{1 8 8 0 - 1 8 8 4}$ & 67,984 & $\mathbf{1 9 2 0}$ & 349,042 \\
\hline $\mathbf{1 8 8 5 - 1 8 8 9}$ & 135,005 & $\mathbf{1 9 2 1}$ & 67,495 \\
\hline $\mathbf{1 8 9 0 - 1 8 9 4}$ & 216,697 & $\mathbf{1 9 2 2}$ & 41,637 \\
\hline $\mathbf{1 8 9 5 - 1 8 9 9}$ & 257,868 & $\mathbf{1 9 2 3}$ & 51,740 \\
\hline $\mathbf{1 9 0 0 - 1 9 0 4}$ & 769,269 & $\mathbf{1 9 2 4}$ & 35,374 \\
\hline $\mathbf{1 9 0 5 - 1 9 0 9}$ & $1,385,342$ & $\mathbf{1 9 2 5}$ & 29,723 \\
\hline $\mathbf{1 9 1 0 - 1 9 1 4}$ & $1,265,535$ & TOTAL & $\mathbf{4 , 8 9 5 , 0 8 8}$ \\
\hline $\begin{array}{l}\text { * with Canada } \\
\text { Sources: } \text { 'Emigrazione Italiana. Legislazione e Statistiche. Accordi Internazionali. } \text { Roma: Remo Sandron } \\
\text { Editore, } 1927, \text { Tav. IV. }\end{array}$ &
\end{tabular}

During the nineties, 604,000 entered the U.S.; only 484,000 appear in the census of 1900 . In the first decade of the nineteenth century, 2,104,000 arrived, but only 1,343,000 were enumerated in 1910. The pattern changed during the following years. Between 1910 and 1920 $1,110,000$ arrived and there were 1,610,000 persons of Italian birth in the 1920 census. By the 1920 s the Italian immigration to the U.S. was a permanent one. It continued in big numbers until the cut off imposed by the Immigration Quota Act in 1921. In fact, only 455,000 Italians entered the U.S. during the twenties following the Immigration Quota Act. In 1930 the census enumerated 1,790,000 Italians. ${ }^{11}$

\subsection{Reasons for Italian immigration into the U.S.}

Several factors caused the boom in the Italian emigration to the United States. First of all the improvement of national and transatlantic transport facilities made the trip affordable: at one point it was cheaper to leave for America than to reach Northern Europe. Secondly, the

\footnotetext{
${ }^{11}$ Glazer \& Moynihan. Beyond the Melting Pot. Cambridge, Massachussets: The M.I.T. University Press, 1964.
} 
economic crisis both in Southern and Northern Italy offered a push to emigrate. Thirdly, there was a growing demand for labor in North America. Last but not least was the fact that in the late nineteenth century Argentina, Brazil, and Uruguay (three nations that attracted millions of Italians before) underwent serious crises connected to rapid economic change and development, and the flow of Italians was deflected to the United States.

As in many other places in the world, Italians in America clustered into groups related to their place of origin. For example, the Sicilian resided in New Orleans, the Neapolitans and Calabrians in Minnesota, and many Northern Italians in California. However, most of the Italians were concentrated on the East Coast, in New York, Boston, Philadelphia, and Baltimore, just where they disembarked. Other groups decided to move away from the towns of arrival and look for new economic opportunities elsewhere. To understand why Italians were so clearly divided in different regional groups, one needs remember that until 1861, Italy was not a united state. Only when Italians arrived in America they did realize what the concept of nation meant. Italians came from a nation that struggled to become a state and at the time of their departure they were still lacking a national consciousness. They most likely identified with their village rather than with Italy. The center of the migrant's concern remained his hometown.

\subsection{Italian and American laws}

The Italian state did little to protect the emigrants during its first years. ${ }^{12}$ The initial negative judgments of the political elite on emigration led to administrative actions aimed at preventing the departure of those lacking adequate financial means and/or a job at their destination. The circolare Menabrea and the circolare Lanza ${ }^{13}$ were promulgated in 1868 and in 1873 respectively. In 1878 the circolare Nicotera mitigated these dispositions. The next law, named after Crispi, was passed in 1888. This law recognized for the first time the freedom to emigrate and paid some attention to the illicit acts of the agents of emigration and

\footnotetext{
${ }^{12}$ Brunialti, Attilio. "L'esodo degli italiani e la legge sell'emigrazione”. In Nuova Antologia di Scienze, Lettere ed Arti, Vol. XVI, C, Terza Serie, pp. 96-114.

${ }^{13}$ Circolare is an official document issued by the government. These two circolari ordered the major offices of the provinces to do as much as possible to prevent illegal emigration and to slow legal ones. In particular the circolare Lanza gave instructions to the mayors to refuse the nulla osta (permit) to emigrate to young males of the age to enter the army, to soldiers not through with army service, to disabled persons, and to anybody with scarce financial means.
} 
the contracts of transportation. This law did not include any direct intervention of the state in the matter of assistance to the emigrants. In 1901 a new law did so. First of all it introduced the vettore as a person legally appointed by a navigation company to recruit emigrants. The vettore substituted for the agente dell'emigrazione, persons that acted by themselves during the first years of the Italian emigration to speculate more or less legally on the need for information and assistance to emigrate. The law also instituted the Commissariato per l'Emigrazione, an organization dependent on the Department of Foreign Affairs. Consuls working for the Commissariato produced inquiries and studies on Italian communities all over the world that were then published in the Bollettino dell'Emigrazione. The aristocratic class extraction of most of the consuls, though, prevented them from having a clear understanding of the problems and conditions of the emigrants. Moreover, the law did not introduce any kind of help for emigrants in the countries of destination, leaving Italy without any sort of international agreement to facilitate the entering of emigrants into foreign labor markets. It left ample ways of action to private associations, though.

The Catholic Church was one of the first to act and help immigrants, both in the United States and in other countries of Italian emigration. The San Raffaele Society, founded by Monsignor Giovanni Battista Scalabrini, opened missions all over the Americas. The "scalabriniani" assisted immigrants both before their departure, helping them in dealing with agents and navigation companies, and at their arrival helping them to find a job and to integrate. $^{14}$

In the United States the situation for the immigrants wasn't that different. Up until the 1930s the state did little to help immigrants to face the many challenges they had in the new country. Once in the United States assistance to the immigrants came mainly from the private sector: religious people connected to the Protestant churches, and laymen belonging to the progressive movement. They assisted immigrants in the search for a job, a house - subtracting them from the hands of the padroni - and to better understand the complicated American laws concerning immigration.

Since the beginning of the great immigration, only the entrepreneurs were favorable to an unlimited arrival of people from Southeast Europe. Others had fears of a different nature: political, because of assumed subversive elements; social, because of assumed criminality;

\footnotetext{
${ }^{14}$ Beccherini, Francesco. Il Fenomeno dell'Emigrazione negli Stati Uniti. Sansepolcro: Tipografia Boncompagni, 1906.
} 
cultural, because of fear of the loss of Anglo-Saxon supremacy; and religious, because the new immigrants were mostly Catholics. Once the need for cheap labor ceased, they had their victory.

To curb the rapid movement of immigrants into the United States the government passed a series of laws. In 1921 the government passed the Immigration Quota Act. It restricted immigration to $3 \%$ of the population of each specific country according to the census of 1910. In 1924 the act was revised to restrict further the percentage to $2 \%$ using the census of 1890. These acts heavily affected Italian immigration. Before the enactment of the first restriction, Italian immigration into the United States in 1921 was 222,260. In 1925 it dropped to 6,203 . 


\section{Chapter II - Provenience}

\subsection{Italians and the mines of Lake Superior}

During the 1880s numerous mines opened on the copper and iron ranges of Lake Superior. The first mines opened in the mid-1840s on the Marquette Iron Range and on the Copper Range of Michigan. Michigan's Menominee Iron Range opened in the 1870s. In 1884 mines were opened on the Vermillion Iron Range in Minnesota and on the Gogebic Iron Range on the border between Michigan and Wisconsin. The Mesabi Iron Range and the Cuyuna Iron Range in Minnesota opened in 1892 and in 1912 respectively. ${ }^{15}$ During the first stage of mining a small work force was employed. In the second stage, an enlarged force labored to open up and test the most promising finds. Most of the workers in the mines were European immigrants. At the beginning British, Scandinavian, Irish and Germans were the most numerous nationalities. Around 1900 the number of Finnish, Croatian, Polish, Slovenian and Italian immigrants grew larger.

The Italian colony of the Upper Peninsula of Michigan is one of the oldest Italian colonies of the central states of the US. ${ }^{16}$ The very first Italians reached the Upper Peninsula of Michigan during the 1860s. They came to work in the copper and iron mines of Houghton, Marquette, Gogebic, and Menominee Counties. After 1880 they started to form significant communities in the region of Lake Superior. Because the earliest Italians were temporary residents ${ }^{17}$, many more Italians reached the area and worked in the mines than are reflected in census reports.

\subsection{Definition of Italian Community}

The goal of this study was to gather data about the number of the Italian immigrants that settled in Houghton County from the Michigan state censuses of 1870, 1880, 1900, 1910, 1920, and 1930 (see Appendix VI-XI). The census of 1890 is not available because it was lost

\footnotetext{
${ }^{15}$ Vecoli, J. Rudolph. “Dalle Marche e dall'Umbria alle miniere del Lago Superiore”. In E. Sori (ed.), Le Marche fuori dalle Marche. Ancona: Proposte e ricerche, 1998, p. 677.

${ }^{16}$ Castigliano, Attilio. “Origine, sviluppo, importanza ed avvenire delle colonie italiane del Nord Michigan e del Nord Minnesota”. In Bollettino dell'Emigrazione Vol. 7 (1913), p. 723.

${ }^{17}$ Temporary because they tended to return to Italy after a while. See Chapter I.
} 
in a fire. The census of 1870 is the first year examined because before that time the presence of Italians in the county is almost irrelevant. ${ }^{18}$ The last year is 1930 because it is the last census available to the public, and it is evident that global recession and Depression slowed emigration from Europe.

Individuals who were born in Italy, individuals who were not born in Italy but with at least one Italian parent or grandparent, individuals with Italian surnames, or individuals with varying nationalities, married to one of the previous, are included in the Italian community.

Previous studies about Italian immigrants to the Upper Peninsula of Michigan stated general patterns about their regions of provenience. According to the works of Professor Rudolph Vecoli and Professor Russell Magnaghi, Italians coming from different regions of Italy settled in different counties of the Upper Peninsula. Gogebic County Italians were mainly from Piedmont, Abruzzo, Sicily and Tyrol ${ }^{19}$. Menominee County Italians mostly came from the province of Venice. In Dickinson County there were people from Tyrol, Abruzzo, and Lombardy. Iron County Italians mostly came from Umbria, Marches, Abruzzo, and Tyrol. Houghton County Italians mostly came from the regions of Piedmont, Tuscany, and Lombardy (see Figure III, p. 26). ${ }^{20}$

In 1912 Attilio Castigliano, Italian consul in Duluth ${ }^{21}$, Minnesota, wrote in a report published in the Bollettino dell'Emigrazione, the official publication of the Commissariato per l'Emigrazione ${ }^{22}$, that

\begin{abstract}
"The Italian colony of the Upper Peninsula of Michigan is one of the oldest Italian colonies of the central states of the North American Federation. In fact our workers, mainly piemontesi and toscani, started to work, in big numbers, in the copper mines of Calumet, Michigan, since the beginning of the mines themselves, that is about fifty years ago [...] Most of the Italians in the Copper Country came from Piemonte and more specifically from Canavese; numerous are
\end{abstract}

\footnotetext{
${ }^{18}$ The census of 1860 enumerates four Italians: Joseph Gatan, Joseph Coppo, Vital Coppo, and Bart Quello.

${ }^{19}$ Tyrol is not an administrative region of Italy, it is an area bordering Austria in which Italian and Austrian culture mixes.

${ }^{20}$ Magnaghi, M. Russell. "Italians in the Upper Peninsula". In James M. Anderson and Iva A. Smith (eds), Ethnic Groups in Michigan Vol. 2, The People of Michigan. Detroit: Ethnos Press, 1983, p. 173.

${ }^{21}$ The Upper Peninsula of Michigan was under the jurisdiction of the Regia Agenzia Consolare (Royal Consular Agency) of Duluth. The Lower Peninsula was under the jurisdiction of the Regia Agenzia Consolare of Detroit.

${ }^{22}$ See Chapter I about Commissariato dell'Emigrazione and Bollettino dell'Emigrazione.
} 
the Toscani (Lucchesi), little the Veneti and very little the Lombardi; Southern Italians are absent". ${ }^{23}$

The sources used to verify these data and to track down the provenience of Italian immigrants to Houghton County have been essentially four: the Ellis Island database that includes ships' manifests indicating the last place of residence of the immigrants, the employment cards of Calumet and Hecla Mining Company kept at Michigan Technological University and Copper Country Archives that indicate the place of birth of the workers, the available issues of the Italian newspaper Il Minatore Italiano (The Italian Miner) edited by Italian immigrants in Calumet starting from 1896 that sometimes included data about local Italians, and the oral interviews taken by Professor Russell Magnaghi during the 1980s with Italian immigrants and descendants of the Upper Peninsula in which people talk about their villages of origin.

It has been possible to establish a region and province of origin for most of the immigrants sometimes even their city and/or their village. Other individuals cannot be identified to this level; because the name of the immigrant on the census is not clearly readable; because the immigrant arrived before 1897 and on the ships' manifests of the Ellis Island database only the country of origin is indicated; or because the immigrant arrived in the US through a port that is not New York and manifests were not accessible. In the case of married women there is a different problem. If they arrived in the United States with their husbands or some years later but already married, their name is on the ships' manifests. If they married after their arrival, because they changed their surname, their name on the censuses does not match that on the ships' manifests. In some cases however, the employment cards indicate the maiden names of the wives of the workers, so it's been possible to track down their provenience.

Children born in the United States from Italian parents were assigned the provenience of the father if both the parents were Italians and the provenience of the Italian parent if one of them was not Italian.

\footnotetext{
${ }^{23}$ Castigliano, Attilio. "Origine, sviluppo, importanza ed avvenire delle colonie italiane del Nord Michigan e del Nord Minnesota”. In Bollettino dell'Emigrazione Vol. 7 (1913), p. 723 and 731.
} 


\subsection{Italians in Houghton County in 1870: numbers and provenience}

In 1870 the Italian community in Houghton County included just twenty-one individuals. Six of them were actually born in Italy, eleven were born in another country but from at least one Italian parent, four were from other nations and married an Italian (one Irish, one Canadian, one American, and one French).

As far as provenience of those born in Italy and their sons, two provinces and two regions are represented: Piemonte and its province Torino, and Toscana and its province Lucca. Nine immigrants were from the province of Torino, five from the province of Lucca, and three of them unknown (the problem with them is the surname and the fact that they came before 1897).

\subsection{Italians in Houghton County in 1880: numbers and provenience}

In 1880 the community numbered 220 individuals. Of these 177 (81\%) were actually born in Italy; thirty-five (16\%) were born in another country from at least one Italian parent. The rest of the community included seven individuals of other nationalities married to Italians (3\%): three Irish, one German, one French, one Canadian, and one American.

The regions and the provinces represented are three, Piemonte (Torino), Toscana (Lucca), and Veneto (Vicenza): ninety-six immigrants (44\%) came from the province of Torino, $60(27 \%)$ from the province of Lucca, and three from the province of Vicenza (1\%). For 54 immigrants $(25 \%)$ no information was available.

\begin{tabular}{|l|c|}
\hline \multicolumn{2}{|c|}{ Table III. Provenience 1880 } \\
\hline Unknown & $\mathbf{5 4}(25 \%)$ \\
\hline Other nationalities & $\mathbf{7}(3 \%)$ \\
\hline Piemonte (province of Torino) & $\mathbf{9 6}(44 \%)$ \\
\hline Toscana (province of Lucca) & $\mathbf{6 0}(27 \%)$ \\
\hline Veneto (province of Vicenza) & $\mathbf{3}(1 \%)$ \\
\hline TOTAL & $\mathbf{2 2 0}$ \\
\hline
\end{tabular}

Table III shows the provenience of the individuals of the community in 1880 . Although it is not of particular use now because the numbers are not that big, it will help to 
stress a specific pattern of Italian immigration to this county through the years. In fact the three regions represented in the table will remain in the first place as the sources of immigration to Houghton County. The table also helps to compare the trend of Italian immigration to Houghton County with that of general emigration from Italy during this period (see Figure I, Chapter I). In fact from 1876 until 1900, the so-called first phase of Italian emigration, most Italian emigrants left from the northern regions of Veneto, Friuli Venezia Giulia, and Piemonte.

Because some names of the 1880 census match names on the census of the following years, the documents yielded specific information about the villages (nowadays probably municipalities) in the province of Torino from which some of them came. The villages are Agliè, Locana Canavese, Pont Canavese, San Giorgio Canavese, San Martino Canavese, and Vialfre'. The pattern that starts to emerge and that will be evident in the following years is the fact that people from the province of Torino were actually coming from a limited area of the province itself called Canavese (see paragraph 2.4.2 of this chapter).

\subsection{Italians in Houghton County in 1900: numbers and provenience}

In 1900 the Italian community reaches 2,789 . The increase was probably already evident in 1890, but there are not data available for that year. Of a total of 2,789 people, 1,891 $(68 \%)$ were born in Italy, 855 (31\%) had at least an Italian parent. The rest of them, thirty-two $(1 \%)$ were from other nations and married an Italian.

With the increase in number also increased the number of provinces and regions of origin. There are in fact ten regions and twenty-one provinces represented.

There is no information about the provenience for 675 Italians (24.2\%). As for the others, as Table IV shows, Torino and Lucca remain the first two provinces, with respectively $1,489(53.3 \%)$ and $434(15.6 \%)$ individuals. The third one is Belluno with forty-nine individuals (1.8\%), followed by Bergamo with thirty-eight (1.4\%) and Imperia with fifteen $(0.5 \%)$. The rest of the provinces with less than fifteen immigrants sum up to seventy individuals $(2.5 \%)$.

Marriages with persons from other nations increased. In fact there are nineteen individuals $(0.7 \%)$ of other nationalities married to Italians (three Canadian, six German, three Irish, three American, one Belgian, one Norwegian, one Scottish, and one Swedish). 
Again there was information about the villages of provenience of many immigrants of the province of Torino, and as in 1880, those villages are all in the area of Canavese. Of a total of 1,489 persons coming from the province of Torino, at least 966 came from thirty-eight different villages of the Canavese area (see APPENDIX VIII, Census of 1900). The remaining 523 were probably from those villages too, but the information available did not help to establish it. On the other hand, immigrants from the province of Lucca came from at least nineteen different villages/cities scattered all over the province, not just from a restricted area. $^{24}$

\begin{tabular}{|c|c|c|c|}
\hline \multicolumn{4}{|c|}{ Table IV. Provenience 1900} \\
\hline Unknown & & & $\begin{array}{c}\mathbf{6 7 5} \\
(24.22 \%) \\
\end{array}$ \\
\hline Other nationalities & & & $\begin{array}{c}19 \\
(0.7 \%)\end{array}$ \\
\hline \multirow{4}{*}{ Piemonte } & Torino & $1,489(15.6 \%)$ & \multirow{4}{*}{$\begin{array}{c}1,493 \\
(53.54 \%)\end{array}$} \\
\hline & Novara & 2 & \\
\hline & Vercelli & 1 & \\
\hline & Cuneo & 1 & \\
\hline \multirow{3}{*}{ Toscana } & Lucca & $434(15.6 \%)$ & \multirow{3}{*}{$\begin{array}{c}\mathbf{4 4 0} \\
(15.8 \%)\end{array}$} \\
\hline & Pistoia & 5 & \\
\hline & Pisa & 1 & \\
\hline \multirow{5}{*}{ Lombardia } & Bergamo & $38(1.4 \%)$ & \multirow{5}{*}{$\begin{array}{c}\mathbf{6 7} \\
(2.41 \%)\end{array}$} \\
\hline & Milano & 10 & \\
\hline & Varese & 8 & \\
\hline & Como & 7 & \\
\hline & Sondrio & 4 & \\
\hline \multirow{3}{*}{ Veneto } & Belluno & $49(1.8 \%)$ & \multirow{3}{*}{$\begin{array}{c}\mathbf{6 2} \\
(2.22 \%)\end{array}$} \\
\hline & Vicenza & 11 & \\
\hline & Treviso & 2 & \\
\hline Liguria & Imperia & $15(0.5 \%)$ & $\begin{array}{c}15 \\
(0.53 \%)\end{array}$ \\
\hline Emilia Romagna & Modena & 7 & $\begin{array}{c}7 \\
(0.2 \%) \\
\end{array}$ \\
\hline Campania & Napoli & 4 & $\begin{array}{c}\mathbf{4} \\
(0.14 \%)\end{array}$ \\
\hline Trentino Alto Adige & Bolzano & 3 & $\begin{array}{c}\mathbf{3} \\
(0.11 \%)\end{array}$ \\
\hline Abruzzo & L'Aquila & 2 & $\begin{array}{c}\mathbf{2} \\
(0.07 \%)\end{array}$ \\
\hline Friuli Venezia Giulia & Udine & 1 & $\begin{array}{c}1 \\
(0.03 \%)\end{array}$ \\
\hline Marche & Ascoli Piceno & 1 & $\begin{array}{c}\mathbf{1} \\
(0.03 \%)\end{array}$ \\
\hline TOTAL & & & 2,789 \\
\hline
\end{tabular}

${ }^{24}$ The province of Torino is about 6,830 square $\mathrm{km}$ whereas the province of Lucca is about 1,772 . 


\subsection{Italians in Houghton County in 1910: numbers and provenience}

In 1910 the Italian community in Houghton County reached its peak with 4,384 people: 2,539 of them (58\%) were actually born in Italy, 1,786 (41\%) had at least one Italian parent, fourteen had at least an Italian grandparent, and twenty-seven were from other nationalities.

\begin{tabular}{|c|c|c|c|}
\hline \multicolumn{4}{|c|}{ Table V. Provenience 1910} \\
\hline Unknown & & & $\begin{array}{c}\mathbf{8 4 3} \\
(19.22 \%)\end{array}$ \\
\hline Other nationalities & & & $\begin{array}{c}\mathbf{2 2} \\
(0.51 \%)\end{array}$ \\
\hline \multirow{4}{*}{ Piemonte } & Torino & $2,201(50.2 \%)$ & \multirow{4}{*}{$\begin{array}{c}\mathbf{2 , 2 0 7} \\
(50.35 \%)\end{array}$} \\
\hline & Vercelli & 4 & \\
\hline & Alessandria & 1 & \\
\hline & Cuneo & 1 & \\
\hline \multirow{6}{*}{ Toscana } & Lucca & $989(22.6 \%)$ & \multirow{6}{*}{$\begin{array}{c}\mathbf{1 , 0 3 2} \\
(23.55 \%)\end{array}$} \\
\hline & Pisa & 13 & \\
\hline & Pistoia & 12 & \\
\hline & Firenze & 8 & \\
\hline & Livorno & 7 & \\
\hline & Massa Carrara & 3 & \\
\hline \multirow{7}{*}{ Lombardia } & Bergamo & $41(0.9 \%)$ & \multirow{7}{*}{$\begin{array}{c}134 \\
(3.1 \%)\end{array}$} \\
\hline & Varese & 39 & \\
\hline & Milano & $27(0.6 \%)$ & \\
\hline & Sondrio & 11 & \\
\hline & Como & 7 & \\
\hline & Brescia & 6 & \\
\hline & Cremona & 3 & \\
\hline \multirow{5}{*}{ Veneto } & Belluno & $61(1.4 \%)$ & \multirow{5}{*}{$\begin{array}{c}\mathbf{8 6} \\
(1.96 \%)\end{array}$} \\
\hline & Vicenza & 14 & \\
\hline & Venezia & 5 & \\
\hline & Verona & 3 & \\
\hline & Treviso & 3 & \\
\hline \multirow{2}{*}{ Molise } & Isernia & 11 & \multirow{2}{*}{$\begin{array}{c}12 \\
(0.27 \%)\end{array}$} \\
\hline & Campobasso & 1 & \\
\hline Campania & Napoli & 10 & $\begin{array}{c}10 \\
(0.22 \%)\end{array}$ \\
\hline \multirow{2}{*}{ Calabria } & Crotone & 7 & \multirow{2}{*}{$\begin{array}{c}9 \\
(0.21 \%)\end{array}$} \\
\hline & Cosenza & 2 & \\
\hline Trentino Alto Adige & Trento & 5 & $\begin{array}{c}\mathbf{5} \\
(0.11 \%)\end{array}$ \\
\hline Valle d'Aosta & Aosta & 5 & $\begin{array}{c}5 \\
(0.11 \%)\end{array}$ \\
\hline Friuli Venezia Giulia & Udine & 4 & $\begin{array}{c}\mathbf{4} \\
(0.09 \%)\end{array}$ \\
\hline Sardegna & Cagliari & 4 & $\begin{array}{c}\mathbf{4} \\
(0.09 \%)\end{array}$ \\
\hline
\end{tabular}




\begin{tabular}{|l|l|c|c|}
\hline Emilia Romagna & Parma & 3 & $\begin{array}{c}\mathbf{3} \\
(0.06 \%)\end{array}$ \\
\hline \multirow{2}{*}{ Lazio } & Roma & 1 & $\begin{array}{c}\mathbf{2} \\
(0.04 \%)\end{array}$ \\
\cline { 2 - 4 } Sicilia & Frosinone & 1 & $\begin{array}{c}\mathbf{2} \\
(0.04 \%)\end{array}$ \\
\hline Umbria & Messina & 2 & $\begin{array}{c}\mathbf{1} \\
(0.02 \%)\end{array}$ \\
\hline Basilicata & Perugia & 1 & $\begin{array}{c}\mathbf{1} \\
(0.02 \%)\end{array}$ \\
\hline Abruzzo & Potenza & 1 & $\begin{array}{c}\mathbf{1} \\
(0.02 \%)\end{array}$ \\
\hline TOTAL & L'Aquila & 1 & $\mathbf{4 , 3 8 4}$ \\
\hline
\end{tabular}

The places of origin increased in variety, accompanying the large increase in the size of the community. The regions represented are fifteen. Compared to 1910, Marche and Liguria do not appear anymore, the new ones are Valle d'Aosta, Lazio, Umbria, Molise, Calabria, Sicilia, Sardegna, and Basilicata.

People for whom there are no data about provenience are 843 (19.2\%). People from other nationalities slightly increase in number, but decrease in percentage $(0.5 \%)$, showing that Italians preferred to marry among themselves when possible, and that the marriage pool had grown sufficiently large to enable this preference.

Torino is again the first province for numbers of immigrants with 2,201 (50.2\%), Lucca follows with 989 (22.6\%). Belluno sixty-one (1.4\%) is still the third, followed by Bergamo with forty-one $(0.9 \%)$, Varese with thirty-nine $(0.9 \%)$, and Milano with twentyseven $(0.6 \%)$. The remaining provinces with less than fifteen immigrants account for $3.7 \%$.

The increase in the variety of provinces confirms the general trend of Italian emigration during this period (see Table II, Chapter I). In fact from 1901 until 1915, the period of the so-called 'great emigration', people start to leave in great numbers from many regions, not only from northern ones. It is important to notice, though, that this is also the time when emigration from Southern Italy tops that from the North. Yet we find just 22 people from southern regions (Calabria, Campania, Basilicata, and Sicilia. The number could increase to 42 if we include 20 individuals coming from central regions (Lazio, Abruzzo, Molise, Sardegna, and Umbria). ${ }^{25}$ One of the reasons why Houghton County does not appear a favorite destination for southern Italians may be the weather, not really attractive for people

\footnotetext{
${ }^{25}$ In some ships' manifests, in fact, central regions are considered South.
} 
used to warm temperature during most of the year. Another reason may be the fact that by that time prejudices about Southern Italians - and Southern Europeans in genera ${ }^{26}$ - were already widespread and companies such as Calumet and Hecla and Quincy may have specifically asked for Northern immigrants when recruiting.

Again the villages of provenience for many immigrants are in the province of Torino, and as in 1880 and 1900 those villages are all in the area of Canavese. On a total of 2,201 immigrants from the province of Torino, 1,703 surely came from at least forty-seven different villages of the area of Canavese. The remaining 498 were probably from the same area, but there is no direct evidence. Those from the province of Lucca came from at least 38 different villages scattered all over the province.

\subsection{Italians in Houghton County in 1920: numbers and provenience}

In 1920 the community counts 4,379 individuals: 2,045 (47\%) were born in Italy, 2,234 (51\%) had at least one Italian parent, fifty-three at least one Italian grandparent, and forty-seven were of different nationalities. The community appears stable compared to 1910 in which the members numbered 4,384.

What changes a little is the proportion of people born in Italy and first generation Americans. In 1910 2,539 out of 4,384 (58\%) were born in Italy whereas in 1920 the number drops to 2045 out of 4,379 (47\%). There are two reasons: fewer Italians arrived in Houghton County, and those that were already here started a family and had children. This trend follows the general trend of Italian emigration in this decade; in fact fewer people were allowed to leave Italy because of the war and military service.

The regions represented are eighteen as in 1910, but Marche takes the place of Umbria. The provinces are forty. The number of people whose provenience is unknown is 878 (20\%). Comparing 1910 to 1920 there are some changes in the percentages of immigrants coming from the two main provinces represented, Torino and Lucca. In 1920 people from the province of Torino decrease to 1,902 (43.4\%). People from the province of Lucca increase to $1,261(28.8 \%)$. Belluno is still the third province with forty-one immigrants $(0.9 \%)$, followed

\footnotetext{
${ }^{26}$ Laughlin H. H. (Statement by). Analysis of America's Modern Melting Pot. Hearing before the Committee on Immigration and Naturalization, House of Representative, LXII Congress, III Session, Washington 1923, Serial 7-C, pp. 725-831.
} 
as in 1910 by Bergamo with thirty-nine (0.9\%). Only three other provinces have more than fifteen immigrants: Varese thirty $(0.7 \%)$, Vicenza twenty-nine $(0.7 \%)$, and Milano eighteen $(0.4 \%)$. The rest of the provinces with less than fifteen immigrants account for $3.3 \%$ of the total. Marriages between Italians and people from other nations increased. There are in fact 39 $(0.9 \%)$ people of different nationalities married to Italians.

The number of provinces represented in 1920 is similar to 1910, thirty-six and forty respectively, but in 1920 there is an increase in the southern ones. The overall number of people from southern regions (Campania, Basilicata, Calabria, and Sicilia) increases to thirtysix. Also the variety of the provinces of the central regions (Toscana, Marche, Lazio, Sardegna, Molise, Abruzzo, Emilia-Romagna) increases in 1920.

\begin{tabular}{|c|c|c|c|}
\hline \multicolumn{4}{|c|}{ Table VI. Provenience 1920} \\
\hline Unknown & & & $\begin{array}{c}878 \\
(20 \%) \\
\end{array}$ \\
\hline Other nationalities & & & $\begin{array}{c}39 \\
(0.9 \%) \\
\end{array}$ \\
\hline \multirow{4}{*}{ Piemonte } & Torino & $1,902(43.4 \%)$ & \multirow{4}{*}{$\begin{array}{c}1,906 \\
(43.52 \%)\end{array}$} \\
\hline & Alessandria & 2 & \\
\hline & Vercelli & 1 & \\
\hline & Cuneo & 1 & \\
\hline \multirow{4}{*}{ Toscana } & Lucca & $1,261(28.8 \%)$ & \multirow{4}{*}{$\begin{array}{c}\mathbf{1 , 2 8 7} \\
(29.4 \%)\end{array}$} \\
\hline & Pisa & 15 & \\
\hline & Firenze & 8 & \\
\hline & Massa Carrara & 3 & \\
\hline \multirow{5}{*}{ Lombardia } & Bergamo & $39(0.9 \%)$ & \multirow{5}{*}{$\begin{array}{c}97 \\
(2.22 \%)\end{array}$} \\
\hline & Varese & $30(0.7 \%)$ & \\
\hline & Milano & $18(0.4 \%)$ & \\
\hline & Sondrio & 8 & \\
\hline & Como & 2 & \\
\hline \multirow{5}{*}{ Veneto } & Belluno & $41(0.9 \%)$ & \multirow{5}{*}{$\begin{array}{c}\mathbf{8 1} \\
(1.85 \%)\end{array}$} \\
\hline & Vicenza & $29(0.7 \%)$ & \\
\hline & Venezia & 6 & \\
\hline & Treviso & 3 & \\
\hline & Verona & 2 & \\
\hline Basilicata & Potenza & 13 & $\begin{array}{c}13 \\
(0.3 \%)\end{array}$ \\
\hline \multirow{3}{*}{ Campania } & Napoli & 6 & \multirow{3}{*}{$\begin{array}{c}\mathbf{1 0} \\
(0.23 \%)\end{array}$} \\
\hline & Benevento & 2 & \\
\hline & Avellino & 2 & \\
\hline \multirow{2}{*}{ Sicilia } & Palermo & 9 & \multirow{2}{*}{$\begin{array}{c}10 \\
(0.23 \%)\end{array}$} \\
\hline & Trapani & 1 & \\
\hline \multirow{3}{*}{ Marche } & Fano & 4 & \multirow{3}{*}{$\begin{array}{c}9 \\
(0.21 \%)\end{array}$} \\
\hline & Ancona & 3 & \\
\hline & Ascoli Piceno & 2 & \\
\hline
\end{tabular}




\begin{tabular}{|c|c|c|c|}
\hline \multirow{2}{*}{ Lazio } & Roma & 7 & \multirow{2}{*}{$\begin{array}{c}\mathbf{8} \\
(0.18 \%)\end{array}$} \\
\hline & Rieti & 1 & \\
\hline Sardegna & Cagliari & 8 & $\begin{array}{c}\mathbf{8} \\
(0.18 \%)\end{array}$ \\
\hline Molise & Campobasso & 6 & $\begin{array}{c}6 \\
(0.14 \%)\end{array}$ \\
\hline Valle d'Aosta & Aosta & 6 & $\begin{array}{c}6 \\
(0.14 \%)\end{array}$ \\
\hline Friuli Venezia Giulia & Trieste & 6 & $\begin{array}{c}6 \\
(0.14 \%)\end{array}$ \\
\hline \multirow{2}{*}{ Abruzzo } & Teramo & 4 & \multirow{2}{*}{$\begin{array}{c}\mathbf{5} \\
(0.12 \%)\end{array}$} \\
\hline & Chieti & 1 & \\
\hline Trentino Alto Adige & Trento & 4 & $\begin{array}{c}4 \\
(0.10 \%)\end{array}$ \\
\hline \multirow{3}{*}{ Calabria } & Crotone & 1 & \multirow{3}{*}{$\begin{array}{c}\mathbf{3} \\
(0.07 \%)\end{array}$} \\
\hline & Catanzaro & 1 & \\
\hline & Cosenza & 1 & \\
\hline Emilia Romagna & Rimini & 3 & $\begin{array}{c}\mathbf{3} \\
(0.07 \%)\end{array}$ \\
\hline TOTAL & & & 4,379 \\
\hline
\end{tabular}

The villages of provenience for many immigrants of the province of Torino, as in 1880, 1900, and 1910, are all in the area of Canavese. Those from the province of Lucca came from villages or cities scattered all over the province.

\subsection{Italians in Houghton County in 1930: numbers and provenience}

In 1930 the community significantly drops to 2,839 people. There are $989(34.8 \%)$ people actually born in Italy, 1,547 (54.5\%) with at least one Italian parent, $190(6.7 \%)$ with at least one Italian grandparent, and 113 (4\%) individuals of different nationalities.

The variety of the provinces and the regions does not diminish though. The regions represented are fifteen (compared to 1920 Basilicata and Valle d'Aosta disappear and Liguria comes back), and the provinces are thirty-three.

Immigrants for whom there are no data available about provenience are $560(19.7 \%)$. Marriages between Italians and non-Italians significantly increase. In fact there are 113 people of different nationalities (3.9\%). Torino remains the first province with 1,240 immigrants (43.7\%), followed as usual by Lucca with 663 (23.3\%). This time Bergamo, with forty-one immigrants (1.4\%), tops Belluno with thirty-seven (1.3\%). Milano and Pistoia follow with 
nineteen $(0.7 \%)$ and eighteen $(0.7 \%)$ individuals respectively. The remaining provinces, each with less than fifteen immigrants, account for $5.2 \%$.

Also in 1930 the villages of provenience of many immigrants of the province of Torino are all in the area of Canavese. Out of 1,240 immigrants coming from the province of Torino, 835 came from at least twenty-eight different villages of the Canavese. Those from the province of Lucca came from at least twenty-six villages/cities scattered all over the province.

\begin{tabular}{|c|c|c|c|}
\hline \multicolumn{4}{|c|}{ Table VII. Provenience 1930} \\
\hline Unknown & & & $\begin{array}{c}\mathbf{5 6 0} \\
(19.7 \%)\end{array}$ \\
\hline Other nationalities & & & $\begin{array}{c}109 \\
(3.9 \%)\end{array}$ \\
\hline \multirow{3}{*}{ Piemonte } & Torino & 1,240 & \multirow{3}{*}{$\begin{array}{c}\mathbf{1 , 2 4 9} \\
(43.99 \%)\end{array}$} \\
\hline & Cuneo & 7 & \\
\hline & Alessandria & 2 & \\
\hline \multirow{6}{*}{ Toscana } & Lucca & 663 & \multirow{6}{*}{$\begin{array}{c}\mathbf{6 9 6} \\
(24.52 \%)\end{array}$} \\
\hline & Pistoia & 18 & \\
\hline & Firenze & 9 & \\
\hline & Pisa & 4 & \\
\hline & Grosseto & 1 & \\
\hline & Massa Carrara & 1 & \\
\hline \multirow{6}{*}{ Lombardia } & Bergamo & 41 & \multirow{6}{*}{$\begin{array}{c}\mathbf{8 6} \\
(3 \%)\end{array}$} \\
\hline & Varese & 22 & \\
\hline & Milano & 19 & \\
\hline & Como & 2 & \\
\hline & Cremona & 1 & \\
\hline & Sondrio & 1 & \\
\hline \multirow{4}{*}{ Veneto } & Belluno & 37 & \multirow{4}{*}{$\begin{array}{c}\mathbf{7 2} \\
(2.54 \%)\end{array}$} \\
\hline & Vicenza & 23 & \\
\hline & Venezia & 11 & \\
\hline & Verona & 1 & \\
\hline \multirow{2}{*}{ Friuli Venezia Giulia } & Trieste & 10 & \multirow{2}{*}{$\begin{array}{c}\mathbf{1 5} \\
(0.53 \%)\end{array}$} \\
\hline & Udine & 5 & \\
\hline \multirow{2}{*}{ Marche } & Ancona & 7 & \multirow{2}{*}{$\begin{array}{c}\mathbf{1 4} \\
(0.5 \%)\end{array}$} \\
\hline & Ascoli Piceno & 7 & \\
\hline Calabria & Cosenza & 9 & $\begin{array}{c}9 \\
(0.32 \%)\end{array}$ \\
\hline Molise & Campobasso & 8 & $\begin{array}{c}\mathbf{8} \\
(0.28 \%)\end{array}$ \\
\hline Trentino Alto Adige & Trento & 8 & $\begin{array}{c}\mathbf{8} \\
(0.28 \%)\end{array}$ \\
\hline Lazio & Roma & 5 & $\begin{array}{c}\mathbf{5} \\
(0.17 \%)\end{array}$ \\
\hline Liguria & Imperia & 2 & $\begin{array}{c}\mathbf{2} \\
(0.07 \%)\end{array}$ \\
\hline Abruzzo & Chieti & 1 & $\begin{array}{c}\mathbf{2} \\
(0.07 \%)\end{array}$ \\
\hline
\end{tabular}




\begin{tabular}{|c|c|c|c|}
\hline \multirow{2}{*}{ Emilia Romagna } & Bologna & 1 & \multirow{2}{*}{$\begin{array}{c}\mathbf{2} \\
(0.07 \%)\end{array}$} \\
\hline & Reggio Emilia & 1 & \\
\hline Campania & Napoli & 1 & $\begin{array}{c}1 \\
(0.03 \%)\end{array}$ \\
\hline Sicilia & Trapani & 1 & $\begin{array}{c}1 \\
(0.03 \%)\end{array}$ \\
\hline TOTAL & & & 2,839 \\
\hline
\end{tabular}

\subsection{Emigration from Toscana and Piemonte}

The data clearly show a predominance of immigrants coming from two single provinces, Torino and Lucca, of two different regions, Piemonte and Toscana.

The maps below (Figure VI and VII) show respectively the position of the Italian regions and the approximate borders of the provinces in each region. Piemonte is one of the Northern regions of Italy, Toscana is considered Central or Northern-central Italy. The city and province of Torino is in the northern part of Piemonte (see map of the provinces, number seven). The city and province of Lucca is in the central part of Toscana, with some of the territories on the Tirrenian Sea (see map of the provinces, number fifty-one). The boundaries of the provinces have been changed through the years and provinces have been added. In both Lucca and Torino, some cities and small villages now belong to new provinces. ${ }^{27}$ Therefore, in dealing with the data, we have to consider that. Nonetheless, municipalities and villages from whom most of the Italian emigrants to the Houghton County came still remain in the same region.

\subsubsection{Lucca}

Lucca is one of the ten provinces of Toscana. It stretches for about 1,772 square kilometers and it is comprised between the Ligurian and Tirrenian Seas, the chain of Appennino Tosco-emiliano, and the valley of Arno.

Nowadays the province itself, besides the city of Lucca, has a population of about 380,000 inhabitants divided among about thirty-five municipalities (see Appendix IV for a complete list).

\footnotetext{
${ }^{27}$ For example in 1923 some villages previously included in the province of Lucca passed to that of Pistoia.
} 


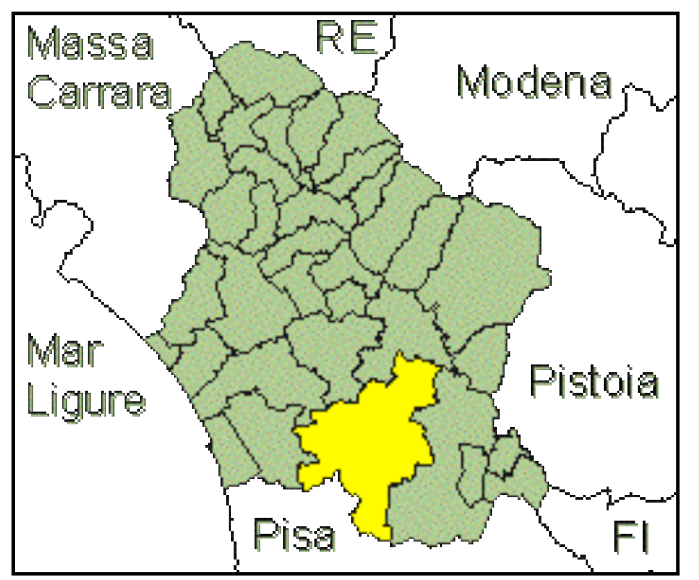

Figure IV. Map of the Province of Lucca showing borders of the city (clearer) and borders of the other municipalities.

The data about the provenience of Italian immigrants to Houghton County show that those who came from the province of Lucca were actually leaving from many villages scattered all over the province.

In 1900 Lucchesi mainly came from the municipalities of Collodi, Matraia, Pariana, and Villa Basilica. In 1910 they came from Collodi, Pescaglia, Matraia, Segromigno, and Santa Maria del Giudice. In 1920 the sources were Capannori, Colle di Compito, Collodi, Matraia, Pescaglia, Santa Maria del Giudice, and Segromigno. In 1930 people came from Collodi, Castelvecchio, Matraia, Pariana, Segromigno, Santa Maria del Giudice, and Ruota.

The reasons that pushed Lucchesi out of Lucca are probably the same reasons discussed in Chapter I - that forced millions of Italians to leave their villages and cities in the late nineteenth and early twentieth century. What remains unclear is what particular factors attracted them to Houghton County. Chain migration accounts for the largest number of them but does not explain why the very first Lucchesi settled here.

\subsubsection{Area of Canavese in the province of Torino}

Torino is the main city of the region of Piemonte, and with its 6,830 square $\mathrm{km}$ of surface is also its biggest province. Nowadays the province itself including the city of Torino counts more than 2,200,000 inhabitants. The Canavese is a northwest area of the province of Torino. It is delimited towards the northwest by the region of Valle d'Aosta, and towards east by the province of Vercelli. It is about 2,000 square kilometers in size, and includes around 
278,000 inhabitants distributed in more than 120 municipalities (see Appendix V for a complete list).

In all the years examined Pont Canavese, San Giorgio Canavese and San Martino Canavese (see following map) were the villages from which most of the immigrants to Houghton County came.

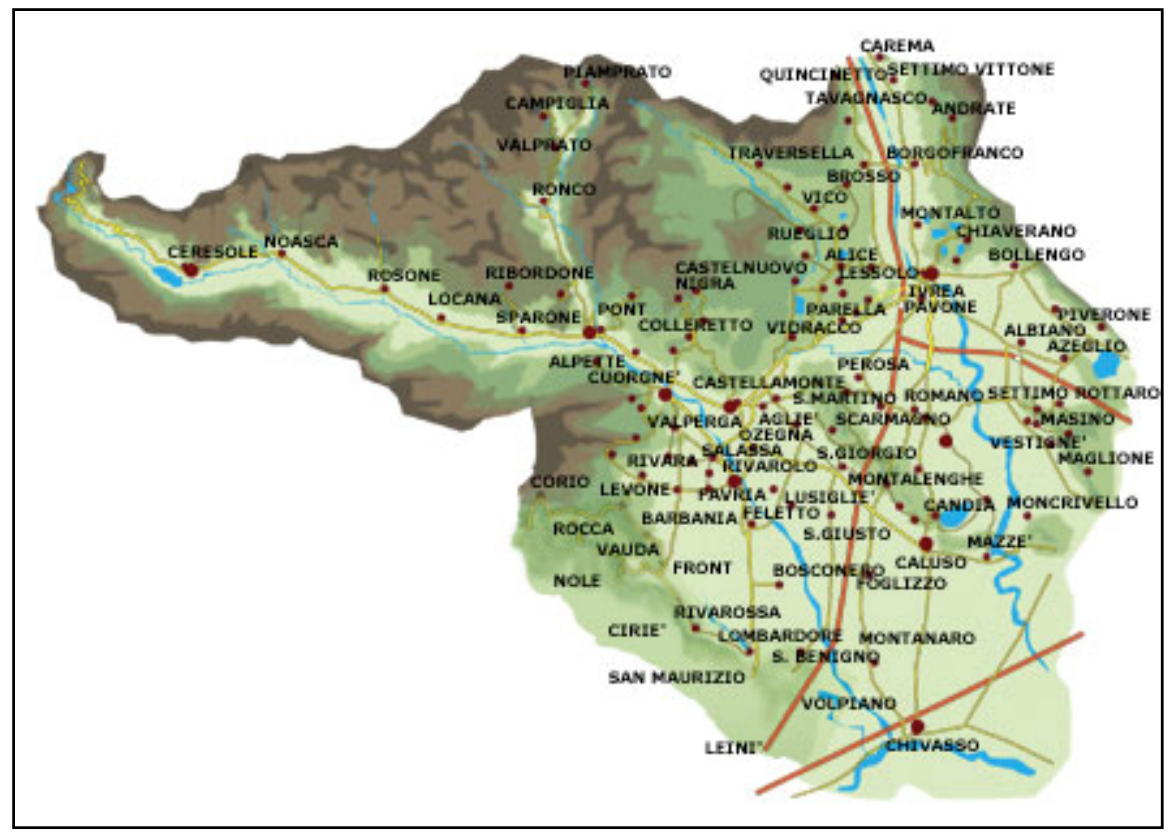

Figure V. Map of the area of Canavese in the province of Torino showing main villages. 


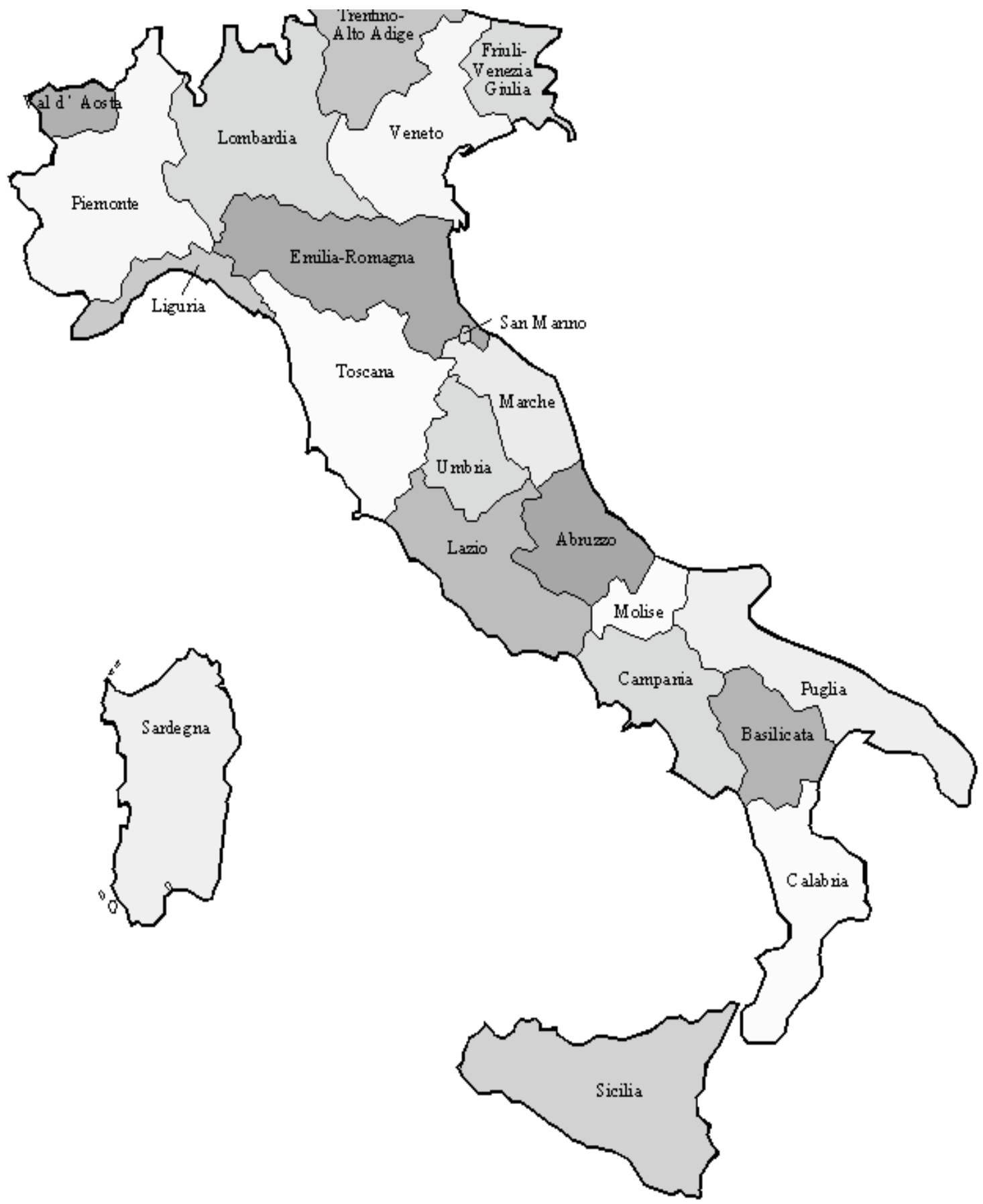

Figure VI. Map of Italy showing borders of the regions. 


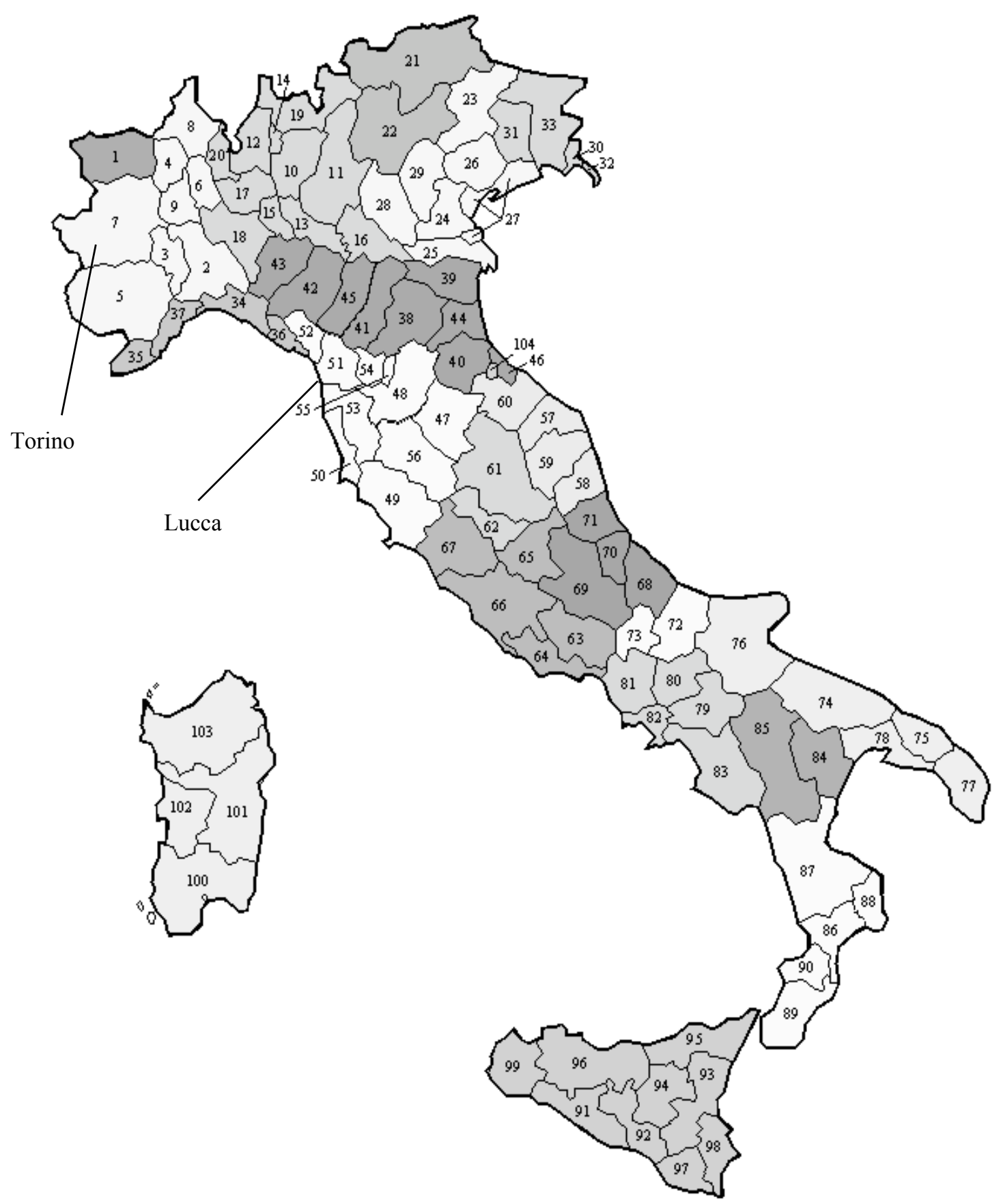

Figure VII. Map of Italy showing borders of the provinces. 


\section{List of Italian provinces divided by region}

\author{
Valle D'Aosta \\ 1. Aosta \\ Piemonte (Piedmont) \\ 2. Alessandria \\ 3. Asti \\ 4. Biella \\ 5. Cuneo \\ 6. Novara \\ 7. Torino \\ 8. Verbania \\ 9. Vercelli
}

\section{Lombardia (Lombardy) \\ 10. Bergamo \\ 11. Brescia \\ 12. Como \\ 13. Cremona \\ 14. Lecco \\ 15. Lodi \\ 16. Mantova \\ 17. Milano \\ 18. Pavia \\ 19. Sondrio \\ 20. Varese}

\section{Trentino Alto Adige}

21. Bolzano

22. Trento

\section{Veneto \\ 23. Belluno \\ 24. Padova \\ 25. Rovigo \\ 26. Treviso \\ 27. Venezia \\ 28. Verona \\ 29. Vicenza}

Friuli Venezia Giulia

30. Gorizia

31. Pordenone

32. Trieste

33. Udine

\section{Liguria}

34. Genova

35. Imperia

36. La Spezia

37. Savona

\section{Emilia Romagna}

38. Bologna

39. Ferrara
40. Forlì

41. Modena

42. Parma

43. Piacenza

44. Ravenna

45. Reggio Emilia

46. Rimini

Toscana (Tuscany)

47. Arezzo

48. Firenze

49. Grosseto

50. Livorno

51. Lucca

52. Massa Carrara

53. Pisa

54. Pistoia

55. Prato

56. Siena

Marche

57. Ancona

58. Ascoli Piceno

59. Macerata

60. Pesaro Urbino

\section{Umbria}

61. Perugia

62. Terni

Lazio

63. Frosinone

64. Latina

65. Rieti

66. Roma

67. Viterbo

\begin{abstract}
Abruzzo
68. Chieti

69. L'Aquila

70. Pescara

71. Teramo
\end{abstract}

Molise

72. Campobasso

73. Isernia

Puglia

74. Bari

75. Brindisi

76. Foggia

77. Lecce

78. Taranto

\section{Campania}

79. Avellino

80. Benevento

81. Caserta

82. Napoli

83. Salerno

\section{Basilicata}

84. Matera

85. Potenza

Calabria

86. Catanzaro

87. Cosenza

88. Crotone

89. Reggio Calabria

90. Vibo Valentia

Sicilia (Sicily)

91. Agrigento

92. Caltanissetta

93. Catania

94. Enna

95. Messina

96. Palermo

97. Ragusa

98. Siracusa

99. Trapani

Sardegna (Sardinia)

100. Cagliari

101. Nuoro

102. Oristano

103. Sassari 


\subsection{Why they came to Houghton County}

Most of the Italian immigrants in Houghton County came from just two provinces in Italy. The reasons for this are not immediately clear. A plausible explanation comes from some descendants of the immigrants themselves who still live in Houghton County or nearby. Doctor John B. Marta, ${ }^{28}$ whose grandparents were among the first settlers from the province of Torino, came up with an interesting story. He said that he did not know all of the reasons why Torinesi came to Houghton County, but that his grandmother told him about the Italian counsel, Attilio Castigliano, contacting people in Italy with the information that he had jobs for about 300 people. He contacted people from the Canavese because he was born in that area and had immigrated to Houghton County earlier. These emigrants sailed from Italy to New York where they boarded a train and were taken to the Upper Peninsula. Many more Torinesi came to join their relatives.

There are no sources to support Marta's explanation and again the fact that the Italian counsel for the Upper Peninsula of Michigan - that was under the jurisdiction of the Regia Agenzia Consolare (Royal Consular Agency) of Duluth - was from the area of Canavese could as well be a consequence of the fact that there were many Torinesi in this area, even before he was elected counsel and recruited more of them.

Other descendants of the first settlers interviewed by Professor Magnaghi gave a similar explanation. In this case the connection between Houghton County and the Canavese is Bartolomeo Quello, one of the very first Italian immigrants to this area. He was from Locana Canavese and apparently brought many Italians from his area to work with him in the woods when he had a lumbering company, and then in the mine when he started to work there. $^{29}$

Another hypothesis that Italians arrived here just because "the train went as far as Keweenaw and they couldn't go any further", but it seems quite anecdotal. ${ }^{30}$

Whatever the reasons for the pioneers to settle in Houghton County, one thing is clear. They were the starters of a chain immigration that followed intertwined regional and familial connections. Evidences of that are on many ships' manifests of the Ellis Island

\footnotetext{
${ }^{28}$ E-mail of John Marta to the author, March $23^{\text {rd }}, 2004$.

${ }^{29}$ Magnaghi, Russell. Interview with Peter and Charles Vitton. Franklin Mine, Houghton, Michigan, 12-101982.

${ }^{30}$ Magnaghi, Russell. Interview with Louis Lombardi. Calumet, Michigan, 12-03-1982.
} 
database. On some of them, in fact, besides giving the exact address of the final destination of the immigrant, it is also indicated if they were going to join relatives and/or friends. Many of them actually did. ${ }^{31}$

So far there are no specific reasons why many Italian immigrants from the province of Lucca settled in Houghton County either. One of the things that they have in common with the people from Canavese is the fact that these two areas have a significant mining tradition, at least by Italian standards. ${ }^{32}$ But the hypothesis that they both came here because of their mining skills is far from being proven. Ships' manifests offer some hints when describing the occupation of the immigrant, but it is not certain if immigrants are declaring the occupation they used to have, or the one they are hoping to have.

\subsection{Comparisons}

The following graphs show a comparison in the composition of the Italian community according to birthplace in the years examined and help to give an idea of the flux of Italian immigrants to Houghton County through the years.

In 1880 the Italian community is considerably small compared to the following years, and it is mainly composed by the so-called pioneers, mostly single men who arrived a few years earlier and had not started families yet. This explains the small percentage of individuals not born in Italy, who are mostly children. In 1900 this percentage almost doubles (31\%) and the percentage of individuals born in Italy decreases proportionally (68\%). Although data from 1890 are missing and a more reliable comparison is not possible, it is likely that the percentage increased because many of the immigrants married and started to have children. Individuals born in Italy are still the majority of the community suggesting that the flux of 'new' Italians to Houghton County is still relevant. In 1910 the community is considerably larger than in 1900, but the percentage of people born in Italy decreases to $58 \%$. This is a first indication that fewer 'new' Italians are reaching the Upper Peninsula. In

\footnotetext{
${ }^{31}$ www.ellisisland.org.

32 In the area of Canavese some metals and minerals have been mined since the Roman period. The production has never reached relevant industrial scale even during the $19^{\text {th }}$ and $20^{\text {th }}$ centuries. Considering the general mining tradition in Italy, however, it would not be wrong calling the Canavese a mining area. The province of Lucca is famous for its quarries of marble. There are also mines of metals and again even though they have never reached a relevant industrial scale of production, the province of Lucca is definitely a mining area.
} 
1920 the percentage of individuals not born in Italy exceeds for the first time that of the people born in Italy suggesting that the flux of newcomers has remarkably decreased. In 1930 the community is considerably smaller than 1920, and the decreasing trend for individuals born in Italy continues. They now represent just $35 \%$ of the community.
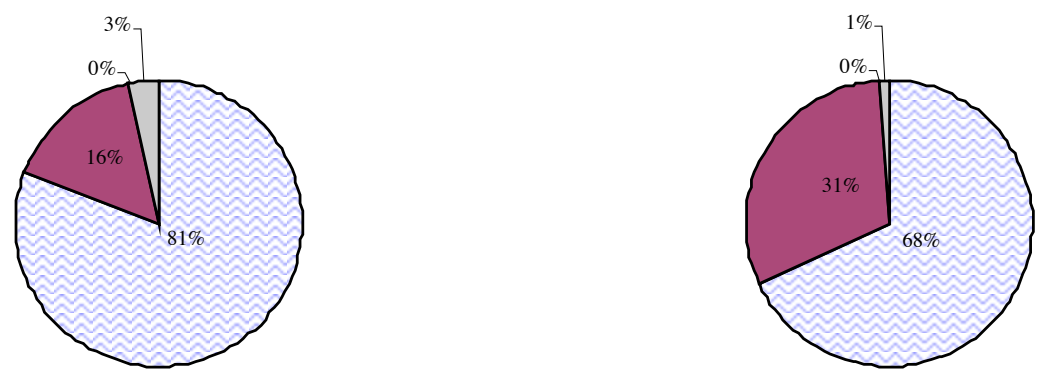

1880

1900
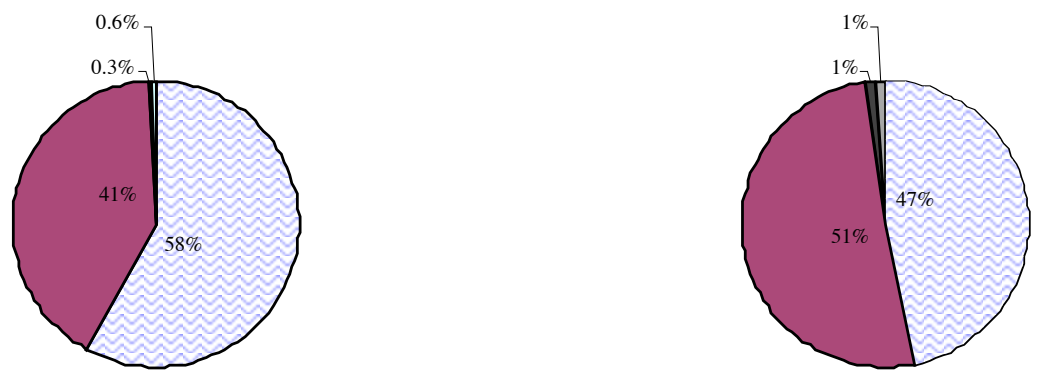

1910

1920

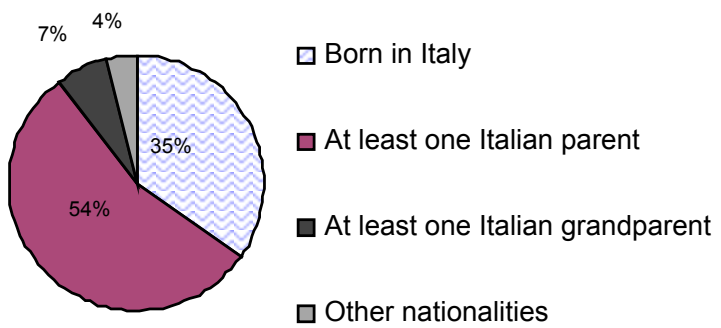

1930

Figure VIII. Composition of Italian community by birth in 1880, 1900, 1910, 1920, and 1930. 
It would have been interesting to determine the number of newcomers for each year examined, but it has not been possible because of a major problem in interpreting the data. The date of arrival in the US indicated in the censuses for each immigrant should refer to the very first time the immigrant reached the United States. In many cases, however, because many Italians were travelling back and forth between Italy and the United States, it refers to the latest time the immigrant returned to the United States. ${ }^{33}$

In interpreting these data we have to consider that censuses 'describe' the population only every ten years. Nothing emerges of what is going on during the years in between. For example, considering just the data from the census one would say that the Italian community in Houghton County reached its peak in 1910. In fact, according to Attilio Castigliano it reached it in 1906-1907, and after these years the number of Italian decreased considerably. ${ }^{34}$

Table VIII. Comparison of the Provenience of Italian Immigrants in 1870, 1880, 1900, 1910, 1920, and 1930 (Only the region with more immigrants are shown).

\begin{tabular}{|l|c|c|c|c|r|}
\hline & Piemonte & Toscana & Lombardia & Veneto & Total Italians \\
\hline $\mathbf{1 8 7 0}$ & $9(43 \%)$ & $5(24 \%)$ & 0 & 0 & 21 \\
\hline $\mathbf{1 8 8 0}$ & $96(44 \%)$ & $60(27 \%)$ & 0 & $3(1 \%)$ & 220 \\
\hline $\mathbf{1 9 0 0}$ & $1,493(53.54 \%)$ & $440(15.8 \%)$ & $67(2.41 \%)$ & $62(2.22 \%)$ & 2,789 \\
\hline $\mathbf{1 9 1 0}$ & $2,207(50.35 \%)$ & $1,302(23.55 \%)$ & $134(3.1 \%)$ & $86(1.96 \%)$ & 4,384 \\
\hline $\mathbf{1 9 2 0}$ & $1,906(43.52 \%)$ & $1,287(29.4 \%)$ & $97(2.22 \%)$ & $81(1.85 \%)$ & 4,379 \\
\hline $\mathbf{1 9 3 0}$ & $1,249(43.99 \%)$ & $696(24.52 \%)$ & $86(3 \%)$ & $72(2.54 \%)$ & 2,839 \\
\hline
\end{tabular}

The data from the employment cards of Calumet and Hecla also suggest big fluxes of Italian immigrants in between the years of the censuses. Many of these cards, in fact, include

\footnotetext{
${ }^{33}$ These data emerged when comparing the information of a single immigrant in the different years. Often the date of arrival indicated in 1900 did not correspond to that indicated in 1910, or to that in 1920. A subsequent look at the ships' manifest confirmed that in many cases the immigrant indicated each time the date of his latest return to the US.

${ }^{34}$ Castigliano, Attilio. "Origine, sviluppo, importanza ed avvenire delle colonie italiane del Nord Michigan e del Nord Minnesota”. In Bollettino dell’Emigrazione Vol. 7 (1913), p. 727.
} 
the date of the first arrival in the US of the immigrants, and they are often in between the years of the census. The cards also show that after 1910 most of the Italian newcomers were arriving from central and southern regions. The data from the censuses show this increase in the number of central-southern Italian immigrants in Houghton County after 1910, but not as much as the employment cards would suggest. 


\section{Chapter III - Occupations}

\subsection{Goals and Methods}

The aim of the following chapter is to learn more about the occupations that Italians immigrants to Houghton County had and to verify if there is a relation between province of origin in Italy and occupation.

Information about the jobs that Italians had in 1870, 1880, 1900, 1910, 1920, and 1930 are from the censuses of the state of Michigan, Houghton County, of the same years. It has not been possible to find anything about 1890 because the census was lost. Several Polk's Directories produced in those years also indicate the occupations of some people, but they do not always match the information found in the censuses.

There are discrepancies in the terminology used by the agents working on collecting the information for the state censuses. In fact a variety of agents worked in a range of townships over several census years. Some of them are quite specific when describing occupations. In some cases they have been as meticulous as to indicate job, place of job, and level of job. In other cases they have been vague; for example they enumerate 'laborer', not indicating what kind of laborer, or where the laborer worked. ${ }^{35}$

The various levels of detail describing occupations are retained in the tables that follow (Table IX, X, XI, XII, XIII, AND XIV). However, in order to compare the results of this analysis with previously assumed ideas about the fields in which Italians were mostly occupied, the occupations were grouped into eleven categories. The latter are large enough to be workable and condense data, but fine enough to capture change. The categories are Mining and related industries, Transportation, Boarding, Building, Farming, Food and drink selling, Professionals and Skilled Tradesmen, General office jobs, General merchandise sale, Unskilled, and Others.

The categories Mining and related, Farming, Construction, and Food and drink selling have been included because previous studies indicated them as the field in which

\footnotetext{
35 This 'flexibility' in the use of terminology is particularly relevant when considering, for example, mining jobs. In fact some of the census takers may have used the term 'miner' to indicate that a person was working in the mines regardless his actual rank; that may have affected data.
} 
most Italians were occupied. ${ }^{36}$ Transportation, General merchandise sale, Professionals and Skilled Tradesmen, Boarding, and General office jobs have been included because the data from the censuses frequently showed Italians working in these sectors, at least in some years. Unskilled includes all those workers with no particular skills or for whom the censuses do not indicate any. Others include those whose occupation does not fit into the previous categories.

\subsection{Occupations in 1870}

In 1870 the census for Houghton County includes six Italian families for a total of twenty-one Italians. As Table IX shows, eight of them have an occupation.

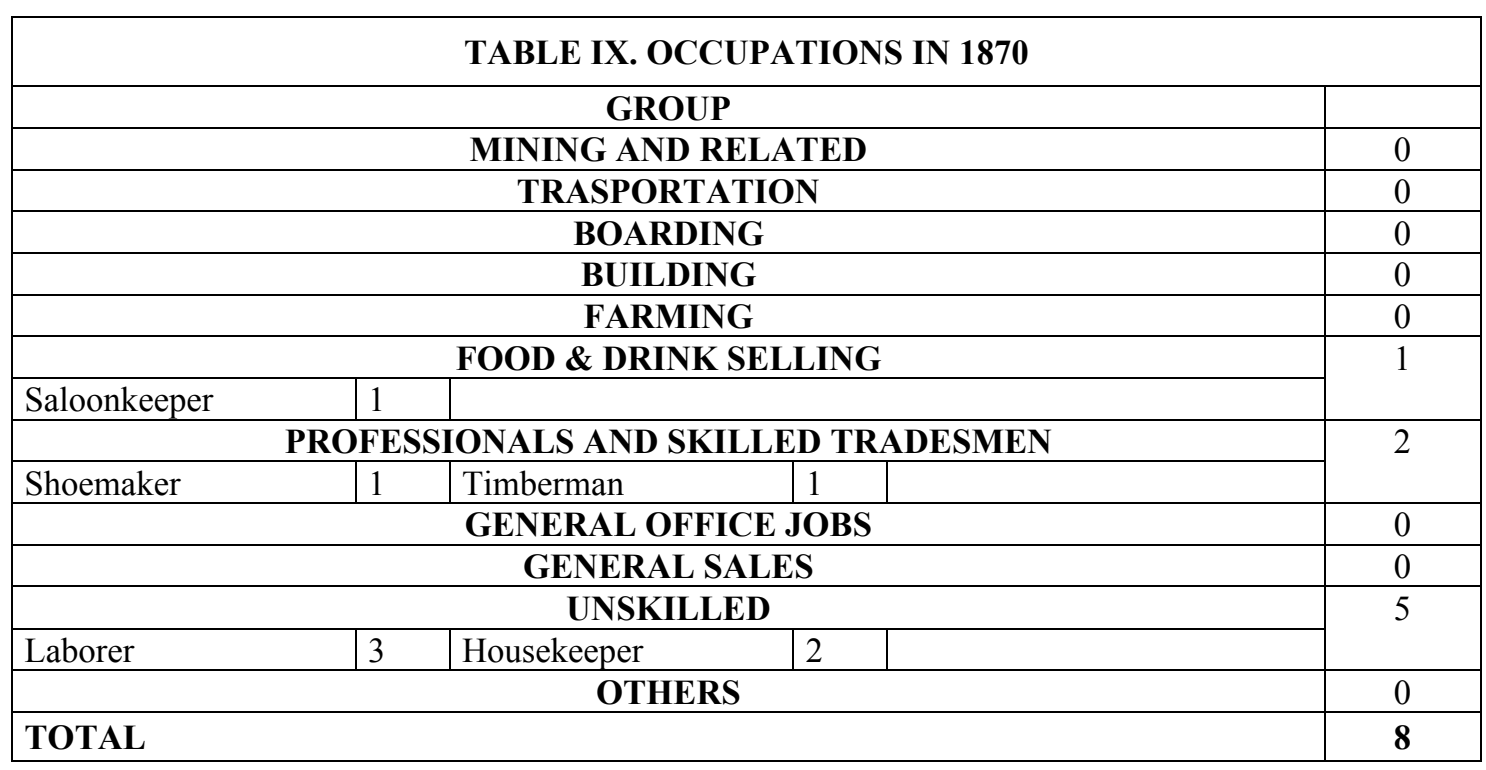

Unless the three laborers, for whom the place of work is not indicated, are mine laborers, there are no Italians working in the mines yet.

No women are indicated as working outside the household. Because the relationships among the persons in the same households are not specified, no boarders are mentioned; therefore it is not possible to say if women are contributing directly to the monetary incomes of the family.

\footnotetext{
${ }^{36}$ Glazer, Nathan and Daniel Moynihan. Beyond the Melting Pot. Cambridge: M.I.T. Press. 1970. According to the work of Glazer and Moynihan Italians arrived in the countries of emigration "as stonecutters, mason, and unskilled laborers, but they remained to become merchants, professional people, and - where opportunity offered $[\ldots]$ - farmers", p. 182.
} 
It is not possible to establish a significant relation between occupation and proveniences because the number of Italians is still too small and their origins not completely clear for this year.

\subsection{Occupations in 1880}

In 1880 the Italian community increases to 220 individuals. According to the census 163 of them work outside the household.

As Table X shows many Italians are working in the mines (60), with a predominance of laborers, thirty-five, over miners, twenty-three.

\begin{tabular}{|c|c|c|c|c|c|}
\hline \multicolumn{6}{|c|}{ TABLE X. OCCUPATIONS IN 1880} \\
\hline \multicolumn{6}{|c|}{ GROUP } \\
\hline \multicolumn{5}{|c|}{ MINING AND RELATED } & \multirow{2}{*}{$\begin{array}{c}\mathbf{6 0} \\
(34.7 \%)\end{array}$} \\
\hline Laborer/copper mine & 35 & Miner/copper mine & 23 & \begin{tabular}{l|l} 
Timberman/copper mine & 2 \\
\end{tabular} & \\
\hline \multicolumn{5}{|c|}{ TRASPORTATION } & 0 \\
\hline \multicolumn{5}{|c|}{ BOARDING } & 0 \\
\hline \multicolumn{5}{|c|}{ BUILDING } & 3 \\
\hline Mason & 3 & & & & $(1.7 \%)$ \\
\hline \multicolumn{5}{|c|}{ FARMING } & 1 \\
\hline Farmer and contractor & 1 & & & & $(0.5 \%)$ \\
\hline \multicolumn{5}{|c|}{ FOOD \& DRINK SELLING } & 8 \\
\hline Saloonkeeper & 6 & Saloon and boarding & 2 & & $(4.5 \%)$ \\
\hline \multicolumn{5}{|c|}{ PROFESSIONALS AND SKILLED TRADESMEN } & 4 \\
\hline Shoemaker & 2 & Timberman & 2 & & $(2.2 \%)$ \\
\hline \multicolumn{5}{|c|}{ GENERAL OFFICE JOBS } & 0 \\
\hline \multicolumn{5}{|c|}{ GENERAL SALES } & 2 \\
\hline Clerk/store & 2 & & & & $(1.5 \%)$ \\
\hline \multicolumn{5}{|c|}{ UNSKILLED } & $\begin{array}{c}\mathbf{8 6} \\
(49.7 \%)\end{array}$ \\
\hline Housekeeper & 24 & Laborer & 62 & & \\
\hline \multicolumn{5}{|c|}{ OTHERS } & 9 \\
\hline Student & 9 & & & & $(5.2 \%)$ \\
\hline \multicolumn{5}{|l|}{ TOTAL } & 173 \\
\hline
\end{tabular}

The category with more people is Unskilled. However it includes sixty-two laborers for which the census does not specify anything, but likely they are mine laborers. In fact the category of Unskilled almost disappears as the enumeration of the work categories gets more specified by the census takers in the following years. 
As in 1870 no women were enumerated as working outside the household. Twentyfour of them are indicated as housekeepers. In 1880 the relations among the members of the same household are indicated in the census, and sixteen households out of twenty-six include boarders. This suggests that the women are making an active contribution to the incomes of the family.

\subsection{Occupations in 1900}

In 1900 the Italian community includes 2,789 members. According to the census 1,680 of them have an occupation.

Most of the Italians are employed either in the mines or in fields strictly connected to them (65.3\%). Trammers are the most numerous, 428, followed by miners, 290 , laborers, 220 , and timber men, seventy-six. These data are not surprising. Given the fact that Italy did not have a prominent mining tradition, Italians occupied low rank positions in the mining job hierarchy, at least during their first years of work. ${ }^{37}$

\begin{tabular}{|c|c|c|c|c|c|c|}
\hline \multicolumn{7}{|c|}{ TABLE XI. OCCUPATIONS IN 1900} \\
\hline \multicolumn{6}{|c|}{ GROUP } & \\
\hline \multicolumn{6}{|c|}{ MINING AND RELATED } & $\begin{array}{c}1,097 \\
(65.3 \%)\end{array}$ \\
\hline Blacksmith/mine & 1 & Laborer/rock house & 24 & Teamster/copper & 2 & \\
\hline Drill boy/mine & 19 & Lander/copper mine & 3 & Timberman/copper & 76 & \\
\hline Engineer/ engine & 2 & Miner/copper mine & 290 & Tender/dry house & 2 & \\
\hline Fireman/engine & 2 & Oiler/engine & 1 & Trammer/copper & 428 & \\
\hline Laborer/mine & 105 & Overseer/copper mine & 1 & Watchman/copper & 18 & \\
\hline Laborer, day/mine & 115 & Pump man/copper mine & 1 & & & \\
\hline Laborer/mill & 6 & Sprinkler/copper mine & 1 & & & \\
\hline \multicolumn{6}{|c|}{ TRASPORTATION } & \multirow{2}{*}{$\begin{array}{c}\mathbf{2} \\
(0.12 \%)\end{array}$} \\
\hline Laborer/railroad & 1 & Driver & 1 & & & \\
\hline \multicolumn{6}{|c|}{ BOARDING } & \multirow{2}{*}{$\begin{array}{c}7 \\
(0.42 \%)\end{array}$} \\
\hline Boarding house keep. & 6 & Landlord & 1 & & & \\
\hline \multicolumn{6}{|c|}{ BUILDING } & \multirow{4}{*}{$\begin{array}{c}48 \\
(2.9 \%)\end{array}$} \\
\hline Carpenter & 5 & Plasterer helper & 1 & Stone cutter & 2 & \\
\hline Contractor & 1 & Painter & 1 & & & \\
\hline Mason & 37 & Stone contractor & 1 & & & \\
\hline
\end{tabular}

\footnotetext{
37 "Besides being locked out of supervisory positions, only one fifth of the [...] Italians killed underground had worked their way up to miners' jobs. More than half of them had risked their lives - and lost them - while still on the very bottom rungs of the wage earning ladder". Lankton, D. Larry. Cradle to Grave: Life and Death at the Lake Superior Copper Mines. Oxford University Press: New York, 1991, p. 113.
} 


\begin{tabular}{|c|c|c|c|c|c|c|}
\hline \multicolumn{6}{|c|}{ FARMING } & \multirow{2}{*}{$\begin{array}{c}\mathbf{2} \\
(0.11 \%)\end{array}$} \\
\hline Laborer/farm & 2 & & & & & \\
\hline \multicolumn{6}{|c|}{ FOOD \& DRINK SELLING } & \multirow{5}{*}{$\begin{array}{c}\mathbf{4 5} \\
(2.68 \%)\end{array}$} \\
\hline Baker & 6 & Grocer & 6 & Peddler/groceries & 2 & \\
\hline Bartender & 7 & Liquor dealer & 2 & & & \\
\hline Butcher & 5 & Salesman & 2 & & & \\
\hline Cook/restaurant & 1 & Saloonkeeper & 14 & & & \\
\hline \multicolumn{6}{|c|}{ PROFESSIONALS AND SKILLED TRADESMEN } & \multirow{6}{*}{$\begin{array}{c}47 \\
(2.79 \%)\end{array}$} \\
\hline Surgeon & 2 & Fireman & 2 & Tailor & 3 & \\
\hline Policeman & 2 & Miller & 2 & Teacher & 1 & \\
\hline Priest & 1 & Sprinkler & 2 & Watch repairer & 1 & \\
\hline Blacksmith & 11 & Machinist & 1 & Teamster & 14 & \\
\hline Dressmaker & 2 & Shoemaker & 3 & & & \\
\hline \multicolumn{6}{|c|}{ GENERAL OFFICE JOBS } & \multirow{3}{*}{$\begin{array}{c}\mathbf{9} \\
(0.53 \%)\end{array}$} \\
\hline Bookkeeper & 3 & Printer & 1 & Appr. newspaper & 1 & \\
\hline Clerk & 3 & Proprietor newspaper & 1 & & & \\
\hline \multicolumn{6}{|c|}{ GENERAL MERCHANDISE SALE } & \multirow{3}{*}{$\begin{array}{c}\mathbf{2 3} \\
(1.3 \%) \\
3\end{array}$} \\
\hline Teamster/store & 1 & Saleslady & 3 & & & \\
\hline Merchant & 6 & Salesman & 13 & & & \\
\hline \multicolumn{6}{|c|}{ UNSKILLED } & \multirow{3}{*}{$\begin{array}{c}43 \\
(2.6 \%)\end{array}$} \\
\hline Servant & 23 & Laborer & 13 & Washwoman & 1 & \\
\hline Housekeeper & 5 & Janitor & 1 & & & \\
\hline \multicolumn{6}{|c|}{ OTHERS } & \multirow{2}{*}{$\begin{array}{c}\mathbf{3 5 7} \\
(21.25 \%) \\
\end{array}$} \\
\hline At school & 328 & Not clear & 28 & Patient & 1 & \\
\hline TOTAL & & & & & & 1,680 \\
\hline
\end{tabular}

The second group in number is Other with 357 individuals, most of whom are students, $328 .^{38}$

Building, Professionals and Skilled Tradesmen, and Food and drink selling follow with relatively small numbers of workers. One important datum to notice in the category of Food and drink selling is the number of saloonkeepers. According to previous studies and the stories of many descendants of the first Italian immigrants, Italians were prominent in Houghton County in the liquor business. In this case, however, they do not appear to be that many: fourteen saloonkeepers and two liquor dealers. The same is true for masons, just thirty-seven, which according to many descendants interviewed by Professor Magnaghi should have been really numerous. ${ }^{39}$

Another interesting datum concerns Farming. The number of workers in this field increases notably over the years, but considering the fact that Italian emigrants were almost

\footnotetext{
${ }^{38}$ In the following years, 1910, 1920, and 1930, the number of students is almost null even though the community is considerably bigger. This may be a consequence of the fact that students were no longer considered 'occupied' by census takers and therefore were not recorded.

${ }^{39}$ Magnaghi, Russell. Interviews with Italian Descendants of the Upper Peninsula of Michigan, Northern Michigan University Archives.
} 
totally countrymen, it is a little surprising to find just two farm laborers. One reason may be that during the first years Italian emigration was temporary and people wanted to earn as much as possible in a short time and then go back to Italy. ${ }^{40}$ Investing in land, therefore, would have been too much of a long-term engagement. Secondly, buying land was not immediately affordable for the ones that had already in mind to stay in the foreign country. Thirdly, Italians could have worked as farm laborers for other landowners, but the salaries in agriculture were fairly low compared to other sectors such as mining. ${ }^{41}$ Another thing to be considered is the short farming season in Upper Michigan that did not allow a steady income all year long for people working in agriculture.

As for women, the census indicates that forty-three of them are working outside the household in 1900. Twenty-three of them are servants, five boarding house keepers, five housekeepers, four salesladies, two dressmakers, two grocers, one milliner, and one washwoman. As for boarding house keepers, it is important to notice that many more Italian households include boarders although the census does not indicate the head nor the wife as boarding house keepers. These data, therefore, are indicative but not conclusive of women's roles.

There seems to be no relation between region of provenience of the Italians and jobs because they are more or less equally distributed in all the groups and jobs.

\subsection{Occupations in 1910}

In 1910 the great increase in the Italian community only partially corresponds to increases of the number of working people. In fact in 1900 the working population - without students who are not included in 1910 - was 48.5\% of the community, whereas in 1910 they represented $42.1 \%$. This is a result of the fact that there are many more families than single men compared to 1900 and many members of the families are children.

The bulk of the Italians are occupied in the mines (72.9\%). The absolute number of miners is higher compared to 1900,365 versus 290 . However most of the Italians still have

\footnotetext{
${ }^{40}$ Di Palma-Castiglione, G. F. "Vari centri italiani negli stati di Indiana, Ohio, Michigan, Minnesota e Wisconsin". In Bollettino dell'Emigrazione Vol. 14 (15 Giugno 1915), p. 27.

${ }^{41}$ Ibidem, p. 26. According to Di Palma-Castiglione the salaries of farm laborers in 1912, ranged between $\$ 18$ and $\$ 25$ a month.
} 
jobs that do not require a great deal of skill such as trammers, 300, laborers, 233, and timbermen, 170.

\begin{tabular}{|c|c|c|c|c|c|c|}
\hline \multicolumn{7}{|c|}{ TABLE XII. OCCUPATIONS IN 1910} \\
\hline \multicolumn{6}{|c|}{ GROUP } & Total \\
\hline \multicolumn{6}{|c|}{ MINING AND RELATED } & \multirow{25}{*}{$\begin{array}{c}1,347 \\
(72.9 \%)\end{array}$} \\
\hline Accountant $/$ mine & 1 & Iron molder helper & 1 & Rock house man & 7 & \\
\hline Bell ringer/mine & 1 & Laborer/copper mine & 233 & Roller fixer/copper mine & 3 & \\
\hline Blacksmith/mine & 5 & Laborer/foundry & 2 & Rope fixer/copper mine & 1 & \\
\hline Brake fixer/mine & 1 & Laborer/rock house & 19 & Shaftman/copper mine & 1 & \\
\hline Car dumper/mine & 2 & Laborer/shaft house & 1 & Sheave tender/mine & 8 & \\
\hline Car filler/mine & 1 & Laborer/smelter & 2 & Shift boss/copper mine & 3 & \\
\hline Car lander/mine & 1 & Laborer/smelter off. & 1 & Skip filler/copper mine & 13 & \\
\hline Carpenter/mine & 2 & Laborer/stamp mill & 9 & Sprinkler/copper mine & 2 & \\
\hline Chute man/mine & 1 & Lander/copper mine & 8 & Stemmer/copper mine & 3 & \\
\hline Day man/mine & 1 & Lander/rock house & 2 & Surface $\mathrm{man} / \mathrm{mine}$ & 6 & \\
\hline Day tender/mine & 1 & Machinist/mine & 9 & Swamper/copper mine & 1 & \\
\hline Disc fixer/mine & 1 & Machinist/stamp mill & 2 & Teamster/copper mine & 2 & \\
\hline Drayman/mine & 3 & Mason/copper mine & 23 & Timber boss/mine & 23 & \\
\hline Drill boy/mine & 17 & Mechanic/mine & 25 & Timber gang/mine & 5 & \\
\hline Engine boy/mine & 1 & Miner/copper mine & 365 & Timberman/copper mine & 142 & \\
\hline Engineer/mine & 4 & Motorman/mine & 3 & Track layer/copper mine & 2 & \\
\hline Engineer/stationary & 2 & Motorman/smelter & 1 & Trammer/copper mine & 296 & \\
\hline Fireman/boiler house & 7 & Odd jobs/copper mine & 5 & Trammer boss/mine & 4 & \\
\hline Fireman/copper mine & 6 & Oiler/engine house & 1 & Truck man/copper mine & 1 & \\
\hline Fireman/smelter & 3 & Painter/copper mine & 1 & Watchman/copper mine & 26 & \\
\hline Fireman/stamp mill & 3 & Picker/copper mine & 4 & Wiper/engine & 3 & \\
\hline Fireman/stationary & 2 & Piper/copper mine & 4 & & & \\
\hline Foreman/mine & 2 & Platman/copper mine & 1 & & & \\
\hline Head runner/mill & 2 & Puffer boy/mine & 3 & & & \\
\hline \multicolumn{6}{|c|}{ TRASPORTATION } & \multirow{4}{*}{$\begin{array}{c}\mathbf{1 6} \\
(0.9 \%)\end{array}$} \\
\hline Boiler man/railroad & 1 & Fireman/railroad & 1 & Odd j./roundhouse & 1 & \\
\hline Brakeman/railroad & 2 & Laborer & 2 & Sec. gang/railroad & 1 & \\
\hline Driver & 4 & Laborer/railroad & 3 & Teamster & 1 & \\
\hline \multicolumn{6}{|c|}{ BOARDING } & \multirow{6}{*}{$\begin{array}{c}\mathbf{1 8} \\
(0.9 \%) \\
\mathbf{2 9} \\
(1.6 \%)\end{array}$} \\
\hline Keeper boarding h. & 16 & Boarding h./prop. & 2 & & & \\
\hline & & BUILDING & & & & \\
\hline Carpenter & 6 & Mason & 13 & Wood chopper & 2 & \\
\hline Carpenter helper & 1 & Painter & 2 & Lumberman & 1 & \\
\hline Contractor/building & 1 & Plasterer & 3 & & & \\
\hline \multicolumn{6}{|c|}{ FARMING } & \multirow{3}{*}{$\begin{array}{c}\mathbf{2 9} \\
(1.6 \%)\end{array}$} \\
\hline Dairy person/farm & 3 & Laborer/farm & 14 & & & \\
\hline Farmer & 11 & Milk peddler/farm & 1 & & & \\
\hline \multicolumn{6}{|c|}{ FOOD \& DRINK SELLING } & \multirow{8}{*}{$\begin{array}{c}\mathbf{1 6 9} \\
(9.1 \%)\end{array}$} \\
\hline Baker & 10 & Delivery person & 7 & Proprietor/store & 12 & \\
\hline Bartender & 18 & Laborer & 8 & Saleslady & 8 & \\
\hline Brewer & 1 & Manager/store & 3 & Salesman & 6 & \\
\hline Brewing co. agent & 1 & Manager/brewery & 2 & Saloonkeeper & 29 & \\
\hline Butcher & 8 & Manufacturer/macaroni & 1 & Sausage maker & 1 & \\
\hline Clerk & 6 & Meat cutter & 1 & Storekeeper & 2 & \\
\hline Grocer/grocery store & 9 & Proprietor/saloon & 13 & Teamster & 23 & \\
\hline
\end{tabular}




\begin{tabular}{|c|c|c|c|c|c|c|}
\hline \multicolumn{6}{|c|}{ PROFESSIONALS AND SKILLED TRADESMEN } & \multirow{9}{*}{$\begin{array}{c}\mathbf{5 7} \\
(3.1)\end{array}$} \\
\hline Blacksmith & 4 & Machine apprentice & 1 & Physician & 1 & \\
\hline Blacksmith helper & 1 & Marshall & 1 & \begin{tabular}{|l|} 
Plumber \\
\end{tabular} & 2 & \\
\hline Clergyman & 1 & Mattress maker & 1 & Priest & 1 & \\
\hline Dressmaker & 11 & Milling apprentice & 1 & Tailor & 14 & \\
\hline Deputy & 1 & Musician & 3 & Umbrella repairer & 1 & \\
\hline Electrician & 1 & Nurse & 2 & Undertaker & 1 & \\
\hline Engineer & 1 & Organizer West. Fed. & 1 & & & \\
\hline Fireman & 3 & Platman & 4 & & & \\
\hline \multicolumn{6}{|c|}{ GENERAL OFFICE JOBS } & \multirow{5}{*}{$\begin{array}{c}\mathbf{2 0} \\
(1.1 \%)\end{array}$} \\
\hline Agent & 1 & Collector/bank & 1 & Secretary/insurance co. & 1 & \\
\hline Bookkeeper & 6 & Employment agent & 2 & Treasurer/village & 1 & \\
\hline Banker & 1 & Interpreter/law office & 1 & Typesetter/printing co. & 3 & \\
\hline Clerk/post office & 1 & Office girl & 1 & Manager/telegraph & 1 & \\
\hline \multicolumn{6}{|c|}{ GENERAL MERCHANDISE SALE } & \multirow{4}{*}{$\begin{array}{c}\mathbf{3 0} \\
(1.6 \%)\end{array}$} \\
\hline Clerk & 4 & Saleslady & 13 & Storekeeper & 1 & \\
\hline Merchant & 2 & Salesman & 5 & & & \\
\hline Peddler & 1 & Store proprietor & 4 & & & \\
\hline \multicolumn{6}{|c|}{ UNSKILLED } & \multirow{5}{*}{$\begin{array}{c}91 \\
(4.9 \%)\end{array}$} \\
\hline Housekeeper & 8 & Laundress & 2 & Waiter & 9 & \\
\hline Ironer/steam laundry & 1 & Maid & 1 & Washwoman & 5 & \\
\hline Janitor & 3 & Odd jobs & 2 & & & \\
\hline Laborer & 21 & Servant & 35 & & & \\
\hline \multicolumn{6}{|c|}{ OTHERS } & \multirow{2}{*}{$\begin{array}{c}\mathbf{4 2} \\
(2.3 \%)\end{array}$} \\
\hline Not clear & 32 & Own income & 10 & & & \\
\hline \multicolumn{6}{|l|}{ TOTAL } & 1,848 \\
\hline
\end{tabular}

The number of Italians in the group of Food and drink selling considerably increases to $169(9.1 \%)$. More specifically, saloonkeepers and proprietors of saloons are forty-two. Bakers and grocers do not increase proportionally.

The third group is Unskilled (4.9\%) and it interestingly includes a majority of women working as servants and housekeepers. In fact the number of women working outside the household in 1910 rises to 129 and besides servants and housekeepers, there are many clerks, salesladies, laundresses, and boarding house keepers.

The fourth group of employed people in 1910 is Professionals and Skilled Tradesmen $(3.1 \%)$, most of whom, twenty-five, are tailors and dressmakers.

As for the group of Building, the number of masons is still below expectations at just thirteen. However there are twenty-three Italian masons working in the mines.

The group of Boarding includes just women, sixteen boarding house keepers and two proprietors. However, as in the previous years, many more households included boarders than the number of boarding houses indicated by the census. 
As in 1900, there seems to be no relation between region of provenience of the Italians and jobs because they are more or less equally distributed in the various sectors and jobs.

\subsection{Occupations in 1920}

In 1920 the Italian community remains stable in number, 4,379, and the percentage of employed (37.1\%) decreases compared to 1910 (42.1\%). Again the reason for that is in the increasing number of children together with the decreasing number of newcomers.

Mining jobs are still prevalent among Italians, although the percentage drops from $72.9 \%$ of 1910 to $66.7 \%$. What increases significantly is the absolute number of miners. More interestingly, for the first time it tops the number of laborers, timbermen, and trammers together. These data show that Italians have moved up in the hierarchy of mining jobs. The reasons may be that many of them have been working in the mines for years by that time, and have acquired those skills allowing them to get better jobs. Secondly, after the strike of 1913 many people left the area for other destinations thus opening positions for others.

The second group is Food and drink selling. What emerges from the data is the fact that liquor dealers and saloon proprietors are remarkably fewer compared to 1910 -seven in 1920 and 42 in 1910 - and people in the food business, on the contrary, have increased. A reason may be found in the fact that this is the period of Prohibition ${ }^{42}$ and even though they continued to deal in alcohol, they did not declare it.

The number of Professionals and Skilled Tradesmen increases from fifty-seven to seventy-one (4.4\%), and the group of Unskilled drops a little bit from 91 (4.9\%) of 1910 to $66(4.1 \%)$. Again the group of Farming does not include many individuals, but the number of farmers goes from eleven in 1910 to twenty-nine in 1920 showing that more people are investing in the land.

Women occupied outside the household in 1920 are, according to the census, 146. For the most part they are salesladies and/or clerks in stores. Many of them, twenty-eight, are also working as servants, maids or housekeepers as in 1920. As for office jobs, there are

\footnotetext{
42 The Eighteenth Amendment to the Constitution of the United States was ratified on January 29, 1919; and, on January 29, 1920, Prohibition became the law of the land.
} 
five bookkeepers, four telephone operators, three stenographers, one type composer and a secretary. They are involved also in businesses; in fact there are six storekeepers and two merchants. Professionals and Skilled Tradesmen are sixteen: seven dressmakers, four teachers, four nurses, three cooks, one seamstress, and one midwife. Boarding house keepers are four but, as in the previous years, these data area minimum figure because many more households include boarders. The remaining twenty-eight have unskilled jobs such as laborers, cleaners, and waiters.

\begin{tabular}{|c|c|c|c|c|c|c|}
\hline \multicolumn{7}{|c|}{ TABLE XIII. OCCUPATIONS IN 1920} \\
\hline \multicolumn{6}{|c|}{ GROUP } & Total \\
\hline \multicolumn{6}{|c|}{ MINING AND RELATED } & \\
\hline Blacksmith/copper mine & 18 & Janitor/mine & 4 & Paycheck/copper mine & 1 & \multirow{28}{*}{$\begin{array}{c}\mathbf{1 0 8 4} \\
(66.7 \%)\end{array}$} \\
\hline Brakeman/copper mine & 1 & Labor agent/mine & 1 & Picker/copper mine & 12 & \\
\hline Captain/copper mine & 1 & Laborer surface/mine & 6 & Pipe fixer/copper mine & 4 & \\
\hline Carpenter/stamp mill & 1 & Laborer/copper mine & 134 & Pump man/copper mine & 6 & \\
\hline Carpenter/copper mine & 6 & Laborer/coal dock/yard & 2 & Rock house man & 1 & \\
\hline Coal passer/copper mine & 2 & Laborer/stamp mill & 5 & Roller fixer/copper mine & 2 & \\
\hline Copper dipper/smelter & 1 & Laborer/foundry & 2 & Saleslady/mine store & 1 & \\
\hline Diamond driller/mine & 1 & Laborer/rock house & 9 & Shaft man/copper mine & 2 & \\
\hline Drayman/copper mine & 3 & Laborer/smelter & 12 & Shift boss/copper mine & 1 & \\
\hline Drill boy/copper mine & 1 & Laborer/stamp mill & 13 & Sprinkler/copper mine & 1 & \\
\hline Drill sharpener/mine & 3 & Lander/copper mine & 3 & Stenographer/mine off. & 1 & \\
\hline Dumper/copper mine & 12 & Machinist/copper mill & 3 & Teamster/copper mine & 4 & \\
\hline Electrician/copper mine & 4 & Machinist/copper mine & 4 & Teamster/coal & 2 & \\
\hline Employment clerk/mine & 1 & Machinist/shaft & 1 & Teamster/drayman mine & 1 & \\
\hline Engine oiler/mine & 1 & Mason/copper mine & 20 & Telephone operator/mine & 1 & \\
\hline Engine wiper/mine & 1 & Mechanic/mine & 1 & Timber boss/mine & 6 & \\
\hline Engineer/copper mine & 2 & Mechanic/foundry & 1 & Timberman/copper mine & 82 & \\
\hline Engineer/stat. engine & 1 & Mill hand/stamp mill & 4 & Timekeeper/copper mine & 1 & \\
\hline Fireman/boiler house & 12 & Mine inspector/mine & 1 & Tool boy/copper mine & 1 & \\
\hline Fireman/copper mine & 3 & Miner/copper mine & 478 & Train man/copper mine & 1 & \\
\hline Fireman/engine house & 4 & Molder/copper mine & 1 & Trammer/copper mine & 11 & \\
\hline Fireman/smelter & 4 & Motorman/copper mine & 11 & Truck driver/mine & 2 & \\
\hline Fireman/stat.engine & 1 & Motorman/rock house & 1 & Washer/copper mill & 1 & \\
\hline Fireman/stat. boiler & 1 & Molder/foundry & 1 & Watchman/copper mine & 8 & \\
\hline Foreman/copper mine & 6 & Not clear/copper mine & 3 & Weigh master/mine & 1 & \\
\hline Furnace mover/smelter & 1 & Oiler/copper mine & 1 & Welder/copper mine & 3 & \\
\hline Helper/smelter & 1 & Operator/gas engine & 1 & Yardman/smelter & 2 & \\
\hline Hoist boy/copper mine & 1 & Painter/copper mine & 1 & & & \\
\hline \multicolumn{6}{|c|}{ TRASPORTATION } & \multirow{9}{*}{$\begin{array}{c}\mathbf{5 7} \\
(3.5 \%)\end{array}$} \\
\hline Boiler maker/railroad & 2 & Driver & 7 & Laborer/railroad & 14 & \\
\hline Boiler wash/railroad & 1 & Engine cleaner/loc. & 2 & Machinist/railroad & 6 & \\
\hline Car repairer/railroad & 1 & Fireman/railroad & 2 & Motorman/railway & 1 & \\
\hline Carpenter/railroad & 1 & Fireman/roundhouse & 1 & Overseer/highway & 1 & \\
\hline Clerk/railroad & 1 & Freight depot/railroad & 2 & Railroad office assistant & 1 & \\
\hline Coach cleaner/railroad & 2 & Helper/railroad & 1 & Teamster/public road & 1 & \\
\hline Conductor/street car & 2 & Laborer & 4 & Trucker/street railroad & 1 & \\
\hline Conductor/street railway & 2 & Laborer/railway & 1 & & & \\
\hline
\end{tabular}




\begin{tabular}{|c|c|c|c|c|c|c|}
\hline \multicolumn{6}{|c|}{ BOARDING } & \multirow{2}{*}{$\begin{array}{c}11 \\
(0.7 \%)\end{array}$} \\
\hline Keeper/boarding h. & 5 & Laborer/hotel & 2 & Servant & 2 & \\
\hline Keeper/hotel & 1 & Proprietor/hotel & 1 & & & \\
\hline \multicolumn{6}{|c|}{ BUILDING } & \multirow{4}{*}{$\begin{array}{c}\mathbf{2 0} \\
(1.2 \%)\end{array}$} \\
\hline Carpenter & 8 & Laborer/saw mill & 1 & Painter & 1 & \\
\hline Contractor & 2 & Logger & 1 & Plasterer & 1 & \\
\hline Laborer/lumber camp & 3 & Mason/building & 2 & Wood chopper & 1 & \\
\hline & & FARMING & & & & \multirow{4}{*}{$\begin{array}{c}42 \\
(2.6 \%)\end{array}$} \\
\hline Dairy person & 1 & Farmer/general farm & 23 & Manager/home dairy & 1 & \\
\hline Farm laborer & 6 & Helper/farm & 1 & & & \\
\hline Farmer/dairy farm & 3 & Laborer/dairy farm & 7 & & & \\
\hline \multicolumn{6}{|c|}{ FOOD \& DRINK SELLING } & \multirow{9}{*}{$\begin{array}{c}149 \\
(9.2 \%)\end{array}$} \\
\hline Baker & 10 & Laborer/store & 3 & Proprietor/restaurant & 1 & \\
\hline Bartender & 1 & Laborer/macaroni fac. & 2 & Proprietor/drink parlor & 7 & \\
\hline Butcher & 8 & Manager/food store & 5 & Saleslady & 13 & \\
\hline Clerk & 13 & Meat cutter & 3 & Salesman & 7 & \\
\hline Cook & 4 & Merchant/food store & 22 & Shopkeeper & 1 & \\
\hline Delivery boy/bakery & 1 & Merchant/macaroni fac. & 1 & Teamster & 16 & \\
\hline Grocer & 2 & Owner/food store & 21 & Waiter & 4 & \\
\hline Helper/food store & 4 & Propr./macaroni fact. & 1 & & & \\
\hline \multicolumn{6}{|c|}{ PROFESSIONALS AND SKILLED TRADESMEN } & \multirow{9}{*}{$\begin{array}{c}71 \\
(4.4 \%)\end{array}$} \\
\hline Apprentice/mach. shop & 2 & Mechanic & 6 & Shoemaker & 7 & \\
\hline Auto mechanic/garage & 2 & Midwife & 1 & Tailor & 8 & \\
\hline Blacksmith & 7 & Missioner/mission & 1 & Teacher & 4 & \\
\hline Dressmaker & 5 & Nurse & 4 & Undertaker & 1 & \\
\hline Electrician & 2 & Plumber & 5 & Veterinary & 1 & \\
\hline Fireman & 2 & Policeman & 1 & Proprietor/printing shop & 1 & \\
\hline Lawyer & 1 & Repairer/auto & 3 & Teamster & 3 & \\
\hline Machinist & 2 & Seamstress & 1 & Turner/mill & 1 & \\
\hline \multicolumn{6}{|c|}{ GENERAL OFFICE JOBS } & \multirow{6}{*}{$\begin{array}{c}33 \\
(2 \%)\end{array}$} \\
\hline Agent/books & 1 & Cashier/insurance & 1 & Telegraph/telephone op. & 5 & \\
\hline Agent/life insurance & 1 & Clerk & 1 & Teller/bank & 1 & \\
\hline Bookkeeper & 7 & Composer/print shop & 1 & Typewriter & 1 & \\
\hline Clerk/bank & 7 & Stenographer & 2 & Manager/print shop & 1 & \\
\hline Secretary & 1 & Printer/shop & 2 & Manager/telegraph off. & 1 & \\
\hline \multicolumn{6}{|c|}{ GENERAL MERCHANDISE SALE } & \multirow{5}{*}{$\begin{array}{c}51 \\
(3.1 \%)\end{array}$} \\
\hline Clerk & 3 & Merchant/store & 4 & Owner/store & 3 & \\
\hline Manager/shop & 2 & Store proprietor & 4 & Peddler & 1 & \\
\hline Saleslady & 17 & Storekeeper & 3 & & & \\
\hline Salesman & 8 & Teamster & 6 & & & \\
\hline \multicolumn{6}{|c|}{ UNSKILLED } & \multirow{4}{*}{$\begin{array}{c}\mathbf{6 6} \\
(4.1 \%)\end{array}$} \\
\hline Cleaner & 2 & Janitor & 6 & Laundress & 6 & \\
\hline Domestic/private family & 2 & Laborer & 15 & Maid & 1 & \\
\hline Housekeeper & 4 & Laborer/factory & 4 & Servant & 26 & \\
\hline \multicolumn{6}{|c|}{ OTHERS } & \multirow{3}{*}{$\begin{array}{c}\mathbf{4 0} \\
(2.5 \%)\end{array}$} \\
\hline Army & 1 & Not clear & 24 & & & \\
\hline At school & 14 & Retired & 1 & & & \\
\hline \multicolumn{6}{|l|}{ TOTAL } & 1,624 \\
\hline
\end{tabular}


The great diversity in the jobs - men's and women's - is both a consequence of the increasing number of the members of the community and the general diversification of jobs during these years. Also in 1920 there seems to be no relation between region of provenience of the Italians and jobs because they are more or less equally distributed in the various sectors and jobs.

\subsection{Occupations in 1930}

In 1930 the community is considerably smaller than in 1910 and 1920, 2,839 rather than 4,384 and 4,379. The percentage of active members is, however, a little higher than in 1920, 38.7\% versus $37.1 \%$.

The percentage of people working for the mines is considerably smaller than 1920 , in fact it decreases from $66.7 \%$ to $49.6 \%$. This is not just a result of Italians changing jobs, but of the general trend of the mining industry declining in these years. Miners are the most numerous, 181, followed by laborers, 131, timbermen and blacksmith, twenty-three and twenty-two respectively. Despite the decrease in the number of individuals, the great of variety of mining jobs remains.

The group of Food and drink selling is greater in percentage (15.3\%) if compared to the previous years. The increase is mostly due to the increase in the number of proprietors and managers of food stores. The third group is Professionals and Skilled Tradesmen (7.2\%). Compared to the previous years, the biggest group is that of teachers, twelve.

The women working in 1930 are 142. Most of them, forty-three are working as salesladies in stores, but many are also involved in office jobs. In fact there are thirteen bookkeepers, six stenographers, four telephone operators, two postmasters, and one type composer at a newspaper. As for other skilled jobs there are eleven teachers, two seamstress, three nurses, and two dressmakers. There are also two managers of boarding houses and four proprietors of stores, even though three of them are actually widows whose former husbands were the original owners of the stores. Three women are indicated as merchants and two as managers of a business activity actually owned by their husbands. As for the remaining, they have unskilled jobs such as servants, laborers in factories, waiters, and janitors. 


\begin{tabular}{|c|c|c|c|c|c|c|}
\hline \multicolumn{7}{|c|}{ TABLE XIV. OCCUPATIONS IN 1930} \\
\hline \multicolumn{6}{|c|}{ GROUP } & \multirow{3}{*}{$\begin{array}{c}\text { Total } \\
\mathbf{5 4 5} \\
(49.6 \%)\end{array}$} \\
\hline \multicolumn{6}{|c|}{ MINING AND RELATED } & \\
\hline Assistant timekeeper/mine & 1 & Fireman/smelter & 2 & Pattern maker & 2 & \\
\hline Bell ringer/copper mine & 1 & Fireman/stationary boiler & 1 & Pipe man/copper mine & 4 & \\
\hline Blacksmith/copper mine & 22 & Floatman/copper mine & 1 & Policeman/copper mine & 2 & \\
\hline Boiler fireman/copper mine & 1 & Foreman/copper mine & 9 & Pump man/copper mine & 9 & \\
\hline Boiler tender/smelter & 1 & Foundry man/copper mine & 5 & Refiner/copper works & 1 & \\
\hline Captain/copper mine & 1 & Furnace man/smelter & 2 & Rock house man & 1 & \\
\hline Car filler/copper mine & 1 & Greaser/steam engine & 1 & Roller repairer/mine & 4 & \\
\hline Caretaker/copper mine & 1 & Head tender/copper mine & 1 & Shaft foreman/mine & 1 & \\
\hline Carpenter/copper mine & 9 & Inspector/copper mine & 1 & Shift captain/mine & 1 & \\
\hline Chute man/copper mine & 2 & Inspector/smelter & 1 & Ship tender/mine & 1 & \\
\hline Construction work/mine & 1 & Laborer/copper mine & 131 & Stationary eng./mine & 4 & \\
\hline Copper refiner/smelter & 1 & Laborer/foundry & 3 & Stationary fireman/mine & 3 & \\
\hline Crane inspector/mine & 1 & Laborer/reclaim plant & 1 & Steelworker/smelter & 1 & \\
\hline Draftsman/copper mine & 1 & Laborer/rock house & 2 & Stenographer/mine & 1 & \\
\hline Drayman/copper mine & 3 & Laborer/smelter & 6 & Stone $\mathrm{mason} / \mathrm{mine}$ & 6 & \\
\hline Drill boy/copper mine & 1 & Laborer/stamp mill & 15 & Tapper/smelter & 1 & \\
\hline Drill sharpener & 3 & Lander/copper mine & 3 & Teamster/copper mine & 2 & \\
\hline Driller/copper mine & 5 & Machinist/copper mine & 4 & Temperature & 1 & \\
\hline Engineer/stamp mill & 1 & Mason/copper mine & 4 & Timberman/copper mine & 23 & \\
\hline Electrician/copper mine & 6 & Mechanic/copper mine & 3 & Timekeeper/copper mine & 4 & \\
\hline Employment clerk/copper & 1 & Miner/copper mine & 181 & Trammer/copper mine & 4 & \\
\hline Engineer/copper mine & 2 & Molder/foundry & 1 & Watchman/copper mine & 4 & \\
\hline Fender/rock crusher & 1 & Motorman/rock house & 1 & & & \\
\hline Fireman/boiler house & 1 & Oiler & 5 & & & \\
\hline Fireman/copper mine & 12 & Painter/copper mine & 1 & & & \\
\hline \multicolumn{6}{|c|}{ TRASPORTATION } & \multirow{8}{*}{$\begin{array}{c}42 \\
(3.8 \%)\end{array}$} \\
\hline Blacksmith/railroad & 1 & Electrician/railroad & 1 & Office worker/railroad & 1 & \\
\hline Brakeman/railroad & 3 & Laborer/street car co. & 1 & Owner/bus line & 1 & \\
\hline Bus an truck driver & 14 & Locomotive engineer & 1 & Proprietor/taxi cab & 1 & \\
\hline Fireman/railroad & 3 & Locomotive fireman & 1 & Stenographer/railroad & 1 & \\
\hline Foreman/freight depot & 1 & Machinist/railroad & 2 & Workman/railroad & 1 & \\
\hline Inspector/railroad & 1 & Manager/bus line & 1 & & & \\
\hline Laborer/railroad & 6 & Motorman/street car & 1 & & & \\
\hline \multicolumn{6}{|c|}{ BOARDING } & \multirow{6}{*}{$\begin{array}{c}\mathbf{4} \\
(0.4 \%) \\
\mathbf{2 3} \\
(2.1 \%)\end{array}$} \\
\hline Manager boarding house & 3 & Proprietor boarding house & 1 & & & \\
\hline \multicolumn{6}{|c|}{ BUILDING } & \\
\hline Carpenter & 2 & Mason & 5 & Proprietor lumber camp & 1 & \\
\hline Engineer/saw mill & 2 & Painter & 6 & Sawyer/saw mill & 1 & \\
\hline Lumber & 3 & Plasterer & 2 & Stone mason & 1 & \\
\hline \multicolumn{6}{|c|}{ FARMING } & \multirow{3}{*}{$\begin{array}{c}39 \\
(3.5 \%)\end{array}$} \\
\hline Dairy man/own farm & 1 & Farmer/fruit farm & 1 & Laborer/farm & 19 & \\
\hline Farmer/dairy farm & 3 & Farmer/general farm & 15 & & & \\
\hline \multicolumn{6}{|c|}{ FOOD \& DRINK SELLING } & \multirow{9}{*}{$\begin{array}{c}\mathbf{1 6 9} \\
(15.3 \%)\end{array}$} \\
\hline Bakery & 19 & Manager/fruit store & 1 & Teamster & 8 & \\
\hline Bartender & 1 & Meat cutter/grocery & 1 & Tester/creamery & 1 & \\
\hline Butcher & 7 & Merchant/dry goods & 1 & Truck driver & 9 & \\
\hline Confectionery/candy store & 15 & Merchant/fruit & 2 & Saleslady & 24 & \\
\hline Delivery person & 6 & Proprietor/macaroni & 1 & Salesman & 11 & \\
\hline Druggist/drug store & 2 & Proprietor/restaurant & 4 & Waiter & 9 & \\
\hline Grocer & 23 & Sausage maker & 1 & & & \\
\hline Laborer & 8 & Soft drink parlor & 15 & & & \\
\hline
\end{tabular}




\begin{tabular}{|c|c|c|c|c|c|c|}
\hline \multicolumn{6}{|c|}{ PROFESSIONALS AND SKILLED TRADESMEN } & \multirow{10}{*}{$\begin{array}{c}79 \\
(7.2 \%)\end{array}$} \\
\hline Apprentice barber & 1 & Dressmaker & 2 & Shoemaker/repairer & 7 & \\
\hline Apprentice plumber & 1 & Electrical engineer & 1 & Seamstress & 2 & \\
\hline Auto mechanic & 5 & Fireman & 2 & Sheriff & 1 & \\
\hline Barber & 4 & Foreman & 2 & Steelworker/contractor & 1 & \\
\hline Beauty operator & 1 & Mechanic auto & 11 & Tailor & 2 & \\
\hline Blacksmith & 5 & Musician/orchestra & 2 & Teacher & 12 & \\
\hline Chauffeur & 2 & Nurse & 3 & Undertaker & 2 & \\
\hline Cigar maker & 1 & Painter & 1 & & & \\
\hline Cook & 2 & Plumber & 6 & & & \\
\hline \multicolumn{6}{|c|}{ GENERAL OFFICE JOBS } & \multirow{7}{*}{$\begin{array}{c}\mathbf{6 1} \\
(5.5 \%)\end{array}$} \\
\hline Assistant cashier/bank & 1 & Composer type /newspaper & 2 & Printer & 4 & \\
\hline Assistant clerk & 1 & Editor/newspaper & 1 & Secretary & 1 & \\
\hline Banker & 1 & Employee/insurance & 2 & Stenographer & 4 & \\
\hline Bookkeeper & 18 & Interpreter/court & 1 & Telephone operator & 2 & \\
\hline Cashier/bank & 2 & Operator telephone & 2 & Teller/bank & 1 & \\
\hline Clerk & 15 & Postmaster & 2 & Timekeeper/telephone & 1 & \\
\hline \multicolumn{6}{|c|}{ GENERAL MERCHANDISE SALE } & \multirow{5}{*}{$\begin{array}{c}\mathbf{5 1} \\
(4.6 \%)\end{array}$} \\
\hline Attendant gas-oil station & 6 & Merchant/general store & 5 & Saleslady & 12 & \\
\hline Manager gas station & 1 & Proprietor/store & 3 & Salesman & 19 & \\
\hline Manager ice rink & 1 & Proprietor gas-oil station & 2 & & & \\
\hline Manager/pool room & 2 & & & & & \\
\hline \multicolumn{6}{|c|}{ UNSKILLED } & \multirow{5}{*}{$\begin{array}{c}\mathbf{7 4} \\
(6.7 \%)\end{array}$} \\
\hline Cleaner & 3 & Janitor & 5 & Presser/dry cleaner & 1 & \\
\hline Chore person & 2 & Laborer & 29 & Servant & 16 & \\
\hline Dishwasher & 1 & Laundress & 6 & & & \\
\hline Housekeeper & 8 & Maid & 1 & & & \\
\hline \multicolumn{6}{|c|}{ OTHERS } & \multirow{2}{*}{$\begin{array}{c}\mathbf{1 4} \\
(1.3 \%)\end{array}$} \\
\hline At school & 1 & Not clear & 10 & Retired & 3 & \\
\hline \multicolumn{6}{|l|}{ TOTAL } & 1,101 \\
\hline
\end{tabular}

\subsection{Comparisons and conclusion}

The following graphs show a comparison of the percentages of workers for each sector through the years. One thing to note in comparing the data shown in the graphs is the scale difference among occupations, in particular between Mining and Related and all the other groups. It is important to remember that in 1880 the members of the Italian community were just 220, few in comparison with the following years. However, the percentages for this year have been included to examine potential trends.

As Figure IX shows, Mining and related has an increasing trend for the first thirty years, from 1880 till 1910. It also corresponds to the increase in the numbers of Italians in Houghton County. In 1920, however, despite the fact that the community has more or less the same number of individuals as in 1910, the percentage of people working for the mines 
starts to decrease. In 1930 it is not the category with the absolute majority of employed persons anymore.

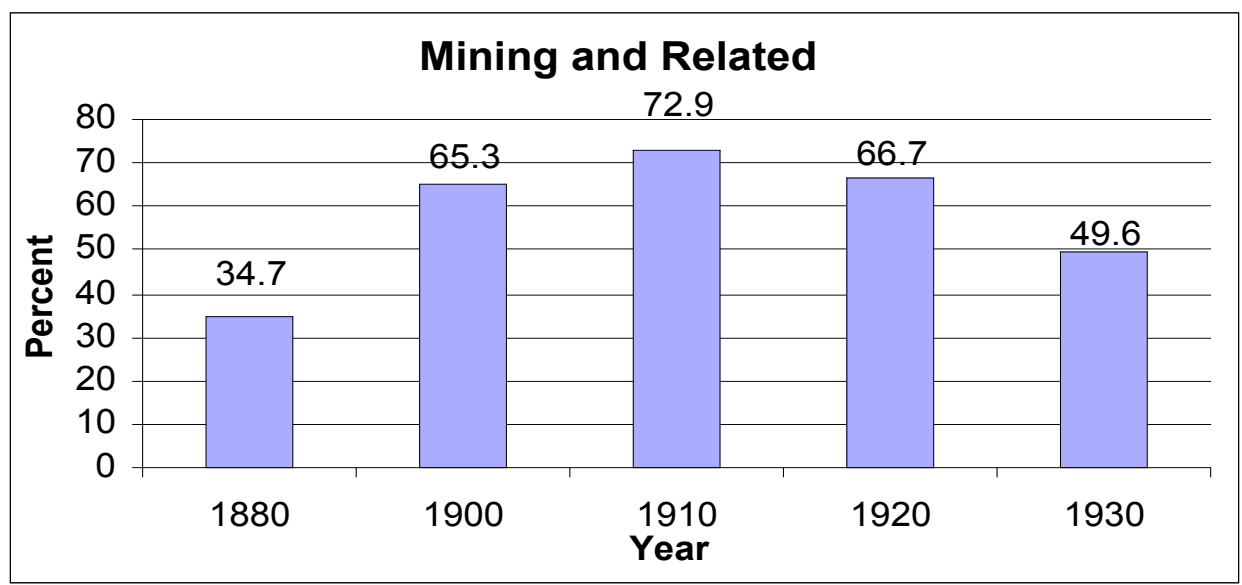

Figure IX. Percentages of workers in Mining and related in 1880, 1900, 1910, 1920, and 1930.

Transportation (Figure X), although with lower absolute numbers of workers than mining in every year, follows a completely different trend. In fact the number of workers increases steadily. Besides the increase in the number of workers for the railroad, what increases is the number of drivers both for taxi companies and for bus companies. ${ }^{43}$

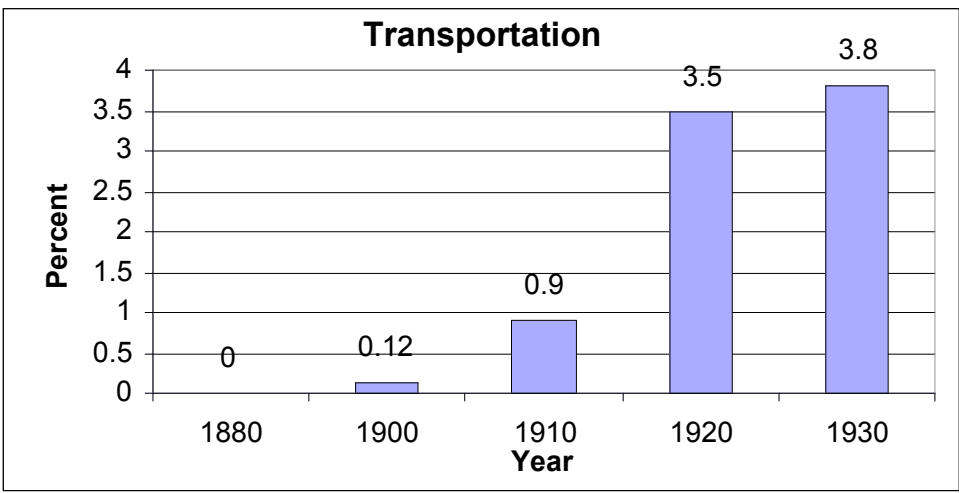

Figure X. Percentages of workers in Transportation in 1880, 1900, 1910, 1920, and 1930.

${ }^{43}$ According to Magnaghi "In the 1920s Caesar Lucchesi pioneered a bus service between South Range and Houghton, and maintained winter schedules by plowing the roads before the state of Michigan started this service". Magnaghi, M. Russell. Italians in Michigan. East Lansing: Michigan State University Press, 2001, p. 22 . 
As Figure XI shows, Boarding reaches its peak in 1910, but as also previously stated, these data are far from being exact because many more households included boarders than the ones in which one member is indicated as boarding house keeper. Probably many individuals did not considered themselves as boarding house keepers when they had other occupations outside the house.

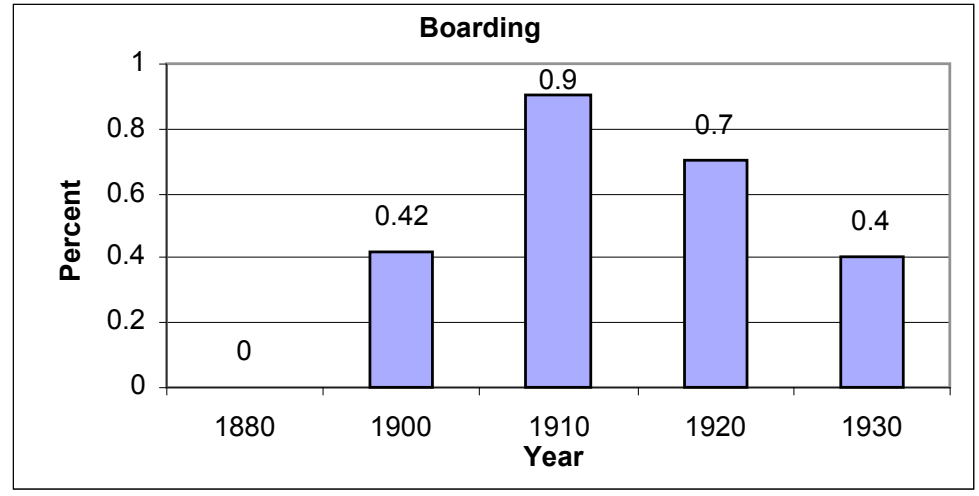

Figure XI. Percentages of workers in Boarding in 1880, 1900, 1910, 1920, and 1930.

Besides the fact that the number of workers in Building (Figure XII) is far lower than what was expected, the trend of the percentages is the strangest. ${ }^{44}$ The small number could be explained by the fact that many of them were working for the mines and are not therefore included in this group. It could also explain the fact that in 1930 the percentage increases; in fact given the decline in mining industry, it is possible that many workers had to find an alternative way to earn their living.

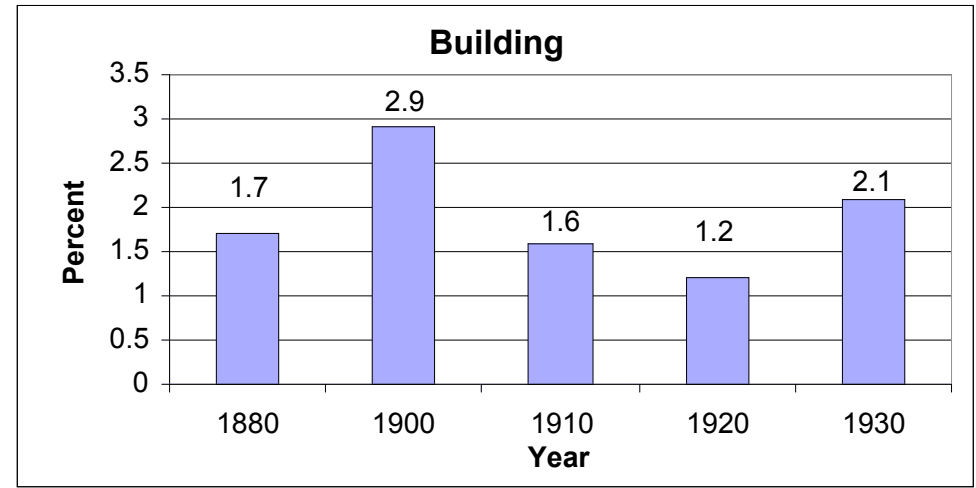

Figure XII. Percentages of workers in Building in 1880, 1900, 1910, 1920, and 1930.

\footnotetext{
${ }^{44}$ This may be a consequence of the small sample.
} 
Taking into consideration that 1880 may give a distorted picture because of the small number of workers, Farming (Figure XIII) has a steadily increasing trend through the years. As previously stated, the reason may be found in the fact that many Italians made up their minds and decided to stay in the United States for good. They did not need to earn as much money as possible in the shortest time possible to go back to Italy, and at the same time they were more willing to invest money in buying farms.

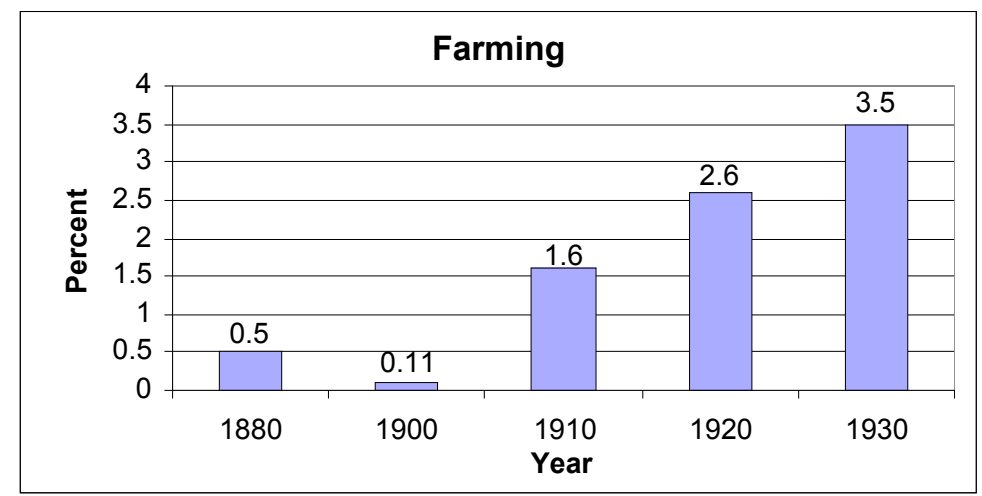

Figure XIII. Percentages of workers in Farming in 1880, 1900, 1910, 1920, and 1930.

Food and drink selling (Figure XIV) has a steady trend through the years, although it is important to note that the number of liquor dealers goes up and down in correspondence with particular moments in the country, such as Prohibition (see previous paragraphs).

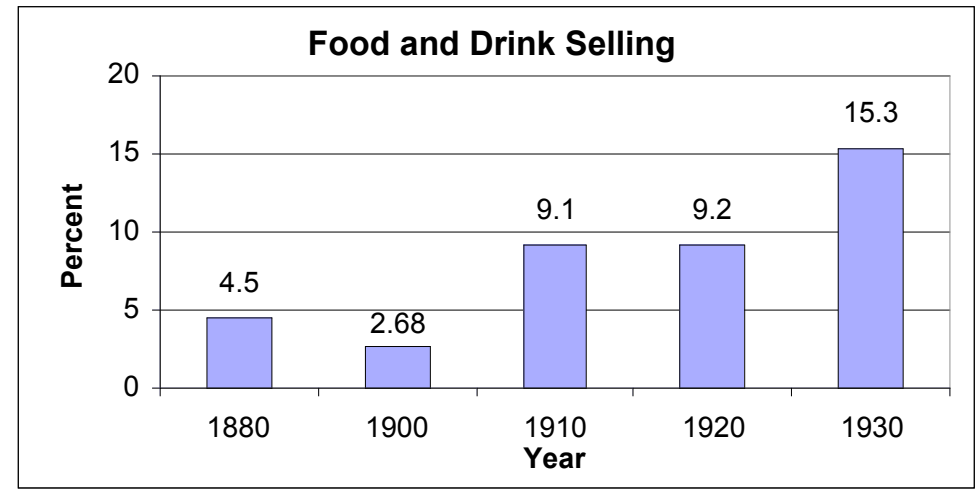

Figure XIV. Percentages of workers in Food and drink selling in 1880, 1900, 1910, 1920, and 1930. 
The percentages of Professionals and Skilled Tradesmen (Figure XV) and General office jobs (Figure XVI) seem to be evolving in accordance with the general developments in society. In fact its increase is a result of two factors: 1) the development of the sector of services, and therefore the appearance of new job categories and 2) the increase in the number of working women, many of whom are involved in office jobs.

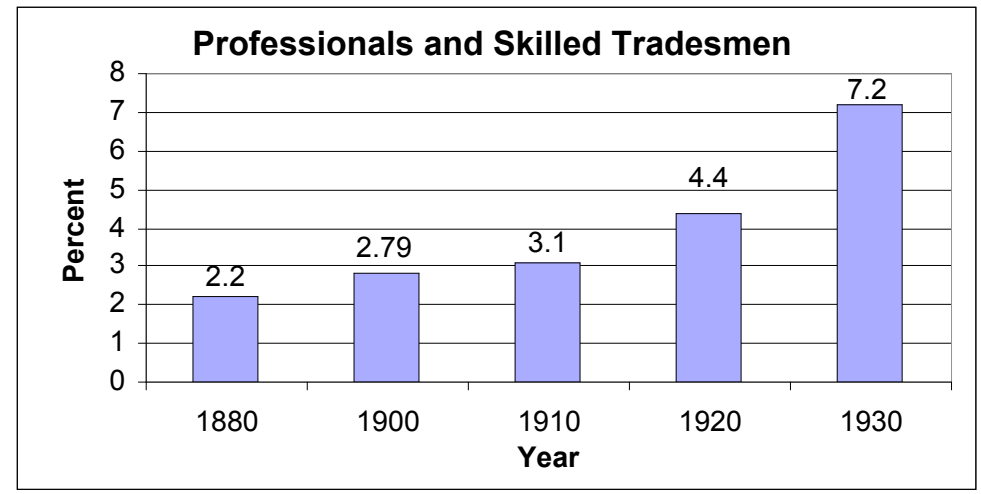

Figure XV. Percentages of workers in Professionals and Skilled Tradesmen in $1880,1900,1910,1920$, and 1930

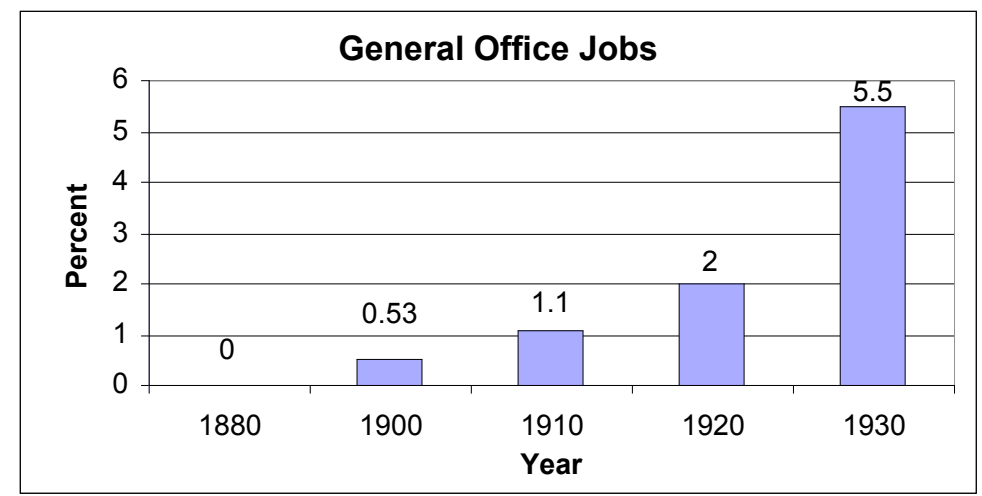

Figure XVI. Percentages of workers in General office jobs in 1880, 1900, 1910, 1920 , and 1930

The same explanation could be true for the General sales sector (Figure XVII). In fact, besides the fact that Italians succeeded in investing their savings and start independent activities such as stores, the number of occupied in this sector increases because many women are working as saleslady in these stores. These data also reflect the growth of the retail sector overall. 


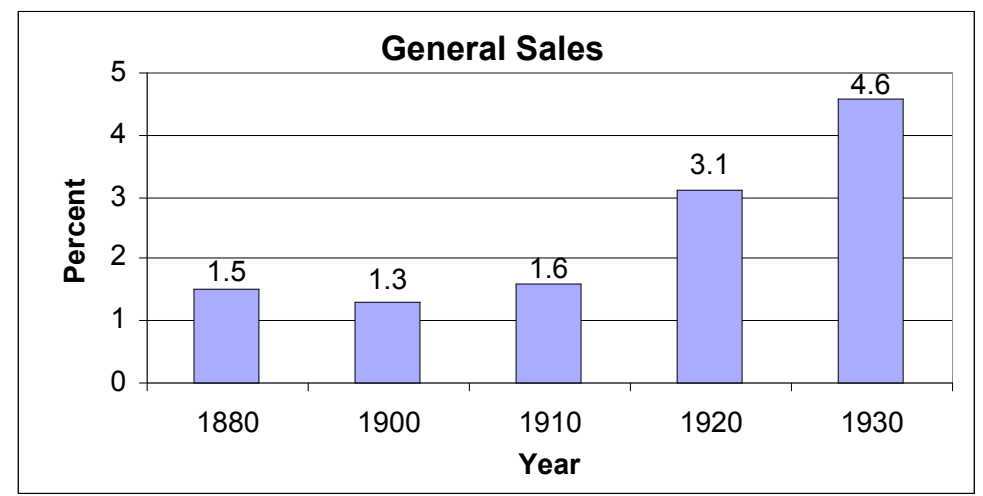

Figure XVII. Percentages of workers in General sales in 1880, 1900, 1910, 1920, and 1930

Unskilled (Figure XVIII), always considering the fact that 1880 includes a great number of laborers whose place of job is not indicated but it is likely that they are mine laborers, has a steady trend. The great increase in the percentage in 1930 is due to the number of unskilled laborers in factories.

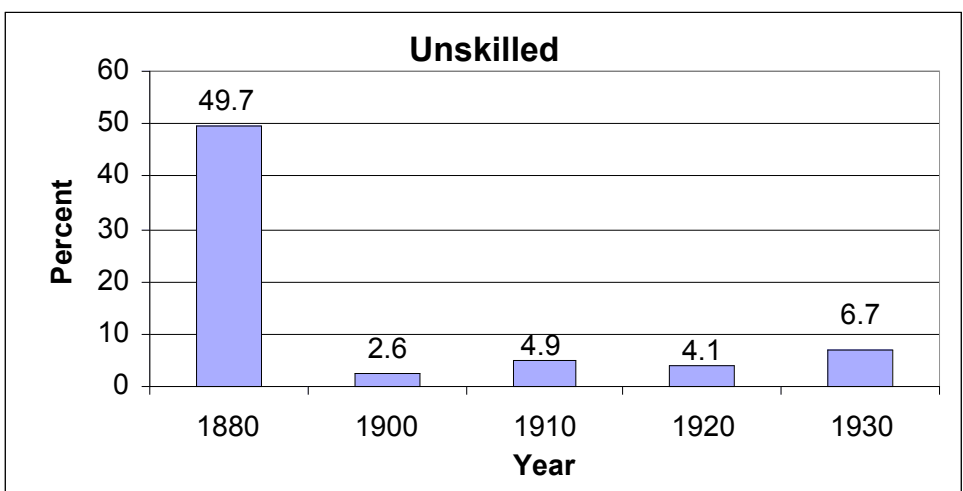

Figure XVIII. Percentages of workers in Unskilled in 1880, 1900, 1910, 1920, and 1930

The final category, Other (Figure XIX), is also consistent through the years although in 1900 it appears to be extremely high, but it is because the census included students in the group of "employed". 


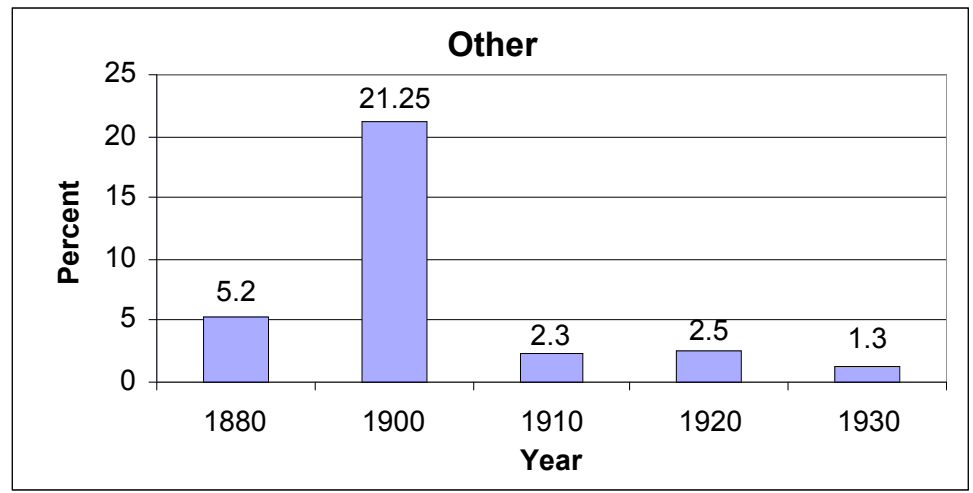

Figure XIX. Percentages of workers in Other in 1880, 1900, 1910, 1920, and 1930.

One last note about occupations is on the relation between provenience and jobs. It appears that they are not related. In fact, considering the two main groups of Italians, Torinesi and Lucchesi, and the sector of occupation with more workers, mining, the percentages indicate that they are equally distributed (in absolute numbers Torinesi are always the larger number of the two groups).

In $190037 \%$ of the Torinesi and 41\% of Lucchesi are working in the mines. In 1910 the percentage is $26 \%$ for the Torinesi and 36\% for the Lucchesi. In $192023 \%$ of the Torinesi are working in the mines are, and 24\% of the Lucchesi. In $193020 \%$ of the Torinesi and $18 \%$ of the Lucchesi were working in the mines (Figure XX).

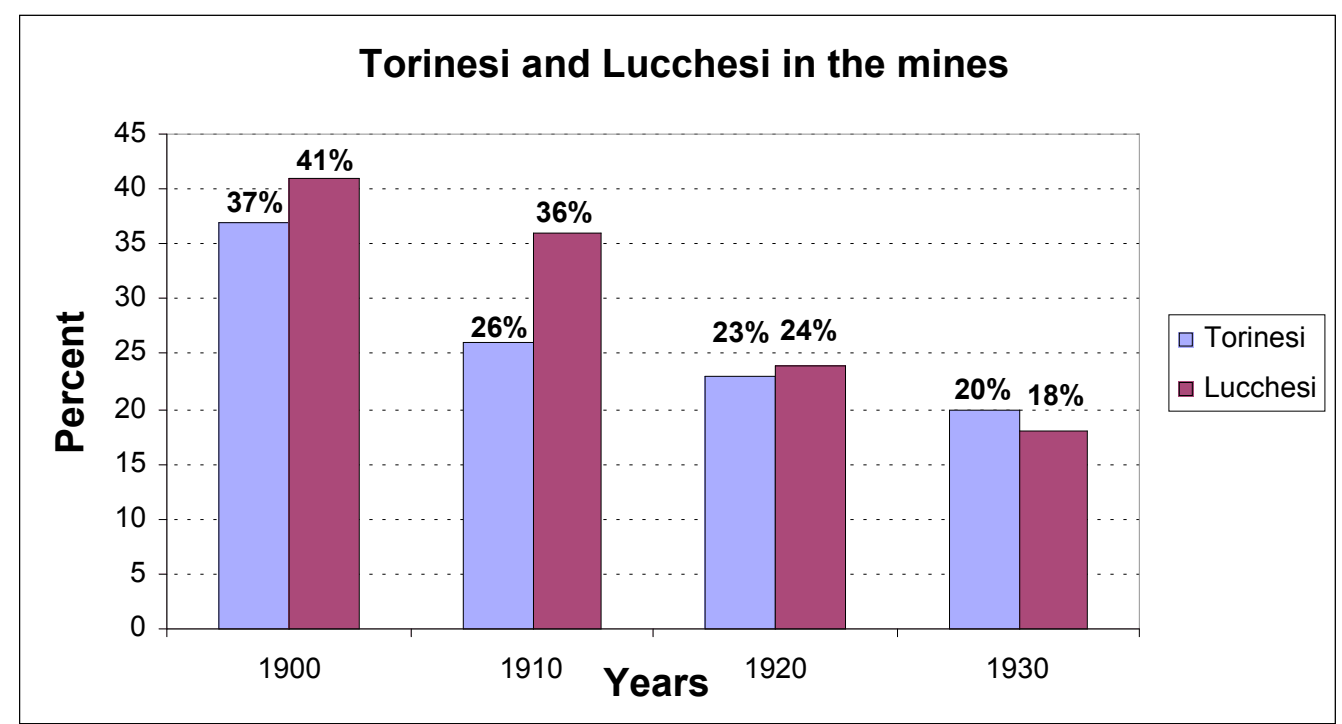

Figure XX. Percentages of Torinesi and Lucchesi working in the mines in 1900, 1910, 1920, and 1930. 


\section{Chapter IV - Settlements}

\subsection{Goals and Methods}

The aim of the following chapter is to learn more about where in Houghton County Italians preferred to settle and to find out whether there was a relation between province of origin and spatial clustering in locations and villages.

The data used to determine the pattern of settlement of Italians in Houghton County are from the censuses of the state of Michigan, Houghton County, of 1870, 1880, 1900, 1910, 1920, and 1930 .

In these censuses, Houghton County is divided into townships and villages. Their boundaries are not the exactly the same in each year however. There exist no known sources defining how long current boundaries have been configured, but it seems that they are/were relatively fluid over larger spans of time. The more significant changes probably occurred during the setting off and/or creation of new townships. Over time, townships have ceded property back and forth, affecting their boundaries. In addition, unincorporated township property was regularly annexed into incorporated villages and cities. However, because changes came slowly and in small degrees for the most part, they should not be significant in affecting comparisons among general patterns of Italian settlement through the years. ${ }^{45}$

Organized or incorporated villages (e.g. Hancock, Calumet, and Laurium) are technically not part of townships because the villages are managed by their own governments. Spatially, however, they can be identified as within townships. Therefore, even when they were incorporated, they have been grouped with the township to allow spatial continuity and help comparisons. South Range Village has been included in Adams Township, Calumet, Laurium, and Red Jacket Village in Calumet Township, Hancock in Hancock Township, Houghton in Portage Township, Lake Linden in Schoolcraft Township, and Hubbell Village in Torch Lake Township.

\footnotetext{
${ }^{45}$ Houghton has grown spatially due to land annexed from the surrounding Portage Township. For instance, that the boundaries between Adams and Stanton townships changed quite dramatically in the early 20th century. One could also look in the minutes of the Houghton County Board of Supervisors, part of the MTU Archives "state archives collection". (RG 89-464, volumes 16-24, 1861-1963). The Board must approve any change in township boundaries, so they are detailed here.
} 
Tables XV-XIX show the distribution, in percentages and absolute numbers, of the Italians over the townships and the villages. Some mining locations are also indicated when there were Italians living there.

After determining numbers and percentages of Italians for each township and village, the patterns of settlement of the two main groups of Italian immigrants, Torinesi and Lucchesi, have been compared to see if they showed different preferences as to townships and villages of residence. ${ }^{46}$ Italians originating in other provinces were excluded from examination because of low absolute numbers.

\subsection{Settlements in 1870}

In 1870 Houghton County is divided into eleven townships: Adams, Baraga, Calumet, Franklin, Hancock, Huron, L’Anse, Portage, Quincy, Schoolcraft, Webster. The Italian community counts just twenty-one individuals. They are concentrated in Franklin Township. ${ }^{47}$ Only one Italian is living in Baraga Township.

\subsection{Settlements in 1880}

In 1880 the division of Houghton County changes a little bit. The townships are seven: Adams, Calumet, Franklin, Hancock, Portage, Quincy, and Schoolcraft. Baraga, Huron, L'Anse, and Webster Township are no longer part of Houghton County.

Italians have increased in number from twenty-one to 220. As Table XIV shows, most of them are living within the territory of Calumet Township: 131 in Red Jacket Village, and twenty-eight in Precinct 2. In Franklin Township there are fifty-four Italians, and seven in Quincy.

\footnotetext{
${ }^{46}$ Many previous studies about Italian immigrants have shown that they were really province or even villageminded in the sense that they tended to congregate with other Italians from the same province or even the same village. See, for example, Park, Robert and Herbert Miller, Old World Traits Transplanted, New York: 1921, pp.146-151.

${ }^{47}$ Italian descendants interviewed by professor Magnaghi remember that the very first Italians in Houghton County settled in Franklin. Magnaghi, M. Russell. Interview with Mike Gemignani, 5-14-1982, Franklin Mine, Michigan.
} 
As for distribution of Torinesi and Lucchesi, the former are clearly clustered in Calumet Township, and more exactly in Red Jacket Village (70\%). The latter are almost equally distributed between Calumet (52\%) and Franklin Township (48\%).

\begin{tabular}{|c|c|c|c|c|c|c|}
\hline \multicolumn{7}{|c|}{ TABLE XV. SETTLEMENTS IN 1880} \\
\hline \multicolumn{2}{|l|}{ TOWNSHIP } & \multirow{2}{*}{$\begin{array}{c}\begin{array}{c}\text { Italians } \\
\text { Total and \% }\end{array} \\
0\end{array}$} & \multicolumn{2}{|c|}{$\begin{array}{c}\text { TORINESI } \\
\text { TOTAL AND \% }\end{array}$} & \multicolumn{2}{|c|}{$\begin{array}{c}\text { Lucchesi } \\
\text { Total and \% }\end{array}$} \\
\hline \multicolumn{2}{|l|}{ ADAMS } & & \multicolumn{2}{|c|}{0} & \multicolumn{2}{|c|}{0} \\
\hline \multicolumn{2}{|l|}{ CALUMET } & \multirow[t]{3}{*}{$\begin{array}{c}159 \\
(72.3 \%)\end{array}$} & \multicolumn{2}{|c|}{$\begin{array}{c}86 \\
(89.5 \%)\end{array}$} & \multicolumn{2}{|c|}{$\begin{array}{c}31 \\
(52 \%)\end{array}$} \\
\hline Red Jacket & 131 & & Red Jacket & $\begin{array}{l}67 \\
(70 \%)\end{array}$ & Red Jacket & $\begin{array}{l}25 \\
(42 \\
\%)\end{array}$ \\
\hline Precinct 2 & 28 & & Precinct 2 & $\begin{array}{l}19 \\
(19.5 \\
\%)\end{array}$ & Precinct 2 & $\begin{array}{l}6 \\
(10 \\
\%)\end{array}$ \\
\hline \multicolumn{2}{|l|}{ FRANKLIN } & $\begin{array}{c}\mathbf{5 4} \\
(24.5 \%)\end{array}$ & \multicolumn{2}{|c|}{$\begin{array}{c}10 \\
(10.5 \%)\end{array}$} & \multicolumn{2}{|c|}{$\begin{array}{c}29 \\
(48 \%) \\
\end{array}$} \\
\hline \multicolumn{2}{|l|}{ HANCOCK } & 0 & \multicolumn{2}{|c|}{0} & \multicolumn{2}{|c|}{0} \\
\hline \multicolumn{2}{|l|}{ PORTAGE } & 0 & \multicolumn{2}{|c|}{0} & \multicolumn{2}{|c|}{0} \\
\hline \multicolumn{2}{|l|}{ QUINCY } & $\begin{array}{c}7 \\
(3.2 \%)\end{array}$ & & & & \\
\hline \multicolumn{2}{|l|}{ SCHOOLCRAFT } & 0 & & & \multicolumn{2}{|c|}{0} \\
\hline \multicolumn{2}{|l|}{ TOTAL IN 1880} & 220 & \multicolumn{2}{|c|}{$\begin{array}{c}96 \\
\text { (43.6\% of the total } \\
\text { Italians) }\end{array}$} & \multicolumn{2}{|c|}{$\begin{array}{c}\mathbf{6 0} \\
\text { (27.3\% of the tota } \\
\text { Italians) }\end{array}$} \\
\hline
\end{tabular}

\subsection{Settlements in 1900}

By 1900 Houghton County is divided into thirteen townships. Seven are the same as 1880 - Adams, Calumet, Franklin, Hancock, Portage, Quincy, and Schoolcraft. The new ones are Chassell, Duncan, Elm River, Laird, Osceola, and Torch Lake. The villages in which Italians are living include Laurium, Red Jacket, Hancock, Houghton, and Lake Linden.

As Table XVI shows, most of the 2,789 Italians residing in Houghton County in 1900 were actually living in Calumet Township - 1,950 (69.9\%). Franklin and Adams Township follow with 461 (16.5\%) and 214 (7.7\%) respectively. In Osceola Township there were 96 Italians (3.44\%). Hancock and Portage Township counted respectively 33 (1.2\%) 
and 17 Italians $(0.6 \%)$. Only one Italian was living in Torch Lake $(0.03 \%)$, as well as in Schoolcraft Township (0.03\%).

Torinesi and Lucchesi represented respectively $53.4 \%$ and $15.6 \%$ of the Italian community in 1900. A careful analysis of the data shows interesting differences between their clustering. ${ }^{48}$ Torinesi clearly prevailed in numbers and in percentage in Calumet Township and in the two villages within its boundaries, Laurium (17.8\%) and Red Jacket $(8.7 \%)$.

\begin{tabular}{|c|c|c|c|c|c|c|}
\hline \multicolumn{7}{|c|}{ TABLE XVI. SETTLEMENTS 1900} \\
\hline \multicolumn{2}{|l|}{ TOWNSHIP } & $\begin{array}{c}\text { Italians } \\
\text { Total and \% }\end{array}$ & \multicolumn{2}{|c|}{$\begin{array}{c}\text { Torinesi } \\
\text { Total and \% }\end{array}$} & \multicolumn{2}{|c|}{$\begin{array}{c}\text { Lucchesi } \\
\text { Total and \% }\end{array}$} \\
\hline \multicolumn{2}{|l|}{ ADAMS } & \multirow{4}{*}{$\begin{array}{c}214 \\
(7.7 \%)\end{array}$} & \multirow{4}{*}{\multicolumn{2}{|c|}{$\begin{array}{c}89 \\
(6 \%)\end{array}$}} & \multirow{4}{*}{\multicolumn{2}{|c|}{$\begin{array}{c}76 \\
(17.5 \%)\end{array}$}} \\
\hline Atlantic Mine Location & 136 & & & & & \\
\hline Baltic Mine Location & 7 & & & & & \\
\hline Rest of the Twp. & 71 & & & & & \\
\hline \multicolumn{2}{|l|}{ CALUMET } & \multirow[t]{4}{*}{$\begin{array}{c}\mathbf{1 , 9 5 0} \\
(69.9 \%)\end{array}$} & \multicolumn{2}{|c|}{$\begin{array}{c}1215 \\
(81.5 \%)\end{array}$} & \multicolumn{2}{|c|}{$\begin{array}{c}146 \\
(33.8 \%)\end{array}$} \\
\hline Laurium Village & 427 & & Laurium Village & $266(17.8 \%)$ & Laurium Village & $17(4 \%)$ \\
\hline Red Jacket Village & 271 & & Red Jacket Village & $129(8.7 \%)$ & Red Jacket Vill. & $56(13 \%)$ \\
\hline Rest of the Twp. & 1,252 & & Rest of the Twp. & $820(55 \%)$ & Rest of Twp. & $73(16.8 \%)$ \\
\hline \multicolumn{2}{|l|}{ CHASSELL } & $\begin{array}{c}\mathbf{0} \\
(0 \%)\end{array}$ & \multicolumn{2}{|l|}{0} & \multicolumn{2}{|l|}{0} \\
\hline \multicolumn{2}{|l|}{ DUNCAN } & $\begin{array}{c}\mathbf{0} \\
(0 \%)\end{array}$ & \multicolumn{2}{|l|}{0} & \multicolumn{2}{|l|}{0} \\
\hline \multicolumn{2}{|l|}{ FRANKLIN } & $\begin{array}{c}461 \\
(16.5 \%)\end{array}$ & \multicolumn{2}{|c|}{$\begin{array}{c}125 \\
(1.2 \%)\end{array}$} & \multicolumn{2}{|c|}{$\begin{array}{c}169 \\
(38.9 \%)\end{array}$} \\
\hline \multicolumn{2}{|l|}{ ELM RIVER } & $\begin{array}{c}\mathbf{0} \\
(0 \%)\end{array}$ & \multicolumn{2}{|c|}{0} & \multicolumn{2}{|c|}{0} \\
\hline \multicolumn{2}{|l|}{ HANCOCK } & \multirow[t]{3}{*}{$\begin{array}{c}33 \\
(1.2 \%)\end{array}$} & \multirow{3}{*}{\multicolumn{2}{|c|}{$\begin{array}{c}18 \\
(1.2 \%)\end{array}$}} & \multirow{3}{*}{\multicolumn{2}{|c|}{0}} \\
\hline Hancock Village & 26 & & & & & \\
\hline Rest of Twp. & 7 & & & & & \\
\hline \multicolumn{2}{|l|}{ LAIRD } & $\begin{array}{c}\mathbf{0} \\
(0 \%)\end{array}$ & \multicolumn{2}{|l|}{0} & \multicolumn{2}{|l|}{0} \\
\hline \multicolumn{2}{|l|}{ OSCEOLA } & $\begin{array}{c}96 \\
(3.44 \%)\end{array}$ & \multicolumn{2}{|c|}{$\begin{array}{c}30 \\
(2 \%)\end{array}$} & \multicolumn{2}{|c|}{$\begin{array}{c}42 \\
(9.6 \%)\end{array}$} \\
\hline PORTAGE & & $\begin{array}{c}17 \\
(0.6 \%)\end{array}$ & $\begin{array}{r}9 \\
(0.6 \%\end{array}$ & & 0 & \\
\hline QUINCY & & $\begin{array}{c}16 \\
(0.6 \%)\end{array}$ & $\begin{array}{c}3 \\
\left(0.2^{\circ}\right.\end{array}$ & & $\begin{array}{r}1 \\
\left(0.2^{2}\right.\end{array}$ & \\
\hline SCHOOLCRAF & & $\begin{array}{c}1 \\
(0.03 \%)\end{array}$ & 0 & & 0 & \\
\hline TORCH LAKE & & $\begin{array}{c}1 \\
(0.03 \%)\end{array}$ & 0 & & 0 & \\
\hline TOTAL IN 190 & & 2,789 & $\begin{array}{r}\mathbf{1 , 4 8} \\
(53.4 \% \text { of the to }\end{array}$ & tal Italians) & (15.6\% of the & otal Italians) \\
\hline
\end{tabular}

\footnotetext{
${ }^{48}$ The comparisons are made among the percentages, not among absolute numbers.
} 
Lucchesi, on the other hand, settled in big percentages in Franklin Township (38.9\%), Calumet Township (33.5\%), and Adams Township (17.5\%). A smaller group was in Osceola Township (9.6\%). They are particularly concentrated in Red Jacket village (13\%).

\subsection{Settlements in 1910}

In 1910 Houghton County includes fourteen townships: Adams, Calumet, Chassell, Duncan, Elm River, Franklin, Hancock, Laird, Osceola, Portage, Quincy, Schoolcraft, Stanton - which was not in the census of 1900 - and Torch Lake. The villages in which Italians are living are seven. Five are the same as in 1900 - Laurium, Red Jacket, Hancock, Houghton, and Lake Linden - and the new ones are Hubbell and South Range.

The numbers of Italians in Houghton County significantly increases from 2,789 to 4,384, and the township in which they were more numerous is still Calumet with 2,405 Italians (54.9\%). This time Adams Township is the second with 730 (16.7\%), followed by Franklin with $668(15.2 \%)$.

Torinesi are 2,201 (50.2\% of the total Italian community) and Lucchesi 989 (22.5\%). As for distribution of Torinesi and Lucchesi, the former are mostly living in Calumet Township (72.3\%), and particularly in Raymbaultown (12.7\%) and Calumet and Hecla Location (11.4\%). The highest concentration is in Laurium village (19.4\%). Compared to 1900, their percentage almost doubles in Adams Township (13,6\%). In fact they are numerous in South Range Village (5.2\%).

Lucchesi, as in 1900, are spread over more townships, in particular Franklin (30\%), Adams (26.2\%), and Calumet (23.2\%). In Calumet Township they are clustered in Raymbaultown (5.2\%) and Calumet and Hecla Location (3.4\%). As far as the villages within the boundaries of Calumet Township, Lucchesi are numerous in Red Jacket (6.6\%). The highest concentration of Lucchesi, however, is in Trimountain (18.8\%). In fact in Trimountain Location the census indicates an "Italian Settlement". There are 118 Italians living there and eighty-nine of them are from the province of Lucca. The rest of them are from other provinces of Toscana, and there are no Torinesi. This may show that people from the same 
region are actually clustering together. ${ }^{49}$ In fact it seems that Sunny Italy (this is the name of the settlement in the recollection of people interviewed by Professor Magnaghi and also according to the censuses of 1920 and 1930) was the place where Toscani having some kind of interest in farming preferred to settle. ${ }^{50}$ On the other hand, farmers from Piemonte clustered in an area of Franklin Township called Farm Section Location in the census of $1920 .^{51}$

\begin{tabular}{|c|c|c|c|c|c|c|}
\hline \multicolumn{7}{|c|}{ TABLE XVII. SETTLEMENTS 1910} \\
\hline \multicolumn{2}{|l|}{ TOWNSHIP } & $\begin{array}{c}\text { Italians } \\
\text { Total and \% }\end{array}$ & \multicolumn{2}{|c|}{$\begin{array}{c}\text { Torinesi } \\
\text { Total and \% }\end{array}$} & \multicolumn{2}{|c|}{$\begin{array}{c}\text { Lucchesi } \\
\text { Total and \% }\end{array}$} \\
\hline \multicolumn{2}{|l|}{ ADAMS } & \multirow[t]{6}{*}{$\begin{array}{c}730 \\
(16.7 \%)\end{array}$} & \multicolumn{2}{|c|}{$\begin{array}{c}300 \\
(13.6 \%)\end{array}$} & \multicolumn{2}{|c|}{$\begin{array}{c}260 \\
(26.2 \%)\end{array}$} \\
\hline Atlantic Mine Location & 41 & & Atlantic Loc. & $14(0.6 \%)$ & Atlantic Loc. & $17(1.7 \%)$ \\
\hline Baltic Mine Location & 164 & & Baltic Loc. & $96(4.4 \%)$ & Baltic Loc. & $25(2.5 \%)$ \\
\hline Painesdale & 89 & & Painesdale & $49(2.2 \%)$ & Painesdale & $16(1.6 \%)$ \\
\hline South Range Village & 150 & & South Range & $114(5.2 \%)$ & South Range & $16(1.6 \%)$ \\
\hline Trimountain & 286 & & Trimountain & $27(1.2 \%)$ & Trimountain & $\begin{array}{l}186 \\
(18.8 \%)\end{array}$ \\
\hline \multicolumn{2}{|l|}{ CALUMET } & \multirow[t]{6}{*}{$\begin{array}{c}2,405 \\
(54.9 \%)\end{array}$} & \multicolumn{2}{|c|}{$\begin{array}{c}1,308 \\
(72.3 \%)\end{array}$} & \multicolumn{2}{|c|}{$\begin{array}{l}180 \\
(23.2 \%)\end{array}$} \\
\hline C. \& H. Location & 366 & & C. \& H. Loc. & $251(11.4 \%)$ & C. \& H. Loc. & $34(3.4 \%)$ \\
\hline Laurium Village & 608 & & Laurium Vill. & $426(19.4 \%)$ & Laurium Village & $34(3.4 \%)$ \\
\hline Raymbaultown & 279 & & Raymbaultown & $279(12.7 \%)$ & Raymbaultown & $52(5.2 \%)$ \\
\hline Red Jacket Village & 268 & & Red Jacket Vill. & $141(6.4 \%)$ & Red Jacket Vill. & $67(6.6 \%)$ \\
\hline Rest of the Twp. & 1,163 & & Rest of the Twp & $490(22.4 \%)$ & Rest of the Twp. & $45(4.5 \%)$ \\
\hline \multicolumn{2}{|l|}{ CHASSELL } & $\begin{array}{c}\mathbf{0} \\
(0 \%)\end{array}$ & \multicolumn{2}{|c|}{0} & \multicolumn{2}{|l|}{0} \\
\hline \multicolumn{2}{|l|}{ DUNCAN } & $\begin{array}{c}\mathbf{0} \\
(0 \%)\end{array}$ & \multicolumn{2}{|c|}{0} & \multicolumn{2}{|l|}{0} \\
\hline \multicolumn{2}{|l|}{ ELM RIVER } & $\begin{array}{c}\mathbf{0} \\
(0 \%)\end{array}$ & \multicolumn{2}{|c|}{0} & \multicolumn{2}{|l|}{0} \\
\hline \multicolumn{2}{|l|}{ FRANKLIN } & $\begin{array}{c}\mathbf{6 6 8} \\
(15.2 \%) \\
\end{array}$ & \multicolumn{2}{|c|}{$\begin{array}{l}155 \\
(7 \%)\end{array}$} & \multicolumn{2}{|c|}{$\begin{array}{c}294 \\
(30 \%)\end{array}$} \\
\hline \multicolumn{2}{|l|}{ HANCOCK } & \multirow[t]{3}{*}{$\begin{array}{c}180 \\
(4.1 \%)\end{array}$} & \multicolumn{2}{|c|}{$\begin{array}{c}57 \\
(2.6 \%)\end{array}$} & \multicolumn{2}{|c|}{$\begin{array}{c}59 \\
(6 \%)\end{array}$} \\
\hline Hancock City & 157 & & Hancock City & $41(1.9 \%)$ & Hancock City & $59(6 \%)$ \\
\hline Rest of Twp. & 23 & & Rest of Twp. & $16(0.7 \%)$ & Rest of Twp. & 0 \\
\hline \multicolumn{2}{|l|}{ LAIRD } & $\begin{array}{c}\mathbf{5} \\
(0.1 \%)\end{array}$ & \multicolumn{2}{|c|}{$\begin{array}{c}4 \\
(0.2 \%)\end{array}$} & \multicolumn{2}{|l|}{0} \\
\hline \multicolumn{2}{|l|}{ OSCEOLA } & \multirow[t]{4}{*}{$\begin{array}{c}157 \\
(3.6 \%)\end{array}$} & 5 & & 51 & \\
\hline Opechee Location & 23 & & & & & \\
\hline Tamarack City & 39 & & & & & \\
\hline Rest of Twp. & 95 & & & & & \\
\hline
\end{tabular}

${ }^{49}$ This is true also for Italians in other parts of the United States. See Glazer, Nathan and Daniel Moynihan. Beyond the Melting Pot. Cambridge: M.I.T. Press. 1970, p. 186.

${ }^{50}$ Magnaghi, Russell. Interview with Louis Lombardi, 12-03-1982. In Lombardi's words they settled there because even though "better soil came off the hills [...but in Sunny Italy the temperature] is 10 or 12 degrees higher than on the hills".

${ }^{51}$ See APPENDIX X, Census of 1920. 


\begin{tabular}{|c|c|c|c|c|}
\hline \multicolumn{2}{|l|}{ PORTAGE } & \multirow{3}{*}{$\begin{array}{c}\mathbf{8 5} \\
(1.9 \%)\end{array}$} & \multirow{3}{*}{$\begin{array}{c}19 \\
(0.8 \%)\end{array}$} & \multirow{3}{*}{$\begin{array}{c}16 \\
(1.6 \%)\end{array}$} \\
\hline Houghton & 18 & & & \\
\hline Hurontown & 67 & & & \\
\hline \multicolumn{2}{|l|}{ QUINCY } & \multirow{4}{*}{$\begin{array}{l}90 \\
(2 \%)\end{array}$} & \multirow{4}{*}{$\begin{array}{c}5 \\
(0.2 \%)\end{array}$} & \multirow{4}{*}{$\begin{array}{c}66 \\
(1.6 \%)\end{array}$} \\
\hline Hardscrabble Location & 57 & & & \\
\hline Limerick Location & 9 & & & \\
\hline Sing Sing Location & 24 & & & \\
\hline \multicolumn{2}{|c|}{ SCHOOLCRAFT } & $\begin{array}{c}9 \\
(0.2 \%)\end{array}$ & $\begin{array}{c}7 \\
(0.3 \%)\end{array}$ & 0 \\
\hline \multicolumn{2}{|l|}{ STANTON } & \multirow{3}{*}{$\begin{array}{l}\mathbf{3 0} \\
(0.7 \%)\end{array}$} & \multirow[t]{3}{*}{0} & \multirow{3}{*}{$\begin{array}{c}5 \\
(0.5 \%)\end{array}$} \\
\hline Beacon Hill & 17 & & & \\
\hline Rest of Twp. & 13 & & & \\
\hline \multicolumn{2}{|c|}{ TORCH LAKE } & \multirow[t]{2}{*}{$\begin{array}{c}\mathbf{2 5} \\
(0.6 \%)\end{array}$} & \multirow[t]{2}{*}{$\begin{array}{c}11 \\
(0.5 \%)\end{array}$} & \multirow[t]{2}{*}{$\begin{array}{c}6 \\
(0.6 \%)\end{array}$} \\
\hline Hubbell Village & 25 & & & \\
\hline TOTAL & & 4,384 & $\begin{array}{c}\mathbf{2 , 2 0 1} \\
\text { (50.2\% of the total Italians) }\end{array}$ & $\begin{array}{c}\mathbf{9 8 9} \\
(22.5 \% \text { of the total Italians) }\end{array}$ \\
\hline
\end{tabular}

\subsection{Settlement in 1920}

In 1920 the various boundaries are the same as in 1910.

The number of Italians in Houghton County is almost the same as in 1910, 4,379. Most of them are still living in Calumet Township (47.6\%), although the percentage decreases compared to 1910. The Italian population significantly increases in Adams Township (23.6\%), and decreases a little in Franklin (12.7\%).

Torinesi are 1,902 (43.4\% of the Italian community), a drop compared to 1910 . They are still concentrated in Calumet Township (70.7\%), Adams (11.8\%), and Franklin (7\%). The highest concentration of Torinesi is again in Laurium village (18.8\%), however they are also numerous in Raymbaultown (10\%), Wolverine (7.2\%), South Range (5.4\%), and Hancock City (3.9\%).

Lucchesi significantly increase to 1,261 (28.8\% of the total Italian community). Adams becomes the first township as far as number of Lucchesi (42.9\%), but they still are numerous in Franklin (20\%), and Calumet (17.2\%). In Franklin Township they are clustered in Pewabic Location (8.1\%) and Franklin Location (7.2\%). As far as villages, Lucchesi are numerous in Hancock (7.2\%), South Range (5.6\%), Red Jacket (5\%), and Laurium (3.9\%). Trimountain remains the location with the highest concentration of Lucchesi $(24.9 \%)$. 
TABLE XVIII. SETTLEMENTS 1920

\begin{tabular}{|c|c|c|c|c|c|c|}
\hline \multicolumn{2}{|l|}{ TOWNSHIP } & $\begin{array}{c}\text { Italians } \\
\text { Total and \% } \\
\end{array}$ & \multicolumn{2}{|c|}{$\begin{array}{c}\text { Torinesi } \\
\text { Total and \% }\end{array}$} & \multicolumn{2}{|c|}{$\begin{array}{c}\text { Lucchesi } \\
\text { Total and \% }\end{array}$} \\
\hline \multicolumn{2}{|l|}{ ADAMS } & \multirow[t]{6}{*}{$\begin{array}{c}\mathbf{1 , 0 3 5} \\
(23.63 \%)\end{array}$} & \multicolumn{2}{|c|}{$\begin{array}{c}225 \\
(11.8 \%) \\
\end{array}$} & \multicolumn{2}{|c|}{$\begin{array}{c}540 \\
(42.9 \%) \\
\end{array}$} \\
\hline Atlantic Mine Loc. & 38 & & Atlantic Loc. & $15(0.8 \%)$ & Atlantic Loc. & $13(1 \%)$ \\
\hline Baltic Mine Location & 211 & & Baltic Loc. & $62(3.2 \%)$ & Baltic Loc. & $94 \quad(7.4 \%)$ \\
\hline Painesdale & 147 & & Painesdale & $35(1.8 \%)$ & Painesdale & $51 \quad(4 \%)$ \\
\hline South Range Village & 215 & & South Range & $102(5.4 \%)$ & South Range & $70 \quad(5.6 \%)$ \\
\hline Trimountain & 424 & & Trimountain & $11(0.6 \%)$ & Trimountain & $312(24.9 \%)$ \\
\hline \multicolumn{2}{|l|}{ CALUMET } & \multirow[t]{6}{*}{$\begin{array}{c}\mathbf{2 , 0 8 7} \\
(47.65 \%)\end{array}$} & \multicolumn{2}{|c|}{$\begin{array}{c}1,346 \\
(70.7 \%)\end{array}$} & \multicolumn{2}{|c|}{$\begin{array}{c}217 \\
(17.2 \%)\end{array}$} \\
\hline Laurium Village & 548 & & Laurium Village & $356(18.8 \%)$ & Laurium & $49(3.9 \%)$ \\
\hline Raymbaultown & 339 & & Raymbaultown & $192(10 \%)$ & Raymbaultown & $47(3.7 \%)$ \\
\hline Red Jacket Village & 161 & & Red Jacket Vill. & $59(3.1 \%)$ & Red Jacket & $63(5 \%)$ \\
\hline Wolverine & 153 & & Wolverine & $138(7.2 \%)$ & Wolverine & $2(0.2 \%)$ \\
\hline Rest of the Twp. & 886 & & Rest of the Twp. & $601(31.6 \%)$ & Rest Twp. & $56(4.4 \%)$ \\
\hline \multicolumn{2}{|l|}{ CHASSELL } & $\begin{array}{c}\mathbf{0} \\
(0 \%)\end{array}$ & \multicolumn{2}{|c|}{0} & \multicolumn{2}{|c|}{0} \\
\hline \multicolumn{2}{|l|}{ DUNCAN } & $\begin{array}{c}\mathbf{4} \\
(0.09 \%) \\
\end{array}$ & \multicolumn{2}{|c|}{$\begin{array}{c}3 \\
(0.15 \%)\end{array}$} & \multicolumn{2}{|c|}{0} \\
\hline \multicolumn{2}{|c|}{ ELM RIVER } & $\begin{array}{c}\mathbf{2} \\
(0.04 \%)\end{array}$ & \multicolumn{2}{|c|}{$\begin{array}{c}1 \\
(0.05 \%)\end{array}$} & \multicolumn{2}{|c|}{0} \\
\hline \multicolumn{2}{|l|}{ FRANKLIN } & \multirow[t]{4}{*}{$\begin{array}{c}\mathbf{5 5 6} \\
(12.7 \%)\end{array}$} & \multicolumn{2}{|c|}{$\begin{array}{c}134 \\
(7.05 \%)\end{array}$} & \multicolumn{2}{|c|}{$\begin{array}{c}252 \\
(20 \%)\end{array}$} \\
\hline Franklin Mine Loc. & 186 & & Franklin Loc. & $45(2.4 \%)$ & Franklin Loc. & $91(7.2 \%)$ \\
\hline Pewabic Location & 149 & & Pewabic Loc. & $15(0.7 \%)$ & Pewabic Loc. & $101(8.1 \%)$ \\
\hline Rest of the Twp. & 221 & & Rest Twp. & $74(3.9 \%)$ & Rest Twp. & $60(4.7 \%)$ \\
\hline \multicolumn{2}{|l|}{ HANCOCK } & \multirow[t]{3}{*}{$\begin{array}{c}\mathbf{2 7 4} \\
(6.3 \%)\end{array}$} & $\begin{array}{r}85 \\
(4.47 \\
\end{array}$ & & & \\
\hline Hancock City & 254 & & Hancock City & $75(3.9 \%)$ & Hancock C. & $(7.2 \%)$ \\
\hline Rest of Twp. & 20 & & Rest of Twp. & $10(0.5 \%)$ & Rest of Twp. & $(0.2 \%)$ \\
\hline LAIRD & & $\begin{array}{c}\mathbf{0} \\
(0 \%)\end{array}$ & 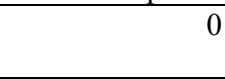 & & & \\
\hline OSCEOLA & & $\begin{array}{c}132 \\
(3.01 \%)\end{array}$ & $\begin{array}{r}44 \\
(2.32\end{array}$ & & & \\
\hline PORTAGE & & $\begin{array}{c}105 \\
(2.4 \%)\end{array}$ & $\begin{array}{r}36 \\
(1.9 \\
\end{array}$ & & & \\
\hline QUINCY & & $\begin{array}{c}91 \\
(2.07 \%)\end{array}$ & $\begin{array}{r}7 \\
(0.36\end{array}$ & & & \\
\hline Hardscrabble Loc. & 62 & & & & & \\
\hline Rest of the Twp. & 29 & & & & & \\
\hline SCHOOLCRA & & $\begin{array}{c}\mathbf{2 8} \\
(0.63 \%)\end{array}$ & $\begin{array}{r}9 \\
(0.47\end{array}$ & & & \\
\hline Lake Linden Vill. & 14 & & & & & \\
\hline Rest of Twp. & 14 & & & & & \\
\hline STANTON & & $\begin{array}{c}\mathbf{3 1} \\
(0.71 \%)\end{array}$ & 0 & & & \\
\hline Beacon Hill & 12 & & & & & \\
\hline Rest of Twp. & 19 & & & & & \\
\hline TORCH LAK & & $\begin{array}{c}34 \\
(0.77)\end{array}$ & $\begin{array}{r}12 \\
(0.63\end{array}$ & & & \\
\hline Hubbell Village & 25 & & & & & \\
\hline Rest of Twp. & 9 & & & & & \\
\hline TOTAL & & 4,379 & $\begin{array}{r}1,90 \\
\text { (43.4\% of the }\end{array}$ & $\begin{array}{l}2 \\
\text { otal Italians) }\end{array}$ & $\begin{array}{r}\mathbf{1}, \\
(28.8 \% \text { of th }\end{array}$ & tal Italians) \\
\hline
\end{tabular}


Seventy-nine Italians are living in Sunny Italy, and sixty-four of them are from the province of Lucca. Lucchesi, as during the previous years, are more distributed over the territory than Piemontesi.

\subsection{Settlements in 1930}

In 1930 the various boundaries are the same as in 1910 and 1920. The Italian community shrinks to 2,839. Calumet is still the township in which most of them are living (54.7\%), followed by Adams (18.9\%), Hancock (9.7\%), and Franklin (8.6\%).

As Table XIX shows most of the Torinesi are in Calumet Township (72.7\%), and smaller percentages live in Adams (11.9\%), and Franklin (6.1\%). They are particularly concentrated in Laurium village (19.7\%), South Range village (5\%), and Franklin Location $(4.8 \%)$.

Adams is still 'the township of Lucchesi'. In fact $36.6 \%$ of them are living there, however, the highest concentration of Lucchesi is no longer in Trimountain but in Hancock City (21\%). Franklin Location (12\%), Trimountain (9.2\%), Painesdale (8.9\%), Baltic Location (8.6\%), and South Range village (7.6\%) follow.

\begin{tabular}{|c|c|c|c|c|c|c|}
\hline \multicolumn{7}{|c|}{ TABLE XVIII. SETTLEMENTS 1930} \\
\hline \multicolumn{2}{|c|}{ TOWNSHIP } & $\begin{array}{l}\text { Italians } \\
\text { Total and } \\
\%\end{array}$ & \multicolumn{2}{|c|}{$\begin{array}{c}\text { Torinesi } \\
\text { Total and \%o }\end{array}$} & \multicolumn{2}{|c|}{$\begin{array}{c}\text { Lucchesi } \\
\text { Total and \% }\end{array}$} \\
\hline \multicolumn{2}{|l|}{ ADAMS } & \multirow[t]{6}{*}{$\begin{array}{c}\mathbf{5 3 7} \\
(18.9 \%)\end{array}$} & \multicolumn{2}{|c|}{$\begin{array}{c}148 \\
(11.9 \%)\end{array}$} & \multicolumn{2}{|c|}{$\begin{array}{c}242 \\
(36.6 \%)\end{array}$} \\
\hline Atlantic Mine Location & 52 & & Atlantic Loc. & $12(1 \%)$ & Atlantic Loc. & $15(2.3 \%)$ \\
\hline Baltic Mine Location & 125 & & Baltic Loc. & $39(3.1 \%)$ & Baltic Loc. & $57(8.6 \%)$ \\
\hline Painesdale & 121 & & Painesdale & $28(2.2 \%)$ & Painesdale & $59(8.9 \%)$ \\
\hline South Range Village & 137 & & South Range & $62(5 \%)$ & South Range & $50(7.6 \%)$ \\
\hline Trimountain & 102 & & Trimountain & $7(0.6 \%)$ & Trimountain & $61(9.2 \%)$ \\
\hline \multicolumn{2}{|l|}{ CALUMET } & $\begin{array}{c}\mathbf{1 , 5 5 3} \\
(54.7 \%)\end{array}$ & \multicolumn{2}{|c|}{$\begin{array}{c}902 \\
(72.7 \%)\end{array}$} & \multicolumn{2}{|c|}{$\begin{array}{c}113 \\
(17.1 \%)\end{array}$} \\
\hline Laurium Village & 460 & & Laurium & $244(19.7 \%)$ & Laurium Village & $27(4.1 \%)$ \\
\hline Calumet Village & 126 & & Calumet & $62(5 \%)$ & Calumet Village & $27(4.1 \%)$ \\
\hline Rest of the Twp. & 913 & & Rest of Twp. & $596(48 \%)$ & Rest of the Twp. & $59(8.9 \%)$ \\
\hline
\end{tabular}




\begin{tabular}{|c|c|c|c|c|c|c|}
\hline \multicolumn{2}{|l|}{ CHASSELL } & $\begin{array}{c}\mathbf{0} \\
(0 \%) \\
\end{array}$ & \multicolumn{2}{|l|}{0} & \multicolumn{2}{|l|}{0} \\
\hline \multicolumn{2}{|l|}{ DUNCAN } & $\begin{array}{c}\mathbf{0} \\
(0 \%)\end{array}$ & \multicolumn{2}{|l|}{0} & \multicolumn{2}{|l|}{0} \\
\hline \multicolumn{2}{|l|}{ ELM RIVER } & $\begin{array}{c}\mathbf{1} \\
(0.03 \%) \\
\end{array}$ & \multicolumn{2}{|l|}{0} & \multicolumn{2}{|l|}{0} \\
\hline \multicolumn{2}{|l|}{ FRANKLIN } & \multirow[t]{4}{*}{$\begin{array}{c}244 \\
(8.6 \%)\end{array}$} & \multicolumn{2}{|c|}{$\begin{array}{c}76 \\
(6.1 \%)\end{array}$} & \multicolumn{2}{|c|}{$\begin{array}{c}102 \\
(15.4 \%) \\
\end{array}$} \\
\hline Franklin Mine Location & 183 & & Franklin Loc. & $60(4.8 \%)$ & Franklin Loc. & $79(12 \%)$ \\
\hline Pewabic Location & 39 & & Pewabic Loc. & $1(0.1 \%)$ & Pewabic Loc. & $18(2.7 \%)$ \\
\hline Rest of the Twp. & 22 & & Rest of the Twp. & $15(1.2 \%)$ & Rest of the Twp. & $5(0.7 \%)$ \\
\hline \multicolumn{2}{|l|}{ HANCOCK } & \multirow[t]{3}{*}{$\begin{array}{c}276 \\
(9.7 \%)\end{array}$} & \multicolumn{2}{|l|}{$\begin{array}{c}49 \\
(4 \%) \\
\end{array}$} & \multicolumn{2}{|c|}{$\begin{array}{c}139 \\
(21 \%)\end{array}$} \\
\hline Hancock City & 269 & & Hancock City & $42(3.4 \%)$ & Hancock City & $139(21 \%)$ \\
\hline Rest of Twp. & 7 & & Rest of Twp. & $7(0.6 \%)$ & Rest of Twp. & 0 \\
\hline \multicolumn{2}{|l|}{ LAIRD } & $\begin{array}{c}\mathbf{0} \\
(0 \%) \\
\end{array}$ & \multicolumn{2}{|l|}{ 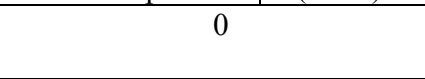 } & \multicolumn{2}{|l|}{ 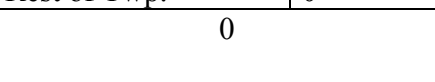 } \\
\hline \multicolumn{2}{|l|}{ OSCEOLA } & $\begin{array}{c}\mathbf{8 1} \\
(2.8 \%) \\
\end{array}$ & \multicolumn{2}{|c|}{$\begin{array}{c}25 \\
(2 \%) \\
\end{array}$} & \multicolumn{2}{|c|}{$\begin{array}{c}19 \\
(2.8 \%) \\
\end{array}$} \\
\hline \multicolumn{2}{|l|}{ PORTAGE } & $\begin{array}{c}\mathbf{5 6} \\
(1.97 \%)\end{array}$ & \multicolumn{2}{|c|}{$\begin{array}{c}16 \\
(1.3 \%)\end{array}$} & \multicolumn{2}{|c|}{$\begin{array}{c}9 \\
(1.3 \%)\end{array}$} \\
\hline Dodgeville & 14 & & Dodgeville & 0 & Dodgeville & 0 \\
\hline Houghton Village & 34 & & Houghton & $10(0.8 \%)$ & Houghton Village & $9(1.3 \%)$ \\
\hline Rest of Twp. & 8 & & Rest of Twp. & $6(0.5 \%)$ & Rest of Twp. & 0 \\
\hline \multicolumn{2}{|l|}{ QUINCY } & $\begin{array}{c}17 \\
(0.6 \%)\end{array}$ & \multicolumn{2}{|l|}{0} & \multicolumn{2}{|c|}{$\begin{array}{c}14 \\
(2.1 \%)\end{array}$} \\
\hline \multicolumn{2}{|c|}{ SCHOOLCRAFT } & \multirow[t]{3}{*}{$\begin{array}{c}19 \\
(0.7 \%)\end{array}$} & \multirow{3}{*}{\multicolumn{2}{|c|}{$\begin{array}{c}6 \\
(0.5 \%)\end{array}$}} & $\begin{array}{r}5 \\
(0.79\end{array}$ & \\
\hline Lake Linden Village & 11 & & & & & \\
\hline Rest of Twp. & 8 & & & & & \\
\hline STANTON & & $\begin{array}{c}\mathbf{2 5} \\
(0.9 \%)\end{array}$ & $\begin{array}{r}9 \\
(0.79\end{array}$ & & $\begin{array}{r}2 \\
\left(0.3^{\circ}\right.\end{array}$ & \\
\hline Beacon Hill & 19 & & & & & \\
\hline Rest of Twp. & 6 & & & & & \\
\hline TORCH LAKI & & $\begin{array}{c}30 \\
(1.1 \%)\end{array}$ & $\begin{array}{r}9 \\
(0.8\end{array}$ & & $\begin{array}{r}18 \\
(2.7 \%\end{array}$ & \\
\hline Hubbell Village & 20 & & & & & \\
\hline Rest of Twp. & 10 & & & & & \\
\hline TOTAL & & 2,839 & $\begin{array}{r}1,24 \\
\text { (of the total }\end{array}$ & alians) & $\begin{array}{r}663 \\
\text { (of the total }\end{array}$ & ians) \\
\hline
\end{tabular}

\subsection{Conclusions}

The following graphs - Figure XXI and XXII - show the change in the numbers of Italians in the townships of Houghton County through the years. Figure XXI includes the three townships - Calumet, Adams, and Franklin - in which the Italian community had an average population above 500. As the graph shows, in each year examined Italians were more numerous in Calumet Township - including the villages within its boundaries. Franklin Township was second in 1880 and 1900, but from 1910 on Adams topped it. 
The decrease in the number of Italians in Calumet and Franklin Township starting in 1910 reflects the decrease in the overall population of Houghton County. In Adams Township the decrease started in 1920.

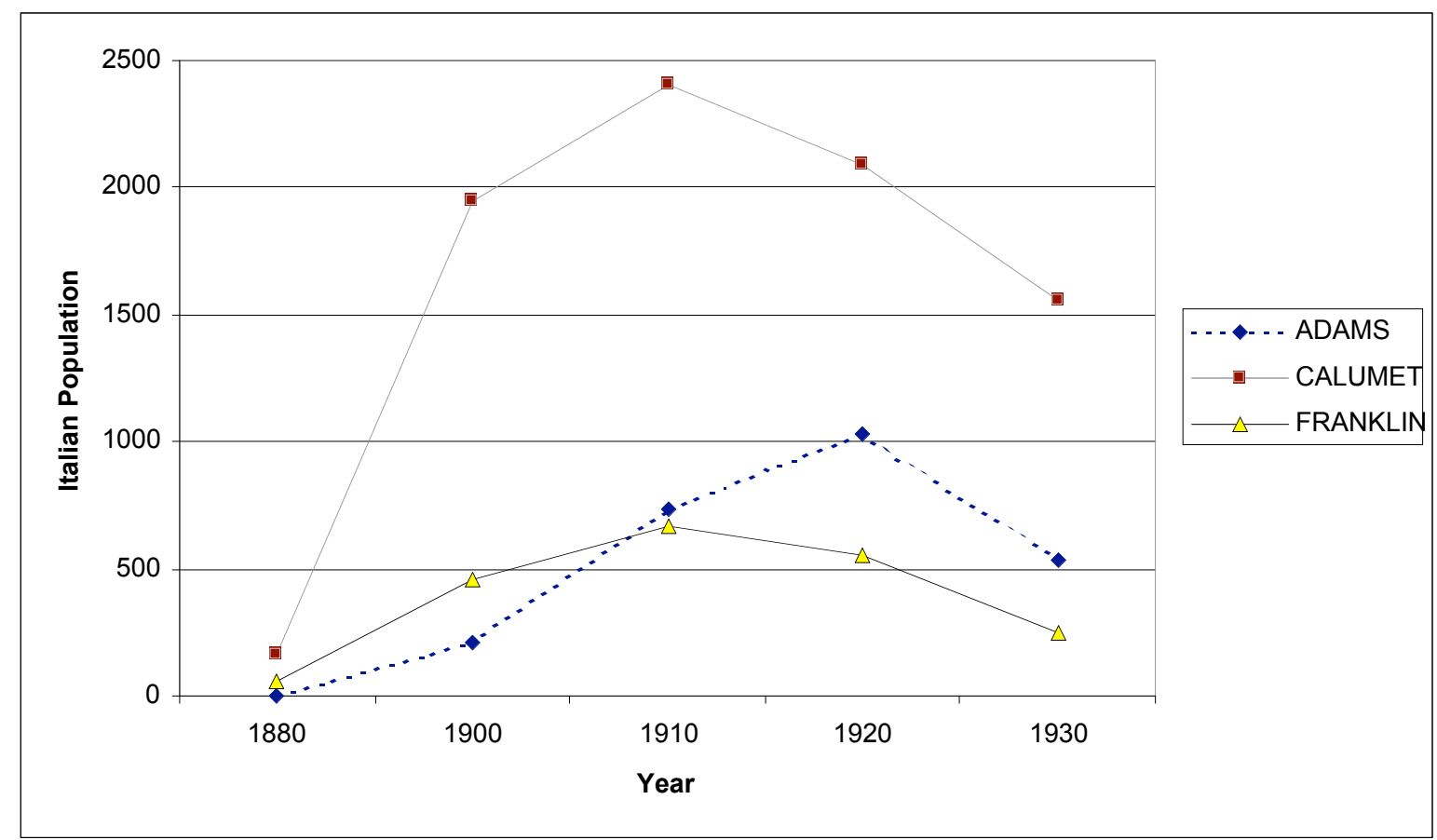

Figure XXI. Number of Italians in Adams, Calumet, and Franklin Township in 1880, 1900, 1910, 1920, and 1930.

Figure XXII show the trend in the Italian population in the remaining townships - all with an average Italian population below 300 people - of Houghton County. Franklin Township is also included to show the concentration of Italians in Franklin, Hancock, and Quincy townships together. Although in these townships the number of Italians is considerably smaller than in the previous three, the graph still shows the same trend: increase in the Italian population from 1880 until 1910 and decrease from that year on. The only anomaly is in Hancock Township. In fact there the Italian population increases also from 1910 until 1920, and it is steady in 1930. However Italians are actually clustering in Hancock city. Apparently, with the population shrinking, Italians tended to concentrate in the city.

As for distribution of Torinesi and Lucchesi across the territory, the data clearly show that they had different preferences. As for townships, most of the Piemontesi throughout the years settled in Calumet Township, whereas Lucchesi spread over different 
townships with peaks in Adams and Franklin Townships in alternate years. As for villages, Laurium appears to be the village of Piemontesi. Lucchesi do not have a village in which their number is high and steady through the years, but a location, Trimountain.

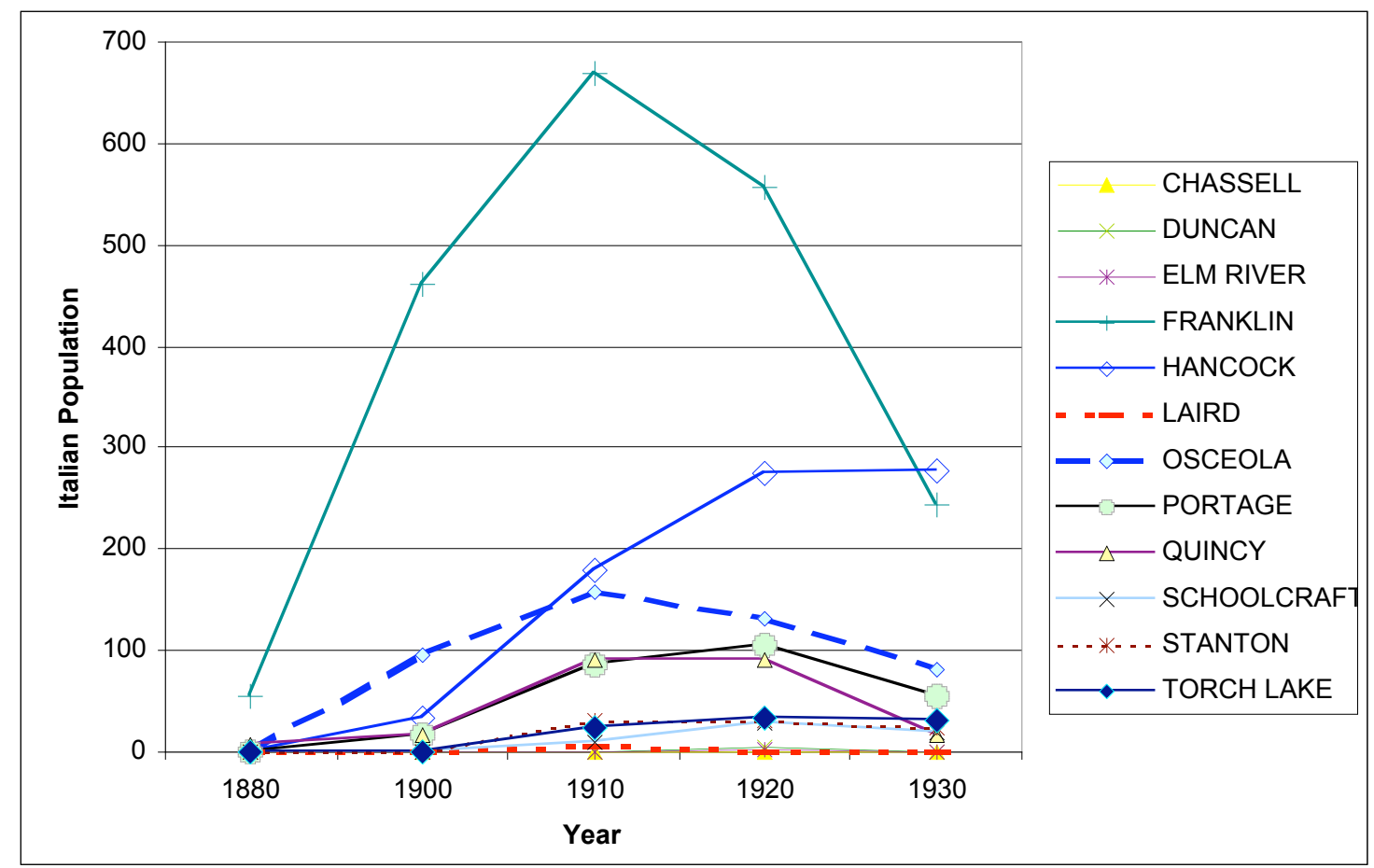

Figure XXII. Number of Italians in the remaining townships in 1880, 1900, 1910, 1920, and 1930.

In conclusion, as far as settlements, Italians appear to follow in Glazer and Moynihan's words "village-mindedness" because "they tended to congregate with others from the same province or even village". 52 This is not surprising for several reasons. First of all there was a language barrier. Standard Italian is a fairly recent achievement. ${ }^{53}$ Immigrants leaving Italy at the end of $19^{\text {th }}$ and beginning of $20^{\text {th }}$ century had not been exposed to it. Coming from different parts of Italy, they spoke such different dialects that often they were not able to understand each other. ${ }^{54}$ Secondly, they did not necessarily think of other

\footnotetext{
${ }^{52}$ Glazer, Nathan and Daniel Moynihan. Beyond the Melting Pot. Cambridge: M.I.T. Press. 1970, p. 186.

${ }^{53}$ The influence of television is probably what allowed the 'birth' of a common language unifying a nation that, from the point of view of dialects, was composed of uncountable smaller 'nations'.

${ }^{54}$ In some recollections of Italian descendants interviewed by Professor Magnaghi, Italians coming from different regions got along very well, but in any kind of gathering "Piemontesi would be by themselves and
} 
regions' Italians as being from their same country. In fact Italy was united in 1861, but its boundaries were not definitive until after World War I.

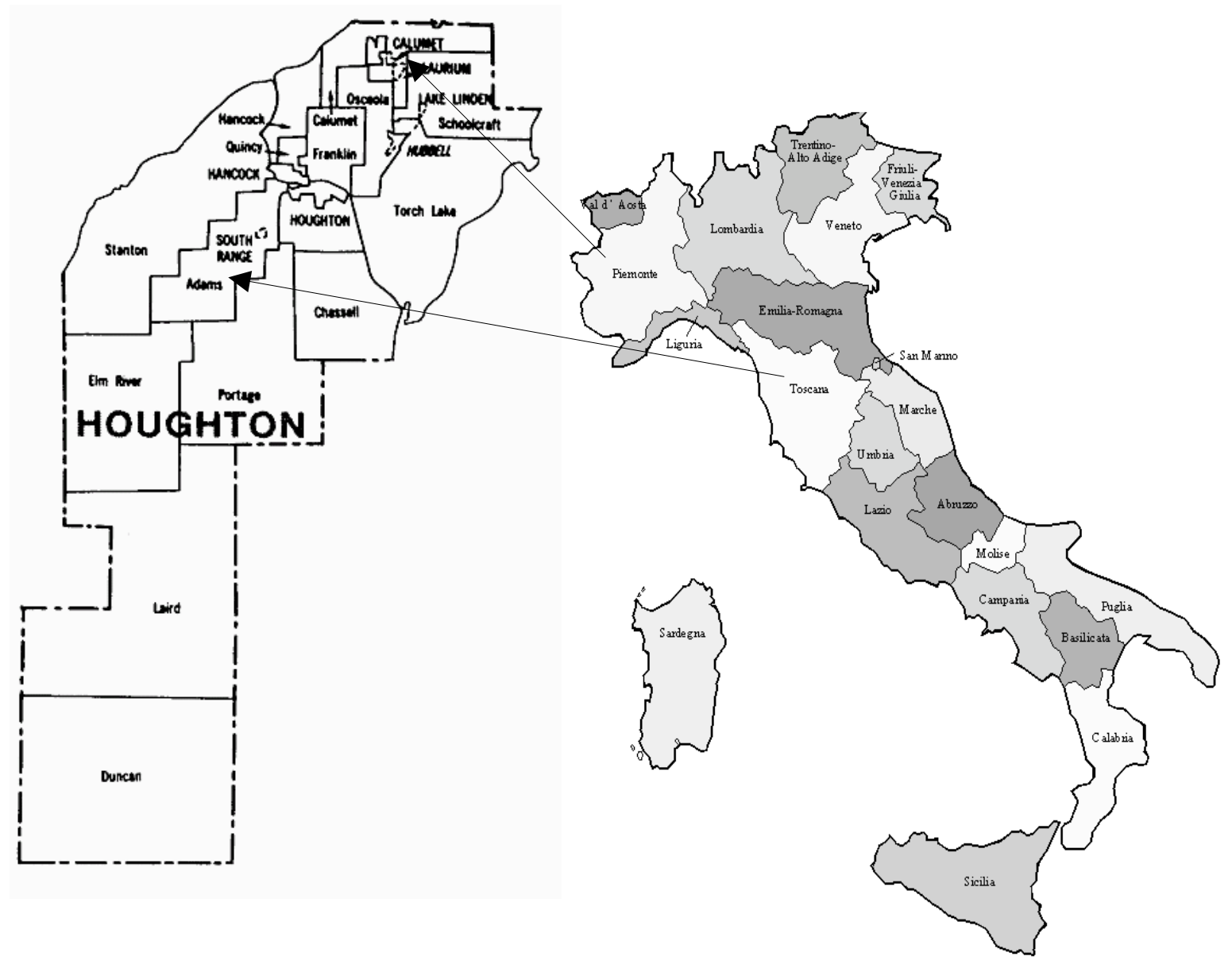

Figure XXIII. Maps of Houghton County and Italy showing the relation between region of origin in Italy and preferred place of settlement of the immigrants in Houghton County. Torinesi preferred Calumet Township and Laurium village. Lucchesi preferred Adams Township and Trimountain location.

Toscani would be by themselves". Magnaghi, M. Russell. Interview with Giovanna Cappo. Baltic, Michigan, 03-16-1982. 


\section{Chapter V - Organizations}

\subsection{Social, cultural, religious, and business organizations}

Despite the regional and province-based Italian settlements, Italians in Houghton County were capable of creating group-wide organizations. They certainly remained linked to their provinces and villages of origins; in fact the very first organizations had the village or the province of origin as common denominators for the members. However, they were able to unite in larger organizations when necessary. The following chapter illustrates the information that it has been possible to gather about some of the organizations that Italians established in Houghton County. Information are from Polk's Directories, personal papers of Professor Russell Magnaghi kept at Central Upper Peninsula and Northern Michigan University Archives, oral interviews taken by Professor Magnaghi, and archival material kept at Michigan Technological University and Copper County Archives.

\subsection{Social organizations: mutual beneficial and mutual help societies}

Mutual beneficial and mutual help societies were very common among Italians of Houghton County. They were not part of the new way of living; in fact mutual beneficial societies (società di mutua beneficenza) and mutual help societies (società di mutuo soccorso) were common in Italy long before the national unification. They most probably derived their model from the 'collegia opificium' of the Roman period, a way of proletarian organization to face problems connected with illnesses, injuries, poverty, wars, and old

age. $^{55}$ These represented a successful way of organization before the appearance of medieval corporations. Actual mutual beneficial and/or help societies first appeared in Italy toward the end of the eighteenth century as voluntary associations among workers to improve moral and material conditions. During the nineteenth century they acquired more specific purposes such as promoting education and welfare.

On the eve of unification, which happened in 1861, Piemonte counted about 115 of these societies, and another ninety-one were spread over Northern Italy, in Lombardia,

\footnotetext{
${ }^{55}$ www.mutuosoccorso.it, 01-30-2004.
} 
Liguria, Veneto, and Emilia-Romagna. These societies had in common a strong professional cohesion, urban locations, and political neutrality, but these changed after 1861.

In 1862, according to a statistic of the Ministero dell'agricoltura, dell'industria e del commercio, there were 443 societies concentrated in Piemonte, Liguria, Lombardia, Toscana, Emilia-Romagna, and Umbria. Principles of solidarity and democracy inspired the foundation of some of them; some others were closer to the moderate political positions of the Italian nobility. This division was further stressed after the constitution of the First International that stressed their different political perspectives. In fact the First International was founded in London in 1864 as the International Workingmen's Association. Karl Marx was a key figure in inspiring its creation and was later chosen as its leader. Its goal was to unite all workers for the purpose of achieving political power along the lines set down by Marx and Friedrich Engels in the Communist Manifesto of 1848.

Some other societies were inspired by Catholic principles. In 1854 the first Catholic society was organized in Genova (Società operaia cattolica italiana) and its bylaws worked as a model for the others. ${ }^{56}$ The main difference between religious and secular societies was the fact that the first ones lacked any interest in social and economic progress of the members. The members of the societies, both religious and secular, were usually required to pay a monthly fee in exchange for a daily allowance in case of illness or work accidents. In the worst case, death, the family of the member received a larger amount of money to face funeral expenses.

Every Italian community in Houghton County had at least a mutual beneficial and/or help society. Often there were many more depending on how big the community was and on other things such as the places of origin of the members, the gender of the members, their civil status, and on the principal purposes of the society. Between the 1870s and the 1930s, more than 40 societies were organized in Houghton County. Each society usually had a group of officials: a president, one or more vice presidents, a secretary and a treasurer. In the case of bigger societies, there were also a vice-secretary and a vice-treasurer, a group of advisers, an executive committee, and an auditor. If one of the aims of the society was to promote naturalization, there was also a secretary of naturalization. There was a committee

${ }^{56}$ www.mutuosoccorso.it, 01-30-2004. 
for health to refer to in case of illness and a committee for death to deal with cases of death. $^{57}$

Thanks to these organizations workers were able to cooperate in confronting the problems of the emerging industrial society before social insurance was available. However the social strength of the 'village' or the 'province' hardly reached a larger scale. In fact most of the societies were organized by and included only individuals from a specific part of Italy, sometimes a province, others even a village.

\subsubsection{Societies in Calumet Township}

The very first Italian society in Houghton County was established in Red Jacket in 1875, the Italian Mutual Beneficial Society (Società Italiana di Mutua Beneficenza). The original members numbered eighty-four. The society was established with the aim of assisting the members and their countrymen in every possible way. As the membership grew and the society had enough money, it was decided to build a hall. The first attempt failed in November 1889 when, after construction had begun and it was nearly completed, a windstorm blew it down. In December of the same year the lodge was incorporated according to the laws of Michigan. In February 1890 the members purchased a lot on Seventh Street in Calumet and in the following years Joseph Wilmus, a contractor, erected the Italian Hall, a three story wooden structure. It lasted until 1908 when, after a celebration, accidental fire broke out and destroyed everything. ${ }^{58}$ Fortunately it was insured with the Italian Fire Insurance Company ${ }^{59}$ for $\$ 14,000$, but the loss was approximately $\$ 27,000$. The members of the Italian Mutual Beneficial Society waited for the Italians belonging to other lodges to join them in the effort to build another hall. Receiving no positive answers, they decided to go on their own and by October of 1908 the 'third' Italian Hall was completed on the same site as the previous one (see Figure XXIII). The dedication took place on October

\footnotetext{
${ }^{57}$ Many societies, besides announcing the date of meetings, also advertised about these committees on the Italian newspapers. For example, Ettore Perrone Society of Calumet, advertised in Il Minatore Italiano of October 13, 1908 that its illness committee was composed of four members: Marta Giuseppe in Calumet, Massoglia Martino in Hecla, G. Gianoglio in Black Hills, and Domenico Motto in Red Jacket. In case of illness the members had to notify the representative in their district within three days of the beginning of their illness or otherwise would lose any right to a claim.

${ }^{58}$ The Hall was rented to St. Joseph's Austrian Society to celebrate its $25^{\text {th }}$ anniversary.

${ }^{59}$ See paragraph 5.4.
} 
10, 1908. It became the meeting place for many of the Italian societies and the focal point for the Italian community itself. However, what made the Italian Hall known nationwide was the so-called Italian Hall Disaster of December 1913. During the 1913 copper miners' strike, members of the Western Federation of Miners decided to organize a Christmas party for the children of the union members on the second floor ballroom of the Italian Hall. Almost 700 children and parents were attending the party on Christmas Eve when somebody allegedly shouted that there was a fire. Seventy-three people died (only three of them were actually Italian) in the attempt to escape the Hall, although there was no fire. Several investigations never found out who caused the panic hollering fire. Nobody was prosecuted.

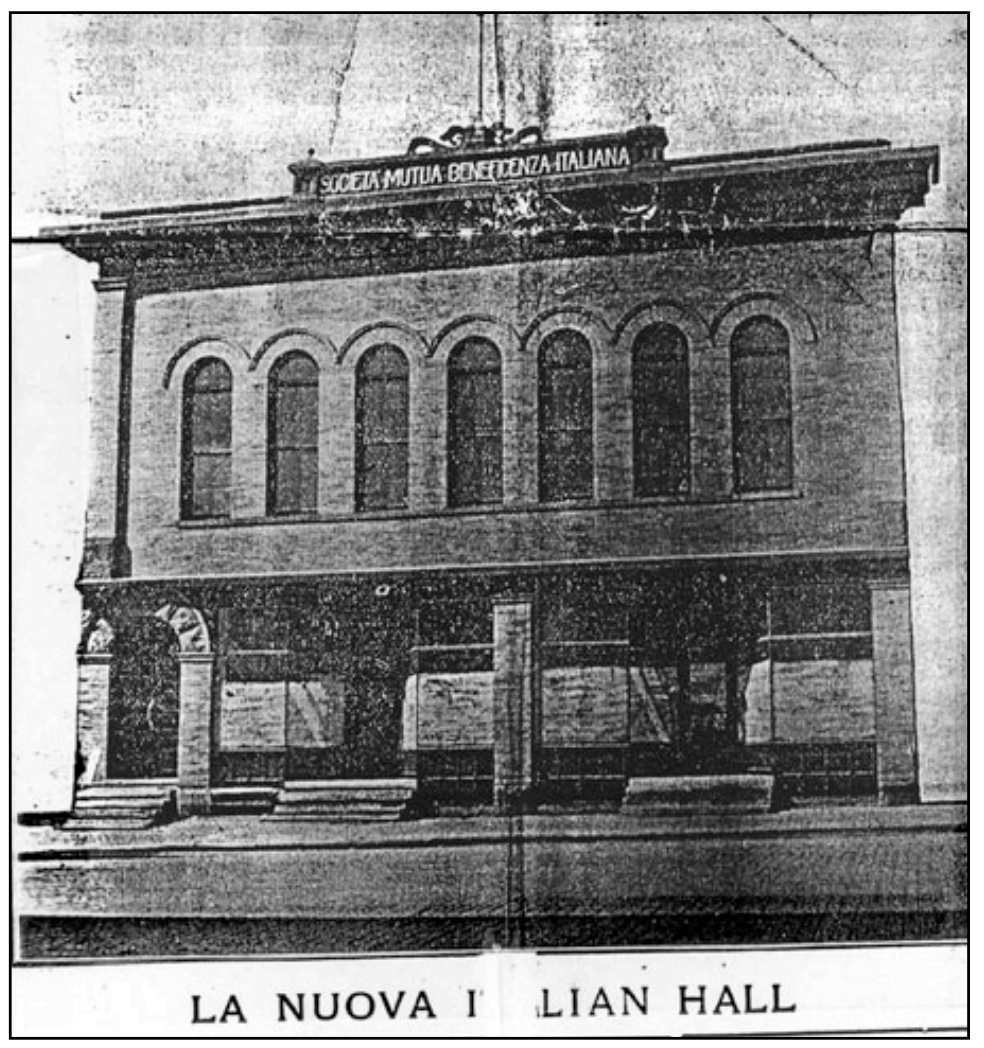

Figure XXIV. The Italian Hall as it appeared on the front page of the special edition of Il Minatore Italiano (October 13, 1908) on the day of the dedication. The article describes the dedication ceremony and introduces many of the persons who attended. 
The Hall continued to be used by the community until the $1960 \mathrm{~s}$, but by the 1970 s it was no longer used for social functions and was in poor condition. In 1984 the Italian Hall was torn down and the Italian Hall Park was established on the same site.

The Italian Mutual Beneficial Society lasted until 1922 when, because of the poor economic conditions and the decrease in the Italian community, they decided to merge with other societies to form the New Italian Beneficial Society of Christopher Columbus (Nuova Società di Beneficenza Italiana Cristoforo Colombo).

In 1895 two other societies were established in Calumet: the Giuseppe Garibaldi Society (Società Giuseppe Garibaldi), and the Christopher Columbus Guard N. 4 (Guardia Cristoforo Colombo No. 4).

In 1897 were organized the Ladies Aid Society of St. Mary's Italian Church (Società Donne Della Chiesa Italiana di Santa Maria) and the Italian Lodge of the Modern Maccabees (Loggia Italiana dei Moderni Maccabei).

In 1907 the Tuscan Society of Giuseppe Giusti (Società Toscana Giuseppe Giusti) was established in Calumet, and in 1908 the Italian Republican Club (Club Repubblicano Italiano).

In 1909 three other societies were organized: the Society of Italian Riflemen (Società Bersaglieri Italiani), the Carlo Botta Society of San Giorgio Canavese (Società Carlo Botta di San Giorgio Canavese), and the Italian Beneficial Society of the Duke of Abruzzi (Società Italiana di Beneficenza Duca degli Abruzzi).

In 1910 three new societies were organized in Calumet: the Giuseppe Garibaldi Bachelor Society (Società Giuseppe Garibaldi Celibi), the Club Alpino among the Residents of Pont Canavese and Locana Canavese (Club Alpino tra i residenti di Pont Canavese e Locana Canavese), and the Supreme Legion of the Knights of Romulus (Legione Suprema dei Cavalieri di Romolo).

The Subordinate Lodge of the Knights of Romulus No. 1 (Loggia Subordinata dei Cavalieri di Romolo No. 1) was organized in 1912. The Sons of Italy Society of Mutual Aid (Società di Mutuo Soccorso Figli d'Italia), and the Daughters or Ladies of the Eternal City (Figlie o Signore Della Città Eterna) were organized in 1915 and 1917 respectively.

In South Hecla the Citizens' League (Lega Cittadina) was established in 1919. In Laurium were established at least six societies. The very first was organized in 1896, the Giuseppe Garibaldi Legion Lodge No. 61 (Legione Giuseppe Garibaldi Loggia No. 61). 
Ettore Perrone Society among the Residents of San Martino Canavese (Società Ettore Perrone di San Martino Canavese) was founded in 1908, and in 1910 the Citizens' League for Mutual Help No. 1 (Lega Cittadina di Mutuo Soccorso No. 1) was formed.

Two other organizations were in Laurium whose date of establishment is not known: Club Alpino and Italian Social Club (Club Sociale Italiano).

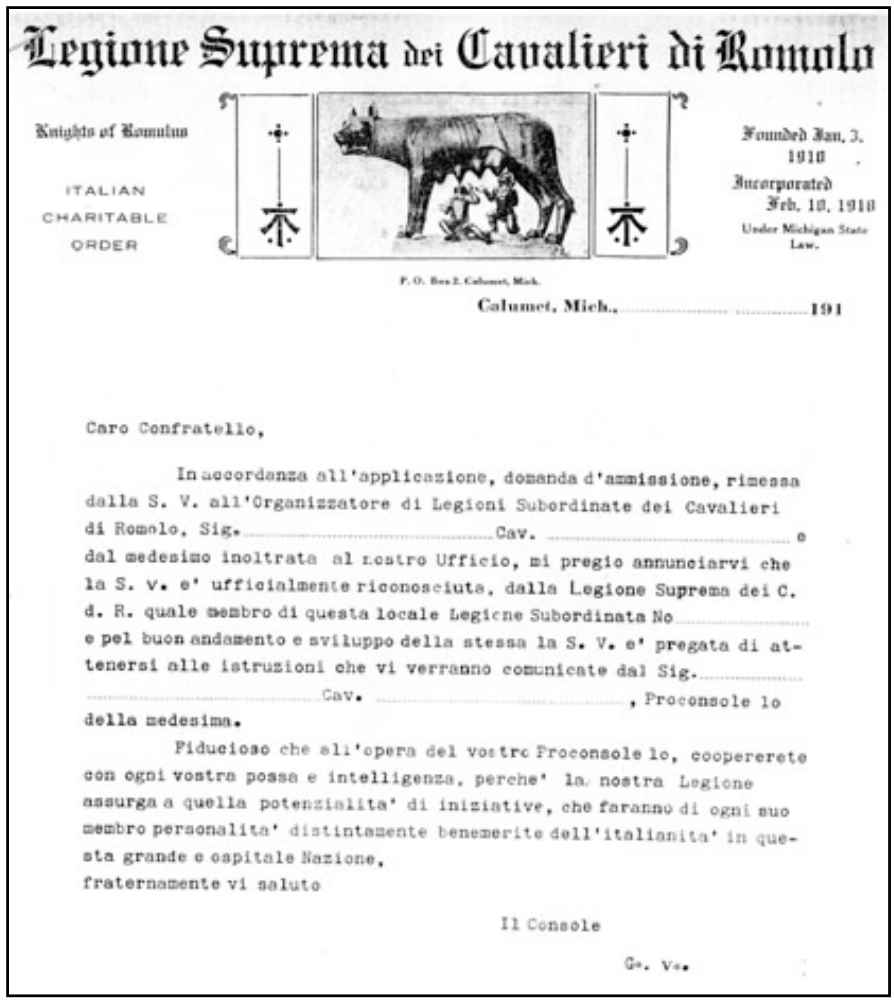

Figure XXV. Copy of the letter sent to the newly accepted members of the Legion of the Knights of Romulus. The letter informs that the person has been officially accepted in the Lodge. The members hope that the new member will do his best to distinguish Italian culture in the new country [Immigration History Research Center at University of Minnesota in Minneapolis]. 


\subsubsection{Societies in Franklin Township}

In Franklin there were five known societies: the Riflemen Society (Società Bersaglieri); the Alessandro Lamarmora Society (Società Alessandro Lamarmora); Young Italy Tuscan Society (Società Toscana Giovane Italia); the Reunited Society of the Sons of Columbus (Società Riunite Figli di Colombo); and the New Industrial Society (Società la Nuova Industria). Their dates of foundation are not known.

Four societies were created in Hancock: the New Industrial Benevolent Society (Nuova Società Industriale di Beneficenza) established in 1903, the Citizens' League No. 2 (Lega Cittadina No. 2) established in 1909 and active until 1937, the Subordinate Lodge of the Knights of Romulus No. 2 (Loggia Subordinata dei Cavalieri di Romolo No. 2) organized in 1912, and the Consorelle Society (Società delle Consorelle).

\subsubsection{Societies in Adams Township}

In South Range numerous societies were organized throughout the years although the dates of foundation are not known for all of them.

The Subordinate Lodge of the Knights of Romulus No. 3 (Loggia Subordinata dei Cavalieri di Romolo No. 3) was established in 1912.

The Daughters of Italy (Figlie d'Italia) was organized in South Range in 1919 by a group of women. Certain criteria were required to be a member of the society, and they remain the same to this day because the society is still active in 2004. A member should be at least sixteen, Catholic, and of course of Italian descent. Starting from 1934 they accepted also members bearing an Italian name even though they are not of Italian descent. The aim of the society was to "unite as much as possible the women living on this side of the Lake, to create the friendship that promotes the normal, material, and national development in this adoptive land far from our Country", 60

Other societies were the Beneficial Society of Italian Hunters (Società di Beneficenza Cacciatori Italiani); the Italian/Tuscan Brotherhood (Fratellanza Italiana/Toscana); the Dante Alighieri Beneficial Society (Società di Beneficenza Dante Alighieri); the Society Pietro Micca of Piedmont (Società Pietro Micca Piemonte); and the

\footnotetext{
${ }^{60}$ Bylaws of the Daughters of Italy, article I.
} 
Citizens' League No. 3 (Lega Cittadina No. 3). As the names of the societies show, many of them had a strong regional identity.

When the membership of some societies grew smaller, they decided to unite and created the United Tuscan Beneficial Societies (Società Toscane Unite di Beneficenza) and The Sons of Italy Beneficial Society (Società di Beneficenza Figli d'Italia), which was

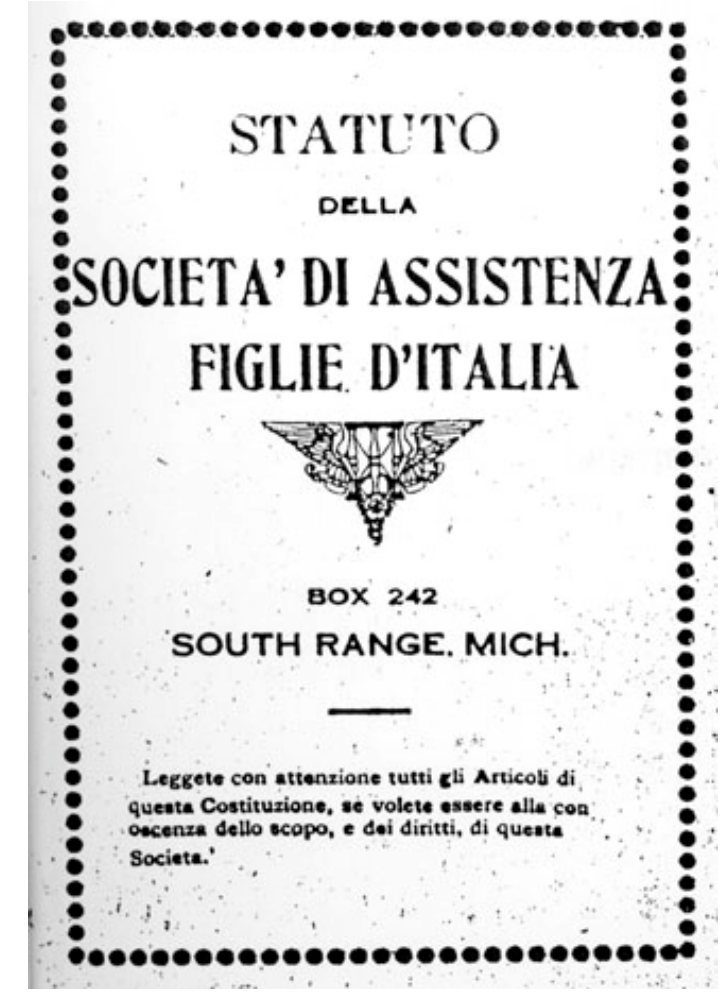

Figure XXVI. Front page of the bylaws of the society of the Daughters of Italy established in South Range in 1919 [Northern Michigan University Archives].

organized in 1918, and combined Beneficial Society of Italian Hunters, Tuscan Brotherhood, United Tuscan Beneficial Society, Dante Alighieri Beneficial Society, and Society Pietro Micca of Piedmont.

There were at least three societies in Trimountain: the Society of Royal Carabineers (Società Carabinieri Reali), the Italian-Tuscan Society (Società Italo-Toscana), and the 
Society Tuscan Youth (Società Gioventù Toscana). The last two reflect the fact that Lucchesi were particularly numerous in the area.

One society is known in Wolverine, the Citizens' League (Lega Cittadina), established in 1919.

Although it has not been possible to find bylaws and written documents for each and every society ${ }^{61}$, many of them, through their names, give us indirect information about their main purposes and, sometimes, about the place of origins of the members. Some of them are named after famous Italian patriots that led the way to the unification of Italy: Pietro Micca, Alessandro Lamarmora, Giuseppe Garibaldi, Carlo Botta, and Ettore Perrone.

Pietro Micca (1677 Sogliano - 1706 Torino) was a Piemontese soldier. During the French siege of Torino (during the Spanish Secessionist war) he was in charge of a mined tunnel. Because some enemies were to enter the city through this tunnel, he blew it up and died to save the city. Alessandro Lamarmora (1799 Torino - ?) was a Piemontese general whose ideas about quick troops with light weapons inspired the organization of the Bersaglieri. Giuseppe Garibaldi (1807 Nice - 1882 Caprera) was an Italian general and nationalist who led 1,000 volunteers in the capture of Sicily and Naples in 1860. His conquest led to the formation of the kingdom of Italy in 1861. Carlo Botta (1766 San Giorgio Canavese - 1837 Paris) was a politician and an historian who followed the ideals of the French Revolution. Ettore Perrone (1789 Torino - 1849 Novara) was a general who played an important role in the riots of 1821 in Piemonte.

Some other societies are named after Italian poets such as Giuseppe Giusti and Dante Alighieri. Giuseppe Giusti (1809 Monsumanno - 1850 Firenze) was a satiric poet very famous during his life. Dante Alighieri (1265 Firenze - 1321 Ravenna) is one of the best Italian poets, and he is considered the 'father' of the Italian language.

Other societies are named after Italian organizations of various kinds such as Giovane Italia, Cacciatori delle Alpi, Bersaglieri, and Carabinieri. The Giovane Italia was a political association organized by Giuseppe Mazzini ${ }^{62}$ in 1831 . Its main ideals were progress and equality. Its members were strong fighters for national unity. The Cacciatori delle Alpi

\footnotetext{
${ }^{61}$ Bylaws have been found for just three societies: the Daughters of Italy; the Colonial Union among the ItaliaAmerican societies of the Counties of Houghton, Keweenaw and Ontonagon; the Italo-American Federation of the societies of the Upper Peninsula of Michigan. See Appendix I and II.

${ }^{62}$ Giuseppe Mazzini (1805-1872) was an Italian patriot and revolutionist that had a decisive role in the Italian struggle for unity.
} 
were volunteer followers of Giuseppe Garibaldi who fought during the second and third Italian Independence War. The Carabinieri is a Corps of the military formed in 1814 that became the first Corps of the Italian army after the unification.

Finally some societies are named after important men in Italian and American history such as Romulus, Christopher Columbus, and the Duke of Abruzzi. Romulus was, according to the legend, the son of Mars and eponymous founder of Rome who, with his twin brother, Remus, was reared and suckled by a wolf. Christopher Columbus (1451 Genova - 1506 Valladolid) was an Italian explorer in the service of Spain who determined that the earth was round and attempted to reach Asia by sailing west from Europe, thereby discovering America in 1492. The Duke of Abruzzi was in fact Luigi Amedeo di Savoia (1873 Madrid - 1933 Duke of Abruzzi village, Somalia). He was an explorer and a general of the navy during World War I.

Despite their different origins, aims, and membership, when the Italian population in Houghton County, and also in other parts of the Upper Peninsula, became smaller, some societies decided to unite. The Colonial Union among the Italian-American Societies of the

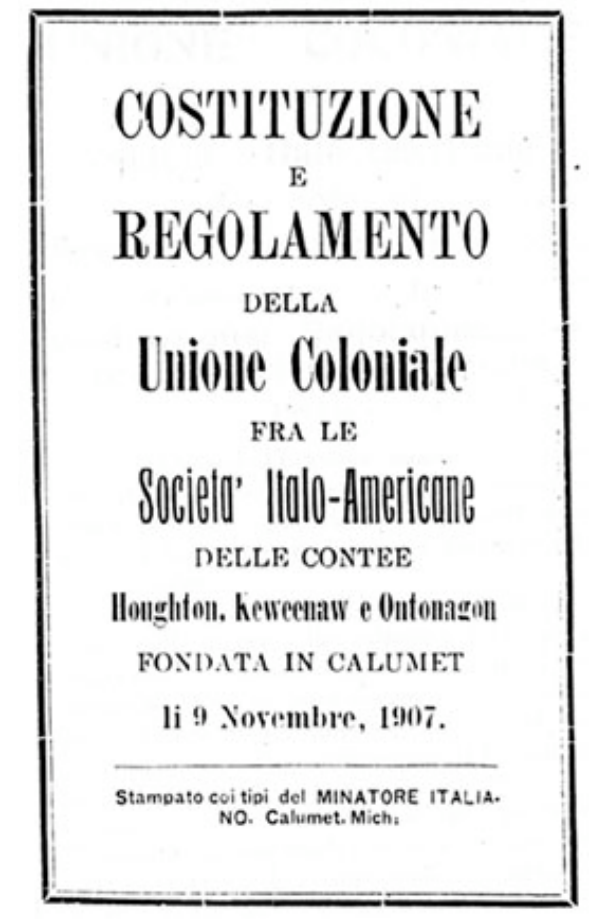

Figure XXVII. Front page of the bylaws of the Colonial Union among the ItalianAmerican societies of the Counties of Houghton, Keweenaw, and Ontonagon established in Calumet in 1907 [Northern Michigan University Archives].

Counties of Houghton, Keweenaw, and Ontonagon (Unione Coloniale fra le Società ItaloAmericane delle contee di Houghton, Keweenaw ed Ontonagon) was founded in Calumet 
on November 9, 1907 (see Figure XXVI). Its aim was to create an association among Italian societies "to help the common interest of the associates and at the same time to promote and support the moral, material, and political development of the Italian nation" in the United States. ${ }^{63}$ The union was composed of the following societies: Ettore Perrone Society and Giuseppe Garibaldi Legion, Lodge No. 61 of Laurium; Italian Mutual Beneficial Society, Christopher Columbus Society No. 4, Club Alpino, Club Carlo Botta, Tuscan Society Giuseppe Giusti, and Giuseppe Garibaldi Bachelor Society of Calumet; Beneficial Society of Italian Hunters of South Range (see bylaws in Appendix I).

The Italo-American Federation of the Societies of the Upper Peninsula of Michigan (Federazione Italo-Americana fra le società italiane dell'Upper Peninsula del Michigan) was founded in Iron Mountain on October 23, 1909 among "all the Italian societies of the Upper Peninsula whose principal aim is mutual help" ${ }^{, 64}$. Its aims were to promote a feeling of brotherhood among all persons of Italian birth and descent, to teach Italian culture and literature, to foster respect for Italian history and institutions, and to induce, assist and encourage all persons of Italian birth to become citizens of the US and actively assume the duties of citizenship (see bylaws in Appendix II). The headquarters of the federation was in Calumet. The associated lodges from Calumet were Tuscan Society Giuseppe Giusti, Italian Mutual Beneficial Society, Christopher Columbus Society No. 4, Club Alpino, Club Carlo Botta, and Giuseppe Garibaldi Bachelor Society. From Laurium the societies were Citizens' League N. 1, Ettore Perrone Society, Giuseppe Garibaldi Society, and Christopher Columbus Society. One associated lodge was from Hancock, Citizens' League N. 2, two from Franklin, Alessandro Lamarmora Society and Young Italy Tuscan Society, and one from South Range, Pietro Micca Society. Other associated lodges were from different counties of the Upper Peninsula. Little is known about the number of the members. ${ }^{65}$

\footnotetext{
${ }^{63}$ See APPENDIX I, Bylaws of the Colonial Union.

${ }^{64}$ See APPENDIX II, Bylaws of the Federation.

${ }^{65}$ In 1922, according to the Federation of the society, Giuseppe Giusti Society had about sixty members; Italian Mutual Beneficial Society about one hundred; Christopher Columbus Society N. 4 about fifty; Club Alpino about eighty; Club Carlo Botta about seventy; Giuseppe Garibaldi Bachelor Society about twenty; Citizens' League N.1 about sixty; Christopher Columbus Society of Laurium about twenty; Ettore Perrone Society about seventy-five; Giuseppe Garibaldi Society of Laurium had about twenty-seven; Citizens' League N. 2 about ninety; Pietro Micca Society about forty.
} 


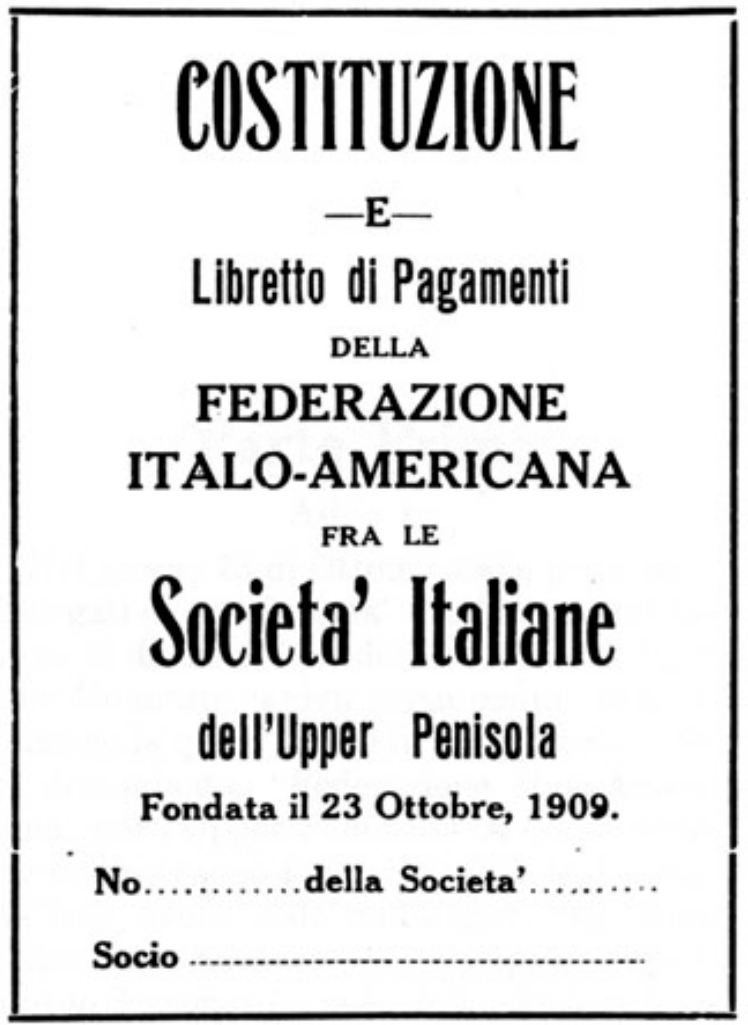

Figure XXVIII. Front page of the bylaws of the Italian-American Federation of the societies of the Upper Peninsula of Michigan established in Iron Mountain in 1909 [Northern Michigan University Archives].

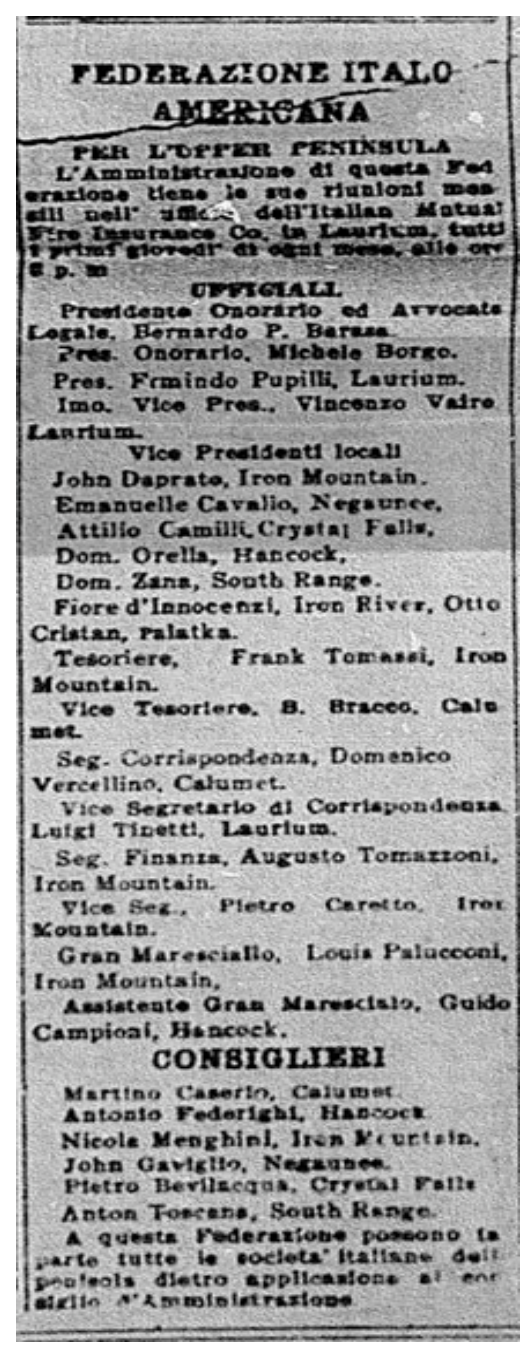

Figure XXIX. Example of announcements published in $\underline{\mathrm{I}}$ Minatore Italiano by Italian societies to inform about the dates of meetings. In this case the society is the Italian-American Federation [Michigan Technological University and Copper Country Archives].

\subsection{Cultural organizations}

Italians also established several periodicals and newspapers in the Italian language. The Pro Nobis (For Us) was the official publication of the Supreme Legion of the Knights of Romulus. It was a monthly review published in Calumet. 
The only available issue known to exist is dated November 1912 (see Figure XXX). It includes information about the Supreme Legion of Calumet and its Subordinate Legions in Hancock, South Range, and Negaunee. The first article is dedicated to introducing the Lodge of the Knights of Romulus. It starts with the history of Romulus and explains that the lodge was named after him because he represents the symbol of the birth of Rome and of the Roman civilization. The article also explains why the members of the lodge felt the need for this publication: promoting Italian culture and showing that, contrary to previous

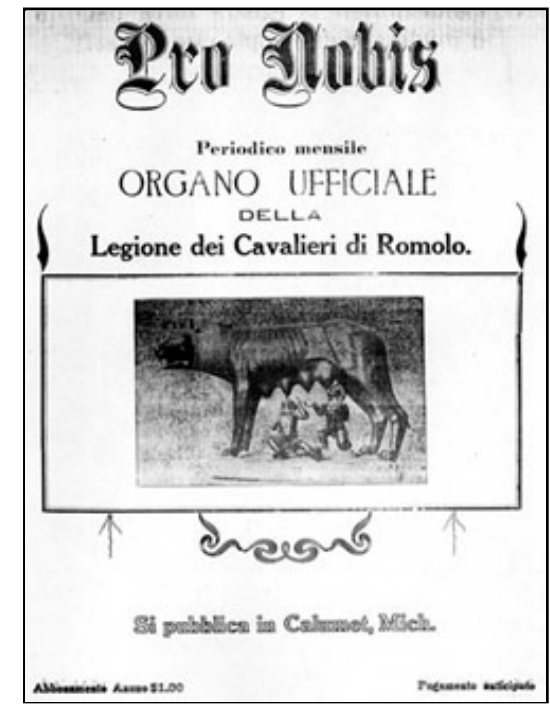

Figure XXX. Front page of Pro Nobis, November 1912 [Immigration History Research Center at University of Minnesota].

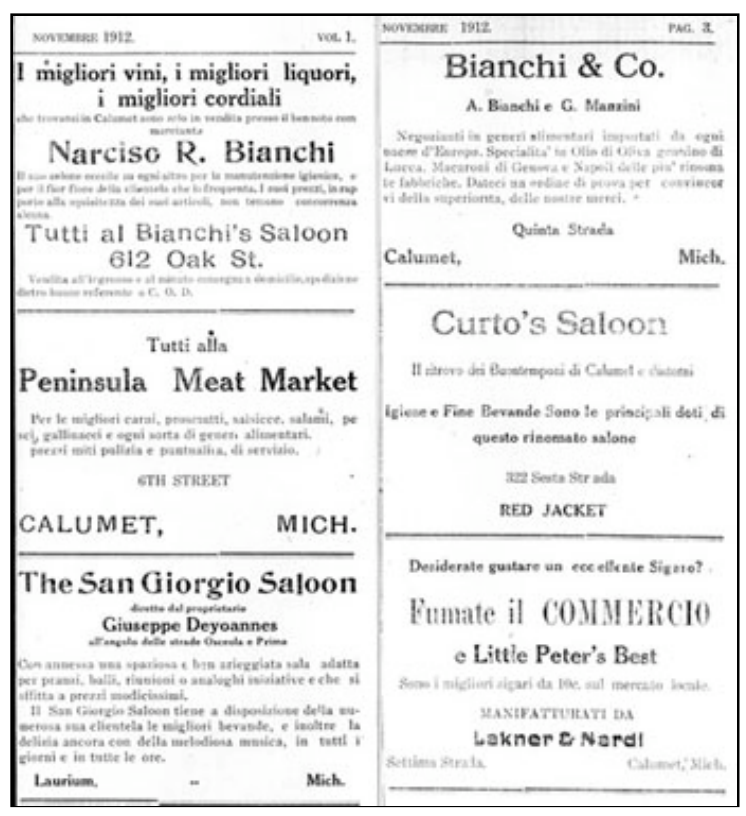

Figure XXXI. Example of advertisements of Italian businesses appearing in the Pro Nobis. [Immigration History Research Center at University of Minnesota].

assumptions, Italians are not 'ignorant'. The actual articles relate Arabic legends about the origin of Libya, electricity and its effects on the development of children, the centenary of the steamship, and medical suggestions. A section is dedicated to quotes and lives of famous Italians. Most part of the publication is filled with advertisements of various businesses (see Figure XXX).

Il Minatore Italiano (The Italian Miner) was established in 1896 and continued publishing into the 1930s, although it is not known when it stopped. It was a daily 


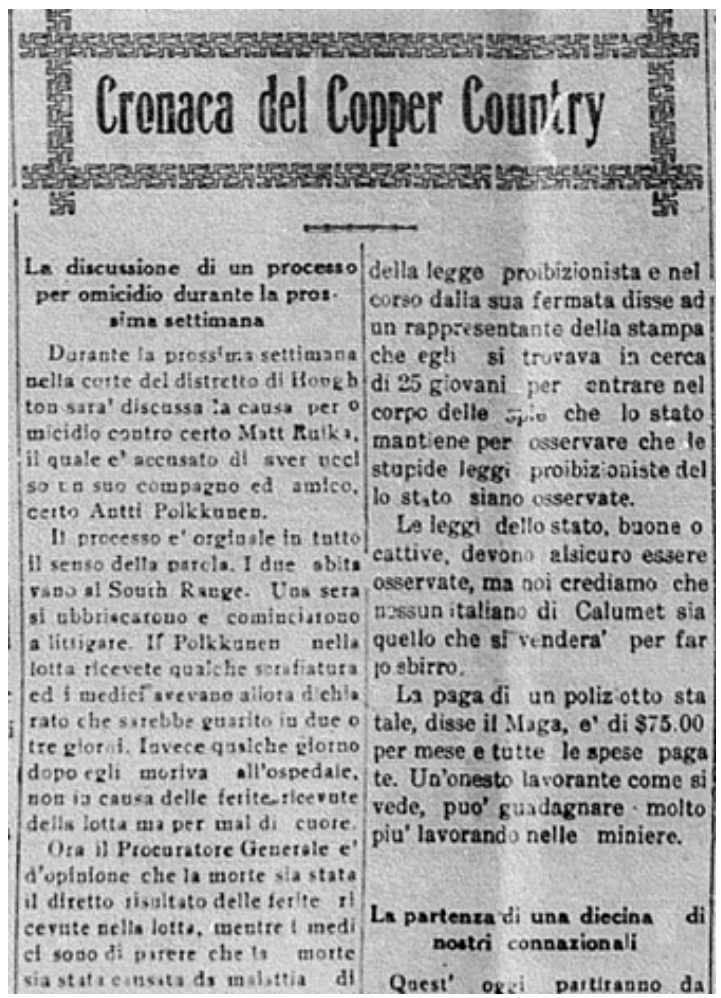

Figure XXXII. Section of Il Minatore Italiano of September 14, 1914 dedicated to the chronicle of the Copper Country. The news concerns a homicide trial in South Range and the departure of some Italians for Italy [Michigan Technological University and Copper Country Archives].

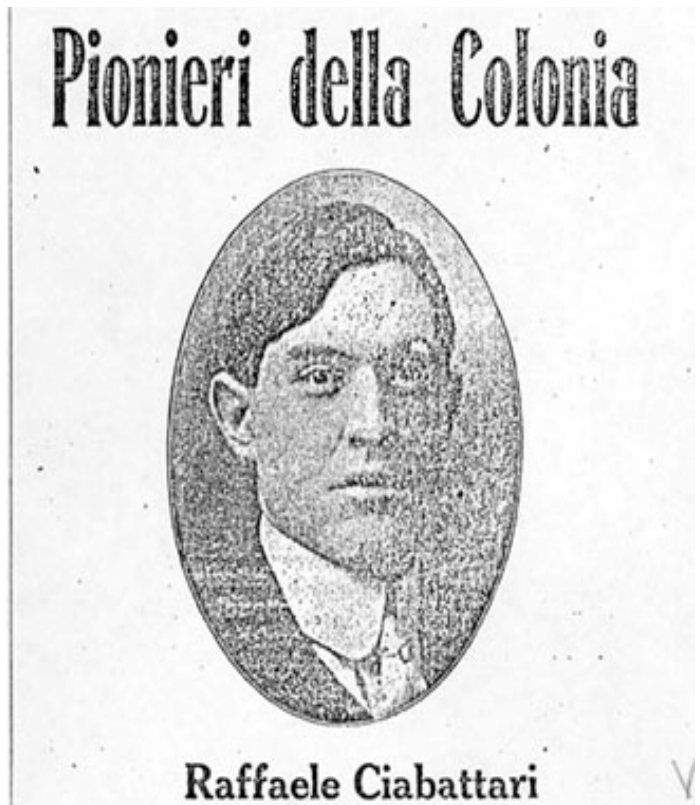

Nacque nella Ridente Toscan il 25 di Felbbraio 1876. Emigro' in questa terra nel 1893 e fu nell'esposizione di Chicago che per primo ebbe occasione di espletare le differenti cittitudini sue personali che accoppiate ad una genialita' indefinita lo spinseto poi in Milwatıkee, quindi in Hancock, sempre apprezzato dovunque per l'affabilita' dei suoi'modi e per l'onesta' del suo agire.

Attualmente, e da parecchi anni, eserisce il "Assembly Buffet" uno dei migliori e meglio frequeutati saloni di quclla cittadina.

Nella colonia italiana di Hancock, l'amico R. Ciabattari gode di una giustificata supremazia giustificatissima date le tante sue relazioni e affinita' di apione colla piu' eletta' so. cięta' indigena di çolą,

Figure XXXIII. Page of the Guida degli Italiani della Copper Country. The picture shows one prominent Italian, Raffaele Ciabattari. He was born in Tuscany and arrived in the United States in 1893. He was the owner of the Assembly Buffet in Hancock. 1910 [Michigan Technological University and Copper Country Archives].

newspaper until 1919. By 1928 it became a tri-weekly. The paper was published in the Tinetti building on Osceola Street in Laurium. The editor was Augusto Marinelli. He was actually Austrian by birth (although all the censuses indicate that he was born in Italy) but of

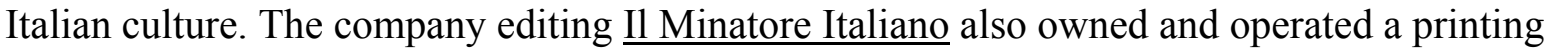
shop, the Miner Publishing Company, which also worked for other Italian organizations. In 
1912 the company edited the Guida degli Italiani Della Copper Country, a valuable guide in Italian which listed most of the Italians living in the region. The guide included a list of all the Italians above the age of fifteen that agreed to be included. In addition, it includes summaries of the lives of some prominent Italians that emerged above the others because of their businesses or involvement in the community (see Figure XXXIII). During the strike of 1913-1914, the newspaper took a strong pro-company stand. ${ }^{66}$ The first page of the newspaper was usually dedicated to the news from the world, with particular focus on those stories regarding Italy. The second page usually had section dedicated to the local news called Cronaca del Copper Country, Chronicle of Copper Country (see Figure XXXII). It also included announcements from the various Italian benevolent societies advertising the places and dates of the meetings. On the third page often appeared either a string of comics or a novel. The fourth page was usually dedicated to in depth accounts of political subjects introduced on the first pages. Advertisements were included in each page regarding Italian products, businesses in Houghton County, companies of transportation, banks, etc.

La Sentinella (The Sentinel) was established probably around 1896 in Red Jacket. Until 1903 it was printed in a shop operated by John Lisa at 407 Seventh Street, Calumet. The editor was Giuseppe Adamo. In 1901 Lisa was both editor and publisher, and the newspaper had a circulation of about 5,000 copies. In 1906 the editor was Teofilo Petriella, a socialist with college training. Around 1907 Petriella left the area to become a strike leader on the Mesabi and Vermillion Ranges. In 1910 the newspaper was published and owned by Luigi Nida and it was still located at 407 Seventh Street. The one surviving issue of the newspaper is dated February 6,1906 and it's filled with socialist ideas. In its later years it was a socialist newspaper. ${ }^{67}$

L'Indipendente (The Independent) was established in 1917 in Hancock. The editor was A. Prati and the corresponding agent was Ermindo Pupilli. It is not known how long the publication lasted. The only available issue is from November 6, 1917. The front page includes news about the Italian fronts of WWI and a section of brief news from all over the world (a criminal case in Minneapolis, the stop to the issuing of passports in Russia, the price of potatoes in Michigan, etc.). The second page includes a section of local news and

\footnotetext{
${ }^{66}$ Although copies of the newspaper for the period of the strike are no longer available, many articles that appeared in the Miners' Bulletin comment and criticize articles that appeared in Il Minatore Italiano.

${ }^{67}$ It has not been possible to locate the issue. This information is from the personal papers of Professor Magnaghi, who at one point had the opportunity to see it.
} 
more news from the world. Page three is almost completely dedicated to articles about war injured and the progresses of medicine in this field. Page four includes part of a novel and news about markets and stocks. All the pages show advertisements of local businesses: D. Giannerini local representative of prestigious fashion houses; Bianchi \& co. grocery shop at 424 Fifth Street in Calumet; Barsotti \& Co. Ice Cream; Caserio \& Massoglia importers of Italian products; Richetta Bros. livery stable; etc. Many banks also advertised in the newspaper: First National Bank and State Savings Bank of Laurium, Calumet State Bank,

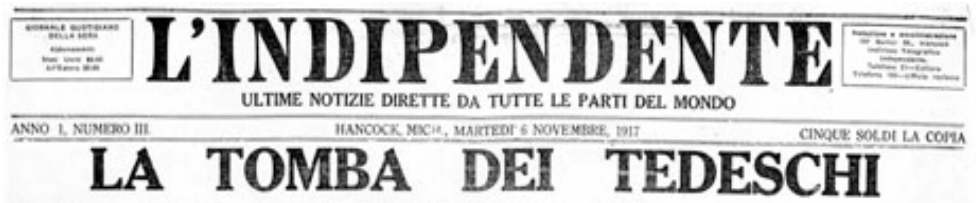

Le linee del Tagliamento non sono ancora sfondate e pagheranno il fio della loro audacia

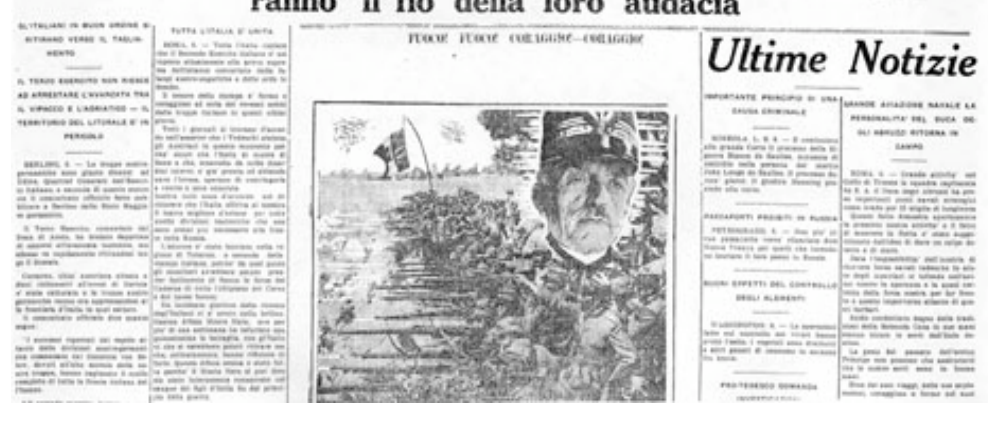

Figure XXXIV. Front page of the only extant issue of L'Indipendente dated November 6, 1917. The headline says "The graveyard of the Germans. The German soldiers on the Tagliamento River are not defeated yet, but they will pay for their daring" [Immigration History Research Center at University of Minnesota].

Superior National Bank of Hancock. Many companies of transportation also published their advertisements on the newspaper, sometimes indicating the prices of their tickets to and from Italy: Transatlantica Italiana (tickets between $\$ 75$ and 80); Sicula Americana; Lloyd Sabaudo (\$75); Navigazione Generale Italiana.

During the 1913-1914 strike the Miners' Bulletin, the Western Federation of Miners' official publication, had one fourth of its copy written in Italian. Ben Goggini (or Gaggini, according to the census), one of the most prolific Italian writers for this newspaper, 
challenged the pro-company stand of Il Minatore Italiano. In all the issues from October 23, 1913 until March 14, 1914 (N. 6 until N. 66), there is a section called Bollettino dei Minatori (see Figure XXXIV) in which the writers criticize the articles of Il Minatore Italiano. More specifically they accuse the editor of that newspaper, Augusto Marinelli, of being a puppet in the hands of the mining companies. The section often includes a list of the names of Italian scabs giving details about their origins, lives, and actions. ${ }^{68}$ Satiric poems, both in Italian and in dialect, about the strike and the workers not adhering to it appear in several issues (see Appendix III). ${ }^{69}$

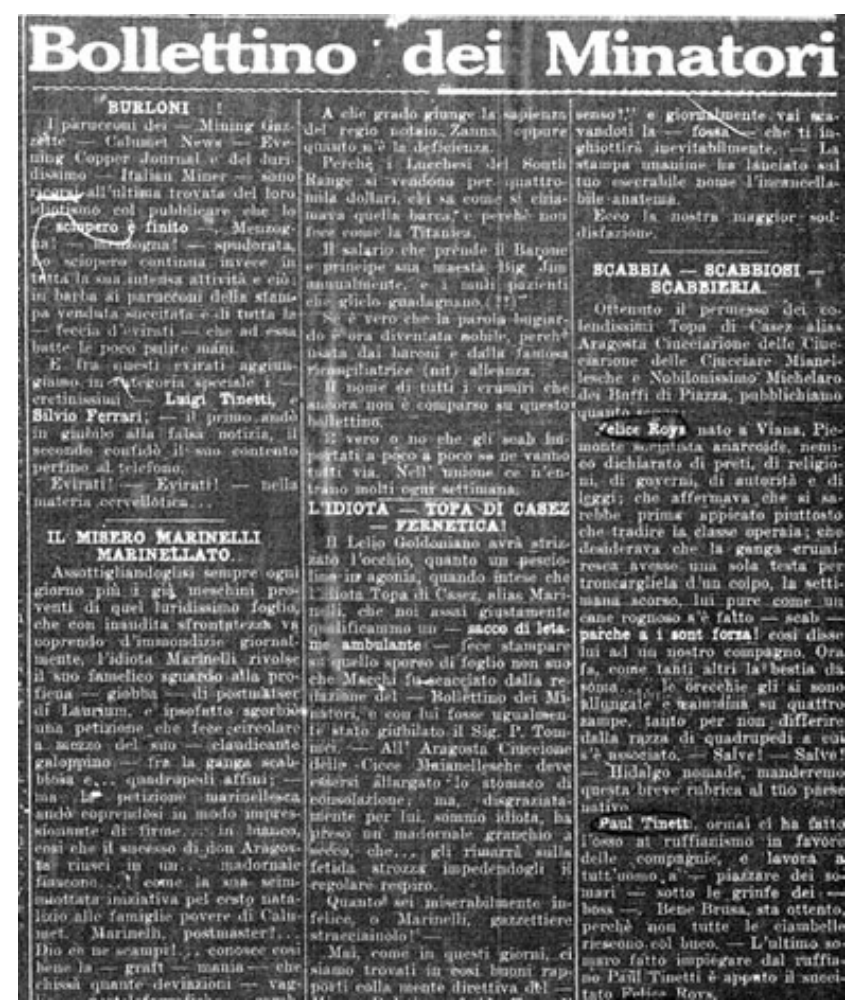

Figure XXXV. Example of Italian section of the Miners' Bulletin as it appeared in each copy of the newspaper [Michigan Technological University and Copper Country Archives].

\footnotetext{
${ }^{68}$ For example on the issue number 63 of 03-25-1914 they write about Carlo Giacoletto of Wolverine. According to the article Giacoletto, after several months of receiving financial support from the union, has become a scab. Cesare Zana is called 'dog of the mine company', as well as Cesare Lucchesi. This kind of article appears in every issue of the Miners' Bulletin and each one includes names of scabs.

${ }^{69}$ For example in the issue number 54 of 01-28-1914 there is a poem dedicated to Guido Locatelli, scab and author of an article against the strike published in Il Minatore Italiano. The poem makes fun of his way of writing and compares him to a donkey. Another poem published in the issue number 24 of 10-07-1913 gives a satiric description of the places of birth of the scabs.
} 
Almost nothing is known about La Democrazia Italiana. It was published in Calumet and lasted a little more than a month, but it is not known when and by whom it was edited.

Italians also formed a dramatic group in Calumet which was particularly active around 1910, the Italian Hall Dramatic Group. A similar group existed in South Range, which offered puppet shows.

\subsection{Religious organizations}

Italian immigrants were mostly, if not totally, Catholics. In Houghton County they founded a church, St. Mary's Church in Calumet in 1897. It served the community until 1966 when it was consolidated with other catholic churches because of the decline in the population. According to some Italian descendants interviewed by Professor Magnaghi, in Calumet there was always an Italian priest. ${ }^{70}$ The censuses also indicate at least one Italian priest each year.

As far as Adams Township, some Italian descendants remember going to church at St. Mary Star of the Sea in Atlantic for many years before the Holy Family Church was built in South Range. These were not Italian churches however, nor did they have Italian priests. ${ }^{71}$

\subsection{Business enterprises}

The Italian Mutual Fire Insurance Company was organized in Laurium in 1898. Michael Richetta was the founder. At first its aim was to help Italians, but through the years the company got bigger and became an insurance company for everybody. In 2000 it was still in business, although with a different name, Great Lakes Mutual Fire Insurance Company (see Figure XXXVI).

In 1910 a group of Italian entrepreneurs from the Upper Peninsula of Michigan formed the Italian Businessmen Association. They intended to secure the cooperation among Italian businessmen throughout the Copper Country by promoting moral and

\footnotetext{
${ }^{70}$ Magnaghi, M. Russell. Interview with Giovanna Cappo. Baltic, Michigan, 03-16-1982.

${ }^{71}$ Ibidem.
} 
business interests. A similar association was organized in Laurium in the same period, the Italian Commercial Club.

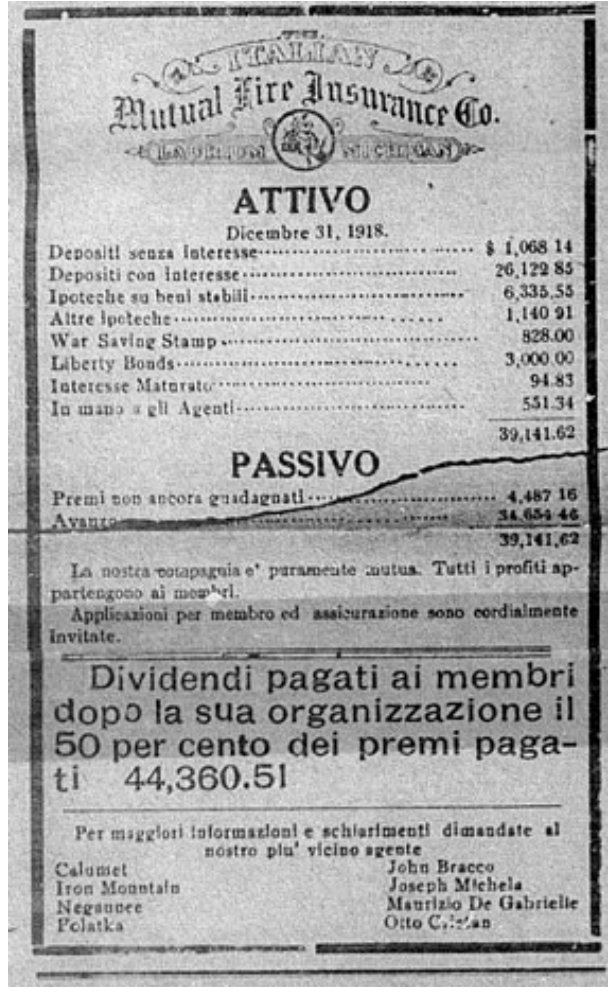

Figure XXXVI. Example of announcement published in Il Minatore Italiano by the Italian Mutual Fire Insurance advertising the assets and the dividends paid in 1918 [Michigan Technological University and Copper Country Archives]. 


\section{Chapter VI - Conclusions}

The main goal of this research was to rediscover the communities of the Italians who lived and worked in Houghton County in order to raise the degree of attention given to the social and cultural heritage that they left. The data and the information gathered have generated a clearer idea of who the Italians that settled in Houghton County were. There is a great deal of information that time and availability has prevented from being used in this work. The results, however, represent a valuable continuum between previous studies and possible future in-depth research projects.

The censuses have helped in determining the number of people that formed the Italian community (born in Italy, of Italian descent, or married to Italians) in the years examined. The first census year in which their number is significant is 1870 ; there were twenty-one. From that year until 1910 the data show an increase in the community; in 1880 there were 220, in 1900 they reached 2,789, and in 1910 there were 4,384. In 1920 the number of Italians remained stable at 4,379. The year 1930 shows a significant decrease to 2,839. Unfortunately there are no available sources to show the number of Italians living in Houghton County in the years in between the census years. In fact, it is likely that their number reached its peak around 1906-1907.

Data showed that most of immigrants came from just two Italian provinces, Torino and Lucca. Although in later years the variety of provinces represented increased, from 1870 until 1930 the Italian community in this county was, and still is in the recollection of the Italian descendants, formed by Torinesi/Piemontesi and Lucchesi/Toscani. Other regions such as Lombardia and Veneto were also represented, but the number of Lombardi and Veneti descendants never reached more than 150. Because it has not been possible to establish regions of origin for many immigrants, further research could look at ships' manifests from ports of arrival other than New York. Determining the port of departure, on the other hand, is not easily done because Italians left from many different Italian and European ports.

Interesting information has been gathered about the jobs that Italians had while living in Houghton County. Previous studies and personal conversations would lead one to believe that Italians in this region were mostly involved in mining jobs, and that many of them were 
liquor dealers, masons, and farmers. Data showed that most Italian workers were in fact employed in the mines. However, the number of Italians that were employed as liquor dealers, farmers, and masons was found to be less significant than previously thought. Dividing the occupations into categories allowed comparisons to be made about the number of Italians working in different fields, and the establishment of employment trends. Further research could compare these trends with those of other ethnic groups of the area.

Census data also showed in what townships and villages Italians preferred to settle. Torinesi descendents showed a preference for Calumet Township and Laurium Village. Adams Township and Trimountain Location were preferred by descendents of Lucchesi. It would be interesting to perform archeological investigations in some of these places to complement data from written documents. A lot more could be discovered about everyday life. Sunny Italy, the Italian settlement in Trimountain, would be the first place to investigate because it was a purely Italian settlement.

Much information has been found about various Italian organizations, such as the benevolent societies. Having access to minute books of some of them would have been of greater help, but unfortunately not much is known about the existence of original documents. If some of them still exist in private possession, hopefully one day they will be donated to public repositories and be accessible by the general public.

Italian newspapers published in Houghton County have also helped in getting to know something about Italian interests, problems, and activities. For some of these newspapers, however, only few issues have been located. It would have been interesting, for example, to have issues of Il Minatore Italiano that covered the period of the 1913-1914 strike. It also would have helped this research to find more issues of other newspapers such as La Sentinella and any issue of La Democrazia Italiana. Again it is likely that private citizens still have some issues and making them available to researchers would provide a great deal of information.

All in all this work reveals several tendencies about Italians that lived in Houghton County between the end of the $19^{\text {th }}$ and the first decades of the $20^{\text {th }}$ century. Many little discoveries, however, represent precious details about the past for some people, researchers and/or Italian descendents, for example. Hopefully this thesis will be a starting point for other scholars to investigate the topic to a greater extent. For example future works could take a closer look at the relation between employment trends of Italians in the three bigger 
mining companies, Calumet \& Hecla, Quincy, and Copper Range, and Italian settlements in Calumet, Quincy, and Adams townships. In fact it would be interesting to know if different companies encouraged or discouraged Italian workers, and possibly changed their preferences at different decades. The databases of the Italian community in 1870, 1880, 1900, 1910, 1920, and 1930 - included as appendixes - also represent a valuable source of information for whoever will be interested in studying family demographics, upward economic mobility, and genealogy. Another interesting work would be comparing Italians to other ethnic groups to see how they differed as for jobs, occupations, settlements, and organization. 
BIBLIOGRAPHY 


\section{BOOKS AND ARTICLES}

AA.VV. Ricordo agli italiani di Iron Mountain, Michigan. Calumet, Michigan: Il Minatore Italiano, 1903. [CSE VD 10 .I7 1903].

Amfitheatrof, Erik. The Children of Columbus. Boston: Little Brown and Company, 1973.

Assante, F. (ed.). Il movimento migratorio italiano dall'unita nazionale ai giorni nostri. Geneva: Librairie Droz, 1978.

Aste, Mario, Jerome Krase, Louise Napolitano-Carman, and Janet E. Worral. Industry, Technology, Labor, and the Italian American Communities. Staten Island, New York: American Italian Historical Association, 1997.

Audenino, P. “The 'Alpine Paradox': Exporting Builders to the World”. In Pozzetta, G. et al. (eds), The Italian Diaspora, Migration Across the Globe. Toronto: Multicultural History Society of Ontario, 1992.

Benvenuti L. Dizionario degli italiani all'estero. Firenze: Tipografia Barbera, 1890.

Bernardy A. A. Italia randagia attraverso gli Stati Uniti. Torino: Fratelli Bocca Editori, 1913.

Bertelli L., Carcagnani G., Rosoli G.F. Migrazioni. Catalogo della biblioteca del CSER. Roma: Centro Studi Emigrazione Roma, 1972.

Bezza, Bruno. Gli italiani fuori d'Italia: gli emigrati italiani nei movimenti operai dei paesi d'adozione, 1880-1940. Milano: ed. F. Angeli, 1983.

Briani, V. Italian Immigrants Abroad: A Bibliography on the Italian Experience outside Italy in Europe, the America, Australia, and Africa. Detroit: B.Ethridge Books, 1979.

Briggs, John W. An Italian Passage: Immigrants to Three American Cities, 1900-1914. New Haven, CT: Yale University Press, 1978.

Brumm, Florence and Magnaghi, Russell M. Immigrants in Iron County, Michigan: 1910 Federal Census. Marquette, MI: Belle Fontaine Press, 1986.

Candeloro, Dominic, Fred L. Gardaphe, and Paolo A. Giordano (eds.) Italian Ethnics: Their Languages, Literature and Lives. Proceedings of the 20th Annual Conference of the American Italian Historical Association. Staten Island, NY: The American Italian Historical Association, 1990.

Cardiello, Pietro. “Michigan”. In Bollettino dell’Emigrazione Vol. 11 (1911), pp. 10-13. 
Castigliano, Attilio. “Origine, sviluppo, importanza ed avvenire delle colonie italiane del nord Michigan e del nord Minnesota". In Bollettino dell'Emigrazione Vol. 7 (1913), pp. 723-39. [Biblioteca Malatestiana Cesena/Biblioteca Societa' Geografica Italiana Roma].

Choldin, Harvey. "Kinship Networks in the Migration Process." In George E. Pozzetta, ed., Immigrant Family Patterns: Demography, Fertility, Housing, Kinship, and Urban Life. New York, NY: Garland Publishing Co., 1991.

Coming to America: American Immigrant Series. New York: Dell, 1981.

Commissariato dell'Emigrazione. Bollettino dell'Emigrazione. (Office of the Commissioner of Emigration. Emigration Bulletin). Rome, Italy: 1902-1927.

Copper County Italian American Bicentennial Celebration Souvenir Booklet. (?) 1976.

Cordasco, Francesco. Italian Americans: A Guide to Information Sources. Detroit: Gale Research Co., c1978.

---------. Italian Mass Emigration: The Exodus of A Latin People. A Bibliographical

Guide To The Bollettino dell'Emigrazione, 1902-1927. Totowa: Rowman \& Littlefield, 1980. [Biblioteca Nazionale Centrale Vittorio Emanuele II, Roma].

---------. Dictionary of American Immigration History. London: The Scarecrow Press, 1990.

Cordasco, Francesco and Michael Vaughn Cordasco. Italians in The United States: An Annotated Bibliography of Doctoral Dissertations Completed at American Universities with a hand list of Selected Published Bibliographies, Related Reference Materials, and Guide Books For Italian Emigrants. Fairview, N.J.: JuniusVaughn Press, 1981.

- The Italian Emigration to the United States, 1880-1930: A Bibliographic Register of Italian Views, Including Selected Numbers from the Italian Commissariat of Emigration, Bollettino dell'Emigrazione. Fairview, N.J.: Junius-Vaughn Press, 1990.

Cordasco, Francesco and Salvatore La Gumina. Italians in the United States; $A$ Bibliography of Reports, Texts, Critical Studies and Related Materials. New York: Oriole Editions, 1972.

Daniels, Roger. Coming to America. New York: Harper Collins Publishers, 1990.

Dillingham. Reports of the Immigration Commission. $61^{\text {st }}$ Congress $2^{\text {nd }}$ Session Senate Document No. 633, Part 17: Copper Mining and Smelting; Part 18: Iron Ore Mining. Washington, D.C.: Government Printing Office, 1911.

Di Palma-Castiglione, G. F. "Vari centri italiani negli stati di Indiana, Ohio, Michigan, Minnesota e Wisconsin”. In Bollettino dell'Emigrazione Vol. 14 (15 Giugno 1915), pp. 7-46. 
Foerster, Robert F. The Italian Emigration of our Times. New York: Arno Press, 1969.

Gans, Herbert. "Tradition and Change in Italo-American Family Structure." In Racial and Ethnic Relations: Selected Readings, Bernard E. Segal ed. New York, NY: Thomas V. Crowell, 1966.

- The Urban Villagers: Group and Class Life in the Life of American Italians. New York: NY: Free Press of Glencoe, 1962.

Gates, B. William Jr. Michigan Copper and Boston Dollars. Cambridge, Massachusetts: Harvard University Press, 1951.

Glazer, Nathan and Daniel Moynihan. Beyond the Melting Pot. Cambridge: M.I.T. Press. 1970.

Glazier, Ira A. and P. William Filby, (eds.). Italians to America: Lists of Passengers Arriving at U.S. Ports, 1880-1899. 6 vols. Wilmington: Scholarly Resources, 1992.

Guida degli Italiani della Copper Country. Laurium, Michigan: Il Minatore Italiano, 1910. Reprinted, Iron Mountain: The Ralph W. Secord Press, 1987. [Available at Michigan Technological University and Copper Country Archives].

Handlin, Oscar. The Uprooted. Boston: Little Brown and Co., 1973.

Heap, William A. The Story of Ellis Island. New Jersey: Prentice-Hall, 1959.

La Gumina, J. Savatore. The Immigrant Speaks. New York: The Center for Migration Studies, 1981.

-------. (ed.). The Italian American Experience: An Encyclopedia. New York: Garland, 2000 .

Lankton, D. Larry. Beyond the Boundaries: Life and Landscape at the Lake Superior Copper Mines, 1840-1875. New York, 1997.

--------. Cradle to Grave: Life and Death at the Lake Superior Copper Mines. New York, 1991.

L'Emigrazione Italiana. Legislazione e Statistiche. Accordi Internazionali. Roma: Remo Sandron Editore, 1927, Tav. IV.

Light, Ivan Hubert. Italians in America: Annotated Guide to New York Times Articles, 1890-1940. Monticello, Ill.: Council of Planning Librarians, 1975.

Magnaghi, M. Russell. Ethnic Organizations in Houghton County. Marquette: Belle Fountaine Press, 1982.

- "Italians in the Upper Peninsula". In Ethnic Groups in Michigan, James M. Anderson and Iva A. Smith, eds., Vol. 2, The People of Michigan. Detroit: Ethnos Press, 1983, pp. 173-176. 
---------. “Italian Newspapers in the Upper Peninsula”. In Harlow's Wooden Man, 19:1 (Winter 1983), pp. 9-11.

---------. Immigrants in Marquette County: 1910 Federal Census. Marquette, Michigan:

Belle Fontaine Press, 1984. [Archives Non-Circulating. Call Number: F575.A1 M3].

- The Gogebic Iron Range's Italian Immigrants. Marquette, MI: Belle Fontaine

Press, 1984, booklet.

--------. "The Italian Literary and Cultural Tradition in Upper Michigan". In Chronicle of the Historical Society of Michigan, 21:1 (Spring 1985), 11-14.

-------. (compiler). Immigrants in Iron County, Michigan: 1910 Federal Census.

Marquette, MI: Belle Fontaine Press, 1986, booklet.

--------. Miners, Merchants, and Midwives: Michigan's Upper Peninsula Italians.

Marquette, Michigan: Belle Fontaine Press, 1987. [Archives Non-Circulating. Call

Number: F575.I8 M33 1987].

-. "On the Road [Internal Migration of Immigrants]". In Harlow's Wooden Man,

33:1 (Winter 1997), 8-11.

---------. Italian Clubs, Societies and Organizations in the State of Michigan, 1873-2000.

Marquette: Belle Fontaine Press, 2000.

---------. Italians in Michigan. [East Lansing: Michigan State University Press, 2001.

[Archives Non-Circulating. Call Number: F575.I8 M34 2001].

"Many Italians Once Worked in the Quincy". In Quincy Mining Gazette (Houghton), 25 August 1973.

Monette, Clarence J. Some Fatal Accidents. Lake Linden, Michigan: C. J. Monette, c1994.

--------. A Brief History of Ahmeek, Michigan. Lake Linden: C. J. Monette, c1981.

Nash, Michael. Conflict and Accommodation: Coal Miners, Steel Workers, and socialism, 1890-1920. Westport, Conn.: Greenwood Press, 1962.

Park, Robert and Herbert Miller. Old World Traits Transplanted. New York: 1921.

Polk's 1912 Calumet, Hancock, Houghton, and Laurium Directory. [Available at Michigan Technological University and Copper Country Archives].

Polk's 1930 Calumet Directory including Hubbell, Lake Linden and Laurium. [Available at Michigan Technological University and Copper Country Archives].

Polk's Houghton County Directory. Years 1897-88, 1899-1900, 1901-02, 1903-04 , 1905 06, 1907-08, and 1916-17. [Available at Michigan Technological University and Copper Country Archives].

Polk's 1930 Houghton \& Hancock City Directory including Atlantic Mine, Baltic, Dollar Bay, Franklin, Painsedale, Quincy, Ripley, South Range, and Trimountain. [Available at Michigan Technological University and Copper Country Archives].

Re, Vittorio. Michigan's Italian Community. A Historical Perspective. Detroit, MI: Wayne State University Press, 1981.

Rosoli, Gianfausto. Un secolo di emigrazione italiana 1876-1976, Roma, Cser, 1978. 
Rozzonelli, C. The Italian Immigration to Michigan: the Piedmontese Experience, 18601920. Minneapolis (Minnesota): Immigration History Research Center (IIHRC), 1991.

Sanfilippo, Matteo (ed). Fonti ecclesiastiche per la storia dell'emigrazione e dei gruppi etnici nel Nord America: gli Stati Uniti (1893-1922). CSE, Studi Emigrazione, XXXII, 120, 1995, pp. 603-768.

Schweitzer, Lynn A. Italian American Collection: a Brief Description. Bibliography. St. Paul: University of Minnesota, 1976. Series: IHRC Ethnic Collection Series; no. 5.

Sturino, Franc. Forging the Chain: Italian Migration to North America, 1880-1930. Toronto: Multicultural History Society of Ontario, 1990.

Thurner, Arthur W. Calumet Copper and People: History of a Michigan Mining Community, 1864-1970. Privately Published, 1974.

---------. Strangers and Sojourners. Detroit: Wayne State University Press, 1994.

Tirabassi, Maddalena. L’emigrazione Italiana. In www.comune.torino.it/infocultura/intercultura/12/12f3.html, 01-30-2004.

Tomasi, Silvano M. (ed.) "Italian American workers, 1880-1920: padrone slaves or primitive rebels". In Perspectives in Italian Immigration and Ethnicity, NY: Center for Migration Studies, 1977.

Tomasi, Silvano M. and Madeline H. Engel. The Italian Experience in the United States. New York, NY: Center for Migration Studies, 1977.

Vecoli, J. Rudolph. Emigranti italiani e movimenti operai negli Stati Uniti. Una riflessione personale su etnicita' e classe sociale. In Journal of the CHA/Revue de la SHC, 1993. - (et al.) Gli italiani negli Stati Uniti. L'emigrazione e l'opera degli italiani negli Stati Uniti d'America. Atti del III Symposium di studi americani. Firenze, 27-29 maggio 1969. Firenze: Istituto di Studi Americani-Università degli Studi di Firenze, 1972. [CSE CA 08.05 .A3 I8]. - (ed.) Italian Immigrants in Rural and Small Town America. Proceedings of the Fourteenth Annual Conference of the American Italian Historical Association. Staten Island, NY: The American Italian Historical Association, 1987.

--------. "The Italian Diaspora, 1876-1976”. In R. Cohen (ed.), The Cambridge Survey of World Migrations. Cambridge-New York: Cambridge U.P., 1995.

---------. 'Dalle Marche e dall'Umbria alle miniere del Lago Superiore”. In E. Sori (ed.), Le Marche fuori dalle Marche. Ancona: Proposte e ricerche, 1998, pp. 677-694. [CSE BA 09 .S7 I-IV].

---------. "The Italian American Press and the Construction of Social Reality, 1850-1920". In J. P. Danky, W. A. Wiegand (eds.) Print culture in a Diverse America. Urbana: University of Illinois Press, 1998. 
Villari, Luigi. Gli Stati Uniti d'America e l'emigrazione italiana. New York: Arno Press, 1975.

Yans-McLaughlin and Marjorie Lightman. Ellis Island and the Peopling of America. New York: The New York Press. 1990.

\section{MEDIA}

Magnaghi, Russell M. "The Italian Heritage in the Upper Peninsula". Slide lecture and video founded by the Paisano Club of Upper Michigan and the Michigan Council for the Humanities, 1983. [Available at Northern Michigan University Archives]. -. Italian Migration to the Upper Peninsula. Interviews. Center for Oral History at Northern Michigan University. [Available at Northern Michigan University Archives. Copies of some interviews are also available at Michigan Technological University and Copper Country Archives].

\section{NEWSPAPERS and REVIEWS}

[Available in microfilm at Michigan Technological University and Copper Country Archives except where noted]

Miners' Bulletin. Hancock, Michigan: Western Federation of Miners.

Torch Lake Times. Lake Linden, Michigan.

The Native Copper Times. Lake Linden, Michigan.

Il Minatore Italiano. Laurium/Calumet, Michigan: Laurium Printing Co.

The Evening Copper Journal. Hancock, Michigan: Hancock Journal Co., 1909-.

Calumet News. Calumet, Michigan: Mining Gazette Company, 1907-.

Copper Country Evening News. Calumet, Michigan: Fred Mackenzie, -1907.

Calumet Conglomerate. Red Jacket (Calumet P. O.), Michigan: Conglomerate Printing Co.

The Evening Journal/Hancock Evening Journal. Hancock, Michigan: Hancock Journal Co.

Mining Gazette/Houghton Mining Gazette/Portage Lake Mining Gazette. Houghton: J. R. Devereux, 1858-.

Nortwestern Mining Journal. Hancock, Michigan: Kibbee \& Wilson. 
The Daily Mining Gazette. Houghton, Michigan: Mining Gazette Company.

The Keweenaw Miner. Mohawk, Michigan: Keweenaw Printing Co.

Miners' Bulletin (1913 Strike).

Lake Superior News and Miners' Journal. Copper Harbor, Michigan: E. D. Burr, 1846-.

Copper: A Weekly Review of the Lake Superior Mines.

Hancock Mining Herald. Hancock, Michigan.

The Independent/L'Indipendente. Calumet, Michigan, November 6, 1917. [Original available at Immigration History Research Center at University of Minnesota. Paper copy available at Michigan Technological University and Copper Country Archives].

Michigan Copper Journal. Hancock, Michigan.

Portage Lake Herald. Hancock, Michigan.

Portage Lake Mining Gazette. Houghton, Michigan.

Pro Nobis (For Us; Knights of Romulus). Calumet, Michigan, November 1912. [Original available at Immigration History Research Center at University of Minnesota. Paper copy available at Michigan Technological University and Copper Country Archives].

Semi-Weekly Copper Journal. Hancock, Michigan.

Wage Slave. Hancock, Michigan.

\section{PERSONAL CORRESPONDENCE}

E-mail of John Marta to the author, March 23rd, 2004.

\section{PRIMARY SOURCES}

Bylaws of the Colonial Union among the Italian-American societies of the Counties of Houghton, Keweenaw, and Ontonagon. Calumet, Michigan, 1907. [Northern Michigan University Archives. Also available at Michigan Technological University and Copper Country Archives].

Bylaws of the society of the Daughters of Italy. South Range, Michigan, 1919. [Northern Michigan University Archives. Also available at Michigan Technological University and Copper Country Archives]. 
Bylaws of the Italian-American Federation of the societies of the Upper Peninsula of Michigan. Iron Mountain, Michigan, 1909. [Northern Michigan University Archives. Also available at Michigan Technological University and Copper Country Archives].

Calumet \& Hecla Mining Company Collection. Employment Records. Michigan Technological University and Copper County Archives. [Michigan Technological University and Copper Country Archives].

Magnaghi, M. Russell's Personal Papers, 1840-1994. Northern Michigan University Archives, UNIV. SERIES 47, 21 Boxes (8.0 linear feet).

Federal Censuses of the State of Michigan.

1870 - 9th Federal Census, Houghton County, Michigan. $1880-10^{\text {th }}$ Federal Census, Houghton County, Michigan. $1900-12^{\text {th }}$ Federal Census, Houghton County, Michigan. $1910-13^{\text {th }}$ Federal Census, Houghton County, Michigan. $1920-14^{\text {th }}$ Federal Census, Houghton County, Michigan. $1930-15^{\text {th }}$ Federal Census, Houghton County, Michigan.

Italian-American Federation of the Upper Peninsula, Calumet, Michigan. Records, 1908-1925. 1 linear foot. [Immigration History Research Center at University of Minnesota Archives].

Quincy Mining Company Collection. RECORD SERIES 6. EMPLOYMENT AND MEDICAL RECORDS, 1851-1988. [Michigan Technological University and Copper Country Archives].

\section{WEB SITES}

www.ellisisland.org, January 2003-January 2004.

www.comune.torino.it/infocultura/intercultura/12/12f3.html, 01-30-2004. www.mutuosoccorso.it, 01-30-2004. 
APPENDIXES 


\begin{abstract}
APPENDIX I
Bylaws of the Colonial Union among the Italian-American Societies of the Counties of Houghton, Keweenaw, and Ontonagon
\end{abstract}




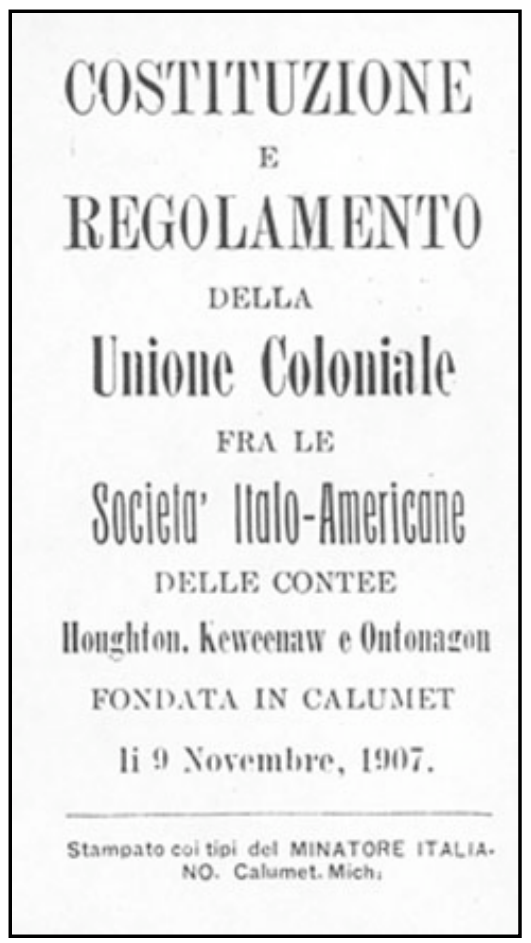




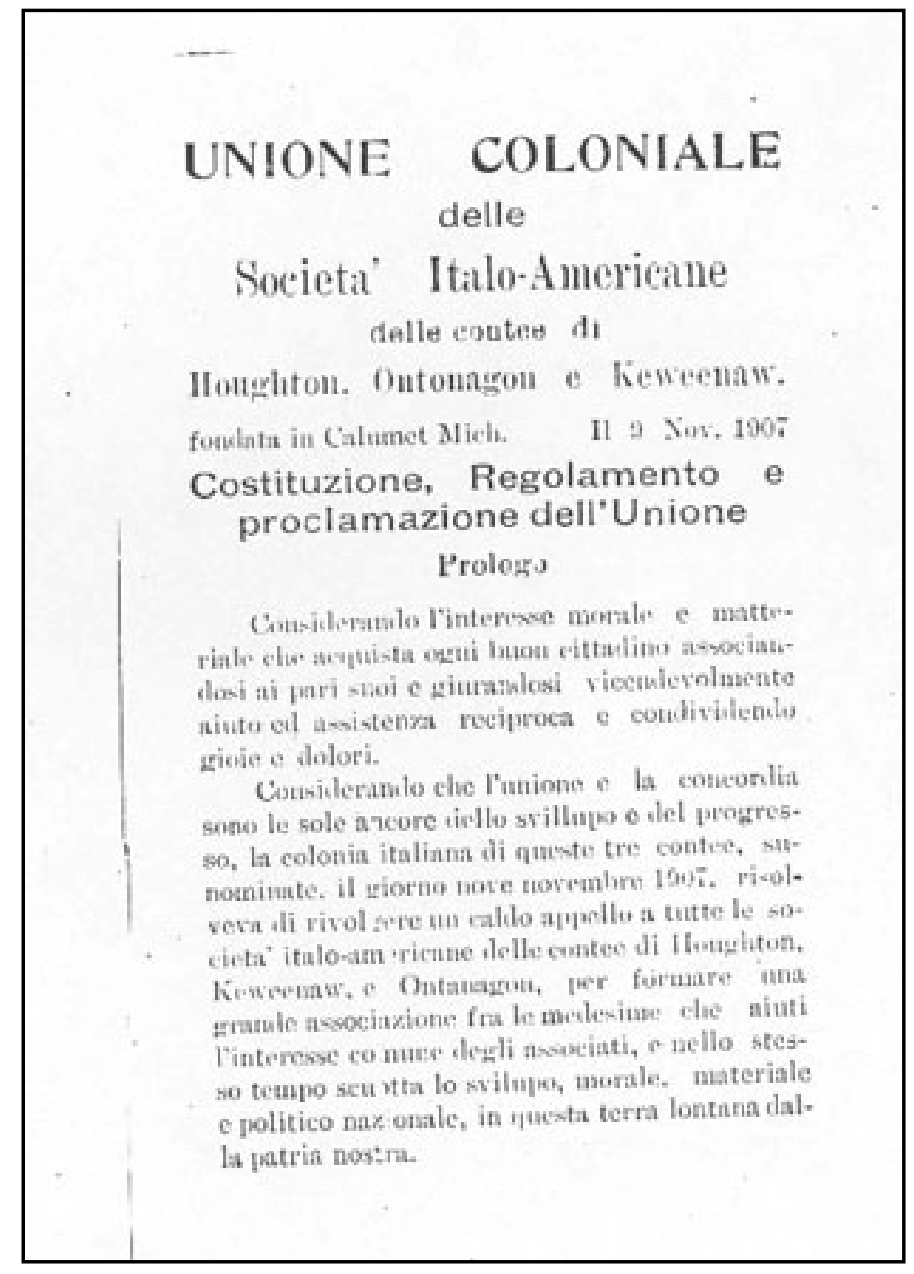

\begin{abstract}
Preface
Considering the moral and material interest that each good citizen gains in being part of associations among peers to help each other [...]. Considering that unity and understanding are the only ways to development and progress [...] the day November 9 1907, the Italian colony of the counties of Houghton, Keweenaw and Ontonagon addresses to all the Italian societies of the same counties to create an association for helping the associates and promote moral, material, and political development in this country.
\end{abstract}




\begin{tabular}{|c|c|}
\hline 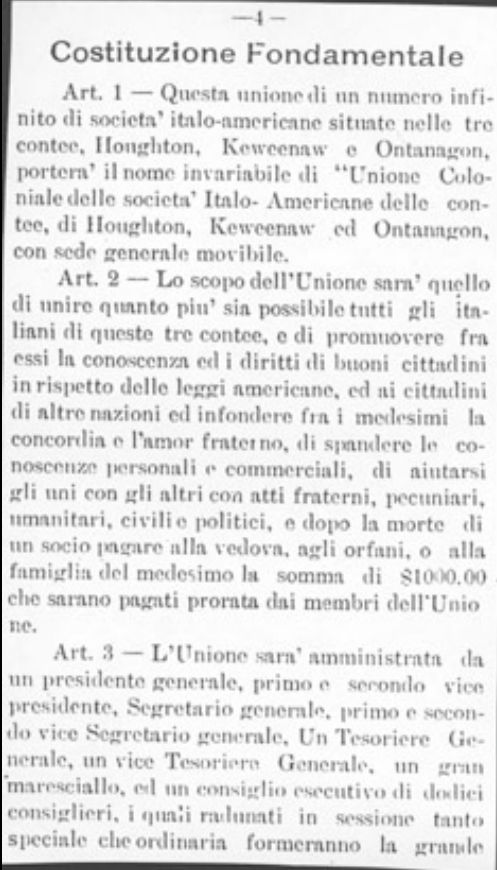 & 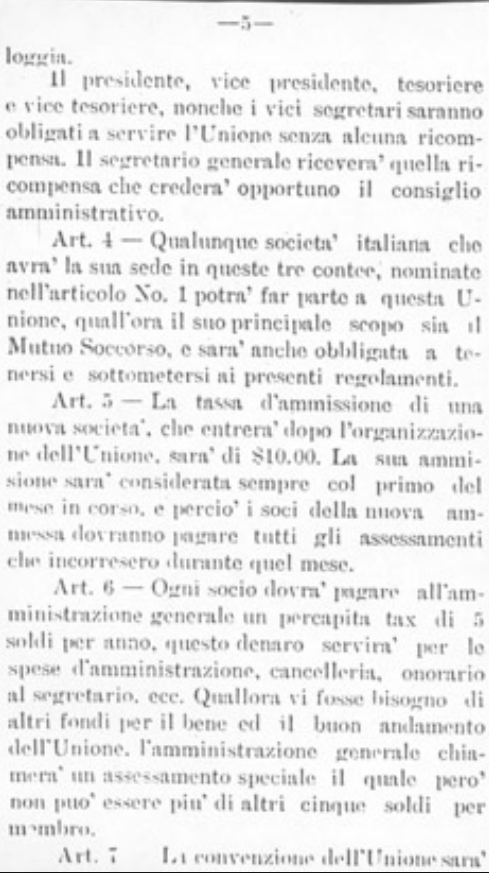 \\
\hline
\end{tabular}

\section{Constitution}

Art. 1 - This union among uncountable Italian-American societies of the counties of Houghton, Keweenaw and Ontonagon will be named "Unione Coloniale delle Società ItaloAmericane delle contee di Houghton, Keweenaw and Ontonagon". The name could not be changed. The headquarters can be changed.

Art. 2 - The aim of the Union will be to unite as much as possible Italians of these three counties, promote the understanding of their citizen rights while respecting American laws [...] and promote mutual help through fraternal, financial, political and civil actions. [...] After the death of a member a widow will receive $\$ 1,000[\ldots]$.

Art. 3 - The administration of the Union will be composed by the General President, the First and Second Vice-President, the General Secretary, the General Treasurer, the Vice-Treasurer, the Great Marshall, and a committee of twelve Advisors [...]. Nobody will be paid for serving the Union but the General Secretary.

Art. 4 - Any Italian society based in one of the three counties can join the Union.

Art. 5 - The admission fee for each society joining after today is $\$ 10$.

Art. 6 - Each member society will pay 5 cents for each one of its member [...]. 


\begin{tabular}{|c|c|}
\hline 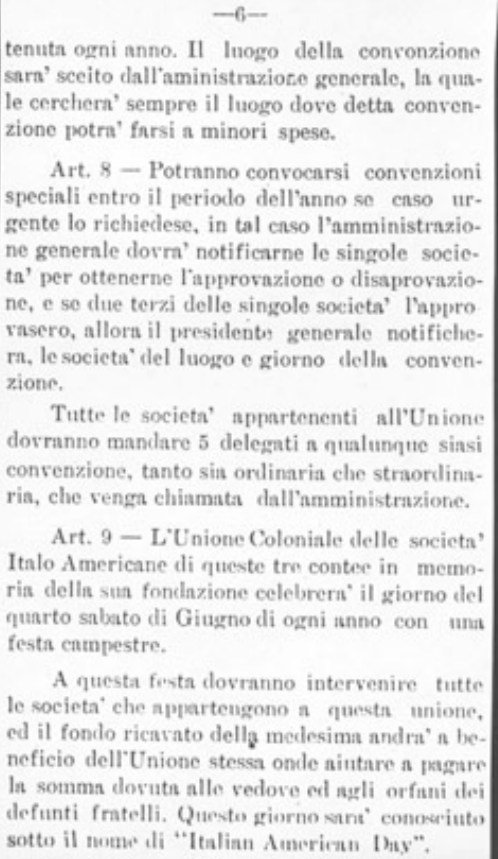 & 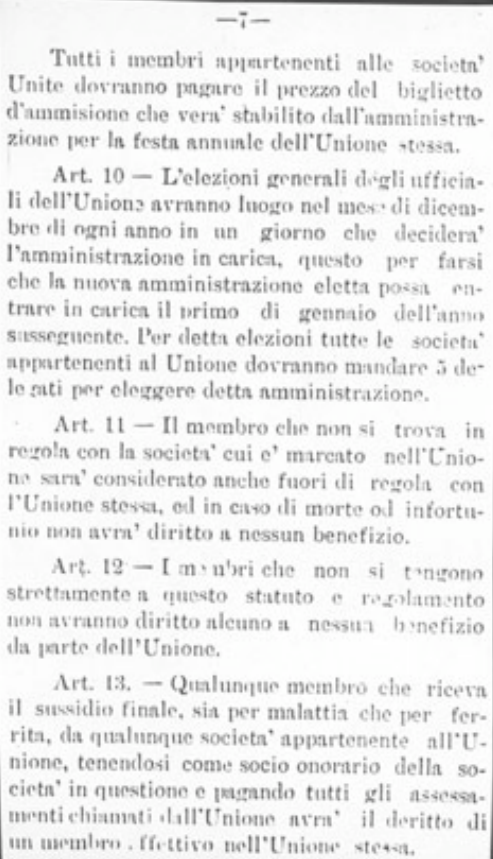 \\
\hline
\end{tabular}

Art. 7. - A convention will be held each year in a place decided by the general administration. Art. 8 - Special conventions could be held during the year in case of need [...]. Each society will send five deputies to the convention [...].

Art. 9 - To remember the day of the organization, this Union will celebrate on each fourth Saturday of the month of June with a field party. Each society will participate at the celebration and the money gathered will be for the Union [...].

Art. 10 - General elections of the Officials will be held each December [...]. Each society will send five deputies.

Art. 11 - Members who are late in their payments will be not considered in case of illness or death.

Art. 12 - Members not acting according to this bylaws will lose any right.

Art. 13 - Any member receiving monetary help for illness or injury [...] will become honorary member if he will continue to pay his fees. 
Doveri e diritti delle singole societa' e dei membri in generale.

Art. 1. - Tutte le socicta" dorranno pagare autecipatamente il pereapita tnx di cinque solili per membro, secompagnato da un raports dei membri in regola con In societa' stessa, o d coloro che si sono iseritti nei registri della medesima per pagare le tasse e gli assessamenti dell't'aione.

Art. 2 - Alla morte di un socio appartenent w al una delle societr' che fanno parte al. l'unioga, il segretario generale chiamern' un as seswam snto di $\$ 10.00 .00$ che sara' pagato Propata dai aci componenti l'Unione.

Irt. 3 - I jarenti legali di un fratello defunto asramo diritto di ricevere dall'Unione lis somm if 81000.00 qual somma sara' ricavati come istto nel precedente articolo.

I membri appartenenti all'Ćnione dovranno dichiarare in iscritto all'ufficio generale det IThione a chi intentono lascian i 81000,00$)$

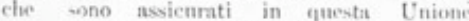

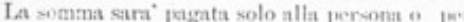
sone if dicate dalliassicurato, It cavo clue le jeresone inilicate dullinssicurato al tempe della sut morte non esistessero pint" l'ammonture sara" pugato ai farenti pis 'prosimi.

Quall'ora un membro di una societa' apuer-

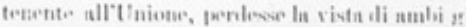

ocelii, o amhi le mani, o anhai i piesli, il segre

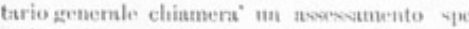
eiale medesimo di quello che chiamereblye per la morte di un confratello, e ifuesto deraso sura

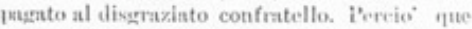
sti arme' al diritto di ricesere dall' C'ni mo la somma di s1000,00. Quel membro che jerrilinoe tunt gamb o un loraccio asra' diritto alla sommus

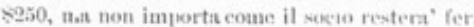
rito I't'nione non sara' responsabile a pin di $\$ 1000,00$ di jagamento.

Art. 1 - La vede genenale dos ra' fornire tntte lo singole societse uno stamyato sulatto ver i muorti menvili, o per reyistrare i sod unmesi, espmbi e morti e membri in regoln I a socinta" che non alempie a mandare regolar mante i suoi nuporti al segretario generale sarn multata di \$.3.00 per le prima volta, per la so. coula $\$ 10,40 \mathrm{r}-$ In terza sara cancelata dai ruoli. Doveri degli Ufficiali

Doseri del Presilente (ivenerale.

Art. 1 - Sara devere del persilitente gene

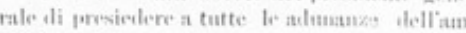
ministmxione cocentiva, di far coverrare t.

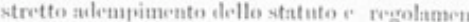
to dell'Unione. Firmera' tutti sli oritini di wate zamento che portino gia' la firma del Sigreta

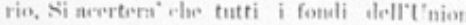

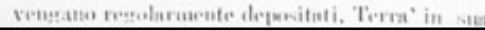

\section{Rights and obligations of the societies and members.}

Art. 1 - All the societies will pay the per capita fees in advance [...] and will present a list of their members [...].

Art. 2 - In case of death of a member the General Secretary will require $\$ 1,000$ to the Union. Art. 3 - The relatives of a dead member will receive \$1,000 [...]. The members of the Union will declare that they renounce to $\$ 1,000$ in favor of the persons indicated by the deceased member $[\ldots]$.

Art. 4 - Each society will receive forms for monthly statements, new members, expulsed members, dead members, and members in truck with payments. Each society will send the form to the Union in order to avoid a fee of $\$ 5$.

\section{Rights and obligations of the Officials. Obligations of the President.}

Art. 1 - The President will chair each meeting and will make sure everybody acts according to the bylaws $[\ldots]$. He will sign each order of payment $[\ldots]$ and will make sure that the funds of the Union be deposited regularly [...]. 


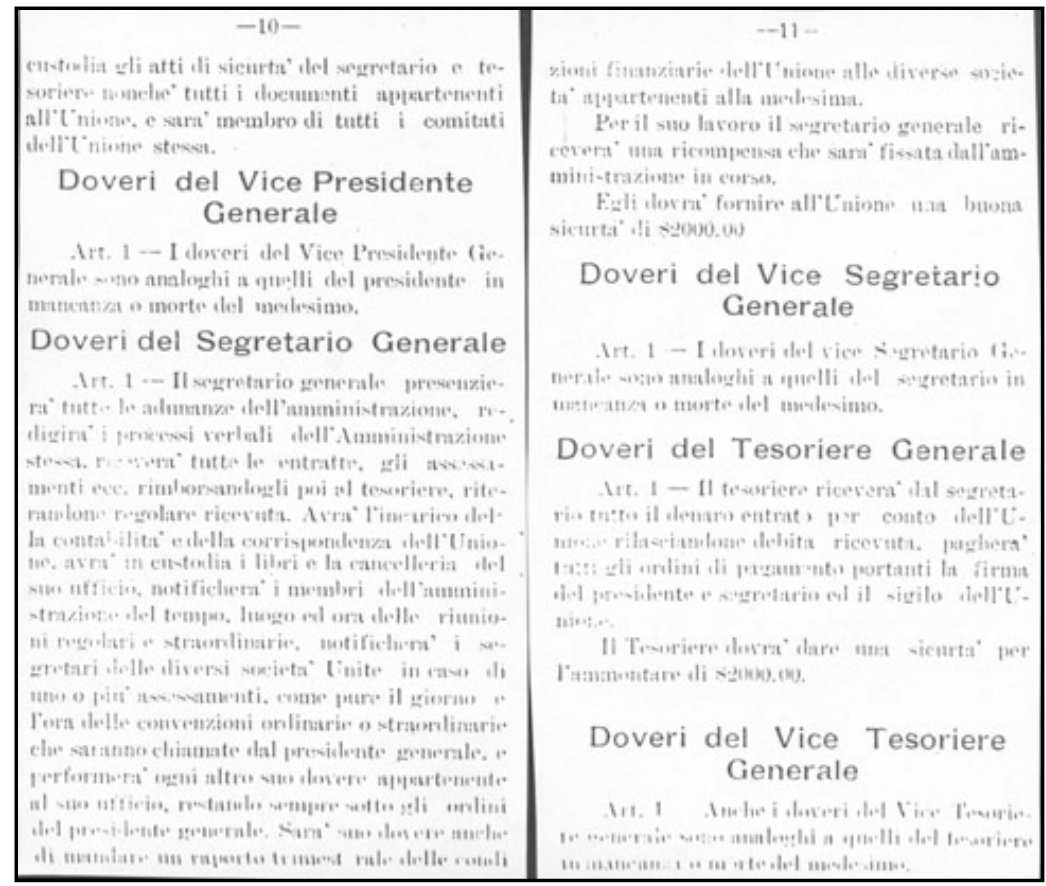

Obligations of the Vice-President.

Art. 1 - The obligations of the Vice-President are the same as the President's [...].

\section{Obligations of the General Secretary.}

Art. 1 - The General Secretary will be present in each meeting, will write the minute book, and will collect the money. He will take care of accounting and will keep the books of the Union $[\ldots]$.

\section{Obligations of the General Vice-Secretary.}

Art. 1 - The obligations of the Vice-Secretary are the same as the Secretary's [...].

\section{Obligations of the General Treasurer.}

Art. 1 - The Treasurer will receive all the money from the Secretary, and will pay every order of payment signed by the President and The Secretary [...]. 


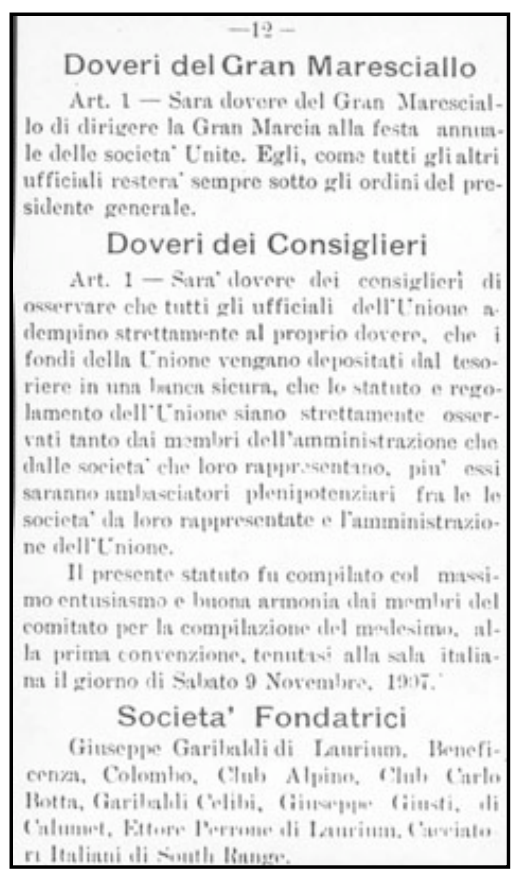

\section{Obligations of the Great Marshall.}

Art. 1 - The Great Marshall will lead the Great Marching during the annual celebration [...].

\section{Obligations of the Advisors.}

Art. 1 - The Advisers will supervise all the Officials of the Union making sure they act according to the bylaws $[\ldots]$. 


\section{APPENDIX II}

Bylaws of the Italian-American Federation of the Societies of the Upper Peninsula of Michigan 


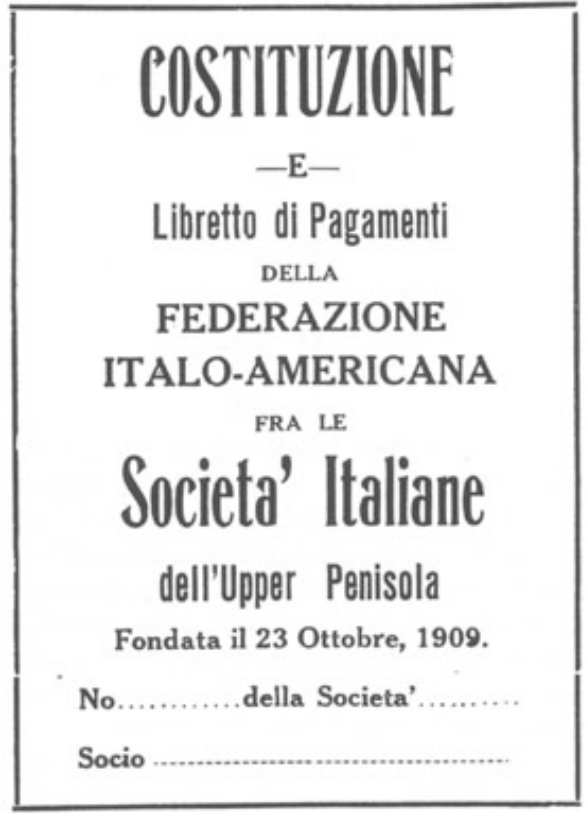

\author{
Constitution \\ And \\ Paycheck Book \\ of the Italian-American Federation \\ among \\ the Societies of the Upper Peninsula
}




\section{Parte Prima}

ART. 1.

Nel giorno 23 di Ottobre 1909, presenti i Delegati di 28 Societa' Italiane aventi sede nelle diverse citta' del Northern Mich., in Iron Mountain veniva organizzata un'associazione la quale venne ir quel giorno stesso denominata, "Federazione Italo-Americana" per l'Upper Peninsula. A detta Federazione potranno far parte tutte le Societa' Italiane aventi sede nell'Upper Peninsula, quando esse abbiano per principale scopo il Mutuo Soccorso. La sede della Federazione sara' stabile in Calumet, Mich.

\section{First Part}

Art. 1

On the day October 23 1909, present the delegates of 28 Italian societies of Northern Michigan, in Iron Mountain was organized an association called "Italian-American Federation" of the Upper Peninsula. The societies allowed to join the federation need to be located in the Upper Peninsula and to have as main goal Mutual Help. The headquarter of the Federation is in Calumet, Michigan 


\begin{tabular}{|c|c|}
\hline $\begin{array}{l}\text { ART. } 2 \text {. } \\
\text { Scopo principale della federazione sara' } \\
\text { quello di conservare fra i membri delle di- } \\
\text { verse societa' Italiane quel continuo contat- } \\
\text { to equella armonia che sono necessari tra } \\
\text { figli di una stessa patria; di promuovere la } \\
\text { Cittadinanza Americana il piu' che sia pos- } \\
\text { sibile; promuovere l'insegnamento della } \\
\text { lingua Italiana sia nelle scuole pubbliche, } \\
\text { sia coadiuvando l'istituzione di scuole pri- } \\
\text { vate; procurare che gli elettori Italiani pro- } \\
\text { cedano uniti e compatti nei casi di elezioni } \\
\text { generali, per modo che la forza del voto } \\
\text { degli Italiani venga apprezzata, e di istitui- } \\
\text { re nel seno delia Federazione una Cassa } \\
\text { Iutuo Soccorso, per aiutare i membri in } \\
\text { caso di infortunio o di morte, e finalmente } \\
\text { di sostenersi gli uni con gli altri con atti } \\
\text { fraterni, umanitari civili e politici. } \\
\text { ART. } 3 \text {. } \\
\text { L'amministrazione della Federazione sa- } \\
\text { ra' composta da un Presidente, un primo } \\
\text { Vice Presidente, ed uno per ogni localita', }\end{array}$ & $\begin{array}{l}\text { un Segretario ed un vice Segretario di cor- } \\
\text { rispondenza, da un Segretario ed un vice } \\
\text { Segretario di finanza, da un Tesoriere e da } \\
\text { un vice Tesoriere, da un Maresciallo ed As- } \\
\text { sistente Maresciale, e da un Consigliere per } \\
\text { ogni localita' e di un numero illimitato di } \\
\text { Presidenti Onorari, i quali verranno scelti } \\
\text { tra quei membri che si siano maggior- } \\
\text { mante distinti nella Federazione o tra i con- } \\
\text { nazionali i quali per meriti speciali abbiano } \\
\text { diritto alla riconoscenza comune. } \\
\text { Tutti questi ufficiali avranno il diritto di } \\
\text { far uso del titolo “Generale". Oltre agli uffi- } \\
\text { ciali suddetti l'amministrazione sara' anco- } \\
\text { ra composta da un deputato per ogni socie- } \\
\text { ta', eletto dalle medesime annualmente al } \\
\text { tempo delle elezione ordinarie, essi saranno } \\
\text { considerati come consiglieri, ed obbligati } \\
\text { d'intervenire alle riunioni che chiamera' il } \\
\text { Vice Presidente locale per i rapporti, per il } \\
\text { benessere ed il buon andamento della Fede. } \\
\text { razione. Finalmente da un comitato per le } \\
\text { operazioni di naturalizzazione per ogni loca- } \\
\text { lita'. Gli ufficiali superiori ed i deputati, i } \\
\text { consiglieri ed i comitati di naturalizzazione } \\
\text { comporranno la Gran Loggia. }\end{array}$ \\
\hline
\end{tabular}

\section{Art. 2}

Principal aim of the federation is to keep in touch the societies and preserve the harmony among sons of the same country; to promote American citizenship as much as possible; to enhance the teaching of the Italian language in public schools [...]; to unity the Italian voters in case of general elections; to organize a Cash of Mutual Help to help the members in case of illness or death.

\section{Art. 3}

The administration of the Federation is composed by a President, one first Vice President, one Vice President for each locations, a Secretary and Vice Secretary of Correspondence, a Secretary and a Vice Secretary of Finance, a Treasurer, a Marshall and an Assistant Marshall, an Advisor for each location and an unlimited number of Honorary Presidents [...]. Moreover, a Deputy for each society will be part of the administration. There will be a Committee of Naturalization. The Officials, the Deputies and the Committee will form the Great Lodge. 


\begin{tabular}{|c|c|}
\hline $\begin{array}{l}\text { ART. } 4 \text {. } \\
\text { Tutti gli ufficiali dovranno prestare la lo- } \\
\text { ro opera gratuitamente, ad eccezione dei se- } \\
\text { gretari, ai quali sara' pagato uno stipendio } \\
\text { in base, al lavoro che la carica medesima } \\
\text { portera' progresso della federazione. Que- } \\
\text { sta verra' fissata ogni anno alla convenzio- } \\
\text { ne. } \\
\text { ART. } 5 \\
\text { Qualunque societa' Italiana avente sede } \\
\text { nell'Upper Peninsula, potra' far parte della } \\
\text { Federazione Italo Americana, qualora il suo } \\
\text { principale scopo sia il Mutuo Soccorso. } \\
\text { Per essere ammessa a far parte della fe- } \\
\text { derazione la societa' dovra' presentare copia } \\
\text { della deliberazione presa dai membri in se- } \\
\text { duta ordinaria o straordinaria, la quale ver- } \\
\text { ra' presentata nella prima riunione del con- } \\
\text { siflio d'amministrazione. } \\
\text { ART. } 6 \text {. } \\
\text { L'ammissione della Societa' verra' consi } \\
\text { derata col primo del mese in corso, coll'obli } \\
\text { g) di presentare l'elenco di tutti i soci in }\end{array}$ & $\begin{array}{l}\text {-7- } \\
\text { regola, coll'ammontare di (10) dieci soldi per } \\
\text { socio a pro percapita. } \\
\text { ART. } 7 \text {. } \\
\text { Tutte le Societa' federate pagheranno un } \\
\text { percapita di } 10 \text { soldi, 'per anno per ogni } \\
\text { membro effettivo la quale tassa andra' a } \\
\text { credito del conto amminastrazione. Da que- } \\
\text { sto conto verranno detratte le spese di can- } \\
\text { celleria, onorario ai segretarii, posta ecc. } \\
\text { per quanto riguarda la Federazione. Nel ca- } \\
\text { so in cui maggiori fondi fossero necessari, } \\
\text { l'amministrazione generale e' autorizzata ha } \\
\text { chiamare un altro percapita l'ammontare } \\
\text { del quale non potra' essere superiore ai die- } \\
\text { ci soldi. } \quad \text { ART. } 8 \text {. } \\
\text { La Federazione Italo Americana avra' o- } \\
\text { gni anno una convenzione alla quale ogni } \\
\text { societa' federata dovra' mandare due dele- } \\
\text { gati. Nel caso una societa' avesse meno di } \\
100 \text { membri, e volesse mandare un solo dele } \\
\text { gato, avra' diritto a farlo. Questo delegato } \\
\text { avra' pero' diritto ad un solo voto. Questa } \\
\text { convenzione dovra' aver luogo due giorni }\end{array}$ \\
\hline
\end{tabular}

\section{Art. 4}

All the Officials have to work for the federation for free, except for the Secretaries [...].

Art. 5

Any Italian society based on the Upper Peninsula of Michigan can become member of the federation, if its main goal is mutual help [...].

Art. 6

The admission of a new society will be effective on the current month. The society has to present a list of the members and 10 cents for each one of them.

\section{Art. 7}

Each society will pay 10 cents per year for each of its members. This amount, subtracted stationery expenses, salaries of the secretaries, and mail expense, will be credited on the administration account $[\ldots]$.

Art. 8

The Federation will held a convention each year. Each society will send two delegates to the convention $[\ldots]$. 


\begin{tabular}{|c|c|}
\hline $\begin{array}{l}\text { prima della festa annuale della Federazione } \\
\text { la quale sara' conosciuta col nome di 'Ita- } \\
\text { lo-American Day." La localita' scelta per la } \\
\text { convenzione avra' pure diritto alla suddetta } \\
\text { festa ufficiale. La localita' verra' fissata } \\
\text { nella convenzione annuale. Le societa' loca- } \\
\text { li, una o piu', prenderanno la festa a loro } \\
\text { carico, cioe' spese e guadagni. } \\
\text { ART. 9. } \\
\text { Potranno convocarsi convenzioni speciali } \\
\text { durante l'anno nel caso, in cui l'urgenza lo } \\
\text { richiedesse. In tal caso lamministrazione } \\
\text { notifichera' il consiglio dei consiglieri i quali } \\
\text { fisseranno la data ed il luogo in cui la mede } \\
\text { sima dovra' aver luogo. Ogni societa' dovra' } \\
\text { mandare a questa convenzione un solo Dele } \\
\text { gato. } \\
\text { In tempo delle elezioni statali e federali } \\
\text { l'amministrazione della Federazione a tem- } \\
\text { po propizio chiamera' una convenzione spe- } \\
\text { ciale. per difendere i diritti dei connaziona. } \\
\text { li alla quale prendera' parte un Delegato } \\
\text { per ogni Societa' Federata, che sara' eletto, } \\
\text { dalla sua Societa', ed il socio che accettera' }\end{array}$ & $\begin{array}{l}\text { - } 9 \text { - } \\
\text { tale carica dovra' sopperira a tutto proprie } \\
\text { spese. } \\
\text { ART. } 10 \text {. } \\
\text { Le societa' attualmente federate dovran- } \\
\text { no mandare un elenco completo di tutti i } \\
\text { membri in regola colla societa'.Si dovra' pu- } \\
\text { re fare menzione se gli stessi sono cittadini- } \\
\text { americani oppure no. Questo dovra' poi esse- } \\
\text { re fatto dalle societa' nuovo ammesse entro } \\
\text { il periodo di due mesi dalla loro ammissione. } \\
\text { Ogni societa' dovra' poi mandare ogni me- } \\
\text { se un elenco dei soci nuovi ammessi, con } \\
\text { tutte le indieazioni summenzionate, non- } \\
\text { che' un elenco dei soci i quali hanno fatto } \\
\text { richiesta del foglio di via, di quelli decessi } \\
\text { o scaduti. } \\
\text { Il membro che faccia richiesta del foglio } \\
\text { di viaggio dovra' provare che trovasi in re- } \\
\text { gola e pagare la tassa di } 10 \text { soldi per averlo } \\
\text { e gli verra' rilasciato dall'amministrazione } \\
\text { locale. } \\
\text { La arT. } 11 \text {. }\end{array}$ \\
\hline
\end{tabular}

Art. 9

Special conventions can be held during the year [...]. In case of state or federal elections a special convention will be held to stand for the rights of Italians [...].

\section{Art. 10}

The societies will send a list of all the members whose payments are taken care of and declare if they are American citizens or not [...].

\section{Art. 11}

The federation will provide the societies with all the forms; applications, receipts, monthly statements, list of the members who did not pay, list of expulsed members, list of new members, etc. 


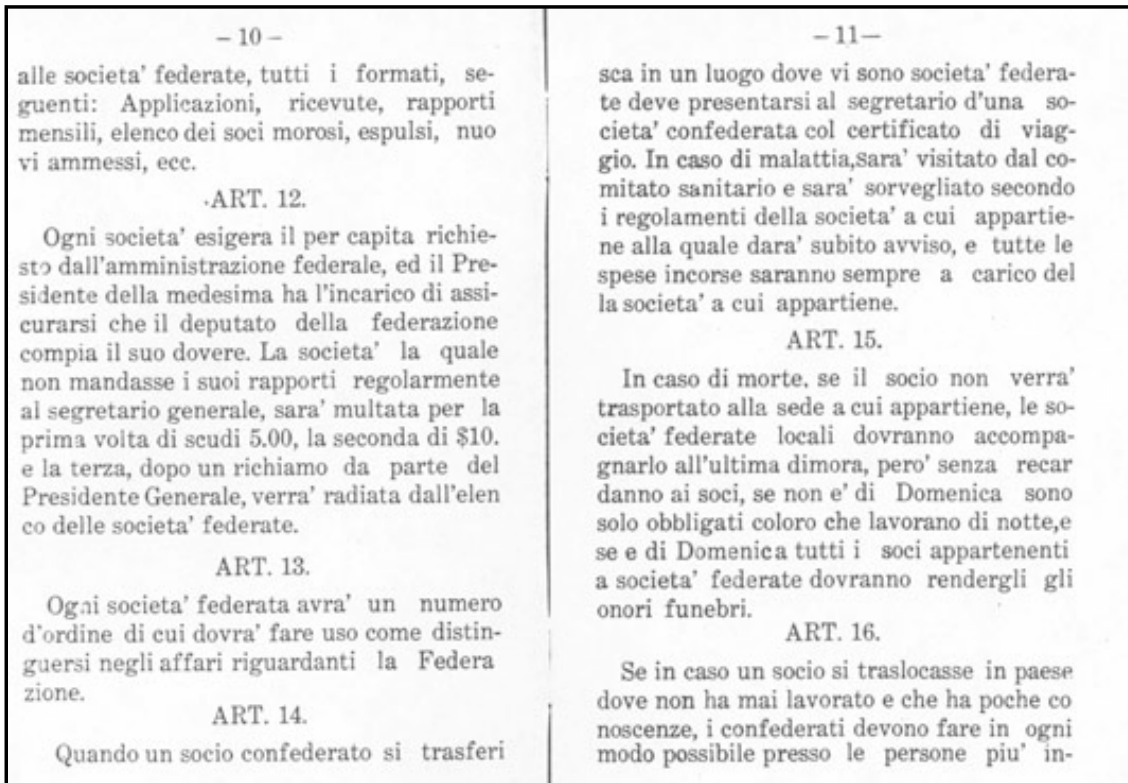

\section{Art. 12}

Each society will collect the per capita fee requested by the federation from the members.[...]. Each society will send reports to the federation [...].

\section{Art. 13}

Each society will be assigned a number to distinguish itself from the others.

\section{Art. 14}

In case a member moves to a place where there is a federate society, he is to refer to it in case of illness [...], but he will receive the money from his society.

\section{Art. 15}

In case of death he will not be brought back to his original place, and the local societies will take care of everything $[\ldots]$.

\section{Art. 16}

If a member moves to a place that he does not know the members of the local societies will do as much as they can to help him find a job. 


$$
-12-
$$

fluenti per farlo impiegare e dargli lavoro.

ART. 17.

Qualora un membro d'una loggia federata dovesse trasportarsi altrove dovra' munirsi del foglio di viaggio e se desidera trasferirsi in un altra societa' federata non regionale, dove ha presa nuova residenza, potra' essere ammesso senza pagare l'ammissione purche' si assogetti alla costituzione sociale e visita medica, se la societa' lo richiede, e dia la sua dimissione dalla societa' che appartiene, e venga trovato meritevole. ART. 18.

Ogni societa' federata che volesse ritirarsi e' obligata a farne avviso al Segretario Generale di Corrispondenza, tre mesi prima della sua scadenza, altrimenti sara' conside rata sempre federata, e sottoposta ed obbligata di pagare tutte lespese che verranno incontrate dalla Federazione.

$$
\text { ART. } 19 .
$$

Doveri degli Ufficiali

Sara' dovere del presidente generale di

\section{$-13-$}

presiedere a tutte le sedute del consiglio d'amministrazione di far osservare lo statuto ed i regolamenti della federazione.

Firmera' tutti gli atti enerenti all'amministrazione della Federazione e tutti i buoni di pagamento che portino gia' la firma del segretario. Avra' cura che i fondi della federazione vengano regolarmente deposita ti, e terra' in sua custodia gli atti di sicurta' del segretario e del tesoriere. Egli avra' diritto di prendere parte a tutti i comitati che saranno nominati per il buon andamento della federazione.

$$
\text { ART. } 20 .
$$

I Vice presidenti avranno gli stessi doveri del presidente, ed il primo vice presidente in sua assenza ne' farra' le veci.

Essi inoltre dovranno sorvegliare che le cose procedano regolarmente nelle localita' di loro residenza.

$$
\text { ATR. } 21 .
$$

Sara' dovere del segretario generale dei riccordi di mandare gli avvisi per le riunio-

\section{Art. 17}

If a member move to a place where there is a federate society he can transfer his membership to that society $[\ldots]$.

\section{Art. 18}

If a society wants to withdraw from the federation, they have to inform the Secretary of Correspondence $[\ldots]$.

\section{Art. 19}

\section{Duties of the Officials}

The President will chair all the meeting of the Officials and will make sure that the bylaws be respected. He will sign every administrative act, and payments [...]. He will make sure that the funds of the federation will be deposited [...].

\section{Art. 20}

The Vice Presidents will have the same duties as the President [...]. They will watch over the federate societies in their locations. 


\begin{tabular}{|c|c|}
\hline $\begin{array}{l}\text { ni del consiglio amministrattivo, tenere la } \\
\text { corrispondenza che gli affari della Federa- } \\
\text { zione ricchiedono, prendere parte alle riu- } \\
\text { nioni, redigere i verbali, preparare i lavori } \\
\text { per le convenzioni, controllare i conti del } \\
\text { segretario di finanza, ed inviare il rapporto } \\
\text { semestrale alle loggie federate. Avra' pure } \\
\text { cura dei libri della Federazione e del tim- } \\
\text { bro sociale, e dovra' dare una cauzione da } \\
\text { fissarsi dal consiglio amministrattivo. } \\
\qquad \text { ART. } 22 \\
\text { Il Segretario Generale di finanza dovra' } \\
\text { tenere la contabilita', ricevere i pagamenti } \\
\text { che gli verranno fatti dalle sorieta' per con } \\
\text { to dei soci, mandare gli avvisi per gli asses- } \\
\text { samenti ai deputati di tutte le societa' fede- } \\
\text { rate versare il denaro al Tesoriere, e fare } \\
\text { tutto quanto che il Presidente gli ordinera' } \\
\text { in attinenza col disbrigo degli affari della } \\
\text { federazione. } \\
\text { Dovra' aver cura degli oggetti dati in sua } \\
\text { consegna. Dovra' procurare una cauzione } \\
\text { che verra' fissata dal consiglio amministra- } \\
\text { tivo. }\end{array}$ & $\begin{array}{l}\text { ART. } 23 \\
\text { I Vice Segretari dovenno fare le veci del } \\
\text { segretario al quale essi sono collegati. in ca } \\
\text { so di temporanea assenza del medesimo. } \\
\text { ART. } 24 \\
\text { Il tesoriere ricevera' dal segretario tutto } \\
\text { il denaro ricevuto per conto della federazio } \\
\text { ne rilasciandone ricevuta, firmera' tutti } \\
\text { gli ordini di pagamento portanti la firma } \\
\text { del Presidente e del Segretario, terra } \\
\text { cura dei rapporti mensili delle banche nel- } \\
\text { le qualı il denaro della federazione verra' } \\
\text { depositato, ed avra' cura di tutto quanto } \\
\text { riguarda l'interesse finanziario dell'insti } \\
\text { tuzıne. Dovra' dare una cauzione da fissa- } \\
\text { rsi alla convenzione annuale. } \\
\text { ART. } 25 \\
\text { Sara' dovere dei cousiglieri di osservare } \\
\text { che tuttigli ufficiali facciano il loro dovere } \\
\text { che i fondi della Federazione vengano depo } \\
\text { sitati in banche sicure, dovranno personal } \\
\text { mente o a mezzo di delegazioni fatte ad al- } \\
\text { tri membri dell'amministrazione, prendere }\end{array}$ \\
\hline
\end{tabular}

\section{Art. 21}

The General Secretary will send out notes to the members of the administration to inform

them about meetings, he will take care of the correspondence, he will organize the conventions, he will check the statements [...] he will keep the books of the Federation [...].

Art. 22

The General Secretary of Finance will take care of accounting [...].

Art. 23

The Vice-Secretaries will take the place of their Secretary in case of his absence.

\section{Art. 24}

The Treasurer will take care of all the money [...] and will sign all the orders of payment [...].

\section{Art. 25}

The Advisors will supervise over the Officials [...]. 


$$
-16-
$$

parte a tutte le riunioni del consiglio amministrativo e dovranno curare che il regolamento venga strettamente osservato. Tut ti gli ufficciali e consiglieri hanno diritto al voto.

ART. 26.

I deputati della federazione dovranno rap presentare la medesima nel sceno della societa' dalla quale sono stati nominati ed attendere a tutte le riunione della societa' stessa, riscuotere le contribuzioni e quan to riguarda alla Cassa Mutuo Soccorso e riscuotere dalla societa' a cui appartengono le tasse per capita della federazione, inscrizione dei nuovi membri, la spedizione delle domande dei nuovi aspiranti alla Cassa di Mutuo Soccorso al segretario generale, tenere il registro dei pagamenti delle contribuzioni, versare mensilmente al segretario generale i fondi riscossi, fare in somma tutto quanto per l'interesse della federa zione richiede nell'interno della societa' alla quale appartengono. Essi verranno nominati annualmente dalla societa' che rappre-

\section{$-17-$}

sentano. I deputati di ogni singola societa' sono pure tenuti di avvisare quei membri in scritti alla Cass. Mutuo Soccorso nella loro lista anche se si trovassero all'estero, ogni volta che verranno chiamate le contribuzioni o percapita.

Le spese di cancelleria e posta incontrate dai deputati verranno pagate dalla federa. zisne ogni anno dietro nota specificata dal deputato, queste spese veranno levate dal fondo percapita.

ART. 27.

La presente costituzione e regolamento della Federazione e Cassa Mutuo Soccorso Italo-Americana dell.Upper Peninsula puo essere modificato ad ogni convenzione an nuale, seconco i bisogni necesari.

Art. 26

The Deputies of the federation will represent the federation itself in each society [...].

Art. 27

This constitution can be modified at every annual convention according to the needs. 


\section{APPENDIX III}

SATIRIC POEMS FROM

THE MINERS' BULLETIN 


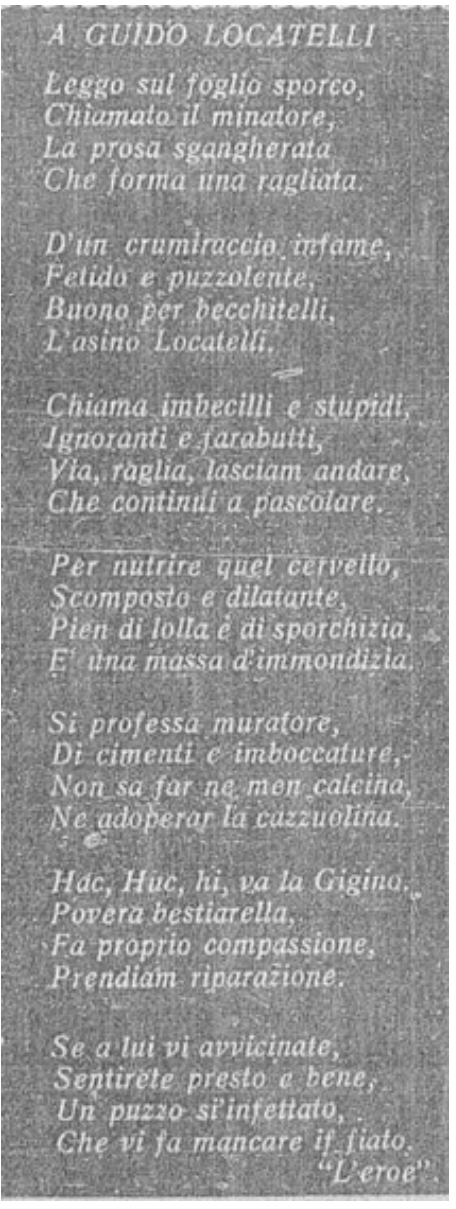

Miners' Bulletin N. 54

01-28-1914

\section{To Guido Locatelli}

I read on the dirty paper

Called the miner

The absurd prose

That compose a donkey speech.

The prose of an infamous scab

Who stinks

And it's good just for 'becchetelli'

The donkey Locatelli.

He calls imbecile and stupid

Ignorant and bastard

Let's let him alone

Let him continue to graze.

To feed that brain

In disorder and confused

Full of dirt

Full of garbage.

He calls himself a mason

Of building

He doesn't even know how to make lime

Nor how to use a trowel.

Hac, Huc, hi, Gigina goes

Poor little animal

We feel pity for him

Let's do something.

If you get close to him

You will feel soon and clearly

That he stinks so much

That takes your breath away. 


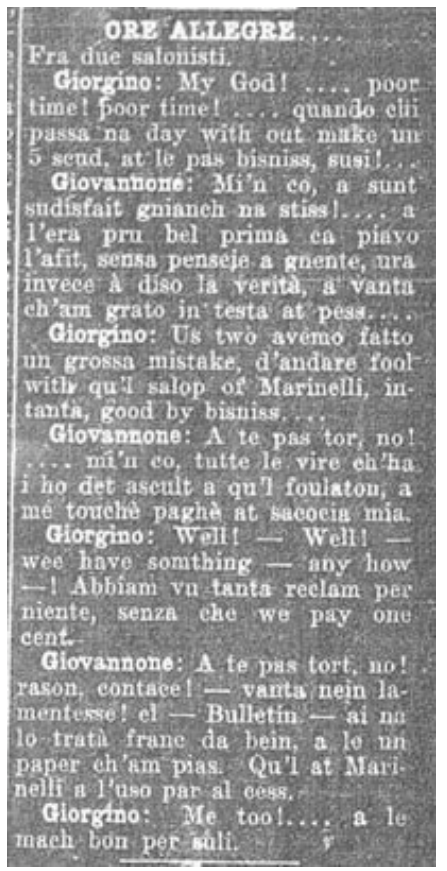

Miners' Bulletin N. 62

\section{Happy Hours}

Two saloon keepers

Little George: My God...poor time poor time! When a day passes by without you earning five cents business ends!

Big John: I'm not satisfied either. It was better before when I had money from renting and I had to think about nothing. Now, to be honest, I have only problems.

Little George: We made a big mistake fooling around with Marinelli....and now we can say goodbye to our businesses...

Big John: You are right. Listening to him I lost money from own my pocket.

Little George: Well! Well! We have something anyhow. We had a lot of publicity without paying any cent.

Big John: You are right, we cannot complain. The Bulletin treated us nicely. I'll use Marinelli's paper as toilet paper.

Little George: Me too. It's good just for that.

03-18-1914 


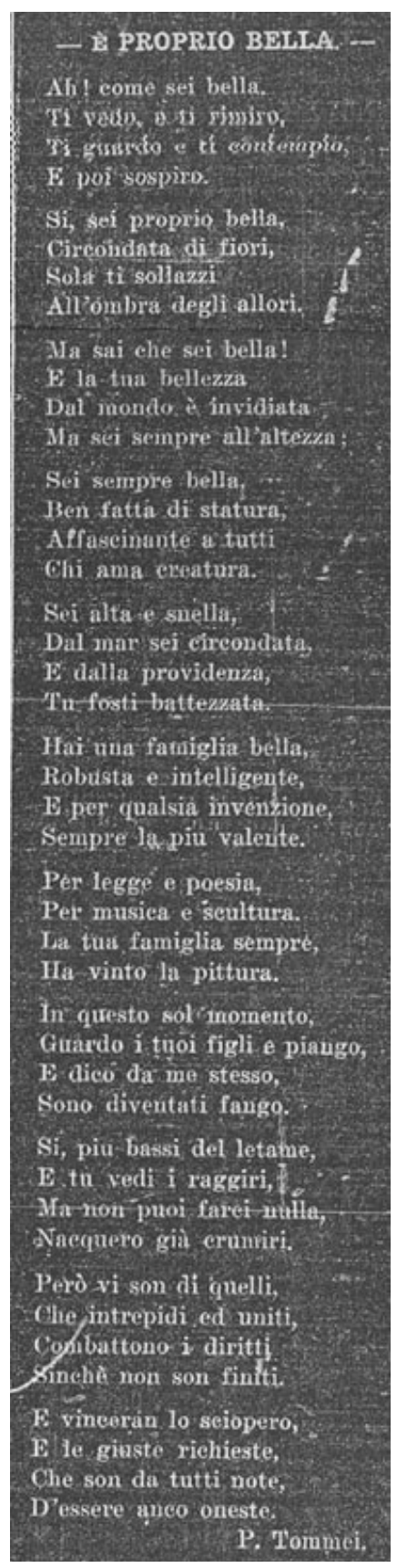

Miners' Bulletin N. 57

02-18-1914

\section{It is really beautiful}

Ah! How beautiful you are I look and look again at you And then I sigh.

Yes you are really beautiful! The world envies your beauty And you always keep up!

You are always beautiful Tall enough Fascinating for everybody Lovely creature.

You are tall and thin Surrounded by the sea You were baptized by providence.

You have a nice family

Strong and smart

Whose inventions are

Always so useful.

Law and poetry, Music and sculpture

Your family master painting.

In this moment

I look at your sons and cry

And I say to myself

They have become mud.

Dirtier than dung

And you can see their dirty acting

But you cant' do anything

They were born scabs.

But some others

Are united and fierce

They stand up for their rights

Till the end.

They will win the strike

And will get what they are asking for And everybody know that

Their requests are fair. 
APPENDIX IV

MAIN MUNICIPALITIES OF THE PROVINCE OF LUCCA 


\section{MAIN MUNICIPALITIES OF THE PROVINCE OF LUCCA}

\begin{tabular}{ll} 
Altopascio & Minucciano \\
Bagni di Lucca & Molazzana \\
Barga & Montecarlo \\
Borgo a Mozzano & Pescaglia \\
Camaiore & Piazza al Serchio \\
Camporgiano & Pietrasanta \\
Capannori & Pieve Fosciana \\
Careggine & Porcari \\
Castelnuovo di Garfagnana & San Romano in Garfagnana \\
Castiglione di Garfagnana & Seravezza \\
Coreglia & Sillano \\
Fabbriche di Vallico & Stazzema \\
Forte dei Marmi & Vagli Sotto \\
Fasciandora & Vergemoli \\
Gallicano & Viareggio \\
Giuncugnano & Villa Basilica \\
Lucca & Villa Collemandina \\
Massarosa & \\
\hline
\end{tabular}


APPENDIX V

MAIN VILLAGES OF THE CANAVESE 


\section{MAIN VILLAGES OF THE CANAVESE FROM WHERE MOST IMMIGRANTS TO HOUGHTON COUNTY CAME}

\begin{tabular}{|c|c|}
\hline Agliè & Ribordone \\
\hline Bairo Canavese & Rivara \\
\hline Barone Canavese & Rivarolo \\
\hline Borgiallo & Romano Canavese \\
\hline Bosconero & Ronco Canavese \\
\hline Candia Canavese & Salassa \\
\hline Castellamonte & San Benigno Canavese \\
\hline Ciriè & San Carlo Canavese \\
\hline Corio & San Colombano Belmonte \\
\hline Cuorgnè & San Giorgio Canavese \\
\hline Foglizzo & San Giusto Canavese \\
\hline Forno & San Martino Canavese \\
\hline Frassinetto & San Maurizio Canavese \\
\hline Ivrea & San Ponso Canavese \\
\hline Lessolo & Scarmagno \\
\hline Levone & Settimo \\
\hline Locana & Sparone \\
\hline Lusigliè & Strambinello \\
\hline Montalenghe & Strambino \\
\hline Orio Canavese & Traversella Valperga \\
\hline Ozegna & Vialfrè \\
\hline Parella & Vistrorio \\
\hline \multicolumn{2}{|l|}{ Pavone Canavese } \\
\hline \multicolumn{2}{|l|}{ Perosa Canavese } \\
\hline \multicolumn{2}{|l|}{ Pertusio } \\
\hline Pont Canavese & \\
\hline Quagliuzzo & \\
\hline
\end{tabular}




\section{APPENDIX VI}

\section{CENSUS of 1870}

\section{LEGEND}

DW: number of dwelling in order of visit.

FA: number of family in order of visit.

RE: real estate.

PE: personal estate.

BIRTH: nation of birth. 


\begin{tabular}{|c|c|c|c|c|c|c|c|c|c|c|c|c|c|}
\hline DW & FA & SURNAME & NAME & AGE & SEX & PROFESSION & $\mathrm{RE}$ & $\mathrm{PE}$ & BIRTH & FATHER FEREIGN & MOTHERFOREIGN & TOWNSHIP & PROVENIENCE \\
\hline 12 & 12 & CRAPPO/COPPO & GAETANO & 46 & $\mathrm{M}$ & WORKING TIMBER & 500 & 1000 & ITALY & YES & YES & BARAGA & TORINO \\
\hline \multirow[t]{6}{*}{136} & 144 & QUELLO & BARTOLEMEW & 30 & M & LABORER & 150 & 150 & ITALY & YES & YES & FRANKLIN & TORINO \\
\hline & & QUELLO & KATE & 25 & M & HOUSEKEEPER & & & IRELAND & YES & YES & FRANKLIN & IRELAND \\
\hline & & QUELLO & KATE & 6 & $\mathrm{~F}$ & & & & MICHIGAN & YES & YES & FRANKLIN & TORINO \\
\hline & & QUELLO & JOHN & 4 & M & & & & MICHIGAN & YES & YES & FRANKLIN & TORINO \\
\hline & & QUELLO & MARY & 2 & $\mathrm{~F}$ & & & & MICHIGAN & YES & YES & FRANKLIN & TORINO \\
\hline & & QUELLO & MICHAEL & 1 & M & & & & MICHIGAN & YES & YES & FRANKLIN & TORINO \\
\hline \multirow[t]{4}{*}{223} & 246 & COPPO & GITAN & 38 & M & LABORER & 800 & 3000 & ITALY & YES & YES & FRANKLIN & TORINO \\
\hline & & COPPO & CATHERINE & 34 & $\mathrm{~F}$ & & & & CANADA & YES & YES & FRANKLIN & CANADA \\
\hline & & COPPO & JOHN & 9 & M & & & & MICHIGAN & YES & YES & FRANKLIN & TORINO \\
\hline & & COPPO & PAUL & 32 & M & LABORER & & & ITALY & YES & YES & FRANKLIN & TORINO \\
\hline \multirow[t]{6}{*}{227} & 250 & PANTERA & JOSEPH & 36 & M & SHOEMAKER & & & ITALY & YES & YES & FRANKLIN & LUCCA \\
\hline & & PANTERA & LUCINDA & 35 & $\mathrm{~F}$ & & & & NEW YORK & NO (NEW YORK) & NO (NEW YORK) & FRANKLIN & USA \\
\hline & & PANTERA & EUGENE & $\begin{array}{l}50 \\
13\end{array}$ & M & & & & WI & YES & NO & FRANKLIN & LUCCA \\
\hline & & PANTERA & $\begin{array}{l}\text { CUGENVE } \\
\text { CHARLES }\end{array}$ & $\frac{13}{7}$ & $\mathrm{M}$ & & & & MICHIGAN & $\begin{array}{l}\text { YES } \\
\text { YES }\end{array}$ & NO & FRANKLIN & LUCCA \\
\hline & & PANTERA & FRANCES & 5 & M & & & & MICHIGAN & YES & NO & FRANKLIN & LUCCA \\
\hline & & PANTERA & MARIA T. & 1 & $\mathrm{~F}$ & & & & MICHIGAN & YES & NO & FRANKLIN & LUCCA \\
\hline \multirow[t]{4}{*}{39} & 43 & ADAMS & JOHN & 38 & M & SALOON KEEPER & 1200 & 400 & ITALY & YES & YES & HANCOCK & \\
\hline & & ADAMS & ANGELINA & 23 & $\mathrm{~F}$ & HOUSE KEEPING & & & FRANCE & YES & YES & HANCOCK & FRANCE \\
\hline & & ADAMS & RICHARD & 5 & M & & & & MICHIGAN & YES & YES & HANCOCK & \\
\hline & & ADAMS & IDA & 1 & $\mathrm{~F}$ & & & & MICHIGAN & YES & YES & HANCOCK & \\
\hline
\end{tabular}




\section{APPENDIX VII}

\section{CENSUS of 1880}

\section{LEGEND}

ID: counter.

DW: number of dwelling in order of visit.

FA: number of family in order of visit.

REL: relation of the person with the head of the household.

PROVENIENCE: place of origin in Italy. These data are not from the census, but form Ellis Island Database, Italian newspapers, and oral interviews. BORN: nation of birth of the person.

FATHER: nation of birth of the father. MOTHER: nation of birth of the mother. TOWNSHIP: township in which the person was living. 


\begin{tabular}{|c|c|c|c|c|c|c|c|c|c|c|c|c|c|}
\hline \begin{tabular}{|l|l|} 
D \\
\end{tabular} & DW & FA & SURNAME & NAME & REL & SE) & AGE & OCCUPATION & \begin{tabular}{|l|} 
PROVENIENCE \\
\end{tabular} & BORN & FATHER & MOTHER & TOWNSHIP \\
\hline 1 & 231 & 272 & COPPO & DOMINICK/GITAN & $\mathrm{H}$ & $M$ & 28 & MINER & TORINO & ITALY & ITALY & ITALY & FRANKLIN \\
\hline 2 & 231 & 272 & COPPO & CATERINA & W & $\mathrm{F}$ & 27 & KEEPING HOUSE & TORINO & ITALY & ITALY & ITALY & FRANKLIN \\
\hline 3 & 231 & 272 & COPPO & MARY & D & $\mathrm{F}$ & 3 & & TORINO & ITALY & ITALY & ITALY & FRANKLIN \\
\hline 4 & 231 & 272 & COPPO & JOHN & $\mathrm{S}$ & M & 1 & & TORINO & ITALY & ITALY & ITALY & FRANKLIN \\
\hline 5 & 231 & 272 & COPPO & ANTONIO & $\mathrm{BR}$ & M & 40 & MINE LABORER & TORINO & ITALY & ITALY & ITALY & FRANKLIN \\
\hline 6 & 231 & 272 & GIOVANO & GEORGE & B & M & 20 & MINE LABORER & & ITALY & ITALY & ITALY & FRANKLIN \\
\hline 7 & 231 & 272 & COPPO & PIETRO & B & M & 27 & MINE LABORER & TORINO & ITALY & ITALY & ITALY & FRANKLIN \\
\hline 8 & 231 & 272 & COPPO & ALLESIO & B & M & 41 & MINE LABORER & TORINO & ITALY & ITALY & ITALY & FRANKLIN \\
\hline 9 & 231 & 272 & COPPO & $\begin{array}{l}\text { LORENZO } \\
\text { LORE }\end{array}$ & $\frac{D}{B}$ & $\mathrm{M}$ & 33 & MINE LABORER & TORINO & ITALY & ITALY & ITALY & FRANKLIN \\
\hline 10 & 231 & 272 & MERTO & ACOLETTO & B & M & 23 & MINE LABORER & & ITALY & ITALY & ITALY & FRANKLIN \\
\hline 11 & 231 & 272 & BIAVA & IACOPO & B & M & 42 & MINE LABORER & VIALFRE'. TO & ITALY & ITALY & ITALY & FRANKLIN \\
\hline 12 & 231 & 272 & COPPO & MILTETTE & B & M & 43 & MINE LABORER & TORINO & ITALY & ITALY & ITALY & FRANKLIN \\
\hline 13 & 285 & 336 & MESENTI & JOSEPH & $\mathrm{B}$ & $M$ & 32 & $\begin{array}{l}\text { MINE LABORER } \\
\text { MIABER }\end{array}$ & & ITALY & ITALY & ITALY & FRANKLIN \\
\hline 14 & 285 & 336 & TREVELLA & JOSEPH & B & M & 19 & MINER & & ITALY & ITALY & ITALY & FRANKLIN \\
\hline 14 & 285 & 336 & GOVETT & THOMAS & $\mathrm{B}$ & M & 25 & MINE LABORER & & ITALY & ITALY & ITALY & FRANKLIN \\
\hline 16 & 285 & 336 & GEORGE & GEORGEWA & B & $\mathrm{M}$ & 20 & MINE LABORER & & ITALY & ITALY & ITALY & FRANKLIN \\
\hline 17 & 285 & 336 & TENNENT & MICHAEL & B & M & 44 & MINE LABORER & & ITALY & ITALY & ITALY & FRANKLIN \\
\hline 18 & 298 & 358 & PANTERA & JOSEPH & $\mathrm{H}$ & M & 46 & SHOEMAKER & LUCCA & ITALY & ITALY & ITALY & FRANKLIN \\
\hline 19 & 298 & 358 & PANTERA & MARIA & W & $\mathrm{F}$ & 45 & KEEPING HOUSE & USA & NEW YORK & NEW YORK & NEW YORK & FRANKLIN \\
\hline 20 & 298 & 358 & PANTERA & CHARLES & s & M & 17 & & LUCCA & WISCONSIN & ITALY & NEW YORK & FRANKLIN \\
\hline 21 & 298 & 358 & PANTERA & FRANK & $\mathrm{s}$ & M & 15 & & LUCCA & MICHIGAN & ITALY & NEW YORK & FRANKLIN \\
\hline 22 & 298 & 358 & PANTERA & MARY & $\mathrm{D}$ & $\mathrm{F}$ & 11 & & LUCCA & MICHIGAN & ITALY & NEW YORK & FRANKLIN \\
\hline$\frac{22}{23}$ & 298 & 358 & PANTERA & WILLIAM & $\frac{\mathrm{U}}{\mathrm{s}}$ & M & 8 & & LUCCA & MICHIGAN & ITALY & NEW YORK & $\begin{array}{l}\text { FRANKLIIN } \\
\text { FRANKLIN }\end{array}$ \\
\hline 24 & 298 & 358 & PANTERA & FREDERICK & $\mathrm{s}$ & M & 5 & & LUCCA & MICHIGAN & ITALY & NEW YORK & FRANKLIN \\
\hline 25 & 298 & 358 & PANTERA & CATHERINE & $\mathrm{D}$ & $\mathrm{F}$ & 4 & & LUCCA & MICHIGAN & ITALY & NEW YORK & FRANKLIN \\
\hline 26 & 298 & 358 & PANTERA & RAFFAELLO & BR & M & 28 & SHOEMAKER & LUCCA & ITALY & ITALY & ITALY & FRANKLIN \\
\hline 27 & 298 & 358 & BIANCHI & ANUGIATO & $\mathrm{B}$ & M & 55 & MINE LABORER & LUCCA & ITALY & ITALY & ITALY & FRANKLIN \\
\hline 28 & 298 & 358 & CHELLINI & GIOVANNI & B & M & 22 & MINE LABORER & LUCCA & ITALY & ITALY & ITALY & FRANKLIN \\
\hline 29 & 298 & 358 & OSTENZI & FRANCESCO & B & M & 30 & MINE LABORER & & ITALY & ITALY & ITALY & FRANKLIN \\
\hline 30 & 298 & 358 & MALFATTI & GIUSEPPE & B & M & 40 & MINE LABORER & LUCCA & ITALY & ITALY & ITALY & FRANKLIN \\
\hline 31 & 298 & 358 & VELLUTINI & GIUSEPPE & B & M & 23 & MINE LABORER & LUCCA & ITALY & ITALY & ITALY & FRANKLIN \\
\hline 32 & 298 & 359 & STEFANI & RAFAELLO & B & M & 25 & MINE LABORER & LUCCA & ITALY & ITALY & ITALY & FRANKLIN \\
\hline 33 & 298 & 359 & STEFANI & CASIMIRO & B & M & 22 & MINE LABORER & LUCCA & ITALY & ITALY & ITALY & FRANKLIN \\
\hline 34 & 298 & 359 & LAZZARI & GISBERTO & $\mathrm{B}$ & M & 21 & MINE LABORER & LUCCA & ITALY & ITALY & ITALY & FRANKLIN \\
\hline 35 & 298 & 359 & PALAGI & SABASTIANO & B & $\mathrm{M}$ & 28 & MINE LABORER & LUCCA & ITALY & ITALY & ITALY & FRANKLIN \\
\hline 36 & 313 & 390 & LENCIONI & NELSON & B & M & 34 & MASON & LUCCA & ITALY & ITALY & ITALY & FRANKLIN \\
\hline $\begin{array}{l}36 \\
37\end{array}$ & 313 & $\begin{array}{l}390 \\
390\end{array}$ & $\begin{array}{l}\text { LENCIUNI } \\
\text { VELLUTINI }\end{array}$ & $\begin{array}{l}\text { NOSEPH } \\
\text { JOSE }\end{array}$ & $\begin{array}{l}\mathrm{B} \\
\mathrm{B}\end{array}$ & $\frac{M}{M}$ & $\begin{array}{l}34 \\
22\end{array}$ & MINE LABORER & LUCCA & $\begin{array}{l}\text { IIALY } \\
\text { ITALY }\end{array}$ & $\begin{array}{l}\text { IIALY } \\
\text { ITALY }\end{array}$ & $\begin{array}{l}\text { ITALY } \\
\text { ITAL }\end{array}$ & $\begin{array}{l}\text { FRANKLIN } \\
\text { FRANKLIN }\end{array}$ \\
\hline 38 & 313 & 390 & GALLIONI & JOSEPH & B & M & 23 & MINE LABORER & & ITALY & ITALY & ITALY & FRANKLIN \\
\hline 39 & 313 & 390 & PALAGI & BASTIAN & B & M & 26 & MINE LABORER & LUCCA & ITALY & ITALY & ITALY & FRANKLIN \\
\hline 40 & 313 & 390 & CHELLINI & JOHN & B & M & 21 & MINE LABORER & LUCCA & ITALY & ITALY & ITALY & FRANKLIN \\
\hline 41 & 313 & 390 & STEFANI & HORATIO & B & M & 25 & MINE LABORER & LUCCA & ITALY & ITALY & ITALY & FRANKLIN \\
\hline 42 & 313 & 390 & LAZZARI & GIBO & B & M & 23 & MINE LABORER & LUCCA & ITALY & ITALY & ITALY & FRANKLIN \\
\hline 43 & 359 & 447 & DELL'ANGELICA & VINCENZO & $\mathrm{H}$ & M & 25 & MINE LABORER & LUCCA & ITALY & ITALY & ITALY & FRANKLIN \\
\hline 44 & 359 & 447 & DELL'ANGELICA & ROSA & W & $\mathrm{F}$ & 27 & STONE MASON & LUCCA & ITALY & ITALY & ITALY & FRANKLIN \\
\hline 45 & 359 & 447 & DELL'ANGELICA & MALANI & $\mathrm{D}$ & $\mathrm{F}$ & 2 & MINE LABORER & LUCCA & ITALY & ITALY & ITALY & FRANKLIN \\
\hline 46 & 359 & 447 & DELL'ANGELICA & MICHAEL & BR & M & 35 & MINE LABORER & LUCCA & ITALY & ITALY & ITALY & FRANKLIN \\
\hline 47 & 359 & 447 & LISSI & MARTIN & B & M & 34 & MINE LABORER & & ITALY & ITALY & ITALY & FRANKLIN \\
\hline 48 & 359 & 447 & GALINI & JOSEPH & B & $\mathrm{M}$ & 24 & MINER & & ITALY & ITALY & ITALY & FRANKLIN \\
\hline 49 & 359 & 447 & GALINI & JAN & B & $\mathrm{M}$ & 24 & MINER & & ITALY & ITALY & ITALY & FRANKLIN \\
\hline $\begin{array}{l}49 \\
50\end{array}$ & $\begin{array}{l}359 \\
359\end{array}$ & $\begin{array}{l}44 ! \\
447\end{array}$ & $\begin{array}{l}\text { GALINI } \\
\text { COLLI }\end{array}$ & $\begin{array}{l}\text { JAN } \\
\text { PETER }\end{array}$ & $\begin{array}{l}\mathrm{B} \\
\mathrm{B}\end{array}$ & $\frac{M}{M}$ & 20 & MINE LABORER & & $\begin{array}{l}\text { IIALY } \\
\text { ITALY }\end{array}$ & $\begin{array}{l}\text { IIALY } \\
\text { ITALY }\end{array}$ & $\begin{array}{l}\text { ITALY } \\
\text { ITALY }\end{array}$ & $\begin{array}{l}\text { FRANKLIN } \\
\text { FRANKLIN }\end{array}$ \\
\hline 51 & 359 & 447 & LENZI/LENCI & NARCISO & B & $\mathrm{M}$ & 34 & STONE MASON & LUCCA & ITALY & ITALY & ITALY & FRANKLIN \\
\hline 52 & 359 & 447 & STEFANI & ORAZIO & B & M & 27 & MINE LABORER & LUCCA & ITALY & ITALY & ITALY & FRANKLIN \\
\hline 53 & 359 & 447 & CESARI & JOSEPH & B & $M$ & 35 & MINE LABORER & LUCCA & ITALY & ITALY & ITALY & FRANKLIN \\
\hline 54 & 359 & 447 & GALOURI & ANGELI & B & M & 27 & MINE LABORER & & ITALY & ITALY & ITALY & FRANKLIN \\
\hline 55 & 135 & 181 & YOTTI & ANGELO & $\mathrm{H}$ & M & 30 & MINER & & ITALY & $\begin{array}{l}\text { ITALY } \\
\end{array}$ & ITALY & QUINCY \\
\hline 56 & 135 & 181 & YOTTI & DELPHINA & w & $\mathrm{F}$ & 17 & KEEPING HOUSE & CANADA & CANADA & CANADA & CANADA & QUINCY \\
\hline 57 & 135 & 181 & YOTTI & ANGELO & s & M & $10 \mathrm{M}$ & & & MICHIGAN & ITALY & CANADA & QUINCY \\
\hline 58 & 135 & 181 & GAVER & PETER & B & M & 30 & MINER & & ITALY & ITALY & ITALY & QUINCY \\
\hline 59 & 135 & 181 & CONTHARIS & PETER & B & M & 28 & MINER & & ITALY & ITALY & ITALY & QUINCY \\
\hline 60 & 135 & 181 & PALAS & AUGUST & $B$ & $M$ & 35 & MINER & & ITALY & ITALY & ITALY & QUINCY \\
\hline 61 & 135 & 181 & MARTINELLO & ABRAM & B & $\mathrm{M}$ & 24 & MINER & & ITALY & ITALY & ITALY & QUINCY \\
\hline 62 & 20 & 29 & DIGHERA & DENNIS & B & M & 31 & LABORER & TORINO & ITALY & ITALY & ITALY & CALUMET 2ND PRECINCT \\
\hline
\end{tabular}




\begin{tabular}{|c|c|c|c|c|c|c|c|c|c|c|c|c|c|}
\hline 63 & 27 & 35 & TINETTI & ANTON & B & M & 35 & LABORER & SAN MARTINO CANAVESE. TO & ITALY & ITALY & ITALY & CALUMET 2ND PRECINCT \\
\hline 64 & 34 & 43 & TORREANO & JOHN & $\mathrm{H}$ & M & 44 & LABORER & AGLIE'. TO & ITALY & ITALY & ITALY & CALUMET 2ND PRECINCT \\
\hline 65 & 34 & 43 & TORREANO & VITTORIA & W & $\mathrm{F}$ & 37 & KEEPING HOUSE & AGLIE'. TO & ITALY & ITALY & ITALY & CALUMET 2ND PRECINCT \\
\hline 66 & 34 & 43 & TORREANO & MARIE & $\mathrm{D}$ & $\mathrm{F}$ & 12 & & AGLIE'. TO & ITALY & ITALY & ITALY & CALUMET 2ND PRECINCT \\
\hline 67 & 34 & 43 & TORREANO & FRANK & s & M & 9 & & AGLIE'. TO & ITALY & ITALY & ITALY & CALUMET 2ND PRECINCT \\
\hline 68 & 34 & 43 & TORREANO & JOSEPH & $\mathrm{s}$ & M & 4 & & AGLIE', TO & MICHIGAN & ITALY & ITALY & CALUMET 2ND PRECINCT \\
\hline 69 & 34 & 43 & TORREANO & KATE & $\mathrm{D}$ & $\mathrm{F}$ & 2 & & AGLIE', TO & MICHIGAN & ITALY & ITALY & CALUMET 2ND PRECINCT \\
\hline 70 & 34 & 43 & CESARI & JOSEPH & B & M & 28 & LABORER & TORINO & ITALY & ITALY & ITALY & CALUMET 2ND PRECINCT \\
\hline 71 & 34 & 43 & CESARI & MARTIN & $\begin{array}{l}\mathrm{D} \\
\mathrm{B}\end{array}$ & $\mathrm{M}$ & 50 & LABORER & TORINO & ITALY & ITALY & $\begin{array}{l}\text { ITALY } \\
\text { ITAL }\end{array}$ & CALUMET 2ND PRECINCT \\
\hline 72 & 34 & 43 & BAGGIORE & PETER & B & M & 28 & LABORER & TORINO & ITALY & ITALY & ITALY & CALUMET 2ND PRECINCT \\
\hline 73 & 34 & 43 & MESSIEO & LAURENCE & B & M & 41 & LABORER & & ITALY & ITALY & ITALY & CALUMET 2ND PRECINCT \\
\hline 74 & 34 & 43 & BESSOLO & BAPTISTE & B & M & 33 & LABORER & TORINO & ITALY & ITALY & ITALY & CALUMET 2ND PRECINCT \\
\hline 75 & 34 & 43 & BESSOLO & CHARLES & B & M & 55 & LABORER & TORINO & ITALY & ITALY & ITALY & CALUMET 2ND PRECINCT \\
\hline 76 & 34 & 43 & BERATORI & $\mathrm{MICH}$. & B & M & 26 & LABORER & TORINO & ITALY & ITALY & ITALY & CALUMET 2ND PRECINCT \\
\hline 77 & 482 & 618 & CARLOTTI & MICHAEL & $\mathrm{H}$ & M & 37 & MINER & LUCCA & ITALY & ITALY & ITALY & CALUMET 2ND PRECINCT \\
\hline 78 & 482 & 618 & CARLOTTI & MARY & W & $\mathrm{F}$ & 37 & KEEPING HOUSE & LUCCA & ITALY & ITALY & ITALY & CALUMET 2ND PRECINCT \\
\hline 79 & 482 & 618 & CARLOTTI & MARY & $\mathrm{D}$ & $\mathrm{F}$ & 12 & & LUCCA & ITALY & ITALY & ITALY & CALUMET 2ND PRECINCT \\
\hline 80 & 482 & 618 & CARLOTTI & FRANK & $\mathrm{s}$ & M & 3 & & LUCCA & MICHIGAN & ITALY & ITALY & CALUMET 2ND PRECINCT \\
\hline 81 & 482 & 618 & CARLOTTI & MICHAEL & $\mathrm{s}$ & M & 6 & & LUCCA & ITALY & ITALY & ITALY & CALUMET 2ND PRECINCT \\
\hline 82 & 482 & 618 & CARLOTTI & AMIDE & $\mathrm{s}$ & M & 8 & & LUCCA & ITALY & ITALY & ITALY & CALUMET 2ND PRECINCT \\
\hline 83 & 482 & 618 & BRICO & PETER & B & M & 35 & LABORER & LULCA & ITALY & ITALY & $\begin{array}{l}\text { ITALY } \\
\end{array}$ & CALUMET 2ND PRECINCT \\
\hline 84 & 537 & 678 & CURTO & MARCO & $\mathrm{H}$ & M & 24 & MINER & TORINO & ITALY & ITALY & ITALY & CALUMET 2ND PRECINCT \\
\hline 85 & 537 & 678 & CURTO & ANGELA & W & $\mathrm{F}$ & 20 & KEEPING HOUSE & TORINO & ITALY & ITALY & ITALY & CALUMET 2ND PRECINCT \\
\hline 86 & 537 & 678 & CURTO & JACOB & $\mathrm{BR}$ & M & 20 & LABORER & TORINO & ITALY & ITALY & ITALY & CALUMET 2ND PRECINCT \\
\hline 87 & 537 & 678 & GUGLIELMINO & ANTONIO & B & M & 34 & MINER & TORINO & ITALY & ITALY & ITALY & CALUMET 2ND PRECINCT \\
\hline 88 & 537 & 678 & GUGLIELMINO & DOMINICK & B & M & 32 & MINER & TORINO & ITALY & ITALY & ITALY & CALUMET 2ND PRECINCT \\
\hline 89 & 537 & 678 & POLA & DOMINICO & B & M & 45 & LABORER & & ITALY & ITALY & ITALY & CALUMET 2ND PRECINCT \\
\hline 90 & 26 & 32 & MEINARDI & DOMINICK & $\mathrm{H}$ & M & 30 & KEEPING SALOON & TORINO & ITALY & ITALY & ITALY & CALUMET 1ST PRECINCT - RED JACKET \\
\hline 91 & 26 & 33 & PASQUINELLI & LEONE & $\mathrm{H}$ & M & 31 & MINER & LUCCA & ITALY & ITALY & ITALY & CALUMET 1ST PRECINCT - RED JACKET \\
\hline 92 & 26 & 33 & PASQUINELLI & PIA & W & $\mathrm{F}$ & 24 & KEEPING HOUSE & LUCCA & ITALY & ITALY & ITALY & CALUMET 1ST PRECINCT - RED JACKET \\
\hline 93 & 26 & 33 & PASQUINELLI & ELIZA & $\mathrm{D}$ & M & 1 & & LUCCA & MICHIGAN & ITALY & ITALY & CALUMET 1ST PRECINCT - RED JACKET \\
\hline 94 & 26 & 34 & QUELLO & BARTHOLOMEW & $\mathrm{H}$ & M & 45 & FARMER \& CONTRACTOR & LOCANA CANAVESE. TO & ITALY & ITALY & ITALY & CALUMET 1ST PRECINCT - RED JACKET \\
\hline 95 & 26 & 34 & QUELLO & KATIE & W & $\mathrm{F}$ & 35 & KEEPING HOUSE & $\begin{array}{l}\text { IRELAND } \\
\text { IRIVAVEL. }\end{array}$ & IRELAND & IRELAND & IRELAND & CALUMET 1ST PRECINCT - RED JACKET \\
\hline 96 & 26 & 34 & QUELLO & KATIE & $\mathrm{D}$ & $\mathrm{F}$ & 16 & AT SCHOOL & LOCANA CANAVESE, TO & MICHIGAN & ITALY & IRELAND & CALUMET 1ST PRECINCT - RED JACKET \\
\hline 97 & 26 & 34 & QUELLO & JOHN & $\mathrm{s}$ & M & 14 & AT SCHOOL & LOCANA CANAVESE. TO & MICHIGAN & ITALY & IRELAND & CALUMET IST PRECINCT - RED JACKET \\
\hline 98 & 26 & 34 & QUELLO & MARY & $\mathrm{D}$ & $\mathrm{F}$ & 12 & AT SCHOOL & LOCANA CANAVESE. TO & MICHIGAN & ITALY & IRELAND & CALUMET 1ST PRECINCT - RED JACKET \\
\hline 99 & 26 & 34 & QUELLO & MICHAEL & $\mathrm{s}$ & M & 10 & AT SCHOOL & LOCANA CANAVESE. TO & MICHIGAN & ITALY & IRELAND & CALUMET 1ST PRECINCT - RED JACKET \\
\hline 100 & 26 & 34 & QUELLO & MARGRET & $\mathrm{D}$ & $\mathrm{F}$ & 8 & AT SCHOOL & LOCANA CANAVESE. TO & MICHIGAN & ITALY & IRELAND & CALUMET 1ST PRECINCT - RED JACKET \\
\hline 101 & 26 & 34 & QUELLO & BARTHOLOMEW & $\mathrm{s}$ & M & 5 & & LOCANA CANAVESE. TO & MICHIGAN & ITALY & IRELAND & CALUMET 1ST PRECINCT - RED JACKET \\
\hline 102 & 26 & 34 & QUELLO & ANNA & $\mathrm{D}$ & $\mathrm{F}$ & 3 & & LOCANA CANAVESE. TO & MICHIGAN & ITALY & IRELAND & CALUMET 1ST PRECINCT - RED JACKET \\
\hline 103 & 26 & 34 & QUELLO & LIZZIE & D & $\mathrm{F}$ & 1 & & LOCANA CANAVESE. TO & MICHIGAN & ITALY & IRELAND & CALUMET 1ST PRECINCT - RED JACKET \\
\hline 104 & 36 & 48 & BORGO & MICHAEL & $\mathrm{H}$ & M & 36 & KEEPING SALOON & VICENZA & ITALY & ITALY & ITALY & CALUMET 1ST PRECINCT - RED JACKET \\
\hline 105 & 36 & 48 & BORGO & KATHERINE & w & $\mathrm{F}$ & 24 & KEEPING HOUSE & IRELAND & IRELAND & IRELAND & IRELAND & CALUMET 1ST PRECINCT - RED JACKET \\
\hline 106 & 36 & 48 & BORGO & JOSEPHINA & $\mathrm{D}$ & $\mathrm{F}$ & 7 & AT SCHOOL & VICENZA & MICHIGAN & ITALY & IRELAND & CALUMET 1ST PRECINCT - RED JACKET \\
\hline 107 & 36 & 48 & BORGO & CESARE & $\mathrm{S}$ & M & 1 & & VICENZA & MICHIGAN & ITALY & IRELAND & CALUMET 1ST PRECINCT - RED JACKET \\
\hline 108 & 43 & 55 & CARAGLIOTTI & LOUIE & $\mathrm{H}$ & M & 52 & KEEPING SALOON & TORINO & ITALY & ITALY & ITALY & CALUMET 1ST PRECINCT - RED JACKET \\
\hline 109 & 43 & 55 & CARAGLIOTTI & MARY & w & $\mathrm{F}$ & 46 & KEEPING HOUSE & TORINO & ITALY & ITALY & ITALY & CALUMET 1ST PRECINCT - RED JACKET \\
\hline 110 & 65 & 82 & BATTISTI & PETER & $\mathrm{H}$ & M & 42 & KEEPING SALOON & & ITALY & ITALY & ITALY & CALUMET 1ST PRECINCT - RED JACKET \\
\hline 111 & 65 & 82 & BATTISTI & NANCETTE & w & $\mathrm{F}$ & 30 & KEEPING SALOON & & ITALY & ITALY & ITALY & CALUMET IST PRECINCT - RED JACKET \\
\hline 112 & 65 & 82 & BATTISTI & LENA & S/D? & $\mathrm{F}$ & 4 & & & MICHIGAN & ITALY & ITALY & CALUMET 1ST PRECINCT - RED JACKET \\
\hline 113 & 65 & 82 & JONTENE & ANDREW & B & M & 32 & MINER & & ITALY & ITALY & ITALY & CALUMET 1ST PRECINCT - RED JACKET \\
\hline 114 & 65 & 82 & GARENO & GEORGE & B & M & 26 & MINER & & ITALY & ITALY & ITALY & CALUMET 1ST PRECINCT - RED JACKET \\
\hline 115 & 98 & 141 & LISA & JAMES & $\mathrm{H}$ & M & 35 & CLERK IN STORE & SAN MARTINO CANAVESE. TO & ITALY & ITALY & ITALY & CALUMET 1ST PRECINCT - RED JACKET \\
\hline 116 & 98 & 141 & LISA & MARTHA E. & W & $\mathrm{F}$ & 28 & KEEPING HOUSE & SAN MARTINO CANAVESE. TO & ITALY & ITALY & ITALY & CALUMET 1ST PRECINCT - RED JACKET \\
\hline 117 & 98 & 141 & LISA & COSTAN E. & S/D? & M & 8 & & SAN MARTINO CANAVESE. TO & ITALY & ITALY & ITALY & CALUMET 1ST PRECINCT - RED JACKET \\
\hline 118 & 98 & 141 & LISA & JOSEPHINE & $\mathrm{D}$ & $\mathrm{F}$ & 6 & & SAN MARTINO CANAVESE. TO & ITALY & ITALY & ITALY & CALUMET 1ST PRECINCT - RED JACKET \\
\hline 119 & 98 & 141 & LISA & JULIA & $\mathrm{D}$ & $\mathrm{F}$ & $7 \mathrm{M}$ & & SAN MARTINO CANAVESE. TO & MICHIGAN & ITALY & ITALY & CALUMET 1ST PRECINCT - RED JACKET \\
\hline 120 & 98 & 141 & LISA & SAVIN & $\mathrm{BR}$ & M & 22 & MINE TIMBERMAN & SAN MARTINO CANAVESE, TO & ITALY & ITALY & ITALY & CALUMET 1ST PRECINCT - RED JACKET \\
\hline 121 & 101 & 149 & CHELI & PETER & $\mathrm{H}$ & M & 40 & MINER & LUCCA & ITALY & ITALY & ITALY & CALUMET 1ST PRECINCT - RED JACKET \\
\hline 122 & 101 & 149 & CHELI & CASIMIRA & W & $\mathrm{F}$ & 31 & KEEPING HOUSE & LUCCA & ITALY & ITALY & ITALY & CALUMET 1ST PRECINCT - RED JACKET \\
\hline 123 & 101 & 149 & CHELI & FREDDIE & $\mathrm{s}$ & M & 5 & & LUCCA & MIC & ITALY & ITALY & CALUMET 1ST PRECINCT - RED JACKET \\
\hline 124 & 101 & 149 & $\mathrm{CHE}$ & LAURENCE & $\mathrm{s}$ & M & 1 & & LUCCA & MICHIGAN & ITALY & ITALY & CALUMET 1ST P \\
\hline 125 & 101 & 149 & BANDONI & JOHN & B & M & 26 & LABORER & LUCCA & ITALY & ITALY & ITALY & CALUMET 1ST PRECINCT - RED JACKET \\
\hline
\end{tabular}




\begin{tabular}{|c|c|c|c|c|c|c|c|c|c|c|c|c|c|}
\hline 126 & 101 & 149 & BANDONI & DAVID & B & M & 28 & LABORER & LUCCA & ITALY & ITALY & ITALY & CALUMET 1ST PRECINCT - RED JACKET \\
\hline 127 & 101 & 149 & CHELI & ANTHONY & B & M & 35 & LABORER & LUCCA & ITALY & ITALY & ITALY & CALUMET IST PRECINCT - RED JACKET \\
\hline 128 & 101 & 149 & CHELI & LAURENCE & B & M & 30 & LABORER & LUCCA & ITALY & ITALY & ITALY & CALUMET 1ST PRECINCT - RED JACKET \\
\hline 129 & 101 & 149 & MICHELINI & CESARE & B & M & 32 & LABORER & LUCCA & ITALY & ITALY & ITALY & CALUMET 1ST PRECINCT - RED JACKET \\
\hline 130 & 101 & 149 & CIOMEI/TOMEI & BERNARD & B & M & 25 & LABORER & LUCCA & ITALY & ITALY & ITALY & CALUMET IST PRECINCT - RED JACKET \\
\hline 131 & 102 & 150 & DE FILIPPI & FRANK & $\mathrm{H}$ & M & 37 & LABORER & SAN GIORGIO CANAVESE. TO & ITALY & ITALY & ITALY & CALUMET 1ST PRECINCT - RED JACKET \\
\hline 132 & 102 & 150 & DE FILIPPI & DOMENICA & W & $\mathrm{F}$ & 24 & KEEPING HOUSE & SAN GIORGIO CANAVESE. TO & ITALY & ITALY & ITALY & CALUMET 1ST PRECINCT - RED JACKET \\
\hline 133 & 102 & 150 & DE FILIPPI & DOMENICO & $\mathrm{s}$ & M & 1 & & SAN GIORGIO CANAVESE. TO & ITALY & ITALY & ITALY & CALUMET 1ST PRECINCT - RED JACKET \\
\hline 134 & 102 & 150 & BINANDO & JOSEPH & B & M & 26 & LABORER & TORINO & ITALY & ITALY & ITALY & CALUMET 1ST PRECINCT - RED JACKET \\
\hline 135 & 102 & 150 & DE FILIPPI & PETER & $\mathrm{B}$ & M & 30 & LABORER & TORINO & ITALY & ITALY & ITALY & CALUMET 1ST PRECINCT - RED JACKET \\
\hline 136 & 102 & 150 & NIGRO/NIGRA & MIKE & B & M & 41 & MINER & TORINO & ITALY & ITALY & ITALY & CALUMET 1ST PRECINCT - RED JACKET \\
\hline 137 & 102 & 150 & MIGLIA/MEAGLIO & CHARLES & B & M & 27 & LABORER & TORINO & ITALY & ITALY & ITALY & CALUMET 1ST PRECINCT - RED JACKET \\
\hline 138 & 102 & 150 & JANTA & JOSEPH & B & M & 31 & LABORER & & ITALY & ITALY & ITALY & CALUMET 1ST PRECINCT - RED JACKET \\
\hline 139 & 102 & 150 & DE FILIPPI & JAMES & B & M & 31 & LABORER & TORINO & ITALY & ITALY & ITALY & CALUMET 1ST PRECINCT - RED JACKET \\
\hline 140 & 102 & 150 & DE FILIPPI & DOMENICO & B & M & 33 & LABORER & TORINO & ITALY & ITALY & ITALY & CALUMET 1ST PRECINCT - RED JACKET \\
\hline 141 & 102 & 150 & NIGRO/NIGRA & JOHN & B & M & 38 & LABORER & TORINO & ITALY & ITALY & ITALY & CALUMET 1ST PRECINCT - RED JACKET \\
\hline 142 & 103 & 151 & DATA/DATTA & JOSEPH & $\mathrm{H}$ & M & 32 & LABORER & TORINO & ITALY & ITALY & ITALY & $\begin{array}{l}\text { CALUMET IST PRECINCT - RED JACKET } \\
\text { T RET }\end{array}$ \\
\hline 143 & 103 & 151 & DATA/DATTA & KATIE & W & $\mathrm{F}$ & 23 & KEEPING HOUSE & TORINO & MICHIGAN & ITALY & ITALY & CALUMET 1ST PRECINCT - RED JACKET \\
\hline 144 & 103 & 151 & DATA/DATTA & MARTHA & $\mathrm{D}$ & $\mathrm{F}$ & 2 & & TORINO & ITALY & ITALY & ITALY & CALUMET 1ST PRECINCT - RED JACKET \\
\hline 145 & 124 & 185 & PEDRETT & SALVESTER & $\mathrm{H}$ & M & 23 & CLERK IN STORE & & ITALY & ITALY & ITALY & CALUMET IST PRECINCT - RED JACKET \\
\hline 146 & 124 & 185 & PEDRETT & MARY & W & $\mathrm{F}$ & 22 & KEEPING HOUSE & IRELAND & MICHIGAN & IRELAND & IRELAND & CALUMET 1ST PRECINCT - RED JACKET \\
\hline 147 & 124 & 185 & PEDRETT & SALVESTER & $\mathrm{s}$ & M & 1 & & & MICHIGAN & ITALY & MICHIGAN & CALUMET 1ST PRECINCT - RED JACKET \\
\hline 148 & 124 & 185 & PEDRETT & JOHNEY & $\mathrm{S}$ & M & $1 \mathrm{M}$ & & & MICHIGAN & ITALY & MICHIGAN & CALUMET 1ST PRECINCT - RED JACKET \\
\hline 149 & 144 & 215 & LENZI & SIELE & $\mathrm{H}$ & M & 31 & KEEPING SALOON & LUCCA & ITALY & ITALY & ITALY & CALUMET 1ST PRECINCT - RED JACKET \\
\hline 150 & 144 & 215 & LENZI & MARY ANN & w & $\mathrm{F}$ & 37 & KEEPING HOUSE & FRANCE & FRANCE & FRANCE & FRANCE & CALUMET 1ST PRECINCT - RED JACKET \\
\hline 151 & 144 & 215 & LENZI & MARY L. & D & $\mathrm{F}$ & 3 & REEFIINU TUUSE & $\begin{array}{l}\text { LUCCA } \\
\text { LUCC }\end{array}$ & MICHIGAN & ITALY & FRANCE & CALUMET 1ST PRECINCT - RED JACKET \\
\hline 152 & 144 & 215 & LENZI & ANTONETTE & $\mathrm{D}$ & $\mathrm{F}$ & $5 \mathrm{M}$ & & LUCCA & MICHIGAN & ITALY & FRANCE & CALUMET 1ST PRECINCT - RED JACKET \\
\hline 153 & 144 & 215 & MASCO & DOMMICK & B & $\mathrm{F}$ & 35 & LABORER & & ITALY & ITALY & ITALY & CALUMET 1ST PRECINCT - RED JACKET \\
\hline 154 & 144 & 215 & MASCO & JOHN & B & $\mathrm{F}$ & 27 & MINER & & ITALY & ITALY & ITALY & $\begin{array}{l}\text { CALUMET IST PRECINCT - RED JACKET } \\
\text { C RET }\end{array}$ \\
\hline 155 & 144 & 215 & PENOLY & PETER & B & $\mathrm{F}$ & 25 & LABORER & & ITALY & ITALY & ITALY & CALUMET 1ST PRECINCT - RED JACKET \\
\hline 156 & 144 & 215 & WETONEY & FREDK & B & $\mathrm{F}$ & 30 & LABORER & & ITALY & ITALY & ITALY & CALUMET 1ST PRECINCT - RED JACKET \\
\hline 157 & 144 & 215 & BENEDIC & JOHN & B & $\mathrm{F}$ & 35 & LABORER & & ITALY & ITALY & ITALY & CALUMET 1ST PRECINCT - RED JACKET \\
\hline 158 & 151 & 226 & VACCHIERI & DOMINIC & $\mathrm{H}$ & M & 21 & LABORER & RIVAROLO. TO & ITALY & ITALY & ITALY & CALUMET 1ST PRECINCT - RED JACKET \\
\hline 159 & 151 & 226 & VACCHIERI & LENA & W & $\mathrm{F}$ & 15 & & RIVAROLO, TO & ITALY & ITALY & ITALY & CALUMET 1ST PRECINCT - RED JACKET \\
\hline 160 & 154 & 232 & MALIO & JAMES & $\mathrm{H}$ & M & 32 & LABORER & & ITALY & ITALY & ITALY & CALUMET 1ST PRECINCT - RED JACKET \\
\hline 161 & 154 & 232 & MALIO & LENA & w & $\mathrm{F}$ & 27 & KEEPING HOUSE & & ITALY & ITALY & ITALY & CALUMET 1ST PRECINCT - RED JACKET \\
\hline 162 & 154 & 232 & CASTATTANO & JACOB & B & M & 30 & MINER & & ITALY & ITALY & ITALY & CALUMET 1ST PRECINCT - RED JACKET \\
\hline 163 & 154 & 232 & MALINO & FRANK & $\frac{D}{B}$ & M & $29 ?$ & LABORER & & ITALY & ITALY & $\begin{array}{l}\text { ITALY } \\
\text { ITA }-19\end{array}$ & CALUMET 1ST PRECINCT - RED JACKET \\
\hline 164 & 154 & 232 & MALINO & MIKE & B & M & 33 & LABORER & & ITALY & ITALY & ITALY & CALUMET 1ST PRECINCT - RED JACKET \\
\hline 165 & 154 & 232 & MALINO & PETER & B & M & $24 ?$ & LABORER & & ITALY & ITALY & ITALY & CALUMET 1ST PRECINCT - RED JACKET \\
\hline 166 & 154 & 232 & PETERANE & PETER & B & M & 36 & LABORER & & ITALY & ITALY & ITALY & CALUMET 1ST PRECINCT - RED JACKET \\
\hline 167 & 154 & 232 & PAOGAONO & JACOB & B & M & 39 & LABORER & & ITALY & ITALY & ITALY & CALUMET 1ST PRECINCT - RED JACKET \\
\hline 168 & 154 & 232 & BASSO & JAMES & B & M & 34 & LABORER & TORINO & ITALY & ITALY & ITALY & CALUMET 1ST PRECINCT - RED JACKET \\
\hline 169 & 175 & 283 & BRACCO & CARLO & $\mathrm{H}$ & M & 37 & KEEPING & TORINO & ITALY & ITALY & ITALY & CALUMET 1ST PRECINCT - RED JACKET \\
\hline 170 & 175 & 283 & BRACCO & MARY & W & $\mathrm{F}$ & 40 & KEEPING HOUSE & TORINO & ITALY & ITALY & ITALY & CALUMET IST PRECINCT - RED JACKET \\
\hline 171 & 175 & 283 & BRACCO & ROSA & D & $\mathrm{F}$ & 10 & AT SCHOOL & TORINO & ITALY & ITALY & ITALY & CALUMET 1ST PRECINCT - RED JACKET \\
\hline 172 & 175 & 283 & GIANDRONE & BATTISTA & B & M & 27 & LABORER & TORINO & ITALY & ITALY & ITALY & CALUMET 1ST PRECINCT - RED JACKET \\
\hline 173 & 175 & 283 & ROSSIO & NATALE & B & M & 20 & LABORER & TORINO & ITALY & ITALY & ITALY & CALUMET 1ST PRECINCT - RED JACKET \\
\hline 174 & 175 & 283 & MICHETTI & VINCENZO & B & M & 27 & LABORER & PONT CANAVESE. TO & ITALY & ITALY & ITALY & CALUMET 1ST PRECINCT - RED JACKET \\
\hline 175 & 175 & 283 & RASTELLO & PETER & B & M & 16 & AT SCHOOL & TORINO & ITALY & ITALY & ITALY & CALUMET 1ST PRECINCT - RED JACKET \\
\hline 176 & 176 & 284 & COPPO & JOHN & $\mathrm{H}$ & M & 35 & MINER & TORINO & ITALY & ITALY & ITALY & CALUMET 1ST PRECINCT - RED JACKET \\
\hline 177 & 176 & 284 & COPPO & MADDALENA & W & $\mathrm{F}$ & 27 & KEEPING HOUSE & TORINO & ITALY & ITALY & ITALY & CALUMET 1ST PRECINCT - RED JACKET \\
\hline 178 & 176 & 284 & COPPO & LENA & D & $\mathrm{F}$ & 3 & & TORINO & ITALY & ITALY & ITALY & CALUMET 1ST PRECINCT - RED JACKET \\
\hline 179 & 176 & 284 & COPPO & JOHN BAPTISTE & $\mathrm{s}$ & M & $8 \mathrm{M}$ & & TORINO & ITALY & ITALY & ITALY & CALUMET 1ST PRECINCT - RED JACKET \\
\hline 180 & 177 & 285 & COPPO & VITALE & $\mathrm{H}$ & M & 37 & KEEPING & TORINO & ITALY & ITALY & ITALY & CALUMET 1ST PRECINCT - RED JACKET \\
\hline 181 & 177 & 285 & COPPO & MARYL. & w & $\mathrm{F}$ & 36 & KEEPING HOUSE & TORINO & ITALY & ITALY & ITALY & CALUMET 1ST PRECINCT - RED JACKET \\
\hline 182 & 177 & 285 & COPPO & KATIE & D & $\mathrm{F}$ & 11 & AT SCHOOL & TORINO & ITALY & ITALY & ITALY & CALUMET 1ST PRECINCT - RED JACKET \\
\hline 183 & 177 & 285 & COPPO & JOSEPH & $\mathrm{S}$ & M & 9 & & TORINO & ITALY & ITALY & ITALY & CALUMET 1ST PRECINCT - RED JACKET \\
\hline 184 & 177 & 285 & COPPO & ANTHONY & $\mathrm{s}$ & M & 6 & & TORINO & ITALY & ITALY & ITALY & CALUMET 1ST PRECINCT - RED JACKET \\
\hline 185 & 177 & 285 & COPPO & $\mathrm{JOHN}$ & $\mathrm{s}$ & M & 5 & & TORINO & ITALY & ITALY & ITALY & CALUMET 1ST PRECINCT - RED JACKET \\
\hline 186 & 177 & 285 & COPPO & ROSIE & D & $\mathrm{F}$ & 2 & & TORINO & ITALY & ITALY & ITALY & CALUMET 1ST PRECINCT - RED JACKET \\
\hline 187 & 177 & 285 & COPPO & PETER & B & M & 27 & LABORER & TORINO & ITALY & ITALY & ITALY & CALUMET 1ST PRECINCT - RED JACKET \\
\hline 188 & 177 & 285 & COPPO & AUGUST & B & M & 31 & LABORER & TORINO & ITALY & ITALY & ITALY & CALUMET 1ST PRECINCT - RED JACKET \\
\hline
\end{tabular}




\begin{tabular}{|c|c|c|c|c|c|c|c|c|c|c|c|c|c|}
\hline 189 & 177 & 285 & COPPO & DOMINIC & B & M & 26 & LABORER & TORINO & ITALY & ITALY & ITALY & CALUMET 1ST PRECINCT - RED JACKET \\
\hline 190 & 177 & 285 & CARETT & CHARLES & B & M & 25 & LABORER & & ITALY & ITALY & ITALY & CALUMET 1ST PRECINCT - RED JACKET \\
\hline 191 & 177 & 285 & CURTO & JOHN & B & M & 25 & LABORER & TORINO & ITALY & ITALY & ITALY & CALUMET 1ST PRECINCT - RED JACKET \\
\hline 192 & 177 & 285 & GIAMPAOLI & JOHN & B & M & 30 & LABORER & LUCCA & ITALY & ITALY & ITALY & CALUMET 1ST PRECINCT - RED JACKET \\
\hline 193 & 231 & 375 & MARTINI & MIKE & $\mathrm{H}$ & M & 30 & TIMBERMAN & LUCCA & ITALY & ITALY & ITALY & CALUMET 1ST PRECINCT - RED JACKET \\
\hline 194 & 231 & 375 & MARTINI & AGNES & W & $\mathrm{F}$ & 23 & KEEPING HOUSE & GERMANY & WURTENBERG & WURTENBERG & WURTENBERG & CALUMET 1ST PRECINCT - RED JACKET \\
\hline 195 & 231 & 375 & MARTINI & AGATA & D & $\mathrm{F}$ & 4 & & LUCCA & MICHIGAN & ITALY & WURTENBERG & CALUMET 1ST PRECINCT - RED JACKET \\
\hline 196 & 231 & 375 & SOLVONI & ANGEL & B & M & 44 & LABORER & & ITALY & ITALY & ITALY & CALUMET 1ST PRECINCT - RED JACKET \\
\hline 197 & 231 & 375 & RAPPA & MEDE & B & M & 29 & LABORER & & ITALY & ITALY & ITALY & CALUMET 1ST PRECINCT - RED JACKET \\
\hline 198 & 231 & 375 & PELLEGRINI & EMIL & B & M & 38 & LABORER & LUCCA & ITALY & ITALY & ITALY & CALUMET 1ST PRECINCT - RED JACKET \\
\hline 199 & 231 & 375 & PELLEGRINI & RAMOLD & B & M & 23 & LABORER & LUCCA & ITALY & ITALY & ITALY & CALUMET 1ST PRECINCT - RED JACKET \\
\hline 200 & 231 & 375 & GEMIGNANI & RONALD & B & M & 29 & LABORER & LUCCA & ITALY & ITALY & ITALY & CALUMET IST PRECINCT - RED JACKET \\
\hline 201 & 231 & 375 & GEMIGNANI & ANTONE & B & M & 42 & LABORER & LUCCA & ITALY & ITALY & ITALY & CALUMET 1ST PRECINCT - RED JACKET \\
\hline 202 & 246 & 295 & BUTORI & FRANK & $\mathrm{H}$ & M & 27 & LABORER & LUCCA & ITALY & ITALY & ITALY & CALUMET 1ST PRECINCT - RED JACKET \\
\hline 203 & 246 & 295 & BUTORI & ASSUNTA & W & $\mathrm{F}$ & 21 & KEEPING HOUSE & LUCCA & ITALY & ITALY & ITALY & CALUMET 1ST PRECINCT - RED JACKET \\
\hline 204 & 246 & 396 & BRACCO & JOSEPH & $\mathrm{H}$ & M & 27 & LABORER & TORINO & ITALY & ITALY & ITALY & CALUMET 1ST PRECINCT - RED JACKET \\
\hline 205 & 246 & 396 & BRACCO & TERESA & W & $\mathrm{F}$ & 26 & KEEPING HOUSE & TORINO & ITALY & ITALY & ITALY & CALUMET 1ST PRECINCT - RED JACKET \\
\hline 206 & 246 & 396 & BRACCO & FRANCIS & $\mathrm{s}$ & M & 3 & & TORINO & MICHIGAN & ITALY & ITALY & CALUMET 1ST PRECINCT - RED JACKET \\
\hline 207 & 246 & 396 & BRACCO & LOUIE & $\mathrm{s}$ & M & $5 \mathrm{M}$ & & TORINO & MICHIGAN & ITALY & ITALY & CALUMET 1ST PRECINCT - RED JACKET \\
\hline 208 & 549 & 612 & TORREANO & JOSEPH & $\mathrm{H}$ & M & 34 & LABORER & TORINO & ITALY & ITALY & ITALY & CALUMET 1ST PRECINCT - RED JACKET \\
\hline 209 & 549 & 612 & TORREANO & VINCENZA & W & $\mathrm{F}$ & 32 & KEEPING HOUSE & TORINO & ITALY & ITALY & ITALY & CALUMET 1ST PRECINCT - RED JACKET \\
\hline 210 & 549 & 612 & TORREANO & KATIE & $\mathrm{D}$ & $\mathrm{F}$ & 14 & & TORINO & FRANCE & ITALY & ITALY & CALUMET 1ST PRECINCT - RED JACKET \\
\hline 211 & 549 & 612 & TORREANO & AMELIA & $\mathrm{D}$ & $\mathrm{F}$ & 2 & & TORINO & MICHIGAN & ITALY & ITALY & CALUMET 1ST PRECINCT - RED JACKET \\
\hline 212 & 549 & 612 & TORREANO & JOHN & $\mathrm{s}$ & M & 1 & & TORINO & MICHIGAN & ITALY & ITALY & CALUMET 1ST PRECINCT - RED JACKET \\
\hline 213 & 709 & 768 & RAOPPLAS & JOHN & B & M & 33 & LABORER & & ITALY & ITALY & ITALY & CALUMET 1ST PRECINCT - RED JACKET \\
\hline 214 & 709 & 768 & VANZAGO & DOMINICK & B & M & 24 & LABORER & & ITALY & ITALY & ITALY & CALUMET IST PRECINCT - RED JACKET \\
\hline 215 & 709 & 768 & BRETT & DOMINICK & B & M & 25 & LABORER & & ITALY & ITALY & ITALY & CALUMET 1ST PRECINCT - RED JACKET \\
\hline 216 & 709 & 768 & POSTE & GEORGE & B & M & 32 & LABORER & & ITALY & ITALY & ITALY & CALUMET 1ST PRECINCT - RED JACKET \\
\hline 217 & 709 & 768 & BESSEL & FRANK & B & M & 42 & MINER & & ITALY & ITALY & ITALY & CALUMET 1ST PRECINCT - RED JACKET \\
\hline 218 & 709 & 768 & VENTURINO & BAZIL & B & M & 31 & MINE TIMBERMAN & & ITALY & ITALY & ITALY & CALUMET 1ST PRECINCT - RED JACKET \\
\hline 219 & 709 & 768 & NIGRO/NIGRA & MIKE & B & M & 42 & MINER & TORINO & ITALY & ITALY & ITALY & CALUMET 1ST PRECINCT - RED JACKET \\
\hline 220 & 709 & 768 & NIGRO/NIGRA & $\mathrm{JOHN}$ & $\mathrm{B}$ & $\mathrm{M}$ & 42 & TIMBERMAN & TORINO & ITALY & ITALY & ITALY & CALUMET 1ST PRECINCT - RED JACKET \\
\hline
\end{tabular}




\section{APPENDIX VIII}

\section{CENSUS of 1900}

\section{LEGEND}

ID: counter.

$\mathbf{N}$ : street number as it appeared on the house.

CIV: marital status. $\mathrm{S}$ indicates that the person is single. $\mathrm{M}$ indicates that the person is married, the number that follows indicates the years of marriage. $\mathrm{W}$ indicates that the person is a widow. REL: relation of the person with the head of the household.

BORN: nation of birth of the person. FATHER: nation of birth of the father. MOTHER: nation of birth of the mother.

NA: indicate if the person has become an U.S. citizen (NA) or is an alien (AL). UN indicates unknown.

PROVENIENCE: place of origin in Italy. These data are not from the census, but form Ellis Island Database, Italian newspapers, Oral interviews.

TOWNSHIP: township in which the person was living. 


\begin{tabular}{|c|c|c|c|c|c|c|c|c|c|c|c|c|c|c|}
\hline ID & $\mathbf{N}$ & SURNAME & NAME & REL & AG & CIV & BORN & FATHER & MOTHER & NA & OCCUPATION & PROVENIENCE & ADDRESS & \begin{tabular}{|c} 
TOWNSHIP \\
\end{tabular} \\
\hline 1 & & CIABATTARI & PIETRO & $\mathrm{H}$ & 28 & M1 & ITALY & ITALY & ITALY & $\mathrm{AL}$ & TIMBERMAN/CM & LUCCA & & ADAMS - ATLANTIC MINE \\
\hline 2 & & CIABATTARI & CLARA & w & 29 & M1 & ITALY & ITALY & ITALY & & & & & ADAMS - ATLANTIC MINE \\
\hline 3 & & PAOLINI & JOSEPH/JOHN & B & 37 & M7 & ITALY & ITALY & ITALY & $\mathrm{AL}$ & DAY LABORER/CM & LUCCA & & ADAMS - ATLANTIC MINE \\
\hline 4 & & MEI & JEROME/JOHN & B & 40 & M3 & ITALY & ITALY & ITALY & AL & DAY LABORER/CM & RUOTA. LU & & ADAMS - ATLANTIC MINE \\
\hline 5 & & BUNDI & $\begin{array}{l}\text { ANGELO } \\
\text { ANGIN }\end{array}$ & $\begin{array}{l}D \\
B\end{array}$ & 25 & M2 & ITALY & ITALY & ITALY & $\mathrm{AL}$ & DAY LABORER/CM & & & ADAMS - ATLANTIC MINE \\
\hline$\frac{5}{6}$ & & CIABATTARI & DOMENICO & B & 26 & M1 & $\begin{array}{l}\text { ITALY } \\
\text { ITALY }\end{array}$ & ITALY & $\begin{array}{l}\text { ITALY } \\
\text { ITAL }\end{array}$ & $\frac{A L}{A L}$ & DAY LABORER/CM & CAPANNORI. LU & & ADAMS - ATLANTIC MINE \\
\hline$\frac{6}{7}$ & & $\begin{array}{l}\text { CIABAIIARI } \\
\text { SERRA }\end{array}$ & $\begin{array}{l}\text { DOHN } \\
\text { JOHICO }\end{array}$ & $\begin{array}{l}\mathrm{B} \\
\mathrm{H}\end{array}$ & 26 & M? & $\begin{array}{l}\text { ITALY } \\
\text { ITALY }\end{array}$ & $\begin{array}{l}\text { IITALY } \\
\text { ITALY }\end{array}$ & $\begin{array}{l}\text { IIALY } \\
\text { ITALY }\end{array}$ & $\begin{array}{l}\mathrm{AL} \\
?\end{array}$ & $\begin{array}{l}\text { DAY LABORER/CM } \\
\text { DABORER IM }\end{array}$ & SAN GIORGIO CANAVESE. TO & & $\begin{array}{l}\text { ADAMS - ATLANANTC MINE } \\
\text { ADAMS ATLANTIC }\end{array}$ \\
\hline 8 & & SERRA & MARY & w & 35 & $?$ & ITALY & ITALY & ITALY & & & & & ADAMS - ATLANTIC MINE \\
\hline 9 & & SERRA & JAMES & s & 10 & $\dot{s}$ & MICHIGAN & ITALY & ITALY & & & SAN GIORGIO CANAVESE. TO & & ADAMS - ATLANTIC MINE \\
\hline 10 & & SERRA & MINNIE & $\mathrm{D}$ & 8 & s & MICHIGAN & ITALY & ITALY & & & SAN GIORGIO CANAVESE. TO & & ADAMS - ATLANTIC MINE \\
\hline 11 & & SERRA & MARY & $\mathrm{D}$ & $8 \mathrm{M}$ & $\frac{1}{4}$ & MICHIGAN & ITALY & ITALY & & & SAN GIORGIO CANAVESE. TO & & ADAMS - ATLANTIC MINE \\
\hline 12 & & CUFFIA & DOMENICO & B & 32 & M5 & ITALY & ITALY & ITALY & UN & TRAMMER/CM & SAN GIORGIO CANAVESE. TO & & ADAMS - ATLANTIC MINE \\
\hline 13 & & PASTORE & DOMENICO & B & 24 & s & ITALY & ITALY & ITALY & UN & DAY LABORER/CM & TORINO & & ADAMS - ATLANTIC MINE \\
\hline 14 & & NOZERO & DOMENICO & B & 41 & M13 & ITALY & ITALY & ITALY & UN & DAY LABORER/CM & SAN MARTINO CANAVESE. TO & & ADAMS - ATLANTIC MINE \\
\hline 15 & & SERRA & ANDRE & B & 40 & M11 & ITALY & ITALY & ITALY & UN & LABORER/ROCK HOUSE & SAN GIORGIO CANAVESE. TO & & ADAMS - ATLANTIC MINE \\
\hline 16 & & CASTELLINA & PAUL & $\mathrm{H}$ & 39 & M11 & ITALY & ITALY & ITALY & NA & DAY LABORER/CM & TORINO & & ADAMS - ATLANTIC MINE \\
\hline 17 & & CASTELLINA & CLARA & w & 31 & M11 & BELGIUM & BELGIUM & BELGIUM & & & BELGIO & & ADAMS - ATLANTIC MINE \\
\hline 18 & & CASTELLINA & ANTONIO & s & 7 & $\mathrm{~S}$ & IOWA & $\begin{array}{l}\text { ITALY } \\
\text { ITI }\end{array}$ & BELGIUM & & AT SCHOOL 10 & TORINO & & ADAMS - ATLANTIC MINE \\
\hline $\begin{array}{l}18 \\
19\end{array}$ & & CASTELLINA & PAUL JR. & $\mathrm{s}$ & $\frac{1}{2}$ & $\begin{array}{l}\mathrm{s} \\
\mathrm{s}\end{array}$ & IOWA & $\begin{array}{l}\text { IITALY } \\
\text { ITALY }\end{array}$ & BELGIUM & & AI SCHOOL IO & TORINO & & ADAMS - ATLANTIC MINE \\
\hline 20 & & $\begin{array}{l}\text { CHIMINO } \\
\text { CIN }\end{array}$ & GIOVANNI & $\mathrm{H}$ & 47 & M21 & ITALY & ITALY & ITALY & & LABORER/ROCK HOUSE & SAN GIORGIO CANAVESE. TO & & ADAMS - ATLANTIC MINE \\
\hline 21 & & CHIMINO & MARGHERITA & w & 40 & M21 & ITALY & ITALY & ITALY & & & & & ADAMS - ATLANTIC MINE \\
\hline 22 & & CHIMINO & GIOVANNI & s & 9 & $\mathrm{~S}$ & ITALY & ITALY & $\begin{array}{l}\text { ITALY } \\
\text { ITAL }\end{array}$ & & AT SCHOOL 10 & SAN GIORGIO CANAVESE. TO & & ADAMS - ATLANTIC MINE \\
\hline 23 & & CHIMINO & GIUSEPPE & s & 7 & s & MICHIGAN & ITALY & ITALY & & AT SCHOOL 10 & SAN GIORGIO CANAVESE. TO & & ADAMS - ATLANTIC MINE \\
\hline 24 & & CHIMINO & GRAZIA & D & $\frac{1}{6}$ & $\frac{2}{2}$ & MICHIGAN & ITALY & ITALY & & AT SCHOOL 10 & SAN GIORGIO CANAVESE. TO & & ADAMS - ATLANTIC MINE \\
\hline 25 & & CHIMINO & MARGHERITA & $\mathrm{D}$ & 1 & $\mathrm{~s}$ & MICHIGAN & $\begin{array}{l}\text { ITALY } \\
\text { ITAL }\end{array}$ & $\begin{array}{l}\text { ITALY } \\
\text { ITAL }\end{array}$ & & & SAN GIORGIO CANAVESE. TO & & ADAMS - ATLANTIC MINE \\
\hline 26 & & NOZERO & PIETRO & B & 45 & M18 & ITALY & ITALY & ITALY & & TRAMMER/CM & SAN MARTINO CANAVESE. TO & & ADAMS - ATLANTIC MINE \\
\hline 27 & & $\mathrm{ClUCCl}$ & NICOLA & $\mathrm{H}$ & 31 & M10 & ITALY & ITALY & ITALY & & LABORER/ROCK HOUSE & MATRAIA. LU & & ADAMS - ATLANTIC MINE \\
\hline 28 & & $\mathrm{ClUCCl}$ & SALOMINA & w & 31 & M10 & ITALY & ITALY & ITALY & & & MATRAIA. LU & & ADAMS - ATLANTIC MINE \\
\hline 29 & & $\mathrm{ClUCCl}$ & AMELIA & $\mathrm{D}$ & 7 & $\mathrm{~s}$ & MICHIGAN & ITALY & ITALY & & AT SCHOOL 10 & MATRAIA. LU & & ADAMS - ATLANTIC MINE \\
\hline 30 & & ClUCCl & GUERRINO & s & 6 & $\mathrm{~s}$ & MICHIGAN & ITALY & ITALY & & AT SCHOOL 10 & MATRAIA, LU & & ADAMS - ATLANTIC MINE \\
\hline 31 & & ClUCCl & PAOLO & $\mathrm{s}$ & 5 & s & MICHIGAN & ITALY & ITALY & & AT SCHOOL 1 & MATRAIA, LU & & ADAMS - ATLANTIC MINE \\
\hline 32 & & CIUCCI & NATALINA & $\mathrm{D}$ & 3 & s & MICHIGAN & ITALY & ITALY & & & MATRAIA. LU & & ADAMS - ATLANTIC MINE \\
\hline 32 & & $\begin{array}{l}\text { CIUCCI } \\
\text { CIUCCI }\end{array}$ & $\begin{array}{l}\text { NAIALINA } \\
\text { EMMA }\end{array}$ & $\begin{array}{l}\mathrm{D} \\
\mathrm{D}\end{array}$ & $\frac{3}{2}$ & 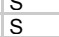 & MICHIGAN & $\begin{array}{l}\text { IITALY } \\
\text { ITALY }\end{array}$ & $\begin{array}{l}\text { IIIALY } \\
\text { ITALY }\end{array}$ & & & $\begin{array}{l}\text { MAIRAIA. LU } \\
\text { MATRAIA LU }\end{array}$ & & $\begin{array}{l}\text { ADAMS - AILANANIC MINE } \\
\text { ADAMS - ATLANTINE }\end{array}$ \\
\hline 34 & & $\mathrm{ClUCCl}$ & ENRICO & s & $4 \mathrm{M}$ & $\mathrm{s}$ & MICHIGAN & ITALY & ITALY & & & MATRAIA. LU & & ADAMS - ATLANTIC MINE \\
\hline 35 & & CIUCCI & ALFREDO & $\mathrm{BR}$ & 35 & M7 & ITALY & ITALY & ITALY & & TRAMMER/CM & MATRAIA. LU & & ADAMS - ATLANTIC MINE \\
\hline 36 & & MINETTI & JOSEPH & $\mathrm{H}$ & 33 & M3 & ITALY & ITALY & ITALY & & DAY LABORER/CM & TORINO & & ADAMS - ATLANTIC MINE \\
\hline 37 & & MINETTI & JOSEPHINA & W & 22 & M3 & FRANCE & FRANCE & FRANCE & & & FRANCE & & ADAMS - ATLANTIC MINE \\
\hline 38 & & MILANO & GIUSEPPE & B & 30 & $\mathrm{~s}$ & ITALY & ITALY & ITALY & & LANDER/CM & COURGNE'. TO & & ADAMS - ATLANTIC MINE \\
\hline 39 & & TOCCOLINI & MASSIMO & $\mathrm{H}$ & 31 & M8 & ITALY & ITALY & ITALY & & TRAMMER/CM & BELLUNO & & ADAMS - ATLANTIC MINE \\
\hline 40 & & TOCCOLINI & NUNCIATTA & W & 26 & M8 & ITALY & ITALY & ITALY & & & BELLUNO & & ADAMS - ATLANTIC MINE \\
\hline 41 & & TOCCOLINI & NATALI & $\mathrm{D}$ & 8 & s & ITALY & ITALY & ITALY & & AT SCHOOL 10 & BELLUNO & & ADAMS - ATLANTIC MINE \\
\hline 42 & & TOCCOLINI & EUGENE & $\mathrm{s}$ & 6 & $\mathrm{~s}$ & ITALY & ITALY & ITALY & & AT SCHOOL 10 & BELLUNO & & ADAMS - ATLANTIC MINE \\
\hline 43 & & TOCCOLINI & ELLEN & $\mathrm{D}$ & 4 & $\mathrm{~s}$ & ITALY & ITALY & ITALY & & & BELLUNO & & ADAMS - ATLANTIC MINE \\
\hline 44 & & TOCCOLINI & AMELIA & $\mathrm{D}$ & 10 & s & MICHIGAN & ITALY & ITALY & & & BELLUNO & & ADAMS - ATLANTIC MINE \\
\hline $\begin{array}{r}44 \\
45\end{array}$ & & ELLENA & JOSEPH & $\mathrm{H}$ & 30 & M12 & ITALY & ITALY & ITALY & AL & LABORER/ROCK HOUSE & SAN GIUSTO CANAVESE.TO & & ADAMS - ATLANTIC MINE \\
\hline$\frac{45}{46}$ & & $\begin{array}{l}\text { ELENA } \\
\text { ELLENA }\end{array}$ & TRACY & $\begin{array}{l}\mathrm{H} \\
\mathrm{W}\end{array}$ & 28 & $\frac{\text { M12 }}{\text { M12 }}$ & $\begin{array}{l}\text { ITALY } \\
\text { ITALY }\end{array}$ & $\begin{array}{l}\text { IIALY } \\
\text { ITALY }\end{array}$ & $\begin{array}{l}\text { IIALY } \\
\text { ITALY }\end{array}$ & $A L$ & LABURER/ROCK HOUSE & $\begin{array}{l}\text { SAN GIUSTO CANAVESE. } \\
\text { SAN GIUSTO CANAVESE. TO }\end{array}$ & & $\begin{array}{l}\text { ADAMS - AILANIIC MINE } \\
\text { ADAMS - ATLANTIC MINE }\end{array}$ \\
\hline 47 & & ELLENA & PETER & s & 10 & $\mathrm{~S}$ & ITALY & ITALY & ITALY & & AT SCHOOL 10 & SAN GIUSTO CANAVESE. TO & & ADAMS - ATLANTIC MINE \\
\hline 48 & & ELLENA & TRACY & $\mathrm{D}$ & 8 & $\mathrm{~s}$ & ITALY & ITALY & ITALY & & AT SCHOOL 10 & SAN GIUSTO CANAVESE. TO & & ADAMS - ATLANTIC MINE \\
\hline 49 & & ELLENA & JOHN & s & $\frac{0}{7}$ & s & MICHIGAN & ITALY & ITALY & & AT SCHOOL 10 & SAN GIUSTO CANAVESE. TO & & ADAMS - ATLANTIC MINE \\
\hline 50 & & ELLENA & MATTI & $\mathrm{s}$ & 6 & s & MICHIGAN & $\begin{array}{l}\text { ITALY } \\
\text { ITALY }\end{array}$ & $\begin{array}{l}\text { ITALY } \\
\text { ITALY }\end{array}$ & & AT SCHOOL 10 & SAN GIUSTO CANAVESE. TO & & ADAMS - ATLANTIC MINE \\
\hline 51 & & ELLENA & TONI & $\mathrm{D}$ & 4 & $\mathrm{~s}$ & MICHIGAN & ITALY & ITALY & & & SAN GIUSTO CANAVESE. TO & & ADAMS - ATLANTIC MINE \\
\hline 52 & & ELLENA & AUGUST & $\mathrm{s}$ & 2 & $\mathrm{~s}$ & MICHIGAN & ITALY & ITALY & & & SAN GIUSTO CANAVESE. TO & & ADAMS - ATLANTIC MINE \\
\hline 53 & & ELLENA & MONICA & $\mathrm{D}$ & 1 & s & MICHIGAN & ITALY & ITALY & & & SAN GIUSTO CANAVESE. TO & & ADAMS - ATLANTIC MINE \\
\hline 54 & & ZANOTTI & KATHERINE & $\mathrm{H}$ & 54 & w & ITALY & ITALY & ITALY & & BOARDING HOUSE KEEPER & CUCEGLIO. TO & & ADAMS - ATLANTIC MINE \\
\hline 55 & & ZANOTTI & VICTOR & s & 23 & s & ITALY & ITALY & ITALY & NA & TRAMMER/CM & CUCEGLIO TO & & ADAMS - ATLANTIC MINE \\
\hline 56 & & ZANA & DOMENICO & SIL & 25 & M2 & ITALY & ITALY & ITALY & $\mathrm{AL}$ & LABORER/ROCK HOUSE & CUCEGLIO. TO & & ADAMS - ATLANTIC MINE \\
\hline 57 & & ZANA & ANTONIA & GD & 11 & S S & MICHIGAN & ITALY & ITALY & & & TORINO & & ADAMS - ATLANTIC MINE \\
\hline 58 & & CUFFIA & BATTISTA & $B$ & 31 & M5 & ITALY & ITALY & ITALY & $\mathrm{AL}$ & LABORER/ROCK HOUSE & TORINO & & ADAMS - ATLANTIC MINE \\
\hline 59 & & ZANOTTI & JOSEPH & B & 34 & S & ITALY & ITALY & ITALY & $A L$ & TRAMMER/CM & TORINO & & ADAMS - ATLANTIC MINE \\
\hline 60 & & ZANOTTI & JAMES & B & 36 & s & ITALY & ITALY & ITALY & $\mathrm{AL}$ & TRAMMER/CM & TORINO & & ADAMS - ATLANTIC MINE \\
\hline
\end{tabular}




\begin{tabular}{|c|c|c|c|c|c|c|c|c|c|c|c|c|}
\hline 61 & NARETTO & ANTONE & B & 40 & S & ITALY & ITALY & ITALY & NA & TIMBERMAN/CM & TORINO & ADAMS - ATLANTIC MINE \\
\hline 62 & RAFFAELLI & RAPHAEL & $\mathrm{H}$ & 37 & M14 & ITALY & ITALY & ITALY & $\mathrm{AL}$ & TRAMMER/CM & LUCCA & ADAMS - ATLANTIC MINE \\
\hline 63 & RAFFAELLI & KATE & W & 32 & M14 & ITALY & ITALY & ITALY & & & LUCCA & ADAMS - ATLANTIC MINE \\
\hline 64 & RAFFAELLI & ANITA & $\mathrm{D}$ & 12 & $\mathrm{~S}$ & ITALY & ITALY & ITALY & & AT SCHOOL 10 & LUCCA & ADAMS - ATLANTIC MINE \\
\hline 65 & RAFFAELLI & ITALO & $\mathrm{s}$ & 10 & s & ITALY & ITALY & ITALY & & AT SCHOOL 10 & LUCCA & ADAMS - ATLANTIC MINE \\
\hline 66 & RAFFAELLI & MINNIE & $\mathrm{D}$ & $4 \mathrm{M}$ & s & ITALY & ITALY & ITALY & & & LUCCA & ADAMS - ATLANTIC MINE \\
\hline 67 & BATTISTONI & AMY & B & 20 & s & ITALY & ITALY & ITALY & & & LUCCA & ADAMS - ATLANTIC MINE \\
\hline 68 & PAOLINI & JOSEPH & B & 28 & s & ITALY & ITALY & ITALY & $\mathrm{AL}$ & DAY LABORER/CM & LUCCA & ADAMS - ATLANTIC MINE \\
\hline $\begin{array}{l}68 \\
69\end{array}$ & CODINI & ERNEST & $\begin{array}{l}\mathrm{B} \\
\mathrm{B}\end{array}$ & $\frac{28}{35}$ & M11 & $\begin{array}{l}\text { ITALY } \\
\text { ITALY }\end{array}$ & $\begin{array}{l}\text { IITALY } \\
\text { ITALY }\end{array}$ & $\begin{array}{l}\text { IIALY } \\
\text { ITALY }\end{array}$ & $A L$ & $\begin{array}{l}\text { DAY LABURER/CMI } \\
\text { DAY LABORER/CM }\end{array}$ & $\begin{array}{l}\text { LUCCA } \\
\text { LUCCA }\end{array}$ & ADAMS - ATLANTIC MINE \\
\hline 70 & BEI & JOHN & B & 21 & $\mathrm{~s}$ & ITALY & ITALY & ITALY & & TRAMMER/CM & LUCCA & ADAMS - ATLANTIC MINE \\
\hline 71 & PAOLINI & ELIX & B & 24 & M5 & ITALY & ITALY & ITALY & & TRAMMER/CM & LUCCA & ADAMS - ATLANTIC MINE \\
\hline 72 & COTTINI & ADOLPH & B & 34 & $\mathrm{~s}$ & ITALY & ITALY & ITALY & & DAY LABORER/CM & LUCCA & ADAMS - ATLANTIC MINE \\
\hline 73 & AMANTI & LORENZO & $\mathrm{H}$ & 28 & M2 & ITALY & ITALY & ITALY & & TRAMMER/CM & LUCCA & ADAMS - ATLANTIC MINE \\
\hline 74 & AMANTI & ANGELINA & w & 22 & M2 & ITALY & ITALY & ITALY & & & LUCCA & ADAMS - ATLANTIC MINE \\
\hline 75 & NELLI & JOSEPH & B & 27 & $\mathrm{~s}$ & ITALY & ITALY & ITALY & & LABORER/ROCK HOUSE & LUCCA & ADAMS - ATLANTIC MINE \\
\hline 76 & RAFFAELLI & DOMINICK & B & 27 & s & ITALY & ITALY & ITALY & & DAY LABORER/CM & LUCCA & ADAMS - ATLANTIC MINE \\
\hline 77 & BERITINA & PAUL & B & 20 & $\mathrm{~s}$ & ITALY & ITALY & ITALY & & DAY LABORER/CM & LUCCA & ADAMS - ATLANTIC MINE \\
\hline 78 & ORSI & GIACINTO & B & 25 & $\mathrm{~s}$ & ITALY & ITALY & ITALY & & TRAMMER/CM & LUCCA & ADAMS - ATLANTIC MINE \\
\hline 79 & GALDINI & PASQUALE & B & 45 & M16 & ITALY & ITALY & ITALY & $\mathrm{AL}$ & DAY LABORER/CM & & ADAMS - ATLANTIC MINE \\
\hline 80 & MEAGLIA & NICK & B & 45 & $\mathrm{~s}$ & ITALY & ITALY & ITALY & & $\begin{array}{l}\text { MINER/CM } \\
\text { MIVI }\end{array}$ & TORINO & ADAMS - ATLANTIC MINE \\
\hline 81 & BERITINA & DUDIMIO & $\begin{array}{l}\mathrm{D} \\
\mathrm{B}\end{array}$ & $\frac{45}{25}$ & s & ITALY & ITALY & ITALY & & TRAMMER/CM & PARIANA. LU & ADAMS - ATLANTIC MINE \\
\hline $\begin{array}{l}81 \\
82\end{array}$ & $\begin{array}{l}\text { BERIIINA } \\
\text { MASSA }\end{array}$ & $\begin{array}{l}\text { ERNANI } \\
\text { ERIMIO }\end{array}$ & $\begin{array}{l}\mathrm{B} \\
\mathrm{H}\end{array}$ & 31 & M9 & $\begin{array}{l}\text { IIALY } \\
\text { ITALY }\end{array}$ & $\begin{array}{l}\text { IIALY } \\
\text { ITALY }\end{array}$ & $\begin{array}{l}\text { IITALY } \\
\text { ITALY }\end{array}$ & & $\begin{array}{l}\text { TRAMMMER/IM } \\
\text { CARPENTER }\end{array}$ & $\begin{array}{l}\text { PARIIANA, LU } \\
\text { CIRIE', TO }\end{array}$ & $\begin{array}{l}\text { ADAMS - AILANANTC MINE } \\
\text { ADAMS - ATLANTINE }\end{array}$ \\
\hline 83 & MASSA & ANGELINA & W & 30 & M9 & ITALY & ITALY & ITALY & & & CIRIE'. TO & ADAMS - ATLANTIC MINE \\
\hline 84 & MASSA & GIOVANNI & $\mathrm{s}$ & 7 & s & ITALY & ITALY & ITALY & & AT SCHOOL 10 & CIRIE'. TO & ADAMS - ATLANTIC MINE \\
\hline 85 & MASSA & DEANAREDO & $\mathrm{s}$ & 6 & $\mathrm{~s}$ & ITALY & ITALY & ITALY & & AT SCHOOL 4 & CIRIE'. TO & ADAMS - ATLANTIC MINE \\
\hline 86 & MASSA & MARIA & $\mathrm{D}$ & 4 & $\mathrm{~s}$ & ITALY & ITALY & ITALY & & & CIRIE'. TO & ADAMS - ATLANTIC MINE \\
\hline 87 & BUSSA & JAMES & B & 23 & $\mathrm{~s}$ & ITALY & ITALY & ITALY & $\mathrm{AL}$ & TRAMMER/CM & TORINO & ADAMS - ATLANTIC MINE \\
\hline 88 & ROCK & JOHN & B & 22 & $\mathrm{~s}$ & ITALY & ITALY & ITALY & NA & TIMBERMAN/CM & TORINO & ADAMS - ATLANTIC MINE \\
\hline 89 & ENRICO & LUIGI & B & 25 & M4 & ITALY & ITALY & ITALY & $\mathrm{AL}$ & TRAMMER/CM & CORIO. TO & ADAMS - ATLANTIC MINE \\
\hline 90 & TINETTI & JAMES & B & 45 & M17 & ITALY & ITALY & ITALY & AL & LABORER/ROCK HOUSE & SAN MARTINO CANAVESE.TO & ADAMS - ATLANTIC MINE \\
\hline 91 & NOZERO & ANTONIO & B & 28 & $\mathrm{~S}$ & ITALY & ITALY & ITALY & $\mathrm{AL}$ & TRAMMER/CM & SAN MARTINO CANAVESE. TO & ADAMS - ATLANTIC MINE \\
\hline 92 & CENTANINO & ANTONE & B & 25 & $\mathrm{~s}$ & ITALY & ITALY & ITALY & PA & TRAMMER/CM & TORINO & ADAMS - ATLANTIC MINE \\
\hline 92 & $\begin{array}{l}\text { CENIANINA } \\
\text { CIABATARI }\end{array}$ & SEBASTIAN & $\begin{array}{l}\mathrm{B} \\
\mathrm{H}\end{array}$ & $\frac{25}{33}$ & M6 & $\begin{array}{l}\text { IITALY } \\
\text { ITALY }\end{array}$ & $\begin{array}{l}\text { IITALY } \\
\text { ITALY }\end{array}$ & $\begin{array}{l}\text { IITALY } \\
\text { ITALY }\end{array}$ & PA & DAY LABORER/CM & SEGROMIGNO. LU & $\begin{array}{l}\text { ADAAMS - AILANTIC MINE } \\
\text { ADAMS ATLANTIC }\end{array}$ \\
\hline 93 & $\begin{array}{l}\text { CIABAIIARI } \\
\text { CIABATARI }\end{array}$ & MARIA & $\begin{array}{ll}H \\
\text { W }\end{array}$ & 33 & M6 & $\begin{array}{l}\text { IIIALY } \\
\text { ITALY }\end{array}$ & $\begin{array}{l}\text { IITALY } \\
\text { ITALY }\end{array}$ & $\begin{array}{l}\text { IITALY } \\
\text { ITALY }\end{array}$ & PA & DAY LABURER/CIM & SEGRUIVIGIVU. LU & $\begin{array}{l}\text { ADAAMS - ATLANTIC MINE } \\
\text { ADATLANS }\end{array}$ \\
\hline 95 & CIABATTARI & IDA & $\mathrm{D}$ & 4 & $\mathrm{~s}$ & MICHIGAN & ITALY & ITALY & & & SEGROMIGNO.LU & ADAMS - ATLANTIC MINE \\
\hline 96 & CIABATTARI & ISABELLA & D & $\frac{4}{2}$ & s & MICHIGAN & $\begin{array}{l}\text { ITALY } \\
\text { ITAL }\end{array}$ & ITALY & & & SEGROMIGNO. LU & ADAMS - ATLANTIC MINE \\
\hline 97 & CIABATTARI & GIULIO & s & 0 & s & MICHIGAN & ITALY & ITALY & & & SEGROMIGNO. LU & ADAMS - ATLANTIC MINE \\
\hline 98 & MASONI & LOUIS & B & 23 & s & ITALY & ITALY & $\begin{array}{l}\text { IIALY } \\
\text { ITALY }\end{array}$ & $\mathrm{AL}$ & TRAMMER/CM & GALICANO LU & ADAMS - ATLANTIC MINE \\
\hline 99 & GIANNINI & ANTONE & $\mathrm{D}$ & 39 & M10 & $\begin{array}{l}\text { ITALY } \\
\text { ITALY }\end{array}$ & ITALY & ITALY & NA & DAY LABORER/CM & LUCCA & ADAMS - ATLANTIC MINE \\
\hline 100 & GIANNINI & KATE & w & 29 & M10 & ITALY & ITALY & ITALY & & & LUCCA & ADAMS - ATLANTIC MINE \\
\hline 101 & GIANNINI & MADELINE & D & 9 & $\mathrm{~s}$ & ITALY & ITALY & ITALY & & AT SCHOOL 6 & LUCCA & ADAMS - ATLANTIC MINE \\
\hline 102 & GIANNINI & JAMES & $\mathrm{s}$ & 7 & $\mathrm{~s}$ & MICHIGAN & ITALY & ITALY & & AT SCHOOL 6 & LUCCA & ADAMS - ATLANTIC MINE \\
\hline 103 & GIANNINI & $\begin{array}{l}\text { KATE } \\
\text { KATE }\end{array}$ & $\mathrm{D}$ & 1 & s & MICHIGAN & ITALY & ITALY & & & LUCCA & ADAMS - ATLANTIC MINE \\
\hline 104 & DE FILIPPI & ANTONIO & B & 35 & M13 & ITALY & ITALY & ITALY & $\mathrm{AL}$ & DAY LABORER/CM & SAN GIUSTO CANAVESE. TO & ADAMS - ATLANTIC MINE \\
\hline 105 & PAOLI & DOMINICK & B & 45 & M18 & ITALY & ITALY & ITALY & $A L$ & DAY LABORER/CM & LUCCA & ADAMS - ATLANTIC MINE \\
\hline 106 & SEBASTI & JOHN & $\mathrm{B}$ & 23 & $\mathrm{~S}$ & ITALY & ITALY & ITALY & $\mathrm{AL}$ & DAY LABORER/CM & & ADAMS - ATLANTIC MINE \\
\hline 106 & $\begin{array}{l}\text { SEBASII } \\
\text { MICHELETTI }\end{array}$ & JOHN & $\begin{array}{l}B \\
B\end{array}$ & 23 & $\begin{array}{l}2 \\
\text { s }\end{array}$ & $\begin{array}{l}\text { IIALY } \\
\text { ITALY }\end{array}$ & $\begin{array}{l}\text { IIALY } \\
\text { ITALY }\end{array}$ & $\begin{array}{l}\text { IITALY } \\
\text { ITALY }\end{array}$ & $\begin{array}{l}\text { AL } \\
\text { NA }\end{array}$ & $\begin{array}{l}\text { DAY LABURER/CM } \\
\text { TRAMMER/CM }\end{array}$ & LUCCA & $\begin{array}{l}\text { ADAMS - AILANIC MINE } \\
\text { ADAMS - ATLANTIC MINE }\end{array}$ \\
\hline 108 & MILANO & PETER & $\mathrm{H}$ & 28 & M2 & ITALY & ITALY & $\begin{array}{l}\text { ITALY } \\
\end{array}$ & $\mathrm{AL}$ & TRAMMER/CM & $\begin{array}{l}\text { TORINO } \\
\text { TOM }\end{array}$ & ADAMS - ATLANTIC MINE \\
\hline 109 & MILANO & MADELINE & W & 24 & M2 & CANADA & CANADA & CANADA & & & CANADA & ADAMS - ATLANTIC MINE \\
\hline 110 & MILANO & JOHN & s & 14 & $\begin{array}{l}\text { NLL } \\
\text { S }\end{array}$ & MICHIGAN & $\begin{array}{l}\text { ITALY } \\
\text { ITALA }\end{array}$ & $\begin{array}{l}\text { CAIVADA } \\
\text { CANADA }\end{array}$ & & & $\begin{array}{l}\text { TAIVADA } \\
\text { TORINO }\end{array}$ & ADAMS - ATLANTIC MINE \\
\hline 111 & MILANO & JOSEPHINE & D & $2 \mathrm{M}$ & $\frac{1}{3}$ & MICHIGAN & ITALY & $\begin{array}{l}\text { CAIVADA } \\
\text { CANADA }\end{array}$ & & & TORINO & ADAMS - ATLANTIC MINE \\
\hline 112 & WALKER & FRANK & B & 25 & $\frac{2}{3}$ & ITALY & ITALY & $\begin{array}{l}\text { ITALY } \\
\text { ITA }\end{array}$ & AL & TRAMMER/CM & & ADAMS - ATLANTIC MINE \\
\hline 113 & $\begin{array}{l}\text { WILVESTRI } \\
\text { SILVER }\end{array}$ & $\begin{array}{l}\text { FRANONIO } \\
\text { ANTONIO }\end{array}$ & $\begin{array}{l}\mathrm{B} \\
\mathrm{B}\end{array}$ & 26 & s & ITALY & ITALY & $\begin{array}{l}\text { ITALY } \\
\text { ITALY }\end{array}$ & $\begin{array}{ll}A L \\
A L\end{array}$ & TRAMMER/CM & & ADAMS - ATLANTIC MINE \\
\hline 114 & LUKO & MICHAEL & B & 31 & $\mathrm{~s}$ & ITALY & ITALY & ITALY & $\mathrm{AL}$ & TRAMMER/CM & & ADAMS - ATLANTIC MINE \\
\hline 115 & BURZIO & ANTONE & B & 25 & s & ITALY & ITALY & ITALY & AL & TRAMMER/CM & TORINO & ADAMS - ATLANTIC MINE \\
\hline 116 & AMPHOLEH & FRANK & B & 27 & s & ITALY & ITALY & ITALY & AL & TRAMMER/CM & & ADAMS - ATLANTIC MINE \\
\hline 117 & MARTINI & MARCO & B & 25 & s & ITALY & ITALY & ITALY & $A L$ & TRAMMER/CM & LUCCA & ADAMS - ATLANTIC MINE \\
\hline 118 & GIAMPAOLI & FRED & B & 24 & $\mathrm{~s}$ & ITALY & ITALY & ITALY & $A L$ & TRAMMER/CM & LUCCA & ADAMS - ATLANTIC MINE \\
\hline 119 & ANITO & LUCHIE & B & 31 & M5 & ITALY & ITALY & ITALY & $\mathrm{AL}$ & TRAMMER/CM & & ADAMS - ATLANTIC MINE \\
\hline 120 & STEFANELLA & MODESTO & B & 24 & 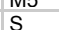 & $\begin{array}{l}\text { IIALY } \\
\text { ITALY }\end{array}$ & $\begin{array}{l}\text { ITALY } \\
\text { ITALY }\end{array}$ & $\begin{array}{l}\text { MALY } \\
\text { ITALY }\end{array}$ & $\begin{array}{l}\text { AL } \\
\text { AL }\end{array}$ & TRAMMER/CM & & ADAMS - ATLANTIC MINE \\
\hline 121 & BOSSIO & $\mathrm{JOHN}$ & B & 27 & $\mathrm{~s}$ & ITALY & ITALY & ITALY & $A L$ & TRAMMER/CM & TORINO & ADAMS - ATLANTIC MINE \\
\hline 122 & REMIGIO & DANEPILLO & $\begin{array}{l}\mathrm{D} \\
\mathrm{B}\end{array}$ & 24 & $\mathrm{~s}$ & ITALY & $\begin{array}{l}\text { ITALY } \\
\text { ITAL }\end{array}$ & ITALY & $\mathrm{AL}$ & TRAMMER/CM & TORINO & ADAMS - ATLANTIC MINE \\
\hline 123 & $\begin{array}{l}\text { REICIOIO } \\
\text { VICI }\end{array}$ & OLIVO & $\mathrm{D}_{\mathrm{H}}$ & 24 & M3 & $\begin{array}{l}\text { ITALY } \\
\text { ITALY }\end{array}$ & ITALY & $\begin{array}{l}\text { MALY } \\
\text { ITALY }\end{array}$ & $\begin{array}{l}\mathrm{AL} \\
\mathrm{AL}\end{array}$ & TRAMMER/CM & VILLA BASILICA. LU & ADAMS - ATLANTIC MINE \\
\hline
\end{tabular}




\begin{tabular}{|c|c|c|c|c|c|c|c|c|c|c|c|c|}
\hline 124 & VICINI & CONCETTA & W & 22 & M3 & ITALY & ITALY & ITALY & & & VILLA BASILICA. LU & ADAMS - ATLANTIC MINE \\
\hline 125 & VICINI & PALMIRA & $\mathrm{D}$ & 1 & $\mathrm{~s}$ & ITALY & ITALY & ITALY & & & VILLA BASILICA. LU & ADAMS - ATLANTIC MINE \\
\hline 126 & POCK/ROCK & PETER & $\mathrm{H}$ & 29 & M0 & ITALY & ITALY & ITALY & PA & TRAMMER/CM & & ADAMS - ATLANTIC MINE \\
\hline 127 & POCK/ROCK & CLARA & W & 24 & M0 & ITALY & ITALY & ITALY & & & & ADAMS - ATLANTIC MINE \\
\hline 128 & MARCHESCHI & MICHELE & B & 20 & $\mathrm{~s}$ & ITALY & ITALY & ITALY & $\mathrm{AL}$ & TRAMMER/CM & LUCCA & ADAMS - ATLANTIC MINE \\
\hline 129 & PIRONNI & ANGELO & B & 24 & $\mathrm{~s}$ & ITALY & ITALY & ITALY & $\mathrm{AL}$ & NOT CLEAR & SEGROMIGNO. LU & ADAMS - ATLANTIC MINE \\
\hline 130 & MARCHESCHI & PETER & B & 46 & M20 & ITALY & ITALY & ITALY & $\mathrm{AL}$ & TRAMMER/CM & LUCCA & ADAMS - ATLANTIC MINE \\
\hline 131 & PARENTI & JOSEPH & B & 41 & M 15 & ITALY & ITALY & ITALY & AL & TRAMMER/CM & CASTELVECCHIO. LU & ADAMS - ATLANTIC MINE \\
\hline 132 & PAOLI & CARLO & B & 24 & M3 & ITALY & ITALY & ITALY & $\mathrm{AL}$ & TRAMMER/CM & CAPANNORI. LU & ADAMS - ATLANTIC MINE \\
\hline 133 & PARENTI & MAURIZIO & B & 21 & $\mathrm{~s}$ & ITALY & ITALY & ITALY & $\mathrm{AL}$ & TRAMMER/CM & CASTELVECCHIO. LU & ADAMS - ATLANTIC MINE \\
\hline 134 & GRISOLANO & TONIE & B & 36 & M12 & ITALY & ITALY & ITALY & $\mathrm{AL}$ & MINER/CM & TORINO & ADAMS - ATLANTIC MINE \\
\hline 135 & VANACHII & FRANK & B & 23 & s & ITALY & ITALY & ITALY & $\mathrm{AL}$ & TRAMMER/CM & & ADAMS - ATLANTIC MINE \\
\hline 136 & MARTINELLI & FRANK & B & 29 & s & ITALY & ITALY & ITALY & $\mathrm{AL}$ & TIMBERMAN/CM & & ADAMS - ATLANTIC MINE \\
\hline 137 & GEDDA & PETER & $\mathrm{H}$ & 34 & M10 & ITALY & ITALY & ITALY & $\mathrm{PA}$ & MINER/CM & TORINO & ADAMS - BALTIC \\
\hline 138 & GEDDA & LORENZINA & W & 27 & M10 & ITALY & ITALY & ITALY & & & TORINO & ADAMS - BALTIC \\
\hline 139 & GEDDA & PETER JR. & s & 8 & S & ITALY & ITALY & ITALY & & AT SCHOOL 10 & TORINO & ADAMS - BALTIC \\
\hline 140 & GEDDA & JOSEPH & $\mathrm{s}$ & 5 & s & MICHIGAN & ITALY & ITALY & & & TORINO & ADAMS - BALTIC \\
\hline 141 & GEDDA & KATE & $\mathrm{D}$ & 4 & $\mathrm{~s}$ & MICHIGAN & ITALY & ITALY & & & TORINO & ADAMS - BALTIC \\
\hline 142 & GEDDA & GEORGE & $\mathrm{s}$ & $\frac{4}{2}$ & s & MICHIGAN & ITALY & ITALY & & & TORINO & ADAMS - BALTIC \\
\hline 143 & GEDDA & ROSA & D & 11 & s & MICHIGAN & ITALY & $\begin{array}{l}\text { ITALY } \\
\text { ITAL }\end{array}$ & & & TORINO & ADAMS - BALTIC \\
\hline 144 & TASNASSIO & DAVID & B & 39 & $\mathrm{~s}$ & ITALY & ITALY & ITALY & NA & TRAMMER/CM & & ADAMS \\
\hline 145 & FLOUTTIE & PETER & B & 28 & $\mathrm{~s}$ & ITALY & ITALY & ITALY & $\mathrm{AL}$ & TIMBERMAN/CM & & ADAMS \\
\hline 146 & FLOUTTIE & JOSEPH & B & 35 & $\mathrm{~s}$ & ITALY & ITALY & ITALY & $A L$ & TRAMMER/CM & & ADAMS \\
\hline 147 & DATA & LORENZO & B & 26 & $\mathrm{~s}$ & ITALY & ITALY & ITALY & $A L$ & TRAMMER/CM & TORINO & ADAMS \\
\hline 148 & DATA & PAUL & B & 18 & $\mathrm{~s}$ & ITALY & ITALY & ITALY & & TRAMMER/CM & TORINO & ADAMS \\
\hline 149 & MILANO & ANTONE & B & 28 & M5 & ITALY & ITALY & ITALY & $\mathrm{AL}$ & TRAMMER/CM & COURGNE'. TO & ADAMS \\
\hline 150 & GARDA & DOMENICO & B & 37 & M8 & ITALY & ITALY & ITALY & $\mathrm{AL}$ & MINER/CM & TORINO & ADAMS \\
\hline 151 & NARETTO & $\begin{array}{l}\text { SANTO } \\
\text { SATO }\end{array}$ & B & 31 & M9 & ITALY & ITALY & ITALY & PA & TRAMMER/CM & TORINO & ADAMS \\
\hline 152 & ROSA & GIACOMO & B & 23 & $\mathrm{~s}$ & ITALY & ITALY & ITALY & $\mathrm{AL}$ & TRAMMER/CM & TORINO & ADAMS \\
\hline 153 & TORREANO & JOHN & B & 26 & $\mathrm{~s}$ & ITALY & ITALY & ITALY & AL & TRAMMER/CM & TORINO & ADAMS \\
\hline 154 & TOSCANA & ANTONE & $\mathrm{H}$ & 23 & M0 & ITALY & ITALY & ITALY & PA & MINER/CM & SAN GIORGIO CANAVESE. TO & ADAMS \\
\hline 155 & TOSCANA & MARY & W & 18 & MO & ITALY & ITALY & ITALY & & & & ADAMS \\
\hline 155 & TOSCANA & MINNIE & $\begin{array}{l}\text { W } \\
\text { D }\end{array}$ & $6 \mathrm{M}$ & IN & MICHIGAN & $\begin{array}{l}\text { IITALY } \\
\text { ITALY }\end{array}$ & $\begin{array}{l}\text { IIALY } \\
\text { ITALY }\end{array}$ & & & SAN GIORGIO CANAVESE. TO & $\begin{array}{l}\text { ADAMIS } \\
\text { ADAMS }\end{array}$ \\
\hline 157 & GIULIANI & FRANK & B & 23 & s & ITALY & ITALY & ITALY & AL & TIMBERMAN/CM & & ADAMS \\
\hline 158 & GRISOLANO & DOMINICK & B & 40 & M13 & ITALY & ITALY & ITALY & $\mathrm{AL}$ & MINER/CM & TORINO & ADAMS \\
\hline 159 & CASTELLANI & ANTONE & B & 35 & S & ITALY & ITALY & ITALY & $\mathrm{AL}$ & TRAMMER/CM & TURIV & ADAMS \\
\hline 160 & ANDRINI & BERNARDO & $\mathrm{H}$ & 28 & M5 & ITALY & ITALY & ITALY & $\mathrm{AL}$ & DAY LABORER/CM & LUCCA & ADAMS \\
\hline 161 & ANDRINI & ROSANNA & W & 28 & M5 & ITALY & ITALY & ITALY & & & LUCCA & ADAMS \\
\hline 162 & ANDRINI & OLGA & $\mathrm{D}$ & 2 & $\mathrm{~s}$ & ITALY & ITALY & ITALY & & & LUCCA & ADAMS \\
\hline 163 & MASCHIRIE & CARLO & B & 23 & $\mathrm{~s}$ & ITALY & ITALY & ITALY & $\mathrm{AL}$ & TRAMMER/CM & & ADAMS \\
\hline 164 & GENSIPPE & WILLIAM & B & 31 & M8 & ITALY & ITALY & ITALY & $\mathrm{AL}$ & DAY LABORER/CM & & ADAMS \\
\hline 165 & BELLONI & RENARDO & B & 30 & M5 & ITALY & ITALY & ITALY & $\mathrm{AL}$ & TRAMMER/CM & & ADAMS \\
\hline 166 & CRECEMETTE & MELLE & B & 37 & M4 & ITALY & ITALY & ITALY & $A L$ & LANDER/CM & & ADAMS \\
\hline 167 & BIANCHI & MARGHERITA & B & 22 & s & ITALY & ITALY & ITALY & & & LUCCA & ADAMS \\
\hline 168 & DATI & NAPOLEON & $\begin{array}{l}D \\
B\end{array}$ & 48 & M22 & ITALY & ITALY & ITALY & $\mathrm{AL}$ & LANDER/CM & LUCCA & ADAMS \\
\hline 169 & BENDONNI & JOSEPH & B & 55 & M25 & ITALY & ITALY & ITALY & $\mathrm{AL}$ & DAY LABORER/CM & LUCCA & ADAMS \\
\hline 170 & ANDREINI & GEORGE & B & 18 & $\mathrm{~S}$ & ITALY & ITALY & ITALY & & DAY LABORER/CM & LUCCA & ADAMS \\
\hline 171 & ANDREINI & ORLANDO & B & 25 & $\mathrm{~s}$ & ITALY & ITALY & ITALY & $\mathrm{AL}$ & DAY LABORER/CM & LUCCA & ADAMS \\
\hline 172 & VOLPI & JOHN & $\begin{array}{l}\mathrm{O} \\
\mathrm{H}\end{array}$ & 33 & M6 & ITALY & ITALY & ITALY & PA & MINER/CM & AGLIE'. TO & ADAMS \\
\hline 173 & VOLPI & KATE & W & 28 & M6 & ITALY & ITALY & ITALY & & & & ADAMS \\
\hline 174 & VOLPI & JOHN & $\mathrm{s}$ & 5 & S & MICHIGAN & ITALY & ITALY & & & AGLIE'. TO & ADAMS \\
\hline 175 & VOLPI & FRED & $\mathrm{s}$ & 3 & $\mathrm{~s}$ & MICHIGAN & ITALY & ITALY & & & AGLIE', TO & ADAMS \\
\hline 176 & VOLPI & ANNIE & D & 1 & s & MICHIGAN & ITALY & ITALY & & & AGLIE', TO & ADAMS \\
\hline 177 & VOLPI & JOSEPHINE & D & $1 \mathrm{M}$ & s & MICHIGAN & ITALY & ITALY & & & AGLIE'. TO & ADAMS \\
\hline 178 & MILANO & ANNIE & B & 9 & s & MICHIGAN & ITALY & ITALY & & & TORINO & ADAMS \\
\hline 179 & MILANO & GIUSEPPINA & B & 23 & s & MICHIGAN & ITALY & ITALY & & & TORINO & ADAMS \\
\hline 180 & BERTOLERO & PAUL & B & 24 & s & ITALY & ITALY & ITALY & AL & TRAMMER/CM & TORINO & ADAMS \\
\hline 181 & BERTOLERO & ARTHUR & B & $\begin{array}{l}24 \\
40\end{array}$ & M17 & ITALY & ITALY & ITALY & $\mathrm{AL}$ & TRAMMER/CM & TORINO & ADAMS \\
\hline 182 & $\begin{array}{l}\text { CASERIO } \\
\text { Coll }\end{array}$ & JOHN & B & 33 & M11 & ITALY & ITALY & ITALY & $\mathrm{AL}$ & TRAMMER/CM & STRAMBINELLO. TO & ADAMS \\
\hline 183 & ANDREINI & JOSEPH & B & 35 & $\mathrm{~S}$ & ITALY & ITALY & ITALY & $A L$ & TRAMMER/CM & LUCCA & ADAMS \\
\hline 184 & ANDREINI & EMIL & B & 31 & s & ITALY & ITALY & ITALY & $A L$ & TRAMMER/CM & CASTAGNARI. LU & ADAMS \\
\hline $\begin{array}{l}104 \\
185\end{array}$ & ROSILE & GIUSEPPE & $\begin{array}{l}\mathrm{D} \\
\mathrm{H}\end{array}$ & 22 & M0 & ITALY & ITALY & $\begin{array}{l}\text { ITALY } \\
\end{array}$ & $A L$ & TRAMMER/CM & SAN GIUSTO CANAVESE. TO & ADAMS \\
\hline 186 & ROSILE & ROSA & W & 16 & M0 & ITALY & ITALY & ITALY & & & SAN GIUSTO CANAVESE. TO & ADAMS \\
\hline
\end{tabular}




\begin{tabular}{|c|c|c|c|c|c|c|c|c|c|c|c|c|c|c|}
\hline 187 & & SUCCA & DOMINICK & B & 35 & M3 & ITALY & ITALY & ITALY & AL & LABORER/ROCK HOUSE & SAN GIUSTO CANAVESE. TO & & ADAMS \\
\hline 188 & & PUCCI & JOSEPH & B & 35 & s & ITALY & ITALY & ITALY & AL & DAY LABORER/CM & LUCCA & & ADAMS \\
\hline 189 & & PEDRO & JOHN/JANO & B & 28 & $\mathrm{~s}$ & ITALY & ITALY & ITALY & AL & TRAMMER/CM & & & ADAMS \\
\hline 190 & & MARTINELLI & FERDINANDO & B & 22 & $\mathrm{~s}$ & ITALY & ITALY & ITALY & $\mathrm{AL}$ & DAY LABORER/CM & & & ADAMS \\
\hline 191 & & DOMINICI & JOHNATAN & B & 30 & M5 & ITALY & ITALY & ITALY & $\mathrm{AL}$ & LABORER/ROCK HOUSE & & & ADAMS \\
\hline 192 & & MILANO & JOSEPH & $\mathrm{H}$ & 28 & M7 & ITALY & ITALY & ITALY & $\mathrm{AL}$ & TRAMMER/CM & COURGNE'. TO & & ADAMS \\
\hline 193 & & MILANO & PETRONILLA & w & 23 & M7 & ITALY & ITALY & ITALY & & & COURGNE'. TO & & ADAMS \\
\hline 194 & & MILANO & KATHERINE & $\mathrm{D}$ & 2 & s & ITALY & ITALY & ITALY & & & COURGNE'. TO & & ADAMS \\
\hline 195 & & BIANUCCI & EUGENE & $\mathrm{H}$ & 36 & M13 & ITALY & ITALY & ITALY & NA & TRAMMER/CM & ALTOPASCIO. LU & & ADAMS \\
\hline 196 & & BIANUCCI & CLORINDA & W & 33 & M13 & ITALY & ITALY & ITALY & & & ALTOPASCIO. LU & & ADAMS \\
\hline 197 & & BIANUCCI & ARTHUR & $\mathrm{s}$ & 11 & $\mathrm{~s}$ & ITALY & ITALY & ITALY & & AT SCHOOL 10 & ALTOPASCIO. LU & & ADAMS \\
\hline 198 & & BIANUCCI & JOSEPH J. & $\mathrm{s}$ & 7 & $\mathrm{~s}$ & ITALY & ITALY & ITALY & & AT SCHOOL 10 & ALTOPASCIO. LU & & ADAMS \\
\hline 199 & & BIANUCCI & GUIDO & $\mathrm{s}$ & 3 & $\mathrm{~s}$ & MICHIGAN & ITALY & ITALY & & & ALTOPASCIO. LU & & ADAMS \\
\hline 200 & & BIANUCCI & JULIUS & $\mathrm{s}$ & 1 & s & MICHIGAN & ITALY & ITALY & & & ALTOPASCIO. LU & & ADAMS \\
\hline 201 & & FOSSEN & JOHN & B & 30 & $\mathrm{~S}$ & ITALY & ITALY & ITALY & $\mathrm{AL}$ & TRAMMER/CM & & & ADAMS \\
\hline 202 & & MONTFORT & JAMES & B & 23 & s & ITALY & ITALY & ITALY & AL & TRAMMER/CM & & & ADAMS \\
\hline 203 & & BIANCHI & JOSEPH & B & 18 & $\mathrm{~s}$ & ITALY & ITALY & ITALY & & TRAMMER/CM & LUCCA & & ADAMS \\
\hline 204 & & ANTONI & LOUIS & B & 31 & $\mathrm{~S}$ & ITALY & ITALY & ITALY & $\mathrm{AL}$ & DAY LABORER/CM & & & ADAMS \\
\hline 205 & & PEDRONI & JOSEPH & B & 22 & s & ITALY & ITALY & ITALY & $\mathrm{AL}$ & DAY LABORER/CM & & & ADAMS \\
\hline 206 & & BATTISTONI & PAUL & $\mathrm{H}$ & 25 & M0 & ITALY & ITALY & ITALY & $\mathrm{AL}$ & TRAMMER/CM & LUCCA & & ADAMS \\
\hline 207 & & BATTISTONI & PERIMIRA & w & 25 & MO & ITALY & ITALY & ITALY & & & & & ADAMS \\
\hline 208 & & BERNARDI & CHARLES & $\mathrm{H}$ & 38 & M13 & ITALY & ITALY & ITALY & $\mathrm{AL}$ & TRAMMER/CM & & & ADAMS \\
\hline 209 & & BERNARDI & JOSEPHINE & W & 33 & M13 & ITALY & ITALY & ITALY & AL & TRAIVIVILK/UIVI & & & ADAMS \\
\hline 210 & & BETTI & PIETRO & $\mathrm{H}$ & 36 & M12 & ITALY & ITALY & ITALY & $\mathrm{AL}$ & TRAMMER/CM & SEGROMIGNO. LU & & ADAMS \\
\hline 211 & & BETTI & MATILDA & w & 30 & M12 & ITALY & ITALY & ITALY & & & SEGROMIGNO. LU & & ADAMS \\
\hline 212 & & BEI & $\mathrm{JOHN}$ & B & 21 & $\mathrm{~s}$ & ITALY & ITALY & ITALY & $\mathrm{AL}$ & TRAMMER/CM & & & ADAMS \\
\hline 213 & & SODINI & ERNESTO & B & 35 & M11 & ITALY & ITALY & ITALY & $A L$ & DAY LABORER/CM & COLLODI. LU & & ADAMS \\
\hline 214 & & CONSANO & FRANK & B & 35 & M3 & ITALY & ITALY & ITALY & AL & LABORER/ROCK HOUSE & PISA & & ADAMS \\
\hline 215 & 3224 & SCUSSEL & JOSEPH & $\mathrm{H}$ & 30 & M7 & ITALY & ITALY & ITALY & $\mathrm{AL}$ & MINER/CM & BELLUNO & TUNNEL ST. & CALUMET - SWEDETOWN \\
\hline 216 & 3224 & SCUSSEL & TERESA & W & 25 & M7 & ITALY & ITALY & ITALY & & & BELLUNO & TUNNEL ST. & CALUMET - SWEDETOWN \\
\hline 217 & 3224 & SCUSSEL & ONORATO & $\mathrm{s}$ & 5 & $\mathrm{~S}$ & ITALY & ITALY & ITALY & & & BELLUNO & TUNNEL ST. & CALUMET - SWEDETOWN \\
\hline 218 & 3224 & SCUSSEL & JOSEPH & $\mathrm{s}$ & 3 & $\mathrm{~S}$ & MICHIGAN & ITALY & ITALY & & & BELLUNO & TUNNEL ST. & CALUMET - SWEDETOWN \\
\hline 219 & 3224 & SCUSSEL & MARY & $\mathrm{D}$ & 2 & $\mathrm{~s}$ & MICHIGAN & ITALY & ITALY & & & BELLUNO & TUNNEL ST. & CALUMET - SWEDETOWN \\
\hline 220 & 3224 & SCUSSEL & JOHN & NEP & 16 & $\mathrm{~s}$ & ITALY & ITALY & ITALY & AL & MINER/CM & BELLUNO & TUNNEL ST. & CALUMET - SWEDETOWN \\
\hline 221 & 3224 & SOMMARIVA & GEORGE & $\mathrm{B}$ & 37 & $\mathrm{~s}$ & ITALY & ITALY & ITALY & $\mathrm{AL}$ & MINER/CM & BELLUNO & TUNNEL ST. & CALUMET - SWEDETOWN \\
\hline 222 & 3224 & SCUSSEL & JOSEPH & NEP & 18 & s & ITALY & ITALY & ITALY & $\mathrm{AL}$ & MINER/CM & BELLUNO & TUNNEL ST. & $\begin{array}{l}\text { CALUMET - SWEDETOWN } \\
\text { CWOE }\end{array}$ \\
\hline 223 & 3224 & MIANA & SYLVESTER & B & 25 & s & ITALY & ITALY & ITALY & $A L$ & MINER/CM & VOLTAGO. BELLUNO & TUNNEL ST. & CALUMET - SWEDETOWN \\
\hline 224 & 3224 & LEMPIO & $\begin{array}{l}\text { ANULET } \\
\text { AN }\end{array}$ & B & 23 & s & ITALY & ITALY & ITALY & $A L$ & MINER/CM & & TUNNEL ST. & CALUMET - SWEDETOWN \\
\hline 225 & 3224 & SCHENA & JOHN & B & 35 & s & ITALY & ITALY & ITALY & $\mathrm{N}$ ? & STONE MASON & RIVAMONTE. BELLUNO & TUNNEL ST. & CALUMET - SWEDETOWN \\
\hline 226 & 3224 & GIUSTI & PAOLO & B & 23 & s & ITALY & ITALY & ITALY & $\mathrm{AL}$ & MINER/CM & LUCCA & TUNNEL ST. & CALUMET - SWEDETOWN \\
\hline 227 & 3224 & SCUSSEL & ATTILIO & BRIL & 30 & s & ITALY & ITALY & ITALY & AL & MINER/CM & BELLUNO & TUNNEL ST. & CALUMET - SWEDETOWN \\
\hline 228 & 3204 & SCUSSEL & JOHN & $\mathrm{H}$ & & M15 & ITALY & ITALY & ITALY & $\mathrm{N} ?$ & MINER/CM & VOLTAGO. BELLUNO & RIDGE ST. & CALUMET - SWEDETOWN \\
\hline 229 & 3204 & SCUSSEL & JOHANNA & W & I & M15 & ITALY & ITALY & ITALY & & & VOLTAGO. BELLUNO & RIDGE ST. & CALUMET - SWEDETOWN \\
\hline 230 & 3204 & SCUSSEL & DICIDURCO & s & 1 & $\mathrm{~s}$ & ITALY & ITALY & ITALY & & DRILL BOY/CM & VOLTAGO. BELLUNO & RIDGE ST. & CALUMET - SWEDETOWN \\
\hline 231 & 3204 & SCUSSEL & ALEXANDER & $\mathrm{s}$ & 1 & $\mathrm{~S}$ & ITALY & ITALY & ITALY & & AT SCHOOL & VOLTAGO. BELLUNO & RIDGE ST. & CALUMET - SWEDETOWN \\
\hline 232 & 3204 & SCUSSEL & FRANK & $\mathrm{s}$ & I & $\mathrm{S}$ & MICHIGAN & ITALY & ITALY & & AT SCHOOL & VOLTAGO. BELLUNO & RIDGE ST. & CALUMET - SWEDETOWN \\
\hline 233 & 3204 & SCUSSEL & JOHN & NEP & i & $\mathrm{s}$ & ITALY & ITALY & ITALY & $\mathrm{AL}$ & MINER/CM & VOLTAGO. BELLUNO & RIDGE ST. & CALUMET - SWEDETOWN \\
\hline 234 & 3204 & SCUSSEL & FRANK & NEP & $\frac{1}{1}$ & s & ITALY & ITALY & ITALY & AL & MINER/CM & VOLTAGO. BELLUNO & RIDGE ST. & CALUMET - SWEDETOWN \\
\hline 235 & 3204 & ZANIN & OLIVO & B & i & s & ITALY & ITALY & ITALY & $\mathrm{AL}$ & BLACKSMITH/CM & RIVAMONTE. BELLUNO & RIDGE ST. & CALUMET - SWEDETOWN \\
\hline 236 & 3204 & GABRIO & SILVIO & B & 1 & s & ITALY & ITALY & ITALY & $\mathrm{AL}$ & MINER/CM & & RIDGE ST. & CALUUET - SWEDETOWN \\
\hline 237 & 3204 & BRENTEL & JOHN & B & 19 & $\mathrm{~s}$ & ITALY & ITALY & ITALY & $\mathrm{AL}$ & DRILL BOY/CM & SOVRAMONTE. BELLUNO & RIDGE ST. & CALUMET - SWEDETOWN \\
\hline 238 & 3204 & BRENTEL & BENJAMIN & B & 16 & s & ITALY & ITALY & ITALY & $\mathrm{AL}$ & DRILL BOY/CM & SOVRAMONTE, BELLUNO & RIDGE ST. & CALUMET - SWEDETOWN \\
\hline 239 & 3103 & NOTARI & JOHN & $\mathrm{H}$ & 40 & M17 & ITALY & ITALY & ITALY & NA & MINER/CM & POPIGLIO. PISTOIA & RIDGE ST. & CALUMET - SWEDETOWN \\
\hline 240 & 3103 & NOTARI & CESIRA & w & 37 & M17 & ITALY & ITALY & ITALY & & & POPIGLIO. PISTOIA & RIDGE ST. & CALUMET - SWEDETOWN \\
\hline 241 & 3103 & NOTARI & ANNIE & D & 12 & $\mathrm{~s}$ & ITALY & ITALY & ITALY & & AT SCHOOL 10 & POPIGLIO. PISTOIA & RIDGE ST. & CALUMET - SWEDETOWN \\
\hline 242 & 3103 & NOTARI & PETER & $\mathrm{s}$ & 7 & $\mathrm{~s}$ & MICHIGAN & ITALY & ITALY & & AT SCHOOL 10 & POPIGLIO. PISTOIA & RIDGE ST. & CALUMET - SWEDETOWN \\
\hline 243 & 3103 & NOTARI & JAMES & $\mathrm{s}$ & 5 & $\mathrm{~S}$ & MICHIGAN & ITALY & ITALY & & AT SCHOOL 3 & POPIGLIO. PISTOIA & RIDGE ST. & CALUMET - SWEDETOWN \\
\hline 244 & 3102 & CONADERA & VIRGILIO & $\mathrm{H}$ & 40 & M? & ITALY & ITALY & ITALY & NA & MINER/CM & RIVAMONTE, BELLUNO & TUNNEL ST. & CALUMET PR. 1 \\
\hline 245 & 3102 & CONADERA & MONICA & w & 40 & M? & ITALY & ITALY & ITALY & & & RIVAMONTE. BELLUNO & TUNNEL ST. & CALUMET PR. 1 \\
\hline 246 & 3102 & CONADERA & ANTONIA & $\mathrm{D}$ & 15 & $\mathrm{~s}$ & ITALY & ITALY & ITALY & & & RIVAMONTE. BELLUNO & TUNNEL ST. & CALUMET PR. 1 \\
\hline 247 & 3102 & CONADERA & CORA & $\mathrm{D}$ & 10 & $\mathrm{~s}$ & ITALY & ITALY & ITALY & & AT SCHOOL & RIVAMONTE. BELLUNO & TUNNEL ST. & CALUMET PR 1 \\
\hline 248 & 3102 & CONADERA & RENA & $\mathrm{D}$ & 3 & s & MICHIGAN & ITALY & ITALY & & & RIVAMONTE. BELLUNO & TUNNEL ST. & CALUMET PR. 1 \\
\hline 249 & 3102 & CONADERA & JOHANINA & $\mathrm{D}$ & 1 & s & MICHIGAN & ITALY & ITALY & & & RIVAMONTE. BELLUNO & TUNNEL ST. & CALUMET PR. 1 \\
\hline
\end{tabular}




\begin{tabular}{|c|c|c|c|c|c|c|c|c|c|c|c|c|c|c|}
\hline \multirow{2}{*}{$\begin{array}{l}250 \\
251\end{array}$} & \multicolumn{2}{|c|}{3102 PICCHI } & \multirow{2}{*}{$\begin{array}{l}\text { LOUIS } \\
\text { CANNELLA }\end{array}$} & \multirow{2}{*}{$\begin{array}{l}\text { H } \\
\text { W }\end{array}$} & \multirow{2}{*}{$\begin{array}{l}26 \\
18\end{array}$} & \multirow{2}{*}{$\begin{array}{l}\text { M3 } \\
\text { M3 }\end{array}$} & \multirow{2}{*}{$\begin{array}{l}\text { ITALY } \\
\text { ITALY }\end{array}$} & \multirow{2}{*}{$\begin{array}{l}\text { ITALY } \\
\text { ITALY }\end{array}$} & \multirow{2}{*}{$\begin{array}{l}\text { ITALY } \\
\text { ITALY }\end{array}$} & \multirow[t]{2}{*}{$\mathrm{AL}$} & MINER/CM & LUCCA & TUNNEL ST. & CALUMET PR. 1 \\
\hline & 3102 & $\mathrm{PICCHI}$ & & & & & & & & & & & TUNNEL ST. & CALUMET PR. 1 \\
\hline 252 & 3102 & $\mathrm{PICCHI}$ & & $\mathrm{s}$ & 0 & s & MICHIGAN & & & & & LUCCA & TUNNEL ST. & CALUMET PR. 1 \\
\hline 253 & 3102 & $\mathrm{PICCHI}$ & ALESSIO & $\mathrm{BR}$ & 23 & s & ITALY & ITALY & ITALY & $\mathrm{AL}$ & MINER/CM & LUCCA & TUNNEL ST. & CALUMET PR. 1 \\
\hline 254 & 1762 & FRANCISCA & JOHN & $\mathrm{H}$ & 40 & M16 & ITALY & ITALY & ITALY & NA & MINER/CM & PEROSA CANAVESE. TO & BOUNDARY ST. & CALUMET PR. 1 \\
\hline 255 & 1762 & FRANCISCA & ANGELA & W & 40 & M16 & ITALY & ITALY & ITALY & & & PEROSA CANAVESE. TO & BOUNDARY ST. & CALUMET PR. 1 \\
\hline 256 & 1762 & FRANCISCA & LOUIS & s & 15 & $\mathrm{~s}$ & ITALY & ITALY & ITALY & & AT SCHOOL & PEROSA CANAVESE. TO & BOUNDARY ST. & CALUMET PR. 1 \\
\hline 257 & 1762 & FRANCISCA & ANGELO & $\mathrm{s}$ & 15 & s & ITALY & ITALY & ITALY & & AT SCHOOL & PEROSA CANAVESE. TO & BOUNDARY ST. & CALUMET PR. 1 \\
\hline 258 & 1762 & FRANCISCA & JOHN & $\mathrm{s}$ & 7 & $\mathrm{~S}$ & ITALY & ITALY & ITALY & & & PEROSA CANAVESE. TO & BOUNDARY ST. & CALUMET PR. 1 \\
\hline 259 & 1762 & FRANCISCA & FRANK & $\mathrm{s}$ & 2 & $\mathrm{~s}$ & ITALY & ITALY & ITALY & & & PEROSA CANAVESE. TO & BOUNDARY ST. & CALUMET PR. 1 \\
\hline 260 & 1547 & BROCCO & ANTONIO & $\mathrm{H}$ & 32 & M9 & ITALY & ITALY & ITALY & NA & MINER/CM & FIORANO. MODENA & HECLA STREET & CALUMET PR. 1 \\
\hline 261 & 1547 & BROCCO & TERESA & W & 30 & M9 & ITALY & ITALY & ITALY & & & FIORANO. MODENA & HECLA STREET & CALUMET PR. 1 \\
\hline 262 & 1547 & BROCCO & MARY & D & 2 & $\mathrm{~s}$ & MICHIGAN & ITALY & ITALY & & & FIORANO. MODENA & HECLA STREET & CALUMET PR. 1 \\
\hline 263 & 1547 & BROCCO & FELIX & $s$ & 4 & $\mathrm{~s}$ & MICHIGAN & ITALY & ITALY & & & FIORANO. MODENA & HECLA STREET & CALUMET PR. 1 \\
\hline 264 & 1547 & BROCCO & JAMES & s & 5 & s & MICHIGAN & ITALY & ITALY & & & FIORANO. MODENA & HECLA STREET & CALUMET PR. 1 \\
\hline 265 & 1547 & BROCCO & JOSEPH & s & 7 & $\mathrm{~s}$ & MICHIGAN & ITALY & ITALY & & & FIORANO, MODENA & HECLA STREET & CALUMET PR. 1 \\
\hline 266 & 1547 & BROCCO & JOHN & $B R$ & 28 & $\mathrm{~S}$ & ITALY & ITALY & ITALY & $\mathrm{AL}$ & LABORER & FIORANO. MODENA & HECLA STREET & CALUMET PR. 1 \\
\hline 267 & 1547 & GALATIN & $\mathrm{MICH}$ & $\mathrm{H}$ & 26 & M0 & ITALY & ITALY & ITALY & $\mathrm{AL}$ & LABORER & & HECLA STREET & CALUMET PR. 1 \\
\hline 268 & 1547 & GALATIN & CESARINA & W & 26 & MO & ITALY & ITALY & ITALY & & & & HECLA STREET & CALUMET PR. 1 \\
\hline 269 & 1547 & MARSOLA & JAMES & $\mathrm{H}$ & 36 & M15 & ITALY & ITALY & ITALY & & & & HECLA STREET & CALUMET PR. 1 \\
\hline 270 & 1547 & MARSOLA & MARIANA & W & 43 & M15 & ITALY & ITALY & ITALY & & & & HECLA STREET & CALUMET PR. 1 \\
\hline 271 & 1547 & MARSOLA & JOHN & s & 6 & $\mathrm{~s}$ & MICHIGAN & ITALY & ITALY & & & & HECLA STREET & CALUMET PR. 1 \\
\hline 272 & 1537 & FERRARI & ALBERT & $\mathrm{H}$ & 28 & M10 & ITALY & ITALY & ITALY & $\mathrm{AL}$ & MINER/CM & PARIANA. LU & HECLA STREET & CALUMET PR. 1 \\
\hline 273 & 1537 & FERRARI & ANNA & W & 27 & M10 & ITALY & ITALY & ITALY & & & PARIANA. LU & HECLA STREET & CALUMET PR. 1 \\
\hline 274 & 1537 & FERRARI & CAMILLA & D & 6 & $\mathrm{~s}$ & MICHIGAN & ITALY & ITALY & & & PARIANA. LU & HECLA STREET & CALUMET PR. 1 \\
\hline 275 & 1537 & FERRARI & MARY & D & 6 & s & MICHIGAN & ITALY & ITALY & & & PARIANA. LU & HECLA STREET & CALUMET PR. 1 \\
\hline 276 & 1537 & FERRARI & ORLAND & $\mathrm{D}$ ? & 5 & $\mathrm{~s}$ & MICHIGAN & ITALY & ITALY & & & PARIANA. LU & HECLA STREET & CALUMET PR. 1 \\
\hline 277 & 1537 & FERRARI & JAMES & s & 4 & s & MICHIGAN & ITALY & ITALY & & & PARIANA, LU & HECLA STREET & CALUMET PR. 1 \\
\hline 278 & 1537 & GUERRA & ANGEL & B & 30 & s & ITALY & ITALY & ITALY & $\mathrm{AL}$ & MINER/CM & LUCCA & HECLA STREET & CALUMET PR. 1 \\
\hline 279 & 1537 & GUERRA & THOMAS & B & 32 & $\mathrm{~s}$ & ITALY & ITALY & ITALY & $\mathrm{AL}$ & MINER/CM & LUCCA & HECLA STREET & CALUMET PR. 1 \\
\hline 280 & 1537 & GUERRA & AUGUST & B & 28 & $\mathrm{~s}$ & ITALY & ITALY & ITALY & $\mathrm{AL}$ & MINER/CM & LUCCA & HECLA STREET & CALUMET PR. 1 \\
\hline 281 & 1537 & VEZZANI & SAM & B & 32 & s & ITALY & ITALY & ITALY & $\mathrm{AL}$ & MINER/CM & PORCARI. LU & HECLA STREET & CALUMET PR. 1 \\
\hline 282 & 1537 & BIANCHI & MARCHESO & B & 20 & $\mathrm{~s}$ & ITALY & ITALY & ITALY & $\mathrm{AL}$ & MINER/CM & LUCCA & HECLA STREET & CALUMET PR. 1 \\
\hline 283 & 1535 & GIOVANNINI & ANTONIO & $\mathrm{H}$ & 35 & M9 & ITALY & ITALY & ITALY & NA & MINER/CM & SAN GIUSTO CANAVESE, TO & HECLA STREET & CALUMET PR. 1 \\
\hline 284 & 1535 & GIOVANNINI & GIUSTA & W & 24 & M9 & ITALY & ITALY & ITALY & UN & & SAN GIUSTO CANAVESE. TO & HECLA STREET & CALUMET PR. 1 \\
\hline 285 & 1535 & GIOVANNINI & TERESA & D & 9 & s & ITALY & ITALY & ITALY & UN & AT SCHOOL 10 & SAN GIUSTO CANAVESE. TO & HECLA STREET & CALUMET PR. 1 \\
\hline 286 & 1535 & GIOVANNINI & ANTONIO & s & 7 & s & MICHIGAN & ITALY & ITALY & & AT SCHOOL 10 & SAN GIUSTO CANAVESE. TO & HECLA STREET & CALUMET PR. 1 \\
\hline 287 & 1535 & GIOVANNINI & VICTOR & $\mathrm{s}$ & 5 & $\mathrm{~s}$ & MICHIGAN & ITALY & ITALY & & & SAN GIUSTO CANAVESE. TO & HECLA STREET & CALUMET PR. 1 \\
\hline 288 & 1535 & GIOVANNINI & DOMENICO & $\mathrm{s}$ & 3 & $\mathrm{~s}$ & MICHIGAN & ITALY & ITALY & & & SAN GIUSTO CANAVESE. TO & HECLA STREET & CALUMET PR. 1 \\
\hline 289 & 1537 & NARDI & ROBERT & $\mathrm{H}$ & 31 & M7 & ITALY & ITALY & ITALY & $\mathrm{AL}$ & MINER/CM & LUCCA & HECLA STREET & CALUMET PR. 1 \\
\hline 290 & 1537 & NARDI & CESERINA & W & 27 & M7 & ITALY & ITALY & ITALY & & & LUCCA & HECLA STREET & CALUMET PR. 1 \\
\hline 291 & 1537 & NARDI & GIULIO & $s$ & 7 & $\mathrm{~s}$ & MICHIGAN & ITALY & ITALY & & & LUCCA & HECLA STREET & CALUMET PR. 1 \\
\hline 292 & 1537 & NARDI & GIULIANA & D & 5 & $\mathrm{~s}$ & MICHIGAN & ITALY & ITALY & & AT SCHOOL 10 & LUCCA & HECLA STREET & CALUMET PR. 1 \\
\hline 293 & 1537 & NARDI & ROCCO & S & 4 & s & MICHIGAN & ITALY & ITALY & & & LUCCA & HECLA STREET & CALUMET PR. 1 \\
\hline 294 & 1537 & NARDI & JOSEPINA & $\mathrm{D}$ & 2 & s & MICHIGAN & ITALY & ITALY & & & LUCCA & HECLA STREET & CALUMET PR. 1 \\
\hline 295 & 1544 & FETTA & JOHN & $\mathrm{H}$ & 41 & M4 & ITALY & ITALY & ITALY & NA & MINER/CM & BAIRO CANAVESE. TO & HECLA STREET & CALUMET PR. 1 \\
\hline 296 & 1544 & FETTA & BARBARA & W & 25 & M4 & ITALY & ITALY & ITALY & & IVIIVERACIII & DAIRU UAIVAVEOE. TO & HECLA STREET & CALUMET PR. 1 \\
\hline 297 & 1544 & FETTA & $\begin{array}{l}\text { DUAY } \\
\text { LUAA }\end{array}$ & D & 2 & S & MICHIGAN & ITALY & ITALY & & & BAIRO CANAVESE. TO & HECLA STREET & CALUMET PR. 1 \\
\hline 298 & 1544 & FETTA & BENJAMIN & s & 2 & s & MICHIGAN & ITALY & ITALY & & & BAIRO CANAVESE. TO & HECLA STREET & CALUMET PR. 1 \\
\hline 299 & 1544 & FORNO & CHRISTINA & SIIL & 21 & $\mathrm{~s}$ & ITALY & ITALY & ITALY & & SERVANT & $\begin{array}{l}\text { TORINO } \\
\text { TOUL TS }\end{array}$ & HECLA STREET & CALUMET PR. 1 \\
\hline 300 & 1686 & RICCA & JOSEPH & $\mathrm{H}$ & 36 & M? & ITALY & ITALY & ITALY & & MINER/CM & TORINO & BOUNDARY ST. & CALUMET PR. 1 \\
\hline 301 & 1686 & RICCA & MARGHERITA & W & 52 & M? & ITALY & ITALY & ITALY & & & & BOUNDARY ST. & CALUMET PR. 1 \\
\hline 302 & 1686 & RICCA & MATTHEW & s & 8 & $\mathrm{~s}$ & ITALY & ITALY & ITALY & & & TORINO & BOUNDARY ST. & CALUMET PR. 1 \\
\hline 303 & 1686 & RICCA & LUCY & $\mathrm{D}$ & 6 & $\mathrm{~s}$ & ITALY & ITALY & ITALY & & & TORINO & BOUNDARY ST. & CALUMET PR. 1 \\
\hline 304 & 1686 & RICCA & MARY & $\mathrm{D}$ & 3 & s & ITALY & ITALY & ITALY & & & TORINO & BOUNDARY ST. & CALUMET PR. 1 \\
\hline 305 & 1686 & RICCA & CALUMBER & $\mathrm{s}$ & $1 \mathrm{M}$ & $\mathrm{s}$ & ITALY & ITALY & ITALY & & & TORINO & BOUNDARY ST. & CALUMET PR. 1 \\
\hline 306 & 1686 & RICCA & ANTON & B & 26 & $?$ & ITALY & ITALY & ITALY & & MINER/CM & TORINO & BOUNDARY ST. & CALUMET PR. 1 \\
\hline 307 & 1602 & VIANI & NICHOLAS & $\mathrm{H}$ & 51 & M30 & ITALY & ITALY & ITALY & $?$ & NOT CLEAR & PORTO MAURIZIO, IMPERIA & RAILROAD ST. & CALUMET PR. 1 \\
\hline 308 & 1602 & VIANI & CATHARINA & w & 49 & M30 & ITALY & ITALY & ITALY & $?$ & & PORTO MAURIZIO. IMPERIA & RAILROAD ST. & CALUMET PR. 1 \\
\hline 309 & 1602 & VIANI & PETER & s & 27 & $\mathrm{~s}$ & ITALY & ITALY & ITALY & $?$ & NOT CLEAR & PORTO MAURIZIO. IMPERIA & RAILROAD ST. & CALUMET PR. 1 \\
\hline 310 & 1602 & VIANI & FRANK & $\mathrm{s}$ & 25 & $\mathrm{~s}$ & ITALY & ITALY & ITALY & $?$ & NOT CLEAR & PORTO MAURIZIO. IMPERIA & RAILROAD ST. & CALUMET PR 1 \\
\hline 311 & 1602 & VIANI & GEORGE & $\mathrm{s}$ & 23 & $\mathrm{~s}$ & ITALY & ITALY & ITALY & $?$ & NOT CLEAR & PORTO MAURIZIO. IMPERIA & RAILROAD ST. & CALUMET PR. 1 \\
\hline 312 & 1604 & GIULIO & ANTON & $\mathrm{H}$ & 42 & M9 & ITALY & ITALY & ITALY & NA & MINER/CM & SAN GIORGIO CANAVESE. TO & RAILROAD ST. & CALUMET PR. 1 \\
\hline
\end{tabular}




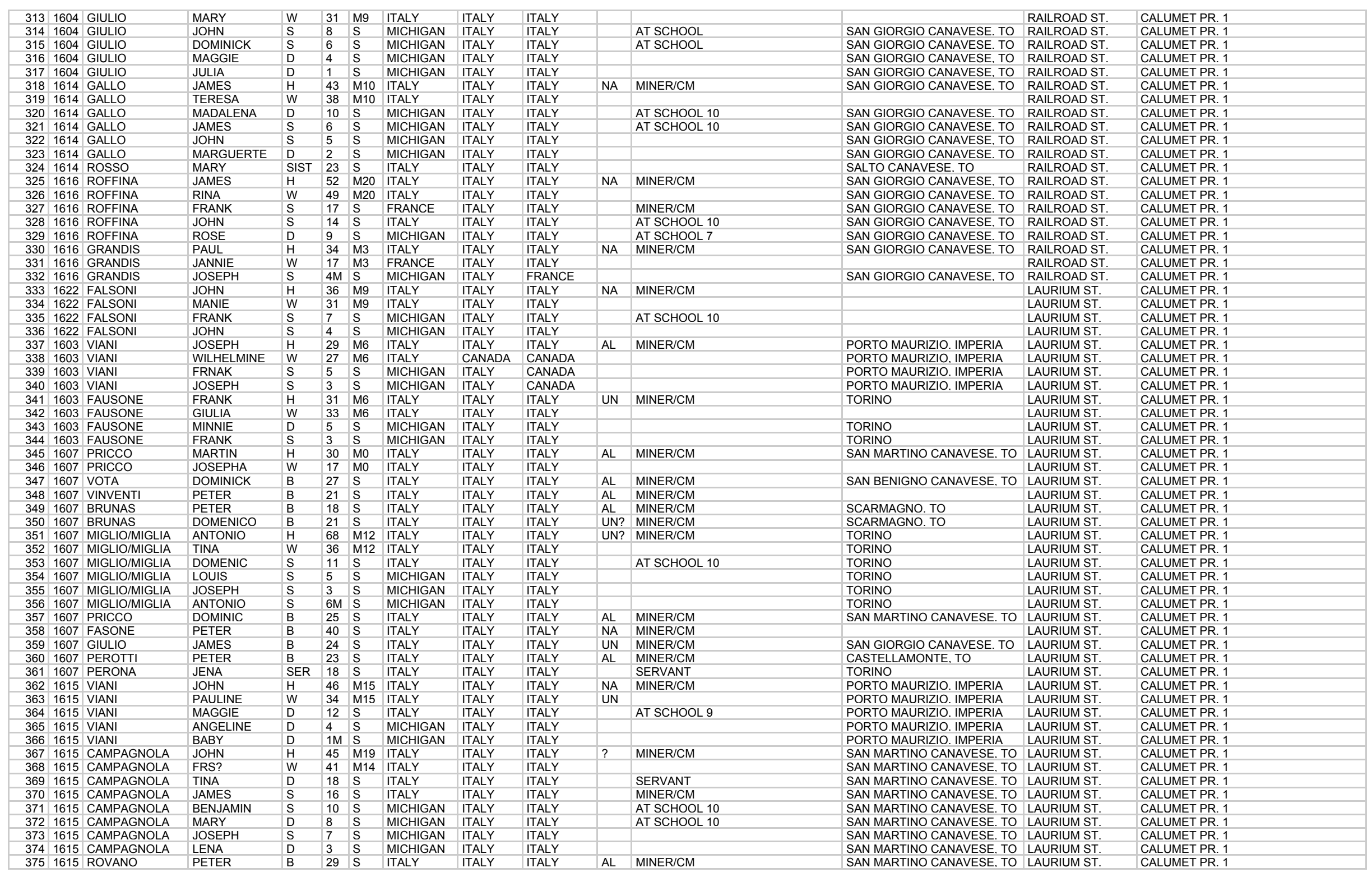




\begin{tabular}{|c|c|c|c|c|c|c|c|c|c|c|c|c|c|c|}
\hline \multirow{2}{*}{$\begin{array}{l}376 \\
377 \\
\end{array}$} & \multirow{2}{*}{\multicolumn{2}{|c|}{1615 ROVANO }} & \multirow{2}{*}{$\begin{array}{l}\text { ANTON } \\
\text { ANTON }\end{array}$} & \multirow{2}{*}{$\begin{array}{l}\mathrm{B} \\
\mathrm{B}\end{array}$} & \multirow{2}{*}{$\begin{array}{l}22 \\
28\end{array}$} & \multirow{2}{*}{\multicolumn{2}{|c|}{\begin{tabular}{l|l}
$S$ & ITALY \\
\end{tabular}}} & \multirow{2}{*}{$\begin{array}{l}\text { ITALY } \\
\text { ITALY }\end{array}$} & \multirow{2}{*}{$\begin{array}{l}\text { ITALY } \\
\text { ITALLY }\end{array}$} & \multirow{2}{*}{$\begin{array}{l}\mathrm{AL} \\
\mathrm{NA}\end{array}$} & \multirow{2}{*}{$\begin{array}{l}\text { MINER/CM } \\
\text { MINER/CM }\end{array}$} & SAN MARTINO CANAVESE. TO & LAURIUM ST. & CALUMET PR. 1 \\
\hline & & BARSICA & & & & $\mathrm{s}$ & & & & & & SAN MARTINO CANAVESE. TO & LAURIUM ST. & CALUMET PR. 1 \\
\hline 378 & 1615 & BARSICA & JOSEPH & B & 21 & $s$ & ITALY & ITALY & ITALY & AL & MINER/CM & SAN MARTINO CANAVESE. TO & LAURIUM ST. & CALUMET PR. 1 \\
\hline 379 & 1615 & TINETTI & PETER & B & 24 & $\mathrm{~s}$ & ITALY & ITALY & ITALY & AL & MINER/CM & SAN MARTINO CANAVESE. TO & LAURIUM ST. & CALUMET PR. 1 \\
\hline 380 & 1615 & TINETTI & MARTIN & B & 28 & $\mathrm{~S}$ & ITALY & ITALY & ITALY & UN & MINER/CM & SAN MARTINO CANAVESE. TO & LAURIUM ST. & CALUMET PR. 1 \\
\hline 381 & 1615 & MASERO & FRANK & B & 28 & $\mathrm{~s}$ & ITALY & ITALY & ITALY & $?$ & MINER/CM & TORINO & LAURIUM ST. & CALUMET PR. 1 \\
\hline 382 & 1615 & SCALA & ANTHONY & B & 28 & $\mathrm{~s}$ & ITALY & ITALY & ITALY & AL & MINER/CM & STRAMBINELLO. TO & LAURIUM ST. & CALUMET PR. 1 \\
\hline 383 & 1615 & CENTANINO & JOHN & B & 24 & $\mathrm{~s}$ & ITALY & ITALY & ITALY & $\mathrm{AL}$ & MINER/CM & TORINO & LAURIUM ST. & CALUMET PR. 1 \\
\hline 384 & 1615 & COPPO & EUGENE & B & 19 & $\mathrm{~s}$ & ITALY & ITALY & ITALY & $\mathrm{AL}$ & MINER/CM & PONT CANAVESE. TO & LAURIUM ST. & CALUMET PR. 1 \\
\hline 385 & 1615 & TINETTI & MICHELE & B & 27 & $\mathrm{~s}$ & ITALY & ITALY & ITALY & $\mathrm{AL}$ & MINER/CM & SAN MARTINO CANAVESE. TO & LAURIUM ST. & CALUMET PR. 1 \\
\hline 386 & 1615 & BRONZO & DOMINIC & B & 24 & $\mathrm{~s}$ & ITALY & ITALY & ITALY & $\mathrm{AL}$ & MINER/CM & SAN MARTINO CANAVESE. TO & LAURIUM ST. & CALUMET PR. 1 \\
\hline 387 & 1617 & DARMA & JOHN & B & 24 & $\mathrm{~s}$ & ITALY & ITALY & ITALY & UN & MINER/CM & & LAURIUM ST. & CALUMET PR. 1 \\
\hline 388 & 1617 & DATA/DATTA & ANTONIO & $\mathrm{H}$ & 27 & M5 & ITALY & ITALY & ITALY & AL & MINER/CM & SAN GIORGIO CANAVESE. TO & $\begin{array}{l}\text { LAURIUM ST. } \\
\text { LAIN }\end{array}$ & CALUMET PR. 1 \\
\hline 389 & 1617 & DATA/DATTA & ANNIE & W & 25 & M5 & ITALY & ITALY & ITALY & & & SAN GIORGIO CANAVESE. TO & LAURIUM ST. & CALUMET PR. 1 \\
\hline 390 & 1617 & DATA/DATTA & GIUSEPPE & s & 3 & $\mathrm{~s}$ & ITALY & ITALY & ITALY & & & SAN GIORGIO CANAVESE. TO & LAURIUM ST. & CALUMET PR. 1 \\
\hline 391 & 1617 & DATA/DATTA & MICHELE & $\mathrm{s}$ & 11 & $\mathrm{~s}$ & ITALY & ITALY & ITALY & & & SAN GIORGIO CANAVESE, TO & LAURIUM ST. & CALUMET PR. 1 \\
\hline 392 & 1617 & DORMIA & ANTON & B & 27 & $\mathrm{~S}$ & ITALY & ITALY & ITALY & UN & MINER/CM & & LAURIUM ST. & CALUMET PR. 1 \\
\hline 393 & 1619 & MARCHETTI & JOHN & $\mathrm{H}$ & 30 & M0 & ITALY & ITALY & ITALY & NA & & LUCCA & LAURIUM ST. & CALUMET PR. 1 \\
\hline 394 & 1619 & MARCHETTI & AURELIA & w & 19 & MO & ITALY & ITALY & ITALY & & & & LAURIUM ST. & CALUMET PR. 1 \\
\hline 395 & 1619 & MASSOGLIA & PETER & $\mathrm{H}$ & 30 & M3 & ITALY & ITALY & ITALY & NA & BLACKSMITH & TORINO & LAURIUM ST. & CALUMET PR. 1 \\
\hline 396 & 1619 & MASSOGLIA & CATHERINA & w & 32 & M3 & ITALY & ITALY & ITALY & & & TORINO & LAURIUM ST. & CALUMET PR. 1 \\
\hline 397 & 1619 & MASSOGLIA & MARIE & $\mathrm{D}$ & 10 & $\mathrm{~s}$ & ITALY & ITALY & ITALY & & AT SCHOOL 10 & TORINO & LAURIUM ST. & CALUMET PR. 1 \\
\hline 398 & 1621 & DE FILIPPI & JOSEPH & $\mathrm{H}$ & 30 & M3 & ITALY & ITALY & ITALY & NA & MINER/CM & SAN GIORGIO CANAVESE. TO & LAURIUM ST. & CALUMET PR. 1 \\
\hline 399 & 1621 & DE FILIPPI & MARIE & W & 20 & M3 & ITALY & ITALY & ITALY & & & & LAURIUM ST. & CALUMET PR. 1 \\
\hline 400 & 1621 & DE FILIPPI & BABYIJULIA & D & $1 \mathrm{M}$ & $\mathrm{s}$ & MICHIGAN & ITALY & ITALY & & & SAN GIORGIO CANAVESE. TO & LAURIUM ST. & CALUMET PR. 1 \\
\hline 401 & 1631 & CAPELLO & JOHN & $\mathrm{H}$ & 67 & M? & ITALY & ITALY & ITALY & & MINER/CM & SAN BENIGNO CANAVESE. TO & ROCKLAND ST. & CALUMET PR. 1 \\
\hline 402 & 1631 & CAPELLO & MARY & W & 41 & $M ?$ & ITALY & ITALY & ITALY & & & SAN BENIGNO CANAVESE. TO & ROCKLAND ST. & CALUMET PR. 1 \\
\hline 403 & 1631 & NIGRA/NIGRO & JOSPEH & B & 39 & $?$ & ITALY & ITALY & ITALY & & MASON & SAN GIUSTO CANAVESE, TO & ROCKLAND ST. & CALUMET PR. 1 \\
\hline 404 & 1631 & VIOLA & PAUL & B & 30 & $?$ & ITALY & ITALY & ITALY & & MINER/CM & SAN BENIGNO CANAVESE. TO & ROCKLAND ST. & CALUMET PR. 1 \\
\hline 405 & 1631 & NOTARIO & $\mathrm{JOHN}$ & B & 28 & $?$ & ITALY & ITALY & ITALY & & MINER/CM & SAN BENIGNO CANAVESE. TO & ROCKLAND ST. & CALUMET PR. 1 \\
\hline 406 & 1631 & BOGGIO & FRANK & B & 32 & $?$ & ITALY & ITALY & ITALY & & MINER/CM & SAN GIUSTO CANAVESE. TO & ROCKLAND ST. & CALUMET PR. 1 \\
\hline 407 & 1631 & BRACCO & PAUL & B & 26 & $?$ & ITALY & ITALY & ITALY & & MINER/CM & TORINO & ROCKLAND ST. & CALUMET PR. 1 \\
\hline 408 & 1631 & BRACCO & MIKE & B & 26 & $?$ & ITALY & ITALY & ITALY & & MINER/CM & TORINO & ROCKLAND ST. & CALUMET PR. 1 \\
\hline 409 & 1631 & SASSO & FRANK & B & 30 & $?$ & ITALY & ITALY & ITALY & & MINER/CM & SAN BENIGNO CANAVESE, TO & ROCKLAND ST. & CALUMET PR. 1 \\
\hline 410 & 1631 & DARMA & JOSEPH & B & 33 & $?$ & ITALY & ITALY & ITALY & & MINER/CM & & ROCKLAND ST. & CALUMET PR. 1 \\
\hline 411 & 1516 & MICHELA & GEORGE & $\mathrm{D}$ & 38 & M10 & $\begin{array}{l}\text { ITALY } \\
\text { ITALY }\end{array}$ & ITALY & ITALY & & MINER/CM & SAN GIORGIO CANAVESE. TO & ROCKLAND ST. & CALUMET PR. 1 \\
\hline 412 & 1516 & MICHELA & VITTORIA & w & 31 & M10 & ITALY & ITALY & ITALY & & & & ROCKLAND ST. & CALUMET PR. 1 \\
\hline 413 & 1516 & MICHELA & DOMENICO & s & 9 & $\mathrm{~s}$ & MICHIGAN & ITALY & ITALY & & & SAN GIORGIO CANAVESE. TO & ROCKLAND ST. & CALUMET PR. 1 \\
\hline 414 & 1516 & MICHELA & JOHN & s & 7 & $\mathrm{~s}$ & MICHIGAN & ITALY & ITALY & & & SAN GIORGIO CANAVESE. TO & ROCKLAND ST. & CALUMET PR. 1 \\
\hline 415 & 1516 & MICHELA & MARY & $\mathrm{D}$ & 6 & $\mathrm{~s}$ & MICHIGAN & ITALY & ITALY & & & SAN GIORGIO CANAVESE. TO & ROCKLAND ST. & CALUMET PR. 1 \\
\hline 416 & 1516 & MICHELA & JOHN & $\mathrm{s}$ & 4 & $\mathrm{~s}$ & MICHIGAN & ITALY & ITALY & & & SAN GIORGIO CANAVESE. TO & ROCKLAND ST. & CALUMET PR. 1 \\
\hline 417 & 1516 & MICHELA & FLORA & D & 3 & s & MICHIGAN & ITALY & ITALY & & & SAN GIORGIO CANAVESE. TO & ROCKLAND ST. & CALUMET PR. 1 \\
\hline 418 & 1516 & MICHELA & ANGELO & $\mathrm{s}$ & 1 & $\mathrm{~S}$ & MICHIGAN & ITALY & ITALY & & & SAN GIORGIO CANAVESE. TO & ROCKLAND ST. & CALUMET PR. 1 \\
\hline 419 & 1303 & CRUGNOLA & CHARLES & H & 38 & M? & ITALY & ITALY & ITALY & & MASON & COMO & ROCKLAND ST. & CALUMET PR. 1 \\
\hline 420 & 1303 & CRUGNOLA & MODESTA & w & 37 & $\mathrm{M} ?$ & ITALY & ITALY & ITALY & & & COMO & ROCKLAND ST. & CALUMET PR. 1 \\
\hline 421 & 1303 & CRUGNOLA & ERMINIA & $\mathrm{D}$ & 9 & $\mathrm{~s}$ & SWITZERLA & ITALY & ITALY & & & COMO & ROCKLAND ST. & CALUMET PR. 1 \\
\hline 422 & 1303 & CRUGNOLA & EMMA & $\mathrm{D}$ & 8 & $\mathrm{~s}$ & SWITZERLA & ITALY & ITALY & & & COMO & ROCKLAND ST. & CALUMET PR. 1 \\
\hline 423 & 1303 & CRUGNOLA & GETANO & $\mathrm{s}$ & 5 & s & MICHIGAN & ITALY & ITALY & & & COMO & ROCKLAND ST. & CALUMET PR. 1 \\
\hline 424 & 1303 & CRUGNOLA & MARY & $\mathrm{D}$ & 3 & s & MICHIGAN & ITALY & ITALY & & & COMO & ROCKLAND ST. & CALUMET PR. 1 \\
\hline 425 & 1303 & CRUGNOLA & LIDIA & D & $1 \mathrm{M}$ & $\mathrm{s}$ & MICHIGAN & ITALY & ITALY & & & COMO & ROCKLAND ST. & CALUMET PR. 1 \\
\hline 426 & 1303 & SCUSSEL & GABRIEL & $\mathrm{H}$ & 38 & M? & ITALY & ITALY & ITALY & & MINER/CM & BELLUNO & ROCKLAND ST. & CALUMET PR. 1 \\
\hline 427 & 1303 & SCUSSEL & JOANNA & W & 28 & M? & ITALY & ITALY & ITALY & & & BELLUNO & ROCKLAND ST. & CALUMET PR. 1 \\
\hline 428 & 1303 & SCUSSEL & DAMIANO & BRIL & 22 & $\mathrm{~s}$ & ITALY & ITALY & ITALY & & MINER/CM & BELLUNO & ROCKLAND ST. & CALUMET PR. 1 \\
\hline 429 & 1306 & PIGNOCCO & CHARLES & $\mathrm{H}$ & 40 & M? & ITALY & ITALY & ITALY & & MINER/CM & SAN GIORGIO CANAVESE. TO & PINE/ROCKLAND & CALUMET PR. 1 \\
\hline 430 & 1306 & PIGNOCCO & SECONDINA & w & 29 & M? & ITALY & ITALY & ITALY & & & & PINE/ROCKLAND & CALUMET PR. 1 \\
\hline 431 & 1306 & PIGNOCCO & JOHN & $\mathrm{s}$ & 9 & $\mathrm{~s}$ & MICHIGAN & ITALY & ITALY & & AT SCHOOL & SAN GIORGIO CANAVESE. TO & PINE/ROCKLAND & CALUMET PR. 1 \\
\hline 432 & 1306 & PIGNOCCO & DOMENICO & $\mathrm{s}$ & 8 & s & MICHIGAN & ITALY & ITALY & & AT SCHOOL & SAN GIORGIO CANAVESE. TO & PINE/ROCKLAND & CALUMET PR. 1 \\
\hline 433 & 1306 & PIGNOCCO & GEORGE & $\mathrm{s}$ & 5 & s & MICHIGAN & ITALY & ITALY & & AT SCHOOL & SAN GIORGIO CANAVESE, TO & PINE/ROCKLAND & CALUMET PR. 1 \\
\hline 434 & 1306 & PIGNOCCO & JULIA & $\mathrm{D}$ & 4 & s & MICHIGAN & ITALY & ITALY & & & SAN GIORGIO CANAVESE. TO & PINE/ROCKLAND & CALUMET PR. 1 \\
\hline 435 & 1304 & VALLE & ANTONIO & $\mathrm{H}$ & 50 & M20 & ITALY & ITALY & ITALY & & MINER/CM & TORINO & PINE/ROCKLAND & CALUMET PR. 1 \\
\hline 436 & 1304 & VALLE & MARY & w & 36 & M20 & ITALY & ITALY & ITALY & & & TORINO & PINE/ROCKLAND & CALUMET PR 1 \\
\hline 437 & 1304 & VALLE & STEPHEN & s & 19 & $\mathrm{~s}$ & ITALY & ITALY & ITALY & & MINER/CM & TORINO & PINE/ROCKLAND & CALUMET PR. 1 \\
\hline 438 & 1304 & VALLE & MARY & $\mathrm{D}$ & 12 & s & MICHIGAN & ITALY & ITALY & & & TORINO & PINE/ROCKLAND & CALUMET PR. 1 \\
\hline
\end{tabular}




\begin{tabular}{|c|c|c|c|c|c|c|c|c|c|c|c|c|c|c|}
\hline 439 & 1318 & BOGGIO & JOHN & $\mathrm{H}$ & 36 & M? & ITALY & ITALY & ITALY & $?$ & MINER/CM & SAN GIUSTO CANAVESE. TO & ROCKLAND ST. & CALUMET PR. 1 \\
\hline 440 & 1318 & BOGGIO & MAGGIE & W & 35 & M? & ITALY & ITALY & ITALY & & & SAN GIUSTO CANAVESE. TO & ROCKLAND ST. & CALUMET PR. 1 \\
\hline 441 & 1318 & BOGGIO & ANTONIO & $s$ & 7 & $\mathrm{~S}$ & ITALY & ITALY & ITALY & & & SAN GIUSTO CANAVESE. TO & ROCKLAND ST. & CALUMET PR. 1 \\
\hline 442 & 1318 & BOGGIO & MAUD & D & 6 & $\mathrm{~s}$ & ITALY & ITALY & ITALY & & & SAN GIUSTO CANAVESE. TO & ROCKLAND ST. & CALUMET PR. 1 \\
\hline 443 & 1318 & BINANDO & JOSEPH & B & 24 & $?$ & ITALY & ITALY & ITALY & & MINER/CM & CORTEREGGIO. TO & ROCKLAND ST. & CALUMET PR. 1 \\
\hline 444 & 1318 & VIGO & MARK & B & 31 & $?$ & ITALY & ITALY & ITALY & & MINER/CM & CORIO. TO & ROCKLAND ST. & CALUMET PR. 1 \\
\hline 445 & 1318 & TRUCCANO & PETER & B & 19 & $?$ & ITALY & ITALY & ITALY & & MINER/CM & CORTEREGGIO. TO & ROCKLAND ST. & CALUMET PR. 1 \\
\hline 446 & 1318 & TRUCCANO & JOHN & B & 23 & $?$ & ITALY & ITALY & ITALY & & MINER/CM & CORTEREGGIO. TO & ROCKLAND ST. & CALUMET PR. 1 \\
\hline 447 & 1318 & CERUTTI & GEORGE & B & 30 & $?$ & ITALY & ITALY & ITALY & & MINER/CM & SAN GIORGIO CANAVESE. TO & ROCKLAND ST. & CALUMET PR. 1 \\
\hline 448 & 1318 & TRUCCANO & JOSEPH & B & 24 & $?$ & ITALY & ITALY & ITALY & & MINER/CM & CORTEREGGIO. TO & ROCKLAND ST. & CALUMET PR. 1 \\
\hline 449 & 1318 & ZONINO & JOHN & B & 38 & $?$ & ITALY & ITALY & ITALY & & MINER/CM & & ROCKLAND ST. & CALUMET PR. 1 \\
\hline 450 & 3940 & CINOTTA & ANGELO & B & 33 & s & ITALY & ITALY & ITALY & NA & MINER/CM & & & CALUMET PR. 1 \\
\hline 451 & 3941 & PICCHIOTTINO & JOSEPH & $\mathrm{H}$ & 39 & M14 & ITALY & ITALY & ITALY & NA & MINER/CM & PONT CANAVESE. TO & & CALUMET PR. 1 \\
\hline 452 & 3941 & PICCHIOTTINO & PAULINE & W & 33 & M14 & ITALY & ITALY & ITALY & & & PONT CANAVESE. TO & & CALUMET PR. 1 \\
\hline 453 & 3941 & PICCHIOTTINO & MINNIE & D & 12 & s & MICHIGAN & ITALY & ITALY & & AT SCHOOL 10 & PONT CANAVESE. TO & & CALUMET PR. 1 \\
\hline 454 & 3941 & PICCHIOTTINO & LENA & $\mathrm{D}$ & 10 & $\mathrm{~s}$ & MICHIGAN & ITALY & ITALY & & AT SCHOOL 10 & PONT CANAVESE, TO & & CALUMET PR. 1 \\
\hline 455 & 3941 & ROLANDO & JOSEPH & B & 24 & $\mathrm{~s}$ & ITALY & ITALY & ITALY & UN & MINER/CM & PONT CANAVESE. TO & & CALUMET PR. 1 \\
\hline 456 & 3941 & ROMANO & JAMES & B & 28 & $\mathrm{~S}$ & ITALY & ITALY & ITALY & UN & MINER/CM & TORINO & & CALUMET PR. 1 \\
\hline 457 & 3941 & ROMANO & JOSEPH & B & 27 & S & ITALY & ITALY & ITALY & UN & MINER/CM & SAN GIORGIO CANAVESE. TO & & CALUMET PR. 1 \\
\hline 458 & 3941 & PICCHIOTTINO & BAPTISTE & $\mathrm{H}$ & 32 & M8 & ITALY & ITALY & ITALY & UN & MINER/CM & PONT CANAVESE. TO & & CALUMET PR. 1 \\
\hline 459 & 3941 & PICCHIOTTINO & MAGGIE & w & 39 & M8 & ITALY & ITALY & ITALY & & & & & CALUMET PR. 1 \\
\hline 460 & 3941 & PICCHIOTTINO & ANGELO & s & 6 & $\mathrm{~s}$ & MICHIGAN & ITALY & ITALY & & AT SCHOOL 10 & PONT CANAVESE, TO & & CALUMET PR. 1 \\
\hline 461 & 3941 & PICCHIOTTINO & LUCY & $\mathrm{D}$ & 5 & $\mathrm{~s}$ & MICHIGAN & ITALY & ITALY & & AT SCHOOL 5 & PONT CANAVESE. TO & & CALUMET PR. 1 \\
\hline 462 & 3941 & PICCHIOTTINO & KATY & $\mathrm{D}$ & 3 & $\mathrm{~s}$ & MICHIGAN & ITALY & ITALY & & & PONT CANAVESE. TO & & CALUMET PR. 1 \\
\hline 463 & 3922 & BASSO & JAMES & $\mathrm{H}$ & 40 & M10 & ITALY & ITALY & ITALY & NA & MINER/CM & SAN GIORGIO CANAVESE. TO & 6TH ST. & CALUMET PR. 1 \\
\hline 464 & 3922 & BASSO & FRANCES & W & 27 & M10 & ITALY & ITALY & ITALY & & DIIIVLTO & & 6TH ST. & CALUMET PR. 1 \\
\hline 465 & 3922 & BASSO & TERESA & D & 8 & $\mathrm{~s}$ & MICHIGAN & ITALY & ITALY & & & SAN GIORGIO CANAVESE. TO & 6TH ST. & CALUMET PR. 1 \\
\hline 466 & 3922 & BASSO & KATY & $\mathrm{D}$ & 7 & $\mathrm{~s}$ & MICHIGAN & ITALY & ITALY & & & SAN GIORGIO CANAVESE, TO & 6TH ST. & CALUMET PR. 1 \\
\hline 467 & 3922 & BASSO & LUCY & D & 5 & $\mathrm{~s}$ & MICHIGAN & ITALY & ITALY & & & SAN GIORGIO CANAVESE. TO & 6TH ST. & CALUMET PR. 1 \\
\hline 468 & 3922 & BASSO & LOUIS & $\mathrm{s}$ & 3 & $\mathrm{~S}$ & MICHIGAN & ITALY & ITALY & & & SAN GIORGIO CANAVESE. TO & 6TH ST. & CALUMET PR. 1 \\
\hline 469 & 3922 & BASSO & JOSEPH & $\mathrm{s}$ & 1 & $\mathrm{~S}$ & MICHIGAN & ITALY & ITALY & & & SAN GIORGIO CANAVESE. TO & 6TH ST. & CALUMET PR. 1 \\
\hline 470 & 3922 & PONSETTO & GIORGIO & B & 33 & $\mathrm{~S}$ & ITALY & ITALY & ITALY & NA & MINER/CM & SAN GIORGIO CANAVESE. TO & 6TH ST. & CALUMET PR. 1 \\
\hline 471 & 3922 & ANDRINA & STEVE & B & 25 & $\mathrm{~s}$ & ITALY & ITALY & ITALY & $\mathrm{AL}$ & MINER/CM & SAN MARTINO CANAVESE. TO & 6TH ST. & CALUMET PR. 1 \\
\hline 472 & 3922 & NORA & ANTONIO & B & 22 & $\mathrm{~s}$ & ITALY & ITALY & ITALY & $\mathrm{AL}$ & MINER/CM & TORINO & 6TH ST. & CALUMET PR. 1 \\
\hline 473 & 3922 & SOSSI/SUSSI & JAMES & B & 22 & $\mathrm{~s}$ & ITALY & ITALY & ITALY & $\mathrm{AL}$ & MINER/CM & VIALFRE'. TO & 6TH ST. & CALUMET PR. 1 \\
\hline 474 & $\mid 3922$ & SOSSI/SUSSI & JOSEPH & $\begin{array}{l}\mathrm{D} \\
\mathrm{B}\end{array}$ & 26 & s & ITALY & ITALY & ITALY & $\begin{array}{ll}\text { AL } \\
\text { AL }\end{array}$ & MINER/CM & $\begin{array}{l}\text { VIALFRE'. TO } \\
\text { VIAFR }\end{array}$ & 6TH ST. & CALUMET PR. 1 \\
\hline 475 & 3922 & RUGONI & $\mathrm{JOHN}$ & B & 34 & M & ITALY & ITALY & ITALY & NA & MINER/CM & & 6TH ST. & CALUMET PR. 1 \\
\hline 476 & 3922 & PAGONI & DOMINICK & B & 22 & s & ITALY & ITALY & ITALY & NA & MINER/CM & & 6TH ST. & CALUMET PR. 1 \\
\hline 477 & 3923 & CURTO & JAMES & $\mathrm{H}$ & 39 & M14 & ITALY & ITALY & ITALY & NA & MINER/CM & TORINO & 6TH ST. & CALUMET PR. 1 \\
\hline 478 & 3923 & CURTO & MARY & W & 31 & M14 & PORTUGAL & PORTUG & PORTUGAL & & & & 6TH ST. & CALUMET PR. 1 \\
\hline 479 & 3923 & CURTO & ANDREW & s & 9 & S & MICHIGAN & ITALY & PORTUGAL & & AT SCHOOL & TORINO & 6TH ST. & CALUMET PR. 1 \\
\hline 480 & 3923 & CURTO & FRANCIS & $\mathrm{s}$ & 6 & $\mathrm{~s}$ & MICHIGAN & ITALY & PORTUGAL & & AT SCHOOL & TORINO & 6TH ST. & CALUMET PR. 1 \\
\hline 481 & 3923 & CURTO & PETER & $\mathrm{s}$ & 2 & $\mathrm{~S}$ & MICHIGAN & ITALY & PORTUGAL & & & TORINO & 6TH ST. & CALUMET PR. 1 \\
\hline 482 & 3923 & CURTO & JOSEPHINE & $\mathrm{D}$ & 2 & $\mathrm{~S}$ & MICHIGAN & ITALY & PORTUGAL & & & TORINO & 6TH ST. & CALUMET PR. 1 \\
\hline 483 & 3923 & BARATONO & PETER & B & 30 & $\mathrm{~S}$ & ITALY & ITALY & ITALY & NA & MINER/CM & TORINO & 6TH ST. & CALUMET PR. 1 \\
\hline 484 & 3923 & GOMETTO & JOSEPH & B & 41 & $\mathrm{~S}$ & ITALY & ITALY & ITALY & NA & MINER/CM & TORINO & 6TH ST. & CALUMET PR. 1 \\
\hline 485 & 3923 & CASTA & PETER & $\mathrm{B}$ & 22 & s & ITALY & ITALY & ITALY & & LABORER & & 6TH ST. & CALUMET PR. 1 \\
\hline 486 & 3923 & BARATONO & MARY & SER & 25 & s & ITALY & ITALY & ITALY & & SERVANT & TORINO & 6TH ST. & CALUMET PR. 1 \\
\hline 487 & & TORREANO & FRED & $\mathrm{H}$ & 40 & M? & ITALY & ITALY & ITALY & NA & NOT CLEAR & TORINO & WOODLAND ST. & CALUMET - LAURIUM \\
\hline 488 & & TORREANO & KATE & w & 32 & $\mathrm{M} ?$ & ITALY & ITALY & ITALY & & & & WOODLAND ST. & CALUMET - LAURIUM \\
\hline 489 & & TORREANO & MARY & $\mathrm{D}$ & 10 & $s$ & MICHIGAN & ITALY & ITALY & & AT SCHOOL 10 & TORINO & WOODLAND ST. & CALUMET - LAURIUM \\
\hline 490 & & TORREANO & TRACEY & $\mathrm{D}$ & 3 & $\mathrm{~s}$ & MICHIGAN & ITALY & ITALY & & & TORINO & WOODLAND ST. & CALUMET - LAURIUM \\
\hline 491 & & TORREANO & JOHN & s & 2 & s & MICHIGAN & ITALY & ITALY & & & TORINO & WOODLAND ST. & CALUMET - LAURIUM \\
\hline 492 & & ZANARDI & CHARLES & $\mathrm{H}$ & 40 & M? & ITALY & ITALY & ITALY & NA & TEAMSTER/CM & BERGAMO & SENECA ST. & CALUMET - LAURIUM \\
\hline 493 & & ZANARDI & JENNIE & W & 29 & M? & ITALY & ITALY & ITALY & & & & SENECA ST. & CALUMET - LAURIUM \\
\hline 494 & & ZANARDI & MARY & D & 7 & $\mathrm{~s}$ & MICHIGAN & ITALY & ITALY & & AT SCHOOL 10 & BERGAMO & SENECA ST. & CALUMET - LAURIUM \\
\hline 495 & & RADEY & ANTONIO & $\mathrm{H}$ & 30 & M5 & ITALY & ITALY & ITALY & $?$ & MINER/CM & & KEARSARGE ST. & CALUMET - LAURIUM \\
\hline 496 & & RADEY & CARIE & W & 22 & M5 & ITALY & ITALY & ITALY & $?$ & & & KEARSARGE ST. & CALUMET - LAURIUM \\
\hline 497 & & RADEY & STEPHEN & s & 3 & $\mathrm{~s}$ & ITALY & ITALY & ITALY & & & & KEARSARGE ST. & CALUMET - LAURIUM \\
\hline 498 & & RADEY & MARY & $\mathrm{D}$ & 3 & s & ITALY & ITALY & ITALY & & & & KEARSARGE ST. & CALUMET - LAURIUM \\
\hline 499 & & FUEK/ROCK & ALBERT & B & 19 & M4 & ITALY & ITALY & ITALY & $\mathrm{AL}$ & PLASTERER HELPER & & KEARSARGE ST. & CALUMET - LAURIUM \\
\hline 500 & & FUEK/ROCK & DOMINICA & B & 53 & M4 & ITALY & ITALY & ITALY & & HOUSEKEEPER & & KEARSARGE ST. & CALUMET - LAURIUM \\
\hline 501 & & FUEK/ROCK & JOSEPH & B & 34 & $?$ & ITALY & ITALY & ITALY & $\mathrm{AL}$ & STONE MASON & & KEARSARGE ST. & CALUMET - LAURIUM \\
\hline
\end{tabular}




\begin{tabular}{|c|c|c|c|c|c|c|c|c|c|c|c|c|c|}
\hline 502 & DELL'ACQUA & ANGELO? & $\mathrm{H}$ & 35 & M2 & ITALY & ITALY & ITALY & NA & BLACKSMITH & CUGGIANO. MILANO & KEARSARGE ST. & CALUMET - LAURIUM \\
\hline 503 & DELL'ACQUA & $?$ & w & 22 & M2 & ITALY & ITALY & ITALY & & & CUGGIANO. MILANO & KEARSARGE ST. & CALUMET - LAURIUM \\
\hline 504 & DELL'ACQUA & MARY & D & 1 & $\mathrm{~s}$ & MICHIGAN & ITALY & ITALY & & & CUGGIANO. MILANO & KEARSARGE ST. & CALUMET - LAURIUM \\
\hline 505 & DELL'ACQUA & ANGELO & $\mathrm{BR}$ & 30 & $?$ & MICHIGAN & ITALY & ITALY & NA & TIMBERMAN/CM & CUGGIANO. MILANO & KEARSARGE ST. & CALUMET - LAURIUM \\
\hline 506 & DELL'ACQUA & PETER & $\mathrm{BR}$ & 28 & $?$ & ITALY & ITALY & ITALY & $\mathrm{AL}$ & BLACKSMITH & CUGGIANO. MILANO & KEARSARGE ST. & CALUMET - LAURIUM \\
\hline 507 & VENEGONE & JOHN & BIL & 23 & $?$ & ITALY & ITALY & ITALY & NA & TIMBERMAN/CM & CUGGIANO. MILANO & KEARSARGE ST. & CALUMET - LAURIUM \\
\hline 508 & BUFFER & DOMINIC & $\mathrm{H}$ & 40 & M16 & ITALY & ITALY & ITALY & NA & MINER/CM & & KEARSARGE ST. & CALUMET - LAURIUM \\
\hline 509 & BUFFER & ANTONIA & W & 36 & M16 & ITALY & ITALY & ITALY & & & & KEARSARGE ST. & CALUMET - LAURIUM \\
\hline 510 & BUFFER & MARY & D & 15 & s & ITALY & ITALY & ITALY & & & & KEARSARGE ST. & CALUMET - LAURIUM \\
\hline 511 & BUFFER & ALEX & $\mathrm{s}$ & 7 & $\mathrm{~s}$ & ILLINOIS & ITALY & ITALY & & & & KEARSARGE ST. & CALUMET - LAURIUM \\
\hline 512 & BUFFER & LIZZIE? & D & 4 & $\mathrm{~s}$ & ILLINOIS & ITALY & ITALY & & & & KEARSARGE ST. & CALUMET - LAURIUM \\
\hline 513 & MASSA & ANTONIO & B & 39 & $\mathrm{~S}$ & ITALY & ITALY & ITALY & $\mathrm{AL}$ & TRAMMER/CM & PONT CANAVESE. TO & KEARSARGE ST. & CALUMET - LAURIUM \\
\hline 514 & PANNE & JOSEPH & B & 29 & $\mathrm{~s}$ & ITALY & ITALY & ITALY & $\mathrm{AL}$ & TRAMMER/CM & & KEARSARGE ST. & CALUMET - LAURIUM \\
\hline 515 & VINCENTI & ANTONE & B & 43 & $\mathrm{~s}$ & ITALY & ITALY & ITALY & $\mathrm{AL}$ & TRAMMER/CM & CASTELLAMONTE. TO & KEARSARGE ST. & CALUMET - LAURIUM \\
\hline 516 & GUIZO? & MARTIN & B & 23 & $\mathrm{~s}$ & ITALY & ITALY & ITALY & AL & TRAMMER/CM & & KEARSARGE ST. & CALUMET - LAURIUM \\
\hline 517 & GALLINATTI & MAURICE & $\mathrm{H}$ & 31 & M6 & ITALY & ITALY & ITALY & PA & WATCHMAN/CM & AGLIE', TO & TAMARACK ST. & CALUMET - LAURIUM \\
\hline 518 & GALLINATTII & LOUISA & W & 25 & M6 & ITALY & ITALY & ITALY & & & & TAMARACK ST. & CALUMET - LAURIUM \\
\hline 519 & GALLINATTI & TRACEY & D & 11 & $\mathrm{~s}$ & MICHIGAN & ITALY & ITALY & & & AGLIE'. TO & TAMARACK ST. & CALUMET - LAURIUM \\
\hline 520 & MIGLIO/MIGLIA & GIORGIO & $\mathrm{H}$ & 28 & M3 & ITALY & ITALY & ITALY & PA & TRAMMER/CM & SAN GIORGIO CANAVESE. TO & TAMARACK ST. & CALUMET - LAURIUM \\
\hline 521 & MIGLIO/MIGLIA & DOMENICA & w & 24 & M3 & ITALY & ITALY & ITALY & & & SAN GIORGIO CANAVESE. TO & TAMARACK ST. & CALUMET - LAURIUM \\
\hline 522 & MIGLIO/MIGLIA & FRS & s & 2 & $\mathrm{~s}$ & MICHIGAN & ITALY & ITALY & & & SAN GIORGIO CANAVESE. TO & TAMARACK ST. & CALUMET - LAURIUM \\
\hline 523 & MIGLIO/MIGLIA & ANTONIA & $\mathrm{D}$ & $3 \mathrm{M}$ & s & MICHIGAN & ITALY & ITALY & & & SAN GIORGIO CANAVESE, TO & TAMARACK ST. & CALUMET - LAURIUM \\
\hline 524 & NIGRA/NIGRO & TONY & $\mathrm{H}$ & 35 & M0 & ITALY & ITALY & ITALY & $\mathrm{AL}$ & STONE MASON & SAN GIUSTO CANAVESE. TO & TAMARACK ST. & CALUMET - LAURIUM \\
\hline 525 & NIGRA/NIGRO & BENEDETTA & W & 30 & MO & ITALY & ITALY & ITALY & & & SAN GIUSTO CANAVESE. TO & TAMARACK ST. & CALUMET - LAURIUM \\
\hline 526 & BESSOLO & DOMINICK & $\mathrm{H}$ & 35 & M12 & ITALY & ITALY & ITALY & PA & TIMBERMAN & BESSOLO CANAVESE. TO & IROQUOIS ST. & CALUMET - LAURIUM \\
\hline 527 & BESSOLO & TRACEY & W & 34 & M12 & ITALY & ITALY & ITALY & & & BESSOLO CANAVESE. TO & IROQUOIS ST. & CALUMET - LAURIUM \\
\hline 528 & BESSOLO & MARYANN & D & 11 & $\mathrm{~s}$ & ITALY & ITALY & ITALY & & AT SCHOOL 2 & BESSOLO CANAVESE. TO & IROQUOIS ST. & CALUMET - LAURIUM \\
\hline 529 & BESSOLO & CHARLES & $\mathrm{s}$ & 8 & $\mathrm{~s}$ & ITALY & ITALY & ITALY & & AT SCHOOL 2 & BESSOLO CANAVESE, TO & IROQUOIS ST. & CALUMET - LAURIUM \\
\hline 530 & BESSOLO & MARY & D & 5 & $\mathrm{~s}$ & ITALY & ITALY & ITALY & & & BESSOLO CANAVESE. TO & IROQUOIS ST. & CALUMET - LAURIUM \\
\hline 531 & BESSOLO & MATT & $\mathrm{BR}$ & 34 & $\mathrm{~s}$ & ITALY & ITALY & ITALY & NA & TIMBER BOSS/CM & BESSOLO CANAVESE. TO & IROQUOIS ST. & CALUMET - LAURIUM \\
\hline 532 & BESSOLO & FELIX & $\mathrm{BR}$ & 31 & $\mathrm{~s}$ & ITALY & ITALY & ITALY & $\mathrm{PA}$ & MINER/CM & BESSOLO CANAVESE. TO & IROQUOIS ST. & CALUMET - LAURIUM \\
\hline 533 & BESSOLO & CHARLES & $\mathrm{BR}$ & 20 & $\mathrm{~s}$ & ITALY & ITALY & ITALY & $?$ & TRAMMER/CM & BESSOLO CANAVESE. TO & IROQUOIS ST. & CALUMET - LAURIUM \\
\hline 534 & GROSSIO & JOHN & $\mathrm{H}$ & 32 & M4 & ITALY & ITALY & ITALY & $?$ & TRAMMER/CM & TORINO & IROQUOIS ST. & CALUMET - LAURIUM \\
\hline 535 & GROSSIO & MARY & W & 25 & M4 & ITALY & ITALY & ITALY & & & TORINO & IROQUOIS ST. & CALUMET - LAURIUM \\
\hline 536 & GROSSIO & JOHN & s & & & MICHIGAN & ITALY & ITALY & & & TORINO & IROQUOIS ST. & CALUMET - LAURIUM \\
\hline 537 & PASTORE & ANTONE & B & 20 & s & ITALY & ITALY & ITALY & AL & TRAMMER/CM & SAN MARTINO CANAVESE. TO & IROQUOIS ST. & CALUMET - LAURIUM \\
\hline 538 & ROVANO & PETER & B & 36 & $\mathrm{~s}$ & ITALY & ITALY & ITALY & NA & TRAMMER/CM & SAN MARTINO CANAVESE. TO & IROQUOIS ST. & CALUMET - LAURIUM \\
\hline 539 & PIANA & AUGUST & B & 25 & $\mathrm{~s}$ & ITALY & ITALY & ITALY & $\mathrm{AL}$ & TRAMMER/CM & TORINO & IROQUOIS ST. & CALUMET - LAURIUM \\
\hline 540 & SAPINO & MARTIN & B & 27 & $\mathrm{~s}$ & ITALY & ITALY & ITALY & $\mathrm{AL}$ & TRAMMER/CM & SAN MARTINO CANAVESE. TO & IROQUOIS ST. & CALUMET - LAURIUM \\
\hline 541 & GARRIE & PETER & B & 28 & $\mathrm{~s}$ & ITALY & ITALY & ITALY & $\mathrm{AL}$ & TRAMMER/CM & & IROQUOIS ST. & CALUMET - LAURIUM \\
\hline 542 & SAPINO & NATALIE & B & 22 & $\mathrm{~S}$ & ITALY & ITALY & ITALY & $\mathrm{AL}$ & TRAMMER/CM & SAN MARTINO CANAVESE. TO & IROQUOIS ST. & \\
\hline 543 & DE FILIPPI & DOMINICO & B & 53 & $\mathrm{~s}$ & ITALY & ITALY & ITALY & NA & TRAMMER/CM & TORINO & IROQUOIS ST. & CALUMET - LAURIUM \\
\hline 544 & ZUCCA & JOSEPH & B & 26 & $\mathrm{~S}$ & ITALY & ITALY & ITALY & $\mathrm{AL}$ & & TORINO & IROQUOIS ST. & CALUMET - LAURIUM \\
\hline 545 & PASTORE & DOMINIC & B & 23 & $\mathrm{~S}$ & ITALY & ITALY & ITALY & $\mathrm{AL}$ & & SAN MARTINO CANAVESE. TO & IROQUOIS ST. & CALUMET - LAURIUM \\
\hline 546 & MARTA & JOHN & $\mathrm{H}$ & 31 & M2 & ITALY & ITALY & ITALY & NA & DAY LABORER/CM & SAN MARTINO CANAVESE. TO & AMYGDALOID ST. & CALUMET - LAURIUM \\
\hline 547 & MARTA & THERESA & w & 34 & M2 & ITALY & ITALY & ITALY & & & & AMYGDALOID ST. & CALUMET - LAURIUM \\
\hline 548 & MARTA & ANNIE & $\mathrm{D}$ & 1 & $\mathrm{~s}$ & MICHIGAN & ITALY & ITALY & & & SAN MARTINO CANAVESE. TO & AMYGDALOID ST. & CALUMET - LAURIUM \\
\hline 549 & ROPPOLO & ANTONIO & $\mathrm{H}$ & 45 & M3 & ITALY & ITALY & ITALY & ?? & N. G. & $\begin{array}{l}\text { PEROSA CANAVESE. TO } \\
\text { PER }\end{array}$ & AMYGDALOID ST. & CALUMET - LAURIUM \\
\hline 550 & ROPPOLO & KATE & W & 28 & M3 & ITALY & ITALY & ITALY & & & PEROSA CANAVESE. TO & AMYGDALOID ST. & CALUMET - LAURIUM \\
\hline 551 & ROPPOLO & MARTIN & s & 1 & $\mathrm{~s}$ & MICHIGAN & ITALY & ITALY & & & PEROSA CANAVESE. TO & AMYGDALOID ST. & CALUMET - LAURIUM \\
\hline 552 & GAGGOLULA & BAPTISTE & $\mathrm{H}$ & 33 & M14 & ITALY & ITALY & ITALY & NA & TIMBERMAN & & AMYGDALOID ST. & CALUMET - LAURIUM \\
\hline 553 & GAGGOLULA & PAULINA & W & 35 & M14 & ITALY & ITALY & ITALY & & & & AMYGDALOID ST. & CALUMET - LAURIUM \\
\hline 554 & GAGGOLULA & FRED & s & 12 & $\mathrm{~s}$ & ITALY & ITALY & ITALY & $\mathrm{AL}$ & AT SCHOOL 10 & & AMYGDALOID ST. & CALUMET - LAURIUM \\
\hline 555 & FORNERIS & ANGELO & $\mathrm{H}$ & 25 & MO & ITALY & ITALY & ITALY & $\mathrm{AL}$ & TEAMSTER/STORE & TORINO & AMYGDALOID ST. & CALUMET - LAURIUM \\
\hline 556 & FORNERIS & ANNA & W & 18 & MO & ITALY & ITALY & ITALY & & & & AMYGDALOID ST. & CALUMET - LAURIUM \\
\hline 557 & BERNARDO & JAMES & B & 35 & $\mathrm{~s}$ & ITALY & ITALY & ITALY & $\mathrm{AL}$ & MINER/CM & PONT CANAVESE. TO & AMYGDALOID ST. & CALUMET - LAURIUM \\
\hline 558 & BERNARDO & TONEY & B & 30 & $\mathrm{~S}$ & ITALY & ITALY & ITALY & $\mathrm{AL}$ & DAY LABORER/CM & PONT CANAVESE. TO & AMYGDALOID ST. & CALUMET - LAURIUM \\
\hline 559 & SOSSI & VINCENT & $\mathrm{H}$ & 30 & M2 & ITALY & ITALY & ITALY & $\mathrm{AL}$ & MINER/CM & TORINO & AMYGDALOID ST. & CALUMET - LAURIUM \\
\hline 560 & sossi & TRACEY & w & 25 & M2 & ITALY & ITALY & ITALY & & & TORINO & AMYGDALOID ST. & CALUMET - LAURIUM \\
\hline 561 & SOSSI & PHELICITA?? & s & $5 \mathrm{M}$ & $\mathrm{s}$ & ITALY & ITALY & ITALY & & & TORINO & AMYGDALOID ST. & CALUMET - LAURIUM \\
\hline 562 & MASCAGNO & FRANCESCO & $\mathrm{H}$ & 31 & M5 & ITALY & ITALY & ITALY & PA & TRAMMER/CM & TORINO & CALEDONIA ST. & CALUMET PR 2 \\
\hline 563 & MASCAGNO & GIACINTA & W & 30 & M5 & ITALY & $\begin{array}{l}\text { IITALY } \\
\text { ITAL }\end{array}$ & $\begin{array}{l}\text { ITALY } \\
\text { ITALY }\end{array}$ & PA & TRAIVIVIER/CIVI & TORINO & $\begin{array}{l}\text { CALEDONIA ST. } \\
\text { CALED S }\end{array}$ & CALUMET PR. 2 \\
\hline 564 & MASCAGNO & MARY & D & 4 & s & ITALY & ITALY & ITALY & & & TORINO & CALEDONIA ST. & CALUMET PR. 2 \\
\hline
\end{tabular}




\begin{tabular}{|c|c|c|c|c|c|c|c|c|c|c|c|c|c|}
\hline 565 & MASCAGNO & MIKE & $\mathrm{s}$ & 1 & $\mathrm{~s}$ & MICHIGAN & ITALY & ITALY & & & TORINO & CALEDONIA ST. & CALUMET PR. 2 \\
\hline 566 & CORA & JOHN & B & 30 & S & ITALY & ITALY & ITALY & PA & TRAMMER/CM & & CALEDONIA ST. & CALUMET PR. 2 \\
\hline 567 & BESSOLO & PETER & B & 22 & $\mathrm{~s}$ & ITALY & ITALY & ITALY & AL & TRAMMER/CM & SAN MARTINO CANAVESE. TO & CALEDONIA ST. & CALUMET PR. 2 \\
\hline 568 & BOBBIO & TONY & B & 31 & $\mathrm{~s}$ & ITALY & ITALY & ITALY & NA & TRAMMER/CM & SAN BENIGNO CANAVESE. TO & CALEDONIA ST. & CALUMET PR. 2 \\
\hline 569 & GRUA & CHARLES & B & 22 & $\mathrm{~S}$ & ITALY & ITALY & ITALY & $\mathrm{AL}$ & TRAMMER/CM & SAN BENIGNO CANAVESE. TO & CALEDONIA ST. & CALUMET PR. 2 \\
\hline 570 & VIANNO & PAUL & B & 23 & s & ITALY & ITALY & ITALY & $\mathrm{AL}$ & TRAMMER/CM & & CALEDONIA ST. & CALUMET PR. 2 \\
\hline 571 & TORETTI & TONY & $\mathrm{H}$ & 55 & W? & ITALY & ITALY & ITALY & NA & SPRINKLER & & CALEDONIA ST. & CALUMET PR. 2 \\
\hline 572 & TORETTI & DOMINICA & $\mathrm{D}$ & 22 & $\mathrm{~s}$ & ITALY & ITALY & ITALY & NA & DAY LABORER/CM & & CALEDONIA ST. & CALUMET PR. 2 \\
\hline 573 & TORETTI & GLADO? & $\mathrm{S}$ & 19 & s & ITALY & ITALY & ITALY & $\mathrm{AL}$ & DRILL BOY/CM & & CALEDONIA ST. & CALUMET PR. 2 \\
\hline 574 & TORETTI & GEORGE & $\mathrm{s}$ & 17 & $\mathrm{~s}$ & ITALY & ITALY & ITALY & & LABORER/CM & & CALEDONIA ST. & CALUMET PR. 2 \\
\hline 575 & TORETTI & FRANK & $\mathrm{s}$ & 17 & $\mathrm{~s}$ & ITALY & ITALY & ITALY & & LABORER/CM & & CALEDONIA ST. & CALUMET PR. 2 \\
\hline 576 & TORETTI & JAMES & s & 15 & $\mathrm{~S}$ & ITALY & ITALY & ITALY & & LABORER/CM & & CALEDONIA ST. & CALUMET PR. 2 \\
\hline 577 & TORETTI & JOHN & s & 11 & S & ITALY & ITALY & ITALY & & AT SCHOOL & & CALEDONIA ST. & CALUMET PR. 2 \\
\hline 578 & INO/TREO & JOHN & B & 28 & M2 & ITALY & ITALY & ITALY & & TRAMMER/CM & & CALEDONIA ST. & CALUMET PR. 2 \\
\hline 579 & INO/TREO & MAGGIE & B & 28 & M2 & ITALY & ITALY & ITALY & & & & CALEDONIA ST. & CALUMET PR. 2 \\
\hline 580 & INO/TREO & FRANK & $\begin{array}{l}\mathrm{D} \\
\mathrm{B}\end{array}$ & 1 & S & MICHIGAN & ITALY & ITALY & & & & CALEDONIA ST. & CALUMET PR. 2 \\
\hline 581 & PELLEGRINI & LAURENCE & $\mathrm{H}$ & 42 & M3 & ITALY & ITALY & ITALY & NA & STONE MASON & LUCCA & CALEDONIA ST. & CALUMET PR. 2 \\
\hline 582 & PELLEGRINI & FANNY & W & 36 & M3 & ITALY & ITALY & ITALY & & & & CALEDONIA ST. & CALUMET PR. 2 \\
\hline 583 & PELLEGRINI & FRANK & s & 14 & $\mathrm{~s}$ & ILLINOIS & ITALY & ITALY & & AT SCHOOL 10 & LUCCA & CALEDONIA ST. & CALUMET PR. 2 \\
\hline 584 & PELLEGRINI & MAUSC? & S & 12 & s & MICHIGAN & ITALY & ITALY & & AT SCHOOL 10 & LUCCA & CALEDONIA ST. & CALUMET PR. 2 \\
\hline 585 & PELLEGRINI & EDITH & $\mathrm{D}$ & 10 & s & MICHIGAN & ITALY & ITALY & & AT SCHOOL 10 & LUCCA & CALEDONIA ST. & CALUMET PR. 2 \\
\hline 586 & PELLEGRINI & RALPH & $\mathrm{s}$ & 8 & $\mathrm{~s}$ & MICHIGAN & ITALY & ITALY & & AT SCHOOL 10 & LUCCA & CALEDONIA ST. & CALUMET PR. 2 \\
\hline 587 & PELLEGRINI & SAMUEL & $\mathrm{s}$ & 1 & $\mathrm{~s}$ & MICHIGAN & ITALY & ITALY & & & LUCCA & CALEDONIA ST. & CALUMET PR. 2 \\
\hline 588 & PELLEGRINI & LOAMI & $\mathrm{D}$ & $8 \mathrm{M}$ & s & MICHIGAN & ITALY & ITALY & & & LUCCA & CALEDONIA ST. & $\begin{array}{l}\text { CALUMET PR. } 2 \\
\text { CALUE }\end{array}$ \\
\hline 589 & CIABATTARI & MICHAEL & $\mathrm{H}$ & 33 & M3 & ITALY & ITALY & ITALY & $\mathrm{AL}$ & DAY LABORER/CM & SEGROMIGNO. LU & CALEDONIA ST. & CALUMET PR. 2 \\
\hline 590 & CIABATTARI & ESMERALDA & W & 22 & M3 & ITALY & ITALY & ITALY & & & SEGROMIGNO. LU & CALEDONIA ST. & CALUMET PR. 2 \\
\hline 591 & CIABATTARI & VINCENZO & s & 1 & s & MICHIGAN & ITALY & ITALY & & & SEGROMIGNO. LU & CALEDONIA ST. & CALUMET PR. 2 \\
\hline 592 & CIABATTARI & FRANK & BR & 23 & s & ITALY & ITALY & ITALY & $\mathrm{AL}$ & TRAMMER/CM & SEGROMIGNO, LU & CALEDONIA ST. & CALUMET PR. 2 \\
\hline 593 & TORREANO & VINCENZA & $\mathrm{H}$ & 49 & w & ITALY & ITALY & ITALY & & BOARDING HOUSE KEEPER & TORINO & ROCKLAND ST. & CALUMET PR. 2 \\
\hline 594 & TORREANO & AMELIA & $\mathrm{D}$ & 22 & s & MICHIGAN & ITALY & ITALY & & HOUSEWORK & TORINO & ROCKLAND ST. & CALUMET PR. 2 \\
\hline 595 & TORREANO & JOHN & $\mathrm{s}$ & 20 & $\mathrm{~S}$ & MICHIGAN & ITALY & ITALY & & BLACKSMITH & TORINO & ROCKLAND ST. & CALUMET PR. 2 \\
\hline 596 & TORREANO & FRED & $\mathrm{s}$ & 19 & $\mathrm{~S}$ & MICHIGAN & ITALY & ITALY & & BLACKSMITH & TORINO & ROCKLAND ST. & CALUMET PR. 2 \\
\hline 597 & TORREANO & JOSEPH & s & 18 & $\mathrm{~s}$ & MICHIGAN & ITALY & ITALY & & PUMP MAN/CM & TORINO & ROCKLAND ST. & CALUMET PR. 2 \\
\hline 598 & TORREANO & PETER & $\mathrm{s}$ & 14 & $\mathrm{~s}$ & MICHIGAN & ITALY & ITALY & & CLERK/CLOTHING & TORINO & ROCKLAND ST. & CALUMET PR. 2 \\
\hline 599 & TORREANO & ADELINA & $\mathrm{D}$ & 11 & $\mathrm{~s}$ & MICHIGAN & ITALY & ITALY & & AT SCHOOL 10 & TORINO & ROCKLAND ST. & CALUMET PR. 2 \\
\hline 600 & PRICCO & DOMINIC & B & 38 & $\mathrm{~s}$ & ITALY & ITALY & ITALY & & MINER/CM & SAN GIOVANNI CANAVESE. & ROCKLAND ST. & CALUMET PR. 2 \\
\hline 601 & TORREANO & ANGELO & $\begin{array}{l}\mathrm{D} \\
\mathrm{B}\end{array}$ & 24 & S & ITALY & ITALY & ITALY & & MINER/CM & $\begin{array}{l}\text { TORINO } \\
\text { TORIVI AIVAVLOL. }\end{array}$ & ROCKLAND ST. & $\begin{array}{l}\text { CALUMET PR. } 2 \\
\text { CALUE }\end{array}$ \\
\hline $\begin{array}{l}601 \\
602\end{array}$ & BESSOLO & $\begin{array}{l}\text { ANGELO } \\
\text { LOUIS }\end{array}$ & $\begin{array}{l}\mathrm{B} \\
\mathrm{B}\end{array}$ & $\begin{array}{l}24 \\
28\end{array}$ & $\begin{array}{l}\mathrm{s} \\
\mathrm{s}\end{array}$ & $\begin{array}{l}\text { IITALY } \\
\text { ITALY }\end{array}$ & $\begin{array}{l}\text { IITALY } \\
\text { ITALY }\end{array}$ & $\begin{array}{l}\text { IIALY } \\
\text { ITALY }\end{array}$ & & MINER/CM & TORINO & ROCKLAND ST. & $\begin{array}{l}\text { CALUMET PR. } 2 \\
\text { CALUETR }\end{array}$ \\
\hline 603 & MAGGARO & $\begin{array}{l}\text { VINCENT } \\
\text { VIN }\end{array}$ & B & 35 & $\mathrm{~s}$ & ITALY & ITALY & ITALY & & MINER/CM & & ROCKLAND ST. & CALUMET PR. 2 \\
\hline 604 & ZUCCO & JAMES & $\begin{array}{l}\mathrm{D} \\
\mathrm{B}\end{array}$ & 31 & $\mathrm{~s}$ & ITALY & ITALY & ITALY & & MINER/CM & TORINO & ROCKLAND ST. & CALUMET PR. 2 \\
\hline 605 & DELUCAS & $\begin{array}{l}\text { DAVID } \\
\text { DAVIS }\end{array}$ & B & 23 & s & ITALY & ITALY & ITALY & & CARPENTER & & ROCKLAND ST. & CALUMET PR. 2 \\
\hline 606 & TAMBELLINI & ANGELO & $\mathrm{H}$ & 30 & M? & ITALY & ITALY & ITALY & $?$ & WATCHMAN/CM & LUCCA & EAST PINE ST. & CALUMET PR. 2 \\
\hline 607 & TAMBELLINI & LIZZIE & W & 31 & M? & MICHIGAN & ITALY & ITALY & & & & EAST PINE ST. & CALUMET PR. 2 \\
\hline 608 & TAMBELLINI & PASCAE & s & 5 & $\mathrm{~s}$ & MICHIGAN & ITALY & MICHIGAN & & & LUCCA & EAST PINE ST. & CALUMET PR. 2 \\
\hline 609 & TAMBELLINI & ARTHUR & $\mathrm{s}$ & 3 & $\mathrm{~s}$ & MICHIGAN & ITALY & MICHIGAN & & & LUCCA & EAST PINE ST. & CALUMET PR. 2 \\
\hline 610 & GEDDA & PETER & $\mathrm{H}$ & 30 & M? & ITALY & ITALY & ITALY & & & TORINO & EAST PINE ST. & CALUMET PR. 2 \\
\hline 611 & GEDDA & KATE & w & 26 & M? & ITALY & ITALY & ITALY & & & TORINO & EAST PINE ST. & CALUMET PR. 2 \\
\hline 612 & GEDDA & ORESTE & s & 4 & S & MICHIGAN & ITALY & ITALY & & & TORINO & EAST PINE ST. & CALUMET PR. 2 \\
\hline 613 & GEDDA & LETIZIA & $\mathrm{s}$ & $\frac{4}{3}$ & s & MICHIGAN & ITALY & ITALY & & & TORINO & EAST PINE ST. & $\begin{array}{l}\text { CALUMET PR. } 2 \\
\text { CALET }\end{array}$ \\
\hline 614 & GEDDA & JOHANNA & $\mathrm{D}$ & $1 \mathrm{M}$ & $\mathrm{s}$ & MICHIGAN & ITALY & ITALY & & & TORINO & EAST PINE ST. & $\begin{array}{l}\text { CALUMET PR. } 2 \\
\text { CALET }\end{array}$ \\
\hline $\begin{array}{l}614 \\
615\end{array}$ & $\begin{array}{l}\text { GEDUA } \\
\text { SCIACERO }\end{array}$ & $\begin{array}{l}\text { AUGUST } \\
\text { AUAST }\end{array}$ & $\mathrm{H}$ & 34 & M9 & $\begin{array}{l}\text { MTALY } \\
\text { ITAN }\end{array}$ & $\begin{array}{l}\text { MALY } \\
\text { ITALY }\end{array}$ & $\begin{array}{l}\text { IIALY } \\
\text { ITALY }\end{array}$ & NA & TRAMMER/CM & TORINO & EAST PINE ST. & $\begin{array}{l}\text { CALUMET PR. } 2 \\
\text { CALUET }\end{array}$ \\
\hline $\begin{array}{l}615 \\
616\end{array}$ & $\begin{array}{l}\text { SCIACERO } \\
\text { SCIACERO }\end{array}$ & $\begin{array}{l}\text { AUGUSI } \\
\text { MARGARETTA }\end{array}$ & $\begin{array}{l}\text { H } \\
W \\
\end{array}$ & 31 & $\begin{array}{l}\text { M99 } \\
\text { M9 }\end{array}$ & $\begin{array}{l}\text { IIALY } \\
\text { GERMANY }\end{array}$ & $\begin{array}{l}\text { MALY } \\
\text { GERMAN }\end{array}$ & $\begin{array}{l}\text { IIALY } \\
\text { GERMANY }\end{array}$ & NA & IRAMIMER/CMM & IORINO & EAST PINE ST. & $\begin{array}{l}\text { CALUME TR. } 2 \\
\text { CALUMET PR. } 2\end{array}$ \\
\hline 617 & SCIACERO & JOHN & s & 6 & $\mathrm{~s}$ & MICHIGAN & ITALY & GERMANY & & AT SCHOOL 5 & TORINO & EAST PINE ST. & CALUMET PR. 2 \\
\hline 618 & SCIACERO & MARY & $\mathrm{D}$ & 4 & $\mathrm{~s}$ & MICHIGAN & ITALY & GERMANY & & & TORINO & EAST PINE ST. & CALUMET PR. 2 \\
\hline 619 & SCIACERO & STEPHEN & $\mathrm{s}$ & 2 & $\mathrm{~s}$ & MICHIGAN & ITALY & GERMANY & & & TORINO & EAST PINE ST. & CALUMET PR. 2 \\
\hline 620 & SCIACERO & ANGELO & $\mathrm{s}$ & $3 \mathrm{M}$ & $\mathrm{s}$ & MICHIGAN & ITALY & GERMANY & & & TORINO & EAST PINE ST. & CALUMET PR. 2 \\
\hline 621 & MARTA & BAPTISTE & $\mathrm{H}$ & 34 & M13 & ITALY & ITALY & ITALY & NA & TIMBERMAN/CM & SAN MARTINO CANAVESE. TO & EAST PINE ST. & CALUMET PR. 2 \\
\hline 622 & MARTA & MARY & w & 27 & M13 & ITALY & ITALY & ITALY & & & & EAST PINE ST. & CALUMET PR. 2 \\
\hline 623 & MARTA & GIOVANNA & $\mathrm{D}$ & 10 & $\mathrm{~s}$ & MICHIGAN & ITALY & ITALY & & AT SCHOOL 10 & SAN MARTINO CANAVESE. TO & EAST PINE ST. & CALUMET PR. 2 \\
\hline 624 & MARTA & JOHN & $\mathrm{s}$ & 6 & $\mathrm{~s}$ & MICHIGAN & ITALY & ITALY & & AT SCHOOL 5 & SAN MARTINO CANAVESE. TO & EAST PINE ST. & CALUMET PR. 2 \\
\hline 625 & MARTA & FRANK & s & 5 & $\mathrm{~s}$ & MICHIGAN & ITALY & ITALY & & & SAN MARTINO CANAVESE. TO & EAST PINE ST. & CALUMET PR 2 \\
\hline 626 & MARTA & MADDALENA & $\mathrm{D}$ & 3 & s & MICHIGAN & ITALY & ITALY & & & SAN MARTINO CANAVESE. TO & EAST PINE ST. & $\begin{array}{l}\text { CALUMET PR. } 2 \\
\text { CALUE }\end{array}$ \\
\hline 627 & MARTA & LEONE & s & $\frac{3}{1}$ & s & MICHIGAN & ITALY & ITALY & & & SAN MARTINO CANAVESE.TO & EAST PINE ST. & CALUMET PR. 2 \\
\hline
\end{tabular}




\begin{tabular}{|c|c|c|c|c|c|c|c|c|c|c|c|c|c|}
\hline 628 & BESSOLO & GIUSEPPE & BIL & 22 & $\mathrm{~s}$ & ITALY & ITALY & ITALY & NA & BLACKSMITH & SCARMAGNO.TO & EAST PINE ST. & CALUMET PR. 2 \\
\hline 629 & CURTO & PETER & B & 27 & S & ITALY & ITALY & ITALY & $\mathrm{AL}$ & TRAMMER/CM & TORINO & EAST PINE ST. & CALUMET PR. 2 \\
\hline 630 & REVELLO & MARTIN & B & 30 & $\mathrm{~s}$ & ITALY & ITALY & ITALY & $\mathrm{AL}$ & TRAMMER/CM & TORINO & EAST PINE ST. & CALUMET PR. 2 \\
\hline 631 & NIGRA/NIGRO & PETRONIA & $\mathrm{H}$ & 30 & W & ITALY & ITALY & ITALY & & BOARDING HOUSE KEEPER & TORINO & CEDAR ST. & CALUMET PR. 2 \\
\hline 632 & PIGNOCCO & KATE & $\mathrm{D}$ & 29 & M6 & ITALY & ITALY & ITALY & & & TORINO & CEDAR ST. & CALUMET PR. 2 \\
\hline 633 & PIGNOCCO & DOMINICK & SIL & 26 & M6 & ITALY & ITALY & ITALY & NA & TRAMMER/CM & SAN GIORGIO CANAVESE. TO & CEDAR ST. & CALUMET PR. 2 \\
\hline 634 & FALCONIERI & MICHELE & $\mathrm{B}$ & 50 & $\mathrm{~s}$ & ITALY & ITALY & ITALY & $\mathrm{AL}$ & TRAMMER/CM & SAN GIORGIO CANAVESE. TO & CEDAR ST. & CALUMET PR. 2 \\
\hline 635 & PURACHIA & JOHN & B & 23 & s & ITALY & ITALY & ITALY & $\mathrm{AL}$ & TRAMMER/CM & CUNEO & CEDAR ST. & CALUMET PR. 2 \\
\hline 636 & SASSO & NICK & B & 28 & $\mathrm{~S}$ & ITALY & ITALY & ITALY & NA & TRAMMER/CM & SAN BENIGNO CANAVESE. TO & CEDAR ST. & CALUMET PR. 2 \\
\hline 637 & FRATTINO & JOHN & B & 31 & $\mathrm{~s}$ & ITALY & ITALY & ITALY & $\mathrm{AL}$ & TRAMMER/CM & & CEDAR ST. & CALUMET PR 2 \\
\hline 638 & GUSSINO & ANGELO & B & 35 & $\mathrm{~s}$ & ITALY & ITALY & ITALY & NA & TRAMMER/CM & & CEDAR ST. & CALUMET PR. 2 \\
\hline 639 & PIGNOCCO & PETER & B & 28 & $\mathrm{~s}$ & ITALY & ITALY & ITALY & PA & TRAMMER/CM & SAN GIORGIO CANAVESE. TO & CEDAR ST. & CALUMET PR. 2 \\
\hline 640 & SUINO & JOSEPH & B & 27 & s & ITALY & ITALY & ITALY & $?$ & TIMBERMAN & TORINO & CEDAR ST. & CALUMET PR. 2 \\
\hline 641 & GALETTO & JOSEPH & $\mathrm{H}$ & 36 & M8 & ITALY & ITALY & ITALY & NA & TIMBER BOSS/CM & SAN GIORGIO CANAVESE. TO & CEDAR ST. & CALUMET PR. 2 \\
\hline 642 & GALETTO & MARY & W & 26 & M8 & ITALY & ITALY & ITALY & & & & CEDAR ST. & CALUMET PR. 2 \\
\hline 643 & GALETTO & JAMES & s & 6 & S & MICHIGAN & ITALY & ITALY & & AT SCHOOL 5 & SAN GIORGIO CANAVESE, TO & CEDAR ST. & CALUMET PR. 2 \\
\hline 644 & GALETTO & MARY & $\mathrm{D}$ & 4 & $\mathrm{~s}$ & MICHIGAN & ITALY & ITALY & & & SAN GIORGIO CANAVESE. TO & CEDAR ST. & CALUMET PR. 2 \\
\hline 645 & GALETTO & PETER & $\mathrm{s}$ & 1 & $\mathrm{~s}$ & MICHIGAN & ITALY & ITALY & & & SAN GIORGIO CANAVESE. TO & CEDAR ST. & CALUMET PR. 2 \\
\hline 646 & BERCHIATTI & MARY & $\mathrm{cOU}$ & 11 & S & MICHIGAN & ITALY & ITALY & & HOUSEWORK & TORINO & CEDAR ST. & CALUMET PR. 2 \\
\hline 647 & ELLINOR & JOSEPH & $\mathrm{H}$ & 30 & M2 & ITALY & ITALY & ITALY & $\mathrm{AL}$ & LABORER/CM & & CEDAR ST. & CALUMET PR. 2 \\
\hline 648 & ELLINOR & DOMINICA & w & 23 & M2 & ITALY & ITALY & ITALY & & & & CEDAR ST. & CALUMET PR. 2 \\
\hline 649 & ELLINOR & JOHN & s & 1 & $\mathrm{~s}$ & MICHIGAN & ITALY & ITALY & & & & CEDAR ST. & CALUMET PR. 2 \\
\hline 650 & CAPELLO & NICK & $\mathrm{H}$ & 39 & M18 & ITALY & ITALY & ITALY & NA & WATCHMAN/CM & SAN BENIGNO CANAVESE. TO & CEDAR ST. & CALUMET PR. 2 \\
\hline 651 & CAPELLO & ANNIE & W & 36 & M18 & $\begin{array}{l}\text { IITALY } \\
\text { ITALY }\end{array}$ & $\begin{array}{l}\text { IITALY } \\
\text { ITALY }\end{array}$ & $\begin{array}{l}\text { ITALY } \\
\text { ITALY }\end{array}$ & INA & WWATCHIVIAIN/CIVI & SAIV DEIVIGINU CAIVAVESE. IO & CEDAR ST. & $\begin{array}{l}\text { CALUMET PR. } 2 \\
\text { CALUE }\end{array}$ \\
\hline 652 & CAPELLO & THOMAS & s & 18 & $\mathrm{~s}$ & ITALY & ITALY & ITALY & & DRILL BOY/CM & SAN BENIGNO CANAVESE. TO & CEDAR ST. & CALUMET PR. 2 \\
\hline 653 & CAPELLO & MINNIE & $\mathrm{D}$ & 12 & $\mathrm{~s}$ & ITALY & ITALY & ITALY & & AT SCHOOL 10 & SAN BENIGNO CANAVESE. TO & CEDAR ST. & CALUMET PR. 2 \\
\hline 654 & CAPELLO & TONY & s & 7 & s & MICHIGAN & ITALY & ITALY & & AT SCHOOL 5 & SAN BENIGNO CANAVESE. TO & CEDAR ST. & CALUMET PR. 2 \\
\hline 655 & CAPELLO & MAGGIE & $\mathrm{D}$ & 5 & s & MICHIGAN & ITALY & ITALY & & & SAN BENIGNO CANAVESE, TO & CEDAR ST. & CALUMET PR. 2 \\
\hline 656 & CAPELLO & MARY & $\mathrm{D}$ & 4 & s & MICHIGAN & ITALY & ITALY & & & SAN BENIGNO CANAVESE. TO & CEDAR ST. & CALUMET PR. 2 \\
\hline 657 & CAPELLO & ANNE & $\mathrm{D}$ & $8 \mathrm{M}$ & s & MICHIGAN & ITALY & ITALY & & & SAN BENIGNO CANAVESE. TO & CEDAR ST. & CALUMET PR. 2 \\
\hline 658 & GAMOLETTO & JOHN & NEP & 22 & $\mathrm{~s}$ & ITALY & ITALY & ITALY & $\mathrm{AL}$ & TRAMMER/CM & SAN BENIGNO CANAVESE. TO & CEDAR ST. & CALUMET PR. 2 \\
\hline 659 & FRATINA & EMILIO & $\mathrm{H}$ & 27 & M? & ITALY & ITALY & ITALY & $\mathrm{AL}$ & TRAMMER/CM & SAN GIORGIO CANAVESE. TO & CEDAR ST. & CALUMET PR. 2 \\
\hline 660 & FRATINA & MARIA & w & 23 & M? & ITALY & ITALY & ITALY & & & SAN GIORGIO CANAVESE. TO & CEDAR ST. & CALUMET PR. 2 \\
\hline 661 & BASSO & LOUIS & $\mathrm{H}$ & 28 & M6 & ITALY & ITALY & ITALY & PA & TRAMMER/CM & SAN GIORGIO CANAVESE, TO & CEDAR ST. & CALUMET PR. 2 \\
\hline 662 & BASSO & JENNIE & w & 25 & M6 & ITALY & ITALY & ITALY & & & SAN GIORGIO CANAVESE. TO & CEDAR ST. & CALUMET PR. 2 \\
\hline 663 & BASSO & JOSEPH & s & 5 & $s$ & MICHIGAN & ITALY & ITALY & & & SAN GIORGIO CANAVESE. TO & CEDAR ST. & CALUMET PR. 2 \\
\hline 664 & BASSO & MAGGIE & $\mathrm{D}$ & 3 & S & MICHIGAN & ITALY & ITALY & & & SAN GIORGIO CANAVESE. TO & CEDAR ST. & $\begin{array}{l}\text { CALUMET PR. } 2 \\
\text { CALUE }\end{array}$ \\
\hline 664 & BASSO & JOAN EVA & $\begin{array}{l}\mathrm{D} \\
\mathrm{D}\end{array}$ & $\frac{3}{1}$ & $\begin{array}{l}\mathrm{s} \\
\mathrm{s}\end{array}$ & MICHIGAN & $\begin{array}{l}\text { IITALY } \\
\text { ITALY }\end{array}$ & $\begin{array}{l}\text { ITALY } \\
\text { ITALY }\end{array}$ & & & SAN GIORGIO CANAVESE. TO & CEDAR ST. & $\begin{array}{l}\text { CALUMET PR. } 2 \\
\text { CALUETR }\end{array}$ \\
\hline 666 & GENDRONI & JOSEPH & $\mathrm{H}$ & 34 & M4 & ITALY & ITALY & ITALY & PA & TRAMMER/CM & TORINO & CEDAR ST. & CALUMET PR. 2 \\
\hline 667 & GENDRONI & JOHANNA & W & 27 & M4 & ITALY & ITALY & ITALY & & & TORINO & CEDAR ST. & CALUMET PR. 2 \\
\hline 668 & GENDRONI & JOSEPH & s & 4 & S & MICHIGAN & ITALY & ITALY & & & TORINO & CEDAR ST. & CALUMET PR. 2 \\
\hline 669 & GENDRONI & PETER & $\mathrm{s}$ & 2 & S & MICHIGAN & ITALY & ITALY & & & TORINO & CEDAR ST. & CALUMET PR. 2 \\
\hline 670 & FIORINA & JOHN & $\mathrm{H}$ & 26 & M3 & ITALY & ITALY & ITALY & PA & TRAMMER/CM & SAN GIUSTO CANAVESE. TO & CEDAR ST. & CALUMET PR. 2 \\
\hline 671 & FIORINA & THERESA & W & 20 & M3 & ITALY & ITALY & ITALY & & & & CEDAR ST. & CALUMET PR. 2 \\
\hline 672 & FIORINA & KATE & $\mathrm{D}$ & 1 & s & MICHIGAN & ITALY & ITALY & & & SAN GIUSTO CANAVESE. TO & CEDAR ST. & CALUMET PR. 2 \\
\hline 673 & DE FILIPPI & JOHN & $\mathrm{H}$ & 46 & M22 & ITALY & ITALY & ITALY & & MINER/CM & SAN GIORGIO CANAVESE. TO & CEDAR ST. & CALUMET PR. 2 \\
\hline 674 & DE FILIPPI & TERESA & w & 40 & M22 & ITALY & ITALY & ITALY & & & SAN GIORGIO CANAVESE. TO & CEDAR ST. & CALUMET PR. 2 \\
\hline 675 & DE FILIPPI & FELIX & s & 20 & s & ITALY & ITALY & ITALY & & MINER/CM & SAN GIORGIO CANAVESE. TO & CEDAR ST. & CALUMET PR. 2 \\
\hline 676 & DE FILIPPI & ANTONIO & s & 14 & $\mathrm{~s}$ & MICHIGAN & ITALY & ITALY & & AT SCHOOL & SAN GIORGIO CANAVESE. TO & CEDAR ST. & $\begin{array}{l}\text { CALUMET PR. } 2 \\
\text { CALUE }\end{array}$ \\
\hline 677 & DE FILIPPI & DOMENICA & $\mathrm{D}$ & 12 & $\mathrm{~s}$ & MICHIGAN & ITALY & ITALY & & AT SCHOOL & SAN GIORGIO CANAVESE. TO & CEDAR ST. & $\begin{array}{l}\text { CALUMET PR. } 2 \\
\text { CALUE }\end{array}$ \\
\hline 678 & DE FILIPPI & GIUSEPPE & s & 10 & s & MICHIGAN & ITALY & ITALY & & AT SCHOOL & SAN GIORGIO CANAVESE.TO & CEDAR ST. & CALUMET PR. 2 \\
\hline $\begin{array}{l}6 / 8 \\
679\end{array}$ & $\begin{array}{l}\text { DEFLIPPI } \\
\text { DE FILIPPI }\end{array}$ & $\begin{array}{l}\text { GIUSEPPE } \\
\text { LENA/GIOV }\end{array}$ & $\begin{array}{l}\mathrm{S} \\
\mathrm{D}\end{array}$ & $\frac{10}{9}$ & 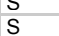 & $\begin{array}{l}\text { MICHIGAN } \\
\text { MICHIGAN }\end{array}$ & $\begin{array}{l}\text { IITALY } \\
\text { ITALY }\end{array}$ & $\begin{array}{l}\text { IIALY } \\
\text { ITALY }\end{array}$ & & $\begin{array}{l}\text { AT SCHOOL } 10 \\
\text { AT SCHOOL }\end{array}$ & SAN GIORGIO CANAVESE, TO & $\begin{array}{l}\text { CEDAR SI. } \\
\text { CEDAR ST. }\end{array}$ & $\begin{array}{l}\text { CALUME TPR. } 2 \\
\text { CALUMET PR. } 2\end{array}$ \\
\hline 680 & DE FILIPPI & ANGELO & s & 7 & $\mathrm{~s}$ & MICHIGAN & ITALY & ITALY & & AT SCHOOL 5 & SAN GIORGIO CANAVESE. TO & CEDAR ST. & CALUMET PR. 2 \\
\hline 681 & DE FILIPPI & GIOVANNI & $\mathrm{s}$ & 5 & s & MICHIGAN & ITALY & ITALY & & AT SCHOOL 5 & SAN GIORGIO CANAVESE. TO & CEDAR ST. & CALUMET PR. 2 \\
\hline 682 & GARLI & JOHN & $\mathrm{H}$ & 22 & M1 & ITALY & ITALY & ITALY & PA & LABORER/CM & & CEDAR ST. & CALUMET PR. 2 \\
\hline 683 & GARLI & MARY & w & 18 & M1 & ITALY & ITALY & ITALY & & & & CEDAR ST. & CALUMET PR. 2 \\
\hline 684 & GARLI & ANGELINA & MO & 43 & w & ITALY & ITALY & ITALY & & HOUSEWORK & & CEDAR ST. & CALUMET PR. 2 \\
\hline 685 & GENARDO & DOMINIC & B & 36 & s & ITALY & ITALY & ITALY & PA & TRAMMER/CM & & CEDAR ST. & CALUMET PR. 2 \\
\hline 686 & CASSALLIO & JOSEPH & B & 22 & $\mathrm{~S}$ & ITALY & ITALY & ITALY & $\mathrm{AL}$ & LABORER/CM & & CEDAR ST. & CALUMET PR. 2 \\
\hline 687 & CASSALLIO & ORNA B ? & B & 31 & s & ITALY & ITALY & ITALY & $\mathrm{AL}$ & TRAMMER/CM & & CEDAR ST. & CALUMET PR. 2 \\
\hline 688 & DE FILIPPI & VICTOR & B & 28 & s & ITALY & ITALY & ITALY & PA & TRAMMER/CM & SAN GIORGIO CANAVESE. TO & CEDAR ST. & CALUMET PR. 2 \\
\hline 689 & DE FILIPPI & LEON & $\begin{array}{l}\mathrm{D} \\
\mathrm{B}\end{array}$ & 30 & s & ITALY & ITALY & ITALY & PA & TRAMMER/CM & SAN GIORGIO CANAVESE. TO & CEDAR ST. & $\begin{array}{l}\text { CALUMET PR. } 2 \\
\text { CALUE }\end{array}$ \\
\hline 690 & PECHIETTO & EMANUEL & B & 21 & s & ITALY & ITALY & ITALY & AL & DRILL BOY/CM & & CEDAR ST. & CALUMET PR. 2 \\
\hline
\end{tabular}




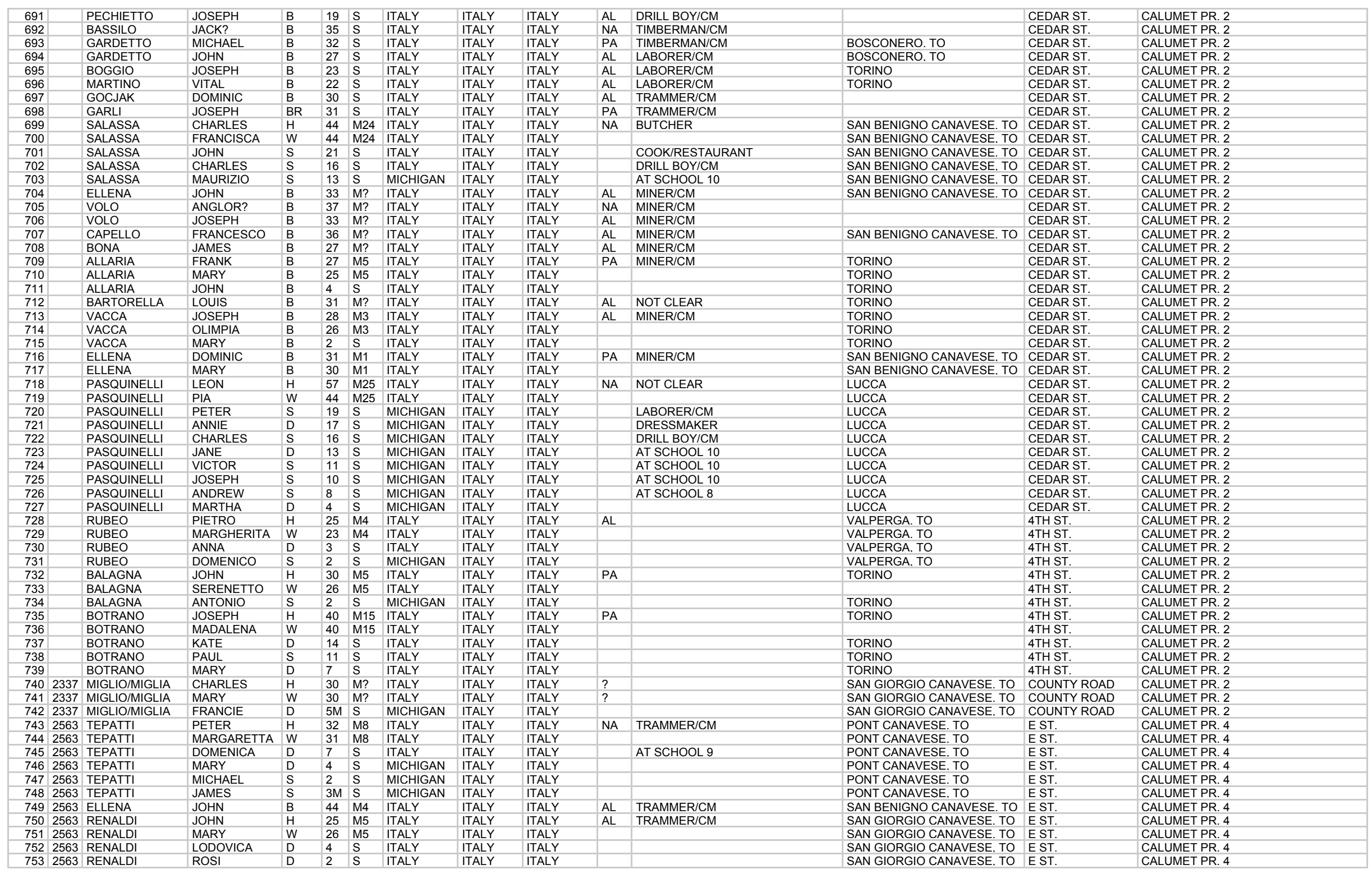




\begin{tabular}{|c|c|c|c|c|c|c|c|c|c|c|c|c|c|c|}
\hline 754 & 2563 & BUTONIO & BOTO & B & 24 & M2 & ITALY & ITALY & ITALY & $\mathrm{AL}$ & TRAMMER/CM & & EST. & CALUMET PR. 4 \\
\hline 755 & 2563 & ANTONIO & PETER & $\mathrm{H}$ & 28 & M7 & ITALY & ITALY & ITALY & NA & MINER/CM & & EST. & CALUMET PR. 4 \\
\hline 756 & 2563 & ANTONIO & COSTANCE & W & 28 & M7 & ITALY & ITALY & ITALY & & & & EST. & CALUMET PR. 4 \\
\hline 757 & 2563 & ANTONIO & JOHN & s & $4 \mathrm{M}$ & $\mathrm{s}$ & ITALY & ITALY & ITALY & & & & EST. & CALUMET PR. 4 \\
\hline 758 & 2567 & SOPPELSA & LOUIS & $\mathrm{H}$ & 41 & M9 & ITALY & ITALY & ITALY & NA & TIMBERMAN/CM & VOLTAGO. BELLUNO & E ST. & CALUMET PR. 4 \\
\hline 759 & 2567 & SOPPELSA & MARY & w & 40 & M9 & GERMANY & GERMAN & GERMANY & & & & E ST. & CALUMET PR. 4 \\
\hline 760 & 2567 & SOPPELSA & MARY & $\mathrm{D}$ & 4 & $\mathrm{~s}$ & MICHIGAN & ITALY & GERMANY & & & VOLTAGO. BELLUNO & E ST. & CALUMET PR. 4 \\
\hline 761 & 2567 & FAUSONE & TRACY & SER & 30 & s & ITALY & ITALY & ITALY & & SERVANT & SAN GIORGIO CANAVESE. TO & E ST. & CALUMET PR. 4 \\
\hline 762 & 2567 & SCHENA & MICHAEL & B & 24 & $\mathrm{~s}$ & ITALY & ITALY & ITALY & AL & TRAMMER/CM & RIVAMONTE. BELLUNO & EST. & CALUMET PR. 4 \\
\hline 763 & 2567 & SCHENA & PETER & B & 20 & $\mathrm{~s}$ & ITALY & ITALY & ITALY & $\mathrm{AL}$ & MINER/CM & RIVAMONTE. BELLUNO & E ST. & CALUMET PR. 4 \\
\hline 764 & 2567 & CONEDERA & JOSEPH & B & 22 & $\mathrm{~s}$ & ITALY & ITALY & ITALY & $\mathrm{AL}$ & MINER/CM & VOLTAGO. BELLUNO & EST. & CALUMET PR. 4 \\
\hline 765 & 2567 & SOMMARIVA & AUGUST & B & 40 & $\mathrm{~s}$ & ITALY & ITALY & ITALY & $\mathrm{AL}$ & TRAMMER/CM & BELLUNO & E ST. & CALUMET PR. 4 \\
\hline 766 & 2567 & SOMMARIVA & LOUIS & B & 32 & $\mathrm{~s}$ & ITALY & ITALY & ITALY & PA & TRAMMER/CM & BELLUNO & E ST. & CALUMET PR. 4 \\
\hline 767 & 2567 & DAL COL & FERMO & B & 25 & s & ITALY & ITALY & ITALY & PA & TRAMMER/CM & BELLUNO & EST. & CALUMET PR. 4 \\
\hline 768 & 2524 & BRACCO & JOHN & $\mathrm{H}$ & 37 & M13 & ITALY & ITALY & ITALY & NA & MINER/CM & TORINO & D ST. & CALUMET PR. 4 \\
\hline 769 & 2524 & BRACCO & DOMENICA & W & 35 & M13 & ITALY & ITALY & ITALY & & & & D ST. & CALUMET PR. 4 \\
\hline 770 & 2524 & BRACCO & JOSEPH & $s$ & 6 & s & MICHIGAN & ITALY & ITALY & & AT SCHOOL 9 & TORINO & D ST. & CALUMET PR. 4 \\
\hline 771 & 2524 & BRACCO & TONY & $\mathrm{s}$ & 4 & $\mathrm{~S}$ & MICHIGAN & ITALY & ITALY & & AT SCHOOL 9 & TORINO & D ST. & CALUMET PR. 4 \\
\hline 772 & 2524 & BRACCO & MADELINE & $\mathrm{D}$ & 2 & S & MICHIGAN & ITALY & ITALY & & & TORINO & D ST. & CALUMET PR. 4 \\
\hline 773 & 2524 & SANDRETTO & JOHN & $\mathrm{H}$ & 24 & M0 & ITALY & ITALY & ITALY & & TRAMMER/CM & PONT CANAVESE. TO & D ST. & CALUMET PR. 4 \\
\hline 774 & 2524 & SANDRETTO & MARY & w & 23 & MO & ITALY & ITALY & ITALY & & & & D ST. & CALUMET PR. 4 \\
\hline 775 & 2524 & REANO CONTO & CARLO & $\mathrm{H}$ & 24 & MO & ITALY & ITALY & ITALY & $\mathrm{AL}$ & TRAMMER/CM & CORTEREGGIO, TO & D ST. & CALUMET PR. 4 \\
\hline 776 & $|2524|$ & REANO CONTO & TERESA & W & 23 & MO & ITALY & ITALY & ITALY & AL & TRAIVIVILK/UIVI & CORTEREGGIO. TO & $\begin{array}{l}\text { D ST. } \\
\text { D T. }\end{array}$ & CALUMET PR. 4 \\
\hline 777 & 2524 & GASPARDO & JOHN & $\mathrm{H}$ & 37 & M6 & ITALY & ITALY & ITALY & & TIMBER BOSS & PONT CANAVESE. TO & D ST. & CALUMET PR. 4 \\
\hline 778 & 2524 & GASPARDO & ANNIE & w & 22 & M6 & ITALY & ITALY & ITALY & & & & DST. & CALUMET PR. 4 \\
\hline 779 & 2524 & GASPARDO & MARY & D & 3 & s & MICHIGAN & ITALY & ITALY & & & PONT CANAVESE. TO & D ST. & CALUMET PR. 4 \\
\hline 780 & 2524 & GASPARDO & ROSIE & D & $3 \mathrm{M}$ & $\mathrm{s}$ & MICHIGAN & ITALY & ITALY & & & PONT CANAVESE. TO & D ST. & CALUMET PR. 4 \\
\hline 781 & 2524 & SANDRETTO & SILVESTRO & $\mathrm{H}$ & 31 & M8 & ITALY & ITALY & ITALY & PA & TRAMMER/CM & PONT CANAVESE, TO & D ST. & CALUMET PR. 4 \\
\hline 782 & 2524 & SANDRETTO & ANGELA & W & 31 & M8 & ITALY & ITALY & ITALY & & & PONT CANAVESE. TO & D ST. & CALUMET PR. 4 \\
\hline 783 & 2524 & SANDRETTO & MINNIE & $\mathrm{D}$ & 7 & $\mathrm{~S}$ & ITALY & ITALY & ITALY & & AT SCHOOL 9 & PONT CANAVESE. TO & D ST. & CALUMET PR. 4 \\
\hline 784 & 2524 & SANDRETTO & ANNIE & $\mathrm{D}$ & 1 & $\mathrm{~s}$ & MICHIGAN & ITALY & ITALY & & & PONT CANAVESE. TO & D ST. & CALUMET PR. 4 \\
\hline 785 & 2524 & SANDRETTO & JAMES & $\mathrm{s}$ & 2 & $\mathrm{~s}$ & MICHIGAN & ITALY & ITALY & & & PONT CANAVESE. TO & D ST. & CALUMET PR. 4 \\
\hline 786 & 2520 & GHIGGIA & DOMINICK & $\mathrm{H}$ & 26 & M1 & ITALY & ITALY & ITALY & NA & MINER/CM & SCARMAGNO. TO & D ST. & CALUMET PR. 4 \\
\hline 787 & 2520 & GHIGGIA & PAULINE & W & 19 & M1 & ITALY & ITALY & ITALY & & & & D ST. & CALUMET PR. 4 \\
\hline 788 & 2520 & GHIGGIA & LOUIS & $\mathrm{H}$ & 60 & M28 & ITALY & ITALY & ITALY & NA & MINER/CM & SCARMAGNO. TO & D ST. & CALUMET PR. 4 \\
\hline 789 & 2520 & GHIGGIA & ANTONIA & W & 46 & M28 & $\begin{array}{l}\text { ITALY } \\
\text { ITAL }\end{array}$ & ITALY & ITALY & INA & IVIINERT/UI & SCARMAGNO. TO & $\begin{array}{l}\text { D ST. } \\
\text { D ST. }\end{array}$ & $\begin{array}{l}\text { CALUMET PR. } 4 \\
\text { CALE }\end{array}$ \\
\hline 790 & 2520 & GHIGGIA & MARY & $\mathrm{D}$ & 17 & $\mathrm{~s}$ & ITALY & ITALY & ITALY & & & SCARMAGNO. TO & D ST. & CALUMET PR. 4 \\
\hline 791 & 2520 & GHIGGIA & MAGGIE & $\mathrm{D}$ & 14 & $\mathrm{~s}$ & ITALY & ITALY & ITALY & & & SCARMAGNO. TO & D ST. & CALUMET PR. 4 \\
\hline 792 & 2520 & GHIGGIA & KATIE & $\mathrm{D}$ & 11 & $\mathrm{~s}$ & ITALY & ITALY & ITALY & & AT SCHOOL 9 & SCARMAGNO. TO & D ST. & CALUMET PR. 4 \\
\hline 793 & 2520 & GHIGGIA & JOHN & $\mathrm{s}$ & 9 & s & ITALY & ITALY & ITALY & & AT SCHOOL 9 & SCARMAGNO. TO & D ST. & CALUMET PR. 4 \\
\hline 794 & 2520 & GARDENA & JOSEPH & B & 37 & M12 & ITALY & ITALY & ITALY & NA & TRAMMER/CM & & D ST. & CALUMET PR. 4 \\
\hline 795 & 2520 & ANSELMO & VINCENZO & B & 34 & $\mathrm{~s}$ & ITALY & ITALY & ITALY & $\mathrm{PA}$ & TRAMMER/CM & BARONE CANAVESE. TO & D ST. & CALUMET PR. 4 \\
\hline 796 & 2520 & TORREANO & JOE & B & 22 & $\mathrm{~S}$ & ITALY & ITALY & ITALY & & MINER/CM & TORINO & D ST. & CALUMET PR. 4 \\
\hline 797 & 2520 & BRUNAS & DOMINICK & B & 37 & s & ITALY & ITALY & ITALY & NA & MINER/CM & SCARMAGNO. TO & D ST. & CALUMET PR. 4 \\
\hline 798 & 2518 & PIANA & JOHN & $\mathrm{H}$ & 36 & M11 & ITALY & ITALY & ITALY & NA & TEAMSTER & TORINO & D ST. & CALUMET PR. 4 \\
\hline 799 & 2518 & PIANA & MARY & w & 33 & M11 & ITALY & ITALY & ITALY & & AT SCHOOL & TORINO & D ST. & CALUMET PR. 4 \\
\hline 800 & 2518 & PIANA & DOMINICK & s & 10 & $\mathrm{~s}$ & MICHIGAN & ITALY & ITALY & & AT SCHOOL 9 & TORINO & D ST. & CALUMET PR. 4 \\
\hline 801 & 2518 & PIANA & FRANK & s & 8 & $\mathrm{~s}$ & MICHIGAN & ITALY & ITALY & & AT SCHOOL 9 & TORINO & D ST. & CALUMET PR. 4 \\
\hline 802 & 2518 & PIANA & JOHN & $\mathrm{s}$ & 4 & s & MICHIGAN & ITALY & ITALY & & AT SCHOOL & TORINO & D ST. & CALUMET PR. 4 \\
\hline 803 & 2518 & RU/AFFANO & FRANK & B & 20 & s & ITALY & ITALY & ITALY & & TRAMMER/CM & & D ST. & CALUMET PR. 4 \\
\hline 804 & 2518 & MARDA & JOHN & B & 25 & $\mathrm{~s}$ & ITALY & ITALY & ITALY & $\mathrm{AL}$ & TRAMMER/CM & & D ST. & CALUMET PR. 4 \\
\hline 805 & 2518 & PONSETTO & BARTOLOMEO & B & 22 & $\mathrm{~s}$ & ITALY & ITALY & ITALY & $\mathrm{AL}$ & TRAMMER/CM & SAN GIORGIO CANAVESE, TO & D ST. & CALUMET PR. 4 \\
\hline 806 & 2508 & LENCIONI & ANGELO & $\mathrm{H}$ & 28 & M9 & ITALY & ITALY & ITALY & & TIMBER BOSS/CM & LUCCA & D ST. & CALUMET PR. 4 \\
\hline 807 & 2508 & LENCIONI & SARAH & W & 28 & M9 & IRELAND & IRELAND & IRELAND & & & IRELAND & D ST. & CALUMET PR. 4 \\
\hline 808 & 2508 & LENCIONI & LOUIS & $\mathrm{s}$ & 8 & $\mathrm{~S}$ & MICHIGAN & ITALY & IRELAND & & AT SCHOOL 9 & LUCCA & D ST. & CALUMET PR. 4 \\
\hline 809 & 2508 & LENCIONI & NETTER & D & 7 & $\mathrm{~s}$ & MICHIGAN & ITALY & IRELAND & & AT SCHOOL 9 & LUCCA & D ST. & CALUMET PR. 4 \\
\hline 810 & 2508 & LENCIONI & MARY & $\mathrm{D}$ & 6 & s & MICHIGAN & ITALY & IRELAND & & AT SCHOOL 6 & LUCCA & D ST. & CALUMET PR. 4 \\
\hline 811 & 2508 & LENCIONI & DOLLIE & $\mathrm{D}$ & 4 & s & MICHIGAN & ITALY & IRELAND & & & LUCCA & D ST. & CALUMET PR. 4 \\
\hline 812 & 2508 & LENCIONI & DALLA & $\mathrm{D}$ & 3 & s & MICHIGAN & ITALY & IRELAND & & & LUCCA & D ST. & CALUMET PR. 4 \\
\hline 813 & 2508 & LENCIONI & ROSA & $\mathrm{D}$ & $6 \mathrm{M}$ & s & MICHIGAN & ITALY & IRELAND & & & LUCCA & D ST. & CALUMET PR. 4 \\
\hline 814 & 2508 & ANTONIOTTI & JOSEPH & B & 25 & s & ITALY & ITALY & ITALY & & & & D ST & CALUMET PR. 4 \\
\hline 815 & 2508 & PERRON & JOHN & B & 25 & s & ITALY & ITALY & ITALY & & & & D ST. & CALUMET PR. 4 \\
\hline 816 & 2505 & FAUSONE & PETER & $\mathrm{H}$ & 38 & M29 & ITALY & ITALY & ITALY & NA & WATCHMAN/CM & TORINO & D ST. & CALUMET PR. 4 \\
\hline
\end{tabular}




\begin{tabular}{|c|c|c|c|c|c|c|c|c|c|c|c|c|c|c|}
\hline 817 & 2505 & FAUSONE & MARY & W & 44 & M29 & ITALY & ITALY & ITALY & & & & D ST. & CALUMET PR. 4 \\
\hline 818 & 2505 & FAUSONE & JOSEPH & s & 25 & S & MICHIGAN & ITALY & ITALY & & DRILL BOY/CM & TORINO & D ST. & CALUMET PR. 4 \\
\hline 819 & 2505 & FAUSONE & JOHN & $\mathrm{s}$ & 7 & $\mathrm{~S}$ & MICHIGAN & ITALY & ITALY & & & TORINO & D ST. & CALUMET PR. 4 \\
\hline 820 & 2507 & ZANARDI & LOUIS & $\mathrm{H}$ & 40 & M3 & ITALY & ITALY & ITALY & & TRAMMER/CM & BERGAMO & D ST. & CALUMET PR. 4 \\
\hline 821 & 2507 & ZANARDI & MAGGIE & W & 28 & M3 & ITALY & ITALY & ITALY & & & BERGAMO & D ST. & CALUMET PR. 4 \\
\hline 822 & 2507 & ZANARDI & CHARLES & S & 1 & $\mathrm{~s}$ & MICHIGAN & ITALY & ITALY & & & BERGAMO & D ST. & CALUMET PR. 4 \\
\hline 823 & 2507 & ZANARDI & JOHN & S & $3 \mathrm{M}$ & $\mathrm{s}$ & MICHIGAN & ITALY & ITALY & & & BERGAMO & D ST. & CALUMET PR. 4 \\
\hline 824 & 2509 & BRUSCA & IVE? & $\mathrm{H}$ & 37 & M7 & ITALY & ITALY & ITALY & NA & MINER/CM & & D ST. & CALUMET PR. 4 \\
\hline 825 & 2509 & BRUSCA & ANTONIA & W & 30 & M7 & ITALY & ITALY & ITALY & INA & 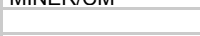 & & D ST. & CALUMET PR. 4 \\
\hline 826 & 2509 & BRUSCA & BATIS & S & 5 & $\mathrm{~S}$ & MICHIGAN & ITALY & ITALY & & AT SCHOOL 9 & & D ST. & CALUMET PR. 4 \\
\hline 827 & 2509 & BRACCO & PETER & B & 32 & $\mathrm{~s}$ & ITALY & ITALY & ITALY & PA & TRAMMER/CM & TORINO & D ST. & CALUMET PR. 4 \\
\hline 828 & 2509 & BAUSANO & JOHN & B & 25 & s & ITALY & ITALY & ITALY & & TRAMMER/CM & TORINO & DST. & CALUMET PR. 4 \\
\hline 829 & 2513 & TAPPERO & JOSEPH & $\mathrm{H}$ & 44 & M14 & ITALY & ITALY & ITALY & $\mathrm{AL}$ & WATCHMAN/CM & SAN GIUSTO CANAVESE. TO & D ST. & CALUMET PR. 4 \\
\hline 830 & 2513 & TAPPERO & DOMENICA & W & 32 & M14 & ITALY & ITALY & ITALY & & & SAN GIUSTO CANAVESE. TO & D ST. & CALUMET PR. 4 \\
\hline 831 & 2513 & TAPPERO & KATIE & $\mathrm{D}$ & 13 & s & ITALY & ITALY & ITALY & & AT SCHOOL 9 & SAN GIUSTO CANAVESE. TO & D ST. & CALUMET PR. 4 \\
\hline 832 & 2513 & TAPPERO & DOMINICK & S & 10 & $\mathrm{~S}$ & ITALY & ITALY & ITALY & & AT SCHOOL 9 & SAN GIUSTO CANAVESE, TO & D ST. & CALUMET PR. 4 \\
\hline 833 & 2513 & TAPPERO & JOHN & $\mathrm{s}$ & 7 & $\mathrm{~s}$ & MICHIGAN & ITALY & ITALY & & AT SCHOOL 9 & SAN GIUSTO CANAVESE. TO & D ST. & CALUMET PR. 4 \\
\hline 834 & 2513 & TAPPERO & JOSEPH & $\mathrm{s}$ & 5 & $\mathrm{~S}$ & MICHIGAN & ITALY & ITALY & & AT SCHOOL 9 & SAN GIUSTO CANAVESE. TO & D ST. & CALUMET PR. 4 \\
\hline 835 & 2513 & TAPPERO & BERTHA MAI & $\mathrm{D}$ & 3 & $\mathrm{~s}$ & MICHIGAN & ITALY & ITALY & & & SAN GIUSTO CANAVESE. TO & D ST. & CALUMET PR. 4 \\
\hline 836 & 2513 & TAPPERO & MARY & $\mathrm{D}$ & $4 \mathrm{M}$ & $\mathrm{s}$ & MICHIGAN & ITALY & ITALY & & & SAN GIUSTO CANAVESE. TO & D ST. & CALUMET PR. 4 \\
\hline 837 & 2513 & TAPPERO & JOSEPH & $\mathrm{H}$ & 28 & M2 & ITALY & ITALY & ITALY & AL & TRAMMER/CM & SAN GIUSTO CANAVESE. TO & D ST. & CALUMET PR. 4 \\
\hline 838 & 2513 & TAPPERO & JOSEPHENA & W & 23 & M2 & ITALY & ITALY & ITALY & & & SAN GIUSTO CANAVESE, TO & D ST. & CALUMET PR. 4 \\
\hline 839 & 2513 & TAPPERO & TRACY & $\mathrm{D}$ & 1 & $\mathrm{~s}$ & MICHIGAN & ITALY & ITALY & & & SAN GIUSTO CANAVESE. TO & D ST. & CALUMET PR. 4 \\
\hline 840 & 2513 & TAPPERO & BARTOLOMEO & $\mathrm{H}$ & 33 & M12 & ITALY & ITALY & ITALY & AL & TIMBERMAN/CM & SAN GIORGIO CANAVESE. TO & D ST. & CALUMET PR. 4 \\
\hline 841 & 2513 & TAPPERO & KATIE & w & 31 & M12 & ITALY & ITALY & ITALY & & & SAN GIORGIO CANAVESE. TO & D ST. & CALUMET PR. 4 \\
\hline 842 & 2513 & TAPPERO & KATIE & $\mathrm{D}$ & 11 & $\mathrm{~s}$ & ITALY & ITALY & ITALY & & AT SCHOOL 9 & SAN GIORGIO CANAVESE. TO & D ST. & CALUMET PR. 4 \\
\hline 843 & 2513 & TAPPERO & JOSEPH & $\mathrm{s}$ & 8 & $\mathrm{~S}$ & ITALY & ITALY & ITALY & & AT SCHOOL 9 & SAN GIORGIO CANAVESE. TO & D ST. & CALUMET PR. 4 \\
\hline 844 & 2513 & TAPPERO & ROSA & $\mathrm{D}$ & 4 & $\mathrm{~s}$ & MICHIGAN & ITALY & ITALY & & & SAN GIORGIO CANAVESE, TO & D ST. & CALUMET PR. 4 \\
\hline 845 & 2513 & TAPPERO & MARY & $\mathrm{D}$ & 2 & $\mathrm{~s}$ & MICHIGAN & ITALY & ITALY & & & SAN GIORGIO CANAVESE. TO & D ST. & CALUMET PR. 4 \\
\hline 846 & 2513 & ALASINA & D. & $\mathrm{H}$ & 32 & M8 & ITALY & ITALY & ITALY & $\mathrm{AL}$ & TRAMMER/CM & TORINO & D ST. & CALUMET PR. 4 \\
\hline 847 & 2513 & ALASINA & ANNIE & W & 32 & M8 & ITALY & ITALY & ITALY & & & & D ST. & CALUMET PR. 4 \\
\hline 848 & 2513 & ALASINA & PETER & $\mathrm{s}$ & 7 & $\mathrm{~s}$ & MICHIGAN & ITALY & ITALY & & AT SCHOOL 9 & TORINO & D ST. & CALUMET PR. 4 \\
\hline 849 & 2517 & ALLARIA & ANTONIO & $\mathrm{H}$ & 26 & M3 & ITALY & ITALY & ITALY & NA & TRAMMER/CM & SAN BENIGNO CANAVESE. TO & D ST. & CALUMET PR. 4 \\
\hline 850 & 2517 & ALLARIA & DOMENICA & W & 26 & M3 & ITALY & ITALY & ITALY & & & & D ST. & CALUMET PR. 4 \\
\hline 851 & 2517 & ALLARIA & DOMENICA & $\mathrm{D}$ & & & MICHIGAN & ITALY & ITALY & & & SAN BENIGNO CANAVESE. TO & D ST. & CALUMET PR. 4 \\
\hline 852 & 2517 & BARINOTTI & FRANK & $\mathrm{H}$ & 31 & M11 & ITALY & ITALY & ITALY & NA & TIMBERMAN/CM & $\begin{array}{l}\text { PONT CANAVESE. TO } \\
\text { POE. }\end{array}$ & $\begin{array}{l}\text { D ST. } \\
\text { D ST }\end{array}$ & CALUMET PR. 4 \\
\hline 853 & 2517 & BARINOTTI & MADDALENA & w & 27 & M11 & ITALY & ITALY & ITALY & & & TORINO & D ST. & CALUMET PR. 4 \\
\hline 854 & 2517 & BARINOTTI & JOHN & s & 8 & $\mathrm{~s}$ & MICHIGAN & ITALY & ITALY & & AT SCHOOL 9 & PONT CANAVESE. TO & D ST. & CALUMET PR. 4 \\
\hline 855 & 2517 & BARINOTTI & MAGGIE & $\mathrm{D}$ & $2 \mathrm{M}$ & $\mathrm{s}$ & MICHIGAN & ITALY & ITALY & & & PONT CANAVESE. TO & D ST. & CALUMET PR. 4 \\
\hline 856 & 2521 & GASPARDO & DOMENICO & $\mathrm{H}$ & 39 & M5 & ITALY & ITALY & ITALY & NA & SPRINKLER/CM & PONT CANAVESE. TO & D ST. & CALUMET PR. 4 \\
\hline 857 & 2521 & GASPARDO & MARIA & w & 30 & M5 & ITALY & ITALY & ITALY & & & & D ST. & CALUMET PR. 4 \\
\hline 858 & 2521 & SARANGIA & MIKE & $\mathrm{H}$ & 37 & M17 & ITALY & ITALY & ITALY & NA & LABORER/CM & PONT CANAVESE. TO & D ST. & CALUMET PR. 4 \\
\hline 859 & 2521 & SARANGIA & MADELINE & W & 37 & M17 & ITALY & ITALY & ITALY & & & PONT CANAVESE. TO & D ST. & CALUMET PR. 4 \\
\hline 860 & 2521 & SARANGIA & JOHN & s & 16 & $\mathrm{~s}$ & ITALY & ITALY & ITALY & & DRILL BOY/CM & PONT CANAVESE. TO & D ST. & CALUMET PR. 4 \\
\hline 861 & 2521 & SARANGIA & MARY & $\mathrm{D}$ & 10 & $\mathrm{~S}$ & MICHIGAN & ITALY & ITALY & & AT SCHOOL 9 & PONT CANAVESE. TO & D ST. & CALUMET PR. 4 \\
\hline 862 & 2521 & SARANGIA & KATIE & $\mathrm{D}$ & 7 & $\mathrm{~S}$ & MICHIGAN & ITALY & ITALY & & AT SCHOOL 9 & PONT CANAVESE. TO & D ST. & CALUMET PR. 4 \\
\hline 863 & 2521 & SARANGIA & PAULINE & $\mathrm{D}$ & 3 & s & MICHIGAN & ITALY & ITALY & & & PONT CANAVESE. TO & D ST. & CALUMET PR. 4 \\
\hline 864 & 2521 & CERETTO & JOHN & $\mathrm{H}$ & 29 & M4 & ITALY & ITALY & ITALY & PA & TRAMMER/CM & PONT CANAVESE. TO & $\begin{array}{l}\text { D ST. } \\
\text { D }\end{array}$ & CALUMET PR. 4 \\
\hline 865 & 2521 & CERETTO & LUCIA & W & 26 & M4 & ITALY & ITALY & ITALY & & & & D ST. & CALUMET PR. 4 \\
\hline 866 & 2521 & CERETTO & FRANCESCA & $\mathrm{D}$ & 3 & s & MICHIGAN & ITALY & ITALY & & & PONT CANAVESE. TO & D ST. & CALUMET PR. 4 \\
\hline 867 & 2521 & CERETTO & JOHANNA & $\mathrm{D}$ & 1 & s & MICHIGAN & ITALY & ITALY & & & PONT CANAVESE. TO & D ST. & CALUMET PR. 4 \\
\hline 868 & 2521 & CERETTO & DOMINICK & B & 27 & M5 & ITALY & ITALY & ITALY & $\mathrm{AL}$ & TRAMMER/CM & PONT CANAVESE, TO & D ST. & CALUMET PR. 4 \\
\hline 869 & 2521 & FEIRA & MARTIN & B & 24 & $\mathrm{~s}$ & ITALY & ITALY & ITALY & & TRAMMER/CM & PONT CANAVESE. TO & D ST. & CALUMET PR. 4 \\
\hline 870 & 2523 & NARDI & MIKE & $\mathrm{H}$ & 33 & M4 & ITALY & ITALY & ITALY & PA & TIMBERMAN/CM & CARMIGLIANO. LU & D ST. & CALUMET PR. 4 \\
\hline 871 & 2523 & NARDI & JULIA & W & 34 & M4 & ITALY & ITALY & ITALY & & & & D ST. & CALUMET PR. 4 \\
\hline 872 & 2523 & NARDI & ALMANDO? & D & 3 & $\mathrm{~s}$ & MICHIGAN & ITALY & ITALY & & & CARMIGLIANO. LU & D ST. & CALUMET PR. 4 \\
\hline 873 & 2523 & NARDI & JENNIE & $\mathrm{D}$ & 1 & s & MICHIGAN & ITALY & ITALY & & & CARMIGLIANO. LU & D ST. & CALUMET PR. 4 \\
\hline 874 & 2523 & NARDI & PETER & B & 39 & M12 & ITALY & ITALY & ITALY & NA & MINER/CM & CARMIGLIANO, LU & D ST. & CALUMET PR. 4 \\
\hline 875 & 2523 & FULEGER & DOMINICK & $\mathrm{H}$ & 35 & M5 & ITALY & ITALY & ITALY & PA & TRAMMER/CM & & D ST. & CALUMET PR. 4 \\
\hline 876 & 2523 & FULEGER & KATIE & W & 25 & M5 & ITALY & ITALY & ITALY & & & & D ST. & CALUMET PR. 4 \\
\hline 877 & 2523 & FULEGER & ROSIE & D & 8 & s & MICHIGAN & ITALY & ITALY & & & & D ST & CALUMET PR. 4 \\
\hline 878 & 2525 & CROTELLA & FRANK & $\mathrm{H}$ & 38 & M5 & ITALY & ITALY & ITALY & NA & TIMBER BOSS/CM & & D ST. & CALUMET PR. 4 \\
\hline 879 & 2525 & CROTELLA & MARY & w & 26 & M5 & ITALY & ITALY & ITALY & & & & D ST. & CALUMET PR. 4 \\
\hline
\end{tabular}




\begin{tabular}{|c|c|c|c|c|c|c|c|c|c|c|c|c|c|c|}
\hline 880 & 2525 & CROTELLA & VINCENCE & $\mathrm{s}$ & 2 & S & MICHIGAN & ITALY & ITALY & & & & D ST. & CALUMET PR. 4 \\
\hline 881 & 2525 & CASTIGLIANO & DOMINICK & B & 30 & M2 & ITALY & ITALY & ITALY & AL & TIMBERMAN/CM & SALASSA. TO & D ST. & CALUMET PR. 4 \\
\hline 882 & 2525 & PAGANUCCI & JAMES & $\mathrm{H}$ & 27 & M1 & ITALY & ITALY & ITALY & PA & TRAMMER/CM & SAN PIETRO. LU & D ST. & CALUMET PR. 4 \\
\hline 883 & 2525 & PAGANUCCI & TRACY & W & 24 & M1 & ITALY & ITALY & ITALY & & & SAN PIETRO. LU & D ST. & CALUMET PR. 4 \\
\hline 884 & 2525 & PAGANUCCI & DOMINICK & s & $2 \mathrm{M}$ & $\mathrm{s}$ & MICHIGAN & ITALY & ITALY & & & SAN PIETRO. LU & D ST. & CALUMET PR. 4 \\
\hline 885 & 2529 & RICCA & ANTONE & $\mathrm{H}$ & 38 & M7 & ITALY & ITALY & ITALY & $?$ & WATCHMAN/CM & TORINO & D ST. & CALUMET PR. 4 \\
\hline 886 & 2529 & RICCA & ANTONIETTA & W & 27 & M7 & ITALY & ITALY & ITALY & & & & D ST. & CALUMET PR. 4 \\
\hline 887 & 2529 & RICCA & VETO & $\mathrm{H}$ & 26 & M? & ITALY & ITALY & ITALY & & TRAMMER/CM & TORINO & D ST. & CALUMET PR. 4 \\
\hline 888 & 2529 & RICCA & PAULINA & W & 24 & M? & ITALY & ITALY & ITALY & & 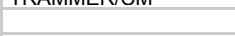 & VALPERGA. TO & $\begin{array}{l}\text { D ST. } \\
\text { DT. }\end{array}$ & CALUMET PR. 4 \\
\hline 889 & 2529 & RICCA & LUCY & $\mathrm{D}$ & 1 & $\mathrm{~s}$ & MICHIGAN & ITALY & ITALY & & & TORINO & D ST. & CALUMET PR. 4 \\
\hline 890 & 2529 & RUATTO & FRANK & B & 22 & $?$ & ITALY & ITALY & ITALY & & TRAMMER/CM & VALPERGA. TO & D ST. & CALUMET PR. 4 \\
\hline 891 & 2476 & BIGANDO & GIORGIO & $\mathrm{H}$ & 43 & M20 & ITALY & ITALY & ITALY & $\mathrm{AL}$ & TIMBERMAN/CM & TORINO & C ST. & CALUMET PR. 4 \\
\hline 892 & 2476 & BIGANDO & VICTORIA & W & 40 & M20 & ITALY & ITALY & ITALY & & & TORINO & C ST. & CALUMET PR. 4 \\
\hline 893 & 2476 & BIGANDO & BATTISTA & s & 17 & $\mathrm{~s}$ & ITALY & ITALY & ITALY & & DRILL BOY/CM & TORINO & C ST. & CALUMET PR. 4 \\
\hline 894 & 2476 & BIGANDO & JOHN & $\mathrm{s}$ & 14 & $\mathrm{~s}$ & ITALY & ITALY & ITALY & & DRILL BOY/CM & TORINO & C ST. & CALUMET PR. 4 \\
\hline 895 & 2476 & BIGANDO & MARY & $\mathrm{D}$ & 11 & $\mathrm{~s}$ & ITALY & ITALY & ITALY & & AT SCHOOL 9 & TORINO & C ST. & CALUMET PR. 4 \\
\hline 896 & 2476 & BIGANDO & PETER & $\mathrm{s}$ & 5 & $\mathrm{~s}$ & ILLINOIS & ITALY & ITALY & & AT SCHOOL 9 & TORINO & C ST. & CALUMET PR. 4 \\
\hline 897 & 2476 & ROSO & PETER & B & 50 & $\mathrm{~S}$ & ITALY & ITALY & ITALY & NA & TRAMMER/CM & TORINO & C ST. & CALUMET PR. 4 \\
\hline 898 & 2476 & COGNONATTO & GIUSEPPE & B & 30 & $\mathrm{~s}$ & ITALY & ITALY & ITALY & $\mathrm{AL}$ & TRAMMER/CM & SAN PONSO CANAVESE. TO & C ST. & CALUMET PR. 4 \\
\hline 899 & 2476 & OTTINO & ANTON & B & 31 & M15 & ITALY & ITALY & ITALY & $\mathrm{AL}$ & TRAMMER/CM & VALPERGA. TO & C ST. & CALUMET PR. 4 \\
\hline 900 & 2476 & VALLERO & PETER & B & 22 & $\mathrm{~s}$ & ITALY & ITALY & ITALY & $\mathrm{AL}$ & TRAMMER/CM & VALPERGA. TO & C ST. & CALUMET PR. 4 \\
\hline 901 & 2476 & COSTA & ANT & $\mathrm{H}$ & 30 & M0 & ITALY & ITALY & ITALY & & TIMBERMAN/CM & TORINO & C ST. & CALUMET PR. 4 \\
\hline 902 & 2476 & COSTA & MARY & W & 22 & MO & ITALY & ITALY & ITALY & & IIVIDERIVIAIVILIVI & 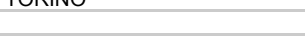 & C ST. & CALUMET PR. 4 \\
\hline 903 & 2472 & ROLANDO & PETER & $\mathrm{H}$ & 66 & M? & ITALY & ITALY & ITALY & NA & DRY HOUSE TENDER & TORINO & C ST. & CALUMET PR. 4 \\
\hline 904 & 2472 & ROLANDO & MARY & w & 53 & M? & ITALY & ITALY & ITALY & & & TORINO & C ST. & CALUMET PR. 4 \\
\hline 905 & 2472 & ROLANDO & JOHN & $\mathrm{s}$ & 17 & $\mathrm{~s}$ & ITALY & ITALY & ITALY & & TIMBERMAN/CM & TORINO & C ST. & CALUMET PR. 4 \\
\hline 906 & 2472 & GUYA & TONY & B & 33 & M9 & ITALY & ITALY & ITALY & $\mathrm{AL}$ & TRAMMER/CM & & C ST. & CALUMET PR. 4 \\
\hline 907 & 2472 & MANIEN & TONY & B & 33 & M10 & ITALY & ITALY & ITALY & AL & LABORER/CM & & C ST. & CALUMET PR. 4 \\
\hline 908 & 2472 & MILANO & DOMINICK & B & 28 & W & ITALY & ITALY & ITALY & $\mathrm{AL}$ & LABORER/CM & TORINO & C ST. & CALUMET PR. 4 \\
\hline 909 & 2468 & SALOTTI & JAMES & $\mathrm{H}$ & 51 & M26 & ITALY & ITALY & ITALY & NA & WATCHMAN/CM & CORIO. TO & C ST. & CALUMET PR. 4 \\
\hline 910 & 2468 & SALOTTI & MINNIE & W & 49 & M26 & ITALY & ITALY & ITALY & & & & C ST. & CALUMET PR. 4 \\
\hline 911 & 2468 & SALOTTI & CHARLIE & s & 12 & $\mathrm{~s}$ & MICHIGAN & ITALY & ITALY & & AT SCHOOL 9 & CORIO. TO & C ST. & CALUMET PR. 4 \\
\hline 912 & 2468 & SALOTTI & MARY & $\mathrm{D}$ & 9 & $\mathrm{~s}$ & MICHIGAN & ITALY & ITALY & & AT SCHOOL 9 & CORIO. TO & C ST. & CALUMET PR. 4 \\
\hline 913 & 2468 & SALOTTI & JOHN & $\mathrm{s}$ & 7 & s & MICHIGAN & ITALY & ITALY & & AT SCHOOL 9 & CORIO, TO & C ST. & CALUMET PR. 4 \\
\hline 914 & 2460 & SANDRETTO & DOMINICK & $\mathrm{H}$ & 27 & M6 & ITALY & ITALY & ITALY & NA & TRAMMER/CM & PONT CANAVESE. TO & C STREET & $\begin{array}{l}\text { CALUMET PR. } 4 \\
\text { CALE }\end{array}$ \\
\hline $\begin{array}{l}914 \\
915\end{array}$ & 2460 & SANDRETTO & MINNIE & w & 24 & M6 & ITALY & ITALY & ITALY & INA & TRAIVIVIER/CIVI & PUVI CANAVESE. TO & C STREET & $\begin{array}{l}\text { CALUMET PR. } 4 \\
\text { CALE }\end{array}$ \\
\hline 916 & 2460 & SANDRETTO & PETER & s & 4 & $\mathrm{~s}$ & ILLINOIS & ITALY & ITALY & & AT SCHOOL 9 & PONT CANAVESE. TO & C STREET & CALUMET PR. 4 \\
\hline 917 & 2460 & SANDRETTO & MARY & $\mathrm{D}$ & 3 & $\mathrm{~S}$ & MICHIGAN & ITALY & ITALY & & & PONT CANAVESE. TO & C STREET & CALUMET PR. 4 \\
\hline 918 & 2460 & SANDRETTO & LUCY & $\mathrm{D}$ & 1 & s & MICHIGAN & ITALY & ITALY & & & PONT CANAVESE. TO & C STREET & CALUMET PR. 4 \\
\hline 919 & 2460 & BALAGNA & ANTONIO & B & 28 & M2 & ITALY & ITALY & ITALY & $\mathrm{AL}$ & DAY LABORER/CM & TORINO & C STREET & CALUMET PR. 4 \\
\hline 920 & 2460 & SANDRETTO & PETER & B & 26 & $\mathrm{~s}$ & ITALY & ITALY & ITALY & & TRAMMER/CM & PONT CANAVESE. TO & C STREET & CALUMET PR. 4 \\
\hline 921 & 2460 & COPPO & DOMINICK & B & 48 & M1 & ITALY & ITALY & ITALY & NA & DRY HOUSE TENDER & PONT CANAVESE. TO & C STREET & CALUMET PR. 4 \\
\hline 922 & 2460 & ROLANDO & FELIX & B & 27 & M1 & ITALY & ITALY & ITALY & & TRAMMER/CM & PONT CANAVESE. TO & C STREET & CALUMET PR. 4 \\
\hline 923 & 2460 & NIPOTE & GIACOMO & B & 27 & $\mathrm{~s}$ & ITALY & ITALY & ITALY & $\mathrm{AL}$ & DAY LABORER/CM & PONT CANAVESE. TO & C STREET & CALUMET PR. 4 \\
\hline 924 & 2460 & GENOVA & FRANK & B & 25 & $\mathrm{~S}$ & ITALY & ITALY & ITALY & $\mathrm{AL}$ & DAY LABORER/CM & & C STREET & CALUMET PR. 4 \\
\hline 925 & 2458 & SOPPELSA & EUGENIO & $\mathrm{H}$ & 40 & M4 & ITALY & ITALY & ITALY & NA & TRAMMER/CM & BELLUNO & C STREET & CALUMET PR. 4 \\
\hline 926 & 2458 & SOPPELSA & ANTONIA & W & 29 & M4 & ITALY & ITALY & ITALY & & & BELLUNO & C STREET & CALUMET PR. 4 \\
\hline 927 & 2458 & SOPPELSA & D/SEMON & BIL & 42 & $\mathrm{~s}$ & MICHIGAN & ITALY & ITALY & PA & WATCHMAN/CM & BELLUNO & C STREET & CALUMET PR. 4 \\
\hline 928 & 2458 & DA CAMPO & $\begin{array}{l}\text { JAMES } \\
\text { JAM }\end{array}$ & $\mathrm{B}$ & 44 & $\mathrm{~s}$ & MICHIGAN & ITALY & ITALY & NA? & TIMBERMAN/CM & BELLUNO & C STREET & CALUMET PR. 4 \\
\hline 929 & 2454 & AIRAUDI & LOUIS & $\mathrm{H}$ & 34 & M5 & ITALY & ITALY & ITALY & $\mathrm{PA}$ & TIMBERMAN/CM & TORINO & C STREET & CALUMET PR. 4 \\
\hline 930 & 2454 & AIRAUDI & DOMENICA & W & 37 & M5 & ITALY & ITALY & ITALY & & & TORINO & C STREET & CALUMET PR. 4 \\
\hline 931 & 2454 & AIRAUDI & ROSA & $\mathrm{D}$ & 4 & $\mathrm{~s}$ & MICHIGAN & ITALY & ITALY & & & TORINO & C STREET & CALUMET PR. 4 \\
\hline 932 & 2454 & AIRAUDI & CHRISTIAN & $\mathrm{s}$ & 2 & $\mathrm{~s}$ & MICHIGAN & ITALY & ITALY & & & TORINO & C STREET & CALUMET PR. 4 \\
\hline 933 & 2454 & BOGGIO & PONZO & $\mathrm{H}$ & 35 & M10 & ITALY & ITALY & ITALY & PA & TRAMMER/CM & SAN PONSO CANAVESE. TO & C STREET & CALUMET PR. 4 \\
\hline 934 & 2454 & BOGGIO & MARY & w & 36 & M10 & ITALY & ITALY & ITALY & & & SAN PONSO CANAVESE. TO & C STREET & CALUMET PR. 4 \\
\hline 935 & 2454 & BOGGIO & LENA & $\mathrm{D}$ & 7 & $\mathrm{~s}$ & MICHIGAN & ITALY & ITALY & & & SAN PONSO CANAVESE. TO & C STREET & CALUMET PR. 4 \\
\hline 936 & 2454 & BOGGIO & JOSEPHINA & $\mathrm{D}$ & 4 & s & MICHIGAN & ITALY & ITALY & & & SAN PONSO CANAVESE. TO & C STREET & CALUMET PR. 4 \\
\hline 937 & 2454 & BOGGIO & ALMANDO & $\mathrm{s}$ & 1 & s & MICHIGAN & ITALY & ITALY & & & SAN PONSO CANAVESE, TO & C STREET & CALUMET PR. 4 \\
\hline 938 & 2457 & PIANA & JOHN & $\mathrm{H}$ & 25 & M3 & ITALY & ITALY & ITALY & $\mathrm{AL}$ & TRAMMER/CM & TORINO & C STREET & CALUMET PR. 4 \\
\hline 939 & 2457 & PIANA & JENNIE & w & 19 & M3 & ILLINOIS & ITALY & ITALY & & & & C STREET & CALUMET PR. 4 \\
\hline 940 & 2457 & PIANA & JOHN & s & $9 \mathrm{M}$ & $\mathrm{s}$ & ILLINOIS & ITALY & ILLINOIS & & & TORINO & C STREET & CALUMET PR. 4 \\
\hline 941 & 2457 & MACARIO & PIETRO & $\mathrm{H}$ & 39 & M14 & ITALY & ITALY & ILLINOIS & NA & TRAMMER/CM & TORINO & C STREET & CALUMET PR. 4 \\
\hline 942 & 2457 & MACARIO & TRACY & W & 31 & M14 & ITALY & ITALY & ILLINOIS & & & TORINO & C STREET & CALUMET PR. 4 \\
\hline
\end{tabular}




\begin{tabular}{|c|c|c|c|c|c|c|c|c|c|c|c|c|c|c|}
\hline \multirow{2}{*}{$\begin{array}{l}943 \\
944 \\
\end{array}$} & 2457 & MACARIO & JOHN & $\mathrm{s}$ & 12 & $\mathrm{~s}$ & ITALY & ITALY & ILLINOIS & & AT SCHOOL 9 & TORINO & C STREET & \multirow{3}{*}{$\begin{array}{l}\text { CALUMET PR. } 4 \\
\text { CALUMET PR. } 4 \\
\text { CALUMET PR. } 4\end{array}$} \\
\hline & 2457 & MACARIO & KATIE & D & 7 & S & MICHIGAN & ITALY & ILLINOIS & & AT SCHOOL 7 & TORINO & C STREET & \\
\hline 945 & 2457 & MACARIO & JOE & $\mathrm{s}$ & 2 & s & MICHIGAN & ITALY & ILLINOIS & & & TORINO & C STREET & \\
\hline 946 & 2477 & PICCHIOTTINO & PAUL & $\mathrm{H}$ & 38 & M16 & ITALY & ITALY & ITALY & $\mathrm{AL}$ & WATCHMAN/CM & PONT CANAVESE. TO & C STREET & CALUMET PR. 4 \\
\hline 947 & 2477 & PICCHIOTTINO & MINNIE & W & 32 & M16 & ITALY & ITALY & ITALY & & & & C STREET & CALUMET PR. 4 \\
\hline 948 & 2477 & PICCHIOTTINO & JOHN & s & 13 & $\mathrm{~s}$ & ITALY & ITALY & ITALY & & AT SCHOOL 9 & PONT CANAVESE. TO & C STREET & CALUMET PR. 4 \\
\hline 949 & 2477 & PICCHIOTTINO & JOSEPH & $\mathrm{s}$ & 5 & $\mathrm{~S}$ & MICHIGAN & ITALY & ITALY & & AT SCHOOL 8 & PONT CANAVESE. TO & C STREET & CALUMET PR. 4 \\
\hline 950 & 2477 & PICCHIOTTINO & ANNIE & $\mathrm{D}$ & 3 & $\mathrm{~S}$ & MICHIGAN & ITALY & ITALY & & & PONT CANAVESE. TO & C STREET & CALUMET PR. 4 \\
\hline 951 & 2477 & PICCHIOTTINO & MARY & $\mathrm{D}$ & $8 \mathrm{M}$ & $\mathrm{s}$ & MICHIGAN & ITALY & ITALY & & & PONT CANAVESE. TO & C STREET & CALUMET PR. 4 \\
\hline 952 & 2477 & FACOLA & BATIS & B & 34 & M1 & ITALY & ITALY & ITALY & AL & TRAMMER/CM & & C STREET & CALUMET PR. 4 \\
\hline 953 & 2477 & CERETTO & JAMES & B & 35 & M1 & ITALY & ITALY & ITALY & $\mathrm{AL}$ & TRAMMER/CM & TORINO & C STREET & CALUMET PR. 4 \\
\hline 954 & 2477 & AUTERO & DOMINICK & B & 30 & $?$ & ITALY & ITALY & ITALY & $\mathrm{AL}$ & TRAMMER/CM & PONT CANAVESE. TO & C STREET & CALUMET PR. 4 \\
\hline 955 & 2477 & ROLANDO & GIACOMO & B & 30 & M1 & ITALY & ITALY & ITALY & $\mathrm{AL}$ & CARPENTER & PONT CANAVESE. TO & C STREET & CALUMET PR. 4 \\
\hline 956 & 2479 & ANTONIONO & BATISTA & $\mathrm{H}$ & 36 & M6 & ITALY & ITALY & ITALY & NA & TIMBERMAN/CM & TORINO & C STREET & CALUMET PR. 4 \\
\hline 957 & 2479 & ANTONIONO & MARIE & W & 36 & M6 & ITALY & ITALY & ITALY & & & TORINO & C STREET & CALUMET PR. 4 \\
\hline 958 & 2479 & ANTONIONO & MARY & $\mathrm{D}$ & 5 & $\mathrm{~S}$ & ITALY & ITALY & ITALY & & AT SCHOOL 9 & TORINO & C STREET & CALUMET PR. 4 \\
\hline 959 & 2479 & ANTONIONO & JOHN & $\mathrm{s}$ & 1 & $\mathrm{~s}$ & MICHIGAN & ITALY & ITALY & & & TORINO & C STREET & CALUMET PR. 4 \\
\hline 960 & 2479 & RICCA & JAMES & B & 31 & $\mathrm{~S}$ & ITALY & ITALY & ITALY & AN & TRAMMER/CM & TORINO & C STREET & CALUMET PR. 4 \\
\hline 961 & 2479 & CARDIO & PETER & B & 29 & s & ITALY & ITALY & ITALY & NA & TRAMMER/CM & & C STREET & CALUMET PR. 4 \\
\hline 962 & 2479 & ENRICO & BATTISTA & B & 31 & M1 & ITALY & ITALY & ITALY & NA & TRAMMER/CM & SCARMAGNO. TO & C STREET & CALUMET PR. 4 \\
\hline 963 & 2479 & ENRICO & JOHN & B & 29 & $\mathrm{~s}$ & ITALY & ITALY & ITALY & $\mathrm{AL}$ & TRAMMER/CM & SCARMAGNO. TO & C STREET & CALUMET PR. 4 \\
\hline 964 & 2479 & ENRICO & GIACOMO & B & 21 & s & ITALY & ITALY & ITALY & $\mathrm{AL}$ & TRAMMER/CM & SCARMAGNO, TO & $\begin{array}{l}\text { C STREET } \\
\text { C TSE }\end{array}$ & CALUMET PR. 4 \\
\hline 965 & 2479 & PASTORE & ANTONIO & B & 22 & $\mathrm{~s}$ & ITALY & ITALY & ITALY & AL & TRAMMER/CM & SAN MARTINO CANAVESE. TO & C STREET & CALUMET PR. 4 \\
\hline 966 & 2479 & GALLINATTI & JOSEPH & B & 30 & M1 & ITALY & ITALY & ITALY & NA & TRAMMER/CM & AGLIE'. TO & C STREET & CALUMET PR. 4 \\
\hline 967. & 2479 & REGIS & LUIGI & B & 38 & M1 & ITALY & ITALY & ITALY & $\mathrm{AL}$ & TRAMMER/CM & SAN GIUSTO CANAVESE. TO & C STREET & CALUMET PR. 4 \\
\hline 968 & 2479 & BARINOTTI & JAMES & B & 36 & M1 & ITALY & ITALY & ITALY & NA & MINER/CM & PONT CANAVESE. TO & C STREET & CALUMET PR. 4 \\
\hline 969 & 2479 & SUCCA & GIORGIO & B & 22 & s & ITALY & ITALY & ITALY & AL & TRAMMER/CM & SAN GIUSTO CANAVESE. TO & C STREET & CALUMET PR. 4 \\
\hline 970 & 2479 & ALLARIA & JOHN & B & 25 & s & ITALY & ITALY & ITALY & NA & MINER/CM & SAN GIORGIO CANAVESE, TO & C STREET & CALUMET PR. 4 \\
\hline 971 & 2426 & COPPO & ANTONIO & $\mathrm{H}$ & 35 & M11 & ITALY & ITALY & ITALY & NA & TIMBERMAN/CM & PONT CANAVESE. TO & B STREET & CALUMET PR. 4 \\
\hline 972 & 2426 & COPPO & MARY & W & 29 & M11 & ITALY & ITALY & ITALY & & & & B STREET & CALUMET PR. 4 \\
\hline 973 & 2426 & COPPO & VETO & $\mathrm{s}$ & 7 & $\mathrm{~s}$ & MICHIGAN & ITALY & ITALY & & AT SCHOOL 9 & PONT CANAVESE. TO & B STREET & CALUMET PR. 4 \\
\hline 974 & 2426 & COPPO & JOHN & $\mathrm{s}$ & 4 & $\mathrm{~S}$ & MICHIGAN & ITALY & ITALY & & AT SCHOOL 9 & PONT CANAVESE. TO & B STREET & CALUMET PR. 4 \\
\hline 975 & 2426 & COPPO & ANNIE & $\mathrm{D}$ & 3 & s & MICHIGAN & ITALY & ITALY & & & PONT CANAVESE. TO & B STREET & CALUMET PR. 4 \\
\hline 976 & 2426 & (GI)JISEPPI & JOSEPH & B & 39 & M1 & ITALY & ITALY & ITALY & $\mathrm{AL}$ & SURGEON & TCUNT UAIVAVLOL. TO & B STREET & CALUMET PR. 4 \\
\hline 977 & 2426 & MICHELETTO & CALCIO & B & 23 & $\mathrm{~s}$ & ITALY & ITALY & ITALY & AL & SURGEON & TORINO & B STREET & CALUMET PR. 4 \\
\hline 978 & 2426 & BLESSENT & ANTONIO & B & 40 & M1 & ITALY & ITALY & ITALY & & TIMBERMAN/CM & SPARONE. TO & B STREET & CALUMET PR. 4 \\
\hline 979 & 2426 & MORECO & RIVO & B & 25 & M1 & ITALY & ITALY & ITALY & $\mathrm{AL}$ & TRAMMER/CM & & B STREET & CALUMET PR. 4 \\
\hline 980 & 2426 & BOGGIO & PETER & B & 28 & M1 & ITALY & ITALY & ITALY & $\mathrm{AL}$ & TIMBERMAN/CM & TORINO & B STREET & CALUMET PR. 4 \\
\hline 981 & 2426 & BOGGIO & BALANO & B & 28 & $\mathrm{~s}$ & ITALY & ITALY & ITALY & $\mathrm{AL}$ & TRAMMER/CM & TORINO & B STREET & CALUMET PR. 4 \\
\hline 982 & 2426 & PASTO & DOMINICK & B & 24 & s & ITALY & ITALY & ITALY & AL & TRAMMER/CM & VERCELLI & B STREET & CALUMET PR. 4 \\
\hline 983 & 2426 & REGIS & JOHN & B & 25 & $\mathrm{~S}$ & ITALY & ITALY & ITALY & AL & TRAMMER/CM & SAN GIUSTO CANAVESE. TO & B STREET & CALUMET PR. 4 \\
\hline 984 & 2406 & AIMONE & DOMINICK & $\mathrm{H}$ & 45 & M9 & ITALY & ITALY & ITALY & NA & WATCHMAN/CM & PONT CANAVESE. TO & C STREET & CALUMET PR. 4 \\
\hline 985 & 2406 & AIMONE & DOMENICA & W & 30 & M9 & ITALY & ITALY & ITALY & & & & C STREET & CALUMET PR. 4 \\
\hline 986 & 2406 & AIMONE & JOHN & s & 6 & $\mathrm{~s}$ & MICHIGAN & ITALY & ITALY & & AT SCHOOL 9 & PONT CANAVESE. TO & C STREET & CALUMET PR. 4 \\
\hline 987 & 2406 & AIMONE & GEORGE & $\mathrm{s}$ & 5 & $\mathrm{~s}$ & MICHIGAN & ITALY & ITALY & & AT SCHOOL 9 & PONT CANAVESE. TO & C STREET & CALUMET PR. 4 \\
\hline 988 & 2406 & AIMONE & KATIE & $\mathrm{D}$ & 3 & $\mathrm{~s}$ & MICHIGAN & ITALY & ITALY & & & PONT CANAVESE. TO & C STREET & CALUMET PR. 4 \\
\hline 989 & 2406 & AIMONE & ANNE & $\mathrm{D}$ & 9M & $\mathrm{s}$ & MICHIGAN & ITALY & ITALY & & & PONT CANAVESE. TO & C STREET & CALUMET PR. 4 \\
\hline 990 & 2406 & ANSELMO & DOMINICK & B & 36 & M1 & ITALY & ITALY & ITALY & $\mathrm{AL}$ & TIMBERMAN/CM & BARONE CANAVESE. TO & C STREET & CALUMET PR. 4 \\
\hline 991 & 2404 & ZANARDI & JOSEPH & $\mathrm{H}$ & 40 & M4 & ITALY & ITALY & ITALY & $\mathrm{AL}$ & WATCHMAN/CM & SELINO. BERGAMO & B STREET & CALUMET PR. 4 \\
\hline 992 & 2404 & ZANARDI & MARY & W & 28 & M4 & ITALY & ITALY & ITALY & & & & B STREET & CALUMET PR. 4 \\
\hline 993 & 2404 & ZANARDI & CHARLES & s & 2 & $\mathrm{~S}$ & MICHIGAN & ITALY & ITALY & & & SELINO. BERGAMO & B STREET & CALUMET PR. 4 \\
\hline 994 & 2404 & ZANARDI & JOSEPH & S & 1 & s & MICHIGAN & ITALY & ITALY & & & SELINO, BERGAMO & & CALUMET PR. 4 \\
\hline 995 & 2404 & TONDINI & JOSEPH & $\mathrm{H}$ & 43 & M12 & ITALY & ITALY & ITALY & $\mathrm{AL}$ & TRAMMER/CM & SELINO. BERGAMO & B STREET & CALUMET PR. 4 \\
\hline 996 & 2404 & TONDINI & MARY & W & 41 & M12 & ITALY & ITALY & ITALY & & & SELINO. BERGAMO & B STREET & CALUMET PR. 4 \\
\hline 997 & 2404 & TONDINI & ORSALA & D & 10 & $\mathrm{~s}$ & ITALY & ITALY & ITALY & & & SELINO. BERGAMO & B STREET & CALUMET PR. 4 \\
\hline 998 & 2404 & TONDINI & JOSEPH & $\mathrm{s}$ & 4 & $\mathrm{~s}$ & ITALY & ITALY & ITALY & & & SELINO. BERGAMO & B STREET & CALUMET PR. 4 \\
\hline 999 & 2404 & FROSIO & LANFRANCO & B & 26 & s & ITALY & ITALY & ITALY & $\mathrm{AL}$ & NOT CLEAR & BERGAMO & B STREET & CALUMET PR. 4 \\
\hline 1000 & 2404 & DEDONATI & ALESSANDRO & B & 30 & M & ITALY & ITALY & ITALY & $\mathrm{AL}$ & TRAMMER/CM & & B STREET & CALUMET PR. 4 \\
\hline 1001 & 2409 & ANTONETTI & BATIS & $\mathrm{H}$ & 41 & M1 & ITALY & ITALY & ITALY & $\mathrm{AL}$ & WATCHMAN/CM & TORINO & B STREET & CALUMET PR. 4 \\
\hline 1002 & 2409 & ANTONETTI & YOSA & W & 42 & M1 & ITALY & ITALY & ITALY & & & TORINO & B STREET & CALUMET PR. 4 \\
\hline 1003 & 2409 & TORNATORE & JOSEPH & $\mathrm{B}$ & 32 & $?$ & ITALY & ITALY & ITALY & PA & WATCHMAN/CM & TORINO & B STREET & CALUMET PR. 4 \\
\hline 1004 & 2409 & BARINOTTI & JOSEPH & $\mathrm{H}$ & 29 & M8 & ITALY & ITALY & ITALY & & MINER/CM & PONT CANAVESE. TO & B STREET & CALUMET PR. 4 \\
\hline 1005 & 2409 & BARINOTTI & MARY & W & 23 & M8 & ITALY & ITALY & ITALY & & & & B STREET & CALUMET PR. 4 \\
\hline
\end{tabular}




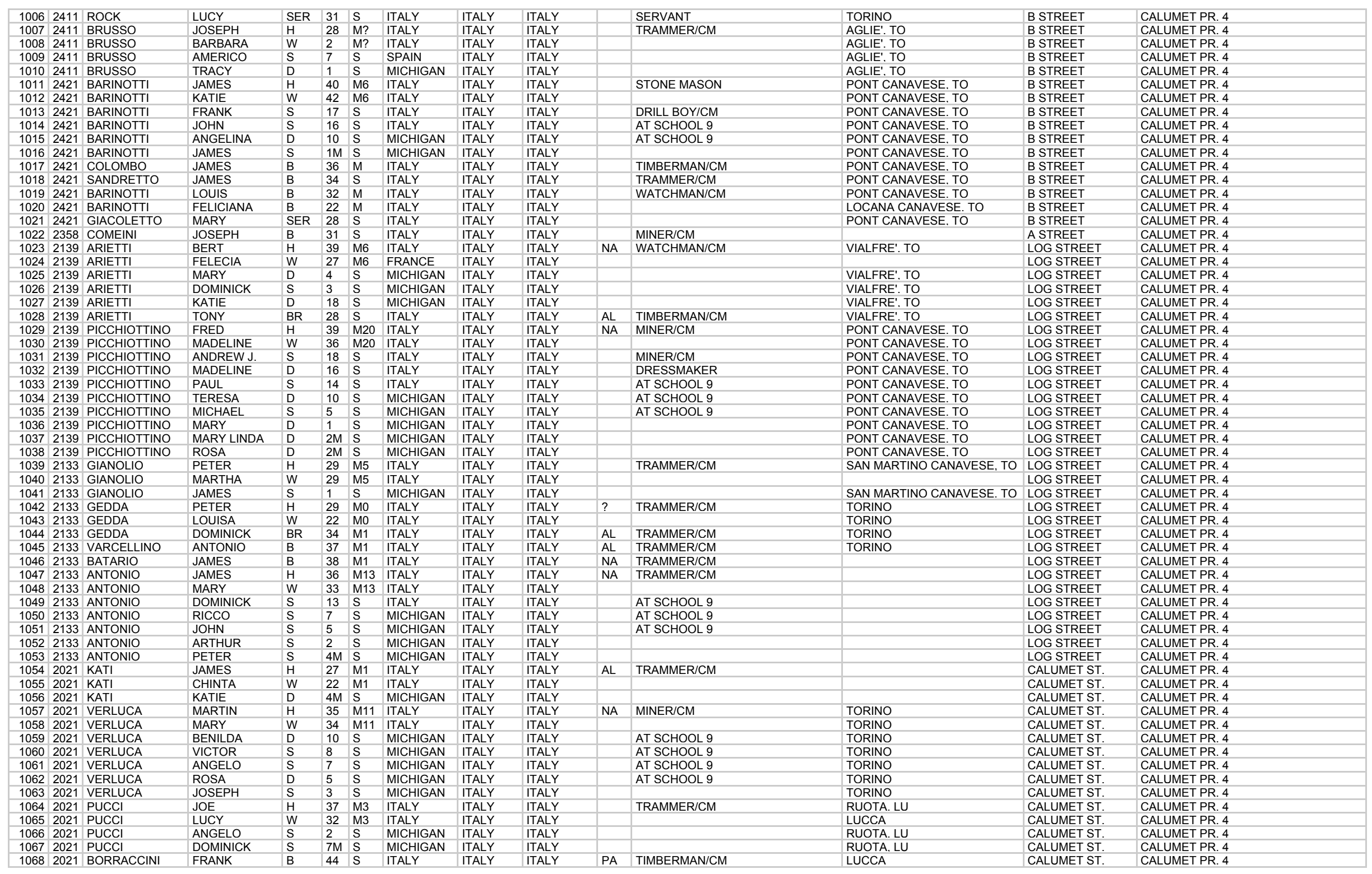




\begin{tabular}{|c|c|c|c|c|c|c|c|c|c|c|c|c|c|c|}
\hline 1069 & 2021 & PUCCI & ANGO & B & 38 & $\mathrm{~s}$ & ITALY & ITALY & ITALY & PA & TRAMMER/CM & LUCCA & CALUMET ST. & CALUMET PR. 4 \\
\hline 1070 & 2013 & PICCHIOTTINO & PETER & $\mathrm{H}$ & 30 & M8 & ITALY & ITALY & ITALY & AL & TIMBERMAN/CM & PONT CANAVESE. TO & CALUMET ST. & CALUMET PR. 4 \\
\hline 1071 & 2013 & PICCHIOTTINO & MARY & W & 26 & M8 & ITALY & ITALY & ITALY & & & & CALUMET ST. & CALUMET PR. 4 \\
\hline 1072 & 2013 & PICCHIOTTINO & JOSEPH & $\mathrm{s}$ & 3 & $\mathrm{~s}$ & MICHIGAN & ITALY & ITALY & & & PONT CANAVESE. TO & CALUMET ST. & CALUMET PR. 4 \\
\hline 1073 & 2013 & PICCHIOTTINO & PAUL & $\mathrm{s}$ & 1 & $\mathrm{~S}$ & MICHIGAN & ITALY & ITALY & & & PONT CANAVESE. TO & CALUMET ST. & CALUMET PR. 4 \\
\hline 1074 & 2013 & BARTONO & JOHN & $\mathrm{H}$ & 33 & M0 & ITALY & ITALY & ITALY & PA & TIMBERMAN/CM & VIALFRE'. TO & CALUMET ST. & CALUMET PR. 4 \\
\hline 1075 & 2013 & BARTONO & ANNIE & w & 23 & MO & ITALY & ITALY & ITALY & & & & CALUMET ST. & CALUMET PR. 4 \\
\hline 1076 & 2013 & BARTONO & DOMINICK & $\mathrm{BR}$ & 23 & $?$ & ITALY & ITALY & ITALY & $\mathrm{AL}$ & DAY LABORER/CM & VIALFRE'. TO & CALUMET ST. & CALUMET PR. 4 \\
\hline 1077 & 2013 & NIGRA/NIGRO & ANTONIO & B & 28 & $?$ & ITALY & ITALY & ITALY & NA & TIMBERMAN/CM & RIVAROLO CANAVESE. TO & CALUMET ST. & CALUMET PR. 4 \\
\hline 1078 & 2009 & TORREANO & VICTORIA & $\mathrm{H}$ & 56 & W & ITALY & ITALY & ITALY & & N. G. & TORINO & CALUMET ST. & CALUMET PR. 4 \\
\hline 1079 & 2009 & TORREANO & JOSEPH & $\mathrm{s}$ & 24 & $\mathrm{~s}$ & MICHIGAN & ITALY & ITALY & & LABORER/SHAFT HOUSE & TORINO & CALUMET ST. & CALUMET PR. 4 \\
\hline 1080 & 2009 & TORREANO & KATE & $\mathrm{D}$ & 22 & $\mathrm{~s}$ & MICHIGAN & ITALY & ITALY & & CLERK/DRY GOODS & TORINO & CALUMET ST. & CALUMET PR. 4 \\
\hline 1081 & 2009 & SANDRETTO & KATE & GD & 9 & $\mathrm{~s}$ & MICHIGAN & ITALY & ITALY & & AT SCHOOL 9 & PONT CANAVESE. TO & CALUMET ST. & CALUMET PR. 4 \\
\hline 1082 & 2009 & TALENTINO & PETER & B & 13 & $\mathrm{~s}$ & ITALY & ITALY & ITALY & & AT SCHOOL 1 & SAN MARTINO CANAVESE. TO & CALUMET ST. & CALUMET PR. 4 \\
\hline 1083 & 2009 & TINETTI & GIOVANNI & B & 27 & $\mathrm{~s}$ & ITALY & ITALY & ITALY & & TIMBERMAN/CM & SAN MARTINO CANAVESE. TO & CALUMET ST. & CALUMET PR. 4 \\
\hline 1084 & 2009 & CIAGNE & MARTIN & B & 30 & $\mathrm{~s}$ & ITALY & ITALY & ITALY & PA & TRAMMER/CM & PONT CANAVESE, TO & CALUMET ST. & CALUMET PR. 4 \\
\hline 1085 & 2009 & CIAGNE & LOUIS & B & 22 & $\mathrm{~s}$ & ITALY & ITALY & ITALY & & TRAMMER/CM & PONT CANAVESE. TO & CALUMET ST. & CALUMET PR. 4 \\
\hline 1086 & 2009 & CIAGNE & MARTIN & B & 25 & $\mathrm{~S}$ & ITALY & ITALY & ITALY & & MINER/CM & PONT CANAVESE. TO & CALUMET ST. & CALUMET PR. 4 \\
\hline 1087 & 2009 & PAGNIUCCI & LOUIS & $\mathrm{H}$ & 29 & M1 & ITALY & ITALY & ITALY & NA & MINER/CM & LUCCA & CALUMET ST. & CALUMET PR. 4 \\
\hline 1088 & 2009 & PAGNIUCCI & CORBINA & w & 17 & M1 & ITALY & ITALY & ITALY & & & LUCCA & CALUMET ST. & CALUMET PR. 4 \\
\hline 1089 & 2020 & CHELI & FREDERIC & $\mathrm{H}$ & 24 & M2 & MICHIGAN & ITALY & ITALY & & OILER/ENGINE & LUCCA & CALUMET ST. & CALUMET PR. 4 \\
\hline 1090 & 2020 & CHELI & ROSA & W & 23 & M2 & MICHIGAN & ITALY & ITALY & & & & CALUMET ST. & CALUMET PR. 4 \\
\hline 1091 & 2020 & CHELI & PETER & s & 1 & $\mathrm{~S}$ & MICHIGAN & MICHIGA & MICHIGAN & & & LUCCA & CALUMET ST. & CALUMET PR. 4 \\
\hline 1092 & 2020 & CIABATTARI & CARLO & $\mathrm{H}$ & 26 & MO & ITALY & ITALY & ITALY & NA & JANITOR & SEGROMIGNO. LU & CALUMET ST. & CALUMET PR. 4 \\
\hline 1093 & 2020 & CIABATTARI & GIORGIA & w & 19 & MO & ITALY & ITALY & ITALY & & & & CALUMET ST. & CALUMET PR. 4 \\
\hline 1094 & 2020 & BLESSENT & JOHN & $\mathrm{H}$ & 28 & M2 & ITALY & ITALY & ITALY & AL & TIMBERMAN/CM & SPARONE. TO & CALUMET ST. & CALUMET PR. 4 \\
\hline 1095 & 2020 & BLESSENT & MARY & W & 28 & M2 & ITALY & ITALY & ITALY & & & STARUIVL. TS & $\begin{array}{l}\text { CALUMET ST. } \\
\text { CTET }\end{array}$ & CALUMET PR. 4 \\
\hline 1096 & 2020 & BLESSENT & JENNIE & $\mathrm{D}$ & 9 & $\mathrm{~s}$ & MICHIGAN & ITALY & ITALY & & AT SCHOOL 9 & SPARONE, TO & CALUMET ST. & CALUMET PR. 4 \\
\hline 1097 & 2034 & LESH & JOSEPH & $\mathrm{H}$ & 33 & M17 & ITALY & ITALY & ITALY & $?$ & TIMBERMAN/CM & TORINO & CALUMET ST. & CALUMET PR. 4 \\
\hline 1098 & 2034 & LESH & JOSEPHINA & W & 45 & M17 & ITALY & ITALY & ITALY & & & TORINO & CALUMET ST. & CALUMET PR. 4 \\
\hline 1099 & 2034 & DAIRO & KATIE & NIEC & 11 & $\mathrm{~S}$ & MICHIGAN & ITALY & ITALY & & AT SCHOOL & TORINO & CALUMET ST. & CALUMET PR. 4 \\
\hline 1100 & 2034 & DAIRO & MARY & NIEC & 4 & $\mathrm{~S}$ & MICHIGAN & ITALY & ITALY & & & TORINO & CALUMET ST. & CALUMET PR. 4 \\
\hline 1101 & 1033 & PISTONO & ANTONIO & $\mathrm{H}$ & 31 & M9 & ITALY & ITALY & ITALY & PA & TIMBERMAN/CM & TORINO & CALUMET ST. & CALUMET PR. 4 \\
\hline 1102 & 1033 & PISTONO & ELLEONOR & W & 28 & M9 & SCOTLAND & SCOTLAN & SCOTLAND & & & SCOTLAND & CALUMET ST. & CALUMET PR. 4 \\
\hline 1103 & 1033 & PISTONO & $\begin{array}{l}\text { WILLIAM } \\
\text { WIA }\end{array}$ & s & 5 & $s$ & PENNSYLV & ITALY & SCOTLAND & & & TORINO & CALUMET ST. & CALUMET PR. 4 \\
\hline 1104 & 1033 & PISTONO & LAWRENCE & s & 4 & s & PENNSYLV & ITALY & SCOTLAND & & & TORINO & CALUMET ST. & CALUMET PR. 4 \\
\hline 1105 & 1033 & PISTONO & MARTHA & $\mathrm{D}$ & & $\mathrm{s}$ & MICHIGAN & ITALY & SCOTLAND & & & TORINO & CALUMET ST. & CALUMET PR. 4 \\
\hline 1106 & 1033 & PISTONO & DOMINICK & $\mathrm{s}$ & $4 \mathrm{M}$ & s & MICHIGAN & ITALY & SCOTLAND & & & TORINO & CALUMET ST. & CALUMET PR. 4 \\
\hline 1107 & 3913 & RICHETTA & THOMAS & $\mathrm{H}$ & 37 & M2 & ITALY & ITALY & $\begin{array}{l}\text { ITALY } \\
\text { ITII }\end{array}$ & PA & MINER/CM & SAN MARTINO CANAVESE. TO & SIXTH STREET & CALUMET PR. 6 \\
\hline 1108 & 3913 & RICHETTA & JOSEPHINE & W & 26 & M2 & ITALY & ITALY & ITALY & & & & SIXTH STREET & CALUMET PR. 6 \\
\hline 1109 & 3913 & RICHETTA & ANTHONY & s & 11 & $\mathrm{~s}$ & MICHIGAN & ITALY & ITALY & & & & SIXTH STREET & CALUMET PR. 6 \\
\hline 1110 & 3913 & RICHETTA & DOMINICK & $\mathrm{H}$ & 18 & M0 & ITALY & ITALY & ITALY & AL & MINER/CM & SAN MARTINO CANAVESE. TO & SIXTH STREET & CALUMET PR. 6 \\
\hline 1111 & 3913 & RICHETTA & MANDE & W & 17 & MO & ITALY & ITALY & ITALY & & & & SIXTH STREET & CALUMET PR. 6 \\
\hline 1112 & 3913 & RICHETTA & PETER & B & 24 & $\mathrm{~s}$ & ITALY & ITALY & ITALY & $\mathrm{AL}$ & MINER/CM & SAN MARTINO CANAVESE. TO & SIXTH STREET & CALUMET PR. 6 \\
\hline 1113 & 3913 & LANCINA & DOMINICK & B & 27 & $\mathrm{~S}$ & ITALY & ITALY & ITALY & $\mathrm{AL}$ & MINER/CM & SAN GIORGIO CANAVESE. TO & SIXTH STREET & CALUMET PR. 6 \\
\hline 1114 & 3913 & BOGGIO & GEORGE & B & 24 & M2 & ITALY & ITALY & ITALY & $\mathrm{AL}$ & MINER/CM & SAN GIORGIO CANAVESE. TO & TEMPLE STREET & CALUMET PR. 6 \\
\hline 1115 & 3913 & LOCATELLI & CIPRIANO & B & 30 & $\mathrm{~s}$ & ITALY & ITALY & ITALY & PA & DAY LABORER/CM & SELINO. BERGAMO & TEMPLE STREET & CALUMET PR. 6 \\
\hline 1116 & 3913 & LANCINA & MIKE & $\begin{array}{l}\mathrm{D} \\
\mathrm{B}\end{array}$ & 19 & s & ITALY & ITALY & ITALY & $F A$ & MINER/CM & SAN GIORGIO CANAVESE. TO & TEMPLE STREET & CALUMET PR. 6 \\
\hline 1117 & 3947 & ROSSIO & MIKE & $\mathrm{H}$ & 46 & M22 & ITALY & ITALY & ITALY & NA & MINER/CM & PONT CANAVESE. TO & TEMPLE STREET & CALUMET PR. 6 \\
\hline 1118 & 3947 & ROSSIO & DOMENICA & w & 40 & M22 & ITALY & ITALY & ITALY & & & PONT CANAVESE. TO & TEMPLE STREET & CALUMET PR. 6 \\
\hline 1119 & 3947 & ROSSIO & MIKE & s & 20 & $\mathrm{~s}$ & ITALY & ITALY & ITALY & & MINER/CM & PONT CANAVESE. TO & TEMPLE STREET & CALUMET PR. 6 \\
\hline 1120 & 3947 & ROSSIO & LUCY & $\mathrm{D}$ & 19 & $\mathrm{~s}$ & ITALY & ITALY & ITALY & & & PONT CANAVESE, TO & TEMPLE STREET & CALUMET PR. 6 \\
\hline 1121 & 3947 & ROSSIO & LENA & $\mathrm{D}$ & 18 & $\mathrm{~s}$ & ITALY & ITALY & ITALY & & & PONT CANAVESE. TO & TEMPLE STREET & CALUMET PR. 6 \\
\hline 1122 & 3947 & ROSSIO & MARTIN & $\mathrm{s}$ & 9 & $\mathrm{~s}$ & MICHIGAN & ITALY & ITALY & & AT SCHOOL 9 & PONT CANAVESE. TO & TEMPLE STREET & CALUMET PR. 6 \\
\hline 1123 & 3947 & ROSSIO & ANNIE & D & 8 & s & MICHIGAN & ITALY & ITALY & & & PONT CANAVESE. TO & TEMPLE STREET & CALUMET PR. 6 \\
\hline 1124 & 3947 & ROSSIO & CHARLIE & $\mathrm{s}$ & 4 & $\mathrm{~S}$ & MICHIGAN & ITALY & ITALY & & & PONT CANAVESE. TO & TEMPLE STREET & CALUMET PR. 6 \\
\hline 1125 & 3947 & CERETTO & ANTONIO & $\mathrm{H}$ & 28 & M2 & ITALY & ITALY & ITALY & NA & MINER/CM & PONT CANAVESE. TO & TEMPLE STREET & CALUMET PR. 6 \\
\hline 1126 & 3947 & CERETTO & MARIA & w & 31 & M2 & ITALY & ITALY & ITALY & & & & TEMPLE STREET & CALUMET PR. 6 \\
\hline 1127 & 3947 & CERETTO & ANTONIO & s & 6 & $\mathrm{~s}$ & MICHIGAN & ITALY & ITALY & & & PONT CANAVESE. TO & TEMPLE STREET & CALUMET PR. 6 \\
\hline 1128 & 3947 & CANAVESIO & JOHN & B & 28 & $\mathrm{~s}$ & ITALY & ITALY & ITALY & PA & MINER/CM & TORINO & TEMPLE STREET & CALUMET PR. 6 \\
\hline 1129 & 3947 & CANAVESIO & LAWRENCE & B & 37 & $\mathrm{~s}$ & ITALY & ITALY & ITALY & PA & MINER/CM & TORINO & TEMPLE STREET & CALUMET PR. 6 \\
\hline 1130 & 3947 & JUERIO & LAWRENCE & B & 17 & s & ITALY & ITALY & ITALY & AL & DAY LABORER/CM & & TEMPLE STREET & CALUMET PR. 6 \\
\hline 1131 & 3950 & LACARLIE & CHARLES & $\mathrm{H}$ & 39 & M6 & ITALY & ITALY & ITALY & PA & TIMBERMAN/CM & & TEMPLE STREET & CALUMET PR. 6 \\
\hline
\end{tabular}




\begin{tabular}{|c|c|c|c|c|c|c|c|c|c|c|c|c|c|c|}
\hline \multirow{2}{*}{$\begin{array}{l}1132 \\
1133\end{array}$} & \multirow{2}{*}{$\begin{array}{l}3950 \mathrm{~L} \\
3950 \mathrm{C}\end{array}$} & \multirow{2}{*}{\begin{tabular}{|l} 
LACARLIE \\
GALLINATTI
\end{tabular}} & \multirow{2}{*}{$\begin{array}{l}\text { JOSEPHINE } \\
\text { JULIUS }\end{array}$} & \multirow{3}{*}{$\begin{array}{l}\text { W } \\
\text { SSO }\end{array}$} & \multirow{2}{*}{\begin{tabular}{l|}
44 \\
23
\end{tabular}} & \multirow{2}{*}{\multicolumn{2}{|c|}{\begin{tabular}{c|c} 
M6 & ITALY \\
S & ITALY
\end{tabular}}} & \multirow{2}{*}{$\begin{array}{l}\text { ITALY } \\
\text { ITALY }\end{array}$} & \multirow{2}{*}{$\begin{array}{l}\text { ITALY } \\
\text { ITALY }\end{array}$} & \multirow[b]{2}{*}{ PA } & & & TEMPLE STREET & CALUMET PR. 6 \\
\hline & & & & & & & & & & & MINER/CM & TORINO & TEMPLE STREET & CALUMET PR. 6 \\
\hline 1134 & 3950 & GALLINATTI & & & 19 & $\mathrm{~s}$ & ITALY & & & & TEAMSTER & TORINO & TEMPLE STREET & CALUMET PR. 6 \\
\hline 1135 & 3950 & GALLINATTI & JAMES & sSO & 8 & $\mathrm{~s}$ & MICHIGAN & ITALY & ITALY & & & TORINO & TEMPLE STREET & CALUMET PR. 6 \\
\hline 1136 & 3950 & LACARLIE & MANDE & $\mathrm{D}$ & 5 & $\mathrm{~S}$ & MICHIGAN & ITALY & ITALY & & & & TEMPLE STREET & CALUMET PR. 6 \\
\hline 1137 & 3951 & ROSSIO & CHARLES & $\mathrm{H}$ & 36 & M7 & ITALY & ITALY & ITALY & NA & MINER/CM & PONT CANAVESE. TO & TEMPLE STREET & CALUMET PR. 6 \\
\hline 1138 & 3951 & ROSSIO & ROSE & W & 38 & M7 & ITALY & ITALY & ITALY & & & & TEMPLE STREET & CALUMET PR. 6 \\
\hline 1139 & 3951 & ROSSIO & FRANK & $\mathrm{BR}$ & 43 & $\mathrm{~s}$ & ITALY & ITALY & ITALY & NA & MINER/CM & PONT CANAVESE. TO & TEMPLE STREET & CALUMET PR. 6 \\
\hline 1140 & 3951 & DE MATTEIS & CARLO & B & 40 & M20 & ITALY & ITALY & ITALY & $\mathrm{AL}$ & MINER/CM & SAN GIORGIO CANAVESE. TO & TEMPLE STREET & CALUMET PR. 6 \\
\hline 1141 & 3951 & VIGO & VINCENZO & B & 34 & M13 & ITALY & ITALY & ITALY & $\mathrm{AL}$ & MINER/CM & CORIO. TO & TEMPLE STREET & CALUMET PR. 6 \\
\hline 1142 & 3948 & SANDRETTO & MICHELE & $\mathrm{H}$ & 34 & M10 & ITALY & ITALY & ITALY & $?$ & TRAMMER/CM & PONT CANAVESE. TO & TEMPLE STREET & CALUMET PR. 6 \\
\hline 1143 & 3948 & SANDRETTO & MARGHERITA & W & 29 & M10 & ITALY & ITALY & ITALY & $?$ & & TORINO & TEMPLE STREET & CALUMET PR. 6 \\
\hline 1144 & 3948 & SANDRETTO & JOHN & $\mathrm{s}$ & 1 & $\mathrm{~s}$ & MICHIGAN & ITALY & ITALY & & & PONT CANAVESE. TO & TEMPLE STREET & CALUMET PR. 6 \\
\hline 1145 & 3948 & SANDRETTO & BARNEY & $\mathrm{s}$ & $2 \mathrm{M}$ & $\mathrm{s}$ & MICHIGAN & ITALY & ITALY & & & PONT CANAVESE. TO & TEMPLE STREET & CALUMET PR. 6 \\
\hline 1146 & 3950 & CURTO & JOHN & $\mathrm{H}$ & 22 & M3 & ITALY & ITALY & ITALY & $?$ & MINER/CM & VIALFRE'. TO & TEMPLE STREET & CALUMET PR. 6 \\
\hline 1147 & 3950 & CURTO & DOMENICA & W & 17 & M3 & ITALY & ITALY & ITALY & $?$ & & & TEMPLE STREET & CALUMET PR. 6 \\
\hline 1148 & 3950 & CURTO & ANGELO & D & 1 & $\mathrm{~s}$ & MICHIGAN & ITALY & ITALY & & & VIALFRE'. TO & TEMPLE STREET & CALUMET PR. 6 \\
\hline 1149 & 3950 & CURTO & CATHERINE & $\mathrm{D}$ & $4 \mathrm{M}$ & $\mathrm{s}$ & MICHIGAN & ITALY & ITALY & & & VIALFRE'. TO & TEMPLE STREET & CALUMET PR. 6 \\
\hline 1150 & 3950 & CHIERI & MARTIN & B & 21 & $\mathrm{~s}$ & ITALY & ITALY & ITALY & $?$ & MINER/CM & TORINO & TEMPLE STREET & CALUMET PR. 6 \\
\hline 1151 & 3950 & CURTO & JAMES & B & 19 & s & MICHIGAN & ITALY & ITALY & & SALESMAN/GROCERIES & TORINO & TEMPLE STREET & CALUMET PR. 6 \\
\hline 1152 & 3967 & MANZETTI & PAUL & $\mathrm{H}$ & 30 & M5 & ITALY & ITALY & ITALY & NA & TRAMMER/CM & PONT CANAVESE. TO & WEDGE STREET & CALUMET PR. 6 \\
\hline 1153 & 3967 & MANZETTI & KATIE & w & 24 & M5 & ITALY & ITALY & ITALY & & & & WEDGE STREET & CALUMET PR. 6 \\
\hline 1154 & 3967 & MANZETTI & JOHAUNA & $\mathrm{D}$ & 3 & $\mathrm{~S}$ & MICHIGAN & ITALY & ITALY & & & PONT CANAVESE. TO & WEDGE STREET & CALUMET PR. 6 \\
\hline 1155 & 3967 & GRINDATTI & PETER & $\mathrm{H}$ & 27 & M3 & ITALY & ITALY & ITALY & PA & TIMBERMAN/CM & PONT CANAVESE. TO & WEDGE STREET & CALUMET PR. 6 \\
\hline 1156 & 3967 & GRINDATTI & KATIE & w & 24 & M3 & ITALY & ITALY & ITALY & & & & WEDGE STREET & CALUMET PR. 6 \\
\hline 1157 & 3967 & GRINDATTI & MARY & $\mathrm{D}$ & 2 & $\mathrm{~s}$ & MICHIGAN & ITALY & ITALY & & & PONT CANAVESE. TO & WEDGE STREET & CALUMET PR. 6 \\
\hline 1158 & 3967 & GRINDATTI & DOMINICK & $\mathrm{s}$ & $3 \mathrm{M}$ & $\mathrm{s}$ & MICHIGAN & ITALY & ITALY & & & PONT CANAVESE. TO & WEDGE STREET & CALUMET PR. 6 \\
\hline 1159 & 3967 & PICCHIOTTINO & FRED & $\mathrm{H}$ & 30 & M10 & ITALY & ITALY & ITALY & NA & TEAMSTER & PONT CANAVESE, TO & WEDGE STREET & CALUMET PR. 6 \\
\hline 1160 & 3967 & PICCHIOTTINO & JENNIE & w & 31 & M10 & ITALY & ITALY & ITALY & & & & WEDGE STREET & CALUMET PR. 6 \\
\hline 1161 & 3967 & PICCHIOTTINO & LUCY & $\mathrm{D}$ & 6 & $\mathrm{~s}$ & MICHIGAN & ITALY & ITALY & & & PONT CANAVESE. TO & WEDGE STREET & CALUMET PR. 6 \\
\hline 1162 & 3967 & PICCHIOTTINO & PETER & $\mathrm{s}$ & 2 & $\mathrm{~S}$ & MICHIGAN & ITALY & ITALY & & & PONT CANAVESE. TO & WEDGE STREET & CALUMET PR. 6 \\
\hline 1163 & 3967 & PICCHIOTTINO & PAULINA & $\mathrm{D}$ & 2 & $\mathrm{~s}$ & MICHIGAN & ITALY & ITALY & & & PONT CANAVESE. TO & WEDGE STREET & CALUMET PR. 6 \\
\hline 1164 & 3967 & GRINDATTI & LUCY & B & 53 & M33 & ITALY & ITALY & ITALY & & NOT CLEAR & PONT CANAVESE. TO & WEDGE STREET & CALUMET PR. 6 \\
\hline 1165 & 3958 & BOBBIO & PAUL & $\mathrm{H}$ & 37 & M2 & ITALY & ITALY & ITALY & PA & TIMBERMAN/CM & SAN BENIGNO CANAVESE, TO & WEDGE STREET & CALUMET PR. 6 \\
\hline 1166 & 3958 & BOBBIO & ORSULA & w & 25 & M2 & ITALY & ITALY & ITALY & & & & WEDGE STREET & CALUMET PR. 6 \\
\hline 1167 & 3958 & BOBBIO & MIKE & $\mathrm{s}$ & $8 \mathrm{M}$ & $\mathrm{s}$ & MICHIGAN & ITALY & ITALY & & & SAN BENIGNO CANAVESE. TO & WEDGE STREET & CALUMET PR. 6 \\
\hline 1168 & 3958 & VECCHIA & LOUIS & B & 34 & $\mathrm{~s}$ & ITALY & ITALY & ITALY & NA & TRAMMER/CM & SAN BENIGNO CANAVESE. TO & WEDGE STREET & CALUMET PR. 6 \\
\hline 1169 & 3958 & VIOLA & JOE & B & 27 & $\mathrm{~S}$ & ITALY & ITALY & ITALY & $\mathrm{PA}$ & TRAMMER/CM & SAN BENIGNO CANAVESE. TO & WEDGE STREET & CALUMET PR. 6 \\
\hline 1170 & 3958 & CERUTTI & DOMINICK & B & 28 & s & ITALY & ITALY & ITALY & $\mathrm{AL}$ & TRAMMER/CM & SAN BENIGNO CANAVESE. TO & WEDGE STREET & CALUMET PR. 6 \\
\hline 1171 & 3958 & CAPELLO & JOE & B & 29 & $\mathrm{~s}$ & ITALY & ITALY & ITALY & $\mathrm{PA}$ & TRAMMER/CM & TORINO & WEDGE STREET & CALUMET PR. 6 \\
\hline 1172 & 3958 & BIANCO & JOE & B & 27 & M3 & ITALY & ITALY & ITALY & AL & TRAMMER/CM & SAN BENIGNO CANAVESE. TO & WEDGE STREET & CALUMET PR. 6 \\
\hline 1173 & 3958 & MORETTO & PAULL & B & 41 & $\mathrm{~s}$ & ITALY & ITALY & ITALY & $\mathrm{AL}$ & TIMBERMAN/CM & SAN BENIGNO CANAVESE. TO & WEDGE STREET & CALUMET PR. 6 \\
\hline 1174 & 3958 & VIOLA & TONY & B & 32 & $\mathrm{~S}$ & ITALY & ITALY & ITALY & NA & TIMBERMAN/CM & SAN BENIGNO CANAVESE. TO & WEDGE STREET & CALUMET PR. 6 \\
\hline 1175 & 3958 & TAPPERO & THOMAS & B & 19 & s & ITALY & ITALY & ITALY & $\mathrm{AL}$ & TIMBERMAN/CM & SAN BENIGNO CANAVESE. TO & WEDGE STREET & CALUMET PR. 6 \\
\hline 1176 & 3960 & GHERNA & JOHN & $\mathrm{H}$ & 42 & M18 & ITALY & ITALY & ITALY & NA & MINER/CM & PONT CANAVESE. TO & WEDGE STREET & CALUMET PR. 6 \\
\hline 1177 & 3960 & GHERNA & THERESA & w & 33 & M18 & ITALY & ITALY & ITALY & & & PONT CANAVESE. TO & WEDGE STREET & CALUMET PR. 6 \\
\hline 1178 & 3960 & GHERNA & JOE & s & 16 & $\mathrm{~s}$ & ITALY & ITALY & ITALY & & MINER/CM & PONT CANAVESE. TO & WEDGE STREET & CALUMET PR. 6 \\
\hline 11179 & 3960 & GHERNA & JOHN & s & 10 & s & ITALY & ITALY & ITALY & & AT SCHOOL 9 & PONT CANAVESE. TO & WEDGE STREET & CALUMET PR. 6 \\
\hline 1180 & 3960 & GHERNA & MANDE & $\mathrm{D}$ & 6 & $\mathrm{~s}$ & MICHIGAN & ITALY & ITALY & & & PONT CANAVESE. TO & WEDGE STREET & CALUMET PR. 6 \\
\hline 1181 & 3960 & GHERNA & FRANK & $\mathrm{s}$ & 3 & $\mathrm{~s}$ & MICHIGAN & ITALY & ITALY & & & PONT CANAVESE. TO & WEDGE STREET & CALUMET PR. 6 \\
\hline 1182 & 3960 & GHERNA & JAMES & $\mathrm{s}$ & 1 & $\mathrm{~s}$ & MICHIGAN & ITALY & ITALY & & & PONT CANAVESE. TO & WEDGE STREET & CALUMET PR. 6 \\
\hline 1183 & 3960 & GHERNA & MARK & $\mathrm{s}$ & $8 \mathrm{M}$ & $\mathrm{s}$ & MICHIGAN & ITALY & ITALY & & & PONT CANAVESE, TO & WEDGE STREET & CALUMET PR. 6 \\
\hline 1184 & 3960 & CURTO & JOHN & B & 36 & s & ITALY & ITALY & ITALY & NA & TIMBERMAN/CM & TORINO & WEDGE STREET & CALUMET PR. 6 \\
\hline 1185 & 3960 & GHERNA & LEWIS & B & 29 & M10 & ITALY & ITALY & ITALY & $\mathrm{AL}$ & TIMBERMAN/CM & PONT CANAVESE. TO & WEDGE STREET & CALUMET PR. 6 \\
\hline 1186 & 3960 & PICCHIOTTINO & GIUSEPPE & $\mathrm{H}$ & 31 & M7 & ITALY & ITALY & ITALY & PA & SALESMAN/DRY GOODS & PONT CANAVESE. TO & WEDGE STREET & CALUMET PR. 6 \\
\hline 1187 & 3960 & PICCHIOTTINO & HANNAH & W & 29 & M7 & NORWAY & NORWAY & NORWAY & & & & WEDGE STREET & CALUMET PR. 6 \\
\hline 1188 & 4018 & LANCINA & BERNARD & $\mathrm{H}$ & 45 & M20 & ITALY & ITALY & ITALY & NA & MASON & SAN GIORGIO CANAVESE. TO & PORTLAND ST. & CALUMET PR. 6 \\
\hline 1189 & 4018 & LANCINA & KATIE & W & 39 & M20 & ITALY & ITALY & ITALY & & & SAN GIORGIO CANAVESE, TO & PORTLAND ST. & CALUMET PR. 6 \\
\hline 1190 & 4018 & LANCINA & DOMINICK & s & 18 & $\mathrm{~s}$ & ITALY & ITALY & ITALY & & MASON & SAN GIORGIO CANAVESE. TO & PORTLAND ST. & CALUMET PR. 6 \\
\hline 1191 & 4018 & LANCINA & JOHN & $\mathrm{s}$ & 13 & $\mathrm{~s}$ & MICHIGAN & ITALY & ITALY & & AT SCHOOL 9 & SAN GIORGIO CANAVESE. TO & PORTLAND ST. & CALUMET PR. 6 \\
\hline 1192 & 4018 & LANCINA & BERNARD & $\mathrm{s}$ & 9 & $\mathrm{~s}$ & MICHIGAN & ITALY & ITALY & & & SAN GIORGIO CANAVESE. TO & PORTLAND ST. & CALUMET PR. 6 \\
\hline 1193 & 4018 & LANCINA & GEORGE & $\mathrm{s}$ & 4 & s & MICHIGAN & ITALY & ITALY & & & SAN GIORGIO CANAVESE. TO & PORTLAND ST. & CALUMET PR. 6 \\
\hline 1194 & 4004 & MOLINARI & ANTHONY & $\mathrm{H}$ & 32 & s & ITALY & ITALY & ITALY & NA & PRIEST & & PORTLAND ST. & CALUMET PR. 6 \\
\hline
\end{tabular}




\begin{tabular}{|c|c|c|c|c|c|c|c|c|c|c|c|c|c|c|c|}
\hline 1195 & 4045 & BOBBIO & PAUL & $\mathrm{H}$ & 40 & M10 & ITALY & ITALY & ITALY & & DAY LABORER/CM & SAN BENIGNO CANAVESE. TO & ACORN STREET & CALUMET PR. 6 & \\
\hline 1196 & 4045 & BOBBIO & JOSEPHINA & W & 34 & M10 & ITALY & ITALY & ITALY & & & & ACORN STREET & CALUMET PR. 6 & \\
\hline 1197 & 4045 & BOBBIO & FELIX & $\mathrm{s}$ & 5 & $\mathrm{~s}$ & MICHIGAN & ITALY & ITALY & & & SAN BENIGNO CANAVESE. TO & ACORN STREET & CALUMET PR. 6 & \\
\hline 1198 & 4045 & ZANOTTI & TONY & B & 26 & s & ITALY & ITALY & ITALY & & DAY LABORER/CM & PEROSA CANAVESE. TO & ACORN STREET & CALUMET PR. 6 & \\
\hline 1199 & 4045 & AIMONETTO & BERNARD & B & 36 & M8 & ITALY & ITALY & ITALY & & TRAMMER/CM & TORINO & ACORN STREET & CALUMET PR. 6 & \\
\hline 1200 & 4045 & BESTONZO & BATTISTA & B & 25 & $\mathrm{~S}$ & ITALY & ITALY & ITALY & & TRAMMER/CM & SAN BENIGNO CANAVESE. TO & ACORN STREET & CALUMET PR. 6 & \\
\hline 1201 & 4045 & BERTINO & MIKE & B & 27 & s & ITALY & ITALY & ITALY & & TRAMMER/CM & SAN BENIGNO CANAVESE. TO & ACORN STREET & CALUMET PR. 6 & \\
\hline 1202 & 4045 & VECCHIA & JOHN & B & 35 & M2 & ITALY & ITALY & ITALY & & TRAMMER/CM & SAN BENIGNO CANAVESE. TO & ACORN STREET & CALUMET PR. 6 & \\
\hline 1203 & 4045 & CRAVERO & GIORGIO & B & 25 & $\mathrm{~S}$ & ITALY & ITALY & ITALY & & DAY LABORER/CM & SAN BENIGNO CANAVESE. TO & ACORN STREET & CALUMET PR. 6 & \\
\hline 1204 & 4045 & NIGRA/NIGRO & GEORGE & B & 28 & $\mathrm{~s}$ & ITALY & ITALY & ITALY & & TRAMMER/CM & SAN BENIGNO CANAVESE. TO & ACORN STREET & CALUMET PR. 6 & \\
\hline 1205 & 4045 & ZANOTTI & TONY & B & 16 & $\mathrm{~s}$ & ITALY & ITALY & ITALY & & MINER/CM & PARELLA. TO & ACORN STREET & CALUMET PR. 6 & \\
\hline 1206 & 4045 & MELCHIOR & DOMINICK & B & 29 & $\mathrm{~s}$ & ITALY & ITALY & ITALY & & TRAMMER/CM & SAN BENIGNO CANAVESE. TO & ACORN STREET & CALUMET PR. 6 & \\
\hline 1207 & 4060 & COPPO & VICTOR & $\mathrm{H}$ & 31 & M8 & ITALY & ITALY & ITALY & & MINER/CM & PONT CANAVESE. TO & ACORN STREET & CALUMET PR. 6 & \\
\hline 1208 & 4060 & COPPO & JENNIE & W & 25 & M8 & ITALY & ITALY & ITALY & & & & ACORN STREET & CALUMET PR. 6 & \\
\hline 1209 & 4060 & COPPO & ANTHONY & $\mathrm{s}$ & 6 & $\mathrm{~S}$ & MICHIGAN & ITALY & ITALY & & & PONT CANAVESE. TO & ACORN STREET & CALUMET PR. 6 & \\
\hline 1210 & 4060 & COPPO & KATE & D & 3 & $\mathrm{~S}$ & MICHIGAN & ITALY & ITALY & & & PONT CANAVESE, TO & ACORN STREET & CALUMET PR. 6 & \\
\hline 1211 & 4060 & COPPO & ERNESTINE & $\mathrm{s}$ & $9 \mathrm{M}$ & $\mathrm{s}$ & MICHIGAN & ITALY & ITALY & & & PONT CANAVESE. TO & ACORN STREET & CALUMET PR. 6 & \\
\hline 1212 & 4045 & CURTO & JAMES & $\mathrm{H}$ & 41 & M3 & ITALY & ITALY & ITALY & NA & TIMBERMAN/CM & TORINO & ACORN STREET & CALUMET PR. 6 & \\
\hline 1213 & 4045 & CURTO & KATIE & w & 36 & M3 & ITALY & ITALY & ITALY & & & & ACORN STREET & CALUMET PR. 6 & \\
\hline 1214 & 4065 & BORNU & CAESAR & $\mathrm{H}$ & 25 & M2 & ITALY & ITALY & ITALY & PA & MINER/CM & & ELM STREET & CALUMET PR. 6 & \\
\hline 1215 & 4065 & BORNU & THERESA & w & 25 & M2 & ITALY & ITALY & ITALY & & & & ELM STREET & CALUMET PR. 6 & \\
\hline 1216 & 4065 & BORNU & KATIE & D & 1 & $\mathrm{~s}$ & MICHIGAN & ITALY & ITALY & & & & ELM STREET & CALUMET PR. 6 & \\
\hline 1217 & 4063 & MICHETTI & JOHN & $\mathrm{H}$ & 44 & M21 & ITALY & ITALY & ITALY & PA & MINER/CM & PONT CANAVESE. TO & ELM STREET & CALUMET PR. 6 & \\
\hline 1218 & 4063 & MICHETTI & MADELINE & W & 40 & M21 & ITALY & ITALY & ITALY & & & PONT CANAVESE. TO & ELM STREET & CALUMET PR. 6 & \\
\hline 1219 & 4063 & MICHETTI & JOHN & $\mathrm{s}$ & 19 & $\mathrm{~S}$ & ITALY & ITALY & ITALY & & DAY LABORER/CM & PONT CANAVESE. TO & ELM STREET & CALUMET PR. 6 & \\
\hline 1220 & 4063 & MICHETTI & DOMINICA & D & 9 & $\mathrm{~s}$ & MICHIGAN & ITALY & ITALY & & & PONT CANAVESE. TO & ELM STREET & CALUMET PR. 6 & \\
\hline 1221 & 4063 & MICHETTI & MIKE & $\mathrm{s}$ & 7 & $\mathrm{~s}$ & MICHIGAN & ITALY & ITALY & & & PONT CANAVESE. TO & ELM STREET & CALUMET PR. 6 & \\
\hline 1222 & 4063 & MICHETTI & PAUL & $\mathrm{s}$ & 5 & $\mathrm{~s}$ & MICHIGAN & ITALY & ITALY & & & PONT CANAVESE, TO & ELM STREET & CALUMET PR. 6 & \\
\hline 1223 & 4063 & MICHETTI & PETER & $\mathrm{s}$ & 3 & $\mathrm{~S}$ & MICHIGAN & ITALY & ITALY & & & PONT CANAVESE. TO & ELM STREET & CALUMET PR. 6 & \\
\hline 1224 & 4063 & MICHETTI & KATE & $\mathrm{D}$ & 2 & $\mathrm{~s}$ & MICHIGAN & ITALY & ITALY & & & PONT CANAVESE. TO & ELM STREET & CALUMET PR. 6 & \\
\hline 1225 & 4063 & MICHETTI & FRED & BR & 38 & M14 & ITALY & ITALY & ITALY & NA & MINER/CM & PONT CANAVESE. TO & ELM STREET & CALUMET PR. 6 & \\
\hline 1226 & 4063 & MICHETTI & JOSEPH & BR & 28 & M7 & ITALY & ITALY & ITALY & $\mathrm{AL}$ & MINER/CM & PONT CANAVESE. TO & ELM STREET & CALUMET PR. 6 & \\
\hline 1227 & 4063 & BROGLIATTI & PROSPERO & $\mathrm{H}$ & 34 & M3 & ITALY & ITALY & ITALY & AL & TRAMMER/CM & ROMANO CANAVESE. TO & ELM STREET & CALUMET PR. 6 & \\
\hline 1228 & 4063 & BROGLIATTI & MARIA & W & 26 & M3 & ITALY & ITALY & ITALY & & 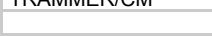 & ROMANO CANAVESE, TO & ELM STREET & CALUMET PR. 6 & \\
\hline 1229 & 4063 & BROGLIATTI & THERESA & D & 1 & s & MICHIGAN & ITALY & ITALY & & & ROMANO CANAVESE. TO & ELM STREET & CALUMET PR. 6 & \\
\hline 1230 & 4065 & NIGRA/NIGRO & ANTON & $\mathrm{H}$ & 39 & M18 & ITALY & ITALY & ITALY & NA & TIMBERMAN/CM & TORINO & ELM STREET & CALUMET PR. 6 & \\
\hline 1231 & 4065 & NIGRAINIGRO & CATERINA & w & 37 & M18 & ITALY & ITALY & ITALY & & & TORINO & ELM STREET & CALUMET PR. 6 & \\
\hline 1232 & 4065 & NIGRA/NIGRO & JOE & $\mathrm{s}$ & 17 & $\mathrm{~s}$ & ITALY & ITALY & ITALY & & DAY LABORER/CM & TORINO & ELM STREET & CALUMET PR. 6 & \\
\hline 1233 & 4065 & NIGRA/NIGRO & FRANCES & $\mathrm{D}$ & 12 & $\mathrm{~s}$ & ITALY & ITALY & ITALY & & AT SCHOOL 9 & TORINO & ELM STREET & CALUMET PR. 6 & \\
\hline 1234 & 4065 & NIGRAINIGRO & KATE & D & 7 & $\mathrm{~S}$ & MICHIGAN & ITALY & ITALY & & & TORINO & ELM STREET & CALUMET PR. 6 & \\
\hline 1235 & 4065 & NIGRA/NIGRO & MAGGIE & D & 3 & $\mathrm{~S}$ & MICHIGAN & ITALY & ITALY & & & TORINO & ELM STREET & CALUMET PR. 6 & \\
\hline 1236 & 4065 & NIGRAINIGRO & GIOVANNI & $\mathrm{s}$ & 1 & $\mathrm{~s}$ & MICHIGAN & ITALY & ITALY & & & TORINO & ELM STREET & CALUMET PR. 6 & \\
\hline 1237 & 4087 & POGGIONE & CHARLES & $\mathrm{H}$ & 37 & M14 & ITALY & ITALY & ITALY & PA & DAY LABORER/CM & SAN GIORGIO CANAVESE. TO & $\mathrm{C}$ ? & CALUMET PR. 6 & \\
\hline 1238 & 4087 & POGGIONE & MANNIE & w & 36 & M14 & ITALY & ITALY & ITALY & & & SAN GIORGIO CANAVESE. TO & C? & CALUMET PR. 6 & \\
\hline 1239 & 4087 & POGGIONE & KATR & $\mathrm{D}$ & 12 & s & ITALY & ITALY & ITALY & & AT SCHOOL 9 & SAN GIORGIO CANAVESE. TO & C? & CALUMET PR. 6 & \\
\hline 1240 & 4087 & POGGIONE & TRACEY & $\mathrm{D}$ & 10 & $\mathrm{~S}$ & ITALY & ITALY & ITALY & & AT SCHOOL 9 & SAN GIORGIO CANAVESE. TO & $\mathrm{C}$ ? & CALUMET PR. 6 & \\
\hline 1241 & 4087 & POGGIONE & CRESCENCE & D & 7 & $\mathrm{~s}$ & MICHIGAN & ITALY & ITALY & & & SAN GIORGIO CANAVESE. TO & C? & CALUMET PR. 6 & \\
\hline 1242 & 4087 & POGGIONE & MARY & $\mathrm{D}$ & 5 & $\mathrm{~S}$ & MICHIGAN & ITALY & ITALY & & & SAN GIORGIO CANAVESE. TO & C? & CALUMET PR. 6 & \\
\hline 1243 & 4087 & POGGIONE & GEORGE & $\mathrm{s}$ & 2 & $\mathrm{~s}$ & MICHIGAN & ITALY & ITALY & & & SAN GIORGIO CANAVESE. TO & C? & CALUMET PR. 6 & \\
\hline 1244 & 4066 & CAPELLO & JOE & $\mathrm{H}$ & 29 & M6 & ITALY & ITALY & ITALY & AL & MINER/CM & SAN BENIGNO CANAVESE. TO & ACORN STREET & CALUMET PR. 6 & \\
\hline 1245 & 4066 & CAPELLO & MARIA & W & 26 & M6 & ITALY & ITALY & ITALY & & & SAN BENIGNO CANAVESE. TO & ACORN STREET & CALUMET PR. 6 & \\
\hline 1246 & 4066 & CAPELLO & GENEVIEVE & D & 5 & $\mathrm{~s}$ & ITALY & ITALY & ITALY & & & SAN BENIGNO CANAVESE, TO & ACORN STREET & CALUMET PR. 6 & \\
\hline 1247 & 4066 & RIVA & ANGELO & B & 25 & $\mathrm{~s}$ & ITALY & ITALY & ITALY & $\mathrm{AL}$ & MINER/CM & SALTO CANAVESE. TO & ACORN STREET & CALUMET PR. 6 & \\
\hline 1248 & 4066 & CONTRATTO & ANDREW & B & 45 & M22 & ITALY & ITALY & ITALY & $\mathrm{AL}$ & MINER/CM & LOCANA CANAVESE. TO & ACORN STREET & CALUMET PR. 6 & \\
\hline 1249 & 4066 & COZARIO-NOVA & JOE & B & 36 & $\mathrm{~s}$ & ITALY & ITALY & ITALY & $\mathrm{PA}$ & TRAMMER/CM & TORINO & ACORN STREET & CALUMET PR. 6 & \\
\hline 1250 & 4066 & TORNATORE & JOE & B & 22 & $\mathrm{~s}$ & ITALY & ITALY & ITALY & $\mathrm{AL}$ & MINER/CM & SAN BENIGNO CANAVESE. TO & ACORN STREET & CALUMET PR. 6 & \\
\hline 1251 & 4066 & CAPELLO & LEWIS & B & 33 & s & ITALY & ITALY & ITALY & NA & TIMBERMAN/CM & SALTO CANAVESE. TO & ACORN STREET & CALUMET PR. 6 & \\
\hline 1252 & 4066 & NOTARIO & TOMMASO & B & 28 & M3 & ITALY & ITALY & ITALY & $\mathrm{AL}$ & MINER/CM & SAN BENIGNO CANAVESE, TO & ACORN STREET & CALUMET PR. 6 & \\
\hline 1253 & 4066 & ROLANDO & STEVEN & $\mathrm{H}$ & 50 & M7 & ITALY & ITALY & ITALY & $\mathrm{PA}$ & STONECUTTER & TORINO & ACORN STREET & CALUMET PR. 6 & \\
\hline 1254 & 4066 & ROLANDO & THERESA & W & 37 & M7 & ITALY & ITALY & ITALY & & & & ACORN STREET & CALUMET PR. 6 & \\
\hline 1255 & 4066 & ROLANDO & JOHN & $\mathrm{s}$ & 2 & $\mathrm{~s}$ & MICHIGAN & ITAI & ITALY & & & TORINO & ACORN STREET & CALUMET & \\
\hline 1256 & 4066 & ROLANDO & TONY & $\mathrm{s}$ & 2 & $\mathrm{~s}$ & MICHIGAN & ITALY & ITALY & & & TORINO & ACORN STREET & CALUMET PR. 6 & \\
\hline 1257 & 4066 & JOGRUNI & JOE & B & 20 & $\mathrm{~s}$ & ITALY & ITALY & ITALY & $\mathrm{AL}$ & & & ACORN STREET & CALUMET PR. 6 & \\
\hline
\end{tabular}




\begin{tabular}{|c|c|c|c|c|c|c|c|c|c|c|c|c|c|c|}
\hline 1258 & 4067 & MIGLIO/MIGLIA & TONY & $\mathrm{H}$ & 41 & M9 & ITALY & ITALY & ITALY & NA & SPRINKLER & SAN GIORGIO CANAVESE. TO & ELM STREET & CALUMET PR. 6 \\
\hline 1259 & 4067 & MIGLIO/MIGLIA & LUISA & W & 28 & M9 & ITALY & ITALY & ITALY & & & SAN GIORGIO CANAVESE. TO & ELM STREET & CALUMET PR. 6 \\
\hline 1260 & 4067 & MIGLIO/MIGLIA & PIERINO & $s$ & 8 & $\mathrm{~s}$ & MICHIGAN & ITALY & ITALY & & & SAN GIORGIO CANAVESE. TO & ELM STREET & CALUMET PR. 6 \\
\hline 1261 & 4067 & MIGLIO/MIGLIA & ANTONIO & $\mathrm{s}$ & 6 & $\mathrm{~s}$ & MICHIGAN & ITALY & ITALY & & & SAN GIORGIO CANAVESE. TO & ELM STREET & CALUMET PR. 6 \\
\hline 1262 & 4067 & GUGLIELMO & JOE & $\mathrm{H}$ & 35 & M1 & ITALY & ITALY & ITALY & PA & NOT CLEAR & MONTELANGHE. TO & ELM STREET & CALUMET PR. 6 \\
\hline 1263 & 4067 & GUGLIELMO & MARY & W & 28 & M1 & ITALY & ITALY & ITALY & & & & ELM STREET & CALUMET PR. 6 \\
\hline 1264 & 4067 & GUGLIELMO & MIKE & s & $1 \mathrm{M}$ & & MICHIGAN & ITALY & ITALY & & & MONTELANGHE. TO & ELM STREET & CALUMET PR. 6 \\
\hline 1265 & 4067 & GUGLIELMO & JOHN & B & 47 & M17 & ITALY & ITALY & ITALY & AL & TRAMMER/CM & MONTELANGHE. TO & ELM STREET & CALUMET PR. 6 \\
\hline 1266 & 4250 & MICHETTI & PAUL & $\mathrm{H}$ & 30 & M4 & ITALY & ITALY & ITALY & NA & MINER/CM & PONT CANAVESE. TO & TENTH STREET & CALUMET PR. 6 \\
\hline 1267 & 4250 & MICHETTI & KATIE & W & 36 & M4 & ITALY & ITALY & ITALY & & & & TENTH STREET & CALUMET PR. 6 \\
\hline 1268 & 4250 & TRIONE & CHARLES & SSO & 10 & $\mathrm{~s}$ & MICHIGAN & ITALY & ITALY & & AT SCHOOL 9 & TORINO & TENTH STREET & CALUMET PR. 6 \\
\hline 1269 & 4250 & TRIONE & PAUL & sso & 9 & $\mathrm{~s}$ & MICHIGAN & ITALY & ITALY & & & TORINO & TENTH STREET & CALUMET PR. 6 \\
\hline 1270 & 4250 & TRIONE & AUGUST & sSO & 7 & $\mathrm{~s}$ & MICHIGAN & ITALY & ITALY & & & TORINO & TENTH STREET & CALUMET PR. 6 \\
\hline 1271 & 4250 & MICHETTI & TOM & B & 33 & M13 & ITALY & ITALY & ITALY & PA & MINER/CM & PONT CANAVESE. TO & TENTH STREET & CALUMET PR. 6 \\
\hline 1272 & 4250 & BONOTTO & LEWIS & B & 24 & s & ITALY & ITALY & ITALY & AL & MINER/CM & SAN BENIGNO CANAVESE. TO & TENTH STREET & CALUMET PR. 6 \\
\hline 1273 & 4250 & NIDA & JOE & B & 27 & $\mathrm{~s}$ & ITALY & ITALY & ITALY & $\mathrm{AL}$ & MINER/CM & RIVAROLO CANAVESE, TO & TENTH STREET & CALUMET PR. 6 \\
\hline 1274 & 4250 & POGGIONI & KATE & B & 61 & W & ITALY & ITALY & ITALY & & & SAN GIORGIO CANAVESE. TO & TENTH STREET & CALUMET PR. 6 \\
\hline 1275 & 4250 & GASPARDO & FRANK & B & 44 & M27 & ITALY & ITALY & ITALY & $\mathrm{AL}$ & MINER/CM & PONT CANAVESE. TO & TENTH STREET & CALUMET PR. 6 \\
\hline 1276 & 4250 & BERSANO & JOHN & B & 30 & $\mathrm{~s}$ & ITALY & ITALY & ITALY & $\mathrm{AL}$ & MINER/CM & SAN MARTINO CANAVESE. TO & TENTH STREET & CALUMET PR. 6 \\
\hline 1277 & 4250 & MANZETTI & FIORENTINO & B & 29 & $\mathrm{~s}$ & ITALY & ITALY & ITALY & $\mathrm{AL}$ & MINER/CM & PONT CANAVESE. TO & TENTH STREET & CALUMET PR. 6 \\
\hline 1278 & 4246 & PIANA & VINCENT & $\mathrm{H}$ & 36 & M13 & ITALY & ITALY & ITALY & PA & TRAMMER/CM & QUAGLIUZZO. TO & TENTH STREET & CALUMET PR. 6 \\
\hline 1279 & 4246 & PIANA & THERESA & w & 36 & M13 & ITALY & ITALY & ITALY & & & QUAGLIUZZO, TO & TENTH STREET & CALUMET PR. 6 \\
\hline 1280 & 4246 & PIANA & MAGGIE & $\mathrm{D}$ & 13 & s & ITALY & ITALY & ITALY & & AT SCHOOL 10 & QUAGLIUZZO. TO & TENTH STREET & CALUMET PR. 6 \\
\hline 1281 & 4246 & PIANA & LIBBIE & $\mathrm{D}$ & 5 & $\mathrm{~s}$ & MICHIGAN & ITALY & ITALY & & & QUAGLIUZZO. TO & TENTH STREET & CALUMET PR. 6 \\
\hline 1282 & 4246 & PIANA & JOHN & $\mathrm{s}$ & 3 & s & MICHIGAN & ITALY & ITALY & & & QUAGLIUZZO. TO & TENTH STREET & CALUMET PR. 6 \\
\hline 1283 & 4242 & DE FILIPPI & DOMINICK & $\mathrm{H}$ & 32 & M8 & ITALY & ITALY & ITALY & NA & MINER/CM & SAN GIORGIO CANAVESE. TO & $\begin{array}{l}\text { TENTH STREET } \\
\text { TET }\end{array}$ & CALUMET PR. 6 \\
\hline 1284 & 4242 & DE FILIPPI & MARY & W & 30 & M8 & ITALY & ITALY & ITALY & & & & TENTH STREET & CALUMET PR. 6 \\
\hline 1285 & 4242 & DE FILIPPI & ANDREW & s & 1 & $\mathrm{~s}$ & MICHIGAN & ITALY & ITALY & & & SAN GIORGIO CANAVESE, TO & TENTH STREET & CALUMET PR. 6 \\
\hline 1286 & 4242 & DE FILIPPI & JOE & $\mathrm{s}$ & 3 & $\mathrm{~s}$ & MICHIGAN & ITALY & ITALY & & & SAN GIORGIO CANAVESE. TO & TENTH STREET & CALUMET PR. 6 \\
\hline 1287 & 4242 & DE FILIPPI & MAGGIE & D & 6 & $\mathrm{~s}$ & MICHIGAN & ITALY & ITALY & & & SAN GIORGIO CANAVESE. TO & TENTH STREET & CALUMET PR. 6 \\
\hline 1288 & 4242 & DE FILIPPI & MARY & $\mathrm{D}$ & 7 & $\mathrm{~s}$ & MICHIGAN & ITALY & ITALY & & & SAN GIORGIO CANAVESE. TO & TENTH STREET & CALUMET PR. 6 \\
\hline 1289 & 4242 & DE FILIPPI & DOMINICK & B & 21 & M2 & ITALY & ITALY & ITALY & PA & TRAMMER/CM & SAN GIORGIO CANAVESE. TO & TENTH STREET & CALUMET PR. 6 \\
\hline 1290 & 4242 & BAUDINO & PIETRO & B & 25 & M4 & ITALY & ITALY & ITALY & PA & TRAMMER/CM & BOSCONERO. TO & TENTH STREET & CALUMET PR. 6 \\
\hline 1291 & 4242 & LITIZZETTO & ANTONIO & B & 37 & MO & ITALY & ITALY & ITALY & PA & TRAMMER/CM & RIVAROLO CANAVESE, TO & TENTH STREET & CALUMET PR. 6 \\
\hline 1292 & 4238 & TEPATTI & JOHN & $\begin{array}{l}\mathrm{D} \\
\mathrm{H}\end{array}$ & 37 & M15 & $\begin{array}{l}\text { ITALY } \\
\text { ITAL }\end{array}$ & ITALY & ITALY & NA & TIMBERMAN/CM & $\begin{array}{l}\text { PONT CANAVESE. TO } \\
\text { PON }\end{array}$ & TENTH STREET & CALUMET PR. 6 \\
\hline 1293 & 4238 & TEPATTI & PAULINA & w & 34 & M15 & ITALY & ITALY & ITALY & & & & TENTH STREET & CALUMET PR. 6 \\
\hline 1294 & 4238 & TEPATTI & TONY & s & 8 & $\mathrm{~s}$ & MICHIGAN & ITALY & ITALY & & & PONT CANAVESE. TO & TENTH STREET & CALUMET PR. 6 \\
\hline 1295 & 4238 & TEPATTI & MARTIN & $\mathrm{s}$ & 6 & $\mathrm{~S}$ & MICHIGAN & ITALY & ITALY & & & PONT CANAVESE. TO & TENTH STREET & CALUMET PR. 6 \\
\hline 1296 & 4238 & TEPATTI & BARNEY & $\mathrm{s}$ & 1 & $\mathrm{~s}$ & MICHIGAN & ITALY & ITALY & & & PONT CANAVESE. TO & TENTH STREET & CALUMET PR. 6 \\
\hline 1297 & 4245 & COPPO & MARTIN & $\mathrm{H}$ & 40 & M24 & ITALY & ITALY & ITALY & NA & MINER/CM & PONT CANAVESE. TO & ELEVENTH ST. & CALUMET PR. 6 \\
\hline 1298 & 4245 & COPPO & KATE & W & 45 & M24 & ITALY & ITALY & ITALY & & & PONT CANAVESE. TO & ELEVENTH ST. & CALUMET PR. 6 \\
\hline 1299 & 4245 & COPPO & LINA & D & 17 & $\mathrm{~s}$ & ITALY & ITALY & ITALY & & & PONT CANAVESE. TO & ELEVENTH ST. & CALUMET PR. 6 \\
\hline 1300 & 4245 & COPPO & MIKE & $\mathrm{s}$ & 14 & $\mathrm{~S}$ & MICHIGAN & ITALY & ITALY & & AT SCHOOL 9 & PONT CANAVESE. TO & ELEVENTH ST. & CALUMET PR. 6 \\
\hline 1301 & 4245 & COPPO & PETER & $\mathrm{s}$ & 6 & $\mathrm{~S}$ & MICHIGAN & ITALY & ITALY & & & PONT CANAVESE. TO & ELEVENTH ST. & CALUMET PR. 6 \\
\hline 1302 & 4245 & CENTANINO & DOMINICK & $\mathrm{H}$ & 28 & M8 & ITALY & ITALY & ITALY & NA & MASON & SAN MARTINO CANAVESE. TO & ELEVENTH ST. & CALUMET PR. 6 \\
\hline 1303 & 4245 & CENTANINO & MAGGIE & w & 27 & M8 & ITALY & ITALY & ITALY & & & & ELEVENTH ST. & CALUMET PR. 6 \\
\hline 1304 & 4245 & CENTANINO & DOMINICK & s & 5 & $\mathrm{~s}$ & MICHIGAN & ITALY & ITALY & & & SAN MARTINO CANAVESE. TO & ELEVENTH ST. & CALUMET PR. 6 \\
\hline 1305 & 4245 & CENTANINO & JOHN & $\mathrm{s}$ & 4 & $\mathrm{~s}$ & MICHIGAN & ITALY & ITALY & & & SAN MARTINO CANAVESE. TO & ELEVENTH ST. & CALUMET PR. 6 \\
\hline 1306 & 4245 & CENTANINO & MARY & $\mathrm{D}$ & 2 & s & MICHIGAN & ITALY & ITALY & & & SAN MARTINO CANAVESE. TO & ELEVENTH ST. & CALUMET PR. 6 \\
\hline 1307 & 4247 & SODER & JOHN & $\mathrm{H}$ & 32 & M7 & ITALY & ITALY & ITALY & PA & MINER/CM & & ELEVENTH ST. & CALUMET PR. 6 \\
\hline 1308 & 4247 & SODER & MARY & W & 37 & M7 & ITALY & ITALY & ITALY & & & & ELEVENTH ST. & CALUMET PR. 6 \\
\hline 1309 & 4261 & STONETTI & FRANK & B & 23 & $\mathrm{~S}$ & ITALY & ITALY & ITALY & $\mathrm{AL}$ & DAY LABORER/CM & & & CALUMET PR. 6 \\
\hline 1310 & 4232 & PISTONATTI & THERESA & $\mathrm{H}$ & 33 & W & ITALY & ITALY & ITALY & & N. G. & TORINO & TENTH STREET & CALUMET PR. 6 \\
\hline 1311 & 4232 & PISTONATTI & JOHN & s & 34 & s? & ITALY & ITALY & ITALY & $\mathrm{AL}$ & MINER/CM & TORINO & TENTH STREET & CALUMET PR. 6 \\
\hline 1312 & 4232 & PISTONATTI & MARY & D & 11 & s & ITALY & ITALY & ITALY & & AT SCHOOL 8 & TORINO & TENTH STREET & CALUMET PR. 6 \\
\hline 1313 & 4232 & PISTONATTI & JOE & $\mathrm{s}$ & 8 & $\mathrm{~s}$ & MICHIGAN & ITALY & ITALY & & & TORINO & TENTH STREET & CALUMET PR. 6 \\
\hline 1314 & 4232 & GARDETTO & GIACOMO & B & 23 & $\mathrm{~S}$ & ITALY & ITALY & ITALY & $\mathrm{AL}$ & TRAMMER/CM & BOSCONERO. TO & TENTH STREET & CALUMET PR. 6 \\
\hline 1315 & 4232 & OURAILU & $\mathrm{JOE}$ & B & 26 & s & ITALY & ITALY & ITALY & $\mathrm{AL}$ & TRAMMER/CM & & TENTH STREET & CALUMET PR. 6 \\
\hline 1316 & 4232 & OURAILU & LOUIS & B & 19 & $\mathrm{~s}$ & ITALY & ITALY & ITALY & & TRAMMER/CM & & TENTH STREET & CALUMET PR. 6 \\
\hline 1317 & 4232 & DE STEFANI & GIOVANNI & $\mathrm{H}$ & 49 & W & ITALY & ITALY & ITALY & PA & WATCHMAN/CM & & TENTH STREET & CALUMET PR. 6 \\
\hline 1318 & 4232 & MELIA/MELIS & JOE & $\mathrm{H}$ & 40 & M7 & ITALY & ITALY & ITALY & $\mathrm{AL}$ & MINER/CM & & TENTH STREET & CALUMET PR. 6 \\
\hline 1319 & 4232 & MELIA/MELIS & CAROLINE & W & 28 & M7 & ITALY & ITALY & ITALY & & & & TENTH STREET & CALUMET PR. 6 \\
\hline 1320 & 4232 & MELIA/MELIS & DOMINICK & s & 6 & $\mathrm{~s}$ & MICHIGAN & ITALY & ITALY & & & & TENTH STREET & CALUMET PR. 6 \\
\hline
\end{tabular}




\begin{tabular}{|c|c|c|c|c|c|c|c|c|c|c|c|c|c|c|}
\hline \multirow{2}{*}{$\begin{array}{l}1321 \\
1322\end{array}$} & \multirow{2}{*}{\begin{tabular}{|l|}
4232 \\
4232
\end{tabular}} & \multirow{2}{*}{$\begin{array}{l}\text { MELIA/MELIS } \\
\text { MELIA/MELIS }\end{array}$} & \multirow{2}{*}{$\begin{array}{l}\text { MARY } \\
\text { VINCENT }\end{array}$} & \multirow{2}{*}{$\begin{array}{l}\mathrm{D} \\
\mathrm{S}\end{array}$} & \multirow{2}{*}{$\begin{array}{l}3 \\
2 \mathrm{M}\end{array}$} & \multirow{2}{*}{$\begin{array}{l}\text { S } \\
\text { S }\end{array}$} & \multirow{2}{*}{$\begin{array}{l}\text { MICHIGAN } \\
\text { MICHIGAN }\end{array}$} & \multirow{2}{*}{$\begin{array}{l}\text { ITALY } \\
\text { ITALY }\end{array}$} & \multirow{2}{*}{$\begin{array}{l}\text { ITALY } \\
\text { ITALY }\end{array}$} & & & & TENTH STREET & CALUMET PR. 6 \\
\hline & & & & & & & & & & 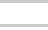 & & & TENTH STREET & CALUMET PR. 6 \\
\hline 1323 & 4234 & & & $\mathrm{H}$ & 36 & M11 & & & & & MASON & SAN GIORGIO CANAVESE. TO & TENTH STREET & CALUMET PR. 6 \\
\hline 1324 & 4234 & CHIMINO & MAGGIE & W & 36 & M11 & ITALY & ITALY & ITALY & & & SAN GIORGIO CANAVESE. TO & TENTH STREET & CALUMET PR. 6 \\
\hline 1325 & 4234 & CHIMINO & JOE & s & 9 & $\mathrm{~s}$ & MICHIGAN & ITALY & ITALY & & & SAN GIORGIO CANAVESE. TO & TENTH STREET & CALUMET PR. 6 \\
\hline 1326 & 4234 & CHIMINO & MARY & $\mathrm{D}$ & 8 & s & MICHIGAN & ITALY & ITALY & & & SAN GIORGIO CANAVESE. TO & TENTH STREET & CALUMET PR. 6 \\
\hline 1327 & 4234 & CHIMINO & VICTORIA & $\mathrm{D}$ & 6 & $\mathrm{~S}$ & MICHIGAN & ITALY & ITALY & & & SAN GIORGIO CANAVESE. TO & TENTH STREET & CALUMET PR. 6 \\
\hline 1328 & 4234 & CHIMINO & PETER & $\mathrm{s}$ & 5 & s & MICHIGAN & ITALY & ITALY & & & SAN GIORGIO CANAVESE. TO & TENTH STREET & CALUMET PR. 6 \\
\hline 1329 & $4234 \mid$ & CHIMINO & KATIE & $\mathrm{D}$ & 3 & s & MICHIGAN & ITALY & ITALY & & & SAN GIORGIO CANAVESE. TO & TENTH STREET & CALUMET PR. 6 \\
\hline 1330 & 4234 & BERNARDO & JOE & B & 31 & $\mathrm{~s}$ & ITALY & ITALY & ITALY & AL & MINER/CM & TORINO & TENTH STREET & CALUMET PR. 6 \\
\hline 1331 & 4234 & VENUTA & JOE & B & 43 & M20 & ITALY & ITALY & ITALY & $\mathrm{PA}$ & MINER/CM & SAN GIORGIO CANAVESE. TO & TENTH STREET & CALUMET PR. 6 \\
\hline 1332 & 4234 & ROMANO & JOE & B & 24 & $\mathrm{~s}$ & ITALY & ITALY & ITALY & AL & MINER/CM & TORINO & TENTH STREET & CALUMET PR. 6 \\
\hline 1333 & 4234 & VINCENTI & ANTONIO & B & 29 & s & ITALY & ITALY & ITALY & AL & MINER/CM & CASTELLAMONTE. TO & TENTH STREET & CALUMET PR. 6 \\
\hline 1334 & 4234 & BANNA/BARINA & DOMINICK & B & 22 & $\mathrm{~s}$ & ITALY & ITALY & ITALY & $\mathrm{AL}$ & MINER/CM & & TENTH STREET & CALUMET PR. 6 \\
\hline 1335 & 4234 & MILLEU & & B & 41 & s & ITALY & ITALY & ITALY & NA & DAY LABORER/CM & & TENTH STREET & CALUMET PR. 6 \\
\hline 1336 & 4234 & GORDINO & FERDINAND & B & 25 & $\mathrm{~S}$ & ITALY & ITALY & ITALY & AL & MINER/CM & TORINO & TENTH STREET & CALUMET PR. 6 \\
\hline 1337 & 4234 & ROMANO & JAMES & B & 30 & $\mathrm{~S}$ & ITALY & ITALY & ITALY & $\mathrm{AL}$ & MINER/CM & AGLIE'. TO & TENTH STREET & CALUMET PR. 6 \\
\hline 1338 & 4234 & TONATTO & GIOVANNI & B & 36 & M3 & ITALY & ITALY & ITALY & $\mathrm{AL}$ & MINER/CM & ROMANO CANAVESE. TO & TENTH STREET & CALUMET PR. 6 \\
\hline 1339 & 4234 & TEPATTI & ANTON & $\mathrm{H}$ & 32 & M1 & ITALY & ITALY & ITALY & PA & MINER/CM & PONT CANAVESE. TO & TENTH STREET & CALUMET PR. 6 \\
\hline 1340 & 4234 & TEPATTI & CELESTINA & w & 22 & M1 & ITALY & ITALY & ITALY & $r M$ & 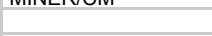 & TUVT UAIVAVLUL. TS & TENTH STREET & CALUMET PR. 6 \\
\hline 1341 & 4234 & TEPATTI & JOHN & s & 10 & $\mathrm{~s}$ & MICHIGAN & ITALY & ITALY & & & PONT CANAVESE. TO & TENTH STREET & CALUMET PR. 6 \\
\hline 1342 & $\mid 4232$ & BASSO & JOE & $\mathrm{H}$ & 25 & M1 & ITALY & ITALY & ITALY & $\mathrm{AL}$ & TRAMMER/CM & SAN GIORGIO CANAVESE, TO & TENTH STREET & CALUMET PR. 6 \\
\hline 1343 & 4232 & BASSO & JACINTA & W & 26 & M1 & ITALY & ITALY & ITALY & & & SAN GIORGIO CANAVESE. TO & TENTH STREET & CALUMET PR. 6 \\
\hline 1344 & 4232 & BASSO & JOE & S & $3 \mathrm{M}$ & $\mathrm{s}$ & MICHIGAN & ITALY & ITALY & & & SAN GIORGIO CANAVESE. TO & TENTH STREET & CALUMET PR. 6 \\
\hline 1345 & 4232 & DE FILIPPI & LOUIS & $\mathrm{H}$ & 36 & M9 & ITALY & ITALY & ITALY & NA & MINER/CM & SAN GIORGIO CANAVESE. TO & TENTH STREET & CALUMET PR. 6 \\
\hline 1346 & 4232 & DE FILIPPI & KATIE & W & 3 & M9 & ITALY & ITALY & ITALY & & & SAN GIORGIO CANAVESE. TO & TENTH STREET & CALUMET PR. 6 \\
\hline 1347 & 4232 & DE FILIPPI & DOMINICA & D & 8 & $\mathrm{~s}$ & MICHIGAN & ITALY & ITALY & & & SAN GIORGIO CANAVESE. TO & TENTH STREET & CALUMET PR. 6 \\
\hline 1348 & 4232 & DE FILIPPI & MARY & D & 6 & $\mathrm{~s}$ & MICHIGAN & ITALY & ITALY & & & SAN GIORGIO CANAVESE, TO & TENTH STREET & CALUMET PR. 6 \\
\hline 1349 & 4232 & RENALDI & JOE & $\mathrm{H}$ & 35 & MO & ITALY & ITALY & ITALY & NA & DAY LABORER/CM & SAN GIORGIO CANAVESE. TO & TENTH STREET & CALUMET PR. 6 \\
\hline 1350 & 4232 & RENALDI & THERESA & W & 35 & MO & ITALY & ITALY & ITALY & & & & TENTH STREET & CALUMET PR. 6 \\
\hline 1351 & 4232 & RENALDI & $\mathrm{JOE}$ & B & 21 & $\mathrm{~s}$ & ITALY & ITALY & ITALY & & MINER/CM & SAN GIORGIO CANAVESE. TO & TENTH STREET & CALUMET PR. 6 \\
\hline 1352 & 4246 & PEILA & MIKE & $\mathrm{H}$ & 36 & M6 & ITALY & ITALY & ITALY & PA & DAY LABORER/CM & PONT CANAVESE. TO & TENTH STREET & CALUMET PR. 6 \\
\hline 1353 & 4246 & PEILA & LUCIA & W & 28 & M6 & ITALY & ITALY & ITALY & & & PONT CANAVESE. TO & TENTH STREET & CALUMET PR. 6 \\
\hline 1354 & 4246 & PEILA & JOHN & s & 2 & s & ITALY & ITALY & ITALY & & & PONT CANAVESE, TO & TENTH STREET & CALUMET PR. 6 \\
\hline 1355 & 4246 & PEILA & JOHN & B & 32 & s & ITALY & ITALY & ITALY & $\mathrm{AL}$ & DAY LABORER/CM & PONT CANAVESE. TO & TENTH STREET & CALUMET PR. 6 \\
\hline 1356 & 4246 & COPPO & JAMES & B & 34 & M7 & ITALY & ITALY & ITALY & $\mathrm{AL}$ & DAY LABORER/CM & PONT CANAVESE. TO & TENTH STREET & CALUMET PR. 6 \\
\hline 1357 & 4253 & BOGGIO & JAMES & $\mathrm{H}$ & 34 & M6 & ITALY & ITALY & ITALY & PA & MINER/CM & TORINO & TENTH STREET & CALUMET PR. 6 \\
\hline 1358 & 4253 & BOGGIO & KATIE & W & 29 & M6 & ITALY & ITALY & ITALY & & & & TENTH STREET & CALUMET PR. 6 \\
\hline 1359 & 4253 & BOGGIO & JOE & $\mathrm{s}$ & 5 & s & MICHIGAN & ITALY & ITALY & & & TORINO & TENTH STREET & CALUMET PR. 6 \\
\hline 1360 & 4253 & BOGGIO & MARY & $\mathrm{D}$ & 1 & s & MICHIGAN & ITALY & ITALY & & & TORINO & TENTH STREET & CALUMET PR. 6 \\
\hline 1361 & 4222 & ANTONIETTI & JOHN & $\mathrm{H}$ & 38 & M12 & ITALY & ITALY & ITALY & NA & MASON & & TENTH STREET & CALUMET PR. 6 \\
\hline 1362 & 4222 & ANTONIETTI & MAGGIE & W & 38 & M12 & ITALY & ITALY & ITALY & & & & TENTH STREET & CALUMET PR. 6 \\
\hline 1363 & 4222 & ANTONIETTI & MARY & D & 10 & $\mathrm{~s}$ & ITALY & ITALY & ITALY & & AT SCHOOL 9 & & TENTH STREET & CALUMET PR. 6 \\
\hline 1364 & 4222 & ANTONIETTI & TILLIE & $\mathrm{D}$ & 9 & $\mathrm{~s}$ & ITALY & ITALY & ITALY & & & & TENTH STREET & CALUMET PR. 6 \\
\hline 1365 & 4222 & ANTONIETTI & SANTINA & $\mathrm{D}$ & 6 & $\mathrm{~s}$ & MICHIGAN & ITALY & ITALY & & & & TENTH STREET & CALUMET PR. 6 \\
\hline 1366 & 4222 & ANTONIETTI & NATALIE & D & 5 & $\mathrm{~S}$ & MICHIGAN & ITALY & ITALY & & & & TENTH STREET & CALUMET PR. 6 \\
\hline 1367 & 4222 & ANTONIETTI & JOE & s & 1 & s & MICHIGAN & ITALY & ITALY & & & & TENTH STREET & CALUMET PR. 6 \\
\hline 1368 & 4222 & REGIS & JOE & $\mathrm{H}$ & 35 & M7 & ITALY & ITALY & ITALY & NA & MINER/CM & SAN GIUSTO CANAVESE. TO & TENTH STREET & CALUMET PR. 6 \\
\hline 1369 & 4222 & REGIS & PAULINA & w & 29 & M7 & ITALY & ITALY & ITALY & & & & TENTH STREET & CALUMET PR. 6 \\
\hline 1370 & 4222 & REGIS & CLOTILDA & $\mathrm{D}$ & 6 & $\mathrm{~s}$ & MICHIGAN & ITALY & ITALY & & & SAN GIUSTO CANAVESE. TO & TENTH STREET & CALUMET PR. 6 \\
\hline 1371 & 4222 & REGIS & FELIX & $\mathrm{s}$ & 4 & $\mathrm{~s}$ & MICHIGAN & ITALY & ITALY & & & SAN GIUSTO CANAVESE. TO & TENTH STREET & CALUMET PR. 6 \\
\hline 1372 & 4222 & REGIS & NATALIE & D & 1 & $\mathrm{~s}$ & MICHIGAN & ITALY & ITALY & & & SAN GIUSTO CANAVESE, TO & TENTH STREET & CALUMET PR. 6 \\
\hline 1373 & 4222 & CARBONATTI/O & VITTORIO & B & 32 & s & ITALY & ITALY & ITALY & PA & BLACKSMITH & PONT CANAVESE. TO & TENTH STREET & CALUMET PR. 6 \\
\hline 1374 & 4246 & MATTI & STEVEN & $\mathrm{H}$ & 44 & M9 & ITALY & ITALY & ITALY & NA & MINER/CM & & TENTH STREET & CALUMET PR. 6 \\
\hline 1375 & 4246 & MATTI & ADVIGI & w & 36 & M9 & ITALY & ITALY & ITALY & & & & TENTH STREET & CALUMET PR. 6 \\
\hline 1376 & 4246 & MATTI & KATIE & D & 6 & $\mathrm{~s}$ & MICHIGAN & ITALY & ITALY & & & & TENTH STREET & CALUMET PR. 6 \\
\hline 1377 & 4246 & MATTI & MANDE & $\mathrm{D}$ & 5 & $\mathrm{~s}$ & MICHIGAN & ITALY & ITALY & & & & TENTH STREET & CALUMET PR. 6 \\
\hline 1378 & 4246 & MATTI & DOMINICK & $\mathrm{s}$ & 3 & s & MICHIGAN & ITALY & ITALY & & & & TENTH STREET & CALUMET PR. 6 \\
\hline 1379 & 4246 & MATTI & MARY & $\mathrm{D}$ & $9 \mathrm{M}$ & $\mathrm{s}$ & MICHIGAN & ITALY & ITALY & & & & TENTH STREET & CALUMET PR. 6 \\
\hline 1380 & 4246 & FEZZIA & MIKE & $\mathrm{H}$ & 34 & M? & ITALY & ITALY & ITALY & $\mathrm{AL}$ & MINER/CM & & TENTH STREET & CALUMET PR. 6 \\
\hline 1381 & 4246 & FEZZIA & PAULINE & w & 29 & MO? & ITALY & ITALY & ITALY & & & & TENTH STREET & CALUMET P \\
\hline 1382 & 4246 & FEZZIA & BATISTE & S & 6 & s & MICHIGAN & ITALY & ITALY & & & & TENTH STREET & CALUMET PR. 6 \\
\hline 1383 & 4246 & FEZZIA & JOE & $\mathrm{s}$ & 4 & $\mathrm{~s}$ & MICHIGAN & ITALY & ITALY & & & & TENTH STREET & CALUMET PR. 6 \\
\hline
\end{tabular}




\begin{tabular}{|c|c|c|c|c|c|c|c|c|c|c|c|c|c|c|}
\hline \multirow{2}{*}{$\begin{array}{l}1384 \\
1385\end{array}$} & \multirow{2}{*}{\begin{tabular}{|l|}
4246 \\
4246
\end{tabular}} & \multirow{2}{*}{$\begin{array}{l}\text { FEZZIA } \\
\text { BALICH }\end{array}$} & \multirow{2}{*}{$\begin{array}{l}\text { PETER } \\
\text { FRANK }\end{array}$} & \multirow{2}{*}{$\begin{array}{l}\text { S } \\
\text { B }\end{array}$} & \multirow{2}{*}{$\begin{array}{l}0 \\
30\end{array}$} & \multirow{2}{*}{\multicolumn{2}{|c|}{\begin{tabular}{l|l} 
S & MICHIGAN \\
$S$ & ITALY
\end{tabular}}} & \multirow{2}{*}{$\begin{array}{l}\text { ITALY } \\
\text { ITALY }\end{array}$} & \multirow{2}{*}{$\begin{array}{l}\text { ITALY } \\
\text { ITALY }\end{array}$} & \multirow[b]{2}{*}{$\mathrm{AL}$} & 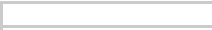 & & TENTH STREET & CALUMET PR. 6 \\
\hline & & & & & & & & & & & MINER/CM & & TENTH STREET & CALUMET PR. 6 \\
\hline 1386 & 4246 & GOIJ & JOE & B & 20 & $\mathrm{~s}$ & ITALY & & & AL & MINER/CM & & TENTH STREET & CALUMET PR. 6 \\
\hline 1387 & 4306 & RIVARA & JAMES & $\mathrm{H}$ & 40 & M11 & ITALY & ITALY & ITALY & NA & DAY LABORER/CM & TORINO & ELEVENTH ST. & CALUMET PR. 6 \\
\hline 1388 & 4306 & RIVARA & MARY & W & 35 & M11 & ITALY & ITALY & ITALY & & & & ELEVENTH ST. & CALUMET PR. 6 \\
\hline 1389 & 4306 & RIVARA & ROSE & $\mathrm{D}$ & 9 & $\mathrm{~s}$ & MICHIGAN & ITALY & ITALY & & & TORINO & ELEVENTH ST. & CALUMET PR. 6 \\
\hline 1390 & 4306 & RIVARA & JAMES & $\mathrm{s}$ & 1 & s & MICHIGAN & ITALY & ITALY & & & TORINO & ELEVENTH ST. & CALUMET PR. 6 \\
\hline 1391 & 4306 & COGNANATTO & DOMINICK & B & 20 & s & ITALY & ITALY & ITALY & & MINER/CM & SAN PONSO CANAVESE. TO & ELEVENTH ST. & CALUMET PR. 6 \\
\hline 1392 & 4306 & CALUSIO & JOHN & $\mathrm{H}$ & 38 & M13 & ITALY & ITALY & ITALY & NA & MINER/CM & SAN GIORGIO CANAVESE. TO & ELEVENTH ST. & CALUMET PR. 6 \\
\hline 1393 & 4306 & CALUSIO & ANNIE & w & 34 & M13 & ITALY & ITALY & ITALY & & & SAN GIORGIO CANAVESE. TO & ELEVENTH ST. & CALUMET PR. 6 \\
\hline 1394 & 4306 & CALUSIO & PETER & $\mathrm{s}$ & 12 & $\mathrm{~s}$ & ITALY & ITALY & ITALY & & AT SCHOOL 9 & SAN GIORGIO CANAVESE. TO & ELEVENTH ST. & CALUMET PR. 6 \\
\hline 1395 & 4306 & CALUSIO & AGNES & $\mathrm{D}$ & 8 & $\mathrm{~s}$ & MICHIGAN & ITALY & ITALY & & & SAN GIORGIO CANAVESE. TO & ELEVENTH ST. & CALUMET PR. 6 \\
\hline 1396 & 4306 & CALUSIO & MARY & $\mathrm{D}$ & 5 & s & MICHIGAN & ITALY & ITALY & & & SAN GIORGIO CANAVESE. TO & ELEVENTH ST. & CALUMET PR. 6 \\
\hline 1397 & 4306 & CALUSIO & ANGELICA & D & 1 & $\mathrm{~s}$ & MICHIGAN & ITALY & ITALY & & & SAN GIORGIO CANAVESE. TO & ELEVENTH ST. & CALUMET PR. 6 \\
\hline 1398 & 4306 & BERCHIATTI & ANGELICA & AC & 6 & $\mathrm{~s}$ & ILLINOIS & ITALY & ITALY & & & PONT CANAVESE. TO & ELEVENTH ST. & CALUMET PR. 6 \\
\hline 1399 & 4879 & GENOVIO & MARTIN & $\mathrm{H}$ & 36 & M15 & ITALY & ITALY & ITALY & NA & MINER/CM & & R. J. SHAFT & CALUMET PR. 6 \\
\hline 1400 & 4879 & GENOVIO & KATIE & W & 34 & M15 & ITALY & ITALY & ITALY & & & & R. J. SHAFT & CALUMET PR. 6 \\
\hline 1401 & 4879 & GENOVIO & MARTHA & $\mathrm{D}$ & 14 & $\mathrm{~s}$ & ITALY & ITALY & ITALY & & AT SCHOOL 9 & & R. J. SHAFT & CALUMET PR. 6 \\
\hline 1402 & 4879 & GENOVIO & JOHN & $\mathrm{s}$ & 11 & s & MICHIGAN & ITALY & ITALY & & & & R. J. SHAFT & CALUMET PR. 6 \\
\hline 1403 & 4879 & GENOVIO & LUCY & $\mathrm{D}$ & 8 & s & MICHIGAN & ITALY & ITALY & & & & R. J. SHAFT & CALUMET PR. 6 \\
\hline 1404 & 4879 & GENOVIO & PIERINA & $\mathrm{D}$ & 5 & s & MICHIGAN & ITALY & ITALY & & & & R. J. SHAFT & CALUMET PR. 6 \\
\hline 1405 & 4879 & GENOVIO & KATIE & $\mathrm{D}$ & 1 & s & MICHIGAN & ITALY & ITALY & & & & R. J. SHAFT & CALUMET PR. 6 \\
\hline 1406 & 4879 & GROSSO & WILLIAM & B & 34 & $\mathrm{~s}$ & ITALY & ITALY & ITALY & NA & MINER/CM & SAN MARTINO CANAVESE. TO & R. J. SHAFT & CALUMET PR. 6 \\
\hline 1407 & 4879 & DENOVIO & JOHN & B & 23 & s & ITALY & ITALY & ITALY & $\mathrm{PA}$ & MINER/CM & & R. J. SHAFT & CALUMET PR. 6 \\
\hline 1408 & 4879 & BRACCO & DOMENICO & B & 42 & M21 & ITALY & ITALY & ITALY & NA & MINER/CM & TORINO & R. J. SHAFT & CALUMET PR. 6 \\
\hline 1409 & 4879 & BRACCO & LUIGI & B & 18 & $\mathrm{~s}$ & ITALY & ITALY & ITALY & & MINER/CM & TORINO & R. J. SHAFT & CALUMET PR. 6 \\
\hline 1410 & 4879 & POSTEI & JOHN & B & 39 & $\mathrm{~s}$ & ITALY & ITALY & ITALY & NA & DAY LABORER/CM & & R. J. SHAFT & CALUMET PR. 6 \\
\hline 1411 & 4879 & CANTELLO & JOHN & B & 29 & $\mathrm{~s}$ & ITALY & ITALY & ITALY & NA & MINER/CM & SAN GIORGIO CANAVESE, TO & R. J. SHAFT & CALUMET PR. 6 \\
\hline 1412 & 4879 & GROSSO & MARTIN & B & 29 & $\mathrm{~s}$ & ITALY & ITALY & ITALY & $\mathrm{AL}$ & MINER/CM & SAN MARTINO CANAVESE. TO & R. J. SHAFT & CALUMET PR. 6 \\
\hline 1413 & 4879 & POSTEI & JOHN & B & 25 & $\mathrm{~s}$ & ITALY & ITALY & ITALY & $\mathrm{AL}$ & MINER/CM & & R. J. SHAFT & CALUMET PR. 6 \\
\hline 1414 & 4879 & DIGHERA & JAMES & B & 30 & $\mathrm{~s}$ & ITALY & ITALY & ITALY & PA & MINER/CM & SAN GIORGIO CANAVESE. TO & R. J. SHAFT & CALUMET PR. 6 \\
\hline 1415 & & BAUSANO & ANTONIO & B & 20 & $\mathrm{~s}$ & ITALY & ITALY & ITALY & $\mathrm{AL}$ & TIMBERMAN/CM & PONT CANAVESE. TO & & CALUMET PR. 7 \\
\hline 1416 & & PICCHIOTTINO & PIETRO & $\mathrm{H}$ & 30 & M7 & ITALY & ITALY & ITALY & $\mathrm{AL}$ & MINER/CM & PONT CANAVESE. TO & & CALUMET PR. 7 \\
\hline 1417 & & PICCHIOTTINO & CATERINA & w & 27 & M7 & ITALY & ITALY & ITALY & & & & & CALUMET PR. 7 \\
\hline 1418 & & PICCHIOTTINO & CATERINA & $\mathrm{D}$ & & & MICHIGAN & ITALY & ITALY & & & PONT CANAVESE. TO & & CALUMET PR. 7 \\
\hline $\begin{array}{l}1410 \\
1419\end{array}$ & & PICCHIOTTINO & JOHN & s & 1 & s & MICHIGAN & ITALY & ITALY & & & PONT CANAVESE. TO & & CALUMET PR. 7 \\
\hline 1420 & & TRUFFA & GIOVANNI & B & 23 & s & ITALY & ITALY & ITALY & $\mathrm{AL}$ & TRAMMER/CM & PONT CANAVESE. TO & & CALUMET PR. 7 \\
\hline 1421 & & BAGONA & JOHN & B & 24 & $\mathrm{~S}$ & ITALY & ITALY & ITALY & $\mathrm{AL}$ & TRAMMER/CM & & & CALUMET PR. 7 \\
\hline 1422 & & VALERIO & JOSEPH & B & 28 & s & ITALY & ITALY & ITALY & $\mathrm{AL}$ & DAY LABORER/CM & TORINO & & CALUMET PR. 7 \\
\hline 1423 & & VETONIO & JOHN & B & 20 & s & ITALY & ITALY & ITALY & $\mathrm{AL}$ & DAY LABORER/CM & & & CALUMET PR. 7 \\
\hline 1424 & & VETISTO & TONY & B & 28 & s & ITALY & ITALY & ITALY & $\mathrm{AL}$ & TRAMMER/CM & & & CALUMET PR. 7 \\
\hline 1425 & & NAGARIO & ANTON & B & 33 & s & ITALY & ITALY & ITALY & $\mathrm{AL}$ & TRAMMER/CM & & & CALUMET PR. 7 \\
\hline 1426 & & LAVORIO & ANTON & B & 36 & $\mathrm{~S}$ & ITALY & ITALY & ITALY & $\mathrm{AL}$ & TRAMMER/CM & & & CALUMET PR. 7 \\
\hline 1427 & & VETUNG & JAMES & B & 40 & $\mathrm{~S}$ & ITALY & ITALY & ITALY & $\mathrm{AL}$ & DAY LABORER/CM & & & CALUMET PR. 7 \\
\hline 1428 & & BAUSANO & PETER & B & 21 & $\mathrm{~S}$ & ITALY & ITALY & ITALY & $\mathrm{AL}$ & TRAMMER/CM & TORINO & & CALUMET PR. 7 \\
\hline 1429 & & BAUSANO & BATISTE & B & 38 & s & ITALY & ITALY & ITALY & $\mathrm{AL}$ & DAY LABORER/CM & TORINO & & CALUMET PR. 7 \\
\hline 1430 & & BALAGNA & PETER & $\mathrm{H}$ & 48 & M22 & ITALY & ITALY & ITALY & PA & OVERSEER/CM & TORINO & & CALUMET PR. 7 \\
\hline 1431 & & BALAGNA & $\begin{array}{l}\text { LUCY } \\
\text { LUER }\end{array}$ & w & 42 & M22 & $\begin{array}{l}\text { ITALY } \\
\text { ITAY }\end{array}$ & ITALY & ITALY & $P A$ & OVERSEER/UIUI & TORINO & & CALUMET PR. 7 \\
\hline 1432 & & COPPO & JOSEPH & B & 48 & $\mathrm{~s}$ & ITALY & ITALY & ITALY & PA & DAY LABORER/CM & PONT CANAVESE. TO & & CALUMET PR. 7 \\
\hline 1433 & & EMBASANO & ANTON & B & 48 & $\mathrm{~s}$ & ITALY & ITALY & ITALY & $\mathrm{AL}$ & LABORER/CM & & & CALUMET PR. 7 \\
\hline 1434 & & JOKEY? & TONY & B & 45 & $\mathrm{~s}$ & ITALY & ITALY & ITALY & $\mathrm{AL}$ & LABORER/CM & & & CALUMET PR. 7 \\
\hline 1435 & & GIACHINO & JOHN & B & 20 & $\mathrm{~S}$ & ITALY & ITALY & ITALY & $\mathrm{AL}$ & LABORER/CM & TORINO & & CALUMET PR. 7 \\
\hline 1436 & & BALAGNA & JOHN & B & 40 & $\mathrm{~s}$ & ITALY & ITALY & ITALY & $\mathrm{AL}$ & LABORER/CM & TORINO & & CALUMET PR. 7 \\
\hline 1437 & & BALAGNA & MIKE & B & 24 & s & ITALY & ITALY & ITALY & $\mathrm{AL}$ & LABORER/CM & TORINO & & CALUMET PR. 7 \\
\hline 1438 & & BALAGNA & CELESTE & B & 26 & s & ITALY & ITALY & ITALY & $\mathrm{AL}$ & LABORER/CM & PONT CANAVESE. TO & & CALUMET PR. 7 \\
\hline 1439 & & PICCHIOTINO & BATISTE & B & 25 & $\mathrm{~s}$ & ITALY & ITALY & ITALY & $\mathrm{AL}$ & LABORER/CM & PONT CANAVESE. TO & & CALUMET PR. 7 \\
\hline 1440 & & COPPO & PETER & B & 45 & s & ITALY & ITALY & ITALY & $\mathrm{AL}$ & LABORER/CM & PONT CANAVESE. TO & & CALUMET PR. 7 \\
\hline 1441 & & ROLANDO & FRANK & B & 40 & s & ITALY & ITALY & ITALY & $\mathrm{AL}$ & LABORER/CM & TORINO & & CALUMET PR. 7 \\
\hline 1442 & & CARLENO & FRANK & B & 18 & $\mathrm{~s}$ & ITALY & ITALY & ITALY & $\mathrm{AL}$ & LABORER/CM & & & CALUMET PR. 7 \\
\hline 1443 & & GIACHINO & MARTIN & B & 28 & s & ITALY & ITALY & ITALY & $\mathrm{AL}$ & LABORER/CM & TORINO & & CALUMET PR. 7 \\
\hline 1444 & & BAUSANO & DOMINICK & B & 40 & s & ITALY & ITALY & ITALY & $\mathrm{AL}$ & LABORER/CM & TORINO & & CALUMET PR. 7 \\
\hline 1445 & & BOSVASO & PETER & B & 44 & s & ITALY & ITALY & ITALY & AL & LABORER/CM & & & CALUMET PR. 7 \\
\hline 1446 & & $\mathrm{RICCl}$ & ANDREW & $\mathrm{H}$ & 35 & M3 & ITALY & ITALY & ITALY & AL & MINER/CM & LUCCA & & CALUMET PR. 7 \\
\hline
\end{tabular}




\begin{tabular}{|c|c|c|c|c|c|c|c|c|c|c|c|c|}
\hline 1447 & $\mathrm{RICCl}$ & JOSEPHINA & W & 32 & M3 & ITALY & ITALY & ITALY & & & & CALUMET PR. 7 \\
\hline 1448 & $\mathrm{RICCl}$ & LOUIS & $\mathrm{s}$ & 6 & $\mathrm{~S}$ & MICHIGAN & ITALY & ITALY & & & LUCCA & CALUMET PR. 7 \\
\hline 1449 & DREASE/DUASE & MARY & B & 50 & $\mathrm{~s}$ & ITALY & ITALY & ITALY & & & & CALUMET PR. 7 \\
\hline 1450 & GIACOLETTO & ANTON & $\mathrm{H}$ & 31 & M9 & ITALY & ITALY & ITALY & AL & MINER/CM & PONT CANAVESE. TO & CALUMET PR. 7 \\
\hline 1451 & GIACOLETTO & LOUISA & W & 26 & M9 & ITALY & ITALY & ITALY & $\mathrm{AL}$ & & & CALUMET PR. 7 \\
\hline 1452 & GIACOLETTTO & JOSEPH & s & 7 & $\mathrm{~s}$ & MICHIGAN & ITALY & ITALY & & AT SCHOOL 10 & PONT CANAVESE. TO & CALUMET PR. 7 \\
\hline 1453 & GIACOLETTO & PETER & $\mathrm{s}$ & 3 & $\mathrm{~s}$ & MICHIGAN & ITALY & ITALY & & & PONT CANAVESE. TO & CALUMET PR. 7 \\
\hline 1454 & GIACOLETTTO & $\mathrm{JOHN}$ & $\mathrm{s}$ & 1 & $\mathrm{~S}$ & MICHIGAN & ITALY & ITALY & & & PONT CANAVESE. TO & CALUMET PR. 7 \\
\hline 1455 & SOLOTTI & LOUIS & B & 42 & $\mathrm{~s}$ & ITALY & ITALY & ITALY & $\mathrm{AL}$ & TRAMMER/CM & TORINO & CALUMET PR. 7 \\
\hline 1456 & GIACOLETTO & JOHN & B & 31 & $\mathrm{~s}$ & ITALY & ITALY & ITALY & $\mathrm{AL}$ & TRAMMER/CM & PONT CANAVESE. TO & CALUMET PR. 7 \\
\hline 1457 & PICCHIOTINO & DOMENICO & B & 22 & $\mathrm{~s}$ & ITALY & ITALY & ITALY & $\mathrm{AL}$ & TRAMMER/CM & PONT CANAVESE. TO & CALUMET PR. 7 \\
\hline 1458 & SANDRETTO & JOHN & B & 23 & $\mathrm{~s}$ & ITALY & ITALY & ITALY & $\mathrm{AL}$ & TRAMMER/CM & PONT CANAVESE. TO & CALUMET PR. 7 \\
\hline 1459 & BERNARDO & JAMES & B & 30 & $\mathrm{~s}$ & ITALY & ITALY & ITALY & $\mathrm{AL}$ & TRAMMER/CM & PONT CANAVESE. TO & CALUMET PR. 7 \\
\hline 1460 & RUSHIA? & JAMES & B & 27 & $\mathrm{~s}$ & ITALY & ITALY & ITALY & $\mathrm{AL}$ & TRAMMER/CM & & CALUMET PR. 7 \\
\hline 1461 & RUSIA & TONY & B & 42 & s & ITALY & ITALY & ITALY & $\mathrm{AL}$ & TRAMMER/CM & & CALUMET PR. 7 \\
\hline 1462 & BAUSANO & LOUIS & $\mathrm{H}$ & 28 & M2 & ITALY & ITALY & ITALY & NA & MINER/CM & TORINO & CALUMET PR. 7 \\
\hline 1463 & BAUSANO & ROSA & W & 26 & M2 & ITALY & ITALY & ITALY & & & & CALUMET PR. 7 \\
\hline 1464 & BAUSANO & PETER & $\mathrm{s}$ & 1 & $\mathrm{~s}$ & MICHIGAN & ITALY & ITALY & & & TORINO & CALUMET PR. 7 \\
\hline 1465 & CURTO & MIKE & $\mathrm{H}$ & 28 & M3 & ITALY & ITALY & ITALY & $\mathrm{AL}$ & MINER/CM & TORINO & CALUMET PR. 7 \\
\hline 1466 & CURTO & ANNIE & w & 19 & M3 & ITALY & ITALY & ITALY & $\mathrm{AL}$ & & & CALUMET PR. 7 \\
\hline 1467 & CHARLEY & MARTIN & B & 22 & $\mathrm{~s}$ & ITALY & ITALY & ITALY & $\mathrm{AL}$ & MINER/CM & & CALUMET PR. 7 \\
\hline 1468 & PICCHIOTTINO & JOSEPH & B & 24 & $\mathrm{~s}$ & ITALY & ITALY & ITALY & $A L$ & TRAMMER/CM & PONT CANAVESE, TO & CALUMET PR. 7 \\
\hline 1469 & MARSEMISE & MARTIN & B & 25 & s & ITALY & ITALY & ITALY & $\mathrm{AL}$ & TRAMMER/CM & & CALUMET PR. 7 \\
\hline 1470 & MARTINO & LEWIS & B & 33 & M10 & ITALY & ITALY & ITALY & $\mathrm{AL}$ & TRAMMER/CM & TORINO & CALUMET PR. 7 \\
\hline 1471 & TAPPERO & TONY & B & 30 & $\mathrm{~s}$ & ITALY & ITALY & ITALY & $\mathrm{AL}$ & TRAMMER/CM & TORINO & CALUMET PR. 7 \\
\hline 1472 & MARESH? & ANCHETO? & B & 24 & $\mathrm{~s}$ & ITALY & ITALY & ITALY & $\mathrm{AL}$ & TRAMMER/CM & & CALUMET PR. 7 \\
\hline 1473 & CESTUAN? & BATISTE & B & 24 & $\mathrm{~s}$ & ITALY & $\begin{array}{l}\text { ITALY } \\
\end{array}$ & $\begin{array}{l}\text { ITALY } \\
\end{array}$ & $A L$ & TRAMMER/CM & & CALUMET PR. 7 \\
\hline 1474 & ALBERTI & JAMES & $\mathrm{H}$ & 39 & M13 & ITALY & ITALY & ITALY & $\mathrm{AL}$ & MINER/CM & TORINO & CALUMET PR. 7 \\
\hline 1475 & ALBERTI & LINDA & W & 34 & M13 & ITALY & ITALY & ITALY & $\mathrm{AL}$ & & TORINO & CALUMET PR. 7 \\
\hline 1476 & ALBERTI & MARY & D & 12 & $\mathrm{~s}$ & ITALY & ITALY & ITALY & $\mathrm{AL}$ & AT SCHOOL 10 & TORINO & CALUMET PR. 7 \\
\hline 1477 & ALBERTI & JOSEPHINE & D & 8 & S & ITALY & ITALY & ITALY & $\mathrm{AL}$ & AT SCHOOL 10 & TORINO & CALUMET PR. 7 \\
\hline 1478 & ALBERTI & JOSEPH & $\mathrm{s}$ & 6 & $\mathrm{~s}$ & ITALY & ITALY & ITALY & $\mathrm{AL}$ & AT SCHOOL 10 & TORINO & CALUMET PR. 7 \\
\hline 1479 & ALBERTI & DOMINICK & $\mathrm{s}$ & 4 & $\mathrm{~s}$ & ITALY & ITALY & ITALY & $\mathrm{AL}$ & & & CALUMET PR. 7 \\
\hline 1480 & ALBERTI & ROSA & $\mathrm{D}$ & 2 & $\mathrm{~s}$ & ITALY & ITALY & ITALY & $\mathrm{AL}$ & & & CALUMET PR. 7 \\
\hline 1481 & ALBERTI & JAMES & $\mathrm{s}$ & 1 & $\mathrm{~s}$ & ITALY & ITALY & ITALY & $A L$ & & & CALUMET PR 7 \\
\hline 1482 & COSTA & PIETRO & B & 23 & s & ITALY & ITALY & $\begin{array}{l}\text { ITALY } \\
\end{array}$ & $A L$ & NOT CLEAR & SPARONE. TO & CALUMET PR. 7 \\
\hline 1483 & DUMOND & CHARLES & $\mathrm{H}$ & 51 & M15 & CANADA & CANADA & CANADA & $?$ & LABORER/CM & CANADA & CALUMET PR. 7 \\
\hline 1484 & DUMOND & JANETTE & w & 52 & M15 & ITALY & ITALY & ITALY & & & & CALUMET PR. 7 \\
\hline 1485 & $\begin{array}{l}\text { TINETTI } \\
\text { TIN }\end{array}$ & TONY & $\mathrm{H}$ & 28 & M1? & ITALY & ITALY & ITALY & AL & LABORER/CM & SAN MARTINO CANAVESE. TO & CALUMET PR. 7 \\
\hline 1486 & TINETTI & TRACY & W & 28 & M1? & ITALY & ITALY & ITALY & $\mathrm{AL}$ & 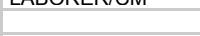 & SAIV IIATI IIIV CAIVAVLOL. TO & CALUMET PR. 7 \\
\hline 1487 & TINETTI & PETER B. & s & 7 & $\mathrm{~s}$ & MICHIGAN & ITALY & ITALY & & AT SCHOOL 10 & SAN MARTINO CANAVESE. TO & CALUMET PR. 7 \\
\hline 1488 & TINETTI & MIKE & $\mathrm{s}$ & 5 & $\mathrm{~s}$ & MICHIGAN & ITALY & ITALY & & & SAN MARTINO CANAVESE. TO & CALUMET PR. 7 \\
\hline 1489 & TINETTI & BATISTE & $\mathrm{s}$ & 2 & $\mathrm{~s}$ & MICHIGAN & ITALY & ITALY & & & SAN MARTINO CANAVESE. TO & CALUMET PR. 7 \\
\hline 1490 & TINETTI & EDA & $\mathrm{D}$ & $2 \mathrm{M}$ & $\mathrm{s}$ & MICHIGAN & ITALY & ITALY & & & SAN MARTINO CANAVESE. TO & CALUMET PR. 7 \\
\hline 1491 & BAUSANO & DOMINICK & B & 22 & $\mathrm{~S}$ & MICHIGAN & ITALY & ITALY & & LABORER/CM & TORINO & CALUMET PR. 7 \\
\hline 1492 & COMETTO & PETER & B & 23 & $\mathrm{~s}$ & MICHIGAN & ITALY & ITALY & & LABORER/CM & TORINO & CALUMET PR. 7 \\
\hline 1493 & BARNATO & DOMINICK & B & 35 & $\mathrm{~s}$ & ITALY & ITALY & ITALY & $\mathrm{AL}$ & MINER/CM & PORTO MAURIZIO. IMPERIA & CALUMET PR. 7 \\
\hline 1494 & BAUSANO & MAGGIE & B & 18 & s & ITALY & ITALY & $\begin{array}{l}\text { ITALY } \\
\text { ITALY }\end{array}$ & $\mathrm{AL}$ & SERVANT & TORINO & CALUMET PR. 7 \\
\hline 1495 & ROLANDO & PAUL & $\mathrm{H}$ & 54 & M21 & ITALY & ITALY & ITALY & $\mathrm{AL}$ & DAY LABORER/CM & PONT CANAVESE. TO & CALUMET PR. 7 \\
\hline 1496 & ROLANDO & TRACEY & w & 61 & M21 & ITALY & ITALY & ITALY & & & PONT CANAVESE. TO & CALUMET PR. 7 \\
\hline 1497 & ROLANDO & ANTON & s & 15 & $\mathrm{~S}$ & ITALY & ITALY & ITALY & & DRILL BOY/CM & PONT CANAVESE. TO & CALUMET PR. 7 \\
\hline 1498 & ROLANDO & ROSA & $\mathrm{D}$ & 9 & $\mathrm{~s}$ & MICHIGAN & ITALY & ITALY & & AT SCHOOL 10 & PONT CANAVESE, TO & CALUMET PR. 7 \\
\hline 1499 & ROLANDO & TRACY & $\mathrm{D}$ & 7 & $\mathrm{~s}$ & MICHIGAN & ITALY & ITALY & & AT SCHOOL 10 & PONT CANAVESE. TO & CALUMET PR. 7 \\
\hline 1500 & ROLANDO & JOSEPHINE & $\mathrm{D}$ & 5 & $\mathrm{~s}$ & MICHIGAN & ITALY & ITALY & & & PONT CANAVESE. TO & CALUMET PR. 7 \\
\hline 1501 & DAL SANTO & JAMES & B & 33 & $\mathrm{~S}$ & ITALY & ITALY & ITALY & $\mathrm{AL}$ & TRAMMER/CM & VICENZA & CALUMET PR. 7 \\
\hline 1502 & SANDRETTO & JOHN & B & 22 & s & ITALY & ITALY & ITALY & & LABORER/CM & PONT CANAVESE. TO & CALUMET PR. 7 \\
\hline 1503 & RASTELLO & GUSTAV? & $\mathrm{H}$ & 48 & M24 & ITALY & ITALY & ITALY & NA & LABORER/CM & PONT CANAVESE. TO & CALUMET PR. 7 \\
\hline 1504 & RASTELLO & DALTRY? & w & 35 & M24 & ITALY & ITALY & ITALY & & & PONT CANAVESE, TO & CALUMET PR. 7 \\
\hline 1505 & RASTELLO & LENA & D & 12 & $\mathrm{~s}$ & ITALY & ITALY & ITALY & & AT SCHOOL 10 & PONT CANAVESE. TO & CALUMET PR. 7 \\
\hline 1506 & RASTELLO & ROSA & $\mathrm{D}$ & 10 & $\mathrm{~s}$ & MICHIGAN & ITALY & ITALY & & AT SCHOOL 10 & PONT CANAVESE. TO & CALUMET PR. 7 \\
\hline 1507 & RASTELLO & MARY & $\mathrm{D}$ & 5 & $\mathrm{~s}$ & MICHIGAN & ITALY & ITALY & & & PONT CANAVESE. TO & CALUMET PR. 7 \\
\hline 1508 & BALMA/BAIMA & DOMENICO & $\mathrm{H}$ & ? & 3 & ITALY & $\begin{array}{l}\text { IITALY } \\
\text { ITAL }\end{array}$ & $\begin{array}{l}\text { ITALY } \\
\text { ITALY }\end{array}$ & & & TORINO & CALUMET PR. 7 \\
\hline 1509 & BALMA/BAIMA & ?? & W & $?$ & & ITALY & ITALY & ITALY & & & TORINO & CALUMET PR. 7 \\
\hline
\end{tabular}




\begin{tabular}{|c|c|c|c|c|c|c|c|c|c|c|c|c|c|c|}
\hline 1510 & & BALMA/BAIMA & JOHN & $\mathrm{s}$ & 10 & & & ITALY & ITALY & & AT SCHOOL & TORINO & & CALUMET PR. 7 \\
\hline 1511 & $?$ & BALMA/BAIMA & JULIO & $\mathrm{s}$ & 8 & & & ITALY & ITALY & & AT SCHOOL & TORINO & & CALUMET PR. 7 \\
\hline 1512 & $?$ & BALMA/BAIMA & DOMENICO & $\mathrm{s}$ & 6 & & & ITALY & ITALY & & & TORINO & & CALUMET PR. 7 \\
\hline 1513 & $?$ & BALMA/BAIMA & ALFRED & $\mathrm{s}$ & 4 & & & ITALY & ITALY & & & TORINO & & CALUMET PR. 7 \\
\hline 1514 & $?$ & BALMA/BAIMA & RUMI & D & 1 & & & ITALY & ITALY & & & TORINO & & CALUMET PR. 7 \\
\hline 1515 & & ELLENA & ANGELO? & $\mathrm{H}$ & 27 & M3 & ITALY & ITALY & ITALY & PA & TRAMMER/CM & TORINO & & CALUMET PR. 7 \\
\hline 1516 & & ELLENA & PAULINA & W & 24 & M3 & ITALY & ITALY & ITALY & & & & & CALUMET PR. 7 \\
\hline 1517 & & ELLENA & MAGGIE & $\mathrm{D}$ & 3 & $\mathrm{~s}$ & MICHIGAN & ITALY & ITALY & & & TORINO & & CALUMET PR. 7 \\
\hline 1518 & & ELLENA & JOHN & $\mathrm{s}$ & 1 & s & MICHIGAN & ITALY & ITALY & & & TORINO & & CALUMET PR. 7 \\
\hline 1519 & & SUCCA & JOSEPH & B & 41 & M19 & ITALY & ITALY & ITALY & $\mathrm{AL}$ & LABORER/ROCK HOUSE & SAN GIUSTO CANAVESE. TO & & CALUMET PR. 7 \\
\hline 1520 & & DEL SANTO & FRANK & B & 28 & $\mathrm{~s}$ & ITALY & ITALY & ITALY & $\mathrm{AL}$ & TRAMMER/CM & VICENZA & & CALUMET PR. 7 \\
\hline 1521 & & BINANDO & CHARLES & B & 24 & $\mathrm{~s}$ & ITALY & ITALY & ITALY & $\mathrm{AL}$ & TRAMMER/CM & TORINO & & CALUMET PR. 7 \\
\hline 1522 & & LUCHISWAY? & JOHN & B & 25 & $\mathrm{~s}$ & ITALY & ITALY & ITALY & $\mathrm{AL}$ & TRAMMER/CM & & & CALUMET PR. 7 \\
\hline 1523 & & RICHETTA & JOSEPH & B & 27 & $\mathrm{~s}$ & ITALY & ITALY & ITALY & $\mathrm{AL}$ & TRAMMER/CM & SAN MARTINO CANAVESE. TO & & CALUMET PR. 7 \\
\hline 1524 & & OSSILANDO & FRED & B & 23 & $\mathrm{~s}$ & ITALY & ITALY & ITALY & AL & TRAMMER/CM & & & CALUMET PR. 7 \\
\hline 1525 & & MORETTO & JOHN & $\mathrm{H}$ & 26 & M0 & ITALY & ITALY & ITALY & AL & TRAMMER/CM & CORTEREGGIO, TO & & CALUMET PR. 7 \\
\hline 1526 & & MORETTO & $? ?$ & W & 18 & MO & ITALY & ITALY & ITALY & $\mathrm{AL}$ & & & & CALUMET PR. 7 \\
\hline 1527 & & BARTENO & FELIX & B & 22 & $\mathrm{~s}$ & ITALY & ITALY & ITALY & $\mathrm{AL}$ & LABORER/CM & & & CALUMET PR. 7 \\
\hline 1528 & & BARTEN & PETER & B & 30 & s & ITALY & ITALY & ITALY & $\mathrm{AL}$ & LABORER/CM & & & CALUMET PR. 7 \\
\hline 1529 & & GARDETTO & ANTON & B & 26 & $\mathrm{~S}$ & ITALY & ITALY & ITALY & $\mathrm{AL}$ & LABORER/CM & BOSCONERO. TO & & CALUMET PR. 7 \\
\hline 1530 & & GERRUCHITA & DOMINICK & B & 22 & $\mathrm{~s}$ & ITALY & ITALY & ITALY & $\mathrm{AL}$ & LABORER/CM & & & CALUMET PR. 7 \\
\hline 1531 & & PISTONETTO & JOHN & B & 28 & $\mathrm{~s}$ & ITALY & ITALY & ITALY & $\mathrm{AL}$ & LABORER/CM & TORINO & & CALUMET PR. 7 \\
\hline 1532 & & BENGHETTO & ANTON & $\begin{array}{l}\mathrm{D} \\
\mathrm{B}\end{array}$ & 34 & s & ITALY & ITALY & ITALY & $\begin{array}{ll}A L \\
A L\end{array}$ & LABORER/CM & TURIVU & & CALUMET PR. 7 \\
\hline 1533 & & GARDETTO & FILIPPO & B & 36 & $\mathrm{~S}$ & ITALY & ITALY & ITALY & $\mathrm{AL}$ & LABORER/CM & BOSCONERO. TO & & CALUMET PR. 7 \\
\hline 1534 & & CANDE & JOHN & B & 31 & s & ITALY & ITALY & ITALY & $A L$ & LABORER/CM & & & CALUMET PR. 7 \\
\hline 1535 & & BACINE & MIKE & $\mathrm{H}$ & 44 & M16 & ITALY & ITALY & ITALY & $\mathrm{AL}$ & TIMBERMAN/CM & & & CALUMET PR. 7 \\
\hline 1536 & & BACINE & MARY & W & 41 & M16 & ITALY & ITALY & ITALY & ML & 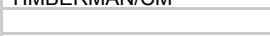 & & & CALUMET PR. 7 \\
\hline 1537 & & BACINE & CHARLEY & s & 16 & s & ITALY & ITALY & ITALY & & DRILL BOY/CM & & & CALUMET PR. 7 \\
\hline 1538 & & BACINE & JENNIE & $\mathrm{D}$ & 14 & $\mathrm{~s}$ & ITALY & ITALY & ITALY & & & & & CALUMET PR. 7 \\
\hline 1539 & & BACINE & DENE & $\mathrm{s}$ & 9 & $\mathrm{~S}$ & ITALY & ITALY & ITALY & & AT SCHOOL 10 & & & CALUMET PR. 7 \\
\hline 1540 & & BACINE & ENOS & $\mathrm{s}$ & 2 & $\mathrm{~S}$ & MICHIGAN & ITALY & ITALY & & & & & CALUMET PR. 7 \\
\hline 1541 & & BACINE & LYDIA & $\mathrm{D}$ & $5 \mathrm{M}$ & $\mathrm{s}$ & MICHIGAN & ITALY & ITALY & & & & & CALUMET PR. 7 \\
\hline 1542 & 316 & VENTURINO & ANGELO & $\mathrm{H}$ & 51 & M20 & ITALY & ITALY & ITALY & & MINER/CM & & CALUMET AVE. & CALUMET - LAURIUM \\
\hline 1543 & 316 & VENTURINO & GESTIE? & w & 38 & M20 & WISCONSI & WISCONS & WISCONSI & & & USA & CALUMET AVE. & CALUMET - LAURIUM \\
\hline 1544 & 316 & VENTURINO & J? & s & 18 & $\mathrm{~s}$ & MICHIGAN & ITALY & WISCONSI & & & & CALUMET AVE. & CALUMET - LAURIUM \\
\hline 1545 & 316 & VENTURINO & MARY & $\mathrm{D}$ & 15 & $\mathrm{~s}$ & MICHIGAN & ITALY & WISCONSI & & & & $\begin{array}{l}\text { CALUMET AVE. } \\
\text { CAUE }\end{array}$ & CALUMET - LAURIUM \\
\hline 1546 & 316 & VENTURINO & ANNE & $\mathrm{D}$ & 13 & $\mathrm{~S}$ & MICHIGAN & ITALY & WISCONSI & & & & CALUMET AVE. & CALUMET - LAURIUM \\
\hline 1547 & 316 & VENTURINO & JOHN & $\mathrm{s}$ & 11 & s & MICHIGAN & ITALY & WISCONSI & & & & CALUMET AVE. & CALUMET - LAURIUM \\
\hline 1548 & 316 & VENTURINO & BART & s & 9 & s & MICHIGAN & ITALY & WISCONSI & & & & CALUMET AVE. & CALUMET - LAURIUM \\
\hline 1549 & & RAFOTTO & BERNARD & $\mathrm{H}$ & 40 & M1 & ITALY & ITALY & ITALY & NA & SALOONKEEPER & & FIRST STREET & CALUMET - LAURIUM \\
\hline 1550 & & RAFOTTO & PERONIA & W & 27 & M1 & ITALY & ITALY & ITALY & & & & FIRST STREET & CALUMET - LAURIUM \\
\hline 1551 & & RAFOTTO & FRANK & s & $3 \mathrm{M}$ & s & MICHIGAN & ITALY & ITALY & & & & FIRST STREET & CALUMET - LAURIUM \\
\hline 1552 & & MARIE & RO? & DOM & 21 & s & ITALY & ITALY & ITALY & & SERVANT & & FIRST STREET & CALUMET - LAURIUM \\
\hline 1553 & & YOTTI & ANGELO & $\mathrm{H}$ & 51 & M22 & ITALY & ITALY & ITALY & & NOT CLEAR & & CALUMET AVE. & CALUMET - LAURIUM \\
\hline 1554 & & YOTTI & DONNA & W & 35 & M22 & CANADA & CANADA & CANADA & & & CANADA & CALUMET AVE. & CALUMET - LAURIUM \\
\hline 1555 & & YOTTI & PETER & s & 14 & $\mathrm{~s}$ & MICHIGAN & ITALY & CANADA & & AT SCHOOL & & CALUMET AVE. & CALUMET - LAURIUM \\
\hline 1556 & & YOTTI & MARNIE & $\mathrm{D}$ & 11 & $\mathrm{~s}$ & MICHIGAN & ITALY & CANADA & & AT SCHOOL & & CALUMET AVE. & CALUMET - LAURIUM \\
\hline 1550 & & YOTTI & ALVINA & $\mathrm{D}$ & 9 & s & MICHIGAN & ITALY & CANADA & & AT SCHOOL & & $\begin{array}{l}\text { CALUMET AVE. } \\
\text { CAUE }\end{array}$ & $\begin{array}{l}\text { CALUMET - LAURIUM } \\
\text { CAUM }\end{array}$ \\
\hline 1558 & & YOTTI & GILBERT & $\mathrm{s}$ & 5 & s & MICHIGAN & ITALY & CANADA & & & & CALUMET AVE. & CALUMET - LAURIUM \\
\hline 1559 & & YOTTI & ANNE & $\mathrm{D}$ & 3 & s & MICHIGAN & ITALY & CANADA & & & & CALUMET AVE. & CALUMET - LAURIUM \\
\hline 1560 & & YOTTI & DELLA & $\mathrm{D}$ & $8 \mathrm{M}$ & s & MICHIGAN & ITALY & CANADA & & & & CALUMET AVE. & CALUMET - LAURIUM \\
\hline 1561 & & MARCO & JOSEPH & $\mathrm{H}$ & 28 & M2 & MICHIGAN & ITALY & ITALY & & BLACKSMITH & TORINO & CALUMET AVE. & CALUMET - LAURIUM \\
\hline 1562 & & MARCO & MAGGIE & W & 25 & M2 & MICHIGAN & ITALY & ITALY & & & & CALUMET AVE. & CALUMET - LAURIUM \\
\hline 1563 & & MARCO & MARY & $\mathrm{D}$ & $5 \mathrm{M}$ & $\mathrm{s}$ & MICHIGAN & MICHIGA & MICHIGAN & & & TORINO & CALUMET AVE. & CALUMET - LAURIUM \\
\hline 1564 & 114 & TORREANO & GIOVANNI & $\mathrm{H}$ & 35 & M11 & ITALY & ITALY & ITALY & NA & MINER/CM & TORINO & FIRST STREET & CALUMET - LAURIUM \\
\hline 1565 & 114 & TORREANO & FELICITA & W & 36 & M11 & ITALY & ITALY & ITALY & & & TORINO & FIRST STREET & CALUMET - LAURIUM \\
\hline 1566 & 114 & TORREANO & CATERINA & $\mathrm{D}$ & 10 & $\mathrm{~s}$ & MICHIGAN & ITALY & ITALY & & AT SCHOOL 10 & TORINO & FIRST STREET & CALUMET - LAURIUM \\
\hline 1567 & 114 & TORREANO & FRANK & s & 8 & $\mathrm{~s}$ & MICHIGAN & ITALY & ITALY & & AT SCHOOL 10 & TORINO & FIRST STREET & CALUMET - LAURIUM \\
\hline 1568 & 114 & TORREANO & MARTIN & $\mathrm{s}$ & 6 & $\mathrm{~S}$ & MICHIGAN & ITALY & ITALY & & AT SCHOOL 10 & TORINO & FIRST STREET & CALUMET - LAURIUM \\
\hline 1569 & 114 & TORREANO & PAULINE & $\mathrm{D}$ & 6 & $\mathrm{~s}$ & MICHIGAN & ITALY & ITALY & & AT SCHOOL 10 & TORINO & FIRST STREET & CALUMET - LAURIUM \\
\hline 1570 & 114 & TORREANO & MARY & $\mathrm{D}$ & 4 & $\mathrm{~s}$ & MICHIGAN & ITALY & ITALY & & & TORINO & FIRST STREET & CALUMET - LAURIUM \\
\hline 1571 & 114 & TORREANO & ANGELO & $\mathrm{s}$ & 2 & s & MICHIGAN & ITALY & ITALY & & & TORINO & FIRST STREET & CALUMET - LAURIUM \\
\hline 1572 & 114 & TAPPERO & JOSEPH & $\mathrm{H}$ & 24 & s & ITALY & ITALY & ITALY & AL & TRAMMER/CM & TORINO & FIRST STREET & CALUMET - LAURIUM \\
\hline
\end{tabular}




\begin{tabular}{|c|c|c|c|c|c|c|c|c|c|c|c|c|c|c|}
\hline 1573 & 114 & NOTARIO & JOSEPH & PART & 32 & S & ITALY & ITALY & ITALY & $\mathrm{AL}$ & TIMBERMAN/CM & TORINO & FIRST STREET & CALUMET - LAURIUM \\
\hline 1574 & 150 & CONTARINI & JOSEPH & $\mathrm{H}$ & 40 & M9 & ITALY & ITALY & ITALY & NA & STONE MASON & TREVISO & OSCEOLA ST. & CALUMET - LAURIUM \\
\hline 1575 & 150 & CONTARINI & SOPHIA & W & 29 & M9 & ITALY & ITALY & ITALY & & & & OSCEOLA ST. & CALUMET - LAURIUM \\
\hline 1576 & 150 & CONTARINI & ARTHUR & s & 11 & $\mathrm{~S}$ & MICHIGAN & ITALY & ITALY & & & TREVISO & OSCEOLA ST. & CALUMET - LAURIUM \\
\hline 1577 & 150 & ZANARDI & PETER & $\mathrm{H}$ & 25 & MO & ITALY & ITALY & ITALY & & N. G. & BERGAMO & OSCEOLA ST. & CALUMET - LAURIUM \\
\hline 1578 & 150 & ZANARDI & CECILIA & W & 16 & MO & ITALY & ITALY & ITALY & & & BERGAMO & OSCEOLA ST. & CALUMET - LAURIUM \\
\hline 1579 & 150 & FAUSONE & PETER & $\mathrm{H}$ & 28 & M7 & ITALY & ITALY & ITALY & PA & MINER/CM & VALPERGA. TO & OSCEOLA ST. & CALUMET - LAURIUM \\
\hline 1580 & 150 & FAUSONE & MARY & W & 22 & M7 & ITALY & ITALY & ITALY & & & VALPERGA. TO & OSCEOLA ST. & CALUMET - LAURIUM \\
\hline 1581 & 150 & FAUSONE & MIKE & s & 5 & S & ITALY & ITALY & ITALY & & & VALPERGA. TO & OSCEOLA ST. & CALUMET - LAURIUM \\
\hline 1582 & 150 & FAUSONE & JOE & $\mathrm{s}$ & 3 & $\mathrm{~S}$ & ITALY & ITALY & ITALY & & & VALPERGA. TO & OSCEOLA ST. & CALUMET - LAURIUM \\
\hline 1583 & 150 & FAUSONE & JAMES & $\mathrm{s}$ & 11 & $\mathrm{~s}$ & MICHIGAN & ITALY & ITALY & & & VALPERGA. TO & OSCEOLA ST. & CALUMET - LAURIUM \\
\hline 1584 & 150 & BALAGNA & JAMES & B & 45 & W & ITALY & ITALY & ITALY & NA & TIMBERMAN/CM & PONT CANAVESE. TO & OSCEOLA ST. & CALUMET - LAURIUM \\
\hline 1585 & 150 & CANAVERA & JOSEPH & B & 40 & M8 & ITALY & ITALY & ITALY & AL & TIMBERMAN/CM & TORINO & OSCEOLA ST. & CALUMET - LAURIUM \\
\hline 1586 & 150 & DOMINICI & CHE?? & $\mathrm{H}$ & 37 & M1 & ITALY & ITALY & ITALY & $\mathrm{AL}$ & POLICEMAN & & OSCEOLA ST. & CALUMET - LAURIUM \\
\hline 1587 & 150 & DOMINICI & FELICITA & W & 24 & M1 & ITALY & ITALY & ITALY & & & & OSCEOLA ST. & CALUMET - LAURIUM \\
\hline 1588 & 150 & FRANCESCO & FRANCESCO & $\mathrm{H}$ & 56 & M11 & ITALY & ITALY & ITALY & PA & WATCH REPAIRER & TORINO & OSCEOLA ST. & CALUMET - LAURIUM \\
\hline 1589 & 150 & FRANCESCO & MARIE & W & 31 & M11 & ITALY & ITALY & ITALY & & & TORINO & OSCEOLA ST. & CALUMET - LAURIUM \\
\hline 1590 & 150 & FRANCESCO & CHESERINE & $\mathrm{D}$ & 9 & $\mathrm{~s}$ & ITALY & ITALY & ITALY & & AT SCHOOL 10 & TORINO & OSCEOLA ST. & CALUMET - LAURIUM \\
\hline 1591 & 150 & FRANCESCO & LAURA & $\mathrm{D}$ & 7 & $\mathrm{~s}$ & ITALY & ITALY & ITALY & & AT SCHOOL 10 & TORINO & OSCEOLA ST. & CALUMET - LAURIUM \\
\hline 1592 & 150 & FRANCESCO & MARIE & $\mathrm{D}$ & 5 & $\mathrm{~s}$ & ITALY & ITALY & ITALY & & & TORINO & OSCEOLA ST. & CALUMET - LAURIUM \\
\hline 1593 & 150 & FRANCESCO & FRED & s & 11 & $\mathrm{~s}$ & MICHIGAN & ITALY & ITALY & & & TORINO & OSCEOLA ST. & CALUMET - LAURIUM \\
\hline 1594 & 150 & FISHER & BERTHA & $\mathrm{D}$ & 16 & s & MICHIGAN & ITALY & ITALY & & & & OSCEOLA ST. & CALUMET - LAURIUM \\
\hline 1595 & 150 & BARTONE & JOHN & $\mathrm{H}$ & 22 & MO & ITALY & ITALY & ITALY & AL & TRAMMER/CM & & OSCEOLA ST. & CALUMET - LAURIUM \\
\hline 1596 & 150 & BARTONE & JENNY & W & 17 & M0 & ITALY & ITALY & ITALY & & & & OSCEOLA ST. & CALUMET - LAURIUM \\
\hline 1597 & 112 & MARINELLI & AUGUST & $\mathrm{H}$ & 28 & M3 & ITALY & ITALY & ITALY & & PROPIETOR/NEWSPAPER & BOLZANO & OSCEOLA ST. & CALUMET - LAURIUM \\
\hline 1598 & 112 & MARINELLI & LENA & W & 23 & M3 & GERMANY & GERMAN & GERMANY & & & GERMANY & OSCEOLA ST. & CALUMET - LAURIUM \\
\hline 1599 & 112 & MARINELLI & DEWEY & s & 1 & $\mathrm{~s}$ & MICHIGAN & MICHIGA & GERMANY & & & BOLZANO & OSCEOLA ST. & CALUMET - LAURIUM \\
\hline 1600 & 112 & PALAZZOLI & RUDOLPH & B & 50 & s & ITALY & ITALY & ITALY & NA & PRINTER & & OSCEOLA ST. & CALUMET - LAURIUM \\
\hline 1601 & 118 & TINETTI & PETER & $\mathrm{H}$ & 38 & M14 & ITALY & ITALY & ITALY & & SALOONKEEPER & SAN MARTINO CANAVESE. TO & OSCEOLA ST. & CALUMET - LAURIUM \\
\hline 1602 & 118 & TINETTI & ANGELINA & w & 35 & M14 & ITALY & ITALY & ITALY & & & & OSCEOLA ST. & CALUMET - LAURIUM \\
\hline 1603 & 118 & TINETTI & JOHANNA & $\mathrm{D}$ & 11 & $\mathrm{~s}$ & MICHIGAN & ITALY & ITALY & & AT SCHOOL 10 & SAN MARTINO CANAVESE. TO & OSCEOLA ST. & CALUMET - LAURIUM \\
\hline 1604 & 118 & TINETTI & TONY & $\mathrm{s}$ & 9 & $\mathrm{~s}$ & ITALY & ITALY & ITALY & & AT SCHOOL 10 & SAN MARTINO CANAVESE. TO & OSCEOLA ST. & CALUMET - LAURIUM \\
\hline 1605 & 118 & TINETTI & MARTHA & $\mathrm{D}$ & 6 & s & ITALY & ITALY & ITALY & & AT SCHOOL 10 & SAN MARTINO CANAVESE. TO & OSCEOLA ST. & CALUMET - LAURIUM \\
\hline 1606 & 118 & TINETTI & JOHN & $\mathrm{s}$ & 2 & s & ITALY & ITALY & ITALY & & & SAN MARTINO CANAVESE, TO & OSCEOLA ST. & CALUMET - LAURIUM \\
\hline 1607 & 118 & SCIACERO & ANTONIO & $\mathrm{H}$ & 27 & M0 & ITALY & ITALY & ITALY & NA & BARTENDER & SAN MARTINO CANAVESE. TO & OSCEOLA ST. & CALUMET - LAURIUM \\
\hline 1608 & 118 & SCIACERO & ANGELICA & w & 21 & M0 & ITALY & ITALY & ITALY & & & & OSCEOLA ST. & CALUMET - LAURIUM \\
\hline 1609 & 118 & PRICCO & $\mathrm{JOHN}$ & $\mathrm{H}$ & 25 & M1 & ITALY & ITALY & ITALY & $\mathrm{AL}$ & TRAMMER/CM & SAN MARTINO CANAVESE. TO & OSCEOLA ST. & CALUMET - LAURIUM \\
\hline 1610 & 118 & PRICCO & ANTONIO & W & 21 & M1 & ITALY & ITALY & ITALY & $\mathrm{ML}_{\mathrm{L}}$ & 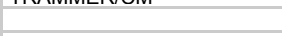 & 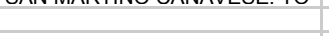 & OSCEOLA ST. & CALUMET - LAURIUM \\
\hline 1611 & 118 & PRICCO & FRANK & $\mathrm{s}$ & $3 \mathrm{M}$ & $\mathrm{s}$ & MICHIGAN & ITALY & ITALY & & & SAN MARTINO CANAVESE. TO & OSCEOLA ST. & CALUMET - LAURIUM \\
\hline 1612 & 120 & BINANDO & JOHN & $\mathrm{H}$ & 37 & M18 & ITALY & ITALY & ITALY & NA & TEAMSTER & SAN GIORGIO CANAVESE. TO & OSCEOLA ST. & CALUMET - LAURIUM \\
\hline 1613 & 120 & BINANDO & LOUISE & W & 37 & M18 & ITALY & ITALY & ITALY & & & SAN GIORGIO CANAVESE. TO & OSCEOLA ST. & CALUMET - LAURIUM \\
\hline 1614 & 120 & BINANDO & PETER & s & 17 & $\mathrm{~s}$ & ITALY & ITALY & ITALY & & TEAMSTER & SAN GIORGIO CANAVESE. TO & OSCEOLA ST. & CALUMET - LAURIUM \\
\hline 1615 & 120 & BINANDO & TONY & $\mathrm{s}$ & 9 & $\mathrm{~s}$ & ITALY & ITALY & ITALY & & AT SCHOOL 10 & SAN GIORGIO CANAVESE. TO & OSCEOLA ST. & CALUMET - LAURIUM \\
\hline 1616 & 120 & BINANDO & TINA & $\mathrm{D}$ & $2 \mathrm{M}$ & $\mathrm{s}$ & MICHIGAN & ITALY & ITALY & & & SAN GIORGIO CANAVESE. TO & OSCEOLA ST. & CALUMET - LAURIUM \\
\hline 1617 & 138 & DELL'ANGELICA & VINCENT & $\mathrm{H}$ & 50 & M24 & ITALY & ITALY & ITALY & NA & MINER/CM & LUCCA & OSCEOLA ST. & CALUMET - LAURIUM \\
\hline 1618 & 138 & DELL'ANGELICA & ROSE & W & 47 & M24 & ITALY & ITALY & ITALY & & & LUCCA & OSCEOLA ST. & CALUMET - LAURIUM \\
\hline 1619 & 138 & DELL'ANGELICA & MELANI & D & 22 & s & FRANCE & ITALY & ITALY & & & LUCCA & OSCEOLA ST. & CALUMET - LAURIUM \\
\hline 1620 & 138 & DELL'ANGELICA & AMERIGO & $\mathrm{s}$ & 20 & s & MICHIGAN & ITALY & ITALY & & MINER/CM & LUCCA & OSCEOLA ST. & CALUMET - LAURIUM \\
\hline 1621 & 138 & DELL'ANGELICA & ADOLPH & s & 16 & s & MICHIGAN & ITALY & ITALY & & MINER/CM & LUCCA & OSCEOLA ST. & CALUMET - LAURIUM \\
\hline 1622 & 138 & DELL'ANGELICA & LOUIS & s & 14 & s & MICHIGAN & ITALY & ITALY & & MINER/CM & LUCCA & OSCEOLA ST. & CALUMET - LAURIUM \\
\hline 1623 & 138 & DELL'ANGELICA & LYDA & $\mathrm{D}$ & 10 & $\mathrm{~s}$ & MICHIGAN & ITALY & ITALY & & AT SCHOOL 10 & LUCCA & OSCEOLA ST. & CALUMET - LAURIUM \\
\hline 1624 & 138 & DELL'ANGELICA & FLORENCE & $\mathrm{D}$ & 5 & $\mathrm{~s}$ & MICHIGAN & ITALY & ITALY & & & LUCCA & OSCEOLA ST. & CALUMET - LAURIUM \\
\hline 1625 & 138 & DELL'ANGELICA & GILBERT & $\mathrm{s}$ & 2 & $\mathrm{~s}$ & MICHIGAN & ITALY & ITALY & & & LUCCA & OSCEOLA ST. & CALUMET - LAURIUM \\
\hline 1626 & 138 & ClUCCI & SAMUEL & B & 39 & s & ITALY & ITALY & ITALY & PA & MINER/CM & LUCCA & OSCEOLA ST. & CALUMET - LAURIUM \\
\hline 1627 & 143 & BINANDO & PETER & $\mathrm{H}$ & 33 & M2 & ITALY & ITALY & ITALY & NA & TEAMSTER/CM & SAN GIORGIO CANAVESE. TO & HECLA STEET & CALUMET - LAURIUM \\
\hline 1628 & 143 & BINANDO & MARY & w & 34 & M2 & ITALY & ITALY & ITALY & & & SAN GIORGIO CANAVESE. TO & HECLA STEET & CALUMET - LAURIUM \\
\hline 1629 & 131 & BIANCO & JOSEPH & $\mathrm{H}$ & 36 & M12 & ITALY & ITALY & ITALY & NA & TIMBERMAN/CM & SCARMAGNO. TO & HECLA STREET & CALUMET - LAURIUM \\
\hline 1630 & 131 & BIANCO & AGREBIA & w & 36 & M12 & ITALY & ITALY & ITALY & & & SCARMAGNO, TO & HECLA STEET & CALUMET - LAURIUM \\
\hline 1631 & 131 & BIANCO & CHARLES & s & 6 & $\mathrm{~s}$ & MICHIGAN & ITALY & ITALY & & AT SCHOOL 10 & SCARMAGNO. TO & HECLA STREET & CALUMET - LAURIUM \\
\hline 1632 & 131 & BIANCO & PETER & $\mathrm{s}$ & 4 & $\mathrm{~s}$ & MICHIGAN & ITALY & ITALY & & & SCARMAGNO. TO & HECLA STEET & CALUMET - LAURIUM \\
\hline 1633 & 131 & BIANCO & ANTON & B & 34 & W & ITALY & ITALY & ITA & NA & TRAMMER/CM & & HECLA STREET & CALUMET - L \\
\hline 1634 & 131 & BIANCO & BERNARD & B & 28 & s & ITALY & ITALY & ITALY & NA & TRAMMER/CM & & HECLA STEET & CALUMET - \\
\hline 1635 & 131 & BIANCO & JOHN & B & 58 & W & ITALY & ITALY & ITALY & $\mathrm{AL}$ & DAY LABORER/CM & TORINO & HECLA STEET & CALUMET - LAURIUM \\
\hline
\end{tabular}




\begin{tabular}{|c|c|c|c|c|c|c|c|c|c|c|c|c|c|c|}
\hline 1636 & 131 & AGREBBI & JOSEPH & B & 27 & $S$ & ITALY & ITALY & ITALY & $\mathrm{AL}$ & TRAMMER/CM & & HECLA STEET & CALUMET - LAURIUM \\
\hline 1637 & 129 & TORREANO & JAMES & $\mathrm{H}$ & 32 & M7 & ITALY & ITALY & ITALY & NA & GROCER & TORINO & HECLA STREET & CALUMET - LAURIUM \\
\hline 1638 & 129 & TORREANO & ANNIE & W & 23 & M7 & ITALY & ITALY & ITALY & & & & HECLA STEET & CALUMET - LAURIUM \\
\hline 1639 & 129 & TORREANO & FRANK & s & 4 & $S$ & MICHIGAN & ITALY & ITALY & & & TORINO & HECLA STREET & CALUMET - LAURIUM \\
\hline 1640 & 129 & TORREANO & JOHN & $\mathrm{s}$ & 1 & s & MICHIGAN & ITALY & ITALY & & & TORINO & HECLA STEET & CALUMET - LAURIUM \\
\hline 1641 & 129 & ACORA & MAGGIE & DOM & 23 & $\mathrm{~S}$ & ITALY & ITALY & ITALY & & & & HECLA STREET & CALUMET - LAURIUM \\
\hline 1642 & 125 & MARCO & GEORGE & $\mathrm{H}$ & 38 & M9 & ITALY & ITALY & ITALY & NA & NOT CLEAR & TORINO & HECLA STEET & CALUMET - LAURIUM \\
\hline 1643 & 125 & MARCO & JOSE & W & 32 & M9 & MICHIGAN & ITALY & ITALY & & & & HECLA STEET & CALUMET - LAURIUM \\
\hline 1644 & 125 & MARCO & LUCY & D & 6 & $S$ & MICHIGAN & ITALY & ITALY & & AT SCHOOL 10 & TORINO & HECLA STEET & CALUMET - LAURIUM \\
\hline 1645 & 125 & MARCO & DOMINICK & $\mathrm{s}$ & 8 & $\mathrm{~S}$ & MICHIGAN & ITALY & ITALY & & AT SCHOOL 10 & TORINO & HECLA STREET & CALUMET - LAURIUM \\
\hline 1646 & 125 & MARCO & FRANK & $\mathrm{s}$ & 4 & $\mathrm{~s}$ & MICHIGAN & ITALY & ITALY & & & TORINO & HECLA STEET & CALUMET - LAURIUM \\
\hline 1647 & 125 & MARCO & PETER & s & 2 & $\mathrm{~s}$ & MICHIGAN & ITALY & ITALY & & & TORINO & HECLA STREET & CALUMET - LAURIUM \\
\hline 1648 & 125 & MARCO & TRACY & $\mathrm{D}$ & 1 & s & MICHIGAN & ITALY & ITALY & & & TORINO & HECLA STEET & CALUMET - LAURIUM \\
\hline 1649 & 125 & $? ?$ & ANTON & DOM & 20 & $\mathrm{~s}$ & ITALY & ITALY & ITALY & & SERVANT & & HECLA STREET & CALUMET - LAURIUM \\
\hline 1650 & 125 & VAIRO & JOSEPH & $\mathrm{H}$ & 28 & M2 & ITALY & ITALY & ITALY & $\mathrm{AL}$ & & LOCANA CANAVESE. TO & HECLA STEET & CALUMET - LAURIUM \\
\hline 1651 & 125 & VAIRO & JOHANNA & W & 21 & M2 & ITALY & ITALY & ITALY & & & LOCANA CANAVESE, TO & HECLA STEET & CALUMET - LAURIUM \\
\hline 1652 & 125 & VAIRO & MARY & D & 1 & $\mathrm{~s}$ & MICHIGAN & ITALY & ITALY & & & LOCANA CANAVESE. TO & HECLA STEET & CALUMET - LAURIUM \\
\hline 1653 & & CONTRATTO & PETER & $\mathrm{H}$ & 41 & M10 & ITALY & ITALY & ITALY & NA & BLACKSMITH & LOCANA CANAVESE. TO & HECLA STREET & CALUMET - LAURIUM \\
\hline 1654 & & CONTRATTO & JENNY & w & 26 & M10 & ITALY & ITALY & ITALY & & & LOCANA CANAVESE. TO & HECLA STEET & CALUMET - LAURIUM \\
\hline 1655 & & CONTRATTO & ANDREW & s & 5 & s & MICHIGAN & ITALY & ITALY & & & LOCANA CANAVESE. TO & HECLA STREET & CALUMET - LAURIUM \\
\hline 1656 & & CONTRATTO & ANNE & $\mathrm{D}$ & 4 & $\mathrm{~s}$ & MICHIGAN & ITALY & ITALY & & & LOCANA CANAVESE. TO & HECLA STEET & CALUMET - LAURIUM \\
\hline 1657 & & CONTRATTO & JAMES & $\mathrm{s}$ & 2 & $\mathrm{~s}$ & MICHIGAN & ITALY & ITALY & & & LOCANA CANAVESE, TO & HECLA STREET & CALUMET - LAURIUM \\
\hline 1658 & & QUELLO & JOHN & B & 28 & $\mathrm{~S}$ & ITALY & ITALY & ITALY & $\mathrm{AL}$ & TIMBERMAN/CM & LOCANA CANAVESE. TO & HECLA STEET & CALUMET - LAURIUM \\
\hline 1659 & & QUELLO & PETER & B & 45 & $\mathrm{~s}$ & ITALY & ITALY & ITALY & $\mathrm{AL}$ & TIMBERMAN/CM & LOCANA CANAVESE. TO & HECLA STREET & CALUMET - LAURIUM \\
\hline 1660 & & QUELLO & DOMINICK & B & 45 & $\mathrm{~s}$ & ITALY & ITALY & ITALY & $\mathrm{AL}$ & TRAMMER/CM & LOCANA CANAVESE. TO & HECLA STEET & CALUMET - LAURIUM \\
\hline 1661 & & TOMASI & ANDREW & B & 36 & $\mathrm{~s}$ & ITALY & ITALY & ITALY & NA & DAY LABORER/CM & LOCANA CANAVESE. TO & HECLA STREET & CALUMET - LAURIUM \\
\hline 1662 & & GIACHERIO & ANTONIO & B & 26 & $\mathrm{~s}$ & ITALY & ITALY & ITALY & AL & DAY LABORER/CM & 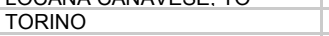 & HECLA STEET & CALUMET - LAURIUM \\
\hline 1663 & & TOMASI & BET & B & 28 & s & ITALY & ITALY & ITALY & $\mathrm{AL}$ & DAY LABORER/CM & TORINO & HECLA STEET & CALUMET - LAURIUM \\
\hline 1664 & & REGIS & JOHN & $\mathrm{H}$ & 38 & MO & ITALY & ITALY & ITALY & NA & TRAMMER/CM & SAN GIUSTO CANAVESE. TO & HECLA STREET & CALUMET - LAURIUM \\
\hline 1665 & & REGIS & LENA & W & 22 & MO & ITALY & ITALY & ITALY & & & & HECLA STEET & CALUMET - LAURIUM \\
\hline 1666 & & REGIS & JOSEPHINE & MO & 57 & W & ITALY & ITALY & ITALY & & & SAN GIUSTO CANAVESE. TO & HECLA STREET & CALUMET - LAURIUM \\
\hline 1667 & & POSTO & LOUIS & B & 25 & s & ITALY & ITALY & ITALY & $\mathrm{AL}$ & TRAMMER/CM & & HECLA STEET & CALUMET - LAURIUM \\
\hline 1668 & & BAGO & PETER & B & 25 & $\mathrm{~s}$ & ITALY & ITALY & ITALY & AL & TRAMMER/CM & TORINO & HECLA STREET & CALUMET - LAURIUM \\
\hline 1669 & & BINANDO & JOSEPH & B & 28 & $\mathrm{~s}$ & ITALY & ITALY & ITALY & $\mathrm{AL}$ & DAY LABORER/CM & CORTEREGGIO, TO & HECLA STEET & CALUMET - LAURIUM \\
\hline 1670 & & RICHETTA & MICHAEL & $\begin{array}{l}\mathrm{D} \\
\mathrm{H}\end{array}$ & 47 & M26 & $\begin{array}{l}\text { ITALY } \\
\text { ITAL }\end{array}$ & ITALY & ITALY & NA & SALOON OWNER & SAN MARTINO CANAVESE. TO & HECLA STEET & $\begin{array}{l}\text { CALUMET - LAURIUM } \\
\text { CAUM }\end{array}$ \\
\hline 1671 & & RICHETTA & DOMENICA & w & 46 & M26 & ITALY & ITALY & ITALY & & & & HECLA STEET & CALUMET - LAURIUM \\
\hline 1672 & & RICHETTA & JAMES & s & 23 & MO & MICHIGAN & ITALY & ITALY & & TEAMSTER & SAN MARTINO CANAVESE. TO & HECLA STREET & CALUMET - LAURIUM \\
\hline 1673 & & RICHETTA & JOSEPHINE & $\mathrm{D}$ & 13 & $\mathrm{~s}$ & MICHIGAN & ITALY & ITALY & & AT SCHOOL 10 & SAN MARTINO CANAVESE. TO & HECLA STEET & CALUMET - LAURIUM \\
\hline 1674 & & RICHETTA & THERESA & $\mathrm{D}$ & 12 & s & MICHIGAN & ITALY & ITALY & & AT SCHOOL & SAN MARTINO CANAVESE. TO & HECLA STREET & CALUMET - LAURIUM \\
\hline 1675 & & RICHETTA & DOMENICA & DIL & 18 & M0 & ITALY & ITALY & ITALY & & & & HECLA STEET & CALUMET - LAURIUM \\
\hline 1676 & & RICHETTA & ANGELO & $\mathrm{BR}$ & 42 & M19 & ITALY & ITALY & ITALY & NA & BARTENDER & SAN MARTINO CANAVESE. TO & HECLA STREET & CALUMET - LAURIUM \\
\hline 1677 & & RICHETTA & VICTORIA & SIL & 41 & M19 & ITALY & ITALY & ITALY & & & SAN MARTINO CANAVESE. TO & HECLA STEET & CALUMET - LAURIUM \\
\hline 1678 & & RICHETTA & JAMES & NEP & 19 & $\mathrm{~s}$ & ITALY & ITALY & ITALY & & DAY LABORER/CM & SAN MARTINO CANAVESE. TO & HECLA STREET & CALUMET - LAURIUM \\
\hline 1679 & & RICHETTA & JOSEPHINE & NIEC & 13 & $\mathrm{~S}$ & MICHIGAN & ITALY & ITALY & & AT SCHOOL 10 & SAN MARTINO CANAVESE. TO & HECLA STEET & CALUMET - LAURIUM \\
\hline 1680 & & RICHETTA & THERESA & NIEC & 7 & $\mathrm{~s}$ & MICHIGAN & ITALY & ITALY & & AT SCHOOL & SAN MARTINO CANAVESE. TO & HECLA STEET & CALUMET - LAURIUM \\
\hline 1681 & & PIANO & JAMES & B & 28 & s & ITALY & ITALY & ITALY & $\mathrm{AL}$ & TRAMMER/CM & TORINO & HECLA STEET & CALUMET - LAURIUM \\
\hline 1682 & & ROVANO & ANTON & B & 35 & M1 & ITALY & ITALY & ITALY & $\mathrm{AL}$ & DAY LABORER/CM & SAN MARTINO CANAVESE. TO & HECLA STREET & CALUMET - LAURIUM \\
\hline 1683 & & ROVANO & DOMINICK & $\begin{array}{l}\mathrm{D} \\
\mathrm{B}\end{array}$ & 46 & M21 & ITALY & ITALY & ITALY & $\mathrm{AL}$ & STONE MASON & SAN MARTINO CANAVESE. TO & HECLA STEET & CALUMET - LAURIUM \\
\hline 1684 & & BRUSSO & MICHELE & $\mathrm{H}$ & 30 & M2 & ITALY & ITALY & ITALY & $\mathrm{AL}$ & SALOONKEEPER & AGLIE'. TO & HECLA STREET & CALUMET - LAURIUM \\
\hline 1685 & & BRUSSO & MARIANNA & w & 34 & M2 & MICHIGAN & ITALY & ITALY & & & & HECLA STEET & CALUMET - LAURIUM \\
\hline 1686 & & BRUSSO & MARY & $\mathrm{D}$ & $9 \mathrm{M}$ & $\mathrm{s}$ & MICHIGAN & ITALY & MICHIGAN & & & AGLIE'. TO & HECLA STREET & CALUMET - LAURIUM \\
\hline 1687 & & GEDDA & PETER & $\mathrm{H}$ & 28 & M4 & ITALY & ITALY & ITALY & $\mathrm{AL}$ & BARTENDER & TORINO & HECLA STEET & CALUMET - LAURIUM \\
\hline 1688 & & GEDDA & ANNE & W & 27 & M4 & ITALY & ITALY & ITALY & & DOARTLINULK & & HECLA STEET & CALUMET - LAURIUM \\
\hline 1689 & & GEDDA & ANNE & $\mathrm{D}$ & 3 & $\mathrm{~s}$ & MICHIGAN & ITALY & ITALY & & & TORINO & HECLA STEET & CALUMET - LAURIUM \\
\hline 1690 & & CRO & ROTILDA & DOM & 18 & $\mathrm{~s}$ & ITALY & ITALY & ITALY & & SERVANT & & HECLA STREET & CALUMET - LAURIUM \\
\hline 1691 & 326 & MARTA & DOMENICO & $\mathrm{H}$ & 36 & M12 & ITALY & ITALY & ITALY & NA & BAKER & TORINO & HECLA STEET & CALUMET - LAURIUM \\
\hline 1692 & 326 & MARTA & KATY & w & 35 & M12 & ITALY & ITALY & ITALY & & & TORINO & HECLA STREET & CALUMET - LAURIUM \\
\hline 1693 & 326 & MARTA & MARTIN & s & 10 & s & MICHIGAN & ITALY & ITALY & & AT SCHOOL 10 & TORINO & HECLA STEET & CALUMET - LAURIUM \\
\hline 1694 & 326 & MARTA & IRENE & $\mathrm{D}$ & 9 & $\mathrm{~S}$ & MICHIGAN & ITALY & ITALY & & AT SCHOOL 10 & TORINO & HECLA STREET & CALUMET - LAURIUM \\
\hline 1695 & 326 & MARTA & ANNE & $\mathrm{D}$ & 8 & $\mathrm{~s}$ & MICHIGAN & ITALY & ITALY & & AT SCHOOL 10 & TORINO & HECLA STEET & CALUMET - LAURIUM \\
\hline 1696 & 326 & MARTA & JOHN & $\mathrm{s}$ & 7 & $\mathrm{~s}$ & MICHIGAN & ITALY & ITALY & & & TORINO & HECLA STREET & CALUMET - LAURIUM \\
\hline 1697 & 326 & MARTA & FERDINANDO & s & 5 & s & MICHIGAN & ITALY & ITALY & & & TORINO & HECLA STEET & CALUMET - LAURIUM \\
\hline 1698 & 326 & MARTA & $\begin{array}{l}\text { MARTA? } \\
\text { M }\end{array}$ & FATH & 74 & W & ITALY & ITALY & ITALY & AL & & TORINO & HECLA STREET & CALUMET - LAURIUM \\
\hline
\end{tabular}




\begin{tabular}{|c|c|c|c|c|c|c|c|c|c|c|c|c|c|c|}
\hline 1699 & 313 & GALLASERO & CHARLES & $\mathrm{H}$ & 39 & M17 & ITALY & ITALY & ITALY & NA & TIMBERMAN/CM & TORINO & HECLA STEET & CALUMET - LAURIUM \\
\hline 1700 & 313 & GALLASERO & ADELAIDE & W & 38 & M17 & ITALY & ITALY & ITALY & & & TORINO & HECLA STREET & CALUMET - LAURIUM \\
\hline 1701 & 313 & GALLASERO & CAROLINE & $\mathrm{D}$ & 11 & s & MICHIGAN & ITALY & ITALY & & AT SCHOOL 10 & TORINO & HECLA STEET & CALUMET - LAURIUM \\
\hline 1702 & 313 & GALLASERO & MANICE & D & 9 & S & MICHIGAN & ITALY & ITALY & & AT SCHOOL 10 & TORINO & HECLA STREET & CALUMET - LAURIUM \\
\hline 1703 & 313 & GALLASERO & PETER & $\mathrm{s}$ & 6 & $\mathrm{~S}$ & MICHIGAN & ITALY & ITALY & & AT SCHOOL 6 & TORINO & HECLA STEET & CALUMET - LAURIUM \\
\hline 1704 & 313 & GALLASERO & MARY & $\mathrm{D}$ & 4 & $\mathrm{~S}$ & MICHIGAN & ITALY & ITALY & & & TORINO & HECLA STREET & CALUMET - LAURIUM \\
\hline 1705 & 313 & GALLASERO & CHARLES & S & 1 & S & MICHIGAN & ITALY & ITALY & & & TORINO & HECLA STEET & CALUMET - LAURIUM \\
\hline 1706 & & SUINO & FRANK & $\mathrm{H}$ & 31 & M3 & ITALY & ITALY & ITALY & $?$ & POLICEMAN & TORINO & HECLA STREET & CALUMET - LAURIUM \\
\hline 1707 & & SUINO & MARY & W & 26 & M3 & ITALY & ITALY & ITALY & & PULICLIVIAIV & 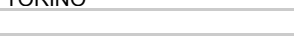 & HECLA STEET & CALUMET - LAURIUM \\
\hline 1708 & & SUINO & BERNARD & s & 2 & $\mathrm{~s}$ & MICHIGAN & ITALY & ITALY & & & TORINO & HECLA STREET & CALUMET - LAURIUM \\
\hline 1709 & & SUINO & GIORGIO & $\mathrm{s}$ & 11 & $\mathrm{~s}$ & MICHIGAN & ITALY & ITALY & & & TORINO & HECLA STEET & CALUMET - LAURIUM \\
\hline 1710 & & SUINO & MARION & NIE & $2 \mathrm{M}$ & $\mathrm{s}$ & MICHIGAN & ITALY & ITALY & & & TORINO & HECLA STREET & CALUMET - LAURIUM \\
\hline 1711 & 221 & FERRARI & SILVIO & $\mathrm{H}$ & 31 & M4 & ITALY & ITALY & ITALY & PA & TRAMMER/CM & LUCCA & KEARSARGE ST. & CALUMET - LAURIUM \\
\hline 1712 & 221 & FERRARI & ILDEGONDA & W & 24 & M4 & ITALY & ITALY & ITALY & & & LUCCA & KEARSARGE ST. & CALUMET - LAURIUM \\
\hline 1713 & 221 & FADERIE & DOMINIC & B & 34 & $s$ & ITALY & ITALY & ITALY & $\mathrm{AL}$ & TRAMMER/CM & & KEARSARGE ST. & CALUMET - LAURIUM \\
\hline 1714 & 221 & FADERIE & AURELIA? & B & 30 & $\mathrm{~s}$ & ITALY & ITALY & ITALY & & & & KEARSARGE ST. & CALUMET - LAURIUM \\
\hline 1715 & & BAGGIORE & FRANK & $\mathrm{H}$ & 32 & M14 & ITALY & ITALY & ITALY & & MINER/CM & TORINO & HECLA STREET & CALUMET - LAURIUM \\
\hline 1716 & & BAGGIORE & KATY & W & 34 & M14 & FRANCE & ITALY & ITALY & & & TORINO & HECLA STREET & CALUMET - LAURIUM \\
\hline 1717 & & BAGGIORE & JOHN & s & 12 & S & MICHIGAN & FRANCE & ITALY & & AT SCHOOL 8 & TORINO & HECLA STREET & CALUMET - LAURIUM \\
\hline 1718 & & BAGGIORE & MARY & $\mathrm{D}$ & 11 & $\mathrm{~S}$ & MICHIGAN & FRANCE & ITALY & & AT SCHOOL 10 & TORINO & HECLA STREET & CALUMET - LAURIUM \\
\hline 1719 & & BAGGIORE & JOSEPH & $\mathrm{s}$ & 9 & $\mathrm{~s}$ & MICHIGAN & FRANCE & ITALY & & AT SCHOOL 10 & TORINO & HECLA STREET & CALUMET - LAURIUM \\
\hline 1720 & & BAGGIORE & DUILIA & $\mathrm{D}$ & 7 & $\mathrm{~s}$ & MICHIGAN & FRANCE & ITALY & & AT SCHOOL 10 & TORINO & HECLA STREET & CALUMET - LAURIUM \\
\hline 1721 & & BAGGIORE & INCENZO & S & 5 & $\mathrm{~s}$ & MICHIGAN & FRANCE & ITALY & & AT SCHOOL 3 & TORINO & HECLA STREET & CALUMET - LAURIUM \\
\hline 1722 & & BAGGIORE & OTTO & $\mathrm{s}$ & 2 & $\mathrm{~s}$ & MICHIGAN & FRANCE & ITALY & & & TORINO & HECLA STREET & CALUMET - LAURIUM \\
\hline 1723 & & BARONE & CHARLES & $\mathrm{H}$ & 42 & M14 & ITALY & ITALY & ITALY & NA & TRAMMER/CM & & $\begin{array}{l}\text { HECLA STREET } \\
\text { HET }\end{array}$ & CALUMET - LAURIUM \\
\hline 1724 & & BARONE & TRACEY & W & 32 & M14 & ITALY & ITALY & ITALY & & & & HECLA STREET & CALUMET - LAURIUM \\
\hline 1725 & & BARONE & LOUIS & s & 7 & $\mathrm{~s}$ & ITALY & ITALY & ITALY & & & & HECLA STREET & CALUMET - LAURIUM \\
\hline 1726 & & BARONE & MARY & $\mathrm{D}$ & 4 & s & ITALY & ITALY & ITALY & & & & HECLA STREET & CALUMET - LAURIUM \\
\hline 1727 & 108 & ZANA & VALENTINO & $\mathrm{H}$ & 40 & M4 & ITALY & ITALY & ITALY & NA & WATCHMAN/CM & TORINO & HECLA STREET & CALUMET - LAURIUM \\
\hline 1728 & 108 & ZANA & TRACY & W & 25 & M4 & ITALY & ITALY & ITALY & & & & HECLA STREET & CALUMET - LAURIUM \\
\hline 1729 & 108 & ZANA & PALMER & $\mathrm{s}$ & 3 & $\mathrm{~s}$ & MICHIGAN & ITALY & ITALY & & & TORINO & HECLA STREET & CALUMET - LAURIUM \\
\hline 1730 & 108 & ZANA & $\mathrm{JOH}$ & $\mathrm{s}$ & $8 \mathrm{M}$ & $\mathrm{s}$ & MICHIGAN & ITALY & ITALY & & & TORINO & HECLA STREET & CALUMET - LAURIUM \\
\hline 1731 & & MARCO & PETER & $\mathrm{H}$ & 30 & M9 & ITALY & ITALY & ITALY & NA & TIMBERMAN/CM & TORINO & FIRST STREET & CALUMET - LAURIUM \\
\hline 1732 & & MARCO & MARGARET & w & 26 & M9 & ITALY & ITALY & ITALY & & & TORINO & FIRST STREET & CALUMET - LAURIUM \\
\hline 1733 & & MARCO & DOMINICK & s & 7 & $\mathrm{~S}$ & ITALY & ITALY & ITALY & & AT SCHOOL 10 & TORINO & FIRST STREET & CALUMET - LAURIUM \\
\hline 1734 & & MARCO & LUCY & $\mathrm{D}$ & 4 & $\mathrm{~s}$ & MICHIGAN & ITALY & ITALY & & & TORINO & FIRST STREET & CALUMET - LAURIUM \\
\hline 1735 & & MARCO & MAUD & $\mathrm{D}$ & 1 & s & MICHIGAN & ITALY & ITALY & & & TORINO & FIRST STREET & CALUMET - LAURIUM \\
\hline 1736 & & CAPELLO & ANTON & $\mathrm{H}$ & 34 & M8 & ITALY & ITALY & ITALY & NA & DAY LABORER/CM & PONT CANAVESE. TO & FIRST STREET & CALUMET - LAURIUM \\
\hline 1737 & & CAPELLO & CLARA & w & 25 & M8 & ITALY & ITALY & ITALY & & & PONT CANAVESE. TO & FIRST STREET & CALUMET - LAURIUM \\
\hline 1738 & & CAPELLO & MARY & $\mathrm{D}$ & 6 & $\mathrm{~s}$ & MICHIGAN & ITALY & ITALY & & AT SCHOOL 5 & PONT CANAVESE. TO & FIRST STREET & CALUMET - LAURIUM \\
\hline 1739 & & CAPELLO & JAMES & $\mathrm{s}$ & 4 & $\mathrm{~S}$ & MICHIGAN & ITALY & ITALY & & & PONT CANAVESE. TO & FIRST STREET & CALUMET - LAURIUM \\
\hline 1740 & & CAPELLO & JOHN & $\mathrm{s}$ & 2 & $\mathrm{~s}$ & MICHIGAN & ITALY & ITALY & & & PONT CANAVESE. TO & FIRST STREET & CALUMET - LAURIUM \\
\hline 1741 & & CAPELLO & JOSEPH & $\mathrm{s}$ & $7 \mathrm{M}$ & $\mathrm{s}$ & MICHIGAN & ITALY & ITALY & & & PONT CANAVESE. TO & FIRST STREET & CALUMET - LAURIUM \\
\hline 1742 & & CAPELLO & MARY & $\mathrm{D}$ & 6 & $\mathrm{~s}$ & MICHIGAN & ITALY & ITALY & & AT SCHOOL 5 & PONT CANAVESE. TO & FIRST STREET & CALUMET - LAURIUM \\
\hline 1743 & & CAPELLO & JAMES & $\mathrm{s}$ & 4 & $\mathrm{~s}$ & MICHIGAN & ITALY & ITALY & & & PONT CANAVESE. TO & FIRST STREET & CALUMET - LAURIUM \\
\hline 1744 & & CAPELLO & JOHN & $\mathrm{s}$ & 2 & $\mathrm{~s}$ & MICHIGAN & ITALY & ITALY & & & PONT CANAVESE. TO & FIRST STREET & CALUMET - LAURIUM \\
\hline 1745 & & CAPELLO & JOSEPH & $\mathrm{s}$ & $7 \mathrm{M}$ & s & MICHIGAN & ITALY & ITALY & & & PONT CANAVESE. TO & FIRST STREET & CALUMET - LAURIUM \\
\hline 1746 & & TONIEZA & ANTON & H & 25 & M2 & ITALY & ITALY & ITALY & PA & TRAMMER/CM & TUUI LAINAVESE. TO & KEARSARGE ST. & $\begin{array}{l}\text { CALUMET - LAURIUM } \\
\text { CAUM }\end{array}$ \\
\hline 1747 & & TONIEZA & $?$ & w & 22 & M2 & ITALY & ITALY & ITALY & & & & KEARSARGE ST. & CALUMET - LAURIUM \\
\hline 1748 & & CONTRATTO & PIETRO & B & 21 & $\mathrm{~s}$ & ITALY & ITALY & ITALY & $\mathrm{AL}$ & TRAMMER/CM & LOCANA CANAVESE. TO & KEARSARGE ST. & CALUMET - LAURIUM \\
\hline 1749 & & TONIEZA & ANDREW & B & 26 & $\mathrm{~s}$ & ITALY & ITALY & ITALY & $\mathrm{AL}$ & TRAMMER/CM & & KEARSARGE ST. & CALUMET - LAURIUM \\
\hline 1750 & & TONIEZA & PETER & B & 25 & $\mathrm{~s}$ & ITALY & ITALY & ITALY & $\mathrm{AL}$ & TRAMMER/CM & & KEARSARGE ST. & CALUMET - LAURIUM \\
\hline 1751 & & TONIEZA & CHAS & B & 22 & $\mathrm{~s}$ & ITALY & ITALY & ITALY & $\mathrm{AL}$ & TRAMMER/CM & & KEARSARGE ST. & CALUMET - LAURIUM \\
\hline 1752 & & VERGA & PETER & $\mathrm{H}$ & 31 & s & ITALY & ITALY & ITALY & PA & SHOEMAKER & TORINO & KEARSARGE ST. & CALUMET - LAURIUM \\
\hline 1753 & 109 & ORLANDO & JOHN & $\mathrm{H}$ & 35 & M3 & ITALY & ITALY & ITALY & NA & TRAMMER/CM & & KEARSARGE ST. & CALUMET - LAURIUM \\
\hline 1754 & 109 & ORLANDO & TRACY & W & 29 & M3 & ITALY & ITALY & ITALY & & & & KEARSARGE ST. & CALUMET - LAURIUM \\
\hline 1755 & & MARTA & PIETRO & $\mathrm{H}$ & 39 & M8 & ITALY & ITALY & ITALY & $?$ & TIMBERMAN/CM & SAN GIOVANNI CANAVESE. & KEARSARGE ST. & CALUMET - LAURIUM \\
\hline 1756 & & MARTA & MARY & w & 24 & M8 & MICHIGAN & ITALY & ITALY & & & & KEARSARGE ST. & CALUMET - LAURIUM \\
\hline 1757 & & MARTA & MINNIE & $\mathrm{D}$ & 7 & $\mathrm{~s}$ & MICHIGAN & ITALY & MICHIGAN & & AT SCHOOL 10 & SAN GIOVANNI CANAVESE. & KEARSARGE ST. & CALUMET - LAURIUM \\
\hline 1758 & & MARTA & TRACY & $\mathrm{D}$ & 5 & s & MICHIGAN & ITALY & MICHIGAN & & AT SCHOOL 2 & SAN GIOVANNI CANAVESE. & KEARSARGE ST. & CALUMET - LAURIUM \\
\hline 1759 & & MARTA & MARY & $\mathrm{D}$ & 3 & $\mathrm{~s}$ & MICHIGAN & ITALY & MICHIGAN & & & SAN GIOVANNI CANAVESE. & KEARSARGE ST. & CALUMET - LAURIUM \\
\hline 1760 & & MARTA & ADELAIDE & $\mathrm{D}$ & $8 \mathrm{M}$ & s & MICHIGAN & ITALY & MICHIGAN & & & SAN GIOVANNI CANAVESE. & KEARSARGE ST. & CALUMET - LAURIUM \\
\hline 1761 & & MARTA & DOMINICK & BR & 31 & M16 & MICHIGAN & ITALY & MICHIGAN & & TRAMMER/CM & SAN GIOVANNI CANAVESE. & KEARSARGE ST. & CALUMET - LAURIUM \\
\hline
\end{tabular}




\begin{tabular}{|c|c|c|c|c|c|c|c|c|c|c|c|c|c|c|}
\hline 1762 & & CHIRI & PAUL & B & 25 & M3 & ITALY & ITALY & ITALY & PA & TRAMMER/CM & TORINO & KEARSARGE ST. & CALUMET - LAURIUM \\
\hline 1763 & & SERIFINO & ANDREW & B & 28 & M4 & ITALY & ITALY & ITALY & PA & TRAMMER/CM & & KEARSARGE ST. & CALUMET - LAURIUM \\
\hline 1764 & & CARBONATTO & CHARLES & $\mathrm{H}$ & 27 & M3 & ITALY & ITALY & ITALY & $\mathrm{AL}$ & WATCHMAN/CM & PONT CANAVESE. TO & KEARSARGE ST. & CALUMET - LAURIUM \\
\hline 1765 & & CARBONATTO & MARY & W & 23 & M3 & ITALY & ITALY & ITALY & & & PONT CANAVESE. TO & KEARSARGE ST. & CALUMET - LAURIUM \\
\hline 1766 & & MARGINETTO & ANDREW & $\mathrm{H}$ & 29 & M6 & ITALY & ITALY & ITALY & NA & STONE MASON & TORINO & KEARSARGE ST. & CALUMET - LAURIUM \\
\hline 1767 & & MARGINETTO & FELICITA & w & 21 & M6 & ITALY & ITALY & ITALY & & & & KEARSARGE ST. & CALUMET - LAURIUM \\
\hline 1768 & & MARGINETTO & BERNARD & $\mathrm{s}$ & 5 & $\mathrm{~s}$ & MICHIGAN & ITALY & ITALY & & AT SCHOOL 4 & TORINO & KEARSARGE ST. & CALUMET - LAURIUM \\
\hline 1769 & & MARGINETTO & PETER & $\mathrm{s}$ & 4 & $\mathrm{~s}$ & MICHIGAN & ITALY & ITALY & & & TORINO & KEARSARGE ST. & CALUMET - LAURIUM \\
\hline 1770 & & MARGINETTO & MARY & D & $7 \mathrm{M}$ & s & MICHIGAN & ITALY & ITALY & & & TORINO & KEARSARGE ST. & CALUMET - LAURIUM \\
\hline 1771 & & PELLEGRINI & LUIGI & B & 24 & s & ITALY & ITALY & ITALY & $\mathrm{AL}$ & NOT CLEAR & CAMAIORE. LU & KEARSARGE ST. & CALUMET - LAURIUM \\
\hline 1772 & & RICHETTA & JOHN & $\mathrm{H}$ & 30 & M4 & ITALY & ITALY & ITALY & NA & DAY LABORER/CM & SAN MARTINO CANAVESE. TO & KEARSARGE ST. & CALUMET - LAURIUM \\
\hline 1773 & & RICHETTA & MARY & w & 22 & M4 & ITALY & ITALY & ITALY & & & SAN MARTINO CANAVESE. TO & KEARSARGE ST. & CALUMET - LAURIUM \\
\hline 1774 & & RICHETTA & ANNIE & $\mathrm{D}$ & 2 & $\mathrm{~s}$ & ITALY & ITALY & ITALY & & & SAN MARTINO CANAVESE. TO & KEARSARGE ST. & CALUMET - LAURIUM \\
\hline 1775 & & TINETTI & PAOLO & $\mathrm{H}$ & 24 & M2 & ITALY & ITALY & ITALY & $\mathrm{AL}$ & TRAMMER/CM & SAN MARTINO CANAVESE. TO & KEARSARGE ST. & CALUMET - LAURIUM \\
\hline 1776 & & TINETTI & EMILY & W & 23 & M2 & ITALY & ITALY & ITALY & & & SAN MARTINO CANAVESE. TO & KEARSARGE ST. & CALUMET - LAURIUM \\
\hline 1777 & & TINETTI & FRANK & $\mathrm{s}$ & 1 & $\mathrm{~s}$ & ITALY & ITALY & ITALY & & & SAN MARTINO CANAVESE, TO & KEARSARGE ST. & CALUMET - LAURIUM \\
\hline 1778 & & TINETTI & JOHN & s & ?M & s & ITALY & ITALY & ITALY & & & SAN MARTINO CANAVESE. TO & KEARSARGE ST. & CALUMET - LAURIUM \\
\hline 1779 & & MASSOGLIA & PETER & ?IL & 52 & M25 & ITALY & ITALY & ITALY & $\mathrm{AL}$ & DAY LABORER/CM & TORINO & KEARSARGE ST. & CALUMET - LAURIUM \\
\hline 1780 & 131 & PERENCHIO & JOSEPH & $\mathrm{H}$ & 35 & M9 & ITALY & ITALY & ITALY & PA & MINER/CM & TORINO & KEARSARGE ST. & CALUMET - LAURIUM \\
\hline 1781 & 131 & PERENCHIO & MARY & w & 28 & M9 & ITALY & ITALY & ITALY & & & TORINO & KEARSARGE ST. & CALUMET - LAURIUM \\
\hline 1782 & 131 & PERENCHIO & ANDREW & s & 7 & $\mathrm{~s}$ & ITALY & ITALY & ITALY & & AT SCHOOL 10 & TORINO & KEARSARGE ST. & CALUMET - LAURIUM \\
\hline 1783 & 131 & PERENCHIO & JOHN & $\mathrm{s}$ & 2 & $\mathrm{~s}$ & ITALY & ITALY & ITALY & & & TORINO & KEARSARGE ST. & CALUMET - LAURIUM \\
\hline 1784 & 131 & ADDA & GEORGE & B & 27 & $\mathrm{~s}$ & ITALY & ITALY & ITALY & PA & TRAMMER/CM & TORINO & KEARSARGE ST. & CALUMET - LAURIUM \\
\hline 1785 & 131 & PERENCHIO & JOHN & B & 25 & s & ITALY & ITALY & ITALY & & DAY LABORER/CM & TORINO & KEARSARGE ST. & CALUMET - LAURIUM \\
\hline 1786 & 137 & FAUSONE & PETER & $\mathrm{H}$ & 39 & M9 & ITALY & ITALY & ITALY & NA & TIMBERMAN/CM & TORINO & KEARSARGE ST. & CALUMET - LAURIUM \\
\hline 1787 & 137 & FAUSONE & MARTA? & W & 28 & M9 & ITALY & ITALY & ITALY & & & & KEARSARGE ST. & CALUMET - LAURIUM \\
\hline 1788 & 137 & FAUSONE & PETER & $\mathrm{s}$ & 7 & $\mathrm{~s}$ & MICHIGAN & ITALY & ITALY & & AT SCHOOL 5 & TORINO & KEARSARGE ST. & CALUMET - LAURIUM \\
\hline 1789 & 137 & FAUSONE & FRANK & $\mathrm{s}$ & 4 & $\mathrm{~s}$ & MICHIGAN & ITALY & ITALY & & & TORINO & KEARSARGE ST. & CALUMET - LAURIUM \\
\hline 1790 & 137 & FAUSONE & DOMINICK & $\mathrm{s}$ & 1 & s & MICHIGAN & ITALY & ITALY & & & TORINO & KEARSARGE ST. & CALUMET - LAURIUM \\
\hline 1791 & 137 & CHERO & LOUIS & $\mathrm{H}$ & 40 & M11 & ITALY & ITALY & ITALY & $\mathrm{AL}$ & TIMBERMAN/CM & & KEARSARGE ST. & CALUMET - LAURIUM \\
\hline 1792 & 137 & CHERO & MARY & W & 39 & M11 & ITALY & ITALY & ITALY & & & & KEARSARGE ST. & CALUMET - LAURIUM \\
\hline 1793 & 137 & CHERO & MARY & $\mathrm{D}$ & 7 & $\mathrm{~s}$ & MICHIGAN & ITALY & ITALY & & AT SCHOOL 5 & & KEARSARGE ST. & CALUMET - LAURIUM \\
\hline 1794 & 137 & CHERO & JOHN & $\mathrm{s}$ & 2 & s & ITALY & ITALY & ITALY & & & & KEARSARGE ST. & CALUMET - LAURIUM \\
\hline 1795 & 137 & CASERIO & TONY & $\mathrm{H}$ & 37 & M20 & ITALY & ITALY & ITALY & NA & DAY LABORER/CM & STRAMBINELLO, TO & KEARSARGE ST. & CALUMET - LAURIUM \\
\hline 1796 & 137 & CASERIO & MAGGIE & w & 40 & M20 & ITALY & ITALY & ITALY & & & STRAMBINELLO. TO & KEARSARGE ST. & CALUMET - LAURIUM \\
\hline 1797 & 137 & $\begin{array}{l}\text { CASERIO } \\
\text { CASER }\end{array}$ & DOMINICK & s & 18 & $s$ & ITALY & ITALY & ITALY & & DRILL BOY/CM & STRAMBINELLO. TO & KEARSARGE ST. & $\begin{array}{l}\text { CALUMET - LAURIUM } \\
\text { CAU }\end{array}$ \\
\hline 1798 & 137 & CASERIO & MARY & $\mathrm{D}$ & 14 & $\mathrm{~s}$ & ITALY & ITALY & ITALY & & AT SCHOOL 10 & STRAMBINELLO. TO & KEARSARGE ST. & CALUMET - LAURIUM \\
\hline 1799 & 137 & CASERIO & JENNIE & $\mathrm{D}$ & 11 & $\mathrm{~s}$ & ITALY & ITALY & ITALY & & AT SCHOOL 10 & STRAMBINELLO. TO & KEARSARGE ST. & CALUMET - LAURIUM \\
\hline 1800 & & BARSANTI & ORLANDO & B & 24 & $\mathrm{~s}$ & ITALY & ITALY & ITALY & $\mathrm{N}$ & SALESMAN & SEGROMIGNO. LU & KEARSARGE ST. & CALUMET - LAURIUM \\
\hline 1801 & 221 & GALLASERO & JOHN & $\mathrm{H}$ & 31 & M12 & ITALY & ITALY & ITALY & PA & TRAMMER/CM & TORINO & TAMARACK ST. & CALUMET - LAURIUM \\
\hline 1802 & 221 & GALLASERO & ROSIE & W & 33 & M12 & ITALY & ITALY & ITALY & & & & TAMARACK ST. & CALUMET - LAURIUM \\
\hline 1803 & 221 & GALLASERO & JAMES & $\mathrm{s}$ & 7 & s & MICHIGAN & ITALY & ITALY & & AT SCHOOL 9 & TORINO & TAMARACK ST. & CALUMET - LAURIUM \\
\hline 1804 & 221 & GALLASERO & DOMINICK & $\mathrm{s}$ & 6 & $\mathrm{~s}$ & MICHIGAN & ITALY & ITALY & & AT SCHOOL 4 & TORINO & TAMARACK ST. & CALUMET - LAURIUM \\
\hline 1805 & 221 & GALLASERO & JOHN & $\mathrm{s}$ & 3 & $\mathrm{~s}$ & MICHIGAN & ITALY & ITALY & & & TORINO & TAMARACK ST. & CALUMET - LAURIUM \\
\hline 1806 & 221 & GALLASERO & MARY & D & 1 & $\mathrm{~s}$ & MICHIGAN & ITALY & ITALY & & & TORINO & TAMARACK ST. & CALUMET - LAURIUM \\
\hline 1807 & & CAPELLO & JAMES & $\mathrm{H}$ & 40 & M12 & ITALY & ITALY & ITALY & NA & STONE CONTRACTOR & SAN BENIGNO CANAVESE. TO & IROQUOIS ST. & CALUMET - LAURIUM \\
\hline 1808 & & CAPELLO & JOHANNA & w & 30 & M12 & ITALY & ITALY & ITALY & & & & IROQUOIS ST. & CALUMET - LAURIUM \\
\hline 1809 & & CAPELLO & DUSOLINA & $\mathrm{D}$ & 11 & $\mathrm{~s}$ & MICHIGAN & ITALY & ITALY & & AT SCHOOL 10 & SAN BENIGNO CANAVESE. TO & IROQUOIS ST. & CALUMET - LAURIUM \\
\hline 1810 & & CAPELLO & JOHN & $\mathrm{s}$ & 10 & $\mathrm{~s}$ & MICHIGAN & ITALY & ITALY & & AT SCHOOL 10 & SAN BENIGNO CANAVESE. TO & IROQUOIS ST. & CALUMET - LAURIUM \\
\hline 1811 & & CAPELLO & LOUIS & $\mathrm{s}$ & 8 & $\mathrm{~s}$ & MICHIGAN & ITALY & ITALY & & AT SCHOOL 10 & SAN BENIGNO CANAVESE. TO & IROQUOIS ST. & CALUMET - LAURIUM \\
\hline 1812 & & CAPELLO & JAMES & $\mathrm{s}$ & 5 & $\mathrm{~s}$ & MICHIGAN & ITALY & ITALY & & & SAN BENIGNO CANAVESE. TO & IROQUOIS ST. & CALUMET - LAURIUM \\
\hline 1813 & & CAPELLO & FRED & $\mathrm{s}$ & 1 & s & MICHIGAN & ITALY & ITALY & & & SAN BENIGNO CANAVESE. TO & IROQUOIS ST. & CALUMET - LAURIUM \\
\hline 1814 & & ZIROTTI & JOHN & $\mathrm{H}$ & 35 & M11 & ITALY & ITALY & ITALY & AL & TRAMMER/CM & 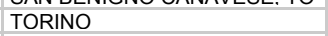 & TAMARACK ST. & CALUMET - LAURIUM \\
\hline 1815 & & ZEROTI-PRICCO & MARY & W & 32 & M11 & ITALY & ITALY & ITALY & & & TORINO & TAMARACK ST. & CALUMET - LAURIUM \\
\hline 1816 & & ZIROTTI & AMELIA & D & 9 & $\mathrm{~s}$ & FRANCE & ITALY & ITALY & & AT SCHOOL 10 & TORINO & TAMARACK ST. & CALUMET - LAURIUM \\
\hline 1817 & & ZIROTTI & GINA & $\mathrm{D}$ & 6 & $\mathrm{~s}$ & FRANCE & ITALY & ITALY & & & TORINO & TAMARACK ST. & CALUMET - LAURIUM \\
\hline 1818 & & ZIROTTI & FREDERICK & $\mathrm{s}$ & 4 & $\mathrm{~s}$ & FRANCE & ITALY & ITALY & & & TORINO & TAMARACK ST. & CALUMET - LAURIUM \\
\hline 1819 & & ZIROTTI & JOHN & $\mathrm{s}$ & 10 & s & MICHIGAN & ITALY & ITALY & & & TORINO & TAMARACK ST. & CALUMET - LAURIUM \\
\hline 1820 & & SENTO & PIETRO & B & 31 & M0 & ITALY & ITALY & ITALY & $\mathrm{AL}$ & TRAMMER/CM & SAN MARTINO CANAVESE. TO & TAMARACK ST. & CALUMET - LAURIUM \\
\hline 1821 & & GIARTI & JOSEPH & B & 30 & $\mathrm{~s}$ & ITALY & ITALY & ITALY & NA & TRAMMER/CM & & TAMARACK ST. & CALUMET - LAURIUM \\
\hline 1822 & & MASSOGLIA & MARTIN & B & 43 & $\mathrm{~s}$ & ITALY & ITALY & ITALY & $\mathrm{AL}$ & & SAN MARTINO CANAVESE. TO & TAMARACK ST. & CALUMET - LAURIUM \\
\hline 1823 & & PRICCO & MARGARET & SIL & 35 & s & ITALY & ITALY & ITALY & & SERVANT & SAN MARTINO CANAVESE. TO & TAMARACK ST. & CALUMET - LAURIUM \\
\hline 1824 & & TERESA & LAURA & $\mathrm{H}$ & 35 & M9 & ITALY & ITALY & ITALY & & WASH WOMAN & TORINO & TAMARACK ST. & CALUMET - LAURIUM \\
\hline
\end{tabular}




\begin{tabular}{|c|c|c|c|c|c|c|c|c|c|c|c|c|c|c|}
\hline 1825 & & TERESA & ALBERT & $\mathrm{s}$ & 7 & $\mathrm{~s}$ & ITALY & ITALY & ITALY & & AT SCHOOL 10 & TORINO & TAMARACK ST. & CALUMET - LAURIUM \\
\hline 1826 & & TERESA & JOSEPH & $\mathrm{s}$ & 5 & S & ITALY & ITALY & ITALY & & & TORINO & TAMARACK ST. & CALUMET - LAURIUM \\
\hline 1827 & & MONTICELLI & JAMES & $\mathrm{H}$ & 25 & M2 & ITALY & ITALY & ITALY & $\mathrm{AL}$ & TRAMMER/CM & MATRAIA. LU & TAMARACK ST. & CALUMET - LAURIUM \\
\hline 1828 & & MONTICELLI & CAROLINE & W & 24 & M2 & ITALY & ITALY & ITALY & & & & TAMARACK ST. & CALUMET - LAURIUM \\
\hline 1829 & & ZEINA/ZEIMA & VENTURE & B & 32 & M7 & ITALY & ITALY & ITALY & $\mathrm{AL}$ & TRAMMER/CM & & TAMARACK ST. & CALUMET - LAURIUM \\
\hline 1830 & & MAZZONI & EMILIO & B & 31 & $\mathrm{~S}$ & ITALY & ITALY & ITALY & $\mathrm{AL}$ & TRAMMER/CM & LUCCA & TAMARACK ST. & CALUMET - LAURIUM \\
\hline 1831 & & LOCATELLI & PETER & B & 25 & s & ITALY & ITALY & ITALY & $\mathrm{AL}$ & STONE MASON & SELINO. BERGAMO & TAMARACK ST. & CALUMET - LAURIUM \\
\hline 1832 & & LUCAS & CENTURIO & $\mathrm{H}$ & 24 & M0 & ITALY & ITALY & ITALY & NA & TRAMMER/CM & & TAMARACK ST. & CALUMET - LAURIUM \\
\hline 1833 & & LUCAS & ANTONIETTE & W & 28 & MO & ITALY & ITALY & ITALY & & T1 & & TAMARACK ST. & CALUMET - LAURIUM \\
\hline 1834 & & LOCATELLI & CARLO & $\mathrm{H}$ & 34 & M3 & ITALY & ITALY & ITALY & $\mathrm{AL}$ & STONE MASON & SELINO. BERGAMO & TAMARACK ST. & CALUMET - LAURIUM \\
\hline 1835 & & LOCATELLI & SEHERINA & W & 23 & M3 & ITALY & ITALY & ITALY & & & SELINO. BERGAMO & TAMARACK ST. & CALUMET - LAURIUM \\
\hline 1836 & & LOCATELLI & AUGUST & s & 2 & $\mathrm{~s}$ & MICHIGAN & ITALY & ITALY & & & SELINO. BERGAMO & TAMARACK ST. & CALUMET - LAURIUM \\
\hline 1837 & & LOCATELLI & MARY & D & 1 & $\mathrm{~S}$ & MICHIGAN & ITALY & ITALY & & & SELINO. BERGAMO & TAMARACK ST. & CALUMET - LAURIUM \\
\hline 1838 & & LOCATELLI & CHARLES & B & 29 & M8 & ITALY & ITALY & ITALY & $\mathrm{AL}$ & TRAMMER/CM & SELINO. BERGAMO & TAMARACK ST. & CALUMET - LAURIUM \\
\hline 1839 & & LUCAS & LEO & B & 24 & $\mathrm{~s}$ & ITALY & ITALY & ITALY & AL & TRAMMER/CM & & TAMARACK ST. & CALUMET - LAURIUM \\
\hline 1840 & & BESSOLO & FRANK & $\mathrm{H}$ & 40 & M10 & ITALY & ITALY & ITALY & NA & MINER/CM & BESSOLO CANAVESE, TO & TAMARACK ST. & CALUMET - LAURIUM \\
\hline 1841 & & BESSOLO & JOSEPHINE & W & 32 & M10 & ITALY & ITALY & ITALY & & & & TAMARACK ST. & CALUMET - LAURIUM \\
\hline 1842 & & BESSOLO & ADAM & s & 9 & $\mathrm{~s}$ & MICHIGAN & ITALY & ITALY & & AT SCHOOL 10 & BESSOLO CANAVESE. TO & TAMARACK ST. & CALUMET - LAURIUM \\
\hline 1843 & & BESSOLO & EVA & $\mathrm{D}$ & 7 & $\mathrm{~s}$ & MICHIGAN & ITALY & ITALY & & AT SCHOOL 10 & BESSOLO CANAVESE. TO & TAMARACK ST. & CALUMET - LAURIUM \\
\hline 1844 & & BESSOLO & DESOHINA & $\mathrm{D}$ & 6 & $\mathrm{~s}$ & MICHIGAN & ITALY & ITALY & & AT SCHOOL 10 & BESSOLO CANAVESE. TO & TAMARACK ST. & CALUMET - LAURIUM \\
\hline 1845 & & BESSOLO & FELIX & B & 28 & $\mathrm{~s}$ & ITALY & ITALY & ITALY & NA & TRAMMER/CM & SCARMAGNO. TO & TAMARACK ST. & CALUMET - LAURIUM \\
\hline 1846 & & TORREANO & JOSEPH & B & 26 & $\mathrm{~s}$ & ITALY & ITALY & ITALY & PA & TRAMMER/CM & TORINO & TAMARACK ST. & CALUMET - LAURIUM \\
\hline 1847 & & CHINO & JOSEP & B & 55 & $\mathrm{~s}$ & ITALY & ITALY & ITALY & NA & DAY LABORER/CM & & TAMARACK ST. & CALUMET - LAURIUM \\
\hline 1848 & & BORGO & DOMINICK & $\mathrm{H}$ & 37 & M12 & ITALY & ITALY & ITALY & $?$ & TEAMSTER & VICENZA & TAMARACK ST. & CALUMET - LAURIUM \\
\hline 1849 & & BORGO & KATRIRMA & w & 28 & M12 & ITALY & ITALY & ITALY & & & & TAMARACK ST. & CALUMET - LAURIUM \\
\hline 1850 & & BORGO & JOHN & $\mathrm{s}$ & 10 & $\mathrm{~s}$ & MICHIGAN & ITALY & ITALY & & AT SCHOOL 10 & VICENZA & TAMARACK ST. & CALUMET - LAURIUM \\
\hline 1851 & & ROLANDO & ANTON & B & 30 & $\mathrm{~s}$ & ITALY & ITALY & ITALY & & TEAMSTER & TORINO & TAMARACK ST. & CALUMET - LAURIUM \\
\hline 1852 & & TESTA & JOHN & $\mathrm{H}$ & 23 & M3 & ITALY & ITALY & ITALY & & MINER/CM & BAIRO CANAVESE, TO & TAMARACK ST. & CALUMET - LAURIUM \\
\hline 1853 & & TESTA & MARY & w & 18 & M3 & ITALY & ITALY & ITALY & & & BAIRO CANAVESE. TO & TAMARACK ST. & CALUMET - LAURIUM \\
\hline 1854 & & VAIRO & VINCENZO & $\mathrm{H}$ & 35 & M10 & ITALY & ITALY & ITALY & NA & STONE MASON & LOCANA CANAVESE. TO & TAMARACK ST. & CALUMET - LAURIUM \\
\hline 1855 & & VAIRO & GIULIA & W & 32 & M10 & ITALY & ITALY & ITALY & & & LOCANA CANAVESE. TO & TAMARACK ST. & CALUMET - LAURIUM \\
\hline 1856 & & VAIRO & DOMENICO & s & 10 & $\mathrm{~s}$ & ITALY & ITALY & ITALY & & AT SCHOOL 10 & LOCANA CANAVESE. TO & TAMARACK ST. & CALUMET - LAURIUM \\
\hline 1857 & & VAIRO & GIOVANNI & $\mathrm{s}$ & 5 & $\mathrm{~s}$ & MICHIGAN & ITALY & ITALY & & & LOCANA CANAVESE. TO & TAMARACK ST. & CALUMET - LAURIUM \\
\hline 1858 & & VAIRO & JENNIE & $\mathrm{D}$ & 2 & $\mathrm{~s}$ & MICHIGAN & ITALY & ITALY & & & LOCANA CANAVESE, TO & TAMARACK ST. & CALUMET - LAURIUM \\
\hline 1859 & & FELETI? & MARY & SER & 30 & s & ITALY & ITALY & ITALY & & SERVANT & & TAMARACK ST. & CALUMET - LAURIUM \\
\hline 1860 & & BUGNI/BAGNI & FRAN? & $\mathrm{H}$ & 32 & M2 & ITALY & ITALY & ITALY & NA & BUTCHER & & TAMARACK ST. & CALUMET - LAURIUM \\
\hline 1861 & & BUGNI/BAGNI & ROSE & W & 20 & M2 & ITALY & ITALY & ITALY & & & & TAMARACK ST. & CALUMET - LAURIUM \\
\hline 1862 & & EMANUEL & LOUIS & B & 28 & $\mathrm{~s}$ & ITALY & ITALY & ITALY & PA & BUTCHER & & TAMARACK ST. & CALUMET - LAURIUM \\
\hline 1863 & & NICHAUS & PETER & $\mathrm{H}$ & 40 & M6 & ITALY & ITALY & ITALY & NA & MINER/CM & & TAMARACK ST. & CALUMET - LAURIUM \\
\hline 1864 & & NICHAUS & KATE & W & 27 & M6 & ITALY & ITALY & ITALY & INA & & & TAMARACK ST. & CALUMET - LAURIUM \\
\hline 1865 & & NICHAUS & MIKE & s & 4 & $\mathrm{~s}$ & MICHIGAN & ITALY & ITALY & & & & TAMARACK ST. & CALUMET - LAURIUM \\
\hline 1866 & & NICHAUS & MAGGIE & D & 3 & $\mathrm{~s}$ & MICHIGAN & ITALY & ITALY & & & & TAMARACK ST. & CALUMET - LAURIUM \\
\hline 1867 & & NICHAUS & JOHN & $\mathrm{s}$ & $2 \mathrm{M}$ & $\mathrm{s}$ & MICHIGAN & ITALY & ITALY & & & & TAMARACK ST. & CALUMET - LAURIUM \\
\hline 1868 & & VAIRO & DOMINICK & $\mathrm{H}$ & 36 & M13 & ITALY & ITALY & ITALY & NA & SALOONKEEPER & LOCANA CANAVESE. TO & TAMARACK ST. & CALUMET - LAURIUM \\
\hline 1869 & & CERUTTI & LUCY & SER & 25 & $\mathrm{~s}$ & ITALY & ITALY & ITALY & & SERVANT & TORINO & TAMARACK ST. & CALUMET - LAURIUM \\
\hline 1870 & & TEPATTI & LORENZO? & B & 34 & s & ITALY & ITALY & ITALY & PA & STONE MASON & PONT CANAVESE. TO & TAMARACK ST. & CALUMET - LAURIUM \\
\hline 1871 & & TEPATTI & FRANCISCO & B & 27 & M3 & ITALY & ITALY & ITALY & PA & DAY LABORER/CM & PONT CANAVESE. TO & TAMARACK ST. & CALUMET - LAURIUM \\
\hline 1872 & & ARDISIN? & FIN? & B & 28 & M3 & ITALY & ITALY & ITALY & $\mathrm{AL}$ & DAY LABORER/CM & & TAMARACK ST. & CALUMET - LAURIUM \\
\hline 1873 & & SONTUTSI & PETER & B & 40 & $\mathrm{~s}$ & ITALY & ITALY & ITALY & NA & MINER/CM & & TAMARACK ST. & CALUMET - LAURIUM \\
\hline 1874 & & CERETATTO & TOMATZI? & B & 22 & $\mathrm{~s}$ & ITALY & ITALY & ITALY & PA & TEAMSTER & & TAMARACK ST. & CALUMET - LAURIUM \\
\hline 1875 & & JANI? & CAROLINO & B & 35 & $\mathrm{~s}$ & ITALY & ITALY & ITALY & NA & STONE MASON & & TAMARACK ST. & CALUMET - LAURIUM \\
\hline 1876 & & GROSEGNE & CAMILLO & B & 37 & s & ITALY & ITALY & ITALY & NA & DAY LABORER/CM & & TAMARACK ST. & CALUMET - LAURIUM \\
\hline 1877 & & CAPELLO & CARLO & $\mathrm{H}$ & 45 & M21 & ITALY & ITALY & ITALY & NA & MINER/CM & COURGNE'. TO & TAMARACK ST. & CALUMET - LAURIUM \\
\hline 1878 & & CAPELLO & MARY & w & 40 & M21 & ITALY & ITALY & ITALY & & & & TAMARACK ST. & CALUMET - LAURIUM \\
\hline 1879 & & LOZI & CAESAR & B & 35 & S & ITALY & ITALY & ITALY & AL & BARTENDER & & TAMARACK ST. & CALUMET - LAURIUM \\
\hline 1880 & & BESSOLO & PETER & $\mathrm{H}$ & 50 & $?$ & ITALY & ITALY & ITALY & NA & MINER/CM & SAN MARTINO CANAVESE. TO & KEARSARGE ST. & CALUMET - LAURIUM \\
\hline 1881 & & BESSOLO & MARY & w & 47 & $?$ & ITALY & ITALY & ITALY & & & SAN MARTINO CANAVESE. TO & KEARSARGE ST. & CALUMET - LAURIUM \\
\hline 1882 & & BESSOLO & PETER J. & s & 17 & $?$ & ALGERIA & ITALY & ITALY & & AT SCHOOL 10 & SAN MARTINO CANAVESE, TO & KEARSARGE ST. & CALUMET - LAURIUM \\
\hline 1883 & & BESSOLO & JAMES & $\mathrm{s}$ & 8 & $?$ & MICHIGAN & ITALY & ITALY & & AT SCHOOL 10 & SAN MARTINO CANAVESE. TO & KEARSARGE ST. & CALUMET - LAURIUM \\
\hline 1884 & & BESSOLO & JOHN & $\mathrm{H}$ & 28 & $?$ & ITALY & ITALY & ITALY & & MINER/CM & BESSOLO CANAVESE. TO & KEARSARGE ST. & CALUMET - LAURIUM \\
\hline 1885 & & BESSOLO & MODESTA & w & 25 & $?$ & ITALY & ITALY & ITALY & & & & KEARSARGE ST. & CALUMET - LAURIUM \\
\hline 1886 & & BESSOLO & MARY & $\mathrm{D}$ & $7 \mathrm{M}$ & $?$ & MICHIGAN & ITALY & ITALY & & & BESSOLO CANAVESE. TO & KEARSARGE ST. & CALUMET - LAURIUM \\
\hline 1887 & 111 & RONMONTTA & $\mathrm{F} ?$ & $\mathrm{H}$ & 40 & M6 & ITALY & ITALY & ITALY & NA & STONE MASON & & LAKE LINDEN & CALUMET - LAURIUM \\
\hline
\end{tabular}




\begin{tabular}{|c|c|c|c|c|c|c|c|c|c|c|c|c|c|c|}
\hline 1888 & 111 & RONMONTTA & ROSA & w & 40 & M6 & ITALY & ITALY & ITALY & & & & LAKE LINDEN & CALUMET - LAURIUM \\
\hline 1889 & 111 & RONMONTTA & CENTINA & $\mathrm{D}$ & 4 & $\mathrm{~s}$ & MICHIGAN & ITALY & ITALY & & & & LAKE LINDEN & CALUMET - LAURIUM \\
\hline 1890 & 111 & RONMONTTA & ROSA & $\mathrm{D}$ & 2 & $\mathrm{~s}$ & MICHIGAN & ITALY & ITALY & & & & LAKE LINDEN & CALUMET - LAURIUM \\
\hline 1891 & 607 & LUCCHESI & FELIX & $\mathrm{H}$ & 28 & M1 & ITALY & ITALY & ITALY & NA & LABORER/CM & LUCCA & PEWABIC ST. & CALUMET - LAURIUM \\
\hline 1892 & 607 & LUCCHESI & EMMA & w & 21 & M1 & ITALY & ITALY & ITALY & & & & PEWABIC ST. & CALUMET - LAURIUM \\
\hline 1893 & 607 & BESSONE & BAT & B & 43 & $\mathrm{~s}$ & ITALY & ITALY & ITALY & PA & LABORER/CM & SAN BENIGNO CANAVESE. TO & PEWABIC ST. & CALUMET - LAURIUM \\
\hline 1894 & 344 & DELL'OSSO & CHARLES? & $\mathrm{H}$ & 40 & $?$ & ITALY & ITALY & ITALY & NA & MERCHANT & 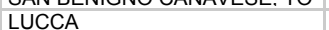 & FIFTH STREET & CALUMET - RED JACKET \\
\hline 1895 & 344 & DELL'OSSO & GIOVANNI & $\mathrm{BR}$ & 29 & $?$ & ITALY & ITALY & ITALY & NA & MERCHANT & $\begin{array}{l}\text { LUCCA } \\
\text { LUCCA }\end{array}$ & $\begin{array}{l}\text { FIFTH STREET } \\
\text { FITET }\end{array}$ & CALUMET - RED JACKET \\
\hline $\begin{array}{l}1895 \\
1896\end{array}$ & $\begin{array}{l}344 \\
344\end{array}$ & $\begin{array}{l}\text { DELLOSSO } \\
\text { BIAGI }\end{array}$ & $\begin{array}{l}\text { GIVNAPHII } \\
\text { JOSEPH }\end{array}$ & $\begin{array}{l}\text { BR } \\
\text { NEP }\end{array}$ & 17 & $?$ & $\begin{array}{l}\text { IITALY } \\
\text { ITALY }\end{array}$ & $\begin{array}{l}\text { IITALY } \\
\text { ITALY }\end{array}$ & $\begin{array}{l}\text { IIALY } \\
\text { ITALY }\end{array}$ & $\begin{array}{l}\text { NA } \\
? ?\end{array}$ & $\begin{array}{l}\text { MUERCHANI } \\
\text { LABORER }\end{array}$ & $\begin{array}{l}\text { LUCCA } \\
\text { LUCCA }\end{array}$ & $\begin{array}{l}\text { FIF IH SIREEI } \\
\text { FIFTH STREET }\end{array}$ & $\begin{array}{l}\text { CALUMET - RED JACKE IACKET } \\
\text { CALUM RE }\end{array}$ \\
\hline 1897 & 344 & FORNERIS & MARIO & $\mathrm{H}$ & 34 & $?$ & ITALY & ITALY & ITALY & NA & MASON & TORINO & FIFTH STREET & CALUMET - RED JACKET \\
\hline 1898 & 344 & FORNERIS & PALMA & w & 25 & $?$ & ITALY & ITALY & ITALY & & & & FIFTH STREET & CALUMET - RED JACKET \\
\hline 1899 & 344 & FORNERIS & TEMISTOCLE & s & $9 \mathrm{M}$ & $?$ & MICHIGAN & ITALY & ITALY & & & TORINO & FIFTH STREET & CALUMET - RED JACKET \\
\hline 1900 & 344 & TANAGLIA & CAMILLO & $\mathrm{H}$ & 40 & $?$ & ITALY & ITALY & ITALY & PA & SALESMAN & & FIFTH STREET & CALUMET - RED JACKET \\
\hline 1901 & 308 & FISHER & JOE & $\mathrm{H}$ & 40 & M7 & ITALY & ITALY & ITALY & $?$ & BUTCHER & & FIFTH STREET & CALUMET - RED JACKET \\
\hline 1902 & 308 & FISHER & KATIE & W & 25 & M7 & MICHIGAN & ITALY & ITALY & & & & FIFTH STREET & CALUMET - RED JACKET \\
\hline 1903 & 308 & FISHER & ROSIE & $\mathrm{D}$ & 5 & $\mathrm{~S}$ & MICHIGAN & ITALY & MICHIGAN & & & & FIFTH STREET & CALUMET - RED JACKET \\
\hline 1904 & 308 & FISHER & TERESA & $\mathrm{D}$ & 2 & $\mathrm{~s}$ & MICHIGAN & ITALY & MICHIGAN & & & & FIFTH STREET & CALUMET - RED JACKET \\
\hline 1905 & 308 & SADO & JOHN & B & 26 & s & ITALY & ITALY & ITALY & AL & DAY LABORER/CM & STRAMBINELLO. TO & FIFTH STREET & CALUMET - RED JACKET \\
\hline 1906 & 304 & TOMEI & PAUL & $\mathrm{H}$ & 26 & M7 & ITALY & ITALY & ITALY & NA & MERCHANT & LUCCA & FIFTH STREET & CALUMET - RED JACKET \\
\hline 1907 & 304 & TOMEI & MARY & w & 24 & M7 & ITALY & ITALY & ITALY & & & LUCCA & FIFTH STREET & CALUMET - RED JACKET \\
\hline 1908 & 304 & TOMEI & FAUSTINO & s & $\begin{array}{l}24 \\
5\end{array}$ & S & MICHIGAN & ITALY & ITALY & & & LUCCA & $\begin{array}{l}\text { FIFTH STREET } \\
\text { FIFT }\end{array}$ & CALUMET - RED JACKET \\
\hline $\begin{array}{l}1908 \\
1909\end{array}$ & $\begin{array}{l}304 \\
304\end{array}$ & $\begin{array}{l}\text { TOMEI } \\
\text { TOMEI }\end{array}$ & OLIVER & $\begin{array}{l}\mathrm{s} \\
\mathrm{s}\end{array}$ & $\begin{array}{l}5 \\
2\end{array}$ & $\begin{array}{l}\mathrm{s} \\
\mathrm{s}\end{array}$ & $\begin{array}{l}\text { MICHGAN } \\
\text { MICHIGAN }\end{array}$ & $\begin{array}{l}\text { IIALY } \\
\text { ITALY }\end{array}$ & $\begin{array}{l}\text { IIALY } \\
\text { ITALY }\end{array}$ & & & $\begin{array}{l}\text { LUCCA } \\
\text { LUCCA }\end{array}$ & $\begin{array}{l}\text { FIFIH SIREEI } \\
\text { FIFTH STREET }\end{array}$ & $\begin{array}{l}\text { CALUME - RED JACKEI I } \\
\text { CALUMET - RED JACKET }\end{array}$ \\
\hline 1910 & 304 & TOMEI & FILOMENA & SIS & 17 & $\mathrm{~s}$ & ITALY & ITALY & ITALY & & SERVANT & LUCCA & FIFTH STREET & CALUMET - RED JACKET \\
\hline 1911 & 251 & CURTO & J. & $\begin{array}{l}\mathrm{H} \\
\mathrm{H}\end{array}$ & 19 & s & MICHIGAN & ITALY & ITALY & & SALESMAN & TORINO & FIFTH STREET & CALUMET - RED JACKET \\
\hline 1912 & 251 & CURTO & DOMINICK & BR & 16 & $\mathrm{~s}$ & MICHIGAN & ITALY & $\begin{array}{l}\text { ITALY } \\
\text { ITALY }\end{array}$ & & SALESMAN & TORINO & $\begin{array}{l}\text { FIFTH STREET } \\
\text { FIF IN }\end{array}$ & CALUMET - RED JACKET \\
\hline 1913 & 251 & CURTO & ANGELO & $\mathrm{BR}$ & 15 & $\mathrm{~s}$ & MICHIGAN & ITALY & ITALY & & AT SCHOOL 10 & TORINO & FIFTH STREET & CALUMET - RED JACKET \\
\hline 1914 & 251 & CURTO & PETER & $\mathrm{BR}$ & 11 & $\mathrm{~s}$ & MICHIGAN & ITALY & ITALY & & AT SCHOOL 10 & TORINO & FIFTH STREET & CALUMET - RED JACKET \\
\hline 1915 & 251 & CURTO & VICTOR & $\mathrm{BR}$ & 13 & $\mathrm{~s}$ & MICHIGAN & ITALY & ITALY & & AT SCHOOL 10 & TORINO & FIFTH STREET & CALUMET - RED JACKET \\
\hline 1916 & 251 & CURTO & MARK & $\mathrm{BR}$ & 1 & $\mathrm{~s}$ & MICHIGAN & ITALY & ITALY & & & TORINO & FIFTH STREET & CALUMET - RED JACKET \\
\hline 1917 & 251 & TOCCOLETTI & ANTONIO & B & 28 & M10 & ITALY & ITALY & ITALY & PA & DAY LABORER/CM & & FIFTH STREET & CALUMET - RED JACKET \\
\hline 1918 & 251 & TROCCO & ANTONIO & B & 26 & M3 & ITALY & ITALY & ITALY & $\mathrm{AL}$ & DAY LABORER/CM & & FIFTH STREET & CALUMET - RED JACKET \\
\hline 1919 & 251 & BARATONO & ALEXANDER & B & 30 & $\mathrm{~s}$ & ITALY & ITALY & ITALY & $A L$ & DAY LABORER/CM & TORINO & FIFTH STREET & CALUMET - RED JACKET \\
\hline 1920 & 251 & CHIRI & MARK & $\begin{array}{l}\mathrm{D} \\
\mathrm{B}\end{array}$ & 18 & s & ITALY & ITALY & ITALY & & DAY LABORER/CM & VIALFRE'. TO & $\begin{array}{l}\text { FIFTH STREET } \\
\text { FITET }\end{array}$ & CALUMET - RED JACKET \\
\hline $\begin{array}{l}1920 \\
1921\end{array}$ & 251 & TOSCOLINO & GIOVANNI & $\begin{array}{l}B \\
B\end{array}$ & $\frac{18}{35}$ & M10 & $\begin{array}{l}\text { ITALY } \\
\text { ITALY }\end{array}$ & $\begin{array}{l}\text { IIALY } \\
\text { ITALY }\end{array}$ & $\begin{array}{l}\text { IIALY } \\
\text { ITALY }\end{array}$ & PA & $\begin{array}{l}\text { DAY LABORER/CM } \\
\text { DAY LARERIM }\end{array}$ & VIALFRE, IO & $\begin{array}{l}\text { FIF IH SIREEI } \\
\text { FIFTH STREET }\end{array}$ & $\begin{array}{l}\text { CALUMET - RED JACKET } \\
\text { CACE I }\end{array}$ \\
\hline 1922 & 343 & PALMINI & MICHAEL & $\mathrm{H}$ & 54 & M11 & ITALY & ITALY & ITALY & NA & DAY LABORER/CM & LUCCA & FIFTH STREET & CALUMET - RED JACKET \\
\hline 1923 & 343 & PALMINI & MINNIE & w & $\begin{array}{l}54 \\
36\end{array}$ & M11 & SWEDEN & SWEDEN & SWEDEN & & & SWEDEN & $\begin{array}{l}\text { FIFTH STREET } \\
\text { FITET }\end{array}$ & CALUMET - RED JACKET \\
\hline 1924 & 343 & PALMINI & MICHAEL & s & 9 & $\mathrm{~S}$ & MINNESOT & ITALY & SWEDEN & & AT SCHOOL 10 & $\begin{array}{l}\text { LUCCA } \\
\text { LUELI }\end{array}$ & $\begin{array}{l}\text { FIFTH STREET } \\
\text { FIFT }\end{array}$ & CALUMET - RED JACKET \\
\hline 1924 & 343 & PERANASO & $\begin{array}{l}\text { WICAAEL } \\
\text { EDWARD }\end{array}$ & B & 29 & $\begin{array}{l}\mathrm{s} \\
\mathrm{s}\end{array}$ & ITALY & $\begin{array}{l}\text { IIIALY } \\
\text { ITALY }\end{array}$ & $\begin{array}{l}\text { ITALY IN } \\
\text { ITAL }\end{array}$ & PA & DAY LABORER/CM & & $\begin{array}{l}\text { FIFTH STREET } \\
\text { FIFTH }\end{array}$ & CALUMET - RED JACKET \\
\hline 1920 & 331 & TOMEI & MODESTO & $\begin{array}{l}\mathrm{D} \\
\mathrm{H}\end{array}$ & 30 & M9 & ITALY & ITALY & ITALY & NA & NOT CLEAR & LUCCA & $\begin{array}{l}\text { FIFTH STREET } \\
\text { FIF STEE }\end{array}$ & $\begin{array}{l}\text { CALUMET - RED JACKET } \\
\text { CAED J }\end{array}$ \\
\hline 1927 & 331 & TOMEI & MAGGIE & W & 26 & M9 & MICHIGAN & CANADA & CANADA & & & LUCUA & $\begin{array}{l}\text { FIFTH STREET } \\
\text { FIFT }\end{array}$ & CALUMET - RED JACKET \\
\hline 1928 & 303 & THIGGI? & MAGGIE & SER & 14 & $\mathrm{~s}$ & ITALY & ITALY & ITALY & & SERVANT & & FIFTH STREET & CALUMET - RED JACKET \\
\hline 1929 & 332 & CURTO & MARCO & $\mathrm{H}$ & 44 & UNK & ITALY & ITALY & ITALY & NA & SALOONKEEPER & TORINO & SIXTH STREET & CALUMET - RED JACKET \\
\hline 1930 & 332 & CURTO & SABINO & $\mathrm{s}$ & 7 & $\mathrm{~s}$ & MICHIGAN & ITALY & ITALY & & SALOONKEEPER & TORINO & SIXTH STREET & CALUMET - RED JACKET \\
\hline 1931 & 332 & CURTO & MARY & $\mathrm{D}$ & 9 & s & MICHIGAN & ITALY & ITALY & & SERVANT & TORINO & SIXTH STREET & CALUMET - RED JACKET \\
\hline 1932 & 332 & DAIRO & TERESA & SER & 32 & s & ITALY & ITALY & ITALY & & DAY LABORER/CM & TORINO & SIXTH STREET & CALUMET - RED JACKET \\
\hline 1933 & 317 & COPPO & ANTHONY & $\mathrm{H}$ & 43 & w & ITALY & ITALY & ITALY & NA & DAY LABORER/CM & PONT CANAVESE. TO & SIXTH STREET & CALUMET - RED JACKET \\
\hline $\begin{array}{l}1933 \\
1934\end{array}$ & 317 & $\begin{array}{l}\text { COPPO } \\
\text { COPPO }\end{array}$ & $\begin{array}{l}\text { ANIHUNY } \\
\text { JOHN }\end{array}$ & $\frac{\mathrm{H}}{\mathrm{H}}$ & $\frac{43}{22}$ & $\begin{array}{l}\mathrm{w} \\
\mathrm{s}\end{array}$ & WISCONSI & $\begin{array}{l}\text { IIALY } \\
\text { ITALY }\end{array}$ & $\begin{array}{l}\text { IIALY } \\
\text { ITALY }\end{array}$ & NA & $\begin{array}{l}\text { DAY LABORERR/M } \\
\text { DAY LABORER/CM }\end{array}$ & PONT CANAVESE. TO & $\begin{array}{l}\text { SIXIASIREEI } \\
\text { SIXTH STREET }\end{array}$ & $\begin{array}{l}\text { CALUMEI - RED JACKEI } \\
\text { CALUMET - RED JACKET }\end{array}$ \\
\hline 1935 & 317 & COPPO & ALBERT & $\mathrm{s}$ & 19 & s & WISCONSI & ITALY & ITALY & & & PONT CANAVESE. TO & SIXTH STREET & CALUMET - RED JACKET \\
\hline 1936 & 317 & COPPO & $\begin{array}{l}\text { KADERI } \\
\text { KATE }\end{array}$ & $\mathrm{D}$ & 17 & s & WISCONSI & ITALY & ITALY & & DAY LABORER/CM & $\begin{array}{l}\text { PONT CANAVESE. TO } \\
\text { PONE }\end{array}$ & SIXTH STREET & CALUMET - RED JACKET \\
\hline 1937 & 317 & COPPO & $\begin{array}{l}\text { NATE } \\
\text { FRED }\end{array}$ & s & 15 & s & WISCONSI & $\begin{array}{l}\text { IITALY } \\
\text { ITALY }\end{array}$ & ITALY & & AT SCHOOL 10 & $\begin{array}{l}\text { PONT CANAVESE. TO } \\
\text { PONE C }\end{array}$ & SIXTH STREET & CALUMET - RED JACKET \\
\hline 1938 & 317 & COPPO & JENNIE & $\mathrm{D}$ & 11 & s & WISCONSI & ITALY & ITALY & & AT SCHOOL 10 & PONT CANAVESE. TO & $\begin{array}{l}\text { SIXTH STREET } \\
\text { SIXTEET }\end{array}$ & $\begin{array}{l}\text { CALUMET - RED JACKET } \\
\text { CACKE }\end{array}$ \\
\hline 1939 & 317 & COPPO & CELESTINA & D & 9 & $\begin{array}{l}\mathrm{s} \\
\mathrm{s}\end{array}$ & WISCONSI & ITALY & ITALY & & & PONT CANAVESE, TO & $\begin{array}{l}\text { SIXTH STREET } \\
\text { SIXTEI }\end{array}$ & $\begin{array}{l}\text { CALUMET - RED JACKET } \\
\text { CACED J }\end{array}$ \\
\hline 1939 & 101 & LISA & JAMES & $\mathrm{H}$ & 56 & M30 & ITALY & $\begin{array}{l}\text { IITALY } \\
\text { ITALY }\end{array}$ & $\begin{array}{l}\text { ITALY } \\
\text { ITALY }\end{array}$ & NA & MERCHANT & SAN MARTINO CANAVESE. TO & $\begin{array}{l}\text { SIXTH STREET } \\
\text { SIREI }\end{array}$ & $\begin{array}{l}\text { CALUMET - RED JACKET } \\
\text { CALED JALE }\end{array}$ \\
\hline 1941 & 101 & LISA & MARTHA & W & 48 & M30 & ITALY & ITALY & ITALY & & & SAN MARTINO CANAVESE. TO & $\begin{array}{l}\text { SIXTH STREET } \\
\end{array}$ & CALUMET - RED JACKET \\
\hline 1942 & 101 & LISA & JOSEPHINE & $\mathrm{D}$ & 25 & $\mathrm{~s}$ & ITALY & ITALY & ITALY & & & SAN MARTINO CANAVESE. TO & SIXTH STREET & CALUMET - RED JACKET \\
\hline 1943 & 101 & LISA & JULIA & $\mathrm{D}$ & 20 & s & MICHIGAN & ITALY & ITALY & & & SAN MARTINO CANAVESE. TO & SIXTH STREET & CALUMET - RED JACKET \\
\hline 1944 & 101 & LISA & MARY & $\mathrm{D}$ & 18 & s & MICHIGAN & ITALY & ITALY & & & SAN MARTINO CANAVESE. TO & SIXTH STREET & CALUMET - RED JACKET \\
\hline 1945 & 101 & LISA & ANGELINE & $\mathrm{D}$ & 16 & s & MICHIGAN & ITALY & ITALY & & AT SCHOOL 10 & SAN MARTINO CANAVESE. TO & SIXTH STREET & $\begin{array}{l}\text { CALUMET - RED JACKET } \\
\text { CACKE }\end{array}$ \\
\hline 1946 & 101 & LISA & ?RENI & $\mathrm{D}$ & 15 & s & MICHIGAN & ITALY & ITALY & & AT SCHOOL 10 & SAN MARTINO CANAVESE TO & SIXTH STREET & CALUMET - RED JACKET \\
\hline 1940 & 101 & LISA & KATIE & $\mathrm{D}$ & 14 & $\begin{array}{l}\mathrm{s} \\
\mathrm{s}\end{array}$ & MICHIGAN & $\begin{array}{l}\text { IIIALY } \\
\text { ITALY }\end{array}$ & $\begin{array}{l}\text { IITALY } \\
\text { ITALY }\end{array}$ & & AT SCHOOL 10 & SAN MARTINO CANAVESE TO & SIXTH STREET & CALUMET - RED JACKET \\
\hline 1948 & 101 & LISA & JAMES & s & 10 & $\mathrm{~s}$ & MICHIGAN & ITALY & ITALY & & AT SCHOOL 10 & SAN MARTINO CANAVESE. TO & SIXTH STREET & $\begin{array}{l}\text { CALUMET - RED JACKET } \\
\text { CAEE }\end{array}$ \\
\hline 1940 & 101 & LISA & JOHN & $\mathrm{BR}$ & 39 & s & ITALY & ITALY & ITALY & PA & $\begin{array}{l}\text { PINTER } \\
\text { PAINTER IO }\end{array}$ & SAN MARTINO CANAVESE.TO & SIXTH STREET & CALUMET - RED JACKET \\
\hline 1950 & 101 & CONTRATTO & MARY & SER & 36 & 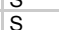 & $\begin{array}{l}\text { MIILY } \\
\text { ITALY }\end{array}$ & ITALY & $\begin{array}{l}\text { IITALY } \\
\text { ITALY }\end{array}$ & & $\begin{array}{l}\text { PAIVIEK } \\
\text { SERVANT }\end{array}$ & LOCANA CANAVESE TO & SIXTH STREET & CALUMET - RED JACKET \\
\hline
\end{tabular}




\begin{tabular}{|c|c|c|c|c|c|c|c|c|c|c|c|c|c|c|}
\hline 1951 & 340 & SUINO & DOMINICK & $\mathrm{H}$ & 38 & M8 & ITALY & ITALY & ITALY & PA & NOT CLEAR & TORINO & SEVENTH ST. & CALUMET - RED JACKET \\
\hline 1952 & 340 & SUINO & KATHERINE & W & 31 & M8 & ITALY & ITALY & ITALY & & & & SEVENTH ST. & CALUMET - RED JACKET \\
\hline 1953 & 340 & SUINO & BERNARD & $\mathrm{s}$ & 6 & $\mathrm{~s}$ & MICHIGAN & ITALY & ITALY & & & TORINO & SEVENTH ST. & CALUMET - RED JACKET \\
\hline 1954 & 340 & SUINO & MARIE & $\mathrm{D}$ & 4 & $\mathrm{~s}$ & MICHIGAN & ITALY & ITALY & & & TORINO & SEVENTH ST. & CALUMET - RED JACKET \\
\hline 1955 & 340 & SUINO & BERNARD & NEP & $2 \mathrm{M}$ & $\mathrm{s}$ & MICHIGAN & ITALY & ITALY & & & TORINO & SEVENTH ST. & CALUMET - RED JACKET \\
\hline 1956 & 340 & NARETTO & MICHAEL & $\mathrm{H}$ & 30 & M9 & ITALY & ITALY & ITALY & NA & BAKER & AGLIE', TO & SEVENTH ST. & CALUMET - RED JACKET \\
\hline 1957 & 340 & NARETTO & CATHERINE & W & 35 & M9 & ITALY & ITALY & ITALY & & & AGLIE', TO & SEVENTH ST. & CALUMET - RED JACKET \\
\hline 1958 & 820 & BORGO & CATHERINE & $\mathrm{H}$ & 44 & M28 & IRELAND & IRELAND & IRELAND & & N. G. & $\begin{array}{l}\text { IRELAND } \\
\text { IRELAN }\end{array}$ & SEVENTH ST. & CALUMET - RED JACKET \\
\hline 1959 & 820 & BORGO & JOSEPHINE & $\mathrm{D}$ & 27 & S & MICHIGAN & ITALY & IRELAND & & T. T. & VICENZA & SEVENTH ST. & CALUMET - RED JACKET \\
\hline 1960 & 820 & BORGO & IDA & D & 23 & $\mathrm{~s}$ & MICHIGAN & ITALY & IRELAND & & MILLER & VICENZA & SEVENTH ST. & CALUMET - RED JACKET \\
\hline 1961 & 820 & BORGO & CESARE & $\mathrm{s}$ & 20 & $\mathrm{~s}$ & MICHIGAN & ITALY & IRELAND & & TEAMSTER & VICENZA & SEVENTH ST. & CALUMET - RED JACKET \\
\hline 1962 & 820 & BORGO & WALTER & $\mathrm{s}$ & 19 & $\mathrm{~s}$ & MICHIGAN & ITALY & IRELAND & & NOT CLEAR & VICENZA & SEVENTH ST. & CALUMET - RED JACKET \\
\hline 1963 & 820 & BORGO & NOBLE & $\mathrm{s}$ & 17 & $\mathrm{~s}$ & MICHIGAN & ITALY & IRELAND & & AT SCHOOL 10 & VICENZA & SEVENTH ST. & CALUMET - RED JACKET \\
\hline 1964 & 820 & BORGO & AGNES & D & 10 & $\mathrm{~s}$ & WISCONSI & ITALY & IRELAND & & AT SCHOOL 10 & VICENZA & SEVENTH ST. & CALUMET - RED JACKET \\
\hline 1965 & 820 & BORGO & REY & S & & $\mathrm{S}$ & WISCONSI & ITALY & IRELAND & & AT SCHOOL 10 & VICENZA & SEVENTH ST. & CALUMET - RED JACKET \\
\hline 1966 & 321 & BANDETTINI & MICHAEL & $\mathrm{H}$ & 66 & M30 & ITALY & ITALY & ITALY & NA & DAY LABORER/CM & LUCCA & SEVENTH ST. & CALUMET - RED JACKET \\
\hline 1967 & 321 & BANDETTINI & EASTER & W & 56 & M30 & ITALY & ITALY & ITALY & & & LUCCA & SEVENTH ST. & CALUMET - RED JACKET \\
\hline 1968 & 321 & BANDETTINI & MARCO & s & 27 & $\mathrm{~s}$ & MICHIGAN & ITALY & ITALY & & SALESMAN & LUCCA & SEVENTH ST. & CALUMET - RED JACKET \\
\hline 1969 & 321 & $\begin{array}{l}\text { PRILY? } \\
\text { PII }\end{array}$ & MARIA & SERV & 27 & $\mathrm{~s}$ & ITALY & ITALY & ITALY & $\mathrm{AL}$ & SERVANT & & SEVENTH ST. & CALUMET - RED JACKET \\
\hline 1970 & 321 & TAMBELLINI & JOE & B & 21 & $\mathrm{~s}$ & ITALY & ITALY & ITALY & $\mathrm{AL}$ & MASON & LUCCA & SEVENTH ST. & CALUMET - RED JACKET \\
\hline 1971 & 321 & MICHELINI & SAM & $\begin{array}{l}\mathrm{D} \\
\mathrm{B}\end{array}$ & 21 & s & ITALY & ITALY & ITALY & $\mathrm{AL}$ & MASON & LUCCA & SEVENTH ST. & CALUMET - RED JACKET \\
\hline 1972 & 321 & PAGANUCCI & JOHN & B & 27 & s & ITALY & ITALY & ITALY & $\mathrm{AL}$ & DAY LABORER/CM & LUCCA & SEVENTH ST. & CALUMET - RED JACKET \\
\hline 1973 & 321 & CIABATTARI & RINALDO & B & 24 & $\mathrm{~S}$ & ITALY & ITALY & ITALY & $\mathrm{AL}$ & TAILOR & SEGROMIGNO. LU & SEVENTH ST. & CALUMET - RED JACKET \\
\hline 1974 & 321 & PAGANUCCI & VINCENT & B & 44 & $\mathrm{~s}$ & ITALY & ITALY & ITALY & $\mathrm{AL}$ & SALESMAN & LUCCA & SEVENTH ST. & CALUMET - RED JACKET \\
\hline 1975 & 321 & BARSOTTI & PETER & B & 16 & $\mathrm{~s}$ & ITALY & ITALY & ITALY & $?$ & SALESMAN & LUCCA & SEVENTH ST. & $\begin{array}{l}\text { CALUMET - RED JACKET } \\
\end{array}$ \\
\hline 1976 & 321 & PENA & PETER & B & 47 & $\mathrm{~s}$ & ITALY & ITALY & ITALY & NA & MERCHANT & & SEVENTH ST. & CALUMET - RED JACKET \\
\hline 1977 & 212 & COIDERO & JOSEPH & $\mathrm{H}$ & 35 & M9 & ITALY & ITALY & ITALY & NA & MERCHANT & & SIXTH STREET & CALUMET - RED JACKET \\
\hline 1978 & 212 & COIDERO & LUCY & W & 25 & M9 & ITALY & ITALY & ITALY & $\mathrm{AL}$ & & & SIXTH STREET & CALUMET - RED JACKET \\
\hline 1979 & 212 & COIDERO & LUCY & D & 8 & $\mathrm{~S}$ & MICHIGAN & ITALY & ITALY & & & & SIXTH STREET & CALUMET - RED JACKET \\
\hline 1980 & 212 & COIDERO & TONY & $\mathrm{s}$ & 7 & $\mathrm{~S}$ & MICHIGAN & ITALY & ITALY & & & & SIXTH STREET & CALUMET - RED JACKET \\
\hline 1981 & 212 & COIDERO & PETER & $\mathrm{s}$ & 5 & $\mathrm{~s}$ & MICHIGAN & ITALY & ITALY & & & & SIXTH STREET & CALUMET - RED JACKET \\
\hline 1982 & 212 & COIDERO & DOMINICK & $\mathrm{s}$ & $4 \mathrm{M}$ & $\mathrm{s}$ & MICHIGAN & ITALY & ITALY & & & & SIXTH STREET & CALUMET - RED JACKET \\
\hline 1983 & 212 & BERCHIATTI & MAGGIE & SER & 13 & s & MICHIGAN & ITALY & ITALY & & & PONT CANAVESE. TO & SIXTH STREET & CALUMET - RED JACKET \\
\hline 1984 & 212 & MICHETTI & VINCENZO & $\begin{array}{l}\mathrm{H} \\
\mathrm{H}\end{array}$ & 47 & M17 & ITALY & ITALY & ITALY & NA & DAY LABORER/CM & PONT CANAVESE, TO & ASH LANE & CALUMET - RED JACKET \\
\hline 1985 & & MICHETTI & LUCIA & w & 38 & M17 & ITALY & ITALY & ITALY & & & & ASH LANE & CALUMET - RED JACKET \\
\hline 1986 & & MICHETTI & JOHN & s & 16 & $\mathrm{~s}$ & MICHIGAN & ITALY & ITALY & & DAY LABORER/CM & PONT CANAVESE. TO & ASH LANE & CALUMET - RED JACKET \\
\hline 1987 & & MICHETTI & MARIA & $\mathrm{D}$ & 14 & $\mathrm{~s}$ & MICHIGAN & ITALY & ITALY & & AT SCHOOL & PONT CANAVESE. TO & ASH LANE & CALUMET - RED JACKET \\
\hline 1988 & & MICHETTI & BESSIE & $\mathrm{D}$ & 10 & $\mathrm{~s}$ & MICHIGAN & ITALY & ITALY & & AT SCHOOL & PONT CANAVESE. TO & ASH LANE & CALUMET - RED JACKET \\
\hline 1989 & & MICHETTI & PETRONILLA & $\mathrm{D}$ & 7 & $\mathrm{~s}$ & MICHIGAN & ITALY & ITALY & & AT SCHOOL & PONT CANAVESE. TO & ASH LANE & CALUMET - RED JACKET \\
\hline 1990 & & MICHETTI & GIUSEPPINA & $\mathrm{D}$ & 5 & $\mathrm{~S}$ & MICHIGAN & ITALY & ITALY & & & PONT CANAVESE. TO & ASH LANE & CALUMET - RED JACKET \\
\hline 1991 & & MICHETTI & LINA & D & 1 & $\mathrm{~S}$ & MICHIGAN & ITALY & ITALY & & & PONT CANAVESE. TO & ASH LANE & CALUMET - RED JACKET \\
\hline 1992 & & LINCALDO? & BERNARD & B & 38 & $\mathrm{~s}$ & ITALY & ITALY & ITALY & PA & DAY LABORER/CM & & ASH LANE & CALUMET - RED JACKET \\
\hline 1993 & & CABANIO & L? & B & 40 & s & ITALY & ITALY & ITALY & $\mathrm{PA}$ & DAY LABORER/CM & & ASH LANE & CALUMET - RED JACKET \\
\hline 1994 & & LORONA/LANZA & JOSEPH & $\mathrm{H}$ & 41 & M10 & ITALY & ITALY & ITALY & $\mathrm{AL}$ & DAY LABORER/CM & & ASH LANE & CALUMET - RED JACKET \\
\hline 1995 & & LORONA/LANZA & $?$ & w & 34 & M10 & ITALY & ITALY & ITALY & & & & ASH LANE & CALUMET - RED JACKET \\
\hline 1996 & & LORONA/LANZA & VINCENT & $\mathrm{s}$ & 3 & S & MICHIGAN & ITALY & ITALY & & & & ASH LANE & CALUMET - RED JACKET \\
\hline 1997 & & LORONA/LANZA & JOHN & s & $7 \mathrm{M}$ & $\mathrm{s}$ & MICHIGAN & ITALY & ITALY & & & & ASH LANE & CALUMET - RED JACKET \\
\hline 1998 & & ARIOLINI & JOSEPH? & $\mathrm{H}$ & 34 & M0 & ITALY & ITALY & ITALY & NA & MINER/CM & & ASH LANE & CALUMET - RED JACKET \\
\hline 1999 & & ARIOLINI & FANNIA? & w & 22 & MO & ITALY & ITALY & ITALY & & & & ASH LANE & CALUMET - RED JACKET \\
\hline 2000 & 217 & RASTELLLO & GIOVANNI B. & $\mathrm{H}$ & 31 & M11 & ITALY & ITALY & ITALY & NA & TAILOR & PONT CANAVESE. TO & SIXTH STREET & CALUMET - RED JACKET \\
\hline 2001 & 217 & RASTELLLO & TERESA & W & 32 & M11 & ITALY & ITALY & ITALY & & & 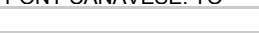 & SIXTH STREET & CALUMET - RED JACKET \\
\hline 2002 & 217 & RASTELLO & DOMENICO & $S$ & 9 & $\mathrm{~s}$ & MICHIGAN & ITALY & ITALY & & & PONT CANAVESE, TO & SIXTH STREET & CALUMET - RED JACKET \\
\hline 2003 & 217 & RASTELLO & MARIA & D & 8 & $\mathrm{~s}$ & MICHIGAN & ITALY & ITALY & & AT SCHOOL 10 & PONT CANAVESE. TO & SIXTH STREET & CALUMET - RED JACKET \\
\hline 2004 & 217 & RASTELLO & ROSA & D & 3 & $\mathrm{~s}$ & MICHIGAN & ITALY & ITALY & & & PONT CANAVESE. TO & SIXTH STREET & CALUMET - RED JACKET \\
\hline 2005 & 217 & RASTELLO & MARIA & $\mathrm{MO}$ & 62 & W & ITALY & ITALY & ITALY & & & PONT CANAVESE. TO & SIXTH STREET & CALUMET - RED JACKET \\
\hline 2006 & 221 & COPPO & VITAL & $\mathrm{H}$ & 58 & M28 & ITALY & ITALY & ITALY & NA & TIMBERMAN & PONT CANAVESE. TO & SIXTH STREET & CALUMET - RED JACKET \\
\hline 2007 & 221 & COPPO & LUCY & w & 55 & M28 & ITALY & ITALY & ITALY & & & & SIXTH STREET & CALUMET - RED JACKET \\
\hline 2008 & 221 & COPPO & MARY & $\mathrm{D}$ & 19 & s & MICHIGAN & ITALY & ITALY & & & PONT CANAVESE, TO & SIXTH STREET & CALUMET - RED JACKET \\
\hline 2009 & 221 & COPPO & PETER & $\mathrm{s}$ & 16 & $\mathrm{~S}$ & MICHIGAN & ITALY & ITALY & & & PONT CANAVESE. TO & SIXTH STREET & CALUMET - RED JACKET \\
\hline 2010 & 221 & COPPO & VICTOR & $\mathrm{s}$ & 11 & $\mathrm{~S}$ & MICHIGAN & ITALY & ITALY & & & PONT CANAVESE. TO & SIXTH STREET & CALUMET - RED JACKET \\
\hline 2011 & 221 & VAIRO & JOSEPH & $\mathrm{H}$ & 40 & M3 & ITALY & ITALY & ITALY & NA & SALOONKEEPER & LOCANA CANAVESE. TO & SIXTH STREET & CALUMET - RED JACKET \\
\hline 2012 & 221 & VAIRO & ANNA & w & 27 & M3 & ITALY & ITALY & ITALY & & & & SIXTH STREET & CALUMET - RED JACKET \\
\hline 2013 & 221 & VAIRO & DOMINICK & $\mathrm{s}$ & 2 & $\mathrm{~s}$ & MICHIGAN & ITALY & ITALY & & & LOCANA CANAVESE. TO & SIXTH STREET & CALUMET - RED JACKET \\
\hline
\end{tabular}




\begin{tabular}{|c|c|c|c|c|c|c|c|c|c|c|c|c|c|c|}
\hline 2014 & 221 & VAIRO & ANDREW & s & $7 \mathrm{M}$ & $\mathrm{s}$ & MICHIGAN & ITALY & ITALY & & & LOCANA CANAVESE. TO & SIXTH STREET & CALUMET - RED JACKET \\
\hline 2015 & 221 & CONTRAD & LUCY & SER & 20 & $\mathrm{~s}$ & ITALY & ITALY & ITALY & & SERVANT & & SIXTH STREET & CALUMET - RED JACKET \\
\hline 2016 & 221 & CATO? & JOHN & B & 25 & $\mathrm{~s}$ & ITALY & ITALY & ITALY & $\mathrm{AL}$ & DAY LABORER/CM & & SIXTH STREET & CALUMET - RED JACKET \\
\hline 2017 & 221 & VERRITO & JAMES & B & 27 & $\mathrm{~S}$ & ITALY & ITALY & ITALY & $\mathrm{AL}$ & DAY LABORER/CM & & SIXTH STREET & CALUMET - RED JACKET \\
\hline 2018 & 221 & BANNO & ANTON & B & 30 & MO & ITALY & ITALY & ITALY & NA & DAY LABORER/CM & & SIXTH STREET & CALUMET - RED JACKET \\
\hline 2019 & 221 & CONTONO & JAMES & B & 30 & MO & ITALY & ITALY & ITALY & $\mathrm{AL}$ & DAY LABORER/CM & & SIXTH STREET & CALUMET - RED JACKET \\
\hline 2020 & 221 & ORSOLANO & $\mathrm{JOHN}$ & B & 23 & MO & ITALY & ITALY & ITALY & $\mathrm{AL}$ & DAY LABORER/CM & SAN GIORGIO CANAVESE. TO & SIXTH STREET & CALUMET - RED JACKET \\
\hline 2021 & 221 & ORSOLANO & JOHAN & $\begin{array}{l}\mathrm{D} \\
\mathrm{B}\end{array}$ & 24 & MO & ITALY & ITALY & ITALY & $\mathrm{AL}$ & DAT LADU RERTUIVI & SAN GIORGIO CANAVESE. TO & SIXTH STREET & $\begin{array}{l}\text { CALUMET - RED JACKET } \\
\text { CACKE }\end{array}$ \\
\hline 2022 & 221 & PICCHIOTTINO & FRED & B & 50 & MO & ITALY & ITALY & ITALY & NA & BARTENDER & PONT CANAVESE. TO & SIXTH STREET & CALUMET - RED JACKET \\
\hline 2023 & 216 & TONDETTI & FRANK & $\mathrm{H}$ & 28 & M4 & ITALY & ITALY & ITALY & & & & SEVENTH ST. & CALUMET - RED JACKET \\
\hline 2024 & 216 & TONDETTI & JOHANNA & w & 20 & M4 & ITALY & ITALY & ITALY & & & & $\begin{array}{l}\text { SEVENTH ST. } \\
\text { STE }\end{array}$ & CALUMET - RED JACKET \\
\hline 2025 & 216 & TONDETTI & KATIE & $\mathrm{D}$ & 2 & $\mathrm{~s}$ & MICHIGAN & ITALY & ITALY & & & & SEVENTH ST. & CALUMET - RED JACKET \\
\hline 2026 & & TAMBELLINI & JOHN & $\mathrm{H}$ & 34 & M3 & ITALY & ITALY & ITALY & & & LUCCA & PORTLAND ST. & CALUMET - RED JACKET \\
\hline 2027 & & TAMBELLINI & CHARLOTTE & W & 25 & M3 & ITALY & ITALY & ITALY & & & LUCCA & PORTLAND ST. & CALUMET - RED JACKET \\
\hline 2028 & & TAMBELLINI & JULIUS & s & 2 & $\mathrm{~s}$ & MICHIGAN & ITALY & ITALY & & & LUCCA & PORTLAND ST. & CALUMET - RED JACKET \\
\hline 2029 & & TAMBELLINI & JENNA & D & $6 \mathrm{M}$ & $\mathrm{s}$ & MICHIGAN & ITALY & ITALY & & & LUCCA & PORTLAND ST. & CALUMET - RED JACKET \\
\hline 2030 & & TAMBELLINI & ALMINDO & B & 27 & $\mathrm{~s}$ & ITALY & ITALY & ITALY & NA & BARTENDER & LUCCA & PORTLAND ST. & CALUMET - RED JACKET \\
\hline 2031 & 113 & POTATO & SYLVESTER & B & 26 & $\mathrm{~s}$ & ITALY & ITALY & ITALY & $\mathrm{PA}$ & DAY LABORER/CM & & SEVENTH ST. & CALUMET - RED JACKET \\
\hline 2032 & 444 & MARTINI & GEORGE & $\mathrm{H}$ & 49 & M21 & ITALY & ITALY & ITALY & NA & LIQUOR DEALER & LUCCA & FIFTH STREET & CALUMET - RED JACKET \\
\hline 2033 & 444 & MARTINI & AGNES & W & 33 & M21 & GERMANY & GERMAN & GERMANY & & & GERMANY & FIFTH STREET & CALUMET - RED JACKET \\
\hline 2034 & 444 & BANDITINI & ETTA & D & 23 & M4 & MICHIGAN & ITALY & GERMANY & & & LUCCA & FIFTH STREET & CALUMET - RED JACKET \\
\hline 2035 & 444 & BANDITINI & JOHN & SIL & 24 & M4 & FRANCE & ITALY & ITALY & NA & LIQUOR SALESMAN & LUCCA & FIFTH STREET & CALUMET - RED JACKET \\
\hline 2036 & 444 & BANDITINI & LYDIA & GD & 1 & $\mathrm{~s}$ & MICHIGAN & FRANCE & MICHIGAN & & & LUCCA & FIFTH STREET & CALUMET - RED JACKET \\
\hline 2037 & 454 & BRACCO & CARLO & $\mathrm{H}$ & 57 & M38 & ITALY & ITALY & ITALY & NA & LANDLORD & TORINO & SIXTH STREET & CALUMET - RED JACKET \\
\hline 2038 & 454 & BRACCO & MARY & w & 61 & M38 & ITALY & ITALY & ITALY & & & & SIXTH STREET & CALUMET - RED JACKET \\
\hline 2039 & 454 & MANFREDINI & JOSEPH & $\mathrm{H}$ & 24 & M3 & ITALY & ITALY & ITALY & & N. G. & LUCCA & SIXTH STREET & CALUMET - RED JACKET \\
\hline 2040 & 454 & MANFREDINI & CAROLINA & w & 20 & M3 & ITALY & ITALY & ITALY & $\mathrm{AL}$ & BOARDING HOUSE KEEPER & LUCCA & SIXTH STREET & CALUMET - RED JACKET \\
\hline 2041 & 454 & MANFREDINI & RINA & $\mathrm{D}$ & 2 & $\mathrm{~s}$ & MICHIGAN & ITALY & ITALY & & & LUCCA & SIXTH STREET & CALUMET - RED JACKET \\
\hline 2042 & 454 & RITZIERI & JACOB & B & 17 & $\mathrm{~S}$ & ITALY & ITALY & ITALY & AL & LABORER/CM & & SIXTH STREET & CALUMET - RED JACKET \\
\hline 2043 & 454 & CHARDALI & JOSEPH & B & 18 & $\mathrm{~S}$ & ITALY & ITALY & ITALY & $\mathrm{AL}$ & LABORER/CM & & SIXTH STREET & CALUMET - RED JACKET \\
\hline 2044 & 454 & MANFREDINI & AMATO & B & 21 & $\mathrm{~s}$ & ITALY & ITALY & ITALY & $\mathrm{AL}$ & LABORER & LUCCA & SIXTH STREET & CALUMET - RED JACKET \\
\hline 2045 & 454 & NARCISO & JACOB & B & 42 & M21 & ITALY & ITALY & ITALY & PA & LABORER/CM & & SIXTH STREET & CALUMET - RED JACKET \\
\hline 2046 & 430 & MESKI & JOHN & B & 55 & $?$ & ITALY & ITALY & ITALY & PA & DAY LABORER/CM & & SIXTH STREET & CALUMET - RED JACKET \\
\hline 2047 & 430 & CROLL & PETER & $\begin{array}{l}\mathrm{O} \\
\mathrm{H}\end{array}$ & 36 & M5 & ITALY & ITALY & ITALY & PA & LABORER/CM & NOVARA & SIXTH STREET & $\begin{array}{l}\text { CALUMET - RED JACKET } \\
\text { CACKE }\end{array}$ \\
\hline 2048 & 430 & CROLL & ANITA & w & 21 & M5 & MICHIGAN & ITALY & ITALY & & & & SIXTH STREET & CALUMET - RED JACKET \\
\hline 2049 & 430 & CROLL & FLORIAN & $\mathrm{D}$ & 3 & $\mathrm{~s}$ & MICHIGAN & ITALY & ITALY & & & NOVARA & SIXTH STREET & CALUMET - RED JACKET \\
\hline 2050 & 511 & MIGLIO/MIGLIA & CARLO & $\mathrm{H}$ & 46 & M20 & ITALY & ITALY & ITALY & NA & TAILOR & SAN GIORGIO CANAVESE. TO & FIFTH STREET & CALUMET - RED JACKET \\
\hline 2051 & 511 & MIGLIO/MIGLIA & TERESA & w & 40 & M20 & ITALY & ITALY & ITALY & & & & $\begin{array}{l}\text { FIFTH STREET } \\
\text { FTH }\end{array}$ & CALUMET - RED JACKET \\
\hline 2052 & 511 & MIGLIO/MIGLIA & MARTA & $\mathrm{D}$ & 17 & $\mathrm{~s}$ & MICHIGAN & ITALY & ITALY & & & SAN GIORGIO CANAVESE. TO & FIFTH STREET & CALUMET - RED JACKET \\
\hline 2053 & 511 & MIGLIO/MIGLIA & JOSEPH & $\mathrm{s}$ & 15 & $\mathrm{~s}$ & MICHIGAN & ITALY & ITALY & & & SAN GIORGIO CANAVESE. TO & FIFTH STREET & CALUMET - RED JACKET \\
\hline 2054 & 511 & MIGLIO/MIGLIA & ANTON & $\mathrm{s}$ & 15 & $\mathrm{~S}$ & MICHIGAN & ITALY & ITALY & & AT SCHOOL 8 & SAN GIORGIO CANAVESE. TO & FIFTH STREET & CALUMET - RED JACKET \\
\hline 2055 & 511 & MIGLIO/MIGLIA & JAMES & $\mathrm{s}$ & 6 & $\mathrm{~s}$ & MICHIGAN & ITALY & ITALY & & AT SCHOOL 8 & SAN GIORGIO CANAVESE. TO & FIFTH STREET & CALUMET - RED JACKET \\
\hline 2056 & 511 & MIGLIO/MIGLIA & TERESA & $\mathrm{D}$ & 3 & $\mathrm{~s}$ & MICHIGAN & ITALY & ITALY & & & SAN GIORGIO CANAVESE. TO & FIFTH STREET & CALUMET - RED JACKET \\
\hline 2057 & 511 & MIGLIO/MIGLIA & MARY & $\mathrm{D}$ & $3 \mathrm{M}$ & $\mathrm{s}$ & MICHIGAN & ITALY & ITALY & & & SAN GIORGIO CANAVESE. TO & FIFTH STREET & CALUMET - RED JACKET \\
\hline 2058 & 511 & VENUTA & JOSEPH & $\mathrm{COU}$ & 34 & M3 & ITALY & ITALY & ITALY & PA & BAKER & SAN GIORGIO CANAVESE. TO & FIFTH STREET & CALUMET - RED JACKET \\
\hline 2059 & 511 & VENUTA & NATALINA & $\mathrm{COU}$ & 23 & M3 & ITALY & ITALY & ITALY & $r M$ & DARLT & SAN GIORGIO CANAVESE. TO & FIFTH STREET & CALUMET - RED JACKET \\
\hline 2060 & 415 & PINOCCHI & PETER & B & 57 & $\mathrm{~s}$ & ITALY & ITALY & ITALY & NA & LABORER BOSS/CM & LUCCA & SIXTH STREET & CALUMET - RED JACKET \\
\hline 2061 & 415 & PINOCCHI & THOMAS & B & 50 & $\mathrm{~s}$ & ITALY & ITALY & ITALY & NA & LABORER/CM & LUCCA & SIXTH STREET & CALUMET - RED JACKET \\
\hline 2062 & 442 & LOCATELLI & JOHN & $\mathrm{H}$ & 41 & M14 & ITALY & ITALY & ITALY & NA & LABORER/CM & SELINO. BERGAMO & SEVENTH ST. & CALUMET - RED JACKET \\
\hline 2063 & 442 & LOCATELLI & KATE & w & 40 & M14 & ITALY & ITALY & ITALY & & & SELINO. BERGAMO & SEVENTH ST. & CALUMET - RED JACKET \\
\hline 2064 & 442 & LOCATELLI & ADELINA & $\mathrm{D}$ & 10 & $\mathrm{~s}$ & ITALY & ITALY & ITALY & & AT SCHOOL 4 & SELINO. BERGAMO & SEVENTH ST. & CALUMET - RED JACKET \\
\hline 2065 & 442 & LOCATELLI & ANNIUS? & $\mathrm{s}$ & 7 & $\mathrm{~S}$ & ITALY & ITALY & ITALY & & AT SCHOOL 5 & SELINO, BERGAMO & SEVENTH ST. & CALUMET - RED JACKET \\
\hline 2066 & 442 & LOCATELLI & MARCH & D & 13 & $\mathrm{~S}$ & ITALY & ITALY & ITALY & & AT SCHOOL 6 & SELINO. BERGAMO & SEVENTH ST. & CALUMET - RED JACKET \\
\hline 2067 & 442 & LOCATELLI & PETER & $\mathrm{s}$ & 1 & $\mathrm{~s}$ & MICHIGAN & ITALY & ITALY & & & SELINO. BERGAMO & SEVENTH ST. & CALUMET - RED JACKET \\
\hline 2068 & 442 & LOCATELLI & THREASA & $\mathrm{D}$ & 5 & $\mathrm{~S}$ & MICHIGAN & ITALY & ITALY & & & SELINO. BERGAMO & SEVENTH ST. & CALUMET - RED JACKET \\
\hline 2069 & 442 & LOCATELLI & LIZZIE & $\mathrm{D}$ & 3 & s & MICHIGAN & ITALY & ITALY & & & SELINO. BERGAMO & SEVENTH ST. & CALUMET - RED JACKET \\
\hline 2070 & 442 & CURTO & PETER & $\mathrm{H}$ & 47 & M22 & ITALY & ITALY & ITALY & PA & TEAMSTER & TORINO & SEVENTH ST. & CALUMET - RED JACKET \\
\hline 2071 & 442 & CURTO & MARY & W & 42 & M22 & ITALY & ITALY & ITALY & & & & SEVENTH ST. & CALUMET - RED JACKET \\
\hline 2072 & 442 & GALETTO & JOHANNA & $\mathrm{D}$ & 21 & M4 & ITALY & ITALY & ITALY & & & TORINO & SEVENTH ST. & CALUMET - RED JACKET \\
\hline 2073 & 442 & CURTO & CHARLES & $\mathrm{s}$ & 10 & s & MICHIGAN & ITALY & ITALY & & AT SCHOOL 6 & TORINO & SEVENTH ST. & CALUMET - RED JACKET \\
\hline 2074 & 442 & CURTO & MARNIE & D & 9 & $\mathrm{~s}$ & MICHIGA & ITALY & & & $\mathrm{OL} 7$ & INO & VENTH ST. & CALUMET - RED JACKET \\
\hline 2075 & 442 & CURTO & KATIE & $\mathrm{D}$ & 8 & $\mathrm{~s}$ & MICHIGAN & ITALY & ITALY & & & & & CALUMET - \\
\hline 2076 & 442 & CURTO & THOMAS & $\mathrm{s}$ & 7 & $\mathrm{~s}$ & MICHIGAN & ITALY & ITALY & & AT SCHOOL 8 & TORINO & SEVENTH ST. & CALUMET - RED JACKET \\
\hline
\end{tabular}




\begin{tabular}{|c|c|c|c|c|c|c|c|c|c|c|c|c|c|c|}
\hline 2077 & 442 & CURTO & ANNA & $\mathrm{D}$ & 2 & $\mathrm{~s}$ & MICHIGAN & ITALY & ITALY & & & TORINO & SEVENTH ST. & CALUMET - RED JACKET \\
\hline 2078 & 442 & CURTO & PETER & $\mathrm{S}$ & $4 \mathrm{M}$ & $\mathrm{S}$ & MICHIGAN & ITALY & ITALY & & & TORINO & SEVENTH ST. & CALUMET - RED JACKET \\
\hline 2079 & 442 & CURTO & JOHN & BR & 45 & s & ITALY & ITALY & ITALY & NA & SALESMAN & TORINO & SEVENTH ST. & CALUMET - RED JACKET \\
\hline 2080 & 440 & VITTON & PETER & $\mathrm{H}$ & 40 & M4 & ITALY & ITALY & ITALY & & BAKER & LOCANA CANAVESE. TO & SEVENTH ST. & CALUMET - RED JACKET \\
\hline 2081 & 440 & VITTON & JOHANNA & w & 28 & M4 & ITALY & ITALY & ITALY & & & LOCANA CANAVESE. TO & SEVENTH ST. & CALUMET - RED JACKET \\
\hline 2082 & 440 & VITTON & JULIUS & $\mathrm{s}$ & 6 & $\mathrm{~S}$ & ITALY & ITALY & ITALY & & & LOCANA CANAVESE. TO & SEVENTH ST. & CALUMET - RED JACKET \\
\hline 2083 & 440 & VITTON & SYLVAN & $\mathrm{s}$ & 4 & $\mathrm{~s}$ & ITALY & ITALY & ITALY & & & LOCANA CANAVESE. TO & SEVENTH ST. & CALUMET - RED JACKET \\
\hline 2084 & 403 & QUELLO & BAT & $\mathrm{H}$ & 58 & M38 & ITALY & ITALY & ITALY & $N A$ & CONTRACTOR & LOCANA CANAVESE. TO & EIGHTH STREET & CALUMET - RED JACKET \\
\hline 2085 & 403 & QUELLO & KATHERINE & W & 56 & M38 & IRELAND & IRELAND & IRELAND & INA & CUVIRACIOR & $\begin{array}{l}\text { IRELAND } \\
\text { IRIVAVESE. IO }\end{array}$ & $\begin{array}{l}\text { EIGHTH STREET } \\
\text { EITHE }\end{array}$ & $\begin{array}{l}\text { CALUMET - RED JACKET } \\
\end{array}$ \\
\hline 2086 & 403 & QUELLO & JOHN & $\mathrm{s}$ & 32 & $\mathrm{~S}$ & MICHIGAN & ITALY & IRELAND & & BUTCHER & LOCANA CANAVESE. TO & EIGHTH STREET & CALUMET - RED JACKET \\
\hline 2087 & 403 & QUELLO & MARGARET & D & 23 & $\mathrm{~s}$ & MICHIGAN & ITALY & IRELAND & & & LOCANA CANAVESE. TO & EIGHTH STREET & CALUMET - RED JACKET \\
\hline 2088 & 403 & QUELLO & BAT & $\mathrm{s}$ & 22 & $\mathrm{~s}$ & MICHIGAN & ITALY & IRELAND & & BOOKKEEPER/BANK & LOCANA CANAVESE. TO & EIGHTH STREET & CALUMET - RED JACKET \\
\hline 2089 & 403 & QUELLO & ANNIE & D & 21 & $\mathrm{~s}$ & MICHIGAN & ITALY & IRELAND & & AT SCHOOL 8 & LOCANA CANAVESE. TO & EIGHTH STREET & CALUMET - RED JACKET \\
\hline 2090 & 403 & QUELLO & ELISABETH & $\mathrm{D}$ & 19 & $\mathrm{~s}$ & MICHIGAN & ITALY & IRELAND & & AT SCHOOL 8 & LOCANA CANAVESE. TO & EIGHTH STREET & CALUMET - RED JACKET \\
\hline 2091 & 403 & QUELLO & ALICE & D & 14 & $\mathrm{~S}$ & MICHIGAN & ITALY & IRELAND & & AT SCHOOL 8 & LOCANA CANAVESE. TO & EIGHTH STREET & CALUMET - RED JACKET \\
\hline 2092 & 409 & GARDETTO & FRANK & $\mathrm{H}$ & 62 & M36 & ITALY & ITALY & ITALY & PA & DAY LABORER/CM & BOSCONERO, TO & SEVENTH ST. & CALUMET - RED JACKET \\
\hline 2093 & 409 & GARDETTO & MARGHERITA & W & 53 & M36 & ITALY & ITALY & ITALY & & GROCER & BOSCONERO. TO & SEVENTH ST. & CALUMET - RED JACKET \\
\hline 2094 & 409 & GARDETTO & MARIA & D & 33 & M15 & ITALY & ITALY & ITALY & & GROCER & BOSCONERO. TO & SEVENTH ST. & CALUMET - RED JACKET \\
\hline 2095 & 409 & GARDETTO & DOMENICO & $\mathrm{s}$ & 30 & M1 & ITALY & ITALY & ITALY & NA & SALOONKEEPER & BOSCONERO. TO & SEVENTH ST. & CALUMET - RED JACKET \\
\hline 2096 & 409 & GARDETTO & GIUSEPPE & SOIL & 29 & $?$ & ITALY & ITALY & ITALY & PA & GROCER & TORINO & SEVENTH ST. & CALUMET - RED JACKET \\
\hline 2097 & 409 & GARDETTO & TERESA & $\mathrm{D}$ & 24 & M1 & ITALY & $\begin{array}{l}\text { ITALY } \\
\text { ITA }\end{array}$ & $\begin{array}{l}\text { ITALY } \\
\text { ITA }\end{array}$ & $F H$ & HRUCEK & BOSCONERO. TO & $\begin{array}{l}\text { SEVENTH ST. } \\
\text { STE }\end{array}$ & $\begin{array}{l}\text { CALUMET - RED JACKET } \\
\end{array}$ \\
\hline 2098 & 409 & GARDETTO & FRANK & GS & $1 \mathrm{M}$ & $\mathrm{s}$ & MICHIGAN & ITALY & ITALY & & & BOSCONERO, TO & SEVENTH ST. & CALUMET - RED JACKET \\
\hline 2099 & 409 & GARDETTO & FRANK G. & GS & 11 & $\mathrm{~s}$ & MICHIGAN & ITALY & ITALY & & AT SCHOOL 7 & BOSCONERO. TO & SEVENTH ST. & CALUMET - RED JACKET \\
\hline 2100 & 409 & GARDETTO & SAVINO & GS & 5 & $\mathrm{~s}$ & MICHIGAN & ITALY & ITALY & & AT SCHOOL 7 & BOSCONERO. TO & SEVENTH ST. & CALUMET - RED JACKET \\
\hline 2101 & 409 & GARDETTO & DOMENICO & GS & 4 & $\mathrm{~S}$ & MICHIGAN & ITALY & ITALY & & & BOSCONERO. TO & SEVENTH ST. & CALUMET - RED JACKET \\
\hline 2102 & 409 & ROCH/ROCK & JOSEPH & B & 30 & $\mathrm{~s}$ & ITALY & ITALY & ITALY & PA & BLACKSMITH & TORINO & SEVENTH ST. & CALUMET - RED JACKET \\
\hline 2103 & 409 & ROCH/ROCK & JOSEPH & B & 24 & $\mathrm{~s}$ & ITALY & ITALY & ITALY & $\mathrm{AL}$ & MINER/CM & TORINO & SEVENTH ST. & CALUMET - RED JACKET \\
\hline 2104 & 409 & SERAZIO & CARLO & B & 23 & $\mathrm{~s}$ & ITALY & ITALY & ITALY & $\mathrm{AL}$ & LABORER/CM & SAN GIORGIO CANAVESE, TO & SEVENTH ST. & CALUMET - RED JACKET \\
\hline 2105 & 409 & CARPENO & GAITANO & B & 40 & s & ITALY & ITALY & ITALY & NA & MASON & & SEVENTH ST. & CALUMET - RED JACKET \\
\hline 2106 & 409 & SANTANERA & DOMINIC & B & 40 & M4 & ITALY & ITALY & ITALY & NA & BOOKKEEPER & & SEVENTH ST. & CALUMET - RED JACKET \\
\hline 2107 & 409 & GARDETTO & MARIA & B & 12 & $\mathrm{~s}$ & ITALY & ITALY & ITALY & & AT SCHOOL 8 & TORINO & SEVENTH ST. & CALUMET - RED JACKET \\
\hline 2108 & 415 & BANDONI & JOHN & $\mathrm{H}$ & 55 & M14 & ITALY & ITALY & ITALY & NA & GROCER & LUCCA & SEVENTH ST. & CALUMET - RED JACKET \\
\hline 2109 & 415 & BANDONI & ANNA & W & 51 & M14 & ITALY & $\begin{array}{l}\text { ITALY } \\
\text { ITAL }\end{array}$ & ITALY & TNA & GRUCEK & LUCCA & $\begin{array}{l}\text { SEVENTH ST. } \\
\text { SEVE }\end{array}$ & CALUMET - RED JACKET \\
\hline 2110 & 415 & CHELI & LAWRENCE & SS & 22 & $\mathrm{~s}$ & MICHIGAN & ITALY & ITALY & & LABORER & LUCCA & SEVENTH ST. & CALUMET - RED JACKET \\
\hline 2111 & 415 & CHELI & EDITH & $\mathrm{SD}$ & 18 & $\mathrm{~s}$ & ITALY & ITALY & ITALY & & SALESLADY & LUCCA & SEVENTH ST. & CALUMET - RED JACKET \\
\hline 2112 & 415 & BANDONI & JENNIE & $\mathrm{D}$ & 12 & $\mathrm{~s}$ & MICHIGAN & ITALY & ITALY & & AT SCHOOL 7 & LUCCA & SEVENTH ST. & CALUMET - RED JACKET \\
\hline 2113 & 415 & BANDONI & MARY & D & 10 & $\mathrm{~s}$ & MICHIGAN & ITALY & ITALY & & AT SCHOOL 7 & LUCCA & SEVENTH ST. & CALUMET - RED JACKET \\
\hline 2114 & 415 & BANDONI & PETER & $\mathrm{s}$ & 5 & $\mathrm{~s}$ & MICHIGAN & ITALY & ITALY & & AT SCHOOL 7 & LUCCA & SEVENTH ST. & CALUMET - RED JACKET \\
\hline 2115 & 415 & BAUDINO & GEORGE & $\mathrm{H}$ & 32 & M8 & ITALY & ITALY & ITALY & PA & LABORER/CM & RIVAROLO CANAVESE. TO & SEVENTH ST. & CALUMET - RED JACKET \\
\hline 2116 & 415 & BAUDINO & MARY & W & 27 & M8 & ITALY & ITALY & ITALY & & & RIVAROLO CANAVESE. TO & SEVENTH ST. & CALUMET - RED JACKET \\
\hline 2117 & 415 & BAUDINO & ANITA & D & 5 & $\mathrm{~s}$ & MICHIGAN & ITALY & ITALY & & AT SCHOOL 6 & RIVAROLO CANAVESE. TO & SEVENTH ST. & CALUMET - RED JACKET \\
\hline 2118 & 415 & BAUDINO & THREASA & D & 2 & $\mathrm{~s}$ & MICHIGAN & ITALY & ITALY & & & RIVAROLO CANAVESE. TO & SEVENTH ST. & CALUMET - RED JACKET \\
\hline 2119 & 415 & BAUDINO & EARNESTINA & D & 1 & s & MICHIGAN & ITALY & ITALY & & & RIVAROLO CANAVESE. TO & SEVENTH ST. & CALUMET - RED JACKET \\
\hline 2120 & 415 & BURTSA & JOHN & $\mathrm{H}$ & 26 & M3 & ITALY & ITALY & ITALY & PA & LABORER/CM & & SEVENTH ST. & CALUMET - RED JACKET \\
\hline 2121 & 415 & BURTSA & MARY & w & 20 & M3 & AUSTRIA & AUSTRIA & AUSTRIA & & & & SEVENTH ST. & CALUMET - RED JACKET \\
\hline 2122 & 415 & BURTSA & MARY & $\mathrm{D}$ & 1 & $\mathrm{~s}$ & MICHIGAN & ITALY & AUSTRIA & & & & SEVENTH ST. & CALUMET - RED JACKET \\
\hline 2123 & 415 & TAMBELLINI & GIOVANNI & $\mathrm{H}$ & 26 & M2 & ITALY & ITALY & ITALY & NA & LABORER/CM & SEGROMIGNO. LU & SEVENTH ST. & CALUMET - RED JACKET \\
\hline 2124 & 415 & TAMBELLINI & NATALINA & W & 20 & M2 & ITALY & ITALY & ITALY & & & & SEVENTH ST. & CALUMET - RED JACKET \\
\hline 2125 & 415 & TAMBELLINI & TERESA & $\mathrm{D}$ & 0 & s & MICHIGAN & ITALY & ITALY & & & SEGROMIGNO. LU & SEVENTH ST. & CALUMET - RED JACKET \\
\hline 2126 & 415 & TAMBELLINI & ALFRED & $\mathrm{BR}$ & 21 & $\mathrm{~s}$ & ITALY & ITALY & ITALY & $\mathrm{AL}$ & DRILL BOYICM & SEGROMIGNO. LU & SEVENTH ST. & CALUMET - RED JACKET \\
\hline 2127 & 415 & BIANCARDI & LOUIS & $\mathrm{H}$ & 68 & S & ITALY & ITALY & ITALY & NA & N. G. & TORINO & SEVENTH ST. & CALUMET - RED JACKET \\
\hline 2128 & 815 & BRACCO & BERNARDO & $\mathrm{H}$ & 45 & M23 & ITALY & ITALY & ITALY & NA & BAKER & PONT CANAVESE, TO & ELM STREET & CALUMET - RED JACKET \\
\hline 2129 & 815 & BRACCO & MARIA & W & 42 & M23 & ITALY & ITALY & ITALY & & & PONT CANAVESE. TO & ELM STREET & CALUMET - RED JACKET \\
\hline 2130 & 815 & BRACCO & GIOVANNI & $\mathrm{s}$ & 22 & $\mathrm{~s}$ & ITALY & ITALY & ITALY & NA & BOOKKEEPER & PONT CANAVESE. TO & ELM STREET & CALUMET - RED JACKET \\
\hline 2131 & 815 & BRACCO & GIUSEPPINA & D & 20 & $\mathrm{~s}$ & ITALY & ITALY & ITALY & & SALESLADY & PONT CANAVESE. TO & ELM STREET & CALUMET - RED JACKET \\
\hline 2132 & 815 & BRACCO & LENA & $\mathrm{D}$ & 18 & $\mathrm{~s}$ & FRANCE & ITALY & ITALY & & SALESLADY & PONT CANAVESE. TO & ELM STREET & CALUMET - RED JACKET \\
\hline 2133 & 815 & BRACCO & CARLO & $\mathrm{s}$ & 16 & $\mathrm{~s}$ & MICHIGAN & ITALY & ITALY & & AT SCHOOL 5 & PONT CANAVESE. TO & ELM STREET & CALUMET - RED JACKET \\
\hline 2134 & 815 & BRACCO & ROSA & $\mathrm{D}$ & 14 & $\mathrm{~s}$ & MICHIGAN & ITALY & ITALY & & AT SCHOOL 7 & PONT CANAVESE, TO & ELM STREET & CALUMET - RED JACKET \\
\hline 2135 & 815 & BRACCO & ANGELO & $\mathrm{s}$ & 9 & $\mathrm{~s}$ & MICHIGAN & ITALY & ITALY & & AT SCHOOL 7 & PONT CANAVESE. TO & ELM STREET & CALUMET - RED JACKET \\
\hline 2136 & 815 & BRACCO & MARIA & $\mathrm{D}$ & 7 & $\mathrm{~s}$ & MICHIGAN & ITALY & ITALY & & AT SCHOOL 7 & PONT CANAVESE. TO & ELM STREET & CALUMET - RED JACKET \\
\hline 2137 & 815 & BRACCO & CRISTINA & D & 5 & s & MICHIGAN & ITALY & ITALY & & AT SCHOOL 4 & PONT CANAVESE. TO & STREET & CALUMET - R \\
\hline 2138 & 815 & SUINO & JOSEPH & B & 36 & s & ITALY & ITALY & ITALY & NA & SALOONKEEPER & TORINO & & CALUMET - RED JACKET \\
\hline 2139 & 815 & WALLS & $\mathrm{JOHN}$ & B & 37 & $\mathrm{~s}$ & ITALY & ITALY & ITALY & NA & SALESMAN & & ELM STREET & CALUMET - RED JACKET \\
\hline
\end{tabular}




\begin{tabular}{|c|c|c|c|c|c|c|c|c|c|c|c|c|c|c|}
\hline 2140 & 815 & WALLS & FRANK & B & 27 & S & ITALY & ITALY & ITALY & PA & SALESMAN & & ELM STREET & CALUMET - RED JACKET \\
\hline 2141 & 825 & COPPO & JOHN & $\mathrm{H}$ & 36 & M1 & ITALY & ITALY & ITALY & NA & LABORER/CM & PONT CANAVESE. TO & SEVENTH ST. & CALUMET - RED JACKET \\
\hline 2142 & 825 & COPPO & KATE & W & 20 & M1 & ITALY & ITALY & ITALY & & & PONT CANAVESE. TO & SEVENTH ST. & CALUMET - RED JACKET \\
\hline 2143 & 825 & WALLZ & HUMBERTO & $\mathrm{H}$ & 32 & M9 & ITALY & ITALY & ITALY & NA & SALOONKEEPER & & SEVENTH ST. & CALUMET - RED JACKET \\
\hline 2144 & 825 & WALLZ & ANNA & W & 28 & M9 & ITALY & ITALY & ITALY & & & & SEVENTH ST. & CALUMET - RED JACKET \\
\hline 2145 & 825 & WALLZ & ANNA & $\mathrm{D}$ & 7 & $\mathrm{~s}$ & MICHIGAN & ITALY & ITALY & & AT SCHOOL 8 & & SEVENTH ST. & CALUMET - RED JACKET \\
\hline 2146 & 825 & WALLZ & MARGARET & $\mathrm{D}$ & 5 & s & MICHIGAN & ITALY & ITALY & & AT SCHOOL 4 & & SEVENTH ST. & CALUMET - RED JACKET \\
\hline 2147 & 825 & WALLZ & JOHN B. & $\mathrm{s}$ & 4 & $\mathrm{~s}$ & MICHIGAN & ITALY & ITALY & & & & SEVENTH ST. & CALUMET - RED JACKET \\
\hline 2148 & 825 & PICCHIOTTINO & DOMINICK & $\mathrm{H}$ & 31 & M6 & ITALY & ITALY & ITALY & NA & SALESMAN & PONT CANAVESE. TO & $\begin{array}{l}\text { SEVENTH ST. } \\
\text { SEVE }\end{array}$ & CALUMET - RED JACKET \\
\hline 2149 & 825 & PICCHIOTTINO & ASUNTA & W & 22 & M6 & ITALY & ITALY & ITALY & & & BERGAMO & SEVENTH ST. & CALUMET - RED JACKET \\
\hline 2150 & 825 & PICCHIOTTINO & LUCY & $\mathrm{D}$ & 4 & $\mathrm{~s}$ & MICHIGAN & ITALY & ITALY & & AT SCHOOL & PONT CANAVESE. TO & SEVENTH ST. & CALUMET - RED JACKET \\
\hline 2151 & 825 & PICCHIOTTINO & CHARLES & $\mathrm{s}$ & 1 & $\mathrm{~s}$ & MICHIGAN & ITALY & ITALY & & 年 & PONT CANAVESE. TO & SEVENTH ST. & CALUMET - RED JACKET \\
\hline 2152 & 825 & BALAGNA & ANTON & B & 50 & s & ITALY & ITALY & ITALY & NA & LABORER/CM & TORINO & SEVENTH ST. & CALUMET - RED JACKET \\
\hline 2153 & 825 & PELARATTI & ATTILIO & B & 18 & s & ITALY & ITALY & ITALY & & DRILL BOY/CM & BERGAMO & SEVENTH ST. & CALUMET - RED JACKET \\
\hline 2154 & 516 & PROCISSI & JOHN & $\mathrm{H}$ & 42 & M20 & ITALY & ITALY & ITALY & NA & MASON/CONTRACTOR & LUCCA & PINE STREET & CALUMET - RED JACKET \\
\hline 2155 & 516 & PROCISSI & MODESTA & W & 41 & M20 & ITALY & ITALY & ITALY & & & LUCCA & PINE STREET & CALUMET - RED JACKET \\
\hline 2156 & 516 & PROCISSI & LADENIA & D & 19 & s & ITALY & ITALY & ITALY & & & LUCCA & PINE STREET & CALUMET - RED JACKET \\
\hline 2157 & 516 & PROCISSI & CARA & $\mathrm{D}$ & 16 & $\mathrm{~S}$ & ITALY & ITALY & ITALY & & & LUCCA & PINE STREET & CALUMET - RED JACKET \\
\hline 2158 & 516 & PROCISSI & LOUIS & $\mathrm{s}$ & 12 & $\mathrm{~s}$ & MICHIGAN & ITALY & ITALY & & AT SCHOOL 7 & LUCCA & PINE STREET & CALUMET - RED JACKET \\
\hline 2159 & 516 & PROCISSI & LAURA & $\mathrm{D}$ & 11 & $\mathrm{~s}$ & MICHIGAN & ITALY & ITALY & & AT SCHOOL 7 & LUCCA & PINE STREET & CALUMET - RED JACKET \\
\hline 2160 & 516 & PROCISSI & PASQUALLI & $\mathrm{s}$ & 9 & $\mathrm{~s}$ & MICHIGAN & ITALY & ITALY & & AT SCHOOL 7 & LUCCA & PINE STREET & CALUMET - RED JACKET \\
\hline 2161 & 516 & PROCISSI & AGNES & $\mathrm{D}$ & 6 & $\mathrm{~s}$ & MICHIGAN & ITALY & ITALY & & AT SCHOOL & LUCCA & PINE STREET & CALUMET - RED JACKET \\
\hline 2162 & 516 & PROCISSI & RAPHEAL & $\mathrm{D}$ & 5 & $\mathrm{~s}$ & MICHIGAN & ITALY & ITALY & & & LUCCA & PINE STREET & CALUMET - RED JACKET \\
\hline 2163 & 516 & PROCISSI & WILBERT & $\mathrm{s}$ & 3 & $\mathrm{~s}$ & MICHIGAN & ITALY & ITALY & & & LUCCA & PINE STREET & CALUMET - RED JACKET \\
\hline 2164 & 516 & PROCISSI & ELEANOR & D & $5 \mathrm{M}$ & $\mathrm{s}$ & MICHIGAN & ITALY & ITALY & & & LUCCA & PINE STREET & $\begin{array}{l}\text { CALUMET - RED JACKET } \\
\end{array}$ \\
\hline 2165 & & PANTERA & JOSEPH & $\mathrm{H}$ & 66 & M37 & ITALY & ITALY & ITALY & NA & PEDLAR/GROCERIES & LUCCA & & FRANKLIN \\
\hline 2166 & & PANTERA & MARIA & W & 65 & M37 & NEW YORK & NEW & NEW YORK & & & USA & & FRANKLIN \\
\hline 2167 & & PANTERA & WILLIAM & s & 28 & s & MICHIGAN & ITALY & NEW YORK & & TEAMSTER & LUCCA & & FRANKLIN \\
\hline 2168 & & PANTERA & FREDERICK & $\mathrm{s}$ & 27 & $\mathrm{~s}$ & MICHIGAN & ITALY & NEW YORK & & BARTENDER & LUCCA & & FRANKLIN \\
\hline 2169 & & PANTERA & JOSEPH & $\mathrm{s}$ & 19 & s & MICHIGAN & ITALY & NEW YORK & & & LUCCA & & FRANKLIN \\
\hline 2170 & & BIANCHI & GIOVANNI & B & 53 & M27 & ITALY & ITALY & ITALY & AL & & LUCCA & & FRANKLIN \\
\hline 2171 & & BARASA & BERNARD & B & 22 & $\mathrm{~s}$ & MICHIGAN & ITALY & MASSACHU & & SALESMAN & TORINO & & FRANKLIN \\
\hline 2172 & & BONINI & ULYSSES & $\mathrm{H}$ & 27 & M1 & ITALY & ITALY & ITALY & AL & TRAMMER/CM & COLLODI. LU & & FRANKLIN \\
\hline 2173 & & BONINI & ANGELINE & w & 22 & M1 & ITALY & ITALY & ITALY & UN & & COLLODI, LU & & FRANKLIN \\
\hline 2174 & & BONINI & BABY & s & $3 \mathrm{M}$ & & MICHIGAN & ITALY & ITALY & & & COLLODI. LU & & FRANKLIN \\
\hline 2175 & & BARSACCI & ANGELO & B & 42 & M8 & ITALY & ITALY & ITALY & $\mathrm{AL}$ & TRAMMER/CM & LUCCA & & FRANKLIN \\
\hline 2176 & & BAGNESCHI & ANDREW & B & 34 & M10 & ITALY & ITALY & ITALY & $\mathrm{AL}$ & TRAMMER/CM & LUCCA & & FRANKLIN \\
\hline 2177 & & CARTI & MICHAEL & B & 36 & $\mathrm{~s}$ & ITALY & ITALY & ITALY & $\mathrm{AL}$ & TRAMMER/CM & & & FRANKLIN \\
\hline 2178 & & CATTINI & GILBERTO & B & 28 & $\mathrm{~s}$ & ITALY & ITALY & ITALY & $\mathrm{AL}$ & DAY LABORER/CM & LUCCA & & FRANKLIN \\
\hline 2179 & & CATTINI & ALERIANO & B & 38 & M12 & ITALY & ITALY & ITALY & $\mathrm{AL}$ & STONE MASON & LUCCA & & FRANKLIN \\
\hline 2180 & & CIABATTARI & ANGELO & $\mathrm{H}$ & 54 & M13 & ITALY & ITALY & ITALY & NA & LABORER/ROCK HOUSE & LUCCA & & FRANKLIN \\
\hline 2181 & & CIABATTARI & NERCE & W & 32 & M13 & ITALY & ITALY & ITALY & & & LUCCA & & FRANKLIN \\
\hline 2182 & & CIABATTARI & MARY & D & 13 & S & ITALY & ITALY & ITALY & & AT SCHOOL 10 & LUCCA & & FRANKLIN \\
\hline 2183 & & CIABATTARI & ELIZABETH & $\mathrm{D}$ & 8 & $\mathrm{~s}$ & MICHIGAN & ITALY & ITALY & & AT SCHOOL 10 & LUCCA & & FRANKLIN \\
\hline 2184 & & CIABATTARI & JULIA & $\mathrm{D}$ & 7 & $\mathrm{~s}$ & MICHIGAN & ITALY & ITALY & & AT SCHOOL 10 & LUCCA & & FRANKLIN \\
\hline 2185 & & CIABATTARI & PETER & $\mathrm{s}$ & 6 & $\mathrm{~s}$ & MICHIGAN & ITALY & ITALY & & AT SCHOOL 10 & LUCCA & & FRANKLIN \\
\hline 2186 & & CIABATTARI & LAWRENCE & $\mathrm{s}$ & 1 & $\mathrm{~s}$ & MICHIGAN & ITALY & ITALY & & & LUCCA & & FRANKLIN \\
\hline 2187 & & CIABATTARI & ORLANDO & $\mathrm{s}$ & $3 \mathrm{M}$ & s & MICHIGAN & ITALY & ITALY & & & LUCCA & & FRANKLIN \\
\hline 2188 & & CIABATTARI & RAFFAELLO & $\mathrm{BR}$ & 25 & s & ITALY & ITALY & ITALY & NA & PEDLAR/GROCERIES & LUCCA & & FRANKLIN \\
\hline 2189 & & CIABATTARI & $\begin{array}{l}\text { ANASIA } \\
\text { P }\end{array}$ & SIIL & 28 & s & ITALY & ITALY & ITALY & & SERVANT & LUCCA & & FRANKLIN \\
\hline 2190 & & GIUSFREDI & ORLANDO & $B$ & 19 & $\mathrm{~s}$ & ITALY & ITALY & ITALY & UN & TRAMMER/CM & SEGROMIGNO. LU & & FRANKLIN \\
\hline 2191 & & FILIANI/FELCARI & OCTAVIO & B & 17 & $\mathrm{~s}$ & ITALY & ITALY & ITALY & UN & & & & FRANKLIN \\
\hline 2192 & & FILIANI/FELCARI & LOWRENCE & B & 31 & M7 & ITALY & ITALY & ITALY & $\mathrm{AL}$ & & & & FRANKLIN \\
\hline 2193 & & ARCHIMEDE & LELCE/LELO & B & 31 & W & ITALY & ITALY & ITALY & $\mathrm{AL}$ & TRAMMER/CM & & & FRANKLIN \\
\hline 2194 & & BRILDI & NAITILI & B & 28 & s & ITALY & ITALY & ITALY & NA & MINER/CM & & & FRANKLIN \\
\hline 2195 & & CHISERA & JOSEPH & B & 33 & M4 & ITALY & ITALY & ITALY & $\mathrm{AL}$ & TRAMMER/CM & & & FRANKLIN \\
\hline 2196 & & ORESTA & PETE & B & 28 & M4 & ITALY & ITALY & ITALY & $\mathrm{AL}$ & LABORER/FARM & & & FRANKLIN \\
\hline 2197 & & PISANI/RINOSI & PAULO & B & 20 & s & ITALY & ITALY & ITALY & UN & TRAMMER/CM & & & FRANKLIN \\
\hline 2198 & & PETRINI & ACHILLE & B & 37 & M16 & ITALY & ITALY & ITALY & $\mathrm{AL}$ & DAY LABORER/CM & LUCCA & & FRANKLIN \\
\hline 2199 & & M? & CASIMIRO & B & 40 & M14 & ITALY & ITALY & ITALY & $\mathrm{AL}$ & TRAMMER/CM & & & FRANKLIN \\
\hline 2200 & & NAPOLI & JOSEPH & B & 25 & M1 & ITALY & ITALY & ITALY & $\mathrm{AL}$ & TRAMMER/CM & & & FRANKLIN \\
\hline 2201 & & MONTICELLI & SAMUEL & $\mathrm{H}$ & 29 & M4 & ITALY & ITALY & ITALY & NA & ENGINEER/STATIONARY ENGINE & LUCCA & & FRANKLIN \\
\hline 2202 & & MONTICELLI & TERESINA & W & 21 & M4 & ITALY & ITALY & ITALY & & & & & FRANKLIN \\
\hline
\end{tabular}




\begin{tabular}{|c|c|c|c|c|c|c|c|c|c|c|c|c|}
\hline 2203 & MONTICELLI & GUIDO & $\mathrm{s}$ & 2 & $S$ & MICHIGAN & ITALY & ITALY & & & LUCCA & FRANKLIN \\
\hline 2204 & MONTICELLI & JENNI & D & $8 \mathrm{M}$ & $\mathrm{S}$ & MICHIGAN & ITALY & ITALY & & & LUCCA & FRANKLIN \\
\hline 2205 & MONTICELLI & AMERIGO & BR & 27 & $\mathrm{~s}$ & ITALY & ITALY & ITALY & $\mathrm{AL}$ & STONE MASON & LUCCA & FRANKLIN \\
\hline 2206 & CELESTE & DOMINICA & B & 28 & $\mathrm{~S}$ & ITALY & ITALY & ITALY & AL & LABORER & & FRANKLIN \\
\hline 2207 & QUILICI & OTTAVIO & B & 27 & $\mathrm{~S}$ & ITALY & ITALY & ITALY & $\mathrm{AL}$ & TRAMMER/CM & MATRAIA. LU & FRANKLIN \\
\hline 2208 & TOMEI & CAESAR & B & 22 & $\mathrm{~S}$ & ITALY & ITALY & ITALY & $\mathrm{AL}$ & LABORER/FARM & LUCCA & FRANKLIN \\
\hline 2209 & CENTANINO & ANSELMINA & SER & 18 & s & ITALY & ITALY & ITALY & & HOUSEKEEPER & TORINO & FRANKLIN \\
\hline 2210 & MALONI & TOBIA & $\mathrm{H}$ & 39 & M8 & ITALY & ITALY & ITALY & $\mathrm{AL}$ & LABORER & & FRANKLIN \\
\hline 2211 & MALONI & MARIA & w & 28 & M8 & ITALY & ITALY & ITALY & UN & & & FRANKLIN \\
\hline 2212 & MALONI & AMERIGO & s & 0 & $\mathrm{~s}$ & MICHIGAN & ITALY & ITALY & & & & FRANKLIN \\
\hline 2213 & ROSSI/ORSI & GIRNI & B & 33 & M4 & ITALY & ITALY & ITALY & $\mathrm{AL}$ & LABORER & & FRANKLIN \\
\hline 2214 & ROSSI/ORSI & FRANK & B & 27 & M3 & ITALY & ITALY & ITALY & PA & LABORER/CM & & FRANKLIN \\
\hline 2215 & TORTI & HIMMA? & B & 32 & M8 & ITALY & ITALY & ITALY & $\mathrm{AL}$ & TRAMMER/CM & & FRANKLIN \\
\hline 2216 & DIFONSO & PASQUALE & B & 48 & M14 & ITALY & ITALY & ITALY & $\mathrm{AL}$ & LABORER/CM & PETTORANO. L'AQUILA & FRANKLIN \\
\hline 2217 & DIFONSO & PASQUALE & B & 37 & M14 & ITALY & ITALY & ITALY & $\mathrm{AL}$ & MINER/CM & PETTORANO. L'AQUILA & FRANKLIN \\
\hline 2218 & MALONI & TOBIA & B & 40 & M10 & ITALY & ITALY & ITALY & $\mathrm{AL}$ & LABORER/CM & & FRANKLIN \\
\hline 2219 & PELIGAN & NICHOLAS & $\mathrm{H}$ & 31 & MO & ITALY & ITALY & ITALY & NA & FIREMAN/STATIONARY ENGINE & & FRANKLIN \\
\hline 2220 & PELIGAN & IS? & W & 18 & M0 & ITALY & ITALY & ITALY & UN & & & FRANKLIN \\
\hline 2221 & $\mathrm{RICCl}$ & EGIDIO & B & 27 & $\mathrm{~s}$ & ITALY & ITALY & ITALY & PA & TRAMMER/CM & LUCCA & FRANKLIN \\
\hline 2222 & LENCI & URVASIO & B & 30 & M2 & ITALY & ITALY & ITALY & NA & LABORER/CM & PESCAGLIA. LU & FRANKLIN \\
\hline 2223 & MICHELE & CREONTE & B & 26 & M3 & ITALY & ITALY & ITALY & $\mathrm{AL}$ & MINER/CM & PESCAGLIA, LU & FRANKLIN \\
\hline 2224 & BAGNESCHI & DANIEL & B & 25 & $\mathrm{~s}$ & ITALY & ITALY & ITALY & AL & MINER/CM & LUCCA & FRANKLIN \\
\hline 2225 & BAGELIO? & VIANI & B & 27 & $\mathrm{~s}$ & ITALY & ITALY & ITALY & $\mathrm{AL}$ & TRAMMER/CM & & FRANKLIN \\
\hline 2226 & RAFFAELLI & ALBERTO & $\mathrm{H}$ & 28 & M5 & ITALY & ITALY & ITALY & $A L$ & TRAMMER/CM & COLLODI. LU & FRANKLIN \\
\hline 2227 & RAFFAELLI & ANGELINA & w & 25 & M5 & ITALY & ITALY & ITALY & UN & & & FRANKLIN \\
\hline 2228 & RAFFAELLI & CLARA & $\mathrm{D}$ & 0 & $\mathrm{~s}$ & MICHIGAN & ITALY & ITALY & & & COLLODI. LU & FRANKLIN \\
\hline 2229 & DEL GHINGARO & LOUIS & B & 22 & $\mathrm{~S}$ & ITALY & ITALY & ITALY & $\mathrm{AL}$ & TRAMMER/CM & LUCCA & FRANKLIN \\
\hline 2230 & DEL GHINGARO & ORAZIO & B & 24 & $\mathrm{~s}$ & ITALY & ITALY & ITALY & $\mathrm{AL}$ & TRAMMER/CM & LUCCA & FRANKLIN \\
\hline 2231 & GIUSFREDI & ALFREDO & B & 24 & $\mathrm{~s}$ & ITALY & ITALY & ITALY & $\mathrm{AL}$ & TRAMMER/CM & LUCCA & FRANKLIN \\
\hline 2232 & ?NTINI & CIALDI? & B & 30 & M7 & ITALY & ITALY & ITALY & $\mathrm{AL}$ & TRAMMER/CM & & FRANKLIN \\
\hline 2233 & GIACOBETTI & DEONISIO & B & 33 & M6 & ITALY & ITALY & ITALY & $\mathrm{AL}$ & TRAMMER/CM & & FRANKLIN \\
\hline 2234 & GEMIGNANI & FRANCESCO & $\mathrm{H}$ & 33 & M2 & ITALY & ITALY & ITALY & $\mathrm{AL}$ & LABORER/CM & PESCAGLIA. LU & FRANKLIN \\
\hline 2235 & GEMIGNANI & TERESA & w & 24 & M2 & ITALY & ITALY & ITALY & UN & & FLOUAULIA.LU & FRANKLIN \\
\hline 2236 & GEMIGNANI & ALADINO & s & 1 & $\mathrm{~s}$ & MICHIGAN & ITALY & ITALY & & & PESCAGLIA, LU & FRANKLIN \\
\hline 2237 & GEMIGNANI & LENA & D & 0 & $\mathrm{~s}$ & MICHIGAN & ITALY & ITALY & & & PESCAGLIA. LU & FRANKLIN \\
\hline 2238 & PATERNI & JOSEPH & B & 30 & $\frac{2}{2}$ & ITALY & ITALY & ITALY & $\mathrm{AL}$ & TRAMMER/CM & MONSAGRATI. LU & FRANKLIN \\
\hline 2239 & RAFFAELLI & PAUL & B & 19 & $\mathrm{~s}$ & ITALY & ITALY & ITALY & $\mathrm{AL}$ & TRAMMER/CM & LUCCA & FRANKLIN \\
\hline 2240 & LENCIONI & ALFREDO & B & 19 & $\mathrm{~s}$ & ITALY & ITALY & ITALY & $\mathrm{AL}$ & TRAMMER/CM & LUCCA & FRANKLIN \\
\hline 2241 & MASSIMO & ANTONY & B & 27 & $\mathrm{~s}$ & ITALY & ITALY & ITALY & $\mathrm{AL}$ & TRAMMER/CM & TORINO & FRANKLIN \\
\hline 2242 & MAZZONI & CHERILINO & B & 28 & $\mathrm{~s}$ & ITALY & ITALY & ITALY & $\mathrm{AL}$ & TRAMMER/CM & LUCCA & FRANKLIN \\
\hline 2243 & MUSANI & RAFFAELLO & B & 25 & $\mathrm{~s}$ & ITALY & ITALY & ITALY & $\mathrm{AL}$ & TRAMMER/CM & & FRANKLIN \\
\hline 2244 & BENEDETTI & TRANQUILLO & B & 27 & s & ITALY & ITALY & ITALY & $\mathrm{AL}$ & TRAMMER/CM & CAMAIORE. LU & FRANKLIN \\
\hline 2245 & GEMIGNANI & JAMES & B & 49 & M19 & ITALY & ITALY & ITALY & NA & STONE MASON & LUCCA & FRANKLIN \\
\hline 2246 & GEMIGNANI & NORMAN & B & 15 & $\mathrm{~s}$ & NEW YORK & ITALY & INDIANA & & AT SCHOOL 2 & LUCCA & FRANKLIN \\
\hline 2247 & MATSOLA & SAMUEL & B & 29 & s & ITALY & ITALY & ITALY & $\mathrm{AL}$ & TRAMMER/CM & & FRANKLIN \\
\hline 2248 & ALLARIA & PIETRO & $\mathrm{H}$ & 52 & M30 & ITALY & ITALY & ITALY & NA & LABORER/CM & SAN GIORGIO CANAVESE. TO & FRANKLIN \\
\hline 2249 & ALLARIA & DOMENICA & w & 52 & M30 & ITALY & ITALY & ITALY & UN & & SAN GIORGIO CANAVESE. TO & FRANKLIN \\
\hline 2250 & ALLARIA & WILLIAM & s & 11 & s & MICHIGAN & ITALY & ITALY & & AT SCHOOL 10 & SAN GIORGIO CANAVESE. TO & FRANKLIN \\
\hline 2251 & PASTORE & FRANK & B & 26 & s & ITALY & $\begin{array}{l}\text { IITALY } \\
\text { ITALY }\end{array}$ & $\begin{array}{l}\text { ITALY } \\
\text { ITAL }\end{array}$ & $\mathrm{AL}$ & TRAMMER/CM & TORINO & $\begin{array}{l}\text { FRANKLIN } \\
\text { FRANKIN }\end{array}$ \\
\hline 2252 & PASTORE & JAMES & B & 22 & $\mathrm{~s}$ & ITALY & ITALY & ITALY & AL & TRAMMER/CM & CUCEGLIO. TO & FRANKLIN \\
\hline 2253 & DE MATTEIS & ANTONIO & B & 25 & $\mathrm{~s}$ & ITALY & ITALY & ITALY & $\mathrm{AL}$ & TRAMMER/CM & SAN GIORGIO CANAVESE. TO & FRANKLIN \\
\hline 2254 & VAIRO & GIN? & $\begin{array}{l}\mathrm{D} \\
\mathrm{B}\end{array}$ & 26 & s & ITALY & ITALY & ITALY & $\mathrm{AL}$ & TRAMMER/CM & $\begin{array}{l}\text { LOCANA CANAVESE, TO } \\
\text { LOTE }\end{array}$ & FRANKLIN \\
\hline 2255 & VITORI & EOVELLI & B & 19 & $\mathrm{~s}$ & ITALY & ITALY & ITALY & $A L$ & TRAMMER/CM & & FRANKLIN \\
\hline 2256 & BARITINI & JOHN & B & 30 & $\mathrm{~s}$ & ITALY & ITALY & ITALY & $\mathrm{AL}$ & TRAMMER/CM & & FRANKLIN \\
\hline 2257 & TALLETTI & PETER & B & 23 & $\mathrm{~S}$ & ITALY & ITALY & ITALY & $\mathrm{AL}$ & TRAMMER/CM & TORINO & FRANKLIN \\
\hline 2258 & DEL BIANCO & VINCENZO & B & 26 & $\mathrm{~S}$ & ITALY & ITALY & ITALY & $\mathrm{AL}$ & TRAMMER/CM & & FRANKLIN \\
\hline 2259 & GIANNE & ANTHONY & B & 25 & s & ITALY & ITALY & ITALY & $\mathrm{AL}$ & TRAMMER/CM & TORINO & FRANKLIN \\
\hline 2260 & TAPPERO & CARLO & B & 30 & W & ITALY & ITALY & ITALY & NA & STONE MASON & SAN GIORGIO CANAVESE, TO & FRANKLIN \\
\hline 2261 & CORTOPASSI & CHERUBINO & $\mathrm{H}$ & 33 & M3 & ITALY & ITALY & ITALY & $\mathrm{AL}$ & LABORER/ROCK HOUSE & LUCCA & FRANKLIN \\
\hline 2262 & CORTOPASSI & DOMENICA & w & 22 & M3 & ITALY & ITALY & ITALY & UN & & & FRANKLIN \\
\hline 2263 & CORTOPASSI & NATALINA & $\mathrm{D}$ & 2 & S & MICHIGAN & ITALY & ITALY & & & LUCCA & FRANKLIN \\
\hline 2264 & ANDREOZZI & JOSEPH & $\mathrm{H}$ & 35 & M9 & ITALY & ITALY & ITALY & AL & FIREMAN/STATIONARY ENGINE & LUCCA & FRANKLIN \\
\hline 2265 & ANDREOZZI & PAULINA & w & 31 & M9 & ITALY & ITALY & ITALY & UN & & LUCCA & FRANKLIN \\
\hline
\end{tabular}




\begin{tabular}{|c|c|c|c|c|c|c|c|c|c|c|c|c|}
\hline 2266 & ANDREOZZI & GABRIELLO & $\mathrm{s}$ & 8 & $\mathrm{~s}$ & ITALY & ITALY & ITALY & UN & AT SCHOOL 10 & LUCCA & FRANKLIN \\
\hline 2267 & ANDREOZZI & FRED & $\mathrm{s}$ & 2 & s & MICHIGAN & ITALY & ITALY & & & LUCCA & FRANKLIN \\
\hline 2268 & ANDREOZZI & ORESTE & BR & 26 & $\mathrm{~S}$ & ITALY & ITALY & ITALY & $\mathrm{AL}$ & TRAMMER/CM & LUCCA & FRANKLIN \\
\hline 2269 & ANDREOZZI & ARMANDO & $\mathrm{BR}$ & 37 & M7 & ITALY & ITALY & ITALY & $\mathrm{AL}$ & TRAMMER/CM & LUCCA & FRANKLIN \\
\hline 2270 & ANDREOZZI & ZELIS & B & 26 & M7 & ITALY & ITALY & ITALY & $\mathrm{AL}$ & TRAMMER/CM & LUCCA & FRANKLIN \\
\hline 2271 & DEMARCA & JAKUM & B & 24 & $\mathrm{~S}$ & ITALY & ITALY & ITALY & $\mathrm{AL}$ & MINER/CM & & FRANKLIN \\
\hline 2272 & BIANCHI & GIUSEPPE & B & 22 & S & ITALY & ITALY & ITALY & $\mathrm{AL}$ & LABORER/CM & LUCCA & FRANKLIN \\
\hline 2273 & FILIPPI & FRED & B & 32 & M6 & ITALY & ITALY & ITALY & $\mathrm{AL}$ & TRAMMER/CM & CAPANNORI. LU & FRANKLIN \\
\hline 2274 & BATTISTONI & $?$ & $\mathrm{H}$ & 49 & M5 & ITALY & ITALY & ITALY & NA & ENGINEER/STATIONARY ENGINE & LUCCA & FRANKLIN \\
\hline 2275 & BATTISTONI & CORINA & W & 26 & M5 & ITALY & ITALY & ITALY & & & LUCCA & FRANKLIN \\
\hline 2276 & MONTICELL & JENNIE & NIEC & $7 \mathrm{M}$ & $\mathrm{s}$ & MICHIGAN & ITALY & ITALY & & & LUCCA & FRANKLIN \\
\hline 2277 & HAMMASCIOTTI & DOMINICK & B & 28 & M5 & ITALY & ITALY & ITALY & $\mathrm{AL}$ & STONE MASON & & FRANKLIN \\
\hline 2278 & JULENCI? & JOSEPH & B & 27 & M5 & ITALY & ITALY & ITALY & $\mathrm{AL}$ & TRAMMER/CM & & FRANKLIN \\
\hline 2279 & MAFFEI & ANIBALE & B & 24 & $\mathrm{~s}$ & ITALY & ITALY & ITALY & $\mathrm{AL}$ & TRAMMER/CM & LUCCA & FRANKLIN \\
\hline 2280 & MISCHI & ANGELO & B & 29 & M3 & ITALY & ITALY & ITALY & AL & TRAMMER/CM & & FRANKLIN \\
\hline 2281 & ANAGRETO & PETER & B & 33 & M6 & ITALY & ITALY & ITALY & $\mathrm{AL}$ & TRAMMER/CM & & FRANKLIN \\
\hline 2282 & MARTINELLO & DANIEL & B & 20 & $\mathrm{~s}$ & ITALY & ITALY & ITALY & $\mathrm{AL}$ & LABORER/CM & & FRANKLIN \\
\hline 2283 & CORTOPASSI & ANGELO & B & 24 & M2 & ITALY & ITALY & ITALY & $\mathrm{AL}$ & TRAMMER/CM & LUCCA & FRANKLIN \\
\hline 2284 & MASSINI & FRANK & B & 43 & M11 & ITALY & ITALY & ITALY & $\mathrm{AL}$ & TRAMMER/CM & & FRANKLIN \\
\hline 2285 & WILLIAM & SAMUEL & B & 31 & s & ITALY & ITALY & ITALY & NA & LABORER/SMELTER & & FRANKLIN \\
\hline 2286 & SCAMBI & ALFREDO & B & 17 & s & ITALY & ITALY & ITALY & $\mathrm{AL}$ & LABORER/CM & SEGROMIGNO. LU & FRANKLIN \\
\hline 2287 & GIONOTTI & ANTHONY & B & 45 & M15 & ITALY & ITALY & ITALY & $\mathrm{AL}$ & STONECUTTER & & FRANKLIN \\
\hline 2288 & PISTRALLI & LOUIS & B & 48 & M27 & ITALY & ITALY & ITALY & PA & LABORER/ROCK HOUSE & & FRANKLIN \\
\hline 2289 & CHELINI & GIUSEPPE & B & 26 & M4 & ITALY & ITALY & ITALY & $\mathrm{AL}$ & TRAMMER/CM & SEGROMIGNO. LU & FRANKLIN \\
\hline 2290 & $\mathrm{RICCl}$ & LOUIS & B & 22 & s & ITALY & ITALY & ITALY & $\mathrm{AL}$ & TRAMMER/CM & LUCCA & FRANKLIN \\
\hline 2291. & $\mathrm{BEI} / \mathrm{A}$ & ARTURO & B & 28 & s & ITALY & ITALY & ITALY & $A L$ & LABORER/CM & LUCCA & FRANKLIN \\
\hline 2292 & GIULI & ANTONIO & B & 28 & M4 & ITALY & ITALY & ITALY & $\mathrm{AL}$ & TRAMMER/CM & & FRANKLIN \\
\hline 2293 & BANDETTINI & PETER & B & 29 & s & ITALY & ITALY & ITALY & NA & SALESMAN & LUCCA & FRANKLIN \\
\hline 2294 & PAOLINI & PAUL & B & 31 & M1 & ITALY & ITALY & ITALY & $\mathrm{AL}$ & TRAMMER/CM & LUCCA & FRANKLIN \\
\hline 2295 & FRANCIONI & LARRY & $\mathrm{H}$ & 29 & M3 & ITALY & ITALY & ITALY & $\mathrm{AL}$ & MINER/CM & LUCCA & FRANKLIN \\
\hline 2296 & FRANCIONI & OLYMPIA & W & 31 & M3 & ITALY & ITALY & ITALY & UN & & LUCCA & FRANKLIN \\
\hline 2297 & GUAVIE/GUAERI & PAUL & B & 28 & $\mathrm{~s}$ & ITALY & ITALY & ITALY & $\mathrm{AL}$ & MINER/CM & & FRANKLIN \\
\hline 2298 & $\mathrm{BOCCl}$ & DOMINICO & B & 28 & s & ITALY & ITALY & ITALY & $\mathrm{AL}$ & TRAMMER/CM & & FRANKLIN \\
\hline 2299 & $\mathrm{BOCCl}$ & CARLO & B & 33 & M7 & ITALY & ITALY & ITALY & $\mathrm{AL}$ & TRAMMER/CM & & FRANKLIN \\
\hline 2300 & FRANCIONI & LOUIS & B & 32 & $\mathrm{~S}$ & ITALY & ITALY & ITALY & NA & TRAMMER/CM & LUCCA & FRANKLIN \\
\hline 2301 & BARSOCCHI & ERNESTO & B & 21 & $\mathrm{~s}$ & ITALY & ITALY & ITALY & $\mathrm{AL}$ & TRAMMER/CM & RUOTA. LU & FRANKLIN \\
\hline 2302 & BANDONI & DOMINICO & B & 48 & s & ITALY & ITALY & ITALY & $A L$ & MINER/CM & LUCCA & FRANKLIN \\
\hline 2303 & $\mathrm{RICCl}$ & FRANK & $\mathrm{H}$ & 30 & M7 & ITALY & ITALY & ITALY & PA & MINER/CM & LUCCA & FRANKLIN \\
\hline 2304 & $\mathrm{RICCl}$ & NATHALINA & W & 25 & M7 & ITALY & ITALY & ITALY & UN & & & FRANKLIN \\
\hline 2305 & ISOLA & EUGENE & B & 29 & M3 & ITALY & ITALY & ITALY & AL & TRAMMER/CM & LUCCA & FRANKLIN \\
\hline 2306 & BANDONI & DOMINICK & B & 27 & M3 & ITALY & ITALY & ITALY & PA & MINER/CM & LUCCA & FRANKLIN \\
\hline 2307 & MILANI & MELO & B & 22 & M1 & ITALY & ITALY & ITALY & $\mathrm{AL}$ & TRAMMER/CM & LUCCA & FRANKLIN \\
\hline 2308 & FORTUNO & AMERIGO & B & 32 & M1 & ITALY & ITALY & ITALY & $\mathrm{AL}$ & TRAMMER/CM & & FRANKLIN \\
\hline 2309 & NICOLI & FRANK & B & 23 & s & ITALY & ITALY & ITALY & $\mathrm{AL}$ & TRAMMER/CM & & FRANKLIN \\
\hline 2310 & CORDONI & FRANK & B & 23 & $\mathrm{~S}$ & ITALY & ITALY & ITALY & $\mathrm{AL}$ & TRAMMER/CM & & FRANKLIN \\
\hline 2311 & TADDEUCCI & AMERIGO & B & 27 & M2 & ITALY & ITALY & ITALY & $\mathrm{AL}$ & TRAMMER/CM & LUCCA & FRANKLIN \\
\hline 2312 & $\mathrm{RICCl}$ & JOSEPH & $\mathrm{s}$ & 3 & $\mathrm{~s}$ & MICHIGAN & ITALY & ITALY & & & LUCCA & FRANKLIN \\
\hline 2313 & DEL GHINGARO & JOHN & H & 35 & M10 & ITALY & ITALY & ITALY & AL & MINER/CM & LUCCA & FRANKLIN \\
\hline 2314 & DEL GHINGARO & ANGELINA & w & 34 & M10 & ITALY & ITALY & ITALY & UN & & & FRANKLIN \\
\hline 2315 & DEL GHINGARO & JULIA & D & $9 \mathrm{M}$ & $\mathrm{s}$ & MICHIGAN & ITALY & ITALY & & & LUCCA & FRANKLIN \\
\hline 2316 & DI VECCHIO & JUST & B & 29 & M8 & ITALY & ITALY & ITALY & PA & STONE MASON & LUCCA & FRANKLIN \\
\hline 2317 & PARENTI & JOHN & B & 25 & s & ITALY & ITALY & ITALY & $\mathrm{AL}$ & TRAMMER/CM & CASTELVECCHIO, LU & FRANKLIN \\
\hline 2318 & MARCHESCHI & FRANK & B & 40 & M12 & ITALY & ITALY & ITALY & $\mathrm{AL}$ & TRAMMER/CM & LUCCA & FRANKLIN \\
\hline 2319 & ANDREINI & MASSIMO & B & 27 & S & ITALY & ITALY & ITALY & $\mathrm{AL}$ & TRAMMER/CM & CAPANNORI. LU & FRANKLIN \\
\hline 2320 & DI VECCHIO & PHILIPH & B & 24 & MO & ITALY & ITALY & ITALY & $\mathrm{AL}$ & TRAMMER/CM & LUCCA & FRANKLIN \\
\hline 2321 & BARTOLOMEI & $J A C O B$ & B & 25 & $\mathrm{~s}$ & ITALY & ITALY & ITALY & $\mathrm{AL}$ & TRAMMER/CM & LUCCA & FRANKLIN \\
\hline 2322 & PEROTTI & FRANK & B & 25 & M3 & ITALY & ITALY & ITALY & $\mathrm{AL}$ & TRAMMER/CM & TORINO & FRANKLIN \\
\hline 2323 & GIACOBETTI & RIALLI & B & 24 & $\mathrm{~s}$ & ITALY & ITALY & ITALY & $\mathrm{AL}$ & LABORER/ROCK HOUSE & & FRANKLIN \\
\hline 2324 & BARTOLOMEO & VINCENT & $\mathrm{H}$ & 37 & M10 & ITALY & ITALY & ITALY & NA & LABORER/ROCK HOUSE & & FRANKLIN \\
\hline 2325 & BARTOLOMEO & MARINO & w & 38 & M10 & ITALY & ITALY & ITALY & UN & & & FRANKLIN \\
\hline 2326 & BARTOLOMEO & ESTHER & $\mathrm{D}$ & 9 & $\mathrm{~s}$ & ITALY & ITALY & ITALY & UN & AT SCHOOL 5 & & FRANKLIN \\
\hline 2327 & BARTOLOMEO & JOSEPH & $\mathrm{s}$ & 5 & s & MICHIGAN & ITALY & ITALY & & & & FRANKLIN \\
\hline 2328 & BARTOLOMEO & ANGELINA & $\mathrm{D}$ & 1 & s & NEW YORK & ITALY & ITALY & & & & FRANKLIN \\
\hline
\end{tabular}




\begin{tabular}{|c|c|c|c|c|c|c|c|c|c|c|c|c|}
\hline 2329 & VENTURELLI & $?$ & B & 23 & $\mathrm{~s}$ & ITALY & ITALY & ITALY & $\mathrm{AL}$ & LABORER/CM & & FRANKLIN \\
\hline 2330 & NOMELLINI & MICHAEL & B & 21 & s & ITALY & ITALY & ITALY & $\mathrm{AL}$ & TRAMMER/CM & LUCCA & FRANKLIN \\
\hline 2331 & BESSOLO & CASPAR & $\mathrm{H}$ & 40 & M12 & ITALY & ITALY & ITALY & NA & MINER/CM & TORINO & FRANKLIN \\
\hline 2332 & BESSOLO & VERONICA & W & 30 & M12 & ITALY & ITALY & ITALY & UN & & TORINO & FRANKLIN \\
\hline 2333 & BESSOLO & ANNIE & D & 7 & $\mathrm{~s}$ & TEXAS & ITALY & ITALY & & AT SCHOOL 10 & TORINO & FRANKLIN \\
\hline 2334 & BESSOLO & PAUL & $\mathrm{s}$ & 5 & s & KANSAS & ITALY & ITALY & & AT SCHOOL 9 & TORINO & FRANKLIN \\
\hline 2335 & SANTORI & ANNIBALE & B & 44 & M10 & ITALY & ITALY & ITALY & $\mathrm{AL}$ & TRAMMER/CM & SEGROMIGNO. LU & FRANKLIN \\
\hline 2336 & LISA & $\mathrm{JOHN}$ & B & 24 & s & ITALY & ITALY & ITALY & $\mathrm{AL}$ & BAKER & SAN MARTINO CANAVESE. TO & FRANKLIN \\
\hline 2337 & RENALDI & BAPTISTE & $\mathrm{H}$ & 36 & M7 & ITALY & ITALY & ITALY & NA & LABORER/CM & SAN GIORGIO CANAVESE. TO & FRANKLIN \\
\hline 2338 & RENALDI & MARION & W & 31 & M7 & ITALY & ITALY & ITALY & UN & & & FRANKLIN \\
\hline 2339 & RENALDI & THERESA & D & 4 & s & MICHIGAN & ITALY & ITALY & & & SAN GIORGIO CANAVESE. TO & FRANKLIN \\
\hline 2340 & RENALDI & JOSEPH & $\mathrm{s}$ & 3 & $\mathrm{~s}$ & MICHIGAN & ITALY & ITALY & & & SAN GIORGIO CANAVESE. TO & FRANKLIN \\
\hline 2341 & RENALDI & JAMES & $\mathrm{s}$ & 1 & $\mathrm{~s}$ & MICHIGAN & ITALY & ITALY & & & SAN GIORGIO CANAVESE. TO & FRANKLIN \\
\hline 2342 & RENALDI & MARIAN & $\mathrm{D}$ & $1 \mathrm{M}$ & $\mathrm{s}$ & MICHIGAN & ITALY & ITALY & & & SAN GIORGIO CANAVESE. TO & FRANKLIN \\
\hline 2343 & ROLANDO & LORENZO & B & 16 & $\mathrm{~s}$ & ITALY & ITALY & ITALY & UN & LABORER/MILL & TORINO & FRANKLIN \\
\hline 2344 & DE FILIPPI & GIOVANNI & B & 25 & $\mathrm{~s}$ & ITALY & ITALY & ITALY & $\mathrm{AL}$ & TRAMMER/CM & SAN GIORGIO CANAVESE, TO & FRANKLIN \\
\hline 2345 & ROLANDO & MICHAEL & B & 43 & M20 & ITALY & ITALY & ITALY & $\mathrm{PA}$ & LABORER/CM & TORINO & FRANKLIN \\
\hline 2346 & GIULIO & GIOVANNI & B & 26 & M3 & ITALY & ITALY & ITALY & $\mathrm{AL}$ & LABORER/CM & SAN GIORGIO CANAVESE. TO & FRANKLIN \\
\hline 2347 & DE FILIPPI & JOHN & B & 32 & $\mathrm{~s}$ & ITALY & ITALY & ITALY & NA & LABORER/CM & SAN GIORGIO CANAVESE. TO & FRANKLIN \\
\hline 2348 & ROMANO & PETER & $\mathrm{H}$ & 34 & M6 & ITALY & ITALY & ITALY & $\mathrm{AL}$ & LABORER/CM & TORINO & FRANKLIN \\
\hline 2349 & ROMANO & MARY & W & 24 & M6 & ITALY & ITALY & $\begin{array}{l}\text { ITALY } \\
\text { ITAL }\end{array}$ & UN & LADOURERTIU & Turivo & FRANKLIN \\
\hline 2350 & ROMANO & WILLIAM & s & 3 & $\mathrm{~s}$ & MICHIGAN & ITALY & ITALY & & & TORINO & FRANKLIN \\
\hline 2351 & ROMANO & ANTONIA & $\mathrm{D}$ & 5 & $\mathrm{~s}$ & ITALY & ITALY & ITALY & & & TORINO & FRANKLIN \\
\hline 2352 & ROMANO & GEORGIA & $\mathrm{D}$ & $5 \mathrm{M}$ & $\mathrm{s}$ & MICHIGAN & ITALY & ITALY & & & TORINO & FRANKLIN \\
\hline 2353 & ROMANO & JOHN & $B R$ & 29 & MO & ITALY & ITALY & ITALY & $\mathrm{AL}$ & LABORER/CM & TORINO & FRANKLIN \\
\hline 2354 & ROMANO & JOSEPH & $\mathrm{BR}$ & 32 & s & ITALY & ITALY & ITALY & $\mathrm{AL}$ & TRAMMER/CM & TORINO & FRANKLIN \\
\hline 2355 & PETRINI & JOHN & B & 24 & $\mathrm{~s}$ & ITALY & ITALY & ITALY & $\mathrm{AL}$ & TRAMMER/CM & TORINO & FRANKLIN \\
\hline 2356 & POGGIONE & JOHN & $\mathrm{H}$ & 31 & M4 & ITALY & ITALY & ITALY & NA & MINER/CM & SAN GIORGIO CANAVESE, TO & FRANKLIN \\
\hline 2357 & POGGIONE & MARY & W & 20 & M4 & ITALY & ITALY & ITALY & UN & & & FRANKLIN \\
\hline 2358 & POGGIONE & JAMES & s & 3 & $\mathrm{~s}$ & MICHIGAN & ITALY & ITALY & & & SAN GIORGIO CANAVESE. TO & FRANKLIN \\
\hline 2359 & POGGIONE & PETER & $\mathrm{s}$ & 1 & $\mathrm{~s}$ & MICHIGAN & ITALY & ITALY & & & SAN GIORGIO CANAVESE. TO & FRANKLIN \\
\hline 2360 & DE LORENZI & PETER & B & 24 & $\mathrm{~s}$ & ITALY & ITALY & ITALY & $\mathrm{AL}$ & LABORER/CM & & FRANKLIN \\
\hline 2361 & FANATO & EUGENE & B & 32 & s & ITALY & ITALY & ITALY & NA & NOT CLEAR & & FRANKLIN \\
\hline 2362 & TERODELPHI & SERAPHINO & B & 23 & M2 & ITALY & ITALY & ITALY & $\mathrm{AL}$ & LABORER/CM & & FRANKLIN \\
\hline 2363 & ALLARIA & DOMINICK & B & 23 & $\mathrm{~s}$ & ITALY & ITALY & ITALY & $\mathrm{AL}$ & LABORER/CM & SAN GIORGIO CANAVESE. TO & FRANKLIN \\
\hline 2364 & ALLARIA & PETER & B & 25 & $\mathrm{~s}$ & ITALY & ITALY & ITALY & $\mathrm{AL}$ & LABORER/CM & SAN GIORGIO CANAVESE. TO & FRANKLIN \\
\hline 2365 & DE FILIPPI & DOMENICO & B & 26 & $\mathrm{~s}$ & ITALY & ITALY & ITALY & AL & TRAMMER/CM & SAN GIORGIO CANAVESE. TO & FRANKLIN \\
\hline 2366 & GALETTO & JOSEPH & B & 27 & $\mathrm{~s}$ & ITALY & ITALY & ITALY & $\mathrm{AL}$ & TRAMMER/CM & SAN GIORGIO CANAVESE. TO & FRANKLIN \\
\hline 2367 & BERTOLARO & LOUIS & B & 26 & s & ITALY & ITALY & ITALY & $\mathrm{AL}$ & TRAMMER/CM & COURGNE'. TO & FRANKLIN \\
\hline 2368 & RAFFAELLI & ATTILIO & $\mathrm{H}$ & 33 & M6 & ITALY & ITALY & ITALY & $\mathrm{NA}$ & LABORER & COLLODI. LU & FRANKLIN \\
\hline 2369 & RAFFAELLI & IDA & W & 25 & M6 & ITALY & ITALY & ITALY & UN & & & FRANKLIN \\
\hline 2370 & RAFFAELLI & ANGELINA & D & 5 & $\mathrm{~s}$ & NEW YORK & ITALY & ITALY & & & COLLODI. LU & FRANKLIN \\
\hline 2371 & RAFFAELLI & GIUSEPPE & $\mathrm{s}$ & 3 & $\mathrm{~s}$ & MICHIGAN & ITALY & ITALY & & & COLLODI. LU & FRANKLIN \\
\hline 2372 & BUCCl & NATALI & B & 26 & $\mathrm{~s}$ & ITALY & ITALY & ITALY & $\mathrm{AL}$ & TRAMMER/CM & TORINO & FRANKLIN \\
\hline 2373 & SAORI/SOARI & NANA & B & 20 & $\mathrm{~s}$ & ITALY & ITALY & ITALY & $\mathrm{AL}$ & TRAMMER/CM & & FRANKLIN \\
\hline 2374 & $\mathrm{BUCCl}$ & ALFRED & B & 26 & s & ITALY & ITALY & ITALY & $\mathrm{AL}$ & TRAMMER/CM & TORINO & FRANKLIN \\
\hline 2375 & ZANOTTI & ANTHONY & $\mathrm{H}$ & 32 & M7 & ITALY & ITALY & ITALY & PA & LABORER/ROCK HOUSE & TORINO & FRANKLIN \\
\hline 2376 & ZANOTTI & ANNIE & w & 31 & M7 & ITALY & ITALY & ITALY & UN & & & FRANKLIN \\
\hline 2377 & ZANOTTI & DOMINICK & $\mathrm{s}$ & 7 & $\mathrm{~s}$ & PENNSYLV & ITALY & ITALY & & AT SCHOOL 8 & TORINO & FRANKLIN \\
\hline 2378 & ZANOTTI & PETER & $\mathrm{s}$ & 5 & $\mathrm{~s}$ & PENNSYLV & ITALY & ITALY & & & TORINO & FRANKLIN \\
\hline 2379 & ZANOTTI & KATHERINE & $\mathrm{D}$ & 2 & $\mathrm{~s}$ & MICHIGAN & ITALY & ITALY & & & TORINO & FRANKLIN \\
\hline 2380 & ZANOTTI & MINNIE & D & 1 & s & MICHIGAN & ITALY & ITALY & & & TORINO & FRANKLIN \\
\hline 2381 & CASPER & FRANK & $\mathrm{H}$ & 30 & M1 & ITALY & ITALY & ITALY & PA & LABORER/CM & & FRANKLIN \\
\hline 2382 & CASPER & MARY & W & 25 & M1 & ITALY & ITALY & ITALY & UN & & & FRANKLIN \\
\hline 2383 & FILIPPI & NICOLAS & s & 3 & $\mathrm{~s}$ & ITALY & ITALY & ITALY & & & & FRANKLIN \\
\hline 2384 & DIXON & GEORGE & B & 26 & s & ITALY & ITALY & ITALY & PA & STONE MASON & & FRANKLIN \\
\hline 2385 & MUZZI & MICHAEL & B & 29 & M5 & ITALY & ITALY & ITALY & $\mathrm{AL}$ & LABORER/CM & & FRANKLIN \\
\hline 2386 & MUZZI & FRANK & B & 30 & $\mathrm{~S}$ & ITALY & ITALY & ITALY & PA & TRAMMER/CM & & FRANKLIN \\
\hline 2387 & COLOMBO & JOSEPH & $\mathrm{H}$ & 40 & M12 & ITALY & ITALY & ITALY & NA & LABORER/ROCK HOUSE & PONT CANAVESE. TO & FRANKLIN \\
\hline 2388 & COLOMBO & MADELINE & w & 31 & M12 & ITALY & ITALY & ITALY & UN & & PONT CANAVESE. TO & FRANKLIN \\
\hline 2389 & COLOMBO & JOSEPHINE & $\mathrm{D}$ & 11 & $\mathrm{~s}$ & MICHIGAN & ITALY & ITALY & & AT SCHOOL 10 & PONT CANAVESE. TO & FRANKLIN \\
\hline 2390 & COLOMBO & JOSEPH & $\mathrm{s}$ & 9 & $\mathrm{~s}$ & MICHIGAN & ITALY & ITALY & & AT SCHOOL 10 & PONT CANAVESE. TO & FRANKLIN \\
\hline 2391 & COLOMBO & MARY & D & 7 & $\mathrm{~s}$ & MICHIGAN & ITALY & ITALY & & AT SCHOOL 10 & PONT CANAVESE. TO & FRANKLIN \\
\hline
\end{tabular}




\begin{tabular}{|c|c|c|c|c|c|c|c|c|c|c|c|c|}
\hline 2392 & COLOMBO & FRIDA & D & 6 & $\mathrm{~s}$ & MICHIGAN & ITALY & ITALY & & AT SCHOOL 10 & PONT CANAVESE. TO & FRANKLIN \\
\hline 2393 & COLOMBO & ABRAHAM & $\mathrm{s}$ & 4 & $\mathrm{~S}$ & MICHIGAN & ITALY & ITALY & & & PONT CANAVESE. TO & FRANKLIN \\
\hline 2394 & COLOMBO & SAMUEL & $\mathrm{s}$ & 2 & $\mathrm{~s}$ & MICHIGAN & ITALY & ITALY & & & PONT CANAVESE. TO & FRANKLIN \\
\hline 2395 & COLOMBO & ANTHONY & $\mathrm{s}$ & $2 \mathrm{M}$ & $S$ & MICHIGAN & ITALY & ITALY & & & PONT CANAVESE. TO & FRANKLIN \\
\hline 2396 & GUENSI & MARY & SIIL & 28 & M3 & ITALY & ITALY & ITALY & UN & & PONT CANAVESE. TO & FRANKLIN \\
\hline 2397 & GUENSI & JOHN & BIL & 36 & M3 & ITALY & ITALY & ITALY & $\mathrm{PA}$ & LABORER/ROCK HOUSE & PONT CANAVESE. TO & FRANKLIN \\
\hline 2398 & GUENSI & PETER & NEP & 2 & $\mathrm{~s}$ & MICHIGAN & ITALY & ITALY & & & PONT CANAVESE. TO & FRANKLIN \\
\hline 2399 & GUENSI & MERL? & NEP & 1 & s & MICHIGAN & ITALY & ITALY & & & PONT CANAVESE. TO & FRANKLIN \\
\hline 2400 & BOSSI & ANTHONY & $\mathrm{H}$ & 37 & M7 & ITALY & ITALY & ITALY & NA & TRAMMER/CM & & FRANKLIN \\
\hline 2401 & BOSSI & LOUISA & W & 30 & M7 & ITALY & ITALY & ITALY & UN & & & FRANKLIN \\
\hline 2402 & BOSSI & JOSEPH & s & 4 & $\mathrm{~s}$ & MICHIGAN & ITALY & ITALY & & & & FRANKLIN \\
\hline 2403 & BOSSI & MAGGIE & $\mathrm{D}$ & 1 & $\mathrm{~s}$ & MICHIGAN & ITALY & ITALY & & & & FRANKLIN \\
\hline 2404 & NARATI & JOHN & B & 34 & M2 & ITALY & ITALY & ITALY & PA & TRAMMER/CM & & FRANKLIN \\
\hline 2405 & BALAGNA & GIACOMO & B & 25 & M4 & ITALY & ITALY & ITALY & $\mathrm{AL}$ & TRAMMER/CM & PONT CANAVESE. TO & FRANKLIN \\
\hline 2406 & BOSSI & JOHN & BR & 23 & $\mathrm{~s}$ & MICHIGAN & ITALY & ITALY & & TRAMMER/CM & & FRANKLIN \\
\hline 2407 & GARINO & FELIX & BIL & 22 & $\mathrm{~s}$ & ITALY & ITALY & ITALY & $\mathrm{AL}$ & TRAMMER/CM & SAN GIORGIO CANAVESE, TO & FRANKLIN \\
\hline 2408 & BARONI & MICHAEL & B & 38 & W & ITALY & ITALY & ITALY & NA & LABORER/ROCK HOUSE & BOLZANO & FRANKLIN \\
\hline 2409 & MEINARDI & SAVINO & $\mathrm{H}$ & 37 & M17 & ITALY & ITALY & ITALY & PA & TEAMSTER & TORINO & FRANKLIN \\
\hline 2410 & MEINARDI & KATE & w & 35 & M17 & ITALY & ITALY & ITALY & UN & & TORINO & FRANKLIN \\
\hline 2411 & MEINARDI & LUCINDA & $\mathrm{D}$ & 16 & s & ITALY & ITALY & ITALY & UN & & TORINO & FRANKLIN \\
\hline 2412 & GOSATTI & ANDREW & $\mathrm{H}$ & 27 & M8 & ITALY & ITALY & ITALY & PA & TRAMMER/CM & BIANZANO. SONDRIO & FRANKLIN \\
\hline 2413 & GOSATTI & MARY & W & 27 & M8 & ITALY & ITALY & ITALY & UN & & BIANZANO, SONDRIO & FRANKLIN \\
\hline 2414 & GOSATTI & ANTHONY & s & 7 & $\mathrm{~s}$ & ITALY & ITALY & ITALY & UN & AT SCHOOL 10 & BIANZANO. SONDRIO & FRANKLIN \\
\hline 2415 & GOSATTI & JOSEPH & $\mathrm{s}$ & 5 & $\mathrm{~s}$ & ITALY & ITALY & ITALY & UN & & BIANZANO. SONDRIO & FRANKLIN \\
\hline 2416 & BIANCHI & PIETRO & $\mathrm{H}$ & 35 & M12 & ITALY & ITALY & ITALY & $\mathrm{AL}$ & BLACKSMITH & COLLODI. LU & FRANKLIN \\
\hline 2417 & BIANCHI & CAROLINA & W & 30 & M12 & ITALY & ITALY & ITALY & UN & & COLLODI. LU & FRANKLIN \\
\hline 2418 & BIANCHI & GIUSEPPE & $\mathrm{s}$ & 12 & $\mathrm{~S}$ & ITALY & ITALY & ITALY & UN & AT SCHOOL 10 & COLLODI. LU & FRANKLIN \\
\hline 2419 & BONINI & PETER & B & 30 & $\mathrm{~s}$ & ITALY & ITALY & ITALY & $\mathrm{AL}$ & LABORER/ROCK HOUSE & COLLODI, LU & FRANKLIN \\
\hline 2420 & BONINI & SEVERINO & B & 25 & MO & ITALY & ITALY & ITALY & $\mathrm{AL}$ & LABORER/CM & COLLODI. LU & FRANKLIN \\
\hline 2421 & BIANCHI & EUGENIO & B & 28 & M4 & ITALY & ITALY & ITALY & $\mathrm{AL}$ & LABORER/CM & LUCCA & FRANKLIN \\
\hline 2422 & CAVALZANI & BERNARD & B & 24 & $\mathrm{~s}$ & ITALY & ITALY & ITALY & $\mathrm{AL}$ & TRAMMER/CM & SAN GENNARO. LU & FRANKLIN \\
\hline 2423 & CIOMEI & THOMAS & B & 22 & $\mathrm{~s}$ & ITALY & ITALY & ITALY & $\mathrm{AL}$ & TRAMMER/CM & COLLODI. LU & FRANKLIN \\
\hline 2424 & FRANCIONI & FRANCES & B & 35 & $\mathrm{~s}$ & ITALY & ITALY & ITALY & PA & LABORER/ROCK HOUSE & LUCCA & FRANKLIN \\
\hline 2425 & FRANCIONI & PAUL & B & 26 & s & ITALY & ITALY & ITALY & $\mathrm{AL}$ & TRAMMER/CM & LUCCA & FRANKLIN \\
\hline 2426 & PUCCl & BATTISTA & B & 32 & M3 & ITALY & ITALY & ITALY & AL & TRAMMER/CM & COLLE DI CAMPITO. LU & FRANKLIN \\
\hline 2427 & FRANCIONI & JOSEPH & $\begin{array}{l}\mathrm{D} \\
\mathrm{H}\end{array}$ & 33 & M2 & ITALY & ITALY & ITALY & $\mathrm{AL}$ & LABORER/CM & LUCCA & FRANKLIN \\
\hline 2428 & FRANCIONI & ESTHER & w & 21 & M2 & ITALY & ITALY & ITALY & UN & & LUCCA & FRANKLIN \\
\hline 2429 & MARCHESCHI & EUGENIO & $\mathrm{B}$ & 24 & W & ITALY & ITALY & ITALY & AL & TRAMMER/CM & LUCCA & FRANKLIN \\
\hline 2430 & MONTICELLO & ORESTE & $\mathrm{H}$ & 35 & M13 & ITALY & ITALY & ITALY & $\mathrm{AL}$ & LABORER/CM & MATRAIA. LU & FRANKLIN \\
\hline 2431 & MONTICELLO & SALOMINA & W & 34 & M13 & ITALY & ITALY & ITALY & $\mathrm{AL}$ & & MATRAIA. LU & FRANKLIN \\
\hline 2432 & MONTICELLO & GIUSEPPE & $\mathrm{s}$ & 9 & $\mathrm{~s}$ & ITALY & ITALY & ITALY & UN & AT SCHOOL 2 & MATRAIA. LU & FRANKLIN \\
\hline 2433 & MONTICELLO & ANGELINA & D & 6 & $\mathrm{~S}$ & ITALY & ITALY & ITALY & $\mathrm{AL}$ & AT SCHOOL 2 & MATRAIA. LU & FRANKLIN \\
\hline 2434 & MONTICELLO & GIORGIO & $\mathrm{s}$ & 7 & $\mathrm{~S}$ & ITALY & ITALY & ITALY & $\mathrm{AL}$ & AT SCHOOL 3 & MATRAIA. LU & FRANKLIN \\
\hline 2435 & MONTICELLO & VINCENZO & $\mathrm{s}$ & 4 & S & ITALY & ITALY & ITALY & $\mathrm{AL}$ & & MATRAIA. LU & FRANKLIN \\
\hline 2436 & GELLI & ORLANDO & B & 25 & $\mathrm{~S}$ & ITALY & ITALY & ITALY & $\mathrm{AL}$ & LABORER/CM & MATRAIA. LU & FRANKLIN \\
\hline 2437 & RICCl & ANGELO & $\mathrm{H}$ & 44 & M3 & ITALY & ITALY & ITALY & $\mathrm{AL}$ & MINER/CM & MATRAIA. LU & FRANKLIN \\
\hline 2438 & RICCI & PICA & w & 28 & M3 & ITALY & ITALY & ITALY & & & MATRAIA. LU & FRANKLIN \\
\hline 2439 & RICCl & ZEIARA & D & $\begin{array}{l}20 \\
10\end{array}$ & $\mathrm{~S}$ & ITALY & ITALY & ITALY & & AT SCHOOL 1 & MATRAIA. LU & FRANKLIN \\
\hline 2440 & $\mathrm{RICCl}$ & ALBERTO & $\mathrm{s}$ & 2 & $\mathrm{~s}$ & MICHIGAN & ITALY & ITALY & & & MATRAIA. LU & FRANKLIN \\
\hline 2441 & $\mathrm{RICCl}$ & MARY & D & 2 & $\mathrm{~s}$ & MICHIGAN & ITALY & ITALY & & & MATRAIA. LU & FRANKLIN \\
\hline 2442 & RICCl & NATALINA & $\mathrm{D}$ & $3 \mathrm{M}$ & $\mathrm{s}$ & MICHIGAN & ITALY & ITALY & & & MATRAIA. LU & FRANKLIN \\
\hline 2443 & SANTORI & AURELIO & B & 27 & M4 & ITALY & ITALY & ITALY & $\mathrm{AL}$ & TRAMMER/CM & SEGROMIGNO, LU & FRANKLIN \\
\hline 2444 & GRADI & NICOLA & B & 27 & $\mathrm{~s}$ & ITALY & ITALY & ITALY & $A L$ & LABORER/ROCK HOUSE & CAPANNORI. LU & FRANKLIN \\
\hline 2445 & GIUSEPPI & PETER & B & 21 & s & ITALY & ITALY & ITALY & $\mathrm{AL}$ & TRAMMER/CM & & FRANKLIN \\
\hline 2446 & SANTORI & EMILIO & B & 34 & M7 & ITALY & ITALY & ITALY & $\mathrm{AL}$ & TRAMMER/CM & SEGROMIGNO. LU & FRANKLIN \\
\hline 2447 & CORTOPASSI & OTTAVIO & B & 34 & $\mathrm{~S}$ & ITALY & ITALY & ITALY & $\mathrm{AL}$ & TRAMMER/CM & LUCCA & FRANKLIN \\
\hline 2448 & SANTORI & AMERIGO & B & 24 & $\mathrm{~S}$ & ITALY & ITALY & ITALY & $\mathrm{AL}$ & LABORER/CM & SEGROMIGNO. LU & FRANKLIN \\
\hline 2449 & SOLONNI & ALIPPIO & B & 45 & s & ITALY & ITALY & ITALY & $\mathrm{AL}$ & TRAMMER/CM & & FRANKLIN \\
\hline 2450 & TUKORI & FRANCIS & B & 28 & M3 & ITALY & ITALY & ITALY & $\mathrm{AL}$ & LABORER/CM & & FRANKLIN \\
\hline 2451 & ROCCHI & EGISTO & B & 29 & $\mathrm{~s}$ & ITALY & ITALY & ITALY & AL & TIMBERMAN/CM & MATRAIA. LU & FRANKLIN \\
\hline 2452 & TADDEUCCI & JOSEPH & B & 23 & s & ITALY & ITALY & ITALY & $\mathrm{AL}$ & TRAMMER/CM & LUCCA & FRANKLIN \\
\hline 2453 & MUNJANNI & AMELIO & B & 29 & M2 & ITALY & ITALY & ITALY & $\mathrm{AL}$ & TRAMMER/CM & & FRANKLIN \\
\hline 2454 & BALCONI & CHARLES & $\mathrm{H}$ & 48 & $\mathrm{M} 21$ & ITALY & ITALY & ITALY & AL & CARPENTER & MERCALLO. VARESE & FRANKLIN \\
\hline
\end{tabular}




\begin{tabular}{|c|c|c|c|c|c|c|c|c|c|c|c|c|}
\hline 2455 & BALCONI & MARY & W & 37 & M21 & ITALY & ITALY & ITALY & UN & & MERCALLO. VARESE & FRANKLIN \\
\hline 2456 & BALCONI & PERINA & $\mathrm{D}$ & 18 & s & ITALY & ITALY & ITALY & UN & AT SCHOOL 10 & MERCALLO. VARESE & FRANKLIN \\
\hline 2457 & BALCONI & CHARLES & $\mathrm{s}$ & 15 & $\mathrm{~s}$ & MICHIGAN & ITALY & ITALY & & APPRENTICE/NEWSPAPER OFFICE & MERCALLO. VARESE & FRANKLIN \\
\hline 2458 & BALCONI & MARY & D & 12 & $\mathrm{~s}$ & MICHIGAN & ITALY & ITALY & & AT SCHOOL 8 & MERCALLO. VARESE & FRANKLIN \\
\hline 2459 & BALCONI & ANTOINETTE & $\mathrm{D}$ & 9 & $\mathrm{~S}$ & MICHIGAN & ITALY & ITALY & & AT SCHOOL 10 & MERCALLO. VARESE & FRANKLIN \\
\hline 2460 & BALCONI & JOSEPHINE & $\mathrm{D}$ & 9 & $\mathrm{~s}$ & MICHIGAN & ITALY & ITALY & & AT SCHOOL 10 & MERCALLO. VARESE & FRANKLIN \\
\hline 2461 & BALCONI & JOSHUA & $\mathrm{s}$ & 3 & s & MICHIGAN & ITALY & ITALY & & & MERCALLO. VARESE & FRANKLIN \\
\hline 2462 & PAOLI & SAMUEL & $\mathrm{H}$ & 32 & M12 & ITALY & ITALY & ITALY & PA & MINER/CM & LUCCA & FRANKLIN \\
\hline 2463 & PAOLI & CAROLINA & W & 28 & M12 & ITALY & ITALY & ITALY & UN & 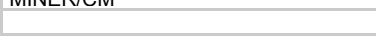 & LUCCA & FRANKLIN \\
\hline 2464 & PAOLI & MARY & $\mathrm{D}$ & 11 & s & ITALY & ITALY & ITALY & UN & AT SCHOOL 10 & LUCCA & FRANKLIN \\
\hline 2465 & PAOLI & ANNIE & $\mathrm{D}$ & 8 & $\mathrm{~s}$ & ITALY & ITALY & ITALY & UN & AT SCHOOL 10 & LUCCA & FRANKLIN \\
\hline 2466 & COLOMBO & ANTHONY & B & 26 & $\mathrm{~s}$ & ITALY & ITALY & ITALY & AL & MINER/CM & PONT CANAVESE. TO & FRANKLIN \\
\hline 2467 & $\mathrm{PUCCl}$ & GIUSEPPE & B & 28 & M4 & ITALY & ITALY & ITALY & $\mathrm{AL}$ & TRAMMER/CM & COLLE DI CAMPIDO. LU & FRANKLIN \\
\hline 2468 & BATTOLINI & FIORE & B & 27 & $\mathrm{~s}$ & ITALY & ITALY & ITALY & $\mathrm{AL}$ & TRAMMER/CM & & FRANKLIN \\
\hline 2469 & RICCA & MARY & $\mathrm{H}$ & 29 & M13 & ITALY & ITALY & ITALY & $?$ & BOARDING HOUSE KEEPER & TORINO & FRANKLIN \\
\hline 2470 & GRISOLANO & BATTISTA & B & 29 & M1 & ITALY & ITALY & ITALY & $?$ & TRAMMER/CM & SPARONE, TO & FRANKLIN \\
\hline 2471 & BERSANO & PETER & B & 25 & $\mathrm{~s}$ & ITALY & ITALY & ITALY & $?$ & TRAMMER/CM & SAN MARTINO CANAVESE. TO & FRANKLIN \\
\hline 2472 & MARCHESA & PETER & B & 27 & $\mathrm{~s}$ & ITALY & ITALY & ITALY & $?$ & TRAMMER/CM & TORINO & FRANKLIN \\
\hline 2473 & ZANOTTI & GIUSEPPE & B & 24 & M12 & ITALY & ITALY & ITALY & $?$ & TRAMMER/CM & TORINO & FRANKLIN \\
\hline 2474 & GROSSO & GIOVANNI & B & 38 & M10 & ITALY & ITALY & ITALY & $?$ & TRAMMER/CM & SAN MARTINO CANAVESE. TO & FRANKLIN \\
\hline 2475 & VACCA & CHARLES & B & 56 & M10 & ITALY & ITALY & ITALY & $?$ & TRAMMER/CM & VALPERGA. TO & FRANKLIN \\
\hline 2476 & PERINO & JOHN & B & 56 & M12 & ITALY & ITALY & ITALY & $?$ & TRAMMER/CM & TORINO & FRANKLIN \\
\hline 2477 & SAVINO & JACOB & $\mathrm{H}$ & 28 & M3 & ITALY & ITALY & ITALY & $?$ & TRAMMER/CM & & FRANKLIN \\
\hline 2478 & SAVINO & SERAPHINA & w & 25 & M3 & ITALY & ITALY & ITALY & $?$ & & & FRANKLIN \\
\hline 2479 & SAVINO & SERAPHINA & $\mathrm{D}$ & 2 & $\mathrm{~s}$ & ITALY & ITALY & ITALY & $?$ & & & FRANKLIN \\
\hline 2480 & SAVINO & MARY & D & $3 \mathrm{M}$ & $\mathrm{s}$ & ITALY & ITALY & ITALY & $?$ & & & FRANKLIN \\
\hline 2481 & GONIAE/GONISI & CHARLES & $\mathrm{H}$ & 33 & M9 & ITALY & ITALY & ITALY & NA & MINER/CM & & FRANKLIN \\
\hline 2482 & GONIAE/GONISI & MARY & W & 34 & M9 & ITALY & ITALY & ITALY & UN & & & FRANKLIN \\
\hline 2483 & GONIAE/GONISI & KATE & $\mathrm{D}$ & 4 & $\mathrm{~s}$ & ITALY & ITALY & ITALY & UN & & & FRANKLIN \\
\hline 2484 & GONIAE/GONISI & MARY & $\mathrm{D}$ & $4 \mathrm{M}$ & $\mathrm{s}$ & MICHIGAN & ITALY & ITALY & & & & FRANKLIN \\
\hline 2485 & DEIRO & JOSEPH & B & 26 & $\mathrm{~s}$ & ITALY & ITALY & ITALY & $\mathrm{AL}$ & TRAMMER/CM & TORINO & FRANKLIN \\
\hline 2486 & DUDICK & JOSEPH & $\mathrm{H}$ & 39 & M1? & ITALY & ITALY & ITALY & $\mathrm{AL}$ & MINER/CM & & FRANKLIN \\
\hline 2487 & DUDICK & JULIA & w & 39 & M1? & ITALY & ITALY & ITALY & UN & & & FRANKLIN \\
\hline 2488 & DUDICK & $\mathrm{BORSCl}$ & $\mathrm{D}$ & 10 & 10 & ITALY & ITALY & ITALY & UN & AT SCHOOL 3 & & FRANKLIN \\
\hline 2489 & GRACCI & LORENZO & $\mathrm{H}$ & 32 & M1 & ITALY & ITALY & ITALY & AL & TRAMMER/CM & & FRANKLIN \\
\hline 2490 & GRACCI & ERMELLINA & w & 27 & M1 & ITALY & ITALY & ITALY & UN & & & FRANKLIN \\
\hline 2491 & GRACCI & ANNIE & $\mathrm{D}$ & 10 & $\mathrm{~s}$ & ITALY & ITALY & ITALY & & & & FRANKLIN \\
\hline 2492 & FRANCIONI & DOMENICO & $\mathrm{B}$ & 37 & s & ITALY & ITALY & ITALY & $\mathrm{AL}$ & TRAMMER/CM & RUOTA. LU & FRANKLIN \\
\hline 2493 & PUCCl & ANGELO & B & 27 & M4 & ITALY & ITALY & ITALY & $\mathrm{AL}$ & TRAMMER/CM & RUOTA. LU & FRANKLIN \\
\hline 2494 & GARI & ANTONIO & B & 30 & MO & ITALY & ITALY & ITALY & $\mathrm{AL}$ & DAY LABORER/CM & & FRANKLIN \\
\hline 2495 & LANDINI & SALVATORE & B & 43 & M17 & ITALY & ITALY & ITALY & $\mathrm{AL}$ & TRAMMER/CM & RUOTA. LU & FRANKLIN \\
\hline 2496 & PAULINO & BAPTISTE & B & 30 & M5 & ITALY & ITALY & ITALY & $\mathrm{AL}$ & TRAMMER/CM & & FRANKLIN \\
\hline 2497 & GELLI & ROMANO & B & 23 & $s$ & ITALY & ITALY & ITALY & $\mathrm{AL}$ & TRAMMER/CM & MATRAIA. LU & FRANKLIN \\
\hline 2498 & PAULINO & CASPAR & B & 34 & M6 & ITALY & ITALY & ITALY & $\mathrm{AL}$ & TRAMMER/CM & & FRANKLIN \\
\hline 2499 & GARI & PETER & B & 27 & $\mathrm{~s}$ & ITALY & ITALY & ITALY & $\mathrm{AL}$ & TRAMMER/CM & & FRANKLIN \\
\hline 2500 & DOLPE & RAPHAEL & B & 26 & s & ITALY & ITALY & ITALY & $\mathrm{AL}$ & TRAMMER/CM & & FRANKLIN \\
\hline 2501 & DEIRO & PAUL & $\mathrm{H}$ & 39 & M15 & FRANCE & FRANCE & FRANCE & NA & MINER/CM & PONT CANAVESE. TO & FRANKLIN \\
\hline 2502 & DEIRO & KATE & W & 32 & M15 & ITALY & ITALY & ITALY & UN & INIINERT/UI & PONT CANAVESE. TO & FRANKLIN \\
\hline 2503 & DEIRO & THOMAS & s & 13 & $\mathrm{~s}$ & ITALY & FRANCE & ITALY & UN & AT SCHOOL 8 & PONT CANAVESE. TO & FRANKLIN \\
\hline 2504 & DEIRO & CARA & $\mathrm{D}$ & 10 & $\mathrm{~s}$ & ITALY & FRANCE & ITALY & UN & AT SCHOOL 2 & PONT CANAVESE. TO & FRANKLIN \\
\hline 2505 & DEIRO & MARY & $\mathrm{D}$ & 8 & $\mathrm{~s}$ & MICHIGAN & FRANCE & ITALY & & AT SCHOOL 2 & PONT CANAVESE. TO & FRANKLIN \\
\hline 2506 & DEIRO & KATE & $\mathrm{D}$ & 10 & s & MICHIGAN & FRANCE & ITALY & & & PONT CANAVESE, TO & FRANKLIN \\
\hline 2507 & LAZZARI & EMILIO & $\mathrm{H}$ & 22 & M0 & ITALY & ITALY & ITALY & $\mathrm{AL}$ & TRAMMER/CM & VILLA BASILICA. LU & FRANKLIN \\
\hline 2508 & LAZZARI & ALIDA & w & 24 & MO & ITALY & ITALY & ITALY & IN & & VILLA BASILICA. LU & FRANKLIN \\
\hline 2509 & FERRARI & LUIGI & B & 20 & s & ITALY & ITALY & ITALY & $\mathrm{AL}$ & TRAMMER/CM & COLLODI. LU & FRANKLIN \\
\hline 2510 & FLOSI & ARMEDO & B & 34 & M11 & ITALY & ITALY & ITALY & $\mathrm{AL}$ & TRAMMER/CM & PARIANA. LU & FRANKLIN \\
\hline 2511 & BARTOLOMEI & JULIUS & B & 19 & $\mathrm{~s}$ & ITALY & ITALY & ITALY & $\mathrm{AL}$ & DAY LABORER/CM & LUCCA & FRANKLIN \\
\hline 2512 & SABATINI & FULTONI? & B & 34 & M7 & ITALY & ITALY & ITALY & $\mathrm{AL}$ & TRAMMER/CM & VILLA BASILICA, LU & FRANKLIN \\
\hline 2513 & SABATINI & ANTONIO & B & 24 & $\mathrm{~s}$ & ITALY & ITALY & ITALY & $\mathrm{AL}$ & DAY LABORER/CM & VILLA BASILICA. LU & FRANKLIN \\
\hline 2514 & SABATINI & EGISTO & B & 21 & s & ITALY & ITALY & ITALY & $\mathrm{AL}$ & LABORER/RAILROAD & VILLA BASILICA. LU & FRANKLIN \\
\hline 2515 & BAZZARINI & RUDOLPH & $\mathrm{H}$ & 31 & M9 & ITALY & ITALY & ITALY & $\mathrm{AL}$ & MINER/CM & LUCCA & FRANKLIN \\
\hline 2516 & BAZZARINI & MARY & w & 28 & M9 & ITALY & ITALY & ITALY & & & & FRANKLIN \\
\hline 2517 & BAZZARINI & ANGELINA & $\mathrm{D}$ & 5 & $\mathrm{~s}$ & MICHIGAN & ITALY & ITALY & & & LUCCA & FRANKLIN \\
\hline
\end{tabular}




\begin{tabular}{|c|c|c|c|c|c|c|c|c|c|c|c|c|}
\hline 2518 & BAZZARINI & ANDREW & $\mathrm{s}$ & 4 & $S$ & MICHIGAN & ITALY & ITALY & & & LUCCA & FRANKLIN \\
\hline 2519 & BAZZARINI & SERAPHINA & D & 1 & $\mathrm{~S}$ & MICHIGAN & ITALY & ITALY & & & LUCCA & FRANKLIN \\
\hline 2520 & PASTORE & GEORGE & $\mathrm{H}$ & 52 & M20 & ITALY & ITALY & ITALY & NA & MINER/CM & TORINO & FRANKLIN \\
\hline 2521 & PASTORE & MARY & W & 45 & M20 & ITALY & ITALY & ITALY & & & & FRANKLIN \\
\hline 2522 & PASTORE & JOSEPH & $\mathrm{s}$ & 19 & $\mathrm{~S}$ & MICHIGAN & ITALY & ITALY & & MINER/CM & TORINO & FRANKLIN \\
\hline 2523 & PASTORE & GEORGE & $\mathrm{s}$ & 15 & $\mathrm{~S}$ & MICHIGAN & ITALY & ITALY & & LABORER/CM & TORINO & FRANKLIN \\
\hline 2524 & PASTORE & MARY & $\mathrm{D}$ & 12 & S & MICHIGAN & ITALY & ITALY & & AT SCHOOL 10 & TORINO & FRANKLIN \\
\hline 2525 & PASTORE & KATHERINE & $\mathrm{D}$ & 10 & $\mathrm{~s}$ & MICHIGAN & ITALY & ITALY & & AT SCHOOL 10 & TORINO & FRANKLIN \\
\hline 2526 & PASTORE & JOHN & $\mathrm{s}$ & 6 & S & MICHIGAN & ITALY & ITALY & & AT SCHOOL 16 & TORINO & FRANKLIN \\
\hline 2527 & PASTORE & PAUL & $\mathrm{s}$ & 4 & S & MICHIGAN & ITALY & ITALY & & & TORINO & FRANKLIN \\
\hline 2528 & MARCO & VINCENZO & B & 24 & $\mathrm{~s}$ & ITALY & ITALY & ITALY & $\mathrm{AL}$ & TRAMMER/CM & TORINO & FRANKLIN \\
\hline 2529 & PERONI & DOMINICK & B & 32 & M8 & ITALY & ITALY & ITALY & $\mathrm{AL}$ & TRAMMER/CM & TORINO & FRANKLIN \\
\hline 2530 & MARGHERIO & JAMES & B & 26 & $\mathrm{~s}$ & ITALY & ITALY & ITALY & $\mathrm{AL}$ & TRAMMER/CM & TORINO & FRANKLIN \\
\hline 2531 & MARCHELLO & GIACOMO & $\mathrm{H}$ & 48 & M13 & ITALY & ITALY & ITALY & NA & MINER/CM & CASTELLAMONTE. TO & FRANKLIN \\
\hline 2532 & MARCHELLO & ANNA & W & 32 & M13 & ITALY & ITALY & ITALY & UN & & & FRANKLIN \\
\hline 2533 & MARCHELLO & ANGELINE & $\mathrm{D}$ & 10 & & MISSOURI & ITALY & ITALY & & AT SCHOOL 10 & CASTELLAMONTE, TO & FRANKLIN \\
\hline 2534 & MARCHELLO & MARY & D & 7 & $\mathrm{~S}$ & MISSOURI & ITALY & ITALY & & AT SCHOOL 10 & CASTELLAMONTE. TO & FRANKLIN \\
\hline 2535 & MARCHELLOO & ROSE & $\mathrm{D}$ & 5 & $\mathrm{~S}$ & MISSOURI & ITALY & ITALY & & & CASTELLAMONTE. TO & FRANKLIN \\
\hline 2536 & MARCHELLO & MICHELE & $\mathrm{s}$ & 3 & $\mathrm{~s}$ & MISSOURI & ITALY & ITALY & & & CASTELLAMONTE. TO & FRANKLIN \\
\hline 2537 & MARCHELLO & JOSEPH & $\mathrm{s}$ & 2 & $\mathrm{~s}$ & MISSOURI & ITALY & ITALY & & & CASTELLAMONTE. TO & FRANKLIN \\
\hline 2538 & MARCHELLO & THOMAS & $\mathrm{s}$ & 1 & $\mathrm{~s}$ & MICHIGAN & ITALY & ITALY & & & CASTELLAMONTE. TO & FRANKLIN \\
\hline 2539 & MARCHELLO & BABY & $\mathrm{s}$ & $1 \mathrm{M}$ & $\mathrm{s}$ & MICHIGAN & ITALY & ITALY & & & CASTELLAMONTE, TO & FRANKLIN \\
\hline 2540 & FORNERIS & GIOVANNI & $\mathrm{H}$ & 38 & M14 & ITALY & ITALY & ITALY & $\mathrm{AL}$ & DAY LABORER/CM & BOSCONERO. TO & FRANKLIN \\
\hline 2541 & FORNERIS & ANTONIA & W & 30 & M14 & ITALY & ITALY & ITALY & UN & & BOSCONERO. TO & FRANKLIN \\
\hline 2542 & FORNERIS & MARY ANN & $\mathrm{D}$ & 10 & $\mathrm{~s}$ & ITALY & ITALY & ITALY & UN & & BOSCONERO. TO & FRANKLIN \\
\hline 2543 & FORNERIS & ANGELINA & $\mathrm{D}$ & 1 & $\mathrm{~s}$ & USA & ITALY & ITALY & & & BOSCONERO. TO & FRANKLIN \\
\hline 2544 & RAPAULO & MARTHA & B & 20 & $\mathrm{~s}$ & ITALY & ITALY & ITALY & $?$ & & & FRANKLIN \\
\hline 2545 & RASTELLO & CHARLES & $\mathrm{H}$ & 37 & M13 & ITALY & ITALY & ITALY & PA & MINER/CM & PONT CANAVESE, TO & FRANKLIN \\
\hline 2546 & RASTELLO & JOSEPHINE & w & 36 & M13 & ITALY & ITALY & ITALY & & & & FRANKLIN \\
\hline 2547 & RASTELLO & PETER & $\mathrm{s}$ & 12 & $\mathrm{~s}$ & MICHIGAN & ITALY & ITALY & & AT SCHOOL 10 & PONT CANAVESE. TO & FRANKLIN \\
\hline 2548 & RASTELLO & ROSE & D & 11 & $\mathrm{~S}$ & MICHIGAN & ITALY & ITALY & & AT SCHOOL 10 & PONT CANAVESE. TO & FRANKLIN \\
\hline 2549 & RASTELLO & JOSEPH & $\mathrm{s}$ & 9 & $\mathrm{~s}$ & MICHIGAN & ITALY & ITALY & & & PONT CANAVESE. TO & FRANKLIN \\
\hline 2550 & RASTELLO & JOHN & $\mathrm{s}$ & 7 & $\mathrm{~s}$ & MICHIGAN & ITALY & ITALY & & & PONT CANAVESE. TO & FRANKLIN \\
\hline 2551 & RASTELLO & LOUISA & $\mathrm{D}$ & 6 & $\mathrm{~s}$ & CALIFORNI & ITALY & ITALY & & & PONT CANAVESE, TO & FRANKLIN \\
\hline 2552 & RASTELLO & CHARLES & $\mathrm{s}$ & 5 & $\mathrm{~s}$ & MICHIGAN & ITALY & ITALY & & & PONT CANAVESE. TO & FRANKLIN \\
\hline 2553 & RASTELLO & FLORENCE & $\mathrm{D}$ & 1 & s & MICHIGAN & ITALY & ITALY & & & PONT CANAVESE. TO & FRANKLIN \\
\hline 2554 & DE PIANTE & ANTOINE & $\mathrm{H}$ & 26 & M2 & ITALY & ITALY & ITALY & $\mathrm{AL}$ & MINER/CM & & FRANKLIN \\
\hline 2555 & DE PIANTE & STELLA & w & 17 & M2 & MICHIGAN & GERMAN & GERMANY & & & GERMANY & FRANKLIN \\
\hline 2556 & DE PIANTE & CHARLES & s & $5 \mathrm{M}$ & $\mathrm{s}$ & MICHIGAN & $\begin{array}{l}\text { ITALY } \\
\end{array}$ & MICHIGAN & & & & FRANKLIN \\
\hline 2557 & DE PIANTE & FERDINAND & $\mathrm{H}$ & 38 & M2 & ITALY & ITALY & ITALY & $\mathrm{AL}$ & MINER/CM & & FRANKLIN \\
\hline 2558 & DE PIANTE & JULIA & W & 35 & M2 & GERMANY & GERMAN & GERMANY & UN & & GERMANY & FRANKLIN \\
\hline 2559 & $\mathrm{ClUCCl}$ & AMATO & $\mathrm{H}$ & 26 & w & ITALY & ITALY & ITALY & $\mathrm{AL}$ & LABORER/CM & LUCCA & FRANKLIN \\
\hline 2560 & GERVA & CARLO & B & 28 & s & ITALY & ITALY & ITALY & UN & TRAMMER/CM & & FRANKLIN \\
\hline 2561 & FINA & CESTILIA & B & 28 & $\mathrm{~s}$ & ITALY & ITALY & ITALY & $\mathrm{AL}$ & TRAMMER/CM & & FRANKLIN \\
\hline 2562 & PILERATI & DOMINICA & SERV & 28 & $\mathrm{~S}$ & ITALY & ITALY & ITALY & $\mathrm{AL}$ & SERVANT & & FRANKLIN \\
\hline 2563 & LANFRI & EUGENE & B & 32 & s & ITALY & ITALY & ITALY & $\mathrm{AL}$ & DAY LABORER/CM & LUCCA & FRANKLIN \\
\hline 2564 & BERNARDI & GIOVANNI & B & 28 & M2 & ITALY & ITALY & ITALY & $A L$ & TRAMMER/CM & & FRANKLIN \\
\hline 2565 & BERNARDI & PIENNA & B & 38 & M9 & ITALY & ITALY & ITALY & $A L$ & TRAMMER/CM & & FRANKLIN \\
\hline 2566 & BARTOLOMEI & ADELMO & B & 26 & $\mathrm{~s}$ & ITALY & ITALY & ITALY & $\mathrm{AL}$ & LABORER/CM & PARIANA. LU & FRANKLIN \\
\hline 2567 & $\begin{array}{l}\text { JACHINT } \\
\text { J }\end{array}$ & PETER & B & 26 & M4 & ITALY & ITALY & ITALY & $A L$ & TRAMMER/CM & & FRANKLIN \\
\hline 2568 & $\mathrm{ClUCCl}$ & AUGUST & B & 26 & $\mathrm{~S}$ & ITALY & ITALY & ITALY & $\mathrm{AL}$ & STAGE DRIVER & LUCCA & FRANKLIN \\
\hline 2569 & PARRAVANO & JOSEPH? & $\mathrm{H}$ & $?$ & M? & ITALY & ITALY & ITALY & & & & FRANKLIN \\
\hline 2570 & PARRAVANO & ANNA? & W & $?$ & M? & SWITZERLA & ITALY & ITALY & & & & FRANKLIN \\
\hline 2571 & PARRAVANO & $?$ & $?$ & $?$ & $\mathrm{~s}$ & MICHIGAN & ITALY & SWITZERLA & & & & FRANKLIN \\
\hline 2572 & PARRAVANO & MARY & D & 13 & $\mathrm{~S}$ & MICHIGAN & ITALY & SWITZERLA & & & & FRANKLIN \\
\hline 2573 & PARRAVANO & ANTONIO & $\mathrm{s}$ & 11 & $\mathrm{~s}$ & MICHIGAN & ITALY & SWITZERLA & & & & FRANKLIN \\
\hline 2574 & PARRAVANO & JOHN & $\mathrm{s}$ & 7 & $\mathrm{~s}$ & MICHIGAN & ITALY & SWITZERLA & & & & FRANKLIN \\
\hline 2575 & PARRAVANO & MARGHERITA & D & 4 & s & MICHIGAN & ITALY & SWITZERLA & & & & FRANKLIN \\
\hline 2576 & ROCCHI & LUIGI & $\mathrm{H}$ & 26 & M3 & ITALY & ITALY & ITALY & $\mathrm{AL}$ & MINER/CM & MATRAIA. LU & FRANKLIN \\
\hline 2577 & ROCCHI & ARGENTINA & w & 26 & M3 & ITALY & ITALY & ITALY & UN & & MATRAIA. LU & FRANKLIN \\
\hline 2578 & ROCCHI & LIZZIE & D & 2 & $\mathrm{~s}$ & MICHIGAN & ITALY & ITALY & & & MATRAIA. LU & FRANKLIN \\
\hline 2579 & ROCCHI & JOSEPH & $\mathrm{s}$ & $4 \mathrm{M}$ & s & MICHIGAN & ITALY & ITALY & & & MATRAIA. LU & FRANKLIN \\
\hline 2580 & GEMIGNANI & GABRIELLO & B & 31 & M6 & ITALY & ITALY & ITALY & $\mathrm{AL}$ & TRAMMER/CM & S. MARIA DEL GIUDICE. LU & FRANKLIN \\
\hline
\end{tabular}




\begin{tabular}{|c|c|c|c|c|c|c|c|c|c|c|c|c|}
\hline 2581 & GIUSFREDI & GABRIELLO & B & 26 & $\mathrm{~S}$ & ITALY & ITALY & ITALY & $\mathrm{AL}$ & TRAMMER/CM & SEGROMIGNO. LU & FRANKLIN \\
\hline 2582 & LENCIONI & GEREMIA & B & 34 & M0 & ITALY & ITALY & ITALY & $\mathrm{AL}$ & TRAMMER/CM & LUCCA & FRANKLIN \\
\hline 2583 & BARTARNI & LOUIS & B & 24 & $\mathrm{~S}$ & ITALY & ITALY & ITALY & $\mathrm{AL}$ & TRAMMER/CM & & FRANKLIN \\
\hline 2584 & LUCCHESI & CESARE & B & 23 & $\mathrm{~S}$ & ITALY & ITALY & ITALY & $\mathrm{AL}$ & TRAMMER/CM & LUCCA & FRANKLIN \\
\hline 2585 & PARMA & JOHN & $\mathrm{H}$ & 37 & M2 & ITALY & ITALY & ITALY & PA & MINER/CM & & FRANKLIN \\
\hline 2586 & PARMA & MARIA & w & 34 & M2 & ITALY & ITALY & ITALY & UN & & & FRANKLIN \\
\hline 2587 & MELENI/MILANO & JOSEPH & $\mathrm{H}$ & 33 & M7 & ITALY & ITALY & ITALY & NA & MINER/CM & & FRANKLIN \\
\hline 2588 & MELENI/MILANO & EMELIA & W & 35 & M7 & ITALY & ITALY & ITALY & UN & & & FRANKLIN \\
\hline 2589 & MELENI/MILANO & PETER & s & 5 & $\mathrm{~s}$ & MONTANA & ITALY & ITALY & & & & FRANKLIN \\
\hline 2590 & MELENI/MILANO & JOHN & $\mathrm{s}$ & 3 & $\mathrm{~s}$ & MONTANA & ITALY & ITALY & & & & FRANKLIN \\
\hline 2591 & $\begin{array}{l}\text { CANTELLO } \\
\text { CA }\end{array}$ & PETER & B & 24 & $\mathrm{~s}$ & ITALY & ITALY & ITALY & UN & MINER/CM & SAN GIORGIO CANAVESE. TO & FRANKLIN \\
\hline 2592 & CERINI & JOHN & B & 28 & M5 & ITALY & ITALY & ITALY & UN & MINER/CM & SAN GIORGIO CANAVESE. TO & FRANKLIN \\
\hline 2593 & CANDIDO & JOHN & B & 20 & $\mathrm{~s}$ & ITALY & ITALY & ITALY & UN & MINER/CM & & FRANKLIN \\
\hline 2594 & RUFINA & ROSE & $\mathrm{H}$ & 36 & w & ITALY & ITALY & ITALY & UN & BOARDING HOUSE KEEPER & SAN GIORGIO CANAVESE. TO & FRANKLIN \\
\hline 2595 & RUFINA & DOMENICO & $\mathrm{s}$ & 6 & $\mathrm{~s}$ & MICHIGAN & ITALY & ITALY & & & SAN GIORGIO CANAVESE. TO & FRANKLIN \\
\hline 2596 & RUFINA & DOMINICA & D & $6 \mathrm{M}$ & $\mathrm{s}$ & MICHIGAN & ITALY & ITALY & & & SAN GIORGIO CANAVESE, TO & FRANKLIN \\
\hline 2597 & DE FILIPPI & GIACOMO & B & 40 & M11 & ITALY & ITALY & ITALY & $\mathrm{AL}$ & MINER/CM & SAN GIORGIO CANAVESE. TO & FRANKLIN \\
\hline 2598 & ROLANDO & JOHN & B & 19 & $\mathrm{~s}$ & ITALY & ITALY & ITALY & PA & MINER/CM & TORINO & FRANKLIN \\
\hline 2599 & CAUCAS & LOUIS & $\mathrm{H}$ & 26 & M? & ITALY & ITALY & ITALY & UN & MINER/CM & & FRANKLIN \\
\hline 2600 & CAUCAS & NOVI & w & 27 & M? & ITALY & ITALY & ITALY & UN & & & FRANKLIN \\
\hline 2601 & BUNNETTI & FRANK & B & 34 & $\mathrm{~s}$ & ITALY & ITALY & ITALY & UN & DAY LABORER/CM & & FRANKLIN \\
\hline 2602 & VALERIO & BAPTISTE & $\begin{array}{l}\mathrm{D} \\
\mathrm{B}\end{array}$ & 22 & s & ITALY & ITALY & ITALY & UN & $\begin{array}{l}\text { CARPENTER } \\
\end{array}$ & TORINO & FRANKLIN \\
\hline 2603 & VITTON & GIACOMO & B & 26 & $\mathrm{~s}$ & ITALY & ITALY & ITALY & UN & TRAMMER/CM & LOCANA CANAVESE. TO & FRANKLIN \\
\hline 2604 & KIARDI & JOHN & B & 17 & $\mathrm{~s}$ & ITALY & ITALY & ITALY & UN & TRAMMER/CM & & FRANKLIN \\
\hline 2605 & VIDETTE & CHARLES & $\mathrm{H}$ & 45 & $M ?$ & ITALY & ITALY & ITALY & PA & TRAMMER/CM & & FRANKLIN \\
\hline 2606 & VIDETTE & CAPPO & W & 25 & M & ITALY & ITALY & ITALY & UN & & & FRANKLIN \\
\hline 2607 & VERGA & DOMENICO & $\mathrm{H}$ & 23 & s & ITALY & ITALY & ITALY & UN & TRAMMER/CM & TORINO & FRANKLIN \\
\hline 2608 & PEDIO & HE? & W & 19 & $\mathrm{~s}$ & ITALY & ITALY & ITALY & UN & DAY LABOREr/CM & & FRANKLIN \\
\hline 2609 & TONALY & TONY & B & 40 & $\mathrm{~S}$ & ITALY & ITALY & ITALY & NA & TIMBERMAN & & FRANKLIN \\
\hline 2610 & RONI & PETER & B & 29 & M4 & ITALY & ITALY & ITALY & $\mathrm{AL}$ & TRAMMER/CM & & FRANKLIN \\
\hline 2611 & RONI & BELINDI & B & 30 & M4 & ITALY & ITALY & ITALY & UN & & & FRANKLIN \\
\hline 2612 & RONI & ANNES & $\mathrm{H}$ & 3 & $\mathrm{~S}$ & ITALY & ITALY & ITALY & UN & & & FRANKLIN \\
\hline 2613 & DEBERINI & JOSEPH & W & 35 & M & ITALY & ITALY & ITALY & AL & TRAMMER/CM & & FRANKLIN \\
\hline 2614 & DEBERINI & JENNIE & D & 27 & M & ITALY & ITALY & ITALY & UN & & & FRANKLIN \\
\hline 2615 & BOTTAN & EARNEST & $\mathrm{H}$ & 31 & M2 & NEW YORK & AUSTRIA & AUSTRIA & & MINER/CM & & FRANKLIN \\
\hline 2616 & BOTTAN & LIBBIE & w & 19 & M2 & ITALY & ITALY & ITALY & UN & & & FRANKLIN \\
\hline 2617 & BOTTAN & MILLIE & D & 11 & $\mathrm{~S}$ & MICHIGAN & NEW & ITALY & & & & FRANKLIN \\
\hline 2618 & COZZI & VALENTINE & B & 28 & s & ITALY & ITALY & ITALY & $\mathrm{AL}$ & TIMBERMAN & UDINE & FRANKLIN \\
\hline 2619 & GIUSFREDI & PORTI & $\mathrm{H}$ & 40 & M10 & ITALY & ITALY & ITALY & NA & MINER/CM & LUCCA & FRANKLIN \\
\hline 2620 & GIUSFREDI & MARY & W & 30 & M10 & ITALY & ITALY & ITALY & UN & & & FRANKLIN \\
\hline 2621 & GIUSFREDI & JOSEPH & s & 7 & $\mathrm{~s}$ & MICHIGAN & ITALY & ITALY & & AT SCHOOL 9 & LUCCA & FRANKLIN \\
\hline 2622 & GIUSFREDI & JOHN & $\mathrm{s}$ & 6 & $\mathrm{~s}$ & MICHIGAN & ITALY & ITALY & & AT SCHOOL 9 & LUCCA & FRANKLIN \\
\hline 2623 & GIUSFREDI & LUCY & D & 4 & S & MICHIGAN & ITALY & ITALY & & & LUCCA & FRANKLIN \\
\hline 2624 & GIUSFREDI & MARY & $\mathrm{D}$ & 2 & $\mathrm{~S}$ & MICHIGAN & ITALY & ITALY & & & LUCCA & FRANKLIN \\
\hline 2625 & GIUSFREDI & MAGGIE & $\mathrm{D}$ & $?$ & s & MICHIGAN & ITALY & ITALY & & & LUCCA & FRANKLIN \\
\hline 2626 & MEOLA & LEONARD & $\mathrm{H}$ & 29 & M6 & ITALY & $\begin{array}{l}\text { ITALY } \\
\end{array}$ & ITALY & NA & TRAMMER/CM & NAPOLI & HANCOCK \\
\hline 2627 & MEOLA & NELLIE & W & 25 & M6 & $\mathrm{OHIO}$ & $\mathrm{OHIO}$ & $\mathrm{OHIO}$ & & & USA & HANCOCK \\
\hline 2628 & MEOLA & MARTY & s & 5 & s & MISSOURI & ITALY & ITALY & & & NAPOLI & HANCOCK \\
\hline 2629 & MEOLA & JONNIE & D & 2 & $\mathrm{~s}$ & $\mathrm{OHIO}$ & ITALY & ITALY & & & NAPOLI & HANCOCK \\
\hline 2630 & MEOLA & BART & $B R$ & 26 & s & ITALY & ITALY & ITALY & UN & LABORER/CM & NAPOLI & HANCOCK \\
\hline 2631 & CADUTI & LEONARDO & B & 38 & M15 & ITALY & ITALY & ITALY & $\mathrm{AL}$ & STONE MASON & ASCOLI PICENO & HANCOCK \\
\hline 2632 & DORIANO & DOMENICO & B & 23 & $\mathrm{~s}$ & ITALY & ITALY & ITALY & $?$ & DAY LABORER/CM & & HANCOCK \\
\hline 2633 & SAWYER & EUGENE & PATI & 48 & M19 & ITALY & ITALY & ITALY & $\mathrm{AL}$ & MINER/CM & & HANCOCK - HANCOCK V. \\
\hline 2634 & VIGNETTO & BATISTI & $\mathrm{H}$ & 33 & M9 & ITALY & ITALY & ITALY & NA & SALOONKEEPER & TORINO & HANCOCK - HANCOCK V. \\
\hline 2635 & VIGNETTO & FELICITA & w & 28 & M9 & ITALY & ITALY & ITALY & & & TORINO & HANCOCK - HANCOCK V. \\
\hline 2636 & VIGNETTO & BATISTE & $\mathrm{D}$ & 8 & $\mathrm{~s}$ & ITALY & ITALY & ITALY & & AT SCHOOL 9 & TORINO & HANCOCK - HANCOCK V. \\
\hline 2637 & VIGNETTO & JOHN & $\mathrm{s}$ & 4 & $\mathrm{~s}$ & UTAH & ITALY & ITALY & & & TORINO & HANCOCK - HANCOCK V. \\
\hline 2638 & VIGNETTO & PETER & $\mathrm{s}$ & 3 & $\mathrm{~s}$ & UTAH & ITALY & ITALY & & & TORINO & HANCOCK - HANCOCK V. \\
\hline 2639 & VIGNETTO & DONIE & $\mathrm{H}$ & 42 & M19 & ITALY & ITALY & ITALY & NA & SALOONKEEPER & TORINO & HANCOCK - HANCOCK V. \\
\hline 2640 & VIGNETTO & CATILDA & W & 34 & M19 & ITALY & ITALY & ITALY & & & & HANCOCK - HANCOCK V. \\
\hline 2641 & VIGNETTO & BASILIO & $\mathrm{s}$ & 17 & $\mathrm{~s}$ & ILLINOIS & ITALY & ITALY & & & TORINO & HANCOCK - HANCOCK V. \\
\hline 2642 & VIGNETTO & NINO & $\mathrm{s}$ & 14 & $\mathrm{~s}$ & ILLINOIS & ITALY & ITALY & & AT SCHOOL 9 & TORINO & HANCOCK - HANCOCK V. \\
\hline 2643 & VIGNETTO & MARY & D & 10 & $\mathrm{~s}$ & ILLINOIS & ITALY & ITALY & & AT SCHOOL 9 & TORINO & HANCOCK - HANCOCK V. \\
\hline
\end{tabular}




\begin{tabular}{|c|c|c|c|c|c|c|c|c|c|c|c|c|c|}
\hline 2644 & & VIGNETTO & BERTHA & $\mathrm{D}$ & 6 & S & ILLINOIS & ITALY & ITALY & & AT SCHOOL 9 & TORINO & HANCOCK - HANCOCK V \\
\hline 2645 & & MINESCUILI & JOSEPHINA & SERV & 17 & s & ITALY & ITALY & ITALY & $?$ & SERVANT & & HANCOCK - HANCOCK V \\
\hline 2646 & & LANGIES & EUGENE & $\mathrm{H}$ & 38 & M2 & ITALY & FRANCE & ITALY & NA & TEACHER/MUSIC & & HANCOCK - HANCOCK V \\
\hline 2647 & & LANGIES & LOUISE & W & 26 & M2 & NORWAY & NORWAY & NORWAY & UN & & NORWAY & HANCOCK - HANCOCK V \\
\hline 2648 & & CASSIDI & ALFONSE & B & 26 & $\mathrm{~S}$ & ITALY & ITALY & ITALY & PA & CLERK & & HANCOCK - HANCOCK V \\
\hline 2649 & & VERGA & JOSEPH & $\mathrm{H}$ & 30 & M9 & ITALY & ITALY & ITALY & $?$ & GROCER & TORINO & HANCOCK - HANCOCK V \\
\hline 2650 & & VERGA & MARY & w & 26 & M9 & ITALY & ITALY & ITALY & & & TORINO & HANCOCK - HANCOCK V \\
\hline 2651 & & VERGA & DOMENICO & $\mathrm{s}$ & 8 & $\mathrm{~s}$ & MICHIGAN & ITALY & ITALY & & AT SCHOOL & TORINO & HANCOCK - HANCOCK V \\
\hline 2652 & & VERGA & MARY & $\mathrm{D}$ & 6 & $\mathrm{~s}$ & MICHIGAN & ITALY & ITALY & & AT GUTIUCL & TORINO & HANCOCK - HANCOCK V \\
\hline 2653 & & VERGA & $?$ & D & 5 & $\mathrm{~s}$ & MICHIGAN & ITALY & ITALY & & & TORINO & HANCOCK - HANCOCK V \\
\hline 2654 & & VERGA & JOSEPH & $\mathrm{s}$ & 3 & $\mathrm{~s}$ & MICHIGAN & ITALY & ITALY & & & TORINO & HANCOCK - HANCOCK V \\
\hline 2655 & & VERGA & MERELO? & $\mathrm{s}$ & 1 & $\mathrm{~s}$ & MICHIGAN & ITALY & ITALY & & & TORINO & HANCOCK - HANCOCK V \\
\hline 2656 & & SCIACERO & ANTON & $\mathrm{H}$ & 26 & M5 & ITALY & ITALY & ITALY & NA & TEAMSTER & SAN MARTINO CANAVESE. TO & HANCOCK - HANCOCK V \\
\hline 2657 & & SCIACERO & MARIA & W & 27 & M5 & ITALY & ITALY & ITALY & & & & HANCOCK - HANCOCK V \\
\hline 2658 & & ANDETTI & ANTON & B & 38 & $\mathrm{~S}$ & ITALY & ITALY & ITALY & NA & SHOEMAKER & & HANCOCK - HANCOCK V \\
\hline 2659 & & MACOCCO & ANTONIO & $\mathrm{H}$ & 35 & M5 & ITALY & ITALY & ITALY & NA & SALOONKEEPER & & OSCEOLA \\
\hline 2660 & & MARTA & PETER & B & 47 & M15 & ITALY & ITALY & ITALY & $\mathrm{AL}$ & MINER/CM & SAN GIOVANNI CANAVESE. & OSCEOLA \\
\hline 2661 & & CONSINA & EMILIO & $\mathrm{H}$ & 39 & M8 & ITALY & ITALY & ITALY & $\mathrm{PA}$ & LABORER/MILL & LUCCA & OSCEOLA \\
\hline 2662 & & CONSINA & AMELIA & w & 29 & M8 & ITALY & ITALY & ITALY & & & LUCCA & OSCEOLA \\
\hline 2663 & & CONSINA & ARTURO & s & 5 & $\mathrm{~s}$ & ITALY & ITALY & ITALY & & & LUCCA & OSCEOLA \\
\hline 2664 & & CONSINA & JOSEPHINA & D & 4 & $\mathrm{~s}$ & MICHIGAN & ITALY & ITALY & & & LUCCA & OSCEOLA \\
\hline 2665 & & CONSINA & ALBERT & s & 2 & $\mathrm{~s}$ & MICHIGAN & ITALY & ITALY & & & LUCCA & OSCEOLA \\
\hline 2666 & & CONSINA & DANTE & $\mathrm{s}$ & 1 & $\mathrm{~s}$ & MICHIGAN & ITALY & ITALY & & & LUCCA & OSCEOLA \\
\hline 2667 & & GANIO & PETER & B & 24 & $\mathrm{~s}$ & ITALY & ITALY & ITALY & & DAY LABORER/CM & & OSCEOLA \\
\hline 2668 & & MIKE & JOSEPH & B & 29 & s & ITALY & ITALY & ITALY & & DAY LABORER/CM & & OSCEOLA \\
\hline 2669 & & CASERIO & CHARLES & $\mathrm{H}$ & 36 & M7 & ITALY & ITALY & ITALY & & LABORER/MILL & TORINO & OSCEOLA \\
\hline 2670 & & CASERIO & MARY & W & 31 & M7 & ITALY & ITALY & ITALY & PA & & TORINO & OSCEOLA \\
\hline 2671 & & CASERIO & AMELIA & D & 6 & S & MICHIGAN & ITALY & ITALY & & & TORINO & OSCEOLA \\
\hline 2672 & & CASERIO & ITALEON? & $\mathrm{s}$ & 4 & $\mathrm{~S}$ & MICHIGAN & ITALY & ITALY & & & TORINO & OSCEOLA \\
\hline 2673 & & CASERIO & NALO & $\mathrm{s}$ & 2 & $\mathrm{~S}$ & MICHIGAN & ITALY & ITALY & & & TORINO & OSCEOLA \\
\hline 2674 & & CASERIO & JUNY? & s & $8 \mathrm{M}$ & S & MICHIGAN & ITALY & ITALY & & & TORINO & OSCEOLA \\
\hline 2675 & & CASERIO & DANTE & BR & 27 & s & ITALY & ITALY & ITALY & AL & LABORER/MILL & TORINO & OSCEOLA \\
\hline 2676 & & CASERIO & JENNY & $\mathrm{SI}$ & 25 & $\mathrm{~s}$ & ITALY & ITALY & ITALY & & & TORINO & OSCEOLA \\
\hline 2677 & & OSTENZI & FRANK & $\mathrm{H}$ & 51 & M20 & ITALY & ITALY & ITALY & PA & N. G. & LUCCA & OSCEOLA \\
\hline 2678 & & OSTENZI & ANGELA & w & 41 & M20 & ITALY & ITALY & ITALY & & & LUCCA & OSCEOLA \\
\hline 2679 & & OSTENZI & AMELIA & D & $\begin{array}{l}41 \\
18\end{array}$ & $s$ & MICHIGAN & ITALY & ITALY & & & LUCCA & OSCEOLA \\
\hline 2680 & & OSTENZI & MARY & $\mathrm{D}$ & 17 & $\mathrm{~s}$ & MICHIGAN & ITALY & ITALY & & & LUCCA & OSCEOLA \\
\hline 2681 & & OSTENZI & PETER & $\mathrm{s}$ & 10 & $\mathrm{~s}$ & MICHIGAN & ITALY & ITALY & & & LUCCA & OSCEOLA \\
\hline 2682 & & OSTENZI & FLORINA? & D & 7 & $\mathrm{~s}$ & MICHIGAN & ITALY & ITALY & & & LUCCA & OSCEOLA \\
\hline 2683 & & OSTENZI & FRED & $\mathrm{s}$ & 4 & $\underline{s}$ & MICHIGAN & $\begin{array}{l}\text { ITALY } \\
\end{array}$ & ITALY & & & LUCCA & OSCEOLA \\
\hline 2684 & & OSTENZI & HUBERT & s & 1 & $\mathrm{~s}$ & MICHIGAN & ITALY & ITALY & & & LUCCA & OSCEOLA \\
\hline 2685 & & LOMBARDERA & ANN & SERV & 21 & s & ITALY & ITALY & ITALY & & SERVANT & LUCCA & OSCEOLA \\
\hline 2686 & & BAIOCCHI & NATALE & $\mathrm{H}$ & 38 & M7 & ITALY & ITALY & ITALY & PA & LABORER/MILL & COLLODI. LU & OSCEOLA \\
\hline 2687 & & BAIOCCHI & MARIA & w & 31 & M7 & ITALY & ITALY & ITALY & & & COLLODI. LU & OSCEOLA \\
\hline 2688 & & BAIOCCHI & ANITA & D & 5 & S & MICHIGAN & ITALY & ITALY & & AT SCHOOL & COLLODI. LU & OSCEOLA \\
\hline 2689 & & BAIOCCHI & GIOIA & D & 4 & $\mathrm{~s}$ & MICHIGAN & ITALY & ITALY & & & COLLODI. LU & OSCEOLA \\
\hline 2690 & & BAIOCCHI & MAIDA & D & 3 & $\mathrm{~s}$ & MICHIGAN & ITALY & ITALY & & & COLLODI. LU & OSCEOLA \\
\hline 2691 & & BAIOCCHI & WILLIAM & $\mathrm{s}$ & 2 & s & MICHIGAN & ITALY & ITALY & & & COLLODI. LU & OSCEOLA \\
\hline 2692 & & BAIOCCHI & CAMILLO & $\mathrm{H}$ & 26 & M1 & ITALY & ITALY & ITALY & PA & LABORER/MILL & COLLODI. LU & OSCEOLA \\
\hline 2693 & & BAIOCCHI & LADY & W & 26 & M1 & ITALY & ITALY & ITALY & & & & OSCEOLA \\
\hline 2694 & & BAIOCCHI & SUFENIA & $\mathrm{D}$ & 0 & $\mathrm{~s}$ & MICHIGAN & ITALY & ITALY & & & COLLODI. LU & OSCEOLA \\
\hline 2695 & & T/IUMERO & AMOS & $\mathrm{H}$ & 33 & M9 & ITALY & ITALY & ITALY & NA & NOT CLEAR & & OSCEOLA \\
\hline 2696 & & T/IUMERO & JENNIE & W & 29 & M9 & MICHIGAN & SCOTLAN & CANADA & & & & OSCEOLA \\
\hline 2697 & & T/IUMERO & RUBY & $\mathrm{D}$ & 8 & $\mathrm{~s}$ & MICHIGAN & ITALY & MICHIGAN & & AT SCHOOL & & OSCEOLA \\
\hline 2698 & & T/IUMERO & ANTHONY & $\mathrm{s}$ & 7 & $\mathrm{~s}$ & MICHIGAN & ITALY & MICHIGAN & & AT SCHOOL & & OSCEOLA \\
\hline 2699 & & T/IUMERO & ESTELLA & D & 4 & s & MICHIGAN & ITALY & MICHIGAN & & & & OSCEOLA \\
\hline 2700 & & T/IUMERO & PRUDENCE & D & 2 & $\mathrm{~s}$ & MICHIGAN & ITALY & MICHIGAN & & & & OSCEOLA \\
\hline 2701 & 308 & MORATTO & T/IOVENY? & $\mathrm{H}$ & 27 & M8 & ITALY & ITALY & ITALY & PA & TIMBERMAN/CM & & OSCEOLA PR. 1 \\
\hline 2702 & 308 & MORATTO & MARY & w & 24 & M8 & ILLINOIS & ILLINOIS & ILLINOIS & & & & OSCEOLA PR. 1 \\
\hline 2703 & 308 & MORATTO & $\mathrm{JOHN}$ & s & 4 & $\mathrm{~s}$ & ILLINOIS & ITALY & ILLINOIS & & & & OSCEOLA PR. 1 \\
\hline 2704 & 308 & MORATTO & JOSEPH & $\mathrm{s}$ & 1 & s & ILLINOIS & ITALY & ILLINOIS & & & & OSCEOLA PR. 1 \\
\hline 2705 & $200 ?$ & AIMONE & BARNEY & $\mathrm{H}$ & 25 & M4 & ITALY & ITALY & ITALY & $\mathrm{AL}$ & MINER/CM & TORINO & OSCEOLA PR. 1 \\
\hline 2706 & $200 ?$ & AIMONE & MARY & w & 25 & M4 & ITALY & ITALY & ITALY & & & TORINO & OSCEOLA PR. 1 \\
\hline
\end{tabular}




\begin{tabular}{|c|c|c|c|c|c|c|c|c|c|c|c|c|c|}
\hline 2707 & $200 ?$ & AIMONE & KATE & $\mathrm{D}$ & 4 & $\mathrm{~s}$ & ILLINOIS & ITALY & ITALY & & & TORINO & OSCEOLA PR. 1 \\
\hline 2708 & $200 ?$ & AIMONE & TERRY/TONY & D & 2 & $\mathrm{~s}$ & ILLINOIS & ITALY & ITALY & & & TORINO & OSCEOLA PR. 1 \\
\hline 2709 & $186 ?$ & BERSON & MARTIN & $\mathrm{H}$ & 28 & MO & ITALY & ITALY & ITALY & $\mathrm{AL}$ & TRAMMER/CM & & OSCEOLA PR. 1 \\
\hline 2710 & $186 ?$ & BERSON & MAGGIE & W & 21 & MO & ITALY & ITALY & ITALY & & & & OSCEOLA PR. 1 \\
\hline 2711 & 50 & SANDRETTO & CON & $\mathrm{H}$ & 44 & M19 & ITALY & ITALY & ITALY & NA & LABORER & PONT CANAVESE. TO & OSCEOLA PR. 3 \\
\hline 2712 & 50 & SANDRETTO & JENIVIAN? & W & 41 & M19 & ITALY & ITALY & ITALY & UN? & & PONT CANAVESE. TO & OSCEOLA PR. 3 \\
\hline 2713 & 50 & SANDRETTO & LENA & D & 16 & s & ITALY & ITALY & ITALY & UN? & & PONT CANAVESE. TO & OSCEOLA PR. 3 \\
\hline 2714 & 50 & SANDRETTO & JENNRI? & $\mathrm{D}$ & 13 & S & ITALY & ITALY & ITALY & UN? & AT SCHOOL & PONT CANAVESE. TO & OSCEOLA PR. 3 \\
\hline 2715 & 50 & SANDRETTO & PETER & s & 13 & $\hat{s}$ & MICHIGAN & ITALY & ITALY & Un? & AT SCHOOL & PONT CANAVESE. TO & OSCEOLA PR. 3 \\
\hline 2716 & 50 & SANDRETTO & LAWRENCE & D & 8 & $\mathrm{~s}$ & MICHIGAN & ITALY & ITALY & & AT SCHOOL & PONT CANAVESE. TO & OSCEOLA PR. 3 \\
\hline 2717 & 50 & SANDRETTO & JENI?? & D & 4 & $\mathrm{~s}$ & MICHIGAN & ITALY & ITALY & & & PONT CANAVESE. TO & OSCEOLA PR. 3 \\
\hline 2718 & 50 & SANDRETTO & LANNI & $\mathrm{s}$ & 3 & $\mathrm{~s}$ & MICHIGAN & ITALY & ITALY & & & PONT CANAVESE. TO & OSCEOLA PR. 3 \\
\hline 2719 & 50 & FALETTI & JOHN & B & 30 & $\mathrm{~s}$ & ITALY & ITALY & ITALY & AL & LABORER & PONT CANAVESE. TO & OSCEOLA PR. 3 \\
\hline 2720 & 50 & GALETTO & PIETRO & B & 41 & M1 & ITALY & ITALY & ITALY & RE? & LABORER/CM & SAN GIORGIO CANAVESE. TO & OSCEOLA PR. 3 \\
\hline 2721 & 50 & GIUSTAT & JOHN & B & 26 & M2 & ITALY & ITALY & ITALY & $\mathrm{AL}$ & LABORER/CM & SALTO CANAVESE. TO & OSCEOLA PR. 3 \\
\hline 2722 & 50 & BARSANTI & PETER & B & 31 & M1 & ITALY & ITALY & ITALY & $\mathrm{AL}$ & LABORER/CM & SEGROMIGNO, LU & OSCEOLA PR. 3 \\
\hline 2723 & 50 & BARSANTI & ALBERT & B & 22 & $\mathrm{~s}$ & ITALY & ITALY & ITALY & $\mathrm{AL}$ & LABORER/CM & SEGROMIGNO. LU & OSCEOLA PR. 3 \\
\hline 2724 & 50 & BARSANTI & ALBERT & B & 22 & $\mathrm{~s}$ & ITALY & ITALY & ITALY & $\mathrm{AL}$ & LABORER/CM & SEGROMIGNO. LU & OSCEOLA PR. 3 \\
\hline 2725 & 50 & GALLASERO & GIACOMO & B & 25 & $\mathrm{~s}$ & ITALY & ITALY & ITALY & $\mathrm{AL}$ & TRAMMER/CM & TORINO & OSCEOLA PR. 3 \\
\hline 2726 & 50 & $\begin{array}{l}\text { JACHINTO } \\
\text { JA }\end{array}$ & SALVIO & B & 22 & $\mathrm{~s}$ & ITALY & ITALY & ITALY & $\mathrm{AL}$ & TRAMMER/CM & & OSCEOLA PR. 3 \\
\hline 2727 & 50 & LANDINI & THOMAS & B & 26 & M6 & ITALY & ITALY & ITALY & AL & FIREMAN & LUCCA & OSCEOLA PR. 3 \\
\hline 2728 & 50 & VERCELLINO & $\begin{array}{l}\text { PETER } \\
\text { PETE }\end{array}$ & $\begin{array}{l}\mathrm{D} \\
\mathrm{H}\end{array}$ & 36 & M5 & ITALY & ITALY & ITALY & $\begin{array}{ll}\mathrm{PL} \\
\mathrm{PA}\end{array}$ & LABORER/CM & PONT CANAVESE, TO & OSCEOLA PR. 3 \\
\hline 2729 & & VERCELLINO & ROSA & W & 34 & M5 & ITALY & ITALY & ITALY & UN & & PONT CANAVESE. TO & OSCEOLA PR. 3 \\
\hline 2730 & & CAVALETTO & LOUIS & BRO & 25 & $\mathrm{~s}$ & ITALY & ITALY & ITALY & AL & LABORER/CM & TORINO & OSCEOLA PR. 3 \\
\hline 2731 & & MARTA & MARTIN & B & 30 & M9 & ITALY & ITALY & ITALY & PA & LABORER/CM & SAN GIOVANNI CANAVESE. & OSCEOLA PR. 3 \\
\hline 2732 & & MARTA & DOMINIK & B & 24 & M6 & ITALY & ITALY & ITALY & AL & LABORER/CM & TORINO & OSCEOLA PR. 3 \\
\hline 2733 & & LANDINI & GIUSEPPE & $\mathrm{H}$ & 27 & M2 & ITALY & ITALY & ITALY & $\mathrm{AL}$ & LABORER/CM & COLLE DI CAMPITO. LU & OSCEOLA PR. 3 \\
\hline 2734 & & LANDINI & ASSUNTA & w & 22 & M2 & ITALY & ITALY & ITALY & UN & & COLLE DI CAMPITO, LU & OSCEOLA PR. 3 \\
\hline 2735 & & LANDINI & ZELMURA & D & 2 & $\mathrm{~s}$ & ITALY & ITALY & ITALY & UN & & COLLE DI CAMPITO. LU & OSCEOLA PR. 3 \\
\hline 2736 & & BARSUCCHI & RAIMONDO & B & 21 & $\mathrm{~S}$ & ITALY & ITALY & ITALY & $\mathrm{AL}$ & LABORER/CM & LUCCA & OSCEOLA PR. 3 \\
\hline 2737 & & GUERRA & FRANCESCO & B & 41 & s & ITALY & ITALY & ITALY & $\mathrm{AL}$ & LABORER/CM & LUCCA & OSCEOLA PR. 3 \\
\hline 2738 & & BARSANTI & ACHILLE & B & 28 & M3 & ITALY & ITALY & ITALY & $A L$ & LABORER/CM & SEGROMIGNO. LU & OSCEOLA PR. 3 \\
\hline 2739 & 47 & FASANA & GIUSEPPE & $\begin{array}{l}\mathrm{D} \\
\mathrm{H}\end{array}$ & 29 & M5 & ITALY & $\begin{array}{l}\text { ITALY } \\
\text { ITA }\end{array}$ & ITALY & $\mathrm{PA}$ & $\begin{array}{l}\text { FIREMAN } \\
\text { FIVI }\end{array}$ & SEORUIIIOTV. LU & OSCEOLA PR. 3 \\
\hline 2740 & 47 & FASANA & ITALIA & w & 29 & M5 & ITALY & ITALY & ITALY & & & & OSCEOLA PR. 3 \\
\hline 2741 & 47 & FASANA & DILETTA & D & 3 & $\mathrm{~s}$ & MICHIGAN & ITALY & ITALY & & & & OSCEOLA PR. 3 \\
\hline 2742 & 47 & FASANA & MIDA & D & 2 & $\mathrm{~s}$ & MICHIGAN & ITALY & ITALY & & & & OSCEOLA PR. 3 \\
\hline 2743 & 47 & FASANA & LENA & $\mathrm{D}$ & 10 & s & MICHIGAN & ITALY & ITALY & & & & OSCEOLA PR. 3 \\
\hline 2744 & 47 & BENI & TONI & $\mathrm{COU}$ & 30 & M6 & ITALY & ITALY & ITALY & AL & LABORER/CM & & OSCEOLA PR. 3 \\
\hline 2745 & 47 & NARDI & GIUSEPPE & $\mathrm{COU}$ & 23 & $\mathrm{~s}$ & ITALY & ITALY & ITALY & $\mathrm{AL}$ & LABORER/CM & LUCCA & OSCEOLA PR. 3 \\
\hline 2746 & 48 & GIANNINI & JULIO & $\mathrm{H}$ & 40 & M12 & ITALY & ITALY & ITALY & NA & MACHINIST & LUCCA & OSCEOLA PR. 3 \\
\hline 2747 & 48 & GIANNINI & CL? & W & 39 & M12 & $\mathrm{OHIO}$ & GERMAN & GERMANY & & & GERMANY & OSCEOLA PR. 3 \\
\hline 2748 & 48 & GIANNINI & $E ?$ & $\mathrm{~s}$ & 10 & $\mathrm{~s}$ & MICHIGAN & ITALY & $\mathrm{OHIO}$ & & AT SCHOOL & LUCCA & OSCEOLA PR. 3 \\
\hline 2749 & 48 & GIANNINI & $\mathrm{JOHN}$ & $\mathrm{s}$ & 9 & $\mathrm{~s}$ & MICHIGAN & ITALY & $\mathrm{OHIO}$ & & AT SCHOOL & LUCCA & OSCEOLA PR. 3 \\
\hline 2750 & 48 & GIANNINI & FRED & $\mathrm{s}$ & 7 & $\mathrm{~s}$ & MICHIGAN & ITALY & $\mathrm{OHIO}$ & & AT SCHOOL & LUCCA & OSCEOLA PR. 3 \\
\hline 2751 & 48 & GIANNINI & INA & D & 5 & $\mathrm{~s}$ & MICHIGAN & ITALY & $\mathrm{OHIO}$ & & AT SCHOOL & LUCCA & OSCEOLA PR. 3 \\
\hline 2752 & 48 & GIANNINI & FRANK & $\mathrm{s}$ & 3 & $\mathrm{~S}$ & MICHIGAN & ITALY & $\mathrm{OHIO}$ & & & LUCCA & OSCEOLA PR. 3 \\
\hline 2753 & 48 & GIANNINI & L? & $\mathrm{D}$ & 2 & $\mathrm{~s}$ & MICHIGAN & ITALY & $\mathrm{OHIO}$ & & & LUCCA & OSCEOLA PR. 3 \\
\hline 2754 & 48 & GIANNINI & $?$ & $\mathrm{~s}$ & 0 & s & MICHIGAN & ITALY & $\mathrm{OHIO}$ & & & LUCCA & OSCEOLAPR. 3 \\
\hline 2755 & +0 & MUSSO & PAOLO & $\mathrm{H}$ & 28 & M2 & ITALY & ITALY & ITALY & AL & STONE MASON & RIVARA CANAVESE. TO & PORTAGE \\
\hline 2756 & & MUSSO & MARION & W & 22 & M2 & ITALY & ITALY & ITALY & & & & PORTAGE \\
\hline 2757 & & MUSSO & MARY & D & 1 & $\mathrm{~S}$ & MICHIGAN & ITALY & ITALY & & & RIVARA CANAVESE. TO & PORTAGE \\
\hline 2758 & & ZOIA & EUGENE & $\mathrm{H}$ & 48 & M19 & ITALY & ITALY & ITALY & UN & SHOEMAKER & CUGGIANO, MILANO & PORTAGE \\
\hline 2759 & & ZOIA & P? & W & 46 & M19 & GERMANY & GERMAN & GERMANY & & & GERMANY & PORTAGE \\
\hline 2760 & & ZOIA & GEORGE & $\mathrm{s}$ & 12 & $\mathrm{~s}$ & CANADA & ITALY & GERMANY & & AT SCHOOL & CUGGIANO. MILANO & PORTAGE \\
\hline 2761 & & ZOIA & ALEXANDER & $\mathrm{s}$ & 10 & $\mathrm{~s}$ & CANADA & ITALY & GERMANY & & AT SCHOOL & CUGGIANO. MILANO & PORTAGE \\
\hline 2762 & & ZOIA & N? & D & 8 & s & CANADA & ITALY & GERMANY & & AT SCHOOL & CUGGIANO. MILANO & PORTAGE \\
\hline 2763 & & PEILA & $?$ & $\mathrm{H}$ & 30 & M2 & ITALY & ITALY & ITALY & $\mathrm{AL}$ & MINER/CM & PONT CANAVESE. TO & PORTAGE \\
\hline 2764 & & PEILA & VITTORIA & W & 24 & M2 & ITALY & ITALY & ITALY & $\mathrm{AL}$ & & PONT CANAVESE, TO & PORTAGE \\
\hline 2765 & & PEILA & CHARLEY & $\mathrm{s}$ & 1 & $\mathrm{~s}$ & ITALY & ITALY & ITALY & & & PONT CANAVESE. TO & PORTAGE \\
\hline 2766 & & PRIGLAH & $?$ & B & 18 & $\mathrm{~s}$ & ITALY & ITALY & ITALY & & TRAMMER/CM & & PORTAGE \\
\hline 2767 & & PETERI & REGINA & B & 23 & s & ITALY & ITALY & ITALY & AL & LABORER & & PORTAGE \\
\hline 2768 & & MIGLIO/MIGLIA & ? & $\mathrm{H}$ & 38 & M19 & ITALY & ITALY & ITALY & N? & STONE MASON & TORINO & PORTAGE \\
\hline 2769 & & MIGLIO/MIGLIA & M. & W & 40 & M19 & ITALY & ITALY & ITALY & & & TORINO & PORTAGE \\
\hline
\end{tabular}




\begin{tabular}{|c|c|c|c|c|c|c|c|c|c|c|c|c|}
\hline 2770 & MIGLIO/MIGLIA & MARNY & D & 14 & $\mathrm{~s}$ & ITALY & ITALY & ITALY & & AT SCHOOL & TORINO & PORTAGE \\
\hline 2771 & MIGLIO/MIGLIA & ROSIE & D & 9 & $\mathrm{~S}$ & MICHIGAN & ITALY & ITALY & & AT SCHOOL & TORINO & PORTAGE \\
\hline 2772 & LOCATELLI & A. & B & 22 & $\mathrm{~s}$ & ITALY & ITALY & ITALY & AL & TRAMMER/CM & SELINO. BERGAMO & QUINCY \\
\hline 2773 & CENTUHIST & FRANK & B & 24 & s & ITALY & ITALY & ITALY & AL & TRAMMER/CM & & QUINCY \\
\hline 2774 & MAGA & GIACOMO & B & 33 & M14 & ITALY & ITALY & ITALY & $\mathrm{PA}$ & TRAMMER/CM & SCARMAGNO. TO & QUINCY \\
\hline 2775 & DE MATTEIS & GUSTAVO & B & 20 & $\mathrm{~s}$ & ITALY & ITALY & ITALY & UN & TRAMMER/CM & SAN GIORGIO CANAVESE. TO & QUINCY \\
\hline 2776 & LOCATELLI & AN & B & 20 & s & ITALY & ITALY & ITALY & UN & TRAMMER/CM & SELINO. BERGAMO & QUINCY \\
\hline 2777 & PAGANIO & JOSEPH & B & 34 & M12 & ITALY & ITALY & ITALY & NA & TRAMMER/CM & & QUINCY \\
\hline 2778 & PAGANIO & JOHN & B & 27 & $\mathrm{~s}$ & ITALY & ITALY & ITALY & $\mathrm{AL}$ & TRAMMER/CM & & QUINCY \\
\hline 2779 & MATANE & JOHN & $\mathrm{B}$ & 27 & M6 & ITALY & ITALY & ITALY & $\mathrm{AL}$ & TRAMMER/CM & & QUINCY \\
\hline 2780 & LOCATELLI & PANE & $\mathrm{B}$ & 34 & $\mathrm{~s}$ & ITALY & ITALY & ITALY & $A L$ & TRAMMER/CM & SELINO. BERGAMO & QUINCY \\
\hline 2781 & DAL COL & ROMANO & $\mathrm{B}$ & 23 & s & ITALY & ITALY & ITALY & $A L$ & TRAMMER/CM & VOLTAGO BELLUNO & QUINCY \\
\hline 2782 & FREDIANI & EUGENIO & B & 38 & M12 & ITALY & ITALY & ITALY & $A L$ & STONE MASON & PARIANA. LU & QUINCY \\
\hline 2783 & LOCATELLI & $\begin{array}{l}\text { PETER } \\
\text { PET }\end{array}$ & B & 36 & $s$ & ITALY & ITALY & ITALY & $A L$ & LABORER/CM & SELINO. BERGAMO & QUINCY \\
\hline 2784 & BAIANI & JAMES & B & 25 & $\mathrm{~s}$ & ITALY & ITALY & ITALY & $A L$ & TRAMMER/CM & & QUINCY \\
\hline 2785 & LOCATELLI & JON & $\begin{array}{l}\mathrm{D} \\
\mathrm{B}\end{array}$ & 20 & s & ITALY & ITALY & ITALY & UN & TRAMMER/CM & SELINO, BERGAMO & QUINCY \\
\hline 2786 & GNECHE & ANGLA & $\begin{array}{l}\mathrm{D} \\
\mathrm{B}\end{array}$ & 43 & M10 & $\begin{array}{l}\text { ITALY } \\
\end{array}$ & ITALY & ITALY & $\mathrm{AL}$ & TRAMMER/CM & & QUINCY \\
\hline 2787 & DE MATTEIS & $\mathrm{JOHN}$ & B & 34 & M6 & ITALY & ITALY & ITALY & $A L$ & TRAMMER/CM & SAN GIORGIO CANAVESE. TO & QUINCY \\
\hline 2788 & VASSANANDO & FRANK & B & 49 & s & ITALY & ITALY & ITALY & $\mathrm{AL}$ & $\begin{array}{l}\text { PATIENT } \\
\text { PAm }\end{array}$ & & SCHOOLCRAFT \\
\hline 2789 & MARKS & LOUIS & B & 33 & $\mathrm{~s}$ & ITALY & ITALY & ITALY & NA & MILLER & & TORCH LAKE \\
\hline
\end{tabular}




\section{APPENDIX IX}

\section{CENSUS of 1910}

\section{LEGEND}

ID: counter.

$\mathbf{N}$ : street number as it appeared on the house.

REL: relation of the person with the head of the household.

BORN: nation of birth of the person.

FATHER: nation of birth of the father.

MOTHER: nation of birth of the mother.

USA: indicate the year of arrival in the U.S.

PROVENIENCE: place of origin in Italy. These data are not from the census, but form Ellis Island Database, Italian newspapers, Oral interviews.

TOWNSHIP: township in which the person was living. 


\begin{tabular}{|c|c|c|c|c|c|c|c|c|c|c|c|c|c|}
\hline$\overline{I D}$ & $\mathbf{N}$ & SURNAME & NAME & REL & AGE & BORN & FATHER & MOTHER & USA & OCCUPATION & PROVENIENCE & ADDRESS & $\begin{array}{l}\text { TOWNSHIP } \\
\end{array}$ \\
\hline 1 & & BIANUCCI & EUGENIO & $\mathrm{H}$ & 46 & ITALY & ITALY & ITALY & 1892 & LABORER/CM & ALTOPASCIO, LU & & ADAMS - ATLANTIC MINE LOCATION \\
\hline 2 & & BIANUCCI & CLORINDA & W & 40 & ITALY & ITALY & ITALY & 1894 & & ALTOPASCIO. LU & & ADAMS - ATLANTIC MINE LOCATION \\
\hline 3 & & BIANUCCI & ARTHUR & s & 19 & ITALY & ITALY & ITALY & 1894 & MACHINIST/CM & ALTOPASCIO. LU & & ADAMS - ATLANTIC MINE LOCATION \\
\hline 4 & & BIANUCCI & JOSEPH & s & 17 & ITALY & ITALY & ITALY & 1894 & MACHINIST/CM & ALTOPASCIO. LU & & ADAMS - ATLANTIC MINE LOCATION \\
\hline 5 & & BIANUCCI & GUIDO & $\mathrm{s}$ & 12 & MICHIGAN & ITALY & ITALY & & & ALTOPASCIO. LU & & ADAMS - ATLANTIC MINE LOCATION \\
\hline 6 & & BIANUCCI & GIULIO & $\mathrm{s}$ & 5 & MICHIGAN & ITALY & ITALY & & & ALTOPASCIO. LU & & ADAMS - ATLANTIC MINE LOCATION \\
\hline 7 & & BIANUCCI & ALFREDO & s & 3 & MICHIGAN & ITALY & ITALY & & & ALTOPASCIO, LU & & ADAMS - ATLANTIC MINE LOCATION \\
\hline 8 & & ClUCCl & NICOLA & $\mathrm{H}$ & 42 & ITALY & ITALY & ITALY & $88 / 90$ & WATCHMAN/CM & MATRAIA. LU & & ADAMS - ATLANTIC MINE LOCATION \\
\hline 9 & & $\mathrm{ClUCCl}$ & SALOMINA & W & 43 & ITALY & ITALY & ITALY & $89 / 90$ & VUATC חIVIATVICIVI & MATRAIA. LU & & ADAMS - ATLANTIC MINE LOCATION \\
\hline 10 & & $\mathrm{ClUCCl}$ & AMELIA & $\mathrm{D}$ & 17 & MICHIGAN & ITALY & ITALY & & & MATRAIA. LU & & ADAMS - ATLANTIC MINE LOCATION \\
\hline 11 & & $\mathrm{ClUCCl}$ & DOMENICO & s & 16 & MICHIGAN & ITALY & ITALY & & NOT CLEAR & MATRAIA. LU & & ADAMS - ATLANTIC MINE LOCATION \\
\hline 12 & & $\mathrm{ClUCCl}$ & PAOLO & $\mathrm{s}$ & 15 & MICHIGAN & ITALY & ITALY & & & MATRAIA. LU & & ADAMS - ATLANTIC MINE LOCATION \\
\hline 13 & & $\mathrm{ClUCCl}$ & NATALINA & $\mathrm{D}$ & 14 & MICHIGAN & ITALY & ITALY & & & MATRAIA. LU & & ADAMS - ATLANTIC MINE LOCATION \\
\hline 14 & & ClUCCI & EMMA & $\mathrm{D}$ & 12 & MICHIGAN & ITALY & ITALY & & & MATRAIA. LU & & ADAMS - ATLANTIC MINE LOCATION \\
\hline 15 & & ClUCCI & ENRICO & $\mathrm{s}$ & 10 & MICHIGAN & ITALY & ITALY & & & MATRAIA. LU & & \\
\hline 16 & & $\mathrm{ClUCCl}$ & ALFREDO & $\mathrm{s}$ & 7 & MICHIGAN & ITALY & ITALY & & & MATRAIA. LU & & ADAMS - ATLANTIC MINE LOCATION \\
\hline 17 & & ClUCCl & PIETRO & $\mathrm{s}$ & 4 & MICHIGAN & ITALY & ITALY & & & MATRAIA. LU & & ADAMS - ATLANTIC MINE LOCATION \\
\hline 18 & & $\mathrm{ROCH} / \mathrm{ROCK}$ & JOHN & $\mathrm{H}$ & 32 & ITALY & ITALY & ITALY & 1905 & TIMBERMAN/CM & RIVAROLO CANAVESE. TO & & ADAMS - ATLANTIC MINE LOCATION \\
\hline 19 & & $\mathrm{ROCH} / \mathrm{ROCK}$ & JENNIE & w & 26 & ITALY & ITALY & ITALY & 1905 & & RIVAROLO CANAVESE. TO & & ADAMS - ATLANTIC MINE LOCATION \\
\hline 20 & & ROCH/ROCK & JOSEPH & s & 3 & MICHIGAN & ITALY & ITALY & & & RIVAROLO CANAVESE. TO & & ADAMS - ATLANTIC MINE LOCATION \\
\hline 21 & & ROCH/ROCK & TERESA & $\mathrm{D}$ & 2 & MICHIGAN & ITALY & ITALY & & & RIVAROLO CANAVESE. TO & & ADAMS - ATLANTIC MINE LOCATION \\
\hline 22 & & ROCH/ROCK & MARIO & s & $4 \mathrm{M}$ & MICHIGAN & ITALY & ITALY & & & RIVAROLO CANAVESE. TO & & ADAMS - ATLANTIC MINE LOCATION \\
\hline 23 & & ROCH/ROCK & PAOLO & $\mathrm{BR}$ & 22 & ITALY & ITALY & ITALY & 1909 & TIMBERMAN/CM & RIVAROLO CANAVESE. TO & & ADAMS - ATLANTIC MINE LOCATION \\
\hline 24 & & $\begin{array}{l}\text { PULAIRTI } \\
\text { PIOLETI }\end{array}$ & DOMENICO & $B$ & 42 & ITALY & ITALY & ITALY & 1901 & 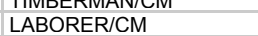 & $\begin{array}{l}\text { TORINO } \\
\text { TO CANAVESE. IO }\end{array}$ & & ADAMS - ATLANTIC MINE LOCATION \\
\hline 25 & & CHIMINO & GIOVANNI & $\mathrm{H}$ & 58 & ITALY & ITALY & ITALY & $85 / 86$ & LABORER/CM & SAN GIORGIO CANAVESE, TO & & ADAMS - ATLANTIC MINE LOCATION \\
\hline 26 & & CHIMINO & MARGHERITA & w & 57 & ITALY & ITALY & ITALY & 1890 & & & & ADAMS - ATLANTIC MINE LOCATION \\
\hline 27 & & CHIMINO & GIOVANNI & s & 19 & MICHIGAN & ITALY & ITALY & & LABORER/CM & SAN GIORGIO CANAVESE. TO & & ADAMS - ATLANTIC MINE LOCATION \\
\hline 28 & & CHIMINO & GIUSEPPE & $\mathrm{s}$ & 17 & MICHIGAN & ITALY & ITALY & & LABORER/CM & SAN GIORGIO CANAVESE. TO & & ADAMS - ATLANTIC MINE LOCATION \\
\hline 29 & & CHIMINO & TERESA & $\mathrm{D}$ & 16 & MICHIGAN & ITALY & ITALY & & & SAN GIORGIO CANAVESE. TO & & ADAMS - ATLANTIC MINE LOCATION \\
\hline 30 & & CHIMINO & MARGHERITA & $\mathrm{D}$ & 11 & MICHIGAN & ITALY & ITALY & & & SAN GIORGIO CANAVESE. TO & & ADAMS - ATLANTIC MINE LOCATION \\
\hline 31 & & ZANOTTI & GIACOMO & B & 45 & ITALY & ITALY & ITALY & 1907 & LABORER/CM & TORINO & & ADAMS - ATLANTIC MINE LOCATION \\
\hline 32 & & BALCONI & ANGELO & $\mathrm{H}$ & 26 & ITALY & ITALY & ITALY & 1905 & LABORER/BREWERY & MERCALLO. VARESE & & ADAMS - ATLANTIC MINE LOCATION \\
\hline 33 & & BALCONI & MARIA & w & 24 & ITALY & ITALY & ITALY & 1906 & & & & ADAMS - ATLANTIC MINE LOCATION \\
\hline 34 & & BALCONI & ENRICO & s & 3 & MICHIGAN & ITALY & ITALY & & & MERCALLO. VARESE & & ADAMS - ATLANTIC MINE LOCATION \\
\hline $\begin{array}{r}34 \\
35\end{array}$ & & BALCONI & ATTILIO & s & $1.6 \mathrm{M}$ & MICHIGAN & ITALY & ITALY & & & MERCALLO. VARESE & & ADAMS - ATLANTIC MINE LOCATION \\
\hline 36 & & BALCONI & GIACOMO & $\mathrm{BR}$ & $\begin{array}{l}1.011 \\
30\end{array}$ & ITALY & ITALY & ITALY & 1903 & LABORER/BREWERY & MERCALLO. VARESE & & ADAMS - ATLANTIC MINE LOCATION \\
\hline 37 & & BALCONI & MARIA & SIL & 26 & ITALY & ITALY & ITALY & 1905 & & MERCALLO, VARESE & & ADAMS - ATLANTIC MINE LOCATION \\
\hline 38 & & BALCONI & RUSSELL & NEP & 7 & ITALY & ITALY & ITALY & 1905 & & MERCALLO. VARESE & & ADAMS - ATLANTIC MINE LOCATION \\
\hline 39 & & BALCONI & STELLA & NIE & $10 \mathrm{M}$ & MICHIGAN & ITALY & ITALY & & & MERCALLO. VARESE & & ADAMS - ATLANTIC MINE LOCATION \\
\hline 40 & & BOLLINI & SERAFINO & B & 31 & ITALY & ITALY & ITALY & 1903 & FIREMAN/SMELTER & MERCALLO. VARESE & & ADAMS - ATLANTIC MINE LOCATION \\
\hline 41 & & MORO & PIETRO & B & 52 & ITALY & ITALY & ITALY & 1882 & TEAMSTER/BREWERY & TORINO & & ADAMS - ATLANTIC MINE LOCATION \\
\hline 42 & $524 ?$ & TAPPERO & BARTOLOMEO & $\mathrm{H}$ & 43 & ITALY & ITALY & ITALY & $1893 ?$ & MINER/CM & SAN GIORGIO CANAVESE. TO & 15TH AVE. & ADAMS - BALTIC MINE LOCATION \\
\hline 43 & $524 ?$ & TAPPERO & $\begin{array}{l}\text { CATERINA } \\
\end{array}$ & w & 42 & ITALY & ITALY & ITALY & 1885 & & SAN GIORGIO CANAVESE, TO & 15TH AVE. & ADAMS - BALTIC MINE LOCATION \\
\hline 44 & $524 ?$ & TAPPERO & GIUSEPPE & s & 18 & ITALY & ITALY & ITALY & 1885 & MINER/CM & SAN GIORGIO CANAVESE. TO & 15TH AVE. & ADAMS - BALTIC MINE LOCATION \\
\hline 45 & $524 ?$ & TAPPERO & ROSA & $\mathrm{D}$ & 14 & MICHIGAN & ITALY & ITALY & & & SAN GIORGIO CANAVESE. TO & 15TH AVE. & ADAMS - BALTIC MINE LOCATION \\
\hline 46 & $524 ?$ & TAPPERO & MARIA & $\mathrm{D}$ & 12 & MICHIGAN & ITALY & ITALY & & & SAN GIORGIO CANAVESE. TO & 15TH AVE. & ADAMS - BALTIC MINE LOCATION \\
\hline 47 & $524 ?$ & BARTOLO(NI) & DOMENICO & B & 21 & ITALY & ITALY & ITALY & $08 ?$ & MASON/CM & & 15TH AVE. & ADAMS - BALTIC MINE LOCATION \\
\hline 48 & $524 ?$ & $\begin{array}{l}\text { FILIPPI } \\
\text { FIIII) }\end{array}$ & MICHELE & $\begin{array}{l}\mathrm{D} \\
\mathrm{B}\end{array}$ & 24 & ITALY & ITALY & ITALY & 1901 & MINER/CM & LUCCA & 15TH AVE. & ADAMS - BALTIC MINE LOCATION \\
\hline 48 & $524 ?$ & CERASCHI & FRANCESCO & $\begin{array}{l}\mathrm{B} \\
\mathrm{B}\end{array}$ & 23 & ITALY & ITALY & ITALY & 1909 & TRAMMER/CM & LUCCA & 15TH AVE. & ADAMS - BALTIC MINE LOCATION \\
\hline 50 & $524 ?$ & POGGIONE & DOMENICO & B & 25 & ITALY & ITALY & ITALY & 1905 & MINER/CM & SAN GIORGIO CANAVESE. TO & 15TH AVE. & ADAMS - BALTIC MINE LOCATION \\
\hline 51 & 522 & $\begin{array}{l}\text { FILIPPI } \\
\text { lat }\end{array}$ & JIM & $\mathrm{B}$ & 20 & ITALY & ITALY & ITALY & 1906 & MINER/MACHINE & LUCCA & 15TH AVE. & ADAMS - BALTIC MINE LOCATION \\
\hline 52 & 522 & BOGGIO & JIM & B & 18 & ITALY & ITALY & ITALY & 1909 & TRAMMER/CM & SAN GIUSTO CANAVESE. TO & 15TH AVE. & ADAMS - BALTIC MINE LOCATION \\
\hline 53 & 522 & VERGA & BARTOLOMEO & B & 25 & ITALY & ITALY & ITALY & 1909 & & SAN GIORGIO CANAVESE. TO & 15TH AVE. & ADAMS - BALTIC MINE LOCATION \\
\hline 54 & 522 & FILIPPI & GIORGIO & B & 23 & ITALY & ITALY & ITALY & 1909 & MINER/MACHINE & LUCCA & 15TH AVE. & ADAMS - BALTIC MINE LOCATION \\
\hline 55 & 522 & POGGIONE & DOMENICO & B & 33 & ITALY & ITALY & ITALY & 1904 & MINER/MACHINE & SAN GIORGIO CANAVESE. TO & 15TH AVE. & ADAMS - BALTIC MINE LOCATION \\
\hline 56 & 524 & BALAGNA & MIKE & $\mathrm{H}$ & 31 & ITALY & ITALY & ITALY & 1903 & MINER/MACHINE & PONT CANAVESE. TO & 15TH AVE. & ADAMS - BALTIC MINE LOCATION \\
\hline 57 & 524 & BALAGNA & ANGELA & w & 31 & ITALY & ITALY & ITALY & 1907 & & PONT CANAVESE. TO & 15TH AVE. & ADAMS - BALTIC MINE LOCATION \\
\hline 58 & 524 & BALAGNA & DOSOLINA & $\mathrm{D}$ & 7 & ITALY & ITALY & ITALY & 1907 & & PONT CANAVESE. TO & 15TH AVE. & ADAMS - BALTIC MINE LOCATION \\
\hline 59 & 524 & BALAGNA & MARY & $\mathrm{D}$ & 2 & MICHIGAN & ITALY & ITALY & & & PONT CANAVESE. TO & 15TH AVE. & ADAMS - BALTIC MINE LOCATION \\
\hline 60 & 524 & BALAGNA & LEGIA & $\mathrm{D}$ & 0 & MICHIGAN & ITALY & ITALY & & & PONT CANAVESE. TO & 15TH AVE. & ADAMS - BALTIC MINE LOCATION \\
\hline 61 & 524 & BERETO & UMBRO & B & 31 & ITALY & ITALY & ITALY & 1906 & MINER/MACHINE & & 15TH AVE. & ADAMS - BALTIC MINE LOCATION \\
\hline 62 & 524 & ALBERTI & DELIDA & SER & 22 & ITALY & ITALY & ITALY & 1909 & SERVANT/PF & & 15TH AVE. & ADAMS - BALTIC MINE LOCATION \\
\hline
\end{tabular}




\begin{tabular}{|c|c|c|c|c|c|c|c|c|c|c|c|c|c|}
\hline $63:$ & 503 & CHIADO & LUIGI & $\mathrm{H}$ & 28 & ITALY & ITALY & ITALY & 1907 & & CORIO. TO & 15TH AVE. & ADAMS - BALTIC MINE LOCATION \\
\hline 64 & 503 & CHIADO & KATE & W & 24 & ITALY & ITALY & ITALY & 1907 & & CORIO. TO & 15TH AVE. & ADAMS - BALTIC MINE LOCATION \\
\hline 65 & 503 & CHIADO & DOMENICO & $s$ & 1 & MICHIGAN & ITALY & ITALY & & & CORIO. TO & 15TH AVE. & ADAMS - BALTIC MINE LOCATION \\
\hline 66 & 503 & ASSALA & DOMENICO & B & 35 & ITALY & ITALY & ITALY & 1904 & TRAMMER/CM & TORINO & 15TH AVE. & ADAMS - BALTIC MINE LOCATION \\
\hline 67 & 503 & $\mathrm{ROCH} / \mathrm{ROCK}$ & JOSEPH & B & 28 & ITALY & ITALY & ITALY & 1906 & MINER/MACHINE & TORINO & 15TH AVE. & ADAMS - BALTIC MINE LOCATION \\
\hline 68 & 503 & MARTA & MARTIN & B & 31 & ITALY & ITALY & ITALY & 1903 & MASON/CM & TORINO & 15TH AVE. & ADAMS - BALTIC MINE LOCATION \\
\hline 69 & 519 & DEL DIN & LUIGI & $\mathrm{H}$ & 42 & ITALY & ITALY & ITALY & 1892 & MASON/UNDERGROUND & RIVAMONTE. BELLUNO & 16TH AVE. & ADAMS - BALTIC MINE LOCATION \\
\hline 70 & 519 & DEL DIN & TERESA & W & 28 & ITALY & ITALY & ITALY & 1906 & & RIVAMONTE. BELLUNO & 16TH AVE. & ADAMS - BALTIC MINE LOCATION \\
\hline 71 & 519 & DEL DIN & JOHNNIE & s & 5 & ITALY & ITALY & ITALY & 1906 & & RIVAMONTE. BELLUNO & 16TH AVE. & ADAMS - BALTIC MINE LOCATION \\
\hline 72 & 519 & DEL DIN & MARTELENA & $\mathrm{D}$ & 3 & MICHIGAN & ITALY & ITALY & & & RIVAMONTE. BELLUNO & 16TH AVE. & ADAMS - BALTIC MINE LOCATION \\
\hline 73 & 519 & DEL DIN & ANTHONY & $\mathrm{s}$ & 1 & MICHIGAN & ITALY & ITALY & & & RIVAMONTE. BELLUNO & 16TH AVE. & ADAMS - BALTIC MINE LOCATION \\
\hline 74 & 519 & MEINARDI & NATALE & B & 28 & ITALY & ITALY & ITALY & 1909 & MASON/UNDERGROUND & RIVAMONTE. BELLUNO & 16TH AVE. & BALTIC MINE LOCATION \\
\hline 75 & 521 & STRINGARI & ANGELO & $\mathrm{H}$ & 36 & ITALY & ITALY & ITALY & 1898 & MINER/UNDERGROUND & BELLUNO & 16TH AVE. & BALTIC MINE LOCATION \\
\hline 76 & 521 & STRINGARI & MASSIMINA & W & 32 & ITALY & ITALY & ITALY & 1898 & & BELLUNO & 16TH AVE. & ADAMS - BALTIC MINE LOCATION \\
\hline 77 & 521 & STRINGARI & LUCIA & $\mathrm{D}$ & 12 & MICHIGAN & ITALY & ITALY & & & BELLUNO & 16TH AVE. & ADAMS - BALTIC MINE LOCATION \\
\hline 78 & 521 & STRINGARI & CHARLIE & $\mathrm{s}$ & 10 & MICHIGAN & ITALY & ITALY & & & BELLUNO & 16TH AVE. & ADAMS - BALTIC MINE LOCATION \\
\hline 79 & 521 & STRINGARI & VICTOR & $\mathrm{s}$ & 1 & MICHIGAN & ITALY & ITALY & & & BELLUNO & 16TH AVE. & ADAMS - BALTIC MINE LOCATION \\
\hline 80 & 521 & PESCATORE & PETER & B & 28 & ITALY & ITALY & ITALY & 1899 & MINER/UNDERGROUND & TORINO & 16TH AVE. & ADAMS - BALTIC MINE LOCATION \\
\hline 81 & 521 & LUDOVICI & COSTANTINO & B & 25 & ITALY & ITALY & ITALY & 1909 & TRAMMER/CM & & 16TH AVE. & ADAMS - BALTIC MINE LOCATION \\
\hline 82 & 521 & TAPPERO & GIORGIO & B & 27 & ITALY & ITALY & ITALY & 1906 & & TORINO & 16TH AVE. & ADAMS - BALTIC MINE LOCATION \\
\hline 83 & 521 & FERRERO & ANGELO & B & 23 & ITALY & ITALY & ITALY & $06 / 07$ & & SAN BENIGNO CANAVESE. TO & 16TH AVE. & ADAMS - BALTIC MINE LOCATION \\
\hline 84 & 521 & DAL COL & FERMO & B & 45 & ITALY & ITALY & ITALY & 1888 & MINER/UNDERGROUND & $\begin{array}{l}\text { BELLUNO } \\
\end{array}$ & 16TH AVE. & ADAMS - BALTIC MINE LOCATION \\
\hline 85 & 521 & MORETTI & $\begin{array}{l}\text { SANTO } \\
\text { SANT }\end{array}$ & $\begin{array}{l}\mathrm{D} \\
\mathrm{B}\end{array}$ & 43 & ITALY & ITALY & ITALY & 1800 & MINER/UNDERGROUND & TAIBON. BELLUNO & 16TH AVE. & ADAMS - BALTIC MINE LOCATION \\
\hline 86 & 521 & MIANA & ANTONIO & B & 40 & ITALY & ITALY & ITALY & $99 / 09$ & TIMBERMAN/CM & VOLTAGO. BELLUNO & 16TH AVE. & ADAMS - BALTIC MINE LOCATION \\
\hline 87 & 523 & BERTOLATTI & JOE & $\mathrm{H}$ & 30 & ITALY & ITALY & ITALY & 1901 & MINER/UNDERGROUND & & 16TH AVE. & ADAMS - BALTIC MINE LOCATION \\
\hline 88 & 523 & BERTOLATTI & MARY & W & 22 & ITALY & ITALY & ITALY & 1906 & & & 16TH AVE. & BALTIC MINE LOCATION \\
\hline 89 & 523 & ANGIETTE & LOUIS & B & 24 & ITALY & ITALY & ITALY & 1906 & MINER/UNDERGROUND & & 16TH AVE. & ADAMS - BALTIC MINE LOCATION \\
\hline 90 & 523 & GUGLIELMETTI & JOHN & B & 41 & ITALY & ITALY & ITALY & 1907 & MASON/UNDERGROUND & TORINO & 16TH AVE. & ADAMS - BALTIC MINE LOCATION \\
\hline 91 & 523 & ROVANO & JOE & B & 25 & ITALY & ITALY & ITALY & 1908 & TRAMMER/CM & TORINO & 16TH AVE. & ADAMS - BALTIC MINE LOCATION \\
\hline 92 & 523 & COLOMBO & JOE & B & 22 & ITALY & ITALY & ITALY & 1909 & TRAMMER/CM & TORINO & 16TH AVE. & ADAMS - BALTIC MINE LOCATION \\
\hline 93 & 523 & COLOMBO & JIM & B & 26 & ITALY & ITALY & ITALY & 1910 & TRAMMER/CM & TORINO & 16TH AVE. & ADAMS - BALTIC MINE LOCATION \\
\hline $94:$ & 523 & ROCHETTI & DOMENICO & B & 38 & ITALY & ITALY & ITALY & 1910 & TRAMMER/CM & & 16TH AVE. & ADAMS - BALTIC MINE LOCATION \\
\hline 95 & 523 & ENRIETTI & DOMENICO & B & 29 & ITALY & ITALY & ITALY & 1906 & MINER/UNDERGROUND & COLLERETTO. TO & 16TH AVE. & ADAMS - BALTIC MINE LOCATION \\
\hline 96 & 526 & MUSSO & CASIMIRO & $\mathrm{H}$ & 29 & ITALY & ITALY & ITALY & 1890 & MINER/UNDERGROUND & $\begin{array}{l}\text { TORINO } \\
\text { TO }\end{array}$ & 16TH AVE. & ADAMS - BALTIC MINE LOCATION \\
\hline 97 & 526 & MUSSO & ROSA & W & 25 & ITALY & ITALY & $\begin{array}{l}\text { ITALY } \\
\text { ITAY }\end{array}$ & 1903 & & THRIVU & 16TH AVE. & ADAMS - BALTIC MINE LOCATION \\
\hline 98 & 526 & MUSSO & CECILIA & $\mathrm{D}$ & 5 & MICHIGAN & ITALY & ITALY & & & TORINO & 16TH AVE. & ADAMS - BALTIC MINE LOCATION \\
\hline 99 & 526 & MUSSO & WILLIAM & s & 2 & MICHIGAN & ITALY & ITALY & & & TORINO & 16TH AVE. & BALTIC MINE LOCATION \\
\hline 100 & 526 & MUSSO & JOHN & s & 1 & MICHIGAN & ITALY & ITALY & & & TORINO & 16TH AVE. & BALTIC MINE LOCATION \\
\hline 101 & 526 & MUSSO & JOE & $\mathrm{COU}$ & 25 & ITALY & ITALY & ITALY & 1909 & TRAMMER/CM & TORINO & 16TH AVE. & BALTIC MINE LOCATION \\
\hline 102 & 526 & BONOVESSO & ALESSIO & B & 28 & ITALY & ITALY & ITALY & 1909 & TRAMMER/CM & & 16TH AVE. & ADAMS - BALTIC MINE LOCATION \\
\hline 103 & 522 & BONO & JOHN & $\mathrm{H}$ & 28 & ITALY & ITALY & ITALY & 1905 & MINER/UNDERGROUND & SAN GIUSTO CANAVESE. TO & 16TH AVE. & ADAMS - BALTIC MINE LOCATION \\
\hline 104 & 522 & BONO & MARY & W & 27 & ITALY & ITALY & ITALY & 1907 & & & 16TH AVE. & ADAMS - BALTIC MINE LOCATION \\
\hline 105 & 522 & BONO & MAGGIE & $\mathrm{D}$ & 2 & MICHIGAN & ITALY & ITALY & & & SAN GIUSTO CANAVESE. TO & 16TH AVE. & ADAMS - BALTIC MINE LOCATION \\
\hline 106 & 522 & BONO & LUISA & $\mathrm{D}$ & 0 & MICHIGAN & ITALY & ITALY & & & SAN GIUSTO CANAVESE. TO & 16TH AVE. & ADAMS - BALTIC MINE LOCATION \\
\hline 107 & 524 & RICCA & TONY & $\mathrm{H}$ & 39 & ITALY & ITALY & ITALY & 1898 & MINER/UNDERGROUND & TORINO & 16TH AVE. & ADAMS - BALTIC MINE LOCATION \\
\hline 108 & 524 & RICCA & MADDALENA & w & 32 & ITALY & ITALY & ITALY & 1906 & & & 16TH AVE. & ADAMS - BALTIC MINE LOCATION \\
\hline 109 & 524 & RICCA & KATE & $\mathrm{D}$ & 7 & MICHIGAN & ITALY & ITALY & & & TORINO & 16TH AVE. & ADAMS - BALTIC MINE LOCATION \\
\hline 110 & 524 & RICCA & TONY & s & 5 & MICHIGAN & ITALY & ITALY & & & TORINO & 16TH AVE. & ADAMS - BALTIC MINE LOCATION \\
\hline 111 & 524 & RICCA & DOMINICK & $\mathrm{s}$ & 2 & MICHIGAN & ITALY & ITALY & & & TORINO & 16TH AVE. & ADAMS - BALTIC MINE LOCATION \\
\hline 112 & 524 & BARTELLONA/BE & $\begin{array}{l}\text { PIETRO } \\
\end{array}$ & B & 25 & ITALY & ITALY & ITALY & $04 / 09$ & TRAMMER/CM & & 16TH AVE. & ADAMS - BALTIC MINE LOCATION \\
\hline 113 & 524 & ROMANO & CARLO & B & 36 & ITALY & ITALY & ITAL & 1907 & TRAMMER/CM & TORINO & 16TH AVE. & BALTIC MINE LOCATION \\
\hline 114 & 524 & NIGRA & TONY & B & 20 & ITALY & ITALY & ITALY & 1909 & TRAMMER/CM & TORINO & 16TH AVE. & BALTIC MINE LOCATION \\
\hline 115 & 524 & GAMBA & JOHN & B & 22 & ITALY & ITALY & ITALY & 1909 & TRAMMER/CM & PASERANO. ALESSANDRIA & HAVE. & ADAMS - BALTIC MINE LOCATION \\
\hline 116 & 520 & DE LAURENTI & JOHN & $\mathrm{H}$ & 35 & ITALY & ITALY & ITALY & 1899 & MINER/MACHINE & CICOGNO. TO & 16TH AVE. & ADAMS - BALTIC MINE LOCATION \\
\hline 117 & 520 & DE LAURENTI & TERESA & W & 27 & ITALY & ITALY & ITALY & 1904 & & & 16TH AVE. & ADAMS - BALTIC MINE LOCATION \\
\hline 118 & 520 & DE LAURENTI & PAOLO & s & 6 & MICHIGAN & ITALY & ITALY & & & CICOGNO. TO & 16TH AVE. & ADAMS - BALTIC MINE LOCATION \\
\hline 119 & 520 & DE LAURENTI & CECILIA & $\mathrm{D}$ & 4 & MICHIGAN & ITALY & ITALY & & & CICOGNO. TO & 16TH AVE. & ADAMS - BALTIC MINE LOCATION \\
\hline 120 & 520 & ENRICO & ANTONIO & B & 21 & ITALY & ITALY & ITALY & 1909 & TRAMMER/CM & SAN GIUSTO CANAVESE, TO & 16TH AVE. & ADAMS - BALTIC MINE LOCATION \\
\hline 121 & 520 & IAVELLO & DOMENICO & B & 22 & ITALY & ITALY & ITALY & 1907 & MASON/CM & SAN GIUSTO CANAVESE. TO & 16TH AVE. & ADAMS - BALTIC MINE LOCATION \\
\hline 122 & 520 & BOGGIO & GIACOMO & B & 20 & ITALY & ITALY & ITALY & 1908 & MASON/CM & SAN GIUSTO CANAVESE. TO & 16TH AVE. & ADAMS - BALTIC MINE LOCATION \\
\hline 123 & 501 & VERGA & JOE & $\mathrm{H}$ & 30 & ITALY & ITALY & ITALY & 1903 & MINER/CM & SAN GIUSTO CANAVESE. TO & 17TH AVE. & ADAMS - BALTIC MINE LOCATION \\
\hline $124:$ & 501 & VERGA & MARY & w & 30 & ITALY & ITALY & ITALY & 1904 & & & 17TH AVE. & ADAMS - BALTIC MINE LOCATION \\
\hline 125 & 501 & VERGA & JENNIE & $\mathrm{D}$ & $9 \mathrm{M}$ & MICHIGAN & ITALY & ITALY & & & SAN GIUSTO CANAVESE. TO & 17TH AVE. & ADAMS - BALTIC MINE LOCATION \\
\hline
\end{tabular}




\begin{tabular}{|c|c|c|c|c|c|c|c|c|c|c|c|c|c|}
\hline 126 & 509 & BIMMIE & JOE & $\mathrm{H}$ & 43 & ITALY & ITALY & ITALY & 1903 & PICKER/CM & & 17TH AVE. & ADAMS - BALTIC MINE LOCATION \\
\hline 127 & 509 & BIMMIE & MARY & w & 42 & ITALY & ITALY & ITALY & 1904 & & & 17TH AVE. & ADAMS - BALTIC MINE LOCATION \\
\hline 128 & 509 & BIMMIE & MINNIE & $\mathrm{D}$ & 17 & ITALY & ITALY & ITALY & 1904 & & & 17TH AVE. & ADAMS - BALTIC MINE LOCATION \\
\hline 129 & 509 & BIMMIE & CRISTINA & $\mathrm{D}$ & 10 & ITALY & ITALY & ITALY & 1904 & & & 17TH AVE. & ADAMS - BALTIC MINE LOCATION \\
\hline 130 & 509 & PAVANIO & JIMMIE & B & 25 & ITALY & ITALY & ITALY & 1906 & PICKER/CM & & 17TH AVE. & ADAMS - BALTIC MINE LOCATION \\
\hline 131 & 210 & MONTICELLI & DOMENICO & $\mathrm{H}$ & 34 & ITALY & ITALY & ITALY & 1905 & MINER/MACHINE & MASSA MARINA. LU & 14TH AVE. & ADAMS - BALTIC MINE LOCATION \\
\hline 132 & 210 & MONTICELLI & PALMIRA & w & 29 & ITALY & ITALY & ITALY & 1905 & & MASSA MARINA. LU & 14TH AVE. & ADAMS - BALTIC MINE LOCATION \\
\hline 133 & 210 & MONTICELLI & LINA & $\mathrm{D}$ & 4 & MICHIGAN & ITALY & ITALY & & & MASSA MARINA. LU & 14TH AVE. & ADAMS - BALTIC MINE LOCATION \\
\hline 134 & 210 & MONTICELLI & ANNETTA & $\mathrm{D}$ & 3 & MICHIGAN & ITALY & ITALY & & & MASSA MARINA. LU & 14TH AVE. & ADAMS - BALTIC MINE LOCATION \\
\hline 135 & 210 & MONTICELLI & MAFALDA & $\mathrm{D}$ & $9 \mathrm{M}$ & MICHIGAN & ITALY & ITALY & & & MASSA MARINA. LU & 14TH AVE. & ADAMS - BALTIC MINE LOCATION \\
\hline 136 & 210 & BARZACHINI & FEDERICO & B & 29 & ITALY & ITALY & ITALY & 1905 & TRAMMER/CM & VICO PISANO. PISA & 14TH AVE. & ADAMS - BALTIC MINE LOCATION \\
\hline 137 & 221 & ANDRINI & ANTONIO & $\mathrm{H}$ & 35 & ITALY & ITALY & ITALY & 1901 & MASON/CM & LUCCA & 14TH AVE. & BALTIC MINE LOCATION \\
\hline 138 & 221 & ANDRINI & FILIDE & W & 31 & ITALY & ITALY & ITALY & 1901 & & LUCCA & 14TH AVE. & ADAMS - BALTIC MINE LOCATION \\
\hline 139 & 221 & ANDRINI & OLGA & $\mathrm{D}$ & 11 & ITALY & ITALY & ITALY & 1901 & & LUCCA & 14TH AVE. & ADAMS - BALTIC MINE LOCATION \\
\hline 140 & 221 & ANDRINI & ACENIO & s & 9 & MICHIGAN & ITALY & ITALY & & & LUCCA & 14TH AVE. & ADAMS - BALTIC MINE LOCATION \\
\hline 141 & 221 & ANDRINI & GINO & $\mathrm{s}$ & 7 & MICHIGAN & ITALY & ITALY & & & LUCCA & 14TH AVE. & ADAMS - BALTIC MINE LOCATION \\
\hline 142 & 221 & ANDRINI & TRACEY & $\mathrm{D}$ & 1 & MICHIGAN & ITALY & ITALY & & & LUCCA & 14TH AVE. & ADAMS - BALTIC MINE LOCATION \\
\hline 143 & 209 & ANDREINI & EMILIO & $\mathrm{H}$ & 41 & ITALY & ITALY & ITALY & 1888 & TIMBERMAN/CM & LUCCA & 14TH AVE. & ADAMS - BALTIC MINE LOCATION \\
\hline 144 & 209 & ANDREINI & MARGHERITA & w & 32 & ITALY & ITALY & ITALY & 1889 & & & 14TH AVE. & ADAMS - BALTIC MINE LOCATION \\
\hline 145 & 209 & ANDREINI & MARIA & $\mathrm{D}$ & 8 & MICHIGAN & ITALY & ITALY & & & LUCCA & 14TH AVE. & ADAMS - BALTIC MINE LOCATION \\
\hline 146 & 209 & ANDREINI & TRACEY & $\mathrm{D}$ & 6 & MICHIGAN & ITALY & ITALY & & & LUCCA & 14TH AVE. & ADAMS - BALTIC MINE LOCATION \\
\hline 147 & 209 & ANDREINI & ERNESTO & s & 5 & MICHIGAN & ITALY & ITALY & & & LUCCA & 14TH AVE. & ADAMS - BALTIC MINE LOCATION \\
\hline 148 & 209 & ANDREINI & WILLIAM & $\mathrm{S}$ & 4 & MICHIGAN & ITALY & ITALY & & & LUCCA & 14TH AVE. & ADAMS - BALTIC MINE LOCATION \\
\hline 149 & 209 & ANDREINI & PAOLO & $\mathrm{s}$ & 1 & MICHIGAN & ITALY & ITALY & & & LUCCA & 14TH AVE. & ADAMS - BALTIC MINE LOCATION \\
\hline 150 & 121 & GEDDA & PIETRO & $\mathrm{H}$ & 42 & ITALY & ITALY & ITALY & 1892 & PIPER/CM & TORINO & 15TH AVE. & BALTIC MINE LOCATION \\
\hline 151 & 121 & GEDDA & LORENZINA & w & 40 & ITALY & ITALY & ITALY & 1892 & & TORINO & 15TH AVE. & BALTIC MINE LOCATION \\
\hline 152 & 121 & GEDDA & PIETRO & $\mathrm{s}$ & 19 & ITALY & ITALY & ITALY & 1892 & LANDER/ROCK HOUSE & TORINO & 15TH AVE. & ADAMS - BALTIC MINE LOCATION \\
\hline 153 & 121 & GEDDA & JOE & $\mathrm{s}$ & 16 & MICHIGAN & ITALY & ITALY & & & TORINO & 15TH AVE. & ADAMS - BALTIC MINE LOCATION \\
\hline 154 & 121 & GEDDA & KATE & $\mathrm{D}$ & 15 & MICHIGAN & ITALY & ITALY & & & TORINO & 15TH AVE. & ADAMS - BALTIC MINE LOCATION \\
\hline 155 & 121 & GEDDA & GIORGIO & $\mathrm{s}$ & 13 & MICHIGAN & ITALY & ITALY & & & TORINO & 15TH AVE. & ADAMS - BALTIC MINE LOCATION \\
\hline 156 & 121 & GEDDA & ROSA & $\mathrm{D}$ & 11 & MICHIGAN & ITALY & ITALY & & & TORINO & 15TH AVE. & ADAMS - BALTIC MINE LOCATION \\
\hline 157 & 121 & GEDDA & MARIA & $\mathrm{D}$ & 9 & MICHIGAN & ITALY & ITALY & & & TORINO & 15TH AVE. & ADAMS - BALTIC MINE LOCATION \\
\hline 158 & 121 & GEDDA & SAM & s & 5 & MICHIGAN & ITALY & ITALY & & & TORINO & 15TH AVE. & ADAMS - BALTIC MINE LOCATION \\
\hline 159 & 121 & GEDDA & ANNIE & $\mathrm{D}$ & 3 & MICHIGAN & ITALY & ITALY & & & TORINO & 15TH AVE. & ADAMS - BALTIC MINE LOCATION \\
\hline 160 & 121 & GEDDA & TRACEY & $\mathrm{D}$ & 0 & MICHIGAN & ITALY & ITALY & & & TORINO & 15TH AVE. & ADAMS - BALTIC MINE LOCATION \\
\hline 161 & 104 & SCALA & DOMENICO & $\mathrm{H}$ & 26 & ITALY & ITALY & ITALY & $05 / 06$ & MINER/MACHINE & TORINO & 13TH AVE. & ADAMS - BALTIC MINE LOCATION \\
\hline 162 & 104 & SCALA & KATE & W & 21 & ITALY & ITALY & ITALY & 1894 & & & 13TH AVE. & ADAMS - BALTIC MINE LOCATION \\
\hline 163 & 104 & SCALA & JOE & s & 1 & MICHIGAN & ITALY & ITALY & & & TORINO & 13TH AVE. & BALTIC MINE LOCATION \\
\hline 164 & 104 & CAPPO & CESARE & B & 22 & ITALY & ITALY & ITALY & 1907 & TIMBERMAN/CM & SAN GIUSTO CANAVESE. TO & 13TH AVE. & BALTIC MINE LOCATION \\
\hline 165 & 104 & ANDRINA & BATTISTA & B & 21 & ITALY & ITALY & ITALY & $06 / 07$ & MINER/MACHINE & SAN MARTINO CANAVESE. TO & 13TH AVE. & ADAMS - BALTIC MINE LOCATION \\
\hline 166 & 104 & LANDI & NATALE & B & 20 & ITALY & ITALY & ITALY & 1907 & TRAMMER/CM & $\begin{array}{l}\text { LOCANA CANAVESE. TO } \\
\text { L }\end{array}$ & 13TH AVE. & ADAMS - BALTIC MINE LOCATION \\
\hline 167 & 104 & CONTO & DOMENICO & B & 26 & ITALY & ITALY & ITALY & 1907 & MASON/CM & SAN GIUSTO CANAVESE. TO & 13TH AVE. & ADAMS - BALTIC MINE LOCATION \\
\hline 168 & 104 & FOGLIA & BARTOLOMEO & B & 22 & ITALY & ITALY & ITALY & 1906 & TIMBERMAN/CM & TORINO & 13TH AVE. & ADAMS - BALTIC MINE LOCATION \\
\hline 169 & 102 & BASSANI & FELICE & $\mathrm{H}$ & 46 & ITALY & ITALY & ITALY & 1892 & TRAMMER BOSS/CM & SAN GIORGIO CANAVESE. TO & 13TH AVE. & ADAMS - BALTIC MINE LOCATION \\
\hline 170 & 102 & BASSANI & TERESA & W & 40 & ITALY & ITALY & ITALY & 1899 & & SAN GIORGIO CANAVESE. TO & 13TH AVE. & ADAMS - BALTIC MINE LOCATION \\
\hline 171 & 102 & BASSO & ANTONIO & B & 46 & ITALY & ITALY & ITALY & 1909 & TRAMMER/CM & SAN GIORGIO CANAVESE. TO & 13TH AVE. & ADAMS - BALTIC MINE LOCATION \\
\hline 172 & 102 & CENTANINO & JOHN & B & 30 & ITALY & ITALY & ITALY & 1900 & MINER/MACHINE & PEROSA CANAVESE. TO & 13TH AVE. & ADAMS - BALTIC MINE LOCATION \\
\hline 173 & 202 & PAOLI & PIETRO & $\begin{array}{l}\mathrm{D} \\
\mathrm{H}\end{array}$ & 40 & ITALY & ITALY & ITALY & 1890 & MINER/MACHINE & $\begin{array}{l}\text { LUCCA } \\
\text { LUAIVAVESE. IO }\end{array}$ & 13TH AVE. & ADAMS - BALTIC MINE LOCATION \\
\hline 174 & 202 & PAOLI & CLARA & w & 31 & ITALY & ITALY & ITALY & 1898 & & & 13TH AVE. & ADAMS - BALTIC MINE LOCATION \\
\hline 175 & 202 & PAOLI & EUGENIA & $\mathrm{D}$ & 7 & MICHIGAN & ITALY & ITALY & & & LUCCA & 13TH AVE. & ADAMS - BALTIC MINE LOCATION \\
\hline 176 & 202 & PAOLI & MAY & $\mathrm{D}$ & 4 & MICHIGAN & ITALY & ITAL & & & LUCCA & 13TH AVE. & BALTIC MINE LOCATION \\
\hline 177 & 202 & PAOLI & FRED & $\mathrm{BR}$ & 31 & ITALY & ITALY & ITALY & 1902 & MINER/MACHINE & LUCCA & 13TH AVE. & BALTIC MINE LOCATION \\
\hline 178 & 204 & FAUSONE & JIMMIE & $\mathrm{H}$ & 40 & ITALY & ITALY & ITALY & 1896 & TIMBERMAN/CM & TORINO & HAVE. & ADAMS - BALTIC MINE LOCATION \\
\hline 179 & 204 & FAUSONE & ANGELA & w & 34 & ITALY & ITALY & ITALY & 1901 & & & 13TH AVE. & ADAMS - BALTIC MINE LOCATION \\
\hline 180 & 204 & FAUSONE & JONNIE & s & 6 & MICHIGAN & ITALY & ITALY & & & TORINO & 13TH AVE. & ADAMS - BALTIC MINE LOCATION \\
\hline 181 & 204 & FAUSONE & JOE & $\mathrm{s}$ & 5 & MICHIGAN & ITALY & ITALY & & & TORINO & 13TH AVE. & ADAMS - BALTIC MINE LOCATION \\
\hline 182 & 204 & FAUSONE & JOHNNIE & s & 4 & MICHIGAN & ITALY & ITALY & & & TORINO & 13TH AVE. & ADAMS - BALTIC MINE LOCATION \\
\hline 183 & 204 & FAUSONE & CATERINA & $\mathrm{D}$ & 2 & MICHIGAN & ITALY & ITALY & & & TORINO & 13TH AVE. & ADAMS - BALTIC MINE LOCATION \\
\hline 184 & 204 & FAUSONE & PETER & $\mathrm{s}$ & $16 \mathrm{M}$ & MICHIGAN & ITALY & ITALY & & & TORINO & 13TH AVE. & ADAMS - BALTIC MINE LOCATION \\
\hline 185 & 206 & MIGLIO/MIGLIA & JOSEPH & $\mathrm{H}$ & 44 & ITALY & ITALY & ITALY & 1890 & MINER/MACHINE & SAN GIORGIO CANAVESE. TO & 13TH AVE. & ADAMS - BALTIC MINE LOCATION \\
\hline 186 & 206 & MIGLIO/MIGLIA & MARIAN & W & 40 & ITALY & ITALY & ITALY & 1891 & IVIVER IVIAC ПIIVE & & 13TH AVE. & ADAMS - BALTIC MINE LOCATION \\
\hline 187 & 206 & MIGLIO/MIGLIA & PETER & s & 15 & MICHIGAN & ITALY & ITALY & & & SAN GIORGIO CANAVESE. TO & 13TH AVE. & ADAMS - BALTIC MINE LOCATION \\
\hline 188 & 206 & MIGLIO/MIGLIA & JOHNNIE & $\mathrm{s}$ & 13 & MICHIGAN & ITALY & ITALY & & & SAN GIORGIO CANAVESE. TO & 13TH AVE. & ADAMS - BALTIC MINE LOCATION \\
\hline
\end{tabular}




\begin{tabular}{|c|c|c|c|c|c|c|c|c|c|c|c|c|c|}
\hline 189 & 206 & MIGLIO/MIGLIA & VITTORIA & D & 8 & MICHIGAN & ITALY & ITALY & & & SAN GIORGIO CANAVESE. TO & 13TH AVE. & ADAMS - BALTIC MINE LOCATION \\
\hline 190 & 206 & MIGLIO/MIGLIA & ANTONIETTA & D & 3 & MICHIGAN & ITALY & ITALY & & & SAN GIORGIO CANAVESE. TO & 13TH AVE. & ADAMS - BALTIC MINE LOCATION \\
\hline 191 & 206 & MIGLIO/MIGLIA & PIETRO & NEP & 18 & MICHIGAN & ITALY & ITALY & & TRAMMER/CM & SAN GIORGIO CANAVESE. TO & 13TH AVE. & ADAMS - BALTIC MINE LOCATION \\
\hline 192 & 206 & CORTESE & AUGUSTO & B & 29 & ITALY & ITALY & ITALY & 1903 & MINER/MACHINE & VALPERGA. TO & 13TH AVE. & ADAMS - BALTIC MINE LOCATION \\
\hline 193 & 208 & PUCCl & JIMMIE & B & 29 & ITALY & ITALY & ITALY & 1904 & LABORER/LIVERY BARN & LUCCA & 13TH AVE. & ADAMS - BALTIC MINE LOCATION \\
\hline 194 & 106 & ZANOTTI & JOSEPH & $\mathrm{H}$ & 36 & ITALY & ITALY & ITALY & 1899 & MINER/MACHINE & TORINO & 13TH AVE. & ADAMS - BALTIC MINE LOCATION \\
\hline 195 & 106 & ZANOTTI & MARGHERITA & w & 33 & ITALY & ITALY & ITALY & 1905 & & TORINO & 13TH AVE. & ADAMS - BALTIC MINE LOCATION \\
\hline 196 & 106 & ZANOTTI & CATERINA & $\mathrm{D}$ & 11 & ITALY & ITALY & ITALY & 1905 & & TORINO & 13TH AVE. & ADAMS - BALTIC MINE LOCATION \\
\hline 197 & 106 & ZANOTTI & FELIX & $\mathrm{s}$ & 3 & MICHIGAN & ITALY & ITALY & & & TORINO & 13TH AVE. & ADAMS - BALTIC MINE LOCATION \\
\hline 198 & 106 & ZANOTTI & GIORGIO & $\mathrm{H}$ & 43 & ITALY & ITALY & ITALY & 1900 & MINER/MACHINE & TORINO & 13TH AVE. & ADAMS - BALTIC MINE LOCATION \\
\hline 199 & 106 & ZANOTTI & MARGHERITA & W & 37 & ITALY & ITALY & ITALY & 1905 & & & 13TH AVE. & ADAMS - BALTIC MINE LOCATION \\
\hline 200 & 106 & ZANOTTI & KATE & D & 18 & MICHIGAN & ITALY & ITALY & & & TORINO & 13TH AVE. & ADAMS - BALTIC MINE LOCATION \\
\hline 201 & 106 & ZANOTTI & ANNA & D & 14 & MICHIGAN & ITALY & ITALY & & & TORINO & 13TH AVE. & ADAMS - BALTIC MINE LOCATION \\
\hline 202 & 106 & ZANOTTI & JOSEPHINE & $\mathrm{D}$ & 11 & MICHIGAN & ITALY & ITALY & & & TORINO & 13TH AVE. & ADAMS - BALTIC MINE LOCATION \\
\hline 203 & 106 & ZANOTTI & FELIX & $\mathrm{s}$ & 4 & MICHIGAN & ITALY & ITALY & & & TORINO & 13TH AVE. & ADAMS - BALTIC MINE LOCATION \\
\hline 204 & 106 & ZANOTTI & PETER & B & 27 & ITALY & ITALY & ITALY & 1906 & MINER/MACHINE & TORINO & 13TH AVE. & ADAMS - BALTIC MINE LOCATION \\
\hline 205 & 106 & ZANOTTI & DOMENICO & B & 37 & ITALY & ITALY & ITALY & 1903 & MINER/MACHINE & PEROSA CANAVESE. TO & 13TH AVE. & ADAMS - BALTIC MINE LOCATION \\
\hline 206 & & TOSCANA & ANTONIO & $\mathrm{H}$ & 34 & ITALY & ITALY & ITALY & 1895 & SALOONKEEPER & SAN GIORGIO CANAVESE. TO & TRIMOUNTAIN AVE. & ADAMS - SOUTH RANGE VILLAGE \\
\hline 207 & & TOSCANA & MARIA & w & 26 & ITALY & ITALY & ITALY & 1890 & & & TRIMOUNTAIN AVE. & ADAMS - SOUTH RANGE VILLAGE \\
\hline 208 & & TOSCANA & MINNIE & $\mathrm{D}$ & 10 & MICHIGAN & ITALY & ITALY & & & SAN GIORGIO CANAVESE. TO & TRIMOUNTAIN AVE. & ADAMS - SOUTH RANGE VILLAGE \\
\hline 209 & & TOSCANA & MARGARET & $\mathrm{D}$ & 8 & MICHIGAN & ITALY & ITALY & & & SAN GIORGIO CANAVESE. TO & TRIMOUNTAIN AVE. & ADAMS - SOUTH RANGE VILLAGE \\
\hline 210 & & TOSCANA & VITAL & $\mathrm{s}$ & 5 & MICHIGAN & ITALY & ITALY & & & SAN GIORGIO CANAVESE, TO & TRIMOUNTAIN AVE. & ADAMS - SOUTH RANGE VILLAGE \\
\hline 211 & & TOSCANA & MARY & $\mathrm{D}$ & 2 & MICHIGAN & ITALY & ITALY & & & SAN GIORGIO CANAVESE. TO & TRIMOUNTAIN AVE. & ADAMS - SOUTH RANGE VILLAGE \\
\hline 212 & & DE BERNARDI & JOHN & B & 24 & ITALY & ITALY & ITALY & 1904 & BARTENDER/SALOON & TORINO & TRIMOUNTAIN AVE. & ADAMS - SOUTH RANGE VILLAGE \\
\hline 213 & & $\begin{array}{l}\text { TEPATTI } \\
\text { TEPTI }\end{array}$ & FILIBERTO & $\mathrm{H}$ & 37 & ITALY & ITALY & ITALY & 1899 & MINER/MACHINE & PONT CANAVESE. TO & TRIMOUNTAIN AVE. & ADAMS - SOUTH RANGE VILLAGE \\
\hline 214 & & TEPATTI & ROSA & W & 41 & ITALY & ITALY & ITALY & 1907 & & PONT CANAVESE. TO & TRIMOUNTAIN AVE. & ADAMS - SOUTH RANGE VILLAGE \\
\hline 215 & & TEPATTI & DOMENICO & $\mathrm{s}$ & 10 & ITALY & ITALY & ITALY & 1907 & & PONT CANAVESE. TO & TRIMOUNTAIN AVE. & ADAMS - SOUTH RANGE VILLAGE \\
\hline 216 & & TEPATTI & ARCHELA & D & 1 & MICHIGAN & ITALY & ITALY & & & PONT CANAVESE, TO & TRIMOUNTAIN AVE. & ADAMS - SOUTH RANGE VILLAGE \\
\hline 217 & & SANTOSI & ANTONIO & $\mathrm{H}$ & 38 & ITALY & ITALY & ITALY & 1902 & MASON/CM & & TRIMOUNTAIN AVE. & ADAMS - SOUTH RANGE VILLAGE \\
\hline 218 & & SANTOSI & TERESA & W & 38 & ITALY & ITALY & ITALY & 1906 & & & TRIMOUNTAIN AVE. & ADAMS - SOUTH RANGE VILLAGE \\
\hline 219 & & SANTOSI & ANNA & D & 9 & ITALY & ITALY & ITALY & 1906 & & & TRIMOUNTAIN AVE. & ADAMS - SOUTH RANGE VILLAGE \\
\hline 220 & & SANTOSI & VITTORINA & $\mathrm{D}$ & 3 & MICHIGAN & ITALY & ITALY & & & & TRIMOUNTAIN AVE. & ADAMS - SOUTH RANGE VILLAGE \\
\hline 221 & & SANTOSI & JOHNNIE & $\mathrm{s}$ & 1 & MICHIGAN & ITALY & ITALY & & & & TRIMOUNTAIN AVE. & ADAMS - SOUTH RANGE VILLAGE \\
\hline 222 & & SANTORI & AMERICO & $\mathrm{H}$ & 33 & ITALY & ITALY & ITALY & 1898 & SALOONKEEPER & SEGROMIGNO, LU & TRIMOUNTAIN AVE. & ADAMS - SOUTH RANGE VILLAGE \\
\hline 223 & & SANTORI & PIERINA & W & 26 & ITALY & ITALY & ITALY & 1886 & SALU IVINETER & SEORUIVIOTIV, LU & $\begin{array}{l}\text { TRIMOUNTAIN AVE. } \\
\text { TIMO }\end{array}$ & ADAMS - SOUTH RANGE VILLAGE \\
\hline 224 & & PAOLI & ARTURO & B & 22 & ITALY & ITALY & ITALY & 1903 & BARTENDER/SALOON & LUCCA & TRIMOUNTAIN AVE. & ADAMS - SOUTH RANGE VILLAGE \\
\hline 225 & & BERLINCIONI & ANDREA & B & 31 & ITALY & ITALY & ITALY & 1906 & BARTENDER/SALOON & FIRENZE & TRIMOUNTAIN AVE. & ADAMS - SOUTH RANGE VILLAGE \\
\hline 226 & & GIACHINO & DOMENICO & $\mathrm{H}$ & 33 & ITALY & ITALY & ITALY & 1910 & TRAMMER/CM & TORINO & TRIMOUNTAIN AVE. & ADAMS - SOUTH RANGE VILLAGE \\
\hline 227 & & GIACHINO & MARY & w & 25 & ITALY & ITALY & ITALY & 1910 & & TORINO & TRIMOUNTAIN AVE. & ADAMS - SOUTH RANGE VILLAGE \\
\hline 228 & & BASSO & LOUIS & $\mathrm{H}$ & 38 & ITALY & ITALY & ITALY & 1888 & MARSHALL/VILLAGE & SAN GIORGIO CANAVESE. TO & TRIMOUNTAIN AVE. & ADAMS - SOUTH RANGE VILLAGE \\
\hline 229 & & BASSO & PETRONILLA & w & 37 & ITALY & ITALY & ITALY & 1893 & & & TRIMOUNTAIN AVE. & ADAMS - SOUTH RANGE VILLAGE \\
\hline 230 & & BASSO & GIUSEPPE & $s$ & 15 & MICHIGAN & ITALY & ITALY & & PUFFER BOY/CM & SAN GIORGIO CANAVESE. TO & TRIMOUNTAIN AVE. & ADAMS - SOUTH RANGE VILLAGE \\
\hline 231 & & BASSO & MARGERITA & D & 14 & MICHIGAN & ITALY & ITALY & & & SAN GIORGIO CANAVESE. TO & TRIMOUNTAIN AVE. & ADAMS - SOUTH RANGE VILLAGE \\
\hline 232 & & BASSO & GIOVANNA & $\mathrm{D}$ & 12 & MICHIGAN & ITALY & ITALY & & & SAN GIORGIO CANAVESE. TO & TRIMOUNTAIN AVE. & ADAMS - SOUTH RANGE VILLAGE \\
\hline 233 & & BASSO & TONY & $\mathrm{s}$ & 6 & MICHIGAN & ITALY & ITALY & & & SAN GIORGIO CANAVESE. TO & TRIMOUNTAIN AVE. & ADAMS - SOUTH RANGE VILLAGE \\
\hline 234 & & BASSO & MINNIE & $\mathrm{D}$ & 2 & MICHIGAN & ITALY & ITALY & & & SAN GIORGIO CANAVESE. TO & TRIMOUNTAIN AVE. & ADAMS - SOUTH RANGE VILLAGE \\
\hline 235 & & MARGHERIO & PIETRO & $\mathrm{H}$ & 26 & ITALY & ITALY & ITALY & 1902 & MINER/MACHINE & SAN GIUSTO CANAVESE. TO & 1ST ST. & ADAMS - SOUTH RANGE VILLAGE \\
\hline 236 & & MARGHERIO & CATERINA & W & 28 & ITALY & ITALY & ITALY & 1902 & IVIIVER/IVIAC RIIVE & SAN GIUSTO CANAVESE. TO & 1ST ST. & ADAMS - SOUTH RANGE VILLAGE \\
\hline 237 & & MARGHERIO & MARIA & $\mathrm{D}$ & 6 & MICHIGAN & ITALY & ITALY & & & SAN GIUSTO CANAVESE. TO & 1ST ST. & ADAMS - SOUTH RANGE VILLAGE \\
\hline 238 & & MARGK & GIUSEPPE & $\mathrm{s}$ & 5 & MICHIGAN & ITALY & ITAL & & & SAN GIUSTO CANAVESE. TO & 1ST ST. & ADAMS - SOUTH RANGE VILLAGE \\
\hline 239 & & MARGHERIO & DOMENICA & $\mathrm{D}$ & 4 & MICHIG & ITALY & ITA & & & E. TO & 1ST ST. & ADAMS - SOUTH RANGE VILLAGE \\
\hline 240 & & MARGHERIO & ANNA & $\mathrm{D}$ & 3 & MICHIGAN & ITAL & ITAL & & & SAN GIUSTO CANAVESE, TO & 1ST ST. & ADAMS - SOUTH RANGE VILLAGE \\
\hline 241 & & MARGHERIO & PIETRO & $\mathrm{s}$ & $3 \mathrm{M}$ & HIGAN & ITA & ITA & & & E.TO & & ADAMS - SOUTH RANGE VILLAGE \\
\hline 242 & & DE MARIA & FRANCESCO & $\mathrm{H}$ & 27 & $\mid$ ITA & ITA & ITA & 1907 & TRAMMER/CM & SAN GIORGIO CANAVESE. TO & 1ST ST. & ADAMS - SOUTH RANGE VILLAGE \\
\hline 243 & & DE MARIA & TERESA & W & 28 & ITALY & ITALY & ITALY & 1907 & & SAN GIORGIO CANAVESE. TO & 1ST ST. & ADAMS - SOUTH RANGE VILLAGE \\
\hline 244 & & DE MARIA & MARY & D & 2 & ITALY & ITALY & ITALY & 1907 & & SAN GIORGIO CANAVESE. TO & 1ST ST. & ADAMS - SOUTH RANGE VILLAGE \\
\hline 245 & & ZANA & DOMENICO & $\mathrm{H}$ & 34 & ITALY & ITALY & ITALY & 1898 & AGENT BREWING CO.NILLAGE & CUCEGLIO. TO & 1ST ST. & ADAMS - SOUTH RANGE VILLAGE \\
\hline 246 & & ZANA & MARIA & w & 35 & ITALY & ITALY & ITALY & 1905 & & CUCEGLIO, TO & 1ST ST. & ADAMS - SOUTH RANGE VILLAGE \\
\hline 247 & & ZANA & ANTONIETTA & $\mathrm{D}$ & 10 & ITALY & ITALY & ITALY & 1905 & & CUCEGLIO. TO & 1ST ST. & ADAMS - SOUTH RANGE VILLAGE \\
\hline 248 & & ZANA & MATIA T. & $\mathrm{D}$ & 3 & MICHIGAN & ITALY & ITALY & & & CUCEGLIO. TO & 1ST ST. & ADAMS - SOUTH RANGE VILLAGE \\
\hline 249 & & ZANA & ELENA & $\mathrm{D}$ & 1 & MICHIGAN & ITALY & ITALY & & & CUCEGLIO. TO & 1ST ST. & ADAMS - SOUTH RANGE VILLAGE \\
\hline 250 & & ZANA & GIUSEPPE & s & 0 & MICHIGAN & ITALY & ITALY & & & CUCEGLIO. TO & 1ST ST. & ADAMS - SOUTH RANGE VILLAGE \\
\hline 251 & & KNUKTLEIN & ENRICHETTA & SIIL & 32 & ITALY & ITALY & ITALY & 1907 & & CUCEGLIO. TO & 1ST ST. & ADAMS - SOUTH RANGE VILLAGE \\
\hline
\end{tabular}




\begin{tabular}{|c|c|c|c|c|c|c|c|c|c|c|c|c|c|}
\hline 252 & & RAFFAELLI & RAFFAELE & $\mathrm{H}$ & 32 & ITALY & ITALY & ITALY & 1898 & DRIVER/BREWING COMPANY & LUCCA & 2ND ST. & ADAMS - SOUTH RANGE VILLAGE \\
\hline 253 & & RAFFAELLI & CATERINA & W & 32 & ITALY & ITALY & ITALY & 1898 & & LUCCA & 2ND ST. & ADAMS - SOUTH RANGE VILLAGE \\
\hline 254 & & RAFFAELLI & AMELIA & $\mathrm{D}$ & 8 & MICHIGAN & ITALY & ITALY & & & LUCCA & 2ND ST. & ADAMS - SOUTH RANGE VILLAGE \\
\hline 255 & & RAFFAELLI & MARIO & $\mathrm{s}$ & 7 & MICHIGAN & ITALY & ITALY & & & LUCCA & 2ND ST. & ADAMS - SOUTH RANGE VILLAGE \\
\hline 256 & & RAFFAELLI & FERRUCCIO & $\mathrm{s}$ & 5 & MICHIGAN & ITALY & ITALY & & & LUCCA & 2ND ST. & ADAMS - SOUTH RANGE VILLAGE \\
\hline 257 & & RAFFAELLI & LENA & $\mathrm{D}$ & 3 & MICHIGAN & ITALY & ITALY & & & LUCCA & 2ND ST. & ADAMS - SOUTH RANGE VILLAGE \\
\hline 258 & 142 & OZZELLO & JOHN & $\mathrm{H}$ & 42 & ITALY & ITALY & ITALY & 1906 & TRAMMER/CM & SAN GIUSTO CANAVESE. TO & 3RD ST. & ADAMS - SOUTH RANGE VILLAGE \\
\hline 259 & 142 & OZZELLO & MARGHERITA & w & 34 & ITALY & ITALY & ITALY & 1907 & & SAN GIUSTO CANAVESE. TO & 3RD ST. & ADAMS - SOUTH RANGE VILLAGE \\
\hline 260 & 142 & OZZELLO & FRANCESCA & $\mathrm{D}$ & 12 & ITALY & ITALY & ITALY & 1907 & & SAN GIUSTO CANAVESE. TO & 3RD ST. & ADAMS - SOUTH RANGE VILLAGE \\
\hline 261 & 142 & OZZELLO & GIUSEPPE & $\mathrm{s}$ & 9 & ITALY & ITALY & ITALY & 1907 & & SAN GIUSTO CANAVESE. TO & 3RD ST. & ADAMS - SOUTH RANGE VILLAGE \\
\hline 262 & 142 & OZZELLO & PIETRO & $\mathrm{s}$ & 1 & MICHIGAN & ITALY & ITALY & & & SAN GIUSTO CANAVESE. TO & 3RD ST. & ADAMS - SOUTH RANGE VILLAGE \\
\hline 263 & 142 & PLASSA & PIETRO & B & 24 & ITALY & ITALY & ITALY & 1905 & TRAMMER/CM & TORINO & 3RD ST. & ADAMS - SOUTH RANGE VILLAGE \\
\hline 264 & 142 & GIACOLETTO & VINCENZO & B & 23 & ITALY & ITALY & ITALY & 1908 & TRAMMER/CM & TORINO & 3RD ST. & ADAMS - SOUTH RANGE VILLAGE \\
\hline 265 & 142 & DEDARIO & PIO & B & 23 & ITALY & ITALY & ITALY & 1906 & BAKER & LOCANA CANAVESE. TO & 3RD ST. & ADAMS - SOUTH RANGE VILLAGE \\
\hline 266 & 142 & GIACOLETTO & VITTORIO & $\mathrm{H}$ & 31 & ITALY & ITALY & ITALY & 1903 & TIMBERMAN/CM & TORINO & 3RD ST. & ADAMS - SOUTH RANGE VILLAGE \\
\hline 267 & 142 & GIACOLETTO & CATERINA & w & 32 & ITALY & ITALY & ITALY & 1903 & & TORINO & 3RD ST. & ADAMS - SOUTH RANGE VILLAGE \\
\hline 268 & 142 & GIACOLETTO & GIUSEPPE & $\mathrm{s}$ & 6 & MICHIGAN & ITALY & ITALY & & & TORINO & 3RD ST. & ADAMS - SOUTH RANGE VILLAGE \\
\hline 269 & 142 & GIACOLETTO & LUCILLA & D & 3 & MICHIGAN & ITALY & ITALY & & & TORINO & 3RD ST. & ADAMS - SOUTH RANGE VILLAGE \\
\hline 270 & 142 & GIACOLETTO & LUISA & $\mathrm{D}$ & $8 \mathrm{M}$ & MICHIGAN & ITALY & ITALY & & & TORINO & 3RD ST. & ADAMS - SOUTH RANGE VILLAGE \\
\hline 271 & 142 & CORGIATTI & BERNARDO & $\mathrm{H}$ & 25 & ITALY & ITALY & ITALY & 1903 & BAKER & PONT CANAVESE. TO & 3RD ST. & ADAMS - SOUTH RANGE VILLAGE \\
\hline 272 & 142 & CORGIATTI & ANNIE & w & 24 & ITALY & ITALY & ITALY & 1903 & & PONT CANAVESE. TO & 3RD ST. & ADAMS - SOUTH RANGE VILLAGE \\
\hline 273 & 142 & CORGIATTI & MARY M. & $\mathrm{D}$ & 2 & MICHIGAN & ITALY & ITALY & & & PONT CANAVESE, TO & 3RD ST. & ADAMS - SOUTH RANGE VILLAGE \\
\hline 274 & $\frac{142}{142}$ & CORGIATTI & LUISA & $\mathrm{D}$ & $7 \mathrm{M}$ & MICHIGAN & ITALY & ITALY & & & PONT CANAVESE. TO & 3RD ST. & ADAMS - SOUTH RANGE VILLAGE \\
\hline 275 & 147 & MANTINO & DOMENICO & $\mathrm{H}$ & 36 & ITALY & ITALY & ITALY & 1900 & TRAMMER/CM & PEROSA CANAVESE. TO & 2ND ST. & ADAMS - SOUTH RANGE VILLAGE \\
\hline 276 & 147 & MANTINO & MARY & w & 36 & ITALY & ITALY & ITALY & 1901 & & & 2ND ST. & ADAMS - SOUTH RANGE VILLAGE \\
\hline 277 & 147 & MANTINO & ALBERTO & s & 4 & MICHIGAN & ITALY & ITALY & & & PEROSA CANAVESE. TO & 2ND ST. & ADAMS - SOUTH RANGE VILLAGE \\
\hline 278 & 147 & MANTINO & CATERINA & D & 13 & MICHIGAN & ITALY & ITALY & & & PEROSA CANAVESE. TO & 2ND ST. & ADAMS - SOUTH RANGE VILLAGE \\
\hline 279 & 147 & MANTINO & LUIGI & $\mathrm{s}$ & 7 & MICHIGAN & ITALY & ITALY & & & PEROSA CANAVESE, TO & 2ND ST. & ADAMS - SOUTH RANGE VILLAGE \\
\hline 280 & & ZANOTTI & ANTONIO & $\mathrm{H}$ & 42 & ITALY & ITALY & ITALY & 1888 & ROCK HOUSE MAN & TORINO & 3RD ST. & ADAMS - SOUTH RANGE VILLAGE \\
\hline 281 & & ZANOTTI & ANNA & W & 42 & ITALY & ITALY & ITALY & 1897 & NOT CLEAR & TORINO & 3RD ST. & ADAMS - SOUTH RANGE VILLAGE \\
\hline 282 & & ZANOTTI & DOMENICO & $\mathrm{s}$ & 17 & ITALY & ITALY & ITALY & 1897 & WIPER/ENGINE & TORINO & 3RD ST. & ADAMS - SOUTH RANGE VILLAGE \\
\hline 283 & & ZANOTTI & PIETRO & $\mathrm{s}$ & 16 & ITALY & ITALY & ITALY & 1897 & WIPER/ENGINE & TORINO & 3RD ST. & ADAMS - SOUTH RANGE VILLAGE \\
\hline 284 & & ZANOTTI & CATERINA & $\mathrm{D}$ & 15 & ITALY & ITALY & ITALY & 1897 & & TORINO & 3RD ST. & ADAMS - SOUTH RANGE VILLAGE \\
\hline 285 & & ZANOTTI & DOMENICA & $\mathrm{D}$ & 12 & MICHIGAN & ITALY & ITALY & & & TORINO & 3RD ST. & ADAMS - SOUTH RANGE VILLAGE \\
\hline 286 & & ZANOTTI & ELLA & $\mathrm{D}$ & 8 & MICHIGAN & ITALY & ITALY & & & TORINO & 3RD ST. & ADAMS - SOUTH RANGE VILLAGE \\
\hline 287 & & ZANOTTI & MARGHERITA & $\mathrm{D}$ & $\frac{0}{6}$ & MICHIGAN & ITALY & ITALY & & & TORINO & 3RD ST. & ADAMS - SOUTH RANGE VILLAGE \\
\hline 288 & & ZANOTTI & GIOVANNI & $\mathrm{s}$ & 4 & MICHIGAN & ITALY & ITALY & & & TORINO & 3RD ST. & ADAMS - SOUTH RANGE VILLAGE \\
\hline 289 & & BLESSENT & MARTINO & B & 41 & ITALY & ITALY & ITALY & 1901 & MINER/MACHINE & SPARONE. TO & 3RD ST. & ADAMS - SOUTH RANGE VILLAGE \\
\hline 290 & & BERTOLOZZI & ATTILIO & $\mathrm{H}$ & 48 & ITALY & ITALY & ITALY & 1898 & DRIVER/BAKERY WAGON & VECOLI. LU & BALTIC AVE. & ADAMS - SOUTH RANGE VILLAGE \\
\hline 291 & & BERTOLOZZI & SITA & W & 39 & ITALY & ITALY & ITALY & 1899 & & VECOLI. LU & BALTIC AVE. & ADAMS - SOUTH RANGE VILLAGE \\
\hline 292 & & BERTOLOZZI & JOHN & $\mathrm{s}$ & 15 & FRANCE & ITALY & ITALY & ???? & & VECOLI. LU & BALTIC AVE. & ADAMS - SOUTH RANGE VILLAGE \\
\hline 293 & & BERTOLOZZI & PAUL & $\mathrm{s}$ & 8 & MICHIGAN & ITALY & ITALY & & & VECOLI. LU & BALTIC AVE. & ADAMS - SOUTH RANGE VILLAGE \\
\hline 294 & & BERTOLOZZI & PETER & $\mathrm{s}$ & 5 & MICHIGAN & ITALY & ITALY & & & VECOLI. LU & BALTIC AVE. & ADAMS - SOUTH RANGE VILLAGE \\
\hline 295 & & BERTOLOZZI & MARY N. & $\mathrm{D}$ & 4 & MICHIGAN & ITALY & ITALY & & & VECOLI. LU & BALTIC AVE. & ADAMS - SOUTH RANGE VILLAGE \\
\hline 296 & & BERTOLOZZI & JENNIE & $\mathrm{D}$ & 3 & MICHIGAN & ITALY & ITALY & & & VECOLI, LU & BALTIC AVE. & ADAMS - SOUTH RANGE VILLAGE \\
\hline 297 & & BERTOLOZZI & ATTILIA & $\mathrm{D}$ & $3 \mathrm{M}$ & MICHIGAN & ITALY & ITALY & & & VECOLI. LU & BALTIC AVE. & ADAMS - SOUTH RANGE VILLAGE \\
\hline 298 & & PASTORE & JOSEPH & $\mathrm{H}$ & 28 & MICHIGAN & ITALY & ITALY & & DRIVER/GROCERY WAGON & TORINO & 2ND ST. & ADAMS - SOUTH RANGE VILLAGE \\
\hline 299 & & PASTORE & MARY & W & 25 & ITALY & ITALY & ITALY & 1889 & DRTVERTORUCERT VUAOUIV & TORIIV & 2ND ST. & ADAMS - SOUTH RANGE VILLAGE \\
\hline 300 & & PASTORE & TERESA & $\mathrm{D}$ & 3 & MICHIGAN & ITALY & ITALY & & & TORINO & 2ND ST. & ADAMS - SOUTH RANGE VILLAGE \\
\hline 301 & & PASTORE & MARY & $\mathrm{sI}$ & 22 & MICHIGAN & ITALY & ITALY & & & TORINO & 2ND ST. & ADAMS - SOUTH RANGE VILLAGE \\
\hline 302 & & PASTORE & MARY & NIE & 3 & MICHIGAN & ITALY & ITAL & & & TORINO & 2ND ST. & ADAMS - SOUTH RANGE VILLAGE \\
\hline 303 & & PASTORE & ANTON & NEP & 1 & MICHIGAN & ITALY & ITALY & & & TORINO & 2ND ST. & ADAMS - SOUTH RANGE VILLAGE \\
\hline 304 & & SANTINO & MIKE & B & 29 & ITALY & ITALY & ITALY & 1900 & TRAMMER/CM & & 3RD ST. & ADAMS - SOUTH RANGE VILLAGE \\
\hline 305 & & AIMONETTO & JIMMIE & B & 38 & ITALY & ITALY & ITALY & 1900 & TRAMMER/CM & SPARONE. TO & 3RD ST. & ADAMS - SOUTH RANGE VILLAGE \\
\hline 306 & & PASTORE & GEORGE & $\mathrm{H}$ & 62 & ITALY & ITALY & ITALY & 1871 & SALOONKEEPER & TORINO & 1ST ST. & ADAMS - SOUTH RANGE VILLAGE \\
\hline 307 & & PASTORE & MARY & W & 56 & ITALY & ITALY & ITALY & 1880 & & & 1ST ST. & ADAMS - SOUTH RANGE VILLAGE \\
\hline 308 & & PASTORE & GEORGE & s & 24 & MICHIGAN & ITALY & ITALY & & DRIVER/GROCERY WAGON & TORINO & 1ST ST. & ADAMS - SOUTH RANGE VILLAGE \\
\hline 309 & & PASTORE & KATE & $\mathrm{D}$ & 21 & MICHIGAN & ITALY & ITALY & & & TORINO & 1ST ST. & ADAMS - SOUTH RANGE VILLAGE \\
\hline 310 & & PASTORE & JOHNNIE & $\mathrm{s}$ & 16 & MICHIGAN & ITALY & ITALY & & & TORINO & 1ST ST. & ADAMS - SOUTH RANGE VILLAGE \\
\hline 311 & & PASTORE & PAUL & $\mathrm{s}$ & 15 & MICHIGAN & ITALY & ITALY & & & TORINO & 1ST ST. & ADAMS - SOUTH RANGE VILLAGE \\
\hline 312 & & VILLA & MAGGIE & $\mathrm{D}$ & 22 & MICHIGAN & ITALY & ITALY & & SERVANT/PF & TORINO & 1ST ST. & ADAMS - SOUTH RANGE VILLAGE \\
\hline 313 & & VOLPI & JOHN & $\mathrm{H}$ & 42 & ITALY & ITALY & ITALY & 1891 & MERCHANT/GENERAL STORE & AGLIE'. TO & 1ST ST. & ADAMS - SOUTH RANGE VILLAGE \\
\hline 314 & & VOLPI & KATE & w & 38 & $\begin{array}{l}\text { ITALY } \\
\text { ITALY }\end{array}$ & ITALY & ITALY & 1892 & & & 1ST ST. & ADAMS - SOUTH RANGE VILLAGE \\
\hline
\end{tabular}




\begin{tabular}{|c|c|c|c|c|c|c|c|c|c|c|c|c|c|}
\hline 315 & & VOLPI & JOHNNIE & $\mathrm{s}$ & 14 & MICHIGAN & ITALY & ITALY & & & AGLIE'. TO & 1ST ST. & ADAMS - SOUTH RANGE VILLAGE \\
\hline 316 & & VOLPI & FREDDIE & $\mathrm{s}$ & 12 & MICHIGAN & ITALY & ITALY & & & AGLIE', TO & 1ST ST. & ADAMS - SOUTH RANGE VILLAGE \\
\hline 317 & & VOLPI & KATE & $\mathrm{D}$ & 11 & MICHIGAN & ITALY & ITALY & & & AGLIE'. TO & 1ST ST. & ADAMS - SOUTH RANGE VILLAGE \\
\hline 318 & & VOLPI & JOSEPHINE & $\mathrm{D}$ & 9 & MICHIGAN & ITALY & ITALY & & & AGLIE'. TO & 1ST ST. & ADAMS - SOUTH RANGE VILLAGE \\
\hline 319 & & VOLPI & JOE & $\mathrm{s}$ & 7 & MICHIGAN & ITALY & ITALY & & & AGLIE', TO & 1ST ST. & ADAMS - SOUTH RANGE VILLAGE \\
\hline 320 & & VOLPI & PETER & $\mathrm{s}$ & 6 & MICHIGAN & ITALY & ITALY & & & AGLIE', TO & $1 \mathrm{ST}$ ST. & ADAMS - SOUTH RANGE VILLAGE \\
\hline 321 & & VOLPI & LIDIA & D & 4 & MICHIGAN & ITALY & ITALY & & & AGLIE', TO & 1ST ST. & ADAMS - SOUTH RANGE VILLAGE \\
\hline 322 & & VOLPI & DOMINIC & s & 2 & MICHIGAN & ITALY & ITALY & & & AGLIE', TO & 1ST ST. & ADAMS - SOUTH RANGE VILLAGE \\
\hline 323 & 109 & ALLEN/ELLENA & AUGUST & $\mathrm{H}$ & 40 & ITALY & ITALY & ITALY & 1891 & SWAMPER/CM & TORINO & 3RD ST. & ADAMS - SOUTH RANGE VILLAGE \\
\hline 324 & 109 & ALLEN/ELLENA & TRACE & w & 38 & ITALY & ITALY & ITALY & 1893 & & TORINO & 3RD ST. & ADAMS - SOUTH RANGE VILLAGE \\
\hline 325 & 109 & ALLEN/ELLENA & PETER & $\mathrm{s}$ & 20 & ITALY & ITALY & ITALY & 1893 & MASON/HOUSE & TORINO & 3RD ST. & ADAMS - SOUTH RANGE VILLAGE \\
\hline 326 & 109 & ALLEN/ELLENA & TRACE & $\mathrm{D}$ & 19 & ITALY & ITALY & ITALY & 1893 & & TORINO & 3RD ST. & ADAMS - SOUTH RANGE VILLAGE \\
\hline 327 & 109 & ALLEN/ELLENA & JOHNNIE & $\mathrm{s}$ & 15 & MICHIGAN & ITALY & ITALY & & & TORINO & 3RD ST. & ADAMS - SOUTH RANGE VILLAGE \\
\hline 328 & 109 & ALLEN/ELLENA & MATTIE & $\mathrm{s}$ & 14 & MICHIGAN & ITALY & ITALY & & & TORINO & 3RD ST. & ADAMS - SOUTH RANGE VILLAGE \\
\hline 329 & 109 & ALLEN/ELLENA & TONY & $\mathrm{s}$ & 13 & MICHIGAN & ITALY & ITALY & & & TORINO & 3RD ST. & ADAMS - SOUTH RANGE VILLAGE \\
\hline 330 & 109 & ALLEN/ELLENA & AUGUST & $\mathrm{s}$ & 13 & MICHIGAN & ITALY & ITALY & & & TORINO & 3RD ST. & ADAMS - SOUTH RANGE VILLAGE \\
\hline 331 & 109 & ALLEN/ELLENA & MAGGIE & D & 12 & MICHIGAN & ITALY & ITALY & & & TORINO & 3RD ST. & ADAMS - SOUTH RANGE VILLAGE \\
\hline 332 & 109 & ALLEN/ELLENA & MARY & $\mathrm{D}$ & 11 & MICHIGAN & ITALY & ITALY & & & TORINO & 3RD ST. & ADAMS - SOUTH RANGE VILLAGE \\
\hline 333 & & ANTONELLI & RALPH & $\mathrm{H}$ & 22 & MICHIGAN & ITALY & ITALY & & DRIVER/WAGON & & 3RD ST. & ADAMS - SOUTH RANGE VILLAGE \\
\hline 334 & & ANTONELLI & ELISABETH & w & 19 & MICHIGAN & ITALY & ITALY & & DAIVLTRVATEOTV & & 3RD ST. & ADAMS - SOUTH RANGE VILLAGE \\
\hline $\begin{array}{l}534 \\
335\end{array}$ & & GRAZIANO & JOHN & B & 36 & ITALY & ITALY & ITALY & 1890 & PICKER/CM & & 1ST ST. & ADAMS - SOUTH RANGE VILLAGE \\
\hline 336 & & EAROLL & JOHN & $\begin{array}{l}D \\
B\end{array}$ & 45 & ITALY & ITALY & ITALY & 1903 & PICKER/CM & & 1ST ST. & ADAMS - SOUTH RANGE VILLAGE \\
\hline 337 & & GALETTO & MIKE & $\mathrm{H}$ & 34 & ITALY & ITALY & ITALY & 1898 & DRAYMAN/VILLAGE & SAN GIORGIO CANAVESE. TO & 1ST ST. & ADAMS - SOUTH RANGE VILLAGE \\
\hline 338 & & GALETTO & PESSILINA & w & 33 & ITALY & ITALY & ITALY & 1899 & & & 1ST ST. & ADAMS - SOUTH RANGE VILLAGE \\
\hline 339 & & GALETTO & KATE & $\mathrm{D}$ & 10 & MICHIGAN & ITALY & ITALY & & & SAN GIORGIO CANAVESE. TO & 1ST ST. & ADAMS - SOUTH RANGE VILLAGE \\
\hline 340 & & GALETTO & GENZIANA & $\mathrm{D}$ & 9 & MICHIGAN & ITALY & ITALY & & & SAN GIORGIO CANAVESE. TO & 1ST ST. & ADAMS - SOUTH RANGE VILLAGE \\
\hline 341 & & GALETTO & VINCENZINA & $\mathrm{D}$ & 7 & MICHIGAN & ITALY & ITALY & & & SAN GIORGIO CANAVESE. TO & 1ST ST. & ADAMS - SOUTH RANGE VILLAGE \\
\hline 342 & & GALETTO & JOSEPH & $\mathrm{s}$ & 3 & MICHIGAN & ITALY & ITALY & & & SAN GIORGIO CANAVESE, TO & 1ST ST. & ADAMS - SOUTH RANGE VILLAGE \\
\hline 343 & 229 & RICCA & MARY & $\mathrm{H}$ & 37 & ITALY & ITALY & ITALY & 1888 & & TORINO & 1ST ST. & ADAMS - SOUTH RANGE VILLAGE \\
\hline 344 & 229 & RICCA & PAULINE & D & 19 & MICHIGAN & ITALY & ITALY & & CLERK/GENERAL STORE & TORINO & 1ST ST./2ND & ADAMS - SOUTH RANGE VILLAGE \\
\hline 345 & 229 & RICCA & JOHN & $\mathrm{s}$ & 13 & MICHIGAN & ITALY & ITALY & & & TORINO & 1ST ST./2ND & ADAMS - SOUTH RANGE VILLAGE \\
\hline 346 & 229 & BOGGIO & ANTONIO & B & 25 & ITALY & ITALY & ITALY & 1907 & TRAMMER/CM & SAN GIUSTO CANAVESE. TO & 1ST ST./2ND & ADAMS - SOUTH RANGE VILLAGE \\
\hline 347 & 229 & BOGGIO & DOMENICO & B & 22 & ITALY & ITALY & ITALY & 1903 & TIMBERMAN/CM & SAN GIUSTO CANAVESE. TO & 1ST ST./2ND & ADAMS - SOUTH RANGE VILLAGE \\
\hline 348 & 229 & BOGGIO & GIOVANNI & B & 43 & ITALY & ITALY & ITALY & 1909 & TRAMMER/CM & SAN GIUSTO CANAVESE, TO & 1ST ST./2ND & ADAMS - SOUTH RANGE VILLAGE \\
\hline 349 & 229 & PETRINI & CARLO & $\mathrm{B}$ & 23 & ITALY & ITALY & ITALY & 1909 & TRAMMER/CM & SAN GIUSTO CANAVESE. TO & 1ST ST./2ND & ADAMS - SOUTH RANGE VILLAGE \\
\hline 350 & 229 & PEDALINO & LUIGI & B & 24 & ITALY & ITALY & ITALY & 1910 & TRAMMER/CM & SAN GIUSTO CANAVESE. TO & 1ST ST./2ND & ADAMS - SOUTH RANGE VILLAGE \\
\hline 351 & 229 & TINETTI & GIACOMO & B & 26 & ITALY & ITALY & ITALY & 1904 & DRIVER/WAGON & CASTELLAMONTE. TO & 1ST ST./2ND & ADAMS - SOUTH RANGE VILLAGE \\
\hline 352 & 229 & IAVELLO & CARLO & B & 28 & ITALY & ITALY & ITALY & 1907 & TRAMMER/CM & SAN GIUSTO CANAVESE. TO & 1ST ST./2ND & ADAMS - SOUTH RANGE VILLAGE \\
\hline 353 & 229 & CAPARELLO & DOMENICO & B & 29 & ITALY & ITALY & ITALY & 1907 & TRAMMER/CM & TORINO & 1ST ST./2ND & ADAMS - SOUTH RANGE VILLAGE \\
\hline 354 & 229 & CERINI & GIOVANNI & B & 40 & ITALY & ITALY & ITALY & 1899 & MINER/MACHINE & SAN GIORGIO CANAVESE. TO & 1ST ST./2ND & ADAMS - SOUTH RANGE VILLAGE \\
\hline 355 & 229 & FAITTO & JIMMIE & B & 34 & ITALY & ITALY & ITALY & 1902 & MINER/MACHINE & & 1ST ST./2ND & ADAMS - SOUTH RANGE VILLAGE \\
\hline 356 & 304 & GIACOLETTO & SAVINO & $\mathrm{H}$ & 39 & ITALY & ITALY & ITALY & 1899 & MINER/CM & PONT CANAVESE. TO & 5TH ST. & ADAMS - TRIMOUNTAIN LOCATION \\
\hline 357 & 304 & GIACOLETTO & SERAFINA & w & 37 & ITALY & ITALY & ITALY & 1899 & & PONT CANAVESE. TO & 5TH ST. & ADAMS - TRIMOUNTAIN LOCATION \\
\hline 358 & 304 & GIACOLETTO & LENA & $\mathrm{D}$ & 12 & ITALY & ITALY & ITALY & 1899 & & PONT CANAVESE. TO & 5TH ST. & ADAMS - TRIMOUNTAIN LOCATION \\
\hline 359 & 304 & GIACOLETTO & MARIA & $\mathrm{D}$ & 10 & MICHIGAN & ITALY & ITALY & & & PONT CANAVESE. TO & 5TH ST. & ADAMS - TRIMOUNTAIN LOCATION \\
\hline 360 & 304 & GIACOLETTO & PAOLO & $\mathrm{s}$ & $1.8 \mathrm{M}$ & MICHIGAN & ITALY & ITALY & & & PONT CANAVESE. TO & 5TH ST. & ADAMS - TRIMOUNTAIN LOCATION \\
\hline 361 & 303 & CANTELLO & JOHN B. & $\mathrm{H}$ & 40 & ITALY & ITALY & ITALY & $89 / 99$ & MINER/CM & SAN GIORGIO CANAVESE. TO & 6TH ST. & ADAMS - TRIMOUNTAIN LOCATION \\
\hline 362 & 303 & CANTELLO & ELISABETH & W & 26 & ITALY & ITALY & ITALY & 1902 & & torino & 6TH ST. & ADAMS - TRIMOUNTAIN LOCATION \\
\hline 363 & 303 & CANTELLO & MARY & $\mathrm{D}$ & 2 & MICHIGAN & ITALY & ITALY & & & SAN GIORGIO CANAVESE. TO & 6TH ST. & ADAMS - TRIMOUNTAIN LOCATION \\
\hline 364 & 302 & MORELLI & JOSEPHAT & $\mathrm{H}$ & 22 & ITALY & ITALY & ITALY & 1905 & MINER/CM & MONTEMAGNO. LU & 6TH ST. & ADAMS - TRIMOUNTAIN LOCATION \\
\hline 365 & 302 & MORELLI & CAROLINE & W & 27 & ITALY & ITALY & ITALY & 1908 & & & 6TH ST. & ADAMS - TRIMOUNTAIN LOCATION \\
\hline 366 & 302 & MORELLI & ARMANDO & $\mathrm{s}$ & $1,3 \mathrm{M}$ & MICHIGAN & ITALY & ITALY & & & MONTEMAGNO, LU & 6TH ST. & ADAMS - TRIMOUNTAIN LOCATION \\
\hline 367 & 302 & MORELLI & BATTISTA & BR & 24 & ITALY & ITALY & ITALY & 1909 & TRAMMER/CM & MONTEMAGNO. LU & 6TH ST. & ADAMS - TRIMOUNTAIN LOCATION \\
\hline 368 & 302 & MEI & CARLO & $\mathrm{COU}$ & 31 & ITALY & ITALY & ITALY & 1905 & TRAMMER/CM & RUOTA. LU & 6TH ST. & ADAMS - TRIMOUNTAIN LOCATION \\
\hline 369 & 317 & MONTICELLI & GIACOMO & $\mathrm{H}$ & 34 & ITALY & ITALY & ITALY & 1892 & MINER/CM & SELINO. BERGAMO & 6TH ST. & ADAMS - TRIMOUNTAIN LOCATION \\
\hline 370 & 317 & MONTICELLI & CAROLINA & w & 33 & ITALY & ITALY & ITALY & 1903 & & SELINO. BERGAMO & 6TH ST. & ADAMS - TRIMOUNTAIN LOCATION \\
\hline 371 & 317 & MONTICELLI & AMEDEO & s & 9 & ITALY & ITALY & ITALY & 1903 & & SELINO. BERGAMO & 6TH ST. & ADAMS - TRIMOUNTAIN LOCATION \\
\hline 372 & 317 & MONTICELLI & EVA & $\mathrm{D}$ & 8 & ITALY & ITALY & ITALY & 1903 & & SELINO, BERGAMO & 6TH ST. & ADAMS - TRIMOUNTAIN LOCATION \\
\hline 373 & 317 & MONTICELLI & ELSA & $\mathrm{D}$ & 6 & MICHIGAN & ITALY & ITALY & & & SELINO. BERGAMO & 6TH ST. & ADAMS - TRIMOUNTAIN LOCATION \\
\hline 374 & 317 & MONTICELLI & ADELINA & $\mathrm{D}$ & 4 & MICHIGAN & ITALY & ITAL & & & SELINO. BERGAMO & 6TH ST. & ADAMS - TRIMOUNTAIN LOCATION \\
\hline 375 & 317 & MONTICELLI & IDA & $\mathrm{D}$ & 2 & MICHIGAN & ITA & ITA & & & SELINO. BERGAMO & 6TH ST. & ADAMS - TRIMOUNTAIN LOCATION \\
\hline 376 & 213 & SANTONI & FRANK & $\mathrm{H}$ & 39 & ITALY & ITALY & ITALY & 1902 & LABORER/ROCK HOUSE & MONTE DI CHIESA. LU & & ADAMS - T \\
\hline 377 & 213 & SANTONI & ANNUNZIATA & W & 35 & ITALY & ITALY & ITALY & 1907 & & & 6TH ST. & ADAMS - TRIMOUNTAIN LOCATION \\
\hline
\end{tabular}




\begin{tabular}{|c|c|c|c|c|c|c|c|c|c|c|c|c|c|}
\hline 378 & 213 & SANTONI & ETA & $\mathrm{D}$ & 13 & MICHIGAN & ITALY & ITALY & & & MONTE DI CHIESA. LU & 6TH ST. & ADAMS - TRIMOUNTAIN LOCATION \\
\hline 379 & 213 & SANTONI & NANDO & $\mathrm{s}$ & 11 & MICHIGAN & ITALY & ITALY & & & MONTE DI CHIESA. LU & 6TH ST. & ADAMS - TRIMOUNTAIN LOCATION \\
\hline 380 & 213 & SANTONI & GIUSTO & $\mathrm{s}$ & 9 & MICHIGAN & ITALY & ITALY & & & MONTE DI CHIESA. LU & 6TH ST. & ADAMS - TRIMOUNTAIN LOCATION \\
\hline 381 & 213 & SANTONI & JAMES & $\mathrm{s}$ & 7 & MICHIGAN & ITALY & ITALY & & & MONTE DI CHIESA. LU & 6TH ST. & ADAMS - TRIMOUNTAIN LOCATION \\
\hline 382 & 213 & SANTONI & FRANK & $\mathrm{s}$ & 3 & MICHIGAN & ITALY & ITALY & & & MONTE DI CHIESA. LU & 6TH ST. & ADAMS - TRIMOUNTAIN LOCATION \\
\hline 383 & 213 & SANTONI & JULIA & D & 2 & MICHIGAN & ITALY & ITALY & & & MONTE DI CHIESA. LU & 6TH ST. & ADAMS - TRIMOUNTAIN LOCATION \\
\hline 384 & 213 & SANTONI & VIOLA & D & $8 \mathrm{M}$ & MICHIGAN & ITALY & ITALY & & & MONTE DI CHIESA. LU & 6TH ST. & ADAMS - TRIMOUNTAIN LOCATION \\
\hline 385 & 213 & GUTTIDO & PETER & B & 20 & ITALY & ITALY & ITALY & 1906 & MINER/CM & LUCCA & 6TH ST. & ADAMS - TRIMOUNTAIN LOCATION \\
\hline 386 & 213 & DEL FRATE & LUIS & B & 18 & ITALY & ITALY & ITALY & 1907 & MINER/CM & LUCCA & 6TH ST. & ADAMS - TRIMOUNTAIN LOCATION \\
\hline 387 & 213 & DI VECCHIO & DIONIGIO & B & 19 & ITALY & ITALY & ITALY & 1907 & TRAMMER/CM & SAN GINESE. LU & 6TH ST. & ADAMS - TRIMOUNTAIN LOCATION \\
\hline 388 & 209 & GIANNECHINI & EUGENIO & $\mathrm{H}$ & 39 & ITALY & ITALY & ITALY & 1902 & MINER/CM & SANTA MARIA AL PIANO. LU & 6TH ST. & ADAMS - TRIMOUNTAIN LOCATION \\
\hline 389 & 209 & GIANNECHINI & TERESA & W & 23 & ITALY & ITALY & ITALY & 1907 & & & 6TH ST. & ADAMS - TRIMOUNTAIN LOCATION \\
\hline 390 & 209 & GIANNECHINI & ALBERT & $\mathrm{s}$ & $1.3 \mathrm{M}$ & MICHIGAN & ITALY & ITALY & & & SANTA MARIA AL PIANO. LU & 6TH ST. & ADAMS - TRIMOUNTAIN LOCATION \\
\hline 391 & 209 & IACOPETTI & ADOLFO & B & 25 & ITALY & ITALY & ITALY & 1905 & TRAMMER/CM & CASTELVECCHIO. LU & 6TH ST. & ADAMS - TRIMOUNTAIN LOCATION \\
\hline 392 & 209 & BERTOLUCCI & AMERIGO & B & 26 & ITALY & ITALY & ITALY & 1908 & MINER/CM & COLLE DI COMPITO. LU & 6TH ST. & ADAMS - TRIMOUNTAIN LOCATION \\
\hline 393 & 202 & TAMBELLINI & WILLIAM & $\mathrm{H}$ & 23 & ITALY & ITALY & ITALY & 1903 & MACHINIST/CM & LUCCA & 6TH ST. & ADAMS - TRIMOUNTAIN LOCATION \\
\hline 394 & 202 & TAMBELLINI & EVA & W & 29 & ITALY & ITALY & ITALY & 1904 & & & 6TH ST. & ADAMS - TRIMOUNTAIN LOCATION \\
\hline 395 & 202 & TAMBELLINI & ELENA & D & 2 & MICHIGAN & ITALY & ITALY & & & LUCCA & 6TH ST. & ADAMS - TRIMOUNTAIN LOCATION \\
\hline 396 & 215 & $\begin{array}{l}\text { PARENTI } \\
\text { PAT }\end{array}$ & VINCENZO & $\mathrm{H}$ & 43 & ITALY & ITALY & ITALY & 1894 & LABORER/CM & CASTELVECCHIO. LU & 7TH ST. & ADAMS - TRIMOUNTAIN LOCATION \\
\hline 397 & 215 & PARENTI & ASSUNTA & W & 41 & ITALY & ITALY & ITALY & 1903 & & CASTELVECCHIO. LU & 7TH ST. & ADAMS - TRIMOUNTAIN LOCATION \\
\hline 398 & 215 & PARENTI & ENRICHETTA & $\mathrm{D}$ & 18 & ITALY & ITALY & ITALY & 1910 & & CASTELVECCHIO. LU & 7TH ST. & ADAMS - TRIMOUNTAIN LOCATION \\
\hline 399 & 215 & PARENTI & MARTA & $\mathrm{D}$ & 16 & ITALY & ITALY & ITALY & 1910 & & CASTELVECCHIO, LU & 7TH ST. & ADAMS - TRIMOUNTAIN LOCATION \\
\hline 400 & 215 & PARENTI & BRUNO & $\mathrm{s}$ & 9 & MICHIGAN & ITALY & ITALY & & & CASTELVECCHIO. LU & 7TH ST. & ADAMS - TRIMOUNTAIN LOCATION \\
\hline 401 & 215 & MAFFEI & ARTURO & B & 27 & ITALY & ITALY & ITALY & 1907 & TRAMMER/CM & LUCCA & 7TH ST. & ADAMS - TRIMOUNTAIN LOCATION \\
\hline 402 & 215 & LOMBARDI & VINCENZO & B & 22 & ITALY & ITALY & ITALY & 1909 & TRAMMER/CM & SAN GINESE. LU & 7TH ST. & ADAMS - TRIMOUNTAIN LOCATION \\
\hline 403 & 215 & PASSI/BUSSI & IACOPO & B & 42 & ITALY & ITALY & ITALY & 1907 & TRAMMER/CM & & 7TH ST. & ADAMS - TRIMOUNTAIN LOCATION \\
\hline 404 & 215 & CAULLA & GIACINTO & B & 24 & ITALY & ITALY & ITALY & $09 / 07$ & TRAMMER/CM & UCRIA. MESSINA & 7TH ST. & ADAMS - TRIMOUNTAIN LOCATION \\
\hline 405 & 215 & CAULLA & PIETRO & B & 21 & ITALY & ITALY & ITALY & 1905 & TRAMMER/CM & UCRIA, MESSINA & 7TH ST. & ADAMS - TRIMOUNTAIN LOCATION \\
\hline 406 & 215 & CINQUINI & NATALE & B & 24 & ITALY & ITALY & ITALY & 1908 & TRAMMER/CM & LUCCA & 7TH ST. & ADAMS - TRIMOUNTAIN LOCATION \\
\hline 407 & 215 & TAMBELLINI & PIETRO & B & 21 & ITALY & ITALY & ITALY & 1907 & TRAMMER/CM & CAPANNORI. LU & 7TH ST. & ADAMS - TRIMOUNTAIN LOCATION \\
\hline 408 & 215 & BELLUOMINI & MICHELE & B & 34 & ITALY & ITALY & ITALY & 1901 & TRAMMER/CM & COLLE DI COMPITO. LU & 7TH ST. & ADAMS - TRIMOUNTAIN LOCATION \\
\hline 409 & 203 & PIERONI & PAOLO & $\mathrm{H}$ & 26 & ITALY & ITALY & ITALY & 1901 & MINER/CM & CAPANNORI. LU & 7TH ST. & ADAMS - TRIMOUNTAIN LOCATION \\
\hline 410 & 203 & PIERONI & CALLINA & W & 20 & ITALY & ITALY & ITALY & 1907 & & & 7TH ST. & ADAMS - TRIMOUNTAIN LOCATION \\
\hline 411 & 203 & PIERONI & GIUSEPPINA & D & $1,6 \mathrm{M}$ & MICHIGAN & ITALY & ITALY & & & CAPANNORI, LU & 7TH ST. & ADAMS - TRIMOUNTAIN LOCATION \\
\hline 412 & 203 & FERRINNIO & GINO & B & 22 & ITALY & ITALY & ITALY & 1904 & TRAMMER/CM & & 7TH ST. & ADAMS - TRIMOUNTAIN LOCATION \\
\hline 413 & 203 & MATUCCI & NICOLA & B & 24 & ITALY & ITALY & ITALY & 1901 & TRAMMER/CM & LUCCA & 7TH ST. & ADAMS - TRIMOUNTAIN LOCATION \\
\hline 414 & 203 & MATUCCI & OLIVO & B & 18 & ITALY & ITALY & ITALY & 1909 & TRAMMER/CM & LUCCA & 7TH ST. & ADAMS - TRIMOUNTAIN LOCATION \\
\hline 415 & 203 & PIERONI & GAULLE & B & 20 & ITALY & ITALY & ITALY & 1909 & TRAMMER/CM & LUCCA & 7TH ST. & ADAMS - TRIMOUNTAIN LOCATION \\
\hline 416 & 203 & NUTINI & PIETRO & $\mathrm{H}$ & 28 & ITALY & ITALY & ITALY & 1903 & MINER/CM & SEGROMIGNO. LU & 8TH ST. & ADAMS - TRIMOUNTAIN LOCATION \\
\hline 417 & 203 & NUTINI & ERSILIA & W & 24 & ITALY & ITALY & ITALY & 1909 & & & 8TH ST. & ADAMS - TRIMOUNTAIN LOCATION \\
\hline 418 & 203 & FIENILI & PASQUALE & B & 33 & ITALY & ITALY & ITALY & 1902 & MINER/CM & GRAGNIANO. LU & 8TH ST. & ADAMS - TRIMOUNTAIN LOCATION \\
\hline 419 & 203 & FIENILI & TERESA & B & 31 & ITALY & ITALY & ITALY & 1909 & & & 8TH ST. & ADAMS - TRIMOUNTAIN LOCATION \\
\hline 420 & 203 & NUTINI & PIETRO & B & 34 & ITALY & ITALY & ITALY & 1902 & MINER/CM & CAPANNORI. LU & 8TH ST. & ADAMS - TRIMOUNTAIN LOCATION \\
\hline 421 & 203 & MONTINI & GABRIELLO & B & 24 & ITALY & ITALY & ITALY & 1907 & TRAMMER/CM & & 8TH ST. & ADAMS - TRIMOUNTAIN LOCATION \\
\hline 422 & 203 & MARTINUCCI & ANGELO & B & 24 & ITALY & ITALY & ITALY & 1903 & MINER/CM & LUCCA & 8TH ST. & ADAMS - TRIMOUNTAIN LOCATION \\
\hline 423 & 203 & MARTINI & RINALDO & B & 23 & ITALY & ITALY & ITALY & 1908 & 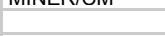 & LUCCA & 8TH ST. & ADAMS - TRIMOUNTAIN LOCATION \\
\hline 424 & 203 & PINI & MARCO & B & 35 & ITALY & ITALY & ITALY & 1906 & MINER/CM & LUCCA & 8TH ST. & ADAMS - TRIMOUNTAIN LOCATION \\
\hline 425 & 203 & NUTINI & RINALDO & B & 26 & ITALY & ITALY & ITALY & 1905 & MINER/CM & CAPANNORI. LU & 8TH ST. & ADAMS - TRIMOUNTAIN LOCATION \\
\hline 426 & 203 & MILANI & PAOLO & B & 29 & ITALY & ITALY & ITALY & 1907 & TRAMMER/CM & GRAGNIANO. LU & 8TH ST. & ADAMS - TRIMOUNTAIN LOCATION \\
\hline 427 & 203 & NELTI & ANGELO & B & 19 & ITALY & ITALY & ITALY & 1908 & TRAMMER/CM & & 8TH ST. & ADAMS - TRIMOUNTAIN LOCATION \\
\hline 428 & 406 & GIULI & ENRICO & $\mathrm{H}$ & 32 & ITALY & ITALY & ITALY & 1901 & LABORER/CM & SAN MARTINO IN FREDDANA. & 4TH ST. & ADAMS - TRIMOUNTAIN LOCATION \\
\hline 429 & 406 & GIULI & ELISABETTA & W & 26 & ITALY & ITALY & ITALY & 1905 & & & 4TH ST. & ADAMS - TRIMOUNTAIN LOCATION \\
\hline 430 & 406 & GIULI & ARGANTE & $\mathrm{s}$ & 4 & MICHIGAN & ITALY & ITALY & & & SAN MARTINO IN FREDDANA. & 4TH ST. & ADAMS - TRIMOUNTAIN LOCATION \\
\hline 431 & 406 & GIULI & RUGGERO & $\mathrm{s}$ & 3 & MICHIGAN & ITALY & ITALY & & & SAN MARTINO IN FREDDANA. & 4TH ST. & ADAMS - TRIMOUNTAIN LOCATION \\
\hline 432 & 406 & GIULI & FERNANDA & $\mathrm{D}$ & $1.6 \mathrm{M}$ & MICHIGAN & ITALY & ITALY & & & SAN MARTINO IN FREDDANA. & 4TH ST. & ADAMS - TRIMOUNTAIN LOCATION \\
\hline 433 & 406 & CORTOPASSI & GIUSEPPE & B & 32 & ITALY & ITALY & ITALY & 1910 & MINER/CM & LUCCA & 4TH ST. & ADAMS - TRIMOUNTAIN LOCATION \\
\hline 434 & 408 & PIERONI & PIETRO & $\mathrm{H}$ & 28 & ITALY & ITALY & ITALY & 1908 & MINER/CM & LUCCA & 4TH ST. & ADAMS - TRIMOUNTAIN LOCATION \\
\hline 435 & 408 & PIERONI & TERESINA & W & 24 & ITALY & ITALY & ITALY & 1908 & & LUCCA & 4TH ST. & ADAMS - TRIMOUNTAIN LOCATION \\
\hline 436 & 408 & PIERONI & GINA & D & 4 & ITALY & ITALY & ITALY & 1908 & & LUCCA & 4TH ST. & ADAMS - TRIMOUNTAIN LOCATION \\
\hline 437 & 408 & PIERONI & ANNA & $\mathrm{D}$ & $7 \mathrm{M}$ & MICHIGAN & ITALY & ITALY & & & LUCCA & 4TH ST. & ADAMS - TRIMOUNTAIN LOCATION \\
\hline 438 & 408 & LORENZO & PIETRO & B & 30 & ITALY & ITA & ITALY & 1910 & R/CM & POTENZA & 4TH ST. & ADAMS - TRIMOUNTAIN LOCATION \\
\hline 439 & 408 & CHINIBARI & PIETRO & B & 18 & ITALY & ITALY & ITALY & 1907 & MINER/CM & & 4TH ST. & ADAMS - TRIMOUNTAIN LOCATION \\
\hline 440 & 408 & PIERONI & NATALE & B & 39 & ITALY & ITALY & ITALY & 1903 & MINER/CM & LUCCA & 4TH ST. & ADAMS - TRIMOUNTAIN LOCATION \\
\hline
\end{tabular}




\begin{tabular}{|c|c|c|c|c|c|c|c|c|c|c|c|c|c|}
\hline 441 & 408 & DEL GHINGARO & GIOVANNI & B & 45 & ITALY & ITALY & ITALY & 1896 & MINER/CM & LUCCA & 4TH ST. & ADAMS - TRIMOUNTAIN LOCATION \\
\hline 442 & 309 & $\mathrm{ClUCCl}$ & MIKE & $\mathrm{H}$ & 36 & ITALY & ITALY & ITALY & 1900 & CARPENTER/CM & LUCCA & 11TH ST. & ADAMS - TRIMOUNTAIN LOCATION \\
\hline 443 & 309 & $\mathrm{ClUCCl}$ & ADELE & W & 29 & ITALY & ITALY & ITALY & 1900 & & LUCCA & 11TH ST. & ADAMS - TRIMOUNTAIN LOCATION \\
\hline 444 & 309 & $\mathrm{ClUCCl}$ & ADELINA & D & 7 & MICHIGAN & ITALY & ITALY & & & LUCCA & 11TH ST. & ADAMS - TRIMOUNTAIN LOCATION \\
\hline 445 & 309 & $\mathrm{CIUCCl}$ & GINO & $\mathrm{s}$ & 3 & MICHIGAN & ITALY & ITALY & & & LUCCA & 11 TH ST. & ADAMS - TRIMOUNTAIN LOCATION \\
\hline 446 & 309 & $\mathrm{ClUCCl}$ & ANNE & D & $3 \mathrm{M}$ & MICHIGAN & ITALY & ITALY & & & LUCCA & 11 TH ST. & ADAMS - TRIMOUNTAIN LOCATION \\
\hline 447 & 309 & STEFANI & UMBERTO & B & 20 & ITALY & ITALY & ITALY & 1909 & MINER/CM & LUCCA & 11TH ST. & ADAMS - TRIMOUNTAIN LOCATION \\
\hline 448 & 309 & PAOLI & GIULIO & B & 20 & ITALY & ITALY & ITALY & 1910 & MINER/CM & SEGROMIGNO. LU & 11 TH ST. & ADAMS - TRIMOUNTAIN LOCATION \\
\hline 449 & 309 & MALLINEN & AMELIO & B & 29 & ITALY & ITALY & ITALY & 1908 & MINER/CM & & 11TH ST. & ADAMS - TRIMOUNTAIN LOCATION \\
\hline 450 & 405 & SALSA & SEBASTIANO & $\mathrm{H}$ & 29 & ITALY & ITALY & ITALY & 1906 & TRAMMER/CM & VERCELLI & 11TH ST. & ADAMS - TRIMOUNTAIN LOCATION \\
\hline 451 & 405 & SALSA & CATERINA & w & 26 & ITALY & ITALY & ITALY & 1907 & & VERCELLI & 11TH ST. & ADAMS - TRIMOUNTAIN LOCATION \\
\hline 452 & 405 & SALSA & MARGHERITA & $\mathrm{D}$ & 4 & ITALY & ITALY & ITALY & 1907 & & VERCELLI & 11TH ST. & ADAMS - TRIMOUNTAIN LOCATION \\
\hline 453 & 405 & SALSA & GIOVANNA & D & $1.6 \mathrm{M}$ & MICHIGAN & ITALY & ITALY & & & VERCELLI & 11TH ST. & ADAMS - TRIMOUNTAIN LOCATION \\
\hline 454 & 405 & NEGRONILDA & PIETRO & $\mathrm{G}$ ? & 26 & ITALY & ITALY & ITALY & 1907 & TRAMMER/CM & & 11TH ST. & ADAMS - TRIMOUNTAIN LOCATION \\
\hline 455 & 405 & VALLINO & GIOVANNI & $\mathrm{H}$ & 31 & ITALY & ITALY & ITALY & 1900 & TRAMMER/CM & SAN GIORGIO CANAVESE. TO & 11TH ST. & ADAMS - TRIMOUNTAIN LOCATION \\
\hline 456 & 405 & VALLINO & TERESA & W & 27 & ITALY & ITALY & ITALY & 1909 & & SAN GIORGIO CANAVESE, TO & 11TH ST. & ADAMS - TRIMOUNTAIN LOCATION \\
\hline 457 & 405 & VALLINO & GIOVANNI & $\mathrm{s}$ & 4 & ITALY & ITALY & ITALY & 1909 & & SAN GIORGIO CANAVESE. TO & 11TH ST. & ADAMS - TRIMOUNTAIN LOCATION \\
\hline 458 & 402 & TAPPERO & GIUSEPPE & $\mathrm{H}$ & 48 & ITALY & ITALY & ITALY & 1890 & MINER/CM & SAN GIUSTO CANAVESE. TO & 11TH ST. & ADAMS - TRIMOUNTAIN LOCATION \\
\hline 459 & 402 & TAPPERO & DOMENICA & w & 43 & ITALY & ITALY & ITALY & 1893 & & SAN GIUSTO CANAVESE. TO & 11TH ST. & ADAMS - TRIMOUNTAIN LOCATION \\
\hline 460 & 402 & TAPPERO & DOMENICO & s & 19 & ITALY & ITALY & ITALY & 1893 & LABORER/CM & SAN GIUSTO CANAVESE. TO & 11TH ST. & ADAMS - TRIMOUNTAIN LOCATION \\
\hline 461 & 402 & TAPPERO & 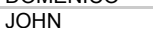 & s & 17 & MICHIGAN & ITALY & ITALY & & PUFFER BOY/CM & SAN GIUSTO CANAVESE. TO & 11TH ST. & ADAMS - TRIMOUNTAIN LOCATION \\
\hline 462 & 402 & TAPPERO & JOSIE & $\mathrm{s}$ & 16 & MICHIGAN & ITALY & ITALY & & & SAN GIUSTO CANAVESE, TO & 11TH ST. & ADAMS - TRIMOUNTAIN LOCATION \\
\hline 463 & 402 & TAPPERO & CHARLIE & $\mathrm{s}$ & 14 & MICHIGAN & ITALY & ITALY & & & SAN GIUSTO CANAVESE. TO & $11 \mathrm{TH}$ ST. & ADAMS - TRIMOUNTAIN LOCATION \\
\hline 464 & 402 & TAPPERO & MARY & $\mathrm{D}$ & 10 & MICHIGAN & ITALY & ITALY & & & SAN GIUSTO CANAVESE. TO & 11TH ST. & ADAMS - TRIMOUNTAIN LOCATION \\
\hline 465 & 402 & TAPPERO & WILLIE & $\mathrm{s}$ & 8 & MICHIGAN & ITALY & ITALY & & & SAN GIUSTO CANAVESE. TO & 11TH ST. & ADAMS - TRIMOUNTAIN LOCATION \\
\hline 466 & 402 & TAPPERO & PETER & $\mathrm{s}$ & 4 & MICHIGAN & ITALY & ITALY & & & SAN GIUSTO CANAVESE. TO & 11TH ST. & ADAMS - TRIMOUNTAIN LOCATION \\
\hline 467 & 412 & SUCCA & GIUSEPPE & $\mathrm{H}$ & 35 & ITALY & ITALY & ITALY & 1900 & PROPRIETOR/CONFECTIONARY & SAN GIUSTO CANAVESE. TO & 11TH ST. & ADAMS - TRIMOUNTAIN LOCATION \\
\hline 468 & 412 & SUCCA & CATERINA & W & 28 & ITALY & ITALY & ITALY & 1904 & & & 11TH ST. & ADAMS - TRIMOUNTAIN LOCATION \\
\hline 469 & 412 & SUCCA & DOLINA & D & 5 & MICHIGAN & ITALY & ITALY & & & SAN GIUSTO CANAVESE. TO & 11TH ST. & ADAMS - TRIMOUNTAIN LOCATION \\
\hline 470 & 412 & SUCCA & PIETRO & $\mathrm{s}$ & 3 & MICHIGAN & ITALY & ITALY & & & SAN GIUSTO CANAVESE. TO & 11TH ST. & ADAMS - TRIMOUNTAIN LOCATION \\
\hline 471 & 412 & SUCCA & MARCELLINA & $\mathrm{SI}$ & 19 & ITALY & ITALY & ITALY & 1907 & SERVANT/PF & SAN GIUSTO CANAVESE. TO & 11TH ST. & ADAMS - TRIMOUNTAIN LOCATION \\
\hline 472 & 412 & SUCCA & FRANCESCO & $\mathrm{BR}$ & 23 & ITALY & ITALY & ITALY & 1906 & MASON/CM & SAN GIUSTO CANAVESE. TO & 11 TH ST. & ADAMS - TRIMOUNTAIN LOCATION \\
\hline 473 & 412 & BERTOLINO & ANTONIO & B & 22 & ITALY & ITALY & ITALY & 1909 & ROCK HOUSE MAN & LOCANA CANAVESE. TO & 11TH ST. & ADAMS - TRIMOUNTAIN LOCATION \\
\hline 474 & 413 & BIANCHI & GIUSEPPE & $\begin{array}{l}\mathrm{O} \\
\mathrm{H}\end{array}$ & 33 & ITALY & ITALY & ITALY & 1903 & MASON/CM & LUCCA & DIVISION ST. & ADAMS - TRIMOUNTAIN LOCATION \\
\hline 475 & 413 & BIANCHI & ISOLA & w & 31 & ITALY & ITALY & ITALY & 1905 & & & DIVISION ST. & ADAMS - TRIMOUNTAIN LOCATION \\
\hline 476 & 413 & BIANCHI & GEMMA & $\mathrm{D}$ & 4 & MICHIGAN & ITALY & ITALY & & & LUCCA & DIVISION ST. & ADAMS - TRIMOUNTAIN LOCATION \\
\hline 477 & 413 & BIANCHI & MARCO & $\mathrm{s}$ & 2 & MICHIGAN & ITALY & ITALY & & & LUCCA & DIVISION ST. & ADAMS - TRIMOUNTAIN LOCATION \\
\hline 478 & 413 & BIANCHI & CELESTINA & D & $1.6 \mathrm{M}$ & MICHIGAN & ITALY & ITALY & & & LUCCA & DIVISION ST. & ADAMS - TRIMOUNTAIN LOCATION \\
\hline 479 & 413 & BIANCHI & BABY & $\mathrm{D}$ & $1 \mathrm{M}$ & MICHIGAN & ITALY & ITALY & & & LUCCA & DIVISION ST. & ADAMS - TRIMOUNTAIN LOCATION \\
\hline 480 & 413 & TONELLI & GIACOMO & B & 54 & ITALY & ITALY & ITALY & 1900 & MASON/CM & & DIVISION ST. & ADAMS - TRIMOUNTAIN LOCATION \\
\hline 481 & 413 & COZZI & VALENTINO & B & 39 & ITALY & ITALY & ITALY & 1901 & MASON/CM & UDINE & DIVISION ST. & ADAMS - TRIMOUNTAIN LOCATION \\
\hline 482 & 413 & MARCHINI & PIETRO & B & 26 & ITALY & ITALY & ITALY & 1907 & MASON/CM & CAPANNORI. LU & DIVISION ST. & ADAMS - TRIMOUNTAIN LOCATION \\
\hline 483 & 413 & PERESAN & DANIELE & B & 48 & ITALY & ITALY & ITALY & 1907 & MASON/CM & & DIVISION ST. & ADAMS - TRIMOUNTAIN LOCATION \\
\hline 484 & 413 & MOSTARDA & GIOVANNI & B & 32 & ITALY & ITALY & ITALY & 1905 & MASON/CM & AULLA. MASSA CARRARA & DIVISION ST. & ADAMS - TRIMOUNTAIN LOCATION \\
\hline 485 & 409 & ANTONIONI & GIUSEPPE & $\mathrm{H}$ & 33 & ITALY & ITALY & ITALY & 1908 & MINER/CM & & COUNTY ROAD & ADAMS - TRIMOUNTAIN LOCATION \\
\hline 486 & 409 & ANTONIONI & DOMENICA & w & 31 & ITALY & ITALY & ITALY & 1908 & & & COUNTY ROAD & ADAMS - TRIMOUNTAIN LOCATION \\
\hline 487 & 409 & ANTONIONI & DOMENICA & $\mathrm{D}$ & $1.2 \mathrm{M}$ & MICHIGAN & ITALY & ITALY & & & & COUNTY ROAD & ADAMS - TRIMOUNTAIN LOCATION \\
\hline 488 & NN & LANDINI & FRANCESCO & $\mathrm{H}$ & 42 & ITALY & ITAL & ITALY & $04 / 09$ & FIREMAN/BOILER HOUSE & COLLE DI COMPITO. LU & IION ST. & ADAMS - TRIMOUNTAIN LOCATION \\
\hline 489 & NN & LANDINI & FANNY & w & 38 & ITALY & ITA & ITALY & 1909 & & COLLE DI COMPITO. LU & SION ST. & ADAMS - TRIMOUNTAIN LOCATION \\
\hline 490 & NN & LANDINI & ALBERT & $\mathrm{s}$ & 17 & ITALY & ITALY & ITALY & 1909 & PUFFER BOY/CM & COLLE DI COMPITO. LU & DIVISION ST. & ADAMS - TRIMOUNTAIN LOCATION \\
\hline 491 & $\mathrm{NN}$ & LANDINI & RAFFAELLO & $\mathrm{s}$ & 14 & ITAL & ITAI & ITALY & 1909 & & COLLE DI COMPITO. LU & DIVISION ST. & ADAMS - TRIMOUNTAIN LOCATION \\
\hline 492 & $\mathrm{NN}$ & PAOLI & CARLO & B & 34 & ITALY & ITALY & ITAL & 1903 & LABORER/CM & COLLE DI COMPITO, LU & DIVISION ST. & ADAMS - TRIMOUNTAIN LOCATION \\
\hline 493 & $\mathrm{NN}$ & MARCHETTI & PAUL & B & 39 & ITALY & ITALY & ITALY & 1906 & MASON/CM & COLLE DI COMPITO. LU & DIVISION ST. & ADAMS - TRIMOUNTAIN LOCATION \\
\hline 494 & NN & PRIAMI & TONY & B & 34 & ITALY & ITALY & ITALY & 1902 & MASON/CM & LUCCA & DIVISION ST. & ADAMS - TRIMOUNTAIN LOCATION \\
\hline 495 & NN & CUSABIO & EUGENIO & $\mathrm{H}$ & 29 & ITALY & ITALY & ITALY & 1901 & MINER/CM & & DIVISION ST. & ADAMS - TRIMOUNTAIN LOCATION \\
\hline 496 & NN & CUSABIO & CAROLINA & w & 27 & ITALY & ITALY & ITALY & 1901 & & & DIVISION ST. & ADAMS - TRIMOUNTAIN LOCATION \\
\hline 497 & NN & CUSABIO & GINO & s & 6 & PA & ITALY & ITALY & & & & DIVISION ST. & ADAMS - TRIMOUNTAIN LOCATION \\
\hline 498 & NN & FINI & JOSEPH & B & 37 & ITALY & ITALY & ITALY & 1906 & LABORER/CM & POPIGLIO, PISTOIA & DIVISION ST. & ADAMS - TRIMOUNTAIN LOCATION \\
\hline 499 & NN & GIANNINI & LUIGI & $\mathrm{H}$ & 30 & ITALY & ITALY & ITALY & 1902 & LABORER/CM & SEGROMIGNO. LU & DIVISION ST. & ADAMS - TRIMOUNTAIN LOCATION \\
\hline 500 & NN & GIANNINI & ROSA & W & 20 & ITALY & ITALY & ITALY & 1908 & & & DIVISION ST. & ADAMS - TRIMOUNTAIN LOCATION \\
\hline 501 & NN & & GIN & s & $6 \mathrm{M}$ & HIGAN & ITA & ITAI & & & SEGROMIGNO. LU & & ADAMS - TRIMOUNTAIN LOCATION \\
\hline 502 & NN & MARCHIONNI & RAFFAELE & $\mathrm{H}$ & 30 & ITALY & ITALY & ITALY & 1905 & MINER/CM & BELLUNO & DIVISION ST. & ADAMS - TRIMOUNTAIN LOCATION \\
\hline 503 & NN & MARCHIONNI & CESIRA & w & 24 & ITALY & ITALY & ITALY & 1909 & & BELLUNO & DIVISION ST. & ADAMS - TRIMOUNTAIN LOCATION \\
\hline
\end{tabular}




\begin{tabular}{|c|c|c|c|c|c|c|c|c|c|c|c|c|c|}
\hline 504 & NN & MARCHIONNI & AURELIO & $\mathrm{s}$ & 5 & ITALY & ITALY & ITALY & 1909 & & BELLUNO & DIVISION ST. & ADAMS - TRIMOUNTAIN LOCATION \\
\hline 505 & $\mathrm{NN}$ & MARCHIONNI & AMELIO & $\mathrm{S}$ & 2 & ITALY & ITALY & ITALY & 1909 & & BELLUNO & DIVISION ST. & ADAMS - TRIMOUNTAIN LOCATION \\
\hline 506 & $\mathrm{NN}$ & MARCHIONNI & RENATO & $\mathrm{s}$ & $1 \mathrm{M}$ & MICHIGAN & ITALY & ITALY & & & BELLUNO & DIVISION ST. & ADAMS - TRIMOUNTAIN LOCATION \\
\hline 507 & $\mathrm{NN}$ & PERNA & GIUSEPPE & $\mathrm{H}$ & 25 & ITALY & ITALY & ITALY & 1906 & MINER/CM & PARIANA. LU & DIVISION ST. & ADAMS - TRIMOUNTAIN LOCATION \\
\hline 508 & NN & PERNA & MARIA & W & 25 & ITALY & ITALY & ITALY & 1906 & & PARIANA. LU & DIVISION ST. & ADAMS - TRIMOUNTAIN LOCATION \\
\hline 509 & NN & PERNA & EUGENIO & s & 2 & MICHIGAN & ITALY & ITALY & & & PARIANA. LU & DIVISION ST. & ADAMS - TRIMOUNTAIN LOCATION \\
\hline 510 & NN & PERNA & BABY & $\mathrm{D}$ & $2 \mathrm{M}$ & MICHIGAN & ITALY & ITALY & & & PARIANA. LU & DIVISION ST. & ADAMS - TRIMOUNTAIN LOCATION \\
\hline 511 & NN & ADAMI & PILADE & B & 25 & ITALY & ITALY & ITALY & 1906 & MINER/CM & VILLA BASILICA. LU & DIVISION ST. & ADAMS - TRIMOUNTAIN LOCATION \\
\hline 512 & NN & ADAMI & GIUSEPPE & $\mathrm{B}$ & 24 & ITALY & ITALY & ITALY & 1907 & TRAMMER/CM & VILLA BASILICA. LU & DIVISION ST. & ADAMS - TRIMOUNTAIN LOCATION \\
\hline 513 & NN & MATTEUCCI & ANSELMO & B & 45 & ITALY & ITALY & ITALY & 1905 & TRAMMER/CM & LUCCA & DIVISION ST. & ADAMS - TRIMOUNTAIN LOCATION \\
\hline 514 & NN & BACCETTI & ADAMO & B & 22 & ITALY & ITALY & ITALY & 1904 & MASON/CM & LUCCA & DIVISION ST. & ADAMS - TRIMOUNTAIN LOCATION \\
\hline 515 & NN & BACCETTI & DOMENICO & B & 35 & ITALY & ITALY & ITALY & 1902 & TRAMMER/CM & LUCCA & DIVISION ST. & ADAMS - TRIMOUNTAIN LOCATION \\
\hline 516 & NN & SIMI & IACOPO & B & 20 & ITALY & ITALY & ITALY & 1907 & TRAMMER/CM & VILLA BASILICA, LU & DIVISION ST. & ADAMS - TRIMOUNTAIN LOCATION \\
\hline 517 & $\mathrm{NN}$ & ADAMI & GIOVANNI & B & 27 & ITALY & ITALY & ITALY & 1906 & TRAMMER/CM & VILLA BASILICA, LU & DIVISION ST. & ADAMS - TRIMOUNTAIN LOCATION \\
\hline 518 & $\mathrm{NN}$ & FLOSI & PASQUALE & $\mathrm{H}$ & 34 & ITALY & ITALY & ITALY & 1901 & MINER/CM & PARIANA. LU & DEPOT ST. & ADAMS - TRIMOUNTAIN LOCATION \\
\hline 519 & $\mathrm{NN}$ & FLOSI & ALONENA? & W & 30 & ITALY & ITALY & ITALY & 1901 & & PARIANA, LU & DEPOT ST. & ADAMS - TRIMOUNTAIN LOCATION \\
\hline 520 & $\mathrm{NN}$ & FLOSI & EVA & D & 4 & ITALY & ITALY & ITALY & 1901 & & PARIANA. LU & DEPOT ST. & ADAMS - TRIMOUNTAIN LOCATION \\
\hline 521 & NN & GIAMPAOLI & FEDERICO & B & 24 & ITALY & ITALY & ITALY & 1910 & TRAMMER/CM & LUCCA & DEPOT ST. & ADAMS - TRIMOUNTAIN LOCATION \\
\hline 522 & NN & FLOSI & PAOLO & B & 22 & ITALY & ITALY & ITALY & 1905 & TRAMMER/CM & PARIANA. LU & DEPOT ST. & ADAMS - TRIMOUNTAIN LOCATION \\
\hline 523 & NN & BATTAGLINI & ETTORE & B & 21 & ITALY & ITALY & ITALY & 1907 & TRAMMER/CM & LUCCA & DEPOT ST. & ADAMS - TRIMOUNTAIN LOCATION \\
\hline 524 & NN & BASSO & GIOVANNI & B & 26 & ITALY & ITALY & ITALY & 1908 & TRAMMER/CM & SAN GIORGIO CANAVESE. TO & DEPOT ST. & ADAMS - TRIMOUNTAIN LOCATION \\
\hline 525 & NN & $\begin{array}{l}\text { DANELLI } \\
\text { DINEL }\end{array}$ & $\begin{array}{l}\text { FILINTO } \\
\text { FIN }\end{array}$ & B & 21 & ITALY & ITALY & ITALY & 1906 & TRAMMER/CM & LUCCA & DEPOT ST. & ADAMS - TRIMOUNTAIN LOCATION \\
\hline 526 & NN & GIANNINI & GIULIO & B & 26 & ITALY & ITALY & ITALY & 1909 & TRAMMER/CM & LUCCA & DEPOT ST. & ADAMS - TRIMOUNTAIN LOCATION \\
\hline 527 & $\mathrm{NN}$ & PERNA & SALVATORE & B & 34 & ITALY & ITALY & ITALY & 1903 & LABORER/CM & PARIANA. LU & DEPOT ST. & ADAMS - TRIMOUNTAIN LOCATION \\
\hline 528 & NN & FERRARI & VITTORIO & $\mathrm{H}$ & 25 & ITALY & ITALY & ITALY & 1902 & TRAMMER/CM & POPIGLIO. PISTOIA & ITALIAN SETTLEMENT & ADAMS - TRIMOUNTAIN LOCATION \\
\hline 529 & NN & FERRARI & MADDALENA & W & 25 & ITALY & ITALY & ITALY & 1908 & & & $\begin{array}{l}\text { ITALIAN SETTLEMENT } \\
\text { ITANE }\end{array}$ & ADAMS - TRIMOUNTAIN LOCATION \\
\hline 530 & NN & FERRARI & BABY & D & $1 \mathrm{M}$ & MICHIGAN & ITALY & ITALY & & & POPIGLIO. PISTOIA & ITALIAN SETTLEMENT & ADAMS - TRIMOUNTAIN LOCATION \\
\hline 531 & $\mathrm{NN}$ & GAGGINI & GUGLIELMO & B & 32 & ITALY & ITALY & ITALY & 1902 & MINER/CM & PITEGLIO, PISTOIA & ITALIAN SETTLEMENT & ADAMS - TRIMOUNTAIN LOCATION \\
\hline 532 & $\mathrm{NN}$ & MANZINI & EMILIO & B & 34 & ITALY & ITALY & ITALY & 1907 & TRAMMER/CM & PITEGLIO. PISTOIA & ITALIAN SETTLEMENT & ADAMS - TRIMOUNTAIN LOCATION \\
\hline 533 & NN & BARTOLOMEI & DIONIGI & B & 24 & ITALY & ITALY & ITALY & 1906 & TRAMMER/CM & LUCCA & ITALIAN SETTLEMENT & ADAMS - TRIMOUNTAIN LOCATION \\
\hline 534 & NN & UGOLINI & EUSEBIO & B & 31 & ITALY & ITALY & ITALY & 1907 & TRAMMER/CM & FIRENZE & ITALIAN SETTLEMENT & ADAMS - TRIMOUNTAIN LOCATION \\
\hline 535 & NN & PELLEGRINI & JACOPO & B & 36 & ITALY & ITALY & ITALY & 1898 & MINER/CM & COLLE DI COMPITO. LU & ITALIAN SETTLEMENT & ADAMS - TRIMOUNTAIN LOCATION \\
\hline 536 & NN & FERRARI & GUGLIELMO & B & 30 & ITALY & ITALY & ITALY & 1907 & TRAMMER/CM & POPIGLIO. PISTOIA & ITALIAN SETTLEMENT & ADAMS - TRIMOUNTAIN LOCATION \\
\hline 537 & NN & PUPILLI & $\begin{array}{l}\text { PIETRO } \\
\text { PIR }\end{array}$ & B & 28 & ITALY & ITALY & ITALY & 1902 & TRAMMER/CM & PITEGLIO, PISTOIA & ITALIAN SETTLEMENT & ADAMS - TRIMOUNTAIN LOCATION \\
\hline 538 & NN & MANNAI & FRANCESCO & B & 24 & ITALY & ITALY & ITALY & 1909 & TRAMMER/CM & MILIS. CAGLIARI & ITALIAN SETTLEMENT & ADAMS - TRIMOUNTAIN LOCATION \\
\hline 539 & NN & PERA & ALBERTO & B & 32 & ITALY & ITALY & ITALY & 1904 & TRAMMER/CM & LUCCA & ITALIAN SETTLEMENT & ADAMS - TRIMOUNTAIN LOCATION \\
\hline 540 & NN & DATI & EUGENIO & $\mathrm{H}$ & 29 & ITALY & ITALY & ITALY & 1905 & LABORER/CM & LUCCA & ITALIAN SETTLEMENT & ADAMS - TRIMOUNTAIN LOCATION \\
\hline 541 & NN & DATI & DUSOLA & w & 24 & ITALY & ITALY & ITALY & 1907 & & LUCCA & ITALIAN SETTLEMENT & ADAMS - TRIMOUNTAIN LOCATION \\
\hline 542 & NN & DATI & DUICE & $\mathrm{D}$ & 5 & ITALY & ITALY & ITALY & 1907 & & LUCCA & $\begin{array}{l}\text { ITALIAN SETTLEMENT } \\
\end{array}$ & ADAMS - TRIMOUNTAIN LOCATION \\
\hline 543 & NN & DATI & RENATO & $\mathrm{s}$ & 2 & MICHIGAN & ITALY & ITALY & & & LUCCA & ITALIAN SETTLEMENT & ADAMS - TRIMOUNTAIN LOCATION \\
\hline 544 & $\mathrm{NN}$ & DORONI & GIOVANNI & B & 24 & ITALY & ITALY & ITALY & 1905 & LABORER/CM & LUCCA & ITALIAN SETTLEMENT & ADAMS - TRIMOUNTAIN LOCATION \\
\hline 545 & $\mathrm{NN}$ & NERI & RAFFAELLO & B & 35 & ITALY & ITALY & ITALY & 1904 & LABORER/CM & & ITALIAN SETTLEMENT & ADAMS - TRIMOUNTAIN LOCATION \\
\hline 546 & NN & DATI & CESARE & B & 26 & ITALY & ITALY & ITALY & 1905 & LABORER/CM & LUCCA & ITALIAN SETTLEMENT & ADAMS - TRIMOUNTAIN LOCATION \\
\hline 547 & NN & BERTOLLI & AMEDEO & B & 33 & ITALY & ITALY & ITALY & 1901 & MINER/CM & LUCCA & ITALIAN SETTLEMENT & ADAMS - TRIMOUNTAIN LOCATION \\
\hline 548 & NN & BERTOLLI & LUIGI & B & 24 & ITALY & ITALY & ITALY & 1906 & MINER/CM & ORBICCIANO. LU & ITALIAN SETTLEMENT & ADAMS - TRIMOUNTAIN LOCATION \\
\hline 549 & NN & BERTOLLI & AUGUSTO & B & 26 & ITALY & ITALY & ITALY & 1907 & LABORER/CM & LUCCA & ITALIAN SETTLEMENT & ADAMS - TRIMOUNTAIN LOCATION \\
\hline 550 & NN & STEFANI & FERDINANDO & B & 23 & ITALY & ITALY & ITALY & 1909 & LABORER/CM & LUCCA & ITALIAN SETTLEMENT & ADAMS - TRIMOUNTAIN LOCATION \\
\hline 551 & $\mathrm{NN}$ & ALAMA & DIONIGIO & $\mathrm{B}$ & 32 & ITALY & ITALY & ITALY & 1906 & LABORER/CM & LOPPEGLIA. LU & ITALIAN SETTLEMENT & ADAMS - TRIMOUNTAIN LOCATION \\
\hline 552 & NN & MARCHESCHI & GIUSEPPE & B & 40 & ITALY & ITALY & ITALY & 1905 & LABORER/CM & LUCCA & ITALIAN SETTLEMENT & ADAMS - TRIMOUNTAIN LOCATION \\
\hline 553 & NN & MANAI & SALVATORE & B & 25 & ITALY & ITALY & ITALY & 1906 & LABORER/CM & LUCCA & ITALIAN SETTLEMENT & ADAMS - TRIMOUNTAIN LOCATION \\
\hline 554 & NN & TADDEUCCI & IACOPO & $\mathrm{H}$ & 31 & ITALY & ITALY & ITALY & 1901 & MINER/CM & LUCCA & $\begin{array}{l}\text { ITALIAN SETTLEMENT } \\
\text { IT }\end{array}$ & ADAMS - TRIMOUNTAIN LOCATION \\
\hline 555 & NN & TADDEUCCI & FERNANDA & W & 27 & ITALY & ITALY & ITALY & 1906 & & & $\begin{array}{l}\text { ITALIAN SETTLEMENT } \\
\text { ITANE }\end{array}$ & ADAMS - TRIMOUNTAIN LOCATION \\
\hline 556 & NN & TADDEUCCI & FRANK & $\mathrm{s}$ & $1.5 \mathrm{M}$ & MICHIGAN & ITALY & ITALY & & & LUCCA & $\begin{array}{l}\text { ITALIAN SETTLEMENT } \\
\end{array}$ & ADAMS - TRIMOUNTAIN LOCATION \\
\hline 557 & NN & TADDEUCCI & LUCILLA & B & 30 & ITALY & ITALY & ITA & 1903 & LABORER/CM & LUCCA & $\begin{array}{l}\text { ITALIAN SETTLEMENT } \\
\text { IT }\end{array}$ & ADAMS - TRIMOUNTAIN LOCATION \\
\hline 558 & NN & QUILICI & EMILIO & B & 25 & ITALY & ITALY & ITALY & 1905 & MINER/CM & LUCCA & ITALIAN SETTLEMENT & ADAMS - TRIMOUNTAIN LOCATION \\
\hline 559 & NN & STEFANI & RAFFAELLO & B & 23 & ITALY & ITALY & ITALY & 1904 & MINER/CM & LUCCA & ITALIAN SETTLEMENT & ADAMS - TRIMOUNTAIN LOCATION \\
\hline 560 & NN & MAFFEI & SELINO & B & 27 & ITALY & ITALY & ITALY & 1906 & MINER/CM & LUCCA & ITALIAN SETTLEMENT & ADAMS - TRIMOUNTAIN LOCATION \\
\hline 561 & NN & STEFANI & GIOVANNI & B & 20 & ITALY & ITALY & ITALY & 1906 & MINER/CM & LUCCA & ITALIAN SETTLEMENT & ADAMS - TRIMOUNTAIN LOCATION \\
\hline 562 & NN & BARNIBBINI & NICOLA & B & 23 & ITALY & ITALY & ITALY & 1909 & LABORER/CM & & ITALIAN SETTLEMENT & ADAMS - TRIMOUNTAIN LOCATION \\
\hline 563 & NN & TOMMEI & ARMANDO & B & 20 & ITALY & ITALY & ITALY & 1907 & LABORER/CM & LUCCA & $\begin{array}{l}\text { ITALIAN SETTLEMENT } \\
\text { IT }\end{array}$ & ADAMS - TRIMOUNTAIN LOCATION \\
\hline 564 & NN & VITTORI & EMILIO & $\mathrm{H}$ & 24 & ITALY & ITALY & ITALY & 1907 & LABORER/CM & SEGNI. ROMA & ITALIAN SETTLEMENT & ADAMS - TRIMOUNTAIN LOCATION \\
\hline 565 & NN & VITTORI & MARY & w & 27 & ITALY & ITALY & ITALY & 1906 & & & ITALIAN SETTLEMENT & ADAMS - TRIMOUNTAIN LOCATION \\
\hline 566 & NN & BARSI & LORENZO & B & 28 & ITALY & ITALY & ITALY & 1905 & TRAMMER/CM & LUCCA & $\begin{array}{l}\text { ITALIAN SETTLEMENT } \\
\text { ITEN }\end{array}$ & ADAMS - TRIMOUNTAIN LOCATION \\
\hline
\end{tabular}




\begin{tabular}{|c|c|c|c|c|c|c|c|c|c|c|c|c|c|}
\hline 567 & NN & DOMIDO & AMANTO & B & 32 & ITALY & ITALY & ITALY & 1907 & TRAMMER/CM & & ITALIAN SETTLEMENT & ADAMS - TRIMOUNTAIN LOCATION \\
\hline 568 & $\mathrm{NN}$ & VITTORI & NARGISO & B & 27 & ITALY & ITALY & ITALY & 1906 & TRAMMER/CM & & ITALIAN SETTLEMENT & ADAMS - TRIMOUNTAIN LOCATION \\
\hline 569 & $\mathrm{NN}$ & TORI & SABBATINO & B & 21 & ITALY & ITALY & ITALY & 1910 & TRAMMER/CM & MONTECARLO. LU & ITALIAN SETTLEMENT & ADAMS - TRIMOUNTAIN LOCATION \\
\hline 570 & $\mathrm{NN}$ & BIANCHI & FRANCESCO & $\mathrm{H}$ & 26 & ITALY & ITALY & ITALY & 1905 & TRAMMER/CM & COLLE DI COMPITO. LU & ITALIAN SETTLEMENT & ADAMS - TRIMOUNTAIN LOCATION \\
\hline 571 & NN & BIANCHI & ONORATA & W & 26 & ITALY & ITALY & ITALY & 1906 & & COLLE DI COMPITO. LU & ITALIAN SETTLEMENT & ADAMS - TRIMOUNTAIN LOCATION \\
\hline 572 & NN & BIANCHI & CELINE & D & 7 & ITALY & ITALY & ITALY & 1906 & & COLLE DI COMPITO. LU & ITALIAN SETTLEMENT & ADAMS - TRIMOUNTAIN LOCATION \\
\hline 573 & NN & BIANCHI & JOSEPH & $\mathrm{s}$ & 6 & ITALY & ITALY & ITALY & 1906 & & COLLE DI COMPITO. LU & ITALIAN SETTLEMENT & ADAMS - TRIMOUNTAIN LOCATION \\
\hline 574 & NN & BIANCHI & JENNE & $\mathrm{D}$ & 3 & MICHIGAN & ITALY & ITALY & & & COLLE DI COMPITO. LU & ITALIAN SETTLEMENT & ADAMS - TRIMOUNTAIN LOCATION \\
\hline 575 & NN & BIANCHI & ANNE & $\mathrm{D}$ & $1.6 \mathrm{M}$ & MICHIGAN & ITALY & ITALY & & & COLLE DI COMPITO. LU & ITALIAN SETTLEMENT & ADAMS - TRIMOUNTAIN LOCATION \\
\hline 576 & NN & $\begin{array}{l}\text { BIANCHI } \\
\text { BIAII }\end{array}$ & BABY & $\mathrm{D}$ & $6 \mathrm{M}$ & MICHIGAN & ITALY & ITALY & & & COLLE DI COMPITO. LU & ITALIAN SETTLEMENT & ADAMS - TRIMOUNTAIN LOCATION \\
\hline 577 & NN & DE SANTI & ALBINO & $\mathrm{H}$ & 30 & ITALY & ITALY & ITALY & 1903 & MINER/CM & CASTELVECCHIO. LU & ITALIAN SETTLEMENT & ADAMS - TRIMOUNTAIN LOCATION \\
\hline 578 & NN & DE SANTI & ELIDE & w & 26 & ITALY & ITALY & ITALY & 1909 & & & ITALIAN SETTLEMENT & ADAMS - TRIMOUNTAIN LOCATION \\
\hline 579 & NN & BARSOCCHI & SEVERINO & B & 24 & ITALY & ITALY & ITALY & 1905 & MINER/CM & LUCCA & ITALIAN SETTLEMENT & ADAMS - TRIMOUNTAIN LOCATION \\
\hline 580 & NN & MARE & PETER & B & 36 & ITALY & ITALY & ITALY & 1902 & TRAMMER/CM & FIRENZE & ITALIAN SETTLEMENT & ADAMS - TRIMOUNTAIN LOCATION \\
\hline 581 & NN & TONELLI & LOUIS & B & 40 & ITALY & ITALY & ITALY & 1903 & LABORER/CM & & ITALIAN SETTLEMENT & ADAMS - TRIMOUNTAIN LOCATION \\
\hline 582 & NN & BARTOLOMEI & $\begin{array}{l}\text { VALENTE } \\
\text { VALE }\end{array}$ & $\mathrm{H}$ & 40 & ITALY & ITALY & ITALY & 1903 & MINER/CM & CASTELVECCHIO, LU & $\begin{array}{l}\text { ITALIAN SETTLEMENT } \\
\text { IT }\end{array}$ & ADAMS - TRIMOUNTAIN LOCATION \\
\hline 583 & NN & BARTOLOMEI & MARY & W & 30 & ITALY & ITALY & ITALY & $08 ?$ & & & $\begin{array}{l}\text { ITALIAN SETTLEMENT } \\
\end{array}$ & ADAMS - TRIMOUNTAIN LOCATION \\
\hline 584 & NN & BARTOLOMEI & DOMENICO & $\mathrm{S}$ & 10 & ITALY & ITALY & ITALY & 1903 & & CASTELVECCHIO. LU & ITALIAN SETTLEMENT & ADAMS - TRIMOUNTAIN LOCATION \\
\hline 585 & NN & BARTOLOMEI & NANDO & $\mathrm{s}$ & 5 & MICHIGAN & ITALY & ITALY & & & CASTELVECCHIO. LU & ITALIAN SETTLEMENT & ADAMS - TRIMOUNTAIN LOCATION \\
\hline 586 & NN & BARTOLOMEI & WILLIAM & $\mathrm{s}$ & $1.6 \mathrm{M}$ & MICHIGAN & ITALY & ITALY & & & CASTELVECCHIO. LU & ITALIAN SETTLEMENT & ADAMS - TRIMOUNTAIN LOCATION \\
\hline 587 & NN & DEL GHINGARO & ORAZIO & $\mathrm{H}$ & 33 & ITALY & ITALY & ITALY & 1899 & MINER/CM & LUCCA & ITALIAN SETTLEMENT & ADAMS - TRIMOUNTAIN LOCATION \\
\hline 588 & NN & DEL GHINGARO & ARMILLIA & w & 27 & ITALY & ITALY & ITALY & 1905 & & & $\begin{array}{l}\text { ITALIAN SETTLEMENT } \\
\text { ITEN }\end{array}$ & ADAMS - TRIMOUNTAIN LOCATION \\
\hline 589 & NN & DEL GHINGARO & FREDERICK & s & $1.6 \mathrm{M}$ & MICHIGAN & ITALY & ITALY & & & LUCCA & $\begin{array}{l}\text { ITALIAN SETTLEMENT } \\
\text { IT }\end{array}$ & ADAMS - TRIMOUNTAIN LOCATION \\
\hline 590 & NN & DEL GHINGARO & BABY & $\mathrm{D}$ & $1 \mathrm{M}$ & MICHIGAN & ITALY & ITALY & & & LUCCA & ITALIAN SETTLEMENT & ADAMS - TRIMOUNTAIN LOCATION \\
\hline 591 & NN & IACOPETTI & CANDELO & B & 34 & ITALY & ITALY & ITALY & 1901 & TRAMMER/CM & CASTELVECCHIO. LU & ITALIAN SETTLEMENT & ADAMS - TRIMOUNTAIN LOCATION \\
\hline 592 & NN & IACOPETTI & FEDERICO & B & 23 & ITALY & ITALY & ITALY & 1907 & TRAMMER/CM & LUCCA & $\begin{array}{l}\text { ITALIAN SETTLEMENT } \\
\text { ITA }\end{array}$ & ADAMS - TRIMOUNTAIN LOCATION \\
\hline 593 & NN & PANATTONI & ROMERO & $\mathrm{B}$ & 22 & ITALY & ITALY & ITALY & 1907 & TRAMMER/CM & LUCCA & ITALIAN SETTLEMENT & ADAMS - TRIMOUNTAIN LOCATION \\
\hline 594 & NN & MARCHESCHI & LORENZO & $\mathrm{H}$ & 32 & ITALY & ITALY & ITALY & 1902 & MINER/CM & LUCCA & $\begin{array}{l}\text { ITALIAN SETTLEMENT } \\
\text { ITAEM }\end{array}$ & ADAMS - TRIMOUNTAIN LOCATION \\
\hline 595 & NN & MARCHESCHI & ELVIRA & W & 32 & ITALY & ITALY & ITALY & 1903 & & LUCCA & ITALIAN SETTLEMENT & ADAMS - TRIMOUNTAIN LOCATION \\
\hline 596 & NN & MARCHESCHI & CARLO & $\mathrm{s}$ & 10 & ITALY & ITALY & ITALY & 1903 & & LUCCA & ITALIAN SETTLEMENT & ADAMS - TRIMOUNTAIN LOCATION \\
\hline 597 & NN & MARCHESCHI & ELISA & $\mathrm{D}$ & 8 & ITALY & ITALY & ITALY & 1903 & & LUCCA & ITALIAN SETTLEMENT & ADAMS - TRIMOUNTAIN LOCATION \\
\hline 598 & NN & MARCHESCHI & GIORGIA & $\mathrm{D}$ & 5 & MICHIGAN & ITALY & ITALY & & & LUCCA & ITALIAN SETTLEMENT & ADAMS - TRIMOUNTAIN LOCATION \\
\hline 599 & NN & MARCHESCHI & FEDERICO & $\mathrm{s}$ & 4 & MICHIGAN & ITALY & ITALY & & & LUCCA & ITALIAN SETTLEMENT & ADAMS - TRIMOUNTAIN LOCATION \\
\hline 600 & NN & MARCHESCHI & BRUNA & D & 2 & MICHIGAN & ITALY & ITALY & & & LUCCA & ITALIAN SETTLEMENT & ADAMS - TRIMOUNTAIN LOCATION \\
\hline 601 & NN & MARCHESCHI & BABY & $\mathrm{s}$ & $8 \mathrm{M}$ & MICHIGAN & ITALY & ITALY & & & LUCCA & $\begin{array}{l}\text { ITALIAN SETTLEMENT } \\
\text { IT }\end{array}$ & ADAMS - TRIMOUNTAIN LOCATION \\
\hline 602 & NN & PIERONI & JOSEPH & $\mathrm{H}$ & 29 & ITALY & ITALY & ITALY & 1907 & MINER/CM & LUCCA & $\begin{array}{l}\text { ITALIAN SETTLEMENT } \\
\text { ITAEN }\end{array}$ & ADAMS - TRIMOUNTAIN LOCATION \\
\hline 603 & NN & PIERONI & ? & W & 23 & $\begin{array}{l}\text { IITALY } \\
\text { ITALY }\end{array}$ & $\begin{array}{l}\text { IITALY } \\
\text { ITALY }\end{array}$ & $\begin{array}{l}\text { ITALY } \\
\text { ITALY }\end{array}$ & 1908 & IVIINER/CINI & LUCCA & ITALIAN SETTLEMENT & ADAMS - TRIMOUNTAIN LOCATION \\
\hline 604 & NN & PIERONI & LOUIS & $\mathrm{s}$ & 2 & ITALY & ITALY & ITALY & 1908 & & LUCCA & ITALIAN SETTLEMENT & ADAMS - TRIMOUNTAIN LOCATION \\
\hline 605 & NN & MARTINUCCI & PETER & B & 21 & ITALY & ITALY & ITALY & 1908 & TRAMMER/CM & LUCCA & $\begin{array}{l}\text { ITALIAN SETTLEMENT } \\
\text { IT }\end{array}$ & ADAMS - TRIMOUNTAIN LOCATION \\
\hline 606 & NN & MARCHESCHI & PARISE & $\mathrm{B}$ & 19 & ITALY & ITALY & ITALY & 1908 & TRAMMER/CM & LUCCA & $\begin{array}{l}\text { ITALIAN SETTLEMENT } \\
\end{array}$ & ADAMS - TRIMOUNTAIN LOCATION \\
\hline 607 & NN & $\begin{array}{l}\text { SIMI } \\
\text { SIACOCII }\end{array}$ & OLINDO & B & 32 & ITALY & ITALY & ITALY & 1910 & MASON/CM & VILLA BASILICA. LU & ITALIAN SETTLEMENT & ADAMS - TRIMOUNTAIN LOCATION \\
\hline 608 & NN & BIANCHI & JOSEPH & B & 23 & ITALY & ITALY & ITALY & 1902 & MINER/CM & LUCCA & $\begin{array}{l}\text { ITALIAN SETTLEMENT } \\
\text { ITEME }\end{array}$ & ADAMS - TRIMOUNTAIN LOCATION \\
\hline 609 & NN & PIERONI & LENY & B & 23 & ITALY & ITALY & ITALY & 1903 & MINER/CM & LUCCA & ITALIAN SETTLEMENT & ADAMS - TRIMOUNTAIN LOCATION \\
\hline 610 & NN & CERVELLI & CORRADO & B & 24 & ITALY & ITALY & ITALY & 1907 & TRAMMER/CM & CAPANNORI. LU & ITALIAN SETTLEMENT & ADAMS - TRIMOUNTAIN LOCATION \\
\hline 611 & NN & PALMERI & FRANK & B & 30 & ITALY & ITALY & ITALY & 1904 & MINER/CM & & ITALIAN SETTLEMENT & ADAMS - TRIMOUNTAIN LOCATION \\
\hline 612 & NN & GIUSTI & UMBERTO & B & 27 & ITALY & ITALY & ITALY & 1909 & TRAMMER/CM & LUCCA & ITALIAN SETTLEMENT & ADAMS - TRIMOUNTAIN LOCATION \\
\hline 613 & NN & PIEROTTI & ORLANDO & $\mathrm{H}$ & 30 & ITALY & ITALY & ITALY & 1906 & MINER/CM & COLLE DI COMPITO. LU & $\begin{array}{l}\text { ITALIAN SETTLEMENT } \\
\end{array}$ & ADAMS - TRIMOUNTAIN LOCATION \\
\hline 614 & NN & PIEROTTI & ROSA & w & 30 & ITALY & ITALY & ITALY & 1907 & & COLLE DI COMPITO. LU & $\begin{array}{l}\text { ITALIAN SETTLEMENT } \\
\text { ITSE }\end{array}$ & ADAMS - TRIMOUNTAIN LOCATION \\
\hline 615 & NN & PIEROTTI & PIETRO & $\mathrm{s}$ & 4 & ITALY & ITALY & ITALY & 1907 & & COLLE DI COMPITO. LU & $\begin{array}{l}\text { ITALIAN SETTLEMENT } \\
\text { ITIEN }\end{array}$ & ADAMS - TRIMOUNTAIN LOCATION \\
\hline $\begin{array}{l}615 \\
616\end{array}$ & NN & $\begin{array}{l}\text { PIEROTIII } \\
\text { PIEROTI }\end{array}$ & LENA & D & $1.6 \mathrm{M}$ & MICHIGAN & $\begin{array}{l}\text { ITALY } \\
\text { ITALY }\end{array}$ & $\begin{array}{l}\text { ITALY } \\
\text { ITALY }\end{array}$ & 1901 & & COLLE DI COMPITO. LU & ITALIAN SETTLEMENT & ADAMS - TRIMOUNTAIN LOCATION \\
\hline 617 & NN & PIEROTTI & ALFIO & B & 25 & ITALY & ITALY & ITALY & 1906 & TRAMMER/CM & COLLE DI COMPITO. LU & $\begin{array}{l}\text { ITALIAN SETTLEMENT } \\
\text { IT }\end{array}$ & ADAMS - TRIMOUNTAIN LOCATION \\
\hline 618 & NN & PIEROTTI & JOSEPH & B & 19 & ITALY & ITALY & ITALY & 1909 & TRAMMER/CM & COLLE DI COMPITO, LU & ITALIAN SETTLEMENT & ADAMS - TRIMOUNTAIN LOCATION \\
\hline 619 & NN & MARCHESCHI & LOUIS & $\mathrm{B}$ & 33 & ITALY & ITALY & ITALY & 1906 & TRAMMER/CM & LUCCA & ITALIAN SETTLEMENT & ADAMS - TRIMOUNTAIN LOCATION \\
\hline 620 & NN & LOMBARDI & ROBERT & $\mathrm{H}$ & 32 & ITALY & ITALY & ITA| & 1907 & R/CM & LUC & IENT & ADAMS - TRIMOUNTAIN LOCATION \\
\hline 621 & NN & MARCHESCHI & GIOVAI & B & 25 & ITA & ITA & ITA| & 1905 & IER/CM & LUCCA & $\begin{array}{l}\text { ITALIAN SETTLEMENT } \\
\text { ITAMEN }\end{array}$ & ADAMS - TRIMOUNTAIN LOCATION \\
\hline 622 & NN & NOVELLI & ANGEL & B & 31 & ITAL & ITAL & ITA & 19 & TRAMMER/CM & CASTELVECCHIO. LU & ITALIAN SETTLEMENT & ADAMS - TRIMOUNTAIN LOCATION \\
\hline 623 & NN & PERA & ADRIANO & B & 35 & ITALY & ITALY & ITALY & 1907 & TRAMMER/CM & VILLA BASILICA. LU & $\begin{array}{l}\text { ITALIAN SETTLEMENT } \\
\text { IT }\end{array}$ & ADAMS - TRIMOUNTAIN LOCATION \\
\hline 624 & NN & MARCHESCHI & AMELIO & B & 32 & ITALY & ITALY & ITALY & 1903 & TRAMMER/CM & LUCCA & ITALIAN SETTLEMENT & ADAMS - TRIMOUNTAIN LOCATION \\
\hline 625 & NN & MARCHESCHI & SATURNO & B & 26 & ITALY & ITALY & ITALY & 1906 & TRAMMER/CM & LUCCA & ITALIAN SETTLEMENT & ADAMS - TRIMOUNTAIN LOCATION \\
\hline 626 & NN & DELVIGNA & LUIGI & B & 26 & ITALY & ITALY & ITALY & 1909 & TRAMMER/CM & LUCCA & $\begin{array}{l}\text { ITALIAN SETTLEMENT } \\
\text { IT }\end{array}$ & ADAMS - TRIMOUNTAIN LOCATION \\
\hline 627 & NN & BARSUGLIO & GIUSEPPE & $\mathrm{H}$ & 34 & ITALY & ITALY & ITALY & 1906 & TRAMMER/CM & LUCCA & ITALIAN SETTLEMENT & ADAMS - TRIMOUNTAIN LOCATION \\
\hline 628 & NN & BARSUGLIO & NUNZIATA & w & 29 & ITALY & ITALY & ITALY & 1907 & & & $\begin{array}{l}\text { ITALIAN SETTLEMENT } \\
\text { ITIEN }\end{array}$ & ADAMS - TRIMOUNTAIN LOCATION \\
\hline $\begin{array}{l}020 \\
629\end{array}$ & NN & BARSUGLIO & MARIO & s & 5 & MICHIGAN & ITALY & ITALY & & & LUCCA & ITALIAN SETTLEMENT & ADAMS - TRIMOUNTAIN LOCATION \\
\hline
\end{tabular}




\begin{tabular}{|c|c|c|c|c|c|c|c|c|c|c|c|c|c|}
\hline 6301 & NN & BARSUGLIO & BRUNO & $\mathrm{s}$ & 2 & MICHIGAN & ITALY & ITALY & & & LUCCA & ITALIAN SETTLEMENT & ADAMS - TRIMOUNTAIN LOCATION \\
\hline 6311 & NN & BARSUGLIO & BABY & D & $9 \mathrm{M}$ & MICHIGAN & ITALY & ITALY & & & LUCCA & ITALIAN SETTLEMENT & ADAMS - TRIMOUNTAIN LOCATION \\
\hline 6321 & NN & MARTINUCCI & ANTONIO & $\mathrm{H}$ & 27 & ITALY & ITALY & ITALY & 1902 & MINER/CM & LUCCA & ITALIAN SETTLEMENT & ADAMS - TRIMOUNTAIN LOCATION \\
\hline 6331 & NN & MARTINUCCI & LISANDRA & W & 25 & ITALY & ITALY & ITALY & 1908 & & LUCCA & ITALIAN SETTLEMENT & ADAMS - TRIMOUNTAIN LOCATION \\
\hline $634 i$ & NN & MARTINUCCI & JOSEPHINA & $\mathrm{D}$ & 3 & ITALY & ITALY & ITALY & 1908 & & LUCCA & ITALIAN SETTLEMENT & ADAMS - TRIMOUNTAIN LOCATION \\
\hline 635 & NN & MARTINUCCI & PIERINA & $\mathrm{D}$ & $1.2 \mathrm{M}$ & MICHIGAN & ITALY & ITALY & & & LUCCA & ITALIAN SETTLEMENT & ADAMS - TRIMOUNTAIN LOCATION \\
\hline 636 & NN & LUCCHYI & JOSEPH & B & 36 & ITALY & ITALY & ITALY & 1898 & TRAMMER/CM & & ITALIAN SETTLEMENT & ADAMS - TRIMOUNTAIN LOCATION \\
\hline 6371 & NN & FINI & PIETRO & B & 42 & ITALY & ITALY & ITALY & 1907 & TRAMMER/CM & FROSINONE & ITALIAN SETTLEMENT & ADAMS - TRIMOUNTAIN LOCATION \\
\hline 638 & NN & PRATALI & NARCISO & B & 26 & ITALY & ITALY & ITALY & 1906 & MINER/CM & LUCCA & $\begin{array}{l}\text { ITALIAN SETTLEMENT } \\
\text { ITAEN }\end{array}$ & ADAMS - TRIMOUNTAIN LOCATION \\
\hline 639 & $\mathrm{NN}$ & NUTI & JOHN & B & 40 & ITALY & ITALY & ITALY & 1907 & TRAMMER/CM & CAPANNORI. LU & ITALIAN SETTLEMENT & ADAMS - TRIMOUNTAIN LOCATION \\
\hline 640 & NN & BIANCHI & BARTOLOMEO & B & 19 & ITALY & ITALY & ITALY & 1909 & TRAMMER/CM & LUCCA & ITALIAN SETTLEMENT & ADAMS - TRIMOUNTAIN LOCATION \\
\hline $641 i$ & NN & PIERONI & TONY & B & 38 & ITALY & ITALY & ITALY & 1905 & TRAMMER/CM & LUCCA & $\begin{array}{l}\text { ITALIAN SETTLEMENT } \\
\end{array}$ & ADAMS - TRIMOUNTAIN LOCATION \\
\hline 642 & & LENCIONI & LUIGI & $\mathrm{H}$ & 33 & ITALY & ITALY & ITALY & 1899 & FIREMAN/CM & LUCCA & & ADAMS - PAINSDALE \\
\hline 643 & & LENCIONI & MESSALINA & W & 31 & ITALY & ITALY & ITALY & 1901 & & & & ADAMS - PAINSDALE \\
\hline 644 & & LENCIONI & MAGGIE & $\mathrm{D}$ & 8 & MICHIGAN & ITALY & ITALY & & & LUCCA & & ADAMS - PAINSDALE \\
\hline 645 & & LENCIONI & JOHN & $\mathrm{s}$ & 7 & MICHIGAN & ITALY & ITALY & & & LUCCA & & ADAMS - PAINSDALE \\
\hline 646 & & LENCIONI & PETRA & s & $3 \mathrm{M}$ & MICHIGAN & ITALY & ITALY & & & LUCCA & & ADAMS - PAINSDALE \\
\hline 647 & & LENCIONI & IDATO & BR & $30 ?$ & ITALY & ITALY & ITALY & 1903 & & LUCCA & & ADAMS - PAINSDALE \\
\hline 648 & & GUIDI & JOSEPH & B & 37 & ITALY & ITALY & ITALY & 1906 & & LUCCA & & ADAMS - PAINSDALE \\
\hline 649 & & TERCHIOLANA & SAMUEL & B & $42 ?$ & ITALY & ITALY & ITALY & $1906 ?$ & LANDER/ROCK HOUSE & & & ADAMS - PAINSDALE \\
\hline 650 & & CIABATTARI & PIETRO & B & 31 & ITALY & ITALY & ITALY & 1908 & ROCK HOUSE MAN & LUCCA & & ADAMS - PAINSDALE \\
\hline 651 & & AMANTI & F? & B & 37 & ITALY & ITALY & ITALY & 1909 & LABORER/CM & LUCCA & & ADAMS - PAINSDALE \\
\hline 652 & & AMANTI & LORENZO & $\begin{array}{l}\mathrm{D} \\
\mathrm{B}\end{array}$ & 32 & ITALY & ITALY & ITALY & 1906 & LABORER/CM & LUCCA & & ADAMS - PAINSDALE \\
\hline 653 & & RENALDI & JAMES & $\mathrm{H}$ & 30 & ITALY & ITALY & ITALY & 1902 & MECHANIC/CM & SAN GIORGIO CANAVESE. TO & & ADAMS - PAINSDALE \\
\hline 654 & & RENALDI & SECONDA & w & 29 & ITALY & ITALY & ITALY & 1903 & & & & ADAMS - PAINSDALE \\
\hline 655 & & RENALDI & ELMA & $\mathrm{D}$ & 4 & MICHIGAN & ITALY & ITALY & & & SAN GIORGIO CANAVESE. TO & & ADAMS - PAINSDALE \\
\hline 656 & & RENALDI & GUY & $\mathrm{s}$ & $1.4 \mathrm{M}$ & MICHIGAN & ITALY & ITALY & & & SAN GIORGIO CANAVESE. TO & & ADAMS - PAINSDALE \\
\hline 657 & & RENALDI & OLIMPIA & $\mathrm{D}$ & 1 & MICHIGAN & ITALY & ITALY & & & SAN GIORGIO CANAVESE, TO & & ADAMS - PAINSDALE \\
\hline 658 & & RENALDI & PIETRO & $\mathrm{H}$ & 32 & ITALY & ITALY & ITALY & $1903 ?$ & LABORER/CM & SAN GIORGIO CANAVESE. TO & & ADAMS - PAINSDALE \\
\hline 659 & & RENALDI & ANNA & W & 32 & ITALY & ITALY & ITALY & 1904 & & & & ADAMS - PAINSDALE \\
\hline 660 & & RENALDI & JAMES & $\mathrm{s}$ & 8 & MICHIGAN & ITALY & ITALY & & & SAN GIORGIO CANAVESE. TO & & ADAMS - PAINSDALE \\
\hline 661 & & RENALDI & FRANK & $\mathrm{D}$ & 5 & MICHIGAN & ITALY & ITALY & & & SAN GIORGIO CANAVESE. TO & & ADAMS - PAINSDALE \\
\hline 662 & & RENALDI & GIULIO & $\mathrm{s}$ & 3 & MICHIGAN & ITALY & ITALY & & & SAN GIORGIO CANAVESE. TO & & ADAMS - PAINSDALE \\
\hline 663 & & RENALDI & TERESINA & $\mathrm{D}$ & 2 & MICHIGAN & ITALY & ITALY & & & SAN GIORGIO CANAVESE, TO & & ADAMS - PAINSDALE \\
\hline 664 & & RICCI & VINCENZO & $\mathrm{H}$ & 34 & ITALY & ITALY & ITALY & 1907 & LABORER/CM & $\begin{array}{l}\text { LUCCA } \\
\text { LUTARES, IO }\end{array}$ & & ADAMS - PAINSDALE \\
\hline 664 & & RICCl & ROSA & W & 33 & ITALY & ITALY & $\begin{array}{l}\text { ITALY } \\
\text { ITAY }\end{array}$ & 1907 & LADUREKRIVIV & LUCCA & & ADAMS - PAINSDALE \\
\hline 666 & & $\mathrm{RICCl}$ & MATILDA & $\mathrm{D}$ & 2 & MICHIGAN & ITALY & ITALY & & & LUCCA & & ADAMS - PAINSDALE \\
\hline 667 & & $\mathrm{RICCl}$ & A. & $\mathrm{D}$ & $1.6 \mathrm{M}$ & MICHIGAN & ITALY & ITALY & & & LUCCA & & PAINSDALE \\
\hline 668 & & $\mathrm{RICCl}$ & OLGA & $\mathrm{D}$ & 1. & MICHIGAN & ITALY & ITALY & & & LUCCA & & ADAMS - PAINSDALE \\
\hline 669 & & GUIDI & EMILIO & B & 35 & ITALY & ITALY & ITALY & 1903 & ROCK HOUSE MAN & MASSA CARRARA & & ADAMS - PAINSDALE \\
\hline 670 & & PIEROTTI & SECONDO & B & 26 & ITALY & ITALY & ITALY & 1907 & ROCK HOUSE MAN & GUBBIO. PERUGIA & & ADAMS - PAINSDALE \\
\hline 671 & & DELVIGNA & GUGLIELMO & B & 27 & ITALY & ITALY & ITALY & 1907 & ROCK HOUSE MAN & LUCCA & & ADAMS - PAINSDALE \\
\hline 672 & & DAILBRAN & DOMENICO & B & 38 & ITALY & ITALY & ITALY & 1904 & ROCK HOUSE MAN & & & ADAMS - PAINSDALE \\
\hline 673 & & OZZELLO & JOSEPH & $\mathrm{H}$ & 41 & ITALY & ITALY & ITALY & 1902 & LABORER/CM & SAN GIUSTO CANAVESE. TO & & ADAMS - PAINSDALE \\
\hline 674 & & OZZELLLO & DOMENICA & W & 31 & ITALY & ITALY & ITALY & 1904 & & & & ADAMS - PAINSDALE \\
\hline 675 & & OZZELLO & MARGHERITA & $\mathrm{D}$ & 10 & MICHIGAN & ITALY & ITALY & & & SAN GIUSTO CANAVESE. TO & & ADAMS - PAINSDALE \\
\hline 676 & & OZZELLO & DOMENICO & s & 9 & MICHIGAN & ITALY & ITALY & & & SAN GIUSTO CANAVESE. TO & & ADAMS - PAINSDALE \\
\hline 677 & & OZZELLO & PIETRO & s & 6 & MICHIGAN & ITALY & ITALY & & & SAN GIUSTO CANAVESE. TO & & ADAMS - PAINSDALE \\
\hline 678 & & REGIS & DOMENICO & $\mathrm{H}$ & 32 & ITALY & ITALY & ITALY & 1902 & FIREMAN/CM & SAN GIUSTO CANAVESE. TO & & ADAMS - PAINSDALE \\
\hline 679 & & REGIS & DOMENICA & w & 27 & ITALY & ITALY & ITALY & 1906 & & & & ADAMS - PAINSDALE \\
\hline 680 & & IAVELLO & CARLO & $\mathrm{H}$ & 29 & ITALY & ITALY & ITALY & 1906 & LABORER/CM & SAN GIUSTO CANAVESE. TO & & ADAMS - PAINSDALE \\
\hline 681 & & IAVELLO & MARY & W & 33 & ITALY & ITALY & ITALY & 1906 & & SAN GIUSTO CANAVESE, TO & & ADAMS - PAINSDALE \\
\hline 682 & & IAVELLO & MARTA & $\mathrm{D}$ & 2 & MICHIGAN & ITALY & ITALY & & & SAN GIUSTO CANAVESE. TO & & ADAMS - PAINSDALE \\
\hline 683 & & MAGNINO & JOHN & $\mathrm{H}$ & 27 & ITALY & ITALY & ITALY & 1905 & MINER/CM & SPARONE. TO & & ADAMS - PAINSDALE \\
\hline 684 & & MAGNINO & MARY & W & 23 & ITALY & ITALY & ITALY & $1907 ?$ & & & & ADAMS - PAINSDALE \\
\hline 685 & & MAGNINO & LUCY & D & $1.2 \mathrm{M}$ & MICHIGAN & ITALY & ITALY & & & SPARONE. TO & & ADAMS - PAINSDALE \\
\hline 686 & & ROMAGNOLI & VINCENZO & B & 27 & ITALY & ITALY & ITALY & 1906 & MINER/CM & & & ADAMS - PAINSDALE \\
\hline 687 & & SUCCA & ANTONIO & B & 28 & ITALY & ITALY & ITALY & 1905 & MINER/CM & TORINO & & ADAMS - PAINSDALE \\
\hline 688 & & MANGONI & GIOVANNI & B & 35 & ITALY & ITALY & ITALY & 1907 & MINER/CM & & & ADAMS - PAINSDALE \\
\hline 689 & & TEPATTI & GIACOMO & B & 40 & ITALY & ITALY & ITALY & 1900 & MINER/CM & PONT CANAVESE. TO & & ADAMS - PAINSDALE \\
\hline 690 & & IAVELLO & ANTONIO & $\begin{array}{l}\mathrm{D} \\
\mathrm{B}\end{array}$ & 27 & ITALY & ITALY & ITALY & 1907 & MINER/CM & SAN GIUSTO CANAVESE. TO & & ADAMS - PAINSDALE \\
\hline 691 & & RENALDI & ANTONIO & $\mathrm{H}$ & 32 & ITALY & ITALY & ITALY & $?$ & MINER/CM & SAN GIORGIO CANAVESE. TO & & ADAMS - PAINSDALE \\
\hline 692 & & RENALDI & MARIA & W & 26 & ITALY & ITALY & ITALY & 1905 & & SAN GIORGIO CANAVESE. TO & & ADAMS - PAINSDALE \\
\hline
\end{tabular}




\begin{tabular}{|c|c|c|c|c|c|c|c|c|c|c|c|c|c|}
\hline 693 & & RENALDI & GIUSEPPE & $\mathrm{s}$ & 3 & MICHIGAN & ITALY & ITALY & & & SAN GIORGIO CANAVESE. TO & & ADAMS - PAINSDALE \\
\hline 694 & & RENALDI & GIUSEPPE & BR & 28 & ITALY & ITALY & ITALY & 1906 & MINER/CM & SAN GIORGIO CANAVESE. TO & & ADAMS - PAINSDALE \\
\hline 695 & & REGIS & JOHN & $\mathrm{H}$ & 33 & ITALY & ITALY & ITALY & 1903 & MINER/CM & SAN GIORGIO CANAVESE. TO & & ADAMS - PAINSDALE \\
\hline 696 & & REGIS & TERESA & W & 30 & ITALY & ITALY & ITALY & 1903 & & SAN GIORGIO CANAVESE. TO & & ADAMS - PAINSDALE \\
\hline 697 & & REGIS & JOSEPH & $\mathrm{s}$ & $1.6 \mathrm{M}$ & MICHIGAN & ITALY & ITALY & & & SAN GIORGIO CANAVESE. TO & & ADAMS - PAINSDALE \\
\hline 698 & & REGIS & GIUSTA & $\mathrm{D}$ & & MICHIGAN & ITALY & ITALY & & & SAN GIORGIO CANAVESE. TO & & ADAMS - PAINSDALE \\
\hline 699 & & POLI/ POL (G) & FRANK & $\mathrm{H}$ & 20 & ITALY & ITALY & ITALY & 1907 & MINER/CM & CREMONA & & ADAMS - PAINSDALE \\
\hline 700 & & POLI/ POL (G) & MARY & w & 25 & ITALY & ITALY & ITALY & 1907 & & CREMONA & & ADAMS - PAINSDALE \\
\hline 701 & & POLI/ POL (G) & CATERINA & $\mathrm{D}$ & 3 & MICHIGAN & ITALY & ITALY & 1501 & & CREMONA & & ADAMS - PAINSDALE \\
\hline 702 & & GIACHINO & JOSEPH & $\mathrm{H}$ & 31 & ITALY & ITALY & ITALY & 1904 & MINER/CM & SPARONE. TO & & ADAMS - PAINSDALE \\
\hline 703 & & GIACHINO & LUISA & w & 29 & ITALY & ITALY & ITALY & 1906 & & & & ADAMS - PAINSDALE \\
\hline 704 & & GIACHINO & LENA & $\mathrm{D}$ & 7 & MICHIGAN & ITALY & ITALY & & & SPARONE. TO & & ADAMS - PAINSDALE \\
\hline 705 & & GIACHINO & LUCIA & D & 3 & MICHIGAN & ITALY & ITALY & & & SPARONE. TO & & ADAMS - PAINSDALE \\
\hline 706 & & GIACHINO & MARY & $\mathrm{D}$ & $1.2 \mathrm{M}$ & MICHIGAN & ITALY & ITALY & & & SPARONE. TO & & ADAMS - PAINSDALE \\
\hline 707 & & GIACHINO & JOSEPH & $\mathrm{s}$ & $1 \mathrm{M}$ & MICHIGAN & ITALY & ITALY & & & SPARONE. TO & & ADAMS - PAINSDALE \\
\hline 708 & 217 & BURGHI & ANTONIO & $\mathrm{H}$ & 35 & ITALY & ITALY & ITALY & 1896 & MINER/CM & PARMA & & ADAMS - PAINSDALE \\
\hline 709 & 217 & BURGHI & GIANNETTA & W & 30 & ITALY & ITALY & ITALY & 1902 & & & & ADAMS - PAINSDALE \\
\hline 710 & 217 & BURGHI & ANNE & D & 4 & MICHIGAN & ITALY & ITALY & & & PARMA & & ADAMS - PAINSDALE \\
\hline 711 & 217 & BURGHI & MARY & $\mathrm{D}$ & 3 & MICHIGAN & ITALY & ITALY & & & PARMA & & ADAMS - PAINSDALE \\
\hline 712 & 217 & NICHLEN & ANDREA & B & 22 & ITALY & ITALY & ITALY & 1901 & MINER/CM & & & ADAMS - PAINSDALE \\
\hline 713 & 217 & BOGGIO & DOMENICO & B & 23 & ITALY & ITALY & ITALY & 1900 & MINER/CM & TORINO & & ADAMS - PAINSDALE \\
\hline 714 & 217 & ANSALDO & MAURIZIO & B & 23 & ITALY & ITALY & ITALY & 1907 & MINER/CM & TORINO & & ADAMS - PAINSDALE \\
\hline 715 & & GUGLIELMINO & FRANK & $\mathrm{H}$ & 31 & ITALY & ITALY & ITALY & 1904 & MINER/CM & SAN GIORGIO CANAVESE. TO & & ADAMS - PAINSDALE \\
\hline 716 & & GUGLIELMINO & CATERINA & w & 28 & ITALY & ITALY & ITALY & 1905 & & & & ADAMS - PAINSDALE \\
\hline 717 & & GUGLIELMINO & FRANK JR. & s & 6 & MICHIGAN & ITALY & ITALY & & & SAN GIORGIO CANAVESE. TO & & ADAMS - PAINSDALE \\
\hline 718 & & GUGLIELMINO & TERESA & $\mathrm{D}$ & 3 & MICHIGAN & ITALY & ITALY & & & SAN GIORGIO CANAVESE. TO & & ADAMS - PAINSDALE \\
\hline 719 & & GUGLIELMINO & DOMENICO & B & 24 & ITALY & ITALY & ITALY & 1906 & MINER/CM & TORINO & & ADAMS - PAINSDALE \\
\hline 720 & & GUGLIELMINO & GIACOMO & B & 24 & ITALY & ITALY & ITALY & 1907 & MINER/CM & TORINO & & ADAMS - PAINSDALE \\
\hline 721 & & LAURENTI & GIACOMO & B & 20 & ITALY & ITALY & ITALY & 1906 & MINER/CM & TORINO & & ADAMS - PAINSDALE \\
\hline 722 & & LAURENTI & MASSIMO & B & 22 & ITALY & ITALY & ITALY & 1908 & MINER/CM & TORINO & & ADAMS - PAINSDALE \\
\hline 723 & & HAKALA & ENRICO & B & 27 & ITALY & ITALY & ITALY & 1904 & MINER/CM & & & ADAMS - PAINSDALE \\
\hline 724 & & BARDISOSNE & ANTONIO & B & 25 & ITALY & ITALY & ITALY & 1907 & MINER/CM & TORINO & & ADAMS - PAINSDALE \\
\hline 725 & & BASSONI & CARLO & B & 23 & ITALY & ITALY & ITALY & 1908 & MINER/CM & LUCCA & & ADAMS - PAINSDALE \\
\hline 726 & & NUCCIO & ANDREA & B & 32 & ITALY & ITALY & ITALY & 1907 & MINER/CM & CANDIA CANAVESE, TO & & ADAMS - PAINSDALE \\
\hline 727 & & ANSELMO & $\begin{array}{l}\text { PIETRO } \\
\text { PIETR }\end{array}$ & B & 25 & ITALY & ITALY & ITALY & 1907 & MINER/CM & PAVONE CANAVESE. TO & & ADAMS - PAINSDALE \\
\hline $\begin{array}{l}727 \\
728\end{array}$ & & SERAZIO & ANTONIO & $\begin{array}{l}\mathrm{D} \\
\mathrm{B}\end{array}$ & 26 & ITALY & ITALY & ITALY & 1906 & MINER/CM & SAN GIORGIO CANAVESE. TO & & ADAMS - PAINSDALE \\
\hline 729 & & GIACHINO & GIUSEPPE & B & 26 & ITALY & ITALY & ITALY & 1908 & MINER/CM & TORINO & & ADAMS - PAINSDALE \\
\hline 730 & & CESARINI & ORLANDO & B & 24 & ITALY & ITALY & ITALY & 1908 & MINER/CM & & & ADAMS - PAINSDALE \\
\hline 731 & 513 & GIANNINI & AUGUSTO & $\mathrm{H}$ & 25 & ITALY & ITALY & ITALY & 1904 & LABORER/ROCK HOUSE & LUCCA & 8TH ST. & CALUMET - RED JACKET VILLAGE \\
\hline 732 & 513 & GIANNINI & OLIMPIA & W & 25 & ITALY & ITALY & ITALY & 1907 & & & 8TH ST. & CALUMET - RED JACKET VILLAGE \\
\hline 733 & 513 & GIANNINI & ELSIE & D & 1 & MICHIGAN & ITALY & ITALY & & & LUCCA & 8TH ST. & CALUMET - RED JACKET VILLAGE \\
\hline 734 & 513 & GIANNINI & PAUL & $\mathrm{s}$ & 0 & MICHIGAN & ITALY & ITALY & & & LUCCA & 8TH ST. & CALUMET - RED JACKET VILLAGE \\
\hline 735 & 513 & GIANNINI & LORENZO & $\mathrm{BR}$ & 22 & ITALY & ITALY & ITALY & 1907 & LABORER/ROCK PLANT & LUCCA & 8TH ST. & CALUMET - RED JACKET VILLAGE \\
\hline 736 & $513 B$ & FERRARI & LUIGI & $\mathrm{H}$ & 30 & ITALY & ITALY & ITALY & 1898 & FIREMAN/STATIONARY BOILER & COLLODI. LU & 8TH ST. & CALUMET - RED JACKET VILLAGE \\
\hline 737 & $513 B$ & FERRARI & MELANIA & W & 28 & ITALY & ITALY & ITALY & 1903 & & & 8TH ST. & CALUMET - RED JACKET VILLAGE \\
\hline 738 & $513 \mathrm{~B}$ & FERRARI & ROSE & $\mathrm{D}$ & 5 & MICHIGAN & ITALY & ITALY & & & COLLODI. LU & 8TH ST. & CALUMET - RED JACKET VILLAGE \\
\hline 739 & $513 B$ & FERRARI & PAUL & s & 3 & MICHIGAN & ITALY & ITALY & & & COLLODI. LU & 8TH ST. & CALUMET - RED JACKET VILLAGE \\
\hline 740 & 812 & PERENCHIO & JOHN B. & H & 35 & ITALY & ITALY & ITALY & 1898 & SALOON/LIQUOR EMPORIUM & PAVONE CANAVESE. TO & W. PINE ST. & CALUMET - RED JACKET VILLAGE \\
\hline 741 & 812 & PERENCHIO & MADE & w & 22 & ITALY & ITALY & ITALY & 1904 & & & W. PINE ST. & CALUMET - RED JACKET VILLAGE \\
\hline 742 & 812 & PERENCHIO & ANDREA & s & 5 & MICHIGAN & ITALY & ITALY & & & PAVONE CANAVESE. TO & W. PINE ST. & CALUMET - RED JACKET VILLAGE \\
\hline 743 & 812 & PERENCHIO & MARGHERITA & $\mathrm{D}$ & 4 & MICHIGAN & ITALY & ITAL & & & E CANAVESE. TO & W. PINE ST. & CALUMET - RED JACKET VILLAGE \\
\hline 744 & 812 & ADDA & GIORGIO & $\mathrm{H}$ & 37 & ITALY & ITALY & ITALY & 1896 & SALOON/LIQUOR EMPORIUM & PAVONE CANAVESE, TO & W. PINE ST. & CALUMET - RED JACKET VILLAGE \\
\hline 745 & 812 & ADDA & MARY & W & 30 & ITALY & ITALY & ITALY & 1906 & & & W. PINE ST. & CALUMET - RED JACKET VILLAGE \\
\hline 746 & 812 & BASTONE & ANTHONY & B & 23 & ITALY & ITALY & ITALY & 1909 & BARTENDER/SALOON & COSENZA & W. PINE ST. & CALUMET - RED JACKET VILLAGE \\
\hline 747 & 812 & GAMARRA & MARY & SER & 28 & ITALY & ITALY & ITALY & 1909 & SERVANT/PF & SAN BENIGNO CANAVESE. TO & W. PINE ST. & CALUMET - RED JACKET VILLAGE \\
\hline 748 & 401 & QUELLO & BARTOLOMEO & $\mathrm{H}$ & 69 & ITALY & ITALY & ITALY & 1865 & & LOCANA CANAVESE. TO & 8TH ST. & CALUMET - RED JACKET VILLAGE \\
\hline 749 & 401 & QUELLO & JOHN & $\mathrm{s}$ & 45 & MICHIGAN & ITALY & IRELAND & & & LOCANA CANAVESE. TO & 8TH ST. & CALUMET - RED JACKET VILLAGE \\
\hline 750 & 401 & QUELLO & BARTOLOMEO & $\mathrm{s}$ & 36 & MICHIGAN & ITALY & IRELAND & & & LOCANA CANAVESE, TO & 8TH ST. & CALUMET - RED JACKET VILLAGE \\
\hline 751 & 401 & QUELLO & MARGHERITA & $\mathrm{D}$ & 35 & MICHIGAN & ITALY & IRELAND & & & LOCANA CANAVESE. TO & 8TH ST. & CALUMET - RED JACKET VILLAGE \\
\hline 752 & 401 & QUELLO & ANNA & $\mathrm{D}$ & 28 & MICHIGAN & ITALY & IRELAND & & & LOCANA CANAVESE. TO & 8TH ST. & CALUMET - RED JACKET VILLAGE \\
\hline 753 & 401 & QUELLO & ELISA & $\mathrm{D}$ & 26 & MICHIGAN & ITALY & IRELAND & & & LOCANA CANAVESE. TO & 8TH ST. & CALUMET - RED JACKET VILLAGE \\
\hline 754 & 401 & QUELLO & ALICE & $\mathrm{D}$ & 20 & MICHIGAN & ITALY & IRELAND & & & LOCANA CANAVESE. TO & 8TH ST. & CALUMET - RED JACKET VILLAGE \\
\hline 755 & 809 & BRACCO & BERNARDO & $\mathrm{H}$ & 56 & ITALY & ITALY & ITALY & $188 ?$ & MERCHANT/BAKERY GRC & PONT CANAVESE. TO & ELM ST. & CALUMET - RED JACKET VILLAGE \\
\hline
\end{tabular}




\begin{tabular}{|c|c|c|c|c|c|c|c|c|c|c|c|c|c|}
\hline 756 & 809 & BRACCO & MARIA & W & 54 & ITALY & ITALY & ITALY & 1883 & & PONT CANAVESE. TO & ELM ST. & CALUMET - RED JACKET VILLAGE \\
\hline 757 & 809 & BRACCO & JOSEPHINE & D & 30 & ITALY & ITALY & ITALY & 1883 & CLERK/BAKERY GROCERY & PONT CANAVESE. TO & ELM ST. & CALUMET - RED JACKET VILLAGE \\
\hline 758 & 809 & BRACCO & CARLO & $\mathrm{s}$ & 26 & MICHIGAN & ITALY & ITALY & & MANAGER/BAKERY GROCERY & PONT CANAVESE. TO & ELM ST. & CALUMET - RED JACKET VILLAGE \\
\hline 759 & 809 & BRACCO & ROSIE & $\mathrm{D}$ & 24 & MICHIGAN & ITALY & ITALY & & CLERK/BAKERY GROCERY & PONT CANAVESE. TO & ELM ST. & CALUMET - RED JACKET VILLAGE \\
\hline 760 & 809 & BRACCO & AUGUSTO/AN & $\mathrm{s}$ & 20 & MICHIGAN & ITALY & ITALY & & & PONT CANAVESE. TO & ELM ST. & CALUMET - RED JACKET VILLAGE \\
\hline 761 & 809 & BRACCO & MARY & $\mathrm{D}$ & 17 & MICHIGAN & ITALY & ITALY & & APPRENTICE/MILLING & PONT CANAVESE. TO & ELM ST. & CALUMET - RED JACKET VILLAGE \\
\hline 762 & 809 & BRACCO & CRISTINA & $\mathrm{D}$ & 14 & MICHIGAN & ITALY & ITALY & & & PONT CANAVESE. TO & ELM ST. & CALUMET - RED JACKET VILLAGE \\
\hline 763 & 809 & COPPO & CATERINA & $\mathrm{H}$ & 29 & MICHIGAN & ITALY & ITALY & & PROPRIETOR/BOARDING HOUSE & & ELM ST. & CALUMET - RED JACKET VILLAGE \\
\hline 764 & 809 & COPPO & ANNA & $\mathrm{D}$ & 7 & MICHIGAN & ITALY & ITALY & & 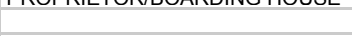 & PONT CANAVESE. TO & ELM ST. & CALUMET - RED JACKET VILLAGE \\
\hline 765 & 809 & COPPO & MARGHERITA & $\mathrm{D}$ & 4 & MICHIGAN & ITALY & ITALY & & & PONT CANAVESE. TO & ELM ST. & CALUMET - RED JACKET VILLAGE \\
\hline 766 & 809 & COPPO & JOHN & $\mathrm{s}$ & 2 & MICHIGAN & ITALY & ITALY & & & PONT CANAVESE. TO & ELM ST. & CALUMET - RED JACKET VILLAGE \\
\hline 767 & 809 & COPPO & ANTONIO & $\mathrm{H}$ & 34 & ITALY & ITALY & ITALY & 1900 & TIMBERMAN/CM & PONT CANAVESE. TO & ELM ST. & CALUMET - RED JACKET VILLAGE \\
\hline 768 & 809 & COPPO & ELSIE & w & 29 & ITALY & ITALY & ITALY & 1902 & & & ELM ST. & CALUMET - RED JACKET VILLAGE \\
\hline 769 & 809 & COPPO & ROSA & D & 6 & MICHIGAN & ITALY & ITALY & & & PONT CANAVESE. TO & ELM ST. & CALUMET - RED JACKET VILLAGE \\
\hline 770 & 809 & COPPO & ALOIS & FA & 68 & ITALY & ITALY & ITALY & 1892 & & PONT CANAVESE. TO & ELM ST. & CALUMET - RED JACKET VILLAGE \\
\hline 771 & 813 & REVELLO & PAUL & $\mathrm{H}$ & 46 & ITALY & ITALY & ITALY & 1890 & PROPRIETOR/LIQUOR SALOON & AGLIE', TO & ELM ST. & CALUMET - RED JACKET VILLAGE \\
\hline 772 & 813 & REVELLO & IDA & W & 22 & ITALY & ITALY & ITALY & 1904 & & & ELM ST. & CALUMET - RED JACKET VILLAGE \\
\hline 773 & 813 & REVELLO & ARTURO & $\mathrm{s}$ & 2 & MICHIGAN & ITALY & ITALY & & & AGLIE'. TO & ELM ST. & CALUMET - RED JACKET VILLAGE \\
\hline 774 & 813 & CATTANEO & MARGHERITA & NIE & 17 & ITALY & ITALY & ITALY & 1907 & & TORINO & ELM ST. & CALUMET - RED JACKET VILLAGE \\
\hline 775 & 825 & BARATONO & ALEX & $\mathrm{H}$ & 31 & ITALY & ITALY & ITALY & 1895 & SALESMAN/GENERAL STORE & TORINO & ELM ST. & CALUMET - RED JACKET VILLAGE \\
\hline 776 & 825 & BARATONO & MADDALENA & w & 28 & ITALY & ITALY & ITALY & 1890 & & & ELM ST. & CALUMET - RED JACKET VILLAGE \\
\hline 777 & 825 & BARATONO & ARTURO & s & 3 & MICHIGAN & ITALY & ITALY & & & TORINO & ELM ST. & CALUMET - RED JACKET VILLAGE \\
\hline 778 & 825 & BARATONO & MARGHERITA & $\mathrm{D}$ & 4 & MICHIGAN & ITALY & ITALY & & & TORINO & ELM ST. & CALUMET - RED JACKET VILLAGE \\
\hline 779 & 825 & PICCHIOTTINO & JOSEPH & $\mathrm{H}$ & 41 & ITALY & ITALY & ITALY & 1889 & ENGINEER/DEPT. STORE & PONT CANAVESE. TO & ELM ST. & CALUMET - RED JACKET VILLAGE \\
\hline 780 & 825 & PICCHIOTTINO & ANNA & w & 39 & ITALY & ITALY & ITALY & 1889 & & PONT CANAVESE. TO & ELM ST. & CALUMET - RED JACKET VILLAGE \\
\hline 781 & 825 & GRINDATTI & LUCY & $\mathrm{H}$ & $53 ?$ & ITALY & ITALY & ITALY & 1899 & & PONT CANAVESE. TO & ELM ST. & CALUMET - RED JACKET VILLAGE \\
\hline 782 & 825 & GRINDATTI & JOHN & $\mathrm{s}$ & 27 & ITALY & ITALY & ITALY & 1899 & ENGINEER/STATIONARY & PONT CANAVESE. TO & ELM ST. & CALUMET - RED JACKET VILLAGE \\
\hline 783 & 825 & GRINDATTI & JOSEPH & $\mathrm{s}$ & 24 & ITALY & ITALY & ITALY & 1899 & BUTCHER/RETAIL MARKET & PONT CANAVESE, TO & ELM ST. & CALUMET - RED JACKET VILLAGE \\
\hline 784 & 825 & GRINDATTI & JAMES & $\mathrm{s}$ & 21 & ITALY & ITALY & ITALY & 1899 & NOT CLEAR & PONT CANAVESE. TO & ELM ST. & CALUMET - RED JACKET VILLAGE \\
\hline 785 & 825 & GRINDATTI & VINCENT & $\mathrm{s}$ & 21 & ITALY & ITALY & ITALY & 1899 & TAILOR APPRENTICE & PONT CANAVESE. TO & ELM ST. & CALUMET - RED JACKET VILLAGE \\
\hline 786 & 415 & CHELI & LORENZO & $\mathrm{H}$ & 31 & MICHIGAN & ITALY & ITALY & & BARTENDER/SALOON & LUCCA & 7TH ST. & CALUMET - RED JACKET VILLAGE \\
\hline 787 & 415 & CHELI & FILOMENA & w & 27 & ITALY & ITALY & ITALY & 1895 & & & 7TH ST. & CALUMET - RED JACKET VILLAGE \\
\hline 788 & 415 & CHELI & EMALINE & $\mathrm{D}$ & 7 & MICHIGAN & MICHIGAN & ITALY & & & LUCCA & 7TH ST. & CALUMET - RED JACKET VILLAGE \\
\hline 789 & 415 & CHELI & ALADINA & $\mathrm{D}$ & 6 & MICHIGAN & MICHIGAN & ITALY & & & LUCCA & 7TH ST. & CALUMET - RED JACKET VILLAGE \\
\hline 790 & 415 & CHELI & FLORENCE & $\mathrm{D}$ & & MICHIGAN & MICHIGAN & ITALY & & & LUCCA & 7TH ST. & CALUMET - RED JACKET VILLAGE \\
\hline 791 & 415 & PIERONI & ORESTE & B & 28 & ITALY & ITALY & ITALY & 1906 & TEAMSTER/LIQUOR & LUCCA & 7TH ST. & CALUMET - RED JACKET VILLAGE \\
\hline 792 & 411 & BANDONI & JOHN & $\mathrm{H}$ & 66 & ITALY & ITALY & ITALY & $75 ?$ & & LUCCA & 7TH ST. & CALUMET - RED JACKET VILLAGE \\
\hline 793 & 411 & BANDONI & CESARINA & W & 62 & ITALY & ITALY & ITALY & $75 ?$ & & LUCCA & 7TH ST. & CALUMET - RED JACKET VILLAGE \\
\hline 794 & 411 & BANDONI & EUGENIA & $\mathrm{D}$ & 23 & MICHIGAN & ITALY & ITALY & & SALESLADY/STORE & LUCCA & 7TH ST. & CALUMET - RED JACKET VILLAGE \\
\hline 795 & 411 & BANDONI & PETER & $\mathrm{s}$ & 15 & MICHIGAN & ITALY & ITALY & & & LUCCA & 7TH ST. & CALUMET - RED JACKET VILLAGE \\
\hline 796 & 411 & GROSSIO & PETER & B & 26 & ITALY & ITALY & ITALY & 1901 & TAILOR & SAN MARTINO CANAVESE. TO & 7TH ST. & CALUMET - RED JACKET VILLAGE \\
\hline 797 & 411 & BIANCHI & NARCISO & $\mathrm{H}$ & 30 & ITALY & ITALY & ITALY & 1898 & SALOON/LIQUOR EMPORIUM & SAN MARTINO IN COLLE. LU & 7TH ST. & CALUMET - RED JACKET VILLAGE \\
\hline 798 & 411 & BIANCHI & IDA & W & 27 & ITALY & ITALY & ITALY & 1898 & & SAN MARTINO IN COLLE. LU & 7TH ST. & CALUMET - RED JACKET VILLAGE \\
\hline 799 & 411 & BIANCHI & JULIO & s & 3 & MICHIGAN & ITALY & ITALY & & & SAN MARTINO IN COLLE. LU & 7TH ST. & CALUMET - RED JACKET VILLAGE \\
\hline 800 & 411 & BIANCHI & CASIMIRA & $\mathrm{D}$ & 1 & MICHIGAN & ITALY & ITALY & & & SAN MARTINO IN COLLE. LU & 7TH ST. & CALUMET - RED JACKET VILLAGE \\
\hline 801 & 411 & PALMINI & MIKE & $\mathrm{H}$ & 65 & ITALY & ITALY & ITALY & 1893 & UMBRELLA REPAIRER & & 7TH ST. & CALUMET - RED JACKET VILLAGE \\
\hline 802 & 411 & PALMINI & MINNIE & W & 47 & SWEDEN & SWEDEN & SWEDEN & 1881 & $\begin{array}{l}\text { NURSE/MIDWIFE } \\
\text { NULA }\end{array}$ & SWEDEN & 7TH ST. & CALUMET - RED JACKET VILLAGE \\
\hline 803 & 411 & PALMINI & PETER & s & 18 & MINNESOT & ITALY & SWEDEN & 1001 & CARPENTER/HOUSE & SWVEDEIN & 7TH ST. & CALUMET - RED JACKET VILLAGE \\
\hline 804 & 407 & PASQUINELLI & PETER & $\mathrm{H}$ & 29 & MICHIGAN & ITALY & ITALY & & PROPRIETOR/SALOON RETAIL & LUCCA & ITALIAN HALL & CALUMET - RED JACKET VILLAGE \\
\hline 805 & 407 & PASQUINELLI & AURELIA & w & 29 & ITALY & ITALY & ITALY & 98/97 & & & ITALIAN H & CALUMET - RED JACKET VILLAGE \\
\hline 806 & 407 & PASQUINELLI & PETER & s & 4 & MICHIGAN & ITALY & ITALY & & & LUCCA & & CALUMET - RED JACKET VILLAGE \\
\hline 807 & 407 & PASQUINELLI & & $\mathrm{D}$ & 6 & MICHIGAN & ITAL & ITA & & & LUCC & ITALIAN H & CALUMET - RED JACKET VILLAGE \\
\hline 808 & 403 & BORGO & DOMENICO & $\mathrm{H}$ & 46 & & & ITA & $81 ?$ & PROPRIETOR/SALOON & VICENZA & & CALUMET - RED JACKET VILLAGE \\
\hline 809 & 403 & BORGO & CATERINA & w & 39 & ITA & ITA & ITA & 1892 & & & 7TH ST. & CALUMET - RED JACKET VILLAGE \\
\hline 810 & 403 & BORGO & JOHN P. & $\mathrm{s}$ & 14 & MICHIGAN & ITAL & ITAI & & MUSICIAN/ORCHESTRA & VICENZA & 7TH ST. & CALUMET - RED JACKET VILLAGE \\
\hline 811 & 403 & GEDDA & JOSEPHINE & NIE & 19 & MICHIGAN & ITALY & ITALY & & SERVANT/PF & TORINO & 7TH ST. & CALUMET - RED JACKET VILLAGE \\
\hline 812 & 509 & ZEI & ZEFFIRO & $\mathrm{H}$ & 28 & ITALY & ITALY & ITALY & 1905 & TAILOR/CUSTOM SHOP & LUCCA & 6TH ST. & CALUMET - RED JACKET VILLAGE \\
\hline 813 & 509 & ZEI & LINA & W & 19 & ITALY & ITALY & ITALY & 1897 & & & 6TH ST. & CALUMET - RED JACKET VILLAGE \\
\hline 814 & 509 & ZEI & ARMANDO & s & $1.6 \mathrm{M}$ & MICHIGAN & ITALY & ITALY & & & LUCCA & 6TH ST. & CALUMET - RED JACKET VILLAGE \\
\hline 815 & 509 & MIGLIO/MIGLIA & CARLO & $\mathrm{H}$ & 57 & ITALY & ITALY & ITALY & 1878 & PROPRIETOR/RETAIL GROCERY & SAN GIORGIO CANAVESE. TO & 6TH ST. & CALUMET - RED JACKET VILLAGE \\
\hline 816 & 509 & MIGLIO/MIGLIA & TERESA & w & 50 & ITALY & ITALY & ITALY & 1888 & & & 6TH ST. & CALUMET - RED JACKET VILLAGE \\
\hline 817 & 509 & MIGLIO/MIGLIA & JOSEPH & s & 25 & MICHIGAN & ITALY & ITALY & & SALESMAN/GROCERY & SAN GIORGIO CANAVESE. TO & 6TH ST. & CALUMET - RED JACKET VILLAGE \\
\hline 818 & 509 & MIGLIO/MIGLIA & GIACOMO & $\mathrm{s}$ & 17 & MICHIGAN & ITALY & ITALY & & BARTENDER/BREWERY & SAN GIORGIO CANAVESE. TO & 6TH ST. & CALUMET - RED JACKET VILLAGE \\
\hline
\end{tabular}




\begin{tabular}{|c|c|c|c|c|c|c|c|c|c|c|c|c|c|}
\hline 819 & 509 & MIGLIO/MIGLIA & TERESA & D & 14 & MICHIGAN & ITALY & ITALY & & & SAN GIORGIO CANAVESE. TO & 6TH ST. & CALUMET - RED JACKET VILLAGE \\
\hline 820 & 509 & MIGLIO/MIGLIA & MARY & $\mathrm{D}$ & 10 & MICHIGAN & ITALY & ITALY & & & SAN GIORGIO CANAVESE. TO & 6TH ST. & CALUMET - RED JACKET VILLAGE \\
\hline 821 & 509 & MIGLIO/MIGLIA & PETER & $\mathrm{s}$ & 8 & MICHIGAN & ITALY & ITALY & & & SAN GIORGIO CANAVESE. TO & 6TH ST. & CALUMET - RED JACKET VILLAGE \\
\hline 822 & 406 & COPPO & EUGENIO & $\mathrm{H}$ & 37 & ITALY & ITALY & ITALY & 1889 & PROPRIETOR/SALOON & PONT CANAVESE. TO & 6TH ST. & CALUMET - RED JACKET VILLAGE \\
\hline 823 & 406 & COPPO & ALADINA & w & 25 & ITALY & ITALY & ITALY & 1901 & & & 6TH ST. & CALUMET - RED JACKET VILLAGE \\
\hline 824 & 406 & COPPO & FRED & s & 6 & MICHIGAN & ITALY & ITALY & & & PONT CANAVESE. TO & 6TH ST. & CALUMET - RED JACKET VILLAGE \\
\hline 825 & 406 & COPPO & $?$ & $\mathrm{~s}$ & 4 & MICHIGAN & ITALY & ITALY & & & PONT CANAVESE. TO & 6TH ST. & $\begin{array}{l}\text { CALUMET - RED JACKET VILLAGE } \\
\text { CEEL }\end{array}$ \\
\hline 826 & 406 & COPPO & MARY & $\mathrm{D}$ & 2 & MICHIGAN & ITALY & ITALY & & SERVANT/PF & PONT CANAVESE. TO & 6TH ST. & CALUMET - RED JACKET VILLAGE \\
\hline 827 & 406 & ZANOLO & MARY & SERV & 21 & ITALY & ITALY & ITALY & 1905 & SERVANT/PF & TORINO & 6TH ST. & CALUMET - RED JACKET VILLAGE \\
\hline 828 & 406 & GIACOLETTO & MARY & SER & 36 & ITALY & ITALY & ITALY & 1899 & SERVANT/PF & PONT CANAVESE. TO & 6TH ST. & CALUMET - RED JACKET VILLAGE \\
\hline 829 & 405 & BALMA/BAIMA & JOSEPH & $\mathrm{H}$ & 42 & ITALY & ITALY & ITALY & 1903 & NOT CLEAR & TORINO & 6TH ST. & CALUMET - RED JACKET VILLAGE \\
\hline 830 & 405 & BALMA/BAIMA & ANGELINA & w & 35 & ITALY & ITALY & ITALY & 1907 & & & 6TH ST. & CALUMET - RED JACKET VILLAGE \\
\hline 831 & 405 & BALMA/BAIMA & MICHELE & s & 5 & MICHIGAN & ITALY & ITALY & & & TORINO & 6TH ST. & CALUMET - RED JACKET VILLAGE \\
\hline 832 & 405 & BALMA/BAIMA & PETER & $\mathrm{s}$ & 3 & MICHIGAN & ITALY & ITALY & & & TORINO & 6TH ST. & CALUMET - RED JACKET VILLAGE \\
\hline 833 & 405 & GIAMPAOLO & ANNA & SER & 17 & ITALY & ITALY & ITALY & 1909 & SERVANT/PF & & 6TH ST. & CALUMET - RED JACKET VILLAGE \\
\hline 834 & 436 & PICCHIOTTINO & PETER & $\mathrm{H}$ & 35 & ITALY & ITALY & ITALY & $92 / 93$ & TEAMSTER/BREWERY & PONT CANAVESE, TO & 6TH ST. & CALUMET - RED JACKET VILLAGE \\
\hline 835 & 436 & PICCHIOTTINO & TERESA & w & 26 & ITALY & ITALY & ITALY & 1903 & & PONT CANAVESE. TO & 6TH ST. & CALUMET - RED JACKET VILLAGE \\
\hline 836 & 436 & PICCHIOTTINO & JOHN & s & 9 & ITALY & ITALY & ITALY & 1903 & & PONT CANAVESE. TO & 6TH ST. & CALUMET - RED JACKET VILLAGE \\
\hline 837 & 436 & PICCHIOTTINO & RICHARD & $\mathrm{s}$ & 5 & MICHIGAN & ITALY & ITALY & & & PONT CANAVESE. TO & 6TH ST. & $\begin{array}{l}\text { CALUMET - RED JACKET VILLAGE } \\
\text { CEELE }\end{array}$ \\
\hline 838 & 436 & PICCHIOTTINO & MARY & $\mathrm{D}$ & 4 & MICHIGAN & ITALY & ITALY & & & PONT CANAVESE. TO & 6TH ST. & $\begin{array}{l}\text { CALUMET - RED JACKET VILLAGE } \\
\text { CEELE }\end{array}$ \\
\hline 839 & 436 & PICCHIOTTINO & $?$ & $\mathrm{D}$ & 2 & MICHIGAN & ITALY & ITALY & & & PONT CANAVESE. TO & 6TH ST. & $\begin{array}{l}\text { CALUMET - RED JACKET VILLAGE } \\
\text { CEEM }\end{array}$ \\
\hline 840 & 436 & BRACCO & MARIA & $\mathrm{H}$ & 46 & ITALY & ITALY & ITALY & 70?? & & & 6TH ST. & CALUMET - RED JACKET VILLAGE \\
\hline 841 & 432 & GHIARDI & ANGELINA & $\mathrm{H}$ & 52 & ITALY & ITALY & ITALY & 1893 & PROPRIETOR/BOARDING HOUSE & SAN MARTINO CANAVESE. TO & 6TH ST. & CALUMET - RED JACKET VILLAGE \\
\hline 842 & 432 & GROSSIO & ZEFIRO & $\mathrm{B}$ & 32 & ITALY & ITALY & ITALY & 1889 & MINER/CM & $\begin{array}{l}\text { TORINO } \\
\text { TOIVAVEL. TO }\end{array}$ & 6TH ST. & CALUMET - RED JACKET VILLAGE \\
\hline 843 & 432 & GIANNINI & LOUIS & B & 25 & ITALY & ITALY & ITALY & 1907 & MINER/CM & LUCCA & 6TH ST. & CALUMET - RED JACKET VILLAGE \\
\hline 844 & 432 & PROLA & MARTINO & B & 21 & ITALY & ITALY & ITALY & 1910 & TIMBERMAN/CM & AGLIE'. TO & 6TH ST. & CALUMET - RED JACKET VILLAGE \\
\hline 845 & 432 & BRUNASSO & STEPHEN & B & 21 & ITALY & ITALY & ITALY & 1909 & TRAMMER/CM & PONT CANAVESE. TO & 6TH ST. & CALUMET - RED JACKET VILLAGE \\
\hline 846 & 432 & REBANDI? & LOUIS & B & 22 & ITALY & ITALY & ITALY & 1910 & TRAMMER/CM & & 6TH ST. & CALUMET - RED JACKET VILLAGE \\
\hline 847 & 432 & GALLO & $\mathrm{JOHN}$ & B & 32 & ITALY & ITALY & ITALY & 1909 & MINER/CM & SAN GIORGIO CANAVESE. TO & 6TH ST. & CALUMET - RED JACKET VILLAGE \\
\hline 848 & 432 & REANO CONTE & BATTISTA & B & 24 & ITALY & ITALY & ITALY & 1908 & TEAMSTER/BREWERY & CORTEREGGIO CANAVESE. TO & 6TH ST. & CALUMET - RED JACKET VILLAGE \\
\hline 849 & 432 & RICCA & PIETRO & B & 34 & ITALY & ITALY & ITALY & 1888 & ODD JOBS/CM & SAN MARTINO CANAVESE. TO & 6TH ST. & CALUMET - RED JACKET VILLAGE \\
\hline 850 & 432 & CAMPAGNOLA & DOMENICO & B & 62 & ITALY & ITALY & ITALY & 1890 & NOT CLEAR & SAN MARTINO CANAVESE. TO & 6TH ST. & CALUMET - RED JACKET VILLAGE \\
\hline 851. & 432 & $\begin{array}{l}\text { TAMIFAGLINIA } \\
\text { TAMBELLINI }\end{array}$ & $\begin{array}{l}\text { ARMIDO } \\
\text { ARIENO }\end{array}$ & B & $\frac{62}{38}$ & $\begin{array}{l}\text { IITALY } \\
\text { ITALY }\end{array}$ & $\begin{array}{l}\text { IITALY } \\
\text { ITALY }\end{array}$ & $\begin{array}{l}\text { ITALY } \\
\text { ITALY }\end{array}$ & 1890 & PROPRIETOR/RETAIL LIQUOR & $\begin{array}{l}\text { LUCCA } \\
\text { LUIINU AINAVESE. IO }\end{array}$ & 5TH ST. & $\begin{array}{l}\text { CALUMET - RED JACKET VILLAGE } \\
\text { CEEETE }\end{array}$ \\
\hline 852 & 405 & TAMBELLINI & ANNA & w & 25 & MICHIGAN & ITALY & ITALY & & & & 5TH ST. & CALUMET - RED JACKET VILLAGE \\
\hline 853 & 405 & TAMBELLINI & $\mathrm{RICH}$ ? & s & 4 & MICHIGAN & ITALY & ITALY & & & LUCCA & 5TH ST. & CALUMET - RED JACKET VILLAGE \\
\hline 854 & 405 & TAMBELLINI & DANTE & s & $\frac{4}{2}$ & MICHIGAN & ITALY & ITALY & & & LUCCA & 5TH ST. & CALUMET - RED JACKET VILLAGE \\
\hline 855 & 405 & TAMBELLINI & CARMELA & MO & 72 & ITALY & ITALY & ITALY & 1907 & & LUCCA & 5TH ST. & CALUMET - RED JACKET VILLAGE \\
\hline 856 & 434 & MARTINI & GEORGE & $\mathrm{H}$ & 59 & ITALY & ITALY & ITALY & 1875 & NOT CLEAR & LUCCA & 5TH ST. & CALUMET - RED JACKET VILLAGE \\
\hline 857 & 434 & MARTINI & AGNES & w & 55 & GERMANY & GERMANY & GERMANY & 1875 & & GERMANY & 5TH ST. & CALUMET - RED JACKET VILLAGE \\
\hline 858 & 434 & MARTINI & ETTA & $\mathrm{D}$ & 32 & MICHIGAN & ITALY & GERMANY & & & LUCCA & 5TH ST. & CALUMET - RED JACKET VILLAGE \\
\hline 859 & 434 & MARTINI & RUDOLPH & NEP & 9 & MICHIGAN & GERMANY & MICHIGAN & & & LUCCA & 5TH ST. & CALUMET - RED JACKET VILLAGE \\
\hline 860 & 419 & CURTO & JOSEPH & $\mathrm{H}$ & 34 & MICHIGAN & ITALY & ITALY & & MANAGER/BREWERY BRANCH & AGLIE'. TO & 7TH ST. & CALUMET - RED JACKET VILLAGE \\
\hline 861 & 419 & CURTO & MARTA & w & 24 & ITALY & ITALY & ITALY & 1895 & & & 7TH ST. & CALUMET - RED JACKET VILLAGE \\
\hline 862 & 419 & CURTO & MARINO & s & 5 & MICHIGAN & ITALY & ITALY & & & AGLIE'. TO & 7TH ST. & CALUMET - RED JACKET VILLAGE \\
\hline 863 & 419 & BRACCO & JOHN & $\mathrm{B}$ & 32 & ITALY & ITALY & ITALY & 1893 & SECRETARY/INSURANCE & TORINO & 7TH ST. & CALUMET - RED JACKET VILLAGE \\
\hline 864 & 419 & $\begin{array}{l}\text { BRACCO } \\
\text { BRACCO }\end{array}$ & FLORENCE & $\begin{array}{l}\mathrm{B} \\
\mathrm{B}\end{array}$ & 28 & MICHIGAN & ITALY & $\begin{array}{l}\text { ITALY } \\
\text { ITAY }\end{array}$ & 1893 & BOOKKEEPER/MEAT MARKET & TORINO & 7TH ST. & CALUMET - RED JACKET VILLAGE \\
\hline 865 & 419 & PERONIO & MARY & SER & 18 & ITALY & ITALY & ITALY & ?? & & TORINO & 7TH ST. & CALUMET - RED JACKET VILLAGE \\
\hline 866 & 411 & BIANCHI & ALBERTO & $\mathrm{H}$ & 44 & ITALY & ITALY & ITALY & 1897 & PROPRIETOR/GROCERY STORE & LUCCA & 7TH ST. & CALUMET - RED JACKET VILLAGE \\
\hline 867 & 411 & BIANCHI & ASSUNTA & w & 44 & ITALY & ITALY & ITALY & 1897 & & & 7TH ST. & CALUMET - RED JACKET VILLAGE \\
\hline 868 & 411 & MANZINI & JOHN & B & 37 & ITALY & ITALY & ITALY & 1903 & PARTNER/GROCERY STORE & PITEGLIO. PISTOIA & 7TH ST. & CALUMET - RED JACKET VILLAGE \\
\hline 869 & 411 & MARCHI & MARCELLINO & B & 33 & ITALY & ITALY & ITALY & 1906 & FIREMAN/STATIONARY ENGINE & COMO & 7TH ST. & CALUMET - RED JACKET VILLAGE \\
\hline 870 & 411 & GAGGINI & FERDINANDO & B & 23 & ITALY & ITALY & ITALY & 1907 & TAILOR & PITEGLIO, PISTOIA & 7TH ST. & CALUMET - RED JACKET VILLAGE \\
\hline 871 & 411 & BIANCHI & JOHN & B & 32 & ITALY & ITALY & ITALY & 1899 & SALESMAN/FRUIT STORE & COLLODI. LU & 7TH ST. & CALUMET - RED JACKET VILLAGE \\
\hline 872 & 411 & CIOMEI & JAMES & B & 30 & ITALY & ITALY & ITALY & 1900 & SALESMAN/FRUIT STORE & COLLODI. LU & 7TH ST. & CALUMET - RED JACKET VILLAGE \\
\hline 873 & 411 & PIERONI & ORESTE & B & 28 & ITALY & ITALY & ITALY & ?? & TEAMSTER/LIQUOR STORE & LUCCA & 7TH ST. & CALUMET - RED JACKET VILLAGE \\
\hline 874 & 411 & LENCIONI & ESILIO & B & 28 & ITALY & ITALY & ITALY & 1898 & BARTENDER/SALOON & LUCCA & 7TH ST. & CALUMET - RED JACKET VILLAGE \\
\hline 875 & $?$ & VERNETTI & JAMES & $\mathrm{H}$ & 38 & ITALY & ITALY & ITALY & 1890 & MINER/CM & TORINO & 6TH ST. & CALUMET - RED JACKET VILLAGE \\
\hline 876 & $?$ & VERNETTI & ANGELINE & w & 33 & ITALY & ITALY & ITALY & 1892 & & & 6TH ST. & $\begin{array}{l}\text { CALUMET - RED JACKET VILLAGE } \\
\text { CEET }\end{array}$ \\
\hline 877 & $?$ & LENZIE & MARION & $\mathrm{H}$ & $? ?$ & FRANCE & FRANCE & FRANCE & 1870 & NOT CLEAR & FRANCE & 6TH ST. & $\begin{array}{l}\text { CALUMET - RED JACKET VILLAGE } \\
\text { CEEETE }\end{array}$ \\
\hline 878 & $?$ & LENZIE & FLORIAN & SS & $? ?$ & MICHIGAN & ITALY & MICHIGAN & & & & 6TH ST. & CALUMET - RED JACKET VILLAGE \\
\hline 879 & $?$ & LENZIE & $\begin{array}{l}\text { LOUISE } \\
\text { LOUI }\end{array}$ & SD & $? ?$ & MICHIGAN & ITALY & MICHIGAN & & & & 6TH ST. & CALUMET - RED JACKET VILLAGE \\
\hline 880 & 330 & TOMMEI & $\begin{array}{l}\text { PALMIRO } \\
\text { PALO }\end{array}$ & $\mathrm{H}$ & 36 & ITALY & $\begin{array}{l}\text { ITALY } \\
\text { ITA }\end{array}$ & ITALY & 1888 & PROPRIETOR/MERCHANDISE & LUCCA & & CALUMET - RED JACKET VILLAGE \\
\hline 881 & 330 & TOMMEI & MARY & w & 33 & ITALY & ITALY & ITALY & 1890 & SALESLADY/MERCHANDISE & & 5TH ST. & CALUMET - RED JACKET VILLAGE \\
\hline
\end{tabular}




\begin{tabular}{|c|c|c|c|c|c|c|c|c|c|c|c|c|c|}
\hline 882 & 330 & TOMMEI & FAUST & $\mathrm{s}$ & 16 & MICHIGAN & ITALY & ITALY & & & LUCCA & 5TH ST. & CALUMET - RED JACKET VILLAGE \\
\hline 883 & 330 & TOMMEI & MARGHERITA & $\mathrm{D}$ & 8 & MICHIGAN & ITALY & ITALY & & & LUCCA & 5TH ST. & CALUMET - RED JACKET VILLAGE \\
\hline 884 & 330 & TOMMEI & PAUL & $\mathrm{s}$ & 5 & MICHIGAN & ITALY & ITALY & & & LUCCA & 5TH ST. & CALUMET - RED JACKET VILLAGE \\
\hline 885 & 341 & BARSOTTI & PETER & $\mathrm{H}$ & 26 & ILLINOIS & ITALY & ITALY & & MANAGER/GROCERY STORE & LUCCA & 5TH ST. & CALUMET - RED JACKET VILLAGE \\
\hline 886 & 341 & BARSOTTI & ARTURO & $\mathrm{BR}$ & 24 & ILLINOIS & ITALY & ITALY & & MERCHANT/GROCERY STORE & LUCCA & 5TH ST. & CALUMET - RED JACKET VILLAGE \\
\hline 887 & 341 & BARSOTTI & GEMMA & $\mathrm{SI}$ & 20 & ILLINOIS & ITALY & ITALY & & CLERK/GROCERY STORE & LUCCA & 5TH ST. & CALUMET - RED JACKET VILLAGE \\
\hline 888 & 341 & BARSOTTI & DUSOLA & $\mathrm{MO}$ & 48 & ITALY & ITALY & ITALY & 1883 & & LUCCA & 5TH ST. & CALUMET - RED JACKET VILLAGE \\
\hline 889 & 331 & VERCELLA & JOHN & $\mathrm{H}$ & 55 & ITALY & ITALY & ITALY & 1880 & BARTENDER/SALOON & SAN MARTINO CANAVESE. TO & 5TH ST. & CALUMET - RED JACKET VILLAGE \\
\hline 890 & 331 & VERCELLA & JOAN & W & 52 & ITALY & ITALY & ITALY & 1881 & 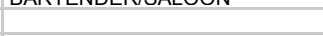 & SAN MARTINO CANAVESE. TO & 5TH ST. & CALUMET - RED JACKET VILLAGE \\
\hline 891 & 331 & VERCELLA & MINNIE & $\mathrm{D}$ & 29 & ITALY & ITALY & ITALY & 1881 & & SAN MARTINO CANAVESE. TO & 5TH ST. & CALUMET - RED JACKET VILLAGE \\
\hline 892 & 329 & VERCELLINO/I & CARLO & $\mathrm{H}$ & 33 & ITALY & ITALY & ITALY & 1906 & PHYSICIAN/PRIVATE WORK & TORINO & 5TH ST. & CALUMET - RED JACKET VILLAGE \\
\hline 893 & 309 & TAMBELLINI & JOHN S. & $\mathrm{H}$ & 35 & ITALY & ITALY & ITALY & 1899 & NOT CLEAR & SEGROMIGNO. LU & 5TH ST. & CALUMET - RED JACKET VILLAGE \\
\hline 894 & 309 & TAMBELLINI & NATALINA & W & 27 & ITALY & ITALY & ITALY & 1899 & & SEGROMIGNO. LU & 5TH ST. & CALUMET - RED JACKET VILLAGE \\
\hline 895 & 309 & TAMBELLINI & TRACY & $\mathrm{D}$ & 9 & MICHIGAN & ITALY & ITALY & & & SEGROMIGNO. LU & 5TH ST. & CALUMET - RED JACKET VILLAGE \\
\hline 896 & 309 & TAMBELLINI & PAUL & $s$ & 7 & MICHIGAN & ITALY & ITALY & & & SEGROMIGNO. LU & 5TH ST. & CALUMET - RED JACKET VILLAGE \\
\hline 897 & 309 & TAMBELLINI & LEONIA & $\mathrm{D}$ & 6 & MICHIGAN & ITALY & ITALY & & & SEGROMIGNO, LU & 5TH ST. & CALUMET - RED JACKET VILLAGE \\
\hline 898 & 309 & TAMBELLINI & PETER & $\mathrm{s}$ & 5 & MICHIGAN & ITALY & ITALY & & & SEGROMIGNO. LU & 5TH ST. & CALUMET - RED JACKET VILLAGE \\
\hline 899 & 322 & CURTO & $\mathrm{JOHN}$ & $\mathrm{H}$ & 32 & ITALY & ITALY & ITALY & 1885 & PROPRIETOR/SALOON & TORINO & 6TH ST. & CALUMET - RED JACKET VILLAGE \\
\hline 900 & 322 & CURTO & DOMENICA & W & 27 & ITALY & ITALY & ITALY & 1896 & & & 6TH ST. & CALUMET - RED JACKET VILLAGE \\
\hline 901 & 322 & CURTO & DOMENICO & $\mathrm{BR}$ & 27 & MICHIGAN & ITALY & ITALY & & SALESMAN/DEPT. STORE & TORINO & 6TH ST. & CALUMET - RED JACKET VILLAGE \\
\hline 902 & 322 & CURTO & PETER & BR & 22 & MICHIGAN & ITALY & ITALY & & & TORINO & 6TH ST. & CALUMET - RED JACKET VILLAGE \\
\hline 903 & 322 & CURTO & MARCO & $\mathrm{BR}$ & 17 & MICHIGAN & ITALY & ITALY & & & TORINO & 6TH ST. & CALUMET - RED JACKET VILLAGE \\
\hline 904 & 322 & MORETTO & CATERINA & $\mathrm{Sl}$ & 19 & MICHIGAN & ITALY & ITALY & & SERVANT/PF & 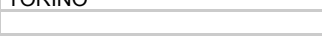 & 6TH ST. & CALUMET - RED JACKET VILLAGE \\
\hline 905 & 322 & CURTO & ANG? & $\mathrm{D}$ & 11 & MICHIGAN & ITALY & ITALY & & & TORINO & 6TH ST. & CALUMET - RED JACKET VILLAGE \\
\hline 906 & 322 & CURTO & CATERINA & $\mathrm{D}$ & 10 & MICHIGAN & ITALY & ITALY & & & TORINO & 6TH ST. & CALUMET - RED JACKET VILLAGE \\
\hline 907 & 322 & CURTO & ANG? & $\mathrm{D}$ & 6 & MICHIGAN & ITALY & ITALY & & & TORINO & 6TH ST. & CALUMET - RED JACKET VILLAGE \\
\hline 908 & 322 & CURTO & CATERINA & $\mathrm{D}$ & $1.7 \mathrm{M}$ & MICHIGAN & ITALY & ITALY & & & TORINO & 6TH ST. & CALUMET - RED JACKET VILLAGE \\
\hline 909 & 326 & BUSANNI & EDWARD & $\mathrm{H}$ & 34 & ITALY & ITALY & ITALY & 1900 & PLASTERER/SHOP & & 6TH ST. & CALUMET - RED JACKET VILLAGE \\
\hline 910 & 326 & BUSANNI & ARTURO & COM & 32 & ITALY & ITALY & ITALY & 1904 & PLASTER ASSISTANT/SHOP & & 6TH ST. & CALUMET - RED JACKET VILLAGE \\
\hline 911 & 326 & BUSANNI & MICHELE & COM & 24 & ITALY & ITALY & ITALY & 1906 & PLASTER ASSISTANT/SHOP & & 6TH ST. & CALUMET - RED JACKET VILLAGE \\
\hline 912 & 301 & LISA & JAMES R. & $\mathrm{H}$ & 64 & ITALY & ITALY & ITALY & 1872 & OWN INCOME & SAN MARTINO CANAVESE. TO & 6TH ST./OAK ST. & CALUMET - RED JACKET VILLAGE \\
\hline 913 & 301 & LISA & MARTA & w & 58 & ITALY & ITALY & ITALY & 1880 & & SAN MARTINO CANAVESE. TO & 6TH ST./OAK ST. & CALUMET - RED JACKET VILLAGE \\
\hline 914 & 301 & LISA & JULIA & $\mathrm{D}$ & 30 & MICHIGAN & ITALY & ITALY & & BOOKKEEPER & SAN MARTINO CANAVESE. TO & 6TH ST./OAK ST. & CALUMET - RED JACKET VILLAGE \\
\hline 915 & 301 & LISA & ANGELINA & $\mathrm{D}$ & 26 & MICHIGAN & ITALY & ITALY & & & SAN MARTINO CANAVESE, TO & 6TH ST./OAK ST. & CALUMET - RED JACKET VILLAGE \\
\hline 916 & 301 & LISA & IRENE & $\mathrm{D}$ & 24 & MICHIGAN & ITALY & $\begin{array}{l}\text { ITALY } \\
\text { ITAY }\end{array}$ & & CLERK/STORE & SAN MARTINO CANAVESE. TO & 6TH ST. IOAK ST. & CALUMET - RED JACKET VILLAGE \\
\hline 917 & 301 & LISA & KATERINA & $\mathrm{D}$ & 22 & MICHIGAN & ITALY & ITALY & & CLERA/STUKE & SAN MARTINO CANAVESE. TO & 6TH ST. IOAK ST. & CALUMET - RED JACKET VILLAGE \\
\hline 918 & 301 & LISA & JAMES & $\mathrm{s}$ & 20 & MICHIGAN & ITALY & ITALY & & & SAN MARTINO CANAVESE. TO & 6TH ST. IOAK ST. & CALUMET - RED JACKET VILLAGE \\
\hline 919 & 213 & PICCHIOTTINO & DOMENICO & $\mathrm{H}$ & 43 & ITALY & ITALY & ITALY & 1886 & PROPRIETOR/SALOON & PONT CANAVESE. TO & 6TH ST. & CALUMET - RED JACKET VILLAGE \\
\hline 920 & 213 & PICCHIOTTINO & ASSUNTA & W & 32 & ITALY & ITALY & ITALY & 1892 & & & 6TH ST. & CALUMET - RED JACKET VILLAGE \\
\hline 921 & 213 & PICCHIOTTINO & CARLO & s & 11 & MICHIGAN & ITALY & ITALY & & & PONT CANAVESE. TO & 6TH ST. & CALUMET - RED JACKET VILLAGE \\
\hline 922 & 213 & PICCHIOTTINO & LUCY & $\mathrm{D}$ & 14 & MICHIGAN & ITALY & ITALY & & & PONT CANAVESE. TO & 6TH ST. & CALUMET - RED JACKET VILLAGE \\
\hline 923 & 213 & PICCHIOTTINO & ALBANO & $\mathrm{COU}$ & 27 & ITALY & ITALY & ITALY & 1906 & MINER/CM & PONT CANAVESE. TO & 6TH ST. & CALUMET - RED JACKET VILLAGE \\
\hline 924 & 213 & BRACCO & GIORGIO & B & 28 & ITALY & ITALY & ITALY & 1905 & MINER/CM & SAN BENIGNO CANAVESE. TO & 6TH ST. & CALUMET - RED JACKET VILLAGE \\
\hline 925 & 213 & VIALE & PIETRO & B & 24 & ITALY & ITALY & ITALY & 1906 & BAKER & SAN BENIGNO CANAVESE. TO & 6TH ST. & CALUMET - RED JACKET VILLAGE \\
\hline 926 & 213 & CONEDERA & ANTONIO & B & 31 & ITALY & ITALY & ITALY & 1896 & BARTENDER/SALOON & VOLTAGO. BELLUNO & 6TH ST. & CALUMET - RED JACKET VILLAGE \\
\hline 927 & 213 & VERNETTI & JOE & B & 40 & ITALY & ITALY & ITALY & 1905 & TAILOR/SHOP & TORINO & 6TH ST. & CALUMET - RED JACKET VILLAGE \\
\hline 928 & 213 & PICCHIOTTINO & ANGELO & $\mathrm{H}$ & 43 & ITALY & ITALY & ITALY & 1899 & MINER/CM & PONT CANAVESE. TO & 6TH ST. & CALUMET - RED JACKET VILLAGE \\
\hline 929 & 213 & PICCHIOTTINO & CATERINA & W & 40 & ITALY & ITALY & ITALY & 1904 & INIINERT/UIMI & PONT CANAVESE. TO & 6TH ST. & CALUMET - RED JACKET VILLAGE \\
\hline 930 & 213 & PICCHIOTTINO & DOMENICO & s & 18 & ITALY & ITALY & ITALY & 1904 & MINER/CM & PONT CANAVESE. TO & 6TH ST. & CALUMET - RED JACKET VILLAGE \\
\hline 931 & 213 & PICCHIOTTINO & CATERINA & $\mathrm{D}$ & 9 & ITALY & ITALY & ITALY & 1904 & & PONT CANAVESE. TO & 6TH ST. & CALUMET - RED JACKET VILLAGE \\
\hline 932 & 213 & PICCHIOTTINO & FRED & $\bar{s}$ & 2 & MICHIGAN & ITAL & ITAI & & & PONT CANAVESE. TO & 6TH ST. & CALUMET - RED JACKET VILLAGE \\
\hline 933 & 213 & PICCHIOTTINO & CARLO & NEP & 26 & MICHIGAN & ITAL & ITAL & & & PONT CANAVESE, TO & 6TH ST. & CALUMET - RED JACKET VILLAGE \\
\hline 934 & 205 & TOMASI & CARLO & $\mathrm{H}$ & 25 & ITALY & ITA & ITA & 1900 & PROPRIETOR/SALOON & LOCANA CANAVESE. TO & 6TH ST. & CALUMET - RED JACKET VILLAGE \\
\hline 935 & 205 & TOMASI & LUISA & W & 24 & ITALY & ITA & ITA & 1900 & & LOCANA CANAVESE. TO & 6TH ST. & CALUMET - RED JACKET VILLAGE \\
\hline 936 & 205 & TOMASI & CARLO & s & 4 & MICHIGAN & ITAL & ITAI & & & LOCANA CANAVESE. TO & 6TH ST. & CALUMET - RED JACKET VILLAGE \\
\hline 937 & 205 & TOMASI & PETER & $\mathrm{s}$ & 2 & MICHIGAN & ITALY & ITALY & & & LOCANA CANAVESE. TO & 6TH ST. & CALUMET - RED JACKET VILLAGE \\
\hline 938 & 205 & TOMASI & ANTONIO & $\mathrm{D}$ & $3 \mathrm{M}$ & MICHIGAN & ITALY & ITALY & & & LOCANA CANAVESE. TO & 6TH ST. & CALUMET - RED JACKET VILLAGE \\
\hline 939 & 205 & GATTA & DOMENICO & B & 32 & ITALY & ITALY & ITALY & 1908 & MINER/CM & LOCANA CANAVESE, TO & 6TH ST. & CALUMET - RED JACKET VILLAGE \\
\hline 940 & 205 & BIANCO & TOMMASO & B & 30 & ITALY & ITALY & ITALY & 1909 & MINER/CM & TORINO & 6TH ST. & CALUMET - RED JACKET VILLAGE \\
\hline 941 & 205 & TOMASI & JAMES & B & 23 & ITALY & ITALY & ITALY & 1909 & MINER/CM & LOCANA CANAVESE. TO & 6TH ST. & CALUMET - RED JACKET VILLAGE \\
\hline 942 & 205 & ROSTAGNO & $\mathrm{JOHN}$ & B & 21 & ITALY & ITALY & ITALY & 1903 & MINER/CM & FORNO RIVARA. TO & 6TH ST. & CALUMET - RED JACKET VILLAGE \\
\hline 943 & 318 & MIGLIO/MIGLIA & TONY & $\mathrm{H}$ & 23 & MICHIGAN & ITALY & ITALY & & BARTENDER/SALOON & SAN GIORGIO CANAVESE. TO & 7TH ST. & CALUMET - RED JACKET VILLAGE \\
\hline 944 & 318 & MIGLIO/MIGLIA & LUCIANA & W & 25 & ITALY & ITALY & ITALY & ???? & & & 7TH ST. & CALUMET - RED JACKET VILLAGE \\
\hline
\end{tabular}




\begin{tabular}{|c|c|c|c|c|c|c|c|c|c|c|c|c|c|}
\hline 945 & 318 & MIGLIO/MIGLIA & CARLO & $\mathrm{s}$ & 4 & SOUTH & ITALY & ITALY & & & SAN GIORGIO CANAVESE. TO & 7TH ST. & CALUMET - RED JACKET VILLAGE \\
\hline 946 & 318 & MACCHI & CARLO & $\mathrm{H}$ & 31 & ITALY & ITALY & ITALY & 1905 & BAKER & MILANO & 7TH ST. & CALUMET - RED JACKET VILLAGE \\
\hline 947 & 318 & MACCHI & ADELE & W & 30 & ITALY & ITALY & ITALY & 1907 & & MILANO & 7TH ST. & CALUMET - RED JACKET VILLAGE \\
\hline 948 & 318 & MACCHI & ANTONIO & s & 7 & ITALY & ITALY & ITALY & 1907 & & MILANO & 7TH ST. & CALUMET - RED JACKET VILLAGE \\
\hline 949 & 318 & MACCHI & ALIDA & $\mathrm{D}$ & 6 & ITALY & ITALY & ITALY & 1907 & & MILANO & 7TH ST. & CALUMET - RED JACKET VILLAGE \\
\hline 950 & 332 & SUINO & DOMENICO & $\mathrm{H}$ & 48 & ITALY & ITALY & ITALY & 1870 & ENGINEER/STATIONARY & TORINO & 7TH ST. & CALUMET - RED JACKET VILLAGE \\
\hline 951 & 332 & SUINO & KATE & w & 40 & ITALY & ITALY & ITALY & 1869 & & & 7TH ST. & CALUMET - RED JACKET VILLAGE \\
\hline 952 & 332 & SUINO & BERNARDO & s & 16 & MICHIGAN & ITALY & ITALY & & & TORINO & 7TH ST. & CALUMET - RED JACKET VILLAGE \\
\hline 953 & 332 & SUINO & MARIE & $\mathrm{D}$ & 14 & MICHIGAN & ITALY & ITALY & & & TORINO & 7TH ST. & CALUMET - RED JACKET VILLAGE \\
\hline 954 & 332 & SUINO & EDWARD & $\mathrm{s}$ & 6 & MICHIGAN & ITALY & ITALY & & & TORINO & 7TH ST. & CALUMET - RED JACKET VILLAGE \\
\hline 955 & 332 & SUINO & KATE & $\mathrm{D}$ & 4 & MICHIGAN & ITALY & ITALY & & & TORINO & 7TH ST. & CALUMET - RED JACKET VILLAGE \\
\hline 956 & 318 & DELL'OSSO & JOSEPH & $\mathrm{H}$ & 32 & ITALY & ITALY & ITALY & 1892 & PROPRIETOR/CONFECTIONARY & LUCCA & BIRCH LANE & CALUMET - RED JACKET VILLAGE \\
\hline 957 & 318 & DELL'OSSO & PAULINE & w & 28 & ITALY & ITALY & ITALY & 1903 & & & BIRCH LANE & CALUMET - RED JACKET VILLAGE \\
\hline 958 & 318 & DELL'OSSO & EMMA & $\mathrm{D}$ & 8 & MICHIGAN & ITALY & ITALY & & & LUCCA & BIRCH LANE & CALUMET - RED JACKET VILLAGE \\
\hline 959 & 318 & DELL'OSSO & LUISA & $\mathrm{D}$ & 6 & MICHIGAN & ITALY & ITALY & & & LUCCA & BIRCH LANE & CALUMET - RED JACKET VILLAGE \\
\hline 960 & 318 & DELL'OSSO & OLGA & $\mathrm{D}$ & 4 & MICHIGAN & ITALY & ITALY & & & LUCCA & BIRCH LANE & CALUMET - RED JACKET VILLAGE \\
\hline 961 & 318 & DELL'OSSO & S? & $\mathrm{s}$ & 2 & MICHIGAN & ITALY & ITALY & & & LUCCA & BIRCH LANE & CALUMET - RED JACKET VILLAGE \\
\hline 962 & 318 & PAGANUCCI & VINCENZO & B & 55 & ITALY & ITALY & ITALY & 1888 & PROPRIETOR/FRUIT STORE & & BIRCH LANE & CALUMET - RED JACKET VILLAGE \\
\hline 963 & 812 & BORGO & MIKE & $\mathrm{H}$ & 67 & ITALY & ITALY & ITALY & 1870 & OWN INCOME & VICENZA & ELM ST. & CALUMET - RED JACKET VILLAGE \\
\hline 964 & 812 & BORGO & CATERINA & w & 57 & IRELAND & IRELAND & IRELAND & 1863 & & IRELAND & ELM ST. & CALUMET - RED JACKET VILLAGE \\
\hline 965 & 812 & BORGO & JOSEPHINE & $\mathrm{D}$ & 35 & MICHIGAN & ITALY & IRELAND & & & VICENZA & ELM ST. & CALUMET - RED JACKET VILLAGE \\
\hline 966 & 812 & BORGO & ADELINA & $\mathrm{D}$ & 31 & MICHIGAN & ITALY & IRELAND & & & VICENZA & ELM ST. & CALUMET - RED JACKET VILLAGE \\
\hline 967 & 812 & BORGO & WALTER & $\mathrm{s}$ & 27 & MICHIGAN & ITALY & IRELAND & & BOOKKEEPER & VICENZA & ELM ST. & CALUMET - RED JACKET VILLAGE \\
\hline 968 & 812 & BORGO & NOBLE & $\mathrm{s}$ & 25 & MICHIGAN & ITALY & IRELAND & & ENGINEER/CM & VICENZA & ELM ST. & CALUMET - RED JACKET VILLAGE \\
\hline 969 & 812 & BORGO & AGNES & $\mathrm{D}$ & 20 & MICHIGAN & ITALY & IRELAND & & NURSE & VICENZA & ELM ST. & CALUMET - RED JACKET VILLAGE \\
\hline 970 & 812 & BORGO & $?$ & $\mathrm{~s}$ & 18 & MICHIGAN & ITALY & IRELAND & & & VICENZA & ELM ST. & CALUMET - RED JACKET VILLAGE \\
\hline 971 & 815 & BARSANTI & FELIX & $\mathrm{H}$ & 36 & ITALY & ITALY & ITALY & 1901 & PROPRIETOR/SALOON & LUCCA & PORTLAND ST. & CALUMET - RED JACKET VILLAGE \\
\hline 972 & 815 & BARSANTI & EUFEMIA & w & 35 & ITALY & ITALY & ITALY & 1906 & & & PORTLAND ST. & CALUMET - RED JACKET VILLAGE \\
\hline 973 & 815 & BARSANTI & JOSEPHINE & $\mathrm{D}$ & 2 & MICHIGAN & ITALY & ITALY & & & LUCCA & PORTLAND ST. & CALUMET - RED JACKET VILLAGE \\
\hline 974 & 815 & BARSANTI & FELIX & $\mathrm{s}$ & $6 \mathrm{M}$ & MICHIGAN & ITALY & ITALY & & & LUCCA & PORTLAND ST. & CALUMET - RED JACKET VILLAGE \\
\hline 975 & 815 & TAMBELLINI & JOSEPH & $\mathrm{H}$ & 39 & ITALY & ITALY & ITALY & 1900 & BARTENDER/SALOON & LUCCA & PORTLAND ST. & CALUMET - RED JACKET VILLAGE \\
\hline 976 & 815 & TAMBELLINI & MACY & w & 33 & ITALY & ITALY & ITALY & 1908 & & & PORTLAND ST. & CALUMET - RED JACKET VILLAGE \\
\hline 977 & 815 & TAMBELLINI & MARY & $\mathrm{D}$ & 11 & MICHIGAN & ITALY & ITALY & & & LUCCA & PORTLAND ST. & CALUMET - RED JACKET VILLAGE \\
\hline 978 & 815 & TAMBELLINI & JOSEPHINE & $\mathrm{D}$ & 4 & MICHIGAN & ITALY & ITALY & & & LUCCA & PORTLAND ST. & CALUMET - RED JACKET VILLAGE \\
\hline 979 & 815 & TAMBELLINI & JENNA & $\mathrm{D}$ & $1.5 \mathrm{M}$ & MICHIGAN & ITALY & ITALY & & & LUCCA & PORTLAND ST. & CALUMET - RED JACKET VILLAGE \\
\hline 980 & 814 & SANDRETTO & LOUIS & $\mathrm{H}$ & 41 & ITALY & ITALY & ITALY & 1892 & PROPRIETOR/SALOON & PONT CANAVESE. TO & PORTLAND ST. & CALUMET - RED JACKET VILLAGE \\
\hline 981 & 814 & SANDRETTO & MARNIE & W & 27 & ITALY & ITALY & ITALY & 1908 & & & PORTLAND ST. & CALUMET - RED JACKET VILLAGE \\
\hline 982 & 814 & SANDRETTO & MARY & $\mathrm{D}$ & 8 & MICHIGAN & ITALY & ITALY & & & PONT CANAVESE. TO & PORTLAND ST. & CALUMET - RED JACKET VILLAGE \\
\hline 983 & 814 & SANDRETTO & LENA & $\mathrm{D}$ & 7 & MICHIGAN & ITALY & ITALY & & & PONT CANAVESE. TO & PORTLAND ST. & CALUMET - RED JACKET VILLAGE \\
\hline 984 & 814 & SANDRETTO & JOHN S. & $\mathrm{s}$ & 5 & MICHIGAN & ITALY & ITALY & & & PONT CANAVESE. TO & PORTLAND ST. & CALUMET - RED JACKET VILLAGE \\
\hline 985 & 814 & BOETTO & LUCY & SIIL & 18 & ITALY & ITALY & ITALY & 1907 & SERVANT/PF & PONT CANAVESE. TO & PORTLAND ST. & CALUMET - RED JACKET VILLAGE \\
\hline 986 & 814 & PAVESIO & ALBINA & SER & 24 & ITALY & ITALY & ITALY & 1909 & SERVANT/PF & PONT CANAVESE. TO & PORTLAND ST. & CALUMET - RED JACKET VILLAGE \\
\hline 987 & 814 & MASSOGLIA & MARY & $\mathrm{H}$ & 29 & ITALY & ITALY & ITALY & 1908 & WASHWOMAN/PF & & PORTLAND ST. & CALUMET - RED JACKET VILLAGE \\
\hline 988 & 814 & MASSOGLIA & PETER & s & 5 & MICHIGAN & ITALY & ITALY & & & SAN MARTINO CANAVESE. TO & PORTLAND ST. & CALUMET - RED JACKET VILLAGE \\
\hline 989 & 814 & MASSOGLIA & JOHN & $\mathrm{s}$ & 3 & ITALY & ITALY & ITALY & & & SAN MARTINO CANAVESE. TO & PORTLAND ST. & CALUMET - RED JACKET VILLAGE \\
\hline 990 & 814 & CURTO & ANGELO & $\mathrm{H}$ & 26 & MICHIGAN & ITALY & ITALY & & MANAGER/BREWERY CO. & TORINO & PORTLAND ST. & CALUMET - RED JACKET VILLAGE \\
\hline 991 & 814 & CURTO & $\begin{array}{ll}\text { LUISA } \\
\text { LUIS }\end{array}$ & W & 22 & MICHIGAN & CANADA & WISCONSI & & IVIAIVASERIDREVERT U. & USA-CANADA & $\begin{array}{l}\text { PORTLAND ST. } \\
\text { POA }\end{array}$ & CALUMET - RED JACKET VILLAGE \\
\hline 992 & 814 & CURTO & BERNA & D & $1.2 \mathrm{M}$ & MICHIGAN & MICHIGAN & MICHIGAN & & & TORINO & $\begin{array}{l}\text { PORTLAND ST. } \\
\text { POA }\end{array}$ & CALUMET - RED JACKET VILLAGE \\
\hline 993 & 814 & CURTO & DOMENICO & $\mathrm{H}$ & 27 & MICHIGAN & ITALY & ITALY & & CLERK/DEPTARTMENT STORE & TORINO & PORTLAND ST. & CALUMET - RED JACKET VILLAGE \\
\hline 994 & 814 & CURTO & MARGHERITA & w & 22 & ITALY & ITALY & ITALY & 1898 & & & PORTLAND ST. & CALUMET - RED JACKET VILLAGE \\
\hline 995 & 117 & TAMBELLINI & JOHN & $\mathrm{H}$ & 46 & ITAL & ITAL & ITA & 1884 & PROPRIETOR/SALOON & LUCCA & RTLAND ST. & CALUMET - RED JACKET VILLAGE \\
\hline 996 & 117 & TAMBELLINI & CELIDE & W & 36 & ITA & ITAL & ITAL & 1897 & & & LAND ST. & CALUMET - RED JACKET VILLAGE \\
\hline 997 & 117 & TAMBELLINI & JULIO & s & 12 & HIGAN & ITA & ITA & & & LUCCA & PO & CALUMET - RED JACKET VILLAGE \\
\hline 998 & 117 & TAMBELLI & & $\mathrm{D}$ & 10 & MICHIGAN & ITA & ITA & & & LUCCA & PORTLAND ST. & CALUMET - RED JACKET VILLAGE \\
\hline 999 & 153 & GALLINATTI & ADOLF & $\mathrm{H}$ & 30 & ITALY & ITALY & ITALY & 1892 & MEAT CUTTER/BUTCHER S. & TORINO & AMYGDALOID ST. & CALUMET - LAURIUM VILLAGE \\
\hline 1000 & 153 & GALLINATTI & MARY & W & 26 & ITALY & ITALY & ITALY & 1892 & & & AMYGDALOID ST. & CALUMET - LAURIUM VILLAGE \\
\hline 1001 & 153 & GALLINATTI & MALVINA & $\mathrm{D}$ & 8 & MICHIGAN & ITALY & ITALY & & & TORINO & AMYGDALOID ST. & CALUMET - LAURIUM VILLAGE \\
\hline 1002 & 153 & ROMANO & ANTON & B & 30 & ITALY & ITALY & ITALY & 1901 & MINER/CM & AGLIE', TO & AMYGDALOID ST. & CALUMET - LAURIUM VILLAGE \\
\hline 1003 & 153 & TOMASI & ANTONIO & B & 26 & ITALY & ITALY & ITALY & 1902 & TEAMSTER/CM & LOCANA CANAVESE. TO & AMYGDALOID ST. & CALUMET - LAURIUM VILLAGE \\
\hline 1004 & 153 & GARDETTO & DOMENICO & $\mathrm{H}$ & 39 & ITALY & ITALY & ITALY & 1886 & TIMBERMAN/CM & BOSCONERO. TO & AMYGDALOID ST. & CALUMET - LAURIUM VILLAGE \\
\hline 1005 & 153 & GARDETTO & MARGHERITA & MO & 64 & ITALY & ITALY & ITALY & 1886 & & BOSCONERO. TO & AMYGDALOID ST. & $\begin{array}{l}\text { CALUMET - LAURIUM VILLAGE } \\
\text { CAULE }\end{array}$ \\
\hline 1006 & 153 & TORREANO & KATE & $\mathrm{H}$ & 41 & ITALY & ITALY & ITALY & 1892 & WASHWOMAN/AT HOME & TORINO & AMYGDALOID ST. & CALUMET - LAURIUM VILLAGE \\
\hline 1007 & 153 & TORREANO & MARY & $\mathrm{D}$ & 20 & ITALY & ITALY & ITALY & 1892 & DRESSMAKER/AT HOME & TORINO & AMYGDALOID ST. & CALUMET - LAURIUM VILLAGE \\
\hline
\end{tabular}




\begin{tabular}{|c|c|c|c|c|c|c|c|c|c|c|c|c|c|}
\hline 1008 & 153 & TORREANO & TRACY & $\mathrm{D}$ & 14 & MICHIGAN & ITALY & ITALY & & & TORINO & AMYGDALOID ST. & CALUMET - LAURIUM VILLAGE \\
\hline 1009 & 153 & TORREANO & $\mathrm{JOHN}$ & $\mathrm{s}$ & 12 & MICHIGAN & ITALY & ITALY & & & TORINO & AMYGDALOID ST. & CALUMET - LAURIUM VILLAGE \\
\hline 1010 & 153 & GIANDRONE & PETER & $\mathrm{H}$ & 32 & ITALY & ITALY & ITALY & 1889 & TIMBERMAN/CM & CUORGNE'. TO & AMYGDALOID ST. & CALUMET - LAURIUM VILLAGE \\
\hline 1011 & 153 & GIANDRONE & FLORENCE & W & 27 & ITALY & ITALY & ITALY & 1889 & & CUORGNE'. TO & AMYGDALOID ST. & CALUMET - LAURIUM VILLAGE \\
\hline 1012 & 153 & GIANDRONE & MARY & $\mathrm{D}$ & 3 & MICHIGAN & ITALY & ITALY & & & CUORGNE'. TO & AMYGDALOID ST. & CALUMET - LAURIUM VILLAGE \\
\hline 1013 & 153 & GIANDRONE & AUGUST & $\mathrm{s}$ & 4 & MICHIGAN & ITALY & ITALY & & & CUORGNE'. TO & AMYGDALOID ST. & CALUMET - LAURIUM VILLAGE \\
\hline 1014 & 225 & LE CHEVALIER & PAUL & $\mathrm{H}$ & 23 & MICHIGAN & FRANCE & FRANCE & & MINER/CM & FRANCE & AMYGDALOID ST. & CALUMET - LAURIUM VILLAGE \\
\hline 1015 & 225 & LE CHEVALIER & MARGUERITE & W & 21 & ITALY & ITALY & ITALY & 1897 & IVIIVERTULIUI & TRAIVUE & AMYGDALOID ST. & $\begin{array}{l}\text { CALUMET - LAURIUM VILLAGE } \\
\text { CALE }\end{array}$ \\
\hline 1016 & 225 & LE CHEVALIER & PAUL JR. & s & 3 & MICHIGAN & MICHIGAN & ITALY & & & & AMYGDALOID ST. & CALUMET - LAURIUM VILLAGE \\
\hline 1017 & 225 & LE CHEVALIER & MARGUERITE & $\mathrm{D}$ & 1 & MICHIGAN & MICHIGAN & ITALY & & & & AMYGDALOID ST. & CALUMET - LAURIUM VILLAGE \\
\hline 1018 & 118 & VAIRO & VINCENZO & $\mathrm{H}$ & 43 & ITALY & ITALY & ITALY & 1888 & CONTRACTOR/BUILDING & LOCANA CANAVESE. TO & SENECA ST. & CALUMET - LAURIUM VILLAGE \\
\hline 1019 & 118 & VAIRO & GIULIA & W & 41 & ITALY & ITALY & ITALY & 1890 & & LOCANA CANAVESE. TO & SENECA ST. & CALUMET - LAURIUM VILLAGE \\
\hline 1020 & 118 & VAIRO & DOMENICO & $s$ & 21 & ITALY & ITALY & ITALY & 1893 & STOREKEEPER/FRUIT & LOCANA CANAVESE. TO & SENECA ST. & CALUMET - LAURIUM VILLAGE \\
\hline 1021 & 118 & VAIRO & GIOVANNI & $\mathrm{s}$ & 15 & MICHIGAN & ITALY & ITALY & & & LOCANA CANAVESE. TO & SENECA ST. & CALUMET - LAURIUM VILLAGE \\
\hline 1022 & 118 & VAIRO & GIOVANNA & $\mathrm{D}$ & 12 & MICHIGAN & ITALY & ITALY & & & LOCANA CANAVESE. TO & SENECA ST. & CALUMET - LAURIUM VILLAGE \\
\hline 1023 & 118 & VAIRO & ELENA & $\mathrm{D}$ & 8 & MICHIGAN & ITALY & ITALY & & & LOCANA CANAVESE, TO & SENECA ST. & CALUMET - LAURIUM VILLAGE \\
\hline 1024 & 118 & PRICCO & DOMINICK & $\mathrm{H}$ & 34 & ITALY & ITALY & ITALY & 1902 & MINER/CM & SAN MARTINO CANAVESE. TO & SENECA ST. & CALUMET - LAURIUM VILLAGE \\
\hline 1025 & 118 & PRICCO & ANGELA & w & 25 & ITALY & ITALY & ITALY & 1905 & & & SENECA ST. & CALUMET - LAURIUM VILLAGE \\
\hline 1026 & 118 & PRICCO & PETER & s & 4 & MICHIGAN & ITALY & ITALY & & & SAN MARTINO CANAVESE. TO & SENECA ST. & CALUMET - LAURIUM VILLAGE \\
\hline 1027 & 118 & PRICCO & JOHN & $\mathrm{s}$ & 2 & MICHIGAN & ITALY & ITALY & & & SAN MARTINO CANAVESE. TO & SENECA ST. & CALUMET - LAURIUM VILLAGE \\
\hline 1028 & 118 & TOMASI & GIACOMO & $\mathrm{H}$ & 26 & ITALY & ITALY & ITALY & 1897 & TEAMSTER/BREWERY & $\begin{array}{l}\text { LOCANA CANAVESE. TO } \\
\text { LOCA }\end{array}$ & SENECA ST. & $\begin{array}{l}\text { CALUMET - LAURIUM VILLAGE } \\
\text { CAUE }\end{array}$ \\
\hline 1029 & 118 & TOMASI & CATERINA & W & 18 & ITALY & ITALY & ITALY & 1903 & & & SENECA ST. & CALUMET - LAURIUM VILLAGE \\
\hline 1030 & 118 & VANILLO & JOSEPH & B & 17 & ITALY & ITALY & ITALY & 1909 & & & SENECA ST. & CALUMET - LAURIUM VILLAGE \\
\hline 1031 & 118 & CATENO & GABRIELE & B & 26 & ITALY & ITALY & ITALY & 1908 & & & SENECA ST. & CALUMET - LAURIUM VILLAGE \\
\hline 1032 & 118 & cosso & GIUSEPPE & $\mathrm{H}$ & 37 & ITALY & ITALY & ITALY & 1888 & TIMBER BOSS/CM & SAN GIORGIO CANAVESE. TO & SENECA ST. & CALUMET - LAURIUM VILLAGE \\
\hline 1033 & 118 & cosso & MARIA & W & 27 & GERMANY & ITALY & ITALY & 1894 & & & SENECA ST. & CALUMET - LAURIUM VILLAGE \\
\hline 1034 & 118 & cosso & STEFANO & $\mathrm{s}$ & 11 & MICHIGAN & GERMANY & ITALY & & & SAN GIORGIO CANAVESE. TO & SENECA ST. & CALUMET - LAURIUM VILLAGE \\
\hline 1035 & 118 & cosso & MARIA & $\mathrm{D}$ & 10 & MICHIGAN & GERMANY & ITALY & & & SAN GIORGIO CANAVESE, TO & SENECA ST. & CALUMET - LAURIUM VILLAGE \\
\hline 1036 & 118 & cosso & DOMENICA & D & 7 & MICHIGAN & GERMANY & ITALY & & & SAN GIORGIO CANAVESE. TO & SENECA ST. & CALUMET - LAURIUM VILLAGE \\
\hline 1037 & 118 & $\operatorname{cosso}$ & DELLA & $\mathrm{D}$ & 4 & MICHIGAN & GERMANY & ITALY & & & SAN GIORGIO CANAVESE. TO & SENECA ST. & CALUMET - LAURIUM VILLAGE \\
\hline 1038 & 118 & $\operatorname{cosso}$ & MARCO & $\mathrm{S}$ & 3 & MICHIGAN & GERMANY & ITALY & & & SAN GIORGIO CANAVESE. TO & SENECA ST. & CALUMET - LAURIUM VILLAGE \\
\hline 1039 & 120 & TOMASI & ANTONIO & $\mathrm{H}$ & 32 & ITALY & ITALY & ITALY & 1891 & DAY MAN/CM & LOCANA CANAVESE. TO & SENECA ST. & CALUMET - LAURIUM VILLAGE \\
\hline 1040 & 120 & TOMASI & ANTONIA & W & 31 & ITALY & ITALY & ITALY & 1896 & Dat iviativicivi & LUCAIVA CAIVAVEOE. TO & SENECA ST. & $\begin{array}{l}\text { CALUMET - LAURIUM VILLAGE } \\
\text { CALE }\end{array}$ \\
\hline 1041 & 120 & TOMASI & GIOVANNA & $\mathrm{D}$ & 9 & MICHIGAN & ITALY & ITALY & & & LOCANA CANAVESE, TO & SENECA ST. & CALUMET - LAURIUM VILLAGE \\
\hline 1042 & 120 & TOMASI & GIACOMO & $\mathrm{s}$ & 8 & MICHIGAN & ITALY & ITALY & & & LOCANA CANAVESE. TO & SENECA ST. & CALUMET - LAURIUM VILLAGE \\
\hline 1043 & 120 & TOMASI & CATERINA & $\mathrm{D}$ & 6 & MICHIGAN & ITALY & ITALY & & & LOCANA CANAVESE. TO & SENECA ST. & CALUMET - LAURIUM VILLAGE \\
\hline 1044 & 120 & TOMASI & ANTONIO JR. & $\mathrm{s}$ & 5 & MICHIGAN & ITALY & ITALY & & & LOCANA CANAVESE. TO & SENECA ST. & CALUMET - LAURIUM VILLAGE \\
\hline 1045 & 120 & TOMASI & VIOLETTA & $\mathrm{D}$ & 1 & MICHIGAN & ITALY & ITALY & & & LOCANA CANAVESE. TO & SENECA ST. & CALUMET - LAURIUM VILLAGE \\
\hline 1046 & 120 & TOMASI & MARGHERITA & $\mathrm{D}$ & $3 \mathrm{M}$ & MICHIGAN & ITALY & ITALY & & & LOCANA CANAVESE. TO & SENECA ST. & CALUMET - LAURIUM VILLAGE \\
\hline 1047 & 120 & BO' & BENEDETTO & B & 22 & ITALY & ITALY & ITALY & 1906 & TRAMMER/CM & TORINO & SENECA ST. & CALUMET - LAURIUM VILLAGE \\
\hline 1048 & 120 & BOETTO & PIETRO & B & 35 & ITALY & ITALY & ITALY & 1908 & TRAMMER/CM & PONT CANAVESE. TO & SENECA ST. & CALUMET - LAURIUM VILLAGE \\
\hline 1049 & 120 & ESTRAMONTE & JAMES & B & 25 & ITALY & ITALY & ITALY & 1908 & TIMBERMAN/CM & LOCANA CANAVESE. TO & SENECA ST. & CALUMET - LAURIUM VILLAGE \\
\hline 1050 & 124 & VIANI & GEORGE & $\mathrm{H}$ & 32 & ITALY & ITALY & ITALY & 1907 & MINER/CM & LUCCA & SENECA ST. & CALUMET - LAURIUM VILLAGE \\
\hline 1051 & 124 & VIANI & LIZZIE & W & 25 & MICHIGAN & ENGLAND & ENGLAND & & & ENGLAND & SENECA ST. & CALUMET - LAURIUM VILLAGE \\
\hline 1052 & 124 & VIANI & KATE & $\mathrm{D}$ & 6 & MICHIGAN & ITALY & MICHIGAN & & & LUCCA & SENECA ST. & CALUMET - LAURIUM VILLAGE \\
\hline 1053 & 124 & VIANI & MARIE & $\mathrm{D}$ & 4 & MICHIGAN & ITALY & MICHIGAN & & & LUCCA & SENECA ST. & CALUMET - LAURIUM VILLAGE \\
\hline 1054 & 124 & VIANI & ELEONOR & $\mathrm{D}$ & 2 & MICHIGAN & ITALY & MICHIGAN & & & LUCCA & ECA ST. & CALUMET - LAURIUM VILLAGE \\
\hline 1055 & 140 & CARBONATTO & VITTORIO & $\mathrm{H}$ & 41 & ITALY & ITALY & ITALY & 1892 & MACHINIST/CM & PONT CANAVESE. TO & & CALUMET - LAURIUM VILLAGE \\
\hline 1056 & 140 & CARBONATTO & MARIA & w & 34 & MICHIGAN & ITALY & ITALY & & & & SEI & CALUMET - LAURIUM VILLAGE \\
\hline 1057 & 140 & CARBONATTO & PHILLYS & $\mathrm{D}$ & 8 & MICHIGAN & ITALY & MICHIGAN & & & PONT CANAVESE. TO & CA ST. & CALUMET - LAURIUM VILLAGE \\
\hline 1058 & 140 & DATTA/DATA & ANTONIO & $\mathrm{H}$ & 36 & ITALY & ITALY & ITALY & 1890 & SURFACE MAN/CM & SAN GIORGIO CANAVESE. TO & A ST. & CALUMET - LAURIUM VILLAGE \\
\hline 1059 & 140 & DATTA/DATA & ANNA & W & 35 & ITALY & ITALY & ITALY & 1890 & & SAN GIORGIO CANAVESE, TO & SENECA ST. & CALUMET - LAURIUM VILLAGE \\
\hline 1060 & 140 & DATTA/DATA & GIUSEPPE & $\mathrm{s}$ & 14 & MICHIGAN & ITALY & ITALY & & & SAN GIORGIO CANAVESE. TO & SENECA ST. & CALUMET - LAURIUM VILLAGE \\
\hline 1061 & 140 & DATTA/DATA & MICHELE & $\mathrm{s}$ & 11 & MICHIGAN & ITALY & ITALY & & & SAN GIORGIO CANAVESE. TO & SENECA ST. & CALUMET - LAURIUM VILLAGE \\
\hline 1062 & 140 & DATTA/DATA & ANTONIO & $\mathrm{s}$ & 9 & MICHIGAN & ITALY & ITALY & & & SAN GIORGIO CANAVESE. TO & SENECA ST. & CALUMET - LAURIUM VILLAGE \\
\hline 1063 & 140 & DE MATTEIS & ANTON & $\mathrm{H}$ & 35 & ITALY & ITALY & ITALY & 1899 & TIMBERMAN/CM & SAN GIORGIO CANAVESE. TO & SENECA ST. & CALUMET - LAURIUM VILLAGE \\
\hline 1064 & 140 & DE MATTEIS & PETRONILLA & w & 29 & ITALY & ITALY & ITALY & UN & & SAN GIORGIO CANAVESE. TO & SENECA ST. & CALUMET - LAURIUM VILLAGE \\
\hline 1065 & 140 & DE MATTEIS & MINNIE & $\mathrm{D}$ & 7 & MICHIGAN & ITALY & ITALY & & & SAN GIORGIO CANAVESE, TO & SENECA ST. & CALUMET - LAURIUM VILLAGE \\
\hline 1066 & 140 & DE MATTEIS & KATE & $\mathrm{D}$ & 5 & MICHIGAN & ITALY & ITALY & & & SAN GIORGIO CANAVESE. TO & SENECA ST. & CALUMET - LAURIUM VILLAGE \\
\hline 1067 & 140 & DE MATTEIS & PHILLYS & $\mathrm{S}$ & 2 & MICHIGAN & ITAL & ITAL & & & SAN GIORGIO CANAVESE. TO & CA ST. & T - LAURIUM VILLA \\
\hline 1068 & 230 & & & $\mathrm{H}$ & 56 & & & & 1897 & & & & \\
\hline 1069 & 230 & CHECCHI & DOMENICA & W & 62 & & ITALY & & 1897 & & & & CALUMET - LAURIUM VILLA \\
\hline 1070 & 230 & MANFREDINI & RINA & $\mathrm{GD}$ & 12 & MICHIGAN & ITALY & ITALY & & & LUCCA & SENECA ST. & CALUMET - LAURIUM VILLAGE \\
\hline
\end{tabular}




\begin{tabular}{|c|c|c|c|c|c|c|c|c|c|c|c|c|c|}
\hline 1071 & 211 & RASTELLO & GIOVANNI & $\mathrm{H}$ & 42 & ITALY & ITALY & ITALY & 1880 & TAILOR & PONT CANAVESE. TO & 1ST ST. & CALUMET - LAURIUM VILLAGE \\
\hline 1072 & 211 & RASTELLO & TERESA & W & 42 & MICHIGAN & ITALY & ITALY & & & PONT CANAVESE. TO & 1ST ST. & CALUMET - LAURIUM VILLAGE \\
\hline 1073 & 211 & RASTELLO & DOMENICO & $\mathrm{s}$ & 18 & MICHIGAN & ITALY & MICHIGAN & & TAILOR & PONT CANAVESE. TO & 1ST ST. & CALUMET - LAURIUM VILLAGE \\
\hline 1074 & 211 & RASTELLO & MARIA & $\mathrm{D}$ & 17 & MICHIGAN & ITALY & MICHIGAN & & & PONT CANAVESE. TO & 1ST ST. & CALUMET - LAURIUM VILLAGE \\
\hline 1075 & 211 & RASTELLO & ROSA & $\mathrm{D}$ & 13 & MICHIGAN & ITALY & MICHIGAN & & & PONT CANAVESE. TO & 1ST ST. & CALUMET - LAURIUM VILLAGE \\
\hline 1076 & 211 & RASTELLO & GIACOMO & s & 9 & MICHIGAN & ITALY & MICHIGAN & & & PONT CANAVESE. TO & 1ST ST. & CALUMET - LAURIUM VILLAGE \\
\hline 1077 & 211 & GALLASERO & GIACOMO & $\mathrm{H}$ & 36 & ITALY & ITALY & ITALY & $89 / 90$ & MERCHANT/GROCERY STORE & PONT CANAVESE. TO & 1ST ST. & CALUMET - LAURIUM VILLAGE \\
\hline 1078 & & GALLASERO & LUCIA & W & 40 & ITALY & ITALY & ITALY & 1902 & 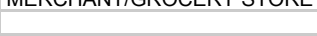 & PONT CANAVESE. TO & 1ST ST. & $\begin{array}{l}\text { CALUMET - LAURIUM VILLAGE } \\
\text { CALE }\end{array}$ \\
\hline 1079 & & GALLASERO & GIOVANNA & $\mathrm{D}$ & 13 & ITALY & ITALY & ITALY & 1902 & & PONT CANAVESE. TO & 1ST ST. & CALUMET - LAURIUM VILLAGE \\
\hline 1080 & & GALLASERO & PIETRO & $\mathrm{S}$ & 12 & ITALY & ITALY & ITALY & 1902 & & PONT CANAVESE. TO & 1ST ST. & CALUMET - LAURIUM VILLAGE \\
\hline 1081 & & GALLASERO & LUCIA & D & $8 \mathrm{M}$ & MICHIGAN & ITALY & ITALY & & & PONT CANAVESE. TO & 1ST ST. & CALUMET - LAURIUM VILLAGE \\
\hline 1082 & & BAZZARONE & SECONDO & BIL & 24 & ITALY & ITALY & ITALY & 1904 & & PONT CANAVESE. TO & 1ST ST. & CALUMET - LAURIUM VILLAGE \\
\hline 1083 & & QUELLO & BATTISTA & $\mathrm{H}$ & 36 & ITALY & ITALY & ITALY & 1890 & SALOONKEEPER & LOCANA CANVESE. TO & 1ST ST. & CALUMET - LAURIUM VILLAGE \\
\hline 1084 & & QUELLO & MARY & W & 34 & ITALY & ITALY & ITALY & 1892 & & & 1ST ST. & CALUMET - LAURIUM VILLAGE \\
\hline 1085 & & QUELLO & JOHN & $\mathrm{s}$ & 9 & MICHIGAN & ITALY & ITALY & & & LOCANA CANVESE. TO & 1ST ST. & CALUMET - LAURIUM VILLAGE \\
\hline 1086 & & QUELLO & MARY & $\mathrm{D}$ & 7 & MICHIGAN & ITALY & ITALY & & & LOCANA CANVESE, TO & 1ST ST. & CALUMET - LAURIUM VILLAGE \\
\hline 1087 & & QUELLO & SILVESTRO & $\mathrm{s}$ & 5 & MICHIGAN & ITALY & ITALY & & & LOCANA CANVESE. TO & 1ST ST. & CALUMET - LAURIUM VILLAGE \\
\hline 1088 & & QUELLO & ELLIS & $\mathrm{D}$ & 2 & MICHIGAN & ITALY & ITALY & & & LOCANA CANVESE. TO & 1ST ST. & CALUMET - LAURIUM VILLAGE \\
\hline 1089 & & PALAZZOLI & RUDOLF & B & 60 & ITALY & ITALY & ITALY & 1880 & TYPESETTER/PRINTING CO. & & 1ST ST. & CALUMET - LAURIUM VILLAGE \\
\hline 1090 & & QUELLO & PETER & $\mathrm{BR}$ & 24 & ITALY & ITALY & ITALY & 1907 & 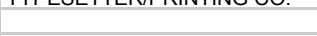 & LOCANA CANVESE. TO & 1ST ST. & CALUMET - LAURIUM VILLAGE \\
\hline 1091 & 216 & PICCHIOTTINO & BATTISTA & $\mathrm{H}$ & 40 & ITALY & $\begin{array}{l}\text { ITALY } \\
\text { ITAL }\end{array}$ & ITALY & 1889 & PLUMBER & PONT CANAVESE. TO & AHMEEK ST. & $\begin{array}{l}\text { CALUMET - LAURIUM VILLAGE } \\
\text { CAUE }\end{array}$ \\
\hline 1092 & 216 & PICCHIOTTINO & MARGHERITA & W & 39 & ITALY & ITALY & ITALY & 1890 & & & AHMEEK ST. & CALUMET - LAURIUM VILLAGE \\
\hline 1093 & 216 & PICCHIOTTINO & ANGELO & $\mathrm{s}$ & 16 & MICHIGAN & ITALY & ITALY & & & PONT CANAVESE. TO & AHMEEK ST. & CALUMET - LAURIUM VILLAGE \\
\hline 1094 & 216 & PICCHIOTTINO & LUCIA & $\mathrm{D}$ & 15 & MICHIGAN & ITALY & ITALY & & & PONT CANAVESE. TO & AHMEEK ST. & CALUMET - LAURIUM VILLAGE \\
\hline 1095 & 216 & PICCHIOTTINO & CATERINA & $\mathrm{D}$ & 13 & MICHIGAN & ITALY & ITALY & & & PONT CANAVESE. TO & AHMEEK ST. & CALUMET - LAURIUM VILLAGE \\
\hline 1096 & 216 & PICCHIOTTINO & ENRICO & s & 7 & MICHIGAN & ITALY & ITALY & & & PONT CANAVESE. TO & AHMEEK ST. & CALUMET - LAURIUM VILLAGE \\
\hline 1097 & 216 & PICCHIOTTINO & GIOVANNI & $\mathrm{s}$ & 6 & MICHIGAN & ITALY & ITALY & & & PONT CANAVESE. TO & AHMEEK ST. & CALUMET - LAURIUM VILLAGE \\
\hline 1098 & 216 & GABANNA & EUGENIO & NEP & 27 & ITALY & ITALY & ITALY & 1890 & ELECTRICIAN/TAMARACK & PONT CANAVESE, TO & AHMEEK ST. & CALUMET - LAURIUM VILLAGE \\
\hline 1099 & 201 & CERUTTI & FRANK & $\mathrm{H}$ & 42 & ITALY & ITALY & ITALY & 1889 & & SAN GIUSTO CANAVESE. TO & AHMEEK ST. & CALUMET - LAURIUM VILLAGE \\
\hline 1100 & 201 & CERUTTI & ELISABETTA & W & 39 & ITALY & ITALY & ITALY & 1889 & & SAN GIUSTO CANAVESE. TO & AHMEEK ST. & CALUMET - LAURIUM VILLAGE \\
\hline 1101 & 201 & CERUTTI & MAGGIE & $\mathrm{D}$ & 17 & MICHIGAN & ITALY & ITALY & & & SAN GIUSTO CANAVESE. TO & AHMEEK ST. & CALUMET - LAURIUM VILLAGE \\
\hline 1102 & 201 & CERUTTI & ROSE & $\mathrm{D}$ & 15 & MICHIGAN & ITALY & ITALY & & & SAN GIUSTO CANAVESE. TO & AHMEEK ST. & CALUMET - LAURIUM VILLAGE \\
\hline 1103 & 201 & CERUTTI & MARY & $\mathrm{D}$ & 9 & MICHIGAN & ITALY & $\begin{array}{l}\text { ITALY } \\
\text { ITAL }\end{array}$ & & & SAN GIUSTO CANAVESE. TO & AHMEEK ST. & $\begin{array}{l}\text { CALUMET - LAURIUM VILLAGE } \\
\text { CALE }\end{array}$ \\
\hline 1104 & 201 & CERUTTI & LIZZIE & $\mathrm{D}$ & 7 & MICHIGAN & ITALY & ITALY & & & SAN GIUSTO CANAVESE, TO & AHMEEK ST. & CALUMET - LAURIUM VILLAGE \\
\hline 1105 & 201 & CERUTTI & FRANCES & $\mathrm{D}$ & 4 & MICHIGAN & ITALY & ITALY & & & SAN GIUSTO CANAVESE. TO & AHMEEK ST. & CALUMET - LAURIUM VILLAGE \\
\hline 1106 & 201 & CERUTTI & FRANK & s & 1 & MICHIGAN & ITALY & ITALY & & & SAN GIUSTO CANAVESE. TO & AHMEEK ST. & CALUMET - LAURIUM VILLAGE \\
\hline 1107 & 201 & CERUTTI & ANTON & $\mathrm{BR}$ & 41 & ITALY & ITALY & ITALY & 1893 & & SAN GIUSTO CANAVESE. TO & AHMEEK ST. & CALUMET - LAURIUM VILLAGE \\
\hline 1108 & 201 & CERUTTI & JOHN & $\mathrm{BR}$ & 38 & ITALY & ITALY & ITALY & 1893 & & SAN GIUSTO CANAVESE. TO & AHMEEK ST. & CALUMET - LAURIUM VILLAGE \\
\hline 1109 & 108 & VAIRO & ANTON & $\mathrm{H}$ & 53 & ITALY & ITALY & ITALY & 1885 & & LOCANA CANAVESE. TO & PEWABIC ST. & CALUMET - LAURIUM VILLAGE \\
\hline 1110 & 108 & VAIRO & MARY & W & 48 & ITALY & ITALY & ITALY & 1885 & & LOCANA CANAVESE. TO & PEWABIC ST. & CALUMET - LAURIUM VILLAGE \\
\hline 1111 & 108 & VAIRO & STEPHEN & $\mathrm{s}$ & 28 & ITALY & ITALY & ITALY & 1885 & & LOCANA CANAVESE. TO & PEWABIC ST. & CALUMET - LAURIUM VILLAGE \\
\hline 1112 & 108 & VAIRO & MARY & $\mathrm{D}$ & 21 & MICHIGAN & ITALY & ITALY & & & LOCANA CANAVESE. TO & PEWABIC ST. & CALUMET - LAURIUM VILLAGE \\
\hline 1113 & 147 & MARINELLI & GIORDANO & $\mathrm{H}$ & 33 & ITALY & ITALY & ITALY & 1908 & & UDINE & IROQUOIS ST. & CALUMET - LAURIUM VILLAGE \\
\hline 1114 & 147 & MARINELLI & DIONURA & W & 23 & ITALY & ITALY & ITALY & 1908 & & UDINE & IROQUOIS ST. & CALUMET - LAURIUM VILLAGE \\
\hline 1115 & 147 & MARINELLI & INEZ & $\mathrm{D}$ & 1 & MICHIGAN & ITALY & ITALY & & & UDINE & $\begin{array}{l}\text { IROQUOIS ST. } \\
\text { IROQ }\end{array}$ & CALUMET - LAURIUM VILLAGE \\
\hline 1116 & 147 & TOGNI & FRANCESCO & $\mathrm{H}$ & 37 & ITALY & ITALY & ITALY & 1909 & & BRENTONICO. TRENTO & IROQUOIS ST. & CALUMET - LAURIUM VILLAGE \\
\hline 1117 & 147 & TOGNI & MARIA & w & 35 & ITALY & ITALY & ITALY & 1909 & & BRENTONICO. TRENTO & IROQUOIS ST. & CALUMET - LAURIUM VILLAGE \\
\hline 1118 & 147 & TOGNI & PIA & $\mathrm{D}$ & 5 & ITALY & ITAL & ITALY & 190 & & BRENTONICO. TRENTO & IROQUOIS ST. & CALUMET - LAURIUM VILLAGE \\
\hline 1119 & 136 & MARTA & JOHN & $\mathrm{H}$ & 43 & ITAL & ITA & ITALY & 1890 & MINER/CM & SAN MARTINO CANAVESE. TO & $\begin{array}{l}\text { IROQUOIS ST. } \\
\text { IROS }\end{array}$ & CALUMET - LAURIUM V \\
\hline 1120 & 136 & MARTA-RUONA & TERESA & w & 44 & ITALY & ITALY & ITALY & 1894 & & & IROQUOIS & CALUMET - LAURIUM VILLAGE \\
\hline 1121 & 136 & MARTA & ANNA & $\mathrm{D}$ & 12 & MICHIGAN & ITALY & ITALY & & & SAN MARTINO CANAVESE. TO & IROQUOIS ST. & CALUMET - LAURIUM VILLAGE \\
\hline 1122 & 136 & MARTA & PETER & S & 10 & MICHIGAN & ITALY & ITALY & & & SAN MARTINO CANAVESE, TO & IROQUOIS ST. & CALUMET - LAURIUM VILLAGE \\
\hline 1123 & 136 & MARTA & TRACY & $\mathrm{D}$ & 3 & MICHIGAN & ITALY & ITALY & & & SAN MARTINO CANAVESE. TO & IROQUOIS ST. & CALUMET - LAURIUM VILLAGE \\
\hline 1124 & 134 & LOCATELLI & PIETRO & $\mathrm{H}$ & 34 & ITALY & ITALY & ITALY & 1900 & MASON/CONTRACTOR & SELINO. BERGAMO & IROQUOIS ST. & CALUMET - LAURIUM VILLAGE \\
\hline 1125 & 134 & LOCATELLI & GIGLIA & W & 28 & ITALY & ITALY & ITALY & 1902 & & & IROQUOIS ST. & CALUMET - LAURIUM VILLAGE \\
\hline 1126 & 134 & LOCATELLI & GIUSEPPINA & $\mathrm{D}$ & 7 & MICHIGAN & ITALY & ITALY & & & SELINO. BERGAMO & IROQUOIS ST. & CALUMET - LAURIUM VILLAGE \\
\hline 1127 & 134 & LOCATELLI & ANSOLING & $\mathrm{D}$ & 6 & MICHIGAN & ITALY & ITALY & & & SELINO. BERGAMO & IROQUOIS ST. & CALUMET - LAURIUM VILLAGE \\
\hline 1128 & 134 & LOCATELLI & ANTONIO & $\mathrm{s}$ & 3 & MICHIGAN & ITALY & ITALY & & & SELINO, BERGAMO & IROQUOIS ST. & CALUMET - LAURIUM VILLAGE \\
\hline 1129 & 134 & LOCATELLI & PIETRO & $\mathrm{S}$ & $10 \mathrm{M}$ & MICHIGAN & ITALY & ITALY & & & SELINO. BERGAMO & IROQUOIS ST. & CALUMET - LAURIUM VILLAGE \\
\hline 1130 & & CERUTTI & JOHN & $\mathrm{H}$ & 45 & ITALY & ITAL & ITAL & 1897 & MASON/CONTRACTOR & SAN GIUSTO CANAVESE & IROQUOIS ST. & CALUMET - LAURIUM VILLAGE \\
\hline 1131 & & CERUT & & W & 44 & & & & 189 & & SAN GIUSTO CANAVES & & CALUMET - LAURIUM VILLAGE \\
\hline 1132 & & CERUTTI & & $\mathrm{D}$ & 19 & & ITALY & & 1899 & SALESLADY/SHOE STORE & SAN GIUSTO CANAVESE. TO & IROQUOIS ST. & CALUMET - LAURIUM VILLAGE \\
\hline 1133 & & CERUTTI & ERMA & D & 16 & ITALY & ITALY & ITALY & 1899 & & SAN GIUSTO CANAVESE. TO & IROQUOIS ST. & CALUMET - LAURIUM VILLAGE \\
\hline
\end{tabular}




\begin{tabular}{|c|c|c|c|c|c|c|c|c|c|c|c|c|c|}
\hline 1134 & & CERUTTI & FRED & $\mathrm{s}$ & 13 & ITALY & ITALY & ITALY & 1899 & & SAN GIUSTO CANAVESE. TO & IROQUOIS ST. & CALUMET - LAURIUM VILLAGE \\
\hline 1135 & & CERUTTI & JOHN & S & 10 & MICHIGAN & ITALY & ITALY & & & SAN GIUSTO CANAVESE. TO & IROQUOIS ST. & CALUMET - LAURIUM VILLAGE \\
\hline 1136 & & BESSOLO & CARLO & $\mathrm{H}$ & 30 & ITALY & ITALY & ITALY & 1899 & SALESMAN/DEPT. STORE & BESSOLO CANAVESE. TO & FLORIDA ST. & CALUMET - LAURIUM VILLAGE \\
\hline 1137 & & BESSOLO & AMELIA & W & 32 & MICHIGAN & ITALY & ITALY & & & & FLORIDA ST. & CALUMET - LAURIUM VILLAGE \\
\hline 1138 & & BESSOLO & ROSA & $\mathrm{D}$ & 8 & MICHIGAN & ITALY & ITALY & & & BESSOLO CANAVESE. TO & FLORIDA ST. & CALUMET - LAURIUM VILLAGE \\
\hline 1139 & & TORREANO & $\mathrm{JOHN}$ & BIL & 30 & MICHIGAN & ITALY & ITALY & & BLACKSMITH/CM & TORINO & FLORIDA ST. & CALUMET - LAURIUM VILLAGE \\
\hline 1140 & 111 & YOTTI & DELPHINE & $\mathrm{H}$ & 47 & CANADA & CANADA & CANADA & & OWN INCOME & CANADA & 1ST ST. & CALUMET - LAURIUM VILLAGE \\
\hline 1141 & 111 & YOTTI & ALVINA & D & 19 & MICHIGAN & ITALY & CANADA & & & & 1ST ST. & CALUMET - LAURIUM VILLAGE \\
\hline 1142 & 111 & YOTTI & GILBERT & $\mathrm{s}$ & 15 & MICHIGAN & ITALY & CANADA & & & & 1ST ST. & CALUMET - LAURIUM VILLAGE \\
\hline 1143 & 111 & YOTTI & DELLA & $\mathrm{D}$ & 10 & MICHIGAN & ITALY & CANADA & & & & 1ST ST. & CALUMET - LAURIUM VILLAGE \\
\hline 1144 & 111 & YOTTI & EDWARD & $\mathrm{s}$ & 8 & MICHIGAN & ITALY & CANADA & & & & 1ST ST. & CALUMET - LAURIUM VILLAGE \\
\hline 1145 & 111 & TOMMEI & EGIDIO & $\mathrm{H}$ & 39 & ITALY & ITALY & ITALY & 1898 & MASON/CONTRACTOR & LUCCA & 1ST ST. & CALUMET - LAURIUM VILLAGE \\
\hline 1146 & 111 & TOMEI & ELISABETH & SER & 36 & ITALY & ITALY & ITALY & 1905 & HOUSEKEEPER/PF & BELLUNO & 1ST ST. & CALUMET - LAURIUM VILLAGE \\
\hline 1147 & 111 & DAL SANTO & ANTONIO & $\mathrm{H}$ & 43 & ITALY & ITALY & ITALY & 1901 & FARMER/FARM & COLTRANA. VICENZA & 1ST ST. & CALUMET - LAURIUM VILLAGE \\
\hline 1148 & 111 & DAL SANTO & GIOVANNI & s & 18 & ITALY & ITALY & ITALY & 1906 & CHOPPER/WOODS & COLTRANA. VICENZA & 1ST ST. & CALUMET - LAURIUM VILLAGE \\
\hline 1149 & 111 & ZORRER & FILIPPO & PART & 44 & ITALY & ITALY & ITALY & 1909 & FARMER/FARM & VICENZA & 1ST ST. & CALUMET - LAURIUM VILLAGE \\
\hline 1150 & 101 & TORREANO & JOHN & $\mathrm{H}$ & 46 & ITALY & ITALY & ITALY & 1885 & SALOONKEEPER & TORINO & OSCEOLA ST. & CALUMET - LAURIUM VILLAGE \\
\hline 1151 & 101 & TORREANO & FELICITA & W & 46 & ITALY & ITALY & ITALY & 1889 & & & OSCEOLA ST. & CALUMET - LAURIUM VILLAGE \\
\hline 1152 & 101 & TORREANO & KATHERINE & $\mathrm{D}$ & 19 & MICHIGAN & ITALY & ITALY & & & TORINO & OSCEOLA ST. & CALUMET - LAURIUM VILLAGE \\
\hline 1153 & 101 & TORREANO & FRANCESCO & s & 18 & MICHIGAN & ITALY & ITALY & & WIPER/ENGINE & TORINO & OSCEOLA ST. & CALUMET - LAURIUM VILLAGE \\
\hline 1154 & 101 & TORREANO & MARTIN & s & 17 & MICHIGAN & ITALY & ITALY & & & TORINO & OSCEOLA ST. & CALUMET - LAURIUM VILLAGE \\
\hline 1155 & 101 & TORREANO & PALOMA & $\mathrm{D}$ & 16 & MICHIGAN & ITALY & ITALY & & & TORINO & OSCEOLA ST. & CALUMET - LAURIUM VILLAGE \\
\hline 1156 & 101 & TORREANO & MARY & $\mathrm{D}$ & 13 & MICHIGAN & ITALY & ITALY & & & TORINO & OSCEOLA ST. & CALUMET - LAURIUM VILLAGE \\
\hline 1157 & 101 & TORREANO & ANGELO & $\mathrm{s}$ & 11 & MICHIGAN & ITALY & ITALY & & & TORINO & OSCEOLA ST. & CALUMET - LAURIUM VILLAGE \\
\hline 1158 & 101 & TORREANO & ELLEN & $\mathrm{D}$ & 4 & MICHIGAN & ITALY & ITALY & & & TORINO & OSCEOLA ST. & CALUMET - LAURIUM VILLAGE \\
\hline 1159 & 111 & TINETTI & PAUL & $\mathrm{H}$ & 34 & ITALY & ITALY & ITALY & 1898 & MERCHANT/GENERAL STORE & SAN MARTINO CANAVESE. TO & OSCEOLA ST. & CALUMET - LAURIUM VILLAGE \\
\hline 1160 & 111 & TINETTI & MADE & W & 34 & ITALY & ITALY & ITALY & 1898 & SALESLADY/GENERAL STORE & SAN MARTINO CANAVESE. TO & OSCEOLA ST. & CALUMET - LAURIUM VILLAGE \\
\hline 1161 & 111 & TINETTI & FRANK & s & 12 & MICHIGAN & ITALY & ITALY & & & SAN MARTINO CANAVESE, TO & OSCEOLA ST. & CALUMET - LAURIUM VILLAGE \\
\hline 1162 & 111 & TINETTI & PETER & $\mathrm{s}$ & 11 & MICHIGAN & ITALY & ITALY & & & SAN MARTINO CANAVESE. TO & OSCEOLA ST. & CALUMET - LAURIUM VILLAGE \\
\hline 1163 & 111 & TINETTI & JOHN & $\mathrm{S}$ & 8 & MICHIGAN & ITALY & ITALY & & & SAN MARTINO CANAVESE. TO & OSCEOLA ST. & CALUMET - LAURIUM VILLAGE \\
\hline 1164 & 111 & TINETTI & ERNEST & $\mathrm{S}$ & 2 & MICHIGAN & ITALY & ITALY & & & SAN MARTINO CANAVESE. TO & OSCEOLA ST. & CALUMET - LAURIUM VILLAGE \\
\hline 1165 & 111 & MASSOGLIA & PETER & B & 61 & ITALY & ITALY & ITALY & 98/99 & SAUSAGE MAKER & SAN MARTINO CANAVESE. TO & OSCEOLA ST. & CALUMET - LAURIUM VILLAGE \\
\hline 1166 & 110 & CURTO & PETER & $\mathrm{H}$ & 57 & ITALY & ITALY & ITALY & 1879 & MERCHANT/GROCERY STORE & TORINO & BOUNDARY ST. & CALUMET - LAURIUM VILLAGE \\
\hline 1167 & 110 & CURTO & MARY & w & 50 & ITALY & ITALY & ITALY & 1889 & & TORINO & BOUNDARY ST. & CALUMET - LAURIUM VILLAGE \\
\hline 1168 & 110 & GALETTO & GIORDANA & $\mathrm{D}$ & 30 & ITALY & ITALY & $\begin{array}{l}\text { ITALY } \\
\text { ITAL }\end{array}$ & 1889 & DRESSMAKER/CLOTHING & TORINO & BOUNDARY ST. & $\begin{array}{l}\text { CALUMET - LAURIUM VILLAGE } \\
\text { CALE }\end{array}$ \\
\hline 1160 & 110 & CURTO & CHARLES & s & 28 & MICHIGAN & ITALY & $\begin{array}{l}\text { ITALY } \\
\text { ITALY }\end{array}$ & 1009 & $\begin{array}{l}\text { LABORER/RAILROAD } \\
\text { LABORE }\end{array}$ & TORINO & BOUNDARY ST. & $\begin{array}{l}\text { CALUMET - LAURIUM VILLAGE } \\
\text { CALE }\end{array}$ \\
\hline 1170 & 110 & CURTO & MARY & $\mathrm{D}$ & 19 & MICHIGAN & ITALY & ITALY & & SALESLADY/GROCERY STORE & TORINO & BOUNDARY ST. & CALUMET - LAURIUM VILLAGE \\
\hline 1171 & 110 & CURTO & KATHERINE & $\mathrm{D}$ & 18 & MICHIGAN & ITALY & ITALY & & & TORINO & BOUNDARY ST. & CALUMET - LAURIUM VILLAGE \\
\hline 1172 & 110 & CURTO & TOMMASO & $\mathrm{s}$ & 17 & MICHIGAN & ITALY & ITALY & & WAITER/CLUBHOUSE & TORINO & BOUNDARY ST. & CALUMET - LAURIUM VILLAGE \\
\hline 1173 & 110 & CURTO & ANNIE & $\mathrm{D}$ & 13 & MICHIGAN & ITALY & ITALY & & & TORINO & BOUNDARY ST. & CALUMET - LAURIUM VILLAGE \\
\hline 1174 & 110 & GALETTO & FRANCESCO & SIN & 39 & ITALY & ITALY & ITALY & 1888 & MINER/CM & TORINO & BOUNDARY ST. & CALUMET - LAURIUM VILLAGE \\
\hline 1175 & 110 & GALETTO & KATHERINE & GD & 10 & MICHIGAN & ITALY & ITALY & & & TORINO & BOUNDARY ST. & CALUMET - LAURIUM VILLAGE \\
\hline 1176 & 110 & GALETTO & MARY & GD & 8 & MICHIGAN & ITALY & ITALY & & & TORINO & BOUNDARY ST. & CALUMET - LAURIUM VILLAGE \\
\hline 1177 & 110 & CURTO & $\mathrm{JOHN}$ & $\mathrm{BR}$ & 55 & ITALY & ITALY & ITALY & 1878 & MANAGER/GROCERY STORE & TORINO & BOUNDARY ST. & CALUMET - LAURIUM VILLAGE \\
\hline 1178 & & FURNO & GIUSEPPE & $\mathrm{H}$ & 42 & ITALY & ITALY & ITALY & 1888 & SALOONKEEPER & AGLIE', TO & 1ST ST. & CALUMET - LAURIUM VILLAGE \\
\hline 1179 & & FURNO & LOUIS & s & 18 & MICHIGAN & ITALY & ITALY & & & AGLIE'. TO & 1ST ST. & CALUMET - LAURIUM VILLAGE \\
\hline 1180 & & TORREANO & JAMES & $\mathrm{H}$ & 41 & ITALY & ITALY & ITALY & 1885 & MERCHANT/GENERAL STORE & TORINO & 1ST ST. & $\begin{array}{l}\text { CALUMET - LAURIUM VILLAGE } \\
\text { CALA }\end{array}$ \\
\hline 1181 & & TORREANO & ANNIE & W & 33 & ITALY & ITALY & $\begin{array}{l}\text { ITALY } \\
\text { ITAL }\end{array}$ & $\begin{array}{l}1000 \\
1888\end{array}$ & SALESLADY/GENERAL STORE & TRIIVU & 1ST ST. & $\begin{array}{l}\text { CALUMET - LAURIUM VILLAGE } \\
\text { CAUE }\end{array}$ \\
\hline 1182 & & TORREANO & FRANK & s & 13 & MICHIGAN & ITALY & ITALY & & & TORINO & 1ST ST. & CALUMET - LAURIUM VILLAGE \\
\hline 1183 & & TORRE, & JOHN & $\mathrm{s}$ & 11 & MICHIGAN & ITALY & ITALY & & & & 1ST ST. & CALUMET - LAURIUM VILLAGE \\
\hline 1184 & & TORREANO & RITA & $\mathrm{D}$ & 8 & MICHIGAN & ITAL & ITA & & & TOR & 1ST & CALUMET - LAURIUM VILLAGE \\
\hline 1185 & & TORREANO & JAMES & s & 6 & MICHIGAN & ITAL & ITAL & & & TORI & 1ST ST. & CALUMET - LAURIUM VILLAGE \\
\hline 1186 & & VERNA & MARY & SER & 22 & ITALY & ITA & ITA & 1904 & SALESLADY/GENERAL STORE & CIRIE'. TO & & CALUMET - LAURIUM VILLAGE \\
\hline 1187 & 410 & GROSSIO & JOHN B. & $\mathrm{H}$ & 42 & ITALY & ITAI & ITA| & 1896 & SALOONKEEPER & TORINO & HECLA ST. & CALUMET - LAURIUM VILLAGE \\
\hline 1188 & 410 & GROSSIO & MARY & W & 35 & MICHIGAN & ITALY & ITALY & & & & HECLA ST. & CALUMET - LAURIUM VILLAGE \\
\hline 1189 & 410 & GROSSIO & MARTIN & s & 16 & ITALY & ITALY & MICHIGAN & 1905 & & TORINO & HECLA ST. & CALUMET - LAURIUM VILLAGE \\
\hline 1190 & 410 & GROSSIO & JOHN & $\mathrm{s}$ & 11 & MICHIGAN & ITALY & MICHIGAN & & & TORINO & HECLA ST. & CALUMET - LAURIUM VILLAGE \\
\hline 1191 & 410 & GUGLIELMINO & DOMENICO & B & 45 & ITALY & ITALY & ITALY & 1895 & LABORER/GENERAL WORK & SAN GIUSTO CANAVESE, TO & HECLA ST. & CALUMET - LAURIUM VILLAGE \\
\hline 1192 & 410 & PASTORE & ANTONIO & B & 33 & ITALY & ITALY & ITALY & 1897 & BARTENDER/SALOON & SAN MARTINO CANAVESE. TO & HECLA ST. & CALUMET - LAURIUM VILLAGE \\
\hline 1193 & 410 & ZUBIENA & GIACOMO & $\begin{array}{l}\mathrm{D} \\
\mathrm{B}\end{array}$ & 30 & ITALY & ITALY & $\begin{array}{l}\text { ITALY } \\
\text { ITAL }\end{array}$ & 1906 & TRAMMER/CM & AGLIE'. TO & HECLA ST. & $\begin{array}{l}\text { CALUMET - LAURIUM VILLAGE } \\
\text { CALE }\end{array}$ \\
\hline 1194 & 410 & OBERTONE & ANTONIO & B & 26 & ITALY & ITALY & ITALY & 1907 & TIMBERMAN/CM & SAN GIORGIO CANAVESE. TO & HECLA ST. & CALUMET - LAURIUM VILLAGE \\
\hline 1195 & 109 & TORREANO & VITTORIA & $\mathrm{H}$ & 66 & ITALY & ITALY & ITALY & 1873 & & TORINO & HECLA ST. & CALUMET - LAURIUM VILLAGE \\
\hline 1196 & 109 & TORREANO & FRANCESCO & S & 38 & ITALY & ITALY & ITALY & 1873 & & TORINO & HECLA ST. & CALUMET - LAURIUM VILLAGE \\
\hline
\end{tabular}




\begin{tabular}{|c|c|c|c|c|c|c|c|c|c|c|c|c|c|}
\hline 1197 & 109 & TORREANO & AGOSTINO & S & 35 & MICHIGAN & ITALY & ITALY & & PAINTER/CM & TORINO & HECLA ST. & CALUMET - LAURIUM VILLAGE \\
\hline 1198 & 109 & TORREANO & JOSEPHINE & DIL & 19 & ITALY & ITALY & ITALY & 1903 & & TORINO & HECLA ST. & CALUMET - LAURIUM VILLAGE \\
\hline 1199 & 109 & TALENTINO & PETER & B & 23 & ITALY & ITALY & ITALY & 1900 & COLLECTOR/BANK & SAN MARTINO CANAVESE. TO & HECLA ST. & CALUMET - LAURIUM VILLAGE \\
\hline 1200 & 131 & BIANCO & JOSEPH & $\mathrm{H}$ & 44 & ITALY & ITALY & ITALY & 1888 & TIMBERMAN/CM & SCARMAGNO. TO & HECLA ST. & CALUMET - LAURIUM VILLAGE \\
\hline 1201 & 131 & BIANCO & MINNIE & w & 44 & ITALY & ITALY & ITALY & 1890 & & & HECLA ST. & CALUMET - LAURIUM VILLAGE \\
\hline 1202 & 131 & BIANCO & JOHN & s & 15 & MICHIGAN & ITALY & ITALY & & & SCARMAGNO. TO & HECLA ST. & CALUMET - LAURIUM VILLAGE \\
\hline 1203 & 131 & BIANCO & PETER & $\mathrm{s}$ & 14 & MICHIGAN & ITALY & ITALY & & & SCARMAGNO. TO & HECLA ST. & CALUMET - LAURIUM VILLAGE \\
\hline 1204 & 131 & BIANCO & PAULINE & $\mathrm{D}$ & 9 & MICHIGAN & ITALY & ITALY & & & SCARMAGNO. TO & HECLA ST. & $\begin{array}{l}\text { CALUMET - LAURIUM VILLAGE } \\
\text { CALE }\end{array}$ \\
\hline 1205 & 131 & MEALLIA & ANTON & B & 39 & ITALY & ITALY & ITALY & 1899 & TRAMMER/CM & BOSCONERO. TO & HECLA ST. & CALUMET - LAURIUM VILLAGE \\
\hline 1206 & 131 & BIANCO & JOSEPH & B & 32 & ITALY & ITALY & ITALY & 1906 & TIMBERMAN/CM & SCARMAGNO. TO & HECLA ST. & CALUMET - LAURIUM VILLAGE \\
\hline 1207 & 131 & BALAGNA & GIACOMO & $\mathrm{H}$ & 51 & ITALY & ITALY & ITALY & 1888 & TIMBERMAN/CM & PONT CANAVESE. TO & HECLA ST. & CALUMET - LAURIUM VILLAGE \\
\hline 1208 & 118 & TINETTI & PETER & $\mathrm{H}$ & 47 & ITALY & ITALY & ITALY & 1883 & TIMBERMAN/CM & SAN MARTINO CANAVESE. TO & OSCEOLA ST. & CALUMET - LAURIUM VILLAGE \\
\hline 1209 & 118 & TINETTI & ANGELINA & W & 45 & ITALY & ITALY & ITALY & 1889 & & & OSCEOLA ST. & CALUMET - LAURIUM VILLAGE \\
\hline 1210 & 118 & TINETTI & JOHANNA & $\mathrm{D}$ & 20 & MICHIGAN & ITALY & ITALY & & & SAN MARTINO CANAVESE. TO & OSCEOLA ST. & CALUMET - LAURIUM VILLAGE \\
\hline 1211 & 118 & TINETTI & ANTONIO & $\mathrm{s}$ & 19 & MICHIGAN & ITALY & ITALY & & DRILL BOY/CM & SAN MARTINO CANAVESE. TO & OSCEOLA ST. & CALUMET - LAURIUM VILLAGE \\
\hline 1212 & 118 & TINETTI & MARTA & $\mathrm{D}$ & 15 & MICHIGAN & ITALY & ITALY & & & SAN MARTINO CANAVESE, TO & OSCEOLA ST. & CALUMET - LAURIUM VILLAGE \\
\hline 1213 & 118 & TINETTI & JOHN & $\mathrm{s}$ & 12 & MICHIGAN & ITALY & ITALY & & & SAN MARTINO CANAVESE. TO & OSCEOLA ST. & CALUMET - LAURIUM VILLAGE \\
\hline 1214 & 118 & TINETTI & MARY & $\mathrm{D}$ & 8 & MICHIGAN & ITALY & ITALY & & & SAN MARTINO CANAVESE. TO & OSCEOLA ST. & CALUMET - LAURIUM VILLAGE \\
\hline 1215 & 118 & TINETTI & MODE & $\mathrm{D}$ & 7 & MICHIGAN & ITALY & ITALY & & & SAN MARTINO CANAVESE. TO & OSCEOLA ST. & CALUMET - LAURIUM VILLAGE \\
\hline 1216 & 118 & TINETTI & IRENE & $\mathrm{D}$ & 5 & MICHIGAN & ITALY & ITALY & & & SAN MARTINO CANAVESE. TO & OSCEOLA ST. & CALUMET - LAURIUM VILLAGE \\
\hline 1217 & 311 & BERSANO & BAPTISTE & $\mathrm{H}$ & 37 & ITALY & ITALY & ITALY & 93/95 & TEAMSTER/BREWERY & SAN MARTINO CANAVESE. TO & 1ST ST. & $\begin{array}{l}\text { CALUMET - LAURIUM VILLAGE } \\
\text { CAUE }\end{array}$ \\
\hline 1218 & 311 & BERSANO & CATHERINE & W & 27 & ITALY & ITALY & ITALY & 1903 & & & 1ST ST. & CALUMET - LAURIUM VILLAGE \\
\hline 1219 & 311 & CAPELLO & ANTONIO & $\mathrm{H}$ & 44 & ITALY & ITALY & ITALY & 1888 & LABORER/CM & SAN BENIGNO CANAVESE. TO & 1ST ST. & CALUMET - LAURIUM VILLAGE \\
\hline 1220 & 311 & CAPELLO & CLARA & W & 34 & ITALY & ITALY & ITALY & 1892 & & & 1ST ST. & CALUMET - LAURIUM VILLAGE \\
\hline 1221 & 311 & CAPELLO & MARY & $\mathrm{D}$ & 15 & MICHIGAN & ITALY & ITALY & & & SAN BENIGNO CANAVESE. TO & 1ST ST. & CALUMET - LAURIUM VILLAGE \\
\hline 1222 & 311 & CAPELLO & JAMES & $s$ & 14 & MICHIGAN & ITALY & ITALY & & & SAN BENIGNO CANAVESE. TO & 1ST ST. & CALUMET - LAURIUM VILLAGE \\
\hline 1223 & 311 & CAPELLO & JOHN & $\mathrm{s}$ & 12 & MICHIGAN & ITALY & ITALY & & & SAN BENIGNO CANAVESE. TO & 1ST ST. & CALUMET - LAURIUM VILLAGE \\
\hline 1224 & 311 & CAPELLO & JOSEPH & $\mathrm{s}$ & 10 & MICHIGAN & ITALY & ITALY & & & SAN BENIGNO CANAVESE, TO & 1ST ST. & CALUMET - LAURIUM VILLAGE \\
\hline 1225 & 311 & CAPELLO & ANNE & D & 7 & MICHIGAN & ITALY & ITALY & & & SAN BENIGNO CANAVESE. TO & 1ST ST. & CALUMET - LAURIUM VILLAGE \\
\hline 1226 & 311 & CAPELLO & THERESA & $\mathrm{D}$ & 5 & MICHIGAN & ITALY & ITALY & & & SAN BENIGNO CANAVESE. TO & 1ST ST. & CALUMET - LAURIUM VILLAGE \\
\hline 1227 & 311 & CAPELLO & VICTOR & s & $10 \mathrm{M}$ & MICHIGAN & ITALY & ITALY & & & SAN BENIGNO CANAVESE. TO & 1ST ST. & CALUMET - LAURIUM VILLAGE \\
\hline 1228 & 311 & GALLASERI & TERESA & MIL & 68 & ITALY & ITALY & ITALY & 1900 & & PONT CANAVESE. TO & 1ST ST. & CALUMET - LAURIUM VILLAGE \\
\hline 1229 & 311 & CARBONATO & CHARLES & $\mathrm{H}$ & 36 & $\begin{array}{l}\text { ITALY } \\
\text { ITAL }\end{array}$ & ITALY & ITALY & 1887 & BUTCHER/OWN SHOP & PONT CANAVESE. TO & 1ST ST. & $\begin{array}{l}\text { CALUMET - LAURIUM VILLAGE } \\
\text { CALE }\end{array}$ \\
\hline 1230 & 311 & CARBONATO & MARY & W & 32 & ITALY & ITALY & ITALY & 1887 & & PONT CANAVESE, TO & 1ST ST. & CALUMET - LAURIUM VILLAGE \\
\hline 1231 & 311 & CARBONATO & PHILLYS & $\mathrm{D}$ & 8 & MICHIGAN & ITALY & ITALY & & & PONT CANAVESE. TO & 1ST ST. & CALUMET - LAURIUM VILLAGE \\
\hline 1232 & 311 & SALICETTI & ANGELO & $\mathrm{H}$ & 38 & ITALY & ITALY & ITALY & 1895 & BUTCHER/OWN SHOP & PEROSA CANAVESE. TO & 1ST ST. & CALUMET - LAURIUM VILLAGE \\
\hline 1233 & 311 & SALICETTI & $\begin{array}{l}\text { PAOLA } \\
\end{array}$ & W & 20 & ITALY & ITALY & ITALY & 1907 & & & 1ST ST. & CALUMET - LAURIUM VILLAGE \\
\hline 1234 & 311 & SALICETTI & ALBERT & $\mathrm{s}$ & 2 & MICHIGAN & ITALY & ITALY & & & PEROSA CANAVESE. TO & 1ST ST. & CALUMET - LAURIUM VILLAGE \\
\hline 1235 & 311 & BERTOLATTO & FRANCESCO & B & 45 & ITALY & ITALY & ITALY & 1895 & LABORER/SALOON & TORINO & 1ST ST. & CALUMET - LAURIUM VILLAGE \\
\hline 1236 & 311 & $\mathrm{BO}^{\prime}$ & THERESA & MIL & 50 & ITALY & ITALY & ITALY & 1909 & & TORINO & 1ST ST. & CALUMET - LAURIUM VILLAGE \\
\hline 1237 & 115 & ROSSIO & MICHAEL & $\mathrm{H}$ & 28 & ITALY & ITALY & ITALY & & FOREMAN/CM & PONT CANAVESE. TO & KEARSARGE ST. & CALUMET - LAURIUM VILLAGE \\
\hline 1238 & 115 & ROSSIO & JULIA & W & 28 & MICHIGAN & ENGLAND & ENGLAND & & & ENGLAND & KEARSARGE ST. & CALUMET - LAURIUM VILLAGE \\
\hline 1239 & 115 & ROSSIO & ERMALINE & $\mathrm{D}$ & 5 & MICHIGAN & ITALY & MICHIGAN & & & PONT CANAVESE. TO & KEARSARGE ST. & CALUMET - LAURIUM VILLAGE \\
\hline 1240 & 115 & ROSSIO & BERNICE & $\mathrm{D}$ & 4 & MICHIGAN & ITALY & MICHIGAN & & & PONT CANAVESE. TO & KEARSARGE ST. & CALUMET - LAURIUM VILLAGE \\
\hline 1241 & 115 & ROSSIO & CLARENCE & $\mathrm{s}$ & $1 \mathrm{M}$ & MICHIGAN & ITALY & MICHIGAN & & & PONT CANAVESE. TO & KEARSARGE ST. & CALUMET - LAURIUM VILLAGE \\
\hline 1242 & 117 & ONORE & BONO & $\mathrm{H}$ & 29 & ITALY & ITALY & ITALY & 1903 & WATCHMAN/CM & STRAMBINELLO. TO & KEARSARGE ST. & CALUMET - LAURIUM VILLAGE \\
\hline 1243 & 117 & ONORE-GALLO & PERINA & W & 26 & ITALY & ITALY & ITALY & 1903 & & STRAMBINELLO. TO & KEARSARGE ST. & CALUMET - LAURIUM VILLAGE \\
\hline 1244 & 117 & ONORE & KATHERINE & D & 5 & MICHIGAN & ITALY & ITALY & & & . TO & EST. & T - LAU \\
\hline 1245 & 117 & ONOP & $\begin{array}{l}\text { JUSTINA } \\
\text { JUL }\end{array}$ & $\mathrm{D}$ & 3 & MICHIGAN & ITALY & ITA & & & O. TO & SARGE ST. & ET - LAU \\
\hline 1246 & 117 & ONORE & PETER & BR & 27 & ITALY & ITALY & ITALY & 1907 & MINER/CM & $-\mathrm{O}$ TO & & ET - LAURIUM VILLAGE \\
\hline 1247 & 117 & CASERIO & MARTINO & $\mathrm{H}$ & 40 & ITA & ITAL & ITAI & & MINER/CM & LLO. TO & KEARSARGE ST. & CALUMET - LAURIUM VILLAGE \\
\hline 1248 & 117 & CASERIO & GIOVANNA & W & 38 & ITAL & ITAL & ITALY & 1906 & & STRAMBINELLO, TO & KEARSARGE ST. & CALUMET - LAURIUM VILLAGE \\
\hline 1249 & 117 & CASERIO & GIUSEPPE & $\mathrm{s}$ & 18 & ITALY & ITALY & ITALY & 1906 & LABORER/CM & STRAMBINELLO. TO & KEARSARGE ST. & CALUMET - LAURIUM VILLAGE \\
\hline 1250 & 117 & CASERIO & MARIA & D & 16 & ITALY & ITALY & ITALY & 1906 & IRONER/STEAM LAUNDRY & STRAMBINELLO. TO & KEARSARGE ST. & CALUMET - LAURIUM VILLAGE \\
\hline 1251 & 117 & GEDDA & PIETRO & B & 21 & ITALY & ITALY & ITALY & 1904 & TAILOR/SHOP & STRAMBINELLO. TO & KEARSARGE ST. & CALUMET - LAURIUM VILLAGE \\
\hline 1252 & 117 & ONORE & GIOVANNI & B & 26 & ITALY & ITALY & ITALY & 1906 & TRAMMER/CM & STRAMBINELLO. TO & KEARSARGE ST. & CALUMET - LAURIUM VILLAGE \\
\hline 1253 & 117 & SCALA & ANTONIO & B & 36 & ITALY & ITALY & ITALY & 1898 & TRAMMER/CM & STRAMBINELLO. TO & KEARSARGE ST. & CALUMET - LAURIUM VILLAGE \\
\hline 1254 & 123 & ROLANDO & ANTONIO & $\mathrm{H}$ & 25 & ITALY & ITALY & ITALY & 1890 & BUTCHER/BUTCHER SHOP & PONT CANAVESE, TO & KEARSARGE ST. & CALUMET - LAURIUM VILLAGE \\
\hline 1255 & 123 & ROLANDO & MADDALENA & W & 25 & ITALY & ITALY & ITALY & 1887 & & & KEARSARGE ST. & CALUMET - LAURIUM VILLAGE \\
\hline 1256 & 123 & ROLANDO & VIRGINIA & $\mathrm{D}$ & 2 & MICHIGAN & ITAL & ITAL & & & PONT CANAVESE. TO & KEARSARGE ST. & CALUMET - LAURIUM VILLAGE \\
\hline 1257 & 123 & LANDO & NZO & $\mathrm{s}$ & $16 \mathrm{M}$ & & & & & & $\mathrm{PO}$ & KEARSARGE ST. & CALUMET - LAURIUM VILLAGE \\
\hline 1258 & 123 & & & $\mathrm{H}$ & 29 & MICHIGAN & GERMANY & GERMAN & & PLUMBER/HARDWARE STORE & GERMANY & & \\
\hline 1259 & 123 & MANTZ & THERESA & w & 30 & ITALY & ITALY & ITALY & 1903 & & & KEARSARGE ST. & CALUMET - LAURIUM VILLAGE \\
\hline
\end{tabular}




\begin{tabular}{|c|c|c|c|c|c|c|c|c|c|c|c|c|c|}
\hline 1260 & 123 & MANTZ & CHARLES & $\mathrm{s}$ & 3 & MICHIGAN & MICHIGAN & ITALY & & & & KEARSARGE ST. & CALUMET - LAURIUM VILLAGE \\
\hline 1261 & 123 & MANTZ & ALOISINO & $\mathrm{S}$ & $14 \mathrm{M}$ & MICHIGAN & MICHIGAN & ITALY & & & & KEARSARGE ST. & CALUMET - LAURIUM VILLAGE \\
\hline 1262 & 123 & ROSSIO & GIUSEPPE & $\mathrm{H}$ & 28 & ITALY & ITALY & ITALY & 1902 & FIREMAN/LAUNDRY & BELMONTE. TRENTO & KEARSARGE ST. & CALUMET - LAURIUM VILLAGE \\
\hline 1263 & 123 & ROSSIO & MARIA & W & 26 & ITALY & ITALY & ITALY & 1906 & & & KEARSARGE ST. & CALUMET - LAURIUM VILLAGE \\
\hline 1264 & 123 & ROSSIO & CATERINA & $\mathrm{D}$ & 2 & MICHIGAN & ITALY & ITALY & & & BELMONTE. TRENTO & KEARSARGE ST. & CALUMET - LAURIUM VILLAGE \\
\hline 1265 & 127 & RICHETTA & GIUSEPPE & $\mathrm{H}$ & 40 & ITALY & ITALY & ITALY & 1890 & CARPENTER/HOUSE & SAN MARTINO CANAVESE. TO & KEARSARGE ST. & CALUMET - LAURIUM VILLAGE \\
\hline 1266 & 127 & RICHETTA & MARIA & W & 32 & ITALY & ITALY & ITALY & 1890 & & SAN MARTINO CANAVESE. TO & KEARSARGE ST. & CALUMET - LAURIUM VILLAGE \\
\hline 1267 & 127 & RICHETTA & ANNETTA & $\mathrm{D}$ & 12 & MICHIGAN & ITALY & ITALY & 1050 & & SAN MARTINO CANAVESE. TO & KEARSARGE ST. & $\begin{array}{l}\text { CALUMET - LAURIUM VILLAGE } \\
\text { CALE }\end{array}$ \\
\hline 1268 & 127 & RICHETTA & ANTONIO & $\mathrm{S}$ & 5 & MICHIGAN & ITALY & ITALY & & & SAN MARTINO CANAVESE. TO & KEARSARGE ST. & CALUMET - LAURIUM VILLAGE \\
\hline 1269 & 131 & ROSSETTO & BARTOLOMEO & $\mathrm{H}$ & 24 & ITALY & ITALY & ITALY & 1907 & TIMBERMAN/CM & TORINO & KEARSARGE ST. & CALUMET - LAURIUM VILLAGE \\
\hline 1270 & 131 & ROSSETTO & FRANCESCA & w & 21 & ITALY & ITALY & ITALY & 1907 & & TORINO & KEARSARGE ST. & CALUMET - LAURIUM VILLAGE \\
\hline 1271 & 131 & ROSSETTO & ANDREW & $\mathrm{s}$ & 2 & MICHIGAN & ITALY & ITALY & & & TORINO & KEARSARGE ST. & CALUMET - LAURIUM VILLAGE \\
\hline 1272 & 131 & PERENCHIO & MARIA & B & 39 & ITALY & ITALY & ITALY & 1897 & OWN INCOME & PAVONE CANAVESE. TO & KEARSARGE ST. & CALUMET - LAURIUM VILLAGE \\
\hline 1273 & 131 & PERENCHIO & ANDREA & B & 17 & ITALY & ITALY & ITALY & 1897 & WAITER/CLUBHOUSE & PAVONE CANAVESE. TO & KEARSARGE ST. & CALUMET - LAURIUM VILLAGE \\
\hline 1274 & 131 & PERENCHIO & GIOVANNI & B & 12 & MICHIGAN & ITALY & ITALY & & & PAVONE CANAVESE. TO & KEARSARGE ST. & CALUMET - LAURIUM VILLAGE \\
\hline 1275 & 131 & PERENCHIO & GAUDENZIO & B & 5 & MICHIGAN & ITALY & ITALY & & & PAVONE CANAVESE, TO & KEARSARGE ST. & CALUMET - LAURIUM VILLAGE \\
\hline 1276 & 139 & CESARE & ANTONIO & $\mathrm{H}$ & 47 & ITALY & ITALY & ITALY & 1883 & LABORER/STREET & SAN MARTINO CANAVESE. TO & KEARSARGE ST. & CALUMET - LAURIUM VILLAGE \\
\hline 1277 & 139 & CESARE & MARGHERITA & W & 47 & ITALY & ITALY & ITALY & 1898 & & SAN MARTINO CANAVESE. TO & KEARSARGE ST. & CALUMET - LAURIUM VILLAGE \\
\hline 1278 & 139 & CESARE & MARIA & $\mathrm{D}$ & 25 & ITALY & ITALY & ITALY & 1898 & DRESSMAKER/AT HOME & SAN MARTINO CANAVESE. TO & KEARSARGE ST. & CALUMET - LAURIUM VILLAGE \\
\hline 1279 & 139 & CESARE & TERESA & $\mathrm{D}$ & 21 & ITALY & ITALY & ITALY & 1898 & DRESSMAKER/AT HOME & SAN MARTINO CANAVESE. TO & KEARSARGE ST. & CALUMET - LAURIUM VILLAGE \\
\hline 1280 & 145 & BASSO & THERESA & B & 17 & MICHIGAN & $\begin{array}{l}\text { ITALY } \\
\text { ITAL }\end{array}$ & ITALY & 1090 & $\begin{array}{l}\text { SERVANT/PF } \\
\text { SEIITIV }\end{array}$ & SAN GIORGIO CANAVESE. TO & 2ND ST. & $\begin{array}{l}\text { CALUMET - LAURIUM VILLAGE } \\
\text { CAUE }\end{array}$ \\
\hline 1281 & 132 & TINETTI & LUIGI & $\mathrm{H}$ & 30 & ITALY & ITALY & ITALY & 1903 & TEAMSTER/BREWERY & SAN MARTINO CANAVESE, TO & HECLA ST. & CALUMET - LAURIUM VILLAGE \\
\hline 1282 & 132 & TINETTI & ROSA & w & 25 & ITALY & ITALY & ITALY & 1903 & & SAN MARTINO CANAVESE. TO & HECLA ST. & CALUMET - LAURIUM VILLAGE \\
\hline 1283 & 132 & TINETTI & MARIA & $\mathrm{D}$ & 4 & MICHIGAN & ITALY & ITALY & & & SAN MARTINO CANAVESE. TO & HECLA ST. & CALUMET - LAURIUM VILLAGE \\
\hline 1284 & 132 & TINETTI & ANNA & $\mathrm{D}$ & 2 & MICHIGAN & ITALY & ITALY & & & SAN MARTINO CANAVESE. TO & HECLA ST. & CALUUMT - LAURIUM VILLAGE \\
\hline 1285 & 132 & TINETTI & NORA & D & $6 \mathrm{M}$ & MICHIGAN & ITALY & ITALY & & & SAN MARTINO CANAVESE. TO & HECLA ST. & CALUMET - LAURIUM VILLAGE \\
\hline 1286 & 132 & VENEGONE & JOHN & $\mathrm{H}$ & 34 & ITALY & ITALY & ITALY & 1892 & TIMBERMAN/CM & CUGGIANO. MILANO & HECLA ST. & CALUMET - LAURIUM VILLAGE \\
\hline 1287 & 132 & VENEGONE & MARY & W & 34 & ITALY & ITALY & ITALY & 1900 & & & HECLA ST. & CALUMET - LAURIUM VILLAGE \\
\hline 1288 & 132 & TINETTI & BATTISTA & $\mathrm{H}$ & 47 & ITALY & ITALY & ITALY & 1885 & WATCHMAN/CM & SAN MARTINO CANAVESE. TO & HECLA ST. & CALUMET - LAURIUM VILLAGE \\
\hline 1289 & 132 & TINETTI & ANTONIA & W & 53 & ITALY & ITALY & ITALY & 1890 & & & HECLA ST. & CALUMET - LAURIUM VILLAGE \\
\hline 1290 & 132 & TINETTI & FRANCESCO & s & 17 & MICHIGAN & ITALY & ITALY & & CARRIAGE BOY/PF & SAN MARTINO CANAVESE. TO & HECLA ST. & CALUMET - LAURIUM VILLAGE \\
\hline 1291 & 132 & TINETTI & GIOVANNI & $\mathrm{s}$ & 15 & MICHIGAN & ITALY & ITALY & & & SAN MARTINO CANAVESE. TO & HECLA ST. & CALUMET - LAURIUM VILLAGE \\
\hline 1292 & 132 & TINETTI & MARIA & $\mathrm{D}$ & 13 & MICHIGAN & ITALY & ITALY & & & SAN MARTINO CANAVESE. TO & HECLA ST. & $\begin{array}{l}\text { CALUMET - LAURIUM VILLAGE } \\
\text { CALE }\end{array}$ \\
\hline 1293 & 132 & TINETTI & ENRICO & $\mathrm{D}$ & $16 \mathrm{M}$ & MICHIGAN & ITALY & ITALY & & & SAN MARTINO CANAVESE, TO & HECLA ST. & CALUMET - LAURIUM VILLAGE \\
\hline 1294 & 130 & MARTA & MARTINO & $\mathrm{H}$ & 31 & ITALY & ITALY & ITALY & 1903 & TEAMSTER/BREWERY & SAN MARTINO CANAVESE. TO & HECLA ST. & CALUMET - LAURIUM VILLAGE \\
\hline 1295 & 130 & MARTA & JOSEPHINE & w & 22 & MICHIGAN & ITALY & ITALY & & & & HECLA ST. & CALUMET - LAURIUM VILLAGE \\
\hline 1296 & 130 & ROVANO & DOMENICO & $\mathrm{H}$ & 24 & ITALY & ITALY & ITALY & 1902 & TIMBER BOSS/CM & SAN MARTINO CANAVESE. TO & HECLA ST. & CALUMET - LAURIUM VILLAGE \\
\hline 1297 & 130 & ROVANO & TERESA & W & 21 & MICHIGAN & ITALY & ITALY & & & & HECLA ST. & CALUMET - LAURIUM VILLAGE \\
\hline 1298 & 130 & ROVANO & FRANCES & $\mathrm{D}$ & 2 & MICHIGAN & ITALY & MICHIGAN & & & SAN MARTINO CANAVESE. TO & HECLA ST. & CALUMET - LAURIUM VILLAGE \\
\hline 1299 & 110 & SUINO & FRANCESCO & $\mathrm{H}$ & 41 & ITALY & ITALY & ITALY & 1890 & MERCHANT/GROCERY STORE & TORINO & HECLA ST. & CALUMET - LAURIUM VILLAGE \\
\hline 1300 & 110 & SUINO & MARIA & W & 36 & ITALY & ITALY & ITALY & $97 / 99$ & SALESLADY/GROCERY STORE & & HECLA ST. & CALUMET - LAURIUM VILLAGE \\
\hline 1301 & 110 & SUINO & BERNARDO & s & 12 & MICHIGAN & ITALY & ITALY & & & TORINO & HECLA ST. & CALUMET - LAURIUM VILLAGE \\
\hline 1302 & 110 & SUINO & GIORGIO & $\mathrm{s}$ & 10 & MICHIGAN & ITALY & ITALY & & & TORINO & HECLA ST. & CALUMET - LAURIUM VILLAGE \\
\hline 1303 & 110 & SUINO & ANGELINA & $\mathrm{D}$ & 7 & MICHIGAN & ITALY & ITALY & & & TORINO & HECLA ST. & CALUMET - LAURIUM VILLAGE \\
\hline 1304 & 110 & ROLANDO & MARY & SER & 19 & MICHIGAN & ITALY & ITALY & & SALESLADY/GROCERY STORE & PONT CANAVESE. TO & HECLA ST. & CALUMET - LAURIUM VILLAGE \\
\hline 1305 & 110 & VAIRO & GIACOMO & $\mathrm{H}$ & 28 & ITALY & ITALY & ITALY & 19001 & SALOONKEEPER & LOCANA CANAVESE. TO & 1ST ST. & CALUMET - LAURIUM VILLAGE \\
\hline 1306 & 110 & VAIRO & ANGELINA & W & 25 & ITALY & ITALY & ITALY & 1908 & & & & CALUMET - LAURIUM VILLAGE \\
\hline 1307 & 110 & GUGLIELMINO & EPPE & B & 28 & & ITA & ITAI & 1906 & TIMBERMAN/CM & SAN GIORGIO CANAVESE. TO & & T - LAL \\
\hline 1308 & 110 & GUGLIELMINO & TISTA & B & 26 & ITA & & ITA & 1907 & TRAMMER/CM & SAN GIORGIO CANAVESE. TO & & ET - LAL \\
\hline 1309 & 110 & $\mathrm{BO}^{\prime}$ & JOHN & $\mathrm{H}$ & 27 & ITA & ITA & ITALY & 1905 & BARTENDER/SALOON & IESE. TO & & JMET - LAU \\
\hline 1310 & 110 & BO'-CRESTO & PAOLINA & W & 25 & & & ITA & 1905 & & & & CALUMET - LAURIUM VILLAGE \\
\hline 1311 & 110 & $\mathrm{BO}^{\prime}$ & THERESA & D & $21 \mathrm{M}$ & MICHIGAN & ITAL & ITAI & & & PONT CANAVESE, TO & 1ST ST. & CALUMET - LAURIUM VILLAGE \\
\hline 1312 & 110 & ROLANDO & JOSEPHINE & SER & 16 & MINNESOT & ITALY & ITALY & & SERVANT/PF & PONT CANAVESE. TO & 1ST ST. & CALUMET - LAURIUM VILLAGE \\
\hline 1313 & 110 & MARTA & BATTISTA & $\mathrm{H}$ & 44 & ITALY & ITALY & ITALY & 1885 & BUTCHER/OWN SHOP & SAN MARTINO CANAVESE. TO & 1ST ST. & CALUMET - LAURIUM VILLAGE \\
\hline 1314 & 110 & MARTA & GIOVANNA & $\mathrm{D}$ & 19 & MICHIGAN & ITALY & ITALY & & & SAN MARTINO CANAVESE. TO & 1ST ST. & CALUMET - LAURIUM VILLAGE \\
\hline 1315 & 110 & MARTA & MADDALENA & $\mathrm{D}$ & 13 & MICHIGAN & ITALY & ITALY & & & SAN MARTINO CANAVESE. TO & 1ST ST. & CALUMET - LAURIUM VILLAGE \\
\hline 1316 & 110 & MARTA & LEONE & $\mathrm{s}$ & 10 & MICHIGAN & ITALY & ITALY & & & SAN MARTINO CANAVESE. TO & 1ST ST. & CALUMET - LAURIUM VILLAGE \\
\hline 1317 & 109 & SCIACERO & DOMENICO & $\mathrm{H}$ & 32 & ITALY & ITALY & ITALY & 1903 & TIMBERMAN/CM & SAN GIOVANNI CANAVESE, TO & TAMARAC & CALUMET - LAURIUM VILLAGE \\
\hline 1318 & 109 & SCIACERO & ANGELINA & W & 33 & ITALY & ITALY & ITALY & 1900 & & & & CALUMET - LAURIUM VILLAGE \\
\hline 1319 & 109 & SCIACERO & & $\mathrm{s}$ & 4 & MICHIGAN & ITAL & ITA & & & SAN GIOVANNI CANAVESE. TO & & RIUM VILLA \\
\hline 1320 & 109 & CERO & CESCO & $\mathrm{s}$ & 2 & IIGAN & & & & & SAN GIOVANNI CANAVESE. TO & & \\
\hline 1321 & 109 & & & B & 46 & & & & $89 / 99$ & & & & \\
\hline 1322 & 109 & ENTANINO & GIOVANNI & B & 23 & ITALY & ITALY & ITALY & 1905 & MINER/CM & PEROSA CANAVESE. TO & TAMARACK ST. & CALUMET - LAURIUM VILLAGE \\
\hline
\end{tabular}




\begin{tabular}{|c|c|c|c|c|c|c|c|c|c|c|c|c|c|}
\hline 1323 & 109 & CENTANINO & ANTONIO & B & 21 & ITALY & ITALY & ITALY & $06 / 07$ & TIMBERMAN/CM & PEROSA CANAVESE. TO & TAMARACK ST. & CALUMET - LAURIUM VILLAGE \\
\hline 1324 & 109 & DE MARIA & CHARLES & B & 37 & ITALY & ITALY & ITALY & 1905 & TIMBERMAN/CM & TORINO & TAMARACK ST. & CALUMET - LAURIUM VILLAGE \\
\hline 1325 & 109 & SCASSA & ROSA & $\mathrm{H}$ & 26 & ITALY & ITALY & ITALY & 1907 & WASHWOMAN/PF & MINAS. CAGLIARI & TAMARACK ST. & CALUMET - LAURIUM VILLAGE \\
\hline 1326 & 109 & SCASSA & CATERINA & $\mathrm{D}$ & 7 & BRASIL & ITALY & ITALY & 1907 & & MINAS. CAGLIARI & TAMARACK ST. & CALUMET - LAURIUM VILLAGE \\
\hline 1327 & 109 & SCASSA & DANTE & s & 5 & BRASIL & ITALY & ITALY & 1907 & & MINAS. CAGLIARI & TAMARACK ST. & CALUMET - LAURIUM VILLAGE \\
\hline 1328 & 123 & SCIACERO & LUIGI & $\mathrm{H}$ & 48 & $\begin{array}{l}\text { ITALY } \\
\text { ITA }\end{array}$ & ITALY & ITALY & 1888 & OWN INCOME & SAN MARTINO CANAVESE. TO & TAMARACK ST. & CALUMET - LAURIUM VILLAGE \\
\hline 1329 & 123 & SCIACERO & MARIA & w & 47 & ITALY & ITALY & ITALY & 1888 & & SAN MARTINO CANAVESE. TO & TAMARACK ST. & CALUMET - LAURIUM VILLAGE \\
\hline 1330 & 123 & SCIACERO & MARIA & $\mathrm{D}$ & 16 & ITALY & ITALY & ITALY & 1896 & & SAN MARTINO CANAVESE. TO & TAMARACK ST. & CALUMET - LAURIUM VILLAGE \\
\hline 1331 & $\frac{120}{123}$ & SCIACERO & GIOVANNI & s & 11 & MICHIGAN & ITALY & ITALY & & & SAN MARTINO CANAVESE, TO & TAMARACK ST. & CALUMET - LAURIUM VILLAGE \\
\hline 1332 & 123 & SCIACERO & FELICE & s & 9 & MICHIGAN & ITALY & ITALY & & & SAN MARTINO CANAVESE. TO & TAMARACK ST. & CALUMET - LAURIUM VILLAGE \\
\hline 1333 & 123 & TINETTI & MARTINO & B & 35 & ITALY & ITALY & ITALY & 1902 & TIMBERMAN/CM & SAN GIOVANNI CANAVESE. TO & TAMARACK ST. & CALUMET - LAURIUM VILLAGE \\
\hline 1334 & 123 & TABACCHI & GIACOMO & B & 20 & ITALY & ITALY & ITALY & 1906 & BLACKSMITH/CM & STRAMBINELLO. TO & TAMARACK ST. & CALUMET - LAURIUM VILLAGE \\
\hline 1335 & 137 & DELL'ACQUA & SAMUEL & $\mathrm{H}$ & 40 & ITALY & ITALY & ITALY & 1890 & MACHINIST/CM & CUGGIANO. MILANO & TAMARACK ST. & CALUMET - LAURIUM VILLAGE \\
\hline 1336 & 137 & DELL'ACQUA & ROSE & w & 33 & ITALY & ITALY & ITALY & 1889 & & & TAMARACK ST. & CALUMET - LAURIUM VILLAGE \\
\hline 1337 & 137 & DELL'ACQUA & MARY & $\mathrm{D}$ & 10 & MICHIGAN & ITALY & ITALY & & & CUGGIANO. MILANO & TAMARACK ST. & CALUMET - LAURIUM VILLAGE \\
\hline 1338 & 137 & DELL'ACQUA & LENA & $\mathrm{D}$ & 7 & MICHIGAN & ITALY & ITALY & & & CUGGIANO, MILANO & TAMARACK ST. & CALUMET - LAURIUM VILLAGE \\
\hline 1339 & 137 & DELL'ACQUA & JOSEPHINE & $\mathrm{D}$ & 3 & MICHIGAN & ITALY & ITALY & & & CUGGIANO. MILANO & TAMARACK ST. & CALUMET - LAURIUM VILLAGE \\
\hline 1340 & 137 & DELL'ACQUA & EDITH & $\mathrm{D}$ & 2 & MICHIGAN & ITALY & ITALY & & & CUGGIANO. MILANO & TAMARACK ST. & CALUMET - LAURIUM VILLAGE \\
\hline 1341 & 137 & BESSOLO & LOUIS & B & 36 & ITALY & ITALY & ITALY & 1896 & MINER/CM & BESSOLO CANAVESE. TO & TAMARACK ST. & CALUMET - LAURIUM VILLAGE \\
\hline 1342 & 104 & CRESTO & BARTOLOMEO & D & 25 & ITALY & ITALY & ITALY & 1904 & BARTENDER/SALOON & CASTELLAMONTE. TO & KEARSARGE ST. & CALUMET - LAURIUM VILLAGE \\
\hline 1343 & 104 & CRESTO & MARIA & w & 28 & ITALY & ITALY & ITALY & 1907 & & & $\begin{array}{l}\text { KEARSARGE ST. } \\
\text { KEARSAR ST }\end{array}$ & CALUMET - LAURIUM VILLAGE \\
\hline $\begin{array}{l}1343 \\
1344\end{array}$ & $\begin{array}{l}104 \\
104\end{array}$ & $\begin{array}{l}\text { CRESIO } \\
\text { CRESTO }\end{array}$ & $\begin{array}{l}\text { MARRAA } \\
\text { MARGHERITA }\end{array}$ & $\begin{array}{l}\mathrm{W} \\
\mathrm{D}\end{array}$ & $8 \mathrm{M}$ & $\begin{array}{l}\text { MALY } \\
\text { MICHIGAN }\end{array}$ & $\begin{array}{l}\text { IIALY } \\
\text { ITALY }\end{array}$ & $\begin{array}{l}\text { IITALY } \\
\text { ITAYY }\end{array}$ & & & CASTELLAMONTE, TO & $\begin{array}{l}\text { KEARSARGE ST. } \\
\text { KEARSARGE ST. }\end{array}$ & $\begin{array}{l}\text { CALUMEI - LAURIUM VILLAGE } \\
\text { CALUMET - LAURIUM VILLAGE }\end{array}$ \\
\hline 1345 & 104 & ROLANDO & ROSE & SER & 17 & MINNESOT & ITALY & ITALY & & SERVANT/PF & PONT CANAVESE. TO & KEARSARGE ST. & CALUMET - LAURIUM VILLAGE \\
\hline 1346 & 104 & CAPELLO & CARLO & $\mathrm{H}$ & 55 & ITALY & ITALY & ITALY & 1884 & LABORER/RAILROAD & CUORGNE'. TO & KEARSARGE ST. & $\begin{array}{l}\text { CALUMET - LAURIUM VILLAGE } \\
\text { CAUE }\end{array}$ \\
\hline 1347 & 104 & CAPELLO & MARIA & w & 50 & ITALY & $\begin{array}{l}\text { ITALY } \\
\text { ITALY }\end{array}$ & ITALY & 1886 & & & KEARSARGE ST. & CALUMET - LAURIUM VILLAGE \\
\hline 1348 & 108 & BAGGIORE & FRANK & $\mathrm{H}$ & 52 & ITALY & ITALY & ITALY & 1880 & TREASURER/VILLAGE & TORINO & TAMARACK ST. & CALUMET - LAURIUM VILLAGE \\
\hline 1349 & 108 & BAGGIORE & KATE & w & 44 & ITALY & ITALY & ITALY & $1877 ?$ & & & TAMARACK ST. & CALUMET - LAURIUM VILLAGE \\
\hline 1350 & 108 & BAGGIORE & JOHN & s & 22 & MICHIGAN & ITALY & ITALY & & LABORER/VILLAGE & TORINO & TAMARACK ST. & CALUMET - LAURIUM VILLAGE \\
\hline 1351 & 108 & BAGGIORE & MARY & $\mathrm{D}$ & 21 & MICHIGAN & ITALY & ITALY & & & TORINO & TAMARACK ST. & CALUMET - LAURIUM VILLAGE \\
\hline 1352 & 108 & BAGGIORE & JOSEPH & s & 19 & MICHIGAN & ITALY & ITALY & & CHAUFFEUR/PF & TORINO & TAMARACK ST. & CALUMET - LAURIUM VILLAGE \\
\hline 1353 & 108 & BAGGIORE & VINCENZO & $\mathrm{s}$ & 15 & MICHIGAN & ITALY & ITALY & & & TORINO & TAMARACK ST. & CALUMET - LAURIUM VILLAGE \\
\hline 1354 & 108 & BAGGIORE & DUILIA & $\mathrm{D}$ & 17 & MICHIGAN & ITALY & ITALY & & & TORINO & TAMARACK ST. & CALUMET - LAURIUM VILLAGE \\
\hline 1355 & 108 & BAGGIORE & OTTO & S & 12 & MICHIGAN & $\begin{array}{l}\text { ITALY } \\
\text { ITALY }\end{array}$ & ITALY & & & TORINO & TAMARACK ST. & CALUMET - LAURIUM VILLAGE \\
\hline 1356 & 100 & BAGGIORE & CAESAR & s & 9 & MICHIGAN & ITALY & ITALY & & & TORINO & TAMARACK ST. & CALUMET - LAURIUM VILLAGE \\
\hline $\begin{array}{l}1356 \\
1357\end{array}$ & $\frac{108}{108}$ & $\begin{array}{l}\text { BAGGIURE } \\
\text { TORREANO }\end{array}$ & $\begin{array}{l}\text { CAESAR } \\
\text { PETER }\end{array}$ & BIL & 24 & $\begin{array}{l}\text { MMICHIGAN } \\
\text { MICHIGAN }\end{array}$ & $\begin{array}{l}\text { IIALY } \\
\text { ITALY }\end{array}$ & $\begin{array}{l}\text { IIALY } \\
\text { ITALY }\end{array}$ & & DEPUTY TREASURER/VILLAGE & TORINO & $\begin{array}{l}\text { IAMARACKK SI. } \\
\text { TAMARACK ST. }\end{array}$ & $\begin{array}{l}\text { CALUMET - LAURIUIUM VILLAGE } \\
\text { CAUET }\end{array}$ \\
\hline 1358 & 104 & DELL'ACQUA & ANGELO & $\begin{array}{l}\mathrm{DIL} \\
\mathrm{H}\end{array}$ & 39 & ITALY & ITALY & ITALY & 1891 & TIMBERMAN/CM & CUGGIANO. MILANO & TAMARACK ST. & CALUMET - LAURIUM VILLAGE \\
\hline 1359 & 104 & DELL'ACQUA & CLARA & W & 29 & ITALY & ITALY & ITALY & 1891 & & CUGGIANO MILANO & TAMARACK ST. & CALUMET - LAURIUM VILLAGE \\
\hline 1360 & 104 & DELL'ACQUA & ORSOLA & $\mathrm{D}$ & 8 & MICHIGAN & ITALY & ITALY & & & CUGGIANO. MILANO & TAMARACK ST. & $\begin{array}{l}\text { CALUMET - LAURIUM VILLAGE } \\
\text { CAUE }\end{array}$ \\
\hline 1361 & 104 & DELL'ACQUA & GAETANO & $\mathrm{s}$ & $\frac{0}{6}$ & MICHIGAN & ITALY & ITALY & & & CUGGIANO. MILANO & TAMARACK ST. & CALUMET - LAURIUM VILLAGE \\
\hline 1362 & 104 & DELL'ACQUA & MARY & $\mathrm{D}$ & $1 \mathrm{M}$ & MICHIGAN & ITALY & ITALY & & & CUGGIANO. MILANO & TAMARACK ST. & CALUMET - LAURIUM VILLAGE \\
\hline 1363 & 104 & $\begin{array}{l}\text { CARCANO } \\
\text { CARC }\end{array}$ & LUIGI & BIL & 31 & ITALY & ITALY & ITALY & 1901 & TRAMMER/CM & CUGGIANO. MILANO & TAMARACK ST. & CALUMET - LAURIUM VILLAGE \\
\hline 1364 & 104 & ROSETTO & CESARE & B & 37 & ITALY & ITALY & ITALY & 1905 & TRAMMER/CM & TORINO & TAMARACK ST. & CALUMET - LAURIUM VILLAGE \\
\hline 1365 & 100 & CAPELLO & JAMES & $\mathrm{H}$ & 52 & ITALY & ITALY & ITALY & 1880 & LABORER/ODD JOBS & SAN BENIGNO CANAVESE. TO & TAMARACK ST. & CALUMET - LAURIUM VILLAGE \\
\hline 1366 & 100 & CAPELLO & JOHANNA & w & 41 & ITALY & ITALY & ITALY & 1888 & & & TAMARACK ST. & CALUMET - LAURIUM VILLAGE \\
\hline 1367 & 100 & CAPELLO & DUSOLINA & $\mathrm{D}$ & 21 & MICHIGAN & ITALY & ITALY & & JANITRESS/DOCTOR'S OFFICE & SAN BENIGNO CANAVESE. TO & TAMARACK ST. & CALUMET - LAURIUM VILLAGE \\
\hline 1368 & 100 & CAPELLO & LOUIS & s & 18 & MICHIGAN & ITALY & ITALY & & $\begin{array}{l}\text { LABORER/RAILROAD } \\
\text { LARL }\end{array}$ & SAN BENIGNO CANAVESE.TO & TAMARACK ST. & CALUMET - LAURIUM VILLAGE \\
\hline 1369 & 100 & CAPELLO & JAMES & s & 15 & MICHIGAN & ITALY & ITALY & & & SAN BENIGNO CANAVESE TO & $\begin{array}{l}\text { TAMARACA S ST. } \\
\text { TAMARACK ST. }\end{array}$ & CALUMET - LAURIUM VILLAGE \\
\hline $\begin{array}{l}1369 \\
1370\end{array}$ & $\begin{array}{l}100 \\
100\end{array}$ & $\begin{array}{l}\text { CAPELLO } \\
\text { CAPELLO }\end{array}$ & $\begin{array}{l}\text { JAMES } \\
\text { FEDELE }\end{array}$ & $\begin{array}{l}2 \\
s\end{array}$ & $\begin{array}{l}15 \\
11\end{array}$ & $\begin{array}{l}\text { MIICIGAN } \\
\text { MICHIGAN }\end{array}$ & $\begin{array}{l}\text { IIALY } \\
\text { ITALY }\end{array}$ & $\begin{array}{l}\text { MALY } \\
\text { ITALY }\end{array}$ & & & $\begin{array}{l}\text { SAN BENIGNO CANAAVESE. } \\
\text { SAN BENIGNO CANAVESE. TO }\end{array}$ & $\begin{array}{l}\text { IAMARAACK SI. } \\
\text { TAMARACK ST. }\end{array}$ & $\begin{array}{l}\text { CALUME - LAURIUM VILLAGE } \\
\text { CALUMET - LAURIUM VILLAGE }\end{array}$ \\
\hline 1371 & 101 & RISO & $\begin{array}{l}\text { TCLELE } \\
\text { JOSEPH }\end{array}$ & $\mathrm{H}$ & 44 & ITALY & ITALY & ITALY & 1890 & LABORER/CM & & TAMARACK ST. & CALUMET - LAURIUM VILLAGE \\
\hline 1372 & 101 & RISO & PAULINE & n & $\begin{array}{l}44 \\
40\end{array}$ & ITALY & $\begin{array}{l}\text { ITALY } \\
\text { ITALY }\end{array}$ & ITALY & 1090 & & & $\begin{array}{l}\text { TAMARACK ST. } \\
\text { TAMARACK }\end{array}$ & CALUMET - LAURIUM VILLAGE \\
\hline 1373 & 101 & RISO & CLOTILDE & D & 16 & MICHIGAN & $\begin{array}{l}\text { ITALY } \\
\text { ITALY }\end{array}$ & ITALY & & SERVANT/PF & & $\begin{array}{l}\text { TANARACh SI. } \\
\text { TAMARACK ST. }\end{array}$ & CALUMET - LAURIUM VILLAGE \\
\hline 1374 & 101 & RISO & FELICE & S & 15 & MICHIGAN & $\begin{array}{l}\text { IITALY } \\
\text { ITALY }\end{array}$ & ITALY & & & & $\begin{array}{l}\text { TAMARACACK ST. } \\
\text { TAMARACK }\end{array}$ & CALUMET - LAURIUM VILLAGE \\
\hline 1375 & 101 & RISO & CRESSON & s & 11 & MICHIGAN & $\begin{array}{l}\text { IITALY } \\
\text { ITALY }\end{array}$ & $\begin{array}{l}\text { ITALY } \\
\text { ITALY }\end{array}$ & & & & $\begin{array}{l}\text { TAMARACACK ST. } \\
\text { TAMARACK ST. }\end{array}$ & CALUMET - LAURIUM VILLAGE \\
\hline 1376 & 101 & RISO & STEVEN & $\mathrm{s}$ & 8 & MICHIGAN & ITALY & ITALY & & & & TAMARACK ST. & CALUMET - LAURIUM VILLAGE \\
\hline 1377 & 101 & RISO & JODIE & $\mathrm{D}$ & 5 & MICHIGAN & ITALY & ITALY & & & & TAMARACK ST. & CALUMET - LAURIUM VILLAGE \\
\hline 1378 & 101 & RISO & ELISABETH & $\mathrm{D}$ & 3 & MICHIGAN & ITALY & ITALY & & & & TAMARACK ST. & CALUMET - LAURIUM VILLAGE \\
\hline 1379 & 104 & FAUSONE & $\begin{array}{l}\text { PETER } \\
\text { PET }\end{array}$ & $\mathrm{H}$ & 48 & ITALY & ITALY & ITALY & 1890 & TRAMMER/CM & TORINO & IROQUOIS ST. & CALUMET - LAURIUM VILLAGE \\
\hline 1380 & 104 & FAUSONE & MARTA & w & 36 & ITALY & ITALY & ITALY & 1891 & & & IROQUOIS ST. & CALUMET - LAURIUM VILLAGE \\
\hline 1381 & 104 & FAUSONE & PETER & s & 16 & MICHIGAN & ITALY & ITALY & & TEAMSTER/BUTCHER SHOP & TORINO & IROQUOIS ST. & CALUMET - LAURIUM VILLAGE \\
\hline 1382 & 104 & FAUSONE & FRANCESCA & $\mathrm{D}$ & 14 & MICHIGAN & ITALY & ITALY & & & TORINO & IROQUOIS ST. & AAUUMFT - AURUMM VIUAG \\
\hline 1383 & 104 & FAUSONE & DOMINICK & S & 11 & MICHIGAN & ITALY & ITALY & & & TORINO & IROQUOIS ST. & CALUMET - LAURIUM VILLAGE \\
\hline 1384 & 104 & FAUSONE & JOHN & s & 9 & MICHIGAN & ITALY & ITALY & & & TORINO & IROQUOIS ST. & CALUMET - LAURIUM VILLAGE \\
\hline $\begin{array}{l}1384 \\
1385\end{array}$ & 104 & FAUSONF & FREDFRICK & s & $\frac{9}{7}$ & IVICHIGAN & MALY & ITAIY & & & TORINO & IROOUOS ST & CALUMT - AURUMM VIUIAGF \\
\hline
\end{tabular}




\begin{tabular}{|c|c|c|c|c|c|c|c|c|c|c|c|c|c|}
\hline 1386 & 104 & FAUSONE & MINE & $\mathrm{D}$ & 4 & MICHIGAN & ITALY & ITALY & & & TORINO & IROQUOIS ST. & CALUMET - LAURIUM VILLAGE \\
\hline 1387 & 110 & GIACOLETTO & MARGARET & $\mathrm{H}$ & 44 & ITALY & ITALY & ITALY & 1901 & & PONT CANAVESE. TO & 1ST ST. & CALUMET - LAURIUM VILLAGE \\
\hline 1388 & 110 & GIACOLETTO & FRANCES & $\mathrm{D}$ & 23 & ITALY & ITALY & ITALY & 1901 & TAILORES/SHOP & PONT CANAVESE. TO & 1ST ST. & CALUMET - LAURIUM VILLAGE \\
\hline 1389 & 110 & GIACOLETTO & JOHN P. & $\mathrm{s}$ & 5 & MICHIGAN & ITALY & ITALY & & & PONT CANAVESE. TO & 1ST ST. & CALUMET - LAURIUM VILLAGE \\
\hline 1390 & 236 & MICHELA & GEORGE & $\mathrm{H}$ & 47 & ITALY & ITALY & ITALY & 1890 & LABORER/VILLAGE & SAN GIORGIO CANAVESE. TO & BOUNDARY ST. & CALUMET - LAURIUM VILLAGE \\
\hline 1391 & 236 & MICHELA & VICTORIA & W & $4 ?$ & ITALY & ITALY & ITALY & 1889 & & & BOUNDARY ST. & CALUMET - LAURIUM VILLAGE \\
\hline 1392 & 236 & MICHELA & DOMINICK & s & 19 & ITALY & ITALY & ITALY & & & SAN GIORGIO CANAVESE. TO & BOUNDARY ST. & CALUMET - LAURIUM VILLAGE \\
\hline 1393 & 236 & MICHELA & JOHN & s & 18 & MICHIGAN & ITALY & ITALY & & & SAN GIORGIO CANAVESE. TO & BOUNDARY ST. & $\begin{array}{l}\text { CALUMET - LAURIUM VILLAGE } \\
\text { LAUM }\end{array}$ \\
\hline 1394 & 236 & MICHELA & MARY & $\mathrm{D}$ & 17 & MICHIGAN & ITALY & ITALY & & & SAN GIORGIO CANAVESE. TO & BOUNDARY ST. & CALUMET - LAURIUM VILLAGE \\
\hline 1395 & 236 & MICHELA & JOSEPHINE & D & 16 & MICHIGAN & ITALY & ITALY & & & SAN GIORGIO CANAVESE. TO & BOUNDARY ST. & CALUMET - LAURIUM VILLAGE \\
\hline 1396 & 236 & MICHELA & GEORGE & $\mathrm{s}$ & 14 & MICHIGAN & ITALY & ITALY & & & SAN GIORGIO CANAVESE. TO & BOUNDARY ST. & CALUMET - LAURIUM VILLAGE \\
\hline 1397 & 236 & MICHELA & ESTELLA & D & 10 & MICHIGAN & ITALY & ITALY & & & SAN GIORGIO CANAVESE. TO & BOUNDARY ST. & CALUMET - LAURIUM VILLAGE \\
\hline 1398 & 236 & MICHELA & VICTOR & $\mathrm{s}$ & 7 & MICHIGAN & ITALY & ITALY & & & SAN GIORGIO CANAVESE. TO & BOUNDARY ST. & CALUMET - LAURIUM VILLAGE \\
\hline 1399 & 236 & MICHELA & MINNIE & $\mathrm{D}$ & 3 & MICHIGAN & ITALY & ITALY & & & SAN GIORGIO CANAVESE. TO & BOUNDARY ST. & CALUMET - LAURIUM VILLAGE \\
\hline 1400 & 236 & MICHELA & ANGELO & $\mathrm{s}$ & 12 & MICHIGAN & ITALY & ITALY & & & SAN GIORGIO CANAVESE. TO & BOUNDARY ST. & CALUMET - LAURIUM VILLAGE \\
\hline 1401 & 243 & CIAGNE & MARTIN & $\mathrm{H}$ & 33 & MICHIGAN & ITALY & ITALY & & SALOONKEEPER & TORINO & OSCEOLA ST. & CALUMET - LAURIUM VILLAGE \\
\hline 1402 & 243 & CIAGNE & ANGELINE & W & 26 & MICHIGAN & ITALY & ITALY & & & & OSCEOLA ST. & CALUMET - LAURIUM VILLAGE \\
\hline 1403 & 243 & CIAGNE & MARIA & $\mathrm{D}$ & 5 & MICHIGAN & MICHIGAN & MICHIGAN & & & TORINO & OSCEOLA ST. & CALUMET - LAURIUM VILLAGE \\
\hline 1404 & 243 & CIAGNE & ANNIE & $\mathrm{D}$ & $6 \mathrm{M}$ & MICHIGAN & MICHIGAN & MICHIGAN & & & TORINO & OSCEOLA ST. & CALUMET - LAURIUM VILLAGE \\
\hline 1405 & 243 & CIAGNE & ADALINE & MO & 52 & ITALY & ITALY & ITALY & 1909 & & TORINO & OSCEOLA ST. & $\begin{array}{l}\text { CALUMET - LAURIUM VILLAGE } \\
\text {. LAUM }\end{array}$ \\
\hline 1406 & 243 & GEDDA & ANNA & MIL & 62 & ITALY & ITALY & ITALY & 1883 & & TORINO & OSCEOLA ST. & CALUMET - LAURIUM VILLAGE \\
\hline 1407 & 243 & GEDDA & LEONA & SIIL & 25 & MICHIGAN & ITALY & ITALY & & SALESLADY/GENERAL STORE & TORINO & OSCEOLA ST. & CALUMET - LAURIUM VILLAGE \\
\hline 1408 & 243 & GEDDA & PETER & $\mathrm{H}$ & 37 & ITALY & ITALY & ITALY & 1886 & SALOONKEEPER & TORINO & OSCEOLA ST. & CALUUMET - LAURIUM VILLAGE \\
\hline 1409 & 243 & GEDDA & JOHANNA & W & 36 & ITALY & ITALY & ITALY & 1893 & & & OSCEOLA ST. & CALUMET - LAURIUM VILLAGE \\
\hline 1410 & 243 & GEDDA & ANNA & $\mathrm{D}$ & 13 & MICHIGAN & ITALY & ITALY & & & TORINO & OSCEOLA ST. & CALUMET - LAURIUM VILLAGE \\
\hline 1411 & 243 & GEDDA & PETER JR. & $\mathrm{s}$ & 7 & MICHIGAN & ITALY & ITALY & & & TORINO & OSCEOLA ST. & CALUMET - LAURIUM VILLAGE \\
\hline 1412 & 243 & GEDDA & TRACEY & $\mathrm{D}$ & 3 & MICHIGAN & ITALY & ITALY & & & TORINO & OSCEOLA ST. & CALUMET - LAURIUM VILLAGE \\
\hline 1413 & 243 & CARLEVATTO & GIOVANNA & MIL & 62 & ITALY & ITALY & ITALY & 1904 & & OZEGNA, TO & OSCEOLA ST. & CALUMET - LAURIUM VILLAGE \\
\hline 1414 & 201 & PRICCO & MARTINO & $\mathrm{H}$ & 40 & ITALY & ITALY & ITALY & 1896 & SALOONKEEPER & SAN MARTINO CANAVESE. TO & HECLA ST. & CALUMET - LAURIUM VILLAGE \\
\hline 1415 & 201 & AIMONETTO & MARIA & SER & 20 & ITALY & ITALY & ITALY & 1909 & SERVANT/PF & SPARONE. TO & HECLA ST. & CALUMET - LAURIUM VILLAGE \\
\hline 1416 & 201 & IERONO & CHARLES & $\mathrm{H}$ & 29 & ITALY & ITALY & ITALY & 1900 & MINER/CM & & HECLA ST. & CALUMET - LAURIUM VILLAGE \\
\hline 1417 & 201 & CONTRATTO & JENNIE & $\mathrm{H}$ & 36 & ITALY & ITALY & ITALY & 1894 & WASHWOMAN/OUT & LOCANA CANAVESE. TO & HECLA ST. & CALUMET - LAURIUM VILLAGE \\
\hline 1418 & 201 & CONTRATTO & JAMES & s & 15 & MICHIGAN & ITALY & ITALY & 1094 & HELPER/FLOWER STORE & LOCANA CANAVESE. TO & HECLA ST. & $\begin{array}{l}\text { CALUMET - LAURIUM VILLAGE } \\
\text { CAUM }\end{array}$ \\
\hline 1419 & 201 & CONTRATTO & ANNA & $\mathrm{D}$ & 13 & MICHIGAN & ITALY & ITALY & & & LOCANA CANAVESE, TO & HECLA ST. & CALUMET - LAURIUM VILLAGE \\
\hline 1420 & 201 & CONTRATTO & ANDREW & $\mathrm{s}$ & 12 & MICHIGAN & ITALY & ITALY & & & LOCANA CANAVESE. TO & HECLA ST. & CALUMET - LAURIUM VILLAGE \\
\hline 1421 & 211 & GALETTO & JOSEPH & $\mathrm{H}$ & 46 & ITALY & ITALY & ITALY & 1897 & MERCHANT/DRY GOODS & SAN GIORGIO CANAVESE. TO & HECLA ST. & CALUMET - LAURIUM VILLAGE \\
\hline 1422 & 211 & GALETTO & MARY & W & 36 & ITALY & ITALY & ITALY & 1890 & & & HECLA ST. & CALUMET - LAURIUM VILLAGE \\
\hline 1423 & 211 & GALETTO & JAMES & $\mathrm{s}$ & 15 & MICHIGAN & ITALY & ITALY & & SALESMAN/DRY GOODS & SAN GIORGIO CANAVESE. TO & HECLA ST. & CALUMET - LAURIUM VILLAGE \\
\hline 1424 & 211 & GALETTO & MARY & $\mathrm{D}$ & 13 & MICHIGAN & ITALY & ITALY & & & SAN GIORGIO CANAVESE. TO & HECLA ST. & CALUMET - LAURIUM VILLAGE \\
\hline 1425 & 211 & GALETTO & PETER & $\mathrm{s}$ & 11 & MICHIGAN & ITALY & ITALY & & & SAN GIORGIO CANAVESE. TO & HECLA ST. & CALUMET - LAURIUM VILLAGE \\
\hline 1426 & 211 & GALETTO & VICTORIA & D & 9 & MICHIGAN & ITALY & ITALY & & & SAN GIORGIO CANAVESE. TO & HECLA ST. & CALUMET - LAURIUM VILLAGE \\
\hline 1427 & 211 & GALETTO & JOSEPH & $\mathrm{s}$ & 7 & MICHIGAN & ITALY & ITALY & & & SAN GIORGIO CANAVESE. TO & HECLA ST. & CALUMET - LAURIUM VILLAGE \\
\hline 1428 & 211 & GALETTO & TRACY & $\mathrm{D}$ & 5 & MICHIGAN & ITALY & ITALY & & & SAN GIORGIO CANAVESE. TO & HECLA ST. & CALUMET - LAURIUM VILLAGE \\
\hline 1429 & 211 & GALETTO & CHARLES & $\mathrm{s}$ & $1.4 \mathrm{M}$ & MICHIGAN & ITALY & ITALY & & & SAN GIORGIO CANAVESE. TO & HECLA ST. & CALUMET - LAURIUM VILLAGE \\
\hline 1430 & 211 & FERAGIO & FRANK & B & 21 & MICHIGAN & ITALY & ITALY & & MINER/CM & & HECLA ST. & CALUMET - LAURIUM VILLAGE \\
\hline 1431 & 221 & ANASTASIA & JOSEPH & $\mathrm{H}$ & 26 & ITALY & ITALY & ITALY & 1900 & CLERGYMAN/LOCAL CHURCH & & HECLA ST. & $\begin{array}{l}\text { CALUMET - LAURIUM VILLAGE } \\
\end{array}$ \\
\hline 1432 & 221 & ANASTASIA & GRACE & W & 24 & PENNSYLV & ITALY & ITALY & & & & HECLA ST. & CALUMET - LAURIUM VILLAGE \\
\hline 1433 & 221 & ANASTASIA & KATHERINE & D & 6 & PENNSYLV & ITAL & PENNSYLV & & & & $\mathrm{HE}$ & ET - LAUF \\
\hline 1434 & 221 & ANASTASIA & PAULINE & $\mathrm{D}$ & 4 & PENNSYLV & ITALY & PENNSYLV & & & & A ST. & ET - LAU \\
\hline 1435 & 221 & ANASTASIA & DAVID & $\mathrm{s}$ & 2 & PENNSYLV & ITALY & PENNSYLV & & & & HECLA & CALUMET - LAURIUM VILLAGE \\
\hline 1436 & 210 & CASTIGLIANO & ATTILIO & $\mathrm{H}$ & 29 & ITA & ITA & ITALY & 1905 & BOOKKEEPER/BANK & VALPERGA. TO & & CALUMET - LAURIUM VILLAGE \\
\hline 1437 & 210 & CASTIGLIANO & CATERINA & W & 22 & ITALY & ITAL & ITALY & 1905 & & VALPERGA, TO & HECLA ST. & CALUMET - LAURIUM VILLAGE \\
\hline 1438 & 210 & CASTIGLIANO & VIRGILIO & $\mathrm{s}$ & 3 & MICHIGAN & ITALY & ITALY & & & VALPERGA. TO & HECLA ST. & CALUMET - LAURIUM VILLAGE \\
\hline 1439 & 210 & CASTIGLIANO & SILVIO & $\mathrm{s}$ & 2 & MICHIGAN & ITALY & ITALY & & & VALPERGA. TO & HECLA ST. & CALUMET - LAURIUM VILLAGE \\
\hline 1440 & 210 & CHIOTTI & MARGHERITA & SIIL & 21 & ITALY & ITALY & ITALY & 1908 & & SAN GIORGIO CANAVESE. TO & HECLA ST. & CALUMET - LAURIUM VILLAGE \\
\hline 1441 & 210 & CASTIGLIANO & TERENZIO & B & 24 & ITALY & ITALY & ITALY & 1909 & BLACKSMITH & VALPERGA. TO & HECLA ST. & CALUMET - LAURIUM VILLAGE \\
\hline 1442 & 208 & NIGRA & ANTONIO & $\mathrm{H}$ & 37 & ITALY & ITALY & ITALY & 1890 & CLERK/RETAIL GROCERY & SAN GIUSTO CANAVESE. TO & HECLA ST. & CALUMET - LAURIUM VILLAGE \\
\hline 1443 & 208 & NIGRA & TERESA & W & 42 & ITALY & ITALY & ITALY & 1890 & 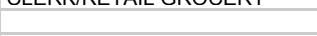 & SAN GIUSTO CANAVESE, TO & HECLA ST. & CALUMET - LAURIUM VILLAGE \\
\hline 1444 & 208 & DAIRO & SABINO & SSON & 17 & MICHIGAN & ITALY & ITALY & & & SAN GIUSTO CANAVESE. TO & HECLA ST. & CALUMET - LAURIUM VILLAGE \\
\hline 1445 & 208 & NIGRA & DOMENICO & $\mathrm{s}$ & 8 & MICHIGAN & ITAL & ITAL & & & CANAVESE. TO & HECLA ST. & LAURIUM VILLAGE \\
\hline 1446 & 208 & & & $\mathrm{D}$ & 6 & HIGAN & & & & & & & \\
\hline 1447 & 211 & & IN/JAMES & $\mathrm{H}$ & 39 & & & & 1892 & LABORER/CM & INAVESE. TO & & E \\
\hline 1448 & 211 & BRONZO & MARIA & W & 39 & ITALY & ITALY & ITALY & 1892 & & SAN MARTINO CANAVESE. TO & KEARSARGE ST. & CALUMET - LAURIUM VILLAGE \\
\hline
\end{tabular}




\begin{tabular}{|c|c|c|c|c|c|c|c|c|c|c|c|c|c|}
\hline 1449 & 211 & BRONZO & GIUSEPPE & S & 16 & MICHIGAN & ITALY & ITALY & & BAKER & SAN MARTINO CANAVESE. TO & KEARSARGE ST. & CALUMET - LAURIUM VILLAGE \\
\hline 1450 & 211 & BRONZO & PIETRO & $\mathrm{COU}$ & 23 & ITALY & ITALY & ITALY & 1907 & LABORER/CM & SAN MARTINO CANAVESE. TO & KEARSARGE ST. & CALUMET - LAURIUM VILLAGE \\
\hline 1451 & 211 & BRONZO & GIUSEPPINA & $\mathrm{COU}$ & 22 & MICHIGAN & ITALY & ITALY & & & SAN MARTINO CANAVESE. TO & KEARSARGE ST. & CALUMET - LAURIUM VILLAGE \\
\hline 1452 & 221 & FERRARI & SILVIO & $\mathrm{H}$ & 42 & ITALY & ITALY & ITALY & 1896 & MINER/CM & LUCCA & KEARSARGE ST. & CALUMET - LAURIUM VILLAGE \\
\hline 1453 & 221 & FERRARI & ILDEGONDA & w & 35 & ITALY & ITALY & ITALY & 1896 & & LUCCA & KEARSARGE ST. & CALUMET - LAURIUM VILLAGE \\
\hline 1454 & 221 & FERRARI & MOLLIE & $\mathrm{D}$ & 7 & MICHIGAN & ITALY & ITALY & & & LUCCA & KEARSARGE ST. & CALUMET - LAURIUM VILLAGE \\
\hline 1455 & 221 & FERRARI & ALBERT & s & 4 & MICHIGAN & ITALY & ITALY & & & LUCCA & KEARSARGE ST. & CALUMET - LAURIUM VILLAGE \\
\hline 1456 & 221 & FERRARI & $\begin{array}{l}\text { HELEN } \\
\text { HELN }\end{array}$ & $\mathrm{D}$ & 1 & MICHIGAN & ITALY & ITALY & & & LUCCA & KEARSARGE ST. & $\begin{array}{l}\text { CALUMET - LAURIUM VILLAGE } \\
\text { CALE }\end{array}$ \\
\hline 1457 & 221 & $\begin{array}{l}\text { PACINI } \\
\text { PANI }\end{array}$ & ARTURO & B & 27 & ITALY & ITALY & ITALY & 1909 & TRAMMER/CM & LUCCA & KEARSARGE ST. & $\begin{array}{l}\text { CALUMET - LAURIUM VILLAGE } \\
\text { CALE }\end{array}$ \\
\hline 1458 & 221 & GALLASERO & JOHN & $\mathrm{H}$ & 42 & ITALY & ITALY & ITALY & 1888 & MINER/CM & PONT CANAVESE. TO & TAMARACK ST. & CALUMET - LAURIUM VILLAGE \\
\hline 1459 & 221 & GALLASERO & ROSA & w & 44 & ITALY & ITALY & ITALY & 1888 & & PONT CANAVESE. TO & TAMARACK ST. & CALUMET - LAURIUM VILLAGE \\
\hline 1460 & 221 & GALLASERO & JAMES & $\mathrm{s}$ & 18 & MICHIGAN & ITALY & ITALY & & MATTRESS MAKER/FACTORY & PONT CANAVESE. TO & TAMARACK ST. & CALUMET - LAURIUM VILLAGE \\
\hline 1461 & 221 & GALLASERO & DOMINICK & $\mathrm{s}$ & 16 & MICHIGAN & ITALY & ITALY & & TAILOR/SHOP & PONT CANAVESE. TO & TAMARACK ST. & CALUMET - LAURIUM VILLAGE \\
\hline 1462 & 221 & GALLASERO & JOHN JR. & $\mathrm{s}$ & 14 & MICHIGAN & ITALY & ITALY & & & PONT CANAVESE. TO & TAMARACK ST. & CALUMET - LAURIUM VILLAGE \\
\hline 1463 & 221 & GALLASERO & MARY & $\mathrm{D}$ & 11 & MICHIGAN & ITALY & ITALY & & & PONT CANAVESE. TO & TAMARACK ST. & CALUMET - LAURIUM VILLAGE \\
\hline 1464 & 221 & GALLASERO & ELSIE & $\mathrm{D}$ & 8 & MICHIGAN & ITALY & ITALY & & & PONT CANAVESE, TO & TAMARACK ST. & CALUMET - LAURIUM VILLAGE \\
\hline 1465 & 221 & GALLASERO & JOSIE & $\mathrm{D}$ & 6 & MICHIGAN & ITALY & ITALY & & & PONT CANAVESE. TO & TAMARACK ST. & CALUMET - LAURIUM VILLAGE \\
\hline 1466 & 221 & GALLASERO & ROSIE & $\mathrm{D}$ & 2 & MICHIGAN & ITALY & ITALY & & & PONT CANAVESE. TO & TAMARACK ST. & CALUMET - LAURIUM VILLAGE \\
\hline 1467 & 211 & FAUSONE & JOHN & $\mathrm{H}$ & 44 & ITALY & ITALY & ITALY & 1885 & FIREMAN/BOILER HOUSE & TORINO & OSCEOLA ST. & CALUMET - LAURIUM VILLAGE \\
\hline 1468 & 211 & FAUSONE & DOMENICA & W & 42 & ITALY & ITALY & ITALY & 1890 & 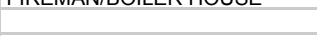 & 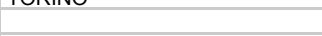 & OSCEOLA ST. & CALUMET - LAURIUM VILLAGE \\
\hline 1469 & 211 & FAUSONE & FRANK & s & 18 & MICHIGAN & ITALY & ITALY & & LABORER/ROCK HOUSE & TORINO & OSCEOLA ST. & CALUMET - LAURIUM VILLAGE \\
\hline 1470 & 211 & FAUSONE & JOHN & $\mathrm{s}$ & 15 & MICHIGAN & ITALY & ITALY & & & TORINO & OSCEOLA ST. & CALUMET - LAURIUM VILLAGE \\
\hline 1471 & 211 & FAUSONE & PETER & $\mathrm{s}$ & 8 & MICHIGAN & ITALY & ITALY & & & TORINO & OSCEOLA ST. & CALUMET - LAURIUM VILLAGE \\
\hline 1472 & 320 & SALOTTI & GIACOMO & $\mathrm{H}$ & 61 & ITALY & ITALY & ITALY & 1885 & WATCHMAN/CM & CORIO. TO & BOUNDARY ST. & CALUMET - LAURIUM VILLAGE \\
\hline 1473 & 320 & SALOTTI & DOMENICA & W & 59 & ITALY & ITALY & ITALY & 1886 & & & BOUNDARY ST. & CALUUMT - LAURIUM VILLAGE \\
\hline 1474 & 320 & SALOTTI & CARLO & $s$ & 23 & MICHIGAN & ITALY & ITALY & & GROCER/GROCERY STORE & CORIO. TO & BOUNDARY ST. & CALUMET - LAURIUM VILLAGE \\
\hline 1475 & 320 & SALOTTI & MARIA & $\mathrm{D}$ & 19 & MICHIGAN & ITALY & ITALY & & & CORIO. TO & BOUNDARY ST. & CALUMET - LAURIUM VILLAGE \\
\hline 1476 & 320 & SALOTTI & GIOVANNI & $\mathrm{s}$ & 17 & MICHIGAN & ITALY & ITALY & & GROCER/GROCERY STORE & CORIO, TO & BOUNDARY ST. & CALUMET - LAURIUM VILLAGE \\
\hline 1477 & 314 & VENTURINO & BASILIO & $\mathrm{H}$ & 61 & ITALY & ITALY & ITALY & 1877 & TIMBERMAN/CM & & BOUNDARY ST. & CALUMET - LAURIUM VILLAGE \\
\hline 1478 & 314 & VENTURINO & GELTRUDE & W & 46 & WISCONSI & GERMANY & GERMANY & & & & BOUNDARY ST. & CALUMET - LAURIUM VILLAGE \\
\hline 1479 & 314 & VENTURINO & ANNIE & $\mathrm{D}$ & 23 & MICHIGAN & ITALY & WISCONSI & & & & BOUNDARY ST. & CALUMET - LAURIUM VILLAGE \\
\hline 1480 & 314 & VENTURINO & JOHN & $\mathrm{s}$ & 21 & MICHIGAN & ITALY & WISCONSI & & MINER/CM & & BOUNDARY ST. & CALUMET - LAURIUM VILLAGE \\
\hline 1481 & 314 & VENTURINO & BERTIE & s & 19 & MICHIGAN & ITALY & WISCONSI & & MINER/CM & & BOUNDARY ST. & $\begin{array}{l}\text { CALUMET - LAURIUM VILLAGE } \\
\text { CALE }\end{array}$ \\
\hline 1482 & 314 & VENTURINO & MIKE & $\mathrm{S}$ & 17 & MICHIGAN & ITALY & WISCONSI & & MINER/CM & & BOUNDARY ST. & CALUMET - LAURIUM VILLAGE \\
\hline 1483 & 314 & VENTURINO & LUCY & D & 14 & MICHIGAN & ITALY & WISCONSI & & & & BOUNDARY ST. & CALUMET - LAURIUM VILLAGE \\
\hline 1484 & 314 & VENTURINO & AUGUST & $\mathrm{s}$ & 11 & MICHIGAN & ITALY & WISCONSI & & & & BOUNDARY ST. & CALUMET - LAURIUM VILLAGE \\
\hline 1485 & 320 & BINANDO & GIOVANNI & $\mathrm{H}$ & 48 & ITALY & ITALY & ITALY & 1889 & LABORER/ODD JOBS & SAN GIORGIO CANAVESE. TO & OSCEOLA ST. & CALUMET - LAURIUM VILLAGE \\
\hline 1486 & 320 & BINANDO & LUISA & W & 46 & ITALY & ITALY & ITALY & 1891 & & SAN GIORGIO CANAVESE. TO & OSCEOLA ST. & CALUMET - LAURIUM VILLAGE \\
\hline 1487 & 320 & BINANDO & PIETRO & $\mathrm{s}$ & 26 & ITALY & ITALY & ITALY & 1891 & MINER/CM & SAN GIORGIO CANAVESE. TO & OSCEOLA ST. & CALUMET - LAURIUM VILLAGE \\
\hline 1488 & 320 & BINANDO & ANTONIO & $\mathrm{S}$ & 19 & ITALY & ITALY & ITALY & 1891 & MINER/CM & SAN GIORGIO CANAVESE. TO & OSCEOLA ST. & CALUMET - LAURIUM VILLAGE \\
\hline 1489 & 320 & BINANDO & LENA & D & 10 & MICHIGAN & ITALY & ITALY & & & SAN GIORGIO CANAVESE. TO & OSCEOLA ST. & CALUMET - LAURIUM VILLAGE \\
\hline 1490 & 338 & BERTASSO & PIETRO & $\mathrm{H}$ & 24 & ITALY & ITALY & ITALY & 1906 & TEAMSTER/BREWERY & PEROSA CANAVESE. TO & OSCEOLA ST. & CALUMET - LAURIUM VILLAGE \\
\hline 1491 & 338 & BERTASSO & TRACY & w & 17 & MINNESOT & ITALY & ITALY & & & & OSCEOLA ST. & CALUMET - LAURIUM VILLAGE \\
\hline 1492 & 325 & RICHETTA & MICHELE & $\mathrm{H}$ & 55 & ITALY & ITALY & ITALY & 1884 & SALOONKEEPER & SAN MARTINO CANAVESE. TO & HECLA ST. & CALUMET - LAURIUM VILLAGE \\
\hline 1493 & 325 & RICHETTA & DOMENICA & W & 54 & ITALY & ITALY & ITALY & 1886 & GALU TURLLT LTI & SAN MARTINO CANAVESE. TO & HECLA ST. & CALUMET - LAURIUM VILLAGE \\
\hline 1494 & 325 & RICHETTA & GIACOMO & s & 33 & ITALY & ITALY & ITALY & 1886 & TEAMSTER/LIVERY BARN & SAN MARTINO CANAVESE. TO & HECLA ST. & CALUMET - LAURIUM VILLAGE \\
\hline 1495 & 325 & RICHETTA & DOMENICA & DIL & 28 & ITALY & ITALY & ITALY & 1900 & & & & CALUMET - LAURIUM VILLAGE \\
\hline 1496 & 325 & RICHETTA & DOMENICA & $\mathrm{GD}$ & 8 & MICHIGAN & ITALY & ITALY & & & SAN MARTINO CANAVESE. TO & $\mathrm{HE}$ & ET - LAURIUM VI \\
\hline 1497 & 325 & RICHETTA & CATERINA & $\mathrm{GD}$ & 6 & MICHIGAN & ITALY & ITALY & & & SAN MARTINO CANAVESE. TO & A ST. & CALUMET - LAUP \\
\hline 1498 & 325 & RICHETTA & MICHELE & GS & $3 \mathrm{M}$ & MICHIGAN & ITALY & ITALY & & & SAN MARTINO CANAVESE. TO & LA ST. & CALUMET - LAURIUM VILLAGE \\
\hline 1499 & 325 & RICHETTA & ANGELO & $\mathrm{H}$ & 51 & ITAL & ITA & ITALY & 1883 & & SAN MARTINO CANAVESE. TO & HECLA S & CALUMET - LAURIUM VILLAGE \\
\hline 1500 & 325 & RICHETTA & VITTORIA & W & 50 & ITAL & ITAL & ITAL & 1886 & & SAN MARTINO CANAVESE, TO & HECLA ST. & CALUMET - LAURIUM VILLAGE \\
\hline 1501 & 325 & RICHETTA & GIACOMO & $\mathrm{s}$ & 29 & ITALY & ITALY & ITALY & 1886 & UNDERTAKER & SAN MARTINO CANAVESE. TO & HECLA ST. & CALUMET - LAURIUM VILLAGE \\
\hline 1502 & 325 & RICHETTA & MARIANNA & DIL & 24 & ITALY & ITALY & ITALY & 1907 & & SAN MARTINO CANAVESE. TO & HECLA ST. & CALUMET - LAURIUM VILLAGE \\
\hline 1503 & 325 & RICHETTA & ANGELO & GS & 1 & MICHIGAN & ITALY & ITALY & & & SAN MARTINO CANAVESE. TO & HECLA ST. & CALUMET - LAURIUM VILLAGE \\
\hline 1504 & 325 & RICHETTA & TERESA & $\mathrm{D}$ & 19 & MICHIGAN & ITALY & ITALY & & HOUSEKEEPER/HOME & SAN MARTINO CANAVESE. TO & HECLA ST. & CALUMET - LAURIUM VILLAGE \\
\hline 1505 & 325 & CENTANINO & GIACOMO & B & 27 & ITALY & ITALY & ITALY & 1907 & LABORER/LIVERY BARN & SAN MARTINO CANAVESE. TO & HECLA ST. & CALUMET - LAURIUM VILLAGE \\
\hline 1506 & 323 & MARTA & CATHERINE & $\mathrm{H}$ & 47 & ITALY & ITALY & ITALY & 1888 & STOREKEEPER/EMPORIUM & TORINO & HECLA ST. & CALUMET - LAURIUM VILLAGE \\
\hline 1507 & 323 & MARTA & MARTIN & s & 20 & MICHIGAN & ITALY & ITALY & & & TORINO & HECLA ST. & CALUMET - LAURIUM VILLAGE \\
\hline 1508 & 323 & MARTA & ANNA & D & 18 & MICHIGAN & ITAL & ITAL & & CLERK/GROCERY STORE & TORINO & HECLA ST. & CALUMET - LAURIUM VILLAGE \\
\hline 1509 & 323 & & & $\mathrm{~s}$ & 16 & & & & & TEAMSTER/GROCERY STORE & & & \\
\hline 1510 & 323 & & & $\mathrm{~s}$ & 15 & & ITALY & & & & & & CALUMET - LAURIUM VILLAC \\
\hline 1511 & 323 & MARTA & EMMA & D & 7 & MICHIGAN & ITALY & ITALY & & & TORINO & HECLA ST. & CALUMET - LAURIUM VILLAGE \\
\hline
\end{tabular}




\begin{tabular}{|c|c|c|c|c|c|c|c|c|c|c|c|c|c|}
\hline 1512 & 323 & MARTA & ADOLF & $s$ & 5 & MICHIGAN & ITALY & ITALY & & & TORINO & HECLA ST. & CALUMET - LAURIUM VILLAGE \\
\hline 1513 & 320 & QUELLO & DOMINICK & $\mathrm{H}$ & 48 & ITALY & ITALY & ITALY & 1886 & SALOONKEEPER & LOCANA CANVESE. TO & HECLA ST. & CALUMET - LAURIUM VILLAGE \\
\hline 1514 & 320 & QUELLO & LUCIA & W & 26 & ITALY & ITALY & ITALY & 1900 & & TORINO & HECLA ST. & CALUMET - LAURIUM VILLAGE \\
\hline 1515 & 320 & QUELLO & ATTILIO & s & 1 & MICHIGAN & ITALY & ITALY & & & LOCANA CANVESE. TO & HECLA ST. & CALUMET - LAURIUM VILLAGE \\
\hline 1516 & 320 & QUELLO & BATTISTA & B & 22 & MICHIGAN & ITALY & ITALY & & MINER/CM & LOCANA CANVESE. TO & HECLA ST. & CALUMET - LAURIUM VILLAGE \\
\hline 1517 & 320 & CALCIO & MARY & SER & 21 & ITALY & ITALY & ITALY & 1908 & SERVANT/PF & PONT CANAVESE. TO & HECLA ST. & CALUMET - LAURIUM VILLAGE \\
\hline 1518 & 320 & PEGUALDO & JAKINO & B & 28 & ITALY & ITALY & ITALY & 1905 & MINER/CM & & HECLA ST. & CALUMET - LAURIUM VILLAGE \\
\hline 1519 & 320 & CONTA & JOHN & $\begin{array}{l}\mathrm{O} \\
\mathrm{H}\end{array}$ & 42 & ITALY & ITALY & ITALY & 1889 & BAKER & TORINO & HECLA ST. & $\begin{array}{l}\text { CALUMET - LAURIUM VILLAGE } \\
\text { CALE }\end{array}$ \\
\hline 1520 & 320 & CONTA & TERESA & W & 33 & ITALY & ITALY & ITALY & 1905 & DARER & 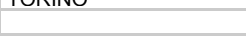 & HECLA ST. & $\begin{array}{l}\text { CALUMET - LAURIUM VILLAGE } \\
\text { CALE }\end{array}$ \\
\hline $1521:$ & 320 & TORREANO & SANTANINI & $\mathrm{H}$ & 32 & ITALY & ITALY & ITALY & 1904 & TEAMSTER/BAKERY & TORINO & HECLA ST. & CALUMET - LAURIUM VILLAGE \\
\hline 1522 & 320 & TORREANO & CAROLINE & W & 29 & ITALY & ITALY & ITALY & 1904 & & TORINO & HECLA ST. & CALUMET - LAURIUM VILLAGE \\
\hline 1523 & 320 & TORREANO & TERESA & $\mathrm{D}$ & 9 & ITALY & ITALY & ITALY & 1904 & & TORINO & HECLA ST. & CALUMET - LAURIUM VILLAGE \\
\hline 1524 & 320 & TORREANO & HUMBERT & s & 6 & MICHIGAN & ITALY & ITALY & & & TORINO & HECLA ST. & CALUMET - LAURIUM VILLAGE \\
\hline 1525 & 320 & TORREANO & JAMES & S & 2 & MICHIGAN & ITALY & ITALY & & & TORINO & HECLA ST. & CALUMET - LAURIUM VILLAGE \\
\hline 1526 & 313 & LUNDY & JOHN & $\mathrm{H}$ & 25 & CANADA & CANADA & CANADA & & BOILER MAN/RAILROAD & CANADA & KEARSARGE ST. & CALUMET - LAURIUM VILLAGE \\
\hline 1527 & 313 & LUNDY & CAROLINE & W & 25 & MICHIGAN & ITALY & ITALY & & & & KEARSARGE ST. & CALUMET - LAURIUM VILLAGE \\
\hline 1528 & 313 & MERLO & GIOVANNI & $\mathrm{H}$ & 25 & ITALY & ITALY & ITALY & 1905 & BAKER & PONT CANAVESE. TO & KEARSARGE ST. & CALUMET - LAURIUM VILLAGE \\
\hline 1529 & 313 & MERLO-ROSSO & TERESA & w & 18 & ITALY & ITALY & ITALY & 1909 & & & KEARSARGE ST. & CALUMET - LAURIUM VILLAGE \\
\hline 1530 & 313 & GALLASERO & CHARLES & $\mathrm{H}$ & 48 & ITALY & ITALY & ITALY & 1883 & FOREMAN/CM & PONT CANAVESE. TO & KEARSARGE ST. & CALUMET - LAURIUM VILLAGE \\
\hline 1531 & 313 & GALLASERO & LUIGIA & W & 39 & ITALY & ITALY & ITALY & 1906 & 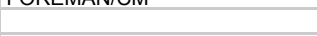 & TCIVT UAIVAVELOL. TS & KEARSARGE ST. & CALUMET - LAURIUM VILLAGE \\
\hline 1532 & 313 & GALLASERO & PETER & s & 17 & MICHIGAN & ITALY & ITALY & & APPRENTICE/MACHINE SHOP & PONT CANAVESE. TO & KEARSARGE ST. & CALUMET - LAURIUM VILLAGE \\
\hline 1533 & 313 & GALLASERO & MARY & $\mathrm{D}$ & 14 & MICHIGAN & ITALY & ITALY & & & PONT CANAVESE, TO & KEARSARGE ST. & CALUMET - LAURIUM VILLAGE \\
\hline 1534 & 313 & GALLASERO & CHARLES & $\mathrm{s}$ & 11 & MICHIGAN & ITALY & ITALY & & & PONT CANAVESE. TO & KEARSARGE ST. & CALUMET - LAURIUM VILLAGE \\
\hline 1535 & 313 & GALLASERO & LOUISE & $\mathrm{D}$ & 9 & MICHIGAN & ITALY & ITALY & & & PONT CANAVESE. TO & KEARSARGE ST. & CALUMET - LAURIUM VILLAGE \\
\hline 1536 & 415 & GASPARDO & JOHN & $\mathrm{H}$ & 46 & ITALY & ITALY & ITALY & 1884 & TIMBERMAN/CM & PONT CANAVESE. TO & OSCEOLA ST. & CALUUMT - LAURIUM VILLAGE \\
\hline 1537 & 415 & GASPARDO & ANNE & W & 33 & ITALY & ITALY & ITALY & 1886 & & PONT CANAVESE. TO & OSCEOLA ST. & CALUMET - LAURIUM VILLAGE \\
\hline 1538 & 415 & GASPARDO & MARY & $\mathrm{D}$ & 14 & MICHIGAN & ITALY & ITALY & & & & OSCEOLA ST. & CALUMET - LAURIUM VILLAGE \\
\hline 1539 & 415 & GASPARDO & ROSE & $\mathrm{D}$ & 10 & MICHIGAN & ITALY & ITALY & & & PONT CANAVESE, TO & OSCEOLA ST. & CALUMET - LAURIUM VILLAGE \\
\hline 1540 & 415 & GASPARDO & MINNIE & D & 8 & MICHIGAN & ITALY & ITALY & & & PONT CANAVESE. TO & OSCEOLA ST. & CALUMET - LAURIUM VILLAGE \\
\hline 1541 & 415 & GASPARDO & JAMES & s & 5 & MICHIGAN & ITALY & ITALY & & & PONT CANAVESE. TO & OSCEOLA ST. & CALUMET - LAURIUM VILLAGE \\
\hline 1542 & 415 & GASPARDO & JOHN & $\mathrm{S}$ & 1 & MICHIGAN & ITALY & ITALY & & & PONT CANAVESE. TO & OSCEOLA ST. & CALUMET - LAURIUM VILLAGE \\
\hline 1543 & 415 & PISTOTTI & SANIO & $\mathrm{H}$ & 31 & ITALY & ITALY & ITALY & 1900 & LABORER/FOUNDRY & TORINO & OSCEOLA ST. & CALUMET - LAURIUM VILLAGE \\
\hline 1544 & 415 & PISTOTTI & JENNIE & W & 19 & MICHIGAN & ITALY & ITALY & 1500 & LADURERTIV UTVURT & 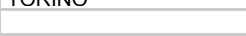 & OSCEOLA ST. & $\begin{array}{l}\text { CALUMET - LAURIUM VILLAGE } \\
\text { CALE }\end{array}$ \\
\hline 1545 & 415 & SERAFINI & ESPERANDIO & $\mathrm{H}$ & 33 & ITALY & ITALY & ITALY & 1903 & & LUCCA & OSCEOLA ST. & CALUMET - LAURIUM VILLAGE \\
\hline 1546 & 415 & SERAFINI & TRACY & W & 28 & ITALY & ITALY & ITALY & 1903 & & LUCCA & OSCEOLA ST. & CALUMET - LAURIUM VILLAGE \\
\hline 1547 & 415 & SERAFINI & MINNIE & s & 7 & ITALY & ITALY & ITALY & 1903 & & LUCCA & OSCEOLA ST. & CALUMET - LAURIUM VILLAGE \\
\hline 1548 & 415 & SERAFINI & JOHN & $\mathrm{s}$ & 5 & MICHIGAN & ITALY & ITALY & & & LUCCA & OSCEOLA ST. & CALUMET - LAURIUM VILLAGE \\
\hline 1549 & 415 & GASPARDO & DOMENICO & B & 25 & ITALY & ITALY & ITALY & 1904 & & PONT CANAVESE. TO & OSCEOLA ST. & CALUMET - LAURIUM VILLAGE \\
\hline 1550 & 415 & SOSSI/SUSSI & JAMES & $\mathrm{H}$ & 31 & ITALY & ITALY & ITALY & 1995 & & VIALFRE'. TO & OSCEOLA ST. & CALUMET - LAURIUM VILLAGE \\
\hline 1551 & 415 & SOSSI/SUSSI & ANGELINE & W & 22 & ITALY & ITALY & ITALY & 1905 & & & OSCEOLA ST. & CALUMET - LAURIUM VILLAGE \\
\hline 1552 & 415 & SOSSI/SUSSI & LUCY & D & 4 & MICHIGAN & ITALY & ITALY & & & VIALFRE'. TO & OSCEOLA ST. & CALUMET - LAURIUM VILLAGE \\
\hline 1553 & 415 & SOSSI/SUSSI & ANTON & $\mathrm{s}$ & 2 & MICHIGAN & ITALY & ITALY & & & VIALFRE'. TO & OSCEOLA ST. & CALUMET - LAURIUM VILLAGE \\
\hline 1554 & 415 & SOSSI/SUSSI & JOSEPH & $\mathrm{BR}$ & 35 & ITALY & ITALY & ITALY & 1891 & & VIALFRE'. TO & OSCEOLA ST. & CALUMET - LAURIUM VILLAGE \\
\hline 1555 & 415 & CERETTO & DOMENICO & $\mathrm{H}$ & 24 & ITALY & ITALY & ITALY & 1906 & & TORINO & OSCEOLA ST. & CALUMET - LAURIUM VILLAGE \\
\hline 1556 & 415 & CERETTO & JANIE & W & 18 & ITALY & ITALY & ITALY & 1903 & & & OSCEOLA ST. & CALUMET - LAURIUM VILLAGE \\
\hline 1557 & 415 & CERETTO & ANGELO & s & 1 & MICHIGAN & ITALY & ITALY & & & TORINO & OSCEOLA ST. & CALUMET - LAURIUM VILLAGE \\
\hline 1558 & 616 & BARSANTI & ORLANDO & $\mathrm{H}$ & & ITALY & ITALY & ITALY & 1891 & BOOKKEEPER/BANK & SEGROMIGNO. LU & LAKE LINDEN AVE. & CALUMET - LAURIUM VILLAGE \\
\hline 1559 & 616 & BARSANTI & KATE & W & & MICHIGAN & ITALY & ITALY & & & & LINDEN AVE. & CALUMET - LAURIUM VILLAGE \\
\hline 1560 & 616 & BARSANTI & ORLANDO & s & & MICHIGAN & ITALY & IIGAN & & & SEGROMIGNO. LU & LAKE LINDEN AVE. & CALUMET - LAURIUM VILLAGE \\
\hline 1561 & 616 & BARSANTI & ARMANDO & $\mathrm{s}$ & ?? & MICHIGAN & ITALY & IIGAN & & & SEGROMIGNO. LU & LAKE LINDEN AVE. & CALUMET - LAURIUM VILLAGE \\
\hline 1562 & 616 & BARSANTI & HUGO & $\mathrm{s}$ & $\because:$ & MICHIGAN & ITALY & MICHIGAN & & & SEGROMIGNO. LU & $\begin{array}{l}\text { LAKE LINDEN AVE. } \\
\text { LINE }\end{array}$ & CALUMET - LAURIUM VILLAGE \\
\hline 1563 & 616 & TORREANO & ADELINA & B & & MICHIGAN & ITALY & MICHIGAN & & & TORINO & LAKE LINDEN AVE. & CALUMET - LAURIUM VILLAGE \\
\hline 1564 & 414 & LOCATELLI & $\mathrm{JOHN}$ & $\mathrm{H}$ & 53 & ITALY & ITALY & ITALY & 1891 & ODD JOBS/VILLAGE & SELINO. BERGAMO & BOUNDARY ST. & CALUMET - LAURIUM VILLAGE \\
\hline 1565 & 414 & LOCATELLI & CATHERINE & W & 52 & ITALY & ITALY & ITALY & 1891 & & SELINO. BERGAMO & BOUNDARY ST. & CALUMET - LAURIUM VILLAGE \\
\hline 1566 & 414 & LOCATELLI & LENA & $\mathrm{D}$ & 21 & ITALY & ITALY & ITALY & 1891 & CLERK/FRUIT STORE & SELINO. BERGAMO & BOUNDARY ST. & CALUMET - LAURIUM VILLAGE \\
\hline 1567 & 414 & LOCATELLI & AGNES & $\mathrm{D}$ & 21 & ITALY & ITALY & ITALY & 1891 & MAID/HOTEL & SELINO. BERGAMO & BOUNDARY ST. & CALUMET - LAURIUM VILLAGE \\
\hline 1568 & 414 & LOCATELLI & SAMUEL & $\mathrm{s}$ & 19 & ITALY & ITALY & ITALY & 1891 & LUMBERMAN/WOOD CAMP & SELINO. BERGAMO & BOUNDARY ST. & CALUMET - LAURIUM VILLAGE \\
\hline 1569 & 414 & LOCATELLI & THERESA & $\mathrm{D}$ & 15 & MICHIGAN & ITALY & ITALY & 1891 & & SELINO, BERGAMO & BOUNDARY ST. & CALUMET - LAURIUM VILLAGE \\
\hline 1570 & 414 & LOCATELLI & LIZIE & $\mathrm{D}$ & 13 & MICHIGAN & ITALY & ITALY & & & SELINO. BERGAMO & BOUNDARY ST. & CALUMET - LAURIUM VILLAGE \\
\hline 1571 & 414 & LOCATELLI & PETER & $\mathrm{S}$ & 10 & MICHIGAN & ITALY & ITAL & & & SELINO. BERGAMO & BOUNDARY ST. & CALUMET - LAURIUM VILLAGE \\
\hline 1572 & 341 & GRISOLANO & ANTHONY & $\mathrm{H}$ & 48 & & & ITA & 1888 & OWN INCOME & TORINO & & \\
\hline 1573 & 341 & GRISOLANO & & w & 42 & & ITALY & & 1890 & & & & URIUM VILLAGE \\
\hline 1574 & 341 & GRISOLANO & HENRY & s & 17 & MICHIGAN & ITALY & ITALY & & DRILL BOY/CM & TORINO & 7TH ST. & CALUMET - LAURIUM VILLAGE \\
\hline
\end{tabular}




\begin{tabular}{|c|c|c|c|c|c|c|c|c|c|c|c|c|c|}
\hline 1575 & 341 & GRISOLANO & JOHN & $\mathrm{s}$ & 16 & MICHIGAN & ITALY & ITALY & & & TORINO & 7TH ST. & CALUMET - LAURIUM VILLAGE \\
\hline 1576 & 341 & GRISOLANO & VICTOR & $\mathrm{S}$ & 13 & MICHIGAN & ITALY & ITALY & & & TORINO & 7TH ST. & CALUMET - LAURIUM VILLAGE \\
\hline 1577 & 341 & GRISOLANO & PETER & $\mathrm{s}$ & 10 & MICHIGAN & ITALY & ITALY & & & TORINO & 7TH ST. & CALUMET - LAURIUM VILLAGE \\
\hline 1578 & 341 & GRISOLANO & ROSE & $\mathrm{D}$ & 6 & MICHIGAN & ITALY & ITALY & & & TORINO & 7TH ST. & CALUMET - LAURIUM VILLAGE \\
\hline 1579 & 341 & GRISOLANO & ANTON & s & 3 & MICHIGAN & ITALY & ITALY & & & TORINO & 7TH ST. & CALUMET - LAURIUM VILLAGE \\
\hline 1580 & 341 & GRISOLANO & MARY & $\mathrm{D}$ & 1 & MICHIGAN & ITALY & ITALY & & & TORINO & 7TH ST. & CALUMET - LAURIUM VILLAGE \\
\hline 1581 & 112 & SIMMONS & JOHN & $\mathrm{H}$ & 31 & MICHIGAN & IRELAND & IRELAND & & BARTENDER/SALOON & IRELAND & IROQUOIS ST. & CALUMET - LAURIUM VILLAGE \\
\hline 1582 & 112 & SIMMONS & MARY & W & 24 & MICHIGAN & ITALY & ITALY & & DARTEIVUERTOALUUTV & INELAIVD & IROQUOIS ST. & $\begin{array}{l}\text { CALUMET - LAURIUM VILLAGE } \\
\text { CAUME }\end{array}$ \\
\hline 1583 & 112 & SIMMONS & JOHN & s & 4 & MICHIGAN & MICHIGAN & MICHIGAN & & & & IROQUOIS ST. & CALUMET - LAURIUM VILLAGE \\
\hline 1584 & 112 & SIMMONS & MARY & D & 2 & MICHIGAN & MICHIGAN & MICHIGAN & & & & IROQUOIS ST. & CALUMET - LAURIUM VILLAGE \\
\hline 1585 & 112 & SIMMONS & ARTHUR & $\mathrm{s}$ & $4 \mathrm{M}$ & MICHIGAN & MICHIGAN & MICHIGAN & & & & IROQUOIS ST. & CALUMET - LAURIUM VILLAGE \\
\hline 1586 & 653 & LUCCHESI & FELIX & $\mathrm{H}$ & 38 & ITALY & ITALY & ITALY & 1891 & MINER/CM & LUCCA & TAMARACK ST. & CALUMET - LAURIUM VILLAGE \\
\hline 1587 & 653 & LUCCHESI & AMELIA & W & 30 & ITALY & ITALY & ITALY & 1897 & & & TAMARACK ST. & CALUMET - LAURIUM VILLAGE \\
\hline 1588 & 653 & LUCCHESI & MATILDA & $\mathrm{D}$ & 9 & MICHIGAN & ITALY & ITALY & & & LUCCA & TAMARACK ST. & CALUMET - LAURIUM VILLAGE \\
\hline 1589 & 653 & LUCCHESI & JOSEPH & s & 8 & MICHIGAN & ITALY & ITALY & & & LUCCA & TAMARACK ST. & CALUMET - LAURIUM VILLAGE \\
\hline 1590 & 653 & LUCCHESI & PETER & $\mathrm{s}$ & 7 & MICHIGAN & ITALY & ITALY & & & LUCCA & TAMARACK ST. & CALUMET - LAURIUM VILLAGE \\
\hline 1591 & 653 & LUCCHESI & RAFFAELE & $\mathrm{s}$ & 5 & MICHIGAN & ITALY & ITALY & & & LUCCA & TAMARACK ST. & CALUMET - LAURIUM VILLAGE \\
\hline 1592 & 653 & LUCCHESI & ANNIE & $\mathrm{D}$ & 2 & MICHIGAN & ITALY & ITALY & & & LUCCA & TAMARACK ST. & CALUMET - LAURIUM VILLAGE \\
\hline 1593 & 653 & LUCCHESI & FELIX & s & $1 \mathrm{M}$ & MICHIGAN & ITALY & ITALY & & & LUCCA & TAMARACK ST. & CALUMET - LAURIUM VILLAGE \\
\hline 1594 & 219 & BARSANTI & PASQUALE & $\mathrm{H}$ & 30 & ITALY & ITALY & ITALY & 1903 & PROPRIETOR/CONFECTIONARY & LUCCA & LAKE LINDEN AVE. & CALUMET - LAURIUM VILLAGE \\
\hline 1595 & 219 & BARSANTI & MARY & W & 23 & ITALY & ITALY & ITALY & 1901 & 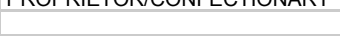 & LUCUA & LAKE LINDEN AVE. & CALUMET - LAURIUM VILLAGE \\
\hline 1596 & 219 & BARSANTI & LAWRENCE & $\mathrm{D}$ & 4 & MICHIGAN & ITALY & ITALY & & & LUCCA & LAKE LINDEN AVE. & CALUMET - LAURIUM VILLAGE \\
\hline 1597 & 219 & BARSANTI & RENA & $\mathrm{D}$ & 3 & MICHIGAN & ITALY & ITALY & & & LUCCA & LAKE LINDEN AVE. & CALUMET - LAURIUM VILLAGE \\
\hline 1598 & 219 & BARSANTI & ALBERTO & $\mathrm{H}$ & 32 & ITALY & ITALY & ITALY & 1895 & PROPRIETOR/CONFECTIONARY & LUCCA & LAKE LINDEN AVE. & CALUMET - LAURIUM VILLAGE \\
\hline 1599 & 219 & BARSANTI & BIANCA & W & 26 & ITALY & ITALY & ITALY & 1886 & & & LAKE LINDEN AVE. & CALUMET - LAURIUM VILLAGE \\
\hline 1600 & 219 & BARSANTI & FLORENCE & $s$ & 6 & MICHIGAN & ITALY & ITALY & & & LUCCA & LAKE LINDEN AVE. & CALUMET - LAURIUM VILLAGE \\
\hline 1601 & 219 & VIGNETTO & JAMES & $\mathrm{H}$ & 35 & ITALY & ITALY & ITALY & 1890 & PROPRIETOR/SALOON & TORINO & LAKE LINDEN AVE. & CALUMET - LAURIUM VILLAGE \\
\hline 1602 & 219 & VIGNETTO & ROSE & W & 33 & FRANCE & ITALY & ITALY & 1881 & & & LAKE LINDEN AVE. & CALUMET - LAURIUM VILLAGE \\
\hline 1603 & 219 & ZANARDI & GIOVANNA & $\mathrm{H}$ & 37 & ITALY & ITALY & ITALY & 1890 & OWN INCOME & BERGAMO & LAKE LINDEN AVE. & CALUMET - LAURIUM VILLAGE \\
\hline 1604 & 219 & ZANARDI & MARY & $\mathrm{D}$ & 17 & MICHIGAN & ITALY & ITALY & & & BERGAMO & LAKE LINDEN AVE. & CALUMET - LAURIUM VILLAGE \\
\hline 1605 & 219 & SCIACERO & GIOVANNI & $\mathrm{H}$ & 39 & ITALY & ITALY & ITALY & 1895 & PROPRIETOR/SALOON & SAN MARTINO CANAVESE. TO & LAKE LINDEN AVE. & CALUMET - LAURIUM VILLAGE \\
\hline 1606 & 219 & SCIACERO & CLARA & SIIL & 37 & ITALY & ITALY & ITALY & 1896 & 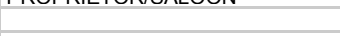 & 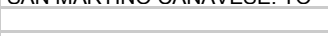 & LAKE LINDEN AVE. & CALUMET - LAURIUM VILLAGE \\
\hline 1607 & 109 & STEFANI & CARLO & $\mathrm{H}$ & 61 & $\begin{array}{l}\text { ITALY } \\
\text { ITAL }\end{array}$ & ITALY & $\begin{array}{l}\text { ITALY } \\
\text { ITAL }\end{array}$ & 1887 & DRAYMAN/CM & LUCCA & ALLOUEZ & CALUMET - FLORIDA LOCATION \\
\hline 1608 & 109 & STEFANI & ELISA & W & 38 & ITALY & ITALY & ITALY & 1908 & & & ALLOUEZ & CALUMET - FLORIDA LOCATION \\
\hline 1609 & 109 & STEFANI & LIZZIE & $\mathrm{D}$ & 16 & MICHIGAN & ITALY & ITALY & & & LUCCA & ALLOUEZ & CALUMET - FLORIDA LOCATION \\
\hline 1610 & 109 & STEFANI & JOHNNIE & $\mathrm{s}$ & 10 & MICHIGAN & ITALY & ITALY & & & LUCCA & ALLOUEZ & CALUMET - FLORIDA LOCATION \\
\hline 1611 & 109 & STEFANI & AMANDA & $\mathrm{s}$ & 8 & MICHIGAN & ITALY & ITALY & & & LUCCA & ALLOUEZ & CALUMET - FLORIDA LOCATION \\
\hline 1612 & 109 & STEFANI & EDITH & $\mathrm{D}$ & 8 & MICHIGAN & ITALY & ITALY & & & LUCCA & ALLOUEZ & CALUMET - FLORIDA LOCATION \\
\hline 1613 & 371 & BRUSSO & GIUSEPPE & $\mathrm{H}$ & 44 & ITALY & ITALY & ITALY & 1888 & WATCHMAN/CM & AGLIE'. TO & QUINCY ST. & CALUMET - FLORIDA LOCATION \\
\hline 1614 & 371 & BRUSSO & BARBARA & W & 37 & ITALY & ITALY & ITALY & 1888 & & AGLIE', TO & QUINCY ST. & CALUMET - FLORIDA LOCATION \\
\hline 1615 & 371 & BRUSSO & AMERIGO & $\mathrm{s}$ & 18 & ITALY & ITALY & ITALY & 1888 & DRILL BOY/CM & AGLIE'. TO & QUINCY ST. & CALUMET - FLORIDA LOCATION \\
\hline 1616 & 371 & BRUSSO & TERESA & $\mathrm{D}$ & 11 & MICHIGAN & ITALY & ITALY & & & AGLIE'. TO & QUINCY ST. & CALUMET - FLORIDA LOCATION \\
\hline 1617 & 371 & BRUSSO & LORENZO & s & 9 & MICHIGAN & ITALY & ITALY & & & AGLIE'. TO & QUINCY ST. & CALUMET - FLORIDA LOCATION \\
\hline 1618 & 371 & BRUSSO & DOMENICO & $\mathrm{s}$ & 7 & MICHIGAN & ITALY & ITALY & & & AGLIE', TO & QUINCY ST. & CALUMET - FLORIDA LOCATION \\
\hline 1619 & 371 & BRUSSO & JOSEPHINE & $\mathrm{D}$ & 5 & MICHIGAN & ITALY & ITALY & & & AGLIE', TO & QUINCY ST. & CALUMET - FLORIDA LOCATION \\
\hline 1620 & 910 & LUCCHESI & JOHN & $\mathrm{H}$ & 46 & ITALY & ITALY & ITALY & 1879 & MINER/CM & LUCCA & ATLANTIC ST. & CALUMET - FLORIDA LOCATION \\
\hline 1621 & 910 & LUCCHESI & MARGHERITA & w & 35 & ITALY & ITALY & ITALY & 1895 & & & ATLANTIC ST. & CALUMET - FLORIDA LOCATION \\
\hline 1622 & 910 & LUCCHESI & CLARA & D & 13 & MICHIGAN & ITALY & ITALY & & & LUCCA & ATLANTIC ST. & CALUMET - FLORIDA LOCATION \\
\hline 1623 & 910 & LUCCHESI & ADELINA & $\mathrm{D}$ & 12 & MICHIGAN & ITALY & ITAL & & & LUCCA & ATLANTIC ST. & CALUMET - FLORIDA LOCATION \\
\hline 1624 & 910 & LUCCHESI & ILEOLA & $\mathrm{D}$ & 11 & MICHIGAN & ITALY & ITAI & & & LUCCA & ATLANTIC ST. & CALUMET - FLORIDA LOCATION \\
\hline 1625 & 910 & LUCCHESI & EMMA & $\mathrm{D}$ & 9 & MICHIGAN & ITALY & ITAI & & & LUCCA & ATLANTIC ST. & CALUMET - FLORIDA LOCATION \\
\hline 1626 & 910 & LUCCHESI & MARY & D & 8 & MICHIGAN & ITALY & ITALY & & & LUCCA & ATLANTIC ST. & CALUMET - FLORIDA LOCATION \\
\hline $1627:$ & 910 & LUCCHESI & SAM & $\mathrm{s}$ & 4 & MICHIGAN & ITALY & ITALY & & & LUCCA & ATLANTIC ST. & CALUMET - FLORIDA LOCATION \\
\hline 1628 & 910 & LUCCHESI & MARGHERITA & D & 2 & MICHIGAN & ITALY & ITALY & & & LUCCA & BUSCH ST. & CALUMET - FLORIDA LOCATION \\
\hline 1629 & 146 & MARINELLI & DOMENICO & $?$ & 25 & ITALY & ITALY & ITALY & $190 ?$ & TRAMMER/CM & & BUSCH ST. & CALUMET - FLORIDA LOCATION \\
\hline 1630 & 146 & MARINELLI & LENA & $?$ & 21 & ITALY & ITALY & ITALY & 1910 & & & BUSCH ST. & CALUMET - FLORIDA LOCATION \\
\hline 1631 & 145 & BOBBIO & ANTONIO & $\mathrm{H}$ & 40 & ITALY & ITALY & ITALY & 1889 & TIMBERMAN/CM & SAN BENIGNO CANAVESE. TO & CALUMET AVE. & CALUMET - C. \& H. LOCATION \\
\hline 1632 & 145 & BOBBIO & MARY & W & 35 & ITALY & ITALY & ITALY & 1900 & HIVIDLTIVIAITICIVI & SAIV DLIVISTIN CAIVAVLOL. TO & CALUMET AVE. & CALUMET - C. \& H. LOCATION \\
\hline 1633 & 145 & BOBBIO & $\mathrm{JOE}$ & s & 8 & MICHIGAN & ITALY & ITALY & & & SAN BENIGNO CANAVESE. TO & CALUMET AVE. & CALUMET - C. \& H. LOCATION \\
\hline 1634 & 145 & BOBBIO & WILLIAM & $\mathrm{s}$ & 6 & MICHIGAN & ITALY & ITAL & & & SAN BENIGNO CANAVESE. TO & CALUMET AVE. & CALUMET - C. \& H. LOCATION \\
\hline 1635 & 386 & IAVELLI & & $\mathrm{H}$ & 32 & & & & 1900 & TRAMMER/CM & BOSCONERO.TC & A ST. & ION \\
\hline 1636 & 386 & & & w & 28 & & ITALY & & 1907 & & & & CALUMET - C. \& H. LOCATION \\
\hline 1637 & 386 & IAVELLI & DEFENDENTE & $\mathrm{s}$ & 2 & MICHIGAN & ITALY & ITALY & & & BOSCONERO. TO & CALEDONIA ST. & CALUMET - C. \& H. LOCATION \\
\hline
\end{tabular}




\begin{tabular}{|c|c|c|c|c|c|c|c|c|c|c|c|c|c|}
\hline 1638 & 386 & IAVELLI & ANTONIO & $\mathrm{H}$ & 38 & ITALY & ITALY & ITALY & 1900 & TIMBERMAN/CM & BOSCONERO. TO & CALEDONIA ST. & CALUMET - C. \& H. LOCATION \\
\hline 1639 & 386 & IAVELLI & ISABELLA & W & 32 & ITALY & ITALY & ITALY & 1905 & & & CALEDONIA ST. & CALUMET - C. \& H. LOCATION \\
\hline 1640 & 386 & IAVELLI & DEFENDENTE & $\mathrm{s}$ & 4 & MICHIGAN & ITALY & ITALY & & & BOSCONERO. TO & CALEDONIA ST. & CALUMET - C. \& H. LOCATION \\
\hline 1641 & 386 & IAVELLI & MARY & D & $10 \mathrm{M}$ & MICHIGAN & ITALY & ITALY & & & BOSCONERO. TO & CALEDONIA ST. & CALUMET - C. \& H. LOCATION \\
\hline 1642 & 385 & MARTA & LORENZO & $\mathrm{H}$ & 32 & ITALY & ITALY & ITALY & 1901 & TIMBERMAN/CM & AGLIE'. TO & CALEDONIA ST. & CALUMET - C. \& H. LOCATION \\
\hline 1643 & 385 & MARTA & FILOMENA & W & 32 & ITALY & ITALY & ITALY & 1903 & & & CALEDONIA ST. & CALUMET - C. \& H. LOCATION \\
\hline 1644 & 385 & MARTA & MARIA & $\mathrm{D}$ & 6 & MICHIGAN & ITALY & ITALY & & & AGLIE'. TO & CALEDONIA ST. & CALUMET - C. \& H. LOCATION \\
\hline 1645 & 385 & MARTA & MASSIMO & s & 4 & MICHIGAN & ITALY & ITALY & & & AGLIE'. TO & CALEDONIA ST. & CALUMET - C. \& H. LOCATION \\
\hline 1646 & 385 & MARTA & ANTONIO & BR & 18 & ITALY & ITALY & ITALY & 1907 & LABORER/CM & AGLIE'. TO & CALEDONIA ST. & CALUMET - C. \& H. LOCATION \\
\hline 1647 & 385 & RICARDI & ANTONIO & $\mathrm{H}$ & 37 & ITALY & ITALY & ITALY & 1898 & TIMBERMAN/CM & SCARMAGNO. TO & CALEDONIA ST. & CALUMET - C. \& H. LOCATION \\
\hline 1648 & 385 & RICARDI & CATERINA & w & 36 & ITALY & ITALY & ITALY & 1902 & & SCARMAGNO. TO & CALEDONIA ST. & CALUMET - C. \& H. LOCATION \\
\hline 1649 & 385 & RICARDI & TONY & $\mathrm{s}$ & 12 & ITALY & ITALY & ITALY & 1902 & & SCARMAGNO. TO & CALEDONIA ST. & CALUMET - C. \& H. LOCATION \\
\hline 1650 & 385 & RICARDI & GIUSEPPINA & $\mathrm{D}$ & 10 & ITALY & ITALY & ITALY & 1902 & & SCARMAGNO. TO & CALEDONIA ST. & CALUMET - C. \& H. LOCATION \\
\hline 1651 & 385 & RICARDI & ELENA & $\mathrm{D}$ & 7 & MICHIGAN & ITALY & ITALY & & & SCARMAGNO. TO & CALEDONIA ST. & CALUMET - C. \& H. LOCATION \\
\hline 1652 & 385 & RICARDI & MIKE & $\mathrm{s}$ & 4 & MICHIGAN & ITALY & ITALY & & & SCARMAGNO. TO & CALEDONIA ST. & CALUMET - C. \& H. LOCATION \\
\hline 1653 & 389 & BOGGIO & PONZIO & $\mathrm{H}$ & 46 & ITALY & ITALY & ITALY & 1888 & WATCHMAN/CM & SAN PONSO CANAVESE, TO & CALEDONIA ST. & CALUMET - C. \& H. LOCATION \\
\hline 1654 & 389 & BOGGIO & MARY & W & 45 & ITALY & ITALY & ITALY & 1892 & & & CALEDONIA ST. & CALUMET - C. \& H. LOCATION \\
\hline 1655 & 389 & BOGGIO & MADDALENA & $\mathrm{D}$ & 16 & MICHIGAN & ITALY & ITALY & & & SAN PONSO CANAVESE. TO & CALEDONIA ST. & CALUMET - C. \& H. LOCATION \\
\hline 1656 & 389 & BOGGIO & GIUSEPPINA & $\mathrm{D}$ & 13 & MICHIGAN & ITALY & ITALY & & & SAN PONSO CANAVESE. TO & CALEDONIA ST. & CALUMET - C. \& H. LOCATION \\
\hline 1657 & 389 & BOGGIO & BARNEY & $\mathrm{s}$ & 11 & MICHIGAN & ITALY & ITALY & & & SAN PONSO CANAVESE. TO & CALEDONIA ST. & CALUMET - C. \& H. LOCATION \\
\hline 1658 & 252 & AIMONE & JOHN & $\mathrm{H}$ & 36 & ITALY & ITALY & ITALY & 1898 & MINER/CM & TORINO & PORTLAND ST. & CALUMET - C. \& H. LOC. - ALLION \\
\hline $\begin{array}{l}1050 \\
1659\end{array}$ & 202 & AIMONE & MARY & W & 35 & ITALY & ITALY & ITALY & 1897 & IVIINERT/UIII & TORIINO & PORTLAND ST. & 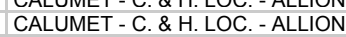 \\
\hline 1660 & & AIMONE & CRISTINA & $\mathrm{D}$ & 16 & MICHIGAN & ITALY & ITALY & & SALESLADYIDRY GOODS & TORINO & PORTLAND ST. & CALUMET - C. \& H. LOC - ALLION \\
\hline 1661 & & AIMONE & ROSIE & $\mathrm{D}$ & 13 & MICHIGAN & ITALY & ITALY & & & TORINO & PORTLAND ST. & CALUMET - C. \& H. LOC. - ALLION \\
\hline 1662 & & AIMONE & JOSEPH & $\mathrm{s}$ & 12 & MICHIGAN & ITALY & ITALY & & & TORINO & PORTLAND ST. & CALUMET - C. \& H. LOC. - ALLION \\
\hline 1663 & & AIMONE & MAGGIE & $\mathrm{D}$ & 2 & MICHIGAN & ITALY & ITALY & & & TORINO & PORTLAND ST. & CALUMET - C. \& H. LOC. - ALLION \\
\hline 1664 & 459 & ANDRINA & JOHN & $\mathrm{H}$ & 26 & ITALY & ITALY & ITALY & 1900 & & SAN MARTINO CANAVESE. TO & CALEDONIA ST. & CALUMET - C. \& H. LOC. - ALLION \\
\hline 1665 & & ANDRINA & DOMENICA & W & 22 & MICHIGAN & ITALY & ITALY & & & & CALEDONIA ST. & CALUMET - C. \& H. LOC. - ALLION \\
\hline 1666 & & ANDRINA & MARTA & D & 3 & MICHIGAN & ITALY & MICHIGAN & & & SAN MARTINO CANAVESE. TO & CALEDONIA ST. & CALUMET - C. \& H. LOC. - ALLION \\
\hline 1667 & & ANDRINA & JOHN & $\mathrm{s}$ & 2 & MICHIGAN & ITALY & MICHIGAN & & & SAN MARTINO CANAVESE. TO & CALEDONIA ST. & CALUMET - C. \& H. LOC. - ALLION \\
\hline 1668 & & DE CAROLI & JOSEPHINE & $\mathrm{H}$ & 55 & ITALY & ITALY & ITALY & 1888 & DRESSMAKER/WORK OUT & TORINO & CALEDONIA ST. & CALUMET - C. \& H. LOC. - ALLION \\
\hline 1669 & & DE CAROLI & MADDALENA & $\mathrm{D}$ & 10 & MICHIGAN & ITALY & ITALY & 1000 & 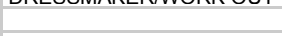 & TORINO & CALEDONIA ST. & CALUMET - C. \& H. LOC. - ALLION \\
\hline 1670 & 858 & FAUSONE & GIUSEPPE & $\mathrm{H}$ & 26 & ITALY & ITALY & ITALY & 1891 & MINER/CM & TORINO & CUMBRIA ST. & CALUMET - C. \& H. LOC. - ALLION \\
\hline 1671 & 858 & FAUSONE & LENA & w & 18 & ITALY & ITALY & ITALY & 1909 & & & CUMBRIA ST. & CALUMET - C. \& H. LOC. - ALLION \\
\hline 1672 & 94 & BESSOLO & FELIX & $\mathrm{H}$ & 41 & ITALY & ITALY & ITALY & 1890 & MINER/CM & SCARMAGNO. TO & EAST PINE ST. & CALUMET - C. \& H. LOC - ALLION \\
\hline 1673 & & BESSOLO & EUGENIA & w & 27 & ITALY & ITALY & ITALY & 1899 & & & EAST PINE ST. & CALUMET - C. \& H. LOC. - ALLION \\
\hline 1674 & & BESSOLO & MARION & $\mathrm{D}$ & 7 & MICHIGAN & ITALY & ITALY & & & SCARMAGNO. TO & EAST PINE ST. & CALUMET - C. \& H. LOC. - ALLION \\
\hline 1675 & & BESSOLO & CARL & $\mathrm{s}$ & 2 & MICHIGAN & ITALY & ITALY & & & SCARMAGNO. TO & EAST PINE ST. & CALUMET - C. \& H. LOC. - ALLION \\
\hline 1676 & & BESSOLO & MARGHERITA & HK & 36 & ITALY & ITALY & ITALY & ?? & HOUSEKEEPER/PF & SCARMAGNO. TO & EAST PINE ST. & CALUMET - C. \& H. LOC. - ALLION \\
\hline 1677 & 625 & DE FILIPPI & DOMENICO & $\mathrm{H}$ & 50 & ITALY & ITALY & ITALY & 1886 & MINER/CM & IVREA. TO & WATER WORKS R. & CALUMET - C. \& H. LOC. - ALLION \\
\hline 1678 & & DE FILIPPI & TERESA & W & 34 & ITALY & ITALY & ITALY & 1892 & & & WATER WORKS R. & CALUMET - C. \& H. LOC. - ALLION \\
\hline 1679 & & DE FILIPPI & BASTIAN & s & 12 & MICHIGAN & ITALY & ITALY & & & IVREA. TO & WATER WORKS R. & CALUMET - C. \& H. LOC. - ALLION \\
\hline 1680 & & DE FILIPPI & JULIUS? & $\mathrm{D}$ & 16 & MICHIGAN & ITALY & ITALY & & SALESLADY/DEPT. STORE & IVREA. TO & WATER WORKS R. & CALUMET - C. \& H. LOC. - ALLION \\
\hline 1681 & & DE FILIPPI & MARY & $\mathrm{D}$ & 10 & MICHIGAN & ITALY & ITALY & & & IVREA. TO & WATER WORKS R. & CALUMET - C. \& H. LOC. - ALLION \\
\hline 1682 & & DE FILIPPI & DOM? & $\mathrm{D}$ & 8 & MICHIGAN & ITALY & ITALY & & & IVREA. TO & WATER WORKS R. & CALUMET - C. \& H. LOC. - ALLION \\
\hline 1683 & & CENA & DOMENICO & B & 24 & ITALY & ITALY & ITALY & 1906 & MINER/CM & SAN BENIGNO CANAVESE. TO & WATER WORKS R. & CALUMET - C. \& H. LOC. - ALLION \\
\hline 1684 & & CAPELLO & BATTISTA & B & 46 & ITALY & ITALY & ITALY & 1906 & MINER/CM & SAN BENIGNO CANAVESE. TO & WATER WORKS R. & CALUMET - C. \& H. LOC. - ALLION \\
\hline 1685 & 730 & PELLEGRINI & LOUIS & $\mathrm{H}$ & 34 & ITALY & ITALY & ITAL & 1900 & MINER/CM & BERGAMO & WATER WORKS R. & CALUMET - C. \& H. LOC. - ALLION \\
\hline 1686 & & PELLEGRINI & FEUDE & w & 34 & ITALY & ITALY & ITALY & 1892 & & & WATER WORKS R. & CALUMET - C. \& H. LOC. - ALLION \\
\hline 1687 & & PELLEGRINI & JOSEPH & s & 4 & MICHIGAN & ITALY & ITALY & & & BERGAMO & WATER WORKS R. & CALUMET - C. \& H. LOC. - ALLION \\
\hline 1688 & & & LEO & $\mathrm{s}$ & $8 \mathrm{M}$ & MICHIGAN & ITALY & ITA & & & BERGAMO & WATER WORKS R. & CALUMET - C. \& H. LOC. - ALLION \\
\hline 1689 & & MASNADO/A & BERNARDO & SS & 16 & MICHIGAN & ITALY & ITAL & & & BERGAMO & WATER WORKS R. & CALUMET - C. \& H. LOC. - ALLION \\
\hline 1690 & & MASNADO/A & MARY & SD & 11 & MICHIGAN & ITALY & ITALY & & & BERGAMO & WATER WORKS R. & CALUMET - C. \& H. LOC. - ALLION \\
\hline 1691 & & MASNADO/A & LINA & SD & 12 & MICHIGAN & ITALY & ITALY & & & BERGAMO & WATER WORKS R. & CALUMET - C. \& H. LOC. - ALLION \\
\hline 1692 & & MASNADO/A & ANTONIO & SS & 14 & MICHIGAN & ITALY & ITALY & & & BERGAMO & WATER WORKS R. & CALUMET - C. \& H. LOC. - ALLION \\
\hline 1693 & & MASNADO/A & PAOLINA & SD & 9 & MICHIGAN & ITALY & ITALY & & & BERGAMO & WATER WORKS R. & CALUMET - C. \& H. LOC. - ALLION \\
\hline 1694 & & ROTTA & ELIA & B & 19 & MICHIGAN & ITALY & ITALY & & & TORINO & WATER WORKS R. & CALUMET - C. \& H. LOC. - ALLION \\
\hline 1695 & & VEDOVATI & JOSEPH & B & 30 & ITALY & ITALY & ITALY & 1908 & MINER/CM & BERGAMO & WATER WORKS R. & CALUMET - C. \& H. LOC. - ALLION \\
\hline 1696 & & BALMA/BAIMA & ANGELINA & $\mathrm{H}$ & 24 & ITALY & ITALY & ITALY & 1900 & & & WATER WORKS R. & CALUMET - C. \& H. LOC. - ALLION \\
\hline 1697 & & BALMA/BAIMA & $\mathrm{JOHN}$ & $\mathrm{s}$ & $9 \mathrm{M}$ & MICHIGAN & ITALY & ITAI & & SERVANT/PF & TORINO & WATER WORKS R. & CALUMET - C. \& H. LOC. - ALLION \\
\hline 1698 & 532 & DE FILIPPI & & $\mathrm{H}$ & 57 & & ITA & ITA & 1882 & MINER/CM & SAN GIORGIO CANAVESE. TO & & C. \& H. LOC. - ALLION \\
\hline 1699 & & & & W & 50 & & & ITALY & 1886 & & & & \\
\hline 1700 & & DE FILIPPI & ANTONIO & $\mathrm{s}$ & 23 & MICHIGAN & ITALY & ITALY & & & SAN GIORGIO CANAVESE. TO & 3RD ST. & CALUMET - C. \& H. LOC. - ALLION \\
\hline
\end{tabular}




\begin{tabular}{|c|c|c|c|c|c|c|c|c|c|c|c|c|c|}
\hline 1701 & & DE FILIPPI & JOSEPH & $\mathrm{s}$ & 20 & MICHIGAN & ITALY & ITALY & & BUTCHER/SHOP & SAN GIORGIO CANAVESE. TO & 3RD ST. & CALUMET - C. \& H. LOC. - ALLION \\
\hline 1702 & & DE FILIPPI & LENA & $\mathrm{D}$ & 19 & MICHIGAN & ITALY & ITALY & & & SAN GIORGIO CANAVESE. TO & 3RD ST. & CALUMET - C. \& H. LOC. - ALLION \\
\hline 1703 & & DE FILIPPI & ANGELO & $\mathrm{s}$ & 17 & MICHIGAN & ITALY & ITALY & & MINER/CM & SAN GIORGIO CANAVESE. TO & 3RD ST. & CALUMET - C. \& H. LOC. - ALLION \\
\hline 1704 & & DE FILIPPI & JOHN & $\mathrm{s}$ & 15 & MICHIGAN & ITALY & ITALY & & & SAN GIORGIO CANAVESE. TO & 3RD ST. & CALUMET - C. \& H. LOC. - ALLION \\
\hline 1705 & 534 & DE FILIPPI & FELICE & $\mathrm{H}$ & 30 & ITALY & ITALY & ITALY & 1896 & MINER/CM & SAN GIORGIO CANAVESE. TO & 3RD ST. & CALUMET - C. \& H. LOC. - ALLION \\
\hline 1706 & & DE FILIPPI & VIRGINIA & w & 30 & ITALY & ITALY & ITALY & 1899 & & & 3RD ST. & CALUMET - C. \& H. LOC. - ALLION \\
\hline 1707 & & DE FILIPPI & JOHN & s & 7 & MICHIGAN & ITALY & ITALY & & & SAN GIORGIO CANAVESE. TO & 3RD ST. & CALUMET - C. \& H. LOC. - ALLION \\
\hline 1708 & & MARTA & MASSIMO & $\mathrm{H}$ & 33 & ITALY & ITALY & ITALY & 1900 & MINER/CM & $\begin{array}{l}\text { TORINO } \\
\text { TOIOIVAVEOL. TO }\end{array}$ & 3RD ST. & CALUMET - C. \& H. LOC. - ALLION \\
\hline 1709 & & MARTA & KATE & W & 23 & ITALY & ITALY & ITALY & 1905 & & TORINO & 3RD ST. & CALUMET - C. \& H. LOC. - ALLION \\
\hline 1710 & & MARTA & MA? & $\mathrm{D}$ & 4 & MICHIGAN & ITALY & ITALY & & & TORINO & 3RD ST. & CALUMET - C. \& H. LOC. - ALLION \\
\hline 1711 & & BRUSSO & MIKE & B & 40 & ITALY & ITALY & ITALY & 1892 & MINER/CM & AGLIE'. TO & 3RD ST. & CALUMET - C. \& H. LOC. - ALLION \\
\hline 1712 & 525 & SADO & JOSEPH & $\mathrm{H}$ & 31 & ITALY & ITALY & ITALY & 1902 & MINER/CM & STRAMBINELLO. TO & 3RD ST./CEDAR ST. & CALUMET - C. \& H. LOC. - ALLION \\
\hline 1713 & & SADO & CATERINA & w & 31 & ITALY & ITALY & ITALY & 1891 & DRESSMAKER/AT HOME & SAN GIORGIO CANAVESE. TO & 3RD ST./CEDAR ST. & CALUMET - C. \& H. LOC. - ALLION \\
\hline 1714 & & NIGRA & PETRONILLA & MOIL & 40 & ITALY & ITALY & ITALY & 1882 & & TORINO & 3RD ST./CEDAR ST. & CALUMET - C. \& H. LOC. - ALLION \\
\hline 1715 & 546 & BATIATTO & JAMES & $\mathrm{H}$ & 32 & ITALY & ITALY & ITALY & 1902 & MINER/CM & & 2ND STREET & CALUMET - C. \& H. LOC. - ALLION \\
\hline 1716 & & BATIATTO & STEFANIA & W & 31 & ITALY & ITALY & ITALY & 1902 & & & 2ND STREET & CALUMET - C. \& H. LOC. - ALLION \\
\hline 1717 & & BATIATTO & TRACA & $\mathrm{D}$ & 3 & MICHIGAN & ITALY & ITALY & & & & 2ND STREET & CALUMET - C. \& H. LOC. - ALLION \\
\hline 1718 & 568 & PONSETTO & G. & $\mathrm{H}$ & 44 & ITALY & ITALY & ITALY & 1891 & TIMBERMAN/CM & SAN GIORGIO CANAVESE. TO & CEDAR ST. & CALUMET - C. \& H. LOC. - ALLION \\
\hline 1719 & & PONSETTO & JENNIE & W & 40 & ITALY & ITALY & ITALY & 1904 & & PONT CANAVESE. TO & CEDAR ST. & CALUMET - C. \& H. LOC. - ALLION \\
\hline 1720 & & RASTELLO & KATE & SIIL & 38 & ITALY & ITALY & ITALY & 1905 & & PONT CANAVESE. TO & CEDAR ST. & CALUMET - C. \& H. LOC. - ALLION \\
\hline 1721 & 566 & BAUDINO & PIETRO & $\mathrm{H}$ & 34 & ITALY & ITALY & ITALY & 1900 & BLACKSMITH/CM & BOSCONERO. TO & CEDAR ST. & CALUMET - C. \& H. LOC. - ALLION \\
\hline 1722 & & BAUDINO & MARIA & W & 31 & ITALY & ITALY & ITALY & 1905 & & BOSCONERO, TO & CEDAR ST. & CALUMET - C. \& H. LOC. - ALLION \\
\hline 1723 & & BAUDINO & ANDREA & s & 11 & ITALY & ITALY & ITALY & 1905 & & BOSCONERO. TO & CEDAR ST. & CALUMET - C. \& H. LOC. - ALLION \\
\hline 1724 & & MIGLIO/MIGLIA & MARY & $\mathrm{H}$ & 39 & ITALY & ITALY & ITALY & 1891 & LAUNDRESS/WORK OUT & SAN GIORGIO CANAVESE. TO & CEDAR ST. & CALUMET - C. \& H. LOC. - ALLION \\
\hline 1725 & & MIGLIO/MIGLIA & FRANCIS & $\mathrm{D}$ & 10 & MICHIGAN & ITALY & ITALY & & & SAN GIORGIO CANAVESE. TO & CEDAR ST. & CALUMET - C. \& H. LOC. - ALLION \\
\hline 1726 & & MIGLIO/MIGLIA & LUCILLE & $\mathrm{D}$ & 9 & MICHIGAN & ITALY & ITALY & & & SAN GIORGIO CANAVESE. TO & CEDAR ST. & CALUMET - C. \& H. LOC. - ALLION \\
\hline 1727 & & MIGLIO/MIGLIA & MARY & $\mathrm{D}$ & 6 & ITALY & ITALY & ITALY & $07 ? ?$ & & SAN GIORGIO CANAVESE. TO & CEDAR ST. & CALUMET - C. \& H. LOC. - ALLION \\
\hline 1728 & 570 & RICCA & BATTISTA & $\mathrm{H}$ & 26 & ITALY & ITALY & ITALY & 1905 & MINER/CM & SAN MARTINO CANAVESE, TO & CEDAR ST. & CALUMET - C. \& H. LOC. - ALLION \\
\hline 1729 & & RICCA & VIRGINIA & W & 26 & ITALY & ITALY & ITALY & 1903 & & & CEDAR ST. & CALUMET - C. \& H. LOC. - ALLION \\
\hline 1730 & & SUINO & GEORGE & SS & 4 & MICHIGAN & ITALY & ITALY & & & TORINO & CEDAR ST. & CALUMET - C. \& H. LOC. - ALLION \\
\hline 1731 & 587 & VIGO & MARCO & $\mathrm{H}$ & 39 & ITALY & ITALY & ITALY & 1897 & LABORER/SHAFT HOUSE & CORIO. TO & CEDAR ST. & CALUMET - C. \& H. LOC. - ALLION \\
\hline 1732 & on & VIGO & KATE & W & 37 & ITALY & ITALY & ITALY & 1900 & 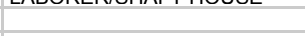 & שO & CEDAR ST. & CALUMET - C. \& H. LOC. - ALLION \\
\hline 1733 & & VIGO & GEORGE & s & 7 & MICHIGAN & ITALY & ITALY & 1500 & & CORIO. TO & CEDAR ST. & CALUMET - C. \& H. LOC. - ALLION \\
\hline 1734 & & VIGO & MARINA & $\mathrm{D}$ & 8 & MICHIGAN & ITALY & ITALY & & & CORIO, TO & CEDAR ST. & CALUMET - C. \& H. LOC. - ALLION \\
\hline 1735 & & SANDRETTO & JOE & $\mathrm{H}$ & 25 & ITALY & ITALY & ITALY & 1908 & TRAMMER/CM & PONT CANAVESE. TO & CEDAR ST. & CALUMET - C. \& H. LOC. - ALLION \\
\hline 1736 & & SANDRETTO & MARY & w & 21 & ITALY & ITALY & ITALY & 1909 & & & CEDAR ST. & CALUMET - C. \& H. LOC. - ALLION \\
\hline $1737:$ & 575 & PASQUINELLI & LEON & $\mathrm{H}$ & 61 & ITALY & ITALY & ITALY & 1876 & LABORER/CM & LUCCA & CEDAR ST. & CALUMET - C. \& H. LOC. - ALLION \\
\hline 1738 & & PASQUINELLI & PIA & W & 53 & ITALY & ITALY & ITALY & 1878 & & LUCCA & CEDAR ST. & CALUMET - C. \& H. LOC. - ALLION \\
\hline 1739 & & PASQUINELLI & CARLO & $\mathrm{s}$ & 25 & MICHIGAN & ITALY & ITALY & & SALOONKEEPER & LUCCA & CEDAR ST. & CALUMET - C. \& H. LOC. - ALLION \\
\hline 1740 & & PASQUINELLI & JANE & $\mathrm{D}$ & 23 & MICHIGAN & ITALY & ITALY & & SALESLADY/BOOKSTORE & LUCCA & CEDAR ST. & CALUMET - C. \& H. LOC. - ALLION \\
\hline 1741 & & PASQUINELLI & JOE & $\mathrm{s}$ & 19 & MICHIGAN & ITALY & ITALY & & LABORER/CM & LUCCA & CEDAR ST. & CALUMET - C. \& H. LOC. - ALLION \\
\hline 1742 & & PASQUINELLI & ANGELO & $\mathrm{s}$ & 18 & MICHIGAN & ITALY & ITALY & & WAITER/RESTAURANT & LUCCA & CEDAR ST. & CALUMET - C. \& H. LOC. - ALLION \\
\hline 1743 & & PASQUINELLI & MARTINA & $\mathrm{D}$ & 13 & MICHIGAN & ITALY & ITALY & & & LUCCA & CEDAR ST. & CALUMET - C. \& H. LOC. - ALLION \\
\hline 1744 & 521 & MICHETTI & & $\mathrm{H}$ & 48 & ITALY & ITALY & ITALY & 1880 & MINER/CM & PONT CANAVESE. TO & 4TH ST. & CALUMET - C. \& H. LOC. - ALLION \\
\hline 1745 & & MICHETTI & JOHANNA & W & 44 & ITALY & ITALY & ITALY & 1891 & 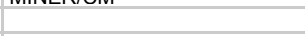 & PONT CANAVESE. TO & 4TH ST. & CALUMET - C. \& H. LOC. - ALLION \\
\hline 1746 & & MICHETTI & LUCILLA & $\mathrm{D}$ & 19 & ITALY & ITALY & ITALY & 1891 & WAITER/RESTAURANT & PONT CANAVESE. TO & 4TH ST. & CALUMET - C. \& H. LOC. - ALLION \\
\hline 1747 & & MICHETTI & ANNE & $\mathrm{D}$ & 10 & ITALY & ITALY & ITAL & 1891 & & PONT CANAVESE. TO & 4TH ST. & CALUMET - C. \& H. LOC. - ALLION \\
\hline 1748 & & MICHETTI & KATE & D & 6 & MICHIGAN & ITALY & ITAL & & & PONT CANAVESE. TO & 4TH ST. & CALUMET - C. \& H. LOC. - ALLION \\
\hline 1749 & & MICHETTI & MADDALENA & $\mathrm{D}$ & 3 & MICHIGAN & ITALY & ITA & & & PONT CANAVESE. TO & ST. & CALUMET - C. \& H. LOC. - ALLION \\
\hline 1750 & 521 & FABRI & ERNEST & $\mathrm{H}$ & 25 & ITALY & ITALY & ITALY & 1904 & LABORER/CM & & 4TH ST. & CALUMET - C. \& H. LOC. - ALLION \\
\hline 1751 & & FABRI & PIA & W & 21 & ITALY & ITAL & ITAI & 1906 & & & 4TH ST. & CALUMET - C. \& H. LOC. - ALLION \\
\hline 1752 & & FABRI & LOUIS & $\mathrm{s}$ & 0 & MICHIGAN & ITALY & ITALY & & & & 4TH ST. & CALUMET - C. \& H. LOC. - ALLION \\
\hline 1753 & 524 & BARATONO & DOMENICO & $\mathrm{H}$ & 31 & ITALY & ITALY & ITALY & 1902 & TIMBERMAN/CM & VIALFRE'. TO & 4TH ST. & CALUMET - C. \& H. LOC. - ALLION \\
\hline 1754 & & BARATONO & ROSE & W & 27 & ITALY & ITALY & ITALY & 1908 & & & 4TH ST. & CALUMET - C. \& H. LOC. - ALLION \\
\hline 1755 & & BARATONO & JAMES & s & 2 & MICHIGAN & ITALY & ITALY & & & VIALFRE'. TO & 4TH ST. & CALUMET - C. \& H. LOC. - ALLION \\
\hline 1756 & 533 & ZAGARI & MARY & $\mathrm{H}$ & 36 & ITALY & ITALY & ITALY & ???? & LAUNDRESS/WORK OUT & & 3RD ST. & CALUMET - C. \& H. LOC. - ALLION \\
\hline 1757 & & ZAGARI & MATTEO & s & 9 & MICHIGAN & CROATIA & ITALY & & & & 3RD ST. & CALUMET - C. \& H. LOC. - ALLION \\
\hline 1758 & & ZAGARI & VENICE & s & 8 & MICHIGAN & CROATIA & ITALY & & & & 3RD ST. & CALUMET - C. \& H. LOC. - ALLION \\
\hline 1759 & 107 & MICHELA & MARIA & SER & 18 & MICHIGAN & ITALY & ITALY & & SERVANT/PF & & CALUMET AVE. & CALUMET - C. \& H. LOCATION \\
\hline 1760 & 1302 & MICHETTI & LUCY & $\mathrm{H}$ & 46 & ITALY & ITALY & ITAL & 1884 & & & ROCKLAND ST. & CALUMET - C. \& H. LOCATION \\
\hline 1761 & 1302 & & & $\mathrm{~s}$ & 25 & IGAN & & & & LABORER/CM & & $\mathrm{RO}$ & CATION \\
\hline 1762 & 1302 & MICHETTI & & $\mathrm{D}$ & 20 & & ITALY & ITALY & & TYPESETTER/PRINTING CO & PONT CANAVESE. TO & ROCKLAND ST. & CALUMET - C. 8 \\
\hline 1763 & 1302 & MICHETTI & SILVIA & $\mathrm{D}$ & 16 & MICHIGAN & ITALY & ITALY & & & PONT CANAVESE. TO & ROCKLAND ST. & CALUMET - C. \& H. LOCATION \\
\hline
\end{tabular}




\begin{tabular}{|c|c|c|c|c|c|c|c|c|c|c|c|c|c|}
\hline 1764 & 1302 & MICHETTI & JOSEPHINE & $\mathrm{D}$ & 15 & MICHIGAN & ITALY & ITALY & & & PONT CANAVESE. TO & ROCKLAND ST. & CALUMET - C. \& H. LOCATION \\
\hline 1765 & 1302 & MICHETTI & LENA & $\mathrm{D}$ & 11 & MICHIGAN & ITALY & ITALY & & & PONT CANAVESE. TO & ROCKLAND ST. & CALUMET - C. \& H. LOCATION \\
\hline 1766 & 1312 & GALLINATTI & MAURIZIO & $\mathrm{H}$ & 40 & ITALY & ITALY & ITALY & 1890 & MINER/CM & TORINO & ROCKLAND ST. & CALUMET - C. \& H. LOCATION \\
\hline 1767 & 1312 & GALLINATTI & LUIGIA & W & 36 & ITALY & ITALY & ITALY & 1891 & & & ROCKLAND ST. & CALUMET - C. \& H. LOCATION \\
\hline 1768 & 1312 & GALLINATTI & TONY & s & 6 & MICHIGAN & ITALY & ITALY & & & TORINO & ROCKLAND ST. & CALUMET - C. \& H. LOCATION \\
\hline 1769 & 1312 & GALLINATTI & ADELINA & $\mathrm{D}$ & 2 & MICHIGAN & ITALY & ITALY & & & TORINO & ROCKLAND ST. & CALUMET - C. \& H. LOCATION \\
\hline 1770 & 1312 & GALLINATTI & FRANK & s & 3 & MICHIGAN & ITALY & ITALY & & & TORINO & ROCKLAND ST. & CALUMET - C. \& H. LOCATION \\
\hline 1771 & 1312 & GALLINATTI & IDA & $\mathrm{D}$ & $17 ?$ & MICHIGAN & ITALY & ITALY & & & TORINO & ROCKLAND ST. & CALUMET - C. \& H. LOCATION \\
\hline 1772 & 1312 & GALLINATTI & MARIA & $\mathrm{D}$ & 0 & MICHIGAN & ITALY & ITALY & & & TORINO & ROCKLAND ST. & CALUMET - C. \& H. LOCATION \\
\hline 1773 & 1303 & CRUGNOLA & CARLO & $\mathrm{H}$ & 42 & ITALY & ITALY & ITALY & 1893 & MASON/CM & COMO & ROCKLAND ST. & CALUMET - C. \& H. LOCATION \\
\hline 1774 & 1303 & CRUGNOLA & MODESTA & w & 47 & ITALY & ITALY & ITALY & 1893 & & COMO & ROCKLAND ST. & CALUMET - C. \& H. LOCATION \\
\hline 1775 & 1303 & CRUGNOLA & ERMINIA & $\mathrm{D}$ & 14 & MICHIGAN & ITALY & ITALY & & & COMO & ROCKLAND ST. & CALUMET - C. \& H. LOCATION \\
\hline 1776 & 1303 & CRUGNOLA & GAETANO & $s$ & 15 & ITALY & ITALY & ITALY & 1893 & & COMO & ROCKLAND ST. & CALUMET - C. \& H. LOCATION \\
\hline 1777 & 1303 & CRUGNOLA & MARY & $\mathrm{D}$ & 12 & MICHIGAN & ITALY & ITALY & & & COMO & ROCKLAND ST. & CALUMET - C. \& H. LOCATION \\
\hline 1778 & 1303 & CRUGNOLA & LIDIA & $\mathrm{D}$ & 9 & MICHIGAN & ITALY & ITALY & & & COMO & ROCKLAND ST. & CALUMET - C. \& H. LOCATION \\
\hline 1779 & 1306 & PIGNOCCO & CARLO & $\mathrm{H}$ & 39 & ITALY & ITALY & ITALY & 1884 & ODD JOBS/CM & SAN GIORGIO CANAVESE, TO & ROCKLAND ST. & CALUMET - C. \& H. LOCATION \\
\hline 1780 & 1306 & PIGNOCCO & LINA & W & 34 & ITALY & ITALY & ITALY & 1889 & & & ROCKLAND ST. & CALUMET - C. \& H. LOCATION \\
\hline 1781 & 1306 & PIGNOCCO & JOHN & s & 20 & MICHIGAN & ITALY & ITALY & & FIREMAN/ROUNDHOUSE & SAN GIORGIO CANAVESE. TO & ROCKLAND ST. & CALUMET - C. \& H. LOCATION \\
\hline 1782 & 1306 & PIGNOCCO & DOMINICK & $\mathrm{s}$ & 18 & MICHIGAN & ITALY & ITALY & & WAITER/CLUB HOUSE & SAN GIORGIO CANAVESE. TO & ROCKLAND ST. & CALUMET - C. \& H. LOCATION \\
\hline 1783 & 1306 & PIGNOCCO & GEORGE & $\mathrm{s}$ & 16 & MICHIGAN & ITALY & ITALY & & NOT CLEAR & SAN GIORGIO CANAVESE. TO & ROCKLAND ST. & CALUMET - C. \& H. LOCATION \\
\hline 1784 & 1306 & PIGNOCCO & JULIA & $\mathrm{D}$ & 14 & MICHIGAN & ITALY & ITALY & & & SAN GIORGIO CANAVESE. TO & ROCKLAND ST. & CALUMET - C. \& H. LOCATION \\
\hline 1785 & 1306 & ROSSO & MARY & $\mathrm{H}$ & 33 & ITALY & ITALY & ITALY & 1892 & & TORINO & ROCKLAND ST. & CALUMET - C. \& H. LOCATION \\
\hline 1786 & 1306 & ROSSO & MARY S. & $\mathrm{D}$ & 9 & MICHIGAN & ITALY & ITALY & & & FOGLIAZZO CANAVESE. TO & ROCKLAND ST. & CALUMET - C. \& H. LOCATION \\
\hline 1787 & 1306 & ROSSO & MAUD & $\mathrm{D}$ & 8 & MICHIGAN & ITALY & ITALY & & & FOGLIAZZO CANAVESE. TO & ROCKLAND ST. & CALUMET - C. \& H. LOCATION \\
\hline 1788 & 1306 & ROSSO & JOHN & $\mathrm{s}$ & 6 & MICHIGAN & ITALY & ITALY & & & FOGLIAZZO CANAVESE. TO & ROCKLAND ST. & CALUMET - C. \& H. LOCATION \\
\hline 1789 & 1306 & ROSSO & MINNIE & $\mathrm{D}$ & 2 & MICHIGAN & ITALY & ITALY & & & FOGLIAZZO CANAVESE. TO & ROCKLAND ST. & CALUMET - C. \& H. LOCATION \\
\hline 1790 & 1306 & ROSSO & LUISA & $\mathrm{D}$ & 0 & MICHIGAN & ITALY & ITALY & & & FOGLIAZZO CANAVESE. TO & ROCKLAND ST. & CALUMET - C. \& H. LOCATION \\
\hline 1791 & 1314 & BRUNO & GIOVANNI & $\mathrm{H}$ & 35 & ITALY & ITALY & ITALY & 1903 & MINER/CM & SALASSA, TO & ROCKLAND ST. & CALUMET - C. \& H. LOCATION \\
\hline 1792 & 1314 & BRUNO & MARGHERITA & W & 29 & ITALY & ITALY & ITALY & 1906 & & SALASSA. TO & ROCKLAND ST. & CALUMET - C. \& H. LOCATION \\
\hline 1793 & 1314 & BRUNO & LENA & $\mathrm{D}$ & 10 & ITALY & ITALY & ITALY & 1906 & & SALASSA. TO & ROCKLAND ST. & CALUMET - C. \& H. LOCATION \\
\hline 1794 & 1314 & BRUNO & ANGELO & $\mathrm{S}$ & 7 & ITALY & ITALY & ITALY & 1906 & & SALASSA. TO & ROCKLAND ST. & CALUMET - C. \& H. LOCATION \\
\hline 1795 & 1314 & BRUNO & V? & $\mathrm{s}$ & $5 \mathrm{M}$ & MICHIGAN & ITALY & ITALY & & & SALASSA. TO & ROCKLAND ST. & CALUMET - C. \& H. LOCATION \\
\hline 1796 & 1310 & ZANOTTI & ANTONIO & $\mathrm{H}$ & 27 & ITALY & ITALY & $\begin{array}{l}\text { ITALY } \\
\text { ITAL }\end{array}$ & 1903 & MINER/CM & PEROSA CANAVESE. TO & ROCKLAND ST. & CALUMET - C. \& H. LOCATION \\
\hline 1797 & 1310 & ZANOTTI & CELESTE & W & 22 & ITALY & ITALY & ITALY & 1903 & & SAN MARTINO CANAVESE, TO & ROCKLAND ST. & CALUMET - C. \& H. LOCATION \\
\hline 1798 & 1310 & ZANOTTI & JOE & s & 6 & MICHIGAN & ITALY & ITALY & & & PEROSA CANAVESE. TO & ROCKLAND ST. & CALUMET - C. \& H. LOCATION \\
\hline 1799 & 1310 & ZANOTTI & MICHAEL & B & 30 & ITALY & ITALY & ITALY & 1902 & MINER/CM & RIVAROLO CANAVESE. TO & ROCKLAND ST. & CALUMET - C. \& H. LOCATION \\
\hline 1800 & 1310 & ZANOTTI & JOE & B & 20 & ITALY & ITALY & ITALY & 1901 & LANDER/CM & RIVAROLO CANAVESE. TO & ROCKLAND ST. & CALUMET - C. \& H. LOCATION \\
\hline 1801 & 1310 & ANDRINA & PIETRO & BRIL & 28 & ITALY & ITALY & ITALY & 1901 & MINER/CM & SAN MARTINO CANAVESE. TO & ROCKLAND ST. & CALUMET - C. \& H. LOCATION \\
\hline 1802 & 1310 & ANDRINA & JOHN & BRIL & 25 & ITALY & ITALY & ITALY & 1905 & TRAMMER/CM & SAN MARTINO CANAVESE. TO & ROCKLAND ST. & CALUMET - C. \& H. LOCATION \\
\hline 1803 & 1310 & BOGGIO & JOHN & B & 40 & ITALY & ITALY & ITALY & 1890 & TIMBER BOSS/CM & TORINO & ROCKLAND ST. & CALUMET - C. \& H. LOCATION \\
\hline 1804 & 1310 & STALINI & ANTONIO & B & 31 & ITALY & ITALY & ITALY & 1900 & TIMBER BOSS/CM & & ROCKLAND ST. & CALUMET - C. \& H. LOCATION \\
\hline 1805 & 1310 & ANDRINA & JOHN & $\mathrm{COU}$ & 20 & ITALY & ITALY & ITALY & 1901 & TRAMMER/CM & SAN MARTINO CANAVESE. TO & ROCKLAND ST. & CALUMET - C. \& H. LOCATION \\
\hline 1806 & 1310 & FOGHINO & MARTIN & B & 27 & ITALY & ITALY & ITALY & 1900 & TRAMMER/CM & SAN MARTINO CANAVESE. TO & ROCKLAND ST. & CALUMET - C. \& H. LOCATION \\
\hline 1807 & 1310 & FUGATTI & UMBERTO & B & 21 & ITALY & ITALY & ITALY & 1905 & TRAMMER/CM & & ROCKLAND ST. & CALUMET - C. \& H. LOCATION \\
\hline 1808 & 326 & PELLEGRINI & LORENZO & $\mathrm{H}$ & 46 & ITALY & ITALY & ITALY & 1885 & MASON/CM & LUCCA & CALEDONIA ST. & CALUMET - C. \& H. LOCATION \\
\hline 1809 & 326 & PELLEGRINI & FANNY & W & 40 & ITALY & ITALY & ITALY & 1897 & & & CALEDONIA ST. & CALUMET - C. \& H. LOCATION \\
\hline 1810 & 326 & PELLEGRINI & S? & s & 12 & MICHIGAN & ITALY & ITALY & & & LUCCA & CALEDONIA ST. & CALUMET - C. \& H. LOCATION \\
\hline 1811 & 326 & PELLEGRINI & ANNIE & & 9 & MICHIGAN & ITALY & ITALY & & & LUCCA & IIA ST. & CALUMET - C. \& H. LOCATION \\
\hline 1812 & 326 & PELLEGRINI & EMMA & $\mathrm{D}$ & 3 & MICHIGAN & ITALY & ITALY & & & LUCCA & NIA ST. & CALUMET - C. \& H. LOCATION \\
\hline 1813 & 326 & PELLEGRINI & RAIMOND & $\mathrm{s}$ & $19 / 4$ & MICHIGAN & ITALY & ITALY & & & LUCCA & DNIA ST. & CALUMET - C. \& H. LOCATION \\
\hline 1814 & 326 & PELLEGRINI & RALPH & $\mathrm{s}$ & 18 & MICHIGAN & ITALY & ITALY & & & LUCCA & DONIA ST. & CALUMET - C. \& H. LOCATION \\
\hline 1815 & 326 & PELLEGRINI & EDITH & $\mathrm{D}$ & 14 & MICHIGAN & ITALY & ITALY & & & LUCCA & CALEDONIA ST. & CALUMET - C. \& H. LOCATION \\
\hline 1816 & 326 & PELLEGRINI & MASSIMO & $\mathrm{s}$ & 22 & MICHIGAN & ITALY & ITALY & & TIMBERMAN/CM & LUCCA & CALEDONIA ST. & CALUMET - C. \& H. LOCATION \\
\hline 1817 & 319 & LANDINI & JOSEPH & $\mathrm{H}$ & 37 & ITALY & ITALY & ITALY & 1898 & FIREMAN/CM & COLLE DI COMPITO. LU & CALEDONIA ST. & CALUMET - C. \& H. LOCATION \\
\hline 1818 & 319 & LANDINI & AMELIA & w & 36 & MICHIGAN & CANADA & CANADA & & & CANADA & CALEDONIA ST. & CALUMET - C. \& H. LOCATION \\
\hline 1819 & 319 & LANDINI & R. & $\mathrm{D}$ & 12 & MICHIGAN & ITALY & MICHIGAN & $?$ & & COLLE DI COMPITO. LU & CALEDONIA ST. & CALUMET - C. \& H. LOCATION \\
\hline 1820 & 319 & LANDINI & FELIX & $\mathrm{s}$ & 9 & MICHIGAN & ITALY & MICHIGAN & & & COLLE DI COMPITO. LU & CALEDONIA ST. & CALUMET - C. \& H. LOCATION \\
\hline 1821 & 319 & $\begin{array}{l}\text { LANDINI } \\
\text { LANI }\end{array}$ & ANNIE & $\mathrm{D}$ & 8 & MICHIGAN & ITALY & MICHIGAN & & & COLLE DI COMPITO, LU & CALEDONIA ST. & CALUMET - C. \& H. LOCATION \\
\hline 1822 & 319 & LANDINI & MARIE & $\mathrm{D}$ & 7 & MICHIGAN & ITALY & MICHIGAN & & & COLLE DI COMPITO. LU & CALEDONIA ST. & CALUMET - C. \& H. LOCATION \\
\hline 1823 & 319 & LANDINI & ANTHONY & S & 3 & MICHIGAN & ITALY & MICHIGAI & & & COLLE DI COMPITO. LU & CALEDONIA ST. & CALUMET - C. \& H. LOCATION \\
\hline 1824 & 319 & LANDI & IELIA & $\mathrm{D}$ & 2 & CHIGA & ITA & & & & MPITO. LU & & CALUMET \\
\hline 1825 & 319 & LANDINI & & $\mathrm{s}$ & $11 \mathrm{M}$ & MICHIGAN & ITALY & MICHIGAN & & & & CALEDONIA ST. & \\
\hline 1826 & 1507 & SILVA & GABRIELE & $\mathrm{H}$ & 29 & ITALY & ITALY & ITALY & 1905 & MINER/CM & PONT CANAVESE. TO & HECLA ST. & CALUMET - C. \& H. LOCATION \\
\hline
\end{tabular}




\begin{tabular}{|c|c|c|c|c|c|c|c|c|c|c|c|c|c|}
\hline 1827 & 1507 & SILVA & MARGHERITA & W & 23 & ITALY & ITALY & ITALY & 1893 & & & HECLA ST. & CALUMET - C. \& H. LOCATION \\
\hline 1828 & 1507 & SILVA & MARY & D & $7 \mathrm{M}$ & MICHIGAN & ITALY & ITALY & & & PONT CANAVESE. TO & HECLA ST. & CALUMET - C. \& H. LOCATION \\
\hline 1829 & 1507 & REGIS & JOHN & $\mathrm{H}$ & 40 & ITALY & ITALY & ITALY & 1891 & MINER/CM & SAN GIUSTO CANAVESE. TO & HECLA ST. & CALUMET - C. \& H. LOCATION \\
\hline 1830 & 1507 & REGIS & ELLEN & W & 33 & ITALY & ITALY & ITALY & 1899 & & & HECLA ST. & CALUMET - C. \& H. LOCATION \\
\hline 1831 & 1507 & REGIS & JOSEPHINE & $\mathrm{D}$ & 9 & MICHIGAN & ITALY & ITALY & & & SAN GIUSTO CANAVESE. TO & HECLA ST. & CALUMET - C. \& H. LOCATION \\
\hline 1832 & 1507 & REGIS & MINNIE & $\mathrm{s}$ & 8 & MICHIGAN & ITALY & ITALY & & & SAN GIUSTO CANAVESE. TO & HECLA ST. & CALUMET - C. \& H. LOCATION \\
\hline 1833 & 1507 & REGIS & GEORGE & $\mathrm{D}$ & 7 & MICHIGAN & ITALY & ITALY & & & SAN GIUSTO CANAVESE. TO & HECLA ST. & CALUMET - C. \& H. LOCATION \\
\hline 1834 & 1509 & FERRARI & JOSEPH & $\mathrm{H}$ & 40 & ITALY & ITALY & ITALY & 1898 & NOT CLEAR & LUCCA & HECLA ST. & CALUMET - C. \& H. LOCATION \\
\hline 1835 & 1509 & FERRARI & MARY & W & 39 & ITALY & ITALY & ITALY & 1896 & INUT CLEAR & LUC $A$ & HECLA ST. & CALUMET - C. \& H. LOCATION \\
\hline 1836 & 1509 & FERRARI & DELIA & $\mathrm{D}$ & 13 & MICHIGAN & ITALY & ITALY & & & LUCCA & HECLA ST. & CALUMET - C. \& H. LOCATION \\
\hline 1837 & 1509 & FERRARI & MIDA & $\mathrm{D}$ & 12 & MICHIGAN & ITALY & ITALY & & & LUCCA & HECLA ST. & CALUMET - C. \& H. LOCATION \\
\hline 1838 & 1509 & FERRARI & LENA & $\mathrm{D}$ & 10 & MICHIGAN & ITALY & ITALY & & & LUCCA & HECLA ST. & CALUMET - C. \& H. LOCATION \\
\hline 1839 & 1509 & FERRARI & $\mathrm{JOHN}$ & $\mathrm{s}$ & 9 & MICHIGAN & ITALY & ITALY & & & LUCCA & HECLA ST. & CALUMET - C. \& H. LOCATION \\
\hline 1840 & 1509 & FERRARI & LEWIS & $\mathrm{s}$ & 7 & MICHIGAN & ITALY & ITALY & & & LUCCA & HECLA ST. & CALUMET - C. \& H. LOCATION \\
\hline 1841 & 1602 & IANO/GANO? & JAMES & $\mathrm{H}$ & 38 & ITALY & ITALY & ITALY & 1897 & LABORER/CM & & LAURIUM ST. & CALUMET - C. \& H. LOCATION \\
\hline 1842 & 1602 & IANO/GANO? & MAGGIE & W & 37 & ITALY & ITALY & ITALY & 1897 & & & LAURIUM ST. & CALUMET - C. \& H. LOCATION \\
\hline 1843 & 1602 & IANO/GANO? & FRANK & $\mathrm{s}$ & 12 & MICHIGAN & ITALY & ITALY & & & & LAURIUM ST. & CALUMET - C. \& H. LOCATION \\
\hline 1844 & 1602 & IANO/GANO? & FRANCIS & $\mathrm{D}$ & 10 & MICHIGAN & ITALY & ITALY & & & & LAURIUM ST. & CALUMET - C. \& H. LOCATION \\
\hline 1845 & 1602 & CERUTTI & GIORGIO & $\mathrm{H}$ & 38 & ITALY & ITALY & ITALY & $86 / 96$ & LABORER/CM & SAN GIORGIO CANAVESE. TO & LAURIUM ST. & CALUMET - C. \& H. LOCATION \\
\hline 1846 & 1602 & CERUTTI & MADDALENA & W & 26 & ITALY & ITALY & ITALY & 1903 & LADO TERTUIVI & 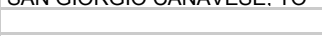 & LAURIUM ST. & CALUMET - C. \& H. LOCATION \\
\hline 1847 & 1602 & CERUTTI & FRANK & s & 6 & MICHIGAN & ITALY & ITALY & & & SAN GIORGIO CANAVESE. TO & LAURIUM ST. & CALUMET - C. \& H. LOCATION \\
\hline 1848 & 1602 & CERUTTI & PETER & $\mathrm{s}$ & 3 & MICHIGAN & ITALY & ITALY & & & SAN GIORGIO CANAVESE, TO & LAURIUM ST. & CALUMET - C. \& H. LOCATION \\
\hline 1849 & 1602 & CERUTTI & DOMINIC & $\mathrm{s}$ & 0 & MICHIGAN & ITALY & ITALY & & & SAN GIORGIO CANAVESE. TO & LAURIUM ST. & CALUMET - C. \& H. LOCATION \\
\hline 1850 & 1602 & GALLINATTI & JULIO & $\mathrm{H}$ & 34 & ITALY & ITALY & ITALY & 1900 & MINER/CM & TORINO & LAURIUM ST. & CALUMET - C. \& H. LOCATION \\
\hline 1851 & 1602 & GALLINATTI & DORA & W & 29 & ITALY & ITALY & ITALY & 1905 & & TORINO & LAURIUM ST. & CALUMET - C. \& H. LOCATION \\
\hline 1852 & 1602 & GALLINATTI & ESTER & $\mathrm{D}$ & 3 & MICHIGAN & ITALY & ITALY & & & TORINO & LAURIUM ST. & CALUMET - C. \& H. LOCATION \\
\hline 1853 & 1604 & GIULIO & ANTONIO & $\mathrm{H}$ & 49 & ITALY & ITALY & ITALY & 1882 & ENGINEER/CM & SAN GIORGIO CANAVESE. TO & LAURIUM ST. & CALUMET - C. \& H. LOCATION \\
\hline 1854 & 1604 & GIULIO & MARY & W & 41 & ITALY & ITALY & ITALY & 1882 & & SAN GIORGIO CANAVESE, TO & LAURIUM ST. & CALUMET - C. \& H. LOCATION \\
\hline 1855 & 1604 & GIULIO & JAMES & $\mathrm{s}$ & 18 & MICHIGAN & ITALY & ITALY & & BUTCHER/SHOP & SAN GIORGIO CANAVESE. TO & LAURIUM ST. & CALUMET - C. \& H. LOCATION \\
\hline 1856 & 1604 & GIULIO & DOMENICO & $\mathrm{S}$ & 16 & MICHIGAN & ITALY & ITALY & & DRILL BOY/CM & SAN GIORGIO CANAVESE. TO & LAURIUM ST. & CALUMET - C. \& H. LOCATION \\
\hline 1857 & 1604 & GIULIO & GIULIA & $\mathrm{D}$ & 12 & MICHIGAN & ITALY & ITALY & & & SAN GIORGIO CANAVESE. TO & LAURIUM ST. & CALUMET - C. \& H. LOCATION \\
\hline 1858 & 1604 & GIULIO & PHYLLIS & $\mathrm{D}$ & 6 & MICHIGAN & ITALY & ITALY & & & SAN GIORGIO CANAVESE. TO & LAURIUM ST. & CALUMET - C. \& H. LOCATION \\
\hline 1859 & 1604 & GIULIO & TONY & s & 3 & MICHIGAN & ITALY & ITALY & & & SAN GIORGIO CANAVESE. TO & LAURIUM ST. & CALUMET - C. \& H. LOCATION \\
\hline 1860 & 1614 & GALLO & JAMES & $\mathrm{H}$ & 53 & ITALY & ITALY & ITALY & 1883 & SPRINKLER/CM & SAN GIORGIO CANAVESE, TO & LAURIUM ST. & CALUMET - C. \& H. LOCATION \\
\hline 1861 & 1614 & GALLO & TERESA & w & 45 & ITALY & ITALY & ITALY & 1891 & & & LAURIUM ST. & CALUMET - C. \& H. LOCATION \\
\hline 1862 & 1614 & GALLO & JOHN & s & 15 & MICHIGAN & ITALY & ITALY & & & SAN GIORGIO CANAVESE. TO & LAURIUM ST. & CALUMET - C. \& H. LOCATION \\
\hline 1863 & 1614 & GALLO & JAMES & $\mathrm{s}$ & 16 & MICHIGAN & ITALY & ITALY & & & SAN GIORGIO CANAVESE. TO & LAURIUM ST. & CALUMET - C. \& H. LOCATION \\
\hline 1864 & 1614 & GALLO & MARGARET & $\mathrm{D}$ & 12 & MICHIGAN & ITALY & ITALY & & & SAN GIORGIO CANAVESE. TO & LAURIUM ST. & CALUMET - C. \& H. LOCATION \\
\hline 1865 & 1614 & GALLO & MARY & $\mathrm{D}$ & 10 & MICHIGAN & ITALY & ITALY & & & SAN GIORGIO CANAVESE. TO & LAURIUM ST. & CALUMET - C. \& H. LOCATION \\
\hline 1866 & 1614 & GALLO & MADELINE & $\mathrm{D}$ & 20 & ITALY & ITALY & ITALY & 91/97 & & SAN GIORGIO CANAVESE. TO & LAURIUM ST. & CALUMET - C. \& H. LOCATION \\
\hline 1867 & 1616 & GRANDIS & PAUL & $\mathrm{H}$ & 42 & ITALY & ITALY & ITALY & $? ? ?$ & TIMBERMAN/CM & SAN GIORGIO CANAVESE. TO & LAURIUM ST. & CALUMET - C. \& H. LOCATION \\
\hline 1868 & 1616 & GRANDIS & TERESA & W & 26 & ITALY & ITALY & ITALY & 1892 & & TORINO & LAURIUM ST. & CALUMET - C. \& H. LOCATION \\
\hline 1869 & 1616 & GRANDIS & CARMINE? & s & 8 & MICHIGAN & ITALY & ITALY & & & SAN GIORGIO CANAVESE. TO & LAURIUM ST. & CALUMET - C. \& H. LOCATION \\
\hline 1870 & 1616 & ROFFINA & JAMES & FAIL & 62 & ITALY & ITALY & ITALY & 1890 & & SAN GIORGIO CANAVESE. TO & LAURIUM ST. & CALUMET - C. \& H. LOCATION \\
\hline 1871 & 1616 & ROFFINA & MINNIE & MOIL & 58 & ITALY & ITALY & ITALY & 1891 & & SAN GIORGIO CANAVESE. TO & LAURIUM ST. & CALUMET - C. \& H. LOCATION \\
\hline 1872 & 1616 & ROFFINA & FRANK & BIL & 28 & ITALY & ITALY & ITALY & 1892 & ODD JOBS/ROUNDHOUSE & SAN GIORGIO CANAVESE. TO & LAURIUM ST. & CALUMET - C. \& H. LOCATION \\
\hline 1873 & 1616 & GRANDIS & JOHN & $\mathrm{BR}$ & 38 & ITALY & ITALY & ITALY & 1900 & TIMBERMAN/CM & SAN GIORGIO CANAVESE. TO & LAURIUM ST. & CALUMET - C. \& H. LOCATION \\
\hline 1874 & 1616 & GRANDIS & CARMEN & NIE & 12 & ITALY & ITALY & ITALY & $?$ & & SAN GIORGIO CANAVESE. TO & LAURIUM ST. & CALUMET - C. \& H. LOCATION \\
\hline 1875 & 1618 & MARCHETTI & JOHN & $\mathrm{H}$ & 40 & ITALY & ITALY & ITALY & 1890 & MACHINIST/CM & & LAURIUM ST. & CALUMET - C. \& H. LOCATION \\
\hline 1876 & 1618 & MARCHETTI & AURELIA & W & 28 & ITALY & ITALY & ITALY & 1898 & & & LAURIUM ST. & CALUMET - C. \& H. LOCATION \\
\hline 1877 & 1618 & MARCHETTI & $\mathrm{JOHN}$ & $\mathrm{s}$ & 4 & MICHIGAN & ITALY & ITALY & & & & LAURIUM ST. & CALUMET - C. \& H. LOCATION \\
\hline 1878 & 1618 & MARTA & LORENZO & B & 20 & ITALY & ITALY & ITALY & 1908 & MINER/CM & AGLIE', TO & LAURIUM ST. & CALUMET - C. \& H. LOCATION \\
\hline 1879 & 1618 & MARTA-SCALA & MARIA & B & 20 & ITALY & ITALY & ITALY & 1909 & SERVANT/PF & & LAURIUM ST. & CALUMET - C. \& H. LOCATION \\
\hline 1880 & 1601 & FAUSONE & FRANK & $\mathrm{H}$ & 41 & ITALY & ITALY & ITALY & 1892 & MINER/CM & TORINO & LAURIUM ST. & CALUMET - C. \& H. LOCATION \\
\hline 1881 & 1601 & FAUSONE & JULIA & w & 41 & ITALY & ITALY & ITALY & 1893 & & & LAURIUM ST. & CALUMET - C. \& H. LOCATION \\
\hline 1882 & 1601 & FAUSONE & MINNIE & $\mathrm{D}$ & 16 & MICHIGAN & ITALY & ITALY & & & TORINO & LAURIUM ST. & CALUMET - C. \& H. LOCATION \\
\hline 1883 & 1601 & FAUSONE & FRANK & $\mathrm{s}$ & 14 & MICHIGAN & ITALY & ITALY & & & TORINO & LAURIUM ST. & CALUMET - C. \& H. LOCATION \\
\hline 1884 & 1601 & FAUSONE & KATIE & $\mathrm{D}$ & 5 & MICHIGAN & ITALY & ITALY & & & TORINO & LAURIUM ST. & CALUMET - C. \& H. LOCATION \\
\hline 1885 & 1603 & BARSICA & ANTONIO & $\mathrm{H}$ & 38 & ITALY & ITALY & ITALY & 1896 & TIMBERMAN/CM & SAN MARTINO CANAVESE. TO & LAURIUM ST. & CALUMET - C. \& H. LOCATION \\
\hline 1886 & 1603 & BARSICA & TERESA & W & 34 & ITALY & ITALY & ITALY & ???? & & PEROSA CANAVESE. TO & LAURIUM ST. & CALUMET - C. \& H. LOCATION \\
\hline 1887 & 1603 & BARSICA & ITALIA & $\mathrm{D}$ & 5 & MICHIGAN & ITA & ITAL & & & SAN MARTINO CANAVESE. TO & & CALUMET \\
\hline 1888 & 1603 & YOTTI & ANTONIO & $\mathrm{H}$ & 31 & ITALY & ITALY & ITALY & 1890 & TIMBERMAN/CM & & & CALUMET - C. \& H. LOCATION \\
\hline 1889 & 1603 & YOTTI & MARY & w & 25 & ITALY & ITALY & ITALY & 1904 & 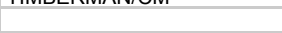 & & LAURIUM ST. & CALUMET - C. \& H. LOCATION \\
\hline
\end{tabular}




\begin{tabular}{|c|c|c|c|c|c|c|c|c|c|c|c|c|c|}
\hline 1890 & 1603 & YOTTI & MARY & $\mathrm{D}$ & 6 & MICHIGAN & ITALY & ITALY & & & & LAURIUM ST. & CALUMET - C. \& H. LOCATION \\
\hline 1891 & 1603 & YOTTI & ROSE & $\mathrm{D}$ & 3 & MICHIGAN & ITALY & ITALY & & & & LAURIUM ST. & CALUMET - C. \& H. LOCATION \\
\hline 1892 & 1603 & YOTTI & DOMENICO & $\mathrm{s}$ & 2 & MICHIGAN & ITALY & ITALY & & & & LAURIUM ST. & CALUMET - C. \& H. LOCATION \\
\hline 1893 & 1607 & MIGLIO/MIGLIA & ANTONIO & $\mathrm{H}$ & 46 & ITALY & ITALY & ITALY & 1891 & MINER/CM & TORINO & LAURIUM ST. & CALUMET - C. \& H. LOCATION \\
\hline 1894 & 1607 & MIGLIO/MIGLIA & KATE & w & 45 & ITALY & ITALY & ITALY & 1893 & & & LAURIUM ST. & CALUMET - C. \& H. LOCATION \\
\hline 1895 & 1607 & MIGLIO/MIGLIA & DOMENICO & s & 28 & MICHIGAN & ITALY & ITALY & & ODD JOBS/CM & TORINO & LAURIUM ST. & CALUMET - C. \& H. LOCATION \\
\hline 1896 & 1607 & MIGLIO/MIGLIA & LOUIS & $\mathrm{s}$ & 17 & MICHIGAN & ITALY & ITALY & & ODD JOBS/BARNS & TORINO & LAURIUM ST. & CALUMET - C. \& H. LOCATION \\
\hline 1897 & 1607 & MIGLIO/MIGLIA & JOE & s & 14 & MICHIGAN & ITALY & ITALY & & UDL NODOIDARTVO & TORINO & LAURIUM ST. & CALUMET - C. \& H. LOCATION \\
\hline 1898 & 1607 & ROFFINA & FRANK & $\mathrm{H}$ & 25 & ITALY & ITALY & ITALY & 94? & TIMBERMAN/CM & SAN GIORGIO CANAVESE. TO & $\begin{array}{l}\text { LAURIUM ST. } \\
\text { LAUR }\end{array}$ & CALUMET - C. \& H. LOCATION \\
\hline 1899 & 1607 & ROFFINA & ORSOLA & W & 31 & ITALY & ITALY & ITALY & 94? & & SAN GIORGIO CANAVESE. TO & LAURIUM ST. & CALUMET - C. \& H. LOCATION \\
\hline 1900 & 1607 & ROFFINA & NEDI & $\mathrm{D}$ & 6 & MICHIGAN & ITALY & ITALY & & & SAN GIORGIO CANAVESE. TO & LAURIUM ST. & CALUMET - C. \& H. LOCATION \\
\hline 1901 & 1607 & ROFFINA & JEANNIE & $\mathrm{D}$ & 4 & MICHIGAN & ITALY & ITALY & & & SAN GIORGIO CANAVESE. TO & LAURIUM ST. & CALUMET - C. \& H. LOCATION \\
\hline 1902 & 1615 & CAMPAGNOLA & JOHN & $\mathrm{H}$ & 55 & ITALY & ITALY & ITALY & 1886 & TIMBER BOSS/CM & SAN MARTINO CANAVESE. TO & LAURIUM ST. & CALUMET - C. \& H. LOCATION \\
\hline 1903 & 1615 & CAMPAGNOLA & FRANCIS & w & 51 & ITALY & ITALY & ITALY & $84 ?$ & & & LAURIUM ST. & CALUMET - C. \& H. LOCATION \\
\hline 1904 & 1615 & CAMPAGNOLA & JAMES & s & 26 & MICHIGAN & ITALY & ITALY & & MINER/CM & SAN MARTINO CANAVESE. TO & LAURIUM ST. & CALUMET - C. \& H. LOCATION \\
\hline 1905 & 1615 & CAMPAGNOLA & BEN & $\mathrm{S}$ & 20 & MICHIGAN & ITALY & ITALY & & MINER/CM & SAN MARTINO CANAVESE, TO & LAURIUM ST. & CALUMET - C. \& H. LOCATION \\
\hline 1906 & 1615 & CAMPAGNOLA & JOE & $\mathrm{s}$ & 16 & MICHIGAN & ITALY & ITALY & & & SAN MARTINO CANAVESE. TO & LAURIUM ST. & CALUMET - C. \& H. LOCATION \\
\hline 1907 & 1615 & CAMPAGNOLA & MARY & $\mathrm{D}$ & 18 & MICHIGAN & ITALY & ITALY & & & SAN MARTINO CANAVESE. TO & LAURIUM ST. & CALUMET - C. \& H. LOCATION \\
\hline 1908 & 1615 & CAMPAGNOLA & LENA & $\mathrm{D}$ & 13 & MICHIGAN & ITALY & ITALY & & & SAN MARTINO CANAVESE. TO & LAURIUM ST. & CALUMET - C. \& H. LOCATION \\
\hline 1909 & 1615 & CENTANINO & TINA & $\mathrm{D}$ & 28 & ITALY & ITALY & ITALY & $84 ?$ & & SAN MARTINO CANAVESE. TO & LAURIUM ST. & CALUMET - C. \& H. LOCATION \\
\hline 1910 & 1615 & ROVANO & DOMENICO & B & 30 & ITALY & ITALY & ITALY & 1902 & MINER/CM & SAN MARTINO CANAVESE. TO & LAURIUM ST. & CALUMET - C. \& H. LOCATION \\
\hline 1911 & 1615 & BRONZO & PETER & B & 28 & ITALY & ITALY & ITALY & 1905 & LABORER/ROCK HOUSE & SAN MARTINO CANAVESE, TO & LAURIUM ST. & CALUMET - C. \& H. LOCATION \\
\hline 1912 & 1615 & RICHETTA & JAMES & B & 26 & ITALY & ITALY & ITALY & 1908 & MINER/CM & SAN MARTINO CANAVESE. TO & LAURIUM ST. & CALUMET - C. \& H. LOCATION \\
\hline 1913 & 1615 & SAPINO & DOMENICO & B & 28 & ITALY & ITALY & ITALY & $00 / 06$ & MINER/CM & SAN MARTINO CANAVESE. TO & LAURIUM ST. & CALUMET - C. \& H. LOCATION \\
\hline 1914 & 1615 & SAPINO & PIETRO & B & 26 & ITALY & ITALY & ITALY & 1906 & MINER/CM & SAN MARTINO CANAVESE. TO & LAURIUM ST. & CALUMET - C. \& H. LOCATION \\
\hline 1915 & 1615 & ALDO & GIACINTO & B & 30 & ITALY & ITALY & ITALY & 1903 & MINER/CM & SAN MARTINO CANAVESE. TO & LAURIUM ST. & CALUMET - C. \& H. LOCATION \\
\hline 1916 & 1615 & GERA & ANTONIO & B & 27 & ITALY & ITALY & ITALY & 1906 & MINER/CM & SAN MARTINO CANAVESE. TO & LAURIUM ST. & CALUMET - C. \& H. LOCATION \\
\hline 1917 & 1617 & CANTELLO & JOE & $\mathrm{H}$ & 30 & ITALY & ITALY & ITALY & 1900 & MINER/CM & SAN GIORGIO CANAVESE, TO & LAURIUM ST. & CALUMET - C. \& H. LOCATION \\
\hline 1918 & 1617 & CANTELLO & MARTINA & W & 29 & ITALY & ITALY & ITALY & $190 ? ?$ & & & LAURIUM ST. & CALUMET - C. \& H. LOCATION \\
\hline 1919 & 1617 & CANTELLO & TONY & s & 8 & MICHIGAN & ITALY & ITALY & & & SAN GIORGIO CANAVESE. TO & LAURIUM ST. & CALUMET - C. \& H. LOCATION \\
\hline 1920 & 1617 & CANTELLO & CRISTINA & $\mathrm{D}$ & 5 & MICHIGAN & ITALY & ITALY & & & SAN GIORGIO CANAVESE. TO & LAURIUM ST. & CALUMET - C. \& H. LOCATION \\
\hline 1921 & 1617 & MARTA & JOHN & $\mathrm{H}$ & 26 & ITALY & ITALY & ITALY & 1906 & MINER/CM & AGLIE'. TO & LAURIUM ST. & CALUMET - C. \& H. LOCATION \\
\hline 1922 & 1617 & MARTA & KATE & W & 27 & ITALY & ITALY & ITALY & $08 ?$ & IVIIIVER CIVI & AULIL. 10 & LAURIUM ST. & CALUMET - C. \& H. LOCATION \\
\hline 1923 & 1617 & MARTA & MARY & $\mathrm{D}$ & 1 & MICHIGAN & ITALY & ITALY & & & AGLIE', TO & LAURIUM ST. & CALUMET - C. \& H. LOCATION \\
\hline 1924 & 1621 & ZUCCA & JOE & $\mathrm{H}$ & 39 & ITALY & ITALY & ITALY & 1898 & TIMBERMAN/CM & TORINO & LAURIUM ST. & CALUMET - C. \& H. LOCATION \\
\hline 1925 & 1621 & ZUCCA & MARY & W & 25 & ITALY & ITALY & ITALY & 1903 & & & LAURIUM ST. & CALUMET - C. \& H. LOCATION \\
\hline 1926 & 1621 & ZUCCA & JOE & s & 3 & MICHIGAN & ITALY & ITALY & & & TORINO & LAURIUM ST. & CALUMET - C. \& H. LOCATION \\
\hline 1927 & 1621 & ZUCCA & JOHN & $\mathrm{s}$ & 2 & MICHIGAN & ITALY & ITALY & & & TORINO & LAURIUM ST. & CALUMET - C. \& H. LOCATION \\
\hline 1928 & 1621 & CASTIGLIANO & DOMENICO & $\mathrm{H}$ & 32 & ITALY & ITALY & ITALY & 1899 & ODD JOBS/CM & SALASSA. TO & LAURIUM ST. & CALUMET - C. \& H. LOCATION \\
\hline 1929 & 1621 & CASTIGLIANO & CATERINA & W & 35 & ITALY & ITALY & ITALY & 1901 & & SALASSA. TO & LAURIUM ST. & CALUMET - C. \& H. LOCATION \\
\hline 1930 & 1621 & CASTIGLIANO & PETER & $\mathrm{s}$ & 15 & ITALY & ITALY & ITALY & 1901 & & SALASSA. TO & LAURIUM ST. & CALUMET - C. \& H. LOCATION \\
\hline 1931 & 1621 & CASTIGLIANO & JENNIE & $\mathrm{D}$ & 11 & ITALY & ITALY & ITALY & 1901 & & SALASSA. TO & LAURIUM ST. & CALUMET - C. \& H. LOCATION \\
\hline 1932 & 1621 & CASTIGLIANO & LOUIS & $\mathrm{s}$ & $9 \mathrm{M}$ & MICHIGAN & ITALY & ITALY & & & SALASSA. TO & LAURIUM ST. & CALUMET - C. \& H. LOCATION \\
\hline 1933 & 1625 & DIGHERA & CAROLINA & $\mathrm{H}$ & 35 & ITALY & ITALY & ITALY & 1900 & & SAN GIORGIO CANAVESE. TO & LAURIUM ST. & CALUMET - C. \& H. LOCATION \\
\hline 1934 & 1625 & DIGHERA & TOM & s & 7 & MICHIGAN & ITALY & ITALY & & & SAN GIORGIO CANAVESE. TO & LAURIUM ST. & CALUMET - C. \& H. LOCATION \\
\hline 1935 & 1625 & DIGHERA & FRANK & $\mathrm{S}$ & 5 & MICHIGAN & ITALY & ITALY & & & SAN GIORGIO CANAVESE. TO & LAURIUM ST. & CALUMET - C. \& H. LOCATION \\
\hline 1936 & 1625 & PERONA & BATTISTA & $\mathrm{H}$ & 27 & ITALY & ITALY & ITALY & 1902 & MINER/CM & PONT CANAVESE. TO & LAURIUM ST. & CALUMET - C. \& H. LOCATION \\
\hline 1937 & 1625 & PERONA & MARY & W & 27 & ITALY & ITALY & ITALY & 1902 & & PONT CANAVESE. TO & LAURIUM ST. & CALUMET - C. \& H. LOCATION \\
\hline 1938 & 1625 & PERONA & VITTORIA & $\mathrm{D}$ & 5 & MICHIGAN & ITALY & ITALY & & & PONT CANAVESE. TO & LAURIUM ST. & \\
\hline 1939 & 1625 & PERONA & JOE & $\mathrm{s}$ & 2 & MICHIGAN & ITALY & ITALY & & & PONT CANAVESE. TO & LAURIUM ST. & CALUMET - C. \& H. LOCATION \\
\hline 1940 & 1625 & PERONA & INES & $\mathrm{D}$ & $11 \mathrm{M}$ & MICHIGAN & ITALY & ITALY & & & PONT CANAVESE. TO & LAURIUM ST. & CALUMET - C. \& H. LOCATION \\
\hline 1941 & 1631 & CAPELLO & JOHN & $\mathrm{H}$ & 47 & ITALY & ITALY & ITALY & 1890 & WATCHMAN/CM & SAN BENIGNO CANAVESE, TO & LAURIUM ST. & CALUMET - C. \& H. LOCATION \\
\hline 1942 & 1631 & CAPELLO & MARY & w & 50 & ITALY & ITALY & ITALY & 1904 & & SAN BENIGNO CANAVESE. TO & LAURIUM ST. & CALUMET - C. \& H. LOCATION \\
\hline 1943 & 1631 & NOTARIO & SIMONE & NEP & 24 & ITALY & ITALY & ITALY & 1904 & MINER/CM & SAN BENIGNO CANAVESE. TO & LAURIUM ST. & CALUMET - C. \& H. LOCATION \\
\hline 1944 & 1631 & NOTARIO & JOHN & NEP & 22 & ITALY & ITALY & ITALY & 1904 & ODD JOBS/CM & SAN BENIGNO CANAVESE. TO & LAURIUM ST. & CALUMET - C. \& H. LOCATION \\
\hline 1945 & 1631 & SCIACERO & MARTIN & $\mathrm{H}$ & 34 & ITALY & ITALY & ITALY & 1900 & TIMBERMAN/CM & SAN MARTINO CANAVESE. TO & LAURIUM ST. & CALUMET - C. \& H. LOCATION \\
\hline 1946 & 1631 & SCIACERO & KATY & W & 33 & ITALY & ITALY & ITALY & 1901 & & & LAURIUM ST. & CALUMET - C. \& H. LOCATION \\
\hline 1947 & 1631 & SCIACERO & ANNE & $\mathrm{D}$ & 5 & MICHIGAN & ITALY & ITALY & & & SAN MARTINO CANAVESE, TO & LAURIUM ST. & CALUMET - C. \& H. LOCATION \\
\hline 1948 & 1631 & SCIACERO & PETER & $\mathrm{S}$ & 3 & MICHIGAN & ITALY & ITALY & & & SAN MARTINO CANAVESE. TO & LAURIUM ST. & CALUMET - C. \& H. LOCATION \\
\hline 1949 & 1722 & FERRETTI & MARY & $\mathrm{H}$ & 37 & ITALY & ITALY & ITALY & 1895 & & & LAURIUM ST. & CALUMET - C. \& H. LOCATION \\
\hline 1950 & 1722 & FERRET & JOSEPH & $\mathrm{s}$ & 5 & MICHIGAN & & ITA & & & & & CALUMET - C. \& H. L \\
\hline 1951 & 1722 & SCIACERO & MASERINA & B & 24 & ITALY & ITALY & ITALY & ?? & & TORINO & & CALUMET - C. \& H. LOCATION \\
\hline 1952 & 1722 & OTAVIO & PETER & B & 29 & ITALY & ITALY & ITALY & ?? & & & LAURIUM ST. & CALUMET - C. \& H. LOCATION \\
\hline
\end{tabular}




\begin{tabular}{|c|c|c|c|c|c|c|c|c|c|c|c|c|c|}
\hline 1953 & 1546 & BARATONO & JOHN & $\mathrm{H}$ & 42 & ITALY & ITALY & ITALY & 1889 & TIMBERMAN/CM & VIALFRE'. TO & HECLA ST. & CALUMET - C. \& H. LOCATION \\
\hline 1954 & 1546 & BARATONO & MARY N. & W & 31 & ITALY & ITALY & ITALY & 1899 & & & HECLA ST. & CALUMET - C. \& H. LOCATION \\
\hline 1955 & 1546 & BARATONO & JAMES & $\mathrm{s}$ & 10 & MICHIGAN & ITALY & ITALY & & & VIALFRE'. TO & HECLA ST. & CALUMET - C. \& H. LOCATION \\
\hline 1956 & 1546 & BARATONO & DOMENICO & $\mathrm{s}$ & 7 & MICHIGAN & ITALY & ITALY & & & VIALFRE'. TO & HECLA ST. & CALUMET - C. \& H. LOCATION \\
\hline 1957 & 1546 & BARATONO & JOSIE & $\mathrm{s}$ & 6 & MICHIGAN & ITALY & ITALY & & & VIALFRE'. TO & HECLA ST. & CALUMET - C. \& H. LOCATION \\
\hline 1958 & 1546 & BARATONO & ARTURO & s & 4 & MICHIGAN & ITALY & ITALY & & & VIALFRE'. TO & HECLA ST. & CALUMET - C. \& H. LOCATION \\
\hline 1959 & 1544 & FETTA & JOHN & $\mathrm{H}$ & 52 & ITALY & ITALY & ITALY & 1887 & MINER/CM & BAIRO CANAVESE. TO & HECLA ST. & CALUMET - C. \& H. LOCATION \\
\hline 1960 & 1544 & FETTA & BARBARA & W & 36 & ITALY & ITALY & ITALY & 1896 & IVIIVERTCIVI & DAIRU UAIVAVEOE. TO & HECLA ST. & CALUMET - C. \& H. LOCATION \\
\hline 1961 & 1544 & FETTA & BENIAMINO & s & 12 & MICHIGAN & ITALY & ITALY & & & BAIRO CANAVESE. TO & HECLA ST. & CALUMET - C. \& H. LOCATION \\
\hline 1962 & 1544 & FETTA & LUCY & $\mathrm{D}$ & 11 & MICHIGAN & ITALY & ITALY & & & BAIRO CANAVESE. TO & HECLA ST. & CALUMET - C. \& H. LOCATION \\
\hline 1963 & 1544 & FETTA & MARY & $\mathrm{D}$ & 10 & MICHIGAN & ITALY & ITALY & & & BAIRO CANAVESE. TO & HECLA ST. & CALUMET - C. \& H. LOCATION \\
\hline 1964 & 1544 & FETTA & LOUIS & $\mathrm{s}$ & 6 & MICHIGAN & ITALY & ITALY & & & BAIRO CANAVESE. TO & HECLA ST. & CALUMET - C. \& H. LOCATION \\
\hline 1965 & 1544 & QUILICO & ANTON & B & 19 & ITALY & ITALY & ITALY & 1907 & TRAMMER/CM & TORINO & HECLA ST. & CALUMET - C. \& H. LOCATION \\
\hline 1966 & 1549 & SANDRETTO & MIKE & $\mathrm{H}$ & 43 & ITALY & ITALY & ITALY & 1890 & WATCHAMN/CM & PONT CANAVESE. TO & HECLA ST. & CALUMET - C. \& H. LOCATION \\
\hline 1967 & 1549 & SANDRETTO & MAGGIE & W & 37 & ITALY & ITALY & ITALY & 1897 & & & HECLA ST. & CALUMET - C. \& H. LOCATION \\
\hline 1968 & 1549 & SANDRETTO & JOHN & $\mathrm{s}$ & 11 & MICHIGAN & ITALY & ITALY & & & PONT CANAVESE, TO & HECLA ST. & CALUMET - C. \& H. LOCATION \\
\hline 1969 & 1549 & SANDRETTO & BARNEY & $\mathrm{s}$ & 9 & MICHIGAN & ITALY & ITALY & & & PONT CANAVESE. TO & HECLA ST. & CALUMET - C. \& H. LOCATION \\
\hline 1970 & 1549 & SANDRETTO & MARY & $\mathrm{D}$ & 8 & MICHIGAN & ITALY & ITALY & & & PONT CANAVESE. TO & HECLA ST. & CALUMET - C. \& H. LOCATION \\
\hline 1971 & 1549 & SANDRETTO & CHARLES & $\mathrm{s}$ & 5 & MICHIGAN & ITALY & ITALY & & & PONT CANAVESE. TO & HECLA ST. & CALUMET - C. \& H. LOCATION \\
\hline 1972 & 1537 & FERRARI & ALBERTO & $\mathrm{H}$ & 40 & ITALY & ITALY & ITALY & 1890 & WATCHMAN/CM & PARIANA. LU & HECLA ST. & CALUMET - C. \& H. LOCATION \\
\hline 1973 & 1537 & FERRARI & ERNESTA & w & 40 & MICHIGAN & ITALY & ITALY & & & & HECLA ST. & CALUMET - C. \& H. LOCATION \\
\hline 1974 & 1537 & FERRARI & CAMILLA & $\mathrm{D}$ & 15 & MICHIGAN & ITALY & MICHIGAN & & & PARIANA, LU & HECLA ST. & CALUMET - C. \& H. LOCATION \\
\hline 1975 & 1537 & FERRARI & MARY & $\mathrm{D}$ & 15 & MICHIGAN & ITALY & MICHIGAN & & & PARIANA. LU & HECLA ST. & CALUMET - C. \& H. LOCATION \\
\hline 1976 & 1537 & FERRARI & SANDRO & $\mathrm{S}$ & 14 & MICHIGAN & ITALY & MICHIGAN & & & PARIANA. LU & HECLA ST. & CALUMET - C. \& H. LOCATION \\
\hline 1977 & 1537 & FERRARI & JENNIE & $\mathrm{D}$ & 13 & MICHIGAN & ITALY & MICHIGAN & & & PARIANA. LU & HECLA ST. & CALUMET - C. \& H. LOCATION \\
\hline 1978 & 1535 & GIOVANNINI & ANTONIO & $\mathrm{H}$ & 43 & ITALY & ITALY & ITALY & 1890 & MINER/CM & SAN GIUSTO CANAVESE. TO & HECLA ST. & CALUMET - C. \& H. LOCATION \\
\hline 1979 & 1535 & GIOVANNINI & GIUSTA & W & 36 & ITALY & ITALY & ITALY & 1891 & & SAN GIUSTO CANAVESE. TO & HECLA ST. & CALUMET - C. \& H. LOCATION \\
\hline 1980 & 1535 & GIOVANNINI & TERESA & $\mathrm{D}$ & 20 & ITALY & ITALY & ITALY & 1891 & DRESSMAKER/TAILORING CO. & SAN GIUSTO CANAVESE, TO & HECLA ST. & CALUMET - C. \& H. LOCATION \\
\hline 1981 & 1535 & GIOVANNINI & ANTONIO & $\mathrm{s}$ & 18 & MICHIGAN & ITALY & ITALY & & MINER/CM & SAN GIUSTO CANAVESE. TO & HECLA ST. & CALUMET - C. \& H. LOCATION \\
\hline 1982 & 1535 & GIOVANNINI & VITTORIO & $\mathrm{S}$ & 16 & MICHIGAN & ITALY & ITALY & & & SAN GIUSTO CANAVESE. TO & HECLA ST. & CALUMET - C. \& H. LOCATION \\
\hline 1983 & 1535 & GIOVANNINI & MINNIE & $\mathrm{D}$ & 14 & MICHIGAN & ITALY & ITALY & & & SAN GIUSTO CANAVESE. TO & HECLA ST. & CALUMET - C. \& H. LOCATION \\
\hline 1984 & 1686 & RICCA & JOSEPH & $\mathrm{H}$ & 45 & ITALY & ITALY & ITALY & $1899 ?$ & MINER/CM & TORINO & LAURIUM ST. & CALUMET - C. \& H. LOCATION \\
\hline 1985 & 1686 & RICCA & MARGHERITA & W & 39 & ITALY & ITALY & $\begin{array}{l}\text { ITALY } \\
\text { ITAL }\end{array}$ & $1899 ?$ & IVIIVERTIUIUI & TORINO & LAURIUM ST. & CALUMET - C. \& H. LOCATION \\
\hline 1986 & 1686 & RICCA & MATTEO & s & 18 & MICHIGAN & ITALY & ITALY & & DRILL BOY/CM & TORINO & LAURIUM ST. & CALUMET - C. \& H. LOCATION \\
\hline 1987 & 1686 & RICCA & LUCY & $\mathrm{D}$ & 16 & MICHIGAN & ITALY & ITALY & & & TORINO & LAURIUM ST. & CALUMET - C. \& H. LOCATION \\
\hline 1988 & 1686 & RICCA & MARY & $\mathrm{D}$ & 12 & MICHIGAN & ITALY & ITALY & & & TORINO & LAURIUM ST. & CALUMET - C. \& H. LOCATION \\
\hline 1989 & 1686 & RICCA & FRANK & $\mathrm{s}$ & 9 & MICHIGAN & ITALY & ITALY & & & TORINO & LAURIUM ST. & CALUMET - C. \& H. LOCATION \\
\hline 1990 & 1686 & RICCA & VERONICA & $\mathrm{D}$ & 5 & MICHIGAN & ITALY & ITALY & & & TORINO & LAURIUM ST. & CALUMET - C. \& H. LOCATION \\
\hline 1991 & 1686 & RICCA & JOE & $\mathrm{s}$ & $5 \mathrm{M}$ & MICHIGAN & ITALY & ITALY & & & TORINO & LAURIUM ST. & CALUMET - C. \& H. LOCATION \\
\hline 1992 & 1762 & FRANCISCA & JOHN & $\mathrm{H}$ & 50 & ITALY & ITALY & ITALY & 1885 & MINER/CM & PEROSA CANAVESE. TO & BOUNDRY ST. & CALUMET - C. \& H. LOCATION \\
\hline 1993 & 1762 & FRANCISCA & ANGELA & W & 51 & ITALY & ITALY & ITALY & 1894 & & PEROSA CANAVESE. TO & BOUNDRY ST. & CALUMET - C. \& H. LOCATION \\
\hline 1994 & 1762 & FRANCISCA & ANGELO & $\mathrm{BR}$ & 21 & ITALY & ITALY & ITALY & 1894 & MINER/CM & PEROSA CANAVESE. TO & BOUNDRY ST. & CALUMET - C. \& H. LOCATION \\
\hline 1995 & 1762 & FRANCISCA & JOHN & $\mathrm{s}$ & 18 & ITALY & ITALY & ITALY & 1894 & MINER/CM & PEROSA CANAVESE. TO & BOUNDRY ST. & CALUMET - C. \& H. LOCATION \\
\hline 1996 & 1762 & FRANCISCA & FRANK & s & 14 & MICHIGAN & ITALY & ITALY & & DRILL BOY/CM & PEROSA CANAVESE. TO & BOUNDRY ST. & CALUMET - C. \& H. LOCATION \\
\hline 1997 & 3120 & TEPATTI & JOHN & $\mathrm{H}$ & 47 & ITALY & ITALY & ITALY & 1886 & MINER/CM & PONT CANAVESE. TO & TUNNEL ST. & CALUMET - SWEDE TOWN \\
\hline 1998 & 3120 & TEPATTI & AUGUSTA & W & 46 & ITALY & ITALY & ITALY & 1902 & & & TUNNEL ST. & CALUMET - SWEDE TOWN \\
\hline 1999 & 3120 & TEPATTI & MARTIN & s & 16 & MICHIGAN & ITALY & ITALY & & SALESMAN/WINE STORE & PONT CANAVESE. TO & TUNNEL ST. & CALUMET - SWEDE TOWN \\
\hline 2000 & 3120 & TEPATTI & BERNARD & $\mathrm{s}$ & 11 & MICHIGAN & ITALY & ITALY & & & PONT CANAVESE. TO & ST. & CALUMET - SWEDE TOWN \\
\hline 2001 & 3120 & TEPATTI & MARY & $\mathrm{D}$ & 4 & MICHIGAN & ITALY & ITALY & & & PONT CANAVESE. TO & TUNNEL ST. & CALUMET - SWEDE TOWN \\
\hline 2002 & 3232 & SOMMARIVA & AUGUST & $\mathrm{H}$ & 52 & ITALY & ITALY & ITALY & 1899 & TIMBERMAN/CM & BELLUNO & & CALUMET - SWEDE TOWN \\
\hline 2003 & 3232 & SOMMARIVA & MARY & W & 47 & ITALY & ITALY & ITALY & 1900 & & BELLUNO & RIDGE ST. & CALUMET - SWEDE TOWN \\
\hline 2004 & 3232 & BELLINGIER & UMBERTO & SSO & 19 & ITALY & ITALY & ITALY & 1904 & TRAMMER/CM & BELLUNO & RIDGE ST. & CALUMET - SWEDE TOWN \\
\hline 2005 & 3232 & SECOLA & $\mathrm{JOHN}$ & B & 19 & ITALY & ITALY & ITALY & 1907 & TRAMMER/CM & & TUNNEL ST. & CALUMET - SWEDE TOWN \\
\hline 2006 & 3232 & SASSO & DOMENICO & B & 25 & ITALY & ITALY & ITALY & 1909 & MINER/CM & RIVAMONTE. BELLUNO & TUNNEL ST. & CALUMET - SWEDE TOWN \\
\hline 2007 & 3103 & NOTARI & JOHN & $\mathrm{H}$ & 57 & ITALY & ITALY & ITALY & 1888 & WATCHMAN/CM & PITEGLIO. PISTOIA & RIDGE ST. & CALUMET - SWEDE TOWN \\
\hline 2008 & 3103 & NOTARI & CESIRA & w & 48 & ITALY & ITALY & ITALY & 1891 & & & RIDGE ST. & CALUMET - SWEDE TOWN \\
\hline 2009 & 3103 & NOTARI & PETER & s & 17 & MICHIGAN & ITALY & ITALY & & & PITEGLIO. PISTOIA & RIDGE ST. & CALUMET - SWEDE TOWN \\
\hline 2010 & 3103 & NOTARI & JAMES & $\mathrm{s}$ & 14 & MICHIGAN & ITALY & ITALY & & & PITEGLIO, PISTOIA & RIDGE ST. & CALUMET - SWEDE TOWN \\
\hline 2011 & 2012 & POGGIONE & DOMENICO & $\mathrm{H}$ & 31 & ITALY & ITALY & ITALY & 1893 & MINER/CM & SAN GIORGIO CANAVESE. TO & CALUMET AVE. & CALUMET - RAYMBAULTOWN \\
\hline 2012 & 2012 & POGGIONE & TERESA & W & 29 & ITALY & ITALY & ITALY & 1906 & & & CALUME & CALUMET - RAYMBAULTOWN \\
\hline 2013 & 2012 & POGGION & KATE & D & 3 & HIGAN & & ITA & & & SAN GIORGIO CANAVESE. TO & TAVE. & CALUMET - RA \\
\hline 2014 & 2012 & POGGIONE & & $\mathrm{s}$ & $6 \mathrm{M}$ & MICHIGAN & ITALY & & & & SAN GIORGIO CANAVESE. TO & UMET AVE. & CAL \\
\hline 2015 & 2012 & VAIRO & JOSEPH & $\mathrm{H}$ & 37 & ITALY & ITALY & ITALY & 1900 & MINER/CM & LOCANA CANAVESE. TO & CALUMET AVE. & CALUMET - RAYMBAULTOWN \\
\hline
\end{tabular}




\begin{tabular}{|c|c|c|c|c|c|c|c|c|c|c|c|c|c|}
\hline 2016 & 2012 & VAIRO & MARY & W & 29 & ITALY & ITALY & ITALY & 1909 & & & CALUMET AVE. & CALUMET - RAYMBAULTOWN \\
\hline 2017 & 2012 & VAIRO & FRANCIS & $s$ & 0 & MICHIGAN & ITALY & ITALY & & & LOCANA CANAVESE. TO & CALUMET AVE. & CALUMET - RAYMBAULTOWN \\
\hline 2018 & 2012 & VAIRO & MARY & D & 12 & MICHIGAN & ITALY & ITALY & & & LOCANA CANAVESE. TO & CALUMET AVE. & CALUMET - RAYMBAULTOWN \\
\hline 2019 & 2012 & VAIRO & JAMES & $\mathrm{s}$ & 10 & MICHIGAN & ITALY & ITALY & & & LOCANA CANAVESE. TO & CALUMET AVE. & CALUMET - RAYMBAULTOWN \\
\hline 2020 & 2012 & VAIRO & PETER & $\mathrm{s}$ & 7 & MICHIGAN & ITALY & ITALY & & & LOCANA CANAVESE. TO & CALUMET AVE. & CALUMET - RAYMBAULTOWN \\
\hline 2021 & 2012 & VAIRO & ANNE & $\mathrm{D}$ & 3 & MICHIGAN & ITALY & ITALY & & & LOCANA CANAVESE. TO & CALUMET AVE. & CALUMET - RAYMBAULTOWN \\
\hline 2022 & 2024 & PRICCO & GIOVANNI & $\mathrm{H}$ & 26 & ITALY & ITALY & ITALY & 1909 & BRAKESMAN/RAILROAD & SAN MARTINO CANAVESE. TO & CALUMET AVE. & CALUMET - RAYMBAULTOWN \\
\hline 2023 & 2024 & PRICCO & TERESA & W & 31 & ITALY & ITALY & ITALY & 1909 & DRAREOIVAIVIRAILRUAD & SAN MARTINO CANAVESE. TO & $\begin{array}{l}\text { CALUMET AVE. } \\
\text { CAUE }\end{array}$ & CALUMET - RAYMBAULTOWN \\
\hline 2024 & 2024 & PRICCO & ANDREA & s & $15 \mathrm{M}$ & MICHIGAN & ITALY & ITALY & & & SAN MARTINO CANAVESE. TO & CALUMET AVE. & CALUMET - RAYMBAULTOWN \\
\hline 2025 & 2024 & BOGGIO & GIORGIO & $\mathrm{H}$ & 26 & ITALY & ITALY & ITALY & 1903 & TEAMSTER/BREWERY & SAN GIORGIO CANAVESE. TO & CALUMET AVE. & CALUMET - RAYMBAULTOWN \\
\hline 2026 & 2024 & BOGGIO & ANITA & w & 26 & ITALY & ITALY & ITALY & 1905 & & & CALUMET AVE. & CALUMET - RAYMBAULTOWN \\
\hline 2027 & 2024 & BOGGIO & IRENE & $\mathrm{D}$ & 3 & MICHIGAN & ITALY & ITALY & & & SAN GIORGIO CANAVESE. TO & CALUMET AVE. & CALUMET - RAYMBAULTOWN \\
\hline 2028 & 2024 & BOGGIO & DOMENICO & $s$ & $20 \mathrm{M}$ & MICHIGAN & ITALY & ITALY & & & SAN GIORGIO CANAVESE. TO & CALUMET AVE. & CALUMET - RAYMBAULTOWN \\
\hline 2029 & 2024 & BARSICA & GIUSEPPE & $\mathrm{H}$ & 31 & ITALY & ITALY & ITALY & 1900 & MINER/CM & TORINO & CALUMET AVE. & CALUMET - RAYMBAULTOWN \\
\hline 2030 & 2024 & BARSICA & MARY & W & 28 & ITALY & ITALY & ITALY & 1906 & & & CALUMET AVE. & CALUMET - RAYMBAULTOWN \\
\hline 2031 & 2024 & BARSICA & JOHN & $\mathrm{s}$ & $12 \mathrm{M}$ & MICHIGAN & ITALY & ITALY & & & TORINO & CALUMET AVE. & CALUMET - RAYMBAULTOWN \\
\hline 2032 & 2024 & CALZO & SECONDO & $\mathrm{H}$ & 34 & ITALY & ITALY & ITALY & 1900 & TRAMMER/CM & & CALUMET AVE. & CALUMET - RAYMBAULTOWN \\
\hline 2033 & 2024 & CALZO & GIUSTINA & W & 24 & ITALY & ITALY & ITALY & 1909 & & & CALUMET AVE. & CALUMET - RAYMBAULTOWN \\
\hline 2034 & 2024 & RANIERI & MARY & SIIL & 21 & ITALY & ITALY & ITALY & 1909 & & & CALUMET AVE. & CALUMET - RAYMBAULTOWN \\
\hline 2035 & 2000 & TAPPERO & JOSEPH & $\mathrm{H}$ & 32 & ITALY & ITALY & ITALY & 1898 & MINER/CM & TORINO & CALUMET AVE. & CALUMET - RAYMBAULTOWN \\
\hline 2036 & 2000 & TAPPERO & AMBRY & W & 20 & MICHIGAN & ITALY & ITALY & 1050 & IVIIIVERTLIVI & 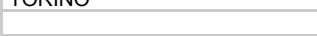 & $\begin{array}{l}\text { CALUMET AVE. } \\
\text { CAUE }\end{array}$ & CALUMET - RAYMBAULTOWN \\
\hline 2037 & 2000 & TAPPERO & TERESA & $\mathrm{D}$ & 2 & MICHIGAN & ITALY & ITALY & & & TORINO & CALUMET AVE. & CALUMET - RAYMBAULTOWN \\
\hline 2038 & 3005 & CORTESE & GIOVANNI & $\mathrm{H}$ & 46 & ITALY & ITALY & ITALY & 1886 & TIMBER BOSS/CM & SALASSA. TO & CALUMET AVE. & CALUMET - RAYMBAULTOWN \\
\hline 2039 & 3005 & CORTESE & ANGELA & w & 35 & ITALY & ITALY & ITALY & 1895 & & & CALUMET AVE. & $\begin{array}{l}\text { CALUMET - RAYMBAULTOWN } \\
\end{array}$ \\
\hline 2040 & 3005 & CORTESE & MAURIZIO & $\mathrm{s}$ & 13 & MICHIGAN & ITALY & ITALY & & & SALASSA. TO & CALUMET AVE. & CALUMET - RAYMBAULTOWN \\
\hline 2041 & 3005 & CORTESE & CAROLINA & D & 11 & MICHIGAN & ITALY & ITALY & & & SALASSA. TO & CALUMET AVE. & CALUMET - RAYMBAULTOWN \\
\hline 2042 & 3005 & CIOCATTO & MAURIZIO & $\mathrm{H}$ & 28 & ITALY & ITALY & ITALY & 1899 & TRAMMER BOSS/CM & VALPERGA. TO & CALUMET AVE. & CALUMET - RAYMBAULTOWN \\
\hline 2043 & 3005 & CIOCATTO & ANGELA & W & 24 & ITALY & ITALY & ITALY & 1900 & & & CALUMET AVE. & CALUMET - RAYMBAULTOWN \\
\hline 2044 & 3005 & CIOCATTO & ANDREINA & D & 2 & MICHIGAN & ITALY & ITALY & & & VALPERGA. TO & CALUMET AVE. & CALUMET - RAYMBAULTOWN \\
\hline 2045 & 3005 & RICCA & VICTOR & $\mathrm{H}$ & 34 & ITALY & ITALY & ITALY & 1897 & TIMBER BOSS/CM & SAN PONSO CANAVESE. TO & CALUMET AVE. & CALUMET - RAYMBAULTOWN \\
\hline 2046 & 3005 & RICCA & PAOLINA & W & 34 & ITALY & ITALY & ITALY & 1898 & & & CALUMET AVE. & CALUMET - RAYMBAULTOWN \\
\hline 2047 & 3005 & RICCA & LUCIA & $\mathrm{D}$ & 11 & MICHIGAN & ITALY & ITALY & & & SAN PONSO CANAVESE. TO & CALUMET AVE. & CALUMET - RAYMBAULTOWN \\
\hline 2048 & 3005 & RICCA & MATTIA & $\mathrm{s}$ & 9 & MICHIGAN & ITALY & ITALY & & & SAN PONSO CANAVESE. TO & $\begin{array}{l}\text { CALUMET AVE. } \\
\text { CAUE }\end{array}$ & CALUMET - RAYMBAULTOWN \\
\hline 2049 & 3005 & RICCA & PETER & $\mathrm{s}$ & 5 & MICHIGAN & ITALY & ITALY & & & SAN PONSO CANAVESE, TO & CALUMET AVE. & CALUMET - RAYMBAULTOWN \\
\hline 2050 & 3005 & RICCA & AUGUST & $\mathrm{s}$ & 3 & MICHIGAN & ITALY & ITALY & & & SAN PONSO CANAVESE. TO & CALUMET AVE. & CALUMET - RAYMBAULTOWN \\
\hline 2051 & 3005 & RICCA & MICHAEL & $\mathrm{s}$ & $7 \mathrm{M}$ & MICHIGAN & ITALY & ITALY & & & SAN PONSO CANAVESE. TO & CALUMET AVE. & CALUMET - RAYMBAULTOWN \\
\hline 2052 & 2001 & LEONATTI & MICHELE & $\mathrm{H}$ & 24 & ITALY & ITALY & ITALY & $00 / 06$ & TIMBERMAN/CM & CASTELLAMONTE. TO & CALUMET AVE. & $\begin{array}{l}\text { CALUMET - RAYMBAULTOWN } \\
\end{array}$ \\
\hline 2053 & 2001 & LEONATTI & FRANCESCA & W & 23 & ITALY & ITALY & ITALY & 1908 & & & CALUMET AVE. & CALUMET - RAYMBAULTOWN \\
\hline 2054 & 2001 & LEONATTI & DOMENICO & $\mathrm{s}$ & $14 \mathrm{M}$ & MICHIGAN & ITALY & ITALY & & & CASTELLAMONTE. TO & CALUMET AVE. & CALUMET - RAYMBAULTOWN \\
\hline 2055 & 2001 & CURTO & JAMES C. & $\mathrm{H}$ & 31 & ITALY & ITALY & ITALY & 1879 & MINER/CM & TORINO & CALUMET AVE. & CALUMET - RAYMBAULTOWN \\
\hline 2056 & 2001 & CURTO & KATE & W & 47 & ITALY & ITALY & ITALY & 1895 & & & CALUMET AVE. & CALUMET - RAYMBAULTOWN \\
\hline 2057 & 2001 & CURTO & JIM? & $\mathrm{s}$ & 9 & MICHIGAN & ITALY & ITALY & & & TORINO & CALUMET AVE. & CALUMET - RAYMBAULTOWN \\
\hline 2058 & 2001 & CURTO & ORSA? & $\mathrm{s}$ & 7 & MICHIGAN & ITALY & ITALY & & & TORINO & CALUMET AVE. & CALUMET - RAYMBAULTOWN \\
\hline 2059 & 2013 & PICCHIOTTINO & PETER & $\mathrm{H}$ & 40 & ITALY & ITALY & ITALY & 1894 & TIMBERMAN/CM & PONT CANAVESE. TO & CALUMET AVE. & CALUMET - RAYMBAULTOWN \\
\hline 2060 & 2013 & PICCHIOTTINO & MARY & W & 36 & MICHIGAN & ITALY & ITALY & & 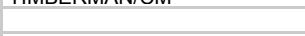 & 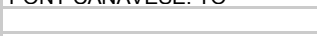 & CALUMET AVE. & CALUMET - RAYMBAULTOWN \\
\hline 2061 & 2013 & PICCHIOTTINO & JOSEPH & $\mathrm{s}$ & 13 & MICHIGAN & ITALY & ITALY & & & PONT CANAVESE. TO & CALUMET AVE. & CALUMET - RAYMBAULTOWN \\
\hline 2062 & 2013 & PICCHIOTTINO & PAUL & $\mathrm{s}$ & 12 & MICHIGAN & ITALY & ITALY & & & PONT CANAVESE. TO & CALUMET AVE. & CALUMET - RAYMBAULTOWN \\
\hline 2063 & 2013 & PICCHIOTTINO & ANGELO & s & 10 & MICHIGAN & ITALY & ITAL & & & PONT CANAVESE. TO & CAL & CALUMET - RAYMBAULTOWN \\
\hline 2064 & 2013 & PICCHIOTTINO & ? & $\mathrm{s}$ & 8 & MICHIGAN & ITALY & ITALY & & & PONT CANAVESE. TO & CAL & CALUMET - RAYMBAULTOWN \\
\hline 2065 & 2013 & PICCHIOTTINO & PETER & $\mathrm{s}$ & 6 & MICHIGAN & ITALY & ITALY & & & NAVESE. TO & CALUMET A & CALUMET - RAYMBAULTOWN \\
\hline 2066 & 2013 & PICCHIOTTINO & LOUIS & $\mathrm{s}$ & 3 & MICHIGAN & ITALY & ITAL & & & PONT CANAVESE. TO & CALUMET AVE. & CALUMET - RAYMBAULTOWN \\
\hline 2067 & 2021 & TOMASI & ANDREA & $\mathrm{H}$ & 42 & ITALY & ITALY & ITALY & 1888 & HELPER/BLACKSMITH SHOP & LOCANA CANAVESE, TO & CALUMET AVE. & CALUMET - RAYMBAULTOWN \\
\hline 2068 & 2021 & TOMASI & MARGHERITA & W & 28 & ITALY & ITALY & ITALY & 1898 & & & CALUMET AVE. & CALUMET - RAYMBAULTOWN \\
\hline 2069 & 2021 & TOMASI & JENNY & D & 8 & MICHIGAN & ITALY & ITALY & & & LOCANA CANAVESE. TO & CALUMET AVE. & CALUMET - RAYMBAULTOWN \\
\hline 2070 & 2021 & TOMASI & LUISA & D & 7 & MICHIGAN & ITALY & ITALY & & & LOCANA CANAVESE. TO & CALUMET AVE. & CALUMET - RAYMBAULTOWN \\
\hline 2071 & 2021 & TOMASI & ANDREA & $\mathrm{s}$ & 5 & MICHIGAN & ITALY & ITALY & & & LOCANA CANAVESE. TO & CALUMET AVE. & CALUMET - RAYMBAULTOWN \\
\hline 2072 & 2021 & TOMASI & MICHAEL & $\mathrm{s}$ & 3 & MICHIGAN & ITALY & ITALY & & & LOCANA CANAVESE. TO & CALUMET AVE. & CALUMET - RAYMBAULTOWN \\
\hline 2073 & 2029 & SCUSSEL & JOSEPH & $\mathrm{H}$ & 28 & ITALY & ITALY & ITALY & 1899 & MINER/CM & BELLUNO & CALUMET AVE. & CALUMET - RAYMBAULTOWN \\
\hline 2074 & 2029 & SCUSSEL & MARGHERITA & W & 25 & ITALY & ITALY & ITALY & 1904 & & & CALUMET AVE. & CALUMET - RAYMBAULTOWN \\
\hline 2075 & 2029 & SCUSSEL & JOSEPH & $\mathrm{s}$ & 5 & MICHIGAN & ITALY & ITAL & & & BELLUNO & CALUMET A & CALUMET - RAYMBAULTOWN \\
\hline 76 & 2029 & SCUSSEL & KATE & D & $14 \mathrm{M}$ & MICHIGAN & ITA & ITA & & & & $\mathrm{Cr}$ & T - RAYMB \\
\hline 77 & 2029 & ZANARDI & TISTA & B & 35 & ITALY & & ITALY & 1890 & TIMBER BOSS/CM & & $\mathrm{CA}$ & CALUME \\
\hline 78 & 2029 & OSTORERO & MAURIZIO & B & 34 & ITALY & ITALY & ITALY & 1906 & TIMBERMAN/CM & GIAVENO. TO & CALUMET AVE. & CALUMET - RAYMBAULTOWN \\
\hline
\end{tabular}




\begin{tabular}{|c|c|c|c|c|c|c|c|c|c|c|c|c|c|}
\hline 2079 & 2029 & OSTORERO & PETER & B & 32 & ITALY & ITALY & ITALY & 1905 & TIMBERMAN/CM & GIAVENO. TO & CALUMET AVE. & CALUMET - RAYMBAULTOWN \\
\hline 2080 & 2029 & PASQUALONI & MICHELE & B & 25 & ITALY & ITALY & ITALY & 1905 & TRAMMER/CM & SPARONE. TO & CALUMET AVE. & CALUMET - RAYMBAULTOWN \\
\hline 2081 & 2029 & RIVA & MAURIZIO & B & 35 & ITALY & ITALY & ITALY & 1899 & MINER/CM & SPARONE. TO & CALUMET AVE. & CALUMET - RAYMBAULTOWN \\
\hline 2082 & 2029 & BOGGIO & MICHELE & B & 32 & ITALY & ITALY & ITALY & 1902 & TIMBERMAN/CM & SAN GIORGIO CANAVESE. TO & CALUMET AVE. & CALUMET - RAYMBAULTOWN \\
\hline 2083 & 2029 & RIVA & JOHN & B & 15 & ITALY & ITALY & ITALY & 1910 & & SPARONE. TO & CALUMET AVE. & CALUMET - RAYMBAULTOWN \\
\hline 2084 & 2029 & CIAGNE & LUIGI & $\mathrm{H}$ & 30 & ITALY & ITALY & ITALY & 1892 & MINER/CM & TORINO & CALUMET AVE. & CALUMET - RAYMBAULTOWN \\
\hline 2085 & 2029 & CIAGNE & DOMENICA & W & 26 & ITALY & ITALY & ITALY & 1901 & & & CALUMET AVE. & CALUMET - RAYMBAULTOWN \\
\hline 2086 & 2029 & CIAGNE & LENA & $\mathrm{D}$ & 5 & MICHIGAN & ITALY & ITALY & 1501 & & TORINO & CALUMET AVE. & CALUMET - RAYMBAULTOWN \\
\hline 2087 & 2563 & TEPATTI & PETER & $\mathrm{H}$ & 42 & ITALY & ITALY & ITALY & 1892 & WATCHMAN/CM & PONT CANAVESE. TO & E ST. & CALUMET - RAYMBAULTOWN \\
\hline 2088 & 2563 & TEPATTI & MAGGIE & W & 41 & ITALY & ITALY & ITALY & 1895 & KEEPER/BOARDING HOUSE & PONT CANAVESE. TO & E ST. & CALUMET - RAYMBAULTOWN \\
\hline 2089 & 2563 & TEPATTI & MINNIE & $\mathrm{D}$ & 17 & ITALY & ITALY & ITALY & 1895 & & PONT CANAVESE. TO & EST. & CALUMET - RAYMBAULTOWN \\
\hline 2090 & 2563 & TEPATTI & MARY & $\mathrm{D}$ & 15 & MICHIGAN & ITALY & ITALY & & & PONT CANAVESE. TO & E ST. & CALUMET - RAYMBAULTOWN \\
\hline 2091 & 2563 & TEPATTI & MASSIMO & s & 14 & MICHIGAN & ITALY & ITALY & & & PONT CANAVESE. TO & EST. & CALUMET - RAYMBAULTOWN \\
\hline 2092 & 2563 & TEPATTI & & $\mathrm{s}$ & & MICHIGAN & ITALY & ITALY & & & PONT CANAVESE. TO & EST. & CALUMET - RAYMBAULTOWN \\
\hline 2093 & 2563 & TEPATTI & PETER & $\mathrm{s}$ & 8 & MICHIGAN & ITALY & ITALY & & & PONT CANAVESE. TO & EST. & CALUMET - RAYMBAULTOWN \\
\hline 2094 & 2563 & TEPATTI & MAGGIE & D & 6 & MICHIGAN & ITALY & ITALY & & & PONT CANAVESE, TO & EST. & CALUMET - RAYMBAULTOWN \\
\hline 2095 & 2563 & GUGLIELMO & LORENZO & B & 19 & ITALY & ITALY & ITALY & 1906 & TRAMMER/CM & SAN GIORGIO CANAVESE. TO & E ST. & CALUMET - RAYMBAULTOWN \\
\hline 2096 & 2563 & GIULIO/GIUGLIO & PETER & B & 26 & ITALY & ITALY & ITALY & 1907 & TRAMMER/CM & SAN GIORGIO CANAVESE. TO & E ST. & CALUMET - RAYMBAULTOWN \\
\hline 2097 & 2563 & OTTINO & ANTONIO & B & 46 & ITALY & ITALY & ITALY & 1897 & WATCHMAN/CM & VALPERGA. TO & EST. & CALUMET - RAYMBAULTOWN \\
\hline 2098 & 2563 & OTTINO & JAMES & B & 22 & ITALY & ITALY & ITALY & 1910 & TRAMMER/CM & SAN GIUSTO CANAVESE. TO & EST. & CALUMET - RAYMBAULTOWN \\
\hline 2099 & 2563 & FORNAREGIO & FRANK & $\begin{array}{l}\mathrm{D} \\
\mathrm{B}\end{array}$ & 22 & ITALY & $\begin{array}{l}\text { ITALY } \\
\text { ITAL }\end{array}$ & ITALY & 1908 & TRAMMER/CM & SAIV OTUS TS CAIVAVESOE. TS & E ST. & CALUMET - RAYMBAULTOWN \\
\hline 2100 & 2563 & BOLLATO & JOSEPH & B & 25 & ITALY & ITALY & ITALY & 1905 & TRAMMER/CM & & E ST. & CALUMET - RAYMBAULTOWN \\
\hline 2101 & 2563 & FUSERO & JAMES & B & 23 & ITALY & ITALY & ITALY & 1905 & TRAMMER/CM & FOGLIAZZO CANAVESE. TO & EST. & CALUMET - RAYMBAULTOWN \\
\hline 2102 & 2524 & BRACCO & $\begin{array}{l}\text { PETER } \\
\text { PETER }\end{array}$ & $\mathrm{H}$ & 41 & ITALY & ITALY & ITALY & 1893 & MINER/CM & 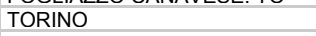 & D ST. & CALUMET - RAYMBAULTOWN \\
\hline 2103 & 2524 & BRACCO & VINCENZA & W & 27 & ITALY & ITALY & ITALY & 1901 & & & DST. & CALUMET - RAYMBAULTOWN \\
\hline 2104 & 2524 & BRACCO & TONY & $s$ & 7 & MICHIGAN & ITALY & ITALY & & & TORINO & D ST. & CALUMET - RAYMBAULTOWN \\
\hline 2105 & 2524 & BRACCO & MADDALENA & $\mathrm{D}$ & 5 & MICHIGAN & ITALY & ITALY & & & TORINO & D ST. & CALUMET - RAYMBAULTOWN \\
\hline 2106 & 2524 & BRACCO & PETER & $\mathrm{s}$ & 4 & MICHIGAN & ITALY & ITALY & & & TORINO & D ST. & CALUMET - RAYMBAULTOWN \\
\hline 2107 & 2521 & BIGANDO & BATTISTA & $\mathrm{H}$ & 27 & ITALY & ITALY & ITALY & $91 / 01$ & PAINTER/WORKING OUT & TORINO & D ST. & CALUMET - RAYMBAULTOWN \\
\hline 2108 & 2521 & BIGANDO & MARY & W & 20 & MICHIGAN & ITALY & ITALY & & & & D ST. & CALUMET - RAYMBAULTOWN \\
\hline 2109 & 2521 & BIGANDO & MONDE & $\mathrm{D}$ & $10 \mathrm{M}$ & MICHIGAN & ITALY & ITALY & & & TORINO & D ST. & CALUMET - RAYMBAULTOWN \\
\hline 2110 & 2521 & SARANGIA & MICHELE & $\mathrm{H}$ & 48 & ITALY & ITALY & ITALY & 1888 & SURFACE MAN/CM & PONT CANAVESE. TO & D ST. & CALUMET - RAYMBAULTOWN \\
\hline 2111 & 2521 & SARANGIA & MADDALENA & W & 48 & ITALY & ITALY & ITALY & 1800 & SURTALE TIATVIUIVI & PUIT CIIAAVEOE. TO & $\begin{array}{l}\text { D ST. } \\
\text { DT. }\end{array}$ & CALUMET - RAYMBAULTOWN \\
\hline 2112 & 2521 & SARANGIA & CATERINA & $\mathrm{D}$ & 17 & MICHIGAN & ITALY & ITALY & & SALESLADY/DEPT. STORE & PONT CANAVESE, TO & D ST. & CALUMET - RAYMBAULTOWN \\
\hline 2113 & 2521 & SARANGIA & PAOLINA & D & 13 & MICHIGAN & ITALY & ITALY & & & PONT CANAVESE. TO & D ST. & CALUMET - RAYMBAULTOWN \\
\hline 2114 & $252 ?$ & BRACCO & JOHN & $\mathrm{H}$ & 45 & ITALY & ITALY & ITALY & 1889 & ROLLER FIXER/CM & TORINO & D ST. & CALUMET - RAYMBAULTOWN \\
\hline 2115 & $252 ?$ & BRACCO & MINNIE & W & 45 & ITALY & ITALY & ITALY & ???? & & TORINO & D ST. & CALUMET - RAYMBAULTOWN \\
\hline 2116 & $252 ?$ & BRACCO & JOSEPH & $\mathrm{s}$ & 17 & MICHIGAN & ITALY & ITALY & & & TORINO & D ST. & CALUMET - RAYMBAULTOWN \\
\hline 2117 & $252 ?$ & BRACCO & TONY & $\mathrm{s}$ & 14 & MICHIGAN & ITALY & ITALY & & & TORINO & D ST. & CALUMET - RAYMBAULTOWN \\
\hline 2118 & $252 ?$ & BRACCO & MARTIN & $\mathrm{S}$ & 13 & MICHIGAN & ITALY & ITALY & & & TORINO & D ST. & CALUMET - RAYMBAULTOWN \\
\hline 2119 & $252 ?$ & BRACCO & MINNIE & D & 10 & MICHIGAN & ITALY & ITALY & & & TORINO & DST. & CALUMET - RAYMBAULTOWN \\
\hline 2120 & $252 ?$ & BRACCO & MARY & $\mathrm{D}$ & 6 & MICHIGAN & ITALY & ITALY & & & TORINO & D ST. & CALUMET - RAYMBAULTOWN \\
\hline 2121 & 2522 & CERETTO & JOHN & $\mathrm{H}$ & 47 & ITALY & ITALY & ITALY & 1896 & TRAMMER/CM & PONT CANAVESE. TO & D ST. & CALUMET - RAYMBAULTOWN \\
\hline 2122 & 2522 & CERETTO & LUCY & W & 36 & ITALY & ITALY & ITALY & 1898 & & & D ST. & CALUMET - RAYMBAULTOWN \\
\hline 2123 & 2522 & CERETTO & FRANCES & $\mathrm{D}$ & 13 & MICHIGAN & ITALY & ITALY & & & PONT CANAVESE. TO & D ST. & CALUMET - RAYMBAULTOWN \\
\hline 2124 & 2522 & CERETTO & ANNA & $\mathrm{D}$ & 10 & MICHIGAN & ITALY & ITALY & & & PONT CANAVESE. TO & D ST. & CALUMET - RAYMBAULTOWN \\
\hline 2125 & 2522 & CERETTO & MARY & D & 8 & MICHIGAN & ITALY & ITALY & & & PONT CANAVESE. TO & D ST. & CALUMET - RAYMBAULTOWN \\
\hline 2126 & 2522 & FEIRA/FERA & JOSEPH & B & 40 & ITALY & ITALY & ITALY & 1906 & TRAMMER/CM & PONT CANAVESE. TO & D ST. & CALUMET - RAYMBAULTOWN \\
\hline 2127 & 2520 & ZANIN & OLIVER & $\mathrm{H}$ & 37 & ITALY & ITALY & ITALY & 1890 & TIMBERMAN/CM & RIVAMONTE. BELLUNO & $\mathrm{DS}$ & CALUMET - RAYMBAULTOWN \\
\hline 2128 & 2520 & ZANIN & ANTONIA & W & 26 & ITALY & ITALY & ITALY & 1890 & & & D ST. & CALUMET - RAYMBAULTOWN \\
\hline 2129 & 2520 & ZANIN & JOHN & $\mathrm{s}$ & 9 & MICHIGAN & ITALY & ITALY & & & RIVAMONTE. BELLUNO & D ST. & CALUMET - RAYMBAULTOWN \\
\hline 2130 & 2520 & ZANIN & ANGELINA & D & 0 & MICHIGAN & ITALY & ITALY & & & RIVAMONTE, BELLUNO & D ST. & CALUMET - RAYMBAULTOWN \\
\hline 2131 & 2520 & ZANIN & ?? & $\mathrm{D}$ & 4 & MICHIGAN & ITALY & ITALY & & & RIVAMONTE. BELLUNO & DST. & CALUMET - RAYMBAULTOWN \\
\hline 2132 & 2520 & ZANIN & GINA & D & 3 & MICHIGAN & ITALY & ITALY & & & RIVAMONTE. BELLUNO & DST. & CALUMET - RAYMBAULTOWN \\
\hline 2133 & 2520 & CONADERA & VIRGILIO & B & 50 & ITALY & ITALY & ITALY & 1890 & MINER/CM & RIVAMONTE. BELLUNO & D ST. & CALUMET - RAYMBAULTOWN \\
\hline 2134 & 2520 & ZANIN & FELIX & B & 22 & ITALY & ITALY & ITALY & 1907 & TRAMMER/CM & RIVAMONTE. BELLUNO & D ST. & CALUMET - RAYMBAULTOWN \\
\hline 2135 & 2520 & ZANIN & JAMES & B & 18 & ITALY & ITALY & ITALY & 1909 & TRAMMER/CM & RIVAMONTE. BELLUNO & D ST. & CALUMET - RAYMBAULTOWN \\
\hline 2136 & 2518 & PIANA & JOHN & $\mathrm{H}$ & 44 & ITALY & ITALY & ITALY & 1882 & TRAMMER/CM & QUAGLIUZZO CANAVESE, TO & D ST. & CALUMET - RAYMBAULTOWN \\
\hline 2137 & 2518 & PIANA & MARY & W & 40 & ITALY & ITALY & ITALY & 1890 & & & D ST. & CALUMET - RAYMBAULTOWN \\
\hline 2138 & 2518 & PIANA & FRANK & $\mathrm{s}$ & 19 & MICHIGAN & ITALY & ITALY & & ROLLER FIXER/CM & QUAGLIUZZO CANAVESE. TO & D ST. & CALUMET - RAYMBAULTOWN \\
\hline 2139 & 2518 & PIANA & $\mathrm{JOH}$ & $\mathrm{s}$ & 16 & MICHIGAN & ITA & ITA & & & QUAGLIUZZO CANAVESE. TO & DS & LTOWN \\
\hline 2140 & 2518 & PIANA & DOMENICO & $\mathrm{s}$ & 8 & MICHIGAN & ITALY & ITALY & & & QUAGLIUZZO CANAVESE. TO & D ST. & CALUMET - RAYMBAULTOWN \\
\hline 2141 & 2518 & PIANA & ANGELINE & D & 8 & MICHIGAN & ITALY & ITALY & & & $\begin{array}{l}\text { QUAGLIUZZO CANAVESE. TO } \\
\text { Q CAVS }\end{array}$ & D ST. & CALUMET - RAYMBAULTOWN \\
\hline
\end{tabular}




\begin{tabular}{|c|c|c|c|c|c|c|c|c|c|c|c|c|c|}
\hline 2142 & 2508 & RENALDI & JOSEPH & $\mathrm{H}$ & 34 & ITALY & ITALY & ITALY & 1900 & MINER/CM & SAN GIORGIO CANAVESE. TO & D ST. & CALUMET - RAYMBAULTOWN \\
\hline 2143 & 2508 & RENALDI & ALBINA & W & 35 & ITALY & ITALY & ITALY & 1901 & & SAN GIORGIO CANAVESE. TO & D ST. & CALUMET - RAYMBAULTOWN \\
\hline 2144 & 2508 & RENALDI & LUCY & $\mathrm{D}$ & 12 & ITALY & ITALY & ITALY & 1901 & & SAN GIORGIO CANAVESE. TO & DST. & CALUMET - RAYMBAULTOWN \\
\hline 2145 & 2508 & RENALDI & JOSEPH & $\mathrm{s}$ & 10 & ITALY & ITALY & ITALY & 1901 & & SAN GIORGIO CANAVESE. TO & D ST. & CALUMET - RAYMBAULTOWN \\
\hline 2146 & 2508 & RENALDI & STEPHEN & $\mathrm{s}$ & 8 & MICHIGAN & ITALY & ITALY & & & SAN GIORGIO CANAVESE. TO & D ST. & CALUMET - RAYMBAULTOWN \\
\hline 2147 & 2508 & RENALDI & CHARLES & $\mathrm{s}$ & 6 & MICHIGAN & ITALY & ITALY & & & SAN GIORGIO CANAVESE. TO & D ST. & CALUMET - RAYMBAULTOWN \\
\hline 2148 & 2508 & RENALDI & NETTIE & $\mathrm{D}$ & 5 & MICHIGAN & ITALY & ITALY & & & SAN GIORGIO CANAVESE. TO & D ST. & CALUMET - RAYMBAULTOWN \\
\hline 2149 & 2508 & RENALDI & ANGELO & s & 2 & MICHIGAN & ITALY & ITALY & & & SAN GIORGIO CANAVESE. TO & $\begin{array}{l}\text { D ST. } \\
\text { DT. }\end{array}$ & CALUMET - RAYMBAULTOWN \\
\hline 2150 & 2506 & PUCCI & JOSEPH & $\mathrm{H}$ & 48 & ITALY & ITALY & ITALY & 1889 & WATCHMAN/CM & RUOTA. LU & D ST. & CALUMET - RAYMBAULTOWN \\
\hline 2151 & 2506 & PUCCI-LANDINI & LUCY & w & 41 & ITALY & ITALY & ITALY & 1896 & & & D ST. & CALUMET - RAYMBAULTOWN \\
\hline 2152 & 2506 & PUCCI & ANGELO & s & 13 & MICHIGAN & ITALY & ITALY & & & RUOTA. LU & D ST. & CALUMET - RAYMBAULTOWN \\
\hline 2153 & 2506 & $\mathrm{PUCCl}$ & DOMENICO & $\mathrm{s}$ & 12 & MICHIGAN & ITALY & ITALY & & & RUOTA. LU & DST. & CALUMET - RAYMBAULTOWN \\
\hline 2154 & 2506 & $\mathrm{PUCCl}$ & EUGENIO & $\mathrm{s}$ & 9 & MICHIGAN & ITALY & ITALY & & & RUOTA. LU & D ST. & CALUMET - RAYMBAULTOWN \\
\hline 2155 & 2506 & $\mathrm{PUCCl}$ & PETER & $\mathrm{s}$ & 6 & MICHIGAN & ITALY & ITALY & & & RUOTA. LU & D ST. & CALUMET - RAYMBAULTOWN \\
\hline 2156 & 2506 & PUCCl & ALBERT & $\mathrm{s}$ & 2 & MICHIGAN & ITALY & ITALY & & & RUOTA. LU & D ST. & CALUMET - RAYMBAULTOWN \\
\hline 2157 & 2128 & VEZZANI & SAMUELE & $\mathrm{H}$ & 42 & ITALY & ITALY & ITALY & 1899 & TIMBERMAN/CM & PORCARI, LU & LOG ST. & CALUMET - RAYMBAULTOWN \\
\hline 2158 & 2128 & VEZZANI & ASSUNTA & W & 44 & ITALY & ITALY & ITALY & 1908 & HOUSEKEEPER/PF & & LOG ST. & CALUMET - RAYMBAULTOWN \\
\hline 2159 & 2128 & VEZZANI & MARCO & s & 4 & MICHIGAN & ITALY & ITALY & & & PORCARI. LU & LOG ST. & CALUMET - RAYMBAULTOWN \\
\hline 2160 & 2128 & VEZZANI & VITTORIA & $\mathrm{D}$ & 2 & MICHIGAN & ITALY & ITALY & & & PORCARI. LU & LOG ST. & CALUMET - RAYMBAULTOWN \\
\hline 2161 & 2133 & GIVOGRE & BATTISTA & $\mathrm{H}$ & 54 & ITALY & ITALY & ITALY & 1889 & MINER/CM & AGLIE'. TO & LOG ST. & CALUMET - RAYMBAULTOWN \\
\hline 2162 & 2133 & GIVOGRE & MARY & W & 50 & ITALY & $\begin{array}{l}\text { ITALY } \\
\text { ITAL }\end{array}$ & ITALY & 1889 & IVIIVERTCIVI & AGLIE', TO & LOG ST. & CALUMET - RAYMBAULTOWN \\
\hline 2163 & 2133 & GIVOGRE & JAMES & s & 10 & MICHIGAN & ITALY & ITALY & & MUSICIAN/ORCHESTRA & AGLIE', TO & LOG ST. & CALUMET - RAYMBAULTOWN \\
\hline 2164 & 2133 & GIVOGRE & CATERINA & $\mathrm{D}$ & 12 & MICHIGAN & ITALY & ITALY & & & AGLIE'. TO & LOG ST. & CALUMET - RAYMBAULTOWN \\
\hline 2165 & 2133 & BARATONO & LORENZO & $\mathrm{H}$ & 29 & ITALY & ITALY & ITALY & 1903 & TEAMSTER/BREWERY & TORINO & LOG ST. & CALUMET - RAYMBAULTOWN \\
\hline 2166 & 2133 & BARATONO & DOMENICA & W & 29 & ITALY & ITALY & ITALY & 1906 & & & LOG ST. & CALUMET - RAYMBAULTOWN \\
\hline 2167 & 2133 & BARATONO & LORENZO & $\mathrm{s}$ & 2 & MICHIGAN & ITALY & ITALY & & & TORINO & LOG ST. & CALUMET - RAYMBAULTOWN \\
\hline 2168 & 2135 & GOGLIO & CARLO & $\mathrm{H}$ & 41 & ITALY & ITALY & ITALY & 1897 & MINER/CM & PONT CANAVESE. TO & LOG ST. & CALUMET - RAYMBAULTOWN \\
\hline 2169 & 2135 & GOGLIO & MARIA & W & 44 & ITALY & ITALY & ITALY & 1897 & & PONT CANAVESE, TO & LOG ST. & CALUMET - RAYMBAULTOWN \\
\hline 2170 & 2135 & GOGLIO & KATE & D & 14 & ITALY & ITALY & ITALY & 1897 & & PONT CANAVESE. TO & LOG ST. & CALUMET - RAYMBAULTOWN \\
\hline 2171 & 2135 & GOGLIO & JOSEPH & $\mathrm{S}$ & 9 & MICHIGAN & ITALY & ITALY & & & PONT CANAVESE. TO & LOG ST. & CALUMET - RAYMBAULTOWN \\
\hline 2172 & 2135 & GOGLIO & FRANK & $\mathrm{S}$ & 6 & MICHIGAN & ITALY & ITALY & & & PONT CANAVESE. TO & LOG ST. & CALUMET - RAYMBAULTOWN \\
\hline 2173 & 2135 & GOGLIO & JOHN & $\mathrm{s}$ & 4 & MICHIGAN & ITALY & ITALY & & & PONT CANAVESE. TO & LOG ST. & CALUMET - RAYMBAULTOWN \\
\hline 2174 & 2135 & BEGA & DOMENICO & $\mathrm{H}$ & 21 & ITALY & ITALY & ITALY & 1908 & TRAMMER/CM & RUNI CAIVAVEOE. TO & LOG ST. & CALUMET - RAYMBAULTOWN \\
\hline 2175 & 2135 & BEGA & TERESA & w & 20 & ITALY & ITALY & ITALY & 1908 & & & LOG ST. & CALUMET - RAYMBAULTOWN \\
\hline 2176 & 2135 & BEGA & LUCY & $\mathrm{D}$ & $3 \mathrm{M}$ & MICHIGAN & ITALY & ITALY & & & & LOG ST. & CALUMET - RAYMBAULTOWN \\
\hline 2177 & 2139 & ARIETTI & BARTOLOMEO & $\mathrm{H}$ & 48 & ITALY & ITALY & ITALY & 1883 & LANDER/CM & VIALFRE'. TO & LOG ST. & CALUMET - RAYMBAULTOWN \\
\hline 2178 & 2139 & ARIETTI & MARY & D & 15 & MICHIGAN & ITALY & ITALY & & & & LOG ST. & CALUMET - RAYMBAULTOWN \\
\hline 2179 & 2139 & ARIETTI & JONNIE & $\mathrm{s}$ & 13 & MICHIGAN & ITALY & ITALY & & & VIALFRE'. TO & LOG ST. & CALUMET - RAYMBAULTOWN \\
\hline 2180 & 2139 & ARIETTI & KATE & $\mathrm{D}$ & 11 & MICHIGAN & ITALY & ITALY & & & VIALFRE'. TO & LOG ST. & CALUMET - RAYMBAULTOWN \\
\hline 2181 & 2139 & ARIETTI & THOMAS & $\mathrm{S}$ & 9 & MICHIGAN & ITALY & ITALY & & & VIALFRE'. TO & LOG ST. & CALUMET - RAYMBAULTOWN \\
\hline 2182 & 2527 & SOMMARIVA & FRANCESCO & $\mathrm{H}$ & 45 & ITALY & ITALY & ITALY & 1890 & TIMBERMAN/CM & VENEZIA & D ST. & CALUMET - RAYMBAULTOWN \\
\hline 2183 & 2527 & SOMMARIVA & ARCANGELA & w & 30 & ITALY & ITALY & ITALY & 1910 & & & D ST. & CALUMET - RAYMBAULTOWN \\
\hline 2184 & 2527 & SOMMARIVA & FRANK & s & 8 & MICHIGAN & ITALY & ITALY & & & VENEZIA & D ST. & CALUMET - RAYMBAULTOWN \\
\hline 2185 & 2527 & SOMMARIVA & NATALINO & $\mathrm{s}$ & 7 & MICHIGAN & ITALY & ITALY & & & VENEZIA & D ST. & CALUMET - RAYMBAULTOWN \\
\hline 2186 & 2527 & SOMMARIVA & JOHANNA & $\mathrm{D}$ & 5 & MICHIGAN & ITALY & ITALY & & & VENEZIA & D ST. & CALUMET - RAYMBAULTOWN \\
\hline 2187 & 2527 & SOMMARIVA & DESIDERIO & $\mathrm{S}$ & $14 \mathrm{M}$ & MICHIGAN & ITALY & ITALY & & & VENEZIA & D ST. & CALUMET - RAYMBAULTOWN \\
\hline 2188 & 2525 & BRUNO & DOMENICO & $\mathrm{H}$ & 29 & ITALY & ITALY & ITALY & 1904 & MINER/CM & SALASSA. TO & D ST. & CALUMET - RAYMBAULTOWN \\
\hline 2189 & 2525 & BRUNO & ADELE & w & 30 & ITALY & ITALY & ITALY & 1906 & & & D ST. & CALUMET - RAYMBAULTOWN \\
\hline 2190 & 2525 & BRUNO & KATE & $\mathrm{D}$ & 2 & MICHIGAN & ITALY & ITALY & & & SALASSA. TO & D ST. & CALUMET - RAYMBAULTOWN \\
\hline 2191 & 2525 & BRUNO & JOSEPH & s & $8 \mathrm{M}$ & MICHIGAN & ITALY & ITALY & & & SALASSA. TO & D ST. & CALUMET - RAYMBAULTOWN \\
\hline 2192 & 2525 & BRUNO & CHARLES & $B R$ & 24 & ITALY & ITALY & ITALY & 1910 & TRAMMER/CM & SALASSA. TO & D ST. & CALUMET - RAYMBAULTOWN \\
\hline 2193 & 2525 & RICCA & MARY & $\mathrm{H}$ & 26 & ITALY & ITALY & ITALY & 1905 & & & D ST. & CALUMET - RAYMBAULTOWN \\
\hline 2194 & 2525 & RICCA & MATTIA & $\mathrm{s}$ & 3 & MICHIGAN & ITALY & ITALY & & & SAN PONsO CANAVESE. TO & D ST. & CALUMET - RAYMBAULTOWN \\
\hline 2195 & 2523 & NARDI & MICHAEL & $\mathrm{H}$ & 43 & ITALY & ITALY & ITALY & 1888 & TIMBER BOSS/CM & LUCCA & DST. & CALUMET - RAYMBAULTOWN \\
\hline 2196 & 2523 & NARDI & GIULIA & W & 34 & ITALY & ITALY & ITALY & 1896 & & & D ST. & CALUMET - RAYMBAULTOWN \\
\hline 2197 & 2523 & NARDI & ARMANDO & s & 13 & MICHIGAN & ITALY & ITALY & & & LUCCA & D ST. & CALUMET - RAYMBAULTOWN \\
\hline 2198 & 2523 & NARDI & VIRGINIA & $\mathrm{D}$ & 12 & MICHIGAN & ITALY & ITALY & & & LUCCA & D ST. & CALUMET - RAYMBAULTOWN \\
\hline 2199 & 2523 & NARDI & EUSEBIA & $\mathrm{D}$ & 9 & MICHIGAN & ITALY & ITALY & & & LUCCA & D ST. & CALUMET - RAYMBAULTOWN \\
\hline 2200 & 2523 & NARDI & JAMES & $\mathrm{s}$ & 8 & MICHIGAN & ITALY & ITALY & & & LUCCA & D ST. & CALUMET - RAYMBAULTOWN \\
\hline 2201 & 2523 & NARDI & FRED & $\mathrm{s}$ & 6 & MICHIGAN & ITALY & ITALY & & & LUCCA & D ST. & CALUMET - RAYMBAULTOWN \\
\hline 2202 & 2523 & NARDI & NATALINA & D & 3 & MICHIGAN & ITAL & ITAL & & & LUCCA & D ST. & CALUMET - RAY \\
\hline 2203 & 2523 & MAZZONI & EUSTACHIO & B & 50 & ITALY & ITALY & ITALY & 1892 & DAY TENDER/CM & LUCCA & D ST. & CALUMET - RAYMBAULTOWN \\
\hline 2204 & 2523 & SALAME & ARTURO & $\mathrm{H}$ & 30 & ITALY & ITALY & ITALY & 1902 & SURFACE MAN/CM & & D ST. & CALUMET - RAYMBAULTOWN \\
\hline
\end{tabular}




\begin{tabular}{|c|c|c|c|c|c|c|c|c|c|c|c|c|c|}
\hline 2205 & 2523 & SALAME & ANNUNZIATA & MO & 56 & ITALY & ITALY & ITALY & 1906 & & & DST. & CALUMET - RAYMBAULTOWN \\
\hline 2206 & 2519 & ORSOLANO & ANTONIO & $\mathrm{H}$ & 42 & ITALY & ITALY & ITALY & 1892 & TIMBERMAN/CM & SAN GIORGIO CANAVESE. TO & DST. & CALUMET - RAYMBAULTOWN \\
\hline 2207 & 2519 & ORSOLANO & ANNA & W & 41 & ITALY & ITALY & ITALY & 1892 & & TORINO & D ST. & CALUMET - RAYMBAULTOWN \\
\hline 2208 & 2519 & ORSOLANO & VICTOR & $\mathrm{s}$ & 17 & MICHIGAN & ITALY & ITALY & & DRILL BOY/CM & SAN GIORGIO CANAVESE. TO & D ST. & CALUMET - RAYMBAULTOWN \\
\hline 2209 & 2519 & ORSOLANO & TONY & $\mathrm{s}$ & 10 & MICHIGAN & ITALY & ITALY & & & SAN GIORGIO CANAVESE. TO & D ST. & CALUMET - RAYMBAULTOWN \\
\hline 2210 & 2519 & ORSOLANO & $\mathrm{C} ?$ & $\mathrm{~s}$ & 7 & MICHIGAN & ITALY & ITALY & & & SAN GIORGIO CANAVESE. TO & D ST. & CALUMET - RAYMBAULTOWN \\
\hline 2211 & 2517 & BARINOTTI & FRANK & $\mathrm{H}$ & 40 & ITALY & ITALY & ITALY & 1889 & DUMP MAN/CM & PONT CANAVESE. TO & $\begin{array}{l}\text { D ST. } \\
\text { DT. }\end{array}$ & CALUMET - RAYMBAULTOWN \\
\hline 2212 & 2517 & BARINOTTI & MADDALENA & W & 37 & ITALY & ITALY & ITALY & 1891 & 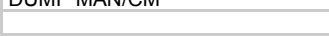 & TUNT CAIVAVEOE. TO & $\begin{array}{l}\text { D ST. } \\
\text { D }\end{array}$ & CALUMET - RAYMBAULTOWN \\
\hline 2213 & 2517 & BARINOTTI & JOHN & s & 18 & MICHIGAN & ITALY & ITALY & & DRILL BOY/CM & PONT CANAVESE. TO & D ST. & CALUMET - RAYMBAULTOWN \\
\hline 2214 & 2517 & BARINOTTI & MAGGIE & D & 10 & MICHIGAN & ITALY & ITALY & & & PONT CANAVESE. TO & D ST. & CALUMET - RAYMBAULTOWN \\
\hline 2215 & 2517 & BARINOTTI & KATE & $\mathrm{D}$ & 7 & MICHIGAN & ITALY & ITALY & & & PONT CANAVESE. TO & D ST. & CALUMET - RAYMBAULTOWN \\
\hline 2216 & 2517 & BARINOTTI & MARY & $\mathrm{D}$ & 5 & MICHIGAN & ITALY & ITALY & & & PONT CANAVESE. TO & D ST. & CALUMET - RAYMBAULTOWN \\
\hline 2217 & 2517 & BARINOTTI & FRANK & $\mathrm{s}$ & 3 & MICHIGAN & ITALY & ITALY & & & PONT CANAVESE. TO & D ST. & CALUMET - RAYMBAULTOWN \\
\hline 2218 & 2517 & SEGHI & DOMENICO & $\mathrm{H}$ & 50 & ITALY & ITALY & ITALY & 1909 & TRAMMER/CM & & D ST. & CALUMET - RAYMBAULTOWN \\
\hline 2219 & 2517 & SEGHI & FILOMENA & W & 23 & ITALY & ITALY & ITALY & 1910 & & & D ST. & CALUMET - RAYMBAULTOWN \\
\hline 2220 & 2515 & BOGGIO & JOHN & $\mathrm{H}$ & 27 & ITALY & ITALY & ITALY & 1907 & TRAMMER/CM & SAN GIUSTO CANAVESE, TO & D ST. & CALUMET - RAYMBAULTOWN \\
\hline 2221 & 2515 & BOGGIO & JOHANNA & W & 25 & ITALY & ITALY & ITALY & 1907 & & SAN GIUSTO CANAVESE. TO & D ST. & CALUMET - RAYMBAULTOWN \\
\hline 2222 & 2515 & BOGGIO & GIORGIO & s & 3 & MICHIGAN & ITALY & ITALY & & & SAN GIUSTO CANAVESE. TO & D ST. & CALUMET - RAYMBAULTOWN \\
\hline 2223 & 2515 & BOGGIO & GIORGIO & $\mathrm{H}$ & 22 & ITALY & ITALY & ITALY & 1907 & TRAMMER/CM & SAN GIUSTO CANAVESE. TO & D ST. & CALUMET - RAYMBAULTOWN \\
\hline 2224 & 2515 & BOGGIO & TERESA & w & 22 & ITALY & ITALY & ITALY & 1910 & TRAIVIVLCR & SAN GIUSTO CANAVESE. TO & $\begin{array}{l}\text { D ST. } \\
\text { DT. }\end{array}$ & CALUMET - RAYMBAULTOWN \\
\hline 2225 & 2515 & BOGGIO & ANNA & $\mathrm{D}$ & 4 & ITALY & ITALY & ITALY & 1910 & & SAN GIUSTO CANAVESE. TO & $\begin{array}{l}\text { D ST. } \\
\text { DT. }\end{array}$ & CALUMET - RAYMBAULTOWN \\
\hline 2226 & 2515 & BOGGIO & EMMA & D & $\frac{4}{2}$ & ITALY & ITALY & ITALY & 1910 & & SAN GIUSTO CANAVESE, TO & $\begin{array}{l}\text { D ST. } \\
\text { D }\end{array}$ & CALUMET - RAYMBAULTOWN \\
\hline 2227 & 2513 & TAPPERO & JOSEPH & $\mathrm{H}$ & 38 & ITALY & ITALY & ITALY & 1898 & TIMBERMAN/CM & SAN GIUSTO CANAVESE. TO & D ST. & CALUMET - RAYMBAULTOWN \\
\hline 2228 & 2513 & TAPPERO & JOSEPHINE & w & 32 & ITALY & ITALY & ITALY & 1898 & & SAN GIUSTO CANAVESE. TO & DST. & CALUMET - RAYMBAULTOWN \\
\hline 2229 & 2513 & TAPPERO & IDA & $\mathrm{D}$ & 11 & MICHIGAN & ITALY & ITALY & & & SAN GIUSTO CANAVESE. TO & D ST. & CALUMET - RAYMBAULTOWN \\
\hline 2230 & 2513 & TAPPERO & JOSEPH & $\mathrm{s}$ & 6 & MICHIGAN & ITALY & ITALY & & & SAN GIUSTO CANAVESE. TO & D ST. & CALUMET - RAYMBAULTOWN \\
\hline 2231 & 2513 & TAPPERO & DANIEL & $\mathrm{s}$ & 5 & MICHIGAN & ITALY & ITALY & & & SAN GIUSTO CANAVESE. TO & D ST. & CALUMET - RAYMBAULTOWN \\
\hline 2232 & 2513 & TAPPERO & MARY & $\mathrm{D}$ & 2 & MICHIGAN & ITALY & ITALY & & & SAN GIUSTO CANAVESE, TO & D ST. & CALUMET - RAYMBAULTOWN \\
\hline 2233 & 2513 & AIMONE & BENEDETTO & B & 26 & ITALY & ITALY & ITALY & ???? & NOT CLEAR & TORINO & D ST. & CALUMET - RAYMBAULTOWN \\
\hline 2234 & 2513 & BARTOLINO & BARTOLOMEO & B & 27 & ITALY & ITALY & ITALY & ???? & NOT CLEAR & & D ST. & CALUMET - RAYMBAULTOWN \\
\hline 2235 & 2513 & TAPPERO & JOSEPH & $\mathrm{H}$ & 52 & ITALY & ITALY & ITALY & 1892 & WATCHMAN/CM & SAN GIUSTO CANAVESE. TO & D ST. & CALUMET - RAYMBAULTOWN \\
\hline 2236 & 2513 & TAPPERO & DOMENICA & W & 43 & ITALY & ITALY & ITALY & 1894 & & 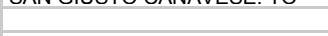 & D ST. & CALUMET - RAYMBAULTOWN \\
\hline 2237 & 2513 & TAPPERO & KATE & D & 23 & MICHIGAN & ITALY & ITALY & 1094 & SALESLADY/GROCERY STORE & SAN GIUSTO CANAVESE. TO & $\begin{array}{l}\text { D ST. } \\
\text { D }\end{array}$ & CALUMET - RAYMBAULTOWN \\
\hline 2238 & 2513 & TAPPERO & DOMENICA & $\mathrm{s}$ & 20 & MICHIGAN & ITALY & ITALY & & DRILL BOYICM & SAN GIUSTO CANAVESE, TO & D ST. & CALUMET - RAYMBAULTOWN \\
\hline 2239 & 2513 & TAPPERO & JOHN & $\mathrm{s}$ & 17 & MICHIGAN & ITALY & ITALY & & DRILL BOY/CM & SAN GIUSTO CANAVESE. TO & D ST. & CALUMET - RAYMBAULTOWN \\
\hline 2240 & 2513 & TAPPERO & JOSEPH & $\mathrm{s}$ & 16 & MICHIGAN & ITALY & ITALY & & & SAN GIUSTO CANAVESE. TO & D ST. & CALUMET - RAYMBAULTOWN \\
\hline 2241 & 2513 & TAPPERO & CARLO & $\mathrm{s}$ & 13 & MICHIGAN & ITALY & ITALY & & & SAN GIUSTO CANAVESE. TO & DST. & CALUMET - RAYMBAULTOWN \\
\hline 2242 & 2513 & TAPPERO & WILLIAM & $\mathrm{s}$ & 8 & MICHIGAN & ITALY & ITALY & & & SAN GIUSTO CANAVESE. TO & D ST. & CALUMET - RAYMBAULTOWN \\
\hline 2243 & 2513 & TAPPERO & MARY & $\mathrm{D}$ & 10 & MICHIGAN & ITALY & ITALY & & & SAN GIUSTO CANAVESE. TO & D ST. & CALUMET - RAYMBAULTOWN \\
\hline 2244 & 2513 & TAPPERO & PETER & $\mathrm{S}$ & 3 & MICHIGAN & ITALY & ITALY & & & SAN GIUSTO CANAVESE. TO & D ST. & CALUMET - RAYMBAULTOWN \\
\hline 2245 & 2513 & MARCHESI & PIETRO & $\mathrm{H}$ & 37 & ITALY & ITALY & ITALY & 1899 & MINER/CM & TORINO & D ST. & CALUMET - RAYMBAULTOWN \\
\hline 2246 & 2513 & MARCHESI & ERNESTA & w & 31 & ITALY & ITALY & ITALY & 1905 & & TORINO & D ST. & CALUMET - RAYMBAULTOWN \\
\hline 2247 & 2513 & MARCHESI & ANNETTA & $\mathrm{D}$ & 4 & MICHIGAN & ITALY & ITALY & & & TORINO & D ST. & CALUMET - RAYMBAULTOWN \\
\hline 2248 & 2509 & GIANOLIO & PIETRO & $\mathrm{H}$ & 39 & ITALY & ITALY & ITALY & 1895 & WATCHMAN/CM & SAN MARTINO CANAVESE. TO & D ST. & CALUMET - RAYMBAULTOWN \\
\hline 2249 & 2509 & GIANOLIO & MARTA & W & 39 & ITALY & ITALY & ITALY & 1895 & 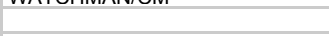 & SAN MARTINO CANAVESE. TO & D ST. & CALUMET - RAYMBAULTOWN \\
\hline 2250 & 2509 & GIANOLIO & JAMES & $\mathrm{s}$ & 12 & MICHIGAN & ITALY & ITALY & & & SAN MARTINO CANAVESE. TO & D ST. & CALUMET - RAYMBAULTOWN \\
\hline 2251 & 2509 & GIANOLIO & BERT & $\mathrm{D}$ & 9 & MICHIGAN & ITALY & ITALY & & & SAN MARTINO CANAVESE. TO & D ST. & CALUMET - RAYMBAULTOWN \\
\hline 2252 & 2509 & GIANOLIO & KATE & $\mathrm{D}$ & $10 \mathrm{M}$ & MICHIGAN & ITALY & ITALY & & & SAN MARTINO CANAVESE. TO & D ST. & CALUMET - RAYMBAULTOWN \\
\hline 2253 & 2509 & MICHETTI & DOMENICO & $\mathrm{H}$ & 26 & ITALY & ITALY & ITALY & 1900 & MINER/CM & PONT CANAVESE. TO & D ST. & CALUMET - RAYMBAULTOWN \\
\hline 2254 & 2476 & BIGANDO & GIORGIO & $\mathrm{H}$ & 54 & ITALY & ITALY & ITALY & 1893 & WATCHMAN/CM & TORINO & C ST. & CALUMET - RAYMBAULTOWN \\
\hline 2255 & 2476 & BIGANDO & VITTORIA & W & 48 & ITALY & ITALY & ITALY & 1892 & & & C ST. & CALUMET - RAYMBAULTOWN \\
\hline 2256 & 2476 & BIGANDO & PETER & $\mathrm{s}$ & 15 & MICHIGAN & ITALY & ITALY & & & TORINO & C ST. & CALUMET - RAYMBAULTOWN \\
\hline 2257 & 2476 & BATTISTI? & JOHN & B & 19 & ITALY & ITALY & ITALY & 1909 & & & C ST. & CALUMET - RAYMBAULTOWN \\
\hline 2258 & 2476 & BATTISTI? & MARY & $\mathrm{D}$ & 21 & ITALY & ITALY & ITALY & 1892 & & TORINO & C ST. & CALUMET - RAYMBAULTOWN \\
\hline 2259 & 2476 & BATTISTI? & FRANK & SOIL & 23 & ITALY & ITALY & ITALY & 1908 & TRAMMER/CM & & C ST. & CALUMET - RAYMBAULTOWN \\
\hline 2260 & 2476 & RIVA & JOHN & B & 26 & ITALY & ITALY & ITALY & 1910 & TRAMMER/CM & RIVARA CANAVESE. TO & C ST. & CALUMET - RAYMBAULTOWN \\
\hline 2261 & 2476 & PERONA & LORENZO & B & 33 & ITALY & ITALY & ITALY & 1895 & TRAMMER/CM & CASTELLAMONTE. TO & C ST. & CALUMET - RAYMBAULTOWN \\
\hline 2262 & 2476 & BRUSSO & ELENA & B & 28 & MICHIGAN & ITALY & ITALY & 1906 & & AGLIE', TO & C ST. & CALUMET - RAYMBAULTOWN \\
\hline 2263 & 2476 & BRUSSO & JOSEPH & $\mathrm{H}$ & 47 & ITALY & ITALY & ITALY & 1887 & ROLLER FIXER/CM & AGLIE'. TO & C ST. & CALUMET - RAYMBAULTOWN \\
\hline 2264 & 2476 & BRUSSO & ANTONIA & W & 40 & ITALY & ITALY & ITALY & 1892 & & & C ST. & - RAYMBAULTOWN \\
\hline 265 & 2476 & BRUSSO & BATTISTA & $\mathrm{s}$ & 15 & MICHIGAN & ITAI & ITAI & & & AGLIE'. TO & C ST. & CALUMET - RAYMBAULTO \\
\hline 266 & 2476 & BRUSSO & MARY & D & 8 & MICHIGAN & ITALY & ITALY & & & & C ST. & CALUMET \\
\hline 67 & 2474 & SCUSSEL & FRANK & $\mathrm{H}$ & 26 & ITALY & ITALY & ITALY & 1900 & MINER/CM & BELLUNO & C ST. & CALUMET - RAYMBAULTOWN \\
\hline
\end{tabular}




\begin{tabular}{|c|c|c|c|c|c|c|c|c|c|c|c|c|c|}
\hline 2268 & 2474 & SCUSSEL & MARGHERITA & W & 22 & ITALY & ITALY & ITALY & 1903 & & & C ST. & CALUMET - RAYMBAULTOWN \\
\hline 2269 & 2474 & SCUSSEL & JOHN & BR & 24 & ITALY & ITALY & ITALY & 1902 & MINER/CM & BELLUNO & C ST. & CALUMET - RAYMBAULTOWN \\
\hline 2270 & 2474 & MATTIELLO & JAMES & $\mathrm{H}$ & 27 & ITALY & ITALY & ITALY & 1905 & TRAMMER/CM & LUCCA & C ST. & CALUMET - RAYMBAULTOWN \\
\hline 2271 & 2474 & MATTIELLO & MARY & W & 22 & ITALY & ITALY & ITALY & 1903 & & & C ST. & CALUMET - RAYMBAULTOWN \\
\hline 2272 & 2474 & MATTIELLO & MARGHERITA & $\mathrm{D}$ & $15 \mathrm{M}$ & MICHIGAN & ITALY & ITALY & & & LUCCA & C ST. & CALUMET - RAYMBAULTOWN \\
\hline 2273 & 2474 & MATTIELLO & ELEONORA & $\mathrm{D}$ & 0 & MICHIGAN & ITALY & ITALY & & & LUCCA & C ST. & CALUMET - RAYMBAULTOWN \\
\hline 2274 & 2474 & MATTIELLO & GEORGE & $\mathrm{BR}$ & 40 & ITALY & ITALY & ITALY & 1909 & TRAMMER/CM & LUCCA & C ST. & CALUMET - RAYMBAULTOWN \\
\hline 2275 & 2474 & COSTA & ANTONIO & $\mathrm{H}$ & 36 & ITALY & ITALY & ITALY & 1900 & MINER/CM & TORINO & C ST. & CALUMET - RAYMBAULTOWN \\
\hline 2276 & 2474 & COSTA & MARIA & W & 33 & ITALY & ITALY & ITALY & 1900 & & TORINO & C ST. & CALUMET - RAYMBAULTOWN \\
\hline 2277 & 2474 & FAVERO & ANTONIO & B & 41 & ITALY & ITALY & ITALY & $90 ? ?$ & LABORER/ODD JOBS & & C ST. & CALUMET - RAYMBAULTOWN \\
\hline 2278 & 2474 & COSTA & MAGGIE & NIE & 8 & ITALY & ITALY & ITALY & 1903 & & TORINO & C ST. & CALUMET - RAYMBAULTOWN \\
\hline 2279 & 2474 & FAVERO & GIOVANNI & B & 8 & MICHIGAN & ITALY & ITALY & & & & C ST. & CALUMET - RAYMBAULTOWN \\
\hline 2280 & 2458 & SOPPELSA & EUGENIO & $\mathrm{H}$ & 50 & ITALY & ITALY & ITALY & 1892 & TIMBERMAN/CM & VOLTAGO. BELLUNO & C ST. & CALUMET - RAYMBAULTOWN \\
\hline 2281 & 2458 & SOPPELSA & ANTONIA & w & 40 & ITALY & ITALY & ITALY & 1897 & & & C ST. & CALUMET - RAYMBAULTOWN \\
\hline 2282 & 2458 & SOPPELSA & EUGENIO & $\mathrm{s}$ & 5 & MICHIGAN & ITALY & ITALY & & & VOLTAGO. BELLUNO & C ST. & CALUMET - RAYMBAULTOWN \\
\hline 2283 & 2458 & SOPPELSA & ANTONIO & $\mathrm{s}$ & 4 & MICHIGAN & ITALY & ITALY & & & VOLTAGO, BELLUNO & C ST. & CALUMET - RAYMBAULTOWN \\
\hline 2284 & 2458 & SOPPELSA & ANDRICA? & $?$ & 2 & MICHIGAN & ITALY & ITALY & & & VOLTAGO. BELLUNO & C ST. & CALUMET - RAYMBAULTOWN \\
\hline 2285 & 2458 & SOPPELSA & LOMO? & $?$ & 0 & MICHIGAN & ITALY & ITALY & & & VOLTAGO. BELLUNO & C ST. & CALUMET - RAYMBAULTOWN \\
\hline 2286 & 2452 & COGNONATTO & BATTISTA & $\mathrm{H}$ & 38 & ITALY & ITALY & ITALY & 1902 & MINER/CM & SAN PONSO CANAVESE. TO & C ST. & CALUMET - RAYMBAULTOWN \\
\hline 2287 & 2452 & COGNONATO & CARLOTTA & w & 33 & ITALY & ITALY & ITALY & 1903 & 年 & 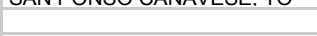 & C ST. & CALUMET - RAYMBAULTOWN \\
\hline 2288 & 2452 & COGNONATTO & MINNIE & $\mathrm{D}$ & 13 & MICHIGAN & ITALY & ITALY & & & SAN PONSO CANAVESE. TO & C ST. & CALUMET - RAYMBAULTOWN \\
\hline 2289 & 2452 & COGNONATTO & TERESA & D & 10 & MICHIGAN & ITALY & ITALY & & & SAN PONSO CANAVESE, TO & $\begin{array}{ll}\text { C ST. } \\
\text { CS. }\end{array}$ & CALUMET - RAYMBAULTOWN \\
\hline 2290 & 2452 & COGNONATTO & MARY & $\mathrm{D}$ & 5 & MICHIGAN & ITALY & ITALY & & & SAN PONSO CANAVESE. TO & C ST. & CALUMET - RAYMBAULTOWN \\
\hline 2291 & 2452 & COGNONATTO & PETER & $\mathrm{s}$ & 2 & MICHIGAN & ITALY & ITALY & & & SAN PONSO CANAVESE. TO & C ST. & CALUMET - RAYMBAULTOWN \\
\hline 2292 & 2452 & GIACHINO & JAMES & $\mathrm{H}$ & 42 & ITALY & ITALY & ITALY & 1890 & MINER/CM & TORINO & C ST. & CALUMET - RAYMBAULTOWN \\
\hline 2293 & 2452 & GIACHINO & MARIANA & W & 36 & ITALY & ITALY & ITALY & 1896 & & & C ST. & CALUMET - RAYMBAULTOWN \\
\hline 2294 & 2452 & GIACHINO & JAMES & s & 11 & MICHIGAN & ITALY & ITALY & & & TORINO & C ST. & CALUMET - RAYMBAULTOWN \\
\hline 2295 & 2452 & GIACHINO & ANNE & $\mathrm{D}$ & 8 & MICHIGAN & ITALY & ITALY & & & TORINO & C ST. & CALUMET - RAYMBAULTOWN \\
\hline 2296 & 2452 & GIACHINO & TOMMIE & $\mathrm{S}$ & 7 & MICHIGAN & ITALY & ITALY & & & TORINO & C ST. & CALUMET - RAYMBAULTOWN \\
\hline 2297 & 2452 & GIACHINO & JOHN & $\mathrm{s}$ & 3 & MICHIGAN & ITALY & ITALY & & & TORINO & C ST. & CALUMET - RAYMBAULTOWN \\
\hline 2298 & 2452 & BESSO & PETER & B & 41 & ITALY & ITALY & ITALY & 1903 & TRAMMER/CM & TORINO & C ST. & CALUMET - RAYMBAULTOWN \\
\hline 2299 & 2452 & GIACHINO & JOHN & B & 27 & ITALY & ITALY & ITALY & 1909 & TRAMMER/CM & TORINO & C ST. & CALUMET - RAYMBAULTOWN \\
\hline 2300 & 2123 & LUCCHESI & LIVIO & B & 22 & ITALY & ITALY & ITALY & 1906 & TIMBERMAN/CM & LUCCA & C ST. & CALUMET - RAYMBAULTOWN \\
\hline 2301 & 2481 & AIMONE & DOMENICO & $\mathrm{H}$ & 54 & ITALY & ITALY & ITALY & 1887 & WATCHAMN/CM & PONT CANAVESE, TO & C ST. & CALUMET - RAYMBAULTOWN \\
\hline 2302 & 2481 & AIMONE & CATERINA & w & 46 & ITALY & ITALY & ITALY & 1891 & & & C ST. & CALUMET - RAYMBAULTOWN \\
\hline 2303 & 2481 & AIMONE & $\mathrm{JOHN}$ & s & 17 & MICHIGAN & ITALY & ITALY & & & PONT CANAVESE. TO & C ST. & CALUMET - RAYMBAULTOWN \\
\hline 2304 & 2481 & AIMONE & GEORGE & $\mathrm{s}$ & 15 & MICHIGAN & ITALY & ITALY & & & PONT CANAVESE. TO & C ST. & CALUMET - RAYMBAULTOWN \\
\hline 2305 & 2481 & AIMONE & KATE & D & 14 & MICHIGAN & ITALY & ITALY & & & PONT CANAVESE. TO & C ST. & CALUMET - RAYMBAULTOWN \\
\hline 2306 & 2481 & AIMONE & ANNE & $\mathrm{D}$ & 11 & MICHIGAN & ITALY & ITALY & & & PONT CANAVESE. TO & C ST. & CALUMET - RAYMBAULTOWN \\
\hline 2307 & 2481 & AIMONE & MARY & $\mathrm{D}$ & 10 & MICHIGAN & ITALY & ITALY & & & PONT CANAVESE. TO & C ST. & CALUMET - RAYMBAULTOWN \\
\hline 2308 & 2481 & PELLA & BENEDETTO & B & 48 & ITALY & ITALY & ITALY & 1888 & TIMBERMAN/CM & & C ST. & CALUMET - RAYMBAULTOWN \\
\hline 2309 & 2481 & BARDA & PIETRO & B & 30 & ITALY & ITALY & ITALY & 1901 & TIMBERMAN/CM & QUAGLIUZZO CANAVESE. TO & C ST. & CALUMET - RAYMBAULTOWN \\
\hline 2310 & 2481 & BARDA & SILVIO & B & 21 & ITALY & ITALY & ITALY & 1907 & TRAMMER/CM & QUAGLIUZZZO CANAVESE. TO & C ST. & CALUMET - RAYMBAULTOWN \\
\hline 2311 & 2481 & AIMONE & BARTOLOMEO & B & 30 & ITALY & ITALY & ITALY & 1908 & TRAMMER/CM & TORINO & C ST. & CALUMET - RAYMBAULTOWN \\
\hline 2312 & 2481 & ROATO & MICHELE & B & 31 & ITALY & ITALY & ITALY & 1909 & TRAMMER/CM & VALPERGA. TO & C ST. & CALUMET - RAYMBAULTOWN \\
\hline 2313 & 2481 & ROATO & WILLIAM & B & 21 & ITALY & ITALY & ITALY & 1909 & TRAMMER/CM & VALPERGA. TO & C ST. & CALUMET - RAYMBAULTOWN \\
\hline 2314 & 2479 & ROSSIO & BATTISTA & $\mathrm{H}$ & 35 & ITALY & ITALY & ITALY & 1900 & TRAMMER/CM & PONT CANAVESE. TO & C ST. & CALUMET - RAYMBAULTOWN \\
\hline 2315 & 2479 & ROSSIO & MARGHERITA & w & 32 & ITALY & ITALY & ITALY & 1902 & & & C ST. & CALUMET - RAYMBAULTOWN \\
\hline 2316 & 2479 & ROSSIO & MARY & $\mathrm{D}$ & 5 & MICHIGAN & ITALY & ITALY & & & PONT CANAVESE. TO & C ST. & CALUMET - RAYMBAULTOWN \\
\hline 2317 & 2479 & ROSSIO & ASSUNTA & $\mathrm{D}$ & 4 & MICHIGAN & ITALY & ITALY & & & PONT CANAVESE. TO & C ST. & CALUMET - RAYMBAULTOWN \\
\hline 2318 & 2479 & ROSSIO & ROSIE & $\mathrm{D}$ & 2 & MICHIGAN & ITALY & ITALY & & & PONT CANAVESE. TO & C ST. & CALUMET - RAYMBAULTOWN \\
\hline 2319 & 2477 & PICCHIOTTINO & PAUL & $\mathrm{H}$ & 48 & ITALY & ITALY & ITALY & 1892 & WATCHMAN/CM & PONT CANAVESE, TO & C ST. & CALUMET - RAYMBAULTOWN \\
\hline 2320 & 2477 & PICCHIOTTINO & MINNIE & W & 42 & ITALY & ITALY & ITALY & UN & & PONT CANAVESE. TO & C ST. & CALUMET - RAYMBAULTOWN \\
\hline 2321 & 2477 & PICCHIOTTINO & JOHN & $\mathrm{s}$ & 22 & MICHIGAN & ITALY & ITALY & & TEAMSTER/BAKERY & PONT CANAVESE. TO & C ST. & CALUMET - RAYMBAULTOWN \\
\hline 2322 & 2477 & PICCHIOTTINO & JOSEPH & $\mathrm{s}$ & 15 & MICHIGAN & ITALY & ITALY & & & PONT CANAVESE. TO & C ST. & CALUMET - RAYMBAULTOWN \\
\hline 2323 & 2477 & PICCHIOTTINO & ANNE & D & 12 & MICHIGAN & ITALY & ITALY & & & PONT CANAVESE. TO & C ST. & CALUMET - RAYMBAULTOWN \\
\hline 2324 & 2477 & PICCHIOTTINO & MARY & $\mathrm{D}$ & 10 & MICHIGAN & ITALY & ITALY & & & PONT CANAVESE. TO & C ST. & CALUMET - RAYMBAULTOWN \\
\hline 2325 & 2477 & PICCHIOTTINO & FRANK & $\mathrm{s}$ & 8 & MICHIGAN & ITALY & ITALY & & & PONT CANAVESE, TO & C ST. & CALUMET - RAYMBAULTOWN \\
\hline 2326 & 2477 & PICCHIOTTINO & GIORGIO & $\mathrm{s}$ & 4 & MICHIGAN & ITALY & ITALY & & & PONT CANAVESE. TO & C ST. & CALUMET - RAYMBAULTOWN \\
\hline 2327 & 2451 & MACARIO & PIETRO & $\mathrm{H}$ & 45 & ITALY & ITALY & ITALY & 1899 & TIMBERMAN/CM & TORINO & C ST. & CALUMET - RAYMBAULTOWN \\
\hline 328 & 2451 & MACARIO & TERESA & W & 38 & ITALY & ITAI & ITALY & 1891 & & TORINO & C ST. & CALUMET - RAYMBAULTOWN \\
\hline 329 & 2451 & MACARIO & JOHN & s & 21 & ITALY & ITALY & ITALY & 1891 & CAR FILLER/CM & TORINO & C ST. & CALUMET - RA \\
\hline 30 & 2451 & MACARIO & CATERINA & $\mathrm{D}$ & 18 & MICHIGAN & ITALY & ITALY & & & TORINO & C ST. & CALUMET - RAYMBAULTOWN \\
\hline
\end{tabular}




\begin{tabular}{|c|c|c|c|c|c|c|c|c|c|c|c|c|c|}
\hline 2331 & 2451 & MACARIO & JOSEPH & $\mathrm{s}$ & 13 & MICHIGAN & ITALY & ITALY & & & TORINO & C ST. & CALUMET - RAYMBAULTOWN \\
\hline 2332 & 2451 & MACARIO & PETER & $\mathrm{S}$ & 10 & MICHIGAN & ITALY & ITALY & & & TORINO & C ST. & CALUMET - RAYMBAULTOWN \\
\hline 2333 & 2451 & MACARIO & MARTIN & $\mathrm{s}$ & $21 ?$ & MICHIGAN & ITALY & ITALY & & & TORINO & C ST. & CALUMET - RAYMBAULTOWN \\
\hline 2334 & 2451 & MACARIO & JOHN & BRIL & 65 & ITALY & ITALY & ITALY & 1891 & & TORINO & C ST. & CALUMET - RAYMBAULTOWN \\
\hline 2335 & 2404 & ZANARDI & JOSEPH & $\mathrm{H}$ & 53 & ITALY & ITALY & ITALY & 1888 & WATCHMAN/CM & SELINO. BERGAMO & B ST. & CALUMET - RAYMBAULTOWN \\
\hline 2336 & 2404 & ZANARDI & MARY & w & 38 & ITALY & ITALY & ITALY & 1897 & & & B ST. & CALUMET - RAYMBAULTOWN \\
\hline 2337 & 2404 & ZANARDI & CARLO & s & 12 & MICHIGAN & ITALY & ITALY & & & SELINO. BERGAMO & B ST. & CALUMET - RAYMBAULTOWN \\
\hline 2338 & 2404 & ZANARDI & JOSEPH & s & 11 & MICHIGAN & ITALY & ITALY & & & SELINO. BERGAMO & B ST. & CALUMET - RAYMBAULTOWN \\
\hline 2339 & 2404 & ZANARDI & JOHN & s & 10 & MICHIGAN & ITALY & ITALY & & & SELINO. BERGAMO & B ST. & CALUMET - RAYMBAULTOWN \\
\hline 2340 & 2406 & CENA & PAUL & $\mathrm{H}$ & 37 & ITALY & ITALY & ITALY & 1904 & TRAMMER/CM & SAN BENIGNO CANAVESE. TO & B ST. & CALUMET - RAYMBAULTOWN \\
\hline 2341 & 2406 & CENA & TERESA & w & 37 & ITALY & ITALY & ITALY & 1904 & & SAN BENIGNO CANAVESE. TO & B ST. & CALUMET - RAYMBAULTOWN \\
\hline 2342 & 2406 & CENA & CARLO & $\mathrm{s}$ & 12 & MICHIGAN & ITALY & ITALY & & & SAN BENIGNO CANAVESE. TO & B ST. & CALUMET - RAYMBAULTOWN \\
\hline 2343 & 2406 & CENA & JOSEPHINE & $\mathrm{D}$ & 11 & MICHIGAN & ITALY & ITALY & & & SAN BENIGNO CANAVESE. TO & B ST. & CALUMET - RAYMBAULTOWN \\
\hline 2344 & 2426 & GAIS & DOMENICO & $\mathrm{H}$ & 24 & ITALY & ITALY & ITALY & 1900 & MINER/CM & TORINO & B ST. & CALUMET - RAYMBAULTOWN \\
\hline 2345 & 2426 & GAIS & MARY & W & 20 & ITALY & ITALY & ITALY & 1909 & & & B ST. & CALUMET - RAYMBAULTOWN \\
\hline 2346 & 2426 & COPPO & ANTONIO & $\mathrm{H}$ & 45 & ITALY & ITALY & ITALY & 1882 & SURFACE MAN/CM & PONT CANAVESE, TO & B ST. & CALUMET - RAYMBAULTOWN \\
\hline 2347 & 2426 & COPPO & MARY & W & 40 & ITALY & ITALY & ITALY & 1890 & & & B ST. & CALUMET - RAYMBAULTOWN \\
\hline 2348 & 2426 & COPPO & VITTORIO & s & 18 & MICHIGAN & ITALY & ITALY & & & PONT CANAVESE. TO & B ST. & CALUMET - RAYMBAULTOWN \\
\hline 2349 & 2426 & COPPO & JOHN & $\mathrm{s}$ & 16 & MICHIGAN & ITALY & ITALY & & & PONT CANAVESE. TO & B ST. & CALUMET - RAYMBAULTOWN \\
\hline 2350 & 2426 & COPPO & ANNA & $\mathrm{D}$ & 14 & MICHIGAN & ITALY & ITALY & & & PONT CANAVESE. TO & $\begin{array}{l}\text { B ST. } \\
\text { ST. }\end{array}$ & CALUMET - RAYMBAULTOWN \\
\hline 2351 & 2426 & COPPO & VITTORIA & $\mathrm{D}$ & 9 & MICHIGAN & $\begin{array}{l}\text { ITALY } \\
\text { ITAL }\end{array}$ & ITALY & & & PONT CANAVESE. TO & B ST. & CALUMET - RAYMBAULTOWN \\
\hline 2352 & 2426 & COPPO & EMILIA & D & 6 & MICHIGAN & $\begin{array}{l}\text { ITALY } \\
\text { ITAL }\end{array}$ & ITALY & & & PONT CANAVESE, TO & B ST. & CALUMET - RAYMBAULTOWN \\
\hline 2353 & 2421 & ALLARIA & JOHN & $\mathrm{H}$ & 34 & ITALY & ITALY & ITALY & 1888 & MINER/CM & SAN GIORGIO CANAVESE. TO & B ST. & CALUMET - RAYMBAULTOWN \\
\hline 2354 & 2421 & ALLARIA & MARY & W & 28 & ITALY & ITALY & ITALY & 1900 & & & B ST. & CALUMET - RAYMBAULTOWN \\
\hline 2355 & 2421 & ALLARIA & NOTA & $\mathrm{D}$ & 8 & MICHIGAN & ITALY & ITALY & & & SAN GIORGIO CANAVESE. TO & BST. & CALUMET - RAYMBAULTOWN \\
\hline 2356 & 2421 & ALLARIA & PETER & s & 6 & MICHIGAN & ITALY & ITALY & & & SAN GIORGIO CANAVESE. TO & B ST. & CALUMET - RAYMBAULTOWN \\
\hline 2357 & 2421 & ALLARIA & JOHN & $\mathrm{s}$ & 4 & MICHIGAN & ITALY & ITALY & & & SAN GIORGIO CANAVESE. TO & B ST. & CALUMET - RAYMBAULTOWN \\
\hline 2358 & 2421 & ALLARIA & TERESA & $\mathrm{D}$ & 15 & MICHIGAN & ITALY & ITALY & & & SAN GIORGIO CANAVESE, TO & BST. & CALUMET - RAYMBAULTOWN \\
\hline 2359 & 2421 & AIMONE & FRANK & $\mathrm{H}$ & 41 & ITALY & ITALY & ITALY & 1903 & TIMBERMAN/CM & PONT CANAVESE. TO & B ST. & CALUMET - RAYMBAULTOWN \\
\hline 2360 & 2421 & AIMONE & CARLOTTA & W & 42 & ITALY & ITALY & ITALY & 1905 & & & B ST. & CALUMET - RAYMBAULTOWN \\
\hline 2361 & 2421 & DIGHERA & GEORGE & BRIL & 37 & ITALY & ITALY & ITALY & 1900 & TIMBERMAN/CM & SAN GIORGIO CANAVESE. TO & B ST. & CALUMET - RAYMBAULTOWN \\
\hline 2362 & 2421 & DIGHERA & MARIA & SIIL & 32 & ITALY & ITALY & ITALY & 1909 & 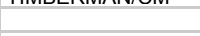 & SAN GIORGIO CANAVESE. TO & B ST. & CALUMET - RAYMBAULTOWN \\
\hline 2363 & 2421 & DE FILIPPI & JOHN & B & 34 & $\begin{array}{l}\text { ITALY } \\
\text { ITAL }\end{array}$ & ITALY & ITALY & 1907 & TRAMMER/CM & SAN GIORGIO CANAVESE. TO & B ST. & CALUMET - RAYMBAULTOWN \\
\hline 2364 & 2421 & COSTORIACO? & EUGENIO & B & 22 & ITALY & ITALY & ITALY & 1910 & TRAMMER/CM & & B ST. & CALUMET - RAYMBAULTOWN \\
\hline 2365 & 2421 & COSTORIACO? & JOHN & B & 23 & ITALY & ITALY & ITALY & 1909 & TRAMMER/CM & & B ST. & CALUMET - RAYMBAULTOWN \\
\hline 2366 & 2421 & COSTORIACO? & DOMENICO & B & 25 & ITALY & ITALY & ITALY & 1909 & TRAMMER/CM & & B ST. & CALUMET - RAYMBAULTOWN \\
\hline 2367 & 2421 & ANDRIETTA & ANTONIO & B & 28 & ITALY & ITALY & ITALY & 1903 & TRAMMER/CM & & B ST. & CALUMET - RAYMBAULTOWN \\
\hline 2368 & 2421 & ANDRIETTA & CATERINA & B & 19 & ITALY & ITALY & ITALY & 1892 & TRAMMER/CM & & B ST. & CALUMET - RAYMBAULTOWN \\
\hline 2369 & 2421 & TORREANO & TERESA & SER & 14 & MICHIGAN & ITALY & ITALY & & & TORINO & B ST. & CALUMET - RAYMBAULTOWN \\
\hline 2370 & 2411 & GASPARDO & DOMENICO & $\mathrm{H}$ & 49 & ITALY & ITALY & ITALY & 1899 & WATCHMAN/CM & PONT CANAVESE. TO & B ST. & CALUMET - RAYMBAULTOWN \\
\hline 2371 & 2411 & GASPARDO & MARY & W & 38 & ITALY & ITALY & ITALY & 1899 & & PONT CANAVESE. TO & B ST. & CALUMET - RAYMBAULTOWN \\
\hline 2372 & 2411 & GASPARDO & JAMES & s & 7 & MICHIGAN & ITALY & ITALY & & & PONT CANAVESE. TO & B ST. & CALUMET - RAYMBAULTOWN \\
\hline 2373 & 2411 & GASPARDO & DOMENICO & $\mathrm{s}$ & 5 & MICHIGAN & ITALY & ITALY & & & PONT CANAVESE. TO & B ST. & CALUMET - RAYMBAULTOWN \\
\hline 2374 & 2411 & CALVETTI & SEVERINO & $\mathrm{H}$ & 32 & ITALY & ITALY & ITALY & 1905 & TIMBERMAN/CM & MILANO & B ST. & CALUMET - RAYMBAULTOWN \\
\hline 2375 & 2411 & CALVETTI & MARIA & W & 26 & ITALY & ITALY & ITALY & 1907 & 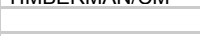 & ivitarve & B ST. & CALUMET - RAYMBAULTOWN \\
\hline 2376 & 2411 & CALVETTI & ROCCO & $\mathrm{s}$ & 6 & MICHIGAN & ITALY & ITALY & & & MILANO & B ST. & CALUMET - RAYMBAULTOWN \\
\hline 2377 & 2411 & CALVETTI & JOHN & $\mathrm{s}$ & 4 & MICHIGAN & ITALY & ITALY & & & MILANO & B ST. & CALUMET - RAYMBAULTOWN \\
\hline 2378 & 2411 & CALVETTI & CATERINA & $\mathrm{D}$ & 2 & MICHIGAN & ITALY & ITALY & & & MILANO & B ST. & CALUMET - RAYMBAULTOWN \\
\hline 2379 & 2411 & CALVETTI & MAULE & $\mathrm{D}$ & $4 \mathrm{M}$ & MICHIGAN & ITALY & ITALY & & & MILANO & B ST. & CALUMET - RAYMBAULTOWN \\
\hline 2380 & 2411 & BETTINI & JOSEPH & B & 31 & ITALY & ITALY & ITALY & 1904 & TRAMMER/CM & & B ST. & CALUMET - RAYMBAULTOWN \\
\hline 2381 & 2411 & RICCA & MICHAEL & B & 28 & ITALY & ITALY & ITALY & $08 ?$ & DISC FIXER/CM & RIVAROLO CANAVESE. TO & B ST. & CALUMET - RAYMBAULTOWN \\
\hline 2382 & 2413 & OSTORERO & PETER & $\mathrm{H}$ & 33 & ITALY & ITALY & ITALY & 1905 & TIMBERMAN/CM & TORINO & B ST. & CALUMET - RAYMBAULTOWN \\
\hline 2383 & 2413 & OSTORERO & TERESA & W & $23 ?$ & ITALY & ITALY & ITALY & 1905 & & TORINO & B ST. & CALUMET - RAYMBAULTOWN \\
\hline 2384 & 2413 & OSTORERO & ANDREW & $\mathrm{s}$ & 3 & MICHIGAN & ITALY & ITALY & & & TORINO & BST. & CALUMET - RAYMBAULTOWN \\
\hline 2385 & 2413 & OSTORERO & PAUL & $\mathrm{s}$ & $2 \mathrm{M}$ & MICHIGAN & ITALY & ITALY & & & TORINO & B ST. & CALUMET - RAYMBAULTOWN \\
\hline 2386 & 2403 & GIULIO & PIETRO & $\mathrm{H}$ & 39 & ITALY & ITALY & ITALY & 1899 & TIMBERMAN/CM & PONT CANAVESE. TO & B ST. & CALUMET - RAYMBAULTOWN \\
\hline 2387 & 2403 & GIULIO & URSULA & W & 35 & ITALY & ITALY & ITALY & 1901 & & & B ST. & CALUMET - RAYMBAULTOWN \\
\hline 2388 & 2403 & GIULIO & MARY & $\mathrm{D}$ & 12 & MICHIGAN & ITALY & ITALY & & & PONT CANAVESE, TO & $\begin{array}{l}\text { B ST. } \\
\text { B }\end{array}$ & CALUMET - RAYMBAULTOWN \\
\hline 2389 & 2403 & GIULIO & MAGGIE & $\mathrm{D}$ & 10 & MICHIGAN & ITALY & ITALY & & & PONT CANAVESE. TO & B ST. & CALUMET - RAYMBAULTOWN \\
\hline 2390 & 2403 & GIULIO & JAMES & S & 8 & MICHIGAN & ITALY & ITALY & & & PONT CANAVESE. TO & B ST. & CALUMET - RAYMBAULTOWN \\
\hline 2391 & 2403 & GIULIO & & $\mathrm{s}$ & 6 & MICHIGAN & ITAL & ITALY & & & PONT CANAVESE. TO & B ST. & CALUMET - RAYMBAULTOWN \\
\hline 2392 & 2403 & MASSOGLIA & DOMENICO & $\mathrm{H}$ & 25 & ITALY & ITALY & ITALY & 1901 & TRAMMER/CM & SAN GIOVANNI CANAVESE. TO & B ST. & CALUMET - RAYMBAULTOWN \\
\hline 2393 & 2403 & MASSOGLIA & HELENA & w & 26 & ITALY & ITALY & ITALY & 1904 & & & $\begin{array}{l}\text { B ST. } \\
\text { ST }\end{array}$ & CALUMET - RAYMBAULTOWN \\
\hline
\end{tabular}




\begin{tabular}{|c|c|c|c|c|c|c|c|c|c|c|c|c|c|}
\hline 2394 & 2403 & MASSOGLIA & ANGELINA & $\mathrm{D}$ & 3 & MICHIGAN & ITALY & ITALY & & & SAN GIOVANNI CANAVESE. TO & B ST. & CALUMET - RAYMBAULTOWN \\
\hline 2395 & 2655 & CURTO & BATTISTA & $\mathrm{H}$ & 33 & ITALY & ITALY & ITALY & 1897 & TIMBERMAN/CM & AGLIE'. TO & UNION ST. & CALUMET - RAYMBAULTOWN \\
\hline 2396 & 2655 & CURTO & DOMENICA & W & 28 & ITALY & ITALY & ITALY & 1900 & & & UNION ST. & CALUMET - RAYMBAULTOWN \\
\hline 2397 & 2655 & CURTO & MASSIMO & s & 2 & MICHIGAN & ITALY & ITALY & & & AGLIE'. TO & UNION ST. & CALUMET - RAYMBAULTOWN \\
\hline 2398 & 2655 & CURTO & JOSEPH & SSON & 4 & MICHIGAN & ITALY & ITALY & & & AGLIE', TO & UNION ST. & CALUMET - RAYMBAULTOWN \\
\hline 2399 & 2655 & CURTO & TOMMASO & s & 7 & MICHIGAN & ITALY & ITALY & & & AGLIE'. TO & UNION ST. & CALUMET - RAYMBAULTOWN \\
\hline 2400 & 2667 & ANTONIETTI & GIORGIO & $\mathrm{H}$ & 38 & ITALY & ITALY & ITALY & 1906 & TIMBERMAN/CM & SAN GENNARO. LU & UNION ST. & CALUMET - RAYMBAULTOWN \\
\hline 2401 & 2667 & ANTONIETTI & GIULIA & W & 28 & ITALY & ITALY & ITALY & 1908 & HIVIDERIVIATVICIVI & SAIV OEIVIVART. LU & UNION ST. & CALUMET - RAYMBAULTOWN \\
\hline 2402 & 2667 & ANTONIETTI & REMO & s & 6 & MICHIGAN & ITALY & ITALY & & & SAN GENNARO. LU & UNION ST. & CALUMET - RAYMBAULTOWN \\
\hline 2403 & 2667 & ANTONIETTI & BRUNA & D & 4 & MICHIGAN & ITALY & ITALY & & & SAN GENNARO. LU & UNION ST. & CALUMET - RAYMBAULTOWN \\
\hline 2404 & 2667 & ANTONIETTI & JAMES & $\mathrm{s}$ & 2 & MICHIGAN & ITALY & ITALY & & & SAN GENNARO. LU & UNION ST. & CALUMET - RAYMBAULTOWN \\
\hline 2405 & 2667 & LUCCHESI & EMILIO & B & 29 & ITALY & ITALY & ITALY & 1907 & TRAMMER/CM & LUCCA & UNION ST. & CALUMET - RAYMBAULTOWN \\
\hline 2406 & 2667 & DONATO & ADOLFO & B & 26 & ITALY & ITALY & ITALY & 1907 & TRAMMER/CM & & UNION ST. & CALUMET - RAYMBAULTOWN \\
\hline 2407 & 2667 & SERAFINI & MAURIZIO & B & 31 & ITALY & ITALY & ITALY & 1907 & TRAMMER/CM & LUCCA & UNION ST. & CALUMET - RAYMBAULTOWN \\
\hline 2408 & 2669 & NARDI & ROBERTO & $\mathrm{H}$ & 39 & ITALY & ITALY & ITALY & 1892 & MINER/CM & LUCCA & UNION ST. & CALUMET - RAYMBAULTOWN \\
\hline 2409 & 2669 & NARDI & CESARINA & W & 35 & ITALY & ITALY & ITALY & 1892 & & LUCCA & UNION ST. & CALUMET - RAYMBAULTOWN \\
\hline 2410 & 2669 & NARDI & $\mathrm{ROBI}$ & $\mathrm{s}$ & $14 / 9$ & MICHIGAN & ITALY & ITALY & & & LUCCA & UNION ST. & CALUMET - RAYMBAULTOWN \\
\hline 2411 & 2669 & NARDI & GIULIA & $\mathrm{D}$ & 15 & MICHIGAN & ITALY & ITALY & & DRESSMAKER/AT HOME & LUCCA & UNION ST. & CALUMET - RAYMBAULTOWN \\
\hline 2412 & 2669 & NARDI & JOSEPHINA & $\mathrm{D}$ & 12 & MICHIGAN & ITALY & ITALY & & & LUCCA & UNION ST. & CALUMET - RAYMBAULTOWN \\
\hline 2413 & 2669 & NARDI & MIKE & $\mathrm{s}$ & 10 & MICHIGAN & ITALY & ITALY & & & LUCCA & UNION ST. & CALUMET - RAYMBAULTOWN \\
\hline 2414 & 2669 & NARDI & CHESTER & s & 7 & MICHIGAN & ITALY & ITALY & & & LUCCA & UNION ST. & CALUMET - RAYMBAULTOWN \\
\hline 2415 & 2669 & NARDI & CECILIA & $\mathrm{D}$ & 4 & MICHIGAN & ITALY & ITALY & & & LUCCA & UNION ST. & CALUMET - RAYMBAULTOWN \\
\hline 2416 & 2669 & NARDI & MIKE & NEP & 19 & MICHIGAN & ITALY & ITALY & & & LUCCA & UNION ST. & CALUMET - RAYMBAULTOWN \\
\hline 2417 & 2669 & SODINI & ARTURO & $\mathrm{H}$ & 29 & ITALY & ITALY & ITALY & 1903 & LABORER/CM & COLLODI. LU & UNION ST. & CALUMET - RAYMBAULTOWN \\
\hline 2418 & 2669 & SODINI & NUNZIATA & MO & 57 & ITALY & ITALY & ITALY & 1906 & & & UNION ST. & CALUMET - RAYMBAULTOWN \\
\hline 2419 & 2675 & PAGNUCCI & LUIGI & $\mathrm{H}$ & & ITALY & ITALY & ITALY & 1890 & MINER/CM & RUOTA. LU & UNION ST. & CALUMET - RAYMBAULTOWN \\
\hline 2420 & 2675 & PAGNUCCI & CHERUBINA & W & & MICHIGAN & ITALY & ITALY & & & & UNION ST. & CALUMET - RAYMBAULTOWN \\
\hline 2421 & 2675 & PAGNUCCI & LIDIA & $\mathrm{D}$ & & MICHIGAN & ITALY & MICHIGAN & & & RUOTA, LU & UNION ST. & CALUMET - RAYMBAULTOWN \\
\hline 2422 & 2675 & PAGNUCCI & PAUL & $\mathrm{s}$ & & MICHIGAN & ITALY & MICHIGAN & & & RUOTA. LU & UNION ST. & CALUMET - RAYMBAULTOWN \\
\hline 2423 & 2675 & PAGNUCCI & LOUIS & $\mathrm{S}$ & & MICHIGAN & ITALY & MICHIGAN & & & RUOTA. LU & UNION ST. & CALUMET - RAYMBAULTOWN \\
\hline 2424 & 2675 & PAGNUCCI & ARTELLIO & $\mathrm{S}$ & & MICHIGAN & ITALY & MICHIGAN & & & RUOTA. LU & UNION ST. & CALUMET - RAYMBAULTOWN \\
\hline 2425 & 2675 & PAGNUCCI & SERAFINO & B & $?$ & ITALY & ITALY & ITALY & 1907 & MINER/CM & RUOTA. LU & UNION ST. & CALUMET - RAYMBAULTOWN \\
\hline 2426 & 2768 & GHIGGIA & DOMENICO & $\begin{array}{l}\mathrm{D} \\
\mathrm{H}\end{array}$ & 36 & $\begin{array}{l}\text { ITALY } \\
\text { ITAL }\end{array}$ & ITALY & $\begin{array}{l}\text { ITALY } \\
\text { ITAL }\end{array}$ & 1890 & MINER/CM & SCARMAGNO. TO & UNION ST. & CALUMET - RAYMBAULTOWN \\
\hline 2427 & 2768 & GHIGGIA & PAOLINA & W & 27 & ITALY & ITALY & ITALY & 1896 & & & UNION ST. & CALUMET - RAYMBAULTOWN \\
\hline 2428 & 2768 & GHIGGIA & LOUIS & s & 3 & MICHIGAN & ITALY & ITALY & & & SCARMAGNO. TO & UNION ST. & CALUMET - RAYMBAULTOWN \\
\hline 2429 & 2760 & LUCCHESI & CENTURIO & $\mathrm{H}$ & 29 & ITALY & ITALY & ITALY & 1894 & TRAMMER/CM & LUCCA & COLUMBIA ST. & CALUMET - RAYMBAULTOWN \\
\hline 2430 & 2760 & LUCCHESI & ANTONIETTA & W & 35 & ITALY & ITALY & ITALY & 1899 & & & COLUMBIA ST. & CALUMET - RAYMBAULTOWN \\
\hline 2431 & 2760 & LUCCHESI & MARY & $\mathrm{D}$ & 7 & MICHIGAN & ITALY & ITALY & & & LUCCA & COLUMBIA ST. & CALUMET - RAYMBAULTOWN \\
\hline 2432 & 2760 & LUCCHESI & PAOLINA & $\mathrm{D}$ & 5 & MICHIGAN & ITALY & ITALY & & & LUCCA & COLUMBIA ST. & CALUMET - RAYMBAULTOWN \\
\hline 2433 & 2760 & LUCCHESI & ANGELINA & $\mathrm{D}$ & 2 & MICHIGAN & ITALY & ITALY & & & LUCCA & COLUMBIA ST. & CALUMET - RAYMBAULTOWN \\
\hline 2434 & 2760 & LUCCHESI & IDA & D & $7 \mathrm{M}$ & MICHIGAN & ITALY & ITALY & & & LUCCA & COLUMBIA ST. & CALUMET - RAYMBAULTOWN \\
\hline 2435 & 2760 & SALANI & FRANCESCO & $\mathrm{H}$ & 33 & ITALY & ITALY & ITALY & 1905 & TIMBERMAN/CM & COLLODI. LU & COLUMBIA ST. & CALUMET - RAYMBAULTOWN \\
\hline 2436 & 2760 & SALANI & PETRONILLA & w & 26 & ITALY & ITALY & ITALY & 1905 & & COLLODI. LU & COLUMBIA ST. & CALUMET - RAYMBAULTOWN \\
\hline 2437 & 2760 & SALANI & CELESTINA & $\mathrm{D}$ & 4 & MICHIGAN & ITALY & ITALY & & & COLLODI. LU & COLUMBIA ST. & CALUMET - RAYMBAULTOWN \\
\hline 2438 & 2758 & PICCHIOTTINO & FRED & $\mathrm{H}$ & 49 & ITALY & ITALY & ITALY & 1886 & LANDER/CM & PONT CANAVESE. TO & COLUMBIA ST. & CALUMET - RAYMBAULTOWN \\
\hline 2439 & 2758 & PICCHIOTTINO & MADELINA & W & 47 & ITALY & ITALY & ITALY & 1889 & & & COLUMBIA ST. & CALUMET - RAYMBAULTOWN \\
\hline 2440 & 2758 & PICCHIOTTINO & PAUL & s & 24 & MICHIGAN & ITALY & ITALY & & FIREMAN/HOSPITAL & PONT CANAVESE. TO & COLUMBIA ST. & CALUMET - RAYMBAULTOWN \\
\hline 2441 & 2758 & PICCHIOTTINO & MADELINE & $\mathrm{D}$ & 27 & MICHIGAN & ITALY & ITALY & & & PONT CANAVESE. TO & COLUMBIA ST. & CALUMET - RAYMBAULTOWN \\
\hline 2442 & 2758 & PICCHIOTTINO & JENNIE & $\mathrm{D}$ & 20 & MICHIGAN & ITALY & ITALY & & & PONT CANAVESE. TO & COLUMBIA ST. & CALUMET - RAYMBAULTOWN \\
\hline 2443 & 2758 & PICCHIOTTINO & MICHAEL & $\mathrm{s}$ & 16 & MICHIGAN & ITALY & ITALY & & & PONT CANAVESE. TO & COLUMBIA ST. & CALUMET - RAYMBAULTOWN \\
\hline 2444 & 2758 & PICCHIOTTINO & MARY & $\mathrm{D}$ & 12 & MICHIGAN & ITALY & ITALY & & & PONT CANAVESE. TO & COLUMBIA ST. & CALUMET - RAYMBAULTOWN \\
\hline 2445 & 2758 & PICCHIOTTINO & ROSE & D & 10 & MICHIGAN & ITALY & ITALY & & & PONT CANAVESE, TO & COLUMBIA ST. & CALUMET - RAYMBAULTOWN \\
\hline 2446 & 2758 & PICCHIOTTINO & GIULIA & $\mathrm{D}$ & 10 & MICHIGAN & ITALY & ITALY & & & PONT CANAVESE. TO & COLUMBIA ST. & CALUMET - RAYMBAULTOWN \\
\hline 2447 & 2758 & PICCHIOTTINO & FLORENCE & D & 7 & MICHIGAN & ITALY & ITALY & & & PONT CANAVESE. TO & COLUMBIA ST. & CALUMET - RAYMBAULTOWN \\
\hline 2448 & 2754 & SAVINO & ENRICO & $\mathrm{H}$ & 27 & ITALY & ITALY & ITALY & 1900 & MINER/CM & SCARMAGNO. TO & COLUMBIA ST. & CALUMET - RAYMBAULTOWN \\
\hline 2449 & 2754 & SAVINO & MARIA & w & 24 & ITALY & ITALY & ITALY & $06 ?$ & & SCARMAGNO. TO & COLUMBIA ST. & CALUMET - RAYMBAULTOWN \\
\hline 2450 & 2754 & SAVINO & ANTONIETTA & $\mathrm{D}$ & 3 & MICHIGAN & ITALY & ITALY & & & SCARMAGNO. TO & COLUMBIA ST. & CALUMET - RAYMBAULTOWN \\
\hline 2451 & 2754 & SAVINO & JAMES & $\mathrm{s}$ & $12 \mathrm{M}$ & MICHIGAN & ITALY & ITALY & & & SCARMAGNO, TO & COLUMBIA ST. & CALUMET - RAYMBAULTOWN \\
\hline 2452 & 2754 & BOGGIO & ALEX & B & 23 & ITALY & ITALY & ITALY & 1910 & LABORER/CM & SAN GIUSTO CANAVESE. TO & COLUMBIA ST. & CALUMET - RAYMBAULTOWN \\
\hline 2453 & 2754 & FRASA & JOHN & B & 28 & ITALY & ITALY & ITALY & 1908 & TRAMMER/CM & & COLUMBIA ST. & CALUMET - RAYMBAULTOWN \\
\hline 2454 & 2754 & PALPERETTI & GIUSEPPE & B & 28 & & ITA & ITALY & 1906 & ERMAN/CM & & & LTOWN \\
\hline 2455 & 2754 & BESSONE & & B & 31 & & ITALY & ITALY & 1906 & TIMBERMAN/CM & SAN BENIGNO CANAVESE. TO & COLUMBIA ST. & CALUMET - RAYMBAULTOWN \\
\hline 2456 & 3573 & LOSCETTO & JAMES & $\mathrm{H}$ & 27 & ITALY & ITALY & ITALY & 1907 & TRAMMER/CM & & COLUMBIA ST. & CALUMET - RAYMBAULTOWN \\
\hline
\end{tabular}




\begin{tabular}{|c|c|c|c|c|c|c|c|c|c|c|c|c|c|}
\hline 2457 & 3573 & LOSCETTO & LOUISE & W & 25 & ITALY & ITALY & ITALY & 1907 & & & COLUMBIA ST. & CALUMET - RAYMBAULTOWN \\
\hline 2458 & 4814 & CENTANINO & DOMENICO & $\mathrm{H}$ & 35 & ITALY & ITALY & ITALY & 1890 & MINER/CM & SAN MARTINO CANAVESE. TO & RED JACKET SHAFT & CALUMET - RED JACKET SHAFT \\
\hline 2459 & 4814 & CENTANINO & MAGGIE & W & 36 & ITALY & ITALY & ITALY & 1891 & & & RED JACKET SHAFT & CALUMET - RED JACKET SHAFT \\
\hline 2460 & 4814 & CENTANINO & DOMENICO & s & 15 & MICHIGAN & ITALY & ITALY & & & SAN MARTINO CANAVESE. TO & RED JACKET SHAFT & CALUMET - RED JACKET SHAFT \\
\hline 2461 & 4814 & CENTANINO & JOHN & $\mathrm{s}$ & 14 & MICHIGAN & ITALY & ITALY & & & SAN MARTINO CANAVESE. TO & RED JACKET SHAFT & CALUMET - RED JACKET SHAFT \\
\hline 2462 & 4814 & CENTANINO & MARY & $\mathrm{D}$ & 12 & MICHIGAN & ITALY & ITALY & & & SAN MARTINO CANAVESE. TO & RED JACKET SHAFT & CALUMET - RED JACKET SHAFT \\
\hline 2463 & 4814 & CENTANINO & LOUISE & $\mathrm{D}$ & $1 \mathrm{M}$ & MICHIGAN & ITALY & ITALY & & & SAN MARTINO CANAVESE. TO & RED JACKET SHAFT & CALUMET - RED JACKET SHAFT \\
\hline 2464 & $\begin{array}{l}4014 \\
4814\end{array}$ & COLOMBO & $\begin{array}{l}\text { JOSEPH } \\
\text { JOSE }\end{array}$ & B & 49 & ITALY & ITALY & ITALY & $90 / 95$ & MINER/CM & 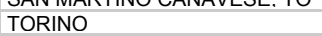 & RED JACKET SHAFT & CALUMET - RED JACKET SHAFT \\
\hline 2465 & $\begin{array}{l}4014 \\
47\end{array}$ & PACINI & MIKE & $\begin{array}{l}\mathrm{O} \\
\mathrm{H}\end{array}$ & 56 & ITALY & ITALY & ITALY & 1893 & LABORER/CM & FIRENZE & $\begin{array}{l}\text { TAMARACK ST. } \\
\text { TART }\end{array}$ & $\begin{array}{l}\text { CALUMET - TAMARACK } \\
\text { CAAT }\end{array}$ \\
\hline 2466 & 47 & PACINI & MARY & w & 53 & ITALY & ITALY & ITALY & 1897 & & FIRENZE & TAMARACK ST. & CALUMET - TAMARACK \\
\hline 2467 & 47 & PACCINI & CHARLES & s & 24 & ITALY & ITALY & ITALY & 1897 & FIREMAN/CM & FIRENZE & TAMARACK ST. & CALUMET - TAMARACK \\
\hline 2468 & 47 & PACCINI & INES & $\mathrm{D}$ & 13 & MICHIGAN & ITALY & ITALY & & & FIRENZE & TAMARACK ST. & CALUMET - TAMARACK \\
\hline 2469 & 4011 & DE FILIPPI & DOMENICO & $\mathrm{H}$ & 40 & ITALY & ITALY & ITALY & 1888 & TIMBER BOSS/CM & TORINO & PORTLAND ST. & CALUMET - YELLOW JACKET \\
\hline 2470 & 4011 & DE FILIPPI & MARY & W & 40 & ITALY & ITALY & ITALY & 1890 & & & PORTLAND ST. & CALUMET - YELLOW JACKET \\
\hline 2471 & 4011 & DE FILIPPI & MARY & $\mathrm{D}$ & 18 & MICHIGAN & ITALY & ITALY & & & TORINO & PORTLAND ST. & CALUMET - YELLOW JACKET \\
\hline 2472 & 4011 & DE FILIPPI & MAGGIE & $\mathrm{D}$ & 17 & MICHIGAN & ITALY & ITALY & & & TORINO & PORTLAND ST. & CALUMET - YELLOW JACKET \\
\hline 2473 & 4011 & DE FILIPPI & JOSEPH & $\mathrm{s}$ & 14 & MICHIGAN & ITALY & ITALY & & & TORINO & PORTLAND ST. & CALUMET - YELLOW JACKET \\
\hline 2474 & 4011 & DE FILIPPI & ERNEST & $\mathrm{s}$ & 9 & MICHIGAN & ITALY & ITALY & & & TORINO & PORTLAND ST. & CALUMET - YELLOW JACKET \\
\hline 2475 & 4011 & DE FILIPPI & FEDERICO & $\mathrm{s}$ & 2 & MICHIGAN & ITALY & ITALY & & & TORINO & PORTLAND ST. & CALUMET - YELLOW JACKET \\
\hline 2476 & 4206 & NAVONE & JOHN & $\mathrm{H}$ & 31 & ITALY & ITALY & ITALY & $190 ?$ & MINER/CM & RIVAROLO CANAVESE. TO & PORTLAND ST. & CALUMET - YELLOW JACKET \\
\hline 2477 & 4206 & NAVONE & MARY & W & 24 & ITALY & $\begin{array}{l}\text { ITALY } \\
\text { ITAL }\end{array}$ & ITALY & 1905 & IVIIIVEN & SAN GIORGIO CANAVESE. TO & $\begin{array}{l}\text { PORTLAND ST. } \\
\text { PON }\end{array}$ & CALUMET - YELLOW JACKET \\
\hline 2478 & 4206 & NAVONE & JULIA & $\mathrm{D}$ & 2 & MICHIGAN & ITALY & ITALY & & & RIVAROLO CANAVESE, TO & PORTLAND ST. & CALUMET - YELLLW JACKET \\
\hline 2479 & 4206 & MANLY & LOUIS & BROT & 25 & ITALY & ITALY & ITALY & 1909 & TIMBERMAN/CM & & PORTLAND ST. & CALUMET - YELLOW JACKET \\
\hline 2480 & 4010 & SILVIONI & ANICETO & $\mathrm{H}$ & 29 & ITALY & ITALY & ITALY & 1905 & PRIEST/CHURCH & & PORTLAND ST. & $\begin{array}{l}\text { CALUMET - YELLOW JACKET } \\
\end{array}$ \\
\hline 2481 & 4010 & LENZINO & DOMENICO & $\mathrm{H}$ & 29 & MICHIGAN & CALIFORNI & CALIFORNI & & MASON/HOUSE & & PORTLAND ST. & CALUMET - YELLOW JACKET \\
\hline 2482 & 4010 & LENZINO & CAROLINA & W & 21 & ITALY & ITALY & ITALY & 1904 & & & PORTLAND ST. & CALUMET - YELLOW JACKET \\
\hline 2483 & 4010 & LENZINO & KATE & $\mathrm{D}$ & 3 & MICHIGAN & CALIFORNI & ITALY & & & & PORTLAND ST. & CALUMET - YELLOW JACKET \\
\hline 2484 & 4010 & LENZINO & GEORGE & $\mathrm{s}$ & $11 \mathrm{M}$ & MICHIGAN & CALIFORNI & ITALY & & & & PORTLAND ST. & CALUMET - YELLOW JACKET \\
\hline 2485 & 4010 & NARETTO & LOUIS & $\mathrm{H}$ & 35 & ITALY & ITALY & ITALY & 1905 & MINER/CM & TORINO & PORTLAND ST. & CALUMET - YELLOW JACKET \\
\hline 2486 & 4010 & NARETTO & MARY & W & 32 & ITALY & ITALY & ITALY & 1905 & & TORINO & PORTLAND ST. & CALUMET - YELLLOW JACKET \\
\hline 2487 & 4022 & ROSAD & FRANK & $\mathrm{H}$ & 26 & ITALY & ITALY & ITALY & 1905 & MINER/CM & & PORTLAND ST. & CALUMET - YELLOW JACKET \\
\hline 2488 & 4022 & ROSAD & FILOMENA & W & 24 & ITALY & ITALY & ITALY & $89 ? ?$ & 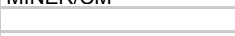 & & PORTLAND ST. & CALUMET - YELLOW JACKET \\
\hline 2489 & 4022 & ROSAD & ERNESTINA & D & 2 & MICHIGAN & ITALY & ITALY & oy:? & & & $\begin{array}{l}\text { PORTLAND ST. } \\
\text { PON }\end{array}$ & CALUMET - YELLOW JACKET \\
\hline 2490 & 4022 & ROSAD & PINO & $\mathrm{S}$ & $3 \mathrm{M}$ & MICHIGAN & ITALY & ITALY & & & & PORTLAND ST. & CALUMET - YELLLOW JACKET \\
\hline 2491 & 4022 & DESANIS?? & JOSEPH & $\mathrm{H}$ & 31 & ITALY & ITALY & ITALY & 1908 & BARTENDER/SALOON & & PORTLAND ST. & CALUMET - YELLOW JACKET \\
\hline 2492 & 4022 & DESANIS?? & MARY & W & 25 & ITALY & ITALY & ITALY & 1908 & & & PORTLAND ST. & CALUMET - YELLOW JACKET \\
\hline 2493 & 4022 & DESANIS?? & DOMENICO & s & 7 & MICHIGAN & ITALY & ITALY & & & & PORTLAND ST. & CALUMET - YELLOW JACKET \\
\hline 2494 & 4022 & DESANIS?? & JAMES & $\mathrm{s}$ & $6 \mathrm{M}$ & MICHIGAN & ITALY & ITALY & & & & PORTLAND ST. & CALUMET - YELLOW JACKET \\
\hline 2495 & 4022 & BONA & CARLO & $\mathrm{H}$ & 35 & ITALY & ITALY & ITALY & 1895 & MINER/CM & & PORTLAND ST. & CALUMET - YELLOW JACKET \\
\hline 2496 & 4022 & BONA & TRACY & W & 35 & ITALY & ITALY & ITALY & 1895 & & & PORTLAND ST. & CALUMET - YELLOW JACKET \\
\hline 2497 & 4022 & BONA & CESARINA & D & 6 & MICHIGAN & ITALY & ITALY & & & & PORTLAND ST. & CALUMET - YELLOW JACKET \\
\hline 2498 & 4022 & BONA & JOSEPH & $\mathrm{s}$ & 5 & MICHIGAN & ITALY & ITALY & & & & PORTLAND ST. & CALUMET - YELLOW JACKET \\
\hline 2499 & 4026 & GASPARDO & FRANK & $\mathrm{H}$ & 57 & ITALY & ITALY & ITALY & 1894 & SPRINKLER/CM & PONT CANAVESE. TO & PORTLAND ST. & CALUMET - YELLOW JACKET \\
\hline 2500 & 4026 & GASPARDO & MARK & S & 38 & ITALY & ITALY & ITALY & 1894 & NOT CLEAR & PONT CANAVESE. TO & PORTLAND ST. & CALUMET - YELLOW JACKET \\
\hline 2501 & 4026 & GASPARDO & MADDALENA & $\mathrm{D}$ & 37 & ITALY & ITALY & ITALY & 1901 & & PONT CANAVESE. TO & PORTLAND ST. & CALUMET - YELLOW JACKET \\
\hline 2502 & 4026 & GASPARDO & DOMENICO & $\mathrm{s}$ & 24 & ITALY & ITALY & ITALY & 1908 & & PONT CANAVESE. TO & PORTLAND ST. & CALUMET - YELLOW JACKET \\
\hline 2503 & 4026 & GASPARDO & TERESA & D & 30 & ITALY & ITALY & ITALY & 1907 & & PONT CANAVESE. TO & PORTLAND ST. & CALUMET - YELLOW JACKET \\
\hline 2504 & 4029 & ZANOTTI & ANTONIO & $\mathrm{H}$ & 26 & ITALY & ITALY & ITALY & 1905 & SHAFTMAN/CM & PARELLA. TO & OAK & CALUMET - YELLOW JACKET \\
\hline 2505 & 4029 & ZANOTTI & MARY & w & 20 & MICHIGAN & ITALY & ITALY & & & TORINO & OAK ST. & CALUMET - YELLLOW JACKET \\
\hline 2506 & 4029 & ZANOTTI & GENEVIEVE & $\mathrm{D}$ & 2 & MICHIGAN & ITALY & ITALY & & & PARELLA. TO & OAK & CALUMET - YELLOW JACKET \\
\hline 2507 & 4029 & ZANOTTI & FRANCIS & $\mathrm{s}$ & $5 \mathrm{M}$ & MICHIGAN & ITALY & ITALY & & & PARELLA. TO & OAK ST. & CALUMET - YELLOW JACKET \\
\hline 2508 & 4029 & ROCH/ROCK & JOHN & B & 34 & ITALY & ITALY & ITALY & 1892 & SALOONKEEPER & RIVAROLO CANAVESE, TO & OAK ST. & CALUMET - YELLLW JACKET \\
\hline 2509 & 4029 & PAPILI/RUPILI & JOSEPH & $\mathrm{H}$ & 29 & ITALY & ITALY & ITALY & 1905 & PIPER/CM & & OAK ST. & CALUMET - YELLOW JACKET \\
\hline 2510 & 4029 & PAPILI/RUPILI & MARY & W & 31 & ITALY & ITALY & ITALY & 1908 & TAILOR & & OAK ST. & CALUMET - YELLOW JACKET \\
\hline 2511 & 4245 & BOBBIO & PAUL & $\mathrm{H}$ & 43 & ITALY & ITALY & ITALY & 1884 & MINER/CM & SAN BENIGNO CANAVESE. TO & ACORN ST. & CALUMET - YELLOW JACKET \\
\hline 2512 & 4245 & BOBBIO & JOSEPHINE & w & 23 & ITALY & ITALY & ITALY & 1890 & & & ACORN ST. & CALUMET - YELLLOW JACKET \\
\hline 2513 & 4245 & BOBBIO & FELIX & s & 7 & MICHIGAN & ITALY & ITALY & & & SAN BENIGNO CANAVESE. TO & ACORN ST. & CALUMET - YELLOW JACKET \\
\hline 2514 & 4245 & BOBBIO & MARY & $\mathrm{D}$ & 2 & MICHIGAN & ITALY & ITALY & & & SAN BENIGNO CANAVESE, TO & ACORN ST. & CALUMET - YELLOW JACKET \\
\hline 2515 & 4245 & BOBBIO & JOHN & $\mathrm{S}$ & 22 & MICHIGAN & ITALY & ITALY & & & SAN BENIGNO CANAVESE. TO & ACORN ST. & CALUMET - YELLOW JACKET \\
\hline 2516 & 4245 & VIOLA & PAUL & B & 38 & ITALY & ITAL & ITAI & 1906 & MINE & & ACORN ST. & CALUMET - YELLOW JACKET \\
\hline 2517 & 4245 & BATINELLO? & & B & 27 & & & & 190 & & & & LW IACK \\
\hline 2518 & 4245 & COPPO & & B & 22 & & ITALY & & 1907 & & PONT CANAVESE. TO & & CALUMET - YELLOW JACKET \\
\hline 2519 & 4245 & VIOLA & DOMENICO & B & $28 ?$ & ITALY & ITALY & ITALY & 1907 & TRAMMER/CM & & ACORN ST. & CALUMET - YELLOW JACKET \\
\hline
\end{tabular}




\begin{tabular}{|c|c|c|c|c|c|c|c|c|c|c|c|c|c|}
\hline 2520 & 4245 & PISTRANZI? & VINCENZO & B & 23 & ITALY & ITALY & ITALY & ??? & TRAMMER/CM & & ACORN ST. & CALUMET - YELLOW JACKET \\
\hline 2521 & 4245 & BARINOTTI & LOUIS & $\mathrm{H}$ & 42 & ITALY & ITALY & ITALY & 1892 & MINER/CM & PONT CANAVESE. TO & ACORN ST. & CALUMET - YELLOW JACKET \\
\hline 2522 & 4245 & BARINOTTI & FELICITA & W & 32 & ITALY & ITALY & ITALY & 1898 & & LOCANA CANAVESE. TO & ACORN ST. & CALUMET - YELLOW JACKET \\
\hline 2523 & 4245 & BARINOTTI & LOUIS JR. & s & 3 & MICHIGAN & ITALY & ITALY & & & PONT CANAVESE. TO & ACORN ST. & CALUMET - YELLOW JACKET \\
\hline 2524 & 4245 & BARINOTTI & JOSEPH & $\mathrm{s}$ & 1 & MICHIGAN & ITALY & ITALY & & & PONT CANAVESE. TO & ACORN ST. & CALUMET - YELLOW JACKET \\
\hline 2525 & 4245 & BARINOTTI & MINNIE & $\mathrm{D}$ & 2 & MICHIGAN & ITALY & ITALY & & & PONT CANAVESE. TO & ACORN ST. & YELLOW JACKET \\
\hline 2526 & 4245 & VERNETTI & MAGGIE & $\mathrm{H}$ & 45 & ITALY & ITALY & ITALY & 1901 & SERVANT/PF & PONT CANAVESE. TO & ACORN ST. & CALUMET - YELLOW JACKET \\
\hline 2527 & 4245 & VERNETTI & ROSE & D & 18 & ITALY & ITALY & ITALY & 1901 & SERVANT/PF & PONT CANAVESE. TO & ACORN ST. & CALUMET - YELLOW JACKET \\
\hline 2528 & 4060 & GRAZIARA?? & $\mathrm{JOHN}$ & $\mathrm{H}$ & 29 & ITALY & ITALY & ITALY & 1908 & LABORER/SHOP & & ACORN ST. & CALUMET - YELLOW JACKET \\
\hline 2529 & 4060 & GRAZIARA?? & MARY & W & 29 & ITALY & ITALY & ITALY & 1909 & & & ACORN ST. & CALUMET - YELLOW JACKET \\
\hline 2530 & 4060 & GRAZIARA?? & JOHN JR. & $\mathrm{s}$ & 8 & ITALY & ITALY & ITALY & 1909 & & & ACORN ST. & CALUMET - YELLOW JACKET \\
\hline 2531 & 4060 & CAPELLO & JOSEPH & $\mathrm{H}$ & 39 & ITALY & ITALY & ITALY & 1898 & TIMBER BOSS/CM & SAN BENIGNO CANAVESE. TO & ACORN ST. & CALUMET - YELLOW JACKET \\
\hline 2532 & 4060 & CAPELLO & MARY & W & 35 & ITALY & ITALY & ITALY & 1899 & & TORINO & ACORN ST. & CALUMET - YELLOW JACKET \\
\hline 2533 & 4060 & CAPELLO & GINETTA & $\mathrm{D}$ & 14 & ITALY & ITALY & ITALY & 1899 & & SAN BENIGNO CANAVESE. TO & ACORN ST. & CALUMET - YELLOW JACKET \\
\hline 2534 & 4060 & $\begin{array}{l}\text { CAPELLLO } \\
\text { CAPELO }\end{array}$ & $\begin{array}{l}\text { LOUIS } \\
\text { LOUIS }\end{array}$ & $\mathrm{BR}$ & $\frac{14}{43}$ & $\begin{array}{l}\text { IITALY } \\
\text { ITALY }\end{array}$ & ITALY & $\begin{array}{l}\text { ITALY } \\
\text { ITALY }\end{array}$ & 1888 & TIMBER BOSS/CM & SAN BENIGNO CANAVESE. TO & ACORN ST. & CALUMET - YELLOW JACKET \\
\hline 2535 & $\begin{array}{l}4000 \\
4060\end{array}$ & $\begin{array}{l}\text { CAPELLO } \\
\text { RICCA }\end{array}$ & FRANK & $\begin{array}{l}B R \\
B\end{array}$ & 39 & $\begin{array}{l}\text { IITALY } \\
\text { ITALY }\end{array}$ & $\begin{array}{l}\text { IITALY } \\
\text { ITAY }\end{array}$ & $\begin{array}{l}\text { IIALY } \\
\text { ITALY }\end{array}$ & $\begin{array}{l}1888{ }^{0} \\
08 ? ?\end{array}$ & TIMBERMAN/CM & $\begin{array}{l}\text { SANBENIGINU CAINAVESE. IO } \\
\text { TORINO }\end{array}$ & ACORN ST. & $\begin{array}{l}\text { CALUME - - YELLLW JACKE I } \\
\text { CALUMET - YELOW JACKT }\end{array}$ \\
\hline 2536 & 4060 & RICCA & JOSEPH & B & 30 & ITALY & ITALY & ITALY & 1905 & TIMBERMAN/CM & TORINO & ACORN ST. & CALUMET - YELLOW JACKET \\
\hline 2537 & 4060 & GEARDI? & JAMES & B & 26 & ITALY & ITALY & ITALY & 1902 & TIMBERMAN/CM & & ACORN ST. & CALUMET - YELLOW JACKET \\
\hline 2538 & 4063 & MICHETTI & JOHN & $\mathrm{H}$ & 50 & ITALY & ITALY & ITALY & 1882 & TIMBERMAN/CM & PONT CANAVESE. TO & ELM ST. & CALUMET - YELLOW JACKET \\
\hline 2539 & 4063 & MICHETTI & MADDALENA & W & 50 & ITALY & ITALY & ITALY & 1888 & & PONT CANAVESE. TO & ELM ST. & CALUMET - YELLOW JACKET \\
\hline 2540 & 4063 & MICHETTI & JOHN & s & 22 & ITALY & ITALY & ITALY & 1888 & LANDER/CM & PONT CANAVESE. TO & ELM ST. & CALUMET - YELLOW JACKET \\
\hline 2541 & 4063 & MICHETTI & MARY & $\mathrm{D}$ & 19 & MICHIGAN & ITALY & ITALY & & & PONT CANAVESE, TO & ELM ST. & CALUMET - YELLOW JACKET \\
\hline 2542 & 4063 & MICHETTI & MICHAEL & $\mathrm{S}$ & 17 & MICHIGAN & ITALY & ITALY & & DRILL BOY/CM & PONT CANAVESE. TO & ELM ST. & CALUMET - YELLOW JACKET \\
\hline 2543 & 4063 & MICHETTI & PAUL & $\mathrm{S}$ & 15 & MICHIGAN & ITALY & ITALY & & & PONT CANAVESE. TO & ELM ST. & CALUMET - YELLOW JACKET \\
\hline 2544 & 4063 & MICHETTI & PETER & $\mathrm{s}$ & 10 & MICHIGAN & ITALY & ITALY & & & PONT CANAVESE. TO & ELM ST. & CALUMET - YELLOW JACKET \\
\hline 2545 & 4063 & MICHETTI & KATE & $\mathrm{D}$ & 12 & MICHIGAN & ITALY & ITALY & & & PONT CANAVESE. TO & ELM ST. & CALUMET - YELLOW JACKET \\
\hline 2546 & 4065 & NIGRA & ANTONIO & $\mathrm{H}$ & 50 & ITALY & ITALY & ITALY & 1887 & TIMBER BOSS/CM & RIVAROLO CANAVESE. TO & ELM ST. & $\begin{array}{l}\text { CALUMET - YELLOW JACKET } \\
\end{array}$ \\
\hline 2547 & 4065 & $\begin{array}{l}\text { NIGRA } \\
\text { NIGRA }\end{array}$ & KATE & W & 48 & $\begin{array}{l}\text { IITALY } \\
\text { ITAL }\end{array}$ & ITALY & $\begin{array}{l}\text { ITALY } \\
\text { ITAL }\end{array}$ & 1890 & HIVIDER BUSS/CIVI & RIVAROLO CANAVESE, TO & ELM ST. & CALUMET - YELLOW JACKET \\
\hline 2548 & 4065 & NIGRA & FRANCIS & $\mathrm{D}$ & 22 & ITALY & ITALY & ITALY & 1890 & DRESSMAKER/AT HOME & RIVAROLO CANAVESE. TO & ELM ST. & CALUMET - YELLOW JACKET \\
\hline 2549 & 4065 & NIGRA & KATE & $\mathrm{D}$ & 17 & MICHIGAN & ITALY & ITALY & & OFFICE GIRL & RIVAROLO CANAVESE. TO & ELM ST. & CALUMET - YELLOW JACKET \\
\hline 2550 & 4065 & NIGRA & MARGHERITA & $\mathrm{D}$ & 13 & MICHIGAN & ITALY & ITALY & & & RIVAROLO CANAVESE. TO & ELM ST. & CALUMET - YELLOW JACKET \\
\hline 2551 & 4065 & NIGRA & JOHN & $\mathrm{s}$ & 11 & MICHIGAN & ITALY & ITALY & & & RIVAROLO CANAVESE. TO & ELM ST. & $\begin{array}{l}\text { CALUMET - YELLOW JACKET } \\
\text { CEEK J }\end{array}$ \\
\hline 2552 & 4065 & BOBBIO & FELIX & B & 23 & ITALY & ITALY & ITALY & 1904 & MINER/CM & SAN BENIGNO CANAVESE. TO & ELM ST. & CALUMET - YELLOW JACKET \\
\hline 2553 & 4065 & MAGA & JOHN & B & 40 & ITALY & ITALY & ITALY & 1880 & TIMBERMAN/CM & SCARMAGNO, TO & ELM ST. & CALUMET - YELLOW JACKET \\
\hline 2554 & 4065 & MUSSATO & ERNEST & B & 36 & ITALY & ITALY & ITALY & 1893 & TIMBERMAN/CM & BAIRO CANAVESE. TO & ELM ST. & CALUMET - YELLOW JACKET \\
\hline 2555 & 4065 & MUSSATO & ORESTE & B & 17 & ITALY & ITALY & ITALY & 1909 & CAR LANDER/CM & BAIRO CANAVESE. TO & ELM ST. & CALUMET - YELLOW JACKET \\
\hline 2556 & 4065 & FENOGLIO & JOHN & B & 28 & ITALY & ITALY & ITALY & 09?? & TIMBER BOSS/CM & TORINO & ELM ST. & CALUMET - YELLOW JACKET \\
\hline 2557 & 4065 & VALLERO & MIKE & B & 27 & ITALY & ITALY & ITALY & 1903 & MINER/CM & TORINO & ELM ST. & CALUMET - YELLOW JACKET \\
\hline 2558 & 4082 & GUGLIELMO & JOSEPH & $\mathrm{H}$ & 43 & ITALY & ITALY & ITALY & 1888 & LABORER/CM & MONTELANGHE. TO & ELM ST. & CALUMET - YELLOW JACKET \\
\hline 2559 & 4082 & GUGLIELMO & MARY & W & 36 & ITALY & ITALY & $\begin{array}{l}\text { ITALY } \\
\text { ITALY }\end{array}$ & 1898 & LABURER/UIVI & TVIUINIELAIVGHE. IO & ELM ST. & CALUMET - YELLOW JACKET \\
\hline 2560 & 4082 & GUGLIELMO & MICHAEL & s & 10 & MICHIGAN & ITALY & ITALY & & & MONTELANGHE. TO & ELM ST. & CALUMET - YELLOW JACKET \\
\hline 2561 & 4082 & MIGLIO/MIGLIA & DOMENICO & B & 32 & ITALY & ITALY & ITALY & 1905 & DRAYMAN/CM & SAN GIORGIO CANAVESE. TO & ELM ST. & CALUMET - YELLOW JACKET \\
\hline 2562 & 4082 & MIGLIO/MIGLIA & MARTINO & B & 49 & ITALY & ITALY & ITALY & 1887 & TIMBERMAN/CM & SAN GIORGIO CANAVESE. TO & ELM ST. & CALUMET - YELLOW JACKET \\
\hline 2563 & 4082 & ROSSIO & TONY & B & 33 & ITALY & ITALY & ITALY & 1901 & TIMBERMAN/CM & & ELM ST. & CALUMET - YELLOW JACKET \\
\hline 2564 & 4082 & BONOTTO & DOMENICO & $\mathrm{H}$ & 30 & ITALY & ITALY & ITALY & 1898 & TIMBERMAN/CM & SAN BENIGNO CANAVESE. TO & ELM ST. & $\begin{array}{l}\text { CALUMET - YELLOW JACKET } \\
\text { CELE }\end{array}$ \\
\hline 2565 & 4082 & BONOTTO & BENEDETTA & W & 26 & ITALY & ITALY & ITALY & 1904 & & & ELM ST. & CALUMET - YELLOW JACKET \\
\hline 2566 & 4082 & BONOTTO & MARIE & $\mathrm{D}$ & 5 & MICHIGAN & ITALY & ITALY & & & SAN BENIGNO CANAVESE. TO & ELM ST. & CALUMET - YELLOW JACKET \\
\hline 2567 & 4085 & BAUDINO & GEORGE & $\mathrm{H}$ & 43 & ITALY & ITALY & ITALY & 1891 & TIMBERMAN/CM & RIVAROLO CANAVESE. TO & ACORN ST. & CALUMET - YELLOW JACKET \\
\hline 2568 & 4085 & BAUDINO & MARY & w & 48 & ITALY & ITALY & ITALY & 1891 & & RIVAROLO CANAVESE. TO & ACORN ST. & CALUMET - YELLOW JACKET \\
\hline 2569 & 4085 & BAUDINO & ANITA & $\mathrm{D}$ & 16 & MICHIGAN & ITALY & ITALY & & & RIVAROLO CANAVESE. TO & ACORN ST. & CALUMET - YELLOW JACKET \\
\hline 2570 & 4085 & BAUDINO & TRACY & $\mathrm{D}$ & 14 & MICHIGAN & ITALY & ITALY & & & RIVAROLO CANAVESE. TO & ACORN ST. & CALUMET - YELLOW JACKET \\
\hline 2571 & 4085 & BAUDINO & ERNESTINE & $\mathrm{D}$ & 12 & MICHIGAN & ITALY & ITALY & & & RIVAROLO CANAVESE, TO & ACORN ST. & CALUMET - YELLOW JACKET \\
\hline 2572 & $\begin{array}{l}4005 \\
4085\end{array}$ & BAUDINO & OLIVER & s & $\frac{12}{9}$ & MICHIGAN & ITALY & ITALY & & & RIVAROLO CANAVESE. TO & ACORN ST. & CALUMET - YELLOW JACKET \\
\hline 2573 & 4085 & BAUDINO & PETER & $\mathrm{s}$ & 7 & MICHIGAN & ITALY & ITALY & & & RIVAROLO CANAVESE. TO & ACORN ST. & CALUMET - YELLOW JACKET \\
\hline 2574 & 4085 & BAUDINO & VIOLET & $\mathrm{D}$ & $22 \mathrm{M}$ & MICHIGAN & ITALY & ITALY & & & RIVAROLO CANAVESE. TO & ACORN ST. & CALUMET - YELLOW JACKET \\
\hline 2575 & 4085 & BAUDINO & ALGISO & NEP & 27 & ITALY & ITALY & ITALY & 1901 & BLACKSMITH/SHOP & RIVAROLO CANAVESE. TO & RN ST. & CALUMET - YELLOW JACKET \\
\hline 2576 & 4085 & BAUDINO & JOHN & NEP & 20 & $\begin{array}{l}\text { ITALY } \\
\text { ITAL }\end{array}$ & ITA & ITALY & 1907 & $\begin{array}{l}\text { TIMBERMAN/CM } \\
\text { TIMER }\end{array}$ & RIVAROLO CANAVESE. TO & SN ST. & CALUMET - YELLOW JA \\
\hline 2577 & $? ? ? ?$ & REANI?TO & JOHN & $\mathrm{H}$ & 25 & ITALY & ITALY & ITALY & 1902 & LABORER/CM & & RN ST. & CALUMET - YELLOW JACKET \\
\hline 2578 & $? ? ?$ & REANI?TO & KATE & W & 22 & ITALY & ITALY & ITALY & $\frac{1902}{1892}$ & & & ACORN ST. & CALUMET - YELLOW JACKET \\
\hline 2579 & ??? & REANI?TO & FRANCES & $\mathrm{D}$ & $3 \mathrm{M}$ & MICHIGAN & ITALY & ITALY & & & & ACORN ST. & $\begin{array}{l}\text { CALUMET - YELLOW JACKET } \\
\text { CAEE }\end{array}$ \\
\hline 2580 & 4087 & POGGIONE & CARLO & $\mathrm{H}$ & 48 & ITALY & ITALY & ITALY & 1889 & LANDER/CM & SAN GIORGIO CANAVESE. TO & ACORN ST. & CALUMET - YELLOW JACKET \\
\hline 2581 & 4087 & POGGIONE & MARY & W & 47 & ITALY & ITALY & ITALY & 1890 & & & ACORN ST. & CALUMET - YELLOW JACKET \\
\hline 2582 & 4087 & POGGIONE & LOUISE & $\mathrm{D}$ & 7 & MICHIGAN & ITALY & ITALY & & & SAN GIORGIO CANAVESE. TO & ACORN ST. & CALUMET - YELLOW JACKET \\
\hline
\end{tabular}




\begin{tabular}{|c|c|c|c|c|c|c|c|c|c|c|c|c|c|}
\hline 2583 & 4087 & POGGIONE & MARY & $\mathrm{D}$ & 16 & MICHIGAN & ITALY & ITALY & & & SAN GIORGIO CANAVESE. TO & ACORN ST. & CALUMET - YELLOW JACKET \\
\hline 2584 & 4087 & POGGIONE & JOSEPH & $\mathrm{s}$ & 12 & MICHIGAN & ITALY & ITALY & & & SAN GIORGIO CANAVESE. TO & ACORN ST. & CALUMET - YELLOW JACKET \\
\hline 2585 & 4087 & POGGIONE & LOUIS & $\mathrm{s}$ & 2 & MICHIGAN & ITALY & ITALY & & & SAN GIORGIO CANAVESE. TO & ACORN ST. & CALUMET - YELLOW JACKET \\
\hline 2586 & 4087 & CASTELLINO & SIMONE & B & 52 & ITALY & ITALY & ITALY & 1888 & TIMBERMAN/CM & TORINO & ACORN ST. & CALUMET - YELLOW JACKET \\
\hline 2587 & 4087 & VIANA/VIANI & JOHN & B & 27 & ITALY & ITALY & ITALY & 1906 & TRAMMER/CM & LUCCA & ACORN ST. & CALUMET - YELLOW JACKET \\
\hline 2588 & 4089 & MICHELINI & SANTINO & $\mathrm{H}$ & 34 & ITALY & ITALY & ITALY & 1889 & LABORER/CM & LUCCA & ACORN ST. & CALUMET - YELLOW JACKET \\
\hline 2589 & 4089 & MICHELINI & ESTER & w & 31 & ITALY & ITALY & ITALY & 1902 & LADO TELT/UIV & LUCUA & ACORN ST. & CALUMET - YELLOW JACKET \\
\hline 2590 & 4089 & MICHELINI & EDITH & $\mathrm{D}$ & 5 & MICHIGAN & ITALY & ITALY & & & LUCCA & ACORN ST. & CALUMET - YELLOW JACKET \\
\hline 2591 & 4089 & MICHELINI & ANGELO & s & 7 & MICHIGAN & ITALY & ITALY & & & LUCCA & ACORN ST. & CALUMET - YELLOW JACKET \\
\hline 2592 & 4089 & MICHELINI & JULIA & D & 4 & MICHIGAN & ITALY & ITALY & & & LUCCA & ACORN ST. & CALUMET - YELLOW JACKET \\
\hline 2593 & 4089 & MICHELINI & JULIO & $\mathrm{s}$ & 2 & MICHIGAN & ITALY & ITALY & & & LUCCA & ACORN ST. & CALUMET - YELLOW JACKET \\
\hline 2594 & 4089 & PAOLI & AURELIO & $\mathrm{D}$ & 31 & ITALY & ITALY & ITALY & 1901 & TAILOR/SHOP & LUCCA & ACORN ST. & CALUMET - YELLOW JACKET \\
\hline 2595 & 4287 & MICHELINI & LORENZO & $\mathrm{H}$ & 31 & ITALY & ITALY & ITALY & 1904 & LABORER/CM & LUCCA & ACORN ST. & CALUMET - YELLOW JACKET \\
\hline 2596 & 4287 & MICHELINI & RACHELE & W & 29 & ITALY & ITALY & ITALY & 1905 & & & ACORN ST. & CALUMET - YELLOW JACKET \\
\hline 2597 & 4287 & MICHELINI & ANGELINA & $\mathrm{D}$ & ?? & MICHIGAN & ITALY & ITALY & & & LUCCA & ACORN ST. & CALUMET - YELLOW JACKET \\
\hline 2598 & 4287 & MICHELINI & EUGENIO & $\mathrm{s}$ & 9 & MICHIGAN & ITALY & ITALY & & & LUCCA & ACORN ST. & CALUMET - YELLOW JACKET \\
\hline 2599 & 4287 & MICHELINI & MARY & D & $10 \mathrm{M}$ & MICHIGAN & ITALY & ITALY & & & LUCCA & ACORN ST. & CALUMET - YELLOW JACKET \\
\hline 2600 & 4222 & ANTONIETTI & JOHN & $\mathrm{H}$ & 47 & ITALY & ITALY & ITALY & 1891 & MASON/HOUSE & & 10TH ST. & CALUMET - YELLOW JACKET \\
\hline 2601 & 4222 & ANTONIETTI & MARGHERITA & w & 47 & ITALY & ITALY & ITALY & 1890 & & & 10TH ST. & CALUMET - YELLOW JACKET \\
\hline 2602 & 4222 & ANTONIETTI & MARIA & $\mathrm{D}$ & 20 & ITALY & ITALY & ITALY & 1893 & SALESLADY/GENERAL STORE & & 10TH ST. & CALUMET - YELLOW JACKET \\
\hline 2603 & 4222 & ANTONIETTI & CLOTILDE & $\mathrm{D}$ & 19 & ITALY & ITALY & ITALY & 1893 & SALEOLAD TU UETERAL STURE & & 10TH ST. & CALUMET - YELLOW JACKET \\
\hline 2604 & 4222 & ANTONIETTI & SANTINA & $\mathrm{D}$ & 16 & MICHIGAN & ITALY & ITALY & & & & 10TH ST. & CALUMET - YELLOW JACKET \\
\hline 2605 & 4222 & ANTONIETTI & ANGELICA & $\mathrm{D}$ & 14 & MICHIGAN & ITALY & ITALY & & & & 10TH ST. & CALUMET - YELLOW JACKET \\
\hline 2606 & 4222 & ANTONIETTI & JOSEPH & $\mathrm{S}$ & 11 & MICHIGAN & ITALY & ITALY & & & & 10TH ST. & CALUMET - YELLOW JACKET \\
\hline 2607 & 4222 & DE MARIA & DOMENICO & B & 27 & ITALY & ITALY & ITALY & 1900 & TIMBERMAN/CM & TORINO & 10TH ST. & CALUMET - YELLOW JACKET \\
\hline 2608 & 4222 & DE MARIA & GILDO & B & 31 & ITALY & ITALY & ITALY & 1901 & CARPENTER/HOUSE & TORINO & 10TH ST. & CALUMET - YELLOW JACKET \\
\hline 2609 & 4232 & MASCAGNO & FRANK & $\mathrm{H}$ & 43 & ITALY & ITALY & ITALY & 1899 & TIMBERMAN/CM & TORINO & 10TH ST. & CALUMET - YELLOW JACKET \\
\hline 2610 & 4232 & MASCAGNO & GIACINTA & w & 38 & ITALY & ITALY & ITALY & 1899 & & TORINO & 10TH ST. & CALUMET - YELLOW JACKET \\
\hline 2611 & 4232 & MASCAGNO & JOANNA & D & 14 & ITALY & ITALY & ITALY & 1899 & & TORINO & 10TH ST. & CALUMET - YELLOW JACKET \\
\hline 2612 & 4232 & MASCAGNO & MICHAEL & $\mathrm{s}$ & 11 & MICHIGAN & ITALY & ITALY & & & TORINO & 10TH ST. & CALUMET - YELLOW JACKET \\
\hline 2613 & 4232 & MASCAGNO & ERNEST & $\mathrm{s}$ & 8 & MICHIGAN & ITALY & ITALY & & & TORINO & 10TH ST. & CALUMET - YELLOW JACKET \\
\hline 2614 & 4232 & PEILA & CARLO & $\mathrm{H}$ & ?? & ITALY & ITALY & ITALY & 1907 & LABORER/CM & CASTELLAMONTE. TO & 10TH ST. & CALUMET - YELLOW JACKET \\
\hline 2615 & 4232 & PEILA & MARY & W & $? ?$ & ITALY & ITALY & ITALY & 1908 & LADU KEK/CIVI & CAOTELLAIVIUIVIE. TO & 10TH ST. & CALUMET - YELLOW JACKET \\
\hline 2616 & 4232 & PEILA & JOSH & s & ?? & MICHIGAN & ITALY & ITALY & & & CASTELLAMONTE, TO & 10TH ST. & CALUMET - YELLOW JACKET \\
\hline 2617 & 4234 & CHIMINO & GIACOMO & $\mathrm{H}$ & 47 & ITALY & ITALY & ITALY & 1890 & MASON/HOUSE & SAN GIORGIO CANAVESE. TO & 10TH ST. & CALUMET - YELLOW JACKET \\
\hline 2618 & 4234 & CHIMINO & MARGHERITA & w & 47 & ITALY & ITALY & ITALY & 1890 & & SAN GIORGIO CANAVESE. TO & 10TH ST. & CALUMET - YELLOW JACKET \\
\hline 2619 & 4234 & CHIMINO & JOSEPH & $\mathrm{s}$ & 20 & MICHIGAN & ITALY & ITALY & & & SAN GIORGIO CANAVESE. TO & 10TH ST. & CALUMET - YELLOW JACKET \\
\hline 2620 & 4234 & CHIMINO & MARY & $\mathrm{D}$ & 18 & MICHIGAN & ITALY & ITALY & & & SAN GIORGIO CANAVESE. TO & 10TH ST. & CALUMET - YELLOW JACKET \\
\hline 2621 & 4234 & CHIMINO & VITTORIA & $\mathrm{D}$ & 17 & MICHIGAN & ITALY & ITALY & & & SAN GIORGIO CANAVESE. TO & 10TH ST. & CALUMET - YELLOW JACKET \\
\hline 2622 & 4234 & CHIMINO & PETER & $\mathrm{S}$ & 16 & MICHIGAN & ITALY & ITALY & & & SAN GIORGIO CANAVESE. TO & 10TH ST. & CALUMET - YELLOW JACKET \\
\hline 2623 & 4234 & CHIMINO & KATE & D & 10 & MICHIGAN & ITALY & ITALY & & & SAN GIORGIO CANAVESE. TO & 10TH ST. & CALUMET - YELLOW JACKET \\
\hline 2624 & 4234 & CHIMINO & JAMES & $\mathrm{s}$ & 9 & MICHIGAN & ITALY & ITALY & & & SAN GIORGIO CANAVESE. TO & 10TH ST. & CALUMET - YELLOW JACKET \\
\hline 2625 & 4234 & CHIMINO & DOMINICK & $\mathrm{s}$ & 6 & MICHIGAN & ITALY & ITALY & & & SAN GIORGIO CANAVESE. TO & 10TH ST. & CALUMET - YELLOW JACKET \\
\hline 2626 & 4234 & BALMA/BAIMA & DOMENICO & B & 32 & ITALY & ITALY & ITALY & 1899 & TIMBERMAN/CM & SAN GIORGIO CANAVESE. TO & 10TH ST. & CALUMET - YELLOW JACKET \\
\hline 2627 & 4234 & BALMA/BAIMA & MICHELE & B & 25 & ITALY & ITALY & ITALY & 1907 & LABORER/CM & TORINO & 10TH ST. & CALUMET - YELLOW JACKET \\
\hline 2628 & 4234 & BERCHIATTI & JOHN & B & 32 & ITALY & ITALY & ITALY & 1905 & PLATMAN & SAN GIORGIO CANAVESE. TO & 10TH ST. & CALUMET - YELLOW JACKET \\
\hline 2629 & 4236 & PICCHIOTTINO & FEDELE & $\mathrm{H}$ & 32 & ITALY & ITALY & ITALY & 1902 & TIMBER BOSS/CM & PONT CANAVESE. TO & 10TH ST. & CALUMET - YELLOW JACKET \\
\hline 2630 & 4236 & PICCHIOTTINO & LUCIA & W & 27 & ITALY & ITALY & ITALY & $02 / 07$ & & PONT CANAVESE. TO & 10TH ST. & CALUMET - YELLOW JACKET \\
\hline 2631 & 4236 & PICCHIOTTINO & MINNIE & D & 2 & ITALY & ITALY & ITALY & & & PONT CANAVESE. TO & 10TH ST. & CALUMET - YELLOW JACKET \\
\hline 2632 & 4236 & DE STEFANI & JOHN & FIL & 60 & ITALY & ITALY & ITALY & $02 ?$ & LABORER/CM & & 10TH ST. & CALUMET - YELLOW JACKET \\
\hline 2633 & 4238 & GERVASSONE & AUGUSTO & $\mathrm{H}$ & 32 & ITALY & ITALY & ITALY & 1893 & TIMBERMAN/CM & VERRES. AOSTA & 10TH ST./11TH ST. & CALUMET - YELLOW JACKET \\
\hline 2634 & 4238 & GERVASSONE & LUIGIA & W & 28 & ITALY & ITALY & ITALY & 1893 & & VERRES, AOSTA & 10TH ST./11TH ST. & CALUMET - YELLOW JACKET \\
\hline 2635 & 4238 & GERVASSONE & KATE & $\mathrm{D}$ & 6 & MICHIGAN & ITALY & ITALY & & & VERRES. AOSTA & 10TH ST./11TH ST. & CALUMET - YELLOW JACKET \\
\hline 2636 & 4238 & GERVASSONE & ERNEST & $\mathrm{s}$ & 3 & MICHIGAN & ITALY & ITALY & & & VERRES. AOSTA & 10TH ST./11TH ST. & CALUMET - YELLOW JACKET \\
\hline 2637 & 4238 & GERVASSONE & VITTORIA & $\mathrm{D}$ & $1.2 \mathrm{M}$ & MICHIGAN & ITALY & ITALY & & & VERRES. AOSTA & 10TH ST./11TH ST. & CALUMET - YELLOW JACKET \\
\hline 2638 & 4238 & AUTERO & DOMENICO & $\mathrm{H}$ & 40 & ITALY & ITALY & ITALY & 1890 & & PONT CANAVESE. TO & 10TH ST./11TH ST. & CALUMET - YELLOW JACKET \\
\hline 2639 & 4238 & AUTERO & MADDALENA & w & 32 & ITALY & ITALY & ITALY & 1900 & & & 10TH ST./11TH ST. & CALUMET - YELLOW JACKET \\
\hline 2640 & 4238 & AUTERO & JOHN & s & 7 & MICHIGAN & ITALY & ITALY & & & PONT CANAVESE, TO & 10TH ST./11TH ST. & CALUMET - YELLOW JACKET \\
\hline 2641 & 4238 & AUTERO & MARY & $\mathrm{D}$ & 2 & MICHIGAN & ITALY & ITALY & & & PONT CANAVESE. TO & 10TH ST./11TH ST. & CALUMET - YELLOW JACKET \\
\hline 2642 & 4238 & AUTERO & JOSEPHINE & $\mathrm{D}$ & 6 & MICHIGAN & ITALY & ITAL & & & PONT CANAVESE. TO & 10TH ST./11TH ST. & CALUMET - YELLOW JACKET \\
\hline & 4238 & PUCCI & CARLO & B & 34 & & & & 1901 & R/CM & LUCCA & & ET - \\
\hline 44 & 4238 & SER & & $\mathrm{H}$ & 38 & ITALY & ITALY & ITALY & 1906 & MINER/CM & SAN GIORGIO CANAVE & & CALUMET - \\
\hline 45 & 4238 & SERRA & MARIA & w & 33 & ITALY & ITALY & ITALY & 1907 & & SAN GIORGIO CANAVESE. TO & 10TH ST./11TH ST. & CALUMET - YELLOW JACKET \\
\hline
\end{tabular}




\begin{tabular}{|c|c|c|c|c|c|c|c|c|c|c|c|c|c|}
\hline 2646 & 4238 & SERRA & KATE & D & 15 & ITALY & ITALY & ITALY & 1907 & SALESLADY/DEPT. STORE & SAN GIORGIO CANAVESE. TO & 10TH ST./11TH ST. & CALUMET - YELLOW JACKET \\
\hline 2647 & 4238 & SERRA & JOSEPH & $\mathrm{s}$ & 13 & ITALY & ITALY & ITALY & 1907 & & SAN GIORGIO CANAVESE. TO & 10TH ST./11TH ST. & CALUMET - YELLOW JACKET \\
\hline 2648 & 4238 & SERRA & EUGENE? & $\mathrm{D}$ & 12 & ITALY & ITALY & ITALY & 1907 & & SAN GIORGIO CANAVESE. TO & 10TH ST./11TH ST. & CALUMET - YELLOW JACKET \\
\hline 2649 & 4238 & SERRA & MARTIN & $\mathrm{s}$ & 8 & ITALY & ITALY & ITALY & 1907 & & SAN GIORGIO CANAVESE. TO & 10TH ST./11TH ST. & CALUMET - YELLOW JACKET \\
\hline 2650 & 4238 & SERRA & IRINA & D & $1.2 \mathrm{M}$ & MICHIGAN & ITALY & ITALY & & & SAN GIORGIO CANAVESE. TO & 10TH ST./11TH ST. & CALUMET - YELLOW JACKET \\
\hline 2651 & 4238 & CASERIO & JAMES & B & 20 & ITALY & ITALY & ITALY & 1906 & TIMBERMAN/CM & STRAMBINELLO. TO & 10TH ST. & CALUMET - YELLOW JACKET \\
\hline 2652 & 4238 & PEROLINO & PETER & B & 20 & ITALY & ITALY & ITALY & 1906 & TIMBERMAN/CM & TORINO & 10TH ST. & CALUMET - YELLOW JACKET \\
\hline 2653 & 4246 & GAMARRA & DOMENICO & $\mathrm{H}$ & 34 & ITALY & ITALY & ITALY & 1903 & MASON/HOUSE & SAN BENIGNO CANAVESE. TO & 10TH ST. & CALUMET - YELLOW JACKET \\
\hline 2654 & 4246 & GAMARRA & MINNIE & W & 28 & ITALY & ITALY & ITALY & $06 / 08$ & & SAN BENIGNO CANAVESE. TO & 10TH ST. & CALUMET - YELLLOW JACKET \\
\hline 2655 & 4246 & GAMARRA & PAT & s & 11 & ITALY & ITALY & ITALY & $06 / 08$ & & SAN BENIGNO CANAVESE. TO & 10TH ST. & CALUMET - YELLOW JACKET \\
\hline 2656 & 4246 & GAMARRA & JAMES & BR & 21 & ITALY & ITALY & ITALY & 1906 & TIMBERMAN/CM & SAN BENIGNO CANAVESE. TO & 10TH ST. & CALUMET - YELLOW JACKET \\
\hline 2657 & 4246 & FRANCIONI & JOHN & B & 28 & ITALY & ITALY & ITALY & 1906 & TIMBERMAN/CM & LUCCA & 10TH ST. & CALUMET - YELLOW JACKET \\
\hline 2658 & $\begin{array}{l}4<40 \\
4246\end{array}$ & ROSSO & ANGELO & $\begin{array}{l}\mathrm{D} \\
\mathrm{B}\end{array}$ & 18 & ITALY & ITALY & $\begin{array}{l}\text { ITALY } \\
\text { ITALY }\end{array}$ & 1906 & TRAMMER/CM & FOGLIAZZO CANAVESE. TO & 10TH ST. & CALUMET - YELLOW JACKET \\
\hline 2659 & 4246 & FALCOMBELLO & BASILIO & B & 36 & ITALY & ITALY & ITALY & 1910 & TRAMMER/CM & SAN BENIGNO CANAVESE. TO & 10TH ST. & CALUMET - YELLOW JACKET \\
\hline 2660 & 4246 & MASERO & FRANK & $\mathrm{H}$ & 39 & ITALY & ITALY & ITALY & 1895 & TIMBERMAN/CM & & 10TH ST. & CALUMET - YELLOW JACKET \\
\hline 2661 & 4246 & MASERO & JOSEPHINE & W & 29 & ITALY & ITALY & ITALY & 1899 & & & 10TH ST. & CALUMET - YELLOW JACKET \\
\hline 2662 & 4246 & MASERO & MARY & $\mathrm{D}$ & 8 & MICHIGAN & ITALY & ITALY & & & & 10TH ST. & CALUMET - YELLOW JACKET \\
\hline 2663 & 4246 & MASERO & JENNIE & $\mathrm{D}$ & 3 & MICHIGAN & ITALY & ITALY & & & & 10TH ST. & CALUMET - YELLOW JACKET \\
\hline 2664 & 4246 & MASERO & ANGELINE & D & 3 & MICHIGAN & ITALY & ITALY & & & & 10TH ST. & CALUMET - YELLOW JACKET \\
\hline 2665 & 4248 & TEPATTI & TONI & $\mathrm{H}$ & 43 & ITALY & ITALY & ITALY & 1893 & PLATMAN/CM & PONT CANAVESE. TO & 10TH ST. & CALUMET - YELLOW JACKET \\
\hline 2666 & 4248 & TEPATTI & CELESTINA & W & 34 & ITALY & ITALY & ITALY & 1898 & & & 10TH ST. & CALUMET - YELLOW JACKET \\
\hline 2667 & 4248 & TEPATTI & JOHN & s & 10 & MICHIGAN & ITALY & ITALY & & & PONT CANAVESE, TO & 10TH ST. & CALUMET - YELLLOW JACKET \\
\hline 2668 & 4248 & TEPATTI & JAMES & $\mathrm{s}$ & 9 & MICHIGAN & ITALY & ITALY & & & PONT CANAVESE. TO & 10TH ST. & CALUMET - YELLOW JACKET \\
\hline 2669 & 4248 & TEPATTI & KATE & $\mathrm{D}$ & 5 & MICHIGAN & ITALY & ITALY & & & PONT CANAVESE. TO & 10TH ST. & CALUMET - YELLOW JACKET \\
\hline 2670 & 4248 & PONA/PERA & VINCENT & $\mathrm{H}$ & 39 & ITALY & ITALY & ITALY & 1899 & LABORER/CM & & 10TH ST. & CALUMET - YELLOW JACKET \\
\hline 2671 & 4248 & PONA/PERA & MARY & w & 39 & ITALY & ITALY & ITALY & 1900 & LADURER/UIVI & & $\begin{array}{l}\text { 10TH ST. } \\
\text { 10T. }\end{array}$ & CALUMET - YELLOW JACKET \\
\hline 2672 & 4248 & PONA/PERA & ERNESTINE & $\mathrm{D}$ & 14 & ITALY & ITALY & ITALY & 1900 & SALESLADY/DEPT. STORE & & 10TH ST. & CALUMET - YELLOW JACKET \\
\hline 2673 & 4248 & PONA/PERA & MARNIE & $\mathrm{D}$ & 11 & MICHIGAN & ITALY & ITALY & & & & 10TH ST. & CALUMET - YELLOW JACKET \\
\hline 2674 & 4248 & PONA/PERA & JAMES & s & 9 & MICHIGAN & ITALY & ITALY & & & & 10TH ST. & $\begin{array}{l}\text { CALUMET - YELLOW JACKET } \\
\end{array}$ \\
\hline 2675 & 4248 & PONA/PERA & VINCENT JR. & $\mathrm{s}$ & 5 & MICHIGAN & ITALY & ITALY & & & & 10TH ST. & CALUMET - YELLOW JACKET \\
\hline 2676 & 4248 & PONA/PERA & CESARE & $\mathrm{s}$ & 3 & MICHIGAN & ITALY & ITALY & & & & 10TH ST. & CALUMET - YELLOW JACKET \\
\hline 2677 & 4248 & PONA/PERA & CARLO & $\mathrm{s}$ & 2 & MICHIGAN & ITALY & ITALY & & & & 10TH ST. & CALUMET - YELLOW JACKET \\
\hline 2678 & 4250 & MICHETTI & PAUL & $\mathrm{H}$ & 40 & ITALY & ITALY & ITALY & 1892 & TIMBER BOSS/CM & PONT CANAVESE. TO & 10TH ST. & CALUMET - YELLOW JACKET \\
\hline 2679 & 4250 & MICHETTI & KATE & W & 44 & ITALY & ITALY & ITALY & ???? & & & 10TH ST. & CALUMET - YELLOW JACKET \\
\hline 2680 & 4250 & MICHETTI & FRED & s & 9 & MICHIGAN & ITALY & ITALY & & & PONT CANAVESE. TO & 10TH ST. & CALUMET - YELLOW JACKET \\
\hline 2681 & 4250 & MICHETTI & OLSON & s & 6 & MICHIGAN & ITALY & ITALY & & & PONT CANAVESE. TO & 10TH ST. & CALUMET - YELLOW JACKET \\
\hline 2682 & 4250 & TRIONE & CARLO & SS & 21 & MICHIGAN & ITALY & ITALY & & ENGINE BOY/CM & TORINO & 10TH ST. & CALUMET - YELLOW JACKET \\
\hline 2683 & 4250 & TRIONE & PAOLO & ss & 19 & MICHIGAN & ITALY & ITALY & & LABORER/CM & TORINO & 10TH ST. & CALUMET - YELLOW JACKET \\
\hline 2684 & 4250 & TRIONE & AUGUSTO & SS & 18 & MICHIGAN & ITALY & ITALY & & LABORER/CM & TORINO & 10TH ST. & CALUMET - YELLOW JACKET \\
\hline 2685 & 4217 & ELLENA & JOHN & $\mathrm{H}$ & 45 & ITALY & ITALY & ITALY & 1900 & TIMBERMAN/CM & SAN BENIGNO CANAVESE. TO & 11TH ST. & CALUMET - YELLOW JACKET \\
\hline 2686 & 4217 & ELLENA & CATERINA & W & 43 & ITALY & ITALY & ITALY & 1903 & & & 11TH ST. & CALUMET - YELLOW JACKET \\
\hline 2687 & 4217 & ELLENA & DOMENICO & $\mathrm{BR}$ & 41 & ITALY & ITALY & ITALY & 1889 & NOT CLEAR & SAN BENIGNO CANAVESE. TO & 11TH ST. & CALUMET - YELLOW JACKET \\
\hline 2688 & 4217 & ELLENA & ANGELO & BR & 29 & ITALY & ITALY & ITALY & 1904 & NOT CLEAR & SAN BENIGNO CANAVESE. TO & 11 TH ST. & CALUMET - YELLOW JACKET \\
\hline 2689 & 4217 & ALLARIA & JOHN & NEP & 21 & ITALY & ITALY & ITALY & 1909 & NOT CLEAR & SAN BENIGNO CANAVESE. TO & 11TH ST. & \\
\hline 2690 & 4238 & SANDRETTO & JAMES & $\mathrm{H}$ & 32 & ITALY & ITALY & ITALY & 1905 & LABORER/CM & PONT CANAVESE. TO & 11TH ST. & CALUMET - YELLLW JACKET \\
\hline 2691 & & SANDRETTO & ANNE & W & 25 & ITALY & ITALY & ITALY & 1906 & & TORINO & 11TH ST. & CALUMET - YELLOW JACKET \\
\hline 2692 & & SANDRETTO & MINNIE & $\mathrm{D}$ & 3 & MICHIGAN & ITALY & ITALY & & & PONT CANAVESE. TO & 11TH ST. & CALUMET - YELLOW JACKET \\
\hline 2693 & & SANDRETTO & MARY & $\mathrm{D}$ & 2 & MICHIGAN & ITALY & ITALY & & & PONT CANAVESE. TO & 11TH ST. & CALUMET - YELLOW JACKET \\
\hline 2694 & & SANDRETTO & ORLANDO & $\mathrm{s}$ & 1 & MICHIGAN & ITALY & ITALY & & & PONT CANAVESE. TO & 11TH ST. & CALUMET - YELLOW JACKET \\
\hline 2695 & & $\begin{array}{l}\text { RASTELLO } \\
\text { RAS }\end{array}$ & ANDREW & $\mathrm{B}$ & 27 & ITALY & ITALY & ITALY & 1908 & LABORER/CM & PONT CANAVESE. TO & 11TH ST. & CALUMET - YELLOW JACKET \\
\hline 2696 & & RASTELLO & JOHN & $\begin{array}{l}\mathrm{D} \\
\mathrm{H}\end{array}$ & 34 & ITALY & ITALY & ITALY & 1906 & LABORER/CM & PONT CANAVESE. TO & 11TH ST. & CALUMET - YELLOW JACKET \\
\hline 2697 & & RASTELLOO & ANGIE & w & 30 & ITALY & ITALY & ITALY & ???? & & PONT CANAVESE, TO & 11TH ST. & CALUMET - YELLOW JACKET \\
\hline 2698 & & RASTELLO & $?$ & s & 3 & MICHIGAN & ITALY & ITALY & & & PONT CANAVESE. TO & 11TH ST. & CALUMET - YELLOW JACKET \\
\hline 2699 & 4245 & MORETTO & JOHN & $\mathrm{H}$ & 31 & ITALY & ITALY & ITALY & 1903 & TIMBERMAN/CM & SAN PONSO CANAVESE. TO & 11TH ST. & $\begin{array}{l}\text { CALUMET - YELLOW JACKET } \\
\end{array}$ \\
\hline 2700 & & MORETTO & MADDALENA & W & 22 & ITALY & ITALY & ITALY & 1904 & & & 11TH ST. & CALUMET - YELLOW JACKET \\
\hline 2701 & & MORETTO & KATE & D & 2 & MICHIGAN & ITALY & ITALY & & & SAN PONSO CANAVESE. TO & 11TH ST. & CALUMET - YELLOW JACKET \\
\hline 2702 & & MORETTO & MINNIE & D & $10 \mathrm{M}$ & MICHIGAN & ITALY & ITALY & & & SAN PONSO CANAVESE. TO & 11TH ST. & CALUMET - YELLOW JACKET \\
\hline 2703 & & (P)AJONI & JOHN & $\mathrm{H}$ & 42 & ITALY & ITALY & ITALY & ???? & TIMBERMAN/CM & & 11TH ST. & CALUMET - YELLOW JACKET \\
\hline 2704 & & (P)AJONI & MARY & W & 46 & ITALY & ITALY & ITALY & 1902 & & & 11TH ST. & CALUMET - YELLOW JACKET \\
\hline 2705 & & (P)AJONI & JOSEPH & s & 7 & MICHIGAN & ITALY & ITALY & & & & 11TH ST. & CALUMET - YELLLOW JACKET \\
\hline 2706 & 42?? & GROSSO & ANTONE & $\mathrm{H}$ & 36 & ITALY & ITALY & ITALY & 1899 & LABORER/CM & MONTELANGHE. TO & $11 \mathrm{TH}$ ST. & CALUMET - YELLOW JACKET \\
\hline 2707 & & GROSSO & TRACY & w & 36 & ITALY & ITALY & ITALY & 1905 & & MONTELANGHE. TO & 11 TH ST. & CALUMET - YELLOW JACKET \\
\hline 2708 & & GROSSO & STEPHEN & s & 12 & ITALY & ITALY & ITALY & 1905 & & MONTELANGHE. TO & 11TH ST. & CALUMET - YELLOW JACKET \\
\hline
\end{tabular}




\begin{tabular}{|c|c|c|c|c|c|c|c|c|c|c|c|c|c|}
\hline 2709 & & GROSSO & DOMENICO & $\mathrm{s}$ & 10 & ITALY & ITALY & ITALY & 1905 & & MONTELANGHE. TO & 11TH ST. & CALUMET - YELLOW JACKET \\
\hline 2710 & & CIABATTARI & TEO & H & 26 & ITALY & ITALY & ITALY & 1902 & LABORER/CM & LUCCA & 11TH ST. & CALUMET - YELLOW JACKET \\
\hline 2711 & & CIABATTARI & MARY & W & 20 & MICHIGAN & GERMANY & GERMANY & & & GERMANY & 11TH ST. & CALUMET - YELLOW JACKET \\
\hline 2712 & & CIABATTARI & & s & 2 & MICHIGAN & MICHIGAN & MICHIGAN & & & LUCCA & 11TH ST. & CALUMET - YELLOW JACKET \\
\hline 2713 & & CIABATTARI & FRANK & $\mathrm{s}$ & $3 \mathrm{M}$ & MICHIGAN & MICHIGAN & MICHIGAN & & & LUCCA & 11TH ST. & CALUMET - YELLOW JACKET \\
\hline 2714 & 4342 & FEIRA & DOMENICO & $\mathrm{H}$ & 31 & ITALY & ITALY & ITALY & 1905 & LABORER/CM & PONT CANAVESE. TO & ACORN ST. & CALUMET - YELLOW JACKET \\
\hline 2715 & 4342 & FEIRA & KATE & W & 27 & ITALY & ITALY & ITALY & 1907 & & PONT CANAVESE. TO & ACORN ST. & CALUMET - YELLOW JACKET \\
\hline 2716 & 4342 & FEIRA & MARY & $\mathrm{D}$ & 6 & ITALY & ITALY & ITALY & 1907 & & PONT CANAVESE. TO & ACORN ST. & CALUMET - YELLOW JACKET \\
\hline 2717 & 4342 & GRIGLIATTI & TOMMASO & $\mathrm{H}$ & 23 & ITALY & ITALY & ITALY & 1905 & TIMBERMAN/CM & SAN BENIGNO CANAVESE. TO & ACORN ST. & CALUMET - YELLLOW JACKET \\
\hline 2718 & 4342 & GRIGLIATTI & ROSE & W & 21 & ITALY & ITALY & ITALY & 1908 & 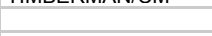 & & ACORN ST. & CALUMET - YELLOW JACKET \\
\hline 2719 & 4342 & GRIGLIATTI & CHARLES & $\mathrm{s}$ & $1.7 \mathrm{M}$ & MICHIGAN & ITALY & ITALY & & & SAN BENIGNO CANAVESE. TO & ACORN ST. & CALUMET - YELLOW JACKET \\
\hline 2720 & 4342 & CRAVERO & LORENZO & B & 25 & ITALY & ITALY & ITALY & 1909 & TRAMMER/CM & SAN BENIGNO CANAVESE. TO & ACORN ST. & CALUMET - YELLOW JACKET \\
\hline 2721 & 4342 & NIGRA & CARLO & B & 26 & ITALY & ITALY & ITALY & 1907 & TRAMMER/CM & SAN BENIGNO CANAVESE. TO & ACORN ST. & CALUMET - YELLOW JACKET \\
\hline 2722 & 4342 & DATTO/DATA & JOHN & B & 21 & ITALY & ITALY & ITALY & 1909 & TRAMMER/CM & SAN GIORGIO CANAVESE. TO & ACORN ST. & CALUMET - YELLOW JACKET \\
\hline 2723 & 4342 & VECCHIA & JOSEPH & B & 25 & ITALY & ITALY & ITALY & 1906 & TRAMMER/CM & SAN BENIGNO CANAVESE. TO & ACORN ST. & CALUMET - YELLOW JACKET \\
\hline 2724 & 4342 & NOTARIO & JOSEPH & B & 20 & ITALY & ITALY & ITALY & 1900 & TRAMMER/CM & SAN BENIGNO CANAVESE, TO & ACORN ST. & CALUMET - YELLOW JACKET \\
\hline 2725 & 4324 & MICHETTI & JOSEPH & $\mathrm{H}$ & 37 & ITALY & ITALY & ITALY & 1891 & PLATMAN & PONT CANAVESE. TO & OAK ST. & CALUMET - YELLOW JACKET \\
\hline 2726 & 4324 & MICHETTI & ORSOLA & W & 38 & ITALY & ITALY & ITALY & 1900 & & PONT CANAVESE. TO & OAK ST. & CALUMET - YELLOW JACKET \\
\hline 2727 & 4324 & MICHETTI & FRED & $\mathrm{s}$ & 13 & ITALY & ITALY & ITALY & 1900 & & PONT CANAVESE. TO & OAK ST. & CALUMET - YELLOW JACKET \\
\hline 2728 & 4324 & MICHETTI & JOHN & $\mathrm{s}$ & 9 & MICHIGAN & ITALY & ITALY & & & PONT CANAVESE. TO & OAK ST. & CALUMET - YELLOW JACKET \\
\hline 2729 & 4324 & MICHETTI & PAUL & $\mathrm{S}$ & 5 & MICHIGAN & ITALY & ITALY & & & PONT CANAVESE. TO & OAK ST. & CALUMET - YELLOW JACKET \\
\hline 2730 & 4324 & MICHETTI & JOSEPH & $\mathrm{s}$ & 2 & MICHIGAN & ITALY & ITALY & & & PONT CANAVESE, TO & OAK ST. & CALUMET - YELLLOW JACKET \\
\hline 2731 & 4313 & DE FILIPPI & JOSEPH & $\mathrm{H}$ & 29 & ITALY & ITALY & ITALY & 1903 & NOT CLEAR & SAN GIORGIO CANAVESE. TO & OAK ST. & CALUMET - YELLOW JACKET \\
\hline 2732 & 4313 & DE FILIPPI & ANGELA & W & 26 & ITALY & ITALY & ITALY & 1905 & TV TI CLLAR & 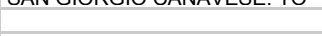 & OAK ST. & CALUMET - YELLOW JACKET \\
\hline 2733 & 4313 & DE FILIPPI & JOSEPH JR. & s & 4 & MICHIGAN & ITALY & ITALY & & & SAN GIORGIO CANAVESE. TO & OAK ST. & CALUMET - YELLOW JACKET \\
\hline 2734 & 4313 & DE FILIPPI & CARLO & $\mathrm{s}$ & $1.2 \mathrm{M}$ & MICHIGAN & ITALY & ITALY & & & SAN GIORGIO CANAVESE. TO & OAK ST. & CALUMET - YELLOW JACKET \\
\hline 2735 & 4313 & DE FILIPPI & MARY & $\mathrm{D}$ & 8 & MICHIGAN & ITALY & ITALY & & & SAN GIORGIO CANAVESE. TO & OAK ST. & CALUMET - YELLOW JACKET \\
\hline 2736 & 4311 & DE FILIPPI & DOMENICO & $\mathrm{H}$ & 31 & ITALY & ITALY & ITALY & 1897 & TIMBER BOSS/CM & SAN GIORGIO CANAVESE, TO & OAK ST. & CALUMET - YELLOW JACKET \\
\hline 2737 & 4311 & SERVOTTI & FRANCA & MIL & $50 ?$ & ITALY & ITALY & ITALY & $89 / 99$ & & & OAK ST. & CALUMET - YELLOW JACKET \\
\hline 2738 & 4306 & CALUSIO & JOHN & $\mathrm{H}$ & 45 & ITALY & ITALY & ITALY & 1887 & TIMBERMAN/CM & SAN GIORGIO CANAVESE. TO & PORTLAND ST. & CALUMET - YELLOW JACKET \\
\hline 2739 & 4306 & CALUSIO & ANNA & W & 43 & ITALY & ITALY & ITALY & 1891 & & SAN GIORGIO CANAVESE. TO & PORTLAND ST. & CALUMET - YELLOW JACKET \\
\hline 2740 & 4306 & CALUSIO & PETER & $\mathrm{s}$ & 22 & ITALY & ITALY & ITALY & 1891 & MINER/CM & SAN GIORGIO CANAVESE. TO & PORTLAND ST. & CALUMET - YELLOW JACKET \\
\hline 2741 & 4306 & CALUSIO & AGNES & $\mathrm{D}$ & 17 & MICHIGAN & ITALY & ITALY & & & SAN GIORGIO CANAVESE. TO & PORTLAND ST. & CALUMET - YELLOW JACKET \\
\hline 2742 & 4306 & CALUSIO & MARY & $\mathrm{D}$ & 16 & MICHIGAN & ITALY & ITALY & & & SAN GIORGIO CANAVESE, TO & PORTLAND ST. & CALUMET - YELLOW JACKET \\
\hline 2743 & 4306 & CALUSIO & ANGELINE & $\mathrm{D}$ & 11 & MICHIGAN & ITALY & ITALY & & & SAN GIORGIO CANAVESE. TO & PORTLAND ST. & CALUMET - YELLOW JACKET \\
\hline 2744 & 4306 & RIVARA & $\begin{array}{l}\text { JAMES } \\
\text { Ja }\end{array}$ & $\mathrm{H}$ & 53 & ITALY & ITALY & ITALY & 1883 & TIMBERMAN/CM & TORINO & PORTLAND ST. & CALUMET - YELLOW JACKET \\
\hline 2745 & 4306 & RIVARA & MARY & W & 45 & ITALY & ITALY & ITALY & 1883 & 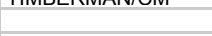 & TORINO & $\begin{array}{l}\text { PORTLAND ST. } \\
\text { POT }\end{array}$ & CALUMET - YELLOW JACKET \\
\hline 2746 & 4306 & RIVARA & JAMES JR. & s & 11 & MICHIGAN & ITALY & ITALY & & & TORINO & PORTLAND ST. & CALUMET - YELLOW JACKET \\
\hline 2747 & 4306 & RIVARA & $\mathrm{JOHN}$ & $\mathrm{S}$ & 7 & MICHIGAN & ITALY & ITALY & & & TORINO & PORTLAND ST. & CALUMET - YELLOW JACKET \\
\hline 2748 & 4306 & RIVARA & KATE & D & 6 & MICHIGAN & ITALY & ITALY & & & TORINO & PORTLAND ST. & CALUMET - YELLOW JACKET \\
\hline 2749 & 4306 & MIGLIO/MIGLIA & DOMENICO & $\mathrm{H}$ & 20 & ITALY & ITALY & ITALY & 1890 & BLACKSMITH/SHOP & CASTELLAMONTE. TO & PORTLAND ST. & CALUMET - YELLOW JACKET \\
\hline 2750 & 4306 & MIGLIO/MIGLIA & ROSE & W & 19 & MICHIGAN & ITALY & ITALY & & & & PORTLAND ST. & CALUMET - YELLOW JACKET \\
\hline 2751 & 3919 & POGGIONE & PETER & $\mathrm{H}$ & 40 & ITALY & ITALY & ITALY & 1890 & BRAKE FIXER/CM & SAN GIORGIO CANAVESE. TO & 6TH ST. & CALUMET - NEWTOWN \\
\hline 2752 & 3919 & POGGIONE & KATE & W & 34 & ITALY & ITALY & ITALY & 1897 & & & 6TH ST. & CALUMET - NEWTOWN \\
\hline 2753 & 3919 & POGGIONE & JOSEPH & $\mathrm{s}$ & 10 & MICHIGAN & ITALY & ITALY & & & SAN GIORGIO CANAVESE. TO & 6TH ST. & CALUMET - NEWTOWN \\
\hline 2754 & 3919 & POGGIONE & KATE & D & 5 & MICHIGAN & ITALY & ITALY & & & SAN GIORGIO CANAVESE. TO & 6TH ST. & CALUMET - NEWTOWN \\
\hline 2755 & 3919 & POGGIONE & DOMENICO & $\mathrm{s}$ & 6 & MICHIGAN & ITALY & ITALY & & & SAN GIORGIO CANAVESE. TO & 6TH ST. & CALUMET - NEWTOWN \\
\hline 2756 & 3919 & POGGIONE & ERNEST & $\mathrm{s}$ & 3 & MICHIGAN & ITALY & ITALY & & & SAN GIORGIO CANAVESE. TO & 6TH ST. & CALUMET - NEWTOWN \\
\hline 2757 & 3919 & POGGIONE & ALBERTO & $\mathrm{s}$ & 2 & MICHIGAN & ITALY & ITALY & & & SAN GIORGIO CANAVESE. TO & 6TH ST. & CALUMET - NEWTOWN \\
\hline 2758 & 3922 & BASSO & JAMES & H & 49 & ITALY & ITALY & ITALY & 1889 & BELL RINGER/CM & SAN GIORGIO CANAVESE. TO & 6TH ST. & CALUMET - NEWTOWN \\
\hline 2759 & 3922 & BASSO & FRANCES & W & 37 & ITALY & ITALY & ITALY & 1890 & & & 6TH ST. & CALUMET - NEWTOWN \\
\hline 2760 & 3922 & BASSO & TERESA & $\mathrm{D}$ & 17 & MICHIGAN & ITALY & ITALY & & SERVANT/PF & SAN GIORGIO CANAVESE, TO & 6TH ST. & CALUMET - NEWTOWN \\
\hline 2761 & 3922 & BASSO & KATE & $\mathrm{D}$ & 16 & MICHIGAN & ITALY & ITALY & & SERVANT/PF & SAN GIORGIO CANAVESE. TO & 6TH ST. & CALUMET - NEWTOWN \\
\hline 2762 & 3922 & BASSO & LUCY & D & 14 & MICHIGAN & ITALY & ITALY & & & SAN GIORGIO CANAVESE. TO & 6TH ST. & CALUMET - NEWTOWN \\
\hline 2763 & 3922 & BASSO & LOUIS & s & 13 & MICHIGA & ITAL & ITALY & & & SAN GIORGIO CANAVESE. TO & 6TH ST. & CALUMET - NEWTOWN \\
\hline 2764 & 3922 & BASSO & JOSEPH & s & 11 & MICHIGAN & ITALY & ITALY & & & SAN GIORGIO CANAVESE. TO & 6TH ST. & CALUMET - NEWTOWN \\
\hline 2765 & 3922 & BASSO & JAMES & $\mathrm{s}$ & 9 & MICHIGAN & ITALY & ITALY & & & SAN GIORGIO CANAVESE. TO & 6TH ST. & CALUMET - NEWTOWN \\
\hline 2766 & 3922 & BASSO & CHARLES & $\mathrm{s}$ & 7 & MICHIGAN & ITALY & ITALY & & & SAN GIORGIO CANAVESE, TO & 6TH ST. & CALUMET - NEWTOWN \\
\hline 2767 & 3922 & BASSO & JOHN & $\mathrm{S}$ & 4 & MICHIGAN & ITALY & ITALY & & & SAN GIORGIO CANAVESE. TO & 6TH ST. & CALUMET - NEWTOWN \\
\hline 2768 & 3922 & BASSO & PETER & $\mathrm{s}$ & 2 & MICHIGAN & ITALY & ITALY & & & SAN GIORGIO CANAVESE. TO & 6TH ST. & CALUMET - NEWTOWN \\
\hline 2769 & 3925 & CURTO & JAMES & $\mathrm{H}$ & 51 & ITALY & ITALY & ITALY & 1889 & TIMBER BOSS/CM & TORINO & 6TH ST. & CALUMET - NEWTOWN \\
\hline 2770 & 3925 & CURTO & MARY & W & 49 & ITALY & ITALY & ITALY & 1898 & & & 6TH ST. & CALUMET - NEWTOWN \\
\hline 2771 & 3925 & CURTO & ANDREW & s & 19 & MICHIGAN & ITALY & ITALY & & TIMBERMAN/CM & TORINO & 6TH ST. & CALUMET - NEWTOWN \\
\hline
\end{tabular}




\begin{tabular}{|c|c|c|c|c|c|c|c|c|c|c|c|c|c|}
\hline 2772 & 3925 & CURTO & FRANCES & $\mathrm{D}$ & 17 & MICHIGAN & ITALY & ITALY & & & TORINO & 6TH ST. & CALUMET - NEWTOWN \\
\hline 2773 & 3925 & CURTO & PETER & $\mathrm{s}$ & 12 & MICHIGAN & ITALY & ITALY & & & TORINO & 6TH ST. & CALUMET - NEWTOWN \\
\hline 2774 & 3925 & CURTO & JOSEPHINE & D & 11 & MICHIGAN & ITALY & ITALY & & & TORINO & 6TH ST. & CALUMET - NEWTOWN \\
\hline 2775 & 3925 & CURTO & JAMES JR. & $\mathrm{s}$ & 7 & MICHIGAN & ITALY & ITALY & & & TORINO & 6TH ST. & CALUMET - NEWTOWN \\
\hline 2776 & 3925 & GAMETRO & JOSEPH & B & 51 & ITALY & ITALY & ITALY & 1905 & LABORER/CM & TORINO & 6TH ST. & CALUMET - NEWTOWN \\
\hline 2777 & 3925 & GAMETRO & JOHN & B & 20 & ITALY & ITALY & ITALY & 1907 & TRAMMER/CM & TORINO & 6TH ST. & CALUMET - NEWTOWN \\
\hline 2778 & 3925 & GAMETRO & TONY & B & 19 & ITALY & ITALY & ITALY & 1908 & TRAMMER/CM & TORINO & 6TH ST. & CALUMET - NEWTOWN \\
\hline 2779 & 3925 & GAMETRO & MICHAEL & $\begin{array}{l}\mathrm{D} \\
\mathrm{B}\end{array}$ & 22 & ITALY & ITALY & ITALY & 1907 & TRAMMER/CM & TORINO & 6TH ST. & CALUMET - NEWTOWN \\
\hline 2780 & 3925 & ANDRINA & $\begin{array}{l}\text { JAMES } \\
\text { JALEL }\end{array}$ & $\begin{array}{l}\mathrm{D} \\
\mathrm{B}\end{array}$ & 24 & ITALY & ITALY & ITALY & 1907 & TRAMMER/CM & VIALFRE'. TO & 6TH ST. & CALUMET - NEWTOWN \\
\hline 2781 & 3925 & ANDRINA & MARCO & B & 22 & ITALY & ITALY & ITALY & ???? & TIMBERMAN/CM & VIALFRE'. TO & 6TH ST. & CALUMET - NEWTOWN \\
\hline 2782 & 3925 & PERLA & MIKE & $\mathrm{H}$ & 48 & ITALY & ITALY & ITALY & 1888 & LABORER/CM & & 6TH ST. & CALUMET - NEWTOWN \\
\hline 2783 & 3925 & PERLA & DOMENICA & D & 16 & ITALY & ITALY & ITALY & ???? & & & 6TH ST. & CALUMET - NEWTOWN \\
\hline 2784 & 3925 & PERLA & JOSEPH & $\mathrm{s}$ & 12 & MICHIGAN & ITALY & ITALY & & & & 6TH ST. & CALUMET - NEWTOWN \\
\hline 2785 & 3925 & PERLA & JOHN & $\mathrm{s}$ & 8 & MICHIGAN & ITALY & ITALY & & & & 6TH ST. & CALUMET - NEWTOWN \\
\hline 2786 & 3925 & PERLA & MIKE & $\mathrm{S}$ & 5 & MICHIGAN & ITALY & ITALY & & & & 6TH ST. & CALUMET - NEWTOWN \\
\hline 2787 & 3925 & PERLA & ERN? & $\mathrm{s}$ & 2 & MICHIGAN & ITALY & ITALY & & & & 6TH ST. & CALUMET - NEWTOWN \\
\hline 2788 & 3941 & PICCHIOTTINO & PAULINE & $\mathrm{H}$ & 43 & ITALY & ITALY & ITALY & 1888 & KEEPER/BOARDING HOUSE & PONT CANAVESE. TO & TEMPLE ST. & CALUMET - NEWTOWN \\
\hline 2789 & 3941 & PICCHIOTTINO & MINNIE & $\mathrm{D}$ & 21 & MICHIGAN & ITALY & ITALY & & & PONT CANAVESE. TO & TEMPLE ST. & CALUMET - NEWTOWN \\
\hline 2790 & 3941 & PICCHIOTTINO & LINA & STEP & 19 & MICHIGAN & ITALY & ITALY & & SALESLADY/GROCERY STORE & PONT CANAVESE. TO & TEMPLE ST. & CALUMET - NEWTOWN \\
\hline 2791 & 3941 & $\begin{array}{l}\text { PASTORE } \\
\end{array}$ & LOUIS & B & 20 & ITALY & ITALY & ITALY & 1901 & TRAMMER/CM & SAN GIUSTO CANAVESE. TO & TEMPLE ST. & CALUMET - NEWTOWN \\
\hline 2792 & 3941 & MARTA & CARLO & $\begin{array}{l}\mathrm{D} \\
\mathrm{H}\end{array}$ & 30 & ITALY & ITALY & $\begin{array}{l}\text { ITALY } \\
\text { ITA }\end{array}$ & 1899 & TIMBERMAN/CM & AGLIE'. TO & TEMPLE ST. & CALUMET - NEWTOWN \\
\hline 2793 & 3941 & MARTA & MADDALENA & w & 21 & ITALY & ITALY & ITALY & 1906 & & & TEMPLE ST. & CALUMET - NEWTOWN \\
\hline 2794 & 3941 & MARTA & MARY & $\mathrm{D}$ & 3 & MICHIGAN & ITALY & ITALY & & & AGLIE'. TO & TEMPLE ST. & CALUMET - NEWTOWN \\
\hline 2795 & 3941 & MARTA & ANGELINA & $\mathrm{D}$ & $1.2 \mathrm{M}$ & MICHIGAN & ITALY & ITALY & & & AGLIE'. TO & TEMPLE ST. & CALUMET - NEWTOWN \\
\hline 2796 & 3942 & PROLA & GIUSEPPE & $\mathrm{H}$ & 35 & ITALY & ITALY & ITALY & 1900 & TIMBER BOSS/CM & AGLIE'. TO & TEMPLE ST. & CALUMET - NEWTOWN \\
\hline 2797 & 3942 & PROLA & GIUSEPPA & W & 32 & ITALY & ITALY & ITALY & 1901 & & AGLIE'. TO & TEMPLE ST. & CALUMET - NEWTOWN \\
\hline 2798 & 3942 & PROLA & TONY & s & 9 & ITALY & ITALY & ITALY & 1901 & & AGLIE', TO & TEMPLE ST. & CALUMET - NEWTOWN \\
\hline 2799 & 3942 & PROLA & $\mathrm{D}$ ? & $\mathrm{s}$ & 7 & MICHIGAN & ITALY & ITALY & & & AGLIE', TO & TEMPLE ST. & CALUMET - NEWTOWN \\
\hline 2800 & 3942 & PROLA & MARTIN & $\mathrm{S}$ & 5 & MICHIGAN & ITALY & ITALY & & & AGLIE'. TO & TEMPLE ST. & CALUMET - NEWTOWN \\
\hline 2801 & 3942 & SANDRETTO & TOMMASO & B & 25 & ITALY & ITALY & ITALY & 1909 & TRAMMER/CM & PONT CANAVESE. TO & TEMPLE ST. & CALUMET - NEWTOWN \\
\hline 2802 & 3942 & SANDRETTO & BATTISTA & B & 38 & ITALY & ITALY & ITALY & 1909 & TRAMMER/CM & PONT CANAVESE. TO & TEMPLE ST. & CALUMET - NEWTOWN \\
\hline 2803 & 3942 & SANDRETTO & TONY & B & 22 & ITALY & ITALY & ITALY & 1909 & TRAMMER/CM & PONT CANAVESE. TO & TEMPLE ST. & CALUMET - NEWTOWN \\
\hline 2804 & 3942 & $\begin{array}{l}\text { PAOLI } \\
\text { PAOT }\end{array}$ & JOHN & B & 39 & ITALY & ITALY & ITALY & 1901 & LABORER/CM & $\begin{array}{l}\text { LUCCA } \\
\text { LUINALS. TO }\end{array}$ & TEMPLE ST. & CALUMET - NEWTOWN \\
\hline 2805 & 3942 & BRACCO & JOHN & B & 24 & ITALY & ITALY & ITALY & 1908 & MINER/CM & SAN BENIGNO CANAVESE, TO & TEMPLE ST. & CALUMET - NEWTOWN \\
\hline 2806 & 3942 & GALLINATTI & DOMENICO & $\mathrm{H}$ & 30 & ITALY & ITALY & ITALY & 1902 & MINER/CM & AGLIE'. TO & TEMPLE ST. & CALUMET - NEWTOWN \\
\hline 2807 & 3942 & GALLINATTI & MARY & w & 25 & ITALY & ITALY & ITALY & 1902 & & AGLIE'. TO & TEMPLE ST. & CALUMET - NEWTOWN \\
\hline 2808 & 3942 & GALLINATTI & TONY & $\mathrm{s}$ & 4 & MICHIGAN & ITALY & ITALY & & & AGLIE'. TO & TEMPLE ST. & CALUMET - NEWTOWN \\
\hline 2809 & 3943 & RICHETTA & TOMMASO & $\mathrm{H}$ & 47 & ITALY & ITALY & ITALY & 1889 & TIMBERMAN/CM & SAN MARTINO CANAVESE. TO & TEMPLE ST. & CALUMET - NEWTOWN \\
\hline 2810 & 3943 & RICHETTA & JOSEPHINE & W & 53 & ITALY & ITALY & ITALY & 9??? & & SAN MARTINO CANAVESE. TO & TEMPLE ST. & CALUMET - NEWTOWN \\
\hline 2811 & 3943 & RICHETTA & ANTHONY & $\mathrm{s}$ & 10 & MICHIGAN & ITALY & ITALY & & & SAN MARTINO CANAVESE. TO & TEMPLE ST. & CALUMET - NEWTOWN \\
\hline 2812 & 3943 & RICHETTA & ANNA & $\mathrm{D}$ & 7 & ITALY & ITALY & ITALY & 1907 & & SAN MARTINO CANAVESE. TO & TEMPLE ST. & CALUMET - NEWTOWN \\
\hline 2813 & 3943 & ROVANO & JAMES & BRIL & 39 & ITALY & ITALY & ITALY & 1891 & MUSICIAN/SALOON & SAN MARTINO CANAVESE. TO & TEMPLE ST. & CALUMET - NEWTOWN \\
\hline 2814 & 3943 & ROVANO & MARY & NIE & 24 & ITALY & ITALY & ITALY & 1910 & TAILOR/SHOP & SAN MARTINO CANAVESE. TO & TEMPLE ST. & CALUMET - NEWTOWN \\
\hline 2815 & 3942 & GRINDATTI & PIETRO & $\mathrm{H}$ & 31 & ITALY & ITALY & ITALY & 1892 & TIMBERMAN/CM & PONT CANAVESE. TO & TEMPLE ST. & CALUMET - NEWTOWN \\
\hline 2816 & 3942 & GRINDATTI & KATE & W & 30 & ITALY & ITALY & ITALY & 1895 & & 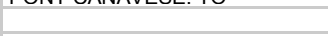 & TEMPLE ST. & CALUMET - NEWTOWN \\
\hline 2817 & 3942 & GRINDATTI & MARY & D & 12 & MICHIGAN & ITALY & ITALY & & & PONT CANAVESE. TO & TEMPLE ST. & CALUMET - NEWTOWN \\
\hline 2818 & 3942 & GRINDATTI & DOMENICO & $\mathrm{s}$ & 10 & MICHIGAN & ITALY & ITALY & & & PONT CANAVESE. TO & TEMPLE ST. & CALUMET - NEWTOWN \\
\hline 2819 & 3942 & POGGIONE & KATE & B & 69 & ITALY & ITALY & ITALY & 1870 & WASHWOMAN/OUT & SAN GIORGIO CANAVESE. TO & TEMPLE ST. & CALUMET - NEWTOWN \\
\hline 2820 & 3947 & GOTTA & JOHN & $\mathrm{H}$ & 35 & ITALY & ITALY & ITALY & 1899 & TIMBERMAN/CM & LOCANA CANAVESE. TO & TEMPLE ST. & CALUMET - NEWTOWN \\
\hline 2821 & 3947 & GOTTA & JOHANNA & w & 24 & ITALY & ITALY & ITALY & 1901 & & & TEMPLE ST. & CALUMET - NEWTOWN \\
\hline 2822 & 3947 & GOTTA & MARIE & D & $1.2 \mathrm{M}$ & MICHIGAN & ITALY & ITALY & & & LOCANA CANAVESE. TO & TEMPLE ST. & CALUMET - NEWTOWN \\
\hline 2823 & 3947 & TOMASI & DOMENICO & B & 42 & ITALY & ITALY & ITALY & 88?? & LABORER/CM & LOCANA CANAVESE, TO & TEMPLE ST. & CALUMET - NEWTOWN \\
\hline 2824 & 3947 & TOMASI & JAMES/JOE & B & 22 & ITALY & ITALY & ITALY & 07 ?? & LABORER/CM & LOCANA CANAVESE. TO & TEMPLE ST. & CALUMET - NEWTOWN \\
\hline 2825 & 3947 & TOMASI & PETER & B & 30 & ITALY & ITALY & ITALY & $06 ? ?$ & TIMBERMAN/CM & LOCANA CANAVESE. TO & TEMPLE ST. & CALUMET - NEWTOWN \\
\hline 2826 & 3947 & GOTTA & PETER & B & 24 & ITALY & ITALY & ITALY & 1903 & TIMBERMAN/CM & LOCANA CANAVESE. TO & TEMPLE ST. & CALUMET - NEWTOWN \\
\hline 2827 & 3947 & QUELLO & JAMES & B & 18 & ITALY & ITALY & ITALY & 1909 & LABORER/CM & LOCANA CANVESE. TO & TEMPLE ST. & CALUMET - NEWTOWN \\
\hline 2828 & 3947 & CERETTO & ANTONIO & $\mathrm{H}$ & 39 & ITALY & ITALY & ITALY & 1895 & TIMBERMAN/CM & PONT CANAVESE. TO & TEMPLE ST. & CALUMET - NEWTOWN \\
\hline 2829 & 3947 & CERETTO & MARIA & W & 42 & ITALY & ITALY & ITALY & 1893 & & & TEMPLE ST. & CALUMET - NEWTOWN \\
\hline 2830 & 3947 & CERETTO & LINA & D & 9 & MICHIGAN & ITALY & ITALY & & & PONT CANAVESE. TO & TEMPLE ST. & CALUMET - NEWTOWN \\
\hline 2831 & 3947 & CERETTO & DELFINA & $\mathrm{D}$ & 4 & MICHIGAN & ITALY & ITALY & & & PONT CANAVESE. TO & TEMPLE ST. & CALUMET - NEWTOWN \\
\hline 2832 & 3947 & CERETTO & JOHANNA & D & 2 & MICHIGAN & ITA & ITA & & & PONT CANAVESE. TO & PLE ST. & T - NEWTOWN \\
\hline 2833 & 3947 & GRILIATI & ANTONIO & SSON & 16 & MICHIGAN & ITALY & ITALY & & & & TEMPLE ST. & CALUMET - NE \\
\hline 2834 & 3949 & BUCHER & ANNA & $\mathrm{H}$ & 36 & SYRIA & SYRIA & SYRIA & 1900 & NOT CLEAR & & TEMPLE ST. & CALUMET - NEWTOWN \\
\hline
\end{tabular}




\begin{tabular}{|c|c|c|c|c|c|c|c|c|c|c|c|c|c|}
\hline 2835 & 3949 & BUCHER & MARIA & $\mathrm{D}$ & 10 & ITALY & ITALY & SYRIA & 1908 & & & TEMPLE ST. & CALUMET - NEWTOWN \\
\hline 2836 & 3949 & BUCHER & SAM & $\mathrm{s}$ & 15 & ITALY & ITALY & SYRIA & 1908 & & & TEMPLE ST. & CALUMET - NEWTOWN \\
\hline 2837 & 3949 & BUCHER & SALY? & D & $6 ?$ & ITALY & ITALY & SYRIA & 1908 & & & TEMPLE ST. & CALUMET \\
\hline 2838 & 3949 & MORETTO & JAMES & $\mathrm{H}$ & 26 & ITALY & ITALY & ITALY & 1902 & TIMBER BOSS/CM & TORINO & TEMPLE ST. & CALUMET \\
\hline 2839 & 3949 & MORETTO & KATE & W & 22 & ITALY & ITALY & ITALY & 1900 & & & TEMPLE ST. & CALUMET - NEWTOWN \\
\hline 2840 & 3950 & CORTESE & MICHAEL & $\mathrm{H}$ & 44 & ITALY & ITALY & ITALY & 1881 & TIMBERMAN/CM & VALPERGA. TO & TEMPLE ST. & CALUMET - NEWTOWN \\
\hline 2841 & 3950 & CORTESE & MARGHERITA & W & 30 & ITALY & ITALY & ITALY & 1899 & & VALPERGA. TO & TEMPLE ST. & CALUMET - NEWTOWN \\
\hline 2842 & 3950 & CORTESE & MAURICE & $\mathrm{s}$ & 5 & MICHIGAN & ITALY & ITALY & & & VALPERGA. TO & TEMPLE ST. & CALUMET - NEWTOWN \\
\hline 2843 & 3950 & VERCELLINO & DOMENICO & B & 27 & ITALY & ITALY & ITALY & 1907 & TIMBERMAN/CM & $\begin{array}{l}\text { TORINO } \\
\text { TOR, TO }\end{array}$ & TEMPLE ST. & CALUMET - NEWTOWN \\
\hline 2844 & 3950 & VERCELLINO & VINCENZO & B & 25 & ITALY & ITALY & ITALY & 1907 & TIMBERMAN/CM & TORINO & TEMPLE ST. & CALUMET - NEWTOWN \\
\hline 2845 & 3953 & GHIARDI & JOHN & $\mathrm{H}$ & 32 & ITALY & ITALY & ITALY & 1894 & BLACKSMITH & SAN MARTINO CANAVESE. TO & TEMPLE ST. & CALUMET - NEWTOWN \\
\hline 2846 & 3953 & GHIARDI & MARTA & W & 27 & ITALY & ITALY & ITALY & ???? & & SAN MARTINO CANAVESE. TO & TEMPLE ST. & CALUMET - NEWTOWN \\
\hline 2847 & 3953 & GHIARDI & ANGELINA & D & & MICHIGAN & ITALY & ITALY & & & SAN MARTINO CANAVESE. TO & TEMPLE ST. & CALUMET - NEWTOWN \\
\hline 2848 & 3953 & GHIARDI & MARTIN & $\mathrm{s}$ & 5 & MICHIGAN & ITALY & ITALY & & & SAN MARTINO CANAVESE. TO & TEMPLE ST. & CALUMET - NEWTOWN \\
\hline 2849 & 3953 & GHIARDI & TRACEY & $\mathrm{D}$ & 3 & MICHIGAN & ITALY & ITALY & & & SAN MARTINO CANAVESE. TO & TEMPLE ST. & CALUMET - NEWTOWN \\
\hline 2850 & 3953 & GHIARDI & ITALIA & D & 1 & MICHIGAN & ITALY & ITALY & & & SAN MARTINO CANAVESE, TO & TEMPLE ST. & CALUMET - NEWTOWN \\
\hline 2851 & 3953 & NARETTO & MIKE & $\mathrm{H}$ & 40 & ITALY & ITALY & ITALY & 1890 & TIMBERMAN/CM & AGLIE'. TO & TEMPLE ST. & CALUMET - NEWTOWN \\
\hline 2852 & 3953 & NARETTO & RITA & W & 41 & ITALY & ITALY & ITALY & 1890 & & AGLIE'. TO & TEMPLE ST. & CALUMET - NEWTOWN \\
\hline 2853 & 3953 & NARETTO & JOSEPHINE & $\mathrm{D}$ & 8 & MICHIGAN & ITALY & ITALY & & & AGLIE', TO & TEMPLE ST. & CALUMET - NEWTOWN \\
\hline 2854 & 3953 & NARETTO & DOMENICO & B & 23 & ITALY & ITALY & ITALY & 1903 & MINER/CM & AGLIE', TO & TEMPLE ST. & CALUMET - NEWTOWN \\
\hline 2855 & 3953 & NARETTO & MIKE & B & 25 & ITALY & ITALY & $\begin{array}{l}\text { ITALY } \\
\text { ITA }\end{array}$ & 1907 & TIMBERMAN/CM & AGLIE'. TO & TEMPLE ST. & CALUMET - NEWTOWN \\
\hline 2856 & 3908 & BOBBIO & PAUL & $\mathrm{H}$ & 49 & ITALY & ITALY & ITALY & 1888 & TIMBERMAN/CM & SAN BENIGNO CANAVESE, TO & WEDGE ST. & CALUMET - NEWTOWN \\
\hline 2857 & 3908 & BOBBIO & ORSOLA & W & 33 & ITALY & ITALY & ITALY & 1888 & & SAN BENIGNO CANAVESE. TO & WEDGE ST. & CALUMET - NEWTOWN \\
\hline 2858 & 3908 & BOBBIO & MIKE & $\mathrm{s}$ & 11 & MICHIGAN & ITALY & ITALY & & & SAN BENIGNO CANAVESE. TO & WEDGE ST. & CALUMET - NEWTOWN \\
\hline 2859 & 3908 & BOBBIO & DOMENICO & $\mathrm{s}$ & 9 & MICHIGAN & ITALY & ITALY & & & SAN BENIGNO CANAVESE. TO & WEDGE ST. & CALUMET - NEWTOWN \\
\hline 2860 & 3908 & BOBBIO & JOHN & $\mathrm{s}$ & 2 & MICHIGAN & ITALY & ITALY & & & SAN BENIGNO CANAVESE. TO & WEDGE ST. & CALUMET - NEWTOWN \\
\hline 2861 & 3908 & MARCHESCHI & DOMENICO & B & 36 & ITALY & ITALY & ITALY & 1890 & MINER/CM & LUCCA & WEDGE ST. & CALUMET - NEWTOWN \\
\hline 2862 & 3908 & CAPELLO & DOMENICO & B & 24 & ITALY & ITALY & ITALY & 1900 & TIMBERMAN/CM & SAN BENIGNO CANAVESE, TO & WEDGE ST. & CALUMET - NEWTOWN \\
\hline 2863 & 3908 & SALASSA & JOSEPH & B & 50 & ITALY & ITALY & ITALY & 1889 & TIMBERMAN/CM & SAN BENIGNO CANAVESE. TO & WEDGE ST. & CALUMET - NEWTOWN \\
\hline 2864 & 3908 & DI VECCHIO & JOSEPH & B & 39 & ITALY & ITALY & ITALY & 1898 & WATCHMAN/CM & LUCCA & WEDGE ST. & CALUMET - NEWTOWN \\
\hline 2865 & 3908 & BRACCO & JOHN & B & 23 & ITALY & ITALY & ITALY & 1906 & TIMBERMAN/CM & TORINO & WEDGE ST. & CALUMET - NEWTOWN \\
\hline 2866 & 3908 & CAPELLO & TONY & B & 29 & ITALY & ITALY & ITALY & 1903 & TRAMMER/CM & SAN BENIGNO CANAVESE. TO & WEDGE ST. & CALUMET - NEWTOWN \\
\hline 2867 & $\begin{array}{l}5900 \\
3908\end{array}$ & BAUDERIO? & DOMENICO & B & 27 & ITALY & ITALY & ITALY & 1908 & TRAMMER/CM & SAIV DEIVISTU LAIVAVEOE. TO & WEDGE ST. & CALUMET - NEWTOWN \\
\hline 2868 & 3939 & MARCHESCHI & CHARLES & $\mathrm{H}$ & 33 & ITALY & ITALY & ITALY & 1897 & TRAMMER/CM & LUCCA & WEDGE ST. & CALUMET - NEWTOWN \\
\hline 2869 & 3939 & MARCHESCHI & ELENA & w & 32 & ITALY & ITALY & ITALY & 1897 & & LUCCA & WEDGE ST. & CALUMET - NEWTOWN \\
\hline 2870 & 3939 & MARCHESCHI & ISABELLA & $\mathrm{D}$ & 5 & MICHIGAN & ITALY & ITALY & & & LUCCA & WEDGE ST. & CALUMET - NEWTOWN \\
\hline 2871 & 3939 & MARCHESCHI & $\begin{array}{l}\text { IRENE } \\
\text { IREN }\end{array}$ & $\mathrm{D}$ & 10 & MICHIGAN & ITALY & ITALY & & & LUCCA & WEDGE ST. & CALUMET - NEWTOWN \\
\hline 2872 & 3939 & GELIACHI & MARCO & $\mathrm{H}$ & 40 & ITALY & ITALY & ITALY & ???? & MINER/CM & & WEDGE ST. & CALUMET - NEWTOWN \\
\hline 2873 & 3939 & GELIACHI & FRANK & $\mathrm{s}$ & 16 & MICHIGAN & ITALY & ITALY & & & & WEDGE ST. & CALUMET - NEWTOWN \\
\hline 2874 & 3939 & GELIACHI & VALENTINA & $\mathrm{S}$ & 19 & MICHIGAN & ITALY & ITALY & & & & WEDGE ST. & CALUMET - NEWTOWN \\
\hline 2875 & 3939 & GELIACHI & VERONA & D & 13 & MICHIGAN & ITALY & ITALY & & & & WEDGE ST. & CALUMET - NEWTOWN \\
\hline 2876 & 3960 & GHERNA & JOHN & $\mathrm{H}$ & 52 & ITALY & ITALY & ITALY & 1890 & LABORER/CM & PONT CANAVESE. TO & WEDGE ST. & CALUMET - NEWTOWN \\
\hline 2877 & 3960 & GHERNA & TERESA & w & 48 & ITALY & ITALY & ITALY & 1892 & & PONT CANAVESE. TO & WEDGE ST. & CALUMET - NEWTOWN \\
\hline 2878 & 3960 & GHERNA & JOHN B. & $\mathrm{s}$ & 21 & ITALY & ITALY & ITALY & 1892 & MANAGER/TELEGRAPH & PONT CANAVESE. TO & WEDGE ST. & CALUMET - NEWTOWN \\
\hline 2879 & 3960 & GHERNA & MAUD M. & $\mathrm{D}$ & 16 & MICHIGAN & ITALY & ITALY & & 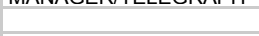 & PONT CANAVESE. TO & WEDGE ST. & CALUMET - NEWTOWN \\
\hline 2880 & 3960 & GHERNA & FRANK & $\mathrm{s}$ & 13 & MICHIGAN & ITALY & ITALY & & & PONT CANAVESE. TO & WEDGE ST. & CALUMET - NEWTOWN \\
\hline 2881 & 3960 & GHERNA & JAMES & $\mathrm{s}$ & 12 & MICHIGAN & ITALY & ITALY & & & PONT CANAVESE. TO & WEDGE ST. & CALUMET - NEWTOWN \\
\hline 2882 & 3960 & GHERNA & MORENO & s & 11 & MICHIGAN & ITALY & ITALY & & & PONT CANAVESE. TO & WEDGE ST. & CALUMET - NEWTOWN \\
\hline 2883 & 3960 & GHERNA & ROBERTO & $\mathrm{s}$ & 8 & MICHIGAN & ITALY & ITALY & & & PONT CANAVESE. TO & WEDGE ST. & CALUMET - NEWTOWN \\
\hline 2884 & 3960 & GHERNA & ISABELLA & $\mathrm{D}$ & 6 & MICHIGAN & ITALY & ITALY & & & PONT CANAVESE. TO & WEDGE ST. & CALUMET - NEWTOWN \\
\hline 2885 & 3960 & PURACHIA & JOHN & B & 39 & ITALY & ITALY & ITALY & 1897 & LABORER/CM & CUNEO & WEDGE ST. & CALUMET - NEWTOWN \\
\hline 2886 & 3960 & CURTO & JOHN & B & 47 & ITALY & ITALY & ITALY & 1886 & TIMBER BOSS/CM & VIALFRE', TO & WEDGE ST. & CALUMET - NEWTOWN \\
\hline 2887 & 3960 & GAIS & JAMES & B & 23 & ITALY & ITALY & ITALY & 1904 & TIMBERMAN/CM & VIALFRE'. TO & WEDGE ST. & CALUMET - NEWTOWN \\
\hline 2888 & 3960 & GHERNA & VITTORIO & B & 21 & ITALY & ITALY & ITALY & 1909 & LABORER/CM & PONT CANAVESE. TO & WEDGE ST. & CALUMET - NEWTOWN \\
\hline 2889 & 3967 & BOGGIO & JOSEPH & $\mathrm{H}$ & 30 & ITALY & ITALY & ITALY & 1902 & TRAMMER/CM & SAN GIUSTO CANAVESE. TO & WEDGE ST. & CALUMET - NEWTOWN \\
\hline 2890 & 3967 & BOGGIO & ANTONIA & W & 23 & ITALY & ITALY & ITALY & 1906 & & & WEDGE ST. & CALUMET - NEWTOWN \\
\hline 2891 & 3967 & BOGGIO & TONY & $\mathrm{s}$ & 1 & MICHIGAN & ITALY & ITALY & & & SAN GIUSTO CANAVESE. TO & WEDGE ST. & CALUMET - NEWTOWN \\
\hline 2892 & 3967 & TOMASI & PETER & $\mathrm{B}$ & 27 & ITALY & ITALY & ITALY & 1906 & TIMBERMAN/CM & LOCANA CANAVESE, TO & WEDGE ST. & CALUMET - NEWTOWN \\
\hline 2893 & 3967 & CONTO & THOMAS & B & 27 & ITALY & ITALY & ITALY & 1907 & TRAMMER/CM & SAN GIORGIO CANAVESE. TO & WEDGE ST. & CALUMET - NEWTOWN \\
\hline 2894 & 3967 & BINANDO & JOHN & B & 35 & ITALY & ITALY & ITALY & 1905 & TIMBERMAN/CM & CORTEREGGIO CANAVESE. TO & WEDGE ST. & CALUMET - NEWTOWN \\
\hline 395 & 3967 & GERRAS & LOUIS & B & 23 & ITALY & ITAI & ITAL & 1901 & TRAMMER/CM & & & CALUMET - NEWTOWN \\
\hline 396 & 3967 & BINANDO & JOSEPH & $\mathrm{H}$ & 38 & ITALY & ITALY & ITALY & 1900 & TIMBERMAN/CM & CORTEREGGIO CANAVESE. TO & WED & CALUMET - NEWTOWN \\
\hline 997 & 3967 & BINANDO & MARY & w & 35 & ITALY & ITALY & ITALY & 1906 & & CORTEREGGIO CANAVESE. TO & WEDGE ST. & CALUMET - NEWTOWN \\
\hline
\end{tabular}




\begin{tabular}{|c|c|c|c|c|c|c|c|c|c|c|c|c|c|}
\hline 2898 & 3967 & BINANDO & PETER & $\mathrm{s}$ & 8 & ITALY & ITALY & ITALY & 1906 & & CORTEREGGIO CANAVESE. TO & WEDGE ST. & CALUMET - NEWTOWN \\
\hline 2899 & 3967 & BINANDO & MICHAEL & $\mathrm{s}$ & 5 & MICHIGAN & ITALY & ITALY & & & CORTEREGGIO CANAVESE. TO & WEDGE ST. & CALUMET - NEWTOWN \\
\hline 2900 & 3967 & BINANDO & JOSEPH & $\mathrm{s}$ & $11 \mathrm{M}$ & MICHIGAN & ITALY & ITALY & & & CORTEREGGIO CANAVESE. TO & WEDGE ST. & CALUMET - NEWTOWN \\
\hline 2901 & 3985 & PISTONATTI & TERESA & $\mathrm{H}$ & 40 & ITALY & ITALY & ITALY & 1891 & DRESSMAKER/AT HOME & BOSCONERO. TO & OSCEOLA ST. & CALUMET - NEWTOWN \\
\hline 2902 & 3985 & CAPELLO & MARY & D & 20 & ITALY & ITALY & ITALY & 1891 & & BOSCONERO. TO & OSCEOLA ST. & CALUMET - NEWTOWN \\
\hline 2903 & 3985 & PISTONATTI & JOSEPH & $\mathrm{s}$ & 18 & MICHIGAN & ITALY & ITALY & & PLATMAN & BOSCONERO. TO & OSCEOLA ST. & CALUMET - NEWTOWN \\
\hline 2904 & 3985 & PISTONATTI & FRANK & BRIL & 33 & ITALY & ITALY & ITALY & 1891 & TIMBERMAN/CM & BOSCONERO. TO & OSCEOLA ST. & CALUMET - NEWTOWN \\
\hline 2905 & 3985 & VIGNA & BATTISTA & B & 20 & ITALY & ITALY & ITALY & 1907 & MINER/CM & SAN BENIGNO CANAVESE. TO & OSCEOLA ST. & CALUMET - NEWTOWN \\
\hline 2906 & 3985 & CAPELLO & TOM & $\begin{array}{l}\mathrm{D} \\
\mathrm{B}\end{array}$ & 28 & ITALY & ITALY & ITALY & 1891 & MINER/CM & SAN BENIGNO CANAVESE. TO & OSCEOLA ST. & CALUMET - NEWTOWN \\
\hline 2907 & 3985 & CAPELLO & TONY & B & 16 & ITALY & ITALY & ITALY & 1909 & PLATMAN & SAN BENIGNO CANAVESE. TO & OSCEOLA ST. & CALUMET - NEWTOWN \\
\hline 2908 & 3985 & ROTA & LORENZO & B & 24 & ITALY & ITALY & ITALY & 1907 & TIMBERMAN/CM & & OSCEOLA ST. & CALUMET - NEWTOWN \\
\hline 2909 & 3985 & ROTA & JOHN & B & 18 & ITALY & ITALY & ITALY & 1907 & TIMBERMAN/CM & & OSCEOLA ST. & CALUMET - NEWTOWN \\
\hline 2910 & 3985 & CAPELLO & NICK & GS & 6 & MICHIGAN & ITALY & ITALY & & & SAN BENIGNO CANAVESE. TO & OSCEOLA ST. & CALUMET - NEWTOWN \\
\hline 2911 & 3985 & CAPELLO & JOHN & GS & 4 & MICHIGAN & ITALY & ITALY & & & SAN BENIGNO CANAVESE. TO & OSCEOLA ST. & CALUMET - NEWTOWN \\
\hline 2912 & 3985 & GAMARRA & DOMENICO & $\mathrm{H}$ & 32 & ITALY & ITALY & ITALY & 1900 & TIMBERMAN/CM & SAN BENIGNO CANAVESE. TO & OSCEOLA ST. & CALUMET - NEWTOWN \\
\hline 2913 & 3985 & GAMARRA & DOMENICA & W & 22 & ITALY & ITALY & ITALY & 1907 & & & OSCEOLA ST. & CALUMET - NEWTOWN \\
\hline 2914 & 3985 & GAMARRA & KATE & D & 2 & MICHIGAN & ITALY & ITALY & & & SAN BENIGNO CANAVESE. TO & OSCEOLA ST. & CALUMET - NEWTOWN \\
\hline 2915 & 3985 & MIGLIO/MIGLIA & JOSEPH & $\mathrm{H}$ & 48 & ITALY & ITALY & ITALY & 1890 & TIMBERMAN/CM & CASTELLAMONTE. TO & OSCEOLA ST. & CALUMET - NEWTOWN \\
\hline 2916 & 3985 & MIGLIO/MIGLIA & CAROLINA & w & 35 & ITALY & ITALY & ITALY & 1893 & 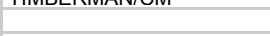 & UAUTLELAIVIUIVIL. TS & OSCEOLA ST. & CALUMET - NEWTOWN \\
\hline 2917 & 3985 & MIGLIO/MIGLIA & DOMENICO & s & 16 & MICHIGAN & ITALY & ITALY & & DRILL BOY/CM & CASTELLAMONTE. TO & OSCEOLA ST. & CALUMET - NEWTOWN \\
\hline 2918 & 3985 & MIGLIO/MIGLIA & MARY & $\mathrm{D}$ & 14 & MICHIGAN & ITALY & ITALY & & & CASTELLAMONTE. TO & OSCEOLA ST. & CALUMET - NEWTOWN \\
\hline 2919 & 3985 & MIGLIO/MIGLIA & JOSEPH JR. & $\mathrm{s}$ & 5 & MICHIGAN & ITALY & ITALY & & & CASTELLAMONTE, TO & OSCEOLA ST. & CALUMET - NEWTOWN \\
\hline 2920 & $39 ? 6$ & ZANOBA & MIKE & $\mathrm{H}$ & 43 & ITALY & ITALY & ITALY & 1893 & LABORER/CM & & OSCEOLA ST. & CALUMET - NEWTOWN \\
\hline 2921 & $39 ? 6$ & ZANOBA & ANNA & W & 35 & ITALY & ITALY & ITALY & $70 / 90$ & & & OSCEOLA ST. & CALUMET - NEWTOWN \\
\hline 2922 & $39 ? 6$ & ZANOBA & MARY & $\mathrm{D}$ & 18 & MICHIGAN & ITALY & ITALY & & SALESLADYIDRY GOODS & & OSCEOLA ST. & CALUMET - NEWTOWN \\
\hline 2923 & $39 ? 6$ & ZANOBA & RITA & D & 16 & MICHIGAN & ITALY & ITALY & & & & OSCEOLA ST. & CALUMET - NEWTOWN \\
\hline 2924 & $39 ? 6$ & ZANOBA & MIKE JR. & $\mathrm{s}$ & 14 & MICHIGAN & ITALY & ITALY & & & & OSCEOLA ST. & CALUMET - NEWTOWN \\
\hline 2925 & $39 ? 6$ & ZANOBA & JOSEPH & $\mathrm{s}$ & 13 & MICHIGAN & ITALY & ITALY & & & & OSCEOLA ST. & CALUMET - NEWTOWN \\
\hline 2926 & $39 ? 6$ & ZANOBA & FRANCES & D & 9 & MICHIGAN & ITALY & ITALY & & & & OSCEOLA ST. & CALUMET - NEWTOWN \\
\hline 2927 & $39 ? 6$ & ZANOBA & JOHN & $\mathrm{s}$ & 7 & MICHIGAN & ITALY & ITALY & & & & OSCEOLA ST. & CALUMET - NEWTOWN \\
\hline 2928 & 3990 & CONTRATTO & ANDREW & $\mathrm{H}$ & 55 & ITALY & ITALY & ITALY & 1893 & WATCHMAN/CM & LOCANA CANAVESE. TO & OSCEOLA ST. & CALUMET - NEWTOWN \\
\hline 2929 & 3990 & CONTRATTO & MARY & W & 42 & ITALY & ITALY & ITALY & 1896 & 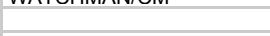 & LUCAIVA URIVAVLUL. TO & OSCEOLA ST. & CALUMET - NEWTOWN \\
\hline 2930 & 3990 & CONTRATTO & PETER & s & 7 & MICHIGAN & ITALY & ITALY & 1050 & & LOCANA CANAVESE. TO & OSCEOLA ST. & CALUMET - NEWTOWN \\
\hline 2931 & 3990 & ROLANDO & JOHN & $\mathrm{H}$ & 27 & ITALY & ITALY & ITALY & 1908 & ROPE FIXER/CM & PONT CANAVESE, TO & OSCEOLA ST. & CALUMET - NEWTOWN \\
\hline 2932 & 3990 & ROLANDO & MARY & w & 22 & ITALY & ITALY & ITALY & 1908 & & PONT CANAVESE. TO & OSCEOLA ST. & CALUMET - NEWTOWN \\
\hline 2933 & 3990 & ROLANDO & MARY & $\mathrm{D}$ & 2 & MICHIGAN & ITALY & ITALY & & & PONT CANAVESE. TO & OSCEOLA ST. & CALUMET - NEWTOWN \\
\hline 2934 & 51 & BINI & ANTONIO & $\mathrm{H}$ & 40 & ITALY & ITALY & ITALY & 1898 & FIREMAN/CM & LUCCA & CEDAR ST. & CALUMET - TAMARACK JR. \\
\hline 2935 & 51 & BINI & ESTER & W & 40 & ITALY & ITALY & ITALY & 1907 & 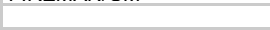 & & CEDAR ST. & CALUMET - TAMARACK JR. \\
\hline 2936 & 51 & BINI & ROSE & $\mathrm{D}$ & 2 & MICHIGAN & ITALY & ITALY & & & LUCCA & CEDAR ST. & CALUMET - TAMARACK JR. \\
\hline 2937 & 51 & LANDINI & TOMMASO & B & 35 & ITALY & ITALY & ITALY & 1898 & FIREMAN/CM & LUCCA & CEDAR ST. & CALUMET - TAMARACK JR. \\
\hline 2938 & 51 & BARSANTI & BRUNO & B & 23 & ITALY & ITALY & ITALY & 1903 & ACCOUNTANT/CM & COLLODI. LU & CEDAR ST. & CALUMET - TAMARACK JR. \\
\hline 2939 & 51 & NARDI & JOSEPH & B & 22 & ITALY & ITALY & ITALY & 1897 & LABORER/CM & PESCIA. LU & CEDAR ST. & CALUMET - TAMARACK JR. \\
\hline 2940 & 51 & MARCHI & AGOSTINO & B & 23 & ITALY & ITALY & ITALY & 1908 & LABORER/CM & VERONA & CEDAR ST. & CALUMET - TAMARACK JR. \\
\hline 2941 & 51 & MARCHI & EUGENIO & B & 37 & ITALY & ITALY & ITALY & 1905 & LABORER/CM & VERONA & CEDAR ST. & CALUMET - TAMARACK JR. \\
\hline 2942 & 51 & CHIPASSORI? & VINCENZO & B & 36 & ITALY & ITALY & ITALY & 1905 & LABORER/CM & & CEDAR ST. & CALUMET - TAMARACK JR. \\
\hline 2943 & 51 & $\begin{array}{l}\text { CHAPPO? } \\
\text { ? }\end{array}$ & ?? & $\mathrm{D}$ & $1.2 \mathrm{M}$ & MICHIGAN & ITALY & ITALY & & LADO TERTIIVI & & CEDAR ST. & CALUMET - TAMARACK JR. \\
\hline 2944 & 14 & PICCHIOTTINO & PETER & $\mathrm{H}$ & 39 & ITALY & ITALY & ITALY & 94/99 & TEAMSTER FIREMAN/CM & PONT CANAVESE. TO & 1ST ST. & CALUMET - WOLVERINE \\
\hline 2945 & 14 & PICCHIOTTINO & CATERINA & w & 38 & ITALY & ITALY & ITALY & $94 / 99$ & & PONT CANAVESE. TO & 1ST ST. & CALUMET - WOLVERINE \\
\hline 2946 & 14 & PICCHIOTTINO & CATERINA & $\mathrm{D}$ & 15 & MICHIGAN & ITALY & ITALY & & & PONT CANAVESE. TO & 1ST ST. & CALUMET - WOLVERINE \\
\hline 2947 & 14 & PICCHIOTTINO & JOHN & $\mathrm{s}$ & 11 & MICHIGAN & ITALY & ITALY & & & PONT CANAVESE. TO & 1ST ST. & CALUMET - WOLVERINE \\
\hline 2948 & 14 & PICCHIOTTINO & $? ?$ & D & 10 & MICHIGAN & ITALY & ITALY & & & PONT CANAVESE. TO & 1ST ST. & CALUMET - WOLVERINE \\
\hline 2949 & 14 & PICCHIOTTINO & LAWRENCE & $\mathrm{S}$ & 2 & MICHIGAN & ITALY & ITALY & & & PONT CANAVESE, TO & 1ST ST. & CALUMET - WOLVERINE \\
\hline 2950 & 14 & BAUSANO & BATTISTA & B & 45 & ITALY & ITALY & ITALY & ???? & MINER/CM & LOCANA CANAVESE. TO & 1ST ST. & CALUMET - WOLVERINE \\
\hline 2951 & 14 & BAUSANO & MAURIZIO & B & 22 & ITALY & ITALY & ITALY & ???? & MINER/CM & LOCANA CANAVESE. TO & 1ST ST. & CALUMET - WOLVERINE \\
\hline 2952 & 14 & BAUSANO & JOHN & B & 24 & ITALY & ITALY & ITALY & 1907 & TIMBER BOSS/CM & LOCANA CANAVESE. TO & 1ST ST. & CALUMET - WOLVERINE \\
\hline 2953 & 14 & BAUSANO & LUIGI & B & 17 & ITALY & ITALY & ITALY & ???? & DRILL BOY/CM & LOCANA CANAVESE. TO & 1ST ST. & CALUMET - WOLVERINE \\
\hline 2954 & 14 & COPPO & NATALE & B & 25 & ITALY & ITALY & ITALY & ???? & TRAMMER/CM & PONT CANAVESE. TO & 1ST ST. & CALUMET - WOLVERINE \\
\hline 2955 & 25 & MICHELA & JOSEPH & $\mathrm{H}$ & 32 & ITALY & ITALY & ITALY & 1907 & WATCHAMN/CM & AGLIE', TO & SMITH AVE. & CALUMET - WOLVERINE \\
\hline 2956 & 25 & MICHELA & DOMENICA & W & 19 & ITALY & ITALY & ITALY & 1907 & & AGLIE', TO & SMITH AVE. & CALUMET - WOLVERINE \\
\hline 2957 & 25 & GALLINATTI & VINCENZO & B & 26 & ITALY & ITALY & ITALY & 1907 & DRAYMAN/CM & TORINO & SMITH AVE. & CALUMET - WOLVERINE \\
\hline 958 & 25 & GALLINAT & MASSIMO & B & 21 & ITA & ITA & ITA & 1907 & SALESMAN/STORE & TORIN & SN & CALUMET - WOLVERINE \\
\hline 959 & 25 & SCAVARDA & JOSEPH & B & 25 & ITALY & ITALY & ITALY & 1905 & NOT CLEAR & TORINO & I AVE. & CALUMET - WOL \\
\hline 60 & 25 & FRISI/FRISE & CARLO & B & 29 & ITALY & ITALY & ITALY & 1903 & MINER/CM & SAN GIORGIO CANAVESE. TO & SMITH AVE. & CALUMET - WOLVERINE \\
\hline
\end{tabular}




\begin{tabular}{|c|c|c|c|c|c|c|c|c|c|c|c|c|c|}
\hline 2961 & 25 & RIASI? & TONIO & B & 31 & ITALY & ITALY & ITALY & 1906 & TIMBER GANG/CM & & SMITH AVE. & CALUMET - WOLVERINE \\
\hline 2962 & 25 & ROSSIO & SEBASTIANO & B & 24 & ITALY & ITALY & ITALY & 1907 & TRAMMER/CM & PONT CANAVESE. TO & SMITH AVE. & CALUMET - WOLVERINE \\
\hline 2963 & 163 & MEINARDI & ANTONIO & $\mathrm{H}$ & 23 & ITALY & ITALY & ITALY & 1907 & TIMBER GANG/CM & TORINO & RAILROAD AVE. & CALUMET - WOLVERINE \\
\hline 2964 & 163 & MEINARDI & LAURA & W & 22 & ITALY & ITALY & ITALY & 1908 & & & RAILROAD AVE. & CALUMET - WOLVERINE \\
\hline 2965 & 163 & BASSOLINI & ANNIE & B & 39 & ITALY & ITALY & ITALY & 1894 & & & RAILROAD AVE. & CALUMET - WOLVERINE \\
\hline 2966 & 163 & BASSOLINI & MINNIE & B & 17 & MICHIGAN & ITALY & ITALY & & & & RAILROAD AVE. & CALUMET - WOLVERINE \\
\hline 2967 & 163 & BASSOLINI & ANNA & B & 9 & MICHIGAN & ITALY & ITALY & & & & RAILROAD AVE. & CALUMET - WOLVERINE \\
\hline 2968 & 163 & ANSELMINO & GIUSEPPE & $\mathrm{H}$ & 40 & ITALY & ITALY & ITALY & 1905 & LABORER/CM & SAN GIUSTO CANAVESE. TO & RAILROAD AVE. & CALUMET - WOLVERINE \\
\hline 2969 & 163 & ANSELMINO & MARGHERITA & W & 38 & ITALY & ITALY & ITALY & 1905 & LADOUALTUIUI & SAN GIUSTO CANAVESE. TO & RAILROAD AVE. & CALUMET - WOLVERINE \\
\hline 2970 & 64 & MASSA & JOSEPH & $\mathrm{H}$ & 31 & ITALY & ITALY & ITALY & 1901 & TIMBER GANG/CM & RIVARA CANAVESE. TO & 2ND ST. & CALUMET - WOLVERINE \\
\hline 2971 & 64 & MASSA & MARGHERITA & W & 29 & ITALY & ITALY & ITALY & 1901 & & RIVARA CANAVESE. TO & 2ND ST. & CALUMET - WOLVERINE \\
\hline 2972 & 64 & MASSA & STEFANO & s & 6 & MICHIGAN & ITALY & ITALY & & & RIVARA CANAVESE. TO & 2ND ST. & CALUMET - WOLVERINE \\
\hline 2973 & 64 & MASSA & JOHN & $\mathrm{s}$ & 4 & MICHIGAN & ITALY & ITALY & & & RIVARA CANAVESE. TO & 2ND ST. & CALUMET - WOLVERINE \\
\hline 2974 & 64 & MASSA & TONY & $\mathrm{s}$ & 2 & MICHIGAN & ITALY & ITALY & & & RIVARA CANAVESE. TO & 2ND ST. & CALUMET - WOLVERINE \\
\hline 2975 & 64 & MASSA & EDWARD & $\mathrm{s}$ & $11 \mathrm{M}$ & MICHIGAN & ITALY & ITALY & & & RIVARA CANAVESE. TO & 2ND ST. & CALUMET - WOLVERINE \\
\hline 2976 & 64 & MASSA & DEFENDENTE & B & 32 & ITALY & ITALY & ITALY & 1910 & MINER/CM & RIVARA CANAVESE, TO & 2ND ST. & CALUMET - WOLVERINE \\
\hline 2977 & 69 & GIACOLETTO & CARLO & $\mathrm{H}$ & 28 & ITALY & ITALY & ITALY & 1905 & TRAMMER/CM & CUORGNE'. TO & CENTRAL ST. & CALUMET - WOLVERINE \\
\hline 2978 & 69 & GIACOLETTO & ANNA & W & 25 & ITALY & ITALY & ITALY & 1907 & & CUORGNE'. TO & CENTRAL ST. & CALUMET - WOLVERINE \\
\hline 2979 & 69 & GIACOLETTO & JENNIE & $\mathrm{D}$ & 6 & ITALY & ITALY & ITALY & 1907 & & CUORGNE'. TO & CENTRAL ST. & CALUMET - WOLVERINE \\
\hline 2980 & 69 & GIACOLETTO & MAURIE? & $?$ & 4 & ITALY & ITALY & ITALY & 1907 & & CUORGNE'. TO & CENTRAL ST. & CALUMET - WOLVERINE \\
\hline 2981 & 69 & GIACOLETTO & CAROLINA & D & $1.6 \mathrm{M}$ & MICHIGAN & ITALY & ITALY & & & CUORGNE'. TO & CENTRAL ST. & CALUMET - WOLVERINE \\
\hline 2982 & 69 & FORNELLI & BARNEY & B & 27 & ITALY & ITALY & ITALY & 1908 & TRAMMER/CM & TORINO & CENTRAL ST. & CALUMET - WOLVERINE \\
\hline 2983 & 69 & SANDRETTO & ANTONIO & B & 25 & ITALY & ITALY & ITALY & 1906 & TRAMMER/CM & PONT CANAVESE. TO & CENTRAL ST. & CALUMET - WOLVERINE \\
\hline 2984 & 69 & GIACOLETTO & JOSEPH & B & 26 & ITALY & ITALY & ITALY & 1908 & TRAMMER/CM & TORINO & CENTRAL ST. & CALUMET - WOLVERINE \\
\hline 2985 & 67 & RICHIARDI & FRANCESCO & $\mathrm{H}$ & 24 & ITALY & ITALY & ITALY & 1908 & MINER/CM & TORINO & CENTRAL ST. & $\begin{array}{l}\text { CALUMET - WOLVERINE } \\
\text { CALT }\end{array}$ \\
\hline 2986 & 67 & RICHIARDI & MARIA & w & 24 & ITALY & ITALY & ITALY & 1908 & & TORINO & CENTRAL ST. & CALUMET - WOLVERINE \\
\hline 2987 & 67 & RICHIARDI & ANGELINA & $\mathrm{D}$ & 4 & ITALY & ITALY & ITALY & 1908 & & TORINO & CENTRAL ST. & CALUMET - WOLVERINE \\
\hline 2988 & 67 & RICHIARDI & JOANNA & $\mathrm{D}$ & 1 & MICHIGAN & ITALY & ITALY & & & TORINO & CENTRAL ST. & CALUMET - WOLVERINE \\
\hline 2989 & 67 & CHIAPERO & ANTONIO & $\mathrm{COU}$ & 22 & ITALY & ITALY & ITALY & 1909 & TRAMMER/CM & CIRIE'. TO & CENTRAL ST. & CALUMET - WOLVERINE \\
\hline 2990 & 67 & GEA & MICHELE & B & 44 & ITALY & ITALY & ITALY & 1906 & TRAMMER/CM & PONT CANAVESE. TO & CENTRAL ST. & CALUMET - WOLVERINE \\
\hline 2991 & 67 & CHIANTELLO & LORENZO & B & 26 & ITALY & ITALY & ITALY & 1908 & TRAMMER/CM & SPARONE. TO & CENTRAL ST. & CALUMET - WOLVERINE \\
\hline 2992 & 67 & PAGLIANO & JOSEPH & B & 23 & ITALY & ITALY & ITALY & 1909 & TRAMMER/CM & SAN BENIGNO CANAVESE. TO & CENTRAL ST. & CALUMET - WOLVERINE \\
\hline 2993 & 67 & VINCENTI & ANDREA & B & 23 & ITALY & ITALY & ITALY & 1909 & TRAMMER/CM & LEVONE CANAVESE. TO & CENTRAL ST. & CALUMET - WOLVERINE \\
\hline 2994 & 108 & MURAO & PAUL & $\mathrm{H}$ & 38 & ITALY & ITALY & ITALY & 1893 & LABORER/ODD JOBS & & CENTRAL ST. & CALUMET - WOLVERINE \\
\hline 2995 & 108 & MURAO & MARION & W & 32 & ITALY & ITALY & $\begin{array}{l}\text { ITALY } \\
\text { ITAY }\end{array}$ & 1896 & LADURER/UDU JUDO & & CENTRAL ST. & CALUMET - WOLVERINE \\
\hline 2996 & 108 & MURAO & MARY & $\mathrm{D}$ & 11 & MICHIGAN & ITALY & ITALY & & & & CENTRAL ST. & CALUMET - WOLVERINE \\
\hline 2997 & 108 & MURAO & CHARLEY & $\mathrm{S}$ & 9 & MICHIGAN & ITALY & ITALY & & & & CENTRAL ST. & CALUMET - WOLVERINE \\
\hline 2998 & 108 & MURAO & KATE & $\mathrm{D}$ & 7 & MICHIGAN & ITALY & ITALY & & & & CENTRAL ST. & CALUMET - WOLVERINE \\
\hline 2999 & 108 & MURAO & HENRY & $\mathrm{s}$ & 3 & MICHIGAN & ITALY & ITALY & & & & CENTRAL ST. & CALUMET - WOLVERINE \\
\hline 3000 & 108 & MURAO & FL? & D & 1.11 & MICHIGAN & ITALY & ITALY & & & & CENTRAL ST. & CALUMET - WOLVERINE \\
\hline 3001 & 58 & GIACOLETTO & ANTONIO & $\mathrm{H}$ & 40 & ITALY & ITALY & ITALY & 1893 & LABORER/CM & PONT CANAVESE. TO & 2ND ST./MINERS ST. & CALUMET - WOLVERINE \\
\hline 3002 & 58 & GIACOLETTO & LUISA & W & 35 & ITALY & ITALY & ITALY & 1895 & & PONT CANAVESE. TO & 2ND ST./MINERS ST. & CALUMET - WOLVERINE \\
\hline 3003 & 58 & GIACOLETTO & JOSEPH & $\mathrm{s}$ & 17 & ITALY & ITALY & ITALY & 1895 & DRILL BOY/CM & PONT CANAVESE. TO & 2ND ST./MINERS ST. & CALUMET - WOLVERINE \\
\hline 3004 & 58 & GIACOLETTO & PETER & $\mathrm{s}$ & 14 & MICHIGAN & ITALY & ITALY & & & PONT CANAVESE. TO & 2ND ST./MINERS ST. & CALUMET - WOLVERINE \\
\hline 3005 & 59 & ROSSIO & JAMES & $\mathrm{H}$ & 35 & ITALY & ITALY & ITALY & 1899 & CARPENTER/HOUSE & PONT CANAVESE. TO & 2ND ST./MINERS ST. & CALUMET - WOLVERINE \\
\hline 3006 & 59 & ROSSIO & M. KATE & w & 36 & ITALY & ITALY & ITALY & 1896 & & & 2ND ST./MINERS ST. & CALUMET - WOLVERINE \\
\hline 3007 & 59 & ROSSIO & $\begin{array}{l}\text { CHARLIE } \\
\text { CHATL }\end{array}$ & s & 16 & MICHIGAN & ITALY & ITALY & & LABORER/PF & PONT CANAVESE. TO & 2ND ST./MINERS ST. & CALUMET - WOLVERINE \\
\hline 3008 & 59 & ROSSIO & JENNIE & $\mathrm{D}$ & 15 & MICHIGAN & ITALY & ITALY & & LADURER/IT & PONT CANAVESE. TO & 2ND ST./MINERS ST. & CALUMET - WOLVERINE \\
\hline 3009 & 59 & ROSSIO & FRANK & $\mathrm{S}$ & 9 & MICHIGAN & ITALY & ITALY & & & PONT CANAVESE. TO & 2ND ST./MINERS ST. & CALUMET - WOLVERINE \\
\hline 3010 & 59 & ROSSIO & MAURICE & $\mathrm{s}$ & 5 & MICHIGAN & ITALY & ITALY & & & PONT CANAVESE. TO & 2ND ST./MINERS ST. & CALUMET - WOLVERINE \\
\hline 3011 & 58 & GIACOLETTO & JOHN & B & 41 & ITALY & ITALY & ITALY & 1892 & TRAMMER/CM & TORINO & 2ND ST./MINERS ST. & CALUMET - WOLVERINE \\
\hline 3012 & 58 & SANDRETTO & MIKE & B & 25 & ITALY & ITALY & ITALY & 1905 & TRAMMER/CM & PONT CANAVESE, TO & 2ND ST./MINERS ST. & CALUMET - WOLVERINE \\
\hline 3013 & 58 & GEA & MIKE & B & 22 & ITALY & ITALY & ITALY & 1907 & LABORER/CM & TORINO & 2ND ST./MINERS ST. & CALUMET - WOLVERINE \\
\hline 3014 & 58 & GALLATE & MARCO? & B & 19 & ITALY & ITALY & ITALY & 1895 & TRAMMER/CM & & 2ND ST./MINERS ST. & CALUMET - WOLVERINE \\
\hline 3015 & 58 & GASPARDO & JOHN & B & 25 & ITALY & ITALY & ITALY & 1903 & TRAMMER/CM & PONT CANAVESE. TO & 2ND ST./MINERS ST. & CALUMET - WOLVERINE \\
\hline 3016 & 58 & GASPARDO & GIACINTO & B & 21 & ITALY & ITALY & ITALY & 1903 & LABORER/CM & PONT CANAVESE. TO & 2ND ST./MINERS ST. & CALUMET - WOLVERINE \\
\hline 3017 & 58 & CERETTO & PIETRO & B & 28 & ITALY & ITALY & ITALY & 1903 & LABORER/CM & PONT CANAVESE. TO & 2ND ST./MINERS ST. & CALUMET - WOLVERINE \\
\hline 3018 & 58 & SANDRETTO & PIETRO & B & 36 & ITALY & ITALY & ITALY & 1898 & TRAMMER/CM & PONT CANAVESE, TO & 2ND ST./MINERS ST. & CALUMET - WOLVERINE \\
\hline 3019 & 53 & BALAGNA & JOHN & $\mathrm{H}$ & 34 & ITALY & ITALY & ITALY & 1897 & LABORER/CM & PONT CANAVESE. TO & 2ND ST./MINERS ST. & CALUMET - WOLVERINE \\
\hline 3020 & 53 & BALAGNA & MARY & W & 34 & ITALY & ITALY & ITALY & 1905 & & & 2ND ST./MINERS ST. & CALUMET - WOLVERINE \\
\hline 3021 & 53 & BALAGNA & AURELIA & $\mathrm{D}$ & 1.?M & MICHIGAN & ITALY & ITALY & & & PONT CANAVESE. TO & 2ND ST./MINERS ST. & CALUMET - WOLVERINE \\
\hline 3022 & 53 & COPPO & FRANK & B & 29 & ITALY & ITALY & ITALY & 1906 & TRAMMER/CM & PONT CANAVESE. TO & 2ND ST./MINERS ST. & CALUMET - WOLVERINE \\
\hline 3023 & 53 & ALBERTANO & MIKE & B & 47 & ITALY & ITALY & ITALY & 1903 & TRAMMER/CM & PONT CANAVESE. TO & 2ND ST./MINERS ST. & CALUMET - WOLVERINE \\
\hline
\end{tabular}




\begin{tabular}{|c|c|c|c|c|c|c|c|c|c|c|c|c|c|}
\hline 3024 & 53 & BALAGNA & MKE & B & 32 & ITALY & ITALY & ITALY & 1907 & TRAMMER/CM & PONT CANAVESE. TO & 2ND ST./MINERS ST. & CALUMET - WOLVERINE \\
\hline 3025 & 53 & BALAGNA & EUGENIO & B & 28 & ITALY & ITALY & ITALY & ???? & TRAMMER/CM & PONT CANAVESE. TO & 2ND ST./MINERS ST. & CALUMET - WOLVERINE \\
\hline 3026 & 53 & SANDRETTO & JOSEPH & B & 34 & ITALY & ITALY & ITALY & 1906 & TRAMMER/CM & PONT CANAVESE. TO & 2ND ST./MINERS ST. & CALUMET - WOLVERINE \\
\hline 3027 & 53 & ALBERTANO & LORENZO & B & 16 & ITALY & ITALY & ITALY & 1909 & DRILL BOY/CM & PONT CANAVESE. TO & 2ND ST./MINERS ST. & CALUMET - WOLVERINE \\
\hline 3028 & 53 & ALBERTANO & GIOVANNI & B & 19 & ITALY & ITALY & ITALY & 1890 & WATCHMAN/CM & PONT CANAVESE. TO & 2ND ST./MINERS ST. & CALUMET - WOLVERINE \\
\hline 3029 & 95 & PICCHIOTTINO & BATTISTA & $\mathrm{H}$ & 36 & ITALY & ITALY & ITALY & 1898 & TIMBER GANG/CM & PONT CANAVESE. TO & 3RD ST. & CALUMET - WOLVERINE \\
\hline 3030 & 95 & PICCHIOTTINO & MADDALENA & W & 24 & ITALY & ITALY & ITALY & 1900 & & & 3RD ST. & CALUMET - WOLVERINE \\
\hline 3031 & 95 & PICCHIOTTINO & RENA & $\mathrm{D}$ & 4 & MICHIGAN & ITALY & ITALY & & & PONT CANAVESE. TO & 3RD ST. & CALUMET - WOLVERINE \\
\hline 3032 & 95 & PICCHIOTTINO & ERNEST & $\mathrm{s}$ & 3 & MICHIGAN & ITALY & ITALY & & & PONT CANAVESE. TO & 3RD ST. & CALUMET - WOLVERINE \\
\hline 3033 & 95 & FALETTI & $\mathrm{JOHN}$ & B & 29 & ITALY & ITALY & ITALY & 1907 & TRAMMER/CM & PONT CANAVESE. TO & 3RD ST. & CALUMET - WOLVERINE \\
\hline 3034 & 95 & FALETTI & CARLO & B & 24 & ITALY & ITALY & ITALY & 1907 & TRAMMER/CM & PONT CANAVESE. TO & 3RD ST. & CALUMET - WOLVERINE \\
\hline 3035 & 95 & RONOLIONE & DOMENICO & B & 25 & ITALY & ITALY & ITALY & 1909 & TRAMMER/CM & & 3RD ST. & CALUMET - WOLVERINE \\
\hline 3036 & 113 & SANDRETTO & BARNEY & $\mathrm{H}$ & 40 & ITALY & ITALY & ITALY & 1900 & MINER/CM & PONT CANAVESE. TO & 4TH ST. & CALUMET - WOLVERINE \\
\hline 3037 & 113 & SANDRETTO & ANGELA & W & 35 & ITALY & ITALY & ITALY & 1901 & & PONT CANAVESE. TO & 4TH ST. & CALUMET - WOLVERINE \\
\hline 3038 & 113 & SANDRETTO & PETER & s & 12 & ITALY & ITALY & ITALY & 1901 & & PONT CANAVESE. TO & 4TH ST. & CALUMET - WOLVERINE \\
\hline 3039 & 113 & SANDRETTO & MARTIN & $\mathrm{s}$ & 7 & MICHIGAN & ITALY & ITALY & & & PONT CANAVESE, TO & 4TH ST. & CALUMET - WOLVERINE \\
\hline 3040 & 113 & SANDRETTO & JOHN & $\mathrm{s}$ & 5 & MICHIGAN & ITALY & ITALY & & & PONT CANAVESE. TO & 4TH ST. & CALUMET - WOLVERINE \\
\hline 3041 & 113 & SANDRETTO & LOUIS & $\mathrm{s}$ & 3 & MICHIGAN & ITALY & ITALY & & & PONT CANAVESE. TO & 4TH ST. & CALUMET - WOLVERINE \\
\hline 3042 & 113 & SANDRETTO & CATERINA & $\mathrm{D}$ & $1.5 \mathrm{M}$ & MICHIGAN & ITALY & ITALY & & & PONT CANAVESE. TO & 4TH ST. & CALUMET - WOLVERINE \\
\hline 3043 & 113 & BALMA/BAIMA & PIETRO & $\mathrm{H}$ & 28 & ITALY & ITALY & ITALY & 1907 & LABORER/CM & CIRIE'. TO & 4TH ST. & CALUMET - WOLVERINE \\
\hline 3044 & 113 & BALMA/BAIMA & DOMENICA & W & 23 & ITALY & ITALY & ITALY & 1908 & & CIRIE'. TO & 4TH ST. & CALUMET - WOLVERINE \\
\hline 3045 & 113 & BALMA/BAIMA & GIADA & D & 2 & ITALY & ITALY & ITALY & 1908 & & CIRIE', TO & 4TH ST. & CALUMET - WOLVERINE \\
\hline 3046 & 113 & BALMA/BAIMA & LUISA & $\mathrm{D}$ & $1.1 \mathrm{M}$ & MICHIGAN & ITALY & ITALY & & & CIRIE'. TO & 4TH ST. & CALUMET - WOLVERINE \\
\hline 3047 & 113 & ROSSIO & ANGELO & $\mathrm{H}$ & 29 & ITALY & ITALY & ITALY & 1907 & TRAMMER/CM & PONT CANAVESE. TO & 4TH ST. & CALUMET - WOLVERINE \\
\hline 3048 & 113 & ROSSIO & TERSILIA & w & $51 ? ?$ & ITALY & ITALY & ITALY & 1909 & & PONT CANAVESE. TO & 4TH ST. & $\begin{array}{l}\text { CALUMET - WOLVERINE } \\
\text { CIIST }\end{array}$ \\
\hline 3049 & 113 & ROSSIO & MARY & $\mathrm{D}$ & 4 & ITALY & ITALY & ITALY & 1909 & & PONT CANAVESE. TO & 4TH ST. & CALUMET - WOLVERINE \\
\hline 3050 & 113 & ROSSIO & PIETRO & $\mathrm{s}$ & $1 \mathrm{M}$ & MICHIGAN & ITALY & ITALY & & & PONT CANAVESE. TO & 4TH ST. & CALUMET - WOLVERINE \\
\hline 3051 & 50 & GIUSTAT & EUGENIO & $\mathrm{H}$ & 33 & ITALY & ITALY & ITALY & 1899 & LABORER/ROCK HOUSE & SALTO CANAVESE, TO & N. 4 SHAFT & CALUMET - WOLVERINE \\
\hline 3052 & 50 & GIUSTAT & GENOVEFFA & W & 32 & ITALY & ITALY & ITALY & 1906 & & SALTO CANAVESE. TO & N. 4 SHAFT & CALUMET - WOLVERINE \\
\hline 3053 & 50 & GIUSTAT & GIUSEPPE & $\mathrm{s}$ & 12 & ITALY & ITALY & ITALY & 1906 & & SALTO CANAVESE. TO & N. 4 SHAFT & CALUMET - WOLVERINE \\
\hline 3054 & 50 & GIUSTAT & ERNEST & $\mathrm{S}$ & 5 & ITALY & ITALY & ITALY & 1906 & & SALTO CANAVESE. TO & N. 4 SHAFT & CALUMET - WOLVERINE \\
\hline 3055 & 50 & GIUSTAT & MARY & $\mathrm{D}$ & 2 & MICHIGAN & ITALY & ITALY & & & SALTO CANAVESE. TO & N. 4 SHAFT & CALUMET - WOLVERINE \\
\hline 3056 & 50 & GIUSTAT & ALEX & s & $1.3 \mathrm{M}$ & MICHIGAN & ITALY & ITALY & & & SALTO CANAVESE. TO & N. 4 SHAFT & CALUMET - WOLVERINE \\
\hline 3057 & 50 & GIUSTAT & JOHN & $\mathrm{BR}$ & 27 & ITALY & ITALY & ITALY & 1906 & LABORER/ROCK HOUSE & SALTO CANAVESE, TO & N. 4 SHAFT & CALUMET - WOLVERINE \\
\hline 3058 & 50 & VERCELLINO & MIKE & B & 39 & ITALY & ITALY & $\begin{array}{l}\text { ITALY } \\
\text { ITAY }\end{array}$ & 1909 & LABORER/ROCK HOUSE & $\begin{array}{l}\text { TORINO } \\
\text { TORAVEL, }\end{array}$ & N. 4 SHAFT & CALUMET - WOLVERINE \\
\hline 3059 & 48 & GRIBALDO & JOSEPH & $\mathrm{H}$ & 41 & ITALY & ITALY & ITALY & 1892 & WATCHMAN/CM & BARONE CANAVESE. TO & N. 4 SHAFT & CALUMET - WOLVERINE \\
\hline 3060 & 48 & GRIBALDO & ROSE & W & 20?? & ITALY & ITALY & ITALY & 1902 & & BARONE CANAVESE. TO & N. 4 SHAFT & CALUMET - WOLVERINE \\
\hline 3061 & 48 & GRIBALDO & ROBERT & s & 19 & ITALY & ITALY & ITALY & 1897 & MACHINIST/CM & BARONE CANAVESE. TO & N. 4 SHAFT & CALUMET - WOLVERINE \\
\hline 3062 & 48 & GRIBALDO & TONY & $\mathrm{s}$ & 17 & ITALY & ITALY & ITALY & 1897 & & BARONE CANAVESE. TO & N. 4 SHAFT & CALUMET - WOLVERINE \\
\hline 3063 & 48 & GRIBALDO & TRACY & $\mathrm{D}$ & 3 & MICHIGAN & ITALY & ITALY & & & BARONE CANAVESE. TO & N. 4 SHAFT & CALUMET - WOLVERINE \\
\hline 3064 & 48 & GRIBALDO & AUSTIN & $\mathrm{D}$ & $10 \mathrm{M}$ & MICHIGAN & ITALY & ITALY & & & BARONE CANAVESE. TO & N. 4 SHAFT & CALUMET - WOLVERINE \\
\hline 3065 & 48 & SANDRETTO & ??? & B & $? ? ?$ & MICHIGAN & ITALY & ITALY & & & PONT CANAVESE. TO & N. 4 SHAFT & CALUMET - WOLVERINE \\
\hline 3066 & 45 & VENEGONE & MAURIZIO & $\mathrm{H}$ & 25 & ITALY & ITALY & ITALY & 1895 & TIMBER GANG/CM & CUGGIANO. MILANO & N. 4 SHAFT & CALUMET - WOLVERINE \\
\hline 3067 & 45 & VENEGONE & ANTONIA & W & 26 & ITALY & ITALY & ITALY & 1904 & & & N. 4 SHAFT & CALUMET - WOLVERINE \\
\hline 3068 & 45 & VENEGONE & MARY & $\mathrm{D}$ & 4 & MICHIGAN & ITALY & ITALY & & & CUGGIANO. MILANO & N. 4 SHAFT & CALUMET - WOLVERINE \\
\hline 3069 & 45 & VENEGONE & A?? & $\mathrm{D}$ & $1.9 \mathrm{M}$ & MICHIGAN & ITALY & ITALY & & & CUGGIANO. MILANO & N. 4 SHAFT & CALUMET - WOLVERINE \\
\hline 3070 & 45 & VENEGONE & JOSEPH & s & $1 \mathrm{M}$ & MICHIGAN & ITALY & ITALY & & & CUGGIANO. MILANO & N. 4 SHAFT & CALUMET - WOLVERINE \\
\hline 3071 & 45 & RICCA & JOHN & B & 29 & ITALY & ITALY & ITALY & 1895 & MINER/CM & TORINO & N. 4 SHAFT & CALUMET - WOLVERINE \\
\hline 3072 & 45 & GATTO & PAUL & B & 28 & ITALY & ITALY & ITALY & 1896 & MINER/CM & & N. 4 SHAFT & CALUMET - WOLVERINE \\
\hline 3073 & 75 & SANDRETTO & COSTANZO & $\mathrm{H}$ & 36 & ITALY & ITALY & ITALY & 1907 & TRAMMER/CM & PONT CANAVESE. TO & WATER ST. & CALUMET - CENTENNIAL \\
\hline 3074 & 75 & SANDRETTO & OTTAVIA & W & 27 & ITALY & ITALY & ITALY & 1909 & & PONT CANAVESE. TO & WATER ST. & CALUMET - CENTENNIAL \\
\hline 3075 & 75 & SANDRETTO & MARINA & $\mathrm{D}$ & 4 & ITALY & ITALY & ITALY & 1909 & & PONT CANAVESE, TO & WATER ST. & \\
\hline 3076 & 75 & SANDRETTO & MARY & $\mathrm{D}$ & 2 & ITALY & ITALY & ITALY & 1909 & & PONT CANAVESE. TO & ER ST. & CALUMET - CENTENNIAL \\
\hline 3077 & 75 & SANDRETTO & ALDA & $\mathrm{D}$ & $1 \mathrm{M}$ & MICHIGAN & ITALY & ITALY & & & PONT CANAVESE. TO & WATER ST. & CALUMET - CENTENNIAL \\
\hline 3078 & 75 & ROLANDO & LUIGI & $\mathrm{H}$ & 48 & ITALY & ITALY & ITALY & 1883 & MINER/CM & PONT CANAVESE. TO & WATER ST. & CALUMET - CENTENNIAL \\
\hline 3079 & 75 & ROLANDO & MARY & W & 46 & ITALY & ITALY & ITALY & 1890 & & & WATER ST. & CALUMET - CENTENNIAL \\
\hline 3080 & 75 & ROLANDO & PIERINA & $\mathrm{D}$ & 14 & MICHIGAN & ITALY & ITALY & & & PONT CANAVESE. TO & WATER ST. & CALUMET - CENTENNIAL \\
\hline 3081 & 75 & ROLANDO & LUISA & $\mathrm{D}$ & 13 & MICHIGAN & ITALY & ITALY & & & PONT CANAVESE, TO & WATER ST. & CALUMET - CENTENNIAL \\
\hline 3082 & 75 & ROLANDO & JOSEPHINE & $\mathrm{D}$ & 11 & MICHIGAN & ITALY & ITALY & & & PONT CANAVESE. TO & WATER ST. & CALUMET - CENTENNIAL \\
\hline 3083 & 30 & MASSE' & GIOVANNI & $\mathrm{H}$ & 40 & ITALY & ITALY & ITALY & 1900 & MINER/CM & PONT CANAVESE. TO & CONGLOMERATE ST. & CALUMET - CENTENNIAL \\
\hline 3084 & 30 & MASSE' & SERAFINA & W & 40 & ITALY & ITALY & ITALY & 1905 & & PONT CANAVESE. TO & CONGLOMERATE ST. & CALUMET - CENTENNIAL \\
\hline 3085 & 30 & MASSE' & PETER & s & 16 & ITALY & ITALY & ITALY & 1905 & & PONT CANAVESE. TO & CONGLOMERATE ST. & CALUMET - CENTENNIAL \\
\hline 3086 & 30 & BARSANTI & GIOVANNI & B & 44 & ITALY & ITALY & ITALY & 1887 & LABORER/CM & LUCCA & CONGLOMERATE ST. & CALUMET - CENTENNIAL \\
\hline
\end{tabular}




\begin{tabular}{|c|c|c|c|c|c|c|c|c|c|c|c|c|c|}
\hline 3087 & 35 & ROLANDO & PAOLO & $\mathrm{H}$ & 55 & ITALY & ITALY & ITALY & 1886 & WATCHMAN/CM & PONT CANAVESE. TO & CONGLOMERATE ST. & CALUMET - CENTENNIAL \\
\hline 3088 & 35 & ROLANDO & TRACY & W & 48 & ITALY & ITALY & ITALY & 1890 & & & CONGLOMERATE ST. & CALUMET - CENTENNIAL \\
\hline 3089 & 35 & ROLANDO & JOSEPHINE & $\mathrm{D}$ & 16 & MINN & ITALY & ITALY & & & PONT CANAVESE. TO & CONGLOMERATE ST. & CALUMET - CENTENNIAL \\
\hline 3090 & $163 \mathrm{M}$ & NIGRA & ANTONIO & $\mathrm{H}$ & 45 & ITALY & ITALY & ITALY & 1897 & STONE MASON/HOUSE & SAN GIUSTO CANAVESE. TO & RAILROAD ST. & CALUMET - CENTENNIAL \\
\hline 3091 & $163 \mathrm{M}$ & NIGRA & BENEDETTA & W & 48 & ITALY & ITALY & ITALY & 1899 & & SAN GIUSTO CANAVESE. TO & RAILROAD ST. & CALUMET - CENTENNIAL \\
\hline 3092 & $163 \mathrm{M}$ & NIGRA & ELISA & $\mathrm{D}$ & 15 & ITALY & ITALY & ITALY & $1905 ?$ & & SAN GIUSTO CANAVESE. TO & RAILROAD ST. & CALUMET - CENTENNIAL \\
\hline 3093 & $163 \mathrm{M}$ & NIGRA & TONY & s & 7 & MICHIGAN & ITALY & ITALY & & & SAN GIUSTO CANAVESE. TO & RAILROAD ST. & CALUMET - CENTENNIAL \\
\hline 3094 & $163 \mathrm{M}$ & NIGRA & JENNA & $\mathrm{D}$ & 5 & MICHIGAN & ITALY & ITALY & & & SAN GIUSTO CANAVESE. TO & RAILROAD ST. & CALUMET - CENTENNIAL \\
\hline 3095 & 134 & ANDRINA & JOHN & $\mathrm{H}$ & 48 & ITALY & ITALY & ITALY & 1890 & LABORER/CM & SAN MARTINO CANAVESE. TO & COUNTY ROAD & CALUMET - N. KEARSARGE \\
\hline 3096 & 134 & ANDRINA & DOMENICA & W & 45 & ITALY & ITALY & ITALY & 1890 & LADO NERTUIVI & SAN MARTINO CANAVESE. TO & COUNTY ROAD & $\begin{array}{l}\text { CALUMET - N. KEARSARGE } \\
\text { CAARA }\end{array}$ \\
\hline 3097 & 134 & ANDRINA & JOHN & $\mathrm{s}$ & 18 & MICHIGAN & ITALY & ITALY & & LABORER/CM & SAN MARTINO CANAVESE. TO & COUNTY ROAD & CALUMET - N. KEARSARGE \\
\hline 3098 & 134 & ANDRINA & JULIUS & $\mathrm{s}$ & 17 & MICHIGAN & ITALY & ITALY & & LABORER/CM & SAN MARTINO CANAVESE. TO & COUNTY ROAD & CALUMET - N. KEARSARGE \\
\hline 3099 & 134 & ANDRINA & DOMENICO & $\mathrm{s}$ & 15 & MICHIGAN & ITALY & ITALY & & & SAN MARTINO CANAVESE. TO & COUNTY ROAD & CALUMET - N. KEARSARGE \\
\hline 3100 & 134 & ANDRINA & ARTHUR & $\mathrm{s}$ & 14 & MICHIGAN & ITALY & ITALY & & & SAN MARTINO CANAVESE. TO & COUNTY ROAD & CALUMET - N. KEARSARGE \\
\hline 3101 & 134 & ANDRINA & MATT & $\mathrm{s}$ & 10 & MICHIGAN & ITALY & ITALY & & & SAN MARTINO CANAVESE. TO & COUNTY ROAD & CALUMET - N. KEARSARGE \\
\hline 3102 & 134 & ANDRINA & ANNE & $\mathrm{D}$ & 9 & MICHIGAN & ITALY & ITALY & & & SAN MARTINO CANAVESE, TO & COUNTY ROAD & CALUMET - N. KEARSARGE \\
\hline 3103 & 134 & ANDRINA & MARY & $\mathrm{D}$ & 8 & MICHIGAN & ITALY & ITALY & & & SAN MARTINO CANAVESE. TO & COUNTY ROAD & CALUMET - N. KEARSARGE \\
\hline 3104 & & GIACOLETTO & ANTONY & $\mathrm{H}$ & 38 & ITALY & ITALY & ITALY & $85 ? ?$ & TIMBERMAN/CM & TORINO & 2ND ST. & CALUMET - COPPER CITY \\
\hline 3105 & & GIACOLETTO & CARA & w & 41 & ITALY & ITALY & ITALY & 1900 & & TORINO & 2ND ST. & CALUMET - COPPER CITY \\
\hline 3106 & & GIACOLETTO & JOHN & s & 19 & ITALY & ITALY & ITALY & 1901 & NOT CLEAR & TORINO & 2ND ST. & CALUMET - COPPER CITY \\
\hline 3107 & & GIACOLETTO & MARY & $\mathrm{D}$ & 17 & ITALY & ITALY & ITALY & 1903 & & TORINO & 2ND ST. & CALUMET - COPPER CITY \\
\hline 3108 & & GIACOLETTO & DOMENICO & s & 16 & ITALY & ITALY & ITALY & 1903 & & TORINO & 2ND ST. & CALUMET - COPPER CITY \\
\hline 3109 & & GIACOLETTO & ANNE & $\mathrm{D}$ & 10 & ITALY & ITALY & ITALY & 1900 & & TORINO & 2ND ST. & CALUMET - COPPER CITY \\
\hline 3110 & & GIACOLETTO & BART & $\mathrm{s}$ & 9 & MICHIGAN & ITALY & ITALY & & & TORINO & 2ND ST. & CALUMET - COPPER CITY \\
\hline 3111 & & GIACOLETTO & JOSEPHINE & $\mathrm{D}$ & 5 & MICHIGAN & ITALY & ITALY & & & TORINO & 2ND ST. & CALUMET - COPPER CITY \\
\hline 3112 & & GIACOLETTO & TRACY & $\mathrm{D}$ & 3 & MICHIGAN & ITALY & ITALY & & & TORINO & 2ND ST. & CALUMET - COPPER CITY \\
\hline 3113 & & ?? & JOSEPH & B & 32 & ITALY & ITALY & ITALY & 1909 & TRAMMER/CM & & & CALUMET - COPPER CITY \\
\hline 3114 & & ALBERTANO & CAMILLO & B & 39 & ITALY & ITALY & ITALY & 1909 & TRAMMER/CM & PONT CANAVESE, TO & & CALUMET - COPPER CITY \\
\hline 3115 & & BERTOLLI & JOHN & B & 39 & ITALY & ITALY & ITALY & 1903 & MASON/CITY & CASTELNUOVO. LU & & CALUMET - COPPER CITY \\
\hline 3116 & & COSCIA & CASPER & B & 37 & ITALY & ITALY & ITALY & 1909 & MASON/CITY & & & CALUMET - COPPER CITY \\
\hline 3117 & & BERTOLLI & MATTEO & B & 44 & ITALY & ITALY & ITALY & $02 ? ?$ & MASON/CITY & CASTELNUOVO. LU & & CALUMET - COPPER CITY \\
\hline 3118 & & MILANI & CHARLES & B & 48 & ITALY & ITALY & ITALY & $03 ? ?$ & MASON/CITY & SESTO CALENDE. VARESE & & CALUMET - COPPER CITY \\
\hline 3119 & & DIARA & PAUL & B & 51 & ITALY & ITALY & ITALY & 85?? & TRUCK MAN/CM & & & CALUMET - COPPER CITY \\
\hline 3120 & & CONTORSTO & PETER & B & 52 & ITALY & ITALY & ITALY & $90 ? ?$ & LABORER/CM & & & CALUMET - COPPER CITY \\
\hline 3121 & & BESNA/BEA & WILLIAM & $\begin{array}{l}\mathrm{D} \\
\mathrm{H}\end{array}$ & 37 & ITALY & ITALY & ITALY & 1902 & TIMBERMAN/CM & & & $\begin{array}{l}\text { CALUMET - COPPER CITY } \\
\text { CAT }\end{array}$ \\
\hline 3122 & & BESNA/BEA & TERESA & W & 24 & ITALY & ITALY & ITALY & 1912 & & & & CALUMET - COPPER CITY \\
\hline 3123 & & COPPO & VALENTINO & $\mathrm{H}$ & 60 & ITALY & ITALY & ITALY & 1878 & BARN BOSS/BREWERY & PONT CANAVESE. TO & & CALUMET - LAKE VIEW \\
\hline 3124 & & COPPO & KATE & W & 57 & ITALY & ITALY & ITALY & 1878 & & PONT CANAVESE. TO & & CALUMET - LAKE VIEW \\
\hline 3125 & & COPPO & LENA & $\mathrm{D}$ & 27 & MICHIGAN & ITALY & ITALY & & HOUSEKEEPER/HOME & PONT CANAVESE. TO & & CALUMET - LAKE VIEW \\
\hline 3126 & & COPPO & PETER & $\mathrm{s}$ & 17 & MICHIGAN & ITALY & ITALY & & BOTTLER/BREWERY & PONT CANAVESE. TO & & CALUMET - LAKE VIEW \\
\hline 3127 & & COPPO & MICHAEL & s & 23 & MICHIGAN & ITALY & ITALY & & TIMBERMAN/CM & PONT CANAVESE. TO & & CALUMET - LAKE VIEW \\
\hline 3128 & & COPPO & VALENTINO & GS & 1 & MICHIGAN & AUSTRIA & MICHIGAN & & & PONT CANAVESE. TO & & CALUMET - LAKE VIEW \\
\hline 3129 & & BERTOLINO & JAMES & $\mathrm{H}$ & 32 & ITALY & ITALY & ITALY & 1905 & FIREMAN/BREWERY & STRAMBINELLO. TO & & CALUMET - LAKE VIEW \\
\hline 3130 & & BERTOLINO & TERESA & W & 25 & ITALY & ITALY & ITALY & 1906 & & STRAMBINELLO. TO & & CALUMET - LAKE VIEW \\
\hline 3131 & & BERTOLINO & DOMENICO & s & 4 & ITALY & ITALY & ITALY & 1906 & & STRAMBINELLO. TO & & CALUMET - LAKE VIEW \\
\hline 3132 & & BIANCHI & $\mathrm{JOHN}$ & B & 29 & ITALY & ITALY & ITALY & 1905 & TEAMSTER/BREWERY & LUCCA & & CALUMET - LAKE VIEW \\
\hline 3133 & & $\begin{array}{l}\text { CASERIO } \\
\text { CAANER }\end{array}$ & JOSEPH & $\begin{array}{l}\mathrm{D} \\
\mathrm{H}\end{array}$ & 31 & ITALY & ITALY & ITALY & 1905 & $\begin{array}{l}\text { LABORER/BREWERY } \\
\text { LABRE }\end{array}$ & STRAMBINELLO. TO & & CALUMET - LAKE VIEW \\
\hline 3134 & & CASERIO & TERESA & w & 35 & ITALY & ITALY & ITALY & 1905 & LADURERIDREVWERT & $\begin{array}{l}\text { STRAMBINELLO. TO } \\
\text { STALE }\end{array}$ & & CALUMET - LAKE VIEW \\
\hline 3135 & & CASERIO & DOMENICO & $\mathrm{s}$ & 2 & MICHIGAN & ITALY & ITALY & & & STRAMBINELLO. TO & & CALUMET - LAKE VIEW \\
\hline 3136 & 7 & CORGIATTI & BARTOLOMEO & B & 31 & ITALY & ITALY & ITALY & 1880 & MINER/CM & PONT CANAVESE. TO & & FRANKLIN \\
\hline 3137 & 17 & CORTOPASSI & CHERUBINO & $\mathrm{H}$ & 45 & ITALY & ITALY & ITALY & 1889 & LABORER/ODD JOBS & LUCCA & & FRANKLIN \\
\hline 3138 & 17 & CORTOPASSI & DOMENICA & W & 32 & ITALY & ITALY & ITALY & 1896 & & & & FRANKLIN \\
\hline 3139 & 17 & CORTOPASSI & NATALINA & $\mathrm{D}$ & 12 & MICHIGAN & ITALY & ITALY & & & LUCCA & & FRANKLIN \\
\hline 3140 & 17 & CORTOPASSI & ANDREW & $\mathrm{s}$ & 8 & MICHIGAN & ITALY & ITALY & & & LUCCA & & FRANKLIN \\
\hline 3141 & 17 & CORTOPASSI & VITTAL & $\mathrm{D}$ & $3 \mathrm{M}$ & MICHIGAN & ITALY & ITALY & & & LUCCA & & FRANKLIN \\
\hline 3142 & & ClUCCl & LUIGI & $\mathrm{H}$ & 43 & ITALY & ITALY & ITALY & 1885 & LABORER/CM & CAPANNORI. LU & & FRANKLIN \\
\hline 3143 & & $\mathrm{ClUCCl}$ & ITALIA & W & 29 & ITALY & ITALY & ITALY & 1908 & & CAPANNORI. LU & & FRANKLIN \\
\hline 3144 & & $\mathrm{ClUCCl}$ & TERSILIA & $\mathrm{D}$ & 5 & ITALY & ITALY & ITALY & 1908 & & CAPANNORI, LU & & FRANKLIN \\
\hline 3145 & & $\mathrm{ClUCCl}$ & ESTER & $\mathrm{D}$ & 4 & ITALY & ITALY & ITALY & 1908 & & CAPANNORI. LU & & FRANKLIN \\
\hline 3146 & & $\mathrm{ClUCCl}$ & CLOTILDE & $\mathrm{D}$ & 1 & MICHIGAN & ITALY & ITALY & & & CAPANNORI. LU & & FRANKLIN \\
\hline 3147 & & BETTINI & $\mathrm{JOHN}$ & $\mathrm{H}$ & 30 & ITALY & ITALY & ITALY & 1903 & & & & FRANKLIN \\
\hline 3148 & & BETTINI & MABEL & W & 30 & ITALY & ITALY & ITALY & 1905 & & & & FRANKLIN \\
\hline 3149 & & BETTINI & JAMES & s & 3 & MICHIGAN & ITALY & ITALY & & & & & FRANKLIN \\
\hline
\end{tabular}




\begin{tabular}{|c|c|c|c|c|c|c|c|c|c|c|c|c|c|}
\hline 3150 & & BETTINI & LENA & D & $11 \mathrm{M}$ & MICHIGAN & ITALY & ITALY & & & & & FRANKLIN \\
\hline 3151 & & BETTINI & LOUIS & BR & 40 & ITALY & ITALY & ITALY & 1905 & LABORER/CM & & & FRANKLIN \\
\hline 3152 & & (T)ATTONI & PASCAL & B & 29 & ITALY & ITALY & ITALY & 1903 & MINER/CM & & & FRANKLIN \\
\hline 3153 & & LEONE & ALEXANDER & B & 27 & ITALY & ITALY & ITALY & 1904 & LABORER/CM & & & FRANKLIN \\
\hline 3154 & & AGRETTI & ANGELO & B & 28 & ITALY & ITALY & ITALY & 1908 & CARPENTER/HOUSE & CAPANNORI. LU & & FRANKLIN \\
\hline 3155 & & GIUSTI & ORLANDO & $\mathrm{H}$ & 31 & ITALY & ITALY & ITALY & 1904 & BLACKSMITH/CM & PISA & & FRANKLIN \\
\hline 3156 & & GIUSTI & MARIA & W & 32 & ITALY & ITALY & ITALY & 1907 & & PISA & & FRANKLIN \\
\hline 3157 & & GIUSTI & ILIO & $s$ & 5 & ITALY & ITALY & ITALY & 1907 & & PISA & & FRANKLIN \\
\hline 3158 & & GIUSTI & PAOLO & $\mathrm{S}$ & 1 & MICHIGAN & ITALY & ITALY & & & PISA & & FRANKLIN \\
\hline 3159 & 64 & TAMBORINI & SANTINO & $\mathrm{H}$ & 42 & ITALY & ITALY & ITALY & 1901 & MINER/CM & & BACK ST. & FRANKLIN \\
\hline 3160 & 64 & TAMBORINI & LUIGIA & W & 28 & ITALY & ITALY & ITALY & 1903 & & & BACK ST. & FRANKLIN \\
\hline 3161 & 64 & TAMBORINI & MARY & $\mathrm{D}$ & 10 & ITALY & ITALY & ITALY & 1903 & & & BACK ST. & FRANKLIN \\
\hline 3162 & $\frac{64}{64}$ & TAMBORINI & JOSEPHINE & $\mathrm{D}$ & 4 & MICHIGAN & ITALY & ITALY & 1900 & & & $\begin{array}{l}\text { BACK ST. } \\
\text { BACK ST }\end{array}$ & FRANKLIN \\
\hline 3163 & 64 & GHIRINGHELLI & MARIA & SIIL & 19 & ITALY & ITALY & ITALY & 1909 & & SESTO CALENDE. VARESE & BACK ST. & FRANKLIN \\
\hline 3164 & 64 & PIEROTTI & ANTHONY & $\mathrm{COU}$ & 36 & ITALY & ITALY & ITALY & 1895 & MINER/CM & LUCCA & BACK ST. & FRANKLIN \\
\hline 3165 & 64 & PIEROTTI & JOSEPH & $\mathrm{COU}$ & 47 & ITALY & ITALY & ITALY & $05 / 06$ & MINER/CM & LUCCA & BACK ST. & FRANKLIN \\
\hline 3166 & 64 & VARALLI & PASQUALE & B & 36 & ITALY & ITALY & ITALY & 1903 & MINER/CM & & BACK ST. & FRANKLIN \\
\hline 3167 & 64 & BASSETTI & INNOCENTE & B & 31 & ITALY & ITALY & ITALY & 1902 & MINER/CM & SESTO CALENDE. VARESE & BACK ST. & FRANKLIN \\
\hline 3168 & 64 & BASSETTI & GIACOMO & B & 25 & ITALY & ITALY & ITALY & 1909 & LABORER/CM & SESTO CALENDE. VARESE & BACK ST. & FRANKLIN \\
\hline 3169 & 73 & PUCCI & JOE & $\mathrm{H}$ & 35 & ITALY & ITALY & ITALY & 1906 & LABORER/CM & LUCCA & BACK ST. & FRANKLIN \\
\hline 3170 & 73 & $\mathrm{PUCCl}$ & EMILIA & W & 39 & ITALY & ITALY & ITALY & 1908 & & LUCCA & BACK ST. & FRANKLIN \\
\hline 3171 & 73 & PUCCl & VALERIO & s & 11 & ITALY & ITALY & ITALY & 1908 & & LUCCA & BACK ST. & FRANKLIN \\
\hline 3172 & 73 & PUCCl & GUIDO & $\mathrm{s}$ & 9 & ITALY & ITALY & ITALY & 1908 & & LUCCA & BACK ST. & FRANKLIN \\
\hline 3173 & 73 & PUCCl & OLINDO & $\mathrm{s}$ & $11 \mathrm{M}$ & MICHIGAN & ITALY & ITALY & & & LUCCA & BACK ST. & FRANKLIN \\
\hline 3174 & 79 & SCOLATI & ALESSANDRO & $\mathrm{H}$ & 45 & ITALY & ITALY & ITALY & 1898 & MINER/CM & SESTO CALENDE. VARESE & $\begin{array}{l}\text { BACK ST. } \\
\text { BAT }\end{array}$ & FRANKLIN \\
\hline 3175 & 79 & SCOLATI & AMELIA & W & 35 & ITALY & ITALY & ITALY & $\begin{array}{l}1090 \\
1900\end{array}$ & IVIINERT/UIVI & SESTO CALENDE. VARESE & $\begin{array}{l}\text { BACK ST. } \\
\text { BACK ST }\end{array}$ & FRANKLIN \\
\hline 3176 & 79 & SCOLATI & CARLO & s & 16 & ITALY & ITALY & ITALY & 1900 & MOTORMAN/CM & SESTO CALENDE. VARESE & BACK ST. & FRANKLIN \\
\hline 3177 & 79 & SCOLATI & MARIO & $\mathrm{s}$ & 6 & MICHIGAN & ITALY & ITALY & & & SESTO CALENDE, VARESE & BACK ST. & FRANKLIN \\
\hline 3178 & 79 & SCOLATI & GIULIA & $\mathrm{D}$ & 4 & MICHIGAN & ITALY & ITALY & & & SESTO CALENDE. VARESE & BACK ST. & FRANKLIN \\
\hline 3179 & 79 & SCOLATI & MARIA & $\mathrm{D}$ & 1 & MICHIGAN & ITALY & ITALY & & & SESTO CALENDE. VARESE & BACK ST. & FRANKLIN \\
\hline 3180 & & CIABATTARI & VITTORIO & $\mathrm{H}$ & 36 & ITALY & ITALY & ITALY & $188 ?$ & LABORER/CM & SEGROMIGNO. LU & BACK ST. & FRANKLIN \\
\hline 3181 & & CIABATTARI & ADELE & W & 23 & ITALY & ITALY & ITALY & 1908 & & & BACK ST. & FRANKLIN \\
\hline 3182 & & CIABATTARI & MIRANDA & D & 1 & MICHIGAN & ITALY & ITALY & & & SEGROMIGNO. LU & BACK ST. & FRANKLIN \\
\hline 3183 & & PIZZI & ZEFFIRO & B & 27 & ITALY & ITALY & ITALY & 1902 & LABORER/CM & MONTEGROSSO, LU & BACK ST. & FRANKLIN \\
\hline 3184 & & SALSINI & LUIGI & B & 23 & ITALY & ITALY & ITALY & 1902 & LABORER/CM & SANTA MARIA DEL GIUDICE. LU & BACK ST. & FRANKLIN \\
\hline 3185 & & LAZZARI & ALFREDO & B & 24 & ITALY & ITALY & ITALY & 1907 & LABORER/CM & LUCCA & BACK ST. & FRANKLIN \\
\hline 3186 & & ROMANINI & DANTE & B & 21 & ITALY & ITALY & ITALY & 1907 & LABORER/CM & LUCCA & BACK ST. & FRANKLIN \\
\hline 3187 & & ROMANINI & RICCARDO & B & 28 & ITALY & ITALY & ITALY & 1903 & LABORER/CM & LUCCA & $\begin{array}{l}\text { BACK ST. } \\
\text { BACK }\end{array}$ & FRANKLIN \\
\hline 3188 & 83 & PUCCl & CARLO & $\mathrm{H}$ & 43 & ITALY & ITALY & ITALY & 1900 & MINER/CM & LUCCA & BACK ST. & FRANKLIN \\
\hline 3189 & 83 & PUCCI & ASSUNTA & w & 43 & ITALY & ITALY & ITALY & 1906 & & LUCCA & BACK ST. & FRANKLIN \\
\hline 3190 & 83 & PUCCl & ARMANDO & $\mathrm{s}$ & 3 & ITALY & ITALY & ITALY & 1906 & & LUCCA & BACK ST. & FRANKLIN \\
\hline 3191 & 83 & PUCCl & RITA & $\mathrm{D}$ & 1 & MICHIGAN & ITALY & ITALY & & & LUCCA & $\begin{array}{l}\text { BACK ST. } \\
\text { ST }\end{array}$ & FRANKLIN \\
\hline 3192 & 83 & MORRO & GUIDO & B & 28 & ITALY & ITALY & ITALY & 1909 & LABORER/CM & & BACK ST. & FRANKLIN \\
\hline 3193 & 83 & CONSANI & SANTE & B & 33 & ITALY & ITALY & ITALY & 1909 & LABORER/CM & PISA & BACK ST. & FRANKLIN \\
\hline 3194 & & MARCO & JOHN & $\mathrm{H}$ & 38 & ITALY & ITALY & ITALY & 1895 & MINER/CM & TORINO & BACK ST. & FRANKLIN \\
\hline 3195 & & MARCO & LENA & W & 28 & ITALY & ITALY & ITALY & 1884 & & & BACK ST. & FRANKLIN \\
\hline 3196 & & MARCO & LUCY & $\mathrm{D}$ & 9 & MICHIGAN & ITALY & ITALY & & & TORINO & BACK ST. & FRANKLIN \\
\hline 3197 & & MARCO & MAYNE & $\mathrm{D}$ & 8 & MICHIGAN & ITALY & ITALY & & & TORINO & BACK ST. & FRANKLIN \\
\hline 3198 & & MARCO & DOMINICK & $\mathrm{s}$ & 7 & MICHIGAN & ITALY & ITALY & & & TORINO & $\begin{array}{l}\text { BACK ST. } \\
\text { BAT }\end{array}$ & FRANKLIN \\
\hline 3199 & & MARCO & NERINA & $\mathrm{D}$ & 6 & MICHIGAN & ITALY & ITALY & & & TORINO & $\begin{array}{l}\text { BACK ST. } \\
\text { BAT }\end{array}$ & FRANKLIN \\
\hline 3200 & & MARCO & MARTINA & D & 4 & MICHIGAN & ITALY & ITALY & & & TORINO & $\begin{array}{l}\text { BACK ST. } \\
\text { BACK }\end{array}$ & FRANKLIN \\
\hline 3201 & & MARCO & ANDREW & s & $\frac{4}{2}$ & MICHIGAN & ITALY & ITALY & & & TORINO & $\begin{array}{l}\text { BACK ST. } \\
\text { BACK }\end{array}$ & FRANKLIN \\
\hline 3202 & 36 & LENCI & GIOSUE' & $\mathrm{H}$ & 27 & ITALY & ITALY & ITALY & 1901 & MINER/CM & SEGROMIGNO. LU & BACK ST. & FRANKLIN \\
\hline 3203 & 36 & LENCI & GIULIA & w & 16 & MICHIGAN & ITALY & ITALY & & & & BACK ST. & FRANKLIN \\
\hline 3204 & 36 & LENCI & TOMMASO & s & 1 & MICHIGAN & ITALY & ITALY & & & SEGROMIGNO. LU & $\begin{array}{l}\text { BACK ST. } \\
\text { BAT }\end{array}$ & FRANKLIN \\
\hline 3205 & 36 & LENCI & TRACEY & D & $3 \mathrm{M}$ & MICHIGAN & ITALY & ITALY & & & SEGROMIGNO. LU & BACK ST. & FRANKLIN \\
\hline 3206 & 36 & LENCI & FIORELLO & $B R$ & 22 & ITALY & ITALY & ITALY & 1906 & LABORER/CM & SEGROMIGNO. LU & BACK ST. & FRANKLIN \\
\hline 3207 & 14 & CERETTO & GIOVANNI & B & 24 & ITALY & ITALY & ITALY & 1900 & BRAKESMAN/RAILROAD & PONT CANAVESE, TO & BACK ST. & FRANKLIN \\
\hline 3208 & 6 & $\mathrm{ROCCHI}$ & LUIGI & $\mathrm{H}$ & 35 & ITALY & ITALY & ITALY & 1891 & SHIFT BOSS/CM & MATRAIA. LU & & FRANKLIN \\
\hline 3209 & 6 & $\mathrm{ROCCHI}$ & ARGENTINA & W & 39 & ITALY & ITALY & ITALY & 1897 & & & & FRANKLIN \\
\hline 3210 & 6 & $\mathrm{ROCCHI}$ & LIZZIE & D & 12 & MICHIGAN & ITALY & ITALY & & & MATRAIA. LU & & FRANKLIN \\
\hline 3211 & 6 & ROCCHI & GIUSEPPE & $\mathrm{s}$ & 10 & MICHIGAN & ITALY & ITALY & & & MATRAIA. LU & & FRANKLIN \\
\hline 3212 & 6 & $\mathrm{ROCCHI}$ & INES & $\mathrm{D}$ & 8 & MICHIGAN & ITALY & ITALY & & & MATRAIA. LU & & FRANKLIN \\
\hline
\end{tabular}




\begin{tabular}{|c|c|c|c|c|c|c|c|c|c|c|c|c|c|}
\hline 3213 & 6 & $\mathrm{ROCCHI}$ & CAROLINA & $\mathrm{D}$ & 6 & MICHIGAN & ITALY & ITALY & & & MATRAIA. LU & & FRANKLIN \\
\hline 3214 & 6 & ROCCHI & FELIX & $\mathrm{s}$ & 2 & MICHIGAN & ITALY & ITALY & & & MATRAIA. LU & & FRANKLIN \\
\hline 3215 & 6 & GUIDOTTI & FRANCESCO & B & 48 & ITALY & ITALY & ITALY & 1907 & LABORER/CM & MATRAIA. LU & & FRANKLIN \\
\hline 3216 & 6 & $\mathrm{ClUCCl}$ & SAMUELE & B & 49 & ITALY & ITALY & ITALY & 1886 & LABORER/CM & LUCCA & & FRANKLIN \\
\hline 3217 & 6 & PIZZI & JAMES & B & 21 & ITALY & ITALY & ITALY & 1908 & LABORER/CM & LUCCA & & FRANKLIN \\
\hline 3218 & 6 & NERI & GINO & B & 23 & ITALY & ITALY & ITALY & 1910 & LABORER/CM & FUCECCHIO. FIRENZE & & FRANKLIN \\
\hline 3219 & 6 & BIANCHI & FLORINDO & B & 24 & ITALY & ITALY & ITALY & 1908 & LABORER/CM & MATRAIA. LU & & FRANKLIN \\
\hline 3220 & & ORELLA & DOMINICK & $\mathrm{H}$ & 29 & ITALY & ITALY & ITALY & 1885 & INTERPRETER/LAW OFFICE & TORINO & & FRANKLIN \\
\hline 3221 & & ORELLA & TERESA & W & 31 & ITALY & ITALY & ITALY & & & & & FRANKLIN \\
\hline 3222 & & ORELLA & BARNEY & $\mathrm{s}$ & 6 & MICHIGAN & ITALY & ITALY & & & TORINO & & FRANKLIN \\
\hline 3223 & & ORELLA & WILLIAM & $\mathrm{s}$ & 3 & MICHIGAN & ITALY & ITALY & & & TORINO & & FRANKLIN \\
\hline 3224 & & ORELLA & MARY & $\mathrm{D}$ & 2 & MICHIGAN & ITALY & ITALY & & & TORINO & & FRANKLIN \\
\hline 3225 & & ORELLA & AGNES & $\mathrm{D}$ & 0 & MICHIGAN & ITALY & ITALY & & & TORINO & & FRANKLIN \\
\hline 3226 & & DE BATTISTI & MARY & MIL & & ITALY & ITALY & ITALY & 1909 & & & & FRANKLIN \\
\hline 3227 & & PANTERA & JOSEPH & $\mathrm{H}$ & 76 & ITALY & ITALY & ITALY & 1856 & PROPRIETOR/CANDY STORE & LUCCA & & FRANKLIN \\
\hline 3228 & & PANTERA & FRANK & $\mathrm{s}$ & 45 & MICHIGAN & ITALY & NEW YORK & & LABORER/ODD JOBS & LUCCA & & FRANKLIN \\
\hline 3229 & & PANTERA & WILLIAM & $\mathrm{s}$ & 38 & MICHIGAN & ITALY & NEW YORK & & WOOD CHOPPER/CAMP & LUCCA & & FRANKLIN \\
\hline 3230 & & PANTERA & FREDERICK & $\mathrm{s}$ & 37 & MICHIGAN & ITALY & NEW YORK & & JANITOR/SCHOOL & LUCCA & & FRANKLIN \\
\hline 3231 & & O'BRIEN & KATHERINE & $\mathrm{D}$ & & MICHIGAN & ITALY & NEW YORK & & 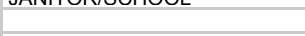 & LUCCA & & FRANKLIN \\
\hline 3232 & & O'BRIEN & WILLIAM & SOIL & & MICHIGAN & MICHIGAN & MICHIGAN & & MOTORMAN/SMELTER & USA & & FRANKLIN \\
\hline 3233 & & O'BRIEN & STANISLAUS & GS & & MICHIGAN & MICHIGAN & MICHIGAN & & & LUCCA & & FRANKLIN \\
\hline 3234 & & O'BRIEN & LUCILE & $\mathrm{GD}$ & & MICHIGAN & MICHIGAN & MICHIGAN & & & LUCCA & & FRANKLIN \\
\hline 3235 & & O'BRIEN & JOSEPH & GS & & MICHIGAN & MICHIGAN & MICHIGAN & & & LUCCA & & FRANKLIN \\
\hline 3236 & 121 & CAMIANO & GIUSEPPE & $\mathrm{H}$ & 43 & ITALY & ITALY & ITALY & 1892 & MINER/CM & TORINO & & FRANKLIN \\
\hline 3237 & 121 & CAMIANO & MARIA & W & 28 & ITALY & ITALY & ITALY & 1903 & & & & FRANKLIN \\
\hline 3238 & 121 & CAMIANO & MARGARET & $\mathrm{D}$ & 3 & MICHIGAN & ITALY & ITALY & & & TORINO & & FRANKLIN \\
\hline 3239 & 121 & CAMIANO & JOHN & $\mathrm{s}$ & 2 & MICHIGAN & ITALY & ITALY & & & TORINO & & FRANKLIN \\
\hline 3240 & 121 & CAMIANO & HELENA & $\mathrm{D}$ & $7 \mathrm{M}$ & MICHIGAN & ITALY & ITALY & & & TORINO & & FRANKLIN \\
\hline 3241 & 121 & MORINO & VITTORIO & B & 39 & ITALY & ITALY & ITALY & 1910 & LABORER/CM & MONCALIERI. TO & & FRANKLIN \\
\hline 3242 & & FREDIANELLI & PIETRO & $\mathrm{H}$ & 29 & ITALY & ITALY & ITALY & 1902 & LABORER/CM & SEGROMIGNO. LU & & FRANKLIN \\
\hline 3243 & & FREDIANELLI & MARIA & W & 26 & ITALY & ITALY & ITALY & 1904 & & SEGROMIGNO. LU & & FRANKLIN \\
\hline 3244 & & FREDIANELLI & ANGELO & s & 5 & MICHIGAN & ITALY & ITALY & & & SEGROMIGNO. LU & & FRANKLIN \\
\hline 3245 & & FREDIANELLI & PIETRO & s & 4 & MICHIGAN & ITALY & ITALY & & & SEGROMIGNO. LU & & FRANKLIN \\
\hline 3246 & & FREDIANELLI & VIOLET & $\mathrm{D}$ & $22 \mathrm{M}$ & MICHIGAN & ITALY & ITALY & & & SEGROMIGNO, LU & & FRANKLIN \\
\hline 3247 & & FREDIANELLI & CATERINA & D & $6 \mathrm{M}$ & MICHIGAN & ITALY & ITALY & & & SEGROMIGNO. LU & & FRANKLIN \\
\hline 3248 & & MENICUCCI & GIULIO & B & 18 & ITALY & ITALY & ITALY & 1909 & LABORER/CM & SEGROMIGNO. LU & & FRANKLIN \\
\hline 3249 & & MATTEUCCI & GIOVANNI & B & 25 & ITALY & ITALY & ITALY & 1909 & LABORER/CM & SEGROMIGNO. LU & & FRANKLIN \\
\hline 3250 & & SERAFINI & LORENZO & B & 19 & ITALY & ITALY & ITALY & 1906 & TIMBERMAN/CM & LUCCA & & FRANKLIN \\
\hline 3251 & & MARTINELLI & PIETRO & B & 17 & ITALY & ITALY & ITALY & 1909 & LABORER/CM & CAPANNORI. LU & & FRANKLIN \\
\hline 3252 & 231 & $\mathrm{ROCCHI}$ & EGIDIO & $\mathrm{H}$ & 38 & ITALY & ITALY & ITALY & 1904 & SHIFT BOSS/CM & MATRAIA. LU & & FRANKLIN \\
\hline 3253 & 231 & $\mathrm{ROCCHI}$ & PHOEBE & W & 31 & GERMANY & GERMANY & GERMANY & 1880 & & GERMANY & & FRANKLIN \\
\hline 3254 & 231 & $\mathrm{ROCCHI}$ & GIUSEPPA & D & 11 & MICHIGAN & ITALY & GERMANY & & & MATRAIA. LU & & FRANKLIN \\
\hline 3255 & 231 & $\mathrm{ROCCHI}$ & TERESA & $\mathrm{D}$ & 9 & MICHIGAN & ITALY & GERMANY & & & MATRAIA. LU & & FRANKLIN \\
\hline 3256 & 231 & $\mathrm{ROCCHI}$ & ALBERTO & $\mathrm{s}$ & 7 & MICHIGAN & ITALY & GERMANY & & & MATRAIA. LU & & FRANKLIN \\
\hline 3257 & 231 & $\mathrm{ROCCHI}$ & TEODORO & $\mathrm{s}$ & 5 & MICHIGAN & ITALY & GERMANY & & & MATRAIA. LU & & FRANKLIN \\
\hline 3258 & 231 & $\mathrm{ROCCHI}$ & ALFONSO & $\mathrm{s}$ & 3 & MICHIGAN & ITALY & GERMANY & & & MATRAIA. LU & & FRANKLIN \\
\hline 3259 & & BOGGIO & PETER & $\mathrm{H}$ & 58 & ITALY & ITALY & ITALY & 1889 & OWN INCOME & TORINO & & FRANKLIN \\
\hline 3260 & & BOGGIO & VERONICA & w & 40 & ITALY & ITALY & ITALY & 1888 & & & & FRANKLIN \\
\hline 3261 & & BESSOLO & ANNIE & SD & 18 & TEXAS & ITALY & ITALY & & & TORINO & & FRANKLIN \\
\hline 3262 & & BESSOLO & PAUL & SSON & 16 & KANSAS & ITALY & ITALY & & MOTORMAN/CM & TORINO & & FRANKLIN \\
\hline 3263 & & BESSOLO & DOMINICK & SSON & 8 & MICHIGAN & ITALY & ITALY & & & TORINO & & FRANKLIN \\
\hline 3264 & & BERTINO & CARLO & BIL & 22 & ITALY & ITALY & ITALY & 1905 & LABORER/CM & RIVARA CANAVESE, TO & & FRANKLIN \\
\hline 3265 & & MORONI & GEROLAMO & B & 22 & ITALY & ITALY & ITALY & 1905 & MINER/CM & SESTO CALENDE. VARESE & & FRANKLIN \\
\hline 3266 & & SALANI & MARIO & B & 19 & ITALY & ITALY & ITALY & 1909 & LABORER/CM & COLLODI. LU & & FRANKLIN \\
\hline 3267 & & GOSATTI & ANDREW SR. & $\mathrm{H}$ & 38 & ITALY & ITALY & ITALY & 1895 & MINER/CM & BIANZANO. SONDRIO & BACK ST. & FRANKLIN \\
\hline 3268 & & GOSATTI & MARY & w & 38 & ITALY & ITALY & ITALY & 1899 & & BIANZANO. SONDRIO & BACK ST. & FRANKLIN \\
\hline 3269 & & GOSATTI & TONY & s & 17 & ITALY & ITALY & ITALY & 1899 & MINER/CM & BIANZANO. SONDRIO & BACK ST. & FRANKLIN \\
\hline 3270 & & GOSATTI & JACOB & $\mathrm{s}$ & 14 & ITALY & ITALY & ITALY & 1899 & LABORER/CM & BIANZANO, SONDRIO & $\begin{array}{l}\text { BACK ST. } \\
\text { BAT }\end{array}$ & FRANKLIN \\
\hline 3271 & & GOSATTI & ANDREW JR. & $\mathrm{s}$ & 8 & MICHIGAN & ITALY & ITALY & & & BIANZANO. SONDRIO & BACK ST. & FRANKLIN \\
\hline 3272 & & GOSATTI & IRINA & $\mathrm{D}$ & 4 & MICHIGAN & ITALY & ITALY & & & BIANZANO. SONDRIO & BACK ST. & FRANKLIN \\
\hline 3273 & & BALTREMME & MICHAEL & B & 31 & ITALY & ITALY & ITALY & 1901 & MINER/CM & & BACK ST. & FRANKLIN \\
\hline 3274 & & BONOMI & JACOB & B & 40 & ITALY & ITALY & ITALY & 1910 & LABORER/CM & & BACK ST. & FRANKLIN \\
\hline 275 & & MARTINO & JAMES & B & 31 & ITALY & ITALY & ITALY & 1892 & LABORER/CM & BOSCONERO. TO & BACK ST. & FRANKLIN \\
\hline
\end{tabular}




\begin{tabular}{|c|c|c|c|c|c|c|c|c|c|c|c|c|c|}
\hline 3276 & & COLOMBO & JOSEPH & $\mathrm{H}$ & 49 & ITALY & ITALY & ITALY & 1888 & LABORER/ROCK HOUSE & PONT CANAVESE. TO & & FRANKLIN \\
\hline 3277 & & COLOMBO & MARY & w & 40 & ITALY & ITALY & ITALY & 1888 & & & & FRANKLIN \\
\hline 3278 & & COLOMBO & JOSEPH & s & 19 & MICHIGAN & ITALY & ITALY & & TEAMSTER/MEAT MARKET & PONT CANAVESE. TO & & FRANKLIN \\
\hline 3279 & & COLOMBO & MARY & $\mathrm{D}$ & 17 & MICHIGAN & ITALY & ITALY & & SERVANT/PF & PONT CANAVESE. TO & & FRANKLIN \\
\hline 3280 & & COLOMBO & PERINA & $\mathrm{D}$ & 16 & MICHIGAN & ITALY & ITALY & & & PONT CANAVESE. TO & & FRANKLIN \\
\hline 3281 & & COLOMBO & ABRAHAM & s & 14 & MICHIGAN & ITALY & ITALY & & & PONT CANAVESE. TO & & FRANKLIN \\
\hline 3282 & & COLOMBO & TONY & $\mathrm{s}$ & 10 & MICHIGAN & ITALY & ITALY & & & PONT CANAVESE. TO & & FRANKLIN \\
\hline 3283 & & COLOMBO & LUCY & $\mathrm{D}$ & 8 & MICHIGAN & ITALY & ITALY & & & PONT CANAVESE. TO & & FRANKLIN \\
\hline 3284 & & COLOMBO & SAMUEL & s & $\frac{0}{5}$ & MICHIGAN & ITALY & ITALY & & & PONT CANAVESE. TO & & FRANKLIN \\
\hline 3285 & & COLOMBO & GEORGE & $\mathrm{s}$ & 3 & MICHIGAN & ITALY & ITALY & & & PONT CANAVESE. TO & & FRANKLIN \\
\hline 3286 & & COLOMBO & LENA & $\mathrm{D}$ & 2 & MICHIGAN & ITALY & ITALY & & & PONT CANAVESE. TO & & FRANKLIN \\
\hline 3287 & & $\mathrm{RICCl}$ & EGIDIO & $\mathrm{H}$ & 37 & ITALY & ITALY & ITALY & 1895 & MINER/CM & LUCCA & & FRANKLIN \\
\hline 3288 & & $\mathrm{RICCl}$ & AMELIA & W & 36 & ITALY & ITALY & ITALY & 1905 & & & & FRANKLIN \\
\hline 3289 & & $\mathrm{RICCl}$ & EMMA & $\mathrm{D}$ & 7 & MICHIGAN & ITALY & ITALY & & & LUCCA & & FRANKLIN \\
\hline 3290 & & $\mathrm{RICCl}$ & JOSEPH & $\mathrm{s}$ & 4 & MICHIGAN & ITALY & ITALY & & & LUCCA & & FRANKLIN \\
\hline 3291 & & $\mathrm{RICCl}$ & MARY & $\mathrm{D}$ & 2 & MICHIGAN & ITALY & ITALY & & & LUCCA & & FRANKLIN \\
\hline 3292 & & $\mathrm{RICCI}$ & ANNIE & $\mathrm{D}$ & $6 \mathrm{M}$ & MICHIGAN & ITALY & ITALY & & & LUCCA & & FRANKLIN \\
\hline 3293 & 64 & CAPELLO & ALESSIO & $\mathrm{H}$ & 37 & ITALY & ITALY & ITALY & 1902 & LABORER/CM & SALTO CANAVESE. TO & BACK ST. & FRANKLIN \\
\hline 3294 & 64 & CAPELLO & ASSUNTA & w & 24 & ITALY & ITALY & ITALY & 1903 & & SALTO CANAVESE. TO & BACK ST. & FRANKLIN \\
\hline 3295 & 64 & CAPELLO & LOUISA & $\mathrm{D}$ & 9 & ITALY & ITALY & ITALY & 1903 & & SALTO CANAVESE. TO & BACK ST. & FRANKLIN \\
\hline 3296 & 64 & CAPELLO & AMALIA & $\mathrm{D}$ & 6 & MICHIGAN & $\begin{array}{l}\text { ITALY } \\
\text { ITA }\end{array}$ & ITALY & 1900 & & SALTO CANAVESE. TO & $\begin{array}{l}\text { BACK ST. } \\
\text { BACK ST }\end{array}$ & FRANKLIN \\
\hline 3297 & 64 & CAPELLO & BEATRICE & $\mathrm{D}$ & 4 & MICHIGAN & ITALY & ITALY & & & SALTO CANAVESE, TO & BACK ST. & FRANKLIN \\
\hline 3298 & 64 & BALAGNA & MICHELE & B & 25 & ITALY & ITALY & ITALY & 1891 & LABORER/CM & PONT CANAVESE. TO & BACK ST. & FRANKLIN \\
\hline 3299 & 64 & CONTO & BATTISTA & B & 26 & ITALY & ITALY & ITALY & $07 / 09$ & TRAMMER/CM & SAN GIORGIO CANAVESE. TO & BACK ST. & FRANKLIN \\
\hline 3300 & 64 & LOCATELLI & JANE & B & 33 & ITALY & ITALY & ITALY & 1906 & LABORER/CM & SELINO. BERGAMO & BACK ST. & FRANKLIN \\
\hline 3301 & 59 & PERONI & DOMINICK JR. & $\mathrm{H}$ & 43 & ITALY & ITALY & ITALY & 1899 & MINER/CM & & BACK ST. & FRANKLIN \\
\hline 3302 & 59 & PERONI & VALERIA & w & 39 & ITALY & ITALY & ITALY & 1902 & & & BACK ST. & FRANKLIN \\
\hline 3303 & 59 & PERONI & JOHN & s & 14 & ITALY & ITALY & ITALY & 1902 & & & BACK ST. & FRANKLIN \\
\hline 3304 & 59 & PERONI & ANTHONY & $\mathrm{S}$ & 13 & ITALY & ITALY & ITALY & 1902 & & & BACK ST. & FRANKLIN \\
\hline 3305 & 59 & PERONI & DOMINICK & $\mathrm{S}$ & 7 & MICHIGAN & ITALY & ITALY & & & & BACK ST. & FRANKLIN \\
\hline 3306 & 59 & PERONI & JAMES & $\mathrm{s}$ & 5 & MICHIGAN & ITALY & ITALY & & & & BACK ST. & FRANKLIN \\
\hline 3307 & 59 & PERONI & JANET & $\mathrm{D}$ & 3 & MICHIGAN & ITALY & ITALY & & & & BACK ST. & FRANKLIN \\
\hline 3308 & 59 & PERONI & DUSOLINA & $\mathrm{D}$ & $1.8 \mathrm{M}$ & ITALY & ITALY & ITALY & & & & $\begin{array}{l}\text { BACK ST. } \\
\text { BACK ST }\end{array}$ & FRANKLIN \\
\hline 3309 & 84 & MICHELETTI & FLORINDO & $\mathrm{H}$ & 31 & ITALY & ITALY & ITALY & 1905 & LABORER/CM & SANTA MARIA DEL GIUDICE, LU & BACK ST. & FRANKLIN \\
\hline 3310 & 84 & MICHELETTI & ARMINDA & w & 32 & ITALY & ITALY & ITALY & 1909 & & SANTA MARIA DEL GIUDICE. LU & BACK ST. & FRANKLIN \\
\hline 3311 & 84 & MICHELETTI & VELIA & $\mathrm{D}$ & 6 & ITALY & ITALY & ITALY & 1909 & & SANTA MARIA DEL GIUDICE. LU & BACK ST. & FRANKLIN \\
\hline 3312 & 84 & MICHELETTI & GIULIA & $\mathrm{D}$ & 0 & MICHIGAN & ITALY & ITALY & & & SANTA MARIA DEL GIUDICE. LU & BACK ST. & FRANKLIN \\
\hline 3313 & & BARSOCCHI & ENRICO & $\mathrm{H}$ & 32 & ITALY & ITALY & ITALY & 1906 & LABORER/CM & LUCCA & BACK ST. & FRANKLIN \\
\hline 3314 & & BARSOCCHI & ASSUNTA & W & 22 & ITALY & ITALY & ITALY & 1906 & KEEPER/BOARDING HOUSE & & BACK ST. & FRANKLIN \\
\hline 3315 & & MAGRI/MASINI & LOUIS & B & 24 & ITALY & ITALY & ITALY & 1905 & LABORER/CM & & BACK ST. & FRANKLIN \\
\hline 3316 & 90 & PRIAMI & ARMENO & $\mathrm{H}$ & 32 & ITALY & ITALY & ITALY & 1900 & CHUTE MAN/CM & LUCCA & BACK ST. & FRANKLIN \\
\hline 3317 & 90 & PRIAMI & EMMA & W & 22 & ITALY & ITALY & ITALY & 1909 & & & BACK ST. & FRANKLIN \\
\hline 3318 & 90 & PUCCl & FRANK & B & 22 & ITALY & ITALY & ITALY & 1906 & TRAMMER/CM & RUOTA. LU & BACK ST. & FRANKLIN \\
\hline 3319 & 90 & PUCCI & GIOVANNI & B & 33 & ITALY & ITALY & ITALY & 1905 & TRAMMER/CM & LUCCA & BACK ST. & FRANKLIN \\
\hline 3320 & 90 & NOVELLINI & MIELO & B & 27 & ITALY & ITALY & ITALY & 1908 & MINER/CM & LUCCA & BACK ST. & FRANKLIN \\
\hline 3321 & 90 & TOMMEI & GIORGIO & B & 25 & ITALY & ITALY & ITALY & 1908 & LABORER/CM & COLLE DI CAMPITO. LU & BACK ST. & FRANKLIN \\
\hline 3322 & 90 & CONSANI & NISIO & B & 27 & ITALY & ITALY & ITALY & 1909 & TRAMMER/CM & CAPANNORI. LU & BACK ST. & FRANKLIN \\
\hline 3323 & 90 & LANDINI & AURELIO & B & 22 & ITALY & ITALY & ITALY & 1909 & TRAMMER/CM & RUOTA. LU & BACK ST. & FRANKLIN \\
\hline 3324 & & ALLARIA & PETER & $\mathrm{H}$ & 63 & ITALY & ITALY & ITALY & 1877 & OWN INCOME & & BACK ST. & FRANKLIN \\
\hline 3325 & & ALLARIA & DOMENICA & w & 63 & ITALY & ITALY & ITALY & 1886 & & SAN GIORGIO CANAVESE. TO & BACK ST. & FRANKLIN \\
\hline 3326 & & ALLARIA & WILLIAM & $\mathrm{s}$ & 22 & MICHIGAN & ITALY & ITALY & & MINER/CM & SAN GIORGIO CANAVESE. TO & BACK ST. & FRANKLIN \\
\hline 3327 & & GIULIO/GIUGLIO & JOHN & B & 36 & ITALY & ITALY & ITALY & 1900 & MINER/CM & & BACK ST. & FRANKLIN \\
\hline 3328 & & GINETTI & LORENZO & B & 22 & ITALY & ITALY & ITALY & 1907 & TRAMMER/CM & SAN GIORGIO CANAVESE. TO & BACK ST. & FRANKLIN \\
\hline 3329 & & DEL BIANCO & VINCENT & B & 36 & ITALY & ITALY & ITALY & 1893 & MINER/CM & & BACK ST. & FRANKLIN \\
\hline 3330 & & TAPPERO & MARCELLINO & $\mathrm{H}$ & 26 & ITALY & ITALY & ITALY & 1906 & MINER/CM & TORINO & BACK ST. & FRANKLIN \\
\hline 3331 & & TAPPERO & ANGELA & w & 23 & ITALY & ITALY & ITALY & 1907 & & & BACK ST. & FRANKLIN \\
\hline 3332 & & TAPPERO & CAROLINE & $\mathrm{D}$ & $9 \mathrm{M}$ & MICHIGAN & ITALY & ITALY & & & TORINO & BACK ST. & FRANKLIN \\
\hline 3333 & & MARCHIANDO & MATIO & B & 42 & ITALY & ITALY & ITALY & 1905 & MINER/CM & TORINO & BACK ST. & FRANKLIN \\
\hline 3334 & & NOVELLI & AUGUSTO & $\mathrm{H}$ & 34 & ITALY & ITALY & ITALY & 1904 & SKIP FILLER/CM & CASTELVECCHIO. LU & BACK ST. & FRANKLIN \\
\hline 3335 & & NOVELLI & VIOLA & w & 25 & ITALY & ITALY & ITALY & 1906 & & & BACK ST. & FRANKLIN \\
\hline 3336 & & NOVELLI & ORLANDO & SSON & & MICHIGAN & ITALY & ITALY & & & & BACK ST. & FRANKLIN \\
\hline 3337 & & DI BENE & PIETRO & B & 30 & ITALY & ITALY & ITALY & 1906 & LABORER/CM & LUCCA & BACK ST. & FRANKLIN \\
\hline 3338 & & DAL SANTO & GIOVANNI & B & 21 & ITALY & ITALY & ITALY & 1906 & LABORER/CM & COLTRANA. VICENZA & BACK ST. & FRANKLIN \\
\hline
\end{tabular}




\begin{tabular}{|c|c|c|c|c|c|c|c|c|c|c|c|c|c|}
\hline 3339 & & GALLI & LUIGI & B & 18 & ITALY & ITALY & ITALY & 1909 & LABORER/CM & CAPANNORI. LU & BACK ST. & FRANKLIN \\
\hline 3340 & 19 & MARTINUCCI & GIACOMO & $\mathrm{H}$ & 30 & ITALY & ITALY & ITALY & 1901 & MINER/CM & COLLE DI COMPITO. LU & BACK ST. & FRANKLIN \\
\hline 3341 & 19 & MARTINUCCI & SELENE & W & 22 & ITALY & ITALY & ITALY & 1906 & KEEPER/BOARDING HOUSE & & BACK ST. & FRANKLIN \\
\hline 3342 & 19 & MARTINUCCI & PETER & $\mathrm{s}$ & 1.10 & MICHIGAN & ITALY & ITALY & & & COLLE DI COMPITO. LU & BACK ST. & FRANKLIN \\
\hline 3343 & 19 & BAGNESCHI & DOMENICO & B & 23 & ITALY & ITALY & ITALY & 1904 & TRAMMER/CM & CASTELVECCHIO. LU & BACK ST. & FRANKLIN \\
\hline 3344 & 19 & MARTINUCCI & ADOLFO & B & 24 & ITALY & ITALY & ITALY & 1903 & MINER/CM & COLLE DI COMPITO. LU & BACK ST. & FRANKLIN \\
\hline 3345 & 19 & BAGNESCHI & GIOVANNI & B & 18 & ITALY & ITALY & ITALY & 1909 & LABORER/CM & CASTELVECCHIO. LU & $\begin{array}{l}\text { BACK ST. } \\
\text { BAT }\end{array}$ & FRANKLIN \\
\hline 3346 & 19 & BAGNESCHI & GIUSEPPE & $\begin{array}{l}\mathrm{D} \\
\mathrm{B}\end{array}$ & 20 & ITALY & ITALY & ITALY & 1907 & TRAMMER/CM & CASTELVECCHIO. LU & BACK ST. & FRANKLIN \\
\hline 3347 & 19 & MARIANI & FIMANTE & $\begin{array}{l}\mathrm{D} \\
\mathrm{B}\end{array}$ & 20 & ITALY & ITALY & ITALY & 1909 & LABORER/CM & CAOTELVECU TIO.LU & $\begin{array}{l}\text { BACK ST. } \\
\text { BAT }\end{array}$ & FRANKLIN \\
\hline 3348 & 19 & GHILARDI & NATALE & B & 21 & ITALY & ITALY & ITALY & 1907 & TRAMMER/CM & CASTELVECCHIO.LU & BACK ST. & FRANKLIN \\
\hline 3349 & 19 & GALLI & SPERANDIO & B & 21 & ITALY & ITALY & ITALY & 1907 & LABORER/CM & CAPANNORI. LU & BACK ST. & FRANKLIN \\
\hline 3350 & 29 & SALANI & ALIPPIO & $\mathrm{H}$ & 35 & ITALY & ITALY & ITALY & 1890 & TRAMMER/CM & COLLODI. LU & & FRANKLIN \\
\hline 3351 & 29 & SALANI & ADA & W & 22 & ITALY & ITALY & ITALY & 1900 & KEEPER/BOARDING HOUSE & & & FRANKLIN \\
\hline 3352 & 29 & SALANI & MARIA & $\mathrm{D}$ & 4 & MICHIGAN & ITALY & ITALY & & & COLLODI. LU & & FRANKLIN \\
\hline 3353 & 29 & SALANI & LOUIS & $\mathrm{s}$ & 2 & MICHIGAN & ITALY & ITALY & & & COLLODI. LU & & FRANKLIN \\
\hline 3354 & 29 & CARMIGIANI & TOMMASO & B & 32 & ITALY & ITALY & ITALY & 1906 & TRAMMER/CM & ALTOPASCIO, LU & & FRANKLIN \\
\hline 3355 & 29 & CARMIGIANI & UGO & B & 22 & ITALY & ITALY & ITALY & 1907 & TRAMMER/CM & ALTOPASCIO. LU & & FRANKLIN \\
\hline 3356 & 29 & MARCHI & ITALO & B & 19 & ITALY & ITALY & ITALY & 1907 & LABORER/CM & PISA & & FRANKLIN \\
\hline 3357 & 29 & VANNI & VINCENT & B & 21 & ITALY & ITALY & ITALY & 1908 & LABORER/CM & BAGNI DI LUCCA. LU & & FRANKLIN \\
\hline 3358 & 29 & GIOVANNETTI & ARCHIMEDE & B & 21 & ITALY & ITALY & ITALY & 1909 & LABORER/CM & LUCCA & & FRANKLIN \\
\hline 3359 & & MONTICELLI & AMERIGO & $\mathrm{H}$ & 39 & ITALY & ITALY & ITALY & 1896 & FIREMAN/BOILER HOUSE & LUCCA & & FRANKLIN \\
\hline 3360 & & MONTICELLI & CHRISTINA & w & 39 & ITALY & ITALY & ITALY & 1900 & & & & FRANKLIN \\
\hline 3361 & & MONTICELLI & ALBERT & $\mathrm{s}$ & 9 & MICHIGAN & ITALY & ITALY & & & LUCCA & & FRANKLIN \\
\hline 3362 & & MONTICELLI & LOUIS & $\mathrm{s}$ & 8 & MICHIGAN & ITALY & ITALY & & & LUCCA & & FRANKLIN \\
\hline 3363 & & MONTICELLI & EMIL & $\mathrm{s}$ & 6 & MICHIGAN & ITALY & ITALY & & & LUCCA & & FRANKLIN \\
\hline 3364 & & MONTICELLI & ATTILIO & s & 4 & MICHIGAN & ITALY & ITALY & & & LUCCA & & FRANKLIN \\
\hline 3365 & & MONTICELLI & SETTIMIO & $\mathrm{s}$ & 1.10 & MICHIGAN & ITALY & ITALY & & & LUCCA & & FRANKLIN \\
\hline 3366 & & RUBERTI & ISIDORO & B & 24 & ITALY & ITALY & ITALY & 1906 & TRAMMER/CM & OGLIANO, TREVISO & & FRANKLIN \\
\hline 3367 & & BALDASSARRE/I & GIUSEPPE & B & 39 & ITALY & ITALY & ITALY & 1900 & TRAMMER/CM & SANTA MARIA DEL GIUDICE. LU & & FRANKLIN \\
\hline 3368 & & CRIVELLINI & GIULIO & B & 28 & ITALY & ITALY & ITALY & 1906 & SKIP FILLER/CM & OGLIANO. TREVISO & & FRANKLIN \\
\hline 3369 & & PENNACCHIOTTI & AMERIGO & B & 25 & ITALY & ITALY & ITALY & 1909 & LABORER/CM & & & FRANKLIN \\
\hline 3370 & & MONTICELLO & ORESTE & $\mathrm{H}$ & 43 & ITALY & ITALY & ITALY & 1882 & KEEPER/SALOON & MATRAIA. LU & & FRANKLIN \\
\hline 3371 & & MONTICELLO & SALOMINA & W & 45 & ITALY & ITALY & ITALY & 1880 & KEEPER/BOARDING HOUSE & DIATRAIA. LU & & FRANKLIN \\
\hline 3372 & & MONTICELLO & GIORGIO & s & 17 & MICHIGAN & ITALY & ITALY & & PAINTER/HOUSE & MATRAIA, LU & & FRANKLIN \\
\hline 3373 & & MONTICELLO & ANGELINA & $\mathrm{D}$ & 16 & MICHIGAN & ITALY & ITALY & & & MATRAIA. LU & & FRANKLIN \\
\hline 3374 & & MONTICELLO & VINCENZO & $\mathrm{s}$ & 12 & ITALY & ITALY & ITALY & 1900 & & MATRAIA. LU & & FRANKLIN \\
\hline 3375 & & MONTICELLO & LORENZO & $\mathrm{s}$ & 8 & MICHIGAN & ITALY & ITALY & & & MATRAIA. LU & & FRANKLIN \\
\hline 3376 & & MONTICELLO & GUIDO & $\mathrm{s}$ & 5 & MICHIGAN & ITALY & ITALY & & & MATRAIA. LU & & FRANKLIN \\
\hline 3377 & & MONTICELLO & FRANK & $\mathrm{s}$ & 3 & MICHIGAN & ITALY & ITALY & & & MATRAIA. LU & & FRANKLIN \\
\hline 3378 & & MONTICELLO & GIOVANNI & $\mathrm{s}$ & $6 \mathrm{M}$ & MICHIGAN & ITALY & ITALY & & & MATRAIA, LU & & FRANKLIN \\
\hline 3379 & & GIANNERINI & DOMENICO & B & 26 & ITALY & ITALY & ITALY & 1900 & NOT CLEAR & MATRAIA. LU & & FRANKLIN \\
\hline 3380 & & BIANCHI & FELICE & B & 35 & ITALY & ITALY & ITALY & 1902 & TRAMMER BOSS/CM & MATRAIA. LU & & FRANKLIN \\
\hline 3381 & & BIANCHI & FRANCESCO & B & 33 & ITALY & ITALY & ITALY & 1908 & TRAMMER/CM & MATRAIA. LU & & FRANKLIN \\
\hline 3382 & & RICCOMINI & NARCISO & B & 34 & ITALY & ITALY & ITALY & 1902 & SKIP FILLER/CM & LUCCA & & FRANKLIN \\
\hline 3383 & & RICCOMINI & MAURIZIO & B & 30 & ITALY & ITALY & ITALY & 1905 & SHEAVE TENDER/CM & LUCCA & & FRANKLIN \\
\hline 3384 & & LENCIONI & GIOVANNI & B & 20 & ITALY & ITALY & ITALY & 1907 & TRAMMER/CM & SEGROMIGNO. LU & & FRANKLIN \\
\hline 3385 & & CASTI & CHERUBINO & B & 23 & ITALY & ITALY & ITALY & 1907 & LABORER/CM & VERONA & & FRANKLIN \\
\hline 3386 & & QUILICI & JOHN & B & 29 & ITALY & ITALY & ITALY & 1905 & SKIP FILLER/CM & LUCCA & & FRANKLIN \\
\hline 3387 & 28 & GEMIGNANI & FRANCESCO & $\mathrm{H}$ & 42 & ITALY & ITALY & ITALY & 1891 & PROPRIETOR/CANDY STORE & PESCAGLIA, LU & & FRANKLIN \\
\hline 3388 & 28 & GEMIGNANI & TERESA & W & 31 & ITALY & ITALY & ITALY & 1898 & & PESCAGLIA. LU & & FRANKLIN \\
\hline 3389 & 28 & GEMIGNANI & ALADINO & $\mathrm{s}$ & 11 & MICHIGAN & ITALY & ITALY & & & PESCAGLIA. LU & & FRANKLIN \\
\hline 3390 & 28 & GEMIGNANI & ITALIA & $\mathrm{D}$ & 9 & MICHIGAN & ITALY & ITALY & & & PESCAGLIA, LU & & FRANKLIN \\
\hline 3391 & 28 & GEMIGNANI & ADOLFO & $\mathrm{s}$ & 7 & MICHIGAN & ITALY & ITALY & & & PESCAGLIA. LU & & FRANKLIN \\
\hline 3392 & & ELPANI & BASTIANO & B & 25 & ITALY & ITALY & ITALY & 1906 & MINER/CM & LOPPEGLIA. LU & & FRANKLIN \\
\hline 3393 & & MARACCINI & NATALE & $\mathrm{H}$ & 23 & ITALY & ITALY & ITALY & 1907 & LABORER/CM & SANTA MARIA DEL GIUDICE. LU & & FRANKLIN \\
\hline 3394 & & MARACCINI & ADELE & w & 23 & ITALY & ITALY & ITALY & $07 / 09$ & & SANTA MARIA DEL GIUDICE. LU & & FRANKLIN \\
\hline 3395 & & MARACCINI & BEPPINA & $\mathrm{D}$ & $2 \mathrm{M}$ & MICHIGAN & ITALY & ITALY & & & SANTA MARIA DEL GIUDICE. LU & & FRANKLIN \\
\hline 3396 & & BARTASANI & RICHARD & B & 24 & ITALY & ITALY & ITALY & 1909 & TRAMMER/CM & & & FRANKLIN \\
\hline 3397 & & MENICUCCI & PIETRO & B & 38 & ITALY & ITALY & ITALY & 1907 & LABORER/CM & LUCCA & & FRANKLIN \\
\hline 3398 & & SAPINO & MARCELLO & $\mathrm{H}$ & 45 & ITALY & ITALY & ITALY & 1896 & MINER/CM & ROSIGNANO. LIVORNO & & FRANKLIN \\
\hline 3399 & & SAPINO & ANNA & W & 44 & ITALY & ITALY & ITALY & 1901 & & ROSIGNANO. LIVORNO & & FRANKLIN \\
\hline 3400 & & SAPINO & ANTONIO & s & 19 & ITALY & ITALY & ITALY & 1901 & MINER/CM & ROSIGNANO. LIVORNO & & FRANKLIN \\
\hline 3401 & & SAPINO & MARIA & $\mathrm{D}$ & 17 & ITALY & ITALY & ITALY & 1901 & & ROSIGNANO. LIVORNO & & FRANKLIN \\
\hline
\end{tabular}




\begin{tabular}{|c|c|c|c|c|c|c|c|c|c|c|c|c|}
\hline 3402 & & SAPINO & CESARE & $\mathrm{s}$ & 15 & ITALY & ITALY & ITALY & 1901 & & ROSIGNANO. LIVORNO & FRANKLIN \\
\hline 3403 & & SAPINO & FRANK & $\mathrm{s}$ & 8 & MICHIGAN & ITALY & ITALY & & & ROSIGNANO. LIVORNO & FRANKLIN \\
\hline 3404 & & SAPINO & MARGHERITA & D & 6 & MICHIGAN & ITALY & ITALY & & & ROSIGNANO. LIVORNO & FRANKLIN \\
\hline 3405 & 41 & GRASSESCHI & TEODORO & $\mathrm{H}$ & 27 & ITALY & ITALY & ITALY & 1908 & TRAMMER/CM & SANTA MARIA DEL GIUDICE. LU & FRANKLIN \\
\hline 3406 & 41 & GRASSESCHI & MARIA & w & 26 & ITALY & ITALY & ITALY & 1909 & & & FRANKLIN \\
\hline 3407 & 41 & GRASSESCHI & RENATO & s & $7 \mathrm{M}$ & MICHIGAN & ITALY & ITALY & & & SANTA MARIA DEL GIUDICE. LU & FRANKLIN \\
\hline 3408 & 41 & FABBRI & $\begin{array}{l}\text { PETER } \\
\text { PETER }\end{array}$ & $\mathrm{B}$ & 25 & ITALY & ITALY & ITALY & 1909 & TRAMMER/CM & LUCCA & FRANKLIN \\
\hline 3409 & 41 & GRASSESCHI & ADOLFO & $\begin{array}{l}\mathrm{D} \\
\mathrm{B}\end{array}$ & 24 & ITALY & ITALY & ITALY & 1907 & LABORER/CM & SANTA MARIA DEL GIUDICE. LU & FRANKLIN \\
\hline 3410 & & VALENTINO & JAMES & $\mathrm{H}$ & 28 & ITALY & ITALY & ITALY & $? ?$ & TIMBERMAN/CM & & FRANKLIN \\
\hline 3411 & & VALENTINO & NATALINA & w & 24 & ITALY & ITALY & ITALY & 1907 & & & FRANKLIN \\
\hline 3412 & & VALENTINO & EMILIO & $\mathrm{s}$ & $1.5 \mathrm{M}$ & MICHIGAN & ITALY & ITALY & & & & FRANKLIN \\
\hline 3413 & & TABARACCI & JOSEPH & $\mathrm{H}$ & 28 & ITALY & ITALY & ITALY & 1897 & LABORER/CM & SANTA MARIA DEL GIUDICE. LU & FRANKLIN \\
\hline 3414 & & TABARACCI & FIDORINA & W & 27 & ITALY & ITALY & ITALY & 1908 & & & FRANKLIN \\
\hline 3415 & & TABARACCI & MARY L. & $\mathrm{D}$ & $9 \mathrm{M}$ & MICHIGAN & ITALY & ITALY & & & SANTA MARIA DEL GIUDICE. LU & FRANKLIN \\
\hline 3416 & 38 & MARACCINI & TELEMACO & $\mathrm{H}$ & 30 & ITALY & ITALY & ITALY & 1905 & TIMBERMAN/CM & SANTA MARIA DEL GIUDICE. LU & FRANKLIN \\
\hline 3417 & 38 & MARACCINI & ELISA & W & 26 & ITALY & ITALY & ITALY & 1906 & & SANTA MARIA DEL GIUDICE, LU & FRANKLIN \\
\hline 3418 & 38 & MARACCINI & ISIDORA & D & 5 & ITALY & ITALY & ITALY & 1906 & & SANTA MARIA DEL GIUDICE. LU & FRANKLIN \\
\hline 3419 & 38 & MARACCINI & IVA & $\mathrm{D}$ & 2 & MICHIGAN & ITALY & ITALY & & & SANTA MARIA DEL GIUDICE. LU & FRANKLIN \\
\hline 3420 & 38 & MARACCINI & MARY & $\mathrm{D}$ & $1.1 \mathrm{M}$ & MICHIGAN & ITALY & ITALY & & & 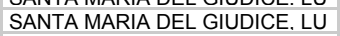 & FRANKLIN \\
\hline 3421 & 38 & BALDASSARRE & ITALO & B & 18 & ITALY & ITALY & ITALY & 1909 & LABORER/CM & LUCCA & FRANKLIN \\
\hline 3422 & 37 & $\mathrm{RICCl}$ & ANGELO & $\mathrm{H}$ & 33 & ITALY & ITALY & ITALY & 1904 & SHEAVE TENDER/CM & MATRAIA, LU & FRANKLIN \\
\hline 3423 & 37 & $\mathrm{RICCl}$ & EMILIA & w & 34 & ITALY & ITALY & ITALY & 1907 & & & FRANKLIN \\
\hline 3424 & 37 & $\mathrm{RICCl}$ & GESUINA & $\mathrm{D}$ & 2 & MICHIGAN & ITALY & ITALY & & & MATRAIA. LU & FRANKLIN \\
\hline 3425 & 37 & RICCl & ANGELINA & $\mathrm{D}$ & $8 \mathrm{M}$ & MICHIGAN & ITALY & ITALY & & & MATRAIA. LU & FRANKLIN \\
\hline 3426 & & $\mathrm{RICCl}$ & CESARE & $\mathrm{H}$ & 33 & ITALY & ITALY & ITALY & 1900 & MINER/CM & MATRAIA. LU & FRANKLIN \\
\hline 3427 & & $\mathrm{RICCl}$ & ALBERTINA & W & 24 & ITALY & ITALY & ITALY & 1909 & & & FRANKLIN \\
\hline 3428 & & $\mathrm{RICCl}$ & DOMENICA & $\mathrm{s}$ & $4 \mathrm{M}$ & MICHIGAN & ITALY & ITALY & & & MATRAIA. LU & FRANKLIN \\
\hline 3429 & & GIOMETTI & RAFFAELE & B & 34 & ITALY & ITALY & ITALY & 1900 & TRAMMER/CM & MASSA MACINAIA, LU & FRANKLIN \\
\hline 3430 & & VIANI & BASILIO & B & 37 & ITALY & ITALY & ITALY & 1896 & SKIP FILLER/CM & MONTE SAN QUIRICO. LU & FRANKLIN \\
\hline 3431 & & $\mathrm{RICCl}$ & ADOLF & B & 27 & ITALY & ITALY & ITALY & 1909 & LABORER/CM & MATRAIA. LU & FRANKLIN \\
\hline 3432 & & $\mathrm{RICCl}$ & LOUIS & B & 17 & ITALY & ITALY & ITALY & 1909 & LABORER/CM & MATRAIA. LU & FRANKLIN \\
\hline 3433 & & $\mathrm{RICCl}$ & ADELE & SER & 20 & ITALY & ITALY & ITALY & 1910 & SERVANT/PF & MATRAIA. LU & FRANKLIN \\
\hline 3434 & & MASINI & ALFREDO & $\mathrm{H}$ & 25 & ITALY & ITALY & ITALY & 1905 & SHEAVE TENDER/CM & MONSACRATI. LU & FRANKLIN \\
\hline 3435 & & MASINI & ASSUNTA & w & 16 & ITALY & ITALY & ITALY & 1908 & KEEPER/BOARDING HOUSE & MONSACRATI, LU & FRANKLIN \\
\hline 3436 & & $\mathrm{RICCl}$ & JOHN & B & 32 & ITALY & ITALY & ITALY & 1907 & SKIP FILLER/CM & MATRAIA. LU & FRANKLIN \\
\hline 3437 & & LANDINI & SALVATORE & B & 52 & ITALY & ITALY & ITALY & $07 / 02$ & STEMMER/CM & RUOTA. LU & FRANKLIN \\
\hline 3438 & & LANDINI & MANDINO & B & 18 & ITALY & ITALY & ITALY & 1909 & STEMMER/CM & LUCCA & FRANKLIN \\
\hline 3439 & & BALDASSARRE/I & JOHN & B & 51 & ITALY & ITALY & ITALY & 1909 & LABORER/CM & LUCCA & FRANKLIN \\
\hline 3440 & & T/G/ZARMACI & EUGENE & B & 28 & ITALY & ITALY & ITALY & 1906 & TRAMMER/CM & & FRANKLIN \\
\hline 3441 & & BALDASSARRE & GIOVANNI & $\mathrm{H}$ & 36 & ITALY & ITALY & ITALY & 1901 & LABORER/CM & SANTA MARIA DEL GIUDICE. LU & FRANKLIN \\
\hline 3442 & & BALDASSARRE & CREUSA & W & 41 & ITALY & ITALY & ITALY & 1909 & & SANTA MARIA DEL GIUDICE. LU & FRANKLIN \\
\hline 3443 & & BALDASSARRE & ASSUNTA & D & 15 & ITALY & ITALY & ITALY & 1909 & & SANTA MARIA DEL GIUDICE. LU & FRANKLIN \\
\hline 3444 & & BALDASSARRE & CARLO & $\mathrm{s}$ & 3 & ITALY & ITALY & ITALY & 1909 & & SANTA MARIA DEL GIUDICE. LU & FRANKLIN \\
\hline 3445 & & BALDASSARRE & CESARE & $\mathrm{s}$ & $6 \mathrm{M}$ & MICHIGAN & ITALY & ITALY & & & SANTA MARIA DEL GIUDICE. LU & FRANKLIN \\
\hline 3446 & & GIUSTI & AMELIO & B & 40 & ITALY & ITALY & ITALY & 1910 & TRAMMER/CM & LUCCA & FRANKLIN \\
\hline 3447 & 42 & GAGLIANI & LUDOVICO & $\mathrm{H}$ & 26 & ITALY & ITALY & ITALY & 1907 & SHEAVE TENDER/CM & CAPANNORI. LU & FRANKLIN \\
\hline 3448 & 42 & GAGLIANI & ANGELINA & w & 22 & ITALY & ITALY & ITALY & 1909 & & CAPANNORI. LU & FRANKLIN \\
\hline 3449 & 42 & GAGLIANI & NELLA & $\mathrm{D}$ & 3 & ITALY & ITALY & ITALY & 1909 & & CAPANNORI. LU & FRANKLIN \\
\hline 3450 & 47 & MANDOLI & G. & $\mathrm{H}$ & 39 & ITALY & ITALY & ITALY & 1906 & LABORER/CM & SEGROMIGNO. LU & FRANKLIN \\
\hline 3451 & 47 & MANDOLI & TERESA & w & 39 & ITALY & ITALY & ITALY & 1908 & & SEGROMIGNO. LU & FRANKLIN \\
\hline 3452 & 47 & MANDOLI & ALADINO & $\mathrm{s}$ & 5 & ITALY & ITALY & ITALY & 1908 & & SEGROMIGNO. LU & FRANKLIN \\
\hline 3453 & 47 & MANDOLI & ANTHONY & $\mathrm{s}$ & 3 & ITALY & ITALY & ITALY & 1908 & & SEGROMIGNO, LU & FRANKLIN \\
\hline 3454 & 47 & RICCOMINI & ARCANGELO & B & 24 & ITALY & ITALY & ITALY & 1909 & TRAMMER/CM & LUCCA & FRANKLIN \\
\hline 3455 & & EVANGELISTA & ANGELO & $\mathrm{H}$ & 31 & ITALY & ITALY & ITALY & 1902 & LABORER/CM & SANTA MARIA DEL GIUDICE. LU & FRANKLIN \\
\hline 3456 & & EVANGELISTA & GERMA & w & 27 & ITALY & ITALY & ITALY & 1906 & & & FRANKLIN \\
\hline 3457 & & EVANGELISTA & FIORINO & s & 3 & MICHIGAN & ITALY & ITALY & & & SANTA MARIA DEL GIUDICE. LU & FRANKLIN \\
\hline 3458 & & EVANGELISTA & EMILIO & $\mathrm{s}$ & 1 & MICHIGAN & ITALY & ITALY & & & SANTA MARIA DEL GIUDICE. LU & FRANKLIN \\
\hline 3459 & & MASSOGLIA & DOMENICO & $\mathrm{H}$ & 39 & ITALY & ITALY & ITALY & 1899 & LABORER/CM & SAN MARTINO CANAVESE, TO & FRANKLIN \\
\hline 3460 & & MASSOGLIA & ANNA & W & 34 & ITALY & ITALY & ITALY & 1905 & & & FRANKLIN \\
\hline 3461 & & MASSOGLIA & FRANK & s & 4 & MICHIGAN & ITALY & ITALY & & & SAN MARTINO CANAVESE. TO & FRANKLIN \\
\hline 3462 & & MASSOGLIA & PIETRO & $\mathrm{s}$ & 4 & MICHIGAN & ITALY & ITALY & & & SAN MARTINO CANAVESE. TO & FRANKLIN \\
\hline 3463 & & SCAVARDA & JAMES & B & 22 & ITALY & ITALY & ITALY & 1905 & LABORER/CM & 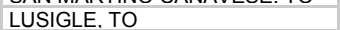 & FRANKLIN \\
\hline 3464 & & BERNO & ANDREA & B & 22 & ITALY & ITALY & ITALY & 1906 & LABORER/CM & SAN MARTINO CANAVESE. TO & FRANKLIN \\
\hline
\end{tabular}




\begin{tabular}{|c|c|c|c|c|c|c|c|c|c|c|c|c|}
\hline 3465 & & MEALLIA & ANTONIO & B & 39 & ITALY & ITALY & ITALY & $00 / 06$ & TRAMMER/CM & BOSCONERO. TO & FRANKLIN \\
\hline 3466 & & ALLARIA & TONY & $\mathrm{H}$ & 37 & ITALY & ITALY & ITALY & 1887 & MINER/CM & SAN BENIGNO CANAVESE. TO & FRANKLIN \\
\hline 3467 & & ALLARIA & DOMENICA & W & 36 & ITALY & ITALY & ITALY & 1897 & & & FRANKLIN \\
\hline 3468 & & ALLARIA & DOMENICA & D & 12 & MICHIGAN & ITALY & ITALY & & & SAN BENIGNO CANAVESE. TO & FRANKLIN \\
\hline 3469 & & ALLARIA & PETER & $\mathrm{s}$ & 9 & MICHIGAN & ITALY & ITALY & & & SAN BENIGNO CANAVESE. TO & FRANKLIN \\
\hline 3470 & & ALLARIA & IRENE & D & 6 & MICHIGAN & ITALY & ITALY & & & SAN BENIGNO CANAVESE. TO & FRANKLIN \\
\hline 3471 & & MORONI & ANGELO & B & 32 & ITALY & ITALY & ITALY & 1909 & MINER/CM & SESTO CALENDE. VARESE & FRANKLIN \\
\hline 3472 & & MORONI & CESARE & B & 26 & ITALY & ITALY & ITALY & 1906 & MINER/CM & SESTO CALENDE. VARESE & FRANKLIN \\
\hline 3473 & & MORONI & SERAFINO & B & 30 & ITALY & ITALY & ITALY & 1908 & TRAMMER/CM & SESTO CALENDE. VARESE & FRANKLIN \\
\hline 3474 & & BASSETTI & DONATO & B & 24 & ITALY & ITALY & ITALY & 1906 & TRAMMER/CM & SESTO CALENDE. VARESE & FRANKLIN \\
\hline 3475 & & LENCIONI & JOSEPH H. & $\mathrm{H}$ & 28 & ITALY & ITALY & ITALY & 1900 & LABORER/ROCK HOUSE & LUCCA & FRANKLIN \\
\hline 3476 & & LENCIONI & ILIANA & W & 28 & ITALY & $\begin{array}{l}\text { ITALY } \\
\text { ITA }\end{array}$ & ITALY & 1903 & LADUURERIRU R TUUSE & LUCUA & FRANKLIN \\
\hline 3477 & & LENCIONI & NIVIA & $\mathrm{D}$ & $\begin{array}{l}20 \\
5\end{array}$ & MICHIGAN & ITALY & $\begin{array}{l}\text { ITALY } \\
\text { ITAL }\end{array}$ & 1900 & & LUCCA & FRANKLIN \\
\hline 3478 & & LENCIONI & ROSE & $\mathrm{D}$ & 3 & MICHIGAN & ITALY & ITALY & & & LUCCA & FRANKLIN \\
\hline 3479 & & LENCIONI & NARCISO & $\mathrm{S}$ & 2 & MICHIGAN & ITALY & ITALY & & & LUCCA & FRANKLIN \\
\hline 3480 & & LENCl & SALVATORE & B & 41 & ITALY & ITALY & ITALY & 1895 & LABORER/CM & TORCIGLIANO, LU & FRANKLIN \\
\hline 3481 & & LENCI & JOHN & B & 32 & ITALY & ITALY & ITALY & 1900 & TIMBER BOSS/CM & PESCAGLIA. LU & FRANKLIN \\
\hline 3482 & & CIARDI & ALIDEO & B & 23 & ITALY & ITALY & ITALY & 1907 & LABORER/ROCK HOUSE & PISA & FRANKLIN \\
\hline 3483 & & STEFANI & LOUIS & B & 30 & ITALY & ITALY & ITALY & 1900 & SHEAVE TENDER/CM & LUCCA & FRANKLIN \\
\hline 3484 & & REALI & ATTILIO & $\mathrm{H}$ & 27 & ITALY & ITALY & ITALY & 1906 & MINER/CM & CALCINATO. BRESCIA & FRANKLIN \\
\hline 3485 & & REALI & ANITA & W & 25 & ITALY & ITALY & ITALY & 1909 & & CALCINATO. BRESCIA & FRANKLIN \\
\hline 3486 & & REALI & AVILIA & $\mathrm{D}$ & 5 & ITALY & ITALY & ITALY & 1909 & & CALCINATO, BRESCIA & FRANKLIN \\
\hline 3487 & & REALI & REPUBBLICA & $\mathrm{D}$ & 4 & ITALY & ITALY & ITALY & 1909 & & CALCINATO. BRESCIA & FRANKLIN \\
\hline 3488 & & REALI & LAICA & $\mathrm{D}$ & 2 & ITALY & ITALY & ITALY & 1909 & & CALCINATO. BRESCIA & FRANKLIN \\
\hline 3489 & & REALI & MATRIGLIANA & $\mathrm{D}$ & $2 \mathrm{M}$ & MICHIGAN & ITALY & ITALY & & & CALCINATO. BRESCIA & FRANKLIN \\
\hline 3490 & & PANICHI & $\begin{array}{l}\text { GOFFREDO } \\
\text { GOFRE }\end{array}$ & B & 36 & ITALY & ITALY & $\begin{array}{l}\text { ITALY } \\
\text { ITAL }\end{array}$ & 1906 & SKIP FILLER/CM & PISA & FRANKLIN \\
\hline 3491 & & PARELLI & MANNEO & B & 26 & ITALY & ITALY & ITALY & 1910 & TRAMMER/CM & & FRANKLIN \\
\hline 3492 & & NATTUCI & PALMIRO & B & 41 & ITALY & ITALY & ITALY & 1910 & TRAMMER/CM & MONTECATINI & FRANKLIN \\
\hline 3493 & & DINELLI & IACOPO & $\mathrm{H}$ & 35 & ITALY & ITALY & ITALY & 1901 & LABORER/CM & MATRAIA. LU & FRANKLIN \\
\hline 3494 & & DINELLI & FILOMENA & W & 29 & ITALY & ITALY & ITALY & 1905 & & MATRAIA. LU & FRANKLIN \\
\hline 3495 & & DINELLI & GIULIANO & $\mathrm{s}$ & 10 & ITALY & ITALY & ITALY & 1905 & & MATRAIA. LU & FRANKLIN \\
\hline 3496 & & DINELLI & GERMANA & $\mathrm{D}$ & 8 & ITALY & ITALY & ITALY & 1905 & & MATRAIA. LU & FRANKLIN \\
\hline 3497 & & DINELLI & PETER & $s$ & 4 & MICHIGAN & ITALY & ITALY & & & MATRAIA. LU & FRANKLIN \\
\hline 3498 & & DINELLI & MARSILIA & D & 4 & MICHIGAN & ITALY & ITALY & & & MATRAIA, LU & FRANKLIN \\
\hline 3499 & & DINELLI & PIERINA & $\mathrm{D}$ & 2 & MICHIGAN & ITALY & ITALY & & & MATRAIA. LU & FRANKLIN \\
\hline 3500 & & DINELLI & SILVIA & $\mathrm{D}$ & $4 \mathrm{M}$ & MICHIGAN & ITALY & ITALY & & & MATRAIA. LU & FRANKLIN \\
\hline 3501 & & VIANI & ULISSE & B & 28 & ITALY & ITALY & ITALY & 1906 & LABORER/CM & LUCCA & FRANKLIN \\
\hline 3502 & & ADAMI & BARTOLOMEO & B & 27 & ITALY & ITALY & ITALY & 1906 & LABORER/CM & VILLA BASILICA. LU & FRANKLIN \\
\hline 3503 & & POILIA & SABBATINO & B & 42 & ITALY & ITALY & ITALY & 1904 & LABORER/CM & & FRANKLIN \\
\hline 3504 & & CALAMARI & UGO & B & 24 & ITALY & ITALY & ITALY & 1907 & LABORER/CM & VILLA BASILICA, LU & FRANKLIN \\
\hline 3505 & & BONINI & WALTER & $\mathrm{H}$ & 35 & ITALY & ITALY & ITALY & 1894 & SKIP FILLER/CM & COLLODI. LU & FRANKLIN \\
\hline 3506 & & BONINI & MARY & W & 24 & ITALY & ITALY & ITALY & 1896 & & & FRANKLIN \\
\hline 3507 & & BONINI & LENA & D & 2 & MICHIGAN & ITALY & ITALY & & & COLLODI. LU & FRANKLIN \\
\hline 3508 & & BONINI & BROSINO & $\mathrm{s}$ & $9 \mathrm{M}$ & MICHIGAN & ITALY & ITALY & & & COLLODI. LU & FRANKLIN \\
\hline 3509 & 28 & BIANCHI & MICHELE & $\mathrm{H}$ & 45 & ITALY & ITALY & ITALY & 1893 & LABORER/CM & MATRAIA. LU & FRANKLIN \\
\hline 3510 & 28 & BIANCHI & CONCETTA & W & 35 & ITALY & ITALY & ITALY & 1905 & KEEPER/BOARDING HOUSE & MATRAIA. LU & FRANKLIN \\
\hline 3511 & 28 & BIANCHI & VICO & s & 8 & ITALY & ITALY & ITALY & 1905 & & MATRAIA. LU & FRANKLIN \\
\hline 3512 & 28 & BIANCHI & BIANCHINA & $\mathrm{D}$ & 4 & MICHIGAN & ITALY & ITALY & & & MATRAIA. LU & FRANKLIN \\
\hline 3513 & 28 & BIANCHI & MARIA & $\mathrm{D}$ & 2 & MICHIGAN & ITALY & ITALY & & & MATRAIA. LU & FRANKLIN \\
\hline 3514 & 28 & BIANCHI & ABRAMO & $\mathrm{COU}$ & 19 & ITALY & ITALY & ITALY & 1907 & SKIP FILLER/CM & CAPANNORI. LU & FRANKLIN \\
\hline 3515 & 28 & VANNUCCI & AURELIO & $\mathrm{B}$ & 27 & ITALY & ITALY & ITALY & 1906 & CAR DUMPER/CM & LUCCA & FRANKLIN \\
\hline 3516 & 28 & BINI & JOHN & B & 20 & ITALY & ITALY & ITALY & 1907 & TRAMMER/CM & & FRANKLIN \\
\hline 3517 & 28 & IMPERATE & PASQUALE & B & 33 & ITALY & ITALY & ITALY & 1909 & TRAMMER/CM & & FRANKLIN \\
\hline 3518 & 28 & ANDREOTTI & GIUSEPPE & B & 24 & ITALY & ITALY & ITALY & 1907 & LABORER/CM & LUCCA & FRANKLIN \\
\hline 3519 & 28 & ANDREOZZI & AMEDEO & $\mathrm{H}$ & 47 & ITALY & ITALY & ITALY & 1901 & MINER/CM & SANTA MARIA DEL GIUDICE. LU & FRANKLIN \\
\hline 3520 & 28 & ANDREOZZI & TERESA & W & 34 & ITALY & ITALY & ITALY & 1909 & KEEPER/BOARDING HOUSE & & FRANKLIN \\
\hline 3521 & 28 & COTTINI & GIOVANNI & B & 31 & ITALY & ITALY & ITALY & 1908 & TRAMMER/CM & COLLODI. LU & FRANKLIN \\
\hline 3522 & 28 & MARIANI & EUGENE & B & 21 & ITALY & ITALY & ITALY & 1909 & TRAMMER/CM & SANTA MARIA DEL GIUDICE, LU & FRANKLIN \\
\hline 3523 & 28 & ANDREOZZI & GABRIELE & $\mathrm{COU}$ & 19 & ITALY & ITALY & ITALY & 1895 & LABORER/CM & LUCCA & FRANKLIN \\
\hline 3524 & 28 & BONINI & GIOVACCHINO & B & 19 & ILLINOIS & ITALY & ITALY & & LABORER/CM & COLLODI. LU & FRANKLIN \\
\hline 3525 & 28 & GEMIGNANI & FRANK & B & 21 & ITALY & ITALY & ITALY & 1909 & TRAMMER/CM & LUCCA & FRANKLIN \\
\hline 3526 & 29 & LOMBARDINI & GERVASO & $\mathrm{H}$ & 29 & ITALY & ITALY & ITALY & 1900 & MINER/CM & & FRANKLIN \\
\hline 3527 & 29 & LOMBARDINI & FANNY & W & 32 & ITALY & ITALY & ITALY & 1903 & & & FRANKLIN \\
\hline
\end{tabular}




\begin{tabular}{|c|c|c|c|c|c|c|c|c|c|c|c|c|}
\hline 3528 & 29 & LOMBARDINI & OLINTO & S & 8 & MICHIGAN & ITALY & ITALY & & & & FRANKLIN \\
\hline 3529 & 29 & LOMBARDINI & JENNIE & $\mathrm{D}$ & 6 & MICHIGAN & ITALY & ITALY & & & & FRANKLIN \\
\hline 3530 & 29 & LOMBARDINI & NIA & $\mathrm{D}$ & 4 & MICHIGAN & ITALY & ITALY & & & & FRANKLIN \\
\hline 3531 & 29 & LOMBARDINI & VELIA & $\mathrm{D}$ & 3 & MICHIGAN & ITALY & ITALY & & & & FRANKLIN \\
\hline 3532 & 29 & LOMBARDINI & ALFRED & $s$ & $1.2 \mathrm{M}$ & MICHIGAN & ITALY & ITALY & & & & FRANKLIN \\
\hline 3533 & 29 & NOVI & FRANCESCO & B & 21 & ITALY & ITALY & ITALY & 1907 & TRAMMER/CM & PESCAGLIA. LU & FRANKLIN \\
\hline 3534 & 29 & SALANI & MENOTTI & B & 27 & ITALY & ITALY & ITALY & $04 / 09$ & TRAMMER/CM & COLLODI. LU & FRANKLIN \\
\hline 3535 & & ADAMI & BIAGIO & $\mathrm{H}$ & 30 & ITALY & ITALY & ITALY & 1904 & TRAMMER/CM & VILLA BASILICA. LU & FRANKLIN \\
\hline 3536 & & ADAMI & IDA & W & 31 & ITALY & ITALY & ITALY & 1905 & & & FRANKLIN \\
\hline 3537 & & ADAMI & BENEDETTO & s & 4 & MICHIGAN & ITALY & ITALY & & & VILLA BASILICA. LU & FRANKLIN \\
\hline 3538 & & ADAMI & GIULIANO & $\mathrm{s}$ & $1.2 \mathrm{M}$ & MICHIGAN & ITALY & ITALY & & & VILLA BASILICA. LU & FRANKLIN \\
\hline 3539 & & ROSETTI & PASQUALE & $\mathrm{H}$ & 35 & ITALY & ITALY & ITALY & 1905 & FIREMAN/BOILER HOUSE & MASNAGO. VARESE & FRANKLIN \\
\hline 3540 & & ROSETTI & ANNE & W & 33 & ITALY & ITALY & $\begin{array}{l}\text { ITALY } \\
\text { ITALY }\end{array}$ & 1905 & KEEPER/BOARDING HOUSE & MASNAGO. VARESE & FRANKLIN \\
\hline 3541 & & ROSETTI & JOHN & s & 8 & ITALY & ITALY & $\begin{array}{l}\text { ITALY } \\
\text { ITAL }\end{array}$ & 1905 & REEF LR/DUARUIIV TUUSE & MASNAGO. VARESE & FRANKLIN \\
\hline 3542 & & ROSETTI & JULIUS & $\mathrm{S}$ & 3 & MICHIGAN & ITALY & ITALY & & & MASNAGO. VARESE & FRANKLIN \\
\hline 3543 & & ROSETTI & SISTINA & $\mathrm{D}$ & 2 & MICHIGAN & ITALY & ITALY & & & MASNAGO, VARESE & FRANKLIN \\
\hline 3544 & & ROSETTI & EMIL & $\mathrm{s}$ & $6 \mathrm{M}$ & MICHIGAN & ITALY & ITALY & & & MASNAGO. VARESE & FRANKLIN \\
\hline 3545 & & $\mathrm{ClCCl}$ & DOMENICO & B & 21 & ITALY & ITALY & ITALY & 1909 & LABORER/CM & & FRANKLIN \\
\hline 3546 & & MATTOCCI & CHARLES & B & 45 & ITALY & ITALY & ITALY & 1910 & TRAMMER/CM & LUCCA & FRANKLIN \\
\hline 3547 & & $\mathrm{COCO}$ & PAUL & B & 19 & ITALY & ITALY & ITALY & 1910 & LABORER/CM & & FRANKLIN \\
\hline 3548 & & DI GREGORIO & TONY & B & 26 & ITALY & ITALY & ITALY & 1910 & LABORER/CM & & FRANKLIN \\
\hline 3549 & & TATTONI & VINCHO & B & 36 & ITALY & ITALY & ITALY & 1892 & STEMMER/CM & & FRANKLIN \\
\hline 3550 & 202 & FEDERIGHI & EMILIO & $\mathrm{H}$ & 42 & ITALY & ITALY & ITALY & 1900 & MINER/CM & LUCCA & FRANKLIN \\
\hline 3551 & 202 & FEDERIGHI & ROSA & W & 31 & ITALY & ITALY & ITALY & 1908 & KEEPER/BOARDING HOUSE & LUCCA & FRANKLIN \\
\hline 3552 & 202 & FEDERIGHI & ANTONIO & s & $8 \mathrm{M}$ & MICHIGAN & ITALY & ITALY & & & LUCCA & FRANKLIN \\
\hline 3553 & 202 & MAGA & DOMINICK & $\mathrm{H}$ & 49 & ITALY & ITALY & ITALY & 1888 & TRAMMER BOSS/CM & SAN MARTINO CANAVESE. TO & FRANKLIN \\
\hline 3554 & & MAGA & MARTA T. & W & 44 & ITALY & ITALY & ITALY & 1905 & KEEPER/BOARDING HOUSE & SAN MARTINO CANAVESE. TO & FRANKLIN \\
\hline 3555 & & MAGA & JOSEPH & $\mathrm{s}$ & 22 & ITALY & ITALY & ITALY & $01 / 07$ & MINER/CM & SAN MARTINO CANAVESE, TO & FRANKLIN \\
\hline 3556 & & MAGA & TERESA & $\mathrm{D}$ & 26 & ITALY & ITALY & ITALY & 1906 & & SAN MARTINO CANAVESE. TO & FRANKLIN \\
\hline 3557 & & MAGA & DOMENICA & $\mathrm{D}$ & 3 & MICHIGAN & ITALY & ITALY & & & SAN MARTINO CANAVESE. TO & FRANKLIN \\
\hline 3558 & & MASSOGLIA & MADDALENA & GD & 3 & MICHIGAN & ITALY & ITALY & & & SAN MARTINO CANAVESE. TO & FRANKLIN \\
\hline 3559 & & MASSOGLIA & TONY & SOIL & 34 & ITALY & ITALY & ITALY & 1906 & LABORER/CM & SAN MARTINO CANAVESE. TO & FRANKLIN \\
\hline 3560 & & LOCATELLI & ALESSANDRO & B & 33 & ITALY & ITALY & ITALY & 1899 & SHIFT BOSS/CM & SELINO. BERGAMO & FRANKLIN \\
\hline 3561 & & PASTORE & JAMES & B & 32 & ITALY & ITALY & ITALY & 1899 & MINER/CM & CUCEGLIO, TO & FRANKLIN \\
\hline 3562 & & NOZERO & LUIGI & B & 24 & ITALY & ITALY & ITALY & 1905 & MINER/CM & SAN MARTINO CANAVESE. TO & FRANKLIN \\
\hline 3563 & & FASANA & EUGENE & B & 22 & ITALY & ITALY & ITALY & 1903 & MINER/CM & TORINO & FRANKLIN \\
\hline 3564 & & ROLANDO & DOMENICO & B & 22 & ITALY & ITALY & ITALY & 1904 & TRAMMER/CM & PONT CANAVESE. TO & FRANKLIN \\
\hline 3565 & & BOGGIO & CARLO & $\begin{array}{l}\mathrm{D} \\
\mathrm{B}\end{array}$ & 33 & ITALY & ITALY & ITALY & 1903 & TRAMMER/CM & TORINO & FRANKLIN \\
\hline 3566 & & MAGRI & LUIGI & $\mathrm{H}$ & 34 & ITALY & ITALY & ITALY & 1905 & TRAMMER/CM & MONSACRATI. LU & FRANKLIN \\
\hline 3567 & & MAGRI & APOLLONIA & W & 32 & ITALY & ITALY & ITALY & 1906 & & MONSACRATI. LU & FRANKLIN \\
\hline 3568 & & MAGRI & GIORGIO & $\mathrm{F}$ & 7 & ITALY & ITALY & ITALY & 1906 & & MONSACRATI. LU & FRANKLIN \\
\hline 3569 & & MAGRI & ARTURO & $\mathrm{F}$ & 6 & ITALY & ITALY & ITALY & 1906 & & MONSACRATI. LU & FRANKLIN \\
\hline 3570 & & MAGRI & PAOLO & $\mathrm{F}$ & $1.8 \mathrm{M}$ & MICHIGAN & ITALY & ITALY & & & MONSACRATI. LU & FRANKLIN \\
\hline 3571 & & MAGRI & IRENE & D & $5 \mathrm{M}$ & MICHIGAN & ITALY & ITALY & & & MONSACRATI. LU & FRANKLIN \\
\hline 3572 & & PANTALEONI & ANGELO & B & 32 & ITALY & ITALY & ITALY & 1904 & TRAMMER/CM & SANTA MARIA DEL GIUDICE. LU & FRANKLIN \\
\hline 3573 & & FREDIANELLI & ALFREDO & $\mathrm{H}$ & 33 & ITALY & ITALY & ITALY & 1900 & SHEAVE TENDER/CM & CAPANNORI. LU & FRANKLIN \\
\hline 3574 & & FREDIANELLI & SESTILIA & w & 24 & ITALY & ITALY & ITALY & 1900 & KEEPER/BOARDING HOUSE & CAPANNORI. LU & FRANKLIN \\
\hline 3575 & & FREDIANELLI & GIUSEPPE & s & 3 & MICHIGAN & ITALY & ITALY & & & CAPANNORI. LU & FRANKLIN \\
\hline 3576 & & FREDIANELLI & LUIGI & $\mathrm{s}$ & 2 & MICHIGAN & ITALY & ITALY & & & CAPANNORI. LU & FRANKLIN \\
\hline 3577 & & LENCIONI & LORENZO & B & 41 & ITALY & ITALY & ITALY & 1900 & LABORER/CM & SEGROMIGNO. LU & FRANKLIN \\
\hline 3578 & & FREDIANELLI & LUIGI & B & 25 & ITALY & ITALY & ITALY & 1907 & LABORER/CM & LUCCA & FRANKLIN \\
\hline 3579 & & PANTALEONI & UMBERTO & B & 22 & ITALY & ITALY & ITALY & 1906 & TIMBERMAN/CM & SANTA MARIA DEL GIUDICE, LU & FRANKLIN \\
\hline 3580 & & MATTEUCCI & ERNEST & B & 24 & ITALY & ITALY & ITALY & 1909 & TRAMMER/CM & LUCCA & FRANKLIN \\
\hline 3581 & & GIANNECHINI & ALEMANNO & B & 19 & ITALY & ITALY & ITALY & 1909 & TRAMMER/CM & LUCCA & \\
\hline 3582 & & CAPELLI & ONORATO & B & 19 & ITALY & ITALY & ITALY & 1909 & TRAMMER/CM & LUCCA & FRANKLIN \\
\hline 3583 & & CAPELLI & GIUSEPPE & B & 18 & ITALY & ITALY & ITALY & 1909 & TRAMMER/CM & LUCCA & FRANKLIN \\
\hline 3584 & & MUGNANI & EMILIO & B & 22 & ITALY & ITALY & ITALY & 1904 & MINER/CM & LUCCA & FRANKLIN \\
\hline 3585 & & STEFANI & EMILIO & $\mathrm{H}$ & 27 & ITALY & ITALY & ITALY & 1904 & TIMBERMAN/CM & SEGROMIGNO, LU & FRANKLIN \\
\hline 3586 & & STEFANI & EMILIA & W & 23 & ITALY & ITALY & ITALY & 1909 & & & FRANKLIN \\
\hline 3587 & & STEFANI & NATALINO & s & $1 \mathrm{M}$ & MICHIGAN & ITALY & ITALY & & & SEGROMIGNO. LU & FRANKLIN \\
\hline 3588 & & STEFANI & GIOVANNI & $\mathrm{BR}$ & 20 & ITALY & ITALY & ITALY & 1907 & LABORER/CM & SEGROMIGNO. LU & FRANKLIN \\
\hline 3589 & & TABARACCI & ANTONIO & B & 26 & ITALY & ITALY & ITALY & 1902 & SHEAVE TENDER/CM & SANTA MARIA DEL GIUDICE. LU & FRANKLIN \\
\hline 3590 & & ANSANO & MENUCINO & B & 46 & ITALY & ITALY & ITALY & 1909 & LABORER/CM & & FRANKLIN \\
\hline
\end{tabular}




\begin{tabular}{|c|c|c|c|c|c|c|c|c|c|c|c|}
\hline 3591 & DE FILIPPI & DOMENICO & $\mathrm{H}$ & 36 & ITALY & ITALY & ITALY & 1906 & MINER/CM & SAN GIORGIO CANAVESE. TO & FRANKLIN \\
\hline 3592 & DE FILIPPI & MARTINA & w & 22 & ITALY & ITALY & ITALY & 1906 & KEEPER/BOARDING HOUSE & SAN GIORGIO CANAVESE. TO & FRANKLIN \\
\hline 3593 & DE FILIPPI & GIULIA & $\mathrm{D}$ & 3 & MICHIGAN & ITALY & ITALY & & & SAN GIORGIO CANAVESE. TO & FRANKLIN \\
\hline 3594 & VERGA & ALESSANDRO & B & 25 & ITALY & ITALY & ITALY & 1906 & MINER/CM & SAN GIORGIO CANAVESE. TO & FRANKLIN \\
\hline 3595 & GOSATTI & ANTONIO & B & 26 & ITALY & ITALY & ITALY & 1909 & TRAMMER/CM & BIANZANO. SONDRIO & FRANKLIN \\
\hline 3596 & GALETTO & JOSEPH & B & 35 & ITALY & ITALY & ITALY & 1890 & LABORER/CM & SAN GIORGIO CANAVESE. TO & FRANKLIN \\
\hline 3597 & GOSATTI & GIOVANNI & B & 22 & ITALY & ITALY & ITALY & 1910 & TRAMMER/CM & BIANZANO. SONDRIO & FRANKLIN \\
\hline 3598 & REGIS & LOUIS & $\begin{array}{l}\mathrm{D} \\
\mathrm{B}\end{array}$ & 42 & ITALY & ITALY & ITALY & 1904 & TRAMMER/CM & SAN GIUSTO CANAVESE. TO & FRANKLIN \\
\hline 3599 & POLINELLI & PIETRO & $\begin{array}{l}\mathrm{D} \\
\mathrm{B}\end{array}$ & 23 & ITALY & ITALY & ITALY & 1908 & TRAMMER/CM & BIANZANO. SONDRIO & FRANKLIN \\
\hline 3600 & COTTONI & GEORGE & $\mathrm{H}$ & 25 & ITALY & ITALY & ITALY & 1908 & MINER/CM & RIVAROLO CANAVESE. TO & FRANKLIN \\
\hline 3601 & COTTONI & FURMIA & w & 24 & ITALY & ITALY & ITALY & 1909 & & RIVAROLO CANAVESE. TO & FRANKLIN \\
\hline 3602 & COTTONI & JOHANNA & $\mathrm{D}$ & 2 & ITALY & ITALY & ITALY & $?$ & & RIVAROLO CANAVESE. TO & FRANKLIN \\
\hline 3603 & COTTONI & LUOISA & $\mathrm{D}$ & $4 \mathrm{M}$ & ITALY & ITALY & ITALY & & & RIVAROLO CANAVESE. TO & FRANKLIN \\
\hline 3604 & DAO & GIUSEPPE & $\mathrm{H}$ & 29 & ITALY & ITALY & ITALY & 1906 & SHEAVE TENDER/CM & CICONIO. TO & FRANKLIN \\
\hline 3605 & DAO & MARIA & W & 21 & ITALY & ITALY & ITALY & 1909 & KEEPER/BOARDING HOUSE & CICONIO. TO & FRANKLIN \\
\hline 3606 & DAO & GIOVANNI & B & 31 & ITALY & ITALY & ITALY & 1904 & LABORER/CM & CICONIO, TO & FRANKLIN \\
\hline 3607 & DAO & ANTONIO & B & 26 & ITALY & ITALY & ITALY & 1907 & TRAMMER/CM & CICONIO.TO & FRANKLIN \\
\hline 3608 & DE LAURENTI & PIETRO & B & 30 & ITALY & ITALY & ITALY & 1904 & MINER/CM & CICONIO. TO & FRANKLIN \\
\hline 3609 & DE LAURENTI & ANGELO & B & 27 & ITALY & ITALY & ITALY & 1905 & LABORER/CM & CICONIO. TO & FRANKLIN \\
\hline 3610 & DE LAURENTI & VITTORIO & B & 22 & ITALY & ITALY & ITALY & 1909 & LABORER/CM & CICONIO. TO & FRANKLIN \\
\hline 3611 & $\begin{array}{l}\text { FERINA } \\
\text { FER }\end{array}$ & GIUSEPPE & $\begin{array}{l}\mathrm{D} \\
\mathrm{B}\end{array}$ & 24 & ITALY & ITALY & ITALY & 1907 & TRAMMER/CM & CICONIO. TO & FRANKLIN \\
\hline 3612 & QUENCI & JOHN & $\mathrm{H}$ & 40 & ITALY & ITALY & ITALY & 1890 & MINER/CM & & FRANKLIN \\
\hline 3613 & QUENCI & MARY & w & 30 & ITALY & ITALY & ITALY & 1897 & & & FRANKLIN \\
\hline 3614 & QUENCI & PETER & $S$ & 12 & MICHIGAN & ITALY & ITALY & & & & FRANKLIN \\
\hline 3615 & QUENCI & MARIO & $\mathrm{s}$ & 11 & MICHIGAN & ITALY & ITALY & & & & FRANKLIN \\
\hline 3616 & QUENCI & JOHN JR. & s & 9 & MICHIGAN & ITALY & ITALY & & & & FRANKLIN \\
\hline 3617 & QUENCI & JOSEPHINE & $\mathrm{D}$ & 7 & MICHIGAN & ITALY & ITALY & & & & FRANKLIN \\
\hline 3618 & QUENCI & JAMES & S & 6 & MICHIGAN & ITALY & ITALY & & & & FRANKLIN \\
\hline 3619 & QUENCI & CAROLINE & D & 4 & MICHIGAN & ITALY & ITALY & & & & FRANKLIN \\
\hline 3620 & QUENCI & IDA & $\mathrm{D}$ & 2 & MICHIGAN & ITALY & ITALY & & & & FRANKLIN \\
\hline 3621 & QUENCI & JOSEPH & S & $6 \mathrm{M}$ & MICHIGAN & ITALY & ITALY & & & & FRANKLIN \\
\hline 3622 & LENCIONI & JOSEPH & $\mathrm{H}$ & 40 & ITALY & ITALY & ITALY & 1898 & LABORER/ROCK HOUSE & LUCCA & FRANKLIN \\
\hline 3623 & LENCIONI & ESTHER & W & 30 & ITALY & ITALY & ITALY & $\begin{array}{l}1090 \\
1898\end{array}$ & LADUNAERIRU R TUOSE & LUCCA & FRANKLIN \\
\hline 3624 & LENCIONI & LIVIA & $\mathrm{D}$ & 10 & MICHIGAN & ITALY & ITALY & & & LUCCA & FRANKLIN \\
\hline 3625 & LENCIONI & IDA & $\mathrm{D}$ & 8 & MICHIGAN & ITALY & ITALY & & & LUCCA & FRANKLIN \\
\hline 3626 & LENCIONI & DOMINICK & $\mathrm{s}$ & 6 & MICHIGAN & ITALY & ITALY & & & LUCCA & FRANKLIN \\
\hline 3627 & LENCIONI & ALDINO & $\mathrm{s}$ & 4 & MICHIGAN & ITALY & ITALY & & & LUCCA & FRANKLIN \\
\hline 3628 & LENCIONI & LENA & $\mathrm{D}$ & $1.9 \mathrm{M}$ & MICHIGAN & ITALY & ITALY & & & LUCCA & FRANKLIN \\
\hline 3629 & PAROLA & PIETRO & $\mathrm{H}$ & 25 & ITALY & ITALY & ITALY & 1907 & MINER/CM & AGLIE'. TO & FRANKLIN \\
\hline 3630 & PAROLA & MARIA & W & 26 & ITALY & ITALY & ITALY & 1906 & & AGLIE', TO & FRANKLIN \\
\hline 3631 & PAROLA & MARIA & D & $1.5 \mathrm{M}$ & MICHIGAN & ITALY & ITALY & & & AGLIE'. TO & FRANKLIN \\
\hline 3632 & FASANA & ADOLFO & B & 26 & ITALY & ITALY & ITALY & 1907 & MINER/CM & TORINO & FRANKLIN \\
\hline 3633 & PAROLA & GIUSEPPE & B & 19 & ITALY & ITALY & ITALY & 1907 & & AGLIE'. TO & FRANKLIN \\
\hline 3634 & CONTO & DOMINICK & $\mathrm{H}$ & 26 & ITALY & ITALY & ITALY & 1899 & & CUCEGLIO. TO & FRANKLIN \\
\hline 3635 & CONTO & KATHERINE & W & 21 & ITALY & ITALY & ITALY & 1909 & & Gentotio. of to & FRANKLIN \\
\hline 3636 & VERBENA & MADDALENA & SER & 23 & ITALY & ITALY & ITALY & 1908 & SERVANT/PF & SAN MARTINO CANAVESE. TO & FRANKLIN \\
\hline 3637 & MESNAGHETTI & PETER & $\mathrm{H}$ & 31 & ITALY & ITALY & ITALY & 1902 & MINER/CM & SESTO CALENDE. VARESE & FRANKLIN \\
\hline 3638 & MESNAGHETTI & VITTORINA & w & 24 & ITALY & ITALY & ITALY & 1906 & & & FRANKLIN \\
\hline 3639 & MESNAGHETTI & CARLO & s & 3 & MICHIGAN & ITALY & ITALY & & & SESTO CALENDE. VARESE & FRANKLIN \\
\hline 3640 & OBERTONE & DOMINICK & $\mathrm{H}$ & 30 & ITALY & ITALY & ITALY & 1903 & MINER/CM & SAN GIORGIO CANAVESE. TO & FRANKLIN \\
\hline 3641 & OBERTONE & MARY & W & 30 & ITALY & ITALY & ITALY & 1902 & & SAN GIORGIO CANAVESE. TO & FRANKLIN \\
\hline 3642 & OBERTONE & FRANK & $\mathrm{s}$ & $2 ?$ & ITALY & ITALY & ITALY & 1908 & & SAN GIORGIO CANAVESE, TO & FRANKLIN \\
\hline 3643 & OBERTONE & MARY & $\mathrm{D}$ & 4 & MICHIGAN & ITALY & ITALY & & & SAN GIORGIO CANAVESE. TO & FRANKLIN \\
\hline 3644 & VERLUCA & MARY & $\mathrm{H}$ & 45 & ITALY & ITALY & ITALY & 1890 & & RIBARDONE. TO & FRANKLIN \\
\hline 3645 & VERLUCA & VICTOR & $\mathrm{s}$ & 18 & MICHIGAN & ITALY & ITALY & & PIPER/CM & RIBARDONE. TO & FRANKLIN \\
\hline 3646 & VERLUCA & ANGELO & $\mathrm{s}$ & 16 & MICHIGAN & ITALY & ITALY & & MOTORMAN/CM & RIBARDONE. TO & FRANKLIN \\
\hline 3647 & VERLUCA & JOSEPH & $\mathrm{s}$ & 13 & MICHIGAN & ITALY & ITALY & & & RIBARDONE. TO & FRANKLIN \\
\hline 3648 & VERLUCA & EMMA & $\mathrm{D}$ & 10 & MICHIGAN & ITALY & ITALY & & & RIBARDONE, TO & FRANKLIN \\
\hline 3649 & FASANA & JOHN & $\mathrm{H}$ & 36 & ITALY & ITALY & ITALY & 1898 & MINER/CM & TORINO & FRANKLIN \\
\hline 3650 & FASANA & TERESA & W & 35 & ITALY & ITALY & ITALY & 1900 & & & FRANKLIN \\
\hline 3651 & FASANA & MARY & $\mathrm{D}$ & 5 & & ITALY & ITALY & & & TORINO & FRANKLIN \\
\hline 3652 & FASANA & CLARA & B & 4 & MICHIGAN & ITALY & ITALY & & & TORINO & FRANKLIN \\
\hline 3653 & FASANA & CATHERINA & B & 2 & MICHIGAN & ITALY & ITALY & & & TORINO & FRANKLIN \\
\hline
\end{tabular}




\begin{tabular}{|c|c|c|c|c|c|c|c|c|c|c|c|}
\hline 3654 & BALAGNA & GIUSEPPE & B & 28 & ITALY & ITALY & ITALY & 1905 & MINER/CM & PONT CANAVESE. TO & FRANKLIN \\
\hline 3655 & ALLARIA & JOSEPH & $\mathrm{H}$ & 31 & ITALY & ITALY & ITALY & 1903 & MINER/CM & TORINO & FRANKLIN \\
\hline 3656 & ALLARIA & MARIA & W & 23 & ITALY & ITALY & ITALY & 1908 & & & FRANKLIN \\
\hline 3657 & ALLARIA & TERESA & $\mathrm{D}$ & $3 \mathrm{M}$ & MICHIGAN & ITALY & ITALY & & & TORINO & FRANKLIN \\
\hline 3658 & POGGIONE & JOHN & $\mathrm{H}$ & 42 & ITALY & ITALY & ITALY & 1888 & SALOONKEEPER & SAN GIORGIO CANAVESE. TO & FRANKLIN \\
\hline 3659 & POGGIONE & MARY & w & 30 & ITALY & ITALY & ITALY & 1885 & & & FRANKLIN \\
\hline 3660 & POGGIONE & JAMES & s & 12 & MICHIGAN & ITALY & ITALY & & & SAN GIORGIO CANAVESE. TO & FRANKLIN \\
\hline & POGGIONE & PETER & $\mathrm{s}$ & 11 & MICHIGAN & ITALY & ITALY & & & SAN GIORGIO CANAVESE. TO & FRANKLIN \\
\hline 3662 & POGGIONE & JOSEPH & s & 9 & MICHIGAN & ITALY & ITALY & & & SAN GIORGIO CANAVESE. TO & FRANKLIN \\
\hline 3663 & POGGIONE & CLARA & $\mathrm{D}$ & 3 & MICHIGAN & ITALY & ITALY & & & SAN GIORGIO CANAVESE. TO & FRANKLIN \\
\hline 3664 & POGGIONE & JOHN JR. & $\mathrm{s}$ & 2 & MICHIGAN & ITALY & ITALY & & & SAN GIORGIO CANAVESE. TO & FRANKLIN \\
\hline 3665 & POGGIONE & FELIX & $\mathrm{S}$ & $5 \mathrm{M}$ & MICHIGAN & ITALY & ITALY & & & SAN GIORGIO CANAVESE. TO & FRANKLIN \\
\hline 3666 & POGGIONE & JOSEPH & BR & 31 & ITALY & ITALY & ITALY & 1897 & TEAMSTER/BUTCHER SHOP & SAN GIORGIO CANAVESE. TO & FRANKLIN \\
\hline 3667 & POGGIONE & JOSEPHINE & SIIL & 22 & MICHIGAN & ITALY & ITALY & & & & FRANKLIN \\
\hline 3668 & TRIGOLO & DOMINICK & $\mathrm{B}$ & 22 & ITALY & ITALY & ITALY & 1905 & LABORER/CM & & FRANKLIN \\
\hline 3669 & TRIGOLO & CAROLINE & B & 19 & ITALY & ITALY & ITALY & 1910 & SERVANT/PF & & FRANKLIN \\
\hline 3670 & CASTELLO & CARLO & B & 20 & ITALY & ITALY & ITALY & 1906 & LABORER/CM & TORINO & FRANKLIN \\
\hline 3671 & MEINARDI & SAVINO & $\mathrm{H}$ & 46 & ITALY & ITALY & ITALY & 1891 & OWN INCOME & TORINO & FRANKLIN \\
\hline 3672 & MEINARDI & CATHERINA & w & 45 & ITALY & ITALY & ITALY & 1891 & & TORINO & FRANKLIN \\
\hline 3673 & BARNA & MICHAEL & $\mathrm{H}$ & 49 & ITALY & ITALY & ITALY & 1887 & LABORER/FOUNDRY & 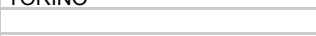 & FRANKLIN \\
\hline 3674 & BARNA & FRANK & s & 12 & MICHIGAN & $\begin{array}{l}\text { ITALY } \\
\text { ITA }\end{array}$ & ITALY & 1000 & LADUKERITU UVURT & & FRANKLIN \\
\hline 3675 & BARNA & JOHN & $\mathrm{s}$ & 7 & MICHIGAN & ITALY & ITALY & & & & FRANKLIN \\
\hline 3676 & CHIABOTTI & MARY & SERV & 25 & ITALY & ITALY & ITALY & 1902 & SERVANT/PF & PONT CANAVESE. TO & FRANKLIN \\
\hline 3677 & ROLANDO & LORENZO & $\mathrm{H}$ & 27 & ITALY & ITALY & ITALY & 1900 & TRACK LAYER/CM & PONT CANAVESE. TO & FRANKLIN \\
\hline 3678 & ROLANDO & MARGHERITA & W & 18 & ITALY & ITALY & ITALY & 1908 & & & FRANKLIN \\
\hline 3679 & ROLANDO & DOMENICO & s & $8 \mathrm{M}$ & MICHIGAN & ITALY & ITALY & & & PONT CANAVESE. TO & FRANKLIN \\
\hline 3680 & RIVA & BATTISTA & $\mathrm{H}$ & 27 & ITALY & ITALY & ITALY & 1902 & MINER/CM & SPARONE. TO & FRANKLIN \\
\hline 3681 & RIVA & BENILDA & w & 20 & ITALY & ITALY & ITALY & 1890 & & & FRANKLIN \\
\hline 3682 & RIVA & GIUSEPPE & $\mathrm{s}$ & 1.11 & MICHIGAN & ITALY & ITALY & & & SPARONE. TO & FRANKLIN \\
\hline 3683 & PIEROTTI & FRANK & $\mathrm{H}$ & 34 & ITALY & ITALY & ITALY & 1900 & LABORER/ROCK HOUSE & CASTELVECCHIO. LU & FRANKLIN \\
\hline 3684 & PIEROTTI & GIANNINA & W & 33 & ITALY & ITALY & ITALY & 1907 & & CASTELVECCHIO. LU & FRANKLIN \\
\hline 3685 & PIEROTTI & PRIMETTA & $\mathrm{D}$ & 14 & ITALY & ITALY & ITALY & 1907 & & CASTELVECCHIO. LU & FRANKLIN \\
\hline 3686 & PIEROTTI & ISOLINA & $\mathrm{D}$ & 1.10 & MICHIGAN & ITALY & ITALY & 1500 & & CASTELVECCHIO. LU & FRANKLIN \\
\hline 3687 & PIEROTTI & MARSILIO & $\mathrm{s}$ & $8 \mathrm{M}$ & MICHIGAN & ITALY & ITALY & & & CASTELVECCHIO, LU & FRANKLIN \\
\hline 3688 & DI VECCHIO & MARTINO & B & 41 & ITALY & ITALY & ITALY & 1905 & FIREMAN/BOILER HOUSE & CAPANNORI. LU & FRANKLIN \\
\hline 3689 & BENVENUTI & ALFONSINO & B & 20 & ITALY & ITALY & ITALY & 1908 & LABORER/CM & & FRANKLIN \\
\hline 3690 & RASTELLO & CHARLES & $\mathrm{H}$ & 48 & ITALY & ITALY & ITALY & 1886 & SALOON/LIQUORS & PONT CANAVESE. TO & FRANKLIN \\
\hline 3691 & RASTELLO & JOSEPHINE & W & 46 & ITALY & ITALY & ITALY & 1887 & & & FRANKLIN \\
\hline 3692 & RASTELLO & PETER & $\mathrm{s}$ & 22 & MICHIGAN & ITALY & ITALY & & NOT CLEAR & PONT CANAVESE. TO & FRANKLIN \\
\hline 3693 & RASTELLOO & ROSE & $\mathrm{D}$ & 20 & MICHIGAN & ITALY & ITALY & & & PONT CANAVESE. TO & FRANKLIN \\
\hline 3694 & RASTELLO & JOSEPH & $\mathrm{s}$ & 18 & MICHIGAN & ITALY & ITALY & & PIPER/CM & PONT CANAVESE. TO & FRANKLIN \\
\hline 3695 & RASTELLO & JOHN & $\mathrm{s}$ & 17 & MICHIGAN & ITALY & ITALY & & LABORER/CM & PONT CANAVESE. TO & FRANKLIN \\
\hline 3696 & RASTELLO & LUIGIA & $\mathrm{D}$ & 15 & CAL & ITALY & ITALY & & & PONT CANAVESE. TO & FRANKLIN \\
\hline 3697 & RASTELLO & CHARLES JR. & $\mathrm{s}$ & 12 & MICHIGAN & ITALY & ITALY & & & PONT CANAVESE. TO & FRANKLIN \\
\hline 3698 & RASTELLO & FLORENCE & $\mathrm{D}$ & 10 & MICHIGAN & ITALY & ITALY & & & PONT CANAVESE. TO & FRANKLIN \\
\hline 3699 & MARCO & JOHN & B & 50 & ITALY & ITALY & ITALY & 1885 & CARPENTER/HOUSE & TORINO & FRANKLIN \\
\hline 3700 & BERTOLERI & PETRONILLA & $\mathrm{H}$ & 29 & ITALY & ITALY & ITALY & 1901 & & & FRANKLIN \\
\hline 3701 & BERTOLERI & LOUIS & s & 6 & UTAH & ITALY & ITALY & & & & FRANKLIN \\
\hline 3702 & BERTOLERI & PETER & $\mathrm{s}$ & 5 & MICHIGAN & ITALY & ITALY & & & & FRANKLIN \\
\hline 3703 & BALAGNA & GIACOMO & B & 34 & ITALY & ITALY & ITALY & 1899 & MINER/CM & PONT CANAVESE. TO & FRANKLIN \\
\hline 3704 & TAMBURINI & PIETRO & B & 36 & ITALY & ITALY & ITALY & 1905 & MINER/CM & VERGIATE. MILANO & FRANKLIN \\
\hline 3705 & PORENELLI & BARTOLOMEO & B & 24 & ITALY & ITALY & ITALY & 1909 & TRAMMER/CM & BIANZANO, SONDRIO & FRANKLIN \\
\hline 3706 & $\mathrm{PUCCl}$ & DOMINICK & $\mathrm{H}$ & 35 & ITALY & ITALY & ITALY & 1898 & MINER/CM & LUCCA & FRANKLIN \\
\hline 3707 & PUCCI & CECILIA & W & 26 & ITALY & ITALY & ITALY & 1905 & & & FRANKLIN \\
\hline 3708 & PUCCl & ARMANDO & s & 3 & MICHIGAN & ITALY & ITALY & & & LUCCA & FRANKLIN \\
\hline 3709 & PUCCI & DOMINICK & $\mathrm{s}$ & 1.10 & MICHIGAN & ITALY & ITALY & & & LUCCA & FRANKLIN \\
\hline 3710 & LANDINI & LUIGI & B & 18 & ITALY & ITALY & ITALY & 1909 & LABORER/CM & RUOTA. LU & FRANKLIN \\
\hline 3711 & BARSOCCHI & ANTHONY & B & 17 & ITALY & ITALY & ITALY & 1909 & & LUCCA & FRANKLIN \\
\hline 3712 & PANICHI & ARMIDO & B & 26 & ITALY & ITALY & ITALY & 1904 & & PISA & FRANKLIN \\
\hline 3713 & MANGNANI & JOSEPH & B & 36 & ITALY & ITALY & ITALY & 1903 & TRAMMER/CM & & FRANKLIN \\
\hline 3714 & GUIDI & PETER & $\mathrm{H}$ & 26 & ITALY & ITALY & ITALY & 1906 & & LUCCA & FRANKLIN \\
\hline 3715 & GUIDI & ROSE & w & 16 & MICHIGAN & ITALY & ITALY & & & & FRANKLIN \\
\hline 3716 & CAVALZANI & ERNEST & $\mathrm{H}$ & 30 & ITALY & ITALY & ITALY & 1903 & MINER/CM & SAN GENNARO. LU & FRANKLIN \\
\hline
\end{tabular}




\begin{tabular}{|c|c|c|c|c|c|c|c|c|c|c|c|c|c|}
\hline 3717 & 9 & CAVALZANI & MARIA & W & 28 & ITALY & ITALY & ITALY & 1905 & & & & FRANKLIN \\
\hline 3718 & 9 & CAVALZANI & CESARA & D & $10 \mathrm{M}$ & MICHIGAN & ITALY & ITALY & & & SAN GENNARO. LU & & FRANKLIN \\
\hline 3719 & 9 & CAVALZANI & BERNARDO & BR & 33 & ITALY & ITALY & ITALY & 1898 & MINER/CM & SAN GENNARO. LU & & FRANKLIN \\
\hline 3720 & 11 & PERA & SAMUEL & $\mathrm{H}$ & 30 & ITALY & ITALY & ITALY & 1902 & MINER/CM & LUCCA & & FRANKLIN \\
\hline 3721 & 11 & PERA & GERTRUDE & w & 24 & ITALY & ITALY & ITALY & 1907 & & & & FRANKLIN \\
\hline 3722 & 11 & PERA & FRANK & s & $1.7 \mathrm{M}$ & MICHIGAN & ITALY & ITALY & & & LUCCA & & FRANKLIN \\
\hline 3723 & 11 & PERA & NELLO & $\mathrm{s}$ & $1 \mathrm{M}$ & MICHIGAN & ITALY & ITALY & & & LUCCA & & FRANKLIN \\
\hline 3724 & 11 & DEL MORO & FRANK & B & 25 & ITALY & ITALY & ITALY & 1908 & SKIP FILLER/CM & PISA & & FRANKLIN \\
\hline 3725 & 11 & PELLEGRINI & MICHAEL & $\begin{array}{l}\mathrm{D} \\
\mathrm{B}\end{array}$ & 24 & ITALY & ITALY & ITALY & 1909 & TRAMMER/CM & PISA & & FRANKLIN \\
\hline 3726 & 11 & PRIAMI & AMERIGO & B & 21 & ITALY & ITALY & ITALY & 1904 & LABORER/CM & RUOTA. LU & & FRANKLIN \\
\hline 3727 & & SCOLATI & PETER & $\mathrm{H}$ & 41 & ITALY & ITALY & ITALY & 1890 & MINER/CM & SESTO CALENDE. VARESE & & FRANKLIN \\
\hline 3728 & & SCOLATI & MARY & w & 32 & ITALY & ITALY & ITALY & 1891 & KEEPER/BOARDING HOUSE & & & FRANKLIN \\
\hline 3729 & & SCOLATI & LOUIS & $s$ & 16 & MICHIGAN & ITALY & ITALY & & LABORER/CM & SESTO CALENDE. VARESE & & FRANKLIN \\
\hline 3730 & & SCOLATI & CHARLES & $\mathrm{s}$ & 10 & MICHIGAN & ITALY & ITALY & & & SESTO CALENDE. VARESE & & FRANKLIN \\
\hline 3731 & & DE FILIPPI & PETER & B & 56 & ITALY & ITALY & ITALY & 1889 & LABORER/CM & TORINO & & FRANKLIN \\
\hline 3732 & & LOCATELLI & ANTONIO & B & 36 & ITALY & ITALY & ITALY & 1901 & MINER/CM & SELINO, BERGAMO & & FRANKLIN \\
\hline 3733 & & MORONI & SAMUEL & B & 38 & ITALY & ITALY & ITALY & 1904 & LABORER/CM & & & FRANKLIN \\
\hline 3734 & & CERBERI & CESARE & B & 32 & ITALY & ITALY & ITALY & 1906 & LABORER/CM & VERGIATE. MILANO & & FRANKLIN \\
\hline 3735 & & SABATTINI & MARTIN & B & 39 & ITALY & ITALY & ITALY & 1890 & LABORER/CM & & & FRANKLIN \\
\hline 3736 & & BONINI & JOHN & B & 22 & ITALY & ITALY & ITALY & 1907 & LABORER/CM & & & FRANKLIN \\
\hline 3737 & & MONTONATI & ENRICO & $\mathrm{O}$ & 41 & ITALY & ITALY & $\begin{array}{l}\text { ITALY } \\
\end{array}$ & 1892 & MINER/CM & SESTO CALENDE. VARESE & & FRANKLIN \\
\hline 3738 & & MONTONATI & TERESA & W & 33 & ITALY & ITALY & ITALY & 1900 & IVIINERT/UIII & OESTU UALEIVUE. VAREOE & & FRANKLIN \\
\hline 3739 & & MONTONATI & OLIMPIA & $\mathrm{D}$ & 8 & MICHIGAN & ITALY & ITALY & & & SESTO CALENDE. VARESE & & FRANKLIN \\
\hline 3740 & & MONTONATI & ALFONSO & $\mathrm{s}$ & 7 & MICHIGAN & ITALY & ITALY & & & SESTO CALENDE. VARESE & & FRANKLIN \\
\hline 3741 & & MONTONATI & ALDO & $\mathrm{s}$ & 6 & MICHIGAN & ITALY & ITALY & & & SESTO CALENDE. VARESE & & FRANKLIN \\
\hline 3742 & & MONTONATI & WILLIAM & $\mathrm{s}$ & $2 \mathrm{M}$ & MICHIGAN & ITALY & ITALY & & & SESTO CALENDE. VARESE & & FRANKLIN \\
\hline 3743 & & NOTARIO & CHARLES & $\mathrm{H}$ & 36 & ITALY & ITALY & ITALY & 1898 & TRAMMER/CM & TORINO & & FRANKLIN \\
\hline 3744 & & NOTARIO & MARY & w & 31 & ITALY & ITALY & ITALY & 1905 & & & & FRANKLIN \\
\hline 3745 & & NOTARIO & ALBERT & s & 4 & MICHIGAN & ITALY & ITALY & & & TORINO & & FRANKLIN \\
\hline 3746 & & NOTARIO & NELLO & $\mathrm{s}$ & 3 & MICHIGAN & ITALY & ITALY & & & TORINO & & FRANKLIN \\
\hline 3747 & & FACHINI & LOUIS & B & 37 & ITALY & ITALY & ITALY & 1904 & TRAMMER/CM & & & FRANKLIN \\
\hline 3748 & & DOLINO & EUGENE & B & 24 & ITALY & ITALY & ITALY & 1909 & TRAMMER/CM & & & FRANKLIN \\
\hline 3749 & & VITTON & BATTISTA & $\mathrm{O}$ & 37 & ITALY & ITALY & ITALY & 1898 & FARMER/DAIRY FARM & LOCANA CANAVESE. TO & & FRANKLIN \\
\hline 3750 & & VITTON & MARY & W & 30 & ITALY & ITALY & ITALY & 1900 & ГАARVIERTLAIRT ГARTV & LUUAIVA CAINAVEOE. TS & & FRANKLIN \\
\hline 3751 & & VITTON & JOHN & s & 8 & MICHIGAN & ITALY & ITALY & & & LOCANA CANAVESE. TO & & FRANKLIN \\
\hline 3752 & & VITTON & FRANCES & $\mathrm{D}$ & 7 & MICHIGAN & ITALY & ITALY & & & LOCANA CANAVESE. TO & & FRANKLIN \\
\hline 3753 & & VITTON & BATTISTA & $\mathrm{s}$ & 5 & MICHIGAN & ITALY & ITALY & & & LOCANA CANAVESE. TO & & FRANKLIN \\
\hline 3754 & & VITTON & ANNE & $\mathrm{D}$ & 4 & MICHIGAN & ITALY & ITALY & & & LOCANA CANAVESE. TO & & FRANKLIN \\
\hline 3755 & & VITTON & JAMES & $\mathrm{s}$ & $5 \mathrm{M}$ & MICHIGAN & ITALY & ITALY & & & LOCANA CANAVESE. TO & & FRANKLIN \\
\hline 3756 & & PERELLI & JAMES & B & 43 & ITALY & ITALY & ITALY & 1904 & LABORER/DAIRY FARM & LOCANA CANAVESE. TO & & FRANKLIN \\
\hline 3757 & & MOGLI & JOHN & B & 20 & OKLAHOMA & ITALY & ITALY & & LABORER/DAIRY FARM & LOCANA CANAVESE. TO & & FRANKLIN \\
\hline 3758 & & MANZETTI & FIORENTINO & B & 37 & $\begin{array}{l}\text { ITALY } \\
\text { ITA }\end{array}$ & ITALY & ITALY & 1892 & LABORER/DAIRY FARM & & & FRANKLIN \\
\hline 3759 & & SOLIVE & GIOVANNI & B & 44 & ITALY & ITALY & ITALY & 1899 & LABORER/DAIRY FARM & LOCANA CANAVESE. TO & & FRANKLIN \\
\hline 3760 & & ESTRAMONTE & CHARLES & B & 30 & ITALY & ITALY & ITALY & 1909 & LABORER/DAIRY FARM & LOCANA CANAVESE. TO & & FRANKLIN \\
\hline 3761 & & ESTRAMONTE & PHILYS & B & 34 & ITALY & ITALY & ITALY & 1906 & LABORER/DAIRY FARM & LOCANA CANAVESE. TO & & FRANKLIN \\
\hline 3762 & $?$ & MARCHETTI & ETTORE & $\mathrm{B}$ & 24 & ITALY & ITALY & ITALY & 1903 & MINER/CM & LUCCA & BACK ST. & FRANKLIN \\
\hline 3763 & $?$ & BARSOCCHI & EMILIO & B & 25 & ITALY & ITALY & ITALY & 1905 & SKIP FILLER/CM & LUCCA & BACK ST. & FRANKLIN \\
\hline 3764 & $?$ & FRANCIONI & GIOVANNI & B & 33 & ITALY & ITALY & ITALY & 1905 & LABORER/CM & LUCCA & BACK ST. & FRANKLIN \\
\hline 3765 & $?$ & LANDINI & ALBINO & B & 19 & ITALY & ITALY & ITALY & 1909 & LABORER/CM & LUCCA & BACK ST. & FRANKLIN \\
\hline 3766 & $?$ & BARSOCCHI & LOUIS & B & 29 & ITALY & ITALY & ITALY & 1905 & LABORER/CM & LUCCA & BACK ST. & FRANKLIN \\
\hline 3767 & $?$ & NATALI & PASQUALE & B & 29 & ITALY & ITALY & ITALY & $08 / 09$ & LABORER/CM & CAPANNORI. LU & $\begin{array}{l}\text { BACK ST. } \\
\text { B. }\end{array}$ & FRANKLIN \\
\hline 3768 & $?$ & GIUSTI & EGISTO & B & 25 & ITALY & ITALY & ITALY & 1908 & TRAMMER/CM & LUCCA & BACK ST. & FRANKLIN \\
\hline 3769 & $?$ & MICHELETTI & ANGELO & B & 38 & ITALY & ITALY & ITALY & 1905 & TRAMMER/CM & SANTA MARIA DEL GIUDICE. LU & BACK ST. & FRANKLIN \\
\hline 3770 & $?$ & MARCONI & GUIDO & B & 29 & ITALY & ITALY & ITALY & 1908 & TRAMMER/CM & PISA & BACK ST. & FRANKLIN \\
\hline 3771 & $?$ & ANDREOZZI & TRANQUILLO & B & 31 & ITALY & ITALY & ITALY & 1905 & SKIP FILLER/CM & LUCCA & BACK ST. & FRANKLIN \\
\hline 3772 & $?$ & TABARACCI & LUDOVICO & B & 31 & ITALY & ITALY & ITALY & 1904 & SKIP FILLER/CM & SANTA MARIA DEL GIUDICE. LU & BACK ST. & FRANKLIN \\
\hline 3773 & $?$ & GRASSESCHI & DANTE & B & 30 & ITALY & ITALY & ITALY & 1910 & TRAMMER/CM & SANTA MARIA DEL GIUDICE. LU & BACK ST. & FRANKLIN \\
\hline 3774 & $?$ & ORLANDO & ANTHONY & B & 36 & ITALY & ITALY & ITALY & 1889 & TEAMSTER/GROCERY STORE & & BACK ST. & FRANKLIN \\
\hline 3775 & $?$ & MELANI & NELLO & $\mathrm{B}$ & 36 & ITALY & ITALY & ITALY & 1904 & MINER/CM & SEGROMIGNO. LU & $\begin{array}{l}\text { BACK ST. } \\
\text { BA }\end{array}$ & FRANKLIN \\
\hline 3776 & $?$ & DELLA RUFFA & ITALO & $\begin{array}{l}\mathrm{D} \\
\mathrm{B}\end{array}$ & 22 & ITALY & ITALY & ITALY & 1908 & LABORER/CM & BORGO A MOZZANO. LU & $\begin{array}{l}\text { BACK ST. } \\
\text { BACK }\end{array}$ & $\begin{array}{l}\text { FRANKLIN } \\
\text { FRANLIN }\end{array}$ \\
\hline 3777 & $?$ & LORENZETTI & RAFFAELE & B & 24 & ITALY & ITALY & ITALY & 1906 & LABORER/CM & LUCCA & BACK ST. & FRANKLIN \\
\hline 3778 & $?$ & GIUSFREDI & ORLANDO & B & 30 & ITALY & ITALY & ITALY & 1901 & FIREMAN/BOILER HOUSE & BORGO A MOZZANO. LU & BACK ST. & FRANKLIN \\
\hline 3779 & $?$ & PUCCETTI & GIACOMO & B & 26 & ITALY & ITALY & ITALY & 1906 & MINER/CM & $\begin{array}{l}\text { LUCCA } \\
\text { LUTLETIN LU }\end{array}$ & BACK ST. & FRANKLIN \\
\hline
\end{tabular}




\begin{tabular}{|c|c|c|c|c|c|c|c|c|c|c|c|c|c|}
\hline 3780 & & $\mathrm{RICCl}$ & LOUIS & B & 32 & ITALY & ITALY & ITALY & 1901 & MINER/CM & LUCCA & BACK ST. & FRANKLIN \\
\hline 3781 & $?$ & POLINELLI & JAMES & B & 30 & ITALY & ITALY & ITALY & 1906 & LABORER/CM & BIANZANO. SONDRIO & BACK ST. & FRANKLIN \\
\hline 3782 & & RUFFINO & ROSA & $\mathrm{H}$ & 41 & ITALY & ITALY & ITALY & 1892 & & & & FRANKLIN \\
\hline 3783 & & PROCISSI & DOMINICK & $\mathrm{s}$ & 16 & MICHIGAN & ITALY & ITALY & & LABORER/ODD JOBS & LUCCA & & FRANKLIN \\
\hline 3784 & & PROCISSI & CONNIE & $\mathrm{D}$ & 11 & MICHIGAN & ITALY & ITALY & & & LUCCA & & FRANKLIN \\
\hline 3785 & & PROCISSI & PETER & $\mathrm{H}$ & 57 & ITALY & ITALY & ITALY & 1880 & LABORER/SMELTING OFFICE & LUCCA & & FRANKLIN \\
\hline 3786 & & PROCISSI & ORSOLA & w & 53 & ITALY & ITALY & ITALY & 1883 & & & & FRANKLIN \\
\hline 3787 & & PROCISSI & LOUISE & B & 20 & MICHIGAN & ITALY & ITALY & & & LUCCA & & FRANKLIN \\
\hline 3788 & & PROCISSI & LOUIS & B & 23 & MICHIGAN & ITALY & ITALY & & & LUCCA & & FRANKLIN \\
\hline 3789 & & BARTONI & CHARLES & $\mathrm{H}$ & 56 & ITALY & ITALY & ITALY & 1884 & CARPENTER/IRON FOUNDRY & & & FRANKLIN \\
\hline 3790 & & BARTONI & VIOLET & w & 47 & ITALY & ITALY & ITALY & 1884 & & & & FRANKLIN \\
\hline 3791 & & BARTONI & CAHRLES JR. & s & 24 & MICHIGAN & ITALY & ITALY & & TYPESETTER/PRINTING CO. & & & FRANKLIN \\
\hline 3792 & & BARTONI & ANTOINETTE & $\mathrm{D}$ & 19 & MICHIGAN & ITALY & ITALY & & & & & FRANKLIN \\
\hline 3793 & & BARTONI & JOSEPHINE & $\mathrm{D}$ & 19 & MICHIGAN & ITALY & ITALY & & & & & FRANKLIN \\
\hline 3794 & & BARTONI & JOSHUA & $\mathrm{s}$ & 13 & MICHIGAN & ITALY & ITALY & & & & & FRANKLIN \\
\hline 3795 & & BARTONI & LOUISE & $\mathrm{D}$ & 7 & MICHIGAN & ITALY & ITALY & & & & & FRANKLIN \\
\hline 3796 & & PERA & PARENA & B & $7 \mathrm{M}$ & MICHIGAN & ITALY & ITALY & & & & & FRANKLIN \\
\hline 3797 & & FERO(NO) & MARTIN & $\mathrm{H}$ & 34 & ITALY & ITALY & ITALY & 1900 & FARMER/FARM & & & FRANKLIN \\
\hline 3798 & & FERO(NO) & MARY & w & 28 & ITALY & ITALY & ITALY & 1906 & & & & FRANKLIN \\
\hline 3799 & & FERO(NO) & JOHANNA & $\mathrm{D}$ & 2 & MICHIGAN & ITALY & ITALY & & & & & FRANKLIN \\
\hline 3800 & & FERO(NO) & DOMINICK & $\mathrm{s}$ & 1 & MICHIGAN & ITALY & ITALY & & & & & FRANKLIN \\
\hline 3801 & & FAUSONE & GILDO & B & 40 & ITALY & ITALY & ITALY & 1890 & LABORER/FARM & TORINO & & FRANKLIN \\
\hline 3802 & & KASPER & JAMES & B & 21 & ITALY & ITALY & ITALY & 1906 & LABORER/FARM & & & FRANKLIN \\
\hline 3803 & & BELESSANA & PETER & B & 18 & ITALY & ITALY & ITALY & 1909 & LABORER/FARM & & & FRANKLIN \\
\hline 3804 & & SANDRETTO & SYLVESTER & $\mathrm{H}$ & 41 & ITALY & ITALY & ITALY & 1894 & DAIRYMAN/DAIRY FARM & PONT CANAVESE. TO & & HANCOCK \\
\hline 3805 & & SANDRETTO & ANGELICA & w & 41 & ITALY & ITALY & ITALY & 1894 & DAIRYWOMAN/DAIRY FARM & PONT CANAVESE. TO & & HANCOCK \\
\hline 3806 & & SANDRETTO & MANNIE & $\mathrm{D}$ & 17 & ITALY & ITALY & ITALY & 1894 & DAIRYWOMAN/DAIRY FARM & PONT CANAVESE. TO & & HANCOCK \\
\hline 3807 & & SANDRETTO & ANNIE & $\mathrm{D}$ & 12 & MICHIGAN & ITALY & ITALY & & & PONT CANAVESE, TO & & HANCOCK \\
\hline 3808 & & SANDRETTO & JAMES & $\mathrm{s}$ & 10 & MICHIGAN & ITALY & ITALY & & & PONT CANAVESE. TO & & HANCOCK \\
\hline 3809 & & MARIATTA & JOHN & B & 30 & ITALY & ITALY & ITALY & 1903 & LABORER/FARM & & & HANCOCK \\
\hline 3810 & & CASTAGNETTO & GIACOMO & B & 45 & ITALY & ITALY & ITALY & 1906 & LABORER/FARM & PONT CANAVESE. TO & & HANCOCK \\
\hline 3811 & & COPPO & MARY & $\mathrm{H}$ & 56 & ITALY & ITALY & ITALY & 1881 & FARMER/FARM & & & HANCOCK \\
\hline 3812 & & COPPO & JOHN & s & 26 & MICHIGAN & ITALY & ITALY & & SALESMAN/GENERAL STORE & PONT CANAVESE. TO & & HANCOCK \\
\hline 3813 & & COPPO & MARION & DIL & 19 & ITALY & ITALY & ITALY & 1906 & HOUSEKEEPER/HOME & PONT CANAVESE, TO & & HANCOCK \\
\hline 3814 & & COPPO & ROSALIA & D & 23 & MICHIGAN & ITALY & ITALY & 1500 & DRESSMAKER/AT HOME & PONT CANAVESE. TO & & HANCOCK \\
\hline 3815 & & COPPO & TRACEY & $\mathrm{D}$ & 17 & MICHIGAN & ITALY & ITALY & & HOUSEKEEPER/HOME & PONT CANAVESE. TO & & HANCOCK \\
\hline 3816 & & COPPO & KATHERINE & $\mathrm{D}$ & 16 & MICHIGAN & ITALY & ITALY & & & PONT CANAVESE. TO & & HANCOCK \\
\hline 3817 & & ERNIETTI & BERNARD & B & 35 & ITALY & ITALY & ITALY & 1890 & LABORER/FARM & & & HANCOCK \\
\hline 3818 & & MORRO & AUGUST & $\mathrm{H}$ & 39 & ITALY & ITALY & ITALY & 1885 & LABORER/WORKING OUT & & & HANCOCK \\
\hline 3819 & & MORRO & JEANNIE & W & 26 & MICHIGAN & CANADA & WISCONSI & & & & & HANCOCK \\
\hline 3820 & & BARVIE & VICTOR & INMA & 60 & ITALY & ITALY & ITALY & $?$ & NOT CLEAR & & & HANCOCK \\
\hline 3821 & & GASPARDO & PAUL & $\mathrm{H}$ & 37 & ITALY & ITALY & ITALY & 1896 & FARMER/FARM & PONT CANAVESE. TO & & HANCOCK \\
\hline 3822 & & GASPARDO & MARY & W & 35 & ITALY & ITALY & ITALY & 1897 & & & & HANCOCK \\
\hline 3823 & & GASPARDO & JOHN & s & 12 & MICHIGAN & ITALY & ITALY & & & PONT CANAVESE. TO & & HANCOCK \\
\hline 3824 & & GASPARDO & ANNE & $\mathrm{D}$ & 10 & MICHIGAN & ITALY & ITALY & & & PONT CANAVESE. TO & & HANCOCK \\
\hline 3825 & & GASPARDO & JAMES & $\mathrm{S}$ & 5 & MICHIGAN & ITALY & ITALY & & & PONT CANAVESE. TO & & HANCOCK \\
\hline 3826 & & VERGA & ORESTE & B & 36 & ITALY & ITALY & ITALY & 1899 & LABORER/FARM & TORINO & & HANCOCK \\
\hline 3827 & 114 & COPPO & & $\mathrm{H}$ & 39 & ITALY & ITALY & ITALY & 1895 & $\begin{array}{l}\text { LABORER/STREET } \\
\text { LARER }\end{array}$ & PONT CANAVESE. TO & FRANKLIN ST. & HANCOCK CITY \\
\hline 3828 & 114 & COPPO & MARY & w & 31 & ITALY & ITALY & ITALY & 1899 & & PONT CANAVESE. TO & FRANKLIN ST. & HANCOCK CITY \\
\hline 3829 & 114 & COPPO & MARK & s & 15 & ITALY & ITALY & ITALY & 1899 & LABORER/STREET & PONT CANAVESE. TO & FRANKLIN ST. & HANCOCK CITY \\
\hline 3830 & 114 & COPPO & JOSEPH & $\mathrm{s}$ & 14 & ITALY & ITALY & ITALY & 1899 & & PONT CANAVESE. TO & FRANKLIN ST. & HANCOCK CITY \\
\hline 3831 & 114 & COPPO & JAMES & $\mathrm{s}$ & 9 & MICHIGAN & ITALY & ITALY & & & PONT CANAVESE, TO & FRANKLIN ST. & HANCOCK CITY \\
\hline 3832 & 114 & COPPO & LOUISE & $\mathrm{D}$ & 7 & MICHIGAN & ITALY & ITALY & & & PONT CANAVESE. TO & FRANKLIN ST. & HANCOCK CITY \\
\hline 3833 & 114 & COPPO & JENNIE & $\mathrm{D}$ & 6 & MICHIGAN & ITALY & ITALY & & & PONT CANAVESE. TO & FRANKLIN ST. & HANCOCK CITY \\
\hline 3834 & 114 & COPPO & TONY & $\mathrm{s}$ & 5 & MICHIGAN & ITALY & ITALY & & & PONT CANAVESE. TO & FRANKLIN ST. & HANCOCK CITY \\
\hline 3835 & 114 & COPPO & ANNE & $\mathrm{D}$ & 4 & MICHIGAN & ITALY & ITALY & & & PONT CANAVESE. TO & FRANKLIN ST. & HANCOCK CITY \\
\hline 3836 & 114 & COPPO & KATE & $\mathrm{D}$ & $1 \mathrm{M}$ & MICHIGAN & ITALY & ITALY & & & PONT CANAVESE. TO & FRANKLIN ST. & HANCOCK CITY \\
\hline 3837 & 114 & VILAS & JOSEPH & B & 25 & ITALY & ITALY & ITALY & ???? & & & FRANKLIN ST. & HANCOCK CITY \\
\hline 3838 & 114 & BOGGIO & JOHN & B & 39 & ITALY & ITALY & ITALY & ???? & & TORINO & FRANKLIN ST. & HANCOCK CITY \\
\hline 3839 & 109 & MEINARDI & JOHN & $\mathrm{H}$ & 35 & ITALY & ITALY & ITALY & 1899 & MERCHANT/RETAIL LIQUOR & SAN GIORGIO CANAVESE. TO & QUINCY ST. & HANCOCK CITY \\
\hline 3840 & 109 & MEINARDI & ANGELINA & W & 28 & ITALY & ITALY & ITALY & 1905 & & & QUINCY ST. & HANCOCK CITY \\
\hline 3841 & 109 & MEINARDI & JOSEPH & s & 4 & MICHIGAN & ITALY & ITALY & & & SAN GIORGIO CANAVESE. TO & QUINCY ST. & HANCOCK CITY \\
\hline 3842 & 410 & PERA & FRANK & $\mathrm{H}$ & 25 & ITALY & ITALY & ITALY & 1903 & SALOONKEEPER & LUCCA & RAVINE ST. & HANCOCK CITY \\
\hline
\end{tabular}




\begin{tabular}{|c|c|c|c|c|c|c|c|c|c|c|c|c|c|}
\hline 3843 & 410 & PERA & MARY & W & 22 & MICHIGAN & ITALY & ITALY & & & & RAVINE ST. & HANCOCK CITY \\
\hline 3844 & 404 & MEOLA & LEONARD & $\mathrm{H}$ & 40 & ITALY & ITALY & ITALY & 1890 & EMPLOYMENT AGENT & NAPOLI & RAVINE ST. & HANCOCK CITY \\
\hline 3845 & 404 & MEOLA & NELLIE & W & 35 & $\mathrm{OHIO}$ & ITALY & ITALY & & & NAPOLI & RAVINE ST. & HANCOCK CITY \\
\hline 3846 & 404 & MEOLA & MARIE & D & 17 & $\mathrm{OHIO}$ & ITALY & $\mathrm{OHIO}$ & & & NAPOLI & RAVINE ST. & HANCOCK CITY \\
\hline 3847 & 238 & PASQUINELLI & VITTORIO & $\mathrm{H}$ & 21 & MICHIGAN & ITALY & ITALY & & WAITER/RESTAURANT & LUCCA & QUINCY ST. & HANCOCK CITY \\
\hline 3848 & 231 & FORNERIS & ANGELO & $\mathrm{H}$ & 37 & ITALY & ITALY & ITALY & 1895 & BUTCHER/MEAT MARKET & TORINO & HANCOCK ST. & HANCOCK CITY \\
\hline 3849 & 231 & FORNERIS & MARIA & w & 38 & ITALY & ITALY & ITALY & 1898 & & & HANCOCK ST. & HANCOCK CITY \\
\hline 3850 & 231 & RONCAGLIONE & JOHN & B & 23 & ITALY & ITALY & ITALY & 1898 & FARMER/FARM & PONT CANAVESE. TO & HANCOCK ST. & HANCOCK CITY \\
\hline 3851 & 231 & MEINARDI & LORENZO & B & 37 & ITALY & ITALY & ITALY & 1895 & MINER/CM & SAN GIORGIO CANAVESE. TO & HANCOCK ST. & HANCOCK CITY \\
\hline 3852 & 231 & LOCATTI & JOSEPH & B & 29 & ITALY & ITALY & ITALY & $03 / 05$ & MINER/CM & MERCENASCO CANAVESE. TO & HANCOCK ST. & HANCOCK CITY \\
\hline 3853 & 231 & BEIONA & CARLO & B & 39 & ITALY & ITALY & ITALY & 1906 & LABORER/COAL DOCK & & HANCOCK ST. & HANCOCK CITY \\
\hline 3854 & 231 & MALFORTE & PRIMO & B & 25 & ITALY & ITALY & ITALY & 1897 & MINER/CM & & HANCOCK ST. & HANCOCK CITY \\
\hline 3855 & 231 & FORNERIS & PERCILLA & SER & 30 & ITALY & ITALY & ITALY & 1910 & SERVANT/BOARDING HOUSE & TORINO & HANCOCK ST. & HANCOCK CITY \\
\hline 3856 & 301 & VIGNETTO & DOMENICO & $\mathrm{H}$ & 52 & ITALY & ITALY & ITALY & 1878 & MERCHANT/RETAIL LIQUOR & TORINO & HANCOCK ST. & HANCOCK CITY \\
\hline 3857 & 301 & VIGNETTO & CLOTILDE & W & 48 & ITALY & ITALY & ITALY & 1881 & & & HANCOCK ST. & HANCOCK CITY \\
\hline 3858 & 301 & VIGNETTO & NUIO & $\mathrm{D}$ & $?$ & ILLINOIS & ITALY & ITALY & & BOOKKEEPER/SALOON & TORINO & HANCOCK ST. & HANCOCK CITY \\
\hline 3859 & 301 & VIGNETTO & MARIA & $\mathrm{D}$ & 21 & ILLINOIS & ITALY & ITALY & & & TORINO & HANCOCK ST. & HANCOCK CITY \\
\hline 3860 & 301 & VIGNETTO & BERTA & $\mathrm{D}$ & 16 & ILLINOIS & ITALY & ITALY & & & TORINO & HANCOCK ST. & HANCOCK CITY \\
\hline 3861 & 301 & VIGNETTO & WILLIAM & s & 8 & MICHIGAN & ITALY & ITALY & & & TORINO & HANCOCK ST. & HANCOCK CITY \\
\hline 3862 & 301 & MONTICELLO/I & GIUSEPPE & B & 26 & ITALY & ITALY & ITALY & 1900 & TEAMSTER/BREWERY & & HANCOCK ST. & HANCOCK CITY \\
\hline 3863 & 301 & DEBERNA & BATTISTA & B & 45 & ITALY & ITALY & ITALY & 1895 & MINER/CM & & HANCOCK ST. & HANCOCK CITY \\
\hline 3864 & 301 & ALBRECCA & MICHAEL & $\begin{array}{l}\mathrm{D} \\
\mathrm{B}\end{array}$ & 41 & ITALY & ITALY & ITALY & 1903 & TAILOR/SHOP & & HANCOCK ST. & HANCOCK CITY \\
\hline 3865 & 301 & AIMONETTO & FRANK & $\mathrm{H}$ & 38 & ITALY & ITALY & ITALY & 1902 & LABORER/SMELTER & TORINO & HANCOCK ST. & HANCOCK CITY \\
\hline 3866 & 301 & AIMONETTO & MARY & W & 24 & ITALY & ITALY & ITALY & 1907 & & & HANCOCK ST. & HANCOCK CITY \\
\hline 3867 & 301 & AIMONETTO & ROSINA & $\mathrm{D}$ & $1.6 \mathrm{M}$ & MICHIGAN & ITALY & ITALY & & & TORINO & HANCOCK ST. & HANCOCK CITY \\
\hline 3868 & 308 & CALSETTA & JOHN & $\mathrm{H}$ & 39 & ITALY & ITALY & ITALY & 1905 & SALOONKEEPER & & RAVINE ST. & HANCOCK CITY \\
\hline 3869 & 308 & CALSETTA & ROSIE & W & 24 & ITALY & ITALY & ITALY & 1907 & & & RAVINE ST. & HANCOCK CITY \\
\hline 3870 & 308 & CALSETTA & JOHN & s & 2 & MICHIGAN & ITALY & ITALY & & & & RAVINE ST. & HANCOCK CITY \\
\hline 3871 & 308 & CALSETTA & ANNA & $\mathrm{D}$ & 6 & MICHIGAN & ITALY & ITALY & & & & RAVINE ST. & HANCOCK CITY \\
\hline 3872 & 234 & PUCCl & LUIGI & B & 31 & ITALY & ITALY & ITALY & 1890 & MERCHANT/RETAIL LIQUOR & LUCCA & QUINCY ST. & HANCOCK CITY \\
\hline 3873 & 425 & MEOLA & BARTOLOMEO & $\mathrm{H}$ & 36 & ITALY & ITALY & ITALY & ???? & EMPLOYMENT AGENT & NAPOLI & QUINCY ST. & HANCOCK CITY \\
\hline 3874 & 425 & MEOLA & JENNIE & w & 35 & ITALY & ITALY & ITALY & ???? & & NAPOLI & QUINCY ST. & HANCOCK CITY \\
\hline 3875 & 425 & MEOLA & VIRGINIA & $\mathrm{D}$ & 8 & MICHIGAN & ITALY & ITALY & & & NAPOLI & QUINCY ST. & HANCOCK CITY \\
\hline 3876 & 425 & MEOLA & TERESA & $\mathrm{D}$ & 7 & MICHIGAN & ITALY & ITALY & & & NAPOLI & QUINCY ST. & HANCOCK CITY \\
\hline 3877 & 425 & MEOLA & LESLIE & $\mathrm{D}$ & 3 & MICHIGAN & ITALY & ITALY & & & NAPOLI & QUINCY ST. & HANCOCK CITY \\
\hline 3878 & 425 & MEOLA & ROSE & $\mathrm{D}$ & $9 \mathrm{M}$ & MICHIGAN & ITALY & ITALY & & & NAPOLI & QUINCY ST. & HANCOCK CITY \\
\hline 3879 & 425 & BRENCAN? & FRANK & B & 28 & ITALY & ITALY & ITALY & 1903 & HELPER/IRON MOLDER & & QUINCY ST. & HANCOCK CITY \\
\hline 3880 & 425 & CORTE & PAUL & B & 20 & ITALY & ITALY & ITALY & 1910 & SECTION GANG/RAILROAD & & QUINCY ST. & HANCOCK CITY \\
\hline 3881 & 425 & CATHCIAE? & DEMETRIO & B & 60 & ITALY & ITALY & ITALY & 1906 & LABORER/CM & & QUINCY ST. & HANCOCK CITY \\
\hline 3882 & 627 & GEDDA & VITTORIO & $\mathrm{H}$ & 36 & ITALY & ITALY & ITALY & 1905 & BAKER & TORINO & PINE ST. & HANCOCK CITY \\
\hline 3883 & 627 & PONSETTO & DOMENICO & B & $?$ & ITALY & ITALY & ITALY & 1903 & BAKER & SAN GIORGIO CANAVESE. TO & PINE ST. & HANCOCK CITY \\
\hline 3884 & 627 & GEDDA & ROSA & SER & 26 & ITALY & ITALY & ITALY & 1907 & SERVANT/PF & TORINO & PINE ST. & HANCOCK CITY \\
\hline 3885 & 625 & PONSETTO & JOHN & $\mathrm{H}$ & 29 & ITALY & ITALY & ITALY & 1906 & BARTENDER/SALOON & SAN GIORGIO CANAVESE. TO & PINE ST. & HANCOCK CITY \\
\hline 3886 & 625 & PONSETTO & ROSE & W & 24 & ITALY & ITALY & ITALY & 1904 & & & PINE ST. & HANCOCK CITY \\
\hline 3887 & 625 & PONSETTO & LOUISE & $\mathrm{D}$ & 2 & MICHIGAN & ITALY & ITALY & & & SAN GIORGIO CANAVESE. TO & PINE ST. & HANCOCK CITY \\
\hline 3888 & 625 & PONSETTO & ANNA & $\mathrm{D}$ & $6 \mathrm{M}$ & MICHIGAN & ITALY & ITALY & & & SAN GIORGIO CANAVESE. TO & PINE ST. & HANCOCK CITY \\
\hline 3889 & 625 & ORSOLANO & CARLO & B & 30 & ITALY & ITALY & ITALY & 1900 & MINER/CM & SAN GIORGIO CANAVESE. TO & PINE ST. & HANCOCK CITY \\
\hline 3890 & 625 & TELLETIVO & MARTIN & B & 35 & ITALY & ITALY & ITALY & 1904 & TEAMSTER/BAKERY & & PINE ST. & HANCOCK CITY \\
\hline 3891 & 625 & DANIELI & FRANK & $\mathrm{H}$ & 24 & ITALY & ITALY & ITALY & 1905 & MINER/CM & RIVAROLO CANAVESE. TO & PINE ST. & HANCOCK CITY \\
\hline 3892 & 625 & DANIELI & JOSIE & W & 20 & ITALY & ITALY & ITALY & 1905 & & RIVAROLO CANAVESE. TO & PINE ST. & HANCOCK CITY \\
\hline 3893 & 625 & DANIELI & ROSIE & $\mathrm{D}$ & $2 \mathrm{M}$ & MICHIGAN & ITALY & ITALY & & & RIVAROLO CANAVESE. TO & PINE ST. & HANCOCK CITY \\
\hline 3894 & 808 & SABBATINI & ANTONIO & $\mathrm{H}$ & 33 & ITALY & ITALY & ITALY & 1900 & LABORER/CM & LUCCA & FRANKLIN ST. & HANCOCK CITY \\
\hline 3895 & 808 & SABBATINI & ELLEN & W & 28 & ITALY & ITALY & ITALY & 1906 & & & FRANKLIN ST. & HANCOCK CITY \\
\hline 3896 & 808 & SABBATINI & MAGGIE & $\mathrm{D}$ & 3 & MICHIGAN & ITALY & ITALY & & & LUCCA & FRANKLIN ST. & HANCOCK CITY \\
\hline 3897 & 808 & SABBATINI & CELIA & $\mathrm{D}$ & 2 & MICHIGAN & ITALY & ITALY & & & LUCCA & FRANKLIN ST. & HANCOCK CITY \\
\hline 3898 & 808 & SABBATINI & MIKE & $\mathrm{s}$ & $7 \mathrm{M}$ & MICHIGAN & ITALY & ITALY & & & LUCCA & FRANKLIN ST. & HANCOCK CITY \\
\hline 3899 & 808 & STORIGGINE & LUCCI & B & 35 & ITALY & ITALY & ITALY & 1900 & MINER/CM & & FRANKLIN ST. & HANCOCK CITY \\
\hline 3900 & 808 & MANESCHI & JULIO & B & 28 & ITALY & ITALY & ITALY & 1905 & MINER/CM & CARRARA & FRANKLIN ST. & HANCOCK CITY \\
\hline 3901 & 711 & GIUSFREDI & GABRIELLO & $\mathrm{H}$ & 38 & ITALY & ITALY & ITALY & 1898 & MERCHANT/OWN ACTIVITY & SEGROMIGNO. LU & FRANKLIN ST. & HANCOCK CITY \\
\hline 3902 & 711 & GIUSFREDI & MATILDA & W & 26 & ITALY & ITALY & ITALY & $\begin{array}{l}1090 \\
1902\end{array}$ & & & FRANKLIN ST. & HANCOCK CITY \\
\hline 3903 & 711 & GIUSFREDI & ROSIE & $\mathrm{D}$ & 5 & MICHIGAN & ITALY & ITALY & & & SEGROMIGNO. LU & FRANKLIN ST. & HANCOCK CITY \\
\hline 3904 & 711 & VIRBIRO & $\mathrm{JOHN}$ & B & 31 & ITALY & ITALY & ITALY & 1902 & TEAMSTER/GROCERY STORE & & FRANKLIN ST. & HANCOCK CITY \\
\hline 3905 & 711 & CIABATTARI & DIEGO & $\mathrm{H}$ & 43 & ITALY & ITALY & ITALY & 1880 & MINER/CM & LUCCA & FRANKLIN ST. & HANCOCK CITY \\
\hline
\end{tabular}




\begin{tabular}{|c|c|c|c|c|c|c|c|c|c|c|c|c|c|}
\hline 3906 & 711 & CIABATTARI & CLELIA & W & 47 & ITALY & ITALY & ITALY & 1888 & & & FRANKLIN ST. & HANCOCK CITY \\
\hline 3907 & 711 & CIABATTARI & EMILIO & $s$ & $5 \mathrm{M}$ & MICHIGAN & ITALY & ITALY & & & LUCCA & FRANKLIN ST. & HANCOCK CITY \\
\hline 3908 & 711 & CIABATTARI & LIZZIE & D & 19 & MICHIGAN & ITALY & ITALY & & & LUCCA & FRANKLIN ST. & HANCOCK CITY \\
\hline 3909 & 711 & CIABATTARI & PIETRO & $\mathrm{s}$ & 15 & MICHIGAN & ITALY & ITALY & & & LUCCA & FRANKLIN ST. & HANCOCK CITY \\
\hline 3910 & 711 & CIABATTARI & LAMEUSIA & $\mathrm{D}$ & 11 & MICHIGAN & ITALY & ITALY & & & LUCCA & FRANKLIN ST. & HANCOCK CITY \\
\hline 3911 & 711 & CIABATTARI & GENE & $\mathrm{s}$ & 9 & MICHIGAN & ITALY & ITALY & & & LUCCA & FRANKLIN ST. & HANCOCK CITY \\
\hline 3912 & NN & NARGISO & ANGELO & $\mathrm{B}$ & 29 & ITALY & ITALY & ITALY & 1907 & MINER/CM & Lour & FRANKLIN ST. & HANCOCK CITY \\
\hline 3913 & 635 & CAMPIONI & GUIDO & $\begin{array}{l}\mathrm{O} \\
\mathrm{H}\end{array}$ & 24 & ITALY & ITALY & ITALY & 1910 & BAKER & LUCCA & ELM ST. & HANCOCK CITY \\
\hline 3914 & 635 & CAMPIONI & MARY & W & 17 & ITALY & ITALY & ITALY & 1902 & & & ELM ST. & HANCOCK CITY \\
\hline 3915 & 1016 & PANTALEONI & VIRGILIO & $\mathrm{H}$ & 28 & ITALY & ITALY & ITALY & 1906 & FIREMAN/SMELTER & LUCCA & MINNESOTA ST. & HANCOCK CITY \\
\hline 3916 & 1016 & PANTALEONI & GIULIA & w & 23 & ITALY & ITALY & ITALY & 1906 & & LUCCA & MINNESOTA ST. & HANCOCK CITY \\
\hline 3917 & 1016 & PANTALEONI & ADELAIDE & D & 3 & MICHIGAN & ITALY & ITALY & & & LUCCA & MINNESOTA ST. & HANCOCK CITY \\
\hline 3918 & 1016 & PANTALEONI & MABLE & $\mathrm{D}$ & $1.2 \mathrm{M}$ & MICHIGAN & ITALY & ITALY & & & LUCCA & MINNESOTA ST. & HANCOCK CITY \\
\hline 3919 & 1016 & PANTALEONI & MICHELE & $\mathrm{BR}$ & 22 & ITALY & ITALY & ITALY & 1909 & FIREMAN/SMELTER & LUCCA & MINNESOTA ST. & HANCOCK CITY \\
\hline 3920 & 1040 & FEDERIGHI & ANTONIO & $\mathrm{H}$ & 42 & ITALY & ITALY & ITALY & 1891 & MERCHANT/GENERAL STORE & LUCCA & SUMMIT ST. & HANCOCK CITY \\
\hline 3921 & 1040 & FEDERIGHI & ENRICA & W & 31 & ITALY & ITALY & ITALY & 1891 & & LUCCA & SUMMIT ST. & HANCOCK CITY \\
\hline 3922 & 1040 & FEDERIGHI & TRESSA & D & 10 & MICHIGAN & ITALY & ITALY & & & LUCCA & SUMMIT ST. & HANCOCK CITY \\
\hline 3923 & 1040 & FEDERIGHI & EMILIO & $\mathrm{s}$ & 7 & MICHIGAN & ITALY & ITALY & & & LUCCA & SUMMIT ST. & HANCOCK CITY \\
\hline 3924 & 1040 & FEDERIGHI & SEBASTIANO & $\mathrm{s}$ & 5 & MICHIGAN & ITALY & ITALY & & & LUCCA & SUMMIT ST. & HANCOCK CITY \\
\hline 3925 & 1040 & FEDERIGHI & DOMENICO & $\mathrm{s}$ & 2 & MICHIGAN & ITALY & ITALY & & & LUCCA & SUMMIT ST. & HANCOCK CITY \\
\hline 3926 & 1040 & MICHELETTI & ALBERTO & $\mathrm{B}$ & 22 & ITALY & ITALY & $\begin{array}{l}\text { ITALY } \\
\text { ITA }\end{array}$ & 1906 & SALESMAN/GROCERY & LUCCA & SUMMIT ST. & HANCOCK CITY \\
\hline 3927 & 1040 & LAVORINI & CARMELA & SER & 20 & ITALY & ITALY & ITALY & 1908 & SERVANT/PF & LUCCA & SUMMIT ST. & HANCOCK CITY \\
\hline 3928 & 1022 & GUELCH/QUILICI & JOHN & $\mathrm{H}$ & 26 & ITALY & ITALY & ITALY & 1898 & MINER/CM & & RIDGE ST. & HANCOCK CITY \\
\hline 3929 & 1022 & GUELCH/QUILICI & MINNIE & w & 24 & MICHIGAN & GERMANY & CANADA & & & & RIDGE ST. & HANCOCK CITY \\
\hline 3930 & 912 & BAY & JOHN & $\mathrm{H}$ & 32 & ITALY & ITALY & ITALY & 1895 & MINER/CM & & PINE ST. & HANCOCK CITY \\
\hline 3931 & 912 & BAY & EMMA & W & 23 & MICHIGAN & FINLAND & FINLAND & & & FINLAND & PINE ST. & HANCOCK CITY \\
\hline 3932 & 1011 & LENCI & URVASIO & $\mathrm{H}$ & 42 & ITALY & ITALY & ITALY & 1895 & MERCHANT/GROCERY STORE & PESCAGLIA. LU & 5TH ST. & HANCOCK CITY \\
\hline 3933 & 1011 & LENCI & ISOLINA & W & 32 & ITALY & ITALY & ITALY & 1903 & & & 5TH ST. & HANCOCK CITY \\
\hline 3934 & 1011 & LENCI & GIORGIO & $\mathrm{s}$ & 7 & MICHIGAN & ITALY & ITALY & & & PESCAGLIA. LU & 5TH ST. & HANCOCK CITY \\
\hline 3935 & 1011 & LENCI & GUIDO & $\mathrm{s}$ & 4 & MICHIGAN & ITALY & ITALY & & & LUCCA & 5TH ST. & HANCOCK CITY \\
\hline 3936 & 1032 & LAVORINI & PIETRO & $\mathrm{H}$ & 46 & ITALY & ITALY & ITALY & 1909 & MANUFACTURER/MACARONI & LUCCA & 5TH ST. & HANCOCK CITY \\
\hline 3937 & 1032 & LAVORINI & MARY & W & 46 & ITALY & ITALY & ITALY & 1909 & 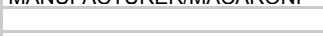 & & 5TH ST. & HANCOCK CITY \\
\hline 3938 & 1032 & LAVORINI & FERRUCCIO & s & 21 & ITALY & ITALY & ITALY & 1909 & LABORER/MACARONI SHOP & LUCCA & 5TH ST. & HANCOCK CITY \\
\hline 3939 & 1032 & FABRI? & GIOVANNI & B & 70 & ITALY & ITALY & ITALY & 1897 & MINER/CM & & 5TH ST. & HANCOCK CITY \\
\hline 3940 & 1032 & ROSI & GIUSEPPE & B & 44 & ITALY & ITALY & ITALY & 1910 & LABORER/MACARONI SHOP & NAPOLI & 5TH ST. & HANCOCK CITY \\
\hline 3941 & 1010 & LUCCHESI & PASQUALE & $\mathrm{H}$ & 20 & ITALY & ITALY & ITALY & $07 / 09$ & LABORER/MACARONI SHOP & LUCCA & INGOT ST. & HANCOCK CITY \\
\hline 3942 & 1010 & PAOLI & OLINTO & $\mathrm{BACH}$ & 19 & ITALY & ITALY & ITALY & $07 / 09$ & WAGON DRIVER/GROCERY & LUCCA & INGOT ST. & HANCOCK CITY \\
\hline 3943 & 1010 & MIGNANI & RAFFAELLO & $\mathrm{H}$ & 39 & ITALY & ITALY & ITALY & 1900 & MERCHANT/GROCERY STORE & & INGOT ST. & HANCOCK CITY \\
\hline 3944 & 1010 & MIGNANI & ADELE & W & 36 & ITALY & ITALY & ITALY & 1900 & & & INGOT ST. & HANCOCK CITY \\
\hline 3945 & 1050 & BATTISTONI & OTTAVIO & B & 62 & ITALY & ITALY & ITALY & 1889 & ENGINEER/CM & LUCCA & INGOT ST. & HANCOCK CITY \\
\hline 3946 & 1050 & BATTISTONI & JOHN & $\mathrm{H}$ & 38 & ITALY & ITALY & ITALY & 1880 & ENGINEER/CM & LUCCA & INGOT ST. & HANCOCK CITY \\
\hline 3947 & 1050 & BATTISTONI & DORINA & w & 37 & ITALY & ITALY & ITALY & 1876 & & & INGOT ST. & HANCOCK CITY \\
\hline 3948 & 1050 & BATTISTONI & JENNY & D & 10 & MICHIGAN & ITALY & ITALY & & & LUCCA & INGOT ST. & HANCOCK CITY \\
\hline 3949 & 1050 & BATTISTONI & WIDO & $\mathrm{s}$ & 12 & MICHIGAN & ITALY & ITALY & & & LUCCA & INGOT ST. & HANCOCK CITY \\
\hline 3950 & 1017 & QUILICI & JOSEPH & $\mathrm{H}$ & 50 & ITALY & ITALY & ITALY & 1907 & PEDLAR/STATUE & LUCCA & INGOT ST. & HANCOCK CITY \\
\hline 3951 & 1017 & QUILICI & TERESA & W & 53 & ITALY & ITALY & ITALY & 1907 & & LUCCA & INGOT ST. & HANCOCK CITY \\
\hline 3952 & 1017 & FRANCIONI & DOMENICO & B & 42 & ITALY & ITALY & ITALY & 1901 & MINER/CM & LUCCA & INGOT ST. & HANCOCK CITY \\
\hline 3953 & 1017 & QUILICI & QUINTO & B & 17 & ITALY & ITALY & ITALY & 1907 & WAGON DRIVER/GROCERY & LUCCA & INGOT ST. & HANCOCK CITY \\
\hline 3954 & 1017 & QUILICI & FRANK & B & 21 & ITALY & ITALY & ITALY & 1907 & MINER/CM & LUCCA & INGOT ST. & HANCOCK CITY \\
\hline 3955 & 1010 & TADDEUCCI & JOSEPH & $\mathrm{H}$ & 33 & ITALY & ITALY & ITALY & 1898 & DRIVER/GROCERY WAGON & LUCCA & ETHEL AVE. & HANCOCK CITY \\
\hline 3956 & 1010 & TADDEUCCI & TRESSA & W & 30 & ITALY & ITALY & ITALY & 1901 & & & ETHEL AVE. & HANCOCK CITY \\
\hline 3957 & 1010 & TADDEUCCI & BRUNA & D & 8 & MICHIGAN & ITALY & ITALY & & & LUCCA & ETHEL AVE. & HANCOCK CITY \\
\hline 3958 & 1010 & TADDEUCCI & JOHN & $\mathrm{s}$ & 7 & MICHIGAN & ITALY & ITALY & & & LUCCA & ETHEL AVE. & HANCOCK CITY \\
\hline 3959 & 1010 & TADDEUCCI & VERIULIA & D & 6 & MICHIGAN & ITALY & ITALY & & & LUCCA & ETHEL AVE. & HANCOCK CITY \\
\hline 3960 & 1010 & TADDEUCCI & ALBERT & $\mathrm{s}$ & 3 & MICHIGAN & ITALY & ITALY & & & LUCCA & ETHEL AVE. & HANCOCK CITY \\
\hline 3961 & 1010 & TADDEUCCI & FRANK & $\mathrm{s}$ & $1.2 \mathrm{M}$ & MICHIGAN & ITALY & ITALY & & & LUCCA & ETHEL AVE. & HANCOCK CITY \\
\hline 3962 & & DI VECCHIO & AUGUSTO & $\mathrm{H}$ & 54 & ITALY & ITALY & ITALY & 1896 & SALOONKEEPER & LUCCA & OPP. CAR BARN & HANCOCK CITY \\
\hline 3963 & $\frac{1}{1}$ & DI VECCHIO & CHARLOTTE & w & 44 & ITALY & ITALY & ITALY & 1900 & OALUUIVILETLK & & OPP. CAR BARN & HANCOCK CITY \\
\hline 3964 & I & DI VECCHIO & IDA & D & 16 & ITALY & ITALY & ITALY & 1900 & & LUCCA & OPP. CAR BARN & HANCOCK CITY \\
\hline 3965 & & DI VECCHIO & CHARITY & $\mathrm{D}$ & 17 & ITALY & ITALY & ITALY & 1900 & SALESLADY/CANDY STORE & LUCCA & OPP. CAR BARN & HANCOCK CITY \\
\hline 3966 & & DI VECCHIO & SANTINA & D & 13 & ITALY & ITALY & ITALY & 1900 & & LUCCA & OPP. CAR BARN & HANCOCK CITY \\
\hline 3967 & & DI VECCHIO & JOSEPH & $\mathrm{s}$ & 8 & MICHIGAN & ITALY & ITALY & & & LUCCA & OPP. CAR BARN & HANCOCK CITY \\
\hline 68 & & DI VECCHIO & JENNY & $\mathrm{D}$ & 7 & MICHIGAN & ITALY & ITALY & & & LUCCA & OPP. CAR BARN & HANCOCK CITY \\
\hline
\end{tabular}




\begin{tabular}{|c|c|c|c|c|c|c|c|c|c|c|c|c|c|}
\hline 3969 & 1014 & PIERONI & JOHN & $\mathrm{H}$ & 37 & ITALY & ITALY & ITALY & 1901 & SALOONKEEPER & LUCCA & OPP. CAR BARN & HANCOCK CITY \\
\hline 3970 & 1014 & PIERONI & ANNA & W & 31 & ITALY & ITALY & ITALY & 1905 & & & OPP. CAR BARN & HANCOCK CITY \\
\hline 3971 & 1014 & CERASOLI & MIKE & B & 22 & ITALY & ITALY & ITALY & 1905 & BARTENDER/SALOON & CAPESTRANO. L'AQUILA & OPP. CAR BARN & HANCOCK CITY \\
\hline 3972 & 1014 & PIERONI & DOMENICO & B & 27 & ITALY & ITALY & ITALY & 1909 & LABORER/STREET & LUCCA & OPP. CAR BARN & HANCOCK CITY \\
\hline 3973 & & MARTOLINI & CARLO & $\mathrm{H}$ & 32 & ITALY & ITALY & ITALY & 1901 & ORGANIZER/WESTERN FED. & & EMERY ST. & HANCOCK CITY \\
\hline 3974 & & MARTOLINI & $?$ & w & 26 & ITALY & ITALY & ITALY & 1904 & & & EMERY ST. & HANCOCK CITY \\
\hline 3975 & & MARTOLINI & ALCESTI & s & 7 & ITALY & ITALY & ITALY & 1904 & & & EMERY ST. & HANCOCK CITY \\
\hline 3976 & & MARTOLINI & OTELLO & $\mathrm{s}$ & 5 & MICHIGAN & ITALY & ITALY & & & & EMERY ST. & HANCOCK CITY \\
\hline 3977 & & RENALDI & BATTISTA & $\mathrm{H}$ & 45 & ITALY & ITALY & ITALY & 1887 & MINER/CM & SAN GIORGIO CANAVESE. TO & INGOT ST. & HANCOCK CITY \\
\hline 3978 & & RENALDI & MARIA & w & 40 & ITALY & ITALY & ITALY & 1895 & & & INGOT ST. & HANCOCK CITY \\
\hline 3979 & & RENALDI & TERESA & $\mathrm{D}$ & 14 & MICHIGAN & ITALY & ITALY & & & SAN GIORGIO CANAVESE. TO & INGOT ST. & HANCOCK CITY \\
\hline 3980 & & RENALDI & JOSEPH & s & 12 & MICHIGAN & ITALY & ITALY & & & SAN GIORGIO CANAVESE. TO & INGOT ST. & HANCOCK CITY \\
\hline 3981 & & RENALDI & JAMES & $\mathrm{s}$ & 11 & MICHIGAN & ITALY & ITALY & & & SAN GIORGIO CANAVESE. TO & INGOT ST. & HANCOCK CITY \\
\hline 3982 & & RENALDI & MARION & $\mathrm{D}$ & 9 & MICHIGAN & ITALY & ITALY & & & SAN GIORGIO CANAVESE. TO & INGOT ST. & HANCOCK CITY \\
\hline 3983 & & RENALDI & MARY & $\mathrm{D}$ & 7 & MICHIGAN & ITALY & ITALY & & & SAN GIORGIO CANAVESE. TO & INGOT ST. & HANCOCK CITY \\
\hline 3984 & & VAIRO & GIUSEPPE & $\mathrm{H}$ & 48 & ITALY & ITALY & ITALY & 1890 & FARMER/FARM & LOCANA CANAVESE, TO & & LAIRD \\
\hline 3985 & & VAIRO & MARIA & W & 31 & ITALY & ITALY & ITALY & ???? & & & & LAIRD \\
\hline 3986 & & VAIRO & DOMENICO & s & 12 & MICHIGAN & ITALY & ITALY & & & LOCANA CANAVESE. TO & & LAIRD \\
\hline 3987 & & VAIRO & MARIA & $\mathrm{D}$ & 9 & MICHIGAN & ITALY & ITALY & & & LOCANA CANAVESE. TO & & LAIRD \\
\hline 3988 & & VAIRO & EDLINA & $\mathrm{D}$ & 1 & MICHIGAN & ITALY & ITALY & & & LOCANA CANAVESE. TO & & LAIRD \\
\hline 3989 & 31 & BRONZINI & ALBERTO & $\mathrm{H}$ & 26 & ITALY & ITALY & ITALY & 1905 & LABORER/CM & & 6TH ST. & OSCEOLA \\
\hline 3990 & 31 & BRONZINI & MARIA & w & 25 & ITALY & ITALY & ITALY & ???? & & & 6TH ST. & OSCEOLA \\
\hline 3991 & 31 & BRONZINI & LEONE & s & 1 & MICHIGAN & ITALY & ITALY & & & & 6TH ST. & OSCEOLA \\
\hline 3992 & 31 & MARSALLI & GIOVANNI & B & 27 & ITALY & ITALY & ITALY & 1906 & LABORER/CM & CONEGLIANO. TREVISO & 6TH ST. & OSCEOLA \\
\hline 3993 & 27 & CECCHI & NARCISO & $\mathrm{H}$ & 52 & ITALY & ITALY & ITALY & $188 ?$ & LANDER/CM & LUCCA & CEDAR ST. & OSCEOLA \\
\hline 3994 & 27 & CECCHI & AMELIA & W & 51 & ITALY & ITALY & ITALY & $05 / 03$ & & & CEDAR ST. & OSCEOLA \\
\hline 3995 & 27 & CECCHI & FRANK & $\mathrm{s}$ & 22 & ITALY & ITALY & ITALY & $05 / 03$ & LABORER/CM & LUCCA & CEDAR ST. & OSCEOLA \\
\hline 3996 & 27 & CECCHI & LUCIA & SER & 15 & ITALY & ITALY & ITALY & $05 / 03$ & SERVANT/PF & LUCCA & CEDAR ST. & OSCEOLA \\
\hline 3997 & 27 & TAMBELLINI & OLINTO & B & 23 & ITALY & ITALY & ITALY & 1904 & FIREMAN/BOILER HOUSE & SEGROMIGNO. LU & CEDAR ST. & OSCEOLA \\
\hline 3998 & 27 & QUILICI & DOMINIC & B & 22 & ITALY & ITALY & ITALY & 1905 & TRACK LAYER/CM & LANNARI. LU & CEDAR ST. & OSCEOLA \\
\hline 3999 & 27 & BIANCHI & MARSILIO & B & 22 & ITALY & ITALY & ITALY & 1909 & LANDER/CM & PORCARI. LU & CEDAR ST. & OSCEOLA \\
\hline 4000 & 27 & BARSANTI & DOMENICO & B & 28 & ITALY & ITALY & ITALY & 1906 & TIMBERMAN/CM & SEGROMIGNO. LU & CEDAR ST. & OSCEOLA \\
\hline 4001 & 27 & BARSANTI & FRED & $\begin{array}{l}\mathrm{D} \\
\mathrm{B}\end{array}$ & 25 & $\begin{array}{l}\text { ITALY } \\
\text { ITAL }\end{array}$ & ITALY & ITALY & 1906 & LABORER/ROCK HOUSE & SEGROMIGNO. LU & CEDAR ST. & OSCEOLA \\
\hline 4002 & 32 & RENALDI & JOSEPH & $\mathrm{H}$ & 30 & ITALY & ITALY & ITALY & 1900 & LANDER/CM & SAN GIORGIO CANAVESE, TO & CHERRY ST. & OSCEOLA \\
\hline 4003 & 32 & RENALDI & MAGGIE & w & 23 & MICHIGAN & ITALY & ITALY & & & & CHERRY ST. & OSCEOLA \\
\hline 4004 & 32 & RENALDI & CHARLES & s & 6 & MICHIGAN & ITALY & ITALY & & & SAN GIORGIO CANAVESE. TO & CHERRY ST. & OSCEOLA \\
\hline 4005 & 32 & RENALDI & MARY & $\mathrm{D}$ & 1 & MICHIGAN & ITALY & ITALY & & & SAN GIORGIO CANAVESE. TO & CHERRY ST. & OSCEOLA \\
\hline 4006 & 32 & BARCHETTI & JOHN & B & 18 & MICHIGAN & ITALY & ITALY & & CLERK/STORE & & CHERRY ST. & OSCEOLA \\
\hline 4007 & 24 & BARSANTI & ACHILLE & $\mathrm{H}$ & 38 & ITALY & ITALY & ITALY & 1909 & LABORER/ROCK HOUSE & SEGROMIGNO. LU & NORTH TAMARACH & OSCEOLA \\
\hline 4008 & 24 & BARSANTI & ASSUNTA & W & 32 & ITALY & ITALY & ITALY & 1909 & & SEGROMIGNO. LU & NORTH TAMARACH & OSCEOLA \\
\hline 4009 & 24 & BARSANTI & ANGELO & $\mathrm{s}$ & 2 & ITALY & ITALY & ITALY & 1909 & & SEGROMIGNO. LU & NORTH TAMARACH & OSCEOLA \\
\hline 4010 & 24 & BARSANTI & PAUL & $\mathrm{s}$ & 1 & MICHIGAN & ITALY & ITALY & & & SEGROMIGNO. LU & NORTH TAMARACH & OSCEOLA \\
\hline 4011 & 24 & BARSANTI & ALFREDO & $\mathrm{H}$ & 34 & ITALY & ITALY & ITALY & $09 / 07$ & LABORER/ROCK HOUSE & SEGROMIGNO. LU & NORTH TAMARACH & OSCEOLA \\
\hline 4012 & 24 & BARSANTI & ASSUNTA & W & 25 & ITALY & ITALY & ITALY & 1909 & & SEGROMIGNO. LU & NORTH TAMARACH & OSCEOLA \\
\hline 4013 & 24 & BARSANTI & ETTORE & s & 5 & ITALY & ITALY & ITALY & 1909 & & SEGROMIGNO. LU & NORTH TAMARACH & OSCEOLA \\
\hline 4014 & 46 & RENALDI & JOSEPH & $\mathrm{H}$ & 40 & ITALY & ITALY & ITALY & 1892 & LABORER/ROCK HOUSE & SAN GIORGIO CANAVESE. TO & NORTH TAMARACH & OSCEOLA \\
\hline 4015 & 46 & RENALDI & TRACY & W & 40 & ITALY & ITALY & ITALY & 1899 & & & NORTH TAMARACH & OSCEOLA \\
\hline 4016 & 46 & RENALDI & JOSEPH & $\mathrm{s}$ & 10 & MICHIGAN & ITALY & ITALY & & & SAN GIORGIO CANAVESE. TO & NORTH TAMARACH & OSCEOLA \\
\hline 4017 & 46 & RENALDI & TRACY & $\mathrm{D}$ & 8 & MICHIGAN & ITALY & ITALY & & & SAN GIORGIO CANAVESE. TO & NORTH TAMARACH & OSCEOLA \\
\hline 4018 & 46 & RENALDI & VITTORIA & $\mathrm{D}$ & 6 & MICHIGAN & ITALY & ITALY & & & SAN GIORGIO CANAVESE. TO & NORTH TAMARACH & OSCEOLA \\
\hline 4019 & 48 & ZANONI & AMATO & $\mathrm{H}$ & 50 & ITALY & ITALY & ITALY & $83 / 93$ & MACHINIST/CM & & NORTH TAMARACH & OSCEOLA \\
\hline 4020 & 48 & ZANONI & CLARA & W & 49 & $\mathrm{OHIO}$ & GERMANY & GERMANY & & & & NORTH TAMARACH & OSCEOLA \\
\hline 4021 & 48 & ZANONI & ERNEST & $\mathrm{s}$ & 21 & MICHIGAN & ITALY & GERMANY & & BLACKSMITH/CM & & NORTH TAMARACH & OSCEOLA \\
\hline 4022 & 48 & ZANONI & JOHN & $\mathrm{s}$ & 19 & MICHIGAN & ITALY & GERMANY & & MACHINIST/CM & & NORTH TAMARACH & OSCEOLA \\
\hline 4023 & 48 & ZANONI & FREDERICK & $\mathrm{s}$ & 17 & MICHIGAN & ITALY & GERMANY & & & & NORTH TAMARACH & OSCEOLA \\
\hline 4024 & 48 & ZANONI & ELENA & $\mathrm{D}$ & 15 & MICHIGAN & ITALY & GERMANY & & & & NORTH TAMARACH & OSCEOLA \\
\hline 4025 & 48 & ZANONI & FRANK & $\mathrm{s}$ & 13 & MICHIGAN & ITALY & GERMANY & & & & NORTH TAMARACH & OSCEOLA \\
\hline 4026 & 48 & ZANONI & ELISABETH & $\mathrm{D}$ & 12 & MICHIGAN & ITALY & GERMANY & & & & NORTH TAMARACH & OSCEOLA \\
\hline 4027 & 48 & ZANONI & ANTONIO & $s$ & 10 & MICHIGAN & ITALY & GERMANY & & & & NORTH TAMARACH & OSCEOLA \\
\hline 4028 & 50 & SANDRETTO & COSTANZO & $\mathrm{H}$ & 53 & ITALY & ITALY & ITALY & 1885 & SURFACE MAN/CM & PONT CANAVESE. TO & & OSCEOLA \\
\hline 4029 & 50 & SANDRET & GENOVEFFA & W & 52 & ITAL & ITA & ITALY & 1888 & & & & EOLA \\
\hline 4030 & 50 & SANDRETTO & & $\mathrm{s}$ & 23 & MICHIGAN & ITALY & ITALY & & SURFACE MAN/CM & PONT CANAVESE. TO & & EOLA \\
\hline 4031 & 50 & SANDRETTO & LORENZO & $\mathrm{s}$ & 19 & MICHIGAN & ITALY & ITALY & & OILER/ENGINE HOUSE & PONT CANAVESE. TO & & OSCEOLA \\
\hline
\end{tabular}




\begin{tabular}{|c|c|c|c|c|c|c|c|c|c|c|c|c|c|}
\hline 4032 & 50 & SANDRETTO & GENOVEFFA & $\mathrm{D}$ & 15 & MICHIGAN & ITALY & ITALY & & & PONT CANAVESE. TO & & OSCEOLA \\
\hline 4033 & 50 & SANDRETTO & LOUIS & $\mathrm{s}$ & 13 & MICHIGAN & ITALY & ITALY & & & PONT CANAVESE. TO & & OSCEOLA \\
\hline 4034 & 50 & SANDRETTO & MARGUERITE & GD & 7 & MICHIGAN & ITALY & ITALY & & & PONT CANAVESE. TO & & OSCEOLA \\
\hline 4035 & 50 & SANDRETTO & SAMUEL & NEP & 34 & ITALY & ITALY & ITALY & 1908 & TIMBERMAN/CM & PONT CANAVESE. TO & & OSCEOLA \\
\hline 4036 & 66 & BERTELLI & JOSEPH & $\mathrm{H}$ & 27 & ITALY & ITALY & ITALY & 1905 & TRAMMER/CM & & CONGLOMERATE ST. & OSCEOLA \\
\hline 4037 & 66 & BERTELLI & SESTILIA & w & 24 & ITALY & ITALY & ITALY & 1907 & & & CONGLOMERATE ST. & OSCEOLA \\
\hline 4038 & 66 & BERTELLI & JOSEPHINE & $\mathrm{D}$ & 5 & MICHIGAN & ITALY & ITALY & & & & CONGLOMERATE ST. & OSCEOLA \\
\hline 4039 & 66 & BERTELLI & LENA & $\mathrm{D}$ & 2 & MICHIGAN & ITALY & ITALY & & & & CONGLOMERATE ST. & OSCEOLA \\
\hline 4040 & 66 & BERTELLI & GIOVANNI & B & 23 & ITALY & ITALY & ITALY & 1909 & LABORER/CM & & CONGLOMERATE ST. & OSCEOLA \\
\hline 4041 & 66 & NAPALLI & JOSEPH & B & 35 & ITALY & ITALY & ITALY & 1909 & LABORER/CM & & CONGLOMERATE ST. & OSCEOLA \\
\hline 4042 & 66 & ROLANDO & PETER & B & 33 & ITALY & ITALY & ITALY & 1906 & LABORER/ROCK HOUSE & PONT CANAVESE. TO & & OSCEOLA \\
\hline 4043 & & COLOMBO & GIACOMO & $\mathrm{H}$ & 45 & ITALY & ITALY & ITALY & $98 ?$ & FARMER/FARM & PONT CANAVESE. TO & & OSCEOLA \\
\hline 4044 & & COLOMBO & GIUSEPPA & W & 38 & ITALY & ITALY & ITALY & 1901 & & PONT CANAVESE. TO & & OSCEOLA \\
\hline 4045 & & COLOMBO & DOROTEA & $\mathrm{D}$ & 16 & ITALY & ITALY & ITALY & 1901 & & PONT CANAVESE. TO & & OSCEOLA \\
\hline 4046 & & COLOMBO & CARLO & $\mathrm{s}$ & 14 & ITALY & ITALY & ITALY & 1901 & & PONT CANAVESE. TO & & OSCEOLA \\
\hline 4047 & & COLOMBO & ERNESTO & $\mathrm{s}$ & 12 & ITALY & ITALY & ITALY & 1901 & & PONT CANAVESE, TO & & OSCEOLA \\
\hline 4048 & & COLOMBO & GIACOMO & $\mathrm{s}$ & 7 & MICHIGAN & ITALY & ITALY & & & PONT CANAVESE. TO & & OSCEOLA \\
\hline 4049 & & COLOMBO & FRANK & $\mathrm{s}$ & 6 & MICHIGAN & ITALY & ITALY & & & PONT CANAVESE. TO & & OSCEOLA \\
\hline 4050 & & COLOMBO & MARIA & $\mathrm{D}$ & 4 & MICHIGAN & ITALY & ITALY & & & PONT CANAVESE. TO & & OSCEOLA \\
\hline 4051 & & COLOMBO & ANGELINA & $\mathrm{D}$ & 3 & MICHIGAN & ITALY & ITALY & & & PONT CANAVESE. TO & & OSCEOLA \\
\hline 4052 & 23 & GIULIO/GUILIO & FRANK & $\mathrm{H}$ & 65 & ITALY & ITALY & ITALY & 1895 & LABORER/CM & SAN GIORGIO CANAVESE. TO & CHURCH ST. & OSCEOLA \\
\hline 4053 & 23 & GIULIO/GUILIO & MARY & W & 45 & ITALY & ITALY & ITALY & 1895 & LADURERKUIVI & SAN GIORGIO CANAVESE, TO & CHURCH ST. & OSCEOLA \\
\hline 4054 & 23 & GIULIO/GUILIO & PASCAL & s & 18 & ITALY & ITALY & ITALY & 1895 & & SAN GIORGIO CANAVESE. TO & CHURCH ST. & OSCEOLA \\
\hline 4055 & 23 & GIULIO/GUILIO & LAURA & $\mathrm{D}$ & 17 & ITALY & ITALY & ITALY & 1895 & & SAN GIORGIO CANAVESE. TO & CHURCH ST. & OSCEOLA \\
\hline 4056 & 23 & GIULIO/GUILIO & MATTIE & $\mathrm{D}$ & 15 & ITALY & ITALY & ITALY & 1895 & & SAN GIORGIO CANAVESE. TO & CHURCH ST. & OSCEOLA \\
\hline 4057 & 23 & GIULIO/GUILIO & FREDERICK & $\mathrm{s}$ & 11 & MICHIGAN & ITALY & ITALY & & & SAN GIORGIO CANAVESE. TO & CHURCH ST. & OSCEOLA \\
\hline 4058 & 23 & SAPINO & JONNY & B & 26 & ITALY & ITALY & ITALY & 1903 & & & CHURCH ST. & OSCEOLA \\
\hline 4059 & 135 & BAUSANO & LOUIS & $\mathrm{H}$ & 30 & ITALY & ITALY & ITALY & 1903 & TIMBERMAN/CM & PONT CANAVESE, TO & MILLIONAIRE ST. & OSCEOLA \\
\hline 4060 & 135 & BAUSANO & ANNIE & W & 30 & ITALY & ITALY & ITALY & 1905 & & & MILLIONAIRE ST. & OSCEOLA \\
\hline 4061 & 135 & BAUSANO & KATIE & $\mathrm{D}$ & 3 & MICHIGAN & ITALY & ITALY & & & PONT CANAVESE. TO & MILLIONAIRE ST. & OSCEOLA \\
\hline 4062 & 135 & BAUSANO & DOMENICO & B & 22 & ITALY & ITALY & ITALY & 1909 & TIMBERMAN/CM & PONT CANAVESE. TO & MILLIONAIRE ST. & OSCEOLA \\
\hline 4063 & 134 & DE FILIPPI & JOSEPH & $\mathrm{H}$ & 39 & ITALY & ITALY & ITALY & 1906 & MINER/CM & SAN GIORGIO CANAVESE. TO & MILLIONAIRE ST. & OSCEOLA \\
\hline 4064 & 134 & DE FILIPPI & MARY & W & 30 & ITALY & ITALY & ITALY & ???? & IVIIVERTCIVI & SAIV SIUROUL CAIVAVEOE. TO & MILLIONAIRE ST. & OSCEOLA \\
\hline 4065 & 134 & DE FILIPPI & JOSEPH & s & $?$ & MICHIGAN & ITALY & ITALY & & & SAN GIORGIO CANAVESE, TO & MILLIONAIRE ST. & OSCEOLA \\
\hline 4066 & 134 & DE FILIPPI & JOHN & $\mathrm{s}$ & $?$ & MICHIGAN & ITALY & ITALY & & & SAN GIORGIO CANAVESE. TO & MILLIONAIRE ST. & OSCEOLA \\
\hline 4067 & 134 & DE FILIPPI & MARY & $\mathrm{D}$ & $?$ & MICHIGAN & ITALY & ITALY & & & SAN GIORGIO CANAVESE. TO & MILLIONAIRE ST. & OSCEOLA \\
\hline 4068 & 134 & GUERRUCCI & EGIDIO & $\mathrm{H}$ & 46 & ITALY & ITALY & ITALY & 1907 & TIMBERMAN/CM & LUCCA & MILLIONAIRE ST. & OSCEOLA \\
\hline 4069 & 134 & GUERRUCCI & MARY & W & 43 & ITALY & ITALY & ITALY & 1910 & & & MILLIONAIRE ST. & OSCEOLA \\
\hline 4070 & 266 & MARCO & DOMINICK & $\mathrm{H}$ & 21 & ITALY & ITALY & ITALY & 1904 & MINER/CM & TORINO & MILLIONAIRE ST. & OSCEOLA \\
\hline 4071 & 266 & MARCO & KATE & $\mathrm{MO}$ & 60 & ITALY & ITALY & ITALY & 1904 & & TORINO & MILLIONAIRE ST. & OSCEOLA \\
\hline 4072 & 266 & MARCO & JOHN & $B R$ & 18 & ITALY & ITALY & ITALY & 1904 & DRILL BOY/CM & TORINO & MILLIONAIRE ST. & OSCEOLA \\
\hline 4073 & 266 & MARCO & LUCY & SI & 16 & ITALY & ITALY & ITALY & 1904 & & TORINO & MILLIONAIRE ST. & OSCEOLA \\
\hline 4074 & 266 & MARCO & FRANK & $\mathrm{BR}$ & 6 & MICHIGAN & ITALY & ITALY & & & TORINO & MILLIONAIRE ST. & OSCEOLA \\
\hline 4075 & 187 & AIMONE & BERNARDO & $\mathrm{H}$ & 36 & ITALY & ITALY & ITALY & 1894 & MINER/CM & FORNO RIVARA. TO & BACK ST. & OSCEOLA \\
\hline 4076 & 187 & AIMONE & MARIA & W & 36 & ITALY & ITALY & ITALY & 1894 & 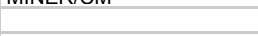 & FORNO RIVARA. TO & BACK ST. & OSCEOLA \\
\hline 4077 & 187 & AIMONE & CATERINA & D & 13 & ILLINOIS & ITALY & ITALY & & & FORNO RIVARA. TO & BACK ST. & OSCEOLA \\
\hline 4078 & 187 & AIMONE & LUCIA & $\mathrm{D}$ & 11 & ILLINOIS & ITALY & ITALY & & & FORNO RIVARA. TO & BACK ST. & OSCEOLA \\
\hline 4079 & 187 & AIMONE & GIORGIO & s & 10 & MICHIGAN & ITALY & ITALY & & & FORNO RIVARA. TO & BACK ST. & OSCEOLA \\
\hline 4080 & 187 & AIMONE & GIOVANNI & $\mathrm{s}$ & 9 & MICHIGAN & ITALY & ITALY & & & FORNO RIVARA. TO & BACK ST. & OSCEOLA \\
\hline 4081 & 187 & AIMONE & MARIA & $\mathrm{D}$ & 5 & ILLINOIS & ITALY & ITALY & & & FORNO RIVARA. TO & BACK ST. & OSCEOLA \\
\hline 4082 & 187 & SCUSSELL & JOSEPH & $\mathrm{H}$ & 39 & ITALY & ITALY & ITALY & 1894 & MINER/CM & BELLUNO & OPECHEE ST. & OSCEOLA - OPECHEE LOCATION \\
\hline 4083 & 187 & SCUSSELL & TERESA & W & 35 & ITALY & ITALY & ITALY & 1895 & & BELLUNO & OPECHEE ST. & OSCEOLA - OPECHEE LOCATION \\
\hline 4084 & 187 & SCUSSELL & ONORATO & $\mathrm{s}$ & 17 & ITALY & ITALY & ITALY & 1895 & DRILL BOY/CM & BELLUNO & OPECHEE ST. & OSCEOLA - OPECHEE LOCATION \\
\hline 4085 & 187 & SCUSSELL & JOHN & $\mathrm{s}$ & 15 & MICHIGAN & ITALY & ITALY & & & BELLUNO & OPECHEE ST. & OSCEOLA - OPECHEE LOCATION \\
\hline 4086 & 187 & SCUSSELL & MARY & $\mathrm{D}$ & 13 & MICHIGAN & ITALY & ITALY & & & BELLUNO & OPECHEE ST. & OSCEOLA - OPECHEE LOCATION \\
\hline 4087 & 187 & SCUSSELL & ANGELO & $\mathrm{s}$ & 11 & MICHIGAN & ITALY & ITALY & & & BELLUNO & OPECHEE ST. & OSCEOLA - OPECHEE LOCATION \\
\hline 4088 & 187 & SCUSSELL & LOUIS & $\mathrm{s}$ & 9 & MICHIGAN & ITALY & ITALY & & & BELLUNO & OPECHEE ST. & OSCEOLA - OPECHEE LOCATION \\
\hline 4089 & 187 & SCUSSELL & ANTON & $\mathrm{s}$ & 7 & MICHIGAN & ITALY & ITALY & & & BELLUNO & OPECHEE ST. & OSCEOLA - OPECHEE LOCATION \\
\hline 4090 & 187 & SCUSSELL & JOSEPH & $\mathrm{S}$ & 5 & MICHIGAN & ITALY & ITALY & & & BELLUNO & OPECHEE ST. & OSCEOLA - OPECHEE LOCATION \\
\hline 4091 & 187 & SCUSSELL & TERESA & $\mathrm{D}$ & 3 & MICHIGAN & ITALY & ITALY & & & BELLUNO & OPECHEE ST. & OSCEOLA - OPECHEE LOCATION \\
\hline 4092 & 187 & MIANA & ASTIANO & B & 35 & -Y & & ITA & 1900 & /CM & VOLTAGO. BELLUNO & OPECHEE ST. & OSCEOLA - OPECHEE LOCATION \\
\hline 4093 & 187 & SCUSSELL & ATTILIO & B & 39 & & ITALY & ITALY & 1900 & LABORER/CM & & OPECHEE ST. & OSCEOLA - OPECHEE LOCATION \\
\hline 4094 & 187 & CHIESA & BATTISTA & B & 26 & ITALY & ITALY & ITALY & 1909 & TRAMMER/CM & CASELLE TORINESE. TO & OPECHEE ST. & OSCEOLA - OPECHEE LOCATION \\
\hline
\end{tabular}




\begin{tabular}{|c|c|c|c|c|c|c|c|c|c|c|c|c|c|}
\hline 4095 & 187 & COMINA & EPIFANIO & B & 18 & ITALY & ITALY & ITALY & 1909 & MINER/CM & VOLTAGO. BELLUNO & OPECHEE ST. & OSCEOLA - OPECHEE LOCATION \\
\hline 4096 & 187 & COMINA & LUIGI & B & 18 & ITALY & ITALY & ITALY & 1909 & TRAMMER/CM & VOLTAGO. BELLUNO & OPECHEE ST. & OSCEOLA - OPECHEE LOCATION \\
\hline 4097 & 184 & TALENTINO & FRANCESCO & $\mathrm{H}$ & 60 & ITALY & ITALY & ITALY & 1903 & LABORER/ROCK HOUSE & PARELLA. TO & OPECHEE ST. & OSCEOLA - OPECHEE LOCATION \\
\hline 4098 & 184 & TALENTINO & DELFINA & W & 44 & MICHIGAN & ITALY & ITALY & & & & OPECHEE ST. & OSCEOLA - OPECHEE LOCATION \\
\hline 4099 & 184 & TALENTINO & PIETRO & s & 23 & MICHIGAN & ITALY & ITALY & & BANKER/BANK & PARELLA. TO & OPECHEE ST. & OSCEOLA - OPECHEE LOCATION \\
\hline 4100 & 184 & TALENTINO & GIACOMO & $\mathrm{s}$ & 17 & MICHIGAN & ITALY & ITALY & & CLERK/POST OFFICE & PARELLA. TO & OPECHEE ST. & OSCEOLA - OPECHEE LOCATION \\
\hline 4101 & 184 & TALENTINO & MANUIS? & $\mathrm{D}$ & 13 & MICHIGAN & ITALY & ITALY & & & PARELLA. TO & OPECHEE ST. & OSCEOLA - OPECHEE LOCATION \\
\hline 4102 & 184 & TALENTINO & VITTORIO & s & 11 & MICHIGAN & ITALY & ITALY & & & PARELLA. TO & OPECHEE ST. & OSCEOLA - OPECHEE LOCATION \\
\hline 4103 & 184 & BORATI & ANTON & B & 32 & ITALY & ITALY & ITALY & 1906 & TRAMMER/CM & & OPECHEE ST. & OSCEOLA - OPECHEE LOCATION \\
\hline 4104 & 184 & PASTORE & DOMENICO & B & 32 & ITALY & ITALY & ITALY & 1898 & LABORER/CM & SAN MARTINO CANAVESE. TO & OPECHEE ST. & OSCEOLA - OPECHEE LOCATION \\
\hline 4105 & 189 & FANTINI & PIETRO & $\mathrm{H}$ & 24 & ITALY & ITALY & ITALY & 1905 & TIMBERMAN/CM & GEROSA. BERGAMO & COUNTY ROAD & OSCEOLA \\
\hline 4106 & 189 & FANTINI & TERESA & W & 26 & ITALY & ITALY & ITALY & $01 / 07$ & & GEROSA. BERGAMO & COUNTY ROAD & OSCEOLA \\
\hline 4107 & & CONSANI & PIETRO & $\mathrm{H}$ & 43 & ITALY & ITALY & ITALY & 1904 & FIREMAN/STAMP MILL & LUCCA & & OSCEOLA - TAMARACK CITY \\
\hline 4108 & & CONSANI & LIZZIE & w & 33 & ITALY & ITALY & ITALY & 1904 & & LUCCA & & OSCEOLA - TAMARACK CITY \\
\hline 4109 & & CONSANI & AMELIA & $\mathrm{D}$ & 7 & ITALY & ITALY & ITALY & 1904 & & LUCCA & & OSCEOLA - TAMARACK CITY \\
\hline 4110 & & CONSANI & CATERINA & $\mathrm{D}$ & 5 & MICHIGAN & ITALY & ITALY & & & LUCCA & & OSCEOLA - TAMARACK CITY \\
\hline 4111 & & CONSANI & PETER & $\mathrm{s}$ & $1.5 \mathrm{M}$ & MICHIGAN & ITALY & ITALY & & & LUCCA & & OSCEOLA - TAMARACK CITY \\
\hline 4112 & & CONSANI & EMILIO & $\mathrm{H}$ & 49 & ITALY & ITALY & ITALY & 1895 & FIREMAN/STAMP MILL & LUCCA & & OSCEOLA - TAMARACK CITY \\
\hline 4113 & & CONSANI & AMELIA & W & 39 & ITALY & ITALY & ITALY & 1895 & & LUCCA & & OSCEOLA - TAMARACK CITY \\
\hline 4114 & & CONSANI & ARTHUR & s & 15 & ITALY & ITALY & ITALY & & & LUCCA & & OSCEOLA - TAMARACK CITY \\
\hline 4115 & & CONSANI & JOSEPHINE & $\mathrm{D}$ & 14 & MICHIGAN & ITALY & ITALY & & & LUCCA & & OSCEOLA - TAMARACK CITY \\
\hline 4116 & & CONSANI & ALBERT & s & 12 & MICHIGAN & ITALY & ITALY & & & LUCCA & & OSCEOLA - TAMARACK CITY \\
\hline 4117 & & CONSANI & FRANK & $\mathrm{s}$ & 11 & MICHIGAN & ITALY & ITALY & & & LUCCA & & OSCEOLA - TAMARACK CITY \\
\hline 4118 & & CONSANI & JOSEPH & $\mathrm{S}$ & 9 & MICHIGAN & ITALY & ITALY & & & LUCCA & & OSCEOLA - TAMARACK CITY \\
\hline 4119 & & CONSANI & PETER & $\mathrm{s}$ & 6 & MICHIGAN & ITALY & ITALY & & & LUCCA & & OSCEOLA - TAMARACK CITY \\
\hline 4120 & & BARACINI & FRANK & B & 45 & ITALY & ITALY & ITALY & 1892 & FIREMAN/STAMP MILL & & & OSCEOLA - TAMARACK CITY \\
\hline 4121 & & PINI & EUGENE & $\mathrm{H}$ & 34 & ITALY & ITALY & ITALY & 1899 & LABORER/STAMP MILL & LUCCA & & OSCEOLA - TAMARACK CITY \\
\hline 4122 & & PINI & LOUISA & W & 32 & ITALY & ITALY & ITALY & 1906 & & & & OSCEOLA - TAMARACK CITY \\
\hline 4123 & & PINI & EUGENE & $\mathrm{s}$ & $1.3 \mathrm{M}$ & MICHIGAN & ITALY & ITALY & & & LUCCA & & OSCEOLA - TAMARACK CITY \\
\hline 4124 & & BAIOCCHI & DANTE & B & 37 & ITALY & ITALY & ITALY & 1899 & & COLLODI. LU & & OSCEOLA - TAMARACK CITY \\
\hline 4125 & & BAIOCCHI & CAMILLO & $\mathrm{H}$ & 37 & ITALY & ITALY & ITALY & 1892 & MACHINIST/STAMP MILL & COLLODI. LU & & OSCEOLA - TAMARACK CITY \\
\hline 4126 & & BAIOCCHI & RADY & W & 34 & ITALY & ITALY & ITALY & 1899 & 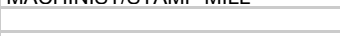 & & & OSCEOLA - TAMARACK CITY \\
\hline 4127 & & BAIOCCHI & ANNE & $\mathrm{D}$ & 9 & MICHIGAN & ITALY & ITALY & $109 y$ & & COLLODI. LU & & OSCEOLA - TAMARACK CITY \\
\hline 4128 & & BAIOCCHI & MARGARET & $\mathrm{D}$ & 8 & MICHIGAN & ITALY & ITALY & & & COLLODI, LU & & OSCEOLA - TAMARACK CITY \\
\hline 4129 & & BAIOCCHI & ELLA & $\mathrm{D}$ & 7 & MICHIGAN & ITALY & ITALY & & & COLLODI. LU & & OSCEOLA - TAMARACK CITY \\
\hline 4130 & & BAIOCCHI & NATALE & $\mathrm{H}$ & 47 & ITALY & ITALY & ITALY & 1889 & MACHINIST/STAMP MILL & COLLODI. LU & & OSCEOLA - TAMARACK CITY \\
\hline 4131 & & BAIOCCHI & MARY & W & 42 & ITALY & ITALY & ITALY & 1893 & & & & OSCEOLA - TAMARACK CITY \\
\hline 4132 & & BAIOCCHI & ANITA & $\mathrm{D}$ & 16 & MICHIGAN & ITALY & ITALY & & & COLLODI. LU & & OSCEOLA - TAMARACK CITY \\
\hline 4133 & & BAIOCCHI & GIOIA & $\mathrm{D}$ & 15 & MICHIGAN & ITALY & ITALY & & & COLLODI. LU & & OSCEOLA - TAMARACK CITY \\
\hline 4134 & & BAIOCCHI & MAIDA & $\mathrm{D}$ & 14 & MICHIGAN & ITALY & ITALY & & & COLLODI. LU & & OSCEOLA - TAMARACK CITY \\
\hline 4135 & & BAIOCCHI & WILLIAM & $\mathrm{s}$ & 13 & MICHIGAN & ITALY & ITALY & & & COLLODI. LU & & OSCEOLA - TAMARACK CITY \\
\hline 4136 & & BAIOCCHI & JENNIE & $\mathrm{D}$ & 9 & MICHIGAN & ITALY & ITALY & & & COLLODI. LU & & OSCEOLA - TAMARACK CITY \\
\hline 4137 & & BAIOCCHI & PAULINA & $\mathrm{D}$ & 8 & MICHIGAN & ITALY & ITALY & & & COLLODI. LU & & OSCEOLA - TAMARACK CITY \\
\hline 4138 & & BAIOCCHI & WALLACE & $\mathrm{s}$ & 6 & MICHIGAN & ITALY & ITALY & & & COLLODI. LU & & OSCEOLA - TAMARACK CITY \\
\hline 4139 & & OSTENZI & FRANK & $\mathrm{H}$ & 62 & ITALY & ITALY & ITALY & 1880 & PROPRIETOR/GROCERY STORE & LUCCA & & OSCEOLA - TAMARACK CITY \\
\hline 4140 & & OSTENZI & MARY & D & 27 & MICHIGAN & ITALY & ITALY & & & LUCCA & & OSCEOLA - TAMARACK CITY \\
\hline 4141 & & OSTENZI & PETER & $\mathrm{s}$ & 20 & MICHIGAN & ITALY & ITALY & & & LUCCA & & OSCEOLA - TAMARACK CITY \\
\hline 4142 & & OSTENZI & FLORINE & $\mathrm{D}$ & 16 & MICHIGAN & ITALY & ITALY & & & LUCCA & & OSCEOLA - TAMARACK CITY \\
\hline 4143 & & OSTENZI & ALFRED & $\mathrm{s}$ & 13 & MICHIGAN & ITALY & ITALY & & & LUCCA & & OSCEOLA - TAMARACK CITY \\
\hline 4144 & & OSTENZI & HUBERT & $\mathrm{s}$ & 11 & MICHIGAN & ITALY & ITALY & & & LUCCA & & OSCEOLA - TAMARACK CITY \\
\hline 4145 & & OSTENZI & MARGARET & $\mathrm{D}$ & 8 & MICHIGAN & ITALY & ITALY & & & LUCCA & & OSCEOLA - TAMARACK CITY \\
\hline 4146 & 40 & MARDA & JOSEPH & $\mathrm{H}$ & 25 & ITALY & ITALY & ITALY & 1907 & MINER/CM & & HURON ST. & PORTAGE - HURONTOWN \\
\hline 4147 & 40 & MARDA & ROSA & W & 20 & ITALY & ITALY & ITALY & 1907 & & & HURON ST. & PORTAGE - HURONTOWN \\
\hline 4148 & 40 & MARDA & SAMUEL & $\mathrm{s}$ & 2 & MICHIGAN & ITALY & ITALY & & & & HURON ST. & PORTAGE - HURONTOWN \\
\hline 4149 & 40 & MARDA & JAMES & $\mathrm{s}$ & $1.1 \mathrm{M}$ & MICHIGAN & ITALY & ITALY & & & & HURON ST. & PORTAGE - HURONTOWN \\
\hline 4150 & & ClUCCl & AUGUST & $\mathrm{H}$ & 35 & ITALY & ITALY & ITALY & 1901 & MINER/CM & LUCCA & DODGEVILLE & PORTAGE - HURONTOWN \\
\hline 4151 & & $\mathrm{CIUCCl}$ & MARY & W & 25 & ITALY & ITALY & ITALY & 1903 & & & DODGEVILLE & PORTAGE - HURONTOWN \\
\hline 4152 & & $\mathrm{ClUCCl}$ & ANNA & $\mathrm{D}$ & 4 & MICHIGAN & ITALY & ITALY & & & LUCCA & DODGEVILLE & PORTAGE - HURONTOWN \\
\hline 4153 & & ClUCCl & LENA & $\mathrm{D}$ & 3 & MICHIGAN & ITALY & ITALY & & & LUCCA & DODGEVILLE & PORTAGE - HURONTOWN \\
\hline 4154 & & BIGONGIARI & LOUIS & $\mathrm{H}$ & 42 & ITALY & ITALY & ITALY & 1902 & LABORER/CM & MATRAIA. LU & DODGEVILLE & PORTAGE - HURONTOWN \\
\hline 4155 & & BIGONG & CESIRA & W & 40 & & ITA & ITAI & 1902 & & MATRAIA. LU & VILLE & PORTAGE - HU \\
\hline 4156 & & BIGONGIARI & CARLO & s & 13 & ITALY & ITALY & ITALY & 1902 & & MATRAIA. LU & DODGEVILLE & PORTAGE - HURONTOWN \\
\hline 4157 & & BIGONGIARI & JOHN & $\mathrm{s}$ & 8 & MICHIGAN & ITALY & ITALY & & & MATRAIA. LU & DODGEVILLE & PORTAGE - HURONTOWN \\
\hline
\end{tabular}




\begin{tabular}{|c|c|c|c|c|c|c|c|c|c|c|c|c|c|}
\hline 4158 & & BIGONGIARI & ACHILLE & $\mathrm{s}$ & 7 & MICHIGAN & ITALY & ITALY & & & MATRAIA. LU & DODGEVILLE & PORTAGE - HURONTOWN \\
\hline 4159 & & BIGONGIARI & MARY & D & 5 & MICHIGAN & ITALY & ITALY & & & MATRAIA. LU & DODGEVILLE & PORTAGE - HURONTOWN \\
\hline 4160 & & FASANA & RALPH & $\mathrm{H}$ & 27 & ITALY & ITALY & ITALY & 1907 & LABORER/CM & TORINO & & PORTAGE - HURONTOWN \\
\hline 4161 & & FASANA & ALICE & W & 28 & ITALY & ITALY & ITALY & 1907 & & TORINO & & PORTAGE - HURONTOWN \\
\hline 4162 & & FASANA & BOUNEAU & s & $19 \mathrm{M}$ & ILLINOIS & ITALY & ITALY & & & TORINO & & PORTAGE - HURONTOWN \\
\hline 4163 & 40 & BERTOLUCCI & ANTONIO & B & 29 & ITALY & ITALY & ITALY & 1908 & LABORER/CM & LUCCA & HURON ST. & PORTAGE - HURONTOWN \\
\hline 4164 & 40 & BUFFONE & ANTONIO & B & 30 & ITALY & ITALY & ITALY & 1908 & LABORER/CM & CARPANZANO. COSENZA & HURON ST. & PORTAGE - HURONTOWN \\
\hline 4165 & 40 & VAGLIATORE & FRANK & B & 34 & ITALY & ITALY & ITALY & 1908 & LABORER/CM & & HURON ST. & PORTAGE - HURONTOWN \\
\hline 4166 & 40 & BELANGO & FELICE & B & 21 & ITALY & ITALY & ITALY & 1905 & LABORER/CM & & 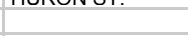 & PORTAGE - HURONTOWN \\
\hline 4167 & & LUIMA/NINA & LEONARD & B & 41 & ITALY & ITALY & ITALY & 1909 & LABORER/CM & & & PORTAGE - HURONTOWN \\
\hline 4168 & & VALENTE & SAM & B & 33 & ITALY & ITALY & ITALY & 1901 & LABORER/CM & CARPINONE. ISERNIA & & PORTAGE - HURONTOWN \\
\hline 4169 & & FUSCHINO & LEONARD & B & 39 & ITALY & ITALY & ITALY & 1901 & LABORER/CM & PESCHE. CAMPOBASSO & & PORTAGE - HURONTOWN \\
\hline 4170 & 29 & PIZZUTI & JOHN & $\mathrm{H}$ & 46 & ITALY & ITALY & ITALY & 1902 & LABORER/CM & CARPINONE. ISERNIA & & PORTAGE - HURONTOWN \\
\hline 4171 & 29 & PIZZUTI-PAONE & FLORINDA & W & 34 & ITALY & ITALY & ITALY & 1905 & & CARPINONE. ISERNIA & & PORTAGE - HURONTOWN \\
\hline 4172 & 29 & PIZZUTI & ROSA & D & 11 & ITALY & ITALY & ITALY & 1905 & & CARPINONE. ISERNIA & HURON ST. & PORTAGE - HURONTOWN \\
\hline 4173 & 29 & PIZZUTI & MARY & $\mathrm{D}$ & 4 & MICHIGAN & ITALY & ITALY & & & CARPINONE, ISERNIA & HURON ST. & PORTAGE - HURONTOWN \\
\hline 4174 & 29 & PIZZUTI & DOMINIQUE & $\mathrm{s}$ & 3 & MICHIGAN & ITALY & ITALY & & & CARPINONE. ISERNIA & HURON ST. & PORTAGE - HURONTOWN \\
\hline 4175 & 29 & PIZZUTI & MARIE & $\mathrm{D}$ & 2 & MICHIGAN & ITALY & ITALY & & & CARPINONE. ISERNIA & HURON ST. & PORTAGE - HURONTOWN \\
\hline 4176 & 29 & PIZZUTI & LEONARD & B & 24 & ITALY & ITALY & ITALY & 1909 & LABORER/CM & CARPINONE. ISERNIA & HURON ST. & PORTAGE - HURONTOWN \\
\hline 4177 & 29 & PIZZUTI & MICHAEL & B & 30 & ITALY & ITALY & ITALY & 1909 & LABORER/CM & CARPINONE. ISERNIA & HURON ST. & PORTAGE - HURONTOWN \\
\hline 4178 & 29 & VALENTE & RALPH & B & 26 & ITALY & ITALY & ITALY & 1904 & LABORER/CM & CARPINONE. ISERNIA & HURON ST. & PORTAGE - HURONTOWN \\
\hline 4179 & 29 & NINA & RAFFAELE & $\begin{array}{l}\mathrm{D} \\
\mathrm{B}\end{array}$ & 44 & ITALY & ITALY & ITALY & 1909 & LABORER/CM & UARTIIVUIVE. IOERTIVIA & HURON ST. & PORTAGE - HURONTOWN \\
\hline 4180 & 29 & VALENTE & MICHAEL & B & 30 & ITALY & ITALY & ITALY & 1903 & LABORER/CM & CARPINONE. ISERNIA & HURON ST. & PORTAGE - HURONTOWN \\
\hline 4181 & 29 & BELANGO & COSMO & B & 43 & ITALY & ITALY & ITALY & 1905 & LABORER/CM & & HURON ST. & PORTAGE - HURONTOWN \\
\hline 4182 & 29 & DEIPEA & JOHN & B & 34 & ITALY & ITALY & ITALY & 1905 & LABORER/CM & & HURON ST. & PORTAGE - HURONTOWN \\
\hline 4183 & 29 & DEALI PUKI & PETER & B & 38 & ITALY & ITALY & ITALY & 1910 & LABORER/CM & & HURON ST. & PORTAGE - HURONTOWN \\
\hline 4184 & 29 & ANDENUCCI & ANTONIO & B & 34 & ITALY & ITALY & ITALY & 1909 & LABORER/CM & & HURON ST. & PORTAGE - HURONTOWN \\
\hline 4185 & 29 & DA CAMPO & ANTONIO & B & 26 & ITALY & ITALY & ITALY & 1903 & LABORER/CM & BELLUNO & HURON ST. & PORTAGE - HURONTOWN \\
\hline 4186 & & $\mathrm{ClUCCl}$ & AUGUST & $\mathrm{H}$ & 36 & ITALY & ITALY & ITALY & 1903 & LABORER/CM & LUCCA & & PORTAGE - HURONTOWN \\
\hline 4187 & & $\mathrm{ClUCCl}$ & BENILDE & W & 26 & ITALY & ITALY & ITALY & 1904 & & & & PORTAGE - HURONTOWN \\
\hline 4188 & & $\mathrm{ClUCCl}$ & ELMER & $\mathrm{s}$ & 3 & MICHIGAN & ITALY & ITALY & & & LUCCA & & PORTAGE - HURONTOWN \\
\hline 4189 & & $\mathrm{ClUCCl}$ & LENA & $\mathrm{D}$ & 1 & MICHIGAN & ITALY & ITALY & & & LUCCA & & PORTAGE - HURONTOWN \\
\hline 4190 & & MONTICELLO/I & BERNARD & B & 22 & ITALY & ITALY & ITALY & 1910 & LABORER/CM & & & PORTAGE - HURONTOWN \\
\hline 4191 & 96 & NOZERO & ANTONIO & $\mathrm{H}$ & 38 & ITALY & ITALY & ITALY & 1894 & LABORER/CM & SAN MARTINO CANAVESE, TO & DODGEVILLE & PORTAGE - HURONTOWN \\
\hline 4192 & 96 & NOZERO & AMELIA & W & 26 & MICHIGAN & ITALY & ITALY & & & & DODGEVILLE & PORTAGE - HURONTOWN \\
\hline 4193 & 96 & NOZERO & MICHAEL & s & 8 & MICHIGAN & ITALY & ITALY & & & SAN MARTINO CANAVESE. TO & DODGEVILLE & PORTAGE - HURONTOWN \\
\hline 4194 & 96 & NOZERO & PETER & $\mathrm{S}$ & 4 & MICHIGAN & ITALY & ITALY & & & SAN MARTINO CANAVESE. TO & DODGEVILLE & PORTAGE - HURONTOWN \\
\hline 4195 & 96 & GUGLIELMINO & JOSEPH & B & 21 & ITALY & ITALY & ITALY & 1906 & LABORER/CM & SAN GIORGIO CANAVESE. TO & DODGEVILLE & PORTAGE - HURONTOWN \\
\hline 4196 & 96 & GRAVES & LOUIS & B & 27 & ITALY & ITALY & ITALY & 1906 & LABORER/CM & & DODGEVILLE & PORTAGE - HURONTOWN \\
\hline 4197 & & BRUSSO & BATTISTA & $\mathrm{H}$ & 27 & ITALY & ITALY & ITALY & 1900 & MINER/CM & TORINO & SUPERIOR MINE & PORTAGE - HURONTOWN \\
\hline 4198 & & BROSSO & MAGGIE & W & 23 & ITALY & ITALY & ITALY & 1907 & & & SUPERIOR MINE & PORTAGE - HURONTOWN \\
\hline 4199 & & COLOMBO & ANTONIO & B & 35 & ITALY & ITALY & ITALY & 1890 & MINER/CM & TORINO & SUPERIOR MINE & PORTAGE - HURONTOWN \\
\hline 4200 & & MASORI & FRANK & B & 23 & ITALY & ITALY & ITALY & 1900 & MINER/CM & & SUPERIOR MINE & PORTAGE - HURONTOWN \\
\hline 4201 & & ANARICO & PETER & B & 28 & ITALY & ITALY & ITALY & 1900 & MINER/CM & & SUPERIOR MINE & PORTAGE - HURONTOWN \\
\hline 4202 & & BORGO & JOSEPH & B & 24 & ITALY & ITALY & ITALY & 1887 & MINER/CM & VICENZA & SUPERIOR MINE & PORTAGE - HURONTOWN \\
\hline 4203 & & PASTORE & JOHN & B & 23 & ITALY & ITALY & ITALY & 1904 & MINER/CM & TORINO & SUPERIOR MINE & PORTAGE - HURONTOWN \\
\hline 4204 & & DENTO & GIORGIO & $\begin{array}{l}\mathrm{D} \\
\mathrm{B}\end{array}$ & 23 & ITALY & ITALY & ITALY & 1907 & MINER/CM & TURIIV & SUPERIOR MINE & PORTAGE - HURONTOWN \\
\hline 4205 & & MASSTERO & JOSEPH & $\begin{array}{l}\mathrm{D} \\
\mathrm{B}\end{array}$ & 25 & ITALY & ITALY & ITALY & $00 / 03$ & MINER/CM & & SUPERIOR MINE & PORTAGE - HURONTOWN \\
\hline 4206 & & BARSOCCHI & FRANCES & B & 22 & NEW YORK & ITALY & ITALY & & HOUSEKEEPER/HOME & LUCCA & & PORTAGE - HURONTOWN \\
\hline 4207 & & BARSOCCHI & ERNEST & B & $1.6 \mathrm{M}$ & MICHIGAN & ITALY & ITALY & & & LUCCA & & PORTAGE - HURONTOWN \\
\hline 4208 & 126 & FALKANES? & PETER & B & 17 & ITALY & ITALY & ITALY & 1906 & WAITER/CAFE' & & & PORTAGE - HURONTOWN \\
\hline 4209 & 128 & FINOGLIO & LOUIS & $\mathrm{H}$ & 28 & ITALY & ITALY & ITALY & 1900 & WAITER/CAFE' & TORINO & & PORTAGE - HURONTOWN \\
\hline 4210 & 128 & FINOGLIO & JOSEPHINE & W & 26 & ITALY & ITALY & ITALY & 1900 & & TORINO & & PORTAGE - HURONTOWN \\
\hline 4211 & 128 & FINOGLIO & KATE & $\mathrm{D}$ & 4 & MICHIGAN & ITALY & ITALY & & & & & PORTAGE - HURONTOWN \\
\hline 4212 & 128 & FINOGLIO & MIKE & $\mathrm{s}$ & 0 & MICHIGAN & ITALY & ITALY & & & TORINO & & PORTAGE - HURONTOWN \\
\hline 4213 & 134 & CARFELLISSA & MIKE & $\mathrm{H}$ & 30 & ITALY & ITALY & ITALY & 1905 & LABORER/BREWERY & & MONTEZUMA & PORTAGE - HOUGHTON \\
\hline 4214 & 134 & CARFELLISSA & JULIA & W & 28 & ITALY & ITALY & ITALY & 1908 & & & MONTEZUMA & PORTAGE - HOUGHTON \\
\hline 4215 & 134 & CARFELLISSA & LENA & $\mathrm{D}$ & 4 & MICHIGAN & ITALY & ITALY & & & & MONTEZUMA & PORTAGE - HOUGHTON \\
\hline 4216 & 134 & CARFELLISSA & MARY & $\mathrm{D}$ & 2 & MICHIGAN & ITALY & ITALY & & & & MONTEZUMA & PORTAGE - HOUGHTON \\
\hline 4217 & 115 & $\begin{array}{l}\text { PANTERA } \\
\text { PAR }\end{array}$ & FRED & B & 32 & MICHIGAN & ITALY & ITALY & & JANITOR/SCHOOL & LUCCA & SHELDEN ST. & PORTAGE - HOUGHTON \\
\hline 4218 & 166 & SCUSSEL & JOHN & $\mathrm{H}$ & 26 & ITALY & ITALY & ITALY & 1898 & WAITER/CAFE' & BELLUNO & & PORTAGE - HOUGHTON \\
\hline 4219 & 166 & SCUSSEL & ANGELINA & w & 25 & ITALY & ITALY & ITALY & 1902 & & & & PORTAGE - HOUGHTON \\
\hline 4220 & 166 & SCUSSEL & $\begin{array}{l}\text { PRIMO } \\
\text { PR }\end{array}$ & s & 6 & MICHIGAN & ITALY & ITALY & & & BELLUNO & & PORTAGE - HOUGHTON \\
\hline
\end{tabular}




\begin{tabular}{|c|c|c|c|c|c|c|c|c|c|c|c|c|c|}
\hline 4221 & 166 & SCUSSEL & MICHELE & $\mathrm{s}$ & 2 & MICHIGAN & ITALY & ITALY & & & BELLUNO & & PORTAGE - HOUGHTON \\
\hline 4222 & 101 & GIULIO & ANTONIO & $\mathrm{H}$ & 38 & ITALY & ITALY & ITALY & 1894 & SALOONKEEPER & SAN GIORGIO CANAVESE. TO & SHELDEN ST. & PORTAGE - HOUGHTON \\
\hline 4223 & 101 & GIULIO & PETRONILLA & W & 31 & ITALY & ITALY & ITALY & 1905 & & & SHELDEN ST. & PORTAGE - HOUGHTON \\
\hline 4224 & 101 & BASSO & GIOVANNI & B & 33 & ITALY & ITALY & ITALY & 1899 & LABORER & SAN GIORGIO CANAVESE. TO & SHELDEN ST. & PORTAGE - HOUGHTON \\
\hline 4225 & 99 & MUSSO/A & $?$ & B & 22 & ITALY & ITALY & ITALY & 1907 & & & & PORTAGE - HOUGHTON \\
\hline 4226 & 99 & FILIPPI & ALBERTO & B & 22 & ITALY & ITALY & ITALY & 1907 & TRAMMER/CM & & & PORTAGE - HOUGHTON \\
\hline 4227 & 100 & CHIOTTI & JAMES & $\mathrm{H}$ & 34 & ITALY & ITALY & ITALY & 1890 & BREWER/BREWERY & SAN GIORGIO CANAVESE. TO & ALBION ST. & PORTAGE - HOUGHTON \\
\hline 4228 & 100 & CHIOTTI & CHRISTINA & W & 32 & ITALY & ITALY & ITALY & 1898 & & & ALBION ST. & PORTAGE - HOUGHTON \\
\hline 4229 & 100 & CHIOTTI & JOHN & s & 6 & MICHIGAN & ITALY & ITALY & & & SAN GIORGIO CANAVESE. TO & ALBION ST. & PORTAGE - HOUGHTON \\
\hline 4230 & 100 & CHIOTTI & KATE & $\mathrm{D}$ & 11 & MICHIGAN & ITALY & ITALY & & & SAN GIORGIO CANAVESE. TO & ALBION ST. & PORTAGE - HOUGHTON \\
\hline 4231 & & GIUSTI & AMEDEO & $\mathrm{H}$ & 33 & ITALY & ITALY & ITALY & 1909 & MINER/CM & LUCCA & & QUINCY - HARDSCRABBLE LOC. \\
\hline 4232 & & GIUSTI & GIULIA & W & 26 & ITALY & ITALY & ITALY & 1906 & & & & QUINCY - HARDSCRABBLE LOC. \\
\hline 4233 & & GIUSTI & GUIDO & $\mathrm{BR}$ & 28 & ITALY & ITALY & ITALY & 1906 & MINER/CM & LUCCA & & QUINCY - HARDSCRABBLE LOC. \\
\hline 4234 & & PANGCHSTI & GIOVANNI & B & 21 & ITALY & ITALY & ITALY & 1909 & MINER/CM & & & QUINCY - HARDSCRABBLE LOC. \\
\hline 4235 & & INNOCENTI & SABATINO & B & 40 & ITALY & ITALY & ITALY & 1909 & MINER/CM & LUCCA & & QUINCY - HARDSCRABBLE LOC. \\
\hline 4236 & & BALDASSARRE & ANNIBALE & B & 25 & ITALY & ITALY & ITALY & 1907 & MINER/CM & LUCCA & & QUINCY - HARDSCRABBLE LOC. \\
\hline 4237 & & STEFANI & LIZZIE & B & 17 & MICHIGAN & ITALY & ITALY & & SERVANT/PF & LUCCA & & QUINCY - HARDSCRABBLE LOC. \\
\hline 4238 & 78 & GIUSTI & ARTURO & $\mathrm{H}$ & 33 & ITALY & ITALY & ITALY & 1899 & MINER/CM & LUCCA & & QUINCY - HARDSCRABBLE LOC. \\
\hline 4239 & 78 & GIUSTI & MARY & w & 30 & ITALY & ITALY & ITALY & 1899 & & LUCCA & & QUINCY - HARDSCRABBLE LOC. \\
\hline 4240 & 78 & GIUSTI & AUGUST & s & 9 & MICHIGAN & ITALY & ITALY & & & LUCCA & & QUINCY - HARDSCRABBLE LOC. \\
\hline 4241 & 78 & GIUSTI & EVELINA & $\mathrm{D}$ & 3 & MICHIGAN & ITALY & ITALY & & & LUCCA & & QUINCY - HARDSCRABBLE LOC. \\
\hline 4242 & 78 & FEDERIGHI & $\begin{array}{l}\text { EMILIO } \\
\text { EMIO }\end{array}$ & B & 19 & ITALY & ITALY & ITALY & 1908 & MINER/CM & LUCCA & & QUINCY - HARDSCRABBLE LOC. \\
\hline 4243 & 78 & PANTALEONI & ITALO & B & 23 & ITALY & ITALY & ITALY & 1906 & MINER/CM & SANTA MARIA DEL GIUDICE. LU & & QUINCY - HARDSCRABBLE LOC. \\
\hline 4244 & 75 & GUERRA & TOMMASO & $\mathrm{H}$ & 40 & ITALY & ITALY & ITALY & 1905 & & LUCCA & & QUINCY - HARDSCRABBLE LOC. \\
\hline 4245 & 75 & GUERRA-MEI & CLEMENTINA & w & 30 & ITALY & ITALY & ITALY & 1905 & & LUCCA & & $\begin{array}{l}\text { QUINCY - HARDSCRABBLE LOC } \\
\text { Q }\end{array}$ \\
\hline 4246 & 75 & GUERRA & $\begin{array}{l}\text { PETER } \\
\end{array}$ & $\mathrm{s}$ & 1.10 & MICHIGAN & ITALY & ITALY & & & LUCCA & & QUINCY - HARDSCRABBLE LOC. \\
\hline 4247 & 74 & FRANCIONI & LOUIS & $\mathrm{H}$ & $?$ & ITALY & ITALY & ITALY & ???? & NOT CLEAR & LUCCA & & QUINCY - HARDSCRABBLE LOC. \\
\hline 4248 & 74 & FRANCIONI & LIDIA & W & $?$ & ITALY & ITALY & ITALY & $? ? ? ?$ & & LUCCA & & QUINCY - HARDSCRABBLE LOC. \\
\hline 4249 & 74 & PISANI & ADOLF & B & $?$ & ITALY & ITALY & ITALY & ???? & NOT CLEAR & SAN CAMIANO. LU & & QUINCY - HARDSCRABBLE LOC. \\
\hline 4250 & 74 & PISANI & GABRIEL & B & $?$ & ITALY & ITALY & ITALY & ???? & NOT CLEAR & SAN CAMIANO. LU & & QUINCY - HARDSCRABBLE LOC. \\
\hline 4251 & 74 & PISANI & PAUL & B & $?$ & ITALY & ITALY & ITALY & ???? & NOT CLEAR & SAN CAMIANO. LU & & QUINCY - HARDSCRABBLE LOC. \\
\hline 4252 & 74 & FANINI & JACOB & B & $?$ & ITALY & ITALY & ITALY & ???? & NOT CLEAR & & & QUINCY - HARDSCRABBLE LOC. \\
\hline 4253 & 74 & MEI & DANTE & B & $?$ & ITALY & ITALY & ITALY & ???? & NOT CLEAR & RUOTA. LU & & QUINCY - HARDSCRABBLE LOC. \\
\hline 4254 & 74 & CHIBORI & LOUIS & B & $?$ & ITALY & ITALY & ITALY & ???? & NOT CLEAR & & & QUINCY - HARDSCRABBLE LOC. \\
\hline 4255 & 74 & GUERRA & PAUL & $\begin{array}{l}\mathrm{D} \\
\mathrm{B}\end{array}$ & $?$ & ITALY & ITALY & $\begin{array}{l}\text { ITALY } \\
\text { ITAY }\end{array}$ & $? ? ? ?$ & NOT CLEAR & LUCCA & & QUINCY - HARDSCRABBLE LOC. \\
\hline 4256 & 75 & PAPATA & FRANK & $\begin{array}{l}\mathrm{D} \\
\mathrm{B}\end{array}$ & 52 & NY & ITALY & ITALY & !?!? & MINER/CM & LUCUA & & QUINCY - HARDSCRABBLE LOC. \\
\hline 4257 & 75 & $\mathrm{PICCl}$ & FRANK & B & 28 & ITALY & ITALY & ITALY & 1909 & MINER/CM & & & QUINCY - HARDSCRABBLE LOC. \\
\hline 4258 & 75 & FAOLA & MARY & B & 19 & ITALY & ITALY & ITALY & 1909 & MINER/CM & & & QUINCY - HARDSCRABBLE LOC. \\
\hline 4259 & 75 & GELLI & ROMANO & B & 33 & ITALY & ITALY & ITALY & 1907 & MINER/CM & MATRAIA. LU & & QUINCY - HARDSCRABBLE LOC. \\
\hline 4260 & 58 & FOLENA & AMATO & $\mathrm{H}$ & 46 & ITALY & ITALY & ITALY & 1889 & MINER/CM & MATRAIA. LU & & QUINCY - HARDSCRABBLE LOC. \\
\hline 4261 & 58 & FOLENA & SANTA & w & 38 & ITALY & ITALY & ITALY & 1901 & & MATRAIA. LU & & QUINCY - HARDSCRABBLE LOC. \\
\hline 4262 & 58 & FOLENA & ONORIO & $s$ & 17 & ITALY & ITALY & ITALY & 1901 & LABORER/CM & MATRAIA. LU & & QUINCY - HARDSCRABBLE LOC. \\
\hline 4263 & 58 & FOLENA & MARIO & $\mathrm{s}$ & 8 & MICHIGAN & ITALY & ITALY & & & MATRAIA. LU & & QUINCY - HARDSCRABBLE LOC. \\
\hline 4264 & 58 & FOLENA & MILLIE & $\mathrm{D}$ & 6 & MICHIGAN & ITALY & ITALY & & & MATRAIA, LU & & QUINCY - HARDSCRABBLE LOC. \\
\hline 4265 & 58 & FOLENA & LUISA & $\mathrm{D}$ & 5 & MICHIGAN & ITALY & ITALY & & & MATRAIA, LU & & QUINCY - HARDSCRABBLE LOC. \\
\hline 4266 & 58 & FOLENA & MABEL & $\mathrm{D}$ & 3 & MICHIGAN & ITALY & ITALY & & & MATRAIA. LU & & QUINCY - HARDSCRABBLE LOC. \\
\hline 4267 & 58 & FOLENA & ADOLF & $\mathrm{s}$ & 2 & MICHIGAN & ITALY & ITALY & & & MATRAIA. LU & & QUINCY - HARDSCRABBLE LOC. \\
\hline 4268 & 58 & FOLENA & ALBERT & $\mathrm{s}$ & $2 \mathrm{M}$ & MICHIGAN & ITALY & ITALY & & & MATRAIA. LU & & QUINCY - HARDSCRABBLE LOC. \\
\hline 4269 & 58 & FOLENA & ABRAM & s & $2 \mathrm{M}$ & MICHIGAN & ITALY & $\begin{array}{l}\text { MALY } \\
\text { ITALY }\end{array}$ & & & MATRAIA. LU & & QUINCY - HARDSCRABBLE LOC. \\
\hline 4270 & 54 & BALDASSARRE & RUGGERO & $\mathrm{H}$ & 38 & ITALY & ITALY & ITALY & 1901 & MINER/CM & SANTA MARIA DEL GIUDICE. LU & & QUINCY - LIMERICK LOCATION \\
\hline 4271 & 54 & BALDASSARRE & LIZZIE & W & 33 & ITALY & ITALY & ITALY & 1906 & & SANTA MARIA DEL GIUDICE. LU & & QUINCY - LIMERICK LOCATION \\
\hline 4272 & 54 & BALDASSARRE & ALADINO & s & 15 & ITALY & ITALY & ITALY & 1906 & MINER/CM & SANTA MARIA DEL GIUDICE, LU & & QUINCY - LIMERICK LOCATION \\
\hline 4273 & 54 & BALDASSARRE & AMELIO & $\mathrm{S}$ & 14 & ITALY & ITALY & ITALY & 1906 & & SANTA MARIA DEL GIUDICE. LU & & QUINCY - LIMERICK LOCATION \\
\hline 4274 & 54 & BALDASSARRE & PAOLA & $\mathrm{D}$ & 9 & ITALY & ITALY & ITALY & 1906 & & SANTA MARIA DEL GIUDICE. LU & & QUINCY - LIMERICK LOCATION \\
\hline 4275 & 54 & BALDASSARRE & EVA & $\mathrm{D}$ & 2 & MICHIGAN & ITALY & ITALY & & & SANTA MARIA DEL GIUDICE. LU & & QUINCY - LIMERICK LOCATION \\
\hline 4276 & 54 & BALDASSARRE & PREMIATA & $\mathrm{D}$ & 2 & MICHIGAN & ITALY & ITALY & & & SANTA MARIA DEL GIUDICE. LU & & QUINCY - LIMERICK LOCATION \\
\hline 4277 & 54 & BALDASSARRE & CRISTIANA & $\mathrm{D}$ & $1.7 \mathrm{M}$ & MICHIGAN & ITALY & ITALY & & & SANTA MARIA DEL GIUDICE. LU & & QUINCY - LIMERICK LOCATION \\
\hline 4278 & 54 & PAPA & JOHN & B & 52 & ITALY & ITALY & ITALY & 1906 & MINER/CM & & & QUINCY - LIMERICK LOCATION \\
\hline 4279 & 56 & PATERNI & LUIGI & $\mathrm{H}$ & 34 & ITALY & ITALY & ITALY & 1896 & MINER/CM & BAGNI DI LUCCA. LU & & QUINCY - HARDSCRABBLE LOC. \\
\hline 4280 & 56 & PATERNI & LINDA & W & 29 & ITALY & ITALY & ITALY & 1901 & & & & QUINCY - HARDSCRABBLE LOC. \\
\hline 4281 & 56 & PATERNI & MARY & $\mathrm{D}$ & 9 & MICHIGAN & ITALY & ITALY & & & BAGNI DI LUCCA. LU & & QUINCY - HARDSCRABBLE LOC. \\
\hline 4282 & 56 & PATERNI & ROSE & $\mathrm{D}$ & 7 & MICHIGAN & ITALY & ITALY & & & BAGNI DI LUCCA. LU & & QUINCY - HARDSCRABBLE LOC. \\
\hline 4283 & 56 & PATERNI & LUISA & $\mathrm{D}$ & 5 & MICHIGAN & ITALY & ITALY & & & BAGNI DI LUCCA. LU & & QUINCY - HARDSCRABBLE LOC. \\
\hline
\end{tabular}




\begin{tabular}{|c|c|c|c|c|c|c|c|c|c|c|c|c|}
\hline 42845 & PATERNI & PAOLO & $\mathrm{s}$ & 3 & MICHIGAN & ITALY & ITALY & & & BAGNI DI LUCCA. LU & & QUINCY - HARDSCRABBLE LOC \\
\hline 42855 & BUGANI & ANNIBALE & B & 25 & ITALY & ITALY & ITALY & 1909 & TRAMMER/CM & & & QUINCY - HARDSCRABBLE LOC \\
\hline 42865 & PATERNI & GIUSEPPE & B & 22 & ITALY & ITALY & ITALY & 1907 & TRAMMER/CM & BAGNI DI LUCCA. LU & & QUINCY - HARDSCRABBLE LOC \\
\hline 42875 & AVELLINI & ANDREA & B & 46 & ITALY & ITALY & ITALY & 1907 & TIMBERMAN/CM & MONSACRATI. LU & & QUINCY - HARDSCRABBLE LOC \\
\hline 42885 & MAGRI & ANGELO & B & 21 & ITALY & ITALY & ITALY & 1907 & LABORER/CM & PIETRASANTA. LU & & QUINCY - HARDSCRABBLE LOC \\
\hline 42895 & ANDREOZZI & CHERUBINO & B & 44 & ITALY & ITALY & ITALY & 1905 & LABORER/CM & SANTA MARIA DEL GIUDICE. LU & & QUINCY - HARDSCRABBLE LOC \\
\hline 42905 & ANDREOZZI & RINALDO & B & 31 & ITALY & ITALY & ITALY & 1901 & TIMBERMAN/CM & SANTA MARIA DEL GIUDICE. LU & & QUINCY - HARDSCRABBLE LOC \\
\hline 42915 & CONSANI & LUIGI & $\begin{array}{l}\mathrm{D} \\
\mathrm{B}\end{array}$ & 35 & ITALY & ITALY & ITALY & 1898 & MINER/CM & LUCCA & & QUINCY - HARDSCRABBLE LOC \\
\hline 42925 & ANDREOZZI & ELIA & B & 33 & ITALY & ITALY & ITALY & 1906 & TRAMMER/CM & LUCCA & & QUINCY - HARDSCRABBLE LOC \\
\hline 42935 & PATERNI & FRANCESCO & B & 22 & ITALY & ITALY & ITALY & 1906 & TRAMMER/CM & LUCCA & & QUINCY - HARDSCRABBLE LOC \\
\hline 42945 & CORSI & LUIGI/UGO & B & 20 & ITALY & ITALY & ITALY & 1905 & TRAMMER/CM & MONSACRATI. LU & & QUINCY - HARDSCRABBLE LOC \\
\hline 42955 & CORSI & EUGENIO & B & 22 & ITALY & ITALY & ITALY & 1907 & LABORER/CM & MONSACRATI. LU & & QUINCY - HARDSCRABBLE LOC \\
\hline 42965 & ROGANI & LUIGI & B & 23 & ITALY & ITALY & ITALY & 1906 & MINER/CM & LUCCA & & QUINCY - HARDSCRABBLE LOC \\
\hline 42979 & RAFFAELLI & FRANCO & $\mathrm{H}$ & 28 & ITALY & ITALY & ITALY & 1903 & MINER/CM & COLLODI. LU & & QUINCY - SING SING LOCATION \\
\hline 42989 & RAFFAELLI & GIULIA & W & 26 & ITALY & ITALY & ITALY & 1907 & & & & QUINCY - SING SING LOCATION \\
\hline 42999 & RAFFAELLI & PAOLINA & D & 2 & MICHIGAN & ITALY & ITALY & & & COLLODI, LU & & QUINCY - SING SING LOCATION \\
\hline 43009 & RAFFAELLI & NUNZIATINA & $\mathrm{D}$ & $2 \mathrm{M}$ & MICHIGAN & ITALY & ITALY & & & COLLODI. LU & & QUINCY - SING SING LOCATION \\
\hline 43019 & BONINI & JULIO & B & 40 & ITALY & ITALY & ITALY & 1908 & MINER/CM & COLLODI. LU & & QUINCY - SING SING LOCATION \\
\hline 43029 & BONINI & ULISSE & B & 40 & ITALY & ITALY & ITALY & 1908 & MINER/CM & COLLODI.LU & & QUINCY - SING SING LOCATION \\
\hline 43039 & PASQUINI & ZACCARIA & B & 27 & ITALY & ITALY & ITALY & 1907 & MINER/CM & GOLLUD, LU & & $\begin{array}{l}\text { QUINCY - SING SING LOCATION } \\
\text { Q }\end{array}$ \\
\hline 43049 & PIZZA & REMO & B & 42 & ITALY & ITALY & ITALY & 1908 & MINER/CM & LUCCA & & QUINCY - SING SING LOCATION \\
\hline 43059 & COTTINI & VIRGILIO & B & 37 & ITALY & ITALY & ITALY & 1906 & MINER/CM & COLLODI, LU & & QUINCY - SING SING LOCATION \\
\hline 43065 & LOMBARDINI & ALFREDO & $\mathrm{H}$ & 33 & ITALY & ITALY & ITALY & 1899 & MINER/CM & & & QUINCY - SING SING LOCATION \\
\hline 43075 & LOMBARDINI & GIUSEPPINA & w & 26 & ILLINOIS & GERMANY & GERMANY & & & GERMANY & & 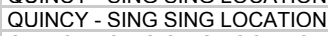 \\
\hline 43085 & LOMBARDINI & MARIA & D & 7 & MICHIGAN & ITALY & ITALY & & & & & QUINCY - SING SING LOCATION \\
\hline 43095 & LOMBARDINI & LUISA & $\mathrm{D}$ & 6 & MICHIGAN & ITALY & ITALY & & & & & QUINCY - SING SING LOCATION \\
\hline 43105 & LOMBARDINI & CARLO & $\mathrm{s}$ & 4 & MICHIGAN & ITALY & ITALY & & & & & QUINCY - SING SING LOCATION \\
\hline 43115 & LOMBARDINI & ALBERTO & $\mathrm{s}$ & $1,2 \mathrm{M}$ & MICHIGAN & ITALY & ITALY & & & & & QUINCY - SING SING LOCATION \\
\hline 43125 & CESARETTI & JOSEPH & $\mathrm{H}$ & 27 & ITALY & ITALY & ITALY & 1900 & MINER/CM & LUCCA & & QUINCY - SING SING LOCATION \\
\hline 43135 & CESARETTI & EMILIA & W & 25 & ITALY & ITALY & ITALY & 1908 & & LUCCA & & QUINCY - SING SING LOCATION \\
\hline 43145 & CESARETTI & TONY & s & $10 \mathrm{M}$ & MICHIGAN & ITALY & ITALY & & & LUCCA & & QUINCY - SING SING LOCATION \\
\hline 4315 & GEDDA & GIOVANNI & $\mathrm{H}$ & 45 & ITALY & ITALY & ITALY & 1902 & FARMER/FARM & SAN MARTINO CANAVESE. TO & & $\begin{array}{l}\text { QUINCY - SING SING LOCATION } \\
\text { Q }\end{array}$ \\
\hline 4316 & GEDDA & MARIA & W & 40 & ITALY & ITALY & ITALY & 1907 & ГARTIVTII PARTIUI & SAIVIVARTIIVU LAIVAVEOS. TO & & QUINCY - SING SING LOCATION \\
\hline 4317 & GEDDA & ROSA & D & 16 & MICHIGAN & ITALY & ITALY & & & SAN MARTINO CANAVESE, TO & & QUINCY - SING SING LOCATION \\
\hline 4318 & GEDDA & GIOVANNA & D & 14 & MICHIGAN & ITALY & ITALY & & & SAN MARTINO CANAVESE. TO & & QUINCY - SING SING LOCATION \\
\hline 4319 & GEDDA & MARTINO & $\mathrm{s}$ & 5 & MICHIGAN & ITALY & ITALY & & & SAN MARTINO CANAVESE. TO & & QUINCY - SING SING LOCATION \\
\hline 4320 & GEDDA & MARIA & $\mathrm{D}$ & 2 & MICHIGAN & ITALY & ITALY & & & SAN MARTINO CANAVESE. TO & & QUINCY - SING SING LOCATION \\
\hline 4321 & ZANOTTI & TONY & $\mathrm{H}$ & 27 & ITALY & ITALY & ITALY & 1907 & MINER/CM & TORINO & & SCHOOLCRAFT \\
\hline 4322 & ZANOTTI & TRACY & W & 20 & ITALY & ITALY & ITALY & 1907 & & & & SCHOOLCRAFT \\
\hline 4323 & SANDRETTO & DOMENICO & $\mathrm{H}$ & 39 & ITALY & ITALY & ITALY & 1885 & MILK PEDLAR/FARM & PONT CANAVESE. TO & & SCHOOLCRAFT \\
\hline 4324 & SANDRETTO & MINNIE & W & 38 & ITALY & ITALY & ITALY & 1880 & & & & SCHOOLCRAFT \\
\hline 4325 & SANDRETTO & PETER & s & 14 & ILLINOIS & ITALY & ITALY & & LABORER/FARM & PONT CANAVESE. TO & & SCHOOLCRAFT \\
\hline 4326 & SANDRETTO & ILARY & $\mathrm{D}$ & 12 & MICHIGAN & ITALY & ITALY & & & PONT CANAVESE. TO & & SCHOOLCRAFT \\
\hline 4327 & SANDRETTO & LUCY & $\mathrm{D}$ & 10 & MICHIGAN & ITALY & ITALY & & & PONT CANAVESE. TO & & SCHOOLCRAFT \\
\hline 4328 & SANDRETTO & JOHN & $\mathrm{s}$ & 6 & MICHIGAN & ITALY & ITALY & & & PONT CANAVESE. TO & & SCHOOLCRAFT \\
\hline 4329 & BALAGNA & FRANK & $\mathrm{B}$ & 18 & MICHIGAN & ITALY & ITALY & & LABORER & PONT CANAVESE. TO & & SCHOOLCRAFT \\
\hline 4330 & ALTOMARE & SANTI & $\mathrm{H}$ & 35 & ITALY & ITALY & ITALY & 1901 & LABORER/STAMP MILL & & BEACON HILL & STANTON - BEACON HILL \\
\hline 4331 & ALTOMARE & CAROLINE & W & 30 & ITALY & ITALY & ITALY & 1904 & & & BEACON HILL & STANTON - BEACON HILL \\
\hline 4332 & ALTOMARE & JOSEPH & $\mathrm{s}$ & 11 & ITALY & ITALY & ITALY & 1904 & & & BEACON HILL & STANTON - BEACON HILL \\
\hline 4333 & ALTOMARE & SALIC & $\mathrm{D}$ & 5 & MICHIGAN & ITALY & ITALY & 1004 & & & BEACON HILL & STANTON - BEACON HILL \\
\hline 4334 & ALTOMARE & TONY & $\mathrm{s}$ & 2 & MICHIGAN & ITALY & ITALY & & & & SN HILL & STANTON - BEACON HILL \\
\hline 4335 & MERABELL & GAETANO & B & 30 & ITALY & ITALY & ITALY & 1905 & LABORER/STAMP MILL & & BEACON HILL & STANTON - BEACON HILL \\
\hline 4336 & PARIANO & FRANK & $\mathrm{H}$ & 33 & ITALY & ITALY & ITALY & 1909 & LABORER/STAMP MILL & COTRONEI. CROTONE & BEACON HILL & STANTON - BEACON HILL \\
\hline 4337 & PARIANO & SERAFINA & W & 32 & ITALY & ITALY & ITALY & 1909 & & COTRONEI. CROTONE & BEACON HILL & STANTON - BEACON HILL \\
\hline 4338 & PARIANO & TRACY & s & 10 & ITALY & ITALY & ITALY & 1909 & & COTRONEI. CROTONE & BEACON HILL & STANTON - BEACON HILL \\
\hline 4339 & PARIANO & MICHAEL & $\mathrm{s}$ & 6 & ITALY & ITALY & ITALY & 1909 & & COTRONEI. CROTONE & BEACON HILL & STANTON - BEACON HILL \\
\hline 4340 & PARIANO & MARY & $\mathrm{D}$ & 3 & ITALY & ITALY & ITALY & 1909 & & COTRONEI. CROTONE & BEACON HILL & STANTON - BEACON HILL \\
\hline 4341 & PARIANO & GIUSEPPE & $\mathrm{s}$ & 2 & ITALY & ITALY & ITALY & 1909 & & COTRONEI, CROTONE & $\begin{array}{l}\text { BEACON HILL } \\
\text { BEA }\end{array}$ & STANTON - BEACON HILL \\
\hline 4342 & PARIANO & GIUSEPPINA & D & 1 & ITALY & ITALY & ITALY & 1909 & & COTRONEI. CROTONE & BEACON HILL & STANTON - BEACON HILL \\
\hline 4343 & BARIALLA & DOMINICK & B & 34 & ITALY & ITALY & ITALY & 1908 & LABORER/STAMP MILL & & BEACON HILL & STANTON - BEACON HILL \\
\hline 4344 & BARIALLA & NICOLAS & B & 32 & ITALY & ITA & ITA & 1909 & LABORER/STAMP MILL & & BEACON HILL & STANTON - BEACON HILL \\
\hline 4345 & PERRONE & SALVATORE & $\mathrm{H}$ & 25 & ITALY & ITALY & ITALY & 1896 & LABORER/STAMP MILL & & BEACON HILL & STANTON - BEACON HILL \\
\hline 4346 & PERRONE & ROSIE & W & 23 & ITALY & ITALY & ITALY & 1900 & & & BEACON HILL & STANTON - BEACON HILL \\
\hline
\end{tabular}




\begin{tabular}{|c|c|c|c|c|c|c|c|c|c|c|c|c|c|}
\hline 4347 & & RIFFALO & JOSEPH & $\mathrm{H}$ & 23 & ITALY & ITALY & ITALY & 1906 & CARPENTER/CARPENTER S. & & EDGEMERE ROAD & STANTON \\
\hline 4348 & & RIFFALO & LENA & W & 22 & ITALY & ITALY & ITALY & 1906 & & & EDGEMERE ROAD & STANTON \\
\hline 4349 & & RIFFALO & KATHERINE & $\mathrm{D}$ & 3 & MICHIGAN & ITALY & ITALY & & & & EDGEMERE ROAD & STANTON \\
\hline 4350 & & RIFFALO & CINDERALLA & $\mathrm{D}$ & 2 & MICHIGAN & ITALY & ITALY & & & & EDGEMERE ROAD & STANTON \\
\hline 4351 & & RIFFALO & GIANO & $\mathrm{s}$ & $4 \mathrm{M}$ & MICHIGAN & ITALY & ITALY & & & & EDGEMERE ROAD & STANTON \\
\hline 4352 & & PARDI & EDITH & SIIL & 15 & ITALY & ITALY & ITALY & 1906 & & & EDGEMERE ROAD & STANTON \\
\hline 4353 & & MARCUS? & FRANK & $\mathrm{H}$ & 24 & ITALY & ITALY & ITALY & 1905 & LABORER/STAMP MILL & & & STANTON \\
\hline 4354 & & MARCUS? & SUSIE & W & 19 & ITALY & ITALY & ITALY & 1907 & & & & STANTON \\
\hline 4355 & & PROCISSI & JOSEPH & $\mathrm{H}$ & 49 & ITALY & ITALY & ITALY & 1899 & HEAD RUNNER/STAMP MILL & LUCCA & FREDA ROAD & STANTON \\
\hline 4356 & & PROCISSI & MARIA & W & 49 & ITALY & ITALY & ITALY & 1899 & & LUCCA & FREDA ROAD & STANTON \\
\hline 4357 & & PROCISSI & PETER & s & 17 & ITALY & ITALY & ITALY & 1899 & HELPER/CARPENTER SHOP & LUCCA & FREDA ROAD & STANTON \\
\hline 4358 & & PROCISSI & PACOL & $\mathrm{s}$ & 14 & ITALY & ITALY & ITALY & 1899 & & LUCCA & FREDA ROAD & STANTON \\
\hline 4359 & & PROCISSI & ELISABETH & $\mathrm{D}$ & 3 & MICHIGAN & ITALY & ITALY & & & LUCCA & FREDA ROAD & STANTON \\
\hline 4360 & & COSTA & LOUIS & $\mathrm{H}$ & 58 & ITALY & ITALY & ITALY & 1884 & HEAD RUNNER/STAMP MILL & TORINO & & TORCH LAKE - HUBBEL \\
\hline 4361 & & COSTA & LOUISE & W & 50 & CANADA & CANADA & CANADA & 1881 & & CANADA & & TORCH LAKE - HUBBEL \\
\hline 4362 & & COSTA & ALICE & $\mathrm{D}$ & 13 & MICHIGAN & ITALY & CA & & & TORINO & & TORCH LAKE - HUBBEL \\
\hline 4363 & & COSTA & ANTHONY & $\mathrm{s}$ & 12 & MICHIGAN & ITALY & CA & & & TORINO & & TORCH LAKE - HUBBEL \\
\hline 4364 & & COSTA & AUGUST & $\mathrm{s}$ & 9 & MICHIGAN & ITALY & CA & & & TORINO & & TORCH LAKE - HUBBEL \\
\hline 4365 & 11 & CHELLINI & JOHN & $\mathrm{H}$ & 49 & ITALY & ITALY & ITALY & 1882 & LABORER/SMELTER & LUCCA & DUNCAN AVE. & TORCH LAKE - HUBBEL \\
\hline 4366 & 11 & CHELLINI & SARAH & W & 40 & ENGLAND & ENGLAND & ENGLAND & 1876 & & ENGLAND & DUNCAN AVE. & TORCH LAKE - HUBBEL \\
\hline 4367 & 11 & CHELLINI & KATIE & $\mathrm{D}$ & 19 & MICHIGAN & ITALY & ENGLAND & & & LUCCA & DUNCAN AVE. & TORCH LAKE - HUBBEL \\
\hline 4368 & 11 & CHELLINI & RALPH & $\mathrm{s}$ & 17 & MICHIGAN & ITALY & ENGLAND & & & LUCCA & DUNCAN AVE. & TORCH LAKE - HUBBEL \\
\hline 4369 & 11 & CHELLINI & MELTON & $\mathrm{s}$ & 16 & MICHIGAN & ITALY & ENGLAND & & & LUCCA & DUNCAN AVE. & TORCH LAKE - HUBBEL \\
\hline 4370 & 11 & CHELLINI & JOHN & s & 14 & MICHIGAN & ITALY & ENGLAND & & & LUCCA & DUNCAN AVE. & TORCH LAKE - HUBBEL \\
\hline 4371 & 11 & CHELLINI & GEORGE & $\mathrm{s}$ & 12 & MICHIGAN & ITALY & ENGLAND & & & LUCCA & DUNCAN AVE. & TORCH LAKE - HUBBEL \\
\hline 4372 & & MICHELE & GOUDIAS & $\mathrm{H}$ & 31 & CANADA & CANADA & CANADA & 1883 & LABORER/STAMP MILL & CANADA & & TORCH LAKE - HUBBEL \\
\hline 4373 & & MICHELE & LUCY & W & 27 & ITALY & ITALY & ITALY & & & & & TORCH LAKE - HUBBEL \\
\hline 4374 & & MICHELE & LAWRENCE & $\mathrm{s}$ & 3 & MICHIGAN & CANADA & ITALY & & & & & TORCH LAKE - HUBBEL \\
\hline 4375 & & MICHELE & CORINE & $\mathrm{D}$ & 2 & MICHIGAN & CANADA & ITALY & & & & & TORCH LAKE - HUBBEL \\
\hline 4376 & & BANDETTINI & ANNA & $\mathrm{H}$ & 26 & MICHIGAN & GERMANY & GERMANY & & STOREKEEPER/CANDY STORE & GERMANY & & TORCH LAKE - HUBBEL \\
\hline 4377 & & BANDETTINI & ARIA & $?$ & 17 & MICHIGAN & ITALY & MICHIGAN & & & & & TORCH LAKE - HUBBEL \\
\hline 4378 & & GASPARDO & BATTISTA & $\mathrm{H}$ & 31 & ITALY & ITALY & ITALY & 1902 & FARMER/FARM & PONT CANAVESE. TO & & TORCH LAKE - HUBBEL \\
\hline 4379 & & GASPARDO & ANTOINETTE & W & 32 & ITALY & ITALY & ITALY & 1902 & & PONT CANAVESE. TO & & TORCH LAKE - HUBBEL \\
\hline 4380 & & GASPARDO & $\begin{array}{l}\text { SERAFINO } \\
\text { SE }\end{array}$ & s & 6 & MICHIGAN & ITALY & ITALY & & & PONT CANAVESE, TO & & TORCH LAKE - HUBBEL \\
\hline 4381 & & GASPARDO & JOHN & $\mathrm{s}$ & 5 & MICHIGAN & ITALY & ITALY & & & PONT CANAVESE. TO & & TORCH LAKE - HUBBEL \\
\hline 4382 & & GASPARDO & JAMES & $\mathrm{s}$ & 3 & MICHIGAN & ITALY & ITALY & & & PONT CANAVESE. TO & & TORCH LAKE - HUBBEL \\
\hline 4383 & & GASPARDO & MARTIN & $\mathrm{s}$ & $18 \mathrm{M}$ & MICHIGAN & ITALY & ITALY & & & PONT CANAVESE. TO & & TORCH LAKE - HUBBEL \\
\hline 4384 & & GASPARDO & SERAFINO & FA & $?$ & ITALY & ITALY & ITALY & 1902 & LABORER/ODD JOBS & PONT CANAVESE. TO & & TORCH LAKE - HUBBEL \\
\hline
\end{tabular}




\section{APPENDIX X}

\section{CENSUS of 1920}

\section{LEGEND}

ID: counter.

$\mathbf{N}$ : street number as it appeared on the house.

REL: relation of the person with the head of the household.

BORN: nation of birth of the person.

FATHER: nation of birth of the father.

MOTHER: nation of birth of the mother.

USA: indicate the year of arrival in the U.S.

PROVENIENCE: place of origin in Italy. These data are not from the census, but form Ellis Island Database, Italian newspapers, Oral interviews.

TOWNSHIP: township in which the person was living. 


\begin{tabular}{|c|c|c|c|c|c|c|c|c|c|c|c|c|c|}
\hline ID & $\mathrm{N}$ & SURNAME & NAME & \begin{tabular}{|l|l} 
REL \\
\end{tabular} & AGE & BORN & FATHER & MOTHER & USA & OCCUPATION & PROVENIENCE & ADDRESS & \begin{tabular}{|c|} 
TOWNSHIP \\
\end{tabular} \\
\hline 1 & ??9 & GUIZZETTI & ANTONIO & $\mathrm{H}$ & 24 & ITALY & ITALY & ITALY & 1896 & TRAMMER/CM & MILANO & & ADAMS - ATLANTIC LOCATION \\
\hline 2 & $? ? 9$ & GUIZZETTI & LEMPI & w & 20 & FINLAND & FINLAND & FINLAND & & & FINLAND & & ADAMS - ATLANTIC LOCATION \\
\hline 3 & $? ? 9$ & GUIZZETTI & ANDREW & s & 1 & MICHIGAN & ITALY & FINLAND & & & MILANO & & ADAMS - ATLANTIC LOCATION \\
\hline 4 & 115 & BIANUCCI & JOSEPH & $\mathrm{H}$ & 27 & ITALY & ITALY & ITALY & UN & MECHANIC/MILL & ALTOPASCIO. LU & & ADAMS - ATLANTIC LOCATION \\
\hline 5 & 115 & BIANUCCI & ESTER & w & 23 & MICHIGAN & MICHIGAN & GERMANY & & & GERMANY & & ADAMS - ATLANTIC LOCATION \\
\hline 6 & 31 & BIANUCCI & EUGENIO & $\mathrm{H}$ & 53 & ITALY & ITALY & ITALY & 1890 & SURFACE LABORER/CM & ALTOPASCIO. LU & & ADAMS - ATLANTIC LOCATION \\
\hline & 31 & BIANUCCI & CLORINDA & w & 47 & ITALY & ITALY & ITALY & 1895 & & ALTOPASCIO, LU & & ADAMS - ATLANTIC LOCATION \\
\hline 8 & 31 & BIANUCCI & ARTURO & $\mathrm{s}$ & 29 & ITALY & ITALY & ITALY & UN & ELECTRICIAN/MILL & ALTOPASCIO. LU & & ADAMS - ATLANTIC LOCATION \\
\hline & 31 & BIANUCCI & GIULIO & s & 15 & MICHIGAN & ITALY & ITALY & & & ALTOPASCIO. LU & & ADAMS - ATLANTIC LOCATION \\
\hline 10 & 31 & BIANUCCI & ALFRED & $\mathrm{s}$ & 13 & MICHIGAN & ITALY & ITALY & & & ALTOPASCIO. LU & & ADAMS - ATLANTIC LOCATION \\
\hline 11 & & $\mathrm{ClUCCl}$ & DOMENICO & SOIL & 26 & MICHIGAN & ITALY & ITALY & & TURNER/MILL & MATRAIA. LU & & ADAMS - ATLANTIC LOCATION \\
\hline 12 & & $\mathrm{ClUCCl}$ & LUCILLA & D & 17 & MICHIGAN & ITALY & ITALY & & & ALTOPASCIO. LU & & ADAMS - ATLANTIC LOCATION \\
\hline 13 & 182 & $\mathrm{ClUCCl}$ & NICOLAS & $\mathrm{H}$ & 51 & ITALY & ITALY & ITALY & 1889 & WATCHMAN/CM & MATRAIA. LU & & ADAMS - ATLANTIC LOCATION \\
\hline 14 & 182 & ClUCCl & MARY & W & 52 & ITALY & ITALY & ITALY & 1891 & & & & ADAMS - ATLANTIC LOCATION \\
\hline 15 & 182 & $\mathrm{ClUCCl}$ & PAUL & $\mathrm{s}$ & 24 & MICHIGAN & ITALY & ITALY & & MECHANIC/FACTORY & MATRAIA, LU & & ADAMS - ATLANTIC LOCATION \\
\hline 16 & 182 & CluCCl & ERIC & $\mathrm{s}$ & 20 & MICHIGAN & ITALY & ITALY & & MECHANIC/FACTORY & MATRAIA. LU & & ADAMS - ATLANTIC LOCATION \\
\hline 17 & 182 & CIUCCI & ALFRED & $\mathrm{s}$ & 17 & MICHIGAN & ITALY & ITALY & & MECHANIC/FACTORY & MATRAIA, LU & & ADAMS - ATLANTIC LOCATION \\
\hline 18 & 182 & ClUCCl & PETER & $\mathrm{s}$ & 13 & MICHIGAN & ITALY & ITALY & & & MATRAIA. LU & & ADAMS - ATLANTIC LOCATION \\
\hline 19 & 175 & CHIMINO & $\mathrm{JOHN}$ & $\mathrm{H}$ & 28 & MICHIGAN & ITALY & ITALY & & MECHANIC/MILL & SAN GIORGIO CANAVESE. TO & & ADAMS - ATLANTIC LOCATION \\
\hline 20 & 175 & CHIMINO & AMELIA & w & 27 & MICHIGAN & ITALY & ITALY & & & & & ADAMS - ATLANTIC LOCATION \\
\hline 21 & 175 & CHIMINO & JOSEPHINE & $\mathrm{D}$ & $6 \mathrm{M}$ & MICHIGAN & MICHIGAN & MICHIGAN & & & SAN GIORGIO CANAVESE. TO & & ADAMS - ATLANTIC LOCATION \\
\hline 22 & 197 & CHIMINO & JOHN & $\mathrm{H}$ & 68 & ITALY & ITALY & ITALY & 1885 & FARMER & SAN GIORGIO CANAVESE. TO & & ADAMS - ATLANTIC LOCATION \\
\hline 23 & 197 & CHIMINO & MARGHERITA & W & 50 & ITALY & $\begin{array}{l}\text { ITALY } \\
\text { ITAL }\end{array}$ & ITALY & $\begin{array}{l}1000 \\
1890\end{array}$ & TARTIILK & SAN GIORGIO CANAVESE. TO & & ADAMS - ATLANTIC LOCATION \\
\hline 24 & 197 & CHIMINO & JOSEPH & $\mathrm{s}$ & 26 & MICHIGAN & ITALY & ITALY & & & SAN GIORGIO CANAVESE. TO & & ADAMS - ATLANTIC LOCATION \\
\hline 25 & 197 & CHIMINO & TERESA & D & 24 & MICHIGAN & ITALY & ITALY & & SALESLADY/STORE & SAN GIORGIO CANAVESE, TO & & ADAMS - ATLANTIC LOCATION \\
\hline 26 & 197 & CHIMINO & MARGHERITA & $\mathrm{D}$ & 21 & MICHIGAN & ITALY & ITALY & & SALESLADYISTORE & SAN GIORGIO CANAVESE. TO & & ADAMS - ATLANTIC LOCATION \\
\hline 27 & & VERNA & JOSEPHINE & $\mathrm{H}$ & 51 & ITALY & ITALY & ITALY & 1904 & & CIRIE'. TO & & ADAMS - ATLANTIC LOCATION \\
\hline 28 & & VERNA & JAMES & s & 19 & ITALY & ITALY & ITALY & 1904 & LABORER/SMELTER & CIRIE'. TO & & ADAMS - ATLANTIC LOCATION \\
\hline 29 & & VERNA & MATILDA & D & 17 & ITALY & ITALY & ITALY & 1904 & & CIRIE'. TO & & ADAMS - ATLANTIC LOCATION \\
\hline 30 & & VERNA & CLYDE & s & 16 & ITALY & ITALY & ITALY & 1904 & LABORER/CM & CIRIE'. TO & & ADAMS - ATLANTIC LOCATION \\
\hline 31 & & VERNA & ANNA & D & 13 & MICHIGAN & ITALY & ITALY & & & CIRIE', TO & & ADAMS - ATLANTIC LOCATION \\
\hline 32 & & CALUSCHI & LUKES & B & 27 & ITALY & ITALY & ITALY & $14 ? ?$ & FIREMAN/SMELTER & & & ADAMS - ATLANTIC LOCATION \\
\hline 33 & & TRAGLIA & ANTONIO & B & 24 & ITALY & ITALY & ITALY & 1906 & FURNACE MOVER/SMELTER & & & ADAMS - ATLANTIC LOCATION \\
\hline 34 & & TAMBORINO & JOHN & $\mathrm{H}$ & 37 & ITALY & ITALY & ITALY & 1911 & MINER/CM & LOCANA CANAVESE. TO & & ADAMS - ATLANTIC LOCATION \\
\hline 35 & & TAMBORINO & JOSEPHINE & W & 25 & ITALY & ITALY & ITALY & 1913 & IVIINER/LIVI & LUCAIVA CAIVAVESE. TO & & ADAMS - ATLANTIC LOCATION \\
\hline 36 & & TAMBORINO & CARLO & $\mathrm{s}$ & 5 & ILLINOIS & ITALY & ITALY & & & LOCANA CANAVESE. TO & & ADAMS - ATLANTIC LOCATION \\
\hline 37 & & TAMBORINO & RENA & $\mathrm{D}$ & 3 & ILLINOIS & ITALY & ITALY & & & LOCANA CANAVESE, TO & & ADAMS - ATLANTIC LOCATION \\
\hline 38 & & BOLLINI & SERAFINO & B & 45 & ITALY & ITALY & ITALY & ?? & LABORER/SMELTER & MERCALLO. VARESE & & ADAMS - ATLANTIC LOCATION \\
\hline 39 & 102 & VENTURINI & FRANK & $\mathrm{H}$ & 53 & ITALY & ITALY & ITALY & 1908 & MINER/CM & CAMAIORE. LU & 12TH AVE. & ADAMS - BALTIC \\
\hline 40 & 102 & VENTURINI & FELICY & W & 40 & ITALY & ITALY & ITALY & 1901 & & & 12TH AVE. & ADAMS - BALTIC \\
\hline 41 & 102 & VENTURINI & EUGENIO & $\mathrm{s}$ & 20 & MICHIGAN & ITALY & ITALY & & NOT CLEAR & CAMAIORE. LU & 12TH AVE. & ADAMS - BALTIC \\
\hline 42 & 102 & VENTURINI & JOSEPHINE? & D & 11 & MICHIGAN & ITALY & ITALY & & & CAMAIORE. LU & 12TH AVE. & ADAMS - BALTIC \\
\hline 43 & 102 & VENTURINI & DOROTHY & D & 9 & MICHIGAN & ITALY & ITALY & & & CAMAIORE, LU & 12TH AVE. & ADAMS - BALTIC \\
\hline 44 & 102 & VENTURINI & GIULIO & $\mathrm{s}$ & 7 & MICHIGAN & ITALY & ITALY & & & CAMAIORE. LU & 12TH AVE. & ADAMS - BALTIC \\
\hline 45 & 102 & VENTURINI & PASQUALE & s & 2 & MICHIGAN & ITALY & ITALY & & & CAMAIORE. LU & 12TH AVE. & ADAMS - BALTIC \\
\hline 46 & 102 & MARCHESCHI & RAFFAELE & B & 35 & ITALY & ITALY & ITALY & 1905 & MINER/CM & COLLE DI COMPITO. LU & 12TH AVE. & ADAMS - BALTIC \\
\hline 47 & 106 & CONSANI & DAVIDE & $\mathrm{H}$ & 29 & ITALY & ITALY & ITALY & 1912 & MINER/CM & LUCCA & 12TH AVE. & ADAMS - BALTIC \\
\hline 48 & 106 & CONSANI & MARY & w & 24 & MICHIGAN & ITALY & ITALY & & & & 12TH AVE. & ADAMS - BALTIC \\
\hline 49 & 106 & CONSANI & AMELIA & D & 7 & MICHIGAN & ITALY & MICHIGAN & & & LUCCA & 12TH AVE. & ADAMS - BALTIC \\
\hline 50 & 106 & CONSANI & EMILIA & $\mathrm{s}$ & 2 & & ITALY & MICHIGAN & & & LUCCA & 12TH AVE. & ADAMS - BALTIC \\
\hline 51 & 106 & CONSANI & LOUIS & $\mathrm{s}$ & 0 & MICHIGAN & ITALY & MICHIGAN & & & LUCCA & 12TH AVE. & ADAMS - BALTIC \\
\hline 52 & 118 & PIZZI & VINCENZO & $\mathrm{H}$ & 34 & ITALY & ITALY & ITALY & 1910 & MINER/CM & PESCAGLIA. LU & 12TH AVE. & ADAMS - BALTIC \\
\hline 53 & 118 & PIZZI & GLORIA & W & 32 & ITALY & ITALY & ITALY & 11?? & & & 12TH AVE. & ADAMS - BALTIC \\
\hline 54 & 118 & PIZZI & BIANCA & D & 6 & MICHIGAN & ITALY & ITALY & & & PESCAGLIA. LU & 12TH AVE. & ADAMS - BALTIC \\
\hline 55 & 118 & PIZZI & ROSINA & D & 5 & MICHIGAN & ITALY & ITALY & & & PESCAGLIA. LU & 12TH AVE. & ADAMS - BALTIC \\
\hline 56 & 118 & PIZZI & ANTONIO & S & 1 & MICHIGAN & ITALY & ITALY & & & PESCAGLIA. LU & 12TH AVE. & ADAMS - BALTIC \\
\hline 57 & 118 & SALSINI & FREDI & B & 30 & ITALY & ITALY & ITALY & 1910 & MINER/CM & PESCAGLIA. LU & 12TH AVE. & ADAMS - BALTIC \\
\hline 58 & 118 & PIZZI & JOSEPH & B & 31 & ITALY & ITALY & ITALY & 1910 & MINER/CM & LUCCA & 12TH AVE. & ADAMS - BALTIC \\
\hline 59 & 118 & ANGELO? & JOSEPH & B & 31 & ITALY & ITALY & ITALY & 1910 & MINER/CM & & 12TH AVE. & ADAMS - BALTIC \\
\hline 60 & 224 & FANELLI & FRANK & $\begin{array}{l}\mathrm{D} \\
\mathrm{H}\end{array}$ & 37 & ITALY & ITALY & ITALY & 1901 & MINER/CM & & 13TH ST. & ADAMS - BALTIC \\
\hline 61 & 224 & FANELLI & GIULIA & W & 27 & ITALY & ITALY & ITALY & 1901 & IVIIVET/LIVI & & 13TH ST. & ADAMS - BALTIC \\
\hline 62 & 224 & FANELLI & LILIA & D & 7 & MICHIGAN & ITALY & ITALY & & & & 13TH ST. & ADAMS - BALTIC \\
\hline
\end{tabular}




\begin{tabular}{|c|c|c|c|c|c|c|c|c|c|c|c|c|c|}
\hline 63 & 224 & FANELLI & PRANDO & $\mathrm{s}$ & 5 & MICHIGAN & ITALY & ITALY & & & & 13TH ST. & ADAMS - BALTIC \\
\hline 64 & 224 & FANELLI & CARLO & $\mathrm{s}$ & 4 & MICHIGAN & ITALY & ITALY & & & & 13TH ST. & ADAMS - BALTIC \\
\hline 65 & 218 & CORTESE & LUIGI & $\mathrm{H}$ & 41 & ITALY & ITALY & ITALY & 1904 & MINER/CM & PARELLA. TO & 13TH ST. & ADAMS - BALTIC \\
\hline 66 & 218 & CORTESE & MARY & w & 36 & ITALY & ITALY & ITALY & 1906 & & & 13TH ST. & ADAMS - BALTIC \\
\hline 67 & 218 & CORTESE & JAMES & s & 11 & MICHIGAN & ITALY & ITALY & & & PARELLA. TO & 13TH ST. & ADAMS - BALTIC \\
\hline 68 & 218 & CORTESE & MADDALENA & $\mathrm{D}$ & 9 & MICHIGAN & ITALY & ITALY & & & PARELLA. TO & 13TH ST. & ADAMS - BALTIC \\
\hline 69 & 218 & CORTESE & AUGUSTO & BR & 40 & ITALY & ITALY & ITALY & 1903 & MINER/CM & PARELLA. TO & 13TH ST. & ADAMS - BALTIC \\
\hline 70 & 218 & ENRIETTI & DOMENICO & B & 36 & ITALY & ITALY & ITALY & 1906 & MINER/CM & TORINO & 13TH ST. & ADAMS - BALTIC \\
\hline 71 & 216 & RENALDI & PETER & $\mathrm{H}$ & 44 & ITALY & ITALY & ITALY & 1903 & MINER/CM & SAN GIORGIO CANAVESE. TO & 13TH ST. & ADAMS - BALTIC \\
\hline 72 & 216 & RENALDI & ANNE & W & 43 & ITALY & ITALY & ITALY & 1904 & & & 13TH ST. & ADAMS - BALTIC \\
\hline 73 & 216 & RENALDI & FRANK & $\mathrm{s}$ & 14 & MICHIGAN & ITALY & ITALY & & & SAN GIORGIO CANAVESE. TO & 13TH ST. & ADAMS - BALTIC \\
\hline 74 & 216 & RENALDI & PETER & $\mathrm{S}$ & 12 & MICHIGAN & ITALY & ITALY & & & SAN GIORGIO CANAVESE. TO & 13TH ST. & ADAMS - BALTIC \\
\hline 75 & 216 & RENALDI & TERESINA & $\mathrm{D}$ & 11 & MICHIGAN & ITALY & ITALY & & & SAN GIORGIO CANAVESE. TO & 13TH ST. & ADAMS - BALTIC \\
\hline 76 & 216 & RENALDI & MATILDE & $\mathrm{D}$ & 9 & MICHIGAN & ITALY & ITALY & & & SAN GIORGIO CANAVESE. TO & 13TH ST. & ADAMS - BALTIC \\
\hline 77 & 216 & RENALDI & PIERINA EDDA & $\mathrm{D}$ & 3 & MICHIGAN & ITALY & ITALY & & & SAN GIORGIO CANAVESE. TO & 13TH ST. & ADAMS - BALTIC \\
\hline 78 & 206 & PIEROZZI & FRANK & $\mathrm{H}$ & 52 & ITALY & ITALY & ITALY & 1907 & MINER/CM & LUCCA & 13TH ST. & ADAMS - BALTIC \\
\hline 79 & 206 & PIEROZZI & AMELIA & W & 42 & ITALY & ITALY & ITALY & 1911 & & & 13TH ST. & ADAMS - BALTIC \\
\hline 80 & 206 & PIEROZZI & LOUIS & $\mathrm{s}$ & 7 & ILLINOIS & ITALY & ITALY & & & LUCCA & 13TH ST. & ADAMS - BALTIC \\
\hline 81 & 206 & PIEROZZI & PAM? & D & 12 & ITALY & ITALY & ITALY & 1911 & & LUCCA & 13TH ST. & ADAMS - BALTIC \\
\hline 82 & 104 & GAIS & TONY & $\mathrm{H}$ & 49 & ITALY & ITALY & ITALY & 1905 & MINER/CM & TORINO & 13TH ST. & ADAMS - BALTIC \\
\hline 83 & 104 & GAIS & $?$ & D & 14 & MICHIGAN & ITALY & ITALY & 1900 & IVIITERTLIVI & TuRivo & 13TH ST. & ADAMS - BALTIC \\
\hline 84 & 104 & GAIS & MONITTA & D & 12 & MICHIGAN & ITALY & ITALY & & & TORINO & 13TH ST. & ADAMS - BALTIC \\
\hline 85 & 104 & GAIS & RINA & D & 9 & MICHIGAN & ITALY & ITALY & & & TORINO & 13TH ST. & ADAMS - BALTIC \\
\hline 86 & 104 & GAIS & COSTANTINA & D & 7 & MICHIGAN & ITALY & ITALY & & & TORINO & 13TH ST. & ADAMS - BALTIC \\
\hline 87 & 104 & ANDREONI & MAURIZIO & $\mathrm{H}$ & 30 & ITALY & ITALY & ITALY & 1913 & MINER/CM & & 13TH ST. & ADAMS - BALTIC \\
\hline 88 & 104 & ANDREONI & LUISA & W & 32 & ITALY & ITALY & ITALY & 1916 & & & 13TH ST. & ADAMS - BALTIC \\
\hline 89 & 104 & ANDREONI & ADA & D & 1 & MICHIGAN & ITALY & ITALY & & & & 13TH ST. & ADAMS - BALTIC \\
\hline 90 & 104 & ANDREONI & EVA & D & $2 \mathrm{M}$ & MICHIGAN & ITALY & ITALY & & & & 13TH ST. & ADAMS - BALTIC \\
\hline 91 & 109 & GIACHINO & JAMES & $\mathrm{H}$ & 38 & ITALY & ITALY & ITALY & 1904 & MINER/CM & TORINO & 14TH ST. & ADAMS - BALTIC \\
\hline 92 & 109 & GIACHINO & TERESA & W & 37 & ITALY & ITALY & ITALY & 1905 & & & 14TH ST. & ADAMS - BALTIC \\
\hline 93 & 109 & GIACHINO & JOSEPH & s & 13 & MICHIGAN & ITALY & ITALY & & & TORINO & 14TH ST. & ADAMS - BALTIC \\
\hline 94 & 109 & GIACHINO & MARY & D & 10 & MICHIGAN & ITALY & ITALY & & & TORINO & 14TH ST. & ADAMS - BALTIC \\
\hline 95 & 109 & GIACHINO & RENA & D & 8 & MICHIGAN & ITALY & ITALY & & & TORINO & 14TH ST. & ADAMS - BALTIC \\
\hline 96 & 109 & GIACHINO & JAMES JR. & s & 6 & MICHIGAN & ITALY & ITALY & & & TORINO & 14TH ST. & ADAMS - BALTIC \\
\hline 97 & 109 & GIACHINO & ANGELA & D & 2 & MICHIGAN & ITALY & ITALY & & & TORINO & 14TH ST. & ADAMS - BALTIC \\
\hline 98 & 109 & BOGGIO & JOHN & B & 48 & ITALY & ITALY & ITALY & 1902 & MINER/CM & TORINO & 14TH ST. & ADAMS - BALTIC \\
\hline 99 & 113 & GENOVESI & OLIVIERO & $\mathrm{H}$ & 27 & ITALY & ITALY & ITALY & 1911 & MINER/CM & VILLA BASILICA. LU & 14TH ST. & ADAMS - BALTIC \\
\hline 100 & 113 & GENOVESI & ANNA & W & 19 & WYOMING & ITALY & ITALY & & & & 14TH ST. & ADAMS - BALTIC \\
\hline 101 & 113 & GENOVESI & WILLIAM & $\mathrm{s}$ & 3 & WYOMING & ITALY & WYOMING & & & VILLA BASILICA. LU & 14TH ST. & ADAMS - BALTIC \\
\hline 102 & 113 & GENOVESI & ARNOLD & $\mathrm{s}$ & $10 \mathrm{M}$ & MICHIGAN & ITALY & WYOMING & & & VILLA BASILICA. LU & 14TH ST. & ADAMS - BALTIC \\
\hline 103 & 115 & BINI & MAURIZIO & $\mathrm{H}$ & 28 & ITALY & ITALY & ITALY & 1911 & MINER/CM & PESCAGLIA. LU & 14TH ST. & ADAMS - BALTIC \\
\hline 104 & 115 & PERFETTI & LUIGI & $\mathrm{H}$ & 40 & ITALY & ITALY & ITALY & 1909 & MINER/CM & LOPPEGLIA. LU & 14TH ST. & ADAMS - BALTIC \\
\hline 105 & 115 & PERFETTI & ARMIDA & w & 35 & ITALY & ITALY & ITALY & 1913 & & LOPPEGLIA. LU & 14TH ST. & ADAMS - BALTIC \\
\hline 106 & 115 & PERFETTI & FLORENCE & D & 10 & ITALY & ITALY & ITALY & 1913 & & LOPPEGLIA. LU & 14TH ST. & ADAMS - BALTIC \\
\hline 107 & 115 & PERFETTI & COSIMO & $\mathrm{s}$ & 5 & MICHIGAN & ITALY & ITALY & & & LOPPEGLIA, LU & 14TH ST. & ADAMS - BALTIC \\
\hline 108 & 115 & PERFETTI & ASSUNTA & D & 4 & MICHIGAN & ITALY & ITALY & & & LOPPEGLIA. LU & 14TH ST. & ADAMS - BALTIC \\
\hline 109 & 115 & PERFETTI & MAFALDA & D & 2 & MICHIGAN & ITALY & ITALY & & & LOPPEGLIA. LU & 14TH ST. & ADAMS - BALTIC \\
\hline 110 & 115 & PERFETTI & MARIO & D & $2 \mathrm{M}$ & MICHIGAN & ITALY & ITALY & & & LOPPEGLIA. LU & 14TH ST. & ADAMS - BALTIC \\
\hline 111 & 117 & BIANCHI & AMOS & $\mathrm{H}$ & 28 & ITALY & ITALY & ITALY & 1913 & MINER/CM & LUCCA & 14TH ST. & ADAMS - BALTIC \\
\hline 112 & 117 & BIANCHI & MARIA & W & 29 & ITALY & ITALY & ITALY & 1913 & & LUCCA & 14TH ST. & ADAMS - BALTIC \\
\hline 113 & 117 & BIANCHI & VEIRI? & BR & 24 & ITALY & ITALY & ITALY & 1912 & MINER/CM & LUCCA & 14TH ST. & ADAMS - BALTIC \\
\hline 114 & 117 & BIANCHI & STEWART & $\mathrm{BR}$ & 23 & ITALY & ITALY & ITALY & 1913 & MINER/CM & LUCCA & 14TH ST. & ADAMS - BALTIC \\
\hline 115 & 121 & RUGONI & FRANCESCO & $\mathrm{H}$ & 33 & ITALY & ITALY & ITALY & 1906 & MINER/CM & MAGGIONO. LU & 14TH ST. & ADAMS - BALTIC \\
\hline 116 & 121 & RUGONI & TEONISTA & W & 33 & ITALY & ITALY & ITALY & 1915 & & LUCCA & 14TH ST. & ADAMS - BALTIC \\
\hline 117 & 121 & RUGONI & JOSEPH & SSO & 12 & ITALY & ITALY & ITALY & 1915 & & LUCCA & 14TH ST. & ADAMS - BALTIC \\
\hline 118 & 121 & RUGONI & IOLANDA & SD & 14 & ITALY & ITALY & ITALY & 1915 & & LUCCA & 14TH ST. & ADAMS - BALTIC \\
\hline 119 & 121 & RUGONI & ARMANDA? & $\mathrm{D}$ & 3 & MICHIGAN & ITALY & ITALY & & & MAGGIONO. LU & 14TH ST. & ADAMS - BALTIC \\
\hline 120 & 121 & RUGONI & ROSIE & D & 1 & MICHIGAN & ITALY & ITALY & & & MAGGIONO, LU & 14TH ST. & ADAMS - BALTIC \\
\hline 121 & 208 & ANDREINI & EMILIO & $\mathrm{H}$ & 50 & ITALY & ITALY & ITALY & 1899 & MINER/CM & LUCCA & 14TH ST. & ADAMS - BALTIC \\
\hline 122 & 208 & ANDREINI & MARGHERITA & W & 40 & ITALY & ITALY & ITALY & 1900 & & & 14TH ST. & ADAMS - BALTIC \\
\hline 123 & 208 & ANDREINI & TERESA & $\mathrm{D}$ & 16 & MICHIGAN & ITALY & ITALY & & & LUCCA & 14TH ST. & ADAMS - BALTIC \\
\hline 124 & 208 & ANDREINI & ERNESTO & $\mathrm{s}$ & 15 & MICHIGAN & ITALY & ITALY & & & LUCCA & 14TH ST. & ADAMS - BALTIC \\
\hline 125 & 208 & ANDREINI & WILLIAM & $\mathrm{s}$ & 13 & MICHIGAN & ITALY & ITALY & & & LUCCA & 14TH ST. & ADAMS - BALTIC \\
\hline
\end{tabular}




\begin{tabular}{|c|c|c|c|c|c|c|c|c|c|c|c|c|c|}
\hline 126 & 208 & ANDREINI & PAUL & $\mathrm{s}$ & 10 & MICHIGAN & ITALY & ITALY & & & LUCCA & 14TH ST. & ADAMS - BALTIC \\
\hline 127 & 208 & ANDREINI & MAFALDA & D & 5 & MICHIGAN & ITALY & ITALY & & & LUCCA & 14TH ST. & ADAMS - BALTIC \\
\hline 128 & 208 & ANDREINI & IOLANDA & D & 3 & MICHIGAN & ITALY & ITALY & & & LUCCA & 14TH ST. & ADAMS - BALTIC \\
\hline 129 & 209 & RAFFAELLI & FEDERICO & $\mathrm{H}$ & 34 & ITALY & ITALY & ITALY & 1901 & MINER/CM & COLLODI. LU & 14TH ST. & ADAMS - BALTIC \\
\hline 130 & 209 & RAFFAELLI & GIULIA & w & 34 & ITALY & ITALY & ITALY & 1906 & & & 14TH ST. & ADAMS - BALTIC \\
\hline 131 & 209 & RAFFAELLI & RENA/IRENE & D & 9 & MICHIGAN & ITALY & ITALY & & & COLLODI. LU & 14TH ST. & ADAMS - BALTIC \\
\hline 132 & 209 & RAFFAELLI & JOSEPH & $\mathrm{s}$ & 8 & MICHIGAN & ITALY & ITALY & & & COLLODI. LU & 14TH ST. & ADAMS - BALTIC \\
\hline 133 & 209 & RAFFAELLI & AMELIA & D & 7 & MICHIGAN & ITALY & ITALY & & & COLLODI. LU & 14TH ST. & ADAMS - BALTIC \\
\hline 134 & 209 & RAFFAELLI & GUIDO & $\mathrm{s}$ & 3 & MICHIGAN & ITALY & ITALY & & & COLLODI. LU & 14TH ST. & ADAMS - BALTIC \\
\hline 135 & 209 & RAFFAELLI & MARIA & D & $11 \mathrm{M}$ & MICHIGAN & ITALY & ITALY & & & COLLODI. LU & 14TH ST. & ADAMS - BALTIC \\
\hline 136 & 209 & DEL GHINGARO & PIETRO & B & 28 & ITALY & ITALY & ITALY & 1907 & MINER/CM & COLLE DI COMPITO. LU & 14TH ST. & ADAMS - BALTIC \\
\hline 137 & 209 & DONATI & AMERIGO & B & 25 & ITALY & ITALY & ITALY & 1911 & MINER/CM & MONTEMAGNO. LU & 14TH ST. & ADAMS - BALTIC \\
\hline 138 & 213 & DINUCCI & GREG & $\mathrm{H}$ & 32 & ITALY & ITALY & ITALY & 1908 & MINER/CM & PESCAGLIA. LU & 14TH ST. & ADAMS - BALTIC \\
\hline $\begin{array}{r}150 \\
139\end{array}$ & 213 & DINUCCI & LENA & w & 26 & ITALY & ITALY & ITALY & 1911 & IVIIIVER/CIVI & FEOUALIA, LU & 14TH ST. & ADAMS - BALTIC \\
\hline 140 & 213 & DINUCCI & RINALDO & s & 70 & MICHIGAN & ITALY & ITALY & 1911 & & PESCAGLIA, LU & 14TH ST. & ADAMS - BALTIC \\
\hline $\begin{array}{l}140 \\
141\end{array}$ & 213 & DINUCCI & ARMANDO & $\frac{s}{s}$ & 4 & MICHIGAN & ITALY & ITALY & & & PESCAGLIA, LU & 14TH ST. & ADAMS - BALTIC \\
\hline 142 & 213 & DINUCCI & ISOLA & $\mathrm{s}$ & 1 & MICHIGAN & ITALY & ITALY & & & PESCAGLIA. LU & 14TH ST. & ADAMS - BALTIC \\
\hline 143 & 213 & BELLOSCHI & OVIDIO & B & 31 & ITALY & ITALY & ITALY & 1913 & MINER/CM & & 14TH ST. & ADAMS - BALTIC \\
\hline 144 & 213 & PIORSI? & PANDO & B & 36 & ITALY & ITALY & ITALY & 1913 & MINER/CM & & 14TH ST. & ADAMS - BALTIC \\
\hline 145 & 213 & MOROTTI & GIULIO & B & 26 & ITALY & ITALY & ITALY & 1911 & MINER/CM & & 14TH ST. & ADAMS - BALTIC \\
\hline 146 & 217 & FAUSONE & JAMES & $\mathrm{H}$ & 50 & ITALY & ITALY & ITALY & $94 / 89$ & MINER/CM & TORINO & 14TH ST. & ADAMS - BALTIC \\
\hline 147 & 217 & FAUSONE & ANGELINA & w & 43 & ITALY & ITALY & ITALY & 1900 & & & 14TH ST. & ADAMS - BALTIC \\
\hline 148 & 217 & FAUSONE & JAMES JR. & s & 18 & MICHIGAN & ITALY & ITALY & & LABORER/CM & TORINO & 14TH ST. & ADAMS - BALTIC \\
\hline 149 & 217 & FAUSONE & JOSEPH & s & 15 & MICHIGAN & ITALY & ITALY & & & TORINO & 14TH ST. & ADAMS - BALTIC \\
\hline 150 & 217 & FAUSONE & JOHN & $\mathrm{s}$ & 13 & MICHIGAN & ITALY & ITALY & & & TORINO & 14TH ST. & ADAMS - BALTIC \\
\hline 151 & 217 & FAUSONE & KATE & $\mathrm{D}$ & 12 & MICHIGAN & ITALY & ITALY & & & TORINO & 14TH ST. & ADAMS - BALTIC \\
\hline 152 & 217 & FAUSONE & $\begin{array}{l}\text { NAIL } \\
\text { PETER }\end{array}$ & s & 10 & MICHIGAN & ITALY & ITALY & & & TORINO & 14TH ST. & ADAMS - BALTIC \\
\hline 152 & 217 & $\begin{array}{l}\text { FAUSUNNE } \\
\text { FAUSONE }\end{array}$ & MARY & D & 8 & MICHIGAN & ITALY & $\begin{array}{l}\text { ITALY } \\
\text { ITAL }\end{array}$ & & & TORINO & 14TH ST. & ADAMS - BALTIC \\
\hline 154 & 217 & FAUSONE & ANTONIO & s & 5 & MICHIGAN & ITALY & ITALY & & & TORINO & 14TH ST. & ADAMS - BALTIC \\
\hline 155 & 221 & GIANNELLI & GIUSEPPE & $\mathrm{H}$ & 41 & ITALY & ITALY & ITALY & 1903 & MINER/CM & LUCCA & 14TH ST. & ADAMS - BALTIC \\
\hline 150 & 221 & GIANNELLI & ANGELA & W & 31 & ITALY & ITALY & ITALY & 1915 & IVIIIVLTV & LUCCA & 14TH ST. & ADAMS - BALTIC \\
\hline 157 & 221 & GIANNELLI & IOLANDA & D & 6 & ITALY & ITALY & ITALY & 1915 & & LUCCA & 14TH ST. & ADAMS - BALTIC \\
\hline 158 & 221 & GIANNELLI & RAFFAELLO & $\mathrm{s}$ & 3 & MICHIGAN & ITALY & ITALY & & & LUCCA & 14TH ST. & ADAMS - BALTIC \\
\hline 159 & 221 & GIANNELLI & GUIDO & $\mathrm{s}$ & 1 & MICHIGAN & ITALY & ITALY & & & LUCCA & 14TH ST. & ADAMS - BALTIC \\
\hline 160 & 221 & MARTINELLI & BERNARDO & B & 31 & ITALY & ITALY & ITALY & 1904 & MINER/CM & CAPANNORI. LU & 14TH ST. & ADAMS - BALTIC \\
\hline 161 & 221 & SCIACONETTI? & GIOVANNI & B & 30 & ITALY & ITALY & ITALY & 1912 & MINER/CM & & 14TH ST. & ADAMS - BALTIC \\
\hline 162 & 221 & PIZZI & ANTONIO & B & 30 & ITALY & ITALY & ITALY & 1913 & MINER/CM & LUCCA & 14TH ST. & ADAMS - BALTIC \\
\hline 163 & 221 & TOMMEI & LUIGI & B & 32 & ITALY & ITALY & ITALY & 1913 & MINER/CM & LUCCA & 14TH ST. & ADAMS - BALTIC \\
\hline 164 & 225 & VALENTINO & JAMES & D & 42 & ITALY & ITALY & ITALY & 1914 & MINER/CM & & 14TH ST. & ADAMS - BALTIC \\
\hline $\begin{array}{r}104 \\
165\end{array}$ & 225 & VALENTINO & NATALINA & W & 32 & $\begin{array}{l}\text { ITALY } \\
\end{array}$ & ITALY & ITALY & 1905 & MINER/CM & & 14TH ST. & ADAMS - BALTIC \\
\hline 166 & 225 & VALENTINO & EMILIO & s & 11 & ITALY & ITALY & ITALY & 1906 & & & 14TH ST. & ADAMS - BALTIC \\
\hline 167 & 225 & VALENTINO & JOSEPH & $\mathrm{s}$ & 9 & MICHIGAN & ITALY & ITALY & & & & 14TH ST. & ADAMS - BALTIC \\
\hline 168 & 225 & VALENTINO & VINCENZO & $\mathrm{s}$ & 7 & MICHIGAN & ITALY & ITALY & & & & 14TH ST. & ADAMS - BALTIC \\
\hline 169 & 225 & VALENTINO & $\begin{array}{l}\text { PETER } \\
\end{array}$ & $\mathrm{s}$ & 5 & MICHIGAN & ITALY & ITALY & & & & 14TH ST. & ADAMS - BALTIC \\
\hline 170 & 220 & GEMIGNANI & ANGELO & $\mathrm{H}$ & 27 & ITALY & ITALY & $\begin{array}{l}\text { ITALY } \\
\text { ITAL }\end{array}$ & 1918 & MINER/CM & LUCCA & 14TH ST. & ADAMS - BALTIC \\
\hline 171 & 220 & GEMIGNANI & EMMA & w & 22 & MICHIGAN & ITALY & ITALY & & & & 14TH ST. & ADAMS - BALTIC \\
\hline 172 & 220 & GEMIGNANI & FRANCIS & s & 3 & MICHIGAN & ITALY & MICHIGAN & & & LUCCA & 14TH ST. & ADAMS - BALTIC \\
\hline 173 & 220 & GEMIGNANI & LEONARDO & s & 1 & MICHIGAN & ITALY & MICHIGAN & & & LUCCA & 14TH ST. & ADAMS - BALTIC \\
\hline 174 & 220 & GEMIGNANI & ENRICO & BR & 29 & ITALY & ITALY & ITALY & 1914 & MINER/CM & LUCCA & 14TH ST. & ADAMS - BALTIC \\
\hline 175 & 216 & BERTODATTI & CARLO & $\mathrm{H}$ & 32 & ITALY & ITALY & ITALY & 1909 & NOT CLEAR & SAN GIORGIO CANAVESE. TO & 14TH ST. & ADAMS - BALTIC \\
\hline 176 & 216 & BERTODATTI & CATERINA & w & 30 & ITALY & ITALY & ITALY & 1910 & & & 14TH ST. & ADAMS - BALTIC \\
\hline 177 & 216 & BERTODATTI & DOMENICO & s & 7 & MICHIGAN & ITALY & ITALY & & & SAN GIORGIO CANAVESE, TO & 14TH ST. & ADAMS - BALTIC \\
\hline 178 & 208 & BARBERIS & DOMENICO & $\mathrm{H}$ & 38 & ITALY & ITALY & ITALY & 1900 & MINER/CM & RIVAROLO CANAVESE. TO & 14TH ST. & ADAMS - BALTIC \\
\hline 179 & 208 & BARBERIS & MARY & w & 33 & ITALY & ITALY & ITALY & 1901 & & & 14TH ST. & ADAMS - BALTIC \\
\hline 180 & 208 & BARBERIS & ANGELO & s & 12 & MICHIGAN & ITALY & ITALY & & & RIVAROLO CANAVESE. TO & 14TH ST. & ADAMS - BALTIC \\
\hline 181 & 208 & BARBERIS & ROSIE & $\mathrm{D}$ & 14 & MICHIGAN & ITALY & $\begin{array}{l}\text { ITALY } \\
\end{array}$ & & & RIVAROLO CANAVESE. TO & 14TH ST. & ADAMS - BALTIC \\
\hline 182 & 208 & BARBERIS & DOMENICO JR. & s & 10 & MICHIGAN & ITALY & ITALY & & & RIVAROLO CANAVESE. TO & 14TH ST. & ADAMS - BALTIC \\
\hline 183 & 208 & BARBERIS & JOHN & s & 8 & MICHIGAN & ITALY & $\begin{array}{l}\text { ITALY } \\
\text { ITAL }\end{array}$ & & & RIVAROLO CANAVESE, TO & 14TH ST. & ADAMS - BALTIC \\
\hline 184 & 110 & MARACCINI & WALTER & $\mathrm{H}$ & 30 & ITALY & ITALY & ITALY & 1907 & MINER/CM & LUCCA & 14TH ST. & ADAMS - BALTIC \\
\hline 185 & 110 & MARACCINI & ROSA & w & 35 & ITALY & ITALY & ITALY & 1906 & & & 14TH ST. & ADAMS - BALTIC \\
\hline 186 & 110 & PUCCI & ENZO & sso & 11 & MICHIGAN & ITALY & ITALY & & & LUCCA & 14TH ST. & ADAMS - BALTIC \\
\hline 187 & 110 & PUCCl & LUISA & SD & 10 & MICHIGAN & ITALY & ITALY & & & LUCCA & 14TH ST. & ADAMS - BALTIC \\
\hline 188 & 110 & PUCCl & OLGA & $\mathrm{SD}$ & 9 & MICHIGAN & ITALY & ITALY & & & LUCCA & 14TH ST. & ADAMS - BALTIC \\
\hline
\end{tabular}




\begin{tabular}{|c|c|c|c|c|c|c|c|c|c|c|c|c|c|}
\hline 189 & 110 & MARACCINI & LUIGI & $\mathrm{s}$ & 6 & MICHIGAN & ITALY & ITALY & & & LUCCA & 14TH ST. & ADAMS - BALTIC \\
\hline 190 & 110 & MARACCINI & VALENTINA & D & 2 & MICHIGAN & ITALY & ITALY & & & LUCCA & 14TH ST. & ADAMS - BALTIC \\
\hline 191 & 121 & IACOPETTI & EMILIO & $\mathrm{H}$ & 41 & ITALY & ITALY & ITALY & 1899 & MINER/CM & LUCCA & 15TH ST. & ADAMS - BALTIC \\
\hline 192 & 121 & IACOPETTI & CARMINA & w & 32 & ITALY & ITALY & ITALY & 1908 & & LUCCA & 15TH ST. & ADAMS - BALTIC \\
\hline 193 & 121 & IACOPETTI & ERNESTINA & D & 11 & ITALY & ITALY & ITALY & 1908 & & LUCCA & 15TH ST. & ADAMS - BALTIC \\
\hline 194 & 121 & IACOPETTI & ARTONISTE & $\mathrm{D}$ & 10 & MICHIGAN & ITALY & ITALY & & & LUCCA & 15TH ST. & ADAMS - BALTIC \\
\hline 195 & 121 & IACOPETTI & ORLANDA? & $\mathrm{D}$ & 8 & MICHIGAN & ITALY & ITALY & & & LUCCA & 15TH ST. & ADAMS - BALTIC \\
\hline 196 & 121 & IACOPETTI & LUIGI & $\mathrm{s}$ & 6 & MICHIGAN & ITALY & ITALY & & & LUCCA & 15TH ST. & ADAMS - BALTIC \\
\hline 197 & 121 & IACOPETTI & URTIMINA & $\mathrm{s}$ & $9 \mathrm{M}$ & MICHIGAN & ITALY & ITALY & & & LUCCA & 15TH ST. & ADAMS - BALTIC \\
\hline 198 & 121 & POLISETINI? & GIUSEPPE & B & 26 & ITALY & ITALY & ITALY & 1912 & MINER/CM & & 15TH ST. & ADAMS - BALTIC \\
\hline 199 & 121 & BERNARDINI & BERTO & B & 31 & ITALY & ITALY & ITALY & 1912 & MINER/CM & & 15TH ST. & ADAMS - BALTIC \\
\hline 200 & 121 & DEL GHINGARO & LIVIO & B & 23 & ITALY & ITALY & ITALY & 1913 & MINER/CM & CASTELVECCHIO. LU & 15TH ST. & ADAMS - BALTIC \\
\hline 201 & 121 & FONTANA & VITTORIO & B & 35 & ITALY & ITALY & ITALY & 1909 & MINER/CM & COLLE DI COMPITO. LU & 15TH ST. & ADAMS - BALTIC \\
\hline 202 & 109 & CAPPO & CESARE & $\mathrm{H}$ & 31 & ITALY & ITALY & ITALY & 1907 & MINER/CM & SAN GIUSTO CANAVESE. TO & 15TH ST. & ADAMS - BALTIC \\
\hline 203 & 109 & CAPPO-MARE & JENNY & W & 22 & ITALY & ITALY & ITALY & 1908 & & & 15TH ST. & ADAMS - BALTIC \\
\hline 204 & 109 & CAPPO & ANNE & $\mathrm{D}$ & 6 & MICHIGAN & ITALY & ITALY & & & SAN GIUSTO CANAVESE, TO & 15TH ST. & ADAMS - BALTIC \\
\hline 205 & 109 & CAPPO & JOSEPH & $\mathrm{s}$ & 4 & MICHIGAN & ITALY & ITALY & & & SAN GIUSTO CANAVESE. TO & 15TH ST. & ADAMS - BALTIC \\
\hline 206 & 109 & CAPPO & LOUIS & $\mathrm{s}$ & $1 \mathrm{M}$ & MICHIGAN & ITALY & ITALY & & & SAN GIUSTO CANAVESE. TO & 15TH ST. & ADAMS - BALTIC \\
\hline 207 & 122 & RICCA & ANTONIO & $\mathrm{H}$ & 30 & ITALY & ITALY & ITALY & 1911 & MINER/CM & SAN MARTINO CANAVESE. TO & 16TH ST. & ADAMS - BALTIC \\
\hline 208 & 122 & RICCA & CATERINA & w & 32 & ITALY & ITALY & ITALY & 1916 & & & 16TH ST. & ADAMS - BALTIC \\
\hline 209 & 115 & RAFFAELLI & FRANK & $\mathrm{H}$ & 34 & ITALY & ITALY & ITALY & 01?? & BAKER/BAKERY & COLLODI. LU & 16TH ST. & ADAMS - BALTIC \\
\hline 210 & 115 & RAFFAELLI & JULIA & w & 32 & ITALY & ITALY & ITALY & 1907 & DANER/DARERT & GOLLUDI. LU S S S & 16TH ST. & ADAMS - BALTIC \\
\hline 211 & 115 & RAFFAELLI & PALMIRA & D & 11 & MICHIGAN & ITALY & ITALY & & & COLLODI. LU & 16TH ST. & ADAMS - BALTIC \\
\hline 212 & 115 & RAFFAELLI & LINA & D & 9 & MICHIGAN & ITALY & ITALY & & & COLLODI. LU & 16TH ST. & ADAMS - BALTIC \\
\hline 213 & 115 & RAFFAELLI & FLORENCE & $\mathrm{D}$ & 8 & MICHIGAN & ITALY & ITALY & & & COLLODI.LU & 16TH ST. & ADAMS - BALTIC \\
\hline 214 & 115 & RAFFAELLI & BRUNO & $\mathrm{s}$ & 7 & MICHIGAN & ITALY & ITALY & & & COLLODI. LU & 16TH ST. & ADAMS - BALTIC \\
\hline 215 & 115 & RAFFAELLI & MARY & D & 6 & MICHIGAN & ITALY & ITALY & & & COLLODI. LU & 16TH ST. & ADAMS - BALTIC \\
\hline 216 & 115 & RAFFAELLI & RAIMONDO & $\mathrm{s}$ & 1 & MICHIGAN & ITALY & ITALY & & & COLLODI, LU & 16TH ST. & ADAMS - BALTIC \\
\hline 217 & & SERRA & MARY & $\mathrm{H}$ & 50 & ITALY & ITALY & ITALY & 1895 & KEEPER/BOARDING HOUSE & SAN GIORGIO CANAVESE. TO & STANTON AVE. & ADAMS - BALTIC \\
\hline 218 & & SERRA & MARY & D & 20 & MICHIGAN & ITALY & ITALY & & $\mathrm{COOK} / \mathrm{PF}$ & SAN GIORGIO CANAVESE. TO & STANTON AVE. & ADAMS - BALTIC \\
\hline 219 & & SERRA & LENA & D & 17 & MICHIGAN & ITALY & ITALY & & & SAN GIORGIO CANAVESE. TO & STANTON AVE. & ADAMS - BALTIC \\
\hline 220 & & FURNO & PETER & B & 25 & ITALY & ITALY & ITALY & 1912 & MINER/CM & TORINO & STANTON AVE. & ADAMS - BALTIC \\
\hline 221 & & TOSATTO & JOHN & B & 27 & ITALY & ITALY & ITALY & 1913 & MINER/CM & TORINO & STANTON AVE. & ADAMS - BALTIC \\
\hline 222 & & GALETTO & STEVEN & B & 42 & ITALY & ITALY & ITALY & 1904 & MINER/CM & SAN GIORGIO CANAVESE, TO & STANTON AVE. & ADAMS - BALTIC \\
\hline 223 & & BECTRINI & MIKE & B & 43 & ITALY & ITALY & ITALY & 1915 & MINER/CM & & STANTON AVE. & ADAMS - BALTIC \\
\hline 224 & & CHIOTTI & JOHN & B & 32 & ITALY & ITALY & ITALY & 1912 & MINER/CM & TORINO & STANTON AVE. & ADAMS - BALTIC \\
\hline 225 & 120 & MARE & LOUIS & $\mathrm{H}$ & 47 & ITALY & ITALY & ITALY & 1905 & MINER/CM & CUORGNE'. TO & 15TH ST. & ADAMS - BALTIC \\
\hline 226 & 120 & MARE & ANNE & w & 42 & ITALY & ITALY & ITALY & 1905 & & CUORGNE'. TO & 15TH ST. & ADAMS - BALTIC \\
\hline 227 & 120 & MARE & MARY & $\mathrm{D}$ & 16 & ITALY & ITALY & ITALY & 1905 & & CUORGNE'. TO & 15TH ST. & ADAMS - BALTIC \\
\hline 228 & 522 & DATA/DATTA & JOHN & $\mathrm{H}$ & 27 & ITALY & ITALY & ITALY & 1907 & MINER/CM & PONT CANAVESE. TO & 16TH ST. & ADAMS - BALTIC \\
\hline 229 & 522 & DATA/DATTA & MARY & W & 28 & ITALY & ITALY & ITALY & 1907 & & PONT CANAVESE. TO & 16TH ST. & ADAMS - BALTIC \\
\hline 230 & 522 & BALMA/BAIMA & JOHN JR. & sso & 13 & MICHIGAN & ITALY & ITALY & & & TORINO & 16TH ST. & ADAMS - BALTIC \\
\hline 231 & 522 & BALMA/BAIMA & LOUIS & sso & 9 & MICHIGAN & ITALY & ITALY & & & TORINO & 16TH ST. & ADAMS - BALTIC \\
\hline 232 & 522 & BALMA/BAIMA & GUIDO & sso & 7 & MICHIGAN & ITALY & ITALY & & & TORINO & 16TH ST. & ADAMS - BALTIC \\
\hline 233 & 522 & DATA/DATTA & TONY & $\mathrm{s}$ & 4 & MICHIGAN & ITALY & ITALY & & & PONT CANAVESE. TO & 16TH ST. & ADAMS - BALTIC \\
\hline 234 & 518 & DEL DIN & LOUIS & $\mathrm{H}$ & 52 & ITALY & ITALY & ITALY & 1892 & MINER/CM & RIVAMONTE. BELLUNO & 16TH ST. & ADAMS - BALTIC \\
\hline 235 & 518 & DEL DIN & TERESA & w & 38 & ITALY & ITALY & ITALY & 1908 & & RIVAMONTE. BELLUNO & 16TH ST. & ADAMS - BALTIC \\
\hline 236 & 518 & DEL DIN & JOHN & s & 14 & ITALY & ITALY & ITALY & 1908 & & RIVAMONTE. BELLUNO & 16TH ST. & ADAMS - BALTIC \\
\hline 237 & 518 & DEL DIN & MADDALENA & D & 12 & MICHIGAN & ITALY & ITALY & & & RIVAMONTE. BELLUNO & 16TH ST. & ADAMS - BALTIC \\
\hline 238 & 518 & DEL DIN & TONY & $\mathrm{s}$ & 11 & MICHIGAN & ITALY & ITALY & & & RIVAMONTE. BELLUNO & 16TH ST. & ADAMS - BALTIC \\
\hline 239 & 509 & ZANOTTI & ROBERTO & $\mathrm{H}$ & 43 & ITALY & ITALY & ITALY & 1906 & MINER/CM & PEROSA. TO & 16TH ST. & ADAMS - BALTIC \\
\hline 240 & 509 & ZANOTTI & MARY & w & 39 & ITALY & ITALY & ITALY & 1909 & & PEROSA, TO & 16TH ST. & ADAMS - BALTIC \\
\hline 241 & 509 & ZANOTTI & LENA & D & 17 & ITALY & ITALY & ITALY & 1909 & CLERK/CANDY STORE & PEROSA. TO & 16TH ST. & ADAMS - BALTIC \\
\hline 242 & 509 & ZANOTTI & FELIX & s & 18 & MICHIGAN & ITALY & ITALY & & & PEROSA. TO & 16TH ST. & ADAMS - BALTIC \\
\hline 243 & 509 & FERNELLIO/S & JOSEPH & $\mathrm{H}$ & 36 & ITALY & ITALY & ITALY & 1903 & MINER/CM & & 16TH ST. & ADAMS - BALTIC \\
\hline 244 & 509 & FERNELLIO/S & MANFARIN? & D & 8 & MINNESOT & ITALY & ITALY & & & & 16TH ST. & ADAMS - BALTIC \\
\hline 245 & 509 & FERNELLIO/S & TONY & s & 5 & MINNESOT & ITALY & ITALY & & & & 16TH ST. & ADAMS - BALTIC \\
\hline 246 & 2 & GIORGINI & ALBERT & w & 27 & ITALY & ITALY & ITALY & 1911 & MINER/CM & & 6TH - EAST OF BALTIC & ADAMS - BALTIC \\
\hline 247 & 2 & GIORGINI & TERESA & $\mathrm{H}$ & 27 & MICHIGAN & ITALY & ITALY & & & & 6TH - EAST OF BALTIC & ADAMS - BALTIC \\
\hline 248 & 2 & GIORGINI & ARMIDA & D & 3 & MICHIGAN & ITALY & MICHIGAN & & & & 6TH - EAST OF BALTIC & ADAMS - BALTIC \\
\hline 249 & 2 & GIORGINI & MERIGA & D & 1 & MICHIGAN & ITALY & MICHIGAN & & & & 6TH - EAST OF BALTIC & ADAMS - BALTIC \\
\hline 250 & NN & GUIDOTTI & PETER & $\mathrm{H}$ & 31 & ITALY & ITALY & ITALY & 1918 & MANAGER/GROCERY STORE & SEGROMIGNO. LU & MAIN ST. & ADAMS - SOUTH RANGE \\
\hline 251 & & GUIDOTTI & CATERINA & w & 30 & $\begin{array}{l}\text { ITALY } \\
\text { ITALY }\end{array}$ & ITALY & ITALY & 1919 & 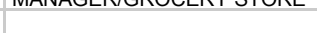 & & MAIN ST. & ADAMS - SOUTH RANGE \\
\hline
\end{tabular}




\begin{tabular}{|c|c|c|c|c|c|c|c|c|c|c|c|c|c|}
\hline 252 & & GUIDOTTI & LAILA & D & 6 & MICHIGAN & ITALY & ITALY & & & SEGROMIGNO. LU & MAIN ST. & ADAMS - SOUTH RANGE \\
\hline 253 & & GUIDOTTI & TERESA & D & 5 & MICHIGAN & ITALY & ITALY & & & SEGROMIGNO. LU & MAIN ST. & ADAMS - SOUTH RANGE \\
\hline 254 & & GUIDOTTI & EGISTO & $\mathrm{s}$ & 4 & MICHIGAN & ITALY & ITALY & & & SEGROMIGNO. LU & MAIN ST. & ADAMS - SOUTH RANGE \\
\hline 255 & & GUIDOTTI & MARIA & $\mathrm{D}$ & $5 \mathrm{M}$ & MICHIGAN & ITALY & ITALY & & & SEGROMIGNO. LU & MAIN ST. & ADAMS - SOUTH RANGE \\
\hline 256 & NN & FERRETTI & ANTONIO & $\mathrm{H}$ & 52 & ITALY & ITALY & ITALY & 1899 & MINER/CM & ASCOLI PICENO & MAIN ST. & ADAMS - SOUTH RANGE \\
\hline 257 & & FERRETTI & ELENA & w & 53 & ITALY & ITALY & ITALY & 1901 & & ASCOLI PICENO & MAIN ST. & ADAMS - SOUTH RANGE \\
\hline 258 & NN & PARIAMI & TONY & $\mathrm{H}$ & 45 & ITALY & ITALY & ITALY & 1901 & MINER/CM & LUCCA & MAIN ST. & ADAMS - SOUTH RANGE \\
\hline 259 & INiv & PARIAMI & MARY & w & 34 & ITALY & ITALY & ITALY & 1908 & IVIIVERTUIVI & LUCUA & MAIN ST. & ADAMS - SOUTH RANGE \\
\hline 260 & & PARIAMI & LOUIS & s & 7 & MICHIGAN & ITALY & ITALY & 1900 & & LUCCA & MAIN ST. & ADAMS - SOUTH RANGE \\
\hline 261 & & PARIAMI & ELISA & $\mathrm{D}$ & 6 & MICHIGAN & ITALY & ITALY & & & LUCCA & MAIN ST. & ADAMS - SOUTH RANGE \\
\hline 262 & & PARIAMI & JOHN & $\mathrm{s}$ & 5 & MICHIGAN & ITALY & ITALY & & & LUCCA & MAIN ST. & ADAMS - SOUTH RANGE \\
\hline 263 & NN & SANTORI & AMERIGO & $\mathrm{H}$ & 43 & ITALY & ITALY & ITALY & UN & PROPRIETOR/BAKERY & SEGROMIGNO. LU & MAIN ST. & ADAMS - SOUTH RANGE \\
\hline 264 & & SANTORI & PRIMA & W & 34 & ITALY & ITALY & ITALY & UN & & & MAIN ST. & ADAMS - SOUTH RANGE \\
\hline 265 & & SANTORI & MATTEO & BR & 30 & ITALY & ITALY & ITALY & 1912 & & SEGROMIGNO. LU & MAIN ST. & ADAMS - SOUTH RANGE \\
\hline 266 & & MATTEUCCI & FRED & B & 32 & ITALY & ITALY & ITALY & 1905 & MANAGER/CANDY STORE & COLLE DI COMPITO. LU & MAIN ST. & ADAMS - SOUTH RANGE \\
\hline 267 & & PORERI & PAUL & B & 38 & ITALY & ITALY & ITALY & 1908 & MINER/CM & & MAIN ST. & ADAMS - SOUTH RANGE \\
\hline 268 & & GENOVESI & DANTE & B & 40 & ITALY & ITALY & ITALY & 1911 & MINER/CM & & MAIN ST. & ADAMS - SOUTH RANGE \\
\hline 269 & NN & STEFANI & JOHN & $\mathrm{H}$ & 44 & ITALY & ITALY & ITALY & 1899 & & VILLA BASILICA. LU & MAIN ST. & ADAMS - SOUTH RANGE \\
\hline 270 & & STEFANI & CAMILLA & w & 31 & ITALY & ITALY & ITALY & 1905 & & & MAIN ST. & ADAMS - SOUTH RANGE \\
\hline 271 & & STEFANI & CHERIDA & D & 13 & MICHIGAN & ITALY & ITALY & & & VILLA BASILICA. LU & MAIN ST. & ADAMS - SOUTH RANGE \\
\hline 272 & & STEFANI & MARY & $\mathrm{D}$ & 4 & MICHIGAN & ITALY & ITALY & & & VILLA BASILICA. LU & MAIN ST. & ADAMS - SOUTH RANGE \\
\hline 273 & & STEFANI & LILLIAN & $\mathrm{D}$ & 8 & MICHIGAN & ITALY & ITALY & & & VILLA BASILICA, LU & MAIN ST. & ADAMS - SOUTH RANGE \\
\hline 274 & & STEFANI & JOSEPH & $\mathrm{s}$ & 6 & MICHIGAN & ITALY & ITALY & & & VILLA BASILICA. LU & MAIN ST. & ADAMS - SOUTH RANGE \\
\hline 275 & & STEFANI & PRIMA & $\mathrm{D}$ & 2 & MICHIGAN & ITALY & ITALY & & & VILLA BASILICA. LU & MAIN ST. & ADAMS - SOUTH RANGE \\
\hline 276 & NN & ANDREINI & JOSEPH & $\mathrm{H}$ & 35 & ITALY & ITALY & ITALY & 1905 & MANAGER/CANDY STORE & CAPANNORI. LU & MAIN ST. & ADAMS - SOUTH RANGE \\
\hline 277 & & ANDREINI & ANGELINE & W & 26 & ITALY & ITALY & ITALY & 13?? & & & MAIN ST. & ADAMS - SOUTH RANGE \\
\hline 278 & & ANDREINI & ELISA & $\mathrm{D}$ & 4 & MICHIGAN & ITALY & ITALY & & & CAPANNORI. LU & MAIN ST. & ADAMS - SOUTH RANGE \\
\hline 279 & & ANDREINI & JOSEPH & $\mathrm{s}$ & 2 & MICHIGAN & ITALY & ITALY & & & CAPANNORI, LU & MAIN ST. & ADAMS - SOUTH RANGE \\
\hline 280 & & ANDREINI & CRISTIAN & $\mathrm{s}$ & 1 & MICHIGAN & ITALY & ITALY & & & CAPANNORI. LU & MAIN ST. & ADAMS - SOUTH RANGE \\
\hline 281 & NN & PROCISSI & PETER & B & 26 & FRANCE & ITALY & ITALY & UN & CLERK/CANDY STORE & LUCCA & MAIN ST. & ADAMS - SOUTH RANGE \\
\hline 282 & & MARTA & MAUDE & B & 23 & MICHIGAN & ITALY & ITALY & & CLERK/CANDY STORE & TORINO & MAIN ST. & ADAMS - SOUTH RANGE \\
\hline 283 & $\mathrm{NN}$ & GIACHINO & ADOLPH? & $\mathrm{H}$ & 44 & ITALY & ITALY & ITALY & 1900 & & TORINO & MAIN ST. & ADAMS - SOUTH RANGE \\
\hline 284 & 6 & SIMONIA & JOSEPH & $\mathrm{H}$ & 53 & ITALY & ITALY & ITALY & UN & PROPRIETOR/FURNITURE SHOP & 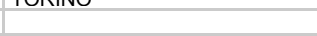 & MAIN ST. & ADAMS - SOUTH RANGE \\
\hline 285 & & SIMONIA & JOHN & $\mathrm{s}$ & 13 & MICHIGAN & AUSTRIA & AUSTRIA & & & & MAIN ST. & ADAMS - SOUTH RANGE \\
\hline 286 & 18 & BASSO & LUIGI & $\mathrm{H}$ & 47 & ITALY & ITALY & ITALY & 1892 & JANITOR/SCHOOL & SAN GIORGIO CANAVESE. TO & MAIN ST. & ADAMS - SOUTH RANGE \\
\hline 287 & & BASSO & NELLI & w & 45 & ITALY & ITALY & ITALY & 1893 & & 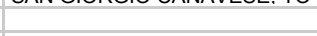 & MAIN ST. & ADAMS - SOUTH RANGE \\
\hline 288 & & BASSO & JOSEPH & $\mathrm{s}$ & 25 & MICHIGAN & ITALY & ITALY & & BANKING/SOUTH RANGE BANK & SAN GIORGIO CANAVESE. TO & MAIN ST. & ADAMS - SOUTH RANGE \\
\hline 289 & & BASSO & JOHANNA & D & 20 & MICHIGAN & ITALY & ITALY & & TEACHER/PUBLIC SCHOOL & SAN GIORGIO CANAVESE. TO & MAIN ST. & ADAMS - SOUTH RANGE \\
\hline 290 & & BASSO & ANTONIO & $\mathrm{s}$ & 15 & MICHIGAN & ITALY & ITALY & & & SAN GIORGIO CANAVESE. TO & MAIN ST. & ADAMS - SOUTH RANGE \\
\hline 291 & & BASSO & LOUISE & D & 12 & MICHIGAN & ITALY & ITALY & & & SAN GIORGIO CANAVESE. TO & MAIN ST. & ADAMS - SOUTH RANGE \\
\hline 292 & 53 & ZANA & DOMENICO & $\mathrm{H}$ & 44 & ITALY & ITALY & ITALY & 1905 & FREIGHT DEPOT/C.R.R. DEPOT & CUCEGLIO. TO & 1ST ST. & ADAMS - SOUTH RANGE \\
\hline 293 & & ZANA & MARY & w & 44 & ITALY & ITALY & ITALY & 1907 & 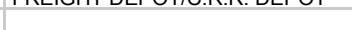 & CUCEGLIO. TO & 1ST ST. & ADAMS SOITH RANGE \\
\hline 294 & & ZANA & ANTONIETTA & D & 20 & MICHIGAN & ITALY & ITALY & & CLERK/GROCERY STORE & CUCEGLIO TO & $1 \mathrm{ST}$ ST & ADAMS SOITH RANGE \\
\hline 295 & & ZANA & TERESA & D & 12 & MICHIGAN & ITALY & ITALY & & CLERAIURUCERT STURE & $\begin{array}{l}\text { CUCEGLLO. } 10 \\
\text { CUCEGLO TO }\end{array}$ & $1 \mathrm{ST}$. & AUAISS-SOITH RANGE \\
\hline 296 & & ZANA & EVA & D & 11 & MICHIGAN & ITALY & ITALY & & & CUCEGLIO TO & 1ST CT. & AUAVIS-SOUITHRANGE \\
\hline 297 & & ZANA & JOSEPH & s & 9 & MICHIGAN & ITALY & ITALY & & & CUCEGLIO. TO & 1ST ST. & ADAMS - SOUTH RANGE \\
\hline 298 & NN & ROMANO & PETER & $\mathrm{H}$ & 53 & ITALY & ITALY & ITALY & 1894 & MINER/CM & TORINO & 1ST ST. & ADAMS - SOUTH RANGE \\
\hline 299 & & ROMANO & MARIE & w & 45 & ITALY & ITALY & ITALY & 1893 & 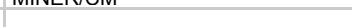 & Normo & 1ST ST. & ADAMS - SOUIH RANGE \\
\hline 300 & & ROMANO & WILLIAM & s & 21 & MICHIGAN & ITALY & ITALY & & MINER/CM & TORINO & 1ST ST & ADAMS - SOUTH RANGE \\
\hline 301 & & ROMANO & JOSEPHINE & $\mathrm{D}$ & 20 & MICHIGAN & ITALY & ITALY & 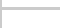 & NOT CLEAR & TORINO & 1 ST ST & ADAMS - SOUTH RANGE \\
\hline 30? & & ROMANO & JOSEPH & s & 15 & MICHIGAN & $\begin{array}{l}\text { IITALY } \\
\text { ITALY }\end{array}$ & $\begin{array}{l}\text { IITALY } \\
\text { ITALY }\end{array}$ & & Jut CLLati & TORINO & 1 ST ST & ADAMS - SOUTH RANGE \\
\hline 303 & & ROMANO & ROSIE & $\mathrm{D}$ & 13 & MICHIGAN & ITALY & ITALY & & & TORINO & 1 ST ST & ADAMS - SOUTH RANGE \\
\hline 304 & & ROMANO & JOHN & $\mathrm{s}$ & 11 & MICHIGAN & ITALY & ITALY & & & TORINO & 1 ST ST & ADAMS - SOUIH RANGE \\
\hline 305 & & ROMANO & ?? & $\mathrm{D}$ & 9 & MICHIGAN & JTALY & ITALY & & & TORINO & 1ST ST. & ADAMS - SOUIHRANGE \\
\hline 306 & & ROMANO & PETER & s & 7 & MICHIANAN & JTALY & ITALY & & & TOPINO & 1 ST ST & ADAMIS - SOUTHR RANGE \\
\hline 307 & 2 & CERUTTI & PETER & H & 63 & TIYYAIV & ITALY & ITAIY & 1808 & & SANGI RGIO CANAYESE TO & ISAITICAYE & ADAMS-SOUTHRANGE \\
\hline 308 & 2 & CERUIII & 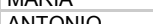 & $u_{c}$ & 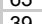 & TALY & TIALY & ITALY & 1090 & RIACKCMUTH & SAIV GIURGI CAAVAVESE. IO & DALTI AVE. & ADAMIS-SOUIHRANGE \\
\hline 309 & & CERUIII & ANIONIO & $a^{3}$ & 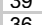 & WALY & IIALY & IIALY & 1090 & BLACKSMIIH & SAIN GIURGIU CANAVESE. IO & BALITCAVE. & ADAMS - SOUITHRANGE \\
\hline 309 & & CEROIIII & GIOVAIVIVI & 5 & 30 & FRAIVLE & 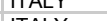 & IITALY & 1898 & NUT CLEAR & SAIN GIURGIU CAINAVESE, IU & BALIT AVE. & ADAMS-SOUIHRANGE \\
\hline 310 & & CERUTTI & JAMES & $\mathrm{s}$ & 36 & FRANCE & ITALY & ITALY & 1898 & NOT CLEAR & SAN GIORGIO CANAVESE. TO & BALTIC AVE. & ADAMS - SOUTH RANGE \\
\hline 311 & & CERUTTI & JOHN & s & 29 & ITALY & ITALY & ITALY & 1898 & NOT CLEAR & SAN GIORGIO CANAVESE. TO & BALTIC AVE. & ADAMS - SOUTH RANGE \\
\hline 312 & & CERUTTI & KATE & DIL & 25 & ITALY & ITALY & ITALY & 1913 & & D CANAVESE. TO & AVE. & ADAMS - SC \\
\hline 313 & & CERUTTI & MARY & GD & 3 & MICHIGAN & ITALY & ITALY & & & SAN GIORGIO CANAVESE. TO & BALTIC AVE. & ADAMS - SOUTH RANGE \\
\hline 314 & & CERUTTI & ANTONIO & GS & 1 & MICHIGAN & ITALY & ITALY & & & SAN GIORGIO CANAVESE. TO & BALTIC AVE. & ADAMS - SOUTH RANGE \\
\hline
\end{tabular}




\begin{tabular}{|c|c|c|c|c|c|c|c|c|c|c|c|c|c|}
\hline 315 & & CERUTTI & VINCENZO & GS & $6 \mathrm{M}$ & MICHIGAN & ITALY & ITALY & & & SAN GIORGIO CANAVESE. TO & BALTIC AVE. & ADAMS - SOUTH RANGE \\
\hline 316 & & DE FILIPPI & $\mathrm{JOHN}$ & B & 44 & ITALY & ITALY & ITALY & 1902 & & SAN GIORGIO CANAVESE. TO & BALTIC AVE. & ADAMS - SOUTH RANGE \\
\hline 317 & & PET??? & LANDO & B & 33 & ITALY & ITALY & ITALY & 1913 & & & BALTIC AVE. & ADAMS - SOUTH RANGE \\
\hline 318 & & CERUTTI & DOMENICO & B & 28 & ITALY & ITALY & ITALY & 1912 & MINER/CM & SAN GIUSTO CANAVESE. TO & BALTIC AVE. & ADAMS - SOUTH RANGE \\
\hline 319 & 33 & SANTORI & ORESTE & $\mathrm{H}$ & 36 & ITALY & ITALY & ITALY & 1909 & & SEGROMIGNO. LU & 1ST ST. & ADAMS - SOUTH RANGE \\
\hline 320 & & SANTORI & ROSA & w & 32 & ITALY & ITALY & ITALY & 1909 & & SEGROMIGNO. LU & 1ST ST. & ADAMS - SOUTH RANGE \\
\hline 321 & & SANTORI & EDO & s & 10 & ITALY & ITALY & ITALY & 1909 & & SEGROMIGNO. LU & 1ST ST. & ADAMS - SOUTH RANGE \\
\hline 322 & & SANTORI & CARLO & s & 7 & CALIFORNI & ITALY & ITALY & 1509 & & SEGROMIGNO. LU & 1ST ST. & ADAMS - SOUTH RANGE \\
\hline 323 & & SANTORI & BONAS & $\mathrm{s}$ & 2 & MICHIGAN & ITALY & ITALY & & & SEGROMIGNO. LU & 1ST ST. & ADAMS - SOUTH RANGE \\
\hline 324 & 33 & LUCCHESI & CESARE & $\mathrm{H}$ & 42 & ITALY & ITALY & ITALY & 1900 & PROPRIETOR/GARAGE & LUCCA & 1ST ST. & ADAMS - SOUTH RANGE \\
\hline 325 & & LUCCHESI & JENNIE & w & 31 & ITALY & ITALY & ITALY & 1902 & & & 1ST ST. & ADAMS - SOUTH RANGE \\
\hline 326 & & LUCCHESI & FRED & s & 12 & MICHIGAN & ITALY & ITALY & & & LUCCA & 1ST ST. & ADAMS - SOUTH RANGE \\
\hline 327 & & LUCCHESI & ANNA & $\mathrm{D}$ & 10 & MICHIGAN & ITALY & ITALY & & & LUCCA & 1ST ST. & ADAMS - SOUTH RANGE \\
\hline 328 & & LUCCHESI & GINO & $\mathrm{s}$ & 6 & MICHIGAN & ITALY & ITALY & & & LUCCA & 1ST ST. & ADAMS - SOUTH RANGE \\
\hline 329 & & LUCCHESI & LEO & $\mathrm{s}$ & 2 & MICHIGAN & ITALY & ITALY & & & LUCCA & 1ST ST. & ADAMS - SOUTH RANGE \\
\hline 330 & 23 & PIZZI & CARLO & $\mathrm{H}$ & 28 & ITALY & ITALY & ITALY & 1910 & PEDDLER & PESCAGLIA, LU & 1ST ST. & ADAMS - SOUTH RANGE \\
\hline 331 & & PIZZI & DALIDA & W & 28 & ITALY & ITALY & ITALY & 1912 & & PESCAGLIA. LU & 1ST ST. & ADAMS - SOUTH RANGE \\
\hline 332 & & PIZZI & MAURO & s & 9 & ITALY & ITALY & ITALY & 1912 & & PESCAGLIA. LU & 1ST ST. & ADAMS - SOUTH RANGE \\
\hline 333 & & PIZZI & LINA & $\mathrm{D}$ & 7 & MICHIGAN & ITALY & ITALY & & & PESCAGLIA. LU & 1ST ST. & ADAMS - SOUTH RANGE \\
\hline 334 & & PIZZI & ANGELO & $\mathrm{s}$ & 5 & MICHIGAN & ITALY & ITALY & & & PESCAGLIA. LU & 1ST ST. & ADAMS - SOUTH RANGE \\
\hline 335 & & PIZZI & MARNA & $\mathrm{D}$ & 4 & MICHIGAN & ITALY & ITALY & & & PESCAGLIA. LU & 1ST ST. & ADAMS - SOUTH RANGE \\
\hline 336 & & FILIPPI & MARIA & SIIL & 22 & ITALY & ITALY & ITALY & 1919 & & LUCCA & 1ST ST. & ADAMS - SOUTH RANGE \\
\hline 337 & & PIZZI & RALPH & $\mathrm{BR}$ & 24 & ITALY & ITALY & ITALY & 1919 & TRUCK DRIVER/GARAGE & PESCAGLIA. LU & 1ST ST. & ADAMS - SOUTH RANGE \\
\hline 338 & & PIZZI & DANIEL & $\mathrm{BR}$ & 27 & ITALY & ITALY & ITALY & 1915 & MINER/CM & PESCAGLIA. LU & 1ST ST. & ADAMS - SOUTH RANGE \\
\hline 339 & & BAIG & JOHN & B & 42 & ITALY & ITALY & ITALY & 1918 & MINER/CM & & 1ST ST. & ADAMS - SOUTH RANGE \\
\hline 340 & 20 & RAFFAELLI & ITALO & $\mathrm{H}$ & 30 & ITALY & ITALY & ITALY & $93 / 13$ & ELECTRICIAN/CM & LUCCA & 1ST ST. & ADAMS - SOUTH RANGE \\
\hline 341 & & RAFFAELLI & ANNA & W & 22 & MICHIGAN & ITALY & ITALY & & & & 1ST ST. & ADAMS - SOUTH RANGE \\
\hline 342 & & RAFFAELLI & $\mathrm{J}$. & $s$ & 3 & MICHIGAN & ITALY & ITALY & & & LUCCA & 1ST ST. & ADAMS - SOUTH RANGE \\
\hline 343 & & RAFFAELLI & CATERINA & D & 1 & MICHIGAN & ITALY & ITALY & & & LUCCA & 1ST ST. & ADAMS - SOUTH RANGE \\
\hline 344 & & RAFFAELLI & MARTIN & $\mathrm{s}$ & $3 \mathrm{M}$ & MICHIGAN & ITALY & ITALY & & & LUCCA & 1ST ST. & ADAMS - SOUTH RANGE \\
\hline 345 & 36 & TOSCANA & ANTON & $\mathrm{H}$ & 43 & ITALY & ITALY & ITALY & 1895 & PROPRIETOR/BAKERY & SAN GIORGIO CANAVESE. TO & 3RD ST. & ADAMS - SOUTH RANGE \\
\hline 346 & & TOSCANA & MARY & w & 37 & ITALY & ITALY & ITALY & 1890 & 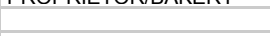 & 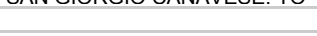 & 3RD ST. & ADAMS - SOUTH RANGE \\
\hline 347 & & TOSCANA & MINNIE & D & 20 & MICHIGAN & ITALY & ITALY & 1090 & NOT CLEAR & SAN GIORGIO CANAVESE. TO & 3RD ST. & ADAMS - SOUTH RANGE \\
\hline 348 & & TOSCANA & MARGHERITA & $\mathrm{D}$ & 18 & MICHIGAN & ITALY & ITALY & & & SAN GIORGIO CANAVESE, TO & 3RD ST. & ADAMS - SOUTH RANGE \\
\hline 349 & & TOSCANA & MARY & $\mathrm{D}$ & 12 & MICHIGAN & ITALY & ITALY & & & SAN GIORGIO CANAVESE. TO & 3RD ST. & ADAMS - SOUTH RANGE \\
\hline 350 & & TOSCANA & KATE & $\mathrm{D}$ & 8 & MICHIGAN & ITALY & ITALY & & & SAN GIORGIO CANAVESE. TO & 3RD ST. & ADAMS - SOUTH RANGE \\
\hline 351 & & TOSCANA & ANNETTE & $\mathrm{D}$ & 5 & MICHIGAN & ITALY & ITALY & & & SAN GIORGIO CANAVESE. TO & 3RD ST. & ADAMS - SOUTH RANGE \\
\hline 352 & 34 & BARSI & ANGELO & $\mathrm{H}$ & 23 & ITALY & ITALY & ITALY & $00 ? ?$ & MINER/CM & LUCCA & 2ND ST. & ADAMS - SOUTH RANGE \\
\hline 353 & & BARSI & FLORA & W & 22 & ITALY & ITALY & ITALY & 1901 & & & 2ND ST. & ADAMS - SOUTH RANGE \\
\hline 354 & & BARSI & GIULIA & D & 2 & MICHIGAN & ITALY & ITALY & & & LUCCA & 2ND ST. & ADAMS - SOUTH RANGE \\
\hline 355 & & BARSI & FRED & FA & 48 & ITALY & ITALY & ITALY & 1908 & MINER/CM & LUCCA & 2ND ST. & ADAMS - SOUTH RANGE \\
\hline 356 & & PINI & FRANK & B & 28 & ITALY & ITALY & ITALY & 1912 & MINER/CM & LUCCA & 2ND ST. & ADAMS - SOUTH RANGE \\
\hline 357 & 32 & BERTOLOZZI & ATTILIO & $\mathrm{H}$ & 56 & ITALY & ITALY & ITALY & 1898 & MINER/CM & VECOLI. LU & 2ND ST. & ADAMS - SOUTH RANGE \\
\hline 358 & & BERTOLOZZI & ZITA & w & 49 & ITALY & ITALY & ITALY & 1900 & & & 2ND ST. & ADAMS - SOUTH RANGE \\
\hline 359 & & BERTOLOZZI & PAUL & s & 19 & MICHIGAN & ITALY & ITALY & & LABORER/ROCK HOUSE & VECOLI. LU & 2ND ST. & ADAMS - SOUTH RANGE \\
\hline 360 & & BERTOLOZZI & PETER & $\mathrm{s}$ & 16 & MICHIGAN & ITALY & ITALY & & & VECOLI. LU & 2ND ST. & ADAMS - SOUTH RANGE \\
\hline 361 & & BERTOLOZZI & LENA & $\mathrm{D}$ & 13 & MICHIGAN & ITALY & ITALY & & & VECOLI. LU & 2ND ST. & ADAMS - SOUTH RANGE \\
\hline 362 & & & JENNIE & $\mathrm{D}$ & 12 & MICHIGAN & ITALY & ITALY & & & VECOLI. LU & 2ND ST. & ADAMS - SOUTH RANGE \\
\hline 363 & & BERTOLOZZI & ATTILIO & $\mathrm{s}$ & 10 & MICHIGAN & ITALY & ITALY & & & VECOLI. LU & 2ND ST. & ADAMS - SOUTH RANGE \\
\hline 364 & & BERTOLOZZI & FLORENCE & $\mathrm{D}$ & 9 & MICHIGAN & ITALY & ITALY & & & VECOLI. LU & 2ND ST. & ADAMS - SOUTH RANGE \\
\hline 365 & 27 & SUCCA & GIORGIO & $\mathrm{H}$ & 43 & ITALY & ITALY & ITALY & 1896 & TEAMSTER/DRAYMAN & TORINO & 3RD ST. & ADAMS - SOUTH RANGE \\
\hline 366 & & SUCCA & JOSEPHINE & W & 33 & ITALY & ITALY & ITALY & 1907 & & & 3RD ST. & ADAMS - SOUTH RANGE \\
\hline 367 & & ROLANDO & JOHN & SSO & 10 & MICHIGAN & ITALY & ITALY & & & PONT CANAVESE. TO & 3RD ST. & ADAMS - SOUTH RANGE \\
\hline 368 & & SUCCA & JOSEPH & $\mathrm{s}$ & 6 & MICHIGAN & ITALY & ITALY & & & TORINO & 3RD ST. & ADAMS - SOUTH RANGE \\
\hline 369 & & SUCCA & PAUL & $\mathrm{s}$ & 2 & MICHIGAN & ITALY & ITALY & & & TORINO & 3RD ST. & ADAMS - SOUTH RANGE \\
\hline 370 & 33 & BALAGNA & MIKE & $\mathrm{H}$ & 44 & ITALY & ITALY & ITALY & 1903 & POLICEMAN/VILLAGE & PONT CANAVESE. TO & 3RD ST. & ADAMS - SOUTH RANGE \\
\hline 371 & & BALAGNA & ANGELA & w & 41 & ITALY & ITALY & ITALY & 1906 & 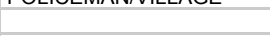 & PONT CANAVESE. TO & 3RD ST. & ADAMS - SOUTH RANGE \\
\hline 372 & & BALAGNA & DUSOLINA & D & 16 & ITALY & ITALY & ITALY & 1906 & DOMESTIC/PF & PONT CANAVESE, TO & 3RD ST. & ADAMS - SOUTH RANGE \\
\hline 373 & & BALAGNA & MARY & $\mathrm{D}$ & 12 & MICHIGAN & ITALY & ITALY & & & PONT CANAVESE. TO & 3RD ST. & ADAMS - SOUTH RANGE \\
\hline 374 & & BALAGNA & LIDIA & D & 9 & MICHIGAN & ITALY & ITALY & & & PONT CANAVESE. TO & 3RD ST. & ADAMS - SOUTH RANGE \\
\hline 375 & & BALAGNA & MADDALENA & $\mathrm{D}$ & 8 & & ITALY & ITAI & & & PONT CANAVESE. TO & 3RD ST. & ADAMS - SOUTH RANGE \\
\hline 376 & & BALAGNA & MIKE JR. & $\mathrm{s}$ & 5 & MICHIGAN & ITALY & ITALY & & & PONT CANAVESE. TO & & ADAMS - SOUTH RANGE \\
\hline 377 & 22 & SANTOSI & ANTONIO & $\mathrm{H}$ & 48 & ITALY & ITALY & ITALY & 1902 & MINER/CM & & 3RD ST. & ADAMS - SOUTH RANGE \\
\hline
\end{tabular}




\begin{tabular}{|c|c|c|c|c|c|c|c|c|c|c|c|c|c|}
\hline 378 & & SANTOSI & TERESA & W & 48 & ITALY & ITALY & ITALY & UN & & & 3RD ST. & ADAMS - SOUTH RANGE \\
\hline 379 & & SANTOSI & VITTORIO & D & 14 & MICHIGAN & ITALY & ITALY & & & & 3RD ST. & ADAMS - SOUTH RANGE \\
\hline 380 & & SANTOSI & JOHN & $\mathrm{s}$ & 12 & MICHIGAN & ITALY & ITALY & & & & 3RD ST. & ADAMS - SOUTH RANGE \\
\hline 381 & 31 & DATA/DATTA & PETER & $\mathrm{H}$ & 29 & ITALY & ITALY & ITALY & 1914 & MINER/CM & PONT CANAVESE. TO & 4TH ST. & ADAMS - SOUTH RANGE \\
\hline 382 & & DATA/DATTA & ANNA & w & 19 & ITALY & ITALY & ITALY & 1905 & & PONT CANAVESE. TO & 4TH ST. & ADAMS - SOUTH RANGE \\
\hline 383 & 36 & RAFFAELLI & RAFFAELLO & $\mathrm{H}$ & 56 & ITALY & ITALY & ITALY & 1895 & LABORER/ROCK HOUSE & LUCCA & CHAMPION AVE. & ADAMS - SOUTH RANGE \\
\hline 384 & 30 & RAFFAELLI & KATE & W & 54 & ITALY & ITALY & ITALY & 1900 & 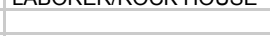 & & CHAMPION AVE. & ADAMS - SOUTH RANGE \\
\hline $\begin{array}{l}504 \\
385\end{array}$ & & RAFFAELLI & AMELIA & $\mathrm{D}$ & 18 & MICHIGAN & ITALY & ITALY & 1900 & & LUCCA & CHAMPION AVE. & ADAMS - SOUTH RANGE \\
\hline 386 & & RAFFAELLI & MARIO & $\mathrm{D}$ & 17 & MICHIGAN & ITALY & ITALY & & TEAMSTER/BAKERY & LUCCA & CHAMPION AVE. & ADAMS - SOUTH RANGE \\
\hline 387 & & RAFFAELLI & FERRUCCIO & $\mathrm{s}$ & 16 & MICHIGAN & ITALY & ITALY & & HELPER/BAKERY & LUCCA & CHAMPION AVE. & ADAMS - SOUTH RANGE \\
\hline 388 & & RAFFAELLI & LENA & $\mathrm{D}$ & 12 & MICHIGAN & ITALY & ITALY & & & LUCCA & CHAMPION AVE. & ADAMS - SOUTH RANGE \\
\hline 389 & 32 & PLASSA & ALBERT & $\mathrm{H}$ & 33 & ITALY & ITALY & ITALY & 1905 & MINER/CM & TORINO & CHAMPION AVE. & ADAMS - SOUTH RANGE \\
\hline 390 & & PLASSA & MARY & W & 31 & MICHIGAN & ITALY & ITALY & & & & CHAMPION AVE. & ADAMS - SOUTH RANGE \\
\hline 391 & & PLASSA & TEODORO & $\mathrm{s}$ & 10 & MICHIGAN & ITALY & ITALY & & & TORINO & CHAMPION AVE. & ADAMS - SOUTH RANGE \\
\hline 392 & & PLASSA & ANGELINA & $\mathrm{D}$ & 8 & MICHIGAN & & ITALY & & & TORINO & CHAMPION AVE. & ADAMS - SOUTH RANGE \\
\hline 393 & & PLASSA & BARTOLOMEO & $\mathrm{s}$ & 7 & MICHIGAN & ITALY & ITALY & & & TORINO & CHAMPION AVE. & ADAMS - SOUTH RANGE \\
\hline 394 & 15 & AIMONETTO & ANTONIO & $\mathrm{H}$ & 48 & ITALY & ITALY & ITALY & UN & MINER/CM & TORINO & & ADAMS - SOUTH RANGE \\
\hline 395 & & AIMONETTO & TERESA & W & 47 & ITALY & ITALY & ITALY & 1890 & & & & ADAMS - SOUTH RANGE \\
\hline 396 & & DE BERNARDI & PETER & STEP & 25 & MINNESOT & ITALY & ITALY & & MINER/CM & TORINO & & ADAMS - SOUTH RANGE \\
\hline 397 & & AIMONETTO & LUISA & D & 17 & MICHIGAN & ITALY & ITALY & & & TORINO & & ADAMS - SOUTH RANGE \\
\hline 398 & & AIMONETTO & TONY & $\mathrm{s}$ & 14 & MICHIGAN & ITALY & ITALY & & & TORINO & & ADAMS - SOUTH RANGE \\
\hline 399 & & AIMONETTO & WILLIAM & $\mathrm{s}$ & 8 & MICHIGAN & ITALY & ITALY & & & TORINO & & ADAMS - SOUTH RANGE \\
\hline 400 & 18 & BENASSI & FELIX & $\mathrm{H}$ & 35 & ITALY & ITALY & ITALY & 1905 & MINER/CM & CAMAIORE. LU & & ADAMS - SOUTH RANGE \\
\hline 401 & & BENASSI & MARY & W & 30 & MICHIGAN & ITALY & ITALY & & 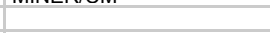 & SAN GIORGIO CANAVESE. TO & & ADAMS - SOUTH RANGE \\
\hline 402 & & BENASSI & MAX & BR & 29 & ITALY & ITALY & ITALY & 1909 & & CAMAIORE. LU & & ADAMS - SOUTH RANGE \\
\hline 403 & 19 & MAGNINO & ROMEO & $\mathrm{H}$ & 41 & ITALY & ITALY & ITALY & UN & MINER/CM & SPARONE. TO & & ADAMS - SOUTH RANGE \\
\hline 404 & & MAGNINO & ANNA & w & 37 & ITALY & ITALY & ITALY & 1900 & & & & ADAMS - SOUTH RANGE \\
\hline 405 & & MAGNINO & JOSEPH & $\mathrm{s}$ & 13 & MICHIGAN & ITALY & ITALY & & & SPARONE, TO & & ADAMS - SOUTH RANGE \\
\hline 406 & & MAGNINO & LENA & D & 12 & MICHIGAN & ITALY & ITALY & & & SPARONE. TO & & ADAMS - SOUTH RANGE \\
\hline 407 & & MAGNINO & ADA & $\mathrm{D}$ & 10 & MICHIGAN & ITALY & ITALY & & & SPARONE. TO & & ADAMS - SOUTH RANGE \\
\hline 408 & & MAGNINO & MARY & D & 2 & MICHIGAN & ITALY & ITALY & & & SPARONE. TO & & ADAMS - SOUTH RANGE \\
\hline 409 & 32 & PIERONI & PETER & $\mathrm{H}$ & 26 & ITALY & ITALY & ITALY & 1910 & MINER/CM & LUCCA & & ADAMS - SOUTH RANGE \\
\hline 410 & 32 & PIERONI & MARY & w & 17 & MICHIGAN & ITALY & ITALY & 1910 & IVIIVERTIUIVI & LUCUA & & ADAMS - SOUTH RANGE \\
\hline 411 & 4 & MIGLIO/MIGLIA & MARIAN & $\mathrm{H}$ & 52 & ITALY & ITALY & ITALY & 1890 & & SAN GIORGIO CANAVESE, TO & & ADAMS - SOUTH RANGE \\
\hline 412 & & MIGLIO/MIGLIA & JOHN & $\mathrm{s}$ & 22 & MICHIGAN & ITALY & ITALY & & MINER/CM & SAN GIORGIO CANAVESE. TO & & ADAMS - SOUTH RANGE \\
\hline 413 & & MIGLIO/MIGLIA & VITTORIO & $\mathrm{D}$ & 17 & MICHIGAN & ITALY & ITALY & & 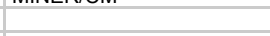 & SAN GIORGIO CANAVESE. TO & & ADAMS - SOUTH RANGE \\
\hline 414 & & MIGLIO/MIGLIA & ANTONIETTA & D & 13 & MICHIGAN & ITALY & ITALY & & & SAN GIORGIO CANAVESE. TO & & ADAMS - SOUTH RANGE \\
\hline 415 & 2 & ZANOTTI & ANTONIO & $\mathrm{H}$ & 52 & ITALY & ITALY & ITALY & 1895 & MINER/CM & TORINO & & ADAMS - SOUTH RANGE \\
\hline 416 & & ZANOTTI & ANNA & W & 52 & ITALY & ITALY & ITALY & 1895 & & TORINO & & ADAMS - SOUTH RANGE \\
\hline 417 & & ZANOTTI & MARGHERITA & D & 16 & MICHIGAN & ITALY & ITALY & & & TORINO & & ADAMS - SOUTH RANGE \\
\hline 418 & & ZANOTTI & JOHN & $\mathrm{S}$ & 14 & MICHIGAN & ITALY & ITALY & & & TORINO & & ADAMS - SOUTH RANGE \\
\hline 419 & & ZANOTTI & DOMENICO & $\mathrm{H}$ & 26 & PENNSYLV & ITALY & ITALY & & MINER/CM & TORINO & & ADAMS - SOUTH RANGE \\
\hline 420 & & ZANOTTI & MARY & w & 22 & MICHIGAN & GERMANY & GERMANY & & & GERMANY & & ADAMS - SOUTH RANGE \\
\hline 421 & 2 & GALETTO & MIKE & $\mathrm{H}$ & 44 & ITALY & ITALY & ITALY & 1898 & MINER/CM & SAN GIORGIO CANAVESE. TO & & ADAMS - SOUTH RANGE \\
\hline 422 & & GALETTO & CESARINA & w & 45 & ITALY & ITALY & ITALY & 1900 & & & & ADAMS - SOUTH RANGE \\
\hline 423 & & GALETTO & VINCENZA & s & 16 & MICHIGAN & ITALY & ITALY & & & SAN GIORGIO CANAVESE. TO & & ADAMS - SOUTH RANGE \\
\hline 424 & & GALETTO & NATALINA & D & 13 & MICHIGAN & ITALY & ITALY & & & SAN GIORGIO CANAVESE. TO & & ADAMS - SOUTH RANGE \\
\hline 425 & & GALETTO & JOSEPH & $\mathrm{s}$ & 11 & MICHIGAN & ITALY & ITALY & & & SAN GIORGIO CANAVESE. TO & & ADAMS - SOUTH RANGE \\
\hline 426 & 4 & LANDINI & JOSEPH & $\mathrm{H}$ & 35 & ITALY & ITALY & ITALY & 1901 & MINER/CM & LUCCA & & ADAMS - SOUTH RANGE \\
\hline 427 & & LANDINI & ANNA & w & 32 & ITALY & ITALY & ITALY & 1899 & & & & ADAMS - SOUTH RANGE \\
\hline 428 & & LANDINI & MARIO & $\mathrm{s}$ & 12 & ILLINOIS & ITALY & ITALY & & & LUCCA & & ADAMS - SOUTH RANGE \\
\hline 429 & & LANDINI & ELMER & $\mathrm{s}$ & 10 & ILLINOIS & ITALY & ITALY & & & LUCCA & & ADAMS - SOUTH RANGE \\
\hline 430 & & LANDINI & ALBERT & $\mathrm{s}$ & 8 & ILLINOIS & ITALY & ITALY & & & LUCCA & & ADAMS - SOUTH RANGE \\
\hline 431 & & LANDINI & LENA & D & 5 & MICHIGAN & ITALY & ITALY & & & LUCCA & & ADAMS - SOUTH RANGE \\
\hline 432 & 6 & RICCA & MARY & $\mathrm{H}$ & 47 & ITALY & ITALY & ITALY & 1898 & & & & ADAMS - SOUTH RANGE \\
\hline 433 & & POLINELLI & JAMES & B & 39 & ITALY & ITALY & ITALY & 1914 & MINER/CM & BIANZANO. SONDRIO & & ADAMS - SOUTH RANGE \\
\hline 434 & & POLINELLI & BARTOLO & B & 36 & ITALY & ITALY & ITALY & 1908 & MINER/CM & BIANZANO. SONDRIO & & ADAMS - SOUTH RANGE \\
\hline 435 & & RICCA & BATTISTA & B & 25 & ITALY & ITALY & ITALY & 1914 & MINER/CM & TORINO & & ADAMS - SOUTH RANGE \\
\hline 436 & & BOGGIO & DOMENICO & B & 32 & ITALY & ITALY & ITALY & UN & MINER/CM & SAN GIUSTO CANAVESE. TO & & ADAMS - SOUTH RANGE \\
\hline 437 & & MARCHETTI & JOHN & B & 29 & ITALY & ITALY & ITALY & 1913 & BAKER/BAKERY & LEONESSA. RIETI & & ADAMS - SOUTH RANGE \\
\hline 438 & & GALETTO & RINA & AC & 13 & MICHIGAN & ITALY & ITALY & & & SAN GIORGIO CANAVESE. TO & & ADAMS - SOUTH RANGE \\
\hline 439 & 12 & ANDRINA & TONY & $\mathrm{H}$ & 43 & ITALY & ITALY & ITALY & 1895 & MINER/CM & TORINO & & ADAMS - S \\
\hline 440 & & ANDRINA & MARY & w & 42 & ITALY & ITALY & ITALY & 1906 & & & & ADAMS - SOUTH RANGE \\
\hline
\end{tabular}




\begin{tabular}{|c|c|c|c|c|c|c|c|c|c|c|c|c|c|}
\hline 441 & & ANDRINA & FRANK & D & 12 & MICHIGAN & ITALY & ITALY & & & TORINO & & ADAMS - SOUTH RANGE \\
\hline 442 & & ANDRINA & MARTINA & D & 14 & MICHIGAN & ITALY & ITALY & & & TORINO & & ADAMS - SOUTH RANGE \\
\hline 443 & 11 & GIOGA & CARLO & $\mathrm{H}$ & 40 & ITALY & ITALY & ITALY & 1900 & MINER/CM & SAN GIUSTO CANAVESE. TO & & ADAMS - SOUTH RANGE \\
\hline 444 & & GIOGA & TERESA & W & 40 & ITALY & ITALY & ITALY & 1900 & & SAN GIUSTO CANAVESE. TO & & ADAMS - SOUTH RANGE \\
\hline 445 & & GIOGA & MARY & D & 15 & ITALY & ITALY & ITALY & 1915 & & SAN GIUSTO CANAVESE. TO & & ADAMS - SOUTH RANGE \\
\hline 446 & & GIOGA & ESTEL & D & 13 & ITALY & ITALY & ITALY & 1913 & & SAN GIUSTO CANAVESE. TO & & ADAMS - SOUTH RANGE \\
\hline 447 & 11 & PETRINI & CARLO & $\mathrm{H}$ & 32 & ITALY & ITALY & ITALY & 1909 & MINER/CM & SAN GIUSTO CANAVESE. TO & & ADAMS - SOUTH RANGE \\
\hline 448 & & PETRINI & MARY & W & 32 & ITALY & ITALY & ITALY & UN & NOT CLEAR & & & ADAMS - SOUTH RANGE \\
\hline 449 & & PETRINI & JOSEPH & s & 4 & MICHIGAN & ITALY & ITALY & & & SAN GIUSTO CANAVESE. TO & & ADAMS - SOUTH RANGE \\
\hline 450 & 10 & CANALLI & MIKE & $\mathrm{H}$ & 42 & ITALY & ITALY & ITALY & 1908 & MINER/CM & & & ADAMS - SOUTH RANGE \\
\hline 451 & & CANALLI & MARGHERITA & W & 40 & ITALY & ITALY & ITALY & 1901 & & & & ADAMS - SOUTH RANGE \\
\hline 452 & & BASSO & JOHN & SSO & 16 & MICHIGAN & ITALY & ITALY & & MINER/CM & SAN GIORGIO CANAVESE. TO & & ADAMS - SOUTH RANGE \\
\hline 453 & & BASSO & CHARLES & sso & 15 & MICHIGAN & ITALY & ITALY & & & SAN GIORGIO CANAVESE. TO & & ADAMS - SOUTH RANGE \\
\hline 454 & & BASSO & JAMES & sso & 13 & MICHIGAN & ITALY & ITALY & & & SAN GIORGIO CANAVESE. TO & & ADAMS - SOUTH RANGE \\
\hline 455 & & BASSO & PETER & sso & 11 & MICHIGAN & ITALY & ITALY & & & SAN GIORGIO CANAVESE. TO & & ADAMS - SOUTH RANGE \\
\hline 456 & & BASSO & JOSEPH & sso & 8 & MICHIGAN & ITALY & ITALY & & & SAN GIORGIO CANAVESE, TO & & ADAMS - SOUTH RANGE \\
\hline 457 & 8 & MASSA & ANTON & $\mathrm{H}$ & 54 & ITALY & ITALY & ITALY & 1893 & FARMER/GENERAL FARM & TORINO & & ADAMS - SOUTH RANGE \\
\hline 458 & & MASSA & MARY & W & 53 & ITALY & ITALY & ITALY & 1891 & & & & ADAMS - SOUTH RANGE \\
\hline 459 & & MASSA & PETER & $\mathrm{AC}$ & 20 & MICHIGAN & ITALY & ITALY & & MINER/CM & TORINO & & ADAMS - SOUTH RANGE \\
\hline 460 & & MASSA & JAMES & $\mathrm{s}$ & 17 & MICHIGAN & ITALY & ITALY & & MINER/CM & TORINO & & ADAMS - SOUTH RANGE \\
\hline 461 & & MASSA & MARGHERITA & $\mathrm{D}$ & 14 & MICHIGAN & ITALY & ITALY & & & TORINO & & ADAMS - SOUTH RANGE \\
\hline 462 & & MASSA & EVA & D & 13 & MICHIGAN & ITALY & ITALY & & & TORINO & & ADAMS - SOUTH RANGE \\
\hline 463 & 0 & MAGNINO & JAMES & $\mathrm{H}$ & 43 & ITALY & ITALY & ITALY & 1906 & FARMER/GENERAL FARM & SPARONE. TO & & ADAMS - SOUTH RANGE \\
\hline 464 & & KISALLI & JOHN & B & 45 & ITALY & ITALY & ITALY & 1911 & MINER/CM & & & ADAMS - SOUTH RANGE \\
\hline 465 & NN & BARTALLI & ARMANDO & $\mathrm{H}$ & 41 & ITALY & ITALY & ITALY & 1902 & MINER/CM & & SUNNY ITALY & ADAMS -TRIMOUNTAIN \\
\hline 466 & & BARTALLI & ANNUNZIATA & W & 24 & ITALY & ITALY & ITALY & 1912 & & & SUNNY ITALY & ADAMS -TRIMOUNTAIN \\
\hline 467 & & BARTALLI & CELESTINA & D & 7 & MICHIGAN & ITALY & ITALY & & & & SUNNY ITALY & ADAMS -TRIMOUNTAIN \\
\hline 468 & & BARTALLI & ANITA & D & 5 & MICHIGAN & ITALY & ITALY & & & & SUNNY ITALY & ADAMS -TRIMOUNTAIN \\
\hline 469 & & VANNUCCI & AURELIO & B & 36 & ITALY & ITALY & ITALY & 1906 & LABORER/CM & LUCCA & SUNNY ITALY & ADAMS -TRIMOUNTAIN \\
\hline 470 & & TADDEUCCI & OLINTO & $\mathrm{H}$ & 32 & ITALY & ITALY & ITALY & 1906 & LABORER/CM & LUCCA & SUNNY ITALY & ADAMS -TRIMOUNTAIN \\
\hline 471 & & TADDEUCCI & IDA & w & 31 & ITALY & ITALY & ITALY & 1916 & & LUCCA & SUNNY ITALY & ADAMS -TRIMOUNTAIN \\
\hline 472 & & TADDEUCCI & GIULIO & s & 6 & ITALY & ITALY & ITALY & 1916 & & LUCCA & SUNNY ITALY & ADAMS -TRIMOUNTAIN \\
\hline 473 & & TADDEUCCI & ADA & $\mathrm{D}$ & 2 & MICHIGAN & ITALY & ITALY & & & LUCCA & SUNNY ITALY & ADAMS -TRIMOUNTAIN \\
\hline 474 & & TADDEUCCI & JACOB & $\mathrm{H}$ & 41 & ITALY & ITALY & ITALY & 1902 & LABORER/CM & LUCCA & SUNNY ITALY & ADAMS -TRIMOUNTAIN \\
\hline 475 & & TADDEUCCI & FERDINANDA & w & 34 & ITALY & ITALY & ITALY & 1906 & & & SUNNY ITALY & ADAMS -TRIMOUNTAIN \\
\hline 476 & & TADDEUCCI & FRANK & s & 12 & MICHIGAN & ITALY & ITALY & & & LUCCA & SUNNY ITALY & ADAMS -TRIMOUNTAIN \\
\hline 477 & & TADDEUCCI & SILVANO & $\mathrm{s}$ & 7 & MICHIGAN & ITALY & ITALY & & & LUCCA & SUNNY ITALY & ADAMS -TRIMOUNTAIN \\
\hline 478 & & STEFANI & RALPH & B & 33 & ITALY & ITALY & ITALY & 1902 & MINER/CM & LUCCA & SUNNY ITALY & ADAMS -TRIMOUNTAIN \\
\hline 479 & & GIAMBASTIANI & PIETRO & B & 28 & ITALY & ITALY & ITALY & 1913 & LABORER/CM & LUCCA & SUNNY ITALY & ADAMS -TRIMOUNTAIN \\
\hline 480 & & GIAMONI & AMERICO & B & 34 & ITALY & ITALY & ITALY & 1912 & MASON/CM & LUCCA & SUNNY ITALY & ADAMS -TRIMOUNTAIN \\
\hline 481 & & MAFFEI & ARTURO & B & 36 & ITALY & ITALY & ITALY & 190 & TRAMMER/CM & LUCCA & SUNNY ITALY & ADAMS -TRIMOUNTAIN \\
\hline 482 & & BARSOCCHI & SEVERINO & B & 33 & ITALY & ITALY & ITALY & 1905 & DUMPER/CM & LUCCA & SUNNY ITALY & ADAMS -TRIMOUNTAIN \\
\hline 483 & & MARCHESCHI & NELLO & $\mathrm{H}$ & 25 & ITALY & ITALY & ITALY & 1911 & MINER/CM & SAN GINESE. LU & SUNNY ITALY & ADAMS -TRIMOUNTAIN \\
\hline 484 & & MARCHESCHI & AMALIA & w & 25 & ITALY & ITALY & ITALY & 1903 & & & SUNNY ITALY & ADAMS -TRIMOUNTAIN \\
\hline 485 & & MARCHESCHI & PAOLINA & D & 1 & MICHIGAN & ITALY & ITALY & & & SAN GINESE. LU & SUNNY ITALY & ADAMS -TRIMOUNTAIN \\
\hline 486 & & GIUSTI & UMBERTO & B & 37 & ITALY & ITALY & ITALY & 1909 & DUMPER/CM & LUCCA & SUNNY ITALY & ADAMS -TRIMOUNTAIN \\
\hline 487 & & MARCHESCHI & LORENZO & B & 41 & ITALY & ITALY & ITALY & 1902 & LABORER/CM & LUCCA & SUNNY ITALY & ADAMS -TRIMOUNTAIN \\
\hline 488 & & PIERONI & JOSEPH & $\mathrm{H}$ & 37 & ITALY & ITALY & ITALY & 1907 & LABORER/CM & CAPANNORI. LU & SUNNY ITALY & ADAMS -TRIMOUNTAIN \\
\hline 489 & & PIERONI & CORINNA & w & 32 & ITALY & ITALY & ITALY & 1906 & & CAPANNORI. LU & SUNNY ITALY & ADAMS -TRIMOUNTAIN \\
\hline 490 & & PIERONI & LOUIS & s & 12 & ITALY & ITALY & ITALY & 1910 & & CAPANNORI. LU & SUNNY ITALY & ADAMS -TRIMOUNTAIN \\
\hline 491 & & PIERONI & CHESTER & $\mathrm{S}$ & 9 & MICHIGAN & ITALY & ITALY & & & CAPANNORI. LU & SUNNY ITALY & ADAMS -TRIMOUNTAIN \\
\hline 492 & & PIERONI & ARMANDO & $\mathrm{s}$ & 7 & MICHIGAN & ITALY & ITALY & & & CAPANNORI, LU & SUNNY ITALY & ADAMS -TRIMOUNTAIN \\
\hline 493 & & PIERONI & GEMMA & $\mathrm{s}$ & 5 & MICHIGAN & ITALY & ITALY & & & CAPANNORI. LU & SUNNY ITALY & ADAMS -TRIMOUNTAIN \\
\hline 494 & & PIERONI & BANGUS & D & 3 & MICHIGAN & ITALY & ITALY & & & CAPANNORI. LU & SUNNY ITALY & ADAMS -TRIMOUNTAIN \\
\hline 495 & & PIERONI & GIACCHINO & s & 2 & MICHIGAN & ITALY & ITALY & & & CAPANNORI. LU & SUNNY ITALY & ADAMS -TRIMOUNTAIN \\
\hline 496 & & PIERONI & ARDUINO & $\mathrm{s}$ & $1 \mathrm{M}$ & MICHIGAN & ITALY & ITALY & & & CAPANNORI. LU & SUNNY ITALY & ADAMS -TRIMOUNTAIN \\
\hline 497 & & BACCETTI & ARDELMO & B & 33 & ITALY & ITALY & ITALY & 1903 & MINER/CM & LUCCA & SUNNY ITALY & ADAMS -TRIMOUNTAIN \\
\hline 498 & & MICHELETTI & FLORINDO & $\mathrm{H}$ & 41 & ITALY & ITALY & ITALY & 1906 & MINER/CM & SANTA MARIA DEL GIUDICE, LU & SUNNY ITALY & ADAMS -TRIMOUNTAIN \\
\hline 499 & & MICHELETTI & ARMIDA & w & 42 & ITALY & ITALY & ITALY & 1910 & & 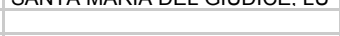 & SUNNY ITALY & ADAMS -TRIMOUNTAIN \\
\hline 500 & & MICHELETTI & GIULIA & $\mathrm{D}$ & 10 & MICHIGAN & ITALY & ITALY & & & SANTA MARIA DEL GIUDICE. LU & SUNNY ITALY & ADAMS -TRIMOUNTAIN \\
\hline 501 & & MICHELETTI & PAPINA & $\mathrm{s}$ & 9 & MICHIGAN & ITALY & ITALY & & & SANTA MARIA DEL GIUDICE. LU & SUNNY ITALY & ADAMS -TRIMOUNTAIN \\
\hline 502 & & MICHELETTI & NELLO & $\mathrm{s}$ & 7 & MICHIGAN & ITALY & ITALY & & & SANTA MARIA DEL GIUDICE. LU & SUNNY ITALY & ADAMS -TRIMOUNTAIN \\
\hline 503 & & MICHELETTI & SABATINO & $\mathrm{s}$ & 3 & MICHIGAN & ITALY & ITALY & & & SANTA MARIA DEL GIUDICE. LU & SUNNY ITALY & ADAMS -TRIMOUNTAIN \\
\hline
\end{tabular}




\begin{tabular}{|c|c|c|c|c|c|c|c|c|c|c|c|c|c|}
\hline 504 & & PIEROTTI & ORLANDO & $\mathrm{H}$ & 40 & ITALY & ITALY & ITALY & 1906 & TRAMMER/CM & COLLE DI COMPITO. LU & SUNNY ITALY & ADAMS -TRIMOUNTAIN \\
\hline 505 & & PIEROTTI & ROSA & W & 39 & ITALY & ITALY & ITALY & 1907 & & COLLE DI COMPITO. LU & SUNNY ITALY & ADAMS -TRIMOUNTAIN \\
\hline 506 & & PIEROTTI & PETER & s & 14 & ITALY & ITALY & ITALY & 1907 & & COLLE DI COMPITO. LU & SUNNY ITALY & ADAMS -TRIMOUNTAIN \\
\hline 507 & & PIEROTTI & LENA & $\mathrm{D}$ & 11 & MICHIGAN & ITALY & ITALY & & & COLLE DI COMPITO. LU & SUNNY ITALY & ADAMS -TRIMOUNTAIN \\
\hline 508 & & PIEROTTI & JOSEPH & $\mathrm{s}$ & 9 & MICHIGAN & ITALY & ITALY & & & COLLE DI COMPITO. LU & SUNNY ITALY & ADAMS -TRIMOUNTAIN \\
\hline 509 & & PIEROTTI & MARY & $\mathrm{D}$ & 8 & MICHIGAN & ITALY & ITALY & & & COLLE DI COMPITO. LU & SUNNY ITALY & ADAMS -TRIMOUNTAIN \\
\hline 510 & & PIEROTTI & JAMES & $\mathrm{D}$ & 6 & MICHIGAN & ITALY & ITALY & & & COLLE DI COMPITO. LU & SUNNY ITALY & ADAMS -TRIMOUNTAIN \\
\hline 511 & & PIEROTTI & ALBERT & s & 1 & MICHIGAN & ITALY & ITALY & & & COLLE DI COMPITO. LU & SUNNY ITALY & ADAMS -TRIMOUNTAIN \\
\hline 512 & & PENNA & JOSEPH & B & 37 & ITALY & ITALY & ITALY & 1913 & MINER/CM & & SUNNY ITALY & ADAMS -TRIMOUNTAIN \\
\hline 513 & & STEFANI & STEFANI & B & 33 & ITALY & ITALY & ITALY & 1906 & MINER/CM & LUCCA & SUNNY ITALY & ADAMS -TRIMOUNTAIN \\
\hline 514 & & LOMBARDI & VITTORIO & $\mathrm{H}$ & 32 & ITALY & ITALY & ITALY & 1909 & FIREMAN/BOILER HOUSE & LUCCA & SUNNY ITALY & ADAMS -TRIMOUNTAIN \\
\hline 515 & & LOMBARDI & MARTA & w & 25 & ITALY & ITALY & ITALY & 1909 & & LUCCA & SUNNY ITALY & ADAMS -TRIMOUNTAIN \\
\hline 516 & & LOMBARDI & LOUIS & $s$ & 7 & MICHIGAN & ITALY & ITALY & & & LUCCA & SUNNY ITALY & ADAMS -TRIMOUNTAIN \\
\hline 517 & & LOMBARDI & FIORINO & $\mathrm{s}$ & 5 & MICHIGAN & ITALY & ITALY & & & LUCCA & SUNNY ITALY & ADAMS -TRIMOUNTAIN \\
\hline 518 & & CALAMASSI & NATALE & B & 24 & ITALY & ITALY & ITALY & 1914 & TRAMMER/CM & FIRENZE & SUNNY ITALY & ADAMS -TRIMOUNTAIN \\
\hline 519 & & MARCHESCHI & AURELIO & B & 41 & ITALY & ITALY & ITALY & 1902 & LABORER/CM & LUCCA & SUNNY ITALY & ADAMS -TRIMOUNTAIN \\
\hline 520 & & ORSI & PIETRO & B & 27 & ITALY & ITALY & ITALY & 1912 & TRAMMER/CM & CAPANNORI. LU & SUNNY ITALY & ADAMS -TRIMOUNTAIN \\
\hline 521 & & MARTINELLI & NATALE & B & 42 & ITALY & ITALY & ITALY & 1912 & TRAMMER/CM & POPIGLIO. PISTOIA & SUNNY ITALY & ADAMS -TRIMOUNTAIN \\
\hline 522 & & LOMBARDI & COSTANTE & B & 31 & ITALY & ITALY & ITALY & 1914 & TRAMMER/CM & LUCCA & SUNNY ITALY & ADAMS -TRIMOUNTAIN \\
\hline 523 & & IACOPETTI & JOHN & B & 19 & ITALY & ITALY & ITALY & 1917 & DUMPER/CM & LUCCA & SUNNY ITALY & ADAMS -TRIMOUNTAIN \\
\hline 524 & & BROSI/BROJI & DISTINTO & B & 22 & ITALY & ITALY & ITALY & 1902 & TIMBERMAN/CM & & SUNNY ITALY & ADAMS -TRIMOUNTAIN \\
\hline 525 & & $\begin{array}{l}\text { PALMIERO } \\
\end{array}$ & FRANK & $\mathrm{H}$ & 39 & ITALY & ITALY & ITALY & 1903 & MINER/CM & & SUNNY ITALY & ADAMS -TRIMOUNTAIN \\
\hline 526 & & PALMIERO & ELISA & W & 24 & ITALY & ITALY & ITALY & 1915 & IVIINERT UIVI & & SUNNY ITALY & ADAMS -TRIMOUNTAIN \\
\hline 527 & & PALMIERO & FRANK & $\mathrm{s}$ & 5 & MICHIGAN & ITALY & ITALY & & & & SUNNY ITALY & ADAMS -TRIMOUNTAIN \\
\hline 528 & & PALMIERO & DOMENICO & s & 2 & MICHIGAN & ITALY & ITALY & & & & SUNNY ITALY & ADAMS -TRIMOUNTAIN \\
\hline 529 & & BARTOLOMEI & VALENTE & $\mathrm{H}$ & 50 & ITALY & ITALY & ITALY & 1902 & FIREMAN/ENGINE HOUSE & CASTELVECCHIO. LU & SUNNY ITALY & ADAMS -TRIMOUNTAIN \\
\hline 530 & & BARTOLOMEI & MARY & W & 40 & ITALY & ITALY & ITALY & 1902 & & CASTELVECCHIO. LU & SUNNY ITALY & ADAMS -TRIMOUNTAIN \\
\hline 531 & & BARTOLOMEI & DOMENICO & s & 21 & ITALY & ITALY & ITALY & 1902 & MINER/CM & CASTELVECCHIO, LU & SUNNY ITALY & ADAMS -TRIMOUNTAIN \\
\hline 532 & & BARTOLOMEI & NANDO & $\mathrm{s}$ & 16 & MICHIGAN & ITALY & ITALY & & & CASTELVECCHIO. LU & SUNNY ITALY & ADAMS -TRIMOUNTAIN \\
\hline 533 & & BARTOLOMEI & LUIGI & $\mathrm{s}$ & 12 & MICHIGAN & ITALY & ITALY & & & CASTELVECCHIO. LU & SUNNY ITALY & ADAMS -TRIMOUNTAIN \\
\hline 534 & & BARTOLOMEI & LUIGI & $\mathrm{H}$ & 35 & ITALY & ITALY & ITALY & 1905 & TRAMMER/CM & PARIANA. LU & SUNNY ITALY & ADAMS -TRIMOUNTAIN \\
\hline 535 & & BARTOLOMEI & NELLA & w & 29 & ITALY & ITALY & ITALY & 1911 & & & SUNNY ITALY & ADAMS -TRIMOUNTAIN \\
\hline 536 & & BARTOLOMEI & LETTA & D & 8 & MICHIGAN & ITALY & ITALY & & & CASTELVECCHIO. LU & SUNNY ITALY & ADAMS -TRIMOUNTAIN \\
\hline 537 & & BARTOLOMEI & RAFFAELLO & s & 6 & MICHIGAN & ITALY & ITALY & & & LUCCA & SUNNY ITALY & ADAMS -TRIMOUNTAIN \\
\hline 538 & & BARTOLOMEI & HALLEN & D & & MICHIGAN & ITALY & ITALY & & & LUCCA & SUNNY ITALY & ADAMS -TRIMOUNTAIN \\
\hline $\begin{array}{l}530 \\
539\end{array}$ & & BARTOLOMEI & ANDELINO & COU & 45 & ITALY & ITALY & ITALY & 1899 & MASON/CM & LUCCA & SUNNY ITALY & ADAMS -TRIMOUNTAIN \\
\hline 540 & & FLOSI & FIORELLO & B & 51 & ITALY & ITALY & ITALY & 1905 & TRAMMER/CM & PARIANA. LU & SUNNY ITALY & ADAMS -TRIMOUNTAIN \\
\hline 541 & & DATI & SEVERINO & B & 45 & ITALY & ITALY & ITALY & 1913 & MOTORMAN/CM & PARIANA. LU & SUNNY ITALY & ADAMS -TRIMOUNTAIN \\
\hline 542 & & BIANCHI & JOSEPH & B & 42 & ITALY & ITALY & ITALY & 1901 & MINER/CM & LUCCA & SUNNY ITALY & ADAMS -TRIMOUNTAIN \\
\hline 543 & & GIAMPAOLI & FEDERICO & B & 34 & ITALY & ITALY & ITALY & 1909 & TRAMMER/CM & CAPANNORI. LU & SUNNY ITALY & ADAMS -TRIMOUNTAIN \\
\hline 544 & & COSSIA & GASPARE & $\mathrm{H}$ & 47 & ITALY & ITALY & ITALY & 1909 & JANITOR/DRAYHOUSE & GOLASECCA. VARESE & 2ND ST. & ADAMS -TRIMOUNTAIN \\
\hline 545 & & COSSIA & MARIANNA & W & 42 & ITALY & ITALY & ITALY & 1917 & & GOLASECCA. VARESE & 2ND ST. & ADAMS -TRIMOUNTAIN \\
\hline 546 & & COSSIA & CARLO & $\mathrm{s}$ & 18 & ITALY & ITALY & ITALY & 1917 & & GOLASECCA. VARESE & 2ND ST. & ADAMS -TRIMOUNTAIN \\
\hline 547 & & COSSIA & JOSEPHINE & D & 15 & ITALY & ITALY & ITALY & 1917 & & GOLASECCA. VARESE & 2ND ST. & ADAMS -TRIMOUNTAIN \\
\hline 548 & & DE SANTI & ALBINO & $\mathrm{H}$ & 40 & ITALY & ITALY & ITALY & 1904 & & CASTELVECCHIO. LU & 2ND ST. & ADAMS -TRIMOUNTAIN \\
\hline 549 & & DE SANTI & ELIDA & w & 36 & ITALY & ITALY & ITALY & 1909 & MINER/CM & & 2ND ST. & ADAMS -TRIMOUNTAIN \\
\hline 550 & & BARRETTE & GIULIO & B & 29 & ITALY & ITALY & ITALY & 1907 & & & 2ND ST. & ADAMS -TRIMOUNTAIN \\
\hline 551 & & NOVELLI & ANGELO & B & 40 & ITALY & ITALY & ITALY & 1912 & DUMPER/CM & LUCCA & 2ND ST. & ADAMS -TRIMOUNTAIN \\
\hline 552 & & DASANBIAGIO & CHERINTO & B & 28 & ITALY & $\begin{array}{l}\text { ITALY } \\
\text { ITALY }\end{array}$ & $\begin{array}{l}\text { ITALY } \\
\text { ITALY }\end{array}$ & 1912 & TRAMMER/CM & LUCCA & 2ND ST. & ADAMS -TRIMOUNTAIN \\
\hline 553 & & PAOLI & FELIX & $\mathrm{H}$ & 32 & ITALY & ITALY & ITALY & 1905 & MINER/CM & SEGROMIGNO. LU & 2ND ST. & ADAMS -TRIMOUNTAIN \\
\hline 554 & & PAOLI & ROSINA & w & 38 & ITALY & ITALY & ITALY & 1910 & LABORER/CM & MATRAIA. LU & 2ND ST. & ADAMS -TRIMOUNTAIN \\
\hline 555 & & PAOLI & MARY & $\mathrm{D}$ & 6 & MICHIGAN & ITALY & ITALY & & & SEGROMIGNO, LU & 2ND ST. & ADAMS -TRIMOUNTAIN \\
\hline 556 & & PAOLI & BERNARDO & $\mathrm{s}$ & 4 & MICHIGAN & ITALY & ITALY & & & SEGROMIGNO. LU & 2ND ST. & ADAMS -TRIMOUNTAIN \\
\hline 557 & & PAOLI & CELSO & $\mathrm{s}$ & 3 & MICHIGAN & ITALY & ITALY & & & SEGROMIGNO. LU & 2ND ST. & ADAMS -TRIMOUNTAIN \\
\hline 558 & & PAOLI & PASQUINA & D & $10 \mathrm{M}$ & MICHIGAN & ITALY & ITALY & & & SEGROMIGNO. LU & 2ND ST. & ADAMS -TRIMOUNTAIN \\
\hline 559 & & $\mathrm{ClUCCl}$ & MCHELE & BRIL & 45 & ITALY & ITALY & ITALY & 1889 & CARPENTER/SHOP & MATRAIA. LU & 2ND ST. & ADAMS -TRIMOUNTAIN \\
\hline 560 & & $\mathrm{RICCl}$ & ALBERTO & B & 22 & MICHIGAN & ITALY & ITALY & & LABORER/CM & MATRAIA. LU & 2ND ST. & ADAMS -TRIMOUNTAIN \\
\hline 561 & & NUTINI & GIUSEPPE & B & 24 & ITALY & ITALY & ITALY & 1917 & TRAMMER/CM & LUCCA & 2ND ST. & ADAMS -TRIMOUNTAIN \\
\hline 562 & & PERGOLA & GIULIO & B & 24 & ITALY & ITALY & ITALY & 1913 & TRAMMER/CM & SEGROMIGNO. LU & 2ND ST. & ADAMS -TRIMOUNTAIN \\
\hline 563 & & TORREANO & TERESA & NUR & 23 & MICHIGAN & ITALY & ITALY & & NURSE & TORINO & 2ND ST. & ADAMS -TRIMOUNTAIN \\
\hline 564 & 203 & LANDINI & FRANK & $\mathrm{H}$ & 52 & ITALY & ITALY & ITALY & 1882 & ENGINEER/STATIONARY & COLLE DI COMPITO. LU & 7TH ST. & ADAMS -TRIMOUNTAIN \\
\hline 565 & & LANDINI & FANNY & W & 48 & ITALY & ITALY & ITALY & 1909 & & COLLE DI COMPITO. LU & 7TH ST. & ADAMS -TRIMOUNTAIN \\
\hline 566 & & LANDINI & ALBERT & s & 25 & ITALY & ITALY & ITALY & 1909 & LABORER/CM & COLLE DI COMPITO. LU & 7TH ST. & ADAMS -TRIMOUNTAIN \\
\hline
\end{tabular}




\begin{tabular}{|c|c|c|c|c|c|c|c|c|c|c|c|c|c|}
\hline 567 & & LANDINI & RALPH & $\mathrm{s}$ & 23 & ITALY & ITALY & ITALY & 1909 & REPAIRMAN/MOTORLANE & COLLE DI COMPITO. LU & 7TH ST. & ADAMS -TRIMOUNTAIN \\
\hline 568 & & LANDINI & NELLA & $\mathrm{D}$ & 17 & ITALY & ITALY & ITALY & 1914 & & COLLE DI COMPITO. LU & 7TH ST. & ADAMS -TRIMOUNTAIN \\
\hline 569 & & LANDINI & BRUNA & D & 7 & MICHIGAN & ITALY & ITALY & & & COLLE DI COMPITO. LU & 7TH ST. & ADAMS -TRIMOUNTAIN \\
\hline 570 & & FRASS & ALBERT & B & 24 & ITALY & ITALY & ITALY & 1913 & MINER/CM & & 7TH ST. & ADAMS -TRIMOUNTAIN \\
\hline 571 & & VIRDIS & BACCHINO & B & 42 & ITALY & ITALY & ITALY & 1915 & MINER/CM & CAGLIARI & 7TH ST. & ADAMS -TRIMOUNTAIN \\
\hline 572 & $? 02$ & NUTINI & PETER & $\mathrm{H}$ & 38 & ITALY & ITALY & ITALY & 1903 & FIREMAN/ENGINE HOUSE & CAPANNORI. LU & 2ND ST. & ADAMS -TRIMOUNTAIN \\
\hline 573 & 102 & NUTINI & CELIA & W & 35 & ITALY & ITALY & ITALY & 1909 & 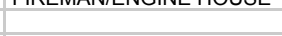 & DAR RIVIVUTI. LU & 2ND ST. & ADAMS -TRIMOUNTAIN \\
\hline 574 & & NUTINI & ALADINO & s & 9 & MICHIGAN & ITALY & ITALY & 1900 & & CAPANNORI. LU & 2ND ST. & ADAMS -TRIMOUNTAIN \\
\hline 575 & & NUTINI & ANNA & $\mathrm{D}$ & 7 & MICHIGAN & ITALY & ITALY & & & CAPANNORI. LU & 2ND ST. & ADAMS -TRIMOUNTAIN \\
\hline 576 & & NUTINI & PELLEGRINO & $\mathrm{s}$ & 5 & MICHIGAN & ITALY & ITALY & & & CAPANNORI. LU & 2ND ST. & ADAMS -TRIMOUNTAIN \\
\hline 577 & & NUTINI & JULIO & $\mathrm{s}$ & 1 & MICHIGAN & ITALY & ITALY & & & CAPANNORI. LU & 2ND ST. & ADAMS -TRIMOUNTAIN \\
\hline 578 & & FIENILI & PASQUALE & $\mathrm{H}$ & 46 & ITALY & ITALY & ITALY & 1903 & LABORER/CM & GRAGNIANO. LU & 2ND ST. & ADAMS -TRIMOUNTAIN \\
\hline 579 & & FIENILI & TERESA & W & 46 & ITALY & ITALY & ITALY & 1910 & & GRAGNIANO. LU & 2ND ST. & ADAMS -TRIMOUNTAIN \\
\hline 580 & & FIENILI & JOSEPH & s & 22 & ITALY & ITALY & ITALY & 1914 & FIREMAN/BOILER HOUSE & GRAGNIANO. LU & 2ND ST. & ADAMS -TRIMOUNTAIN \\
\hline 581 & & FIENILI & ANNA & $\mathrm{D}$ & 8 & MICHIGAN & ITALY & ITALY & & & GRAGNIANO. LU & 2ND ST. & ADAMS -TRIMOUNTAIN \\
\hline 582 & & CATELLI & GUIDO & $\mathrm{COU}$ & 23 & ITALY & ITALY & ITALY & 1912 & MINER/CM & SEGROMIGNO, LU & 2ND ST. & ADAMS -TRIMOUNTAIN \\
\hline 583 & & GEMIGNANI & RAFFAELLO & B & 25 & ITALY & ITALY & ITALY & 1912 & MINER/CM & LUCCA & 2ND ST. & ADAMS -TRIMOUNTAIN \\
\hline 584 & & NUTINI & ORLANDO & B & 30 & ITALY & ITALY & ITALY & 1906 & MINER/CM & CAPANNORI. LU & 2ND ST. & ADAMS -TRIMOUNTAIN \\
\hline 585 & & SIMI & JACOB & $\mathrm{H}$ & 30 & ITALY & ITALY & ITALY & 1908 & MINER/CM & VILLA BASILICA. LU & 2ND ST. & ADAMS -TRIMOUNTAIN \\
\hline 586 & & SIMI & MARY & w & 26 & ITALY & ITALY & ITALY & 1913 & & & 2ND ST. & ADAMS -TRIMOUNTAIN \\
\hline 587 & & SIMI & GENO & s & 4 & MICHIGAN & ITALY & ITALY & & & VILLA BASILICA. LU & 2ND ST. & ADAMS -TRIMOUNTAIN \\
\hline 588 & & SIMI & JOSEPH & $\mathrm{s}$ & 1 & MICHIGAN & ITALY & ITALY & & & VILLA BASILICA, LU & 2ND ST. & ADAMS -TRIMOUNTAIN \\
\hline 589 & & SIMI & ONORATO & $\mathrm{COU}$ & 32 & ITALY & ITALY & ITALY & 1911 & MINER/CM & VILLA BASILICA. LU & 2ND ST. & ADAMS -TRIMOUNTAIN \\
\hline 590 & & ADAMI & JOHN & B & 31 & ITALY & ITALY & ITALY & 1911 & MINER/CM & VILLA BASILICA. LU & 2ND ST. & ADAMS -TRIMOUNTAIN \\
\hline 591 & & MAFFEI & DAVID & B & UN & ITALY & ITALY & ITALY & 1903 & MASON/CM & LUCCA & 2ND ST. & ADAMS -TRIMOUNTAIN \\
\hline 592 & & CERCHIARI & ARTURO & B & 26 & ITALY & ITALY & ITALY & 1911 & MINER/CM & VILLA BASILICA. LU & 2ND ST. & ADAMS -TRIMOUNTAIN \\
\hline 593 & & ADAMI & BARTOLOMEO & B & 38 & ITALY & ITALY & ITALY & 1906 & LABORER/CM & VILLA BASILICA. LU & 2ND ST. & ADAMS -TRIMOUNTAIN \\
\hline 594 & & MARCHESCHI & ORESTE & $\mathrm{H}$ & 36 & ITALY & ITALY & ITALY & 1913 & LABORER/CM & LUCCA & 2ND ST. & ADAMS -TRIMOUNTAIN \\
\hline 595 & & MARCHESCHI & CAROLINA & W & 36 & ITALY & ITALY & ITALY & 1915 & & LUCCA & 2ND ST. & ADAMS -TRIMOUNTAIN \\
\hline 596 & & MARCHESCHI & OLINTO & $\mathrm{s}$ & 11 & ITALY & ITALY & ITALY & 1915 & & LUCCA & 2ND ST. & ADAMS -TRIMOUNTAIN \\
\hline 597 & & MARCHESCHI & FANNY & D & 9 & ITALY & ITALY & ITALY & 1915 & & LUCCA & 2ND ST. & ADAMS -TRIMOUNTAIN \\
\hline 598 & & MARCHESCHI & EVO & $\mathrm{s}$ & 3 & MICHIGAN & ITALY & ITALY & & & LUCCA & 2ND ST. & ADAMS -TRIMOUNTAIN \\
\hline 599 & & URBANI & MARIANO & B & 29 & ITALY & ITALY & ITALY & 1913 & DUMPER/CM & VERONA & 2ND ST. & ADAMS -TRIMOUNTAIN \\
\hline 600 & & MILANI & JOSEPH & B & 26 & ITALY & ITALY & ITALY & 1911 & TRAMMER/CM & MILANO & 2ND ST. & ADAMS -TRIMOUNTAIN \\
\hline 601 & & BIANCHINI & PETER & B & 30 & ITALY & ITALY & ITALY & 1911 & TRAMMER/CM & & 2ND ST. & ADAMS -TRIMOUNTAIN \\
\hline 602 & & LAZAREVICH & DANTE & B & 21 & ITALY & ITALY & ITALY & 1915 & TRAMMER/CM & & 2ND ST. & ADAMS -TRIMOUNTAIN \\
\hline 603 & & NAPOLI & JOSEPH & B & 45 & ITALY & ITALY & ITALY & 1896 & TRAMMER/CM & & 2ND ST. & ADAMS -TRIMOUNTAIN \\
\hline 604 & & FIENILI & ANNIBALE & $\mathrm{H}$ & 32 & ITALY & ITALY & ITALY & 1910 & LABORER/CM & GRAGNIANO. LU & 2ND ST. & ADAMS -TRIMOUNTAIN \\
\hline 605 & & FIENILI & MARIA & W & 31 & ITALY & ITALY & ITALY & 1914 & & & 2ND ST. & ADAMS -TRIMOUNTAIN \\
\hline 606 & & FIENILI & MARINO & $\mathrm{S}$ & 3 & MICHIGAN & ITALY & ITALY & & & GRAGNIANO. LU & 2ND ST. & ADAMS -TRIMOUNTAIN \\
\hline 607 & & FIENILI & AGOSTINO & BR & 24 & ITALY & ITALY & ITALY & 1912 & TRAMMER/CM & GRAGNIANO. LU & 2ND ST. & ADAMS -TRIMOUNTAIN \\
\hline 608 & & FIENILI & CESARE & $\mathrm{BR}$ & 31 & ITALY & ITALY & ITALY & 1911 & TRAMMER/CM & GRAGNIANO. LU & 2ND ST. & ADAMS -TRIMOUNTAIN \\
\hline 609 & & MILANI & OTTAVIO & B & 23 & ITALY & ITALY & ITALY & 1913 & TRAMMER/CM & CAPANNORI. LU & 2ND ST. & ADAMS -TRIMOUNTAIN \\
\hline 610 & & CAMPIONI & CARLO & B & 31 & ITALY & ITALY & ITALY & 1913 & MINER/CM & LUCCA & 2ND ST. & ADAMS -TRIMOUNTAIN \\
\hline 611 & & $\mathrm{PIZZI}$ & PIETRO & B & 32 & ITALY & ITALY & ITALY & 1905 & TRAMMER/CM & PESCAGLIA. LU & 2ND ST. & ADAMS -TRIMOUNTAIN \\
\hline 612 & & LEONELLI & VITTORIO & B & 28 & ITALY & ITALY & ITALY & 1913 & MASON/CM & TLUUAOLIA, LU & 2ND ST. & ADAMS -TRIMOUNTAIN \\
\hline 613 & & CORTOPASSI & GIUSEPPE & B & 39 & ITALY & ITALY & ITALY & 1909 & TRAMMER/CM & LUCCA & 2ND ST. & ADAMS -TRIMOUNTAIN \\
\hline 614 & & MARTINELLI & GIOVANNI & B & 51 & ITALY & ITALY & ITALY & 1912 & TRAMMER/CM & LUCCA & 2ND ST. & ADAMS -TRIMOUNTAIN \\
\hline 615 & & PAOLI & GIULIO & $\mathrm{H}$ & 30 & ITALY & ITALY & ITALY & 1909 & LABORER/CM & SEGROMIGNO. LU & 2ND ST. & ADAMS -TRIMOUNTAIN \\
\hline 616 & & PAOLI & TISBE & W & 28 & ITALY & ITALY & ITALY & 1912 & & & 2ND ST. & ADAMS -TRIMOUNTAIN \\
\hline 617 & & PAOLI & BELINDA & D & 3 & MICHIGAN & ITALY & ITALY & & & SEGROMIGNO. LU & 2ND ST. & ADAMS -TRIMOUNTAIN \\
\hline 618 & & PAOLI & ANGELINA & D & 2 & MICHIGAN & ITALY & ITALY & & & SEGROMIGNO, LU & 2ND ST. & ADAMS -TRIMOUNTAIN \\
\hline 619 & & PAOLI & GUIDO & $\mathrm{s}$ & $7 \mathrm{M}$ & MICHIGAN & ITALY & ITALY & & & SEGROMIGNO. LU & 2ND ST. & ADAMS -TRIMOUNTAIN \\
\hline 620 & & MONTICELLI & CARLO & B & 30 & ITALY & ITALY & ITALY & 1912 & TRAMMER/CM & MATRAIA. LU & 2ND ST. & ADAMS -TRIMOUNTAIN \\
\hline 621 & 25 & FERRARI & VITTORIO & $\mathrm{H}$ & 38 & ITALY & ITALY & ITALY & 1908 & LABORER/CM & POPIGLIO. PISTOIA & 2ND ST. & ADAMS -TRIMOUNTAIN \\
\hline 622 & & FERRARI & MADDALENA & w & 35 & ITALY & ITALY & ITALY & 1908 & & & 2ND ST. & ADAMS -TRIMOUNTAIN \\
\hline 623 & & FERRARI & ALDO & s & 8 & MICHIGAN & ITALY & ITALY & & & POPIGLIO. PISTOIA & 2ND ST. & ADAMS -TRIMOUNTAIN \\
\hline 624 & & FERRARI & FEDE & $\mathrm{D}$ & 6 & MICHIGAN & ITALY & ITALY & & & POPIGLIO, PISTOIA & 2ND ST. & ADAMS -TRIMOUNTAIN \\
\hline 625 & 208 & PIERONI & SAMUEL & $\mathrm{H}$ & 28 & ITALY & ITALY & ITALY & 1906 & MINER/CM & COLLE DI COMPITO. LU & 7TH ST. & ADAMS -TRIMOUNTAIN \\
\hline 626 & & PIERONI & MARY & W & 27 & ITALY & ITALY & ITALY & 1914 & & & 7TH ST. & ADAMS -TRIMOUNTAIN \\
\hline 627 & & PIERONI & CLARA & $\mathrm{D}$ & 4 & MICHIGAN & ITALY & ITALY & & & COLLE DI COMPITO. LU & 7TH ST. & ADAMS -TRIMOUNTAIN \\
\hline 628 & & PIERONI & NORA & $\mathrm{D}$ & 2 & MICHIGAN & ITALY & ITALY & & & COLLE DI COMPITO. LU & 7TH ST. & ADAMS -TRIMOUNTAIN \\
\hline 629 & & PIERONI & ORNI & $\mathrm{s}$ & $5 \mathrm{M}$ & MICHIGAN & ITALY & ITALY & & & COLLE DI COMPITO. LU & 7TH ST. & ADAMS -TRIMOUNTAIN \\
\hline
\end{tabular}




\begin{tabular}{|c|c|c|c|c|c|c|c|c|c|c|c|c|c|}
\hline 630 & 206 & CANI & PAUL & $\mathrm{H}$ & 29 & ITALY & ITALY & ITALY & 1914 & MINER/CM & FANO & 7TH ST. & ADAMS -TRIMOUNTAIN \\
\hline 631 & & CANI & BRUNA & w & 18 & MICHIGAN & ITALY & ITALY & & & & 7TH ST. & ADAMS -TRIMOUNTAIN \\
\hline 632 & & CANI & MICHELINA & D & $10 \mathrm{M}$ & MICHIGAN & ITALY & ITALY & & & FANO & 7TH ST. & ADAMS -TRIMOUNTAIN \\
\hline 633 & 207 & FLOSI & MASSIMO & $\mathrm{H}$ & 36 & ITALY & ITALY & ITALY & 1905 & MINER/CM & PARIANA. LU & 5TH ST. & ADAMS -TRIMOUNTAIN \\
\hline 634 & & FLOSI & ANGELINA & w & 34 & ITALY & ITALY & ITALY & 1913 & & PARIANA, LU & 5TH ST. & ADAMS -TRIMOUNTAIN \\
\hline 635 & & FLOSI & PASQUINA & $\mathrm{D}$ & 13 & ITALY & ITALY & ITALY & 1913 & & PARIANA, LU & 5TH ST. & ADAMS -TRIMOUNTAIN \\
\hline 636 & & FLOSI & SANTINA & $\mathrm{D}$ & 4 & MICHIGAN & ITALY & ITALY & & & PARIANA. LU & 5TH ST. & ADAMS -TRIMOUNTAIN \\
\hline 637 & 207 & MATTEUCCI & OLIDE & $\mathrm{H}$ & 27 & ITALY & ITALY & ITALY & 1909 & MINER/CM & COLLE DI COMPITO. LU & 5TH ST. & ADAMS -TRIMOUNTAIN \\
\hline 638 & 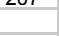 & MATTEUCCI & MARY & W & 26 & ITALY & ITALY & ITALY & 1913 & 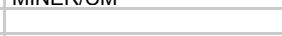 & 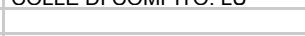 & 5TH ST. & ADAMS -TRIMOUNTAIN \\
\hline 639 & & MATTEUCCI & DANTE & $\mathrm{s}$ & 6 & MICHIGAN & ITALY & ITALY & & & COLLE DI COMPITO. LU & 5TH ST. & ADAMS -TRIMOUNTAIN \\
\hline 640 & & MATTEUCCI & PETER & $\mathrm{s}$ & 4 & MICHIGAN & ITALY & ITALY & & & COLLE DI COMPITO. LU & 5TH ST. & ADAMS -TRIMOUNTAIN \\
\hline 641 & 303 & SPIZZI & FASTINO & $\mathrm{H}$ & 34 & ITALY & ITALY & ITALY & 1909 & TRAMMER/CM & MILANO & & ADAMS -TRIMOUNTAIN \\
\hline 642 & & SPIZZI & VITTORIA & W & 30 & ITALY & ITALY & ITALY & 1914 & & MILANO & & ADAMS -TRIMOUNTAIN \\
\hline 643 & & SPIZZI & GINO & $s$ & 4 & MICHIGAN & ITALY & ITALY & & & MILANO & & ADAMS -TRIMOUNTAIN \\
\hline 644 & & SPIZZI & MARIO & $\mathrm{s}$ & 3 & MICHIGAN & ITALY & ITALY & & & MILANO & & ADAMS -TRIMOUNTAIN \\
\hline 645 & 314 & GARNERO & GILDO & $\mathrm{H}$ & 38 & ITALY & ITALY & ITALY & 1911 & FIREMAN/ENGINE HOUSE & & 5TH ST. & ADAMS -TRIMOUNTAIN \\
\hline 646 & & GARNERO & LUCY & W & 32 & ITALY & ITALY & ITALY & 1911 & & & 5TH ST. & ADAMS -TRIMOUNTAIN \\
\hline 647 & & GARNERO & MARY & $\mathrm{D}$ & 7 & MICHIGAN & ITALY & ITALY & & & & 5TH ST. & ADAMS -TRIMOUNTAIN \\
\hline 648 & 312 & GIUSFREDI & ARMANDO & $\mathrm{H}$ & 35 & ITALY & ITALY & ITALY & 1912 & & LUCCA & 5TH ST. & ADAMS -TRIMOUNTAIN \\
\hline 649 & & GIUSFREDI & IDA & w & 35 & ITALY & ITALY & ITALY & 1915 & & & 5TH ST. & ADAMS -TRIMOUNTAIN \\
\hline 650 & & GIUSFREDI & ANGELO & s & 1 & MICHIGAN & ITALY & ITALY & & & LUCCA & 5TH ST. & ADAMS -TRIMOUNTAIN \\
\hline 651 & 207 & LUPETTI & VIRGILIO & $\mathrm{H}$ & 34 & ITALY & ITALY & ITALY & 1912 & MINER/CM & CALCI, PISA & 6TH AVE. & ADAMS -TRIMOUNTAIN \\
\hline 652 & & $\begin{array}{l}\text { LUPETTI } \\
\text { LUTI }\end{array}$ & ANGELINA & w & 28 & ITALY & ITALY & ITALY & 1912 & & CALCI. PISA & 6TH AVE. & ADAMS -TRIMOUNTAIN \\
\hline 653 & & LUPETTI & MARGHERITA & $\mathrm{D}$ & 9 & ITALY & ITALY & ITALY & 1912 & & CALCI. PISA & 6TH AVE. & ADAMS -TRIMOUNTAIN \\
\hline 654 & & LUPETTI & EDO & $\mathrm{s}$ & 3 & MICHIGAN & ITALY & ITALY & & & CALCI. PISA & 6TH AVE. & ADAMS -TRIMOUNTAIN \\
\hline 655 & & LUPETTI & AGOSTINO & $\mathrm{s}$ & $10 \mathrm{M}$ & MICHIGAN & ITALY & ITALY & & & CALCI. PISA & 6TH AVE. & ADAMS -TRIMOUNTAIN \\
\hline 656 & 211 & QUILICI & JOHN & $\mathrm{H}$ & 35 & ITALY & ITALY & ITALY & 1900 & MINER/CM & LUCCA & 6TH AVE. & ADAMS -TRIMOUNTAIN \\
\hline 657 & & QUILICI & MARY & w & 35 & MICHIGAN & GERMANY & CANADA & & & GERMANY & 6TH AVE. & ADAMS -TRIMOUNTAIN \\
\hline 658 & & QUILICI & MERCEDES & $\mathrm{D}$ & 8 & MICHIGAN & ITALY & ITALY & & & LUCCA & 6TH AVE. & ADAMS -TRIMOUNTAIN \\
\hline 659 & & QUILICI & FLORENCE & $\mathrm{D}$ & 7 & MICHIGAN & ITALY & ITALY & & & LUCCA & 6TH AVE. & ADAMS -TRIMOUNTAIN \\
\hline 660 & & QUILICI & CORINNA & D & 3 & MICHIGAN & ITALY & ITALY & & & LUCCA & 6TH AVE. & ADAMS -TRIMOUNTAIN \\
\hline 661 & & QUILICI & PAUL & s & 2 & MICHIGAN & ITALY & ITALY & & & LUCCA & 6TH AVE. & ADAMS -TRIMOUNTAIN \\
\hline 662 & & QUILICI & JEAN & D & $8 \mathrm{M}$ & MICHIGAN & ITALY & ITALY & & & LUCCA & 6TH AVE. & ADAMS -TRIMOUNTAIN \\
\hline 663 & 313 & MATTEUCCI & NICOLA & $\mathrm{H}$ & 34 & ITALY & ITALY & ITALY & 1902 & MINER/CM & COLLE DI COMPITO, LU & 6TH AVE. & ADAMS -TRIMOUNTAIN \\
\hline 664 & & MATTEUCCI & IDA & w & 34 & ITALY & ITALY & ITALY & 1912 & & COLLE DI COMPITO. LU & 6TH AVE. & ADAMS -TRIMOUNTAIN \\
\hline 604 665 & & MATTEUCCI & FILOMENA & D & 9 & ITALY & ITALY & ITALY & 1912 & & COLLE DI COMPITO. LU & $\begin{array}{l}\text { GTH AVE. } \\
\text { 6TH AVE. }\end{array}$ & ADAMS -TRIMOUNTAIN \\
\hline 666 & & MATTEUCCI & GUERRINO & $\mathrm{s}$ & 7 & MICHIGAN & ITALY & ITALY & & & COLLE DI COMPITO. LU & 6TH AVE. & ADAMS -TRIMOUNTAIN \\
\hline 667 & & MATTEUCCI & TEPPA? & $\mathrm{s}$ & 6 & MICHIGAN & ITALY & ITALY & & & COLLE DI COMPITO. LU & 6TH AVE. & ADAMS -TRIMOUNTAIN \\
\hline 668 & & MATTEUCCI & VASCO & $\mathrm{s}$ & 2 & MICHIGAN & ITALY & ITALY & & & COLLE DI COMPITO. LU & 6TH AVE. & ADAMS -TRIMOUNTAIN \\
\hline 669 & & MATTEUCCI & BRUNA & D & $8 \mathrm{M}$ & MICHIGAN & ITALY & ITALY & & & COLLE DI COMPITO. LU & 6TH AVE. & ADAMS -TRIMOUNTAIN \\
\hline 670 & & CERVELLI & FRANCESCO & B & 36 & ITALY & ITALY & ITALY & 1902 & MINER/CM & CAPANNORI. LU & 6TH AVE. & ADAMS -TRIMOUNTAIN \\
\hline 671 & 315 & MARIANI & FRED & $\mathrm{H}$ & 27 & ITALY & ITALY & ITALY & 1907 & MINER/CM & CAPANNORI. LU & 6TH AVE. & ADAMS -TRIMOUNTAIN \\
\hline 672 & & MARIANI & SANTINA & w & 24 & ITALY & ITALY & ITALY & 1914 & & & 6TH AVE. & ADAMS -TRIMOUNTAIN \\
\hline 673 & & MARIANI & MARIO & s & 5 & MICHIGAN & ITALY & ITALY & & & CAPANNORI. LU & 6TH AVE. & ADAMS -TRIMOUNTAIN \\
\hline 674 & & MARIANI & LIDA & D & $4 \mathrm{M}$ & MICHIGAN & ITALY & ITALY & & & CAPANNORI. LU & 6TH AVE. & ADAMS -TRIMOUNTAIN \\
\hline 675 & 314 & NELLI & GUIDO & $\mathrm{H}$ & 32 & ITALY & ITALY & ITALY & 1910 & MINER/CM & VALPROMARO. LU & 6TH AVE. & ADAMS -TRIMOUNTAIN \\
\hline 676 & & NELLI & GIORGIA & w & 30 & ITALY & ITALY & ITALY & 1912 & IVIIVERA LIVI & VALTRUUVIARU. LU & 6TH AVE. & ADAMS -TRIMOUNTAIN \\
\hline 677 & & NELLI & OVIDIO & s & 5 & MICHIGAN & ITALY & ITALY & & & VALPROMARO. LU & $\begin{array}{l}\text { 6TH AVE. } \\
\text { GTH A }\end{array}$ & ADAMS -TRIMOUNTAIN \\
\hline 678 & & NELLI & NIILO & $\mathrm{s}$ & 1 & MICHIGAN & ITALY & ITALY & & & VALPROMARO. LU & 6TH AVE. & ADAMS -TRIMOUNTAIN \\
\hline 679 & & NELLI & ALMA & $\mathrm{D}$ & $2 \mathrm{M}$ & MICHIGAN & ITALY & ITALY & & & VALPROMARO. LU & 6TH AVE. & ADAMS -TRIMOUNTAIN \\
\hline 680 & & NELLI & GIORGIO & BR & 35 & ITALY & ITALY & ITALY & 1911 & TRAMMER/CM & VALPROMARO. LU & 6TH AVE. & ADAMS -TRIMOUNTAIN \\
\hline 681 & & NERI & ROMEO & $\mathrm{B}$ & 25 & ITALY & ITALY & ITALY & 1902 & TRAMMER/CM & & 6TH AVE. & ADAMS -TRIMOUNTAIN \\
\hline 682 & & MEI & CARLO & B & 41 & ITALY & ITALY & ITALY & 1914 & MASON/CM & RUOTA. LU & 6TH AVE. & ADAMS -TRIMOUNTAIN \\
\hline 683 & & NATALI & CINQUIN & B & 34 & ITALY & ITALY & ITALY & 1914 & MASON/CM & & 6TH AVE. & ADAMS -TRIMOUNTAIN \\
\hline 684 & & GOASELLI & DOMENICO & B & 35 & ITALY & ITALY & ITALY & 1914 & NOT CLEAR & & 6TH AVE. & ADAMS -TRIMOUNTAIN \\
\hline 685 & 209 & GIANNECHINI & EUGENIO & $\mathrm{H}$ & 44 & ITALY & ITALY & ITALY & 1902 & MINER/CM & SANTA MARIA AL PIANO. LU & 7TH AVE. & ADAMS -TRIMOUNTAIN \\
\hline 686 & & GIANNECHINI & TERESA & w & 33 & ITALY & ITALY & ITALY & 1908 & & & 7TH AVE. & ADAMS -TRIMOUNTAIN \\
\hline 687 & & GIANNECHINI & ALBERTO & s & 12 & MICHIGAN & ITALY & ITALY & & & SANTA MARIA AL PIANO, LU & 7TH AVE. & ADAMS -TRIMOUNTAIN \\
\hline 688 & & GIANNECHINI & MARIO & $\mathrm{s}$ & 9 & MICHIGAN & ITALY & ITALY & & & SANTA MARIA AL PIANO. LU & 7TH AVE. & ADAMS -TRIMOUNTAIN \\
\hline 689 & & GIANNECHINI & NELLA & $\mathrm{D}$ & 7 & MICHIGAN & ITALY & ITALY & & & SANTA MARIA AL PIANO. LU & 7TH AVE. & ADAMS -TRIMOUNTAIN \\
\hline 690 & & GIANNECHINI & JEAN & $\mathrm{D}$ & 3 & MICHIGAN & ITALY & ITALY & & & SANTA MARIA AL PIANO. LU & 7TH AVE. & ADAMS -TRIMOUNTAIN \\
\hline 691 & & ?ROGIOVANNI & GIUSEPPE & B & 36 & ITALY & ITALY & ITALY & 1907 & TRAMMER/CM & & 7TH AVE. & ADAMS -TRIMOUNTAIN \\
\hline 692 & & BERTOLUCCI & AMERICO & B & 36 & ITALY & ITALY & ITALY & 1911 & MINER/CM & COLLE DI CAMPITO. LU & 7TH AVE. & ADAMS -TRIMOUNTAIN \\
\hline
\end{tabular}




\begin{tabular}{|c|c|c|c|c|c|c|c|c|c|c|c|c|c|}
\hline 693 & 211 & QUILICI & AMILDO & $\mathrm{H}$ & 34 & ITALY & ITALY & ITALY & 1905 & FIREMAN/BOILER HOUSE & LUCCA & 7TH AVE. & ADAMS -TRIMOUNTAIN \\
\hline 694 & & QUILICI & ROSA & w & 30 & ITALY & ITALY & ITALY & 1911 & & & 7TH AVE. & ADAMS -TRIMOUNTAIN \\
\hline 695 & & QUILICI & ANNA & D & 7 & MICHIGAN & ITALY & ITALY & & & LUCCA & 7TH AVE. & ADAMS -TRIMOUNTAIN \\
\hline 696 & & QUILICI & JOSEPH & $\mathrm{s}$ & 4 & MICHIGAN & ITALY & ITALY & & & LUCCA & 7TH AVE. & ADAMS -TRIMOUNTAIN \\
\hline 697 & & LUCCHESI & SAM & B & 30 & ITALY & ITALY & ITALY & 1912 & TRAMMER/CM & LUCCA & 7TH AVE. & ADAMS -TRIMOUNTAIN \\
\hline 698 & & GIAMMATTEI & FELICE & B & 28 & ITALY & ITALY & ITALY & 1914 & TRAMMER/CM & & 7TH AVE. & ADAMS -TRIMOUNTAIN \\
\hline 699 & & BERTUCCl & LOUIS & B & 37 & ITALY & ITALY & ITALY & 1912 & MINER/CM & FABBIANO MONTANINO. LU & 7TH AVE. & ADAMS -TRIMOUNTAIN \\
\hline 700 & & MARCHESCHI & FRED & B & 26 & ITALY & ITALY & ITALY & 1912 & TRAMMER/CM & LUCCA & 7TH AVE. & ADAMS -TRIMOUNTAIN \\
\hline 701 & 215 & PARENTI & VINCENZO & $\mathrm{H}$ & 50 & ITALY & ITALY & ITALY & 1896 & MINER/CM & CASTELVECCHIO. LU & 7TH AVE. & ADAMS -TRIMOUNTAIN \\
\hline 702 & & PARENTI & ASSUNTA & W & 50 & ITALY & ITALY & ITALY & 1900 & & & 7TH AVE. & ADAMS -TRIMOUNTAIN \\
\hline 703 & & PARENTI & BRUNO & $\mathrm{s}$ & 18 & MICHIGAN & ITALY & ITALY & & LABORER/FACTORY & CASTELVECCHIO. LU & 7TH AVE. & ADAMS -TRIMOUNTAIN \\
\hline 704 & & DI VECCHIO & LEO & B & 28 & ITALY & ITALY & ITALY & 1907 & BLACKSMITH/CM & BENEVENTO & 7TH AVE. & ADAMS -TRIMOUNTAIN \\
\hline 705 & & GIAMPAOLI & ALESSANDRO & B & 30 & ITALY & ITALY & ITALY & 1912 & MASON/CM & CAPANNORI. LU & 7TH AVE. & ADAMS -TRIMOUNTAIN \\
\hline 706 & & PATINGA??E & VINCENZO & B & 23 & ITALY & ITALY & ITALY & 1914 & TRAMMER/CM & & 7TH AVE. & ADAMS -TRIMOUNTAIN \\
\hline 707 & & MONTI & DELOVINO? & B & 35 & ITALY & ITALY & ITALY & 1913 & DUMPER/CM & & 7TH AVE. & ADAMS -TRIMOUNTAIN \\
\hline 708 & & SABBATINI & GIUSTO & B & 40 & ITALY & ITALY & ITALY & 1900 & TRAMMER/CM & LUCCA & 7TH AVE. & ADAMS -TRIMOUNTAIN \\
\hline 709 & 307 & MORELLI & BATTISTA & $\mathrm{H}$ & 33 & ITALY & ITALY & ITALY & 1909 & MASON/CM & MONTEMAGNO. LU & 7TH AVE. & ADAMS -TRIMOUNTAIN \\
\hline 710 & & MORELLI & MARGHERITA & W & 29 & ITALY & ITALY & ITALY & 1913 & & & 7TH AVE. & ADAMS -TRIMOUNTAIN \\
\hline 711 & & MORELLI & BRUNA & D & 2 & MICHIGAN & ITALY & ITALY & & & MONTEMAGNO. LU & 7TH AVE. & ADAMS -TRIMOUNTAIN \\
\hline 712 & & MARGHERIO & $\mathrm{JOHN}$ & $\mathrm{H}$ & 36 & ITALY & ITALY & ITALY & 1905 & MASON/CM & SAN GIUSTO CANAVESE. TO & 7TH AVE. & ADAMS -TRIMOUNTAIN \\
\hline 713 & & MARGHERIO & MARY & w & 31 & ITALY & ITALY & ITALY & 1906 & & & 7TH AVE. & ADAMS -TRIMOUNTAIN \\
\hline 714 & & MARGHERIO & ANTONIO & s & 5 & MICHIGAN & ITALY & ITALY & & & SAN GIUSTO CANAVESE, TO & 7TH AVE. & ADAMS -TRIMOUNTAIN \\
\hline 715 & & MARGHERIO & JOHN & s & 4 & MICHIGAN & ITALY & ITALY & & & SAN GIUSTO CANAVESE. TO & 7TH AVE. & ADAMS -TRIMOUNTAIN \\
\hline 716 & 317 & MONTICELLI & JAMES & $\mathrm{H}$ & 44 & ITALY & ITALY & ITALY & 1893 & MINER/CM & LUCCA & 7TH AVE. & ADAMS -TRIMOUNTAIN \\
\hline 717 & & MONTICELLI & AMEDEO & $\mathrm{s}$ & 18 & MICHIGAN & ITALY & ITALY & & TOOL BOYICM & LUCCA & 7TH AVE. & ADAMS -TRIMOUNTAIN \\
\hline 718 & & MONTICELLI & EVA & $\mathrm{D}$ & 17 & ITALY & ITALY & ITALY & 1902 & 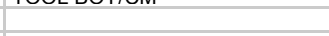 & LUCCA & 7TH AVE. & ADAMS -TRIMOUNTAIN \\
\hline 719 & & MONTICELLI & ELSIE & D & 15 & MICHIGAN & ITALY & ITALY & & & LUCCA & 7TH AVE. & ADAMS -TRIMOUNTAIN \\
\hline 720 & & MONTICELLI & ADELINA & D & 13 & MICHIGAN & ITALY & ITALY & & & LUCCA & 7TH AVE. & ADAMS -TRIMOUNTAIN \\
\hline 721 & & MONTICELLI & EDITH & D & 11 & MICHIGAN & ITALY & ITALY & & & LUCCA & 7TH AVE. & ADAMS -TRIMOUNTAIN \\
\hline 722 & & MONTICELLI & LOUIS & NEP & 28 & ITALY & ITALY & ITALY & 1914 & TRAMMER/CM & LUCCA & 7TH AVE. & ADAMS -TRIMOUNTAIN \\
\hline 723 & 320 & GIANNINI & LOUIS & $\mathrm{H}$ & 40 & ITALY & ITALY & ITALY & 1906 & CARPENTER/HOUSE & SEGROMIGNO. LU & 7TH AVE. & ADAMS -TRIMOUNTAIN \\
\hline 724 & & GIANNINI & ROSA & w & 30 & ITALY & ITALY & ITALY & 1906 & & SEGROMIGNO. LU & 7TH AVE. & ADAMS -TRIMOUNTAIN \\
\hline 725 & & GIANNINI & GENO & s & 10 & MICHIGAN & ITALY & ITALY & & & SEGROMIGNO. LU & 7TH AVE. & ADAMS -TRIMOUNTAIN \\
\hline 726 & & GIANNINI & ORAZIO? & $\mathrm{s}$ & 6 & MICHIGAN & ITALY & ITALY & & & SEGROMIGNO, LU & 7TH AVE. & ADAMS -TRIMOUNTAIN \\
\hline 727 & & GIANNINI & LORENZO & s & 3 & MICHIGAN & ITALY & ITALY & & & SEGROMIGNO. LU & 7TH AVE. & ADAMS -TRIMOUNTAIN \\
\hline 728 & & GIANNINI & ELSIE & $\mathrm{D}$ & 1 & MICHIGAN & $\begin{array}{l}\text { ITALY } \\
\end{array}$ & $\begin{array}{l}\text { ITALY } \\
\end{array}$ & & & SEGROMIGNO. LU & 7TH AVE. & ADAMS -TRIMOUNTAIN \\
\hline 729 & 310 & SABBATINI & ANTONIO & $\mathrm{H}$ & 43 & ITALY & ITALY & ITALY & 1899 & LABORER/CM & LUCCA & 7TH AVE. & ADAMS -TRIMOUNTAIN \\
\hline 730 & & SABBATINI & ELLEN & w & 37 & AUSTRIA & AUSTRIA & AUSTRIA & 1905 & & & 7TH AVE. & ADAMS -TRIMOUNTAIN \\
\hline 731 & & SABBATINI & MARGHERITA & $\mathrm{D}$ & 12 & MICHIGAN & ITALY & AUSTRIA & & & LUCCA & 7TH AVE. & ADAMS -TRIMOUNTAIN \\
\hline 732 & & SABBATINI & ELSIE & D & 11 & MICHIGAN & ITALY & AUSTRIA & & & LUCCA & 7TH AVE. & ADAMS -TRIMOUNTAIN \\
\hline 733 & & SABBATINI & MIKE & $\mathrm{s}$ & 9 & MICHIGAN & ITALY & AUSTRIA & & & LUCCA & 7TH AVE. & ADAMS -TRIMOUNTAIN \\
\hline 734 & & SABBATINI & MARIO & $\mathrm{s}$ & 8 & MICHIGAN & ITALY & AUSTRIA & & & LUCCA & 7TH AVE. & ADAMS -TRIMOUNTAIN \\
\hline 735 & & SABBATINI & JOSEPHINE & D & 6 & MICHIGAN & ITALY & AUSTRIA & & & LUCCA & 7TH AVE. & ADAMS -TRIMOUNTAIN \\
\hline 736 & & PANTALEONI & ANGELO & B & 42 & ITALY & ITALY & ITALY & 1903 & MINER/CM & SANTA MARIA DEL GIUDICE. LU & 7TH AVE. & ADAMS -TRIMOUNTAIN \\
\hline 737 & & CHENET & JOSEPH & B & 45 & ITALY & ITALY & ITALY & 1915 & MINER/CM & & 7TH AVE. & ADAMS -TRIMOUNTAIN \\
\hline 738 & & GIULIANELLI & JACOB & B & 45 & ITALY & ITALY & ITALY & 1916 & TRAMMER/CM & & 7TH AVE. & ADAMS -TRIMOUNTAIN \\
\hline 739 & 304 & TAMBELLINI & AMERTANO & $\mathrm{H}$ & 31 & ITALY & ITALY & ITALY & 1904 & MINER/CM & LUCCA & 7TH AVE. & ADAMS -TRIMOUNTAIN \\
\hline 740 & & TAMBELLINI & EVA & w & 38 & ITALY & ITALY & ITALY & 1904 & MIDWIFE/GENERAL PRACTICE & LUCCA & 7TH AVE. & ADAMS -TRIMOUNTAIN \\
\hline 741 & & TAMBELLINI & $\begin{array}{l}\text { LVA } \\
\text { HELLEN }\end{array}$ & $\begin{array}{l}\text { W } \\
\text { D }\end{array}$ & $\frac{38}{12}$ & MICHIGAN & $\begin{array}{l}\text { ITALY } \\
\text { ITALY }\end{array}$ & $\begin{array}{l}\text { ITALY } \\
\text { ITALY }\end{array}$ & 1904 & TVILWVIFE/GEIVERAL PRAC ILE & LUCCA & $\begin{array}{l}\text { 7TH AVE. } \\
\text { 7TH AVE. }\end{array}$ & ADAMS -TRIMOUNTAIN \\
\hline 742 & 212 & $\begin{array}{l}\text { PIERONI } \\
\end{array}$ & LOUIS & $\mathrm{H}$ & 33 & ITALY & ITALY & ITALY & 1899 & MINER/CM & LUCCA & 7TH AVE. & ADAMS -TRIMOUNTAIN \\
\hline 743 & & PIERONI & FILOMENA & W & 33 & ITALY & ITALY & ITALY & 1912 & & & 7TH AVE. & ADAMS -TRIMOUNTAIN \\
\hline 744 & & PIERONI & ALFI & s & 4 & MICHIGAN & ITALY & ITALY & & & LUCCA & 7TH AVE. & ADAMS -TRIMOUNTAIN \\
\hline 745 & & PIERONI & LALA & D & 3 & MICHIGAN & ITALY & ITALY & & & LUCCA & 7TH AVE. & ADAMS -TRIMOUNTAIN \\
\hline 746 & & PIERONI & ALOISA & D & 1 & MICHIGAN & ITALY & ITALY & & & LUCCA & 7TH AVE. & ADAMS -TRIMOUNTAIN \\
\hline 747 & & BIANCHI & PIETRO & B & 40 & ITALY & ITALY & ITALY & 1912 & TRAMMER/CM & LUCCA & 7TH AVE. & ADAMS -TRIMOUNTAIN \\
\hline 748 & 210 & DEL GHINGARO & LUIGI & $\mathrm{H}$ & 41 & ITALY & ITALY & ITALY & 1898 & TRAMMER/CM & CASTELVECCHIO. LU & 7TH AVE. & ADAMS -TRIMOUNTAIN \\
\hline 749 & & DEL GHINGARO & AURELIA & w & 38 & ITALY & ITALY & ITALY & 1903 & & & 7TH AVE. & ADAMS -TRIMOUNTAIN \\
\hline 750 & & DEL GHINGARO & FRED & s & 13 & MICHIGAN & ITALY & ITALY & & & CASTELVECCHIO, LU & 7TH AVE. & ADAMS -TRIMOUNTAIN \\
\hline 751 & & DEL GHINGARO & INES & D & 9 & MICHIGAN & ITALY & ITALY & & & CASTELVECCHIO. LU & 7TH AVE. & ADAMS -TRIMOUNTAIN \\
\hline 752 & & DEL GHINGARO & UGO & s & 7 & MICHIGAN & ITALY & ITALY & & & CASTELVECCHIO. LU & 7TH AVE. & ADAMS -TRIMOUNTAIN \\
\hline 753 & & DEL GHINGARO & NELLA & D & 6 & MICHIGAN & ITALY & ITALY & & & CASTELVECCHIO. LU & 7TH AVE. & ADAMS -TRIMOUNTAIN \\
\hline 754 & & CINQUINI & PIETRO & B & 30 & ITALY & ITALY & ITALY & 1912 & TRAMMER/CM & LUCCA & 7TH AVE. & ADAMS -TRIMOUNTAIN \\
\hline 755 & & PACE & GIUSEPPE & B & 35 & ITALY & ITALY & ITALY & 1914 & TRAMMER/CM & CUSTONACI. TRAPANI & 7TH AVE. & ADAMS -TRIMOUNTAIN \\
\hline
\end{tabular}




\begin{tabular}{|c|c|c|c|c|c|c|c|c|c|c|c|c|c|}
\hline 756 & & BARTOLOMEI & CANDIDO & B & UN & ITALY & ITALY & ITALY & 1910 & TRAMMER/CM & PARIANA. LU & 7TH AVE. & ADAMS -TRIMOUNTAIN \\
\hline 757 & 206 & SANTONI & FRANK & $\mathrm{H}$ & 50 & ITALY & ITALY & ITALY & 1903 & LABORER/CM & MONTE DI CHIESA. LU & 8TH AVE & ADAMS -TRIMOUNTAIN \\
\hline 758 & & SANTONI & ANNUNZIATA & W & 43 & ITALY & ITALY & ITALY & 1906 & & MONTE DI CHIESA. LU & 8TH AVE & ADAMS -TRIMOUNTAIN \\
\hline 759 & & SANTONI & EUGENIO & $\mathrm{s}$ & 16 & ITALY & ITALY & ITALY & 1906 & LABORER/CM & MONTE DI CHIESA. LU & 8TH AVE & ADAMS -TRIMOUNTAIN \\
\hline 760 & & SANTONI & FRANK & $\mathrm{s}$ & 13 & MICHIGAN & ITALY & ITALY & & & MONTE DI CHIESA, LU & 8TH AVE & ADAMS -TRIMOUNTAIN \\
\hline 761 & & SANTONI & GIULIA & D & 11 & MICHIGAN & ITALY & ITALY & & & MONTE DI CHIESA, LU & 8TH AVE & ADAMS -TRIMOUNTAIN \\
\hline 762 & & SANTONI & BIANCHINA & D & 10 & MICHIGAN & ITALY & ITALY & & & MONTE DI CHIESA. LU & 8TH AVE & ADAMS -TRIMOUNTAIN \\
\hline 763 & & SANTONI & PIERINA & $\mathrm{D}$ & 8 & MICHIGAN & ITALY & ITALY & & & MONTE DI CHIESA. LU & 8TH AVE & ADAMS -TRIMOUNTAIN \\
\hline 764 & & SANTONI & IRMA & D & 5 & MICHIGAN & ITALY & ITALY & & & MONTE DI CHIESA. LU & 8TH AVE & ADAMS -TRIMOUNTAIN \\
\hline 765 & & SANTONI & ATCHIO? & $\mathrm{s}$ & 3 & MICHIGAN & ITALY & ITALY & & & MONTE DI CHIESA. LU & 8TH AVE & ADAMS -TRIMOUNTAIN \\
\hline 766 & 202 & MANAI & SALVATORE & $\mathrm{H}$ & 38 & ITALY & ITALY & ITALY & 1907 & MASON/CM & LUCCA & 8TH AVE & ADAMS -TRIMOUNTAIN \\
\hline 767 & & MANAI-MURRO & GIOVANNA & w & 27 & ITALY & ITALY & ITALY & 1912 & & CAGLIARI & 8TH AVE & ADAMS -TRIMOUNTAIN \\
\hline 768 & & MANAI & NORMA & D & 6 & MICHIGAN & ITALY & ITALY & & & LUCCA & 8TH AVE & ADAMS -TRIMOUNTAIN \\
\hline 769 & & MURRO & ELISA & SIIL & 22 & ITALY & ITALY & ITALY & 1920 & SERVANT/BOARDING HOUSE & CAGLIARI & 8TH AVE & ADAMS -TRIMOUNTAIN \\
\hline 770 & & NUTINI & FIORE & B & 25 & ITALY & ITALY & ITALY & 1910 & TRAMMER/CM & LUCCA & 8TH AVE & ADAMS -TRIMOUNTAIN \\
\hline 771 & & CORTOPASSI & DANTE & B & 32 & ITALY & ITALY & ITALY & 1910 & TRAMMER/CM & LUCCA & 8TH AVE & ADAMS -TRIMOUNTAIN \\
\hline 772 & & CORTOPASSI & OLIVIERO & B & 29 & ITALY & ITALY & ITALY & 1910 & TRAMMER/CM & LUCCA & 8TH AVE & ADAMS -TRIMOUNTAIN \\
\hline 773 & & RUFFA & ITALO & B & 33 & ITALY & ITALY & ITALY & 1908 & MASON/CM & & 8TH AVE & ADAMS -TRIMOUNTAIN \\
\hline 774 & 408 & PILLONI & GIUSEPPE & $\mathrm{H}$ & 41 & ITALY & ITALY & ITALY & 1913 & MASON/CM & MILIS. CAGLIARI & 8TH AVE & ADAMS -TRIMOUNTAIN \\
\hline 775 & & PILLONI-MANAI & PAOLINA & w & 41 & ITALY & ITALY & ITALY & 1919 & & MILIS. CAGLIARI & 8TH AVE & ADAMS -TRIMOUNTAIN \\
\hline 776 & & PILLONI & CAMILLA & D & 14 & ITALY & ITALY & ITALY & 1919 & & MILIS. CAGLIARI & 8TH AVE & ADAMS -TRIMOUNTAIN \\
\hline 777 & & PILLONI & RACHELE & $\mathrm{D}$ & 10 & ITALY & ITALY & ITALY & 1919 & & MILIS, CAGLIARI & 8TH AVE & ADAMS -TRIMOUNTAIN \\
\hline 778 & & PILLONI & EPIFANIO & s & 7 & ITALY & ITALY & ITALY & 1919 & & MILIS. CAGLIARI & 8TH AVE & ADAMS -TRIMOUNTAIN \\
\hline 779 & 308 & GIUSTI & GINO & $\mathrm{H}$ & 22 & ITALY & ITALY & ITALY & 1913 & DUMPER/CM & LUCCA & 8TH AVE & ADAMS -TRIMOUNTAIN \\
\hline 780 & & GIUSTI & MARY & w & 20 & MICHIGAN & ITALY & ITALY & & & & 8TH AVE & ADAMS -TRIMOUNTAIN \\
\hline 781 & & GIUSTI & ARMANDA & D & 1 & MICHIGAN & ITALY & ITALY & & & LUCCA & 8TH AVE & ADAMS -TRIMOUNTAIN \\
\hline 782 & 305 & $\mathrm{RICCl}$ & JOSEPH & $\mathrm{H}$ & 38 & ITALY & ITALY & ITALY & 1899 & MINER/CM & LUCCA & 9TH AVE. & ADAMS -TRIMOUNTAIN \\
\hline 783 & & $\mathrm{RICCl}$ & ASSUNTA & W & 24 & ITALY & ITALY & ITALY & 1909 & & & 9TH AVE. & ADAMS -TRIMOUNTAIN \\
\hline 784 & & RICCI & JOSEPHINE & D & 10 & MICHIGAN & ITALY & ITALY & & & LUCCA & 9TH AVE. & ADAMS -TRIMOUNTAIN \\
\hline 785 & & RICCI & PETER & s & 9 & MICHIGAN & ITALY & ITALY & & & LUCCA & 9TH AVE. & ADAMS -TRIMOUNTAIN \\
\hline 786 & & $\mathrm{RICCl}$ & FRANK & $\mathrm{s}$ & 5 & MICHIGAN & ITALY & ITALY & & & LUCCA & 9TH AVE. & ADAMS -TRIMOUNTAIN \\
\hline 787 & & $\mathrm{RICCl}$ & LORDINE & D & 1 & MICHIGAN & ITALY & ITALY & & & LUCCA & 9TH AVE. & ADAMS -TRIMOUNTAIN \\
\hline 788 & 401 & MILANI & JOHN & $\mathrm{H}$ & 29 & ITALY & ITALY & ITALY & 1913 & MASON/CM & LUCCA & 9TH AVE. & ADAMS -TRIMOUNTAIN \\
\hline 789 & & MILANI & DUSOLINA & w & 27 & ITALY & ITALY & ITALY & 1913 & & LUCCA & 9TH AVE. & ADAMS -TRIMOUNTAIN \\
\hline 790 & & MILANI & ANNA & D & 5 & MICHIGAN & ITALY & ITALY & & & LUCCA & 9TH AVE. & ADAMS -TRIMOUNTAIN \\
\hline 791 & & MILANI & MARIA & $\mathrm{D}$ & 3 & MICHIGAN & ITALY & ITALY & & & LUCCA & $\begin{array}{l}\text { 9TH AVE. } \\
\text { 9TH }\end{array}$ & ADAMS -TRIMOUNTAIN \\
\hline 792 & & MILANI & JOSEPH & $\mathrm{s}$ & 1 & MICHIGAN & ITALY & ITALY & & & LUCCA & 9TH AVE. & ADAMS -TRIMOUNTAIN \\
\hline 793 & 402 & ADAMI & JOSEPH & $\mathrm{H}$ & 33 & ITALY & ITALY & ITALY & 1905 & MINER/CM & VILLA BASILICA. LU & 9TH AVE. & ADAMS -TRIMOUNTAIN \\
\hline 794 & & ADAMI & CESIRA & w & 34 & ITALY & ITALY & ITALY & 1908 & & & 9TH AVE. & ADAMS -TRIMOUNTAIN \\
\hline 795 & & ADAMI & RENATO & $\mathrm{s}$ & 10 & MICHIGAN & ITALY & ITALY & & & VILLA BASILICA. LU & 9TH AVE. & ADAMS -TRIMOUNTAIN \\
\hline 796 & & ADAMI & DESIDERIO & $\mathrm{s}$ & 7 & MICHIGAN & ITALY & ITALY & & & VILLA BASILICA. LU & 9TH AVE. & ADAMS -TRIMOUNTAIN \\
\hline 797 & & ADAMI & SESTILIO & $\mathrm{s}$ & $6 \mathrm{M}$ & MICHIGAN & ITALY & ITALY & & & VILLA BASILICA. LU & 9TH AVE. & ADAMS -TRIMOUNTAIN \\
\hline 798 & & CARCHIA & VITTORIO & B & 30 & ITALY & ITALY & ITALY & 1911 & MINER/CM & & 9TH AVE. & ADAMS -TRIMOUNTAIN \\
\hline 799 & & ADAMI & ONORATO & B & 32 & ITALY & ITALY & ITALY & 1910 & MINER/CM & VILLA BASILICA. LU & 9TH AVE. & ADAMS -TRIMOUNTAIN \\
\hline 800 & & MARTIS & ANTONIO & B & 50 & ITALY & ITALY & ITALY & 1912 & TRAMMER/CM & & 9TH AVE. & ADAMS -TRIMOUNTAIN \\
\hline 801 & 406 & GIULI & HENRY & $\mathrm{H}$ & 41 & ITALY & ITALY & ITALY & 1901 & FIREMAN/BOILER HOUSE & SAN MARTINO IN FREDDANA. & 9TH AVE. & ADAMS -TRIMOUNTAIN \\
\hline 802 & & GIULI & ELISABETH & W & 37 & ITALY & ITALY & ITALY & 1905 & & SAIV IVIARTIIV IIN TREDLAIVA. & $\begin{array}{l}\text { 9TH AVE. } \\
\text { 9TH }\end{array}$ & ADAMS -TRIMOUNTAIN \\
\hline 803 & & GIULI & ARGANTE & $\begin{array}{l}\mathrm{s} \\
\mathrm{u}\end{array}$ & 13 & MICHIGAN & ITALY & ITALY & 1900 & & SAN MARTINO IN FREDDANA. & $\begin{array}{l}\text { 9TH AVE. } \\
\text { 9TH }\end{array}$ & ADAMS -TRIMOUNTAIN \\
\hline 804 & & GIULI & RUGGERO & $\mathrm{s}$ & 11 & MICHIGAN & ITALY & ITALY & & & SAN MARTINO IN FREDDANA. & 9TH AVE. & ADAMS -TRIMOUNTAIN \\
\hline 805 & & GIULI & FERNANDA & $\mathrm{D}$ & 10 & MICHIGAN & ITALY & ITALY & & & SAN MARTINO IN FREDDANA. & 9TH AVE. & ADAMS -TRIMOUNTAIN \\
\hline 806 & & GIULI & RENATO & $\mathrm{s}$ & 7 & MICHIGAN & ITALY & ITALY & & & SAN MARTINO IN FREDDANA. & 9TH AVE. & ADAMS -TRIMOUNTAIN \\
\hline 807 & & GIULI & ALADINO & $\mathrm{s}$ & 5 & MICHIGAN & ITALY & ITALY & & & SAN MARTINO IN FREDDANA, & 9TH AVE. & ADAMS -TRIMOUNTAIN \\
\hline 808 & & GIULI & DOMENICO & $\mathrm{s}$ & 2 & MICHIGAN & ITALY & ITALY & & & SAN MARTINO IN FREDDANA, & 9TH AVE. & ADAMS -TRIMOUNTAIN \\
\hline 809 & & GIULI & LUIGI & $\mathrm{s}$ & $\mathrm{O}$ & MICHIGAN & ITALY & ITALY & & & SAN MARTINO IN FREDDANA. & 9TH AVE. & ADAMS -TRIMOUNTAIN \\
\hline 810 & 1009 & SEPPATERRENO & JOSEPH & $\mathrm{H}$ & 22 & ITALY & ITALY & ITALY & 1913 & MINER/CM & ROMA & 4TH AVE. & ADAMS -TRIMOUNTAIN \\
\hline 811 & & SEPPATERRENO & AGNES & W & 24 & MICHIGAN & SWITZERLA & SWITZERLA & & & SWITZERLAND & 4TH AVE. & ADAMS -TRIMOUNTAIN \\
\hline 812 & 404 & BACCETTI & JOSEPH & $\mathrm{H}$ & 39 & ITALY & ITALY & ITALY & 1900 & MASON/CM & MASSA MACINAIA. LU & 7TH AVE. & ADAMS -TRIMOUNTAIN \\
\hline 813 & & BACCETTI & GENO & s & 15 & MICHIGAN & ITALY & ITALY & & & MASSA MACINAIA, LU & 7TH AVE. & ADAMS -TRIMOUNTAIN \\
\hline 814 & & BACCETTI & BIMBO & $\mathrm{s}$ & 12 & MICHIGAN & ITALY & ITALY & & & MASSA MACINAIA. LU & 7TH AVE. & ADAMS -TRIMOUNTAIN \\
\hline 815 & & BACCETTI & SUSAN & $\mathrm{D}$ & 10 & MICHIGAN & ITALY & ITALY & & & MASSA MACINAIA. LU & 7TH AVE. & ADAMS -TRIMOUNTAIN \\
\hline 816 & & BACCETTI & ALBERTO & $\mathrm{s}$ & 8 & MICHIGAN & ITALY & ITALY & & & MASSA MACINAIA. LU & 7TH AVE. & ADAMS -TRIMOUNTAIN \\
\hline 817 & 301 & PIERONI & PAUL & $\mathrm{H}$ & 35 & ITALY & ITALY & ITALY & 1903 & FIREMAN/BOILER HOUSE & CAPANNORI. LU & 11TH AVE. & ADAMS -TRIMOUNTAIN \\
\hline 818 & & PIERONI & NICOLE & w & 29 & ITALY & ITALY & ITALY & 1908 & & & 11TH AVE. & ADAMS -TRIMOUNTAIN \\
\hline
\end{tabular}




\begin{tabular}{|c|c|c|c|c|c|c|c|c|c|c|c|c|c|}
\hline 819 & & PIERONI & JOSEPHINE & D & 11 & MICHIGAN & ITALY & ITALY & & & CAPANNORI. LU & 11TH AVE. & ADAMS -TRIMOUNTAIN \\
\hline 820 & & PIERONI & VERDINO & s & 10 & MICHIGAN & ITALY & ITALY & & & CAPANNORI. LU & 11TH AVE. & ADAMS -TRIMOUNTAIN \\
\hline 821 & & PIERONI & ANNA & D & 5 & MICHIGAN & ITALY & ITALY & & & CAPANNORI. LU & 11TH AVE. & ADAMS -TRIMOUNTAIN \\
\hline 822 & 307 & BIANCHI & FRANCESCO & $\mathrm{H}$ & 35 & ITALY & ITALY & ITALY & 1905 & MINER/CM & COLLE DI COMPITO. LU & 11TH AVE. & ADAMS -TRIMOUNTAIN \\
\hline 823 & & BIANCHI & ONORATA & w & 35 & ITALY & ITALY & ITALY & 1906 & & COLLE DI COMPITO. LU & 11TH AVE. & ADAMS -TRIMOUNTAIN \\
\hline 824 & & BIANCHI & CHELDEA & D & 16 & ITALY & ITALY & ITALY & 1906 & SERVANT/PF & COLLE DI COMPITO. LU & 11TH AVE. & ADAMS -TRIMOUNTAIN \\
\hline 825 & & BIANCHI & JOSEPH & $\mathrm{s}$ & 15 & ITALY & ITALY & ITALY & 1906 & & COLLE DI COMPITO. LU & 11TH AVE. & ADAMS -TRIMOUNTAIN \\
\hline 826 & & BIANCHI & GIACOMINA & D & 13 & MICHIGAN & ITALY & ITALY & & & COLLE DI COMPITO. LU & 11TH AVE. & ADAMS -TRIMOUNTAIN \\
\hline 827 & & BIANCHI & ANNA & $\mathrm{D}$ & 11 & MICHIGAN & ITALY & ITALY & & & COLLE DI COMPITO. LU & 11TH AVE. & ADAMS -TRIMOUNTAIN \\
\hline 828 & & BIANCHI & BRUNA & D & 10 & MICHIGAN & ITALY & ITALY & & & COLLE DI COMPITO. LU & 11TH AVE. & ADAMS -TRIMOUNTAIN \\
\hline 829 & & BIANCHI & ELISA & $\mathrm{D}$ & 8 & MICHIGAN & ITALY & ITALY & & & COLLE DI COMPITO. LU & 11TH AVE. & ADAMS -TRIMOUNTAIN \\
\hline 830 & & BIANCHI & JOSEPHINE & D & 5 & MICHIGAN & ITALY & ITALY & & & COLLE DI COMPITO. LU & 11TH AVE. & ADAMS -TRIMOUNTAIN \\
\hline 831 & & BIANCHI & LIDA & D & 2 & MICHIGAN & ITALY & ITALY & & & COLLE DI COMPITO. LU & 11TH AVE. & ADAMS -TRIMOUNTAIN \\
\hline 832 & & BIANCHI & NORMA & D & 1 & MICHIGAN & ITALY & ITALY & & & COLLE DI COMPITO. LU & 11TH AVE. & ADAMS -TRIMOUNTAIN \\
\hline 833 & 405 & SIMI & OLINTO & $\mathrm{H}$ & 42 & ITALY & ITALY & ITALY & 1900 & MASON/CM & VILLA BASILICA. LU & 11TH AVE. & ADAMS -TRIMOUNTAIN \\
\hline 834 & & SIMI & ISABELLA & W & 31 & ITALY & ITALY & ITALY & 1911 & & VILLA BASILICA, LU & 11TH AVE. & ADAMS -TRIMOUNTAIN \\
\hline 835 & & SIMI & LOUIS & $\mathrm{s}$ & 12 & ITALY & ITALY & ITALY & 1911 & & VILLA BASILICA. LU & 11TH AVE. & ADAMS -TRIMOUNTAIN \\
\hline 836 & & SIMI & CALISTO & $\mathrm{s}$ & 11 & ITALY & ITALY & ITALY & 1911 & & VILLA BASILICA. LU & 11TH AVE. & ADAMS -TRIMOUNTAIN \\
\hline 837 & & SIMI & TOSCA & D & 8 & MICHIGAN & ITALY & ITALY & & & VILLA BASILICA. LU & 11TH AVE. & ADAMS -TRIMOUNTAIN \\
\hline 838 & & SIMI & ANTONIETTA & D & 6 & MICHIGAN & ITALY & ITALY & & & VILLA BASILICA. LU & 11TH AVE. & ADAMS -TRIMOUNTAIN \\
\hline 839 & & SIMI & ALINO & $\mathrm{s}$ & 3 & MICHIGAN & ITALY & ITALY & & & VILLA BASILICA. LU & 11TH AVE. & ADAMS -TRIMOUNTAIN \\
\hline 840 & 407 & TAPPERO & JOSEPH & $\mathrm{H}$ & 64 & ITALY & ITALY & ITALY & 1890 & LABORER/CM & SAN GIUSTO CANAVESE, TO & 11TH AVE. & ADAMS -TRIMOUNTAIN \\
\hline 841 & 40 & TAPPERO & MINNIE & W & 54 & ITALY & ITALY & ITALY & 1892 & LADU RER/UIVI & SAN GIUSTO CANAVESE. TO & 11TH AVE. & ADAMS -TRIMOUNTAIN \\
\hline 842 & & TAPPERO & DOMINICK & $\mathrm{s}$ & 29 & ITALY & ITALY & ITALY & 1890 & TRAMMER/CM & SAN GIUSTO CANAVESE. TO & 11TH AVE. & ADAMS -TRIMOUNTAIN \\
\hline 843 & & TAPPERO & JOSEPH & $\mathrm{s}$ & 26 & MICHIGAN & ITALY & ITALY & & LABORER/CM & SAN GIUSTO CANAVESE. TO & 11TH AVE. & ADAMS -TRIMOUNTAIN \\
\hline 844 & & TAPPERO & CARLO & $\mathrm{s}$ & 23 & MICHIGAN & ITALY & ITALY & & TRAMMER/CM & SAN GIUSTO CANAVESE. TO & 11TH AVE. & ADAMS -TRIMOUNTAIN \\
\hline 845 & & TAPPERO & WILLIAM & $\mathrm{s}$ & 18 & MICHIGAN & ITALY & ITALY & & TRAMMER/CM & SAN GIUSTO CANAVESE. TO & 11TH AVE. & ADAMS -TRIMOUNTAIN \\
\hline 846 & & TAPPERO & PETER & $\mathrm{s}$ & 15 & MICHIGAN & ITALY & ITALY & & & SAN GIUSTO CANAVESE, TO & 11TH AVE. & ADAMS -TRIMOUNTAIN \\
\hline 847 & 302 & BERTACCHI & GAETANO & $\mathrm{H}$ & 38 & ITALY & ITALY & ITALY & 1907 & DUMPER/CM & VALPROMARO. LU & 11TH AVE. & ADAMS -TRIMOUNTAIN \\
\hline 848 & & BERTACCHI & ZORAIDE & W & 36 & ITALY & ITALY & ITALY & 1908 & & VALPROMARO. LU & 11TH AVE. & ADAMS -TRIMOUNTAIN \\
\hline 849 & & BERTACCHI & ZAIRA & D & 13 & ITALY & ITALY & ITALY & 1908 & & VALPROMARO. LU & 11TH AVE. & ADAMS -TRIMOUNTAIN \\
\hline 850 & & BERTACCHI & EVELINA & D & 7 & ITALY & ITALY & ITALY & 1912 & & VALPROMARO. LU & 11TH AVE. & ADAMS -TRIMOUNTAIN \\
\hline 851 & & BERTACCHI & EDO & $\mathrm{s}$ & 5 & MICHIGAN & ITALY & ITALY & & & VALPROMARO. LU & 11TH AVE. & ADAMS -TRIMOUNTAIN \\
\hline 852 & 313 & MALLEGNI & JOSEPH & $\mathrm{H}$ & 37 & ITALY & ITALY & ITALY & 1913 & TRAMMER/CM & PIEVE CAMAIORE, LU & 12TH AVE & ADAMS -TRIMOUNTAIN \\
\hline 853 & & MALLEGNI & ELENA & w & 41 & ITALY & ITALY & ITALY & 1915 & & PIEVE CAMAIORE. LU & 12TH AVE & ADAMS -TRIMOUNTAIN \\
\hline 854 & & MALLEGNI & ANGELO & s & $\frac{41}{10}$ & ITALY & ITALY & ITALY & 1915 & & PIEVE CAMAIORE. LU & 12TH AVE & ADAMS -TRIMOUNTAIN \\
\hline 855 & & MALLEGNI & EMIL & s & 7 & ITALY & ITALY & ITALY & 1915 & & PIEVE CAMAIORE. LU & 12TH AVE & ADAMS -TRIMOUNTAIN \\
\hline 856 & & MALLEGNI & AMALIA & D & 3 & MICHIGAN & ITALY & ITALY & & & PIEVE CAMAIORE. LU & 12TH AVE & ADAMS -TRIMOUNTAIN \\
\hline 857 & & MALLEGNI & ALADINO & $\mathrm{s}$ & $6 \mathrm{M}$ & MICHIGAN & ITALY & ITALY & & & PIEVE CAMAIORE. LU & 12TH AVE & ADAMS -TRIMOUNTAIN \\
\hline 858 & & SIMI & TOMMASO & B & 35 & ITALY & $\begin{array}{l}\text { ITALY } \\
\text { ITA }\end{array}$ & ITALY & 1913 & DUMPER/CM & VILLA BASILICA. LU & 12TH AVE & ADAMS -TRIMOUNTAIN \\
\hline 859 & 315 & BARSUGLIO & GIUSEPPE & $\mathrm{H}$ & 43 & ITALY & ITALY & ITALY & 1906 & TRAMMER/CM & LUCCA & 12TH AVE & ADAMS -TRIMOUNTAIN \\
\hline 860 & & BARSUGLIO & ANNUNZIATA & W & 38 & ITALY & ITALY & ITALY & 1907 & & LUCCA & 12TH AVE & ADAMS -TRIMOUNTAIN \\
\hline 861 & & BARSUGLIO & MARIO & $\mathrm{s}$ & 14 & ITALY & ITALY & ITALY & 1907 & & LUCCA & 12TH AVE & ADAMS -TRIMOUNTAIN \\
\hline 862 & & BARSUGLIO & BRUNO & $\mathrm{s}$ & 11 & MICHIGAN & ITALY & ITALY & & & LUCCA & 12TH AVE & ADAMS -TRIMOUNTAIN \\
\hline 863 & & BARSUGLIO & DINA & D & 10 & MICHIGAN & ITALY & ITALY & & & LUCCA & 12TH AVE & ADAMS -TRIMOUNTAIN \\
\hline 864 & & BARSUGLIO & TOSCA & D & 8 & MICHIGAN & ITALY & ITALY & & & LUCCA & 12TH AVE & ADAMS -TRIMOUNTAIN \\
\hline 865 & & BARSUGLIO & FREDI & $\mathrm{s}$ & 5 & MICHIGAN & ITALY & ITALY & & & LUCCA & 12TH AVE & ADAMS -TRIMOUNTAIN \\
\hline 866 & 413 & LENCIONI & JOSEPH & $\mathrm{H}$ & 52 & ITALY & ITALY & ITALY & 1898 & LABORER/CM & LUCCA & 12TH AVE & ADAMS -TRIMOUNTAIN \\
\hline 867 & 415 & LENCIONI & ESTER & W & 39 & ITALY & $\begin{array}{l}\text { ITALY } \\
\text { ITAL }\end{array}$ & ITALY & 1898 & LABURER/CIMI & LUCCA & 12TH AVE & ADAMS -TRIMOUNTAIN \\
\hline 868 & & LENCIONI & LAVIA & D & 19 & MICHIGAN & ITALY & ITALY & & SALESLADY/DEPT. STORE & LUCCA & 12TH AVE & ADAMS -TRIMOUNTAIN \\
\hline 869 & & LENCIONI & EDITH & $\mathrm{D}$ & 17 & MICHIGAN & ITALY & ITALY & & & LUCCA & 12TH AVE & ADAMS -TRIMOUNTAIN \\
\hline 870 & & LENCIONI & DOMENICO & $\mathrm{s}$ & 16 & MICHIGAN & ITALY & ITALY & & LABORER/CM & LUCCA & 12TH AVE & ADAMS -TRIMOUNTAIN \\
\hline 871 & & LENCIONI & ALADINO & $\mathrm{s}$ & 13 & MICHIGAN & ITALY & ITALY & & & LUCCA & 12TH AVE & ADAMS -TRIMOUNTAIN \\
\hline 872 & & LENCIONI & LENA & D & 10 & MICHIGAN & ITALY & ITALY & & & LUCCA & 12TH AVE & ADAMS -TRIMOUNTAIN \\
\hline 873 & & LENCIONI & ANTONIO & $\mathrm{s}$ & 7 & MICHIGAN & ITALY & ITALY & & & LUCCA & 12TH AVE & ADAMS -TRIMOUNTAIN \\
\hline 874 & 908 & CORTOPASSI & SALVATORE & $\mathrm{H}$ & 26 & ITALY & ITALY & ITALY & 1910 & MINER/CM & PESCAGLIA, LU & 6TH AVE & ADAMS -TRIMOUNTAIN \\
\hline 875 & & CORTOPASSI & CESIRA & w & 26 & ITALY & ITALY & ITALY & 1912 & & LUCCA & 6TH AVE & ADAMS -TRIMOUNTAIN \\
\hline 876 & & IACOPETTI & LENA & $\mathrm{SD}$ & 6 & MICHIGAN & ITALY & ITALY & & & LUCCA & 6TH AVE & ADAMS -TRIMOUNTAIN \\
\hline 877 & & CORTOPASSI & MARY & $\mathrm{D}$ & 0 & MICHIGAN & ITALY & ITALY & & & PESCAGLIA. LU & 6TH AVE & ADAMS -TRIMOUNTAIN \\
\hline 878 & & FRANCIONI & DOMENICO & B & 67 & ITALY & ITALY & ITALY & 1899 & TRAMMER/CM & RUOTA. LU & 6TH AVE & ADAMS -TRIMOUNTAIN \\
\hline 879 & 414 & GAGGINI & WILLIAM & $\mathrm{H}$ & 40 & ITALY & ITALY & ITALY & 1902 & MINER/CM & PITEGLIO. PISTOIA & $\begin{array}{l}\text { 9TH AVE. } \\
\text { 9TH }\end{array}$ & ADAMS -TRIMOUNTAIN \\
\hline 880 & & GAGGINI & TERESA & W & 30 & ITALY & ITALY & ITALY & 1911 & & & 9TH AVE. & ADAMS -TRIMOUNTAIN \\
\hline 881 & & GAGGINI & ALMA & D & 7 & MICHIGAN & ITALY & ITALY & & & PITEGLIO. PISTOIA & 9TH AVE. & ADAMS -TRIMOUNTAIN \\
\hline
\end{tabular}




\begin{tabular}{|c|c|c|c|c|c|c|c|c|c|c|c|c|c|}
\hline 882 & & GAGGINI & GILDA & $\mathrm{D}$ & 6 & MICHIGAN & ITALY & ITALY & & & PITEGLIO. PISTOIA & 9TH AVE. & ADAMS -TRIMOUNTAIN \\
\hline 883 & & GAGGINI & SELINA & D & 1 & MICHIGAN & ITALY & ITALY & & & PITEGLIO. PISTOIA & 9TH AVE. & ADAMS -TRIMOUNTAIN \\
\hline 884 & 903 & IACOPETTI & CANDELO & $\mathrm{H}$ & 40 & ITALY & ITALY & ITALY & 1901 & TRAMMER/CM & CASTELVECCHIO. LU & DIVISION ST. & ADAMS -TRIMOUNTAIN \\
\hline 885 & & IACOPETTI & CARLOTTA & W & 37 & ITALY & ITALY & ITALY & 1912 & & & DIVISION ST. & ADAMS -TRIMOUNTAIN \\
\hline 886 & & IACOPETTI & CATERINA & $\mathrm{D}$ & 5 & MICHIGAN & ITALY & ITALY & & & CASTELVECCHIO. LU & DIVISION ST. & ADAMS -TRIMOUNTAIN \\
\hline 887 & & IACOPETTI & JOSEPH & $\mathrm{s}$ & 4 & MICHIGAN & ITALY & ITALY & & & CASTELVECCHIO. LU & DIVISION ST. & ADAMS -TRIMOUNTAIN \\
\hline 888 & & PAPUCCI & ORESTE & B & 32 & ITALY & ITALY & ITALY & 1912 & DUMPER/CM & CAPANNORI. LU & DIVISION ST. & ADAMS -TRIMOUNTAIN \\
\hline 889 & 136 & GUGLIELMINO & FRANK & $\mathrm{H}$ & 40 & ITALY & ITALY & ITALY & 1904 & MINER/CM & SAN GIORGIO CANAVESE. TO & KEARSAGE ST. & ADAMS - PAINSDALE \\
\hline 890 & & GUGLIELMINO & KATE & W & 37 & MICHIGAN & ITALY & MICHIGAN & & & SAN GIORGIO CANAVESE. TO & KEARSAGE ST. & ADAMS - PAINSDALE \\
\hline 891 & & GUGLIELMINO & FRANK & s & 15 & ITALY & ITALY & MICHIGAN & 1907 & SALESMAN/GENERAL STORE & SAN GIORGIO CANAVESE. TO & KEARSAGE ST. & ADAMS - PAINSDALE \\
\hline 892 & & GUGLIELMINO & TERESA & $\mathrm{D}$ & 12 & MICHIGAN & ITALY & MICHIGAN & & & SAN GIORGIO CANAVESE. TO & KEARSAGE ST. & ADAMS - PAINSDALE \\
\hline 893 & & GUGLIELMINO & JOSEPH & $\mathrm{s}$ & 7 & MICHIGAN & ITALY & MICHIGAN & & & SAN GIORGIO CANAVESE. TO & KEARSAGE ST. & ADAMS - PAINSDALE \\
\hline 894 & 135 & BARONI & FELIX & $\mathrm{H}$ & 54 & ITALY & ITALY & ITALY & 1894 & DRAYMAN/CM & LUCCA & KEARSAGE ST. & ADAMS - PAINSDALE \\
\hline 895 & & BARONI & TERESA & W & 48 & ITALY & ITALY & ITALY & 1903 & & & KEARSAGE ST. & ADAMS - PAINSDALE \\
\hline 896 & & GILLETTI & FRANK & B & 51 & ITALY & ITALY & ITALY & 1884 & & & KEARSAGE ST. & ADAMS - PAINSDALE \\
\hline 897 & & IAVELLI & JOSEPH & B & 42 & ITALY & ITALY & ITALY & 1907 & COAL PASSER/CM & TORINO & KEARSAGE ST. & ADAMS - PAINSDALE \\
\hline 898 & & IAVELLI & VITTORIO & B & 40 & ITALY & ITALY & ITALY & 1899 & COAL PASSER/CM & & KEARSAGE ST. & ADAMS - PAINSDALE \\
\hline 899 & 137 & BALDASSARRI & GIUSEPPE & $\mathrm{H}$ & 45 & ITALY & ITALY & ITALY & 1901 & PICKER/CM & SANTA MARIA DEL GIUDICE. LU & IROQUOIS ST. & ADAMS - PAINSDALE \\
\hline 900 & & BALDASSARRI & CREUSA & w & 50 & ITALY & ITALY & ITALY & 1909 & & SANTA MARIA DEL GIUDICE. LU & IROQUOIS ST. & ADAMS - PAINSDALE \\
\hline 901 & & BALDASSARRI & CARLO & s & 12 & ITALY & ITALY & ITALY & 1909 & & SANTA MARIA DEL GIUDICE. LU & IROQUOIS ST. & ADAMS - PAINSDALE \\
\hline 902 & & BALDASSARRI & ANTONIO & s & 10 & MICHIGAN & ITALY & ITALY & & & SANTA MARIA DEL GIUDICE. LU & IROQUOIS ST. & ADAMS - PAINSDALE \\
\hline 903 & & DAPEZZI & PIETRO & B & 26 & ITALY & ITALY & ITALY & 1914 & DUMPER/CM & & IROQUOIS ST. & ADAMS - PAINSDALE \\
\hline 904 & 116 & RENALDI & TONY & $\mathrm{H}$ & 38 & ITALY & ITALY & ITALY & 1898 & MINER/CM & SAN GIORGIO CANAVESE. TO & FOREST ST. & ADAMS - PAINSDALE \\
\hline 905 & & RENALDI & MARY & W & 36 & MICHIGAN & ITALY & ITALY & & & & FOREST ST. & ADAMS - PAINSDALE \\
\hline 906 & & RENALDI & JOSEPH & $\mathrm{s}$ & 14 & MICHIGAN & ITALY & ITALY & & & SAN GIORGIO CANAVESE. TO & FOREST ST. & ADAMS - PAINSDALE \\
\hline 907 & & RENALDI & MARY & D & 9 & MICHIGAN & ITALY & ITALY & & & SAN GIORGIO CANAVESE. TO & FOREST ST. & ADAMS - PAINSDALE \\
\hline 908 & & RENALDI & RENA & $\mathrm{D}$ & 2 & MICHIGAN & ITALY & ITALY & & & SAN GIORGIO CANAVESE. TO & FOREST ST. & ADAMS - PAINSDALE \\
\hline 909 & & BESAT & MARTIN & B & 48 & ITALY & ITALY & ITALY & 1906 & MINER/CM & & FOREST ST. & ADAMS - PAINSDALE \\
\hline 910 & & BESAT & MATTEO & B & 44 & ITALY & ITALY & ITALY & 1916 & MINER/CM & & FOREST ST. & ADAMS - PAINSDALE \\
\hline 911 & 144 & BORGO & ANTONIO & $\mathrm{H}$ & 42 & ITALY & ITALY & ITALY & 1897 & MINER/CM & ARSIERI. VICENZA & FOREST ST. & ADAMS - PAINSDALE \\
\hline 912 & & BORGO & JENNIE & w & 36 & ITALY & ITALY & ITALY & 1903 & & & FOREST ST. & ADAMS - PAINSDALE \\
\hline 913 & & BORGO & MARIE & D & 13 & MICHIGAN & ITALY & ITALY & & & ARSIERI. VICENZA & FOREST ST. & ADAMS - PAINSDALE \\
\hline 914 & & BORGO & MARA & $\mathrm{D}$ & 12 & MICHIGAN & ITALY & ITALY & & & ARSIERI. VICENZA & FOREST ST. & ADAMS - PAINSDALE \\
\hline 915 & 144 & CERETTO & BARTOLOMEO & $\mathrm{H}$ & 28 & ITALY & ITALY & ITALY & 1912 & FIREMAN/CM & TORINO & FOREST ST. & ADAMS - PAINSDALE \\
\hline 916 & & CERETTO & ROSA & w & 29 & ITALY & ITALY & ITALY & 1919 & & & FOREST ST. & ADAMS - PAINSDALE \\
\hline 917 & 142 & BALAGNA & JAMES & $\mathrm{H}$ & 44 & ITALY & ITALY & ITALY & 1897 & PICKER/CM & PONT CANAVESE. TO & FOREST ST. & ADAMS - PAINSDALE \\
\hline 918 & & BALAGNA & JENNIE & W & 37 & ITALY & ITALY & ITALY & 1901 & & & FOREST ST. & ADAMS - PAINSDALE \\
\hline 919 & & BARTOLARO & LOUIS & sso & 17 & MICHIGAN & ITALY & ITALY & & MACHINIST/CM & & FOREST ST. & ADAMS - PAINSDALE \\
\hline 920 & & BARTOLARO & PETER & SSO & 15 & MICHIGAN & ITALY & ITALY & & LABORER/HOTEL & & FOREST ST. & ADAMS - PAINSDALE \\
\hline 921 & & BALAGNA & FELIX & $\mathrm{s}$ & 7 & MICHIGAN & ITALY & ITALY & & & PONT CANAVESE. TO & FOREST ST. & ADAMS - PAINSDALE \\
\hline 922 & & BALAGNA & SELINA & D & 5 & MICHIGAN & ITALY & ITALY & & & PONT CANAVESE. TO & FOREST ST. & ADAMS - PAINSDALE \\
\hline 923 & & BALAGNA & MARY & D & 4 & MICHIGAN & ITALY & ITALY & & & PONT CANAVESE. TO & FOREST ST. & ADAMS - PAINSDALE \\
\hline 924 & 143 & IAVELLO & CARLO & $\mathrm{H}$ & 39 & ITALY & ITALY & ITALY & 1897 & MINER/CM & SAN GIUSTO CANAVESE. TO & FOREST ST. & ADAMS - PAINSDALE \\
\hline 925 & & IAVELLO & MARIA & w & 43 & ITALY & ITALY & ITALY & 1907 & & & FOREST ST. & ADAMS - PAINSDALE \\
\hline 926 & & IAVELLO & MARTINA & D & 16 & MICHIGAN & ITALY & ITALY & & & SAN GIUSTO CANAVESE. TO & FOREST ST. & ADAMS - PAINSDALE \\
\hline 927 & & IAVELLO & JOHN & $\mathrm{s}$ & 10 & MICHIGAN & ITALY & ITALY & & & SAN GIUSTO CANAVESE. TO & FOREST ST. & ADAMS - PAINSDALE \\
\hline 928 & & IAVELLO & TERESA & D & 6 & MICHIGAN & ITALY & ITALY & & & SAN GIUSTO CANAVESE. TO & FOREST ST. & ADAMS - PAINSDALE \\
\hline 929 & & IAVELLO & MARY & D & 3 & MICHIGAN & ITALY & ITALY & & & SAN GIUSTO CANAVESE. TO & FOREST ST. & ADAMS - PAINSDALE \\
\hline 930 & 172 & POLUSI & NICOLAS & $\mathrm{H}$ & 27 & ITALY & ITALY & ITALY & 1903 & MECHANIC/CM & & FOREST ST. & ADAMS - PAINSDALE \\
\hline 931 & & POLUSI & COSTANCE? & W & 27 & MICHIGAN & ITALY & MICHIGAN & & & & FOREST ST. & ADAMS - PAINSDALE \\
\hline 932 & & POLUSI & JAMES & s & 1 & MICHIGAN & ITALY & ITALY & & & & FOREST ST. & ADAMS - PAINSDALE \\
\hline 933 & & POLUSI & MARY & SI & 12 & MICHIGAN & ITALY & ITALY & & & & FOREST ST. & ADAMS - PAINSDALE \\
\hline 934 & 167 & FERONDI & GIULIO & $\mathrm{H}$ & 30 & ITALY & ITALY & ITALY & 1907 & MINER/CM & & CONGRESS ST. & ADAMS - PAINSDALE \\
\hline 935 & & FERONDI & TINA & W & 20 & ITALY & ITALY & ITALY & UN & & & CONGRESS ST. & ADAMS - PAINSDALE \\
\hline 936 & & FERONDI & ELISA & D & 1 & MICHIGAN & ITALY & ITALY & & & & CONGRESS ST. & ADAMS - PAINSDALE \\
\hline 937 & 165 & SIGNORONI & JOSEPH & $\mathrm{H}$ & 29 & MICHIGAN & ITALY & ITALY & & TRAMMER/CM & TORINO & CONCORD ST. & ADAMS - PAINSDALE \\
\hline 938 & & SIGNORONI & MARIA & w & 25 & ITALY & ITALY & ITALY & 1916 & & & CONCORD ST. & ADAMS - PAINSDALE \\
\hline 939 & & SIGNORONI & RINALDO & s & 1 & MICHIGAN & MICHIGAN & MICHIGAN & & & TORINO & CONCORD ST. & ADAMS - PAINSDALE \\
\hline 940 & 141 & IAVELLO & ANTONIO & $\mathrm{H}$ & 37 & ITALY & ITALY & ITALY & 1915 & TRAMMER/CM & SAN GIORGIO CANAVESE. TO & CONCORD ST. & ADAMS - PAINSDALE \\
\hline 941 & & IAVELLO & GENOVEFFA & W & 34 & ITALY & ITALY & ITALY & 1916 & & & CONCORD ST. & ADAMS - PAINSDALE \\
\hline 942 & & IAVELLO & E & $\mathrm{s}$ & 9 & ITALY & ITA & ITA & 1916 & & SAN GIORGIO CANAVESE. TO & CONCORD ST. & ADAMS - PAINSDALE \\
\hline 943 & 124 & MASINI & LOUIS & $\mathrm{H}$ & 28 & ITALY & ITALY & ITALY & 1910 & MINER/CM & LUCCA & ONTONAGON ST. & ADAMS - PAINSDALE \\
\hline 944 & & MASINI & LENA & w & UN & CROATIA & ITALY & ITALY & 1916 & & CROATIA & ONTONAGON ST. & ADAMS - PAINSDALE \\
\hline
\end{tabular}




\begin{tabular}{|c|c|c|c|c|c|c|c|c|c|c|c|c|c|}
\hline 945 & & MASINI & AMERICO & $\mathrm{S}$ & 2 & MICHIGAN & ITALY & CROATIA & & & LUCCA & ONTONAGON ST. & ADAMS - PAINSDALE \\
\hline 946 & 159 & GHENZONI & JOSEPH & $\mathrm{H}$ & 33 & ITALY & ITALY & ITALY & 1907 & MINER/CM & & BALTIC ST. & ADAMS - PAINSDALE \\
\hline 947 & & GHENZONI & TERESA & W & 31 & ITALY & ITALY & ITALY & 1906 & & & BALTIC ST. & ADAMS - PAINSDALE \\
\hline 948 & & GHENZONI & G? & s & 7 & MICHIGAN & ITALY & ITALY & & & & BALTIC ST. & ADAMS - PAINSDALE \\
\hline 949 & 158 & BIANCHI & BASILIO & H & 43 & ITALY & ITALY & ITALY & 1909 & LABORER/ROCK HOUSE & PORCARI. LU & BALTIC ST. & ADAMS - PAINSDALE \\
\hline 950 & & BIANCHI & CARMELA & w & 38 & ITALY & ITALY & ITALY & 1912 & 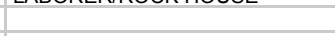 & 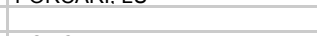 & BALTIC ST. & ADAMS - PAINSDALE \\
\hline 951 & & BIANCHI & SANTE & $\mathrm{s}$ & 6 & MICHIGAN & ITALY & ITALY & & & PORCARI. LU & BALTIC ST. & ADAMS - PAINSDALE \\
\hline 952 & & BIANCHI & SALVATORE & s & 4 & MICHIGAN & ITALY & ITALY & & & PORCARI. LU & BALTIC ST. & ADAMS - PAINSDALE \\
\hline 953 & & BIANCHI & MARY & s & 2 & MICHIGAN & ITALY & ITALY & & & PORCARI. LU & BALTIC ST. & ADAMS - PAINSDALE \\
\hline 954 & & PIZZI & REGOLO & B & 27 & ITALY & ITALY & ITALY & 1905 & MINER/CM & LUCCA & BALTIC ST. & ADAMS - PAINSDALE \\
\hline 955 & & PARLOMINI & GAETANO? & B & 28 & ITALY & ITALY & ITALY & 1912 & MOTORMAN/CM & & BALTIC ST. & ADAMS - PAINSDALE \\
\hline 956 & & FOBA..? & PETER & B & 38 & ITALY & ITALY & ITALY & 1916 & PICKER/CM & & BALTIC ST. & ADAMS - PAINSDALE \\
\hline 957 & 150 & OBERTONE & DOMENICO & $\mathrm{H}$ & 40 & ITALY & ITALY & ITALY & 1902 & MINER/CM & SAN GIORGIO CANAVESE. TO & BALTIC ST. & ADAMS - PAINSDALE \\
\hline 958 & & OBERTONE & MARY & W & 39 & ITALY & ITALY & ITALY & 1902 & & SAN GIORGIO CANAVESE. TO & BALTIC ST. & ADAMS - PAINSDALE \\
\hline 959 & & OBERTONE & MARY & D & 14 & MICHIGAN & ITALY & ITALY & & & SAN GIORGIO CANAVESE. TO & BALTIC ST. & ADAMS - PAINSDALE \\
\hline 960 & & OBERTONE & FRANK & $\mathrm{s}$ & 11 & MICHIGAN & ITALY & ITALY & & & SAN GIORGIO CANAVESE, TO & BALTIC ST. & ADAMS - PAINSDALE \\
\hline 961 & 154 & MANZINI & EMILIO & $\mathrm{H}$ & 31 & ITALY & ITALY & ITALY & 1913 & MOTORMAN/CM & LUCCA & BALTIC ST. & ADAMS - PAINSDALE \\
\hline 962 & & PIZZI & ADOLFO & B & 35 & ITALY & ITALY & ITALY & 1909 & MOTORMAN/CM & PESCAGLIA. LU & BALTIC ST. & ADAMS - PAINSDALE \\
\hline 963 & & MANZINI & JENNIE & W & 31 & ITALY & ITALY & ITALY & 1913 & & LUCCA & BALTIC ST. & ADAMS - PAINSDALE \\
\hline 964 & & MANZINI & NIZO & $\mathrm{s}$ & 6 & ITALY & ITALY & ITALY & 1913 & & LUCCA & BALTIC ST. & ADAMS - PAINSDALE \\
\hline 965 & & MANZINI & DOMENICO & $\mathrm{s}$ & 4 & MICHIGAN & ITALY & ITALY & & & LUCCA & BALTIC ST. & ADAMS - PAINSDALE \\
\hline 966 & & MANZINI & DONNA & D & 2 & MICHIGAN & ITALY & ITALY & & & LUCCA & BALTIC ST. & ADAMS - PAINSDALE \\
\hline 967 & & MANZINI & FRANK & $\mathrm{s}$ & 1 & MICHIGAN & ITALY & ITALY & & & LUCCA & BALTIC ST. & ADAMS - PAINSDALE \\
\hline 968 & & BASTOLGI & ANGELA & SIIL & 23 & ITALY & ITALY & ITALY & 1919 & & & BALTIC ST. & ADAMS - PAINSDALE \\
\hline 969 & 195? & CANARICCI & JOHN & $\mathrm{H}$ & 39 & ITALY & ITALY & ITALY & 1913 & NOT CLEAR & RIMINI & & ADAMS - PAINSDALE \\
\hline 970 & 100 & CANARICCI & MARY & W & 40 & ITALY & ITALY & ITALY & $?$ & & RIMINI & & ADAMS - PAINSDALE \\
\hline 971 & & CANARICCI & $?$ & $?$ & $?$ & ITALY & ITALY & ITALY & $?$ & & RIMINI & & ADAMS - PAINSDALE \\
\hline 972 & & FERAIA? & $?$ & B & 25 & ITALY & ITALY & ITALY & 1912 & NOT CLEAR & & & ADAMS - PAINSDALE \\
\hline 973 & & TINTORI & ANGELA & B & 25 & ITALY & ITALY & ITALY & 1912 & NOT CLEAR & LUCCA & & ADAMS - PAINSDALE \\
\hline 974 & 179 & BALAGNA & MIKE & $\mathrm{H}$ & 34 & ITALY & ITALY & ITALY & 1891 & TRAMMER/CM & PONT CANAVESE. TO & & ADAMS - PAINSDALE \\
\hline 975 & & BALAGNA & MARY & W & 29 & ITALY & ITALY & ITALY & 1911 & & & & ADAMS - PAINSDALE \\
\hline 976 & & BALAGNA & ALVISA & D & 4 & MICHIGAN & ITALY & ITALY & & & PONT CANAVESE. TO & & ADAMS - PAINSDALE \\
\hline 977 & 107 & BERTOLLI & GINO & $\mathrm{H}$ & 32 & ITALY & ITALY & ITALY & 1911 & MINER/CM & ORBICCIANO. LU & ADAMS ST. & ADAMS - PAINSDALE \\
\hline 978 & & BERTOLLI & ROSA & W & 35 & ITALY & ITALY & ITALY & 1914 & & ORBICCIANO, LU & ADAMS ST. & ADAMS - PAINSDALE \\
\hline 979 & & BERTOLLI & RONNA & $\mathrm{s}$ & 11 & ITALY & ITALY & ITALY & 1914 & & ORBICCIANO. LU & ADAMS ST. & ADAMS - PAINSDALE \\
\hline 980 & & BERTOLLI & SALLIE & $\mathrm{D}$ & 9 & ITALY & ITALY & ITALY & 1914 & & ORBICCIANO. LU & ADAMS ST. & ADAMS - PAINSDALE \\
\hline 981 & & BERTOLLI & RALPH & s & 0 & MICHIGAN & ITALY & ITALY & & & ORBICCIANO. LU & ADAMS ST. & ADAMS - PAINSDALE \\
\hline 982 & & BERTOLLI & JAMES & BR & 34 & ITALY & ITALY & ITALY & 1907 & MINER/CM & ORBICCIANO. LU & ADAMS ST. & ADAMS - PAINSDALE \\
\hline 983 & 133 & DEL FRATE & LUIGI & $\mathrm{H}$ & 28 & ITALY & ITALY & ITALY & 1907 & FIREMAN/STATIONARY BOILER & LUCCA & ADAMS ST. & ADAMS - PAINSDALE \\
\hline 984 & & DEL FRATE & LILIAN & W & 23 & MICHIGAN & ITALY & ITALY & & & & ADAMS ST. & ADAMS - PAINSDALE \\
\hline 985 & & DEL FRATE & EUGENE & $\mathrm{s}$ & 3 & MICHIGAN & ITALY & MICHIGAN & & & LUCCA & ADAMS ST. & ADAMS - PAINSDALE \\
\hline 986 & & DEL FRATE & MARIA & D & 1 & MICHIGAN & ITALY & MICHIGAN & & & LUCCA & ADAMS ST. & ADAMS - PAINSDALE \\
\hline 987 & 8 & (?)ARACONI & $?$ & $\mathrm{H}$ & 44 & ITALY & ITALY & ITALY & 1908 & MOTORMAN/CM & & 2ND AVE. & ADAMS - PAINSDALE \\
\hline 988 & & (?)ARACONI & ERINA & W & 35 & ITALY & ITALY & ITALY & 1908 & & & 2ND AVE. & ADAMS - PAINSDALE \\
\hline 989 & & (D)IGNENE? & $?$ & B & 27 & ITALY & ITALY & ITALY & 1909 & & & 2ND AVE. & ADAMS - PAINSDALE \\
\hline 990 & & TEPPONI? & $?$ & B & 24 & ITALY & ITALY & ITALY & 1913 & PICKER/CM & & 2ND AVE. & ADAMS - PAINSDALE \\
\hline 991 & & CARAZZA? & RENALDO? & B & 31 & ITALY & ITALY & ITALY & 1917 & PICKER/CM & & 2ND AVE. & ADAMS - PAINSDALE \\
\hline 992 & & ? & & B & 31 & ITALY & ITALY & ITALY & 1913 & PICKER/CM & & 2ND AVE. & ADAMS - PAINSDALE \\
\hline 993 & & MICHELETTI & SANTE & $\begin{array}{l}\mathrm{B} \\
\mathrm{B}\end{array}$ & 43 & $\begin{array}{l}\text { ITALY } \\
\text { ITAL }\end{array}$ & $\begin{array}{l}\text { ITALY } \\
\text { ITALY }\end{array}$ & ITALY & 1904 & PICKER/CM & LUCCA & $\begin{array}{l}\text { 2ND AVE. } \\
\text { 2ND. }\end{array}$ & ADAMS - PAINSDALE \\
\hline 994 & & MICHELETTI & ? & B & $?$ & ITALY & ITALY & ITALY & $? ?$ & & LUCCA & 2ND AVE. & ADAMS - PAINSDALE \\
\hline 995 & 12 & $\mathrm{RICCl}$ & DINCIN? & $\mathrm{H}$ & $?$ & ITALY & ITALY & ITALY & $? ?$ & MINER/CM & LUCCA & 2ND AVE. & ADAMS - PAINSDALE \\
\hline 996 & & RICCl & ROSE: & W & 42 & ITALY & ITALY & ITALY & 1906 & & LUCCA & 2ND AVE. & ADAMS - PAINSDALE \\
\hline 997 & & RICCl & MATILDA & D & 12 & MICHIGAN & ITALY & ITALY & & & LUCCA & 2ND AVE. & ADAMS - PAINSDALE \\
\hline 998 & & RICCI & ATTILIO & $\mathrm{s}$ & 11 & MICHIGAN & ITALY & ITALY & & & LUCCA & 2ND AVE. & ADAMS - PAINSDALE \\
\hline 999 & & RICCI & $?$ & D & 9 & MICHIGAN & ITALY & ITALY & & & LUCCA & 2ND AVE. & ADAMS - PAINSDALE \\
\hline 1000 & & RICCI & JOHN & $\mathrm{s}$ & $9 \mathrm{M}$ & MICHIGAN & ITALY & ITALY & & & LUCCA & 2ND AVE. & ADAMS - PAINSDALE \\
\hline 1001 & 21 & $\mathrm{RICCl}$ & FRANCO & $\mathrm{H}$ & 31 & ITALY & ITALY & ITALY & 1913 & MOTORMAN/CM & LUCCA & 2ND ST. & ADAMS - PAINSDALE \\
\hline 1002 & & $\mathrm{RICCl}$ & GIUSEPPINA & w & 30 & ITALY & ITALY & ITALY & 1913 & & LUCCA & 2ND ST. & ADAMS - PAINSDALE \\
\hline 1003 & 15 & VETTORI & EMILIO & $\mathrm{H}$ & $34 ?$ & ITALY & ITALY & ITALY & 1907 & & MONTECARLO. LU & 2ND ST. & ADAMS - PAINSDALE \\
\hline 1004 & & VETTORI & MARY & w & 37 & ITALY & ITALY & ITALY & 1906 & & & 2ND ST. & ADAMS - PAINSDALE \\
\hline 1005 & & VETTORI & ATTILIO & s & 10 & MICHIGAN & ITALY & ITALY & & PICKER/CM & MONTECARLO. LU & 2ND ST. & ADAMS - PAINSDALE \\
\hline 1006 & & VETTORI & ALADINO & $\mathrm{s}$ & 4 & MICHIGAN & ITALY & ITALY & & & MONTECARLO. LU & 2ND ST. & ADAMS - PAINSDALE \\
\hline 1007 & & VETTORI & JOHN & $\mathrm{s}$ & 2 & MICHIGAN & ITALY & ITALY & & & MONTECARLO. LU & 2ND ST. & ADAMS - PAINSDALE \\
\hline
\end{tabular}




\begin{tabular}{|c|c|c|c|c|c|c|c|c|c|c|c|c|c|}
\hline 1008 & & PAOLI & ATTILIO & B & 21 & ITALY & ITALY & ITALY & 1915 & DUMPER/CM & CAPANNORI. LU & 2ND ST. & ADAMS - PAINSDALE \\
\hline 1009 & & TORE? & SALVATORE & B & 32 & ITALY & ITALY & ITALY & 1910 & TRAMMER/CM & & 2ND ST. & ADAMS - PAINSDALE \\
\hline 1010 & & LENCIONI & ALFREDO & B & 40 & ITALY & ITALY & ITALY & 1913 & FIREMAN/STATIONARY & LUCCA & 2ND ST. & ADAMS - PAINSDALE \\
\hline 1011 & 11 & PEROTTI & FRANK & $\mathrm{H}$ & 44 & ITALY & ITALY & ITALY & 1907 & PICKER/CM & TORINO & 1ST ST. & ADAMS - PAINSDALE \\
\hline 1012 & & PEROTTI & JENNY & w & 42 & ITALY & ITALY & ITALY & 1908 & & & 1ST ST. & ADAMS - PAINSDALE \\
\hline 1013 & & PEROTTI & LINA & D & 11 & MICHIGAN & ITALY & ITALY & & & TORINO & 1ST ST. & ADAMS - PAINSDALE \\
\hline 1014 & & GIUSTI & ORLANDO & B & 40 & ITALY & ITALY & ITALY & 1919 & BLACKSMITH/SHOP & LUCCA & 1ST ST. & ADAMS - PAINSDALE \\
\hline 1015 & & GIUSTI & ILIO & B & 17 & ITALY & ITALY & ITALY & 1907 & BLACKSMITH/SHOP & PISA & 1ST ST. & ADAMS - PAINSDALE \\
\hline 1016 & & FUSO & ANGELO & $\begin{array}{l}\mathrm{D} \\
\mathrm{B}\end{array}$ & 32 & ITALY & ITALY & ITALY & 1914 & $\begin{array}{l}\text { PICKER/CM } \\
\text { PIOTU }\end{array}$ & & 1ST ST. & ADAMS - PAINSDALE \\
\hline 1017 & & PIERONI & ANTONIO & B & 45 & ITALY & ITALY & ITALY & 1905 & TRAMMER/CM & LUCCA & 1ST ST. & ADAMS - PAINSDALE \\
\hline 1018 & & FANA? & ALFREDO & B & 35 & ITALY & ITALY & ITALY & 1912 & PICKER/CM & & 1ST ST. & ADAMS - PAINSDALE \\
\hline 1019 & & SAMANECHI? & ORDELLI? & B & 33 & ITALY & ITALY & ITALY & 1906 & TRAMMER/CM & & 1ST ST. & ADAMS - PAINSDALE \\
\hline 1020 & 32 & PELLEGRINI & ANGELA & $\mathrm{H}$ & 26 & SWEDEN & SWEDEN & SWEDEN & 1911 & & LUCCA & 2ND ST. & ADAMS - PAINSDALE \\
\hline 1021 & & PELLEGRINI & GIOIA & D & 3 & MICHIGAN & ITALY & SWEDEN & & & LUCCA & 2ND ST. & ADAMS - PAINSDALE \\
\hline 1022 & & PELLEGRINI & CLARA & D & 2 & MICHIGAN & ITALY & SWEDEN & & & LUCCA & 2ND ST. & ADAMS - PAINSDALE \\
\hline 1023 & & REI & JUST & B & 26 & ITALY & ITALY & ITALY & 1912 & PICKER/CM & & 2ND ST. & ADAMS - PAINSDALE \\
\hline 1024 & & DELLE & PETER & B & 32 & ITALY & ITALY & ITALY & 1908 & & & 2ND ST. & ADAMS - PAINSDALE \\
\hline 1025 & $?$ & RIVA & LOUIS & $\mathrm{H}$ & 40 & ITALY & ITALY & ITALY & UN & MINER/CM & TORINO & & ADAMS - PAINSDALE \\
\hline 1026 & & RIVA & JOHANNA & w & 30 & ITALY & ITALY & ITALY & 1909 & & & & ADAMS - PAINSDALE \\
\hline 1027 & & RIVA & ANGELO & $\mathrm{s}$ & 8 & MICHIGAN & ITALY & ITALY & & & TORINO & & ADAMS - PAINSDALE \\
\hline 1028 & & RIVA & JULIA & $\mathrm{D}$ & 6 & MICHIGAN & ITALY & ITALY & & & TORINO & & ADAMS - PAINSDALE \\
\hline $\begin{array}{l}1029 \\
1029\end{array}$ & & ? & ? & B & $\begin{array}{l}0 \\
28\end{array}$ & ITALY & ITALY & ITALY & 1913 & TRAMMER/CM & TURIIV & & ADAMS - PAINSDALE \\
\hline 1030 & & GESSILING? & & B & 25 & ITALY & ITALY & ITALY & 1913 & WELDER/CM & & & ADAMS - PAINSDALE \\
\hline 1031 & & ? $11 / 114 / 24-28$ & & B & 33 & ITALY & ITALY & ITALY & $?$ & MINER/CM & & & ADAMS - PAINSDALE \\
\hline 1032 & & $?$ & & B & 26 & ITALY & ITALY & ITALY & $?$ & PIPE MAN/CM & & & ADAMS - PAINSDALE \\
\hline 1033 & 8 & CAVAGNETTO & FRANK & B & 37 & ITALY & ITALY & ITALY & 1905 & TIMBERMAN/CM & VIVERONE. BIELLA & & ADAMS - PAINSDALE \\
\hline 1034 & 12 & RUGONI & ANGELO & B & 34 & ITALY & ITALY & ITALY & 1906 & WELDER/CM & LUCCA & & ADAMS - PAINSDALE \\
\hline 1035 & 12 & RAI-PRA & MARCELLINO & B & 37 & ITALY & ITALY & ITALY & 1914 & WELDER/CM & & & ADAMS - PAINSDALE \\
\hline 1036 & 1302 & MICHETTI & JOHN & $\mathrm{H}$ & 34 & MICHIGAN & ITALY & ITALY & & MINER/CM & PONT CANAVESE. TO & ROCKLAND ST. & CALUMET \\
\hline 1037 & & MICHETTI & LUCY & w & 57 & ITALY & ITALY & ITALY & 1895 & & & ROCKLAND ST. & $\begin{array}{l}\text { CALUMET } \\
\end{array}$ \\
\hline 1038 & & MICHETTI & JOSEPHINE & si & 24 & MICHIGAN & MICHIGAN & ITALY & & SALESLADY/DEPT. STORE & PONT CANAVESE. TO & ROCKLAND ST. & CALUMET \\
\hline 1039 & & MICHETTI & LENA & SI & 20 & MICHIGAN & MICHIGAN & ITALY & & TEACHER/PUBLIC SCHOOL & PONT CANAVESE. TO & ROCKLAND ST. & CALUMET \\
\hline 1040 & & GALLO & LUCY & NIEC & 3 & MICHIGAN & MICHIGAN & ITALY & & 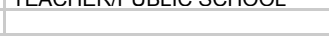 & PONT CANAVESE. TO & ROCKLAND ST. & CALUMET \\
\hline 1041 & 1303 & CRUGNOLA & CARLO & $\mathrm{H}$ & 59 & ITALY & ITALY & ITALY & 1893 & MASON/CM & COMO & ROCKLAND ST. & CALUMET \\
\hline 1042 & & CRUGNOLA & MODESTA & W & 58 & ITALY & ITALY & ITALY & 1893 & & COMO & ROCKLAND ST. & CALUMET \\
\hline 1043 & & AZZI & PETER & SOIL & 32 & ITALY & ITALY & ITALY & 1906 & MINER/CM & MASSA CARRARA & ROCKLAND ST. & CALUMET \\
\hline 1044 & & AZZI & ERNEST & NEP & 7 & MICHIGAN & ITALY & ITALY & & 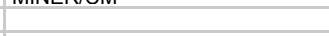 & MASSA CARRARA & ROCKLAND ST. & $\begin{array}{l}\text { CALUMET } \\
\text { CALE }\end{array}$ \\
\hline 1045 & 1306 & PIGNOCCO & CARLO & $\mathrm{H}$ & 57 & ITALY & ITALY & ITALY & 1884 & WAITER/PRIVATE CLUB & SAN GIORGIO CANAVESE. TO & ROCKLAND ST. & CALUMET \\
\hline 1046 & & PIGNOCCO & SECONDINA & W & 48 & MICHIGAN & ITALY & ITALY & & SALESLADY/FRUIT STORE & & ROCKLAND ST. & CALUMET \\
\hline 1047 & & PIGNOCCO & DOMENICO & $\mathrm{s}$ & 27 & MICHIGAN & ITALY & MICHIGAN & & ELECTRICIAN/CM & SAN GIORGIO CANAVESE. TO & ROCKLAND ST. & CALUMET \\
\hline 1048 & & PIGNOCCO & GIORGIO & $\mathrm{s}$ & 25 & MICHIGAN & ITALY & MICHIGAN & & & SAN GIORGIO CANAVESE. TO & ROCKLAND ST. & CALUMET \\
\hline 1049 & & PIGNOCCO & GIULIA & D & 23 & MICHIGAN & ITALY & MICHIGAN & & & SAN GIORGIO CANAVESE. TO & ROCKLAND ST. & CALUMET \\
\hline 1050 & 1306 & ROSSO & MARY & $\mathrm{H}$ & 44 & ITALY & ITALY & ITALY & 1899 & & TORINO & ROCKLAND ST. & CALUMET \\
\hline 1051 & & ROSSO & MARY & D & 19 & MICHIGAN & ITALY & ITALY & & HOUSEKEEPER/PF & TORINO & ROCKLAND ST. & CALUMET \\
\hline 1052 & & ROSSO & MADDALENA & D & 17 & MICHIGAN & ITALY & ITALY & & OPERATOR/TELEPHONE & TORINO & ROCKLAND ST. & CALUMET \\
\hline 1053 & & ROSSO & JOHN & $\mathrm{s}$ & 15 & MICHIGAN & ITALY & ITALY & & DELIVERY BOY/BAKERY SHOP & TORINO & ROCKLAND ST. & CALUMET \\
\hline 1054 & & ROSSO & MINNIE & D & 11 & MICHIGAN & ITALY & ITALY & & & TORINO & ROCKLAND ST. & CALUMET \\
\hline 1055 & & ROSSO & LOUISE & $\mathrm{D}$ & 10 & MICHIGAN & ITALY & ITALY & & & TORINO & ROCKLAND ST. & $\begin{array}{l}\text { CALUMET } \\
\end{array}$ \\
\hline 1056 & 1310 & ZANOTTI & ANTON & $\mathrm{H}$ & 45 & ITALY & ITALY & ITALY & 1897 & & PEROSA. TO & ROCKLAND ST. & CALUMET \\
\hline 1057 & & ZANOTTI & CELESTE & w & 39 & ITALY & ITALY & ITALY & 1905 & & SAN MARTINO CANAVESE. TO & ROCKLAND ST. & CALUMET \\
\hline 1058 & & ZANOTTI & JOSEPH & $\mathrm{s}$ & 16 & ITALY & ITALY & ITALY & $? ?$ & & PEROSA. TO & ROCKLAND ST. & CALUMET \\
\hline 1059 & 1312 & LANDI & NATALE & $\mathrm{H}$ & $? 9$ & ITALY & ITALY & ITALY & 1916 & MINER/CM & LOCANA CANAVESE, TO & ROCKLAND ST. & CALUMET \\
\hline 1060 & & LANDI & CATERINA & W & $2 ?$ & ITALY & ITALY & ITALY & 1919 & & & ROCKLAND ST. & CALUMET \\
\hline 1061 & & CONEDERA & ANTONIO & $\mathrm{H}$ & 46 & ITALY & ITALY & ITALY & 1895 & MINER/CM & BELLUNO & ROCKLAND ST. & CALUMET \\
\hline 1062 & & CONEDERA & DOMENICA & w & 29 & ITALY & ITALY & ITALY & 1911 & & & ROCKLAND ST. & CALUMET \\
\hline 1063 & 1314 & BRUSSO & GIORGIO & H & 58 & ITALY & ITALY & ITALY & 1887 & MINER/CM & AGLIE'. TO & ROCKLAND ST. & CALUMET \\
\hline 1064 & & BRUSSO & ANTONIA & W & 50 & ITALY & ITALY & ITALY & 1893 & & & ROCKLAND ST. & CALUMET \\
\hline 1065 & & BRUSSO & MARY & D & 17 & MICHIGAN & ITALY & ITALY & & NOT CLEAR & AGLIE', TO & ROCKLAND ST. & CALUMET \\
\hline 1066 & 1330 & BIGANDO & BAT & $\mathrm{H}$ & 36 & ITALY & ITALY & ITALY & 1887 & TEAMSTER/CM & TORINO & ROCKLAND ST. & CALUMET \\
\hline 1067 & & BIGANDO & MARY & W & 27 & MICHIGAN & ITALY & ITALY & & & & ROCKLAND ST. & CALUMET \\
\hline 1068 & & BIGANDO & MAUD & D & 16 & MICHIGAN & ITALY & MICHIGAN & & & TORINO & CKLAND & CALUMET \\
\hline 1069 & & BIGANDO & GEORGE & $\mathrm{s}$ & 7 & MICHIGAN & ITALY & MICHIGAN & & & TORINO & ROCKLAND ST. & CALUMET \\
\hline 1070 & & BIGANDO & VITTORIA & D & 5 & MICHIGAN & ITALY & MICHIGAN & & & TORINO & ROCKLAND ST. & CALUMET \\
\hline
\end{tabular}




\begin{tabular}{|c|c|c|c|c|c|c|c|c|c|c|c|c|c|}
\hline 1071 & 1328 & MARTA & JOHN & $\mathrm{H}$ & 35 & ITALY & ITALY & ITALY & 1906 & MINER/CM & AGLIE'. TO & ROCKLAND ST. & CALUMET \\
\hline 1072 & & MARTA & CATERINA & W & 31 & ITALY & ITALY & ITALY & 1908 & & & ROCKLAND ST. & CALUMET \\
\hline 1073 & & MARTA & MARY & D & 10 & MICHIGAN & ITALY & ITALY & & & AGLIE'. TO & ROCKLAND ST. & CALUMET \\
\hline 1074 & & MARTA & MAX & $\mathrm{s}$ & 8 & MICHIGAN & ITALY & ITALY & & & AGLIE'. TO & ROCKLAND ST. & CALUMET \\
\hline 1075 & & MARTA & JOHN & $\mathrm{s}$ & 6 & MICHIGAN & ITALY & ITALY & & & AGLIE', TO & ROCKLAND ST. & CALUMET \\
\hline 1076 & & MARTA & IRENE & D & 4 & MICHIGAN & ITALY & ITALY & & & AGLIE'. TO & ROCKLAND ST. & CALUMET \\
\hline 1077 & $134 ?$ & BOBBIO & MARY & $\mathrm{H}$ & 44 & ITALY & ITALY & ITALY & 1899 & & SAN BENIGNO CANAVESE. TO & ROCKLAND ST. & $\begin{array}{l}\text { CALUMET } \\
\text { CALE }\end{array}$ \\
\hline 1078 & $104:$ & BOBBIO & WILLIAM & s & $\frac{44}{15}$ & MICHIGAN & ITALY & ITALY & 1095 & WOOD CHOPPER/LUMBER CO. & SAN BENIGNO CANAVESE. TO & ROCKLAND ST. & CALUMET \\
\hline 1079 & 1392 & RISSIO & JOSEPH & $\mathrm{H}$ & 56 & ITALY & ITALY & ITALY & 1889 & MINER/CM & SAN BENIGNO CANAVESE. TO & ROCKLAND ST. & CALUMET \\
\hline 1080 & & RISSIO & PAULINE & W & 45 & ITALY & ITALY & ITALY & 1892 & & & ROCKLAND ST. & CALUMET \\
\hline 1081 & & RISSIO & CRESENZA & D & 21 & MICHIGAN & ITALY & ITALY & & SALESLADY/FRUIT STORE & SAN BENIGNO CANAVESE. TO & ROCKLAND ST. & CALUMET \\
\hline 1082 & & RISSIO & STEFANO & $\mathrm{S}$ & 17 & MICHIGAN & ITALY & ITALY & & HELPER/FRUIT STORE & SAN BENIGNO CANAVESE. TO & ROCKLAND ST. & CALUMET \\
\hline 1083 & & RISSIO & SADIE & $\mathrm{D}$ & 15 & MICHIGAN & ITALY & ITALY & & & SAN BENIGNO CANAVESE. TO & ROCKLAND ST. & CALUMET \\
\hline 1084 & & RISSIO & ELISABETH & $\mathrm{D}$ & 12 & MICHIGAN & ITALY & ITALY & & & SAN BENIGNO CANAVESE. TO & ROCKLAND ST. & CALUMET \\
\hline 1085 & & RISSIO & DOMENICO & $\mathrm{s}$ & 9 & MICHIGAN & ITALY & ITALY & & & SAN BENIGNO CANAVESE. TO & ROCKLAND ST. & CALUMET \\
\hline 1086 & 1550 & ZUBIENA & JOHN & $\mathrm{H}$ & UN & ITALY & ITALY & ITALY & 1911 & MINER/CM & PEROSA, TO & HECLA ST. & CALUMET \\
\hline 1087 & & ZUBIENA & MADDALENA & W & UN & ITALY & ITALY & ITALY & 1911 & & PEROSA. TO & HECLA ST. & CALUMET \\
\hline 1088 & & ZUBIENA & FRANK & $\mathrm{s}$ & 7 & MICHIGAN & ITALY & ITALY & & & PEROSA. TO & HECLA ST. & $\begin{array}{l}\text { CALUMET } \\
\end{array}$ \\
\hline 1089 & & ZUBIENA & KATE & D & 4 & MICHIGAN & ITALY & ITALY & & & PEROSA. TO & HECLA ST. & $\begin{array}{l}\text { CALUMET } \\
\end{array}$ \\
\hline 1090 & & ZUBIENA & JULIA & D & 4 & MICHIGAN & ITALY & ITALY & & & PEROSA. TO & HECLA ST. & $\begin{array}{l}\text { CALUMET } \\
\end{array}$ \\
\hline 1091 & 1548 & GIANNINI & LAURENCE & $\mathrm{H}$ & 33 & ITALY & ITALY & ITALY & 1900 & LABORER/ROCK HOUSE & LUCCA & HECLA ST. & CALUMET \\
\hline 1092 & 1540 & GIANNINI & CAMILLE & W & 25 & MICHIGAN & $\begin{array}{l}\text { ITALY } \\
\text { ITAL }\end{array}$ & ITALY & 1900 & LADURER/KUU K TUUSE & LUCCA & HECLA ST. & CALUMET \\
\hline 1093 & & GIANNINI & GIUSTINE & D & $4 ?$ & MICHIGAN & ITALY & MICHIGAN & & & LUCCA & HECLA ST. & CALUMET \\
\hline 1094 & 1546 & BARATONO & JOHN & $\mathrm{H}$ & 53 & ITALY & ITALY & ITALY & 1891 & MINER/CM & VIALFRE'. TO & HECLA ST. & CALUMET \\
\hline 1095 & & BARATONO & JAMES & $\mathrm{s}$ & 19 & MICHIGAN & ITALY & ITALY & & & VIALFRE'. TO & HECLA ST. & CALUMET \\
\hline 1096 & & BARATONO & JOSEPH & $\mathrm{s}$ & 15 & MICHIGAN & ITALY & ITALY & & & VIALFRE'. TO & HECLA ST. & CALUMET \\
\hline 1097 & & BARATONO & ENRICO & $\mathrm{s}$ & 13 & MICHIGAN & ITALY & ITALY & & & VIALFRE'. TO & HECLA ST. & CALUMET \\
\hline 1098 & 1549 & SANDRETTO & MIKE & $\mathrm{H}$ & 53 & ITALY & ITALY & ITALY & 1889 & LABORER/ROCK HOUSE & PONT CANAVESE, TO & HECLA ST. & CALUMET \\
\hline 1099 & & SANDRETTO & MARGARET & W & 49 & ITALY & ITALY & ITALY & 1897 & & & HECLA ST. & CALUMET \\
\hline 1100 & & SANDRETTO & JOHN & s & 21 & MICHIGAN & ITALY & ITALY & & & PONT CANAVESE. TO & HECLA ST. & $\begin{array}{l}\text { CALUMET } \\
\end{array}$ \\
\hline 1101 & & SANDRETTO & BARNEY & $\mathrm{s}$ & 19 & MICHIGAN & ITALY & ITALY & & & PONT CANAVESE. TO & HECLA ST. & $\begin{array}{l}\text { CALUMET } \\
\end{array}$ \\
\hline 1102 & & SANDRETTO & CARLO & $\mathrm{s}$ & 15 & MICHIGAN & ITALY & ITALY & & & PONT CANAVESE. TO & HECLA ST. & CALUMET \\
\hline 1103 & & SANDRETTO & MARY & D & 18 & MICHIGAN & ITALY & ITALY & & & PONT CANAVESE. TO & HECLA ST. & $\begin{array}{l}\text { CALUMET } \\
\end{array}$ \\
\hline 1104 & $15 ? ?$ & GASPARDO & DOMENICO & $\mathrm{H}$ & $\begin{array}{l}10 \\
59\end{array}$ & ITALY & ITALY & ITALY & 1886 & MINER/CM & PONT CANAVESE, TO & HECLA ST. & CALUMET \\
\hline 1105 & & GASPARDO & MARIA & w & 50 & ITALY & ITALY & ITALY & 1900 & & & HECLA ST. & CALUMET \\
\hline 1106 & & GASPARDO & JAMES & s & 17 & MICHIGAN & ITALY & ITALY & & & PONT CANAVESE. TO & HECLA ST. & CALUMET \\
\hline 1107 & & GASPARDO & DOMENICO & $\mathrm{s}$ & 15 & MICHIGAN & ITALY & ITALY & & & PONT CANAVESE. TO & HECLA ST. & CALUMET \\
\hline 1108 & 1546 & FETTA & JOHN & $\mathrm{H}$ & 64 & ITALY & ITALY & ITALY & 1889 & MINER/CM & BAIRO CANAVESE. TO & HECLA ST. & CALUMET \\
\hline 1109 & & FETTA-FURNO & BARBARA & W & 44 & ITALY & ITALY & ITALY & 1896 & & & HECLA ST. & CALUMET \\
\hline 1110 & & FETTA & BENJAMIN & $\mathrm{s}$ & 22 & MICHIGAN & ITALY & ITALY & & LABORER/AUTO FACTORY & BAIRO CANAVESE. TO & HECLA ST. & CALUMET \\
\hline 1111 & & FETTA & LUCY & D & 21 & MICHIGAN & ITALY & ITALY & & SALESLADY/DEPT. STORE & BAIRO CANAVESE. TO & HECLA ST. & CALUMET \\
\hline 1112 & & FETTA & MARY & D & 19 & MICHIGAN & ITALY & ITALY & & & BAIRO CANAVESE. TO & HECLA ST. & $\begin{array}{l}\text { CALUMET } \\
\end{array}$ \\
\hline 1113 & & FETTA & LOUIS & $\mathrm{s}$ & 15 & MICHIGAN & ITALY & ITALY & & TEAMSTER/BUTCHER & BAIRO CANAVESE. TO & HECLA ST. & CALUMET \\
\hline 1114 & 1587 & FERRARI & ALBERTO & $\mathrm{H}$ & 48 & ITALY & ITALY & ITALY & 1889 & CARPENTER/CARPENTER SH. & LUCCA & HECLA ST. & CALUMET \\
\hline 1115 & & FERRARI & ERNESTA & W & 47 & ITALY & ITALY & ITALY & 1892 & & & HECLA ST. & CALUMET \\
\hline 1116 & & LENCIONI & MARY & D & 26 & MICHIGAN & ITALY & ITALY & 1002 & & LUCCA & HECLA ST. & CALUMET \\
\hline 1117 & & LENCIONI & NATALE & SOIL & 26 & MICHIGAN & ITALY & ITALY & & MINER/CM & LUCCA & HECLA ST. & CALUMET \\
\hline 1118 & 1535 & GIOVANNINI & ANTONIO & $\mathrm{H}$ & 46 & ITALY & ITALY & ITALY & 1891 & LABORER & SAN GIUSTO CANAVESE. TO & HECLA ST. & CALUMET \\
\hline 1119 & & GIOVANNINI & JESSE & W & 49 & ITALY & ITALY & ITALY & 1890 & & & HECLA ST. & CALUMET \\
\hline 1120 & & GIOVANNINI & TERESA & $\mathrm{D}$ & 26 & MICHIGAN & ITALY & ITALY & & SALESLADY/DEPT. STORE & SAN GIUSTO CANAVESE. TO & HECLA ST. & CALUMET \\
\hline 1121 & & GIOVANNINI & VINCENZO & $\mathrm{S}$ & 24 & MICHIGAN & ITALY & ITALY & & SALESMAN/DEPT. STORE & SAN GIUSTO CANAVESE. TO & HECLA ST. & CALUMET \\
\hline 1122 & & GIOVANNINI & MINNIE & D & 21 & MICHIGAN & ITALY & ITALY & & SALESLADYISHOE STORE & SAN GIUSTO CANAVESE, TO & HECLA ST. & CALUMET \\
\hline 1123 & 1546 & GUERRA & FRANK & $\mathrm{H}$ & 55 & ITALY & ITALY & ITALY & 1891 & & LUCCA & HECLA ST. & CALUMET \\
\hline 1124 & & GUERRA & ESTER & W & 43 & ITALY & ITALY & ITALY & 1899 & & & HECLA ST. & CALUMET \\
\hline 1125 & & GUERRA & JOHN & s & 19 & MICHIGAN & ITALY & ITALY & & & LUCCA & HECLA ST. & CALUMET \\
\hline 1126 & 3232 & SOMMARIVA & AUGUSTO & $\mathrm{H}$ & 61 & ITALY & ITALY & ITALY & 1890 & TEAMSTER/CM & BELLUNO & RIDGE ST. & CALUMET \\
\hline 1127 & & SOMMARIVA & MARY & w & 57 & ITALY & ITALY & ITALY & 1900 & & & RIDGE ST. & CALUMET \\
\hline 1128 & & SOMMARIVA & NICO & $\mathrm{s}$ & 17 & MICHIGAN & ITALY & ITALY & & LABORER/CM & BELLUNO & RIDGE ST. & $\begin{array}{l}\text { CALUMET } \\
\end{array}$ \\
\hline 1129 & & SOMMARIVA & LUIGI & $\mathrm{s}$ & 15 & MICHIGAN & ITALY & ITALY & & & BELLUNO & RIDGE ST. & $\begin{array}{l}\text { CALUMET } \\
\end{array}$ \\
\hline 1130 & & SOMMARIVA & $\mathrm{JOHN}$ & $\mathrm{s}$ & 13 & MICHIGAN & ITALY & ITALY & & & BELLUNO & RIDGE ST. & CALUMET \\
\hline 1131 & 3103 & NOTARI & JOHN & $\mathrm{H}$ & 61 & ITALY & ITA & ITALY & 1887 & TIMBERMAN/CM & POPIGLIO. PISTOIA & RIDGE ST. & $\begin{array}{l}\text { CALUMET } \\
\end{array}$ \\
\hline 1132 & & NOTARI & CESIRA & W & 58 & ITALY & ITALY & ITALY & 1891 & & & RIDGE ST. & CALUMET \\
\hline 1133 & 3120 & TEPATTI & JOHN & $\mathrm{H}$ & 57 & ITALY & ITALY & ITALY & 1888 & LABORER/CM & PONT CANAVESE. TO & TUNNEL ST. & CALUMET \\
\hline
\end{tabular}




\begin{tabular}{|c|c|c|c|c|c|c|c|c|c|c|c|c|c|}
\hline 1134 & & TEPATTI & ANGELA & W & 57 & ITALY & ITALY & ITALY & 1891 & & & TUNNEL ST. & CALUMET \\
\hline 1135 & & TEPATTI & BARNEY & $\mathrm{s}$ & 21 & MICHIGAN & ITALY & ITALY & & MACHINIST/MACHINIST SHOP & PONT CANAVESE. TO & TUNNEL ST. & CALUMET \\
\hline 1136 & & TEPATTI & MARY & D & 18 & MICHIGAN & ITALY & ITALY & & & PONT CANAVESE. TO & TUNNEL ST. & CALUMET \\
\hline 1137 & 3023 & BRACCO & ANTONIO & $\mathrm{H}$ & 55 & ITALY & ITALY & ITALY & 1889 & LABORER/RAILROAD & TORINO & OSCEOLA ROAD & CALUMET \\
\hline 1138 & & BRACCO & TERESA & w & 53 & ITALY & ITALY & ITALY & 1894 & & & OSCEOLA ROAD & CALUMET \\
\hline 1139 & & BRACCO & JAMES & s & 34 & MICHIGAN & ITALY & ITALY & & BOILER MAKER/ROUNDHOUSE & TORINO & OSCEOLA ROAD & CALUMET \\
\hline 1140 & & BRACCO & FELIX & $\mathrm{s}$ & 23 & MICHIGAN & ITALY & ITALY & & BOILER MAKER/ROUNDHOUSE & TORINO & OSCEOLA ROAD & $\begin{array}{l}\text { CALUMET } \\
\text { CALE }\end{array}$ \\
\hline 1141 & & BRACCO & CATERINA & DIL & 20 & MICHIGAN & ITALY & ITALY & & & & OSCEOLA ROAD & CALUMET \\
\hline 1142 & & BRACCO & ANTONIO & S & 14 & MICHIGAN & ITALY & ITALY & & & TORINO & OSCEOLA ROAD & CALUMET \\
\hline 1143 & 1686 & RICCA & JOSEPH & $\mathrm{H}$ & 46 & ITALY & ITALY & ITALY & 1887 & SHIFT BOSS/CM & TORINO & LAURIUM ST. & CALUMET \\
\hline 1144 & & RICCA & MARGHERITA & w & 49 & ITALY & ITALY & ITALY & 1889 & & & LAURIUM ST. & CALUMET \\
\hline 1145 & & RICCA & FRANK & $\mathrm{s}$ & 19 & MICHIGAN & ITALY & ITALY & & RAILROAD OFFICE ASSISTANT & TORINO & LAURIUM ST. & CALUMET \\
\hline 1146 & & RICCA & VERONICA & $\mathrm{D}$ & 15 & MICHIGAN & ITALY & ITALY & & & TORINO & LAURIUM ST. & CALUMET \\
\hline 1147 & & RICCA & JOSEPH & $\mathrm{s}$ & 9 & MICHIGAN & ITALY & ITALY & & & TORINO & LAURIUM ST. & CALUMET \\
\hline 1148 & & HILL & LUCILLE & $\mathrm{D}$ & 23 & MICHIGAN & ITALY & ITALY & & & TORINO & LAURIUM ST. & CALUMET \\
\hline 1149 & 1677 & BARANSAKI & STEVE & $\mathrm{H}$ & 28 & MICHIGAN & RUSSIA & RUSSIA & & MINER/CM & RUSSIA & LAURIUM ST. & CALUMET \\
\hline 1150 & & BARANSAKI & FRANCIS & W & 24 & MICHIGAN & ITALY & ITALY & & & & LAURIUM ST. & CALUMET \\
\hline 1151 & & BARANSAKI & STEVE & $\mathrm{s}$ & $11 \mathrm{M}$ & MICHIGAN & MICHIGAN & MICHIGAN & & & & LAURIUM ST. & CALUMET \\
\hline 1152 & 1676 & OZZELLO & JOSEPH & $\mathrm{H}$ & 32 & ITALY & ITALY & ITALY & 1910 & TIMBER BOSS/CM & SAN GIUSTO CANAVESE. TO & LAURIUM ST. & $\begin{array}{l}\text { CALUMET } \\
\text { CALE }\end{array}$ \\
\hline 1153 & 00 & OZZELLO & MARY & W & 24 & MICHIGAN & ITALY & MICHIGAN & 1010 & 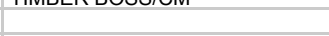 & TORINO & LAURIUM ST. & CALUMET \\
\hline 1154 & & OZZELLO & BERNARDO & s & 6 & MICHIGAN & ITALY & MICHIGAN & & & SAN GIUSTO CANAVESE. TO & LAURIUM ST. & CALUMET \\
\hline 1155 & & OZZELLO & TEODORO & $\frac{5}{s}$ & ? & MICHIGAN & $\begin{array}{l}\text { ITALY } \\
\text { ITAL }\end{array}$ & MICHIGAN & & & SAN GIUSTO CANAVESE, TO & $\begin{array}{l}\text { LAURIUM ST. } \\
\text { LAU }\end{array}$ & $\begin{array}{l}\text { CALUMET } \\
\text { CAUILT }\end{array}$ \\
\hline 1156 & & OZZELLO & VERNA & $\mathrm{D}$ & $11 \mathrm{M}$ & MICHIGAN & ITALY & MICHIGAN & & & SAN GIUSTO CANAVESE. TO & LAURIUM ST. & CALUMET \\
\hline 1157 & 1662 & LE CHEVALIER & PAUL & $\mathrm{H}$ & 33 & MICHIGAN & FRANCE & FRANCE & & MACHINIST/MACHINE SHAFT & FRANCE & LAURIUM ST. & CALUMET \\
\hline 1158 & & LE CHEVALIER & MARGHERITA & W & 31 & ITALY & ITALY & ITALY & 1893 & & & LAURIUM ST. & CALUMET \\
\hline 1159 & & LE CHEVALIER & PAUL JR. & $\mathrm{s}$ & 13 & MICHIGAN & MICHIGAN & ITALY & & & & LAURIUM ST. & CALUMET \\
\hline 1160 & & LE CHEVALIER & GIULIO & $\mathrm{s}$ & 8 & MICHIGAN & MICHIGAN & ITALY & & & & LAURIUM ST. & CALUMET \\
\hline 1161 & & LE CHEVALIER & ERNIE & $\mathrm{s}$ & 7 & MICHIGAN & MICHIGAN & ITALY & & & & LAURIUM ST. & CALUMET \\
\hline 1162 & & LE CHEVALIER & JOSEPH & $\mathrm{S}$ & 6 & MICHIGAN & MICHIGAN & ITALY & & & & LAURIUM ST. & CALUMET \\
\hline 1163 & & LE CHEVALIER & ANGELINA & D & 3 & MICHIGAN & MICHIGAN & ITALY & & & & LAURIUM ST. & $\begin{array}{l}\text { CALUMET } \\
\end{array}$ \\
\hline 1164 & & LE CHEVALIER & PETER & $\mathrm{s}$ & $11 \mathrm{M}$ & MICHIGAN & MICHIGAN & ITALY & & & & LAURIUM ST. & $\begin{array}{l}\text { CALUMET } \\
\end{array}$ \\
\hline 1165 & 1631 & CAPELLO & JOHN & $\mathrm{H}$ & 56 & ITALY & ITALY & ITALY & 1890 & LABORER/CM & SAN BENIGNO CANAVESE. TO & LAURIUM ST. & CALUMET \\
\hline 1166 & 1001 & CAPELLO & MARY & w & 62 & ITALY & ITALY & ITALY & 1890 & LADURER/UIUI & SAN BENIGNO CANAVESE. TO & LAURIUM ST. & CALUMET \\
\hline 1167 & 1626 & BENEITONE & DOMENICO & $\mathrm{H}$ & 39 & ITALY & ITALY & ITALY & 1907 & MINER/CM & SALASSA, TO & LAURIUM ST. & CALUMET \\
\hline 1168 & & BENEITONE & MARY & w & 23 & MICHIGAN & ITALY & ITALY & & & & LAURIUM ST. & CALUMET \\
\hline 1169 & & BENEITONE & MONICA & D & 2 & MICHIGAN & ITALY & ITALY & & & SALASSA. TO & LAURIUM ST. & CALUMET \\
\hline 1170 & & BENEITONE & LUIGI & $\mathrm{s}$ & 1 & MICHIGAN & ITALY & ITALY & & & SALASSA. TO & LAURIUM ST. & CALUMET \\
\hline 1171 & 1629 & ONORE & BONO & $\mathrm{H}$ & 39 & ITALY & ITALY & ITALY & 1903 & PUMP MAN/CM & STRAMBELLO. TO & LAURIUM ST. & CALUMET \\
\hline 1172 & & ONORE & CESARINA & W & 36 & ITALY & ITALY & ITALY & 1903 & & STRAMBELLO. TO & LAURIUM ST. & CALUMET \\
\hline 1173 & & ONORE & CATERINA & $\mathrm{D}$ & 15 & MICHIGAN & ITALY & ITALY & & & STRAMBELLO. TO & LAURIUM ST. & CALUMET \\
\hline 1174 & & ONORE & JOSEPHINE & D & 12 & MICHIGAN & ITALY & ITALY & & & STRAMBELLO. TO & LAURIUM ST. & CALUMET \\
\hline 1175 & & ONORE & ALMA & D & 7 & MICHIGAN & ITALY & ITALY & & & STRAMBELLO. TO & LAURIUM ST. & CALUMET \\
\hline 1176 & & ONORE & STEVEN & $\mathrm{s}$ & 5 & MICHIGAN & ITALY & ITALY & & & STRAMBELLO. TO & LAURIUM ST. & CALUMET \\
\hline 1177 & & BAUDINO & MADELINE & $\mathrm{H}$ & 26 & ITALY & ITALY & ITALY & 1916 & & & LAURIUM ST. & CALUMET \\
\hline 1178 & & BAUDINO & JOHN & $\mathrm{s}$ & 3 & MICHIGAN & ITALY & ITALY & & & RIVAROLO CANAVESE. TO & LAURIUM ST. & CALUMET \\
\hline 1179 & 1624 & BIANCIOTTO & GIULIO & $\mathrm{H}$ & 38 & ITALY & ITALY & ITALY & 1906 & MINER/CM & TORINO & LAURIUM ST. & CALUMET \\
\hline 1180 & & BIANCIOTTO & ANNA & w & 36 & ITALY & ITALY & ITALY & 1907 & & & LAURIUM ST. & CALUMET \\
\hline 1181 & & BIANCIOTTO & CATERINA & D & 11 & MICHIGAN & ITALY & ITALY & & & TORINO & LAURIUM ST. & CALUMET \\
\hline 1182 & & BIANCIOTTO & MAUD & $\mathrm{D}$ & 8 & MICHIGAN & ITALY & ITALY & & & TORINO & LAURIUM ST. & CALUMET \\
\hline 1183 & & BIANCIOTTO & THOMAS & $\mathrm{s}$ & 7 & MICHIGAN & ITALY & ITALY & & & TORINO & LAURIUM ST. & CALUMET \\
\hline 1184 & 1621 & SCIACERO & MARTIN & $\mathrm{H}$ & 43 & ITALY & ITALY & ITALY & 1898 & MINER/CM & SAN MARTINO CANAVESE. TO & LAURIUM ST. & CALUMET \\
\hline 1185 & & SCIACERO & CATERINA & W & 42 & ITALY & ITALY & ITALY & 1901 & & & LAURIUM ST. & CALUMET \\
\hline 1186 & & SCIACERO & ANNA & $\mathrm{D}$ & $25 ?$ & MICHIGAN & ITALY & ITALY & & & SAN MARTINO CANAVESE. TO & LAURIUM ST. & CALUMET \\
\hline 1187 & & SCIACERO & PETER & $\mathrm{S}$ & 12 & MICHIGAN & ITALY & ITALY & & & SAN MARTINO CANAVESE. TO & LAURIUM ST. & CALUMET \\
\hline 1188 & & CASTIGLIANO & DOMENICO & $\mathrm{H}$ & 50 & ITALY & ITALY & ITALY & 1899 & LABORER/CM & SALASSA. TO & LAURIUM ST. & CALUMET \\
\hline 1189 & & CASTIGLIANO & CATERINA & w & 45 & ITALY & ITALY & ITALY & 1900 & & SALASSA. TO & LAURIUM ST. & CALUMET \\
\hline 1190 & & CASTIGLIANO & JENNIE & D & 21 & ITALY & ITALY & ITALY & 1900 & & SALASSA. TO & LAURIUM ST. & CALUMET \\
\hline 1191 & & CASTIGLIANO & LUIGI & $\mathrm{s}$ & 10 & MICHIGAN & ITALY & ITALY & & & SALASSA, TO & LAURIUM ST. & $\begin{array}{l}\text { CALUMET } \\
\end{array}$ \\
\hline 1192 & & CASTIGLIANO & LINA & $\mathrm{D}$ & 8 & MICHIGAN & ITALY & ITALY & & & SALASSA. TO & LAURIUM ST. & CALUMET \\
\hline 1193 & 1619 & BIANCO & ANTONIO & $\mathrm{H}$ & 53 & ITALY & ITALY & ITALY & 1890 & MINER/CM & LOCANA CANAVESE. TO & LAURIUM ST. & CALUMET \\
\hline 1194 & & BIANCO & ANNA & W & 40 & ITALY & ITA & ITALY & 1900 & & & LAURIUM ST. & CALUMET \\
\hline 1195 & & MARTA & JOHN & SOIL & 31 & ITALY & ITALY & ITALY & 1913 & MINER/CM & TORINO & LAURIUM ST. & CALUMET \\
\hline 1196 & & MARTA & MINNIE & D & 25 & MICHIGAN & ITALY & ITALY & & & LOCANA CANAVESE. TO & LAURIUM ST. & CALUMET \\
\hline
\end{tabular}




\begin{tabular}{|c|c|c|c|c|c|c|c|c|c|c|c|c|c|}
\hline 1197 & & MARTA & BARBARA & GD & 4 & MICHIGAN & ITALY & ITALY & & & LOCANA CANAVESE. TO & LAURIUM ST & CALUMET \\
\hline 1198 & 1616 & GRANDIS & PAUL & $\mathrm{H}$ & 54 & ITALY & ITALY & ITALY & 1890 & MINER/CM & SAN GIORGIO CANAVESE. TO & LAURIUM ST. & CALUMET \\
\hline 1199 & & GRANDIS & TERESA & W & 36 & ITALY & ITALY & ITALY & 1891 & & & LAURIUM ST & CALUMET \\
\hline 1200 & & GRANDIS & CARMEN & D & 17 & MICHIGAN & ITALY & ITALY & & SALESLADY/GROCERY STORE & SAN GIORGIO CANAVESE. TO & LAURIUM ST. & $\begin{array}{l}\text { CALUMET } \\
\end{array}$ \\
\hline 1201 & & GRANDIS & PAUL JR. & $\mathrm{s}$ & 7 & MICHIGAN & ITALY & ITALY & & & SAN GIORGIO CANAVESE. TO & LAURIUM ST. & CALUMET \\
\hline 1202 & & ONORE & JOHN & B & 36 & ITALY & ITALY & ITALY & 1906 & MINER/CM & STRAMBINELLO. TO & LAURIUM ST. & CALUMET \\
\hline 1203 & & ONORE & IRENE & B & 8 & MICHIGAN & ITALY & ITALY & & & STRAMBINELLO. TO & LAURIUM ST. & $\begin{array}{l}\text { CALUMET } \\
\text { CALE }\end{array}$ \\
\hline 1204 & & ONORE & TRACY & B & 7 & MICHIGAN & ITALY & ITALY & & & STRAMBINELLO. TO & LAURIUM ST. & CALUMET \\
\hline 1205 & & ONORE & JAMES & $\begin{array}{l}\mathrm{D} \\
\mathrm{B}\end{array}$ & 6 & MICHIGAN & ITALY & ITALY & & & STRAMBINELLO. TO & LAURIUM ST & CALUMET \\
\hline 1206 & & ANSIE & MARY & AUNT & 77 & ITALY & ITALY & ITALY & 1891 & & & LAURIUM ST & CALUMET \\
\hline 1207 & 1615 & CENTANINO & JOHN & $\mathrm{H}$ & 43 & ITALY & ITALY & ITALY & 1890 & LABORER/CM & SAN MARTINO CANAVESE. TO & LAURIUM ST & $\begin{array}{l}\text { CALUMET } \\
\end{array}$ \\
\hline 1208 & & CENTANINO & DOMENICA & W & 38 & ITALY & ITALY & ITALY & 1887 & & & LAURIUM ST. & CALUMET \\
\hline 1209 & & CENTANINO & DOMENICO & $\mathrm{s}$ & 17 & MICHIGAN & ITALY & ITALY & & & SAN MARTINO CANAVESE. TO & LAURIUM ST & CALUMET \\
\hline 1210 & & CAMPAGNOLA & BEN & $\mathrm{H}$ & 38 & ITALY & ITALY & ITALY & 1914 & MINER/CM & SAN MARTINO CANAVESE. TO & LAURIUM ST. & CALUMET \\
\hline 1211 & & CAMPAGNOLA & REGINA & W & 24 & MICHIGAN & ITALY & ITALY & & & & LAURIUM ST. & CALUMET \\
\hline 1212 & & CAMPAGNOLA & JAMES & $\mathrm{H}$ & 36 & ITALY & ITALY & ITALY & 1889 & MINER/CM & SAN MARTINO CANAVESE, TO & LAURIUM ST. & CALUMET \\
\hline 1213 & & CAMPAGNOLA & DOMENICA & W & 32 & ITALY & ITALY & ITALY & 1907 & & & LAURIUM ST & CALUMET \\
\hline 1214 & & CAMPAGNOLA & FRANCES & D & 12 & MICHIGAN & ITALY & ITALY & & & SAN MARTINO CANAVESE. TO & LAURIUM ST. & CALUMET \\
\hline 1215 & & CAMPAGNOLA & MARY & $\mathrm{D}$ & 8 & MICHIGAN & ITALY & ITALY & & & SAN MARTINO CANAVESE. TO & LAURIUM ST. & CALUMET \\
\hline 1216 & & BRONZO & DOMENICO & B & 30 & ITALY & ITALY & ITALY & 1914 & TIMBERMAN/CM & SAN MARTINO CANAVESE. TO & LAURIUM ST. & CALUMET \\
\hline 1217 & & COPPO & ERNEST & B & 29 & ITALY & ITALY & ITALY & 1914 & TIMBERMAN/CM & PONT CANAVESE. TO & LAURIUM ST. & $\begin{array}{l}\text { CALUMET } \\
\end{array}$ \\
\hline 1218 & 1614 & BRONZO & JOSEPH & $\mathrm{H}$ & 38 & ITALY & ITALY & ITALY & 1914 & MINER/CM & SAN MARTINO CANAVESE, TO & LAURIUM ST. & CALUMET \\
\hline 1219 & & BRONZO & MARY & w & 28 & MICHIGAN & ITALY & ITALY & & & & LAURIUM ST & CALUMET \\
\hline 1220 & & BRONZO & ANTONIO & s & 3 & MICHIGAN & ITALY & MICHIGAN & & & SAN MARTINO CANAVESE. TO & LAURIUM ST. & CALUMET \\
\hline 1221 & & BRONZO & MARY & D & 1 & MICHIGAN & ITALY & MICHIGAN & & & SAN MARTINO CANAVESE. TO & LAURIUM ST. & CALUMET \\
\hline 1222 & & GALLO & JAMES & $\mathrm{H}$ & $60 ?$ & ITALY & ITALY & ITALY & 1887 & WATCHMAN/CM & SAN GIORGIO CANAVESE. TO & LAURIUM ST & CALUMET \\
\hline 1223 & & GALLO & JENNIE & W & 62 & ITALY & ITALY & ITALY & 1890 & & & LAURIUM ST. & CALUMET \\
\hline 1224 & & GALLO & MARY & $\mathrm{D}$ & 19 & MICHIGAN & ITALY & ITALY & & & SAN GIORGIO CANAVESE, TO & LAURIUM ST. & CALUMET \\
\hline 1225 & 1617 & ROVANO & JOHN & $\mathrm{H}$ & 40 & ITALY & ITALY & ITALY & 1914 & MINER/CM & SAN MARTINO CANAVESE. TO & LAURIUM ST. & CALUMET \\
\hline 1226 & & ROVANO & MINNIE & W & 33 & ITALY & ITALY & ITALY & 1914 & & SAN MARTINO CANAVESE. TO & LAURIUM ST. & $\begin{array}{l}\text { CALUMET } \\
\end{array}$ \\
\hline 1227 & & ROVANO & PETER & $\mathrm{s}$ & $4 ?$ & MICHIGAN & ITALY & ITALY & & & SAN MARTINO CANAVESE. TO & LAURIUM ST. & $\begin{array}{l}\text { CALUMET } \\
\end{array}$ \\
\hline 1228 & 1617 & CAMPAGNOLA & JAMES & $\mathrm{H}$ & 29 & ITALY & ITALY & ITALY & 1905 & MINER/CM & SAN MARTINO CANAVESE. TO & LAURIUM ST. & CALUMET \\
\hline 1229 & & CAMPAGNOLA & ANGELA & W & 20 & MICHIGAN & ITALY & ITALY & & & & LAURIUM ST. & $\begin{array}{l}\text { CALUMET } \\
\end{array}$ \\
\hline 1230 & 1607 & MIGLIO/MIGLIA & CARRIE & $\mathrm{H}$ & 55 & ITALY & ITALY & ITALY & 1892 & & TORINO & LAURIUM ST. & CALUMET \\
\hline 1231 & & MIGLIO/MIGLIA & LOUIS & s & 24 & MICHIGAN & ITALY & ITALY & & LABORER/CM & TORINO & LAURIUM ST & CALUMET \\
\hline 1232 & & MIGLIO/MIGLIA & JOSEPH & $\mathrm{s}$ & 23 & MICHIGAN & ITALY & ITALY & & MINER/CM & TORINO & LAURIUM ST & CALUMET \\
\hline 1233 & & MIGLIO/MIGLIA & JENNIE & DIL & 26 & MICHIGAN & ITALY & ITALY & & & & LAURIUM ST & CALUMET \\
\hline 1234 & & MIGLIO/MIGLIA & CARRIE & $\mathrm{GD}$ & 3 & MICHIGAN & ITALY & ITALY & & & TORINO & LAURIUM ST & CALUMET \\
\hline 1235 & & PEROTTI & PETER & B & 44 & ITALY & ITALY & ITALY & 1897 & TIMBERMAN/CM & CASTELLAMONTE. TO & LAURIUM ST. & CALUMET \\
\hline 1236 & 1604 & GIULIO & ANTONIO & $\mathrm{H}$ & 60 & ITALY & ITALY & ITALY & 1884 & LABORER/CM & SAN GIORGIO CANAVESE. TO & LAURIUM ST. & CALUMET \\
\hline 1237 & & GIULIO & MARY & W & 57 & ITALY & ITALY & ITALY & 1891 & & & LAURIUM ST. & CALUMET \\
\hline 1238 & & GIULIO & DOMENICO & $\mathrm{s}$ & 25 & MICHIGAN & ITALY & ITALY & & TEAMSTER/CM & SAN GIORGIO CANAVESE. TO & LAURIUM ST. & CALUMET \\
\hline 1239 & & GIULIO & GIULIA & D & 21 & MICHIGAN & ITALY & ITALY & & SALESLADY/GROCERY STORE & SAN GIORGIO CANAVESE. TO & LAURIUM ST & CALUMET \\
\hline 1240 & & GIULIO & PHILLIES & $\mathrm{D}$ & 20 & MICHIGAN & ITALY & ITALY & & & SAN GIORGIO CANAVESE. TO & LAURIUM ST. & $\begin{array}{l}\text { CALUMET } \\
\end{array}$ \\
\hline 1241 & & GIULIO & ANTONIO & $\mathrm{s}$ & 13 & MICHIGAN & ITALY & ITALY & & & SAN GIORGIO CANAVESE. TO & LAURIUM ST. & CALUMET \\
\hline 1242 & & GIULIO & MARGHERITA & $\mathrm{D}$ & 12 & MICHIGAN & ITALY & ITALY & & & SAN GIORGIO CANAVESE. TO & LAURIUM ST & CALUMET \\
\hline 1243 & 1603 & BARSICA & ANTONIO & $\mathrm{H}$ & 48 & ITALY & ITALY & ITALY & 1897 & MINER/CM & SAN MARTINO CANAVESE. TO & LAURIUM ST & CALUMET \\
\hline 1244 & & BARSICA & DOMENICA & w & 45 & ITALY & ITALY & ITALY & 93?? & 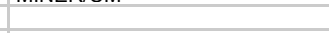 & & LAURIUM ST & $\begin{array}{l}\text { CALUMET } \\
\end{array}$ \\
\hline 1245 & & BARSICA & CATERINA & D & 14 & MICHIGAN & ITALY & ITALY & & & SAN MARTINO CANAVESE. TO & LAURIUM ST. & CALUMET \\
\hline 1246 & & BARSICA & FRANCESCO & B & 32 & ITALY & ITALY & ITALY & 11?? & MINER/CM & SAN MARTINO CANAVESE. TO & LAURIUM ST & CALUMET \\
\hline 1247 & & SAPINO & DOMENICO & B & 30 & ITALY & ITALY & ITALY & 1905 & MINER/CM & SAN MARTINO CANAVESE. TO & LAURIUM ST & CALUMET \\
\hline 1248 & & PANKNECHLIN & ANDREA & B & 44 & ITALY & ITALY & ITALY & 1910 & LABORER/CM & & LAURIUM ST. & CALUMET \\
\hline 1249 & 1601 & FAUSONE & FRANK & $\mathrm{H}$ & 52 & ITALY & ITALY & ITALY & 1913 & LABORER/CM & TORINO & LAURIUM ST. & CALUMET \\
\hline 1250 & 1601 & FAUSONE & GIULIA & W & 54 & ITALY & ITALY & ITALY & 1919 & & & LAURIUM ST. & CALUMET \\
\hline 1251 & & FAUSONE & MINNIE & D & 25 & MICHIGAN & ITALY & ITALY & & & TORINO & LAURIUM ST. & CALUMET \\
\hline 1252 & & FAUSONE & FRANK & $\mathrm{s}$ & 23 & MICHIGAN & ITALY & ITALY & & SHIPPING CLERK & TORINO & LAURIUM ST. & CALUMET \\
\hline 1253 & & FAUSONE & CATERINA & D & 14 & MICHIGAN & ITALY & ITALY & & & TORINO & LAURIUM ST. & CALUMET \\
\hline 1254 & 1618 & QUELLO & PETER & $\mathrm{H}$ & 34 & ITALY & ITALY & ITALY & 1910 & LABORER/CM & LOCANA CANAVESE, TO & LAURIUM ST. & $\begin{array}{l}\text { CALUMET } \\
\end{array}$ \\
\hline 1255 & 00 & QUELLO & MARIA & D & 6 & MICHIGAN & ITALY & ITALY & 1010 & LADURLTIUIU & LOCANA CANAVESE. TO & LAURIUM ST & CALUMET \\
\hline 1256 & & QUELLO & PIERINA & D & 5 & MICHIGAN & ITALY & ITALY & & & LOCANA CANAVESE. TO & LAURIUM ST. & CALUMET \\
\hline 1257 & & QUELLO & JOHN & $\mathrm{s}$ & 4 & MICHIGAN & ITALY & ITALY & & & LOCANA CANAVESE. TO & LAURIUM ST. & CALUMET \\
\hline 1258 & & & BACC? & $\mathrm{BR}$ & 51 & ITALY & ITA & ITALY & $13 ? ?$ & BLACKSMITH & LOCANA CANAVESE, TO & LAURIUM ST. & CALUMET \\
\hline 1259 & 1622 & MUSSATO & ORESTE & $\mathrm{H}$ & 26 & ITALY & ITALY & ITALY & 1910 & $\begin{array}{l}\text { TIMBERMAN/CM } \\
\text { TIM }\end{array}$ & BAIRO CANAVESE TO & LAURIUM ST. & CALUMET \\
\hline
\end{tabular}




\begin{tabular}{|c|c|c|c|c|c|c|c|c|c|c|c|c|c|}
\hline 1260 & & MUSSATO & DOMENICA & W & 34 & ITALY & ITALY & ITALY & 1909 & & TORINO & LAURIUM ST. & CALUMET \\
\hline 1261 & & ROVANO & ANTONIO & SSO & 12 & ITALY & ITALY & ITALY & 1909 & & TORINO & LAURIUM ST. & CALUMET \\
\hline 1262 & & ROVANO & DOMENICO & B & 26 & ITALY & ITALY & ITALY & 1910 & TIMBERMAN/CM & SAN MARTINO CANAVESE. TO & LAURIUM ST. & CALUMET \\
\hline 1263 & & MUSSATO & ERNEST & B & 46 & ITALY & ITALY & ITALY & 1880 & TIMBERMAN/CM & BAIRO CANAVESE. TO & LAURIUM ST. & CALUMET \\
\hline 1264 & 1628 & DIGHERA & CAROLINA & $\mathrm{H}$ & 46 & ITALY & ITALY & ITALY & 1880 & & TORINO & LAURIUM ST. & CALUMET \\
\hline 1265 & & DIGHERA & THOMAS & $\mathrm{s}$ & 16 & MICHIGAN & ITALY & ITALY & & TEAMSTER/GARBAGE & SAN GIORGIO CANAVESE. TO & LAURIUM ST. & CALUMET \\
\hline 1266 & & DIGHERA & FRANK & $\mathrm{s}$ & 15 & MICHIGAN & ITALY & ITALY & & & SAN GIORGIO CANAVESE. TO & LAURIUM ST. & $\begin{array}{l}\text { CALUMET } \\
\end{array}$ \\
\hline 1267 & & MANTINO & DOMENICO & $\mathrm{H}$ & 46 & ITALY & ITALY & ITALY & 1890 & TIMBERMAN/CM & PEROSA. TO & BOUNDARY ST & $\begin{array}{l}\text { CALUMET } \\
\end{array}$ \\
\hline 1268 & & MANTINO & MARIA & W & 46 & ITALY & ITALY & ITALY & 1891 & 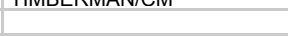 & TERUOA. TO & BOUNDARY ST & CALUMET \\
\hline 1269 & & MANTINO & TRACY & $\mathrm{D}$ & 9 & MICHIGAN & ITALY & ITALY & & & PEROSA. TO & BOUNDARY ST & CALUMET \\
\hline 1270 & 1762 & FRANCISCA & JOHN & $\mathrm{H}$ & 63 & ITALY & ITALY & ITALY & 1885 & MINER/CM & PEROSA. TO & BOUNDARY ST & $\begin{array}{l}\text { CALUMET } \\
\end{array}$ \\
\hline 1271 & & FRANCISCA & ANGELINA & W & 65 & ITALY & ITALY & ITALY & 1895 & & PEROSA. TO & BOUNDARY ST & CALUMET \\
\hline 1272 & & FRANCISCA & JOHN & $\mathrm{s}$ & 27 & ITALY & ITALY & ITALY & 1895 & MINER/CM & PEROSA. TO & BOUNDARY ST & CALUMET \\
\hline 1273 & $17 ? ?$ & PISTOTTI & MARIO & $\mathrm{H}$ & 40 & ITALY & ITALY & ITALY & 1900 & LABORER/CM & SPARONE. TO & BOUNDARY ST & CALUMET \\
\hline 1274 & & PISTOTTI & ANGELA & W & 30 & MICHIGAN & ITALY & ITALY & & & & BOUNDARY ST & CALUMET \\
\hline 1275 & & PISTOTTI & MARIA & D & 2 & MICHIGAN & ITALY & MICHIGAN & & & SPARONE, TO & BOUNDARY ST & CALUMET \\
\hline 1276 & & PISTOTTI & CATERINA & D & 6 & MICHIGAN & ITALY & MICHIGAN & & & SPARONE. TO & BOUNDARY ST & CALUMET \\
\hline 1277 & 1724 & SAPINO & ANTONIO & $\mathrm{H}$ & 35 & ITALY & ITALY & ITALY & 1907 & MINER/CM & SAN MARTINO CANAVESE. TO & BOUNDARY ST & CALUMET \\
\hline 1278 & & SAPINO & JENNIE & W & 35 & ITALY & ITALY & ITALY & 1910 & & & BOUNDARY ST & CALUMET \\
\hline 1279 & & SAPINO & CATHERINE & $\mathrm{D}$ & 7 & MICHIGAN & ITALY & ITALY & & & SAN MARTINO CANAVESE. TO & BOUNDARY ST & CALUMET \\
\hline 1280 & 1722 & SCIACERO & LUIGI & $\mathrm{H}$ & 59 & ITALY & ITALY & ITALY & 1890 & MINER/CM & SAN MARTINO CANAVESE. TO & BOUNDARY ST & CALUMET \\
\hline 1281 & 1122 & SCIACERO & MARIA & w & 58 & ITALY & ITALY & ITALY & 1894 & MIIINER/CIVI & SAIV IVIARTIINU CAIVAVESE. TO & BOUNDARY ST & CALUMET \\
\hline 1282 & & SCIACERO & JOHN & $\mathrm{s}$ & 21 & MICHIGAN & ITALY & ITALY & & LABORER/CM & SAN MARTINO CANAVESE. TO & BOUNDARY ST & CALUMET \\
\hline 1283 & & SCIACERO & PHILLIES & D & 19 & MICHIGAN & ITALY & ITALY & & & SAN MARTINO CANAVESE. TO & BOUNDARY ST & $\begin{array}{l}\text { CALUMET } \\
\end{array}$ \\
\hline 1284 & 1798 & GIVOGRE & JAMES & $\mathrm{H}$ & 28 & MICHIGAN & ITALY & ITALY & & PAYCHECKICM & AGLIE'. TO & & CALUMET \\
\hline 1285 & & GIVOGRE & CATHERINE & W & 25 & MICHIGAN & ITALY & ITALY & & & & & CALUMET \\
\hline 1286 & 1790 & COLOMBO & MIKE & $\mathrm{H}$ & 29 & ITALY & ITALY & ITALY & 1908 & MINER/CM & TORINO & CEMETERY ST. & CALUMET \\
\hline 1287 & & COLOMBO & TRACY & W & 26 & ITALY & ITALY & ITALY & 1911 & & & CEMETERY ST. & CALUMET \\
\hline 1288 & & COLOMBO & JOHN & $\mathrm{S}$ & 5 & MICHIGAN & ITALY & ITALY & & & TORINO & CEMETERY ST. & CALUMET \\
\hline 1289 & & COLOMBO & JOSEPH & BR & 32 & ITALY & ITALY & ITALY & 1910 & MINER/CM & TORINO & CEMETERY ST. & $\begin{array}{l}\text { CALUMET } \\
\end{array}$ \\
\hline 1290 & 1788 & GIVOGRE & BATTISTA & $\mathrm{H}$ & 63 & ITALY & ITALY & ITALY & 1889 & JANITOR/WASHER ROOM & AGLIE'. TO & CEMETERY ST. & $\begin{array}{l}\text { CALUMET } \\
\end{array}$ \\
\hline 1291 & & GIVOGRE & MARY & W & 60 & ITALY & ITALY & ITALY & 1889 & & & CEMETERY ST. & CALUMET \\
\hline 1292 & 515 & SOSSI/SUSSI & JAMES & $\mathrm{H}$ & 41 & ITALY & ITALY & ITALY & 1898 & MINER/CM & VIALFRE'. TO & 4TH ST. & CALUMET - BLUE JACKET \\
\hline 1293 & 010 & SOSSI/SUSSI & ANGELINA & W & $\begin{array}{l}41 \\
34 \\
4\end{array}$ & ITALY & ITALY & ITALY & 1903 & IVIIIVER/UIVI & VIALTKE. TO & 4TH ST. & CALUMET - BLUE JACKET \\
\hline 1294 & & SOSSI/SUSSI & LUCY & $\mathrm{D}$ & 14 & MICHIGAN & ITALY & ITALY & & & VIALFRE'. TO & 4TH ST. & CALUMET - BLUE JACKET \\
\hline 1295 & & SOSSI/SUSSI & ANTONIO & $\mathrm{s}$ & 11 & MICHIGAN & ITALY & ITALY & & & VIALFRE'. TO & 4TH ST. & CALUMET - BLUE JACKET \\
\hline 1296 & & SOSSI/SUSSI & CATERINA & $\mathrm{D}$ & 8 & MICHIGAN & ITALY & ITALY & & & VIALFRE'. TO & 4TH ST. & CALUMET - BLUE JACKET \\
\hline 1297 & & SOSSI/SUSSI & MIKE & $\mathrm{s}$ & 7 & MICHIGAN & ITALY & ITALY & & & VIALFRE'. TO & 4TH ST. & CALUMET - BLUE JACKET \\
\hline 1298 & & SOSSI/SUSSI & JOSEPH & BR & 45 & ITALY & ITALY & ITALY & 1902 & MOTORMAN/ROCK HOUSE & VIALFRE'. TO & 4TH ST. & CALUMET - BLUE JACKET \\
\hline 1299 & 521 & MICHETTI & FRED & $\mathrm{H}$ & 58 & ITALY & ITALY & ITALY & 1896 & MINER/CM & PONT CANAVESE. TO & 4TH ST. & CALUMET - BLUE JACKET \\
\hline 1300 & & MICHETTI & JOHANNA & W & 54 & ITALY & ITALY & ITALY & 1900 & & PONT CANAVESE. TO & 4TH ST. & CALUMET - BLUE JACKET \\
\hline 1301 & & MICHETTI & ANNA & D & 20 & ITALY & ITALY & ITALY & 1900 & WAITRESS/RESTAURANT & PONT CANAVESE. TO & 4TH ST. & CALUMET - BLUE JACKET \\
\hline 1302 & & MICHETTI & KATE & D & 16 & MICHIGAN & ITALY & ITALY & & & PONT CANAVESE. TO & 4TH ST. & CALUMET - BLUE JACKET \\
\hline 1303 & & MICHETTI & MAUD & D & 13 & MICHIGAN & ITALY & ITALY & & & PONT CANAVESE. TO & 4TH ST. & CALUMET - BLUE JACKET \\
\hline 1304 & 524 & BARATONO & DOMENICO & $\mathrm{H}$ & 42 & ITALY & ITALY & ITALY & 1900 & MINER/CM & VIALFRE'. TO & 4TH ST. & CALUMET - BLUE JACKET \\
\hline 1305 & 024 & BARATONO & ROSA & w & 38 & ITALY & ITALY & ITALY & 1909 & 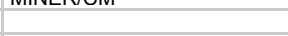 & 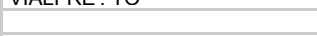 & 4TH ST. & CALUMET - BLUE JACKET \\
\hline 1306 & & BARATONO & JAMES & $\mathrm{s}$ & 11 & MICHIGAN & ITALY & ITALY & & & VIALFRE'. TO & 4TH ST. & CALUMET - BLUE JACKET \\
\hline 1307 & & BARATONO & ANNE & D & 5 & MICHIGAN & ITALY & ITALY & & & VIALFRE'. TO & 4TH ST. & CALUMET - BLUE JACKET \\
\hline 1308 & 520 & GAMARRA & JAMES & $\mathrm{H}$ & 36 & ITALY & ITALY & ITALY & 1907 & TIMBERMAN/CM & SAN BENIGNO CANAVESE. TO & 4TH ST. & CALUMET - BLUE JACKET \\
\hline 1309 & & GAMARRA & MARY & W & 29 & COLORADO & ITALY & ITALY & & & & 4TH ST. & CALUMET - BLUE JACKET \\
\hline 1310 & & GAMARRA & NORBERT & $\mathrm{s}$ & 6 & MICHIGAN & ITALY & COLORADO & & & SAN BENIGNO CANAVESE. TO & 4TH ST. & CALUMET - BLUE JACKET \\
\hline 1311 & & GAMARRA & WINFRED & $\mathrm{s}$ & $6 \mathrm{M}$ & MICHIGAN & ITALY & COLORADO & & & SAN BENIGNO CANAVESE, TO & 4TH ST. & CALUMET - BLUE JACKET \\
\hline 1312 & 573 & PASQUINELLI & PIA & $\mathrm{H}$ & 67 & ITALY & ITALY & ITALY & 1878 & & & CEDAR ST. & CALUMET - BLUE JACKET \\
\hline 1313 & & PASQUINELLI & CARLO & $\mathrm{S}$ & 34 & MICHIGAN & ITALY & ITALY & & TEAMSTER/HARDWARE & LUCCA & CEDAR ST. & CALUMET - BLUE JACKET \\
\hline 1314 & & PASQUINELLI & VITTORIO & $\mathrm{s}$ & 31 & MICHIGAN & ITALY & ITALY & & SALESMAN & LUCCA & CEDAR ST. & CALUMET - BLUE JACKET \\
\hline 1315 & & PASQUINELLI & MARTA & D & 23 & MICHIGAN & ITALY & ITALY & & SALESLADY/SHOE STORE & LUCCA & CEDAR ST. & CALUMET - BLUE JACKET \\
\hline 1316 & 587 & VIGO & MARCO & $\mathrm{H}$ & 57 & ITALY & ITALY & ITALY & 1899 & LABORER/CM & CORIO. TO & CEDAR ST. & CALUMET - BLUE JACKET \\
\hline 1317 & & VIGO & CATERINA & W & 47 & ITALY & ITALY & ITALY & 1901 & & & CEDAR ST. & CALUMET - BLUE JACKET \\
\hline 1318 & & VIGO & MARNIE & D & 17 & MICHIGAN & ITALY & ITALY & & & CORIO. TO & CEDAR ST. & CALUMET - BLUE JACKET \\
\hline 1319 & & VIGO & GEORGE & $\mathrm{s}$ & 16 & MICHIGAN & ITALY & ITALY & & & CORIO. TO & CEDAR ST. & CALUMET - BLUE JACKET \\
\hline 1320 & & & MIKE & B & 38 & ITALY & ITA & ITALY & 1903 & & SAN GIORGIO & CEDAR ST. & CALUMET - BLUE JACKET \\
\hline 1321 & & CAMINO & ANTONIO & B & 37 & ITALY & ITALY & ITALY & 1906 & TIMBERMAN/CM & SAN BENIGNO CANAVESE. TO & CEDAR ST. & CALUMET \\
\hline 1322 & & TRENTO & ATTILIO & B & 20 & ITALY & ITALY & ITALY & 1917 & TRAMMER/CM & & CEDAR ST. & CALUMET - BLUE JACKET \\
\hline
\end{tabular}




\begin{tabular}{|c|c|c|c|c|c|c|c|c|c|c|c|c|c|}
\hline 1323 & & MARCON & VITTORIO & B & 28 & ITALY & ITALY & ITALY & 1913 & TIMBERMAN/CM & TREVISO & CEDAR ST. & CALUMET - BLUE JACKET \\
\hline 1324 & 566 & PONSETTO & GIORGIO & $\mathrm{H}$ & 58 & ITALY & ITALY & ITALY & 1890 & LABORER/CM & SAN GIORGIO CANAVESE. TO & CEDAR ST. & CALUMET - BLUE JACKET \\
\hline 1325 & & PONSETTO & JENNIE & W & 57 & ITALY & ITALY & ITALY & 1904 & & & CEDAR ST. & CALUMET - BLUE JACKET \\
\hline 1326 & 532 & DE FILIPPI & JOHN & $\mathrm{H}$ & 67 & ITALY & ITALY & ITALY & 1882 & NOT CLEAR/CM & SAN GIORGIO CANAVESE. TO & 3RD ST. & CALUMET - BLUE JACKET \\
\hline 1327 & & DE FILIPPI & TRACY & w & 60 & ITALY & ITALY & ITALY & 1897 & & & 3RD ST. & CALUMET - BLUE JACKET \\
\hline 1328 & & DE FILIPPI & LINA & D & 28 & MICHIGAN & ITALY & ITALY & & & SAN GIORGIO CANAVESE. TO & 3RD ST. & CALUMET - BLUE JACKET \\
\hline 1329 & & DE FILIPPI & ANGELO & $\mathrm{s}$ & 26 & MICHIGAN & ITALY & ITALY & & MINER/CM & SAN GIORGIO CANAVESE. TO & 3RD ST. & CALUMET - BLUE JACKET \\
\hline 1330 & & DE FILIPPI & JOHN & s & 24 & ITALY & ITALY & ITALY & 1898 & MINER/CM & SAN GIORGIO CANAVESE. TO & 3RD ST. & CALUMET - BLUE JACKET \\
\hline 1331 & 534 & DE FILIPPI & PETER & $\mathrm{H}$ & 40 & ITALY & ITALY & ITALY & 1896 & MINER/CM & SAN GIORGIO CANAVESE. TO & 3RD ST. & CALUMET - BLUE JACKET \\
\hline 1332 & & DE FILIPPI & PASQUINA & W & 41 & ITALY & ITALY & ITALY & 1899 & & & 3RD ST. & CALUMET - BLUE JACKET \\
\hline 1333 & & DE FILIPPI & JOHN & $\mathrm{s}$ & 17 & MICHIGAN & ITALY & ITALY & & & SAN GIORGIO CANAVESE. TO & 3RD ST. & CALUMET - BLUE JACKET \\
\hline 1334 & & NARRA-GEDDA & LUCIA & $\mathrm{H}$ & 28 & ITALY & ITALY & ITALY & 1912 & & TORINO & 3RD ST. & CALUMET - BLUE JACKET \\
\hline 1335 & & NARRA & ANDREW & $\mathrm{s}$ & 6 & MICHIGAN & ITALY & ITALY & & & PONT CANAVESE. TO & 3RD ST. & CALUMET - BLUE JACKET \\
\hline 1336 & 525 & SADO & JOSEPH & $\mathrm{H}$ & 41 & ITALY & ITALY & ITALY & 1900 & CARPENTER/CM & STRAMBINELLO. TO & 3RD ST. & CALUMET - BLUE JACKET \\
\hline 1337 & & SADO & CATERINA & w & 39 & ITALY & ITALY & ITALY & 1892 & & & 3RD ST. & CALUMET - BLUE JACKET \\
\hline 1338 & & DE FILIPPI & ANTONIO & $\mathrm{H}$ & 33 & MICHIGAN & ITALY & ITALY & & MOTORMAN/CM & SAN GIORGIO CANAVESE, TO & 3RD ST. & CALUMET - BLUE JACKET \\
\hline 1339 & & DE FILIPPI & MARY & W & 24 & MICHIGAN & POLAND & POLAND & & & SAN GIORGIO CANAVESE. TO & 3RD ST. & CALUMET - BLUE JACKET \\
\hline 1340 & 570 & RICCA & BATTISTA & $\mathrm{H}$ & 34 & ITALY & ITALY & ITALY & 1905 & LABORER/CM & RIVAROLO CANAVESE. TO & WATER WORKS & CALUMET - BLUE JACKET \\
\hline 1341 & & RICCA & VIRGINIA & W & 34 & MICHIGAN & ITALY & ITALY & & & & WATER WORKS & CALUMET - BLUE JACKET \\
\hline 1342 & & RICCA & GEORGE & s & 13 & MICHIGAN & ITALY & MICHIGAN & & & RIVAROLO CANAVESE. TO & WATER WORKS & CALUMET - BLUE JACKET \\
\hline 1343 & 625 & DE FILIPPI & DOMENICO & $\mathrm{H}$ & 62 & ITALY & ITALY & ITALY & 1888 & & TORINO & WATER WORKS & CALUMET - BLUE JACKET \\
\hline 1344 & 020 & DE FILIPPI & TOSCA & W & 44 & $\begin{array}{l}\text { ITALY } \\
\text { ITAL }\end{array}$ & $\begin{array}{l}\text { ITALY } \\
\text { ITAL }\end{array}$ & ITALY & UN & & TORIIN & WATER WORKS & CALUMET - BLUE JACKET \\
\hline 1345 & 730 & PELLEGRINI & LUIGI & $\mathrm{H}$ & 47 & ITALY & ITALY & ITALY & 1893 & MINER/CM & BERGAMO & WATER WORKS & CALUMET - BLUE JACKET \\
\hline 1346 & & PELLEGRINI & GILDA & W & 47 & ITALY & ITALY & ITALY & 1892 & & PESCAGLIA. LU & WATER WORKS & CALUMET - BLUE JACKET \\
\hline 1347 & & PELLEGRINI & BEN & SSO & 24 & MICHIGAN & ITALY & ITALY & & & PESCAGLIA. LU & WATER WORKS & CALUMET - BLUE JACKET \\
\hline 1348 & & PELLEGRINI & MARION & SD & 20 & MICHIGAN & ITALY & ITALY & & & PESCAGLIA. LU & WATER WORKS & CALUMET - BLUE JACKET \\
\hline 1349 & & PELLEGRINI & JOSEPH & $\mathrm{s}$ & 14 & MICHIGAN & ITALY & ITALY & & & BERGAMO & WATER WORKS & CALUMET - BLUE JACKET \\
\hline 1350 & & PELLEGRINI & LEO & $\mathrm{s}$ & 11 & MICHIGAN & ITALY & ITALY & & & BERGAMO & WATER WORKS & CALUMET - BLUE JACKET \\
\hline 1351 & & PELLEGRINI & MARGHERITA & D & 9 & MICHIGAN & ITALY & ITALY & & & BERGAMO & WATER WORKS & CALUMET - BLUE JACKET \\
\hline 1352 & 74 & RICARDI & ANTONIO & $\mathrm{H}$ & 46 & ITALY & ITALY & ITALY & 1899 & MINER/CM & SCARMAGNO. TO & EAST PINE ST. & CALUMET \\
\hline 1353 & & RICARDI & CATERINA & W & 45 & ITALY & ITALY & ITALY & 1903 & & & EAST PINE ST. & $\begin{array}{l}\text { CALUMET } \\
\end{array}$ \\
\hline 1354 & & RICARDI & MIKE & $\mathrm{s}$ & 14 & MICHIGAN & ITALY & ITALY & & & SCARMAGNO. TO & EAST PINE ST. & CALUMET \\
\hline 1355 & & RICARDI & MARY & D & 3 & MICHIGAN & ITALY & ITALY & & & SCARMAGNO. TO & EAST PINE ST. & $\begin{array}{l}\text { CALUMET } \\
\end{array}$ \\
\hline 1356 & & RICARDI & ANGELINA & D & 17 & MICHIGAN & ITALY & ITALY & & & SCARMAGNO, TO & EAST PINE ST. & CALUMET \\
\hline 1357 & & ALLEN & AGOSTINO & SIL & 29 & ITALY & ITALY & ITALY & 1914 & TRAMMER/CM & & EAST PINE ST. & CALUMET \\
\hline 1358 & & ALLEN & JOSEPHINE & D & 20 & ITALY & ITALY & ITALY & 1903 & & BAIRO CANAVESE. TO & EAST PINE ST. & CALUMET \\
\hline 1359 & 82 & FAUSONE & JOSEPH & $\mathrm{H}$ & 37 & ITALY & ITALY & ITALY & 1890 & MINER/CM & TORINO & EAST PINE ST. & $\begin{array}{l}\text { CALUMET } \\
\end{array}$ \\
\hline 1360 & & FAUSONE & LENA & W & 27 & ITALY & ITALY & ITALY & 1909 & & & EAST PINE ST. & CALUMET \\
\hline 1361 & & FAUSONE & PETER & $\mathrm{s}$ & 9 & MICHIGAN & ITALY & ITALY & & & TORINO & EAST PINE ST. & CALUMET \\
\hline 1362 & & FAUSONE & JOSEPH & $\mathrm{S}$ & 8 & MICHIGAN & ITALY & ITALY & & & TORINO & EAST PINE ST. & CALUMET \\
\hline 1363 & & FAUSONE & ATTILIO & $\mathrm{S}$ & 1 & MICHIGAN & ITALY & ITALY & & & TORINO & EAST PINE ST. & CALUMET \\
\hline 1364 & 94 & GALETTO & PETER & $\mathrm{H}$ & 36 & ITALY & ITALY & ITALY & 1907 & MINER/CM & SAN GIORGIO CANAVESE. TO & EAST PINE ST. & CALUMET \\
\hline 1365 & & GALETTO & CATERINA & W & 36 & ITALY & ITALY & ITALY & 1907 & & SAN GIORGIO CANAVESE. TO & EAST PINE ST. & CALUMET \\
\hline 1366 & & GALETTO & JOHN & $\mathrm{s}$ & 11 & MICHIGAN & ITALY & ITALY & & & SAN GIORGIO CANAVESE. TO & EAST PINE ST. & CALUMET \\
\hline 1367 & & GALETTO & NETTIE & $\mathrm{D}$ & 9 & MICHIGAN & ITALY & ITALY & & & SAN GIORGIO CANAVESE. TO & EAST PINE ST. & CALUMET \\
\hline 1368 & & GALETTO & JOSEPH & $\mathrm{s}$ & 6 & MICHIGAN & ITALY & ITALY & & & SAN GIORGIO CANAVESE. TO & EAST PINE ST. & CALUMET \\
\hline 1369 & 63 & $\mathrm{FIO}$ & TEODORO & $\mathrm{H}$ & 29 & ITALY & ITALY & ITALY & 1909 & MINER/CM & ROMANO CANAVESE. TO & MINE ST. & CALUMET \\
\hline 1370 & & FIO-DE FILIPPI & VINCENZA & w & 26 & MICHIGAN & ITALY & ITALY & & & TORINO & MINE ST. & $\begin{array}{l}\text { CALUMET } \\
\end{array}$ \\
\hline 1371 & & FIO & JOHN & $\mathrm{s}$ & 5 & MICHIGAN & ITALY & MICHIGAN & & & ROMANO CANAVESE. TO & MINE ST. & CALUMET \\
\hline 1372 & & $\mathrm{FIO}$ & DOMENICO & $\mathrm{s}$ & $10 \mathrm{M}$ & MICHIGAN & ITALY & MICHIGAN & & & ROMANO CANAVESE. TO & MINE ST. & CALUMET \\
\hline 1373 & 57 & RUFFINA & FRANK & $\mathrm{H}$ & 39 & ITALY & ITALY & ITALY & 1899 & TIMBERMAN/CM & SAN GIORGIO CANAVESE. TO & MINE ST. & CALUMET \\
\hline 1374 & & RUFFINA & MARY & W & 41 & ITALY & ITALY & ITALY & 1899 & & SAN GIORGIO CANAVESE, TO & MINE ST. & CALUMET \\
\hline 1375 & & RUFFINA & NETTIE & $\mathrm{D}$ & 15 & MICHIGAN & ITALY & ITALY & & & SAN GIORGIO CANAVESE. TO & MINE ST. & CALUMET \\
\hline 1376 & & RUFFINA & JENNIE & D & 13 & MICHIGAN & ITALY & ITALY & & & SAN GIORGIO CANAVESE. TO & MINE ST. & CALUMET \\
\hline 1377 & & RUFFINA & BARTOLOMEO & $\bar{s}$ & 9 & MICHIGAN & ITALY & ITALY & & & SAN GIORGIO CANAVESE. TO & MINE ST. & CALUMET \\
\hline 1378 & 171 & TOMMEI & ANGELO & H & 48 & ITALY & ITALY & ITALY & 1898 & MASON/CM & LUCCA & CALUMET AVE. & CALUMET \\
\hline 1379 & & TOMMEI & ELISABETH & w & 47 & ITALY & ITALY & ITALY & 1907 & & & CALUMET AVE. & CALUMET \\
\hline 1380 & 177 & CANTELLO & BATTISTA JR. & $\mathrm{H}$ & 50 & ITALY & ITALY & ITALY & 1888 & MINER/CM & SAN GIORGIO CANAVESE, TO & CALUMET AVE. & $\begin{array}{l}\text { CALUMET } \\
\end{array}$ \\
\hline 1381 & & CANTELLO & ELISABETH & W & 46 & ITALY & ITALY & ITALY & 1902 & & & CALUMET AVE. & CALUMET \\
\hline 1382 & & CANTELLO & MARY & D & 13 & MICHIGAN & ITALY & ITALY & & & SAN GIORGIO CANAVESE. TO & CALUMET AVE. & CALUMET \\
\hline 1383 & & CANTELLO & GEORGE & $\mathrm{s}$ & 4 & MICHIGAN & ITALY & ITALY & & & SAN GIORGIO CANAVESE. TO & CALUMET AVE. & $\begin{array}{l}\text { CALUMET } \\
\end{array}$ \\
\hline 1384 & 184 & VALLERO & MARY & $\mathrm{H}$ & 32 & ITALY & ITALY & ITALY & 1916 & & VALPERGA. TO & CALUMET AVE. & $\begin{array}{l}\text { CALUMET } \\
\end{array}$ \\
\hline 1385 & & VALLERO & JOHN & $\mathrm{s}$ & 15 & ITALY & ITALY & ITALY & 1916 & & VALPERGA. TO & CALUMET AVE. & CALUMET \\
\hline
\end{tabular}




\begin{tabular}{|c|c|c|c|c|c|c|c|c|c|c|c|c|c|}
\hline 1386 & & VALLERO & LUISA & $\mathrm{D}$ & 10 & MICHIGAN & ITALY & ITALY & & & VALPERGA. TO & CALUMET AVE. & CALUMET \\
\hline 1387 & 191 & cosso & GIUSEPPE & $\mathrm{H}$ & 46 & ITALY & ITALY & ITALY & 1896 & TIMBERMAN/CM & SAN GIORGIO CANAVESE. TO & CALUMET AVE. & CALUMET \\
\hline 1388 & & cosso & MARY & W & 37 & ITALY & ITALY & ITALY & 1896 & & SAN GIORGIO CANAVESE. TO & CALUMET AVE. & CALUMET \\
\hline 1389 & & cosso & MARY & D & 19 & MICHIGAN & ITALY & ITALY & & TELEPHONE OPERATOR/CM & SAN GIORGIO CANAVESE. TO & CALUMET AVE. & $\begin{array}{l}\text { CALUMET } \\
\end{array}$ \\
\hline 1390 & & cosso & MINNIE & $\mathrm{D}$ & 17 & MICHIGAN & ITALY & ITALY & & & SAN GIORGIO CANAVESE. TO & CALUMET AVE. & CALUMET \\
\hline 1391 & & cosso & DELLA & $\mathrm{D}$ & 13 & MICHIGAN & ITALY & ITALY & & & SAN GIORGIO CANAVESE. TO & CALUMET AVE. & CALUMET \\
\hline 1392 & & cosso & MARK & $\mathrm{s}$ & 11 & MICHIGAN & ITALY & ITALY & & & SAN GIORGIO CANAVESE. TO & CALUMET AVE. & $\begin{array}{l}\text { CALUMET } \\
\text { CALE }\end{array}$ \\
\hline 1393 & 160 & CORNALE & DOMENICO & $\mathrm{H}$ & 30 & ITALY & ITALY & ITALY & 1914 & MINER/CM & VICENZA & CALUMET AVE. & CALUMET \\
\hline 1394 & 100 & CORNALE & KATE & W & 28 & ITALY & ITALY & ITALY & 1907 & IVIIVESAR LIVI & TORINO & CALUMET AVE. & CALUMET \\
\hline 1395 & & CORNALE & JAMES & s & 7 & MICHIGAN & ITALY & ITALY & & & VICENZA & CALUMET AVE. & CALUMET \\
\hline 1396 & & CORNALE & DOMENICO & $\mathrm{s}$ & 2 & MICHIGAN & ITALY & ITALY & & & VICENZA & CALUMET AVE. & $\begin{array}{l}\text { CALUMET } \\
\end{array}$ \\
\hline 1397 & 134 & LANDINI & ZERNINA & SER & 21 & ITALY & ITALY & ITALY & 1908 & SERVANT/PF & LUCCA & CALUMET AVE. & CALUMET \\
\hline 1398 & $25 ?$ & TORREANO & JOHN & $\mathrm{H}$ & 43 & MICHIGAN & ITALY & ITALY & & BLACKSMITH/CM & TORINO & ROCKLAND ST. & CALUMET \\
\hline 1399 & & TORREANO & CLARA & W & 31 & MICHIGAN & MICHIGAN & MICHIGAN & & & USA & ROCKLAND ST. & CALUMET \\
\hline 1400 & & TORREANO & CATERINA & D & 8 & MICHIGAN & MICHIGAN & MICHIGAN & & & TORINO & ROCKLAND ST. & CALUMET \\
\hline 1401 & & TORREANO & ELEONORA & D & 7 & MICHIGAN & MICHIGAN & MICHIGAN & & & TORINO & ROCKLAND ST. & CALUMET \\
\hline 1402 & & TORREANO & RAIMONDO & $\mathrm{s}$ & 5 & MICHIGAN & MICHIGAN & MICHIGAN & & & TORINO & ROCKLAND ST. & $\begin{array}{l}\text { CALUMET } \\
\end{array}$ \\
\hline 1403 & 276 & BESSOLO & FELIX & $\mathrm{H}$ & 57 & ITALY & ITALY & ITALY & 1892 & TIMBER BOSS/CM & SCARMAGNO. TO & ROCKLAND ST. & $\begin{array}{l}\text { CALUMET } \\
\end{array}$ \\
\hline 1404 & & BESSOLO & MARIANNA & D & 17 & MICHIGAN & ITALY & ITALY & & & SCARMAGNO. TO & ROCKLAND ST. & $\begin{array}{l}\text { CALUMET } \\
\end{array}$ \\
\hline 1405 & & BESSOLO & CARLO & $\mathrm{s}$ & 12 & MICHIGAN & ITALY & ITALY & & & SCARMAGNO. TO & ROCKLAND ST. & $\begin{array}{l}\text { CALUMET } \\
\end{array}$ \\
\hline 1406 & & BESSOLO & VITTORIA & $\mathrm{D}$ & 9 & MICHIGAN & ITALY & ITALY & & & SCARMAGNO. TO & ROCKLAND ST. & CALUMET \\
\hline 1407 & 319 & $\begin{array}{l}\text { LANDINI } \\
\text { LANDI }\end{array}$ & JOSEPH & $\mathrm{H}$ & 46 & ITALY & ITALY & ITALY & 1900 & FIREMAN/BOILER HOUSE & COLLE DI COMPITO, LU & CALEDONIA ST. & CALUMET \\
\hline 1408 & & LANDINI & AMELIA & w & 45 & MICHIGAN & ITALY & ITALY & & & & CALEDONIA ST. & CALUMET \\
\hline 1409 & & LANDINI & ANNA & D & 18 & MICHIGAN & ITALY & MICHIGAN & & SERVANT/PF & COLLE DI COMPITO. LU & CALEDONIA ST. & CALUMET \\
\hline 1410 & & LANDINI & FELIX & $\mathrm{s}$ & 18 & MICHIGAN & ITALY & MICHIGAN & & LABORER/CM & COLLE DI COMPITO. LU & CALEDONIA ST. & CALUMET \\
\hline 1411 & & LANDINI & MARIA & $\mathrm{D}$ & 17 & MICHIGAN & ITALY & MICHIGAN & & SERVANT/PF & COLLE DI COMPITO. LU & CALEDONIA ST. & CALUMET \\
\hline 1412 & & LANDINI & ANTONIO & $\mathrm{s}$ & 13 & MICHIGAN & ITALY & MICHIGAN & & & COLLE DI COMPITO. LU & CALEDONIA ST. & CALUMET \\
\hline 1413 & & LANDINI & AMELIA & D & 12 & MICHIGAN & ITALY & MICHIGAN & & & COLLE DI COMPITO, LU & CALEDONIA ST. & CALUMET \\
\hline 1414 & & LANDINI & PAUL & $\mathrm{S}$ & 10 & MICHIGAN & ITALY & MICHIGAN & & & COLLE DI COMPITO. LU & CALEDONIA ST. & CALUMET \\
\hline 1415 & & LANDINI & MELISSA & D & 9 & MICHIGAN & ITALY & MICHIGAN & & & COLLE DI COMPITO. LU & CALEDONIA ST. & $\begin{array}{l}\text { CALUMET } \\
\end{array}$ \\
\hline 1416 & & LANDINI & RAFFAELE & $\mathrm{s}$ & 7 & MICHIGAN & ITALY & MICHIGAN & & & COLLE DI COMPITO. LU & CALEDONIA ST. & $\begin{array}{l}\text { CALUMET } \\
\end{array}$ \\
\hline 1417 & & LANDINI & VERONICA & D & 5 & MICHIGAN & ITALY & MICHIGAN & & & COLLE DI COMPITO. LU & CALEDONIA ST. & CALUMET \\
\hline 1418 & 319 & BALAGNA & MARY & $\mathrm{H}$ & 44 & ITALY & ITALY & ITALY & 1905 & & PONT CANAVESE. TO & CALEDONIA ST. & $\begin{array}{l}\text { CALUMET } \\
\end{array}$ \\
\hline 1419 & 30 & BALAGNA & ORELLA & $\mathrm{D}$ & 12 & MICHIGAN & MICHIGAN & ITALY & 1905 & & PONT CANAVESE, TO & CALEDONIA ST. & CALUMET \\
\hline 1420 & & BALAGNA & JOSEPH & s & 10 & MICHIGAN & MICHIGAN & ITALY & & & PONT CANAVESE. TO & CALEDONIA ST. & CALUMET \\
\hline 1421 & & BALAGNA & VITTORIA & $\mathrm{D}$ & 7 & MICHIGAN & MICHIGAN & ITALY & & & PONT CANAVESE. TO & CALEDONIA ST. & CALUMET \\
\hline 1422 & 326 & PELLEGRINI & LORENZO & $\mathrm{H}$ & 62 & ITALY & ITALY & ITALY & 1888 & MASON/CM & LUCCA & CALEDONIA ST. & CALUMET \\
\hline 1423 & & PELLEGRINI & FANNIE & W & 52 & ITALY & ITALY & ITALY & 1889 & & & CALEDONIA ST. & CALUMET \\
\hline 1424 & & PELLEGRINI & CECIL & $\mathrm{s}$ & 21 & MICHIGAN & ITALY & ITALY & & & LUCCA & CALEDONIA ST. & CALUMET \\
\hline 1425 & & PELLEGRINI & EUGENIA & $\mathrm{D}$ & 13 & MICHIGAN & ITALY & ITALY & & & LUCCA & CALEDONIA ST. & CALUMET \\
\hline 1426 & & JAMES & ALBERT & SOIL & 21 & MICHIGAN & ENGLAND & ENGLAND & & PIPE MAN/CM & ENGLAND & CALEDONIA ST. & CALUMET \\
\hline 1427 & & PELLEGRINI & ANNA & $\mathrm{D}$ & 19 & MICHIGAN & ITALY & ITALY & & & LUCCA & CALEDONIA ST. & $\begin{array}{l}\text { CALUMET } \\
\end{array}$ \\
\hline 1428 & 333 & BEAUCHAMP & JOHN & $\mathrm{H}$ & 42 & MICHIGAN & CANADA & USA & & BRAKEMAN/CM & CANADA-USA & CALEDONIA ST. & CALUMET \\
\hline 1429 & & BEAUCHAMP & MALINIE & W & 40 & ITALY & ITALY & ITALY & 1882 & & & CALEDONIA ST. & $\begin{array}{l}\text { CALUMET } \\
\end{array}$ \\
\hline 1430 & & BEAUCHAMP & HILARY & $\mathrm{s}$ & 12 & MICHIGAN & MICHIGAN & ITALY & & & & CALEDONIA ST. & CALUMET \\
\hline 1431 & & BEAUCHAMP & RUTH & D & 10 & MICHIGAN & MICHIGAN & ITALY & & & & CALEDONIA ST. & $\begin{array}{l}\text { CALUMET } \\
\end{array}$ \\
\hline 1432 & & BEAUCHAMP & CRESCENE & D & 8 & MICHIGAN & MICHIGAN & ITALY & & & & CALEDONIA ST. & CALUMET \\
\hline 1433 & & BEAUCHAMP & ROBERT & s & 4 & MICHIGAN & MICHIGAN & ITALY & & & & CALEDONIA ST. & CALUMET \\
\hline 1434 & 363 & MEALLIA & ANTONIO & $\mathrm{H}$ & 48 & ITALY & ITALY & ITALY & 1899 & MINER/CM & BOSCONERO. TO & CALEDONIA ST. & CALUMET \\
\hline 1435 & & MEALLIA & GIOVANNA & w & 38 & $\begin{array}{l}\text { ITALY } \\
\text { ITAL }\end{array}$ & ITALY & ITALY & 1908 & & & CALEDONIA ST. & $\begin{array}{l}\text { CALUMET } \\
\end{array}$ \\
\hline 1436 & & MEALLIA & LUCY & $\mathrm{D}$ & 7 & MICHIGAN & ITALY & ITALY & & & BOSCONERO. TO & CALEDONIA ST. & CALUMET \\
\hline 1437 & & MEALLIA & DOMENICO & $\mathrm{S}$ & 5 & MICHIGAN & ITALY & ITALY & & & BOSCONERO, TO & CALEDONIA ST. & $\begin{array}{l}\text { CALUMET } \\
\end{array}$ \\
\hline 1438 & & MEALLIA & MARTINO & $\mathrm{s}$ & 3 & MICHIGAN & ITALY & ITALY & & & BOSCONERO. TO & CALEDONIA ST. & CALUMET \\
\hline 1439 & 381 & MARCHETTI & JOHN & $\mathrm{H}$ & 50 & ITALY & ITALY & ITALY & $96 / 98$ & BLACKSMITH/CM & & CALEDONIA ST. & CALUMET \\
\hline 1440 & & MARCHETTI & AURELIA & w & 40 & ITALY & ITALY & ITALY & 1900 & & & CALEDONIA ST. & CALUMET \\
\hline 1441 & & MARCHETTI & JOHN & s & 14 & MICHIGAN & ITALY & ITALY & & & & CALEDONIA ST. & CALUMET \\
\hline 1442 & & ZUBIENA & ANGELO & B & 31 & ITALY & ITALY & ITALY & 1913 & TRAMMER/CM & PEROSA. TO & CALEDONIA ST. & CALUMET \\
\hline 1443 & & ZUBIENA & CESARE & B & 32 & ITALY & ITALY & ITALY & 1909 & TIMBERMAN/CM & TORINO & CALEDONIA ST. & $\begin{array}{l}\text { CALUMET } \\
\end{array}$ \\
\hline 1444 & & MARTA & LORENZO & B & 36 & ITALY & ITALY & ITALY & 1907 & TRAMMER/CM & AGLIE'. TO & CALEDONIA ST. & CALUMET \\
\hline 1445 & 389 & BOGGIO & PONZIO & $\mathrm{H}$ & 57 & ITALY & ITALY & ITALY & UN & MINER/CM & SAN PONSO CANAV & CALEDONIA ST. & CALUMET \\
\hline 1446 & & BOGGIO & MARY & W & 57 & ITALY & ITALY & ITALY & UN & & SAN PONSO CANA & CALEDONIA ST. & $\begin{array}{l}\text { CALUMET } \\
\end{array}$ \\
\hline 1447 & & BOGGIO & JOSEPHINE & D & 23 & MICHIGAN & ITALY & ITALY & & TEL. OPERATOR/TEL. CO & SAN PONSO CANAVESE. TO & CALEDONIA ST. & CALUMET \\
\hline 1448 & 424 & RICCA & VITTORIO & $\mathrm{H}$ & 44 & ITALY & ITALY & ITALY & 1898 & MINER/CM & SAN PONSO CANAVESE. TO & CALEDONIA ST. & CALUMET \\
\hline
\end{tabular}




\begin{tabular}{|c|c|c|c|c|c|c|c|c|c|c|c|c|c|}
\hline 1449 & & RICCA & PAULINE & W & 44 & ITALY & ITALY & ITALY & 1898 & & SAN PONSO CANAVESE. TO & CALEDONIA ST. & CALUMET \\
\hline 1450 & & RICCA & MATTEO & $s$ & 19 & MICHIGAN & ITALY & ITALY & & TRAMMER/CM & SAN PONSO CANAVESE. TO & CALEDONIA ST. & CALUMET \\
\hline 1451 & & RICCA & AUGUSTO & $\mathrm{s}$ & 18 & MICHIGAN & ITALY & ITALY & & & SAN PONSO CANAVESE. TO & CALEDONIA ST. & CALUMET \\
\hline 1452 & & RICCA & MIKE & $\mathrm{s}$ & 10 & MICHIGAN & ITALY & ITALY & & & SAN PONSO CANAVESE. TO & CALEDONIA ST. & CALUMET \\
\hline 1453 & & DELONGECHINI & LUCIA & $\mathrm{D}$ & 20 & MICHIGAN & ITALY & ITALY & & & SAN PONSO CANAVESE. TO & CALEDONIA ST. & $\begin{array}{l}\text { CALUMET } \\
\text { CALE }\end{array}$ \\
\hline 1454 & & DELONGECHINI & VITTORIO & GS & 1 & MICHIGAN & MICHIGAN & MICHIGAN & & & SAN PONSO CANAVESE. TO & CALEDONIA ST. & CALUMET \\
\hline 1455 & 386 & $\begin{array}{l}\text { IAVELLI } \\
\text { IAVI }\end{array}$ & ANTONIO & $\mathrm{H}$ & 46 & ITALY & ITALY & ITALY & 1900 & TIMBERMAN/CM & BOSCONERO. TO & CALEDONIA ST. & CALUMET \\
\hline 1456 & & IAVELLI & ISABELLE & W & 41 & ITALY & ITALY & ITALY & 1905 & & & CALEDONIA ST. & CALUMET \\
\hline 1457 & & IAVELLI & DEFENDENTE & s & 14 & MICHIGAN & ITALY & ITALY & & & BOSCONERO. TO & CALEDONIA ST. & CALUMET \\
\hline 1458 & & IAVELLI & MARY & $\mathrm{D}$ & 11 & MICHIGAN & ITALY & ITALY & & & BOSCONERO. TO & CALEDONIA ST. & CALUMET \\
\hline 1459 & & IAVELLI & JOHN & $\mathrm{BR}$ & 42 & ITALY & ITALY & ITALY & 1900 & TIMBERMAN/CM & BOSCONERO. TO & CALEDONIA ST. & CALUMET \\
\hline 1460 & & IAVELLI & KATE & SIIL & 38 & ITALY & ITALY & ITALY & 1907 & & BOSCONERO. TO & CALEDONIA ST. & CALUMET \\
\hline 1461 & & IAVELLI & DEFENDENTE & NEP & 12 & MICHIGAN & ITALY & ITALY & & & BOSCONERO. TO & CALEDONIA ST. & CALUMET \\
\hline 1462 & & IAVELLI & MARY & NIEC & 8 & MICHIGAN & ITALY & ITALY & & & BOSCONERO. TO & CALEDONIA ST. & CALUMET \\
\hline 1463 & & IAVELLI & MARION & NIEC & 4 & MICHIGAN & ITALY & ITALY & & & BOSCONERO. TO & CALEDONIA ST. & CALUMET \\
\hline 1464 & 346 & NIGRA & ANTHONY & $\mathrm{H}$ & 45 & ITALY & ITALY & ITALY & 1895 & TIMBER BOSS/CM & TORINO & CALEDONIA ST. & CALUMET \\
\hline 1465 & & NIGRA & ELENA & $\mathrm{D}$ & 17 & MICHIGAN & ITALY & ITALY & & & & CALEDONIA ST. & CALUMET \\
\hline 1466 & 440 & CAPELLO & ANTONIO & $\mathrm{H}$ & 27 & MICHIGAN & ITALY & ITALY & & FIREMAN/BOILER HOUSE & SAN BENIGNO CANAVESE. TO & CALEDONIA ST. & CALUMET \\
\hline 1467 & & CAPELLO & VIRGINIA & w & 23 & MICHIGAN & ITALY & ITALY & & & & CALEDONIA ST. & CALUMET \\
\hline 1468 & & CAPELLO & ANNA & $\mathrm{D}$ & 3 & MICHIGAN & MICHIGAN & MICHIGAN & & & SAN BENIGNO CANAVESE. TO & CALEDONIA ST. & CALUMET \\
\hline 1469 & & CAPELLO & MARGARET & $\mathrm{D}$ & 1 & MICHIGAN & MICHIGAN & MICHIGAN & & & SAN BENIGNO CANAVESE. TO & CALEDONIA ST. & CALUMET \\
\hline 1470 & & CAPELLO & JULIA & $\mathrm{D}$ & $1 \mathrm{M}$ & MICHIGAN & MICHIGAN & MICHIGAN & & & SAN BENIGNO CANAVESE, TO & CALEDONIA ST. & CALUMET \\
\hline 1471 & 474 & (A)CLEK & ANTONIO & $\mathrm{H}$ & 37 & ITALY/SLOV & ITALY & ITALY & 1909 & MINER/CM & & CALEDONIA ST. & CALUMET \\
\hline 1472 & titi & (A)CLEK & JOSEPHINE & W & 27 & YUGOSLAV & YUGOSLAV & YUGOSLAV & 1911 & & & CALEDONIA ST. & CALUMET \\
\hline 1473 & & (A)CLEK & JOSEPHINE & $\mathrm{D}$ & 7 & MICHIGAN & ITALY & YUGOSLAV & & & & CALEDONIA ST. & CALUMET \\
\hline 1474 & & (A)CLEK & ANTONIO & s & 6 & MICHIGAN & ITALY & YUGOSLAV & & & & CALEDONIA ST. & CALUMET \\
\hline 1475 & & (A)CLEK & FRANK & $\mathrm{s}$ & 5 & MICHIGAN & ITALY & YUGOSLAV & & & & CALEDONIA ST. & CALUMET \\
\hline 1476 & & (A)CLEK & JOHN & $\mathrm{s}$ & 3 & MICHIGAN & ITALY & YUGOSLAV & & & & CALEDONIA ST. & CALUMET \\
\hline 1477 & & (A)CLEK & DOROTHY & D & 2 & MICHIGAN & ITALY & YUGOSLAV & & & & CALEDONIA ST. & CALUMET \\
\hline 1478 & & (A)CLEK & ANNA & $\mathrm{D}$ & $1 \mathrm{M}$ & MICHIGAN & ITALY & YUGOSLAV & & & & CALEDONIA ST. & CALUMET \\
\hline 1479 & 461 & ANDRINA & JOHN & $\mathrm{H}$ & 36 & ITALY & ITALY & ITALY & 1902 & MINER/CM & SAN MARTINO CANAVESE. TO & CALEDONIA ST. & CALUMET \\
\hline 1480 & to & ANDRINA & MARNIE & w & 32 & MICHIGAN & ITALY & MICHIGAN & $100 \mathrm{c}$ & 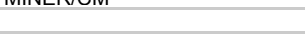 & 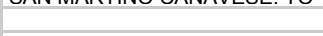 & CALEDONIA ST. & CALUMET \\
\hline 1481 & & ANDRINA & MARTHA & D & 13 & MICHIGAN & ITALY & MICHIGAN & & & SAN MARTINO CANAVESE. TO & CALEDONIA ST. & CALUMET \\
\hline 1482 & & ANDRINA & JOHN & $\mathrm{s}$ & 12 & MICHIGAN & ITALY & MICHIGAN & & & SAN MARTINO CANAVESE, TO & CALEDONIA ST. & CALUMET \\
\hline 1483 & & ANDRINA & ERNEST & s & 7 & MICHIGAN & ITALY & MICHIGAN & & & SAN MARTINO CANAVESE. TO & CALEDONIA ST. & CALUMET \\
\hline 1484 & ?? & MICHELINI & CESARE & $\mathrm{H}$ & 33 & ITALY & ITALY & ITALY & 1904 & MINER/CM & LUCCA & CALEDONIA ST. & CALUMET \\
\hline 1485 & & MICHELINI & TILLIE & W & 27 & ITALY & ITALY & ITALY & 1894 & & & CALEDONIA ST. & CALUMET \\
\hline 1486 & & MICHELINI & ALFREDO & $\mathrm{s}$ & 6 & MICHIGAN & ITALY & ITALY & & & LUCCA & CALEDONIA ST. & CALUMET \\
\hline 1487 & & MICHELINI & CATERINA & $\mathrm{D}$ & 2 & MICHIGAN & ITALY & ITALY & & & LUCCA & CALEDONIA ST. & CALUMET \\
\hline 1488 & 883 & TORRINO & DOM & $\mathrm{H}$ & 44 & ITALY & ITALY & ITALY & 1900 & TRAMMER/CM & TORINO & CAMBRIA ST. & CALUMET \\
\hline 1489 & & TORRINO & MARY & W & 36 & ITALY & ITALY & ITALY & 1913 & & & CAMBRIA ST. & CALUMET \\
\hline 1490 & & TORRINO & JAMES & s & 6 & OKLAOMA & ITALY & ITALY & & & TORINO & CAMBRIA ST. & CALUMET \\
\hline 1491 & & TORRINO & DOMENICO & $\mathrm{s}$ & 4 & OKLAOMA & ITALY & ITALY & & & TORINO & CAMBRIA ST. & CALUMET \\
\hline 1492 & & TORRINO & JOSEPH & $\mathrm{s}$ & 1 & MICHIGAN & ITALY & ITALY & & & TORINO & CAMBRIA ST. & CALUMET \\
\hline 1493 & 888 & ROTA & ELIA & $\mathrm{H}$ & 28 & ITALY & ITALY & ITALY & 1908 & MINER/CM & BERGAMO & CAMBRIA ST. & CALUMET \\
\hline 1494 & & ROTA & NATALINA & W & 22 & MICHIGAN & ITALY & ITALY & & & BERGAMO & CAMBRIA ST. & CALUMET \\
\hline 1495 & & ROTA & MARGARET & $\mathrm{D}$ & 2 & MICHIGAN & ITALY & MICHIGAN & & & BERGAMO & CAMBRIA ST. & CALUMET \\
\hline 1496 & & ROTA & BERNARDO & s & 5 & MICHIGAN & ITALY & MICHIGAN & & & BERGAMO & CAMBRIA ST. & CALUMET \\
\hline 1497 & 2024 & ALLARIA & JOHN & $\mathrm{H}$ & 43 & ITALY & ITALY & ITALY & 1881 & MINE INSPECTOR/CM & SAN GIORGIO CANAVESE. TO & CALUMET AVE. & CALUMET - RAYMBAULTOWN \\
\hline 1498 & & ALLARIA & MARY & W & 36 & ITALY & ITALY & ITALY & 1900 & & & CALUMET AVE. & CALUMET - RAYMBAULTOWN \\
\hline 1499 & & ALLARIA & NATH & $\mathrm{D}$ & 17 & MICHIGAN & ITALY & ITALY & & BOOKKEEPER/COAL OFFICE & SAN GIORGIO CANAVESE. TO & CALUMET AVE. & CALUMET - RAYMBAULTOWN \\
\hline 1500 & & ALLARIA & PETER & $\mathrm{s}$ & 15 & MICHIGAN & ITALY & ITALY & & & SAN GIORGIO CANAVESE, TO & CALUMET AVE. & CALUMET - RAYMBAULTOWN \\
\hline 1501 & & ALLARIA & $\mathrm{JOHN}$ & $\mathrm{s}$ & 13 & MICHIGAN & ITALY & ITALY & & & SAN GIORGIO CANAVESE. TO & CALUMET AVE. & CALUMET - RAYMBAULTOWN \\
\hline 1502 & & ALLARIA & ALAN & D & 11 & MICHIGAN & ITALY & ITALY & & & SAN GIORGIO CANAVESE. TO & CALUMET AVE. & CALUMET - RAYMBAULTOWN \\
\hline 1503 & 2033 & ZANARDI & BATTISTA & $\mathrm{H}$ & 52 & ITALY & ITALY & ITALY & 1890 & MINER/CM & BERGAMO & CALUMET AVE. & CALUMET - RAYMBAULTOWN \\
\hline 1504 & & ZANARDI & MARY & w & 37 & ITALY & ITALY & ITALY & 1903 & & & CALUMET AVE. & CALUMET - RAYMBAULTOWN \\
\hline 1505 & & ZANARDI & CARL & $\mathrm{s}$ & 6 & MICHIGAN & ITALY & ITALY & & TIMBERMAN/CM & BERGAMO & CALUMET AVE. & CALUMET - RAYMBAULTOWN \\
\hline 1506 & & PASQUALONE & MIKE & BIL & 34 & ITALY & ITALY & ITALY & 1907 & FOREMAN/CM & SPARONE, TO & CALUMET AVE. & CALUMET - RAYMBAULTOWN \\
\hline 1507 & 2029 & MENEGUZZO & JOSEPH & $\mathrm{H}$ & 34 & ITALY & ITALY & ITALY & 1901 & & COSTO BONARTO. TRIESTE & CALUMET AVE. & CALUMET - RAYMBAULTOWN \\
\hline 1508 & & MENEGUZZO & LUCINDA? & W & 29 & ITALY & ITALY & ITALY & 1904 & & & CALUMET AVE. & CALUMET - RAYMBAULTOWN \\
\hline 1509 & & MENEGUZZ & & s & 8 & HIGAN & ITAL & & & & COSTO BONARTO. TRIESTE & CALUMET AVE. & CALUMET - RAYMBAULTOWN \\
\hline 1510 & & EEGUZZO & & $\mathrm{D}$ & 6 & MICHIGAN & ITALY & & & & COSTO BONARTO. TRIESTE & CALUMET AVE. & CALUMET - RAYMBAULTOWN \\
\hline 1511 & & MENEGUZZO & MARY & $\mathrm{D}$ & 5 & MICHIGAN & ITALY & ITALY & & & COSTO BONARTO. TRIESTE & CALUMET AVE. & CALUMET - RAYMBAULTOWN \\
\hline
\end{tabular}




\begin{tabular}{|c|c|c|c|c|c|c|c|c|c|c|c|c|c|}
\hline 1512 & & MENEGUZZO & JOSEPH & $\mathrm{s}$ & 3 & MICHIGAN & ITALY & ITALY & & & COSTO BONARTO. TRIESTE & CALUMET AVE. & CALUMET - RAYMBAULTOWN \\
\hline 1513 & & MENEGUZZO & PETER & $\mathrm{s}$ & 1 & MICHIGAN & ITALY & ITALY & & & COSTO BONARTO. TRIESTE & CALUMET AVE. & CALUMET - RAYMBAULTOWN \\
\hline 1514 & 2021 & TOMASI & ANDREA & $\mathrm{H}$ & 56 & ITALY & ITALY & ITALY & 1885 & BLACKSMITH/CM & LOCANA CANAVESE. TO & CALUMET AVE. & CALUMET - RAYMBAULTOWN \\
\hline 1515 & & TOMASI & MARGHERITA & W & 39 & ITALY & ITALY & ITALY & 1891 & & & CALUMET AVE. & CALUMET - RAYMBAULTOWN \\
\hline 1516 & & TOMASI & JENNIE & D & 18 & MICHIGAN & ITALY & ITALY & & SERVANT/PF & LOCANA CANAVESE. TO & CALUMET AVE. & CALUMET - RAYMBAULTOWN \\
\hline 1517 & & TOMASI & LOUISE & $\mathrm{D}$ & 17 & MICHIGAN & ITALY & ITALY & & SERVANT/PF & LOCANA CANAVESE. TO & CALUMET AVE. & CALUMET - RAYMBAULTOWN \\
\hline 1518 & & TOMASI & ANTONIO & $\mathrm{s}$ & 15 & MICHIGAN & ITALY & ITALY & & & LOCANA CANAVESE. TO & CALUMET AVE. & CALUMET - RAYMBAULTOWN \\
\hline 1519 & & TOMASI & MIKE & $\mathrm{s}$ & 12 & MICHIGAN & ITALY & ITALY & & & LOCANA CANAVESE. TO & CALUMET AVE. & CALUMET - RAYMBAULTOWN \\
\hline 1520 & & TOMASI & MARGHERITA & $\mathrm{D}$ & 8 & MICHIGAN & ITALY & ITALY & & & LOCANA CANAVESE. TO & CALUMET AVE. & CALUMET - RAYMBAULTOWN \\
\hline 1521 & & TOMASI & PETER & $\mathrm{s}$ & 5 & MICHIGAN & ITALY & ITALY & & & LOCANA CANAVESE. TO & CALUMET AVE. & CALUMET - RAYMBAULTOWN \\
\hline 1522 & & TOMASI & PAUL & $\mathrm{s}$ & 5 & MICHIGAN & ITALY & ITALY & & & LOCANA CANAVESE. TO & CALUMET AVE. & $\begin{array}{l}\text { CALUMET - RAYMBAULTOWN } \\
\text { CATE }\end{array}$ \\
\hline 1523 & 2013 & RIVA & MORRIS & $\mathrm{H}$ & 45 & ITALY & ITALY & ITALY & 1898 & MINER/CM & SPARONE. TO & CALUMET AVE. & CALUMET - RAYMBAULTOWN \\
\hline 1524 & & RIVA & ROSE & W & 43 & ITALY & ITALY & ITALY & 1910 & & TORINO & CALUMET AVE. & CALUMET - RAYMBAULTOWN \\
\hline 1525 & & RIVA & FRANK & $\mathrm{s}$ & 8 & MICHIGAN & ITALY & ITALY & & & SPARONE. TO & CALUMET AVE. & CALUMET - RAYMBAULTOWN \\
\hline 1526 & & RIVA & MADDALENA & $\mathrm{D}$ & 4 & MICHIGAN & ITALY & ITALY & & & SPARONE. TO & CALUMET AVE. & CALUMET - RAYMBAULTOWN \\
\hline 1527 & 2124 & BRACCO & PETER & $\mathrm{H}$ & 52 & ITALY & ITALY & ITALY & 1892 & MINER/CM & TORINO & RAYMBAULT ST. & CALUMET - RAYMBAULTOWN \\
\hline 1528 & & BRACCO & VINCENZA & W & 37 & ITALY & ITALY & ITALY & 1900 & & & RAYMBAULT ST. & CALUMET - RAYMBAULTOWN \\
\hline 1529 & & BRACCO & ANTONIO & $\mathrm{s}$ & 17 & MICHIGAN & ITALY & ITALY & & TIMBERMAN/CM & TORINO & RAYMBAULT ST. & CALUMET - RAYMBAULTOWN \\
\hline 1530 & & BRACCO & MADDALENA & $\mathrm{D}$ & 15 & MICHIGAN & ITALY & ITALY & & DRESSMAKER/AT HOME & TORINO & RAYMBAULT ST. & CALUMET - RAYMBAULTOWN \\
\hline 1531 & & BRACCO & PETER & $\mathrm{s}$ & 14 & MICHIGAN & ITALY & ITALY & & & TORINO & RAYMBAULT ST. & CALUMET - RAYMBAULTOWN \\
\hline 1532 & & BRACCO & MARY & D & 8 & MICHIGAN & ITALY & ITALY & & & TORINO & RAYMBAULT ST. & CALUMET - RAYMBAULTOWN \\
\hline 1533 & & BRACCO & JOSEPH & $\mathrm{s}$ & 6 & MICHIGAN & ITALY & ITALY & & & TORINO & RAYMBAULT ST. & CALUMET - RAYMBAULTOWN \\
\hline 1534 & 2236 & BALINA & AUGUSTA & $\mathrm{H}$ & 31 & ITALY & ITALY & ITALY & 1905 & KEEPER/BOARDING HOUSE & MERCALLO. VARESE & RAYMBAULT ST. & CALUMET - RAYMBAULTOWN \\
\hline 1535 & & BALINA & JOHN & $\mathrm{s}$ & 10 & MICHIGAN & ITALY & ITALY & & & MERCALLO. VARESE & RAYMBAULT ST. & CALUMET - RAYMBAULTOWN \\
\hline 1536 & & VECHOVACI & JOSEPH & B & 44 & ITALY & ITALY & ITALY & 1901 & MISSIONER/ITALIAN MISSION & & RAYMBAULT ST. & CALUMET - RAYMBAULTOWN \\
\hline 1537 & & BARATA & ANTONIO & B & 42 & ITALY & ITALY & ITALY & 1905 & TRAMMER/CM & & RAYMBAULT ST. & CALUMET - RAYMBAULTOWN \\
\hline 1538 & & ENRICO & BATTISTA & B & 51 & ITALY & ITALY & ITALY & 1893 & MINER/CM & SCARMAGNO. TO & RAYMBAULT ST. & CALUMET - RAYMBAULTOWN \\
\hline 1539 & 2001 & CURTO & JAMES & $\mathrm{H}$ & 61 & ITALY & ITALY & ITALY & 1888 & LABORER/CM & TORINO & CALUMET AVE. & CALUMET - RAYMBAULTOWN \\
\hline 1540 & & CURTO & KATE & W & 56 & ITALY & ITALY & ITALY & 1894 & & & CALUMET AVE. & CALUMET - RAYMBAULTOWN \\
\hline 1541 & & CURTO & $\mathrm{JOHN}$ & $\mathrm{s}$ & 19 & MICHIGAN & ITALY & ITALY & & MACHINIST HELPER/RAILROAD & TORINO & CALUMET AVE. & CALUMET - RAYMBAULTOWN \\
\hline 1542 & & CURTO & CARLO/UGO & $\mathrm{s}$ & 17 & MICHIGAN & ITALY & ITALY & & TIMBERMAN/CM & TORINO & CALUMET AVE. & CALUMET - RAYMBAULTOWN \\
\hline 1543 & 2142 & COGNONATTO & BATTISTA & $\mathrm{H}$ & 48 & ITALY & ITALY & ITALY & 1902 & MINER/CM & SAN PONSO CANAVESE. TO & RAYMBAULT ST. & CALUMET - RAYMBAULTOWN \\
\hline 1544 & & COGNONATTO & CARLOTTA & W & 43 & ITALY & ITALY & ITALY & 1902 & & SAN PONSO CANAVESE. TO & RAYMBAULT ST. & CALUMET - RAYMBAULTOWN \\
\hline 1545 & & COGNONATTO & MARY & D & 15 & MICHIGAN & ITALY & ITALY & & & SAN PONSO CANAVESE, TO & RAYMBAULT ST. & CALUMET - RAYMBAULTOWN \\
\hline 1546 & & COGNONATTO & PETER & $\mathrm{s}$ & 11 & MICHIGAN & ITALY & ITALY & & & SAN PONSO CANAVESE. TO & RAYMBAULT ST. & CALUMET - RAYMBAULTOWN \\
\hline 1547 & & COGNONATTO & JOSEPH & $\mathrm{s}$ & 9 & MICHIGAN & ITALY & ITALY & & & SAN PONSO CANAVESE. TO & RAYMBAULT ST. & $\begin{array}{l}\text { CALUMET - RAYMBAULTOWN } \\
\text { N }\end{array}$ \\
\hline 1548 & 2148 & $\mathrm{PICCHI}$ & PETER & $\mathrm{H}$ & 19 & MICHIGAN & ITALY & ITALY & & TIMBERMAN/CM & SEGROMIGNO. LU & RAYMBAULT ST. & CALUMET - RAYMBAULTOWN \\
\hline 1549 & 270 & PICCHI-NARDI & EMILIA & W & 19 & MICHIGAN & ITALY & ITALY & & 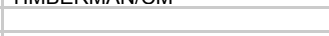 & LUCCA & RAYMBAULT ST. & CALUMET - RAYMBAULTOWN \\
\hline 1550 & & $\mathrm{PICCHI}$ & LUIGI & $\mathrm{s}$ & 1 & MICHIGAN & MICHIGAN & MICHIGAN & & & SEGROMIGNO. LU & RAYMBAULT ST. & CALUMET - RAYMBAULTOWN \\
\hline 1551 & & ZEI & LORENZO & $\mathrm{H}$ & 30 & ITALY & ITALY & ITALY & 1911 & MINER/CM & SEGROMIGNO. LU & RAYMBAULT ST. & CALUMET - RAYMBAULTOWN \\
\hline 1552 & & ZEI & AMELIA & W & 37 & ITALY & ITALY & ITALY & 1912 & & & RAYMBAULT ST. & CALUMET - RAYMBAULTOWN \\
\hline 1553 & & $\mathrm{PICCHI}$ & VIRGILIO & sSO & 18 & MICHIGAN & ITALY & ITALY & & TIMBERMAN/CM & SEGROMIGNO. LU & RAYMBAULT ST. & CALUMET - RAYMBAULTOWN \\
\hline 1554 & & ZEI & UGO & $\mathrm{s}$ & 7 & MICHIGAN & ITALY & ITALY & & & LUCCA & RAYMBAULT ST. & CALUMET - RAYMBAULTOWN \\
\hline 1555 & & PROLA & JOHN & B & 25 & ITALY & ITALY & ITALY & 1914 & TRAMMER/CM & TORINO & RAYMBAULT ST. & CALUMET - RAYMBAULTOWN \\
\hline 1556 & 2135 & GOGLIO/GIULIO & CARLO & $\mathrm{H}$ & 50 & ITALY & ITALY & ITALY & 1898 & MINER/CM & PONT CANAVESE. TO & RAYMBAULT ST. & CALUMET - RAYMBAULTOWN \\
\hline 1557 & 200 & GOGLIO/GIULIO & KATE & W & 48 & ITALY & ITALY & ITALY & 1905 & 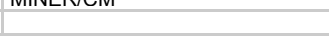 & TUIVT UAIVAVLOL. TO & RAYMBAULT ST. & CALUMET - RAYMBAULTOWN \\
\hline 1558 & & GOGLIO/GIULIO & JOSEPH & $\mathrm{s}$ & 19 & MICHIGAN & ITALY & ITALY & & LABORER/CM & PONT CANAVESE. TO & RAYMBAULT ST. & CALUMET - RAYMBAULTOWN \\
\hline 1559 & & GOGLIO/GIULIO & FRANK? & $\mathrm{s}$ & 16 & MICHIGAN & ITALY & ITALY & & MOTORMAN/CM & PONT CANAVESE. TO & JLT ST. & IET - RAYN \\
\hline 1560 & & GOGLIO/GIULIO & JOHN & $\mathrm{s}$ & 14 & MICHIGAN & ITAL & ITALY & & & PONT CANAVESE. TO & RAYMBAULT ST. & CALUMET - RAYMB \\
\hline 1561 & 2133 & ROSSIO & BATTISTA & $\mathrm{H}$ & 45 & ITALY & ITA & ITALY & 1899 & MINER/CM & PONT CANAVESE. TO & JLT ST. & CALUMET - RAY \\
\hline 1562 & & ROSSIO & MARGHERITA & W & 42 & ITALY & ITALY & ITAI & 1902 & & & AULT ST. & CALUMET - RAYMBAULTOWN \\
\hline 1563 & & ROSSIO & MARY & D & 15 & MICHIGAN & ITALY & ITAI & & & PONT CANAVESE, TO & RAYMBAULT ST. & CALUMET - RAYMBAULTOWN \\
\hline 1564 & & ROSSIO & ROSA & D & 11 & MICHIGAN & ITALY & ITALY & & & PONT CANAVESE. TO & RAYMBAULT ST. & CALUMET - RAYMBAULTOWN \\
\hline 1565 & & ROSSIO & TONY & $\mathrm{s}$ & 8 & MICHIGAN & ITALY & ITALY & & & PONT CANAVESE. TO & RAYMBAULT ST. & CALUMET - RAYMBAULTOWN \\
\hline 1566 & & ROSSIO & MARTA & D & 5 & MICHIGAN & ITALY & ITALY & & & PONT CANAVESE. TO & RAYMBAULT ST. & CALUMET - RAYMBAULTOWN \\
\hline 1567 & & ROSSIO & ANDREINA & D & 3 & MICHIGAN & ITALY & ITALY & & & PONT CANAVESE. TO & RAYMBAULT ST. & CALUMET - RAYMBAULTOWN \\
\hline 1568 & & ROSSIO & LUIGI & D & 1 & MICHIGAN & ITALY & ITALY & & & PONT CANAVESE. TO & RAYMBAULT ST. & CALUMET - RAYMBAULTOWN \\
\hline 1569 & 2209 & POMA & VINCENZO & $\mathrm{H}$ & 50 & ITALY & ITALY & ITALY & 1898 & MINER/CM & IVREA, TO & MIDDLE ST. & CALUMET - RAYMBAULTOWN \\
\hline 1570 & & POMA & MARY & W & 49 & ITALY & ITALY & ITALY & 1900 & & IVREA. TO & MIDDLE ST. & CALUMET - RAYMBAULTOWN \\
\hline 1571 & & POMA & MARGHERITA & D & 20 & ITALY & ITALY & ITALY & 1900 & & IVREA. TO & MIDDLE ST. & CALUMET - RAYMBAULTOWN \\
\hline 1572 & & & VINCENZO & $\mathrm{s}$ & 16 & & ITA & ITA & & NOT CLEAR/CM & IVREA. TO & & CALUMET - RAYMBAULTOWN \\
\hline 1573 & & & & $\mathrm{~s}$ & 14 & & ITA & & & & A. TO & & $\mathrm{CA}$ \\
\hline 1574 & & POMA & CARLO & $\mathrm{s}$ & 11 & MICHIGAN & ITALY & ITALY & & & IVREA. TO & MIDDLE ST. & CALUMET - RAYMBAULTOWN \\
\hline
\end{tabular}




\begin{tabular}{|c|c|c|c|c|c|c|c|c|c|c|c|c|c|}
\hline 1575 & 2621 & SCUSSEL & JOSEPH & $\mathrm{H}$ & 34 & ITALY & ITALY & ITALY & 1900 & MINER/CM & AGORDO. BELLUNO & LIBERTY ST. & CALUMET - RAYMBAULTOWN \\
\hline 1576 & & SCUSSEL & JOSEPHINE & W & 22 & ITALY & ITALY & ITALY & 1915 & & & LIBERTY ST. & CALUMET - RAYMBAULTOWN \\
\hline 1577 & & SCUSSEL & JOHANNA & D & 2 & MICHIGAN & ITALY & ITALY & & & AGORDO. BELLUNO & LIBERTY ST. & CALUMET - RAYMBAULTOWN \\
\hline 1578 & & SCUSSEL & JOHN & $\mathrm{s}$ & 1 & MICHIGAN & ITALY & ITALY & & & AGORDO. BELLUNO & LIBERTY ST. & CALUMET - RAYMBAULTOWN \\
\hline 1579 & 2322 & CENA & PAUL & $\mathrm{H}$ & 47 & ITALY & ITALY & ITALY & 1904 & FIREMAN/CM & SAN BENIGNO CANAVESE. TO & A ST. & CALUMET - RAYMBAULTOWN \\
\hline 1580 & & CENA & TERESA & w & 45 & ITALY & ITALY & ITALY & 1904 & & SAN BENIGNO CANAVESE. TO & A ST. & CALUMET - RAYMBAULTOWN \\
\hline 1581 & & CENA & CARLO & s & 22 & ITALY & ITALY & ITALY & 1904 & LABORER/CM & SAN BENIGNO CANAVESE. TO & A ST. & CALUMET - RAYMBAULTOWN \\
\hline 1582 & & CENA & JOSEPHINE & D & 20 & ITALY & ITALY & ITALY & 1904 & & SAN BENIGNO CANAVESE. TO & A ST. & CALUMET - RAYMBAULTOWN \\
\hline 1583 & 2362 & CIAGNE & LOUIS & $\mathrm{H}$ & 41 & ITALY & ITALY & ITALY & 1893 & MINER/CM & TORINO & A ST. & CALUMET - RAYMBAULTOWN \\
\hline 1584 & & CIAGNE & MARNIE & W & 37 & ITALY & ITALY & ITALY & 1901 & & & A ST. & CALUMET - RAYMBAULTOWN \\
\hline 1585 & & CIAGNE & LINA & $\mathrm{D}$ & 15 & MICHIGAN & ITALY & ITALY & & & TORINO & A ST. & CALUMET - RAYMBAULTOWN \\
\hline 1586 & & CIAGNE & ELENA & $\mathrm{D}$ & 7 & MICHIGAN & ITALY & ITALY & & & TORINO & AST. & CALUMET - RAYMBAULTOWN \\
\hline 1587 & 2356 & GIOVETTI & CARLO & $\mathrm{H}$ & 37 & ITALY & ITALY & ITALY & 1911 & TIMBERMAN/CM & SAN GIORGIO CANAVESE. TO & AST. & CALUMET - RAYMBAULTOWN \\
\hline 1588 & & GIOVETTI & TERESA & W & 35 & ITALY & ITALY & ITALY & 1912 & & SAN GIORGIO CANAVESE. TO & AST. & CALUMET - RAYMBAULTOWN \\
\hline 1589 & & GIOVETTI & JOSEPH & $\mathrm{s}$ & 8 & ITALY & ITALY & ITALY & 1912 & & SAN GIORGIO CANAVESE. TO & AST. & CALUMET - RAYMBAULTOWN \\
\hline 1590 & 2403 & TAPPERO & BATTISTA & $\mathrm{H}$ & 41 & ITALY & ITALY & ITALY & 1901 & MINER/CM & TORINO & A ST. & CALUMET - RAYMBAULTOWN \\
\hline 1591 & & TAPPERO & STEFANIA & W & 39 & ITALY & ITALY & ITALY & 1901 & & TORINO & A ST. & CALUMET - RAYMBAULTOWN \\
\hline 1592 & & TAPPERO & TERESA & D & 13 & MICHIGAN & ITALY & ITALY & & & TORINO & A ST. & CALUMET - RAYMBAULTOWN \\
\hline 1593 & & TAPPERO & KATE & $\mathrm{D}$ & 8 & MICHIGAN & ITALY & ITALY & & & TORINO & A ST. & CALUMET - RAYMBAULTOWN \\
\hline 1594 & & TAPPERO & JOSEPHINE & $\mathrm{D}$ & 3 & MICHIGAN & ITALY & ITALY & & & TORINO & A ST. & CALUMET - RAYMBAULTOWN \\
\hline 1595 & 2419 & RICCA & MIKE & $\mathrm{H}$ & 38 & ITALY & ITALY & ITALY & 1905 & MINER/CM & RIVAROLO CANAVESE. TO & B ST. & CALUMET - RAYMBAULTOWN \\
\hline 1596 & & RICCA & MARY & W & 39 & ITALY & ITALY & ITALY & $02 / 12$ & & & B ST. & CALUMET - RAYMBAULTOWN \\
\hline 1597 & & RICCA & JOHN & $\mathrm{s}$ & 13 & MICHIGAN & ITALY & ITALY & & & RIVAROLO CANAVESE. TO & B ST. & CALUMET - RAYMBAULTOWN \\
\hline 1598 & 2421 & DIGHERA & GIORGIO & $\mathrm{H}$ & 46 & ITALY & ITALY & ITALY & 1899 & MINER/CM & SAN GIORGIO CANAVESE. TO & B ST. & CALUMET - RAYMBAULTOWN \\
\hline 1599 & & DIGHERA & MARY & W & 42 & ITALY & ITALY & ITALY & 1909 & & & BST. & CALUMET - RAYMBAULTOWN \\
\hline 1600 & & DIGHERA & JENNIE & D & 9 & MICHIGAN & ITALY & ITALY & & & SAN GIORGIO CANAVESE. TO & B ST. & CALUMET - RAYMBAULTOWN \\
\hline 1601 & & DIGHERA & DOMENICO & $\mathrm{S}$ & 7 & MICHIGAN & ITALY & ITALY & & & SAN GIORGIO CANAVESE. TO & B ST. & CALUMET - RAYMBAULTOWN \\
\hline 1602 & & DIGHERA & ELSIE & D & 1 & MICHIGAN & ITALY & ITALY & & & SAN GIORGIO CANAVESE, TO & B ST. & CALUMET - RAYMBAULTOWN \\
\hline 1603 & & AIMONE & FRANK & $\mathrm{H}$ & 51 & ITALY & ITALY & ITALY & 1902 & TIMBERMAN/CM & PONT CANAVESE. TO & B ST. & CALUMET - RAYMBAULTOWN \\
\hline 1604 & & AIMONE & CARLOTTA & W & 52 & ITALY & ITALY & ITALY & 1904 & & & B ST. & CALUMET - RAYMBAULTOWN \\
\hline 1605 & 2423 & MASSE' & JOHN & $\mathrm{H}$ & 51 & ITALY & ITALY & ITALY & 1897 & MINER/CM & PONT CANAVESE. TO & B ST. & CALUMET - RAYMBAULTOWN \\
\hline 1606 & & MASSE' & SERAFINA & W & 52 & ITALY & ITALY & ITALY & 1905 & & PONT CANAVESE. TO & B ST. & CALUMET - RAYMBAULTOWN \\
\hline 1607 & & MASSE' & PETER & $\mathrm{s}$ & 25 & ITALY & ITALY & ITALY & 1905 & MINER/CM & PONT CANAVESE. TO & B ST. & CALUMET - RAYMBAULTOWN \\
\hline 1608 & 2926 & COPPO & ANTONIO & $\mathrm{H}$ & 56 & ITALY & ITALY & ITALY & 1881 & LABORER/CM & PONT CANAVESE, TO & B ST. & CALUMET - RAYMBAULTOWN \\
\hline 1609 & & COPPO & MARY & w & 50 & ITALY & ITALY & ITALY & 1882 & & & B ST. & CALUMET - RAYMBAULTOWN \\
\hline 1610 & & COPPO & JOHN & $\mathrm{s}$ & 24 & MICHIGAN & ITALY & ITALY & & ROLLER FIXER/CM & PONT CANAVESE. TO & B ST. & CALUMET - RAYMBAULTOWN \\
\hline 1611 & & COPPO & VITTORIO & D & 18 & MICHIGAN & ITALY & ITALY & & & PONT CANAVESE. TO & B ST. & CALUMET - RAYMBAULTOWN \\
\hline 1612 & & COPPO & AMELIA & D & 15 & MICHIGAN & ITALY & ITALY & & & PONT CANAVESE. TO & B ST. & CALUMET - RAYMBAULTOWN \\
\hline 1613 & 2467 & MACARIO & PETER & $\mathrm{H}$ & 58 & ITALY & ITALY & ITALY & 1890 & WATCHMAN/CM & TORINO & C ST. & CALUMET - RAYMBAULTOWN \\
\hline 1614 & & MACARIO & TRACY & W & 48 & ITALY & ITALY & ITALY & 1890 & & TORINO & C ST. & CALUMET - RAYMBAULTOWN \\
\hline 1615 & & MACARIO & JOHN & $\mathrm{s}$ & 29 & ITALY & ITALY & ITALY & 1890 & TIMBERMAN/CM & TORINO & C ST. & CALUMET - RAYMBAULTOWN \\
\hline 1616 & & MACARIO & JOSEPH & $\mathrm{s}$ & 21 & MICHIGAN & ITALY & ITALY & & & TORINO & C ST. & CALUMET - RAYMBAULTOWN \\
\hline 1617 & & MACARIO & PETER & $\mathrm{s}$ & 18 & MICHIGAN & ITALY & ITALY & & ROLLER FIXER/CM & TORINO & C ST. & CALUMET - RAYMBAULTOWN \\
\hline 1618 & & MACARIO & MARTIN & $\mathrm{s}$ & 14 & MICHIGAN & ITALY & ITALY & & & TORINO & C ST. & CALUMET - RAYMBAULTOWN \\
\hline 1619 & & MACARIO & CARLO & $\mathrm{s}$ & 10 & MICHIGAN & ITALY & ITALY & & & TORINO & C ST. & CALUMET - RAYMBAULTOWN \\
\hline 1620 & & MACARIO & CHESTER & $\mathrm{s}$ & 5 & MICHIGAN & ITALY & ITALY & & & TORINO & C ST. & CALUMET - RAYMBAULTOWN \\
\hline 1621 & 2477 & PETITTI & FRANK & $\mathrm{H}$ & 33 & ITALY & ITALY & ITALY & 1906 & MINER/CM & SAN PONSO CANAVESE. TO & C ST. & CALUMET - RAYMBAULTOWN \\
\hline 1622 & & PETITTI & MARY & w & 31 & ITALY & ITALY & ITALY & 1892 & & SAN PONSO CANAVESE. TO & C ST. & CALUMET - RAYMBAULTOWN \\
\hline 1623 & & PETITTI & JOHN & s & 14 & ITALY & ITALY & ITALY & 1908 & & SAN PONSO CANAVESE. TO & C ST. & CALUMET - RAYMBAULTOWN \\
\hline 1624 & & PETITTI & JOHN & $\mathrm{BR}$ & 27 & ITALY & ITALY & ITALY & 1906 & MINER/CM & SAN PONSO CANAVESE. TO & C ST. & CALUMET - RAYMBAULTOWN \\
\hline 1625 & & PETITTI & JOSEPH & $\mathrm{BR}$ & 40 & ITALY & ITALY & ITALY & 1906 & MINER/CM & SAN PONSO CANAVESE. TO & C ST. & CALUMET - RAYMBAULTOWN \\
\hline 1626 & & BIGA & DOMENICO & B & 30 & ITALY & ITALY & ITALY & 1908 & MINER/CM & FOGLIZZO CANAVESE, TO & C ST. & CALUMET - RAYMBAULTOWN \\
\hline 1627 & & BIGA & LUCY & B & 10 & MICHIGAN & ITALY & ITALY & & & FOGLIZZO CANAVESE. TO & C ST. & CALUMET - RAYMBAULTOWN \\
\hline 1628 & & CHIANTORETTO & JOHN & B & 31 & MICHIGAN & ITALY & ITALY & & & CASTELLAMONTE. TO & C ST. & CALUMET - RAYMBAULTOWN \\
\hline 1629 & & SELINA & PETER & B & 29 & ITALY & ITALY & ITALY & 1914 & MINER/CM & & C ST. & CALUMET - RAYMBAULTOWN \\
\hline 1630 & 2481 & AIMONE & DOMENICO & $\mathrm{H}$ & 65 & ITALY & ITALY & ITALY & 1886 & MINER/CM & PONT CANAVESE. TO & C ST. & CALUMET - RAYMBAULTOWN \\
\hline 1631 & & AIMONE & DOMENICA & w & 44 & ITALY & ITALY & ITALY & 1890 & & & C ST. & CALUMET - RAYMBAULTOWN \\
\hline 1632 & & AIMONE & JOHN & $\mathrm{s}$ & 24 & MICHIGAN & ITALY & ITALY & & TIMBERMAN/CM & PONT CANAVESE, TO & C ST. & CALUMET - RAYMBAULTOWN \\
\hline 1633 & & BARDA & PETER & B & 40 & ITALY & ITALY & ITALY & 1901 & TIMBERMAN/CM & TORINO & C ST. & CALUMET - RAYMBAULTOWN \\
\hline 1634 & & ACCORNERO & GIORGIO & B & $?$ & ITALY & ITALY & ITALY & 1915 & TRAMMER/CM & MANFRINO. ALESSANDRIA & C ST. & CALUMET - RAYMBAULTOWN \\
\hline 1635 & & MACARIO & VITTORIO & B & 38 & ITALY & ITA & ITALY & 1905 & TIMBERMAN/CM & TORINO & C ST. & CALUMET - RAYMBAULTOWN \\
\hline 1636 & & ACCORNERO & GIORGIO & AC & 18 & MICHIGAN & ITALY & ITALY & & & MANFRINO. ALESSANDRIA & C ST. & CALUMET - RAYMBAULTOWN \\
\hline 1637 & 2476 & ZOZO & JOHN & $\mathrm{H}$ & 31 & ITALY & ITALY & ITALY & 1913 & TRAMMER/CM & CASTELGOMBERTO. VI & C ST. & CALUMET - RAYMBAULTOWN \\
\hline
\end{tabular}




\begin{tabular}{|c|c|c|c|c|c|c|c|c|c|c|c|c|c|}
\hline 1638 & & ZOZO & ANNA & W & 20 & MICHIGAN & ITALY & ITALY & & & & C ST. & CALUMET - RAYMBAULTOWN \\
\hline 1639 & & ZOZO & JOHN JR. & $\mathrm{s}$ & 1 & MICHIGAN & ITALY & MICHIGAN & & & CASTELGOMBERTO. VI & C ST. & CALUMET - RAYMBAULTOWN \\
\hline 1640 & & BELLINGIER & ROBERTO & B & 28 & ITALY & ITALY & ITALY & 1913 & MINER/CM & BELLUNO & C ST. & CALUMET - RAYMBAULTOWN \\
\hline 1641 & & BIGANDO & GIORGIO & $\mathrm{H}$ & 64 & ITALY & ITALY & ITALY & 1894 & PUMPMAN/CM & TORINO & C ST. & CALUMET - RAYMBAULTOWN \\
\hline 1642 & & BIGANDO & VITTORIA & W & 55 & ITALY & ITALY & ITALY & 1894 & & & C ST. & CALUMET - RAYMBAULTOWN \\
\hline 1643 & & SCIACERO & JOHN & B & 25 & ITALY & ITALY & ITALY & 1904 & TRAMMER/CM & TORINO & C ST. & CALUMET - RAYMBAULTOWN \\
\hline 1644 & 2474 & MATTIELLO & JOHN & $\mathrm{H}$ & 39 & ITALY & ITALY & ITALY & 1902 & FOREMAN/CM & CASTELGOMBERTO. VI & C ST. & CALUMET - RAYMBAULTOWN \\
\hline 1645 & & MATTIELLO & MARY & W & 31 & ITALY & ITALY & ITALY & 1902 & & CASTELGOMBERTO. VI & C ST. & CALUMET - RAYMBAULTOWN \\
\hline 1646 & & MATTIELLO & MARGHERITA & $\mathrm{D}$ & 11 & MICHIGAN & ITALY & ITALY & & & CASTELGOMBERTO. VI & C ST. & CALUMET - RAYMBAULTOWN \\
\hline 1647 & & MATTIELLO & ELEONORA & $\mathrm{D}$ & 7 & MICHIGAN & ITALY & ITALY & & & CASTELGOMBERTO. VI & C ST. & CALUMET - RAYMBAULTOWN \\
\hline 1648 & & MATTIELLO & JOHN & $\mathrm{s}$ & 5 & MICHIGAN & ITALY & ITALY & & & CASTELGOMBERTO. VI & C ST. & CALUMET - RAYMBAULTOWN \\
\hline 1649 & & MATTIELLO & MARY & $\mathrm{D}$ & $3 \mathrm{M}$ & MICHIGAN & ITALY & ITALY & & & CASTELGOMBERTO. VI & C ST. & CALUMET - RAYMBAULTOWN \\
\hline 1650 & & CARLOTO & LENA & B & 4 & MICHIGAN & ITALY & ITALY & & & & C ST. & CALUMET - RAYMBAULTOWN \\
\hline 1651 & & FIORAZZO & ORLANDO & B & 45 & ITALY & ITALY & ITALY & 1913 & JANITOR/CM & CASTELGOMBERTO. VI & C ST. & CALUMET - RAYMBAULTOWN \\
\hline 1652 & & D'AGOSTINI & FRANK & B & 38 & ITALY & ITALY & ITALY & 1902 & TRAMMER/CM & BELLUNO & C ST. & CALUMET - RAYMBAULTOWN \\
\hline 1653 & & BELLINGIER & ROBERT & B & 28 & ITALY & ITALY & ITALY & 1904 & TRAMMER/CM & BELLUNO & C ST. & CALUMET - RAYMBAULTTOWN \\
\hline 1654 & 2458 & SOPPELSA & ANTONIO & $\mathrm{H}$ & 50 & ITALY & ITALY & ITALY & 1896 & & BELLUNO & C ST. & CALUMET - RAYMBAULTOWN \\
\hline 1655 & & SOPPELSA & EUGENIO & $\mathrm{s}$ & 16 & MICHIGAN & ITALY & ITALY & & JANITOR & BELLUNO & C ST. & CALUMET - RAYMBAULTOWN \\
\hline 1656 & & SOPPELSA & ANTONIO & $\mathrm{s}$ & 14 & MICHIGAN & ITALY & ITALY & & & BELLUNO & C ST. & CALUMET - RAYMBAULTOWN \\
\hline 1657 & & SOPPELSA & HENRY & $\mathrm{s}$ & 11 & MICHIGAN & ITALY & ITALY & & & BELLUNO & C ST. & CALUMET - RAYMBAULTOWN \\
\hline 1658 & & SOPPELSA & LOUIS & $\mathrm{s}$ & 9 & MICHIGAN & ITALY & ITALY & & & BELLUNO & C ST. & CALUMET - RAYMBAULTOWN \\
\hline 1659 & 2452 & GIACHINO & JAMES & $\mathrm{H}$ & 52 & ITALY & ITALY & ITALY & 1890 & MINER/CM & TORINO & C ST. & CALUMET - RAYMBAULTOWN \\
\hline 1660 & 2402 & GIACHINO & ANNA & W & 48 & ITALY & ITALY & ITALY & 1896 & IVIINERT UIVI & TURIVU & $\begin{array}{ll}\text { C ST. } \\
\text { C ST. }\end{array}$ & CALUMET - RAYMBAULTOWN \\
\hline 1661 & & GIACHINO & JAMES JR. & s & 20 & MICHIGAN & ITALY & ITALY & & PIPE MAN/CM & TORINO & C ST. & CALUMET - RAYMBAULTOWN \\
\hline 1662 & & GIACHINO & DOMINIC & $\mathrm{s}$ & 17 & MICHIGAN & ITALY & ITALY & & TIMBERMAN/CM & TORINO & C ST. & CALUMET - RAYMBAULTOWN \\
\hline 1663 & & GIACHINO & JOHN & $\mathrm{s}$ & 11 & MICHIGAN & ITALY & ITALY & & & TORINO & C ST. & CALUMET - RAYMBAULTOWN \\
\hline 1664 & & GIACHINO & PETER & $\mathrm{s}$ & 9 & MICHIGAN & ITALY & ITALY & & & TORINO & C ST. & CALUMET - RAYMBAULTOWN \\
\hline 1665 & & MAGINN & JACOB & $\mathrm{H}$ & 21 & MICHIGAN & ITALY & ITALY & & MINER/CM & & C ST. & CALUMET - RAYMBAULTOWN \\
\hline 1666 & & MAGINN & ANNA & W & 18 & MICHIGAN & ITALY & ITALY & & & & C ST. & CALUMET - RAYMBAULTOWN \\
\hline 1667 & & MAGINN & ANGELINA & D & 2 & MICHIGAN & MICHIGAN & MICHIGAN & & & & C ST. & CALUMET - RAYMBAULTOWN \\
\hline 1668 & & MAGINN & JOSEPH & $\mathrm{s}$ & $9 \mathrm{M}$ & MICHIGAN & MICHIGAN & MICHIGAN & & & & C ST. & CALUMET - RAYMBAULTOWN \\
\hline 1669 & 2450 & PASQUALONE & BATTISTA & $\mathrm{H}$ & 26 & ITALY & ITALY & ITALY & 1912 & TIMBERMAN/CM & SPARONE. TO & C ST. & CALUMET - RAYMBAULTTOWN \\
\hline 1670 & & PASQUALONE & MARY & w & 19 & ITALY & ITALY & ITALY & 1916 & & & C ST. & CALUMET - RAYMBAULTOWN \\
\hline 1671 & & PASQUALONE & NATALINA & D & 1 & MICHIGAN & ITALY & ITALY & & & SPARONE, TO & C ST. & CALUMET - RAYMBAULTOWN \\
\hline 1672 & & PASQUALONE & MARY & D & $1 \mathrm{M}$ & MICHIGAN & ITALY & ITALY & & & SPARONE. TO & C ST. & CALUMET - RAYMBAULTOWN \\
\hline 1673 & 2507 & COPPO & VALENTINO & $\mathrm{H}$ & 70 & ITALY & $\begin{array}{l}\text { ITALY } \\
\text { ITAL }\end{array}$ & $\begin{array}{l}\text { ITALY } \\
\text { ITAL }\end{array}$ & 1878 & & PONT CANAVESE. TO & C ST. & CALUMET - RAYMBAULTOWN \\
\hline 1674 & & COPPO & KATE & w & 65 & ITALY & ITALY & ITALY & 1880 & & PONT CANAVESE. TO & C ST. & CALUMET - RAYMBAULTOWN \\
\hline 1675 & & COPPO & LINA & $\mathrm{D}$ & 38 & ITALY & ITALY & ITALY & 1880 & & PONT CANAVESE. TO & C ST. & CALUMET - RAYMBAULTOWN \\
\hline 1676 & & COPPO & MIKE & $\mathrm{s}$ & 34 & MICHIGAN & ITALY & ITALY & & MINER/CM & PONT CANAVESE. TO & C ST. & CALUMET - RAYMBAULTOWN \\
\hline 1677 & & COPPO & PETER & s & 26 & MICHIGAN & ITALY & ITALY & & MINER/CM & PONT CANAVESE. TO & C ST. & CALUMET - RAYMBAULTOWN \\
\hline 1678 & & COPPO & VALENTINO & GS & 11 & MICHIGAN & ITALY & ITALY & & & PONT CANAVESE. TO & C ST. & \\
\hline 1679 & 2509 & ROLANDO & JOHN & $\mathrm{H}$ & 36 & ITALY & ITALY & ITALY & 1887 & MINER/CM & PONT CANAVESE. TO & C ST. & CALUMET - RAYMBAULTOWN \\
\hline 1680 & & ROLANDO & MARY & w & 30 & ITALY & ITALY & ITALY & 1892 & & & C ST. & CALUMET - RAYMBAULTOWN \\
\hline 1681 & & ROLANDO & MARY & D & 11 & MICHIGAN & ITALY & ITALY & & & PONT CANAVESE. TO & C ST. & CALUMET - RAYMBAULTTOWN \\
\hline 1682 & & ROLANDO & PETER & s & 9 & MICHIGAN & ITALY & ITALY & & & PONT CANAVESE. TO & C ST. & CALUMET - RAYMBAULTTOWN \\
\hline 1683 & & ROLANDO & ROSE & D & 7 & MICHIGAN & ITALY & ITALY & & & PONT CANAVESE. TO & C ST. & CALUMET - RAYMBAULTOWN \\
\hline 1684 & 2513 & TAPPERO & JOSEPH & $\mathrm{H}$ & 48 & ITALY & ITALY & ITALY & 1895 & LABORER/CM & SAN GIUSTO CANAVESE. TO & D ST. & CALUMET - RAYMBAULTOWN \\
\hline 1685 & & TAPPERO & JOSEPHINE & w & 32 & ITALY & ITALY & ITALY & 1895 & & SAN GIUSTO CANAVESE. TO & D ST. & CALUMET - RAYMBAULTOWN \\
\hline 1686 & & TAPPERO & JOSEPH JR. & $\mathrm{s}$ & 16 & MICHIGAN & ITALY & ITALY & & HOIST BOY/CM & SAN GIUSTO CANAVESE. TO & D ST. & CALUMET - RAYMBAULTOWN \\
\hline 1687 & & TAPPERO & EUGENE & $\mathrm{s}$ & 14 & MICHIGAN & ITALY & ITALY & & & SAN GIUSTO CANAVESE. TO & D ST. & CALUMET - RAYMBAULTOWN \\
\hline 1688 & & TAPPERO & MARY & $\mathrm{D}$ & 12 & MICHIGAN & ITALY & ITALY & & & SAN GIUSTO CANAVESE. TO & D ST. & CALUMET - RAYMBAULTOWN \\
\hline 1689 & & TAPPERO & CARLO & $\mathrm{s}$ & 9 & MICHIGAN & ITALY & ITALY & & & SAN GIUSTO CANAVESE, TO & $\begin{array}{l}\text { D ST. } \\
\text { DT. }\end{array}$ & CALUMET - RAYMBAULTOWN \\
\hline 1690 & & TAPPERO & KATE & $\mathrm{D}$ & 6 & MICHIGAN & ITALY & ITALY & & & SAN GIUSTO CANAVESE. TO & D ST. & CALUMET - RAYMBAULTOWN \\
\hline 1691 & 2545 & COSTA & BATTISTA & $\mathrm{H}$ & 35 & ITALY & ITALY & ITALY & 1900 & MINER/CM & TORINO & D ST. & CALUMET - RAYMBAULTOWN \\
\hline 1692 & & COSTA & MARY & w & 33 & ITALY & ITALY & ITALY & 1913 & & & D ST. & CALUMET - RAYMBAULTOWN \\
\hline 1693 & & COSTA & DOMENICO & $\mathrm{s}$ & 4 & MICHIGAN & ITALY & ITALY & & & TORINO & D ST. & CALUMET - RAYMBAULTOWN \\
\hline 1694 & & COSTA & LIZ & D & 3 & MICHIGAN & ITALY & ITALY & & & TORINO & D ST. & CALUMET - RAYMBAULTOWN \\
\hline 1695 & & COSTA & PETER & s & 1 & MICHIGAN & ITALY & ITALY & & & TORINO & D ST. & CALUMET - RAYMBAULTTOWN \\
\hline 1696 & & VERCELLINO & DOMENICO & $\mathrm{H}$ & 36 & ITALY & ITALY & ITALY & 1906 & TIMBERMAN/CM & TORINO & D ST. & CALUMET - RAYMBAULTOWN \\
\hline 1697 & & VERCELLINO & TRACY & w & 27 & ITALY & ITALY & ITALY & 1912 & & & D ST. & CALUMET - RAYMBAULTOWN \\
\hline 1698 & & VERCELLINO & ROSE & $\mathrm{D}$ & 5 & MICHIGAN & ITALY & ITALY & & & TORINO & D ST. & CALUMET - RAYMBAULTOWN \\
\hline 1699 & & VERCELLINO & VINCENZO & BR & 33 & ITALY & ITALY & ITALY & 1909 & TIMBERMAN/CM & TORINO & D ST. & CALUMET - RAYMBAULTOWN \\
\hline 1700 & 2517 & BARINOTTI & FRANK & $\mathrm{H}$ & 48 & ITALY & ITALY & ITALY & 1901 & MINER/CM & PONT CANAVESE. TO & D ST. & CALUMET - RAYMBAULTOWN \\
\hline
\end{tabular}




\begin{tabular}{|c|c|c|c|c|c|c|c|c|c|c|c|c|c|}
\hline 1701 & & BARINOTTI & MADDALENA & W & 35 & ITALY & ITALY & ITALY & 1912 & & & D ST. & CALUMET - RAYMBAULTOWN \\
\hline 1702 & & BARINOTTI & KATE & D & 17 & MICHIGAN & ITALY & ITALY & & OPERATOR/TELEPHONE & PONT CANAVESE. TO & D ST. & CALUMET - RAYMBAULTOWN \\
\hline 1703 & & BARINOTTI & MARY & D & 14 & MICHIGAN & ITALY & ITALY & & & PONT CANAVESE. TO & DST. & CALUMET - RAYMBAULTOWN \\
\hline 1704 & & BARINOTTI & FRANK & $\mathrm{s}$ & 13 & MICHIGAN & ITALY & ITALY & & & PONT CANAVESE. TO & D ST. & CALUMET - RAYMBAULTOWN \\
\hline 1705 & & BARINOTTI & EMMA & D & 5 & MICHIGAN & ITALY & ITALY & & & PONT CANAVESE. TO & D ST. & CALUMET - RAYMBAULTOWN \\
\hline 1706 & & BARINOTTI & IRENE & D & 3 & MICHIGAN & ITALY & ITALY & & & PONT CANAVESE. TO & D ST. & CALUMET - RAYMBAULTOWN \\
\hline 1707 & & BARINOTTI & JAMES & $\mathrm{s}$ & 2 & MICHIGAN & ITALY & ITALY & & & PONT CANAVESE. TO & D ST. & CALUMET - RAYMBAULTOWN \\
\hline 1708 & 2519 & ORSOLANO & ANNA & $\mathrm{H}$ & 50 & ITALY & ITALY & ITALY & 1892 & & TORINO & D ST. & CALUMET - RAYMBAULTOWN \\
\hline 1709 & 2010 & ORSOLANO & CLEMENT & $\mathrm{s}$ & 16 & MICHIGAN & ITALY & ITALY & 1002 & BAKER/BAKERY & SAN GIORGIO CANAVESE. TO & D ST. & CALUMET - RAYMBAULTOWN \\
\hline 1710 & 2521 & BOGGIO & DOMENICO & $\mathrm{H}$ & 37 & ITALY & ITALY & ITALY & 1903 & TIMBERMAN/CM & TORINO & D ST. & CALUMET - RAYMBAULTOWN \\
\hline 1711 & & BOGGIO & MINNIE & W & 27 & ITALY & ITALY & ITALY & 1899 & & & D ST. & CALUMET - RAYMBAULTOWN \\
\hline 1712 & & BOGGIO & JOSEPH & $\mathrm{s}$ & 9 & MICHIGAN & ITALY & ITALY & & & TORINO & D ST. & CALUMET - RAYMBAULTOWN \\
\hline 1713 & & BOGGIO & PETER & $\mathrm{s}$ & 2 & MICHIGAN & ITALY & ITALY & & & TORINO & D ST. & CALUMET - RAYMBAULTOWN \\
\hline 1714 & 2523 & NARDI & MIKE & $\mathrm{H}$ & 53 & ITALY & ITALY & ITALY & 1896 & MINER/CM & CARMIGNANO. LU & D ST. & CALUMET - RAYMBAULTOWN \\
\hline 1715 & & NARDI & JULIA & W & 49 & ITALY & ITALY & ITALY & 1896 & & CARMIGNANO. LU & D ST. & CALUMET - RAYMBAULTOWN \\
\hline 1716 & & NARDI & VIRGINIA & $\mathrm{D}$ & 21 & MICHIGAN & ITALY & ITALY & & SERVANT/PF & CARMIGNANO, LU & D ST. & CALUMET - RAYMBAULTOWN \\
\hline 1717 & & NARDI & FELICE & $\mathrm{s}$ & 18 & MICHIGAN & ITALY & ITALY & & TIMBERMAN/CM & CARMIGNANO. LU & D ST. & CALUMET - RAYMBAULTOWN \\
\hline 1718 & & NARDI & FEDERICO & $\mathrm{s}$ & 15 & MICHIGAN & ITALY & ITALY & & TEAMSTER/GROCERY STORE & CARMIGNANO. LU & D ST. & CALUMET - RAYMBAULTOWN \\
\hline 1719 & & NARDI & LINA & D & 13 & MICHIGAN & ITALY & ITALY & & & CARMIGNANO. LU & D ST. & CALUMET - RAYMBAULTOWN \\
\hline 1720 & & NARDI & ELEONOR & D & $?$ & MICHIGAN & ITALY & ITALY & & & CARMIGNANO. LU & D ST. & CALUMET - RAYMBAULTOWN \\
\hline 1721 & 2527 & PERUZZO & FRANK & $\mathrm{H}$ & 29 & ITALY & ITALY & ITALY & 1913 & TIMBERMAN/CM & $\begin{array}{l}\text { VICENZA } \\
\end{array}$ & D ST. & CALUMET - RAYMBAULTOWN \\
\hline 1722 & & PERUZZO & MINNIE & w & 23 & ITALY & ITALY & ITALY & 1913 & & VICENZA & D ST. & CALUMET - RAYMBAULTOWN \\
\hline 1723 & & PERUZZO & ANGELA & D & 3 & MICHIGAN & ITALY & ITALY & & & VICENZA & D ST. & CALUMET - RAYMBAULTOWN \\
\hline 1724 & & PERUZZO & ELEONORA & $\mathrm{D}$ & 2 & MICHIGAN & ITALY & ITALY & & & VICENZA & D ST. & CALUMET - RAYMBAULTOWN \\
\hline 1725 & 2506 & PUCCl & JOSEPH & $\mathrm{H}$ & 58 & ITALY & ITALY & ITALY & 1888 & WATCHMAN/CM & RUOTA. LU & D ST. & CALUMET - RAYMBAULTOWN \\
\hline 1726 & & PUCCI-LANDINI & LUCIA & W & 53 & ITALY & ITALY & ITALY & 1895 & & & D ST. & CALUMET - RAYMBAULTOWN \\
\hline 1727 & & PUCCI & EUGENE & $\mathrm{s}$ & 18 & MICHIGAN & ITALY & ITALY & & LABORER/CM & RUOTA. LU & D ST. & CALUMET - RAYMBAULTOWN \\
\hline 1728 & & PUCCI & PETER & $\mathrm{s}$ & 17 & MICHIGAN & ITALY & ITALY & & & RUOTA, LU & D ST. & CALUMET - RAYMBAULTOWN \\
\hline 1729 & & PUCCI & ALBERT & $\mathrm{s}$ & 12 & MICHIGAN & ITALY & ITALY & & & RUOTA. LU & D ST. & CALUMET - RAYMBAULTOWN \\
\hline 1730 & & PUCCl & ALMA & D & 10 & MICHIGAN & ITALY & ITALY & & & RUOTA. LU & D ST. & CALUMET - RAYMBAULTOWN \\
\hline 1731 & 2508 & IMPERATE & PASQUALE & $\mathrm{H}$ & 46 & ITALY & ITALY & ITALY & 1905 & MINER/CM & TORINO & D ST. & CALUMET - RAYMBAULTOWN \\
\hline 1732 & & IMPERATE & LIZ & w & 35 & ITALY & ITALY & ITALY & 1910 & & & D ST. & CALUMET - RAYMBAULTOWN \\
\hline 1733 & & IMPERATE & REST? & $\mathrm{s}$ & 8 & MICHIGAN & ITALY & ITALY & & & TORINO & D ST. & CALUMET - RAYMBAULTOWN \\
\hline 1734 & & IMPERATE & LEONA & D & 7 & MICHIGAN & ITALY & ITALY & & & TORINO & D ST. & CALUMET - RAYMBAULTOWN \\
\hline 1735 & & IMPERATE & AMERIGO & $\mathrm{s}$ & 6 & MICHIGAN & ITALY & ITALY & & & TORINO & D ST. & CALUMET - RAYMBAULTOWN \\
\hline 1736 & & IMPERATE & AMELIA & $\mathrm{D}$ & 5 & MICHIGAN & ITALY & ITALY & & & TORINO & D ST. & CALUMET - RAYMBAULTOWN \\
\hline 1737 & & FRENARDI & RAFFAELLO & B & 32 & ITALY & ITALY & ITALY & 1912 & TRAMMER/CM & & D ST. & CALUMET - RAYMBAULTOWN \\
\hline 1738 & 2514 & SOMMARIVA & FRANK & $\mathrm{H}$ & 54 & ITALY & ITALY & ITALY & 1890 & MINER/CM & VENEZIA & D ST. & CALUMET - RAYMBAULTOWN \\
\hline 1739 & & SOMMARIVA & ARCANGELA & W & 39 & ITALY & ITALY & ITALY & 1900 & & & D ST. & CALUMET - RAYMBAULTOWN \\
\hline 1740 & & SOMMARIVA & FRANK & $\mathrm{s}$ & 19 & MICHIGAN & ITALY & ITALY & & BOOKKEEPER/GARAGE & VENEZIA & D ST. & CALUMET - RAYMBAULTOWN \\
\hline 1741 & & SOMMARIVA & NAT & $\mathrm{s}$ & 17 & MICHIGAN & ITALY & ITALY & & LABORER/AUTO FACTORY & VENEZIA & D ST. & CALUMET - RAYMBAULTOWN \\
\hline 1742 & & SOMMARIVA & JOHANNA & D & 14 & MICHIGAN & ITALY & ITALY & & & VENEZIA & D ST. & CALUMET - RAYMBAULTOWN \\
\hline 1743 & & SOMMARIVA & JAMES & $\mathrm{s}$ & 11 & MICHIGAN & ITALY & ITALY & & & VENEZIA & D ST. & CALUMET - RAYMBAULTOWN \\
\hline 1744 & & SOMMARIVA & DOMENICO & $\mathrm{s}$ & 9 & MICHIGAN & ITALY & ITALY & & & VENEZIA & D ST. & CALUMET - RAYMBAULTOWN \\
\hline 1745 & 2518 & PIANA & JOHN & $\mathrm{H}$ & 50 & ITALY & ITALY & ITALY & 1890 & FIREMAN/RAILROAD & QUAGLIUZZO CANAVESE. TO & D ST. & CALUMET - RAYMBAULTOWN \\
\hline 1746 & & PIANA & MARY & w & 49 & ITALY & ITALY & ITALY & 1891 & & & D ST. & CALUMET - RAYMBAULTOWN \\
\hline 1747 & & PIANA & DOMENICO & $\mathrm{s}$ & 18 & MICHIGAN & ITALY & ITALY & & WIPER/RAILROAD & QUAGLIUZZO CANAVESE. TO & D ST. & CALUMET - RAYMBAULTOWN \\
\hline 1748 & & PIANA & ANGELA & D & 18 & MICHIGAN & ITALY & ITALY & & & QUAGLIUZZO CANAVESE. TO & D ST. & CALUMET - RAYMBAULTOWN \\
\hline 1749 & 2526 & ZANIN & OLIVER & $\mathrm{H}$ & 45 & ITALY & ITALY & ITALY & 1895 & MINER/CM & RIVAMONTE. BELLUNO & D ST. & CALUMET - RAYMBAULTOWN \\
\hline 1750 & & ZANIN & ANTONIA & w & 35 & ITALY & ITALY & ITALY & 1895 & & RIVAMONTE. BELLUNO & D ST. & CALUMET - RAYMBAULTOWN \\
\hline 1751 & & ZANIN & JOSEPH & $\mathrm{s}$ & 18 & MICHIGAN & ITALY & ITALY & & MINER/CM & RIVAMONTE. BELLUNO & D ST. & CALUMET - RAYMBAULTOWN \\
\hline 1752 & & ZANIN & ?? & $\mathrm{D}$ & 14 & MICHIGAN & ITALY & ITALY & & & RIVAMONTE, BELLUNO & D ST. & CALUMET - RAYMBAULTOWN \\
\hline 1753 & & ZANIN & FRANCE & D & 12 & MICHIGAN & ITALY & ITALY & & & RIVAMONTE. BELLUNO & D ST. & CALUMET - RAYMBAULTOWN \\
\hline 1754 & & ZANIN & ROSE & D & 8 & MICHIGAN & ITALY & ITALY & & & RIVAMONTE. BELLUNO & D ST. & CALUMET - RAYMBAULTOWN \\
\hline 1755 & & ZANIN & MARIE & D & 3 & MICHIGAN & ITALY & ITALY & & & RIVAMONTE. BELLUNO & D ST. & CALUMET - RAYMBAULTOWN \\
\hline 1756 & & ZANIN & JAMES & BR & 21 & MICHIGAN & ITALY & ITALY & & MINER/CM & RIVAMONTE. BELLUNO & D ST. & CALUMET - RAYMBAULTOWN \\
\hline 1757 & 2528 & CERETTO & JOHN & $\mathrm{H}$ & 49 & ITALY & ITALY & ITALY & 1895 & MINER/CM & PONT CANAVESE. TO & D ST. & CALUMET - RAYMBAULTOWN \\
\hline 1758 & & CERETTO & LUCIA & w & 46 & ITALY & ITALY & ITALY & 1897 & & & D ST. & CALUMET - RAYMBAULTOWN \\
\hline 1759 & & CERETTO & MARY & D & 16 & MICHIGAN & ITALY & ITALY & & & PONT CANAVESE. TO & D ST. & CALUMET - RAYMBAULTOWN \\
\hline 1760 & & CERETTO & JOSEPH & $\mathrm{s}$ & 9 & MICHIGAN & ITALY & ITALY & & & PONT CANAVESE. TO & D ST. & CALUMET - RAYMBAULTOWN \\
\hline 1761 & 2524 & BRACCO & JOHN & $\mathrm{H}$ & 55 & ITALY & ITALY & ITALY & 1889 & MINER/CM & TORINO & D ST. & CALUMET - RAYMBAULTOWN \\
\hline 1762 & & BRACCO & MINNIE & w & 55 & ITALY & ITALY & ITALY & 1891 & & & D ST. & CALUMET - RAYMBAULTOWN \\
\hline 1763 & & BRACCO & JOSEPH & $\mathrm{s}$ & 26 & MICHIGAN & ITALY & ITALY & & CLERK/RAILROAD & TORINO & D ST. & CALUMET - RAYMBAULTOWN \\
\hline
\end{tabular}




\begin{tabular}{|c|c|c|c|c|c|c|c|c|c|c|c|c|c|}
\hline 1764 & & BRACCO & ANTONIO & $\mathrm{s}$ & 24 & MICHIGAN & ITALY & ITALY & & PIPE FIXER/CM & TORINO & D ST. & CALUMET - RAYMBAULTOWN \\
\hline 1765 & & BRACCO & MADDALENA & D & 22 & MICHIGAN & ITALY & ITALY & & & TORINO & DST. & CALUMET - RAYMBAULTOWN \\
\hline 1766 & & BRACCO & MINNIE & D & 19 & MICHIGAN & ITALY & ITALY & & SALESLADY/DRY GOODS STORE & TORINO & D ST. & CALUMET - RAYMBAULTOWN \\
\hline 1767 & & BRACCO & MARY & D & 16 & MICHIGAN & ITALY & ITALY & & & TORINO & D ST. & CALUMET - RAYMBAULTOWN \\
\hline 1768 & 2563 & TEPATTI & PETER & $\mathrm{H}$ & 52 & ITALY & ITALY & ITALY & 1897 & TIMBERMAN/CM & PONT CANAVESE. TO & EST. & CALUMET - RAYMBAULTOWN \\
\hline 1769 & & TEPATTI & MARGHERITA & w & 55 & ITALY & ITALY & ITALY & 1895 & & & EST. & CALUMET - RAYMBAULTOWN \\
\hline 1770 & & TEPATTI & JAMES & s & 20 & MICHIGAN & ITALY & ITALY & & TRAMMER/CM & PONT CANAVESE. TO & EST. & CALUMET - RAYMBAULTOWN \\
\hline 1771 & & TEPATTI & PETER & $\mathrm{s}$ & 17 & MICHIGAN & ITALY & ITALY & & TIMBERMAN/CM & PONT CANAVESE. TO & E ST. & CALUMET - RAYMBAULTOWN \\
\hline 1772 & & TEPATTI & MARGHERITA & $\mathrm{D}$ & 14 & MICHIGAN & ITALY & ITALY & & IIVIDERTIATIVIC TVI & PONT CANAVESE. TO & E ST. & CALUMET - RAYMBAULTOWN \\
\hline 1773 & & BORIGLIONE & JOSEPH & B & 36 & ITALY & ITALY & ITALY & 1910 & TRAMMER/CM & VALPERGA. TO & E ST. & CALUMET - RAYMBAULTOWN \\
\hline 1774 & 2636 & $\begin{array}{l}\text { POLIUTO } \\
\end{array}$ & DOMENICO & $\mathrm{H}$ & 35 & ITALY & ITALY & ITALY & 1905 & TIMBERMAN/CM & SAN MARTINO CANAVESE. TO & UNION ST. & $\begin{array}{l}\text { CALUMET - RAYMBAULTOWN } \\
\text { CATE }\end{array}$ \\
\hline 1775 & & POLIUTO & KATE & W & 26 & MICHIGAN & ITALY & ITALY & & & & UNION ST. & CALUMET - RAYMBAULTOWN \\
\hline 1776 & & POLIUTO & ANGELA & D & 5 & MICHIGAN & ITALY & MICHIGAN & & & SAN MARTINO CANAVESE. TO & UNION ST. & CALUMET - RAYMBAULTOWN \\
\hline 1777 & & POLIUTO & TRACY & $\mathrm{s}$ & 3 & MICHIGAN & ITALY & MICHIGAN & & & SAN MARTINO CANAVESE. TO & UNION ST. & CALUMET - RAYMBAULTOWN \\
\hline 1778 & 2667 & ANTONETTI & GIORGIO & $\mathrm{H}$ & 45 & ITALY & ITALY & ITALY & 1905 & MINER/CM & SAN GENNARO. LU & UNION ST. & CALUMET - RAYMBAULTOWN \\
\hline 1779 & & ANTONETTI & JULIA & W & 35 & ITALY & ITALY & ITALY & 1906 & & SAN GENNARO, LU & UNION ST. & CALUMET - RAYMBAULTOWN \\
\hline 1780 & & ANTONETTI & ROMO & $\mathrm{s}$ & 15 & ITALY & ITALY & ITALY & 1906 & & SAN GENNARO. LU & UNION ST. & CALUMET - RAYMBAULTOWN \\
\hline 1781 & & ANTONETTI & BRUNA & D & 13 & ITALY & ITALY & ITALY & 1906 & & SAN GENNARO. LU & UNION ST. & CALUMET - RAYMBAULTOWN \\
\hline 1782 & & ANTONETTI & GINO & $\mathrm{s}$ & 12 & MICHIGAN & ITALY & ITALY & & & SAN GENNARO. LU & UNION ST. & CALUMET - RAYMBAULTOWN \\
\hline 1783 & 2655 & CURTO & BATTISTA & $\mathrm{H}$ & 44 & ITALY & ITALY & ITALY & 1897 & TIMBERMAN/CM & VIALFRE'. TO & UNION ST. & CALUMET - RAYMBAULTOWN \\
\hline 1784 & 2000 & CURTO & DOMENICA & W & 37 & ITALY & ITALY & ITALY & 1901 & TIVIDERIVIATICHI & VIALTRE. TO & UNION ST. & CALUMET - RAYMBAULTOWN \\
\hline 1785 & & CURTO & JOHN & $\mathrm{s}$ & 16 & MICHIGAN & ITALY & ITALY & & HELPER/GROCERY STORE & VIALFRE', TO & UNION ST. & CALUMET - RAYMBAULTOWN \\
\hline 1786 & & CURTO & JOSEPH & S & 14 & MICHIGAN & ITALY & ITALY & & & VIALFRE'. TO & UNION ST. & CALUMET - RAYMBAULTOWN \\
\hline 1787 & & CURTO & MAX & $\mathrm{s}$ & 11 & MICHIGAN & ITALY & ITALY & & & VIALFRE'. TO & UNION ST. & CALUMET - RAYMBAULTOWN \\
\hline 1788 & & PIETROPAOLI & ANGELO & B & 25 & ITALY & ITALY & ITALY & 1913 & TIMBERMAN/CM & VERONA & UNION ST. & CALUMET - RAYMBAULTOWN \\
\hline 1789 & 2754 & TONARELLI & NARCISO & $\mathrm{H}$ & 39 & ITALY & ITALY & ITALY & 1902 & MINER/CM & PESCIA. LU & COLUMBIA ST. & CALUMET - RAYMBAULTOWN \\
\hline 1790 & & TONARELLI & CONSILIA & W & 35 & ITALY & ITALY & ITALY & 1913 & & PESCIA, LU & COLUMBIA ST. & CALUMET - RAYMBAULTOWN \\
\hline 1791 & & TONARELLI & ITALIA & $\mathrm{D}$ & 9 & ITALY & ITALY & ITALY & 1913 & & PESCIA, LU & COLUMBIA ST. & CALUMET - RAYMBAULTOWN \\
\hline 1792 & & TONARELLI & B? & D & 5 & MICHIGAN & ITALY & ITALY & & & PESCIA. LU & COLUMBIA ST. & CALUMET - RAYMBAULTOWN \\
\hline 1793 & & TONARELLI & ELEONORA & D & 4 & MICHIGAN & ITALY & ITALY & & & PESCIA. LU & COLUMBIA ST. & CALUMET - RAYMBAULTOWN \\
\hline 1794 & & TONARELLI & DANTE & $\mathrm{s}$ & $10 \mathrm{M}$ & MICHIGAN & ITALY & ITALY & & & PESCIA. LU & COLUMBIA ST. & CALUMET - RAYMBAULTOWN \\
\hline 1795 & & VALENTINI & PETER & B & 28 & ITALY & ITALY & ITALY & 1913 & TRAMMER/CM & LUCCA & COLUMBIA ST. & CALUMET - RAYMBAULTOWN \\
\hline 1796 & & BARONI & PAUL & B & 27 & ITALY & ITALY & ITALY & 1919 & & CASTELVECCHIO. LU & COLUMBIA ST. & CALUMET - RAYMBAULTOWN \\
\hline 1797 & 2758 & GASPARDO & SERAFINO & $\mathrm{H}$ & 43 & ITALY & ITALY & ITALY & 1903 & TIMBERMAN/CM & $\begin{array}{l}\text { SPARONE, TO } \\
\text { SPAU }\end{array}$ & COLUMBIA ST. & CALUMET - RAYMBAULTOWN \\
\hline 1798 & & GASPARDO & TRACY & w & 37 & ITALY & ITALY & ITALY & 1904 & & SPARONE. TO & COLUMBIA ST. & CALUMET - RAYMBAULTOWN \\
\hline 1799 & & GASPARDO & MINNIE & D & 16 & ITALY & ITALY & ITALY & 1904 & & SPARONE. TO & COLUMBIA ST. & CALUMET - RAYMBAULTOWN \\
\hline 1800 & & GASPARDO & JOHN & $\mathrm{s}$ & 14 & MICHIGAN & ITALY & ITALY & & & SPARONE. TO & COLUMBIA ST. & CALUMET - RAYMBAULTOWN \\
\hline 1801 & & GASPARDO & DOMENICO & BR & 35 & ITALY & ITALY & ITALY & 1904 & TIMBERMAN/CM & SPARONE. TO & COLUMBIA ST. & CALUMET - RAYMBAULTOWN \\
\hline 1802 & 2760 & LUCCHESI & ANTONIETTA & $\mathrm{H}$ & 47 & ITALY & ITALY & ITALY & 1901 & & LUCCA & COLUMBIA ST. & CALUMET - RAYMBAULTOWN \\
\hline 1803 & & LUCCHESI & PAULINE & $\mathrm{D}$ & 14 & MICHIGAN & ITALY & ITALY & & & LUCCA & COLUMBIA ST. & CALUMET - RAYMBAULTOWN \\
\hline 1804 & & LUCCHESI & ANGELA & D & 12 & MICHIGAN & ITALY & ITALY & & & LUCCA & COLUMBIA ST. & CALUMET - RAYMBAULTOWN \\
\hline 1805 & & LUCCHESI & EDA & D & 10 & MICHIGAN & ITALY & ITALY & & & LUCCA & COLUMBIA ST. & CALUMET - RAYMBAULTOWN \\
\hline 1806 & & LUCCHESI & ORLANDO & $\mathrm{s}$ & 7 & MICHIGAN & ITALY & ITALY & & & LUCCA & COLUMBIA ST. & CALUMET - RAYMBAULTOWN \\
\hline 1807 & & LUCCHESI & GISELDO & BRIL & 33 & ITALY & ITALY & ITALY & 1901 & TRAMMER/CM & LUCCA & COLUMBIA ST. & CALUMET - RAYMBAULTOWN \\
\hline 1808 & 2669 & NARDI & ROBERTO & $\mathrm{H}$ & 51 & ITALY & ITALY & ITALY & 1891 & MINER/CM & LUCCA & UNION ST. & CALUMET - RAYMBAULTOWN \\
\hline 1809 & 2000 & NARDI & CESIRA & w & 46 & ITALY & ITALY & ITALY & 1891 & IVIIIVLTN UIVI & LUCCA & UNION ST. & CALUMET - RAYMBAULTOWN \\
\hline 1810 & & NARDI & GIULIA & D & 25 & MICHIGAN & ITALY & ITALY & & & LUCCA & UNION ST. & CALUMET - RAYMBAULTOWN \\
\hline 1811 & & NARDI & NELLO & $\mathrm{s}$ & 20 & MICHIGAN & ITAL & ITALY & & TIMBERMAN/CM & LUCCA & UNION ST. & CALUMET - RAYMBAULTOWN \\
\hline 1812 & & NARDI & CHESTER & $\mathrm{s}$ & 17 & MICHIGAN & ITAL & ITALY & & AUTO MECHANIC/GARAGE & LUCCA & UNION ST. & CALUMET - RAYMB \\
\hline 1813 & & NARDI & LIZ & $\mathrm{D}$ & 14 & MICHIGAN & ITALY & ITALY & & & LUCCA & UNION ST. & CALUMET - RAYMBAULTOWN \\
\hline 1814 & & LENCIONI & LUIGI & $\mathrm{cOU}$ & 27 & MICHIGAN & ITALY & ITALY & & MINER/CM & LUCCA & UNION ST. & CALUMET - RAYMBAULTOWN \\
\hline 1815 & & PIERCECCHI & CARLO & $\mathrm{H}$ & 27 & ITALY & ITALY & ITALY & 1911 & MINER/CM & LUCCA & UNION ST. & CALUMET - RAYMBAULTOWN \\
\hline 1816 & & PIERCECCHI & JOSEPHINE & W & 21 & MICHIGAN & ITALY & ITALY & & & & UNION ST. & CALUMET - RAYMBAULTOWN \\
\hline 1817 & & PIERCECCHI & ROBERTO & $\mathrm{s}$ & 2 & MICHIGAN & ITALY & MICHIGAN & & & LUCCA & UNION ST. & CALUMET - RAYMBAULTOWN \\
\hline 1818 & & NARDI & MARION & NIEC & 1 & MICHIGAN & ITALY & MICHIGAN & & & LUCCA & UNION ST. & CALUMET - RAYMBAULTOWN \\
\hline 1819 & 2768 & GHIGGIA & DOMENICO & $\mathrm{H}$ & 46 & ITALY & ITALY & ITALY & 1889 & MINER/CM & SCARMAGNO. TO & COLUMBIA ST. & CALUMET - RAYMBAULTOWN \\
\hline 1820 & & GHIGGIA & PAULINE & w & 39 & ITALY & ITALY & ITALY & 1891 & & & COLUMBIA ST. & CALUMET - RAYMBAULTOWN \\
\hline 1821 & & GHIGGIA & JAMES & $\mathrm{s}$ & 13 & MICHIGAN & ITALY & ITALY & & & SCARMAGNO, TO & COLUMBIA ST. & CALUMET - RAYMBAULTOWN \\
\hline 1822 & & GHIGGIA & JOHN & $\mathrm{s}$ & 7 & MICHIGAN & ITALY & ITALY & & & SCARMAGNO. TO & COLUMBIA ST. & CALUMET - RAYMBAULTOWN \\
\hline 1823 & 2464 & ZANARDI & MARY & $\mathrm{H}$ & 49 & ITALY & ITALY & ITALY & 1896 & & BERGAMO & B ST. & CALUMET - RAYMBAULTOWN \\
\hline 1824 & & ZANARDI & CHARLES & $\mathrm{s}$ & 22 & MICHIGAN & ITA & ITALY & & & BERGAMO & B ST. & CALUMET - RAYMBAULTOWN \\
\hline 1825 & & RACE & CESARE & B & 32 & ITALY & ITALY & ITALY & 1911 & & & B ST. & CA \\
\hline 1826 & 2133 & DAIRO & JOSEPHINE & $\mathrm{H}$ & 52 & ITALY & ITALY & ITALY & 1900 & LAUNDRESS/HOME & TORINO & COLUMBIA ST. & CALUMET - RAYMBAULTOWN \\
\hline
\end{tabular}




\begin{tabular}{|c|c|c|c|c|c|c|c|c|c|c|c|c|c|}
\hline 1827 & & DAIRO & PETER & $\mathrm{s}$ & 15 & MICHIGAN & ITALY & ITALY & & HELPER/FARM & TORINO & COLUMBIA ST. & CALUMET - RAYMBAULTOWN \\
\hline 1828 & & DAIRO & JOHN & $\mathrm{s}$ & 13 & MICHIGAN & ITALY & ITALY & & & TORINO & COLUMBIA ST. & CALUMET - RAYMBAULTOWN \\
\hline 1829 & & DAIRO & ALBERT & $\mathrm{s}$ & 12 & MICHIGAN & ITALY & ITALY & & & TORINO & RAYMBAULT ST. & CALUMET - RAYMBAULTOWN \\
\hline 1830 & & DAIRO & ANNA & $\mathrm{D}$ & 7 & MICHIGAN & ITALY & ITALY & & & TORINO & RAYMBAULT ST. & CALUMET - RAYMBAULTOWN \\
\hline 1831 & 3005 & CIOCATTO & MAURIZIO & $\mathrm{H}$ & 37 & ITALY & ITALY & ITALY & 1898 & MINER/CM & VALPERGA. TO & MINE ST. & CALUMET - RAYMBAULTOWN \\
\hline 1832 & & CIOCATTO & ANGELINE & w & 33 & ITALY & ITALY & ITALY & 1906 & & & MINE ST. & CALUMET - RAYMBAULTOWN \\
\hline 1833 & & CIOCATTO & ANDREINA & D & 12 & MICHIGAN & ITALY & ITALY & & & VALPERGA. TO & MINE ST. & CALUMET - RAYMBAULTOWN \\
\hline 1834 & & CIOCATTO & JOSEPHINA & $\mathrm{D}$ & 9 & MICHIGAN & ITALY & ITALY & & & VALPERGA. TO & MINE ST. & CALUMET - RAYMBAULTOWN \\
\hline 1835 & & CIOCATTO & ANGELINA & D & 7 & MICHIGAN & ITALY & ITALY & & & VALPERGA. TO & MINE ST. & CALUMET - RAYMBAULTOWN \\
\hline 1836 & 4028 & ZANOTTI & ANTONIO & $\mathrm{H}$ & 36 & ITALY & ITALY & ITALY & 1899 & PUMP MAN/CM & PARELLA. TO & OAK ST. & CALUMET \\
\hline 1837 & & ZANOTTI & MARY T. & w & 30 & MICHIGAN & ITALY & ITALY & & & & OAK ST. & CALUMET \\
\hline 1838 & & ZANOTTI & GENEVIEVE & $\mathrm{D}$ & 12 & MICHIGAN & MICHIGAN & ITALY & & & PARELLA. TO & OAK ST. & CALUMET \\
\hline 1839 & & ZANOTTI & FRANCIS & $\mathrm{s}$ & 10 & MICHIGAN & MICHIGAN & ITALY & & & PARELLA. TO & OAK ST. & CALUMET \\
\hline 1840 & & ZANOTTI & JOSEPH & $\mathrm{s}$ & $8 \mathrm{M}$ & MICHIGAN & MICHIGAN & ITALY & & & PARELLA. TO & OAK ST. & CALUMET \\
\hline 1841 & 4324 & MICHETTI & JOSEPH & $\mathrm{H}$ & 47 & ITALY & ITALY & ITALY & 1897 & MINER/CM & PONT CANAVESE. TO & OAK ST. & CALUMET \\
\hline 1842 & & MICHETTI & MARY & W & 48 & ITALY & ITALY & ITALY & 1899 & & PONT CANAVESE, TO & OAK ST. & CALUMET \\
\hline 1843 & & MICHETTI & FRED & $\mathrm{s}$ & 22 & ITALY & ITALY & ITALY & 1899 & LABORER/CM & PONT CANAVESE. TO & OAK ST. & CALUMET \\
\hline 1844 & & MICHETTI & $\mathrm{JOHN}$ & $\mathrm{s}$ & 18 & MICHIGAN & ITALY & ITALY & & LABORER/CM & PONT CANAVESE. TO & OAK ST. & CALUMET \\
\hline 1845 & & MICHETTI & PAUL & $\mathrm{s}$ & 16 & MICHIGAN & ITALY & ITALY & & DRILL BOYICM & PONT CANAVESE. TO & OAK ST. & $\begin{array}{l}\text { CALUMET } \\
\end{array}$ \\
\hline 1846 & & MICHETTI & JOSEPH & $\mathrm{s}$ & 12 & MICHIGAN & ITALY & ITALY & & & PONT CANAVESE. TO & OAK ST. & $\begin{array}{l}\text { CALUMET } \\
\text { CALO }\end{array}$ \\
\hline 1847 & & MICHETTI & JOHANNA & D & 8 & MICHIGAN & ITALY & ITALY & & & PONT CANAVESE. TO & OAK ST. & CALUMET \\
\hline 1848 & 4311 & LEONATTI & FRANCES & $\mathrm{H}$ & 52 & ITALY & ITALY & ITALY & 1904 & MINER/CM & CASTELLAMONTE, TO & OAK ST. & CALUMET \\
\hline 1849 & & LEONATTI & LOUIS & s & 39 & ITALY & ITALY & ITALY & 1907 & TIMBERMAN/CM & CASTELLAMONTE. TO & OAK ST. & CALUMET \\
\hline 1850 & & LEONATTI & DOMINICK & $\mathrm{s}$ & 29 & ITALY & ITALY & ITALY & 1907 & & CASTELLAMONTE. TO & OAK ST. & CALUMET \\
\hline 1851 & & LEONATTI & ERNESTINE & DIL & 24 & ITALY & ITALY & ITALY & 1899 & TIMBERMAN/CM & CASTELLAMONTE. TO & OAK ST. & CALUMET \\
\hline 1852 & & DE FILIPPI & DOMINICK & SOIL & 40 & ITALY & ITALY & ITALY & ???? & LAUDER/CM & TORINO & OAK ST. & CALUMET \\
\hline 1853 & 4313 & DE FILIPPI & JOSEPH & $\mathrm{H}$ & 40 & ITALY & ITALY & ITALY & 1903 & & SAN GIORGIO CANAVESE. TO & OAK ST. & CALUMET \\
\hline 1854 & & DE FILIPPI & ANGELINE & W & 37 & ITALY & ITALY & ITALY & 1904 & & & OAK ST. & CALUMET \\
\hline 1855 & & DE FILIPPI & JOSEPH & $\mathrm{s}$ & 14 & MICHIGAN & ITALY & ITALY & & & SAN GIORGIO CANAVESE. TO & OAK ST. & CALUMET \\
\hline 1856 & & DE FILIPPI & CARLO & $\mathrm{s}$ & 11 & MICHIGAN & ITALY & ITALY & & & SAN GIORGIO CANAVESE. TO & OAK ST. & $\begin{array}{l}\text { CALUMET } \\
\end{array}$ \\
\hline 1857 & & DE FILIPPI & ALBERTO & $\mathrm{s}$ & 9 & MICHIGAN & ITALY & ITALY & & & SAN GIORGIO CANAVESE. TO & OAK ST. & $\begin{array}{l}\text { CALUMET } \\
\end{array}$ \\
\hline 1858 & & DE FILIPPI & ERNESTO & $\mathrm{s}$ & 6 & MICHIGAN & ITALY & ITALY & & & SAN GIORGIO CANAVESE. TO & OAK ST. & CALUMET \\
\hline 1859 & & ALLARINA & MATT & B & 27 & ITALY & ITALY & ITALY & 1913 & MINER/CM & SAN GIORGIO CANAVESE. TO & OAK ST. & CALUMET \\
\hline 1860 & 4018 & LANCINA & ANDREA & $\mathrm{H}$ & 62 & ITALY & ITALY & ITALY & 1881 & NOT CLEAR & SAN GIORGIO CANAVESE, TO & PORTLAND ST. & CALUMET \\
\hline 1861 & & LANCINA & ELISABETTA & w & 54 & ITALY & ITALY & ITALY & 1888 & & & PORTLAND ST. & CALUMET \\
\hline 1862 & & LANCINA & ALINA & D & 29 & MICHIGAN & ITALY & ITALY & & & SAN GIORGIO CANAVESE. TO & PORTLAND ST. & CALUMET \\
\hline 1863 & & LANCINA & MARTA & $\mathrm{D}$ & 20 & MICHIGAN & ITALY & ITALY & & & SAN GIORGIO CANAVESE. TO & PORTLAND ST. & CALUMET \\
\hline 1864 & 4020 & NARETTO & MIKE & $\mathrm{H}$ & 49 & ITALY & ITALY & ITALY & 1890 & MINER/CM & AGLIE'. TO & PORTLAND ST. & CALUMET \\
\hline 1865 & & NARETTO & CATERINA & W & 57 & ITALY & ITALY & ITALY & 1890 & & AGLIE'. TO & PORTLAND ST. & CALUMET \\
\hline 1866 & & NARETTO & JOSEPHINE & $\mathrm{D}$ & 17 & MICHIGAN & ITALY & ITALY & & & AGLIE'. TO & PORTLAND ST. & CALUMET \\
\hline 1867 & 4022 & CASERIO & JOSEPH & $\mathrm{H}$ & 49 & ITALY & ITALY & ITALY & 1904 & LABORER/CM & STRAMBINELLO. TO & PORTLAND ST. & CALUMET \\
\hline 1868 & & CASERIO & TRACY & w & 46 & ITALY & ITALY & ITALY & 1906 & & & PORTLAND ST. & CALUMET \\
\hline 1869 & & CASERIO & DOMINICK & $\mathrm{s}$ & 12 & MICHIGAN & ITALY & ITALY & & & STRAMBINELLO. TO & PORTLAND ST. & CALUMET \\
\hline 1870 & 4022 & MERLO & LUIGI & $\mathrm{H}$ & 36 & ITALY & ITALY & ITALY & 1909 & MINER/CM & PEROSA. TO & PORTLAND ST. & $\begin{array}{l}\text { CALUMET } \\
\end{array}$ \\
\hline 1871 & & MERLO & MARIANNA & W & 28 & ITALY & ITALY & ITALY & 1912 & & TORINO & PORTLAND ST. & CALUMET \\
\hline 1872 & & MERLO & LUDOVICA & D & 4 & MICHIGAN & ITALY & ITALY & & & PEROSA. TO & PORTLAND ST. & CALUMET \\
\hline 1873 & & MERLO & VITTORIA & D & 2 & MICHIGAN & ITALY & ITALY & & & PEROSA. TO & PORTLAND ST. & CALUMET \\
\hline 1874 & 4026 & GASPARDO & MARCO & $\mathrm{H}$ & 48 & ITALY & ITALY & ITALY & 1893 & LABORER/CM & PONT CANAVESE. TO & PORTLAND ST. & $\begin{array}{l}\text { CALUMET } \\
\end{array}$ \\
\hline 1875 & & GASPARDO & MADDALENA & w & 46 & ITALY & ITALY & ITALY & 1901 & & & PORTLAND ST. & $\begin{array}{l}\text { CALUMET } \\
\end{array}$ \\
\hline 1876 & & GASPARDO & DOMENICO & $\mathrm{BR}$ & 33 & ITALY & ITALY & ITALY & 1908 & SHAFT MAN/CM & PONT CANAVESE. TO & PORTLAND ST. & CALUMET \\
\hline 1877 & & VERCELLINO & PIETRO & $\mathrm{H}$ & 26 & ITALY & ITALY & ITALY & 1910 & TIMBERMAN/CM & SAN MARTINO CANAVESE. TO & PORTLAND ST. & CALUMET \\
\hline 1878 & & VERCELLINO & MARY & W & 30 & ITALY & ITALY & ITALY & 1897 & & & PORTLAND ST. & CALUMET \\
\hline 1879 & 4009 & RICCA & PETER & $\mathrm{H}$ & 42 & ITALY & ITALY & ITALY & 1899 & CARPENTER/CM & SAN MARTINO CANAVESE. TO & PORTLAND ST. & CALUMET \\
\hline 1880 & & RICCA & MARY & W & 42 & ITALY & ITALY & ITALY & 1906 & & SAN MARTINO CANAVESE. TO & PORTLAND ST. & CALUMET \\
\hline 1881 & & SERRA & JOSEPH & SSO & 23 & ITALY & ITALY & ITALY & 1906 & DRILL SHARPENER/CM & SAN GIORGIO CANAVESE. TO & PORTLAND ST. & CALUMET \\
\hline 1882 & & SERRA & MARTIN & SSO & 18 & ITALY & ITALY & ITALY & 1906 & CAR REPAIRER/RAILROAD & SAN GIORGIO CANAVESE. TO & PORTLAND ST. & CALUMET \\
\hline 1883 & & SERRA & IRMA & $\mathrm{SD}$ & 10 & MICHIGAN & ITALY & ITALY & & & SAN GIORGIO CANAVESE. TO & PORTLAND ST. & CALUMET \\
\hline 1884 & & CASERIO & KATE & $\mathrm{SD}$ & 24 & ITALY & ITALY & ITALY & 1906 & & SAN GIORGIO CANAVESE, TO & PORTLAND ST. & $\begin{array}{l}\text { CALUMET } \\
\end{array}$ \\
\hline 1885 & & CASERIO & JAMES & SOIL & 28 & ITALY & ITALY & ITALY & 1907 & AGENT/LIFE INSURANCE & STRAMBINELLO. TO & PORTLAND ST. & $\begin{array}{l}\text { CALUMET } \\
\end{array}$ \\
\hline 1886 & & CASERIO & HILLARI & GS & 6 & MICHIGAN & ITALY & ITALY & & & SAN GIORGIO CANAVESE. TO & PORTLAND ST. & CALUMET \\
\hline 1887 & & CASERIO & ANDREW & GS & 2 & MICHIGAN & ITA & ITALY & & & SAN GIORGIO CANAVESE. TO & RTLAND ST. & $\begin{array}{l}\text { CALUMET } \\
\end{array}$ \\
\hline 1888 & & DE MURI & $\mathrm{JOH}$ & B & 26 & ITALY & ITALY & & 1913 & MINER/CM & VICENZA & PORTLAND ST. & $\begin{array}{l}\text { CALUMET } \\
\end{array}$ \\
\hline 1889 & & SERRA & ANDREW & B & 49 & ITALY & ITALY & ITALY & 1906 & LABORER/CM & SAN GIORGIO CANAVESE. TO & PORTLAND ST. & CALUMET \\
\hline
\end{tabular}




\begin{tabular}{|c|c|c|c|c|c|c|c|c|c|c|c|c|c|}
\hline 1890 & 4011 & BIGANDO & JOHN & $\mathrm{H}$ & 34 & ITALY & ITALY & ITALY & 1891 & PAINTER/HOUSE & TORINO & PORTLAND ST. & CALUMET \\
\hline 1891 & & BIGANDO & MARY & w & 27 & MICHIGAN & ITALY & ITALY & & & & PORTLAND ST. & CALUMET \\
\hline 1892 & & BIGANDO & LOUISE & $\mathrm{D}$ & 9 & MICHIGAN & ITALY & MICHIGAN & & & TORINO & PORTLAND ST. & CALUMET \\
\hline 1893 & & BIGANDO & $\mathrm{JOHN}$ & $\mathrm{s}$ & 5 & MICHIGAN & ITALY & MICHIGAN & & & TORINO & PORTLAND ST. & CALUMET \\
\hline 1894 & 4002 & RICHETTA & JOSEPHINE & SERV & 30 & ITALY & ITALY & ITALY & 1916 & SERVANT/PF & SAN MARTINO CANAVESE. TO & PORTLAND ST. & CALUMET \\
\hline 1895 & 4306 & LEONATTI & MIKE & H & 36 & ITALY & ITALY & ITALY & 1906 & LABORER/CM & $\begin{array}{l}\text { CASTELLAMONTE. TO } \\
\text { C. }\end{array}$ & PORTLAND ST. & CALUMET \\
\hline 1896 & 4000 & LEONATTI & FRANCES & W & 33 & ITALY & ITALY & ITALY & 1908 & 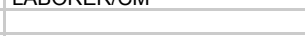 & TORINO & PORTLAND ST. & CALUMET \\
\hline 1897 & & LEONATTI & DOMENICO & s & 10 & MICHIGAN & ITALY & ITALY & 1900 & & CASTELLAMONTE. TO & PORTLAND ST. & CALUMET \\
\hline 1898 & & LEONATTI & ANTONIO & $\mathrm{s}$ & 5 & MICHIGAN & ITALY & ITALY & & & CASTELLAMONTE. TO & PORTLAND ST. & CALUMET \\
\hline 1899 & & LEONATTI & LIZ & D & 9M & MICHIGAN & ITALY & ITALY & & & CASTELLAMONTE. TO & PORTLAND ST. & CALUMET \\
\hline 1900 & & BOGGIO & GIORGIO & BIL & 31 & ITALY & ITALY & ITALY & 1913 & BAKER/BAKERY & SAN PONSO CANAVESE. TO & PORTLAND ST. & $\begin{array}{l}\text { CALUMET } \\
\text { CALE }\end{array}$ \\
\hline 1901 & & BALMA/BAIMA & DOMENICO & $\mathrm{H}$ & 43 & ITALY & ITALY & ITALY & 1900 & LABORER/CM & SAN GIORGIO CANAVESE. TO & PORTLAND ST. & CALUMET \\
\hline 1902 & & BALMA/BAIMA & MIKE & $s$ & 3 & MICHIGAN & ITALY & ITALY & & & SAN GIORGIO CANAVESE. TO & PORTLAND ST. & CALUMET \\
\hline 1903 & & BALMA/BAIMA & MATT & $\mathrm{s}$ & 2 & MICHIGAN & ITALY & ITALY & & & SAN GIORGIO CANAVESE. TO & PORTLAND ST. & CALUMET \\
\hline 1904 & & SALIVA & MARY & SER & 20 & ITALY & ITALY & ITALY & 1919 & HOUSEKEEPER/PF & & PORTLAND ST. & CALUMET \\
\hline 1905 & & CURTO & PETER & $\mathrm{H}$ & 66 & ITALY & ITALY & ITALY & 1879 & WATCHMAN/CM & TORINO & PORTLAND ST. & CALUMET \\
\hline 1906 & & POGGIONE & KATE & $\mathrm{H}$ & 80 & ITALY & ITALY & ITALY & 1888 & & SAN GIORGIO CANAVESE. TO & PORTLAND ST. & CALUMET \\
\hline 1907 & 4206 & NAVONE & MARIANNE & $\mathrm{H}$ & 34 & ITALY & ITALY & ITALY & 1903 & & SAN GIORGIO CANAVESE. TO & 10TH ST. & CALUMET \\
\hline 1908 & & NAVONE & LUDOVICA & D & 12 & MICHIGAN & ITALY & ITALY & & & RIVAROLO CANAVESE. TO & 10TH ST. & CALUMET \\
\hline 1909 & & NAVONE & DOMENICO & $\mathrm{s}$ & 9 & MICHIGAN & ITALY & ITALY & & & RIVAROLO CANAVESE. TO & 10TH ST. & CALUMET \\
\hline 1910 & & ZANOTTI & JOSEPH & B & 29 & ITALY & ITALY & ITALY & 1906 & MINER/CM & LUSIGLIE. TO & 10TH ST. & CALUMET \\
\hline 1911 & 4222 & ANTONIETTI & JOHN & $\mathrm{H}$ & 57 & ITALY & ITALY & ITALY & 1891 & MASON/CM & & 10TH ST. & CALUMET \\
\hline 1912 & & ANTONIETTI & MARGHERITA & w & 57 & ITALY & ITALY & ITALY & 1893 & & & 10TH ST. & CALUMET \\
\hline 1913 & & CLARA & DOMENICO & B & 33 & ITALY & ITALY & ITALY & 1908 & MINER/CM & SAN BENIGNO CANAVESE. TO & 10TH ST. & CALUMET \\
\hline 1914 & & CLARA & JOHN & B & 32 & ITALY & ITALY & ITALY & 1910 & MINER/CM & SAN BENIGNO CANAVESE. TO & 10TH ST. & CALUMET \\
\hline 1915 & 4225 & RENALDI & JOSEPH & $\mathrm{H}$ & 40 & ITALY & ITALY & ITALY & 1900 & CARPENTER/RAILROAD & SAN GIORGIO CANAVESE. TO & 10TH ST. & CALUMET \\
\hline 1916 & & RENALDI & MARGHERITA & W & 33 & MICHIGAN & ITALY & ITALY & & & & 10TH ST. & CALUMET \\
\hline 1917 & & RENALDI & $\mathrm{N} ?$ & $\mathrm{~s}$ & 2 & MICHIGAN & ITALY & MICHIGAN & & & SAN GIORGIO CANAVESE, TO & 10TH ST. & CALUMET \\
\hline 1918 & & RENALDI & GILBERT & $\mathrm{s}$ & $11 \mathrm{M}$ & MICHIGAN & ITALY & MICHIGAN & & & SAN GIORGIO CANAVESE. TO & 10TH ST. & CALUMET \\
\hline 1919 & & VERNETTI & MARGHERITA & $\mathrm{H}$ & 54 & ITALY & ITALY & ITALY & 1901 & & PONT CANAVESE. TO & 10TH ST. & CALUMET \\
\hline 1920 & & DE MARIA & KATE & GD & 8 & MICHIGAN & ITALY & ITALY & & & SAN GIORGIO CANAVESE. TO & 10TH ST. & CALUMET \\
\hline 1921 & 4232 & MASCAGNO & FRANK & $\mathrm{H}$ & 50 & ITALY & ITALY & ITALY & 1898 & MINER/CM & TORINO & 10TH ST. & CALUMET \\
\hline 1922 & 420 & MASCAGNO & GIACINTA & w & 48 & ITALY & ITALY & ITALY & $\begin{array}{l}1090 \\
1898\end{array}$ & IVIIIVERT IIVI & TORINO & 10TH ST. & CALUMET \\
\hline 1923 & & MASCAGNO & MIKE & s & 21 & MICHIGAN & ITALY & ITALY & & BOILER WASHER/RAILROAD & TORINO & 10TH ST. & CALUMET \\
\hline 1924 & & MASCAGNO & ERNEST & $\mathrm{s}$ & 17 & MICHIGAN & ITALY & ITALY & & & TORINO & 10TH ST. & CALUMET \\
\hline 1925 & & NOTARIO & JOSEPHINE & $\mathrm{D}$ & 23 & ITALY & ITALY & ITALY & 1898 & & TORINO & 10TH ST. & CALUMET \\
\hline 1926 & & NOTARIO & JOSEPH & SOIL & 29 & ITALY & ITALY & ITALY & 1909 & MINER/CM & TORINO & 10TH ST. & CALUMET \\
\hline 1927 & 4234 & CHIMINO & JAMES & H & 57 & ITALY & ITALY & ITALY & 1890 & MASON/HOUSE & SAN GIORGIO CANAVESE. TO & 10TH ST. & CALUMET \\
\hline 1928 & & CHIMINO & MARGHERITA & w & 58 & ITALY & ITALY & ITALY & 1890 & & SAN GIORGIO CANAVESE. TO & 10TH ST. & CALUMET \\
\hline 1929 & & CHIMINO & PETER & $\mathrm{s}$ & 24 & MICHIGAN & ITALY & ITALY & & REPAIRER/MOTOR SHOP & SAN GIORGIO CANAVESE. TO & 10TH ST. & CALUMET \\
\hline 1930 & & CHIMINO & KATE & D & 23 & MICHIGAN & ITALY & ITALY & & BOOKKEEPER & SAN GIORGIO CANAVESE. TO & 10TH ST. & CALUMET \\
\hline 1931 & & CHIMINO & DOMINIC & $\mathrm{s}$ & 15 & MICHIGAN & ITALY & ITALY & & & SAN GIORGIO CANAVESE. TO & 10TH ST. & CALUMET \\
\hline 1932 & 4236 & GRANDIS & JOHN & $\mathrm{H}$ & 49 & ITALY & ITALY & ITALY & 1900 & TIMBERMAN/CM & SAN GIORGIO CANAVESE. TO & 10TH ST. & CALUMET \\
\hline 1933 & & GRANDIS & MAGGIE & w & 45 & ITALY & ITALY & ITALY & 1899 & & & 10TH ST. & CALUMET \\
\hline 1934 & 4238 & GERVASSONE & AUGUST & $\mathrm{H}$ & 44 & ITALY & ITALY & ITALY & 1903 & MINER/CM & VERRES. AOSTA & 10TH ST. & CALUMET \\
\hline 1935 & 4200 & GERVASSONE & LUISA & w & 39 & ITALY & ITALY & ITALY & 1903 & IVIIIVLT TIVI & VERRES. AOSTA & 10TH ST. & CALUMET \\
\hline 1936 & & GERVASSONE & KATE & D & 15 & MICHIGAN & ITALY & ITALY & & & VERRES. AOSTA & 10TH ST. & CALUMET \\
\hline 1937 & & GERVASSONE & ERNEST & s & 13 & MICHIGAN & ITALY & ITALY & & & VERRES. AOSTA & 10TH ST. & CALUMET \\
\hline 1938 & & GERVASSONE & VITTORIO & $\mathrm{s}$ & 11 & MICHIGAN & ITALY & ITALY & & & VERRES. AOSTA & 10TH ST. & CALUMET \\
\hline 1939 & & GERVASSONE & IRMA & D & 7 & MICHIGAN & ITALY & ITALY & & & VERRES. AOSTA & 10TH ST. & CALUMET \\
\hline 1940 & 4242 & VALENTI & ANTONIO & $\mathrm{H}$ & 43 & ITALY & ITALY & ITALY & 1906 & MINER/CM & & 10TH ST. & CALUMET \\
\hline 1941 & & VALENTI & CLARA & W & 44 & ITALY & ITALY & ITALY & 1906 & & & 10TH ST. & CALUMET \\
\hline 1942 & & VALENTI & MARIA & D & 16 & ITALY & ITALY & ITALY & 1906 & & & 10TH ST. & CALUMET \\
\hline 1943 & & VALENTI & GIULIA & D & 10 & MICHIGAN & ITALY & ITALY & & & & 10TH ST. & CALUMET \\
\hline 1944 & & VALENTI & LORNA & D & 8 & MICHIGAN & ITALY & ITALY & & & & 10TH ST. & CALUMET \\
\hline 1945 & 4246 & GHIARDI & $\mathrm{JOHN}$ & H & 41 & ITALY & ITALY & ITALY & 1901 & MINER/CM & SAN MARTINO CANAVESE. TO & 10TH ST. & CALUMET \\
\hline 1946 & & GHIARDI & DOMENICA & w & 34 & ITALY & ITALY & ITALY & 1911 & & & 10TH ST. & CALUMET \\
\hline 1947 & & GHIARDI & KATE & D & 6 & MICHIGAN & ITALY & ITALY & & & SAN MARTINO CANAVESE, TO & 10TH ST. & CALUMET \\
\hline 1948 & & GHIARDI & EMMA & D & 2 & MICHIGAN & ITALY & ITALY & & & SAN MARTINO CANAVESE. TO & 10TH ST. & CALUMET \\
\hline 1949 & 4248 & TEPATTI & ANTONIO & $\mathrm{H}$ & 52 & ITALY & ITALY & ITALY & 1890 & PUMP MAN/CM & PONT CANAVESE. TO & 10TH ST. & CALUMET \\
\hline 1950 & & TEPATTI & CELESTINA & W & 42 & ITALY & ITALY & ITALY & 1898 & & & 10TH ST. & CALUMET \\
\hline 1951 & & TEPATTI & JAMES & s & 18 & MICHIGAN & ITALY & ITALY & & BLACKSMITH/SHOP & PONT CANAVESE. TO & 10TH ST. & CALUMET \\
\hline 1952 & & TEPATTI & KATE & D & 14 & MICHIGAN & ITALY & ITALY & & & PONT CANAVESE. TO & 10TH ST. & CALUMET \\
\hline
\end{tabular}




\begin{tabular}{|c|c|c|c|c|c|c|c|c|c|c|c|c|c|}
\hline \multirow{2}{*}{$\begin{array}{l}195 \\
195\end{array}$} & & TEPATTI & CRISTINA & D & 6 & MICHIGAN & ITALY & ITALY & & & PONT CANAVESE. TO & 10TH ST. & CALUMET \\
\hline & 4250 & MICHETTI & PAUL & $\mathrm{H}$ & 49 & ITALY & ITALY & ITALY & 1890 & FOREMAN/CM & PONT CANAVESE. TO & 10TH ST. & CALUMET \\
\hline 1955 & & MICHETTI & CATERINA & W & 56 & ITALY & ITALY & ITALY & $85 / 95$ & & PONT CANAVESE. TO & 10TH ST. & CALUMET \\
\hline 1956 & & MICHETTI & FRED & $\mathrm{s}$ & 18 & MICHIGAN & ITALY & ITALY & & CASHIER/BANK & PONT CANAVESE. TO & 10TH ST. & CALUMET \\
\hline 1957 & & MICHETTI & THOMAS & s & 15 & MICHIGAN & ITALY & ITALY & & & PONT CANAVESE. TO & 10TH ST. & CALUMET \\
\hline 1958 & & MANZETTI & PIERANTONIO & BRIL & 48 & ITALY & ITALY & ITALY & 1896 & TIMBERMAN/CM & PONT CANAVESE. TO & 10TH ST. & $\begin{array}{l}\text { CALUMET } \\
\end{array}$ \\
\hline 1959 & 4060 & COPPO & $\begin{array}{l}\text { VITTORIO } \\
\text { VIT }\end{array}$ & $\mathrm{H}$ & 51 & ITALY & ITALY & ITALY & 1889 & MINER/CM & PONT CANAVESE. TO & ACORN ST. & CALUMET \\
\hline 1960 & 4000 & COPPO & JEANNIE & w & 42 & ITALY & ITALY & ITALY & 1892 & HIIIVLNTUIVI & 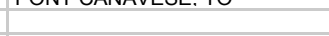 & ACORN ST. & CALUMET \\
\hline 1961 & & COPPO & ERNESTINE & D & 19 & MICHIGAN & ITALY & ITALY & & SALESMAN/DEPT. STORE & PONT CANAVESE. TO & ACORN ST. & CALUMET \\
\hline 1962 & & COPPO & ENRICO & s & 13 & MICHIGAN & ITALY & ITALY & & SALEOIVIAIVIUETT. STOKE & PONT CANAVESE. TO & ACORN ST. & CALUMET \\
\hline 1963 & & COPPO & ISABEL & D & 11 & MICHIGAN & ITALY & ITALY & & & PONT CANAVESE. TO & ACORN ST. & $\begin{array}{l}\text { CALUMET } \\
\end{array}$ \\
\hline 1964 & & COPPO & RALPH & $\mathrm{s}$ & 8 & MICHIGAN & ITALY & ITALY & & & PONT CANAVESE. TO & ACORN ST. & CALUMET \\
\hline 1965 & 4066 & CAPELLO & JOSEPH & $\mathrm{H}$ & 49 & ITALY & ITALY & ITALY & 1894 & MINER/CM & SAN BENIGNO CANAVESE. TO & ACORN ST. & CALUMET \\
\hline 1966 & & CAPELLO & MARIA & w & 45 & ITALY & ITALY & ITALY & 1895 & & & ACORN ST. & CALUMET \\
\hline 1967 & & CAPELLO & LOUIS & BR & 52 & ITALY & ITALY & ITALY & 1889 & MINER/CM & SAN BENIGNO CANAVESE. TO & ACORN ST. & CALUMET \\
\hline 1968 & & BIGANDO & PETER & $\mathrm{H}$ & 24 & ILLINOIS & ITALY & ITALY & & MINER/CM & TORINO & ACORN ST. & CALUMET \\
\hline 1969 & & BIGANDO & GINEVRA & W & 24 & ITALY & ITALY & ITALY & 1915 & & TORINO & ACORN ST. & CALUMET \\
\hline 1970 & & BIGANDO & DELPHINE & D & $8 \mathrm{M}$ & MICHIGAN & ITALY & ITALY & & & TORINO & ACORN ST. & $\begin{array}{l}\text { CALUMET } \\
\end{array}$ \\
\hline 1971 & 4035 & PONTELLO & FRANK & $\mathrm{H}$ & 53 & ITALY & ITALY & ITALY & 1889 & MASON/BUILDING CO. & & ACORN ST. & CALUMET \\
\hline 1972 & & PONTELLO & MARY & w & 48 & ITALY & ITALY & ITALY & 1889 & & & ACORN ST. & CALUMET \\
\hline 1973 & & PONTELLO & ROSE & $\mathrm{D}$ & 18 & MICHIGAN & ITALY & ITALY & & TEACHER/PUBLIC SCHOOL & & ACORN ST. & CALUMET \\
\hline 1974 & & PONTELLO & CLARA & $\mathrm{D}$ & 16 & MICHIGAN & ITALY & ITALY & & & & ACORN ST. & $\begin{array}{l}\text { CALUMET } \\
\end{array}$ \\
\hline 1975 & & PONTELLO & AGNESE & $\mathrm{D}$ & 15 & MICHIGAN & ITALY & ITALY & & & & ACORN ST. & $\begin{array}{l}\text { CALUMET } \\
\end{array}$ \\
\hline 1976 & 4045 & BOBBIO & PAUL & $\mathrm{H}$ & 59 & ITALY & ITALY & ITALY & 1895 & MINER/CM & SAN BENIGNO CANAVESE. TO & ACORN ST. & $\begin{array}{l}\text { CALUMET } \\
\end{array}$ \\
\hline 1977 & & BOBBIO & KATE & w & 38 & ITALY & ITALY & ITALY & 1915 & & & ACORN ST. & $\begin{array}{l}\text { CALUMET } \\
\end{array}$ \\
\hline 1978 & & BOBBIO & MARY & D & 16 & MICHIGAN & ITALY & ITALY & & & SAN BENIGNO CANAVESE. TO & ACORN ST. & CALUMET \\
\hline 1979 & & BOBBIO & JOHN & $\mathrm{s}$ & 11 & MICHIGAN & ITALY & ITALY & & & SAN BENIGNO CANAVESE. TO & ACORN ST. & $\begin{array}{l}\text { CALUMET } \\
\end{array}$ \\
\hline 1980 & & BOBBIO & MARIAN & $\mathrm{D}$ & 2 & MICHIGAN & ITALY & ITALY & & & SAN BENIGNO CANAVESE, TO & ACORN ST. & CALUMET \\
\hline 1981 & 4049 & VIOLA & PAUL & $\mathrm{H}$ & 29 & ITALY & ITALY & ITALY & 1905 & LABORER/CM & SAN BENIGNO CANAVESE. TO & ACORN ST. & CALUMET \\
\hline 1982 & & VIOLA & CRESCENCE & W & 24 & MICHIGAN & ITALY & ITALY & & & & ACORN ST. & CALUMET \\
\hline 1983 & & VIOLA & ANGELO & $\mathrm{s}$ & 4 & MICHIGAN & ITALY & ITALY & & & SAN BENIGNO CANAVESE. TO & ACORN ST. & CALUMET \\
\hline 1984 & 4082 & GUGLIELMO & JOSEPH & $\mathrm{H}$ & 55 & ITALY & ITALY & ITALY & 1887 & LANDER/CM & MONTELANGHE. TO & ELM ST. & $\begin{array}{l}\text { CALUMET } \\
\end{array}$ \\
\hline 1985 & & GUGLIELMO & MARNIE & w & 45 & ITALY & ITALY & ITALY & 1898 & & 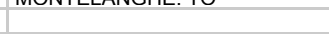 & ELM ST. & CALUMET \\
\hline 1986 & & GUGLIELMO & MIKE & $\mathrm{s}$ & 19 & MICHIGAN & ITALY & ITALY & & TELEGRAPH OPERATOR/OFFICE & MONTELANGHE, TO & ELM ST. & $\begin{array}{l}\text { CALUMET } \\
\end{array}$ \\
\hline 1987 & & TRIONE & AUGUST & $\mathrm{H}$ & 27 & MICHIGAN & ITALY & ITALY & & MINER/CM & TORINO & ELM ST. & $\begin{array}{l}\text { CALUMET } \\
\end{array}$ \\
\hline 1988 & & TRIONE & LOUISE & w & 23 & CALIFORNI & ITALY & ITALY & & & & ELM ST. & CALUMET \\
\hline 1989 & & TRIONE & KATE & D & 2 & MICHIGAN & MICHIGAN & CALIFORNI & & & TORINO & ELM ST. & CALUMET \\
\hline 1990 & 4086 & FRANCONE & JOHN & $\mathrm{H}$ & 30 & ITALY & ITALY & ITALY & 1906 & & SAN BENIGNO CANAVESE. TO & ELM ST. & CALUMET \\
\hline 1991 & & FRANCONE & MARY & w & 29 & MICHIGAN & ITALY & ITALY & & & & ELM ST. & $\begin{array}{l}\text { CALUMET } \\
\end{array}$ \\
\hline 1992 & & FRANCONE & JOHANNA & $\mathrm{D}$ & 7 & MICHIGAN & ITALY & MICHIGAN & & & SAN BENIGNO CANAVESE. TO & ELM ST. & $\begin{array}{l}\text { CALUMET } \\
\end{array}$ \\
\hline 1993 & & FRANCONE & MIKE & $\mathrm{s}$ & 5 & MICHIGAN & ITALY & MICHIGAN & & & SAN BENIGNO CANAVESE. TO & ELM ST. & CALUMET \\
\hline 1994 & 4063 & MICHETTI & JOHN & $\mathrm{H}$ & 64 & ITALY & ITALY & ITALY & 1882 & MINER/CM & PONT CANAVESE. TO & ELM ST. & CALUMET \\
\hline 1995 & & MICHETTI & MADDALENA & W & 60 & ITALY & ITALY & ITALY & 1888 & & & ELM ST. & CALUMET \\
\hline 1996 & & MICHETTI & MIKE & $\mathrm{s}$ & 27 & MICHIGAN & ITALY & ITALY & & NOT CLEAR & PONT CANAVESE. TO & ELM ST. & CALUMET \\
\hline 1997 & & MICHETTI & PAUL & s & 24 & MICHIGAN & ITALY & ITALY & & AUTO MECHANIC/GARAGE & PONT CANAVESE. TO & ELM ST. & $\begin{array}{l}\text { CALUMET } \\
\end{array}$ \\
\hline 1998 & & KERNICK & KATE & $\mathrm{D}$ & 21 & MICHIGAN & ITALY & ITALY & & & PONT CANAVESE. TO & ELM ST. & CALUMET \\
\hline 1999 & & KERNICK & KATE & GD & $10 \mathrm{M}$ & MICHIGAN & ITALY & ITALY & & & PONT CANAVESE. TO & ELM ST. & CALUMET \\
\hline 2000 & 4065 & NIGRA & ANTONIO & $\mathrm{H}$ & 60 & ITALY & ITALY & ITALY & 1885 & TIMBERMAN/CM & RIVAROLO CANAVESE. TO & ELM ST. & $\begin{array}{l}\text { CALUMET } \\
\text { CALUME }\end{array}$ \\
\hline 2001 & & NIGRA & KATE & w & 57 & ITALY & ITALY & ITALY & 1890 & & & ELM ST. & $\begin{array}{l}\text { CALUMET } \\
\end{array}$ \\
\hline 2002 & & NIGRA & JOHN & s & 20 & MICHIGAN & ITALY & ITALY & & SPRINKLER/STREET & RIVAROLO CANAVESE. TO & ELM ST. & $\begin{array}{l}\text { CALUMET } \\
\end{array}$ \\
\hline 2003 & 4067 & MIGLIO/MIGLIA & TONY & $\mathrm{H}$ & 60 & ITALY & ITALY & ITALY & 1882 & LABORER/RAILROAD & SAN GIORGIO CANAVESE. TO & ELM ST. & $\begin{array}{l}\text { CALUMET } \\
\end{array}$ \\
\hline 2004 & 4085 & BAUDINO & GIORGIO & $\mathrm{H}$ & 52 & ITALY & ITALY & ITALY & 1891 & MINER/CM & RIVAROLO CANAVESE, TO & E. CONE ST. & CALUMET \\
\hline 2005 & & BAUDINO & MARY & W & 50 & ITALY & ITALY & ITALY & 1891 & & RIVAROLO CANAVESE. TO & E. CONE ST. & CALUMET \\
\hline 2006 & & BAUDINO & TERESA & D & 23 & MICHIGAN & ITALY & ITALY & & NURSE & RIVAROLO CANAVESE. TO & E. CONE ST. & $\begin{array}{l}\text { CALUMET } \\
\end{array}$ \\
\hline 2007 & & BAUDINO & ERNESTINA & D & 20 & MICHIGAN & ITALY & ITALY & & SALESLADY/MUSIC STORE & RIVAROLO CANAVESE. TO & E. CONE ST. & CALUMET \\
\hline 2008 & & BAUDINO & OLIVER & $\mathrm{s}$ & 18 & MICHIGAN & ITALY & ITALY & & COACH CLEANER/RAILROAD & RIVAROLO CANAVESE. TO & E. CONE ST. & CALUMET \\
\hline 2009 & & BAUDINO & PETER & $\mathrm{s}$ & 16 & MICHIGAN & ITALY & ITALY & & & RIVAROLO CANAVESE. TO & E. CONE ST. & CALUMET \\
\hline 2010 & & BAUDINO & VIOLET & D & 11 & MICHIGAN & ITALY & ITALY & & & RIVAROLO CANAVESE, TO & E. CONE ST. & CALUMET \\
\hline 2011 & & BAUDINO & CARLO & $\mathrm{H}$ & 36 & ITALY & ITALY & ITALY & 1901 & BLACKSMITH/CM & RIVAROLO CANAVESE. TO & E. CONE ST. & CALUMET \\
\hline 2012 & & BAUDINO & ROSE & W & 26 & ITALY & ITALY & ITALY & 1910 & & & E. CONE ST. & CALUMET \\
\hline 2013 & & BAUDINO & JAMES & $\mathrm{s}$ & 5 & MICHIGAN & ITALY & ITALY & & & RIVAROLO CANAVESE. TO & E. CONE ST. & CALUMET \\
\hline 2014 & & BAUDINO & FRANK & $\mathrm{s}$ & $10 \mathrm{M}$ & MICHIGAN & ITALY & ITALY & & & RIVAROLO CANAVESE. TO & E. CONE ST. & CALUMET \\
\hline 2015 & 4089 & MICHELINI & SANTINO & $\mathrm{H}$ & 44 & ITALY & ITALY & ITALY & 1899 & TIMBERMAN/CM & LUCCA & E. CONE ST. & $\begin{array}{l}\text { CALUMET } \\
\end{array}$ \\
\hline
\end{tabular}




\begin{tabular}{|c|c|c|c|c|c|c|c|c|c|c|c|c|c|}
\hline 2016 & & MICHELINI & ESTER & W & 42 & ITALY & ITALY & ITALY & 1902 & & & E. CONE ST. & CALUMET \\
\hline 2017 & & MICHELINI & ANGELO & $s$ & 16 & MICHIGAN & ITALY & ITALY & & & LUCCA & E. CONE ST. & CALUMET \\
\hline 2018 & & MICHELINI & IDA & $\mathrm{D}$ & 14 & MICHIGAN & ITALY & ITALY & & & LUCCA & E. CONE ST. & CALUMET \\
\hline 2019 & & MICHELINI & GIULIA & $\mathrm{D}$ & 12 & MICHIGAN & ITALY & ITALY & & & LUCCA & E. CONE ST. & CALUMET \\
\hline 2020 & & MICHELINI & GIULIO & s & 12 & MICHIGAN & ITALY & ITALY & & & LUCCA & E. CONE ST. & CALUMET \\
\hline 2021 & 4089 & RASTELLO & ANDREA & $\mathrm{H}$ & 40 & ITALY & ITALY & ITALY & 1907 & LABORER/CM & PONT CANAVESE. TO & E. CONE ST. & CALUMET \\
\hline 2022 & 400 & RASTELLO & CELESTE & w & 39 & ITALY & ITALY & ITALY & 1913 & LADU TALTUIUI & PONT CANAVESE. TO & E. CONE ST. & CALUMET \\
\hline 2023 & & RASTELLO & LORENZO & s & 15 & ITALY & ITALY & ITALY & 1913 & & PONT CANAVESE. TO & E. CONE ST. & CALUMET \\
\hline 2024 & & RASTELLO & ELISA & $\mathrm{D}$ & 10 & MICHIGAN & ITALY & ITALY & & & PONT CANAVESE. TO & E. CONE ST. & CALUMET \\
\hline 2025 & 4351 & ZANOTTI & MIKE & $\mathrm{H}$ & 36 & ITALY & ITALY & ITALY & 1901 & MINER/CM & TORINO & PINE ST. & CALUMET \\
\hline 2026 & & ZANOTTI & MARY & w & 32 & ITALY & ITALY & ITALY & 1913 & KEEPER/BOARDING HOUSE & & PINE ST. & CALUMET \\
\hline 2027 & & ZANOTTI & ANGELINA & D & 6 & MICHIGAN & ITALY & ITALY & & & TORINO & PINE ST. & CALUMET \\
\hline 2028 & & ZANOTTI & BEN & $\mathrm{s}$ & 1 & MICHIGAN & ITALY & ITALY & & & TORINO & PINE ST. & CALUMET \\
\hline 2029 & & ZANOTTI & JOHN & $\mathrm{BR}$ & 27 & ITALY & ITALY & ITALY & 1909 & MINER/CM & TORINO & PINE ST. & CALUMET \\
\hline 2030 & & MAGA & JOHN & B & 52 & ITALY & ITALY & ITALY & 1887 & MINER/CM & RIVAROLO CANAVESE. TO & PINE ST. & CALUMET \\
\hline 2031 & & MIGLIO/MIGLIA & JOHN & B & 38 & ITALY & ITALY & ITALY & 1905 & MINER/CM & TORINO & PINE ST. & CALUMET \\
\hline 2032 & & AUTERO & MARTIN & B & 29 & ITALY & ITALY & ITALY & 1913 & MINER/CM & PONT CANAVESE. TO & PINE ST. & CALUMET \\
\hline 2033 & 4347 & FRICCl & DOMENICO & $\mathrm{H}$ & 40 & ITALY & ITALY & ITALY & 1904 & MINER/CM & & W. CONE ST. & CALUMET \\
\hline 2034 & & FRICCl & KATE & w & 40 & ITALY & ITALY & ITALY & 1906 & & & W. CONE ST. & CALUMET \\
\hline 2035 & & FRICCl & MARY & D & 10 & ITALY & ITALY & ITALY & 1906 & & & W. CONE ST. & CALUMET \\
\hline 2036 & & FRICCI & LUISA & $\mathrm{D}$ & 8 & MICHIGAN & ITALY & ITALY & & & & W. CONE ST. & CALUMET \\
\hline 2037 & & FRICCl & PETER & s & 6 & MICHIGAN & ITALY & ITALY & & & & W. CONE ST. & CALUMET \\
\hline 2038 & 4211 & GRIGLIATTI & THOMAS & $\mathrm{H}$ & 32 & ITALY & ITALY & ITALY & 1905 & FIREMAN/BOILER HOUSE & SAN BENIGNO CANAVESE. TO & 11TH ST. & CALUMET \\
\hline 2039 & & GRIGLIATTI & ROSE & W & 30 & ITALY & ITALY & ITALY & 1905 & & SAN BENIGNO CANAVESE. TO & 11TH ST. & CALUMET \\
\hline 2040 & & GRIGLIATTI & CARLO & $s$ & 11 & MICHIGAN & ITALY & ITALY & & & SAN BENIGNO CANAVESE. TO & 11TH ST. & CALUMET \\
\hline 2041 & & GRIGLIATTI & BATTISTA & $\mathrm{s}$ & 9 & MICHIGAN & ITALY & ITALY & & & SAN BENIGNO CANAVESE. TO & 11TH ST. & CALUMET \\
\hline 2042 & 4213 & ALASINA & DOMENICO & $\mathrm{H}$ & 46 & ITALY & ITALY & ITALY & 1887 & LABORER/GARDEN & SAN GIORGIO CANAVESE. TO & 11TH ST. & CALUMET \\
\hline 2043 & & ALASINA & DOMENICA & W & 34 & ITALY & ITALY & ITALY & 1903 & & & 11TH ST. & CALUMET \\
\hline 2044 & & ALASINA & TERESA & D & 15 & MICHIGAN & ITALY & ITALY & & & SAN GIORGIO CANAVESE. TO & 11TH ST. & CALUMET \\
\hline 2045 & & ALASINA & FRANK & $\mathrm{s}$ & 11 & ITALY & ITALY & ITALY & 1911 & & SAN GIORGIO CANAVESE. TO & 11TH ST. & CALUMET \\
\hline 2046 & & ALASINA & ANTONIO & $\mathrm{s}$ & 4 & SOUTH & ITALY & ITALY & & & SAN GIORGIO CANAVESE. TO & 11TH ST. & CALUMET \\
\hline 2047 & & ALASINA & MARY & $\mathrm{D}$ & 2 & MICHIGAN & ITALY & ITALY & & & SAN GIORGIO CANAVESE. TO & 11TH ST. & CALUMET \\
\hline 2048 & & GALLO & DAVID & $\mathrm{H}$ & 25 & MINNESOT & ITALY & ITALY & & ELECTRICIAN/CM & $\begin{array}{l}\text { TORINO } \\
\text { TORIVAVE. TO }\end{array}$ & 11TH ST. & CALUMET \\
\hline 2049 & & GALLO & JOSEPHINE & W & 26 & ITALY & ITALY & ITALY & 1917 & & & 11TH ST. & CALUMET \\
\hline 2050 & & GALLO & MARIA & $\mathrm{D}$ & $5 \mathrm{M}$ & MICHIGAN & MINNESOT & ITALY & & & TORINO & 11TH ST. & CALUMET \\
\hline 2051 & 4217 & ELLENA & JOHN & $\mathrm{H}$ & 52 & ITALY & ITALY & ITALY & 1900 & LABORER/CM & SAN BENIGNO CANAVESE. TO & 11TH ST. & CALUMET \\
\hline 2052 & & ELLENA & KATE & w & 52 & ITALY & ITALY & ITALY & 1902 & 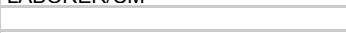 & & 11 TH ST. & CALUMET \\
\hline 2053 & & ELLENA & ANGELO & $\mathrm{BR}$ & 40 & ITALY & ITALY & ITALY & 1904 & LABORER/CM & SAN BENIGNO CANAVESE. TO & 11TH ST. & CALUMET \\
\hline 2054 & 4323 & ZUBIENA & DOMENICO & $\mathrm{H}$ & 33 & ITALY & ITALY & ITALY & 1885 & MINER/CM & TORINO & 11TH ST. & CALUMET \\
\hline 2055 & & ZUBIENA & MADDALENA & W & 29 & ITALY & ITALY & ITALY & 1892 & & & 11TH ST. & CALUMET \\
\hline 2056 & & ZUBIENA & JOHN & $\mathrm{s}$ & 3 & MICHIGAN & ITALY & ITALY & & & TORINO & 11TH ST. & CALUMET \\
\hline 2057 & 4337 & MUSSATO & TERESA & $\mathrm{H}$ & 56 & ITALY & ITALY & ITALY & 1902 & & & 11TH ST. & CALUMET \\
\hline 2058 & 4245 & NEPOTE & JAMES & $\mathrm{H}$ & 46 & ITALY & ITALY & ITALY & 1898 & CARPENTER/SHOP & PONT CANAVESE. TO & 11TH ST. & CALUMET \\
\hline 2059 & & NEPOTE & JAMES JR. & s & 14 & MICHIGAN & ITALY & ITALY & & & PONT CANAVESE. TO & 11TH ST. & CALUMET \\
\hline 2060 & & NEPOTE & PAUL & $\mathrm{s}$ & 12 & MICHIGAN & ITALY & ITALY & & & PONT CANAVESE. TO & 11TH ST. & CALUMET \\
\hline 2061 & & NEPOTE & MARY & $\mathrm{D}$ & 9 & MICHIGAN & ITALY & ITALY & & & PONT CANAVESE. TO & 11TH ST. & CALUMET \\
\hline 2062 & & NEPOTE & LORENZO & $\mathrm{s}$ & 4 & MICHIGAN & ITALY & ITALY & & & PONT CANAVESE. TO & 11TH ST. & CALUMET \\
\hline 2063 & 4253 & GROSSO & ANTONIO & $\mathrm{H}$ & 45 & ITALY & ITALY & ITALY & 1899 & TRUCK MAN/CM & MONTELANGHE. TO & 11TH ST. & CALUMET \\
\hline 2064 & & GROSSO & TERESA & w & 45 & ITALY & ITALY & ITALY & 1902 & 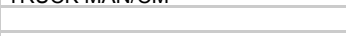 & MONTELANGHE. TO & 11TH ST. & CALUMET \\
\hline 2065 & & GROSSO & STEPHEN & $s$ & 21 & ITALY & ITALY & ITALY & 1902 & WAITER/CLUBHOUSE & MONTELANGHE. TO & 11TH ST. & CALUMET \\
\hline 2066 & & GROSSO & DOMENICO & $\mathrm{s}$ & 20 & ITALY & ITALY & ITALY & 1902 & WAITER/CLUBHOUSE & MONTELANGHE. TO & 11TH ST. & CALUMET \\
\hline 2067 & & CATTINI & JOSEPHINE & $\mathrm{H}$ & 53 & ITALY & ITALY & ITALY & 1905 & & SAN GIORGIO CANAVESE, TO & 11TH ST. & CALUMET \\
\hline 2068 & & CATTINI & ANTONIO & $\mathrm{s}$ & 22 & ITALY & ITALY & ITALY & 1905 & TIMBERMAN/CM & SAN GIORGIO CANAVESE. TO & 11TH ST. & CALUMET \\
\hline 2069 & 3994 & FREEMAN & WILLIAM & $\mathrm{H}$ & 37 & MICHIGAN & SWEDEN & SWEDEN & & PLUMBER & SWEDEN & SCOTT ST. & CALUMET \\
\hline 2070 & & FREEMAN & KATE & w & 32 & ITALY & ITALY & ITALY & 1905 & & & SCOTT ST. & CALUMET \\
\hline 2071 & & FREEMAN & EDWARD & s & 3 & MICHIGAN & MICHIGAN & ITALY & & & & SCOTT ST. & CALUMET \\
\hline 2072 & 3996 & RICHETTA & THOMAS & $\mathrm{H}$ & 56 & ITALY & ITALY & ITALY & 1889 & MINER/CM & SAN MARTINO CANAVESE. TO & SCOTT ST. & CALUMET \\
\hline 2073 & & RICHETTA & JOSEPHINE & W & 46 & ITALY & ITALY & ITALY & 1898 & IVIIIVENCIVI & SAN MARTINO CANAVESE, TO & SCOTT ST. & CALUMET \\
\hline 2074 & & RICHETTA & ANNA & D & 16 & ITALY & ITALY & ITALY & 1907 & & SAN MARTINO CANAVESE. TO & SCOTT ST. & CALUMET \\
\hline 2075 & 3990 & CONTRATTO & MARY & $\mathrm{H}$ & 59 & ITALY & ITALY & ITALY & 1896 & & LOCANA CANAVESE. TO & OSCEOLA R & CALUMET \\
\hline 2076 & & CONTRATTO & PETER & $\mathrm{s}$ & 17 & MICHIGAN & ITALY & ITALY & & TYPEWRITER/BROKERS OFFICE & LOCANA CANAVESE. TO & OSCEOLAR & CALUMET \\
\hline 2077 & 3985 & PISTONETTI & FRANK & $\mathrm{H}$ & 42 & ITALY & ITALY & ITALY & 1891 & TIMBERMAN/CM & BOSCONERO. TO & OSCEOLA ROAD & CALUMET \\
\hline 2078 & & PISTONETTI & DOROTHY & w & 66 & ITALY & ITALY & ITALY & 1888 & & & OSCEOLA ROAD & CALUMET \\
\hline
\end{tabular}




\begin{tabular}{|c|c|c|c|c|c|c|c|c|c|c|c|c|c|}
\hline 2079 & & PISTONETTI & MARGHERITA & $\mathrm{D}$ & 6 & MICHIGAN & ITALY & ITALY & & & BOSCONERO. TO & OSCEOLA ROAD & CALUMET \\
\hline 2080 & & PISTONETTI & JOSEPHINE & D & 1 & MICHIGAN & ITALY & ITALY & & & BOSCONERO. TO & OSCEOLA ROAD & CALUMET \\
\hline 2081 & & COLOMBO & ERNEST & BIL & 21 & ITALY & ITALY & ITALY & 1888 & LABORER/RAILROAD & TORINO & OSCEOLA ROAD & CALUMET \\
\hline 2082 & & PAGANUCCI & MADDALENA & $\mathrm{H}$ & 69 & FRANCE & FRANCE & FRANCE & 1889 & & FRANCE & OSCEOLA ROAD & CALUMET \\
\hline 2083 & & PAGANUCCI & VINCENZO & s & 33 & FRANCE & ITALY & FRANCE & 1889 & MECHANIC/FOUNDRY & LUCCA & OSCEOLA ROAD & CALUMET \\
\hline 2084 & 3982 & MIGLIO/MIGLIA & JOSEPH & $\mathrm{H}$ & 59 & ITALY & ITALY & ITALY & 1890 & SPRINKLER/CM & CASTELLAMONTE. TO & OSCEOLA ROAD & CALUMET \\
\hline 2085 & 0002 & MIGLIO/MIGLIA & CAROLINE & W & 46 & ITALY & ITALY & ITALY & 1893 & 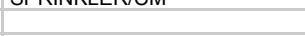 & UAOTLELAIVIOTVIL. TS & OSCEOLA ROAD & CALUMET \\
\hline 2086 & & MIGLIO/MIGLIA & MARY & D & 22 & MICHIGAN & ITALY & ITALY & 1090 & SALESLADY & CASTELLAMONTE. TO & OSCEOLA ROAD & CALUMET \\
\hline 2087 & & CAPOSELLO & GEORGE & $\mathrm{H}$ & ?? & ITALY & ITALY & ITALY & 1882 & LABORER & 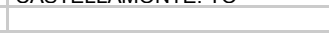 & OSCEOLA ROAD & CALUMET \\
\hline 2088 & 3930 & GHERNA & JOHN & $\mathrm{H}$ & 62 & ITALY & ITALY & ITALY & ???? & MINER/CM & PONT CANAVESE. TO & WEDGE ST. & CALUMET \\
\hline 2089 & & GHERNA & TERESA & W & 52 & MICHIGAN & ITALY & ITALY & & & 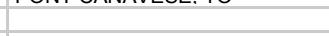 & WEDGE ST. & $\begin{array}{l}\text { CALUMET } \\
\end{array}$ \\
\hline 2090 & & GHERNA & JAMES & $\mathrm{s}$ & 21 & MICHIGAN & ITALY & ITALY & & MOULDER/FOUNDRY & PONT CANAVESE. TO & WEDGE ST. & CALUMET \\
\hline 2091 & & GHERNA & ISABEL & $\mathrm{D}$ & 15 & MICHIGAN & ITALY & ITALY & & & PONT CANAVESE. TO & WEDGE ST. & CALUMET \\
\hline 2092 & & GHERNA & VITTORIO & B & 29 & ITALY & ITALY & ITALY & 1906 & MINER/CM & PONT CANAVESE. TO & WEDGE ST. & CALUMET \\
\hline 2093 & & CURTO & JOHN & B & 56 & ITALY & ITALY & ITALY & 1886 & MINER/CM & VIALFRE'. TO & WEDGE ST. & CALUMET \\
\hline 2094 & 3959 & PASQUINELLI & PETER & $\mathrm{H}$ & 38 & MICHIGAN & ITALY & ITALY & & LABORER/CM & LUCCA & WEDGE ST. & CALUMET \\
\hline 2095 & & PASQUINELLI & AURELIA & W & 39 & ITALY & ITALY & ITALY & 1900 & & & WEDGE ST. & CALUMET \\
\hline 2096 & & PASQUINELLI & LOUISE & D & 16 & MICHIGAN & MICHIGAN & ITALY & & COOK/RESTAURANT & LUCCA & WEDGE ST. & CALUMET \\
\hline 2097 & & PASQUINELLI & PETER & $\mathrm{s}$ & 14 & MICHIGAN & MICHIGAN & ITALY & & & LUCCA & WEDGE ST. & $\begin{array}{l}\text { CALUMET } \\
\end{array}$ \\
\hline 2098 & & PASQUINELLI & CARLO & $\mathrm{s}$ & 8 & MICHIGAN & MICHIGAN & ITALY & & & LUCCA & WEDGE ST. & CALUMET \\
\hline 2099 & 3958 & BONOTTO & DOMENICO & $\mathrm{H}$ & 40 & ITALY & ITALY & ITALY & 1899 & MINER/CM & SAN BENIGNO CANAVESE. TO & WEDGE ST. & CALUMET \\
\hline 2100 & 3000 & BONOTTO & BENEDETTA & w & 36 & $\begin{array}{l}\text { ITALY } \\
\text { ITALY }\end{array}$ & $\begin{array}{l}\text { ITALY } \\
\text { ITAL }\end{array}$ & ITALY & 1902 & MIIINER/CIVI & SAIV DEIVISTUN CAIVAVESE. TO & WEDGE ST. & CALUMET \\
\hline 2101 & & BONOTTO & MINNIE & D & 15 & MICHIGAN & ITALY & ITALY & & & SAN BENIGNO CANAVESE. TO & WEDGE ST. & CALUMET \\
\hline 2102 & & BONOTTO & KATE & $\mathrm{D}$ & 9 & MICHIGAN & ITALY & ITALY & & & SAN BENIGNO CANAVESE. TO & WEDGE ST. & CALUMET \\
\hline 2103 & & BONOTTO & JOHN & $\mathrm{s}$ & 7 & MICHIGAN & ITALY & ITALY & & & SAN BENIGNO CANAVESE. TO & WEDGE ST. & CALUMET \\
\hline 2104 & & BONOTTO & BART & $\mathrm{S}$ & 6 & MICHIGAN & ITALY & ITALY & & & SAN BENIGNO CANAVESE. TO & WEDGE ST. & CALUMET \\
\hline 2105 & & BONOTTO & VINCENZO & BR & 24 & ITALY & ITALY & ITALY & 1912 & MINER/CM & SAN BENIGNO CANAVESE. TO & WEDGE ST. & CALUMET \\
\hline 2106 & & FRANCIONI & THOMAS & B & 32 & ITALY & ITALY & ITALY & 1913 & MINER/CM & LUCCA & WEDGE ST. & CALUMET \\
\hline 2107 & 3967 & BOGGIO & JOSEPH & $\mathrm{H}$ & 40 & ITALY & ITALY & ITALY & 1903 & LABORER/RAILROAD & SAN GIUSTO CANAVESE. TO & WEDGE ST. & CALUMET \\
\hline 2108 & & BOGGIO & ANTONIA & W & 34 & ITALY & ITALY & ITALY & 1906 & & & WEDGE ST. & $\begin{array}{l}\text { CALUMET } \\
\end{array}$ \\
\hline 2109 & & BOGGIO & ANTONIO & $\mathrm{s}$ & 10 & MICHIGAN & ITALY & ITALY & & & SAN GIUSTO CANAVESE. TO & WEDGE ST. & CALUMET \\
\hline 2110 & & BOGGIO & CARLO & $\mathrm{s}$ & 7 & MICHIGAN & ITALY & ITALY & & & SAN GIUSTO CANAVESE. TO & WEDGE ST. & CALUMET \\
\hline 2111 & & QUELLO & JAMES & $\mathrm{H}$ & 27 & ITALY & ITALY & ITALY & 1908 & MINER/CM & LOCANA CANAVESE. TO & WEDGE ST. & CALUMET \\
\hline 2112 & & QUELLO & MARY & w & 21 & ITALY & ITALY & ITALY & 1899 & & & WEDGE ST. & CALUMET \\
\hline 2113 & & QUELLO & JAMES & $\mathrm{s}$ & 2 & MICHIGAN & ITALY & ITALY & & & LOCANA CANAVESE. TO & WEDGE ST. & CALUMET \\
\hline 2114 & & QUELLO & JOSEPH & $\mathrm{s}$ & $11 \mathrm{M}$ & MICHIGAN & ITALY & ITALY & & & LOCANA CANAVESE. TO & WEDGE ST. & CALUMET \\
\hline 2115 & 3941 & PICCHIOTTINO & PAULINE & $\mathrm{H}$ & 53 & ITALY & ITALY & ITALY & 1887 & & & TEMPLE ST. & CALUMET \\
\hline 2116 & setit & PICCHIOTTINO & MINNIE & D & 30 & MICHIGAN & ITALY & ITALY & 100 & COACH CLEANER/RAILROAD & PONT CANAVESE. TO & TEMPLE ST. & CALUMET \\
\hline 2117 & & QUELLO & JOSEPH & GS & 3 & MICHIGAN & ITALY & ITALY & & & LOCANA CANAVESE. TO & TEMPLE ST. & CALUMET \\
\hline 2118 & & CHERUBINI & UGO & $\mathrm{H}$ & 26 & ITALY & ITALY & ITALY & 1913 & TIMBERMAN/CM & SASSOFERRATO. ANCONA & TEMPLE ST. & CALUMET \\
\hline 2119 & & CHERUBINI & LINA & W & 29 & MICHIGAN & ITALY & ITALY & & & & TEMPLE ST. & CALUMET \\
\hline 2120 & & CHERUBINI & ESTER & D & 2 & MICHIGAN & ITALY & MICHIGAN & & & SASSOFERRATO. ANCONA & TEMPLE ST. & CALUMET \\
\hline 2121 & & CHERUBINI & FRANK & $\mathrm{s}$ & $5 \mathrm{M}$ & MICHIGAN & ITALY & MICHIGAN & & & SASSOFERRATO. ANCONA & TEMPLE ST. & CALUMET \\
\hline 2122 & 3942 & VID/TRECCANI & SAM & $\mathrm{H}$ & 51 & ITALY & ITALY & ITALY & 1898 & MINER/CM & & TEMPLE ST. & CALUMET \\
\hline 2123 & & VID/TRECCANI & ARMANDO & s & 14 & MICHIGAN & ITALY & ITALY & 1000 & 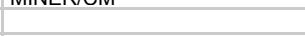 & & TEMPLE ST. & CALUMET \\
\hline 2124 & & VID/TRECCANI & ASSUNTA & $\mathrm{SI}$ & 52 & ITALY & ITALY & ITALY & 1909 & & & TEMPLE ST. & CALUMET \\
\hline 2125 & & LUCCHESI & LOUIS & B & 37 & ITALY & ITALY & ITALY & 1903 & LABORER/RAILROAD & LUCCA & TEMPLE ST. & CALUMET \\
\hline 2126 & & MIGLIO/MIGLIA & DOMENICO & $\mathrm{H}$ & 25 & MICHIGAN & ITALY & ITALY & & CARPENTER/SHOP & CASTELLAMONTE. TO & TEMPLE ST. & CALUMET \\
\hline 2127 & & MIGLIO/MIGLIA & ALICE & w & 24 & MICHIGAN & ITALY & ITALY & & & & TEMPLE ST. & $\begin{array}{l}\text { CALUMET } \\
\end{array}$ \\
\hline 2128 & & MIGLIO/MIGLIA & JOSEPH & $\mathrm{s}$ & 2 & MICHIGAN & MICHIGAN & MICHIGAN & & & CASTELLAMONTE. TO & TEMPLE ST. & CALUMET \\
\hline 2129 & 3347 & CERETTO & ANTONIO & $\mathrm{H}$ & 48 & ITALY & ITALY & ITALY & 1893 & MINER/CM & PONT CANAVESE. TO & TEMPLE ST. & CALUMET \\
\hline 2130 & & CERETTO & MARY & W & 53 & ITALY & ITALY & ITALY & 1893 & & PONT CANAVESE, TO & TEMPLE ST. & CALUMET \\
\hline 2131 & & CERETTO & DELPHINE & $\mathrm{D}$ & 14 & MICHIGAN & ITALY & ITALY & & & PONT CANAVESE. TO & TEMPLE ST. & CALUMET \\
\hline 2132 & & CERETTO & JENNIE & D & 12 & MICHIGAN & ITALY & ITALY & & & PONT CANAVESE. TO & TEMPLE ST. & CALUMET \\
\hline 2133 & & CERETTO & MARY & $\bar{D}$ & 9 & MICHIGAN & ITALY & ITALY & & & PONT CANAVESE. TO & TEMPLE ST. & CALUMET \\
\hline 2134 & & PISTONETTI & TERESA & $\mathrm{H}$ & 53 & ITALY & ITALY & ITALY & 1893 & LAUNDRESS/HOME & BOSCONERO. TO & TEMPLE ST. & CALUMET \\
\hline 2135 & & CAPELLO & MARGHERITA & $\mathrm{D}$ & 29 & ITALY & ITALY & ITALY & 1893 & CLEANER/PF & SAN BENIGNO CANAVESE. TO & TEMPLE ST. & CALUMET \\
\hline 2136 & & CAPELLO & NICK & GS & 13 & MICHIGAN & ITALY & ITALY & & & SAN BENIGNO CANAVESE, TO & TEMPLE ST. & $\begin{array}{l}\text { CALUMET } \\
\end{array}$ \\
\hline 2137 & & CAPELLO & JOHN & GS & 11 & MICHIGAN & ITALY & ITALY & & & SAN BENIGNO CANAVESE. TO & TEMPLE ST. & CALUMET \\
\hline 2138 & & CAPELLO & ANNA & GD & 6 & MICHIGAN & ITALY & ITALY & & & SAN BENIGNO CANAVESE. TO & TEMPLE ST. & CALUMET \\
\hline 2139 & 3945 & PEILA & MIKE & $\mathrm{H}$ & 56 & ITALY & ITALY & ITALY & 1888 & LABORER & PONT CANAVESE. TO & TEMPLE ST. & $\begin{array}{l}\text { CALUMET } \\
\end{array}$ \\
\hline 2140 & & PEILA & JOSEPH & $\mathrm{s}$ & 21 & MICHIGAN & ITALY & ITALY & & & PONT CANAVESE. TO & TEMPLE ST. & CALUMET \\
\hline 2141 & & PEILA & ERNEST & $\mathrm{s}$ & 12 & MICHIGAN & ITALY & ITALY & & & PONT CANAVESE. TO & TEMPLE ST. & CALUMET \\
\hline
\end{tabular}




\begin{tabular}{|c|c|c|c|c|c|c|c|c|c|c|c|c|c|}
\hline 2142 & 3950 & DATA/DATTA & JOHN & $\mathrm{H}$ & 45 & ITALY & ITALY & ITALY & 1895 & MINER/CM & PONT CANAVESE. TO & TEMPLE ST. & CALUMET \\
\hline 2143 & & DATA/DATTA & JENNIE & W & 39 & ITALY & ITALY & ITALY & 1906 & & & TEMPLE ST. & CALUMET \\
\hline 2144 & & DATA/DATTA & MARNIE & D & 10 & MICHIGAN & ITALY & ITALY & & & PONT CANAVESE. TO & TEMPLE ST. & CALUMET \\
\hline 2145 & 3957 & TOMASI & PETER & $\mathrm{H}$ & 38 & ITALY & ITALY & ITALY & 1905 & TIMBERMAN/CM & LOCANA CANAVESE. TO & TEMPLE ST. & CALUMET \\
\hline 2146 & & TOMASI & MARY & w & 40 & ITALY & ITALY & ITALY & 1914 & MANAGER/HOME DAIRY & LOCANA CANAVESE. TO & TEMPLE ST. & CALUMET \\
\hline 2147 & & TOMASI & TONY & s & 16 & ITALY & ITALY & ITALY & 1914 & & LOCANA CANAVESE. TO & TEMPLE ST. & CALUMET \\
\hline 2148 & & TOMASI & TERESA & D & 15 & ITALY & ITALY & ITALY & 1914 & & LOCANA CANAVESE. TO & TEMPLE ST. & CALUMET \\
\hline 2149 & & TOMASI & MARY & $\mathrm{D}$ & 4 & MICHIGAN & ITALY & ITALY & & & LOCANA CANAVESE. TO & TEMPLE ST. & CALUMET \\
\hline 2150 & & BROVAS? & FRANK & B & 33 & ITALY & ITALY & ITALY & 1913 & TIMBERMAN/CM & LUU AIVA UAIVAVEOE. TO & TEMPLE ST. & CALUMET \\
\hline 2151 & 3953 & GHIARDI & JOHN & $\mathrm{H}$ & 42 & ITALY & ITALY & ITALY & 1893 & BLACKSMITH/CM & SAN MARTINO CANAVESE. TO & TEMPLE ST. & CALUMET \\
\hline 2152 & & GHIARDI & MARTA & w & 36 & MICHIGAN & ITALY & ITALY & & & & TEMPLE ST. & CALUMET \\
\hline 2153 & & GHIARDI & ANGELINE & $\mathrm{D}$ & 16 & MICHIGAN & ITALY & ITALY & & & SAN MARTINO CANAVESE. TO & TEMPLE ST. & CALUMET \\
\hline 2154 & & GHIARDI & MARTIN & $\mathrm{s}$ & 14 & MICHIGAN & ITALY & ITALY & & & SAN MARTINO CANAVESE. TO & TEMPLE ST. & CALUMET \\
\hline 2155 & & GHIARDI & TERESA & $\mathrm{D}$ & 12 & MICHIGAN & ITALY & ITALY & & & SAN MARTINO CANAVESE. TO & TEMPLE ST. & CALUMET \\
\hline 2156 & & GHIARDI & ITALIA & $\mathrm{D}$ & 10 & MICHIGAN & ITALY & ITALY & & & SAN MARTINO CANAVESE. TO & TEMPLE ST. & CALUMET \\
\hline 2157 & 3915 & PERA & TONY & $\mathrm{H}$ & 36 & ITALY & ITALY & ITALY & 1905 & MINER/CM & & 6TH ST. & CALUMET \\
\hline 2158 & & PERA & DOMENICA & w & 32 & ITALY & ITALY & ITALY & 1913 & & & 6TH ST. & CALUMET \\
\hline 2159 & & PERA & $\mathrm{JOHN}$ & s & $5 \mathrm{M}$ & MICHIGAN & ITALY & ITALY & & & & 6TH ST. & CALUMET \\
\hline 2160 & & BINANDO & JOSEPH & $\mathrm{H}$ & 46 & ITALY & ITALY & ITALY & 1899 & LABORER/CM & CORTEREGGIO CANAVESE. TO & 6TH ST. & CALUMET \\
\hline 2161 & & BINANDO & MARY & w & 47 & ITALY & ITALY & ITALY & 1900 & & & 6TH ST. & CALUMET \\
\hline 2162 & & BINANDO & MIKE & s & 16 & MICHIGAN & ITALY & ITALY & & LABORER/CM & CORTEREGGIO CANAVESE. TO & 6TH ST. & CALUMET \\
\hline 2163 & & BINANDO & JOSEPH & $\mathrm{s}$ & 11 & MICHIGAN & ITALY & ITALY & & & CORTEREGGIO CANAVESE, TO & 6TH ST. & CALUMET \\
\hline 2164 & & BINANDO & MARY & $\mathrm{D}$ & 7 & MICHIGAN & ITALY & ITALY & & & CORTEREGGIO CANAVESE. TO & 6TH ST. & CALUMET \\
\hline 2165 & 3922 & POGLIANO & JOHN & $\mathrm{H}$ & 55 & ITALY & ITALY & ITALY & 1888 & TRAMMER/CM & SAN GIORGIO CANAVESE. TO & 6TH ST. & CALUMET \\
\hline 2166 & & POGLIANO & MADDALENA & w & 59 & ITALY & ITALY & ITALY & 1900 & & & 6TH ST. & CALUMET \\
\hline 2167 & 3925 & CURTO & JAMES & $\mathrm{H}$ & 58 & ITALY & ITALY & ITALY & 1879 & FOREMAN/CM & VIALFRE'. TO & 6TH ST. & CALUMET \\
\hline 2168 & & CURTO & MARY & w & 48 & PORTUGAL & ITALY & ITALY & 1879 & & VIALFRE'. TO & 6TH ST. & CALUMET \\
\hline 2169 & & CURTO & ANDREW & $s$ & 29 & MICHIGAN & ITALY & PORTUGAL & & TIMBERMAN/CM & VIALFRE', TO & 6TH ST. & CALUMET \\
\hline 2170 & & CURTO & JAMES & $\mathrm{S}$ & 17 & MICHIGAN & ITALY & PORTUGAL & & & VIALFRE'. TO & 6TH ST. & CALUMET \\
\hline 2171 & 3926 & GRINDATTI & PETER & $\mathrm{H}$ & 47 & ITALY & ITALY & ITALY & 1892 & MINER/CM & PONT CANAVESE. TO & 6TH ST. & CALUMET \\
\hline 2172 & & GRINDATTI & KATE & W & 43 & ITALY & ITALY & ITALY & 1896 & & & 6TH ST. & CALUMET \\
\hline 2173 & & GRINDATTI & MARY & $\mathrm{D}$ & 21 & MICHIGAN & ITALY & ITALY & & & PONT CANAVESE. TO & 6TH ST. & CALUMET \\
\hline 2174 & 3950 & BANDETTINI & ANGELO & $\mathrm{H}$ & 52 & ITALY & ITALY & ITALY & 1888 & WATCHMAN/CM & LUCCA & SCOTT ST. & CALUMET \\
\hline 2175 & & BANDETTINI & LIZ & W & 41 & MICHIGAN & ITALY & ITALY & & & & SCOTT ST. & CALUMET \\
\hline 2176 & & BANDETTINI & PASCAL & s & 25 & MICHIGAN & ITALY & MICHIGAN & & & LUCCA & SCOTT ST. & CALUMET \\
\hline 2177 & & BANDETTINI & ATTILIO & $\mathrm{s}$ & 23 & MICHIGAN & ITALY & MICHIGAN & & & LUCCA & SCOTT ST. & CALUMET \\
\hline 2178 & & BANDETTINI & ANGELINA & $\mathrm{D}$ & 11 & MICHIGAN & ITALY & MICHIGAN & & & LUCCA & SCOTT ST. & CALUMET \\
\hline 2179 & 4562 & COPPO & MARTIN & $\mathrm{H}$ & 39 & ITALY & ITALY & ITALY & 1903 & PUMP MAN/CM & PONT CANAVESE. TO & R. J. SHAFT & CALUMET \\
\hline 2180 & & COPPO & EUGENIA & W & 32 & ITALY & ITALY & ITALY & 1905 & & & R. J. SHAFT & CALUMET \\
\hline 2181 & & COPPO & JOHN & s & 12 & MICHIGAN & ITALY & ITALY & & & PONT CANAVESE. TO & R. J. SHAFT & CALUMET \\
\hline 2182 & & COPPO & ENRICO & $\mathrm{s}$ & 8 & MICHIGAN & ITALY & ITALY & & & PONT CANAVESE. TO & R. J. SHAFT & CALUMET \\
\hline 2183 & & COPPO & EDWARD & $\mathrm{s}$ & 6 & MICHIGAN & ITALY & ITALY & & & PONT CANAVESE. TO & R. J. SHAFT & CALUMET \\
\hline 2184 & & COPPO & MARTIN & $\mathrm{s}$ & 4 & MICHIGAN & ITALY & ITALY & & & PONT CANAVESE. TO & R. J. SHAFT & CALUMET \\
\hline 2185 & & COPPO & MARY & $\mathrm{D}$ & 2 & MICHIGAN & ITALY & ITALY & & & PONT CANAVESE. TO & R. J. SHAFT & CALUMET \\
\hline 2186 & & PIOLETTI & $\mathrm{JOHN}$ & B & 59 & ITALY & ITALY & ITALY & 1898 & LABORER/CM & CORIO. TO & R. J. SHAFT & CALUMET \\
\hline 2187 & 4810 & PACINI & CELESTINO & $\mathrm{H}$ & 35 & ITALY & ITALY & ITALY & 1895 & PUMP MAN/CM & POPIGLIO. PISTOIA & R. J. SHAFT & CALUMET \\
\hline 2188 & & PACINI & ELENA & W & 29 & ITALY & ITALY & ITALY & 1916 & & & R. J. SHAFT & CALUMET \\
\hline 2189 & & PACINI & LIDIA & $\mathrm{D}$ & 2 & MICHIGAN & ITALY & ITALY & & & POPIGLIO. PISTOIA & R. J. SHAFT & CALUMET \\
\hline 2190 & 4986 & RASTELLO & CARLO & $\mathrm{H}$ & 57 & ITALY & ITALY & ITALY & 1887 & SHOEMAKER/OWN SHOP & PONT CANAVESE. TO & WATER WORKS & CALUMET \\
\hline 2191 & & RASTELLO & JOSEPHINE & w & 56 & ITALY & ITALY & ITALY & 1887 & & PONT CANAVESE. TO & WATER WORKS & CALUMET \\
\hline 2192 & & RASTELLO & PETER & s & 32 & MICHIGAN & ITALY & ITALY & & BLACKSMITH/SHOP & PONT CANAVESE. TO & WATER WORKS & CALUMET \\
\hline 2193 & & RASTELLO & ROSE & $\mathrm{D}$ & 29 & MICHIGAN & ITALY & ITALY & & & PONT CANAVESE, TO & WATER WORKS & CALUMET \\
\hline 2194 & & RASTELLO & JOSEPH & $\mathrm{s}$ & 28 & MICHIGAN & ITALY & ITALY & & FIREMAN & PONT CANAVESE. TO & WATER WORKS & CALUMET \\
\hline 2195 & & RASTELLO & $\mathrm{JOHN}$ & $\mathrm{s}$ & 26 & MICHIGAN & ITALY & ITALY & & LABORER/RAILROAD & PONT CANAVESE. TO & WATER WORKS & CALUMET \\
\hline 2196 & & RASTELLO & FLORENCE & $\mathrm{D}$ & 20 & MICHIGAN & ITALY & ITALY & & & PONT CANAVESE. TO & WATER WORKS & CALUMET \\
\hline 2197 & & RASTELLO & AGNES & DIN & 23 & MICHIGAN & ITALY & ITALY & & & PONT CANAVESE. TO & WATER WORKS & CALUMET \\
\hline 2198 & & DAIRO & PAUL & $\mathrm{H}$ & 58 & ITALY & ITALY & ITALY & 1889 & LABORER/LUMBER CAMP & TORINO & LAKE VIEW & CALUMET \\
\hline 2199 & & DAIRO & KATE & w & 65 & ITALY & ITALY & ITALY & 1891 & & TORINO & LAKE VIEW & CALUMET \\
\hline 2200 & & DAIRO & THOMAS & s & 30 & ITALY & ITALY & ITALY & 1891 & TEAMSTER/PUBLIC ROAD & TORINO & LAKE VIEW & CALUMET \\
\hline 2201 & & DAIRO & KATE M. & $\mathrm{D}$ & 19 & MICHIGAN & ITALY & ITALY & & & TORINO & LAKE VIEW & CALUMET \\
\hline 2202 & 4306 & RIVARA & JAMES & $\mathrm{H}$ & 59 & ITALY & ITALY & ITALY & 1893 & MINER/CM & TORINO & 11TH ST. & CALUMET \\
\hline 2203 & & RIVARA & KATE & D & 14 & ITALY & ITALY & ITALY & UN & & TORINO & 11TH ST. & CALUMET \\
\hline 2204 & 3928 & PURACHIA & JOHN & B & 48 & ITALY & ITALY & ITALY & 1895 & LANDER/CM & CUNEO & 11TH ST. & CALUMET \\
\hline
\end{tabular}




\begin{tabular}{|c|c|c|c|c|c|c|c|c|c|c|c|c|c|}
\hline 2205 & 41 & CESARETTI & JOSEPH & $\mathrm{H}$ & 33 & ITALY & ITALY & ITALY & 1902 & MINER/CM & CARMIGLIANO. LU & COUNTY ROAD & CALUMET - TAMARACK \\
\hline 2206 & & CESARETTI & EMILIA & W & 34 & ITALY & ITALY & ITALY & 1908 & & & COUNTY ROAD & CALUMET - TAMARACK \\
\hline 2207 & & CESARETTI & TONY & $\mathrm{s}$ & 10 & MICHIGAN & ITALY & ITALY & & & CARMIGLIANO. LU & COUNTY ROAD & CALUMET - TAMARACK \\
\hline 2208 & & CESARETTI & MARY & D & 9 & MICHIGAN & ITALY & ITALY & & & CARMIGLIANO. LU & COUNTY ROAD & CALUMET - TAMARACK \\
\hline 2209 & & CESARETTI & DIANA & D & 7 & MICHIGAN & ITALY & ITALY & & & CARMIGLIANO. LU & COUNTY ROAD & CALUMET - TAMARACK \\
\hline 2210 & & CESARETTI & BRUNA & D & 5 & MICHIGAN & ITALY & ITALY & & & CARMIGLIANO. LU & COUNTY ROAD & CALUMET - TAMARACK \\
\hline 2211 & & CESARETTI & BESSCO? & $\mathrm{s}$ & 4 & MICHIGAN & ITALY & ITALY & & & CARMIGLIANO. LU & COUNTY ROAD & CALUMET - TAMARACK \\
\hline 2212 & & CESARETTI & PAUL & s & $4 \mathrm{M}$ & MICHIGAN & ITALY & ITALY & & & CARMIGLIANO. LU & COUNTY ROAD & CALUMET - TAMARACK \\
\hline 2213 & 47 & PACINI & MIKE & $\mathrm{H}$ & 64 & ITALY & ITALY & ITALY & 1890 & MINER/CM & POPIGLIO. PISTOIA & COUNTY ROAD & CALUMET - TAMARACK \\
\hline 2214 & & PACINI & MARY & W & 62 & ITALY & ITALY & ITALY & 1897 & & & COUNTY ROAD & CALUMET - TAMARACK \\
\hline 2215 & & PACINI & INES & $\mathrm{D}$ & 22 & MICHIGAN & ITALY & ITALY & & SALESLADY/DEPT. STORE & POPIGLIO. PISTOIA & COUNTY ROAD & CALUMET - TAMARACK \\
\hline 2216 & 75 & ROLANDO & JAMES & $\mathrm{H}$ & 58 & ITALY & ITALY & ITALY & 1882 & & PONT CANAVESE. TO & COUNTY ROAD & CALUMET - CENTENNIIAL \\
\hline 2217 & & ROLANDO & MARY & W & 56 & ITALY & ITALY & ITALY & 1890 & & & COUNTY ROAD & CALUMET - CENTENNIAL \\
\hline 2218 & & ROLANDO & JOSEPHINE & D & 20 & MICHIGAN & ITALY & ITALY & & DRESSMAKER/AT HOME & PONT CANAVESE. TO & COUNTY ROAD & CALUMET - CENTENNIAL \\
\hline 2219 & 302 & MASI & FRANK & $\mathrm{H}$ & 30 & ITALY & ITALY & ITALY & 1909 & LABORER/CM & ROMA & & CALUMET - COPPER CITY \\
\hline 2220 & & MASI & LUISE & W & 20 & FRANCE & ITALY & ITALY & 1919 & & & & CALUMET - COPPER CITY \\
\hline 2221 & 316 & ROTA & $\mathrm{JOHN}$ & $\mathrm{H}$ & 30 & ITALY & ITALY & ITALY & 1906 & MINER/CM & SAN BENIGNO CANAVESE. TO & & CALUMET - COPPER CITY \\
\hline 2222 & & ROTA & CHARLOTTE & W & 26 & ITALY & ITALY & ITALY & 1910 & & & & CALUMET - COPPER CITY \\
\hline 2223 & & ROTA & JOHN & $\mathrm{s}$ & 1 & MICHIGAN & ITALY & ITALY & & & SAN BENIGNO CANAVESE. TO & & CALUMET - COPPER CITY \\
\hline 2224 & 110 & BESNA/BEA & WILLIAM & $\mathrm{H}$ & 46 & ITALY & ITALY & ITALY & 1902 & LABORER/CM & & & CALUMET - COPPER CITY \\
\hline 2225 & & BESNA/BEA & TRACY & W & 33 & ITALY & ITALY & ITALY & 1912 & & & & CALUMET - COPPER CITY \\
\hline 2226 & & BESNA/BEA & JOSEPH & s & 10 & MICHIGAN & ITALY & ITALY & & & & & CALUMET - COPPER CITY \\
\hline 2227 & & BESNA/BEA & MARY & D & 8 & MICHIGAN & ITALY & ITALY & & & & & CALUMET - COPPER CITY \\
\hline 2228 & 315 & GIACOLETTO & ANTONIO & $\mathrm{H}$ & 48 & ITALY & ITALY & ITALY & 1896 & LABORER/CM & TORINO & & CALUMET - COPPER CITY \\
\hline 2229 & & GIACOLETTO & MINNIE & W & 51 & ITALY & ITALY & ITALY & 1901 & & TORINO & & CALUMET - COPPER CITY \\
\hline 2230 & & GIACOLETTO & JOSEPHINE & $\mathrm{D}$ & 15 & MICHIGAN & ITALY & ITALY & & & TORINO & & CALUMET - COPPER CITY \\
\hline 2231 & & GIACOLETTO & TRACY & $\mathrm{D}$ & 13 & MICHIGAN & ITALY & ITALY & & & TORINO & & CALUMET - COPPER CITY \\
\hline 2232 & & GIACOLETTO & CRISTINA & D & 7 & MICHIGAN & ITALY & ITALY & & & TORINO & & CALUMET - COPPER CITY \\
\hline 2233 & & GOTTA & PETER & B & 33 & ITALY & ITALY & ITALY & 1907 & TRAMMER/CM & LOCANA CANAVESE. TO & & CALUMET - COPPER CITY \\
\hline 2234 & & ALBERTANO & CAMILLO & B & 48 & ITALY & ITALY & ITALY & 1907 & MINER/CM & PONT CANAVESE. TO & & CALUMET - COPPER CITY \\
\hline 2235 & & LENCIONI & FRANK & B & 28 & ITALY & ITALY & ITALY & 1911 & MINER/CM & LUCCA & & CALUMET - COPPER CITY \\
\hline 2236 & 232 & RONCAGLIA & MARTIN & $\mathrm{H}$ & 34 & ITALY & ITALY & ITALY & UN & TRAMMER/CM & FRASSINETTO. TO & WOLWERINE ST. & CALUMET - WOLVERINE \\
\hline 2237 & 202 & RONCAGLIA & MARY & W & 33 & ITALY & ITALY & ITALY & UN & 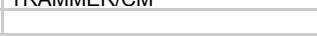 & FRASSINETTO. TO & WOLWERINE ST. & $\begin{array}{l}\text { CALUMET - WOLVERINE } \\
\text { COLVR }\end{array}$ \\
\hline 2238 & & RONCAGLIA & MARNIE & D & 6 & MICHIGAN & ITALY & ITALY & & & FRASSINETTO, TO & WOLWERINE ST. & CALUMET - WOLVERINE \\
\hline 2239 & & RONCAGLIA & IOLANDA & $\mathrm{D}$ & 4 & MICHIGAN & ITALY & ITALY & & & FRASSINETTO. TO & WOLWERINE ST. & CALUMET - WOLVERINE \\
\hline 2240 & & RONCAGLIA & STAPHEN & $\mathrm{s}$ & 1 & MICHIGAN & ITALY & ITALY & & & FRASSINETTO. TO & WOLWERINE ST. & CALUMET - WOLVERINE \\
\hline 2241 & 230 & BALMA/BAIMA & $\begin{array}{l}\text { PETER } \\
\text { PET }\end{array}$ & $\mathrm{H}$ & 37 & ITALY & ITALY & ITALY & 1908 & MINER/CM & CIRIE'. TO & WOLWERINE ST. & CALUMET - WOLVERINE \\
\hline 2242 & 200 & BALMA/BAIMA & VERONICA & W & 33 & ITALY & ITALY & ITALY & 1908 & 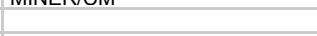 & CIRIE'. TO & WOLWERINE ST. & CALUMET - WOLVERINE \\
\hline 2243 & & BALMA/BAIMA & URSULA & D & 12 & ITALY & ITALY & ITALY & 1908 & & CIRIE'. TO & WOLWERINE ST. & CALUMET - WOLVERINE \\
\hline 2244 & & BALMA/BAIMA & VIRGINIA & $\mathrm{D}$ & 10 & MICHIGAN & ITALY & ITALY & & & CIRIE'. TO & WOLWERINE ST. & CALUMET - WOLVERINE \\
\hline 2245 & & BALMA/BAIMA & MARY & D & 9 & MICHIGAN & ITALY & ITALY & & & CIRIE'. TO & WOLWERINE ST. & CALUMET - WOLVERINE \\
\hline 2246 & & BALMA/BAIMA & JOSEPH & $\mathrm{s}$ & 8 & MICHIGAN & ITALY & ITALY & & & CIRIE'. TO & WOLWERINE ST. & CALUMET - WOLVERINE \\
\hline 2247 & & BALMA/BAIMA & WILL & $\mathrm{s}$ & 6 & MICHIGAN & ITALY & ITALY & & & CIRIE'. TO & WOLWERINE ST. & CALUMET - WOLVERINE \\
\hline 2248 & & BALMA/BAIMA & ELENA & $\mathrm{D}$ & 5 & MICHIGAN & ITALY & ITALY & & & CIRIE'. TO & WOLWERINE ST. & CALUMET - WOLVERINE \\
\hline 2249 & 163. & BIROCCO & GIUSTO & $\mathrm{H}$ & 33 & ITALY & ITALY & ITALY & 1908 & TIMBERMAN/CM & SAN GIUSTO CANAVESE. TO & RAILROAD ST. & CALUMET - WOLVERINE \\
\hline 2250 & 100. & BIROCCO & ELENA & w & 29 & ITALY & ITALY & ITALY & UN & 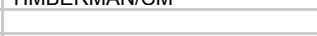 & 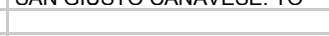 & RAILROAD ST. & CALUMET - WOLVERINE \\
\hline 2251 & & BIROCCO & JESSIE & $\mathrm{s}$ & 5 & MICHIGAN & ITALY & ITALY & & & SAN GIUSTO CANAVESE. TO & RAILROAD ST. & CALUMET - WOLVERINE \\
\hline 2252 & & BIROCCO & ANTONIO & $\mathrm{s}$ & 4 & MICHIGAN & ITALY & ITALY & & & SAN GIUSTO CANAVESE. TO & RAILROAD ST. & CALUMET - WOLVERINE \\
\hline 2253 & 191 & MARTINA & $\begin{array}{l}\text { PETER } \\
\end{array}$ & $\mathrm{H}$ & 24 & ITALY & ITALY & ITALY & 1912 & TIMBERMAN/CM & TORINO & RAILROAD ST. & CALUMET - WOLVERINE \\
\hline 2254 & & MARTINA & KATE & W & 17 & MICHIGAN & ITALY & ITALY & & & & RAILROAD ST. & CALUMET - WOLVERINE \\
\hline 2255 & & CRESTO & BARTOLOMEO & $\mathrm{H}$ & 30 & ITALY & ITALY & ITALY & 1904 & MANAGER/STATION & CASTELLAMONTE. TO & RAILROAD ST. & CALUMET - WOLVERINE \\
\hline 2256 & & CRESTO & MARY & W & 31 & ITALY & ITALY & ITALY & 1908 & & & RAILROAD ST. & CALUMET - WOLVERINE \\
\hline 2257 & & CRESTO & MARGHERITA & $\mathrm{D}$ & 11 & MICHIGAN & ITALY & ITALY & & & CASTELLAMONTE. TO & RAILROAD ST. & CALUMET - WOLVERINE \\
\hline 2258 & & CRESTO & LUCILLE & D & 8 & MICHIGAN & ITALY & ITALY & & & CASTELLAMONTE. TO & RAILROAD ST. & CALUMET - WOLVERINE \\
\hline 2259 & & CRESTO & KATE & D & 6 & MICHIGAN & ITALY & ITALY & & & CASTELLAMONTE. TO & RAILROAD ST. & CALUMET - WOLVERINE \\
\hline 2260 & $42 ?$ & FALETTI & CARLO & $\mathrm{H}$ & 34 & ITALY & ITALY & ITALY & 1907 & TRAMMER/CM & PONT CANAVESE. TO & RAILROAD ST. & CALUMET - WOLVERINE \\
\hline 2261 & & FALETTI & JENNIE & w & 31 & ITALY & ITALY & ITALY & 1907 & & PONT CANAVESE. TO & RAILROAD ST. & CALUMET - WOLVERINE \\
\hline 2262 & & FALETTI & MARY & $\mathrm{D}$ & 4 & MICHIGAN & ITALY & ITALY & & & PONT CANAVESE, TO & RAILROAD ST. & CALUMET - WOLVERINE \\
\hline 2263 & & FALETTI & LUCY & D & 3 & MICHIGAN & ITALY & ITALY & & & PONT CANAVESE. TO & RAILROAD ST. & CALUMET - WOLVERINE \\
\hline 2264 & & BAUSANO & LUIGI & B & 28 & ITALY & ITALY & ITALY & 1907 & TIMBERMAN/CM & LOCANA CANAVESE. TO & RAILROAD ST. & CALUMET - WOLVERINE \\
\hline 2265 & 14 & PICCHIOTTINO & PIETRO & $\mathrm{H}$ & 49 & ITA & ITA & ITALY & 1895 & TIMBER BOSS/CM & PONT CANAVESE. TO & 1ST ST. & CALUMET - WOLVERINE \\
\hline 2266 & & PICCHIOTTINO & CATERINA & w & 48 & ITALY & ITALY & ITALY & 1897 & & & & CALUMET \\
\hline 2267 & & PICCHIOTTINO & GIOVANNI & $\mathrm{s}$ & 21 & MICHIGAN & ITALY & ITALY & & COMPRESSOR ENGINEER/CM & PONT CANAVESE. TO & $1 \mathrm{ST}$ ST. & CALUMET - WOLVERINE \\
\hline
\end{tabular}




\begin{tabular}{|c|c|c|c|c|c|c|c|c|c|c|c|c|c|}
\hline 2268 & & PICCHIOTTINO & HENRIETTE & $\mathrm{D}$ & 19 & MICHIGAN & ITALY & ITALY & & & PONT CANAVESE. TO & 1ST ST. & CALUMET - WOLVERINE \\
\hline 2269 & & PICCHIOTTINO & LORENZO & $\mathrm{s}$ & 12 & MICHIGAN & ITALY & ITALY & & & PONT CANAVESE. TO & 1ST ST. & CALUMET - WOLVERINE \\
\hline 2270 & & PICCHIOTTINO & ELEONORA & D & 8 & MICHIGAN & ITALY & ITALY & & & PONT CANAVESE. TO & 1ST ST. & CALUMET - WOLVERINE \\
\hline 2271 & & COPPO & JOSEPH & B & 26 & ITALY & ITALY & ITALY & 1913 & TIMBERMAN/CM & PONT CANAVESE. TO & 1ST ST. & CALUMET - WOLVERINE \\
\hline 2272 & & COPPO & NATALE & B & 32 & ITALY & ITALY & ITALY & 1910 & TIMBERMAN/CM & PONT CANAVESE. TO & $1 \mathrm{ST}$ ST. & CALUMET - WOLVERINE \\
\hline 2273 & & BAUSANO & MAURIZIO & B & 28 & ITALY & ITALY & ITALY & 1910 & MINER/CM & PONT CANAVESE. TO & 1 ST ST. & CALUMET - WOLVERINE \\
\hline 2274 & & SANDRETTO & THOMAS & B & 25 & ITALY & ITALY & ITALY & 1913 & TIMBERMAN/CM & PONT CANAVESE. TO & 1ST ST. & CALUMET - WOLVERINE \\
\hline 2275 & & SANDRETTO & ANTONIO & $\begin{array}{l}\mathrm{D} \\
\mathrm{B}\end{array}$ & 27 & ITALY & ITALY & ITALY & 1913 & TIMBERMAN/CM & PONT CANAVESE. TO & 1ST ST. & CALUMET - WOLVERINE \\
\hline 2276 & 1 & SILVA & GABRIELE & $\mathrm{H}$ & 48 & ITALY & ITALY & ITALY & 1906 & TEAMSTER/CM & PONT CANAVESE. TO & $\begin{array}{l}\text { SMITH AVE. } \\
\text { SME }\end{array}$ & CALUMET - WOLVERINE \\
\hline 2277 & & SILVA & MARY & $\mathrm{D}$ & 11 & MICHIGAN & ITALY & ITALY & & & PONT CANAVESE. TO & SMITH AVE. & CALUMET - WOLVERINE \\
\hline 2278 & & ANSELMINO & MAGGIE & HK & 47 & ITALY & ITALY & ITALY & 1905 & HOUSEKEEPER/PF & TORINO & SMITH AVE. & CALUMET - WOLVERINE \\
\hline 2279 & & SILVA & JOHN & BR & 36 & ITALY & ITALY & ITALY & 1904 & TRAMMER/CM & PONT CANAVESE. TO & SMITH AVE. & CALUMET - WOLVERINE \\
\hline 2280 & 67 & FALETTI & JOHN & $\mathrm{H}$ & 38 & ITALY & ITALY & ITALY & 1909 & TRAMMER/CM & PONT CANAVESE. TO & CENTER ST. & CALUMET - WOLVERINE \\
\hline 2281 & & FALETTI & PAULINE & w & 34 & ITALY & ITALY & ITALY & 1919 & & & CENTER ST. & CALUMET - WOLVERINE \\
\hline 2282 & 69 & RASTELLO & JOHN & $\mathrm{H}$ & 44 & ITALY & ITALY & ITALY & 1909 & MINER/CM & PONT CANAVESE. TO & CENTER ST. & CALUMET - WOLVERINE \\
\hline 2283 & & RASTELLO & LUCY & W & 45 & ITALY & ITALY & ITALY & 1910 & & PONT CANAVESE, TO & CENTER ST. & CALUMET - WOLVERINE \\
\hline 2284 & & RASTELLO & $?$ & $\mathrm{~s}$ & 12 & ITALY & ITALY & ITALY & 1910 & & PONT CANAVESE. TO & CENTER ST. & CALUMET - WOLVERINE \\
\hline 2285 & & RASTELLO & JOHN & $\mathrm{s}$ & 8 & MICHIGAN & ITALY & ITALY & & & PONT CANAVESE. TO & CENTER ST. & CALUMET - WOLVERINE \\
\hline 2286 & & RASTELLO & CARLO & $\mathrm{s}$ & 4 & MICHIGAN & ITALY & ITALY & & & PONT CANAVESE. TO & CENTER ST. & CALUMET - WOLVERINE \\
\hline 2287 & & SANDRETTO & JOSEPH & B & 42 & ITALY & ITALY & ITALY & 1911 & TIMBERMAN/CM & PONT CANAVESE. TO & CENTER ST. & CALUMET - WOLVERINE \\
\hline 2288 & & FALETTI & JOHN & B & 39 & ITALY & ITALY & ITALY & 1910 & TRAMMER/CM & PONT CANAVESE. TO & CENTER ST. & CALUMET - WOLVERINE \\
\hline 2289 & 62 & SANDRETTO & ? & $\mathrm{H}$ & 38 & $\begin{array}{l}\text { ITALY } \\
\text { ITAL }\end{array}$ & ITALY & ITALY & 1905 & TIMBERMAN/CM & PONT CANAVESE, TO & CENTER ST. & CALUMET - WOLVERINE \\
\hline 2290 & & SANDRETTO & KATE & w & 38 & ITALY & ITALY & ITALY & 1912 & & & CENTER ST. & CALUMET - WOLVERINE \\
\hline 2291 & & SANDRETTO & BATTISTA & s & 6 & MICHIGAN & ITALY & ITALY & & & PONT CANAVESE. TO & CENTER ST. & CALUMET - WOLVERINE \\
\hline 2292 & & SANDRETTO & LUIGI & $\mathrm{s}$ & 5 & MICHIGAN & ITALY & ITALY & & & PONT CANAVESE. TO & CENTER ST. & CALUMET - WOLVERINE \\
\hline 2293 & & SANDRETTO & MADDALENA & $\mathrm{D}$ & 4 & MICHIGAN & ITALY & ITALY & & & PONT CANAVESE. TO & CENTER ST. & CALUMET - WOLVERINE \\
\hline 2294 & & SANDRETTO & JOSEPH & $\mathrm{s}$ & 3 & MICHIGAN & ITALY & ITALY & & & PONT CANAVESE. TO & CENTER ST. & CALUMET - WOLVERINE \\
\hline 2295 & & SANDRETTO & MIKE & $\mathrm{s}$ & 1 & MICHIGAN & ITALY & ITALY & & & PONT CANAVESE, TO & CENTER ST. & CALUMET - WOLVERINE \\
\hline 2296 & 59 & MASSA & JOSEPH & $\mathrm{H}$ & 49 & ITALY & ITALY & ITALY & 1900 & MINER/CM & RIVARA. TO & 2ND ST. & CALUMET - WOLVERINE \\
\hline 2297 & & MASSA & MARGHERITA & W & 39 & ITALY & ITALY & ITALY & 1902 & & & 2ND ST. & CALUMET - WOLVERINE \\
\hline 2298 & & MASSA & JOHN & $\mathrm{s}$ & 14 & MICHIGAN & ITALY & ITALY & & & RIVARA. TO & 2ND ST. & CALUMET - WOLVERINE \\
\hline 2299 & & MASSA & TONY & $\mathrm{s}$ & 12 & MICHIGAN & ITALY & ITALY & & & RIVARA. TO & 2ND ST. & CALUMET - WOLVERINE \\
\hline 2300 & & MASSA & EDWARD & $\mathrm{s}$ & 10 & MICHIGAN & ITALY & ITALY & & & RIVARA. TO & 2ND ST. & CALUMET - WOLVERINE \\
\hline 2301 & & MASSA & FRANCIS & D & 9 & MICHIGAN & ITALY & ITALY & & & RIVARA, TO & 2ND ST. & CALUMET - WOLVERINE \\
\hline 2302 & & MASSA & DEFENDENTE & $\mathrm{s}$ & 7 & MICHIGAN & ITALY & ITALY & & & RIVARA. TO & 2ND ST. & CALUMET - WOLVERINE \\
\hline 2303 & & MASSA & MARY & D & 6 & MICHIGAN & ITALY & ITALY & & & RIVARA. TO & 2ND ST. & CALUMET - WOLVERINE \\
\hline 2304 & & MASSA & MORRIS & s & 4 & MICHIGAN & ITALY & ITALY & & & RIVARA. TO & 2ND ST. & CALUMET - WOLVERINE \\
\hline 2305 & & MASSA & JOSEPHINE & D & 2 & MICHIGAN & ITALY & ITALY & & & RIVARA. TO & 2ND ST. & CALUMET - WOLVERINE \\
\hline 2306 & & FUSI & $?$ & B & 36 & ITALY & ITALY & ITALY & 1906 & TRAMMER/CM & & 2ND ST. & CALUMET - WOLVERINE \\
\hline 2307 & & AUTERO & JOHN & B & 32 & ITALY & ITALY & ITALY & 1911 & LABORER/CM & PONT CANAVESE. TO & 2ND ST. & CALUMET - WOLVERINE \\
\hline 2308 & & GIACOLETTO & JOHN & B & 51 & ITALY & ITALY & ITALY & 1890 & TRAMMER/CM & TORINO & 2ND ST. & CALUMET - WOLVERINE \\
\hline 2309 & 58 & GIACOLETTO & ANTONIO & $\mathrm{H}$ & 50 & ITALY & ITALY & ITALY & 1895 & MINER/CM & TORINO & 2ND ST. & CALUMET - WOLVERINE \\
\hline 2310 & & GIACOLETTO & LOUISE & w & 45 & ITALY & ITALY & ITALY & 1896 & OWNER/CONFECTIONARY ST. & TORINO & 2ND ST. & CALUMET - WOLVERINE \\
\hline 2311 & & GIACOLETTO & JOSEPH & $\mathrm{s}$ & 27 & ITALY & ITALY & ITALY & 1896 & MINER/CM & TORINO & 2ND ST. & CALUMET - WOLVERINE \\
\hline 2312 & & GIACOLETTO & JOHN & s & 21 & MICHIGAN & ITALY & ITALY & & TIMBERMAN/CM & TORINO & 2ND ST. & CALUMET - WOLVERINE \\
\hline 2313 & & GIACOLETTO & $\begin{array}{l}\text { VICTOR } \\
\end{array}$ & $\mathrm{s}$ & 15 & MICHIGAN & ITALY & ITALY & & & TORINO & 2ND ST. & CALUMET - WOLVERINE \\
\hline 2314 & & GIACOLETTO & DOMENICO & $\mathrm{S}$ & 14 & MICHIGAN & ITALY & ITALY & & & TORINO & 2ND ST. & CALUMET - WOLVERINE \\
\hline 2315 & & GIACOLETTO & LOUIS & s & 7 & MICHIGAN & ITALY & ITALY & & & TORINO & 2ND ST. & CALUMET - WOLVERINE \\
\hline 2316 & 94 & GIACOLETTO & CARLO & $\mathrm{H}$ & 36 & ITALY & ITALY & ITALY & 1907 & TRAMMER/CM & CIRIE'. TO & 3RD ST. & CALUMET - WOLVERINE \\
\hline 2317 & & GIACOLETTO & ANNA & W & 35 & ITALY & ITALY & ITALY & 1908 & & CIRIE'. TO & 3RD ST. & CALUMET - WOLVERINE \\
\hline 2318 & & GIACOLETTO & MARNIE & D & 13 & ITALY & ITALY & ITALY & 1909 & & CIRIE'. TO & 3RD ST. & CALUMET - WOLVERINE \\
\hline 2319 & & GIACOLETTO & CLARA & $\mathrm{D}$ & 11 & MICHIGAN & ITALY & ITALY & & & CIRIE', TO & 3RD ST. & CALUMET - WOLVERINE \\
\hline 2320 & & GIACOLETTO & KATE & D & 9 & MICHIGAN & ITALY & ITALY & & & CIRIE'. TO & 3RD ST. & CALUMET - WOLVERINE \\
\hline 2321 & & GIACOLETTO & MARY & D & 7 & MICHIGAN & ITALY & ITALY & & & CIRIE'. TO & 3RD ST. & CALUMET - WOLVERINE \\
\hline 2322 & & GIACOLETTO & DOMENICO & $\mathrm{s}$ & 4 & MICHIGAN & ITALY & ITALY & & & TORINO & 3RD ST. & CALUMET - WOLVERINE \\
\hline 2323 & 95 & PICCHIOTTINO & BATTISTA & H & 45 & ITALY & ITALY & ITALY & 1901 & MINER/CM & PONT CANAVESE. TO & 3RD ST. & CALUMET - WOLVERINE \\
\hline 2324 & & PICCHIOTTINO & MARY & w & 33 & ITALY & ITALY & ITALY & 1904 & & & 3RD ST. & CALUMET - WOLVERINE \\
\hline 2325 & & PICCHIOTTINO & RINA & D & 15 & MICHIGAN & ITALY & ITALY & & & PONT CANAVESE, TO & 3RD ST. & CALUMET - WOLVERINE \\
\hline 2326 & & PICCHIOTTINO & ERNEST & $s$ & 14 & MICHIGAN & ITALY & ITALY & & & PONT CANAVESE. TO & 3RD ST. & CALUMET - WOLVERINE \\
\hline 2327 & & PICCHIOTTINO & GINO & $\mathrm{s}$ & 10 & MICHIGAN & ITALY & ITALY & & & PONT CANAVESE. TO & 3RD ST. & CALUMET - WOLVERINE \\
\hline 2328 & & MERLO & SABINO & B & 27 & ITALY & ITA & ITALY & 1913 & MINER/CM & PONT CANAVESE. TO & 3RD ST. & CALUMET - WC \\
\hline 2329 & 97 & AUTERO & DOMENICO & $\mathrm{H}$ & 50 & ITALY & ITALY & ITALY & 1897 & TIMBERMAN/CM & PONT CANAVESE. TO & 3RD ST. & CALUMET - WOLVE \\
\hline 2330 & & AUTERO & MONDINA & W & 42 & ITALY & ITALY & ITALY & 1901 & & & 3RD ST. & CALUMET - WOLVERINE \\
\hline
\end{tabular}




\begin{tabular}{|c|c|c|c|c|c|c|c|c|c|c|c|c|c|}
\hline 2331 & & AUTERO & JOHN & $\mathrm{s}$ & 17 & MICHIGAN & ITALY & ITALY & & & PONT CANAVESE. TO & 3RD ST. & CALUMET - WOLVERINE \\
\hline 2332 & & AUTERO & ANGELINA & D & 15 & MICHIGAN & ITALY & ITALY & & & PONT CANAVESE. TO & 3RD ST. & CALUMET - WOLVERINE \\
\hline 2333 & & AUTERO & MARY & D & 11 & MICHIGAN & ITALY & ITALY & & & PONT CANAVESE. TO & 3RD ST. & CALUMET - WOLVERINE \\
\hline 2334 & & POLATO & LOUIS & B & 36 & ITALY & ITALY & ITALY & 1913 & TRAMMER/CM & & 3RD ST. & CALUMET - WOLVERINE \\
\hline 2335 & 113 & SANDRETTO & JOHN & $\mathrm{H}$ & 50 & ITALY & ITALY & ITALY & 1912 & & PONT CANAVESE. TO & 4TH ST. & CALUMET - WOLVERINE \\
\hline 2336 & & SANDRETTO & MIKE & s & 18 & MICHIGAN & ITALY & ITALY & & HELPER/MINERAL RAILROAD & PONT CANAVESE. TO & 4TH ST. & CALUMET - WOLVERINE \\
\hline 2337 & & SANDRETTO & LOUIS & $\mathrm{s}$ & 13 & MICHIGAN & ITALY & ITALY & & & PONT CANAVESE. TO & 4TH ST. & CALUMET - WOLVERINE \\
\hline 2338 & & SANDRETTO & KATE & D & 11 & MICHIGAN & ITALY & ITALY & & & PONT CANAVESE. TO & 4TH ST. & CALUMET - WOLVERINE \\
\hline 2339 & & SANDRETTO & MARY & D & 9 & MICHIGAN & ITALY & ITALY & & & PONT CANAVESE. TO & 4TH ST. & CALUMET - WOLVERINE \\
\hline 2340 & & SANDRETTO & ANNE & D & 6 & MICHIGAN & ITALY & ITALY & & & PONT CANAVESE. TO & 4TH ST. & CALUMET - WOLVERINE \\
\hline 2341 & & SANDRETTO & PAUL & $\mathrm{s}$ & 3 & MICHIGAN & ITALY & ITALY & & & PONT CANAVESE. TO & 4TH ST. & CALUMET - WOLVERINE \\
\hline 2342 & & SANDRETTO & BARNEY & $\mathrm{s}$ & 1 & MICHIGAN & ITALY & ITALY & & & PONT CANAVESE. TO & 4TH ST. & CALUMET - WOLVERINE \\
\hline 2343 & 114 & MICHELA & JOE & $\mathrm{H}$ & 42 & ITALY & ITALY & ITALY & 1901 & LABORER/ROCK HOUSE & SAN GIORGIO CANAVESE. TO & 4TH ST. & CALUMET - WOLVERINE \\
\hline 2344 & & MICHELA & MARION & w & 42 & ITALY & ITALY & ITALY & 1907 & & & 4TH ST. & CALUMET - WOLVERINE \\
\hline 2345 & & MICHELA & CLARA & STEP & 9 & MICHIGAN & ITALY & ITALY & & & SAN GIORGIO CANAVESE. TO & 4TH ST. & CALUMET - WOLVERINE \\
\hline 2346 & & MICHELA & JOE & STEP & 4 & MICHIGAN & ITALY & ITALY & & & SAN GIORGIO CANAVESE, TO & 4TH ST. & CALUMET - WOLVERINE \\
\hline 2347 & & MICHELA & DOMENICO & $\mathrm{s}$ & 1 & MICHIGAN & ITALY & ITALY & & & SAN GIORGIO CANAVESE. TO & 4TH ST. & CALUMET - WOLVERINE \\
\hline 2348 & 50 & GIUSTAT & EUGENIO & $\mathrm{H}$ & 44 & ITALY & ITALY & ITALY & 1899 & LABORER/ROCK HOUSE & SALTO CANAVESE. TO & N. 4 LOCATION & CALUMET - WOLVERINE \\
\hline 2349 & & GIUSTAT & GENEVIEVE & W & 43 & ITALY & ITALY & ITALY & 1906 & & SALTO CANAVESE. TO & N. 4 LOCATION & CALUMET - WOLVERINE \\
\hline 2350 & & GIUSTAT & ERNEST & $\mathrm{s}$ & 15 & ITALY & ITALY & ITALY & 1906 & & SALTO CANAVESE. TO & N. 4 LOCATION & CALUMET - WOLVERINE \\
\hline 2351 & & GIUSTAT & MARY & $\mathrm{D}$ & 12 & MICHIGAN & ITALY & ITALY & & & SALTO CANAVESE. TO & N. 4 LOCATION & CALUMET - WOLVERINE \\
\hline 2352 & & GIUSTAT & MARGHERITA & D & $\frac{12}{5}$ & MICHIGAN & $\begin{array}{l}\text { ITALY } \\
\text { ITAL }\end{array}$ & ITALY & & & SALTO CANAVESE, TO & N. 4 LOCATION & CALUMET - WOLVERINE \\
\hline 2353 & 43 & GRIBALDO & JOSEPH & $\mathrm{H}$ & 52 & ITALY & ITALY & ITALY & 1913 & DRAYMAN/CM & TORINO & N. 4 LOCATION & CALUMET - WOLVERINE \\
\hline 2354 & & GRIBALDO & ROSE & W & 34 & ITALY & ITALY & ITALY & 1913 & & TORINO & N. 4 LOCATION & CALUMET - WOLVERINE \\
\hline 2355 & & GRIBALDO & TRACY & $\mathrm{D}$ & 16 & MICHIGAN & ITALY & ITALY & & & TORINO & N. 4 LOCATION & CALUMET - WOLVERINE \\
\hline 2356 & & GRIBALDO & IRENE & D & 8 & MICHIGAN & ITALY & ITALY & & & TORINO & N. 4 LOCATION & CALUMET - WOLVERINE \\
\hline 2357 & & GRIBALDO & BARBARA & D & 6 & MICHIGAN & ITALY & ITALY & & & TORINO & N. 4 LOCATION & CALUMET - WOLVERINE \\
\hline 2358 & & GRIBALDO & LOUIS & $\mathrm{s}$ & 5 & MICHIGAN & ITALY & ITALY & & & TORINO & N. 4 LOCATION & CALUMET - WOLVERINE \\
\hline 2359 & & GRIBALDO & JOHN & $\mathrm{S}$ & 3 & MICHIGAN & ITALY & ITALY & & & TORINO & N. 4 LOCATION & CALUMET - WOLVERINE \\
\hline 2360 & & SANDRETTO & CARLO & B & 31 & ITALY & ITALY & ITALY & 1913 & MINER/CM & PONT CANAVESE. TO & N. 4 LOCATION & CALUMET - WOLVERINE \\
\hline 2361 & 45 & SERRA & UMBERTO & $\mathrm{H}$ & 30 & ITALY & ITALY & ITALY & $10 ? ?$ & TRAMMER/CM & TORINO & & CALUMET - WOLVERINE \\
\hline 2362 & & SERRA & MINNIE & W & 21 & ITALY & ITALY & ITALY & 1906 & & & & CALUMET - WOLVERINE \\
\hline 2363 & & SERRA & PETER & $\mathrm{s}$ & 3 & MICHIGAN & ITALY & ITALY & & & TORINO & & CALUMET - WOLVERINE \\
\hline 2364 & 7 & ROSSIO & ANGELO & $\mathrm{H}$ & 35 & ITALY & ITALY & ITALY & 1907 & TRAMMER/CM & PONT CANAVESE, TO & N. 4 LOCATION & CALUMET - WOLVERINE \\
\hline 2365 & & ROSSIO & TERSILIA & w & 41 & ITALY & ITALY & ITALY & 1909 & & PONT CANAVESE. TO & N. 4 LOCATION & CALUMET - WOLVERINE \\
\hline 2366 & & ROSSIO & MARIA & D & 14 & ITALY & ITALY & ITALY & 1909 & & PONT CANAVESE. TO & N. 4 LOCATION & CALUMET - WOLVERINE \\
\hline 2367 & & ROSSIO & CARLO & $\mathrm{s}$ & 4 & MICHIGAN & ITALY & ITALY & & & PONT CANAVESE. TO & N. 4 LOCATION & CALUMET - WOLVERINE \\
\hline 2368 & & ROSSIO & ANNA & D & 3 & MICHIGAN & ITALY & ITALY & & & PONT CANAVESE. TO & N. 4 LOCATION & CALUMET - WOLVERINE \\
\hline 2369 & & ROSSIO & SEBASTIANO & BR & 32 & ITALY & ITALY & ITALY & 1907 & TIMBERMAN/CM & PONT CANAVESE. TO & N. 4 LOCATION & CALUMET - WOLVERINE \\
\hline 2370 & & TOMASI & DOMENICO & $\mathrm{H}$ & 37 & ITALY & ITALY & ITALY & 1911 & TIMBERMAN/CM & LOCANA CANAVESE. TO & N. 4 LOCATION & CALUMET - WOLVERINE \\
\hline 2371 & & TOMASI & JOSEPHINE & W & 36 & ITALY & ITALY & ITALY & 1911 & & LOCANA CANAVESE. TO & N. 4 LOCATION & CALUMET - WOLVERINE \\
\hline 2372 & & TOMASI & MARY & D & 7 & MICHIGAN & ITALY & ITALY & & & LOCANA CANAVESE. TO & N. 4 LOCATION & CALUMET - WOLVERINE \\
\hline 2373 & & TOMASI & CARLO & $\mathrm{s}$ & 4 & MICHIGAN & ITALY & ITALY & & & LOCANA CANAVESE. TO & N. 4 LOCATION & CALUMET - WOLVERINE \\
\hline 2374 & & TOMASI & JOSEPHINE & $\mathrm{D}$ & $18 \mathrm{M}$ & MICHIGAN & ITALY & ITALY & & & LOCANA CANAVESE. TO & N. 4 LOCATION & CALUMET - WOLVERINE \\
\hline 2375 & & TOMASI & DOMENICO & $\mathrm{s}$ & $?$ & MICHIGAN & ITALY & ITALY & & & LOCANA CANAVESE. TO & N. 4 LOCATION & CALUMET - WOLVERINE \\
\hline 2376 & 11 & MARTINA & BATTISTA & $\mathrm{H}$ & 49 & ITALY & ITALY & ITALY & 1911 & TRAMMER/CM & CIRIE'. TO & N. 4 LOCATION & CALUMET - WOLVERINE \\
\hline 2377 & & MARTINA & TRACY & w & 50 & ITALY & ITALY & ITALY & 1915 & & CIRIE'. TO & N. 4 LOCATION & CALUMET - WOLVERINE \\
\hline 2378 & & MARTINA & JOSEPH & S & 15 & ITALY & ITALY & ITALY & 1915 & & CIRIE'. TO & N. 4 LOCATION & CALUMET - WOLVERINE \\
\hline 2379 & & FERRARIS & JAMES & D IN & 28 & ITALY & ITALY & ITALY & 1913 & & LUCCA & N. 4 LOCATION & CALUMET - WOLVERINE \\
\hline 2380 & & FERRARIS & IDA & D & 26 & ITALY & ITALY & ITALY & 1915 & & LUCCA & N. 4 LOCATION & CALUMET - WOLVERINE \\
\hline 2381 & & BROGLIATTO & JOHN & B & 28 & ITALY & ITALY & ITALY & UN & TIMBERMAN/CM & SALTO CANAVESE. TO & N. 4 LOCATION & CALUMET - WOLVERINE \\
\hline 2382 & 38 & SANDRETTO & COSTANCE & $\mathrm{H}$ & 45 & ITALY & ITALY & ITALY & 1907 & TRAMMER/CM & PONT CANAVESE, TO & N. 4 LOCATION & CALUMET - WOLVERINE \\
\hline 2383 & & SANDRETTO & OTTAVIA & W & 38 & ITALY & ITALY & ITALY & 1909 & & PONT CANAVESE. TO & N. 4 LOCATION & CALUMET - WOLVERINE \\
\hline 2384 & & SANDRETTO & MARINO & $\mathrm{s}$ & 15 & ITALY & ITALY & ITALY & 1909 & & PONT CANAVESE. TO & N. 4 LOCATION & CALUMET - WOLVERINE \\
\hline 2385 & & SANDRETTO & MARY & D & 13 & ITALY & ITALY & ITALY & 1909 & & PONT CANAVESE. TO & N. 4 LOCATION & CALUMET - WOLVERINE \\
\hline 2386 & & SANDRETTO & ALDO & $\mathrm{s}$ & 10 & MICHIGAN & ITALY & ITALY & & & PONT CANAVESE. TO & N. 4 LOCATION & CALUMET - WOLVERINE \\
\hline 2387 & & SANDRETTO & KATE & D & 6 & MICHIGAN & ITALY & ITALY & & & PONT CANAVESE. TO & N. 4 LOCATION & CALUMET - WOLVERINE \\
\hline 2388 & & SANDRETTO & ERNEST & $\mathrm{s}$ & 2 & MICHIGAN & ITALY & ITALY & & & PONT CANAVESE, TO & N. 4 LOCATION & CALUMET - WOLVERINE \\
\hline 2389 & 35 & SANDRETTO & DOMENICO & $\mathrm{H}$ & 26 & ITALY & ITALY & ITALY & 1912 & TIMBERMAN/CM & PONT CANAVESE. TO & 2ND ST. & CALUMET - KEARSARGE \\
\hline 2390 & & SANDRETTO & MARY & W & 21 & ITALY & ITALY & ITALY & 1916 & & & 2ND ST. & CALUMET - KEARSARGE \\
\hline 2391 & & SANDRETTO & ANGELINA & D & 1 & MICHIGAN & ITA & ITALY & & & PONT CANAVESE. TO & 2ND ST. & CALUMET - KEARSARGE \\
\hline 2392 & 2 & GALLINATTI & VINCENZO & $\mathrm{H}$ & 46 & ITALY & ITALY & ITALY & 1907 & DRAYMAN/CM & TORINO & & CALUMET - KEARS \\
\hline 2393 & & GALLINATTI & DELPHINE & w & 36 & ITALY & ITALY & ITALY & 1912 & & TORINO & & CALUMET - KEARSARGE \\
\hline
\end{tabular}




\begin{tabular}{|c|c|c|c|c|c|c|c|c|c|c|c|c|c|}
\hline 2394 & & GALLINATTI & BATTISTA & $\mathrm{s}$ & 16 & ITALY & ITALY & ITALY & 1912 & & TORINO & & CALUMET - KEARSARGE \\
\hline 2395 & & GALLINATTI & JOSEPH & $\mathrm{s}$ & 6 & MICHIGAN & ITALY & ITALY & & & TORINO & & CALUMET - KEARSARGE \\
\hline 2396 & & GALLINATTI & MARY & $\mathrm{D}$ & 4 & MICHIGAN & ITALY & ITALY & & & TORINO & & CALUMET - KEARSARGE \\
\hline 2397 & & GALLINATTI & DOMENICO & $\mathrm{s}$ & 1 & MICHIGAN & ITALY & ITALY & & & TORINO & & CALUMET - KEARSARGE \\
\hline 2398 & & FRESE & CARLO & B & 39 & ITALY & ITALY & ITALY & 1903 & TRAMMER/CM & & & CALUMET - KEARSARGE \\
\hline 2399 & & DAIRO & PAUL & $\mathrm{H}$ & 55 & ITALY & ITALY & ITALY & 1891 & LOGGER/TIMBER CAMP & TORINO & & CALUMET - KEARSARGE \\
\hline 2400 & & DAIRO & KATE & w & 60 & ITALY & ITALY & ITALY & 1891 & & TORINO & & CALUMET - KEARSARGE \\
\hline 2401 & & DAIRO & THOMAS & s & 30 & ITALY & ITALY & ITALY & 1893 & DRIVER/SUPPLY COMPANY & TORINO & & CALUMET - KEARSARGE \\
\hline 2402 & & DAIRO & KATE & D & 19 & MICHIGAN & ITALY & ITALY & & HOUSEKEEPER/HOME & TORINO & & CALUMET - KEARSARGE \\
\hline 2403 & & PERONA & BATTISTA & $\mathrm{H}$ & 37 & ITALY & ITALY & ITALY & 1902 & FARMER & PONT CANAVESE. TO & & CALUMET - KEARSARGE \\
\hline 2404 & & PERONA & MARY & w & 37 & ITALY & ITALY & ITALY & 1903 & & & & CALUMET - KEARSARGE \\
\hline 2405 & & PERONA & VITTORIA & $\mathrm{D}$ & 14 & MICHIGAN & ITALY & ITALY & & MAID/HOME & PONT CANAVESE. TO & & CALUMET - KEARSARGE \\
\hline 2406 & & PERONA & JOSEPH & $\mathrm{s}$ & 12 & MICHIGAN & ITALY & ITALY & & & PONT CANAVESE. TO & & CALUMET - KEARSARGE \\
\hline 2407 & & PERONA & LUCY & D & 10 & MICHIGAN & ITALY & ITALY & & & PONT CANAVESE. TO & & CALUMET - KEARSARGE \\
\hline 2408 & & PERONA & FRANK & $\mathrm{S}$ & 4 & MICHIGAN & ITALY & ITALY & & & PONT CANAVESE. TO & & CALUMET - KEARSARGE \\
\hline 2409 & & COPPO & VALENTINO & $\mathrm{H}$ & 61 & ITALY & ITALY & ITALY & 1890 & LABORER/PUBLIC ROAD & PONT CANAVESE, TO & & CALUMET - KEARSARGE \\
\hline 2410 & & COPPO & KATE & W & 65 & ITALY & ITALY & ITALY & 1890 & & PONT CANAVESE. TO & & CALUMET - KEARSARGE \\
\hline 2411 & & COPPO & LINA & D & 40 & ITALY & ITALY & ITALY & 1890 & & PONT CANAVESE. TO & & CALUMET - KEARSARGE \\
\hline 2412 & & COPPO & VALENTINO & $\mathrm{s}$ & 12 & MICHIGAN & ITALY & ITALY & & & PONT CANAVESE. TO & & CALUMET - KEARSARGE \\
\hline 2413 & 158 & FURNO & LOUIS & $\mathrm{H}$ & 26 & ITALY & ITALY & ITALY & 1895 & TELLER/BANK & AGLIE'. TO & WILLOW AVE. & CALUMET - LAURIUM \\
\hline 2414 & & FURNO & MARY & W & 26 & MICHIGAN & ITALY & ITALY & & & & WILLOW AVE. & CALUMET - LAURIUM \\
\hline 2415 & 154 & CATTANEO & MARTA & $\mathrm{H}$ & 49 & ITALY & ITALY & ITALY & 1890 & & & WILLOW AVE. & CALUMET - LAURIUM \\
\hline 2416 & & CATTANEO & JOHN & s & 21 & ITALY & ITALY & ITALY & 1900 & DRIVER/EXPRESS WAGON & & WILLOW AVE. & CALUMET - LAURIUM \\
\hline 2417 & 150 & SALASSA & CARLO & $\mathrm{H}$ & 35 & ITALY & ITALY & ITALY & 1889 & SALESMAN/MONUMENT & SAN BENIGNO CANAVESE. TO & WILLOW AVE. & CALUMET - LAURIUM \\
\hline 2418 & & SALASSA & ROSE & W & 31 & MICHIGAN & ITALY & ITALY & & & TORINO & WILLOW AVE. & CALUMET - LAURIUM \\
\hline 2419 & & SALASSA & ERNEST & $\mathrm{s}$ & 11 & MICHIGAN & ITALY & MICHIGAN & & & SAN BENIGNO CANAVESE. TO & WILLOW AVE. & CALUMET - LAURIUM \\
\hline 2420 & 150 & SUINO & FRANK & $\mathrm{H}$ & 51 & ITALY & ITALY & ITALY & 1890 & LABORER/CM & CORIO. TO & WOODLAND AVE. & CALUMET - LAURIUM \\
\hline 2421 & & SUINO & MARY & W & 45 & ITALY & ITALY & ITALY & 1891 & & & WOODLAND AVE. & CALUMET - LAURIUM \\
\hline 2422 & & SUINO & BERNARDO & $\mathrm{s}$ & 21 & MICHIGAN & ITALY & ITALY & & LABORER/CM & CORIO. TO & WOODLAND AVE. & CALUMET - LAURIUM \\
\hline 2423 & & SUINO & GEORGE & $\mathrm{s}$ & 20 & MICHIGAN & ITALY & ITALY & & BLACKSMITH/CM & CORIO. TO & WOODLAND AVE. & CALUMET - LAURIUM \\
\hline 2424 & & SUINO & ANGELINE & D & 17 & MICHIGAN & ITALY & ITALY & & & CORIO. TO & WOODLAND AVE. & CALUMET - LAURIUM \\
\hline 2425 & 134 & ADDA & GIORGIO & $\mathrm{H}$ & 46 & ITALY & ITALY & ITALY & 1896 & SHOEMAKER & & WOODLAND AVE. & CALUMET - LAURIUM \\
\hline 2426 & 104 & ADDA & MARY & w & 38 & ITALY & ITALY & ITALY & 1906 & ОПUЕIVIARER & & WOODLAND AVE. & CALUMET - LAURIUM \\
\hline 2427 & 108 & $\begin{array}{l}\text { VAIRO } \\
\text { VADA }\end{array}$ & GIULIA & $\mathrm{H}$ & 51 & ITALY & ITALY & ITALY & 1904 & & LOCANA CANAVESE, TO & SENECA ST. & $\begin{array}{l}\text { CALUMET - LAURIUM } \\
\text { CAUM }\end{array}$ \\
\hline 2428 & & VAIRO & HELEN & $\mathrm{D}$ & 17 & MICHIGAN & ITALY & ITALY & & & LOCANA CANAVESE. TO & SENECA ST. & CALUMET - LAURIUM \\
\hline 2429 & & VAIRO & JULIA & $\mathrm{D}$ & 7 & MICHIGAN & ITALY & ITALY & & & LOCANA CANAVESE. TO & SENECA ST. & CALUMET - LAURIUM \\
\hline 2430 & 112 & PRICCO & DOMENICO & $\mathrm{H}$ & 43 & ITALY & ITALY & ITALY & 1896 & MINER/CM & SAN MARTINO CANAVESE. TO & SENECA ST. & CALUMET - LAURIUM \\
\hline 2431 & & PRICCO & ANGELA & W & 35 & ITALY & ITALY & ITALY & 1905 & & SAN MARTINO CANAVESE. TO & SENECA ST. & CALUMET - LAURIUM \\
\hline 2432 & & PRICCO & PETER & $\mathrm{s}$ & 13 & MICHIGAN & ITALY & ITALY & & & SAN MARTINO CANAVESE. TO & SENECA ST. & CALUMET - LAURIUM \\
\hline 2433 & & PRICCO & JOHN & $\mathrm{S}$ & 11 & MICHIGAN & ITALY & ITALY & & & SAN MARTINO CANAVESE. TO & SENECA ST. & CALUMET - LAURIUM \\
\hline 2434 & & PRICCO & DOMENICO & $\mathrm{S}$ & 3 & MICHIGAN & ITALY & ITALY & & & SAN MARTINO CANAVESE. TO & SENECA ST. & CALUMET - LAURIUM \\
\hline 2435 & & RICHETTA & JAMES & BIL & 24 & ITALY & ITALY & ITALY & 1916 & MINER/CM & SAN MARTINO CANAVESE. TO & SENECA ST. & CALUMET - LAURIUM \\
\hline 2436 & 118 & MASSOGLIA & FRANK & $\mathrm{H}$ & 31 & ITALY & ITALY & ITALY & 1908 & RETAIL MERCHANT/GROCERY & TORINO & SENECA ST. & CALUMET - LAURIUM \\
\hline 2437 & & MASSOGLIA & MARY & W & 25 & ITALY & ITALY & ITALY & 1915 & & & SENECA ST. & CALUMET - LAURIUM \\
\hline 2438 & & MASSOGLIA & MARTIN & $\mathrm{s}$ & 3 & MICHIGAN & ITALY & ITALY & & & TORINO & SENECA ST. & CALUMET - LAURIUM \\
\hline 2439 & & PANATTONI & RALPH & $\mathrm{H}$ & 30 & ITALY & ITALY & ITALY & 1905 & MINER/CM & MONTECARLO. LU & SENECA ST. & CALUMET - LAURIUM \\
\hline 2440 & & PANATTONI & DELLA & w & 23 & MICHIGAN & ITALY & ITALY & & & & SENECA ST. & CALUMET - LAURIUM \\
\hline 2441 & & PANATTONI & CAROLINE & D & 6 & MICHIGAN & ITALY & ITALY & & & MONTECARLO. LU & SENECA ST. & CALUMET - LAURIUM \\
\hline 2442 & & PANATTONI & FLORENCE & $\mathrm{D}$ & 5 & MICHIGAN & ITALY & ITALY & & & MONTECARLO. LU & SENECA ST. & CALUMET - LAURIUM \\
\hline 2443 & & PANATTONI & DOROTHY & D & $7 \mathrm{M}$ & MICHIGAN & ITALY & ITALY & & & MONTECARLO. LU & SENECA ST. & CALUMET - LAURIUM \\
\hline 2444 & 120 & TOMASI & ANTONIO & $\mathrm{H}$ & 42 & ITALY & ITALY & ITALY & 1900 & & LOCANA CANAVESE. TO & SENECA ST. & CALUMET - LAURIUM \\
\hline 2445 & & TOMASI & ANTONIA & W & 41 & ITALY & ITALY & ITALY & 1902 & & & SENECA ST. & CALUMET - LAURIUM \\
\hline 2446 & & TOMASI & JENNIE & $\mathrm{D}$ & 18 & MICHIGAN & ITALY & ITALY & & STENOGRAPHER/CM OFFICE & LOCANA CANAVESE. TO & SENECA ST. & CALUMET - LAURIUM \\
\hline 2447 & & TOMASI & JAMES & $\mathrm{s}$ & 17 & MICHIGAN & ITALY & ITALY & & DRILL SHARPENER/CM & LOCANA CANAVESE. TO & SENECA ST. & CALUMET - LAURIUM \\
\hline 2448 & & TOMASI & KATE & D & 16 & MICHIGAN & ITALY & ITALY & & TAILORESS/TAILOR SHOP & LOCANA CANAVESE. TO & SENECA ST. & CALUMET - LAURIUM \\
\hline 2449 & & TOMASI & TONY & $\mathrm{s}$ & 14 & MICHIGAN & ITALY & ITALY & & & LOCANA CANAVESE. TO & SENECA ST. & CALUMET - LAURIUM \\
\hline 2450 & & TOMASI & IOLANDA & D & 11 & MICHIGAN & ITALY & ITALY & & & LOCANA CANAVESE. TO & SENECA ST. & CALUMET - LAURIUM \\
\hline 2451 & & TOMASI & MARGHERITA & D & 10 & MICHIGAN & ITALY & ITALY & & & LOCANA CANAVESE, TO & SENECA ST. & CALUMET - LAURIUM \\
\hline 2452 & & TOMASI & FLORENCE & $\mathrm{D}$ & 3 & MICHIGAN & ITALY & ITALY & & & LOCANA CANAVESE. TO & SENECA ST. & CALUMET - LAURIUM \\
\hline 2453 & & CONTRATTO & TONY & NEP & 12 & MICHIGAN & ITALY & ITALY & & & LOCANA CANAVESE. TO & SENECA ST. & CALUMET - LAURIUM \\
\hline 2454 & 122 & ALDO & CINTO & $\mathrm{H}$ & 39 & ITA & ITA & ITA & 1904 & MINER/CM & NO CANAVESE. & SENECA ST. & CALUMET - LAURIUM \\
\hline 2455 & & ALDO & KATE & w & 27 & ITALY & ITALY & ITALY & 1904 & 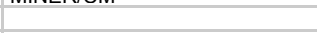 & SAN MARTINO CANAVESE. TO & SENECA ST. & CALUMET - L \\
\hline 2456 & & ALDO & PETER & $\mathrm{s}$ & 3 & MICHIGAN & ITALY & ITALY & & & SAN MARTINO CANAVESE. TO & SENECA ST. & CALUMET - LAURIUM \\
\hline
\end{tabular}




\begin{tabular}{|c|c|c|c|c|c|c|c|c|c|c|c|c|c|}
\hline 2457 & 124 & VIANI/A & GIORGIO & $\mathrm{H}$ & 40 & ITALY & ITALY & ITALY & 1889 & OVERSEER/HIGHWAY & & SENECA ST. & CALUMET - LAURIUM \\
\hline 2458 & & VIANI/A & KATE & $\mathrm{D}$ & 17 & MICHIGAN & ITALY & ITALY & & & & SENECA ST. & CALUMET - LAURIUM \\
\hline 2459 & & VIANI/A & MARY & $\mathrm{D}$ & 13 & MICHIGAN & ITALY & ITALY & & & & SENECA ST. & CALUMET - LAURIUM \\
\hline 2460 & & VIANI/A & ELEONOR & $\mathrm{D}$ & 11 & MICHIGAN & ITALY & ITALY & & & & SENECA ST. & CALUMET - LAURIUM \\
\hline 2461 & 140 & DATA/DATTA & ANTON & $\mathrm{H}$ & 45 & ITALY & ITALY & ITALY & 1889 & FOREMAN/CM & SAN GIORGIO CANAVESE. TO & SENECA ST. & CALUMET - LAURIUM \\
\hline 2462 & & DATA/DATTA & ANNA & w & 44 & ITALY & ITALY & ITALY & 1890 & & & SENECA ST. & CALUMET - LAURIUM \\
\hline 2463 & & DATA/DATTA & MIKE & s & 20 & MICHIGAN & ITALY & ITALY & & DRILL SHARPENER/CM & SAN GIORGIO CANAVESE. TO & SENECA ST. & CALUMET - LAURIUM \\
\hline 2464 & & $\begin{array}{l}\text { DATA/DATTA } \\
\text { DATA }\end{array}$ & TONY & s & 18 & MICHIGAN & ITALY & ITALY & & NOT CLEAR & SAN GIORGIO CANAVESE. TO & SENECA ST. & $\begin{array}{l}\text { CALUMET - LAURIUM } \\
\text { CAUM }\end{array}$ \\
\hline 2465 & & DATA/DATTA & MIKE & $\mathrm{COU}$ & 30 & ITALY & ITALY & ITALY & 1912 & TIMBERMAN/CM & SAN GIORGIO CANAVESE. TO & SENECA ST. & CALUMET - LAURIUM \\
\hline 2466 & 142 & FAUSONE & DOMENICA & $\mathrm{H}$ & 52 & ITALY & ITALY & ITALY & 1891 & & TORINO & SENECA ST. & CALUMET - LAURIUM \\
\hline 2467 & & FAUSONE & PETER & s & 18 & MICHIGAN & ITALY & ITALY & & MACHINIST HELPER/RAILROAD & TORINO & SENECA ST. & CALUMET - LAURIUM \\
\hline 2468 & 230 & CHECCHI & JOSEPH & $\mathrm{H}$ & 70 & ITALY & ITALY & ITALY & 1892 & & & SENECA ST. & CALUMET - LAURIUM \\
\hline 2469 & & CHECCHI & DOMENICA & w & 74 & ITALY & ITALY & ITALY & 1891 & & & SENECA ST. & CALUMET - LAURIUM \\
\hline 2470 & 217 & PICCHIOTTINO & BATTISTA & $\mathrm{H}$ & 51 & ITALY & ITALY & ITALY & 1885 & PLUMBER/OWN SHOP & PONT CANAVESE. TO & AHMEEK ST. & CALUMET - LAURIUM \\
\hline 2471 & & PICCHIOTTINO & MARGHERITA & W & 49 & ITALY & ITALY & ITALY & 1892 & & & AHMEEK ST. & CALUMET - LAURIUM \\
\hline 2472 & & PICCHIOTTINO & ANGELO & $\mathrm{s}$ & 25 & MINNESOT & ITALY & ITALY & & PLUMBER/GENERAL PRACTICE & PONT CANAVESE, TO & AHMEEK ST. & CALUMET - LAURIUM \\
\hline 2473 & & PICCHIOTTINO & LUCY & $\mathrm{D}$ & 24 & MICHIGAN & ITALY & ITALY & & TEL. OPERATOR/TEL. COMPANY & PONT CANAVESE. TO & AHMEEK ST. & CALUMET - LAURIUM \\
\hline 2474 & & PICCHIOTTINO & KATE & $\mathrm{D}$ & 22 & MICHIGAN & ITALY & ITALY & & NOT CLEAR & PONT CANAVESE. TO & AHMEEK ST. & CALUMET - LAURIUM \\
\hline 2475 & & PICCHIOTTINO & ENRICO & $\mathrm{s}$ & 15 & MICHIGAN & ITALY & ITALY & & NOT CLEAR & PONT CANAVESE. TO & AHMEEK ST. & CALUMET - LAURIUM \\
\hline 2476 & & PICCHIOTTINO & JOHN & $\mathrm{s}$ & 13 & MICHIGAN & ITALY & ITALY & & & PONT CANAVESE. TO & AHMEEK ST. & CALUMET - LAURIUM \\
\hline 2477 & & PICCHIOTTINO & PAULINE & $\mathrm{D}$ & 8 & MICHIGAN & ITALY & ITALY & & & PONT CANAVESE. TO & AHMEEK ST. & CALUMET - LAURIUM \\
\hline 2478 & & COPPO & KATE & $\mathrm{COU}$ & 25 & MICHIGAN & ITALY & ITALY & & BOOKKEEPER/INSURANCE & PONT CANAVESE, TO & AHMEEK ST. & CALUMET - LAURIUM \\
\hline 2479 & 208 & TORREANO & KATE & $\mathrm{H}$ & 50 & ITALY & ITALY & ITALY & 1892 & & TORINO & AHMEEK ST. & CALUMET - LAURIUM \\
\hline 2480 & & TORREANO & JOHN & $\mathrm{s}$ & 21 & MICHIGAN & ITALY & ITALY & & MACHINIST/RAILROAD & TORINO & AHMEEK ST. & CALUMET - LAURIUM \\
\hline 2481 & 136 & CARBONATTO & MARY & $\mathrm{H}$ & 42 & MICHIGAN & ITALY & ITALY & & LAUNDRESS/PF & TORINO & AHMEEK ST. & CALUMET - LAURIUM \\
\hline 2482 & & CARBONATTO & PHYLLIS & $\mathrm{D}$ & 19 & MICHIGAN & ITALY & ITALY & & & PONT CANAVESE. TO & AHMEEK ST. & CALUMET - LAURIUM \\
\hline 2483 & 40 & CONTRATTO & JENNIE & $\mathrm{H}$ & 45 & ITALY & ITALY & ITALY & 1899 & LAUNDRESS/OUT & LOCANA CANAVESE. TO & & CALUMET - LAURIUM \\
\hline 2484 & & CONTRATTO & ANDREW & $\mathrm{s}$ & 23 & MICHIGAN & ITALY & ITALY & & MOLDER/CM & LOCANA CANAVESE, TO & & CALUMET - LAURIUM \\
\hline 2485 & & CONTRATTO & ANNA & D & 23 & MICHIGAN & ITALY & ITALY & & & LOCANA CANAVESE. TO & & CALUMET - LAURIUM \\
\hline 2486 & & CONTRATTO & JAMES & $\mathrm{s}$ & 21 & MICHIGAN & ITALY & ITALY & & ELECTRICIAN/CM & LOCANA CANAVESE. TO & & CALUMET - LAURIUM \\
\hline 2487 & 100 & VALLE & ANTON & $\mathrm{H}$ & 60 & ITALY & ITALY & ITALY & 1887 & & SAN GIORGIO CANAVESE. TO & PEWABIC ST. & CALUMET - LAURIUM \\
\hline 2488 & 0 & VALLE & MARY & w & 56 & ITALY & ITALY & ITALY & 1887 & & SAN GIORGIO CANAVESE. TO & PEWABIC ST. & CALUMET - LAURIUM \\
\hline 2489 & & VALLE & $\begin{array}{l}\text { STEVEN } \\
\text { STAVI }\end{array}$ & s & 37 & $\begin{array}{l}\text { ITALY } \\
\text { ITAL }\end{array}$ & ITALY & ITALY & 1887 & MACHINIST/RAILROAD & SAN GIORGIO CANAVESE. TO & PEWABIC ST. & CALUMET - LAURIUM \\
\hline 2490 & 112 & ZIROTTI & $\begin{array}{l}\text { SOHN } \\
\text { JOHN }\end{array}$ & $\mathrm{H}$ & 55 & ITALY & ITALY & ITALY & 1911 & MASON/CM & 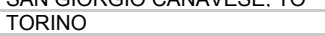 & PEVVADIC ST. & CALUMET - LAURIUM \\
\hline 2491 & & ZIROTTI & MARY & w & 51 & ITALY & ITALY & ITALY & 1911 & & TORINO & & CALUMET - LAURIUM \\
\hline 2492 & & ZIROTTI & AMELIA & $\mathrm{D}$ & 26 & ITALY & ITALY & ITALY & 1911 & SALESLADY/DEPT. STORE & TORINO & & CALUMET - LAURIUM \\
\hline 2493 & 126 & SAVINI & TRACY & $\mathrm{H}$ & 55 & FRANCE & ITALY & ITALY & 1892 & & TORINO & IROQUOIS ST. & CALUMET - LAURIUM \\
\hline 2494 & & SAVINI & JOSEPH & $\mathrm{s}$ & 24 & ITALY & ITALY & FRANCE & 1892 & MANAGER/ICE RINK & TORINO & IROQUOIS ST. & CALUMET - LAURIUM \\
\hline 2495 & 136 & MARTA & JOHN & H & 51 & ITALY & ITALY & ITALY & 1890 & LABORER/CM & SAN MARTINO CANAVESE. TO & IROQUOIS ST. & CALUMET - LAURIUM \\
\hline 2496 & & MARTA-RUONA & TRACY & W & 51 & ITALY & ITALY & ITALY & 1895 & & & IROQUOIS ST. & CALUMET - LAURIUM \\
\hline 2497 & & MARTA & TRACY & D & 18 & MICHIGAN & ITALY & ITALY & & & SAN MARTINO CANAVESE. TO & IROQUOIS ST. & CALUMET - LAURIUM \\
\hline 2498 & & MARTA & JOHN & $\mathrm{s}$ & 10 & MICHIGAN & ITALY & ITALY & & & SAN MARTINO CANAVESE. TO & IROQUOIS ST. & CALUMET - LAURIUM \\
\hline 2499 & 138 & BARONI & UGO & $\mathrm{H}$ & 30 & ITALY & ITALY & ITALY & 1913 & LABORER/CM & ROMA & IROQUOIS ST. & CALUMET - LAURIUM \\
\hline 2500 & & BARONI & ANGELINE & w & 38 & ITALY & ITALY & ITALY & 1913 & & ROMA & IROQUOIS ST. & CALUMET - LAURIUM \\
\hline 2501 & & BARONI & CAMILLO & s & 5 & MICHIGAN & ITALY & ITALY & & & ROMA & IROQUOIS ST. & CALUMET - LAURIUM \\
\hline 2502 & & BARONI & CARMELO & $\mathrm{s}$ & 3 & MICHIGAN & ITALY & ITALY & & & ROMA & IROQUOIS ST. & CALUMET - LAURIUM \\
\hline 2503 & & BARONI & CESARINA & $\mathrm{D}$ & 1 & MICHIGAN & ITALY & ITALY & & & ROMA & IROQUOIS ST. & CALUMET - LAURIUM \\
\hline 2504 & 150 & MARTY & JOSEPH & $\mathrm{H}$ & 37 & MICHIGAN & LUXEMBUR & LUXEMBUR & & PLUMBER & LUXEMBOURG & FLORIDA ST. & CALUMET - LAURIUM \\
\hline 2505 & & MARTY & TRACY & w & 39 & ITALY & ITALY & ITALY & 1905 & & & FLORIDA ST. & CALUMET - LAURIUM \\
\hline 2506 & & MARTY & CHARLES & s & 12 & MICHIGAN & ITALY & ITALY & & & & FLORIDA ST. & CALUMET - LAURIUM \\
\hline 2507 & & MARTY & LOUIS & $\mathrm{s}$ & 10 & MICHIGAN & ITALY & ITALY & & & & FLORIDA ST. & CALUMET - LAURIUM \\
\hline 2508 & & MARTY & KATE & $\mathrm{D}$ & 9 & MICHIGAN & ITALY & ITALY & & & & FLORIDA ST. & CALUMET - LAURIUM \\
\hline 2509 & & MARTY & JOHN & $\mathrm{s}$ & 5 & MICHIGAN & ITALY & ITALY & & & & FLORIDA ST. & CALUMET - LAURIUM \\
\hline 2510 & & MARTY & WILLIAM & $\mathrm{s}$ & $3 \mathrm{M}$ & MICHIGAN & ITALY & ITALY & & & & FLORIDA ST. & CALUMET - LAURIUM \\
\hline 2511 & 146 & MARINELLI & AUGUSTO & $\mathrm{H}$ & 47 & ITALY & ITALY & ITALY & 1893 & PROPRIETOR/PRINTING SHOP & TRENTO & FLORIDA ST. & CALUMET - LAURIUM \\
\hline 2512 & & MARINELLI & MADDALENA & w & 41 & GERMANY & GERMANY & GERMANY & 1887 & & GERMANY & FLORIDA ST. & CALUMET - LAURIUM \\
\hline 2513 & & MARINELLI & D. & s & 21 & MICHIGAN & ITALY & GERMANY & & PRINTER/SHOP & TRENTO & FLORIDA ST. & CALUMET - LAURIUM \\
\hline 2514 & & MARINELLI & WALTER & $\mathrm{s}$ & 19 & MICHIGAN & ITALY & GERMANY & & LABORER/AUTO FACTORY & TRENTO & FLORIDA ST. & CALUMET - LAURIUM \\
\hline 2515 & & MARINELLI & VIRGILIO & $\mathrm{s}$ & 15 & MICHIGAN & ITALY & GERMANY & & TEAMSTER/MEAT MARKET & TRENTO & FLORIDA ST. & CALUMET - LAURIUM \\
\hline 2516 & 100 & BARSANTI & ALBERTO & $\mathrm{H}$ & 39 & ITALY & ITALY & ITALY & 1897 & PROPRIETOR/FRUIT STORE & LUCCA & FLORIDA ST. & CALUMET - LAURIUM \\
\hline 2517 & & BARSANTI & $\mathrm{CC}$ & W & 35 & ITA & ITA & ITA & 1884 & & & RIDA ST. & CALUMET - LAURIUM \\
\hline 2518 & & BARSANTI & ORENCE & $\mathrm{D}$ & 16 & MICHIGAN & ITALY & ITALY & & & LUCC & & CALUMET - LAURIUM \\
\hline 2519 & & BESSALO & CARLO & B & 46 & ITALY & ITALY & ITALY & 1898 & CLERK/BANK & BESSOLO CANAVESE. TO & FLORIDA ST. & CALUMET - LAURIUM \\
\hline
\end{tabular}




\begin{tabular}{|c|c|c|c|c|c|c|c|c|c|c|c|c|c|}
\hline 2520 & 80 & GALLASERO & JAMES & $\mathrm{H}$ & 45 & ITALY & ITALY & ITALY & 1890 & PROPRIETOR/GROCERY STORE & PONT CANAVESE. TO & 1ST ST. & CALUMET - LAURIUM \\
\hline 2521 & & GALLASERO & LUCY & W & 56 & ITALY & ITALY & ITALY & 1906 & & PONT CANAVESE. TO & 1ST ST. & CALUMET - LAURIUM \\
\hline 2522 & & GALLASERO & JEANNE & D & 22 & ITALY & ITALY & ITALY & 1906 & SALESLADY/GROCERY STORE & PONT CANAVESE. TO & 1ST ST. & CALUMET - LAURIUM \\
\hline 2523 & & BAGGIORE & SECONDO & BRIL & 35 & ITALY & ITALY & ITALY & 1907 & MINER/CM & TORINO & 1ST ST. & CALUMET - LAURIUM \\
\hline 2524 & 78 & RASTELLO & JOHN B. & $\mathrm{H}$ & 52 & ITALY & ITALY & ITALY & 1888 & TAILOR/OWN SHOP & PONT CANAVESE. TO & 1ST ST. & CALUMET - LAURIUM \\
\hline 2525 & & RASTELLO & TERESA & w & 53 & ITALY & ITALY & ITALY & 1890 & & & 1ST ST. & CALUMET - LAURIUM \\
\hline 2526 & & RASTELLO & DOMENICO & s & 28 & MICHIGAN & ITALY & ITALY & & TAILOR/TAILOR SHOP & PONT CANAVESE. TO & 1 ST ST. & CALUMET - LAURIUM \\
\hline 2527 & & RASTELLO & MARY & MO & 82 & ITALY & ITALY & ITALY & 90/99 & & PONT CANAVESE. TO & 1ST ST. & CALUMET - LAURIUM \\
\hline 2528 & 104 & LUCCHESI & DELIA & SER & 20 & MICHIGAN & ITALY & ITALY & & SERVANT/PF & LUCCA & & CALUMET - LAURIUM \\
\hline 2529 & 114 & QUELLO & MICHELE & $\mathrm{H}$ & 48 & MICHIGAN & ITALY & IRELAND & & MEAT CUTTER/OWN SHOP & LOCANA CANAVESE. TO & & CALUMET - LAURIUM \\
\hline 2530 & & QUELLO & CHIOTTE & W & 35 & USA & UTAH & UTAH & & & USA & & CALUMET - LAURIUM \\
\hline 2531 & & QUELLO & ALBERTA & $\mathrm{D}$ & 8 & MICHIGAN & MICHIGAN & USA & & & LOCANA CANAVESE. TO & & CALUMET - LAURIUM \\
\hline 2532 & 108 & BRONZO & PETER & $\mathrm{H}$ & 32 & ITALY & ITALY & ITALY & 1906 & TRAMMER/CM & SAN MARTINO CANAVESE. TO & CALUMET ST. & CALUMET - LAURIUM \\
\hline 2533 & & BRONZO & MARY & W & 26 & MICHIGAN & ITALY & ITALY & & & & CALUMET ST. & CALUMET - LAURIUM \\
\hline 2534 & & BRONZO & RENA & D & 7 & MICHIGAN & ITALY & MICHIGAN & & & SAN MARTINO CANAVESE. TO & CALUMET ST. & CALUMET - LAURIUM \\
\hline 2535 & & BRONZO & ARLINE & $\mathrm{D}$ & $10 \mathrm{M}$ & MICHIGAN & ITALY & MICHIGAN & & & SAN MARTINO CANAVESE, TO & CALUMET ST. & CALUMET - LAURIUM \\
\hline 2536 & & FOLLO & MARTIN & $\mathrm{H}$ & 32 & ITALY & ITALY & ITALY & 1911 & TEAMSTER/COAL YARD & SAN MARTINO CANAVESE. TO & CALUMET ST. & CALUMET - LAURIUM \\
\hline 2537 & & FOLLO & MARY & W & 30 & ITALY & ITALY & ITALY & 1914 & & & CALUMET ST. & CALUMET - LAURIUM \\
\hline 2538 & & FOLLO & JOHN & $\mathrm{s}$ & 5 & MICHIGAN & ITALY & ITALY & & & SAN MARTINO CANAVESE. TO & CALUMET ST. & CALUMET - LAURIUM \\
\hline 2539 & 129 & GIULIO & JOHN & $\mathrm{H}$ & 27 & MICHIGAN & ITALY & ITALY & & TRUCK DRIVER/CM & SAN GIORGIO CANAVESE. TO & OSCEOLA ST. & CALUMET - LAURIUM \\
\hline 2540 & & GIULIO & VITTORIA & W & 24 & ITALY & ITALY & ITALY & 1897 & & & OSCEOLA ST. & CALUMET - LAURIUM \\
\hline 2541 & & GIULIO & MARY & $\mathrm{D}$ & 2 & MICHIGAN & MICHIGAN & ITALY & & & SAN GIORGIO CANAVESE, TO & OSCEOLA ST. & CALUMET - LAURIUM \\
\hline 2542 & 125 & BORGO & DOMENICO & $\mathrm{H}$ & 60 & ITALY & ITALY & ITALY & 1881 & NOT CLEAR & VICENZA & OSCEOLA ST. & CALUMET - LAURIUM \\
\hline 2543 & & BORGO & KATE & w & 49 & ITALY & ITALY & ITALY & 1882 & & & OSCEOLA ST. & CALUMET - LAURIUM \\
\hline 2544 & & BORGO & JOHN & $\mathrm{s}$ & 29 & MICHIGAN & ITALY & ITALY & & & VICENZA & OSCEOLA ST. & CALUMET - LAURIUM \\
\hline 2545 & 115 & TINETTI & LOUIS & $\mathrm{H}$ & 39 & ITALY & ITALY & ITALY & 1902 & MOTORMAN/STREET RAILWAY & SAN MARTINO CANAVESE. TO & OSCEOLA ST. & CALUMET - LAURIUM \\
\hline 2546 & & TINETTI & ROSE & W & 34 & ITALY & ITALY & ITALY & 1902 & & SAN MARTINO CANAVESE. TO & OSCEOLA ST. & CALUMET - LAURIUM \\
\hline 2547 & & TINETTI & MARY & D & 13 & MICHIGAN & ITALY & ITALY & & & SAN MARTINO CANAVESE, TO & OSCEOLA ST. & CALUMET - LAURIUM \\
\hline 2548 & & TINETTI & ANNE & D & 11 & MICHIGAN & ITALY & ITALY & & & SAN MARTINO CANAVESE. TO & OSCEOLA ST. & CALUMET - LAURIUM \\
\hline 2549 & & TINETTI & LAURA & D & 10 & MICHIGAN & ITALY & ITALY & & & SAN MARTINO CANAVESE. TO & OSCEOLA ST. & CALUMET - LAURIUM \\
\hline 2550 & & TINETTI & FRANK & $\mathrm{s}$ & 5 & MICHIGAN & ITALY & ITALY & & & SAN MARTINO CANAVESE. TO & OSCEOLA ST. & CALUMET - LAURIUM \\
\hline 2551 & & TINETTI & PETER & $\mathrm{s}$ & 3 & MICHIGAN & ITALY & ITALY & & & SAN MARTINO CANAVESE. TO & OSCEOLA ST. & CALUMET - LAURIUM \\
\hline 2552 & & TINETTI & EDITH & D & 1 & MICHIGAN & ITALY & ITALY & & & SAN MARTINO CANAVESE. TO & OSCEOLA ST. & CALUMET - LAURIUM \\
\hline 2553 & 113 & ROVANO & MARTIN & $\mathrm{H}$ & 40 & ITALY & ITALY & ITALY & 1902 & LABORER/ROCK HOUSE & SAN MARTINO CANAVESE, TO & OSCEOLA ST. & CALUMET - LAURIUM \\
\hline 2554 & 110 & ROVANO & MARY & w & 40 & ITALY & ITALY & ITALY & 1903 & KEEPER/BOARDING HOUSE & DAIVIVATR IIIVU CAIVAVEOE, TO & OSCEOLA ST. & CALUMET - LAURIUM \\
\hline 2555 & & BARINOTTI & JOHN & B & 29 & MICHIGAN & ITALY & ITALY & 1900 & FIREMAN/RAILROAD & PONT CANAVESE. TO & OSCEOLA ST. & CALUMET - LAURIUM \\
\hline 2556 & & QUELLO & BATTISTA & B & 31 & ITALY & ITALY & ITALY & 1913 & BLACKSMITH/CM & LOCANA CANAVESE. TO & OSCEOLA ST. & CALUMET - LAURIUM \\
\hline 2557 & & CERESERO & BATTISTA & B & 30 & ITALY & ITALY & ITALY & 1913 & TRAMMER/CM & MEZZANILE. TO & OSCEOLA ST. & CALUMET - LAURIUM \\
\hline 2558 & & CARBONATTO & VITTORIO & B & 52 & ITALY & ITALY & ITALY & 1890 & MACHINIST/CM & PONT CANAVESE. TO & OSCEOLA ST. & CALUMET - LAURIUM \\
\hline 2559 & & SCIACERO & PETER & B & 24 & ITALY & ITALY & ITALY & 1913 & FIREMAN/BOILER HOUSE & SAN GIOVANNI CANAVESE. TO & OSCEOLA ST. & CALUMET - LAURIUM \\
\hline 2560 & & PEROLINO & PETER & B & 33 & ITALY & ITALY & ITALY & 1906 & MEAT MERCHANT/OWN SHOP & TORINO & OSCEOLA ST. & CALUMET - LAURIUM \\
\hline 2561 & & CASTAGNETTO & SECONDO & B & 31 & ITALY & ITALY & ITALY & 1910 & MINER/CM & PONT CANAVESE. TO & OSCEOLA ST. & CALUMET - LAURIUM \\
\hline 2562 & 109 & TINETTI & PAUL & $\mathrm{H}$ & 44 & ITALY & ITALY & ITALY & 1899 & MERCHANT/CONFECTIONARY & SAN MARTINO CANAVESE. TO & OSCEOLA ST. & CALUMET - LAURIUM \\
\hline 2563 & & TINETTI & MADDALENA & W & 44 & ITALY & ITALY & ITALY & 1899 & & SAN MARTINO CANAVESE. TO & OSCEOLA ST. & CALUMET - LAURIUM \\
\hline 2564 & & TINETTI & FRANK & $\mathrm{s}$ & 20 & MICHIGAN & ITALY & ITALY & & MERCHANT/BOOK STORE & SAN MARTINO CANAVESE. TO & OSCEOLA ST. & CALUMET - LAURIUM \\
\hline 2565 & & TINETTI & JOHN & $\mathrm{s}$ & 17 & MICHIGAN & ITALY & ITALY & & SALESMAN/CONFECTIONARY & SAN MARTINO CANAVESE. TO & OSCEOLA ST. & CALUMET - LAURIUM \\
\hline 2566 & & TINETTI & ERNEST & $\mathrm{s}$ & 11 & MICHIGAN & ITALY & ITALY & & & SAN MARTINO CANAVESE. TO & OSCEOLA ST. & CALUMET - LAURIUM \\
\hline 2567 & 105 & TORREANO & JOHN & $\mathrm{H}$ & 56 & ITALY & ITALY & ITALY & 1885 & PROPRIETOR/SALOON & TORINO & OSCEOLA ST. & CALUMET - LAURIUM \\
\hline 2568 & & TORREANO & PHYLLIS & w & 55 & ITALY & ITALY & ITALY & 1890 & & & OSCEOLA ST. & CALUMET - LAURIUM \\
\hline 2569 & & TORREANO & MARTIN & s & 26 & MICHIGAN & ITALY & ITALY & & CHAUFFEUR/PF & TORINO & OSCEOLA ST. & CALUMET - LAURIUM \\
\hline 2570 & & TORREANO & PAULINE & $\mathrm{D}$ & 25 & MICHIGAN & ITALY & ITALY & & & TORINO & OSCEOLA ST. & CALUMET - LAURIUM \\
\hline 2571 & & TORREANO & ELLEN & $\mathrm{D}$ & 14 & MICHIGAN & ITALY & ITALY & & & TORINO & OSCEOLA ST. & CALUMET - LAURIUM \\
\hline 2572 & 105 & TINETTI & PAUL & $\mathrm{H}$ & 20 & MICHIGAN & ITALY & ITALY & & MERCHANT/CONFECTIONARY & TORINO & OSCEOLA ST. & CALUMET - LAURIUM \\
\hline 2573 & & TINETTI & DORA & w & 22 & MICHIGAN & ITALY & ITALY & & & & OSCEOLA ST. & CALUMET - LAURIUM \\
\hline 2574 & 63 & TORREANO & JAMES & $\mathrm{H}$ & 52 & ITALY & ITALY & ITALY & 1883 & RETAIL MERCHANT/GROCERY & TORINO & 1ST. ST. & CALUMET - LAURIUM \\
\hline 2575 & & TORREANO & ANNE & w & 43 & ITALY & ITALY & ITALY & 1889 & & & 1ST. ST. & CALUMET - LAURIUM \\
\hline 2576 & & TORREANO & FRANK & s & 23 & MICHIGAN & ITALY & ITALY & & TEAMSTER/GROCERY STORE & TORINO & 1ST. ST. & CALUMET - LAURIUM \\
\hline 2577 & & TORREANO & JOHN & $\mathrm{s}$ & 21 & MICHIGAN & ITALY & ITALY & & TEAMSTER/GROCERY STORE & TORINO & 1ST. ST. & CALUMET - LAURIUM \\
\hline 2578 & & TORREANO & RITA & $\mathrm{D}$ & 18 & MICHIGAN & ITALY & ITALY & & SALESLADY/GROCERY STORE & TORINO & 1ST. ST. & CALUMET - LAURIUM \\
\hline 2579 & & TORREANO & JAMES & s & 16 & MICHIGAN & ITALY & ITALY & & TEAMSTER/FRUIT STORE & TORINO & 1ST. ST. & CALUMET - LAURIUM \\
\hline 2580 & & CONTRATTO & KATE & SER & 16 & MICHIGAN & ITALY & ITALY & & SERVANT/PF & LOCANA CANAVESE. TO & 1ST. ST. & CALUMET - LAURIUM \\
\hline 2581 & 116 & TINETTI & PETER & $\mathrm{H}$ & 58 & ITALY & ITALY & ITALY & 1883 & LABORER/CM & SAN MARTINO CANAVESE. TO & OSCEOLA ST. & CALUMET - LAURIUM \\
\hline 2582 & & TINETTI & ANGELINE & w & 54 & ITALY & ITALY & ITALY & 1888 & & & OSCEOLA ST. & CALUMET - LAURIUM \\
\hline
\end{tabular}




\begin{tabular}{|c|c|c|c|c|c|c|c|c|c|c|c|c|c|}
\hline 2583 & & TINETTI & JOHANNA & $\mathrm{D}$ & 29 & MICHIGAN & ITALY & ITALY & & DRESSMAKER/OUT & SAN MARTINO CANAVESE. TO & OSCEOLA ST. & CALUMET - LAURIUM \\
\hline 2584 & & TINETTI & ANTON & $\mathrm{s}$ & 28 & MICHIGAN & ITALY & ITALY & & DIMOND DRILLER/CM & SAN MARTINO CANAVESE. TO & OSCEOLA ST. & CALUMET - LAURIUM \\
\hline 2585 & & TINETTI & MARY & D & 18 & MICHIGAN & ITALY & ITALY & & CLERK/RETAIL GROCERY & SAN MARTINO CANAVESE. TO & OSCEOLA ST. & CALUMET - LAURIUM \\
\hline 2586 & & TINETTI & JOHN & $\mathrm{s}$ & 22 & MICHIGAN & ITALY & ITALY & & TIMBERMAN/CM & SAN MARTINO CANAVESE. TO & OSCEOLA ST. & CALUMET - LAURIUM \\
\hline 2587 & & TINETTI & MAUDE & D & 16 & MICHIGAN & ITALY & ITALY & & & SAN MARTINO CANAVESE. TO & OSCEOLA ST. & CALUMET - LAURIUM \\
\hline 2588 & 136 & DELL'ANGELICA & ROSE & $\mathrm{H}$ & 67 & ITALY & ITALY & ITALY & $77 / 79$ & & & OSCEOLA ST. & CALUMET - LAURIUM \\
\hline 2589 & & DELL'ANGELICA & GILBERT & s & 23 & MICHIGAN & ITALY & ITALY & & LABORER/CM & LUCCA & OSCEOLA ST. & CALUMET - LAURIUM \\
\hline 2590 & & PUPILLI & ERMINDO & SOIL & 35 & ITALY & ITALY & ITALY & 1906 & MINER/CM & FIRENZE & OSCEOLA ST. & CALUMET - LAURIUM \\
\hline 2591 & & PUPILLI & LINA & D & 29 & MICHIGAN & ITALY & ITALY & 1000 & & LUCCA & OSCEOLA ST. & CALUMET - LAURIUM \\
\hline 2592 & 146 & TENAGLIA & CAMILLO & $\mathrm{H}$ & 62 & ITALY & ITALY & ITALY & 1895 & AGENT/BOOKS & & & CALUMET - AHMEEK \\
\hline 2593 & 126 & BIANCO & MINNIE & $\mathrm{H}$ & 55 & ITALY & ITALY & ITALY & 1894 & LAUNDRESS/OUT & TORINO & HECLA ST. & CALUMET - LAURIUM \\
\hline 2594 & & BIANCO & JOHN & $\mathrm{S}$ & 25 & MICHIGAN & ITALY & ITALY & & LABORER SURFACE/CM & SCARMAGNO. TO & HECLA ST. & CALUMET - LAURIUM \\
\hline 2595 & & BIANCO & GENEVIEVE & DIL & 25 & MICHIGAN & ITALY & ITALY & & & & HECLA ST. & CALUMET - LAURIUM \\
\hline 2596 & & BIANCO & PAULINE & $\mathrm{D}$ & 19 & MICHIGAN & ITALY & ITALY & & & SCARMAGNO. TO & HECLA ST. & CALUMET - LAURIUM \\
\hline 2597 & 125 & ZUBIENA & JAMES & $\mathrm{H}$ & 48 & ITALY & ITALY & ITALY & 1906 & RETAIL MERCHANT/GROCERY & TORINO & HECLA ST. & CALUMET - LAURIUM \\
\hline 2598 & & ZUBIENA & JOHN & $\mathrm{S}$ & 24 & ITALY & ITALY & ITALY & 1911 & TEAMSTER/GROCERY STORE & TORINO & HECLA ST. & CALUMET - LAURIUM \\
\hline 2599 & & ZUBIENA & KATE & D & 22 & ITALY & ITALY & ITALY & 1911 & & TORINO & HECLA ST. & CALUMET - LAURIUM \\
\hline 2600 & & ZUBIENA & MARY & D & 20 & ITALY & ITALY & ITALY & 1911 & SALESLADY/GROCERY STORE & TORINO & HECLA ST. & CALUMET - LAURIUM \\
\hline 2601 & 111 & VAIRO & DOMENICO & $\mathrm{H}$ & 29 & ITALY & ITALY & ITALY & 1890 & NOT CLEAR & LOCANA CANAVESE. TO & HECLA ST. & CALUMET - LAURIUM \\
\hline 2602 & & VAIRO & BIANCA & w & 29 & MICHIGAN & ITALY & ITALY & & & & HECLA ST. & CALUMET - LAURIUM \\
\hline 2603 & & VAIRO & LIZ & $\mathrm{D}$ & 2 & MICHIGAN & ITALY & MICHIGAN & & & LOCANA CANAVESE. TO & HECLA ST. & CALUMET - LAURIUM \\
\hline 2604 & & VAIRO & ROBERT & $\mathrm{s}$ & 1 & MICHIGAN & ITALY & MICHIGAN & & & LOCANA CANAVESE, TO & HECLA ST. & CALUMET - LAURIUM \\
\hline 2605 & 109 & TALENTINO & FRANK & $\mathrm{H}$ & 74 & ITALY & ITALY & ITALY & 1901 & & PARELLA. TO & HECLA ST. & CALUMET - LAURIUM \\
\hline 2606 & & TALENTINO & DELFINA & w & 53 & ITALY & ITALY & ITALY & 1903 & & PARELLA. TO & HECLA ST. & CALUMET - LAURIUM \\
\hline 2607 & & TALENTINO & PETER & $\mathrm{s}$ & 32 & ITALY & ITALY & ITALY & 1901 & CLERK/BANK & PARELLA. TO & HECLA ST. & CALUMET - LAURIUM \\
\hline 2608 & & TALENTINO & MARINO & $\mathrm{s}$ & 22 & ITALY & ITALY & ITALY & 1903 & & PARELLA. TO & HECLA ST. & CALUMET - LAURIUM \\
\hline 2609 & 238 & MICHELA & GEORGE & $\mathrm{H}$ & 57 & ITALY & ITALY & ITALY & 1887 & LABORER/LAURIUM VILLAGE & SAN GIORGIO CANAVESE. TO & CALUMET ST. & CALUMET - LAURIUM \\
\hline 2610 & & MICHELA & VITTORIA & W & 50 & ITALY & ITALY & ITALY & 1890 & & & CALUMET ST. & CALUMET - LAURIUM \\
\hline 2611 & & MICHELA & VITTORIO & $\mathrm{s}$ & 15 & MICHIGAN & ITALY & ITALY & & CLEANER/DRY CLEANING & SAN GIORGIO CANAVESE. TO & CALUMET ST. & CALUMET - LAURIUM \\
\hline 2612 & & MICHELA & MINNIE & D & 12 & MICHIGAN & ITALY & ITALY & & & SAN GIORGIO CANAVESE. TO & CALUMET ST. & CALUMET - LAURIUM \\
\hline 2613 & 247 & CIAGNE & MARTIN & $\mathrm{H}$ & 43 & ITALY & ITALY & ITALY & 1896 & PROPRIETOR/HOTEL & TORINO & OSCEOLA ST. & CALUMET - LAURIUM \\
\hline 2614 & & CIAGNE & ANGELINA & w & 33 & MICHIGAN & ITALY & ITALY & & COOK/HOTEL & & OSCEOLA ST. & CALUMET - LAURIUM \\
\hline 2615 & & CIAGNE & ADELINA & $\mathrm{D}$ & 14 & MICHIGAN & ITALY & MICHIGAN & & & TORINO & OSCEOLA ST. & CALUMET - LAURIUM \\
\hline 2616 & & CIAGNE & ANNA & $\mathrm{D}$ & 11 & MICHIGAN & ITALY & MICHIGAN & & & TORINO & OSCEOLA ST. & CALUMET - LAURIUM \\
\hline 2617 & & CIAGNE & ELANA & $\mathrm{D}$ & 7 & MICHIGAN & ITALY & MICHIGAN & & & TORINO & OSCEOLA ST. & CALUMET - LAURIUM \\
\hline 2618 & & CIAGNE & ARTURO & $\mathrm{s}$ & 5 & MICHIGAN & ITALY & MICHIGAN & & & TORINO & OSCEOLA ST. & CALUMET - LAURIUM \\
\hline 2619 & 64 & SALOTTI & CARLO & $\mathrm{H}$ & 32 & MICHIGAN & ITALY & ITALY & & PROPRIETOR/CONFECTIONARY & TORINO & 3RD ST. & CALUMET - LAURIUM \\
\hline 2620 & & SALOTTI & TILLIE & w & 25 & MICHIGAN & ITALY & ITALY & & CLERK/CONFECTIONARY & & 3RD ST. & CALUMET - LAURIUM \\
\hline 2621 & 209 & TORREANO & JOHN & $\mathrm{H}$ & 42 & ITALY & ITALY & ITALY & 1902 & BAKER/OWN SHOP & TORINO & HECLA ST. & CALUMET - LAURIUM \\
\hline 2622 & & TORREANO & CAROLINA & W & 30 & ITALY & ITALY & ITALY & 1903 & & & HECLA ST. & CALUMET - LAURIUM \\
\hline 2623 & & TORREANO & JAMES & $\mathrm{s}$ & 19 & MICHIGAN & ITALY & ITALY & & & TORINO & HECLA ST. & CALUMET - LAURIUM \\
\hline 2624 & & TORREANO & UMBERTO & $\mathrm{s}$ & 15 & MICHIGAN & ITALY & ITALY & & WASHER/COPPER MILL & TORINO & HECLA ST. & CALUMET - LAURIUM \\
\hline 2625 & & TORREANO & LUIGI & $\mathrm{s}$ & 7 & MICHIGAN & ITALY & ITALY & & & TORINO & HECLA ST. & CALUMET - LAURIUM \\
\hline 2626 & & TORREANO & PIERINA & D & 17 & MICHIGAN & ITALY & ITALY & & & TORINO & HECLA ST. & CALUMET - LAURIUM \\
\hline 2627 & & CONTA & JOHN & $\mathrm{H}$ & 51 & ITALY & ITALY & ITALY & 1888 & BAKER/BAKERY & TORINO & HECLA ST. & CALUMET - LAURIUM \\
\hline 2628 & & CONTA & TERESA & w & 40 & ITALY & ITALY & ITALY & 1905 & & & HECLA ST. & CALUMET - LAURIUM \\
\hline 2629 & & CONTA & MARY & $\mathrm{D}$ & 8 & MICHIGAN & ITALY & ITALY & & & TORINO & HECLA ST. & CALUMET - LAURIUM \\
\hline 2630 & & CONTA & JOHN & $\mathrm{s}$ & 5 & MICHIGAN & ITALY & ITALY & & & TORINO & HECLA ST. & CALUMET - LAURIUM \\
\hline 2631 & 328 & TAMBORINO & DOMENICO & $\mathrm{H}$ & 35 & ITALY & $\begin{array}{l}\text { ITALY } \\
\text { ITAL }\end{array}$ & $\begin{array}{l}\text { ITALY } \\
\text { ITAL }\end{array}$ & 1913 & PROPRIETOR/MEAT MARKET & LOCANA CANAVESE. TO & OSCEOLA ST. & CALUMET - LAURIUM \\
\hline 2632 & & TAMBORINO- & MARY & W & 32 & ITALY & ITALY & ITALY & 1919 & & LOCANA CANAVESE. TO & OSCEOLA ST. & CALUMET - LAURIUM \\
\hline 2633 & & TAMBORINO & $\mathrm{JOHN}$ & $\mathrm{s}$ & 10 & ITALY & ITALY & ITALY & 1919 & & LOCANA CANAVESE. TO & OSCEOLA ST. & CALUMET - LAURIUM \\
\hline 2634 & & TAMBORINO & MARY & $\mathrm{D}$ & 7 & ITALY & ITALY & ITALY & 1919 & & LOCANA CANAVESE, TO & OSCEOLA ST. & CALUMET - LAURIUM \\
\hline 2635 & 325 & RICHETTA & JAMES & $\mathrm{H}$ & 42 & ITALY & ITALY & ITALY & 1886 & PROPRIETOR/LIVERY & SAN MARTINO CANAVESE. TO & HECLA ST. & CALUMET - LAURIUM \\
\hline 2636 & & RICHETTA & DOMENICA & W & 37 & ITALY & ITALY & ITALY & 1900 & & & HECLA ST. & CALUMET - LAURIUM \\
\hline 2637 & & RICHETTA & MINNIE & D & 17 & MICHIGAN & ITALY & ITALY & & & SAN MARTINO CANAVESE. TO & HECLA ST. & CALUMET - LAURIUM \\
\hline 2638 & & RICHETTA & KATE & D & 14 & MICHIGAN & ITALY & ITALY & & & SAN MARTINO CANAVESE. TO & HECLA ST. & CALUMET - LAURIUM \\
\hline 2639 & & RICHETTA & MIKE & $\mathrm{s}$ & 10 & MICHIGAN & ITALY & ITALY & & & SAN MARTINO CANAVESE. TO & HECLA ST. & CALUMET - LAURIUM \\
\hline 2640 & & CENTANINO & JAMES & BIL & 36 & ITALY & ITALY & ITALY & 1906 & ROCK HOUSE MAN & SAN MARTINO CANAVESE, TO & HECLA ST. & CALUMET - LAURIUM \\
\hline 2641 & & MORETTO & PAUL & B & 53 & ITALY & ITALY & ITALY & 1891 & STABLE HAND/LIVERY & & HECLA ST. & CALUMET - LAURIUM \\
\hline 2642 & & RICHETTA & JAMES & $\mathrm{H}$ & 38 & ITALY & ITALY & ITALY & 1886 & UNDERTAKER & SAN MARTINO CANAVESE. TO & HECLA ST. & CALUMET - LAURIUM \\
\hline 2643 & & RICHETTA & MARION & w & 32 & ITALY & ITALY & ITALY & 1906 & & SAN MARTINO CANAVESE. TO & HECLA ST. & CALUMET - LAURIUM \\
\hline 2644 & & RICHETTA & ANGELA & s & 11 & MICHIGAN & ITALY & ITALY & & & SAN MARTINO CANAVESE. TO & HECLA ST. & CALUMET - LAURIUM \\
\hline 2645 & & RICHETTA & VITTORIA & MO & 60 & ITALY & ITALY & ITALY & 1886 & & SAN MARTINO CANAVESE. TO & HECLA ST. & CALUMET - LAURIUM \\
\hline
\end{tabular}




\begin{tabular}{|c|c|c|c|c|c|c|c|c|c|c|c|c|c|}
\hline 2646 & 323 & MARTA & KATE & $\mathrm{H}$ & 56 & ITALY & ITALY & ITALY & 1888 & PROPRIETOR/BAKERY & SAN GIOVANNI CANAVESE. TO & HECLA ST. & CALUMET - LAURIUM \\
\hline 2647 & & MARTA & JOHN & s & 26 & MICHIGAN & ITALY & ITALY & & BAKER/BAKERY & TORINO & HECLA ST. & CALUMET - LAURIUM \\
\hline 2648 & & MARTA & LOUIS & $\mathrm{s}$ & 25 & MICHIGAN & ITALY & ITALY & & TEAMSTER/BAKERY & TORINO & HECLA ST. & CALUMET - LAURIUM \\
\hline 2649 & & MARTA & ESUMA? & D & 17 & MICHIGAN & ITALY & ITALY & & & TORINO & HECLA ST. & CALUMET - LAURIUM \\
\hline 2650 & & MARTA & ADOLFO & $\mathrm{s}$ & 15 & MICHIGAN & ITALY & ITALY & & & TORINO & HECLA ST. & CALUMET - LAURIUM \\
\hline 2651 & 320 & SALOTTI & JAMES & $\mathrm{H}$ & 70 & ITALY & ITALY & ITALY & 1883 & & CORIO. TO & CALUMET ST. & CALUMET - LAURIUM \\
\hline 2652 & & ADAMS & MATTHEW & SOIL & 27 & MICHIGAN & AUSTRIA & AUSTRIA & & MINER/CM & AUSTRIA & CALUMET ST. & CALUMET - LAURIUM \\
\hline 2653 & & ADAMS & MARY & $\mathrm{D}$ & 27 & MICHIGAN & ITALY & ITALY & & & CORIO. TO & CALUMET ST. & CALUMET - LAURIUM \\
\hline 2654 & & ADAMS & ROBERT & GS & $7 \mathrm{M}$ & MICHIGAN & ITALY & ITALY & & & CORIO. TO & CALUMET ST. & CALUMET - LAURIUM \\
\hline 2655 & 306 & RENALDI & $\mathrm{JOHN}$ & $\mathrm{H}$ & 45 & ITALY & ITALY & ITALY & 1895 & MANAGER/GROCERY STORE & SAN GIORGIO CANAVESE. TO & OSCEOLA ST. & CALUMET - LAURIUM \\
\hline 2656 & & RENALDI & MARY & w & 45 & ITALY & ITALY & ITALY & 1895 & 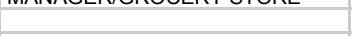 & SAN GIORGIO CANAVESE. TO & OSCEOLA ST. & CALUMET - LAURIUM \\
\hline 2657 & & RENALDI & JOSEPH & s & 15 & MICHIGAN & ITALY & ITALY & 1095 & TEAMSTER/GROCERY STORE & SAN GIORGIO CANAVESE. TO & OSCEOLA ST. & CALUMET - LAURIUM \\
\hline 2658 & & RENALDI & MARGHERITA & $\mathrm{D}$ & 11 & MICHIGAN & ITALY & ITALY & & & SAN GIORGIO CANAVESE. TO & OSCEOLA ST. & CALUMET - LAURIUM \\
\hline 2659 & & MARTA & ROSE & $\mathrm{D}$ & 22 & ITALY & ITALY & ITALY & 1895 & & TORINO & OSCEOLA ST. & CALUMET - LAURIUM \\
\hline 2660 & & MARTA & ROBERT & GS & 4 & MICHIGAN & ITALY & ITALY & & & TORINO & OSCEOLA ST. & CALUMET - LAURIUM \\
\hline 2661 & 415 & MERLO & JOHN & $\mathrm{H}$ & 36 & ITALY & ITALY & ITALY & 1905 & BAKER/BAKERY & RIVAROLO CANAVESE, TO & OSCEOLA ST. & CALUMET - LAURIUM \\
\hline 2662 & & MERLO-ROSSO & TERESA & W & 30 & ITALY & ITALY & ITALY & 1909 & CANDY STORE/AT HOME & & OSCEOLA ST. & CALUMET - LAURIUM \\
\hline 2663 & & MERLO & MARY & $\mathrm{D}$ & 9 & MICHIGAN & ITALY & ITALY & & & RIVAROLO CANAVESE. TO & OSCEOLA ST. & CALUMET - LAURIUM \\
\hline 2664 & & MERLO & CARLO & $\mathrm{s}$ & 8 & MICHIGAN & ITALY & ITALY & & & RIVAROLO CANAVESE. TO & OSCEOLA ST. & CALUMET - LAURIUM \\
\hline 2665 & & MERLO & ANTONIO & $\mathrm{s}$ & 6 & MICHIGAN & ITALY & ITALY & & & RIVAROLO CANAVESE. TO & OSCEOLA ST. & CALUMET - LAURIUM \\
\hline 2666 & & MERLO & EUGENIA & $\mathrm{D}$ & 4 & MICHIGAN & ITALY & ITALY & & & RIVAROLO CANAVESE. TO & OSCEOLA ST. & CALUMET - LAURIUM \\
\hline 2667 & & MERLO & MANNIE & $\mathrm{D}$ & 2 & MICHIGAN & ITALY & ITALY & & & RIVAROLO CANAVESE, TO & OSCEOLA ST. & CALUMET - LAURIUM \\
\hline 2668 & 418 & O'CONNOR & AMELIA & $\mathrm{H}$ & 37 & MICHIGAN & ITALY & ITALY & & NURSE & & OSCEOLA ST. & CALUMET - LAURIUM \\
\hline 2669 & & O'CONNOR & FRANCIS & $\mathrm{s}$ & 13 & MICHIGAN & MICHIGAN & MICHIGAN & & & & OSCEOLA ST. & CALUMET - LAURIUM \\
\hline 2670 & & O'CONNOR & CLEMENTE & $\mathrm{s}$ & 11 & MICHIGAN & MICHIGAN & MICHIGAN & & & & OSCEOLA ST. & CALUMET - LAURIUM \\
\hline 2671 & & O'CONNOR & MARTIN & s & 9 & MICHIGAN & MICHIGAN & MICHIGAN & & & & OSCEOLA ST. & CALUMET - LAURIUM \\
\hline 2672 & 320 & BINANDO & JOHN & $\mathrm{H}$ & 58 & ITALY & ITALY & ITALY & 1890 & LABORER/COAL YARD & SAN GIORGIO CANAVESE. TO & OSCEOLA ST. & CALUMET - LAURIUM \\
\hline 2673 & 141 & GRINDATTI & VINCENZO & $\mathrm{H}$ & 29 & ITALY & ITALY & ITALY & 1900 & TAILOR/TAILOR SHOP & PONT CANAVESE, TO & OSCEOLA ST. & CALUMET - LAURIUM \\
\hline 2674 & & GRINDATTI & CLARA & w & 29 & MICHIGAN & CANADA & CANADA & & & CANADA & OSCEOLA ST. & CALUMET - LAURIUM \\
\hline 2675 & 109 & SCIACERO & DOMENICO & $\mathrm{H}$ & 41 & ITALY & ITALY & ITALY & 1886 & TIMBERMAN/CM & IVREA. TO & TAMARACK ST. & CALUMET - LAURIUM \\
\hline 2676 & & SCIACERO & ANGELA & W & 42 & ITALY & ITALY & ITALY & UN & & IVREA. TO & TAMARACK ST. & CALUMET - LAURIUM \\
\hline 2677 & & SCIACERO & PHYLLIS & $\mathrm{D}$ & 13 & MICHIGAN & ITALY & ITALY & & & IVREA. TO & TAMARACK ST. & CALUMET - LAURIUM \\
\hline 2678 & & SCIACERO & FRANK & $\mathrm{s}$ & 12 & MICHIGAN & ITALY & ITALY & & & IVREA. TO & TAMARACK ST. & CALUMET - LAURIUM \\
\hline 2679 & 123 & MASSOGLIA & DOMENICO & $\mathrm{H}$ & 35 & ITALY & ITALY & ITALY & 1904 & MINER/CM & SAN GIOVANNI CANAVESE, TO & TAMARACK ST. & CALUMET - LAURIUM \\
\hline 2680 & & MASSOGLIA & LENA & w & 36 & ITALY & ITALY & ITALY & 1914 & & & TAMARACK ST. & CALUMET - LAURIUM \\
\hline 2681 & & MASSOGLIA & ANGELINA & $\mathrm{D}$ & 13 & MICHIGAN & ITALY & ITALY & & & SAN GIOVANNI CANAVESE. TO & TAMARACK ST. & CALUMET - LAURIUM \\
\hline 2682 & & MASSOGLIA & JOHN & $\mathrm{s}$ & 9 & MICHIGAN & ITALY & ITALY & & & SAN GIOVANNI CANAVESE. TO & TAMARACK ST. & CALUMET - LAURIUM \\
\hline 2683 & & MASSOGLIA & LUCY & $\mathrm{D}$ & 7 & MICHIGAN & ITALY & ITALY & & & SAN GIOVANNI CANAVESE. TO & TAMARACK ST. & CALUMET - LAURIUM \\
\hline 2684 & & MASSOGLIA & JOSEPH & $\mathrm{s}$ & 5 & MICHIGAN & ITALY & ITALY & & & SAN GIOVANNI CANAVESE. TO & TAMARACK ST. & CALUMET - LAURIUM \\
\hline 2685 & 221 & GALLASERO & JOHN & $\mathrm{H}$ & 52 & ITALY & ITALY & ITALY & 1889 & JANITOR/BANK & PONT CANAVESE. TO & TAMARACK ST. & CALUMET - LAURIUM \\
\hline 2686 & & GALLASERO & ROSA & w & 52 & ITALY & ITALY & ITALY & 1892 & & & TAMARACK ST. & CALUMET - LAURIUM \\
\hline 2687 & & GALLASERO & MARY & D & 20 & MICHIGAN & ITALY & ITALY & & CLERK/GROCERY STORE & PONT CANAVESE. TO & TAMARACK ST. & CALUMET - LAURIUM \\
\hline 2688 & & GALLASERO & ELSIE & $\mathrm{D}$ & 18 & MICHIGAN & ITALY & ITALY & & SERVANT/PF & PONT CANAVESE. TO & TAMARACK ST. & CALUMET - LAURIUM \\
\hline 2689 & & GALLASERO & JOSEPH & $\mathrm{s}$ & 16 & MICHIGAN & ITALY & ITALY & & & PONT CANAVESE. TO & TAMARACK ST. & CALUMET - LAURIUM \\
\hline 2690 & & GALLASERO & ROSE & $\mathrm{D}$ & 11 & MICHIGAN & ITALY & ITALY & & & PONT CANAVESE. TO & TAMARACK ST. & CALUMET - LAURIUM \\
\hline 2691 & 233 & DE FILIPPI & MARY & SERV & 17 & ITALY & ITALY & ITALY & 1906 & SERVANT/PF & TORINO & TAMARACK ST. & CALUMET - LAURIUM \\
\hline 2692 & 124 & VENEGONE & JOHN & $\mathrm{H}$ & 43 & ITALY & ITALY & ITALY & 1904 & TIMBERMAN/CM & CUGGIANO. MILANO & KEARSARGE ST. & CALUMET - LAURIUM \\
\hline 2693 & & VENEGONE & MARY & w & 43 & ITALY & ITALY & ITALY & 1901 & & & KEARSARGE ST. & CALUMET - LAURIUM \\
\hline 2694 & & VENEGONE & FLORENCE & D & 5 & MICHIGAN & ITALY & ITALY & & & CUGGIANO. MILANO & KEARSARGE ST. & CALUMET - LAURIUM \\
\hline 2695 & & RICHETTA & PETER & $\mathrm{H}$ & 43 & ITALY & ITALY & ITALY & 1901 & TIMBERMAN/CM & SAN MARTINO CANAVESE. TO & KEARSARGE ST. & CALUMET - LAURIUM \\
\hline 2696 & & RICHETTA & LOUIS & W & 37 & ITALY & ITALY & ITALY & 1906 & & & KEARSARGE ST. & CALUMET - LAURIUM \\
\hline 2697 & & RICHETTA & ANTHONY & s & 13 & MICHIGAN & ITALY & ITALY & & & SAN MARTINO CANAVESE, TO & KEARSARGE ST. & CALUMET - LAURIUM \\
\hline 2698 & & RICHETTA & JOSEPH & s & 8 & MICHIGAN & ITALY & ITALY & & & SAN MARTINO CANAVESE. TO & KEARSARGE ST. & CALUMET - LAURIUM \\
\hline 2699 & & RICHETTA & ANNETTE & $\mathrm{D}$ & 5 & MICHIGAN & ITALY & ITALY & & & SAN MARTINO CANAVESE. TO & RGE ST. & CALUMET - LAURIUM \\
\hline 2700 & 110 & $\mathrm{BO}^{\prime}$ & JOHN & $\mathrm{H}$ & 38 & ITALY & ITALY & ITALY & 1903 & TEAMSTER/TOWNSHIP & PONT CANAVESE. TO & & CALUMET - LAURIUM \\
\hline 2701 & & $\mathrm{BO}^{\prime}$ & PALMA & W & 36 & ITALY & ITALY & ITALY & 1911 & & & RSARGE ST. & CALUMET - LAURIUM \\
\hline 2702 & & BO' & TRACY & D & 11 & MICHIGAN & ITALY & ITALY & & & PONT CANAVESE. TO & KEARSARGE ST. & CALUMET - LAURIUM \\
\hline 2703 & & BO' & JOHN & $\mathrm{s}$ & 7 & MICHIGAN & ITALY & ITALY & & & PONT CANAVESE, TO & KEARSARGE ST. & CALUMET - LAURIUM \\
\hline 2704 & 104 & CAPELLO & CARLO & $\mathrm{H}$ & 64 & ITALY & ITALY & ITALY & 1884 & FIREMAN/ROUNDHOUSE & CUORGNE'. TO & KEARSARGE ST. & CALUMET - LAURIUM \\
\hline 2705 & & CAPELLO & MARIA & W & 62 & ITALY & ITALY & ITALY & 1886 & & CUORGNE'. TO & KEARSARGE ST. & CALUMET - LAURIUM \\
\hline 2706 & & BONGERA & KATE & $\mathrm{H}$ & 43 & ITALY & ITALY & ITALY & 1910 & & SALASSA. TO & KEARSARGE ST. & CALUMET - LAURIUM \\
\hline 2707 & & BONGERA & ANTONIA & $\mathrm{D}$ & 18 & ITALY & ITALY & ITALY & 1910 & MACHINE OPERATOR/STOCKING & SALASSA. TO & KEARSARGE ST. & CALUMET - LAURIUM \\
\hline 2708 & & BONGERA & MARIA & $\mathrm{D}$ & 11 & ITALY & ITALY & ITALY & 1910 & & SALASSA. TO & KEARSARGE ST. & CALUMET - LAURIUM \\
\hline
\end{tabular}




\begin{tabular}{|c|c|c|c|c|c|c|c|c|c|c|c|c|c|}
\hline 2709 & & ROSSIO & JAMES & $\mathrm{H}$ & 44 & ITALY & ITALY & ITALY & 1900 & CARPENTER/CM & PONT CANAVESE. TO & KEARSARGE ST. & CALUMET - LAURIUM \\
\hline 2710 & & ROSSIO & KATE & W & 46 & ITALY & ITALY & ITALY & 1902 & & PONT CANAVESE. TO & KEARSARGE ST. & CALUMET - LAURIUM \\
\hline 2711 & & ROSSIO & CARLO & $\mathrm{s}$ & 26 & ITALY & ITALY & ITALY & $81 / 91$ & CONDUCTOR/STREET RAILWAY & PONT CANAVESE. TO & KEARSARGE ST. & CALUMET - LAURIUM \\
\hline 2712 & & ROSSIO & FRANK & $\mathrm{s}$ & 17 & MICHIGAN & ITALY & ITALY & & TEAMSTER/GENERAL STORE & PONT CANAVESE. TO & KEARSARGE ST. & CALUMET - LAURIUM \\
\hline 2713 & & ROSSIO & MORRIS & $\mathrm{s}$ & 15 & MICHIGAN & ITALY & ITALY & & TEAMSTER/GENERAL STORE & PONT CANAVESE. TO & KEARSARGE ST. & CALUMET - LAURIUM \\
\hline 2714 & & ROSSIO & LUCY & D & 7 & MICHIGAN & ITALY & ITALY & & & PONT CANAVESE. TO & KEARSARGE ST. & CALUMET - LAURIUM \\
\hline 2715 & & COSTA & FERDINANDO & B & 28 & ITALY & ITALY & ITALY & 1911 & MACHINIST/RAILROAD SHOP & TORINO & KEARSARGE ST. & CALUMET - LAURIUM \\
\hline 2716 & & SANDRETTO & PETER & $\mathrm{B}$ & $\begin{array}{ll}20 \\
47\end{array}$ & ITALY & ITALY & ITALY & 1897 & LABORER/DAY WORK & PONT CANAVESE. TO & KEARSARGE ST. & CALUMET - LAURIUM \\
\hline 2717 & & MASSA & CARLO & B & 41 & ITALY & ITALY & ITALY & 1917 & MINER/CM & TORINO & KEARSARGE ST. & CALUMET - LAURIUM \\
\hline 2718 & 100 & QUELLO & BAT & $\mathrm{H}$ & 46 & ITALY & ITALY & ITALY & 1890 & PROPRIETOR/SOFT DRINK & LOCANA CANAVESE. TO & KEARSARGE ST. & CALUMET - LAURIUM \\
\hline 2719 & & QUELLO & MARY & w & 44 & ITALY & ITALY & ITALY & 1910 & & & KEARSARGE ST. & CALUMET - LAURIUM \\
\hline 2720 & & QUELLO & JOHN & $\mathrm{s}$ & 18 & MICHIGAN & ITALY & ITALY & & & LOCANA CANAVESE. TO & KEARSARGE ST. & CALUMET - LAURIUM \\
\hline 2721 & & QUELLO & MARY & $\mathrm{D}$ & 17 & MICHIGAN & ITALY & ITALY & & & LOCANA CANAVESE. TO & KEARSARGE ST. & CALUMET - LAURIUM \\
\hline 2722 & & QUELLO & SILVIO & $\mathrm{s}$ & 16 & MICHIGAN & ITALY & ITALY & & LABORER/CM & LOCANA CANAVESE. TO & KEARSARGE ST. & CALUMET - LAURIUM \\
\hline 2723 & & QUELLO & ALICE & $\mathrm{D}$ & 12 & MICHIGAN & ITALY & ITALY & & & LOCANA CANAVESE. TO & KEARSARGE ST. & CALUMET - LAURIUM \\
\hline 2724 & & QUELLO & JAMES & $\mathrm{S}$ & 5 & MICHIGAN & ITALY & ITALY & & & LOCANA CANAVESE, TO & KEARSARGE ST. & CALUMET - LAURIUM \\
\hline 2725 & $47 ?$ & MARTA & BATTISTA & $\mathrm{H}$ & 55 & ITALY & ITALY & ITALY & 1885 & PROPRIETOR/MEAT MARKET & SAN MARTINO CANAVESE. TO & KEARSARGE ST. & CALUMET - LAURIUM \\
\hline 2726 & & TINETTI & GIOVANNA & D & 30 & MICHIGAN & ITALY & ITALY & & & SAN MARTINO CANAVESE. TO & KEARSARGE ST. & CALUMET - LAURIUM \\
\hline 2727 & & TINETTI & MARTIN & SOIL & 49 & ITALY & ITALY & ITALY & 1888 & MINER/CM & SAN GIOVANNI CANAVESE. TO & KEARSARGE ST. & CALUMET - LAURIUM \\
\hline 2728 & & MARTA & JOHN & S & 26 & MICHIGAN & ITALY & ITALY & 1000 & IVIITLETCIVI & SAN MARTINO CANAVESE. TO & KEARSARGE ST. & CALUMET - LAURIUM \\
\hline 2729 & 105 & DELL'ACQUA & MARY & $\mathrm{H}$ & 20 & MICHIGAN & ITALY & ITALY & & SALESLADY/FRUIT STORE & CUGGIANO. MILANO & KEARSARGE ST. & CALUMET - LAURIUM \\
\hline 2730 & 100 & DELL'ACQUA & LINA & SI & 17 & MICHIGAN & $\begin{array}{l}\text { ITALY } \\
\text { ITAL }\end{array}$ & ITALY & & SALESLADY/FRUIT STORE & CUGGIANO, MILANO & KEARSARGE ST. & CALUMET - LAURIUM \\
\hline 2731 & & DELL'ACQUA & JOSEPHINE & SI & 13 & MICHIGAN & ITALY & ITALY & & & CUGGIANO. MILANO & KEARSARGE ST. & CALUMET - LAURIUM \\
\hline 2732 & & DELL'ACQUA & EDITH & SI & 12 & MICHIGAN & ITALY & ITALY & & & CUGGIANO. MILANO & KEARSARGE ST. & CALUMET - LAURIUM \\
\hline 2733 & & DELL'ACQUA & ROSE & SI & 9 & MICHIGAN & ITALY & ITALY & & & CUGGIANO. MILANO & KEARSARGE ST. & CALUMET - LAURIUM \\
\hline 2734 & 109 & SULLIVAN & THOMAS & H & 27 & NEW YORK & NEW YORK & NEW YORK & & LABORER/CM & USA & KEARSARGE ST. & CALUMET - LAURIUM \\
\hline 2735 & & SULLIVAN & MARY & W & 20 & ITALY & ITALY & ITALY & ???? & & & KEARSARGE ST. & CALUMET - LAURIUM \\
\hline 2736 & & SULLIVAN & ESELIA? & $\mathrm{D}$ & 2 & MICHIGAN & NEW YORK & NEW YORK & & & & KEARSARGE ST. & CALUMET - LAURIUM \\
\hline 2737 & 111 & ROLANDO & JOHN & $\mathrm{H}$ & 54 & ITALY & ITALY & ITALY & 1890 & SHOEMAKER & PONT CANAVESE. TO & KEARSARGE ST. & CALUMET - LAURIUM \\
\hline 2738 & & ROLANDO & TRACY & W & 47 & ITALY & ITALY & ITALY & 1900 & & & KEARSARGE ST. & CALUMET - LAURIUM \\
\hline 2739 & & ROLANDO & JOHN & $\mathrm{s}$ & 18 & MICHIGAN & ITALY & ITALY & & PLUMBER/HARDWARE STORE & PONT CANAVESE. TO & KEARSARGE ST. & CALUMET - LAURIUM \\
\hline 2740 & & ROLANDO & KATE & $\mathrm{D}$ & 13 & MICHIGAN & ITALY & ITALY & & & PONT CANAVESE. TO & KEARSARGE ST. & CALUMET - LAURIUM \\
\hline 2741 & 115 & TRIONE & PAUL & $\mathrm{H}$ & 28 & MICHIGAN & ITALY & ITALY & & MANAGER/DRY GOODS & TORINO & KEARSARGE ST. & $\begin{array}{l}\text { CALUMET - LAURIUM } \\
\text { CAUM }\end{array}$ \\
\hline 2742 & 170 & TRIONE & KATE & W & 26 & MICHIGAN & $\begin{array}{l}\text { ITALY } \\
\text { ITAL }\end{array}$ & ITALY & & 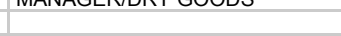 & TURIINO & KEARSARGE ST. & CALUMET - LAURIUM \\
\hline 2743 & & TRIONE & GENEVIEVE & D & 5 & MICHIGAN & MICHIGAN & MICHIGAN & & & TORINO & KEARSARGE ST. & CALUMET - LAURIUM \\
\hline 2744 & 119 & CASERIO & MARTINO & $\mathrm{H}$ & 50 & ITALY & ITALY & ITALY & 1899 & TIMBER BOSS/CM & STRAMBINELLO. TO & KEARSARGE ST. & CALUMET - LAURIUM \\
\hline 2745 & & CASERIO & JOHANNA & W & 49 & ITALY & ITALY & ITALY & 1906 & & STRAMBINELLO. TO & KEARSARGE ST. & CALUMET - LAURIUM \\
\hline 2746 & & CASERIO & JOSEPH & s & 28 & ITALY & ITALY & ITALY & 1906 & MERCHANT/GENRAL STORE & STRAMBINELLO. TO & KEARSARGE ST. & CALUMET - LAURIUM \\
\hline 2747 & & CASERIO & MARY & DIL & 26 & MICHIGAN & ITALY & ITALY & & & STRAMBINELLO. TO & KEARSARGE ST. & CALUMET - LAURIUM \\
\hline 2748 & & CASERIO & MARTIN & GS & 3 & MICHIGAN & ITALY & ITALY & & & STRAMBINELLO. TO & KEARSARGE ST. & CALUMET - LAURIUM \\
\hline 2749 & & CASERIO & JOHN & GD & $6 \mathrm{M}$ & MICHIGAN & ITALY & ITALY & & & STRAMBINELLO. TO & KEARSARGE ST. & CALUMET - LAURIUM \\
\hline 2750 & 123 & ROLANDO & TONY & $\mathrm{H}$ & 35 & ITALY & ITALY & ITALY & 1890 & MEAT CUTTER/REATIL MARKET & PONT CANAVESE. TO & KEARSARGE ST. & CALUMET - LAURIUM \\
\hline 2751 & & ROLANDO & LINA & W & 35 & ITALY & ITALY & ITALY & $87 / 97$ & & & KEARSARGE ST. & CALUMET - LAURIUM \\
\hline 2752 & & ROLANDO & VIRGINIA & D & 12 & MICHIGAN & ITALY & ITALY & & & PONT CANAVESE. TO & KEARSARGE ST. & CALUMET - LAURIUM \\
\hline 2753 & & ROLANDO & COSTANCE & $\mathrm{D}$ & 11 & MICHIGAN & ITALY & ITALY & & & PONT CANAVESE. TO & KEARSARGE ST. & CALUMET - LAURIUM \\
\hline 2754 & & ROLANDO & PAUL & $\mathrm{s}$ & 5 & MICHIGAN & ITALY & ITALY & & & PONT CANAVESE. TO & KEARSARGE ST. & CALUMET - LAURIUM \\
\hline 2755 & & ROLANDO & PETER & s & 3 & MICHIGAN & ITALY & ITALY & & & PONT CANAVESE. TO & KEARSARGE ST. & CALUMET - LAURIUM \\
\hline 2756 & & ROLANDO & TONY & $\mathrm{s}$ & 1 & MICHIGAN & ITALY & ITALY & & & AVESE. TO & EST. & CALUMET - LAI \\
\hline 2757 & 121 & SANDRETTO & PETER & $\mathrm{H}$ & 31 & MICHIGAN & ITALY & ITALY & & LABORER/CM & PONT CANAVESE. TO & RSARGE ST. & CALUMET - LAL \\
\hline 2758 & 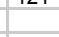 & SANDRETTO & IRENE & w & 19 & MICHIGAN & ITALY & ITALY & & 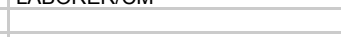 & & EST. & CALUMET - LAURIUM \\
\hline 2759 & & SANDRETTO & PETER & $\mathrm{S}$ & 2 & MICHIGAN & MICHIGAN & MICHIGAN & & & PONT CANAVESE. TO & KEARSARGE ST. & CALUMET - LAURIUM \\
\hline 2760 & & SANDRETTO & GENEVIEVE & $\mathrm{D}$ & 9M & MICHIGAN & MICHIGAN & MICHIGAN & & & PONT CANAVESE, TO & KEARSARGE ST. & CALUMET - LAURIUM \\
\hline 2761 & 127 & RICHETTA & ANTONIO & $\mathrm{H}$ & 30 & ITALY & ITALY & ITALY & $00 ? ?$ & LABORER/DAY WORK & SAN MARTINO CANAVESE. TO & KEARSARGE ST. & CALUMET - LAURIUM \\
\hline 2762 & & RICHETTA & TERESA & W & 27 & MICHIGAN & ITALY & ITALY & & & & KEARSARGE ST. & CALUMET - LAURIUM \\
\hline 2763 & & RICHETTA & MARION & D & 4 & MICHIGAN & ITALY & MICHIGAN & & & SAN MARTINO CANAVESE. TO & KEARSARGE ST. & CALUMET - LAURIUM \\
\hline 2764 & & RICHETTA & VITTORIA & D & 2 & MICHIGAN & ITALY & MICHIGAN & & & SAN MARTINO CANAVESE. TO & KEARSARGE ST. & CALUMET - LAURIUM \\
\hline 2765 & 129 & ANDRINA & DOMENICO & $\mathrm{H}$ & 57 & ITALY & ITALY & ITALY & 1892 & & TORINO & KEARSARGE ST. & CALUMET - LAURIUM \\
\hline 2766 & 120 & ANDRINA & HECTOR & $\mathrm{s}$ & 23 & MICHIGAN & ITALY & ITALY & 1002 & MINER/CM & TORINO & KEARSARGE ST. & CALUMET - LAURIUM \\
\hline 2767 & & ANDRINA & MARY & D & 17 & MICHIGAN & ITALY & ITALY & & & TORINO & KEARSARGE ST. & CALUMET - LAURIUM \\
\hline 2768 & 131 & PERENCHIO & MARIA & $\mathrm{H}$ & 48 & ITALY & ITAL & ITALY & 1896 & & PAVONE CA & KEARSARGE ST. & CALUMET - LAURIUM \\
\hline 2769 & & PERENCH & & $\mathrm{s}$ & 21 & HIGAN & ITA & ITA & & & PAVONE & ET. & IUM \\
\hline 2770 & & PERENCHIO & $\mathrm{GAl}$ & $\mathrm{s}$ & 15 & MICHIGAN & ITA & & & & AVESE. TO & AARGE ST. & IUM \\
\hline 2771 & 140 & SAPINO & PETER & $\mathrm{H}$ & 35 & ITALY & ITALY & ITALY & 1905 & MINER/CM & SAN MARTINO CANAVESE. TO & HECLA ST. & CALUMET - LAURIUM \\
\hline
\end{tabular}




\begin{tabular}{|c|c|c|c|c|c|c|c|c|c|c|c|c|c|}
\hline 2772 & & SAPINO & ANNE & W & 21 & ITALY & ITALY & ITALY & 1902 & & & HECLA ST. & CALUMET - LAURIUM \\
\hline 2773 & & SAPINO & DOMENICO & $\mathrm{s}$ & 2 & MICHIGAN & ITALY & ITALY & & & SAN MARTINO CANAVESE. TO & HECLA ST. & CALUMET - LAURIUM \\
\hline 2774 & & FERRARI & JOSEPH & $\mathrm{H}$ & 49 & ITALY & ITALY & ITALY & 1893 & FIREMAN/BOILER HOUSE & LUCCA & HECLA ST. & CALUMET - LAURIUM \\
\hline 2775 & & FERRARI & ITALIA & W & 49 & ITALY & ITALY & ITALY & 1898 & & & HECLA ST. & CALUMET - LAURIUM \\
\hline 2776 & & FERRARI & LOUIS & s & 17 & MICHIGAN & ITALY & ITALY & & TEAMSTER/GROCERY & LUCCA & HECLA ST. & CALUMET - LAURIUM \\
\hline 2777 & & NARDI & ARMANDO & $\mathrm{H}$ & 23 & MICHIGAN & ITALY & ITALY & & MINER/CM & LUCCA & HECLA ST. & CALUMET - LAURIUM \\
\hline 2778 & & NARDI-FERRARI & MIDA & W & 22 & MICHIGAN & ITALY & ITALY & & & & HECLA ST. & CALUMET - LAURIUM \\
\hline 2779 & 134 & TORREANO & FRANK & $\mathrm{H}$ & 28 & MICHIGAN & ITALY & ITALY & & OPERATOR/GAS ENGINE & TORINO & HECLA ST. & CALUMET - LAURIUM \\
\hline 2780 & 104 & TORREANO & SOFIA & W & 28 & MICHIGAN & CANADA & CANADA & & UTEKATUN UAD EIVUIIVE & CANADA & HECLA ST. & CALUMET - LAURIUM \\
\hline 2781 & & TORREANO & JOHN & s & 1 & MICHIGAN & MICHIGAN & MICHIGAN & & & TORINO & HECLA ST. & CALUMET - LAURIUM \\
\hline 2782 & & BALAGNA & HOMER & BIL & 30 & MICHIGAN & ITALY & ITALY & & CARPENTER & PONT CANAVESE. TO & HECLA ST. & CALUMET - LAURIUM \\
\hline 2783 & 132 & TINETTI & BATTISTA & $\mathrm{H}$ & 56 & ITALY & ITALY & ITALY & 1885 & LABORER & SAN MARTINO CANAVESE. TO & HECLA ST. & CALUMET - LAURIUM \\
\hline 2784 & & TINETTI & ANTONIETTA & W & 42 & ITALY & ITALY & ITALY & 1890 & & & HECLA ST. & CALUMET - LAURIUM \\
\hline 2785 & & TINETTI & FRANK & $\mathrm{s}$ & 26 & MICHIGAN & ITALY & ITALY & & & SAN MARTINO CANAVESE. TO & HECLA ST. & CALUMET - LAURIUM \\
\hline 2786 & & TINETTI & ENRICO & $\mathrm{S}$ & 11 & MICHIGAN & ITALY & ITALY & & & SAN MARTINO CANAVESE. TO & HECLA ST. & CALUMET - LAURIUM \\
\hline 2787 & & TINETTI & TERESA & D & 9 & MICHIGAN & ITALY & ITALY & & & SAN MARTINO CANAVESE, TO & HECLA ST. & CALUMET - LAURIUM \\
\hline 2788 & & TINETTI & EMMA & D & 2 & MICHIGAN & ITALY & ITALY & & & SAN MARTINO CANAVESE. TO & HECLA ST. & CALUMET - LAURIUM \\
\hline 2789 & 130 & MARTA & MARTIN & $\mathrm{H}$ & 41 & ITALY & ITALY & ITALY & 1906 & LABORER/ROCK HOUSE & SAN GIOVANNI CANAVESE. TO & HECLA ST. & CALUMET - LAURIUM \\
\hline 2790 & & MARTA & JOSEPHINE & W & 31 & MICHIGAN & ITALY & ITALY & & & & HECLA ST. & CALUMET - LAURIUM \\
\hline 2791 & & MARTA & TERESA & $\mathrm{D}$ & 7 & MICHIGAN & ITALY & MICHIGAN & & & SAN GIOVANNI CANAVESE. TO & HECLA ST. & CALUMET - LAURIUM \\
\hline 2792 & & MARTA & PETER & $\mathrm{s}$ & 4 & MICHIGAN & ITALY & MICHIGAN & & & SAN GIOVANNI CANAVESE. TO & HECLA ST. & CALUMET - LAURIUM \\
\hline 2793 & 128 & ROVANO & DOMENICO & $\mathrm{H}$ & 34 & ITALY & ITALY & ITALY & 1902 & MINER/CM & SAN MARTINO CANAVESE, TO & HECLA ST. & CALUMET - LAURIUM \\
\hline 2794 & & ROVANO & TERESA & W & 30 & MICHIGAN & ITALY & ITALY & & 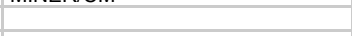 & & HECLA ST. & CALUMET - LAURIUM \\
\hline 2795 & & ROVANO & FRANCIS & D & 11 & MICHIGAN & ITALY & MICHIGAN & & & SAN MARTINO CANAVESE. TO & HECLA ST. & CALUMET - LAURIUM \\
\hline 2796 & & ROVANO & MONICA & $\mathrm{D}$ & 7 & MICHIGAN & ITALY & MICHIGAN & & & SAN MARTINO CANAVESE. TO & HECLA ST. & CALUMET - LAURIUM \\
\hline 2797 & & ROVANO & JOSEPH & $\mathrm{s}$ & 3 & MICHIGAN & ITALY & MICHIGAN & & & SAN MARTINO CANAVESE. TO & HECLA ST. & CALUMET - LAURIUM \\
\hline 2798 & & ROVANO & MARIA & $\mathrm{D}$ & $6 \mathrm{M}$ & MICHIGAN & ITALY & MICHIGAN & & & SAN MARTINO CANAVESE. TO & HECLA ST. & CALUMET - LAURIUM \\
\hline 2799 & 116 & YOTTI & DELPHINE & $\mathrm{H}$ & 55 & CANADA & CANADA & CANADA & 1879 & & CANADA & 1ST ST. & CALUMET - LAURIUM \\
\hline 2800 & & YOTTI & EDWARD & $\mathrm{S}$ & 18 & MICHIGAN & ITALY & CANADA & & MEAT CUTTER/RETAIL MARKET & & 1ST ST. & CALUMET - LAURIUM \\
\hline 2801 & 114 & SERRA & MIKE & $\mathrm{H}$ & 56 & ITALY & ITALY & ITALY & 1888 & MINER/CM & SAN GIORGIO CANAVESE. TO & 1ST ST. & CALUMET - LAURIUM \\
\hline 2802 & & SERRA & MADDALENA & W & 56 & ITALY & ITALY & ITALY & 1889 & & SAN GIORGIO CANAVESE. TO & 1ST ST. & CALUMET - LAURIUM \\
\hline 2803 & 71 & YOTTI & PETER & $\mathrm{H}$ & 34 & MICHIGAN & ITALY & ITALY & & LABORER/FREIGHT DEPOT & & 1ST ST. & CALUMET - LAURIUM \\
\hline 2804 & & YOTTI & MARY & W & 36 & MICHIGAN & ITALY & ITALY & & & & 1ST ST. & CALUMET - LAURIUM \\
\hline 2805 & & YOTTI & HENRY & $\mathrm{s}$ & 10 & MICHIGAN & MICHIGAN & MICHIGAN & & & & 1ST ST. & CALUMET - LAURIUM \\
\hline 2806 & & YOTTI & VALERIE & $\mathrm{D}$ & 8 & MICHIGAN & MICHIGAN & MICHIGAN & & & & 1ST ST. & CALUMET - LAURIUM \\
\hline 2807 & & YOTTI & EVELYN & D & 6 & MICHIGAN & MICHIGAN & MICHIGAN & & & & 1ST ST. & CALUMET - LAURIUM \\
\hline 2808 & & YOTTI & LAWRENCE & $\mathrm{S}$ & 4 & MICHIGAN & MICHIGAN & MICHIGAN & & & & 1ST ST. & CALUMET - LAURIUM \\
\hline 2809 & & YOTTI & MARIE & D & 1 & MICHIGAN & MICHIGAN & MICHIGAN & & & & 1 ST ST. & CALUMET - LAURIUM \\
\hline 2810 & 75 & SALICETTI & ANGELO & $\mathrm{H}$ & 48 & ITALY & ITALY & ITALY & 1892 & PROPRIETOR/MEAT MARKET & PEROSA. TO & 1ST ST. & CALUMET - LAURIUM \\
\hline 2811 & & SALICETTI & PAOLA & W & 39 & ITALY & ITALY & ITALY & 1887 & & & 1ST ST. & CALUMET - LAURIUM \\
\hline 2812 & & SALICETTI & ALBERT & $\mathrm{s}$ & 11 & MICHIGAN & ITALY & ITALY & & & PEROSA. TO & 1ST ST. & CALUMET - LAURIUM \\
\hline 2813 & & SALICETTI & NICK & $\mathrm{s}$ & 4 & MICHIGAN & ITALY & ITALY & & & PEROSA. TO & 1ST ST. & CALUMET - LAURIUM \\
\hline 2814 & & CARBONATTO & CARLO & $\mathrm{H}$ & 46 & ITALY & ITALY & ITALY & 1898 & PROPRIETOR/MEAT MARKET & PONT CANAVESE. TO & 1ST ST. & CALUMET - LAURIUM \\
\hline 2815 & & CARBONATTO & MARIA & W & 43 & ITALY & ITALY & ITALY & 1898 & & PONT CANAVESE. TO & 1 ST ST. & CALUMET - LAURIUM \\
\hline 2816 & & CARBONATTO & PHYLLIS & D & 17 & MICHIGAN & ITALY & ITALY & & & PONT CANAVESE. TO & 1ST ST. & CALUMET - LAURIUM \\
\hline 2817 & 218 & BRONZO & JOHN & $\mathrm{H}$ & 48 & ITALY & ITALY & ITALY & UN & LABORER/CM & SAN MARTINO CANAVESE. TO & 1ST ST. & CALUMET - LAURIUM \\
\hline 2818 & & BRONZO & MARY & w & 48 & ITALY & ITALY & ITALY & UN & & SAN MARTINO CANAVESE. TO & 1ST ST. & CALUMET - LAURIUM \\
\hline 2819 & & BRONZO & JOSEPH & $\mathrm{s}$ & 26 & MICHIGAN & ITALY & ITALY & & BLACKSMITH/CM & SAN MARTINO CANAVESE. TO & 1ST ST. & CALUMET - LAURIUM \\
\hline 2820 & & BRONZO & PETER & B & 33 & ITALY & ITALY & ITALY & $07 / 17$ & LABORER/CM & SAN MARTINO CANAVESE. TO & 1ST ST. & CALUMET - LAURIUM \\
\hline 2821 & & BRONZO & JOSEPHINE & B & 32 & MICHIGAN & ITALY & ITALY & & & SAN MARTINO CANAVESE. TO & KEARSARGE ST. & CALUMET - LAURIUM \\
\hline & 221 & FERRARI & SILVIO & $\mathrm{H}$ & 52 & ITALY & ITALY & ITALY & 1896 & MINER/CM & LUCCA & KEARSARGE ST. & CALUMET - LAURIUM \\
\hline 2823 & & FERRARI & MARIA & W & 32 & ITALY & ITALY & ITALY & 1902 & & & KEARSARGE ST. & CALUMET - LAURIUM \\
\hline 2824 & & FERRARI & LEONELLO & $\mathrm{D}$ & 16 & MICHIGAN & ITALY & ITALY & & SALESLADY/DRY GOODS RETAIL & LUCCA & KEARSARGE ST. & CALUMET - LAURIUM \\
\hline 2825 & & FERRARI & ALBERT & $\mathrm{s}$ & 14 & MICHIGAN & ITALY & ITALY & & & LUCCA & KEARSARGE ST. & CALUMET - LAURIUM \\
\hline 2826 & & FERRARI & ELENA & D & 11 & MICHIGAN & ITALY & ITALY & & & LUCCA & KEARSARGE ST. & CALUMET - LAURIUM \\
\hline 2827 & & FERRARI & GUIDO & $\mathrm{s}$ & $10 \mathrm{M}$ & MICHIGAN & ITALY & ITALY & & & LUCCA & KEARSARGE ST. & CALUMET - LAURIUM \\
\hline 2828 & 233 & BARSANTI & PASCAL & $\mathrm{H}$ & 39 & ITALY & ITALY & ITALY & 1903 & PROPRIETOR/FRUIT STORE & LUCCA & KEARSARGE ST. & CALUMET - LAURIUM \\
\hline 2829 & & BARSANTI & MARY & W & 31 & ITALY & ITALY & ITALY & 1911 & & & KEARSARGE ST. & CALUMET - LAURIUM \\
\hline 2830 & & BARSANTI & LORENZO & $\mathrm{s}$ & 13 & MICHIGAN & ITALY & ITALY & & & LUCCA & KEARSARGE ST. & CALUMET - LAURIUM \\
\hline 2831 & & BARSANTI & RINA & $\mathrm{D}$ & 12 & MICHIGAN & ITALY & ITALY & & & LUCCA & KEARSARGE ST. & CALUMET - LAURIUM \\
\hline 2832 & & BARSANTI & ELSIE & D & 9 & MICHIGAN & ITA & ITAI & & & LUCCA & KEARSARG & URIUM \\
\hline 2833 & 337 & ZEI & ZEFFIRO & $\mathrm{H}$ & 28 & ITALY & ITALY & ITALY & 1905 & TAILOR & LUCCA & KEARSARGE ST. & CALUMET - LAURIUM \\
\hline 2834 & & ZEI & DINA & W & 28 & ITALY & ITALY & ITALY & 1897 & & & KEARSARGE ST. & CALUMET - LAURIUM \\
\hline
\end{tabular}




\begin{tabular}{|c|c|c|c|c|c|c|c|c|c|c|c|c|c|}
\hline 2835 & & ZEI & ARMANDO & $\mathrm{s}$ & 1 & MICHIGAN & ITALY & ITALY & & & LUCCA & KEARSARGE ST. & CALUMET - LAURIUM \\
\hline 2836 & 208 & NIGRA & TERESA & $\mathrm{H}$ & 50 & ITALY & ITALY & ITALY & 1900 & & SAN GIUSTO CANAVESE. TO & HECLA ST. & CALUMET - LAURIUM \\
\hline 2837 & & NIGRA & DOMENICO & $\mathrm{s}$ & 18 & MICHIGAN & ITALY & ITALY & & & SAN GIUSTO CANAVESE. TO & HECLA ST. & CALUMET - LAURIUM \\
\hline 2838 & & NIGRA & MARIA & D & 16 & MICHIGAN & ITALY & ITALY & & & SAN GIUSTO CANAVESE. TO & HECLA ST. & CALUMET - LAURIUM \\
\hline 2839 & & NIGRA & RINO & $\mathrm{s}$ & 9 & MICHIGAN & ITALY & ITALY & & & SAN GIUSTO CANAVESE. TO & HECLA ST. & CALUMET - LAURIUM \\
\hline 2840 & & DIERO & SABINO & B & 26 & MICHIGAN & ITALY & ITALY & & LABORER/AUTO SHOP & SAN GIUSTO CANAVESE. TO & KEARSARGE ST. & CALUMET - LAURIUM \\
\hline 2841 & 315 & LOCATELLI & GUIDO & $\mathrm{H}$ & 36 & ITALY & ITALY & ITALY & 1910 & MINER/CM & SELINO. BERGAMO & KEARSARGE ST. & CALUMET - LAURIUM \\
\hline 2842 & 年 & LOCATELLI & KATE & W & 33 & ITALY & ITALY & ITALY & 1911 & IVIIVERTULIVI & SELINU. DERUAAIVIO & KEARSARGE ST. & CALUMET - LAURIUM \\
\hline 2843 & & LOCATELLI & LETIZIA & $\mathrm{D}$ & 6 & MICHIGAN & ITALY & ITALY & & & SELINO. BERGAMO & KEARSARGE ST. & CALUMET - LAURIUM \\
\hline 2844 & & LOCATELLI & EMIL & $\mathrm{s}$ & 4 & MICHIGAN & ITALY & ITALY & & & SELINO. BERGAMO & KEARSARGE ST. & CALUMET - LAURIUM \\
\hline 2845 & & LOCATELLI & MARIO & $\mathrm{s}$ & 3 & MICHIGAN & ITALY & ITALY & & & SELINO. BERGAMO & KEARSARGE ST. & CALUMET - LAURIUM \\
\hline 2846 & & GALLASSERO & CARLO & $\mathrm{H}$ & 58 & ITALY & ITALY & ITALY & 95?? & LABORER/CM & PONT CANAVESE. TO & KEARSARGE ST. & CALUMET - LAURIUM \\
\hline 2847 & & GALLASSERO & LUISA & W & 50 & ITALY & ITALY & ITALY & 1907 & & & KEARSARGE ST. & CALUMET - LAURIUM \\
\hline 2848 & & GALLASSERO & CARLO & $\mathrm{s}$ & 21 & MICHIGAN & ITALY & ITALY & & CLERK/EMPLOYMENT OFFICE & PONT CANAVESE. TO & KEARSARGE ST. & CALUMET - LAURIUM \\
\hline 2849 & & GALLASSERO & LUISA & $\mathrm{D}$ & 19 & MICHIGAN & ITALY & ITALY & & $\begin{array}{l}\text { SERVANT/PF } \\
\end{array}$ & PONT CANAVESE. TO & KEARSARGE ST. & CALUMET - LAURIUM \\
\hline 2850 & 324 & RICHETTA & JOSEPH & $\mathrm{H}$ & 46 & ITALY & ITALY & ITALY & 1895 & MINER/CM & SAN MARTINO CANAVESE, TO & HECLA ST. & CALUMET - LAURIUM \\
\hline 2851 & & RICHETTA & DOMENICA & $\mathrm{s}$ & 11 & MICHIGAN & ITALY & ITALY & & & SAN MARTINO CANAVESE. TO & HECLA ST. & CALUMET - LAURIUM \\
\hline 2852 & & RICHETTA & LOUIS & $\mathrm{s}$ & 10 & MICHIGAN & ITALY & ITALY & & & SAN MARTINO CANAVESE. TO & HECLA ST. & CALUMET - LAURIUM \\
\hline 2853 & & RICHETTA & FRANK & $\mathrm{s}$ & 9 & MICHIGAN & ITALY & ITALY & & & SAN MARTINO CANAVESE. TO & HECLA ST. & CALUMET - LAURIUM \\
\hline 2854 & & RICHETTA & JENNIE & D & 7 & MICHIGAN & ITALY & ITALY & & & SAN MARTINO CANAVESE. TO & HECLA ST. & CALUMET - LAURIUM \\
\hline 2855 & & RICHETTA & MAUDE & $\mathrm{D}$ & 4 & MICHIGAN & ITALY & $\begin{array}{l}\text { ITALY } \\
\text { ITA }\end{array}$ & & & SAN MARTINO CANAVESE. TO & HECLA ST. & CALUMET - LAURIUM \\
\hline 2856 & & BERTANO & TERESA & SER & 25 & ITALY & ITALY & ITALY & 1915 & SERVANT/PF & & HECLA ST. & CALUMET - LAURIUM \\
\hline 2857 & 320 & QUELLO & DOMENICO & $\mathrm{H}$ & 55 & ITALY & ITALY & ITALY & 1904 & PROPRIETOR/SOFT DRINK & LOCANA CANAVESE. TO & HECLA ST. & CALUMET - LAUUIUM \\
\hline 2858 & & QUELLO & LUCY & W & 35 & ITALY & ITALY & ITALY & 1903 & & & HECLA ST. & CALUMET - LAURIUM \\
\hline 2859 & & QUELLO & ATTILIO & $\mathrm{s}$ & 11 & MICHIGAN & ITALY & ITALY & & & LOCANA CANAVESE. TO & HECLAST. & CALUMET - LAURIUM \\
\hline 2860 & & QUELLO & MARIANNE & D & 9 & MICHIGAN & ITALY & ITALY & & & LOCANA CANAVESE. TO & HECLA ST. & CALUMET - LAURIUM \\
\hline 2861 & & QUELLO & JAMES & $\mathrm{s}$ & 7 & MICHIGAN & ITALY & ITALY & & & LOCANA CANAVESE. TO & HECLA ST. & CALUMET - LAURIUM \\
\hline 2862 & & QUELLO & LUCY & D & 5 & MICHIGAN & ITALY & ITALY & & & LOCANA CANAVESE, TO & HECLA ST. & CALUMET - LAURIUM \\
\hline 2863 & & QUELLO & DOMENICO & $\mathrm{s}$ & 4 & MICHIGAN & ITALY & ITALY & & & LOCANA CANAVESE. TO & HECLA ST. & CALUMET - LAURIUM \\
\hline 2864 & 213 & RICHETTA & MARY & B & 2 & MICHIGAN & ITALY & ITALY & & & SAN MARTINO CANAVESE. TO & & CALUMET - LAURIUM \\
\hline 2865 & 104 & NOZERO & DOMENICO & $\mathrm{H}$ & 30 & ITALY & ITALY & ITALY & 1904 & MINER/CM & SAN MARTINO CANAVESE. TO & TAMARACK ST. & CALUMET - LAURIUM \\
\hline 2866 & & NOZERO & MONICA & w & 34 & MICHIGAN & ITALY & ITALY & & & & TAMARACK ST. & CALUMET - LAURIUM \\
\hline 2867 & & NOZERO & GERTRUDE & D & 15 & MICHIGAN & ITALY & MICHIGAN & & & SAN MARTINO CANAVESE. TO & TAMARACK ST. & CALUMET - LAURIUM \\
\hline 2868 & 106 & LOCATELLI & PETER & $\mathrm{H}$ & 44 & ITALY & ITALY & ITALY & 1902 & CONTRACTOR/MASON WORK & SELINO, BERGAMO & TAMARACK ST. & CALUMET - LAURIUM \\
\hline 2869 & & LOCATELLI & JULIA & w & 39 & ITALY & ITALY & ITALY & 1902 & & SELINO. BERGAMO & TAMARACK ST. & CALUMET - LAURIUM \\
\hline 2870 & & LOCATELLI & JOSEPHINE & $\mathrm{D}$ & 16 & MICHIGAN & ITALY & ITALY & & DOMESTIC/PF & SELINO. BERGAMO & TAMARACK ST. & CALUMET - LAURIUM \\
\hline 2871 & & LOCATELLI & SELMA & $\mathrm{D}$ & 15 & MICHIGAN & ITALY & ITALY & & 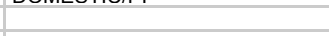 & SELINO. BERGAMO & TAMARACK ST. & CALUMET - LAURIUM \\
\hline 2872 & & LOCATELLI & ANTONIO & $\mathrm{s}$ & 12 & MICHIGAN & ITALY & ITALY & & & SELINO. BERGAMO & TAMARACK ST. & CALUMET - LAURIUM \\
\hline 2873 & & LOCATELLI & PETER & $\mathrm{s}$ & 11 & MICHIGAN & ITALY & ITALY & & & SELINO. BERGAMO & TAMARACK ST. & CALUMET - LAURIUM \\
\hline 2874 & & LOCATELLI & MARY & D & 7 & MICHIGAN & ITALY & ITALY & & & SELINO. BERGAMO & TAMARACK ST. & CALUMET - LAURIUM \\
\hline 2875 & & LOCATELLI & ENRICA & D & 5 & MICHIGAN & ITALY & ITALY & & & SELINO. BERGAMO & TAMARACK ST. & CALUMET - LAURIUM \\
\hline 2876 & 104 & BAGGIORE & FRANK & $\mathrm{H}$ & 62 & ITALY & ITALY & ITALY & 1880 & LABORER/RAILROAD YARD & TORINO & TAMARACK ST. & CALUMET - LAURIUM \\
\hline 2877 & & BAGGIORE & KATE & w & 52 & ITALY & ITALY & ITALY & 1880 & & TORINO & TAMARACK ST. & CALUMET - LAURIUM \\
\hline 2878 & & BAGGIORE & JOHN & s & 32 & MICHIGAN & ITALY & ITALY & & & TORINO & TAMARACK ST. & CALUMET - LAURIUM \\
\hline 2879 & & BAGGIORE & MARY & D & 30 & MICHIGAN & ITALY & ITALY & & & TORINO & TAMARACK ST. & CALUMET - LAURIUM \\
\hline 2880 & & BAGGIORE & JOSEPH & $\mathrm{s}$ & 29 & MICHIGAN & ITALY & ITALY & & CHAUFFEUR/PF & TORINO & TAMARACK ST. & CALUMET - LAURIUM \\
\hline 2881 & & BAGGIORE & DUILIA & D & 27 & MICHIGAN & ITALY & ITALY & & NURSE & TORINO & TAMARACK ST. & CALUMET - LAURIUM \\
\hline 2882 & & BAGGIORE & WILLIAM & $\mathrm{s}$ & 25 & MICHIGAN & ITALY & ITALY & & SALESMAN/GENERAL STORE & TORINO & TAMARACK ST. & CALUMET - LAURIUM \\
\hline 2883 & & BAGGIORE & ATTILIO & $\mathrm{s}$ & 22 & MICHIGAN & ITALY & ITALY & & & TORINO & TAMARACK ST. & CALUMET - LAURIUM \\
\hline 2884 & & BAGGIORE & CESARE & $\mathrm{s}$ & 19 & MICHIGAN & ITALY & ITALY & & & TORINO & TAMARACK ST. & CALUMET - LAURIUM \\
\hline 2885 & & TORREANO & PETER & B & 34 & MICHIGAN & ITALY & ITALY & & SALESMAN/GENERAL STORE & TORINO & TAMARACK ST. & CALUMET - LAURIUM \\
\hline 2886 & 116 & GALETTO & JOSEPH & $\mathrm{H}$ & 53 & ITALY & ITALY & ITALY & 88?? & MERCHANT/GENERAL STORE & SAN GIORGIO CANAVESE, TO & TAMARACK ST. & CALUMET - LAURIUM \\
\hline 2887 & & GALETTO & MARY & W & 45 & ITALY & ITALY & ITALY & 1894 & & & TAMARACK ST. & CALUMET - LAURIUM \\
\hline 2888 & & GALETTO & MARY & D & 23 & MICHIGAN & ITALY & ITALY & & SALESLADY/GENERAL STORE & SAN GIORGIO CANAVESE. TO & TAMARACK ST. & CALUMET - LAURIUM \\
\hline 2889 & & GALETTO & VITTORIA & D & 19 & MICHIGAN & ITALY & ITALY & & & SAN GIORGIO CANAVESE. TO & TAMARACK ST. & CALUMET - LAURIUM \\
\hline 2890 & & GALETTO & JOSEPH & s & 16 & MICHIGAN & ITALY & ITALY & & TEAMSTER/GENERAL STORE & SAN GIORGIO CANAVESE. TO & TAMARACK ST. & CALUMET - LAURIUM \\
\hline 2891 & & GALETTO & TERESA & D & 14 & MICHIGAN & ITALY & ITALY & & & SAN GIORGIO CANAVESE. TO & TAMARACK ST. & CALUMET - LAURIUM \\
\hline 2892 & & GALETTO & CARLO & s & 11 & MICHIGAN & ITALY & ITALY & & & SAN GIORGIO CANAVESE, TO & TAMARACK ST. & CALUMET - LAURIUM \\
\hline 2893 & & GALETTO & ANGELINA & $\mathrm{D}$ & 8 & MICHIGAN & ITALY & ITALY & & & SAN GIORGIO CANAVESE. TO & TAMARACK ST. & CALUMET - LAURIUM \\
\hline 2894 & 124 & TABACCHI & JAMES & $\mathrm{H}$ & 30 & ITALY & ITALY & ITALY & 1905 & BLACKSMITH/CM & STRAMBINELLO. TO & PEWABIC ST. & CALUMET - LAURIUM \\
\hline 395 & & TABACCHI & & W & 27 & HIGAN & ITAI & & & & & PEWABI & CALUMET - LAURIUM \\
\hline 996 & & TABACCHI & & s & 4 & MICHIGAN & ITALY & $\mathrm{MICl}$ & & & STRAMBINELLO. TO & PEWABIC ST. & CALUM \\
\hline 997 & & TABACCHI & ROBERT & $\mathrm{s}$ & 3 & MICHIGAN & ITALY & MICHIGAN & & & STRAMBINELLO. TO & PEWABIC ST. & CALUMET - LAURIUM \\
\hline
\end{tabular}




\begin{tabular}{|c|c|c|c|c|c|c|c|c|c|c|c|c|c|}
\hline 2898 & 208 & NIGRA & KATE & SER & 23 & MICHIGAN & ITALY & ITALY & & SERVANT/PF & TORINO & PEWABIC ST. & CALUMET - LAURIUM \\
\hline 2899 & 240 & GALLASERO & ELSIE & SERV & 18 & MICHIGAN & ITALY & ITALY & & SERVANT/PF & PONT CANAVESE. TO & & CALUMET - LAURIUM \\
\hline 2900 & 91 & CAPELLO & JOHANNA & $\mathrm{H}$ & 52 & ITALY & ITALY & ITALY & 1890 & & & 1ST ST. & CALUMET - LAURIUM \\
\hline 2901 & & CAPELLO & JAMES & $\mathrm{s}$ & 23 & MICHIGAN & ITALY & ITALY & & APPRENTICE/MACHINE SHOP & SAN BENIGNO CANAVESE. TO & 1ST ST. & CALUMET - LAURIUM \\
\hline 2902 & & CAPELLO & FRED & $\mathrm{s}$ & 21 & MICHIGAN & ITALY & ITALY & & PLASTERER & SAN BENIGNO CANAVESE. TO & 1ST ST. & CALUMET - LAURIUM \\
\hline 2903 & & CAPELLO & JOHN & $\mathrm{s}$ & 19 & MICHIGAN & ITALY & ITALY & & APPRENTICE/MACHINE SHOP & SAN BENIGNO CANAVESE. TO & 1ST ST. & CALUMET - LAURIUM \\
\hline 2904 & 104 & FAUSONE & PETER & $\mathrm{H}$ & 59 & ITALY & ITALY & ITALY & 1880 & 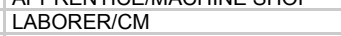 & $\begin{array}{l}\text { TORINO } \\
\text { TOIVAVLU. TO }\end{array}$ & IROQUOIS ST. & CALUMET - LAURIUM \\
\hline 2905 & 104 & FAUSONE & MARTA & W & 47 & ITALY & ITALY & ITALY & 1890 & LADURER/UIVI & TORIVU & IROQUOIS ST. & CALUMET - LAURIUM \\
\hline 2906 & & FAUSONE & PETER & s & 26 & MICHIGAN & ITALY & ITALY & & BUTCHER/MEAT MARKET & TORINO & IROQUOIS ST. & CALUMET - LAURIUM \\
\hline 2907 & & FAUSONE & JOHN & $\mathrm{s}$ & 19 & MICHIGAN & ITALY & ITALY & & SALESMAN/STORE & TORINO & IROQUOIS ST. & CALUMET - LAURIUM \\
\hline 2908 & & FAUSONE & DOMENICO & $\mathrm{s}$ & 21 & MICHIGAN & ITALY & ITALY & & MACHINIST/ROUNDHOUSE & TORINO & $\begin{array}{l}\text { IROQUOIS ST. } \\
\text { IROQ }\end{array}$ & CALUMET - LAURIUM \\
\hline 2909 & & FAUSONE & FRED & $\mathrm{s}$ & 17 & MICHIGAN & ITALY & ITALY & & BUTCHER/MEAT MARKET & TORINO & IROQUOIS ST. & CALUMET - LAURIUM \\
\hline 2910 & & FAUSONE & MINNIE & D & 14 & MICHIGAN & ITALY & ITALY & & STUDENT & TORINO & IROQUOIS ST. & CALUMET - LAURIUM \\
\hline 2911 & 107 & RICHETTA & MARTA & $\mathrm{H}$ & 40 & ITALY & ITALY & ITALY & UN & & TORINO & FLORIDA ST. & CALUMET - LAURIUM \\
\hline 2912 & & RICHETTA & TONY & $\mathrm{s}$ & 18 & MICHIGAN & ITALY & ITALY & & CLERK/DRUG STORE & SAN MARTINO CANAVESE. TO & FLORIDA ST. & CALUMET - LAURIUM \\
\hline 2913 & & RICHETTA & DOMENICO & $\mathrm{s}$ & 15 & MICHIGAN & ITALY & ITALY & & CLERKIDRUG STORE & SAN MARTINO CANAVESE, TO & FLORIDA ST. & CALUMET - LAURIUM \\
\hline 2914 & & RICHETTA & ANNE & D & 5 & MICHIGAN & ITALY & ITALY & & & SAN MARTINO CANAVESE. TO & FLORIDA ST. & CALUMET - LAURIUM \\
\hline 2915 & 99 & BARSANTI & GUIDO & $\mathrm{H}$ & 23 & ITALY & ITALY & ITALY & 1912 & MERCHANT/FRUIT STORE & LUCCA & 1ST ST. & CALUMET - LAURIUM \\
\hline 2916 & & BARSANTI & EMALINA? & W & 27 & ITALY & ITALY & ITALY & 1903 & & & 1ST ST. & CALUMET - LAURIUM \\
\hline 2917 & & BARSANTI & ELSIE & $\mathrm{D}$ & 6 & MICHIGAN & ITALY & ITALY & & & LUCCA & 1ST ST. & CALUMET - LAURIUM \\
\hline 2918 & & BARSANTI & JOHN & s & 4 & MICHIGAN & ITALY & ITALY & & & LUCCA & 1ST ST. & CALUMET - LAURIUM \\
\hline 2919 & & BARSANTI & LAWRENCE & FA & 71 & ITALY & ITALY & ITALY & 1912 & & LUCCA & 1ST ST. & CALUMET - LAURIUM \\
\hline 2920 & & BARSANTI & ALSIE & MO & 70 & ITALY & ITALY & ITALY & 1912 & & LUCCA & 1ST ST. & CALUMET - LAURIUM \\
\hline 2921 & & BARSANTI & $\mathrm{E}$ ? & $\mathrm{BR}$ & 25 & ITALY & ITALY & ITALY & 1912 & LABORER/CM & LUCCA & 1ST ST. & CALUMET - LAURIUM \\
\hline 2922 & 143 & SCIACERO & JOHN & $\mathrm{H}$ & 49 & ITALY & ITALY & ITALY & 1891 & STOREKEEPER & SAN MARTINO CANAVESE. TO & LAKE LINDEN AV. & CALUMET - LAURIUM \\
\hline 2923 & & SCIACERO & CLAIRE & SI & 47 & ITALY & ITALY & ITALY & UN & & SAN MARTINO CANAVESE. TO & LAKE LINDEN AV. & CALUMET - LAURIUM \\
\hline 2924 & 101 & VIGNETTO & JAMES & $\mathrm{H}$ & 46 & ITALY & ITALY & ITALY & 1890 & & TORINO & HECLA ST. & CALUMET - LAURIUM \\
\hline 2925 & & VIGNETTO & ROSE & W & 43 & FRANCE & FRANCE & FRANCE & 1880 & & FRANCE & HECLA ST. & CALUMET - LAURIUM \\
\hline 2926 & 151 & LUCCHESI & FELIX & $\mathrm{H}$ & 50 & ITALY & ITALY & ITALY & 1890 & MINER/CM & LUCCA & TAMARACK ST. & CALUMET - LAURIUM \\
\hline 2927 & & LUCCHESI & AMELIA & W & 40 & ITALY & ITALY & ITALY & UN & & LUCCA & TAMARACK ST. & CALUMET - LAURIUM \\
\hline 2928 & & LUCCHESI & MATILDA & $\mathrm{D}$ & 19 & MICHIGAN & ITALY & ITALY & & & LUCCA & TAMARACK ST. & CALUMET - LAURIUM \\
\hline 2929 & & LUCCHESI & JOSEPH & $\mathrm{s}$ & 17 & MICHIGAN & ITALY & ITALY & & TRAMMER/CM & LUCCA & TAMARACK ST. & CALUMET - LAURIUM \\
\hline 2930 & & LUCCHESI & PETER & $\mathrm{s}$ & 16 & MICHIGAN & ITALY & ITALY & & BAKER/BAKERY & LUCCA & TAMARACK ST. & CALUMET - LAURIUM \\
\hline 2931 & & LUCCHESI & RAFFAELLO & s & 15 & MICHIGAN & ITALY & ITALY & & DAREKRIDANERT & LUCCA & TAMARACK ST. & CALUMET - LAURIUM \\
\hline 2932 & & LUCCHESI & ANNE & $\mathrm{D}$ & 12 & MICHIGAN & ITALY & ITALY & & & LUCCA & TAMARACK ST. & CALUMET - LAURIUM \\
\hline 2933 & & LUCCHESI & FELIX & $\mathrm{s}$ & 10 & MICHIGAN & ITALY & ITALY & & & LUCCA & TAMARACK ST. & CALUMET - LAURIUM \\
\hline 2934 & 138 & SCUSSEL & DESIDERIO & $\mathrm{H}$ & 32 & ITALY & ITALY & ITALY & 1890 & MINER/CM & VOLTAGO. BELLUNO & KEARSARGE ST. & $\begin{array}{l}\text { CALUMET - LAUUIUM } \\
\end{array}$ \\
\hline 2935 & & SCUSSEL & ANNE & W & 30 & ITALY & ITALY & ITALY & UN & & VOLTAGO. BELLUNO & KEARSARGE ST. & CALUMET - LAURIUM \\
\hline 2936 & & SCUSSEL & IRENE & D & 10 & UTAH & ITALY & ITALY & & & VOLTAGO. BELLUNO & KEARSARGE ST. & CALUMET - LAURIUM \\
\hline 2937 & & SCUSSEL & ALICE & D & 9 & UTAH & ITALY & ITALY & & & VOLTAGO. BELLUNO & KEARSARGE ST. & CALUMET - LAURIUM \\
\hline 2938 & & SCUSSEL & AROLD & $\mathrm{S}$ & 4 & UTAH & ITALY & ITALY & & & VOLTAGO. BELLUNO & KEARSARGE ST. & CALUMET - LAURIUM \\
\hline 2939 & 413 & LOCATELLI & $\mathrm{JOHN}$ & $\mathrm{H}$ & 62 & ITALY & ITALY & ITALY & 1882 & LABORER/VILLAGE & SELINO. BERGAMO & LAKE LINDEN AV. & CALUMET - LAURIUM \\
\hline 2940 & & LOCATELLI & KATE & w & 60 & ITALY & ITALY & ITALY & 1894 & & SELINO. BERGAMO & LAKE LINDEN AV. & CALUMET - LAURIUM \\
\hline 2941 & & LOCATELLI & ITALIA? & D & 30 & ITALY & ITALY & ITALY & 1894 & & SELINO. BERGAMO & LAKE LINDEN AV. & CALUMET - LAURIUM \\
\hline 2942 & & LOCATELLI & ELISABETH & D & 23 & MICHIGAN & ITALY & ITALY & & & SELINO. BERGAMO & LAKE LINDEN AV. & CALUMET - LAURIUM \\
\hline 2943 & 5 & LUCCHESI & SAM & $\mathrm{H}$ & 55 & ITALY & ITALY & ITALY & 1879 & WATCHMAN/CM & LUCCA & HANCOCK ST. & CALUMET - LAURIUM \\
\hline 2944 & & LUCCHESI & MARGHERITA & W & 45 & ITALY & ITALY & ITALY & 1885 & & & HANCOCK ST. & CALUMET - LAURIUM \\
\hline 2945 & & LUCCHESI & MARY & D & 18 & MICHIGAN & ITALY & ITALY & & & LUCCA & HANCOCK ST. & CALUMET - LAURIUM \\
\hline 2946 & & LUCCHESI & EMMA & $\mathrm{D}$ & 17 & MICHIGAN & ITALY & ITALY & & COMPOSER/PRINT SHOP & LUCCA & HANCOCK ST. & CALUMET - LAURIUM \\
\hline 2947 & & LUCCHESI & SAM & $\mathrm{s}$ & 14 & MICHIGAN & ITALY & ITALY & & & LUCCA & HANCOCK ST. & CALUMET - LAURIUM \\
\hline 2948 & & LUCCHESI & MARGHERITA & $\mathrm{D}$ & 12 & MICHIGAN & ITALY & ITALY & & & LUCCA & HANCOCK ST. & CALUMET - LAURIUM \\
\hline 2949 & & LUCCHESI & ELEONOR & $\mathrm{D}$ & 5 & MICHIGAN & ITALY & ITALY & & & LUCCA & HANCOCK ST. & CALUMET - LAURIUM \\
\hline 2950 & 563 & MACMILLAIS & THERENCE & $\mathrm{H}$ & 33 & SCOTLAND & SCOTLAND & SCOTLAND & 1912 & CONDUCTOR/STREET RAILWAY & SCOTLAND & WYANDETTE ST. & CALUMET - LAURIUM \\
\hline 2951 & & PASQUINI & MARNIE & W & 26 & MICHIGAN & ITALY & ITALY & & & LUCCA & WYANDETTE ST. & CALUMET - LAURIUM \\
\hline 2952 & & PASQUINI & SERAPHINE & SIIL & 23 & MICHIGAN & ITALY & ITALY & & & LUCCA & WYANDETTE ST. & CALUMET - LAURIUM \\
\hline 2953 & 431 & BRUSSO & JOSEPH & $\mathrm{H}$ & 52 & ITALY & ITALY & ITALY & 1898 & MINER/CM & AGLIE'. TO & QUINCY ST. & CALUMET - LAURIUM \\
\hline 2954 & & BRUSSO & BARBARA & w & 49 & ITALY & ITALY & ITALY & 1898 & & AGLIE', TO & QUINCY ST. & CALUMET - LAURIUM \\
\hline 2955 & & BRUSSO & DOMENICO & s & 16 & MICHIGAN & ITALY & ITALY & & TRAMMER/CM & AGLIE', TO & QUINCY ST. & CALUMET - LAURIUM \\
\hline 2956 & & BRUSSO & JOSEPHINE & $\mathrm{D}$ & 14 & MICHIGAN & ITALY & ITALY & & & AGLIE', TO & QUINCY ST. & CALUMET - LAURIUM \\
\hline 2957 & 109 & STEFANI & ANGELA & $\mathrm{H}$ & 48 & ITALY & ITALY & ITALY & $08 / 11$ & & LUCCA & ALLOUEZ ST. & CALUMET \\
\hline 958 & & & ARMANDO & SSO & 19 & HIGAN & ITA & ITA & & LABORER/COAL DOCK & & DUEZ ST. & CAL \\
\hline 59 & & STEFANI & & $\mathrm{D}$ & 10 & MICHIGAN & ITALY & ITALY & & & LUCCA & & CALUM \\
\hline 60 & & STEFANI & STEVE & $\mathrm{s}$ & 8 & MICHIGAN & ITALY & ITALY & & & LUCCA & ALLOUEZ ST. & CALUMET - LAURIUM \\
\hline
\end{tabular}




\begin{tabular}{|c|c|c|c|c|c|c|c|c|c|c|c|c|c|}
\hline 2961 & & STEFANI & ANNE & $\mathrm{D}$ & 5 & MICHIGAN & ITALY & ITALY & & & LUCCA & ALLOUEZ ST. & CALUMET - LAURIUM \\
\hline 2962 & 120 & GHERNA & JOHN & $\mathrm{H}$ & 31 & ITALY & ITALY & ITALY & 1892 & MANAGER/TELEGRAPH OFFICE & TORINO & 5TH ST. & CALUMET - RED JACKET \\
\hline 2963 & & GHERNA & MARJORIE & W & 28 & MICHIGAN & ENGLAND & ENGLAND & & & ENGLAND & 5TH ST. & CALUMET - RED JACKET \\
\hline 2964 & & GHERNA & MARJORIE & $\mathrm{D}$ & 9 & MICHIGAN & ITALY & MICHIGAN & & & TORINO & 5TH ST. & CALUMET - RED JACKET \\
\hline 2965 & 120 & GRINDATTI & JAMES & $\mathrm{H}$ & 29 & ITALY & ITALY & ITALY & 1898 & MINER/CM & PONT CANAVESE. TO & 5TH ST. & CALUMET - RED JACKET \\
\hline 2966 & & GRINDATTI & MARGHERITA & w & 28 & NEW YORK & NEW YORK & IRELAND & & & USA-IRELAND & 5TH ST. & CALUMET - RED JACKET \\
\hline 2967 & 510 & TAMBELLINI & JOHN & $\mathrm{H}$ & 53 & ITALY & ITALY & ITALY & 1894 & PROPRIETOR/SOFT DRINK & LUCCA & PORTLAND ST. & CALUMET - RED JACKET \\
\hline 2968 & 30 & TAMBELLINI & CICLIDE & w & 44 & ITALY & ITALY & ITALY & 1895 & FRUR & LUCUA & PORTLAND ST. & CALUMET - RED JACKET \\
\hline 2969 & & TAMBELLINI & JEAN & $\mathrm{D}$ & 20 & MICHIGAN & ITALY & ITALY & & & LUCCA & PORTLAND ST. & CALUMET - RED JACKET \\
\hline 2970 & & TAMBELLINI & GIULIANO & $\mathrm{s}$ & 21 & MICHIGAN & ITALY & ITALY & & CLERK/SOFT DRINK & LUCCA & PORTLAND ST. & CALUMET - RED JACKET \\
\hline 2971 & & TAMBELLINI & UGO & $\mathrm{s}$ & 9 & MICHIGAN & ITALY & ITALY & & & LUCCA & PORTLAND ST. & CALUMET - RED JACKET \\
\hline 2972 & 206 & ORICIL/E & JAMES & $\mathrm{H}$ & 33 & MICHIGAN & USA & IRELAND & & VETERINARY & USA-IRELAND & 6TH ST. & CALUMET - RED JACKET \\
\hline 2973 & & ORICIL/E & KATE & W & 29 & MICHIGAN & ITALY & ITALY & & CASHIER/INSURANCE & & 6TH ST. & CALUMET - RED JACKET \\
\hline 2974 & 322 & FORSTER & WILLIAM & $\mathrm{H}$ & 33 & MICHIGAN & GERMANY & GERMANY & & MERCHANT/STORE & GERMANY & 5TH ST. & CALUMET - RED JACKET \\
\hline 2975 & & FORSTER & JANE & W & 32 & MICHIGAN & ITALY & ITALY & & & & 5TH ST. & CALUMET - RED JACKET \\
\hline 2976 & & FORSTER & NADINE & D & 7 & MICHIGAN & MICHIGAN & MICHIGAN & & & & 5TH ST. & CALUMET - RED JACKET \\
\hline 2977 & & FORSTER & WILLIAM & $\mathrm{s}$ & 5 & MICHIGAN & MICHIGAN & MICHIGAN & & & & 5TH ST. & CALUMET - RED JACKET \\
\hline 2978 & & CURTO & MINNIE & B & 35 & MICHIGAN & MICHIGAN & MICHIGAN & & SALESLADY & VIALFRE'. TO & 5TH ST. & CALUMET - RED JACKET \\
\hline 2979 & 330 & TOMMEI & PAUL & $\mathrm{H}$ & 46 & ITALY & ITALY & ITALY & 1888 & RETAIL MERCHANT/GROCERY & LUCCA & 5TH ST. & CALUMET - RED JACKET \\
\hline 2980 & & TOMMEI & MARY & w & 44 & ITALY & ITALY & ITALY & 1889 & & & 5TH ST. & CALUMET - RED JACKET \\
\hline 2981 & & TOMMEI & MARGHERITA & D & 19 & MICHIGAN & ITALY & ITALY & & & LUCCA & 5TH ST. & CALUMET - RED JACKET \\
\hline 2982 & & TOMMEI & PAUL & $\mathrm{s}$ & 14 & MICHIGAN & ITALY & ITALY & & & LUCCA & 5TH ST. & CALUMET - RED JACKET \\
\hline 2983 & 341 & BARSOTTI & DUSOLA & $\mathrm{H}$ & 59 & ITALY & ITALY & ITALY & 1883 & & LUCCA & 5TH ST. & CALUMET - RED JACKET \\
\hline 2984 & & BARSOTTI & PASQUALE & $\mathrm{s}$ & 35 & ILLINOIS & ITALY & ITALY & & MERCHANT/GROCERY & LUCCA & 5TH ST. & CALUMET - RED JACKET \\
\hline 2985 & & BARSOTTI & ARTURO & $\mathrm{s}$ & 33 & ILLINOIS & ITALY & ITALY & & MERCHANT/GROCERY & LUCCA & 5TH ST. & CALUMET - RED JACKET \\
\hline 2986 & & BARSOTTI & JENNIE & DIL & 32 & MICHIGAN & ITALY & ITALY & & & & 5TH ST. & CALUMET - RED JACKET \\
\hline 2987 & & BARSOTTI & ERCOLE & GS & 8 & MICHIGAN & ITALY & ITALY & & & LUCCA & 5TH ST. & CALUMET - RED JACKET \\
\hline 2988 & 331 & PALMINI & MIKE & $\mathrm{H}$ & 75 & ITALY & ITALY & ITALY & 1893 & GRINDER/STREET & & 6TH ST. & CALUMET - RED JACKET \\
\hline 2989 & 318 & GIACOLETTO & FRANCE & B & 33 & ITALY & ITALY & ITALY & 1900 & TAILORESS/TAILOR SHOP & TORINO & 6TH ST. & CALUMET - RED JACKET \\
\hline 2990 & 320 & VENTURINO & BLANCHE & $\mathrm{H}$ & 37 & WISCONSI & WISCONSI & WISCONSI & & & USA & 6TH ST. & CALUMET - RED JACKET \\
\hline 2991 & & VENTURINO & JOHN & $\mathrm{HU}$ & 29 & MICHIGAN & ITALY & WISCONSI & & MINER/CM & & 6TH ST. & CALUMET - RED JACKET \\
\hline 2992 & & COPPO & JOHN & $\mathrm{H}$ & 44 & MICHIGAN & ITALY & ITALY & & LAWYER/PRIVATE PRACTICE & PONT CANAVESE. TO & 6TH ST. & CALUMET - RED JACKET \\
\hline 2993 & & COPPO & MARY & SIST & 39 & MICHIGAN & ITALY & ITALY & & 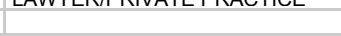 & PONT CANAVESE. TO & 6TH ST. & CALUMET - RED JACKET \\
\hline 2994 & & RASTELLO & COSTANCE & $\mathrm{H}$ & 68 & ITALY & ITALY & ITALY & 1880 & LABORER/STREET & PONT CANAVESE, TO & 6TH ST. & CALUMET - RED JACKET \\
\hline 2995 & & RASTELLO & KATE & w & 66 & ITALY & ITALY & ITALY & 1890 & & PONT CANAVESE. TO & 6TH ST. & CALUMET - RED JACKET \\
\hline 2996 & & RASTELLO & ROSE & $\mathrm{D}$ & 30 & ITALY & ITALY & ITALY & 1890 & SERVANT/HOTEL & PONT CANAVESE. TO & 6TH ST. & CALUMET - RED JACKET \\
\hline 2997 & 301 & LISA & MARTA & $\mathrm{H}$ & 68 & ITALY & ITALY & ITALY & 1879 & & SAN MARTINO CANAVESE. TO & 6TH ST. & CALUMET - RED JACKET \\
\hline 2998 & & LISA & JULIA & D & 38 & MICHIGAN & ITALY & ITALY & & CLERICAL WORK/BANK & SAN MARTINO CANAVESE. TO & 6TH ST. & CALUMET - RED JACKET \\
\hline 2999 & 331 & SUINO & CATERINA & $\mathrm{H}$ & 51 & ITALY & ITALY & ITALY & 1880 & & TORINO & 7TH ST. & CALUMET - RED JACKET \\
\hline 3000 & & SUINO & MARIA & $\mathrm{D}$ & 29 & MICHIGAN & ITALY & ITALY & & SECRETARY/HIGH SCHOOL & TORINO & 7TH ST. & CALUMET - RED JACKET \\
\hline 3001 & & SUINO & EDWARD & $\mathrm{S}$ & 18 & MICHIGAN & ITALY & ITALY & & & TORINO & 7TH ST. & CALUMET - RED JACKET \\
\hline 3002 & & SUINO & KATE & D & 17 & MICHIGAN & ITALY & ITALY & & & TORINO & 7TH ST. & CALUMET - RED JACKET \\
\hline 3003 & 808 & QUILICI & DOMENICO & $\mathrm{H}$ & 30 & ITALY & ITALY & ITALY & 1905 & TEAMSTER/HARDWARE & LANNARI. LU & PORTLAND ST. & CALUMET - RED JACKET \\
\hline 3004 & & QUILICI & JENNIE & w & 29 & ITALY & ITALY & ITALY & 1916 & & & PORTLAND ST. & CALUMET - RED JACKET \\
\hline 3005 & & QUILICI & MINNIE & D & 3 & MICHIGAN & ITALY & ITALY & & & LANNARI, LU & PORTLAND ST. & CALUMET - RED JACKET \\
\hline 3006 & & QUILICI & IOLANDA & $\mathrm{D}$ & $11 \mathrm{M}$ & MICHIGAN & ITALY & ITALY & & & LANNARI. LU & PORTLAND ST. & CALUMET - RED JACKET \\
\hline 3007 & & QUILICI & MINNIE & MO & 70 & ITALY & ITALY & ITALY & 1916 & & LANNARI. LU & PORTLAND ST. & CALUMET - RED JACKET \\
\hline 3008 & 812 & CURTO & DOMENICA & $\mathrm{H}$ & 39 & ITALY & ITALY & ITALY & 1896 & & & PORTLAND ST. & CALUMET - RED JACKET \\
\hline 3009 & & CURTO & ANGELINA & $\mathrm{D}$ & 21 & MICHIGAN & ITALY & ITALY & & SALESLADY/GENERAL STORE & TORINO & & CALUMET - RED JACKET \\
\hline 3010 & & CURTO & KATE & D & 20 & MICHIGAN & ITALY & ITALY & & SALESLADY/GENERAL STORE & TORINO & PORTLAND ST. & RED JACKET \\
\hline 3011 & & CURTO & VITTORIA & D & 16 & MICHIGAN & ITALY & ITALY & & & TORINO & PORTLAND ST. & CALUMET - RED JACKET \\
\hline 3012 & & CURTO & JULIA & D & 11 & MICHIGAN & ITALY & ITALY & & & TORINO & PORTLAND ST. & CALUMET - RED JACKET \\
\hline 3013 & 811 & TAMBELLINI & JOHN & $\mathrm{H}$ & 45 & ITALY & ITALY & ITALY & 1892 & TEAMSTER/WHOLESALE & SEGROMIGNO. LU & SCOTT ST. & CALUMET - RED JACKET \\
\hline 3014 & & TAMBELLINI & NATALINA & W & 37 & ITALY & ITALY & ITALY & 1898 & & & SCOTT ST. & CALUMET - RED JACKET \\
\hline 3015 & & TAMBELLINI & TRACY & D & 19 & MICHIGAN & ITALY & ITALY & & SALESMAN/FRUIT STORE & SEGROMIGNO. LU & SCOTT ST. & CALUMET - RED JACKET \\
\hline 3016 & & TAMBELLINI & PETER & s & 18 & MICHIGAN & ITALY & ITALY & & BAGGE MAN/RAILROAD & SEGROMIGNO. LU & SCOTT ST. & CALUMET - RED JACKET \\
\hline 3017 & & TAMBELLINI & LEONIA & D & 15 & MICHIGAN & ITALY & ITALY & & SALESLADY/FRUIT STORE & SEGROMIGNO. LU & SCOTT ST. & CALUMET - RED JACKET \\
\hline 3018 & & TAMBELLINI & PETER & s & 14 & MICHIGAN & ITALY & ITALY & & & SEGROMIGNO, LU & SCOTT ST. & CALUMET - RED JACKET \\
\hline 3019 & & DIANDA & JENNA & $\mathrm{H}$ & 29 & ILLINOIS & ITALY & ITALY & & & LUCCA & OAK ST. & CALUMET - RED JACKET \\
\hline 3020 & & DIANDA & VINCENZO & $\mathrm{s}$ & 3 & MICHIGAN & ITALY & ILLINOIS & & & LUCCA & OAK ST. & CALUMET \\
\hline 3021 & & DIANDA & MARJORIE & $\mathrm{D}$ & $7 \mathrm{M}$ & HIIGAN & ITA & NOIS & & & LUCCA & OAK ST. & CALUMET \\
\hline 3022 & 9 & KLENO?? & JULIA & $\mathrm{H}$ & 64 & ITALY & ITALY & ITALY & 1886 & & & 9TH ST. & CALUMET - RED JACKET \\
\hline 3023 & & KLENO?? & EDWARD & $\mathrm{s}$ & 18 & MICHIGAN & ITALY & ITALY & & CONDUCTOR/STREET CAR & & 9TH ST. & CALUMET - RED JACKET \\
\hline
\end{tabular}




\begin{tabular}{|c|c|c|c|c|c|c|c|c|c|c|c|c|c|}
\hline 3024 & 812 & BORGO & MIKE & $\mathrm{H}$ & 76 & ITALY & ITALY & ITALY & 1890 & & VICENZA & 9TH ST. & CALUMET - RED JACKET \\
\hline 3025 & & BORGO & KATE & w & 65 & IRELAND & IRELAND & IRELAND & & & IRELAND & 9TH ST. & CALUMET - RED JACKET \\
\hline 3026 & & BORGO & JOSEPHINE & $\mathrm{D}$ & 45 & MICHIGAN & ITALY & IRELAND & & & VICENZA & 9TH ST. & CALUMET - RED JACKET \\
\hline 3027 & & BORGO & ADELAIDE & D & 42 & MICHIGAN & ITALY & IRELAND & & & VICENZA & 9TH ST. & CALUMET - RED JACKET \\
\hline 3028 & 5734 & CIABATTARI & TEODORO & $\mathrm{H}$ & 31 & ITALY & ITALY & ITALY & 1902 & LABORER/STEAM RAILROAD & LUCCA & 8TH ST. & CALUMET - RED JACKET \\
\hline 3029 & & CIABATTARI & CAROLA & w & 29 & MICHIGAN & ITALY & ITALY & & & & 8TH ST. & CALUMET - RED JACKET \\
\hline 3030 & & CIABATTARI & TEODORO & s & 12 & MICHIGAN & ITALY & MICHIGAN & & & LUCCA & 8TH ST. & CALUMET - RED JACKET \\
\hline 3031 & & CIABATTARI & ASDALE? & $\mathrm{s}$ & 10 & MICHIGAN & ITALY & MICHIGAN & & & LUCCA & 8TH ST. & CALUMET - RED JACKET \\
\hline 3032 & & CIABATTARI & FLORENCE & D & 7 & MICHIGAN & ITALY & MICHIGAN & & & LUCCA & 8TH ST. & CALUMET - RED JACKET \\
\hline 3033 & & CIABATTARI & VENNATTA & $\mathrm{D}$ & 5 & MICHIGAN & ITALY & MICHIGAN & & & LUCCA & 8TH ST. & CALUMET - RED JACKET \\
\hline 3034 & & CIABATTARI & PAUL & $\mathrm{s}$ & 2 & MICHIGAN & ITALY & MICHIGAN & & & LUCCA & 8TH ST. & CALUMET - RED JACKET \\
\hline 3035 & 430 & MARTINI & GEORGE & $\mathrm{H}$ & 69 & ITALY & ITALY & ITALY & 1874 & RETIRED & LUCCA & 9TH ST. & CALUMET - RED JACKET \\
\hline 3036 & & MARTINI & ETTA & D & 43 & MICHIGAN & ITALY & ITALY & & & LUCCA & 9TH ST. & CALUMET - RED JACKET \\
\hline 3037 & 401 & TAMBELLINI & ARMIDO & $\mathrm{H}$ & 47 & ITALY & ITALY & ITALY & 1895 & CONFECTIONARY STORE & LUCCA & 5TH ST. & CALUMET - RED JACKET \\
\hline 3038 & & TAMBELLINI & ANNE & W & 37 & MICHIGAN & ITALY & ITALY & & & & 5TH ST. & CALUMET - RED JACKET \\
\hline 3039 & & TAMBELLINI & ED & s & 13 & MICHIGAN & ITALY & MICHIGAN & & & LUCCA & 5TH ST. & CALUMET - RED JACKET \\
\hline 3040 & & TAMBELLINI & DANTE & $\mathrm{s}$ & 12 & MICHIGAN & ITALY & MICHIGAN & & & LUCCA & 5TH ST. & CALUMET - RED JACKET \\
\hline 3041 & & TAMBELLINI & $\mathrm{M} ?$ & D & 9 & MICHIGAN & ITALY & MICHIGAN & & & LUCCA & 5TH ST. & CALUMET - RED JACKET \\
\hline 3042 & & TAMBELLINI & FLOYD & $\mathrm{s}$ & 7 & MICHIGAN & ITALY & MICHIGAN & & & LUCCA & 5TH ST. & CALUMET - RED JACKET \\
\hline 3043 & 421 & BIANCHI & JOHN & $\mathrm{H}$ & 46 & ITALY & ITALY & ITALY & 1902 & OWNER/GROCERY STORE & COLLODI. LU & 5TH ST. & CALUMET - RED JACKET \\
\hline 3044 & & BIANCHI & EUFEMIA & w & 35 & ITALY & ITALY & ITALY & 1916 & & & 5TH ST. & CALUMET - RED JACKET \\
\hline 3045 & & BIANCHI & PETER & $\mathrm{s}$ & 2 & MICHIGAN & ITALY & ITALY & & & COLLODI, LU & 5TH ST. & CALUMET - RED JACKET \\
\hline 3046 & & BIANCHI & MARY & $\mathrm{D}$ & $6 \mathrm{M}$ & MICHIGAN & ITALY & ITALY & & & COLLODI.LU & 5TH ST. & $\begin{array}{l}\text { CALUMET - RED JACKET } \\
\text { CACKE }\end{array}$ \\
\hline 3047 & & TOMMEI & EUGENE & B & 40 & ITALY & ITALY & ITALY & 1901 & OWNER/FRUIT STORE & LUCCA & 5TH ST. & CALUMET - RED JACKET \\
\hline 3048 & & LUCCHESI & MARY & SER & 18 & MICHIGAN & ITALY & ITALY & & SERVANT/PF & LUCCA & 5TH ST. & $\begin{array}{l}\text { CALUMET - RED JACKET } \\
\end{array}$ \\
\hline 3049 & 426 & BALAGNA & CLEMENTE & $\mathrm{H}$ & 39 & ITALY & ITALY & ITALY & 1900 & CARPENTER/HOUSE & PONT CANAVESE. TO & 6TH ST. & CALUMET - RED JACKET \\
\hline 3050 & & BALAGNA & LENA & w & 32 & ITALY & ITALY & ITALY & ???? & & & 6TH ST. & CALUMET - RED JACKET \\
\hline 3051 & & BALAGNA & MARIE & $\mathrm{D}$ & 9 & MICHIGAN & ITALY & ITALY & & & PONT CANAVESE, TO & 6TH ST. & CALUMET - RED JACKET \\
\hline 3052 & 432 & PICCHIOTTINO & PETER & $\mathrm{H}$ & 44 & ITALY & ITALY & ITALY & 1905 & LABORER/CM & PONT CANAVESE. TO & 6TH ST. & CALUMET - RED JACKET \\
\hline 3053 & & PICCHIOTTINO & TERESA & W & 35 & ITALY & ITALY & ITALY & 1905 & & PONT CANAVESE. TO & 6TH ST. & CALUMET - RED JACKET \\
\hline 3054 & & PICCHIOTTINO & JOHN & $\mathrm{s}$ & 18 & MICHIGAN & ITALY & ITALY & & LABORER/FOUNDRY & PONT CANAVESE. TO & 6TH ST. & CALUMET - RED JACKET \\
\hline 3055 & & PICCHIOTTINO & RICHARD & $\mathrm{s}$ & 16 & MICHIGAN & ITALY & ITALY & & LABORER/CM & PONT CANAVESE. TO & 6TH ST. & CALUMET - RED JACKET \\
\hline 3056 & & PICCHIOTTINO & MARY & D & 14 & MICHIGAN & ITALY & ITALY & & & PONT CANAVESE. TO & 6TH ST. & CALUMET - RED JACKET \\
\hline 3057 & & PICCHIOTTINO & GIULIA & $\mathrm{D}$ & 12 & MICHIGAN & ITALY & ITALY & & & PONT CANAVESE, TO & 6TH ST. & CALUMET - RED JACKET \\
\hline 3058 & & PICCHIOTTINO & TEODORO & BR & 35 & ITALY & ITALY & ITALY & 1907 & TAILOR/OWN SHOP & PONT CANAVESE. TO & 6TH ST. & CALUMET - RED JACKET \\
\hline 3059 & 400 & VAIRO & DOMENICO & $\mathrm{H}$ & 30 & ITALY & ITALY & ITALY & 1900 & $\begin{array}{l}\text { KEEPER/HOTEL } \\
\text { KEEPET }\end{array}$ & LOCANA CANAVESE. TO & 6TH ST. & CALUMET - RED JACKET \\
\hline 3060 & & VAIRO-PERONA & MARY & w & 26 & ITALY & ITALY & ITALY & 1908 & & TORINO & 6TH ST. & CALUMET - RED JACKET \\
\hline 3061 & & VAIRO & DOMENICO & s & 7 & MICHIGAN & ITALY & ITALY & & & LOCANA CANAVESE. TO & 6TH ST. & CALUMET - RED JACKET \\
\hline 3062 & & VAIRO & MARY & $\mathrm{D}$ & 6 & MICHIGAN & ITALY & ITALY & & & LOCANA CANAVESE. TO & 6TH ST. & CALUMET - RED JACKET \\
\hline 3063 & & PERONA & MARGHERITA & SIIL & 24 & ITALY & ITALY & ITALY & 1915 & & TORINO & 6TH ST. & CALUMET - RED JACKET \\
\hline 3064 & & ZUBIENA & JOHN & B & 37 & ITALY & ITALY & ITALY & 1900 & HELPER/HOTEL & TORINO & 6TH ST. & CALUMET - RED JACKET \\
\hline 3065 & 403 & MIGLIO/MIGLIA & CARLO & $\mathrm{H}$ & 66 & ITALY & ITALY & ITALY & 1878 & OWNER/GROCERY STORE & SAN GIORGIO CANAVESE. TO & 7TH ST. & CALUMET - RED JACKET \\
\hline 3066 & & MIGLIO/MIGLIA & TERESA & w & 56 & ITALY & ITALY & ITALY & 1891 & & & 7TH ST. & CALUMET - RED JACKET \\
\hline 3067 & & MIGLIO/MIGLIA & JOSEPH & s & 35 & MICHIGAN & ITALY & ITALY & & TEAMSTER/GROCERY STORE & SAN GIORGIO CANAVESE. TO & 7TH ST. & CALUMET - RED JACKET \\
\hline 3068 & & MIGLIO/MIGLIA & PETER & $\mathrm{s}$ & 17 & MICHIGAN & ITALY & ITALY & & & SAN GIORGIO CANAVESE. TO & 7TH ST. & CALUMET - RED JACKET \\
\hline 3069 & & MIGLIO/MIGLIA & TERESA & D & 23 & MICHIGAN & ITALY & ITALY & & & SAN GIORGIO CANAVESE. TO & 7TH ST. & CALUMET - RED JACKET \\
\hline 3070 & 405 & TAMBELLINI & JOSEPH & $\mathrm{H}$ & 50 & ITALY & ITALY & ITALY & 1900 & OWNER/SOFT DRINK & LUCCA & 7TH ST. & CALUMET - RED JACKET \\
\hline 3071 & 400 & TAMBELLINI & TERESA & w & 43 & ITALY & ITALY & ITALY & 1910 & OWVIVERTOUT T LRIIVN & LUCCA & 7TH ST. & CALUMET - RED JACKET \\
\hline 3072 & & TAMBELLINI & MARY & D & 20 & ITALY & ITALY & ITALY & 1909 & & LUCCA & 7TH ST. & CALUMET - RED JACKET \\
\hline 3073 & & TAMBELLINI & JOSEPHINE & B & 14 & ITALY & ITALY & ITALY & 1909 & & LUCCA & 7TH ST. & CALUMET - RED JACKET \\
\hline 3074 & & TAMBELLINI & JEAN & B & 12 & MICHIGAN & ITALY & ITALY & & & LUCCA & 7TH ST. & CALUMET - RED JACKET \\
\hline 3075 & & TAMBELLINI & CRISTINA & B & 9 & MICHIGAN & ITALY & ITALY & & & LUCCA & 7TH ST. & CALUMET - RED JACKET \\
\hline 3076 & & TAMBELLINI & INES & B & 8 & MICHIGAN & ITALY & ITALY & & & LUCCA & 7TH ST. & CALUMET - RED JACKET \\
\hline 3077 & & BARSANTI & FRED & B & 36 & ITALY & ITALY & ITALY & 1911 & LABORER/STEAM RAILROAD & SEGROMIGNO. LU & 7TH ST. & CALUMET - RED JACKET \\
\hline 3078 & 411 & GIANNINI & AUGUSTO & $\mathrm{H}$ & 34 & ITALY & ITALY & ITALY & 1905 & LABORER/STEAM RAILROAD & LUCCA & 7TH ST. & CALUMET - RED JACKET \\
\hline 3079 & & GIANNINI & SANTINA & w & 33 & ITALY & ITALY & ITALY & 1907 & & & 7TH ST. & CALUMET - RED JACKET \\
\hline 3080 & & GIANNINI & ELSIE & D & 11 & MICHIGAN & ITALY & ITALY & & & LUCCA & 7TH ST. & CALUMET - RED JACKET \\
\hline 3081 & & GIANNINI & PAUL & s & 10 & MICHIGAN & ITALY & ITALY & & & LUCCA & 7TH ST. & CALUMET - RED JACKET \\
\hline 3082 & & GIANNINI & LILA & D & 5 & MICHIGAN & ITALY & ITALY & & & LUCCA & 7TH ST. & CALUMET - RED JACKET \\
\hline 3083 & & BANDONE & CASIMIRO & $\mathrm{H}$ & 72 & ITALY & ITALY & ITALY & UN & & & 7TH ST. & CALUMET - RED JACKET \\
\hline 3084 & & CHELI & LOUIS & B & 30 & ITALY & ITALY & ITALY & & MINER/CM & LUCCA & 7TH ST. & CALUMET - RED JACKET \\
\hline 3085 & 415 & CHELI & FILOMENA & $\mathrm{H}$ & 34 & ITALY & ITALY & ITALY & 1900 & & LUCCA & 7TH ST. & CALUMET - RED JACKET \\
\hline 3086 & 410 & CHELI & EUMALINE & $\mathrm{D}$ & 17 & MICHIGAN & ITALY & ITALY & & CLERK/DEPTARTMENT STORE & LUCCA & 7TH ST. & CALUMET - RED JACKET \\
\hline
\end{tabular}




\begin{tabular}{|c|c|c|c|c|c|c|c|c|c|c|c|c|c|}
\hline 3087 & & CHELI & ALADINA & $\mathrm{D}$ & 16 & MICHIGAN & ITALY & ITALY & & CLERK/FRUIT STORE & LUCCA & 7TH ST. & CALUMET - RED JACKET \\
\hline 3088 & & CHELI & FLORENCE & D & 14 & MICHIGAN & ITALY & ITALY & & & LUCCA & 7TH ST. & CALUMET - RED JACKET \\
\hline 3089 & & CHELI & INES & D & 5 & MICHIGAN & ITALY & ITALY & & & LUCCA & 7TH ST. & CALUMET - RED JACKET \\
\hline 3090 & 415 & BIANCHI & NARCISO & $\mathrm{H}$ & 41 & ITALY & ITALY & ITALY & 1898 & OWNER/STORE & LUCCA & 7TH ST. & CALUMET - RED JACKET \\
\hline 3091 & & BIANCHI & EDITH & W & 36 & ITALY & ITALY & ITALY & 1887 & & & 7TH ST. & CALUMET - RED JACKET \\
\hline 3092 & & BIANCHI & GIULIO & s & 13 & MICHIGAN & ITALY & ITALY & & & LUCCA & 7TH ST. & CALUMET - RED JACKET \\
\hline 3093 & & BIANCHI & MYRA & D & 11 & MICHIGAN & ITALY & ITALY & & & LUCCA & 7TH ST. & CALUMET - RED JACKET \\
\hline 3094 & & BIANCHI & REYNOLD & $\mathrm{s}$ & 7 & MICHIGAN & ITALY & ITALY & & & LUCCA & 7TH ST. & CALUMET - RED JACKET \\
\hline 3095 & & BIANCHI & MARIE & $\mathrm{D}$ & 0 & MICHIGAN & ITALY & ITALY & & & LUCCA & 7TH ST. & CALUMET - RED JACKET \\
\hline 3096 & 809 & BRACCO & BERNARDO & $\mathrm{H}$ & 66 & ITALY & ITALY & ITALY & 1883 & HELPER/BAKERY & PONT CANAVESE. TO & ELM ST. & CALUMET - RED JACKET \\
\hline 3097 & & BRACCO & MARIA & W & 64 & ITALY & ITALY & ITALY & 1885 & & & ELM ST. & CALUMET - RED JACKET \\
\hline 3098 & & BRACCO & CRISTINA & $\mathrm{D}$ & 23 & MICHIGAN & ITALY & ITALY & & STENOGRAPHER & PONT CANAVESE. TO & ELM ST. & CALUMET - RED JACKET \\
\hline 3099 & 811 & BRACCO & CARLO & $\mathrm{H}$ & 35 & MICHIGAN & ITALY & ITALY & & LABORER/GARAGE & PONT CANAVESE. TO & ELM ST. & CALUMET - RED JACKET \\
\hline 3100 & & BRACCO & AGNESE & W & 34 & MICHIGAN & GERMANY & MICHIGAN & & & USA-GERMANY & ELM ST. & CALUMET - RED JACKET \\
\hline 3101 & & BRACCO & LEONARD & $\mathrm{s}$ & 8 & MICHIGAN & MICHIGAN & MICHIGAN & & & PONT CANAVESE. TO & ELM ST. & CALUMET - RED JACKET \\
\hline 3102 & & POWLAR & PAUL & $\mathrm{H}$ & 38 & MICHIGAN & GERMANY & GERMANY & & OWNER/GARAGE & GERMANY & ELM ST. & CALUMET - RED JACKET \\
\hline 3103 & & POWLAR & ROSE & W & 32 & MICHIGAN & ITALY & ITALY & & & & ELM ST. & CALUMET - RED JACKET \\
\hline 3104 & & POWLAR & MARION & D & 2 & MICHIGAN & MICHIGAN & MICHIGAN & & & & ELM ST. & CALUMET - RED JACKET \\
\hline 3105 & 825 & BARATONO & ALEX & $\mathrm{H}$ & 41 & ITALY & ITALY & ITALY & 1902 & OWNER/DEPTARTMENT STORE & TORINO & & CALUMET - RED JACKET \\
\hline 3106 & & BARATONO & MADDALENA & w & 37 & ITALY & ITALY & ITALY & 1891 & & & & CALUMET - RED JACKET \\
\hline 3107 & & BARATONO & MARGHERITA & D & 14 & MICHIGAN & ITALY & ITALY & & & TORINO & & CALUMET - RED JACKET \\
\hline 3108 & & BARATONO & ANTONIO & $\mathrm{s}$ & 13 & MICHIGAN & $\begin{array}{l}\text { ITALY } \\
\text { ITAL }\end{array}$ & ITALY & & & TORINO & & CALUMET - RED JACKET \\
\hline 3109 & & GRINDATTI & JOSEPH & $\mathrm{H}$ & 34 & ITALY & ITALY & ITALY & 1900 & BUTCHER/MEAT MARKET & PONT CANAVESE. TO & & CALUMET - RED JACKET \\
\hline 3110 & & GRINDATTI & HEZEL & w & 31 & MICHIGAN & ITALY & ITALY & & & & & CALUMET - RED JACKET \\
\hline 3111 & & GRINDATTI & ELEONORA & D & 6 & MICHIGAN & ITALY & MICHIGAN & & & PONT CANAVESE. TO & & CALUMET - RED JACKET \\
\hline 3112 & & GRINDATTI & WILLIAM & $\mathrm{s}$ & 2 & MICHIGAN & ITALY & MICHIGAN & & & PONT CANAVESE. TO & & CALUMET - RED JACKET \\
\hline 3113 & & MIGLIO/MIGLIA & MARY & $\mathrm{H}$ & 49 & ITALY & ITALY & ITALY & 1891 & SERVANT/PF & & & CALUMET - RED JACKET \\
\hline 3114 & & MIGLIO/MIGLIA & FRANCIS & D & 20 & MICHIGAN & ITALY & ITALY & & BOOKKEEPER/DEPT. STORE & SAN GIORGIO CANAVESE, TO & & CALUMET - RED JACKET \\
\hline 3115 & & MIGLIO/MIGLIA & LUCIA & D & 18 & MICHIGAN & ITALY & ITALY & & CLERK/DEPTARTMENT STORE & SAN GIORGIO CANAVESE. TO & & CALUMET - RED JACKET \\
\hline 3116 & & MIGLIO/MIGLIA & MARY & D & 15 & MICHIGAN & ITALY & ITALY & & SERVANT/PF & SAN GIORGIO CANAVESE. TO & & CALUMET - RED JACKET \\
\hline 3117 & 409 & QUELLO & JOHN & $\mathrm{H}$ & 50 & MICHIGAN & ITALY & ITALY & & CONTRACTOR/ESTATE & LOCANA CANAVESE. TO & 8TH ST. & CALUMET - RED JACKET \\
\hline 3118 & & QUELLO & MARGHERITA & SI & 45 & MICHIGAN & ITALY & ITALY & & 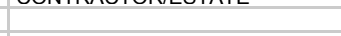 & LOCANA CANAVESE. TO & 8TH ST. & CALUMET - RED JACKET \\
\hline 3119 & & QUELLO & ANNA & $\mathrm{SI}$ & 37 & MICHIGAN & ITALY & ITALY & & TEACHER/PUBLIC SCHOOL & LOCANA CANAVESE. TO & 8TH ST. & CALUMET - RED JACKET \\
\hline 3120 & & QUELLO & BAT & BR & $44 ?$ & MICHIGAN & ITALY & ITALY & & CASHIER/BANK & LOCANA CANAVESE, TO & 8TH ST. & CALUMET - RED JACKET \\
\hline 3121 & & QUELLO & LIZZIE & $\mathrm{SI}$ & 36 & MICHIGAN & ITALY & ITALY & & & LOCANA CANAVESE. TO & 8TH ST. & CALUMET - RED JACKET \\
\hline 3122 & & QUELLO & ELISE & si & 34 & MICHIGAN & ITALY & ITALY & & & LOCANA CANAVESE. TO & 8TH ST. & CALUMET - RED JACKET \\
\hline 3123 & & CURTO & JAMES & $\mathrm{H}$ & 32 & ILLINOIS & ITALY & ITALY & & FARMER/GENERAL FARM & TORINO & & DUNCAN \\
\hline 3124 & & CURTO & CARA & W & 32 & WISCONSI & KENTUKY & KENTUKY & & 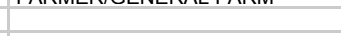 & USA & & DUNCAN \\
\hline 3125 & & CURTO & BLANCHE & $\mathrm{D}$ & 5 & WISCONSI & ILLINOIS & WISCONSI & & & TORINO & & DUNCAN \\
\hline 3126 & & CURTO & BERNIE & $\mathrm{D}$ & 0 & MICHIGAN & ILLINOIS & WISCONSI & & & TORINO & & DUNCAN \\
\hline 3127 & & REGIS & JOSEPH & B & 22 & ITALY & ITALY & ITALY & 1902 & SKIDDER/LUMBER CAMP & TORINO & & ELM RIVER - BLANC LOCATION \\
\hline 3128 & & FREDERIC & DONO & B & 38 & ITALY & ITALY & ITALY & 1906 & LABORER/LUMBER CAMP & & & ELM RIVER \\
\hline 3129 & & BALCONI & CARLO & $\mathrm{H}$ & 68 & ITALY & ITALY & ITALY & 1883 & CARPENTER/CONTRACTOR & MERCALLO. VARESE & PEWABIC ST. & FRANKLIN \\
\hline 3130 & & BALCONI & MARY & W & 56 & ITALY & ITALY & ITALY & 1883 & & MERCALLO. VARESE & PEWABIC ST. & FRANKLIN \\
\hline 3131 & & BALCONI & CARLO & s & 31 & MICHIGAN & ITALY & ITALY & & PRINTER/SHOP & MERCALLO. VARESE & PEWABIC ST. & FRANKLIN \\
\hline 3132 & & BALCONI & LOUISE & D & 17 & MICHIGAN & ITALY & ITALY & & & MERCALLO. VARESE & PEWABIC ST. & FRANKLIN \\
\hline 3133 & & BALCONI & PERINA & GD & 10 & MICHIGAN & ITALY & ITALY & & & MERCALLO. VARESE & PEWABIC ST. & FRANKLIN \\
\hline 3134 & & PROCISSI & PETER & $\mathrm{H}$ & 66 & ITALY & ITALY & ITALY & 1881 & LABORER/SMELTER & LUCCA & FRANKLIN ST. & FRANKLIN \\
\hline 3135 & & PROCISSI & ORSOLA & w & 62 & ITALY & ITALY & ITALY & 1887 & & & FRANKLIN ST. & FRANKLIN \\
\hline 3136 & & FEIRA & MARTINO & $\mathrm{H}$ & 44 & ITALY & ITALY & ITALY & 1890 & FARMER & PONT CANAVESE. TO & FRONT ST. & FRANKLIN \\
\hline 3137 & & FEIRA & MARIA & W & 38 & ITALY & ITALY & ITALY & 1900 & & & FRONT ST. & FRANKLIN \\
\hline 3138 & & FEIRA & GIOVANNA & $\mathrm{D}$ & 12 & MICHIGAN & ITALY & ITALY & & & PONT CANAVESE, TO & FRONT ST. & FRANKLIN \\
\hline 3139 & & FEIRA & DOMENICO & $\mathrm{s}$ & 11 & MICHIGAN & ITALY & ITALY & & & PONT CANAVESE. TO & FRONT ST. & FRANKLIN \\
\hline 3140 & & FEIRA & GIACOMO & $\mathrm{S}$ & 9 & MICHIGAN & ITALY & ITALY & & & PONT CANAVESE. TO & FRONT ST. & FRANKLIN \\
\hline 3141 & & FEIRA & GIUSEPPE & s & 7 & MICHIGAN & ITALY & ITALY & & & PONT CANAVESE. TO & FRONT ST. & FRANKLIN \\
\hline 3142 & & FEIRA & VITTORIA & D & 4 & MICHIGAN & ITALY & ITALY & & & PONT CANAVESE. TO & FRONT ST. & FRANKLIN \\
\hline 3143 & & FEIRA & MARIA & D & $18 \mathrm{M}$ & MICHIGAN & ITALY & ITALY & & & PONT CANAVESE. TO & FRONT ST. & FRANKLIN \\
\hline 3144 & & GASPARDO & SERAFINO & B & 72 & ITALY & ITALY & ITALY & 1899 & LABORER/FARM & PONT CANAVESE, TO & FRONT ST. & FRANKLIN \\
\hline 3145 & & FAUSONE & GILDO & B & 55 & ITALY & ITALY & ITALY & 1894 & LABORER/SAW MILL & TORINO & FRONT ST. & FRANKLIN \\
\hline 3146 & 506 & LOCATELLI & ANDREW & $\mathrm{H}$ & 42 & ITALY & ITALY & ITALY & 1898 & CAPTAIN/CM & SELINO. BERGAMO & LOWER PEV & FRANKLIN - PEWABI \\
\hline 3147 & & LOCATELLI & & W & 28 & & ITA & ITALY & 1912 & & & LOWER PE & FRANKLIN - PE \\
\hline 3148 & & LOCATELLI & LUDIVIA & $\mathrm{s}$ & 7 & MICHIGAN & ITALY & & & & SELINO. BERGAMO & LOWER PEWABIC & FRANKLIN - PEWAE \\
\hline 3149 & & LOCATELLI & JOHN & $\mathrm{s}$ & 5 & MICHIGAN & ITALY & ITALY & & & SELINO. BERGAMO & LOWER PEWABIC & FRANKLIN - PEWABIC LOC. \\
\hline
\end{tabular}




\begin{tabular}{|c|c|c|c|c|c|c|c|c|c|c|c|c|c|}
\hline 3150 & & LOCATELLI & ISOLINA & D & 3 & MICHIGAN & ITALY & ITALY & & & SELINO. BERGAMO & LOWER PEWABIC & FRANKLIN - PEWABIC LOC. \\
\hline 3151 & 537 & PROLA & PETER & $\mathrm{H}$ & 34 & ITALY & ITALY & ITALY & 1908 & MINER/CM & AGLIE'. TO & LOWER PEWABIC & FRANKLIN - PEWABIC LOC. \\
\hline 3152 & & PROLA & MARY & W & 35 & ITALY & ITALY & ITALY & 1905 & & & LOWER PEWABIC & FRANKLIN - PEWABIC LOC. \\
\hline 3153 & & PROLA & MARY & D & 11 & MICHIGAN & ITALY & ITALY & & & AGLIE'. TO & LOWER PEWABIC & FRANKLIN - PEWABIC LOC. \\
\hline 3154 & & PROLA & JOSEPH & $\mathrm{S}$ & 9 & MICHIGAN & ITALY & ITALY & & & AGLIE'. TO & LOWER PEWABIC & FRANKLIN - PEWABIC LOC. \\
\hline 3155 & & PROLA & JOSEPH & BR & 28 & ITALY & ITALY & ITALY & 1907 & MINER/CM & AGLIE'. TO & LOWER PEWABIC & FRANKLIN - PEWABIC LOC. \\
\hline 3156 & 533 & MAGA & JOSEPH & $\mathrm{H}$ & 31 & ITALY & ITALY & ITALY & 1894 & SHAFT MAN/CM & SAN MARTINO CANAVESE. TO & LOWER PEWABIC & FRANKLIN - PEWABIC LOC. \\
\hline 3157 & & MAGA & HELEN & W & 28 & ITALY & ITALY & ITALY & 1910 & & & LOWER PEWABIC & FRANKLIN - PEWABIC LOC. \\
\hline 3158 & & MAGA & DOMENICO & $\mathrm{s}$ & 9 & MICHIGAN & ITALY & ITALY & & & SAN MARTINO CANAVESE. TO & LOWER PEWABIC & FRANKLIN - PEWABIC LOC. \\
\hline 3159 & & MAGA & JOHN & $\mathrm{s}$ & 5 & MICHIGAN & ITALY & ITALY & & & SAN MARTINO CANAVESE. TO & LOWER PEWABIC & FRANKLIN - PEWABIC LOC. \\
\hline 3160 & & MAGA & VINCENZO & $\mathrm{s}$ & 2 & MICHIGAN & ITALY & ITALY & & & SAN MARTINO CANAVESE. TO & LOWER PEWABIC & FRANKLIN - PEWABIC LOC. \\
\hline 3161 & & TINETTI & GIOVANNA & $\mathrm{COU}$ & 28 & MICHIGAN & ITALY & ITALY & & DRESS MAKER & TORINO & LOWER PEWABIC & FRANKLIN - PEWABIC LOC. \\
\hline 3162 & 546 & MARACCINI & SETTIMO & $\mathrm{H}$ & 42 & ITALY & ITALY & ITALY & UN & TIMBER BOSS/CM & SANTA MARIA DEL GIUDICE. LU & LOWER PEWABIC & FRANKLIN - PEWABIC LOC. \\
\hline 3163 & & MARACCINI & ELISA & W & 35 & ITALY & ITALY & ITALY & 1906 & & SANTA MARIA DEL GIUDICE. LU & LOWER PEWABIC & FRANKLIN - PEWABIC LOC. \\
\hline 3164 & & MARACCINI & ISOLINA & $\mathrm{D}$ & 15 & ITALY & ITALY & ITALY & 1906 & & SANTA MARIA DEL GIUDICE. LU & LOWER PEWABIC & FRANKLIN - PEWABIC LOC. \\
\hline 3165 & & MARACCINI & EVA/IVA & $\mathrm{D}$ & 12 & MICHIGAN & ITALY & ITALY & & & SANTA MARIA DEL GIUDICE, LU & LOWER PEWABIC & FRANKLIN - PEWABIC LOC. \\
\hline 3166 & & MARACCINI & MARY & D & 11 & MICHIGAN & ITALY & ITALY & & & SANTA MARIA DEL GIUDICE. LU & LOWER PEWABIC & FRANKLIN - PEWABIC LOC. \\
\hline 3167 & & MARACCINI & FRED & $\mathrm{s}$ & 9 & MICHIGAN & ITALY & ITALY & & & SANTA MARIA DEL GIUDICE. LU & LOWER PEWABIC & FRANKLIN - PEWABIC LOC. \\
\hline 3168 & & MARACCINI & JOSEPH & $\mathrm{s}$ & 5 & MICHIGAN & ITALY & ITALY & & & SANTA MARIA DEL GIUDICE. LU & LOWER PEWABIC & FRANKLIN - PEWABIC LOC. \\
\hline 3169 & & MARACCINI & BRUNA & D & 3 & MICHIGAN & ITALY & ITALY & & & SANTA MARIA DEL GIUDICE. LU & LOWER PEWABIC & FRANKLIN - PEWABIC LOC. \\
\hline 3170 & & MARACCINI & PETER & $\mathrm{s}$ & $3 \mathrm{M}$ & MICHIGAN & ITALY & ITALY & & & SANTA MARIA DEL GIUDICE. LU & LOWER PEWABIC & FRANKLIN - PEWABIC LOC. \\
\hline 3171 & & BENETTI & BIAGIO & B & 33 & ITALY & ITALY & ITALY & 1911 & TRAMMER/CM & CAPANNORI, LU & LOWER PEWABIC & FRANKLIN - PEWABIC LOC. \\
\hline 3172 & & MARACCINI & JOSEPH & BR & 52 & ITALY & ITALY & ITALY & 1912 & TIMBERMAN/CM & SANTA MARIA DEL GIUDICE. LU & LOWER PEWABIC & FRANKLIN - PEWABIC LOC. \\
\hline 3173 & & BURATTI & LEITA & SD & 3 & ARIZONA & ITALY & ITALY & & & & LOWER PEWABIC & FRANKLIN - PEWABIC LOC. \\
\hline 3174 & & BURATTI & NELLO & SSO & 1 & ARIZONA & ITALY & ITALY & & & & LOWER PEWABIC & FRANKLIN - PEWABIC LOC. \\
\hline 3175 & 599 & CESARE & FRANK & $\mathrm{H}$ & 27 & ITALY & ITALY & ITALY & 1906 & MOTORMAN/CM & TORINO & LOWER PEWABIC & FRANKLIN - PEWABIC LOC. \\
\hline 3176 & 399 & $\begin{array}{l}\text { CESARE } \\
\text { CESARE }\end{array}$ & NARCISA & W & 27 & ITALY & ITALY & ITALY & 1911 & IVIOT ORIVIAIV/CIVI & TORIINU & LOWER PEWABIC & FRANKLIN - PEWABIC LOC. \\
\hline 3177 & & CESARE & LIDIA & $\mathrm{D}$ & 3 & MICHIGAN & ITALY & ITALY & & & TORINO & LOWER PEWABIC & FRANKLIN - PEWABIC LOC. \\
\hline 3178 & & CESARE & ILLY & D & 2 & MICHIGAN & ITALY & ITALY & & & TORINO & LOWER PEWABIC & FRANKLIN - PEWABIC LOC. \\
\hline 3179 & & CESARE & EDO & s & 10 & MICHIGAN & ITALY & ITALY & & & TORINO & LOWER PEWABIC & FRANKLIN - PEWABIC LOC. \\
\hline 3180 & & CESARE & PISIA & BR & 24 & ITALY & ITALY & ITALY & 1904 & MINER/CM & TORINO & LOWER PEWABIC & FRANKLIN - PEWABIC LOC. \\
\hline 3181 & & CIABATTARI & VITTORIO & B & 44 & ITALY & ITALY & ITALY & 1900 & LABORER/CM & SEGROMIGNO. LU & LOWER PEWABIC & FRANKLIN - PEWABIC LOC. \\
\hline 3182 & 588 & FEDERIGHI & DOMENICO & $\mathrm{H}$ & 55 & ITALY & ITALY & ITALY & 1880 & LABORER/CM & LUCCA & LOWER PEWABIC & FRANKLIN - PEWABIC LOC. \\
\hline 3183 & & FEDERIGHI & TECLA & W & 53 & ITALY & ITALY & ITALY & 1902 & & & LOWER PEWABIC & FRANKLIN - PEWABIC LOC. \\
\hline 3184 & & FEDERIGHI & CHESTER & s & 14 & MICHIGAN & ITALY & ITALY & & & LUCCA & LOWER PEWABIC & FRANKLIN - PEWABIC LOC. \\
\hline 3185 & & FEDERIGHI & DOMENICO & $\mathrm{s}$ & 12 & MICHIGAN & ITALY & ITALY & & & LUCCA & LOWER PEWABIC & FRANKLIN - PEWABIC LOC. \\
\hline 3186 & 586 & PISANI & ABRAMO & $\mathrm{H}$ & 34 & ITALY & ITALY & ITALY & UN & MINER/CM & LUCCA & LOWER PEWABIC & FRANKLIN - PEWABIC LOC. \\
\hline 3187 & 000 & PISANI & MARISA & W & 29 & ITALY & ITALY & ITALY & 1909 & IVIIIVERT LIVI & LUCUA & LOWER PEWABIC & FRANKLIN - PEWABIC LOC. \\
\hline 3188 & & PISANI & ITALO & $\mathrm{s}$ & 9 & MICHIGAN & ITALY & ITALY & & & LUCCA & LOWER PEWABIC & FRANKLIN - PEWABIC LOC. \\
\hline 3189 & & PISANI & FORTUNATO & $\mathrm{s}$ & 8 & MICHIGAN & ITALY & ITALY & & & LUCCA & LOWER PEWABIC & FRANKLIN - PEWABIC LOC. \\
\hline 3190 & & PISANI & ANNE & D & 5 & MICHIGAN & ITALY & ITALY & & & LUCCA & LOWER PEWABIC & FRANKLIN - PEWABIC LOC. \\
\hline 3191 & & PISANI & LENA & D & 3 & MICHIGAN & ITALY & ITALY & & & LUCCA & LOWER PEWABIC & FRANKLIN - PEWABIC LOC. \\
\hline 3192 & & PISANI & OLGA & D & 2 & MICHIGAN & ITALY & ITALY & & & LUCCA & LOWER PEWABIC & FRANKLIN - PEWABIC LOC. \\
\hline 3193 & 580 & $\mathrm{ROCCHI}$ & EGISTO & $\mathrm{H}$ & 48 & ITALY & ITALY & ITALY & 1893 & MINER/CM & MATRAIA. LU & LOWER PEWABIC & FRANKLIN - PEWABIC LOC. \\
\hline 3194 & & ROCCHI & PHOEBE & W & 41 & GERMANY & ITALY & ITALY & 1918 & & & LOWER PEWABIC & FRANKLIN - PEWABIC LOC. \\
\hline 3195 & & $\mathrm{ROCCHI}$ & ALBERT & $\mathrm{s}$ & 17 & MICHIGAN & ITALY & GERMANY & & CARPENTER/CM & MATRAIA. LU & LOWER PEWABIC & FRANKLIN - PEWABIC LOC. \\
\hline 3196 & & ROCCHI & THEO & $\mathrm{s}$ & 14 & MICHIGAN & ITALY & GERMANY & & & MATRAIA. LU & LOWER PEWABIC & FRANKLIN - PEWABIC LOC. \\
\hline 3197 & & ROCCHI & ABRAMO & $\mathrm{s}$ & 12 & MICHIGAN & ITALY & GERMANY & & & MATRAIA. LU & LOWER PEWABIC & FRANKLIN - PEWABIC LOC. \\
\hline 3198 & & ROCCHI & WILL & $\mathrm{s}$ & 9 & MICHIGAN & ITALY & GERMANY & & & MATRAIA. LU & LOWER PEWABIC & FRANKLIN - PEWABIC LOC. \\
\hline 3199 & & ROCCHI & CARLO & $\mathrm{s}$ & 7 & MICHIGAN & ITALY & GERMANY & & & MATRAIA. LU & LOWER PEWABIC & FRANKLIN - PEWABIC LOC. \\
\hline 3200 & & ROCCHI & EVELINA & $\mathrm{D}$ & 3 & MICHIGAN & ITALY & GERMANY & & & MATRAIA. LU & LOWER PEWABIC & FRANKLIN - PEWABIC LOC. \\
\hline 3201 & 575 & TOMMEI & ANGELO & $\mathrm{H}$ & 30 & ITALY & ITALY & ITALY & 1913 & MINER/CM & LUCCA. LO & LOWER PEWABIC & FRANKLIN - PEWABIC LOC. \\
\hline 3202 & & TOMMEI & VITTORIA & w & 17 & ITALY & ITALY & ITALY & 1917 & & & LOWER PEWABIC & FRANKLIN - PEWABIC LOC. \\
\hline 3203 & 548 & BAGNESCHI & DOMENICO & $\mathrm{H}$ & 33 & ITALY & ITA & ITALY & UN & LABORER/CM & CASTELVECCHIO.LU & UPPER PEWABIC & FRANKLIN - PEWABIC LOC. \\
\hline 3204 & & BAGNESCHI & GIULIA & W & 26 & ITALY & ITALY & ITALY & 1910 & & & UPPER PEWABIC & FRANKLIN - PEWABIC LOC. \\
\hline 3205 & & BAGNESCHI & LETA & D & 7 & MICHIGAN & ITALY & ITALY & & & CASTELVECCHIO.LU & UPPER PEWABIC & FRANKLIN - PEWABIC LOC. \\
\hline 3206 & & BAGNESCHI & JAMES & $\mathrm{s}$ & 5 & MICHIGAN & ITALY & ITALY & & & CASTELVECCHIO. LU & UPPER PEWABIC & FRANKLIN - PEWABIC LOC. \\
\hline 3207 & & BAGNESCHI & STEFANO & $\mathrm{s}$ & 2 & MICHIGAN & ITALY & ITALY & & & CASTELVECCHIO, LU & UPPER PEWABIC & FRANKLIN - PEWABIC LOC. \\
\hline 3208 & & BUCCELLA & DINA & $\mathrm{COU}$ & 13 & ITALY & ITALY & ITALY & 1910 & & & UPPER PEWABIC & FRANKLIN - PEWABIC LOC. \\
\hline 3209 & 551 & CIABATTARI & LOUIS & $\mathrm{H}$ & 23 & ITALY & ITALY & ITALY & 1911 & LABORER/CM & LUCCA & UPPER PEWABIC & FRANKLIN - PEWABIC LOC. \\
\hline 3210 & & CIABATTARI & CESARINA & W & 19 & MICHIGAN & ITALY & ITALY & & & & UPPER PEWABIC & FRANKLIN - PEWABIC LOC. \\
\hline 3211 & & CIABATTARI & GINO & $\mathrm{s}$ & $1 \mathrm{M}$ & MICHIGAN & ITALY & ITALY & & & LUCCA & UPPER PEWABIC & FRANKLIN - PEWABIC LOC. \\
\hline 3212 & 555 & FREDIANELLI & ALFREDO & $\mathrm{H}$ & 42 & ITALY & ITALY & ITALY & 1899 & LABORER/CM & CAPANNORI. LU & UPPER PEWABIC & FRANKLIN - PEWABIC LOC. \\
\hline
\end{tabular}




\begin{tabular}{|c|c|c|c|c|c|c|c|c|c|c|c|c|c|}
\hline 3213 & & FREDIANELLI & SESTILIA & W & 33 & ITALY & ITALY & ITALY & 1899 & & CAPANNORI. LU & UPPER PEWABIC & FRANKLIN - PEWABIC LOC. \\
\hline 3214 & & FREDIANELLI & JOSEPH & s & 13 & MICHIGAN & ITALY & ITALY & & & CAPANNORI. LU & UPPER PEWABIC & FRANKLIN - PEWABIC LOC. \\
\hline 3215 & & FREDIANELLI & LOUIS & $\mathrm{s}$ & 12 & MICHIGAN & ITALY & ITALY & & & CAPANNORI. LU & UPPER PEWABIC & FRANKLIN - PEWABIC LOC. \\
\hline 3216 & & FREDIANELLI & ANSELMO & $\mathrm{BR}$ & 37 & ITALY & ITALY & ITALY & 1899 & TRAMMER/CM & CAPANNORI. LU & UPPER PEWABIC & FRANKLIN - PEWABIC LOC. \\
\hline 3217 & & LUCIANI & DANTE & B & 29 & ITALY & ITALY & ITALY & 1912 & LABORER/CM & & UPPER PEWABIC & FRANKLIN - PEWABIC LOC. \\
\hline 3218 & 565 & BETTINI & $\mathrm{JOHN}$ & $\mathrm{H}$ & 47 & ITALY & ITALY & ITALY & 1903 & LABORER/CM & & UPPER PEWABIC & FRANKLIN - PEWABIC LOC. \\
\hline 3219 & 000 & BETTINI & MABEL & W & 45 & ITALY & ITALY & ITALY & 1905 & LADU ALRUIUI & & UPPER PEWABIC & FRANKLIN - PEWABIC LOC. \\
\hline 3220 & & BETTINI & GINO & s & 13 & MICHIGAN & ITALY & ITALY & 1900 & & & UPPER PEWABIC & FRANKLIN - PEWABIC LOC. \\
\hline 3221 & & BETTINI & LENA & $\mathrm{D}$ & 10 & MICHIGAN & ITALY & ITALY & & & & UPPER PEWABIC & FRANKLIN - PEWABIC LOC. \\
\hline 3222 & & BETTINI & ORLANDA & D & 8 & MICHIGAN & ITALY & ITALY & & & & UPPER PEWABIC & FRANKLIN - PEWABIC LOC. \\
\hline 3223 & & BETTINI & NELLO & $\mathrm{s}$ & 6 & MICHIGAN & ITALY & ITALY & & & & UPPER PEWABIC & FRANKLIN - PEWABIC LOC. \\
\hline 3224 & & BETTINI & BRUNO & $\mathrm{D}$ & 4 & MICHIGAN & ITALY & ITALY & & & & UPPER PEWABIC & FRANKLIN - PEWABIC LOC. \\
\hline 3225 & 590 & FREDIANELLI & PETER & $\mathrm{H}$ & 39 & ITALY & ITALY & ITALY & 1901 & FIREMAN/BOILER HOUSE & SEGROMIGNO. LU & UPPER PEWABIC & FRANKLIN - PEWABIC LOC. \\
\hline 3226 & & FREDIANELLI & MARY & W & 37 & ITALY & ITALY & ITALY & 1903 & & & UPPER PEWABIC & FRANKLIN - PEWABIC LOC. \\
\hline 3227 & & FREDIANELLI & NELLO & $\mathrm{s}$ & 15 & MICHIGAN & ITALY & ITALY & & & SEGROMIGNO. LU & UPPER PEWABIC & FRANKLIN - PEWABIC LOC. \\
\hline 3228 & & FREDIANELLI & BALLO & $\mathrm{s}$ & 13 & MICHIGAN & ITALY & ITALY & & & SEGROMIGNO, LU & UPPER PEWABIC & FRANKLIN - PEWABIC LOC. \\
\hline 3229 & & FREDIANELLI & LETHIA & D & 11 & MICHIGAN & ITALY & ITALY & & & SEGROMIGNO. LU & UPPER PEWABIC & FRANKLIN - PEWABIC LOC. \\
\hline 3230 & & FREDIANELLI & CAROLINA & $\mathrm{D}$ & 10 & MICHIGAN & ITALY & ITALY & & & SEGROMIGNO. LU & UPPER PEWABIC & FRANKLIN - PEWABIC LOC. \\
\hline 3231 & & FREDIANELLI & JOSEPH & $\mathrm{s}$ & 8 & MICHIGAN & ITALY & ITALY & & & SEGROMIGNO. LU & UPPER PEWABIC & FRANKLIN - PEWABIC LOC. \\
\hline 3232 & & FREDIANELLI & GINO & $\mathrm{s}$ & 6 & MICHIGAN & ITALY & ITALY & & & SEGROMIGNO. LU & UPPER PEWABIC & FRANKLIN - PEWABIC LOC. \\
\hline 3233 & & FREDIANELLI & BRUNO & $\mathrm{s}$ & 4 & MICHIGAN & ITALY & ITALY & & & SEGROMIGNO. LU & UPPER PEWABIC & FRANKLIN - PEWABIC LOC. \\
\hline 3234 & & FREDIANELLI & JENNA & $\mathrm{D}$ & 2 & MICHIGAN & ITALY & ITALY & & & SEGROMIGNO, LU & UPPER PEWABIC & FRANKLIN - PEWABIC LOC. \\
\hline 3235 & & FREDIANELLI & SATGO & $\mathrm{s}$ & $3 \mathrm{M}$ & MICHIGAN & ITALY & ITALY & & & SEGROMIGNO. LU & UPPER PEWABIC & FRANKLIN - PEWABIC LOC. \\
\hline 3236 & 584 & DINELLI & PETER & $\mathrm{H}$ & 38 & ITALY & ITALY & ITALY & 1912 & MINER/CM & MATRAIA. LU & UPPER PEWABIC & FRANKLIN - PEWABIC LOC. \\
\hline 3237 & & DINELLI & ARMENIA & W & 34 & ITALY & ITALY & ITALY & 1913 & & MATRAIA. LU & UPPER PEWABIC & FRANKLIN - PEWABIC LOC. \\
\hline 3238 & & DINELLI & ALBERT & $s$ & 12 & ITALY & ITALY & ITALY & 1913 & & MATRAIA. LU & UPPER PEWABIC & FRANKLIN - PEWABIC LOC. \\
\hline 3239 & & DINELLI & NORMA & D & 7 & ITALY & ITALY & ITALY & 1913 & & MATRAIA. LU & UPPER PEWABIC & FRANKLIN - PEWABIC LOC. \\
\hline 3240 & & DINELLI & ERNESTINA & D & 6 & MICHIGAN & ITALY & ITALY & & & MATRAIA, LU & UPPER PEWABIC & FRANKLIN - PEWABIC LOC. \\
\hline 3241 & & DINELLI & LILIA & D & 5 & MICHIGAN & ITALY & ITALY & & & MATRAIA. LU & UPPER PEWABIC & FRANKLIN - PEWABIC LOC. \\
\hline 3242 & & DINELLI & DORA & D & 4 & MICHIGAN & ITALY & ITALY & & & MATRAIA. LU & UPPER PEWABIC & FRANKLIN - PEWABIC LOC. \\
\hline 3243 & & DINELLI & ROLANDO & $\bar{s}$ & 1 & MICHIGAN & ITALY & ITALY & & & MATRAIA. LU & UPPER PEWABIC & FRANKLIN - PEWABIC LOC. \\
\hline 3244 & & GEMIGNANI & JOSEPH & B & UN & ITALY & ITALY & ITALY & UN & MINER/CM & LUCCA & UPPER PEWABIC & FRANKLIN - PEWABIC LOC. \\
\hline 3245 & 582 & GEMIGNANI & EUGENIO & H & 43 & ITALY & ITALY & ITALY & 1901 & MINER/CM & LUCCA & UPPER PEWABIC & FRANKLIN - PEWABIC LOC. \\
\hline 3246 & 302 & GEMIGNANI & ROSA & w & 30 & ITALY & ITALY & ITALY & 1911 & & LUCCA & UPPER PEWABIC & FRANKLIN - PEWABIC LOC. \\
\hline 3247 & & GEMIGNANI & ADELE & $\mathrm{D}$ & 11 & ITALY & ITALY & ITALY & 1911 & & LUCCA & UPPER PEWABIC & FRANKLIN - PEWABIC LOC. \\
\hline 3248 & & GEMIGNANI & BERNICE & $\mathrm{D}$ & 6 & MICHIGAN & ITALY & ITALY & & & LUCCA & UPPER PEWABIC & FRANKLIN - PEWABIC LOC. \\
\hline 3249 & & GEMIGNANI & FRANCIS & $\mathrm{s}$ & 2 & MICHIGAN & ITALY & ITALY & & & LUCCA & UPPER PEWABIC & FRANKLIN - PEWABIC LOC. \\
\hline 3250 & & GEMIGNANI & LOUIS & s & $8 \mathrm{M}$ & MICHIGAN & ITALY & ITALY & & & LUCCA & UPPER PEWABIC & FRANKLIN - PEWABIC LOC. \\
\hline 3251 & & BERTACCHI & JOHN & BIL & 28 & ITALY & ITALY & ITALY & 1901 & MINER/CM & ORBICCIANO. LU & UPPER PEWABIC & FRANKLIN - PEWABIC LOC. \\
\hline 3252 & 196 & GRISOLANO & $\mathrm{JOHN}$ & $\mathrm{H}$ & 25 & MICHIGAN & ITALY & ITALY & & MINER/CM & TORINO & UPPER PEWABIC & FRANKLIN - PEWABIC LOC. \\
\hline 3253 & & GRISOLANO & CLARA & w & 26 & MICHIGAN & NEW YORK & ENGLAND & & & USA-ENGLAND & UPPER PEWABIC & FRANKLIN - PEWABIC LOC. \\
\hline 3254 & ?? & MASINI & ALFRED & $\mathrm{H}$ & 35 & ITALY & ITALY & ITALY & UN & CARPENTER/CM & MONSAGRATI. LU & UPPER PEWABIC & FRANKLIN - PEWABIC LOC. \\
\hline 3255 & & MASINI & MONTONI & w & 26 & ITALY & ITALY & ITALY & 1908 & & & UPPER PEWABIC & FRANKLIN - PEWABIC LOC. \\
\hline 3256 & & MASINI & AMELIA & D & 9 & MICHIGAN & ITALY & ITALY & & & MONSAGRATI. LU & UPPER PEWABIC & FRANKLIN - PEWABIC LOC. \\
\hline 3257 & & MASINI & JOSEPH & s & 7 & MICHIGAN & ITALY & ITALY & & & MONSAGRATI. LU & UPPER PEWABIC & FRANKLIN - PEWABIC LOC. \\
\hline 3258 & & MASINI & ALADINA & D & 6 & MICHIGAN & ITALY & ITALY & & & MONSAGRATI. LU & UPPER PEWABIC & FRANKLIN - PEWABIC LOC. \\
\hline 3259 & & MASINI & BALIA & D & 5 & MICHIGAN & ITALY & ITALY & & & MONSAGRATI. LU & UPPER PEWABIC & FRANKLIN - PEWABIC LOC. \\
\hline 3260 & 359 & GEMIGNANI & ADOLFO & $\mathrm{H}$ & 47 & ITALY & ITALY & ITALY & 1916 & MINER/CM & PESCAGLIA. LU & UPPER PEWABIC & FRANKLIN - PEWABIC LOC. \\
\hline 3261 & & GEMIGNANI & ASSUNTA & w & 44 & ITALY & ITALY & ITALY & 1916 & & PESCAGLIA. LU & UPPER PEWABIC & FRANKLIN - PEWABIC LOC. \\
\hline 3262 & & GEMIGNANI & GINO & $\mathrm{s}$ & 15 & ITALY & ITALY & ITALY & 1916 & SHOEMAKER/GENERAL SHOP & PESCAGLIA. LU & UPPER PEWABIC & FRANKLIN - PEWABIC LOC. \\
\hline 3263 & & GEMIGNANI & FERNANDA & D & 14 & ITALY & ITALY & ITALY & 1916 & & PESCAGLIA. LU & UPPER PEWABIC & FRANKLIN - PEWABIC LOC. \\
\hline 3264 & & ROSSI & JOSEPH & GS & 1 & MICHIGAN & ITALY & ITALY & & & & UPPER PEWABIC & FRANKLIN - PEWABIC LOC. \\
\hline 3265 & & ROSSI & BERNARDO & SOIL & 23 & ITALY & ITALY & ITALY & 1916 & MINER/CM & & UPPER PEWABIC & FRANKLIN - PEWABIC LOC. \\
\hline 3266 & 258 & $\mathrm{RICCl}$ & ANGELA & $\mathrm{H}$ & 42 & ITALY & ITALY & ITALY & 1907 & & MATRAIA. LU & UPPER PEWABIC & FRANKLIN - PEWABIC LOC. \\
\hline 3267 & & $\mathrm{RICCl}$ & GIUSEPPINA & D & 12 & MICHIGAN & ITALY & ITALY & & & MATRAIA. LU & UPPER PEWABIC & FRANKLIN - PEWABIC LOC. \\
\hline 3268 & & $\mathrm{RICCl}$ & MARY & D & 11 & MICHIGAN & ITALY & ITALY & & & MATRAIA. LU & UPPER PEWABIC & FRANKLIN - PEWABIC LOC. \\
\hline 3269 & & $\mathrm{RICCl}$ & ROSIE & D & 8 & MICHIGAN & ITALY & ITALY & & & MATRAIA. LU & UPPER PEWABIC & FRANKLIN - PEWABIC LOC. \\
\hline 3270 & & $\mathrm{RICCl}$ & ANNA & D & 3 & MICHIGAN & ITALY & ITALY & & & MATRAIA, LU & UPPER PEWABIC & FRANKLIN - PEWABIC LOC. \\
\hline 3271 & & QUILICI & JOHN & B & 39 & ITALY & ITALY & ITALY & 1905 & MINER/CM & LUCCA & UPPER PEWABIC & FRANKLIN - PEWABIC LOC. \\
\hline 3272 & STA & PATERNI & LUIGI & $\mathrm{H}$ & 45 & ITALY & ITALY & ITALY & 1896 & MINER/CM & BAGNI DI LUCCA. LU & BACK ST. & FRANKLIN - PEWABIC LOC. \\
\hline 3273 & & PATERNI & ZELINDA & w & 38 & ITALY & ITALY & ITALY & 1901 & & & BACK ST. & FRANKLIN - PEWABIC LOC. \\
\hline 3274 & & PATERNI & MARIA & D & 18 & MICHIGAN & ITALY & ITALY & & & BAGNI DI LUCCA. LU & BACK ST. & FRANKLII \\
\hline 3275 & & PATERNI & ROSINA & D & 16 & MICHIGAN & ITALY & ITALY & & & BAGNI DI LUCCA. LU & BACK ST. & FRANKLIN - PEWABIC LOC. \\
\hline
\end{tabular}




\begin{tabular}{|c|c|c|c|c|c|c|c|c|c|c|c|c|c|}
\hline 3276 & & PATERNI & LOUISA & $\mathrm{D}$ & 14 & MICHIGAN & ITALY & ITALY & & & BAGNI DI LUCCA. LU & BACK ST. & FRANKLIN - PEWABIC LOC. \\
\hline 3277 & & PATERNI & PAOLINO & $\mathrm{s}$ & 12 & MICHIGAN & ITALY & ITALY & & & BAGNI DI LUCCA. LU & BACK ST. & FRANKLIN - PEWABIC LOC. \\
\hline 3278 & & PATERNI & GIORGIO & $\mathrm{s}$ & 4 & MICHIGAN & ITALY & ITALY & & & BAGNI DI LUCCA. LU & BACK ST. & FRANKLIN - PEWABIC LOC. \\
\hline 3279 & & CORSI & GINO & B & 33 & ITALY & ITALY & ITALY & 1886 & MINER/CM & MONSACRATI. LU & BACK ST. & FRANKLIN - PEWABIC LOC. \\
\hline 3280 & & PATERNI & GIUSEPPE & B & 26 & ITALY & ITALY & ITALY & 1915 & LABORER/CM & BAGNI DI LUCCA. LU & BACK ST. & FRANKLIN - PEWABIC LOC. \\
\hline 3281 & & BINI & PIETRO & B & 39 & ITALY & ITALY & ITALY & UN & MINER/CM & & BACK ST. & FRANKLIN - PEWABIC LOC. \\
\hline 3282 & & PATERNI & ANGELO & B & 24 & ITALY & ITALY & ITALY & UN & MINER/CM & BAGNI DI LUCCA. LU & BACK ST. & FRANKLIN - PEWABIC LOC. \\
\hline 3283 & & NELLI & GIUSEPPE & B & 25 & ITALY & ITALY & ITALY & UN & LABORER/CM & LUCCA & BACK ST. & FRANKLIN - PEWABIC LOC. \\
\hline 3284 & & DECOTTI & LUIGI & B & 27 & ITALY & ITALY & ITALY & UN & MINER/CM & & $\begin{array}{l}\text { BACK ST. } \\
\text { BAT }\end{array}$ & FRANKLIN - PEWABIC LOC. \\
\hline 3285 & & PATERNI & ANGELO & B & 28 & ITALY & ITALY & ITALY & UN & LABORER/CM & BAGNI DI LUCCA. LU & BACK ST. & FRANKLIN - PEWABIC LOC. \\
\hline 3286 & & CONSANI & PASQUINIO & B & 27 & ITALY & ITALY & ITALY & 1912 & LABORER/CM & LUCCA & BACK ST. & FRANKLIN - PEWABIC LOC. \\
\hline 3287 & & CONSANI & GIUSEPPE & B & 28 & ITALY & ITALY & ITALY & 1911 & MINER/CM & LUCCA & BACK ST. & FRANKLIN - PEWABIC LOC. \\
\hline 3288 & 252 & PIZZI & CESARE & $\mathrm{H}$ & 42 & ITALY & ITALY & ITALY & 1900 & LABORER/CM & MONSAGRATI, LU & BACK ST. & FRANKLIN - PEWABIC LOC. \\
\hline 3289 & & PIZZI & ALBERTINA & W & 33 & ITALY & ITALY & ITALY & 1900 & & MONSAGRATI. LU & BACK ST. & FRANKLIN - PEWABIC LOC. \\
\hline 3290 & & PIZZI & DOMENICO & $\mathrm{s}$ & 10 & MICHIGAN & ITALY & ITALY & & & MONSAGRATI. LU & BACK ST. & FRANKLIN - PEWABIC LOC. \\
\hline 3291 & & PIZZI & GERINANA & $\mathrm{D}$ & 9 & MICHIGAN & ITALY & ITALY & & & MONSAGRATI, LU & BACK ST. & FRANKLIN - PEWABIC LOC. \\
\hline 3292 & & PIZZI & DINA & D & 6 & MICHIGAN & ITALY & ITALY & & & MONSAGRATI. LU & BACK ST. & FRANKLIN - PEWABIC LOC. \\
\hline 3293 & & PIZZI & OTELLA & $\mathrm{D}$ & 3 & MICHIGAN & ITALY & ITALY & & & MONSAGRATI. LU & BACK ST. & FRANKLIN - PEWABIC LOC. \\
\hline 3294 & & PIZZI & VINCENZO & $\mathrm{COU}$ & 41 & ITALY & ITALY & ITALY & 1903 & TRAMMER/CM & MONSAGRATI. LU & BACK ST. & FRANKLIN - PEWABIC LOC. \\
\hline 3295 & & BRUSORIO & CARLO & $\mathrm{H}$ & 35 & ITALY & ITALY & ITALY & 1909 & MINER/CM & MILANO & & FRANKLIN - COBURNTOWN \\
\hline 3296 & & BRUSORIO & IRENE & w & 31 & ITALY & ITALY & ITALY & 1912 & & & & FRANKLIN - COBURNTOWN \\
\hline 3297 & & BRUSORIO & MARIO & s & 6 & MICHIGAN & ITALY & ITALY & & & MILANO & & FRANKLIN - COBURNTOWN \\
\hline 3298 & & BRUSORIO & LOUIS & s & 4 & MICHIGAN & ITALY & ITALY & & & MILANO & & FRANKLIN - COBURNTOWN \\
\hline 3299 & & BRUSORIO & AMELIA & $\mathrm{D}$ & 1 & MICHIGAN & ITALY & ITALY & & & MILANO & & FRANKLIN - COBURNTOWN \\
\hline 3300 & & BERCETTI & CARLO & B & 41 & ITALY & ITALY & ITALY & 1913 & TRAMMER/CM & & & FRANKLIN - COBURNTOWN \\
\hline 3301 & & GOSATTI & TONY & B & 35 & ITALY & ITALY & ITALY & 1909 & MINER/CM & BIANZANO. SONDRIO & & FRANKLIN - COBURNTOWN \\
\hline 3302 & & PATRI & CAMBRINE & $\mathrm{H}$ & 37 & ITALY & ITALY & ITALY & 1907 & MINER/CM & 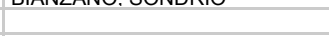 & & FRANKLIN - COBURNTOWN \\
\hline 3303 & & PATRI & MARY & w & 36 & ITALY & ITALY & ITALY & 1916 & & & & FRANKLIN - COBURNTOWN \\
\hline 3304 & & PATRI & VALENTINA & D & 12 & ITALY & ITALY & ITALY & 1916 & & & & FRANKLIN - COBURNTOWN \\
\hline 3305 & & PATRI & ANGELINA & $\mathrm{D}$ & 3 & MICHIGAN & ITALY & ITALY & & & & & FRANKLIN - COBURNTOWN \\
\hline 3306 & & PATRI & PETER & $\mathrm{s}$ & 8 & ITALY & ITALY & ITALY & 1916 & & & & FRANKLIN - COBURNTOWN \\
\hline 3307 & & PAOLINI & ROMEO & B & 36 & ITALY & ITALY & ITALY & UN & TRAMMER/CM & & & FRANKLIN - COBURNTOWN \\
\hline 3308 & & POGGIONE & JOHN & $\mathrm{H}$ & 51 & ITALY & ITALY & ITALY & 1888 & FARMER & SAN GIORGIO CANAVESE. TO & & FRANKLIN - COBURNTOWN \\
\hline 3309 & & POGGIONE & MARY & w & 40 & ITALY & ITALY & ITALY & 1885 & & & & FRANKLIN - COBURNTOWN \\
\hline 3310 & & POGGIONE & JAMES & $\mathrm{s}$ & 23 & MICHIGAN & ITALY & ITALY & & FIREMAN/ENGINE HOUSE & SAN GIORGIO CANAVESE. TO & & FRANKLIN - COBURNTOWN \\
\hline 3311 & & POGGIONE & $\begin{array}{l}\text { PETER } \\
\text { PETER }\end{array}$ & $\frac{3}{2}$ & 21 & MICHIGAN & ITALY & ITALY & & FIREIVIAIVIEIVUINE MUUSE & SAN GIORGIO CANAVESE. TO & & FRANKLIN - COBURNTOWN \\
\hline 3312 & & POGGIONE & JOSEPH & $\mathrm{s}$ & 19 & MICHIGAN & ITALY & ITALY & & & SAN GIORGIO CANAVESE. TO & & FRANKLIN - COBURNTOWN \\
\hline 3313 & & POGGIONE & CLARA & $\mathrm{D}$ & 13 & MICHIGAN & ITALY & ITALY & & & SAN GIORGIO CANAVESE. TO & & FRANKLIN - COBURNTOWN \\
\hline 3314 & & POGGIONE & $\mathrm{JOHN}$ & $\mathrm{s}$ & 11 & MICHIGAN & ITALY & ITALY & & & SAN GIORGIO CANAVESE. TO & & FRANKLIN - COBURNTOWN \\
\hline 3315 & & POGGIONE & FELIX & s & 10 & MICHIGAN & ITALY & ITALY & & & SAN GIORGIO CANAVESE. TO & & FRANKLIN - COBURNTOWN \\
\hline 3316 & & POGGIONE & JOSEPHINE & SIIL & 31 & MICHIGAN & ITALY & ITALY & & & SAN GIORGIO CANAVESE. TO & & FRANKLIN - COBURNTOWN \\
\hline 3317 & & MANZETTA & LENA & SER & 19 & ITALY & ITALY & ITALY & 1905 & SERVANT/PF & PONT CANAVESE. TO & & FRANKLIN - COBURNTOWN \\
\hline 3318 & & PICCHIOTTINO & JOHN & $\mathrm{H}$ & 33 & ITALY & ITALY & ITALY & 1893 & CLERK/GROCERY STORE & PONT CANAVESE. TO & & FRANKLIN - COBURNTOWN \\
\hline 3319 & & PICCHIOTTINO & MARY & w & 27 & MICHIGAN & ITALY & ITALY & & & & & FRANKLIN - COBURNTOWN \\
\hline 3320 & & PICCHIOTTINO & MONDINA & D & 3 & MICHIGAN & ITALY & MICHIGAN & & & PONT CANAVESE. TO & & FRANKLIN - COBURNTOWN \\
\hline 3321 & & COLOMBO & JOSEPH & $\mathrm{H}$ & 59 & ITALY & ITALY & ITALY & 1888 & LABORER/CM & TORINO & & FRANKLIN - COBURNTOWN \\
\hline 3322 & & COLOMBO & MADDALENA & w & 58 & ITALY & ITALY & ITALY & 1888 & & TORINO & & FRANKLIN - COBURNTOWN \\
\hline 3323 & & COLOMBO & JOSEPH & s & 29 & MICHIGAN & ITALY & ITALY & & BUTCHER/MEAT MARKET & TORINO & & FRANKLIN - COBURNTOWN \\
\hline 3324 & & COLOMBO & ABRAMO & s & 24 & MICHIGAN & $\begin{array}{l}\text { ITALY } \\
\text { ITALY }\end{array}$ & $\begin{array}{l}\text { ITALY } \\
\text { ITALY }\end{array}$ & & TEAMSTER/MEAT GROCERY & TORINO & & FRANKLIN - COBURNTOWN \\
\hline 3325 & & COLOMBO & ANTONIO & $\mathrm{s}$ & 19 & MICHIGAN & ITALY & ITALY & & BUTCHER/MEAT MARKET & TORINO & & FRANKLIN - COBURNTOWN \\
\hline 3326 & & COLOMBO & LUCY & $\mathrm{D}$ & 18 & MICHIGAN & ITALY & ITALY & & & TORINO & & FRANKLIN - COBURNTOWN \\
\hline 3327 & & COLOMBO & SAM & $\mathrm{s}$ & 15 & MICHIGAN & ITALY & ITALY & & & TORINO & & FRANKLIN - COBURNTOWN \\
\hline 3328 & & COLOMBO & GEORGE & s & 13 & MICHIGAN & ITALY & ITALY & & & TORINO & & FRANKLIN - COBURNTOWN \\
\hline 3329 & & COLOMBO & LINA & D & 11 & MICHIGAN & ITALY & ITALY & & & TORINO & & FRANKLIN - COBURNTOWN \\
\hline 3330 & & COLOMBO & JOHN & s & 9 & MICHIGAN & ITALY & ITALY & & & TORINO & & FRANKLIN - COBURNTOWN \\
\hline 3331 & & GOSATTI & JOHN & $\mathrm{H}$ & 30 & ITALY & ITALY & ITALY & 1910 & MINER/CM & BIANZANO. SONDRIO & & FRANKLIN - COBURNTOWN \\
\hline 3332 & & GOSATTI & TERESA & w & 30 & ITALY & ITALY & ITALY & 1912 & & & & FRANKLIN - COBURNTOWN \\
\hline 3333 & & GOSATTI & NUNZIA & s & 3 & MICHIGAN & ITALY & ITALY & & & BIANZANO, SONDRIO & & FRANKLIN - COBURNTOWN \\
\hline 3334 & & GOSATTI & JOSEPHINE & D & 2 & MICHIGAN & ITALY & ITALY & & & BIANZANO. SONDRIO & & FRANKLIN - COBURNTOWN \\
\hline 3335 & & MARCHIONE & ANTHONY & B & 24 & ITALY & ITALY & ITALY & 1913 & MINER/CM & & & FRANKLIN - COBURNTOWN \\
\hline 3336 & & MARCHIONE & MATT & B & 51 & ITALY & ITALY & ITALY & 1905 & LABORER/CM & & & FRANKLIN - COBURNTOWN \\
\hline 3337 & & CERUTTI & JOSEPH & B & 32 & ITALY & ITALY & ITALY & 1908 & MINER/CM & SAN GIORGIO CANAVESE. TO & & FRANKLIN - COBURNTOWN \\
\hline 3338 & & GIUNTONAL & AMBROGIO & B & 28 & ITALY & ITALY & ITALY & 1912 & LABORER/CM & & & FRANKLIN - COBURNTOWN \\
\hline
\end{tabular}




\begin{tabular}{|c|c|c|c|c|c|c|c|c|c|c|c|c|c|}
\hline 3339 & & GOSATTI & ANDREW & B & 57 & ITALY & ITALY & ITALY & 1897 & MINER/CM & BIANZANO. SONDRIO & & FRANKLIN - COBURNTOWN \\
\hline 3340 & & GOSATTI & ANDREW & B & 17 & MICHIGAN & ITALY & ITALY & & LABORER/CM & BIANZANO. SONDRIO & & FRANKLIN - COBURNTOWN \\
\hline 3341 & & VELIE & JOSEPH & B & 30 & ITALY & ITALY & ITALY & 1914 & MINER/CM & & & FRANKLIN - COBURNTOWN \\
\hline 3342 & & GANELLINI & JAMES & B & 26 & ITALY & ITALY & ITALY & 1913 & MINER/CM & TORINO & & FRANKLIN - COBURNTOWN \\
\hline 3343 & & ORTENZI & JOHN & $\mathrm{H}$ & 56 & ITALY & ITALY & ITALY & 1890 & MINER/CM & LUCCA & & FRANKLIN - COBURNTOWN \\
\hline 3344 & & ORTENZI & MARY & w & 46 & ITALY & ITALY & ITALY & 1899 & & & & FRANKLIN - COBURNTOWN \\
\hline 3345 & & ORTENZI & PETER & s & 23 & MICHIGAN & ITALY & ITALY & & BUTCHER/MEAT MARKET & LUCCA & & FRANKLIN - COBURNTOWN \\
\hline 3346 & & ORTENZI & MARIO & s & 20 & MICHIGAN & ITALY & ITALY & & ARMY & LUCCA & & FRANKLIN - COBURNTOWN \\
\hline 3347 & & ORTENZI & JOHN & $\hat{s}$ & 19 & MICHIGAN & ITALY & ITALY & & BUTCHER/MEAT MARKET & LUCCA & & FRANKLIN - COBURNTOWN \\
\hline 3348 & & ORTENZI & JOSEPHINE & D & 17 & MICHIGAN & ITALY & ITALY & & & LUCCA & & FRANKLIN - COBURNTOWN \\
\hline 3349 & & ORTENZI & JAMES & $\mathrm{s}$ & 15 & MICHIGAN & ITALY & ITALY & & BUTCHER/MEAT MARKET & LUCCA & & FRANKLIN - COBURNTOWN \\
\hline 3350 & & ORTENZI & CAROLINA & $\mathrm{D}$ & 13 & MICHIGAN & ITALY & ITALY & & & LUCCA & & FRANKLIN - COBURNTOWN \\
\hline 3351 & & ORTENZI & EDITH & D & 11 & MICHIGAN & ITALY & ITALY & & & LUCCA & & FRANKLIN - COBURNTOWN \\
\hline 3352 & & ORTENZI & JOSEPH & $\mathrm{s}$ & 10 & MICHIGAN & ITALY & ITALY & & & LUCCA & & FRANKLIN - COBURNTOWN \\
\hline 3353 & & ORTENZI & MARY & $\mathrm{D}$ & 4 & MICHIGAN & ITALY & ITALY & & & LUCCA & & FRANKLIN - COBURNTOWN \\
\hline 3354 & & DAO & JOHN & $\mathrm{H}$ & 40 & ITALY & ITALY & ITALY & 1905 & MINER/CM & CICONIO, TO & & FRANKLIN - COBURNTOWN \\
\hline 3355 & & DAO & DOEMNICA & W & 28 & ITALY & ITALY & ITALY & 1912 & & & & FRANKLIN - COBURNTOWN \\
\hline 3356 & & DAO & KATE & D & 6 & MICHIGAN & ITALY & ITALY & & & CICONIO.TO & & FRANKLIN - COBURNTOWN \\
\hline 3357 & & FERINA & PETER & $\mathrm{H}$ & 31 & ITALY & ITALY & ITALY & 1914 & MINER/CM & TORINO & & FRANKLIN - COBURNTOWN \\
\hline 3358 & & FERINA & MARY & w & 26 & ITALY & ITALY & ITALY & 1915 & & & & FRANKLIN - COBURNTOWN \\
\hline 3359 & & FASANA & TERESA & $\mathrm{H}$ & 46 & ITALY & ITALY & ITALY & UN & & TORINO & & FRANKLIN - COBURNTOWN \\
\hline 3360 & & FASANA & MARY & D & $\begin{array}{l}40 \\
14\end{array}$ & MICHIGAN & ITALY & ITALY & Uiv & & TORINO & & FRANKLIN - COBURNTOWN \\
\hline 3361 & & FASANA & CLARA & $\mathrm{D}$ & 13 & MICHIGAN & ITALY & ITALY & & & TORINO & & FRANKLIN - COBURNTOWN \\
\hline 3362 & & FASANA & KATE & $\mathrm{D}$ & 12 & MICHIGAN & ITALY & ITALY & & & TORINO & & FRANKLIN - COBURNTOWN \\
\hline 3363 & & FASANA & JOHANNA & D & 9 & MICHIGAN & ITALY & ITALY & & & TORINO & & FRANKLIN - COBURNTOWN \\
\hline 3364 & & MANZETTI & LORENZO & $\mathrm{H}$ & 52 & ITALY & ITALY & ITALY & 1901 & LABORER/CM & PONT CANAVESE. TO & & FRANKLIN - SUNSHINE LOC. \\
\hline 3365 & & MANZETTI & MARY & W & 51 & ITALY & ITALY & ITALY & 1906 & & & & FRANKLIN - SUNSHINE LOC. \\
\hline 3366 & & MANZETTI & KATE & $\mathrm{D}$ & 11 & MICHIGAN & ITALY & ITALY & & & PONT CANAVESE, TO & & FRANKLIN - SUNSHINE LOC. \\
\hline 3367 & & MANZETTI & LORENZO & $\mathrm{S}$ & 10 & MICHIGAN & ITALY & ITALY & & & PONT CANAVESE. TO & & FRANKLIN - SUNSHINE LOC. \\
\hline 3368 & & BLESSENT & $\mathrm{JOHN}$ & $\mathrm{H}$ & 48 & ITALY & ITALY & ITALY & 1885 & FARMER & SPARONE. TO & & FRANKLIN - ARCADIAN MINE \\
\hline 3369 & & BLESSENT & MADDALENA & W & 35 & ITALY & ITALY & ITALY & 1909 & & & & FRANKLIN - ARCADIAN MINE \\
\hline 3370 & & BLESSENT & LUCY & D & 8 & MICHIGAN & ITALY & ITALY & & & SPARONE. TO & & FRANKLIN - ARCADIAN MINE \\
\hline 3371 & & BLESSENT & JOHANNA & $\mathrm{D}$ & 6 & MICHIGAN & ITALY & ITALY & & & SPARONE. TO & & FRANKLIN - ARCADIAN MINE \\
\hline 3372 & & BLESSENT & MARNIE & D & 5 & MICHIGAN & ITALY & ITALY & & & SPARONE, TO & & FRANKLIN - ARCADIAN MINE \\
\hline 3373 & & BLESSENT & PETER & s & 3 & MICHIGAN & ITALY & ITALY & & & SPARONE. TO & & FRANKLIN - ARCADIAN MINE \\
\hline 3374 & & BLESSENT & BATTISTA & $\mathrm{s}$ & 1 & MICHIGAN & ITALY & ITALY & & & SPARONE. TO & & FRANKLIN - ARCADIAN MINE \\
\hline 3375 & & GASPARDO & JAMES & $\mathrm{H}$ & 30 & ITALY & ITALY & ITALY & 1906 & DAIRY BUSINESS & PONT CANAVESE. TO & & FRANKLIN - ARCADIAN MINE \\
\hline 3376 & & GASPARDO & FRANCIS & W & 22 & ITALY & ITALY & ITALY & 1899 & & & & FRANKLIN - ARCADIAN MINE \\
\hline 3377 & & GASPARDO & JOHANNA & $\mathrm{D}$ & 5 & MICHIGAN & ITALY & ITALY & & & PONT CANAVESE. TO & & FRANKLIN - ARCADIAN MINE \\
\hline 3378 & & GASPARDO & BATTISTA & $\mathrm{S}$ & 4 & MICHIGAN & ITALY & ITALY & & & PONT CANAVESE. TO & & FRANKLIN - ARCADIAN MINE \\
\hline 3379 & & GASPARDO & JOHN & $\mathrm{S}$ & 1 & MICHIGAN & ITALY & ITALY & & & PONT CANAVESE. TO & & FRANKLIN - ARCADIAN MINE \\
\hline 3380 & & BLESSENT & MARTIN & B & 45 & ITALY & ITALY & ITALY & 1910 & LABORER/DAIRY BUSINESS & SPARONE. TO & & FRANKLIN - ARCADIAN MINE \\
\hline 3381 & 716 & FRANCHINI & FILIPPO & $\mathrm{H}$ & 25 & ITALY & ITALY & ITALY & 1911 & MINER/CM & FANO & COUNTY ROAD & FRANKLIN - MESNARD LOC. \\
\hline 3382 & & FRANCHINI & MARY & w & 23 & MICHIGAN & ITALY & ITALY & & & & COUNTY ROAD & FRANKLIN - MESNARD LOC. \\
\hline 3383 & & FRANCHINI & ROBERT & $\mathrm{s}$ & 1 & MICHIGAN & ITALY & MICHIGAN & & & FANO & COUNTY ROAD & FRANKLIN - MESNARD LOC. \\
\hline 3384 & 449 & MUGGIANI & FRANK & $\mathrm{H}$ & 30 & ITALY & ITALY & ITALY & 1910 & MINER/CM & Tivo & & FRANKLIN - MESNARD LOC. \\
\hline 3385 & & MUGGIANI & PAULINA & w & 33 & ITALY & ITALY & ITALY & 1913 & & & & FRANKLIN - MESNARD LOC. \\
\hline 3386 & & MUGGIANI & ARGENTINA & $\mathrm{D}$ & 6 & MICHIGAN & ITALY & ITALY & & & & & FRANKLIN - MESNARD LOC. \\
\hline 3387 & & MUGGIANI & GINA & $\mathrm{s}$ & 5 & MICHIGAN & ITALY & ITALY & & & & & FRANKLIN - MESNARD LOC. \\
\hline 3388 & & MUGGIANI & DORA & D & 2 & MICHIGAN & ITALY & ITALY & & & & & FRANKLIN - MESNARD LOC. \\
\hline 3389 & & BERSOCHI & ALGUNU & B & 28 & ITALY & ITALY & ITALY & 1913 & MINER/CM & & & FRANKLIN - MESNARD LOC. \\
\hline 3390 & & MAIANI/MOIANI & GIUSEPPE & B & 36 & ITALY & ITALY & ITALY & 1909 & MINER/CM & & & FRANKLIN - MESNARD LOC. \\
\hline 3391 & 751 & NOVELLI & AUGUSTO & $\mathrm{H}$ & 45 & ITALY & ITALY & ITALY & 1904 & TRAMMER/CM & CASTELVECCHIO. LU & & FRANKLIN - MESNARD LOC. \\
\hline 3392 & & NOVELLI & VIOLA & W & 35 & ITALY & ITALY & ITALY & 1906 & & & & FRANKLIN - MESNARD LOC. \\
\hline 3393 & & NOVELLI & ORLANDO & S & 12 & MICHIGAN & ITALY & ITALY & & & CASTELVECCHIO. LU & & FRANKLIN - MESNARD LOC. \\
\hline 3394 & & SALANI & NUNZIATA & MIL & 66 & ITALY & ITALY & ITALY & 1906 & & LUCCA & & FRANKLIN - MESNARD LOC. \\
\hline 3395 & 743 & STEFANI & FELIX & $\mathrm{H}$ & 39 & MICHIGAN & ITALY & ITALY & & MINER/CM & LUCCA & & FRANKLIN - MESNARD LOC. \\
\hline 3396 & & STEFANI & DOVINA & W & 25 & MICHIGAN & ITALY & ITALY & & & & & FRANKLIN - MESNARD LOC. \\
\hline 3397 & & STEFANI & LENA & D & 3 & MICHIGAN & ITALY & ITALY & & & LUCCA & & FRANKLIN - MESNARD LOC. \\
\hline 3398 & 740 & CORTOPASSI & PETER & $\mathrm{H}$ & 29 & ITALY & ITALY & ITALY & 1911 & MINER/CM & LUCCA & & FRANKLIN - MESNARD LOC. \\
\hline 3399 & & CORTOPASSI & BERTA & W & 27 & ITALY & ITALY & ITALY & 1912 & & & & FRANKLIN - MESNARD LOC. \\
\hline 3400 & & CORTOPASSI & JOHN & s & 6 & MICHIGAN & ITALY & ITALY & & & LUCCA & & FRANKLIN - MESNARD LOC. \\
\hline 3401 & & CORTOPASSI & INES & D & 4 & MICHIGAN & ITALY & ITALY & & & LUCCA & & FRANKLIN - MESNARD LOC. \\
\hline
\end{tabular}




\begin{tabular}{|c|c|c|c|c|c|c|c|c|c|c|c|c|}
\hline 3402 & & CORTOPASSI & MARIO & $\mathrm{s}$ & 1 & MICHIGAN & ITALY & ITALY & & & LUCCA & FRANKLIN - MESNARD LOC. \\
\hline 3403 & 739 & PIZZI & LOUIS & $\mathrm{H}$ & 28 & ITALY & ITALY & ITALY & 1909 & MINER/CM & LUCCA & FRANKLIN - MESNARD LOC. \\
\hline 3404 & & PIZZI & ANITA & W & 24 & ITALY & ITALY & ITALY & 1911 & & & FRANKLIN - MESNARD LOC. \\
\hline 3405 & & PIZZI & LETA & D & 6 & MICHIGAN & ITALY & ITALY & & & LUCCA & FRANKLIN - MESNARD LOC. \\
\hline 3406 & & PIZZI & JOSEPH & $\mathrm{s}$ & 3 & MICHIGAN & ITALY & ITALY & & & LUCCA & FRANKLIN - MESNARD LOC. \\
\hline 3407 & & PIZZI & JAMES & $\mathrm{s}$ & 1 & MICHIGAN & ITALY & ITALY & & & LUCCA & FRANKLIN - MESNARD LOC. \\
\hline 3408 & & PIZZI & ZELINDA & $\mathrm{D}$ & $4 \mathrm{M}$ ? & MICHIGAN & ITALY & ITALY & & & LUCCA & FRANKLIN - MESNARD LOC. \\
\hline 3409 & 776 & MONTANARI & HENRY & $\mathrm{H}$ & 50 & ITALY & ITALY & ITALY & 1894 & MINER/CM & & FRANKLIN - MESNARD LOC. \\
\hline 3410 & & MONTANARI & TERESA & w & 42 & ITALY & ITALY & ITALY & 1900 & & & FRANKLIN - MESNARD LOC. \\
\hline 3411 & & MONTANARI & OLIMPIA & $\mathrm{D}$ & 18 & MICHIGAN & ITALY & ITALY & & & & FRANKLIN - MESNARD LOC. \\
\hline 3412 & & MONTANARI & ALFONSO & $\mathrm{s}$ & 17 & MICHIGAN & ITALY & ITALY & & WEIGH MASTER/CM & & FRANKLIN - MESNARD LOC. \\
\hline 3413 & & MONTANARI & ALDO & $\mathrm{S}$ & 16 & MICHIGAN & ITALY & ITALY & & & & FRANKLIN - MESNARD LOC. \\
\hline 3414 & & MONTANARI & WILLIAM & $\mathrm{s}$ & 10 & MICHIGAN & ITALY & ITALY & & & & FRANKLIN - MESNARD LOC. \\
\hline 3415 & & MONTANARI & RAYMOND & $\mathrm{s}$ & 5 & MICHIGAN & ITALY & ITALY & & & & FRANKLIN - MESNARD LOC. \\
\hline 3416 & 772 & NOVI & CARLO & $\mathrm{H}$ & 28 & ITALY & ITALY & ITALY & 1910 & MINER/CM & PESCAGLIA, LU & FRANKLIN - MESNARD LOC. \\
\hline 3417 & & NOVI & ROSIE & W & 24 & MICHIGAN & ITALY & ITALY & & & & FRANKLIN - MESNARD LOC. \\
\hline 3418 & & NOVI & LOUIS & $\mathrm{s}$ & $8 \mathrm{M}$ & MICHIGAN & ITALY & ITALY & & & PESCAGLIA. LU & FRANKLIN - MESNARD LOC. \\
\hline 3419 & 767 & TINETTI & MARTINO & $\mathrm{H}$ & 46 & ITALY & ITALY & ITALY & 1890 & MINER/CM & SAN MARTINO CANAVESE. TO & FRANKLIN - MESNARD LOC. \\
\hline 3420 & & TINETTI & ENRICHETTA & W & 42 & ITALY & ITALY & ITALY & 1907 & & & FRANKLIN - MESNARD LOC. \\
\hline 3421 & & TINETTI & TERESA & $\mathrm{D}$ & 8 & MICHIGAN & ITALY & ITALY & & & SAN MARTINO CANAVESE. TO & FRANKLIN - MESNARD LOC. \\
\hline 3422 & & TINETTI & MARGHERITA & $\mathrm{D}$ & 7 & MICHIGAN & ITALY & ITALY & & & SAN MARTINO CANAVESE. TO & FRANKLIN - MESNARD LOC. \\
\hline 3423 & & TINETTI & MARY & D & 5 & MICHIGAN & ITALY & ITALY & & & SAN MARTINO CANAVESE, TO & FRANKLIN - MESNARD LOC. \\
\hline 3424 & & TINETTI & ANTONIO & $\mathrm{s}$ & 2 & MICHIGAN & ITALY & ITALY & & & SAN MARTINO CANAVESE. TO & FRANKLIN - MESNARD LOC. \\
\hline 3425 & 771 & PROLA & MARTIN & $\mathrm{H}$ & 31 & ITALY & ITALY & ITALY & 1906 & MINER/CM & AGLIE'. TO & FRANKLIN - MESNARD LOC. \\
\hline 3426 & & PROLA & LOUIS & W & 30 & ITALY & ITALY & ITALY & 1913 & & & FRANKLIN - MESNARD LOC. \\
\hline 3427 & 768 & NATALI & CARLO & $\mathrm{H}$ & 45 & ITALY & ITALY & ITALY & 1889 & MINER/CM & & FRANKLIN - MESNARD LOC. \\
\hline 3428 & & NATALI & MARY & W & 40 & ITALY & ITALY & ITALY & 1905 & & & FRANKLIN - MESNARD LOC. \\
\hline 3429 & & NATALI & ALBERT & $\mathrm{s}$ & 14 & MICHIGAN & ITALY & ITALY & & & & FRANKLIN - MESNARD LOC. \\
\hline 3430 & & NATALI & NELLO & $\mathrm{S}$ & 13 & MICHIGAN & ITALY & ITALY & & & & FRANKLIN - MESNARD LOC. \\
\hline 3431 & & NATALI & LETO & $\mathrm{s}$ & 9 & MICHIGAN & ITALY & ITALY & & & & FRANKLIN - MESNARD LOC. \\
\hline 3432 & & NATALI & LENA & D & 4 & MICHIGAN & ITALY & ITALY & & & & FRANKLIN - MESNARD LOC. \\
\hline 3433 & 768 & VIVENO/VIVINO & CARLO & B & 40 & ITALY & ITALY & ITALY & 1915 & MINER/CM & & FRANKLIN - MESNARD LOC. \\
\hline 3434 & 765 & FORNERIS & BATTISTA & $\mathrm{H}$ & 32 & ITALY & ITALY & ITALY & 1910 & LABORER/CM & SAN GIORGIO CANAVESE. TO & FRANKLIN - MESNARD LOC. \\
\hline 3435 & 766 & CAVALZANI & ERNEST & $\mathrm{H}$ & 40 & ITALY & ITALY & ITALY & 1906 & MINER/CM & SAN GENNARO, LU & FRANKLIN - MESNARD LOC. \\
\hline 3436 & & CAVALZANI & MARY & w & 36 & ITALY & ITALY & ITALY & 1907 & & & FRANKLIN - MESNARD LOC. \\
\hline 3437 & & CAVALZANI & CESARA & D & 10 & MICHIGAN & ITALY & ITALY & & & SAN GENNARO. LU & FRANKLIN - MESNARD LOC. \\
\hline 3438 & & CAVALZANI & JOHN & $\mathrm{s}$ & 9 & MICHIGAN & ITALY & ITALY & & & SAN GENNARO. LU & FRANKLIN - MESNARD LOC. \\
\hline 3439 & & CAVALZANI & ANNE & D & 7 & MICHIGAN & ITALY & ITALY & & & SAN GENNARO. LU & FRANKLIN - MESNARD LOC. \\
\hline 3440 & 767 & ROVENZO & TONY & $\mathrm{H}$ & 37 & ITALY & ITALY & ITALY & 1907 & MINER/CM & & FRANKLIN - MESNARD LOC. \\
\hline 3441 & & ROVENZO & TERESA & W & 32 & ITALY & ITALY & ITALY & 1910 & & & FRANKLIN - MESNARD LOC. \\
\hline 3442 & & ROVENZO & JOSEPH & $\mathrm{s}$ & 6 & MICHIGAN & ITALY & ITALY & & & & FRANKLIN - MESNARD LOC. \\
\hline 3443 & SCS & $\mathrm{RICCl}$ & EGIDIO & $\mathrm{H}$ & 46 & MICHIGAN & ITALY & ITALY & & LABORER/CM & LUCCA & FRANKLIN - MESNARD LOC. \\
\hline 3444 & & RICCI & AMELIA & W & 45 & MICHIGAN & ITALY & ITALY & & & Lovon & FRANKLIN - MESNARD LOC. \\
\hline 3445 & & RICCl & EMMA & D & 17 & MICHIGAN & MICHIGAN & MICHIGAN & & & LUCCA & FRANKLIN - MESNARD LOC. \\
\hline 3446 & & RICCl & JOSEPH & $\mathrm{s}$ & 14 & MICHIGAN & MICHIGAN & MICHIGAN & & & LUCCA & FRANKLIN - MESNARD LOC. \\
\hline 3447 & & RICCl & MARY & D & 12 & MICHIGAN & MICHIGAN & MICHIGAN & & & LUCCA & FRANKLIN - MESNARD LOC. \\
\hline 3448 & & RICCl & ANNE & $\mathrm{D}$ & 10 & MICHIGAN & MICHIGAN & MICHIGAN & & & LUCCA & FRANKLIN - MESNARD LOC. \\
\hline 3449 & & RICCl & JACOB & s & 8 & MICHIGAN & MICHIGAN & MICHIGAN & & & LUCCA & FRANKLIN - MESNARD LOC. \\
\hline 3450 & & RICCl & NICOLAS & $\mathrm{s}$ & 6 & MICHIGAN & MICHIGAN & MICHIGAN & & & LUCCA & \\
\hline 3451 & & RICCl & VERONICA & D & 4 & MICHIGAN & MICHIGAN & MICHIGAN & & & LUCCA & FRANKLIN - MESNARD LOC. \\
\hline 3452 & & $\mathrm{RICCl}$ & LIZ & D & 2 & MICHIGAN & MICHIGAN & MICHIGAN & & & LUCCA & FRANKLIN - MESNARD LOC. \\
\hline 3453 & 42 & PERA & IOLANDA & $\mathrm{H}$ & 34 & ITALY & ITALY & ITALY & 1907 & & & FRANKLIN - FRANKLIN MINE \\
\hline 3454 & & PERA & FRANK & $\mathrm{s}$ & 11 & MICHIGAN & ITALY & ITALY & & & & FRANKLIN - FRANKLIN MINE \\
\hline 3455 & & PERA & NELLO & $\mathrm{S}$ & 10 & MICHIGAN & ITALY & ITALY & & & & FRANKLIN - FRANKLIN MINE \\
\hline 3456 & & PERA & PERENA & D & 8 & MICHIGAN & ITALY & ITALY & & & & FRANKLIN - FRANKLIN MINE \\
\hline 3457 & & PERA & LETO & $\bar{s}$ & 6 & MICHIGAN & ITALY & ITALY & & & & FRANKLIN - FRANKLIN MINE \\
\hline 3458 & & PERA & DINA & D & 4 & MICHIGAN & ITALY & ITALY & & & & FRANKLIN - FRANKLIN MINE \\
\hline 3459 & & CROVICHI & GIULIO & B & 30 & ITALY & ITALY & ITALY & 1912 & MINER/CM & & FRANKLIN - FRANKLIN MINE \\
\hline 3460 & & BELAGRUNI & ARMANDO & B & 29 & ITALY & ITALY & ITALY & 1913 & MINER/CM & & FRANKLIN - FRANKLIN MINE \\
\hline 3461 & 442 & BONINI & CESARE & $\mathrm{H}$ & 31 & ITALY & ITALY & ITALY & 1901 & TRAMMER/CM & COLLODI. LU & FRANKLIN - FRANKLIN MINE \\
\hline 3462 & & BONINI & LIDIA & W & 23 & MICHIGAN & ITALY & ITALY & & & & FRANKLIN - FRANKLIN MINE \\
\hline 3463 & & BONINI & ANNE & $\mathrm{D}$ & 5 & MICHIGAN & ITALY & MICHIGAN & & & COLLODI. LU & FRANKLIN - FRANKLIN MINE \\
\hline 3464 & & BONINI & MARY & D & 3 & MICHIGAN & ITALY & MICHIGAN & & & COLLODI. LU & FRANKLIN - FRANKLIN MINE \\
\hline
\end{tabular}




\begin{tabular}{|c|c|c|c|c|c|c|c|c|c|c|c|c|}
\hline 3465 & & BONINI & WILL & $\mathrm{s}$ & 2 & MICHIGAN & ITALY & MICHIGAN & & & COLLODI. LU & FRANKLIN - FRANKLIN MINE \\
\hline 3466 & & BONINI & FLORA & D & $4 \mathrm{M}$ & MICHIGAN & ITALY & MICHIGAN & & & COLLODI. LU & FRANKLIN - FRANKLIN MINE \\
\hline 3467 & & BONINI & ANGELO & BR & 23 & ITALY & ITALY & ITALY & UN & LABORER/CM & COLLODI. LU & FRANKLIN - FRANKLIN MINE \\
\hline 3468 & 411 & FILIPPI & GIOVANNI & $\mathrm{H}$ & 34 & ITALY & ITALY & ITALY & UN & MINER/CM & PESCAGLIA. LU & FRANKLIN - FRANKLIN MINE \\
\hline 3469 & & FILIPPI & ISABELLA & w & 37 & ITALY & ITALY & ITALY & 1912 & & & FRANKLIN - FRANKLIN MINE \\
\hline 3470 & & FILIPPI & GINO & s & 9 & MICHIGAN & ITALY & ITALY & & & PESCAGLIA. LU & FRANKLIN - FRANKLIN MINE \\
\hline 3471 & & FILIPPI & ANNE & $\mathrm{D}$ & 3 & MICHIGAN & ITALY & ITALY & & & PESCAGLIA. LU & FRANKLIN - FRANKLIN MINE \\
\hline 3472 & & FILIPPI & GINA & $\mathrm{D}$ & $6 \mathrm{M}$ & MICHIGAN & ITALY & ITALY & & & PESCAGLIA. LU & FRANKLIN - FRANKLIN MINE \\
\hline 3473 & 410 & LAZZARI & ALFRED & $\mathrm{H}$ & 33 & ITALY & ITALY & ITALY & 1907 & MINER/CM & LUCCA & FRANKLIN - FRANKLIN MINE \\
\hline 3474 & & LAZZARI & ANGELINA & W & 26 & MICHIGAN & ITALY & ITALY & & & & FRANKLIN - FRANKLIN MINE \\
\hline 3475 & & LAZZARI & ED & $\mathrm{s}$ & 8 & MICHIGAN & ITALY & MICHIGAN & & & LUCCA & FRANKLIN - FRANKLIN MINE \\
\hline 3476 & & LAZZARI & SALOMINA & $\mathrm{D}$ & 3 & MICHIGAN & ITALY & MICHIGAN & & & LUCCA & FRANKLIN - FRANKLIN MINE \\
\hline 3477 & & LAZZARI & DANIEL & $\mathrm{s}$ & ?M & MICHIGAN & ITALY & MICHIGAN & & & LUCCA & FRANKLIN - FRANKLIN MINE \\
\hline 3478 & & MONTICELLI & LAWRENCE & BIL & 18 & MICHIGAN & ITALY & ITALY & & LABORER/CM & LUCCA & FRANKLIN - FRANKLIN MINE \\
\hline 3479 & 445 & PUCCI & SALVATORE & $\mathrm{H}$ & 39 & ITALY & ITALY & ITALY & 1907 & MINER/CM & RUOTA. LU & FRANKLIN - FRANKLIN MINE \\
\hline 3480 & & PUCCI & ANNA & W & 30 & ITALY & ITALY & ITALY & 1911 & & & FRANKLIN - FRANKLIN MINE \\
\hline 3481 & & PUCCl & BIANCA & D & 7 & MICHIGAN & ITALY & ITALY & & & RUOTA. LU & FRANKLIN - FRANKLIN MINE \\
\hline 3482 & & PUCCl & BRUNA & $\mathrm{D}$ & 5 & MICHIGAN & ITALY & ITALY & & & RUOTA. LU & FRANKLIN - FRANKLIN MINE \\
\hline 3483 & & PUCCI & DORA & $\mathrm{D}$ & 1 & MICHIGAN & ITALY & ITALY & & & RUOTA. LU & FRANKLIN - FRANKLIN MINE \\
\hline 3484 & 447 & RENALDI & JACOB & $\mathrm{H}$ & 46 & ITALY & ITALY & ITALY & 1905 & MINER/CM & SAN GIORGIO CANAVESE. TO & FRANKLIN - FRANKLIN MINE \\
\hline 3485 & & RENALDI & FILOMENA & w & 41 & ITALY & ITALY & ITALY & 1908 & & & FRANKLIN - FRANKLIN MINE \\
\hline 3486 & & RENALDI & WILLIAM & $\mathrm{s}$ & 19 & MICHIGAN & ITALY & ITALY & & LABORER/CM & SAN GIORGIO CANAVESE, TO & FRANKLIN - FRANKLIN MINE \\
\hline 3487 & & RENALDI & PETER & $\mathrm{s}$ & 12 & MICHIGAN & ITALY & ITALY & & & SAN GIORGIO CANAVESE. TO & FRANKLIN - FRANKLIN MINE \\
\hline 3488 & & RENALDI & DOMENICO & $\mathrm{s}$ & 7 & MICHIGAN & ITALY & ITALY & & & SAN GIORGIO CANAVESE. TO & FRANKLIN - FRANKLIN MINE \\
\hline 3489 & & RENALDI & GERMANA & $\mathrm{D}$ & 18 & MICHIGAN & ITALY & ITALY & & LABORER/DRY GOODS STORE & SAN GIORGIO CANAVESE. TO & FRANKLIN - FRANKLIN MINE \\
\hline 3490 & & RENALDI & MARCELLA & D & 13 & MICHIGAN & ITALY & ITALY & & & & FRANKLIN - FRANKLIN MINE \\
\hline 3491 & & RENALDI & PIERINA & $\mathrm{D}$ & 12 & MICHIGAN & ITALY & ITALY & & & SAN GIORGIO CANAVESE. TO & FRANKLIN - FRANKLIN MINE \\
\hline 3492 & & RENALDI & SILVIA & $\mathrm{D}$ & 10 & MICHIGAN & ITALY & ITALY & & & SAN GIORGIO CANAVESE, TO & FRANKLIN - FRANKLIN MINE \\
\hline 3493 & & RENALDI & OTTAVIA & D & 6 & MICHIGAN & ITALY & ITALY & & & SAN GIORGIO CANAVESE. TO & FRANKLIN - FRANKLIN MINE \\
\hline 3494 & & RENALDI & DORA & $\mathrm{D}$ & 4 & MICHIGAN & ITALY & ITALY & & & SAN GIORGIO CANAVESE. TO & FRANKLIN - FRANKLIN MINE \\
\hline 3495 & & RENALDI & VIOLA & D & $6 \mathrm{M}$ & MICHIGAN & ITALY & ITALY & & & SAN GIORGIO CANAVESE. TO & FRANKLIN - FRANKLIN MINE \\
\hline 3496 & 446 & BONINI & BALDO & $\mathrm{H}$ & 44 & ITALY & ITALY & ITALY & 1890 & LABORER/CM & COLLODI. LU & FRANKLIN - FRANKLIN MINE \\
\hline 3497 & & BONINI & MARY & w & 34 & ITALY & ITALY & ITALY & 1896 & & & FRANKLIN - FRANKLIN MINE \\
\hline 3498 & & BONINI & LINA & D & 13 & MICHIGAN & ITALY & ITALY & & & COLLODI, LU & FRANKLIN - FRANKLIN MINE \\
\hline 3499 & & BONINI & AMBROSA & $\mathrm{s}$ & 10 & MICHIGAN & ITALY & ITALY & & & COLLODI. LU & FRANKLIN - FRANKLIN MINE \\
\hline 3500 & & BONINI & AMERIGA & $\mathrm{D}$ & 8 & MICHIGAN & ITALY & ITALY & & & COLLODI. LU & FRANKLIN - FRANKLIN MINE \\
\hline 3501 & & BONINI & ANGELO & $\mathrm{s}$ & 6 & MICHIGAN & ITALY & ITALY & & & COLLODI. LU & FRANKLIN - FRANKLIN MINE \\
\hline 3502 & & BONINI & $\begin{array}{l}\text { IRENE } \\
\end{array}$ & $\mathrm{D}$ & 4 & MICHIGAN & ITALY & ITALY & & & COLLODI. LU & FRANKLIN - FRANKLIN MINE \\
\hline 3503 & & BONINI & UGO & s & 1 & MICHIGAN & ITALY & ITALY & & & COLLODI. LU & FRANKLIN - FRANKLIN MINE \\
\hline 3504 & & CRATOCTARI & EUGENIO & BIL & 20 & MICHIGAN & ITALY & ITALY & & LABORER/CM & & FRANKLIN - FRANKLIN MINE \\
\hline 3505 & & GHERA & FIL? & B & 31 & ITALY & ITALY & ITALY & 1912 & LABORER/CM & VILLA BASILICA. LU & FRANKLIN - FRANKLIN MINE \\
\hline 3506 & 407 & CIABATTARI & ADELE & $\mathrm{H}$ & 33 & ITALY & ITALY & ITALY & UN & & LUCCA & FRANKLIN - FRANKLIN MINE \\
\hline 3507 & & CIABATTARI & MIRANDA & D & 10 & MICHIGAN & ITALY & ITALY & & & LUCCA & FRANKLIN - FRANKLIN MINE \\
\hline 3508 & & CIABATTARI & ARGOTEN & D & 9 & MICHIGAN & ITALY & ITALY & & & LUCCA & FRANKLIN - FRANKLIN MINE \\
\hline 3509 & & CIABATTARI & IOLANDA & D & 7 & MICHIGAN & ITALY & ITALY & & & LUCCA & FRANKLIN - FRANKLIN MINE \\
\hline 3510 & & CIABATTARI & GINO & $\mathrm{s}$ & 5 & MICHIGAN & ITALY & ITALY & & & LUCCA & FRANKLIN - FRANKLIN MINE \\
\hline 3511 & & LENCIONI & PETER & B & 29 & ITALY & ITALY & ITALY & 1912 & TRAMMER/CM & LUCCA & FRANKLIN - FRANKLIN MINE \\
\hline 3512 & & LENCIONI & JULIANO & B & 28 & ITALY & ITALY & ITALY & 1912 & LABORER/CM & LUCCA & FRANKLIN - FRANKLIN MINE \\
\hline 3513 & $?$ & BIAGI & GERMANO & $\mathrm{H}$ & 40 & ITALY & ITALY & ITALY & 1905 & MINER/CM & SEGROMIGNO. LU & FRANKLIN - FRANKLIN MINE \\
\hline 3514 & & BIAGI & GIULIA & D & 16 & ITALY & ITALY & ITALY & 1911 & & SEGROMIGNO. LU & FRANKLIN - FRANKLIN MINE \\
\hline 3515 & & BIAGI & BENEDETTO & $\mathrm{s}$ & 13 & MICHIGAN & ITALY & ITALY & & & SEGROMIGNO. LU & FRANKLIN - FRANKLIN MINE \\
\hline 3516 & & BIAGI & WILL & $\mathrm{s}$ & 11 & MICHIGAN & ITALY & ITALY & & & SEGROMIGNO, LU & FRANKLIN - FRANKLIN MINE \\
\hline 3517 & & BIAGI & GIULIA & $\mathrm{D}$ & 6 & MICHIGAN & ITALY & ITALY & & & SEGROMIGNO. LU & FRANKLIN - FRANKLIN MINE \\
\hline 3518 & 480 & CARMINI/NINI & ANTONIO & $\mathrm{H}$ & 30 & ITALY & ITALY & ITALY & 1909 & MINER/CM & & FRANKLIN - FRANKLIN MINE \\
\hline 3519 & & CARMINI/NINI & GIULIA & w & 26 & ITALY & ITALY & ITALY & 1909 & & & FRANKLIN - FRANKLIN MINE \\
\hline 3520 & & CARMINI? & ROSIE & D & 3 & MICHIGAN & ITALY & ITALY & & & & FRANKLIN - FRANKLIN MINE \\
\hline 3521 & 472 & MASSOGLIA & DOMENICO & $\mathrm{H}$ & 49 & ITALY & ITALY & ITALY & 1900 & LABORER/CM & SAN MARTINO CANAVESE. TO & FRANKLIN - FRANKLIN MINE \\
\hline 3522 & & MASSOGLIA & ANNA & w & 49 & ITALY & ITALY & ITALY & 1906 & & & FRANKLIN - FRANKLIN MINE \\
\hline 3523 & & MASSOGLIA & FRANK & $\mathrm{s}$ & 13 & MICHIGAN & ITALY & ITALY & & & SAN MARTINO CANAVESE. TO & FRANKLIN - FRANKLIN MINE \\
\hline 3524 & & MASSOGLIA & PETER & $\mathrm{s}$ & 13 & MICHIGAN & ITALY & ITALY & & & SAN MARTINO CANAVESE. TO & FRANKLIN - FRANKLIN MINE \\
\hline 3525 & & MASSOGLIA & ANNE & D & 6 & MICHIGAN & ITALY & ITALY & & & SAN MARTINO CANAVESE. TO & FRANKLIN - FRANKLIN MINE \\
\hline 3526 & & MARTA & MARTIN & B & 40 & ITALY & ITALY & ITALY & 1902 & MINER/CM & SAN MARTINO CANAVESE. TO & FRANKLIN - FRANKLIN MINE \\
\hline 3527 & & SCAVARDA & JAMES & B & 32 & ITALY & ITALY & ITALY & 1904 & MINER/CM & LUSIGLIE. TO & FRANKLIN - FRANKLIN MINE \\
\hline
\end{tabular}




\begin{tabular}{|c|c|c|c|c|c|c|c|c|c|c|c|c|c|}
\hline 3528 & & BURLO & BERNARD & B & 26 & ITALY & ITALY & ITALY & 1911 & MINER/CM & LUSIGLIE. TO & & FRANKLIN - FRANKLIN MINE \\
\hline 3529 & & MORONI & SANTINO & B & 49 & ITALY & ITALY & ITALY & 1900 & LABORER/CM & & & FRANKLIN - FRANKLIN MINE \\
\hline 3530 & & REGIS & JAMES & B & 23 & ITALY & ITALY & ITALY & 1910 & MINER/CM & SAN GIORGIO CANAVESE. TO & & FRANKLIN - FRANKLIN MINE \\
\hline 3531 & 469 & PANTERA & FRED & $\mathrm{H}$ & 46 & MICHIGAN & ITALY & ITALY & & STOREKEEPER & LUCCA & & FRANKLIN - FRANKLIN MINE \\
\hline 3532 & 476 & NOMELLINI & MIKE & $\mathrm{H}$ & 40 & ITALY & ITALY & ITALY & 1910 & MINER/CM & LUCCA & & FRANKLIN - FRANKLIN MINE \\
\hline 3533 & & NOMELLINI & SANTA & W & 30 & ITALY & ITALY & ITALY & 1912 & & & & FRANKLIN - FRANKLIN MINE \\
\hline 3534 & & NOMELLINI & PAOLINO & $\mathrm{s}$ & 4 & MICHIGAN & ITALY & ITALY & & & LUCCA & & FRANKLIN - FRANKLIN MINE \\
\hline 3535 & & NOMELLINI & PARAGRENA & D & 10 & MICHIGAN & ITALY & ITALY & & & LUCCA & & FRANKLIN - FRANKLIN MINE \\
\hline 3536 & & MARTINUCCI & EGIDIO & B & 28 & ITALY & ITALY & ITALY & 1912 & MINER/CM & LUCCA & & FRANKLIN - FRANKLIN MINE \\
\hline 3537 & & GIULIANI & ALFREDO & B & 26 & ITALY & ITALY & ITALY & 1910 & TRAMMER/CM & & & FRANKLIN - FRANKLIN MINE \\
\hline 3538 & 478 & ALLARIA & TONY & $\mathrm{H}$ & 46 & ITALY & ITALY & ITALY & 1891 & SURFACE LABORER/CM & SAN BENIGNO CANAVESE. TO & & FRANKLIN - FRANKLIN MINE \\
\hline 3539 & 410 & ALLARIA & MINNIE & W & 46 & ITALY & ITALY & ITALY & 1897 & OURTALE LADUREK/UIVI & SAIV DEIVIUTVU CAIVAVEOE. TO & & FRANKLIN - FRANKLIN MINE \\
\hline 3540 & & ALLARIA & NOTA & D & 21 & MICHIGAN & ITALY & ITALY & & SERVANT/RESIDENCE & SAN BENIGNO CANAVESE. TO & & FRANKLIN - FRANKLIN MINE \\
\hline 3541 & & ALLARIA & PETER & $\mathrm{s}$ & 19 & MICHIGAN & ITALY & ITALY & & & SAN BENIGNO CANAVESE. TO & & FRANKLIN - FRANKLIN MINE \\
\hline 3542 & & ALLARIA & IRENE & $\mathrm{D}$ & 15 & MICHIGAN & ITALY & ITALY & & & SAN BENIGNO CANAVESE. TO & & FRANKLIN - FRANKLIN MINE \\
\hline 3543 & 488 & LENCIONI & JOSEPH & $\mathrm{H}$ & 39 & ITALY & ITALY & ITALY & 1901 & SURFACE LABORER/CM & LUCCA & & FRANKLIN - FRANKLIN MINE \\
\hline 3544 & & LENCIONI & JULIANA & w & 39 & ITALY & ITALY & ITALY & $16 ? ?$ & 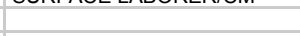 & & & FRANKLIN - FRANKLIN MINE \\
\hline 3545 & & LENCIONI & ROSIE & $\mathrm{D}$ & 11 & MICHIGAN & ITALY & ITALY & & & LUCCA & & FRANKLIN - FRANKLIN MINE \\
\hline 3546 & & LENCIONI & LENA & D & 8 & MICHIGAN & ITALY & ITALY & & & LUCCA & & FRANKLIN - FRANKLIN MINE \\
\hline 3547 & & LENCIONI & NARCISO & $\mathrm{s}$ & 10 & MICHIGAN & ITALY & ITALY & & & LUCCA & & FRANKLIN - FRANKLIN MINE \\
\hline 3548 & & LENCIONI & ACHILLE & $\mathrm{s}$ & 9 & MICHIGAN & ITALY & ITALY & & & LUCCA & & FRANKLIN - FRANKLIN MINE \\
\hline 3549 & & LENCIONI & ANGELO & $\mathrm{s}$ & 6 & MICHIGAN & ITALY & ITALY & & & LUCCA & & FRANKLIN - FRANKLIN MINE \\
\hline 3550 & ST? & GEMIGNANI & FRANCESCO & $\mathrm{H}$ & 51 & ITALY & ITALY & ITALY & 1911 & STOREKEEPER & PESCAGLIA. LU & & FRANKLIN - FRANKLIN MINE \\
\hline 3551 & & GEMIGNANI & AGNES & B & 21 & ITALY & ITALY & ITALY & 1915 & & TLUUAULIA, LU & & FRANKLIN - FRANKLIN MINE \\
\hline 3552 & & GEMIGNANI & FRANK & B & 40 & ITALY & ITALY & ITALY & 1905 & MINER/CM & & & FRANKLIN - FRANKLIN MINE \\
\hline 3553 & & GEMIGNANI & ALADINO & $\mathrm{s}$ & 21 & MICHIGAN & ITALY & ITALY & & LABORER/CM & PESCAGLIA, LU & & FRANKLIN - FRANKLIN MINE \\
\hline 3554 & & GEMIGNANI & ADOLFO & $\mathrm{s}$ & 18 & MICHIGAN & ITALY & ITALY & & LABORER/CM & PESCAGLIA. LU & & FRANKLIN - FRANKLIN MINE \\
\hline 3555 & 471 & EVANGELISTA & AUGUSTO & $\mathrm{H}$ & 40 & ITALY & ITALY & ITALY & 1902 & MINER/CM & SANTA MARIA DEL GIUDICE, LU & COUNTY ROAD & FRANKLIN - FRANKLIN MINE \\
\hline 3556 & & EVANGELISTA & JENNIE & w & 35 & ITALY & ITALY & ITALY & 1905 & & & COUNTY ROAD & FRANKLIN - FRANKLIN MINE \\
\hline 3557 & & EVANGELISTA & FIORINO & s & 13 & MICHIGAN & ITALY & ITALY & & & SANTA MARIA DEL GIUDICE. LU & COUNTY ROAD & FRANKLIN - FRANKLIN MINE \\
\hline 3558 & & EVANGELISTA & EMILIO & $\mathrm{s}$ & 11 & MICHIGAN & ITALY & ITALY & & & SANTA MARIA DEL GIUDICE. LU & COUNTY ROAD & FRANKLIN - FRANKLIN MINE \\
\hline 3559 & & EVANGELISTA & GIORGIO & $\mathrm{s}$ & 7 & MICHIGAN & ITALY & ITALY & & & SANTA MARIA DEL GIUDICE. LU & COUNTY ROAD & FRANKLIN - FRANKLIN MINE \\
\hline 3560 & & EVANGELISTA & HELEN & D & 3 & MICHIGAN & ITALY & ITALY & & & SANTA MARIA DEL GIUDICE. LU & COUNTY ROAD & FRANKLIN - FRANKLIN MINE \\
\hline 3561 & 303 & SALANI & ALIFIO & $\mathrm{H}$ & 44 & ITALY & ITALY & ITALY & 1890 & LABORER BOSS/CM & COLLODI, LU & & FRANKLIN - FRANKLIN MINE \\
\hline 3562 & & SALANI & ADA & w & 22 & ITALY & ITALY & ITALY & 1900 & & & & FRANKLIN - FRANKLIN MINE \\
\hline 3563 & & SALANI & MARY & D & 15 & MICHIGAN & ITALY & ITALY & & & COLLODI. LU & & FRANKLIN - FRANKLIN MINE \\
\hline 3564 & & SALANI & LOUISE & s & 7 & MICHIGAN & ITALY & ITALY & & & COLLODI. LU & & FRANKLIN - FRANKLIN MINE \\
\hline 3565 & & SALANI & ANTONIO & s & $4 \mathrm{M}$ & MICHIGAN & ITALY & ITALY & & & COLLODI. LU & & FRANKLIN - FRANKLIN MINE \\
\hline 3566 & 311 & SALANI & LOUIS & $\mathrm{H}$ & 36 & ITALY & ITALY & ITALY & 1905 & MINER/CM & SANTA MARIA DEL GIUDICE. LU & & FRANKLIN - FRANKLIN MINE \\
\hline 3567 & & SALANI & PERINA & W & 26 & MICHIGAN & ITALY & ITALY & & & & & FRANKLIN - FRANKLIN MINE \\
\hline 3568 & & SALANI & PETER & $\mathrm{s}$ & 6 & MICHIGAN & ITALY & MICHIGAN & & & SANTA MARIA DEL GIUDICE. LU & & FRANKLIN - FRANKLIN MINE \\
\hline 3569 & & SALANI & ROSE & $\mathrm{D}$ & 4 & MICHIGAN & ITALY & MICHIGAN & & & SANTA MARIA DEL GIUDICE. LU & & FRANKLIN - FRANKLIN MINE \\
\hline 3570 & & CARAMELLA & MARIO & B & 21 & ITALY & ITALY & ITALY & 1914 & SURFACE LABORER/CM & & & FRANKLIN - FRANKLIN MINE \\
\hline 3571 & & SALANI & MARIO & $\mathrm{COU}$ & 36 & ITALY & ITALY & ITALY & 1912 & MINER/CM & SANTA MARIA DEL GIUDICE. LU & & FRANKLIN - FRANKLIN MINE \\
\hline 3572 & 314 & FOLENA & AMATO & $\mathrm{H}$ & 56 & ITALY & ITALY & ITALY & 1888 & LABORER/CM & MATRAIA. LU & & FRANKLIN - FRANKLIN MINE \\
\hline 3573 & & FOLENA & SANTINA & w & 48 & ITALY & ITALY & ITALY & 1900 & & & & FRANKLIN - FRANKLIN MINE \\
\hline 3574 & & FOLENA & MARIO & $\mathrm{s}$ & 17 & MICHIGAN & ITALY & ITALY & & LABORER/CM & MATRAIA. LU & & FRANKLIN - FRANKLIN MINE \\
\hline 3575 & & FOLENA & MINNIE & D & 15 & MICHIGAN & ITALY & ITALY & & LABORER & MATRAIA. LU & & FRANKLIN - FRANKLIN MINE \\
\hline 3576 & & FOLENA & LOUISA & D & 13 & MICHIGAN & ITALY & ITALY & & LABORER/LAUNDRY & MATRAIA. LU & & FRANKLIN - FRANKLIN MINE \\
\hline 3577 & & FOLENA & MAPEL & D & 12 & MICHIGAN & ITALY & ITALY & & & MATRAIA. LU & & FRANKLIN - FRANKLIN MINE \\
\hline 3578 & & FOLENA & ADOLF & $\mathrm{s}$ & 10 & MICHIGAN & ITALY & ITALY & & & MATRAIA. LU & & FRANKLIN - FRANKLIN MINE \\
\hline 3579 & & FOLENA & $\begin{array}{l}\text { ALFRED } \\
\text { ALRT }\end{array}$ & $\frac{s}{s}$ & 8 & MICHIGAN & ITALY & ITALY & & & MATRAIA, LU & & FRANKLIN - FRANKLIN MINE \\
\hline 3580 & & FOLENA & ABRAMO & $\mathrm{s}$ & 6 & MICHIGAN & ITALY & ITALY & & & MATRAIA. LU & & FRANKLIN - FRANKLIN MINE \\
\hline 3581 & & FOLENA & ROSIE & $\mathrm{D}$ & 3 & MICHIGAN & ITALY & ITALY & & & MATRAIA. LU & & FRANKLIN - FRANKLIN MINE \\
\hline 3582 & 321 & MARCO & JOHN & $\mathrm{H}$ & 46 & ITALY & ITALY & ITALY & 1890 & MINER/CM & TORINO & & FRANKLIN - FRANKLIN MINE \\
\hline 3583 & & MARCO & LENA & w & 38 & ITALY & ITALY & ITALY & 1885 & & & & FRANKLIN - FRANKLIN MINE \\
\hline 3584 & & MARCO & LUCY & D & 20 & MICHIGAN & ITALY & ITALY & & LABORER/LAUNDRY & TORINO & & FRANKLIN - FRANKLIN MINE \\
\hline 3585 & & MARCO & MARNIE & D & 18 & MICHIGAN & ITALY & ITALY & & CLERK/DRY GOODS STORE & TORINO & & FRANKLIN - FRANKLIN MINE \\
\hline 3586 & & MARCO & DOMENICO & $\mathrm{s}$ & 17 & MICHIGAN & ITALY & ITALY & & LABORER/BUTCHER SHOP & TORINO & & FRANKLIN - FRANKLIN MINE \\
\hline 3587 & & MARCO & PERINA & D & 14 & MICHIGAN & ITALY & ITALY & & & TORINO & & FRANKLIN - FRANKLIN MINE \\
\hline 3588 & & MARCO & MATINA & D & 12 & MICHIGAN & ITALY & ITALY & & & TORINO & & FRANKLIN - FRANKLIN MINE \\
\hline 3589 & & MARCO & ANDREW & $\mathrm{s}$ & 9 & MICHIGAN & ITALY & ITALY & & & TORINO & & FRANKLIN - FRANKLIN MINE \\
\hline 3590 & & MARCO & BESSIE & $\mathrm{D}$ & 8 & MICHIGAN & ITALY & ITALY & & & TORINO & & FRANKLIN - FRANKLIN MINE \\
\hline
\end{tabular}




\begin{tabular}{|c|c|c|c|c|c|c|c|c|c|c|c|c|c|}
\hline 3591 & & MARCO & GIORGIO & $\mathrm{s}$ & 6 & MICHIGAN & ITALY & ITALY & & & TORINO & & FRANKLIN - FRANKLIN MINE \\
\hline 3592 & & MARCO & MAGGIE & D & 5 & MICHIGAN & ITALY & ITALY & & & TORINO & & FRANKLIN - FRANKLIN MINE \\
\hline 3593 & & MARCO & MIKE & $\mathrm{s}$ & 2 & MICHIGAN & ITALY & ITALY & & & TORINO & & FRANKLIN - FRANKLIN MINE \\
\hline 3594 & 328 & AROTO & JOHN & $\mathrm{H}$ & 33 & ITALY & ITALY & ITALY & 1900 & TRAMMER/CM & & & FRANKLIN - FRANKLIN MINE \\
\hline 3595 & & AROTO & LENA & w & 33 & ITALY & ITALY & ITALY & 1901 & & & & FRANKLIN - FRANKLIN MINE \\
\hline 3596 & & AROTO & GINO & s & 15 & ITALY & ITALY & ITALY & 1901? & & & & FRANKLIN - FRANKLIN MINE \\
\hline 3597 & & AROTO & NELLIE & $\mathrm{D}$ & 13 & ITALY & ITALY & ITALY & $1901 ?$ & & & & FRANKLIN - FRANKLIN MINE \\
\hline 3598 & & AROTO & LOUIE & $\mathrm{s}$ & 8 & MICHIGAN & ITALY & ITALY & & & & & FRANKLIN - FRANKLIN MINE \\
\hline 3599 & & AROTO & GENA & $\mathrm{D}$ & $\frac{0}{7}$ & MICHIGAN & ITALY & ITALY & & & & & FRANKLIN - FRANKLIN MINE \\
\hline 3600 & & AROTO & ASTU? & $\mathrm{s}$ & 6 & MICHIGAN & ITALY & ITALY & & & & & FRANKLIN - FRANKLIN MINE \\
\hline 3601 & & AROTO & GIORGIO & $\mathrm{s}$ & 3 & MICHIGAN & ITALY & ITALY & & & & & FRANKLIN - FRANKLIN MINE \\
\hline 3602 & & PRIAMI & FEDERICO & B & 30 & ITALY & ITALY & ITALY & 1913 & MINER/CM & LUCCA & & FRANKLIN - FRANKLIN MINE \\
\hline 3603 & $?$ & PERTINO & CARLO & $\mathrm{H}$ & 32 & ITALY & ITALY & ITALY & 1903 & MINER/CM & & & FRANKLIN - FRANKLIN MINE \\
\hline 3604 & & PERTINO & MARY & W & 29 & ITALY & ITALY & ITALY & 1912 & & & & FRANKLIN - FRANKLIN MINE \\
\hline 3605 & & PERTINO & KATE & $\mathrm{D}$ & 6 & MICHIGAN & ITALY & ITALY & & & & & FRANKLIN - FRANKLIN MINE \\
\hline 3606 & & PERTINO & DOMENICO & $\mathrm{s}$ & 5 & MICHIGAN & ITALY & ITALY & & & & & FRANKLIN - FRANKLIN MINE \\
\hline 3607 & & PERTINO & ANGELINA & D & 2 & MICHIGAN & ITALY & ITALY & & & & & FRANKLIN - FRANKLIN MINE \\
\hline 3608 & & CONTO & $\mathrm{JOHN}$ & B & 40 & ITALY & ITALY & ITALY & 1900 & MINER/CM & TORINO & & FRANKLIN - FRANKLIN MINE \\
\hline 3609 & 350 & BALDASSARRI & RICHARD & $\mathrm{H}$ & 35 & ITALY & ITALY & ITALY & 1907 & LABORER/CM & LUCCA & & FRANKLIN - FRANKLIN MINE \\
\hline 3610 & & BALDASSARRI & SETINA & w & 25 & ITALY & ITALY & ITALY & 1900 & & & & FRANKLIN - FRANKLIN MINE \\
\hline 3611 & & BALDASSARRI & HAZEL & $\mathrm{D}$ & 2 & MICHIGAN & ITALY & ITALY & & & LUCCA & & FRANKLIN - FRANKLIN MINE \\
\hline 3612 & & SALINI & MENOTTI & B & 36 & ITALY & ITALY & ITALY & 1909 & LABORER/CM & LUCCA & & FRANKLIN - FRANKLIN MINE \\
\hline 3613 & 349 & MARTINELLI & PETER & $\mathrm{H}$ & 27 & ITALY & ITALY & ITALY & 1909 & MINER/CM & CAPANNORI. LU & & FRANKLIN - FRANKLIN MINE \\
\hline 3614 & & MARTINELLI & ALAIDE & W & 25 & ITALY & ITALY & ITALY & 1909 & & CAPANNORI. LU & & FRANKLIN - FRANKLIN MINE \\
\hline 3615 & & MARTINELLI & ANZANO & $s$ & $6 \mathrm{M}$ & MICHIGAN & ITALY & ITALY & & & CAPANNORI. LU & & FRANKLIN - FRANKLIN MINE \\
\hline 3616 & 348 & DAIRO & TONY & $\mathrm{H}$ & 35 & ITALY & ITALY & ITALY & 1914 & TRAMMER/CM & TORINO & & FRANKLIN - FRANKLIN MINE \\
\hline 3617 & & DAIRO & ANTONIETTA & W & 24 & ITALY & ITALY & ITALY & 1914 & & TORINO & & FRANKLIN - FRANKLIN MINE \\
\hline 3618 & & DAIRO & GIUSTINO & $\mathrm{s}$ & 6 & MICHIGAN & ITALY & ITALY & & & TORINO & & FRANKLIN - FRANKLIN MINE \\
\hline 3619 & 347 & CIABATTARI & LORENZO & $\mathrm{H}$ & 21 & MICHIGAN & ITALY & ITALY & & LABORER/CM & LUCCA & & FRANKLIN - FRANKLIN MINE \\
\hline 3620 & & CIABATTARI & DELFINA & SMO & 42 & ITALY & ITALY & ITALY & 1905 & & LUCCA & & FRANKLIN - FRANKLIN MINE \\
\hline 3621 & & CIABATTARI & EMIL & $\mathrm{BR}$ & 11 & MICHIGAN & ITALY & ITALY & & & LUCCA & & FRANKLIN - FRANKLIN MINE \\
\hline 3622 & 492 & BALDASSARRI & JOSEPH & $\mathrm{H}$ & 60 & ITALY & ITALY & ITALY & 1909 & MINER/CM & LUCCA & BACK ST? & FRANKLIN - FRANKLIN MINE \\
\hline 3623 & 492 & BALDASSARRI & TERESA & w & 53 & ITALY & ITALY & ITALY & 1909 & IVIIVERATLIVI & LUCCA & BACK ST? & FRANKLIN - FRANKLIN MINE \\
\hline 3624 & 493 & MARACCINI & NATALE & $\mathrm{H}$ & 33 & ITALY & ITALY & ITALY & 1907 & MINER/CM & SANTA MARIA DEL GIUDICE, LU & & FRANKLIN - FRANKLIN MINE \\
\hline 3625 & & MARACCINI & ADELE & w & 34 & ITALY & ITALY & ITALY & 1908 & & & & FRANKLIN - FRANKLIN MINE \\
\hline 3626 & & MARACCINI & JOSEPHINE & $\mathrm{D}$ & 10 & MICHIGAN & ITALY & ITALY & & & SANTA MARIA DEL GIUDICE. LU & & FRANKLIN - FRANKLIN MINE \\
\hline 3627 & & MARACCINI & CORINTA & $\mathrm{D}$ & 7 & MICHIGAN & ITALY & ITALY & & & SANTA MARIA DEL GIUDICE. LU & & FRANKLIN - FRANKLIN MINE \\
\hline 3628 & & MARACCINI & GINA & D & 3 & MICHIGAN & ITALY & ITALY & & & SANTA MARIA DEL GIUDICE. LU & & FRANKLIN - FRANKLIN MINE \\
\hline 3629 & & MAGA & CLEMENTE & B & 33 & ITALY & ITALY & ITALY & 1914 & TRAMMER/CM & SCARMAGNO. TO & & FRANKLIN - FRANKLIN MINE \\
\hline 3630 & & MATTETTI & SANTINO & B & 27 & ITALY & ITALY & ITALY & 1911 & LABORER/CM & & & FRANKLIN - FRANKLIN MINE \\
\hline 3631 & & BASSANI & VINCENZO & B & 22 & ITALY & ITALY & ITALY & 1915 & LABORER/CM & & & FRANKLIN - FRANKLIN MINE \\
\hline 3632 & 6 & CIUCCl & ITALIA & $\mathrm{H}$ & 40 & ITALY & ITALY & ITALY & 1908 & & LUCCA & & FRANKLIN \\
\hline 3633 & & $\mathrm{ClUCCl}$ & CECILIA & D & 13 & ITALY & ITALY & ITALY & 1908 & & CAPANNORI. LU & & FRANKLIN \\
\hline 3634 & & $\mathrm{ClUCCl}$ & ESTER & D & 12 & ITALY & ITALY & ITALY & 1908 & AT SCHOOL & CAPANNORI. LU & & FRANKLIN \\
\hline 3635 & & $\mathrm{ClUCCl}$ & CLOTILDE & D & 10 & MICHIGAN & ITALY & ITALY & & AT SCHOOL & CAPANNORI. LU & & FRANKLIN \\
\hline 3636 & & $\mathrm{ClUCCl}$ & RUSSEL & $\mathrm{s}$ & 8 & MICHIGAN & ITALY & ITALY & & AT SCHOOL & CAPANNORI. LU & & FRANKLIN \\
\hline 3637 & & CluCCl & MARY & D & 4 & MICHIGAN & ITALY & ITALY & & & CAPANNORI. LU & & FRANKLIN \\
\hline 3638 & & CIUCCI & JOSEPH & $\mathrm{s}$ & 3 & MICHIGAN & ITALY & ITALY & & & CAPANNORI. LU & & FRANKLIN \\
\hline 3639 & & CIUCCI & PETER & $\mathrm{s}$ & 3 & MICHIGAN & ITALY & ITALY & & & CAPANNORI. LU & & FRANKLIN \\
\hline 3640 & & $\mathrm{CIUCCl}$ & NORMAND & $\mathrm{s}$ & 1 & MICHIGAN & ITALY & ITALY & & & CAPANNORI. LU & & FRANKLIN \\
\hline 3641 & & $\mathrm{ClUCCl}$ & RAYMOND & $\mathrm{s}$ & 1 & MICHIGAN & ITALY & ITALY & & & CAPANNORI. LU & & FRANKLIN \\
\hline 3642 & & GIANNERINI & DOMENICO & B & 34 & ITALY & ITALY & ITALY & 1900 & MANAGER/PRINT SHOP & MATRAIA, LU & & FRANKLIN \\
\hline 3643 & & CORTOPASSI & CHERUBINO & $\mathrm{H}$ & 48 & ITALY & ITALY & ITALY & UN & LABORER/BLACKSMITH SHOP & LUCCA & & FRANKLIN \\
\hline 3644 & & CORTOPASSI & DOMENICA & W & 41 & ITALY & ITALY & ITALY & UN & & LUCCA & & FRANKLIN \\
\hline 3645 & & CORTOPASSI & ANDREA & s & 17 & MICHIGAN & ITALY & ITALY & & AT SCHOOL & LUCCA & & FRANKLIN \\
\hline 3646 & & CORTOPASSI & VICTOR & s & 9 & MICHIGAN & ITALY & ITALY & & AT SCHOOL & LUCCA & & FRANKLIN \\
\hline 3647 & & BETTINI & GIUSEPPE & $\mathrm{H}$ & 32 & ITALY & ITALY & ITALY & 1909 & LABORER/CM & & & FRANKLIN \\
\hline 3648 & & BETTINI & $\begin{array}{l}\text { VIRGINIA } \\
\text { VIN }\end{array}$ & w & 34 & ITALY & ITALY & ITALY & UN & & & & FRANKLIN \\
\hline 3649 & & BETTINI & DARIO & D & 6 & MICHIGAN & ITALY & ITALY & & AT SCHOOL & & & FRANKLIN \\
\hline 3650 & & BETTINI & OSCAR & $\mathrm{s}$ & 5 & MICHIGAN & ITALY & ITALY & & & & & FRANKLIN \\
\hline 3651 & & BETTINI & GIULIO & s & $6 \mathrm{M}$ & MICHIGAN & ITALY & ITALY & & & & & FRANKLIN \\
\hline 3652 & & AGRETTI & ANGELO & $\mathrm{H}$ & 38 & ITALY & ITALY & ITALY & 1908 & CARPENTER/CM & & & FRANKLIN \\
\hline 3653 & & AGRETTI & CESIRA & W & 45 & ITALY & ITALY & ITALY & 1919 & 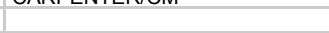 & CAPANNORI. LU & & FRANKLIN \\
\hline
\end{tabular}




\begin{tabular}{|c|c|c|c|c|c|c|c|c|c|c|c|c|c|}
\hline 3654 & & AGRETTI & VITTORIA & D & 18 & ITALY & ITALY & ITALY & 1919 & & CAPANNORI. LU & & FRANKLIN \\
\hline 3655 & & AGRETTI & ORESTE & $\mathrm{s}$ & 16 & ITALY & ITALY & ITALY & 1919 & & CAPANNORI. LU & & FRANKLIN \\
\hline 3656 & 1 & GIUSTI & ORLANDO (WID. & $\mathrm{H}$ & 40 & ITALY & ITALY & ITALY & 1908 & BLACKSMITH/CM & PISA & & FRANKLIN - FARM SECTION \\
\hline 3657 & & GIUSTI & ILIO & $\mathrm{s}$ & 16 & ITALY & ITALY & ITALY & 1906 & SCHOOL & PISA & & FRANKLIN - FARM SECTION \\
\hline 3658 & & GIUSTI & PAUL & $\mathrm{s}$ & 12 & MICHIGAN & ITALY & ITALY & & SCHOOL & PISA & & FRANKLIN - FARM SECTION \\
\hline 3659 & & GIUSTI & ESTER & D & 6 & MICHIGAN & ITALY & ITALY & & SCHOOL & PISA & & FRANKLIN - FARM SECTION \\
\hline 3660 & & GIUSTI & GIORGIO & $\mathrm{s}$ & 4 & MICHIGAN & ITALY & ITALY & & & PISA & & FRANKLIN - FARM SECTION \\
\hline 3661 & & GIUSTI & FREDY & s & 1 & MICHIGAN & ITALY & ITALY & & & PISA & & FRANKLIN - FARM SECTION \\
\hline 3662 & & MARCHESCHI & BRUNETTA & SER & 22 & ITALY & ITALY & ITALY & 1902 & SERVANT/PF & LUCCA & & FRANKLIN - FARM SECTION \\
\hline 3663 & 1 & VITTON & BATTISTA & $\mathrm{H}$ & 49 & ITALY & ITALY & ITALY & 1905 & FARMER/DAIRY FARM & TORINO & & FRANKLIN - FARM SECTION \\
\hline 3664 & & VITTON & MARY & w & 39 & ITALY & ITALY & ITALY & 1899 & & TORINO & & FRANKLIN - FARM SECTION \\
\hline 3665 & & VITTON & JOHN & $\mathrm{s}$ & 17 & ITALY & ITALY & ITALY & 1901 & LABORER/DAIRY FARM & TORINO & & FRANKLIN - FARM SECTION \\
\hline 3666 & & VITTON & FRANCES & D & 16 & MICHIGAN & ITALY & ITALY & & & TORINO & & FRANKLIN - FARM SECTION \\
\hline 3667 & & VITTON & BATTISTA & $\mathrm{s}$ & 14 & MICHIGAN & ITALY & ITALY & & LABORER/DAIRY FARM & TORINO & & FRANKLIN - FARM SECTION \\
\hline 3668 & & VITTON & ANNA & $\mathrm{D}$ & 12 & MICHIGAN & ITALY & ITALY & & SCHOOL & TORINO & & FRANKLIN - FARM SECTION \\
\hline 3669 & & VITTON & JAMES & $\mathrm{s}$ & 10 & MICHIGAN & ITALY & ITALY & & SCHOOL & TORINO & & FRANKLIN - FARM SECTION \\
\hline 3670 & & VITTON & CATERINA & D & 8 & MICHIGAN & ITALY & ITALY & & SCHOOL & TORINO & & FRANKLIN - FARM SECTION \\
\hline 3671 & & VITTON & MARY & $\mathrm{D}$ & 5 & MICHIGAN & ITALY & ITALY & & & TORINO & & FRANKLIN - FARM SECTION \\
\hline 3672 & & VITTON & PETER & $\mathrm{s}$ & 3 & MICHIGAN & ITALY & ITALY & & & TORINO & & FRANKLIN - FARM SECTION \\
\hline 3673 & & MANTINO & JAMES & B & 43 & ITALY & ITALY & ITALY & 1909 & LABORER/DAIRY FARM & TORINO & & FRANKLIN - FARM SECTION \\
\hline 3674 & & BIROCCO & CHARLES & B & 37 & ITALY & ITALY & ITALY & 1907 & LABORER/DAIRY FARM & SAN GIUSTO CANAVESE. TO & & FRANKLIN - FARM SECTION \\
\hline 3675 & & MONTO & EMILIO & B & 33 & ITALY & ITALY & ITALY & 1903 & LABORER/DAIRY FARM & & & FRANKLIN - FARM SECTION \\
\hline 3676 & & BIRETTO/LLO & BATTISTA & B & 32 & ITALY & ITALY & ITALY & UN & LABORER/DAIRY FARM & & & FRANKLIN - FRANKLIN MINE \\
\hline 3677 & 1 & GASPARDO & JOHN & $\mathrm{H}$ & 56 & ITALY & ITALY & ITALY & 1884 & FARMER/DAIRY FARM & PONT CANAVESE. TO & & FRANKLIN - FRANKLIN MINE \\
\hline 3678 & & GASPARDO & ANNA & w & 42 & ITALY & ITALY & ITALY & UN & & PONT CANAVESE. TO & & FRANKLIN - FRANKLIN MINE \\
\hline 3679 & & GASPARDO & MINNIE & $\mathrm{D}$ & 17 & MICHIGAN & ITALY & ITALY & & & PONT CANAVESE. TO & & FRANKLIN - FRANKLIN MINE \\
\hline 3680 & & GASPARDO & GIACOMO & $\mathrm{s}$ & 16 & MICHIGAN & ITALY & ITALY & & FARMER/DAIRY FARM & PONT CANAVESE. TO & & FRANKLIN - FRANKLIN MINE \\
\hline 3681 & & GASPARDO & GIOVANNI & $\mathrm{s}$ & 12 & MICHIGAN & ITALY & ITALY & & & PONT CANAVESE, TO & & FRANKLIN - FRANKLIN MINE \\
\hline 3682 & & GASPARDO & ANNA & D & 4 & MICHIGAN & ITALY & ITALY & & & PONT CANAVESE. TO & & FRANKLIN - FRANKLIN MINE \\
\hline 3683 & & DEIRO & PETER & B & 16 & MICHIGAN & ITALY & ITALY & & & PONT CANAVESE. TO & & FRANKLIN - FARM SECTION \\
\hline 3684 & I & BINCOHNION & JOSEPH & B & UN & ITALY & ITALY & ITALY & UN & FARMER & & & FRANKLIN - FARM SECTION \\
\hline 3685 & 119 & PONTI & MIKE & $\mathrm{H}$ & 21 & ITALY & ITALY & ITALY & 1914 & MINER/CM & BENEVENTO & HANCOK AVE. & HANCOCK CITY \\
\hline 3686 & & PONTI & MARY & w & 22 & MICHIGAN & IRELAND & NEW YORK & & & USA-IRELAND & HANCOK AVE. & HANCOCK CITY \\
\hline 3687 & 103 & BIANCHI & JOHN & $\mathrm{H}$ & 42 & ITALY & ITALY & ITALY & 1899 & PROPRIETOR/FRUIT STORE & COLLODI, LU & QUINCY ST. & HANCOCK CITY \\
\hline 3688 & & BIANCHI & KATE & w & 32 & ITALY & ITALY & ITALY & 1919 & & & QUINCY ST. & HANCOCK CITY \\
\hline 3689 & 319 & DIANDA & ANGELO & $\mathrm{H}$ & 32 & ITALY & ITALY & ITALY & 1909 & PROPRIETOR/FRUIT STORE & LUCCA & QUINCY ST. & HANCOCK CITY \\
\hline 3690 & & DIANDA & CARA & w & 25 & MICHIGAN & ITALY & ITALY & & & & QUINCY ST. & HANCOCK CITY \\
\hline 3691 & & DIANDA & KATE & D & 8 & MICHIGAN & ITALY & MICHIGAN & & & LUCCA & QUINCY ST. & HANCOCK CITY \\
\hline 3692 & & DIANDA & ANGELO & $\mathrm{D}$ & 6 & MICHIGAN & ITALY & MICHIGAN & & & LUCCA & QUINCY ST. & HANCOCK CITY \\
\hline 3693 & 305 & MICHELOTTI & IACOPO & $\mathrm{H}$ & 33 & ITALY & ITALY & ITALY & 1902 & PROPRIETOR/RESTAURANT & SANTA MARIA DEL GIUDICE. LU & QUINCY ST. & HANCOCK CITY \\
\hline 3694 & & MICHELOTTI & MARY & W & 29 & ITALY & ITALY & ITALY & 1896 & & & QUINCY ST. & HANCOCK CITY \\
\hline 3695 & & MICHELOTTI & $\mathrm{JOE}$ & BR & 24 & ITALY & ITALY & ITALY & 1893 & COOK/RESTAURANT & SANTA MARIA DEL GIUDICE. LU & QUINCY ST. & HANCOCK CITY \\
\hline 3696 & & MICHELOTTI & PILADE & BR & 28 & ITALY & ITALY & ITALY & 1908 & SALESMAN/RESTAURANT & SANTA MARIA DEL GIUDICE. LU & QUINCY ST. & HANCOCK CITY \\
\hline 3697 & 323 & MEOLA & LEONARD & $\mathrm{H}$ & 49 & ITALY & ITALY & ITALY & 1889 & LABOR AGENT/CM & AVELLINO & QUINCY ST. & HANCOCK CITY \\
\hline 3698 & & MEOLA & NELLY & w & 44 & $\mathrm{OHIO}$ & $\mathrm{OHIO}$ & KANSAS & & & USA & QUINCY ST. & HANCOCK CITY \\
\hline 3699 & & MEOLA & MARY & D & 22 & $\mathrm{OHIO}$ & ITALY & $\mathrm{OHIO}$ & & SALESLADY/MINING STORE & AVELLINO & QUINCY ST. & HANCOCK CITY \\
\hline 3700 & 319 & DIANDA & ANGELO & $\mathrm{H}$ & 38 & ITALY & ITALY & ITALY & UN & PROPRIETOR/CONFECTIONARY & LUCCA & QUINCY ST. & HANCOCK CITY \\
\hline 3701 & & DIANDA & CARA & w & 28 & ITALY & ITALY & ITALY & UN & & LUCCA & QUINCY ST. & HANCOCK CITY \\
\hline 3702 & & DIANDA & ANGELO & $\mathrm{D}$ & 9 & MICHIGAN & ITALY & ITALY & & & LUCCA & QUINCY ST. & HANCOCK CITY \\
\hline 3703 & & DIANDA & CATERINA & $\mathrm{D}$ & 7 & MICHIGAN & ITALY & ITALY & & & LUCCA & QUINCY ST. & HANCOCK CITY \\
\hline 3704 & 528 & GRASSESCHI & ALFREDO & $\mathrm{H}$ & 29 & ITALY & ITALY & ITALY & 1907 & SHOEMAKER/OWN SHOP & LUCCA & JCY ST. & HANCOCK CITY \\
\hline 3705 & & GRASSESCHI & $\begin{array}{l}\text { LENA } \\
\text { LEN }\end{array}$ & w & 21 & ITALY & ITALY & ITALY & 1916 & & & QUI & HANCOCK CITY \\
\hline 3706 & & GRASSESCHI & SPERANZA & D & 1 & MONTANA & ITALY & ITALY & & & LUCCA & QUINCY ST. & HANCOCK CITY \\
\hline 3707 & & GRASSESCHI & MAURIZIO & $S$ & $8 \mathrm{M}$ & MONTANA & ITALY & ITALY & & & LUCCA & QUINCY ST. & HANCOCK CITY \\
\hline 3708 & & GRASSESCHI & THOMAS & BR & 32 & ITALY & ITALY & ITALY & 1914 & SHOEMAKER/OWN SHOP & LUCCA & QUINCY ST. & HANCOCK CITY \\
\hline 3709 & 502 & BRONZINI & ALBERT & $\mathrm{H}$ & 35 & ITALY & ITALY & ITALY & 1905 & MERCHANT/CANDY STORE & & QUINCY ST. & HANCOCK CITY \\
\hline 3710 & & BRONZINI & ELENA & w & 35 & ITALY & ITALY & ITALY & 1909 & & & QUINCY ST. & HANCOCK CITY \\
\hline 3711 & & BRONZINI & RENATO & s & 9 & MICHIGAN & ITALY & ITALY & & & & QUINCY ST. & HANCOCK CITY \\
\hline 3712 & & BRONZINI & LIDA & D & 7 & MICHIGAN & ITALY & ITALY & & & & QUINCY ST. & HANCOCK CITY \\
\hline 3713 & & BRONZINI & RENA & D & 6 & MICHIGAN & ITALY & ITALY & & & & QUINCY ST. & HANCOCK CITY \\
\hline 3714 & & MARSALLI & JOHN & B & 37 & ITALY & ITALY & ITALY & 1906 & SALES & CONEGLIANO. TREVISO & QUINCY ST. & HANCOCK CITY \\
\hline 3715 & & FREDIANELLI & LUIGI & B & 34 & ITALY & ITALY & ITALY & 1907 & ANT/FRUIT & LUCCA & QUINCY ST. & HANCOCK CITY \\
\hline 716 & 310 & GROSSIO & CARLO & $\mathrm{B}$ & 28 & ITALY & ITALY & ITALY & 1894 & CONDUCTOR/STREET CAR & TORINO & QUINCY ST. & HANCOCK CITY \\
\hline
\end{tabular}




\begin{tabular}{|c|c|c|c|c|c|c|c|c|c|c|c|c|c|}
\hline 3717 & 320 & MEINARDI & JOHN & $\mathrm{H}$ & 44 & ITALY & ITALY & ITALY & 1889 & RETAIL MERCHANT/GROCERY & SAN GIORGIO CANAVESE. TO & QUINCY ST. & HANCOCK CITY \\
\hline 3718 & & MEINARDI & VITTORIA & W & 40 & ITALY & ITALY & ITALY & 1905 & & & QUINCY ST. & \\
\hline 3719 & & MEINARDI & JOE & $\mathrm{s}$ & 13 & MICHIGAN & ITALY & ITALY & & & SAN GIORGIO CANAVESE. TO & QUINCY ST. & HANCOCK CITY \\
\hline 3720 & 309 & COTTINI & PETER & B & 18 & MICHIGAN & ITALY & ITALY & & CLERK/BANK & COLLODI. LU & RAVINE ST. & HANCOCK CITY \\
\hline 3721 & 301 & VIGNETTO & MARY & $\mathrm{H}$ & 30 & WISCONSI & ITALY & ITALY & & & TORINO & RAVINE ST. & HANCOCK CITY \\
\hline 3722 & & VIGNETTO & BERTHA & SI & 26 & ILLINOIS & ITALY & ITALY & & STENOGRAPHER/INSURANCE & TORINO & RAVINE ST. & HANCOCK CITY \\
\hline 3723 & & VIGNETTO & WILL & BR & 19 & MICHIGAN & ITALY & ITALY & & & TORINO & RAVINE ST. & HANCOCK CITY \\
\hline 3724 & & VIGNETTO & ANGELO & $\mathrm{H}$ & 32 & ILLINOIS & ITALY & ITALY & & PROPRIETOR/POOL ROOM & TORINO & RAVINE ST. & HANCOCK CITY \\
\hline 3725 & & VIGNETTO & FLORENCE & W & 32 & MICHIGAN & MICHIGAN & MICHIGAN & & & TORINO & RAVINE ST. & HANCOCK CITY \\
\hline 3726 & 305 & FORNERIS & ANGELO & $\mathrm{H}$ & 46 & ITALY & ITALY & ITALY & 1894 & KEEPER/BOARDING HOUSE & TORINO & WATER ST. & HANCOCK CITY \\
\hline 3727 & & FORNERIS & MARY & W & 52 & ITALY & ITALY & ITALY & 1904 & 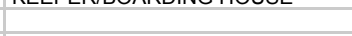 & Thinive & WATER ST. & HANCOCK CITY \\
\hline 3728 & & FORNERIS & MARIAN & D & 18 & MICHIGAN & ITALY & ITALY & 1504 & ENGINE CLEANER/LOCOMOTIVE & TORINO & WATER ST. & HANCOCK CITY \\
\hline 3729 & & BESSOLO & CARLO & NEP & 16 & MICHIGAN & ITALY & ITALY & & LABORER/BAKERY & TORINO & WATER ST. & HANCOCK CITY \\
\hline 3730 & & VANIATO & LUIGI & B & 21 & MICHIGAN & ITALY & ITALY & & MINER/CM & & WATER ST. & HANCOCK CITY \\
\hline 3731 & & SANDRETTO & ANNA & B & 22 & ITALY & ITALY & ITALY & 1913 & ENGINE WIPER/CM & PONT CANAVESE. TO & WATER ST. & HANCOCK CITY \\
\hline 3732 & & ORBINA/DEBINA & JOE & B & 56 & ITALY & ITALY & ITALY & 1891 & LABORER/IRON FOUNDRY & & WATER ST. & HANCOCK CITY \\
\hline 3733 & 304 & PETUGGILLE & JOE & $\mathrm{H}$ & 21 & ITALY & ITALY & ITALY & 1912 & SALESMAN/CANDY STORE & & QUINCY ST. & HANCOCK CITY \\
\hline 3734 & 494 & NORMALI & GINO & $\mathrm{H}$ & 28 & ITALY & ITALY & ITALY & 1907 & FIREMAN/LAUNDRY & CASTELGOMBERTO. VI & HANCOCK ST. & HANCOCK CITY \\
\hline 3735 & & NORMALI & CAMILLA & W & 27 & MICHIGAN & ITALY & ITALY & & & & HANCOCK ST. & HANCOCK CITY \\
\hline 3736 & & NORMALI & GINO & $\mathrm{s}$ & 7 & MICHIGAN & ITALY & MICHIGAN & & & CASTELGOMBERTO. VI & HANCOCK ST. & HANCOCK CITY \\
\hline 3737 & & NORMALI & ALBERT & $\mathrm{s}$ & 5 & MICHIGAN & ITALY & MICHIGAN & & & CASTELGOMBERTO. VI & HANCOCK ST. & HANCOCK CITY \\
\hline 3738 & & GASPARDO & JOHN & B & 35 & ITALY & ITALY & ITALY & 1899 & ENGINE CLEANER/EL. PLANT & PONT CANAVESE, TO & HANCOCK ST. & HANCOCK CITY \\
\hline 3739 & 632 & GEDDA & PETER & $\mathrm{H}$ & 31 & ITALY & ITALY & ITALY & 1904 & TAILOR & STRAMBINELLO. TO & WATER ST. & HANCOCK CITY \\
\hline 3740 & & GEDDA & MARY & w & 30 & ITALY & ITALY & ITALY & 1904 & & STRAMBINELLO. TO & WATER ST. & HANCOCK CITY \\
\hline 3741 & & GEDDA & MARIANA & D & 3 & MICHIGAN & ITALY & ITALY & & & STRAMBINELLO. TO & WATER ST. & HANCOCK CITY \\
\hline 3742 & & GEDDA & JOHN & $\mathrm{s}$ & 1 & MICHIGAN & ITALY & ITALY & & & STRAMBINELLO. TO & WATER ST. & HANCOCK CITY \\
\hline 3743 & 859 & GIACOLETTO & SAVINO & $\mathrm{H}$ & 48 & ITALY & ITALY & ITALY & 1899 & MACHINIST/CM & PONT CANAVESE. TO & PINE ST. & HANCOCK CITY \\
\hline 3744 & & GIACOLETTO & SERAFINA & W & 45 & ITALY & ITALY & ITALY & 1899 & & PONT CANAVESE, TO & PINE ST. & HANCOCK CITY \\
\hline 3745 & & GIACOLETTO & PAUL & s & 11 & MICHIGAN & ITALY & ITALY & & & PONT CANAVESE. TO & PINE ST. & HANCOCK CITY \\
\hline 3746 & & GIACOLETTO & ANGELINA & $\mathrm{D}$ & 7 & MICHIGAN & ITALY & ITALY & & & PONT CANAVESE. TO & PINE ST. & HANCOCK CITY \\
\hline 3747 & & BALDASSARRI & ALADINO & SOIL & 24 & ITALY & ITALY & ITALY & UN & TRAIN MAN/CM & LUCCA & PINE ST. & HANCOCK CITY \\
\hline 3748 & & BALDASSARRI & MARY & $\mathrm{D}$ & 19 & MICHIGAN & ITALY & ITALY & & & LUCCA & PINE ST. & HANCOCK CITY \\
\hline 3749 & & BALDASSARRI & WILL & GS & 1 & MICHIGAN & ITALY & ITALY & & & LUCCA & PINE ST. & HANCOCK CITY \\
\hline 3750 & 833 & DE BERNARDI & RALPH & B & 4 & MICHIGAN & ITALY & MICHIGAN & & & TORINO & FRANKLIN ST. & HANCOCK CITY \\
\hline 3751 & 715 & GROSSIO & JAMES & $\mathrm{H}$ & 50 & ITALY & ITALY & ITALY & 1894 & YARDMAN/ SMELTER & TORINO & PINE ST. & HANCOCK CITY \\
\hline 3752 & & GROSSIO & MARY & w & 34 & ITALY & ITALY & ITALY & 1907 & & & PINE ST. & HANCOCK CITY \\
\hline 3753 & & GROSSIO & JOSEPH & s & 13 & MICHIGAN & ITALY & ITALY & & & TORINO & PINE ST. & HANCOCK CITY \\
\hline 3754 & & GROSSIO & JOHN & $\mathrm{s}$ & 11 & MICHIGAN & ITALY & ITALY & & & TORINO & PINE ST. & HANCOCK CITY \\
\hline 3755 & & PIANA & JAMES & B & 31 & ITALY & ITALY & ITALY & 1911 & MINER/CM & TORINO & PINE ST. & HANCOCK CITY \\
\hline 3756 & & MOTTA & JAMES & B & 30 & ITALY & ITALY & ITALY & UN & LABORER/SMELTER & & PINE ST. & HANCOCK CITY \\
\hline 3757 & & MOZZO & LOUIS & B & 33 & ITALY & ITALY & ITALY & UN & MINER/CM & & PINE ST. & HANCOCK CITY \\
\hline 3758 & 715 & GEDDA & VITTORIO & $\mathrm{H}$ & 46 & ITALY & ITALY & ITALY & UN & PROPRIETOR/BAKERY & TORINO & PINE ST. & HANCOCK CITY \\
\hline 3759 & & GEDDA & ROSA & W & 26 & ITALY & ITALY & ITALY & 1907 & & TORINO & PINE ST. & HANCOCK CITY \\
\hline 3760 & & GEDDA & MADDALENA & D & 7 & MICHIGAN & ITALY & ITALY & & & TORINO & PINE ST. & HANCOCK CITY \\
\hline 3761 & & GEDDA & ANGELINA & $\mathrm{D}$ & 6 & MICHIGAN & ITALY & ITALY & & & TORINO & PINE ST. & HANCOCK CITY \\
\hline 3762 & & GEDDA & JOHN & $\mathrm{s}$ & 3 & MICHIGAN & ITALY & ITALY & & & TORINO & PINE ST. & HANCOCK CITY \\
\hline 3763 & & MICHELETTI & DANTE & B & 31 & ITALY & ITALY & ITALY & 1906 & TEAMSTER/BAKERY & SANTA MARIA DEL GIUDICE. LU & PINE ST. & HANCOCK CITY \\
\hline 3764 & 811 & $\mathrm{ROCH}$ & JOHN & $\mathrm{H}$ & 42 & ITALY & ITALY & ITALY & 1893 & GROCER/GROCERY STORE & RIVAROLO CANAVESE. TO & WARREN ST. & HANCOCK CITY \\
\hline 3765 & & $\mathrm{ROCH}$ & EUGENIA & w & 35 & ITALY & ITALY & ITALY & 1905 & & & WARREN ST. & HANCOCK CITY \\
\hline 3766 & & $\mathrm{ROCH}$ & JOSEPH & s & 13 & MICHIGAN & ITALY & ITALY & & & RIVAROLO CANAVESE. TO & WARREN ST. & HANCOCK CITY \\
\hline 3767 & & $\mathrm{ROCH}$ & TERESA & $\mathrm{D}$ & 11 & MICHIGAN & ITALY & ITALY & & & RIVAROLO CANAVESE. TO & WARREN ST. & HANCOCK CITY \\
\hline 3768 & & $\mathrm{ROCH}$ & MARIO & s & 10 & MICHIGAN & ITALY & ITALY & & & RIVAROLO CANAVESE, TO & WARREN ST. & HANCOCK CITY \\
\hline 3769 & & $\mathrm{ROCH}$ & JOSEPH & BR & 37 & ITALY & ITALY & ITALY & 1905 & GROCER/GROCERY STORE & RIVAROLO CANAVESE. TO & WARREN ST. & HANCOCK CITY \\
\hline 3770 & 803 & GIULIO & ANTONIO & $\mathrm{H}$ & 47 & ITALY & ITALY & ITALY & 1893 & PROPRIETOR/SOFT DRINK & SAN GIORGIO CANAVESE. TO & HANCOCK ST. & HANCOCK CITY \\
\hline 3771 & & GIULIO & NELLIE & W & 42 & ITALY & ITALY & ITALY & 1893 & & SAN GIORGIO CANAVESE. TO & HANCOCK ST. & HANCOCK CITY \\
\hline 3772 & & GIULIO & MARY & D & 9 & MICHIGAN & ITALY & ITALY & & & SAN GIORGIO CANAVESE. TO & HANCOCK ST. & HANCOCK CITY \\
\hline 3773 & 815 & ANDRIZZI & FRANK & $\mathrm{H}$ & 19 & MICHIGAN & ITALY & ITALY & & TEAMSTER/LAUNDRY & & HANCOCK ST. & HANCOCK CITY \\
\hline 3774 & & ANDRIZZI & ELEN & W & 19 & MICHIGAN & MICHIGAN & MICHIGAN & & & & HANCOCK ST. & HANCOCK CITY \\
\hline 3775 & & ANDRIZZI & FRANK & $\mathrm{s}$ & 0 & MICHIGAN & MICHIGAN & MICHIGAN & & & & HANCOCK ST. & HANCOCK CITY \\
\hline 3776 & 101 & FASANA & MIKE & B & 25 & ITALY & ITALY & ITALY & 1898 & PROPRIETOR/SOFT DRINK & & MILL STREET & HANCOCK CITY \\
\hline 3777 & & FASANA & TERESA & B & 19 & ITALY & ITALY & ITALY & 1903 & & & LAKE STREET & HANCOCK CITY \\
\hline 3778 & 212 & REVELLO & PAUL & $\mathrm{H}$ & 55 & ITALY & ITALY & ITALY & 1890 & & AGLIE'. TO & LAKE STREET & HANCOCK CITY \\
\hline 3779 & & REVELLO & IDA & w & 31 & ITALY & ITALY & ITALY & 1904 & & & LAKE STREET & HANCOCK CITY \\
\hline
\end{tabular}




\begin{tabular}{|c|c|c|c|c|c|c|c|c|c|c|c|c|c|}
\hline 3780 & & REVELLO & ARTURO & S & 12 & MICHIGAN & ITALY & ITALY & & & AGLIE'. TO & LAKE STREET & HANCOCK CITY \\
\hline 3781 & & REVELLO & AMALIA & D & 6 & MICHIGAN & ITALY & ITALY & & & AGLIE'. TO & LAKE STREET & HANCOCK CITY \\
\hline 3782 & & REVELLO & LOUIS & $\mathrm{s}$ & 4 & MICHIGAN & ITALY & ITALY & & & AGLIE'. TO & LAKE STREET & HANCOCK CITY \\
\hline 3783 & 800 & LENCI & FIORELLO & $\mathrm{H}$ & 33 & ITALY & ITALY & ITALY & 1906 & MINER/CM & SEGROMIGNO. LU & QUINCY ST. & HANCOCK CITY \\
\hline 3784 & & LENCI & LIZZIE & W & 27 & ITALY & ITALY & ITALY & 1906 & & SEGROMIGNO. LU & QUINCY ST. & HANCOCK CITY \\
\hline 3785 & 941 & SODINI & RUGGERO & $\mathrm{H}$ & 39 & ITALY & ITALY & ITALY & 1901 & MINER/CM & COLLODI. LU & INGOT ST. & HANCOCK CITY \\
\hline 3786 & & SODINI & MILDRED & W & 28 & MICHIGAN & ENGLAND & ENGLAND & & & ENGLAND & INGOT ST. & HANCOCK CITY \\
\hline 3787 & & SODINI & RAYMOND & $s$ & 5 & MICHIGAN & ITALY & MICHIGAN & & & COLLODI. LU & INGOT ST. & HANCOCK CITY \\
\hline 3788 & & SODINI & MARY & D & 2 & MICHIGAN & ITALY & MICHIGAN & & & COLLODI. LU & INGOT ST. & HANCOCK CITY \\
\hline 3789 & 1024 & BELCARI & GIUSEPPE & $\mathrm{H}$ & 48 & ITALY & ITALY & ITALY & 1900 & LABORER/CM & & PROSPECT ST. & HANCOCK CITY \\
\hline 3790 & & BELCARI & EUGENE & W & 48 & ITALY & ITALY & ITALY & 1900 & & & PROSPECT ST. & HANCOCK CITY \\
\hline 3791 & & BELCARI & ANGELO & s & 8 & MICHIGAN & ITALY & ITALY & & & & PROSPECT ST. & HANCOCK CITY \\
\hline 3792 & 1035 & LENCIONI & LOUIS & $\mathrm{H}$ & 43 & ITALY & ITALY & ITALY & 1900 & MERCHANT/GROCERY & LUCCA & $\begin{array}{l}\text { ETHEL AVE. } \\
\text { ETH. }\end{array}$ & HANCOCK CITY \\
\hline 3793 & 1030 & LENCIONI & NATALINA & W & 41 & ITALY & ITALY & ITALY & 1902 & 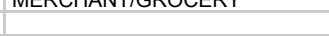 & LUCCA & ETHEL AVE. & HANCOCK CITY \\
\hline 3794 & & LENCIONI & MARY & $\mathrm{D}$ & 17 & MICHIGAN & ITALY & ITALY & & & LUCCA & ETHEL AVE. & HANCOCK CITY \\
\hline 3795 & & LENCIONI & JOHN & $\mathrm{s}$ & 15 & MICHIGAN & ITALY & ITALY & & LABORER/ELECTRIC RAILWAY & LUCCA & ETHEL AVE. & HANCOCK CITY \\
\hline 3796 & & LENCIONI & PETER & $\mathrm{s}$ & 10 & MICHIGAN & ITALY & ITALY & & 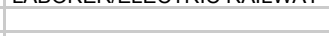 & LUCCA & ETHEL AVE. & HANCOCK CITY \\
\hline 3797 & & LENCIONI & GENEVIEVE & $\mathrm{D}$ & 1 & MICHIGAN & ITALY & ITALY & & & LUCCA & ETHEL AVE. & HANCOCK CITY \\
\hline 3798 & & VITTORI & GINO & B & 26 & ITALY & ITALY & ITALY & 1901 & BLACKSMITH/CM & FIRENZE & ETHEL AVE. & HANCOCK CITY \\
\hline 3799 & 1031 & PERONI & JOHN & $\mathrm{H}$ & 45 & ITALY & ITALY & ITALY & 1900 & MERCHANT/GROCERY & & ETHEL AVE. & HANCOCK CITY \\
\hline 3800 & & PERONI & ANNE & W & 35 & ITALY & ITALY & ITALY & 1903 & & & ETHEL AVE. & HANCOCK CITY \\
\hline 3801 & & SOCCHI & PETER & B & 46 & ITALY & ITALY & ITALY & 1910 & MINER/CM & & ETHEL AVE. & HANCOCK CITY \\
\hline 3802 & & CEOCCIA/DI & JOSEPH & B & 44 & ITALY & ITALY & ITALY & 1910 & LABORER/CM & & ETHEL AVE. & HANCOCK CITY \\
\hline 3803 & & BIANCHI & JOSEPH & B & 27 & ITALY & ITALY & ITALY & 1915 & LABORER/CM & LUCCA & ETHEL AVE. & HANCOCK CITY \\
\hline 3804 & & DEL BIANCO & VINCENT & B & 43 & ITALY & ITALY & ITALY & UN & LABORER/CM & & ETHEL AVE. & HANCOCK CITY \\
\hline 3805 & & LORENZO & A & B & 46 & ITALY & ITALY & ITALY & 1910 & LABORER/CM & & ETHEL AVE. & HANCOCK CITY \\
\hline 3806 & 1027 & DI VECCHIO & GINO & $\begin{array}{l}\mathrm{B} \\
\mathrm{H}\end{array}$ & $\begin{array}{l}46 \\
49\end{array}$ & ITALY & $\begin{array}{l}\text { ITALY } \\
\text { ITALY }\end{array}$ & ITALY & 1897 & LABORER/CM & LUCCA & ETHEL AVE. & HANCOCK CITY \\
\hline 3807 & & DI VECCHIO & CARLETTA & w & 54 & ITALY & ITALY & ITALY & 1901 & MERCHANT/GROCERY & LUCCA & ETHEL AVE. & HANCOCK CITY \\
\hline 3808 & & DI VECCHIO & IDA & $\mathrm{D}$ & 23 & ITALY & ITALY & ITALY & 1901 & & LUCCA & ETHEL AVE. & HANCOCK CITY \\
\hline 3809 & & DI VECCHIO & SANTINA & $\mathrm{D}$ & 21 & ITALY & ITALY & ITALY & 1901 & SALESLADY/GROCERY & LUCCA & ETHEL AVE. & HANCOCK CITY \\
\hline 3810 & & DI VECCHIO & JENNIE & D & 16 & MICHIGAN & ITALY & ITALY & & & LUCCA & ETHEL AVE. & HANCOCK CITY \\
\hline 3811 & 1019 & TADDEUCCI & JOSEPH & $\mathrm{H}$ & 43 & ITALY & ITALY & ITALY & 1897 & MINER/CM & LUCCA & ETHEL AVE. & HANCOCK CITY \\
\hline 3812 & & TADDEUCCI & TRACY & W & 40 & ITALY & ITALY & ITALY & 1899 & & & ETHEL AVE. & HANCOCK CITY \\
\hline 3813 & & TADDEUCCI & JOHN & $\mathrm{s}$ & 17 & MICHIGAN & ITALY & ITALY & & MOTORMAN/CM & LUCCA & ETHEL AVE. & HANCOCK CITY \\
\hline 3814 & & TADDEUCCI & AMELIA & $\mathrm{D}$ & 15 & MICHIGAN & ITALY & ITALY & & & LUCCA & ETHEL AVE. & HANCOCK CITY \\
\hline 3815 & & TADDEUCCI & ALBERT & $\mathrm{s}$ & 13 & MICHIGAN & ITALY & ITALY & & & LUCCA & ETHEL AVE. & HANCOCK CITY \\
\hline 3816 & & TADDEUCCI & FRANK & s & 11 & MICHIGAN & ITALY & ITALY & & & LUCCA & ETHEL AVE. & HANCOCK CITY \\
\hline 3817 & & TADDEUCCI & RAYMOND & $\mathrm{s}$ & 7 & MICHIGAN & ITALY & ITALY & & & LUCCA & ETHEL AVE. & HANCOCK CITY \\
\hline 3818 & & TADDEUCCI & $\mathrm{RICCHIO}$ & $\mathrm{s}$ & 4 & MICHIGAN & ITALY & ITALY & & & LUCCA & ETHEL AVE. & HANCOCK CITY \\
\hline 3819 & & TADDEUCCI & MARY & $\mathrm{D}$ & 4 & MICHIGAN & ITALY & ITALY & & & LUCCA & ETHEL AVE. & HANCOCK CITY \\
\hline 3820 & 1050 & BATTISTONI & OTTO & $\mathrm{H}$ & 72 & ITALY & ITALY & ITALY & 1880 & LABORER/CM & LUCCA & INGOT ST. & HANCOCK CITY \\
\hline 3821 & & BATTISTONI & LEONA & w & 46 & ITALY & ITALY & ITALY & 1895 & & & INGOT ST. & HANCOCK CITY \\
\hline 3822 & & DONATI & JENNIE & D & 20 & MICHIGAN & ITALY & ITALY & & & LUCCA & INGOT ST. & HANCOCK CITY \\
\hline 3823 & & DONATI & ALBERT & SOIL & 29 & ITALY & ITALY & ITALY & 1912 & BLACKSMITH/CM & & INGOT ST. & HANCOCK CITY \\
\hline 3824 & & DONATI & BEN & GS & 2 & MICHIGAN & ITALY & ITALY & & & LUCCA & INGOT ST. & HANCOCK CITY \\
\hline 3825 & & MONTICELLI & SAMUEL & PART & 71 & ITALY & ITALY & ITALY & 1892 & ENGINEER/CM & LUCCA & INGOT ST. & HANCOCK CITY \\
\hline 3826 & & MONTICELLI & WILL & PAR' $^{\prime}$ & 22 & MICHIGAN & ITALY & ITALY & & BLACKSMITH/CM & LUCCA & INGOT ST. & HANCOCK CITY \\
\hline 3827 & 1032 & LAVORINI & PIETRO & $\mathrm{H}$ & 57 & ITALY & ITALY & ITALY & 1908 & MERCHANT/MACCARONI FAC. & LUCCA & INGOT ST. & HANCOCK CITY \\
\hline 3828 & & LAVORINI & MARIA & w & 58 & ITALY & ITALY & ITALY & 1909 & LABORER/MACCARONI FAC. & LUCCA & INGOT ST. & HANCOCK CITY \\
\hline 3829 & & LAVORINI & FERRUCCIO & $\mathrm{s}$ & 31 & ITALY & ITALY & ITALY & 1906 & LABORER/MACCARONI FAC. & LUCCA & INGOT ST. & HANCOCK CITY \\
\hline 3830 & & VITTORI & CAMILLA & $\mathrm{D}$ & 30 & ITALY & ITALY & ITALY & 1908 & & LUCCA & INGOT ST. & HANCOCK CITY \\
\hline 3831 & & $\begin{array}{l}\text { VITTORI } \\
\text { VITOR }\end{array}$ & SETTIMIO & SOIL & 28 & ITALY & ITALY & ITALY & 1915 & MINER/CM & FIRENZE & INGOT ST. & HANCOCK CITY \\
\hline 3832 & 1018 & PAOLI & RALPH & $\mathrm{H}$ & 40 & ITALY & ITALY & ITALY & 1896 & MERCHANT/GROCERY & LUCCA & INGOT ST. & HANCOCK CITY \\
\hline 3833 & & PAOLI & IDA & w & 36 & ITALY & ITALY & ITALY & 1899 & & & INGOT ST. & HANCOCK CITY \\
\hline 3834 & & PAOLI & LUISA & $\mathrm{D}$ & 2 & MICHIGAN & ITALY & ITALY & & & LUCCA & INGOT ST. & HANCOCK CITY \\
\hline 3835 & & PAOLI & LOUIS & $\mathrm{s}$ & $7 \mathrm{M}$ & MICHIGAN & ITALY & ITALY & & & LUCCA & INGOT ST. & HANCOCK CITY \\
\hline 3836 & & PAOLI & ARTURO & BR & 52 & ITALY & ITALY & ITALY & 1900 & MERCHANT/GROCERY & LUCCA & INGOT ST. & HANCOCK CITY \\
\hline 3837 & 1215 & LENCI & URVASIO & $\mathrm{H}$ & 50 & ITALY & ITALY & ITALY & 1904 & MERCHANT/GROCERY & PESCAGLIA, LU & SUMMIT ST. & HANCOCK CITY \\
\hline 3838 & & LENCI & ISOLA & W & 44 & ITALY & ITALY & ITALY & 1904 & & PESCAGLIA. LU & SUMMIT ST. & HANCOCK CITY \\
\hline 3839 & & LENCI & GEORGE & s & 16 & ITALY & ITALY & ITALY & 1904 & & PESCAGLIA. LU & SUMMIT ST. & HANCOCK CITY \\
\hline 3840 & & LENCI & JOHN & $\mathrm{s}$ & 13 & MICHIGAN & ITALY & ITALY & & & PESCAGLIA. LU & SUMMIT ST. & HANCOCK CITY \\
\hline 3841 & 440 & MANESCHI & JULIO & $\mathrm{H}$ & 38 & ITALY & ITALY & ITALY & 1902 & TIMBERMAN/CM & CARRARA & 4TH ST. & HANCOCK CITY \\
\hline 3842 & & MANESCHI & ANGELINA & w & 40 & ITALY & ITALY & ITALY & 1900 & & LUCCA & 4TH ST. & HANCOCK CITY \\
\hline
\end{tabular}




\begin{tabular}{|c|c|c|c|c|c|c|c|c|c|c|c|c|c|}
\hline 3843 & & BARBIERI & PRIMETTA & SD & 18 & ITALY & ITALY & ITALY & 1900 & LAUNDRESS/LAUNDRY & LUCCA & 4TH ST. & HANCOCK CITY \\
\hline 3844 & & BARBIERI & GINO & sso & 17 & ITALY & ITALY & ITALY & 1900 & LABORER/CM & LUCCA & 4TH ST. & HANCOCK CITY \\
\hline 3845 & 1144 & ClUCCl & AUGUSTO & $\mathrm{H}$ & 47 & ITALY & ITALY & ITALY & 1904 & LABORER/SMELTER & LUCCA & SUMMIT ST. & HANCOCK CITY \\
\hline 3846 & & $\mathrm{ClUCCl}$ & BASILIDE & W & 36 & ITALY & ITALY & ITALY & 1904 & & LUCCA & SUMMIT ST. & HANCOCK CITY \\
\hline 3847 & & $\mathrm{ClUCCl}$ & GALALINI & $\mathrm{D}$ & 14 & MICHIGAN & ITALY & ITALY & & & LUCCA & SUMMIT ST. & HANCOCK CITY \\
\hline 3848 & & $\mathrm{ClUCCl}$ & ELMER & $\mathrm{s}$ & 12 & MICHIGAN & ITALY & ITALY & & & LUCCA & SUMMIT ST. & HANCOCK CITY \\
\hline 3849 & & ClUCCl & BERNARDO & $\mathrm{s}$ & 5 & MICHIGAN & ITALY & ITALY & & & LUCCA & SUMMIT ST. & HANCOCK CITY \\
\hline 3850 & & AMERIGO & GABRIEL & B & 47 & ITALY & ITALY & ITALY & 1890 & & LUCU & SUMMIT ST. & HANCOCK CITY \\
\hline 3851 & ?? & SCOLARI & JOSEPH & $\mathrm{H}$ & 35 & ITALY & ITALY & ITALY & 1901 & MINER/CM & VICENZA & 3RD ST. & HANCOCK CITY \\
\hline 3852 & & SCOLARI & IRENE & W & 33 & MICHIGAN & ITALY & ITALY & & & & 3RD ST. & HANCOCK CITY \\
\hline 3853 & 1011 & BALCONI & MIKE & $\mathrm{H}$ & 36 & ITALY & ITALY & ITALY & 1907 & LABORER/SMELTER & MERCALLO. VARESE & ELM ST. & HANCOCK CITY \\
\hline 3854 & & BALCONI & ROSALIA & W & 32 & MICHIGAN & ITALY & ITALY & & & & ELM ST. & HANCOCK CITY \\
\hline 3855 & & BALCONI & LOUIS & $\mathrm{D}$ & 7 & MICHIGAN & ITALY & MICHIGAN & & & MERCALLO. VARESE & ELM ST. & HANCOCK CITY \\
\hline 3856 & & BALCONI & SICILIA & $\mathrm{D}$ & 4 & MICHIGAN & ITALY & MICHIGAN & & & MERCALLO. VARESE & ELM ST. & HANCOCK CITY \\
\hline 3857 & & BALCONI & ERNESTINA & $\mathrm{D}$ & $4 \mathrm{M}$ & MICHIGAN & ITALY & MICHIGAN & & & MERCALLO. VARESE & ELM ST. & HANCOCK CITY \\
\hline 3858 & & ENRIETTI & BERNARDO & B & 44 & ITALY & ITALY & ITALY & 1890 & LABORER/FARM & TORINO & ELM ST. & HANCOCK CITY \\
\hline 3859 & & COPPO & JOHN & $\mathrm{H}$ & 36 & MICHIGAN & ITALY & ITALY & & TRUCKER/STREET RAILROAD & PONT CANAVESE. TO & ELM ST. & HANCOCK CITY \\
\hline 3860 & & COPPO & MARION & W & 34 & ITALY & ITALY & ITALY & 1905 & & & ELM ST. & HANCOCK CITY \\
\hline 3861 & & COPPO & FRANK & $\mathrm{s}$ & 7 & MICHIGAN & MICHIGAN & ITALY & & & PONT CANAVESE. TO & ELM ST. & HANCOCK CITY \\
\hline 3862 & & COPPO & PAUL & $\mathrm{s}$ & 6 & MICHIGAN & MICHIGAN & ITALY & & & PONT CANAVESE. TO & ELM ST. & HANCOCK CITY \\
\hline 3863 & 1013 & GIACHINO & JOSEPH & $\mathrm{H}$ & 31 & ITALY & ITALY & ITALY & 1905 & LABORER/STAMP MILL & SPARONE. TO & ELM ST. & HANCOCK CITY \\
\hline 3864 & & GIACHINO & MINNIE & w & 26 & ITALY & ITALY & ITALY & 1897 & & & ELM ST. & HANCOCK CITY \\
\hline 3865 & & GIACHINO & CATERINA & $\mathrm{D}$ & 6 & MICHIGAN & ITALY & ITALY & & & SPARONE. TO & ELM ST. & HANCOCK CITY \\
\hline 3866 & & GIACHINO & ANGELINA & $\mathrm{D}$ & 4 & MICHIGAN & ITALY & ITALY & & & SPARONE. TO & ELM ST. & HANCOCK CITY \\
\hline 3867 & 915 & PARINI/PARMI & JOHN & $\mathrm{H}$ & 57 & ITALY & ITALY & ITALY & 1890 & LABORER/CITY STREET & & SUMMIT ST. & HANCOCK CITY \\
\hline 3868 & & PARINI/PARMI & MARY & W & 52 & ITALY & ITALY & ITALY & 1897 & & & SUMMIT ST. & HANCOCK CITY \\
\hline 3869 & & PARINI/PARMI & JOSEPHINE & $\mathrm{D}$ & 18 & MICHIGAN & ITALY & ITALY & & & & SUMMIT ST. & HANCOCK CITY \\
\hline 3870 & 1038 & FEDERIGHI & IRENE & $\mathrm{H}$ & 41 & ITALY & ITALY & ITALY & 1899 & MERCHANT/GROCERY & LUCCA & SUMMIT ST. & HANCOCK CITY \\
\hline 3871 & & FEDERIGHI & TERESA & D & 18 & MICHIGAN & ITALY & ITALY & & & LUCCA & SUMMIT ST. & HANCOCK CITY \\
\hline 3872 & & FEDERIGHI & EMIL & $\mathrm{s}$ & 16 & MICHIGAN & ITALY & ITALY & & & LUCCA & SUMMIT ST. & HANCOCK CITY \\
\hline 3873 & & FEDERIGHI & SEBASTIANO & $\mathrm{s}$ & 14 & MICHIGAN & ITALY & ITALY & & & LUCCA & SUMMIT ST. & HANCOCK CITY \\
\hline 3874 & & FEDERIGHI & JOHN & $\mathrm{s}$ & 8 & MICHIGAN & ITALY & ITALY & & & LUCCA & SUMMIT ST. & HANCOCK CITY \\
\hline 3875 & & FEDERIGHI & PAULINE & $\mathrm{D}$ & 5 & MICHIGAN & ITALY & ITALY & & & LUCCA & SUMMIT ST. & HANCOCK CITY \\
\hline 3876 & & FEDERIGHI & MARION & D & 2 & MICHIGAN & ITALY & ITALY & & & LUCCA & SUMMIT ST. & HANCOCK CITY \\
\hline 3877 & 1012 & LORENZETTI & JOHN & $\mathrm{H}$ & 32 & ITALY & ITALY & ITALY & 1910 & LABORER/SMELTER & LUCCA & SUMMIT ST. & HANCOCK CITY \\
\hline 3878 & & LORENZETTI & TERESA & w & 32 & ITALY & ITALY & ITALY & 1910 & & LUCCA & SUMMIT ST. & HANCOCK CITY \\
\hline 3879 & & LORENZETTI & GIULIO & $\mathrm{s}$ & 7 & MICHIGAN & ITALY & ITALY & & & LUCCA & SUMMIT ST. & HANCOCK CITY \\
\hline 3880 & & LORENZETTI & DANTE & $\mathrm{s}$ & 5 & MICHIGAN & ITALY & ITALY & & & LUCCA & SUMMIT ST. & HANCOCK CITY \\
\hline 3881 & & LORENZETTI & OLGA & $\mathrm{D}$ & $5 \mathrm{M}$ & MICHIGAN & ITALY & ITALY & & & LUCCA & SUMMIT ST. & HANCOCK CITY \\
\hline 3882 & & BERLINCIONI & LEANDRO & B & 41 & ITALY & ITALY & ITALY & 1907 & SALESMAN/GROCERY STORE & FIRENZE & SUMMIT ST. & HANCOCK CITY \\
\hline 3883 & & LORENZETTI & RALPH & BR & 35 & ITALY & ITALY & ITALY & 1903 & MINER/CM & LUCCA & SUMMIT ST. & HANCOCK CITY \\
\hline 3884 & 1035 & ORELLA & DOMENICO & $\mathrm{H}$ & 39 & ITALY & ITALY & ITALY & 1886 & BOOKKEEPER/BANK & TORINO & MINNESOTA ST. & HANCOCK CITY \\
\hline 3885 & & ORELLA & TERESA & w & 41 & ITALY & ITALY & ITALY & 1901 & & & MINNESOTA ST. & HANCOCK CITY \\
\hline 3886 & & ORELLA & BERNARDO & s & 16 & MICHIGAN & ITALY & ITALY & & & TORINO & MINNESOTA ST. & HANCOCK CITY \\
\hline 3887 & & ORELLA & WILLIAM & $\mathrm{s}$ & 13 & MICHIGAN & ITALY & ITALY & & & TORINO & MINNESOTA ST. & HANCOCK CITY \\
\hline 3888 & & ORELLA & MARY & D & 12 & MICHIGAN & ITALY & ITALY & & & TORINO & MINNESOTA ST. & HANCOCK CITY \\
\hline 3889 & & ORELLA & AGNES & D & 10 & MICHIGAN & ITALY & ITALY & & & TORINO & MINNESOTA ST. & HANCOCK CITY \\
\hline 3890 & 1002 & CAMPIONI & GUIDO & $\mathrm{H}$ & 33 & ITALY & ITALY & ITALY & 1909 & PROPRIETOR/MACCARONI FAC. & LUCCA & MINNESOTA ST. & HANCOCK CITY \\
\hline 3891 & & CAMPIONI & MABEL & w & 26 & ITALY & ITALY & ITALY & 1907 & & & MINNESOTA ST. & HANCOCK CITY \\
\hline 3892 & & CAMPIONI & COSTANTINA & $\mathrm{D}$ & 8 & MICHIGAN & ITALY & ITALY & & & LUCCA & MINNESOTA ST. & HANCOCK CITY \\
\hline 3893 & & CAMPIONI & JOSEPH & $\mathrm{s}$ & 7 & MICHIGAN & ITALY & ITALY & & & LUCCA & MINNESOTA ST. & HANCOCK CITY \\
\hline 3894 & & CAMPIONI & LOUIS & $\mathrm{s}$ & 5 & MICHIGAN & ITALY & ITALY & & & LUCCA & MINNESOTA ST. & HANCOCK CITY \\
\hline 3895 & & CAMPIONI & PAUL & $\mathrm{s}$ & 5 & MICHIGAN & ITALY & ITALY & & & LUCCA & MINNESOTA ST. & HANCOCK CITY \\
\hline 3896 & 1124 & RENALDI & BATTISTA & $\mathrm{H}$ & 56 & ITALY & ITALY & ITALY & 1886 & MINER/CM & SAN GIORGIO CANAVESE. TO & SEGOBIC ST. & HANCOCK CITY \\
\hline 3897 & & RENALDI & MARY & w & 51 & ITALY & ITALY & ITALY & 1895 & & & SEGOBIC ST. & HANCOCK CITY \\
\hline 3898 & & RENALDI & TERESA & D & 28 & MICHIGAN & ITALY & ITALY & & SALESLADY/DEPT. STORE & SAN GIORGIO CANAVESE. TO & SEGOBIC ST. & HANCOCK CITY \\
\hline 3899 & & RENALDI & JOSEPH & s & 21 & MICHIGAN & ITALY & ITALY & & LABORER/RAILROAD & SAN GIORGIO CANAVESE. TO & SEGOBIC ST. & HANCOCK CITY \\
\hline 3900 & & RENALDI & JAMES & s & 20 & MICHIGAN & ITALY & ITALY & & LABORER/RAILROAD & SAN GIORGIO CANAVESE, TO & SEGOBIC ST. & HANCOCK CITY \\
\hline 3901 & & RENALDI & MARION & D & 18 & MICHIGAN & ITALY & ITALY & & & SAN GIORGIO CANAVESE. TO & SEGOBIC ST. & HANCOCK CITY \\
\hline 3902 & & RENALDI & MARY & $\mathrm{D}$ & 16 & MICHIGAN & ITALY & ITALY & & & SAN GIORGIO CANAVESE. TO & SEGOBIC ST. & HANCOCK CITY \\
\hline 903 & & RENALDI & VITTORIA & $\mathrm{D}$ & 14 & MICHIGAN & ITALY & ITALY & & STUDENT & SAN GIORGIO CANAVES & SEGOBIC ST. & HANCOCK CITY \\
\hline 3904 & & RENALDI & ERNEST & $\mathrm{s}$ & 12 & MICHIGAN & ITALY & ITALY & & & SAN GIORGIO CANAVESE. TO & SEGOBIC ST. & HANCOCK CITY \\
\hline 3905 & & RENALDI & ARTHUR & $\mathrm{s}$ & 12 & MICHIGAN & ITALY & ITALY & & & SAN GIORGIO CANAVESE. TO & SEGOBIC ST. & HANCOCK CITY \\
\hline
\end{tabular}




\begin{tabular}{|c|c|c|c|c|c|c|c|c|c|c|c|c|c|}
\hline 3906 & & RENALDI & GENEVIEVE & $\mathrm{D}$ & 9 & MICHIGAN & ITALY & ITALY & & & SAN GIORGIO CANAVESE. TO & SEGOBIC ST. & HANCOCK CITY \\
\hline 3907 & 1211 & $\mathrm{RICCl}$ & FRANK & $\mathrm{H}$ & 25 & ITALY & ITALY & ITALY & 1912 & PAINTER/CM & LUCCA & INGOT ST. & HANCOCK CITY \\
\hline 3908 & & $\mathrm{RICCl}$ & RITA & W & 20 & MICHIGAN & ITALY & ITALY & & & & INGOT ST. & HANCOCK CITY \\
\hline 3909 & & $\mathrm{RICCl}$ & JOHN & $\mathrm{s}$ & $9 \mathrm{M}$ & MICHIGAN & ITALY & MICHIGAN & & & LUCCA & INGOT ST. & HANCOCK CITY \\
\hline 3910 & 811 & MUGNANI & RAFFAELLO & $\mathrm{H}$ & 45 & ITALY & ITALY & ITALY & 1904 & MERCHANT/GROCERY & & ETHEL AVE. & HANCOCK CITY \\
\hline 3911 & & MUGNANI & ADELIA & w & 40 & ITALY & ITALY & ITALY & 1904 & & & ETHEL AVE. & HANCOCK CITY \\
\hline 3912 & & DI PUCCIO & VIRGILIO & B & 24 & ITALY & ITALY & ITALY & 1913 & TEAMSTER/GROCERY STORE & CAPANNORI. LU & ETHEL AVE. & HANCOCK CITY \\
\hline 3913 & 1300 & GALLINATTI & JOHN & $\mathrm{O}$ & 46 & ITALY & ITALY & ITALY & 1889 & MINER/CM & TORINO & CEDAR ST. & HANCOCK CITY \\
\hline 3914 & & GALLINATTI & ANSELMINA & W & 37 & ITALY & ITALY & ITALY & $98 / 99$ & & & CEDAR ST. & HANCOCK CITY \\
\hline 3915 & & GALLINATTI & JOSEPHINE & D & 18 & MICHIGAN & ITALY & ITALY & & & TORINO & CEDAR ST. & HANCOCK CITY \\
\hline 3916 & & GALLINATTI & MOSES & $\mathrm{s}$ & 15 & MICHIGAN & ITALY & ITALY & & & TORINO & CEDAR ST. & HANCOCK CITY \\
\hline 3917 & 522 & BESSOLO & PAUL & $\mathrm{H}$ & 25 & KANSAS & ITALY & ITALY & & CHAUFFEUR/PF & TORINO & EMMA ST. & HANCOCK CITY \\
\hline 3918 & & BESSOLO & VERONICA & MO & 49 & ITALY & ITALY & ITALY & 1887 & & TORINO & EMMA ST. & HANCOCK CITY \\
\hline 3919 & & BESSOLO & DOMENICO & $B R$ & 18 & MICHIGAN & ITALY & ITALY & & DRIVER & TORINO & EMMA ST. & HANCOCK CITY \\
\hline 3920 & & MASONI & JERRY & $\mathrm{H}$ & 31 & ITALY & ITALY & ITALY & 1909 & FIREMAN/SMELTER & & EMMA ST. & HANCOCK CITY \\
\hline 3921 & & MASONI & AUDIE & W & 27 & MICHIGAN & ITALY & ITALY & & & & EMMA ST. & HANCOCK CITY \\
\hline 3922 & & MASONI & CAROLINE & D & 8 & MICHIGAN & ITALY & ITALY & & & & EMMA ST. & HANCOCK CITY \\
\hline 3923 & & BALCONI & JOHN & $\mathrm{H}$ & 32 & ITALY & ITALY & ITALY & 1914 & FIREMAN/SMELTER & MERCALLO. VARESE & ATLANTIC AVE. & HANCOCK CITY \\
\hline 3924 & & BALCONI & MARY & W & 25 & ITALY & ITALY & ITALY & 1916 & & MERCALLO. VARESE & ATLANTIC AVE. & HANCOCK CITY \\
\hline 3925 & & BALCONI & JOSEPHINE & D & 5 & ITALY & ITALY & ITALY & 1916 & & MERCALLO. VARESE & ATLANTIC AVE. & HANCOCK CITY \\
\hline 3926 & & BALCONI & JULIO & s & 2 & MICHIGAN & ITALY & ITALY & 1910 & & MERCALLO. VARESE & ATLANTIC AVE. & HANCOCK CITY \\
\hline 3927 & & BALCONI & PIERINA & D & $4 \mathrm{M}$ & MICHIGAN & ITALY & ITALY & & & MERCALLO, VARESE & ATLANTIC AVE. & HANCOCK CITY \\
\hline 3928 & & AMATO & FRANK & $\mathrm{H}$ & 48 & ITALY & ITALY & ITALY & 1892 & FIREMAN/SMELTER & & QUINCY ST. & HANCOCK CITY \\
\hline 3929 & & AMATO & CAROLINA & w & 52 & ITALY & ITALY & ITALY & 1907 & & & QUINCY ST. & HANCOCK CITY \\
\hline 3930 & & AMATO & TERESA & D & 12 & MICHIGAN & ITALY & ITALY & & & & QUINCY ST. & HANCOCK CITY \\
\hline 3931 & & AMATO & FRANK & $s$ & 8 & MICHIGAN & ITALY & ITALY & & & & QUINCY ST. & HANCOCK CITY \\
\hline 3932 & & AMATO & MATILDA & D & $2 \mathrm{M}$ & MICHIGAN & ITALY & ITALY & & & & QUINCY ST. & HANCOCK CITY \\
\hline 3933 & & FASANA & ADOLF & $\mathrm{H}$ & 35 & ITALY & ITALY & ITALY & 1908 & LABORER/SMELTER & TORINO & SPRUCE ST. & HANCOCK CITY \\
\hline 3934 & & FASANA & MAUD & W & 35 & ITALY & ITALY & ITALY & 1909 & & & SPRUCE ST. & HANCOCK CITY \\
\hline 3935 & & FASANA & MIKE & s & 9 & MICHIGAN & ITALY & ITALY & & & TORINO & SPRUCE ST. & HANCOCK CITY \\
\hline 3936 & & FASANA & ANTONIO & $\mathrm{s}$ & $?$ & MICHIGAN & ITALY & ITALY & & & TORINO & SPRUCE ST. & HANCOCK CITY \\
\hline 3937 & & FASANA & FRANK & $\mathrm{s}$ & 7 & MICHIGAN & ITALY & ITALY & & & TORINO & SPRUCE ST. & HANCOCK CITY \\
\hline 3938 & & PIONI & DOMENICO & B & 49 & ITALY & ITALY & ITALY & 1905 & LABORER/SMELTER & TORIIN & SPRUCE ST. & HANCOCK CITY \\
\hline 3939 & & SANDRETTO & SYLVESTER & $\mathrm{H}$ & 50 & ITALY & ITALY & ITALY & 1890 & FARMER/GENERAL FARM & PONT CANAVESE, TO & CONE ROAD & HANCOCK \\
\hline 3940 & & SANDRETTO & ANGELICA & w & 52 & ITALY & ITALY & ITALY & 1900 & & & CONE ROAD & HANCOCK \\
\hline 3941 & & SANDRETTO & JAMES & s & 19 & MICHIGAN & ITALY & ITALY & & LABORER/GENRAL FARM & PONT CANAVESE. TO & CONE ROAD & HANCOCK \\
\hline 3942 & & GASPARDO & PAUL & $\mathrm{H}$ & 44 & ITALY & ITALY & ITALY & 1900 & FARMER/GENERAL FARM & PONT CANAVESE. TO & CONE ROAD & HANCOCK \\
\hline 3943 & & GASPARDO & MARY & W & 45 & ITALY & ITALY & ITALY & 1903 & & & CONE ROAD & HANCOCK \\
\hline 3944 & & GASPARDO & BATTISTA & $\mathrm{s}$ & 15 & MICHIGAN & ITALY & ITALY & & & PONT CANAVESE. TO & CONE ROAD & HANCOCK \\
\hline 3945 & & GASPARDO & ANNA & $\mathrm{D}$ & 14 & MICHIGAN & ITALY & ITALY & & & PONT CANAVESE. TO & CONE ROAD & HANCOCK \\
\hline 3946 & & GASPARDO & MARY & D & 13 & MICHIGAN & ITALY & ITALY & & & PONT CANAVESE. TO & CONE ROAD & HANCOCK \\
\hline 3947 & & GASPARDO & DOMENICO & $\mathrm{s}$ & 11 & MICHIGAN & ITALY & ITALY & & & PONT CANAVESE. TO & CONE ROAD & HANCOCK \\
\hline 3948 & & GASPARDO & JOHANNA & D & 5 & MICHIGAN & ITALY & ITALY & & & PONT CANAVESE. TO & CONE ROAD & HANCOCK \\
\hline 3949 & & PUCCI & GIUSEPPE & $\mathrm{H}$ & 33 & ITALY & ITALY & ITALY & 1910 & FARMER/GENERAL FARM & CAPANNORI. LU & SALO ROAD & HANCOCK \\
\hline 3950 & & PUCCI & TERESA & W & 29 & MICHIGAN & ITALY & ITALY & 1070 & 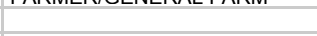 & & SALO ROAD & HANCOCK \\
\hline 3951 & & PUCCI & RUDOLPH & $\mathrm{s}$ & 4 & MICHIGAN & ITALY & MICHIGAN & & & CAPANNORI. LU & SALO ROAD & HANCOCK \\
\hline 3952 & & PUCCI & JOSEPH & $\mathrm{s}$ & 2 & MICHIGAN & ITALY & MICHIGAN & & & CAPANNORI. LU & SALO ROAD & HANCOCK \\
\hline 3953 & & MORO & AUGUSTO & $\mathrm{H}$ & 50 & ITALY & ITALY & ITALY & UN & FARMER/GENERAL FARM & TORINO & SALO ROAD & HANCOCK \\
\hline 3954 & & MORO & VIRGINIA & w & 36 & MICHIGAN & ITALY & ITALY & & & & SALO ROAD & HANCOCK \\
\hline 3955 & & MORO & MODESTO & $\mathrm{s}$ & 3 & MICHIGAN & ITALY & MICHIGAN & & & TORINO & SALO ROAD & HANCOCK \\
\hline 3956 & 32 & CATTINI & $\mathrm{JOHN}$ & $\mathrm{H}$ & 30 & ITALY & ITALY & ITALY & 1908 & MINER/CM & TORINO & CHESTNUST ST. & OSCEOLA - TAMARACH TOWN \\
\hline 3957 & & CATTINI & AMELIA & W & 40 & ITALY & ITALY & ITALY & 1902 & & TORINO & CHESTNUST ST. & OSCEOLA - TAMARACH TOWN \\
\hline 3958 & & CATTINI & ERTOLO & $\mathrm{s}$ & 11 & ITALY & ITALY & ITALY & 1912 & & TORINO & CHESTNUST ST. & OSCEOLA - TAMARACH TOWN \\
\hline 3959 & & CATTINI & EJANO & $\mathrm{s}$ & 9 & MICHIGAN & ITALY & ITALY & & & TORINO & CHESTNUST ST. & OSCEOLA - TAMARACH TOWN \\
\hline 3960 & & CATTINI & TOSCA & D & 5 & MICHIGAN & ITALY & ITALY & & & TORINO & CHESTNUST ST. & OSCEOLA - TAMARACH TOWN \\
\hline 3961 & & CATTINI & VIRGINIA & D & 1 & MICHIGAN & ITALY & ITALY & & & TORINO & CHESTNUST ST. & OSCEOLA - TAMARACH TOWN \\
\hline 3962 & 31 & GAILICE & LOUIS & $\mathrm{H}$ & 26 & ITALY & ITALY & ITALY & 1910 & LABORER SURFACE/CM & & CHESTNUST ST. & OSCEOLA - TAMARACH TOWN \\
\hline 3963 & & GAILICE & LENA & W & 24 & ITALY & ITALY & ITALY & 1913 & & & CHESTNUST ST. & OSCEOLA - TAMARACH TOWN \\
\hline 3964 & & GAILICE & LOLA & D & 4 & MICHIGAN & ITALY & ITALY & & & & CHESTNUST ST. & OSCEOLA - TAMARACH TOWN \\
\hline 3965 & & GAILICE & ONESTO & $\mathrm{s}$ & $?$ & MICHIGAN & ITALY & ITALY & & & & CHESTNUST ST. & OSCEOLA - TAMARACH TOWN \\
\hline 3966 & 31 & CECCHI & NARCISO & $\mathrm{H}$ & 61 & ITALY & ITA & ITALY & 1892 & MINER/CM & LUCCA & $\begin{array}{l}\text { CEDAR ST. } \\
\end{array}$ & OSCEOLA - TAMARACH TOWN \\
\hline 3967 & & CECCHI & EMILIA & w & 60 & ITALY & ITALY & ITALY & $00 ? ?$ & & & CEDAR ST. & OSCEOLA - TAMARACH TOWN \\
\hline 3968 & & LUCCHESI & LOUIS & $\mathrm{H}$ & 35 & ITALY & ITALY & ITALY & $00 ? ?$ & BARTENER/SALOON & LUCCA & CEDAR ST. & OSCEOLA - TAMARACH TOWN \\
\hline
\end{tabular}




\begin{tabular}{|c|c|c|c|c|c|c|c|c|c|c|c|c|c|}
\hline 3969 & & LUCCHESI & LOUISE & W & 28 & ITALY & ITALY & ITALY & 1905 & DRESSMAKER/AT HOME & & CEDAR ST. & OSCEOLA - TAMARACH TOWN \\
\hline 3970 & & LUCCHESI & UGO & s & 8 & ILLINOIS & ITALY & ITALY & & & LUCCA & CEDAR ST. & OSCEOLA - TAMARACH TOWN \\
\hline 3971 & & LUCCHESI & IRENE & D & 4 & ILLINOIS & ITALY & ITALY & & & LUCCA & CEDAR ST. & OSCEOLA - TAMARACH TOWN \\
\hline 3972 & & LUCCHESI & KATHLEN & D & $7 \mathrm{M}$ & MICHIGAN & ITALY & ITALY & & & LUCCA & CEDAR ST. & OSCEOLA - TAMARACH TOWN \\
\hline 3973 & 26 & $\mathrm{BINI}$ & TONY & $\mathrm{H}$ & 50 & ITALY & ITALY & ITALY & 1897 & MINER/CM & LUCCA & BEECH ST. & OSCEOLA - TAMARACH TOWN \\
\hline 3974 & & BINI & ESTER & w & 51 & ITALY & ITALY & ITALY & 1906 & & & BEECH ST. & OSCEOLA - TAMARACH TOWN \\
\hline 3975 & & BINI & ROSA & D & 15 & MICHIGAN & ITALY & ITALY & & & LUCCA & BEECH ST. & OSCEOLA - TAMARACH TOWN \\
\hline 3976 & & BINI & LENA & $\mathrm{D}$ & 5 & MICHIGAN & ITALY & ITALY & & & LUCCA & BEECH ST. & OSCEOLA - TAMARACH TOWN \\
\hline 3977 & & POLLASTRINI & ALFRED & B & 32 & ITALY & ITALY & ITALY & 1903 & MINER/CM & COLLODI. LU & BEECH ST. & OSCEOLA - TAMARACH TOWN \\
\hline 3978 & 25 & BATTUELLO & ANTONIO & $\mathrm{H}$ & 29 & ITALY & ITALY & ITALY & 1913 & MINER/CM & LUSIGLIE. TO & BEECH ST. & OSCEOLA - TAMARACH TOWN \\
\hline 3979 & & BATTUELLO & CAROLINA & w & 29 & ITALY & ITALY & ITALY & 1917 & & LUSIGLIE. TO & BEECH ST. & OSCEOLA - TAMARACH TOWN \\
\hline 3980 & & BATTUELLO & DOMENICO & D & 6 & ITALY & ITALY & ITALY & 1917 & & LUSIGLIE. TO & BEECH ST. & OSCEOLA - TAMARACH TOWN \\
\hline 3981 & & BATTUELLO & ANTONIO & $\mathrm{s}$ & 2 & MICHIGAN & ITALY & ITALY & & & LUSIGLIE. TO & BEECH ST. & OSCEOLA - TAMARACH TOWN \\
\hline 3982 & & BATTUELLO & TRACY & $\mathrm{D}$ & 1 & MICHIGAN & ITALY & ITALY & & & LUSIGLIE. TO & BEECH ST. & OSCEOLA - TAMARACH TOWN \\
\hline 3983 & 50 & SANDRETTO & COSTANZO & $\mathrm{H}$ & 66 & ITALY & ITALY & ITALY & 1888 & ENGINE OILER/CM & PONT CANAVESE. TO & & OSCEOLA - N. TAMARACH \\
\hline 3984 & & SANDRETTO & GENEVIEVE & W & 62 & ITALY & ITALY & ITALY & 1889 & & & & OSCEOLA - N. TAMARACH \\
\hline 3985 & & SANDRETTO & LORENZO & $\mathrm{s}$ & 26 & MICHIGAN & ITALY & ITALY & & BAKER & PONT CANAVESE. TO & & OSCEOLA - N. TAMARACH \\
\hline 3986 & & SANDRETTO & MARGHERITA & D & 17 & MICHIGAN & ITALY & ITALY & & & PONT CANAVESE. TO & & OSCEOLA - N. TAMARACH \\
\hline 3987 & 46 & RENALDI & JOSEPH & $\mathrm{H}$ & 54 & ITALY & ITALY & ITALY & 1894 & CARPENETR/STAMP MILL & SAN GIORGIO CANAVESE. TO & & OSCEOLA - N. TAMARACH \\
\hline 3988 & 40 & RENALDI & TERESA & W & 54 & ITALY & ITALY & ITALY & 1900 & 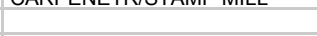 & 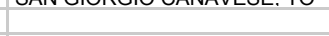 & & OSCEOLA - N. TAMARACH \\
\hline 3989 & & RENALDI & JOSEPH & $\mathrm{s}$ & 19 & MICHIGAN & ITALY & ITALY & & & SAN GIORGIO CANAVESE. TO & & OSCEOLA - N. TAMARACH \\
\hline 3990 & & RENALDI & VITTORIA & D & 16 & MICHIGAN & ITALY & ITALY & & & SAN GIORGIO CANAVESE, TO & & OSCEOLA - N. TAMARACH \\
\hline 3991 & & TADDEUCCI & HENRY & $\mathrm{H}$ & 46 & ITALY & ITALY & ITALY & 1904 & MERCHANT/CONFECTIONARY & LUCCA & STONE ST. & OSCEOLA \\
\hline 3992 & & TADDEUCCI & LIVIA & w & 45 & ITALY & ITALY & ITALY & 1908 & & LUCCA & STONE ST. & OSCEOLA \\
\hline 3993 & & TADDEUCCI & VALENTINA & D & 20 & ITALY & ITALY & ITALY & 1908 & & LUCCA & STONE ST. & OSCEOLA \\
\hline 3994 & & TADDEUCCI & DANTE & $\mathrm{s}$ & 16 & ITALY & ITALY & ITALY & 1908 & & LUCCA & STONE ST. & OSCEOLA \\
\hline 3995 & & PASTORE & DOMENICO & $\mathrm{H}$ & 42 & ITALY & ITALY & ITALY & 1898 & JANITOR/MINE OFFICE & SAN MARTINO CANAVESE. TO & STONE ST. & OSCEOLA \\
\hline 3996 & 270 & MAZZOLINI & OTTAVIO & $\mathrm{H}$ & 39 & ITALY & ITALY & ITALY & 1906 & TRAMMER/FOREMAN & TORINO & STONE ST. & OSCEOLA \\
\hline 3997 & & MAZZOLINI & DOMENICA & W & 33 & ITALY & ITALY & ITALY & 1912 & & TORINO & STONE ST. & OSCEOLA \\
\hline 3998 & & MAZZOLINI & JOSEPHINE & D & 11 & ITALY & ITALY & ITALY & 1912 & & TORINO & STONE ST. & OSCEOLA \\
\hline 3999 & & MAZZOLINI & MARY & D & 5 & MICHIGAN & ITALY & ITALY & & & TORINO & STONE ST. & OSCEOLA \\
\hline 4000 & & MAZZOLINI & CATERINA & D & 4 & MICHIGAN & ITALY & ITALY & & & TORINO & STONE ST. & OSCEOLA \\
\hline 4001 & 233 & CERU & GIUSEPPE & $\mathrm{H}$ & 31 & ITALY & ITALY & ITALY & 1912 & MINER/CM & LUCCA & COUNTY ROAD & OSCEOLA \\
\hline 4002 & & CERU & ERMELLINA & w & 29 & ITALY & ITALY & ITALY & 1914 & & LUCCA & COUNTY ROAD & OSCEOLA \\
\hline 4003 & & CERU & LINO & $\mathrm{s}$ & 9 & ITALY & ITALY & ITALY & 1914 & & LUCCA & COUNTY ROAD & OSCEOLA \\
\hline 4004 & & CERU & PAOLINO & $\mathrm{s}$ & 7 & ITALY & ITALY & ITALY & 1914 & & LUCCA & COUNTY ROAD & OSCEOLA \\
\hline 4005 & & CERU & GINO & $\mathrm{S}$ & 4 & MICHIGAN & ITALY & ITALY & & & LUCCA & COUNTY ROAD & OSCEOLA \\
\hline 4006 & & CERU & PLINO & $\mathrm{s}$ & 2 & MICHIGAN & ITALY & ITALY & & & LUCCA & COUNTY ROAD & OSCEOLA \\
\hline 4007 & & CERU & NELLA & D & $11 \mathrm{M}$ & MICHIGAN & ITALY & ITALY & & & LUCCA & COUNTY ROAD & OSCEOLA \\
\hline 4008 & & CERU & ABRAMO & $\mathrm{BR}$ & 37 & ITALY & ITALY & ITALY & 1913 & MINER/CM & LUCCA & COUNTY ROAD & OSCEOLA \\
\hline 4009 & 1 & FANTINI & PETER & $\mathrm{H}$ & 34 & ITALY & ITALY & ITALY & 1904 & MINER/CM & BERGAMO & MILLIONAIRE ST. & OSCEOLA \\
\hline 4010 & & FANTINI & TERESA & w & 36 & ITALY & ITALY & ITALY & 1901 & & & MILLIONAIRE ST. & OSCEOLA \\
\hline 4011 & & FANTINI & ANGELO & $\mathrm{s}$ & 9 & MICHIGAN & ITALY & ITALY & & & BERGAMO & MILLIONAIRE ST. & OSCEOLA \\
\hline 4012 & & FANTINI & VENTINI & $\mathrm{s}$ & $5 \mathrm{M}$ & MICHIGAN & ITALY & ITALY & & & BERGAMO & MILLIONAIRE ST. & OSCEOLA \\
\hline 4013 & 82 & DE FILIPPI & JOE & $\mathrm{H}$ & 59 & ITALY & ITALY & ITALY & 1892 & MINER/CM & SAN GIORGIO CANAVESE. TO & MILLIONAIRE ST. & OSCEOLA \\
\hline 4014 & & DE FILIPPI & MARY & W & 40 & ITALY & ITALY & ITALY & 1895 & & & MILLIONAIRE ST. & OSCEOLA \\
\hline 4015 & & DE FILIPPI & JOHN & $\mathrm{s}$ & 12 & MICHIGAN & ITALY & ITALY & & & SAN GIORGIO CANAVESE. TO & MILLIONAIRE ST. & OSCEOLA \\
\hline 4016 & & DE FILIPPI & LENA & D & 9 & MICHIGAN & ITALY & ITALY & & & SAN GIORGIO CANAVESE. TO & MILLIONAIRE ST. & OSCEOLA \\
\hline 4017 & & DE FILIPPI & RENA & $\mathrm{D}$ & 7 & MICHIGAN & ITALY & ITALY & & & SAN GIORGIO CANAVESE. TO & MILLIONAIRE ST. & OSCEOLA \\
\hline 4018 & & DE FILIPPI & PETER & $\mathrm{s}$ & 5 & MICHIGAN & ITALY & ITALY & & & SAN GIORGIO CANAVESE. TO & MILLIONAIRE ST. & OSCEOLA \\
\hline 4019 & & DE FILIPPI & GIORGIO & $\mathrm{s}$ & 1 & MICHIGAN & ITALY & ITALY & & & SAN GIORGIO CANAVESE. TO & MILLIONAIRE ST. & OSCEOLA \\
\hline 4020 & & DE FILIPPI & JONNIE & $\mathrm{s}$ & $3 \mathrm{M}$ & MICHIGAN & ITALY & ITALY & & & SAN GIORGIO CANAVESE, TO & MILLIONAIRE ST. & OSCEOLA \\
\hline 4021 & 98 & BAGATINII & ANGELO & $\mathrm{H}$ & 43 & ITALY & ITALY & ITALY & 1894 & MOTORMAN/CM & BELLUNO & MILLIONAIRE ST. & OSCEOLA \\
\hline 4022 & & BAGATINII & ELISABETTA & W & 34 & ITALY & ITALY & ITALY & 1881 & & BELLUNO & MILLIONAIRE ST. & OSCEOLA \\
\hline 4023 & & BAGATINII & EDWARD & s & 17 & PENNSYLV & ITALY & ITALY & & & BELLUNO & MILLIONAIRE ST. & OSCEOLA \\
\hline 4024 & & BAGATINII & ANNE & D & 15 & WISCONSI & ITALY & ITALY & & & BELLUNO & MILLIONAIRE ST. & OSCEOLA \\
\hline 4025 & & BAGATINII & LOUISE & D & 12 & WISCONSI & ITALY & ITALY & & & BELLUNO & MILLIONAIRE ST. & OSCEOLA \\
\hline 4026 & & BAGATINII & EMILIA & D & 10 & MICHIGAN & ITALY & ITALY & & & BELLUNO & MILLIONAIRE ST. & OSCEOLA \\
\hline 4027 & 203 & GEMIGNANI & EGIDIO & $\mathrm{H}$ & 57 & ITALY & ITALY & ITALY & 1903 & MINER/CM & & CHURCH ST. & OSCEOLA \\
\hline 4028 & & GEMIGNANI & MARIA & W & 50 & ITALY & ITALY & ITALY & 1899 & & & CHURCH ST. & OSCEOLA \\
\hline 4029 & & GEMIGNANI & JOHN & $\mathrm{s}$ & 8 & MICHIGAN & ITA & ITALY & & & & CHURCH ST. & OSCEOLA \\
\hline 4030 & & MARACONI? & & B & 46 & ITALY & ITALY & ITALY & 1913 & MINER// & & CHURCH ST. & \\
\hline 4031 & & COLOMBO & JAMES & $\mathrm{H}$ & 54 & ITALY & ITALY & ITALY & 1897 & FARMER/GENERAL FARM & PONT CANAVESE. TO & & OSCEOLA - WOODLAND \\
\hline
\end{tabular}




\begin{tabular}{|c|c|c|c|c|c|c|c|c|c|c|c|c|c|}
\hline 4032 & & COLOMBO & JOSEPHINE & W & 44 & ITALY & ITALY & ITALY & 1900 & & & & OSCEOLA - WOODLAND \\
\hline 4033 & & COLOMBO & JAMES & $\mathrm{s}$ & 18 & MICHIGAN & ITALY & ITALY & & & PONT CANAVESE. TO & & OSCEOLA - WOODLAND \\
\hline 4034 & & COLOMBO & FRANK & $\mathrm{s}$ & 15 & MICHIGAN & ITALY & ITALY & & & PONT CANAVESE. TO & & OSCEOLA - WOODLAND \\
\hline 4035 & & COLOMBO & MARY & D & 14 & MICHIGAN & ITALY & ITALY & & & PONT CANAVESE. TO & & OSCEOLA - WOODLAND \\
\hline 4036 & & COLOMBO & JOHN & D & 13 & MICHIGAN & ITALY & ITALY & & & PONT CANAVESE. TO & & OSCEOLA - WOODLAND \\
\hline 4037 & & BALAGNA & FRANK & $\mathrm{H}$ & 28 & MICHIGAN & ITALY & ITALY & & FARMER & PONT CANAVESE. TO & & OSCEOLA - WOODLAND \\
\hline 4038 & & BALAGNA & CAROLINE & W & 27 & ITALY & ITALY & ITALY & 1916 & & & & OSCEOLA - WOODLAND \\
\hline 4039 & & BALAGNA & JOHN & $\mathrm{s}$ & $8 \mathrm{M}$ & MICHIGAN & MICHIGAN & ITALY & & & PONT CANAVESE. TO & & OSCEOLA - WOODLAND \\
\hline 4040 & & BALAGNA & CLARION & B & 39 & ITALY & ITALY & ITALY & ???? & FARMER HAND/FARM & PONT CANAVESE. TO & & OSCEOLA - WOODLAND \\
\hline 4041 & & BALAGNA & LINA & B & 32 & ITALY & ITALY & ITALY & ???? & FARMER HAND/FARM & PONT CANAVESE. TO & & OSCEOLA - WOODLAND \\
\hline 4042 & & BALAGNA & MAY & D & 9 & MICHIGAN & ITALY & ITALY & & & PONT CANAVESE. TO & & OSCEOLA - WOODLAND \\
\hline 4043 & & BAUSANO & LOUIS & $\mathrm{H}$ & 39 & ITALY & ITALY & ITALY & 1902 & FARMER/GENERAL FARM & PONT CANAVESE. TO & & OSCEOLA - OSCEOLA LOC. \\
\hline 4044 & & BAUSANO & ANNE & w & 41 & ITALY & ITALY & ITALY & 1902 & & PONT CANAVESE. TO & & OSCEOLA - OSCEOLA LOC. \\
\hline 4045 & & BAUSANO & KATE & D & 13 & MICHIGAN & ITALY & ITALY & & & PONT CANAVESE. TO & & OSCEOLA - OSCEOLA LOC. \\
\hline 4046 & & BAUSANO & JOHN & $\mathrm{s}$ & 7 & MICHIGAN & ITALY & ITALY & & & PONT CANAVESE. TO & & OSCEOLA - OSCEOLA LOC. \\
\hline 4047 & & SALSINI & LOUIS & $\mathrm{H}$ & 32 & ITALY & ITALY & ITALY & 1909 & LABORER/CM & SANTA MARIA DEL GIUDICE, LU & MINE ST. & OSCEOLA - TAMARACH CITY \\
\hline 4048 & & SALSINI & JOSEPHINE & W & 23 & MICHIGAN & ITALY & ITALY & & & & MINE ST. & OSCEOLA - TAMARACH CITY \\
\hline 4049 & & SALSINI & RITA & D & 2 & MICHIGAN & ITALY & MICHIGAN & & & & MINE ST. & OSCEOLA - TAMARACH CITY \\
\hline 4050 & & SALSINI & ARLEEEN & D & $3 \mathrm{M}$ & MICHIGAN & ITALY & MICHIGAN & & & SANTA MARIA DEL GIUDICE. LU & MINE ST. & OSCEOLA - TAMARACH CITY \\
\hline 4051 & & CONSANI & EMILIO & $\mathrm{H}$ & 58 & ITALY & ITALY & ITALY & 1895 & & LUCCA & MINE ST. & OSCEOLA - TAMARACH CITY \\
\hline 4052 & & CONSANI & ARTURO & $\mathrm{s}$ & 24 & ITALY & ITALY & ITALY & UN & MACHINIST/CM & LUCCA & MINE ST. & OSCEOLA - TAMARACH CITY \\
\hline 4053 & & CONSANI & $?$ & $\mathrm{COU}$ & 40 & ITALY & ITALY & ITALY & 1906 & SERVANT/PF & LUCCA & MINE ST. & OSCEOLA - TAMARACH CITY \\
\hline 4054 & & BONANNI & EUGENE & B & 32 & ITALY & ITALY & ITALY & 1909 & LABORER/CM & LUCCA & MINE ST. & OSCEOLA - TAMARACH CITY \\
\hline 4055 & & ROMANINI & EUGENIO & B & 33 & ITALY & ITALY & ITALY & 1909 & LABORER/CM & SANTA MARIA DEL GIUDICE. LU & MINE ST. & OSCEOLA - TAMARACH CITY \\
\hline 4056 & & OSTENZI & FRANK & $\mathrm{H}$ & 77 & ITALY & ITALY & ITALY & 1880 & & LUCCA & MINE ST. & OSCEOLA - TAMARACH CITY \\
\hline 4057 & & OSTENZI & MARY & D & 36 & MICHIGAN & ITALY & ITALY & & & LUCCA & MINE ST. & OSCEOLA - TAMARACH CITY \\
\hline 4058 & & OSTENZI & FRED & $\mathrm{s}$ & 22 & MICHIGAN & $\begin{array}{l}\text { ITALY } \\
\text { ITAL }\end{array}$ & ITALY & & LABORER/SMELTER & LUCCA & MINE ST. & OSCEOLA - TAMARACH CITY \\
\hline 4059 & & OSTENZI & HUBERT & $\mathrm{s}$ & 20 & MICHIGAN & ITALY & ITALY & & LABORER/CM & LUCCA & MINE ST. & OSCEOLA - TAMARACH CITY \\
\hline 4060 & & OSTENZI & MARGARET & D & 10 & MICHIGAN & ITALY & ITALY & & & LUCCA & MINE ST. & OSCEOLA - TAMARACH CITY \\
\hline 4061 & & BAIOCCHI & NATALE & $\mathrm{H}$ & 50 & MICHIGAN & ITALY & ITALY & & MACHINIST/COPPER MILL & COLLODI. LU & MINE ST. & OSCEOLA - TAMARACH MILL \\
\hline 4062 & & BAIOCCHI & MARY & W & 52 & ITALY & ITALY & ITALY & UN & 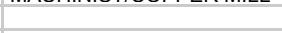 & & MINE ST. & OSCEOLA - TAMARACH MILL \\
\hline 4063 & & BAIOCCHI & PAULINA & D & 18 & MICHIGAN & MICHIGAN & ITALY & & & COLLODI. LU & MINE ST. & OSCEOLA - TAMARACH MILL \\
\hline 4064 & & BAIOCCHI & LOUIS & $\mathrm{s}$ & 17 & MICHIGAN & MICHIGAN & ITALY & & & COLLODI. LU & MINE ST. & OSCEOLA - TAMARACH MILL \\
\hline 4065 & & BAIOCCHI & CAMILLO & $\mathrm{H}$ & 46 & ITALY & ITALY & ITALY & 1889 & MACHINIST/COPPER MILL & COLLODI, LU & MINE ST. & OSCEOLA - TAMARACH MILL \\
\hline 4066 & & BAIOCCHI & LOUISE & W & 45 & ITALY & ITALY & ITALY & $89 / 90$ & & COLLODI. LU & MINE ST. & OSCEOLA - TAMARACH MILL \\
\hline 4067 & & BAIOCCHI & ELLA & $\mathrm{D}$ & 16 & MICHIGAN & ITALY & ITALY & & & COLLODI. LU & MINE ST. & OSCEOLA - TAMARACH MILL \\
\hline 4068 & & BAIOCCHI & DOROTHY & $\mathrm{D}$ & 6 & MICHIGAN & ITALY & ITALY & & & COLLODI. LU & MINE ST. & OSCEOLA - TAMARACH MILL \\
\hline 4069 & & PINI & EUGENIO & $\mathrm{H}$ & 45 & ITALY & ITALY & ITALY & 1899 & LABORER/COPPER MILL & LUCCA & BACK ST. & OSCEOLA - TAMARACH MILL \\
\hline 4070 & & PINI & EUGENIO & $\mathrm{s}$ & 11 & MICHIGAN & ITALY & ITALY & & & LUCCA & BACK ST. & OSCEOLA - TAMARACH MILL \\
\hline 4071 & & PINI & GEORGE & $\mathrm{s}$ & 10 & MICHIGAN & ITALY & ITALY & & & LUCCA & BACK ST. & OSCEOLA - TAMARACH MILL \\
\hline 4072 & & PINI & ALFRED & $\mathrm{s}$ & 5 & MICHIGAN & ITALY & ITALY & & & LUCCA & BACK ST. & \\
\hline 4073 & & PINI & PETER & $\mathrm{s}$ & $10 \mathrm{M}$ & MICHIGAN & ITALY & ITALY & & & LUCCA & BACK ST. & OSCEOLA - TAMARACH MILL \\
\hline 4074 & & BERTOLINI & ANCHISE & $\mathrm{H}$ & 30 & MICHIGAN & ITALY & ITALY & & LABORER/COPPER MILL & CALCI. PISA & BACK ST. & OSCEOLA - TAMARACH MILL \\
\hline 4075 & & BERTOLINI & ANITA & W & 23 & MICHIGAN & ITALY & ITALY & & & & BACK ST. & \\
\hline 4076 & & BERTOLINI & MARY & D & 2 & MICHIGAN & MICHIGAN & MICHIGAN & & & CALCI. PISA & BACK ST. & OSCEOLA - TAMARACH MILL \\
\hline 4077 & & BERTOLINI & AMERIGO & BR & 32 & MICHIGAN & ITALY & ITALY & & LABORER/COPPER MILL & CALCI. PISA & BACK ST. & OSCEOLA - TAMARACH MILL \\
\hline 4078 & & GATTOLIN & FRANK & $\mathrm{H}$ & 37 & ITALY & ITALY & ITALY & 1900 & MACHINIST/COPPER MILL & & BACK ST. & OSCEOLA - TAMARACH MILL \\
\hline 4079 & & GATTOLIN & MARY & w & 30 & ITALY & ITALY & ITALY & 1900 & & & BACK ST. & OSCEOLA - TAMARACH MILL \\
\hline 4080 & & GATTOLIN & TRACY & $\mathrm{D}$ & 8 & MICHIGAN & ITALY & ITALY & & & & BACK ST. & OSCEOLA - TAMARACH MILL \\
\hline 4081 & & GATTOLIN & JOHN & $\mathrm{s}$ & 6 & MICHIGAN & ITALY & ITALY & & & & BACK ST. & OSCEOLA - TAMARACH MILL \\
\hline 4082 & & GATTOLIN & SYLVESTER & s & 5 & MICHIGAN & ITALY & ITALY & & & & BACK ST. & OSCEOLA - TAMARACH MILL \\
\hline 4083 & & LOCATELLI & SAM & $\mathrm{H}$ & 28 & ITALY & ITALY & ITALY & 1893 & JANITOR/SCHOOL & SELINO, BERGAMO & & OSCEOLA \\
\hline 4084 & & LOCATELLI & ROSE & w & 24 & CANADA & CANADA & CANADA & 1900 & & CANADA & & OSCEOLA \\
\hline 4085 & & LOCATELLI & FRANCIS & $\mathrm{s}$ & 4 & MICHIGAN & ITALY & CANADA & & & SELINO. BERGAMO & & OSCEOLA \\
\hline 4086 & & LOCATELLI & ROBERT & $\mathrm{s}$ & 2 & MICHIGAN & ITALY & CANADA & & & SELINO. BERGAMO & & OSCEOLA \\
\hline 4087 & & LOCATELLI & KATE & D & 0 & MICHIGAN & ITALY & CANADA & & & SELINO. BERGAMO & & OSCEOLA \\
\hline 4088 & 34 & CALAMARI & UGO & $\mathrm{H}$ & 34 & ITALY & ITALY & ITALY & 1905 & TRAMMER/CM & VILLA BASILICA. LU & & PORTAGE \\
\hline 4089 & & CALAMARI & CRISTINA & W & 35 & ITALY & ITALY & ITALY & 1911 & & VILLA BASILICA, LU & & PORTAGE \\
\hline 4090 & & LESBO & LEON & $\mathrm{s}$ & 15 & ITALY & ITALY & ITALY & 1911 & & SAN MARTINO CANAVESE. TO & & PORTAGE \\
\hline 4091 & & CALAMARI & WILL & $\mathrm{s}$ & 12 & ITALY & ITALY & ITALY & 1911 & & VILLA BASILICA. LU & & PORTAGE \\
\hline 4092 & & CALAMARI & GIULIA & D & 4 & MICHIGAN & ITALY & ITALY & & & VILLA BASILICA. LU & & PORTAGE \\
\hline 4093 & & CALAMARI & DOMENICO & $\mathrm{s}$ & 1 & MICHIGAN & ITALY & ITALY & & & VILLA BASILICA. LU & & PORTAGE \\
\hline 4094 & & LENCIONI & SEBASTIANO & B & 26 & ITALY & ITALY & ITALY & 1912 & TRAMMER/CM & LUCCA & & PORTAGE \\
\hline
\end{tabular}




\begin{tabular}{|c|c|c|c|c|c|c|c|c|c|c|c|c|c|}
\hline 4095 & & PIZZUTI & JOHN & $\mathrm{H}$ & 55 & ITALY & ITALY & ITALY & 1900 & LABORER/CM & CAMPOBASSO & MAIN ST. & PORTAGE - PERKINSVILLE \\
\hline 4096 & & PIZZUTI & L? & w & 44 & ITALY & ITALY & ITALY & 1906 & & & MAIN ST. & PORTAGE - PERKINSVILLE \\
\hline 4097 & & PIZZUTI & MARY & D & 14 & MICHIGAN & ITALY & ITALY & & & CAMPOBASSO & MAIN ST. & PORTAGE - PERKINSVILLE \\
\hline 4098 & & PIZZUTI & WILL & $\mathrm{s}$ & 13 & MICHIGAN & ITALY & ITALY & & & CAMPOBASSO & MAIN ST. & PORTAGE - PERKINSVILLE \\
\hline 4099 & & PIZZUTI & LISETTA & D & 11 & MICHIGAN & ITALY & ITALY & & & CAMPOBASSO & MAIN ST. & PORTAGE - PERKINSVILLE \\
\hline 4100 & & PIZZUTI & JOHN & s & 6 & MICHIGAN & ITALY & ITALY & & & CAMPOBASSO & MAIN ST. & PORTAGE - PERKINSVILLE \\
\hline 4101 & & PIZZUTI & ROBERT & $\mathrm{s}$ & $4 \mathrm{M}$ & MICHIGAN & ITALY & ITALY & & & CAMPOBASSO & MAIN ST. & PORTAGE - PERKINSVILLE \\
\hline 4102 & & LULLO & ANGELO & B & 52 & ITALY & ITALY & ITALY & 1888 & TRAMMER/CM & & MAIN ST. & PORTAGE - PERKINSVILLE \\
\hline 4103 & & PELLICCIOTTI & JOSEPH & B & 30 & ITALY & ITALY & ITALY & 1914 & TRAMMER/CM & & MAIN ST. & PORTAGE - PERKINSVILLE \\
\hline 4104 & & MEOLA & BATTISTA & $\mathrm{H}$ & 45 & ITALY & ITALY & ITALY & 1900 & FIREMAN/CM & NAPOLI & FREDERICK ST. & PORTAGE - PERKINSVILLE \\
\hline 4105 & & MEOLA & JENNIE & w & 45 & ITALY & ITALY & ITALY & 1902 & & & FREDERICK ST. & PORTAGE - PERKINSVILLE \\
\hline 4106 & & MEOLA & LOLLI & D & 12 & MICHIGAN & ITALY & ITALY & & & NAPOLI & FREDERICK ST. & PORTAGE - PERKINSVILLE \\
\hline 4107 & & MEOLA & ROSANNA & D & 10 & MICHIGAN & ITALY & ITALY & & & NAPOLI & FREDERICK ST. & PORTAGE - PERKINSVILLE \\
\hline 4108 & & MEOLA & MARY ANN & D & 9 & MICHIGAN & ITALY & ITALY & & & NAPOLI & FREDERICK ST. & PORTAGE - PERKINSVILLE \\
\hline 4109 & & MEOLA & PAULINE & D & 3 & MICHIGAN & ITALY & ITALY & & & NAPOLI & FREDERICK ST. & PORTAGE - PERKINSVILLE \\
\hline 4110 & & MEOLA & OTTAVIA & D & 1 & MICHIGAN & ITALY & ITALY & & & NAPOLI & FREDERICK ST. & PORTAGE - PERKINSVILLE \\
\hline 4111 & & JANCO/A & ANTONIO & $\mathrm{H}$ & 25 & ITALY & ITALY & ITALY & 1911 & TRAMMER/CM & 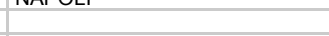 & FREDERICK ST. & PORTAGE - PERKINSVILLE \\
\hline 4112 & & JANCO/A & INES & w & 17 & MICHIGAN & ITALY & ITALY & & & & FREDERICK ST. & PORTAGE - PERKINSVILLE \\
\hline 4113 & & JANCO/A & DOROTHY & $\mathrm{D}$ & $4 \mathrm{M}$ & MICHIGAN & ITALY & MICHIGAN & & & & FREDERICK ST. & PORTAGE - PERKINSVILLE \\
\hline 4114 & 137 & BORGONSOLI & JOHN & B & 31 & ITALY & ITALY & ITALY & 1905 & MINER/CM & CHIETI & & PORTAGE - HURONTOWN \\
\hline 4115 & & BONAGGIO & JAMES & $\mathrm{H}$ & UN & ITALY & ITALY & ITALY & 1900 & FARMER/GENERAL FARM & Uritur & HURON MILL R. & PORTAGE \\
\hline 4116 & & BONAGGIO & MARY & W & 35 & ITALY & ITALY & ITALY & 1913 & & & HURON MILL R. & PORTAGE \\
\hline 4117 & & BONAGGIO & MARY & $\mathrm{D}$ & 4 & MICHIGAN & ITALY & ITALY & & & & HURON MILL R. & PORTAGE \\
\hline 4118 & & ZANOTTI & JAMES & SERV & 55 & ITALY & ITALY & ITALY & 1917 & SERVANT/PF & TORINO & HURON MILL R. & PORTAGE \\
\hline 4119 & & GIOVANNINI & GINO/JAMES & $\mathrm{H}$ & 38 & ITALY & ITALY & ITALY & 1907 & CAR MAN/CONE YARD & SAN GIUSTO CANAVESE. TO & & PORTAGE \\
\hline 4120 & & GIOVANNINI & ROSA & w & 38 & ITALY & ITALY & ITALY & 1908 & & & & PORTAGE \\
\hline 4121 & & GIACOLETTO & VITTORIO & $\mathrm{H}$ & 41 & ITALY & ITALY & ITALY & 1903 & NOT CLEAR & TORINO & & PORTAGE - MANDERFIELD LOC \\
\hline 4122 & & GIACOLETTO & CATERINA & w & 42 & ITALY & ITALY & ITALY & 1903 & & Terimo & & PORTAGE - MANDERFIELD LOC \\
\hline 4123 & & GIACOLETTO & JOSEPH & $\mathrm{s}$ & 15 & MICHIGAN & ITALY & ITALY & & & TORINO & & PORTAGE - MANDERFIELD LOC \\
\hline 4124 & & GIACOLETTO & LUCY & $\mathrm{D}$ & 13 & MICHIGAN & ITALY & ITALY & & & TORINO & & PORTAGE - MANDERFIELD LOC \\
\hline 4125 & & GIACOLETTO & JOSEPHINE & $\mathrm{D}$ & 11 & MICHIGAN & ITALY & ITALY & & & TORINO & & PORTAGE - MANDERFIELD LOC \\
\hline 4126 & & GIACOLETTO & PAUL & $\mathrm{s}$ & 8 & MICHIGAN & ITALY & ITALY & & & TORINO & & PORTAGE - MANDERFIELD LOC \\
\hline 4127 & & GIACOLETTO & JOHN & $\mathrm{s}$ & 2 & MICHIGAN & ITALY & ITALY & & & TORINO & & PORTAGE - MANDERFIELD LOC \\
\hline 4128 & & FAVALA & ANTONIO & $\mathrm{H}$ & 33 & ITALY & ITALY & ITALY & 1906 & MINER/CM & Torins & & PORTAGE - MANDERFIELD LOC \\
\hline 4129 & & FAVALA & KATE & W & 26 & ITALY & ITALY & ITALY & 1911 & & & & PORTAGE - MANDERFIELD LOC \\
\hline 4130 & & FAVALA & JOSEPH & $\mathrm{s}$ & 5 & MICHIGAN & ITALY & ITALY & & & & & PORTAGE - MANDERFIELD LOC \\
\hline 4131 & & FAVALA & MERNIE & $\mathrm{D}$ & 4 & MICHIGAN & ITALY & ITALY & & & & & PORTAGE - MANDERFIELD LOC \\
\hline 4132 & & OZZELLO & JOSEPH & $\mathrm{H}$ & 57 & ITALY & ITALY & ITALY & 1902 & TIMBERMAN/CM & SAN GIUSTO CANAVESE. TO & & PORTAGE - MANDERFIELD LOC \\
\hline 4133 & & OZZELLO & MINNIE & W & 41 & ITALY & ITALY & ITALY & 1903 & & SAN GIUSTO CANAVESE. TO & & PORTAGE - MANDERFIELD LOC \\
\hline 4134 & & OZZELLLO & DOMENICO & $\mathrm{s}$ & 18 & FRANCE & ITALY & ITALY & 1903 & & SAN GIUSTO CANAVESE. TO & & PORTAGE - MANDERFIELD LOC \\
\hline 4135 & & OZZELLO & PETER & $\mathrm{s}$ & 14 & MICHIGAN & ITALY & ITALY & & & SAN GIUSTO CANAVESE. TO & & PORTAGE - MANDERFIELD LOC \\
\hline 4136 & & OZZELLO & CATHERINE & D & 7 & MICHIGAN & ITALY & ITALY & & & SAN GIUSTO CANAVESE. TO & & PORTAGE - MANDERFIELD LOC \\
\hline 4137 & & CENTANINO & JOHN & B & 42 & ITALY & ITALY & ITALY & 1900 & TIMBERMAN/CM & SAN MARTINO CANAVESE. TO & & PORTAGE - MANDERFIELD LOC \\
\hline 4138 & & CENTANINO & MARTIN & B & 32 & ITALY & ITALY & ITALY & 1910 & MINER/CM & SAN MARTINO CANAVESE. TO & & PORTAGE - MANDERFIELD LOC \\
\hline 4139 & & RUFFOLO & JOSEPH & $\mathrm{H}$ & 33 & ITALY & ITALY & ITALY & 1909 & SHOEMAKER/OWN SHOP & & HURON ST. & PORTAGE - DODGEVILLE \\
\hline 4140 & 94 & BERTOLUCCI & ANTONIO & $\mathrm{H}$ & 37 & ITALY & ITALY & ITALY & 1906 & MINER/CM & LUCCA & COUNTY ROAD & PORTAGE - DODGEVILLE \\
\hline 4141 & & BERTOLUCCI & ANGELINA & w & 38 & ITALY & ITALY & ITALY & 1906 & & LUCCA & COUNTY ROAD & PORTAGE - DODGEVILLE \\
\hline 4142 & & BERTOLUCCI & JOHN & $\mathrm{s}$ & 14 & ITALY & ITALY & ITALY & 1906 & & LUCCA & COUNTY ROAD & PORTAGE - DODGEVILLE \\
\hline 4143 & & BERTOLUCCI & DOMENICO & $\mathrm{s}$ & 7 & MICHIGAN & ITALY & ITALY & & & LUCCA & COUNTY ROAD & PORTAGE - DODGEVILLE \\
\hline 4144 & & BERTOLUCCI & VITTORIO & $\mathrm{s}$ & 6 & MICHIGAN & ITALY & ITALY & & & LUCCA & COUNTY ROAD & PORTAGE - DODGEVILLE \\
\hline 4145 & & BORTALUZI & MARY & D & 4 & MICHIGAN & ITALY & ITALY & & & & COUNTY ROAD & PORTAGE - DODGEVILLE \\
\hline 4146 & & BORTALUZI & VITTORIA & D & 1 & MICHIGAN & ITALY & ITALY & & & & COUNTY ROAD & PORTAGE - DODGEVILLE \\
\hline 4147 & 105 & NOZERO & ANTONIO & $\mathrm{H}$ & 47 & ITALY & ITALY & ITALY & 1894 & NOT CLEAR/CM & SAN MARTINO CANAVESE. TO & COUNTY ROAD & PORTAGE - DODGEVILLE \\
\hline 4148 & & NOZERO & ANGELINA & w & 36 & ITALY & ITALY & ITALY & 1902 & & & COUNTY ROAD & PORTAGE - DODGEVILLE \\
\hline 4149 & & NOZERO & MIKE & s & 17 & MICHIGAN & ITALY & ITALY & & NOT CLEAR & SAN MARTINO CANAVESE. TO & COUNTY ROAD & PORTAGE - DODGEVILLE \\
\hline 4150 & & NOZERO & PETER & $\hat{s}$ & 14 & MICHIGAN & ITALY & ITALY & & IVUT CLEAR & SAN MARTINO CANAVESE. TO & COUNTY ROAD & PORTAGE - DODGEVILLE \\
\hline 4151 & & DE ROSA & IPPOLITO & B & 34 & ITALY & ITALY & ITALY & 1909 & TRAMMER/CM & COSENZA & COUNTY ROAD & PORTAGE - DODGEVILLE \\
\hline 4152 & & DE FILIPPI & PETER & B & 49 & ITALY & ITALY & ITALY & 1893 & TRAMMER/CM & TORINO & COUNTY ROAD & PORTAGE - DODGEVILLE \\
\hline 4153 & 130 & FEBBO & JOSEPH & $\mathrm{H}$ & 31 & ITALY & ITALY & ITALY & 1905 & MINER/CM & TERAMO & & PORTAGE - DODGEVILLE \\
\hline 4154 & 100 & FEBBO & MADDALENA & W & 33 & & ITA & IT & 1904 & & & & PORTAGE - DODGEVILLE \\
\hline 4155 & & FEBBO & MARY & D & 14 & MICHIGAN & ITALY & ITALY & & & TERAMO & & PORTAGE - DODGEVILLE \\
\hline 4156 & & FEBBO & LENA & $\mathrm{D}$ & 11 & MICHIGAN & ITALY & ITALY & & & TERAMO & & PORTAGE - DODGEVILLE \\
\hline 4157 & & FEBBO & JOSEPH & $\mathrm{s}$ & 9 & MICHIGAN & ITALY & ITALY & & & TERAMO & & PORTAGE - DODGEVILLE \\
\hline
\end{tabular}




\begin{tabular}{|c|c|c|c|c|c|c|c|c|c|c|c|c|c|}
\hline 4158 & & BRAGOGNOLO & OLINDO & B & 23 & ITALY & ITALY & ITALY & 1912 & MINER/CM & TREVISO & & PORTAGE - DODGEVILLE \\
\hline 4159 & & MADI??IMS & SALVADOR & B & 34 & ITALY & ITALY & ITALY & 1912 & TRAMMER/CM & & & PORTAGE - DODGEVILLE \\
\hline 4160 & & DANIELE & FRANK & $\mathrm{H}$ & 34 & ITALY & ITALY & ITALY & 1905 & TRAMMER/CM & RIVAROLO CANAVESE. TO & & PORTAGE - DODGEVILLE \\
\hline 4161 & & DANIELE & FILIPPA & W & 31 & ITALY & ITALY & ITALY & 1905 & SHOPKEEPER/FRUIT STORE & RIVAROLO CANAVESE. TO & & PORTAGE - DODGEVILLE \\
\hline 4162 & & DANIELE & ANGELINA & D & 10 & MICHIGAN & ITALY & ITALY & & & RIVAROLO CANAVESE. TO & & PORTAGE - DODGEVILLE \\
\hline 4163 & & DANIELE & KATE & $\mathrm{D}$ & 8 & MICHIGAN & ITALY & ITALY & & & RIVAROLO CANAVESE. TO & & PORTAGE - DODGEVILLE \\
\hline 4164 & & DANIELE & MADDALENA & $\mathrm{D}$ & 7 & MICHIGAN & ITALY & ITALY & & & RIVAROLO CANAVESE. TO & & PORTAGE - DODGEVILLE \\
\hline 4165 & & DANIELE & MARGARET & $\mathrm{D}$ & 5 & MICHIGAN & ITALY & ITALY & & & RIVAROLO CANAVESE. TO & & PORTAGE - DODGEVILLE \\
\hline 4166 & & DANIELE & FRANK & s & $6 \mathrm{M}$ & MICHIGAN & ITALY & ITALY & & & RIVAROLO CANAVESE. TO & & PORTAGE - DODGEVILLE \\
\hline 4167 & & DE FILIPPI & ROSI & SIIL & 19 & ITALY & ITALY & ITALY & 1919 & SERVANT/HOME & TORINO & & PORTAGE - DODGEVILLE \\
\hline 4168 & 3 & BRUSSO & BATTISTA & $\mathrm{H}$ & 37 & ITALY & ITALY & ITALY & 1904 & FOREMAN/COPPER MILL & AGLIE'. TO & & PORTAGE - I.R. MILL LOCATION \\
\hline 4169 & & BRUSSO & MARGHERITA & W & 33 & ITALY & ITALY & ITALY & 1907 & & & & PORTAGE - I.R. MILL LOCATION \\
\hline 4170 & & BRUSSO & DOMENICO & $s$ & 9 & MICHIGAN & ITALY & ITALY & & & AGLIE'. TO & & PORTAGE - I.R. MILL LOCATION \\
\hline 4171 & & BRUSSO & ESTER & $\mathrm{D}$ & 6 & MICHIGAN & ITALY & ITALY & & & AGLIE'. TO & & PORTAGE - I.R. MILL LOCATION \\
\hline 4172 & & BRUSSO & BARTOLOMEO & $\mathrm{s}$ & 5 & MICHIGAN & ITALY & ITALY & & & AGLIE'. TO & & PORTAGE - I.R. MILL LOCATION \\
\hline 4173 & 238 & CHIOTTI & JAMES & $\mathrm{H}$ & 46 & ITALY & ITALY & ITALY & 1891 & LABORER/POWER PLANT & SAN GIORGIO CANAVESE, TO & ALBION ST. & PORTAGE - HOUGHTON \\
\hline 4174 & & CHIOTTI & CINTA & W & 42 & ITALY & ITALY & ITALY & 1891 & & SAN GIORGIO CANAVESE. TO & ALBION ST. & PORTAGE - HOUGHTON \\
\hline 4175 & & CHIOTTI & CATERINA & $\mathrm{D}$ & 19 & MICHIGAN & ITALY & ITALY & & TIMEKEEPER/CM & SAN GIORGIO CANAVESE. TO & ALBION ST. & PORTAGE - HOUGHTON \\
\hline 4176 & & CHIOTTI & JOHN & s & 15 & MICHIGAN & ITALY & ITALY & & & SAN GIORGIO CANAVESE. TO & ALBION ST. & PORTAGE - HOUGHTON \\
\hline 4177 & 311 & CHIOMINATTI & MARY & SER & 20 & MICHIGAN & ITALY & ITALY & & SERVANT/PF & & ALBION ST. & PORTAGE - HOUGHTON \\
\hline 4178 & 277 & $\begin{array}{l}\text { PANTERA } \\
\end{array}$ & JOSEPH & $\mathrm{H}$ & 39 & MICHIGAN & ITALY & NEW YORK & & SALESMAN/WHOLESALE MEAT & LUCCA & CALVERLEY ST. & PORTAGE - HOUGHTON \\
\hline 4179 & & PANTERA & ANNA & W & 34 & MICHIGAN & NEW YORK & IRELAND & & SALESIVIAIVIVUTULESALE IVILAI & USA-IRELAND & CALVERLEY ST. & PORTAGE - HOUGHTON \\
\hline 4180 & & PANTERA & MABLE & $\mathrm{D}$ & 13 & MICHIGAN & MICHIGAN & MICHIGAN & & & LUCCA & CALVERLEY ST. & PORTAGE - HOUGHTON \\
\hline 4181 & & PANTERA & CHARLES & $\mathrm{s}$ & 11 & MICHIGAN & MICHIGAN & MICHIGAN & & & LUCCA & CALVERLEY ST. & PORTAGE - HOUGHTON \\
\hline 4182 & & PANTERA & LOUIS & $\mathrm{s}$ & 9 & MICHIGAN & MICHIGAN & MICHIGAN & & & LUCCA & CALVERLEY ST. & PORTAGE - HOUGHTON \\
\hline 4183 & & PANTERA & COLETTA & $\mathrm{D}$ & 5 & MICHIGAN & MICHIGAN & MICHIGAN & & & LUCCA & CALVERLEY ST. & PORTAGE - HOUGHTON \\
\hline 4184 & & PANTERA & ALWYN & $\mathrm{D}$ & 3 & MICHIGAN & MICHIGAN & MICHIGAN & & & LUCCA & CALVERLEY ST. & PORTAGE - HOUGHTON \\
\hline 4185 & & PANTERA & $\mathrm{JOHN}$ & $\mathrm{s}$ & 1 & MICHIGAN & MICHIGAN & MICHIGAN & & & LUCCA & CALVERLEY ST. & PORTAGE - HOUGHTON \\
\hline 4186 & & PANTERA & WILLIS & $\mathrm{BR}$ & 48 & MICHIGAN & ITALY & NEW YORK & & & LUCCA & CALVERLEY ST. & PORTAGE - HOUGHTON \\
\hline 4187 & 89 & GIANNIS? & ROBERT & B & 13 & ITALY & ITALY & ITALY & UN & & & & PORTAGE - HOUGHTON \\
\hline 4188 & & MONNIE & PETER & B & 12 & MICHIGAN & ITALY & ITALY & & & & & PORTAGE - HOUGHTON \\
\hline 4189 & 422 & MALFORTI & DOSILLA & $\mathrm{H}$ & 40 & ITALY & ITALY & ITALY & 1910 & NOT CLEAR & LUCCA & MONTEZUMA ST. & PORTAGE - HOUGHTON \\
\hline 4190 & $4<2$ & MALFORTI & GIORDANO & s & ? & MICHIGAN & ITALY & ITALY & 1510 & TVUT CLEAR & LUCCA & MONTEZUMA ST. & PORTAGE - HOUGHTON \\
\hline 4191 & & MALFORTI & JENNIE & $\mathrm{D}$ & 9 & MICHIGAN & ITALY & ITALY & & & LUCCA & MONTEZUMA ST. & PORTAGE - HOUGHTON \\
\hline 4192 & & MALFORTI & CATERINA & $\mathrm{D}$ & 7 & MICHIGAN & ITALY & ITALY & & & LUCCA & MONTEZUMA ST. & PORTAGE - HOUGHTON \\
\hline 4193 & 209 & PORTILE & FELIX & B & 26 & ITALY & ITALY & ITALY & 1915 & MINER/CM & & SIMPSON ST. & QUINCY \\
\hline 4194 & 107 & COTTINI & EUGENIO & B & 28 & ITALY & ITALY & ITALY & 1909 & MINER/CM & COLLODI. LU & FRENCHTOWN & QUINCY - FRENCH TOWN \\
\hline 4195 & 110 & COTTINI & JOSEPH & $\mathrm{H}$ & 49 & ITALY & ITALY & ITALY & 1902 & MINER/CM & COLLODI. LU & FRENCHTOWN & QUINCY - FRENCH TOWN \\
\hline 4196 & & COTTINI & GIORGIANA & W & 36 & ITALY & ITALY & ITALY & 1907 & & & FRENCHTOWN & QUINCY - FRENCH TOWN \\
\hline 4197 & & COTTINI & LEO & s & 16 & MICHIGAN & ITALY & ITALY & & MINER/CM & COLLODI, LU & FRENCHTOWN & QUINCY - FRENCH TOWN \\
\hline 4198 & & COTTINI & ADAM & $\mathrm{s}$ & 11 & MICHIGAN & ITALY & ITALY & & & COLLODI. LU & FRENCHTOWN & QUINCY - FRENCH TOWN \\
\hline 4199 & & COTTINI & HUGO & $\mathrm{s}$ & 11 & MICHIGAN & ITALY & ITALY & & & COLLODI. LU & FRENCHTOWN & QUINCY - FRENCH TOWN \\
\hline 4200 & & COTTINI & MARGHERITA & $\mathrm{D}$ & 7 & MICHIGAN & ITALY & ITALY & & & COLLODI. LU & FRENCHTOWN & QUINCY - FRENCH TOWN \\
\hline 4201 & & COTTINI & JOSEPHINE & $\mathrm{D}$ & 5 & MICHIGAN & ITALY & ITALY & & & COLLODI. LU & FRENCHTOWN & QUINCY - FRENCH TOWN \\
\hline 4202 & & COTTINI & IRENE & $\mathrm{D}$ & 3 & MICHIGAN & ITALY & ITALY & & & COLLODI, LU & FRENCHTOWN & QUINCY - FRENCH TOWN \\
\hline 4203 & & COTTINI & FRANCES & MO & 74 & ITALY & ITALY & ITALY & 1902 & & COLLODI. LU & FRENCHTOWN & QUINCY - FRENCH TOWN \\
\hline 4204 & 153 & MATTEUCCI & ERNEST & $\mathrm{H}$ & 31 & ITALY & ITALY & ITALY & 1909 & MINER/CM & COLLE DI COMPITO. LU & & QUINCY - LIMERICK LOCATION \\
\hline 4205 & & MATTEUCCI & ANNE & w & 37 & MASSACHU & IRELAND & IRELAND & & & IRELAND & & QUINCY - LIMERICK LOCATION \\
\hline 4206 & 149 & MANDOLI & GIROLAMO & $\mathrm{H}$ & 50 & ITALY & ITALY & ITALY & 1906 & MINER/CM & LUCCA & & QUINCY - LIMERICK LOCATION \\
\hline 4207 & & MANDOLI & TERESA & W & 50 & ITALY & ITALY & ITALY & 1909 & & LUCCA & & QUINCY - LIMERICK LOCATION \\
\hline 4208 & & MANDOLI & ALADINO & s & 15 & ITALY & ITALY & ITALY & 1909 & & LUCCA & & QUINCY - LIMERICK LOCATION \\
\hline 4209 & & MANDOLI & TONY & $\mathrm{s}$ & 13 & ITALY & ITALY & ITALY & 1909 & & LUCCA & & QUINCY - LIMERICK LOCATION \\
\hline 4210 & & QUILICI & OTTAVIO & $\mathrm{H}$ & 46 & ITALY & ITALY & ITALY & 1897 & FIREMAN/BOILER HOUSE & MATRAIA. LU & & QUINCY - LIMERICK LOCATION \\
\hline 4211 & & QUILICI & ARGENTINA & W & 36 & ITALY & ITALY & ITALY & 1910 & & MATRAIA. LU & & QUINCY - LIMERICK LOCATION \\
\hline 4212 & & QUILICI & GIUSEPPINA & $\mathrm{D}$ & 6 & MICHIGAN & ITALY & ITALY & & & MATRAIA. LU & & QUINCY - LIMERICK LOCATION \\
\hline 4213 & & QUILICI & GIOVANNI & $\mathrm{s}$ & 2 & MICHIGAN & ITALY & ITALY & & & MATRAIA. LU & & QUINCY - LIMERICK LOCATION \\
\hline 4214 & 227 & PARIN & ARTURO & $\mathrm{H}$ & 38 & ITALY & ITALY & ITALY & 1903 & MINER/CM & LANNARI. LU & & QUINCY - HARDSCRABBLE \\
\hline 4215 & & PARIN & AGATA & w & 41 & ITALY & ITALY & ITALY & 1913 & & LANNARI, LU & & QUINCY - HARDSCRABBLE \\
\hline 4216 & & PARIN & CORINNA & D & 17 & ITALY & ITALY & ITALY & 1913 & & LANNARI. LU & & QUINCY - HARDSCRABBLE \\
\hline 4217 & & PARIN & PETER & $\mathrm{s}$ & 3 & MICHIGAN & ITALY & ITALY & & & LANNARI. LU & & QUINCY - HARDSCRABBLE \\
\hline 4218 & & PAR & & $\mathrm{s}$ & 2 & MICHIGA & ITA & & & & & & QUINCY - HARDSCRABBLE \\
\hline 4219 & & PARIN & & $\mathrm{s}$ & 1 & MICHIGAN & ITALY & & & & JARI. LU & & QUINCY - HARDSCRABBLE \\
\hline 4220 & 225 & BERNACCHI & EMILIO & $\mathrm{H}$ & 33 & ITALY & ITALY & ITALY & 1911 & MINER/CM & LUCCA & & QUINCY - HARDSCRABBLE \\
\hline
\end{tabular}




\begin{tabular}{|c|c|c|c|c|c|c|c|c|c|c|c|c|}
\hline 4221 & & BERNACCHI & MARY & W & 27 & ITALY & ITALY & ITALY & 1915 & & & QUINCY - HARDSCRABBLE \\
\hline 4222 & & BERNACCHI & ELEONORA & D & 5 & MICHIGAN & ITALY & ITALY & & & LUCCA & QUINCY - HARDSCRABBLE \\
\hline 4223 & & BERNACCHI & FRANK & $\mathrm{s}$ & $10 \mathrm{M}$ & MICHIGAN & ITALY & ITALY & & & LUCCA & QUINCY - HARDSCRABBLE \\
\hline 4224 & & SALANI & GIULIO & B & 33 & ITALY & ITALY & ITALY & 1907 & MINER/CM & & QUINCY - HARDSCRABBLE \\
\hline 4225 & 226 & GIUSTI & ARTURO & $\mathrm{H}$ & 43 & ITALY & ITALY & ITALY & 1900 & MINER/CM & LUCCA & QUINCY - HARDSCRABBLE \\
\hline 4226 & & GIUSTI & MARY & w & 40 & ITALY & ITALY & ITALY & 1906 & & & QUINCY - HARDSCRABBLE \\
\hline 4227 & & GIUSTI & EVELINE & D & 12 & MICHIGAN & ITALY & ITALY & & & LUCCA & QUINCY - HARDSCRABBLE \\
\hline 4228 & & GIUSTI & RINALDO & $\mathrm{s}$ & 10 & MICHIGAN & ITALY & ITALY & & & LUCCA & QUINCY - HARDSCRABBLE \\
\hline 4229 & & GIUSTI & GINA & D & 9 & MICHIGAN & ITALY & ITALY & & & LUCCA & QUINCY - HARDSCRABBLE \\
\hline 4230 & & GIUSTI & MINNIE & $\mathrm{D}$ & 7 & MICHIGAN & ITALY & ITALY & & & LUCCA & QUINCY - HARDSCRABBLE \\
\hline 4231 & & GIUSTI & ANNE & D & 6 & MICHIGAN & ITALY & ITALY & & & LUCCA & QUINCY - HARDSCRABBLE \\
\hline 4232 & & GIUSTI & CRISTIANA & D & 2 & MICHIGAN & ITALY & ITALY & & & LUCCA & QUINCY - HARDSCRABBLE \\
\hline 4233 & 232 & GRASSESCHI & DANTE & $\mathrm{H}$ & 41 & ITALY & ITALY & ITALY & 1908 & MINER/CM & SANTA MARIA DEL GIUDICE. LU & QUINCY - HARDSCRABBLE \\
\hline 4234 & & GRASSESCHI & AMINA & W & 30 & ITALY & ITALY & ITALY & 1910 & & SANTA MARIA DEL GIUDICE. LU & QUINCY - HARDSCRABBLE \\
\hline 4235 & & GRASSESCHI & GIULIO & $\mathrm{s}$ & 11 & ITALY & ITALY & ITALY & 1910 & & SANTA MARIA DEL GIUDICE. LU & QUINCY - HARDSCRABBLE \\
\hline 4236 & & GRASSESCHI & TONY & $\mathrm{S}$ & 10 & ITALY & ITALY & ITALY & 1910 & & SANTA MARIA DEL GIUDICE, LU & QUINCY - HARDSCRABBLE \\
\hline 4237 & & GRASSESCHI & LANDO & $\mathrm{s}$ & 8 & MICHIGAN & ITALY & ITALY & & & SANTA MARIA DEL GIUDICE. LU & QUINCY - HARDSCRABBLE \\
\hline 4238 & & GRASSESCHI & GORDIO & $\mathrm{s}$ & 4 & MICHIGAN & ITALY & ITALY & & & SANTA MARIA DEL GIUDICE. LU & QUINCY - HARDSCRABBLE \\
\hline 4239 & & GRASSESCHI & NELLIO & $\mathrm{s}$ & 3 & MICHIGAN & ITALY & ITALY & & & SANTA MARIA DEL GIUDICE. LU & QUINCY - HARDSCRABBLE \\
\hline 4240 & & GRASSESCHI & JOSEPH & $\mathrm{s}$ & 2 & MICHIGAN & ITALY & ITALY & & & SANTA MARIA DEL GIUDICE. LU & QUINCY - HARDSCRABBLE \\
\hline 4241 & & GRASSESCHI & LUCIA & $\mathrm{D}$ & $10 \mathrm{M}$ & MICHIGAN & ITALY & ITALY & & & SANTA MARIA DEL GIUDICE. LU & QUINCY - HARDSCRABBLE \\
\hline 4242 & & STOCHE & GIUSEPPE & B & 35 & ITALY & ITALY & ITALY & 1913 & MINER/CM & SAIVIA TIARTA UEL GIUDULE. LU & QUINCY - HARDSCRABBLE \\
\hline 4243 & 234 & GIUSTI & AMEDEO & $\mathrm{H}$ & 27 & ITALY & ITALY & ITALY & 1908 & MINER/CM & LUCCA & QUINCY - HARDSCRABBLE \\
\hline 4244 & & GIUSTI & VELIA & W & 18 & ITALY & ITALY & ITALY & 1918 & & & QUINCY - HARDSCRABBLE \\
\hline 4245 & 205 & DECESHERE & EUGENIO & $\mathrm{H}$ & 22 & ITALY & ITALY & ITALY & 1905 & MINER/CM & & QUINCY - HARDSCRABBLE \\
\hline 4246 & & DECESHERE & VITTORIA & W & 20 & ITALY & ITALY & ITALY & 1916 & & & QUINCY - HARDSCRABBLE \\
\hline 4247 & & DECESHERE & BRUNA & $\mathrm{D}$ & $8 \mathrm{M}$ & MICHIGAN & ITALY & ITALY & & & & QUINCY - HARDSCRABBLE \\
\hline 4248 & 206 & BALDASSARRI & RUGGERO & $\mathrm{H}$ & 48 & ITALY & ITALY & ITALY & 1901 & MINER/CM & SANTA MARIA DEL GIUDICE, LU & QUINCY - HARDSCRABBLE \\
\hline 4249 & & BALDASSARRI & LIZZIE & W & 47 & ITALY & ITALY & ITALY & 1906 & & SANTA MARIA DEL GIUDICE. LU & QUINCY - HARDSCRABBLE \\
\hline 4250 & & BALDASSARRI & AMELIO & $\mathrm{s}$ & 22 & ITALY & ITALY & ITALY & 1906 & MINER/CM & SANTA MARIA DEL GIUDICE. LU & QUINCY - HARDSCRABBLE \\
\hline 4251 & & BALDASSARRI & EVA & D & 12 & MICHIGAN & ITALY & ITALY & & & SANTA MARIA DEL GIUDICE. LU & QUINCY - HARDSCRABBLE \\
\hline 4252 & & BALDASSARRI & PREMATA & D & 12 & MICHIGAN & ITALY & ITALY & & & SANTA MARIA DEL GIUDICE. LU & QUINCY - HARDSCRABBLE \\
\hline 4253 & & BALDASSARRI & CRISTIANA & $\mathrm{D}$ & 11 & MICHIGAN & ITALY & ITALY & & & SANTA MARIA DEL GIUDICE. LU & QUINCY - HARDSCRABBLE \\
\hline 4254 & & BALDASSARRI & OLGA & D & 9 & MICHIGAN & ITALY & ITALY & & & $\begin{array}{l}\text { SANTA MARIA DEL GIUDICE, LU } \\
\text { SA DE }\end{array}$ & QUINCY - HARDSCRABBLE \\
\hline 4255 & & BEDINI & EMANUELE & B & 39 & ITALY & ITALY & ITALY & 1913 & MINER/CM & & QUINCY - HARDSCRABBLE \\
\hline 4256 & & PRATER & GIULIO & B & 34 & ITALY & ITALY & ITALY & 1913 & MINER/CM & & QUINCY - HARDSCRABBLE \\
\hline 4257 & & PACCIONE & ARTURO & B & 38 & ITALY & ITALY & ITALY & 1912 & MINER/CM & & QUINCY - HARDSCRABBLE \\
\hline 4258 & 210 & BAGNESCHI & JOSEPH & $\mathrm{H}$ & 28 & ITALY & ITALY & ITALY & 1906 & MINER/CM & CASTELVECCHIO. LU & QUINCY - HARDSCRABBLE \\
\hline 4259 & & BAGNESCHI & CELIDA & W & 25 & ITALY & ITALY & ITALY & 1915 & & & QUINCY - HARDSCRABBLE \\
\hline 4260 & & BAGNESCHI & DINA & $\mathrm{D}$ & 2 & MICHIGAN & ITALY & ITALY & & & CASTELVECCHIO.LU & QUINCY - HARDSCRABBLE \\
\hline 4261 & & BAGNESCHI & ERNEST & $\mathrm{S}$ & 1 & MICHIGAN & ITALY & ITALY & & & CASTELVECCHIO. LU & QUINCY - HARDSCRABBLE \\
\hline 4262 & & BALDASSARRI & ANNIBALE & B & 35 & ITALY & ITALY & ITALY & 1907 & MINER/CM & SANTA MARIA DEL GIUDICE. LU & QUINCY - HARDSCRABBLE \\
\hline 4263 & & TABARACCI & HEZEL & MOIL & 52 & ITALY & ITALY & ITALY & 1915 & & LUCCA & QUINCY - HARDSCRABBLE \\
\hline 4264 & 208 & CIRNANTE & MARIANO & $\mathrm{H}$ & 29 & ITALY & ITALY & ITALY & 1909 & MINER/CM & & QUINCY - HARDSCRABBLE \\
\hline 4265 & & CIRNANTE & CORINA & W & 22 & ITALY & ITALY & ITALY & 1915 & & & QUINCY - HARDSCRABBLE \\
\hline 4266 & & CIRNANTE & DIANA & D & 3 & MICHIGAN & ITALY & ITALY & & & & QUINCY - HARDSCRABBLE \\
\hline 4267 & & CIRNANTE & SARA & $\mathrm{D}$ & $2 \mathrm{M}$ & MICHIGAN & ITALY & ITALY & & & & QUINCY - HARDSCRABBLE \\
\hline 4268 & 204 & FEDERIGHI & EMILIO & $\mathrm{H}$ & 50 & ITALY & ITALY & ITALY & 1893 & MINER/CM & LUCCA & QUINCY - HARDSCRABBLE \\
\hline 4269 & & FEDERIGHI & ROSE & w & 40 & ITALY & ITALY & ITALY & 1908 & 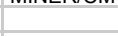 & Lovon & QUINCY - HARDSCRABBLE \\
\hline 4270 & & FEDERIGHI & TONY & $\mathrm{s}$ & 10 & MICHIGAN & ITALY & ITALY & & & LUCCA & QUINCY - HARDSCRABBLE \\
\hline 4271 & & FEDERIGHI & JOSEPHINE & D & 8 & MICHIGAN & ITALY & ITALY & & & LUCCA & OUINCY - HARDSCRABBLE \\
\hline 4272 & & FEDERIGHI & EMILIA & D & 7 & MICHIGAN & ITALY & ITALY & & & LUCCA & QUINCY - HARDSCRABBLE \\
\hline 4273 & & FEDERIGHI & RICCARDA & D & 6 & MICHIGAN & ITALY & ITALY & & & LUCCA & QUINCY - HARDSCRABBLE \\
\hline 4274 & & FEDERIGHI & SEBASTIANO & $\mathrm{S}$ & 5 & MICHIGAN & ITALY & ITALY & & & LUCCA & OUNCY HABDSCRABRIF \\
\hline 4275 & & GIUSTI & EGISTO & B & 34 & ITALY & ITALY & ITALY & 1908 & MINER/CM & LUCCA & OUINCY HARDSCRABRIF \\
\hline 4276 & & GRISOLANO & TONY & $\mathrm{H}$ & 52 & ITALY & ITALY & ITALY & 1890 & EAPMAER & $\begin{array}{ll}\text { LUCLA } \\
\text { TOPINO }\end{array}$ & 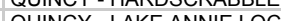 \\
\hline 4277 & & GRISOLANO & MARY & w & 50 & ITALY & ITALY & ITALY & 1892 & FARTILEK & TURIINo & OUINCY - LAKE ANNIE LOC \\
\hline 4278 & & GRISOLANO & RICKO & $\mathrm{s}$ & 26 & MICHIGAN & ITALY & ITALY & & MINER/CM & TORINO & QUINCY - LAKE ANNIE LOC \\
\hline 4279 & & GRISOLANO & ROSE & D & 13 & MICHIGAN & ITALY & ITALY & & & TORINO & QUINCY - LAKE ANNIE LOC \\
\hline 4280 & & GRISOLANO & TONY & $\mathrm{s}$ & 12 & MICHIGAN & ITALY & ITALY & & & TORINO & OUINCY - LAKE ANNIE LOC \\
\hline 4281 & & GRISOLANO & MARY & D & 10 & MICHIGAN & ITALY & ITALY & & & TORINO & QUINCY - LAKE ANNIE LOC \\
\hline 4282 & & GRISOLANO & JENNIE & $\mathrm{D}$ & 8 & MICHIGAN & ITALY & ITALY & & & TORINO & QUINCY - LAKE ANNIE L \\
\hline 4283 & & GRISOLANO & FRANK & $\mathrm{s}$ & 6 & MICHIGAN & ITALY & ITALY & & & TORINO & QUINCY - LAKE ANNIE LOC \\
\hline
\end{tabular}




\begin{tabular}{|c|c|c|c|c|c|c|c|c|c|c|c|c|}
\hline 4284 & SANDRETTO & DOMENICO & $\mathrm{H}$ & 49 & ITALY & ITALY & ITALY & 1888 & FARMER/GENERAL FARM & PONT CANAVESE. TO & N. CALUMET R. & SCHOOLCRAFT \\
\hline 4285 & SANDRETTO & MINNIE & W & 44 & ITALY & ITALY & ITALY & 1894 & & & N. CALUMET R. & SCHOOLCRAFT \\
\hline 4286 & SANDRETTO & MARY & D & 23 & MICHIGAN & ITALY & ITALY & & & PONT CANAVESE. TO & N. CALUMET R. & SCHOOLCRAFT \\
\hline 4287 & SANDRETTO & LUCILLE & D & 20 & MICHIGAN & ITALY & ITALY & & SEAMSTRESS/WORKING OUT & PONT CANAVESE. TO & N. CALUMET R. & SCHOOLCRAFT \\
\hline 4288 & SANDRETTO & $\mathrm{JOHN}$ & $\mathrm{s}$ & 14 & MICHIGAN & ITALY & ITALY & & & PONT CANAVESE. TO & N. CALUMET R. & SCHOOLCRAFT \\
\hline 4289 & VARIO & JOSEPH & H & 47 & ITALY & ITALY & ITALY & 1899 & LABORER/COPPER MILL & LOCANA CANAVESE. TO & ROCK VALLEY R. & SCHOOLCRAFT \\
\hline 4290 & VARIO & MARY & w & 40 & ITALY & ITALY & ITALY & 1909 & 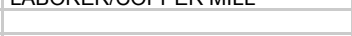 & LECAIVA CAIVAVLOL. TO & ROCK VALLEY R. & SCHOOLCRAFT \\
\hline 4291 & VARIO & PETER & s & 18 & MICHIGAN & ITALY & ITALY & & LABORER/COPPER MILL & LOCANA CANAVESE. TO & ROCK VALLEY R. & SCHOOLCRAFT \\
\hline 4292 & VARIO & ANNE & D & 13 & MICHIGAN & ITALY & ITALY & & & LOCANA CANAVESE. TO & ROCK VALLEY R. & SCHOOLCRAFT \\
\hline 4293 & VARIO & FRANCIS & D & 9 & MICHIGAN & ITALY & ITALY & & & LOCANA CANAVESE. TO & ROCK VALLEY R. & SCHOOLCRAFT \\
\hline 4294 & VARIO & DOMENICO & $\mathrm{s}$ & $2 \mathrm{M}$ & MICHIGAN & ITALY & ITALY & & & LOCANA CANAVESE. TO & & SCHOOLCRAFT \\
\hline 4295 & TURNER & AMOS & $\mathrm{H}$ & 50 & ITALY & ITALY & ITALY & 1893 & FARMER/GENERAL FARM & LUCCA & & SCHOOLCRAFT \\
\hline 4296 & TURNER & KATE & W & 30 & ITALY & ITALY & ITALY & 1914 & & & & SCHOOLCRAFT \\
\hline 4297 & TURNER & AMOS & $\mathrm{s}$ & 1 & MICHIGAN & ITALY & ITALY & & TEAMSTER/COAL COMPANY & LUCCA & & SCHOOLCRAFT \\
\hline 4298 & MICHEL & GODIAS & $\mathrm{H}$ & 43 & CANADA & CANADA & CANADA & 1881 & & CANADA & FIRST ST. & SCHOOLCRAFT - LAKE LINDEN \\
\hline 4299 & MICHEL & LUCY & W & 38 & ITALY & ITALY & ITALY & 1883 & & & FIRST ST. & SCHOOLCRAFT - LAKE LINDEN \\
\hline 4300 & MICHEL & LAWRENCE & s & 13 & MICHIGAN & ITALY & ITALY & & & & FIRST ST. & SCHOOLCRAFT - LAKE LINDEN \\
\hline 4301 & MICHEL & CORINNE & D & 12 & MICHIGAN & ITALY & ITALY & & & & FIRST ST. & SCHOOLCRAFT - LAKE LINDEN \\
\hline 4302 & MICHEL & LUCILLE & $\mathrm{D}$ & 8 & MICHIGAN & ITALY & ITALY & & & & FIRST ST. & SCHOOLCRAFT - LAKE LINDEN \\
\hline 4303 & MICHEL & MELBA & $\mathrm{D}$ & 6 & MICHIGAN & ITALY & ITALY & & & & FIRST ST. & SCHOOLCRAFT - LAKE LINDEN \\
\hline 4304 & MICHEL & EDWARD & $\mathrm{s}$ & 4 & MICHIGAN & ITALY & ITALY & & & & FIRST ST. & SCHOOLCRAFT - LAKE LINDEN \\
\hline 4305 & MICHEL & ALLAN & s & 1 & MICHIGAN & ITALY & ITALY & & & & FIRST ST. & SCHOOLCRAFT - LAKE LINDEN \\
\hline 4306 & TURNER & JEANETTE & $\mathrm{H}$ & $4 ?$ & MICHIGAN & SCOTLAND & CANADA & & & SCOTLAND-CANADA & CENTER ST. & SCHOOLCRAFT - LAKE LINDEN \\
\hline 4307 & TURNER & RUBY & D & 27 & MICHIGAN & ITALY & MICHIGAN & & CLERK/GENERAL STORE & LUCCA & CENTER ST. & SCHOOLCRAFT - LAKE LINDEI \\
\hline 4308 & TURNER & ANTON & $\mathrm{s}$ & 25 & MICHIGAN & ITALY & MICHIGAN & & REPAIRER/GARAGE & LUCCA & CENTER ST. & SCHOOLCRAFT - LAKE LINDEN \\
\hline 4309 & TURNER & ESTELA & $\mathrm{D}$ & 22 & MICHIGAN & ITALY & MICHIGAN & & BOOKKEEPER/GROCERY & LUCCA & CENTER ST. & SCHOOLCRAFT - LAKE LINDEN \\
\hline 4310 & TURNER & RANDOLPH & $\mathrm{s}$ & 18 & MICHIGAN & ITALY & MICHIGAN & & ELECTRICIAN/ELECTRICAL CO. & LUCCA & CENTER ST. & SCHOOLCRAFT - LAKE LINDEN \\
\hline 4311 & TURNER & VIOLET & D & 14 & MICHIGAN & ITALY & MICHIGAN & & & LUCCA & CENTER ST. & SCHOOLCRAFT - LAKE LINDEN \\
\hline 4312 & BALCONI & JAMES & $\mathrm{H}$ & 40 & ITALY & ITALY & ITALY & 1900 & FARMER/GENERAL FARM & MERCALLO. VARESE & & STANTON \\
\hline 4313 & BALCONI & MARIEL & W & 36 & ITALY & ITALY & ITALY & 1904 & & MERCALLO. VARESE & & STANTON \\
\hline 4314 & BALCONI & RUSSELL & $\mathrm{s}$ & 18 & ITALY & ITALY & ITALY & 1904 & & MERCALLO. VARESE & & STANTON \\
\hline 4315 & BALCONI & STELLA & D & 10 & MICHIGAN & ITALY & ITALY & & & MERCALLO. VARESE & & STANTON \\
\hline 4316 & BALCONI & WILLIAM & $\mathrm{s}$ & 3 & MICHIGAN & ITALY & ITALY & & & MERCALLO. VARESE & & STANTON \\
\hline 4317 & BALCONI & ANGELO & $\mathrm{H}$ & 36 & ITALY & ITALY & ITALY & 1904 & FARMER/GENERAL FARM & MERCALLO, VARESE & & STANTON \\
\hline 4318 & BALCONI & MARIA & w & 33 & ITALY & ITALY & ITALY & 1905 & & MERCALLO. VARESE & & STANTON \\
\hline 4319 & BALCONI & HENRY & s & 13 & MICHIGAN & ITALY & ITALY & & & MERCALLO. VARESE & & STANTON \\
\hline 4320 & BALCONI & ATTILIO & $\mathrm{s}$ & 11 & MICHIGAN & ITALY & ITALY & & & MERCALLO. VARESE & & STANTON \\
\hline 4321 & HOYMATTI & LOUIS & $\mathrm{H}$ & 32 & ITALY & ITALY & ITALY & 1907 & LABORER/POULTRY FARM & & & STANTON \\
\hline 4322 & HOYMATTI & ARMIDA & W & 35 & ITALY & ITALY & ITALY & 1913 & & & & STANTON \\
\hline 4323 & HOYMATTI & ARGENTINA & D & $?$ & MICHIGAN & ITALY & ITALY & & & & & STANTON \\
\hline 4324 & HOYMATTI & EMMA & $\mathrm{s}$ & 3 & MICHIGAN & ITALY & ITALY & & & & & STANTON \\
\hline 4325 & HOYMATTI & NATALIE & D & 1 & MICHIGAN & ITALY & ITALY & & & & & STANTON \\
\hline 4326 & GRECO & SALVATORE & $\mathrm{H}$ & 33 & ITALY & ITALY & ITALY & 1903 & MILL HAND/COPPER STAMP MILL & PALERMO & & STANTON - BEACON HILL LOC \\
\hline 4327 & GRECO & VITTORIA & w & 37 & ITALY & ITALY & ITALY & 1904 & & PALERMO & & STANTON - BEACON HILL LOC \\
\hline 4328 & GRECO & CARLO & $\mathrm{s}$ & 12 & MICHIGAN & ITALY & ITALY & & & PALERMO & & STANTON - BEACON HILL LOC \\
\hline 4329 & GRECO & FRANK & $\mathrm{s}$ & 10 & MICHIGAN & ITALY & ITALY & & & PALERMO & & STANTON - BEACON HILL LOC \\
\hline 4330 & GRECO & LOTTIR & $\mathrm{D}$ & 8 & MICHIGAN & ITALY & ITALY & & & PALERMO & & STANTON - BEACON HILL LOC \\
\hline 4331 & GRECO & IRENE & D & 6 & MICHIGAN & ITALY & ITALY & & & PALERMO & & STANTON - BEACON HILL LOC \\
\hline 4332 & GRECO & HELAN & D & 4 & MICHIGAN & ITALY & ITALY & & & PALERMO & & STANTON - BEACON HILL LOC \\
\hline 4333 & GRECO & RAFFAELE & $\mathrm{s}$ & 2 & MICHIGAN & ITALY & ITALY & & & PALERMO & & STANTON - BEACON HILL LOC \\
\hline 4334 & GRECO & TONY & $\mathrm{s}$ & $2 \mathrm{M}$ & MICHIGAN & ITALY & ITALY & & & PALERMO & & STANTON - BEACON HILL LOC \\
\hline 4335 & POERIA & TONY & $\mathrm{H}$ & 33 & ITALY & ITALY & ITALY & 1913 & MILL HAND/COPPER STAMP MILL & & & STANTON - BEACON HILL LOC \\
\hline 4336 & PARIANO & JOHN & B & 28 & ITALY & ITALY & ITALY & 1910 & MILL HAND/COPPER STAMP MILL & COTRONEI. CROTONE & & STANTON - BEACON HILL LOC \\
\hline 4337 & ALBI & FRED & B & 26 & ITALY & ITALY & ITALY & 1913 & MILL HAND/COPPER STAMP MILL & & & STANTON - BEACON HILL LOC \\
\hline 4338 & PROCISSI & JOE & $\mathrm{H}$ & 60 & ITALY & ITALY & ITALY & 1898 & LABORER/STAMP MILL & LUCCA & & STANTON - FREDA LOCATION \\
\hline 4339 & PROCISSI & MARIA & w & 58 & ITALY & ITALY & ITALY & 1898 & LABORER/STAMP MILL & LUCCA & & STANTON - FREDA LOCATION \\
\hline 4340 & PROCISSI & LIZ & D & 13 & MICHIGAN & ITALY & ITALY & & LABORER/STAMP MILL & LUCCA & & STANTON - FREDA LOCATION \\
\hline 4341 & TARUSCIO & SAM & B & 29 & ITALY & ITALY & ITALY & 1912 & LABORER/STAMP MILL & CATANZARO & & STANTON - FREDA LOCATION \\
\hline 4342 & FASONE & PETER & B & 61 & ITALY & ITALY & ITALY & 1888 & LABORER/STAMP MILL & & & STANTON - FREDA LOCATION \\
\hline 4343 & MONTICELLO/I & LOUIS & $\mathrm{H}$ & 28 & ITALY & ITALY & ITALY & 1896 & COPPER DIPPER/SMELTER & & & MELLONSVILLE \\
\hline 4344 & MONTICELLO/I & & W & 27 & MICHIGAN & ITA & ITALY & & & & & MELLONSVILLE \\
\hline 4345 & MONTICELLO/I & ELDOCH & $\mathrm{s}$ & 7 & MICHIGAN & ITALY & MICHIGAN & & & & & \\
\hline 4346 & LENCI & JOHN & $\mathrm{H}$ & 42 & ITALY & ITALY & ITALY & 1890 & LABORER/STAMP MILL & PESCAGLIA. LU & GREGORY ST. & TORCH LAKE - HUBBEL VILL. \\
\hline
\end{tabular}




\begin{tabular}{|c|c|c|c|c|c|c|c|c|c|c|c|c|}
\hline 4347 & LENCI-MATRONI & GENERINA & W & 26 & ITALY & ITALY & ITALY & 1890 & & PESCAGLIA. LU & GREGORY ST & TORCH LAKE - HUBBEL VILL \\
\hline 4348 & LENCI & SERAFINA & D & 6 & MICHIGAN & ITALY & ITALY & & & PESCAGLIA. LU & GREGORY ST & TORCH LAKE - HUBBEL VILL \\
\hline 4349 & LENCI & AMELIA & D & 4 & MICHIGAN & ITALY & ITALY & & & PESCAGLIA. LU & GREGORY ST & TORCH LAKE - HUBBEL VILL \\
\hline 4350 & LENCI & ROBERT & $\mathrm{S}$ & 1 & MICHIGAN & ITALY & ITALY & & & PESCAGLIA. LU & GREGORY ST & TORCH LAKE - HUBBEL VILL \\
\hline 4351 & FATTI & MARIO & B & 30 & ITALY & ITALY & ITALY & 1912 & LABORER/STAMP MILL & & GREGORY ST & TORCH LAKE - HUBBEL VILL \\
\hline 4352 & MONTICELLI & ORESTE & $\mathrm{H}$ & 52 & ITALY & ITALY & ITALY & 1881 & LABORER/SMELTER & MATRAIA. LU & GREGORY ST & TORCH LAKE - HUBBEL VILL \\
\hline 4353 & MONTICELLI & GUIDO & s & 16 & MICHIGAN & ITALY & ITALY & & & MATRAIA. LU & GREGORY ST & TORCH LAKE - HUBBEL VILL \\
\hline 4354 & MONTICELLI & FRANK & $\mathrm{s}$ & 12 & MICHIGAN & ITALY & ITALY & & & MATRAIA. LU & GREGORY ST & TORCH LAKE - HUBBEL VILL \\
\hline 4355 & MONTICELLI & JOHN & $\hat{s}$ & 11 & MICHIGAN & ITALY & ITALY & & & MATRAIA. LU & GREGORY ST & TORCH LAKE - HUBBEL VILL \\
\hline 4356 & LANDINI & GABRIEL & B & 45 & ITALY & ITALY & ITALY & 1890 & LABORER/STAMP MILL & RUOTA. LU & GREGORY ST & TORCH LAKE - HUBBEL VILL \\
\hline 4357 & SERODUCCI & ADOLFO & B & 27 & ITALY & ITALY & ITALY & 1912 & LABORER/STAMP MILL & & GREGORY ST & TORCH LAKE - HUBBEL VILL \\
\hline 4358 & LENCl & ETTORE & B & 20 & ITALY & ITALY & ITALY & 1912 & LABORER/STAMP MILL & PESCAGLIA. LU & GREGORY ST & TORCH LAKE - HUBBEL VILL \\
\hline 4359 & CHELLINI & JOHN & $\mathrm{H}$ & 60 & ITALY & ITALY & ITALY & 1881 & WATCHMAN/CM & LUCCA & EUCLID ST. & TORCH LAKE - HUBBEL VILL \\
\hline 4360 & CHELLINI & SARA & W & 41 & ENGLAND & ENGLAND & ENGLAND & 1895 & & ENGLAND & EUCLID ST. & TORCH LAKE - HUBBEL VILL \\
\hline 4361 & CHELLINI & ALFRED & $\mathrm{s}$ & 27 & MICHIGAN & ITALY & ENGLAND & & HELPER/SMELTER & LUCCA & EUCLID ST. & TORCH LAKE - HUBBEL VILL \\
\hline 4362 & CHELLINI & MLTON & $\mathrm{S}$ & 25 & MICHIGAN & ITALY & ENGLAND & & LABORER & LUCCA & EUCLID ST. & TORCH LAKE - HUBBEL VILL \\
\hline 4363 & CHELLINI & JOHN & $\mathrm{s}$ & 23 & MICHIGAN & ITALY & ENGLAND & & LABORER & LUCCA & EUCLID ST. & TORCH LAKE - HUBBEL VILL \\
\hline 4364 & CHELLINI & GIORGIO & $\mathrm{s}$ & 21 & MICHIGAN & ITALY & ENGLAND & & LABORER/SMELTER & LUCCA & EUCLID ST. & TORCH LAKE - HUBBEL VILL \\
\hline 4365 & COSTA & LOUIS & $\mathrm{H}$ & 68 & ITALY & ITALY & $\begin{array}{l}\text { ITALY } \\
\text { ITA }\end{array}$ & 1890 & LABORER/STAMP MILL & TORINO & EUCLID ST. & TORCH LAKE - HUBBEL VILL \\
\hline 4366 & COSTA & LUCIA & W & 60 & CANADA & ITALY & ITALY & 1892 & & & EUCLID ST. & TORCH LAKE - HUBBEL VILL \\
\hline 4367 & COSTA & ANTONIO & $\mathrm{s}$ & 22 & MICHIGAN & & CANADA & & LABORER/STAMP MILL & & & TORCH LAKE - HUBBEL VILL \\
\hline 4368 & COSTA & AUGUSTO & $\frac{3}{s}$ & 19 & MICHIGAN & $\begin{array}{l}\text { ITALY } \\
\text { ITAL }\end{array}$ & CANADA & & LADURERTS I AIVIF TILLL & TORINO & EUCLID ST. & TORCH LAKE - HUBBEL VILL \\
\hline 4369 & TANALLO & JOSEPH & $\mathrm{H}$ & 40 & ITALY & ITALY & ITALY & 1902 & LABORER & & & TORCH LAKE - HUBBEL VILL \\
\hline 4370 & SCOLA & SANTO & $\mathrm{H}$ & 39 & ITALY & ITALY & ITALY & 1913 & LABORER & BELLUNO & & TORCH LAKE - HUBBEL VILL \\
\hline 4371 & GASPARDO & BATTISTA & $\mathrm{H}$ & 40 & ITALY & ITALY & ITALY & 1892 & FARMER & PONT CANAVESE. TO & & TORCH LAKE - BOAT JACK \\
\hline 4372 & GASPARDO & ANTONIETTA & W & 40 & ITALY & ITALY & ITALY & 1892 & & PONT CANAVESE. TO & & TORCH LAKE - BOAT JACK \\
\hline 4373 & GASPARDO & SERAFINO & s & 15 & MICHIGAN & ITALY & ITALY & & & PONT CANAVESE. TO & & TORCH LAKE - BOAT JACK \\
\hline 4374 & GASPARDO & JOHN & $\mathrm{S}$ & 14 & MICHIGAN & ITALY & ITALY & & & PONT CANAVESE, TO & & TORCH LAKE - BOAT JACK \\
\hline 4375 & GASPARDO & JAMES & $\mathrm{S}$ & 12 & MICHIGAN & ITALY & ITALY & & & PONT CANAVESE. TO & & TORCH LAKE - BOAT JACK \\
\hline 4376 & GASPARDO & MARCO & $\mathrm{s}$ & 11 & MICHIGAN & ITALY & ITALY & & & PONT CANAVESE. TO & & TORCH LAKE - BOAT JACK \\
\hline 4377 & GASPARDO & DOMENICO & $\mathrm{s}$ & 8 & MICHIGAN & ITALY & ITALY & & & PONT CANAVESE. TO & & TORCH LAKE - BOAT JACK \\
\hline 4378 & GASPARDO & JOHANNA & D & 5 & MICHIGAN & ITALY & ITALY & & & PONT CANAVESE. TO & & TORCH LAKE - BOAT JACK \\
\hline 4379 & GASPARDO & MARY TRACY & $\mathrm{D}$ & $4 \mathrm{M}$ & MICHIGAN & ITALY & ITALY & & & PONT CANAVESE. TO & & TORCH LAKE - BOAT JACK \\
\hline
\end{tabular}




\section{APPENDIX XI}

\section{CENSUS of 1930}

\section{LEGEND}

ID: counter.

$\mathbf{N}$ : street number as it appeared on the house.

REL: relation of the person with the head of the household.

BORN: nation of birth of the person.

FATHER: nation of birth of the father.

MOTHER: nation of birth of the mother.

USA: indicate the year of arrival in the U.S.

PROVENIENCE: place of origin in Italy. These data are not from the census, but form Ellis Island Database, Italian newspapers, Oral interviews.

TOWNSHIP: township in which the person was living. 


\begin{tabular}{|c|c|c|c|c|c|c|c|c|c|c|c|c|c|}
\hline ID & $\mathrm{N}$ & SURNAME & NAME & REL & AGE & BORN & FATHER & MOTHER & USA & OCCUPATION & \begin{tabular}{|l} 
PROVENIENCE \\
\end{tabular} & ADDRESS & \begin{tabular}{|l} 
TOWNSHIF \\
\end{tabular} \\
\hline 1 & 138 & GIUSFREDI & IDA & $\mathrm{H}$ & 44 & ITALY & ITALY & ITALY & 1914 & & LUCCA & ADAMS ST. & ADAMS - PAINSDALE \\
\hline 2 & & GIUSFREDI & SIGFRIDO & $\mathrm{s}$ & 18 & MICHIGAN & ITALY & ITALY & & & LUCCA & ADAMS ST. & ADAMS - PAINSDALE \\
\hline 3 & & GIUSFREDI & ANGELO & $\mathrm{s}$ & 11 & MICHIGAN & ITALY & ITALY & & & LUCCA & ADAMS ST. & ADAMS - PAINSDALE \\
\hline 4 & 135 & ANDREINA & ERNEST & $\mathrm{H}$ & 25 & MICHIGAN & ITALY & ITALY & & MINER/CM & TORINO & ADAMS ST. & ADAMS - PAINSDALE \\
\hline 5 & & ANDREINA & MARGARET & w & 22 & MICHIGAN & MICHIGAN & MICHIGAN & & & USA & ADAMS ST. & ADAMS - PAINSDALE \\
\hline 6 & & ANDREINA & $\begin{array}{l}\text { PATRICIA } \\
\text { PAT }\end{array}$ & D & 3 & MICHIGAN & MICHIGAN & MICHIGAN & & & TORINO & ADAMS ST. & ADAMS - PAINSDALE \\
\hline 7 & 160 & BACCETTI & JOSEPH & $\mathrm{H}$ & 50 & ITALY & ITALY & ITALY & 1902 & & MASSA MACINAIA, LU & BALTIC ST. & ADAMS - PAINSDALE \\
\hline 8 & & BACCETTI & MARTON & s & 23 & MICHIGAN & ITALY & ITALY & & LABORER/CM & MASSA MACINAIA. LU & BALTIC ST. & ADAMS - PAINSDALE \\
\hline 9 & & BACCETTI & $\begin{array}{l}\text { SUSIE } \\
\text { SUS }\end{array}$ & $\mathrm{D}$ & 20 & MICHIGAN & ITALY & ITALY & & LADURER/UIUI & MASSA MACINAIA. LU & BALTIC ST. & ADAMS - PAINSDALE \\
\hline 10 & & BACCETTI & ALBERT & S & 20 & MICHIGAN & ITALY & ITALY & & & MASSA MACINAIA. LU & BALTIC ST. & ADAMS - PAINSDALE \\
\hline 11 & & BACCETTI & DOLLY & DIL & 18 & MICHIGAN & FINLAND & FINLAND & & & FINLAND & BALTIC ST. & ADAMS - PAINSDALE \\
\hline 12 & & BACCETTI & MARJORY & $\mathrm{GD}$ & $3 \mathrm{M}$ & MICHIGAN & MICHIGAN & MICHIGAN & & & MASSA MACINAIA. LU & BALTIC ST. & ADAMS - PAINSDALE \\
\hline 13 & 132 & PIZZI & PETER & $\mathrm{H}$ & 42 & ITALY & ITALY & ITALY & $00 / 05$ & & PESCAGLIA. LU & CONCORD ST. & ADAMS - PAINSDALE \\
\hline 14 & & PIZZI & JEMINA & w & 28 & MICHIGAN & ITALY & ITALY & & & & CONCORD ST. & ADAMS - PAINSDALE \\
\hline 15 & & PIZZI & GENA & D & 6 & MICHIGAN & ITALY & MICHIGAN & & & PESCAGLIA. LU & CONCORD ST. & ADAMS - PAINSDALE \\
\hline 16 & 142 & TADDEUCCI & OLINTO & $\mathrm{H}$ & 40 & ITALY & ITALY & ITALY & 1908 & MINER/CM & LUCCA & CONCORD ST. & ADAMS - PAINSDALE \\
\hline 17 & & TADDEUCCI & IDA & w & 39 & ITALY & ITALY & ITALY & 1916 & & LUCCA & CONCORD ST. & ADAMS - PAINSDALE \\
\hline 18 & & TADDEUCCI & GIULIO & $\mathrm{s}$ & 16 & ITALY & ITALY & ITALY & 1916 & & LUCCA & CONCORD ST. & ADAMS - PAINSDALE \\
\hline 19 & & TADDEUCCI & ADA & D & 12 & MICHIGAN & ITALY & ITALY & & & LUCCA & CONCORD ST. & ADAMS - PAINSDALE \\
\hline 20 & & TADDEUCCI & ALVIDA & $\mathrm{D}$ & 8 & MICHIGAN & ITALY & ITALY & & & LUCCA & CONCORD ST. & ADAMS - PAINSDALE \\
\hline 21 & & TADDEUCCI & ROLANDO & s & $\frac{0}{5}$ & MICHIGAN & ITALY & ITALY & & & LUCCA & CONCORD ST. & ADAMS - PAINSDALE \\
\hline 22 & & TADDEUCCI & MARISA & $\mathrm{D}$ & 4 & MICHIGAN & ITALY & ITALY & & & LUCCA & CONCORD ST. & ADAMS - PAINSDALE \\
\hline 23 & & PIZZI & REGOLO & $\mathrm{B}$ & 39 & ITALY & ITALY & $\begin{array}{l}\text { ITALY } \\
\text { ITAL }\end{array}$ & 1916 & MINER/CM & LUCCA & CONCORD ST. & ADAMS - PAINSDALE \\
\hline 24 & 118 & BIANCHI & BASILIO & $\mathrm{H}$ & 52 & ITALY & ITALY & ITALY & 1909 & LABORER/CM & PORCARI. LU & ONTONAGON ST. & ADAMS - PAINSDALE \\
\hline 25 & & BIANCHI & CARMELA & w & 48 & ITALY & ITALY & ITALY & 1912 & & & ONTONAGON ST. & ADAMS - PAINSDALE \\
\hline 26 & & BIANCHI & DANTE & s & 17 & MICHIGAN & ITALY & ITALY & & LABORER/CM & PORCARI. LU & ONTONAGON ST. & ADAMS - PAINSDALE \\
\hline 27 & & BIANCHI & SALVATORE & $\mathrm{s}$ & 14 & MICHIGAN & ITALY & ITALY & & & PORCARI. LU & ONTONAGON ST. & ADAMS - PAINSDALE \\
\hline 28 & & BIANCHI & MARY & $\mathrm{D}$ & 13 & MICHIGAN & ITALY & ITALY & & & PORCARI. LU & ONTONAGON ST. & ADAMS - PAINSDALE \\
\hline 29 & & BIANCHI & LENA & D & 6 & MICHIGAN & ITALY & ITALY & & & PORCARI. LU & ONTONAGON ST. & ADAMS - PAINSDALE \\
\hline 30 & & PALADINI & GAETANO & B & 41 & ITALY & ITALY & ITALY & 1913 & MINER/CM & MILANO & ONTONAGON ST. & ADAMS - PAINSDALE \\
\hline 31 & & PALADINI & JOSEPH & B & 48 & ITALY & ITALY & ITALY & 1900 & MINER/CM & CARPINETTI, REGGIO EMILIA & ONTONAGON ST. & ADAMS - PAINSDALE \\
\hline 32 & & NOMELLINI & MICHAEL & B & 51 & ITALY & ITALY & ITALY & 1903 & MINER/CM & CASTELSERENO. GROSSETO & ONTONAGON ST. & ADAMS - PAINSDALE \\
\hline 33 & 121 & BERTOLLI & JOHN & $\mathrm{H}$ & 41 & ITALY & ITALY & ITALY & 1911 & MINER/CM & ORBICCIANO. LU & ONTONAGON ST. & ADAMS - PAINSDALE \\
\hline 34 & & BERTOLLI & ADORA & w & 41 & ITALY & ITALY & ITALY & 1914 & & ORBICCIANO. LU & ONTONAGON ST. & ADAMS - PAINSDALE \\
\hline $\begin{array}{r}34 \\
35\end{array}$ & & BERTOLLI & JAMES & $\begin{array}{l}\mathrm{s} \\
\mathrm{u}\end{array}$ & 21 & ITALY & ITALY & ITALY & 1914 & MINER/CM & ORBICCIANO. LU & ONTONAGON ST. & ADAMS - PAINSDALE \\
\hline 36 & & BERTOLLI & DOROTHY & $\mathrm{D}$ & 19 & ITALY & ITALY & ITALY & 1914 & & ORBICCIANO. LU & ONTONAGON ST. & ADAMS - PAINSDALE \\
\hline 37 & & BERTOLLI & RALPH & B & 9 & MICHIGAN & ITALY & ITALY & & & ORBICCIANO, LU & ONTONAGON ST. & ADAMS - PAINSDALE \\
\hline 38 & & MOROTTI & JULIS & B & 36 & ITALY & ITALY & ITALY & 1912 & LABORER/CM & ORBICCIANO. LU & ONTONAGON ST. & ADAMS - PAINSDALE \\
\hline 39 & & MATTEUCCI & DOMENICO & B & 60 & ITALY & ITALY & ITALY & 1902 & LABORER/CM & LUCCA & ONTONAGON ST. & ADAMS - PAINSDALE \\
\hline 40 & & PUCCl & JOHN & B & 53 & ITALY & ITALY & ITALY & 1902 & LABORER/CM & LUCCA & ONTONAGON ST. & ADAMS - PAINSDALE \\
\hline 41 & & MARTINELLI & ATTILIO & B & 38 & ITALY & ITALY & ITALY & 1907 & LABORER/CM & ALTOPASCIO. LU & ONTONAGON ST. & ADAMS - PAINSDALE \\
\hline 42 & & BERTOLLI & LUIGI & B & 43 & ITALY & ITALY & ITALY & 1907 & LABORER/CM & ORBICCIANO. LU & ONTONAGON ST. & ADAMS - PAINSDALE \\
\hline 43 & & BRESCHI & ALADINO & B & 23 & PENNSYLVAN & ITALY & ITALY & & LABORER/CM & PISTOIA & ONTONAGON ST. & ADAMS - PAINSDALE \\
\hline 44 & 122 & BARTOLOMEI & ERBERTO & $\mathrm{H}$ & 37 & ITALY & ITALY & ITALY & 1911 & BLACKSMITH/BLACKSMITH SHOP & LUCCA & ONTONAGON ST. & ADAMS - PAINSDALE \\
\hline 45 & & BARTOLOMEI & ROSE & w & 38 & ITALY & ITALY & ITALY & 1919 & & & ONTONAGON ST. & ADAMS - PAINSDALE \\
\hline 46 & & BARTOLOMEI & TRACY & $\mathrm{D}$ & 9 & MICHIGAN & ITALY & ITALY & & & LUCCA & ONTONAGON ST. & ADAMS - PAINSDALE \\
\hline 47 & & BARTOLOMEI & ALFRED & BR & 28 & ITALY & ITALY & ITALY & 1925 & MACHINIST/CM & LUCCA & ONTONAGON ST. & ADAMS - PAINSDALE \\
\hline 48 & 166 & GOSATTI & JOHN & $\mathrm{H}$ & 40 & ITALY & ITALY & ITALY & 1910 & MINER/CM & BIANZANO. BERGAMO & 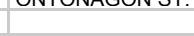 & ADAMS - PAINSDALE \\
\hline 49 & & GOSATTI & TRACY & w & 40 & ITALY & ITALY & ITALY & 1910 & & BIANZANO. BERGAMO & & ADAMS - PAINSDALE \\
\hline 50 & & GOSATTI & NUNZIA & D & 13 & MICHIGAN & ITALY & ITALY & & & BIANZANO. BERGAMO & & ADAMS - PAINSDALE \\
\hline 51 & & ANDRINA & FRANK & B & 39 & ITALY & ITALY & ITALY & 1923 & LABORER/CM & TORINO & & ADAMS - PAINSDALE \\
\hline 52 & & CIAGNE & GIOVANNI & $\mathrm{B}$ & 43 & $\begin{array}{l}\text { ITALY } \\
\end{array}$ & $\begin{array}{l}\text { ITALY } \\
\end{array}$ & ITA & 1921 & LABORER/CM & TORINO & & ADAMS - PAINSDALE \\
\hline 53 & & CASTELLANELLI & JAMES & B & 36 & ITALY & ITALY & ITA & 1913 & ORER/CM & BRIANZONE. SONDRIO & & ADAMS - PAINSDALE \\
\hline 54 & & FEIRA & GIOVANNI & B & $33 ?$ & ITALY & ITALY & ITALY & 1901 & LABORER/CM & TORINO & & ADAMS - PAINSDALE \\
\hline 55 & 154 & PIZZI & VINCENZO & $\mathrm{H}$ & 45 & ITALY & ITALY & ITALY & 1909 & LABORER/CM & PESCAGLIA. LU & FOREST ST. & ADAMS - PAINSDALE \\
\hline 56 & & PIZZI & TERESA & W & 43 & ITALY & ITALY & ITALY & 1911 & & & FOREST ST. & ADAMS - PAINSDALE \\
\hline 57 & & PIZZI & BIANCA & D & 16 & MICHIGAN & ITALY & ITALY & & & PESCAGLIA, LU & FOREST ST. & ADAMS - PAINSDALE \\
\hline 58 & & PIZZI & ROSA & $\mathrm{D}$ & 15 & MICHIGAN & ITALY & ITALY & & & PESCAGLIA. LU & FOREST ST. & ADAMS - PAINSDALE \\
\hline 59 & & PIZZI & ANTONIO & s & 12 & MICHIGAN & ITALY & ITALY & & & PESCAGLIA. LU & FOREST ST. & ADAMS - PAINSDALE \\
\hline 60 & & BRUNELLO & ALESSANDRO & B & 36 & ITALY & ITALY & ITALY & 1920 & LABORER/CM & FAGAGNA. UDINE & FOREST ST. & ADAMS - PAINSDALE \\
\hline 61 & & RIVA & $\begin{array}{l}\text { BATTISTA } \\
\text { BTS }\end{array}$ & B & 30 & ITALY & ITALY & ITALY & 1921 & LABORER/CM & $\begin{array}{l}\text { TORINO } \\
\text { TORO }\end{array}$ & FOREST ST. & ADAMS - PAINSDALE \\
\hline 62 & & BIAGGINI & MARIO & B & 44 & ITALY & ITALY & ITALY & 1896 & LABORER/CM & TORINO & FOREST ST. & ADAMS - PAINSDALE \\
\hline
\end{tabular}




\begin{tabular}{|c|c|c|c|c|c|c|c|c|c|c|c|c|c|}
\hline 63 & & SCOTTON & JOHN & B & 37 & ITALY & ITALY & ITALY & 1921 & LABORER/CM & & FOREST ST. & ADAMS - PAINSDALE \\
\hline 64 & 146 & $\begin{array}{l}\text { IAVELLO } \\
\end{array}$ & CARLO & $\mathrm{H}$ & 45 & ITALY & ITALY & ITALY & 1904 & LABORER/CM & SAN GIUSTO CANAVESE. TO & FOREST ST. & ADAMS - PAINSDALE \\
\hline 65 & 140 & IAVELLO & MARY & W & 50 & ITALY & ITALY & ITALY & 1903 & & 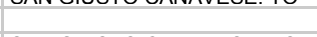 & FOREST ST. & ADAMS - PAINSDALE \\
\hline 66 & & IAVELLO & JOHN & $\mathrm{s}$ & 19 & MICHIGAN & ITALY & ITALY & & & SAN GIUSTO CANAVESE. TO & FOREST ST. & ADAMS - PAINSDALE \\
\hline 67 & & IAVELLLO & TRACY & $\mathrm{D}$ & 17 & MICHIGAN & ITALY & ITALY & & & SAN GIUSTO CANAVESE. TO & FOREST ST. & ADAMS - PAINSDALE \\
\hline 68 & & IAVELLO & MARY & $\mathrm{D}$ & 13 & MICHIGAN & ITALY & ITALY & & & SAN GIUSTO CANAVESE. TO & FOREST ST. & ADAMS - PAINSDALE \\
\hline 69 & & IAVELLO & CATERINE & D & 10 & MICHIGAN & ITALY & ITALY & & & SAN GIUSTO CANAVESE. TO & FOREST ST. & ADAMS - PAINSDALE \\
\hline 70 & 144 & MICHELETTI & FLORINDO & $\mathrm{H}$ & 51 & ITALY & ITALY & ITALY & 1905 & LABORER/CM & SANTA MARIA DEL GIUDICE. & FOREST ST. & ADAMS - PAINSDALE \\
\hline 71 & & MICHELETTI & ARMIDA & W & 52 & ITALY & ITALY & ITALY & 1909 & & & FOREST ST. & ADAMS - PAINSDALE \\
\hline 72 & & MICHELETTI & TONY & $\mathrm{s}$ & 7 & MICHIGAN & ITALY & ITALY & & & SANTA MARIA DEL GIUDICE. & FOREST ST. & ADAMS - PAINSDALE \\
\hline 73 & & MICHELETTI & SABATINO & $\mathrm{s}$ & 14 & MICHIGAN & ITALY & ITALY & & & SANTA MARIA DEL GIUDICE. & FOREST ST. & ADAMS - PAINSDALE \\
\hline 74 & & RICHETTA & JOHN & $\mathrm{H}$ & 29 & ITALY & ITALY & ITALY & 1905 & FIREMAN/CM & SAN MARTINO CANAVESE. TO & FOREST ST. & ADAMS - PAINSDALE \\
\hline 75 & & RICHETTA & GIULIA & W & 20 & MICHIGAN & ITALY & ITALY & & & & FOREST ST. & ADAMS - PAINSDALE \\
\hline 76 & & RICHETTA & JOSEPH & $\mathrm{s}$ & 3 & MICHIGAN & ITALY & MICHIGAN & & & SAN MARTINO CANAVESE. TO & FOREST ST. & ADAMS - PAINSDALE \\
\hline 77 & & RICHETTA & JENNE? & s & 2 & MICHIGAN & ITALY & MICHIGAN & & & SAN MARTINO CANAVESE. TO & FOREST ST. & ADAMS - PAINSDALE \\
\hline 78 & 142 & BALAGNA & JAMES & $\mathrm{H}$ & 56 & ITALY & ITALY & ITALY & 1887 & DRAYMAN/CM & PONT CANAVESE, TO & FOREST ST. & ADAMS - PAINSDALE \\
\hline 79 & 142 & BALAGNA & NELLI & W & 50 & ITALY & ITALY & ITALY & 1908 & Liverivinivitur & 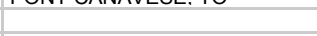 & FOREST ST. & ADAMS - PAINSDALE \\
\hline 80 & & BALAGNA & FELIX & $\mathrm{s}$ & 17 & MICHIGAN & ITALY & ITALY & & OILER/CM & PONT CANAVESE. TO & FOREST ST. & ADAMS - PAINSDALE \\
\hline 81 & & BALAGNA & DELIA & D & 16 & MICHIGAN & ITALY & ITALY & & & PONT CANAVESE. TO & FOREST ST. & ADAMS - PAINSDALE \\
\hline 82 & & BALAGNA & MARY & $\mathrm{D}$ & 15 & MICHIGAN & ITALY & ITALY & & & PONT CANAVESE. TO & FOREST ST. & ADAMS - PAINSDALE \\
\hline 83 & 151 & GAGGINI & TRACY & $\mathrm{H}$ & 38 & ITALY & ITALY & ITALY & 1911 & & PITEGLIO. PISTOIA & HIGHLAND ST & ADAMS - PAINSDALE \\
\hline 84 & & GAGGINI & ALINA & D & 18 & MICHIGAN & ITALY & ITALY & & & PITEGLIO, PISTOIA & HIGHLAND ST & ADAMS - PAINSDALE \\
\hline 85 & & GAGGINI & SELINA & $\mathrm{D}$ & 11 & MICHIGAN & ITALY & ITALY & & & PITEGLIO. PISTOIA & HIGHLAND ST & ADAMS - PAINSDALE \\
\hline 86 & & GAGGINI & GILDA & $\mathrm{D}$ & 17 & MICHIGAN & ITALY & ITALY & & & PITEGLIO. PISTOIA & HIGHLAND ST & ADAMS - PAINSDALE \\
\hline 87 & & KRIEBER & ERNEST & SOIL & 26 & ILLINOIS & GERMANY & GERMANY & & LABORER/CM & GERMANY & HIGHLAND ST & ADAMS - PAINSDALE \\
\hline 88 & & NARETTO & PETER & B & 29 & ITALY & ITALY & ITALY & 1921 & LABORER/CM & TORINO & IROQUOIS ST. & ADAMS - PAINSDALE \\
\hline 89 & & STOCCHERO & ANTONIO & B & 46 & ITALY & ITALY & ITALY & 1905 & & CREMONA & IROQUOIS ST. & ADAMS - PAINSDALE \\
\hline 90 & & PETRAYRONEI & GIUSEPPE & B & 47 & ITALY & ITALY & ITALY & 1907 & LABORER/CM & & $\begin{array}{l}\text { IROQUOIS ST. } \\
\text { IRO }\end{array}$ & ADAMS - PAINSDALE \\
\hline 91 & 8 & MARCONI & JOSEPH & $\mathrm{H}$ & 54 & ITALY & ITALY & ITALY & 1909 & LABORER/CM & & IROQUOIS ST. & ADAMS - PAINSDALE \\
\hline 92 & & MARCONI & ELENA & W & 41 & ITALY & ITALY & ITALY & 1909 & & & IROQUOIS ST. & ADAMS - PAINSDALE \\
\hline 93 & & MARCONI & PETER & $\begin{array}{l}\mathrm{s} \\
\mathrm{i}\end{array}$ & 8 & MICHIGAN & ITALY & ITALY & & & & IROQUOIS ST. & ADAMS - PAINSDALE \\
\hline 94 & & COTTINI & DANTE & B & 25 & ITALY & ITALY & ITALY & 1921 & & COLLODI. LU & IROQUOIS ST. & ADAMS - PAINSDALE \\
\hline 95 & & BARSANTI & PETER & B & 48 & ITALY & ITALY & ITALY & 1905 & LABORER/CM & LUCCA & $\begin{array}{l}\text { IROQUOIS ST. } \\
\text { IRO }\end{array}$ & ADAMS - PAINSDALE \\
\hline 96 & $18 ?$ & BAGNESCHI & AUGUST & $\mathrm{H}$ & 39 & ITALY & ITALY & ITALY & 1911 & LABORER/CM & LUCCA & 2ND ST. & ADAMS - PAINSDALE \\
\hline 97 & & BAGNESCHI & LILIAN & W & 26 & MICHIGAN & ITALY & ITALY & & & & 2ND ST. & ADAMS - PAINSDALE \\
\hline 98 & & BAGNESCHI & MARY & D & 8 & MICHIGAN & ITALY & MICHIGAN & & & LUCCA & 2ND ST. & ADAMS - PAINSDALE \\
\hline 99 & & BAGNESCHI & WILLIAM & $\mathrm{s}$ & 6 & MICHIGAN & ITALY & MICHIGAN & & & LUCCA & 2ND ST. & ADAMS - PAINSDALE \\
\hline 100 & & BAGNESCHI & LUCILLE & D & 4 & MICHIGAN & ITALY & MICHIGAN & & & LUCCA & 2ND ST. & ADAMS - PAINSDALE \\
\hline 101 & & BAGNESCHI & ROSS & $\mathrm{s}$ & 1 & MICHIGAN & ITALY & MICHIGAN & & & LUCCA & 2ND ST. & ADAMS - PAINSDALE \\
\hline 102 & 38 & LENCl & ORLANDO & $\mathrm{H}$ & 36 & ITALY & ITALY & ITALY & 1927 & BLACKSMITH/CM & LUCCA & & ADAMS - PAINSDALE \\
\hline 103 & & LENCl & ROSE & w & 35 & ITALY & ITALY & ITALY & 1927 & & & & ADAMS - PAINSDALE \\
\hline & 4 & ENRIETTI & FRANK & $\mathrm{H}$ & 49 & ITALY & ITALY & ITALY & 1911 & LABORER/CM & TORINO & 2ND ST. & ADAMS - PAINSDALE \\
\hline 105 & & $\begin{array}{l}\text { ENRIETTI } \\
\text { ENIE }\end{array}$ & ORSOLINA & W & 48 & ITALY & ITALY & ITALY & 1920 & LADURERK/UIVI & TORINO & 2ND ST. & ADAMS - PAINSDALE \\
\hline 106 & & $\begin{array}{l}\text { ENRIETTI } \\
\text { ENIE }\end{array}$ & LOUISE & D & $\begin{array}{l}40 \\
19\end{array}$ & ITALY & ITALY & ITALY & 1920 & & TORINO & 2ND ST. & ADAMS - PAINSDALE \\
\hline 107 & & ENRIETTI & MARY & D & 8 & MICHIGAN & ITALY & ITALY & & & TORINO & 2ND ST. & ADAMS - PAINSDALE \\
\hline 108 & & VETTORI & EMILIO & $\mathrm{H}$ & 44 & ITALY & ITALY & ITALY & 1907 & FIREMAN/CM & MONTECARLO. LU & HUBBEL ST. & ADAMS - PAINSDALE \\
\hline 109 & & VETTORI & MARIA & W & 47 & ITALY & ITALY & ITALY & 1906 & & & HUBBEL ST. & ADAMS - PAINSDALE \\
\hline 110 & & VETTORI & ATTILIO & $\mathrm{s}$ & 19 & MICHIGAN & ITALY & ITALY & & MECHANIC & MONTECARLO. LU & HUBBEL ST. & ADAMS - PAINSDALE \\
\hline 111 & & VETTORI & ALADINO & s & 14 & MICHIGAN & ITALY & ITALY & & & MONTECARLO. LU & HUBBEL ST. & ADAMS - PAINSDALE \\
\hline 112 & & VETTORI & JOHN & s & 13 & MICHIGAN & ITALY & ITALY & & & MONTECARLO. LU & HUBBEL ST. & ADAMS - PAINSDALE \\
\hline 113 & & VETTORI & GINO & $\mathrm{s}$ & 8 & MICHIGAN & ITALY & ITALY & & & MONTECARLO. LU & HUBBEL ST. & ADAMS - PAINSDALE \\
\hline 114 & & LENCIONI & EVANGELISTA & B & 50 & ITALY & ITALY & ITALY & 1913 & FIREMAN/CM & SEGROMIGNO, LU & HUBBEL ST. & ADAMS - PAINSDALE \\
\hline 115 & & DATI & SEVERINO & B & 35 & ITALY & ITALY & ITALY & 1913 & LABORER/CM & PARIANA. LU & HUBBEL ST. & ADAMS - PAINSDALE \\
\hline 116 & & AMANTI & DORINDA & B & 50 & ITALY & ITALY & ITALY & 1907 & LABORER/CM & LUCCA & HUBBEL ST. & ADAMS - PAINSDALE \\
\hline 117 & 156 & FEIRA & DOMENICO & $\begin{array}{l}\mathrm{D} \\
\mathrm{H}\end{array}$ & 32 & ITALY & ITALY & ITALY & 1921 & LABORER/CM & TORINO & HUBBEL ST. & ADAMS - PAINSDALE \\
\hline 118 & 130 & FEIRA & JOSEPH & W & 31 & ITALY & $\begin{array}{l}\text { ITALY } \\
\end{array}$ & ITALY & 1923 & & & HUBBEL ST. & ADAMS - PAINSDALE \\
\hline 119 & & FEIRA & MARY & D & $\frac{31}{6}$ & MICHIGAN & ITALY & ITALY & & & TORINO & HUBBEL ST. & ADAMS - PAINSDALE \\
\hline 120 & & FEIRA & DOMENICO & $\mathrm{D}$ & 3 & MICHIGAN & ITALY & ITALY & & & TORINO & HUBBEL ST. & ADAMS - PAINSDALE \\
\hline 121 & & FEIRA & IRMA & D & 1 & MICHIGAN & ITALY & ITALY & & & TORINO & HUBBEL ST. & ADAMS - PAINSDALE \\
\hline 122 & 211 & LASIA & BALDO & $\mathrm{H}$ & 47 & ITA & ITALY & ITALY & 1906 & MINER/CM & & 6TH ST. & ADAMS - TRIMOUNTAIN \\
\hline 123 & & LASIA & MINNIE & W & 42 & ITALY & ITALY & ITALY & 03 ?? & & & 6TH ST. & ADAMS - TRIMOUNTAIN \\
\hline 124 & & LASIA & JOHN & $\mathrm{s}$ & 17 & SOUTH & ITALY & ITALY & & & & 6TH ST. & ADAMS - TRIMOUNTAIN \\
\hline 125 & & LASIA & ALDO & s & 10 & SOUTH & ITALY & ITALY & & & & 6TH ST. & ADAMS - TRIMOUNTAIN \\
\hline
\end{tabular}




\begin{tabular}{|c|c|c|c|c|c|c|c|c|c|c|c|c|c|}
\hline 126 & & LASIA & ARTHUR & $\mathrm{s}$ & 6 & SOUTH & ITALY & ITALY & & & & 6TH ST. & ADAMS - TRIMOUNTAIN \\
\hline 127 & 35 & NUTINI & PETER & $\mathrm{H}$ & 29 & ITALY & ITALY & ITALY & 1921 & MINER/CM & CAPANNORI. LU & 6TH ST. & ADAMS - TRIMOUNTAIN \\
\hline 128 & & NUTINI & JENNIE & W & 23 & MICHIGAN & ITALY & ITALY & & & & 6TH ST. & ADAMS - TRIMOUNTAIN \\
\hline 129 & & NUTINI & TENACE & D & 4 & MICHIGAN & ITALY & MICHIGAN & & & CAPANNORI. LU & 6TH ST. & ADAMS - TRIMOUNTAIN \\
\hline 130 & & NUTINI & ORLANDO & $\mathrm{BR}$ & $48 ?$ & ITALY & ITALY & ITALY & 1919 & LABORER/CM & CAPANNORI. LU & 6TH ST. & ADAMS - TRIMOUNTAIN \\
\hline 131 & 306 & GIULI/GUILI & HENRY & $\mathrm{H}$ & 51 & ITALY & ITALY & ITALY & 1903 & FIREMAN/CM & LUCCA & 6TH ST. & ADAMS - TRIMOUNTAIN \\
\hline 132 & & GIULI/GUILI & ELISABETH & w & 49 & ITALY & ITALY & ITALY & 1907 & & & 6TH ST. & ADAMS - TRIMOUNTAIN \\
\hline 133 & & GIULI/GUILI & ARGANTE & s & 25 & MICHIGAN & ITALY & ITALY & & CARPENTER/CM & LUCCA & 6TH ST. & ADAMS - TRIMOUNTAIN \\
\hline 134 & & GIULI/GUILI & JEDO & $\mathrm{s}$ & 24 & MICHIGAN & ITALY & ITALY & & BLACKSMITH/CM & LUCCA & 6TH ST. & ADAMS - TRIMOUNTAIN \\
\hline 135 & & GIULI/GUILI & MARCO & $\mathrm{s}$ & 17 & MICHIGAN & ITALY & ITALY & & & LUCCA & 6TH ST. & ADAMS - TRIMOUNTAIN \\
\hline 136 & & GIULI/GUILI & DOMENICO & $\mathrm{s}$ & 12 & MICHIGAN & ITALY & ITALY & & & LUCCA & 6TH ST. & ADAMS - TRIMOUNTAIN \\
\hline 137 & & GIULI/GUILI & LOUIS & $\mathrm{s}$ & 10 & MICHIGAN & ITALY & ITALY & & & LUCCA & 6TH ST. & ADAMS - TRIMOUNTAIN \\
\hline 138 & 209 & & EUGENIO & $\mathrm{H}$ & 50 & ITALY & ITALY & ITALY & 1901 & MINER/CM & SANTA MARIA AL PIANO. LU & 7TH AVE. & ADAMS - TRIMOUNTAIN \\
\hline 139 & & GIANNECHINI & & w & 41 & ITALY & ITALY & ITALY & 1908 & & & 7TH AVE. & ADAMS - TRIMOUNTAIN \\
\hline 140 & & & ALLEN & s & 21 & MICHIGAN & ITALY & ITALY & & LABORER/CM & SANTA MARIA AL PIANO. LU & 7TH AVE. & ADAMS - TRIMOUNTAIN \\
\hline 141 & & GIANNECHINI & MARIO & $\mathrm{S}$ & 19 & MICHIGAN & ITALY & ITALY & & LABORER/CM & SANTA MARIA AL PIANO, LU & 7TH AVE. & ADAMS - TRIMOUNTAIN \\
\hline 142 & & GIANNECHINI & NELLA & $\mathrm{D}$ & 17 & MICHIGAN & ITALY & ITALY & & & SANTA MARIA AL PIANO. LU & 7TH AVE. & ADAMS - TRIMOUNTAIN \\
\hline 143 & & GIANNECHINI & GINA & $\mathrm{D}$ & 13 & MICHIGAN & ITALY & ITALY & & & SANTA MARIA AL PIANO. LU & 7TH AVE. & ADAMS - TRIMOUNTAIN \\
\hline 144 & 211 & NERI & AMOS & $\mathrm{H}$ & 45 & ITALY & ITALY & ITALY & 1910 & FIREMAN/CM & FUCECCHIO. FIRENZE & 7TH AVE. & ADAMS - TRIMOUNTAIN \\
\hline 145 & & NERI & ERNESTA & W & 42 & ITALY & ITALY & ITALY & 1911 & & FUCECCHIO. FIRENZE & 7TH AVE. & ADAMS - TRIMOUNTAIN \\
\hline 146 & & TADDEUCCI & LEA & $\mathrm{D}$ & 18 & ITALY & ITALY & ITALY & 1911 & & FUCECCHIO. FIRENZE & 7TH AVE. & ADAMS - TRIMOUNTAIN \\
\hline $\begin{array}{r}140 \\
147\end{array}$ & & TADDEUCCI & FRANK & SOIL & 21 & MICHIGAN & ITALY & ITALY & & BLACKSMITH/CM & $\begin{array}{l}\text { LUCCA } \\
\text { LUIV. FIRENLE }\end{array}$ & 7TH AVE. & ADAMS - TRIMOUNTAIN \\
\hline 148 & & NERI & RALPH & s & 19 & MICHIGAN & ITALY & ITALY & & & FUCECCHIO. FIRENZE & 7TH AVE. & ADAMS - TRIMOUNTAIN \\
\hline 149 & & NERI & INES? & $\mathrm{D}$ & 15 & MICHIGAN & ITALY & ITALY & & & FUCECCHIO. FIRENZE & 7TH AVE. & ADAMS - TRIMOUNTAIN \\
\hline 150 & & NERI & OMERO & $\mathrm{s}$ & 13 & MICHIGAN & ITALY & ITALY & & & FUCECCHIO. FIRENZE & 7TH AVE. & ADAMS - TRIMOUNTAIN \\
\hline 151 & & NERI & JANE & $\mathrm{D}$ & 10 & MICHIGAN & ITALY & ITALY & & & FUCECCHIO. FIRENZE & 7TH AVE. & ADAMS - TRIMOUNTAIN \\
\hline 152 & & NERI & GINO & $\mathrm{BR}$ & 43 & ITALY & ITALY & ITALY & 1911 & LABORER/CM & FUCECCHIO. FIRENZE & 7TH AVE. & ADAMS - TRIMOUNTAIN \\
\hline 153 & & FLOSI & LORENZO & B & 51 & ITALY & ITALY & ITALY & 1921 & MINER/CM & LUCCA & 7TH AVE. & ADAMS - TRIMOUNTAIN \\
\hline 154 & 216 & GARNERO & GILDO & $\mathrm{H}$ & 48 & ITALY & ITALY & ITALY & 1910 & ENGINEER MACHINERY/CM & TORINO & 7TH AVE. & ADAMS - TRIMOUNTAIN \\
\hline 155 & & GARNERO & LUCY & W & 42 & ITALY & ITALY & ITALY & 1912 & & & 7TH AVE. & ADAMS - TRIMOUNTAIN \\
\hline 156 & & GARNERO & MARY & $\mathrm{D}$ & 17 & MICHIGAN & ITALY & ITALY & & & TORINO & 7TH AVE. & ADAMS - TRIMOUNTAIN \\
\hline 157 & 303 & PIERONI & SAM & $\mathrm{H}$ & 38 & ITALY & ITALY & ITALY & 1905 & LABORER/CM & COLLE DI COMPITO. LU & DIVISION ST. & ADAMS - TRIMOUNTAIN \\
\hline 158 & 300 & PIERONI & MARY & W & 38 & ITALY & $\begin{array}{l}\text { ITALY } \\
\text { ITAL }\end{array}$ & ITALY & 1914 & LADURER/UIVI & LOLLE DT C UVITITU. LO & DIVISION ST. & ADAMS - TRIMOUNTAIN \\
\hline 159 & & PIERONI & CLARA & D & 14 & MICHIGAN & ITALY & ITALY & & & COLLE DI COMPITO, LU & DIVISION ST. & ADAMS - TRIMOUNTAIN \\
\hline 160 & & PIERONI & NICA & $\mathrm{D}$ & 13 & MICHIGAN & ITALY & ITALY & & & COLLE DI COMPITO. LU & DIVISION ST. & ADAMS - TRIMOUNTAIN \\
\hline 161 & & PIERONI & ERNEST & $\mathrm{s}$ & 10 & MICHIGAN & ITALY & ITALY & & & COLLE DI COMPITO. LU & DIVISION ST. & ADAMS - TRIMOUNTAIN \\
\hline 162 & & PIERONI & GILLIAN & $\mathrm{D}$ & 4 & MICHIGAN & ITALY & ITALY & & & COLLE DI COMPITO. LU & DIVISION ST. & ADAMS - TRIMOUNTAIN \\
\hline 163 & & PIERONI & GLORIA & D & $5 \mathrm{M}$ & MICHIGAN & ITALY & ITALY & & & COLLE DI COMPITO. LU & DIVISION ST. & ADAMS - TRIMOUNTAIN \\
\hline 164 & 307 & CORTOPASSI & SALVATORE & $\mathrm{H}$ & 38 & ITALY & ITALY & ITALY & 1911 & LABORER/CM & PESCAGLIA. LU & DIVISION ST. & ADAMS - TRIMOUNTAIN \\
\hline 165 & & CORTOPASSI & LUCIA & W & 37 & ITALY & ITALY & ITALY & 1912 & & & DIVISION ST. & ADAMS - TRIMOUNTAIN \\
\hline 166 & & CORTOPASSI & LENA & D & 16 & MICHIGAN & ITALY & ITALY & & & PESCAGLIA. LU & DIVISION ST. & ADAMS - TRIMOUNTAIN \\
\hline 167 & & CORTOPASSI & NORMA & $\mathrm{D}$ & 9 & MICHIGAN & ITALY & ITALY & & & PESCAGLIA, LU & DIVISION ST. & ADAMS - TRIMOUNTAIN \\
\hline 168 & & CORTOPASSI & JOHN & $\mathrm{s}$ & 7 & MICHIGAN & ITALY & ITALY & & & PESCAGLIA. LU & DIVISION ST. & ADAMS - TRIMOUNTAIN \\
\hline 169 & & CORTOPASSI & EDIA & $\mathrm{D}$ & $?$ & MICHIGAN & ITALY & ITALY & & & PESCAGLIA. LU & DIVISION ST. & ADAMS - TRIMOUNTAIN \\
\hline 170 & & DASANBIAGIO & $\mathrm{JOHN}$ & B & 30 & ITALY & ITALY & ITALY & 1920 & MINER/CM & COLLE DI COMPITO. LU & DIVISION ST. & ADAMS - TRIMOUNTAIN \\
\hline 171 & 311 & QUILICI & ANDELMO & $\mathrm{H}$ & 43 & ITALY & ITALY & ITALY & 1906 & FIREMAN/CM & LUCCA & DIVISION ST. & ADAMS - TRIMOUNTAIN \\
\hline 172 & & QUILICI & FAUSTINA & w & 33 & ITALY & ITALY & ITALY & 1922 & & & DIVISION ST. & ADAMS - TRIMOUNTAIN \\
\hline 173 & & QUILICI & ANNE & D & 17 & MICHIGAN & ITALY & ITALY & & & LUCCA & DIVISION ST. & ADAMS - TRIMOUNTAIN \\
\hline 174 & & QUILICI & JOSEPH & $\mathrm{s}$ & 14 & MICHIGAN & ITALY & ITALY & & & LUCCA & DIVISION ST. & \\
\hline 175 & & QUILICI & DORIA & $\mathrm{D}$ & 7 & MICHIGAN & ITALY & ITALY & & & LUCCA & DIVISION ST. & ADAMS - TRIMOUNTAIN \\
\hline 176 & 402 & MARGHERIO & JOHN & $\mathrm{H}$ & 46 & ITALY & ITALY & ITALY & 1907 & & SAN GIUSTO CANAVESE. TO & 15TH AVE. & ADAMS - TRIMOUNTAIN \\
\hline 177 & & MARGHERIO & MARY & W & 41 & ITALY & ITALY & ITALY & 1907 & & SAN GIUSTO CANAVESE, TO & 15TH AVE. & ADAMS - TRIMOUNTAIN \\
\hline 178 & & MARGHERIO & TONY & $\mathrm{s}$ & 16 & MICHIGAN & ITALY & ITALY & & LABORER/CM & SAN GIUSTO CANAVESE. TO & 15TH AVE. & ADAMS - TRIMOUNTAIN \\
\hline 179 & & MARGHERIO & JOHN & $\mathrm{s}$ & 14 & MICHIGAN & ITALY & ITALY & & & SAN GIUSTO CANAVESE. TO & 15TH AVE. & ADAMS - TRIMOUNTAIN \\
\hline 180 & & MARGHERIO & MENNIE & D & 8 & MICHIGAN & ITALY & ITALY & & & SAN GIUSTO CANAVESE. TO & 15TH AVE. & ADAMS - TRIMOUNTAIN \\
\hline 181 & 203 & NELLI & GIORGIO & $\mathrm{H}$ & 45 & ITALY & ITALY & ITALY & 1911 & MINER/CM & VALPROMARO. LU & 15TH AVE. & ADAMS - TRIMOUNTAIN \\
\hline 182 & & NELLI & SANTINA & W & 41 & ITALY & ITALY & ITALY & 1916 & & VALPROMARO. LU & 15TH AVE. & ADAMS - TRIMOUNTAIN \\
\hline 183 & & NELLI & GINO & s & 18 & ITALY & ITALY & ITALY & 1916 & & VALPROMARO, LU & 15TH AVE. & ADAMS - TRIMOUNTAIN \\
\hline 184 & & NELLI & PETER & $\mathrm{s}$ & 10 & MICHIGAN & ITALY & ITALY & & & VALPROMARO. LU & 15TH AVE. & ADAMS - TRIMOUNTAIN \\
\hline 185 & & NELLI & GIORGIA & $\mathrm{D}$ & 7 & MICHIGAN & ITALY & ITALY & & & VALPROMARO. LU & 15TH AVE. & ADAMS - TRIMOUNTAIN \\
\hline 186 & & FREDIANELLI & LOUIS & B & 45 & ITALY & ITALY & ITALY & 1907 & RER/CM & LUCCA & 15TH AVE. & ADAMS - TRIMOUNTAIN \\
\hline 187 & & CORTOPASSI & JOSEPH & B & 50 & ITALY & ITALY & ITALY & 1906 & & LUCCA & 15TH AVE. & ADAMS - TRIMOUNTAIN \\
\hline 188 & 701 & ADAMI & JOHN & $\mathrm{H}$ & 42 & ITALY & ITALY & ITALY & 1912 & MINER/CM & VILLA BASILICA. LU & 15TH AVE. & ADAMS - TRIMOUNTAIN \\
\hline
\end{tabular}




\begin{tabular}{|c|c|c|c|c|c|c|c|c|c|c|c|c|c|}
\hline 189 & & ADAMI & FLORA & W & 30 & ITALY & ITALY & ITALY & 1920 & & & 15TH AVE. & ADAMS - TRIMOUNTAIN \\
\hline 190 & & ADAMI & PRIMO & s & 7 & MICHIGAN & ITALY & ITALY & & & VILLA BASILICA. LU & 15TH AVE. & ADAMS - TRIMOUNTAIN \\
\hline 191 & & ADAMI & ELISABETH & D & 6 & MICHIGAN & ITALY & ITALY & & & VILLA BASILICA. LU & 15TH AVE. & ADAMS - TRIMOUNTAIN \\
\hline 192 & & ADAMI & DARIO & $\mathrm{s}$ & 4 & MICHIGAN & ITALY & ITALY & & & VILLA BASILICA. LU & 15TH AVE. & ADAMS - TRIMOUNTAIN \\
\hline 193 & & FIORELLI & ANDREW & B & 49 & ITALY & ITALY & ITALY & 1913 & LABORER/CM & & 15TH AVE. & ADAMS - TRIMOUNTAIN \\
\hline 194 & & CALAMASSI & JOSEPH & B & 44 & ITALY & ITALY & ITALY & 1907 & MINER/CM & FIRENZE & 15TH AVE. & ADAMS - TRIMOUNTAIN \\
\hline 195 & 711 & DE SANTI & ALBINO & $\mathrm{H}$ & 50 & ITALY & ITALY & ITALY & 1909 & WATCHMAN/CM & CASTELVECCHIO. LU & 15TH AVE. & ADAMS - TRIMOUNTAIN \\
\hline 196 & & DE SANTI & ELIDE & W & 45 & ITALY & ITALY & ITALY & 1919 & & & 15TH AVE. & ADAMS - TRIMOUNTAIN \\
\hline 197 & $311 ?$ & TADDEUCCI & JOSEPH & $\mathrm{H}$ & 50 & ITALY & ITALY & ITALY & 1903 & MINER/CM & LUCCA & 15TH AVE. & ADAMS - TRIMOUNTAIN \\
\hline 198 & & TADDEUCCI & FERNANDA & w & 45 & ITALY & ITALY & ITALY & 1904 & & & 15TH AVE. & ADAMS - TRIMOUNTAIN \\
\hline 199 & & TADDEUCCI & SALVATORE & s & 17 & MICHIGAN & ITALY & ITALY & & & LUCCA & 15TH AVE. & ADAMS - TRIMOUNTAIN \\
\hline 200 & & TADDEUCCI & ALFRED & $\mathrm{s}$ & 10 & MICHIGAN & ITALY & ITALY & & & LUCCA & 15TH AVE. & ADAMS - TRIMOUNTAIN \\
\hline 201 & & BARSOCCHI & SEVERINO & B & 49 & ITALY & ITALY & ITALY & 1904 & MINER/CM & LUCCA & 15TH AVE. & ADAMS - TRIMOUNTAIN \\
\hline 202 & & QUILICI & ALBERT & B & 41 & ITALY & ITALY & ITALY & 1920 & LABORER/CM & LUCCA & 15TH AVE. & ADAMS - TRIMOUNTAIN \\
\hline 203 & & MAFFEI & ARTURO & B & 47 & ITALY & ITALY & ITALY & 1923 & & LUCCA & 15TH AVE. & ADAMS - TRIMOUNTAIN \\
\hline 204 & & GIAMONI & AMERIGO & B & 45 & ITALY & ITALY & ITALY & 1917 & MINER/CM & LUCCA & 15TH AVE. & ADAMS - TRIMOUNTAIN \\
\hline 205 & & GIAMBASTIANI & PETER & B & 39 & ITALY & ITALY & ITALY & 1917 & MINER/CM & LUCCA & 15TH AVE. & ADAMS - TRIMOUNTAIN \\
\hline 206 & 4 & LOMBARDI & VITTORIO & $\mathrm{H}$ & 43 & ITALY & ITALY & ITALY & 1909 & FIREMAN/CM & LUCCA & 15TH AVE. & ADAMS - TRIMOUNTAIN \\
\hline 207 & & LOMBARDI & MARTA & W & 36 & ITALY & ITALY & ITALY & 1910 & & & 15TH AVE. & ADAMS - TRIMOUNTAIN \\
\hline 208 & & LOMBARDI & LOUIS & s & 18 & MICHIGAN & ITALY & ITALY & & OILER/CM & LUCCA & 15TH AVE. & ADAMS - TRIMOUNTAIN \\
\hline 209 & & LOMBARDI & FIORINO & $\mathrm{s}$ & 15 & MICHIGAN & ITALY & ITALY & & & LUCCA & 15TH AVE. & ADAMS - TRIMOUNTAIN \\
\hline 210 & & LOMBARDI & ELSIE & $\mathrm{D}$ & 10 & MICHIGAN & ITALY & ITALY & & & LUCCA & 15TH AVE. & ADAMS - TRIMOUNTAIN \\
\hline 211 & & LOMBARDI & MARY & $\mathrm{D}$ & 5 & MICHIGAN & ITALY & ITALY & & & LUCCA & 15TH AVE. & ADAMS - TRIMOUNTAIN \\
\hline 212 & & LOMBARDI & MARGHERITA & $\mathrm{D}$ & 4 & MICHIGAN & ITALY & ITALY & & & LUCCA & 15TH AVE. & ADAMS - TRIMOUNTAIN \\
\hline 213 & 5 & BARTOLOMEI & GIULIO & $\mathrm{H}$ & 43 & ITALY & ITALY & ITALY & 1911 & MINER/CM & LUCCA & 15TH AVE. & ADAMS - TRIMOUNTAIN \\
\hline 214 & 2 & PALMERO & FRANK & $\mathrm{H}$ & 49 & ITALY & ITALY & ITALY & 1904 & LABORER/CM & & 15TH AVE. & ADAMS - TRIMOUNTAIN \\
\hline 215 & & PALMERO & ELISABETH & w & 35 & ITALY & ITALY & ITALY & 1914 & & & 15TH AVE. & ADAMS - TRIMOUNTAIN \\
\hline 216 & & PALMERO & FRANK & s & 16 & MICHIGAN & ITALY & ITALY & & & & 15TH AVE. & ADAMS - TRIMOUNTAIN \\
\hline 217 & & PALMERO & DOMENICO & $\mathrm{S}$ & 14 & MICHIGAN & ITALY & ITALY & & & & 15TH AVE. & ADAMS - TRIMOUNTAIN \\
\hline 218 & & PALMERO & ANNE & $\mathrm{D}$ & 7 & MICHIGAN & ITALY & ITALY & & & & 15TH AVE. & ADAMS - TRIMOUNTAIN \\
\hline 219 & & PALMERO & ALBERT & $\mathrm{s}$ & 5 & MICHIGAN & ITALY & ITALY & & & & 15TH AVE. & ADAMS - TRIMOUNTAIN \\
\hline 220 & & PALMERO & ALDO & $\mathrm{s}$ & 3 & MICHIGAN & ITALY & ITALY & & & & 15TH AVE. & ADAMS - TRIMOUNTAIN \\
\hline 221 & 4 & $\mathrm{CIUCCl}$ & CASPER & $\mathrm{H}$ & 58 & ITALY & ITALY & ITALY & 1909 & & LUCCA & 15TH AVE. & ADAMS - TRIMOUNTAIN \\
\hline 222 & & $\mathrm{ClUCCl}$ & MARION & W & 52 & ITALY & ITALY & ITALY & 1917 & & & 15TH AVE. & ADAMS - TRIMOUNTAIN \\
\hline 223 & 710 & PERGOLA & GIULIO & $\mathrm{H}$ & 36 & ITALY & ITALY & ITALY & 1915 & MINER/CM & SEGROMIGNO. LU & & ADAMS - TRIMOUNTAIN \\
\hline 224 & 114 & ROMANINI & ERIC & $\mathrm{H}$ & 48 & ITALY & ITALY & ITALY & 1905 & MINER/CM & LUCCA & 12TH AVE. & ADAMS - BALTIC \\
\hline 225 & & ROMANINI & TERESA & W & 40 & ITALY & ITALY & ITALY & 1912 & & & 12TH AVE. & ADAMS - BALTIC \\
\hline 226 & & NELLI & OVIDIO & ss & 16 & MICHIGAN & ITALY & ITALY & & & MONSACRATI. LU & 12TH AVE. & ADAMS - BALTIC \\
\hline 227 & & NELLI & NILO & ss & 13 & MICHIGAN & ITALY & ITALY & & & MONSACRATI. LU & 12TH AVE. & ADAMS - BALTIC \\
\hline 228 & & NELLI & ALMA & SD & 10 & MICHIGAN & ITALY & ITALY & & & MONSACRATI. LU & 12TH AVE. & ADAMS - BALTIC \\
\hline 229 & & ROMANINI & ALBERT & s & 4 & MICHIGAN & ITALY & ITALY & & & LUCCA & 12TH AVE. & ADAMS - BALTIC \\
\hline 230 & & MARTINELLI & BERNARD & B & 41 & ITALY & ITALY & ITALY & 1924 & & CAPANNORI. LU & 12TH AVE. & ADAMS - BALTIC \\
\hline 231 & & PIZZI & JOSEPH & B & 40 & ITALY & ITALY & ITALY & 1911 & MINER/CM & LUCCA & 12TH AVE. & ADAMS - BALTIC \\
\hline 232 & & COMINA & BATTISTA & B & 40 & ITALY & ITALY & ITALY & 1911 & MINER/CM & BELLUNO & 12TH AVE. & ADAMS - BALTIC \\
\hline 233 & 122 & GIACOLETTO & VITTORIO & $\mathrm{H}$ & 51 & ITALY & ITALY & ITALY & 1903 & MINER/CM & TORINO & 13TH AVE. & ADAMS - BALTIC \\
\hline 234 & 122 & GIACOLETTO & KATE & W & 52 & ITALY & ITALY & ITALY & 1903 & & TORINO & 13TH AVE. & ADAMS - BALTIC \\
\hline 235 & & GIACOLETTO & LUCILLE & $\mathrm{D}$ & 23 & MICHIGAN & ITALY & ITALY & & TEACHER/PUBLIC SCHOOL & TORINO & 13TH AVE. & ADAMS - BALTIC \\
\hline 236 & & GIACOLETTO & PAUL & $\mathrm{s}$ & 18 & MICHIGAN & ITALY & ITALY & & & TORINO & 13TH AVE. & ADAMS - BALTIC \\
\hline 237 & & GIACOLETTO & JOHN & $\mathrm{s}$ & 15 & MICHIGAN & ITALY & ITALY & & & TORINO & 13TH AVE. & ADAMS - BALTIC \\
\hline 238 & 220 & CERUTTI & ANTONIO & $\mathrm{H}$ & 38 & ITALY & ITALY & ITALY & 1924 & LABORER/CM & SAN GIORGIO CANAVESE. TO & 13TH AVE. & ADAMS - BALTIC \\
\hline 239 & & CERUTTI & KATE & W & 38 & SOUTH & ITALY & ITALY & & & & 13TH AVE. & ADAMS - BALTIC \\
\hline 240 & & CERUTTI & IRMA & D & 8 & SOUTH & ITALY & SOUTH & & & SAN GIORGIO CANAVESE, TO & 13TH AVE. & ADAMS - BALTIC \\
\hline 241 & & CERUTTI & GIORGIO & $\mathrm{s}$ & 7 & SOUTH & ITALY & SOUTH & & & SAN GIORGIO CANAVESE. TO & 13TH AVE. & ADAMS - BALTIC \\
\hline 242 & & CERUTTI & ALMA & D & 5 & MICHIGAN & ITALY & SOUTH & & & SAN GIORGIO CANAVESE. TO & 13TH AVE. & ADAMS - BALTIC \\
\hline 243 & & CERUTTI & JOHN & $\mathrm{s}$ & 4 & MICHIGAN & ITALY & SOUTH & & & SAN GIORGIO CANAVESE. TO & 13TH AVE. & ADAMS - BALTIC \\
\hline 244 & 218 & CORTESE & LUIGI & $\mathrm{H}$ & 52 & ITALY & ITALY & ITALY & 1894 & MINER/CM & PARELLA. TO & 13TH AVE. & ADAMS - BALTIC \\
\hline 245 & & CORTESE & MARY & W & 47 & ITALY & ITALY & ITALY & 1898 & & & 13TH AVE. & ADAMS - BALTIC \\
\hline 246 & & CORTESE & JAMES & s & 21 & MICHIGAN & ITALY & ITALY & & SALESMAN/GASOLINE STATION & PARELLA, TO & 13TH AVE. & ADAMS - BALTIC \\
\hline 247 & & CORTESE & MADDALENA & $\mathrm{D}$ & 20 & MICHIGAN & ITALY & ITALY & & SALESMAN/CANDY STORE & PARELLA. TO & 13TH AVE. & ADAMS - BALTIC \\
\hline 248 & 217 & FAUSONE & JAMES & $\mathrm{H}$ & 61 & ITALY & ITALY & ITALY & 1893 & MINER/CM & TORINO & 13TH AVE. & ADAMS - BALTIC \\
\hline 249 & & FAUSONE & ANGELA & w & 55 & ITALY & ITALY & ITALY & 1901 & & & 13TH AVE. & ADAMS - BALTIC \\
\hline 250 & & FAUSONE & JAMES & $\mathrm{s}$ & 27 & MICHIGAN & ITALY & ITALY & & & TORINO & 13TH AVE. & ADAMS - BALTIC \\
\hline 251 & & FAUSONE & JOSEPH & $\mathrm{s}$ & 25 & MICHIGAN & ITALY & ITALY & & MECHANIC/MECHANICAL & TORINO & 13TH AVE. & ADAMS - BALTIC \\
\hline
\end{tabular}




\begin{tabular}{|c|c|c|c|c|c|c|c|c|c|c|c|c|c|}
\hline 252 & & FAUSONE & ASTLER & $\mathrm{s}$ & 21 & MICHIGAN & ITALY & ITALY & & MINER/CM & TORINO & 13TH AVE. & ADAMS - BALTIC \\
\hline 253 & & FAUSONE & MARY & D & 18 & MICHIGAN & ITALY & ITALY & & & TORINO & 13TH AVE. & ADAMS - BALTIC \\
\hline 254 & & FAUSONE & ANTON & $\mathrm{s}$ & 15 & MICHIGAN & ITALY & ITALY & & & TORINO & 13TH AVE. & ADAMS - BALTIC \\
\hline 255 & 116 & PIEROZZI & FRANK & $\mathrm{H}$ & 62 & ITALY & ITALY & ITALY & 1907 & MINER/CM & LUCCA & 13TH AVE. & ADAMS - BALTIC \\
\hline 256 & & PIEROZZI & AMELIA & W & 52 & ITALY & ITALY & ITALY & 1911 & & & 13TH AVE. & ADAMS - BALTIC \\
\hline 257 & & PIEROZZI & LOUIS & $\mathrm{s}$ & 17 & MICHIGAN & ITALY & ITALY & & & LUCCA & 13TH AVE. & ADAMS - BALTIC \\
\hline 258 & & PIEROZZI & JOHN & $\mathrm{s}$ & 11 & MICHIGAN & ITALY & ITALY & & & LUCCA & 13TH AVE. & ADAMS - BALTIC \\
\hline 259 & 102 & DEL GHINGARO & FRANK & $\mathrm{H}$ & 27 & MICHIGAN & ITALY & ITALY & & MINER/CM & LUCCA & 13TH AVE. & ADAMS - BALTIC \\
\hline 260 & 102 & DEL GHINGARO & PAULINE & w & 23 & MICHIGAN & ITALY & ITALY & & 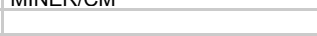 & LUCUA & 13TH AVE. & ADAMS - BALTIC \\
\hline 261 & & DEL GHINGARO & JUAN & s & 2 & MICHIGAN & MICHIGAN & MICHIGAN & & & LUCCA & 13TH AVE. & ADAMS - BALTIC \\
\hline 262 & & DEL GHINGARO & FRANK & $\mathrm{s}$ & 1 & MICHIGAN & MICHIGAN & MICHIGAN & & & LUCCA & 13TH AVE. & ADAMS - BALTIC \\
\hline 263 & 111 & GENOVESI & OLIVIERO & $\mathrm{H}$ & 35 & ITALY & ITALY & ITALY & 1911 & LABORER/CM & VILLA BASILICA, LU & 14TH AVE. & ADAMS - BALTIC \\
\hline 264 & & GENOVESI & ANNA & W & 30 & WYOMING & ITALY & ITALY & & & & 14TH AVE. & ADAMS - BALTIC \\
\hline 265 & & GENOVESI & WILLIAM & $\mathrm{s}$ & 14 & WYOMING & ITALY & ITALY & & & VILLA BASILICA. LU & 14TH AVE. & ADAMS - BALTIC \\
\hline 266 & & GENOVESI & ARNOLD & $\mathrm{s}$ & 11 & MICHIGAN & ITALY & ITALY & & & VILLA BASILICA, LU & 14TH AVE. & ADAMS - BALTIC \\
\hline 267 & & GENOVESI & EDITH & D & 6 & MICHIGAN & ITALY & ITALY & & & VILLA BASILICA, LU & 14TH AVE. & ADAMS - BALTIC \\
\hline 268 & & GENOVESI & ILARIA & D & 2 & MICHIGAN & ITALY & ITALY & & & VILLA BASILICA. LU & 14TH AVE. & ADAMS - BALTIC \\
\hline 269 & 113 & PERFETTI & LOUIS & $\mathrm{H}$ & 49 & ITALY & ITALY & ITALY & 1910 & LABORER/CM & LOPPEGLIA. LU & 14TH AVE. & ADAMS - BALTIC \\
\hline 270 & & PERFETTI & ARMIDA & W & 45 & ITALY & ITALY & ITALY & 1913 & & & 14TH AVE. & ADAMS - BALTIC \\
\hline 271 & & PERFETTI & CASIMIRO & $\mathrm{s}$ & 15 & MICHIGAN & ITALY & ITALY & & & LOPPEGLIA. LU & 14TH AVE. & ADAMS - BALTIC \\
\hline 272 & & PERFETTI & ASSUNTA & $\mathrm{D}$ & 14 & MICHIGAN & ITALY & ITALY & & & LOPPEGLIA. LU & 14TH AVE. & ADAMS - BALTIC \\
\hline 273 & & PERFETTI & MAFALDA & D & 13 & MICHIGAN & ITALY & ITALY & & & $\begin{array}{l}\text { LOPPEGLIA, LU } \\
\text { LOPE }\end{array}$ & 14TH AVE. & ADAMS - BALTIC \\
\hline 274 & & PERFETTI & MARIO & $\mathrm{s}$ & 10 & MICHIGAN & ITALY & ITALY & & & LOPPEGLIA. LU & 14TH AVE. & ADAMS - BALTIC \\
\hline 275 & & PERFETTI & EVA & D & 4 & MICHIGAN & ITALY & ITALY & & & LOPPEGLIA. LU & 14TH AVE. & ADAMS - BALTIC \\
\hline 276 & 117 & COMINA & DAVID & $\mathrm{H}$ & 39 & ITALY & ITALY & ITALY & 1914 & LABORER/CM & BELLUNO & 14TH AVE. & ADAMS - BALTIC \\
\hline 277 & & COMINA & AMELIA & $\mathrm{D}$ & 16 & MICHIGAN & ITALY & ITALY & & & & 14TH AVE. & ADAMS - BALTIC \\
\hline 278 & & COMINA & JOSEPH & $\mathrm{s}$ & 13 & MICHIGAN & ITALY & ITALY & & & BELLUNO & 14TH AVE. & ADAMS - BALTIC \\
\hline 279 & & COMINA & MARGHERITA & D & 9 & MICHIGAN & ITALY & ITALY & & & BELLUNO & 14TH AVE. & ADAMS - BALTIC \\
\hline 280 & & COMINA & ANGELO & $\mathrm{S}$ & 7 & MICHIGAN & ITALY & ITALY & & & BELLUNO & 14TH AVE. & ADAMS - BALTIC \\
\hline 281 & & COMINA & ROSE & $\mathrm{D}$ & 3 & MICHIGAN & ITALY & ITALY & & & BELLUNO & 14TH AVE. & ADAMS - BALTIC \\
\hline 282 & 205 & ANDREINI & EMILIO & $\mathrm{H}$ & 63 & ITALY & ITALY & ITALY & 1898 & & CASTAGNARI. LU & 14TH AVE. & ADAMS - BALTIC \\
\hline 283 & & ANDREINI & MARGHERITA & W & 53 & ITALY & ITALY & ITALY & 1900 & & & 14TH AVE. & ADAMS - BALTIC \\
\hline 284 & & ANDREINI & $\begin{array}{l}\text { PAUL } \\
\text { PALTIA }\end{array}$ & s & 21 & MICHIGAN & $\begin{array}{l}\text { ITALY } \\
\text { ITAL }\end{array}$ & ITALY & 1500 & DRIVER BUS/AUTOMOBILE & CASTAGNARI. LU & 14TH AVE. & ADAMS - BALTIC \\
\hline 285 & & ANDREINI & MAFALDA & D & 15 & MICHIGAN & ITALY & ITALY & & & CASTAGNARI, LU & 14TH AVE. & ADAMS - BALTIC \\
\hline 286 & & ANDREINI & IOLANDA & $\mathrm{D}$ & 13 & MICHIGAN & ITALY & ITALY & & & CASTAGNARI. LU & 14TH AVE. & ADAMS - BALTIC \\
\hline 287 & & ANDREINI & WILLIAM & $\mathrm{s}$ & 23 & MICHIGAN & ITALY & ITALY & & DRIVER BUS/AUTOMOBILE & CASTAGNARI. LU & 14TH AVE. & ADAMS - BALTIC \\
\hline 288 & & ANDREINI & MELBA & $\mathrm{D}$ & 16 & MICHIGAN & ITALY & ITALY & & & CASTAGNARI. LU & 14TH AVE. & ADAMS - BALTIC \\
\hline 289 & 204 & RAFFAELLI & GIULIA & $\mathrm{H}$ & 44 & ITALY & ITALY & ITALY & 1906 & & LUCCA & 14TH AVE. & ADAMS - BALTIC \\
\hline 290 & & RAFFAELLI & IRENE & D & 20 & MICHIGAN & ITALY & ITALY & & SERVANT/PF & & 14TH AVE. & ADAMS - BALTIC \\
\hline 291 & & RAFFAELLI & JOSEPH & $\mathrm{s}$ & 19 & MICHIGAN & ITALY & ITALY & & LABORER/CM & LUCCA & 14TH AVE. & ADAMS - BALTIC \\
\hline 292 & & RAFFAELLI & GUIDO & $\mathrm{s}$ & 14 & MICHIGAN & ITALY & ITALY & & & LUCCA & 14TH AVE. & ADAMS - BALTIC \\
\hline 293 & & RAFFAELLI & MARIE & D & 11 & MICHIGAN & ITALY & ITALY & & & LUCCA & 14TH AVE. & ADAMS - BALTIC \\
\hline 294 & & RAFFAELLI & ROSE & D & 9 & MICHIGAN & ITALY & ITALY & & & LUCCA & 14TH AVE. & ADAMS - BALTIC \\
\hline 295 & 213 & VENTURINI & FRANK & $\mathrm{H}$ & 46 & ITALY & ITALY & ITALY & 1908 & LABORER/CM & CAMAIORE. LU & 14TH AVE. & ADAMS - BALTIC \\
\hline 296 & & VENTURINI & CHOCHE & W & 51 & ITALY & ITALY & ITALY & 1901 & & & 14TH AVE. & ADAMS - BALTIC \\
\hline 297 & & VENTURINI & JULIO & s & 17 & MICHIGAN & ITALY & ITALY & & & CAMAIORE. LU & 14TH AVE. & ADAMS - BALTIC \\
\hline 298 & & VENTURINI & PASQUALE & $\mathrm{s}$ & 12 & MICHIGAN & ITALY & ITALY & & & CAMAIORE. LU & 14TH AVE. & ADAMS - BALTIC \\
\hline 299 & 225 & VALENTINI & JAMES & $\mathrm{H}$ & 48 & ITALY & ITALY & ITALY & 1906 & MINER/CM & ASCOLI PICENO & 14TH AVE. & ADAMS - BALTIC \\
\hline 300 & & VALENTINI & NATALINA & W & 46 & ITALY & ITALY & ITALY & 1910 & & & 14TH AVE. & ADAMS - BALTIC \\
\hline 301 & & VALENTINI & EMILIO & s & 21 & MICHIGAN & ITALY & ITALY & & LABORER/CM & ASCOLI PICENO & 14TH AVE. & ADAMS - BALTIC \\
\hline 302 & & VALENTINI & JOSEPH & $\mathrm{s}$ & 20 & MICHIGAN & ITALY & ITALY & & LABORER/CM & ASCOLI PICENO & 14TH AVE. & ADAMS - BALTIC \\
\hline 303 & & VALENTINI & GINO & $\mathrm{s}$ & 18 & MICHIGAN & ITALY & ITALY & & LABORER/CM & ASCOLI PICENO & 14TH AVE. & ADAMS - BALTIC \\
\hline 304 & & VALENTINI & PETER & $\mathrm{s}$ & 14 & MICHIGAN & ITALY & ITALY & & & ASCOLI PICENO & 14TH AVE. & ADAMS - BALTIC \\
\hline 305 & & VALENTINI & DOROTHY & D & 10 & MICHIGAN & ITALY & ITALY & & & ASCOLI PICENO & 14TH AVE. & ADAMS - BALTIC \\
\hline 306 & & VALENTINI & JULIO & $\mathrm{s}$ & 4 & MICHIGAN & ITALY & ITALY & & & ASCOLI PICENO & 14TH AVE. & ADAMS - BALTIC \\
\hline 307 & 208 & BARBERIS & DOMENICO & $\mathrm{H}$ & 49 & ITALY & ITALY & ITALY & 1901 & MINER/CM & RIVAROLO CANAVESE. TO & 14TH AVE. & ADAMS - BALTIC \\
\hline 308 & & BARBERIS & MARY & W & 44 & ITALY & ITALY & ITALY & 1901 & & RIVAROLO CANAVESE. TO & 14TH AVE. & ADAMS - BALTIC \\
\hline 309 & & BARBERIS & ANGELO & $\mathrm{s}$ & 23 & MICHIGAN & ITALY & ITALY & & LABORER/CM & RIVAROLO CANAVESE, TO & 14TH AVE. & ADAMS - BALTIC \\
\hline 310 & & BARBERIS & DOMENICO & $\mathrm{s}$ & 21 & MICHIGAN & ITALY & ITALY & & LABORER/CM & RIVAROLO CANAVESE. TO & 14TH AVE. & ADAMS - BALTIC \\
\hline 311 & & BARBERIS & $\mathrm{JOHN}$ & $\mathrm{s}$ & 18 & MICHIGAN & ITALY & ITALY & & LABORER/CM & RIVAROLO CANAVESE. TO & 14TH AVE. & ADAMS - BALTIC \\
\hline 312 & 122 & GIANNELLI & JOHN & $\mathrm{H}$ & 40 & ITALY & ITALY & ITALY & 1915 & MANAGER/BOARDING HOUSE & CASTAGNORI. LU & 14TH AVE. & ADAMS - BALTIC \\
\hline 313 & & GIANNELLI & IOLANDA & D & 17 & ITALY & ITALY & ITALY & 1915 & & CASTAGNORI. LU & 14TH AVE. & ADAMS - BALTIC \\
\hline 314 & & GIANNELLI & RALPH & $\mathrm{s}$ & 14 & MICHIGAN & ITALY & ITALY & & & CASTAGNORI. LU & 14TH AVE. & ADAMS - BALTIC \\
\hline
\end{tabular}




\begin{tabular}{|c|c|c|c|c|c|c|c|c|c|c|c|c|c|}
\hline 315 & & GIANNELLI & GUIDO & $\mathrm{s}$ & 11 & MICHIGAN & ITALY & ITALY & & & CASTAGNORI. LU & 14TH AVE. & ADAMS - BALTIC \\
\hline 316 & & GIANNELLI & IRMA & $\mathrm{D}$ & 7 & MICHIGAN & ITALY & ITALY & & & CASTAGNORI. LU & 14TH AVE. & ADAMS - BALTIC \\
\hline 317 & & D'AGOSTINI & FRANK & B & 43 & ITALY & ITALY & ITALY & 1899 & MINER/CM & BELLUNO & 14TH AVE. & ADAMS - BALTIC \\
\hline 318 & 109 & CAPPO & CESARE & $\mathrm{H}$ & 41 & ITALY & ITALY & ITALY & 1906 & FOREMAN/CM & SAN GIUSTO CANAVESE. TO & 15TH AVE. & ADAMS - BALTIC \\
\hline 319 & & CAPPO & JENNIE & w & 31 & ITALY & ITALY & ITALY & 1909 & & & 15TH AVE. & ADAMS - BALTIC \\
\hline 320 & & CAPPO & ANNA & D & 16 & MICHIGAN & ITALY & ITALY & & & SAN GIUSTO CANAVESE. TO & 15TH AVE. & ADAMS - BALTIC \\
\hline 321 & & CAPPO & JOSEPH & $\mathrm{s}$ & 14 & MICHIGAN & ITALY & ITALY & & & SAN GIUSTO CANAVESE. TO & 15TH AVE. & ADAMS - BALTIC \\
\hline 322 & & CAPPO & DARIO & $\mathrm{s}$ & 10 & MICHIGAN & ITALY & ITALY & & & SAN GIUSTO CANAVESE. TO & 15TH AVE. & ADAMS - BALTIC \\
\hline 323 & & CAPPO & CESARE & $\mathrm{S}$ & 6 & MICHIGAN & ITALY & ITALY & & & SAN GIUSTO CANAVESE. TO & 15TH AVE. & ADAMS - BALTIC \\
\hline 324 & & CAPPO & EUGENIO & $\mathrm{s}$ & 5 & MICHIGAN & ITALY & ITALY & & & SAN GIUSTO CANAVESE. TO & 15TH AVE. & ADAMS - BALTIC \\
\hline 325 & 121 & DATTO & PAUL? & $\mathrm{H}$ & 37 & MICHIGAN & ITALY & ITALY & & LABORER/CM & TORINO & 15TH AVE. & ADAMS - BALTIC \\
\hline 326 & & DATTO & MARY & w & 43 & ITALY & ITALY & ITALY & 1908 & & TORINO & 15TH AVE. & ADAMS - BALTIC \\
\hline 327 & & BALMA/BAIMA & JOHN & sso & 23 & ITALY & ITALY & ITALY & 1908 & LABORER/CM & TORINO & 15TH AVE. & ADAMS - BALTIC \\
\hline 328 & & BALMA/BAIMA & LOUIS & sso & & MICHIGAN & ITALY & ITALY & & LABORER/CM & TORINO & 15TH AVE. & ADAMS - BALTIC \\
\hline 329 & & BALMA/BAIMA & GUIDO & sSO & 17 & MICHIGAN & ITALY & ITALY & & & TORINO & 15TH AVE. & ADAMS - BALTIC \\
\hline 330 & & DATTO & TONY & s & 14 & MICHIGAN & MICHIGAN & ITALY & & & TORINO & 15TH AVE. & ADAMS - BALTIC \\
\hline 331 & 161 & RAFFAELLI & FRANK & $\mathrm{H}$ & 47 & ITALY & ITALY & ITALY & 1903 & MINER/CM & COLLODI. LU & 16TH AVE. & ADAMS - BALTIC \\
\hline 332 & & RAFFAELLI & JULIA & W & 45 & ITALY & ITALY & ITALY & 1903 & & COLLODI. LU & 16TH AVE. & ADAMS - BALTIC \\
\hline 333 & & RAFFAELLI & LENA & $\mathrm{D}$ & 20 & MICHIGAN & ITALY & ITALY & & SERVANT/PF & COLLODI. LU & 16TH AVE. & ADAMS - BALTIC \\
\hline 334 & & RAFFAELLI & BRUNO & $\mathrm{s}$ & 18 & MICHIGAN & ITALY & ITALY & & & COLLODI. LU & 16TH AVE. & ADAMS - BALTIC \\
\hline 335 & & RAFFAELLI & MARIA & $\mathrm{D}$ & 16 & MICHIGAN & ITALY & ITALY & & & COLLODI. LU & 16TH AVE. & ADAMS - BALTIC \\
\hline 336 & & RAFFAELLI & FRED & $\mathrm{s}$ & 13 & MICHIGAN & ITALY & ITALY & & & COLLODI, LU & 16TH AVE. & ADAMS - BALTIC \\
\hline 337 & & RAFFAELLI & JOSEPHINE & $\mathrm{D}$ & 11 & MICHIGAN & ITALY & ITALY & & & COLLODI. LU & 16TH AVE. & ADAMS - BALTIC \\
\hline 338 & & RAFFAELLI & JULIO & $\mathrm{s}$ & 5 & MICHIGAN & ITALY & ITALY & & & COLLODI. LU & 16TH AVE. & ADAMS - BALTIC \\
\hline 339 & 224 & CARLI & FRED & $\mathrm{H}$ & 50 & ITALY & ITALY & ITALY & 1911 & MINER/CM & CAPANNORI. LU & 16TH AVE. & ADAMS - BALTIC \\
\hline 340 & & CARLI & ERMINIA & W & 42 & ITALY & ITALY & ITALY & 1911 & & CAPANNORI. LU & 16TH AVE. & ADAMS - BALTIC \\
\hline 341 & & CARLI & RITA & $\mathrm{D}$ & 17 & ILLINOIS & ITALY & ITALY & & & CAPANNORI. LU & 16TH AVE. & ADAMS - BALTIC \\
\hline 342 & & CARLI & ALFRED & $\mathrm{s}$ & 15 & ILLINOIS & ITALY & ITALY & & & CAPANNORI, LU & 16TH AVE. & ADAMS - BALTIC \\
\hline 343 & & CARLI & EMIL & $\mathrm{S}$ & 13 & MICHIGAN & ITALY & ITALY & & & CAPANNORI. LU & 16TH AVE. & ADAMS - BALTIC \\
\hline 344 & & CARLI & MATT & $\mathrm{s}$ & 6 & MICHIGAN & ITALY & ITALY & & & CAPANNORI. LU & 16TH AVE. & ADAMS - BALTIC \\
\hline 345 & 226 & SERRA & MARY & $\mathrm{H}$ & 64 & ITALY & ITALY & ITALY & 1895 & & & 16TH AVE. & ADAMS - BALTIC \\
\hline 346 & & SERRA & LENA & D & 27 & MICHIGAN & ITALY & ITALY & 1000 & & SAN GIORGIO CANAVESE. TO & 16TH AVE. & ADAMS - BALTIC \\
\hline 347 & & FURNO & PETER & B & 35 & ITALY & $\begin{array}{l}\text { ITALY } \\
\text { ITA }\end{array}$ & ITALY & 1913 & MINER/CM & $\begin{array}{l}\text { TORINO } \\
\text { TORIVAVE. TO }\end{array}$ & 16TH AVE. & ADAMS - BALTIC \\
\hline 348 & & CENTANINO & JOSEPH & B & 52 & ITALY & $\begin{array}{l}\text { ITALY } \\
\text { ITAL }\end{array}$ & ITALY & 1901 & MINER/CM & SAN MARTINO CANAVESE, TO & 16TH AVE. & ADAMS - BALTIC \\
\hline 349 & & GUIZZETTI & ANTONIO & $\mathrm{H}$ & 34 & ITALY & ITALY & ITALY & 1897 & MINER/CM & MILANO & 1ST ST. & ADAMS - ATLANTIC MINE \\
\hline 350 & & GUIZZETTI & LEMPI & w & 29 & FINLAND & FINLAND & FINLAND & 1909 & & FINLAND & 1ST ST. & ADAMS - ATLANTIC MINE \\
\hline 351 & & GUIZZETTI & ANDREW & s & 11 & MICHIGAN & ITALY & ITALY & & & MILANO & 1ST ST. & ADAMS - ATLANTIC MINE \\
\hline 352 & & GUIZZETTI & JOHN & $\mathrm{s}$ & 10 & MICHIGAN & ITALY & ITALY & & & MILANO & 1ST ST. & ADAMS - ATLANTIC MINE \\
\hline 353 & & GUIZZETTI & ANITA & $\mathrm{D}$ & 6 & MICHIGAN & ITALY & ITALY & & & MILANO & 1ST ST. & ADAMS - ATLANTIC MINE \\
\hline 354 & & GUIZZETTI & MARY & $\mathrm{D}$ & 4 & MICHIGAN & ITALY & ITALY & & & MILANO & 1ST ST. & ADAMS - ATLANTIC MINE \\
\hline 355 & & BIANUCCI & JOSEPH & $\mathrm{H}$ & 38 & ITALY & ITALY & ITALY & 1894 & ELECTRICAL ENGINEER/SAW & ALTOPASCIO. LU & 2ND ST. & ADAMS - ATLANTIC MINE \\
\hline 356 & & BIANUCCI & ESTER & w & 33 & MICHIGAN & POLAND & POLAND & & & & 2ND ST. & ADAMS - ATLANTIC MINE \\
\hline 357 & & BIANUCCI & FLORENCE & $\mathrm{D}$ & 10 & MICHIGAN & ITALY & MICHIGAN & & & ALTOPASCIO. LU & 2ND ST. & ADAMS - ATLANTIC MINE \\
\hline 358 & 70 & LAITY & RICHARD & $\mathrm{H}$ & 47 & ENGLAND & ENGLAND & ENGLAND & 1904 & MINER/CM & ENGLAND & 2ND ST. & ADAMS - ATLANTIC MINE \\
\hline 359 & & LAITY & ANTONIETTA & w & 34 & ITALY & ITALY & ITALY & 1897 & 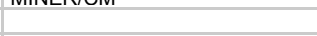 & LTV ULTIVD & 2ND ST. & ADAMS - ATLANTIC MINE \\
\hline 360 & & LAITY & ELEONOR & $\mathrm{D}$ & 14 & MICHIGAN & ITALY & ITALY & & & & 2ND ST. & ADAMS - ATLANTIC MINE \\
\hline 361 & & LAITY & DOROTHY & D & 13 & MICHIGAN & ITALY & ITALY & & & & 2ND ST. & ADAMS - ATLANTIC MINE \\
\hline 362 & 31 & BIANUCCI & FLORINDA & $\mathrm{H}$ & 64 & ITALY & ITALY & ITALY & 1894 & & & FRONT ST. & ADAMS - ATLANTIC MINE \\
\hline 363 & & BIANUCCI & ARTHUR & $\mathrm{s}$ & 40 & ITALY & ITALY & ITALY & 1894 & AUTO REPAIRER MAN/BUS & ALTOPASCIO. LU & FRONT ST. & ADAMS - ATLANTIC MINE \\
\hline 364 & & BIANUCCI & ALFRED & $\mathrm{s}$ & 23 & MICHIGAN & ITALY & ITALY & & ENGINEER/SAW MILL & ALTOPASCIO. LU & FRONT ST. & ADAMS - ATLANTIC MINE \\
\hline 365 & $191 ?$ & $\mathrm{ClUCCl}$ & ALFRED & $\mathrm{H}$ & 27 & MICHIGAN & ITALY & ITALY & & LABORER/HANDLE FACTORY & MATRAIA. LU & & ADAMS - ATLANTIC MINE \\
\hline 366 & & $\mathrm{ClUCCl}$ & FLORENCE & W & 20 & ITALY & ITALY & ITALY & $14 ? ?$ & & & & ADAMS - ATLANTIC MINE \\
\hline 367 & & $\mathrm{ClUCCl}$ & ALFRED & $\mathrm{s}$ & 0 & MICHIGAN & MICHIGAN & ITALY & & & MATRAIA. LU & & ADAMS - ATLANTIC MINE \\
\hline 368 & 175 & CHIMINO & JOHN & $\mathrm{H}$ & 38 & MICHIGAN & ITALY & ITALY & & LUMBER GRADER/SAW MILL & SAN GIORGIO CANAVESE. TO & & ADAMS - ATLANTIC MINE \\
\hline 369 & & CHIMINO & AMELIA & w & 37 & MICHIGAN & ITALY & ITALY & & & & & ADAMS - ATLANTIC MINE \\
\hline 370 & & CHIMINO & JOSEPHINE & $\mathrm{D}$ & 10 & MICHIGAN & MICHIGAN & MICHIGAN & & & SAN GIORGIO CANAVESE. TO & & ADAMS - ATLANTIC MINE \\
\hline 371 & & CHIMINO & DAVID & $\mathrm{s}$ & 4 & MICHIGAN & MICHIGAN & MICHIGAN & & & SAN GIORGIO CANAVESE. TO & & ADAMS - ATLANTIC MINE \\
\hline 372 & 181 & BIANUCCI & GUIDO & $\mathrm{H}$ & 31 & MICHIGAN & ITALY & ITALY & & BUS DRIVER & ALTOPASCIO, LU & & ADAMS - ATLANTIC MINE \\
\hline 373 & 101 & BIANUCCI & NATALIE & W & 33 & MICHIGAN & ITALY & ITALY & & DOS DAIVLK & & & ADAMS - ATLANTIC MINE \\
\hline 374 & & BIANUCCI & ALFRED & s & 11 & MICHIGAN & MICHIGAN & MICHIGAN & & & ALTOPASCIO. LU & & ADAMS - ATLANTIC MINE \\
\hline 375 & & BIANUCCI & EVANGELINE & D & 8 & MICHIGAN & MICHIGAN & MICHIGAN & & & ALTOPASCIO. LU & & \\
\hline 376 & 180 & GEMIGNANI & ANGELO & $\mathrm{H}$ & 36 & ITALY & ITALY & ITALY & 1911 & LUMBER PILER/LUMBER MILL & LUCCA & & ADAMS - ATLANTIC MINE \\
\hline 377 & & GEMIGNANI & EMMA & W & 32 & MICHIGAN & ITALY & ITALY & & & & & ADAMS - ATLANTIC MINE \\
\hline
\end{tabular}




\begin{tabular}{|c|c|c|c|c|c|c|c|c|c|c|c|c|c|}
\hline 378 & & GEMIGNANI & FRANCES & $\mathrm{s}$ & 13 & MICHIGAN & ITALY & MICHIGAN & & & LUCCA & & ADAMS - ATLANTIC MINE \\
\hline 379 & & GEMIGNANI & LEONARD & $\mathrm{s}$ & 11 & MICHIGAN & ITALY & MICHIGAN & & & LUCCA & & ADAMS - ATLANTIC MINE \\
\hline 380 & & GEMIGNANI & GLORIA & $\mathrm{D}$ & 6 & MICHIGAN & ITALY & MICHIGAN & & & LUCCA & & ADAMS - ATLANTIC MINE \\
\hline 381 & 183 & $\mathrm{ClUCCl}$ & NICK & $\mathrm{H}$ & 62 & ITALY & ITALY & ITALY & 1889 & LABORER/HANDLE FACTORY & MATRAIA. LU & & ADAMS - ATLANTIC MINE \\
\hline 382 & & $\mathrm{CIUCCl}$ & MARY & W & 63 & ITALY & ITALY & ITALY & 1890 & & & & ADAMS - ATLANTIC MINE \\
\hline 383 & & $\mathrm{ClUCCl}$ & PAUL & s & 34 & MICHIGAN & ITALY & ITALY & & LABORER/HANDLE FACTORY & MATRAIA. LU & & ADAMS - ATLANTIC MINE \\
\hline 384 & 197 & CHIMINO & TERESA & $\mathrm{H}$ & 35 & MICHIGAN & ITALY & ITALY & & STENOGRAPHER/RAILROAD & & & ADAMS - ATLANTIC MINE \\
\hline 385 & & CHIMINO & JOSEPH & BR & 37 & MICHIGAN & ITALY & ITALY & & & SAN GIORGIO CANAVESE. TO & & ADAMS - ATLANTIC MINE \\
\hline 386 & & BALCONI & ANGELO & $\mathrm{H}$ & 45 & ITALY & ITALY & ITALY & 1910 & COPPER REFINER/C SMELTER & MERCALLO. VARESE & & ADAMS - ATLANTIC MINE \\
\hline 387 & & BALCONI & TERESA & W & 38 & ITALY & ITALY & ITALY & 1925 & & & & ADAMS - ATLANTIC MINE \\
\hline 388 & & BALCONI & HENRY & s & 23 & MICHIGAN & ITALY & ITALY & & & MERCALLO. VARESE & & ADAMS - ATLANTIC MINE \\
\hline 389 & & BALCONI & JOHN & $\mathrm{s}$ & 17 & ITALY & ITALY & ITALY & 1928 & & MERCALLO. VARESE & & ADAMS - ATLANTIC MINE \\
\hline 390 & & MARZETTA & PETER & $\mathrm{H}$ & 50 & ITALY & ITALY & ITALY & 1912 & FIREMAN/SMELTER & MERCALLO. VARESE & & ADAMS - ATLANTIC MINE \\
\hline 391 & & MARZETTA & REGINA & w & 42 & ITALY & ITALY & ITALY & 1920 & & & & ADAMS - ATLANTIC MINE \\
\hline 392 & & FASANA & ADOLFO & $\mathrm{H}$ & 46 & ITALY & ITALY & ITALY & 1907 & BOILER TENDER/C SMELTER & TORINO & & ADAMS - ATLANTIC MINE \\
\hline 393 & & FASANA & MADDALENA & W & 45 & ITALY & ITALY & ITALY & 1908 & & & & ADAMS - ATLANTIC MINE \\
\hline 394 & & FASANA & MIKE & $\mathrm{s}$ & 19 & MICHIGAN & ITALY & ITALY & & SALESMAN/GROCERY STORE & TORINO & & ADAMS - ATLANTIC MINE \\
\hline 395 & & FASANA & FRANK & $\mathrm{s}$ & 17 & MICHIGAN & ITALY & ITALY & & & TORINO & & ADAMS - ATLANTIC MINE \\
\hline 396 & & FASANA & ANTONIETTA & $\mathrm{D}$ & 10 & MICHIGAN & ITALY & ITALY & & & TORINO & & ADAMS - ATLANTIC MINE \\
\hline 397 & & TAMBORINO & PETER & $\mathrm{H}$ & 56 & ITALY & ITALY & ITALY & 1911 & LABORER/SMELTER & LOCANA CANAVESE. TO & & ADAMS - ATLANTIC MINE \\
\hline 398 & & TAMBORINO & MARY & W & 46 & ITALY & ITALY & ITALY & 1915 & & LOCANA CANAVESE. TO & & ADAMS - ATLANTIC MINE \\
\hline 399 & & TAMBORINO & PETER JR & s & 19 & ITALY & ITALY & ITALY & 1915 & & LOCANA CANAVESE, TO & & ADAMS - ATLANTIC MINE \\
\hline 400 & & TAMBORINO & PAOLINA & $\mathrm{D}$ & 13 & MICHIGAN & ITALY & ITALY & & & LOCANA CANAVESE. TO & & ADAMS - ATLANTIC MINE \\
\hline 401 & 61 & PAOLI & FELIX & $\mathrm{H}$ & 42 & ITALY & ITALY & ITALY & 1905 & SALESMAN/GROCERY STORE & SEGROMIGNO. LU & TRIMOUNTAIN AV. & ADAMS - SOUTH RANGE \\
\hline 402 & & PAOLI & ROSE & W & 46 & ITALY & ITALY & ITALY & 1910 & & & TRIMOUNTAIN AV. & ADAMS - SOUTH RANGE \\
\hline 403 & & PAOLI & MARY & $\mathrm{D}$ & 16 & MICHIGAN & ITALY & ITALY & & & SEGROMIGNO. LU & TRIMOUNTAIN AV. & ADAMS - SOUTH RANGE \\
\hline 404 & & PAOLI & BERNARD & $\mathrm{s}$ & 15 & MICHIGAN & ITALY & ITALY & & & SEGROMIGNO. LU & TRIMOUNTAIN AV. & ADAMS - SOUTH RANGE \\
\hline 405 & & PAOLI & GELSO & $\mathrm{s}$ & 13 & MICHIGAN & ITALY & ITALY & & & SEGROMIGNO, LU & TRIMOUNTAIN AV. & ADAMS - SOUTH RANGE \\
\hline 406 & & PAOLI & PASQUINA & D & 11 & MICHIGAN & ITALY & ITALY & & & SEGROMIGNO. LU & TRIMOUNTAIN AV. & ADAMS - SOUTH RANGE \\
\hline 407 & 53 & PASTORE & GEORGE & $\mathrm{H}$ & 41 & MICHIGAN & ITALY & ITALY & & OPERATOR/BAKERY & TORINO & TRIMOUNTAIN AV. & ADAMS - SOUTH RANGE \\
\hline 408 & & PASTORE & PAULINE & W & 39 & MICHIGAN & ITALY & ITALY & & & & TRIMOUNTAIN AV. & ADAMS - SOUTH RANGE \\
\hline 409 & & PASTORE & MARIE & $\mathrm{D}$ & 18 & MICHIGAN & MICHIGAN & MICHIGAN & & & TORINO & TRIMOUNTAIN AV. & ADAMS - SOUTH RANGE \\
\hline 410 & & PASTORE & GEORGE & $\mathrm{s}$ & 16 & MICHIGAN & MICHIGAN & MICHIGAN & & & TORINO & TRIMOUNTAIN AV. & ADAMS - SOUTH RANGE \\
\hline 411 & & PASTORE & IRENE & $\mathrm{D}$ & 14 & MICHIGAN & MICHIGAN & MICHIGAN & & & TORINO & TRIMOUNTAIN AV. & ADAMS - SOUTH RANGE \\
\hline 412 & 38 & SANTORI & MATT & $\mathrm{H}$ & 40 & ITALY & ITALY & ITALY & 1912 & OPERATOR/CANDY AND CIGARS & SEGROMIGNO. LU & TRIMOUNTAIN AV. & ADAMS - SOUTH RANGE \\
\hline 413 & & SANTORI & MARISA & w & 29 & ITALY & ITALY & ITALY & 1920 & 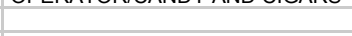 & & TRIMOUNTAIN AV. & ADAMS - SOUTH RANGE \\
\hline 414 & & SANTORI & ANGELINA & $\mathrm{D}$ & 7 & MICHIGAN & ITALY & ITALY & & & SEGROMIGNO. LU & TRIMOUNTAIN AV. & ADAMS - SOUTH RANGE \\
\hline 415 & & SANTORI & LUCILLE & $\mathrm{D}$ & 4 & MICHIGAN & ITALY & ITALY & & & SEGROMIGNO. LU & TRIMOUNTAIN AV. & ADAMS - SOUTH RANGE \\
\hline 416 & 44 & PRIAMI & ANTONIO & $\mathrm{H}$ & 55 & ITALY & ITALY & ITALY & 1902 & LABORER/CM & LUCCA & STANTON AVE. & ADAMS - SOUTH RANGE \\
\hline 417 & & PRIAMI & MARIA & W & 43 & FRANCE & ITALY & ITALY & 1908 & & & STANTON AVE. & ADAMS - SOUTH RANGE \\
\hline 418 & & PRIAMI & LOUIS & s & 17 & MICHIGAN & ITALY & FRANCE & & BLACKSMITH/CM & LUCCA & STANTON AVE & ADAMS - SOUTH RANGE \\
\hline 419 & & PRIAMI & ELSIE & $\mathrm{D}$ & 15 & MICHIGAN & ITALY & FRANCE & & 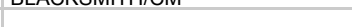 & LUCCA & STANTON AVE & ADAMS SOITH RANGE \\
\hline 420 & & PRIAMI & JOHN & $\mathrm{s}$ & 14 & MICHIGAN & ITALY & FRANCE & & & LUCCA & $\begin{array}{l}\text { STANTON AVE. } \\
\text { STANO }\end{array}$ & ADAMS - SOUTH RANGE \\
\hline 421 & 31 & PIERINI & PAOLO & $\mathrm{H}$ & 45 & ITALY & ITALY & ITALY & 1906 & OPERATOR/CANDY AND CIGARS & LUCCA & STANTON AVE. & ADAMS - SOUTH RANGE \\
\hline 422 & & SIMONINI & JOSEPH & $\mathrm{H}$ & 63 & ITALY & ITALY & ITALY & 1890 & SALESMAN/SECOND HAND & LUCCA & STANTON AVE. & ADAMS - SOUTH RANGE \\
\hline 423 & & MONTINO & JAMES & $\mathrm{H}$ & 48 & ITALY & ITALY & ITALY & 1910 & $\begin{array}{l}\text { LABORER/CM } \\
\text { LARE }\end{array}$ & TORINO & TRIMOUNTAIN AV. & ADAMS - SOUTH RANGE \\
\hline 424 & 18 & BASSO & LOUIS & $\mathrm{H}$ & 58 & ITALY & ITALY & ITALY & 1892 & JANITORIPUBLIC SCHOOL & SAN GIORGIO CANAVESE. TO & TRIMOUNTAIN AV. & ADAMS - SOUTH RANGE \\
\hline 425 & & BASSO & NELLIE & w & 56 & ITALY & ITALY & ITAL & 1893 & & & TRIMOUNTAIN AV. & ADAMS - SOUTH RANGE \\
\hline 426 & & CERUTTI & MARIA & $\mathrm{H}$ & 73 & ITAL & ITA & ITALY & 1900 & & & BALTIC AVE. & ADAMS - SOUTH RANGE \\
\hline 427 & & CERUTTI & ANTON & $\mathrm{s}$ & 48 & FRANCE & ITALY & ITA & 1898 & DRILL SHARPENER/BLACKSMITH & SAN GIORGIO CANAVESE. TO & BALTIC AVE. & ADAMS - SOUTH RANGE \\
\hline 428 & & CERUTTI & JAMES & s & 45 & FRANCE & ITALY & ITAL & 1898 & BUTCHER/MEAT MARKET & SAN GIORGIO CANAVESE. TO & BALTIC AVE. & ADAMS - SOUTH RANGE \\
\hline 429 & & CERUTTI & CATERINA & DIL & 36 & ITALY & ITALY & ITALY & 1913 & CLERK/MEAT MARKET & SAN GIORGIO CANAVESE, TO & BALTIC AVE. & ADAMS - SOUTH RANGE \\
\hline 430 & & CERUTTI & MARY & GD & 13 & MICHIGAN & FRANCE & ITALY & & & SAN GIORGIO CANAVESE. TO & BALTIC AVE. & ADAMS - SOUTH RANGE \\
\hline 431 & & CERUTTI & TONY & GS & 11 & MICHIGAN & FRANCE & ITALY & & & SAN GIORGIO CANAVESE. TO & BALTIC AVE. & ADAMS - SOUTH RANGE \\
\hline 432 & & DE FILIPPI & JOHN & B & 52 & ITALY & ITALY & ITALY & 1901 & MASON/BUILDING & SAN GIORGIO CANAVESE. TO & BALTIC AVE. & ADAMS - SOUTH RANGE \\
\hline 433 & 13 & MAGNINO & JAMES & $\mathrm{H}$ & 57 & ITALY & ITALY & ITALY & 1900 & FARMER/FRUIT FARM & SPARONE. TO & 1ST ST. & ADAMS - SOUTH RANGE \\
\hline 434 & & MAGNINO & ANNA & W & 47 & ITALY & ITALY & ITALY & 1905 & & & 1ST ST. & ADAMS - SOUTH RANGE \\
\hline 435 & & MAGNINO & JOSEPH & $\mathrm{s}$ & 23 & MICHIGAN & ITALY & ITALY & & FIREMAN/SMELTER BOILER & SPARONE, TO & 1ST ST. & ADAMS - SOUTH RANGE \\
\hline 436 & & MAGNINO & EDITH & $\mathrm{D}$ & 20 & MICHIGAN & ITALY & ITALY & & SALESWOMAN & SPARONE. TO & 1ST ST. & ADAMS - SOUTH RANGE \\
\hline 437 & & MAGNINO & MARY & $\mathrm{D}$ & 12 & MICHIGAN & ITALY & ITALY & & & SPARONE. TO & 1ST ST. & ADAMS - SOUTH RANGE \\
\hline 438 & 5 & GIACHINO & JAMES & $\mathrm{H}$ & 48 & & ITA & ITA & 1905 & PUMPMAN/ELECTRIC PUMP & TORINO & 1ST ST. & ADAMS - SOUTH RANGE \\
\hline 439 & & & & W & 47 & & ITALY & ITALY & 1906 & & & & \\
\hline 440 & & GIACHINO & MARY & $\mathrm{D}$ & 20 & MICHIGAN & ITALY & ITALY & & SALESWOMAN/DRY GOODS & TORINO & 1ST ST. & ADAMS - SOUTH RANGE \\
\hline
\end{tabular}




\begin{tabular}{|c|c|c|c|c|c|c|c|c|c|c|c|c|c|}
\hline 441 & & GIACHINO & JUNE & $\mathrm{D}$ & 19 & MICHIGAN & ITALY & ITALY & & & TORINO & 1ST ST. & ADAMS - SOUTH RANGE \\
\hline 442 & & GIACHINO & JAMES & $\mathrm{s}$ & 16 & MICHIGAN & ITALY & ITALY & & & TORINO & 1ST ST. & ADAMS - SOUTH RANGE \\
\hline 443 & & GIACHINO & ANNA & D & 12 & MICHIGAN & ITALY & ITALY & & & TORINO & 1ST ST. & ADAMS - SOUTH RANGE \\
\hline 444 & & GIACHINO & EDITH & D & 9 & MICHIGAN & ITALY & ITALY & & & TORINO & 1ST ST. & ADAMS - SOUTH RANGE \\
\hline 445 & 30 & PASTORE & MARIA & $\mathrm{H}$ & 73 & ITALY & ITALY & ITALY & 1880 & & & TRIMOUNTAIN AV. & ADAMS - SOUTH RANGE \\
\hline 446 & 34 & ANDREINI & JOSEPH & $\mathrm{H}$ & 45 & ITALY & ITALY & ITALY & 1900 & OPERATOR/CONFECTIONARY & CAPANNORI. LU & TRIMOUNTAIN AV. & ADAMS - SOUTH RANGE \\
\hline 447 & & ANDREINI & ANGELINA & w & 37 & ITALY & ITALY & ITALY & 1913 & & & TRIMOUNTAIN AV. & ADAMS - SOUTH RANGE \\
\hline 448 & & ANDREINI & ALSIE & $\mathrm{D}$ & 14 & MICHIGAN & ITALY & ITALY & & & CAPANNORI. LU & TRIMOUNTAIN AV. & ADAMS - SOUTH RANGE \\
\hline 449 & & ANDREINI & JOSEPH & $\mathrm{S}$ & 13 & MICHIGAN & ITALY & ITALY & & & CAPANNORI. LU & TRIMOUNTAIN AV. & ADAMS - SOUTH RANGE \\
\hline 450 & & ANDREINI & EUGENE & $\mathrm{s}$ & 11 & MICHIGAN & ITALY & ITALY & & & CAPANNORI. LU & TRIMOUNTAIN AV. & ADAMS - SOUTH RANGE \\
\hline 451 & 34 & SUCCA & JOHN & $\mathrm{H}$ & 34 & ITALY & ITALY & ITALY & 1912 & MANAGER/POOL ROOM & TORINO & TRIMOUNTAIN AV. & ADAMS - SOUTH RANGE \\
\hline 452 & 34 & LAZZARI & CORRADO & $\mathrm{H}$ & 34 & ITALY & ITALY & ITALY & 1921 & MECHANIC/AUTOMOBILE & LUCCA & TRIMOUNTAIN AV. & ADAMS - SOUTH RANGE \\
\hline 453 & & LAZZARI & MARIA & W & 34 & ITALY & ITALY & ITALY & 1929 & DRESSMAKER/OWN SHOP & & TRIMOUNTAIN AV. & ADAMS - SOUTH RANGE \\
\hline 454 & & LAZZARI & ENZO & $s$ & 9 & ITALY & ITALY & ITALY & 1929 & & LUCCA & TRIMOUNTAIN AV. & ADAMS - SOUTH RANGE \\
\hline 455 & & LUCCHESI & CESARE & $\mathrm{H}$ & 52 & ITALY & ITALY & ITALY & 1900 & OWNER/BUS LINE & LUCCA & 1ST ST. & ADAMS - SOUTH RANGE \\
\hline 456 & & LUCCHESI & GELSOMINA & W & 42 & ITALY & ITALY & ITALY & 1902 & MANAGER/BUS LINE & & 1ST ST. & ADAMS - SOUTH RANGE \\
\hline 457 & & LUCCHESI & FRED & $\mathrm{s}$ & 21 & MICHIGAN & ITALY & ITALY & & MECHANIC/BUS LINE & LUCCA & 1ST ST. & ADAMS - SOUTH RANGE \\
\hline 458 & & LUCCHESI & ANNA & $\mathrm{D}$ & 20 & MICHIGAN & ITALY & ITALY & & CLERK/BUS LINE & LUCCA & 1ST ST. & ADAMS - SOUTH RANGE \\
\hline 459 & & LUCCHESI & GINO & $\mathrm{s}$ & 16 & MICHIGAN & ITALY & ITALY & & & LUCCA & 1ST ST. & ADAMS - SOUTH RANGE \\
\hline 460 & & LUCCHESI & LINA & $\mathrm{s}$ & 13 & MICHIGAN & ITALY & ITALY & & & LUCCA & 1ST ST. & ADAMS - SOUTH RANGE \\
\hline 461 & & LUCCHESI & BRUNO & $\mathrm{s}$ & 10 & MICHIGAN & ITALY & ITALY & & & LUCCA & 1ST ST. & ADAMS - SOUTH RANGE \\
\hline 462 & & LUCCHESI & NORMA & $\mathrm{D}$ & 7 & MICHIGAN & ITALY & ITALY & & & LUCCA & 1ST ST. & ADAMS - SOUTH RANGE \\
\hline 463 & & IAVELLO & CATERINA & $\mathrm{H}$ & 37 & ITALY & ITALY & ITALY & 1910 & & SAN GIUSTO CANAVESE. TO & 1ST ST. & ADAMS - SOUTH RANGE \\
\hline 464 & & IAVELLO & JOSEPH & $\mathrm{s}$ & 16 & MICHIGAN & ITALY & ITALY & & & SAN GIUSTO CANAVESE. TO & 1ST ST. & ADAMS - SOUTH RANGE \\
\hline 465 & & IAVELLO & MINNIE & $\mathrm{D}$ & 14 & MICHIGAN & ITALY & ITALY & & & SAN GIUSTO CANAVESE. TO & 1ST ST. & ADAMS - SOUTH RANGE \\
\hline 466 & & VERCELLINO & JOSEPH & B & 56 & ITALY & ITALY & ITALY & 1904 & LUMBERMAN/LUMBER CO. & TORINO & 1ST ST. & ADAMS - SOUTH RANGE \\
\hline 467 & 23 & PIZZI & RALPH & $\mathrm{H}$ & 33 & ITALY & ITALY & ITALY & 1912 & MECHANIC/AUTOMOBILE & PESCAGLIA. LU & 1ST ST. & ADAMS - SOUTH RANGE \\
\hline 468 & & PIZZI & ROSE & W & 27 & MICHIGAN & ITALY & ITALY & & & & 1ST ST. & ADAMS - SOUTH RANGE \\
\hline 469 & & PIZZI & DAVID & $\mathrm{s}$ & 7 & MICHIGAN & ITALY & MICHIGAN & & & PESCAGLIA. LU & 1ST ST. & ADAMS - SOUTH RANGE \\
\hline 470 & & PASTORE & JOHN & $\mathrm{H}$ & 36 & MICHIGAN & ITALY & ITALY & & OPERATOR/BARBER SHOP & TORINO & 1ST ST. & ADAMS - SOUTH RANGE \\
\hline 471 & & PASTORE & PAULINE & W & 30 & MICHIGAN & ITALY & ITALY & & & & 1ST ST. & ADAMS - SOUTH RANGE \\
\hline 472 & & PASTORE & JOE & s & 14 & MICHIGAN & MICHIGAN & MICHIGAN & & & TORINO & 1ST ST. & ADAMS - SOUTH RANGE \\
\hline 473 & 46 & RUGANI & FRANK & $\mathrm{H}$ & 44 & ITALY & ITALY & ITALY & 1906 & OPERATOR/GROCERY STORE & & TRIMOUNTAIN AV. & ADAMS - SOUTH RANGE \\
\hline 474 & 40 & RUGANI & TEOPISTA & w & 43 & ITALY & $\begin{array}{l}\text { ITALY } \\
\text { ITA }\end{array}$ & ITALY & 1915 & UTERATUR/ORUCERT STURE & LUCCA & TRIMOUNTAIN AV. & ADAMS - SOUTH RANGE \\
\hline 475 & & FINI & IOLANDA & SD & 23 & ITALY & ITALY & ITALY & 1915 & SALESWOMAN/GROCERY STORE & LUCCA & TRIMOUNTAIN AV. & ADAMS - SOUTH RANGE \\
\hline 476 & & RUGANI & ALMANDO & s & 13 & MICHIGAN & ITALY & ITALY & & 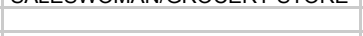 & LUCCA & TRIMOUNTAIN AV. & ADAMS - SOUTH RANGE \\
\hline 477 & & RUGANI & ROSINA & $\mathrm{D}$ & 12 & MICHIGAN & ITALY & ITALY & & & LUCCA & TRIMOUNTAIN AV. & ADAMS - SOUTH RANGE \\
\hline 478 & & RUGANI & GINA & D & 9 & MICHIGAN & ITALY & ITALY & & & LUCCA & TRIMOUNTAIN AV. & ADAMS - SOUTH RANGE \\
\hline 479 & 66 & ROMANO & PETER & $\mathrm{H}$ & 64 & ITALY & ITALY & ITALY & 1896 & & TORINO & TRIMOUNTAIN AV. & ADAMS - SOUTH RANGE \\
\hline 480 & & ROMANO & WILL & $\mathrm{s}$ & 33 & MICHIGAN & ITALY & ITALY & & OPERATOR/BOWLING ALLEY & TORINO & TRIMOUNTAIN AV. & ADAMS - SOUTH RANGE \\
\hline 481 & & ROMANO & JOSEPH & $\mathrm{S}$ & 26 & MICHIGAN & ITALY & ITALY & & OPERATOR & TORINO & TRIMOUNTAIN AV. & ADAMS - SOUTH RANGE \\
\hline 482 & & ROMANO & BATTISTA & $\mathrm{s}$ & 22 & MICHIGAN & ITALY & ITALY & & MINER/CM & TORINO & TRIMOUNTAIN AV. & ADAMS - SOUTH RANGE \\
\hline 483 & & ROMANO & ROSE & $\mathrm{D}$ & 21 & MICHIGAN & ITALY & ITALY & & & TORINO & TRIMOUNTAIN AV. & ADAMS - SOUTH RANGE \\
\hline 484 & & ROMANO & NATALINA & $\mathrm{D}$ & 19 & MICHIGAN & ITALY & ITALY & & SALESWOMAN/CANDY STORE & TORINO & TRIMOUNTAIN AV. & ADAMS - SOUTH RANGE \\
\hline 485 & & ROMANO & PETER JR. & s & 17 & MICHIGAN & ITALY & ITALY & & & TORINO & TRIMOUNTAIN AV. & ADAMS - SOUTH RANGE \\
\hline 486 & 20 & RAFFAELLI & ITALO & SOIL & 40 & ITALY & ITALY & ITALY & 1897 & CHAUFFEUR/TRUCK & LUCCA & 1ST ST & ADAMS - SOUTH RANGE \\
\hline 487 & & RAFFAELLI & ANNA & $\mathrm{D}$ & 33 & MICHIGAN & SWITZERLAN & MICHIGAN & & & LUCCA & 1ST ST & ADAMS - SOUTH RANGE \\
\hline 488 & & RAFFAELLI & KATE & GD & 12 & MICHIGAN & ITALY & MICHIGAN & & & LUCCA & 1ST ST & ADAMS - SOUTH RANGE \\
\hline 489 & & RAFFAELLI & MARTIN & GS & 10 & MICHIGAN & ITALY & MICHIGAN & & & LUCCA & 1ST ST & ADAMS - SOUTH RANGE \\
\hline 490 & & RAFFAELLI & MARIE & $\mathrm{GD}$ & 9 & MICHIGAN & ITALY & MICHIGAN & & & LUCCA & 1ST ST & ADAMS - SOUTH RANGE \\
\hline 491 & & RAFFAELLI & JOAN & $\mathrm{GD}$ & 7 & MICHIGAN & ITALY & MICHIGAN & & & LUCCA & 1ST ST & ADAMS - SOUTH RANGE \\
\hline 492 & & RAFFAELLI & DOROTHY & GD & 5 & MICHIGAN & ITALY & MICHIGAN & & & LUCCA & 1ST ST & ADAMS - SOUTH RANGE \\
\hline 493 & & RAFFAELLI & FLOYD & GS & 1 & MICHIGAN & ITALY & MICHIGAN & & & LUCCA & 1ST ST & ADAMS - SOUTH RANGE \\
\hline 494 & 15 & BASSO & JOSEPH & $\mathrm{H}$ & 35 & MICHIGAN & ITALY & MICHIGAN & & CASHIER/BANK & SAN GIORGIO CANAVESE. TO & 2ND ST. & ADAMS - SOUTH RANGE \\
\hline 495 & & BASSO & MARGHERITA & w & 34 & MICHIGAN & GERMANY & MICHIGAN & & & USA-GERMANY & 2ND ST. & ADAMS - SOUTH RANGE \\
\hline 496 & 14 & GUZZITTI & MARIA & $\mathrm{H}$ & 58 & ITALY & ITALY & ITALY & 1898 & COOK/BOARDING HOUSE & & 2ND ST./1ST ST. & ADAMS - SOUTH RANGE \\
\hline 497 & & SUCCA & ANTON & B & 45 & ITALY & ITALY & ITALY & 1905 & LABORER/CM & TORINO & 2ND ST./1ST ST. & ADAMS - SOUTH RANGE \\
\hline 498 & & SUCCA & GIUSEPPE & B & 40 & ITALY & ITALY & ITALY & 1907 & LABORER/CM & TORINO & 2ND ST./1ST ST. & ADAMS - SOUTH RANGE \\
\hline 499 & 9 & BENASSI & FELIX & $\mathrm{H}$ & 44 & ITALY & ITALY & ITALY & 1905 & LABORER/CM & CAMAIORE. LU & 1ST ST & ADAMS - SOUTH RANGE \\
\hline 500 & & BENASSI & MARY & W & 40 & MICHIGAN & ITALY & ITALY & & & & 1ST ST & ADAMS - SOUTH RANGE \\
\hline 501 & & BENAS & AN & $\mathrm{D}$ & 20 & MICHIGAN & ITA & HIGAN & & & CAMAIOR & 1ST ST & ADAMS - SOUTH RANGE \\
\hline 502 & & & & FIL & 76 & & & & 1885 & & CALLO. VARESE & & ADAMS - S \\
\hline 503 & 4 & ANDRINA & ANTONIO & $\mathrm{H}$ & 53 & ITALY & ITALY & ITALY & 1899 & LABORER/CM & TORINO & 1ST ST & ADAMS - SOUTH RANGE \\
\hline
\end{tabular}




\begin{tabular}{|c|c|c|c|c|c|c|c|c|c|c|c|c|c|}
\hline 504 & & ANDRINA & MARY & W & 50 & ITALY & ITALY & ITALY & 1905 & & & 1ST ST & ADAMS - SOUTH RANGE \\
\hline 505 & & ANDRINA & FRANCES & $\mathrm{D}$ & 22 & ITALY & ITALY & ITALY & & POSTMISTRESS/BALTIC P.O. & TORINO & 1ST ST & ADAMS - SOUTH RANGE \\
\hline 5067 & 7 & PLASSA & ALBERT & $\mathrm{H}$ & 43 & ITALY & ITALY & ITALY & 1905 & OPERATOR/BAKERY SHOP & TORINO & 2ND ST. & ADAMS - SOUTH RANGE \\
\hline 507 & & PLASSA & MARIE & W & 42 & MICHIGAN & ITALY & ITALY & & & & 2ND ST. & ADAMS - SOUTH RANGE \\
\hline 508 & & PLASSA & TEO & SS & 21 & MICHIGAN & ITALY & MICHIGAN & & BAKER/BAKERY & & 2ND ST. & ADAMS - SOUTH RANGE \\
\hline 509 & & PLASSA & ANGELINE & $\mathrm{D}$ & 18 & MICHIGAN & ITALY & MICHIGAN & & & TORINO & 2ND ST. & ADAMS - SOUTH RANGE \\
\hline 510 & & PLASSA & ALBERT & $\mathrm{s}$ & 17 & MICHIGAN & ITALY & MICHIGAN & & BAKER/BAKERY & TORINO & 2ND ST. & ADAMS - SOUTH RANGE \\
\hline 5116 & 6 & BOGGIO & DOMENICO & $\mathrm{H}$ & 43 & ITALY & ITALY & ITALY & 1902 & ROCK HOUSE MAN/CM & SAN GIUSTO CANAVESE. TO & 2ND ST. & ADAMS - SOUTH RANGE \\
\hline 512 & & BOGGIO & MARY & W & 57 & ITALY & ITALY & ITALY & 1888 & 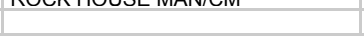 & SAN GIUSTO CANAVESE. TO & 2ND ST. & ADAMS - SOUTH RANGE \\
\hline 5133 & 32 & BERTOLOZZI & ATTILIO & $\mathrm{H}$ & 68 & ITALY & ITALY & ITALY & 1900 & NOT CLEAR & LUCCA & 2ND ST. & ADAMS - SOUTH RANGE \\
\hline 514 & & BERTOLOZZI & ZITA & w & 58 & ITALY & ITALY & ITALY & 1901 & & & 2ND ST. & ADAMS - SOUTH RANGE \\
\hline 515 & & BERTOLOZZI & LENA & D & 22 & MICHIGAN & ITALY & ITALY & & & LUCCA & 2ND ST. & ADAMS - SOUTH RANGE \\
\hline 516 & & BERTOLOZZI & ATTILIA & $\mathrm{D}$ & 18 & MICHIGAN & ITALY & ITALY & & & LUCCA & 2ND ST. & ADAMS - SOUTH RANGE \\
\hline 517 & & BERTOLOZZI & GLORIA & $\mathrm{D}$ & 17 & MICHIGAN & ITALY & ITALY & & & LUCCA & 2ND ST. & ADAMS - SOUTH RANGE \\
\hline 5183 & 36 & TOSCANA & ANTONIO & $\mathrm{H}$ & 53 & ITALY & ITALY & ITALY & 1895 & OPERATOR/CANDY & SAN GIORGIO CANAVESE. TO & 3RD ST. & ADAMS - SOUTH RANGE \\
\hline 519 & & TOSCANA & MARIA & W & 47 & ITALY & ITALY & ITALY & 1891 & & & 3RD ST. & ADAMS - SOUTH RANGE \\
\hline 520 & & TOSCANA & MARGHERITA & D & 28 & MICHIGAN & ITALY & ITALY & & TEACHER/PUBLIC SCHOOL & SAN GIORGIO CANAVESE. TO & 3RD ST. & ADAMS - SOUTH RANGE \\
\hline 521 & & TOSCANA & MARY & $\mathrm{D}$ & 22 & MICHIGAN & ITALY & ITALY & & TEACHER/PUBLIC SCHOOL & SAN GIORGIO CANAVESE. TO & 3RD ST. & ADAMS - SOUTH RANGE \\
\hline 522 & & TOSCANA & CATERINA & $\mathrm{D}$ & 18 & MICHIGAN & ITALY & ITALY & & & SAN GIORGIO CANAVESE. TO & 3RD ST. & ADAMS - SOUTH RANGE \\
\hline 523 & & TOSCANA & ANNETTE & D & 16 & MICHIGAN & ITALY & ITALY & & & SAN GIORGIO CANAVESE. TO & 3RD ST. & ADAMS - SOUTH RANGE \\
\hline 5243 & 33 & BALAGNA & ANGELA & $\mathrm{H}$ & 54 & ITALY & ITALY & ITALY & 1906 & & PONT CANAVESE. TO & 3RD ST. & ADAMS - SOUTH RANGE \\
\hline 525 & & BALAGNA & LIDIA & $\mathrm{D}$ & 20 & MICHIGAN & ITALY & ITALY & & & PONT CANAVESE, TO & 3RD ST. & ADAMS - SOUTH RANGE \\
\hline 526 & & BALAGNA & MIKE & $\mathrm{s}$ & 15 & MICHIGAN & ITALY & ITALY & & & PONT CANAVESE. TO & 3RD ST. & ADAMS - SOUTH RANGE \\
\hline 527 & & CHIANTEL & JOHN & B & 38 & ITALY & ITALY & ITALY & 1912 & LABORER/CM & SPARONE. TO & 3RD ST. & ADAMS - SOUTH RANGE \\
\hline 528 & & COSTA & JAMES & B & 38 & ITALY & ITALY & ITALY & 1922 & LABORER/CM & TORINO & 3RD ST. & ADAMS - SOUTH RANGE \\
\hline 529 & & CANOVA & FRANK & B & 36 & CANADA & ITALY & ITALY & 1916 & LABORER/CM & BERGAMO & 3RD ST. & ADAMS - SOUTH RANGE \\
\hline 5302 & 27 & SUCCA & JOSEPHINE & $\mathrm{H}$ & 43 & ITALY & ITALY & ITALY & 1905 & & TORINO & 3RD ST. & ADAMS - SOUTH RANGE \\
\hline 531 & & ROLANDO & $\mathrm{JOHN}$ & $\mathrm{s}$ & 21 & MICHIGAN & ITALY & ITALY & & TRUCK DRIVER/US MAIL & PONT CANAVESE, TO & 3RD ST. & ADAMS - SOUTH RANGE \\
\hline 532 & & ROLANDO & JOSEPH & $\mathrm{s}$ & 16 & MICHIGAN & ITALY & ITALY & & & PONT CANAVESE. TO & 3RD ST. & ADAMS - SOUTH RANGE \\
\hline 533 & & ROLANDO & PAUL & s & 12 & MICHIGAN & ITALY & ITALY & & & PONT CANAVESE. TO & 3RD ST. & ADAMS - SOUTH RANGE \\
\hline 534 & & SUCCA & JAMES & BRIL & 36 & ITALY & ITALY & ITALY & 1905 & OPERATOR/DRAY LINE & TORINO & 3RD ST. & ADAMS - SOUTH RANGE \\
\hline 5353 & 36 & RAFFAELLI & RALPH & $\mathrm{H}$ & 67 & ITALY & ITALY & ITALY & 1894 & NOT CLEAR & LUCCA & 3RD ST. & ADAMS - SOUTH RANGE \\
\hline 536 & & RAFFAELLI & CATERINA & w & 64 & ITALY & ITALY & ITALY & 1898 & & & 3RD ST. & ADAMS - SOUTH RANGE \\
\hline 537 & & AIMONETTO & PETER & $\mathrm{H}$ & 44 & ITALY & ITALY & ITALY & 1907 & TIMBERMAN/CM & TORINO & 3RD ST. & ADAMS - SOUTH RANGE \\
\hline 5381 & 108 & BORGO & DOMENICO & $\mathrm{H}$ & 69 & ITALY & ITALY & ITALY & 1881 & SALESMAN & VICENZA & CALUMET AVE. & CALUMET - LAURIUM VILLAGE \\
\hline 539 & & BORGO & KATE & w & 58 & ITALY & ITALY & ITALY & 1882 & & VICENZA & CALUMET AVE. & CALUMET - LAURIUM VILLAGE \\
\hline 5401 & 105 & TORREANO & JOHN & $\mathrm{H}$ & 66 & ITALY & ITALY & ITALY & 1884 & & TORINO & OSCEOLA ST. & CALUMET - LAURIUM VILLAGE \\
\hline 541 & & TORREANO & FELICITA & w & 66 & ITALY & ITALY & ITALY & 1888 & & & OSCEOLA ST. & CALUMET - LAURIUM VILLAGE \\
\hline 542 & & TORREANO & ANGELO & $\mathrm{s}$ & 31 & MICHIGAN & ITALY & ITALY & & ELECTRICAL & TORINO & OSCEOLA ST. & CALUMET - LAURIUM VILLAGE \\
\hline 543 & & TORREANO & ELLEN & $\mathrm{D}$ & 24 & MICHIGAN & ITALY & ITALY & & BOOKKEEPER/DOCTOR'S OFFICE & TORINO & OSCEOLA ST. & CALUMET - LAURIUM VILLAGE \\
\hline 5441 & 109 & TINETTI & PAUL & $\mathrm{H}$ & 54 & ITALY & ITALY & ITALY & 1889 & MERCHANT/RETAIL GROCERY & SAN MARTINO CANAVESE. TO & OSCEOLA ST. & CALUMET - LAURIUM VILLAGE \\
\hline 545 & & TINETTI & MADDALENA & w & 53 & ITALY & ITALY & ITALY & 1889 & & SAN MARTINO CANAVESE. TO & OSCEOLA ST. & CALUMET - LAURIUM VILLAGE \\
\hline 546 & & TINETTI & FRANK & s & 31 & MICHIGAN & ITALY & ITALY & & PROPRIETOR/ELECTRICAL & SAN MARTINO CANAVESE. TO & OSCEOLA ST. & CALUMET - LAURIUM VILLAGE \\
\hline 5471 & 132 & BALAGNA & MARY & $\mathrm{H}$ & 54 & ITALY & ITALY & ITALY & 1905 & & PONT CANAVESE. TO & OSCEOLA ST. & CALUMET - LAURIUM VILLAGE \\
\hline 548 & & BALAGNA & JOSEPH & $\mathrm{s}$ & 20 & MICHIGAN & ITALY & ITALY & & MERCHANT/BLACKSMITH SHOP & PONT CANAVESE. TO & OSCEOLA ST. & CALUMET - LAURIUM VILLAGE \\
\hline 549 & & BALAGNA & VITTORIA & $\mathrm{D}$ & 18 & MICHIGAN & ITALY & ITALY & & 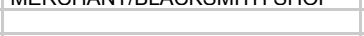 & PONT CANAVESE. TO & OSCEOLA ST. & CALUMET - LAURIUM VILLAGE \\
\hline 5501 & 118 & TINETTI & PETER & $\mathrm{H}$ & 68 & ITALY & ITALY & ITALY & 1888 & LABORER/COUNTY ROAD & SAN MARTINO CANAVESE. TO & OSCEOLA ST. & CALUMET - LAURIUM VILLAGE \\
\hline 551 & & TINETTI & ANGELINA & w & 64 & ITALY & ITALY & ITALY & 1890 & & & OSCEOLA ST. & CALUMET - LAURIUM VILLAGE \\
\hline 5526 & 61 & TORREANO & MADDALENA & $\mathrm{H}$ & 32 & MICHIGAN & ITALY & ITALY & & DRESSMAKER & TORINO & 1ST ST. & CALUMET - LAURIUM VILLAGE \\
\hline 553 & & TORREANO & ANNA & D & 5 & MICHIGAN & MICHIGAN & MICHIGAN & & & TORINO & 1ST ST. & CALUMET - LAURIUM VILLAGE \\
\hline 554 & & TORREANO & FRANCES & $\mathrm{s}$ & 3 & MICHIGAN & MICHIGAN & MICHIGAN & & & TORINO & 1ST ST. & CALUMET - LAURIUM VILLAGE \\
\hline 5556 & 63 & TORREANO & JAMES & $\mathrm{H}$ & 61 & ITALY & ITALY & ITALY & 1885 & MERCHANT/RETAIL GROCERY & TORINO & 1ST ST. & CALUMET - LAURIUM VILLAGE \\
\hline 556 & & TORREANO & RITA & $\mathrm{D}$ & 28 & MICHIGAN & ITALY & ITALY & & SALESLADY/GROCERY & TORINO & 1ST ST. & CALUMET - LAURIUM VILLAGE \\
\hline 5571 & 111 & TALENTINO & FRANK & $\mathrm{H}$ & 84 & ITALY & ITALY & ITALY & 1899 & & PARELLA. TO & HECLA ST. & CALUMET - LAURIUM VILLAGE \\
\hline 558 & & TALENTINO & DELPHINE & w & 63 & ITALY & ITALY & ITALY & 1903 & & & HECLA ST. & CALUMET - LAURIUM VILLAGE \\
\hline 5591 & 125 & ZUBIENA & JAMES & $\mathrm{H}$ & 59 & ITALY & ITALY & ITALY & 1905 & MERCHANT/RETAIL GROCERY & TORINO & HECLA ST. & CALUMET - LAURIUM VILLAGE \\
\hline 560 & & ZUBIENA & JOHN & s & 34 & ITALY & ITALY & ITALY & 1912 & TEAMSTER/RETAIL GROCERY & TORINO & HECLA ST. & CALUMET - LAURIUM VILLAGE \\
\hline 561 & & ZUBIENA & JULIA & DIL & 31 & MICHIGAN & ITALY & ITALY & & & & HECLA ST. & CALUMET - LAURIUM VILLAGE \\
\hline 562 & & ZUBIENA & JAMES & GS & $8 \mathrm{M}$ & MICHIGAN & ITALY & MICHIGAN & & & TORINO & HECLA ST. & CALUMET - LAURIUM VILLAGE \\
\hline 5631 & 129 & BIANCO & MINNIE & $\mathrm{H}$ & 65 & ITALY & ITAL & ITALY & 1891 & & SCARMAGNO. 7 & HECLA ST. & CALUMET - LAURIUM VILLAGE \\
\hline 564 & & BIANCO & PAULINE & D & 28 & IIGAN & & & & APPARTMENTS & SCARMAGNO. TO & LA ST. & CALUMET - LAURIUM VILLAGE \\
\hline 565 & & MENZIES & ES & $\mathrm{H}$ & 34 & MICHIGAN & MICHIGAN & HIGAN & & MECHANIC/CM & & & \\
\hline 566 & & MENZIES & MARY & W & 34 & MICHIGAN & ITALY & ITALY & & & CASTELLAMONTE. TO & 1ST ST. & CALUMET - LAURIUM VILLAGE \\
\hline
\end{tabular}




\begin{tabular}{|c|c|c|c|c|c|c|c|c|c|c|c|c|c|}
\hline 567 & & CATTANEO & MARTA & MIL & 60 & ITALY & ITALY & ITALY & 1901 & & CASTELLAMONTE. TO & 1ST ST. & CALUMET - LAURIUM VILLAGE \\
\hline 568 & 140 & NARDI & ARMANDO & $\mathrm{H}$ & 33 & MICHIGAN & ITALY & ITALY & & MOTORMAN/ELECTRIC STREET & LUCCA & HECLA ST. & CALUMET - LAURIUM VILLAGE \\
\hline 569 & & NARDI & MAIDA & W & 32 & MICHIGAN & ITALY & ITALY & & & LUCCA & HECLA ST. & CALUMET - LAURIUM VILLAGE \\
\hline 570 & & NARDI & GERALDINE & $\mathrm{D}$ & 9 & MICHIGAN & MICHIGAN & MICHIGAN & & & LUCCA & HECLA ST. & CALUMET - LAURIUM VILLAGE \\
\hline 571 & 134 & RICHETTA & JOSEPH & $\mathrm{H}$ & 56 & ITALY & ITALY & ITALY & 1895 & LABORER/CM & SAN MARTINO CANAVESE. TO & HECLA ST. & CALUMET - LAURIUM VILLAGE \\
\hline 572 & & RICHETTA & JENNIE & $\mathrm{D}$ & 18 & MICHIGAN & ITALY & ITALY & & & SAN MARTINO CANAVESE. TO & HECLA ST. & CALUMET - LAURIUM VILLAGE \\
\hline 573 & & RICHETTA & DOMENICO & $\mathrm{s}$ & 21 & MICHIGAN & ITALY & ITALY & & TEAMSTER/CM & SAN MARTINO CANAVESE. TO & HECLA ST. & CALUMET - LAURIUM VILLAGE \\
\hline 574 & & RICHETTA & LOUIS & $\mathrm{s}$ & 20 & MICHIGAN & ITALY & ITALY & & BAKER/BAKERY & SAN MARTINO CANAVESE. TO & HECLA ST. & CALUMET - LAURIUM VILLAGE \\
\hline 575 & & RICHETTA & FRANK & $\mathrm{S}$ & 19 & MICHIGAN & ITALY & ITALY & & LABORER/CM & SAN MARTINO CANAVESE. TO & HECLA ST. & CALUMET - LAURIUM VILLAGE \\
\hline 576 & & RICHETTA & MAUD & $\mathrm{D}$ & 14 & MICHIGAN & ITALY & ITALY & & & SAN MARTINO CANAVESE. TO & HECLA ST. & CALUMET - LAURIUM VILLAGE \\
\hline 577 & 128 & ROVANO & DOMENICO & $\mathrm{H}$ & 44 & ITALY & ITALY & ITALY & 1903 & MINER/CM & SAN MARTINO CANAVESE. TO & HECLA ST. & CALUUMET - LAURIUM VILLAGE \\
\hline 578 & & ROVANO & TERESA & W & 40 & MICHIGAN & ITALY & ITALY & & & SAN MARTINO CANAVESE. TO & HECLA ST. & CALUMET - LAURIUM VILLAGE \\
\hline 579 & & ROVANO & MONICA & $\mathrm{D}$ & 17 & MICHIGAN & MICHIGAN & MICHIGAN & & SALESLADY/BAKERY & SAN MARTINO CANAVESE. TO & HECLA ST. & CALUMET - LAURIUM VILLAGE \\
\hline 580 & & ROVANO & JOSEPH & $\mathrm{s}$ & 14 & MICHIGAN & MICHIGAN & MICHIGAN & & & SAN MARTINO CANAVESE. TO & HECLA ST. & CALUMET - LAURIUM VILLAGE \\
\hline 581 & & ROVANO & MARIA & $\mathrm{D}$ & 10 & MICHIGAN & MICHIGAN & MICHIGAN & & & SAN MARTINO CANAVESE. TO & HECLA ST. & CALUMET - LAURIUM VILLAGE \\
\hline 582 & & ROVANO & MIKE & $\mathrm{s}$ & 4 & MICHIGAN & MICHIGAN & MICHIGAN & & & SAN MARTINO CANAVESE, TO & HECLA ST. & CALUMET - LAURIUM VILLAGE \\
\hline 583 & 114 & MICHETTI & PAUL & $\mathrm{H}$ & 35 & MICHIGAN & ITALY & ITALY & & FOREMAN/AUTOMOBILE & PONT CANAVESE. TO & HECLA ST. & CALUMET - LAURIUM VILLAGE \\
\hline 584 & & MICHETTI & MARY & W & 33 & MICHIGAN & ITALY & ITALY & & & & HECLA ST. & CALUMET - LAURIUM VILLAGE \\
\hline 585 & & MICHETTI & PAUL & s & 1 & MICHIGAN & MICHIGAN & MICHIGAN & & & PONT CANAVESE. TO & HECLA ST. & CALUMET - LAURIUM VILLAGE \\
\hline 586 & 110 & GERA & ANTON & $\mathrm{H}$ & 46 & MICHIGAN & ITALY & ITALY & & MERCHANT/CANDY STORE & SAN MARTINO CANAVESE. TO & HECLA ST. & CALUMET - LAURIUM VILLAGE \\
\hline 587 & & GERA & JAMANICA & W & 43 & MICHIGAN & ITALY & ITALY & & & & HECLA ST. & CALUMET - LAURIUM VILLAGE \\
\hline 588 & & GERA & JOHN & s & 10 & MICHIGAN & MICHIGAN & MICHIGAN & & & SAN MARTINO CANAVESE, TO & HECLA ST. & CALUMET - LAURIUM VILLAGE \\
\hline 589 & 71 & YOTTI & PETER & $\mathrm{H}$ & 43 & MICHIGAN & ITALY & CANADA & & FOREMAN/FREIGHT DEPOT & & $1 \mathrm{ST}$ ST. & CALUMET - LAURIUM VILLAGE \\
\hline 590 & & YOTTI & MARY & W & 44 & MICHIGAN & CANADA & CANADA & & & CANADA & 1ST ST. & $\begin{array}{l}\text { CALUMET - LAURIUM VILLAGE } \\
\text { LAUE }\end{array}$ \\
\hline 591 & & YOTTI & HENRY & s & 20 & MICHIGAN & MICHIGAN & MICHIGAN & & RECEIVING CLERK/AUTOMOBILE & & 1ST ST. & CALUMET - LAURIUM VILLAGE \\
\hline 592 & & YOTTI & VALERIE & $\mathrm{D}$ & 17 & MICHIGAN & MICHIGAN & MICHIGAN & & 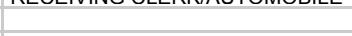 & & 1ST ST. & CALUMET - LAURIUM VILLAGE \\
\hline 593 & & YOTTI & EVELYN & $\mathrm{D}$ & 16 & MICHIGAN & MICHIGAN & MICHIGAN & & & & 1ST ST. & CALUMET - LAURIUM VILLAGE \\
\hline 594 & & YOTTI & LAWRENCE & $\mathrm{s}$ & 14 & MICHIGAN & MICHIGAN & MICHIGAN & & & & 1ST ST. & CALUMET - LAURIUM VILLAGE \\
\hline 595 & & YOTTI & MARIE & D & 10 & MICHIGAN & MICHIGAN & MICHIGAN & & & & 1ST ST. & CALUMET - LAURIUM VILLAGE \\
\hline 596 & & YOTTI & A? & $\mathrm{s}$ & 5 & MICHIGAN & MICHIGAN & MICHIGAN & & & & 1ST ST. & CALUMET - LAURIUM VILLAGE \\
\hline 597 & 73 & BERSANO & BATTISTA & $\mathrm{H}$ & 57 & ITALY & ITALY & ITALY & 1890 & SAUSAGE MAKER/MEAT MARKET & SAN MARTINO CANAVESE. TO & 1ST ST. & CALUMET - LAURIUM VILLAGE \\
\hline 598 & & BERSANO & KATY & W & 47 & ITALY & ITALY & ITALY & 1904 & & & 1ST ST. & CALUMET - LAURIUM VILLAGE \\
\hline 599 & 75 & CARBONATTO & CHARLES & $\mathrm{H}$ & 56 & ITALY & ITALY & ITALY & 1898 & NOT CLEAR & PONT CANAVESE. TO & KEARSARGE ST. & CALUMET - LAURIUM VILLAGE \\
\hline 600 & & CARBONATTO & MARY & W & 53 & ITALY & ITALY & ITALY & 1898 & & PONT CANAVESE, TO & KEARSARGE ST. & CALUMET - LAURIUM VILLAGE \\
\hline 601 & & CARBONATTO & PHYLLIS & $\mathrm{D}$ & 27 & MICHIGAN & ITALY & ITALY & & TEACHER/PUBLIC SCHOOL & PONT CANAVESE. TO & KEARSARGE ST. & CALUMET - LAURIUM VILLAGE \\
\hline 602 & 109 & ALDO & GIACINTO & $\mathrm{H}$ & 50 & MICHIGAN & ITALY & ITALY & & MINER/CM & SAN MARTINO CANAVESE. TO & KEARSARGE ST. & CALUMET - LAURIUM VILLAGE \\
\hline 603 & & ALDO & KATY & W & 37 & MICHIGAN & ITALY & ITALY & & & & KEARSARGE ST. & CALUMET - LAURIUM VILLAGE \\
\hline 604 & & ALDO & PETER & s & 13 & MICHIGAN & MICHIGAN & MICHIGAN & & & SAN MARTINO CANAVESE. TO & KEARSARGE ST. & CALUMET - LAURIUM VILLAGE \\
\hline 605 & 111 & ROLANDO & JOHN & $\mathrm{H}$ & 64 & MICHIGAN & ITALY & ITALY & & SHOEMAKER/OWN SHOP & $\begin{array}{l}\text { PONT CANAVESE. TO } \\
\text { POV }\end{array}$ & KEARSARGE ST. & CALUMET - LAURIUM VILLAGE \\
\hline 606 & III & ROLANDO & TERESA & w & 57 & MICHIGAN & ITALY & ITALY & & 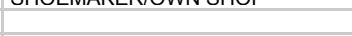 & & KEARSARGE ST. & CALUMET - LAURIUM VILLAGE \\
\hline 607 & & ROLANDO & JOHN & $\mathrm{s}$ & 29 & MICHIGAN & MICHIGAN & MICHIGAN & & PLUMBER/WHAREHOUSE STORE & PONT CANAVESE. TO & KEARSARGE ST. & \\
\hline 608 & & ROLANDO & KATE & D & 24 & MICHIGAN & MICHIGAN & MICHIGAN & & & PONT CANAVESE. TO & KEARSARGE ST. & CALUMET - LAURIUM VILLAGE \\
\hline 609 & 115 & MASNADA & BEN & $\mathrm{H}$ & 35 & MICHIGAN & ITALY & ITALY & & FOREMAN/CM & BERGAMO & KEARSARGE ST. & CALUMET - LAURIUM VILLAGE \\
\hline 610 & & MASNADA & LENA & W & 27 & MICHIGAN & ITALY & ITALY & & & & KEARSARGE ST. & CALUMET - LAURIUM VILLAGE \\
\hline 611 & & DELL'ACQUA & JOSEPHINE & SIIL & 23 & MICHIGAN & ITALY & ITALY & & WAITRESS/TEA ROOM & CUGGIANO. MILANO & KEARSARGE ST. & CALUMET - LAURIUM VILLAGE \\
\hline 612 & 117 & MASSOGLIA & FRANK & $\mathrm{H}$ & 41 & ITALY & ITALY & ITALY & 1906 & MERCHANT/GENERAL STORE & TORINO & KEARSARGE ST. & CALUMET - LAURIUM VILLAGE \\
\hline 613 & & MASSOGLIA & MARY & W & 36 & ITALY & ITALY & ITALY & 1906 & & TORINO & KEARSARGE ST. & CALUMET - LAURIUM VILLAGE \\
\hline 614 & & MASSOGLIA & MARTIN & s & 13 & MICHIGAN & ITALY & ITALY & & & TORINO & KEARSARGE ST. & CALUMET - LAURIUM VILLAGE \\
\hline 615 & & CASERIO & MARTIN & FIL & 61 & ITALY & ITALY & ITALY & 1898 & MINER/CM & STRAMBINELLO. TO & KEARSARGE ST. & CALUMET - LAURIUM VILLAGE \\
\hline 616 & 123 & ROLANDO & TONY & $\mathrm{H}$ & 45 & ITALY & ITALY & ITAL & 1890 & BUTCHER/MEAT MARKET & PONT CANAVESE. TO & KEARSARGE ST. & CALUMET - LAURIUM VILLAGE \\
\hline 617 & & ROLANDO & LENA & w & 46 & ITAL & ITAL & ITAL & 1887 & & & SARGE ST. & CALUMET - LAURIUM VILLAGE \\
\hline 618 & & ROLAN & VIRGINIA & $\mathrm{D}$ & 22 & IGAN & ITALY & ITAL & & SALESLADY/GIFT SHOP & PONT CANAVESE, TO & ARGE ST. & CALUMET - LAURIUM VILLAGE \\
\hline 619 & & ROLANL & CONSTANCE & $\mathrm{s}$ & 21 & GAN & ITA & ITA & & & IVESE. TO & EST. & CALUMET - LAURIUM VILLAGE \\
\hline 620 & & ROLANL & PAUL & $\mathrm{s}$ & 16 & & ITA & ITA & & TEAMSTER/MEAT MARKET & PONT CANAVESE. TO & RSARGE ST. & CALUMET - LAURIUM VILLAGE \\
\hline 621 & & ROLANDO & PETER & $\mathrm{s}$ & 14 & MICHIGA & ITAL & ITAL & & & PONT CANAVESE. TO & KEARSARGE ST. & CALUMET - LAURIUM VILLAGE \\
\hline 622 & & ROLANDO & TONY & s & 11 & MICHIGAN & ITALY & ITALY & & & PONT CANAVESE. TO & KEARSARGE ST. & CALUMET - LAURIUM VILLAGE \\
\hline 623 & & SANDRETTO & CONSTANCE & FIL & 74 & ITALY & ITALY & ITALY & 1887 & & PONT CANAVESE. TO & KEARSARGE ST. & CALUMET - LAURIUM VILLAGE \\
\hline 624 & 127 & CONTRATTO & JOHANNA & $\mathrm{H}$ & 55 & ITALY & ITALY & ITALY & 1894 & & & KEARSARGE ST. & CALUMET - LAURIUM VILLAGE \\
\hline 625 & & CONTRATTO & ANDREW & $\mathrm{s}$ & 35 & MICHIGAN & ITALY & ITALY & & LABORER/RAILROAD & LOCANA CANAVESE. TO & KEARSARGE ST. & CALUMET - LAURIUM VILLAGE \\
\hline 626 & & CONTRATTO & JAMES & $\mathrm{s}$ & 31 & MICHIGAN & ITALY & ITALY & & MUSICIAN/ORCHESTRA & LOCANA CANAVESE. TO & KEARSARGE ST. & CALUMET - LAURIUM VILLAGE \\
\hline 627 & 131 & SAVINI & TERESA & $\mathrm{H}$ & 66 & ITALY & ITALY & ITALY & 1892 & & TORINO & KEARSARGE ST. & CALUMET - LAURIUM VILLAGE \\
\hline 628 & & SAVINI & JOSEPH & s & 34 & ITALY & ITALY & ITALY & 1898 & ICE RINK & TORINO & KEARSARGE ST. & CALUMET - LAURIUM VILLAGE \\
\hline 629 & 142 & DEIRO & CHARLES & $\mathrm{H}$ & 40 & ITALY & ITALY & ITALY & 1908 & MASON/BUILDING & SALTO CANAVESE. TO & KEARSARGE ST. & CALUMET - LAURIUM VILLAGE \\
\hline
\end{tabular}




\begin{tabular}{|c|c|c|c|c|c|c|c|c|c|c|c|c|c|}
\hline 630 & & DEIRO & JULIA & W & 38 & MICHIGAN & ITALY & ITALY & & & & KEARSARGE ST. & CALUMET - LAURIUM VILLAGE \\
\hline 631 & & DEIRO & ANNE & $\mathrm{D}$ & 8 & MICHIGAN & ITALY & MICHIGAN & & & SALTO CANAVESE. TO & KEARSARGE ST. & CALUMET - LAURIUM VILLAGE \\
\hline 632 & & DEIRO & VIRGINIA & D & 7 & MICHIGAN & ITALY & MICHIGAN & & & SALTO CANAVESE. TO & KEARSARGE ST. & CALUMET - LAURIUM VILLAGE \\
\hline 633 & & DEIRO & CARL & $\mathrm{s}$ & 5 & MICHIGAN & ITALY & MICHIGAN & & & SALTO CANAVESE. TO & KEARSARGE ST. & CALUMET - LAURIUM VILLAGE \\
\hline 634 & & DEIRO & SHIRLEY & D & 3 & MICHIGAN & ITALY & MICHIGAN & & & SALTO CANAVESE. TO & KEARSARGE ST. & CALUMET - LAURIUM VILLAGE \\
\hline 635 & 132 & DUGAN/DEIGAN & THOMAS & $\mathrm{H}$ & 29 & MICHIGAN & MICHIGAN & NORTH & & MECHANIC/AUTOMOBILE & IRELAND & KEARSARGE ST. & CALUMET - LAURIUM VILLAGE \\
\hline 636 & & DUGAN/DEIGAN & MARY & W & 28 & MICHIGAN & ITALY & ITALY & & & & KEARSARGE ST. & CALUMET - LAURIUM VILLAGE \\
\hline 637 & & DUGAN/DEIGAN & KATE & D & 8 & MICHIGAN & MICHIGAN & MICHIGAN & & & & KEARSARGE ST. & CALUMET - LAURIUM VILLAGE \\
\hline 638 & & DUGAN/DEIGAN & NORMA & D & 7 & MICHIGAN & MICHIGAN & MICHIGAN & & & & KEARSARGE ST. & CALUMET - LAURIUM VILLAGE \\
\hline 639 & & DUGAN/DEIGAN & COREEN & $\mathrm{D}$ & 5 & MICHIGAN & MICHIGAN & MICHIGAN & & & & KEARSARGE ST. & CALUMET - LAURIUM VILLAGE \\
\hline 640 & 128 & CASERIO & JOSEPH & $\mathrm{H}$ & 38 & ITALY & ITALY & ITALY & 1907 & MERCHANT/DRY GOODS STORE & STRAMBINELLO. TO & KEARSARGE ST. & CALUMET - LAURIUM VILLAGE \\
\hline 641 & & CASERIO & MARY & W & 36 & MICHIGAN & ITALY & ITALY & & & & KEARSARGE ST. & CALUMET - LAURIUM VILLAGE \\
\hline 642 & & CASERIO & MARTIN & $\mathrm{s}$ & 13 & MICHIGAN & ITALY & MICHIGAN & & & STRAMBINELLO. TO & KEARSARGE ST. & CALUMET - LAURIUM VILLAGE \\
\hline 643 & & CASERIO & JOHN & $\mathrm{s}$ & 10 & MICHIGAN & ITALY & MICHIGAN & & & STRAMBINELLO. TO & KEARSARGE ST. & CALUMET - LAURIUM VILLAGE \\
\hline 644 & 124 & RICHETTA & PETER & $\mathrm{H}$ & 54 & ITALY & ITALY & ITALY & 1899 & MINER/CM & SAN MARTINO CANAVESE. TO & KEARSARGE ST. & CALUMET - LAURIUM VILLAGE \\
\hline 645 & & RICHETTA & LOUISE & W & 48 & ITALY & ITALY & ITALY & 1906 & & & KEARSARGE ST. & CALUMET - LAURIUM VILLAGE \\
\hline 646 & & RICHETTA & ANTHONY & $\mathrm{s}$ & 23 & MICHIGAN & ITALY & ITALY & & SALESMAN & SAN MARTINO CANAVESE. TO & KEARSARGE ST. & CALUMET - LAURIUM VILLAGE \\
\hline 647 & & RICHETTA & JOSEPH & $\mathrm{s}$ & 18 & MICHIGAN & ITALY & ITALY & & SALESMAN/TEA COMPANY & SAN MARTINO CANAVESE. TO & KEARSARGE ST. & CALUMET - LAURIUM VILLAGE \\
\hline 648 & & RICHETTA & ANNETTE & D & 16 & MICHIGAN & ITALY & ITALY & & & SAN MARTINO CANAVESE. TO & KEARSARGE ST. & CALUMET - LAURIUM VILLAGE \\
\hline 649 & 106 & VAIRO & JAMES & $\mathrm{H}$ & 48 & ITALY & ITALY & ITALY & 1901 & PROPRIETOR/SOFT DRINK & LOCANA CANAVESE. TO & KEARSARGE ST. & CALUMET - LAURIUM VILLAGE \\
\hline 650 & & VAIRO & ANGELINE & W & 44 & ITALY & ITALY & ITALY & 1908 & & & KEARSARGE ST. & CALUMET - LAURIUM VILLAGE \\
\hline 651 & & VAIRO & JAMES & $\mathrm{s}$ & 19 & MICHIGAN & ITALY & ITALY & & BOOKKEPER/BANK & LOCANA CANAVESE, TO & KEARSARGE ST. & CALUMET - LAURIUM VILLAGE \\
\hline 652 & & VAIRO & JOSEPH & $\mathrm{s}$ & 17 & MICHIGAN & ITALY & ITALY & & USHER/THEATRE & LOCANA CANAVESE. TO & KEARSARGE ST. & CALUMET - LAURIUM VILLAGE \\
\hline 653 & & CAPELLO & KATE/MARY & $\mathrm{H}$ & 70 & ITALY & ITALY & ITALY & ???? & & CUORGNE'. TO & KEARSARGE ST. & CALUMET - LAURIUM VILLAGE \\
\hline 654 & 101 & COSTA & BATTISTA & $\mathrm{H}$ & 47 & MICHIGAN & ITALY & ITALY & & MINER/CM & CUNEO & TAMARACK ST. & CALUMET - LAURIUM VILLAGE \\
\hline 655 & & COSTA & MARY & w & 45 & MICHIGAN & ITALY & ITALY & & & CUNEO & TAMARACK ST. & CALUMET - LAURIUM VILLAGE \\
\hline 656 & & COSTA & DOMENICO & $\mathrm{s}$ & 14 & MICHIGAN & ITALY & ITALY & & & CUNEO & TAMARACK ST. & CALUMET - LAURIUM VILLAGE \\
\hline 657 & & COSTA & LIZ & $\mathrm{D}$ & 13 & MICHIGAN & ITALY & ITALY & & & CUNEO & $\begin{array}{l}\text { TAMARACK ST. } \\
\text { TAT }\end{array}$ & CALUMET - LAURIUM VILLAGE \\
\hline 658 & & COSTA & PETER & s & 12 & MICHIGAN & ITALY & ITALY & & & CUNEO & TAMARACK ST. & CALUMET - LAURIUM VILLAGE \\
\hline 659 & & COSTA & LAWRENCE & $\mathrm{s}$ & 5 & MICHIGAN & ITALY & ITALY & & & CUNEO & TAMARACK ST. & CALUMET - LAURIUM VILLAGE \\
\hline 660 & & COSTA & ANTON & $\mathrm{s}$ & 5 & MICHIGAN & ITALY & ITALY & & & CUNEO & TAMARACK ST. & CALUMET - LAURIUM VILLAGE \\
\hline 661 & 109 & SCIACERO & DOMENICO & $\mathrm{H}$ & 50 & ITALY & ITALY & ITALY & 1902 & MINER/CM & SAN GIOVANNI CANAVESE. & TAMARACK ST. & CALUMET - LAURIUM VILLAGE \\
\hline 662 & & SCIACERO & ANGELINA & W & 50 & ITALY & ITALY & ITALY & $00 / 06$ & & & TAMARACK ST. & CALUMET - LAURIUM VILLAGE \\
\hline 663 & 111 & WILSON & GEORGE & $\mathrm{H}$ & 28 & MICHIGAN & MICHIGAN & MICHIGAN & & SALESMAN/GROCERY STORE & USA & TAMARACK ST. & CALUMET - LAURIUM VILLAGE \\
\hline 664 & & WILSON & PHYLLIS & w & 24 & MICHIGAN & ITALY & ITALY & & & & TAMARACK ST. & CALUMET - LAURIUM VILLAGE \\
\hline 665 & 119 & MASSOGLIA & DOMENICO & $\mathrm{H}$ & 47 & ITALY & ITALY & ITALY & 1901 & LABORER/FOUNDRY CM & SAN GIOVANNI CANAVESE. & TAMARACK ST. & CALUMET - LAURIUM VILLAGE \\
\hline 666 & & MASSOGLIA & LINA & w & 47 & ITALY & ITALY & ITALY & 1903 & & & TAMARACK ST. & CALUMET - LAURIUM VILLAGE \\
\hline 667 & & MASSOGLIA & JOHN & s & 19 & MICHIGAN & ITALY & ITALY & & TIMEKEEPER/TELEPHONE CO. & SAN GIOVANNI CANAVESE. & TAMARACK ST. & CALUMET - LAURIUM VILLAGE \\
\hline 668 & & MASSOGLIA & LUCY & D & 18 & MICHIGAN & ITALY & ITALY & & 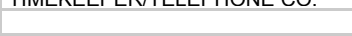 & SAN GIOVANNI CANAVESE. & TAMARACK ST. & $\begin{array}{l}\text { CALUMET - LAURIUM VILLAGE } \\
\end{array}$ \\
\hline 669 & & MASSOGLIA & JOSEPH & $\mathrm{s}$ & 16 & MICHIGAN & ITALY & ITALY & & & SAN GIOVANNI CANAVESE. & TAMARACK ST. & CALUMET - LAURIUM VILLAGE \\
\hline 670 & & MASSOGLIA & DOEMNICO & $\mathrm{s}$ & 8 & MICHIGAN & ITALY & ITALY & & & SAN GIOVANNI CANAVESE. & TAMARACK ST. & CALUMET - LAURIUM VILLAGE \\
\hline 671 & 118 & GALETTO & JOSEPH & $\mathrm{H}$ & 66 & FRANCE & ITALY & ITALY & 1889 & MERCHANT/RETAIL GROCERY & SAN GIORGIO CANAVESE. TO & TAMARACK ST. & CALUMET - LAURIUM VILLAGE \\
\hline 672 & & GALETTO & MARY & W & 56 & ITALY & ITALY & ITALY & 1891 & SALESLADY/GROCERY & & TAMARACK ST. & CALUMET - LAURIUM VILLAGE \\
\hline 673 & & GALETTO & CHARLES & $\mathrm{s}$ & 21 & MICHIGAN & ITALY & ITALY & & WAITER/CONFECTIONARY & & TAMARACK ST. & CALUMET - LAURIUM VILLAGE \\
\hline 674 & & GALETTO & ANGELINA & D & 18 & MICHIGAN & ITALY & ITAI & & WAITRESS/CONFECTIONARY & SAN GIORGIO CANAVESE. TO & TAMARACK ST. & CALUMET - LAURIUM VILLAGE \\
\hline 675 & 108 & LOCATELLI & PETER & $\mathrm{H}$ & 53 & ITALY & ITALY & ITALY & 1899 & MASON/INDEPENDENT & SELINO. BERGAMO & TAMARACK ST. & CALUMET - LAURIUM VILLAGE \\
\hline 676 & & LOCATELLI & JULIA & w & 48 & ITALY & ITALY & ITALY & 1902 & & & TAMARACK ST. & CALUMET - LAURIUM VILLAGE \\
\hline 677 & & LOCATELLI & ANTHONY & $\mathrm{s}$ & 23 & MICHIGAN & ITALY & ITALY & & MASON/BUILDING CONTRACTOR & SELINO. BERGAMO & TAMARACK ST. & CALUMET - LAURIUM VILLAGE \\
\hline 678 & & LOCATELLI & PETER & $\mathrm{s}$ & 21 & MICHIGAN & ITALY & ITALY & & MASON/BUILDING CONTRACTOR & SELINO. BERGAMO & TAMARACK ST. & CALUMET - LAURIUM VILLAGE \\
\hline 679 & & LOCATELLI & MARY & $\mathrm{D}$ & 17 & MICHIGAN & ITALY & ITALY & & SALESLADYIGROCERY & SELINO. BERGAMO & TAMARACK ST. & CALUMET - LAURIUM VILLAGE \\
\hline 680 & & LOCATELLI & EMMA & $\mathrm{D}$ & 15 & MICHIGAN & ITALY & ITALY & & & SELINO. BERGAMO & TAMARACK ST. & $\begin{array}{l}\text { CALUMET - LAURIUM VILLAGE } \\
\end{array}$ \\
\hline 681 & 106 & ADAM & PETER & $\mathrm{H}$ & 35 & MICHIGAN & AUSTRIA & AUSTRIA & & CHAUFFEUR/PF & AUSTRIA & TAMARACK ST. & CALUMET - LAURIUM VILLAGE \\
\hline 682 & & ADAM & JOSEPHINE & w & 27 & MICHIGAN & ITALY & ITALY & & & & TAMARACK ST. & CALUMET - LAURIUM VILLAGE \\
\hline 683 & 103 & GRISOLANO & RICHARD & $\mathrm{H}$ & 36 & & ITA & & & MINER/CM & TORINO & K ST. & CALUN \\
\hline 684 & & GRISOLANO & $\mathrm{BEL}$ & w & 34 & & USA & NOIS & & & & $\begin{array}{l}\text { RACK ST. } \\
\text { RAC }\end{array}$ & CALUM \\
\hline 685 & 103 & TINETTI & LO & $\mathrm{H}$ & 49 & & ITALY & & 1903 & ASSISTANT CASHIER/BANK & N MARTINO CANAVE: & & CALUMET - LAURIUM VILLAGE \\
\hline 686 & & TINETTI & ROSE & W & 45 & ITALY & ITALY & ITAI & 1903 & & SAN MARTINO CANAVESE. TO & PEWABIC ST. & CALUMET - LAURIUM VILLAGE \\
\hline 687 & & TINETTI & MARY & D & 23 & MICHIGAN & ITALY & ITALY & & WAITRESS/CANDY STORE & SAN MARTINO CANAVESE, TO & PEWABIC ST. & CALUMET - LAURIUM VILLAGE \\
\hline 688 & & TINETTI & LAURA & D & 21 & MICHIGAN & ITALY & ITALY & & STENOGRAPHER/CM & SAN MARTINO CANAVESE. TO & PEWABIC ST. & CALUMET - LAURIUM VILLAGE \\
\hline 689 & & TINETTI & FRANK & s & 15 & MICHIGAN & ITALY & ITALY & & & SAN MARTINO CANAVESE. TO & PEWABIC ST. & CALUMET - LAURIUM VILLAGE \\
\hline 690 & & TINETTI & PETER & $\mathrm{s}$ & 13 & MICHIGAN & ITALY & ITALY & & & SAN MARTINO CANAVESE. TO & PEWABIC ST. & CALUMET - LAURIUM VILLAGE \\
\hline 691 & & TINETTI & EDITH & $\mathrm{D}$ & 12 & MICHIGAN & ITALY & ITALY & & & SAN MARTINO CANAVESE. TO & PEWABIC ST. & CALUMET - LAURIUM VILLAGE \\
\hline 692 & & TINETTI & ARTHUR & $\mathrm{s}$ & 8 & MICHIGAN & ITALY & ITALY & & & SAN MARTINO CANAVESE. TO & PEWABIC ST. & CALUMET - LAURIUM VILLAGE \\
\hline
\end{tabular}




\begin{tabular}{|c|c|c|c|c|c|c|c|c|c|c|c|c|c|}
\hline 693 & & TINETTI & MARJORIE & $\mathrm{D}$ & 6 & MICHIGAN & ITALY & ITALY & & & SAN MARTINO CANAVESE. TO & PEWABIC ST. & CALUMET - LAURIUM VILLAGE \\
\hline 694 & 109 & SANARD & HOMER & $\mathrm{H}$ & 33 & MICHIGAN & CANADA & MICHIGAN & & ELECTRICIAN/CM & CANADA-USA & PEWABIC ST. & CALUMET - LAURIUM VILLAGE \\
\hline 695 & & SANARD & NOTA & W & 27 & MICHIGAN & ITALY & ITALY & & & & PEWABIC ST. & CALUMET - LAURIUM VILLAGE \\
\hline 696 & 112 & BESSOLO & CHARLES & $\mathrm{H}$ & 50 & ITALY & ITALY & ITALY & 1897 & BOOKKEEPER/BANK & TORINO & PEWABIC ST. & CALUMET - LAURIUM VILLAGE \\
\hline 697 & & BESSOLO & MYRTLE & W & 38 & MICHIGAN & MICHIGAN & IRELAND & & & USA-IRELAND & PEWABIC ST. & CALUMET - LAURIUM VILLAGE \\
\hline 698 & 91 & CAPPELLO & JOHANNA & $\mathrm{H}$ & 61 & ITALY & ITALY & ITALY & 1887 & & SAN BENIGNO CANAVESE. TO & 1ST ST. & CALUMET - LAURIUM VILLAGE \\
\hline 699 & & CAPPELLO & DUSOLINA & $\mathrm{D}$ & 41 & MICHIGAN & ITALY & ITALY & & STENOGRAPHER/HOTEL & SAN BENIGNO CANAVESE. TO & 1ST ST. & CALUMET - LAURIUM VILLAGE \\
\hline 700 & & CAPPELLO & JAMES & $\mathrm{s}$ & 33 & MICHIGAN & ITALY & ITALY & & MACHINIST/ROUND HOUSE & SAN BENIGNO CANAVESE. TO & 1ST ST. & CALUMET - LAURIUM VILLAGE \\
\hline 701 & & CAPPELLO & FRED & $\mathrm{S}$ & 30 & MICHIGAN & ITALY & ITALY & & PLASTERER/CONTRACTOR & SAN BENIGNO CANAVESE. TO & 1ST ST. & CALUMET - LAURIUM VILLAGE \\
\hline 702 & 113 & BARNOTTI & JOHN & $\mathrm{H}$ & 38 & MICHIGAN & ITALY & ITALY & & LOCOMOTIVE ENGINEER/STEAM & PONT CANAVESE. TO & IROQUOIS ST. & CALUMET - LAURIUM VILLAGE \\
\hline 703 & & BARNOTTI & MAUD & w & 39 & MICHIGAN & CANADA & MICHIGAN & & & & IROQUOIS ST. & CALUMET - LAURIUM VILLAGE \\
\hline 704 & 132 & JOHNSON & EDGAR & $\mathrm{H}$ & 29 & MICHIGAN & SWEDEN & SWEDEN & & CASHIER/BANK & SWEDEN & IROQUOIS AT. & CALUMET - LAURIUM VILLAGE \\
\hline 705 & & JOHNSON & MARY & W & 26 & MICHIGAN & ITALY & ITALY & & & & IROQUOIS AT. & CALUMET - LAURIUM VILLAGE \\
\hline 706 & 133 & TEPATTI & KATE & SER & 24 & MICHIGAN & ITALY & ITALY & & SERVANT/PF & PONT CANAVESE. TO & WILLOW AVE. & CALUMET - LAURIUM VILLAGE \\
\hline 707 & 163 & COPPO & LENA & SER & 61 & ITALY & ITALY & ITALY & $90 / 93$ & SERVANT/PF & PONT CANAVESE. TO & WOODLAND AVE. & CALUMET - LAURIUM VILLAGE \\
\hline 708 & 155 & SANDRI & ADOLPH & B & 20 & MICHIGAN & ITALY & ITALY & & LABORER/CM & & WOODLAND AVE. & CALUMET - LAURIUM VILLAGE \\
\hline 709 & 64 & GIULIO & DOMENICO & $\mathrm{H}$ & 35 & MICHIGAN & ITALY & ITALY & & PROPRIETOR/SOFT DRINK & SAN GIORGIO CANAVESE. TO & 1ST ST. & CALUMET - LAURIUM VILLAGE \\
\hline 710 & & GIULIO & KATE & W & 31 & ITALY & ITALY & ITALY & 1911 & & & 1ST ST. & CALUMET - LAURIUM VILLAGE \\
\hline 711 & 114 & QUELLO & MICHAEL & $\mathrm{H}$ & 55 & MICHIGAN & ITALY & ITALY & & SALESMAN & LOCANA CANAVESE. TO & WOODLAND AVE. & CALUMET - LAURIUM VILLAGE \\
\hline 712 & & QUELLO & CHARLOTTE & w & 48 & MICHIGAN & NEW JERSEY & VERMONT & & & & WOODLAND AVE. & CALUMET - LAURIUM VILLAGE \\
\hline 713 & & QUELLO & $\begin{array}{l}\text { ROBERT } \\
\text { ROR }\end{array}$ & s & 17 & MICHIGAN & MICHIGAN & WISCONSIN & & & LOCANA CANAVESE. TO & WOODLAND AVE. & CALUMET - LAURIUM VILLAGE \\
\hline 714 & 146 & SALASSA & CHARLES & $\mathrm{H}$ & 45 & ITALY & ITALY & ITALY & 1888 & AGENT/LIFE INSURANCE & SAN BENIGNO CANAVESE, TO & WOODLAND AVE. & CALUMET - LAURIUM VILLAGE \\
\hline 715 & & SALASSA & ROSE & W & 41 & MICHIGAN & ITALY & ITALY & & & TORINO & WOODLAND AVE. & CALUMET - LAURIUM VILLAGE \\
\hline 716 & & SALASSA & ERNEST & s & 21 & MICHIGAN & ITALY & MICHIGAN & & CALLISTER- & SAN BENIGNO CANAVESE. TO & WOODLAND AVE. & CALUMET - LAURIUM VILLAGE \\
\hline 717 & 148 & SUINO & BERNARD & $\mathrm{H}$ & 31 & MICHIGAN & ITALY & ITALY & & CARPENTER/CM & CORIO. TO & WOODLAND AVE. & CALUMET - LAURIUM VILLAGE \\
\hline 718 & & SUINO & MARY & W & 27 & MICHIGAN & ITALY & ITALY & & & & WOODLAND AVE. & CALUMET - LAURIUM VILLAGE \\
\hline 719 & & SUINO & MARIE & $\mathrm{D}$ & 6 & MICHIGAN & MICHIGAN & MICHIGAN & & & CORIO. TO & WOODLAND AVE. & CALUMET - LAURIUM VILLAGE \\
\hline 720 & & SUINO & FRANK & $\mathrm{s}$ & 2 & MICHIGAN & MICHIGAN & MICHIGAN & & & CORIO, TO & WOODLAND AVE. & CALUMET - LAURIUM VILLAGE \\
\hline 721 & 150 & SUINO & FRANK & $\mathrm{H}$ & 61 & ITALY & ITALY & ITALY & 1890 & JANITOR/BANK & CORIO. TO & WOODLAND AVE. & CALUMET - LAURIUM VILLAGE \\
\hline 722 & & SUINO & MARIA & W & 57 & ITALY & ITALY & ITALY & 1897 & & & WOODLAND AVE. & CALUMET - LAURIUM VILLAGE \\
\hline 723 & & SUINO & GEORGE & s & 31 & MICHIGAN & ITALY & ITALY & & CARPENTER/CM & CORIO. TO & WOODLAND AVE. & CALUMET - LAURIUM VILLAGE \\
\hline 724 & & SUINO & LUCILLE & DIL & 22 & ITALY & ITALY & ITALY & 1917 & SALESLADY/GROCERY & & WOODLAND AVE. & CALUMET - LAURIUM VILLAGE \\
\hline 725 & 135 & BAUDINO & $\begin{array}{l}\text { PETER } \\
\text { PET }\end{array}$ & $\mathrm{H}$ & 26 & MICHIGAN & ITALY & ITALY & & SALESMAN/RETAIL HARDWARE & RIVAROLO CANAVESE. TO & AMYGDALOID ST. & CALUMET - LAURIUM VILLAGE \\
\hline 726 & & BAUDINO & ELEONOR & w & 22 & MICHIGAN & ITALY & MICHIGAN & & & & AMYGDALOID ST. & CALUMET - LAURIUM VILLAGE \\
\hline 727 & 108 & VAIRO & DOMENICO & $\mathrm{H}$ & 39 & ITALY & ITALY & ITALY & 1901 & SALESMAN & LOCANA CANAVESE. TO & SENECA ST. & CALUUMET - LAURIUM VILLAGE \\
\hline 728 & & VAIRO & BLANCHE & W & 39 & MICHIGAN & ITALY & ITALY & & & & ECA ST. & CALUMET - LAURIUM VILLAGE \\
\hline 729 & & VAIRO & BETTY & $\mathrm{D}$ & 12 & MICHIGAN & ITALY & MICHIGAN & & & LOCANA CANAVESE. TO & SENECA ST. & CALUMET - LAURIUM VILLAGE \\
\hline 730 & & VAIRO & ROBERT & $\mathrm{s}$ & 11 & MICHIGAN & ITALY & MICHIGAN & & & LOCANA CANAVESE. TO & SENECA ST. & CALUMET - LAURIUM VILLAGE \\
\hline 731 & & VAIRO & PEGGY & D & 3 & MICHIGAN & ITALY & MICHIGAN & & & LOCANA CANAVESE. TO & SENECA ST. & CALUMET - LAURIUM VILLAGE \\
\hline 732 & 112 & PRICCO & DOMENICO & $\mathrm{H}$ & 54 & ITALY & ITALY & ITALY & 1895 & MINER/CM & SAN MARTINO CANAVESE. TO & SENECA ST. & CALUMET - LAURIUM VILLAGE \\
\hline 733 & & PRICCO & ANGELINE & W & 45 & ITALY & ITALY & ITALY & 1905 & & & SENECA ST. & CALUMET - LAURIUM VILLAGE \\
\hline 734 & & PRICCO & DOMENICO & s & 14 & MICHIGAN & ITALY & ITALY & & & SAN MARTINO CANAVESE. TO & SENECA ST. & CALUMET - LAURIUM VILLAGE \\
\hline 735 & 116 & PANATTONI & RALPH & $\mathrm{H}$ & 42 & ITALY & ITALY & ITALY & 1902 & PROPRIETOR/RESTAURANT & MONTECARLO. LU & SENECA ST. & CALUMET - LAURIUM VILLAGE \\
\hline 736 & & PANATTONI & DELLA & W & 32 & MICHIGAN & ITALY & ITALY & & & & SENECA ST. & CALUMET - LAURIUM VILLAGE \\
\hline 737 & & PANATTONI & CAROLINA & $\mathrm{D}$ & 16 & MICHIGAN & ITALY & MICHIGAN & & & MONTECARLO. LU & SENECA ST. & CALUMET - LAURIUM VILLAGE \\
\hline 738 & & PANATTONI & FLORENCE & $\mathrm{D}$ & 15 & MICHIGAN & ITALY & MICHIGAN & & & MONTECARLO. LU & SENECA ST. & $\begin{array}{l}\text { CALUMET - LAURIUM VILLAGE } \\
\end{array}$ \\
\hline 739 & & PANATTONI & DOROTHY & $\mathrm{D}$ & 10 & & ITALY & & & & & ECA ST. & CALUMET - LAURIUM VILLAGE \\
\hline 740 & & PANATTONI & MAF & $\mathrm{D}$ & 8 & IGAN & ITA & & & & & A ST. & CALUMET - LAURIUM VIL \\
\hline 741 & 124 & VIANI & RGE & $\mathrm{H}$ & 53 & & ITA & ITAL & UNK & NOT CLEAR & PORTO MAURIZIO. IMPERIA & A ST. & CALUMET - LAURIUM VIL \\
\hline 742 & & SULLIVAN & JOHN & SOIL & 23 & HIGAN & ITA & ITA & & FIREMAN/STEAM RAILROAD & & A ST. & CALUMET - LAURIUM V \\
\hline 743 & & SULLIVAN & MAF & $\mathrm{D}$ & 24 & MICHIGAN & ITA & ITA & & & PORTO MAURIZIO. IMPERIA & & CALUMET - LAURIUM VILLAGE \\
\hline 744 & 126 & TOMASI & ANTONIA & $\mathrm{H}$ & 51 & ITALY & ITA & ITA & 1900 & SERVANT/PF & LOCANA CANAVESE, TO & SENECA ST. & CALUMET - LAURIUM VILLAGE \\
\hline 745 & & TOMASI & IOLANDA/ANT & $\mathrm{D}$ & 21 & MICHIGAN & ITA & ITAI & & SALESLADY/DEPARTMENT & LOCANA CANAVESE. TO & SENECA ST. & CALUMET - LAURIUM VILLAGE \\
\hline 746 & & TOMASI & FLORENCE & D & 13 & MICHIGAN & ITALY & ITALY & & & LOCANA CANAVESE. TO & SENECA ST. & CALUMET - LAURIUM VILLAGE \\
\hline 747 & 140 & DATTO/DATA & ANTON & $\mathrm{H}$ & 67 & ITALY & ITALY & ITALY & 1890 & MINER/CM & SAN GIORGIO CANAVESE. TO & SENECA ST. & CALUMET - LAURIUM VILLAGE \\
\hline 748 & & DATTO/DATA & ANNA & w & 65 & ITALY & ITALY & ITALY & 1892 & & SAN GIORGIO CANAVESE. TO & SENECA ST. & CALUMET - LAURIUM VILLAGE \\
\hline 749 & & DATTO/DATA & ANTON & $\mathrm{s}$ & 28 & MICHIGAN & ITALY & ITALY & & PIPE FIXER/CM & SAN GIORGIO CANAVESE. TO & SENECA ST. & CALUMET - LAURIUM VILLAGE \\
\hline 750 & 142 & TORREANO & KATE & $\mathrm{H}$ & 62 & ITALY & ITALY & ITALY & 1893 & & TORINO & SENECA ST. & CALUMET - LAURIUM VILLAGE \\
\hline 751 & 142 & TORREANO & JOHN & $\mathrm{s}$ & 30 & MICHIGAN & ITALY & ITALY & 1000 & RAILROAD & TORINO & SENECA ST. & $\begin{array}{l}\text { CALUMET - LAURIUM VILLAGE } \\
\end{array}$ \\
\hline 752 & 230 & MANFREDINI & IREN & $\mathrm{H}$ & 31 & MICHIGAN & ITALY & ITA & & ADYIDEPARTM & & SENECA ST. & CALUMET - LAURIUM VILLAGE \\
\hline 753 & 217 & & & $\mathrm{H}$ & 62 & & & & 1886 & PLUMBER/OWN SHOP & PONT CANAVESE. TO & & CALUMET - LAURIUM VILLAGE \\
\hline 754 & & PICCHIOTTINO & MARGHER & W & 59 & & ITALY & & 1893 & & & & \\
\hline 755 & & PICCHIOTTINO & PAULINE & $\mathrm{D}$ & 18 & MICHIGAN & ITALY & ITALY & & & PONT CANAVESE. TO & AHMEEK ST. & CALUMET - LAURIUM VILLAGE \\
\hline
\end{tabular}




\begin{tabular}{|c|c|c|c|c|c|c|c|c|c|c|c|c|c|}
\hline 756 & 139 & GALASSERO & JAMES & $\mathrm{H}$ & 56 & ITALY & ITALY & ITALY & 1900 & MINER/CM & PONT CANAVESE. TO & AHMEEK ST. & CALUMET - LAURIUM VILLAGE \\
\hline 757 & & GALASSERO & LUCY & W & 60 & ITALY & ITALY & ITALY & 1905 & & & AHMEEK ST. & CALUMET - LAURIUM VILLAGE \\
\hline 758 & & PETERSON & WALTER & SOIL & 30 & MICHIGAN & SWEDEN & SWEDEN & & PAINTER/ODD JOBS & SWEDEN & AHMEEK ST. & CALUMET - LAURIUM VILLAGE \\
\hline 759 & & PETERSON & JENNIE & $\mathrm{D}$ & 32 & MICHIGAN & ITALY & ITALY & & SALESLADY/GIFT SHOP & PONT CANAVESE. TO & AHMEEK ST. & CALUMET - LAURIUM VILLAGE \\
\hline 760 & & PETERSON & JAMES & GS & 1 & MICHIGAN & ITALY & ITALY & & & PONT CANAVESE. TO & AHMEEK ST. & CALUMET - LAURIUM VILLAGE \\
\hline 761 & 80 & BOGGIO & JOHN & $\mathrm{H}$ & 47 & ITALY & ITALY & ITALY & 1900 & MERCHANT/RETAIL GROCERY & TORINO & 1ST ST. & CALUMET - LAURIUM VILLAGE \\
\hline 762 & & BOGGIO & AMALIA & W & 45 & ITALY & ITALY & ITALY & 1906 & SALESLADYIGROCERY & & 1ST ST. & CALUMET - LAURIUM VILLAGE \\
\hline 763 & & BOGGIO & SANDRINE & $\mathrm{D}$ & 22 & MICHIGAN & ITALY & ITALY & & SALESLADY/GROCERY & TORINO & 1ST ST. & CALUMET - LAURIUM VILLAGE \\
\hline 764 & & BOGGIO & JOHN & s & 20 & MICHIGAN & ITALY & ITALY & & PLASTERER/ODD JOBS & TORINO & 1ST ST. & $\begin{array}{l}\text { CALUMET - LAURIUM VILLAGE } \\
\text { CAUM }\end{array}$ \\
\hline 765 & & BOGGIO & MARY & $\mathrm{D}$ & 18 & MICHIGAN & ITALY & ITALY & & SALESLADY/GROCERY & TORINO & 1ST ST. & CALUMET - LAURIUM VILLAGE \\
\hline 766 & & BOGGIO & MIKE & $\mathrm{s}$ & 16 & MICHIGAN & ITALY & ITALY & & & TORINO & 1ST ST. & CALUMET - LAURIUM VILLAGE \\
\hline 767 & & BOGGIO & ROSE & D & 12 & MICHIGAN & ITALY & ITALY & & & TORINO & 1ST ST. & CALUMET - LAURIUM VILLAGE \\
\hline 768 & 139 & KLANSECK & LOUIS & $\mathrm{H}$ & 50 & ITALY & ITALY & ITALY & 1901 & & & N. PEWABIC ST. & CALUMET - LAURIUM VILLAGE \\
\hline 769 & & KLANSECK & ANNA & W & 44 & YUGOSLAVIA & YUGOSLAVIA & YUGOSLAVIA & 1907 & & YUGOSLAVIA & N. PEWABIC ST. & CALUMET - LAURIUM VILLAGE \\
\hline 770 & & KLANSECK & JOSEPH & s & 17 & MICHIGAN & ITALY & YUGOSLAVIA & & MESSENGER BOY/TELEGRAPH & & N. PEWABIC ST. & CALUMET - LAURIUM VILLAGE \\
\hline 771 & & KLANSECK & ANGELINE & D & 15 & MICHIGAN & ITALY & YUGOSLAVIA & & & & N. PEWABIC ST. & CALUMET - LAURIUM VILLAGE \\
\hline 772 & & KLANSECK & LOUISE & $\mathrm{D}$ & 14 & MICHIGAN & ITALY & YUGOSLAVIA & & & & N. PEWABIC ST. & CALUMET - LAURIUM VILLAGE \\
\hline 773 & & KLANSECK & ROSE & $\mathrm{D}$ & 12 & MICHIGAN & ITALY & YUGOSLAVIA & & & & N. PEWABIC ST. & CALUMET - LAURIUM VILLAGE \\
\hline 774 & & KLANSECK & RUTH & $\mathrm{D}$ & 11 & MICHIGAN & ITALY & YUGOSLAVIA & & & & N. PEWABIC ST. & CALUMET - LAURIUM VILLAGE \\
\hline 775 & & KLANSECK & ELISABETH & $\mathrm{D}$ & 8 & MICHIGAN & ITALY & YUGOSLAVIA & & & & N. PEWABIC ST. & CALUMET - LAURIUM VILLAGE \\
\hline 776 & & KLANSECK & JOHN & $\mathrm{s}$ & 6 & MICHIGAN & ITALY & YUGOSLAVIA & & & & N. PEWABIC ST. & CALUMET - LAURIUM VILLAGE \\
\hline 777 & & KLANSECK & BERENICE & $\mathrm{D}$ & 4 & MICHIGAN & ITALY & YUGOSLAVIA & & & & N. PEWABIC ST. & CALUMET - LAURIUM VILLAGE \\
\hline 778 & 100 & VALLE & TONY & $\mathrm{H}$ & 73 & ITALY & ITALY & ITALY & 1887 & MINER/CM & SAN GIORGIO CANAVESE. TO & N. PEWABIC ST. & CALUMET - LAURIUM VILLAGE \\
\hline 779 & & VALLE & MARY & W & 68 & ITALY & ITALY & ITALY & 1887 & & SAN GIORGIO CANAVESE. TO & N. PEWABIC ST. & CALUMET - LAURIUM VILLAGE \\
\hline 780 & & VALLE & STEPHEN & $\mathrm{s}$ & 39 & ITALY & ITALY & ITALY & 1893 & LABORER/CM & SAN GIORGIO CANAVESE. TO & N. PEWABIC ST. & CALUMET - LAURIUM VILLAGE \\
\hline 781 & 124 & DE FILIPPI & JOSEPH & $\mathrm{H}$ & ?? & MICHIGAN & ITALY & ITALY & & TIMBERMAN/CM & TORINO & N. PEWABIC ST. & CALUMET - LAURIUM VILLAGE \\
\hline 782 & & DE FILIPPI & STELLA & W & ?? & MICHIGAN & MICHIGAN & POLAND & & & USA-POLAND & N. PEWABIC ST. & CALUMET - LAURIUM VILLAGE \\
\hline 783 & 133 & AIRANDI & MADDALENA & SER & 32 & ITALY & ITALY & ITALY & & & & N. PEWABIC ST. & CALUMET - LAURIUM VILLAGE \\
\hline 784 & & FONTANA & JOHN & B & 13 & MICHIGAN & ITALY & MICHIGAN & & & & N. PEWABIC ST. & CALUMET - LAURIUM VILLAGE \\
\hline 785 & & FONTANA & LOUIS & B & 10 & MICHIGAN & ITALY & MICHIGAN & & & & N. PEWABIC ST. & CALUMET - LAURIUM VILLAGE \\
\hline 786 & 109 & BORGO & CESARE & $\mathrm{H}$ & 61 & MICHIGAN & ITALY & MICHIGAN & & SALESMAN/WHOLESALE & VICENZA & N. IROQUOIS ST. & CALUMET - LAURIUM VILLAGE \\
\hline 787 & & BORGO & DONARDA & W & 48 & CANADA & SCOTLAND & CANADA & & & CANADA-SCOTLAND & N. IROQUOIS ST. & CALUMET - LAURIUM VILLAGE \\
\hline 788 & & BORGO & DANIEL & s & 24 & MICHIGAN & ITALY & CANADA & & CARETAKER/CM & VICENZA & N. IROQUOIS ST. & CALUMET - LAURIUM VILLAGE \\
\hline 789 & & BORGO & KATE & $\mathrm{D}$ & 20 & MICHIGAN & ITALY & CANADA & & BOOKKEEPER/RETAIL & VICENZA & N. IROQUOIS ST. & CALUMET - LAURIUM VILLAGE \\
\hline 790 & & BORGO & HELEN & $\mathrm{D}$ & 19 & MICHIGAN & ITALY & CANADA & & & VICENZA & N. IROQUOIS ST. & CALUMET - LAURIUM VILLAGE \\
\hline 791 & & BORGO & ROBERT & $\mathrm{s}$ & 11 & MICHIGAN & ITALY & CANADA & & & VICENZA & N. IROQUOIS ST. & CALUMET - LAURIUM VILLAGE \\
\hline 792 & & BORGO & ALICE & $\mathrm{D}$ & 10 & MICHIGAN & ITALY & CANADA & & & VICENZA & N. IROQUOIS ST. & CALUMET - LAURIUM VILLAGE \\
\hline 793 & 129 & CAPELLI & LORENZO & $\mathrm{H}$ & 46 & ITALY & ITALY & ITALY & 1898 & MINER/CM & CAPIZZONE. BERGAMO & N. IROQUOIS ST. & CALUMET - LAURIUM VILLAGE \\
\hline 794 & & CAPELLI & ADA & W & 40 & MICHIGAN & ITALY & ITALY & & & & N. IROQUOIS ST. & CALUMET - LAURIUM VILLAGE \\
\hline 795 & & CAPELLI & HENRY & $\mathrm{s}$ & 20 & MICHIGAN & ITALY & MICHIGAN & & TIMBERMAN/CM & CAPIZZONE. BERGAMO & N. IROQUOIS ST. & CALUMET - LAURIUM VILLAGE \\
\hline 796 & 136 & MARTA & TERESA & $\mathrm{H}$ & 64 & ITALY & ITALY & ITALY & 1895 & & SAN MARTINO CANAVESE. TO & N. IROQUOIS ST. & CALUMET - LAURIUM VILLAGE \\
\hline 797 & & MARTA & JOHN & $\mathrm{s}$ & 19 & MICHIGAN & ITALY & ITALY & & MECHANIC/CM & SAN MARTINO CANAVESE. TO & N. IROQUOIS ST. & CALUMET - LAURIUM VILLAGE \\
\hline 798 & 217 & MANTZ & JOSEPH & $\mathrm{H}$ & 46 & ILLINOIS & GERMANY & GERMANY & & PLUMBER/HARDWARE & GERMANY & N. FLORIDA ST. & CALUMET - LAURIUM VILLAGE \\
\hline 799 & & MANTZ & TRACY & W & 49 & ITALY & ITALY & ITALY & 1903 & & & N. FLORIDA ST. & CALUMET - LAURIUM VILLAGE \\
\hline 800 & & MANTZ & CHARLES & s & 22 & MICHIGAN & ITALY & ITALY & & PLUMBER/HARDWARE & & N. FLORIDA ST. & CALUMET - LAURIUM VILLAGE \\
\hline 801 & & MANTZ & LOUIS & $\mathrm{s}$ & 21 & MICHIGAN & ITALY & ITALY & & PATTERN MAKER/CM FOUNDRY & & N. FLORIDA ST. & $\begin{array}{l}\text { CALUMET - LAURIUM VILLAGE } \\
\end{array}$ \\
\hline 802 & & MANTZ & APPOLLONIA & $\mathrm{D}$ & 19 & MICHIGAN & ITALY & ITALY & & TICKET SELLER/THEATRE & & N. FLORIDA ST. & CALUMET - LAURIUM VILLAGE \\
\hline 803 & & MANTZ & JOSEPH & $\mathrm{s}$ & 15 & MICHIGAN & ITALY & ITA & & & & DA ST. & CALUMET - LAURIUM VILLAGE \\
\hline 804 & & MANTZ & WILLIAM & $\mathrm{s}$ & 10 & MICHIGAN & ITA & ITA & & & & A ST. & CALUMET - LAURIUM VILLAGE \\
\hline 805 & 100 & BARSANTI & ALBERT & $\mathrm{H}$ & 50 & ITALY & ITA & ITA & 1896 & MERCHANT/FRUIT & LUCCA & ORIDA ST. & CALUMET - LAURIUM V \\
\hline 806 & & BARSANTI & CORINNE & W & 46 & & ITALY & ITALY & 1896 & & LUCCA & N. FLORIDA ST. & CALUMET - LAURIUM VILLAGE \\
\hline 807 & & UDELL & ROY & SOIL & 27 & MICHIGAN & MICHIGAN & MICHIGAN & & SALESMAN/ELECTRIC & USA & N. FLORIDA ST. & CALUMET - LAURIUM VILLAGE \\
\hline 808 & & UDELL & FLORENCE & $\mathrm{D}$ & 26 & MICHIGAN & ITALY & ITALY & & & LUCCA & N. FLORIDA ST. & CALUMET - LAURIUM VILLAGE \\
\hline 809 & 142 & MARINELLI & AUGUST & $\mathrm{H}$ & 68 & ITALY & ITALY & ITALY & 1890 & EDITOR/NEWSPAPER & TRENTO & N. FLORIDA ST. & CALUMET - LAURIUM VILLAGE \\
\hline 810 & & MARINELLI & LENA & w & 52 & GERMANY & GERMANY & GERMANY & 1886 & & GERMANY & N. FLORIDA ST. & CALUMET - LAURIUM VILLAGE \\
\hline 811 & 206 & MARINELLI & VIRGIL & $\mathrm{H}$ & 25 & MICHIGAN & ITALY & GERMANY & & MOLDER/CM FOUNDRY & TRENTO & N. FLORIDA ST. & CALUMET - LAURIUM VILLAGE \\
\hline 812 & & MARINELLI & CORINNE & W & 22 & MICHIGAN & CANADA & ITALY & & & & N. FLORIDA ST. & CALUMET - LAURIUM VILLAGE \\
\hline 813 & & MARINELLI & ROBERT & $\mathrm{s}$ & 5 & MICHIGAN & MICHIGAN & MICHIGAN & & & TRENTO & N. FLORIDA ST. & CALUMET - LAURIUM VILLAGE \\
\hline 814 & & MARINELLI & JAMES & $\mathrm{s}$ & 2 & MICHIGAN & MICHIGAN & MICHIGAN & & & TRENTO & N. FLORIDA ST. & CALUMET - LAURIUM VILLAGE \\
\hline 815 & 111 & GIULIO & JOHN & $\mathrm{H}$ & 37 & MICHIGAN & ITALY & ITALY & & BUTCHER/MEAT MARKET & SAN GIORGIO CANAVESE. TO & AHMEEK ST. & CALUMET - LAURIUM VILLAGE \\
\hline 816 & & GIULIO & LUDOVICA & w & 34 & & ITALY & & 1900 & & & EEK ST. & CALUMET - LAURIUM VILLAGE \\
\hline 817 & & GIULIO & & D & 12 & & & & & & SAN GIORGIO CANAVESE. TO & & CALUMET - LAURIUM VILLAC \\
\hline 818 & & GIULIO & ILONSET? & $\mathrm{s}$ & 1 & MICHIGAN & MICHIGAN & ITALY & & & SAN GIORGIO CANAVESE. TO & AHMEEK ST. & CALUMET - LAURIUM VILLAGE \\
\hline
\end{tabular}




\begin{tabular}{|c|c|c|c|c|c|c|c|c|c|c|c|c|c|}
\hline 819 & 309 & MERLO & JOHN & $\mathrm{H}$ & 46 & ITALY & ITALY & ITALY & 1900 & & RIVAROLO CANAVESE. TO & ISLE ROYALE ST. & CALUMET - LAURIUM VILLAGE \\
\hline 820 & & MERLO-ROSSO & TERESA & W & 40 & ITALY & ITALY & ITALY & 1908 & BAKER/BAKERY & & ISLE ROYALE ST. & CALUMET - LAURIUM VILLAGE \\
\hline 821 & & MERLO & MARY & $\mathrm{D}$ & 20 & MICHIGAN & ITALY & ITALY & & TEACHER/PUBLIC SCHOOL & RIVAROLO CANAVESE. TO & ISLE ROYALE ST. & CALUMET - LAURIUM VILLAGE \\
\hline 822 & & MERLO & CHARLES & $\mathrm{s}$ & 18 & MICHIGAN & ITALY & ITALY & & FENDER/ROCK CRUSHER & RIVAROLO CANAVESE. TO & ISLE ROYALE ST. & CALUMET - LAURIUM VILLAGE \\
\hline 823 & & MERLO & ANTHONY & $\mathrm{s}$ & 16 & MICHIGAN & ITALY & ITALY & & & RIVAROLO CANAVESE. TO & ISLE ROYALE ST. & CALUMET - LAURIUM VILLAGE \\
\hline 824 & & MERLO & EGENA & $\mathrm{D}$ & 14 & MICHIGAN & ITALY & ITALY & & & RIVAROLO CANAVESE. TO & ISLE ROYALE ST. & CALUMET - LAURIUM VILLAGE \\
\hline 825 & & MERLO & MARNIE & $\mathrm{D}$ & 12 & MICHIGAN & ITALY & ITALY & & & RIVAROLO CANAVESE. TO & ISLE ROYALE ST. & CALUMET - LAURIUM VILLAGE \\
\hline 826 & 205 & CONTEMPELETS & SECONDO & $\mathrm{H}$ & 42 & ITALY & ITALY & ITALY & 1910 & & & ISLE ROYALE ST. & CALUMET - LAURIUM VILLAGE \\
\hline 827 & 221 & TRIONE & PAUL & $\mathrm{H}$ & 38 & MICHIGAN & ITALY & ITALY & & MANAGER/DEPARTMENT STORE & TORINO & IROQUOIS ST. & CALUMET - LAURIUM VILLAGE \\
\hline 828 & & TRIONE & KATE & w & 36 & MICHIGAN & ITALY & ITALY & & & & IROQUOIS ST. & CALUMET - LAURIUM VILLAGE \\
\hline 829 & & TRIONE & DOMINICA & $\mathrm{D}$ & 11 & MICHIGAN & MICHIGAN & MICHIGAN & & & TORINO & IROQUOIS ST. & CALUMET - LAURIUM VILLAGE \\
\hline 830 & & TRIONE & GORDON & $\mathrm{s}$ & 7 & MICHIGAN & MICHIGAN & MICHIGAN & & & TORINO & IROQUOIS ST. & CALUMET - LAURIUM VILLAGE \\
\hline 831 & 233 & BARSANTI & PASCAL & $\mathrm{H}$ & 50 & ITALY & ITALY & ITALY & 1903 & MANAGER/FRUIT STORE & LUCCA & KEARSARGE ST. & CALUMET - LAURIUM VILLAGE \\
\hline 832 & & BARSANTI & MARY & W & 42 & ITALY & ITALY & ITALY & 1901 & & & KEARSARGE ST. & CALUMET - LAURIUM VILLAGE \\
\hline 833 & & BARSANTI & LAWRENCE & $\mathrm{s}$ & 24 & MICHIGAN & ITALY & ITALY & & MANAGER/GAS STATION & LUCCA & KEARSARGE ST. & CALUMET - LAURIUM VILLAGE \\
\hline 834 & & BARSANTI & RINA & D & 23 & MICHIGAN & ITALY & ITALY & & & LUCCA & KEARSARGE ST. & CALUMET - LAURIUM VILLAGE \\
\hline 835 & & BARSANTI & ELSIE & $\mathrm{D}$ & 19 & MICHIGAN & ITALY & ITALY & & & LUCCA & KEARSARGE ST. & CALUMET - LAURIUM VILLAGE \\
\hline 836 & 237 & ZEI & ARMANDO & $\mathrm{H}$ & 46 & ITALY & ITALY & ITALY & 1906 & TAILOR/OWN SHOP & LUCCA & KEARSARGE ST. & CALUMET - LAURIUM VILLAGE \\
\hline 837 & & ZEI & DONA & W & 38 & ITALY & ITALY & ITALY & 1896 & & & KEARSARGE ST. & CALUMET - LAURIUM VILLAGE \\
\hline 838 & & ZEI & ARMANDO & $\mathrm{s}$ & 21 & MICHIGAN & ITALY & ITALY & & DELIVERY BOY/GROCERY STORE & LUCCA & KEARSARGE ST. & CALUMET - LAURIUM VILLAGE \\
\hline 839 & & ZEI & JEAN & $\mathrm{D}$ & 1 & MICHIGAN & ITALY & ITALY & & & LUCCA & KEARSARGE ST. & CALUMET - LAURIUM VILLAGE \\
\hline 840 & 208 & SINA & VINCENT & $\mathrm{H}$ & 29 & MICHIGAN & ITALY & ITALY & & BLACKSMITH/FOR HIMSELF & & HECLA ST. & CALUMET - LAURIUM VILLAGE \\
\hline 841 & & SINA & SINE & w & 24 & MICHIGAN & ITALY & ITALY & & & & HECLA ST. & CALUMET - LAURIUM VILLAGE \\
\hline 842 & & SINA & MARIA & $\mathrm{D}$ & 1 & MICHIGAN & MICHIGAN & MICHIGAN & & & & HECLA ST. & CALUMET - LAURIUM VILLAGE \\
\hline 843 & 315 & GALLASERO & CHARLES & $\mathrm{H}$ & 68 & ITALY & ITALY & ITALY & 1885 & JANITOR/BANK & PONT CANAVESE. TO & HECLA ST. & CALUMET - LAURIUM VILLAGE \\
\hline 844 & & GALLASERO & LOUISE & W & 60 & ITALY & ITALY & ITALY & 1908 & & TORINO & HECLA ST. & CALUMET - LAURIUM VILLAGE \\
\hline 845 & 427 & DUNLOP & WILL & $\mathrm{H}$ & 25 & SCOTLAND & SCOTLAND & SCOTLAND & 1906 & DRIVER/TRUCK & SCOTLAND & HECLA ST. & CALUMET - LAURIUM VILLAGE \\
\hline 846 & & DUNLOP & EVELYN & W & 21 & MICHIGAN & ITALY & ITALY & & & & HECLA ST. & CALUMET - LAURIUM VILLAGE \\
\hline 847 & & DUNLOP & MARION & D & $3 \mathrm{M}$ & MICHIGAN & SCOTLAND & MICHIGAN & & & & HECLA ST. & CALUMET - LAURIUM VILLAGE \\
\hline 848 & & DUNLOP & WILL & $\mathrm{s}$ & 4 & MICHIGAN & SCOTLAND & MICHIGAN & & & & HECLA ST. & CALUMET - LAURIUM VILLAGE \\
\hline 849 & 437 & CALVETTI & JOHN & $\mathrm{H}$ & 24 & MICHIGAN & ITALY & ITALY & & CLERK/OFFICE & MILANO & HECLA ST. & CALUMET - LAURIUM VILLAGE \\
\hline 850 & & CALVETTI & MARION & W & 22 & MICHIGAN & MICHIGAN & MICHIGAN & & & & HECLA ST. & CALUMET - LAURIUM VILLAGE \\
\hline 851 & & CALVETTI & MARION & $\mathrm{D}$ & 1 & MICHIGAN & MICHIGAN & MICHIGAN & & & MILANO & HECLA ST. & CALUMET - LAURIUM VILLAGE \\
\hline 852 & REA & BARTRONE & GABRIEL & $\mathrm{H}$ & 22 & ITALY & ITALY & ITALY & 1916 & DRILLER/CM & & OSCEOLA ST. & CALUMET - LAURIUM VILLAGE \\
\hline 853 & & BARTRONE & MARCELLA & w & 18 & MICHIGAN & ENGLAND & ENGLAND & & & ENGLAND & OSCEOLA ST. & CALUMET - LAURIUM VILLAGE \\
\hline 854 & 324 & SLUSARZYK & JOHN & $\mathrm{H}$ & 38 & MICHIGAN & POLAND & POLAND & & MECHANIC/AUTOMOBILE & POLAND & OSCEOLA ST. & CALUMET - LAURIUM VILLAGE \\
\hline 855 & & SLUSARZYK & MINNIE & W & 27 & MICHIGAN & ITALY & ITALY & & & & OSCEOLA ST. & CALUMET - LAURIUM VILLAGE \\
\hline 856 & 322 & BALAGNA & CLEMENT & $\mathrm{H}$ & 50 & ITALY & ITALY & ITALY & 1900 & CARPENTER/FOR HIMSELF & PONT CANAVESE. TO & OSCEOLA ST. & CALUMET - LAURIUM VILLAGE \\
\hline 857 & & BALAGNA & NANDA & W & 42 & ITALY & ITALY & ITALY & $80 / 90$ & & & OSCEOLA ST. & CALUMET - LAURIUM VILLAGE \\
\hline 858 & & BALAGNA & MARIE & $\mathrm{D}$ & 20 & MICHIGAN & ITALY & ITALY & & & PONT CANAVESE. TO & OSCEOLA ST. & CALUMET - LAURIUM VILLAGE \\
\hline 859 & & BALAGNA & ANNA & D & 8 & MICHIGAN & ITALY & ITALY & & & PONT CANAVESE. TO & OSCEOLA ST. & CALUMET - LAURIUM VILLAGE \\
\hline 860 & 312 & TAMBORINO & DOMENICO & $\mathrm{H}$ & 46 & ITALY & ITALY & ITALY & 1913 & BUTCHER/FOR HIMSELF & LOCANA CANAVESE. TO & OSCEOLA ST. & CALUMET - LAURIUM VILLAGE \\
\hline 861 & & TAMBORINO & MARIE & W & 42 & ITALY & ITALY & ITALY & 1918 & & & OSCEOLA ST. & CALUMET - LAURIUM VILLAGE \\
\hline 862 & & TAMBORINO & JOHN & s & 19 & MICHIGAN & ITALY & ITALY & & & LOCANA CANAVESE. TO & OSCEOLA ST. & CALUMET - LAURIUM VILLAGE \\
\hline 863 & & TAMBORINO & MARY & $\mathrm{D}$ & 16 & MICHIGAN & ITALY & ITALY & & & LOCANA CANAVESE. TO & OSCEOLA ST. & CALUMET - LAURIUM VILLAGE \\
\hline 864 & & TAMBORINO & ERNEST & $\mathrm{s}$ & 9 & MICHIGAN & ITALY & ITALY & & & LOCANA CANAVESE. TO & OSCEOLA ST. & $\begin{array}{l}\text { CALUMET - LAURIUM VILLAGE } \\
\end{array}$ \\
\hline 865 & & TAMBORINO & LUCY & D & 6 & MICHIGAN & ITALY & ITALY & & & LOCANA CANAVESE. TO & & CALUMET - LAURIUM VILLAGE \\
\hline 866 & & TAMBORINO & MARION & REL & 6 & MICHIGAN & ITALY & ITAL & & & LOCANA CANAVESE. TO & & CALUMET - LAL \\
\hline 867 & & TAMBORINO & JENNY & $\mathrm{D}$ & 5 & MICHIGAN & ITA & ITA & & & LOCANA CANAVESE. TO & $\mathrm{OSC}$ & CALUMET - LAURI \\
\hline 868 & 310 & O'CONNOR & AMALIA & $\mathrm{H}$ & 47 & IIGAN & ITALY & ITA & & NURSE/TRAINER & & A ST. & CALUMET - LAURIUM VILLAGE \\
\hline 869 & & O'CONNOR & FRANCIS & $\mathrm{s}$ & 23 & MICHIGAN & MICHIGAN & MICHIGAN & & SALESMAN/GENERAL STORE & & & CALUMET - LAURIUM VILLAGE \\
\hline 870 & & O'CONNOR & CLEMENT & $\mathrm{s}$ & 21 & MICHIGAN & MICHIGAN & MICHIGA & & BARBER/BARBER SHOP & & OSCEOLA ST. & CALUMET - LAURIUM VILLAGE \\
\hline 871 & & O'CONNOR & MARTIN & $\mathrm{s}$ & 19 & MICHIGAN & MICHIGAN & MICHIGAN & & CLERK/BANK & & OSCEOLA ST. & CALUMET - LAURIUM VILLAGE \\
\hline 872 & & O'CONNOR & MARY & $\mathrm{SI}$ & 45 & MICHIGAN & ITALY & ITALY & & & & OSCEOLA ST. & CALUMET - LAURIUM VILLAGE \\
\hline 873 & & O'CONNOR & MARGHERITA & SI & 27 & MICHIGAN & ITALY & ITALY & & & & OSCEOLA ST. & CALUMET - LAURIUM VILLAGE \\
\hline 874 & & O'CONNOR & HUBBELL & BR & 29 & MICHIGAN & ITALY & ITALY & & FLOATMAN/ COPPER MILL & & OSCEOLA ST. & CALUMET - LAURIUM VILLAGE \\
\hline 875 & 65 & SALOTTI & CHARLES & $\mathrm{H}$ & 42 & MICHIGAN & ITALY & ITALY & & OPERATOR/GROCERY STORE & TORINO & 3RD ST. & CALUMET - LAURIUM VILLAGE \\
\hline 876 & & SALOTTI & TULLIE & W & 36 & MICHIGAN & ITALY & ITALY & & & & 3RD ST. & CALUMET - LAURIUM VILLAGE \\
\hline 877 & & SALOTTI & RITA & $\mathrm{D}$ & 5 & MICHIGAN & MICHIGA & MICHIGAN & & & TORINO & 3RD ST. & CALUMET - LAURIUM VILLAGE \\
\hline 878 & & SALOTTI & CHARLES & $\mathrm{s}$ & 2 & MICHIGAN & MICHIGAN & MICHIGAN & & & TORINO & 3RD ST. & CALUMET - LAURIUM VILLAGE \\
\hline 879 & 307 & & TOLOMEO & $\mathrm{H}$ & 55 & & ITA & ITA & & OPERATOR/SOFT DRINK PARLOR & LOCANA CANAVESE. TO & HECLA ST. & CALUUMET - LAURIUM VILLAGE \\
\hline 880 & & & & W & 54 & ITALY & ITALY & ITALY & 1899 & & & & CALUMET - LAURIUM VILLAC \\
\hline 881 & & QUELLO & JAMES & $\mathrm{s}$ & 15 & MICHIGAN & ITALY & ITALY & & & LOCANA CANAVESE. TO & HECLA ST. & CALUMET - LAURIUM VILLAGE \\
\hline
\end{tabular}




\begin{tabular}{|c|c|c|c|c|c|c|c|c|c|c|c|c|c|}
\hline 882 & 315 & O'BRIEN & JAMES & $\mathrm{H}$ & 36 & MICHIGAN & CANADA & MICHIGAN & & OPERATOR/POOL ROOM & CANADA & HECLA ST. & CALUMET - LAURIUM VILLAGE \\
\hline 883 & & O'BRIEN & JENNE & W & 29 & MICHIGAN & ITALY & ITALY & & & & HECLA ST. & CALUMET - LAURIUM VILLAGE \\
\hline 884 & & O'BRIEN & JAMES & $\mathrm{s}$ & 15 & MICHIGAN & MICHIGAN & MICHIGAN & & & & HECLA ST. & CALUMET - LAURIUM VILLAGE \\
\hline 885 & & O'BRIEN & PAUL & $\mathrm{S}$ & 4 & MICHIGAN & MICHIGAN & MICHIGAN & & & & HECLA ST. & CALUMET - LAURIUM VILLAGE \\
\hline 886 & & O'BRIEN & BARBARA & $\mathrm{D}$ & $8 \mathrm{M}$ & MICHIGAN & MICHIGAN & MICHIGAN & & & & HECLA ST. & CALUMET - LAURIUM VILLAGE \\
\hline 887 & 323 & MARTA & CATERINA & $\mathrm{H}$ & 67 & ITALY & ITALY & ITALY & 1885 & & TORINO & HECLA ST. & CALUMET - LAURIUM VILLAGE \\
\hline 888 & & MARTA & JOHN & s & 36 & MICHIGAN & ITALY & ITALY & & BAKER/FOR HIMSELF & TORINO & HECLA ST. & CALUMET - LAURIUM VILLAGE \\
\hline 889 & & MARTA & MARY & DIL & 20 & MICHIGAN & ITALY & ITALY & & & TORINO & HECLA ST. & CALUMET - LAURIUM VILLAGE \\
\hline 890 & & MARTA & ADOLFO & $\mathrm{s}$ & 25 & MICHIGAN & ITALY & ITALY & & BAKER/FOR HIMSELF & TORINO & HECLA ST. & CALUMET - LAURIUM VILLAGE \\
\hline 891 & 325 & RICHETTA & JAMES & $\mathrm{H}$ & 52 & MICHIGAN & ITALY & ITALY & 1886 & MANAGER/SOFT DRINK PARLOR & SAN MARTINO CANAVESE. TO & HECLA ST. & CALUMET - LAURIUM VILLAGE \\
\hline 892 & & RICHETTA & DOMENICO & w & 48 & MICHIGAN & ITALY & ITALY & 1900 & & & HECLA ST. & CALUMET - LAURIUM VILLAGE \\
\hline 893 & & RICHETTA & MIKE & $s$ & 20 & MICHIGAN & MICHIGAN & MICHIGAN & & SALESWOMAN/GENERAL STORE & SAN MARTINO CANAVESE. TO & HECLA ST. & CALUMET - LAURIUM VILLAGE \\
\hline 894 & & RICHETTA & KATE & $\mathrm{D}$ & 25 & MICHIGAN & MICHIGAN & MICHIGAN & & & SAN MARTINO CANAVESE. TO & HECLA ST. & CALUMET - LAURIUM VILLAGE \\
\hline 895 & 333 & RICHETTA & JAMES & $\mathrm{H}$ & 49 & MICHIGAN & ITALY & ITALY & 1886 & UNDERTAKER/FOR HIMSELF & SAN MARTINO CANAVESE. TO & HECLA ST. & CALUMET - LAURIUM VILLAGE \\
\hline 896 & & RICHETTA & MARION & W & 43 & MICHIGAN & ITALY & ITALY & 1906 & & & HECLA ST. & CALUMET - LAURIUM VILLAGE \\
\hline 897 & & RICHETTA & ANGELO & $\mathrm{S}$ & 21 & MICHIGAN & MICHIGAN & MICHIGAN & & TEAMSTER & SAN MARTINO CANAVESE, TO & HECLA ST. & CALUMET - LAURIUM VILLAGE \\
\hline 898 & & RICHETTA & VITTORIA & MO & 70 & ITALY & ITALY & ITALY & 1906 & & & HECLA ST. & CALUMET - LAURIUM VILLAGE \\
\hline 899 & & BOGATNERI & ELISABETH & B & 51 & MICHIGAN & ITALY & ITALY & & & & HECLA ST. & CALUMET - LAURIUM VILLAGE \\
\hline 900 & 320 & QUELLO & DOMENICO & $\mathrm{H}$ & 65 & ITALY & ITALY & ITALY & 1892 & OPERATOR/SOFT DRINK PARLOR & LOCANA CANAVESE. TO & HECLA ST. & CALUMET - LAURIUM VILLAGE \\
\hline 901 & & QUELLO & LUCY & W & 48 & ITALY & ITALY & ITALY & 1899 & & LOCANA CANAVESE. TO & HECLA ST. & CALUMET - LAURIUM VILLAGE \\
\hline 902 & & QUELLO & ATTILIO & s & 21 & MICHIGAN & ITALY & ITALY & & FIREMAN/STEAM RAILROAD & LOCANA CANAVESE. TO & HECLA ST. & CALUMET - LAURIUM VILLAGE \\
\hline 903 & & QUELLO & MARION & $\mathrm{D}$ & 19 & MICHIGAN & ITALY & ITALY & & FIREIVIAIVIS ILAIVI RAILROAD & LOCANA CANAVESE, TO & HECLA ST. & CALUMET - LAURIUM VILLAGE \\
\hline 904 & & QUELLO & JAMES & $\mathrm{s}$ & 18 & MICHIGAN & ITALY & ITALY & & & LOCANA CANAVESE. TO & HECLA ST. & CALUMET - LAURIUM VILLAGE \\
\hline 905 & & QUELLO & LUCY & $\mathrm{D}$ & 16 & MICHIGAN & ITALY & ITALY & & & LOCANA CANAVESE. TO & HECLA ST. & CALUMET - LAURIUM VILLAGE \\
\hline 906 & & QUELLO & DOMENICO & $\mathrm{s}$ & 14 & MICHIGAN & ITALY & ITALY & & & LOCANA CANAVESE. TO & HECLA ST. & CALUMET - LAURIUM VILLAGE \\
\hline 907 & & QUELLO & ERNEST & $\mathrm{s}$ & 13 & MICHIGAN & ITALY & ITALY & & & LOCANA CANAVESE. TO & HECLA ST. & CALUMET - LAURIUM VILLAGE \\
\hline 908 & & QUELLO & GERALDINE & GD & 1 & MICHIGAN & MICHIGAN & MICHIGAN & & & LOCANA CANAVESE. TO & HECLA ST. & CALUMET - LAURIUM VILLAGE \\
\hline 909 & 209 & TORREANO & JOHN & $\mathrm{H}$ & 52 & MICHIGAN & ITALY & ITALY & & BAKER & TORINO & HECLA ST. & CALUMET - LAURIUM VILLAGE \\
\hline 910 & & TORREANO & CAROLINA & W & 50 & ITALY & ITALY & ITALY & 1902 & & & HECLA ST. & CALUMET - LAURIUM VILLAGE \\
\hline 911 & & TORREANO & PIERINA & $\mathrm{D}$ & 30 & MICHIGAN & MICHIGAN & ITALY & & & TORINO & HECLA ST. & CALUMET - LAURIUM VILLAGE \\
\hline 912 & & TORREANO & JAMES & $\mathrm{s}$ & 21 & MICHIGAN & MICHIGAN & ITALY & & HELPER/BAKER & TORINO & HECLA ST. & CALUMET - LAURIUM VILLAGE \\
\hline 913 & & TORREANO & LOUIS & $\mathrm{s}$ & 18 & MICHIGAN & MICHIGAN & ITALY & & & TORINO & HECLA ST. & CALUMET - LAURIUM VILLAGE \\
\hline 914 & 313 & MARALLO & DOMENICO & $\mathrm{H}$ & 39 & ITALY & ITALY & ITALY & 1900 & DRILLER/CM & CHIETI & OSCEOLA ST. & CALUMET - LAURIUM VILLAGE \\
\hline 915 & & MARALLO & PAULINE & w & 36 & MICHIGAN & ITALY & ITALY & & & & OSCEOLA ST. & CALUMET - LAURIUM VILLAGE \\
\hline 916 & & MARALLO & FELICITA & $\mathrm{D}$ & 1 & MICHIGAN & ITALY & MICHIGAN & & & CHIETI & OSCEOLA ST. & CALUMET - LAURIUM VILLAGE \\
\hline 917 & 315 & ROVANO & JAMES & $\mathrm{H}$ & 41 & ITALY & ITALY & ITALY & 1907 & DRILLER/CM & PRANZALITO. TO & OSCEOLA ST. & CALUMET - LAURIUM VILLAGE \\
\hline 918 & & ROVANO-FEIRA & PIERINA & W & 38 & MICHIGAN & ITALY & ITALY & & & TORINO & OSCEOLA ST. & CALUMET - LAURIUM VILLAGE \\
\hline 919 & & ROVANO & TERESA & D & 16 & MICHIGAN & ITALY & MICHIGAN & & & PRANZALITO. TO & OSCEOLA ST. & CALUMET - LAURIUM VILLAGE \\
\hline 920 & & ROVANO & DOMENICO & $\mathrm{s}$ & 11 & MICHIGAN & ITALY & MICHIGAN & & & PRANZALITO. TO & OSCEOLA ST. & CALUMET - LAURIUM VILLAGE \\
\hline 921 & & ROVANO & BARBARA & $\mathrm{D}$ & 3 & MICHIGAN & ITALY & MICHIGAN & & & PRANZALITO. TO & OSCEOLA ST. & CALUMET - LAURIUM VILLAGE \\
\hline 922 & & FEIRA & TERESA & MIL & 70 & ITALY & ITALY & ITALY & 1886 & & TORINO & OSCEOLA ST. & CALUMET - LAURIUM VILLAGE \\
\hline 923 & 335 & RICHETTA & ANTHONY & $\mathrm{H}$ & 40 & ITALY & ITALY & ITALY & 1907 & MOTORMAN/ROCK HOUSE & SAN MARTINO CANAVESE. TO & OSCEOLA ST. & CALUMET - LAURIUM VILLAGE \\
\hline 924 & & RICHETTA & TERESA & W & 37 & MICHIGAN & ITALY & ITALY & & & & OSCEOLA ST. & CALUMET - LAURIUM VILLAGE \\
\hline 925 & & RICHETTA & MARION & $\mathrm{D}$ & 15 & MICHIGAN & ITALY & MICHIGAN & & & SAN MARTINO CANAVESE. TO & OSCEOLA ST. & CALUMET - LAURIUM VILLAGE \\
\hline 926 & & RICHETTA & VICTORIA & $\mathrm{D}$ & 13 & MICHIGAN & ITALY & MICHIGAN & & & SAN MARTINO CANAVESE. TO & OSCEOLA ST. & CALUMET - LAURIUM VILLAGE \\
\hline 927 & & RICHETTA & JOSEPHINE & $\mathrm{D}$ & 6 & MICHIGAN & ITALY & MICHIGAN & & & SAN MARTINO CANAVESE. TO & OSCEOLA ST. & CALUMET - LAURIUM VILLAGE \\
\hline 928 & 320 & ADAMS & MATTHEW & $\mathrm{H}$ & 38 & MICHIGAN & AUSTRIA & AUSTRIA & & DRILLER/CM & AUSTRIA & CALUMET ST. & CALUMET - LAURIUM VILLAGE \\
\hline 929 & & ADAMS-SALOTTI & MARY & w & 38 & MICHIGAN & ITALY & ITALY & & & CORIO. TO & CALUMET ST. & CALUMET - LAURIUM VILLAGE \\
\hline 930 & & ADAMS & ROBERT & $\mathrm{s}$ & 10 & MICHIGAN & MICHIGAN & MICHIGAN & & & CORIO. TO & CALUMET ST. & CALUMET - LAURIUM VILLAGE \\
\hline 931 & & ADAMS & PAUL & s & 4 & MICHIGAN & MICHIGAN & MICHIGAN & & & CORIO. TO & CALUMET ST. & CALUMET - LAURIUM VILLAGE \\
\hline 932 & & SALOTTI & JAMES & FIL & 81 & ITALY & ITA & ITALY & $83 / 93$ & & CORIO. TO & CALUMET ST. & CALUMET - LAURIUM VILLAGE \\
\hline 933 & 415 & MERLO & JOHN & $\mathrm{H}$ & 45 & ITALY & ITALY & ITALY & 1901 & BAKER/BAKERY & TORINO & OSCEOLA ST. & CALUMET - LAURIUM VILLAGE \\
\hline 934 & & MERLO & SAM & $\mathrm{BR}$ & 30 & ITALY & ITALY & ITALY & 1908 & DRILLER/CM & TORINO & OSCEOLA ST. & CALUMET - LAURIUM VILLAGE \\
\hline 935 & 68 & CURTO & DOMENICA & $\mathrm{H}$ & 47 & ITALY & ITALY & ITALY & 1897 & & TORINO & 3RD ST. & CALUMET - LAURIUM VILLAGE \\
\hline 936 & & CURTO & ANGELINE & D & 31 & MICHIGAN & MICHIGAN & ITALY & & NOT CLEAR & TORINO & 3RD ST. & CALUMET - LAURIUM VILLAGE \\
\hline 937 & & CURTO & VITTORIA & $\mathrm{D}$ & 25 & MICHIGAN & MICHIGAN & ITALY & & BOOKKEEPER/GENERAL STORE & TORINO & 3RD ST. & CALUMET - LAURIUM VILLAGE \\
\hline 938 & & CURTO & JENNA & $\mathrm{D}$ & 21 & MICHIGAN & MICHIGAN & ITALY & & STENOGRAPHER/OFFICE & TORINO & 3RD ST. & CALUMET - LAURIUM VILLAGE \\
\hline 939 & 230 & BORGO & JOHN & $\mathrm{H}$ & 39 & MICHIGAN & ITALY & ITALY & & SALESMAN/COMMERCIAL & VICENZA & CALUMET ST. & CALUMET - LAURIUM VILLAGE \\
\hline 940 & 200 & BORGO & MARIE & w & 44 & MICHIGAN & ITALY & ITALY & & SALESWOMAN/GENERAL STORE & & CALUMET ST. & $\begin{array}{l}\text { CALUMET - LAURIUM VILLAGE } \\
\end{array}$ \\
\hline 941 & 226 & BESSOLO & JOHN & $\mathrm{H}$ & 32 & MICHIGAN & ITALY & ITAL & & BLACKSMITH/CM & TORINO & CALUMET ST. & CALUMET - LAURIUM VILLAGE \\
\hline 942 & & BESSO & TERESA & w & 25 & & & & & & & TST. & CALUMET - LAURIUM VILLAGE \\
\hline 943 & & BESSOLO & ILLE & D & 5 & & & & & & & MET ST. & E \\
\hline 944 & & BESSOLO & JEAN & $\mathrm{D}$ & 2 & MICHIGAN & MICHIGAN & MICHIGAN & & & TORINO & CALUMET ST. & CALUMET - LAURIUM VILLAGE \\
\hline
\end{tabular}




\begin{tabular}{|c|c|c|c|c|c|c|c|c|c|c|c|c|c|}
\hline 945 & 204 & GRINDATTI & VICTOR & $\mathrm{H}$ & 40 & ITALY & ITALY & ITALY & 1900 & TAILOR & PONT CANAVESE. TO & CALUMET ST. & CALUMET - LAURIUM VILLAGE \\
\hline 946 & & GRINDATTI & CLARA & W & 38 & MICHIGAN & ITALY & ITALY & & & & CALUMET ST. & CALUMET - LAURIUM VILLAGE \\
\hline 947 & & GRINDATTI & ROBERT & $\mathrm{s}$ & 10 & MICHIGAN & ITALY & MICHIGAN & & & PONT CANAVESE. TO & CALUMET ST. & CALUMET - LAURIUM VILLAGE \\
\hline 948 & & GRINDATTI & BETTY & $\mathrm{D}$ & 5 & MICHIGAN & ITALY & MICHIGAN & & & PONT CANAVESE. TO & CALUMET ST. & CALUMET - LAURIUM VILLAGE \\
\hline 949 & 411 & ERNEST & CARL & $\mathrm{H}$ & 24 & MICHIGAN & FINLAND & FINLAND & & DRIVER/TRUCK & FINLAND & HECLA ST. & CALUMET - LAURIUM VILLAGE \\
\hline 950 & & ERNEST & MARIE & W & 22 & MICHIGAN & ITALY & ITALY & & & & HECLA ST. & CALUMET - LAURIUM VILLAGE \\
\hline 951 & & ERNEST & M? & $\mathrm{D}$ & 2 & MICHIGAN & MICHIGAN & MICHIGAN & & & & HECLA ST. & CALUMET - LAURIUM VILLAGE \\
\hline & 101 & VIGNETTO & JIM & $\mathrm{H}$ & 55 & ITALY & ITALY & ITALY & 1889 & TREASURER/ITALIAN MUTUAL & TORINO & HECLA ST. & CALUMET - LAURIUM VILLAGE \\
\hline 953 & 101 & VIGNETTO & ROSE & W & 63 & FRANCE & FRANCE & FRANCE & 1890 & 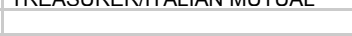 & 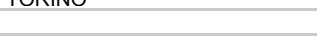 & HECLA ST. & $\begin{array}{l}\text { CALUMET - LAURIUM VILLAGE } \\
\text { CAUM }\end{array}$ \\
\hline 954 & 102 & GIULIO & ANTON & $\mathrm{H}$ & 24 & MICHIGAN & ITALY & ITALY & & LABORER/CM & SAN GIORGIO CANAVESE. TO & KEARSARGE ST. & CALUMET - LAURIUM VILLAGE \\
\hline 955 & & GIULIO & MARGHERITA & w & 23 & MICHIGAN & POLAND & POLAND & & & POLAND & KEARSARGE ST. & CALUMET - LAURIUM VILLAGE \\
\hline 956 & 110 & RASTELLO & JOSEPH & $\mathrm{H}$ & 38 & MICHIGAN & ITALY & ITALY & & SOLICITOR/STEAM LAUNDRY & PONT CANAVESE. TO & KEARSARGE ST. & CALUMET - LAURIUM VILLAGE \\
\hline 957 & & RASTELLO & AGNES & W & 32 & MICHIGAN & POLAND & POLAND & & & POLAND & KEARSARGE ST. & CALUMET - LAURIUM VILLAGE \\
\hline 958 & & RASTELLO & EVELYN & $\mathrm{D}$ & 9 & MICHIGAN & MICHIGAN & MICHIGAN & & & PONT CANAVESE. TO & KEARSARGE ST. & CALUMET - LAURIUM VILLAGE \\
\hline 959 & & RASTELLO & M? & $\mathrm{s}$ & 6 & MICHIGAN & MICHIGAN & MICHIGAN & & & PONT CANAVESE. TO & KEARSARGE ST. & CALUMET - LAURIUM VILLAGE \\
\hline 960 & & RASTELLO & BERNICE & D & 2 & MICHIGAN & MICHIGAN & MICHIGAN & & & PONT CANAVESE, TO & KEARSARGE ST. & CALUMET - LAURIUM VILLAGE \\
\hline 961 & 151 & LUCCHESI & FELIX & $\mathrm{H}$ & 59 & ITALY & ITALY & ITALY & 1891 & MACHINIST/CM & LUCCA & S. TAMARACK ST. & CALUMET - LAURIUM VILLAGE \\
\hline 962 & & LUCCHESI & AMELIA & W & 51 & ITALY & ITALY & ITALY & 1898 & & & S. TAMARACK ST. & CALUMET - LAURIUM VILLAGE \\
\hline 963 & & LUCCHESI & MATILDA & $\mathrm{D}$ & 29 & MICHIGAN & ITALY & ITALY & & & LUCCA & S. TAMARACK ST. & CALUMET - LAURIUM VILLAGE \\
\hline 964 & & LUCCHESI & FELIX JR.?? & $\mathrm{s}$ & 24 & MICHIGAN & ITALY & ITALY & & BRAKESMAN/STEAM RAILROAD & LUCCA & S. TAMARACK ST. & CALUMET - LAURIUM VILLAGE \\
\hline 965 & & LUCCHESI & ANNA & $\mathrm{D}$ & 22 & MICHIGAN & ITALY & ITALY & & & LUCCA & S. TAMARACK ST. & CALUMET - LAURIUM VILLAGE \\
\hline 966 & & LUCCHESI & PETER & $\mathrm{s}$ & 20 & MICHIGAN & ITALY & ITALY & & & LUCCA & S. TAMARACK ST. & CALUMET - LAURIUM VILLAGE \\
\hline 967 & 139 & LEIDI & FRANK & $\mathrm{H}$ & 30 & ITALY & ITALY & ITALY & 1923 & TIMBERMAN/CM & BERGAMO & S. PEWABIC ST. & CALUMET - LAURIUM VILLAGE \\
\hline 968 & & LEIDI & AGNES & W & 34 & SCOTLAND & SCOTLAND & SCOTLAND & 1925 & & SCOTLAND & S. PEWABIC ST. & CALUMET - LAURIUM VILLAGE \\
\hline 969 & & LEIDI & RUTH & $\mathrm{D}$ & 4 & MICHIGAN & ITALY & SCOTLAND & & & BERGAMO & S. PEWABIC ST. & CALUMET - LAURIUM VILLAGE \\
\hline 970 & 122 & ALLARIA & JOHN & $\mathrm{H}$ & 53 & ITALY & ITALY & ITALY & 1883 & INSPECTOR/CM & SAN GIORGIO CANAVESE. TO & S. PEWABIC ST. & CALUMET - LAURIUM VILLAGE \\
\hline 971 & & ALLARIA & MARY & w & 47 & ITALY & ITALY & ITALY & 1890 & & & S. PEWABIC ST. & CALUMET - LAURIUM VILLAGE \\
\hline 972 & & ALLARIA & PETER & s & 25 & MICHIGAN & ITALY & ITALY & & MINER/CM & SAN GIORGIO CANAVESE, TO & S. PEWABIC ST. & CALUMET - LAURIUM VILLAGE \\
\hline 973 & & ALLARIA & JACK/JOHN & $\mathrm{S}$ & 23 & MICHIGAN & ITALY & ITALY & & ELECTRICIAN/CM & SAN GIORGIO CANAVESE. TO & S. PEWABIC ST. & CALUMET - LAURIUM VILLAGE \\
\hline 974 & & ALARIA & JEAN? & $\mathrm{D}$ & 21 & MICHIGAN & ITALY & ITALY & & & SAN GIORGIO CANAVESE. TO & S. PEWABIC ST. & CALUMET - LAURIUM VILLAGE \\
\hline 975 & 146 & LUCCHESI & JOSEPH & $\mathrm{H}$ & 27 & MICHIGAN & ITALY & ITALY & & MINER/CM & LUCCA & S. IROQUOIS ST. & CALUMET - LAURIUM VILLAGE \\
\hline 976 & & LUCCHESI & ANNA & W & 21 & MICHIGAN & ITALY & ITALY & & & & S. IROQUOIS ST. & CALUMET - LAURIUM VILLAGE \\
\hline 977 & 102 & SCIACERO & JOHN & $\mathrm{H}$ & 31 & MICHIGAN & ITALY & ITALY & & BLACKSMITH/CM & TORINO & S. IROQUOIS ST. & CALUMET - LAURIUM VILLAGE \\
\hline 978 & & SCIACERO & VIRGINIA & w & 24 & MICHIGAN & MICHIGAN & MICHIGAN & & & USA & S. IROQUOIS ST. & CALUMET - LAURIUM VILLAGE \\
\hline 979 & 410 & NARDI & CHILI? & $\mathrm{H}$ & 48 & ITALY & ITALY & ITALY & 1898 & MINER/CM & LUCCA & 7TH ST. & CALUMET - LAURIUM VILLAGE \\
\hline 980 & 450 & MULLIN & JOHN & $\mathrm{H}$ & 34 & MICHIGAN & CANADA & MINNESOTA & & BRAKESMAN/STEAM RAILROAD & CANADA-USA & CALUMET ST. & CALUMET - LAURIUM VILLAGE \\
\hline 981 & & MULLIN & TERESA & W & 34 & MICHIGAN & ITALY & ITALY & & & & CALUMET ST. & CALUMET - LAURIUM VILLAGE \\
\hline 982 & & MULLIN & MARY & D & 11 & MICHIGAN & MICHIGAN & MICHIGAN & & & & CALUMET ST. & CALUMET - LAURIUM VILLAGE \\
\hline 983 & & MULLIN & TERESA & D & 10 & MICHIGAN & MICHIGAN & MICHIGAN & & & & CALUMET ST. & CALUMET - LAURIUM VILLAGE \\
\hline 984 & 412 & LOCATELLI & $\mathrm{JOHN}$ & $\mathrm{H}$ & 72 & ITALY & ITALY & ITALY & 1894 & CLEANER/CITY STREET & SELINO. BERGAMO & CALUMET ST. & CALUMET - LAURIUM VILLAGE \\
\hline 985 & & LOCATELLI & KATE & w & 70 & ITALY & ITALY & ITALY & 1896 & & & CALUMET ST. & CALUMET - LAURIUM VILLAGE \\
\hline 986 & & AIMONE & JOHN & BIL & 36 & MICHIGAN & ITALY & ITALY & & PROPRIETOR/SOFT DRINK & TORINO & ALLOUEZ ST. & CALUMET - LAURIUM VILLAGE \\
\hline 987 & & AIMONE & MAE & sl & 31 & MICHIGAN & POLAND & POLAND & & & POLAND & ALLOUEZ ST. & CALUMET - LAURIUM VILLAGE \\
\hline 988 & 431 & BRUSSO & JOSEPH & $\mathrm{H}$ & 64 & ITALY & ITALY & ITALY & 1899 & MINER/CM & AGLIE', TO & S. QUINCY ST. & CALUMET - LAURIUM VILLAGE \\
\hline 989 & & BRUSSO & BARBARA & W & 59 & ITALY & ITALY & ITALY & 1899 & & AGLIE', TO & S. QUINCY ST. & CALUMET - LAURIUM VILLAGE \\
\hline 990 & & BRUSSO & LAWRENCE & s & 28 & MICHIGAN & ITALY & ITALY & & MINER/CM & AGLIE'. TO & S. QUINCY ST. & $\begin{array}{l}\text { CALUMET - LAURIUM VILLAGE } \\
\end{array}$ \\
\hline 991 & & BRUSSO & JOSEPHINE & $\mathrm{D}$ & 24 & MICHIGAN & ITALY & ITALY & & & AGLIE'. TO & S. QUINCY ST. & CALUMET - LAURIUM VILLAGE \\
\hline 992 & 563 & MC MILLAN & THOMAS & $\mathrm{H}$ & 39 & SCOTLAND & SCOTLAND & TLAND & & SALESMAN/ELECTRICAL STORE & LAND & WYANDOTTE ST. & CALUUMET - LAURIUM VILLAGE \\
\hline 993 & & MC MILLAN & MAIME & w & 37 & MICHIGAN & ITALY & ITA & & BOOKKEEPER/CONFECTIONARY & LUCCA & OTTE ST. & CALUMET - LAURIUM VILLAGE \\
\hline 994 & & PASQUINI & JOHN & FIL & 72 & $\begin{array}{r}-Y \\
\text { S }\end{array}$ & ITALY & ITAL & UNK & & & $\begin{array}{l}\text { DTTE ST. } \\
\text { DT. }\end{array}$ & CALUMET - LAURIUM VILLAGE \\
\hline 995 & 503 & TORREANO & JAMES & $\mathrm{H}$ & 26 & MICHIGAN & ITALY & ITAL & & TEAMSTER/GROCERY STORE & TORINO & LAKE LINDEN & CALUMET - LAURIUM VILLAGE \\
\hline 996 & & TORREANO & MARGHERITA & W & 26 & MICHIGAN & ILLINOIS & MICHIGAN & & & & LAKE LINDEN & CALUMET - LAURIUM VILLAGE \\
\hline 997 & & TORREANO & ROBERT & $\mathrm{s}$ & 5 & MICHIGAN & MICHIGAN & MICHIGAN & & & TORINO & LAKE LINDEN & CALUMET - LAURIUM VILLAGE \\
\hline 998 & 340 & BARONI & UGO & $\mathrm{H}$ & 41 & ITALY & ITALY & ITALY & 1914 & PROPRIETOR/RESTAURANT & ROMA & 5TH ST. & CALUMET - CALUMET V. \\
\hline 999 & & BARONI & ANGELINA & w & 43 & ITALY & ITALY & ITALY & 1914 & & ROMA & 5TH ST. & CALUMET - CALUMET V. \\
\hline 1000 & & BARONI & CAMILLO & s & 15 & MICHIGAN & ITALY & ITALY & & & ROMA & 5TH ST. & CALUMET - CALUMET V. \\
\hline 1001 & & BARONI & CARMELO & $\mathrm{s}$ & 12 & MICHIGAN & ITALY & ITALY & & & ROMA & 5TH ST. & CALUMET - CALUMET V. \\
\hline 1002 & & BARONI & CESARINA & $\mathrm{D}$ & 10 & MICHIGAN & ITALY & ITALY & & & ROMA & 5TH ST. & CALUMET - CALUMET V. \\
\hline 1003 & 510 & TAMBELLINI & JOHN & $\mathrm{H}$ & 67 & ITALY & ITALY & ITALY & 1884 & RETIRED & LUCCA & PORTLAI & CALUMET - CALUMET V. \\
\hline 1004 & & TAMB & MARY & W & 54 & ITALY & ITALY & ITAL & 1897 & & & PORTL & CALUMET - CALUMET V. \\
\hline 1005 & & & & $\mathrm{~s}$ & 20 & & & & & & & & CALUMET - CALUMET V. \\
\hline 1006 & & & & D & 28 & & & & & & & VD ST. & $\mathrm{CA}$ \\
\hline 1007 & 510 & TAMBELLINI & JULIO & $\mathrm{H}$ & 32 & MICHIGAN & ITALY & ITALY & & PROPRIETOR/TAXI CAB & LUCCA & PORTLAND ST. & CALUMET - CALUMET V. \\
\hline
\end{tabular}




\begin{tabular}{|c|c|c|c|c|c|c|c|c|c|c|c|c|c|}
\hline 1008 & & TAMBELLINI & HAZEL & W & 32 & MICHIGAN & MICHIGAN & MICHIGAN & & & USA & PORTLAND ST. & CALUMET - CALUMET V. \\
\hline 1009 & & TAMBELLINI & JOHN & s & 6 & MICHIGAN & MICHIGAN & MICHIGAN & & & LUCCA & PORTLAND ST. & CALUMET - CALUMET V. \\
\hline 1010 & 512 & CURTO & JAMES & $\mathrm{H}$ & 66 & MICHIGAN & ITALY & ITALY & & CARPENTER/CM & TORINO & PORTLAND ST. & CALUMET - CALUMET V. \\
\hline 1011 & & CURTO & MARY & W & 60 & POLAND & POLAND & POLAND & & & POLAND & PORTLAND ST. & CALUMET - CALUMET V. \\
\hline 1012 & & CURTO & ANDREW & $\mathrm{s}$ & 40 & MICHIGAN & MICHIGAN & POLAND & & CAPTAIN/CM & TORINO & PORTLAND ST. & CALUMET - CALUMET V. \\
\hline 1013 & 206 & BARSANTI & GUIDO & $\mathrm{H}$ & 40 & ITALY & ITALY & ITALY & 1912 & PROPRIETOR/SOFT DRINK & LUCCA & 6TH ST. & CALUMET - CALUMET V. \\
\hline 1014 & & BARSANTI & ERMILLA & W & 37 & ITALY & ITALY & ITALY & 1913 & & & 6TH ST. & CALUMET - CALUMET V. \\
\hline 1015 & & BARSANTI & ELSIE & D & 15 & MICHIGAN & ITALY & ITALY & & & LUCCA & 6TH ST. & CALUMET - CALUMET V. \\
\hline 1016 & & BARSANTI & JOHN & $\mathrm{s}$ & 14 & MICHIGAN & ITALY & ITALY & & & LUCCA & 6TH ST. & CALUMET - CALUMET V. \\
\hline 1017 & & BARSANTI & CLARA & $\mathrm{D}$ & 10 & MICHIGAN & ITALY & ITALY & & & LUCCA & 6TH ST. & CALUMET - CALUMET V. \\
\hline 1018 & & BARSANTI & ROBERT & s & 8 & MICHIGAN & ITALY & ITALY & & & LUCCA & 6TH ST. & CALUMET - CALUMET V. \\
\hline 1019 & & BARSANTI & LAWRENCE & FIL & 83 & ITALY & ITALY & ITALY & 1913 & RETIRED & LUCCA & 6TH ST. & CALUMET - CALUMET V. \\
\hline 1020 & 400 & $\begin{array}{l}\text { DAROA } \\
\text { VAIRO }\end{array}$ & DOMENICO & $\mathrm{H}$ & $\frac{50}{40}$ & ITALY & ITALY & ITALY & 1901 & PROPRIETOR/BOARDING HOUSE & LOCANA CANAVESE. TO & 6TH ST. & CALUMET - CALUMET V. \\
\hline 1021 & 400 & $\begin{array}{l}\text { VAIRO } \\
\text { VAIRO }\end{array}$ & MARY & w & 37 & ITALY & ITALY & ITALY & 1905 & FRURRILTURIDUAREIVU HUUSE & LUUAIVA CAIVAVESE. IO & 6TH ST. & CALUMET - CALUMET V. \\
\hline 1022 & & VAIRO & DOMENICO & $\mathrm{s}$ & 16 & MICHIGAN & ITALY & ITALY & & & LOCANA CANAVESE. TO & 6TH ST. & CALUMET - CALUMET V. \\
\hline 1023 & & VAIRO & MARIE & RELA & 15 & MICHIGAN & ITALY & ITALY & & & LOCANA CANAVESE, TO & 6TH ST. & CALUMET - CALUMET V. \\
\hline 1024 & & NOTARIO & SAM & B & 43 & ITALY & ITALY & ITALY & 1904 & CLERK/SOFT DRINK PARLOR & SAN BENIGNO CANAVESE. TO & 6TH ST. & CALUMET - CALUMET V. \\
\hline 1025 & 509 & RASTELLO & PETER & H & 41 & MICHIGAN & ITALY & ITALY & & PROPRIETOR/SOFT DRINK & PONT CANAVESE. TO & 6TH ST. & CALUMET - CALUMET V. \\
\hline 1026 & & RASTELLO & ROSE & w & 40 & MICHIGAN & ITALY & ITALY & & & & 6TH ST. & CALUMET - CALUMET V. \\
\hline 1027 & 421 & MANZINI & JOHN & $\mathrm{H}$ & 57 & ITALY & ITALY & ITALY & 1914 & PROPRIETOR/GROCERY STORE & PITEGLIO. PISTOIA & 5TH ST. & CALUMET - CALUMET V. \\
\hline 1028 & & MANZINI & EUFEMIA & W & 45 & ITALY & ITALY & ITALY & 1916 & & & 5TH ST. & CALUMET - CALUMET V. \\
\hline 1029 & & MANZINI & PETER & s & 11 & MICHIGAN & ITALY & ITALY & & & PITEGLIO, PISTOIA & 5TH ST. & CALUMET - CALUMET V. \\
\hline 1030 & & MANZINI & MARY & D & 10 & MICHIGAN & ITALY & ITALY & & & PITEGLIO. PISTOIA & 5TH ST. & $\begin{array}{l}\text { CALUMET - CALUMET V. } \\
\end{array}$ \\
\hline 1031 & & MANZINI & OLIVER & $\mathrm{s}$ & 9 & MICHIGAN & ITALY & ITALY & & & PITEGLIO. PISTOIA & 5TH ST. & CALUMET - CALUMET V. \\
\hline 1032 & & MANZINI & GINO & $\mathrm{s}$ & 8 & MICHIGAN & ITALY & ITALY & & & PITEGLIO. PISTOIA & 5TH ST. & CALUMET - CALUMET V. \\
\hline 1033 & 436 & PICCHIOTTINO & PETER & $\mathrm{H}$ & 54 & ITALY & ITALY & ITALY & 1901 & PROPRIETOR/SOFT DRINK & PONT CANAVESE. TO & 6TH ST. & CALUMET - CALUMET V. \\
\hline 1034 & 430 & $\begin{array}{l}\text { PICCHIOTTINO } \\
\text { PICHO }\end{array}$ & TRACY & W & $\begin{array}{l}54 \\
46\end{array}$ & ITALY & $\begin{array}{l}\text { ITALY } \\
\text { ITAL }\end{array}$ & $\begin{array}{l}\text { ITALY } \\
\text { ITAL }\end{array}$ & 1904 & 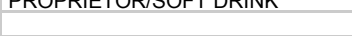 & PUNI CAINAVESE. TO & 6TH ST. & CALUMET - CALUMET V. \\
\hline 1035 & & PICCHIOTTINO & MARY & D & 24 & MICHIGAN & ITALY & ITALY & & & PONT CANAVESE, TO & 6TH ST. & CALUMET - CALUMET V. \\
\hline 1036 & & PICCHIOTTINO & JULIA & $\mathrm{D}$ & 22 & MICHIGAN & ITALY & ITALY & & & PONT CANAVESE. TO & 6TH ST. & CALUMET - CALUMET V. \\
\hline 1037 & 408 & BARATONO & LAWRENCE & B & 49 & ITALY & ITALY & ITALY & 1900 & TEAMSTER/BAKERY & TORINO & 6TH ST. & CALUMET - CALUMET V. \\
\hline 1038 & 404 & RASTELLO & JOHN & $\mathrm{H}$ & 37 & MICHIGAN & ITALY & ITALY & & PLUMBER/PLUMBING & PONT CANAVESE. TO & 6TH ST. & CALUMET - CALUMET V. \\
\hline 1039 & & RASTELLO & ROSIE & W & 21 & MICHIGAN & ENGLAND & ENGLAND & & & ENGLAND & 6TH ST. & CALUMET - CALUMET V. \\
\hline 1040 & & RASTELLO & JOSEPHINE & D & 2 & MICHIGAN & MICHIGAN & MICHIGAN & & & PONT CANAVESE. TO & 6TH ST. & CALUMET - CALUMET V. \\
\hline 1041 & & RASTELLO & CARLO & $\mathrm{s}$ & 4 & MICHIGAN & MICHIGAN & MICHIGAN & & & PONT CANAVESE, TO & 6TH ST. & CALUMET - CALUMET V. \\
\hline 1042 & & RASTELLO & ROSE & $\mathrm{D}$ & $4 \mathrm{M}$ & MICHIGAN & MICHIGAN & MICHIGAN & & & PONT CANAVESE. TO & 6TH ST. & CALUMET - CALUMET V. \\
\hline 1043 & 217 & COPPO & MARY & $\mathrm{H}$ & 49 & MICHIGAN & ITALY & ITALY & & SALESWOMAN/DEPARTMENT & TORINO & 6TH ST. & CALUMET - CALUMET V. \\
\hline 1044 & $217 \mathrm{~B}$ & RASTELLO & DOMENICO & $\mathrm{H}$ & 76 & ITALY & ITALY & ITALY & 1890 & & PONT CANAVESE. TO & 6TH ST. & CALUMET - CALUMET V. \\
\hline 1045 & & RASTELLO & COSTANCE & w & 78 & ITALY & ITALY & ITALY & 1890 & & PONT CANAVESE. TO & 6TH ST. & CALUMET - CALUMET V. \\
\hline 1046 & & RASTELLO & ROSA & D & 40 & ITALY & ITALY & ITALY & 1890 & JANITOR/APARTMENT BUILDING & PONT CANAVESE. TO & 6TH ST. & CALUMET - CALUMET V. \\
\hline 1047 & 613 & CIOMEI & EUGENIO & B & 51 & ITALY & ITALY & ITALY & 1903 & PROPRIETOR/CONFECTIONARY & LUCCA & OAK ST. & CALUMET - CALUMET V. \\
\hline 1048 & 615 & DIANDA & $\begin{array}{l}\text { JAMMA } \\
\text { JAM }\end{array}$ & $\mathrm{H}$ & 39 & ILLINOIS & ITALY & ITALY & & PROPRIETOR/CONFECTIONARY & & OAK ST. & CALUMET - CALUMET V. \\
\hline 1049 & & DIANDA & VINCENT & $\mathrm{s}$ & 13 & MICHIGAN & ITALY & ILLINOIS & & & LUCCA & OAK ST. & CALUMET - CALUMET V. \\
\hline 1050 & & DIANDA & MARJORY & D & 10 & MICHIGAN & ITALY & ILLINOIS & & & LUCCA & OAK ST. & CALUMET - CALUMET V. \\
\hline 1051 & 301 & LISA & MARTA & $\mathrm{H}$ & 79 & ITALY & ITALY & ITALY & 1880 & & SAN MARTINO CANAVESE. TO & 6TH ST. & CALUMET - CALUMET V. \\
\hline 1052 & & LISA & JOSEPHINE & $\mathrm{D}$ & 54 & ITALY & ITALY & ITALY & 1880 & & SAN MARTINO CANAVESE. TO & 6TH ST. & CALUMET - CALUMET V. \\
\hline 1053 & & LISA & JULIA & D & 47 & MICHIGAN & ITALY & ITALY & & BOOKKEEPER/BANK & SAN MARTINO CANAVESE. TO & 6TH ST. & CALUMET - CALUMET V. \\
\hline 1054 & 332 & SUINO & MARY & $\mathrm{H}$ & 34 & MICHIGAN & ITALY & ITALY & & STENOGRAPHER & TORINO & 7TH ST. & CALUMET - CALUMET V. \\
\hline 1055 & & SUINO & KATE & $\mathrm{SI}$ & 24 & MICHIGAN & ITALY & ITALY & & TEACHER/PUBLIC SCHOOL & TORINO & 7TH ST. & CALUMET - CALUMET V. \\
\hline 1056 & 405 & RASTELLO & CHARLES & $\mathrm{H}$ & 33 & ITALY & ITALY & ITALY & & & PONT CANAVESE. TO & 6TH ST. & CALUMET - CALUMET V. \\
\hline 1057 & & RASTELLO & AlLI & w & 32 & MICHIGAN & ITALY & ITALY & & PROPRIETOR/SHOES REPAIR & & 6TH ST. & CALUMET - CALUMET V. \\
\hline 1058 & & RASTELLO & ELRITA & D & $\frac{32}{11}$ & MICHIGAN & ITALY & MICHIGAN & & PRUTRIETURIORUEO RETAIR & PONT CANAVESE. TO & 6TH ST. & CALUMET - CALUMET V. \\
\hline 1059 & & RASTELLO & CHARLES JR. & s & 8 & MICHIGAN & ITALY & MICHIGAN & & & PONT CANAVESE, TO & 6TH ST. & CALUMET - CALUMET V. \\
\hline 1060 & & RASTELLO & PETER & $\mathrm{s}$ & 2 & MICHIGAN & ITALY & MICHIGAN & & & PONT CANAVESE. TO & 6TH ST. & CALUMET - CALUMET V. \\
\hline 1061 & & RASTELLO & MARJORIE & $\mathrm{D}$ & 1 & MICHIGAN & ITA & MICHIGAN & & & PONT CANAVESE. TO & 6TH ST. & CALUMET - CALUMET V. \\
\hline 1062 & 434 & MARTINI & OTTAVIO & B & 45 & ITA & ITA & ITALY & 1907 & MINER/CM & & & CALUMET - CALL \\
\hline 1063 & 418 & SCIACERO & ANGE & SER & 45 & ITA & ITA & ITA & 1907 & SERVANT/PF & TORINO & 8TH ST. & CALUMET - CALUMET V. \\
\hline 1064 & 403 & MIGLIO/MIGLIA & TERESA & $\mathrm{H}$ & 68 & ITALY & ITALY & ITALY & 79?? & & SAN GIORGIO CANAVESE. TO & 7TH ST. & CALUMET - CALUMET V. \\
\hline 1065 & & MIGLIO/MIGLIA & JOSEPH & S & 45 & MICHIGAN & ITALY & ITALY & & MANAGER/GROCERY STORE & SAN GIORGIO CANAVESE, TO & 7TH ST. & CALUMET - CALUMET V. \\
\hline 1066 & & MIGLIO/MIGLIA & TERESA & D & 34 & MICHIGAN & ITALY & ITALY & & JANITORESS/CITY HALL & SAN GIORGIO CANAVESE. TO & 7TH ST. & CALUMET - CALUMET V. \\
\hline 1067 & & MIGLIO/MIGLIA & PETER & s & 28 & MICHIGAN & ITALY & ITALY & & BILL POSTER/THEATRE & SAN GIORGIO CANAVESE. TO & 7TH ST. & CALUMET - CALUMET V. \\
\hline 1068 & 407 & TRIONE & AUGUST & $\mathrm{H}$ & 37 & MICHIGAN & ITALY & ITALY & & JANITOR/STORE BUILDING & TORINO & 7TH ST. & CALUMET - CALUMET V. \\
\hline 1069 & & TRIONE & LOUISE & w & 34 & CALIFORNIA & ITALY & ITALY & & & & 7TH ST. & CALUMET - CALUMET V. \\
\hline 1070 & & TRIONE & KATE & D & 12 & MICHIGAN & MICHIGAN & CALIFORNIA & & & TORINO & 7TH ST. & CALUMET - CALUMET V \\
\hline
\end{tabular}




\begin{tabular}{|c|c|c|c|c|c|c|c|c|c|c|c|c|c|}
\hline 1071 & 411 & BIANCHI & NARCISO & $\mathrm{H}$ & 52 & ITALY & ITALY & ITALY & 1897 & PROPRIETOR/SOFT DRINK & LUCCA & 7TH ST. & CALUMET - CALUMET V \\
\hline 1072 & & BIANCHI & EDITH & w & 47 & ITALY & ITALY & ITALY & 1885 & & LUCCA & 7TH ST. & CALUMET - CALUMET V \\
\hline 1073 & & BIANCHI & JULIO & $\mathrm{s}$ & 23 & MICHIGAN & ITALY & ITALY & & FOUNDRY MAN/CM & LUCCA & 7TH ST. & CALUMET - CALUMET V \\
\hline 1074 & & BIANCHI & CARLO & $\mathrm{s}$ & 18 & MICHIGAN & ITALY & ITALY & & & LUCCA & 7TH ST. & CALUMET - CALUMET V \\
\hline 1075 & & BIANCHI & ELISABETH & $\mathrm{D}$ & 10 & MICHIGAN & ITALY & ITALY & & & LUCCA & 7TH ST. & CALUMET - CALUMET V \\
\hline 1076 & & BANDONI & CASIMIRA & MOIL & 83 & ITALY & ITALY & ITALY & 1885 & & LUCCA & 7TH ST. & CALUMET - CALUMET V \\
\hline 1077 & 415 & SOPPELSA & ANTONIO & $\mathrm{H}$ & 24 & MICHIGAN & ITALY & ITALY & & TRUCK DRIVER/ICE CREAM & BELLUNO & 7TH ST. & CALUMET - CALUMET V \\
\hline 1078 & & SOPPELSA & FRANCESCA & W & 20 & MICHIGAN & SLOVENIA & SLOVENIA & & & SLOVENIA & 7TH ST. & CALUMET - CALUMET V \\
\hline 1079 & & SOPPELSA & TRACY & D & $2 \mathrm{M}$ & MICHIGAN & MICHIGAN & MICHIGAN & & & BELLUNO & 7TH ST. & CALUMET - CALUMET V \\
\hline 1080 & 415 & PICCHIOTTINO & JOHN & $\mathrm{H}$ & 28 & ITALY & ITALY & ITALY & 1902 & MECHANIC/AUTO GARAGE & PONT CANAVESE. TO & 7TH ST. & CALUMET - CALUMET V \\
\hline 1081 & & PICCHIOTTINO & SALLY & w & 26 & MICHIGAN & ITALY & ITALY & & & & 7TH ST. & CALUMET - CALUMET V \\
\hline 1082 & & PICCHIOTTINO & JEAN & D & $1 \mathrm{M}$ & MICHIGAN & ITALY & MICHIGAN & & & PONT CANAVESE. TO & 7TH ST. & CALUMET - CALUMET V \\
\hline 1083 & 443 & TAMBELLINI & ARMIDO & $\mathrm{H}$ & 58 & ITALY & ITALY & ITALY & 1890 & PROPRIETOR/GAS FILLING & LUCCA & 8TH ST. & CALUMET - CALUMET V \\
\hline 1084 & & TAMBELLINI & ANNA & w & 46 & MICHIGAN & ITALY & ITALY & & & & 8TH ST. & CALUMET - CALUMET V \\
\hline 1085 & & TAMBELLINI & EDWARD & $\mathrm{s}$ & 24 & MICHIGAN & ITALY & MICHIGAN & & BLACKSMITH/CM & LUCCA & 8TH ST. & CALUMET - CALUMET V \\
\hline 1086 & & TAMBELLINI & DANTE & $\mathrm{S}$ & 22 & MICHIGAN & ITALY & MICHIGAN & & LABORER/GAS STATION & LUCCA & 8TH ST. & CALUMET - CALUMET V \\
\hline 1087 & & TAMBELLINI & ARMIDA & D & 19 & MICHIGAN & ITALY & MICHIGAN & & & LUCCA & 8TH ST. & CALUMET - CALUMET V \\
\hline 1088 & & TAMBELLINI & FLOYD & $\bar{s}$ & 17 & MICHIGAN & ITALY & MICHIGAN & & ATTENDANT/GAS STATION & LUCCA & 8TH ST. & CALUMET - CALUMET V \\
\hline 1089 & 211 & BIANCHI & JOHN & $\mathrm{H}$ & 48 & ITALY & ITALY & ITALY & 1905 & RETIRED & LUCCA & 7TH ST. & CALUMET - CALUMET V \\
\hline 1090 & & BIANCHI & GLORIDIA? & w & 35 & ITALY & ITALY & ITALY & 1926 & & & 7TH ST. & CALUMET - CALUMET V \\
\hline 1091 & & BIANCHI & MARY & $\mathrm{D}$ & 2 & MICHIGAN & ITALY & ITALY & & & LUCCA & 7TH ST. & CALUMET - CALUMET V \\
\hline 1092 & 812 & BORGO & JOSEPHINE & $\mathrm{H}$ & 56 & MICHIGAN & ITALY & ITALY & & & & ELM ST. & CALUMET - CALUMET V \\
\hline 1093 & 012 & BORGO & INA & SI & 52 & MICHIGAN & ITALY & ITALY & & & VICENZA & ELM ST. & CALUMET - CALUMET V \\
\hline 1094 & & BORGO & NOBLE & BR & 45 & MICHIGAN & ITALY & ITALY & & SALESMAN/ELECTRICAL STORE & VICENZA & ELM ST. & CALUMET - CALUMET V \\
\hline 1095 & 825 & BARATONO & ALEX & $\mathrm{H}$ & 51 & ITALY & ITALY & ITALY & 1896 & SALESMAN/LIFE INSURANCE & TORINO & ELM ST. & CALUMET - CALUMET V \\
\hline 1096 & & BARATONO & MADDALENA & W & 48 & ITALY & ITALY & ITALY & 1890 & & TORINO & ELM ST. & CALUMET - CALUMET V \\
\hline 1097 & & BARATONO & ARTHUR & $\mathrm{s}$ & 27 & MICHIGAN & ITALY & ITALY & & CLERK/GARAGE & TORINO & ELM ST. & CALUMET - CALUMET V \\
\hline 1098 & 813 & MIGLIO/MIGLIA & MARY & $\mathrm{H}$ & 59 & ITALY & ITALY & ITALY & 1891 & & TORINO & ELM ST. & CALUMET - CALUMET V \\
\hline 1099 & & MIGLIO/MIGLIA & MARY & D & 36 & ITALY & ITALY & ITALY & 1907 & BEAUTY OPERATOR/BEAUTY & SAN GIORGIO CANAVESE. TO & ELM ST. & CALUMET - CALUMET V \\
\hline 1100 & & MIGLIO/MIGLIA & RAYMOND & $\bar{s}$ & 3 & MICHIGAN & ITALY & ITALY & & & SAN GIORGIO CANAVESE. TO & ELM ST. & CALUMET - CALUMET V \\
\hline 1101 & 809 & BRACCO & BERNARD & $\mathrm{H}$ & 85 & ITALY & ITALY & ITALY & 1882 & PROPRIETOR/BAKERY & PONT CANAVESE. TO & ELM ST. & CALUMET - CALUMET V \\
\hline 1102 & & BRACCO & MARIE & w & 73 & ITALY & ITALY & ITALY & 1883 & & PONT CANAVESE. TO & ELM ST. & CALUMET - CALUMET V \\
\hline 1103 & & BRACCO & CRISTINA & $\mathrm{D}$ & 34 & MICHIGAN & ITALY & ITALY & & CLERK/BAKERY & PONT CANAVESE. TO & ELM ST. & CALUMET - CALUMET V \\
\hline 1104 & & VIALE & PETER & SOIL & 46 & MICHIGAN & ITALY & ITALY & & BAKER/BAKERY & TORINO & ELM ST. & CALUMET - CALUMET V \\
\hline 1105 & & VIALE & JOSEPHINE & $\mathrm{D}$ & 48 & ITALY & ITALY & ITALY & 1885 & CLERK/BAKERY & PONT CANAVESE. TO & ELM ST. & CALUMET - CALUMET V \\
\hline 1106 & & VIALE & EDMOND & GS & 17 & MICHIGAN & MICHIGAN & ITALY & & & PONT CANAVESE. TO & ELM ST. & CALUMET - CALUMET V \\
\hline 1107 & 401 & QUELLO & JOHN & $\mathrm{H}$ & 58 & MICHIGAN & ITALY & IRELAND & & PROPRIETOR/LUMBER CAMP & LOCANA CANAVESE. TO & 8TH ST. & CALUMET - CALUMET V \\
\hline 1108 & & QUELLO & BATTISTA & BR & 50 & MICHIGAN & ITALY & IRELAND & & BANKER/BANKING & LOCANA CANAVESE. TO & 8TH ST. & CALUMET - CALUMET V \\
\hline 1109 & & QUELLO & MARGHERITA & $\mathrm{SI}$ & 52 & MICHIGAN & ITALY & IRELAND & & BOOKKEEPER/LUMBER CO. & LOCANA CANAVESE. TO & 8TH ST. & CALUMET - CALUMET V \\
\hline 1110 & & QUELLO & ANNA & Si & 47 & MICHIGAN & ITALY & IRELAND & & TEACHER/PUBLIC SCHOOL & LOCANA CANAVESE. TO & 8TH ST. & CALUMET - CALUMET V \\
\hline 1111 & & QUELLO & ALICE & SI & 40 & MICHIGAN & ITALY & IRELAND & & & LOCANA CANAVESE. TO & 8TH ST. & CALUMET - CALUMET V \\
\hline 1112 & $409 /$ & GRINDATTI & ANTONIO & $\mathrm{H}$ & 37 & MICHIGAN & ITALY & ITALY & & TRUCK DRIVER/OIL COMPANY & PONT CANAVESE. TO & 8TH ST. & CALUMET - CALUMET V \\
\hline 1113 & & GRINDATTI & HELEN & w & 27 & MICHIGAN & MICHIGAN & MICHIGAN & & & USA & 8TH ST. & CALUMET - CALUMET V \\
\hline 1114 & & GRINDATTI & GLORIA & D & 7 & MICHIGAN & MICHIGAN & MICHIGAN & & & PONT CANAVESE. TO & 8TH ST. & CALUMET - CALUMET V \\
\hline 1115 & & GRINDATTI & HELEN & $\mathrm{D}$ & 4 & MICHIGAN & MICHIGAN & MICHIGAN & & & PONT CANAVESE. TO & 8TH ST. & CALUMET - CALUMET V \\
\hline 1116 & & GRINDATTI & ANTONY & $\mathrm{s}$ & 1 & MICHIGAN & MICHIGAN & MICHIGAN & & & PONT CANAVESE. TO & 8TH ST. & CALUMET - CALUMET V \\
\hline 1117 & 510 & BRACCO & CHARLES & $\mathrm{H}$ & 46 & MICHIGAN & ITALY & ITALY & & CHAUFFEUR/PF & PONT CANAVESE. TO & 8TH ST. & CALUMET - CALUMET V \\
\hline 1118 & & BRACCO & AGNES & w & 45 & MICHIGAN & GERMANY & GERMANY & & & GERMANY & 8TH ST. & CALUMET - CALUMET V \\
\hline 1119 & & BRACCO & BERNARD J. & s & 18 & MICHIGAN & MICHIGAN & MICHIGAN & & & PONT CANAVESE. TO & 8TH ST. & CALUMET - CALUMET V \\
\hline 1120 & & BRACCO & ROSALYN & $\mathrm{D}$ & 9 & MICHIGAN & MICHIGAN & MICHIGAN & & & PONT CANAVESE. TO & 8TH ST. & CALUMET - CALUMET V \\
\hline 1121 & 516 & FORSTER & JANE & $\mathrm{H}$ & 42 & MICHIGAN & ITALY & ITALY & & & & 7TH ST. & CALUMET - CALUMET V \\
\hline 1122 & 00 & FORSTER & WILLIAM & s & 14 & MICHIGAN & MICHIGAN & MICHIGAN & & & & 7TH ST. & CALUMET - CALUMET V \\
\hline 1123 & & FORSTER & LEONARD & $\mathrm{s}$ & 9 & MICHIGAN & MICHIGAN & MICHIGAN & & & & 7TH ST. & CALUMET - CALUMET V \\
\hline 1124 & & PERONA & BATTISTA & $\mathrm{H}$ & 48 & ITALY & ITALY & ITALY & 1902 & FARMER/DAIRY FARM & PONT CANAVESE. TO & & CALUMET \\
\hline 1125 & & PERONA & MARY & w & 48 & ITALY & ITALY & ITALY & 1903 & & PONT CANAVESE. TO & & CALUMET \\
\hline 1126 & & PERONA & MARY & D & 24 & MICHIGAN & ITALY & ITALY & & & PONT CANAVESE. TO & & CALUMET \\
\hline 1127 & & PERONA & FRANK & $\mathrm{s}$ & 14 & MICHIGAN & ITALY & ITALY & & & PONT CANAVESE. TO & & CALUMET \\
\hline 1128 & & PERONA & JOHN B. & $\mathrm{s}$ & 9 & MICHIGAN & ITALY & ITALY & & & PONT CANAVESE, TO & & CALUMET \\
\hline 1129 & & DAIRO & THOMAS & $\mathrm{H}$ & 40 & ITALY & ITALY & ITALY & 93?? & FIREMAN/STATIONARY BOILER & TORINO & & $\begin{array}{l}\text { CALUMET } \\
\end{array}$ \\
\hline 1130 & & DAIRO & PAUL & $\mathrm{H}$ & 69 & FRANCE & ITALY & ITALY & 1885 & & TORINO & & CALUMET \\
\hline 1131 & 3980 & BANDETTINI & ANGELO & $\mathrm{H}$ & 64 & ITALY & ITALY & ITALY & 1890 & POLICEMAN/CM & LUCCA & SC & CALUMET \\
\hline 1132 & & BANDETTINI & ELISABETH & w & 51 & MICHIGAN & ITALY & ITALY & & & & & CALUMET \\
\hline 1133 & & BANDETTINI & $\begin{array}{l}\text { ANGELO } \\
\text { ANGL }\end{array}$ & s & 8 & MICHIGAN & ITALY & MICHIGAN & & & LUCCA & SCOTT ST & CALUMET \\
\hline
\end{tabular}




\begin{tabular}{|c|c|c|c|c|c|c|c|c|c|c|c|c|c|}
\hline 1134 & 3997 & PERONELLI & ANTON & H & 54 & MICHIGAN & ITALY & ITALY & & MINER/CM & LUCCA & SCOTT ST. & CALUMET \\
\hline 1135 & & PERONELLI & MARY & W & 52 & MICHIGAN & ITALY & ITALY & & & & SCOTT ST. & CALUMET \\
\hline 1136 & & PERONELLI & JOHN & $\mathrm{s}$ & 13 & MICHIGAN & MICHIGAN & MICHIGAN & & & LUCCA & SCOTT ST. & CALUMET \\
\hline 1137 & 3994 & LENCIONI & EGIDIO & $\mathrm{H}$ & 49 & ITALY & ITALY & ITALY & 1920 & BLACKSMITH/CM & LUCCA & SCOTT ST. & CALUMET \\
\hline 1138 & & LENCIONI & DIVA & w & 33 & ITALY & ITALY & ITALY & 1920 & & LUCCA & SCOTT ST. & CALUMET \\
\hline 1139 & & LENCIONI & MARY & D & 9 & MICHIGAN & ITALY & ITALY & & & LUCCA & SCOTT ST. & CALUMET \\
\hline 1140 & 3982 & MIGLIO/MIGLIA & KATE & $\mathrm{H}$ & 59 & ITALY & ITALY & ITALY & 1893 & & & 6TH ST. & CALUMET \\
\hline 1141 & & MIGLIO/MIGLIA & MARY & D & 33 & MICHIGAN & ITALY & ITALY & & SALESWOMAN/CONFECTIONARY & CASTELLAMONTE. TO & 6TH ST. & CALUMET \\
\hline 1142 & 3982 & GRINDATTI & LUCY & $\mathrm{H}$ & $84 ?$ & ITALY & ITALY & ITALY & 1900 & 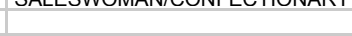 & PONT CANAVESE. TO & OSCEOLA ROAD & CALUMET \\
\hline 1143 & 3983 & BALAGNA & FRANK & $\mathrm{H}$ & 37 & MICHIGAN & ITALY & ITALY & & MINER/CM & PONT CANAVESE. TO & OSCEOLA ROAD & CALUMET \\
\hline 1144 & & BALAGNA & CAROLINA & w & 36 & ITALY & ITALY & ITALY & 1917 & & & OSCEOLA ROAD & CALUMET \\
\hline 1145 & & BALAGNA & JAMES & $\mathrm{S}$ & $10 / 6$ & MICHIGAN & MICHIGAN & ITALY & & & PONT CANAVESE. TO & OSCEOLA ROAD & CALUMET \\
\hline 1146 & & BALAGNA & LAWRENCE & s & 7 & MICHIGAN & MICHIGAN & ITALY & & & PONT CANAVESE. TO & OSCEOLA ROAD & CALUMET \\
\hline 1147 & 3985 & COLOMBO & JAMES & $\mathrm{H}$ & 68 & ITALY & ITALY & ITALY & 92/97 & & PONT CANAVESE. TO & OSCEOLA ROAD & CALUMET \\
\hline 1148 & & COLOMBO & JOSEPHINE & W & 55 & ITALY & ITALY & ITALY & 1894 & & PONT CANAVESE. TO & OSCEOLA ROAD & CALUMET \\
\hline 1149 & & COLOMBO & FRANK & $\mathrm{s}$ & 25 & MICHIGAN & ITALY & ITALY & & CARPENTER/CM & PONT CANAVESE, TO & OSCEOLA ROAD & CALUMET \\
\hline 1150 & 3960 & GHERNA & TERESA & $\mathrm{H}$ & 68 & ITALY & ITALY & ITALY & 1892 & & PONT CANAVESE. TO & WEDGE ST. & CALUMET \\
\hline 1151 & & GHERNA & MARCO & s & 30 & MICHIGAN & ITALY & ITALY & & LABORER/CM & PONT CANAVESE. TO & WEDGE ST. & CALUMET \\
\hline 1152 & & MICHETTI & THOMAS & BIL & 25 & MICHIGAN & ITALY & ITALY & & BLACKSMITH/CM & PONT CANAVESE. TO & WEDGE ST. & CALUMET \\
\hline 1153 & & MICHETTI & ISABELLE & $\mathrm{SI}$ & 25 & MICHIGAN & ITALY & ITALY & & SALESWOMAN/DRY GOODS & PONT CANAVESE. TO & WEDGE ST. & CALUMET \\
\hline 1154 & & PURACHIA & JOHN & B & 59 & ITALY & ITALY & ITALY & UN & MINER/CM & TORINO & WEDGE ST. & CALUMET \\
\hline 1155 & 3959 & PASQUINELLI & PETER & $\begin{array}{l}\mathrm{D} \\
\mathrm{H}\end{array}$ & 48 & MICHIGAN & ITALY & ITALY & Un & LABORER & LUCCA & WEDGE ST. & $\begin{array}{l}\text { CALUMET } \\
\text { CALIVI }\end{array}$ \\
\hline 1156 & & PASQUINELLI & EMILIA & w & 49 & ITALY & ITALY & ITALY & 1901 & & & WEDGE ST. & CALUMET \\
\hline 1157 & & PASQUINELLI & LUCY & D & 21 & MICHIGAN & MICHIGAN & ITALY & & & LUCCA & WEDGE ST. & CALUMET \\
\hline 1158 & & PASQUINELLI & CHARLES & S & 19 & MICHIGAN & MICHIGAN & ITALY & & LABORER/CM & LUCCA & WEDGE ST. & CALUMET \\
\hline 1159 & 3958 & BONOTTO & DOMENICO & $\mathrm{H}$ & 51 & ITALY & ITALY & ITALY & 1900 & MINER/CM & SAN BENIGNO CANAVESE. TO & WEDGE ST. & CALUMET \\
\hline 1160 & & BONOTTO & MINNIE & D & 23 & MICHIGAN & ITALY & ITALY & & SALESWOMAN/DRY GOODS & & WEDGE ST. & CALUMET \\
\hline 1161 & & BONOTTO & CATHERINE & $\mathrm{D}$ & 18 & MICHIGAN & ITALY & ITALY & & & SAN BENIGNO CANAVESE, TO & WEDGE ST. & CALUMET \\
\hline 1162 & & BONOTTO & JOHN & $\mathrm{s}$ & 16 & MICHIGAN & ITALY & ITALY & & & SAN BENIGNO CANAVESE. TO & WEDGE ST. & CALUMET \\
\hline 1163 & & BONOTTO & ALBERT & s & 15 & MICHIGAN & ITALY & ITALY & & & SAN BENIGNO CANAVESE. TO & WEDGE ST. & CALUMET \\
\hline 1164 & 3967 & QUELLO & JAMES & $\mathrm{H}$ & 35 & ITALY & ITALY & ITALY & $01 / 09$ & MINER/CM & LOCANA CANAVESE. TO & WEDGE ST. & CALUMET \\
\hline 1165 & $63 ? ?$ & QUELLO & MARY & w & 31 & ITALY & ITALY & ITALY & 1900 & & LOCANA CANAVESE. TO & WEDGE ST. & CALUMET \\
\hline 1166 & $00: ?$ & QUELLO & JAMES & s & 13 & MICHIGAN & ITALY & ITALY & 1900 & & LOCANA CANAVESE. TO & WEDGE ST. & CALUMET \\
\hline 1167 & & QUELLO & JOSEPH & $\underline{s}$ & 11 & MICHIGAN & ITALY & ITALY & & & LOCANA CANAVESE, TO & WEDGE ST. & CALUMET \\
\hline 1168 & & QUELLO & MARY & D & 9 & MICHIGAN & ITALY & ITALY & & & LOCANA CANAVESE. TO & WEDGE ST. & CALUMET \\
\hline 1169 & & QUELLO & JOHANNA & $\mathrm{D}$ & 6 & MICHIGAN & ITALY & ITALY & & & LOCANA CANAVESE. TO & WEDGE ST. & CALUMET \\
\hline 1170 & & QUELLO & ROBERT & $\mathrm{s}$ & 5 & MICHIGAN & ITALY & ITALY & & & LOCANA CANAVESE. TO & WEDGE ST. & CALUMET \\
\hline 1171 & & QUELLO & FRANCIS & D & 1 & MICHIGAN & ITALY & ITALY & & & LOCANA CANAVESE. TO & WEDGE ST. & CALUMET \\
\hline 1172 & 3934 & GHERNA & VICTOR & $\mathrm{H}$ & 34 & ITALY & ITALY & ITALY & 1906 & MINER/CM & PONT CANAVESE. TO & TEMPLE ST. & CALUMET \\
\hline 1173 & & GHERNA & ANGELINA & W & 23 & MICHIGAN & ITALY & ITALY & & & & TEMPLE ST. & CALUMET \\
\hline 1174 & & GHERNA & ANTON & $\mathrm{s}$ & 5 & MICHIGAN & ITALY & MICHIGAN & & & PONT CANAVESE. TO & TEMPLE ST. & CALUMET \\
\hline 1175 & & GHERNA & JAMES & s & 1 & MICHIGAN & ITALY & MICHIGAN & & & PONT CANAVESE. TO & TEMPLE ST. & CALUMET \\
\hline 1176 & 3937 & DAILY & ANNE & $\mathrm{H}$ & 72 & MICHIGAN & ITALY & ITALY & & & & TEMPLE ST. & CALUMET \\
\hline 1177 & & DAILY & WILLIAM & s & 41 & MICHIGAN & MICHIGAN & MICHIGAN & & MINER/CM & & TEMPLE ST. & CALUMET \\
\hline 1178 & 3941 & CHERUBINI & UGO & $\mathrm{H}$ & 34 & ITALY & ITALY & ITALY & 1913 & CARPENTER/CM & SASSOFERRATO. ANCONA & TEMPLE ST. & CALUMET \\
\hline 1179 & & CHERUBINI & LINA & W & 39 & MICHIGAN & ITALY & ITALY & 1010 & & $\begin{array}{l}\text { PONT CANAVESE. TO } \\
\end{array}$ & TEMPLE ST. & CALUMET \\
\hline 1180 & & CHERUBINI & ESTER & D & 12 & MICHIGAN & ITALY & MICHIGAN & & & SASSOFERRATO. ANCONA & TEMPLE ST. & CALUMET \\
\hline 1181 & & CHERUBINI & $\mathrm{F}$ ? & s & 10 & MICHIGAN & ITALY & MICHIGAN & & & SASSOFERRATO. ANCONA & TEMPLE ST. & CALUMET \\
\hline 1182 & & CHERUBINI & PAOLINA & D & 8 & MICHIGAN & ITALY & MICHIGAN & & & SASSOFERRATO. ANCONA & TEMPLE ST. & CALUMET \\
\hline 1183 & & CHERUBINI & GENEVIEVE & D & 6 & MICHIGAN & ITALY & MICHIGAN & & & SASSOFERRATO. ANCONA & TEMPLE ST. & CALUMET \\
\hline 1184 & & CHERUBINI & CATHERINE & D & 4 & MICHIGAN & ITALY & MICHIGAN & & & SASSOFERRATO. ANCONA & TEMPLE ST. & CALUMET \\
\hline 1185 & & CHERUBINI & JOSEPH & $\mathrm{s}$ & 1 & MICHIGAN & ITALY & MICHIGAN & & & SASSOFERRATO, ANCONA & TEMPLE ST. & CALUMET \\
\hline 1186 & & PICCHIOTTINO & PAULINE & MOIL & 63 & ITALY & ITALY & ITALY & 1897 & & PONT CANAVESE. TO & TEMPLE ST. & CALUMET \\
\hline 1187 & 3942 & VEZZANI & SAM & $\mathrm{H}$ & $?$ & ITALY & ITALY & ITALY & 1888 & MINER/CM & PORCARI. LU & TEMPLE ST. & CALUMET \\
\hline 1188 & & VEZZANI & ASSUNTA & MO & 60 & ITALY & ITALY & ITALY & $10 ? ?$ & & PORCARI. LU & TEMPLE ST. & CALUMET \\
\hline 1189 & 3944 & POMA & VINCENT & $\mathrm{H}$ & 60 & ITALY & ITALY & ITALY & 1905 & MASON/CM & IVREA. TO & TEMPLE ST. & CALUMET \\
\hline 1190 & & POMA & VINCENT & s & 26 & MICHIGAN & ITALY & ITALY & & MASON/CM & IVREA. TO & TEMPLE ST. & CALUMET \\
\hline 1191 & & POMA & CHARLES & s & 22 & MICHIGAN & ITALY & ITALY & & LABORER/FUEL GARAGE & IVREA, TO & TEMPLE ST. & CALUMET \\
\hline 1192 & 3947 & CERETTO & ANTON & $\mathrm{H}$ & $?$ & ITALY & ITALY & ITALY & 1897 & MINER/CM & PONT CANAVE & TEMPLE ST. & CALUMET \\
\hline 1193 & & CERETTO & MARY & W & 69 & ITALY & ITALY & ITALY & 1894 & & PONT CANAVESE. TO & TEMPLE ST. & CALUMET \\
\hline 1194 & & CERETTO & JENNIE & D & 22 & HIGAN & ITA & ITA & & SALESWOMAN/DRY GOODS & PONT CANAVESE. TO & TEMPLE ST. & CALUMET \\
\hline 1195 & & CERETTO & MARY & $\mathrm{D}$ & 20 & MICHIGAN & ITALY & ITALY & & BOOKKEEPER/LUMBER CO. & PONT CANAVESE. TO & PLE ST. & CALUMET \\
\hline 1196 & 3950 & GOTTA & JOHN & $\mathrm{H}$ & 55 & ITALY & ITALY & ITALY & 1900 & MINER/CM & LOCANA CANAVESE. TO & TEMPLE ST. & CALUMET \\
\hline
\end{tabular}




\begin{tabular}{|c|c|c|c|c|c|c|c|c|c|c|c|c|c|}
\hline 1197 & & GOTTA & MARY & $\mathrm{D}$ & 21 & MICHIGAN & ITALY & ITALY & & & & TEMPLE ST. & CALUMET \\
\hline 1198 & 3951 & TOMASI & PETER & $\mathrm{H}$ & 44 & ITALY & ITALY & ITALY & 1906 & MINER/CM & LOCANA CANAVESE. TO & TEMPLE ST. & CALUMET \\
\hline 1199 & & TOMASI & MARY & W & 50 & ITALY & ITALY & ITALY & 1915 & & LOCANA CANAVESE. TO & TEMPLE ST. & CALUMET \\
\hline 1200 & & TOMASI & TONY & $\mathrm{s}$ & 26 & ITALY & ITALY & ITALY & 1915 & LABORER/RAILROAD & LOCANA CANAVESE. TO & TEMPLE ST. & CALUMET \\
\hline 1201 & & TOMASI & MARY & D & 19 & MICHIGAN & ITALY & ITALY & & & LOCANA CANAVESE. TO & TEMPLE ST. & CALUMET \\
\hline 1202 & 3926 & GRINDATTI & KATE & $\mathrm{H}$ & 53 & ITALY & ITALY & ITALY & 1895 & & TORINO & DAM ST. & CALUMET \\
\hline 1203 & & CURTO & ZELLO/JOHN & B & 64 & ITALY & ITALY & ITALY & 1884 & MINER/CM & TORINO & DAM ST. & CALUMET \\
\hline 1204 & & GATTO/GATA & PETER & $\mathrm{B}$ & 38 & ITALY & ITALY & ITALY & 1910 & MINER/CM & LOCANA CANAVESE. TO & DAM ST. & CALUMET \\
\hline 1205 & 3914 & BARATONO & FELICIA & $\mathrm{H}$ & 49 & ITALY & ITALY & ITALY & 1917 & MANAGER/BOARDING HOUSE & TORINO & DAM ST. & CALUMET \\
\hline 1206 & & CREVALA & GIACINTO & B & 48 & ITALY & ITALY & ITALY & 1903 & MINER/CM & & NEW TOWN ST & CALUMET \\
\hline 1207 & & CREVALA & DOMENICO & B & 22 & MICHIGAN & ITALY & ITALY & & MINER/CM & & NEW TOWN ST. & CALUMET \\
\hline 1208 & & BERTOLOTTI & BURT & B & 65 & ITALY & ITALY & ITALY & 1906 & & TORINO & NEW TOWN ST. & CALUMET \\
\hline 1209 & & PERCENSCHAN & MARTIN & B & UN & ITALY & ITALY & ITALY & 1910 & MINER/CM & & NEW TOWN ST & CALUMET \\
\hline 1210 & & LEONE & JOHN & B & 48 & ITALY & ITALY & ITALY & 1909 & MINER/CM & TORINO & NEW TOWN ST & CALUMET \\
\hline 1211 & & GRANDELIS & PETER & B & 51 & ITALY & ITALY & ITALY & 1910 & MINER/CM & BELLUNO & NEW TOWN ST & CALUMET \\
\hline 1212 & & BARATONO & PETER & $\mathrm{S}$ & 12 & MICHIGAN & ITALY & ITALY & & & VIALFRE', TO & NEW TOWN ST. & CALUMET \\
\hline 1213 & & BARATONO & MARY & D & 12 & MICHIGAN & ITALY & ITALY & & & VIALFRE'. TO & NEW TOWN ST & CALUMET \\
\hline 1214 & 3953 & PISTONETTI & TERESA & $\mathrm{H}$ & 65 & ITALY & ITALY & ITALY & 1910 & & BOSCONERO. TO & NEW TOWN ST. & CALUMET \\
\hline 1215 & 587 & VIGO & CATHERINE & $\mathrm{H}$ & 58 & ITALY & ITALY & ITALY & 1910 & & BOSCONERO. TO & CEDAR ST. & CALUMET \\
\hline 1216 & & VIGO & MARNIE & D & 27 & MICHIGAN & ITALY & ITALY & 1010 & TEACHER/PUBLIC SCHOOL & CORIO. TO & CEDAR ST. & CALUMET \\
\hline 1217 & & VIGO & GEORGE & $\mathrm{s}$ & 26 & MICHIGAN & ITALY & ITALY & & DRUGGIST/DRUG STORE & CORIO. TO & CEDAR ST. & CALUMET \\
\hline 1218 & 566 & PONSETTO & GIORGIO & $\mathrm{H}$ & 64 & ITALY & ITALY & ITALY & 1910 & MINER/CM & SAN GIORGIO CANAVESE, TO & CEDAR ST. & CALUMET \\
\hline 1219 & & PONSETTO & JENNE & w & 60 & ITALY & ITALY & ITALY & 1904 & & TORINO & CEDAR ST. & CALUMET \\
\hline 1220 & 563 & SALACK & ANTON & $\mathrm{H}$ & 47 & ITALY & ITALY & ITALY & 1909 & MINER/CM & & CEDAR ST. & CALUMET \\
\hline 1221 & & SALACK & JACKINE & W & 36 & YUGOSLAVIA & YUGOSLAVIA & YUGOSLAVIA & & & YUGOSLAVIA & CEDAR ST. & CALUMET \\
\hline 1222 & & SALACK & JOSEPHINE & $\mathrm{D}$ & 17 & MICHIGAN & ITALY & YUGOSLAVIA & & & & CEDAR ST. & CALUMET \\
\hline 1223 & & SALACK & FRANK & $\mathrm{s}$ & 15 & MICHIGAN & ITALY & YUGOSLAVIA & & & & CEDAR ST. & CALUMET \\
\hline 1224 & & SALACK & JOHN & s & 13 & MICHIGAN & ITALY & YUGOSLAVIA & & & & CEDAR ST. & CALUMET \\
\hline 1225 & & SALACK & DOROTHY & D & 12 & MICHIGAN & ITALY & YUGOSLAVIA & & & & CEDAR ST. & CALUMET \\
\hline 1226 & & SALACK & ANNA & $\mathrm{D}$ & 10 & MICHIGAN & ITALY & YUGOSLAVIA & & & & CEDAR ST. & CALUMET \\
\hline 1227 & & SALACK & MATT & S & 4 & MICHIGAN & ITALY & YUGOSLAVIA & & & & CEDAR ST. & CALUMET \\
\hline 1228 & 515 & SOSSI/SUSSI & JAMES & $\mathrm{H}$ & 51 & ITALY & ITALY & ITALY & 1895 & MINER/CM & VIALFRE'. TO & 4TH ST. & CALUMET \\
\hline 1229 & 010 & SOSSI/SUSSI & ANGELINE & W & 41 & ITALY & ITALY & ITALY & 1902 & IVIIIVER/ IVI & VIALTRE. TO & 4TH ST. & CALUMET \\
\hline 1230 & & SOSSI/SUSSI & ANTON & $\begin{array}{l}\text { s } \\
\text { to }\end{array}$ & $\begin{array}{l}41 \\
21\end{array}$ & MICHIGAN & $\begin{array}{l}\text { ITALY } \\
\end{array}$ & ITALY & & PRINTER/PRINTING CO. & VIALFRE', TO & 4TH ST. & CALUMET \\
\hline 1231 & & SOSSI/SUSSI & MIKE & s & 17 & MICHIGAN & ITALY & ITALY & & & VIALFRE'. TO & 4TH ST. & CALUMET \\
\hline 1232 & & SOSSI/SUSSI & JOSEPH & s & 7 & MICHIGAN & ITALY & ITALY & & & VIALFRE'. TO & 4TH ST. & CALUMET \\
\hline 1233 & & SOSSI/SUSSI & JOSEPH & BR & 55 & ITALY & ITALY & ITALY & 1889 & LABORER/CM & VIALFRE'. TO & 4TH ST. & CALUMET \\
\hline 1234 & 520 & GAMARRA & JAMES & $\mathrm{H}$ & 46 & ITALY & ITALY & ITALY & 1907 & MINER/CM & SAN BENIGNO CANAVESE. TO & 4TH ST. & CALUMET \\
\hline 1235 & & GAMARRA & MARY & W & 36 & COLORADO & ITALY & ITALY & & & & 4TH ST. & CALUMET \\
\hline 1236 & & GAMARRA & NORBERTO & $\mathrm{s}$ & 17 & MICHIGAN & ITALY & COLORADO & & & SAN BENIGNO CANAVESE. TO & 4TH ST. & CALUMET \\
\hline 1237 & & GAMARRA & WINIFRED & D & 10 & MICHIGAN & ITALY & COLORADO & & & SAN BENIGNO CANAVESE. TO & 4TH ST. & CALUMET \\
\hline 1238 & & GAMARRA & DOROTHY & D & 7 & MICHIGAN & ITALY & COLORADO & & & SAN BENIGNO CANAVESE. TO & 4TH ST. & CALUMET \\
\hline 1239 & 521 & MICHETTI & FRED & $\mathrm{H}$ & 68 & ITALY & ITALY & ITALY & 1890 & MINER/CM & PONT CANAVESE. TO & 4TH ST. & CALUMET \\
\hline 1240 & & MICHETTI & MAUD & D & 24 & MICHIGAN & ITALY & ITALY & & SALESWOMAN/DRY GOODS & PONT CANAVESE. TO & 4TH ST. & CALUMET \\
\hline 1241 & & BOGGIO & FRANK & SOIL & 28 & ITALY & ITALY & ITALY & 1903 & MINER/CM & TORINO & 4TH ST. & CALUMET \\
\hline 1242 & & BOGGIO & CATERINA & $\mathrm{D}$ & 26 & MICHIGAN & ITALY & ITALY & 1000 & 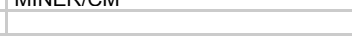 & TORINO & 4TH ST. & CALUMET \\
\hline 1243 & & BOGGIO & ROSINA & GD & 5 & WISCONSIN & ITALY & ITALY & & & TORINO & 4TH ST. & CALUMET \\
\hline 1244 & & BOGGIO & JOHANNA & GD & 3 & WISCONSIN & ITALY & ITALY & & & TORINO & 4TH ST. & CALUMET \\
\hline 1245 & & BOGGIO & GEORGE & GS & 1 & MICHIGAN & ITALY & ITALY & & & TORINO & 4TH ST. & CALUMET \\
\hline 1246 & 524 & BARATONO & DOMENICO & $\mathrm{H}$ & 52 & ITALY & ITALY & ITALY & 1899 & MINER/CM & VIALFRE'. TO & 4TH ST. & CALUMET \\
\hline 1247 & & BARATONO & ROSE & W & 48 & ITALY & ITALY & ITALY & 1902 & & & 4TH ST. & CALUMET \\
\hline 1248 & & BARATONO & ANNA & D & 15 & MICHIGAN & ITALY & ITALY & & & VIALFRE', TO & 4TH ST. & CALUMET \\
\hline 1249 & & BARATONO & LUCILLE & D & 9 & MICHIGAN & ITALY & ITALY & & & VIALFRE'. TO & 4TH ST. & CALUMET \\
\hline 1250 & 524 & BARATONO & JAMES & $\mathrm{H}$ & 21 & MICHIGAN & ITALY & ITALY & & MASON/CM & VIALFRE'. TO & 4TH ST. & CALUMET \\
\hline 1251 & & BARATONO & ELSIE & w & 18 & MICHIGAN & ITALY & ITALY & & & & 4TH ST. & CALUMET \\
\hline 1252 & 532 & DE FILIPPI & ANTON & H & 42 & MICHIGAN & ITALY & ITALY & & NOT CLEAR & TORINO & 3RD ST. & CALUMET \\
\hline 1253 & & DE FILIPPI & MARIE & w & 34 & MICHIGAN & POLAND & POLAND & & & POLAND & 3RD ST. & CALUMET \\
\hline 1254 & & DE FILIPPI & JEAN & D & 8 & MICHIGAN & MICHIGAN & MICHIGAN & & & TORINO & 3RD ST. & CALUMET \\
\hline 1255 & & DE FILIPPI & MARY & $\mathrm{D}$ & 3 & MICHIGAN & MICHIGAN & MICHIGAN & & & TORINO & 3RD ST. & CALUMET \\
\hline 1256 & 534 & DE FILIPPI & FELIX & $\mathrm{H}$ & 51 & ITALY & ITALY & ITALY & 1896 & MINER/CM & TORINO & 3RD ST. & CALUMET \\
\hline 1257 & & DE FILIPPI & VIRGINIA & w & 51 & ITALY & ITALY & ITALY & 1899 & & TORINO & 3RD ST. & CALUM \\
\hline 1258 & & DE FILIPPI & JOHN & s & 27 & MICHIGAN & ITALY & ITALY & & & TORINO & 3RD ST. & CALUMET \\
\hline 1259 & & NARRA-GEDDA & LUCIA & $\mathrm{H}$ & 44 & ITALY & ITALY & ITALY & 1912 & SALESWOMAN/CONFECTIONARY & TORINO & 3RD ST. & CALUMET \\
\hline
\end{tabular}




\begin{tabular}{|c|c|c|c|c|c|c|c|c|c|c|c|c|c|}
\hline 1260 & & NARRA & ANDREW & $\mathrm{s}$ & 17 & MICHIGAN & ITALY & ITALY & & & PONT CANAVESE. TO & 3RD ST. & CALUMET \\
\hline 1261 & 730 & PELLEGRINI & LUIGI & $\mathrm{H}$ & 59 & ITALY & ITALY & ITALY & 1905 & LABORER/CM & BERGAMO & WATERWORK ST. & CALUMET \\
\hline 1262 & & PELLEGRINI & IDA & W & 55 & ITALY & ITALY & ITALY & 1893 & & & WATERWORK ST. & CALUMET \\
\hline 1263 & & PELLEGRINI & JOSEPH & $\mathrm{s}$ & 22 & MICHIGAN & ITALY & ITALY & & BLACKSMITH/CM & BERGAMO & WATERWORK ST. & CALUMET \\
\hline 1264 & & PELLEGRINI & LEO & $\mathrm{s}$ & 20 & MICHIGAN & ITALY & ITALY & & & BERGAMO & WATERWORK ST. & CALUMET \\
\hline 1265 & & PELLEGRINI & MARGHERITA & D & 17 & MICHIGAN & ITALY & ITALY & & & BERGAMO & WATERWORK ST. & CALUMET \\
\hline 1266 & 4862 & COPPO & EUGENIA & $\mathrm{H}$ & 46 & ITALY & ITALY & ITALY & 1906 & & PONT CANAVESE. TO & R. J. SHAFT & CALUMET \\
\hline 1267 & 4002 & COPPO & HENRY & s & 18 & MICHIGAN & ITALY & ITALY & 1900 & LABORER/CM & PONT CANAVESE. TO & R. J. SHAFT & CALUMET \\
\hline 1268 & & COPPO & EDWARD & $\underline{s}$ & 16 & MICHIGAN & ITALY & ITALY & & LADURER/UIVI & PONT CANAVESE. TO & R. J. SHAFT & CALUMET \\
\hline 1269 & & COPPO & MARTIN & s & 14 & MICHIGAN & ITALY & ITALY & & & PONT CANAVESE. TO & R. J. SHAFT & CALUMET \\
\hline 1270 & & COPPO & MARY & D & 12 & MICHIGAN & ITALY & ITALY & & & PONT CANAVESE. TO & R. J. SHAFT & CALUMET \\
\hline 1271 & & COPPO & WILLIAM & S & 9 & MICHIGAN & ITALY & ITALY & & & PONT CANAVESE. TO & R. J. SHAFT & CALUMET \\
\hline 1272 & & COPPO & FRANCIS & $\mathrm{s}$ & 6 & MICHIGAN & ITALY & ITALY & & & PONT CANAVESE. TO & R. J. SHAFT & CALUMET \\
\hline 1273 & 4851 & PACINI & CARLO & $\mathrm{H}$ & 45 & ITALY & ITALY & ITALY & 1895 & PUMPMAN/CM & PUPILLO. PISTOIA & OAK ST. & CALUMET \\
\hline 1274 & & PACINI & HELEN & W & 39 & ITALY & ITALY & ITALY & 1916 & & & OAK ST. & CALUMET \\
\hline 1275 & & PACINI & LIDIA & D & 12 & MICHIGAN & ITALY & ITALY & & & PUPILLO, PISTOIA & OAK ST. & CALUMET \\
\hline 1276 & & PACINI & CARLO & $\mathrm{s}$ & 6 & MICHIGAN & ITALY & ITALY & & & PUPILLO. PISTOIA & OAK ST. & CALUMET \\
\hline 1277 & 4324 & MICHETTI & JOSEPH & $\mathrm{H}$ & 58 & ITALY & ITALY & ITALY & 96?? & MINER/CM & PONT CANAVESE. TO & OAK ST. & CALUMET \\
\hline 1278 & & MICHETTI & JOSEPH JR & $\mathrm{s}$ & 22 & MICHIGAN & ITALY & ITALY & & MINER/CM & PONT CANAVESE. TO & OAK ST. & CALUMET \\
\hline 1279 & & MICHETTI & JOHANNA & $\mathrm{D}$ & 18 & MICHIGAN & ITALY & ITALY & & & PONT CANAVESE. TO & OAK ST. & CALUMET \\
\hline 1280 & 4311 & LEONATTI & LOUIS & $\mathrm{H}$ & 41 & ITALY & ITALY & ITALY & 1908 & MINER/CM & CASTELLAMONTE. TO & OAK ST. & CALUMET \\
\hline 1281 & 4011 & LEONATTI & JOSEPHINE & W & 41 & MICHIGAN & ITALY & ITALY & 1900 & MVIINERTCIOI & UASIELLAIVIUIVIE. IO & OAK ST. & $\begin{array}{l}\text { CALUMET } \\
\text { CALIE }\end{array}$ \\
\hline 1282 & & LEONATTI & FRANCIS & MO & 70 & ITALY & ITALY & ITALY & 1909 & & CASTELLAMONTE. TO & OAK ST. & CALUMET \\
\hline 1283 & 4219 & BALMA/BAIMA & DOMENICO & $\mathrm{H}$ & 53 & MICHIGAN & ITALY & ITALY & & MINER/CM & SAN GIORGIO CANAVESE. TO & OAK ST. & CALUMET \\
\hline 1284 & & BALMA/BAIMA & MARY & W & $29 ?$ & ITALY & ITALY & ITALY & 1920 & & & OAK ST. & CALUMET \\
\hline 1285 & & BALMA/BAIMA & MIKE & $\mathrm{s}$ & 14 & MICHIGAN & MICHIGAN & ITALY & & & SAN GIORGIO CANAVESE. TO & OAK ST. & CALUMET \\
\hline 1286 & & BALMA/BAIMA & MATT & $\mathrm{s}$ & 12 & MICHIGAN & MICHIGAN & ITALY & & & SAN GIORGIO CANAVESE. TO & OAK ST. & CALUMET \\
\hline 1287 & & BALMA/BAIMA & ALBERT & $\mathrm{s}$ & 8 & MICHIGAN & MICHIGAN & ITALY & & & SAN GIORGIO CANAVESE, TO & OAK ST. & CALUMET \\
\hline 1288 & 4029 & ZANOTTI & ANTONIO & $\mathrm{H}$ & 49 & ITALY & ITALY & ITALY & 1899 & MINER/CM & PARELLA. TO & OAK ST. & CALUMET \\
\hline 1289 & & ZANOTTI & MARY & w & 40 & MICHIGAN & ITALY & ITALY & & & & OAK ST. & CALUMET \\
\hline 1290 & & ZANOTTI & G & D & 23 & MICHIGAN & ITALY & MICHIGAN & & & PARELLA. TO & OAK ST. & CALUMET \\
\hline 1291 & & ZANOTTI & FRANCIS & $\mathrm{s}$ & 20 & MICHIGAN & ITALY & MICHIGAN & & TIMBERMAN/CM & PARELLA. TO & OAK ST. & CALUMET \\
\hline 1292 & & ZANOTTI & GERVASO & $\mathrm{s}$ & 11 & MICHIGAN & ITALY & MICHIGAN & & & PARELLA. TO & OAK ST. & CALUMET \\
\hline 1293 & 4022 & MERLO & LOUIS & $\mathrm{H}$ & 46 & ITALY & ITALY & ITALY & 1909 & MINER/CM & PEROSA, TO & PORTLAND ST. & CALUMET \\
\hline 1294 & & MERLO & MARIANNA & w & 38 & ITALY & ITALY & ITALY & 1914 & & TORINO & PORTLAND ST. & CALUMET \\
\hline 1295 & & MERLO & LUDOVICA & D & 14 & MICHIGAN & ITALY & ITALY & & & PEROSA. TO & PORTLAND ST. & CALUMET \\
\hline 1296 & & MERLO & VITTORIA & $\mathrm{D}$ & 12 & MICHIGAN & ITALY & ITALY & & & PEROSA. TO & PORTLAND ST. & CALUMET \\
\hline 1297 & & MERLO & HERMAN & $\mathrm{D}$ & 9 & MICHIGAN & ITALY & ITALY & & & PEROSA. TO & PORTLAND ST. & CALUMET \\
\hline 1298 & & SOSSI/SUSSI & JOSEPH & B & 39 & ITALY & ITALY & ITALY & 1914 & MINER/CM & TORINO & PORTLAND ST. & CALUMET \\
\hline 1299 & 4022 & CASERIO & JOSEPH & $\mathrm{H}$ & 51 & ITALY & ITALY & ITALY & 1908 & LABORER/FOUNDRY CM & STRAMBINELLO. TO & PORTLAND ST. & CALUMET \\
\hline 1300 & & CASERIO & TERESA & W & 55 & ITALY & ITALY & ITALY & 1909 & & TORINO & PORTLAND ST. & CALUMET \\
\hline 1301 & & CASERIO & DOMENICO & s & 22 & MICHIGAN & ITALY & ITALY & & LABORER/FOUNDRY CM & STRAMBINELLO. TO & PORTLAND ST. & CALUMET \\
\hline 1302 & 4026 & GASPARDO & DOMENICO & $\mathrm{H}$ & 59 & ITALY & ITALY & ITALY & 1894 & MINER/CM & PONT CANAVESE. TO & PORTLAND ST. & CALUMET \\
\hline 1303 & & GASPARDO & MAUD & w & 57 & ITALY & ITALY & ITALY & 1898 & & & PORTLAND ST. & CALUMET \\
\hline 1304 & & GASPARDO & MARCO & B & 43 & ITALY & ITALY & ITALY & 1909 & MINER/CM & PONT CANAVESE. TO & PORTLAND ST. & CALUMET \\
\hline 1305 & 4011 & VERCELLINO & PETER & $\mathrm{H}$ & 36 & ITALY & ITALY & ITALY & 1910 & MINER/CM & SAN MARTINO CANAVESE. TO & PORTLAND ST. & CALUMET \\
\hline 1306 & & VERCELLINO & MARY & w & 40 & ITALY & ITALY & ITALY & 1896 & & TORINO & PORTLAND ST. & CALUMET \\
\hline 1307 & 4206 & $\begin{array}{l}\text { NAVONE } \\
\text { NAN }\end{array}$ & MARIANNA & $\mathrm{H}$ & 44 & ITALY & ITALY & ITALY & 1906 & MAID/PF & SAN GIORGIO CANAVESE. TO & 10TH ST. & CALUMET \\
\hline 1308 & & NAVONE & LUDOVICA & D & 21 & MICHIGAN & ITALY & ITALY & & MAID/PF & RIVAROLO CANAVESE. TO & 10TH ST. & CALUMET \\
\hline 1309 & & NAVONE & DOMENICO & $\mathrm{s}$ & 19 & MICHIGAN & ITALY & ITALY & & FOUNDRY MAN/CM & RIVAROLO CANAVESE. TO & 10TH ST. & CALUMET \\
\hline 1310 & 4003 & GRECO & FRANCIS & $\mathrm{H}$ & 46 & ITALY & ITALY & ITALY & 1912 & CLERGY MAN/CAT. CHURCH & & PORTLAND ST. & CALUMET \\
\hline 1311 & & GRECO & LOUISE & SER & 28 & MICHIGAN & ITALY & ITALY & & & & PORTLAND ST. & CALUMET \\
\hline 1312 & 4049 & VERNETTI & MARGHERITA & $\mathrm{H}$ & 63 & ITALY & ITALY & ITALY & 1901 & & PONT CANAVESE. TO & 10TH ST. & CALUMET \\
\hline 1313 & & DE MARIA & CATERINA & GD & 18 & MICHIGAN & ITALY & ITALY & & BOOKKEEPER/GASOLINE PLANT & TORINO & 10TH ST. & CALUMET \\
\hline 1314 & 4066 & CAPELLO & JOSEPH & $\mathrm{H}$ & 59 & ITALY & ITALY & ITALY & 1900 & MINER/CM & SAN BENIGNO CANAVESE. TO & 10TH ST. & CALUMET \\
\hline 1315 & & CAPELLO & MARY & w & 52 & ITALY & ITALY & ITALY & 1901 & & & 10TH ST. & CALUMET \\
\hline 1316 & $281 \mathrm{~A}$ & BENNY & FRANCIS & $\mathrm{H}$ & 32 & MICHIGAN & ENGLAND & ENGLAND & & MINER/CM & ENGLAND & 10TH ST. & CALUMET \\
\hline 1317 & & BENNY & GENIVA & W & 30 & ITALY & $\begin{array}{l}\text { ITALY } \\
\text { ITA }\end{array}$ & ITALY & 1901 & & & 10TH ST. & CALUMET \\
\hline 1318 & 4232 & MASCAGNO & FRANK & $\mathrm{H}$ & 60 & ITALY & ITALY & ITALY & 1898 & MINER/CM & TORINO & 10TH ST. & CALUMET \\
\hline 1319 & & MASCAGNO & GIACINTA & W & 60 & ITALY & ITALY & ITALY & 1898 & & TORINO & 10TH ST. & CALUMET \\
\hline 1320 & & MASCAGNO & ERNEST & $\mathrm{s}$ & 25 & MICHIGAN & ITA & ITALY & & LABORER & TORINO & 10TH ST. & CALUN \\
\hline 1321 & & MASCAGNO & MIKE & $\mathrm{s}$ & 30 & MICHIGAN & ITALY & ITALY & & BLACKSMITH/STEAM RAILI & TORINO & 10TH ST. & CALUMET \\
\hline 1322 & & NOTARIO & JOSEPH & $\mathrm{H}$ & 39 & ITALY & ITALY & ITALY & 1910 & FIREMAN/BOILER HOUSE & SAN BENIGNO CANAVESE. TO & 10TH ST. & CALUMET \\
\hline
\end{tabular}




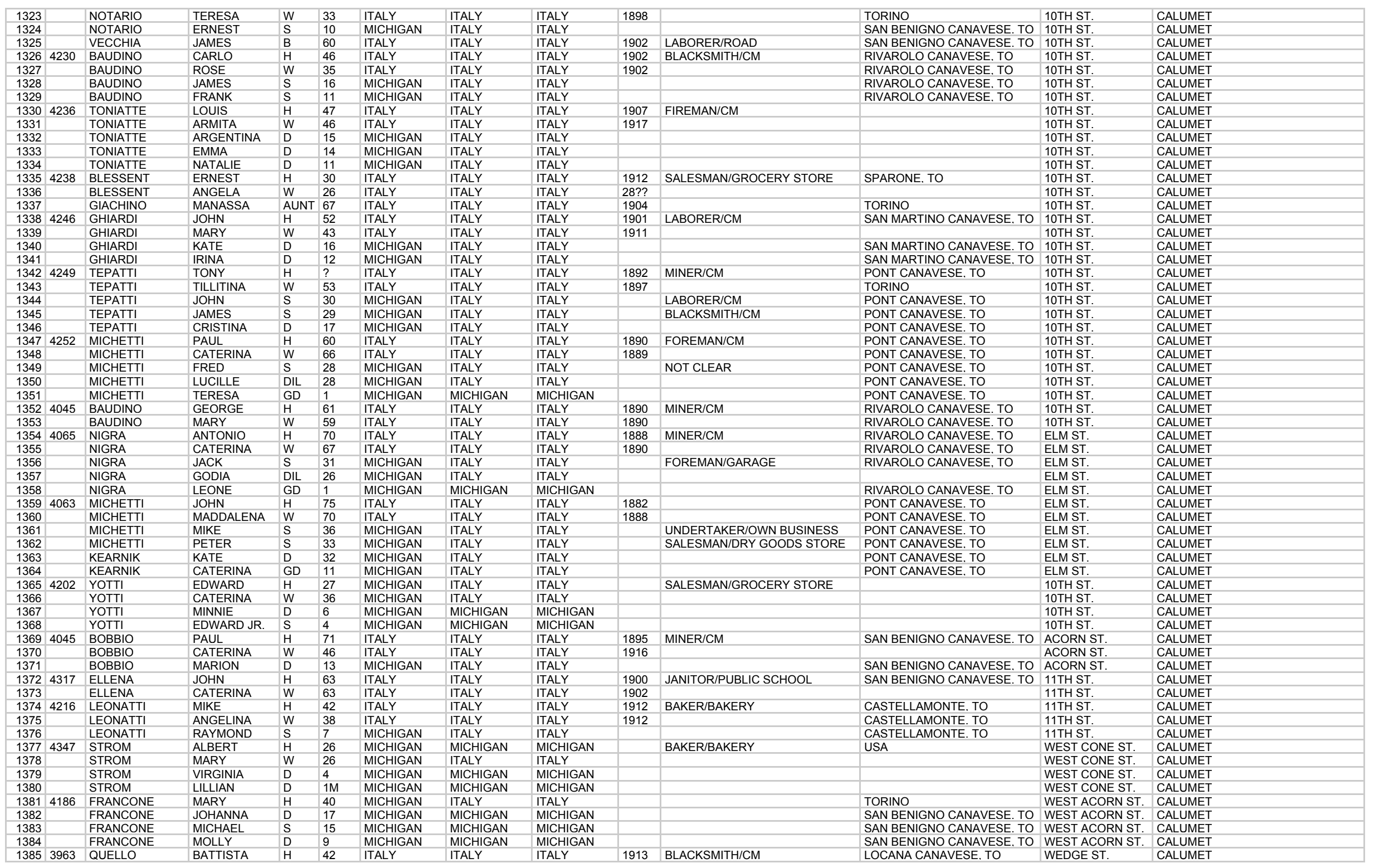




\begin{tabular}{|c|c|c|c|c|c|c|c|c|c|c|c|c|c|}
\hline 1386 & & QUELLO & VIRGINIA & W & 40 & MICHIGAN & ITALY & ITALY & & & & WEDGE ST. & CALUMET \\
\hline 1387 & & QUELLO & JOSEPH & $\mathrm{s}$ & 13 & MICHIGAN & ITALY & MICHIGAN & & & LOCANA CANAVESE. TO & WEDGE ST. & CALUMET \\
\hline 1388 & & QUELLO & MARIE & $\mathrm{D}$ & 9 & MICHIGAN & ITALY & MICHIGAN & & & LOCANA CANAVESE. TO & WEDGE ST. & CALUMET \\
\hline 1389 & & QUELLO & JOHN & $\mathrm{s}$ & 6 & MICHIGAN & ITALY & MICHIGAN & & & LOCANA CANAVESE. TO & WEDGE ST. & CALUMET \\
\hline 1390 & & QUELLO & MARTIN & s & 3 & MICHIGAN & ITALY & MICHIGAN & & & LOCANA CANAVESE. TO & WEDGE ST. & CALUMET \\
\hline 1391 & & QUELLO & PAULINE & $\mathrm{D}$ & 3 & MICHIGAN & ITALY & MICHIGAN & & & LOCANA CANAVESE. TO & WEDGE ST. & CALUMET \\
\hline 1392 & 3924 & NEPOTE & JAMES & $\mathrm{H}$ & 25 & MICHIGAN & ITALY & ITALY & & LABORER/CM & PONT CANAVESE. TO & 6TH ST. & CALUMET \\
\hline 1393 & & NEPOTE & MARY & $\mathrm{SI}$ & 20 & MICHIGAN & ITALY & ITALY & & & PONT CANAVESE. TO & 6TH ST. & CALUMET \\
\hline 1394 & & NEPOTE & LAWRENCE & $\mathrm{BR}$ & 15 & MICHIGAN & ITALY & ITALY & & & PONT CANAVESE. TO & 6TH ST. & $\begin{array}{l}\text { CALUMET } \\
\text { CALUE The }\end{array}$ \\
\hline 1395 & 1179 & BRILL & HENRY & $\mathrm{H}$ & 37 & MICHIGAN & WISCONSIN & WISCONSIN & & CHORE MAN/PF & USA & CEMETERY ST. & CALUMET \\
\hline 1396 & & BRILL & CATHERINE & w & 27 & MICHIGAN & ITALY & ITALY & & & & CEMETERY ST. & CALUMET \\
\hline 1397 & 1785 & KOLB & JOHN & $\mathrm{H}$ & 41 & MICHIGAN & GERMANY & MICHIGAN & & ROLLER REPAIRER/CM & GERMANY & CEMETERY ST. & CALUMET \\
\hline 1398 & & KOLB & CLARA & W & 33 & MICHIGAN & ITALY & ITALY & & & & CEMETERY ST. & CALUMET \\
\hline 1399 & & KOLB & ALBERTINA & $\mathrm{D}$ & 13 & MICHIGAN & MICHIGAN & MICHIGAN & & & & CEMETERY ST. & CALUMET \\
\hline 1400 & & KOLB & JOHN & $\mathrm{S}$ & 11 & MICHIGAN & MICHIGAN & MICHIGAN & & & & CEMETERY ST. & CALUMET \\
\hline 1401 & & KOLB & ELEONOR & D & 9 & MICHIGAN & MICHIGAN & MICHIGAN & & & & CEMETERY ST. & CALUMET \\
\hline 1402 & 1686 & RICCA & JOSEPH & $\mathrm{H}$ & 66 & ITALY & ITALY & ITALY & 1887 & STATIONARY FIREMAN/CM & TORINO & LAURIUM ST. & CALUMET \\
\hline 1403 & & RICCA & MARGHERITA & W & 62 & ITALY & ITALY & ITALY & 1888 & & TORINO & LAURIUM ST. & CALUMET \\
\hline 1404 & & RICCA & VERONICA & D & 23 & MICHIGAN & ITALY & ITALY & & & TORINO & LAURIUM ST. & CALUMET \\
\hline 1405 & & RICCA & JOSEPH JR. & $\mathrm{s}$ & 19 & MICHIGAN & ITALY & ITALY & & FOUNDRY MAN/CM & TORINO & LAURIUM ST. & CALUMET \\
\hline 1406 & 1535 & GIOVANNINI & GIUSTA & $\mathrm{H}$ & 60 & ITALY & ITALY & ITALY & 1891 & & TORINO & HECLA ST. & CALUMET \\
\hline 1407 & 1000 & GIOVANNINI & TERESA & $\mathrm{D}$ & 38 & ITALY & ITALY & ITALY & 1891 & CLERK/DRY GOODS STORE & SAN GIUSTO CANAVESE, TO & HECLA ST. & $\begin{array}{l}\text { CALUMET } \\
\text { CALIVI }\end{array}$ \\
\hline 1408 & & GIOVANNINI & VITTORIO & $\mathrm{s}$ & 34 & MICHIGAN & ITALY & ITALY & & STATIONARY ENGINEER/CM & SAN GIUSTO CANAVESE. TO & HECLA ST. & CALUMET \\
\hline 1409 & & GIOVANNINI & MINNIE & $\mathrm{D}$ & 31 & MICHIGAN & ITALY & ITALY & & & SAN GIUSTO CANAVESE. TO & HECLA ST. & CALUMET \\
\hline 1410 & 1537 & FERRARI & ERNESTINE & $\mathrm{H}$ & 58 & ITALY & ITALY & ITALY & 1894 & & LUCCA & HECLA ST. & CALUMET \\
\hline 1411 & 1545 & SPEHAR & RUDOLPH & H & 32 & MICHIGAN & AUSTRIA & AUSTRIA & & INSPECTOR/SMELTER & AUSTRIA & HECLA ST. & CALUMET \\
\hline 1412 & & SPEHAR & LUCILLE & w & 31 & MICHIGAN & ITALY & ITALY & & & & HECLA ST. & CALUMET \\
\hline 1413 & & SPEHAR & RUDOLPH J. & $\mathrm{s}$ & 7 & MICHIGAN & MICHIGAN & MICHIGAN & & & & HECLA ST. & CALUMET \\
\hline 1414 & 1549 & SANDRETTO & MIKE & $\mathrm{H}$ & 65 & ITALY & ITALY & ITALY & 1890 & LABORER/CM & PONT CANAVESE. TO & HECLA ST. & CALUMET \\
\hline 1415 & & SANDRETTO & MARGHERITA & w & 59 & ITALY & ITALY & ITALY & 1896 & & PONT CANAVESE. TO & HECLA ST. & CALUMET \\
\hline 1416 & & SANDRETTO & BERNARD & S & 30 & MICHIGAN & ITALY & ITALY & & LABORER/CM & PONT CANAVESE. TO & HECLA ST. & CALUMET \\
\hline 1417 & & SANDRETTO & FLORENCE & DIL & 25 & MICHIGAN & ITALY & ITALY & & & & HECLA ST. & CALUMET \\
\hline 1418 & & SANDRETTO & JEAN & GD & 2 & MICHIGAN & ITALY & ITALY & & & PONT CANAVESE. TO & HECLA ST. & CALUMET \\
\hline 1419 & & SANDRETTO & FRANCIS & GS & 1 & MICHIGAN & ITALY & ITALY & & & PONT CANAVESE, TO & HECLA ST. & CALUMET \\
\hline 1420 & & ROUX & MARY & $\mathrm{D}$ & 28 & MICHIGAN & ITALY & ITALY & & & PONT CANAVESE. TO & HECLA ST. & CALUMET \\
\hline 1421 & & ROUX & ARTHUR & SOIL & 27 & MICHIGAN & CANADA & MICHIGAN & & BLACKSMITH/CM & CANADA-USA & HECLA ST. & CALUMET \\
\hline 1422 & 1551 & OZZELLO & JOSEPH & $\mathrm{H}$ & 42 & ITALY & ITALY & ITALY & 1909 & MINER/CM & SAN GIUSTO CANAVESE. TO & HECLA ST. & CALUMET \\
\hline 1423 & & OZZELLO & MARY & w & 34 & MICHIGAN & ITALY & ITALY & & & TORINO & HECLA ST. & CALUMET \\
\hline 1424 & & OZZELLO & BERNARD & $\mathrm{s}$ & 16 & MICHIGAN & ITALY & MICHIGAN & & & SAN GIUSTO CANAVESE. TO & HECLA ST. & CALUMET \\
\hline 1425 & & OZZELLO & TED & $\mathrm{S}$ & 14 & MICHIGAN & ITALY & MICHIGAN & & & SAN GIUSTO CANAVESE. TO & HECLA ST. & CALUMET \\
\hline 1426 & & OZZELLO & VERNA & D & 11 & MICHIGAN & ITALY & MICHIGAN & & & SAN GIUSTO CANAVESE. TO & HECLA ST. & CALUMET \\
\hline 1427 & & OZZELLO & CORINNA & D & 4 & MICHIGAN & ITALY & MICHIGAN & & & SAN GIUSTO CANAVESE. TO & HECLA ST. & CALUMET \\
\hline 1428 & & OZZELLOO & BERNICE & $\mathrm{D}$ & 2 & MICHIGAN & ITALY & MICHIGAN & & & SAN GIUSTO CANAVESE. TO & HECLA ST. & CALUMET \\
\hline 1429 & & OZZELLO & ANTONIETTA & $\mathrm{D}$ & $7 \mathrm{M}$ & MICHIGAN & ITALY & MICHIGAN & & & SAN GIUSTO CANAVESE. TO & HECLA ST. & CALUMET \\
\hline 1430 & 1555 & BRACCO & ANTONY & $\mathrm{H}$ & 34 & MICHIGAN & ITALY & ITALY & & TIMEKEEPER/CM & TORINO & HECLA ST. & CALUMET \\
\hline 1431 & 1000 & BRACCO & LIZ & W & 33 & MICHIGAN & ITALY & ITALY & & HIVILALLTLRU IU & Tumine & HECLA ST. & CALUMET \\
\hline 1432 & & BRACCO & JOHN & $\mathrm{s}$ & 7 & MICHIGAN & MICHIGAN & MICHIGAN & & & TORINO & HECLA ST. & CALUMET \\
\hline 1433 & & BRACCO & KATE & D & 5 & MICHIGAN & MICHIGAN & MICHIGAN & & & TORINO & HECLA ST. & CALUMET \\
\hline 1434 & & BRACCO & GERARD & $\mathrm{s}$ & $3 \mathrm{M}$ & MICHIGAN & MICHIGAN & MICHIGAN & & & TORINO & HECLA ST. & CALUMET \\
\hline 1435 & 1561 & GIANNINI & LAWRENCE & $\mathrm{H}$ & 42 & ITALY & ITALY & ITALY & 1908 & LABORER/ROCK HOUSE & LUCCA & HECLA ST. & CALUMET \\
\hline 1436 & & GIANNINI & CAMILLA & W & 35 & MICHIGAN & ITALY & ITALY & & & LUCCA & HECLA ST. & CALUMET \\
\hline 1437 & & GIANNINI & ERNESTINA & $\mathrm{D}$ & 15 & MICHIGAN & ITALY & MICHIGAN & & & LUCCA & HECLA ST. & CALUMET \\
\hline 1438 & & GIANNINI & LIZ & D & 9 & MICHIGAN & ITALY & MICHIGAN & & & LUCCA & HECLA ST. & CALUMET \\
\hline 1439 & 1564 & RICCA & MATTHEW & $\mathrm{H}$ & 38 & MICHIGAN & ITALY & ITALY & & FOREMAN/CM & TORINO & HECLA ST. & CALUMET \\
\hline 1440 & & RICCA & GERTRUDE & w & 37 & MICHIGAN & MICHIGAN & MICHIGAN & & & USA & HECLA ST. & CALUMET \\
\hline 1441 & & RICCA & MARGHERITA & D & 15 & MICHIGAN & MICHIGAN & MICHIGAN & & & TORINO & HECLA ST. & CALUMET \\
\hline 1442 & & RICCA & JOSEPH & $\mathrm{s}$ & 13 & MICHIGAN & MICHIGAN & MICHIGAN & & & TORINO & HECLA ST. & CALUMET \\
\hline 1443 & & RICCA & DOROTHY & D & 11 & MICHIGAN & MICHIGAN & MICHIGAN & & & TORINO & HECLA ST. & CALUMET \\
\hline 1444 & & RICCA & MARY & $\mathrm{D}$ & 9 & MICHIGAN & MICHIGAN & MICHIGAN & & & TORINO & HECLA ST. & CALUMET \\
\hline 1445 & & RICCA & LAVERNE & D & 2 & MICHIGAN & MICHIGAN & MICHIGAN & & & TORINO & HECLA ST. & CALUMET \\
\hline 1446 & & RICCA & WILHELMINE & D & 1 & MICHIGAN & MICHIGAN & MICHIGAN & & & TORINO & HECLA ST. & CALUM \\
\hline 1447 & 1552 & LENCIONI & NATALE & $\mathrm{H}$ & 37 & MICHIGAN & ITALY & MICHIGAN & & FOREMAN/CM & LUCCA & HECLA ST. & CALUMET \\
\hline 1448 & & LENCIONI & MARY & W & 35 & MICHIGAN & ITALY & ITALY & & & & HECLA ST. & CALUMET \\
\hline
\end{tabular}




\begin{tabular}{|c|c|c|c|c|c|c|c|c|c|c|c|c|c|}
\hline 1449 & & LENCIONI & DELPHINE & $\mathrm{D}$ & 9 & MICHIGAN & MICHIGAN & ITALY & & & LUCCA & HECLA ST. & CALUMET \\
\hline 1450 & & LENCIONI & ANGELO & $\mathrm{s}$ & 8 & MICHIGAN & MICHIGAN & ITALY & & & LUCCA & HECLA ST. & CALUMET \\
\hline 1451 & & LENCIONI & LEONTINA & $\mathrm{D}$ & $3 \mathrm{M}$ & MICHIGAN & MICHIGAN & ITALY & & & LUCCA & HECLA ST. & CALUMET \\
\hline 1452 & & LENCIONI & ANGELO & $B R$ & 29 & MICHIGAN & ITALY & MICHIGAN & & LABORER/CM & LUCCA & HECLA ST. & CALUMET \\
\hline 1453 & 1546 & BARATONO & JAMES & $\mathrm{H}$ & 29 & MICHIGAN & ITALY & ITALY & & MECHANIC/GARAGE & VIALFRE'. TO & HECLA ST. & $\begin{array}{l}\text { CALUMET } \\
\end{array}$ \\
\hline 1454 & & BARATONO & VIOLET & w & 28 & MICHIGAN & CANADA & ENGLAND & & & CANADA-ENGLAND & HECLA ST. & CALUMET \\
\hline 1455 & & BARATONO & ROBERT & s & 7 & MICHIGAN & MICHIGAN & MICHIGAN & & & VIALFRE'. TO & HECLA ST. & CALUMET \\
\hline 1456 & & BARATONO & ARTHUR & BR & 21 & MICHIGAN & ITALY & ITALY & & STONE MASON/CM & VIALFRE'. TO & HECLA ST. & $\begin{array}{l}\text { CALUMET } \\
\text { Cont }\end{array}$ \\
\hline 1457 & 1540 & ZUBIENA & JAMES & $\mathrm{H}$ & 44 & ITALY & ITALY & ITALY & 1911 & MINER/CM & TORINO & HECLA ST. & $\begin{array}{l}\text { CALUMET } \\
\text { CALI T }\end{array}$ \\
\hline 1458 & & ZUBIENA & MADELINE & w & 43 & ITALY & ITALY & ITALY & 1911 & & TORINO & HECLA ST. & CALUMET \\
\hline 1459 & & ZUBIENA & FRANK & s & 17 & MICHIGAN & ITALY & ITALY & & LABORER/CM & TORINO & HECLA ST. & CALUMET \\
\hline 1460 & & ZUBIENA & JULIA & D & 13 & MICHIGAN & ITALY & ITALY & & & TORINO & HECLA ST. & CALUMET \\
\hline 1461 & & ZUBIENA & CATERINE & $\mathrm{D}$ & 13 & MICHIGAN & ITALY & ITALY & & & TORINO & HECLA ST. & CALUMET \\
\hline 1462 & & ZUBIENA & CESARE & $\mathrm{s}$ & 5 & MICHIGAN & ITALY & ITALY & & & TORINO & HECLA ST. & CALUMET \\
\hline 1463 & 1538 & LE CHEVALIER & PAUL & $\mathrm{H}$ & 43 & MICHIGAN & FRANCE & FRANCE & & CONSTRUCTION WORK/CM & FRANCE & HECLA ST. & CALUMET \\
\hline 1464 & & LE CHEVALIER & MARGHERITA & W & 41 & ITALY & ITALY & ITALY & 1892 & & & HECLA ST. & CALUMET \\
\hline 1465 & & LE CHEVALIER & JULIUS & $\mathrm{s}$ & 19 & MICHIGAN & ITALY & MICHIGAN & & TRUCK DRIVER/DRAY LINE & & HECLA ST. & CALUMET \\
\hline 1466 & & LE CHEVALIER & EMIL & s & 17 & MICHIGAN & ITALY & MICHIGAN & & & & HECLA ST. & CALUMET \\
\hline 1467 & & LE CHEVALIER & JOSEPH & $\mathrm{s}$ & 16 & MICHIGAN & ITALY & MICHIGAN & & HELPER/ELECTRIC SHOP & & HECLA ST. & CALUMET \\
\hline 1468 & & LE CHEVALIER & ANGELINE & $\mathrm{D}$ & 14 & MICHIGAN & ITALY & MICHIGAN & & & & HECLA ST. & CALUMET \\
\hline 1469 & & LE CHEVALIER & PETER & $\mathrm{s}$ & 11 & MICHIGAN & ITALY & MICHIGAN & & & & HECLA ST. & CALUMET \\
\hline 1470 & & LE CHEVALIER & ANTONY & $\frac{s}{s}$ & 9 & MICHIGAN & ITALY & MICHIGAN & & & & HECLA ST. & $\begin{array}{l}\text { CALUMET } \\
\text { CALIVI }\end{array}$ \\
\hline 1471 & & LE CHEVALIER & ROSEMARY & D & 7 & MICHIGAN & ITALY & MICHIGAN & & & & HECLA ST. & CALUMET \\
\hline 1472 & & LE CHEVALIER & FRANCIS & $\mathrm{s}$ & 4 & MICHIGAN & ITALY & MICHIGAN & & & & HECLA ST. & CALUMET \\
\hline 1473 & & LE CHEVALIER & MARY & D & $2 \mathrm{M}$ & MICHIGAN & ITALY & MICHIGAN & & & & HECLA ST. & CALUMET \\
\hline 1474 & & LE CHEVALIER & LORAINE & $\mathrm{D}$ & $2 \mathrm{M}$ & MICHIGAN & ITALY & MICHIGAN & & & & HECLA ST. & CALUMET \\
\hline 1475 & 1195 & RISSIO & JOSEPH & $\mathrm{H}$ & 45 & ITALY & ITALY & ITALY & 1890 & MINER/CM & SAN BENIGNO CANAVESE. TO & LAKE LINDEN & CALUMET \\
\hline 1476 & & RISSIO & PAULINE & w & 59 & ITALY & ITALY & ITALY & 1893 & & TORINO & LAKE LINDEN & CALUMET \\
\hline 1477 & & RISSIO & SADIE & D & 24 & MICHIGAN & ITALY & ITALY & & SALESLADY/GROCERY & SAN BENIGNO CANAVESE. TO & LAKE LINDEN & CALUMET \\
\hline 1478 & & RISSIO & LIZ & $\mathrm{D}$ & 22 & MICHIGAN & ITALY & ITALY & & SALESLADY/GROCERY & SAN BENIGNO CANAVESE. TO & LAKE LINDEN & CALUMET \\
\hline 1479 & & RISSIO & ARNOLD & s & 19 & MICHIGAN & ITALY & ITALY & & TEAMSTER/GROCERY STORE & SAN BENIGNO CANAVESE. TO & LAKE LINDEN & CALUMET \\
\hline 1480 & 1418 & MORETTO & JAMES & $\mathrm{H}$ & 45 & ITALY & ITALY & ITALY & 1901 & FOREMAN/CM & TORINO & ROCKLAND ST. & CALUMET \\
\hline 1481 & 1410 & MORETTO & CATERINE & W & 42 & ITALY & ITALY & ITALY & 1903 & PUREIVIAIVICIII & TORINO & ROCKLAND ST. & CALUMET \\
\hline 1482 & & MORETTO & CATERINE & D & 15 & MICHIGAN & ITALY & ITALY & & & TORINO & ROCKLAND ST. & CALUMET \\
\hline 1483 & & MORETTO & MAUD & D & 13 & MICHIGAN & ITALY & ITALY & & & TORINO & ROCKLAND ST. & CALUMET \\
\hline 1484 & & MORETTO & JAMES & $\mathrm{s}$ & 11 & MICHIGAN & ITALY & ITALY & & & TORINO & ROCKLAND ST. & CALUMET \\
\hline 1485 & & MORETTO & EVELYN & D & 8 & MICHIGAN & ITALY & ITALY & & & TORINO & ROCKLAND ST. & CALUMET \\
\hline 1486 & & MORETTO & LUCILLE & D & 5 & MICHIGAN & ITALY & ITALY & & & TORINO & ROCKLAND ST. & CALUMET \\
\hline 1487 & 1374 & FALARDEAU & ARTHUR & H & 31 & MICHIGAN & CANADA & CANADA & & INSPECTOR/RAILROAD & CANADA & ROCKLAND ST. & CALUMET \\
\hline 1488 & & FALARDEAU & CATERINE & w & 32 & MICHIGAN & ITALY & ITALY & & & TORINO & ROCKLAND ST. & CALUMET \\
\hline 1489 & & FALARDEAU & WILLIAM & $\mathrm{S}$ & 9 & MICHIGAN & MICHIGAN & MICHIGAN & & & TORINO & ROCKLAND ST. & CALUMET \\
\hline 1490 & & GIVOGRE & MARY & MIL & 70 & ITALY & ITALY & ITALY & 1899 & & TORINO & ROCKLAND ST. & CALUMET \\
\hline 1491 & 1330 & BIGANDO & BATTISTA & $\mathrm{H}$ & 46 & ITALY & ITALY & ITALY & 1891 & PAINTER/INTERIOR DECORATOR & TORINO & ROCKLAND ST. & CALUMET \\
\hline 1492 & & BIGANDO & MARY & w & 39 & MICHIGAN & ITALY & ITALY & & & & ROCKLAND ST. & CALUMET \\
\hline 1493 & & BIGANDO & MAUD & $\mathrm{D}$ & 21 & MICHIGAN & ITALY & MICHIGAN & & & TORINO & ROCKLAND ST. & CALUMET \\
\hline 1494 & & BIGANDO & GEORGE & s & 18 & MICHIGAN & ITALY & MICHIGAN & & & TORINO & ROCKLAND ST. & CALUMET \\
\hline 1495 & & BIGANDO & VITTORIO & D & 16 & MICHIGAN & ITALY & MICHIGAN & & & TORINO & ROCKLAND ST. & CALUMET \\
\hline 1496 & 1332 & GIVOGRE & JAMES & $\mathrm{H}$ & 36 & MICHIGAN & ITALY & ITALY & & EMPLOYMENT CLERK/CM & AGLIE'. TO & ROCKLAND ST. & CALUMET \\
\hline 1497 & & GIVOGRE & KATE & W & 37 & MICHIGAN & ITALY & ITALY & & 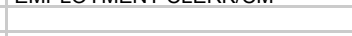 & & ROCKLAND ST. & CALUMET \\
\hline 1498 & & GIVOGRE & JACK & $\mathrm{s}$ & 9 & MICHIGAN & MICHIGAN & MICHIGAN & & & AGLIE'. TO & ROCKLAND ST. & CALUMET \\
\hline 1499 & & GIVOGRE & JAMES & $S$ & 8 & MICHIGAN & MICHIGAN & MICHIGAN & & & AGLIE', TO & ROCKLAND ST. & CALUMET \\
\hline 1500 & & SARANGIA & MICHAEL & FIL & 67 & ITALY & ITALY & ITALY & 1887 & DRAYMAN/CM & TORINO & ROCKLAND ST. & CALUMET \\
\hline 1501 & CLUB & BANDETTINI & JOHN & $\mathrm{H}$ & 54 & FRANCE & ITALY & ITALY & 1883 & & LUCCA & CALUMET AVE. & CALUMET \\
\hline 1502 & 1154 & BATTUELLO & ANTON & $\mathrm{H}$ & 40 & ITALY & ITALY & ITALY & 1914 & MACHINIST/CM & LUSIGLIE. TO & CALUMET AVE. & CALUMET \\
\hline 1503 & & BATTUELLO & CAROLINE & w & 40 & ITALY & ITALY & ITALY & 1917 & & LUSIGLIE. TO & CALUMET AVE. & CALUMET \\
\hline 1504 & & BATTUELLO & MARNIE & D & 16 & ITALY & ITALY & ITALY & 1917 & CLERK/BAKERY & LUSIGLIE. TO & CALUMET AVE. & CALUMET \\
\hline 1505 & & BATTUELLO & ANTON & s & 12 & MICHIGAN & ITALY & ITALY & & & LUSIGLIE. TO & CALUMET AVE. & CALUMET \\
\hline 1506 & & BATTUELLO & TERESA & D & 11 & MICHIGAN & ITALY & ITALY & & & LUSIGLIE, TO & CALUMET AVE. & CALUMET \\
\hline 1507 & & BATTUELLO & MARY & D & 10 & MICHIGAN & ITALY & ITALY & & & LUSIGLIE. TO & CALUMET AVE. & CALUMET \\
\hline 1508 & & BATTUELLO & MARTIN & S & 9 & MICHIGAN & ITALY & ITALY & & & LUSIGLIE. TO & CALUMET AVE. & CALUMET \\
\hline 1509 & & BATTUELLO & JOSEPH & s & 8 & MICHIGAN & ITA & ITA & & & LUSIGLIE. TO & CALUMET AVE. & CALUMET \\
\hline 1510 & & BATTUELLO & BEN & s & 6 & MICHIGAN & ITALY & & & & LUSIGLIE. TO & CALUMET AVE. & CALUMET \\
\hline 1511 & & BATTUELLO & MAFALDA & D & 4 & MICHIGAN & ITALY & ITALY & & & LUSIGLIE. TO & CALUMET AVE. & CALUMET \\
\hline
\end{tabular}




\begin{tabular}{|c|c|c|c|c|c|c|c|c|c|c|c|c|c|}
\hline 1512 & & BATTUELLO & FRANCIS & $\mathrm{s}$ & 2 & MICHIGAN & ITALY & ITALY & & & LUSIGLIE. TO & CALUMET AVE. & CALUMET \\
\hline 1513 & 2013 & RIVA & MARTIN & $\mathrm{H}$ & 30 & ITALY & ITALY & ITALY & 1907 & MINER/CM & RIBARDONE. TO & CALUMET AVE. & CALUMET \\
\hline 1514 & & RIVA & MARY & W & 19 & MICHIGAN & ITALY & ITALY & & & & CALUMET AVE. & CALUMET \\
\hline 1515 & & RIVA & MARTIN & $\mathrm{s}$ & $3 \mathrm{M}$ & MICHIGAN & ITALY & MICHIGAN & & & RIBARDONE. TO & CALUMET AVE. & CALUMET \\
\hline 1516 & 2021 & TOMASI & ANDREW & $\mathrm{H}$ & 64 & ITALY & ITALY & ITALY & 1888 & BLACKSMITH/CM & LOCANA CANAVESE. TO & CALUMET AVE. & CALUMET \\
\hline 1517 & & TOMASI & MARGHERITA & w & 47 & ITALY & ITALY & ITALY & 1897 & & TORINO & CALUMET AVE. & CALUMET \\
\hline 1518 & & TOMASI & MICHAEL & $\mathrm{s}$ & 22 & MICHIGAN & ITALY & ITALY & & BLACKSMITH/CM & LOCANA CANAVESE. TO & CALUMET AVE. & CALUMET \\
\hline 1519 & & TOMASI & MARGHERITA & $\mathrm{D}$ & 17 & MICHIGAN & ITALY & ITALY & & & LOCANA CANAVESE. TO & CALUMET AVE. & CALUMET \\
\hline 1520 & & TOMASI & PAUL & $\mathrm{s}$ & 15 & MICHIGAN & ITALY & ITALY & & & LOCANA CANAVESE. TO & CALUMET AVE. & CALUMET \\
\hline 1521 & & TOMASI & PETER & $\mathrm{s}$ & 15 & MICHIGAN & ITALY & ITALY & & & LOCANA CANAVESE. TO & CALUMET AVE. & CALUMET \\
\hline 1522 & 2029 & MENEGUZZO & JOSEPH & $\mathrm{H}$ & 43 & ITALY & ITALY & ITALY & 1904 & DRILL SHARPENER/CM & COSTO BONARTO. TRIESTE & CALUMET AVE. & CALUMET \\
\hline 1523 & & MENEGUZZO & ROMILDA & W & 43 & ITALY & ITALY & ITALY & 1904 & & COSTO BONARTO. TRIESTE & CALUMET AVE. & CALUMET \\
\hline 1524 & & MENEGUZZO & JOHN & $s$ & 18 & MICHIGAN & ITALY & ITALY & & DRILL SHARPENER/CM & COSTO BONARTO. TRIESTE & CALUMET AVE. & CALUMET \\
\hline 1525 & & MENEGUZZO & ANNA & $\mathrm{D}$ & 17 & MICHIGAN & ITALY & ITALY & & SERVANT/PF & COSTO BONARTO. TRIESTE & CALUMET AVE. & CALUMET \\
\hline 1526 & & MENEGUZZO & MARY & $\mathrm{D}$ & 14 & MICHIGAN & ITALY & ITALY & & & COSTO BONARTO. TRIESTE & CALUMET AVE. & CALUMET \\
\hline 1527 & & MENEGUZZO & JOSEPH & $\mathrm{S}$ & 13 & MICHIGAN & ITALY & ITALY & & & COSTO BONARTO, TRIESTE & CALUMET AVE. & CALUMET \\
\hline 1528 & & MENEGUZZO & PETER & $\mathrm{s}$ & 11 & MICHIGAN & ITALY & ITALY & & & COSTO BONARTO. TRIESTE & CALUMET AVE. & CALUMET \\
\hline 1529 & & MENEGUZZO & FRANK & $\mathrm{s}$ & 8 & MICHIGAN & ITALY & ITALY & & & COSTO BONARTO. TRIESTE & CALUMET AVE. & CALUMET \\
\hline 1530 & & MENEGUZZO & ANTON & $\mathrm{s}$ & 5 & MICHIGAN & ITALY & ITALY & & & COSTO BONARTO. TRIESTE & CALUMET AVE. & CALUMET \\
\hline 1531 & & MENEGUZZO & LOUIS & $\mathrm{s}$ & 1 & MICHIGAN & ITALY & ITALY & & & COSTO BONARTO. TRIESTE & CALUMET AVE. & CALUMET \\
\hline 1532 & 2033 & ZANARDI & MARY & $\mathrm{H}$ & 48 & ITALY & $\begin{array}{l}\text { ITALY } \\
\text { ITA }\end{array}$ & $\begin{array}{l}\text { ITALY } \\
\text { ITAL }\end{array}$ & 1903 & & 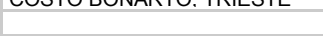 & $\begin{array}{l}\text { CALUMET AVE. } \\
\text { CAUME }\end{array}$ & CALUMET \\
\hline 1533 & & ZANARDI & CARL & s & 16 & MICHIGAN & ITALY & ITALY & & & BERGAMO & CALUMET AVE. & CALUMET \\
\hline 1534 & & FONTANA & PAUL & B & 42 & ITALY & ITALY & ITALY & 1909 & MINER/CM & SAN BENIGNO CANAVESE. TO & CALUMET AVE. & CALUMET \\
\hline 1535 & & CHIANTARETTO & JOHN & B & 42 & ITALY & ITALY & ITALY & 1914 & TIMBERMAN/CM & FOGLIZZO. TO & CALUMET AVE. & CALUMET \\
\hline 1536 & 2038 & KIGER & RALPH & $\mathrm{H}$ & 28 & INDIANA & INDIANA & INDIANA & & MINER/CM & USA & & CALUMET \\
\hline 1537 & & KIGER & JOSEPHINE & W & 28 & ILLINOIS & ITALY & ITALY & & & & & CALUMET \\
\hline 1538 & 2100 & GRISOLANO & TONY & B & 23 & MICHIGAN & ITALY & ITALY & & TRAMMER/CM & TORINO & LOG ST. & CALUMET \\
\hline 1539 & & MARASCHIN & ANTON & B & 42 & ITALY & ITALY & ITALY & 1907 & MINER/CM & VICENZA & LOG ST. & CALUMET \\
\hline 1540 & 2113 & RIEDEL & EUGENE & $\mathrm{H}$ & 24 & GERMANY & GERMANY & GERMANY & 1923 & MINER/CM & GERMANY & LOG ST. & CALUMET \\
\hline 1541 & & RIEDEL & JENNE & W & 27 & MICHIGAN & ITALY & ITALY & & & & LOG ST. & CALUMET \\
\hline 1542 & & RIEDEL & EUGENIA & s & 2 & MICHIGAN & GERMANY & MICHIGAN & & & & LOG ST. & CALUMET \\
\hline 1543 & 2124 & SIMON & JOHN & $\mathrm{H}$ & 25 & NEW JERSEY & AUSTRIA & AUSTRIA & & MINER/CM & AUSTRIA & LOG ST. & CALUMET \\
\hline 1544 & 2124 & SIMON & LOUISE & W & 26 & MICHIGAN & ITALY & ITALY & & IVIINERATLIVI & AUOIRIA & LOG ST. & CALUMET \\
\hline 1545 & 2136 & BALMA/BAIMA & JOHN & $\mathrm{H}$ & 20 & MICHIGAN & ITALY & ITALY & & LABORER/CM & TORINO & LOG ST. & CALUMET \\
\hline 1546 & & VEDOVATI & JOSEPH & B & 55 & ITALY & ITALY & ITALY & 1907 & STONE MASON/CONTRACTOR & BERGAMO & LOG ST. & CALUMET \\
\hline 1547 & 2135 & POGGIONE & JENNIE & $\mathrm{H}$ & 45 & ITALY & ITALY & ITALY & 1906 & & & LOG ST. & CALUMET \\
\hline 1548 & & POGGIONE & CATERINE & D & 22 & MICHIGAN & MICHIGAN & ITALY & & & SAN GIORGIO CANAVESE. TO & LOG ST. & CALUMET \\
\hline 1549 & & POGGIONE & JOSEPH & $\mathrm{s}$ & 20 & MICHIGAN & MICHIGAN & ITALY & & LABORER/CM & SAN GIORGIO CANAVESE. TO & LOG ST. & CALUMET \\
\hline 1550 & & POGGIONE & VIRGINIA & $\mathrm{D}$ & 14 & MICHIGAN & MICHIGAN & ITALY & & 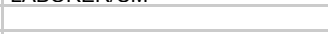 & SAN GIORGIO CANAVESE. TO & LOG ST. & CALUMET \\
\hline 1551 & 2217 & CONEDER & CAROLINO & $\mathrm{H}$ & 29 & MICHIGAN & ITALY & ITALY & & MINER/CM & BELLUNO & MIDDLE ST. & CALUMET \\
\hline 1552 & & CONEDER & MARY & W & 30 & ITALY & ITALY & ITALY & 1929 & & & MIDDLE ST. & CALUMET \\
\hline 1553 & & CONEDER & JOHANNA & $\mathrm{D}$ & 7 & MICHIGAN & MICHIGAN & ITALY & & & BELLUNO & MIDDLE ST. & CALUMET \\
\hline 1554 & & CONEDER & MONICA & $\mathrm{D}$ & 5 & MICHIGAN & MICHIGAN & ITALY & & & BELLUNO & MIDDLE ST. & CALUMET \\
\hline 1555 & 2202 & BAGATTINI & EDWARD & $\mathrm{H}$ & 27 & PENNSYLVAN & ITALY & ITALY & & STATIONARY ENGINEER/CM & CONDINO. TRENTO & MIDDLE ST. & CALUMET \\
\hline 1556 & & BAGATTINI & THERESA & W & 25 & MICHIGAN & CZECHOSLO & CZECHOSLO & & GIf & CZECHOSLOVAKIA & MIDDLE ST. & CALUMET \\
\hline 1557 & & BAGATTINI & GLORIA & $\mathrm{D}$ & 3 & MICHIGAN & PENNSYLVAN & MICHIGAN & & & CONDINO. TRENTO & MIDDLE ST. & CALUMET \\
\hline 1558 & & BAGATTINI & EDWARD & s & 4 & MICHIGAN & PENNSYLVAN & MICHIGAN & & & CONDINO. TRENTO & MIDDLE ST. & CALUMET \\
\hline 1559 & & BAGATTINI & ANGELO & FA & 50 & ITALY & ITALY & ITALY & 1896 & MINER/CM & CONDINO. TRENTO & MIDDLE ST. & CALUMET \\
\hline 1560 & 2374 & ZUBIENA & JOHN & $\mathrm{H}$ & 37 & ITALY & ITALY & ITALY & 1921 & MINER/CM & AGLIE'. TO & A ST. & CALUMET \\
\hline 1561 & & ZUBIENA & ANGELINA & W & 33 & ITALY & ITALY & ITALY & 1906 & & PONT CANAVESE. TO & A ST. & CALUMET \\
\hline 1562 & & ZUBIENA & LUCILLE & $\mathrm{D}$ & 6 & MICHIGAN & ITALY & ITALY & & & AGLIE' TO & A ST. & CALUMET \\
\hline 1563 & & ZUBIENA & TERESA & $\mathrm{D}$ & 5 & MICHIGAN & ITALY & ITALY & & & AGLIE', TO & A ST. & CALUMET \\
\hline 1564 & & ZUBIENA & MARY & $\mathrm{D}$ & 4 & MICHIGAN & ITALY & ITALY & & & AGLIE'. TO & A ST. & CALUMET \\
\hline 1565 & & ZUBIENA & DOMENICO & $\mathrm{s}$ & 2 & MICHIGAN & ITALY & ITALY & & & AGLIE'. TO & A ST. & CALUMET \\
\hline 1566 & 2356 & MASSOGLIA & $\mathrm{JOHN}$ & $\mathrm{H}$ & 23 & MICHIGAN & ITALY & ITALY & & PIPE MAN/CM & SAN MARTINO CANAVESE. TO & A ST. & CALUMET \\
\hline 1567 & & MASSOGLIA & LUCILLE & w & 21 & MICHIGAN & CANADA & CANADA & & & CANADA & A ST. & CALUMET \\
\hline 1568 & & MASSOGLIA & SHIRLEY & $\mathrm{D}$ & 3 & MICHIGAN & MICHIGAN & MICHIGAN & & & SAN MARTINO CANAVESE. TO & A ST. & CALUMET \\
\hline 1569 & & MASSOGLIA & DOROTHY & $\mathrm{D}$ & 2 & MICHIGAN & MICHIGAN & MICHIGAN & & & SAN MARTINO CANAVESE, TO & A ST. & CALUMET \\
\hline 1570 & 2350 & MACARIO & PETER & BIL & 27 & MICHIGAN & ITALY & ITALY & & MINER/CM & 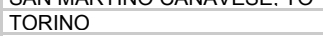 & A ST. & CALUMET \\
\hline 1571 & 2426 & RASTELLO & JOHN & $\mathrm{H}$ & 54 & ITALY & ITALY & ITALY & 1906 & MINER/CM & PONT CANAVESE. TO & B ST. & CALUMET \\
\hline 1572 & & RASTELLO & LUCY & w & 54 & & ITALY & ITALY & 1908 & & & B ST. & CALUMET \\
\hline 1573 & & RASTELLO & JOHN & $\mathrm{s}$ & 18 & MICHIGAN & ITALY & ITALY & & LABORER ODD JOBS/VILLAGE & PONT CANAVESE. TO & & CALUMET \\
\hline 1574 & & RASTELLO & CHARLES & $\mathrm{s}$ & 14 & MICHIGAN & ITALY & ITALY & & & PONT CANAVESE. TO & $\begin{array}{l}\text { B ST. } \\
\text { B. }\end{array}$ & CALUMET \\
\hline
\end{tabular}




\begin{tabular}{|c|c|c|c|c|c|c|c|c|c|c|c|c|c|}
\hline 1575 & 2416 & DESIATTO & JOHN & $\mathrm{H}$ & 45 & ITALY & ITALY & ITALY & 1911 & TIMBERMAN/CM & TORINO & B ST. & CALUMET \\
\hline 1576 & & DESIATTO & MAUD & W & 42 & ITALY & ITALY & ITALY & 1921 & & & B ST. & CALUMET \\
\hline 1577 & & DESIATTO & MARY & $\mathrm{D}$ & 8 & MICHIGAN & ITALY & ITALY & & & TORINO & B ST. & CALUMET \\
\hline 1578 & & DESIATTO & PAULINE & $\mathrm{D}$ & 6 & MICHIGAN & ITALY & ITALY & & & TORINO & B ST. & CALUMET \\
\hline 1579 & & DESIATTO & JOHN & $\mathrm{s}$ & 2 & MICHIGAN & ITALY & ITALY & & & TORINO & B ST. & CALUMET \\
\hline 1580 & 2404 & ZANARDI & CARLO & $\mathrm{H}$ & 31 & MICHIGAN & ITALY & ITALY & & SALESMAN/AUTOMOBILE & BERGAMO & B ST. & CALUMET \\
\hline 1581 & & ZANARDI & ROSE & W & 24 & MICHIGAN & ITALY & ITALY & & & & B ST. & CALUMET \\
\hline 1582 & & ZANARDI & JOSEPH & $\mathrm{BR}$ & 30 & MICHIGAN & ITALY & ITALY & & FACTORY WORKER/AUTO PLANT & BERGAMO & B ST. & CALUMET \\
\hline 1583 & 2402 & DUSA & ANTON & $\mathrm{H}$ & 44 & ITALY & ITALY & ITALY & 1913 & $\begin{array}{l}\text { TRAMMER/CM } \\
\text { TRERIAU TLINI }\end{array}$ & DERUAIVIU & $\begin{array}{l}\text { B ST. } \\
\text { B ST. }\end{array}$ & CALUMET \\
\hline 1584 & & DUSA & MARY & w & 32 & YUGOSLAVIA & YUGOSLAVIA & YUGOSLAVIA & & & YUGOSLAVIA & B ST. & CALUMET \\
\hline 1585 & & DUSA & ANGELINE & $\mathrm{D}$ & 10 & MICHIGAN & ITALY & YUGOSLAVIA & & & & B ST. & CALUMET \\
\hline 1586 & & DUSA & JOSEPHINE & D & 9 & MICHIGAN & ITALY & YUGOSLAVIA & & & & B ST. & CALUMET \\
\hline 1587 & & DUSA & ANTON JR. & $s$ & 7 & MICHIGAN & ITALY & YUGOSLAVIA & & & & B ST. & CALUMET \\
\hline 1588 & & DUSA & CAROLINE & D & 5 & MICHIGAN & ITALY & YUGOSLAVIA & & & & B ST. & CALUMET \\
\hline 1589 & 2419 & RICCA & MICHAEL & $\mathrm{H}$ & 47 & ITALY & ITALY & ITALY & 1908 & PIPE MAN/CM & RIVAROLO CANAVESE. TO & B ST. & CALUMET \\
\hline 1590 & & RICCAA & MARY & W & 49 & ITALY & ITALY & ITALY & 1902 & & & B ST. & CALUMET \\
\hline 1591 & & RICCA & LAWRENCE & $\mathrm{s}$ & 9 & MICHIGAN & ITALY & ITALY & & & RIVAROLO CANAVESE. TO & B ST. & CALUMET \\
\hline 1592 & 2423 & MASSE' & JOHN & $\mathrm{H}$ & 60 & ITALY & ITALY & ITALY & 1900 & STONE MASON/CM & PONT CANAVESE. TO & B ST. & CALUMET \\
\hline 1593 & & MASSE' & SERAFINA & w & 61 & ITALY & ITALY & ITALY & 1907 & & & B ST. & CALUMET \\
\hline 1594 & 2481 & AIMONE & MINNIE & $\mathrm{H}$ & 59 & ITALY & ITALY & ITALY & 1891 & & TORINO & C ST. & CALUMET \\
\hline 1595 & 2401 & ACCORNERO & JOHN & SOIL & 41 & ITALY & $\begin{array}{l}\text { ITALY } \\
\text { ITA }\end{array}$ & $\begin{array}{l}\text { ITALY } \\
\text { ITAL }\end{array}$ & 1913 & TRAMMER/CM & ALESSANDRIA & C ST. & CALUMET \\
\hline 1596 & & ACCORNERO & GEORGE & GS & 12 & MICHIGAN & ITALY & ITALY & & & ALESSANDRIA & C ST. & CALUMET \\
\hline 1597 & 2477 & PETITTI & MARY & $\mathrm{H}$ & 42 & ITALY & ITALY & ITALY & 1891 & & TORINO & C ST. & CALUMET \\
\hline 1598 & & PETITTI & CATERINA & MIL & 72 & ITALY & ITALY & ITALY & 1920 & & SAN PONSO CANAVESE. TO & C ST. & CALUMET \\
\hline 1599 & & PETITTI & JOSEPH & BIL & 51 & ITALY & ITALY & ITALY & 1904 & MINER/CM & SAN PONSO CANAVESE. TO & C ST. & CALUMET \\
\hline 1600 & 2471 & BARDA & SILVIO & $\mathrm{H}$ & 41 & ITALY & ITALY & ITALY & 1907 & TIMBERMAN/CM & QUAGLIUZZO. TO & C ST. & CALUMET \\
\hline 1601 & & BARDA & CATERINA & w & 33 & MICHIGAN & ITALY & ITALY & & & & C ST. & CALUMET \\
\hline 1602 & & BARDA & MINNIE & $\mathrm{D}$ & 17 & MICHIGAN & ITALY & MICHIGAN & & & QUAGLIUZZO, TO & C ST. & CALUMET \\
\hline 1603 & & BARDA & ROMEO & $\mathrm{S}$ & 11 & MICHIGAN & ITALY & MICHIGAN & & & QUAGLIUZZO. TO & C ST. & CALUMET \\
\hline 1604 & & BARDA & JOHN & $\mathrm{s}$ & 5 & MICHIGAN & ITALY & MICHIGAN & & & QUAGLIUZZO. TO & C ST. & CALUMET \\
\hline 1605 & & BARDA & DOMENICO & s & 3 & MICHIGAN & ITALY & MICHIGAN & & & QUAGLIUZZO. TO & C ST. & CALUMET \\
\hline 1606 & 2451 & MACARIO & PETER & $\mathrm{H}$ & 68 & MICHIGAN & ITALY & ITALY & & WATCHMAN/CM & TORINO & C ST. & CALUMET \\
\hline 1607 & 2401 & MACARIO & TERESA & W & 59 & MICHIGAN & ITALY & ITALY & & VUATC RIVIATVICIVI & TURIVU & C ST. & CALUMET \\
\hline 1608 & & MACARIO & CARLO & s & 20 & MICHIGAN & MICHIGAN & MICHIGAN & & MINER/CM & TORINO & C ST. & CALUMET \\
\hline 1609 & & MACARIO & VINCENZO & $\mathrm{s}$ & 17 & MICHIGAN & MICHIGAN & MICHIGAN & & & TORINO & C ST. & CALUMET \\
\hline 1610 & 2450 & PASQUALONE & BATTISTA & $\mathrm{H}$ & 41 & ITALY & ITALY & ITALY & 1912 & MINER/CM & SPARONE. TO & C ST. & CALUMET \\
\hline 1611 & & PASQUALONE & MARY & W & 31 & ITALY & ITALY & ITALY & 1916 & & & C ST. & CALUMET \\
\hline 1612 & & PASQUALONE & MADDALENA & $\mathrm{D}$ & 12 & MICHIGAN & ITALY & ITALY & 1010 & & SPARONE. TO & C ST. & CALUMET \\
\hline 1613 & & PASQUALONE & MARY & D & 10 & MICHIGAN & ITALY & ITALY & & & SPARONE. TO & C ST. & CALUMET \\
\hline 1614 & & PASQUALONE & LUCILLE & $\mathrm{D}$ & 7 & MICHIGAN & ITALY & ITALY & & & SPARONE. TO & C ST. & CALUMET \\
\hline 1615 & 2452 & GIACHINO & JAMES & $\mathrm{H}$ & 61 & ITALY & ITALY & ITALY & 1897 & TIMBERMAN/CM & TORINO & C ST. & CALUMET \\
\hline 1616 & & GIACHINO & ANNE & w & 55 & ITALY & ITALY & ITALY & 1900 & & & C ST. & CALUMET \\
\hline 1617 & & GIACHINO & JAMES JR. & s & 29 & MICHIGAN & ITALY & ITALY & & LABORER/CM & TORINO & C ST. & CALUMET \\
\hline 1618 & & GIACHINO & JOHN & $\mathrm{s}$ & 22 & MICHIGAN & ITALY & ITALY & & LABORER/CM & TORINO & C ST. & CALUMET \\
\hline 1619 & & GIACHINO & PETER & $\mathrm{s}$ & 20 & MICHIGAN & ITALY & ITALY & & LABORER/CM & TORINO & C ST. & CALUMET \\
\hline 1620 & 2474 & MATTIELLO & JOHN & $\mathrm{H}$ & 48 & ITALY & ITALY & ITALY & 1903 & TIMBERMAN/CM & CASTELGOMBERTO. VICENZA & C ST. & CALUMET \\
\hline 1621 & & MATTIELLO & NORA & $\mathrm{D}$ & 17 & MICHIGAN & ITALY & ITALY & & & CASTELLOMBERTO. VICENZA & C ST. & CALUMET \\
\hline 1622 & & MATTIELLO & JOHN & $\mathrm{s}$ & 15 & MICHIGAN & ITALY & ITALY & & & CASTELGOMBERTO. VICENZA & C ST. & CALUMET \\
\hline 1623 & & MATTIELLO & CLARA & $\mathrm{D}$ & 10 & MICHIGAN & ITALY & ITALY & & & CASTELLOMBERTO. VICENZA & C ST. & CALUMET \\
\hline 1624 & & TOMASI & MARGHERITA & $\mathrm{D}$ & 21 & MICHIGAN & ITALY & ITALY & & & $\begin{array}{l}\text { LOCANA CANAVESE. TO } \\
\end{array}$ & C ST. & CALUMET \\
\hline 1625 & & TOMASI & ANDREA & SOIL & 23 & MICHIGAN & ITALY & ITALY & & TIMBERMAN/CM & & C ST. & CALUMET \\
\hline 1626 & & TOMASI & GLORIA & GD & 4 & MICHIGAN & MICHIGAN & MICHIGAN & & & LOCANA CANAVESE, TO & C ST. & CALUMET \\
\hline 1627 & & TOMASI & ANDREW & GS & 3 & MICHIGAN & MICHIGAN & MICHIGAN & & & LOCANA CANAVESE. TO & C ST. & CALUMET \\
\hline 1628 & 2476 & BIGANDO & VITTORIA & $\mathrm{H}$ & 70 & ITALY & ITALY & ITALY & 1884 & & TORINO & C ST. & CALUMET \\
\hline 1629 & & GASPARDO & JOHANNA & SER & 16 & MICHIGAN & ITALY & ITALY & & SERVANT/PF & PONT CANAVESE. TO & C ST. & CALUMET \\
\hline 1630 & & SPAGNETTI & MARY & SER & 18 & ITALY & ITALY & ITALY & 1914 & SERVANT/PF & TORINO & C ST. & CALUMET \\
\hline 1631 & 2527 & CERETTO & JOHN & $\mathrm{H}$ & 59 & ITALY & ITALY & ITALY & 1896 & MINER/CM & PONT CANAVESE. TO & D ST. & CALUMET \\
\hline 1632 & 2027 & CERETTO & LUCIA & W & 55 & ITALY & ITALY & ITALY & 1898 & & PONT CANAVESE, TO & D ST. & CALUMET \\
\hline 1633 & & CERETTO & MARY & $\mathrm{D}$ & 25 & MICHIGAN & ITALY & ITALY & & TEACHER/PUBLIC SCHOOL & PONT CANAVESE. TO & D ST. & CALUMET \\
\hline 1634 & & CERETTO & JOSEPH & $\mathrm{s}$ & 18 & MICHIGAN & ITALY & ITALY & & BLACKSMITH/CM & PONT CANAVESE. TO & D ST. & CALUMET \\
\hline 1635 & 2521 & BOGGIO & DOMENICO & $\mathrm{H}$ & 47 & ITALY & ITALY & ITALY & 1903 & PUMP MAN/CM & TORINO & DST. & CALUMET \\
\hline 1636 & & BOGGIO & MINNIE & W & 37 & ITALY & ITALY & ITALY & 1896 & & TORINO & D ST. & CALUMET \\
\hline 1637 & & BOGGIO & JOSEPH & s & 19 & MICHIGAN & ITALY & ITALY & & ROLLER REPAIRER/CM & TORINO & D ST. & CALUMET \\
\hline
\end{tabular}




\begin{tabular}{|c|c|c|c|c|c|c|c|c|c|c|c|c|c|}
\hline 1638 & & BOGGIO & PETER & $\mathrm{s}$ & 15 & MICHIGAN & ITALY & ITALY & & & TORINO & D ST. & CALUMET \\
\hline 1639 & & BOGGIO & DOMENICO & $\mathrm{s}$ & 2 & MICHIGAN & ITALY & ITALY & & & TORINO & DST. & CALUMET \\
\hline 1640 & 2519 & ORSOLANO & ANNE & $\mathrm{H}$ & 61 & ITALY & ITALY & ITALY & 1895 & & TORINO & D ST. & CALUMET \\
\hline 1641 & 2517 & BARINOTTI & FRANK & $\mathrm{H}$ & 61 & ITALY & ITALY & ITALY & 1890 & PUMP MAN/CM & PONT CANAVESE. TO & D ST. & CALUMET \\
\hline 1642 & & BARINOTTI & MADDALENA & w & 45 & ITALY & ITALY & ITALY & 1912 & & & D ST. & CALUMET \\
\hline 1643 & & BARINOTTI & FRANK & s & 23 & MICHIGAN & ITALY & ITALY & & LOCOMOTIVE FIREMAN/STEAM & PONT CANAVESE. TO & D ST. & CALUMET \\
\hline 1644 & & BARINOTTI & IRENE & $\mathrm{D}$ & 14 & MICHIGAN & ITALY & ITALY & & & PONT CANAVESE. TO & D ST. & CALUMET \\
\hline 1645 & & BARINOTTI & JAMES & $\mathrm{s}$ & 12 & MICHIGAN & ITALY & ITALY & & & PONT CANAVESE. TO & D ST. & $\begin{array}{l}\text { CALUMET } \\
\text { CAM }\end{array}$ \\
\hline 1646 & 2515 & VERCELLINO & DOMENICO & $\mathrm{H}$ & 46 & ITALY & ITALY & ITALY & 1906 & TIMBERMAN/CM & TORINO & D ST. & $\begin{array}{l}\text { CALUMET } \\
\end{array}$ \\
\hline 1647 & & VERCELLINO & TERESA & w & 37 & ITALY & ITALY & ITALY & 1913 & & & D ST. & CALUMET \\
\hline 1648 & & VERCELLINO & ROSE & $\mathrm{D}$ & 15 & MICHIGAN & ITALY & ITALY & & & TORINO & D ST. & $\begin{array}{l}\text { CALUMET } \\
\end{array}$ \\
\hline 1649 & 2513 & IMPERATE & PASQUALE & $\mathrm{H}$ & 54 & ITALY & ITALY & ITALY & 1910 & MINER/CM & TORINO & D ST. & CALUMET \\
\hline 1650 & & IMPERATE & LIZ & W & 45 & ITALY & ITALY & ITALY & 1910 & & TORINO & D ST. & CALUMET \\
\hline 1651 & & IMPERATE & GINO & s & & MICHIGAN & ITALY & ITALY & & & TORINO & D ST. & CALUMET \\
\hline 1652 & & IMPERATE & LEONA & $\mathrm{D}$ & 16 & MICHIGAN & ITALY & ITALY & & & TORINO & D ST. & CALUMET \\
\hline 1653 & & IMPERATE & AMERIGO & $\mathrm{S}$ & 15 & MICHIGAN & ITALY & ITALY & & & TORINO & D ST. & CALUMET \\
\hline 1654 & & IMPERATE & SAM & $\mathrm{s}$ & 14 & MICHIGAN & ITALY & ITALY & & & TORINO & D ST. & CALUMET \\
\hline 1655 & & IMPERATE & AMELIA & D & 13 & MICHIGAN & ITALY & ITALY & & & TORINO & D ST. & CALUMET \\
\hline 1656 & & RENALE & NELLO & BIL & 46 & ITALY & ITALY & ITALY & 1914 & TIMBERMAN/CM & & D ST. & CALUMET \\
\hline 1657 & 2509 & ROLANDO & JOHN B. & $\mathrm{H}$ & 46 & ITALY & ITALY & ITALY & 1887 & MINER/CM & PONT CANAVESE. TO & D ST. & CALUMET \\
\hline 1658 & 2009 & ROLANDO & MARY & W & 40 & ITALY & $\begin{array}{l}\text { ITALY } \\
\text { ITAL }\end{array}$ & $\begin{array}{l}\text { ITALY } \\
\end{array}$ & 1891 & & PONT CANAVESE. TO & $\begin{array}{l}\text { D ST. } \\
\text { D T. }\end{array}$ & CALUMET \\
\hline 1659 & & ROLANDO & PETER & s & 19 & MICHIGAN & ITALY & ITALY & & TIMBERMAN/CM & PONT CANAVESE, TO & D ST. & CALUMET \\
\hline 1660 & & ROLANDO & ROSE & $\mathrm{D}$ & 17 & ITALY & ITALY & ITALY & 1912 & & PONT CANAVESE. TO & D ST. & CALUMET \\
\hline 1661 & & COPPO & MIKE & $\mathrm{COU}$ & 44 & MICHIGAN & ITALY & ITALY & & MINER/CM & PONT CANAVESE. TO & D ST. & $\begin{array}{l}\text { CALUMET } \\
\end{array}$ \\
\hline 1662 & 2507 & MAGNINO & JACOB & $\mathrm{H}$ & 38 & MICHIGAN & ITALY & ITALY & & MINER/CM & SPARONE. TO & D ST. & CALUMET \\
\hline 1663 & & MAGNINO & ANNA & W & 28 & MICHIGAN & ITALY & ITALY & & & & D ST. & CALUMET \\
\hline 1664 & & MAGNINO & ANGELINE & $\mathrm{D}$ & 12 & MICHIGAN & MICHIGAN & MICHIGAN & & & SPARONE. TO & D ST. & CALUMET \\
\hline 1665 & & MAGNINO & JOSEPH & $\mathrm{s}$ & 10 & MICHIGAN & MICHIGAN & MICHIGAN & & & SPARONE, TO & D ST. & CALUMET \\
\hline 1666 & 2506 & DAIRO & GIUSEPPINA & $\mathrm{H}$ & 60 & ITALY & ITALY & ITALY & 1899 & & TORINO & D ST. & CALUMET \\
\hline 1667 & & DAIRO & JOHN & S & 24 & MICHIGAN & ITALY & ITALY & & MINER/CM & TORINO & D ST. & $\begin{array}{l}\text { CALUMET } \\
\end{array}$ \\
\hline 1668 & & DAIRO & ANNE & $\mathrm{D}$ & 18 & MICHIGAN & ITALY & ITALY & & & TORINO & D ST. & CALUMET \\
\hline 1669 & 2512 & BRACCO & PETER & $\mathrm{H}$ & 61 & ITALY & ITALY & ITALY & 1898 & MINER/CM & TORINO & D ST. & CALUMET \\
\hline 1670 & 2012 & BRACCO & MARY & W & 47 & $\begin{array}{l}\text { ITALY } \\
\text { ITA }\end{array}$ & $\begin{array}{l}\text { ITALY } \\
\text { ITAL }\end{array}$ & ITALY & $\begin{array}{l}1090 \\
1901\end{array}$ & IVIIIVERT IVI & TURIVU & $\begin{array}{l}\text { D ST. } \\
\text { D T. }\end{array}$ & CALUMET \\
\hline 1671 & & BRACCO & ANTONY & s & 27 & MICHIGAN & ITALY & ITALY & & MINER/CM & TORINO & D ST. & CALUMET \\
\hline 1672 & & BRACCO & PETER & $\mathrm{s}$ & 23 & MICHIGAN & ITALY & ITALY & & BLACKSMITH/CM & TORINO & D ST. & CALUMET \\
\hline 1673 & & BRACCO & MARY & $\mathrm{D}$ & 18 & MICHIGAN & ITALY & ITALY & & & TORINO & DST. & $\begin{array}{l}\text { CALUMET } \\
\end{array}$ \\
\hline 1674 & & BRACCO & JOSEPH & s & 16 & MICHIGAN & ITALY & ITALY & & & TORINO & D ST. & CALUMET \\
\hline 1675 & & BRACCO & ANTONY JR. & GS & 9 & MICHIGAN & ITALY & ITALY & & & TORINO & D ST. & CALUMET \\
\hline 1676 & 2514 & SOMMARIVA & FRANK & $\mathrm{H}$ & 64 & ITALY & ITALY & ITALY & 1890 & LABORER/CM & VENEZIA & D ST. & CALUMET \\
\hline 1677 & & SOMMARIVA & MARY & W & 48 & ITALY & ITALY & ITALY & 1901 & & & D ST. & CALUMET \\
\hline 1678 & & SOMMARIVA & FRANK & $\mathrm{s}$ & 28 & MICHIGAN & ITALY & ITALY & & SALESMAN/AUTOMOBILE & VENEZIA & D ST. & CALUMET \\
\hline 1679 & & SOMMARIVA & NATALE & $\mathrm{s}$ & 27 & MICHIGAN & ITALY & ITALY & & LABORER/CM & VENEZIA & D ST. & CALUMET \\
\hline 1680 & & SOMMARIVA & JOHANNA & $\mathrm{D}$ & 25 & MICHIGAN & ITALY & ITALY & & & VENEZIA & D ST. & CALUMET \\
\hline 1681 & & SOMMARIVA & GAIO & $\mathrm{s}$ & 21 & MICHIGAN & ITALY & ITALY & & & VENEZIA & D ST. & CALUMET \\
\hline 1682 & & SOMMARIVA & DOMENICO & s & 19 & MICHIGAN & ITALY & ITALY & & & VENEZIA & D ST. & CALUMET \\
\hline 1683 & & SOMMARIVA & ANGELINA & DIL & 26 & MICHIGAN & ITALY & ITALY & & & VLIVLLIA & $\begin{array}{l}\text { D ST. } \\
\text { D }\end{array}$ & CALUMET \\
\hline 1684 & 2518 & PIANA & DOMENICO & $\mathrm{H}$ & 28 & MICHIGAN & ITALY & ITALY & & OILER/CM & TORINO & D ST. & CALUMET \\
\hline 1685 & 2520 & ZANIN & OLIVER & $\mathrm{H}$ & 51 & ITALY & ITALY & ITALY & 1898 & MINER/CM & RIVAMONTE. BELLUNO & D ST. & $\begin{array}{l}\text { CALUMET } \\
\end{array}$ \\
\hline 1686 & & ZANIN & ANNE & w & 44 & ITALY & ITALY & ITALY & 1897 & & & D ST. & $\begin{array}{l}\text { CALUMET } \\
\end{array}$ \\
\hline 1687 & & ZANIN & ROSE & $\mathrm{D}$ & 18 & MICHIGAN & ITALY & ITALY & & & RIVAMONTE. BELLUNO & D ST. & CALUMET \\
\hline 1688 & & ZANIN & MARY & $\mathrm{D}$ & 14 & MICHIGAN & ITALY & ITALY & & & RIVAMONTE. BELLUNO & D ST. & CALUMET \\
\hline 1689 & 2522 & LANFRANCHI & JOSEPH & $\mathrm{H}$ & 24 & ITALY & ITALY & ITALY & 1922 & MINER/CM & MILANO & D ST. & CALUMET \\
\hline 1690 & & LANFRANCHI & ROSE & W & 20 & MICHIGAN & ITALY & ITALY & & & & D ST. & CALUMET \\
\hline 1691 & & LANFRANCHI & ANGELINE & $\mathrm{D}$ & 0 & MICHIGAN & ITALY & MICHIGAN & & & MILANO & D ST. & CALUMET \\
\hline 1692 & 2524 & BRACCO & $\mathrm{JOHN}$ & $\mathrm{H}$ & 65 & ITALY & ITALY & ITALY & 1888 & LANDER/CM & TORINO & D ST. & CALUMET \\
\hline 1693 & & BRACCO & JOSEPH & S & 35 & MICHIGAN & ITALY & ITALY & & OFFICE WORKER/RAILROAD & TORINO & D ST. & CALUMET \\
\hline 1694 & & BRACCO & MARY & $\mathrm{D}$ & 26 & MICHIGAN & ITALY & ITALY & & & TORINO & D ST. & CALUMET \\
\hline 1695 & 2563 & TEPATTI & PETER & $\mathrm{H}$ & 61 & ITALY & ITALY & ITALY & 1891 & WATCHMAN/CM & PONT CANAVESE, TO & E ST. & CALUMET \\
\hline 1696 & & TEPATTI & MARGHERITA & W & 60 & ITALY & ITALY & ITALY & 1896 & & & E ST. & CALUMET \\
\hline 1697 & & TEPATTI & MARGHERITA & $\mathrm{D}$ & 24 & MICHIGAN & ITALY & ITALY & & & PONT CANAVESE. TO & E ST. & CALUMET \\
\hline 1698 & 2622 & ZANIN & JOHN & $\mathrm{H}$ & 28 & MICHIGAN & ITALY & MICHIGAN & & TIMBERMAN/CM & RIVAMONTE. BELLUNO & LIBER & $\begin{array}{l}\text { CALUMET } \\
\end{array}$ \\
\hline 1699 & & ZANIN & ROSE & w & 25 & MICHIGAN & CANADA & MICHIGAN & & & USA-CANADA & LIBERT & CALUMET \\
\hline 1700 & & ZANIN & JEAN & $\mathrm{D}$ & 6 & MICHIGAN & MICHIGAN & MICHIGAN & & & RIVAMONTE. BELLUNO & LIBERTY ST. & CALUMET \\
\hline
\end{tabular}




\begin{tabular}{|c|c|c|c|c|c|c|c|c|c|c|c|c|c|}
\hline 1701 & & ZANIN & JOHN & $S$ & 4 & MICHIGAN & MICHIGAN & MICHIGAN & & & RIVAMONTE. BELLUNO & LIBERTY ST. & CALUMET \\
\hline 1702 & & ZANIN & ROBERT & $\mathrm{S}$ & 3 & MICHIGAN & MICHIGAN & MICHIGAN & & & RIVAMONTE. BELLUNO & LIBERTY ST. & CALUMET \\
\hline 1703 & & ZANIN & KENNETH & $\mathrm{s}$ & 1 & MICHIGAN & MICHIGAN & MICHIGAN & & & RIVAMONTE. BELLUNO & LIBERTY ST. & CALUMET \\
\hline 1704 & 2621 & MACARIO & JOHN & $\mathrm{H}$ & 40 & ITALY & ITALY & ITALY & 1890 & CAR FILLER/CM & TORINO & LIBERTY ST. & CALUMET \\
\hline 1705 & & MACARIO & APOLONIA & w & 44 & MICHIGAN & GERMANY & GERMANY & & & GERMANY & LIBERTY ST. & CALUMET \\
\hline 1706 & & MACARIO & MARGHERITA & D & 8 & MICHIGAN & ITALY & MICHIGAN & & & TORINO & LIBERTY ST. & CALUMET \\
\hline 1707 & 2647 & COLOMBO & MIKE & $\mathrm{H}$ & 39 & ITALY & ITALY & ITALY & 1907 & MINER/CM & TORINO & UNION ST. & CALUMET \\
\hline 1708 & & COLOMBO & TERESA & W & 34 & ITALY & ITALY & ITALY & 1910 & & & UNION ST. & CALUMET \\
\hline 1709 & & COLOMBO & JOHN & s & 15 & ITALY & ITALY & ITALY & 1970 & & TORINO & UNION ST. & CALUMET \\
\hline 1710 & & COLOMBO & ANNE & D & 0 & ITALY & ITALY & ITALY & & & TORINO & UNION ST. & $\begin{array}{l}\text { CALUMET } \\
\end{array}$ \\
\hline 1711 & & COLOMBO & JOSEPH & $\mathrm{BR}$ & 41 & ITALY & ITALY & ITALY & 1910 & MINER/CM & TORINO & UNION ST. & CALUMET \\
\hline 1712 & 2653 & ZANIN & JAMES & $\mathrm{H}$ & 39 & ITALY & ITALY & ITALY & 1909 & LABORER/CM & RIVAMONTE. BELLUNO & UNION ST. & CALUMET \\
\hline 1713 & & ZANIN & RINA & w & 33 & ITALY & ITALY & ITALY & & & 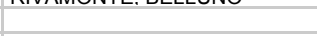 & UNION ST. & CALUMET \\
\hline 1714 & & ZANIN & GENA & D & 7 & ITALY & ITALY & ITALY & & & RIVAMONTE. BELLUNO & UNION ST. & CALUMET \\
\hline 1715 & & ZANIN & ROSE & $\mathrm{D}$ & 5 & ITALY & ITALY & ITALY & & & RIVAMONTE. BELLUNO & UNION ST. & CALUMET \\
\hline 1716 & & ZANIN & GLORIA & $\mathrm{D}$ & 3 & ITALY & ITALY & ITALY & & & RIVAMONTE, BELLUNO & UNION ST. & CALUMET \\
\hline 1717 & & CONADERA & ANTON & BIL & 22 & ITALY & ITALY & ITALY & 1922 & MINER/CM & BELLUNO & UNION ST. & CALUMET \\
\hline 1718 & 2655 & CURTO & BATTISTA & $\mathrm{H}$ & 53 & ITALY & ITALY & ITALY & 1898 & ROLLER REPAIRER/CM & VIALFRE'. TO & UNION ST. & CALUMET \\
\hline 1719 & & CURTO & MINNIE & w & 48 & ITALY & ITALY & ITALY & 1902 & & TORINO & UNION ST. & CALUMET \\
\hline 1720 & & CURTO & MASSIMO & s & 22 & MICHIGAN & ITALY & ITALY & & PAINTER/CM & VIALFRE'. TO & UNION ST. & CALUMET \\
\hline 1721 & & CURTO & $\mathrm{E}$ ? & DIL & 20 & MICHIGAN & ITALY & ITALY & & & & UNION ST. & CALUMET \\
\hline 1722 & & CURTO & KENNETH & GS & 1 & MICHIGAN & ITALY & ITALY & & & VIALFRE', TO & UNION ST. & CALUMET \\
\hline 1723 & & PIETROPAOLI & ANGELO & B & 33 & ITALY & ITALY & ITALY & 1915 & MINER/CM & VERONA & UNION ST. & $\begin{array}{l}\text { CALUMET } \\
\text { Cont }\end{array}$ \\
\hline 1724 & 2666 & LENCIONI & $\begin{array}{l}\text { AOUIS } \\
\text { LOULU }\end{array}$ & $\mathrm{H}$ & 39 & MICHIGAN & ITALY & MICHIGAN & 1915 & MINER/CM & $\begin{array}{l}\text { VERUNA } \\
\text { LUCCA }\end{array}$ & UNION ST. & $\begin{array}{l}\text { CALUVIEI } \\
\text { CALUMET }\end{array}$ \\
\hline 1725 & & LENCIONI & LETTIE & w & 33 & MICHIGAN & FINLAND & NORWAY & & & FINLAND-NORWAY & UNION ST. & CALUMET \\
\hline 1726 & & LENCIONI & EVELYN & D & 9 & MICHIGAN & MICHIGAN & MICHIGAN & & & LUCCA & UNION ST. & CALUMET \\
\hline 1727 & & LENCIONI & LOUIS & $\mathrm{s}$ & 7 & MICHIGAN & MICHIGAN & MICHIGAN & & & LUCCA & UNION ST. & CALUMET \\
\hline 1728 & 2667 & ANTONETTI & GEORGE & $\mathrm{H}$ & 53 & ITALY & ITALY & ITALY & 1904 & STATIONARY FIREMAN/CM & SAN GENNARO, LU & UNION ST. & CALUMET \\
\hline 1729 & & ANTONETTI & JULIA & w & 40 & ITALY & ITALY & ITALY & 1904 & & SAN GENNARO. LU & UNION ST. & CALUMET \\
\hline 1730 & & ANTONETTI & GINO & $\mathrm{s}$ & 22 & MICHIGAN & ITALY & ITALY & & & SAN GENNARO. LU & UNION ST. & CALUMET \\
\hline 1731 & 2669 & NARDI & NELLO & $\mathrm{H}$ & 28 & MICHIGAN & ITALY & ITALY & & CARPENTER/CM & LUCCA & UNION ST. & CALUMET \\
\hline 1732 & & NARDI & FRANCES & w & 26 & MICHIGAN & ITALY & ITALY & & & & UNION ST. & CALUMET \\
\hline 1733 & & NARDI & LOUIS & s & 8 & MICHIGAN & MICHIGAN & MICHIGAN & & & LUCCA & UNION ST. & CALUMET \\
\hline 1734 & & NARDI & DOLORES & $\mathrm{D}$ & 6 & MICHIGAN & MICHIGAN & MICHIGAN & & & LUCCA & UNION ST. & CALUMET \\
\hline 1735 & & NARDI & ELISABETH & $\mathrm{D}$ & & MICHIGAN & MICHIGAN & MICHIGAN & & & LUCCA & UNION ST. & CALUMET \\
\hline 1736 & & NARDI & ELISABETH & $\mathrm{H}$ & 58 & ITALY & ITALY & ITALY & 1894 & & LUCCA & UNION ST. & CALUMET \\
\hline 1737 & & NARDI & JULIA & D & 33 & MICHIGAN & ITALY & ITALY & & & LUCCA & UNION ST. & CALUMET \\
\hline 1738 & & NARDI & MARION & $\overline{G D}$ & 12 & MICHIGAN & ITALY & ITALY & & & LUCCA & UNION ST. & CALUMET \\
\hline 1739 & & NARDI & JULIO & GS & 10 & MICHIGAN & ITALY & ITALY & & & LUCCA & UNION ST. & CALUMET \\
\hline 1740 & 2679 & MASSE' & PETER & $\mathrm{H}$ & 36 & ITALY & ITALY & ITALY & 1906 & MINER/CM & PONT CANAVESE. TO & UNION ST. & CALUMET \\
\hline 1741 & & MASSE' & MADDALENA & w & 24 & ITALY & ITALY & ITALY & 1927 & & & UNION ST. & CALUMET \\
\hline 1742 & & MASSE' & ADELINA & D & 1 & MICHIGAN & ITALY & ITALY & & & PONT CANAVESE. TO & UNION ST. & CALUMET \\
\hline 1743 & 2656 & POLIUTO & DOMENICO & $\mathrm{H}$ & 45 & ITALY & ITALY & ITALY & 1908 & FOUNDRY MAN/CM & SAN MARTINO CANAVESE. TO & UNION ST. & CALUMET \\
\hline 1744 & & POLIUTO & CATERINA & w & 37 & MICHIGAN & ITALY & ITALY & & & TORINO & UNION ST. & CALUMET \\
\hline 1745 & & POLIUTO & ANGELO & s & 16 & MICHIGAN & ITALY & MICHIGAN & & & SAN MARTINO CANAVESE. TO & UNION ST. & CALUMET \\
\hline 1746 & & POLIUTO & TERESA & $\mathrm{D}$ & 14 & MICHIGAN & ITALY & MICHIGAN & & & SAN MARTINO CANAVESE. TO & UNION ST. & CALUMET \\
\hline 1747 & & POLIUTO & ERNEST & $\mathrm{s}$ & 10 & MICHIGAN & ITALY & MICHIGAN & & & SAN MARTINO CANAVESE. TO & UNION ST. & CALUMET \\
\hline 1748 & 2654 & PIERCECCHI & CARL & $\mathrm{H}$ & 37 & ITALY & ITALY & ITALY & 1912 & TEAMSTER/GROCERY STORE & LUCCA & UNION ST. & $\begin{array}{l}\text { CALUMET } \\
\text { CALIE }\end{array}$ \\
\hline 1749 & & PIERCECCHI & JOSEPHINE & w & 32 & MICHIGAN & ITALY & ITALY & & & LUCCA & UNION ST. & CALUMET \\
\hline 1750 & & PIERCECCHI & ROBERT & s & 12 & MICHIGAN & ITALY & MICHIGAN & & & LUCCA & UNION ST. & CALUMET \\
\hline 1751 & & PIERCECCHI & CHESTER & $\mathrm{s}$ & 3 & MICHIGAN & ITALY & MICHIGAN & & & LUCCA & UNION ST. & $\begin{array}{l}\text { CALUMET } \\
\text { Cont }\end{array}$ \\
\hline 1752 & 2754 & BARONI & PAUL & $\mathrm{H}$ & 38 & ITALY & ITALY & ITALY & 1920 & SALESMAN/ALUMINIUM & CASTELVECCHIO, LU & COLUMBIA ST. & CALUMET \\
\hline 1753 & & BARONI & VILMA & w & 25 & MICHIGAN & CROATIA & CROATIA & & & CROATIA & COLUMBIA ST. & CALUMET \\
\hline 1754 & & BARONI & PAUL & $\mathrm{s}$ & $9 \mathrm{M}$ & MICHIGAN & MICHIGAN & MICHIGAN & & & CASTELVECCHIO. LU & COLUMBIA ST. & CALUMET \\
\hline 1755 & 2758 & GASPARDO & TERESA (WID. & $\mathrm{H}$ & 47 & ITALY & ITALY & ITALY & 1904 & & TORINO & COLUMBIA ST. & CALUMET \\
\hline 1756 & & GASPARDO & DOMENICO & BIL & 45 & ITALY & ITALY & ITALY & 1904 & & SPARONE. TO & COLUMBIA ST. & CALUMET \\
\hline 1757 & 2760 & LUCCHESI & ANTONIETTA & $\mathrm{H}$ & 50 & ITALY & ITALY & ITALY & 1900 & & LUCCA & COLUMBIA ST. & CALUMET \\
\hline 1758 & & LUCCHESI & ANGELINA & D & 22 & MICHIGAN & ITALY & ITALY & & SALESLADY/BAKERY & LUCCA & COLUMBIA ST. & CALUMET \\
\hline 1759 & & LUCCHESI & ORLANDO & S & 18 & MICHIGAN & ITALY & ITALY & & LABORER/CM & LUCCA & COLUMBIA ST. & CALUMET \\
\hline 1760 & 2768 & GHIGGIA & DOMENICO & $\mathrm{H}$ & 56 & ITALY & ITALY & ITALY & 1896 & MINER/CM & SCARMAGNO. TO & COLUMBIA ST. & CALUMET \\
\hline 1761 & & GHIGGIA & PAULINE & w & 49 & ITALY & ITALY & ITALY & 1896 & & SCARMAGNO. TO & COLUMBIA ST. & CALUMET \\
\hline 1762 & & GHIGGIA & JOHN & s & 16 & MICHIGAN & ITALY & ITALY & & PAINTER ERRAND BOY/INTERIOR & SCARMAGNO. TO & COLUMBIA ST. & CALUMET \\
\hline 1763 & 3202 & PETITTI & JOHN & $\mathrm{H}$ & 39 & ITALY & $\begin{array}{l}\text { ITALY } \\
\text { ITAL }\end{array}$ & ITALY & 1910 & LANDER/CM & SAN PONSO CANAVESE. TO & RIDGE ST. & CALUMET \\
\hline
\end{tabular}




\begin{tabular}{|c|c|c|c|c|c|c|c|c|c|c|c|c|c|}
\hline 1764 & & PETITTI & PEARL & W & 28 & MICHIGAN & POLAND & POLAND & & & POLAND & RIDGE ST. & CALUMET \\
\hline 1765 & & PETITTI & FRANK & $\mathrm{s}$ & 9 & MICHIGAN & ITALY & MICHIGAN & & & SAN PONSO CANAVESE. TO & RIDGE ST. & CALUMET \\
\hline 1766 & & PETITTI & JOHN & $\mathrm{s}$ & 7 & MICHIGAN & ITALY & MICHIGAN & & & SAN PONSO CANAVESE. TO & RIDGE ST. & CALUMET \\
\hline 1767 & & PETITTI & AGNES & $\mathrm{D}$ & 7 & MICHIGAN & ITALY & MICHIGAN & & & SAN PONSO CANAVESE. TO & RIDGE ST. & CALUMET \\
\hline 1768 & & PETITTI & LAWRENCE & $\mathrm{s}$ & 5 & MICHIGAN & ITALY & MICHIGAN & & & SAN PONSO CANAVESE. TO & RIDGE ST. & CALUMET \\
\hline 1769 & & PETITTI & PEARL & $\mathrm{D}$ & 4 & MICHIGAN & ITALY & MICHIGAN & & & SAN PONSO CANAVESE. TO & RIDGE ST. & CALUMET \\
\hline 1770 & 3225 & BELLINGIER & ROBERT & $\mathrm{H}$ & 38 & MICHIGAN & ITALY & ITALY & & MINER/CM & BELLUNO & RIDGE ST. & CALUMET \\
\hline 1771 & & BELLINGIER & IDA & w & 38 & FINLAND & FINLAND & FINLAND & & & FINLAND & RIDGE ST. & CALUMET \\
\hline 1772 & & DE BERNARDI & LOUIS & SB & 25 & MICHIGAN & ITALY & ITALY & & TIMBERMAN/CM & LOCANA CANAVESE. TO & RIDGE ST. & CALUMET \\
\hline 1773 & 3103 & NOTARI & JOHN & $\mathrm{H}$ & 71 & ITALY & ITALY & ITALY & 1889 & PUMP MAN/CM & PUPILLO. PISTOIA & SKI ST. & CALUMET \\
\hline 1774 & & NOTARI & CESIRA & w & 69 & ITALY & ITALY & ITALY & 1890 & & & SKI ST. & CALUMET \\
\hline 1775 & & NOTARI & PETER & $\mathrm{s}$ & 37 & MICHIGAN & ITALY & ITALY & & STEELWORKER/CONTRACTOR & PUPILLO. PISTOIA & SKI ST. & CALUMET \\
\hline 1776 & & NOTARI & JAMES & $\mathrm{s}$ & 35 & MICHIGAN & ITALY & ITALY & & LABORER/AUTO FACTORY & PUPILLO. PISTOIA & SKI ST. & CALUMET \\
\hline 1777 & 3107 & GALLASERO & JAMES & $\mathrm{H}$ & 37 & MICHIGAN & ITALY & ITALY & & MINER/CM & PONT CANAVESE. TO & SKI ST. & CALUMET \\
\hline 1778 & & GALLASERO & INGA & W & 38 & MICHIGAN & FINLAND & FINLAND & & & FINLAND & SKI ST. & CALUMET \\
\hline 1779 & & GALLASERO & ROBERT & $\mathrm{s}$ & 15 & MICHIGAN & MICHIGAN & MICHIGAN & & & PONT CANAVESE, TO & SKI ST. & CALUMET \\
\hline 1780 & 1306 & TORREANO & JOHN & $\mathrm{H}$ & 31 & MICHIGAN & ITALY & ITALY & & TEAMSTER/GROCERY STORE & TORINO & ROCKLAND ST. & CALUMET \\
\hline 1781 & & TORREANO & JULIA & w & 33 & MICHIGAN & ITALY & ITALY & & & & ROCKLAND ST. & CALUMET \\
\hline 1782 & & TORREANO & JAMES & $\mathrm{s}$ & 7 & MICHIGAN & MICHIGAN & MICHIGAN & & & TORINO & ROCKLAND ST. & CALUMET \\
\hline 1783 & & PIGNOCCO & SECONDINA & MIL & 58 & ITALY & ITALY & ITALY & 1889 & & SAN GIORGIO CANAVESE. TO & ROCKLAND ST. & CALUMET \\
\hline 1784 & 1310 & BIANCO & ANTONIO & $\mathrm{H}$ & 63 & ITALY & ITALY & ITALY & 1890 & BELL RINGER/CM & $\begin{array}{l}\text { LOCANA CANAVESE. TO } \\
\text { LOCS }\end{array}$ & ROCKLAND ST. & CALUMET \\
\hline 1785 & 1010 & BIANCO & ANNE & W & 49 & ITALY & ITALY & ITALY & 1899 & DELL RIVUERT/UIVI & LOCANA CANAVESE, TO & ROCKLAND ST. & $\begin{array}{l}\text { CALUMET } \\
\text { CALIVI }\end{array}$ \\
\hline 1786 & 1312 & MARTA & JOHN & $\mathrm{H}$ & 46 & ITALY & ITALY & ITALY & 1906 & SHAFT FOREMAN/CM & AGLIE'. TO & ROCKLAND ST. & CALUMET \\
\hline 1787 & & MARTA & CATERINA & w & 42 & BRASIL & ITALY & ITALY & 1908 & & TORINO & ROCKLAND ST. & CALUMET \\
\hline 1788 & & MARTA & MARY & $\mathrm{D}$ & 20 & MICHIGAN & ITALY & BRASIL & & & AGLIE'. TO & ROCKLAND ST. & CALUMET \\
\hline 1789 & & MARTA & M? & $\mathrm{s}$ & 19 & MICHIGAN & ITALY & BRASIL & & FOUNDRY MAN/CM & AGLIE'. TO & ROCKLAND ST. & CALUMET \\
\hline 1790 & & MARTA & JOHN & $\mathrm{s}$ & 17 & MICHIGAN & ITALY & BRASIL & & DELIVERY BOY/GROCERY STORE & AGLIE'. TO & ROCKLAND ST. & CALUMET \\
\hline 1791 & & MARTA & IRENE & D & 15 & MICHIGAN & ITALY & BRASIL & & & AGLIE', TO & ROCKLAND ST. & CALUMET \\
\hline 1792 & & MARTA & TEO & $\mathrm{s}$ & 2 & MICHIGAN & ITALY & BRASIL & & & AGLIE'. TO & ROCKLAND ST. & CALUMET \\
\hline 1793 & 1309 & MANZARDO & ANTONIO & $\mathrm{H}$ & 43 & ITALY & ITALY & ITALY & 1906 & MINER/CM & VENEZIA & ROCKLAND ST. & CALUMET \\
\hline 1794 & & MANZARDO & LAURA & w & 42 & ITALY & ITALY & ITALY & 1908 & & & ROCKLAND ST. & CALUMET \\
\hline 1795 & & MANZARDO & DOMENICO & $\mathrm{s}$ & 15 & MICHIGAN & ITALY & ITALY & & & VENEZIA & ROCKLAND ST. & CALUMET \\
\hline 1796 & & MANZARDO & JAMES & $\mathrm{s}$ & 12 & MICHIGAN & ITALY & ITALY & & & VENEZIA & ROCKLAND ST. & CALUMET \\
\hline 1797 & & MANZARDO & FRANCES & $\mathrm{D}$ & 8 & MICHIGAN & ITALY & ITALY & & & VENEZIA & ROCKLAND ST. & CALUMET \\
\hline 1798 & & MANZARDO & FRANK & s & $9 \mathrm{M}$ & MICHIGAN & ITALY & ITALY & & & VENEZIA & ROCKLAND ST. & CALUMET \\
\hline 1799 & & ANYETTI & BERNARD & UNCL & 54 & ITALY & ITALY & ITALY & 1895 & & & ROCKLAND ST. & CALUMET \\
\hline 1800 & 1303 & CRUGNOLA & CARLO & $\mathrm{H}$ & 68 & ITALY & ITALY & ITALY & 1893 & STONE MASON/CM & COMO & ROCKLAND ST. & CALUMET \\
\hline 1801 & & CRUGNOLA & MODESTA & W & 67 & ITALY & ITALY & ITALY & 1893 & & COMO & ROCKLAND ST. & CALUMET \\
\hline 1802 & 1504 & FREEMAN & WILLIAM & H & 48 & MICHIGAN & SWEDEN & SWEDEN & & PLUMBER/HARDWARE & SWEDEN & HECLA ST. & CALUMET \\
\hline 1803 & & FREEMAN & CATHERINE & W & 42 & ITALY & ITALY & ITALY & 1905 & & & HECLA ST. & CALUMET \\
\hline 1804 & & FREEMAN & EDWARD & $\mathrm{s}$ & 14 & MICHIGAN & MICHIGAN & ITALY & & & & HECLA ST. & CALUMET \\
\hline 1805 & & FREEMAN & ANNETTE & D & 8 & MICHIGAN & MICHIGAN & ITALY & & & & HECLA ST. & CALUMET \\
\hline 1806 & 1506 & CHELI & FRED & $\mathrm{H}$ & 54 & MICHIGAN & ITALY & ITALY & & STATIONARY ENGINEER/CM & LUCCA & HECLA ST. & CALUMET \\
\hline 1807 & & CHELI & ROSE & w & 52 & MICHIGAN & ITALY & ITALY & & & & HECLA ST. & CALUMET \\
\hline 1808 & 1523 & ROSSO & MARY & $\mathrm{H}$ & 56 & ITALY & ITALY & ITALY & 1898 & JANITRESS/OFFICE BUILDING & TORINO & HECLA ST. & CALUMET \\
\hline 1809 & 1020 & ROSSO & LOUISE & $\mathrm{D}$ & 20 & MICHIGAN & ITALY & ITALY & 1050 & OPERATOR/TELEPHONE & TORINO & HECLA ST. & CALUMET \\
\hline 1810 & & SANDRETTO & MARIE & $\mathrm{D}$ & 29 & MICHIGAN & ITALY & ITALY & & SERVANT/PF & PONT CANAVESE. TO & HECLA ST. & CALUMET \\
\hline 1811 & 1517 & O'CONNELL & WILL & $\mathrm{H}$ & 26 & MICHIGAN & IRELAND & IRELAND & & BRAKEMAN/RAILROAD & IRELAND & HECLA ST. & CALUMET \\
\hline 1812 & & O'CONNELL & MARY & w & 24 & ITALY & ITALY & ITALY & 1907 & & & HECLA ST. & CALUMET \\
\hline 1813 & 1511 & PIGNOCCO & DOMENICO & $\mathrm{H}$ & 34 & MICHIGAN & ITALY & ITALY & & WAITER/CLUB HOUSE & SAN GIORGIO CANAVESE. TO & HECLA ST. & CALUMET \\
\hline 1814 & & PIGNOCCO & HUMALEN & W & 27 & MICHIGAN & MICHIGAN & ITALY & & & & HECLA ST. & CALUMET \\
\hline 1815 & & PIGNOCCO & FLORENCE & D & 6 & MICHIGAN & MICHIGAN & MICHIGAN & & & SAN GIORGIO CANAVESE, TO & HECLA ST. & CALUMET \\
\hline 1816 & 1509 & FERRARI & LOUIS & $\mathrm{H}$ & 50 & ITALY & ITALY & ITALY & 1903 & STATIONARY FIREMAN/CM & COLLODI. LU & HECLA ST. & CALUMET \\
\hline 1817 & & FERRARI & MELANIA & W & 48 & ITALY & ITALY & ITALY & 1904 & & & HECLA ST. & CALUMET \\
\hline 1818 & & FERRARI & PAUL & s & 18 & MICHIGAN & ITALY & ITALY & & APPRENTICE/BARBER SHOP & COLLODI. LU & HECLA ST. & CALUMET \\
\hline 1819 & & FERRARI & ADELINE & D & 16 & MICHIGAN & ITALY & ITALY & & & COLLODI. LU & HECLA ST. & CALUMET \\
\hline 1820 & 1507 & REGIS & JOHN & $\mathrm{H}$ & 60 & ITALY & ITALY & ITALY & 1891 & LABORER/CM & SAN GIUSTO CANAVESE. TO & HECLA ST. & CALUMET \\
\hline 1821 & & REGIS & ELLEN & W & 53 & ITALY & ITALY & ITALY & 1897 & & & HECLA ST. & CALUMET \\
\hline 1822 & & REGIS & MINNIE & D & 26 & MICHIGAN & ITALY & ITALY & & COMPOSER-TYPE/NEWSPAPER & SAN GIUSTO CANAVESE. TO & HECLA ST. & CALUMET \\
\hline 1823 & & REGIS & GEORGE & $\mathrm{s}$ & 25 & MICHIGAN & ITALY & ITALY & & CARPENTER/CM & SAN GIUSTO CANAVESE. TO & HECLA ST. & CALUMET \\
\hline 1824 & & REGIS & JOHN & s & 15 & MICHIGAN & ITA & ITAL & & & SAN GIUSTO CANAVESE. TO & HECLA ST. & \\
\hline 1825 & & REGIS & ANTONIETTA & DIL & 19 & MICHIGAN & ITALY & ITALY & & & SAN GIUSTO CANAVESE. TO & HECLA ST. & CALUMET \\
\hline 1826 & 1604 & GIULIO & ANTONIO & $\mathrm{H}$ & 71 & ITALY & ITALY & ITALY & 1883 & & SAN GIORGIO CANAVESE. TO & LAURIUM ST. & CALUMET \\
\hline
\end{tabular}




\begin{tabular}{|c|c|c|c|c|c|c|c|c|c|c|c|c|c|}
\hline 1827 & & GIULIO & MARY & W & 61 & ITALY & ITALY & ITALY & 1891 & & & LAURIUM ST. & CALUMET \\
\hline 1828 & & GIULIO & MARGHERITA & D & 21 & MICHIGAN & ITALY & ITALY & & SALESLADY/DRY GOODS STORE & SAN GIORGIO CANAVESE. TO & LAURIUM ST. & CALUMET \\
\hline 1829 & & WADE & PHYLLIS & $\mathrm{D}$ & 25 & MICHIGAN & ITALY & ITALY & & & SAN GIORGIO CANAVESE. TO & LAURIUM ST. & CALUMET \\
\hline 1830 & & WADE & JOHN & SOIL & 26 & MICHIGAN & MICHIGAN & MICHIGAN & & MINER/CM & USA & LAURIUM ST. & CALUMET \\
\hline 1831 & 1614 & GALLO & TERESA (WID. & $\mathrm{H}$ & 69 & ITALY & ITALY & ITALY & 1892 & & TORINO & LAURIUM ST. & CALUMET \\
\hline 1832 & & ZUBIENA & DOMENICO & $\mathrm{H}$ & 43 & ITALY & ITALY & ITALY & 1903 & ELECTRICIAN HELPER/CM & TORINO & LAURIUM ST. & CALUMET \\
\hline 1833 & & ZUBIENA & MADDALENA & w & 38 & ITALY & ITALY & ITALY & 1892 & & & LAURIUM ST. & CALUMET \\
\hline 1834 & & ZUBIENA & JAMES & s & 1 & MICHIGAN & ITALY & ITALY & & & TORINO & LAURIUM ST. & CALUMET \\
\hline 1835 & 1616 & GRANDIS & JOHN & $\mathrm{H}$ & 58 & ITALY & ITALY & ITALY & 1902 & & SAN GIORGIO CANAVESE. TO & LAURIUM ST. & CALUMET \\
\hline 1836 & & GRANDIS & MARGHERITA & w & 55 & ITALY & ITALY & ITALY & 1900 & & & LAURIUM ST. & CALUMET \\
\hline 1837 & 1618 & QUELLO & PETER & $\mathrm{H}$ & 43 & ITALY & ITALY & ITALY & 1907 & LABORER/TOWNSHIP & LOCANA CANAVESE. TO & LAURIUM ST. & CALUMET \\
\hline 1838 & & QUELLO & MARY & W & 39 & ITALY & ITALY & ITALY & 1921 & & & LAURIUM ST. & CALUMET \\
\hline 1839 & & QUELLO & MARIE & $\mathrm{D}$ & 16 & MICHIGAN & ITALY & ITALY & & & LOCANA CANAVESE. TO & LAURIUM ST. & CALUMET \\
\hline 1840 & & QUELLO & PIERINA & D & 15 & MICHIGAN & ITALY & ITALY & & & LOCANA CANAVESE. TO & LAURIUM ST. & CALUMET \\
\hline 1841 & & QUELLO & $\mathrm{JOHN}$ & $\mathrm{s}$ & 14 & MICHIGAN & ITALY & ITALY & & & LOCANA CANAVESE. TO & LAURIUM ST. & CALUMET \\
\hline 1842 & & QUELLO & LOUIS & $\mathrm{S}$ & 6 & MICHIGAN & ITALY & ITALY & & & LOCANA CANAVESE, TO & LAURIUM ST. & CALUMET \\
\hline 1843 & & QUELLO & HENRY & $\mathrm{s}$ & 2 & MICHIGAN & ITALY & ITALY & & & LOCANA CANAVESE. TO & LAURIUM ST. & CALUMET \\
\hline 1844 & 1622 & RICCA & VITTORIO & $\mathrm{H}$ & 53 & ITALY & ITALY & ITALY & 1900 & MINER/CM & SAN PONSO CANAVESE. TO & LAURIUM ST. & CALUMET \\
\hline 1845 & & RICCA & PAULINE & w & 53 & ITALY & ITALY & ITALY & 1900 & & SAN PONSO CANAVESE. TO & LAURIUM ST. & CALUMET \\
\hline 1846 & & RICCA & AUGUST & $\mathrm{s}$ & 22 & MICHIGAN & ITALY & ITALY & & LABORER/CM & SAN PONSO CANAVESE. TO & LAURIUM ST. & CALUMET \\
\hline 1847 & & RICCA & VITTORIO & $\mathrm{s}$ & 10 & MICHIGAN & ITALY & ITALY & & & SAN PONSO CANAVESE. TO & LAURIUM ST. & CALUMET \\
\hline 1848 & & RICCA & PAULINE & D & 8 & MICHIGAN & ITALY & ITALY & & & SAN PONSO CANAVESE, TO & $\begin{array}{l}\text { LAURIUM ST. } \\
\text { LAUT }\end{array}$ & CALUMET \\
\hline 1849 & 1624 & SAPINO/ & PETER & $\mathrm{H}$ & 45 & AFRICA & ITALY & ITALY & 1905 & STONE MASON/CM & SAN MARTINO CANAVESE. TO & LAURIUM ST. & CALUMET \\
\hline 1850 & & SAPINO/ & ANNA & w & 31 & ITALY & ITALY & ITALY & 1902 & & & LAURIUM ST. & CALUMET \\
\hline 1851 & & SAPINO/ & DOMENICO & $\mathrm{S}$ & 12 & MICHIGAN & AFRICA & ITALY & & & SAN MARTINO CANAVESE. TO & LAURIUM ST. & CALUMET \\
\hline 1852 & & SAPINO/ & JOHN & B & 30 & ITALY & ITALY & ITALY & 1923 & SALESMAN/ELECTRIC & SAN MARTINO CANAVESE. TO & LAURIUM ST. & CALUMET \\
\hline 1853 & 1626 & BENEITONE & DOMENICO & $\mathrm{H}$ & 43 & ITALY & ITALY & ITALY & 1905 & MINER/CM & SALASSA. TO & LAURIUM ST. & CALUMET \\
\hline 1854 & & BENEITONE & MARY & W & 33 & MICHIGAN & ITALY & ITALY & & & & LAURIUM ST. & CALUMET \\
\hline 1855 & & BENEITONE & MONICA & D & 12 & MICHIGAN & ITALY & MICHIGAN & & & SALASSA. TO & LAURIUM ST. & CALUMET \\
\hline 1856 & & BENEITONE & LOUIS & S & 10 & MICHIGAN & ITALY & MICHIGAN & & & SALASSA. TO & LAURIUM ST. & CALUMET \\
\hline 1857 & & BENEITONE & BATTISTA & s & 9 & MICHIGAN & ITALY & MICHIGAN & & & SALASSA. TO & LAURIUM ST. & CALUMET \\
\hline 1858 & & BENEITONE & RAYMOND & $\mathrm{s}$ & 5 & MICHIGAN & ITALY & MICHIGAN & & & SALASSA. TO & LAURIUM ST. & CALUMET \\
\hline 1859 & 1641 & SCUSSEL & JOSEPH & $\mathrm{H}$ & 43 & ITALY & $\begin{array}{l}\text { ITALY } \\
\end{array}$ & ITALY & 1901 & MINER/CM & AGORDO. BELLUNO & LAURIUM ST. & CALUMET \\
\hline 1860 & & SCUSSEL & JOSEPHINE & w & 33 & ITALY & ITALY & ITALY & 1916 & & & LAURIUM ST. & CALUMET \\
\hline 1861 & & SCUSSEL & JOHANNA & D & 12 & MICHIGAN & ITALY & ITALY & & & AGORDO. BELLUNO & LAURIUM ST. & CALUMET \\
\hline 1862 & & SCUSSEL & JOSEPH JR. & $\mathrm{s}$ & 11 & MICHIGAN & ITALY & ITALY & & & AGORDO. BELLUNO & LAURIUM ST. & CALUMET \\
\hline 1863 & & SCUSSEL & ELVIRA & D & 10 & MICHIGAN & ITALY & ITALY & & & AGORDO. BELLUNO & LAURIUM ST. & CALUMET \\
\hline 1864 & 1633 & SCUSSEL & FRANK & $\mathrm{H}$ & 46 & ITALY & ITALY & ITALY & 1903 & MINER/CM & BELLUNO & LAURIUM ST. & CALUMET \\
\hline 1865 & & SCUSSEL & MARGHERITA & W & 41 & ITALY & ITALY & ITALY & 1903 & & PARELLA. TO & LAURIUM ST. & CALUMET \\
\hline 1866 & & SCUSSEL & FRANK JR. & s & 19 & MICHIGAN & ITALY & ITALY & & ASSISTANT TIMEKEEPER/CM & BELLUNO & LAURIUM ST. & CALUMET \\
\hline 1867 & & SCUSSEL & JOSEPHINE & D & 18 & MICHIGAN & ITALY & ITALY & & TEAHCER/PIANO MUSIC & BELLUNO & LAURIUM ST. & CALUMET \\
\hline 1868 & & SCUSSEL & DELPHINE & D & 16 & MICHIGAN & ITALY & ITALY & & & BELLUNO & LAURIUM ST. & CALUMET \\
\hline 1869 & & SCUSSEL & HENRY & $\mathrm{s}$ & 14 & MICHIGAN & ITALY & ITALY & & NEWSBOYIDAILY PAPER & BELLUNO & LAURIUM ST. & CALUMET \\
\hline 1870 & & SCUSSEL & MARIO & s & 9 & MICHIGAN & ITALY & ITALY & & & BELLUNO & LAURIUM ST. & CALUMET \\
\hline 1871 & 1631 & CAPELLO & MARY & $\mathrm{H}$ & 71 & ITALY & ITALY & ITALY & 1890 & & & LAURIUM ST. & CALUMET \\
\hline 1872 & 1001 & NOTARI & SAM & NEP & 40 & ITALY & ITALY & ITALY & 1905 & BARTENDER/SOFT DRINK & PISTOIA & LAURIUM ST. & CALUMET \\
\hline 1873 & 1629 & ONORE & BONO & $\mathrm{H}$ & 47 & ITALY & ITALY & ITALY & 1903 & MINER/CM & STRAMBINELLO. TO & LAURIUM ST. & CALUMET \\
\hline 1874 & & ONORE & PIERINA & w & 46 & ITALY & ITALY & ITALY & 1903 & & STRAMBINELLO. TO & LAURIUM ST. & CALUMET \\
\hline 1875 & & ONORE & ALMA & D & 17 & MICHIGAN & ITALY & ITALY & & & STRAMBINELLO. TO & LAURIUM ST. & CALUMET \\
\hline 1876 & & ONORE & STEPHEN & $\mathrm{s}$ & 15 & MICHIGAN & ITALY & ITALY & & & STRAMBINELLO. TO & LAURIUM ST. & CALUMET \\
\hline 1877 & 1625 & SAPINO & DOMENICO & $\mathrm{H}$ & 47 & ITALY & ITALY & ITALY & 1915 & TIMBERMAN/CM & SAN MARTINO CANAVESE. TO & LAURIUM ST. & CALUMET \\
\hline 1878 & & SAPINO & MARIA & W & 37 & ITALY & ITALY & ITALY & 1915 & & SAN MARTINO CANAVESE, TO & LAURIUM ST. & CALUMET \\
\hline 1879 & & SAPINO & ANGELINA & $\mathrm{D}$ & 15 & ITALY & ITALY & ITALY & 1915 & & SAN MARTINO CANAVESE. TO & LAURIUM ST. & CALUMET \\
\hline 1880 & 1621 & ROSSIO & ANGELO & $\mathrm{H}$ & 48 & ITALY & ITALY & ITALY & 1907 & LABORER/CM & PONT CANAVESE. TO & LAURIUM ST. & CALUMET \\
\hline 1881 & & ROSSIO & TERSILIA & w & 51 & ITALY & ITALY & ITALY & 1907 & & PONT CANAVESE. TO & LAURIUM ST. & CALUMET \\
\hline 1882 & & ROSSIO & CHARLES & s & 15 & MICHIGAN & ITALY & ITALY & & & PONT CANAVESE. TO & LAURIUM ST. & CALUMET \\
\hline 1883 & & ROSSIO & ANNE & $\mathrm{D}$ & 13 & MICHIGAN & ITALY & ITALY & & & PONT CANAVESE. TO & LAURIUM ST. & CALUMET \\
\hline 1884 & 1617 & ROVANO & JOHN & $\mathrm{H}$ & 50 & ITALY & ITALY & ITALY & 1914 & LABORER/CM & SAN MARTINO CANAVESE, TO & LAURIUM ST. & CALUMET \\
\hline 1885 & & ROVANO & MINNIE & W & 44 & ITALY & ITALY & ITALY & 1913 & & & LAURIUM ST. & CALUMET \\
\hline 1886 & & ROVANO & PETER & $\mathrm{s}$ & 16 & MICHIGAN & ITALY & ITALY & & & SAN MARTINO CANAVESE. TO & LAURIUM ST. & CALUMET \\
\hline 1887 & & ROVANO & JAMES & $\mathrm{s}$ & 6 & MICHIGAN & ITALY & ITALY & & & SAN MARTINO CANAVESE. TO & LAURIUM ST. & CALUN \\
\hline 1888 & 1615 & CENTANINO & DOMENICA & $\mathrm{H}$ & 47 & ITALY & ITALY & ITALY & 1889 & SEAMSTRESS/DRESS & TORINO & LAURIUM ST. & CALUMET \\
\hline 1889 & & CENTANINO & DOMENICO & $\mathrm{s}$ & 26 & MICHIGAN & ITALY & ITALY & & ATTENDANT/GAS AND OIL & SAN MARTINO CANAVESE. TO & LAURIUM ST. & CALUMET \\
\hline
\end{tabular}




\begin{tabular}{|c|c|c|c|c|c|c|c|c|c|c|c|c|c|}
\hline 1890 & 1613 & CARBONATTO & VITTORIO & $\mathrm{H}$ & 61 & ITALY & ITALY & ITALY & 1891 & MACHINIST/CM & PONT CANAVESE. TO & LAURIUM ST. & CALUMET \\
\hline 1891 & & CARBONATTO & MARY & W & 53 & ITALY & ITALY & ITALY & 1891 & & PONT CANAVESE. TO & LAURIUM ST. & CALUMET \\
\hline 1892 & 1607 & MIGLIO/MIGLIA & MINNIE & $\mathrm{H}$ & 55 & ITALY & ITALY & ITALY & 1893 & & TORINO & LAURIUM ST. & CALUMET \\
\hline 1893 & & MIGLIO/MIGLIA & LOUIS & $\mathrm{S}$ & 34 & MICHIGAN & ITALY & ITALY & & STATIONARY ENGINEER/CM & TORINO & LAURIUM ST. & CALUMET \\
\hline 1894 & 1603 & BARISICA & FRANK & $\mathrm{H}$ & 43 & ITALY & ITALY & ITALY & 1911 & MINER/CM & SAN MARTINO CANAVESE. TO & LAURIUM ST. & CALUMET \\
\hline 1895 & & BARISICA & TERESA & W & 34 & ITALY & ITALY & ITALY & 1916 & & & LAURIUM ST. & CALUMET \\
\hline 1896 & & BARISICA & CATERINA & $\mathrm{D}$ & 9 & MICHIGAN & ITALY & ITALY & & & SAN MARTINO CANAVESE. TO & LAURIUM ST. & CALUMET \\
\hline 1897 & 1601 & FAUSONE & FRANK & $\mathrm{H}$ & 61 & ITALY & ITALY & ITALY & 1892 & STONE MASON/CM & $\begin{array}{l}\text { TORINO } \\
\text { TOR CAVES. TO }\end{array}$ & LAURIUM ST. & CALUMET \\
\hline 1898 & & FAUSONE & JULIA & W & 64 & ITALY & ITALY & ITALY & 1894 & & TORINO & LAURIUM ST. & $\begin{array}{l}\text { CALUMET } \\
\end{array}$ \\
\hline 1899 & & FAUSONE & MINNIE & $\mathrm{D}$ & 35 & MICHIGAN & ITALY & ITALY & & & TORINO & LAURIUM ST. & CALUMET \\
\hline 1900 & & FAUSONE & FRANK & $\mathrm{s}$ & 33 & MICHIGAN & ITALY & ITALY & & ATTENDANT/GAS AND OIL & TORINO & LAURIUM ST. & $\begin{array}{l}\text { CALUMET } \\
\end{array}$ \\
\hline 1901 & & FAUSONE & CATERINA & $\mathrm{D}$ & 24 & MICHIGAN & ITALY & ITALY & & BOOKKEEPER/MUSIC STORE & TORINO & LAURIUM ST. & CALUMET \\
\hline 1902 & 1718 & DIGHERA & THOMAS & $\mathrm{H}$ & 26 & MICHIGAN & ITALY & ITALY & & BLACKSMITH/CM & SAN GIORGIO CANAVESE. TO & BOUNDARY ST. & CALUMET \\
\hline 1903 & & DIGHERA & FRANK & $\mathrm{BR}$ & 25 & MICHIGAN & ITALY & ITALY & & PATTERN MAKER/CM & SAN GIORGIO CANAVESE. TO & BOUNDARY ST. & CALUMET \\
\hline 1904 & 1722 & SCIACERO & LOUIS & $\mathrm{H}$ & 68 & ITALY & ITALY & ITALY & 1889 & MINER/CM & SAN MARTINO CANAVESE. TO & BOUNDARY ST. & CALUMET \\
\hline 1905 & & SCIACERO & MARY & W & 67 & ITALY & ITALY & ITALY & 1889 & & SAN MARTINO CANAVESE, TO & BOUNDARY ST. & CALUMET \\
\hline 1906 & & SCIACERO & JOHN & BIL & 58 & ITALY & ITALY & ITALY & 1892 & & SAN MARTINO CANAVESE. TO & BOUNDARY ST. & CALUMET \\
\hline 1907 & & SULLIVAN & EVELYN & $\mathrm{GD}$ & 12 & MICHIGAN & ITALY & ITALY & & & SALASSA. TO & BOUNDARY ST. & CALUMET \\
\hline 1908 & 1724 & SAPINO & ANTON & $\mathrm{H}$ & 45 & ITALY & ITALY & ITALY & 1912 & STONE MASON/CM & SAN MARTINO CANAVESE. TO & BOUNDARY ST. & CALUMET \\
\hline 1909 & 1124 & SAPINO & ANGELINE & w & 45 & ITALY & ITALY & ITALY & 1913 & 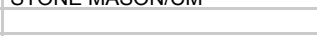 & SAIV IIATS IIN CAIVAVLOL. TO & BOUNDARY ST. & CALUMET \\
\hline 1910 & & SAPINO & CATERINA & $\mathrm{D}$ & 16 & MICHIGAN & ITALY & ITALY & & & SAN MARTINO CANAVESE. TO & BOUNDARY ST. & CALUMET \\
\hline 1911 & & SAPINO & LAWRENCE & $\mathrm{s}$ & 10 & MICHIGAN & ITALY & ITALY & & & SAN MARTINO CANAVESE, TO & BOUNDARY ST. & CALUMET \\
\hline 1912 & 1756 & PISTOTTI & LOUIS & $\mathrm{H}$ & 48 & ITALY & ITALY & ITALY & 1897 & BLACKSMITH HELPER/CM & SPARONE. TO & BOUNDARY ST. & CALUMET \\
\hline 1913 & & PISTOTTI & ANGELINA & W & 39 & MICHIGAN & ITALY & ITALY & & & & BOUNDARY ST. & CALUMET \\
\hline 1914 & & PISTOTTI & CATERINA & $\mathrm{D}$ & 16 & MICHIGAN & ITALY & MICHIGAN & & & SPARONE. TO & BOUNDARY ST. & CALUMET \\
\hline 1915 & & PISTOTTI & MARIA & $\mathrm{D}$ & 12 & MICHIGAN & ITALY & MICHIGAN & & & SPARONE. TO & BOUNDARY ST. & CALUMET \\
\hline 1916 & & PISTOTTI & LOUIS & $\mathrm{s}$ & 10 & MICHIGAN & ITALY & MICHIGAN & & & SPARONE. TO & BOUNDARY ST. & CALUMET \\
\hline 1917 & & PISTOTTI & BERNARD & $\mathrm{s}$ & 2 & MICHIGAN & ITALY & MICHIGAN & & & SPARONE, TO & BOUNDARY ST. & CALUMET \\
\hline 1918 & 1762 & FRANCISCA & ANGELINA & $\mathrm{H}$ & 76 & ITALY & ITALY & ITALY & 1894 & & PEROSA. TO & BOUNDARY ST. & CALUMET \\
\hline 1919 & & FRANCISCA & JOHN & S & 35 & ITALY & ITALY & ITALY & 1894 & LABORER/CM & PEROSA. TO & BOUNDARY ST. & CALUMET \\
\hline 1920 & 2100 & SPINATO & GERVASIO & B & 32 & ITALY & ITALY & ITALY & 1914 & LABORER/CM & MONTECCHIO. VICENZA & LOG ST. & CALUMET \\
\hline 1921 & 73 & SANDRETTO & DOMENICO & $\mathrm{H}$ & 37 & ITALY & ITALY & ITALY & 1911 & NOT CLEAR & PONT CANAVESE. TO & AMYGDALOID ST. & CALUMET - CENTENNIAL LOC. \\
\hline 1922 & 10 & SANDRETTO & MARY & W & 32 & ITALY & $\begin{array}{l}\text { ITALY } \\
\text { ITA }\end{array}$ & ITALY & 1915 & IVUT CLEAR & FUIVI CAIVAVEOE. TO & AMYGDALOID ST. & $\begin{array}{l}\text { CALUMET - CENTENNIAL LOC. } \\
\text {. CEN }\end{array}$ \\
\hline 1923 & & SANDRETTO & ANGELINE & $\mathrm{D}$ & 11 & MICHIGAN & ITALY & ITALY & & & PONT CANAVESE, TO & AMYGDALOID ST. & CALUMET - CENTENNIAL LOC. \\
\hline 1924 & & SANDRETTO & MARIE & D & 10 & MICHIGAN & ITALY & ITALY & & & PONT CANAVESE. TO & AMYGDALOID ST. & CALUMET - CENTENNIAL LOC. \\
\hline 1925 & & SANDRETTO & TERESA & $\mathrm{D}$ & 6 & MICHIGAN & ITALY & ITALY & & & PONT CANAVESE. TO & AMYGDALOID ST. & CALUMET - CENTENNIAL LOC. \\
\hline 1926 & & BOSSA & PAUL & B & 46 & ITALY & ITALY & ITALY & 1909 & LABORER/CM & TORINO & AMYGDALOID ST. & CALUMET - CENTENNIAL LOC. \\
\hline 1927 & 888 & ROTA & ELIA & $\mathrm{H}$ & 38 & ITALY & ITALY & ITALY & $85 / 95$ & MERCHANT/WHOLEALE & BERGAMO & CAMBRIA ST. & 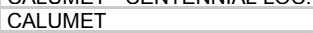 \\
\hline 1928 & & ROTA-MASNADA & NATALINA & W & 32 & MICHIGAN & ITALY & ITALY & & & TORINO & CAMBRIA ST. & CALUMET \\
\hline 1929 & & ROTA & BERNARDO & $\mathrm{s}$ & 15 & MICHIGAN & ITALY & MICHIGAN & & & BERGAMO & CAMBRIA ST. & CALUMET \\
\hline 1930 & & ROTA & MARGE & D & 12 & MICHIGAN & ITALY & MICHIGAN & & & BERGAMO & CAMBRIA ST. & CALUMET \\
\hline 1931 & & ROTA & LAVERNE & $\mathrm{D}$ & 9 & MICHIGAN & ITALY & MICHIGAN & & & BERGAMO & CAMBRIA ST. & CALUMET \\
\hline 1932 & 461 & ANDRINA & JOHN & $\mathrm{H}$ & 45 & ITALY & ITALY & ITALY & 1900 & ROLLER REPAIRER/CM & SAN MARTINO CANAVESE. TO & CALEDONIA ST. & CALUMET \\
\hline 1933 & & ANDRINA & MARNIE & W & 43 & MICHIGAN & ITALY & ITALY & & & & CALEDONIA ST. & CALUMET \\
\hline 1934 & & ANDRINA & JOHN & s & 22 & MICHIGAN & ITALY & MICHIGAN & & PAINTER/HOUSE & SAN MARTINO CANAVESE. TO & CALEDONIA ST. & CALUMET \\
\hline 1935 & & ANDRINA & ERNEST & $\mathrm{s}$ & 17 & MICHIGAN & ITALY & MICHIGAN & & WAITER/CLUB HOUSE & SAN MARTINO CANAVESE. TO & CALEDONIA ST. & CALUMET \\
\hline 1936 & 476 & STELLA & PETER & $\mathrm{H}$ & 40 & ITALY & ITALY & ITALY & 1917 & MINER/CM & VICENZA & CALEDONIA ST. & CALUMET \\
\hline 1937 & & STELLA & VITTORIA & w & 31 & ITALY & ITALY & ITALY & 1923 & & & CALEDONIA ST. & CALUMET \\
\hline 1938 & & STELLA & DOMENICO & $\mathrm{s}$ & 6 & MINNESOTA & ITALY & ITALY & & & VICENZA & CALEDONIA ST. & $\begin{array}{l}\text { CALUMET } \\
\text { CAM }\end{array}$ \\
\hline 1939 & & STELLA & MARIE & $\mathrm{D}$ & 0 & MINNESOTA & ITALY & ITALY & & & VICENZA & CALEDONIA ST. & CALUMET \\
\hline 1940 & 436 & PANYAN & $\mathrm{JOHN}$ & $\mathrm{H}$ & 27 & MICHIGAN & YUGOSLAVIA & YUGOSLAVIA & & MINER/CM & YUGOSLAVIA & CALEDONIA ST. & CALUMET \\
\hline 1941 & & PANYAN & ANGELINE & W & 27 & MICHIGAN & ITALY & ITALY & & & & CALEDONIA ST. & $\begin{array}{l}\text { CALUMET } \\
\end{array}$ \\
\hline 1942 & 833 & FABIAN & ANTON & $\mathrm{H}$ & 55 & ITALY & ITALY & ITALY & 1913 & MINER/CM & & ROCKLAND ST. & CALUMET \\
\hline 1943 & & FABIAN & ANTONIA & W & 40 & ITALY & ITALY & ITALY & 1913 & & & ROCKLAND ST. & CALUMET \\
\hline 1944 & & FABIAN & ALBIN & S & 16 & MICHIGAN & ITALY & ITALY & & & & ROCKLAND ST. & CALUMET \\
\hline 1945 & & FABIAN & RUDOLPH & $\mathrm{s}$ & 15 & MICHIGAN & ITALY & ITALY & & & & ROCKLAND ST. & CALUMET \\
\hline 1946 & & FABIAN & MILAN & $\mathrm{s}$ & 14 & MICHIGAN & ITALY & ITALY & & & & ROCKLAND ST. & CALUMET \\
\hline 1947 & & FABIAN & MARY & D & 12 & MICHIGAN & ITALY & ITALY & & & & ROCKLAND ST. & $\begin{array}{l}\text { CALUMET } \\
\end{array}$ \\
\hline 1948 & & FABIAN & FRANK & $\mathrm{s}$ & 10 & MICHIGAN & ITALY & ITALY & & & & ROCKLAND ST. & CALUMET \\
\hline 1949 & & FABIAN & ANTON & $\mathrm{s}$ & 9 & MICHIGAN & ITALY & ITAL & & & & ROCKLAND ST. & CALUMET \\
\hline 1950 & & FABIAN & & $\mathrm{s}$ & 4 & & ITA & & & & & D ST. & ET \\
\hline 1951 & & FABIAN & & D & 2 & HIGAN & ITALY & & & & & & $\begin{array}{l}\text { CALUMET } \\
\end{array}$ \\
\hline 1952 & 837 & POMA & JAMES & $\mathrm{H}$ & 28 & MICHIGAN & ITALY & ITALY & & LABORER/CM & TRAPANI & ROCKLAND ST. & CALUMET \\
\hline
\end{tabular}




\begin{tabular}{|c|c|c|c|c|c|c|c|c|c|c|c|c|c|}
\hline 1953 & & POMA & HILDA & W & 26 & MICHIGAN & FINLAND & FINLAND & & & FINLAND & ROCKLAND ST. & CALUMET \\
\hline 1954 & 92 & GALETTO & PETER & $\mathrm{H}$ & 46 & ITALY & ITALY & ITALY & 1907 & MINER/CM & SAN GIORGIO CANAVESE. TO & EAST PINE ST. & CALUMET \\
\hline 1955 & & GALETTO & KATE & W & 50 & ITALY & ITALY & ITALY & 1907 & & SAN GIORGIO CANAVESE. TO & EAST PINE ST. & CALUMET \\
\hline 1956 & & GALETTO & JOHN & $\mathrm{s}$ & 20 & MICHIGAN & ITALY & ITALY & & MINER/CM & SAN GIORGIO CANAVESE. TO & EAST PINE ST. & CALUMET \\
\hline 1957 & & GALETTO & MATHE & D & 18 & MICHIGAN & ITALY & ITALY & & SERVANT/PF & SAN GIORGIO CANAVESE. TO & EAST PINE ST. & CALUMET \\
\hline 1958 & & GALETTO & JOSEPH & s & 15 & MICHIGAN & ITALY & ITALY & & & SAN GIORGIO CANAVESE. TO & EAST PINE ST. & CALUMET \\
\hline 1959 & 84 & FAUSONE & JOSEPH & $\mathrm{H}$ & 47 & ITALY & ITALY & ITALY & 1890 & LABORER/TOWNSHIP & TORINO & EAST PINE ST. & CALUMET \\
\hline 1960 & 04 & FAUSONE & PETER & s & 20 & MICHIGAN & ITALY & ITALY & 1090 & MINER/CM & TURIIN & EAST PINE ST. & CALUMET \\
\hline 1961 & & FAUSONE & JOSEPH & $\underline{s}$ & 18 & MICHIGAN & ITALY & ITALY & & DELIVERY MAN/MEAT MARKET & TORINO & EAST PINE ST. & CALUMET \\
\hline 1962 & & FAUSONE & ATTILIO & $\mathrm{s}$ & 11 & MICHIGAN & ITALY & ITALY & & NEWSBOY/STREET & TORINO & EAST PINE ST. & CALUMET \\
\hline 1963 & 74 & RICARDI & ANTONIO & $\mathrm{H}$ & 56 & ITALY & ITALY & ITALY & 1899 & PUMP MAN/CM & SCARMAGNO. TO & EAST PINE ST. & CALUMET \\
\hline 1964 & & RICARDI & KATE & W & 55 & ITALY & ITALY & ITALY & 1902 & & TORINO & EAST PINE ST. & CALUMET \\
\hline 1965 & & RICARDI & MARY & D & 13 & MICHIGAN & ITALY & ITALY & & & SCARMAGNO. TO & EAST PINE ST. & CALUMET \\
\hline 1966 & 68 & ALLEGRA & AUGUST & $\mathrm{H}$ & 39 & ITALY & ITALY & ITALY & 1915 & BOILER FIREMAN/CM & TORINO & EAST PINE ST. & CALUMET \\
\hline 1967 & & ALLEGRA & JOSEPHINE & W & 30 & ITALY & ITALY & ITALY & 1902 & & TORINO & EAST PINE ST. & CALUMET \\
\hline 1968 & & ALLEGRA & KATE & D & 6 & MICHIGAN & ITALY & ITALY & & & TORINO & EAST PINE ST. & CALUMET \\
\hline 1969 & & ALLEGRA & ROBERT & $\mathrm{s}$ & 4 & MICHIGAN & ITALY & ITALY & & & TORINO & EAST PINE ST. & CALUMET \\
\hline 1970 & 328 & VENEGONI & JOHN & $\mathrm{H}$ & 53 & ITALY & ITALY & ITALY & 1900 & TIMBERMAN/CM & CUGGIANO. MILANO & CHURCH ST. & CALUMET \\
\hline 1971 & & VENEGONI & MARY & w & 56 & ITALY & ITALY & ITALY & 1901 & & & CHURCH ST. & CALUMET \\
\hline 1972 & 176 & PELLEGRINI & CECIL & $\mathrm{H}$ & 29 & MICHIGAN & ITALY & ITALY & & MASON/CM & LUCCA & CALUMET AVE. & CALUMET \\
\hline 1973 & 180 & PELLEGRINI & URSULA & W & 22 & ITALY & ITALY & ITALY & 1908 & 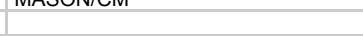 & CIRIE'. TO & $\begin{array}{l}\text { CALUMET AVE. } \\
\text { CALE. }\end{array}$ & CALUMET \\
\hline 1974 & & PELLEGRINI & ROBERT & $\begin{array}{l}\mathrm{s} \\
\mathrm{v}\end{array}$ & 3 & MICHIGAN & MICHIGAN & ITALY & 1900 & & LUCCA & $\begin{array}{l}\text { CALUMET AVE. } \\
\text { CALE. }\end{array}$ & CALUMET \\
\hline 1975 & & BALMA/BAIMA & JOSEPH & BRIL & 19 & MICHIGAN & ITALY & ITALY & & LABORER/CM & CIRIE'. TO & CALUMET AVE. & CALUMET \\
\hline 1976 & & BALMA/BAIMA & ELENA & SIIL & 15 & MICHIGAN & ITALY & ITALY & & & CIRIE'. TO & CALUMET AVE. & CALUMET \\
\hline 1977 & 105 & BRACCO & JOHN & BRIL & 52 & ITALY & ITALY & ITALY & 1883 & SECRETARY/LIFE INSURANCE & TORINO & CALUMET AVE. & CALUMET \\
\hline 1978 & & BRACCO & FLORENCE & SIIL & 49 & MICHIGAN & GERMANY & MICHIGAN & & & TORINO & CALUMET AVE. & CALUMET \\
\hline 1979 & 191 & cosso & JOSEPH & $\mathrm{H}$ & 57 & ITALY & ITALY & ITALY & 1893 & PUMP MAN/CM & SAN GIORGIO CANAVESE. TO & CALUMET AVE. & CALUMET \\
\hline 1980 & & cosso & MARY & w & 47 & ITALY & ITALY & ITALY & 1887 & & & CALUMET AVE. & CALUMET \\
\hline 1981 & & cosso & MINNIE & D & 27 & SWEDEN & ITALY & ITALY & & OPERATOR/TELEPHONE & SAN GIORGIO CANAVESE. TO & CALUMET AVE. & CALUMET \\
\hline 1982 & & cosso & DELA & $\mathrm{D}$ & 25 & MICHIGAN & ITALY & SW & & SALESLADY/CLOTHING & SAN GIORGIO CANAVESE. TO & CALUMET AVE. & CALUMET \\
\hline 1983 & & cosso & MARK & $\mathrm{D}$ & 22 & MICHIGAN & ITALY & SW & & STENOGRAPHER & SAN GIORGIO CANAVESE. TO & CALUMET AVE. & CALUMET \\
\hline 1984 & 293 & SANDRETTO & JOHN & $\mathrm{H}$ & 25 & MICHIGAN & ITALY & ITALY & & LABORER/CM & PONT CANAVESE. TO & ROCKLAND ST. & CALUMET \\
\hline 1985 & & SANDRETTO & ANNA & W & 23 & PENNSYLVAN & PENNSYLVAN & PENNSYLVAN & & & & ROCKLAND ST. & CALUMET \\
\hline 1986 & 286 & BESSOLO & FELIX & $\mathrm{H}$ & 60 & MICHIGAN & ITALY & ITALY & & FOREMAN/CM & SCARMAGNO, TO & ROCK LAND ST. & CALUMET \\
\hline 1987 & & BAULA/BRULA & FRANK & SOIL & 24 & AUSTRIA & AUSTRIA & AUSTRIA & & PIPE FIXER/CM & AUSTRIA & ROCK LAND ST. & CALUMET \\
\hline 1988 & & BAULA/BRULA & VITTORIA & D & 19 & MICHIGAN & MICHIGAN & ITALY & & & SCARMAGNO. TO & ROCK LAND ST. & CALUMET \\
\hline 1989 & 319 & LANDINI & JOSEPH & $\mathrm{H}$ & 57 & ITALY & ITALY & ITALY & 1897 & FIREMAN/CM & COLLE DI COMPITO. LU & CALEDONIA ST. & CALUMET \\
\hline 1990 & & LANDINI & ANTHONY & $\mathrm{s}$ & 23 & MICHIGAN & ITALY & ITALY & & DELIVERY MAN/MEAT AND & COLLE DI COMPITO. LU & CALEDONIA ST. & CALUMET \\
\hline 1991 & & LANDINI & RALPH & $\mathrm{s}$ & 17 & MICHIGAN & ITALY & ITALY & & & COLLE DI COMPITO. LU & CALEDONIA ST. & CALUMET \\
\hline 1992 & & LANDINI & VERONICA & $\mathrm{D}$ & 15 & MICHIGAN & ITALY & ITALY & & & COLLE DI COMPITO. LU & CALEDONIA ST. & CALUMET \\
\hline 1993 & & LA POINTE & CERMISA & D & 31 & ITALY & ITALY & ITALY & 1900 & & COLLE DI COMPITO. LU & CALEDONIA ST. & CALUMET \\
\hline 1994 & & LA POINTE & JEAN & $\mathrm{GD}$ & 1 & MICHIGAN & ITALY & ITALY & & & COLLE DI COMPITO. LU & CALEDONIA ST. & CALUMET \\
\hline 1995 & 333 & TABACCHI & JAMES R. & $\mathrm{H}$ & 40 & ITALY & ITALY & ITALY & 1905 & MECHANIC-DRILL MACHINE/CM & STRAMBINELLO. TO & CALEDONIA ST. & CALUMET \\
\hline 1996 & & TABACCHI & MARY & w & 37 & MICHIGAN & ITALY & ITALY & & & & CALEDONIA ST. & CALUMET \\
\hline 1997 & & TABACCHI & GILBERT & s & 15 & MICHIGAN & ITALY & MICHIGAN & & NEWSBOYIDAILY PAPER & STRAMBINELLO. TO & CALEDONIA ST. & CALUMET \\
\hline 1998 & & TABACCHI & ROBERT & $\mathrm{s}$ & 13 & MICHIGAN & ITALY & MICHIGAN & & TVLVODO TIUAILT TARLI & STRAMBINELLO. TO & CALEDONIA ST. & CALUMET \\
\hline 1999 & 385 & MARCHETTI & JOHN & $\mathrm{H}$ & 59 & ITALY & ITALY & ITALY & 1890 & BLACKSMITH/BLACKSMITH SHOP & LUCCA & CALEDONIA ST. & CALUMET \\
\hline 2000 & & MARCHETTI & AURELIA & w & 47 & ITALY & ITALY & ITALY & 1898 & & LUCCA & CALEDONIA ST. & CALUMET \\
\hline 2001 & & REGIS & JOSEPH & B & 22 & MICHIGAN & ITALY & ITALY & & MINER/CM & SAN GIUSTO CANAVESE. TO & CALEDONIA ST. & CALUMET \\
\hline 2002 & 387 & TINETTI & VITTORIA & $\mathrm{H}$ & 56 & ITALY & ITALY & ITALY & 1904 & & TORINO & CALEDONIA ST. & CALUMET \\
\hline 2003 & & TINETTI & CONSTANCE & $\mathrm{s}$ & 26 & COLORADO & ITALY & ITALY & & BAKER/BAKERY & TORINO & CALEDONIA ST. & CALUMET \\
\hline 2004 & & TINETTI & JOHN & $\mathrm{s}$ & 23 & COLORADO & ITALY & ITALY & & MINER/CM & TORINO & CALEDONIA ST. & CALUMET \\
\hline 2005 & & TINETTI & VITTORIO & $\mathrm{s}$ & 21 & MICHIGAN & ITALY & ITALY & & MINER/CM & TORINO & CALEDONIA ST. & CALUMET \\
\hline 2006 & & BO' & PALMA & $\mathrm{H}$ & 48 & ITALY & ITALY & ITALY & 1907 & HOUSE MAID/PF & TORINO & CALEDONIA ST. & CALUMET \\
\hline 2007 & & $\mathrm{BO}^{\prime}$ & TERESA & D & 21 & MICHIGAN & ITALY & ITALY & & & TORINO & CALEDONIA ST. & CALUMET \\
\hline 2008 & & BO' & JOHN & $\mathrm{s}$ & 17 & MICHIGAN & ITALY & ITALY & & APPRENTICE/PLUMBER & TORINO & CALEDONIA ST. & CALUMET \\
\hline 2009 & 388 & BOGGIO & FUNZIO & $\mathrm{H}$ & 67 & ITALY & ITALY & ITALY & 1883 & PUMPMAN/CM & SAN PONSO CANAVESE. TO & CALEDONIA ST. & CALUMET \\
\hline 2010 & & BOGGIO & MARY & W & 67 & ITALY & ITALY & ITALY & 1883 & & SAN PONSO CANAVESE, TO & CALEDONIA ST. & CALUMET \\
\hline 2011 & 386 & IAVELLI & ANTONIO & $\mathrm{H}$ & 58 & ITALY & ITALY & ITALY & & CARPENTER/CM & BOSCONERO. TO & CALEDONIA ST. & CALUMET \\
\hline 2012 & & IAVELLI & ISABEL & W & 52 & ITALY & ITALY & ITALY & & & & CALEDONIA ST. & CALUMET \\
\hline 2013 & & IAVEI & JOHN & $\mathrm{H}$ & 53 & ITA & ITA & ITA & 1901 & MINER/CM & BOSCONERO. TO & IA ST. & $\mathrm{CA}$ \\
\hline 2014 & & IAVELLI & KATE & w & 48 & & ITALY & ITALY & 1906 & 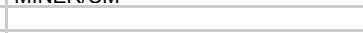 & BOSCONERO. TO & CALEDONIA ST. & CALUMET \\
\hline 2015 & & IAVELLI & MARY & D & 18 & MICHIGAN & ITALY & ITALY & & & BOSCONERO. TO & CALEDONIA ST. & CALUMET \\
\hline
\end{tabular}




\begin{tabular}{|c|c|c|c|c|c|c|c|c|c|c|c|c|c|}
\hline 2016 & & IAVELLI & MARION & $\mathrm{D}$ & 14 & MICHIGAN & ITALY & ITALY & & & BOSCONERO. TO & CALEDONIA ST. & CALUMET \\
\hline 2017 & 305 & MARESCHINI & JOHN & B & 23 & WISCONSIN & ITALY & ITALY & & MINER/CM & & STABLE ST. & CALUMET \\
\hline 2018 & 311 & DOWER & JOHN & $\mathrm{H}$ & 35 & MICHIGAN & MICHIGAN & MICHIGAN & & SMELTER & USA & STABLE ST. & CALUMET \\
\hline 2019 & & DOWER & MARY & W & 33 & MICHIGAN & ITALY & ITALY & & & & STABLE ST. & CALUMET \\
\hline 2020 & & SANDRETTO & JOHN & BRIL & 24 & MICHIGAN & ITALY & ITALY & & CRANE INSPECTOR/CM & PONT CANAVESE. TO & STABLE ST. & CALUMET \\
\hline 2021 & 359 & PACINI & MIKE & $\mathrm{H}$ & 77 & ITALY & ITALY & ITALY & 1886 & & PISTOIA & CALEDONIA ST. & CALUMET \\
\hline 2022 & & PACINI & MARY & w & 72 & ITALY & ITALY & ITALY & 1893 & & FINLAND & CALEDONIA ST. & CALUMET \\
\hline 2023 & 284 & YINARRI & WILLIAM & $\mathrm{H}$ & 25 & MICHIGAN & FINLAND & MICHIGAN & & AUTO REPAIRER/GARAGE & FINLAND & COUNTY ROAD & CALUMET \\
\hline 2024 & & YINARRI & DELPHINE & W & 24 & MICHIGAN & ITALY & ITALY & & & & COUNTY ROAD & CALUMET \\
\hline 2025 & & YINARRI & DOLORES & $\mathrm{D}$ & 1 & MICHIGAN & MICHIGAN & MICHIGAN & & & & COUNTY ROAD & CALUMET \\
\hline 2026 & 99 & ROST & ANTHONY & $\mathrm{H}$ & 42 & MICHIGAN & MICHIGAN & MICHIGAN & & POLICEMAN/CM & USA & COUNTY ROAD & CALUMET \\
\hline 2027 & & ROST & EDITH & W & 39 & MICHIGAN & ITALY & ITALY & & & & COUNTY ROAD & CALUMET \\
\hline 2028 & & ROST & LAWRENCE & $s$ & 18 & MICHIGAN & MICHIGAN & MICHIGAN & & BLACKSMITH/DRILL SHOP & & COUNTY ROAD & CALUMET \\
\hline 2029 & & ROST & FRANK & $\mathrm{s}$ & 15 & MICHIGAN & MICHIGAN & MICHIGAN & & NEWSBOY/DELIVERY PAPER & & COUNTY ROAD & CALUMET \\
\hline 2030 & & ROST & RAYMOND & $\mathrm{s}$ & 14 & MICHIGAN & MICHIGAN & MICHIGAN & & NEWSBOY/WEEKLY PAPER & & COUNTY ROAD & CALUMET \\
\hline 2031 & 58 & GALLINATTI & VINCENZO & $\mathrm{H}$ & 56 & ITALY & ITALY & ITALY & 1907 & PUMP MAN/CM & TORINO & 2ND ST. & CALUMET - KEARSARGE LOC. \\
\hline 2032 & & GALLINATTI & DELFINA & W & 47 & ITALY & ITALY & ITALY & 1912 & & & 2ND ST. & CALUMET - KEARSARGE LOC. \\
\hline 2033 & & GALLINATTI & JOSEPH & s & 16 & MICHIGAN & ITALY & ITALY & & & TORINO & 2ND ST. & CALUMET - KEARSARGE LOC. \\
\hline 2034 & & GALLINATTI & MARY & $\mathrm{D}$ & 13 & MICHIGAN & ITALY & ITALY & & & TORINO & 2ND ST. & CALUMET - KEARSARGE LOC. \\
\hline 2035 & & GALLINATTI & DERRICK & s & 11 & MICHIGAN & ITALY & ITALY & & & TORINO & 2ND ST. & CALUMET - KEARSARGE LOC. \\
\hline 2036 & 310 & FALLETTI & CARLO & $\mathrm{H}$ & 43 & ITALY & ITALY & ITALY & 1908 & TIMBERMAN/CM & PONT CANAVESE. TO & RAILROAD ST. & CALUMET - KEARSARGE LOC. \\
\hline 2037 & 310 & $\begin{array}{l}\text { FALLETTI } \\
\text { FALE }\end{array}$ & JOHANNA & w & 42 & ITALY & ITALY & ITALY & 1913 & IIVIDERIVIATVICIVI & TUNI CAIVAVESE. IO & $\begin{array}{l}\text { RAILROAD ST. } \\
\text { RALR }\end{array}$ & CALUMET - KEARSARGE LOC. \\
\hline 2038 & & FALLETTI & MARY & $\mathrm{D}$ & 13 & MICHIGAN & ITALY & ITALY & & & PONT CANAVESE. TO & RAILROAD ST. & CALUMET - KEARSARGE LOC. \\
\hline 2039 & & FALLETTI & LUCY & $\mathrm{D}$ & 12 & MICHIGAN & ITALY & ITALY & & & PONT CANAVESE. TO & RAILROAD ST. & CALUMET - KEARSARGE LOC. \\
\hline 2040 & & FALLETTI & BEN & $\mathrm{s}$ & 11 & MICHIGAN & ITALY & ITALY & & & PONT CANAVESE. TO & RAILROAD ST. & CALUMET - KEARSARGE LOC. \\
\hline 2041 & & FALLETTI & DOMENICO & s & 8 & MICHIGAN & ITALY & ITALY & & & PONT CANAVESE. TO & RAILROAD ST. & CALUMET - KEARSARGE LOC. \\
\hline 2042 & & FALLETTI & JENNIE & $\mathrm{D}$ & 1 & MICHIGAN & ITALY & ITALY & & & PONT CANAVESE. TO & RAILROAD ST. & CALUMET - KEARSARGE LOC. \\
\hline 2043 & & FALLETTI & CHARLES & $\mathrm{s}$ & $2 \mathrm{M}$ & MICHIGAN & ITALY & ITALY & & & PONT CANAVESE, TO & RAILROAD ST. & CALUMET - KEARSARGE LOC. \\
\hline 2044 & & CAPOSSO & JOHN & B & 48 & ITALY & ITALY & ITALY & 1910 & TIMBERMAN/CM & BOLOGNA & RAILROAD ST. & CALUMET - KEARSARGE LOC. \\
\hline 2045 & 138 & CRESTO & BARTOLOMEO & $\mathrm{H}$ & 43 & ITALY & ITALY & ITALY & 1905 & MERCHANT/CANDY STORE & CASTELLAMONTE. TO & WOLVERINE ST. & CALUMET - KEARSARGE LOC. \\
\hline 2046 & & CRESTO & MARY & w & 53 & ITALY & ITALY & ITALY & 1907 & & & WOLVERINE ST. & CALUMET - KEARSARGE LOC. \\
\hline 2047 & & CRESTO & MARGHERITA & $\mathrm{D}$ & 20 & MICHIGAN & ITALY & ITALY & & SALESLADY/CANDY STORE & CASTELLAMONTE. TO & WOLVERINE ST. & CALUMET - KEARSARGE LOC. \\
\hline 2048 & & CRESTO & $\begin{array}{l}\text { CAMILLO } \\
\text { CAM }\end{array}$ & s & 19 & MICHIGAN & $\begin{array}{l}\text { ITALY } \\
\end{array}$ & ITALY & & DRILL BOY/CM & CASTELLAMONTE. TO & WOLVERINE ST. & $\begin{array}{l}\text { CALUMET - KEARSARGE LOC. } \\
\text {. }\end{array}$ \\
\hline 2049 & & CRESTO & KATE & $\mathrm{D}$ & 16 & MICHIGAN & $\begin{array}{l}\text { ITALY } \\
\end{array}$ & ITALY & & LRILL DUTICIVI & CASTELLAMONTE, TO & WOLVERINE ST. & $\begin{array}{l}\text { CALUMET - KEARSARGE LOC. } \\
\text {. KEARE }\end{array}$ \\
\hline 2050 & 166 & SILVA & JOHN & $\mathrm{H}$ & 47 & ITALY & ITALY & ITALY & 1903 & FOREMAN/CM & PONT CANAVESE. TO & RAILROAD ST. & CALUMET - KEARSARGE LOC. \\
\hline 2051 & & SILVA-NIGRA & MARGHERITA & w & 59 & ITALY & ITALY & ITALY & 1904 & & TORINO & RAILROAD ST. & CALUMET - KEARSARGE LOC. \\
\hline 2052 & & SILVA & GABRIEL & $\mathrm{H}$ & 49 & ITALY & ITALY & ITALY & 1904 & TIMBERMAN/CM & PONT CANAVESE. TO & RAILROAD ST. & CALUMET - KEARSARGE LOC. \\
\hline 2053 & & SILVA-MARTA & MARGHERITA & W & 29 & MICHIGAN & ITALY & ITALY & & & TORINO & RAILROAD ST. & CALUMET - KEARSARGE LOC. \\
\hline 2054 & & SILVA & ANTONIO & $s$ & 7 & MICHIGAN & ITALY & MICHIGAN & & & PONT CANAVESE. TO & RAILROAD ST. & CALUMET - KEARSARGE LOC. \\
\hline 2055 & & SILVA & TRACY & $\mathrm{D}$ & 5 & MICHIGAN & ITALY & MICHIGAN & & & PONT CANAVESE. TO & RAILROAD ST. & CALUMET - KEARSARGE LOC. \\
\hline 2056 & & SILVA & ELISABETH & D & 3 & MICHIGAN & ITALY & MICHIGAN & & & PONT CANAVESE. TO & RAILROAD ST. & CALUMET - KEARSARGE LOC. \\
\hline 2057 & 167 & RONCAGLIONE & $\mathrm{JOHN}$ & $\mathrm{H}$ & 43 & MICHIGAN & ITALY & ITALY & & LABORER/CM & PONT CANAVESE. TO & RAILROAD ST. & CALUMET - KEARSARGE LOC. \\
\hline 2058 & 194 & BIROCCO & AUGUST & $\mathrm{H}$ & 43 & ITALY & ITALY & ITALY & 1906 & TIMBERMAN/CM & SAN GIUSTO CANAVESE. TO & RAILROAD ST. & CALUMET - KEARSARGE LOC. \\
\hline 2059 & & BIROCCO & ELENA & w & 35 & ITALY & ITALY & ITALY & 1905 & & & RAILROAD ST. & CALUMET - KEARSARGE LOC. \\
\hline 2060 & & BIROCCO & JESSIE & $\mathrm{D}$ & 16 & MICHIGAN & ITALY & ITALY & & & SAN GIUSTO CANAVESE. TO & RAILROAD ST. & CALUMET - KEARSARGE LOC. \\
\hline 2061 & & BIROCCO & ANTHONY & $\mathrm{s}$ & 14 & MICHIGAN & ITALY & ITALY & & & SAN GIUSTO CANAVESE. TO & $\begin{array}{l}\text { RAILROAD ST. } \\
\text { R. }\end{array}$ & CALUMET - KEARSARGE LOC. \\
\hline 2062 & 108 & GRIBALDO & JOSEPH & $\mathrm{H}$ & 62 & ITALY & ITALY & ITALY & 1893 & DRAYMAN/CM & TORINO & CENTER ST. & CALUMET - KEARSARGE LOC. \\
\hline 2063 & & GRIBALDO & ROSE & w & 45 & ITALY & ITALY & ITALY & 1902 & & & & CALUMET - KEARSARGE LOC. \\
\hline 2064 & & GRIBALDO & TERESA & $\mathrm{D}$ & 23 & IIGAN & ITALY & ITAL & & SALESLADYIDEPARTMENT & TORINO & CENTER ST. & CALUMET - KEARSARGE LOC. \\
\hline 2065 & & GRIBALDO & $\begin{array}{l}\text { IRANE } \\
\text { IRAN }\end{array}$ & $\mathrm{D}$ & 18 & MICHIGAN & ITALY & ITALY & & DRAFTSMAN/CM & TORINO & रST. & CALUMET - KEARSARGE LOC. \\
\hline 2066 & & GRIBALDO & BARBARA & $\mathrm{D}$ & 17 & MICHIGAN & ITALY & ITAI & & & TORINO & CENTER ST. & CALUMET - KEARSARGE LOC. \\
\hline 2067 & & GRIBALDO & LOUIS & $s$ & 15 & MICHIGAN & ITAL & ITALY & & & TORINO & CENTER ST. & CALUMET - KEARSARGE LOC. \\
\hline 2068 & & GRIBALDO & JOHN & $\mathrm{s}$ & 13 & MICHIGAN & ITALY & ITALY & & NEWSBOY/DAILY PAPER & TORINO & CENTER ST. & CALUMET - KEARSARGE LOC. \\
\hline 2069 & & GRIBALDO & GEORGE & $\mathrm{s}$ & 8 & MICHIGAN & ITALY & ITALY & & & TORINO & CENTER ST. & CALUMET - KEARSARGE LOC. \\
\hline 2070 & & SANDRETTO & CHARLES & B & 47 & ITALY & ITALY & ITALY & 1908 & MINER/CM & PONT CANAVESE. TO & CENTER ST. & CALUMET - KEARSARGE LOC. \\
\hline 2071 & 90 & GIACOLETTO & VICTOR & $\mathrm{H}$ & 26 & MICHIGAN & ITALY & ITALY & & FOREMAN/CM & TORINO & MINE ST.? & CALUMET - KEARSARGE LOC. \\
\hline 2072 & & GIACOLETTO & WILMA & w & 21 & MICHIGAN & FINLAND & FINLAND & & & FINLAND & MINE ST.? & CALUMET - KEARSARGE LOC. \\
\hline 2073 & & GIACOLETTO & KENNETH & s & 2 & MICHIGAN & MICHIGAN & MICHIGAN & & & TORINO & MINE ST.? & CALUMET - KEARSARGE LOC. \\
\hline 2074 & & GIACOLETTO & VITTORIA & $\mathrm{D}$ & $11 \mathrm{M}$ & MICHIGAN & MICHIGAN & MICHIGAN & & & TORINO & MINE ST.? & CALUMET - KEARSARGE LOC. \\
\hline 2075 & 58 & GIACOLETTO & LOUISE & $\mathrm{H}$ & 53 & ITALY & ITALY & ITALY & 1896 & MERCHANT/CANDY STORE & TORINO & WATER ST. & CALUMET - KEARSARGE LOC. \\
\hline 2076 & & GIACC & & $\mathrm{s}$ & 36 & ITA & ITA & ITA & 1896 & & & R ST. & CALUMET - KEARSARGE LOC. \\
\hline 2077 & & GIACOLETTO & DERRICK & $\mathrm{s}$ & 23 & MICHIGAN & ITALY & & & TEAMSTER/CM & & & CALUMET - $\mathrm{K}$ \\
\hline 2078 & & GIACOLETTO & LOUIS & $\mathrm{s}$ & 17 & MICHIGAN & ITALY & ITALY & & & TORINO & WATER ST. & CALUMET - KEARSARGE LOC. \\
\hline
\end{tabular}




\begin{tabular}{|c|c|c|c|c|c|c|c|c|c|c|c|c|c|}
\hline 2079 & 113 & SANDRETTO & JOHN & $\mathrm{H}$ & 60 & ITALY & ITALY & ITALY & 1900 & FARMER/FARM & PONT CANAVESE. TO & 4TH ST. & CALUMET - KEARSARGE LOC. \\
\hline 2080 & & SANDRETTO & MIKE & $\mathrm{s}$ & 27 & MICHIGAN & ITALY & ITALY & & SALESMAN/AUTOMOBILE & PONT CANAVESE. TO & 4TH ST. & CALUMET - KEARSARGE LOC. \\
\hline 2081 & & SANDRETTO & LOUIS & $\mathrm{s}$ & 23 & MICHIGAN & ITALY & ITALY & & LABORER/ROCK HOUSE & PONT CANAVESE. TO & 4TH ST. & CALUMET - KEARSARGE LOC. \\
\hline 2082 & & SANDRETTO & ANNA & D & 16 & MICHIGAN & ITALY & ITALY & & & PONT CANAVESE. TO & 4TH ST. & CALUMET - KEARSARGE LOC. \\
\hline 2083 & & SANDRETTO & PAUL & $\mathrm{s}$ & 13 & MICHIGAN & ITALY & ITALY & & & PONT CANAVESE. TO & 4TH ST. & CALUMET - KEARSARGE LOC. \\
\hline 2084 & & SANDRETTO & BARNEY & $\mathrm{s}$ & 11 & MICHIGAN & ITALY & ITALY & & & PONT CANAVESE. TO & 4TH ST. & CALUMET - KEARSARGE LOC. \\
\hline 2085 & & THERRIAN & CLEARENCE & $\mathrm{H}$ & 23 & MICHIGAN & MICHIGAN & MICHIGAN & & OILER/STATIONARY ENGINE & USA UNTU & & CALUMET \\
\hline 2086 & & THERRIAN & INES & W & 18 & MICHIGAN & ITALY & ITALY & & & & & CALUMET \\
\hline 2087 & 206 & FARAONE & JOHN & $\mathrm{H}$ & 25 & MICHIGAN & ITALY & ITALY & & MERCHANT/CONFECTIONARY & & ALLOUEZ ST. & CALUMET - COPPER CITY \\
\hline 2088 & & FARAONE & AGNESE & w & 26 & MICHIGAN & CROATIA & CROATIA & & & CROATIA & ALLOUEZ ST. & CALUMET - COPPER CITY \\
\hline 2089 & & FARAONE & ANTHONY & $\mathrm{s}$ & 2 & MICHIGAN & MICHIGAN & MICHIGAN & & & & ALLOUEZ ST. & CALUMET - COPPER CITY \\
\hline 2090 & & FARAONE & ANGELA & $\mathrm{D}$ & 1 & MICHIGAN & MICHIGAN & MICHIGAN & & & & ALLOUEZ ST. & CALUMET - COPPER CITY \\
\hline 2091 & & BOMBARJI & TONY & B & 29 & MICHIGAN & ITALY & ITALY & & SAWYER/LUMBER MILL & & & ELM RIVER - DONKEN TOWN \\
\hline 2092 & & VITTON & BATTISTA & $\mathrm{H}$ & 69 & ITALY & ITALY & ITALY & 1899 & FARMER/DAIRY FARM & TORINO & & FRANKLIN - FARM SECTION \\
\hline 2093 & & VITTON & MARY & W & 49 & ITALY & ITALY & ITALY & 1901 & & & & FRANKLIN - FARM SECTION \\
\hline 2094 & & VITTON & ANNA & D & 21 & MICHIGAN & ITALY & ITALY & & LABORER/DAIRY FARM & TORINO & & FRANKLIN - FARM SECTION \\
\hline 2095 & & VITTON & JANE & $\mathrm{s}$ & 20 & MICHIGAN & ITALY & ITALY & & & TORINO & & FRANKLIN - FARM SECTION \\
\hline 2096 & & VITTON & CATERINA & $\mathrm{D}$ & 18 & MICHIGAN & ITALY & ITALY & & & TORINO & & FRANKLIN - FARM SECTION \\
\hline 2097 & & VITTON & MARY & $\mathrm{D}$ & 15 & MICHIGAN & ITALY & ITALY & & & TORINO & & FRANKLIN - FARM SECTION \\
\hline 2098 & & VITTON & PETER & $\mathrm{s}$ & 12 & MICHIGAN & ITALY & ITALY & & & TORINO & & FRANKLIN - FARM SECTION \\
\hline 2099 & & VITTON & LUCILLE & $\mathrm{D}$ & 9 & MICHIGAN & ITALY & ITALY & & & TORINO & & FRANKLIN - FARM SECTION \\
\hline 2100 & & VITTON & CARLO & $\mathrm{s}$ & 7 & MICHIGAN & ITALY & ITALY & & & TORINO & & FRANKLIN - FARM SECTION \\
\hline 2101 & & SIMONETTI & FILIPPO & B & 52 & ITALY & ITALY & ITALY & 1904 & LABORER/DAIRY FARM & & & FRANKLIN - FARM SECTION \\
\hline 2102 & & PANTALEONI & ANGELO & B & 52 & ITALY & ITALY & ITALY & 1903 & LABORER/DAIRY FARM & LUCCA & & FRANKLIN - FARM SECTION \\
\hline 2103 & & MICHELETTI & GEO & B & 19 & MICHIGAN & ITALY & ITALY & & LABORER/DAIRY FARM & SANTA MARIA DEL GIUDICE. & & FRANKLIN - FARM SECTION \\
\hline 2104 & & MICHELETTI & NANDO & B & 18 & MICHIGAN & ITALY & ITALY & & LABORER/DAIRY FARM & SANTA MARIA DEL GIUDICE. & & FRANKLIN - FARM SECTION \\
\hline 2105 & & GALETTO & STEFANO & B & 50 & ITALY & ITALY & ITALY & 1896 & LABORER/DAIRY FARM & SAN GIORGIO CANAVESE. TO & & FRANKLIN - FARM SECTION \\
\hline 2106 & & GASPARDO & JOHN & $\mathrm{H}$ & 67 & ITALY & ITALY & ITALY & 1884 & FARMER/DAIRY FARM & PONT CANAVESE, TO & & FRANKLIN - FARM SECTION \\
\hline 2107 & & GASPARDO & ANNE & W & 52 & ITALY & ITALY & ITALY & 1884 & & PONT CANAVESE. TO & & FRANKLIN - FARM SECTION \\
\hline 2108 & & GASPARDO & JAMES & s & 25 & MICHIGAN & ITALY & ITALY & & LABORER/DAIRY FARM & PONT CANAVESE. TO & & FRANKLIN - FARM SECTION \\
\hline 2109 & & GASPARDO & JOHN & $\mathrm{s}$ & 21 & MICHIGAN & ITALY & ITALY & & LABORER/DAIRY FARM & PONT CANAVESE. TO & & FRANKLIN - FARM SECTION \\
\hline 2110 & & GASPARDO & ANNITA & $\mathrm{D}$ & 14 & MICHIGAN & ITALY & ITALY & & & PONT CANAVESE. TO & & FRANKLIN - FARM SECTION \\
\hline 2111 & & BURATTI & ANTONIO & B & 53 & ITALY & ITALY & ITALY & 1910 & LABORER/DAIRY FARM & TORINO & & FRANKLIN - FARM SECTION \\
\hline 2112 & & BENTINO & CHARLES & $\mathrm{H}$ & 42 & ITALY & ITALY & ITALY & 1912 & MINER/CM & TORINO & & FRANKLIN - FRANKLIN MINE LOC. \\
\hline 2113 & & BENTINO & MARY & w & 39 & ITALY & ITALY & ITALY & 1912 & & TORINO & & FRANKLIN - FRANKLIN MINE LOC. \\
\hline 2114 & & BENTINO & DOMENICO & s & 14 & MICHIGAN & ITALY & ITALY & & & TORINO & & FRANKLIN - FRANKLIN MINE LOC. \\
\hline 2115 & & BENTINO & ANNE & $\mathrm{D}$ & 12 & MICHIGAN & ITALY & ITALY & & & TORINO & & FRANKLIN - FRANKLIN MINE LOC. \\
\hline 2116 & & BALDASSARRI & RICHARD & $\mathrm{H}$ & 44 & ITALY & ITALY & ITALY & 1911 & LABORER/CM & LUCCA & & FRANKLIN - FRANKLIN MINE LOC. \\
\hline 2117 & & BALDASSARRI & MARY & W & 34 & ITALY & ITALY & ITALY & 1913 & & & & FRANKLIN - FRANKLIN MINE LOC. \\
\hline 2118 & & BALDASSARRI & HAZEL & $\mathrm{D}$ & 12 & MICHIGAN & ITALY & ITALY & & & LUCCA & & FRANKLIN - FRANKLIN MINE LOC. \\
\hline 2119 & & BALDASSARRI & JOSEPH & $\mathrm{s}$ & 4 & MICHIGAN & ITALY & ITALY & & & LUCCA & & FRANKLIN - FRANKLIN MINE LOC. \\
\hline 2120 & & BALDASSARRI & MARGHERITA & D & 2 & MICHIGAN & ITALY & ITALY & & & LUCCA & & FRANKLIN - FRANKLIN MINE LOC. \\
\hline 2121 & & MARCO & LEDA & $\mathrm{H}$ & 42 & ITALY & ITALY & ITALY & $89 ?$ & & TORINO & & FRANKLIN - FRANKLIN MINE LOC. \\
\hline 2122 & & MARCO & ANDREW & $\mathrm{s}$ & 21 & MICHIGAN & ITALY & ITALY & & NOT CLEAR & TORINO & & FRANKLIN - FRANKLIN MINE LOC. \\
\hline 2123 & & MARCO & GEORGE & $\mathrm{s}$ & 19 & MICHIGAN & ITALY & ITALY & & & TORINO & & FRANKLIN - FRANKLIN MINE LOC. \\
\hline 2124 & & MARCO & MARGHERITA & D & 14 & MICHIGAN & ITALY & ITALY & & & TORINO & & FRANKLIN - FRANKLIN MINE LOC. \\
\hline 2125 & & MARCO & MIKE & $\mathrm{s}$ & 11 & MICHIGAN & ITALY & ITALY & & & TORINO & & FRANKLIN - FRANKLIN MINE LOC. \\
\hline 2126 & & PRIAMI & FEDERIGO & $\mathrm{H}$ & 40 & ITALY & ITALY & ITALY & 1913 & MINER/CM & LUCCA & & FRANKLIN - FRANKLIN MINE LOC. \\
\hline 2127 & & PRIAMI & FLAVIANA & w & 39 & ITALY & ITALY & ITALY & 1900 & & & & FRANKLIN - FRANKLIN MINE LOC. \\
\hline 2128 & & PRIAMI & LAWRENCE & $\mathrm{s}$ & 8 & MICHIGAN & ITALY & ITALY & & & LUCCA & & FRANKLIN - FRANKLIN MINE LOC. \\
\hline 2129 & & PRIAMI & MIKE & $\mathrm{s}$ & 6 & MICHIGAN & ITALY & ITALY & & & LUCCA & & FRANKLIN - FRANKLIN MINE LOC. \\
\hline 2130 & & PRIAMI & KATE & $\mathrm{D}$ & 5 & MICHIGAN & ITALY & ITALY & & & LUCCA & & FRANKLIN - FRANKLIN MINE LOC. \\
\hline 2131 & & PRIAMI & MARY & D & 4 & MICHIGAN & ITALY & ITALY & & & LUCCA & & FRANKLIN - FRANKLIN MINE LOC. \\
\hline 2132 & & CORALLE & MARIO & $\mathrm{H}$ & 38 & ITALY & ITALY & ITALY & 1916 & WORKMAN/STEAM RAILROAD & & & FRANKLIN - FRANKLIN MINE LOC. \\
\hline 2133 & & CORALLE & JOAN & w & 27 & ITALY & ITALY & ITALY & 1918 & & & & FRANKLIN - FRANKLIN MINE LOC. \\
\hline 2134 & & CORALLE & DELFINA & $\mathrm{D}$ & 2 & MICHIGAN & ITALY & ITALY & & & & & FRANKLIN - FRANKLIN MINE LOC. \\
\hline 2135 & & CORALLE & ELLEN & $\mathrm{D}$ & $5 \mathrm{M}$ & MICHIGAN & ITALY & ITALY & & & & & FRANKLIN - FRANKLIN MINE LOC. \\
\hline 2136 & & RICCOMINI & MARCO & $\mathrm{H}$ & 50 & ITALY & ITALY & ITALY & 1910 & CHUTEMAN/CM & LUCCA & & FRANKLIN - FRANKLIN MINE LOC. \\
\hline 2137 & & RICCOMINI & AGNESE & W & 39 & ITALY & ITALY & ITALY & 1915 & & & & FRANKLIN - FRANKLIN MINE LOC. \\
\hline 2138 & & BONINI & BALDO & $\mathrm{H}$ & 55 & ITALY & ITALY & ITALY & 1898 & MINER/CM & COLLODI. LU & & FRANKLIN - FRANKLIN MINE LOC. \\
\hline 2139 & & BONINI & AMBROSE & $\mathrm{s}$ & 20 & MICHIGAN & ITALY & ITAL & & DELIVERY MAN/GROCERY & LU & & FRANKLIN - FRANKLIN MINE LOC \\
\hline 2140 & & BONINI & MARIE & D & 18 & MICHIGAN & & & & & COLLODI. LU & & FRANKLIN - FRANKLIN MINE LOC \\
\hline 2141 & & BONINI & ANGELO & $\mathrm{s}$ & 16 & MICHIGAN & ITALY & ITALY & & & COLLODI. LU & & FRANKLIN - FRANKLIN MINE LOC \\
\hline
\end{tabular}




\begin{tabular}{|c|c|c|c|c|c|c|c|c|c|c|c|c|}
\hline 2142 & & BONINI & IRENE & $\mathrm{D}$ & 13 & MICHIGAN & ITALY & ITALY & & & COLLODI. LU & FRANKLIN - FRANKLIN MINE LOC \\
\hline 2143 & & BONINI & $\begin{array}{l}\text { UGO } \\
U D H O\end{array}$ & $\frac{S}{s}$ & 11 & MICHIGAN & ITALY & ITALY & & & COLLODI. LU & FRANKLIN - FRANKLIN MINE LOC \\
\hline 2144 & & $\begin{array}{l}\text { BONINI } \\
\text { BONINI }\end{array}$ & $\begin{array}{l}\text { LUDHIO } \\
\text { JOSEPH }\end{array}$ & $\begin{array}{l}s \\
s\end{array}$ & $\frac{8}{4}$ & $\begin{array}{l}\text { MICHIGAN } \\
\text { MICHIGAN }\end{array}$ & ITALY & $\begin{array}{l}\text { ITALY } \\
\text { ITALY }\end{array}$ & & & COLLODI. LU & FRANKLIN - FRANKLIN MINE LOC \\
\hline 2145 & & $\begin{array}{l}\text { BONINI } \\
\text { GROWSI }\end{array}$ & $\begin{array}{l}\text { JOSEPH } \\
\text { OTTO }\end{array}$ & $\mathrm{S}$ & $\begin{array}{l}4 \\
38\end{array}$ & $\begin{array}{l}\text { MICHIGAN } \\
\text { ITALY }\end{array}$ & ITALY & ITALY & & & COLLODI. LU & FRANKLIN - FRANKLIN MINE LOC \\
\hline 2146 & & GROWSI & $\begin{array}{l}\text { OTTO } \\
\text { BRUNA }\end{array}$ & & 38 & ITALY & ITALY & ITALY & 1908 & WAITER/SODA SHOP & & FRANKLIN - FRANKLIN MINE LOC \\
\hline 2147 & & GROWSI & BRUNA & W & 33 & ITALY & ITALY & ITALY & 1916 & & & FRANKLIN - FRANKLIN MINE LOC \\
\hline 2148 & & $\mathrm{RICCl}$ & ANGELA & $\mathrm{H}$ & 53 & ITALY & ITALY & ITALY & 1908 & & LUCCA & FRANKLIN - FRANKLIN MINE LOC \\
\hline 2149 & & $\mathrm{RICCl}$ & JOSEPHINE & D & 21 & MICHIGAN & ITALY & ITALY & & WRAPPER/BUTTER FACTORY & MATRAIA. LU & FRANKLIN - FRANKLIN MINE LOC \\
\hline 2150 & & $\mathrm{RICCl}$ & MARY & D & 20 & MICHIGAN & ITALY & ITALY & & & MATRAIA. LU & FRANKLIN - FRANKLIN MINE LOC \\
\hline 2151 & & RICCI & ROSE & D & 17 & MICHIGAN & ITALY & ITALY & & WRAPPER/BUTTER FACTORY & MATRAIA. LU & FRANKLIN - FRANKLIN MINE LOC \\
\hline 2152 & & $\mathrm{RICCl}$ & ANNE & $\mathrm{D}$ & 12 & MICHIGAN & ITALY & ITALY & & & MATRAIA. LU & FRANKLIN - FRANKLIN MINE LOC \\
\hline 2153 & & QUILICI & JOHN & B & 49 & ITALY & ITALY & ITALY & 1901 & MINER/CM & LUCCA & FRANKLIN - FRANKLIN MINE LOC \\
\hline 2154 & & MONTICELLI & AMERICO & $\mathrm{H}$ & 57 & ITALY & ITALY & ITALY & 1896 & LABORER/CM & LUCCA & FRANKLIN - FRANKLIN MINE LOC \\
\hline 2155 & & MONTICELLI & SILVIA & W & 48 & ITALY & ITALY & ITALY & 1919 & & & FRANKLIN - FRANKLIN MINE LOC \\
\hline 2156 & & MONTICELLI & ATTILIO & $\mathrm{s}$ & 22 & MICHIGAN & ITALY & ITALY & & SMITH/BLACKSMITH SHOP & LUCCA & FRANKLIN - FRANKLIN MINE LOC \\
\hline 2157 & & MONTICELLI & EMIL & $\mathrm{S}$ & 25 & MICHIGAN & ITALY & ITALY & & LABORER/CM & LUCCA & FRANKLIN - FRANKLIN MINE LOC \\
\hline 2158 & & MONTICELLI & SAM & $\mathrm{s}$ & 21 & MICHIGAN & ITALY & ITALY & & LABORER/CM & LUCCA & FRANKLIN - FRANKLIN MINE LOC \\
\hline 2159 & & MONTICELLI & JOSEPH & $\mathrm{S}$ & 8 & MICHIGAN & ITALY & ITALY & & & LUCCA & FRANKLIN - FRANKLIN MINE LOC \\
\hline 2160 & & MONTICELLI & ROSE & D & 7 & MICHIGAN & ITALY & ITALY & & & LUCCA & FRANKLIN - FRANKLIN MINE LOC \\
\hline 2161 & & SABATINI & THOMAS & B & 33 & ITALY & ITALY & ITALY & 1913 & LABORER/AUTO FACTORY & VILLA BASILICA. LU & FRANKLIN - FRANKLIN MINE LOC \\
\hline 2162 & & GRASSESCHI & DANTE & $\mathrm{H}$ & 50 & ITALY & ITALY & ITALY & 1905 & PROPRIETOR/CANDY STORE & SANTA MARIA DEL GIUDICE. & FRANKLIN - FRANKLIN MINE LOC \\
\hline 2163 & & GRASSESCHI & ANNE & W & 40 & ITALY & ITALY & ITALY & 1911 & & SANTA MARIA DEL GIUDICE, & FRANKLIN - FRANKLIN MINE LOC \\
\hline 2164 & & GRASSESCHI & JULIO & $\mathrm{s}$ & 22 & ITALY & ITALY & ITALY & 1911 & & SANTA MARIA DEL GIUDICE. & FRANKLIN - FRANKLIN MINE LOC \\
\hline 2165 & & GRASSESCHI & TONY & $\mathrm{s}$ & 21 & ITALY & ITALY & ITALY & 1911 & FIREMAN/CM & SANTA MARIA DEL GIUDICE. & FRANKLIN - FRANKLIN MINE LOC \\
\hline 2166 & & GRASSESCHI & ORLANDO & $\mathrm{s}$ & 18 & ITALY & ITALY & ITALY & & FIREMAN/CM & SANTA MARIA DEL GIUDICE. & FRANKLIN - FRANKLIN MINE LOC \\
\hline 2167 & & GRASSESCHI & GERARDO & $\mathrm{s}$ & 15 & ITALY & ITALY & ITALY & & & SANTA MARIA DEL GIUDICE. & FRANKLIN - FRANKLIN MINE LOC \\
\hline 2168 & & GRASSESCHI & NATALINO & $\mathrm{s}$ & 13 & ITALY & ITALY & ITALY & & & SANTA MARIA DEL GIUDICE. & FRANKLIN - FRANKLIN MINE LOC \\
\hline 2169 & & GRASSESCHI & DIVINO & $\mathrm{s}$ & 12 & ITALY & ITALY & ITALY & & & SANTA MARIA DEL GIUDICE, & FRANKLIN - FRANKLIN MINE LOC \\
\hline 2170 & & GRASSESCHI & LALIA & D & 11 & ITALY & ITALY & ITALY & & & SANTA MARIA DEL GIUDICE. & FRANKLIN - FRANKLIN MINE LOC \\
\hline 2171 & & GRASSESCHI & LEDA & $\mathrm{D}$ & 10 & ITALY & ITALY & ITALY & & & SANTA MARIA DEL GIUDICE. & FRANKLIN - FRANKLIN MINE LOC \\
\hline 2172 & & STORNO & GIUSEPPE & B & 45 & ITALY & ITALY & ITALY & 1913 & LABORER/CM & & FRANKLIN - FRANKLIN MINE LOC \\
\hline 2173 & & BOCCHISI & RICCARDO & B & 44 & ITALY & ITALY & ITALY & 1907 & LABORER/CM & & FRANKLIN - FRANKLIN MINE LOC \\
\hline 2174 & & GEMIGNANI & JOSEPH & $\mathrm{H}$ & 62 & ITALY & ITALY & ITALY & 1891 & PROPRIETOR/CANDY STORE & LUCCA & FRANKLIN - FRANKLIN MINE LOC \\
\hline 2175 & & GEMIGNANI & ALADINO & s & 30 & MICHIGAN & ITALY & ITALY & & GREASER/STEAM ENGINE & LUCCA & FRANKLIN - FRANKLIN MINE LOC \\
\hline 2176 & & GEMIGNANI & MABLE & DIL & 26 & MICHIGAN & ITALY & ITALY & & & LUCCA & FRANKLIN - FRANKLIN MINE LOC \\
\hline 2177 & & GEMIGNANI & MIKE & GS & 3 & MICHIGAN & ITALY & ITALY & & & LUCCA & FRANKLIN - FRANKLIN MINE LOC \\
\hline 2178 & & BONINI & CESARE & $\mathrm{H}$ & 42 & ITALY & ITALY & ITALY & 1901 & LABORER/CM & COLLODI. LU & FRANKLIN - FRANKLIN MINE LOC \\
\hline 2179 & & BONINI & LIDIA & W & 32 & MICHIGAN & ITALY & ITALY & & & & FRANKLIN - FRANKLIN MINE LOC \\
\hline 2180 & & BONINI & ANNE & $\mathrm{s}$ & 15 & MICHIGAN & ITALY & MICHIGAN & & & COLLODI. LU & FRANKLIN - FRANKLIN MINE LOC \\
\hline 2181 & & BONINI & MARY & $\mathrm{D}$ & 14 & MICHIGAN & ITALY & MICHIGAN & & & COLLODI. LU & FRANKLIN - FRANKLIN MINE LOC \\
\hline 2182 & & BONINI & WILL & $s$ & 12 & MICHIGAN & ITALY & MICHIGAN & & & COLLODI. LU & FRANKLIN - FRANKLIN MINE LOC \\
\hline 2183 & & BONINI & FLORINDA & D & 10 & MICHIGAN & ITALY & MICHIGAN & & & COLLODI. LU & FRANKLIN - FRANKLIN MINE LOC \\
\hline 2184 & & BONINI & WILBERT & $\mathrm{S}$ & 8 & MICHIGAN & ITALY & MICHIGAN & & & COLLODI. LU & FRANKLIN - FRANKLIN MINE LOC \\
\hline 2185 & & BONINI & JAMES & $\mathrm{S}$ & 6 & MICHIGAN & ITALY & MICHIGAN & & & COLLODI. LU & FRANKLIN - FRANKLIN MINE LOC \\
\hline 2186 & & BONINI & LEVIA & $\mathrm{D}$ & 3 & MICHIGAN & ITALY & MICHIGAN & & & COLLODI. LU & FRANKLIN - FRANKLIN MINE LOC \\
\hline 2187 & 484 & LENCIONI & JOSEPH & $\mathrm{H}$ & 48 & ITALY & ITALY & ITALY & 1910 & LABORER/CM & LUCCA & FRANKLIN - FRANKLIN MINE LOC \\
\hline 2188 & & LENCIONI & ULIANA & W & 48 & ITALY & ITALY & ITALY & 1903 & & & FRANKLIN - FRANKLIN MINE LOC \\
\hline 2189 & & LENCIONI & CESARE & s & 21 & MICHIGAN & ITALY & ITALY & & BOOKKEEPER/STORE & LUCCA & FRANKLIN - FRANKLIN MINE LOC \\
\hline 2190 & & LENCIONI & ABILE & $\mathrm{s}$ & 17 & MICHIGAN & ITALY & ITALY & & ATTENDANT/OIL STATION & LUCCA & FRANKLIN - FRANKLIN MINE LOC \\
\hline 2191 & & LENCIONI & FLORENCE & $\mathrm{D}$ & 16 & MICHIGAN & ITALY & ITALY & & & LUCCA & FRANKLIN - FRANKLIN MINE LOC \\
\hline 2192 & & LENCIONI & ANGELO & $\mathrm{s}$ & 14 & MICHIGAN & ITALY & ITALY & & & LUCCA & FRANKLIN - FRANKLIN MINE LOC \\
\hline 2193 & 472 & MASSOGLIA & DOMENICO & $\mathrm{H}$ & 59 & ITALY & ITALY & ITALY & 1898 & LABORER/CM & TORINO & FRANKLIN - FRANKLIN MINE LOC \\
\hline 2194 & & MASSOGLIA & FRANK & $\mathrm{s}$ & 23 & MICHIGAN & ITALY & ITALY & & SALESMAN/STORE & TORINO & FRANKLIN - FRANKLIN MINE LOC \\
\hline 2195 & & MASSOGLIA & ANNE & $\mathrm{D}$ & 16 & MICHIGAN & ITALY & ITALY & & & TORINO & FRANKLIN - FRANKLIN MINE LOC \\
\hline 2196 & 460 & PICCHIOTTINO & JOHN & $\mathrm{H}$ & 43 & ITALY & ITALY & ITALY & 1883 & SALESMAN/GROCERY STORE & PONT CANAVESE. TO & FRANKLIN - FRANKLIN MINE LOC \\
\hline 2197 & & PICCHIOTTINO & MARY & W & 37 & MICHIGAN & ITALY & ITALY & & & & FRANKLIN - FRANKLIN MINE LOC \\
\hline 2198 & & PICCHIOTTINO & MADDALENA & D & 14 & MICHIGAN & ITALY & MICHIGAN & & & PONT CANAVESE. TO & FRANKLIN - FRANKLIN MINE LOC \\
\hline 2199 & & PICCHIOTTINO & PAULINE & D & 9 & MICHIGAN & ITALY & MICHIGAN & & & PONT CANAVESE, TO & FRANKLIN - FRANKLIN MINE LOC \\
\hline 2200 & 407 & CONSANI & TONY & $\mathrm{H}$ & 39 & ITALY & ITALY & ITALY & 1912 & LABORER/CM & LUCCA & FRANKLIN - FRANKLIN MINE LOC \\
\hline 2201 & & CONSANI & JULIA & W & 37 & ITALY & ITALY & ITALY & 1921 & & & FRANKLIN - FRANKLIN MINE LOC \\
\hline 2202 & & CONSANI & ROSE & D & 13 & MICHIGAN & ITALY & ITALY & & & LUCCA & FRANKLIN - FRANKLIN MINE LOC \\
\hline 2203 & & CONSANI & VINCENZO & $\mathrm{s}$ & 8 & MICHIGAN & ITALY & ITALY & & & LUCCA & FRANKLIN - FRANKLIN MINE LOC \\
\hline 2204 & & CONSANI & EDITH & D & 3 & MICHIGAN & ITALY & ITALY & & & LUCCA & FRANKLIN - FRANKLIN MINE LOC \\
\hline
\end{tabular}




\begin{tabular}{|c|c|c|c|c|c|c|c|c|c|c|c|c|c|}
\hline 2205 & & MARTINUCCI & EGIDIO & B & 37 & MICHIGAN & ITALY & ITALY & & LABORER/CM & LUCCA & & FRANKLIN - FRANKLIN MINE LOC \\
\hline 2206 & & GHERNA & FELICE & B & 34 & ITALY & ITALY & ITALY & 1912 & LABORER/CM & PONT CANAVESE. TO & & FRANKLIN - FRANKLIN MINE LOC \\
\hline 2207 & 408 & BERTACCHI & ACHILLE & $\mathrm{H}$ & 33 & ITALY & ITALY & ITALY & 1920 & BARBER/OWN SHOP & ORBICCIANO. LU & US 41 & FRANKLIN - FRANKLIN MINE LOC \\
\hline 2208 & & BERTACCHI & JENNIE & W & 33 & ITALY & ITALY & ITALY & 1922 & & & US 41 & FRANKLIN - FRANKLIN MINE LOC \\
\hline 2209 & & BERTACCHI & EMMA & D & 6 & MICHIGAN & ITALY & ITALY & & & ORBICCIANO. LU & US 41 & FRANKLIN - FRANKLIN MINE LOC \\
\hline 2210 & & BERTACCHI & LOUIS & $\mathrm{s}$ & 5 & MICHIGAN & ITALY & ITALY & & & ORBICCIANO. LU & US 41 & FRANKLIN - FRANKLIN MINE LOC \\
\hline 2211 & 412 & PERA & GERTRUDE & $\mathrm{H}$ & 45 & ITALY & ITALY & ITALY & 1907 & & LUCCA & US 41 & FRANKLIN - FRANKLIN MINE LOC \\
\hline 2212 & & PERA & FRANK & s & 21 & MICHIGAN & ITALY & ITALY & & LABORER/WHOLESALE FRUIT & LUCCA & US 41 & FRANKLIN - FRANKLIN MINE LOC \\
\hline 2213 & & PERA & NELLO & $\mathrm{s}$ & 20 & MICHIGAN & ITALY & ITALY & & BARBER/BARBER SHOP & LUCCA & US 41 & FRANKLIN - FRANKLIN MINE LOC \\
\hline 2214 & & PERA & PIERINA & $\mathrm{D}$ & $?$ & MICHIGAN & ITALY & ITALY & & ASSISTANT HOUSE KEEPER/AT & LUCCA & US 41 & FRANKLIN - FRANKLIN MINE LOC \\
\hline 2215 & & PERA & LUDIO & $\mathrm{S}$ & 15 & MICHIGAN & ITALY & ITALY & & & LUCCA & US 41 & FRANKLIN - FRANKLIN MINE LOC \\
\hline 2216 & & PERA & ANNE & D & 14 & MICHIGAN & ITALY & ITALY & & & LUCCA & US 41 & FRANKLIN - FRANKLIN MINE LOC \\
\hline 2217 & 720 & GOSATTI & ANDREW & $\mathrm{H}$ & 27 & MICHIGAN & ITALY & ITALY & & ELECTRICIAN/CM & BIANZANO. BERGAMO & US 41 & FRANKLIN - FRANKLIN MINE LOC \\
\hline 2218 & & GOSATTI & MARY & W & 22 & MICHIGAN & ITALY & ITALY & & & & US 41 & FRANKLIN - FRANKLIN MINE LOC \\
\hline 2219 & & GOSATTI & JOSEPH & $\mathrm{s}$ & 1 & MICHIGAN & ITALY & MICHIGAN & & & BIANZANO. BERGAMO & US 41 & FRANKLIN - FRANKLIN MINE LOC \\
\hline 2220 & & GOSATTI & PAUL & $\mathrm{s}$ & $2 \mathrm{M}$ & MICHIGAN & ITALY & MICHIGAN & & & BIANZANO, BERGAMO & US 41 & FRANKLIN - FRANKLIN MINE LOC \\
\hline 2221 & & $\mathrm{RICCl}$ & JACOB & BRIL & 18 & MICHIGAN & ITALY & MICHIGAN & & LABORER/CM & LUCCA & US 41 & FRANKLIN - FRANKLIN MINE LOC \\
\hline 2222 & 738 & COLOMBO & JOSEPH & $\mathrm{H}$ & 38 & MICHIGAN & ITALY & ITALY & & MANAGER/OWN GROCERY & TORINO & US 41 & FRANKLIN - FRANKLIN MINE LOC \\
\hline 2223 & & COLOMBO & ELMA & W & 31 & MICHIGAN & FINLAND & SWEDEN & & & FINLAND-SWEDEN & US 41 & FRANKLIN - FRANKLIN MINE LOC \\
\hline 2224 & & QUINCY & JOHN & $\mathrm{H}$ & 66 & ITALY & ITALY & ITALY & 1890 & & & US 41 & FRANKLIN - FRANKLIN MINE LOC \\
\hline 2225 & & QUINCY & MARY & W & 57 & ITALY & ITALY & ITALY & 1897 & & & US 41 & FRANKLIN - FRANKLIN MINE LOC \\
\hline 2226 & & QUINCY & JAMES & s & 25 & MICHIGAN & ITALY & ITALY & & BUTCHER/MEAT MARKET & & US 41 & FRANKLIN - FRANKLIN MINE LOC \\
\hline 2227 & & QUINCY & JOSEPH & $\mathrm{s}$ & 20 & MICHIGAN & ITALY & ITALY & & LABORER/RAILROAD & & US 41 & FRANKLIN - FRANKLIN MINE LOC \\
\hline 2228 & & QUINCY & MARY & D & 7 & MICHIGAN & ITALY & ITALY & & & & US 41 & FRANKLIN - FRANKLIN MINE LOC \\
\hline 2229 & & BO' & JOHN & $\mathrm{H}$ & 50 & ITALY & ITALY & ITALY & 1912 & MINER/CM & PONT CANAVESE. TO & & FRANKLIN - FRANKLIN MINE LOC \\
\hline 2230 & & $\mathrm{BO}^{\prime}$ & MARY & W & 38 & ITALY & ITALY & ITALY & 1912 & & & & FRANKLIN - FRANKLIN MINE LOC \\
\hline 2231 & & BO' & KATE & $\mathrm{D}$ & 16 & MICHIGAN & ITALY & ITALY & & & PONT CANAVESE. TO & & FRANKLIN - FRANKLIN MINE LOC \\
\hline 2232 & & BO' & TINO & $\mathrm{s}$ & 10 & MICHIGAN & ITALY & ITALY & & & PONT CANAVESE, TO & & FRANKLIN - FRANKLIN MINE LOC \\
\hline 2233 & & BRUSORIO & CARLO & $\mathrm{H}$ & 46 & ITALY & ITALY & ITALY & 1909 & MINER/CM & MILANO & & FRANKLIN - FRANKLIN MINE LOC \\
\hline 2234 & & BRUSORIO & IRENE & W & 41 & ITALY & ITALY & ITALY & 1912 & & & & FRANKLIN - FRANKLIN MINE LOC \\
\hline 2235 & & BRUSORIO & MARIA & s & 17 & MICHIGAN & ITALY & ITALY & & & MILANO & & FRANKLIN - FRANKLIN MINE LOC \\
\hline 2236 & & BRUSORIO & LOUIS & $\mathrm{s}$ & 14 & MICHIGAN & ITALY & ITALY & & & MILANO & & FRANKLIN - FRANKLIN MINE LOC \\
\hline 2237 & & BRUSORIO & AMELIA & $\mathrm{D}$ & 12 & MICHIGAN & ITALY & ITALY & & & MILANO & & FRANKLIN - FRANKLIN MINE LOC \\
\hline 2238 & & BRUSORIO & AMOS? & $\mathrm{s}$ & 8 & MICHIGAN & ITALY & ITALY & & & MILANO & & FRANKLIN - FRANKLIN MINE LOC \\
\hline 2239 & & BRUSORIO & ALDO & $\mathrm{s}$ & 5 & MICHIGAN & ITALY & ITALY & & & MILANO & & FRANKLIN - FRANKLIN MINE LOC \\
\hline 2240 & & BRUSORIO & ALFIO & $\mathrm{s}$ & 2 & MICHIGAN & ITALY & ITALY & & & MILANO & & FRANKLIN - FRANKLIN MINE LOC \\
\hline 2241 & & COLOMBO & JOSEPH & $\mathrm{H}$ & 69 & ITALY & ITALY & ITALY & 1888 & WATCHMAN/CM & TORINO & & FRANKLIN - FRANKLIN MINE LOC \\
\hline 2242 & & COLOMBO & MADDALENA & W & 63 & ITALY & ITALY & ITALY & 1888 & & TORINO & & FRANKLIN - FRANKLIN MINE LOC \\
\hline 2243 & & COLOMBO & JAMES & $\mathrm{s}$ & 25 & MICHIGAN & ITALY & ITALY & & LABORER/CM & TORINO & & FRANKLIN - FRANKLIN MINE LOC \\
\hline 2244 & & COLOMBO & GEORGE & $\mathrm{s}$ & 23 & MICHIGAN & ITALY & ITALY & & PRINTER/NEWSPAPER & TORINO & & FRANKLIN - FRANKLIN MINE LOC \\
\hline 2245 & & COLOMBO & LENA & D & 22 & MICHIGAN & ITALY & ITALY & & BOOKKEEPER/GROCERY STORE & TORINO & & FRANKLIN - FRANKLIN MINE LOC \\
\hline 2246 & & COLOMBO & JOHN & $\mathrm{s}$ & 19 & MICHIGAN & ITALY & ITALY & & PRINTER/NEWSPAPER & TORINO & & FRANKLIN - FRANKLIN MINE LOC \\
\hline 2247 & & BALMA/BAIMA & MARION & $\mathrm{H}$ & 46 & MICHIGAN & ITALY & ITALY & & & TORINO & & FRANKLIN - FRANKLIN MINE LOC \\
\hline 2248 & & BALMA/BAIMA & JAMES & S & 18 & MICHIGAN & MICHIGAN & ITALY & & BARBER HELP/BARBER SHOP & TORINO & & FRANKLIN - FRANKLIN MINE LOC \\
\hline 2249 & & BALMA/BAIMA & ETTORE & $\mathrm{s}$ & 16 & MICHIGAN & MICHIGAN & ITALY & & BUTCHER HELPIBUTCHER SHOP & TORINO & & FRANKLIN - FRANKLIN MINE LOC \\
\hline 2250 & & BALMA/BAIMA & ANGELINA & D & 13 & MICHIGAN & MICHIGAN & ITALY & & & TORINO & & FRANKLIN - FRANKLIN MINE LOC \\
\hline 2251 & & BALMA/BAIMA & ALBERT & $\mathrm{s}$ & 11 & MICHIGAN & MICHIGAN & ITALY & & & TORINO & & FRANKLIN - FRANKLIN MINE LOC \\
\hline 2252 & & BALMA/BAIMA & EDWARD & $\mathrm{s}$ & 9 & MICHIGAN & MICHIGAN & ITALY & & & TORINO & & FRANKLIN - FRANKLIN MINE LOC \\
\hline 2253 & & POGGIONE & JOHN & $\mathrm{H}$ & 61 & ITALY & ITALY & ITALY & 1886 & FARMER/GENERAL FARM & & & FRANKLIN - FRANKLIN MINE LOC \\
\hline 2254 & & POGGIONE & MARY & W & 50 & ITALY & ITALY & ITALY & 1886 & & SAN GIORGIO CANAVESE. TO & & FRANKLIN - FRANKLIN MINE LOC \\
\hline 2255 & & POGGIONE & PETER & $\mathrm{s}$ & 30 & MICHIGAN & ITALY & ITALY & & TRUCK DRIVER/FREIGHT & SAN GIORGIO CANAVESE. TO & & FRANKLIN - FRANKLIN MINE LOC \\
\hline 2256 & & POGGIONE & JOHN & $\mathrm{S}$ & 21 & MICHIGAN & ITALY & ITALY & & TRUCK DRIVER/FRUIT & SAN GIORGIO CANAVESE, TO & & FRANKLIN - FRANKLIN MINE LOC \\
\hline 2257 & & POGGIONE & FELIX & $\mathrm{s}$ & 20 & MICHIGAN & ITALY & ITALY & & LABORER/MEAT MARKET & SAN GIORGIO CANAVESE. TO & & FRANKLIN - FRANKLIN MINE LOC \\
\hline 2258 & & NATALI & CARLO & $\mathrm{H}$ & 58 & ITALY & ITALY & ITALY & 1898 & LABORER/CM & & & FRANKLIN - FRANKLIN MINE LOC \\
\hline 2259 & & NATALI & MARY & W & 50 & ITALY & ITALY & ITALY & 1900 & & & & FRANKLIN - FRANKLIN MINE LOC \\
\hline 2260 & & NATALI & ALBERT & s & 24 & MICHIGAN & ITALY & ITALY & & BOOKKEEPER/BANK & & & FRANKLIN - FRANKLIN MINE LOC \\
\hline 2261 & & NATALI & NELLO & $\mathrm{s}$ & 23 & MICHIGAN & ITALY & ITALY & & SALESMAN/GROCERY STORE & & & FRANKLIN - FRANKLIN MINE LOC \\
\hline 2262 & & NATALI & LENA & $\mathrm{D}$ & 13 & MICHIGAN & ITALY & ITALY & & & & & FRANKLIN - FRANKLIN MINE LOC \\
\hline 2263 & & $\mathrm{RICCl}$ & EGIDIO & $\mathrm{H}$ & 57 & ITALY & ITALY & ITALY & 1886 & LABORER/CM & LUCCA & & FRANKLIN - FRANKLIN MINE LOC \\
\hline 2264 & & $\mathrm{RICCl}$ & ROSE & W & 47 & ITALY & ITALY & ITALY & 1886 & & LUCCA & & FRANKLIN - FRANKLIN MINE LOC \\
\hline 2265 & & RICCI & & $\mathrm{s}$ & 17 & HIGAN & ITALY & ITA & & & LUCCA & & FRANKLIN - FRANKLIN MINE LOC \\
\hline 2266 & & RICCl & VERONICA & $\mathrm{D}$ & 14 & MICHIGAN & ITALY & ITALY & & & LUCCA & & FRANKLIN - FRANKLIN MI \\
\hline 2267 & & $\mathrm{RICCl}$ & LIZ & D & 12 & MICHIGAN & ITALY & ITALY & & & LUCCA & & FRANKLIN - FRANKLIN MINE LOC \\
\hline
\end{tabular}




\begin{tabular}{|c|c|c|c|c|c|c|c|c|c|c|c|}
\hline 2268 & $\mathrm{RICCl}$ & $F ?$ & S & 6 & MICHIGAN & ITALY & ITALY & & & LUCCA & FRANKLIN - FRANKLIN MINE LOC. \\
\hline 2269 & ANDREOZZI & TRANQUILLO & $\mathrm{H}$ & 49 & ITALY & ITALY & ITALY & 1905 & CHUTEMAN/CM & LUCCA & FRANKLIN - PEWABIC TOWN \\
\hline 2270 & ANDREOZZI & ROSE & W & 41 & ITALY & ITALY & ITALY & 1912 & & & FRANKLIN - PEWABIC TOWN \\
\hline 2271 & GEMIGNANI & BERNICE & SD & 16 & MICHIGAN & ITALY & ITALY & & & LUCCA & FRANKLIN - PEWABIC TOWN \\
\hline 2272 & GEMIGNANI & FRANK & SS & 13 & MICHIGAN & ITALY & ITALY & & & LUCCA & FRANKLIN - PEWABIC TOWN \\
\hline 2273 & GEMIGNANI & LOUISE & SS & 11 & MICHIGAN & ITALY & ITALY & & & LUCCA & FRANKLIN - PEWABIC TOWN \\
\hline 2274 & MARINI & ASSUNTA & $\mathrm{H}$ & 35 & ITALY & ITALY & ITALY & 1908 & & & FRANKLIN - PEWABIC TOWN \\
\hline 2275 & MARINI & AMELIA & D & 19 & MICHIGAN & ITALY & ITALY & & SERVANT/CLUB HOUSE & & FRANKLIN - PEWABIC TOWN \\
\hline 2276 & MARINI & JOSEPH & $\mathrm{s}$ & 17 & MICHIGAN & ITALY & ITALY & & TRUCK DRIVER/COUNTY ROAD & & FRANKLIN - PEWABIC TOWN \\
\hline 2277 & MARINI & ALADINA & $\mathrm{D}$ & 16 & MICHIGAN & ITALY & ITALY & & STARCHER/STEAM LAUNDRY & & FRANKLIN - PEWABIC TOWN \\
\hline 2278 & MARINI & AMELIA & $\mathrm{D}$ & 15 & MICHIGAN & ITALY & ITALY & & & & FRANKLIN - PEWABIC TOWN \\
\hline 2279 & MARINI & GABRIEL & $\bar{s}$ & 10 & MICHIGAN & ITALY & ITALY & & & & FRANKLIN - PEWABIC TOWN \\
\hline 2280 & MARINI & ALFRED & s & 7 & MICHIGAN & ITALY & ITALY & & & & FRANKLIN - PEWABIC TOWN \\
\hline 2281 & MANDOLIO & ANTONIO & BR & 23 & ITALY & ITALY & ITALY & 1909 & LABORER/CM & & FRANKLIN - PEWABIC TOWN \\
\hline 2282 & BECIA & JOSEPH & $\mathrm{H}$ & 37 & ITALY & ITALY & ITALY & 1926 & LABORER/CM & UDINE & FRANKLIN - PEWABIC TOWN \\
\hline 2283 & BECIA & EVELINA & W & 25 & ITALY & ITALY & ITALY & 1926 & & UDINE & FRANKLIN - PEWABIC TOWN \\
\hline 2284 & BECIA & JOSEPH & $\mathrm{s}$ & 2 & MICHIGAN & ITALY & ITALY & & & UDINE & FRANKLIN - PEWABIC TOWN \\
\hline 2285 & BECIA & ENZO & $\mathrm{s}$ & 1 & MICHIGAN & ITALY & ITALY & & & UDINE & FRANKLIN - PEWABIC TOWN \\
\hline 2286 & LEOZZANO & J? & $\mathrm{H}$ & 26 & MEXICO & MEXICO & MEXICO & 1925 & MINER/CM & MEXICO & FRANKLIN - PEWABIC TOWN \\
\hline 2287 & LEOZZANO & VITTORIA & W & 29 & ITALY & ITALY & ITALY & 1914 & MANAGER/BOARDING HOUSE & & FRANKLIN - PEWABIC TOWN \\
\hline 2288 & DIESANI & BRUNA & SD & 11 & MICHIGAN & MEXICO & MICHIGAN & & & & FRANKLIN - PEWABIC TOWN \\
\hline 2289 & DIESANI & BRUNO & SSO & 8 & MICHIGAN & MEXICO & MICHIGAN & & & & FRANKLIN - PEWABIC TOWN \\
\hline 2290 & MAGENETTI & PETER & B & 25 & ITALY & ITALY & ITALY & 1921 & LABORER/CM & & FRANKLIN - PEWABIC TOWN \\
\hline 2291 & PIZZI & VINCENZO & B & 57 & ITALY & ITALY & ITALY & 1902 & SHIP TENDER/CM & LUCCA & FRANKLIN - PEWABIC TOWN \\
\hline 2292 & PRICCO & FRANCESCO & B & 30 & MICHIGAN & ITALY & ITALY & & LABORER/CM & TORINO & FRANKLIN - PEWABIC TOWN \\
\hline 2293 & FILIPPI & ISABEL & $\mathrm{B}$ & 38 & ITALY & ITALY & ITALY & 1903 & LADURER/CIVI & TURIIN & FRANKLIN - PEWABIC TOWN \\
\hline 2294 & FILIPPI & GINA & S & 19 & ITALY & ITALY & ITALY & 1903 & LABORER/COUNTY ROAD & PESCAGLIA. LU & FRANKLIN - PEWABIC TOWN \\
\hline 2295 & FILIPPI & ANNE & $\mathrm{D}$ & 13 & MICHIGAN & ITALY & ITALY & & & PESCAGLIA, LU & FRANKLIN - PEWABIC TOWN \\
\hline 2296 & FILIPPI & GENA & D & 10 & MICHIGAN & ITALY & ITALY & & & PESCAGLIA. LU & FRANKLIN - PEWABIC TOWN \\
\hline 2297 & FILIPPI & ROLANDO & $\mathrm{s}$ & 8 & MICHIGAN & ITALY & ITALY & & & PESCAGLIA. LU & FRANKLIN - PEWABIC TOWN \\
\hline 2298 & FILIPPI & MARY & D & 8 & MICHIGAN & ITALY & ITALY & & & PESCAGLIA. LU & FRANKLIN - PEWABIC TOWN \\
\hline 2299 & $\mathrm{ROCCHI}$ & EGISTO & $\mathrm{H}$ & 58 & ITALY & ITALY & ITALY & 99?? & SHIFTS CAPTAIN/CM & MATRAIA. LU & FRANKLIN - PEWABIC TOWN \\
\hline 2300 & $\mathrm{ROCCHI}$ & PHOEBE & W & 51 & ITALY & ITALY & ITALY & 1880 & & & FRANKLIN - PEWABIC TOWN \\
\hline 2301 & $\mathrm{ROCCHI}$ & ALBERT & $\mathrm{s}$ & 27 & MICHIGAN & ITALY & ITALY & & ELECTRICIAN/CM & MATRAIA, LU & FRANKLIN - PEWABIC TOWN \\
\hline 2302 & $\mathrm{ROCCHI}$ & TEODORO & s & 25 & MICHIGAN & ITALY & ITALY & & ELECTRICIAN/CM & MATRAIA. LU & FRANKLIN - PEWABIC TOWN \\
\hline 2303 & ROCCHI & WILL & $\mathrm{s}$ & 19 & MICHIGAN & ITALY & ITALY & & LABORER/CM & MATRAIA. LU & FRANKLIN - PEWABIC TOWN \\
\hline 2304 & ROCCHI & CHARLES & $\mathrm{s}$ & 17 & MICHIGAN & ITALY & ITALY & & & MATRAIA. LU & FRANKLIN - PEWABIC TOWN \\
\hline 2305 & ROCCHI & EVELYN & $\mathrm{D}$ & 12 & MICHIGAN & ITALY & ITALY & & & MATRAIA. LU & FRANKLIN - PEWABIC TOWN \\
\hline 2306 & $\mathrm{ROCCHI}$ & ELLA & DIL & 22 & MICHIGAN & ITALY & ITALY & & & MATRAIA. LU & FRANKLIN - PEWABIC TOWN \\
\hline 2307 & ROCCHI & ALBERT & GS & 2 & MICHIGAN & ITALY & ITALY & & & MATRAIA. LU & FRANKLIN - PEWABIC TOWN \\
\hline 2308 & PROCISSI & PETER & $\mathrm{H}$ & 75 & ITALY & ITALY & ITALY & 1883 & LABORER/CM & LUCCA & FRANKLIN - RIPLEY TOWN \\
\hline 2309 & PROCISSI & ORSOLA & w & 72 & ITALY & ITALY & ITALY & 1883 & & LUCCA & FRANKLIN - RIPLEY TOWN \\
\hline 2310 & MANZETTI & LORENZO & $\mathrm{H}$ & 62 & ITALY & ITALY & ITALY & 1907 & FARMER/GENERAL FARM & PONT CANAVESE. TO & FRANKLIN - FRANKLIN MINE LOC. \\
\hline 2311 & MANZETTI & MARY & W & 62 & ITALY & ITALY & ITALY & 1906 & & PONT CANAVESE. TO & FRANKLIN - FRANKLIN MINE LOC. \\
\hline 2312 & MANZETTI & KATE & $\mathrm{D}$ & 21 & MICHIGAN & ITALY & ITALY & & FOLDER/LAUNDRY & PONT CANAVESE. TO & FRANKLIN - FRANKLIN MINE LOC. \\
\hline 2313 & BLESSENT & JOHN & $\mathrm{H}$ & 58 & ITALY & ITALY & ITALY & 1896 & FARMER/GENERAL FARM & SPARONE. TO & FRANKLIN - FRANKLIN MINE LOC. \\
\hline 2314 & BLESSENT & MADDALENA & w & 46 & ITALY & ITALY & ITALY & 1909 & & & FRANKLIN - FRANKLIN MINE LOC. \\
\hline 2315 & BLESSENT & LUCILLE & D & 19 & MICHIGAN & ITALY & ITALY & & FOLDER/STEAM LAUNDRY & SPARONE. TO & FRANKLIN - FRANKLIN MINE LOC. \\
\hline 2316 & BLESSENT & JOHANNA & $\mathrm{D}$ & 17 & MICHIGAN & ITALY & ITALY & & & SPARONE. TO & FRANKLIN - FRANKLIN MINE LOC. \\
\hline 2317 & BLESSENT & MARIE & $\mathrm{D}$ & 15 & MICHIGAN & ITALY & ITALY & & & SPARONE. TO & FRANKLIN - FRANKLIN MINE LOC. \\
\hline 2318 & BLESSENT & PETER & $\bar{s}$ & 14 & MICHIGAN & ITALY & ITALY & & & SPARONE. TO & FRANKLIN - FRANKLIN MINE LOC. \\
\hline 2319 & BLESSENT & BATTISTA & $\mathrm{s}$ & 11 & MICHIGAN & ITALY & ITALY & & & SPARONE, TO & FRANKLIN - FRANKLIN MINE LOC. \\
\hline 2320 & BLESSENT & MARY & D & 9 & MICHIGAN & ITALY & ITALY & & & SPARONE. TO & FRANKLIN - FRANKLIN MINE LOC. \\
\hline 2321 & FEIRA & MARTIN & $\mathrm{H}$ & 54 & ITALY & ITALY & ITALY & & FARMER/GENERAL FARM & PONT CANAVESE. TO & FRANKLIN - FRANKLIN MINE LOC. \\
\hline 2322 & FEIRA & MARY & W & 48 & MICHIGAN & ITALY & ITALY & & & & FRANKLIN - FRANKLIN MINE LOC. \\
\hline 2323 & FEIRA & JOHANNE & D & 22 & MICHIGAN & ITALY & MICHIGAN & & & PONT CANAVESE. TO & FRANKLIN - FRANKLIN MINE LOC. \\
\hline 2324 & FEIRA & DOMENICO & $\mathrm{s}$ & 21 & MICHIGAN & ITALY & MICHIGAN & & LABORER/GENERAL FARM & PONT CANAVESE. TO & FRANKLIN - FRANKLIN MINE LOC. \\
\hline 2325 & FEIRA & JAMES & $\mathrm{s}$ & 19 & MICHIGAN & ITALY & MICHIGAN & & LABORER/GENERAL FARM & PONT CANAVESE, TO & FRANKLIN - FRANKLIN MINE LOC. \\
\hline 2326 & FEIRA & JOSEPH & $\mathrm{s}$ & 17 & MICHIGAN & ITALY & MICHIGAN & & LABORER/GENERAL FARM & PONT CANAVESE. TO & FRANKLIN - FRANKLIN MINE LOC. \\
\hline 2327 & FEIRA & VITTORIA & D & 14 & MICHIGAN & ITALY & MICHIGAN & & & PONT CANAVESE. TO & FRANKLIN - FRANKLIN MINE LOC. \\
\hline 2328 & FEIRA & MARY & D & 11 & MICHIGAN & ITALY & MICHIGAN & & & PONT CANAVESE. TO & FRANKLIN - FRANKLIN MINE LOC. \\
\hline 2329 & FEIRA & ROSE & D & 10 & MICHIGAN & ITALY & MICHIGAN & & & PONT CANAVESE. TO & FRANKLIN - FRANKLIN MINE LOC. \\
\hline 2330 & GASPARDO & JAMES & $\mathrm{H}$ & 41 & ITALY & ITALY & ITALY & 1906 & FARMER/GENERAL FARM & PONT CANAVESE. TO & FRANKLIN - FRANKLIN MINE LOC \\
\hline
\end{tabular}




\begin{tabular}{|c|c|c|c|c|c|c|c|c|c|c|c|c|c|}
\hline 2331 & & GASPARDO & FRANCIS & W & 33 & ITALY & ITALY & ITALY & 1898 & & & & FRANKLIN - FRANKLIN MINE LOC. \\
\hline 2332 & & GASPARDO & JOHANNA & $\mathrm{D}$ & 16 & MICHIGAN & ITALY & ITALY & & & PONT CANAVESE. TO & & FRANKLIN - FRANKLIN MINE LOC. \\
\hline 2333 & & GASPARDO & BATTISTA & $\mathrm{s}$ & 14 & MICHIGAN & ITALY & ITALY & & & PONT CANAVESE. TO & & FRANKLIN - FRANKLIN MINE LOC. \\
\hline 2334 & & GASPARDO & JOHN & $\mathrm{s}$ & 12 & MICHIGAN & ITALY & ITALY & & & PONT CANAVESE. TO & & FRANKLIN - FRANKLIN MINE LOC. \\
\hline 2335 & & BLESSENT & MARTIN & B & 62 & ITALY & ITALY & ITALY & 1898 & LABORER/GENERAL FARM & SPARONE. TO & & FRANKLIN - FRANKLIN MINE LOC. \\
\hline 2336 & 107 & SANTORI & AMERICO & $\mathrm{H}$ & 53 & ITALY & ITALY & ITALY & 1892 & PROPRIETOR/RESTAURANT & SEGROMIGNO. LU & ETHEL AVE. & HANCOCK CITY \\
\hline 2337 & tor & SANTORI & ROSE & w & 35 & ITALY & ITALY & ITALY & 1929 & 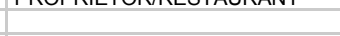 & 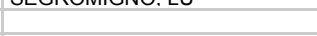 & ETHEL AVE. & HANCOCK CITY \\
\hline 2338 & 300 & NATALI & NELLO & H & 23 & MICHIGAN & ITALY & ITALY & 1929 & MERCHANT/SOFT DRINK & & ETMEL AVE. & HANCOCK CITY \\
\hline 2339 & 000 & NATALI & MARGHERITA & W & 22 & MICHIGAN & ITALY & ITALY & & 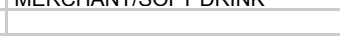 & & & HANCOCK CITY \\
\hline 2340 & & NATALI & JEDO & $\mathrm{BR}$ & 17 & MICHIGAN & ITALY & ITALY & & PORTER/SOFT DRINK PARLOR & & & HANCOCK CITY \\
\hline 2341 & 308 & BONINI & JAMES & $\mathrm{H}$ & 38 & ITALY & ITALY & ITALY & UNK & MERCHANT/SOFT DRINK & LUCCA & RESERVATION & HANCOCK CITY \\
\hline 2342 & & BONINI & STELLA & W & 34 & MICHIGAN & ITALY & ITALY & & & Lector & RESERVATION & HANCOCK CITY \\
\hline 2343 & & BONINI & RUTH & $\mathrm{D}$ & 17 & MICHIGAN & ITALY & MICHIGAN & & & LUCCA & RESERVATION & HANCOCK CITY \\
\hline 2344 & & BONINI & ALBERTA & D & 15 & MICHIGAN & ITALY & MICHIGAN & & & LUCCA & RESERVATION & HANCOCK CITY \\
\hline 2345 & & BONINI & LILLIAN & D & 12 & MICHIGAN & ITALY & MICHIGAN & & & LUCCA & RESERVATION & HANCOCK CITY \\
\hline 2346 & & BONINI & ALBERT & $\mathrm{S}$ & 10 & MICHIGAN & ITALY & MICHIGAN & & & LUCCA & RESERVATION & HANCOCK CITY \\
\hline 2347 & & BONINI & JAMES & $\mathrm{s}$ & 7 & MICHIGAN & ITALY & MICHIGAN & & & LUCCA & RESERVATION & HANCOCK CITY \\
\hline 2348 & & BONINI & ELEONOR & $\mathrm{D}$ & 5 & MICHIGAN & ITALY & MICHIGAN & & & LUCCA & RESERVATION & HANCOCK CITY \\
\hline 2349 & & MONTICELLI & ALBERT & B & 28 & MICHIGAN & ITALY & ITALY & & TRAMMER/CM & LUCCA & RESERVATION & HANCOCK CITY \\
\hline 2350 & 311 & VENCATO & LOUIS & $\mathrm{H}$ & 34 & ITALY & ITALY & ITALY & 1913 & OILER/ENGINE HOUSE & VICENZA & WATER ST. & HANCOCK CITY \\
\hline 2351 & & VENCATO & ANNE & w & 31 & MICHIGAN & ITALY & ITALY & & & TORINO & WATER ST. & HANCOCK CITY \\
\hline 2352 & & VENCATO & DOMENICO & $\begin{array}{l}\mathrm{s} \\
\mathrm{v}\end{array}$ & $\begin{array}{l}9 \\
3 \\
3\end{array}$ & MICHIGAN & ITALY & MICHIGAN & & & VICENZA & WATER ST. & HANCOCK CITY \\
\hline 2353 & & VENCATO & JAMES & s & 5 & MICHIGAN & ITALY & MICHIGAN & & & VICENZA & WATER ST. & HANCOCK CITY \\
\hline 2354 & & SANDRETTO & ANGELINA & MOIL & 62 & ITALY & ITALY & ITALY & 1897 & & TORINO & WATER ST. & HANCOCK CITY \\
\hline 2355 & 320 & PIZZI & JOHN & $\mathrm{H}$ & 35 & ITALY & ITALY & ITALY & 1916 & MERCHANT/CANDIES \& SHOES & LUCCA & HANCOCK AVE & HANCOCK CITY \\
\hline 2356 & & PIZZI-BIANCHI & ANNA & W & 29 & MICHIGAN & ITALY & ITALY & & & LUCCA & HANCOCK AVE & HANCOCK CITY \\
\hline 2357 & & PIZZI & ALICE & $\mathrm{D}$ & 7 & ILLINOIS & ITALY & MICHIGAN & & & LUCCA & HANCOCK AVE. & HANCOCK CITY \\
\hline 2358 & & PIZZI & JOHN & $\mathrm{s}$ & 4 & ILLINOIS & ITALY & MICHIGAN & & & LUCCA & HANCOCK AVE. & HANCOCK CITY \\
\hline 2359 & 322 & MEINARDI & JOHN & $\mathrm{H}$ & 55 & ITALY & ITALY & ITALY & 96?? & MERCHANT/GROCERY & SAN GIORGIO CANAVESE. TO & QUINCY ST. & HANCOCK CITY \\
\hline 2360 & & MEINARDI & VITTORIA & w & 57 & ITALY & ITALY & ITALY & 1905 & & & QUINCY ST. & HANCOCK CITY \\
\hline 2361 & & MEINARDI & JOSEPH & s & 24 & MICHIGAN & ITALY & ITALY & & CLERK/GROCERY & SAN GIORGIO CANAVESE. TO & QUINCY ST. & HANCOCK CITY \\
\hline 2362 & 319 & DIANDA & ANGELO & $\mathrm{H}$ & 49 & ITALY & ITALY & ITALY & 1901 & PROPRIETOR/SOFT DRINK & LUCCA & QUINCY ST. & HANCOCK CITY \\
\hline 2363 & 019 & DIANDA & CORA & W & 39 & ITALY & ITALY & ITALY & 1892 & 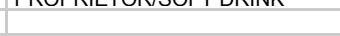 & LUCCA & QUINCY ST. & HANCOCK CITY \\
\hline 2364 & & DIANDA & ANGELINA & D & 20 & MICHIGAN & ITALY & ITALY & & & LUCCA & QUINCY ST. & HANCOCK CITY \\
\hline 2365 & & DIANDA & KATE & D & 17 & MICHIGAN & ITALY & ITALY & & & LUCCA & QUINCY ST. & HANCOCK CITY \\
\hline 2366 & & DIANDA & ALFRED & $\mathrm{s}$ & 6 & MICHIGAN & ITALY & ITALY & & & LUCCA & QUINCY ST. & HANCOCK CITY \\
\hline 2367 & & DIANDA & ROBERT & s & 1 & MICHIGAN & ITALY & ITALY & & & LUCCA & QUINCY ST. & HANCOCK CITY \\
\hline 2368 & 323 & MEOLA & LEONARD & $\mathrm{H}$ & 61 & ITALY & ITALY & ITALY & 1889 & INTERPRETER/COURT & NAPOLI & QUINCY ST. & HANCOCK CITY \\
\hline 2369 & & MEOLA & NELLIS & w & 55 & $\mathrm{OHIO}$ & MISSOURI & KANSAS & & & USA & QUINCY ST. & HANCOCK CITY \\
\hline 2370 & 309 & VIGNETTO & ANGELO & $\mathrm{H}$ & 43 & MICHIGAN & ITALY & ITALY & & MERCHANT/CONFECTIONARY & TORINO & RAVINE ST. & HANCOCK CITY \\
\hline 2371 & & VIGNETTO & FLORENCE & W & 42 & MICHIGAN & MICHIGAN & ENGLAND & & & ENGLAND & RAVINE ST. & HANCOCK CITY \\
\hline 2372 & 309 & KRAMER & FRED & $\mathrm{H}$ & 25 & GERMANY & GERMANY & GERMANY & ???? & MECHANIC/AUTOMOBILE & GERMANY & RAVINE ST. & HANCOCK CITY \\
\hline 2373 & & KRAMER & MARGHERITA & w & 25 & MICHIGAN & ITALY & ITALY & & & & RAVINE ST. & HANCOCK CITY \\
\hline 2374 & & KRAMER & CARLOTTA & D & 3 & MICHIGAN & GERMANY & MICHIGAN & & & USA-GERMANY & RAVINE ST. & HANCOCK CITY \\
\hline 2375 & 413 & MICHELETTI & DANTE & B & 42 & ITALY & ITALY & ITALY & 1906 & COOK/RESTAURANT & SANTA MARIA DEL GIUDICE. & QUINCY ST. & HANCOCK CITY \\
\hline 2376 & 605 & NUTINI & PETER & $\mathrm{H}$ & 47 & ITALY & ITALY & ITALY & 1903 & PROPRIETOR/RESTAURANT & CAPANNORI. LU & HANCOCK AVE & HANCOCK CITY \\
\hline 2377 & & NUTINI & CELIA & w & 44 & ITALY & ITALY & ITALY & 1909 & & & HANCOCK AVE & HANCOCK CITY \\
\hline 2378 & & NUTINI & ALADINO & $\mathrm{s}$ & 18 & MICHIGAN & ITALY & ITALY & & TRUCK DRIVER/GROCERY & CAPANNORI. LU & HANCOCK AVE & HANCOCK CITY \\
\hline 2379 & & NUTINI & ANNA & $\mathrm{D}$ & 16 & MICHIGAN & ITAI & ITAI & & & CAPANNORI. LU & HANCOCK AVE & HANCOCK CITY \\
\hline 2380 & & NUTINI & PAUL & s & 15 & MICHIGAN & ITALY & ITALY & & & CAPANNORI. LU & HANCOCK AVE. & HANCOCK CITY \\
\hline 2381 & & NUTINI & JULIO & $\mathrm{s}$ & 12 & MICHIGAN & ITALY & ITALY & & & CAPANNORI. LU & HANCOCK AVE & HANCOCK CITY \\
\hline 2382 & & NUTINI & NELLO & s & 4 & MICHIGAN & ITALY & ITALY & & & CAPANNORI, LU & HANCOCK AVE & HANCOCK CITY \\
\hline 2383 & 621 & $\mathrm{ClUCCl}$ & STEFANO & $\mathrm{H}$ & 51 & ITALY & ITALY & ITALY & 1908 & & CAPANNORI. LU & HANCOCK AVE. & HANCOCK CITY \\
\hline 2384 & & $\mathrm{ClUCCl}$ & CECILIA & D & 23 & MICHIGAN & ITALY & ITALY & & & CAPANNORI. LU & HANCOCK AVE & HANCOCK CITY \\
\hline 2385 & & $\mathrm{ClUCCl}$ & ESTER & $\mathrm{D}$ & 22 & MICHIGAN & ITALY & ITALY & & WRAPPER/CIGAR FACTORY & CAPANNORI. LU & HANCOCK AVE & HANCOCK CITY \\
\hline 2386 & & $\mathrm{ClUCCl}$ & MATILDA & $\mathrm{D}$ & 19 & MICHIGAN & ITALY & ITALY & & TESTER/CREAMERY & CAPANNORI. LU & HANCOCK AVE & HANCOCK CITY \\
\hline 2387 & & $\mathrm{ClUCCl}$ & RUSSELL & s & 18 & MICHIGAN & ITALY & ITALY & & LABORER/CREAMERY & CAPANNORI. LU & HANCOCK AVE & HANCOCK CITY \\
\hline 2388 & & $\mathrm{CIUCCl}$ & MARY & $\mathrm{D}$ & 14 & MICHIGAN & ITALY & ITALY & & & CAPANNORI, LU & HANCOCK AVE. & HANCOCK CITY \\
\hline 2389 & & $\mathrm{ClUCCl}$ & PETER & s & 13 & MICHIGAN & ITALY & ITALY & & & CAPANNORI. LU & HANCOCK AVE & HANCOCK CITY \\
\hline 2390 & & $\mathrm{ClUCCl}$ & JOSEPH & s & 13 & MICHIGAN & ITALY & ITAI & & & CAPANNORI. LU & HANCOCK AVE & DCK CITY \\
\hline 2391 & & ClUCCl & NORN & $\mathrm{s}$ & 11 & MICHIGAN & ITA & & & & CAPANNORI. LU & JCK AVE & K CITY \\
\hline 2392 & 718 & VILLANELLI & & $\mathrm{H}$ & 40 & & ITALY & ITALY & 1912 & PROPRIETOR/GAS STATION & & QUINCY ST. & \\
\hline 2393 & & VILLANELLI & VINCENZA & W & 29 & ITALY & ITALY & ITALY & 1920 & & & QUINCY ST. & HANCOCK CITY \\
\hline
\end{tabular}




\begin{tabular}{|c|c|c|c|c|c|c|c|c|c|c|c|c|c|}
\hline 2394 & & VILLANELLI & LAURA & D & 8 & MICHIGAN & ITALY & ITALY & & & & QUINCY ST. & HANCOCK CITY \\
\hline 2395 & & VILLANELLI & ARMANDO & $\mathrm{s}$ & 7 & MICHIGAN & ITALY & ITALY & & & & QUINCY ST. & HANCOCK CITY \\
\hline 2396 & & VILLANELLI & HELEN & D & 1 & MICHIGAN & ITALY & ITALY & & & & QUINCY ST. & HANCOCK CITY \\
\hline 2397 & & MARIANI & EVERET & NEP & 20 & MICHIGAN & ITALY & ITALY & & ATTENDANT/GAS STATION & & QUINCY ST. & HANCOCK CITY \\
\hline 2398 & 500 & DANIELE & FRANK & $\mathrm{H}$ & 45 & ITALY & ITALY & ITALY & 1917 & LABORER/WHOLESALE FRUIT & RIVAROLO CANAVESE. TO & QUINCY ST. & HANCOCK CITY \\
\hline 2399 & & DANIELE & MINNIE & w & 41 & ITALY & ITALY & ITALY & 1917 & & RIVAROLO CANAVESE. TO & QUINCY ST. & HANCOCK CITY \\
\hline 2400 & & DANIELE & ANTONIETTA & D & 20 & MICHIGAN & ITALY & ITALY & & & RIVAROLO CANAVESE. TO & QUINCY ST. & HANCOCK CITY \\
\hline 2401 & & DANIELE & MADDALENA & $\mathrm{D}$ & 16 & MICHIGAN & ITALY & ITALY & & & RIVAROLO CANAVESE. TO & QUINCY ST. & HANCOCK CITY \\
\hline 2402 & & DANIELE & MARGHERITA & $\mathrm{D}$ & 15 & MICHIGAN & ITALY & ITALY & & & RIVAROLO CANAVESE. TO & QUINCY ST. & HANCOCK CITY \\
\hline 2403 & & DANIELE & AGNES & $\mathrm{D}$ & 8 & MICHIGAN & ITALY & ITALY & & & RIVAROLO CANAVESE. TO & QUINCY ST. & HANCOCK CITY \\
\hline 2404 & & DANIELE & RUTH & $\mathrm{D}$ & 3 & MICHIGAN & ITALY & ITALY & & & RIVAROLO CANAVESE. TO & QUINCY ST. & HANCOCK CITY \\
\hline 2405 & & O'BRIEN & JOHN & SOIL & 23 & MICHIGAN & MICHIGAN & MICHIGAN & & LABORER/WHOLESALE FRUIT & USA & QUINCY ST. & HANCOCK CITY \\
\hline 2406 & & O'BRIEN & KATE & D & 18 & MICHIGAN & ITALY & ITALY & & & RIVAROLO CANAVESE. TO & QUINCY ST. & HANCOCK CITY \\
\hline 2407 & 423 & GIULIO & ANTONIO & $\mathrm{H}$ & 57 & ITALY & ITALY & ITALY & 1903 & SHOEMAKER/SHOE FACTORY & SAN GIORGIO CANAVESE. TO & HANCOCK AVE. & HANCOCK CITY \\
\hline 2408 & & GIULIO & PETRONILLA & W & 51 & ITALY & ITALY & ITALY & 1905 & & & HANCOCK AVE. & HANCOCK CITY \\
\hline 2409 & & DELBRIDGE & MARY & D & 19 & MICHIGAN & ITALY & ITALY & & & SAN GIORGIO CANAVESE, TO & HANCOCK AVE. & HANCOCK CITY \\
\hline 2410 & & DELBRIDGE & ANTON & GS & $3 \mathrm{M}$ & MICHIGAN & MICHIGAN & MICHIGAN & & & SAN GIORGIO CANAVESE. TO & HANCOCK AVE. & HANCOCK CITY \\
\hline 2411 & & GIULIO & ANNETTE & $\mathrm{D}$ & 8 & MICHIGAN & ITALY & ITALY & & & SAN GIORGIO CANAVESE. TO & HANCOCK AVE. & HANCOCK CITY \\
\hline 2412 & 429 & PATERNI & ANGELO & SOIL & 39 & ITALY & ITALY & ITALY & 1913 & FIREMAN/ELECTRIC POWER & LUCCA & SCOTT ST. & HANCOCK CITY \\
\hline 2413 & & PATERNI & HATTIE & D & 40 & MICHIGAN & GERMANY & MICHIGAN & & & USA-GERMANY & SCOTT ST. & HANCOCK CITY \\
\hline 2414 & & PATERNI & CARL & GS & 2 & MICHIGAN & ITALY & MICHIGAN & & & LUCCA & SCOTT ST. & HANCOCK CITY \\
\hline 2415 & 715 & GEDDA & VITTORIO & $\mathrm{H}$ & 55 & ITALY & ITALY & ITALY & 1892 & BAKER & TORINO & PINE ST. & HANCOCK CITY \\
\hline 2416 & 710 & GEDDA & ROSE & W & 36 & ITALY & ITALY & ITALY & 1908 & DARER & TURIIN & PINE ST. & HANCOCK CITY \\
\hline 2417 & & GEDDA & MADDALENA & $\mathrm{D}$ & 17 & MICHIGAN & ITALY & ITALY & & SALESLADY/BAKERY & TORINO & PINE ST. & HANCOCK CITY \\
\hline 2418 & & GEDDA & ANGELINA & D & 16 & MICHIGAN & ITALY & ITALY & & & TORINO & PINE ST. & HANCOCK CITY \\
\hline 2419 & & GEDDA & JOHN & $\mathrm{s}$ & 13 & MICHIGAN & ITALY & ITALY & & & TORINO & PINE ST. & HANCOCK CITY \\
\hline 2420 & & GEDDA & ISABELLA & D & 2 & MICHIGAN & ITALY & ITALY & & & TORINO & PINE ST. & HANCOCK CITY \\
\hline 2421 & 317 & MONTICELLO & LOUIS & $\mathrm{H}$ & 26 & MICHIGAN & ITALY & ITALY & & MINER/CM & & WRIGHT ST. & HANCOCK CITY \\
\hline 2422 & & MONTICELLO & EBBA & W & 25 & MICHIGAN & FINLAND & FINLAND & & & & WRIGHT ST. & HANCOCK CITY \\
\hline 2423 & & MONTICELLO & RALPH & s & 5 & MICHIGAN & MICHIGAN & MICHIGAN & & & & WRIGHT ST. & HANCOCK CITY \\
\hline 2424 & & MONTICELLO & CLARENCE & S & 2 & MICHIGAN & MICHIGAN & MICHIGAN & & & & WRIGHT ST. & HANCOCK CITY \\
\hline 2425 & 710 & ZUCCO & JAMES & $\mathrm{H}$ & 60 & ITALY & ITALY & ITALY & 1884 & LABORER/CM & TORINO & WEALTON ST. & HANCOCK CITY \\
\hline 2426 & & ZUCCO & MARY & W & 46 & ITALY & ITALY & ITALY & 1903 & & & WEALTON ST. & HANCOCK CITY \\
\hline 2427 & & MATTO & JAMES & B & 41 & ITALY & ITALY & ITALY & 1905 & LABORER/CM & & WEALTON ST. & HANCOCK CITY \\
\hline 2428 & & GASPARDO & \begin{tabular}{|l} 
JAVES \\
JOHN
\end{tabular} & B & $\frac{41}{22}$ & MICHIGAN & ITALY & ITALY & 1900 & TRUCK DRIVER/CREAMERY & PONT CANAVESE. TO & WEALTON ST. & HANCOCK CITY \\
\hline 2429 & 714 & $\begin{array}{l}\text { FASANA } \\
\end{array}$ & TERESA & $\mathrm{H}$ & 56 & ITALY & ITALY & ITALY & 1900 & & TORINO & ELM ST. & HANCOCK CITY \\
\hline 2430 & & FASANA & MARY & D & 25 & MICHIGAN & ITALY & ITALY & & ASSISTANT CLERK/DRY & TORINO & ELM ST. & HANCOCK CITY \\
\hline 2431 & & FASANA & CLARA & $\mathrm{D}$ & 24 & MICHIGAN & ITALY & ITALY & & SALESLADYIDEPARTMENT & TORINO & ELM ST. & HANCOCK CITY \\
\hline 2432 & & FASANA & KATE & D & 22 & MICHIGAN & ITALY & ITALY & & SEAMSTRESSIFUR SHOP & TORINO & ELM ST. & HANCOCK CITY \\
\hline 2433 & & FASANA & JOHANNA & $\mathrm{D}$ & 19 & MICHIGAN & ITALY & ITALY & & & TORINO & ELM ST. & HANCOCK CITY \\
\hline 2434 & 811 & $\mathrm{ROCH}$ & JOE & $\mathrm{H}$ & 48 & ITALY & ITALY & ITALY & 1904 & PROPRIETOR/GROCERY STORE & RIVAROLO CANAVESE. TO & WARREN AVE. & HANCOCK CITY \\
\hline 2435 & & $\mathrm{ROCH}$ & KATE & w & 40 & MICHIGAN & ITALY & ITALY & & & & WARREN AVE. & HANCOCK CITY \\
\hline 2436 & & $\mathrm{ROCH}$ & LOUISELLE & D & 9 & MICHIGAN & ITALY & MICHIGAN & & & RIVAROLO CANAVESE. TO & WARREN AVE. & HANCOCK CITY \\
\hline 2437 & 800 & GEMIGNANI & ADOLFO & $\mathrm{H}$ & 56 & ITALY & ITALY & ITALY & 1901 & SHOEMAKER/SHOP & LUCCA & QUINCY ST. & HANCOCK CITY \\
\hline 2438 & & GEMIGNANI & ASSUNTA & w & 55 & ITALY & ITALY & ITALY & 1916 & & LUCCA & QUINCY ST. & HANCOCK CITY \\
\hline 2439 & & GEMIGNANI & GINO & s & 26 & ITALY & ITALY & ITALY & 1916 & SHOEMAKER/SHOP & LUCCA & QUINCY ST. & HANCOCK CITY \\
\hline 2440 & & GEMIGNANI & BLANCHE & $\mathrm{D}$ & 20 & MICHIGAN & ITALY & ITALY & & & LUCCA & QUINCY ST. & HANCOCK CITY \\
\hline 2441 & & GEMIGNANI & GLORIA & GD & 1 & MICHIGAN & ITALY & ITALY & & & LUCCA & QUINCY ST. & HANCOCK CITY \\
\hline 2442 & & GEMIGNANI & RUDOLPH & GS & $?$ & MICHIGAN & ITALY & ITALY & & & LUCCA & QUINCY ST. & HANCOCK CITY \\
\hline 2443 & & GEMIGNANI & BALDO & $\mathrm{s}$ & 19 & ITALY & ITALY & ITALY & 1916 & SHOEMAKER/SHOP & LUCCA & QUINCY ST. & HANCOCK CITY \\
\hline 2444 & & GROSSI & JOSEPH & $\mathrm{B}$ & 11 & MICHIGAN & ITA & ITALY & & & & QUINCY ST. & HANCOCK CITY \\
\hline 2445 & 716 & OSTI/ORTI & MARY & $\mathrm{B}$ & 35 & MICHIGAN & ITALY & ITALY & & MANAGER/DEPARTMENT STORE & & HANCOCK AVE. & HANCOCK CITY \\
\hline 2446 & 205 & MANDOLI & ALADINO & $\mathrm{H}$ & 25 & ITALY & ITALY & ITALY & 1906 & $\begin{array}{l}\text { PRINTER/PRINT SHOP } \\
\text { PI }\end{array}$ & LUCCA & WATER ST. & HANCOCK CITY \\
\hline 2447 & & MANDOLI & BERTHE & w & ?? & MICHIGAN & MICHIGAN & MICHIGAN & & & USA & WATER ST. & HANCOCK CITY \\
\hline 2448 & & MANDOLI & ROBERT & $\mathrm{s}$ & ?? & MICHIGAN & ITALY & MICHIGAN & & & LUCCA & WATER ST. & HANCOCK CITY \\
\hline 2449 & 715 & KRATT & ERNEST & $\mathrm{H}$ & 37 & MICHIGAN & GERMANY & GERMANY & & AGENT/LIFE INSURANCE & GERMANY & WATER ST. & HANCOCK CITY \\
\hline 2450 & & KRATT & MARGHERITA & w & 34 & MICHIGAN & ITALY & ITALY & & & & WATER ST. & HANCOCK CITY \\
\hline 2451 & & KRATT & PATRICIA & $\mathrm{D}$ & 5 & MICHIGAN & MICHIGAN & MICHIGAN & & & & WATER ST. & HANCOCK CITY \\
\hline 2452 & & KRATT & MARCELLA & $\mathrm{D}$ & 3 & MICHIGAN & MICHIGAN & MICHIGAN & & & & WATER ST. & HANCOCK CITY \\
\hline 2453 & 713 & LENCI & FIORELLO & $\mathrm{H}$ & 44 & ITALY & ITALY & ITALY & 1904 & LABORER/SMELTER & SEGROMIGNO. LU & ELM ST. & HANCOCK CITY \\
\hline 2454 & 713 & LENCI & LIZZIE & W & $\begin{array}{l}44 \\
38\end{array}$ & MICHIGAN & ITALY & ITALY & 1904 & LADURERTONIELILR & SEGRUIVIOTVO. LU & ELM ST. & HANCOCK CITY \\
\hline 2455 & & BOAINI & GEORGE & SSO & 2 & MICHIGAN & ITALY & MICHIGAN & & & & ELM ST. & HANCOCK CITY \\
\hline 2456 & & BALCONI & JOHN & $\mathrm{H}$ & 42 & ITALY & ITALY & ITALY & 1915 & REFINER/COPPER WORKS & MERCALLO. VARESE & ATLANTIC ST. & HANCOCK CITY \\
\hline
\end{tabular}




\begin{tabular}{|c|c|c|c|c|c|c|c|c|c|c|c|c|c|}
\hline 2457 & & BALCONI & MARIA & w & 34 & ITALY & ITALY & ITALY & 1915 & & MERCALLO. VARESE & ATLANTIC ST. & HANCOCK CITY \\
\hline 2458 & & BALCONI & JOSEPHINE & $\mathrm{D}$ & 16 & ITALY & ITALY & ITALY & 1915 & & MERCALLO. VARESE & ATLANTIC ST. & HANCOCK CITY \\
\hline 2459 & & BALCONI & CHARLIE & $\mathrm{s}$ & 12 & MICHIGAN & ITALY & ITALY & & & MERCALLO. VARESE & ATLANTIC ST. & HANCOCK CITY \\
\hline 2460 & & BALCONI & PIERINA & $\mathrm{D}$ & 10 & MICHIGAN & ITALY & ITALY & & & MERCALLO. VARESE & ATLANTIC ST. & HANCOCK CITY \\
\hline 2461 & & BALCONI & ROSE & $\mathrm{D}$ & 7 & MICHIGAN & ITALY & ITALY & & & MERCALLO. VARESE & ATLANTIC ST. & HANCOCK CITY \\
\hline 2462 & & BALCONI & DANTE & s & 5 & MICHIGAN & ITALY & ITALY & & & MERCALLO. VARESE & ATLANTIC ST. & HANCOCK CITY \\
\hline 2463 & & BALCONI & ELEONOR & $\mathrm{D}$ & 3 & MICHIGAN & ITALY & ITALY & & & MERCALLO. VARESE & ATLANTIC ST. & HANCOCK CITY \\
\hline 2464 & & BALCONI & VIRGINIA & $\mathrm{D}$ & 2 & MICHIGAN & ITALY & ITALY & & & MERCALLO. VARESE & ATLANTIC ST. & HANCOCK CITY \\
\hline 2465 & & AMATO & FRANK & $\mathrm{H}$ & 60 & ITALY & ITALY & ITALY & 1899 & LABORER/SMELTER & CASTROVILLARI. COSENZA & QUINCY ST. & HANCOCK CITY \\
\hline 2466 & & AMATO & CAROLINE & W & 43 & ITALY & ITALY & ITALY & 1907 & & & QUINCY ST. & HANCOCK CITY \\
\hline 2467 & & AMATO & FRANK & s & 19 & MICHIGAN & ITALY & ITALY & & & CASTROVILLARI. COSENZA & QUINCY ST. & HANCOCK CITY \\
\hline 2468 & & AMATO & MATILDA & $\mathrm{D}$ & 13 & MICHIGAN & ITALY & ITALY & & & CASTROVILLARI. COSENZA & QUINCY ST. & HANCOCK CITY \\
\hline 2469 & & BERTOLOZZI & TERESA & $\mathrm{D}$ & 21 & MICHIGAN & ITALY & ITALY & & & CASTROVILLARI. COSENZA & QUINCY ST. & HANCOCK CITY \\
\hline 2470 & & BERTOLOZZI & PAULINE & GD & $8 \mathrm{M}$ & MICHIGAN & MICHIGAN & ITALY & & & CASTROVILLARI. COSENZA & QUINCY ST. & HANCOCK CITY \\
\hline 2471 & 522 & PAYNE & FRANK & $\mathrm{H}$ & 65 & MICHIGAN & SCOTLAND & GERMANY & & LABORER/CITY & SCOTLAND-GERMANY & EMMA ST. & HANCOCK CITY \\
\hline 2472 & & PAYNE & VERONICA & W & 60 & ITALY & ITALY & ITALY & 1880 & & & EMMA ST. & HANCOCK CITY \\
\hline 2473 & 1317 & QUENZI & JOHN & $\mathrm{H}$ & 29 & MICHIGAN & ITALY & ITALY & & BUTCHER/MEAT MARKET & & QUINCY ST. & HANCOCK CITY \\
\hline 2474 & & QUENZI & BEATRICE & w & 22 & MICHIGAN & CANADA & CANADA & & & & QUINCY ST. & HANCOCK CITY \\
\hline 2475 & & BARONI & MIKE & $\mathrm{H}$ & 26 & ITALY & ITALY & ITALY & 1921 & BUS DRIVER/BUS CO. & LUCCA & ANTONY? ST. & HANCOCK CITY \\
\hline 2476 & & BARONI & HELEN & w & 27 & ITALY & ITALY & ITALY & 1929 & & & ANTONY? ST. & HANCOCK CITY \\
\hline 2477 & & BARONI & IDA & $\mathrm{D}$ & 2 & MICHIGAN & ITALY & $\begin{array}{l}\text { ITALY } \\
\text { ITLY }\end{array}$ & 1929 & & LUCCA & ANTONY? ST. & HANCOCK CITY \\
\hline 2478 & 1035 & ORELLA & DOMENICO & $\mathrm{H}$ & 49 & ITALY & ITALY & ITALY & 1887 & TELLER/BANK & TORINO & MINNESOTA ST. & HANCOCK CITY \\
\hline 2479 & & ORELLA & TERESA & w & 51 & ITALY & ITALY & ITALY & 1902 & & & MINNESOTA ST. & HANCOCK CITY \\
\hline 2480 & & ORELLA & BERNARD & $s$ & 26 & MICHIGAN & ITALY & ITALY & & & TORINO & MINNESOTA ST. & HANCOCK CITY \\
\hline 2481 & & ORELLA & MARY & $\mathrm{D}$ & 22 & MICHIGAN & ITALY & ITALY & & & TORINO & MINNESOTA ST. & HANCOCK CITY \\
\hline 2482 & & ORELLA & AGNES & $\mathrm{D}$ & 20 & MICHIGAN & ITALY & ITALY & & & TORINO & MINNESOTA ST. & HANCOCK CITY \\
\hline 2483 & 1002 & CAMPIONI & GUIDO & $\mathrm{H}$ & 43 & ITALY & ITALY & ITALY & 1909 & GROCER/GENERAL & LUCCA & MINNESOTA ST. & HANCOCK CITY \\
\hline 2484 & & CAMPIONI & MABEL & w & 36 & ITALY & ITALY & ITALY & 1907 & & & MINNESOTA ST. & HANCOCK CITY \\
\hline 2485 & & CAMPIONI & CONSTANCE & D & 18 & MICHIGAN & ITALY & ITALY & & & LUCCA & MINNESOTA ST. & HANCOCK CITY \\
\hline 2486 & & CAMPIONI & JOSEPH & $\bar{s}$ & 17 & MICHIGAN & ITALY & ITALY & & & LUCCA & MINNESOTA ST. & HANCOCK CITY \\
\hline 2487 & & CAMPIONI & LOUIS & s & 15 & MICHIGAN & ITALY & ITALY & & & LUCCA & MINNESOTA ST. & HANCOCK CITY \\
\hline 2488 & & CAMPIONI & PAUL & $\mathrm{s}$ & 11 & MICHIGAN & ITALY & ITALY & & & LUCCA & MINNESOTA ST. & HANCOCK CITY \\
\hline 2489 & 1029 & IAVELLO & MARTINA & NUR & 21 & MICHIGAN & ITALY & ITALY & & NURSE & TORINO & WATER ST. & HANCOCK CITY \\
\hline 2490 & 1025 & GHIARDI & MARY & NUR & 18 & MICHIGAN & $\begin{array}{l}\text { ITALY } \\
\end{array}$ & $\begin{array}{l}\text { ITALY } \\
\text { ITAL }\end{array}$ & & NURSE & SAN MARTINO CANAVESE, TO & WATER ST. & HANCOCK CITY \\
\hline 2491 & 921 & PAOLI & RALPH & $\mathrm{H}$ & 43 & ITALY & ITALY & ITALY & 1896 & PROPRIETOR/WHOLESALE FRUIT & LUCCA & RAILROAD AVE. & HANCOCK CITY \\
\hline 2492 & & PAOLI & IDA & w & 43 & ITALY & ITALY & ITALY & 1916 & & & RAILROAD AVE. & HANCOCK CITY \\
\hline 2493 & & PAOLI & LOUISE & $\mathrm{D}$ & 12 & MICHIGAN & ITALY & ITALY & & & LUCCA & RAILROAD AVE. & HANCOCK CITY \\
\hline 2494 & & PAOLI & LOUIS & $\mathrm{s}$ & 10 & MICHIGAN & ITALY & ITALY & & & LUCCA & RAILROAD AVE. & HANCOCK CITY \\
\hline 2495 & & PAOLI & RALPH & $\mathrm{s}$ & 5 & MICHIGAN & ITALY & ITALY & & & LUCCA & RAILROAD AVE. & HANCOCK CITY \\
\hline 2496 & 951 & $\mathrm{RICCl}$ & FRANK & $\mathrm{H}$ & 40 & ITALY & ITALY & ITALY & 1913 & MECHANIC/AUTO REPAIR & LUCCA & RAILROAD AVE. & HANCOCK CITY \\
\hline 2497 & & $\mathrm{RICCl}$ & RITA & W & 29 & MICHIGAN & MICHIGAN & MASSACHUS & & & USA & RAILROAD AVE. & HANCOCK CITY \\
\hline 2498 & & $\mathrm{RICCl}$ & MARY & $\mathrm{D}$ & 9 & MICHIGAN & ITALY & MICHIGAN & & & LUCCA & RAILROAD AVE. & HANCOCK CITY \\
\hline 2499 & & RICCI & DONALD & $\mathrm{s}$ & 1 & MICHIGAN & ITALY & MICHIGAN & & & LUCCA & RAILROAD AVE. & HANCOCK CITY \\
\hline 2500 & 1025 & PAOLI & JULIO & $\mathrm{H}$ & 39 & ITALY & ITALY & ITALY & 1909 & CLERK/WHOLESALE FRUIT & SEGROMIGNO. LU & RAILROAD AVE. & HANCOCK CITY \\
\hline 2501 & & PAOLI & TISBE & W & 38 & ITALY & ITALY & ITALY & 1910 & 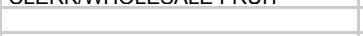 & 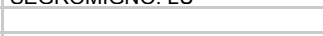 & RAILROAD AVE. & HANCOCK CITY \\
\hline 2502 & & PAOLI & LINDA & $\mathrm{D}$ & 14 & MICHIGAN & ITALY & ITALY & & & SEGROMIGNO. LU & RAILROAD AVE. & HANCOCK CITY \\
\hline 2503 & & PAOLI & ANGELINA & $\mathrm{D}$ & 13 & MICHIGAN & ITALY & ITALY & & & SEGROMIGNO. LU & RAILROAD AVE. & HANCOCK CITY \\
\hline 2504 & & PAOLI & GUIDO & s & 11 & MICHIGAN & ITALY & ITALY & & & SEGROMIGNO. LU & RAILROAD AVE. & HANCOCK CITY \\
\hline 2505 & & PAOLI & LEDA & $\mathrm{D}$ & 7 & MICHIGAN & ITALY & ITALY & & & SEGROMIGNO. LU & RAILROAD AVE. & HANCOCK CITY \\
\hline 2506 & & PAOLI & JULIA & $\mathrm{D}$ & 3 & MICHIGAN & ITALY & ITALY & & & SEGROMIGNO. LU & RAILROAD AVE. & HANCOCK CITY \\
\hline 2507 & & PAOLI & ROSE & $\mathrm{s}$ & 1 & MICHIGAN & ITALY & ITALY & & & SEGROMIGNO. LU & RAILROAD AVE. & HANCOCK CITY \\
\hline 2508 & 1038 & FEDERIGHI & IRENE & $\mathrm{H}$ & 50 & ITALY & ITALY & ITALY & 1900 & STOREKEEPER/GROCERY & LUCCA & SUMMIT ST. & HANCOCK CITY \\
\hline 2509 & & FEDERIGHI & SEBASTIANO & $\mathrm{s}$ & 24 & MICHIGAN & ITALY & ITALY & & TRUCK DRIVER/SAUSAGE & LUCCA & SUMMIT ST. & HANCOCK CITY \\
\hline 2510 & & FEDERIGHI & JOHN & $\mathrm{s}$ & 18 & MICHIGAN & ITALY & ITALY & & CLERK/OIL STATION & LUCCA & SUMMIT ST. & HANCOCK CITY \\
\hline 2511 & & FEDERIGHI & SABINA & $\mathrm{D}$ & 15 & MICHIGAN & ITALY & ITALY & & & LUCCA & SUMMIT ST. & HANCOCK CITY \\
\hline 2512 & & FEDERIGHI & MARY & $\mathrm{D}$ & 12 & MICHIGAN & ITALY & ITALY & & & LUCCA & SUMMIT ST. & HANCOCK CITY \\
\hline 2513 & 1012 & LORENZETTI & JOHN & $\mathrm{H}$ & 42 & ITALY & ITALY & ITALY & 1910 & FURNACE TENDER/SMELTER & LUCCA & SUMMIT ST. & HANCOCK CITY \\
\hline 2514 & 012 & LORENZETTI & TERESA & W & 42 & ITALY & ITALY & ITALY & 1910 & PROPRIETOR/GROCERY STORE & LUCCA & SUMMIT ST. & HANCOCK CITY \\
\hline 2515 & & LORENZETTI & JULIO & s & 17 & MICHIGAN & ITALY & ITALY & & DISHWASHER/RESTAURANT & LUCCA & SUMMIT ST. & HANCOCK CITY \\
\hline 2516 & & LORENZETTI & DANTE & s & 15 & MICHIGAN & ITALY & ITALY & & AUTO MECHANIC/AUTO REPAIR & LUCCA & SUMMIT ST. & HANCOCK CITY \\
\hline 2517 & & LORENZETTI & OLGA & $\mathrm{D}$ & 10 & MICHIGAN & ITALY & ITA & & & LUCCA & TST. & CITY \\
\hline 2518 & & LORENZETTI & ROSIE & $\mathrm{D}$ & 6 & MICHIGAN & ITALY & & & & & SUMMIT ST. & HANCOCK CITY \\
\hline 2519 & & LORENZETTI & RALPH & $\mathrm{BR}$ & 44 & ITALY & ITALY & ITALY & 1907 & MINER/CM & LUCCA & SUMMIT ST. & HANCOCK CITY \\
\hline
\end{tabular}




\begin{tabular}{|c|c|c|c|c|c|c|c|c|c|c|c|c|c|}
\hline 2520 & 915 & PARMA & JOHN & $\mathrm{H}$ & 62 & ITALY & ITALY & ITALY & 1899 & JANITOR/PUBLIC SCHOOL & & SUMMIT ST. & HANCOCK CITY \\
\hline 2521 & & GABRIELLI & AMERICO & B & 52 & ITALY & ITALY & ITALY & 1897 & MINER/CM & LUCCA & SUMMIT ST. & HANCOCK CITY \\
\hline 2522 & & PAONE & DOMENICO & B & 58 & ITALY & ITALY & ITALY & 1900 & LABORER/SMELTER & TORINO & SUMMIT ST. & HANCOCK CITY \\
\hline 2523 & 1013 & BALCONI & MIKE & $\mathrm{H}$ & 43 & ITALY & ITALY & ITALY & 1906 & TAPPER/SMELTER & MERCALLO. VARESE & ELM ST. & HANCOCK CITY \\
\hline 2524 & & BALCONI & ROSE & w & 42 & MICHIGAN & ITALY & ITALY & & & & ELM ST. & HANCOCK CITY \\
\hline 2525 & & BALCONI & MARY & D & 17 & MICHIGAN & ITALY & MICHIGAN & & & MERCALLO. VARESE & ELM ST. & HANCOCK CITY \\
\hline 2526 & & BALCONI & CECILIA & D & 14 & MICHIGAN & ITALY & MICHIGAN & & & MERCALLO. VARESE & ELM ST. & HANCOCK CITY \\
\hline 2527 & & BALCONI & ERNESTINE & $\mathrm{D}$ & 10 & MICHIGAN & ITALY & MICHIGAN & & & MERCALLO. VARESE & ELM ST. & HANCOCK CITY \\
\hline 2528 & & BALCONI & PAUL & $\mathrm{s}$ & 6 & MICHIGAN & ITALY & MICHIGAN & & & MERCALLO. VARESE & ELM ST. & HANCOCK CITY \\
\hline 2529 & 811 & SANTORI & ORESTE & $\mathrm{H}$ & 46 & ITALY & ITALY & ITALY & 1889 & GROCER/GROCERY STORE & SEGROMIGNO. LU & ETHEL AVE. & HANCOCK CITY \\
\hline 2530 & & SANTORI & ROSE & w & 42 & ITALY & ITALY & ITALY & 1909 & & SEGROMIGNO. LU & ETHEL AVE. & HANCOCK CITY \\
\hline 2531 & & SANTORI & EDWARD & $\mathrm{s}$ & 20 & ITALY & ITALY & ITALY & 1909 & & SEGROMIGNO. LU & ETHEL AVE. & HANCOCK CITY \\
\hline 2532 & & SANTORI & CHARLES & s & 17 & MICHIGAN & ITALY & ITALY & & & SEGROMIGNO. LU & ETHEL AVE. & HANCOCK CITY \\
\hline 2533 & & SANTORI & VANDO & $\mathrm{s}$ & 12 & MICHIGAN & ITALY & ITALY & & & SEGROMIGNO. LU & ETHEL AVE. & HANCOCK CITY \\
\hline 2534 & 1124 & RENALDI & MARION & $\mathrm{H}$ & 60 & ITALY & ITALY & ITALY & 1895 & CONFECTIONARY/CANDY STORE & SAN GIORGIO CANAVESE. TO & SIGSBEE ST. & HANCOCK CITY \\
\hline 2535 & & RENALDI & JOSEPH & $\mathrm{S}$ & 32 & MICHIGAN & ITALY & ITALY & & MACHINIST/STEAM RAILROAD & SAN GIORGIO CANAVESE, TO & SIGSBEE ST. & HANCOCK CITY \\
\hline 2536 & & RENALDI & GENEVIEVE & D & 19 & MICHIGAN & ITALY & ITALY & & HOUSEWORK/HOME & SAN GIORGIO CANAVESE. TO & SIGSBEE ST. & HANCOCK CITY \\
\hline 2537 & 1012 & ANDREOTTI & JOSEPH & $\mathrm{H}$ & 44 & ITALY & ITALY & ITALY & 1907 & MINER/CM & CAMILLIANO. LU & ETHEL AVE. & HANCOCK CITY \\
\hline 2538 & & ANDREOTTI & ANGELINA & w & 44 & ITALY & ITALY & ITALY & 1910 & & & ETHEL AVE. & HANCOCK CITY \\
\hline 2539 & & ANDREOTTI & LOUIS & $\mathrm{s}$ & 18 & MICHIGAN & ITALY & ITALY & & PAINTER/PAINTING & CAMILLIANO. LU & ETHEL AVE. & HANCOCK CITY \\
\hline 2540 & & ANDREOTTI & ALDO & $\hat{s}$ & 16 & MICHIGAN & ITALY & ITALY & & TAIIVIERTIFAIIVIIINO & CAMILLIANO. LU & ETHEL AVE. & HANCOCK CITY \\
\hline 2541 & 1215 & LENCl & ISOLA & $\mathrm{H}$ & 56 & ITALY & ITALY & ITALY & $05 / 08$ & & LUCCA & SUMMIT ST. & HANCOCK CITY \\
\hline 2542 & & LENCI & ALBERT & s & 10 & MICHIGAN & ITALY & ITALY & & & PESCAGLIA. LU & SUMMIT ST. & HANCOCK CITY \\
\hline 2543 & 1032 & LAVORINI & FERRUCCIO & $\mathrm{H}$ & 40 & ITALY & ITALY & ITALY & 1906 & PROPRIETOR/MACARONI & LUCCA & INGOT ST. & HANCOCK CITY \\
\hline 2544 & & LAVORINI & FILOMENA & W & 31 & ITALY & ITALY & ITALY & 1920 & & SEGROMIGNO. LU & INGOT ST. & HANCOCK CITY \\
\hline 2545 & & LAVORINI & MARY & $\mathrm{D}$ & 9 & MICHIGAN & ITALY & ITALY & & & LUCCA & INGOT ST. & HANCOCK CITY \\
\hline 2546 & & LAVORINI & TERESA & D & 6 & MICHIGAN & ITALY & ITALY & & & LUCCA & INGOT ST. & HANCOCK CITY \\
\hline 2547 & & LAVORINI & MARIA & MO & 69 & ITALY & ITALY & ITALY & 1906 & & LUCCA & INGOT ST. & HANCOCK CITY \\
\hline 2548 & 1018 & LAZZARI & ALFRED & $\mathrm{H}$ & 43 & ITALY & ITALY & ITALY & 1901 & TRUCK DRIVER/WHOLESALE & LUCCA & RAILROAD AV & HANCOCK CITY \\
\hline 2549 & & LAZZARI & ANGELINA & w & 36 & MICHIGAN & ITALY & ITALY & & & & RAILROAD AV & HANCOCK CITY \\
\hline 2550 & & LAZZARI & ED & $\mathrm{s}$ & 18 & MICHIGAN & ITALY & MICHIGAN & & TRUCK DRIVER/WHOLESALE & LUCCA & RAILROAD AV & HANCOCK CITY \\
\hline 2551 & & LAZZARI & MARIE & $\mathrm{D}$ & 17 & MICHIGAN & ITALY & MICHIGAN & & SALESLADYICANDY KITCHEN & LUCCA & RAILROAD AV & HANCOCK CITY \\
\hline 2552 & & LAZZARI & DANIEL & $\mathrm{s}$ & 10 & MICHIGAN & ITALY & MICHIGAN & & SALEOLAD TIUAIVUT RITU TEIV & LUCCA & RAILROAD AV & HANCOCK CITY \\
\hline 2553 & & LAZZARI & GEORGE & $\underline{s}$ & 9 & MICHIGAN & ITALY & MICHIGAN & & & LUCCA & RAILROAD AV & HANCOCK CITY \\
\hline 2554 & 1018 & MONTICELLI & FRANK & $\mathrm{H}$ & 22 & MICHIGAN & ITALY & ITALY & & LABORER/STAMP MILL & MATRAIA. LU & SUMMIT ST. & HANCOCK CITY \\
\hline 2555 & & MONTICELLI & FLORENCE & w & 20 & MICHIGAN & CANADA & CANADA & & 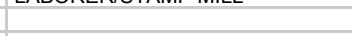 & CANADA & $\begin{array}{l}\text { SUMMIT ST. } \\
\text { SUT }\end{array}$ & HANCOCK CITY \\
\hline 2556 & & MONTICELLI & SHIRLEY & D & 0 & MICHIGAN & MICHIGAN & MICHIGAN & & & MATRAIA. LU & SUMMIT ST. & HANCOCK CITY \\
\hline 2557 & 1018 & NOZERO & JOSEPH & $\mathrm{H}$ & 32 & MICHIGAN & ITALY & ITALY & & MINER/CM & SAN MARTINO CANAVESE. TO & SUMMIT ST. & HANCOCK CITY \\
\hline 2558 & & NOZERO & NELLIE & W & 23 & MICHIGAN & ITALY & ITALY & & & & SUMMIT ST. & HANCOCK CITY \\
\hline 2559 & 940 & MANESCHI & JULIO & $\mathrm{H}$ & 49 & ITALY & ITALY & ITALY & 1905 & MINER/CM & CARRARA & 4TH ST. & HANCOCK CITY \\
\hline 2560 & & MANESCHI & CAROLINA & W & 49 & ITALY & ITALY & ITALY & 1908 & & LUCCA & 4TH ST. & HANCOCK CITY \\
\hline 2561 & & BARBIERI & GINO & sso & 27 & BRASIL & ITALY & ITALY & 1908 & FIREMAN/MEAT MARKET & LUCCA & 4TH ST. & HANCOCK CITY \\
\hline 2562 & 923 & SALANI & ALFIO & $\mathrm{H}$ & 55 & ITALY & ITALY & ITALY & 1890 & MINER/CM & COLLODI. LU & ETHEL ST. & HANCOCK CITY \\
\hline 2563 & & SALANI & ADA & w & 42 & ITALY & ITALY & ITALY & 1900 & & & ETHEL ST. & HANCOCK CITY \\
\hline 2564 & & SALANI & ADAMO & s & 10 & MICHIGAN & ITALY & ITALY & & & COLLODI, LU & ETHEL ST. & HANCOCK CITY \\
\hline 2565 & & SALANI & LOUIS & $\mathrm{s}$ & 18 & MICHIGAN & ITALY & ITALY & & CARETAKER/GAS STATION & COLLODI. LU & ETHEL ST. & HANCOCK CITY \\
\hline 2566 & & SALANI & DOROTHY & DIL & 19 & MICHIGAN & ITALY & ITALY & & & COLLODI. LU & ETHEL ST. & HANCOCK CITY \\
\hline 2567 & 935 & MUGINONRI & RALPH & $\mathrm{H}$ & 55 & ITALY & ITALY & ITALY & 1900 & CLERK/GROCERY STORE & & ETHEL ST. & HANCOCK CITY \\
\hline 2568 & & MUGINONRI & ADELE & w & 50 & ITALY & ITALY & ITALY & 1905 & & & ETHEL ST. & HANCOCK CITY \\
\hline 2569 & & DI PUCCIO & VIRGILIO & NEP & 34 & ITALY & ITALY & ITALY & 1915 & PROPRIETOR/GROCERY STORE & CAPANNORI. LU & ETHEL ST. & HANCOCK CITY \\
\hline 2570 & 1009 & CARTACCI & JOSEPH & $\mathrm{H}$ & 41 & ITALY & ITALY & ITALY & 1913 & LABORER/SMELTER & & ETHEL ST. & HANCOCK CITY \\
\hline 2571 & & CARTACCI & GIOCONDA & W & 34 & ITALY & ITALY & ITALY & 1920 & & & ETHEL ST. & HANCOCK CITY \\
\hline 2572 & & CARTACCI & ANGIOLINA & $\mathrm{D}$ & 9 & ITALY & ITALY & ITALY & 1920 & & & ETHEL ST. & HANCOCK CITY \\
\hline 2573 & & CARTACCI & EDITH & D & 2 & MICHIGAN & ITALY & ITALY & & & & ETHEL ST. & HANCOCK CITY \\
\hline 2574 & 1015 & NOMELLINI & MIKE & $\mathrm{H}$ & 51 & ITALY & ITALY & ITALY & 1913 & MINER/CM & LUCCA & ETHEL ST. & HANCOCK CITY \\
\hline 2575 & & NOMELLINI & PRIMETTA & w & 41 & ITALY & ITALY & ITALY & 1913 & & LUCCA & ETHEL ST. & HANCOCK CITY \\
\hline 2576 & & NOMELLINI & PELLEGRINO & $\mathrm{s}$ & 20 & ITALY & ITALY & ITALY & 1913 & TRUCK DRIVER/TAXI COMPANY & LUCCA & ETHEL ST. & HANCOCK CITY \\
\hline 2577 & & NOMELLINI & PAOLINO & s & 14 & MICHIGAN & ITALY & ITALY & & & LUCCA & ETHEL ST. & HANCOCK CITY \\
\hline 2578 & & NOMELLINI & VASCO & s & 10 & MICHIGAN & ITALY & ITALY & & & LUCCA & ETHEL ST. & HANCOCK CITY \\
\hline 2579 & 1019 & TADDEUCCI & JOSEPH & $\mathrm{H}$ & 53 & ITALY & ITALY & ITALY & ???7 & PROPRIETOR/SOFT DRINK & LUCCA & ETHEL ST. & HANCOCK CITY \\
\hline 2580 & & TADDEUCCI & TERESA & w & 50 & ITALY & ITA & ITALY & 1899 & & & L ST. & HANCOCK CITY \\
\hline 2581 & & TADDEUCCI & JOHN & s & 27 & MICHIGAN & ITALY & ITALY & & LABORER/PAPER FACTORY & LUCCA & ETHEL ST. & HANCOCK CITY \\
\hline 2582 & & TADDEUCCI & FRANK & $\mathrm{s}$ & 20 & MICHIGAN & ITALY & ITALY & & AUTO REPAIRER/GARAGE & LUCCA & ETHEL ST. & HANCOCK CITY \\
\hline
\end{tabular}




\begin{tabular}{|c|c|c|c|c|c|c|c|c|c|c|c|c|c|}
\hline 2583 & & TADDEUCCI & RAIMONDO & $\mathrm{s}$ & 17 & MICHIGAN & ITALY & ITALY & & & LUCCA & ETHEL ST. & HANCOCK CITY \\
\hline 2584 & & TADDEUCCI & $\mathrm{RICCO}$ & $\mathrm{s}$ & 14 & MICHIGAN & ITALY & ITALY & & & LUCCA & ETHEL ST. & HANCOCK CITY \\
\hline 2585 & & TADDEUCCI & MARY & $\mathrm{D}$ & 14 & MICHIGAN & ITALY & ITALY & & & LUCCA & ETHEL ST. & HANCOCK CITY \\
\hline 2586 & & TADDEUCCI & AMELIA & $\mathrm{D}$ & 23 & MICHIGAN & ITALY & ITALY & & CIGAR MAKER/CIGAR FACTORY & LUCCA & ETHEL ST. & HANCOCK CITY \\
\hline 2587 & 1031 & GUIDI & JOSEPH & $\mathrm{H}$ & 52 & ITALY & ITALY & ITALY & 1905 & PROPRIETOR/GROCERY STORE & LUCCA & ETHEL ST. & HANCOCK CITY \\
\hline 2588 & 1052 & DONATI & ALBERT & $\mathrm{H}$ & 38 & ITALY & ITALY & ITALY & 1911 & TRUCK DRIVER/WHOLESALE & LUCCA & INGOT ST. & HANCOCK CITY \\
\hline 2589 & & DONATI & JENNIE & W & 30 & MICHIGAN & ITALY & ITALY & & & LUCCA & INGOT ST. & HANCOCK CITY \\
\hline 2590 & & DONATI & BEN & s & 12 & MICHIGAN & ITALY & MICHIGAN & & & LUCCA & INGOT ST. & HANCOCK CITY \\
\hline 2591 & & DONATI & ALBERT & S & 9 & MICHIGAN & ITALY & MICHIGAN & & & LUCCA & INGOT ST. & HANCOCK CITY \\
\hline 2592 & 1053 & BATTISTONI & CORA & $\mathrm{H}$ & 56 & ITALY & ITALY & ITALY & 1895 & PROPRIETOR/GROCERY STORE & LUCCA & INGOT ST. & HANCOCK CITY \\
\hline 2593 & & MONTICELLO & SAM & BIL & 56 & ITALY & ITALY & ITALY & 1891 & ENGINEER/CM & LUCCA & INGOT ST. & HANCOCK CITY \\
\hline 2594 & 1230 & FREDIANELLI & ALFREDO & $\mathrm{H}$ & 53 & ITALY & ITALY & ITALY & 1900 & LABORER/STREET CAR CO. & CAPANNORI. LU & SUMMIT ST. & HANCOCK CITY \\
\hline 2595 & & FREDIANELLI & SESTILIA & W & 44 & ITALY & ITALY & ITALY & 1900 & & CAPANNORI. LU & SUMMIT ST. & HANCOCK CITY \\
\hline 2596 & & FREDIANELLI & JOSEPH & s & 23 & MICHIGAN & ITALY & ITALY & & LABORER/ODD JOBS & CAPANNORI. LU & SUMMIT ST. & HANCOCK CITY \\
\hline 2597 & & FREDIANELLI & LOUIS & $\mathrm{s}$ & 22 & MICHIGAN & ITALY & ITALY & & TRUCK DRIVER/GROCERY & CAPANNORI. LU & SUMMIT ST. & HANCOCK CITY \\
\hline 2598 & 1113 & SABBATINI & ANTON & $\mathrm{H}$ & 53 & ITALY & ITALY & ITALY & 1903 & LABORER/AUTO FACTORY & LUCCA & SUMMIT ST. & HANCOCK CITY \\
\hline 2599 & & SABBATINI & ELLEN & W & 47 & ITALY & ITALY & ITALY & 1905 & & & SUMMIT ST. & HANCOCK CITY \\
\hline 2600 & & SABBATINI & ELSIE & $\mathrm{D}$ & 21 & MICHIGAN & ITALY & ITALY & & HELPER/RADIO FACTORY & LUCCA & SUMMIT ST. & HANCOCK CITY \\
\hline 2601 & & SABBATINI & MIKE & s & 20 & MICHIGAN & ITALY & ITALY & & PAINTER/CONTRACTOR & LUCCA & SUMMIT ST. & HANCOCK CITY \\
\hline 2602 & & SABBATINI & MARIO & s & 18 & MICHIGAN & ITALY & ITALY & & AUTO MECHANIC/GARAGE & LUCCA & SUMMIT ST. & HANCOCK CITY \\
\hline 2603 & & SABBATINI & JOSEPHINE & $\mathrm{D}$ & 17 & MICHIGAN & ITALY & ITALY & & 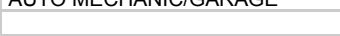 & LUCCA & SUMMIT ST. & HANCOCK CITY \\
\hline 2604 & & SABBATINI & FRANCES & $\mathrm{D}$ & 9 & MICHIGAN & ITALY & ITALY & & & LUCCA & SUMMIT ST. & HANCOCK CITY \\
\hline 2605 & & SANDRETTO & SILVESTRO & $\mathrm{H}$ & 61 & ITALY & ITALY & ITALY & 1892 & FARMER/GENERAL FARM & & CAMEL ROAD & HANCOCK \\
\hline 2606 & & SANDRETTO & JAMES & $\mathrm{s}$ & 30 & MICHIGAN & ITALY & ITALY & & & PONT CANAVESE. TO & CAMEL ROAD & HANCOCK \\
\hline 2607 & & GASPARDO & PAUL & $\mathrm{H}$ & 55 & ITALY & ITALY & ITALY & 1899 & FARMER/GENERAL FARM & PONT CANAVESE. TO & CAMEL ROAD & HANCOCK \\
\hline 2608 & & GASPARDO & MARY & W & 55 & ITALY & ITALY & ITALY & 1903 & & PONT CANAVESE. TO & CAMEL ROAD & HANCOCK \\
\hline 2609 & & GASPARDO & MARY & $\mathrm{D}$ & 23 & MICHIGAN & ITALY & ITALY & & & PONT CANAVESE. TO & CAMEL ROAD & HANCOCK \\
\hline 2610 & & GASPARDO & DOMENICO & $\mathrm{s}$ & 22 & MICHIGAN & ITALY & ITALY & & & PONT CANAVESE, TO & CAMEL ROAD & HANCOCK \\
\hline 2611 & & GASPARDO & JOHANNA & D & 15 & MICHIGAN & ITALY & ITALY & & & PONT CANAVESE. TO & CAMEL ROAD & HANCOCK \\
\hline 2612 & & CAPELLO & ANDREW & $\mathrm{H}$ & 36 & MICHIGAN & ITALY & ITALY & & FIREMAN/CM & SAN BENIGNO CANAVESE. TO & MAPLE ST. & OSCEOLA - TAMARACK \\
\hline 2613 & & CAPELLO & VIRGINIA & w & 33 & MICHIGAN & ITALY & ITALY & & & g & MAPLE ST. & OSCEOLA - TAMARACK \\
\hline 2614 & & CAPELLO & ANNA & $\mathrm{D}$ & 13 & MICHIGAN & MICHIGAN & MICHIGAN & & & SAN BENIGNO CANAVESE. TO & MAPLE ST. & OSCEOLA - TAMARACK \\
\hline 2615 & & CAPELLO & MARGHERITA & $\mathrm{D}$ & 12 & MICHIGAN & MICHIGAN & MICHIGAN & & & SAN BENIGNO CANAVESE. TO & MAPLE ST. & OSCEOLA - TAMARACK \\
\hline 2616 & & CAPELLO & JULIA & $\mathrm{D}$ & 10 & MICHIGAN & MICHIGAN & MICHIGAN & & & SAN BENIGNO CANAVESE, TO & MAPLE ST. & OSCEOLA - TAMARACK \\
\hline 2617 & & CAPELLO & ALBERT & $\mathrm{s}$ & 9 & MICHIGAN & MICHIGAN & MICHIGAN & & & SAN BENIGNO CANAVESE. TO & MAPLE ST. & OSCEOLA - TAMARACK \\
\hline 2618 & & CAPELLO & GEORGE & $\mathrm{s}$ & 4 & MICHIGAN & MICHIGAN & MICHIGAN & & & SAN BENIGNO CANAVESE. TO & MAPLE ST. & OSCEOLA - TAMARACK \\
\hline 2619 & & CAPELLO & JOSEPHINE & $\mathrm{D}$ & 2 & MICHIGAN & MICHIGAN & MICHIGAN & & & SAN BENIGNO CANAVESE. TO & MAPLE ST. & OSCEOLA - TAMARACK \\
\hline 2620 & & CAPELLO & PETER & $\mathrm{s}$ & $4 \mathrm{M}$ & MICHIGAN & MICHIGAN & MICHIGAN & & & SAN BENIGNO CANAVESE. TO & MAPLE ST. & OSCEOLA - TAMARACK \\
\hline 2621 & & PICCIANO & MIKE & $\mathrm{H}$ & 36 & MICHIGAN & ITALY & ITALY & & MINER/CM & CAMPOBASSO & 3RD ST. & OSCEOLA - TAMARACK \\
\hline 2622 & & PICCIANO & MARY & W & 35 & MICHIGAN & ITALY & ITALY & & & & 3RD ST. & OSCEOLA - TAMARACK \\
\hline 2623 & & PICCIANO & JOSEPH & s & 15 & MICHIGAN & MICHIGAN & MICHIGAN & & & CAMPOBASSO & 3RD ST. & OSCEOLA - TAMARACK \\
\hline 2624 & & PICCIANO & CECIL & $\mathrm{D}$ & 13 & MICHIGAN & MICHIGAN & MICHIGAN & & & CAMPOBASSO & 3RD ST. & OSCEOLA - TAMARACK \\
\hline 2625 & & PICCIANO & MARY & $\mathrm{D}$ & 12 & MICHIGAN & MICHIGAN & MICHIGAN & & & CAMPOBASSO & 3RD ST. & OSCEOLA - TAMARACK \\
\hline 2626 & & PICCIANO & LAWRENCE & s & 9 & MICHIGAN & MICHIGAN & MICHIGAN & & & CAMPOBASSO & 3RD ST. & OSCEOLA - TAMARACK \\
\hline 2627 & & PICCIANO & DOMENICO & $\mathrm{s}$ & 7 & MICHIGAN & MICHIGAN & MICHIGAN & & & CAMPOBASSO & 3RD ST. & OSCEOLA - TAMARACK \\
\hline 2628 & & PICCIANO & GLORIA & $\mathrm{D}$ & 3 & MICHIGAN & MICHIGAN & MICHIGAN & & & CAMPOBASSO & 3RD ST. & OSCEOLA - TAMARACK \\
\hline 2629 & & PICCIANO & VIOLA & $\mathrm{D}$ & 1 & MICHIGAN & MICHIGAN & MICHIGAN & & & CAMPOBASSO & 3RD ST. & OSCEOLA - TAMARACK \\
\hline 2630 & & FANTINI & PETER & $\mathrm{H}$ & 45 & ITALY & ITALY & ITALY & 1904 & MINER/CM & GEROSA. BERGAMO & & OSCEOLA - OSCEOLA LOC. \\
\hline 2631 & & FANTINI & TERESA & W & 45 & ITALY & ITALY & ITALY & 1900 & & & & OSCEOLA - OSCEOLA LOC. \\
\hline 2632 & & FANTINI & ANGELA & s & 20 & MICHIGAN & ITALY & ITALY & & LABORER/CM & GEROSA. BERGAMO & & OSCEOLA - OSCEOLA LC \\
\hline 2633 & & FANTINI & LOUIS & $\mathrm{s}$ & 11 & MICHIGAN & ITALY & ITALY & & & GEROSA. BERGAMO & & OSCEOLA - OSCEOLA LOC. \\
\hline 2634 & & FANTINI & ANGELINA & $\mathrm{D}$ & 3 & MICHIGAN & ITALY & ITALY & & & GEROSA, BERGAMO & & OSCEOLA - OSCEOLA LOC. \\
\hline 2635 & & DE FILIPPI & JOE & $\mathrm{H}$ & 60 & ITALY & ITALY & ITALY & 1891 & & SAN GIORGIO CANAVESE. TO & & OSCEOLA - OSCEOLA LOC. \\
\hline 2636 & & DE FILIPPI & MARY & W & 50 & ITALY & ITALY & ITALY & 1896 & & & & OSCEOLA - OSCEOLA LOC. \\
\hline 2637 & & DE FILIPPI & LINA & $\mathrm{D}$ & 19 & MICHIGAN & ITALY & ITALY & & LABORER/CM & SAN GIORGIO CANAVESE. TO & & OSCEOLA - OSCEOLA LOC. \\
\hline 2638 & & DE FILIPPI & PETER & $\bar{s}$ & 13 & MICHIGAN & ITALY & ITALY & & SERVANT/PF & SAN GIORGIO CANAVESE. TO & & OSCEOLA - OSCEOLA LOC. \\
\hline 2639 & & DE FILIPPI & GEORGE & $\mathrm{s}$ & 12 & MICHIGAN & ITALY & ITALY & & & SAN GIORGIO CANAVESE. TO & & OSCEOLA - OSCEOLA LOC. \\
\hline 2640 & & DE FILIPPI & JAMES & s & 10 & MICHIGAN & ITALY & ITALY & & & SAN GIORGIO CANAVESE, TO & & OSCEOLA - OSCEOLA LOC. \\
\hline 2641 & & PUCCl & SALVATORE & $\mathrm{H}$ & 50 & ITALY & ITALY & ITALY & 1900 & MINER/CM & RUOTA. LU & & OSCEOLA - OSCEOLA LOC. \\
\hline 2642 & & $\mathrm{PUCCl}$ & ANNA & w & 38 & ITALY & ITALY & ITALY & 1900 & & RUOTA. LU & & OSCEOLA - OSCEOLA LOC. \\
\hline 2643 & & $\mathrm{PUCCl}$ & BLANCHE & D & 16 & MIC & ITAL & ITA & & & LU & & OSCEOLA - OSCEOLA LOC. \\
\hline 2644 & & PUCCI & BRUNA & $\mathrm{s}$ & 14 & MICHIGAN & ITALY & & & & RUOTA. LU & & OSCEOLA - OSCEOLA L \\
\hline 2645 & & PUCCI & DOROTHY & $\mathrm{D}$ & 12 & MICHIGAN & ITALY & ITALY & & & RUOTA, LU & & OSCEOLA - OSCEOLA LOC. \\
\hline
\end{tabular}




\begin{tabular}{|c|c|c|c|c|c|c|c|c|c|c|c|c|c|}
\hline 2646 & & PUCCI & RAY & $\mathrm{s}$ & 8 & MICHIGAN & ITALY & ITALY & & & RUOTA. LU & & OSCEOLA - OSCEOLA LOC. \\
\hline 2647 & & TADDEUCCI & ENRICO & $\mathrm{H}$ & 58 & ITALY & ITALY & ITALY & 1904 & STOREKEEPER/GROCERY & LUCCA & & OSCEOLA - OSCEOLA LOC. \\
\hline 2648 & & TADDEUCCI & LIVIA & W & 56 & ITALY & ITALY & ITALY & 1907 & & & & OSCEOLA - OSCEOLA LOC. \\
\hline 2649 & & BRUSSO & JOSEPH & $\mathrm{H}$ & 63 & ITALY & ITALY & ITALY & 1898 & FARMER/GENERAL FARM & AGLIE'. TO & & OSCEOLA - OSCEOLA LOC. \\
\hline 2650 & & BRUSSO & BARBARA & w & 60 & ITALY & ITALY & ITALY & 1898 & & AGLIE', TO & & OSCEOLA - OSCEOLA LOC. \\
\hline 2651 & & BRUSSO & DOMENICO & $\mathrm{s}$ & 27 & MICHIGAN & ITALY & ITALY & & FARMER/GENERAL FARM & AGLIE', TO & & OSCEOLA - OSCEOLA LOC. \\
\hline 2652 & & BRUSSO & LUCILLE & DIL & 21 & MICHIGAN & ITALY & ITALY & & & & & OSCEOLA - OSCEOLA LOC. \\
\hline 2653 & & BRUSSO & & GS & 1 & MICHIGAN & ITALY & ITALY & & & AGLIE'. TO & & OSCEOLA - OSCEOLA LOC. \\
\hline 2654 & & BAUSANO & LOUIS & $\mathrm{H}$ & 49 & ITALY & ITALY & ITALY & 1901 & FARMER/GENERAL FARM & PONT CANAVESE. TO & & OSCEOLA - OSCEOLA LOC. \\
\hline 2655 & & BAUSANO & ANNA & w & 52 & ITALY & ITALY & ITALY & 1902 & & & & OSCEOLA - OSCEOLA LOC. \\
\hline 2656 & & BAUSANO & KATE & $\mathrm{D}$ & 25 & MICHIGAN & ITALY & ITALY & & SERVANT/PF & PONT CANAVESE. TO & & OSCEOLA - OSCEOLA LOC. \\
\hline 2657 & & BAUSANO & JOHN & $\mathrm{s}$ & 18 & MICHIGAN & ITALY & ITALY & & LABORER/GENERAL FARM & PONT CANAVESE. TO & & OSCEOLA - OSCEOLA LOC. \\
\hline 2658 & & CERETTO & DOMENICO & $\mathrm{H}$ & 57 & ITALY & ITALY & ITALY & 1896 & FARMER/GENERAL FARM & TORINO & & OSCEOLA - OSCEOLA LOC. \\
\hline 2659 & & CERETTO & TERESA & W & 61 & ITALY & ITALY & ITALY & 1906 & & & & OSCEOLA - OSCEOLA LOC. \\
\hline 2660 & & CERETTO & TRACY & $\mathrm{D}$ & 18 & MICHIGAN & ITALY & ITALY & & STUDENT & TORINO & & OSCEOLA - OSCEOLA LOC. \\
\hline 2661 & & CERETTO & ROSE & D & 17 & MICHIGAN & ITALY & ITALY & & HOUSE WORK/HOME & TORINO & & OSCEOLA - OSCEOLA LOC. \\
\hline 2662 & & LOCATELLI & SAM & $\mathrm{H}$ & 38 & ITALY & ITALY & ITALY & 1894 & JANITOR/PUBLIC SCHOOL & SELINO. BERGAMO & 5TH ST. & OSCEOLA - DOLLAR BAY TOWN \\
\hline 2663 & & LOCATELLI & ROSE & W & 34 & ITALY & ITALY & ITALY & 1900 & & & 5TH ST. & OSCEOLA - DOLLAR BAY TOWN \\
\hline 2664 & & LOCATELLI & FRANCIS & $\mathrm{s}$ & 14 & MICHIGAN & ITALY & ITALY & & & SELINO. BERGAMO & 5TH ST. & OSCEOLA - DOLLAR BAY TOWN \\
\hline 2665 & & LOCATELLI & ROBERT & $\mathrm{s}$ & 13 & MICHIGAN & ITALY & ITALY & & & SELINO. BERGAMO & 5TH ST. & OSCEOLA - DOLLAR BAY TOWN \\
\hline 2666 & & LOCATELLI & KATE & $\mathrm{D}$ & 10 & MICHIGAN & ITALY & ITALY & & & SELINO. BERGAMO & 5TH ST. & OSCEOLA - DOLLAR BAY TOWN \\
\hline 2667 & & LOCATELLI & BETTY & $\mathrm{D}$ & 7 & MICHIGAN & ITALY & ITALY & & & SELINO, BERGAMO & 5TH ST. & OSCEOLA - DOLLAR BAY TOWN \\
\hline 2668 & & LOCATELLI & JAMES & $\mathrm{s}$ & 1 & MICHIGAN & ITALY & ITALY & & & SELINO. BERGAMO & 5TH ST. & OSCEOLA - DOLLAR BAY TOWN \\
\hline 2669 & & BONANNI & EMIL & $\mathrm{H}$ & 43 & ITALY & ITALY & ITALY & $01 / 07$ & LABORER/STAMP MILL & LUCCA & & OSCEOLA - TAMARACK MILLS \\
\hline 2670 & & BONANNI & MARY & w & 28 & MICHIGAN & ITALY & ITALY & & & & & OSCEOLA - TAMARACK MILLS \\
\hline 2671 & & BONANNI & LAWRENCE & s & 6 & MICHIGAN & ITALY & MICHIGAN & & & LUCCA & & OSCEOLA - TAMARACK MILLS \\
\hline 2672 & & BONANNI & ALFRED & $\mathrm{s}$ & 3 & MICHIGAN & ITALY & MICHIGAN & & & LUCCA & & OSCEOLA - TAMARACK MILLS \\
\hline 2673 & & PINI & EUGENE & $\mathrm{H}$ & 53 & ITALY & ITALY & ITALY & 1900 & LABORER/STAMP MILL & LUCCA & & OSCEOLA - TAMARACK MILLS \\
\hline 2674 & & PINI & AMELIA & W & 49 & ITALY & ITALY & ITALY & 83?? & & & & OSCEOLA - TAMARACK MILLS \\
\hline 2675 & & PINI & EUGENE & s & 21 & MICHIGAN & ITALY & ITALY & & LABORER/STAMP MILL & LUCCA & & OSCEOLA - TAMARACK MILLS \\
\hline 2676 & & PINI & GEORGE & $\mathrm{s}$ & 19 & MICHIGAN & ITALY & ITALY & & & LUCCA & & OSCEOLA - TAMARACK MILLS \\
\hline 2677 & & PINI & ALFRED & $\mathrm{s}$ & 15 & MICHIGAN & ITALY & ITALY & & & LUCCA & & OSCEOLA - TAMARACK MILLS \\
\hline 2678 & & PINI & PETER & $\mathrm{s}$ & 11 & MICHIGAN & ITALY & ITALY & & & LUCCA & & OSCEOLA - TAMARACK MILLS \\
\hline 2679 & & BAIOCCHI & NATALE & $\mathrm{H}$ & 67 & ITALY & ITALY & ITALY & 1893 & LABORER/STAMP MILL & COLLODI, LU & & OSCEOLA - TAMARACK MILLS \\
\hline 2680 & & BAIOCCHI & MARY & w & 63 & ITALY & ITALY & ITALY & 1893 & & COLLODI.LU & & OSCEOLA - TAMARACK MILLS \\
\hline 2681 & & BAIOCCHI & CAMILLO & $\mathrm{H}$ & 57 & ITALY & ITALY & ITALY & 1893 & LABORER/STAMP MILL & COLLODI. LU & & OSCEOLA - TAMARACK MILLS \\
\hline 2682 & & BAIOCCHI & LOTTIE & W & 55 & ITALY & ITALY & ITALY & 1899 & & & & OSCEOLA - TAMARACK MILLS \\
\hline 2683 & & BAIOCCHI & DOROTHY & $\mathrm{D}$ & 16 & MICHIGAN & ITALY & ITALY & & & COLLODI. LU & & OSCEOLA - TAMARACK MILLS \\
\hline 2684 & & BERTOLINI & AMERIGO & $\mathrm{H}$ & 43 & ITALY & ITALY & ITALY & 1911 & LABORER/STAMP MILL & CALCI. PISA & & OSCEOLA - TAMARACK MILLS \\
\hline 2685 & & BERTOLINI & LOTHIE & W & 35 & ITALY & ITALY & ITALY & 1920 & & & & OSCEOLA - TAMARACK MILLS \\
\hline 2686 & & BERTOLINI & ELENA & D & 5 & MICHIGAN & ITALY & ITALY & & & CALCI. PISA & & OSCEOLA - TAMARACK MILLS \\
\hline 2687 & & BERTOLINI & MARY & $\mathrm{D}$ & 3 & MICHIGAN & ITALY & ITALY & & & CALCI. PISA & & OSCEOLA - TAMARACK MILLS \\
\hline 2688 & & BERTOLINI & GERTRUDE & $\mathrm{D}$ & 1 & MICHIGAN & ITALY & ITALY & & & CALCI. PISA & & OSCEOLA - TAMARACK MILLS \\
\hline 2689 & & CONANSONI & CAMILLO & $\mathrm{H}$ & 68 & ITALY & ITALY & ITALY & 1895 & & & & OSCEOLA - TAMARACK MILLS \\
\hline 2690 & & VAIRO & PETER & $\mathrm{H}$ & 27 & MICHIGAN & ITALY & ITALY & & LABORER/RECLAIM PLANT & LOCANA CANAVESE. TO & & OSCEOLA - TAMARACK MILLS \\
\hline 2691 & & VAIRO & MILDRED & W & 23 & MICHIGAN & MICHIGAN & MICHIGAN & & LFDURLNTIRLCLAIVI TLAIVI & USA & & OSCEOLA - TAMARACK MILLS \\
\hline 2692 & & VAIRO & ROBERT & $\mathrm{s}$ & 1 & MICHIGAN & MICHIGAN & MICHIGAN & & & LOCANA CANAVESE. TO & & OSCEOLA - TAMARACK MLLS \\
\hline 2693 & 138 & GARBOLINO & DOMENICO & $\mathrm{H}$ & 38 & MICHIGAN & ITALY & ITALY & & BAKER/BAKERY & TORINO & SHELDON ST. & PORTAGE - HOUGHTON VILLAGE \\
\hline 2694 & & GARBOLINO & RUTH & w & 28 & MICHIGAN & ENGLAND & ENGLAND & & & ENGLAND & SHELDON ST. & PORTAGE - HOUGHTON VILLAGE \\
\hline 2695 & & GARBOLINO & PHILLIS & $\mathrm{D}$ & 12 & MICHIGAN & MICHIGAN & MICHIGAN & & & TORINO & SHELDON ST. & PORTAGE - HOUGHTON VILLAGE \\
\hline 2696 & & GARBOLINO & JUNE & $\mathrm{D}$ & 11 & MICHIGAN & MICHIGAN & MICHIGAN & & & TORINO & SHELDON ST. & PORTAGE - HOUGHTON VILLAGE \\
\hline 2697 & 118 & MALFORTI & TERSILIA & $\mathrm{H}$ & 37 & ITALY & ITALY & ITALY & 1910 & CHORE WOMAN/ODD JOBS & LUCCA & MONTEZUMA ST. & PORTAGE - HOUGHTON VILLAGE \\
\hline 2698 & & MALFORTI & CASIRA & $\mathrm{D}$ & 18 & MICHIGAN & ITALY & ITALY & & BOOKKEEPER/PLUMBING SHOP & LUCCA & MONTEZUMA ST. & PORTAGE - HOUGHTON VILLAGE \\
\hline 2699 & & MALFORTI & KATE & D & 17 & MICHIGAN & ITALY & ITALY & & & LUCCA & MONTEZUMA ST. & PORTAGE - HOUGHTON VILLAGE \\
\hline 2700 & & MALFORTI & JEROME & $\mathrm{s}$ & 14 & MICHIGAN & ITALY & ITALY & & & LUCCA & MONTEZUMA ST. & PORTAGE - HOUGHTON VILLAGE \\
\hline 2701 & 99 & FRANCISCO & FRANK & $\mathrm{H}$ & 32 & MICHIGAN & ITALY & ITALY & & SHERIFF/HOUGHTON COUNTY & TORINO & ALBION ST. & PORTAGE - HOUGHTON VILLAGE \\
\hline 2702 & & FRANCISCO & MARY & w & 30 & ITALY & ITALY & ITALY & 1904 & & & ALBION ST. & PORTAGE - HOUGHTON VILLAGE \\
\hline 2703 & & FRANCISCO & GERARD & $\mathrm{s}$ & 4 & MICHIGAN & MICHIGAN & ITALY & & & TORINO & ALBION ST. & PORTAGE - HOUGHTON VILLAGE \\
\hline 2704 & & BALAGNA & MARY & SER & 22 & MICHIGAN & ITALY & ITALY & & SERVANT/PF & PONT CANAVESE. TO & ALBION ST. & PORTAGE - HOUGHTON VILLAGE \\
\hline 2705 & & KDELETA & STEVE & INMA & 50 & ITALY & ITALY & ITALY & 1898 & LABORER/CM & RUIVI CAIVAVESE. IO & ALBION ST. & PORTAGE - HOUGHTON VILLAGE \\
\hline 2706 & 170 & PADUAN & NICOLA & $\mathrm{H}$ & 37 & ITALY & ITALY & ITALY & 1903 & ELECTRICIAN/RAILROAD & & MEMORIAL ROAD & PORTAGE - HOUGHTON VILLAGE \\
\hline 2707 & & PADUAN & ORTESNIA & W & 38 & MICHIGAN & MICHIGAN & MICHIGAN & & & USA & MEMORIAL ROAD & PORTAGE - HOUGHTON VILLAGE \\
\hline 2708 & & PADUAN & JAMES & $\mathrm{s}$ & 11 & MICHIGAN & ITALY & MICHIGAN & & & & MEMORIAL ROAD & PORTAGE - HOUGHTON VILLAGE \\
\hline
\end{tabular}




\begin{tabular}{|c|c|c|c|c|c|c|c|c|c|c|c|c|c|}
\hline 2709 & & PADUAN & WILL & S & 9 & MICHIGAN & ITALY & MICHIGAN & & & & MEMORIAL ROAD & PORTAGE - HOUGHTON VILLAGE \\
\hline 2710 & & PADUAN & JOHN & $\mathrm{s}$ & 8 & MICHIGAN & ITALY & MICHIGAN & & & & MEMORIAL ROAD & PORTAGE - HOUGHTON VILLAGE \\
\hline 2711 & 147 & MAJOR & HENRY & $\mathrm{H}$ & 31 & MICHIGAN & MICHIGAN & MICHIGAN & & METER READER/WATER DEPOT & USA & & PORTAGE - HOUGHTON VILLAGE \\
\hline 2712 & & MAJOR & ANGELINA & w & 27 & MICHIGAN & ITALY & ITALY & & & & & PORTAGE - HOUGHTON VILLAGE \\
\hline 2713 & 285 & BARAGGIONE & JAMES & $\mathrm{H}$ & 51 & ITALY & ITALY & ITALY & 1902 & MANAGER/GROCERY STORE & SAN GIORGIO CANAVESE. TO & EDWARDS ST. & PORTAGE - HOUGHTON VILLAGE \\
\hline 2714 & & BARAGGIONE & LUCIA & w & 43 & ITALY & ITALY & ITALY & 1911 & & & EDWARDS ST. & PORTAGE - HOUGHTON VILLAGE \\
\hline 2715 & & BARAGGIONE & MARY & D & 15 & MICHIGAN & ITALY & ITALY & & & SAN GIORGIO CANAVESE. TO & EDWARDS ST. & PORTAGE - HOUGHTON VILLAGE \\
\hline 2716 & 702 & VIGO & GEORGE & $\mathrm{H}$ & 26 & MICHIGAN & ITALY & ITALY & & MERCHANT/RETAIL DRUGS & TORINO & EDWARDS ST. & PORTAGE - HOUGHTON VILLAGE \\
\hline 2717 & 80 & POGGIONE & JAMES & $\mathrm{H}$ & 32 & MICHIGAN & ITALY & ITALY & & NOT CLEAR & SAN GIORGIO CANAVESE. TO & ALBION ST. & PORTAGE - HOUGHTON VILLAGE \\
\hline 2718 & & POGGIONE & EDITH & w & 29 & MICHIGAN & MICHIGAN & ENGLAND & & & ENGLAND-USA & HURON ST. & PORTAGE - HOUGHTON VILLAGE \\
\hline 2719 & 93 & SODINI & ROGER & $\mathrm{H}$ & 49 & ITALY & ITALY & ITALY & UNK & PROPRIETOR/CIGAR-TABACCO & COLLODI. LU & HURON ST. & PORTAGE - HOUGHTON VILLAGE \\
\hline 2720 & & SODINI & MILDRED & w & 37 & MICHIGAN & ENGLAND & ENGLAND & & & ENGLAND & HURON ST. & PORTAGE - HOUGHTON VILLAGE \\
\hline 2721 & & SODINI & TONY & $\mathrm{s}$ & 15 & MICHIGAN & ITALY & MICHIGAN & & & COLLODI. LU & HURON ST. & PORTAGE - HOUGHTON VILLAGE \\
\hline 2722 & & SODINI & MARIE & D & 13 & MICHIGAN & ITALY & MICHIGAN & & & COLLODI. LU & HURON ST. & PORTAGE - HOUGHTON VILLAGE \\
\hline 2723 & 27 & RAFFAELLI & MARIO & $\mathrm{H}$ & 26 & MICHIGAN & ITALY & ITALY & & CHAUFFEUR/BUS CO. & LUCCA & MONTEZUMA ST. & PORTAGE - HOUGHTON VILLAGE \\
\hline 2724 & & RAFFAELLI & FILLIS & W & 25 & MICHIGAN & ITALY & ITALY & & & & MONTEZUMA ST. & PORTAGE - HOUGHTON VILLAGE \\
\hline 2725 & & RAFFAELLI & ED & $\mathrm{s}$ & 2 & MICHIGAN & MICHIGAN & MICHIGAN & & & LUCCA & MONTEZUMA ST. & PORTAGE - HOUGHTON VILLAGE \\
\hline 2726 & 119 & BUGNI & PETER & B & 26 & MICHIGAN & ITALY & FRANCE & & TEACHER/PUBLIC SCHOOL & & COLLEGE AVE. & PORTAGE - HOUGHTON VILLAGE \\
\hline 2727 & 86 & FANT & ALFONSO & B & 32 & ITALY & ITALY & ITALY & 1923 & MINER/CM & BERGAMO & & PORTAGE - DODGEVILLE TOWN \\
\hline 2728 & & NOZERO & MIKE & $\mathrm{H}$ & 27 & MICHIGAN & ITALY & ITALY & & DELIVERY MAN/RETAIL & SAN MARTINO CANAVESE. TO & & PORTAGE - DODGEVILLE TOWN \\
\hline 2729 & & NOZERO & PEARL & w & 26 & MICHIGAN & CANADA & MICHIGAN & & POSTMASTER/POST OFFICE & CANADA-USA & & PORTAGE - DODGEVILLE TOWN \\
\hline 2730 & & NOZERO & MARY & D & 6 & MICHIGAN & MICHIGAN & MICHIGAN & & & SAN MARTINO CANAVESE, TO & & PORTAGE - DODGEVILLE TOWN \\
\hline 2731 & & RUFFOLO & JOSEPH & $\mathrm{H}$ & 43 & ITALY & ITALY & ITALY & 1909 & SHOEMAKER/OWN SHOP & COSENZA & & PORTAGE - DODGEVILLE TOWN \\
\hline 2732 & & RUFFOLO & GENEVIEVE & W & 34 & ITALY & ITALY & ITALY & 1921 & & & & PORTAGE - DODGEVILLE TOWN \\
\hline 2733 & & RUFFOLO & JOSEPHINE & D & 4 & MICHIGAN & ITALY & ITALY & & & COSENZA & & PORTAGE - DODGEVILLE TOWN \\
\hline 2734 & & RUFFOLO & MIKE & $\mathrm{s}$ & 2 & MICHIGAN & ITALY & ITALY & & & COSENZA & & PORTAGE - DODGEVILLE TOWN \\
\hline 2735 & & RUFFOLO & VINCENZINA & D & 1 & MICHIGAN & ITALY & ITALY & & & COSENZA & & PORTAGE - DODGEVILLE TOWN \\
\hline 2736 & & NOZERO & PETER & $\mathrm{H}$ & 24 & MICHIGAN & ITALY & ITALY & & MEAT CUTTER/RETAIL GROCERY & SAN MARTINO CANAVESE, TO & & PORTAGE - DODGEVILLE TOWN \\
\hline 2737 & & NOZERO & EVA & W & 22 & MICHIGAN & MICHIGAN & MICHIGAN & & & USA & & PORTAGE - DODGEVILLE TOWN \\
\hline 2738 & & NOZERO & ANTON & $\mathrm{H}$ & 57 & ITALY & ITALY & ITALY & 1901 & MERCHANT/RETAIL GROCERY & SAN MARTINO CANAVESE. TO & & PORTAGE - DODGEVILLE TOWN \\
\hline 2739 & & NOZERO & ANGELINA & w & 47 & ITALY & ITALY & ITALY & 1901 & BOOKKEEPER/RETAIL GROCERY & SAN MARTINO CANAVESE. TO & & PORTAGE - DODGEVILLE TOWN \\
\hline 2740 & & DE FILIPPI & PETER & B & 60 & ITALY & ITALY & ITALY & 1893 & $\begin{array}{l}\text { JANITOR/RETAIL GROCERY } \\
\end{array}$ & TORINO & & PORTAGE - DODGEVILLE TOWN \\
\hline 2741 & & OLSON & JULIUS & $\mathrm{H}$ & 35 & MINNESOTA & USA & USA & & SERVICE MAN/ELECTRIC LIGHT & USA & & PORTAGE \\
\hline 2742 & & OLSON & MARY & w & 28 & MICHIGAN & ITALY & ITALY & & & & & PORTAGE \\
\hline 2743 & & LA BELLE & JOSEPH & $\mathrm{H}$ & 32 & MICHIGAN & CANADA & NEW YORK & & FIREMAN/STEAM RAILROAD & CANADA-USA & & PORTAGE \\
\hline 2744 & & LA BELLE & JOSEPHINE & w & 28 & MICHIGAN & ITALY & ITALY & & & & & PORTAGE \\
\hline 2745 & & LA BELLE & BERNARD & s & 8 & MICHIGAN & MICHIGAN & MICHIGAN & & & & & PORTAGE \\
\hline 2746 & & LA BELLE & MARIE & $\mathrm{D}$ & 6 & MICHIGAN & MICHIGAN & MICHIGAN & & & & & PORTAGE \\
\hline 2747 & & LA BELLE & FRANCES & $\mathrm{D}$ & 5 & MICHIGAN & MICHIGAN & MICHIGAN & & & & & PORTAGE \\
\hline 2748 & & LA BELLE & JOAN & $\mathrm{D}$ & 1 & MICHIGAN & MICHIGAN & MICHIGAN & & & & & PORTAGE \\
\hline 2749 & 158 & PUCCI & JOSEPH & $\mathrm{H}$ & 24 & MONTANA & ITALY & ITALY & & LANDER/CM & LUCCA & & QUINCY - LIMERICK LOC. \\
\hline 2750 & & PUCCl & ISABELLE & w & 20 & MICHIGAN & MICHIGAN & MICHIGAN & & & USA & & QUINCY - LIMERICK LOC. \\
\hline 2751 & & PUCCI & JOSEPH & s & 3 & MICHIGAN & MICHIGAN & MICHIGAN & & & LUCCA & & QUINCY - LIMERICK LOC. \\
\hline 2752 & & PUCCI & KATE & $\mathrm{D}$ & 2 & MICHIGAN & MICHIGAN & MICHIGAN & & & LUCCA & & QUINCY - LIMERICK LOC. \\
\hline 2753 & 153 & PIZZI & CESARE & $\mathrm{H}$ & 52 & ITALY & ITALY & ITALY & 1900 & TIMBERMAN/CM & MONSACRATI, LU & & QUINCY - LIMERICK LOC. \\
\hline 2754 & & PIZZI & ALBERTINA & W & 44 & ITALY & ITALY & ITALY & 1909 & MIVIDETIVIAIVI IVI & & & QUINCY - LIMERICK LOC. \\
\hline 2755 & & PIZZI & DOMENICO & $\frac{\mathrm{s}}{\mathrm{w}}$ & 20 & MICHIGAN & ITALY & ITALY & & PAINTER/GARAGE & MONSACRATI. LU & & QUINCY - LIMERICK LOC. \\
\hline 2756 & & PIZZI & CESARINA & D & 19 & MICHIGAN & ITALY & ITALY & & TELEPHONE & MONSACRATI. LU & & QUINCY - LIMERICK LOC. \\
\hline 2757 & & PIZZI & DINA & $\mathrm{D}$ & 16 & MICHIG & ITALY & ITALY & & & MONSACRATI. LU & & QUINCY - LIMERICK LOC. \\
\hline 2758 & & PIZZI & ADELINA & $\mathrm{D}$ & 13 & MICHIGAN & ITA & ITA & & & MONSACRATI, LU & & QUINCY - LIMERICK LOC. \\
\hline 2759 & & PIZZI & ADOLFO & $\mathrm{BR}$ & 47 & ITALY & & & 1909 & LABORER/CM & PESCAGLIA. LU & & QUINCY - LIMERICK LOC. \\
\hline 2760 & 241 & PATERNI & LOUIS & $\mathrm{H}$ & 53 & & ITA & & 1897 & LABORER/CM & BAGNI DI LUCCA, LU & & QUINCY - LIMERICK LOC. \\
\hline 2761 & & PATERNI & ZELINDA & W & 46 & & ITAI & ITA & 1901 & & & & QUINCY - LIMERICK LOC. \\
\hline 2762 & & PATERNI & MARY & D & 27 & MICHIGAN & ITALY & ITALY & & PRESSER/DRY CLEANING & BAGNI DI LUCCA. LU & & QUINCY - LIMERICK LOC. \\
\hline 2763 & & PATERNI & LOUISE & $\mathrm{D}$ & 26 & MICHIGAN & ITALY & ITALY & & TELEPHONE & BAGNI DI LUCCA. LU & & QUINCY - LIMERICK LOC. \\
\hline 2764 & & PATERNI & PAUL & $\mathrm{s}$ & 23 & MICHIGAN & ITALY & ITALY & & BOOKKEEPER/STEAM RAILROAD & BAGNI DI LUCCA. LU & & QUINCY - LIMERICK LOC. \\
\hline 2765 & & PATERNI & GEORGE & s & 14 & MICHIGAN & ITALY & ITALY & & & BAGNI DI LUCCA. LU & & QUINCY - LIMERICK LOC. \\
\hline 2766 & & TURNER & AUBURN & $\mathrm{H}$ & 35 & MICHIGAN & ITALY & MICHIGAN & & PROPRIETOR/PUBLIC GARAGE & LUCCA & MINE ST. & SCHOOLCRAFT - LAKE LINDEN V. \\
\hline 2767 & & TURNER & RUTH & W & 32 & MICHIGAN & MICHIGAN & MICHIGAN & & & USA & MINE ST. & SCHOOLCRAFT - LAKE LINDEN V. \\
\hline 2768 & & TURNER & BETTY & $\mathrm{D}$ & $\frac{6}{6}$ & MICHIGAN & MICHIGAN & MICHIGAN & & & LUCCA & MINE ST. & SCHOOLCRAFT - LAKE LINDEN V. \\
\hline 2769 & & TURNER & AUBURN & $\frac{\mathrm{N}}{\mathrm{s}}$ & $1 \mathrm{M}$ & MICHIGAN & MICHIGAN & MICHIGAN & & & LUCCA & MINE ST. & SCHOOLCRAFT - LAKE LINDEN V. \\
\hline 2770 & & GARO & & $\mathrm{H}$ & 32 & MIC & MICHIGAN & MICHIG & & STEELWORKER/SMELTER & USA & FRONT ST. & SCHOOLCRAFT - LAKE LINDEN V. \\
\hline 2771 & & GARON & FLORENCE & w & 29 & MICHIGAN & ITALY & ITALY & & & & FRONT ST. & SCHOOLCRAFT - LAKE LINDEN V. \\
\hline
\end{tabular}




\begin{tabular}{|c|c|c|c|c|c|c|c|c|c|c|c|c|c|}
\hline 2772 & & GARON & JOSEPH & $\mathrm{s}$ & 9 & MICHIGAN & MICHIGAN & MICHIGAN & & & & FRONT ST. & SCHOOLCRAFT - LAKE LINDEN V. \\
\hline 2773 & & GARON & A? & $\mathrm{D}$ & 1 & MICHIGAN & MICHIGAN & MICHIGAN & & & & FRONT ST. & SCHOOLCRAFT - LAKE LINDEN V. \\
\hline 2774 & & VAIRO & JOSEPH & $\mathrm{H}$ & 56 & ITALY & ITALY & ITALY & $84 / 94$ & LABORER/STAMP MILL & LOCANA CANAVESE. TO & MINE ST. & SCHOOLCRAFT - LAKE LINDEN V. \\
\hline 2775 & & VAIRO & MARY & W & 50 & MICHIGAN & ITALY & ITALY & & & & MINE ST. & SCHOOLCRAFT - LAKE LINDEN V. \\
\hline 2776 & & VAIRO & DOMENICO & s & 10 & MICHIGAN & ITALY & MICHIGAN & & & LOCANA CANAVESE. TO & MINE ST. & SCHOOLCRAFT - LAKE LINDEN V. \\
\hline 2777 & & ROSSIO & MIKE & $\mathrm{H}$ & 49 & ITALY & ITALY & ITALY & 1882 & DAIRY MAN/OWN FARM & TORINO & N. PINE LAKE R. & SCHOOLCRAFT \\
\hline 2778 & & ROSSIO & FLORIDA & w & 38 & MICHIGAN & CANADA & CANADA & & & CANADA & N. PINE LAKE R. & SCHOOLCRAFT \\
\hline 2779 & & ROSSIO & BERNIE & D & 24 & MICHIGAN & ITALY & MICHIGAN & & TEACHER/DISTRICT SCHOOL & TORINO & N. PINE LAKE R. & SCHOOLCRAFT \\
\hline 2780 & & ROSSIO & MARGHERITA & $\mathrm{D}$ & 18 & MICHIGAN & ITALY & MICHIGAN & & 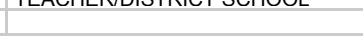 & TORINO & N. PINE LAKE R. & SCHOOLCRAFT \\
\hline 2781 & & ROSSIO & JAMES & s & 17 & MICHIGAN & ITALY & MICHIGAN & & LABORER/DAIRY FARM & TORINO & N. PINE LAKE R. & SCHOOLCRAFT \\
\hline 2782 & & TURNER & AMOS & $\mathrm{H}$ & 60 & ITALY & ITALY & ITALY & 1887 & FARMER/GENERAL FARM & LUCCA & & SCHOOLCRAFT \\
\hline 2783 & & TURNER & KATE & w & 39 & ITALY & ITALY & ITALY & 1914 & & & & SCHOOLCRAFT \\
\hline 2784 & & TURNER & AMOS & s & 12 & ITALY & ITALY & ITALY & & & LUCCA & & SCHOOLCRAFT \\
\hline 2785 & & BALCONI & JAMES & $\mathrm{H}$ & 49 & ITALY & ITALY & ITALY & 1905 & FURNACE MAN/SMELTER & MERCALLO. VARESE & & STANTON \\
\hline 2786 & & BALCONI & MARY & W & 46 & ITALY & ITALY & ITALY & 1905 & & MERCALLO. VARESE & & STANTON \\
\hline 2787 & & BALCONI & WILL & $\mathrm{s}$ & 14 & MICHIGAN & ITALY & ITALY & & & MERCALLO, VARESE & & STANTON \\
\hline 2788 & & CAMPIONI & CARLO & PATI & 42 & ITALY & ITALY & ITALY & 1920 & & LUCCA & & STANTON \\
\hline 2789 & & GRANDIS & JOHN & PATI & 60 & ITALY & ITALY & ITALY & 1900 & & SAN GIORGIO CANAVESE. TO & & STANTON \\
\hline 2790 & & NARDI & JOSEPH & INMA & 53 & ITALY & ITALY & ITALY & 1900 & & PESCIA. LU & & STANTON \\
\hline 2791 & 8 & MARMIELLE & LOUIS & $\mathrm{H}$ & 48 & ITALY & ITALY & ITALY & 1903 & LABORER/STAMP MILL & & & STANTON - BEACON HILL TOWN \\
\hline 2792 & & MARMIELLE & EMILIA & w & 38 & ITALY & ITALY & ITALY & 1905 & & & & STANTON - BEACON HILL TOWN \\
\hline 2793 & & MARMIELLE & ANNE & D & 6 & MICHIGAN & ITALY & ITALY & & & & & STANTON - BEACON HILL TOWN \\
\hline 2794 & & MARMIELLE & DOROTHY & D & 5 & MICHIGAN & ITALY & ITALY & & & & & STANTON - BEACON HILL TOWN \\
\hline 2795 & & MARMIELLE & AUGUST & $\mathrm{s}$ & 3 & MICHIGAN & ITALY & ITALY & & & & & STANTON - BEACON HILL TOWN \\
\hline 2796 & & SIPPI & JOE & B & 23 & ITALY & ITALY & ITALY & 1906 & LABORER/STAMP MILL & & & STANTON - BEACON HILL TOWN \\
\hline 2797 & & PAUBAROY & ADOLFO & B & $20 ?$ & ITALY & ITALY & ITALY & $1904 ?$ & LABORER/STAMP MILL & & & STANTON - BEACON HILL TOWN \\
\hline 2798 & 4 & VITTON & CHARLES & H & 28 & MICHIGAN & ITALY & ITALY & & ELECTRICAL ENGINEER/STAMP & TORINO & & STANTON - BEACON HILL TOWN \\
\hline 2799 & & VITTON & MINNIE & w & 28 & MICHIGAN & ITALY & ITALY & & & & & STANTON - BEACON HILL TOWN \\
\hline 2800 & 24 & MANCO & JAMES & $\mathrm{H}$ & 39 & ITALY & ITALY & ITALY & 1912 & LABORER/STAMP MILL & TORINO & & STANTON - BEACON HILL TOWN \\
\hline 2801 & & MANCO & MUIA & w & 28 & WISCONSIN & ITALY & ITALY & & & & & STANTON - BEACON HILL TOWN \\
\hline 2802 & & MANCO & WILHELMINA & D & 8 & WISCONSIN & ITALY & WISCONSIN & & & TORINO & & STANTON - BEACON HILL TOWN \\
\hline 2803 & & MANCO & JAMES & $\mathrm{s}$ & 6 & WISCONSIN & ITALY & WISCONSIN & & & TORINO & & STANTON - BEACON HILL TOWN \\
\hline 2804 & & MANCO & FUE & $\mathrm{s}$ & 4 & MICHIGAN & ITALY & WISCONSIN & & & TORINO & & STANTON - BEACON HILL TOWN \\
\hline 2805 & & JETTY & ORANGENCE & B & 20 & MICHIGAN & ITALY & ITALY & & LABORER/STAMP MILL & & & STANTON - BEACON HILL TOWN \\
\hline 2806 & 11 & OZZELLO & JOSEPH & $\mathrm{H}$ & 66 & ITALY & ITALY & ITALY & 1902 & LABORER/STAMP MILL & SAN GIUSTO CANAVESE. TO & & STANTON - BEACON HILL TOWN \\
\hline 2807 & & OZZELLO & MINNIE & w & 50 & ITALY & ITALY & ITALY & 1903 & & & & STANTON - BEACON HILL TOWN \\
\hline 2808 & & OZZELLO & PETE & s & 24 & MICHIGAN & ITALY & ITALY & & LABORER/STAMP MILL & SAN GIUSTO CANAVESE. TO & & STANTON - BEACON HILL TOWN \\
\hline 2809 & & OZZELLO & C? & $\mathrm{D}$ & 19 & MICHIGAN & ITALY & ITALY & & & SAN GIUSTO CANAVESE. TO & & STANTON - BEACON HILL TOWN \\
\hline 2810 & ? & BESSOLO & KATE & SER & 23 & MICHIGAN & ITALY & ITALY & & SERVANT/PF & TORINO & & TORCH LAKE \\
\hline 2811 & & SALSINI & LOUIS & $\mathrm{H}$ & 42 & ITALY & ITALY & ITALY & 1907 & HEAD TENDER/CM & SANTA MARIA DEL GIUDICE. & GRANT AVE. & TORCH LAKE - HUBBELL \\
\hline 2812 & & SALSINI & JOSEPHINE & w & 33 & MICHIGAN & ITALY & ITALY & & & & GRANT AVE. & TORCH LAKE - HUBBELL \\
\hline 2813 & & SALSINI & ROSE & D & 12 & MICHIGAN & ITALY & MICHIGAN & & & SANTA MARIA DEL GIUDICE. & GRANT AVE. & TORCH LAKE - HUBBELL \\
\hline 2814 & & SALSINI & A? & $\mathrm{D}$ & 10 & MICHIGAN & ITALY & MICHIGAN & & & SANTA MARIA DEL GIUDICE. & GRANT AVE. & TORCH LAKE - HUBBELL \\
\hline 2815 & & SALSINI & DORA & $\mathrm{D}$ & 8 & MICHIGAN & ITALY & MICHIGAN & & & SANTA MARIA DEL GIUDICE. & GRANT AVE. & TORCH LAKE - HUBBELL \\
\hline 2816 & & SALSINI & ROBERT & s & 7 & MICHIGAN & ITALY & MICHIGAN & & & SANTA MARIA DEL GIUDICE. & GRANT AVE. & TORCH LAKE - HUBBELL \\
\hline 2817 & & SALSINI & LOUIS JR. & $\underline{s}$ & 5 & MICHIGAN & ITALY & MICHIGAN & & & SANTA MARIA DEL GIUDICE. & GRANT AVE. & TORCH LAKE - HUBBELL \\
\hline 2818 & & CONSANI & $\begin{array}{l}\text { ARTHUR } \\
\text { ARTH }\end{array}$ & $\mathrm{H}$ & 35 & ITALY & ITALY & ITALY & 1898 & MERCHANT/RETAIL GROCERY & LUCCA & EDDY ST. & TORCH LAKE - HUBBELL \\
\hline 2819 & & CONSA & LUCY & w & 28 & MICHIGAN & ITALY & ITALY & & & & EDDY ST. & TORCH LAKE - HUBBELL \\
\hline 2820 & & CONSA & GEORGE & s & 5 & MICHIGAN & ITALY & MICHIGAN & & & LUCCA & EDDY ST. & TORCH LAKE - HUBBELL \\
\hline 2821 & & CONSA & LUCILLE & $\mathrm{D}$ & 2 & MICHIG & ITAI & MICHIGAN & & & LUCCA & OY ST. & TORCH LAKE - HUBBELL \\
\hline 2822 & & MONTI & LAWRENCE & $\mathrm{H}$ & 28 & MICHIGAN & ITA & ITALY & & TEMPERATURE TAKER/SMELTER & & CAN? & TORCH LAKE - HUB \\
\hline 2823 & & MONTICELLI & LINA & W & 29 & & ITA & ITA & 1904 & & LUCCA & DUNCAN? & TORCH LAKE - HUBBELL \\
\hline 2824 & & MONTICELLI & LAWRENCE & $\mathrm{s}$ & 2 & HIGAN & MICHIGAN & & & & LUCCA & DUNCAN? & TORCH LAKE - HUBBELL \\
\hline 2825 & & MONTICELLI & MARY & $\mathrm{D}$ & 0 & MICHIGAN & MICHIGAN & ITALY & & & LUCCA & DUNCAN? & TORCH LAKE - HUBBELL \\
\hline 2826 & & LENCI & JOHN & $\mathrm{H}$ & 52 & ITALY & ITALY & ITALY & 1901 & BLACKSMITH/COPPER MILL & PESCAGLIA, LU & GRANT ST. & TORCH LAKE - HUBBELL \\
\hline 2827 & & LENCI & GENERINA & w & 38 & ITALY & ITALY & ITALY & 1912 & & PESCAGLIA. LU & GRANT ST. & TORCH LAKE - HUBBELL \\
\hline 2828 & & LENCI & SERAFINA & D & 16 & MICHIGAN & ITALY & ITALY & & & PESCAGLIA. LU & GRANT ST. & TORCH LAKE - HUBBELL \\
\hline 2829 & & LENCI & AMELIA & $\mathrm{D}$ & 14 & MICHIGAN & ITALY & ITALY & & & PESCAGLIA, LU & GRANT ST. & TORCH LAKE - HUBBELL \\
\hline 2830 & & LENCI & RALFA & $\mathrm{D}$ & 5 & MICHIGAN & ITALY & ITALY & & & PESCAGLIA LU & GRANT ST & TORCH LAKE - HUBBELL \\
\hline 2831 & & GASPARDO & BATTISTA & $\mathrm{H}$ & 50 & MICHIGAN & ITALY & ITALY & & FARMER/GENERAL FARM & PONT CANAVESE. TO & LAKE LINDEN & TORCH LAKE \\
\hline 2832 & & GASPARDO & MARIA & W & 40 & MICHIGAN & ITALY & ITALY & & & & LAKE LINDEN & TORCH LAKE \\
\hline 2833 & & & SERAFINO & s & 25 & & & & & RM & & LAKE LINDEN & TORCH LAKE \\
\hline 2834 & & GASPARDO & JAMES & $\mathrm{s}$ & 22 & MICHIGAN & MICHIGAN & MICHIGAN & & LABORER/GENERAL FARM & PONT CANAVESE. TO & LAKE LINDEN & TORCH LAKE \\
\hline
\end{tabular}




\begin{tabular}{|c|c|c|c|c|c|c|c|c|c|c|c|}
\hline 2835 & GASPARDO & MARTIN & $\mathrm{s}$ & 21 & MICHIGAN & MICHIGAN & MICHIGAN & LABORER/GENERAL FARM & PONT CANAVESE. TO & LAKE LINDEN & TORCH LAKE \\
\hline 2836 & GASPARDO & JENNIE & $\mathrm{s}$ & 18 & MICHIGAN & MICHIGAN & MICHIGAN & LABORER/GENERAL FARM & PONT CANAVESE. TO & LAKE LINDEN & TORCH LAKE \\
\hline 2837 & GASPARDO & JOHANNA & D & 15 & MICHIGAN & MICHIGAN & MICHIGAN & LABORER & PONT CANAVESE. TO & LAKE LINDEN & TORCH LAKE \\
\hline 2838 & GASPARDO & MARY & $\mathrm{D}$ & 6 & MICHIGAN & MICHIGAN & MICHIGAN & & PONT CANAVESE. TO & LAKE LINDEN & TORCH LAKE \\
\hline 2839 & GASPARDO & ANGELO & $\mathrm{s}$ & 6 & MICHIGAN & MICHIGAN & MICHIGAN & & PONT CANAVESE. TO & LAKE LINDEN & TORCH LAKE \\
\hline
\end{tabular}

